NBS

PUBLICATIONS

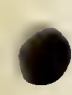

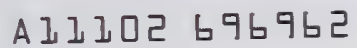

NATL INST OF STANDARDS \& TECH R.I.C

A11102696962

NBS SPECIAL PUBLICATION 400-79

U.S. DEPARTMENT OF COMMERCE/National Bureau of Standards

Senticundudor Menstrement Technolegy.

Results of the Monte Carte

Calculation of One- and

Two-Dimensional Distributions

of Particles and Damage:

Ion Implanted Dopants in Silicon

\title{
John Albers
}


he National Bureau of Standards' was established by an act of Congress on March 3, 1901. The Bureau's overall goal is to strengthen and advance the nation's science and technology and facilitate their effective application for public benefit. To this end, the Bureau conducts research to assure international competitiveness and leadership of U.S industry, science arid technology. NBS work involves development and transfer of measurements, standards and related science and technology, in support of continually improving U.S. productivity, product quality and reliability, innovation and underlying science and engineering. The Bureau's technical work is performed by the National Measurement Laboratory, the National Engineering Laboratory, the Institute for Computer Sciences and Technology, and the Institute for Materials Science and Engineering.

\section{The National Measurement Laboratory}

Provides the national system of physical and chemical measurement; coordinates the system with measurement systems of other nations and furnishes essential services leading to accurate and uniform physical and chemical measurement throughout the Nation's scientific community, industry, and commerce; provides advisory and research services to other Government agencies; conducts physical and chemical research; develops, produces, and distributes Standard Reference Materials; provides calibration services; and manages the National Standard Reference Data System. The Laboratory consists of the following centers:
- Basic Standards ${ }^{2}$

- Radiation Research

- Chemical Physics

- Analytical Chemistry

\section{The National Engineering Laboratory}

Provides technology and technical services to the public and private sectors to address national needs and to solve national problems; conducts research in engineering and applied science in support of these efforts; builds and maintains competence in the necessary disciplines required to carry out this research and technical service; develops engineering data and measurement capabilities; provides engineering measurement traceability services; develops test methods and proposes engineering standards and code changes; develops and proposes new engineering practices; and develops and improves mechanisms to transfer results of its research to the ultimate user. The Laboratory consists of the following centers:
- Applied Mathematics

- Electronics and Electrical Engineering ${ }^{2}$

- Manufacturing Engineering

- Building Technology

- Fire Research

- Chemical Engineering ${ }^{3}$

\section{The Institute for Computer Sciences and Technology}

Conducts research and provides scientific and technical services to aid Federal agencies in the selection, acquisition, application, and use of computer technology to improve effectiveness and economy in Government operations in accordance with Public Law 89-306 (40 U.S.C. 759), relevant Executive Orders, and other directives; carries out this mission by managing the Federal Information Processing Standards Program, developing Federal ADP standards guidelines, and managing Federal participation in ADP voluntary standardization acțivities; provides scientific and technological advisory services and assistance to Federal agencies; and provides the technical foundation for computer-related policies of the Federal Government. The Institute consists of the following divisions:
- Information Systems Engineering

- Systems and Software Technology

- Computer Security

- Systems and Network Architecture

- Advanced Computer Systems

\section{The Institute for Materials Science and Engineering}

Conducts research and provides measurements, data, standards, reference materials, quantitative understanding and other technical information fundamental to the processing, structure, properties and performance of materials; addresses the scientific basis for new advanced materials technologies; plans research around cross-cutting scientific themes such as nondestructive evaluation and phase diagram development; oversees Bureau-wide technical programs in nuclear reactor radiation research and nondestructive evaluation; and broadly disseminates generic technical information resulting from its programs. The Institute consists of the following Divisions:

\footnotetext{
'Headquarters and Laboratories at Gaithersburg, MD, unless otherwise noted; mailing address Gaithersburg, MD 20899

${ }^{2}$ Some divisions within the center are located at Boulder, CO 80303.

'Located at Boulder, CO, with some elements at Gaithersburg, MD
} 


\section{Results of the Monte Carlo Calculation of One- and Two-Dimensional Distributions of Particles and Damage: Ion Implanted Dopants in Silicon}

John Albers

Semiconductor Electronics Division

Center for Electronics and Electrical Engineering

National Engineering Laboratory

National Bureau of Standards

Gaithersburg, MD 20899

September 1987

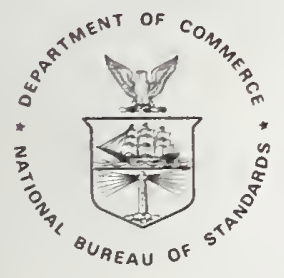

Sponsored by:

Defense Advanced Research Projects Agency 1400 Wilson Boulevard Arlington, VA 22209

U.S. DEPARTMENT OF COMMERCE, Clarence J. Brown, Acting Secretary NATIONAL BUREAU OF STANDARDS, Ernest Ambler, Director 
Library of Congress Catalog Card Number: 87-619864 National Bureau of Standards Special Publication 400-79

Natl. Bur. Stand. (U.S.), Spec. Publ. 400-79, 671 pages (Sept. 1987) CODEN:XNBSAV 
Table of Contents

Page

Abstract ......................... 1

Introduction . . . . . . . . . . . . . . . . . . 2

The Biersack-Haggmark Monte Carlo Code . . . . . . . . . . . . . . . . . . . 4

Two-Body Classical Scattering Theory . . . . . . . . . . . . . . 5

MAGIC Formula for Elastic Scattering . . . . . . . . . . . . . . 9

Displacement Damage . . . . . . . . . . . . . . . . . 11

Electronic Energy Loss . . . . . . . . . . . . . . . . . 13

Combined Effects . . . . . . . . . . . . . . . . . . . 16

Discussion of How the Code Works . . . . . . . . . . . . . . 16

Elements Used in Calculation . . . . . . . . . . . . . . . . . . . . . . 18

One-Dimensional Results for a Uniform Target . . . . . . . . . . . . . . . . 19

Two-Dimensional Results for a Masked Target . . . . . . . . . . . . . 21

Parameterization of Particle Distributions . . . . . . . . . . . . . . . 22

Summary and Conclusions . . . . . . . . . . . . . . . . 23

Acknowledgments . . . . . . . . . . . . . . . . . . . 23

References ....................... . . 24

Appendix A. Tables of Polynomial Fitting Coefficients . . . . . . . . . . . 36

Appendix B. One-Dimensional and Two-Dimensional Distributions . . . . . . 52

Boron . . . . . . . . . . . . . . . . 52

Carbon ......................... 87

Nitrogen . . . . . . . . . . . . . . . . . 125

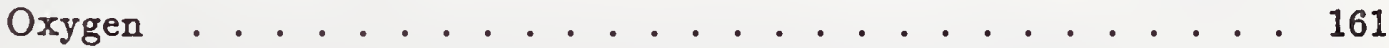

Aluminum . . . . . . . . . . . . . . . . . 196

Silicon . . . . . . . . . . . . . . . 235

Phosphorus . . . . . . . . . . . . . . . . 274

Sulfur . . . . . . . . . . . . . . . 313

Gallium . . . . . . . . . . . . . . . . 354

Germanium . . . . . . . . . . . . 395

Arsenic . . . . . . . . . . . . . . 436

Indium . . . . . . . . . . . . . . 480

Tin . . . . . . . . . . . . . . 518

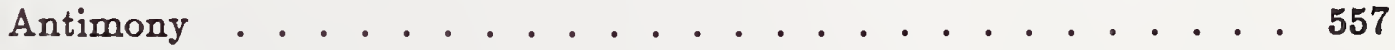


Table of Contents (continued)

Platinum . . . . . . . . . . . . . . 596

Gold . . . . . . . . . . . . . . 632

List of Tables

Page

1. Multiplicative Factor for Calculating Epsilon from Energy (eV) for Various Elements Incident Upon Silicon . . . . . . . . . . . . . . . . 27

2. Values of MAGIC Parameters for Moliere and Krypton-Carbon Potentials . . . . 28

List of Figures

Page

1. Scattering geometry in the laboratory and center of mass frame . . . . . . 29

2. Plot of Moliere and the krypton-carbon screening functions . . . . . . . 30

3. Scattering by the Moliere potential as depicted by the Biersack and Haggmark MAGIC formula . . . . . . . . . . . . . . . . . . 30

4. Scattering by the krypton-carbon potential as depicted by the Biersack and Haggmark MAGIC formula . . . . . . . . . . . . . . 31

5. Comparison of the scattering angle vs. impact parameter determined by the Moliere and the krypton carbon potential for $\epsilon=10^{-1}$. . . . . . . . . . 31

6. Comparison of the scattering angle vs. impact parameter determined by the Moliere and the krypton carbon potential for $\epsilon=10^{-2}$. . . . . . . . . . 32

7. Recoil figure, $\gamma$, as a function of the mass ratio, $M_{2} / M_{1} \ldots \ldots$. . . . . . . 32

8. Electronic stopping for boron and arsenic in silicon as determined by eqs $(39-47)$. . . . . . . . . . . . . . . . . 33

9. Electronic stopping for boron and arsenic in silicon over the range of energies from $10 \mathrm{keV}$ to $1 \mathrm{MeV}$. . . . . . . . . . . . . 33

10. Energy loss as a function of energy . . . . . . . . . . . . . . 34

11. Average energy per primary resulting from $75-\mathrm{keV}$ boron in silicon . . . . . . 34

12. Average energy per primary resulting from $300-\mathrm{keV}$ silicon in silicon . . . . . 35

13. Average energy per primary resulting from $100-\mathrm{keV}$ arsenic in silicon . . . . 35 


\title{
Semiconductor Measurement Technology: \\ Results of the Monte Carlo Calculation of One- and \\ Two-Dimensional Distributions of Particles and Damage: \\ Ion Implanted Dopants in Silicon
}

\author{
John Albers \\ Semiconductor Electronics Division \\ National Bureau of Standards \\ Gaithersburg, Maryland 20899
}

\begin{abstract}
The TRansport of Ions in Matter (TRIM) Monte Carlo code was used to calculate the two-dimensional distributions of particles, primary damage, and electronic and nuclear energy loss for implantation of a line beam source into silicon targets. Approximate twodimensional distributions of the Frenkel pairs (vacancy-interstitial) created by the primary displacement damage of the target atoms were calculated by means of the Kinchin-Pease equation. These particle, damage, and energy loss distributions allowed for the calculation of the one-dimensional distributions of these quantities for implantation into unmasked targets. A superposition technique was used to construct the two-dimensional particle and approximate Frenkel pairs distributions for implantation past a mask edge. The energetic ions used in the calculations were in two groups: those used as intentional dopants in silicon device fabrication and those which either limited lifetime or acted as gettering sites. The particle distributions were parameterized by means of standard polynomial fitting techniques.
\end{abstract}

Key words: displacement damage, electronic energy loss, Frenkel pairs, interstitials, ion implantation, Monte Carlo calculation, nuclear energy loss, silicon device fabrication, twodimensional distributions, vacancies. 


\section{Introduction}

With the present thrust towards fabricating submicron VLSI devices comes an ever increasing need to understand and control two-dimensional dopant impurity distributions and two-dimensional damage distributions. Ion implantation is the primary method for controlled introduction of dopants into semiconductor materials for VLSI applications. The control over the particular dopant as well as over the energy and dose has made implantation an indispensable tool for the fabrication of micron and submicron size structures.

When an energetic ion enters a solid, it slows down and eventually comes to rest as a result of its interaction with the nuclei and electrons of the solid. The interactions with the nuclei and the electrons take place simultaneously but, for purposes of computational modeling, are treated as more or less separate and independent events. The two-body elastic collisions with the core ions are responsible for the lateral scattering as well as primary displacement damage which, in turn, is responsible for vacancy-interstitial or Frenkel pair production through collision cascades. The inelastic scattering of the incident ions by the electrons in the solid contributes to the slowing down but does not enter into lateral scattering or damage production due to the very small mass of the electron compared with that of ions. The two-dimensional distributions of the implanted ions and of the implantation-induced damage play key roles in the physical understanding of the subsequent device processing. This is especially the case for subsequent thermal annealing where the ion-implantation induced damage may enhance the diffusion of the dopants by impurity-vacancy and/or impurity-interstitial interactions.

The two principal techniques which are available for performing ion implantation calculations might be referred to as probabilistic and stochastic (or mechanistic). Both of these techniques assume that the electronic and nuclear stopping of the incident ion by the electrons and nuclei of the solid may be treated as separate events. Also, they both assume that the target is random, i.e., they both neglect any crystalline order in the target. Fi- 
nally, with both computational techniques, the beam current and dose are assumed to be below the point where the incident ions encounter each other in their journey in the solid.

In the probabilistic category, the single-particle distribution function (of the incident ions) or its moments are obtained from the differential or integral form of the Boltzmann transport equation of statistical mechanics. Derivations of this equation for classical [1] or quantum [2] systems clearly show that this equation contains the effects of all possible sequences of separated binary collisions. Hence, the effects of three-body collisions (and higher) are neglected. This appears to be an adequate description for all but very low energies. In the stochastic category, exemplary of the Monte Carlo technique, the trajectories of a large number of incident ions are followed through the random sequence of elastic binary collisions (with the target nuclei) and inelastic electronic energy loss (with the target electrons) and the information about particle positions and energy loss is stored in twodimensional array form. The array information is then used to compute the corresponding distribution functions.

Each of these techniques has strengths and weaknesses. In principle, both techniques should yield the same information.

The probabilistic approach is exemplified by the original LSS [3] calculations and the book by Gibbons et al. [4]. These numerically describe the distribution in terms of its first few moments. In principle, higher moments could be calculated. The four-moment calculation of Winterbon $[5,6]$ is an extension of the LSS calculations and provides good agreement with the SIMS profiles for boron implants into amorphous silicon as obtained by Hofker [7]. There, a Pearson IV equation is used to obtain the distribution from the first four moments.

The probabilistic approach can be used, in principle, to obtain the two- or three-dimensional distribution itself or the set of cross moments from this distribution [8]. While information about the lateral distribution can be obtained, the calculation of the full two- or three- 
dimensional distribution from the cross moments is difficult due to the lack of two- and three-dimensional analogs of the Pearson system of distributions.

The stochastic or mechanistic approach is exemplified by the MARLOWE code of Robinson [9] and the TRIM (TRansport of Ions in Matter) Monte Carlo code of Biersack and Haggmark [10]. For the work presented in this paper, the TRIM code was used to follow the three-dimensional trajectories of the energetic incident ions as well as to calculate the primary displacement energy transferred to the lattice ions. Hence, the two-dimensional distribution of implanted ions and primary displacement damage can be calculated for a line source of fixed incident energy and for a given number of incident particles (usually on the order of $10^{4}$ ). In addition, the distribution of secondary displacement damage was calculated approximately by using the Kinchin-Pease equation. The two-dimensional distributions resulting from a line source can be used to calculate the two-dimensional distributions for the case of implantation past a mask edge by means of superposition of these two-dimensional beams along a partially blocked surface which represents the effect of a sufficiently thick absorbing mask.

\section{The Biersack-Haggmark Monte Carlo Code}

The basic assumption in the version of the TRIM code used in this calculation is that the target is random, i.e., the incident ion beam does not sense any crystallographic order in the target. This can be experimentally achieved by either preamorphizing the target or by suitably tilting the target away from open crystallographic axes. A tilt angle of 7 degrees is often quoted, but the appropriate value may differ from this depending upon the species being used in the implantation. The effects of the implantation process (ion ranges, lattice damage, etc.) are then described in terms of isolated two-body elastic collisions between the energetic ion and the ions of the solid and the viscous drag (inelastic scattering) on the incident ion caused by the electron gas of the target. Each of these contributions to the slowing down process will be discussed in the next sections. The term "Monte Carlo" is applied to the code since the mean free path between the two-body collisions and the 
collision geometry are determined by means of a random number generator. The use of a random number generator for the calculation of both of these quantities is in keeping with the assumed randomness of the target.

\section{Two-Body Classical Scattering Theory}

The elastic scattering of the incident energetic ion by the target nuclei is most easily calculated by means of classical scattering theory. The term "elastic scattering" refers to a collision process where the kinetic energy is conserved. The justification of the use of classical scattering theory is based upon the fact that the partners in the collision are much more massive than the electron and the energies are typically in the range from about 100 $\mathrm{eV}$ to about $500 \mathrm{keV}$. In this situation, it is not necessary to use the Schroedinger equation and the $T$ matrix (Lippmann-Schwinger equation) [11] to describe the scattering event. With this in mind, consider the situation of two structureless particles depicted in the top part of figure 1 . The target ion of mass, $M_{2}$, and atomic number, $Z_{2}$, is initially at rest in the laboratory frame of reference. The energetic incident ion of mass, $M_{1}$, and atomic number, $Z_{1}$, and kinetic energy, $E$, is scattered by the stationary particle via a conservative, spherically symmetric, two-body potential. As the potential is conservative and spherically symmetric, the Newtonian (or Lagrangian) equations for the two-body system may be rewritten in terms of an equation for the center of mass of the system and an equation for a reduced mass particle moving in the center of mass system [12]. The reader is referred to reference 12 for a detailed discussion of the two-body problem in classical mechanics. The present calculation is not concerned with a detailed description of the scattering process but rather with the determination of the asymptotic behavior of the two particles. In particular, the elastic energy lost by the energetic ion and the direction of its final velocity vector relative to its initial velocity vector are of prime interest. These can be determined from the asymptotic scattering angle in the center of mass coordinate system. The scattering event in the center of mass system is depicted in the bottom portion of figure 1 . There, $P$ is the impact parameter, $r_{0}$ is the distance of closest approach, and $E_{r}=E /\left(1+M_{1} / M_{2}\right)$ is the relative energy in the center of mass system. In the 
laboratory frame, the angles, $\Phi^{\prime}$ and $\Phi^{\prime \prime}$, and the energies, $E^{\prime}$ and $E^{\prime \prime}$, are determined from the conservation laws for energy and momentum as

$$
\begin{gathered}
\tan \Phi^{\prime}=\frac{M_{1} \sin \theta}{M_{1}+M_{2} \cos \theta}, \\
\Phi^{\prime \prime}=\frac{\pi-\theta}{2}, \\
E^{\prime}=E-T,
\end{gathered}
$$

and

$$
E^{\prime \prime}=T
$$

where the elastic energy transfer or recoil energy, $T$, is

$$
T=\gamma E \sin ^{2}\left(\frac{\theta}{2}\right)
$$

where the recoil factor, $\gamma$, is given by

$$
\gamma=4 \frac{M_{1} M_{2}}{\left(M_{1}+M_{2}\right)^{2}} .
$$

From eqs (1) to (5), it is clear that the scattering angle, $\theta$, in the center of mass coordinate system is of central importance. The asymptotic scattering angle in the center of mass system is determined from integrating the classical equations of motion and is given by

$$
\theta\left(P, E_{r} ; V(r)\right)=\pi-2 P \int_{r_{0}}^{\infty} \frac{d r}{r^{2}\left(1-\frac{P^{2}}{r^{2}}-\frac{V(r)}{E_{r}}\right)^{1 / 2}} .
$$

The notation, $\theta\left(P, E_{r} ; V(r)\right)$, indicates that the scattering angle depends upon the variables, $P, E_{r}$, and upon the function, $V(r)$. The distance of closest approach, $r_{0}$, is determined from the solution of the equation

$$
\left(1-\frac{P^{2}}{r_{0}^{2}}-\frac{V\left(r_{0}\right)}{E_{r}}\right)=0 .
$$

The solution, $r_{0}$, may be obtained numerically by means of the Newton method with a few iterations. In the above, $V(r)$ is the spherically symmetric two-body potential which 
is of the general form of the screened Coulomb potential. This is written as a product of the Coulomb potential and a screening function in the form

$$
V(r)=\frac{Z_{1} Z_{2} e^{2}}{r} \phi(r)
$$

where $\phi(r)$ is the screening function. In its simplest form, the screening function may be written as

$$
\phi(r)=\exp (-r / a)
$$

where $a$ is a screening length which determines the cut-off of the function. In its most general form, the screening function may be written as a sum of normalized, weighted exponential terms

$$
\phi(r)=\sum_{i=1}^{n} \omega_{i} \exp \left(-b_{i} r / a\right),
$$

where the normalization of the weighting coefficients is expressed as

$$
\sum_{i=1}^{n} \omega_{i}=1
$$

The two particular forms of the general screening function used in ion implantation calculations are the Moliere [13] and the krypton-carbon [14] type. The Moliere screening function is given by

$$
\phi(r)=0.35 \exp (-0.3 r / a)+0.55 \exp (-1.2 r / a)+0.1 \exp (-6 r / a)
$$

while the krypton-carbon potential is given by

$$
\phi(r)=0.191 \exp (-0.279 r / a)+0.474 \exp (-0.637 r / a)+0.335 \exp (-1.919 r / a) .
$$

These two forms of the screening function are presented in figure 2. More recently, Biersack and Ziegler [15] have proposed a universal screening function which is similar to the krypton-carbon screening function. In the analysis and calculation of the effects of energetic ions on solids, it is convenient to make use of the natural length and energy units which appear in the equations. In particular, the screening length provides a natural unit 
of length while the Coulomb part of the screened Coulomb potential provides a natural unit of energy. The screening length used in the calculations was proposed by Firsov [16] and is given by

$$
a=\frac{0.8853 a_{0}}{\left(Z_{1}^{1 / 2}+Z_{2}^{1 / 2}\right)^{2 / 3}}
$$

where $a_{0}=0.529 \mathrm{~A}$ is the Bohr radius. This length occurs naturally in the expression for the screening function given by eq (9) which may be rewritten as

$$
V(R)=\frac{Z_{1} Z_{2} e^{2}}{a R} \phi(R)
$$

In terms of the scaled energy, it is convenient to define a dimensionless energy variable, $\epsilon$, by

$$
\epsilon=\frac{a E_{r}}{Z_{1} Z_{2} e^{2}}=\frac{a}{Z_{1} Z_{2} e^{2}\left(1+M_{1} / M_{2}\right)} E
$$

It is possible to write the above equation as $\epsilon=F E$, where $E$ is the energy is $\mathrm{eV}$. Table 1 contains the listing of the factor, $F$, for a number of ions incident upon silicon. This will be useful in the consideration of the relative effects of nuclear and electronic stopping.

A typical energetic ion may require hundreds if not thousands of two-body elastic collisions before it comes to rest. This would mean that the scattering integral in eq (7) would have to be evaluated a large number of times in order to properly treat the elastic scattering processes. This repeated evaluation would then become the rate limiting step in the calculation. Robinson [17] has used Gauss-Legendre and Gauss-Mehler quadrature techniques in order to evaluate the scattering integral as well as other integrals involved in classical two-body scattering by a central potential. This has been performed for the Moliere screening potential as well as a number of other screening functions. He has presented the results in tabular form for a range of impact parameters and energies and for a number of screening functions. While the repeated evaluation of the scattering integral in the slowing down process is time-consuming, the entry of the results of the Robinson tables into a data set for interpolation is also time-consuming. In addition, extensive tables would use a considerable amount of memory. The answer lies in the use of what Biersack and Haggmark call the "MAGIC" formula. 


\section{MAGIC Formula for Elastic Scattering}

The speed and efficiency of the TRIM code is achieved by the use of a fitted algebraic relation between the scattering angle and the impact parameter and the energy (determined from the Robinson tables). This MAGIC formula is obtained by expressing the scattering angle in terms of a five-parameter expression which reduces to the Rutherford scattering result for large $\epsilon$ and then by determining the parameters by a least-square fit to the results of the Robinson tables. Hence, once the impact parameter and the energy are given, the scattering angle is determined by an algebraic expression which contains by construction the results of the Robinson evaluation of the scattering integral. The construction of the MAGIC formula proceeds as follows. First, make use of the transformation to dimensionless length and energy units. The transformed lengths are

$$
\begin{gathered}
B=P / a, \\
R_{0}=r_{0} / a, \\
R_{r}=\rho / a \\
\Delta=\delta / a
\end{gathered}
$$

where $\rho$ and $\delta$ are related to the scattering geometry. In particular, $\rho$ may be written as

$$
\rho=\frac{2\left(E_{r}-V\left(r_{0}\right)\right)}{-V^{\prime}\left(r_{0}\right)}
$$

where $V^{\prime}\left(r_{0}\right)$ is the derivative of the potential evaluated at the distance of closest approach. In terms of these reduced variables, it is possible to write the equation for the scattering angle as

$$
\cos \frac{\theta}{2}=\frac{B+R_{r}+\Delta}{R_{0}+R_{r}}
$$

The function, $\Delta$, is fitted by means of the required asymptotic behavior to the Rutherford scattering at large $\epsilon$ and the tabular results of Robinson. This leads to the expression

$$
\Delta=A \frac{R_{0}-B}{1+G},
$$


where

$$
\begin{gathered}
A=2 \alpha \epsilon B^{\beta} \\
G=\gamma\left\{\left(1+A^{2}\right)^{1 / 2}-A\right\}^{-1} \\
\alpha=1+C_{1} \epsilon^{-1 / 2} \\
\beta=\frac{C_{2}+\epsilon^{1 / 2}}{C_{3}+\epsilon^{1 / 2}}
\end{gathered}
$$

and

$$
\gamma=\frac{C_{4}+\epsilon}{C_{5}+\epsilon} .
$$

Table 2 contains the listing of the coefficients, $\left\{C_{i}\right\}$, for both the Moliere and the kryptoncarbon potentials. Figures 3 and 4 contain the results of the MAGIC formula for the Moliere and the krypton-carbon potentials, respectively. In these figures, the function $\sin ^{2}\left(\frac{\theta}{2}\right)$ is presented as a function of the reduced impact parameter for a range of reduced energy values. It is clear from these figures that for $\epsilon \geq 1$, elastic scattering is important at only small relative impact parameters. Figures 5 and 6 contain a comparison of the results for two values of the reduced energy. These differences are traceable to the forms of the two screening functions. For $\epsilon>10$, the result for Rutherford scattering, i.e.,

$$
\sin ^{2}\left(\frac{\theta}{2}\right)=\left[1+(2 \epsilon B)^{2}\right]^{-1}
$$

is used in the calculation.

The original TRIM calculations discussed by Biersack and Haggmark made use of the Moliere form of the potential. The results which they obtained for antimony on silicon indicate that the nuclear potential is somewhat too strong as antimony experimentally gets further into the silicon target than predicted by TRIM. The krypton-carbon potential is softer than the Moliere. With this in mind, the krypton-carbon potential was used to generate the data contained in this report.

Once the asymptotic scattering angle is determined from the impact parameter, the energy and the specific form of the potential from the algebraic formula, the elastic energy 
transferred to the lattice atom is determined from the equation

$$
T=\gamma E \sin ^{2}\left(\frac{\theta}{2}\right)
$$

where the recoil factor, $\gamma$, is given by

$$
\gamma=4 \frac{M_{1} M_{2}}{\left(M_{1}+M_{2}\right)^{2}} .
$$

Figure 7 represents the recoil factor, $\gamma$, as a function of the mass ratio, $M_{2} / M_{1}$. Notice that this function peaks for equal masses and falls off to about 0.3 when the mass ratio (or its inverse) is about 10. The nuclear energy transfer determined from eq (31) is responsible for lateral scattering and primary displacement damage. The lateral scattering arises from the elastic scattering where the direction of the energetic ion is turned away from its original incident direction. The primary displacement damage arises from the situation where the energy transferred, $T$, to the target atom is larger than the binding energy holding the target atom to a "lattice" site.

\section{Displacement Damage}

The version of TRIM used in these calculations does not follow the trajectory of the lattice atom which has been displaced by the collision with the energetic ion. In other words, collision cascades which tend to spread out the secondary displacement damage are not explicitly considered. This would lead to a significant increase in the computation time. By way of example, typical calculations with $10^{4}$ incident ions takes anywhere from 1 to 4 hours on a minicomputer. The CPU time increases with incident ion energy and also for incident ions which are smaller in mass than the target. Hence, calculations of boron into silicon usually fall into the longer CPU time range while calculations of arsenic into silicon usually take less than $1 \mathrm{CPU}$ hour. Following the primary recoil would increase the total CPU time by factors in the range of 10 to 100 . However, it is possible to obtain approximate information about the effects of collision cascades and the secondary damage in the form of vacancy-interstitial or Frenkel pairs. The original discussion of the secondary 
damage created during the recoil and slowing down of the primary displaced ion in a solid was presented in a classic paper by Kinchin and Pease [18]. However, over the intervening 20 years estimates of the secondary damage caused by collision cascades have undergone a certain amount of revision. For the present purposes, the modified Kinchin-Pease equation [19] is used to determine the approximate secondary damage caused by the displaced lattice atom. The physical picture of the damage production is one where the ion which is knocked off a "lattice" site moves off as an interstitial and leaves behind a vacancy in the "lattice". This pair of defects is referred to as a Frenkel pair. The behavior of the vacancies and the interstitials is not addressed by the TRIM code. There are a number of processes in which these defects can participate. The vacancy and the interstitial may recombine to give rise to a substitutional ion. This would reduce the defect density in the material. For the purposes of semiconductor device fabrication, this process is devoutly to be wished. However, the vacancies and/or interstitials may coalesce among themselves to form macrodefects which may have to be removed by annealing. Clearly, these topics are of great scientific and technological importance and form the basis of the study of defects.

The assumption which is made at this point is that the Kinchin-Pease equation may be used to calculate the defect (Frenkel pair) distribution. The interstitial is assumed to stay in the same volume element as the vacancy. This volume element is usually on the order of about $100 \AA$ on a side. The fate of the vacancies and interstitials is not considered but the distributions determined by this procedure can be used as the initial conditions for investigations of defects. Within the framework of the Kinchin-Pease equation, the defect producing energy, $E_{d p}$, is determined from the energy transferred, $T$, to the primary displacement according to equation

$$
E_{d p}=\frac{T}{1+k_{d} g\left(\epsilon_{d}\right)}
$$

where

$$
\begin{gathered}
k_{d}=0.1334 Z_{2}^{2 / 3} M_{2}^{-1 / 2}, \\
g\left(\epsilon_{d}\right)=\epsilon_{d}+0.40244 \epsilon_{d}^{3 / 4}+\epsilon_{d}^{1 / 6}
\end{gathered}
$$


and

$$
\epsilon_{d}=0.01024 Z_{2}^{-7 / 3} T
$$

The total number, $\nu$, of Frenkel pairs produced by the primary nuclear energy transfer were determined from

$$
\nu=1
$$

for $E_{D} \leq E_{d p}<2.5 E_{D}$ and

$$
\nu=\frac{0.8 E_{d p}}{2 E_{D}}
$$

for $E_{d p} \geq 2.5 E_{D} . E_{D}$ is the displacement energy which is approximately $25 \mathrm{eV}$ for silicon.

The total number of displaced atoms is assumed to be in the form of vacancy-interstitial pairs. That is to say, the displaced atom leaves behind a vacancy in the lattice while moving away from it to form an interstitial. While actual implantation conditions, i.e., dose rate and target temperature, may provide enough thermal energy for the vacancy and interstitial to recombine or even to form extended defects, it is assumed that the Frenkel pair is a stable configuration at very low temperatures. The subsequent behavior of the vacancy and interstitial is not explicitly considered in the TRIM code. The validity of the approximation that the vacancies and interstitials stay in the same volume element as the primary collision may in some sense be tested by evaluating the average energy transferred to the primary. If this average energy is large enough such that the range of the displaced primary is on the order of the step increment used in the calculation, then the calculated Frenkel pair distribution will tend to be much shallower that the actual Frenkel pair distribution.

\section{Electronic Energy Loss}

In between the two-body collisions described above, the energetic ion is assumed to experience a velocity-dependent viscous drag caused by the electron gas in the target. This electron gas may be viewed as arising from the outer valence electrons of the target atoms. This situation is physically reasonable at low ion energies where the analogy to viscous 
drag seems correct. For large ion energies (but below relativistic energies), the viscous drag may be envisioned as decreasing. The calculations of Lindhard [20] and Firsov [16] for the energy loss are used in TRIM in the low energy regime. In general, the inelastic energy loss of an ion traveling a distance $L$ in a material with atomic density (number density) equal to $N$ may be written as

$$
\Delta E_{e}=L N S_{e}(E)
$$

where $S_{e}(E)$ is the electronic stopping. In the low energy region,

$$
S_{e}(E)=S_{L}=k E^{q}
$$

where the Lindhard theory predicts that

$$
k=k_{L}=\frac{1.212 Z_{1}^{7 / 6} Z_{2}}{\left(Z_{1}^{2 / 3}+Z_{2}^{2 / 3}\right)^{3 / 2} M_{1}^{1 / 2}}
$$

and

$$
q=1 / 2
$$

More generally, $k$ and $q$ may differ from the above values and may be functions of the ion energy. In the high energy regime, the electronic stopping is diminished. In this region, the electronic stopping may be described by the Bethe-Block theory. The Bethe-Block electronic stopping is represented by

$$
S_{B}=\frac{8 \pi Z_{1}^{2} e^{4}}{I_{0} \epsilon_{B}} \ln \left(\epsilon_{B}+1+C / \epsilon_{B}\right)
$$

where

$$
\epsilon_{B}=\frac{2 m_{e} v^{2}}{Z_{2} I_{0}}
$$

and where

$$
I_{0}=12+7 Z_{2}^{-1}
$$

for $Z_{2}<13$, and

$$
I_{0}=9.76+58.5 Z_{2}^{-1.18}
$$


for $Z_{2} \geq 13$. In eq (43), $C=5$ for $Z_{1}>3$. For $Z_{1}<3$, the value, $C=100 Z_{1} / Z_{2}$, is used. The two energy regions are connected by means of an expression introduced by Biersack [21]. This allows for a smooth transition of the electronic stopping from the low-energy regime described by Lindhard and the high-energy regime described by Bethe-Block. In particular, this connection is given by

$$
S_{e}=\left(S_{L}^{-1}+S_{B}^{-1}\right)^{-1}
$$

For the purposes of discussing the various contributions to the electronic stopping which are currently feasible in silicon device fabrication, figure 8 contains the electronic stopping for a light ion like boron and a heavy ion like arsenic incident on a silicon target at normal silicon densities. The curve for boron shows both the low-energy region and the highenergy region as well as the transition region obtained by using the Biersack connection. The curve peaks at 2 to $3 \mathrm{MeV}$. The boron curve is labeled with $k / k l=1.5$ as it was calculated with a correction to the classical Lindhard expression. This will be discussed shortly. For the case of arsenic, there is no peak region even up to $10 \mathrm{MeV}$. Figure 9 is an expanded version of the previous figure in the range of energies from $10 \mathrm{keV}$ up to 1 $\mathrm{MeV}$. If these curves were replotted as stopping vs. $E^{1 / 2}$, the curves would be linear over most of the range of the plot. Most implantations used in semiconductor device fabrication are done in the range of energies from about $10 \mathrm{keV}$ up to about $400 \mathrm{keV}$. Hence, for the energies considered in these calculations (up to $400 \mathrm{keV}$ ), the energies may be considered as low. That is to say, the Lindhard expression could be used directly. However, for purposes of generality, the calculations were performed with the more general Biersack connection.

In the above discussion, the notion of the use of a corrected Lindhard coefficient for the electronic stopping was introduced. This is based upon a number of observations made on the profiles of well-channeled implants of a number of species in silicon targets. In particular, deviations from the model are known to exist $[22,23]$ in terms of oscillations in the electronic stopping. These may be included in the TRIM code in terms of a correction factor to the low-energy electronic stopping. The inelastic electronic stopping is assumed 
not to contribute to either lateral scattering or displacement damage due to the very small mass of the electron compared with the mass of the energetic ion.

Combined Effects

The nuclear and electronic contributions to the stopping are presented in figure 10. Also shown is the combined effect, $S_{\text {tot }}(\epsilon)$, which is obtained from adding the two stopping terms, i.e.,

$$
S_{\text {tot }}(\epsilon)=S_{e}(\epsilon)+S_{n}(\epsilon)
$$

For large $\epsilon$, the electronic stopping dominates, whereas the nuclear stopping is important for small values of $\epsilon$. This can be understood from the results of figures 3 and 4 . For large values of $\epsilon$, elastic nuclear scattering is effective only for small values of the impact parameter. From the evaluation of the factor relating the kinetic energy of the energetic ion and the $\epsilon$ value (see table 1), light mass ions will be stopped mainly by the electronic term, while heavy mass ions will be stopped mainly by the nuclear term. This will be discussed in more detail in the section related to the interpretation of the stopping terms.

\section{Discussion of How the Code Works}

Having described the two components of the stopping of the energetic ion, the typical sequence of the stopping of an individual ion is now discussed. The target is divided into a number of two-dimensional boxes with equal length sides predetermined by the user. In particular, this length is the depth increment entered in the input data file. It is important that this length is not too small such that only a few particles will be in any bin and hence contribute to noise fluctuations in the data. On the other hand, the length should not be too big; otherwise, there will be insufficient detail in the one- and two-dimensional data. The judicious choice of this length comes from experience in the use of the code and is based upon such things as the mass and energy of the incident ion as well as the number of histories used in the simulation. For silicon targets and $10^{4}$ histories, this length is typically on the order of $100 \AA$. The ion is assumed to be normally incident on the top surface of the target. The trajectory of an individual ion which is incident on the target is followed 
through the sequence of binary collisions with the core ions as well as the viscous drag with the electron gas along the path between the collisions with the core ions. If the energy transferred to the lattice ions is below the displacement energy for silicon, the collision is considered to excite the phonons. If the energy transferred is above the displacement energy, the Kinchin-Pease model is used to calculate the approximate number of local vacancy-interstitial pairs created. These calculations follow the incident ion in the target until its energy falls below a predetermined energy (usually $5 \mathrm{eV}$ ) or until it emerges from the back surface of the target. Also, incident ions which are backscattered from the top surface may be followed. This calculation is repeated for each of the ions incident upon the target until the total number of particles in the beam have been considered. For the total of all of the ion histories, the number of stopped ions, primary displacements, Frenkel pairs, and the energy lost to phonon excitation, displacement damage, and electronic excitation are accumulated in two-dimensional arrays corresponding to the locations in the two-dimensional grid. The code follows the collision sequence in three dimensions. In order to obtain the two-dimensional results from a line source and to reduce the effects of the noise resulting in the outer regions of the distribution, two steps were carried out with the data. First, the results which arise from scattering out of the $x-y$ plane were projected into the plane. This simulates the two-dimensional results arising from a line source where contributions from planes to the right and left of the plane of interest would be added. Second, the resulting two-dimensional results were added symmetrically about the incident beam line. The added numbers were then placed on either side of the incident beam line. The results which arise along the incident beam line were simply doubled. This in effect doubles the number of particles with the added smoothing of the results away from incident beam line. The basis of this is founded on the argument that the target is spatially isotropic due to the assumption of a random target and that the two-body potential is spherically symmetric.

The primary focus of the present calculations is to present the two-dimensional distribution of implanted ions as well as an approximate two-dimensional distribution of vacancy- 
interstitial pairs for a single line beam. In addition, the one-dimensional distribution of stopped ions, primary damage, approximate Frenkel pairs, electronic energy loss, phonon excitation energy loss, and displacement damage energy loss is also discussed. The effects of implantation past a mask edge upon the two-dimensional distributions of implanted ions and vacancy-interstitial pairs were obtained by superimposing a number of line beams. As previously mentioned, the validity of the approximate calculation of the local Frenkel pair distributions was investigated from the point of view of the average primary displacement energy.

\section{Elements Used in Calculation}

The TRIM code does not distinguish any one element from any other as to choice of incident energetic ions. In principle, the calculations can be performed on any ion incident upon any target. For the purposes of silicon device technology, there are a small number of incident ions which are of interest. These fall into two categories, those which act as donors or acceptors and those which act to limit lifetime or are gettering sites. This requirement limits the number of incident ions of interest to about sixteen. These include: aluminum, antimony, arsenic, boron, carbon, gallium, germanium, gold, indium, nitrogen, oxygen, phosphorous, platinum, silicon, sulfur, and tin. The only exception to the requirements for either of the two categories is silicon which is included in order to provide an estimate of displacement damage and amorphization when silicon is implanted into silicon.

The one- and two-dimensional plots are contained in Appendix B under the names of the elements. These results were obtained from the TRIM runs for energies from 25 $\mathrm{keV}$ to $400 \mathrm{keV}$ in increments of $25 \mathrm{keV}$. Hence for each element, sixteen values of the energy were used. For each value of the energy, four figures were prepared. One figure contains the energy loss probability densities for electronic, displacement damage, and phonon excitation while another contains the probability densities for atoms, primary displacements, and approximate Frenkel pairs. The two remaining figures present the results of particle and approximate Frenkel pair distributions (normalized to the peak 
value of the former) for implantation past a mask edge.

\section{One-Dimensional Results for a Uniform Target}

The TRIM code can be used to investigate the relative effects of the mass of the incident ion upon things like the displacement damage and electronic energy loss. The information which is of prime interest and which is presented in the figures are the energy loss probability densities for electronic, displacement damage, and phonon excitation and the probability densities for atoms, primary displacements, and approximate Frenkel pairs. These figures were obtained as follows. The one-dimensional data for each of these quantities were obtained by summing the two-dimensional data along each depth step. The one-dimensional data for the implanted atoms were then summed over the depth to obtained a normalization constant. The one-dimensional data for each of these quantities were then divided by this normalization constant to construct the corresponding probability densities. For the case of the implanted ions, the resulting probability density is normalized to unity, whereas the normalization for the other quantities represents the average effect due to a single implanted ion on each of these quantities. For example, the normalization of the primary displacement and the approximate Frenkel pair probability densities gives the average number of primary displacements and approximate Frenkel pairs due to a single incident ion. Likewise, the normalization of the electronic, displacement damage, and phonon excitation probability densities determines the average energy lost to each of these three mechanisms by a typical incident ion. The reason for the magnitude of the probability densities comes from the use of the depth unit in $\mu m$. One of the useful features of the use of the probability densities in this form is that when they are multiplied by the dose, they yield physical densities for each of the quantities considered.

It is of interest to consider the effects of incident ion energy and mass on these unnormalized probability densities. In particular, the effect of the mass of the incident ion is easy to investigate. This can be done by considering three different situations and three different incident ions: $75-\mathrm{keV}$ boron $\left(M_{1}<M_{2}\right), 300-\mathrm{keV}$ silicon $\left(M_{1}=M_{2}\right)$, and 100-keV arsenic 
$\left(M_{1}>M_{2}\right)$. This will serve to introduce the reader to the various figures but also present the kinds of information which can be obtained from these figures. The figures are labelled by the atomic symbol of the incident ion, the incident beam energy, and an index ranging from 1 to 4. With this in mind, the figures of particular interest at this time are B75$(1,2,3,4)$, SI300-(1,2,3,4), and AS100-(1,2,3,4). These are to be found in the figure listings which are presented by increasing atomic number of the incident ion.

First consider the case of $75-\mathrm{keV}$ boron $\left(M_{1}<M_{2}\right)$. Figure B75-1 contains the plots of the energy loss probability densities, while figure B75-2 contains the plots of the probability densities for atoms, primary displacement damage, and approximate Frenkel pairs. From figure B75-1, it is clear that phonon excitation is a relatively small contribution to the slowing down of the incident boron. Electronic energy loss dominates displacement damage over most of the depth. This is part of the reason why boron has great difficulty in amorphizing silicon. It might be argued that the energy loss due to electronic modes goes into the production of heat which would provide a mechanism for the vacancy interstitial reaction and the production of a substitutional silicon. The average energy transferred to a primary displacement may be calculated by dividing the energy loss probability density by the probability density for primary displacement damage. The results of this calculation are contained in figure 11 and represents the average energy lost by the boron atom to primary displacement damage. The maximum in this curve lies at about $0.5 \mathrm{keV}$. The approximate range for silicon into silicon (the primary scattered into the target) at this energy is about $0.0005 \mu \mathrm{m}$. This would indicate that the approximate Frenkel pair densities calculated by the Kinchin-Pease equation should be reasonably accurate.

In going from small mass to equal mass, the probability densities presented in figures SI300-1 and SI300-2 arise for the case of 300-keV Si into Si. Again, the phonon excitation is small compared with the electronic and displacement damage contributions to the energy loss. Also, the electronic energy loss is not as dominant when compared with displacement damage as was seen in the case of the boron implant. This is due to the change in mass in 
going from boron to silicon incident ions. The approximate Frenkel pair probability density increases by about a factor of 5 . This along with the decreased contribution to electronic energy loss indicates that amorphization of silicon by silicon can be accomplished much more easily than by boron. The results of the calculation of the average energy transferred to the primary displacement are contained in figure 12. The maximum in the curve is at about $3 \mathrm{keV}$ where the silicon-into-silicon range is about $0.005 \mu \mathrm{m}$. This would have the effect of pushing the interstitial densities deeper into the target. Within the confines of the method used, this would probably cause the Frenkel pair density to become somewhat more peaked and somewhat deeper than shown in figure SI300-2.

Finally, figures AS100-1 and AS100-2 contain the probability density results for a $100-\mathrm{keV}$ arsenic implant into silicon. Here, the displacement damage energy loss completely dominates other energy losses. In addition, the approximate Frenkel pair probability density is up by an order of magnitude over the corresponding boron results. This points to the relative ease with which the much more massive arsenic ion can damage the silicon lattice to complete amorphization. Figure 13 contains the results of the average energy transferred to a primary displacement. Here, as with the previous example of silicon, the maximum in the curve appears at several $\mathrm{keV}$. This would also tend to push the Frenkel pair densities somewhat deeper into the material. This discussion contains the salient features for the interpretation of the results presented in the figures for each of the incident ions in silicon.

\section{Two-Dimensional Results for a Masked Target}

In this section, the results of the calculation of the two-dimensional particle and approximate damage distributions for implantation past a mask edge are presented and discussed. These distributions are constructed by superposition of the two-dimensional line beam results. This is carried out as follows. Starting from a fixed distance from the edge of a rectangular mesh, i.e., at the first step past the edge of the perfectly absorbing mask, the two-dimensional line beam results for the implanted ions and approximate Frenkel pairs are entered into a two-dimensional array. The line beam is moved over by one-step incre- 
ment and added to the results stored in the corresponding two-dimensional arrays. The line beam is moved over one more step and added again. This process is repeated until the line beam is far from the mask edge. The resulting two two-dimensional arrays (for implanted particles and approximate Frenkel pairs) should then give a reasonably accurate description of these two quantities for implantation past a mask edge.

Figures B75-3 and B75-4 contain the unnormalized two-dimensional particle and approximate Frenkel pair contours for $75-\mathrm{keV}$ boron implanted into silicon. The two-dimensional particle contours show significant penetration under the mask edge. Enhanced diffusion of boron in the presence of the displacement damage could further complicate the situation indicated above.

Figures SI300-3 and SI300-4 contain the particle and Frenkel pair contours for 300-keV silicon implanted into silicon. Figures AS100-3 and AS100-4 contain the particle and Frenkel pair contours for $100-\mathrm{keV}$ arsenic implanted into silicon. By comparison with the boron results, arsenic does not penetrate as far under the mask. However, there is significantly more damage as indicated by the Frenkel pair contours.

\section{Parameterization of Particle Distributions}

In order to make the results of this investigation useful in process modeling, the distributions were parameterized in terms of standard polynomial fitting techniques. The results of these fitting procedures are presented in tabular form in Appendix $A$ for the various elements. It is important to use these polynomial coefficients only over the range of depth quoted in the tables $\left(X_{l}\right.$ and $\left.X_{u}\right)$, as extension past these points can be lead to oscillations which are unphysical. In order to construct the particle distributions from the polynomial coefficients, the following expression is used:

$$
f(X)=\exp \left\{C_{0}+C_{1} X+C_{2} X^{2}+C_{3} X^{3}+C_{4} X^{4}\right\}
$$




\section{Summary and Conclusions}

The TRIM Monte Carlo code can be used in a straightforward manner to obtain information about the energy loss mechanisms and the particle and damage distributions in one dimension (for a uniform target) and in two dimensions (for a masked target). The results are useful for determining particle and damage profiles [24]. These in turn provide easily interpreted input for two-dimensional diffusion/annealing codes to study enhanced diffusion under mask edges. The approximate Frenkel pair probability density when multiplied by the dose give Frenkel pair number distributions which can provide insight into the formation of amorphized or heavily damaged layers. The electronic energy loss information may be used to investigate the thermal effects which arise during implantation. These are specifically related to partial implantation annealing due to the choice of dose rates. The creation of Frenkel pairs along with subsequent condensation into extended defects or the complete removal of implantation-induced damage (depending upon dose rates and/or target temperature) might be more easily understood in light of the results of the TRIM calculations. The validity of the use of the approximate Frenkel pair densities determined from the use of the Kinchin-Pease equation has to be examined in light of the average primary energy. A more complete investigation of this validity and possible corrections would make use of not the average energy per se but rather the energy transferred to each of the primaries and the approximate ranges of silicon into silicon at each of these energies. In addition, extension of these calculations to other semiconductor materials is of considerable interest. This is especially the case in III-V materials. All of these are presently under investigation.

\section{Acknowledgments}

The author would like to thank Dave Myers of Sandia National Laboratories and Charles L. Wilson of the National Bureau of Standards for a number of useful discussions. The basis of the partial test of the validity of the use of the approximate Frenkel pairs was suggested by David Brice of Sandia National Laboratories. The author also thanks Jay Marchiando 
o.: the National Bureau of Standards for his assistance in generating the two-dimensional glots. Finally, the author would like to thank E. Jane Walters for her outstanding job of laying out the final report.

\section{References}

[1] J. T. Hynes and J. M. Deutch, "Nonequilibrium Problems-Projection Operator Techniques", Chapter in Treatise in Physical Chemistry, Vol. VIIB, Mathematical Methods, E. Henderson, Ed., Academic Press, New York (1973).

[2] J. Albers and J. M. Deutch, "On the Rate Equation Description of Spectral Lines", Chem. Phys. 1, 89-98 (1973).

[3] J. Lindhard, M. Scharff, and H. E. Schiott, "Range Concepts and Heavy Ion Ranges", K. Dan. Vidensk. Selsk. Mat. Fys. Medd. 33, No. 14, 1-39 (1963).

[4] J. F. Gibbons, W. S. Johnson, and S. W. Mylroie, Projected Range Statistics, 2nd Edition, Dowden, Stroudsburg (1975).

[5] K. B. Winterbon, Ion Implantation Range and Energy Deposition Distributions Volume 2-Low Incident Ion Energies, Plenum, New York (1975).

[6] K. B. Winterbon, "Computing Moments of Implanted-Ion Range and Energy Distributions", Atomic Energy of Canada Limited Report AECL-5536 (1976).

[7] W. K. Hofker, "Implantation of Boron in Silicon", Phillips Research Reports Supplement No 8, 1975.

[8] K. B. Winterbon, Peter Sigmund, and J. B. Sanders, "Spatial Distribution of Energy Deposited by Atomic Particles in Elastic Collisions", K. Dan. Vidensk. Selsk. Mat. Fys. Medd. 37, No. 14, 1-73 (1970).

[9] M. T. Robinson, "MARLOWE Binary Collision Cascade Simulation Program Version 12", July 1, 1984, Oak Ridge National Lab. 
[10] J. P. Biersack and L. G. Haggmark, "A Monte Carlo Computer Program for the Transport of Energetic Ions in Amorphous Targets", Nucl. Instrum. Methods 174, 257-269 (1980).

[11] L. S. Rodberg and R. M. Thaler, Introduction to the Quantum Theory of Scattering, Academic Press, New York (1967).

[12] H. Goldstein, Classical Mechanics, Addison-Wesley, Reading, Mass. (1950).

[13] G. Moliere, "Theorie der Streuung schneller galadener Teilchen I Einzelstreuung am abgeschirmten Coulomb-Feld", Z. Naturforsch. A2, 133 (1947).

[14] W. Wilson, L. Haggmark, and J. Biersack, "Calculation of Nuclear Stopping, Ranges, and Straggling in the Low-Energy Region", Phys. Rev. B 15, 2458 (1977).

[15] J. P. Biersack and J. F. Ziegler, "The Stopping and Range of Ions in Solids", Chapter in Ion Implantation Techniques, Editors: H. Ryssel and H. Glawischnig, SpringerVerlag, New York (1982).

[16] O. B. Firsov, "A Quantitative Interpretation of the Mean Electron Excitation Energy in Atomic Collisions", Soviet Physics JETP 36, 1517-1523 (1959).

[17] M. T. Robinson, "Tables of Classical Scattering Integrals", Oak Ridge National Laboratory Report ORNL-4556 UC-34-Physics (Sept. 1970).

[18] G. H. Kinchin and R. S. Pease, "The Displacement of Atoms in Solids by Radiation", Reports on Progress in Physics 18, 1-51 (1955).

[19] M. J. Norgett, M. T. Robinson, and I. M. Torrens, "A Proposed Method of Calculating Displacement Dose Rates", Nuclear Engineering Design 33, 50 (1974).

[20] J. Lindhard and A. Winther, "Stopping Power of Electron Gas and Equipartition Rule", K. Dan. Vidensk. Selsk. Mat. Fys. Medd. 34, No. 4, 1-21 (1964). 
[21] J. Biersack and D. Fink: In Atomic Collisions in Solids, Vol.2, Plenum Press, New York, 1975.

[22] F. H. Eisen, "Channeling of Medium-Mass Ions Through Silicon", Can. J. Phys. 46, 561 (1968).

[23] A. El-Hoshy and J. F. Gibbons, "Periodic Dependence of the Electronic Stopping Cross Section for Energetic Heavy Ions in Solids", Phys. Rev. 173, 454 (1968).

[24] J. Albers, "Monte Carlo Calculation of One- and Two-Dimensional Particle and Damage Distributions for Ion Implanted Dopants in Silicon", IEEE Trans. Electron Devices ED-32, 1930 (1985) and IEEE Trans. Computer-Aided Design CAD-4, 374 (1985). 
TABLE 1. Multiplicative Factor for Calculating Epsilon from Energy (eV) For Various Elements Incident Upon Silicon

$\begin{array}{cccc}\text { Element } & \text { Atomic Number } & \text { Atomic Mass } & \text { Factor }(1 / \mathrm{eV}) \\ \text { Hydrogen } & 1 & & \\ \text { Helium } & 2 & 1.008 & 7.9476979 \mathrm{E}-04 \\ \text { Boron } & 5 & 4.003 & 3.4066846 \mathrm{E}-04 \\ \text { Carbon } & 6 & 10.810 & 1.0185441 \mathrm{E}-04 \\ \text { Nitrogen } & 7 & 12.010 & 8.0435129 \mathrm{E}-05 \\ \text { Oxygen } & 8 & 14.007 & 6.4331936 \mathrm{E}-05 \\ \text { Aluminum } & 13 & 15.990 & 5.2748001 \mathrm{E}-05 \\ \text { Silicon } & 14 & 26.980 & 2.4115909 \mathrm{E}-05 \\ \text { Phosphorus } & 15 & 28.086 & 2.1685439 \mathrm{E}-05 \\ \text { Sulfur } & 16 & 30.970 & 1.9029347 \mathrm{E}-05 \\ \text { Gallium } & 31 & 32.060 & 1.7324581 \mathrm{E}-05 \\ \text { Germanium } & 32 & 69.720 & 4.8626148 \mathrm{E}-06 \\ \text { Arsenic } & 33 & 72.590 & 4.5474035 \mathrm{E}-06 \\ \text { Indium } & 49 & 74.920 & 4.2832517 \mathrm{E}-06 \\ \text { Tin } & 50 & 114.820 & 1.9138947 \mathrm{E}-06 \\ \text { Antimony } & 51 & 118.690 & 1.8181523 \mathrm{E}-06 \\ \text { Gold } & 79 & 121.750 & 1.7385657 \mathrm{E}-06 \\ & & 196.967 & 6.7665627 \mathrm{E}-07\end{array}$


TABLE 2. Values of MAGIC Parameters for Moliere and Krypton-Carbon Potentials.

$\begin{array}{ccc}\text { Parameter } & \text { Moliere } & \text { Krypton-carbon } \\ \text { C1 } & & \\ \text { C2 } & 0.6743 & 0.1166 \\ \text { C3 } & 0.009611 & 0.006913 \\ \text { C4 } & 0.005175 & 0.7887 \\ \text { C5 } & 10.00 & 10.79 \\ & 6.314 & 17.16\end{array}$



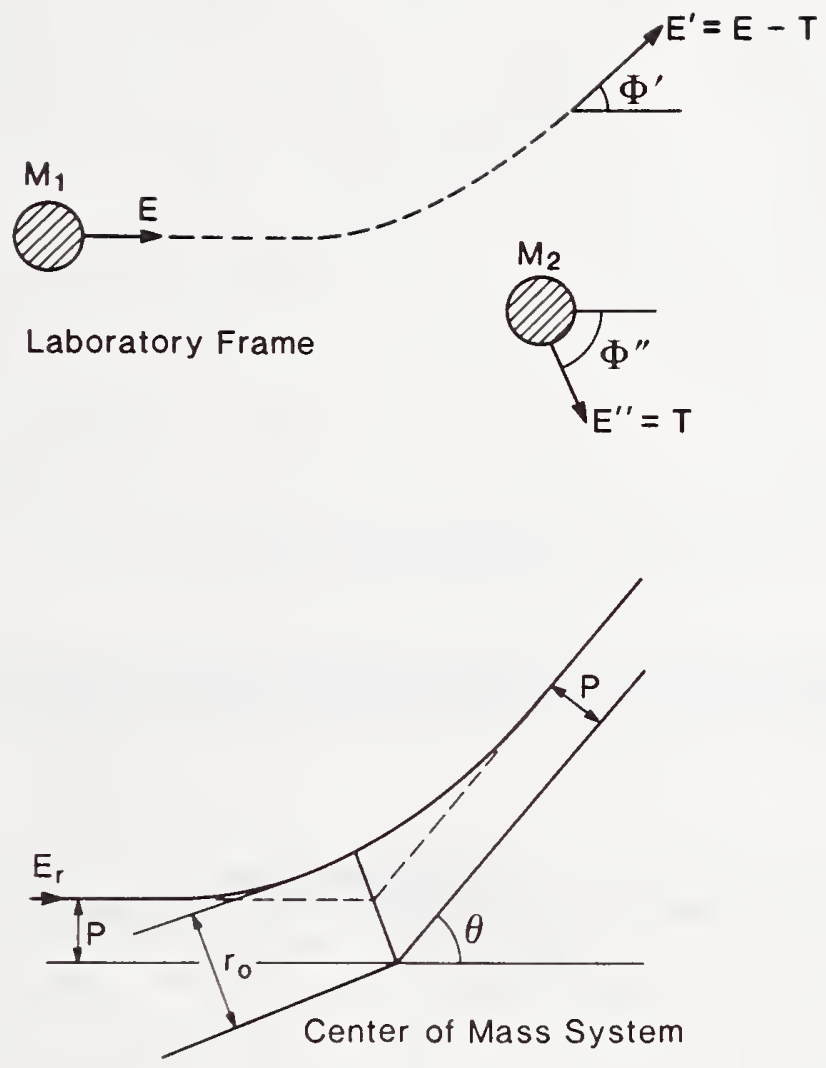

Figure 1. Scattering geometry in the laboratory and center of mass frame. 


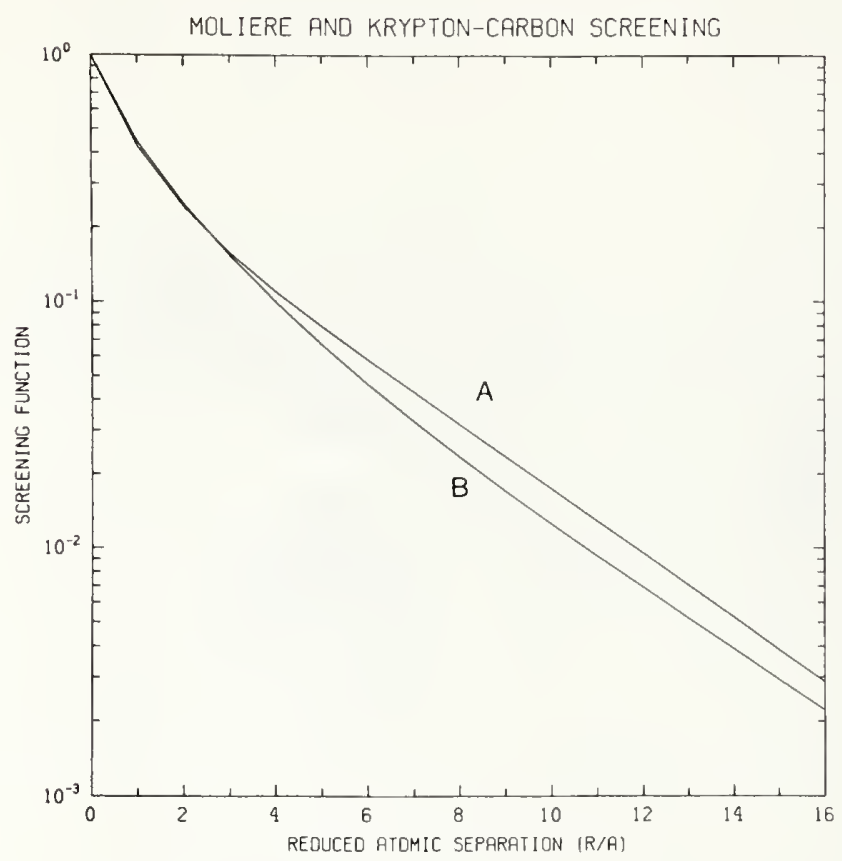

Figure 2. Plot of the Moliere (A) and the krypton-carbon (B) screening functions.

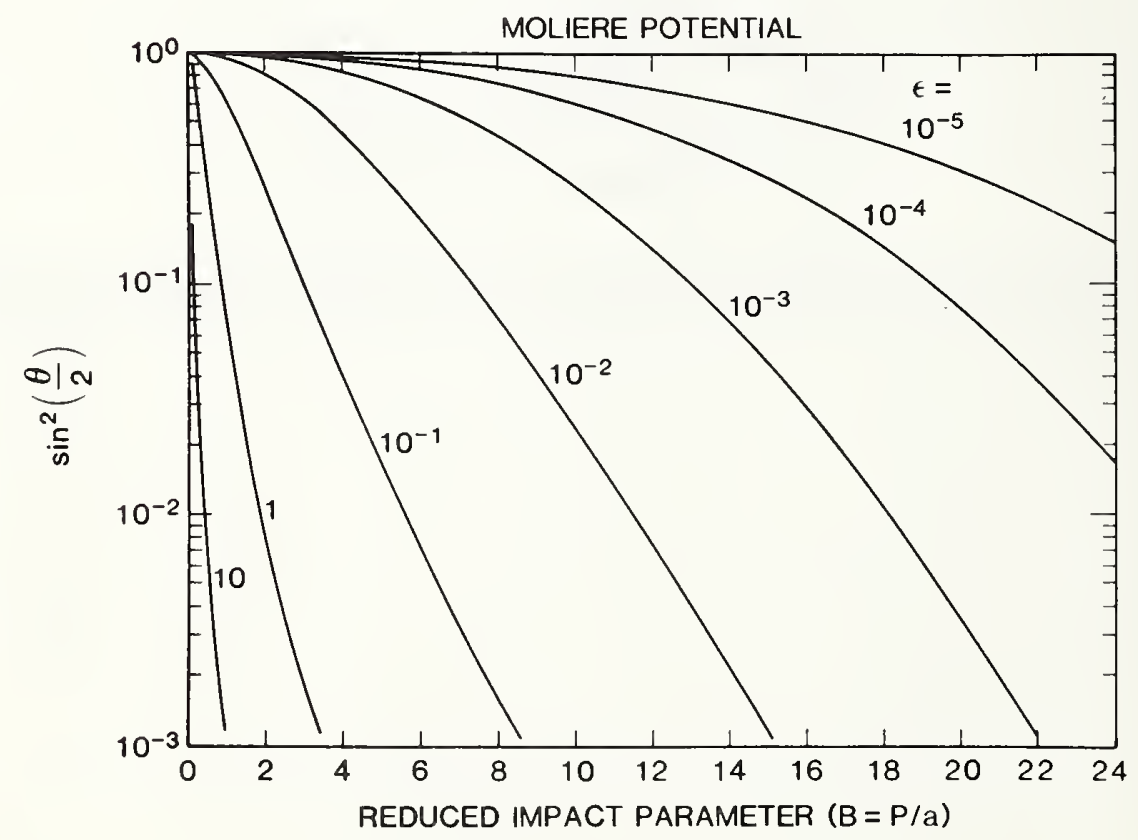

Figure 3. Scattering by the Moliere potential as depicted by the Biersack and Haggmark MAGIC formula. 


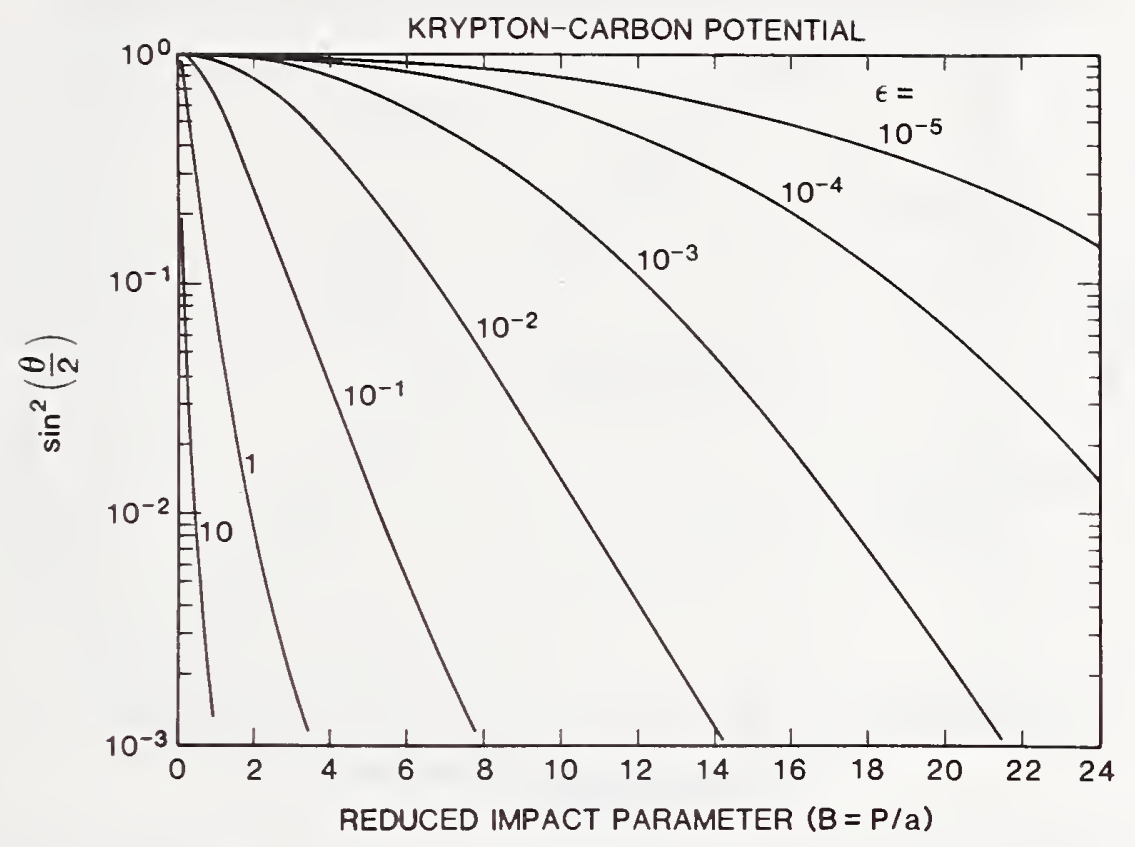

Figure 4. Scattering by the krypton-carbon potential as depicted by the Biersack and Haggmark MAGIC formula.

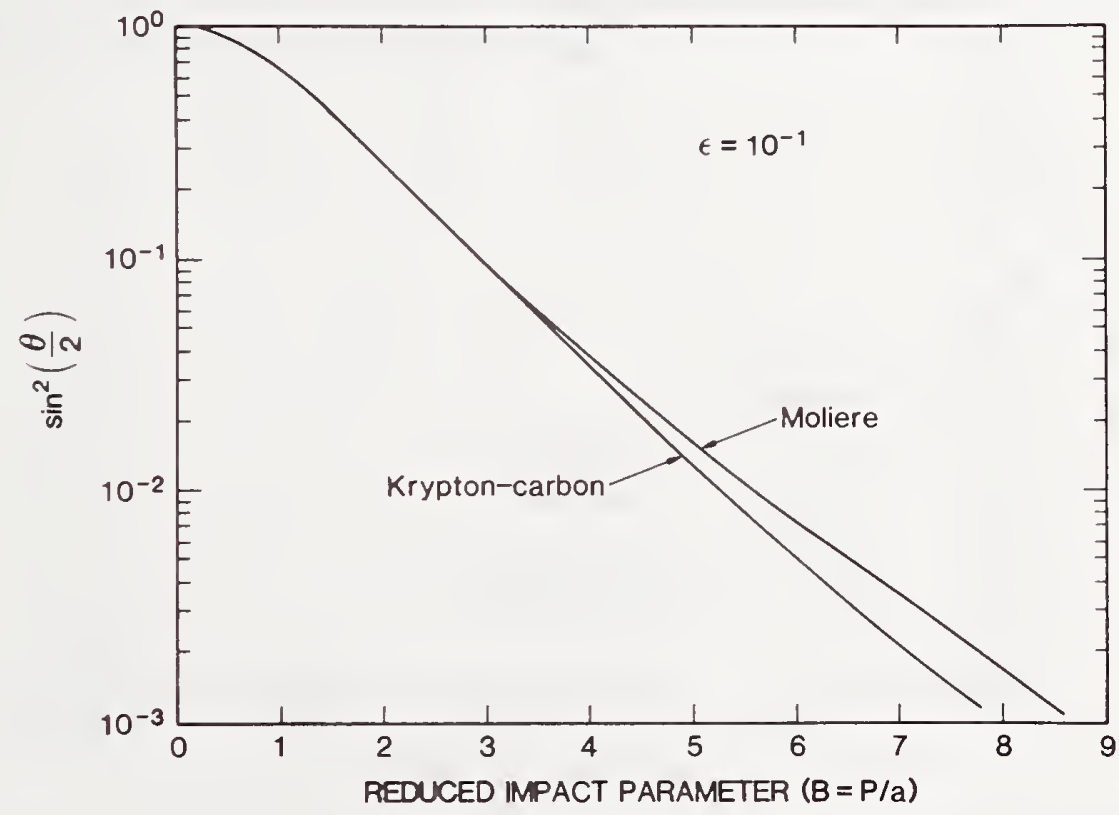

Figure 5. Comparison of the scattering angle vs. impact parameter determined by the Moliere and the krypton carbon potential (both from MAGIC formula) for $\epsilon=10^{-1}$. 


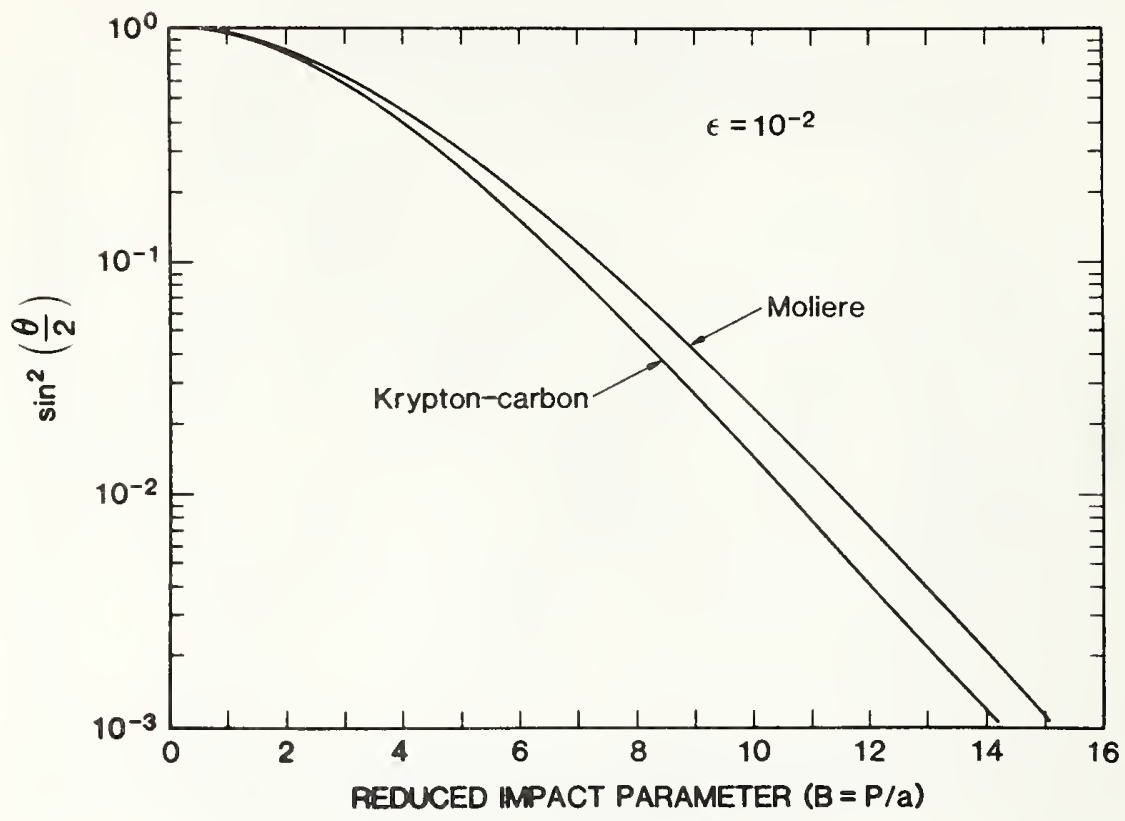

Figure 6. Comparison of the scattering angle vs. impact parameter determined by the Moliere and the krypton carbon potential (both from MAGIC formula) for $\epsilon=10^{-2}$.

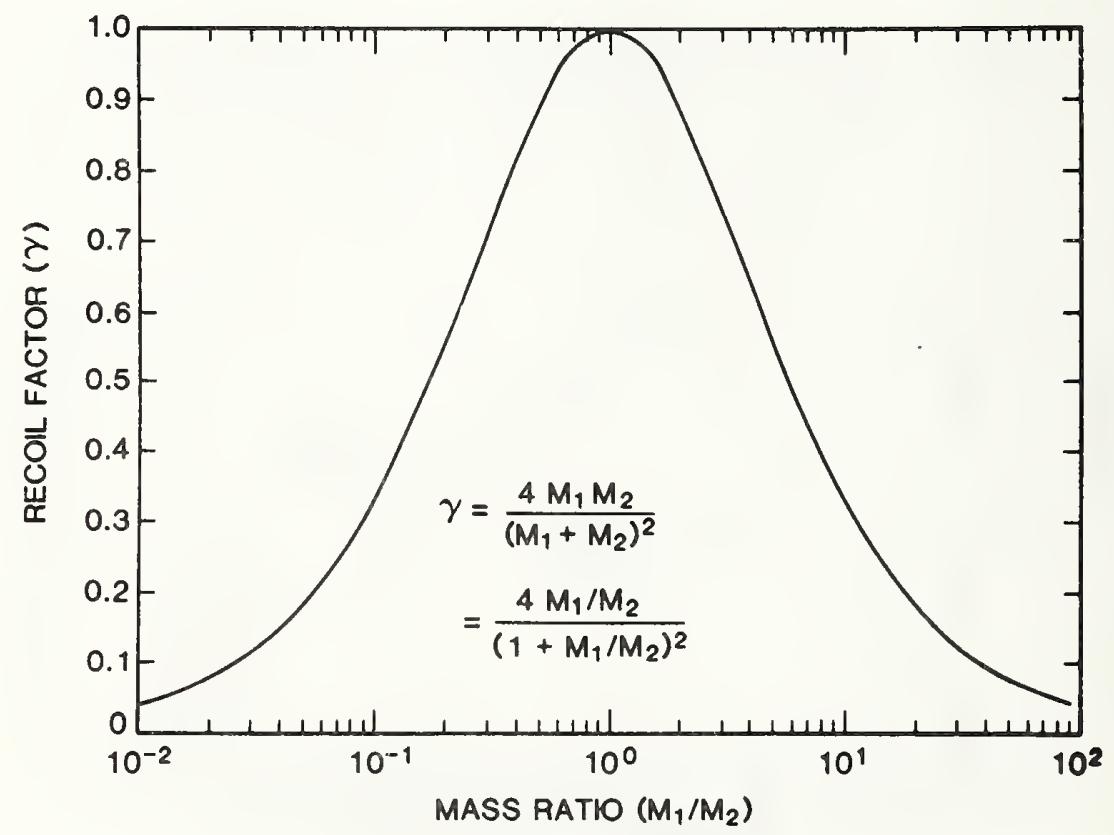

Figure 7. Recoil factor, $\gamma$, as a function of the mass ratio, $M_{2} / M_{1}$. 


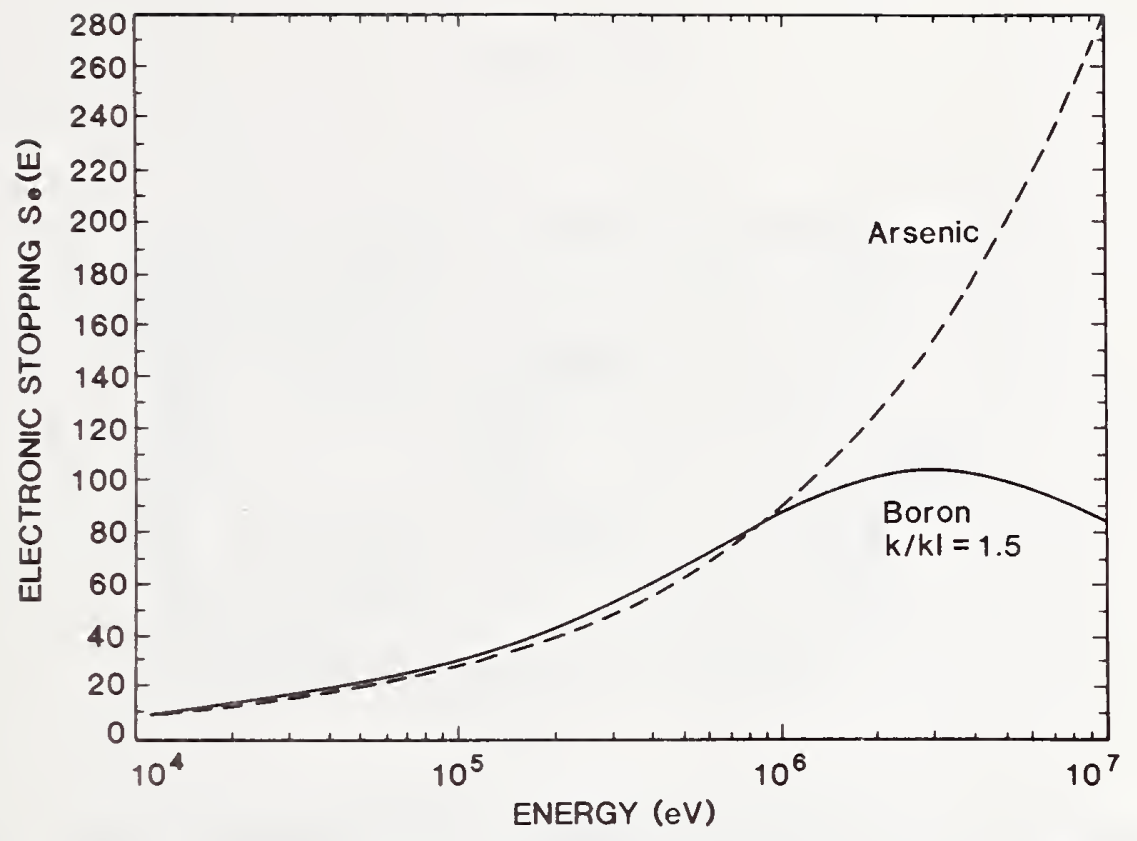

Figure 8. Electronic stopping for boron and arsenic in silicon as determined by eqs (39-47). Notice that the peak of the electronic stopping for boron occurs at about $3 \mathrm{MeV}$. The peak occurs where the Bethe-Block form of the electronic stopping overtakes the Lindhard form of the stopping. The corresponding peak for arsenic occurs outside the upper range of the figure $(10 \mathrm{MeV})$. Both of these energies are outside the usual range of energies used in the ion implantation of semiconductor structures.

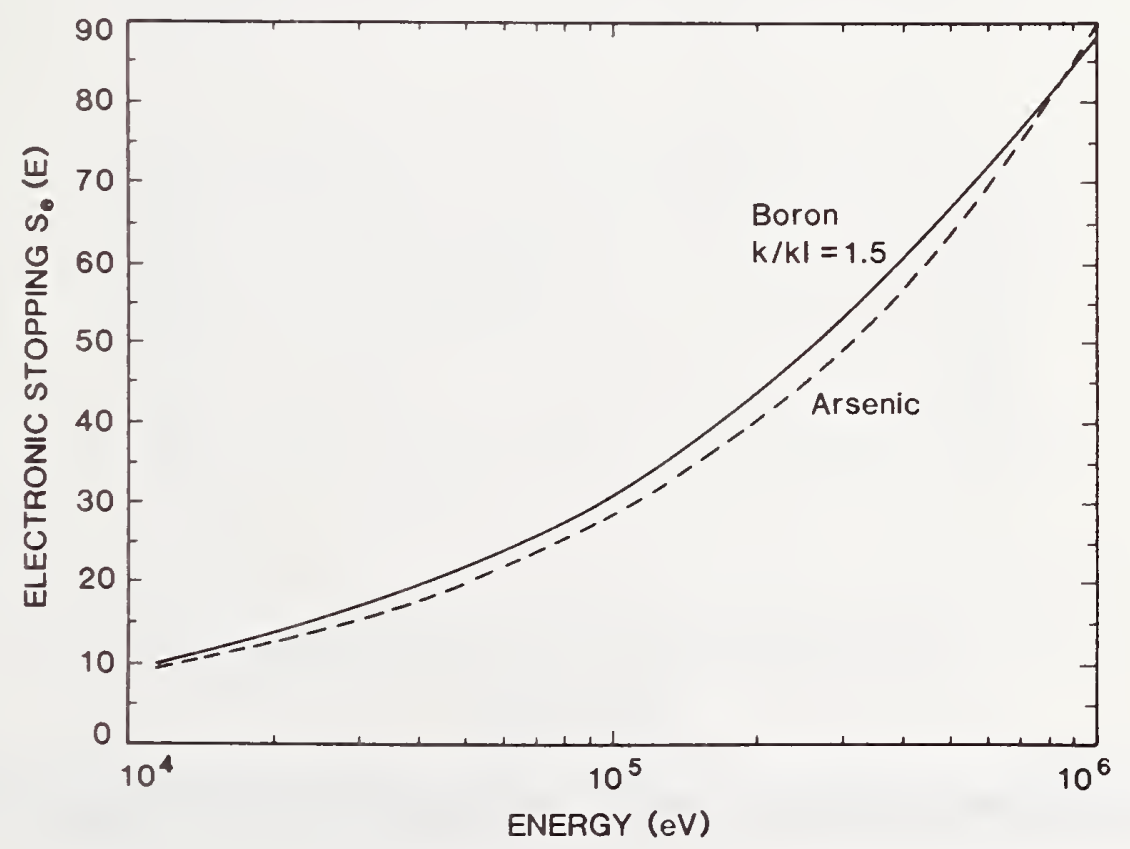

Figure 9. Electronic stopping for boron and arsenic in silicon over the range of energies from $10 \mathrm{keV}$ to $1 \mathrm{MeV}$. 


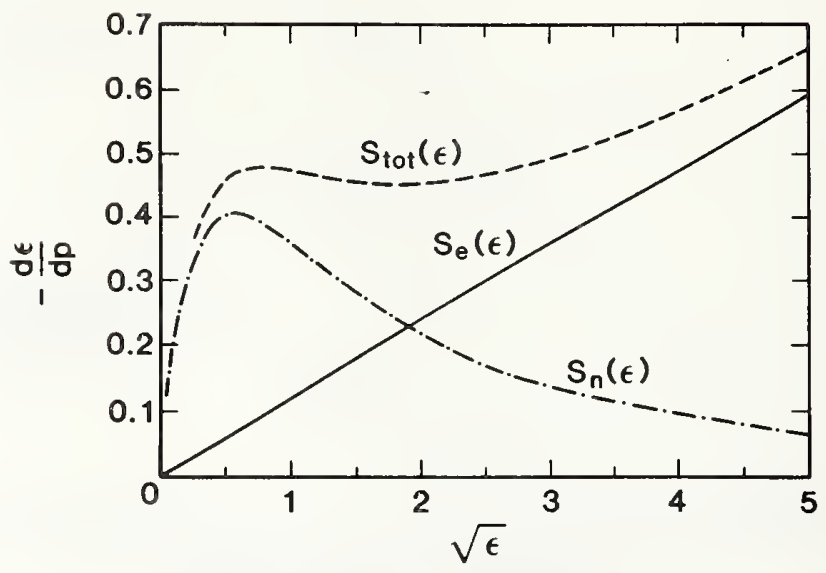

Figure 10. Energy loss as a function of energy. The electronic, nuclear, and composite energy loss curves are shown in this figure.

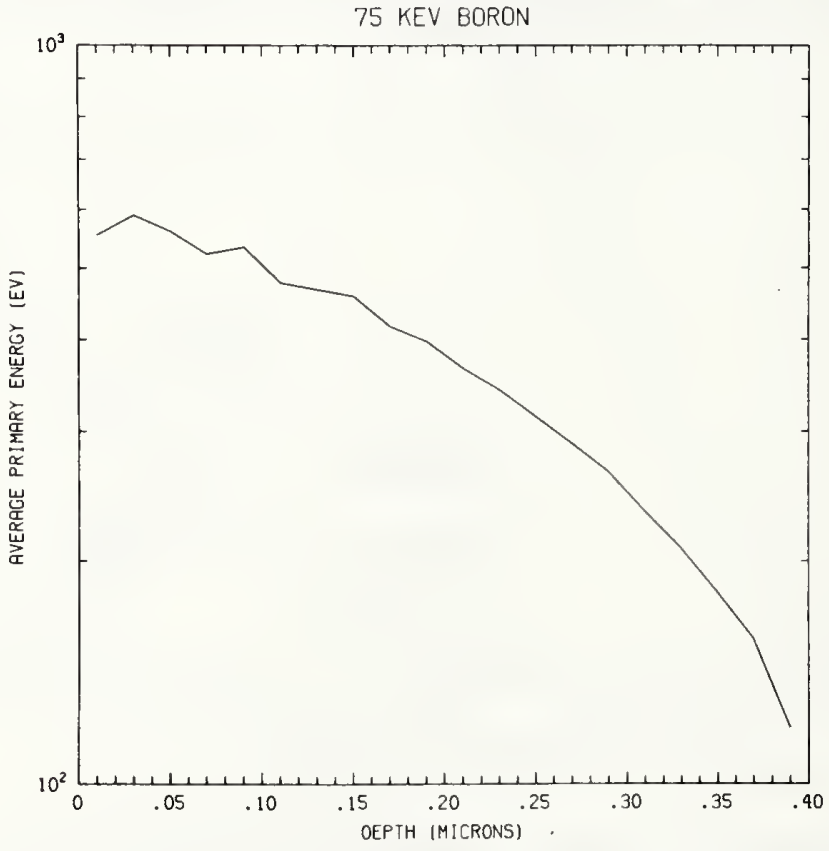

Figure 11. Average energy per primary resulting from $75-\mathrm{keV}$ boron in silicon. 


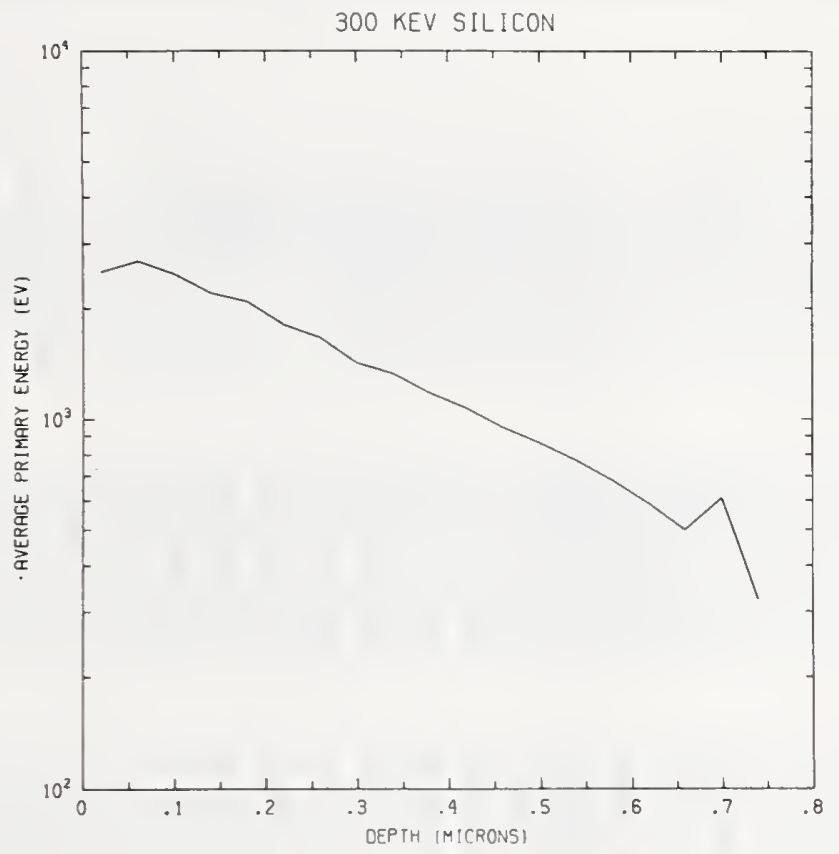

Figure 12. Average energy per primary resulting from $300-\mathrm{keV}$ silicon in silicon.

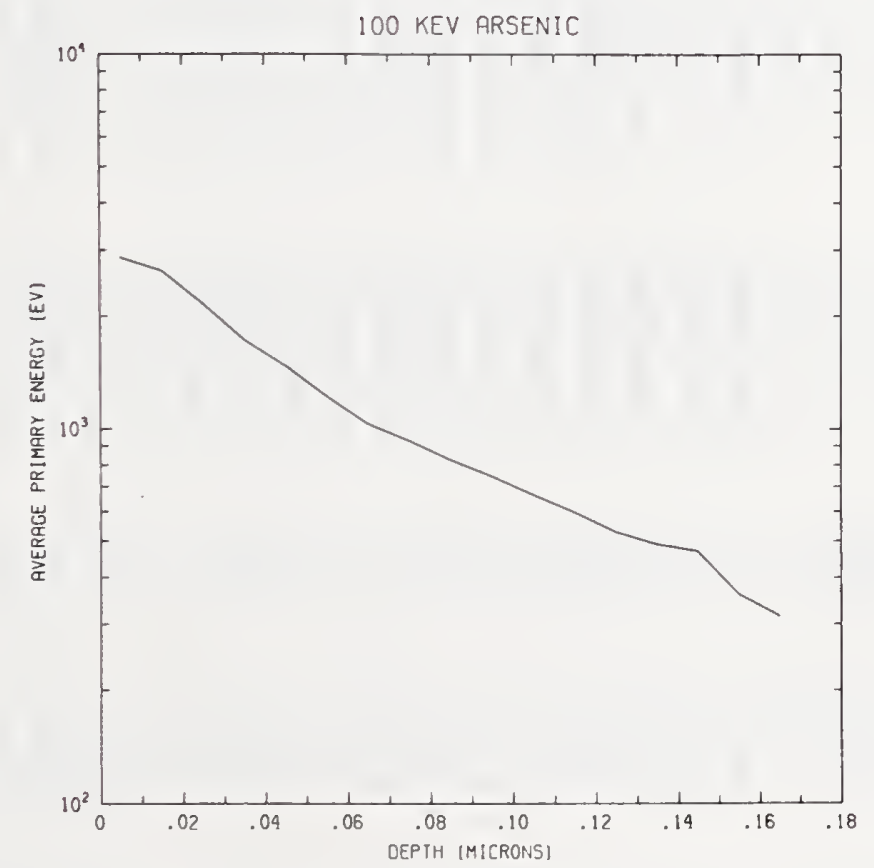

Figure 13. Average energy per primary resulting from $100-\mathrm{keV}$ arsenic in silicon. 
Appendix A. Tables of Polynomial Fitting Coefficients

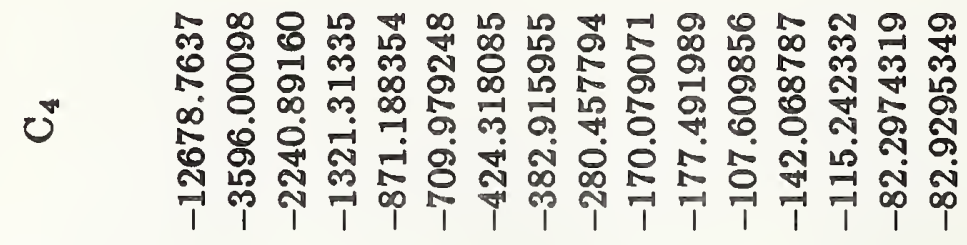

용ㅇㅇㅇㅛ 규

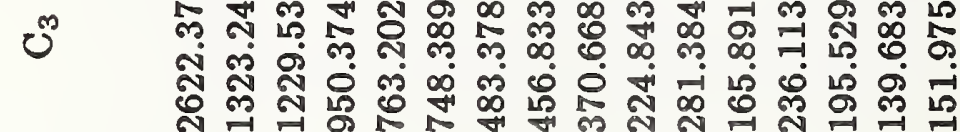

U

舟吕

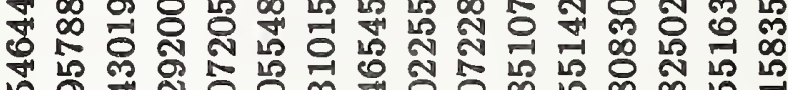
年

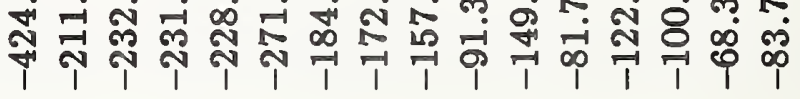

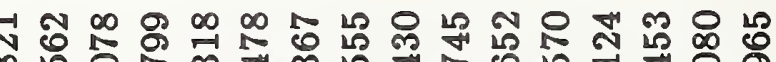

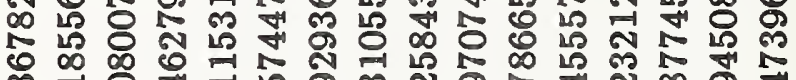
กุ แ

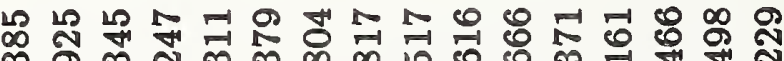
กั 건

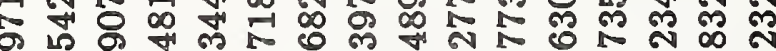

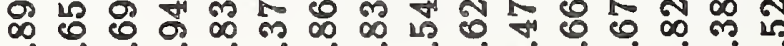

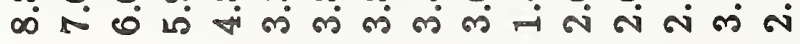

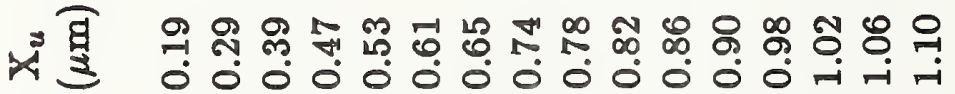

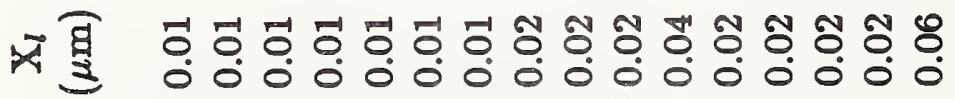

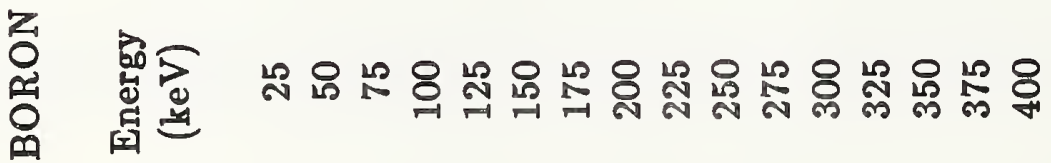




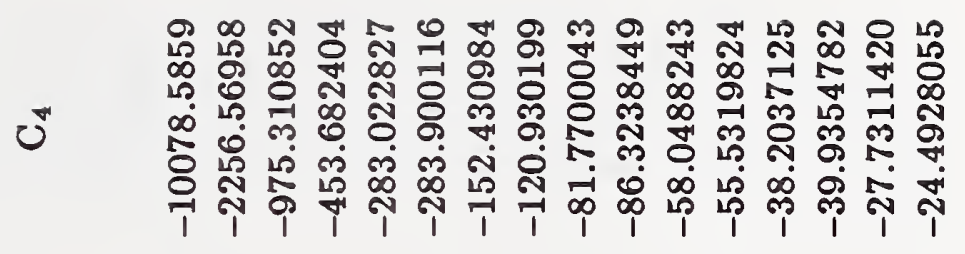

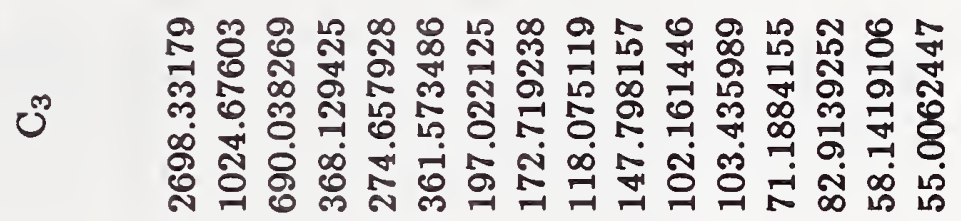

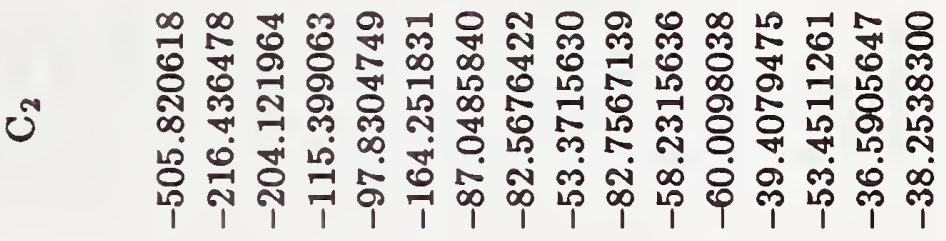

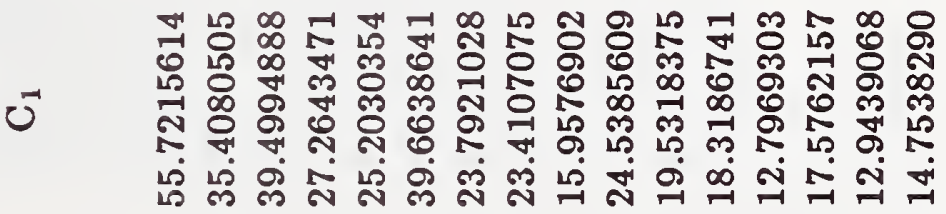

웍 స

ن $\quad$ N 品

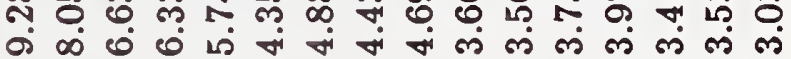

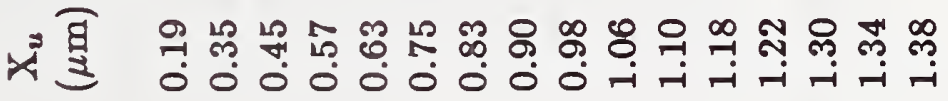

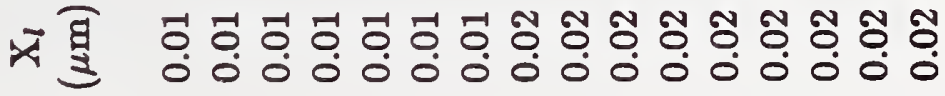

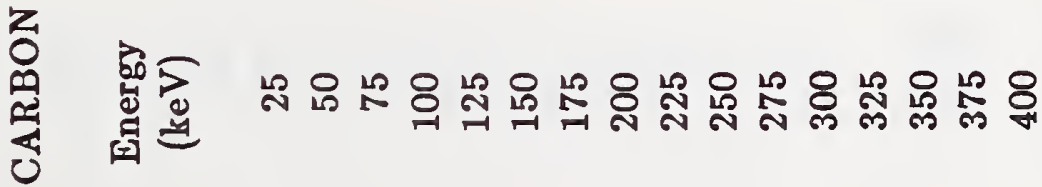




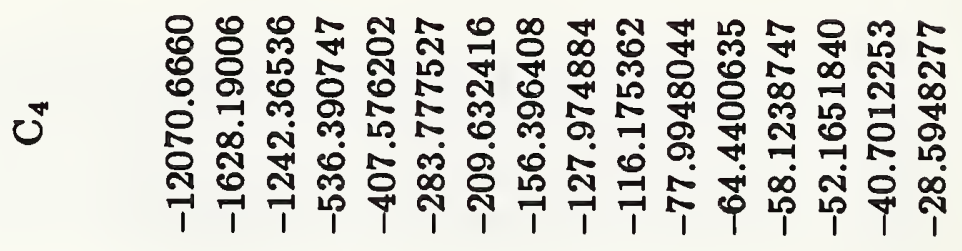

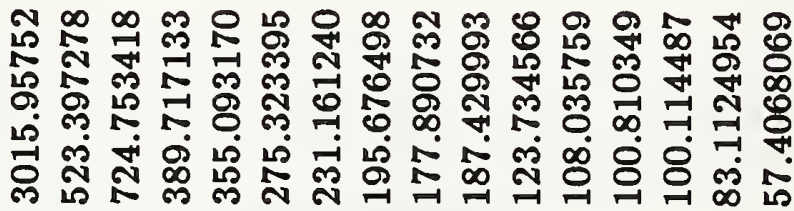

ㄴ ง

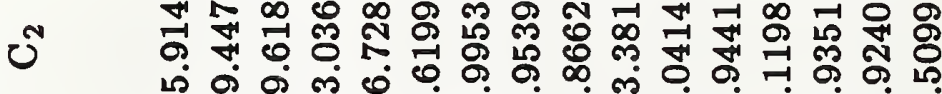
బூ

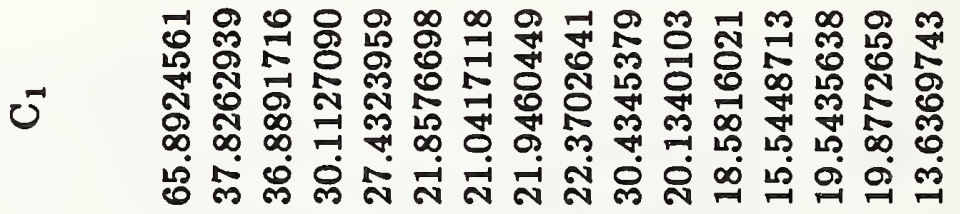

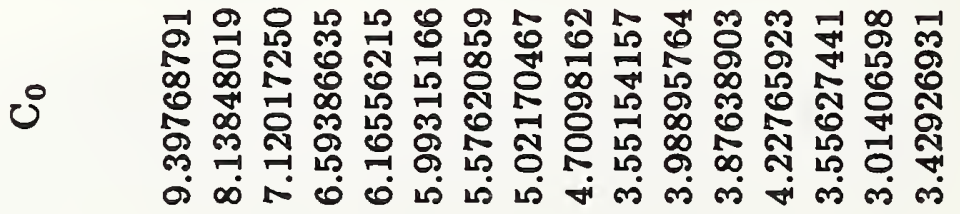

ঋ

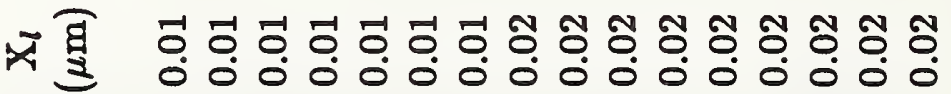

疍

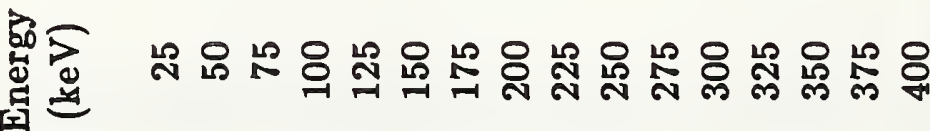



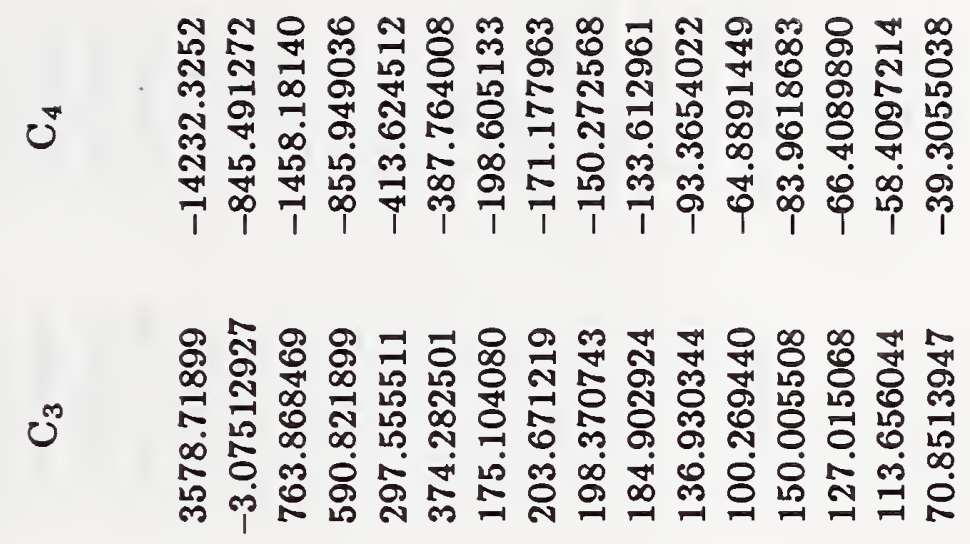

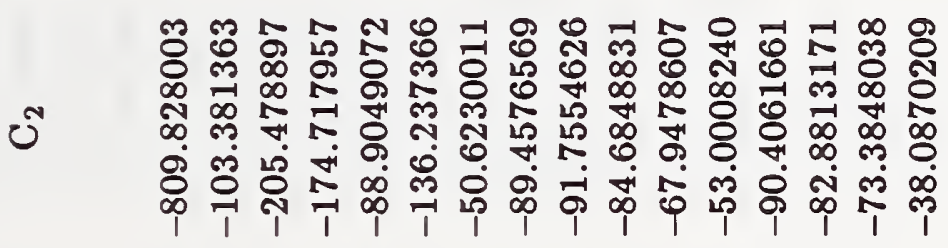
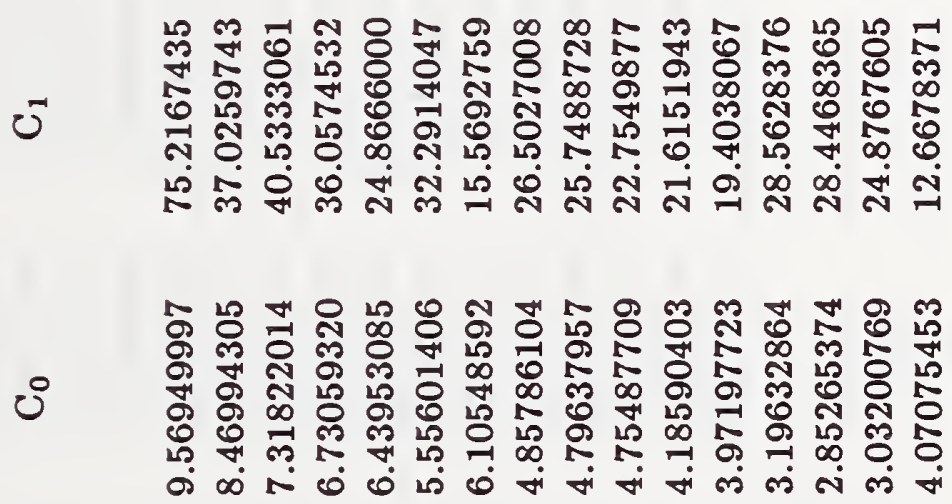

テ

交䚁

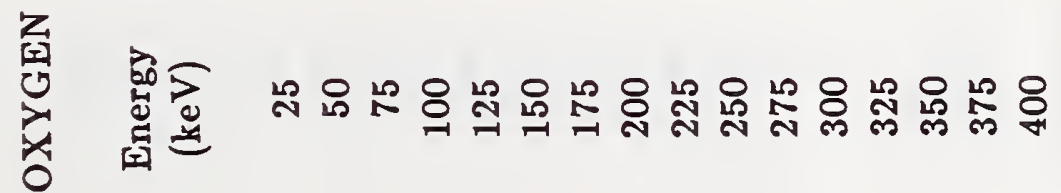




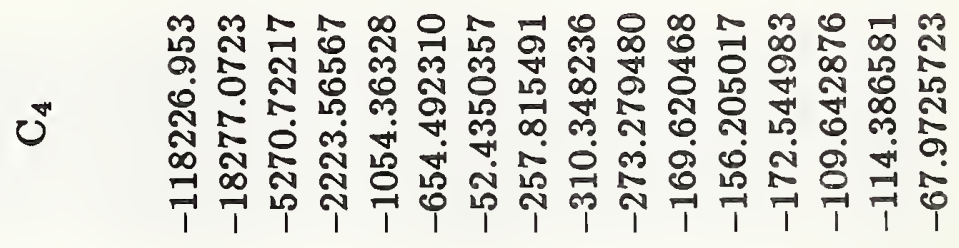

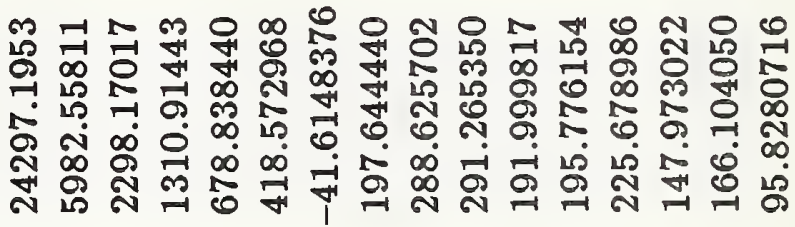

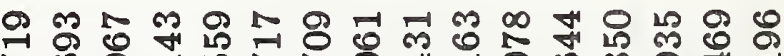
둥요

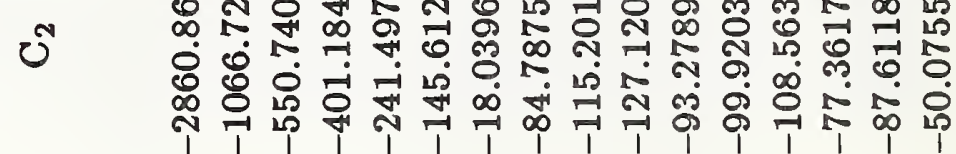

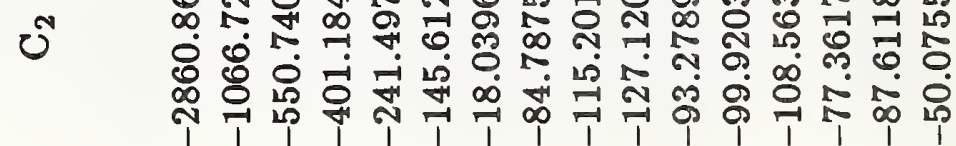

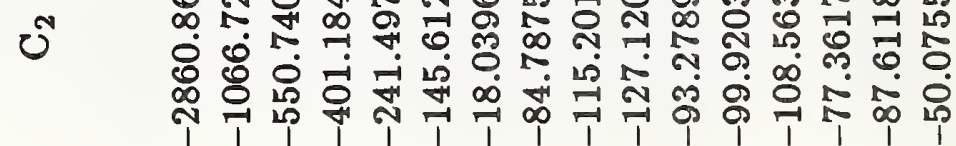
등요 요 证

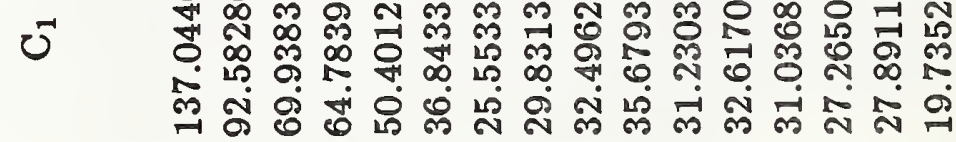

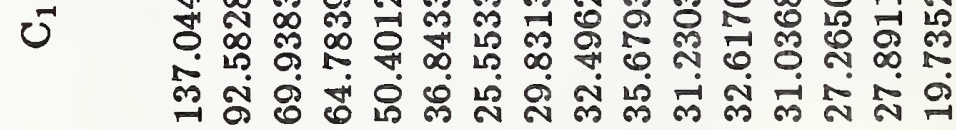

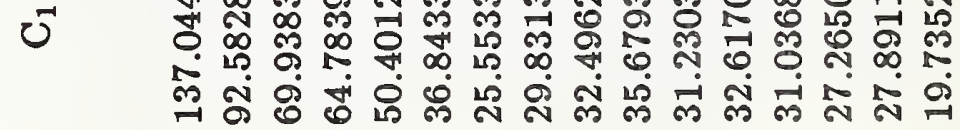

सㅍำ

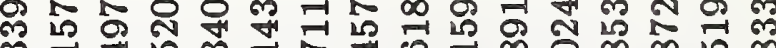
范

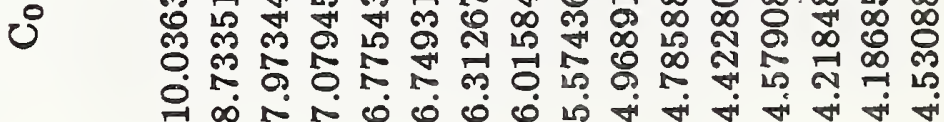

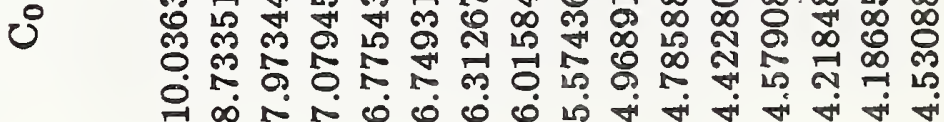

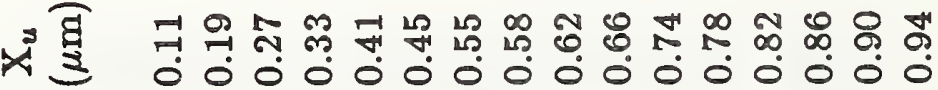

स

曷点 


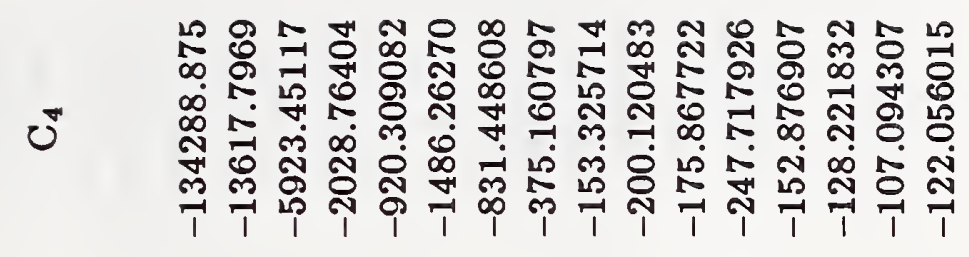

익 등

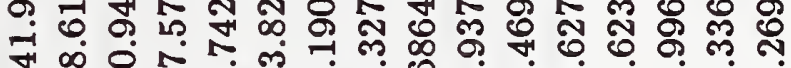
ฟ

น ก 4 i

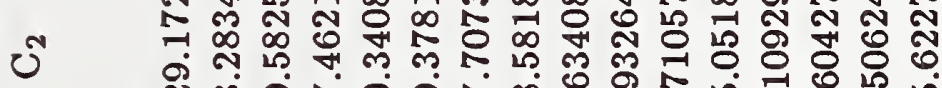
งิ

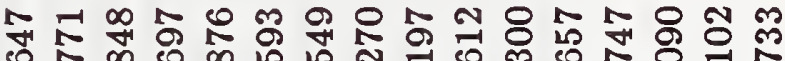

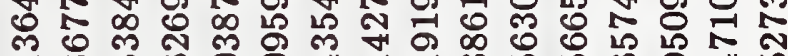

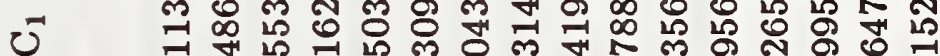
$\therefore$ ఫั

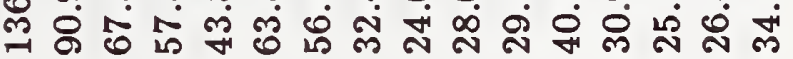

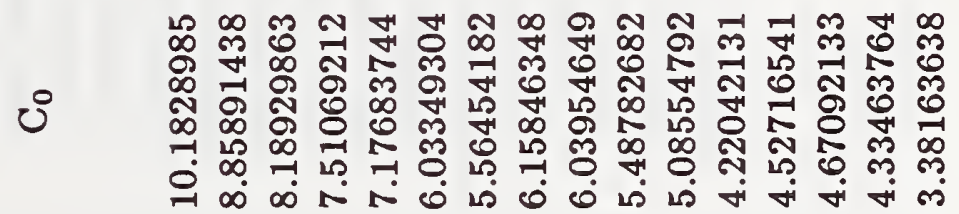

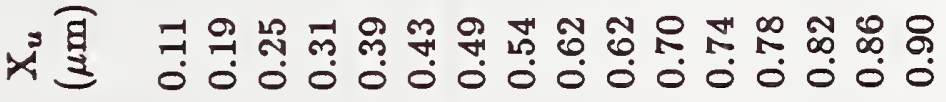

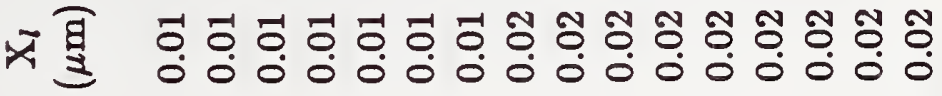

党 
닉 กิ J $\quad$ N ஜ 光年

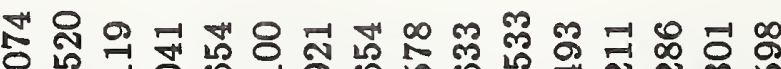

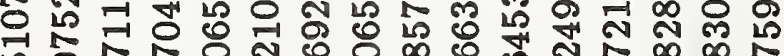
น 总

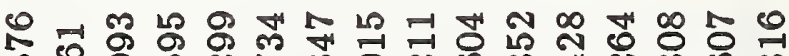
מ

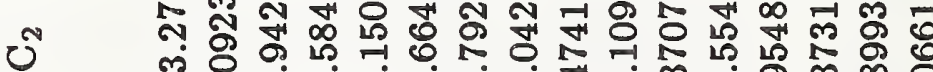
ต 을 m

옹 ஓ m

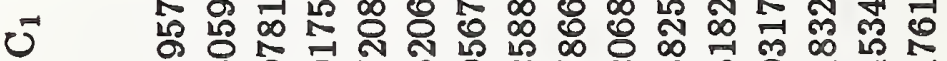
今 웅 -

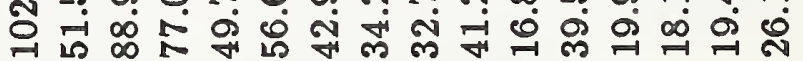

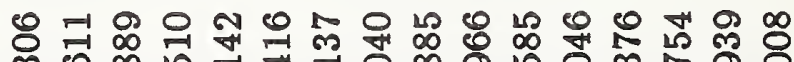
m $\infty$ 山

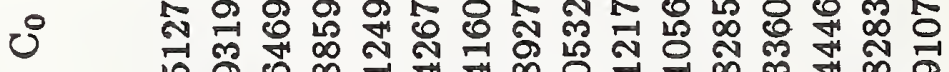

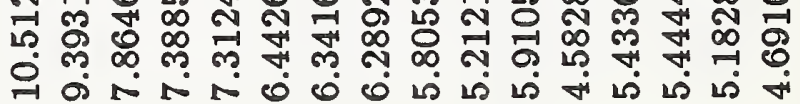

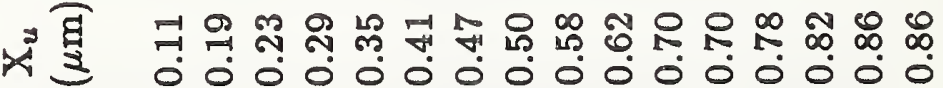

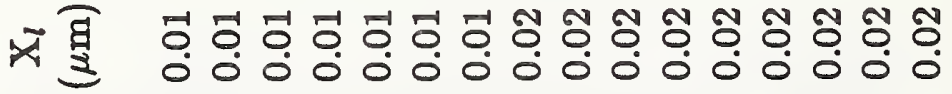

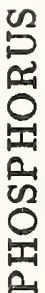

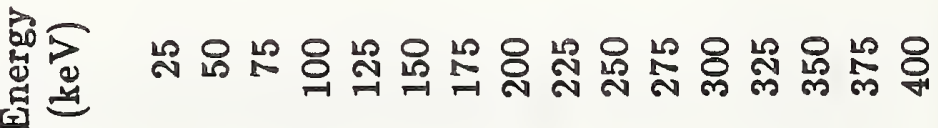




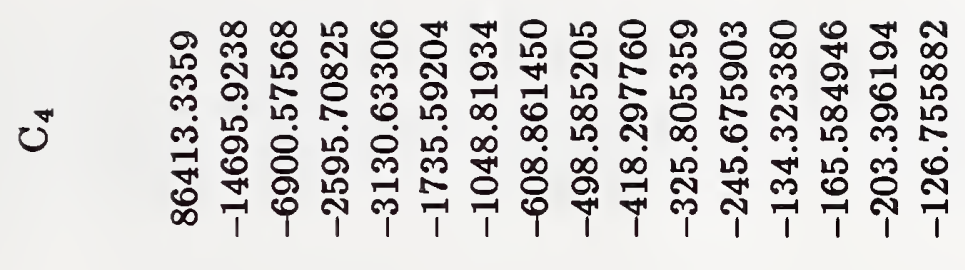

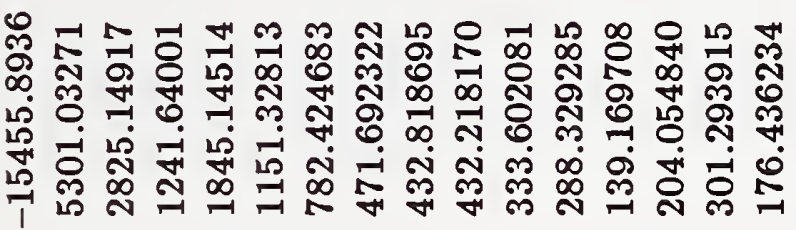

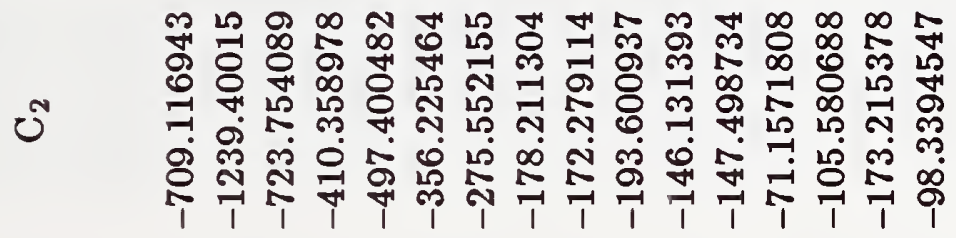

노 ¥ 눈 ๑ $\infty$ 車

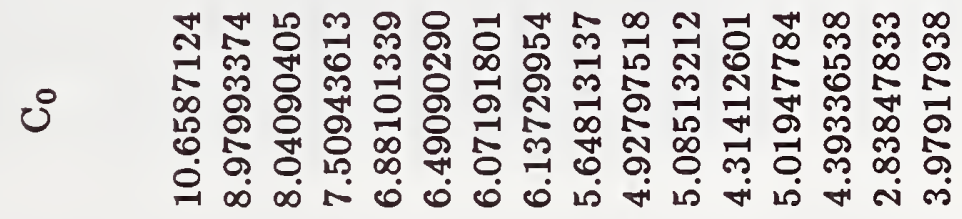

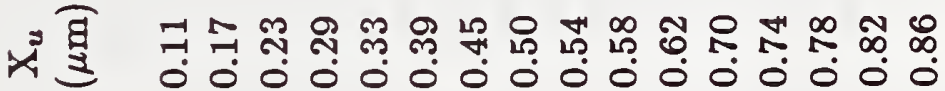

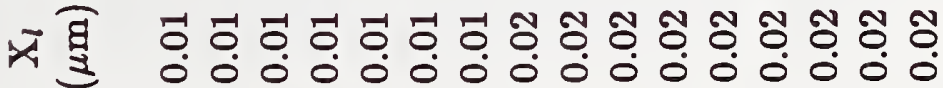

点

离通 


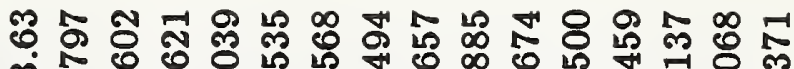
๓

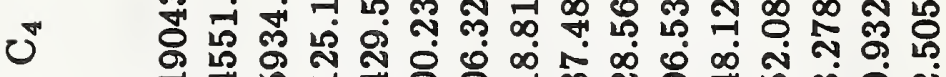

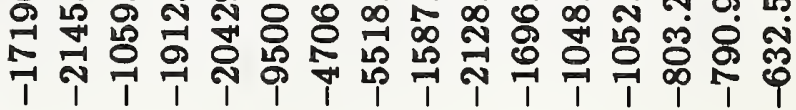

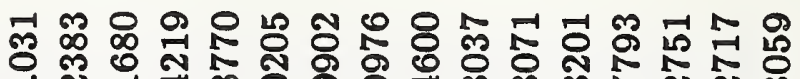
ำ 궁 \& 品 实

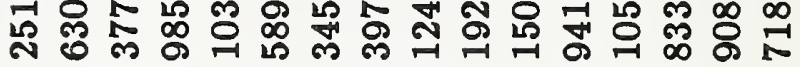
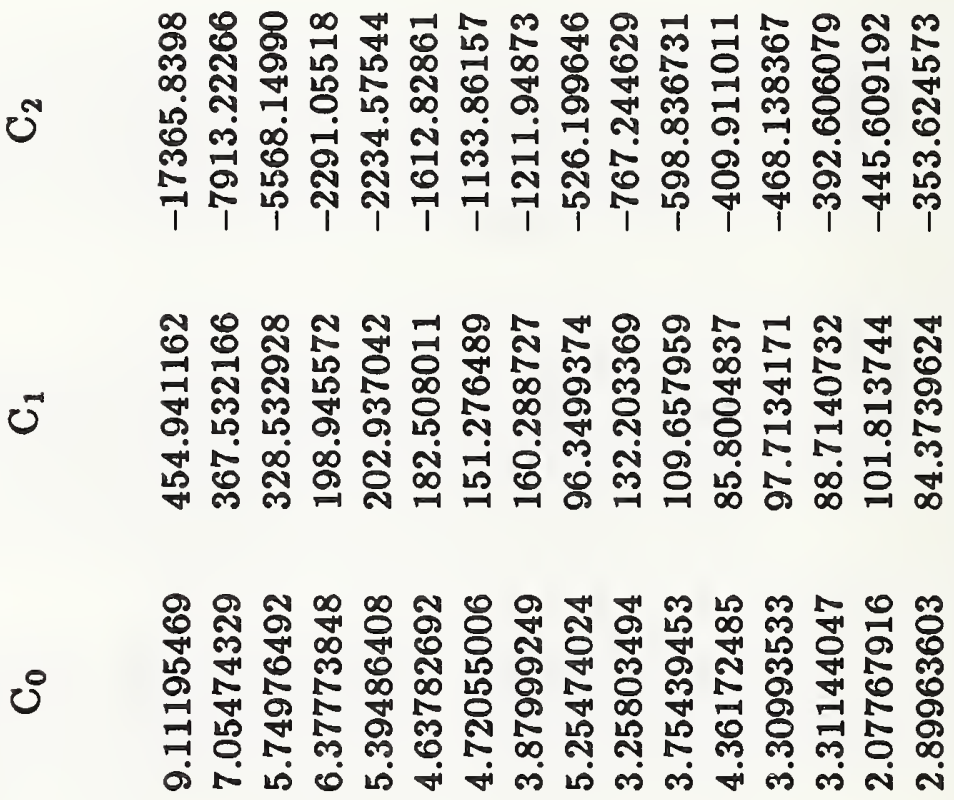

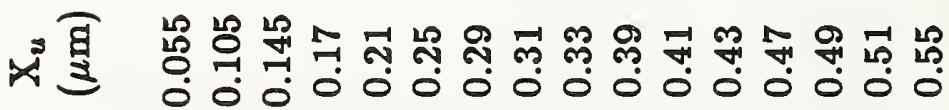

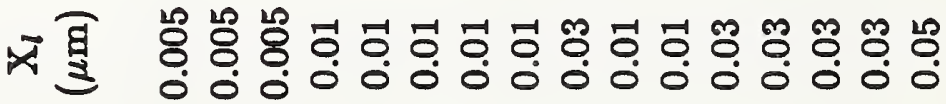

夠造

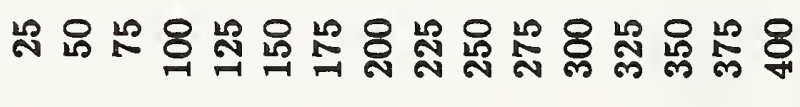



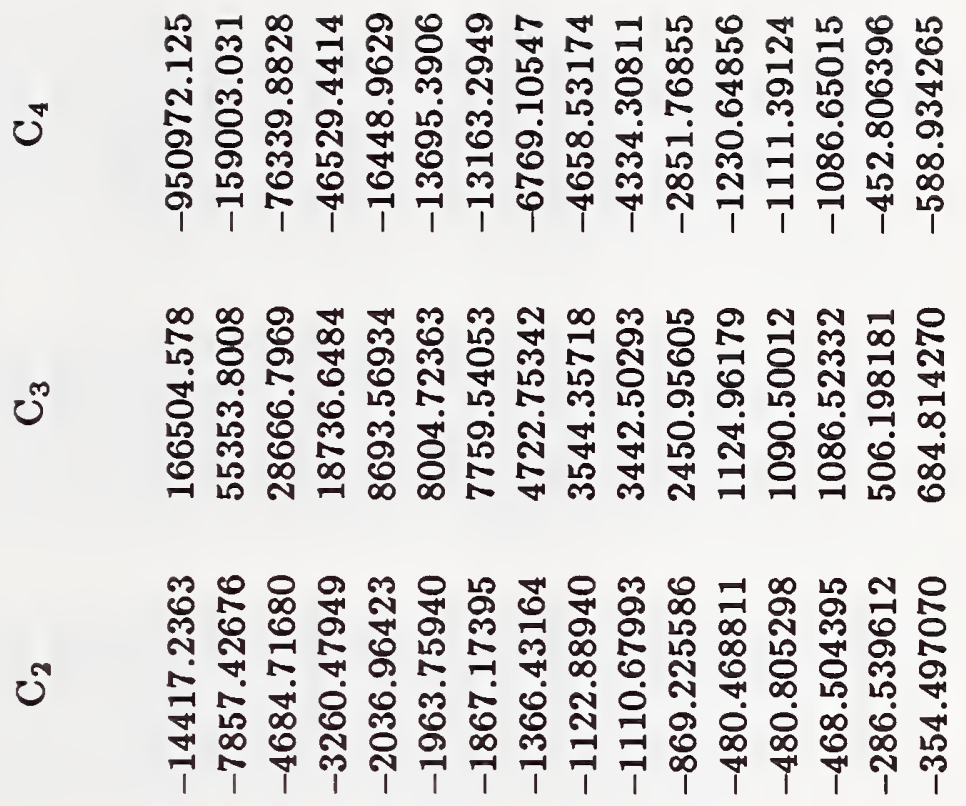

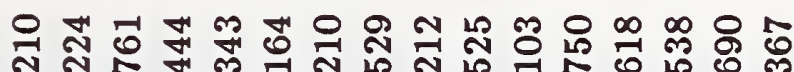
స్ สิ సิ m $\rightarrow$ ต

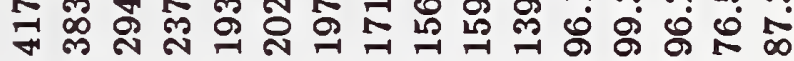

두 ஜ 구 สิน శึ

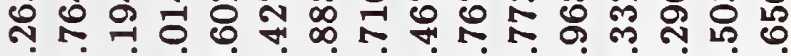
क 0 क 0 म

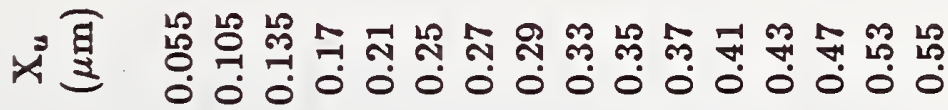

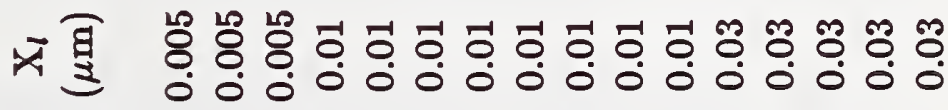

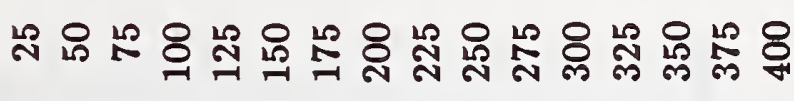



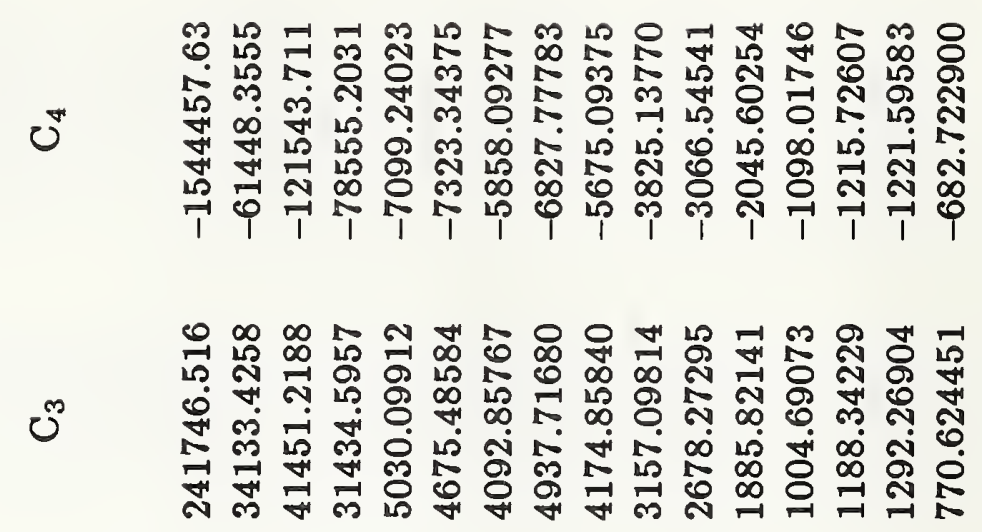

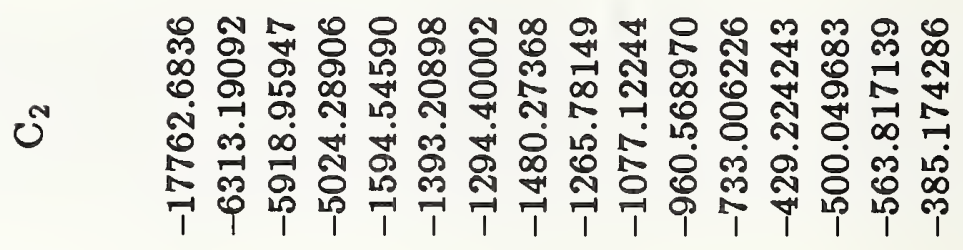

ஜ ชิ

v

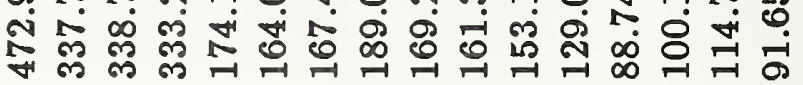

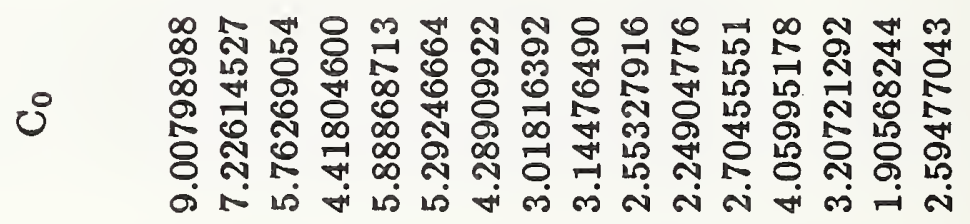

サ

天夏

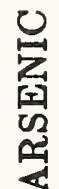

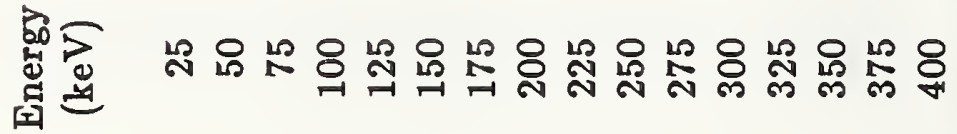




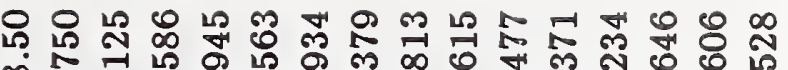
車哭 \% ర 员 o

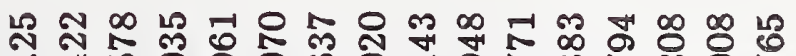
눈 म

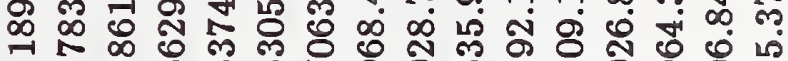

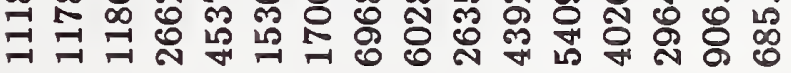

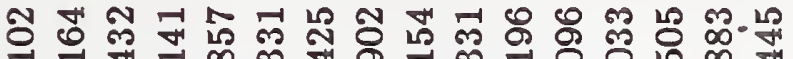
7 $\rightarrow$ J ৩ $\quad 0$ 过 o

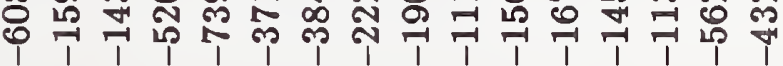

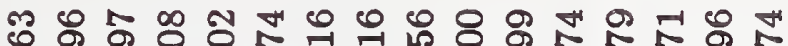

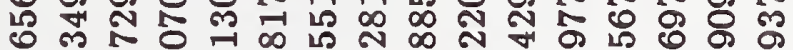

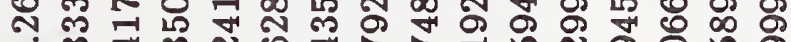

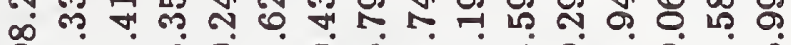

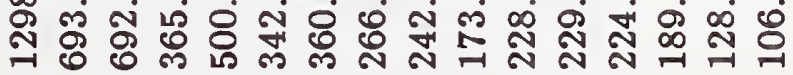

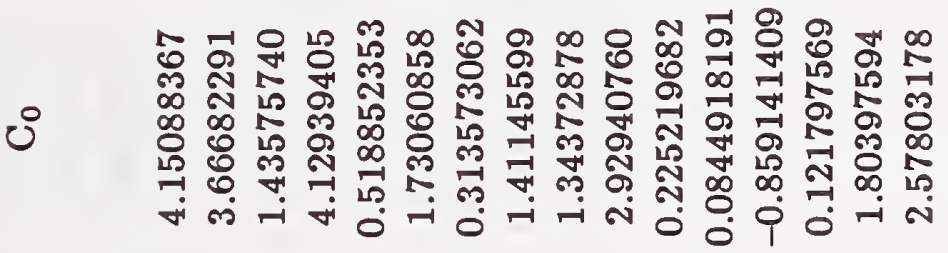

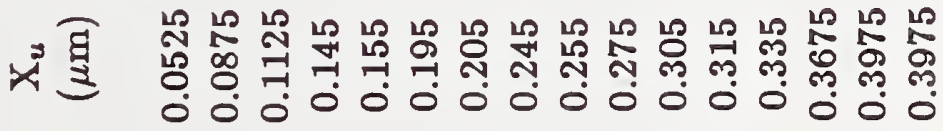

ব得

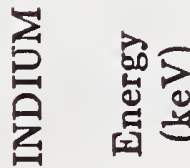

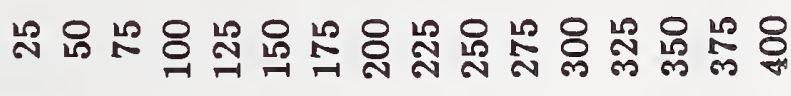


유 눙

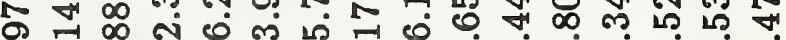

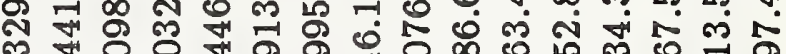
ज † Ұ

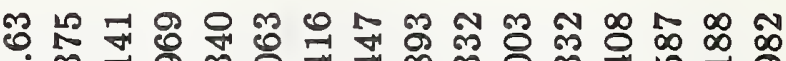
宛 o $\begin{aligned} & \infty \\ & 0\end{aligned}$

o $\begin{aligned} & \infty \\ & 0\end{aligned}$

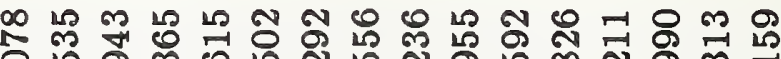

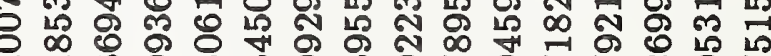

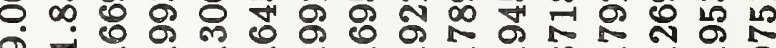

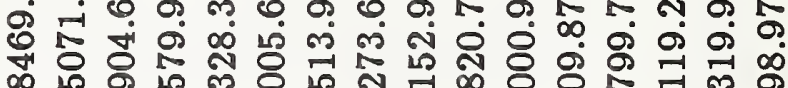

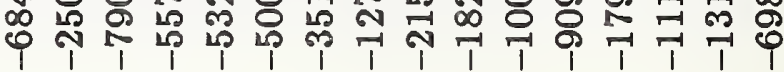

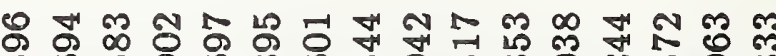

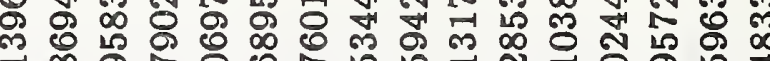

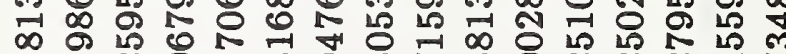
๙

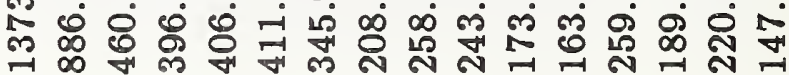

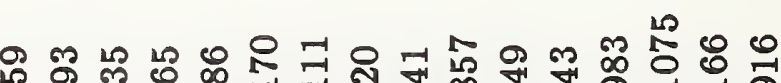
น

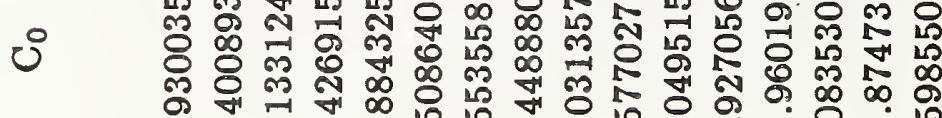

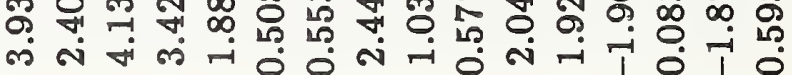
m

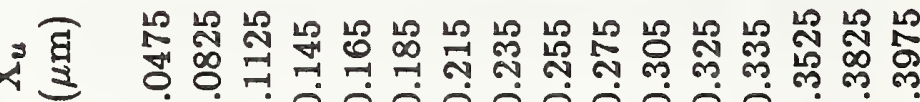

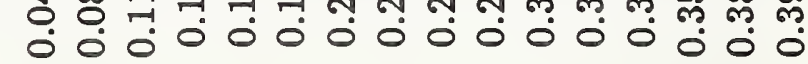

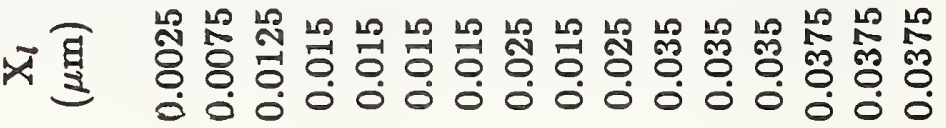

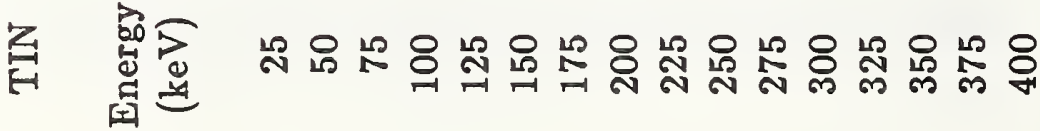



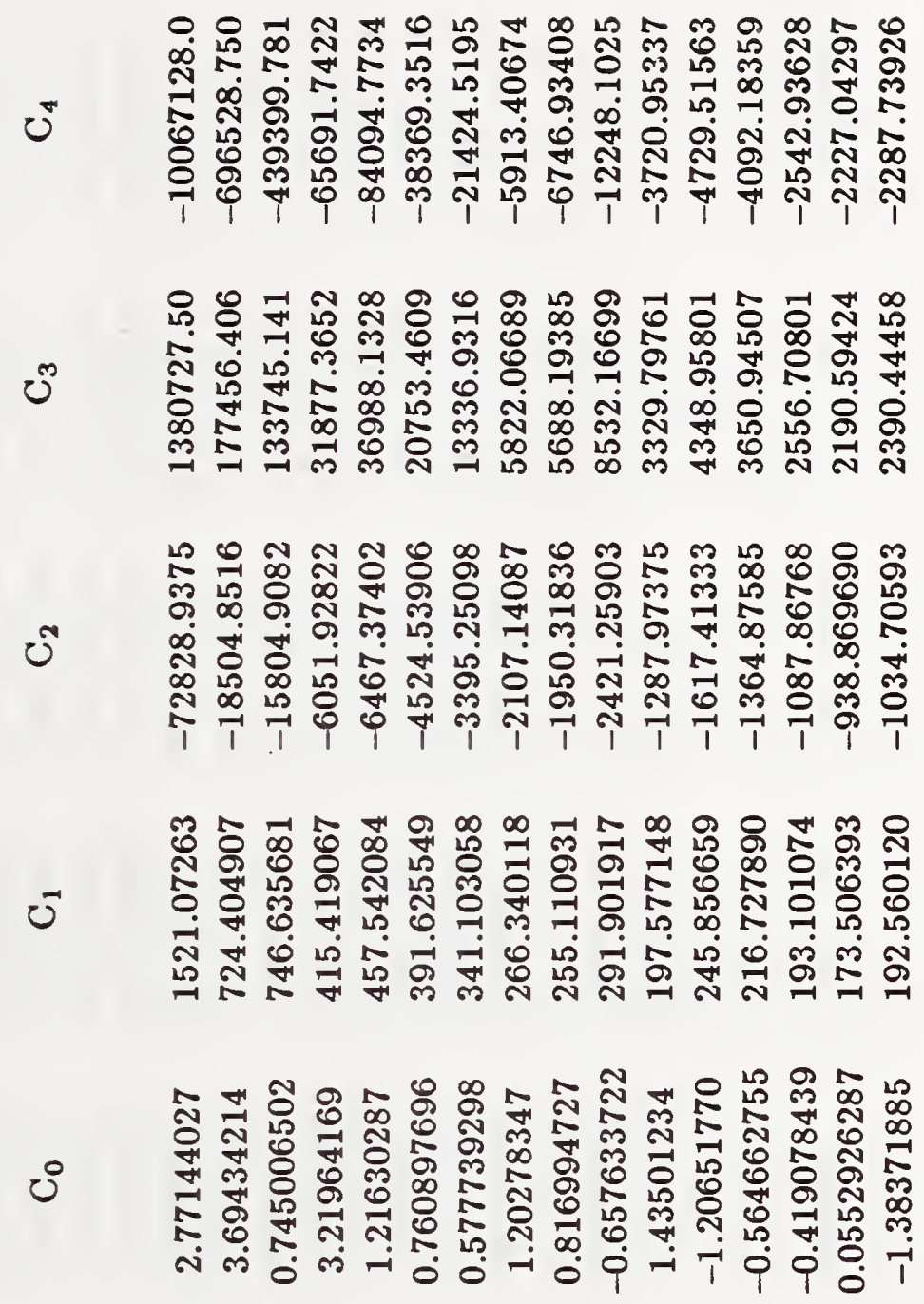

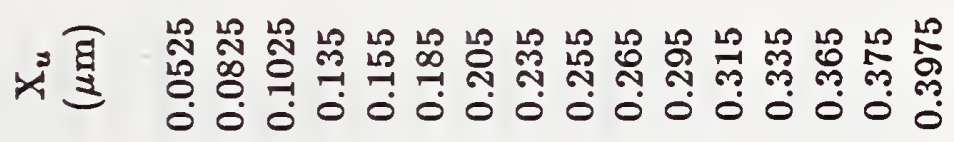

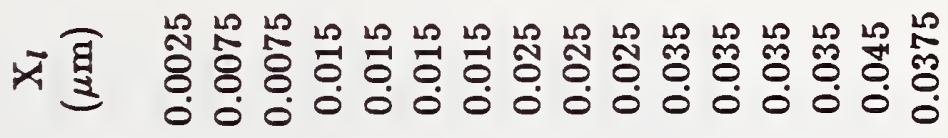

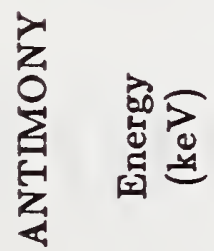

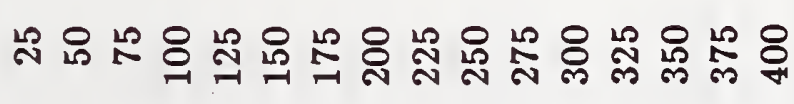



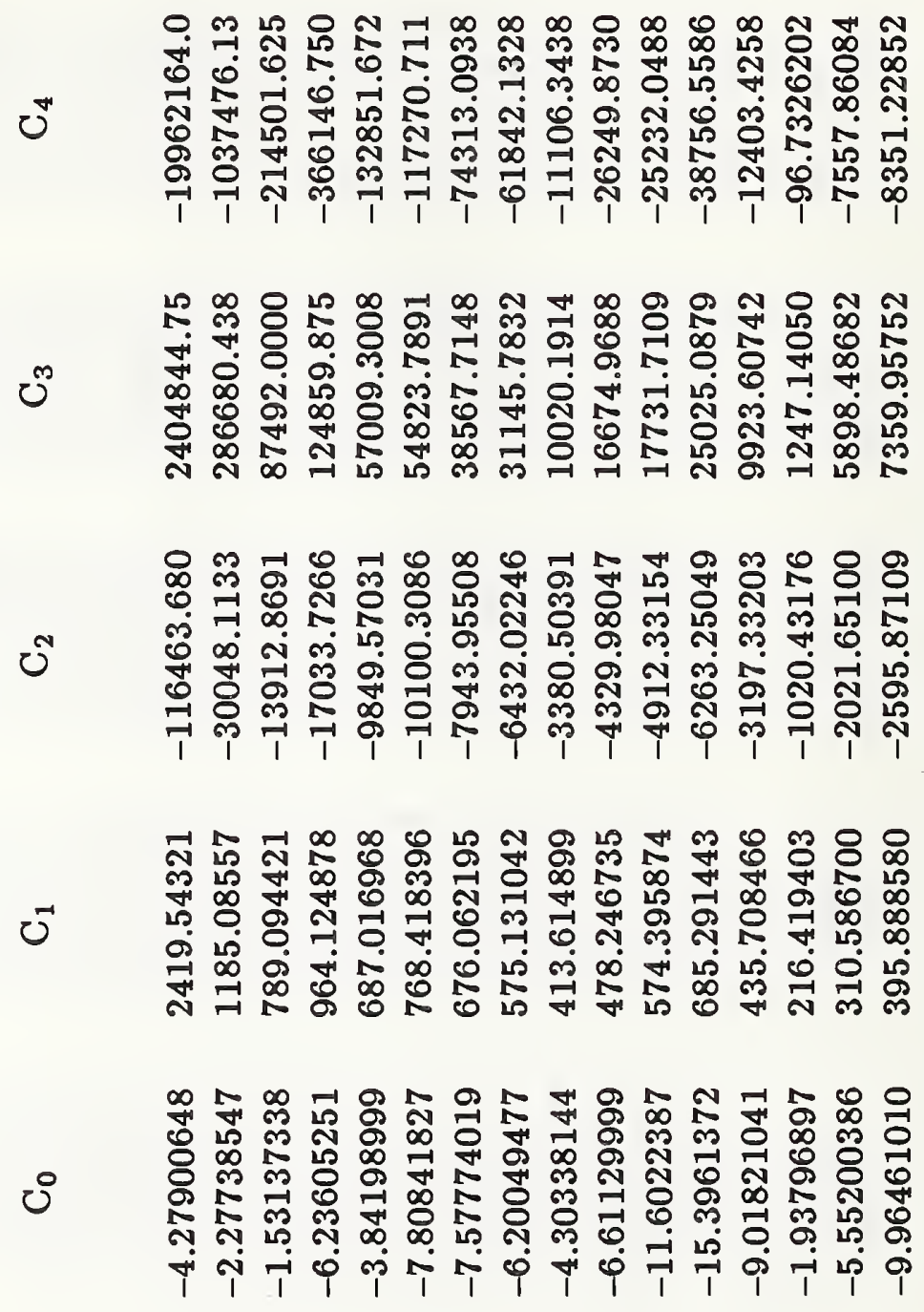

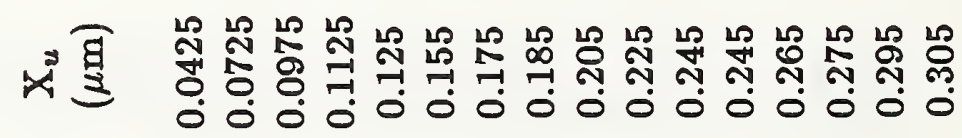

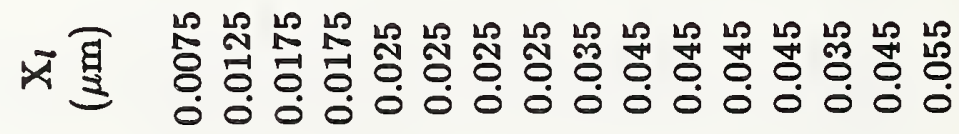

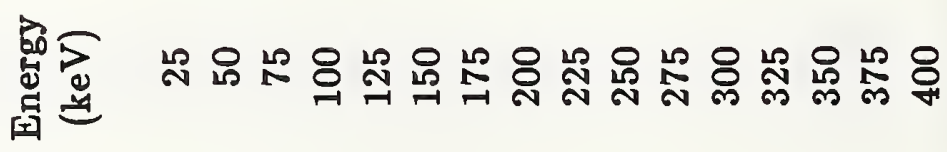



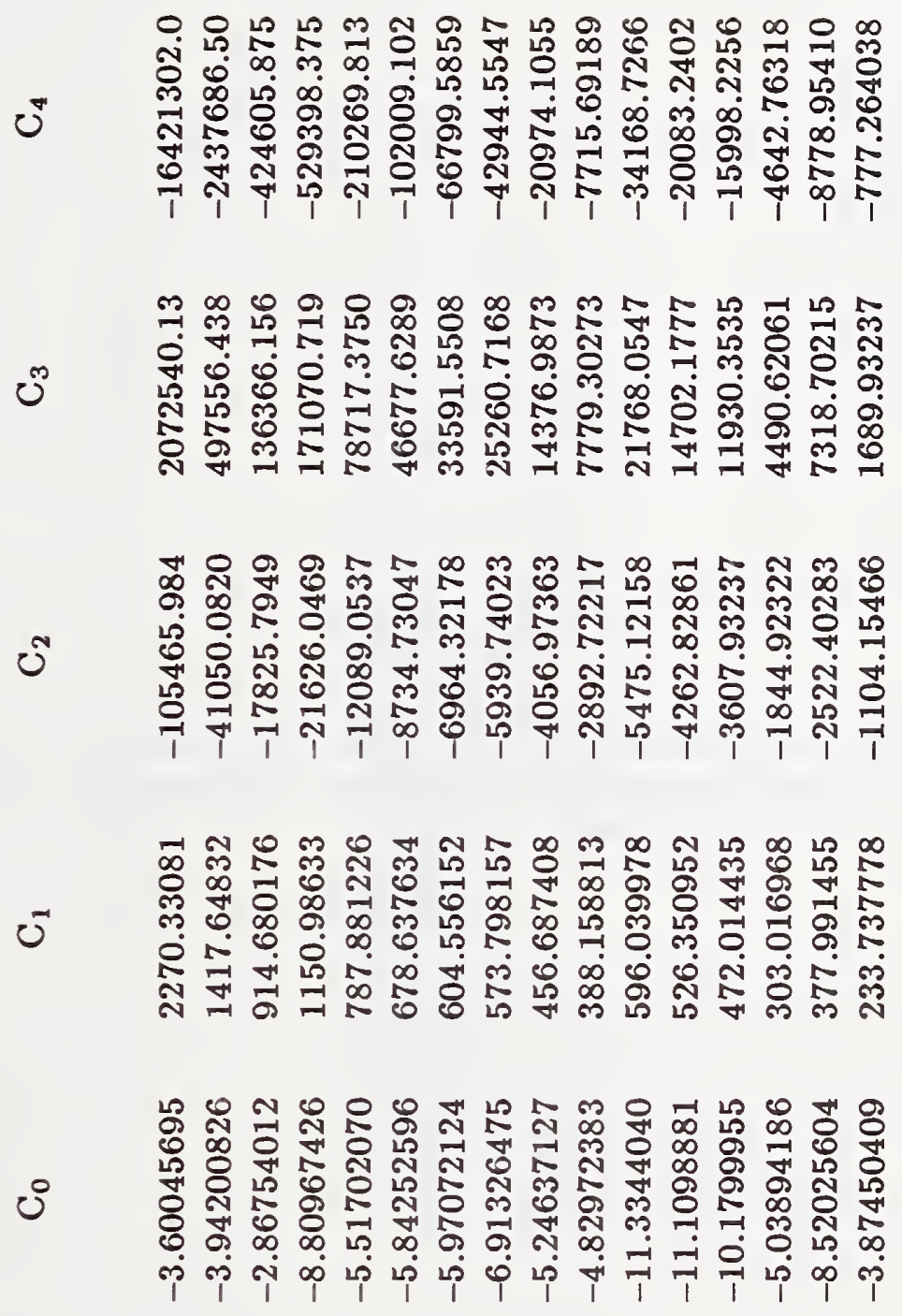

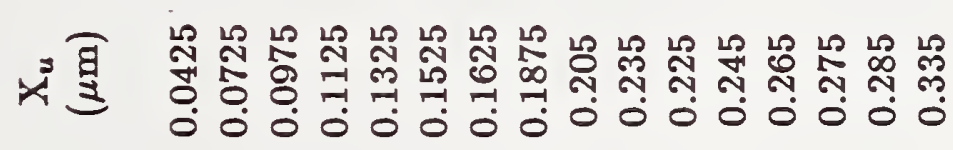

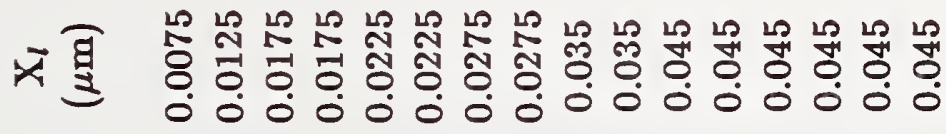

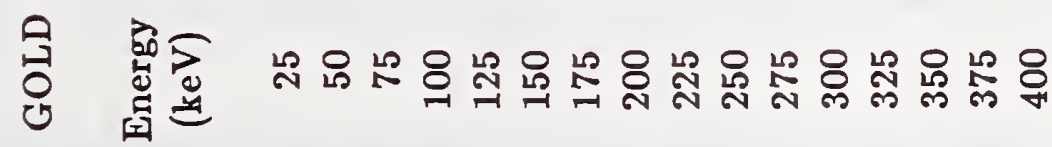


Appendix B. One-Dimensional and Two-Dimensional Distributions

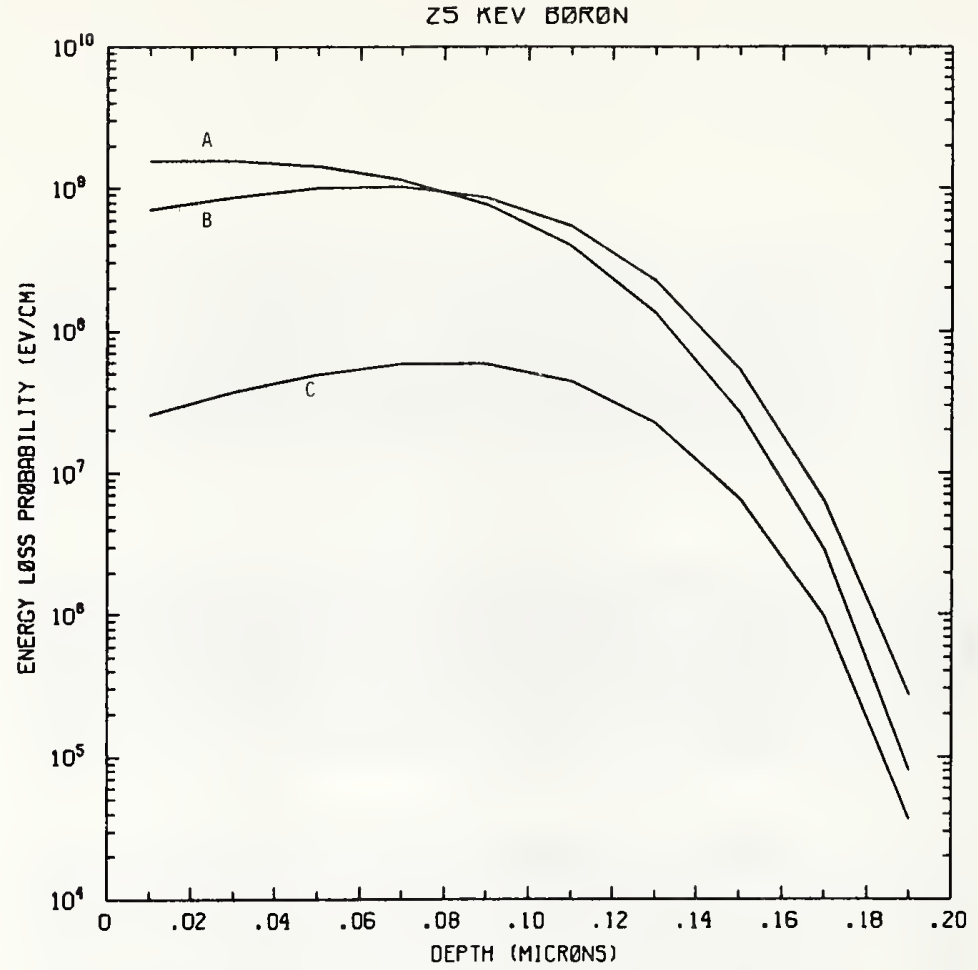

Figure B25-1 One-dimensional probability distributions for energy loss due to electronic excitation (A), displacement damage(B), and phonon excitation (C) for $25 \mathrm{keV}$ boron normally incident on a silicon target.

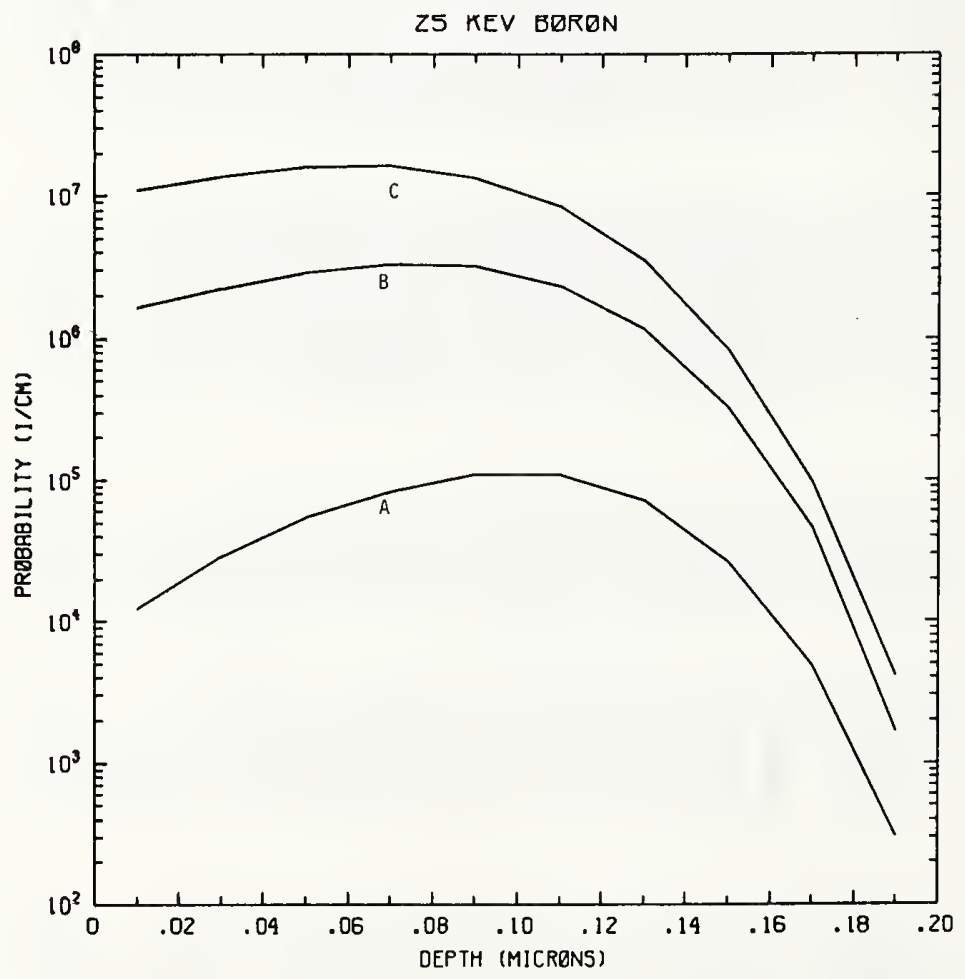

Figure B25-2 One-dimensional probability distributions for implanted boron (A), primary silicon displacements (B), and Frenkel pairs (C) for $25 \mathrm{keV}$ boron normally incident on a silicon target. 
MASK

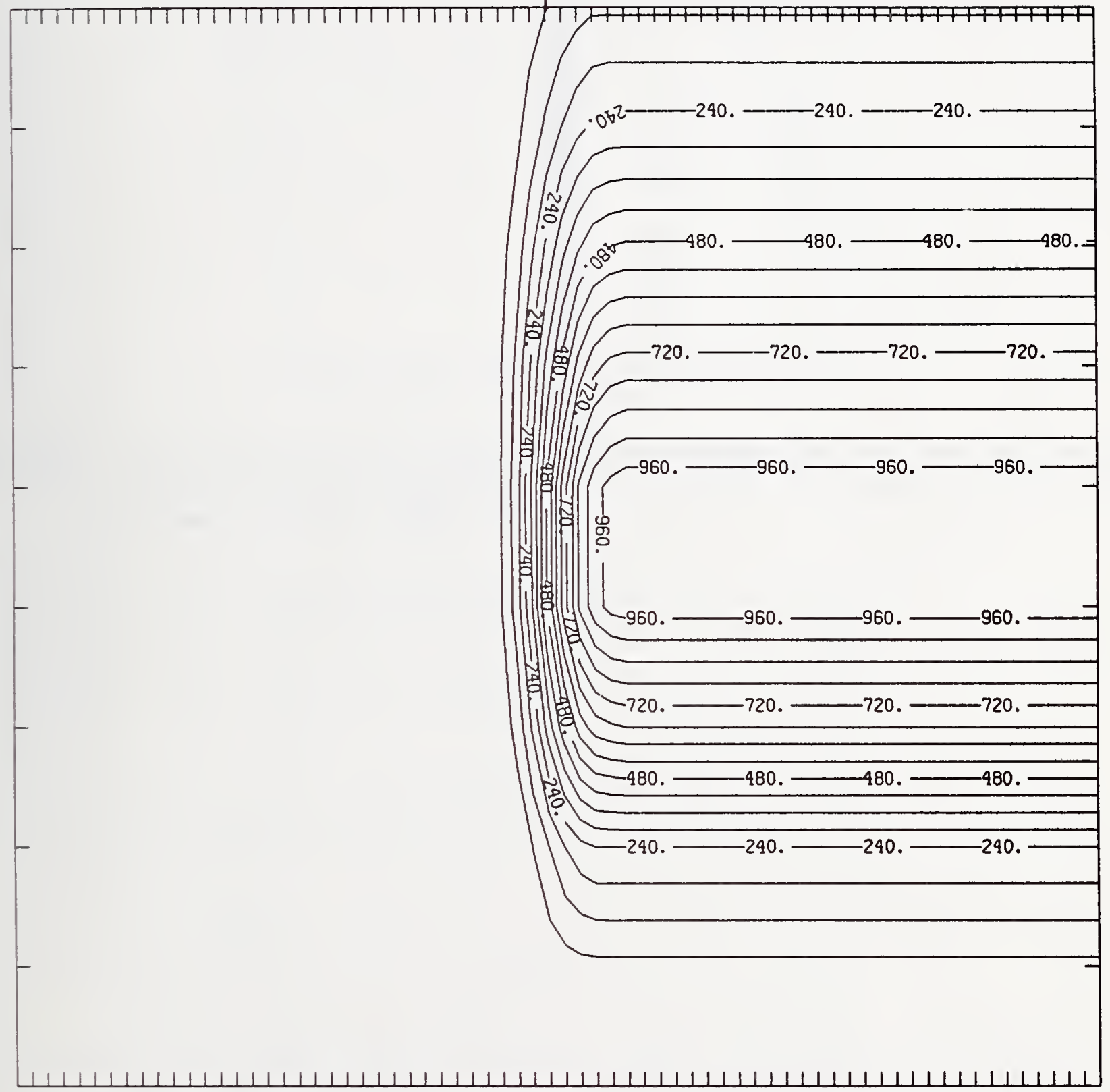

Figure B25-3 Two-dimensional distribution of $25 \mathrm{keV}$ boron implanted past a mask edge. The length increment (distance between tick marks) is $0.02 \mu \mathrm{m}$ and the scale factor is 1000 . 


\section{MASK}

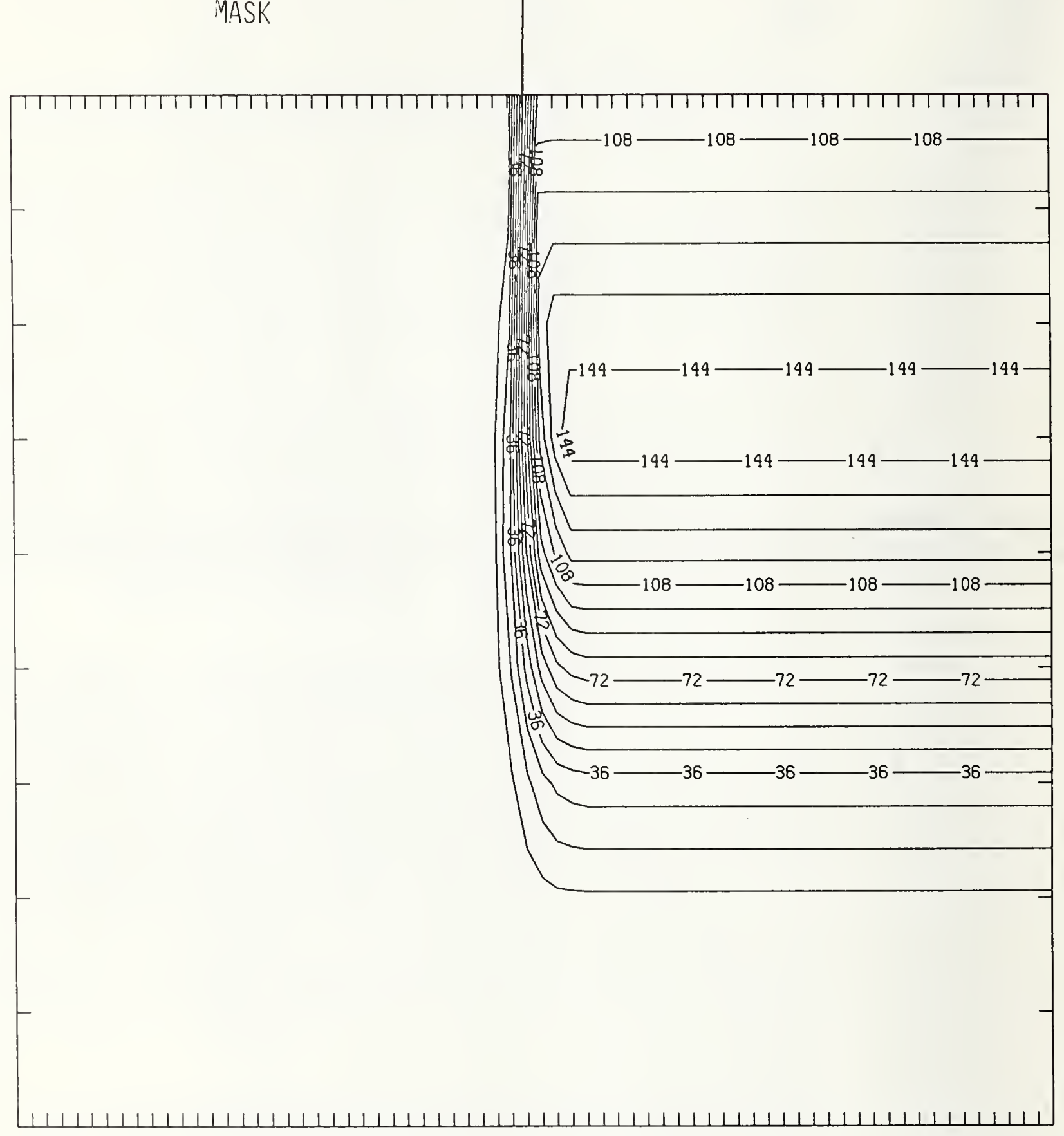

Figure B25-4 Two-dimensional distribution of Frenkel pairs created by $25 \mathrm{keV}$ boron implanted past a mask edge. The length increment (distance between tick marks) is $0.02 \mu \mathrm{m}$ and the scale factor is 1 . 
SD KEV BERDN

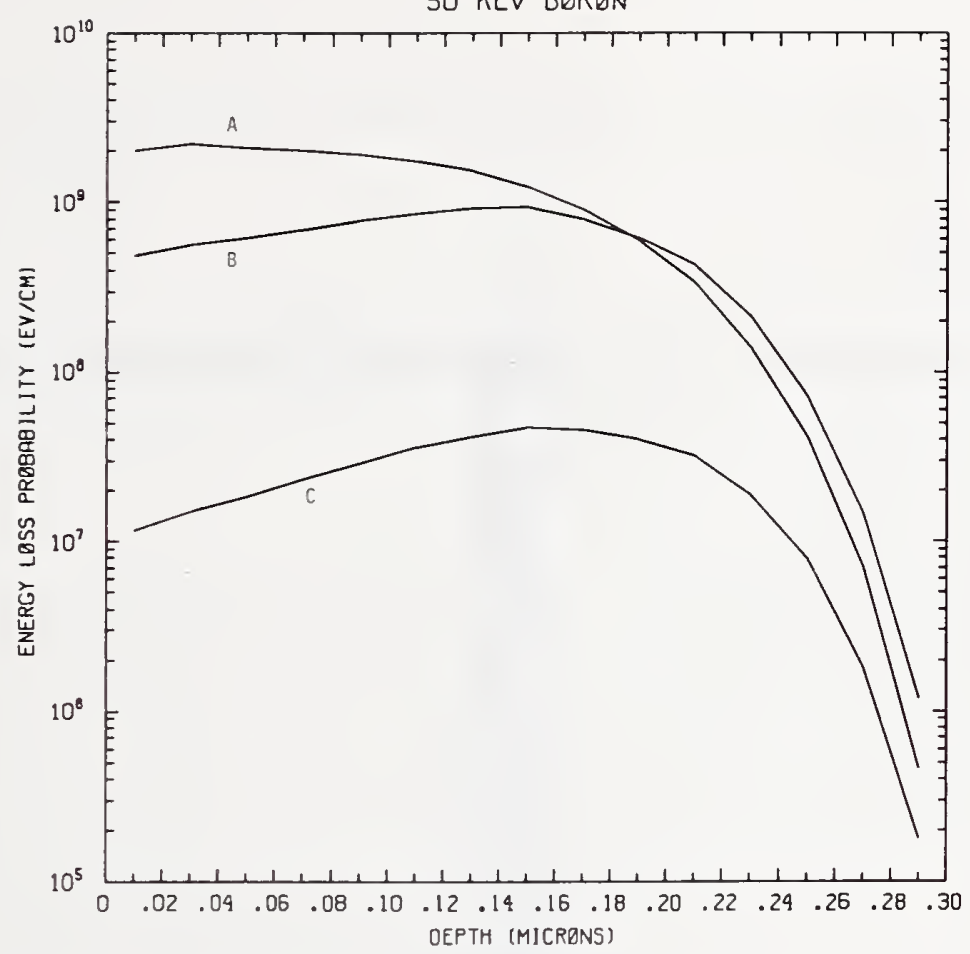

Figure B50-1 One-dimensional probability distributions for energy loss due to electronic excitation (A), displacement damage(B), and phonon excitation (C) for $50 \mathrm{keV}$ boron normally incident on a silicon target.

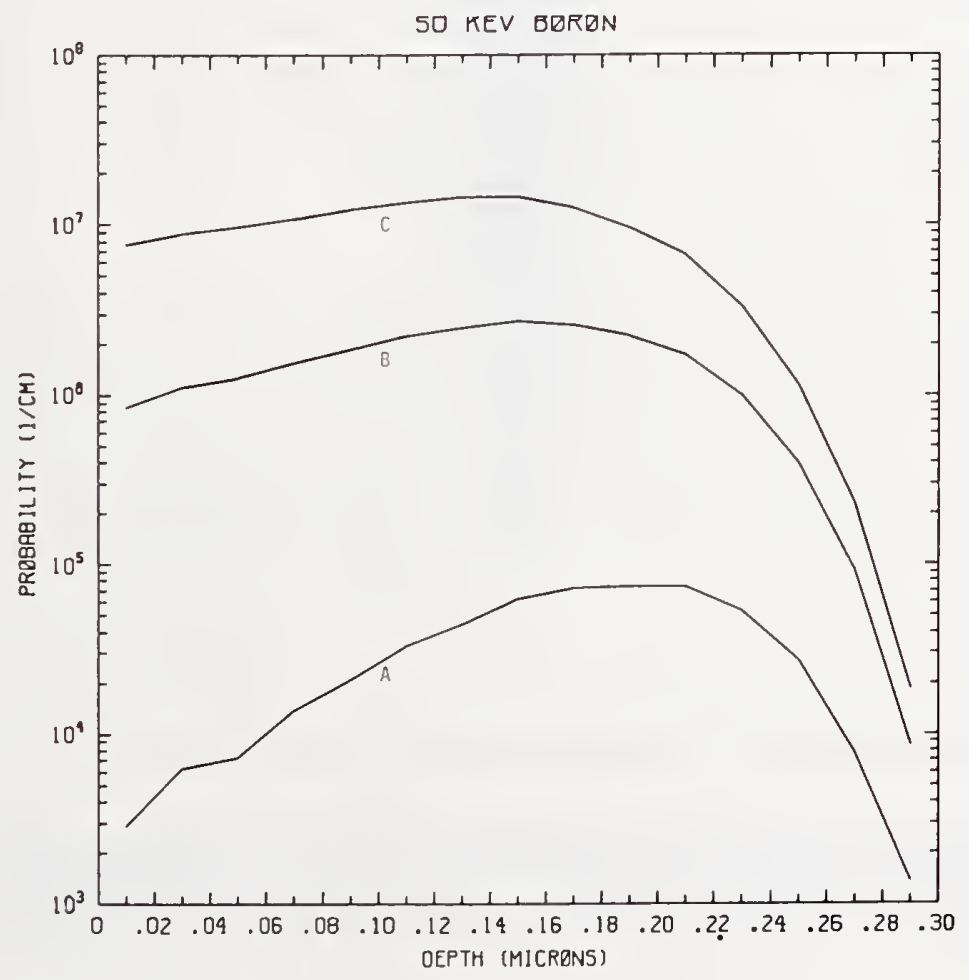

Figure B50-2 One-dimensional probability distributions for implanted boron (A), primary silicon displacements (B), and Frenkel pairs (C) for $50 \mathrm{keV}$ boron normally incident on a silicon target. 


\section{MASK}

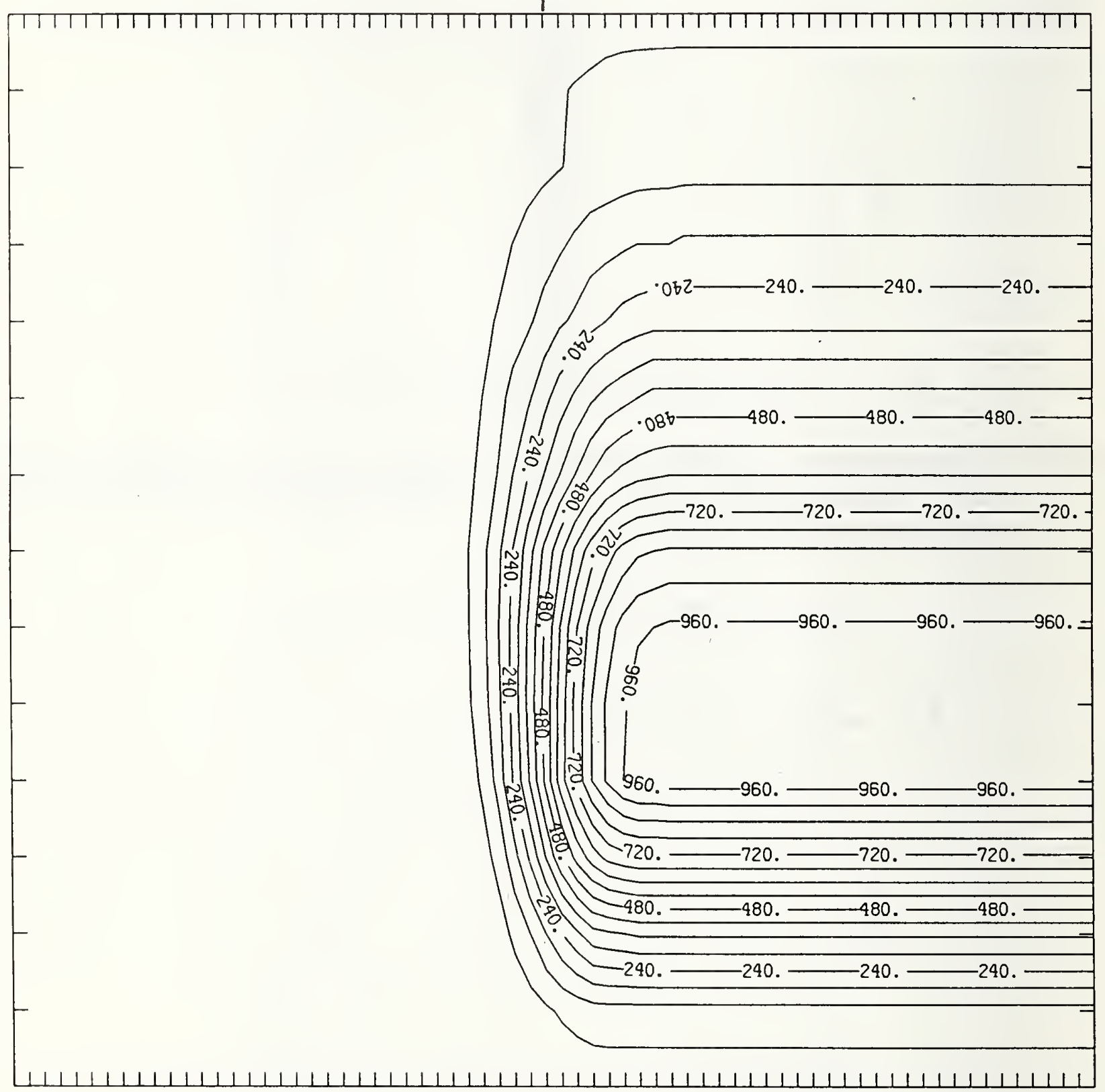

Figure B50-3 Two-dimensional distribution of $50 \mathrm{keV}$ boron implanted past a mask edge. The length increment (distance between tick marks) is $0.02 \mu \mathrm{m}$ and the scale factor is 1000 . 


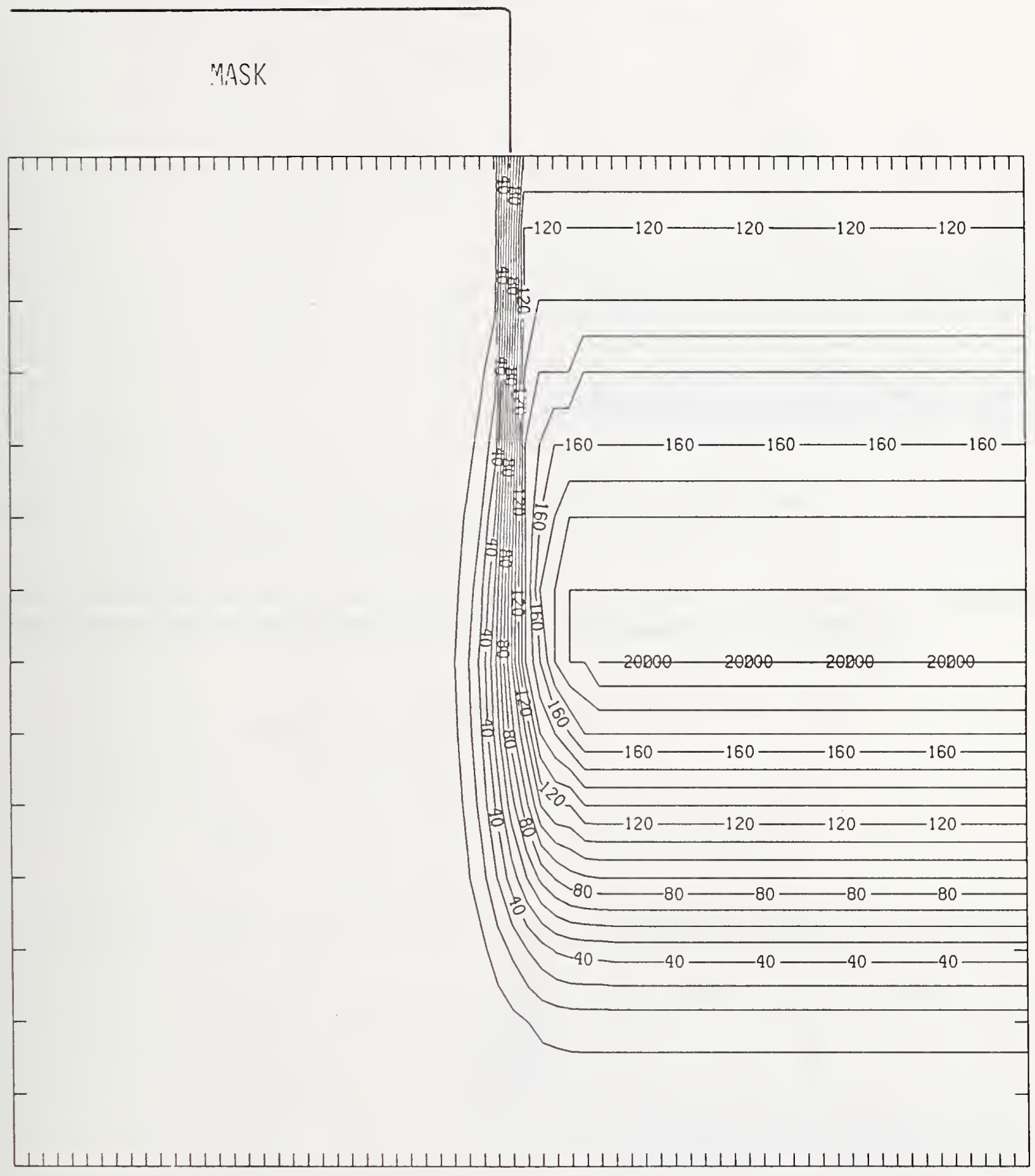

Figure B50-4 Two-dimensional distribution of Frenkel pairs created by $50 \mathrm{keV}$ boron implanted past a mask edge. The length increment (distance between tick marks) is $0.02 \mu \mathrm{m}$ and the scale factor is 1 . 


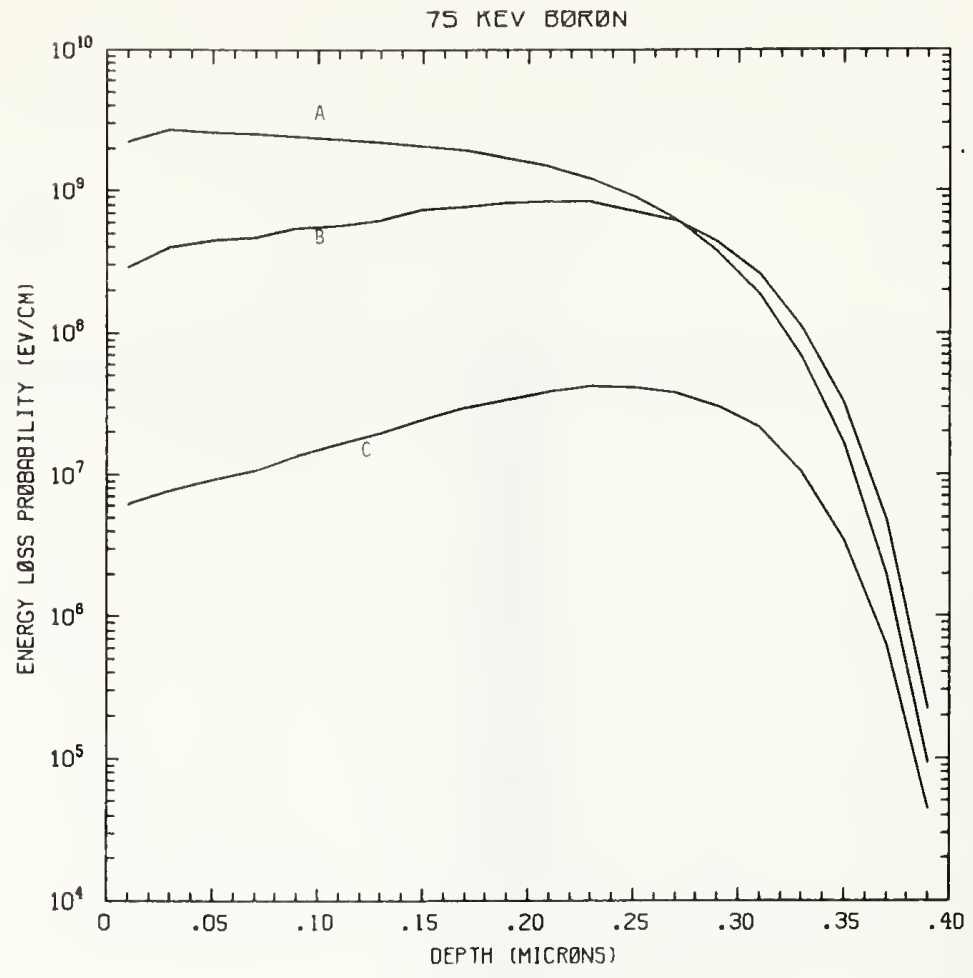

Figure B75-1 One-dimensional probability distributions for energy loss due to electronic excitation (A), displacement damage(B), and phonon excitation (C) for $75 \mathrm{keV}$ boron normally incident on a silicon target.

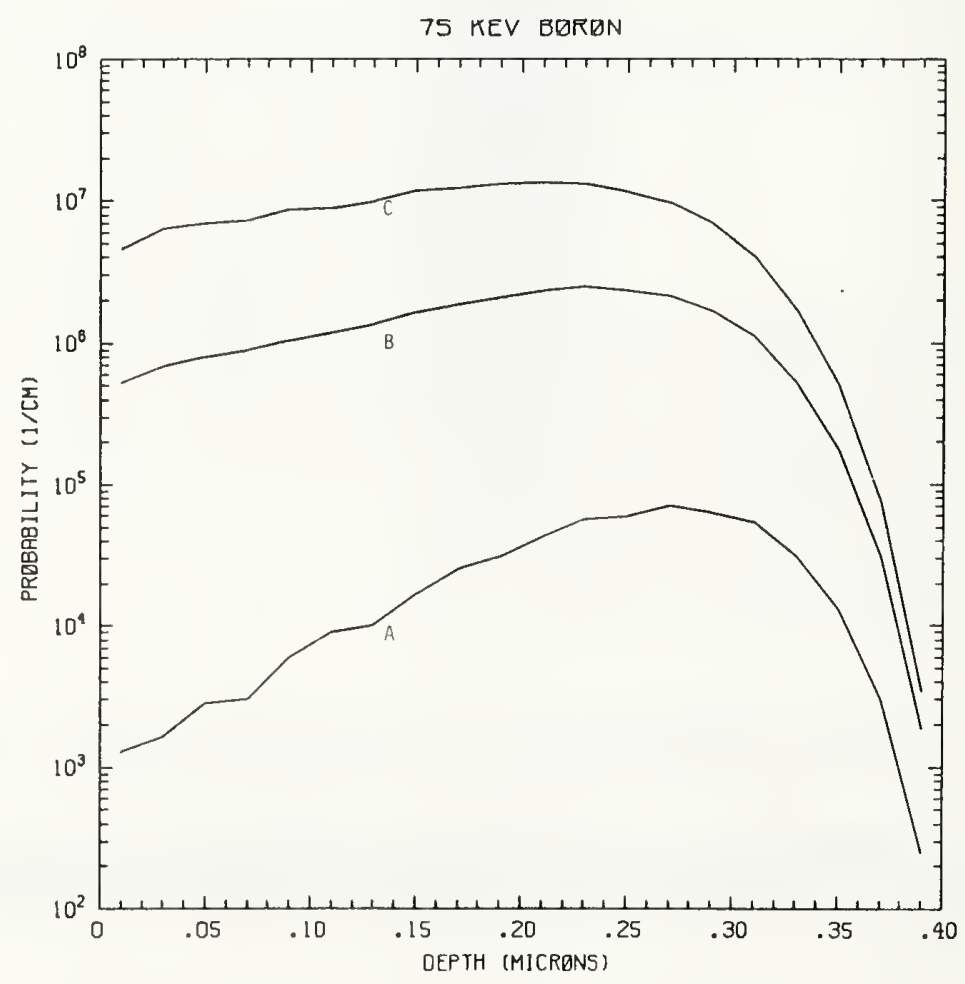

Figure B75-2 One-dimensional probability distributions for implanted boron (A), primary silicon displacements (B), and Frenkel pairs (C) for $75 \mathrm{keV}$ boron normally incident on a silicon target. 


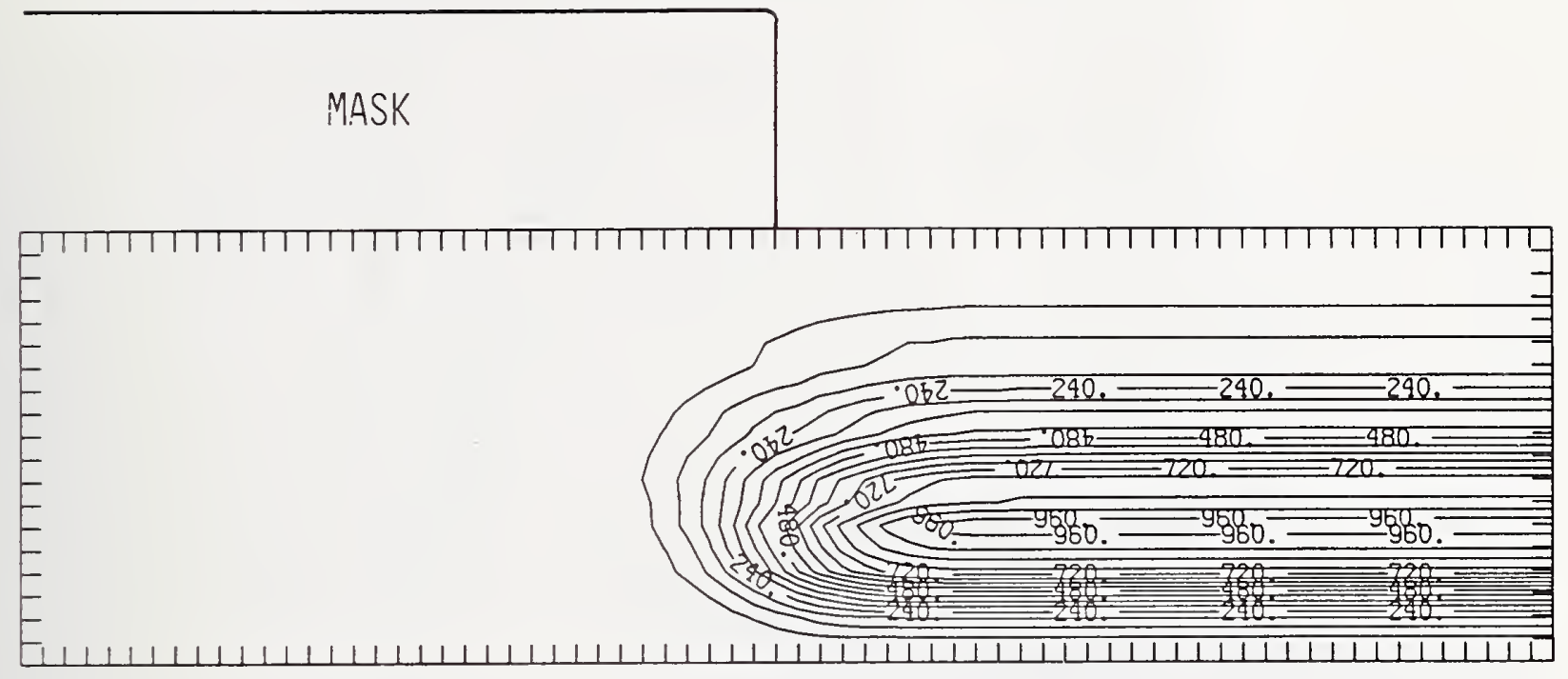

Figure B75-3 Two-dimensional distribution of $75 \mathrm{keV}$ boron implanted past a mask edge. The length increment (distance between tick marks) is $0.02 \mu \mathrm{m}$ and the scale factor is 1000 .

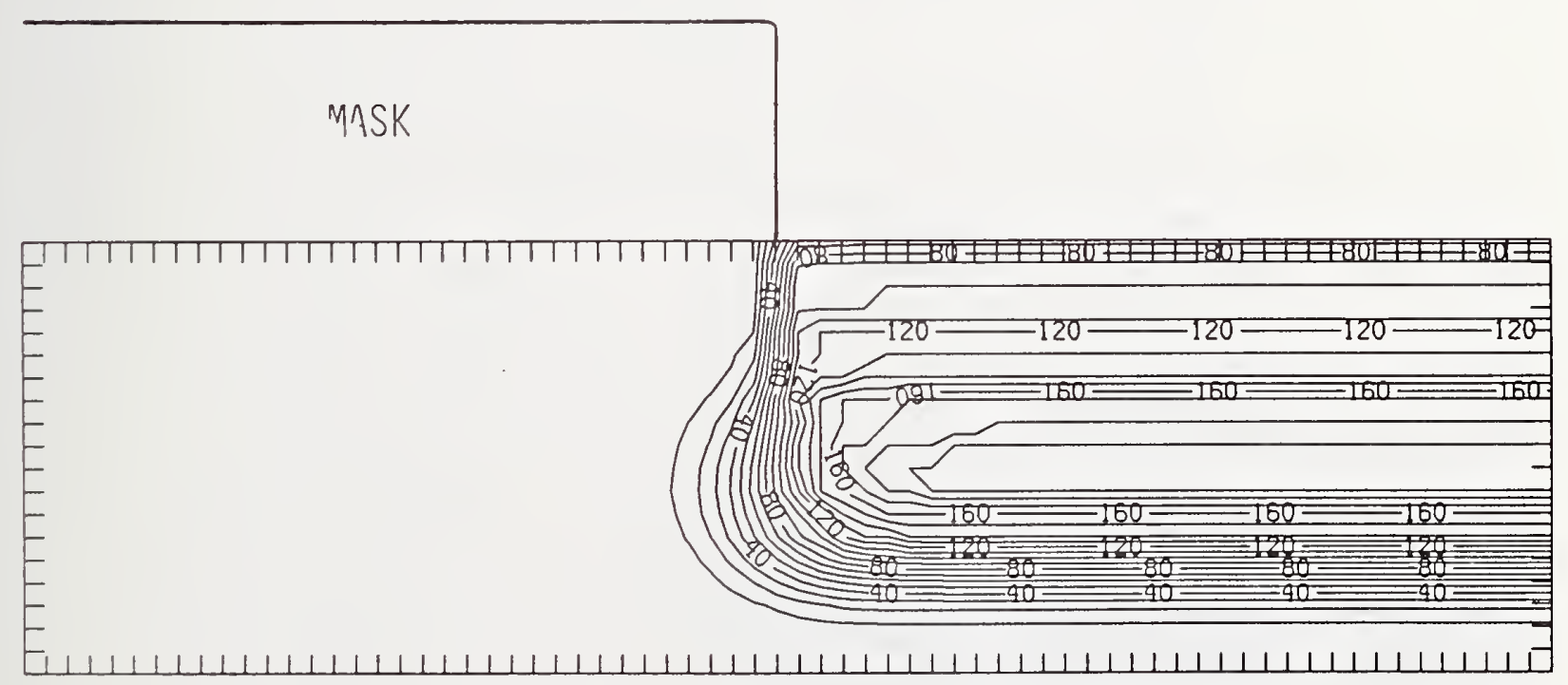

Figure B75-4 Two-dimensional distribution of Frenkel pairs created by $75 \mathrm{keV}$ boron implanted past a mask edge. The length increment (distance between tick marks) is $0.02 \mu \mathrm{m}$ and the scale factor is 1 . 


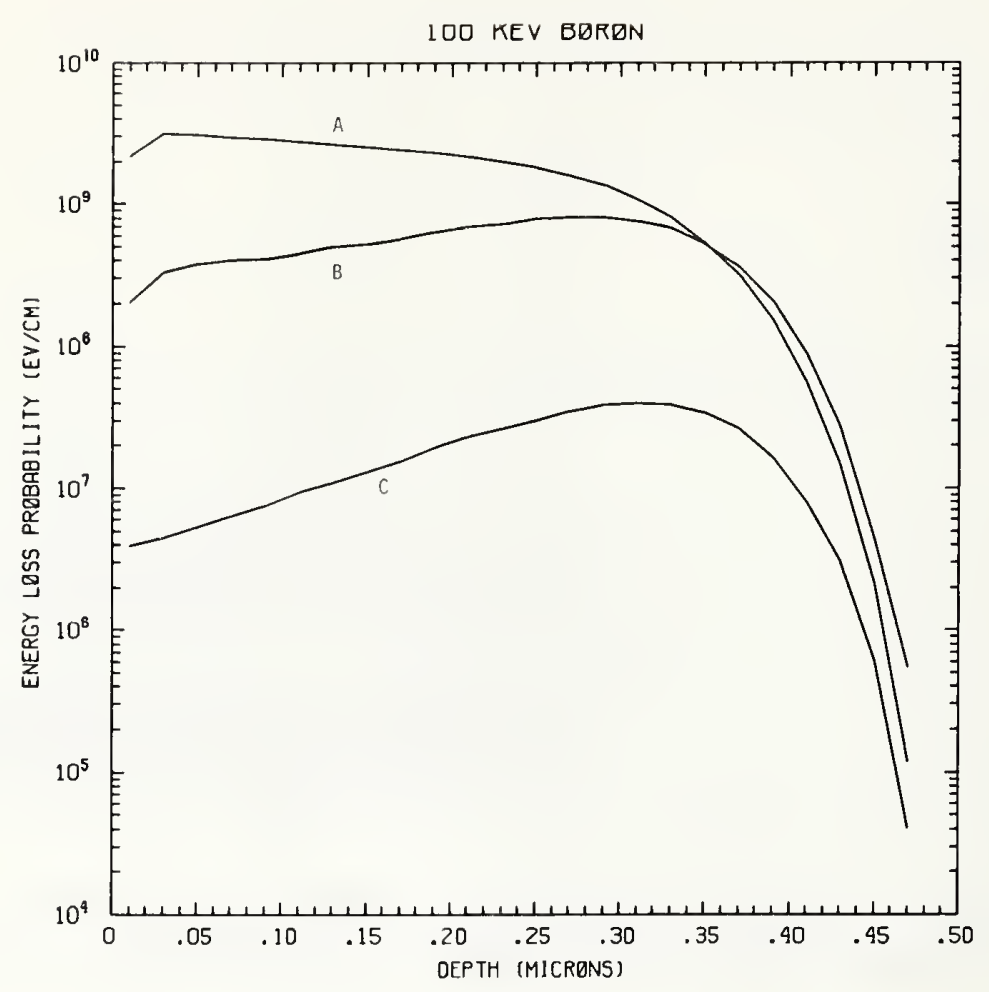

Figure B100-1 One-dimensional probability distributions for energy loss due to electronic excitation (A), displacement damage(B), and phonon excitation (C) for $100 \mathrm{keV}$ boron normally incident on a silicon target.

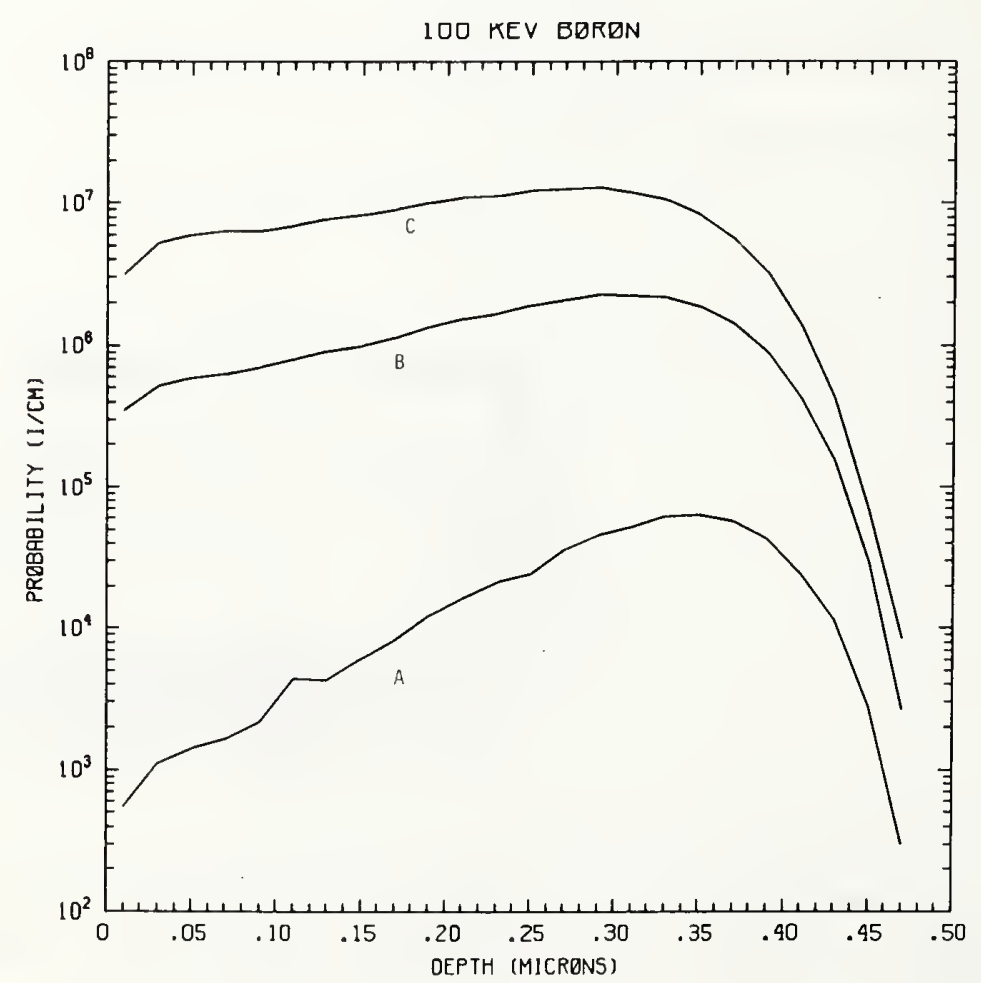

Figure B100-2 One-dimensional probability distributions for implanted boron (A), primary silicon displacements (B), and Frenkel pairs (C) for $100 \mathrm{keV}$ boron normally incident on a silicon target. 


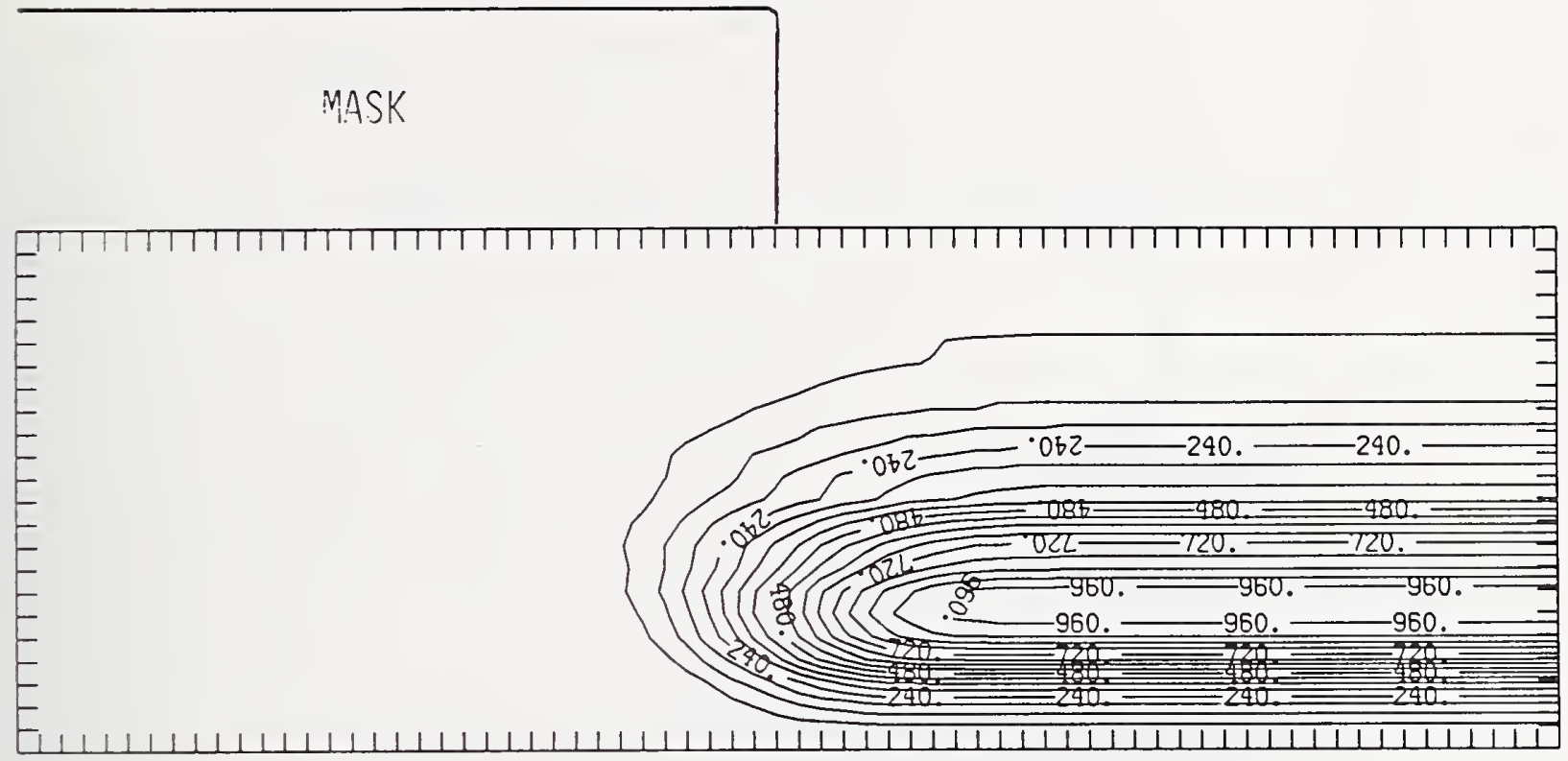

Figure B100-3 Two-dimensional distribution of $100 \mathrm{keV}$ boron implanted past a mask edge. The length increment (distance between tick marks) is $0.02 \mu \mathrm{m}$ and the scale factor is 1000 .

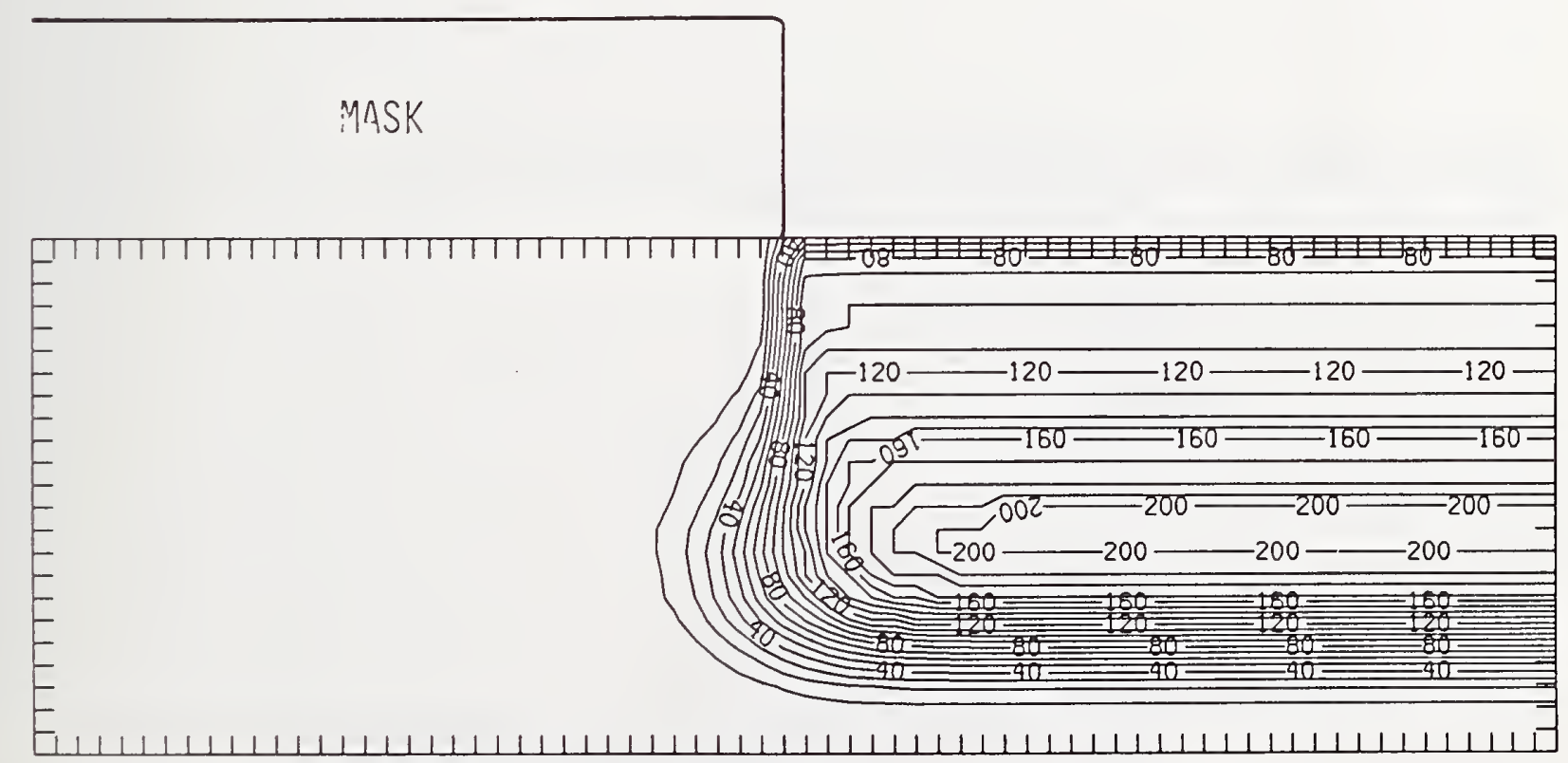

Figure B100-4 Two-dimensional distribution of Frenkel pairs created by $100 \mathrm{keV}$ boron implanted past a mask edge. The length increment (distance between tick marks) is $0.02 \mu \mathrm{m}$ and the scale factor is 1 . 


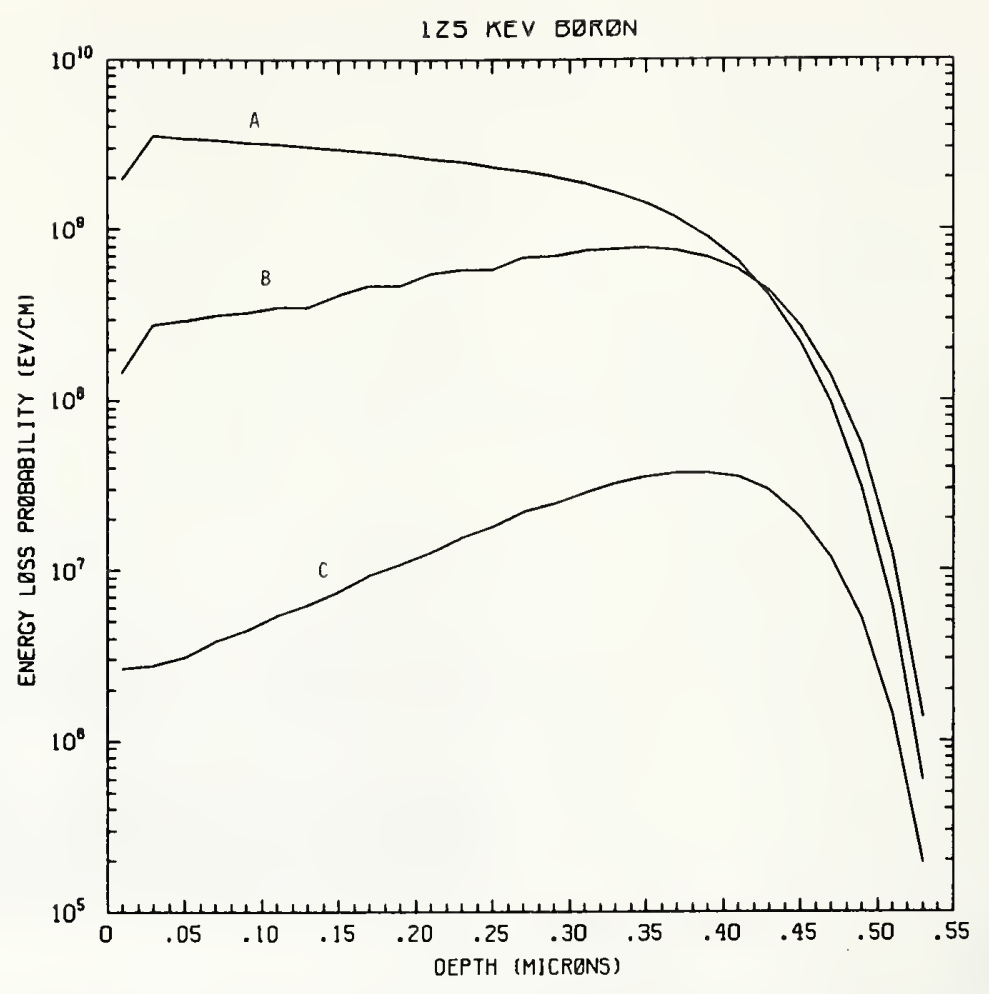

Figure B125-1 One-dimensional probability distributions for energy loss due to electronic excitation (A), displacement damage(B), and phonon excitation (C) for $125 \mathrm{keV}$ boron normally incident on a silicon target.

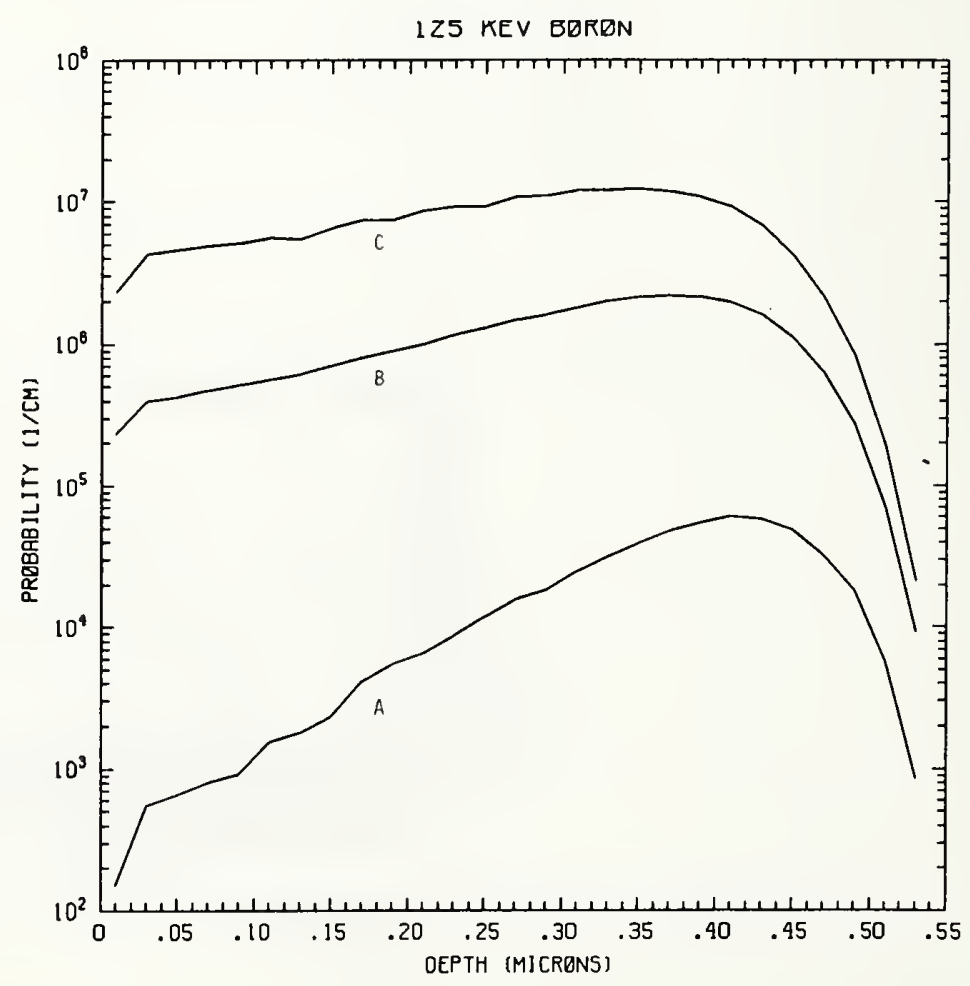

Figure B125-2 One-dimensional probability distributions for implanted boron (A), primary silicon displacements (B), and Frenkel pairs (C) for $125 \mathrm{keV}$ boron normally incident on a silicon target. 


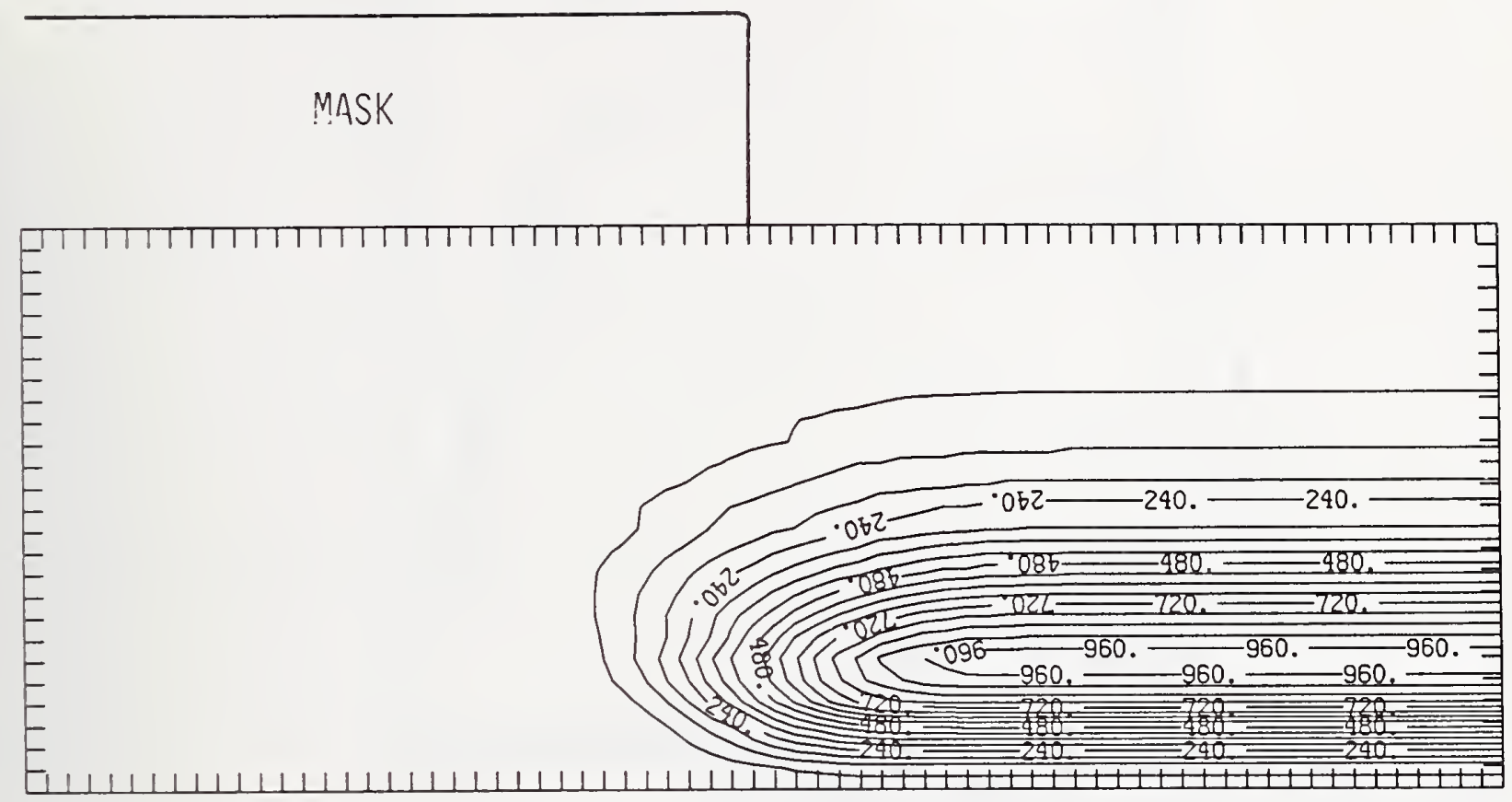

Figure B125-3 Two-dimensional distribution of $125 \mathrm{keV}$ boron implanted past a mask edge. The length increment (distance between tick marks) is $0.02 \mu \mathrm{m}$ and the scale factor is 1000 .

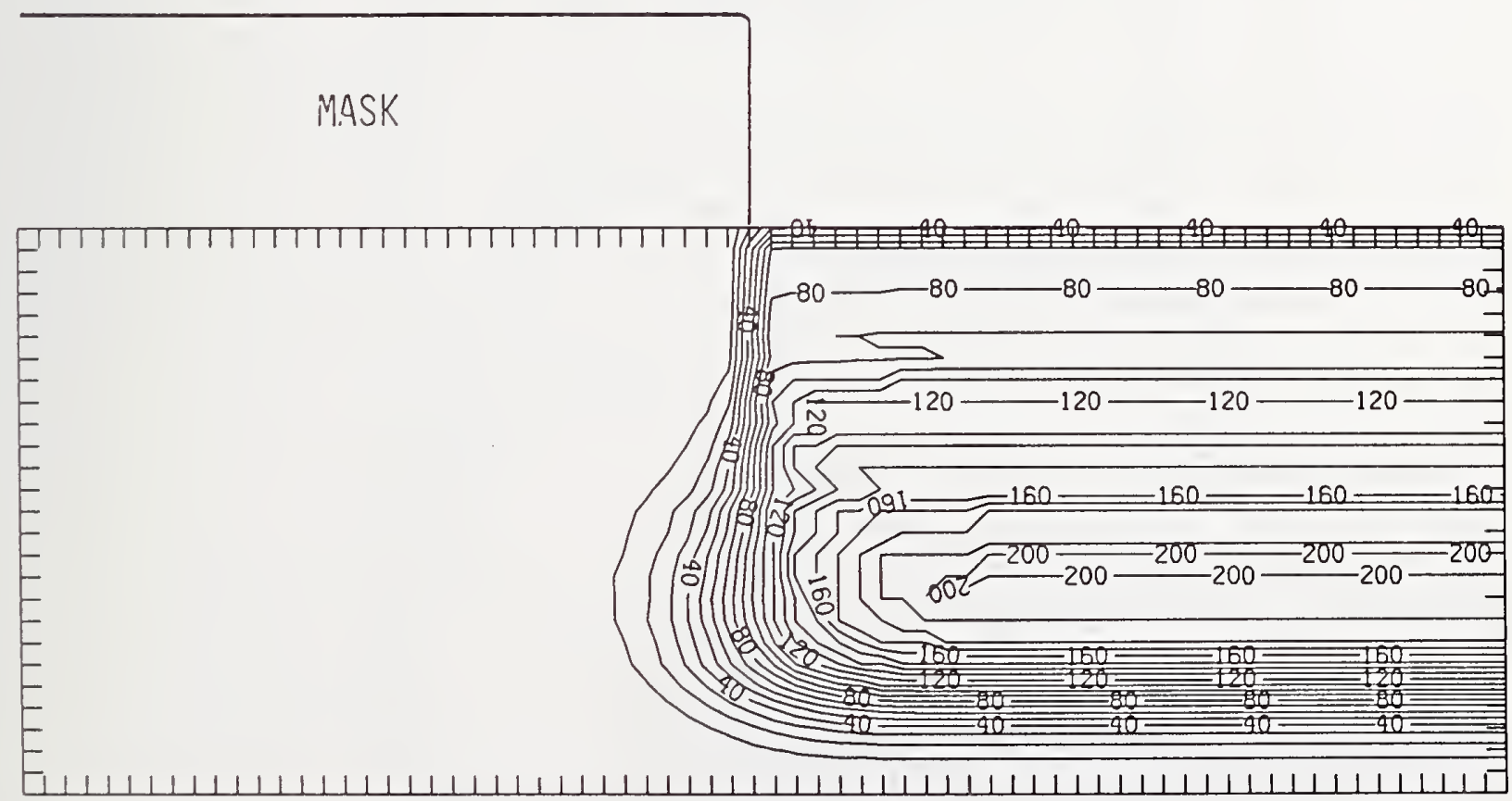

Figure B125-4 Two-dimensional distribution of Frenkel pairs created by $125 \mathrm{keV}$ boron implanted past a mask edge. The length increment (distance between tick marks) is $0.02 \mu \mathrm{m}$ and the scale factor is 1 . 
150 KEV BDREN

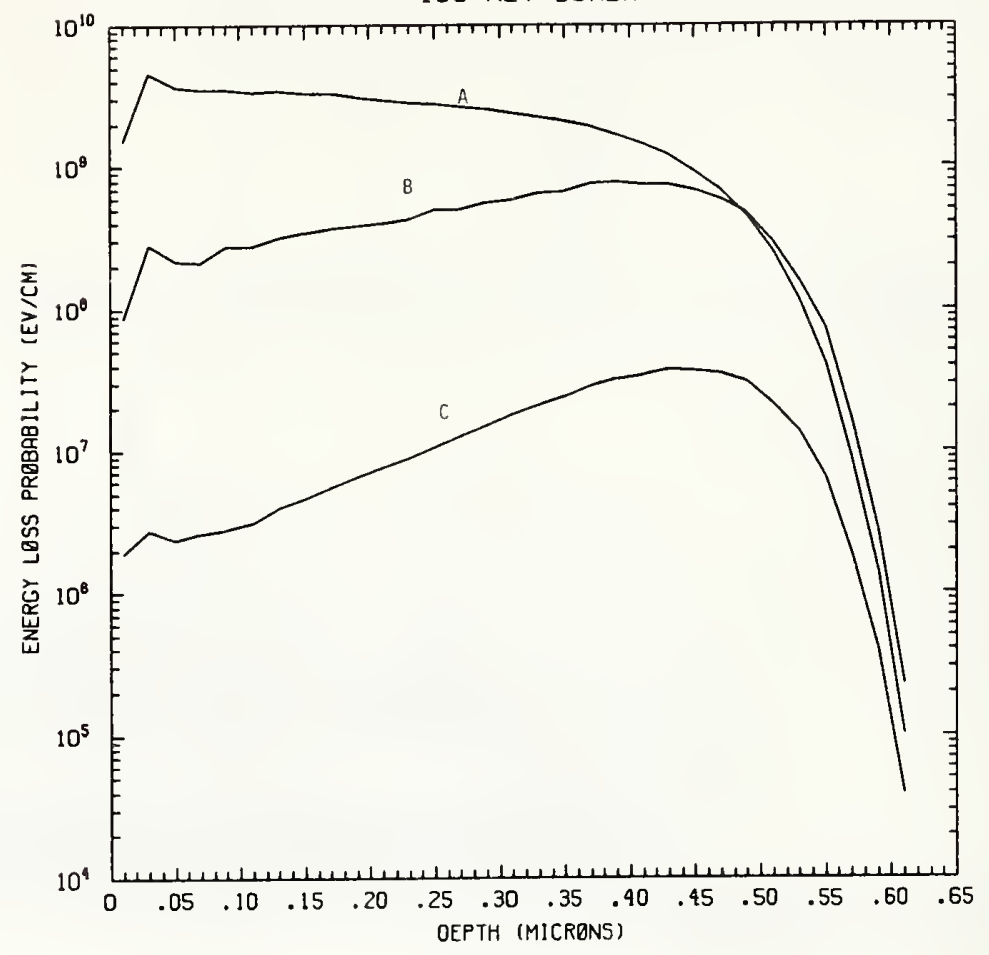

Figure B150-1 One-dimensional probability distributions for energy loss due to electronic excitation (A), displacement damage(B), and phonon excitation (C) for $150 \mathrm{keV}$ boron normally incident on a silicon target.

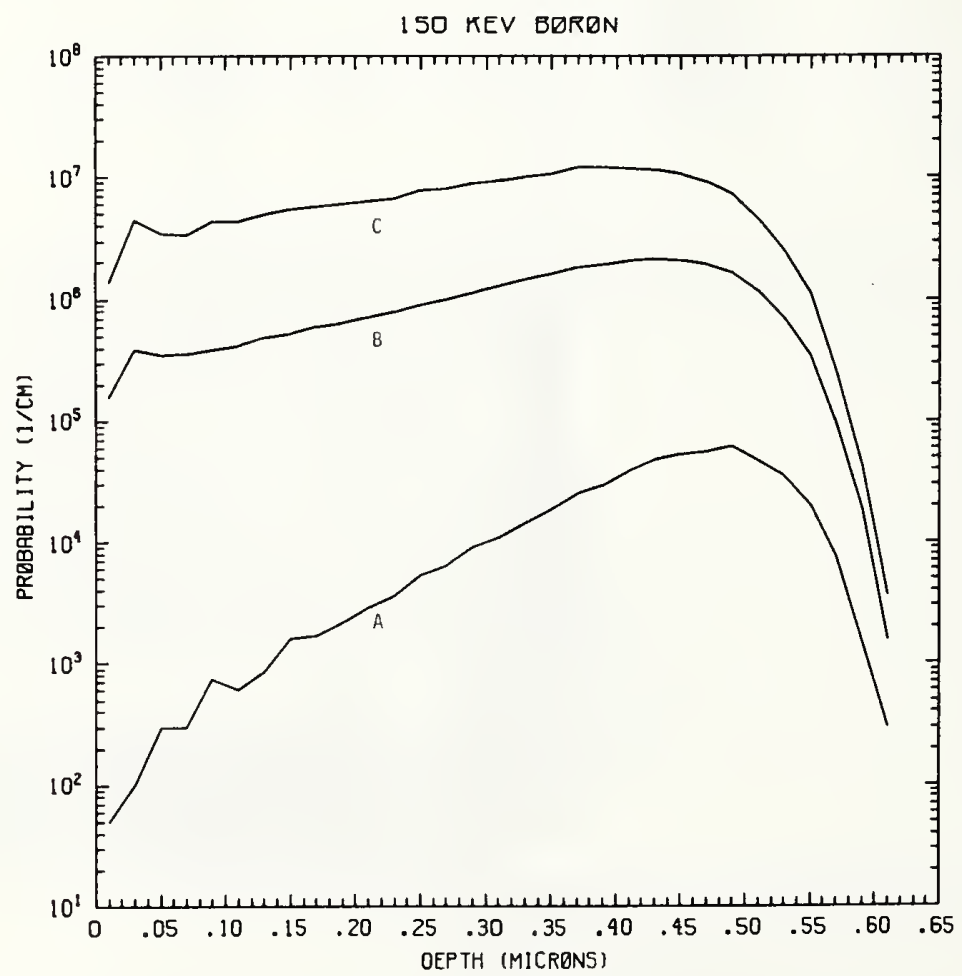

Figure B150-2 One-dimensional probability distributions for implanted boron (A), primary silicon displacements (B), and Frenkel pairs (C) for $150 \mathrm{keV}$ boron normally incident on a silicon target. 
MASK

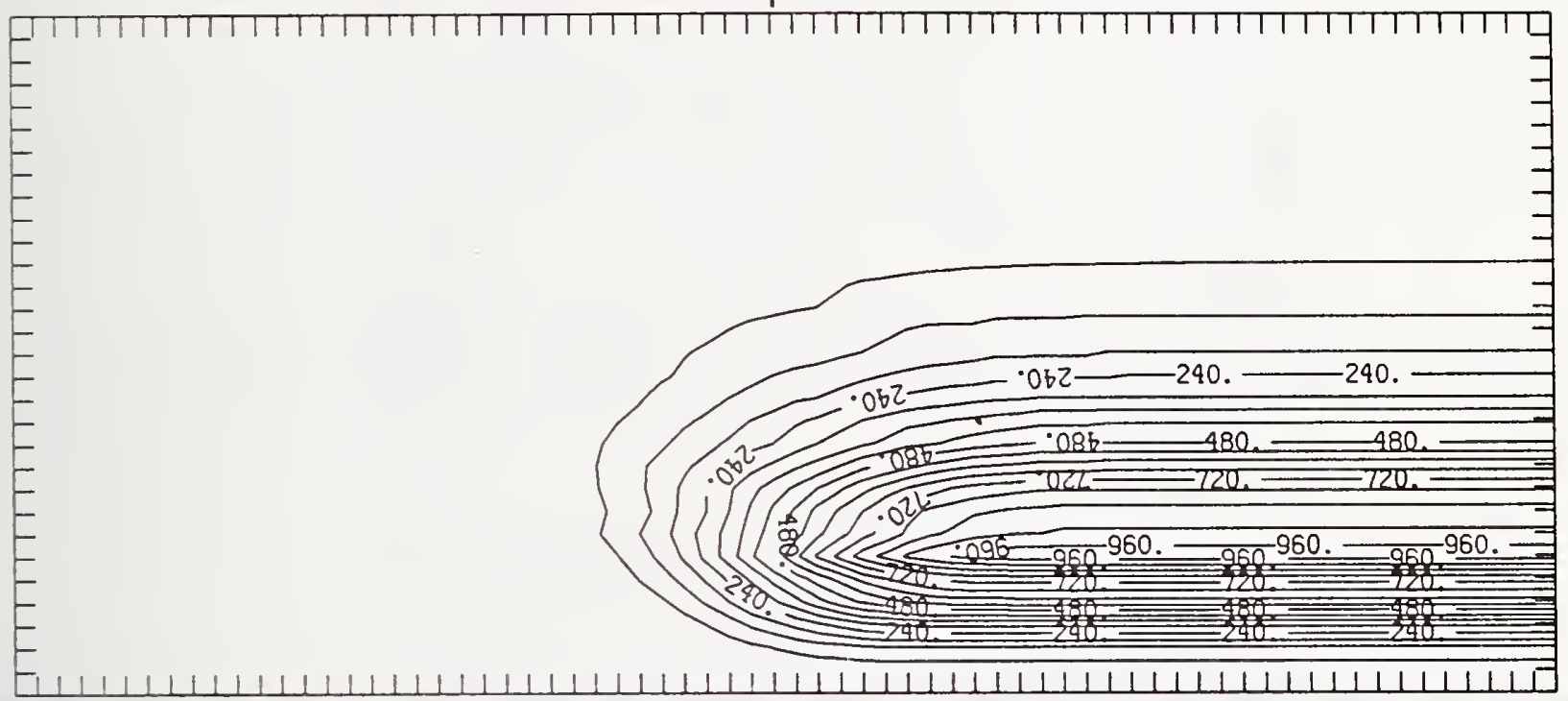

Figure B150-3 Two-dimensional distribution of $150 \mathrm{keV}$ boron implanted past a mask edge. The length increment (distance between tick marks) is $0.02 \mu \mathrm{m}$ and the scale factor is 1000 .

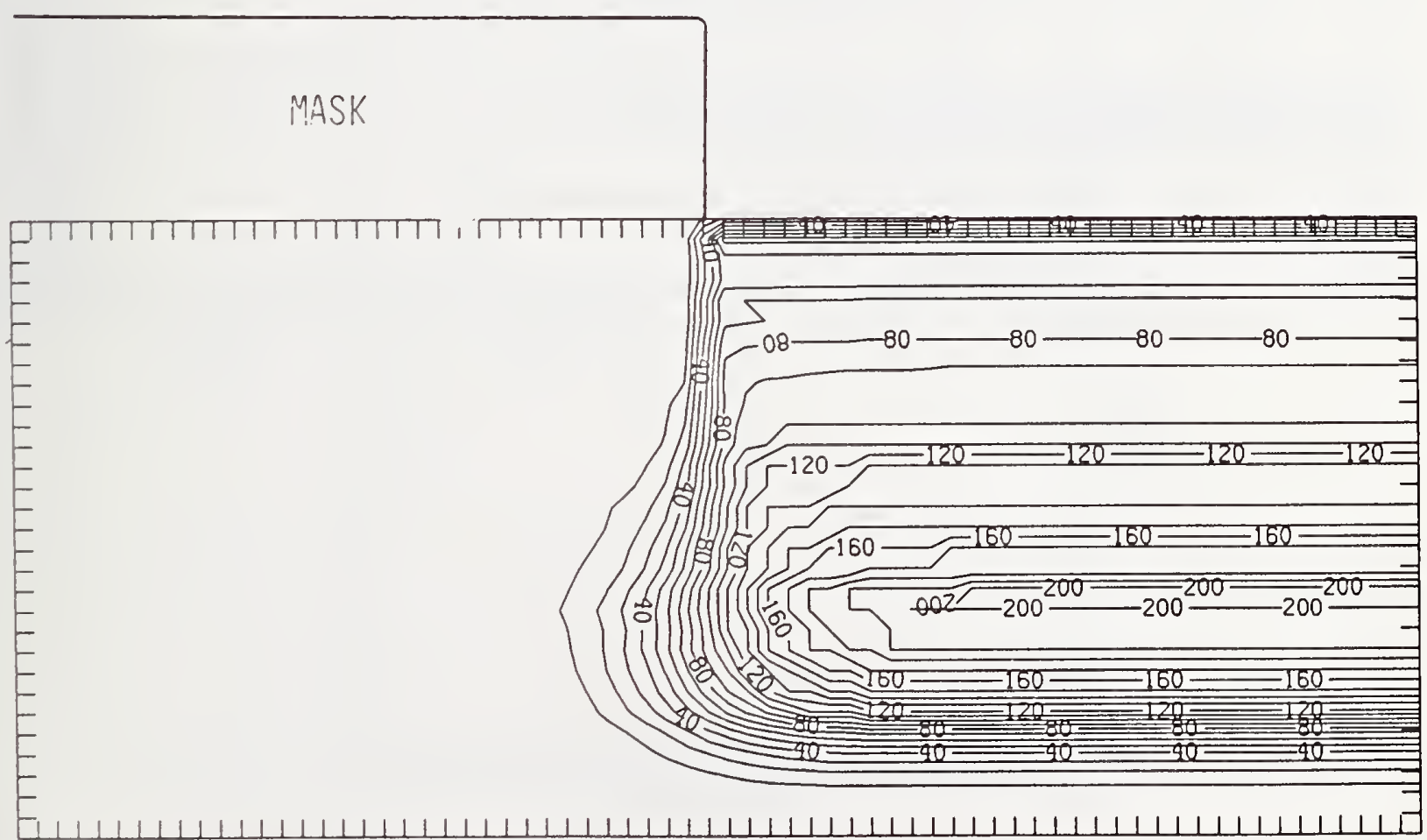

Figure B150-4 Two-dimensional distribution of Frenkel pairs created by $150 \mathrm{keV}$ boron implanted past a mask edge. The length increment (distance between tick marks) is $0.02 \mu \mathrm{m}$ and the scale factor is 1 . 
175 KEV BORON

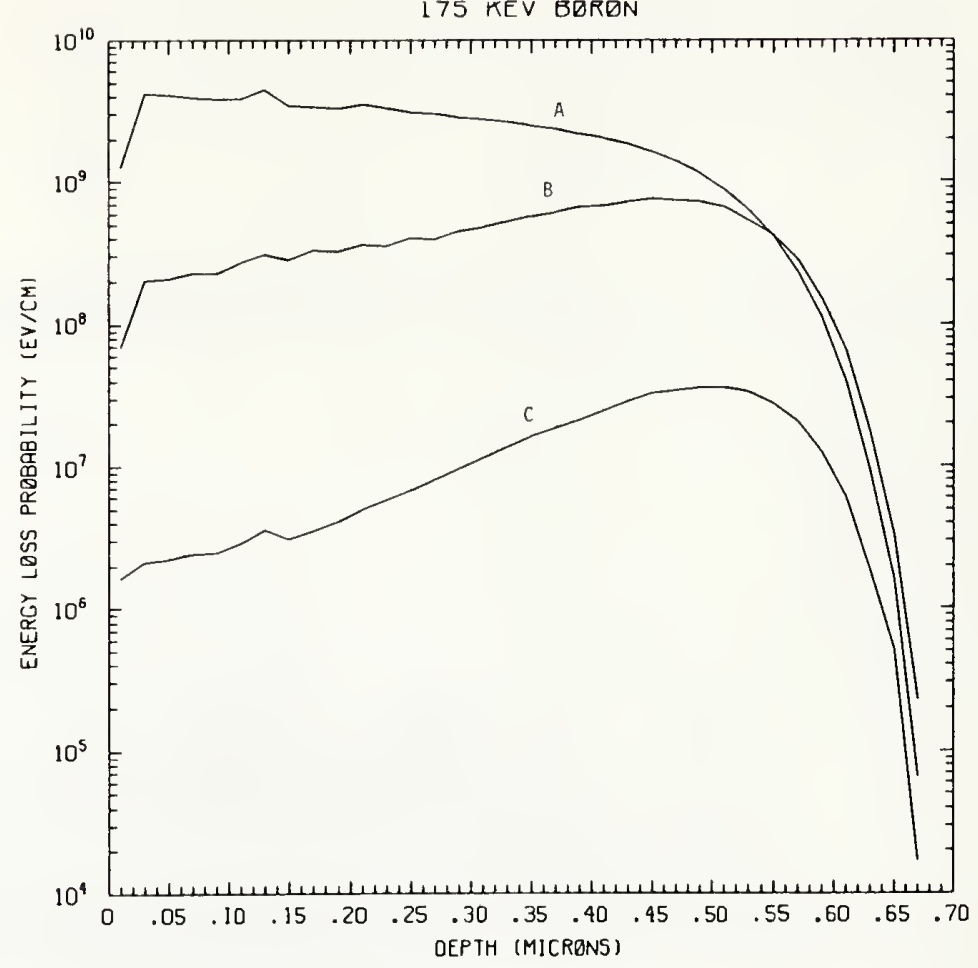

Figure B175-1 One-dimensional probability distributions for energy loss due to electronic excitation (A), displacement damage(B), and phonon excitation (C) for $175 \mathrm{keV}$ boron normally incident on a silicon target.

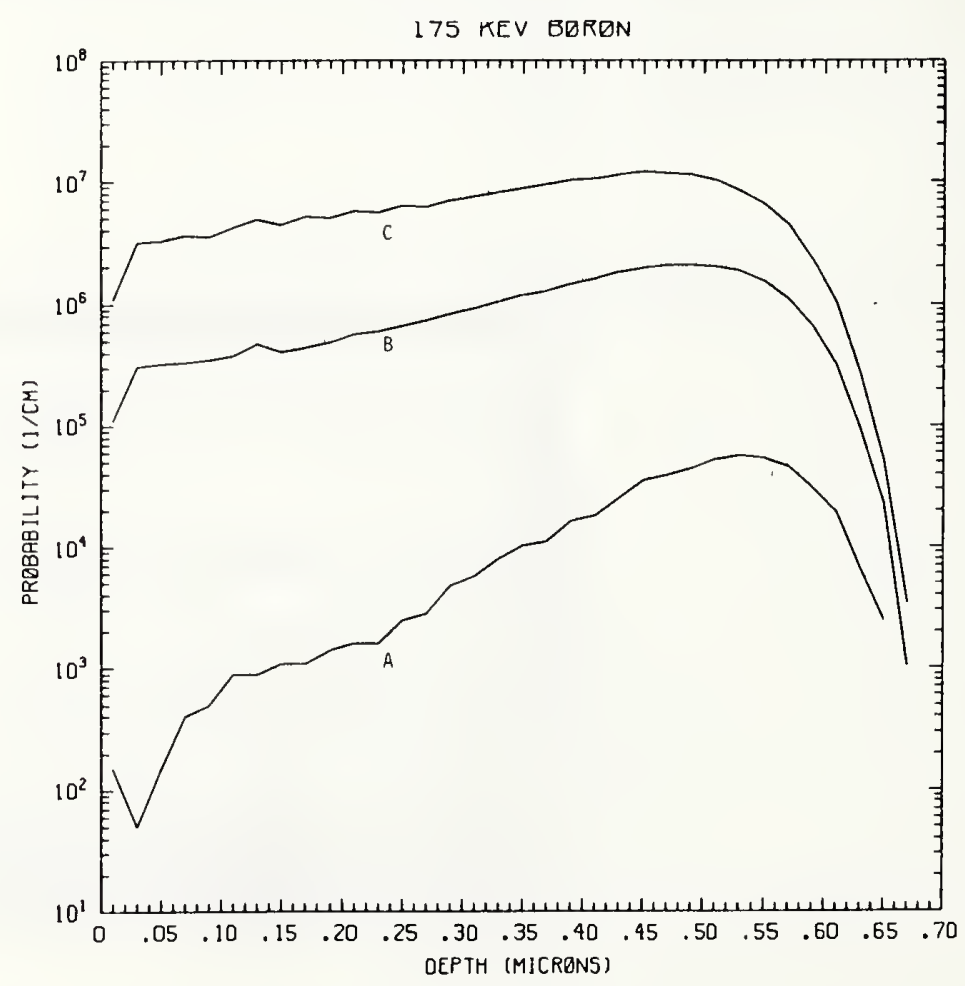

Figure B175-2 One-dimensional probability distributions for implanted boron (A), primary silicon displacements (B), and Frenkel pairs (C) for $175 \mathrm{keV}$ boron normally incident on a silicon target. 


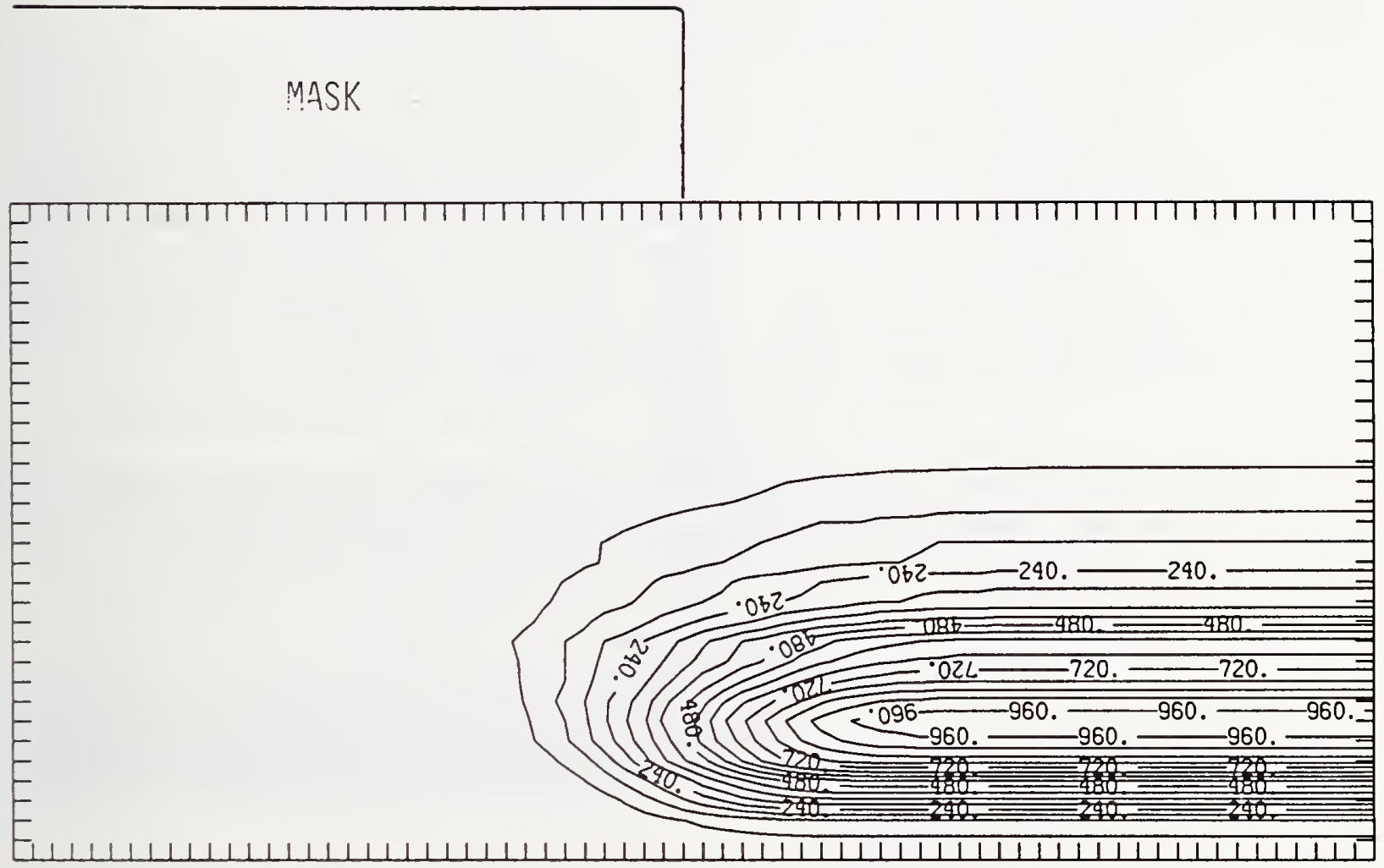

Figure B175-3 Two-dimensional distribution of $175 \mathrm{keV}$ boron implanted past a mask edge. The length increment (distance between tick marks) is $0.02 \mu \mathrm{m}$ and the scale factor is 1000 . 


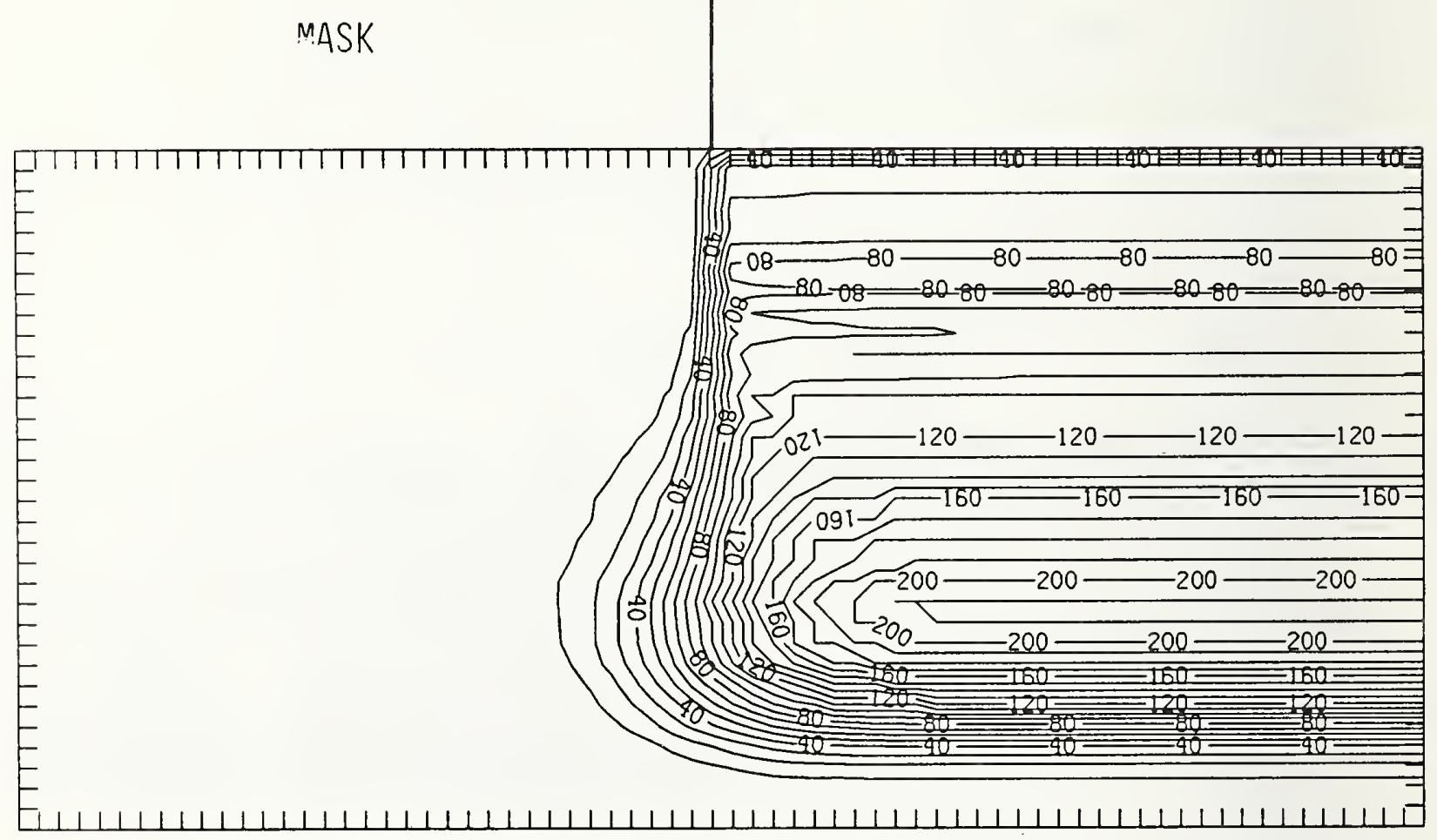

Figure B175-4 Two-dimensional distribution of Frenkel pairs created by $175 \mathrm{keV}$ boron implanted past a mask edge. The length increment (distance between tick marks) is $0.02 \mu \mathrm{m}$ and the scale factor is 1 . 


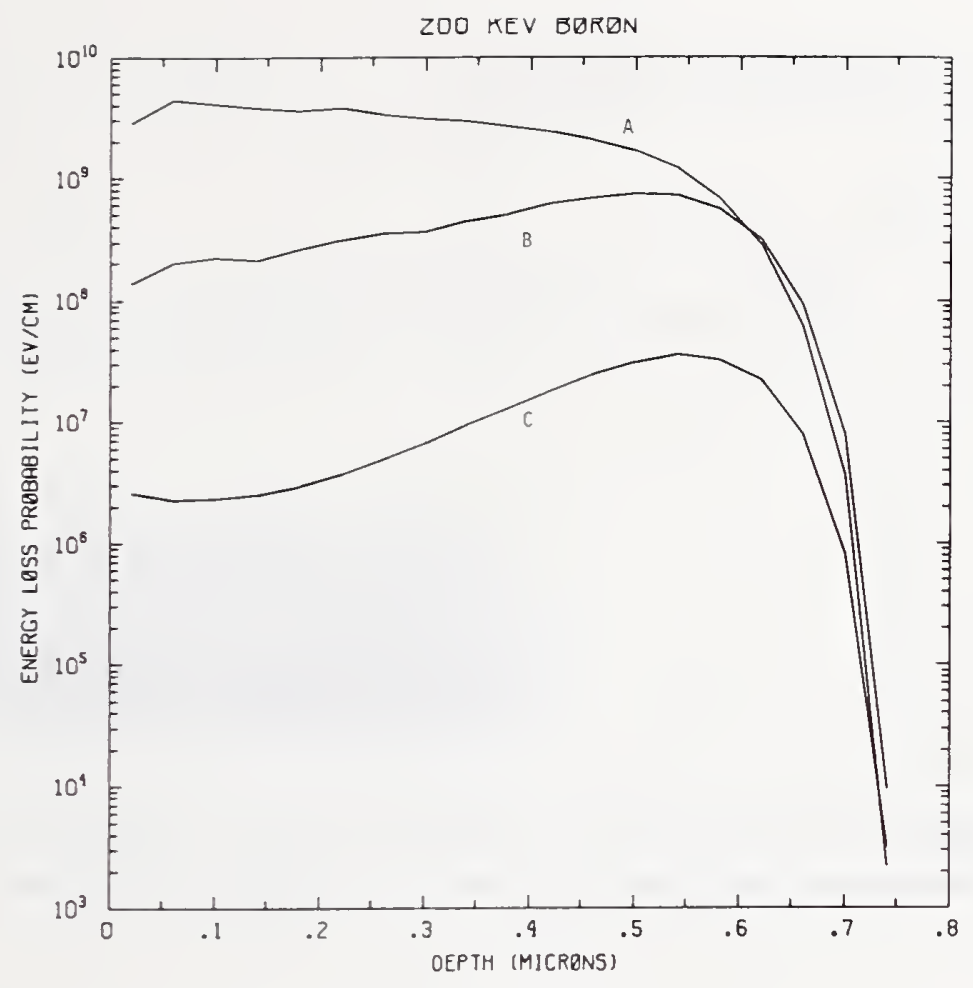

Figure B200-1 One-dimensional probability distributions for energy loss due to electronic excitation (A), displacement damage(B), and phonon excitation (C) for $200 \mathrm{keV}$ boron normally incident on a silicon target.

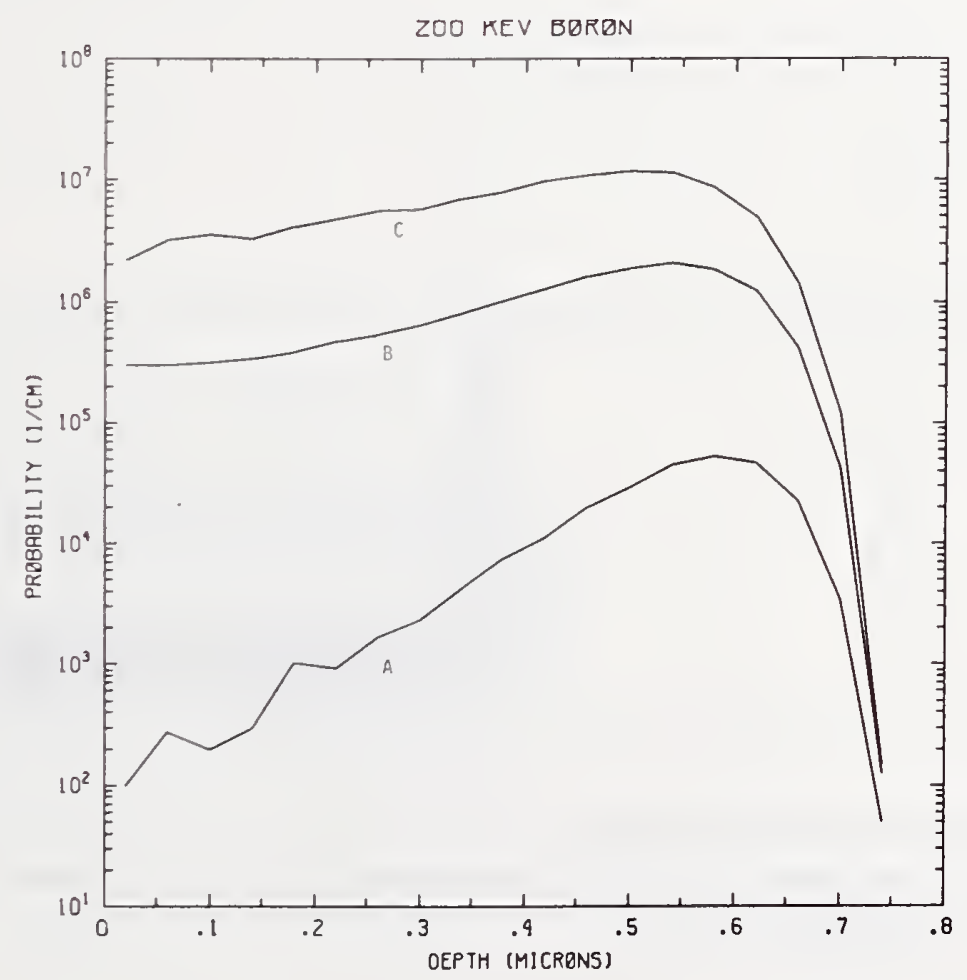

Figure B200-2 One-dimensional probability distributions for implanted boron (A), primary silicon displacements (B), and Frenkel pairs (C) for $200 \mathrm{keV}$ boron normally incident on a silicon target. 


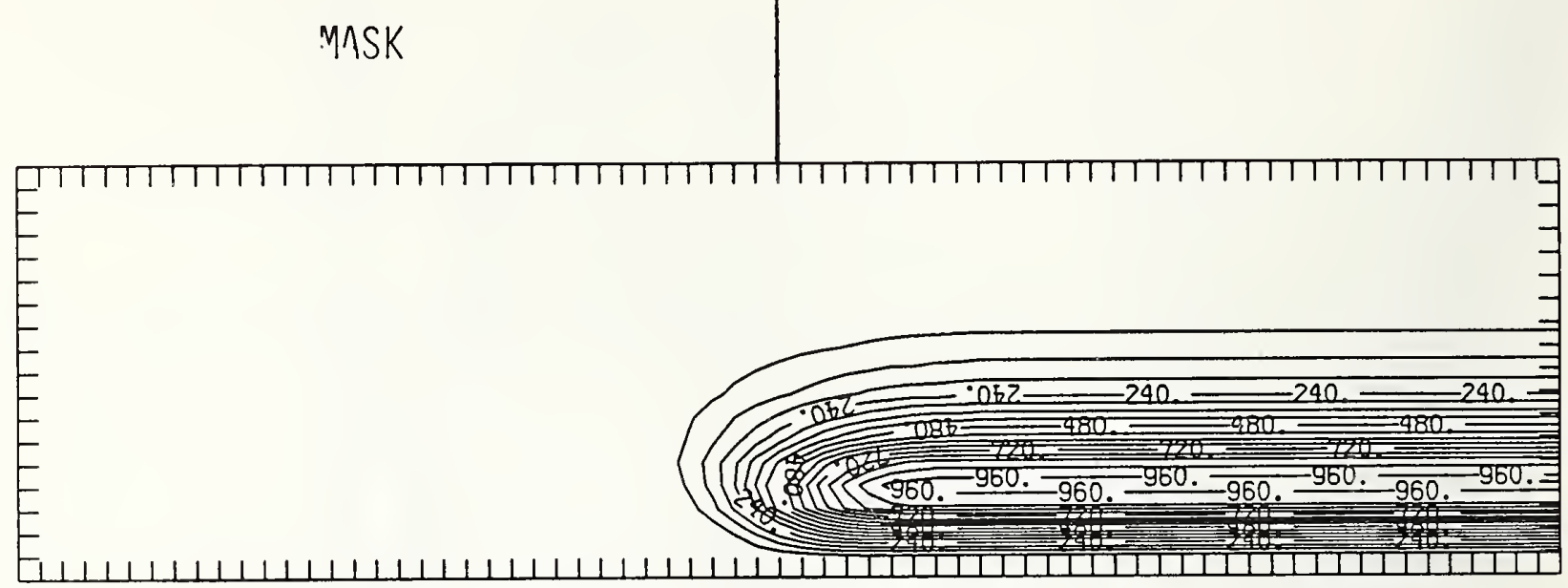

Figure B200-3 Two-dimensional distribution of $200 \mathrm{keV}$ boron implanted past a mask edge. The length increment (distance between tick marks) is $0.04 \mu \mathrm{m}$ and the scale factor is 1000.

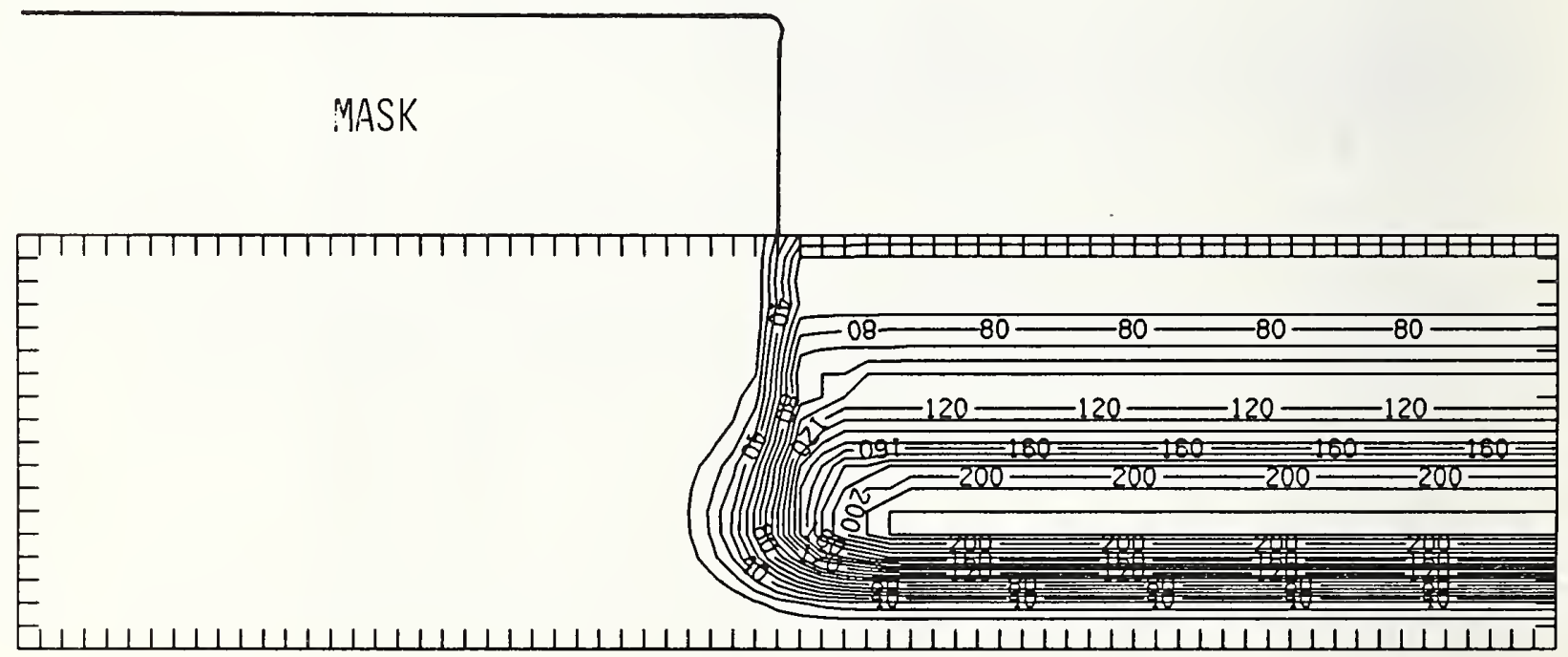

Figure B200-4 Two-dimensional distribution of Frenkel pairs created by $200 \mathrm{keV}$ boron implanted past a mask edge. The length increment (distance between tick marks) is $0.04 \mu \mathrm{m}$ and the scale factor is 1 . 


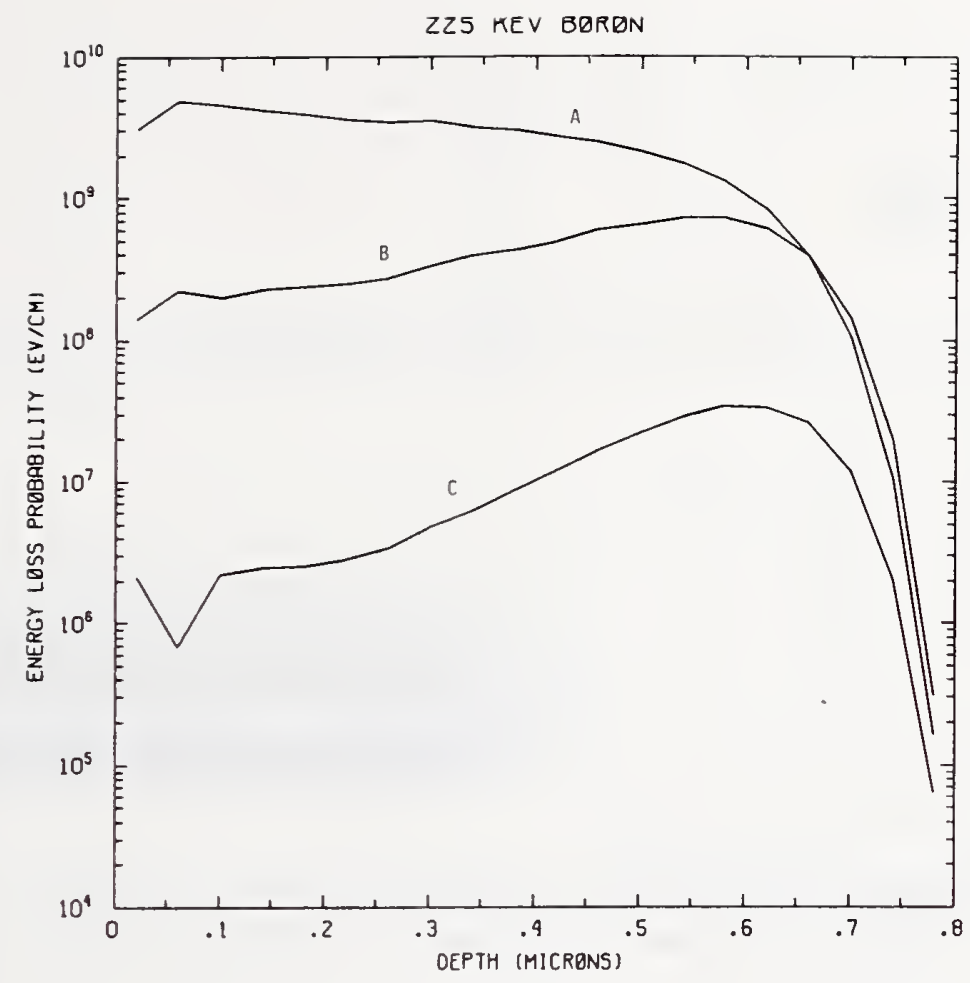

Figure B225-1 One-dimensional probability distributions for energy loss due to electronic excitation (A), displacement damage(B), and phonon excitation (C) for $225 \mathrm{keV}$ boron normally incident on a silicon target.

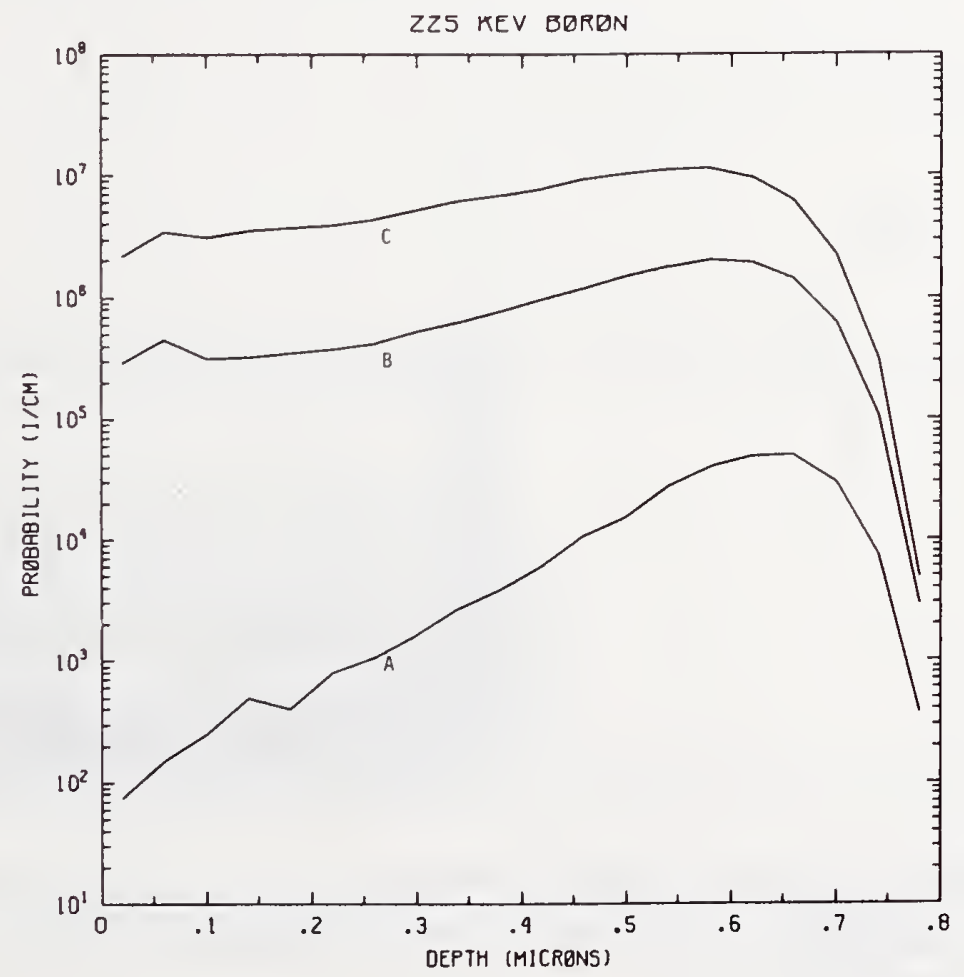

Figure B225-2 One-dimensional probability distributions for implanted boron (A), primary silicon displacements (B), and Frenkel pairs (C) for $225 \mathrm{keV}$ boron normally incident on a silicon target. 


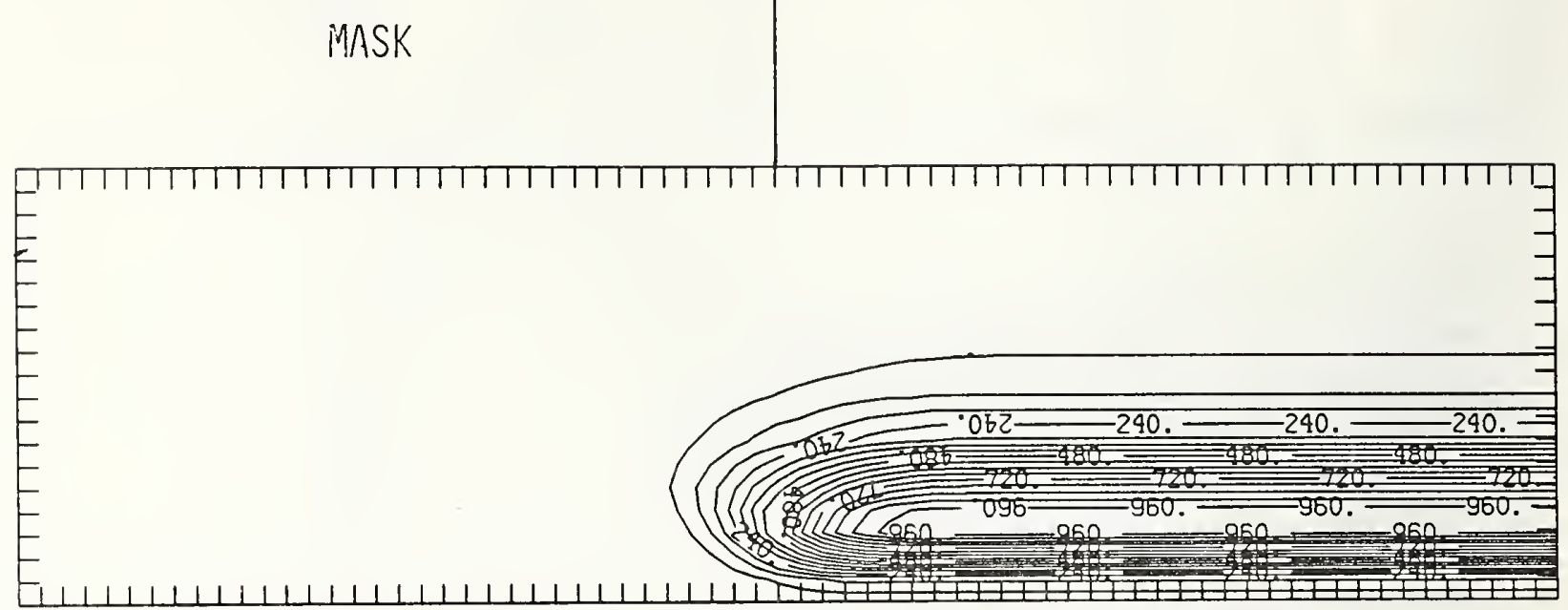

Figure B225-3 Two-dimensional distribution of $225 \mathrm{keV}$ boron implanted past a mask edge. The length increment (distance between tick marks) is $0.04 \mu \mathrm{m}$ and the scale factor is 1000 .

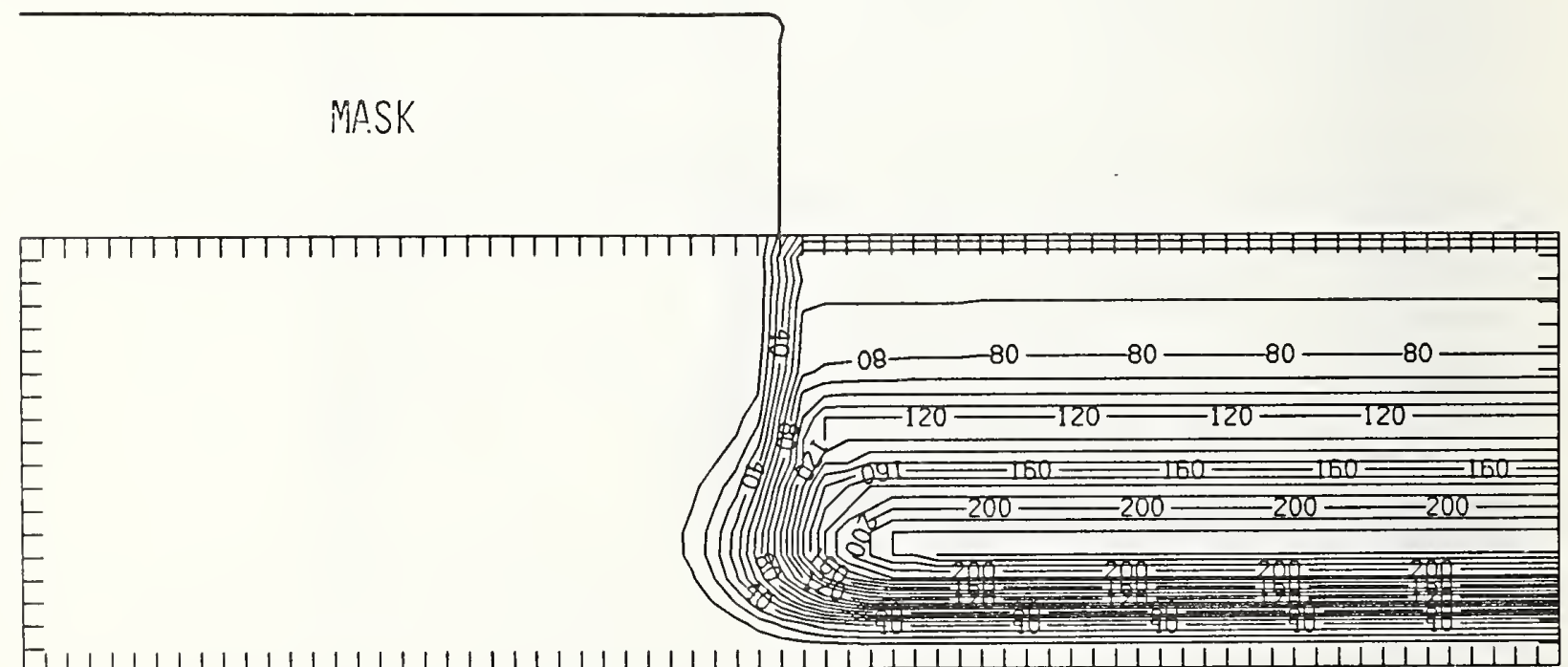

Figure B225-4 Two-dimensional distribution of Frenkel pairs created by $225 \mathrm{keV}$ boron implanted past a mask edge. The length increment (distance between tick marks) is $0.04 \mu \mathrm{m}$ and the scale factor is 1 . 


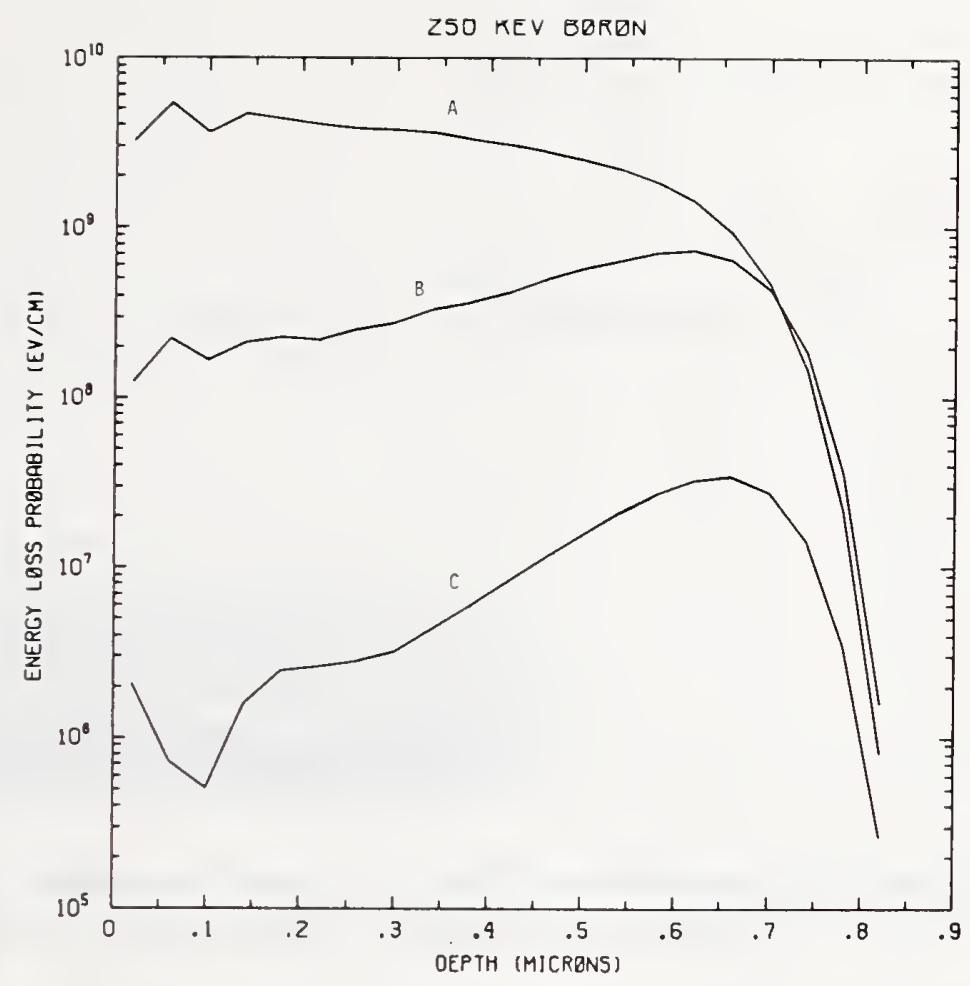

Figure B250-1 One-dimensional probability distributions for energy loss due to electronic excitation (A), displacement damage(B), and phonon excitation (C) for $250 \mathrm{keV}$ boron normally incident on a silicon target.

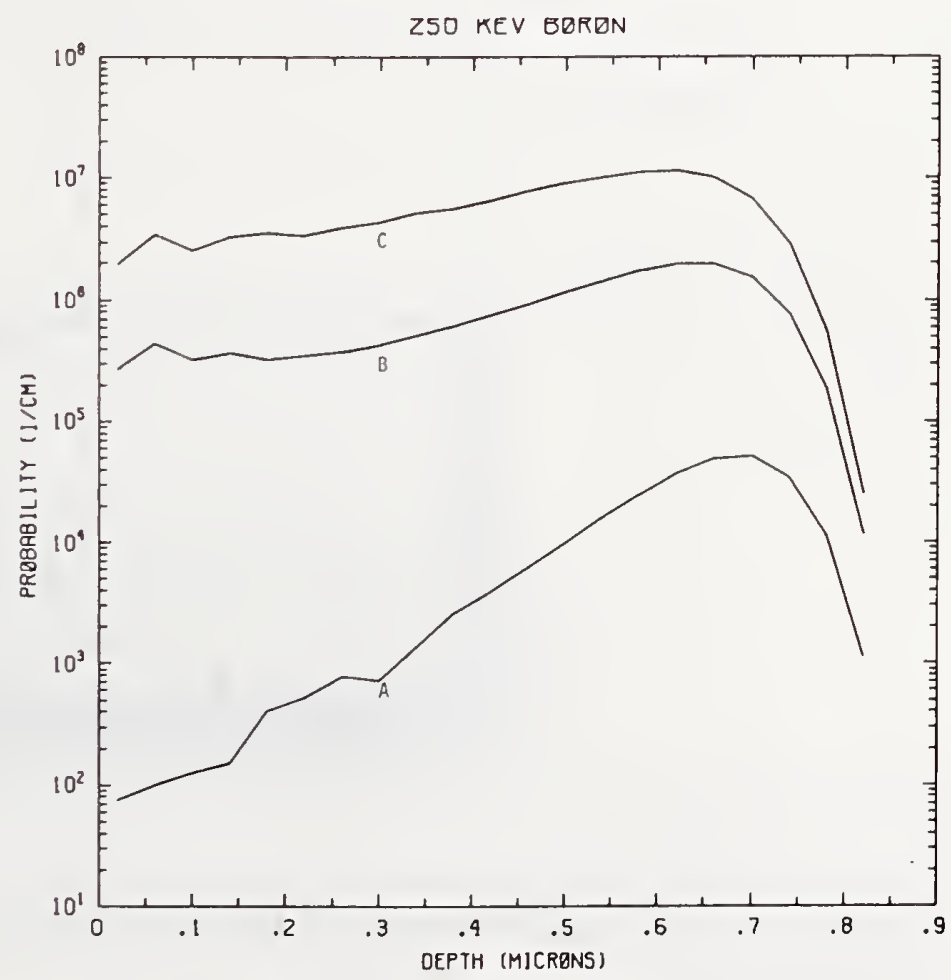

Figure B250-2 One-dimensional probability distributions for implanted boron (A), primary silicon displacements (B), and Frenkel pairs (C) for $250 \mathrm{keV}$ boron normally incident on a silicon target. 


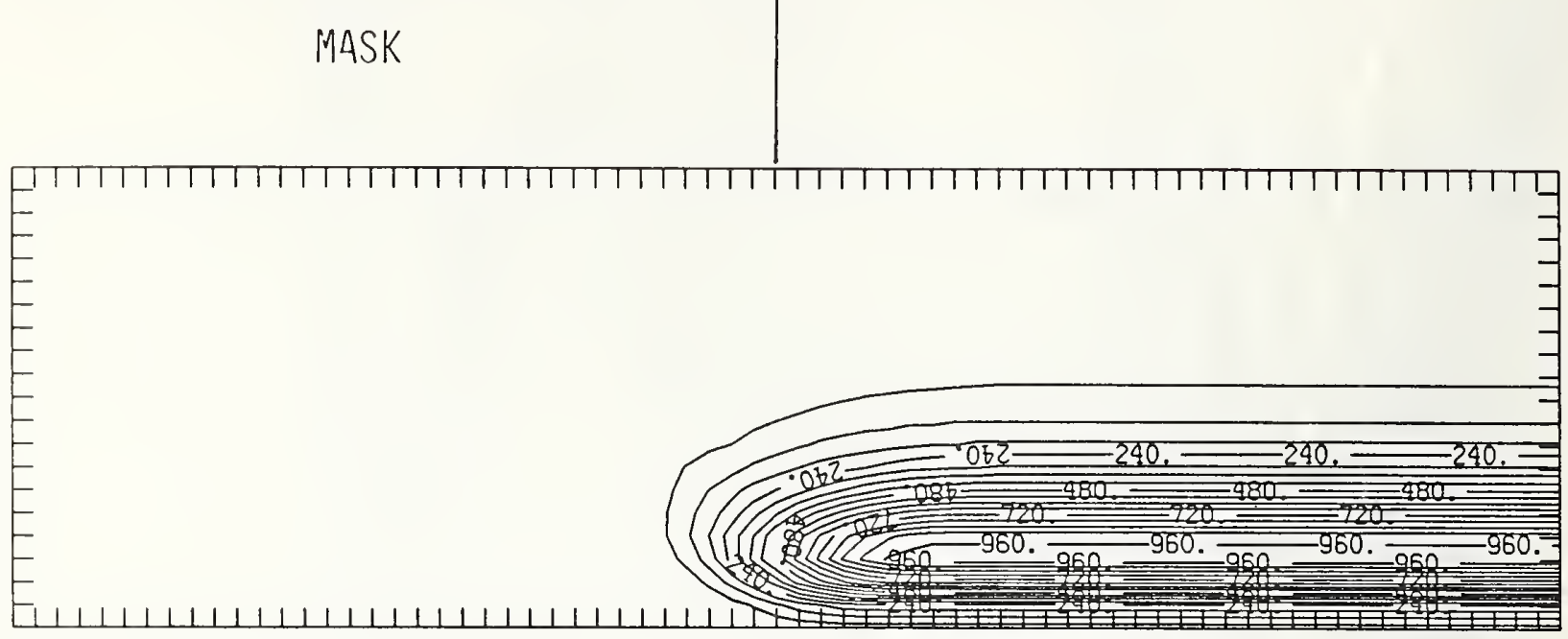

Figure B250-3 Two-dimensional distribution of $250 \mathrm{keV}$ boron implanted past a mask edge. The length increment (distance between tick marks) is $0.04 \mu \mathrm{m}$ and the scale factor is 1000 .

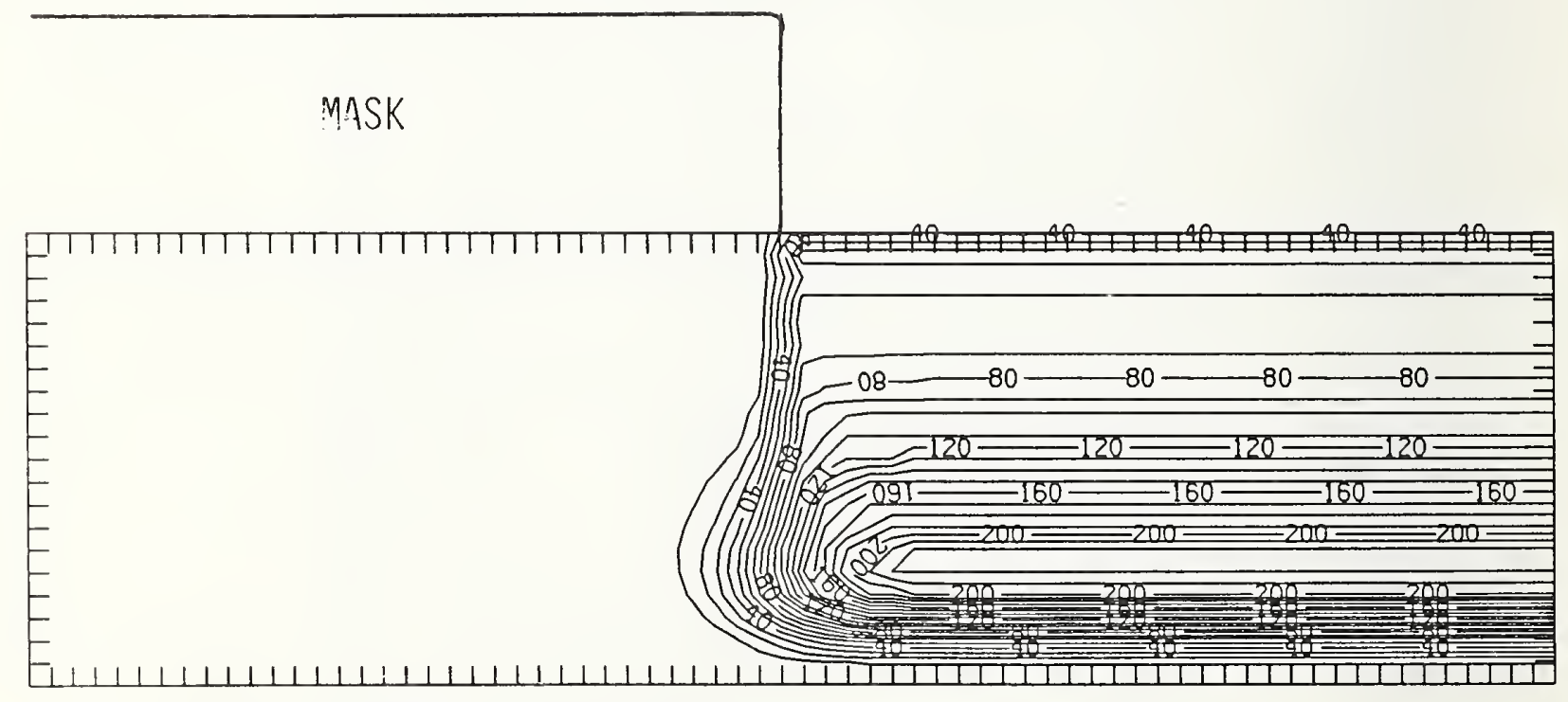

Figure B250-4 Two-dimensional distribution of Frenkel pairs created by $250 \mathrm{keV}$ boron implanted past a mask edge. The length increment (distance between tick marks) is $0.04 \mu \mathrm{m}$ and the scale factor is 1. 


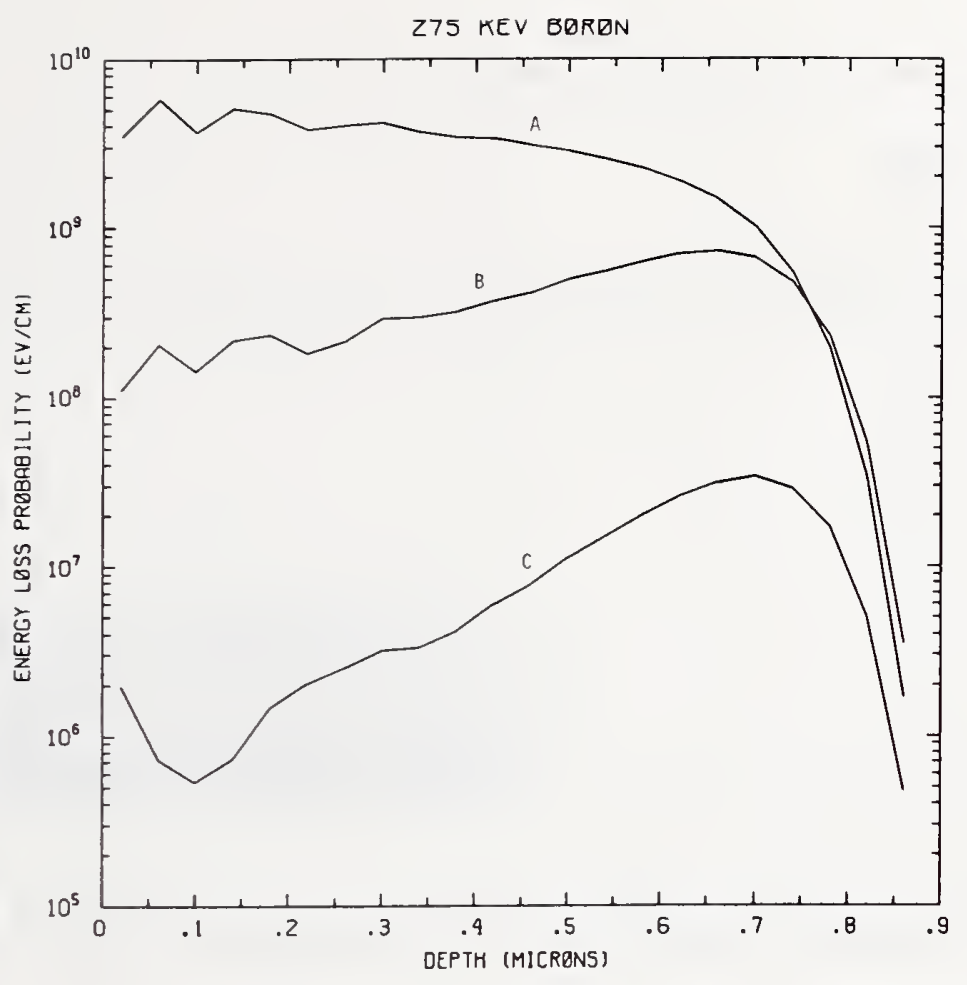

Figure B275-1 One-dimensional probability distributions for energy loss due to electronic excitation (A), displacement damage(B), and phonon excitation (C) for $275 \mathrm{keV}$ boron normally incident on a silicon target.

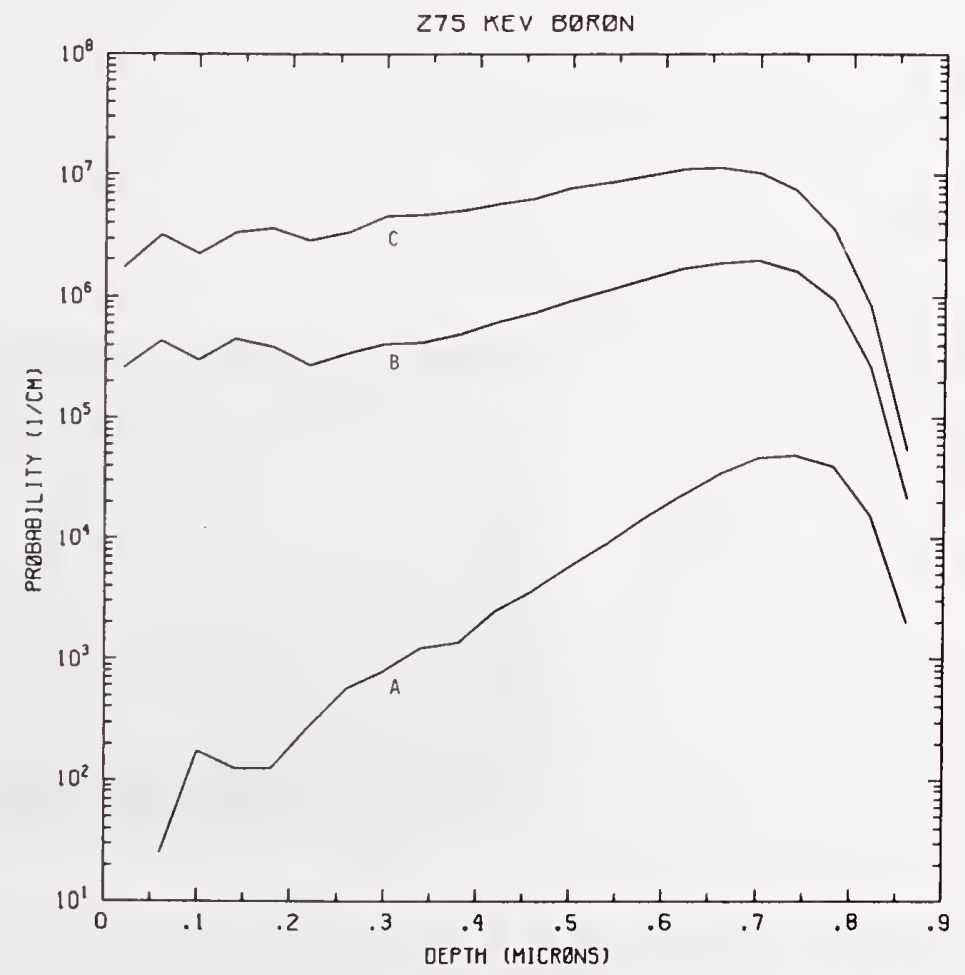

Figure B275-2 One-dimensional probability distributions for implanted boron (A), primary silicon displacements (B), and Frenkel pairs (C) for $275 \mathrm{keV}$ boron normally incident on a silicon target. 


\section{MASK}

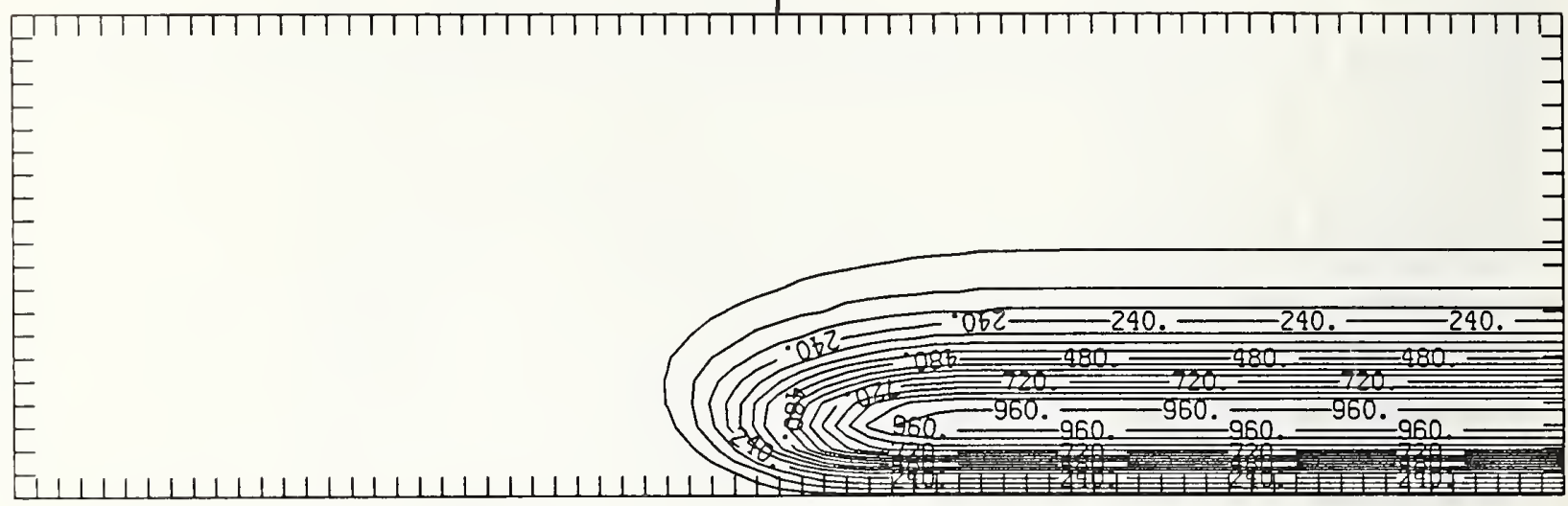

Figure B275-3 Two-dimensional distribution of $275 \mathrm{keV}$ boron implanted past a mask edge. The length increment (distance between tick marks) is $0.04 \mu \mathrm{m}$ and the scale factor is 1000 .

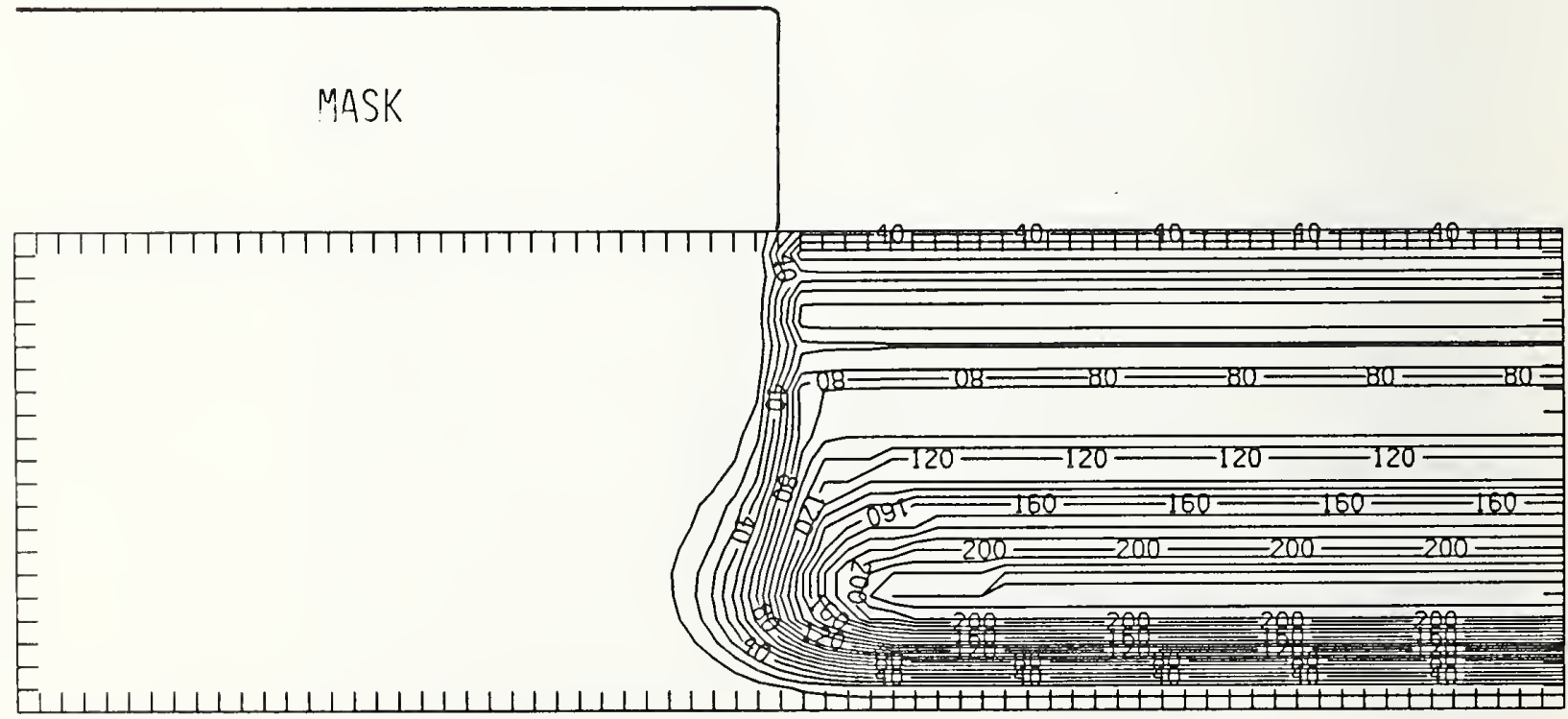

Figure B275-4 Two-dimensional distribution of Frenkel pairs created by $275 \mathrm{keV}$ boron implanted past a mask edge. The length increment (distance between tick marks) is $0.04 \mu \mathrm{m}$ and the scale factor is 1 . 


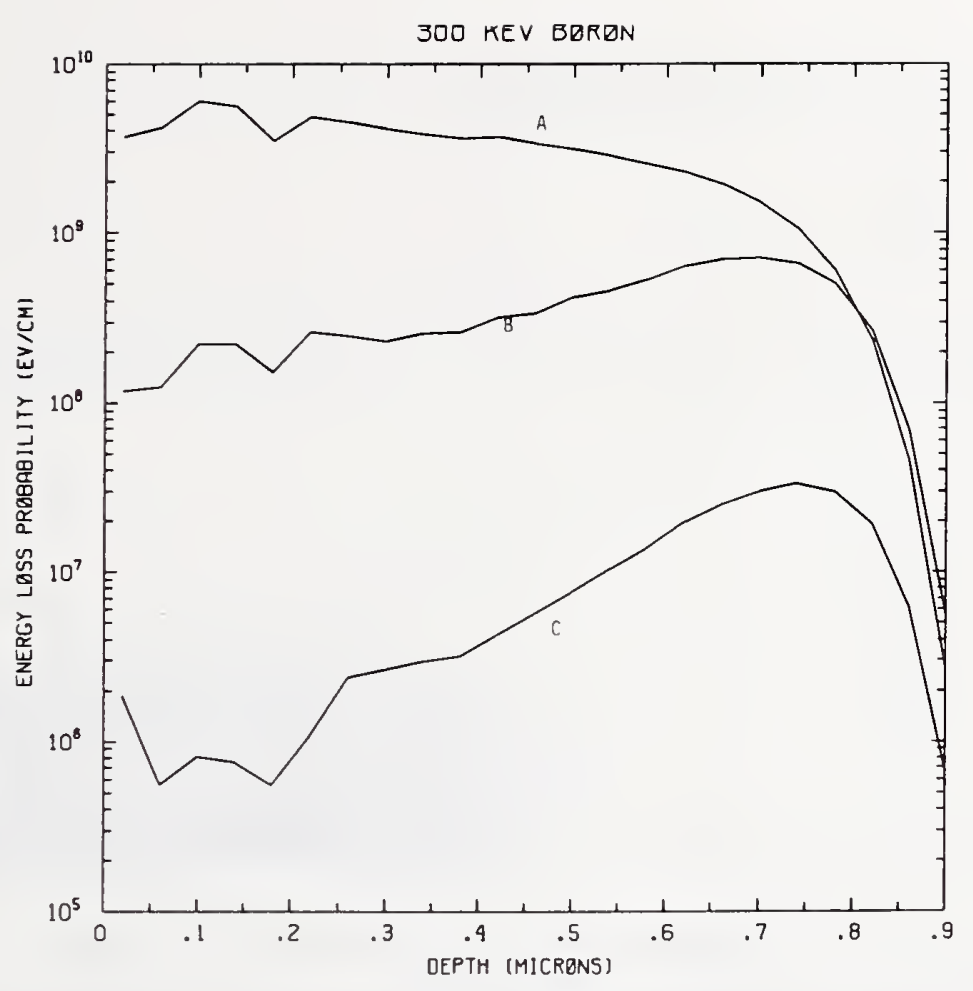

Figure B300-1 One-dimensional probability distributions for energy loss due to electronic excitation (A), displacement damage(B), and phonon excitation (C) for $300 \mathrm{keV}$ boron normally incident on a silicon target.

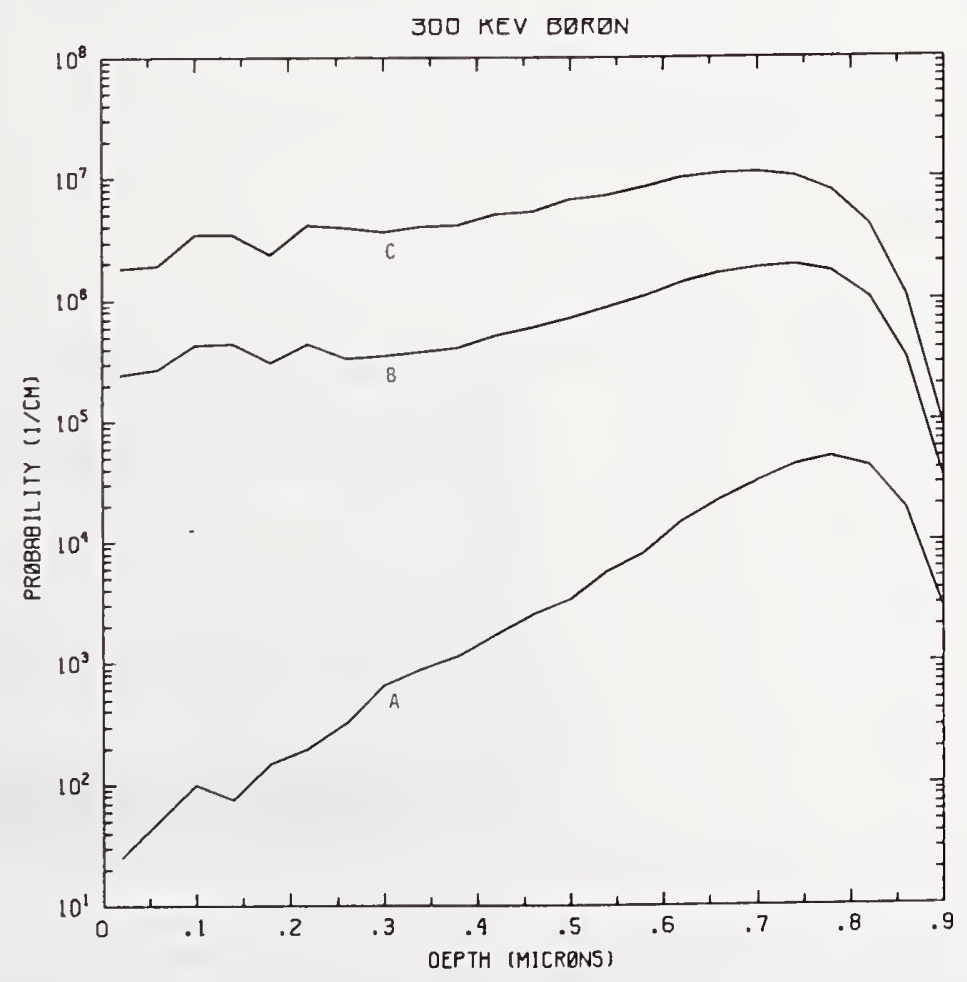

Figure B300-2 One-dimensional probability distributions for implanted boron (A), primary silicon displacements (B), and Frenkel pairs (C) for $300 \mathrm{keV}$ boron normally incident on a silicon target. 


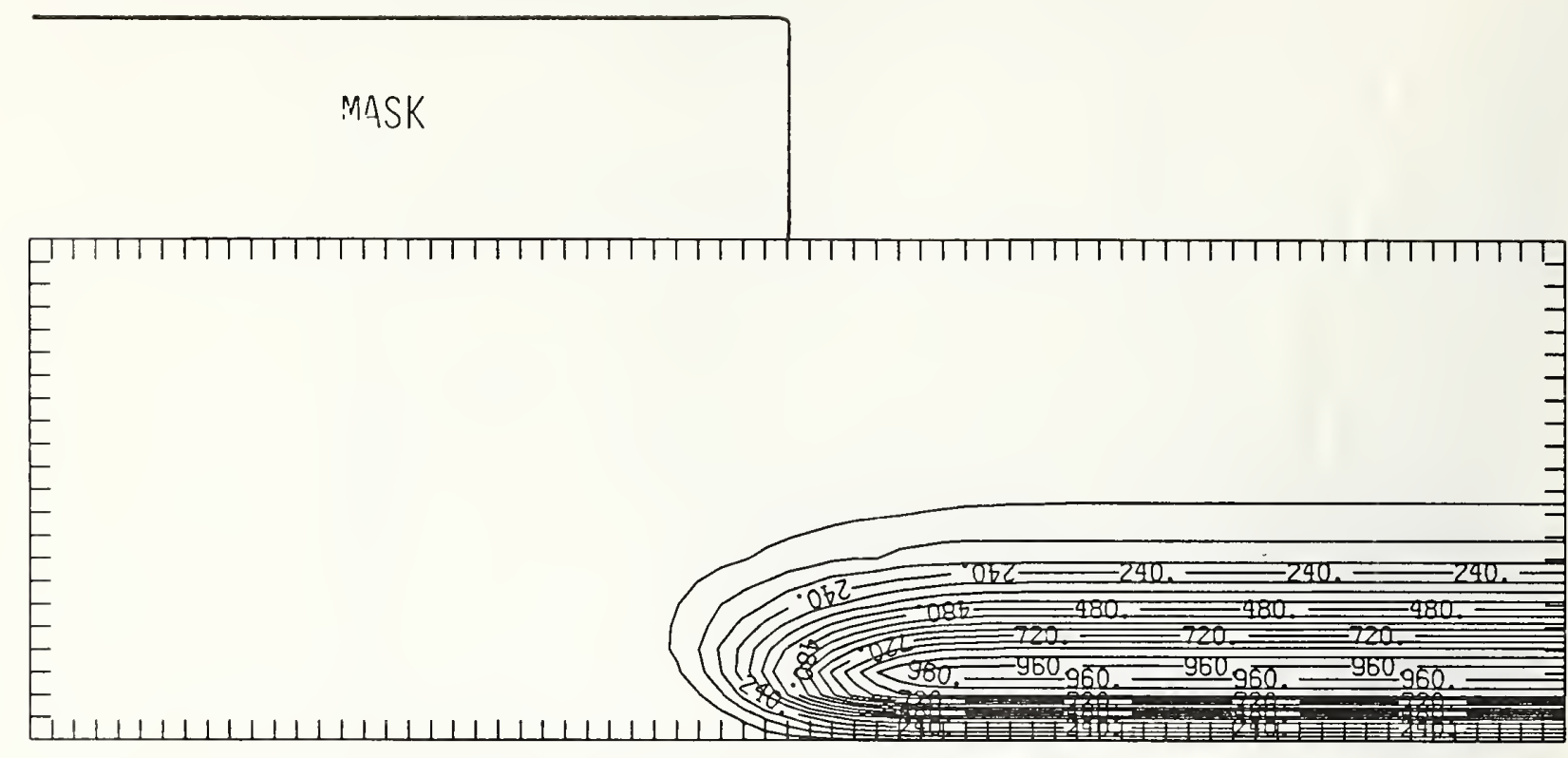

Figure B300-3 Two-dimensional distribution of $300 \mathrm{keV}$ boron implanted past a mask edge. The length increment (distance between tick marks) is $0.04 \mu \mathrm{m}$ and the scale factor is 1000 .

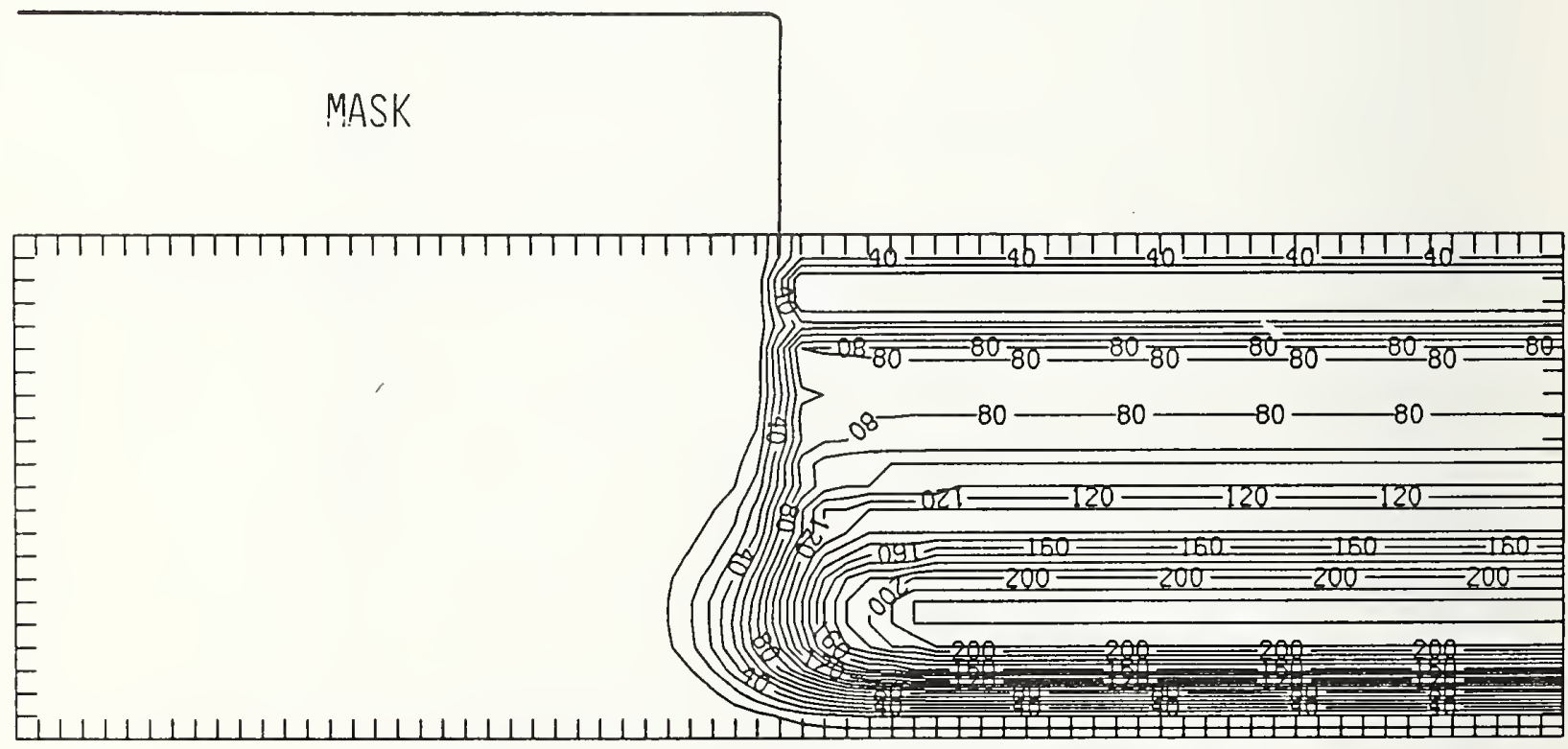

Figure B300-4 Two-dimensional distribution of Frenkel pairs created by $300 \mathrm{keV}$ boron implanted past a mask edge. The length increment (distance between tick marks) is $0.04 \mu \mathrm{m}$ and the scale factor is 1 . 


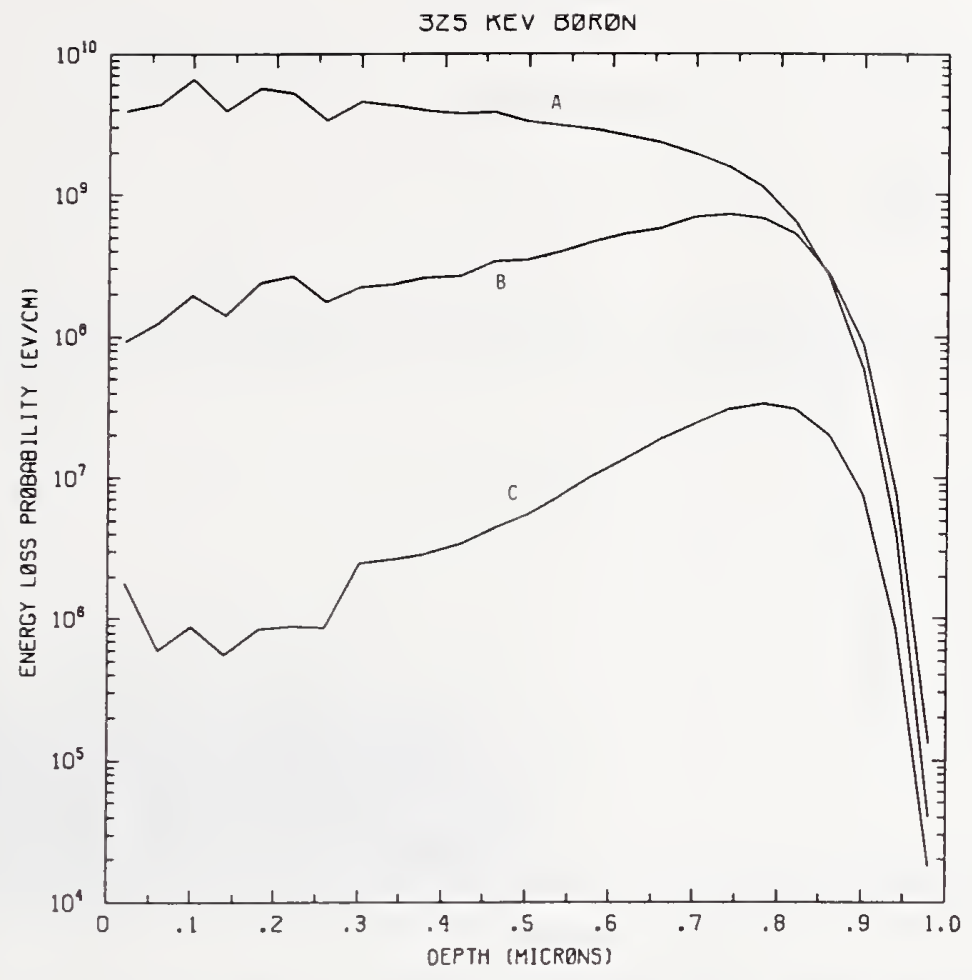

Figure B325-1 One-dimensional probability distributions for energy loss due to electronic excitation (A), displacement damage(B), and phonon excitation (C) for $325 \mathrm{keV}$ boron normally incident on a silicon target.

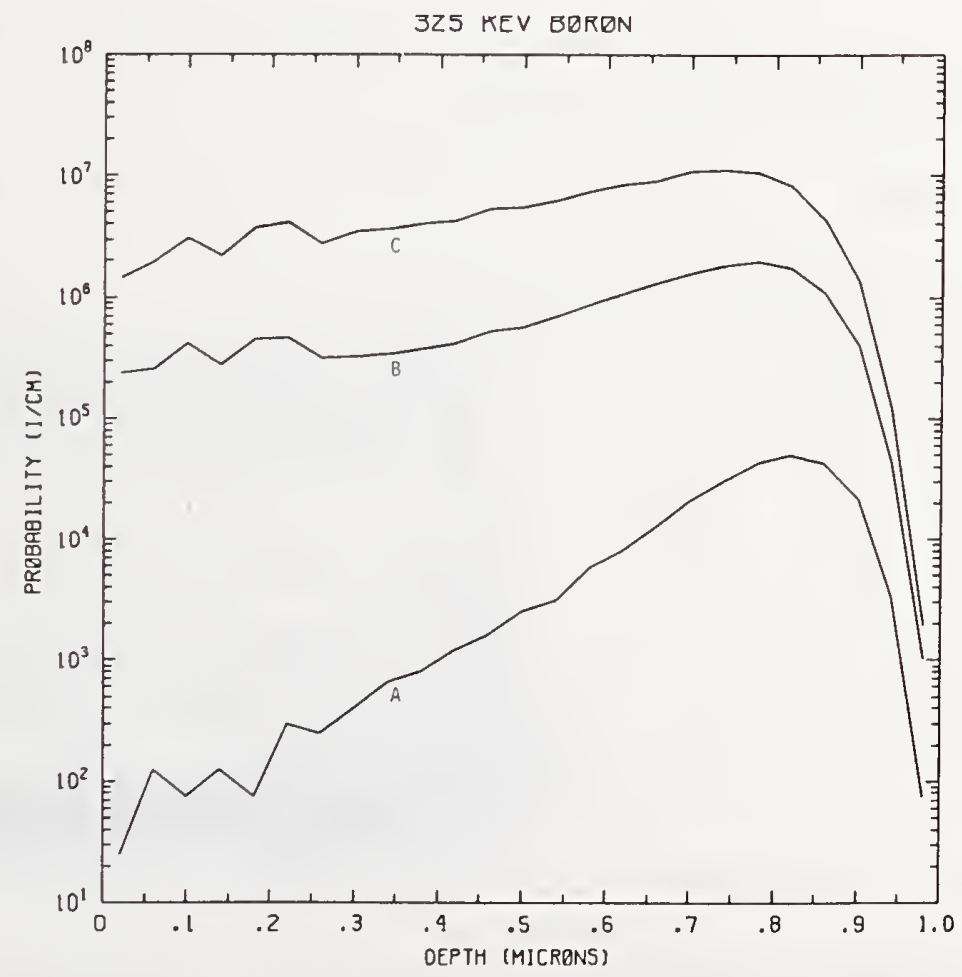

Figure B325-2 One-dimensional probability distributions for implanted boron (A), primary silicon displacements (B), and Frenkel pairs (C) for $325 \mathrm{keV}$ boron normally incident on a silicon target. 


\section{MASK}

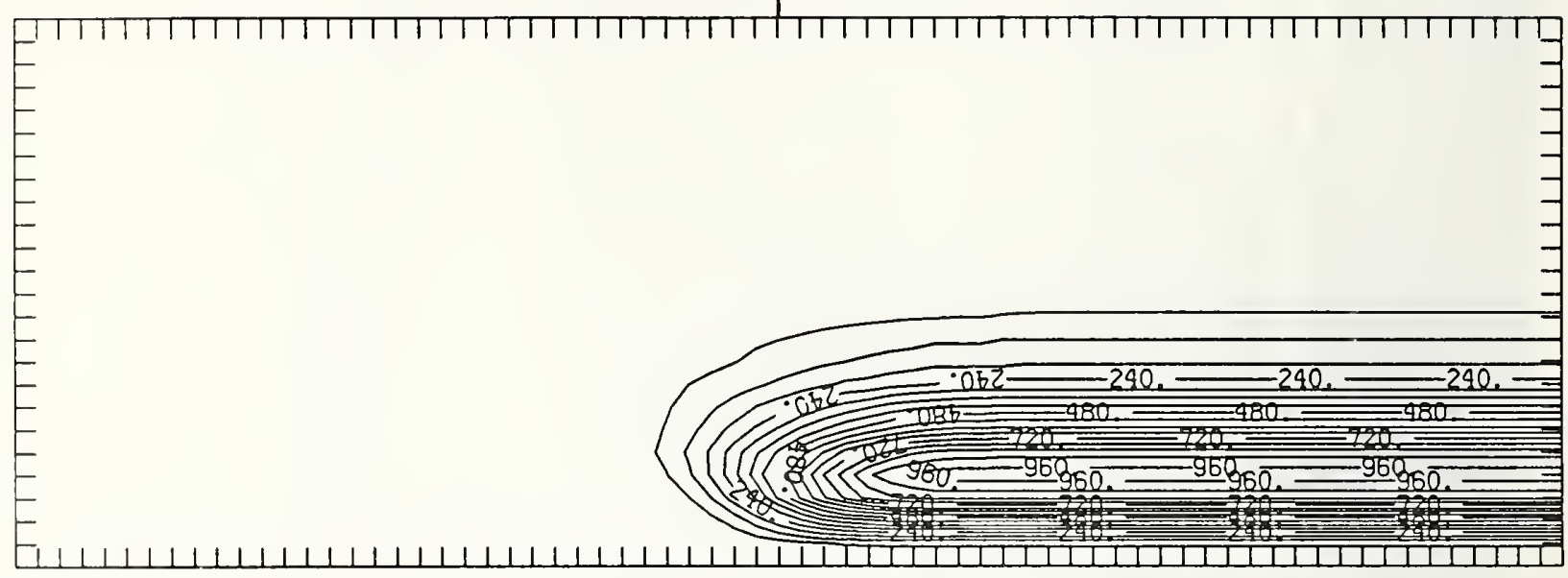

Figure B325-3 Two-dimensional distribution of $325 \mathrm{keV}$ boron implanted past a mask edge. The length increment (distance between tick marks) is $0.04 \mu \mathrm{m}$ and the scale factor is 1000 .

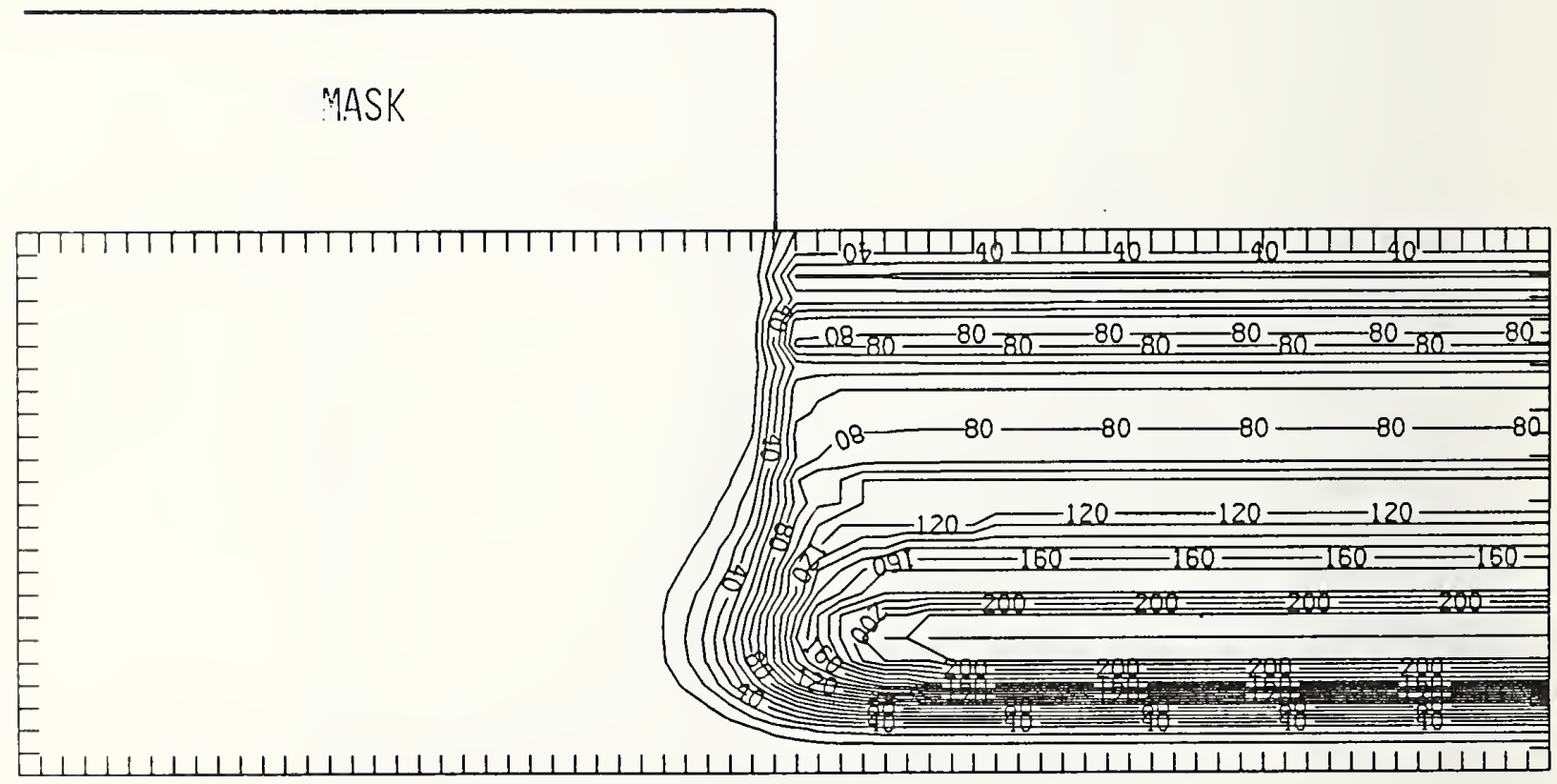

Figure B325-4 Two-dimensional distribution of Frenkel pairs created by $325 \mathrm{keV}$ boron implanted past a mask edge. The length increment (distance between tick marks) is $0.04 \mu \mathrm{m}$ and the scale factor is 1 . 


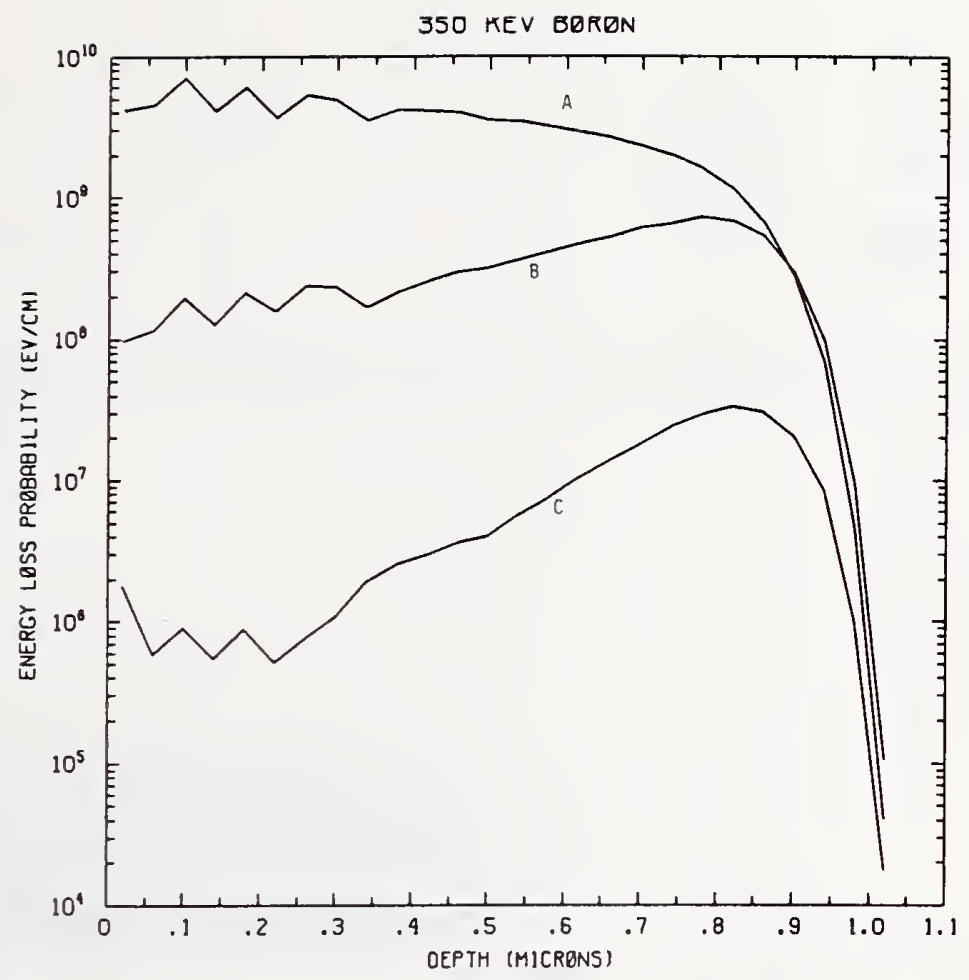

Figure B350-1 One-dimensional probability distributions for energy loss due to electronic excitation (A), displacement damage(B), and phonon excitation (C) for $350 \mathrm{keV}$ boron normally incident on a silicon target.

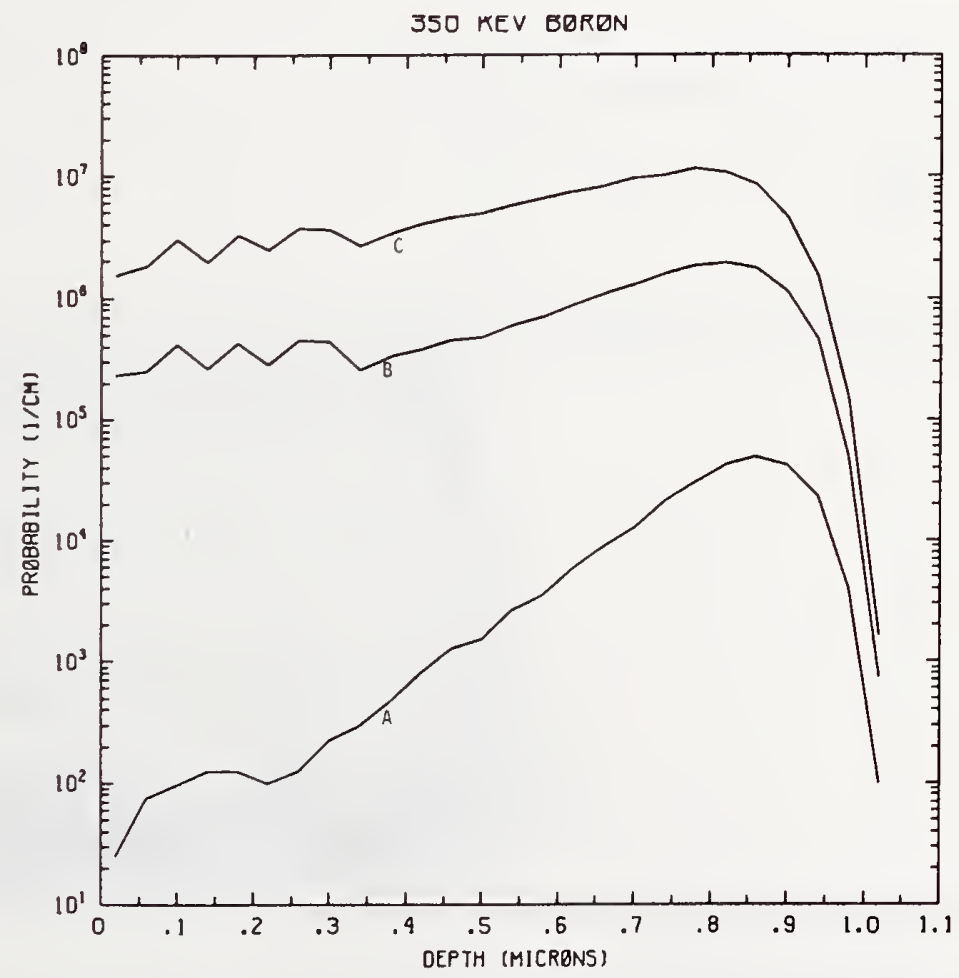

Figure B350-2 One-dimensional probability distributions for implanted boron (A), primary silicon displacements (B), and Frenkel pairs (C) for $350 \mathrm{keV}$ boron normally incident on a silicon target. 
MASK

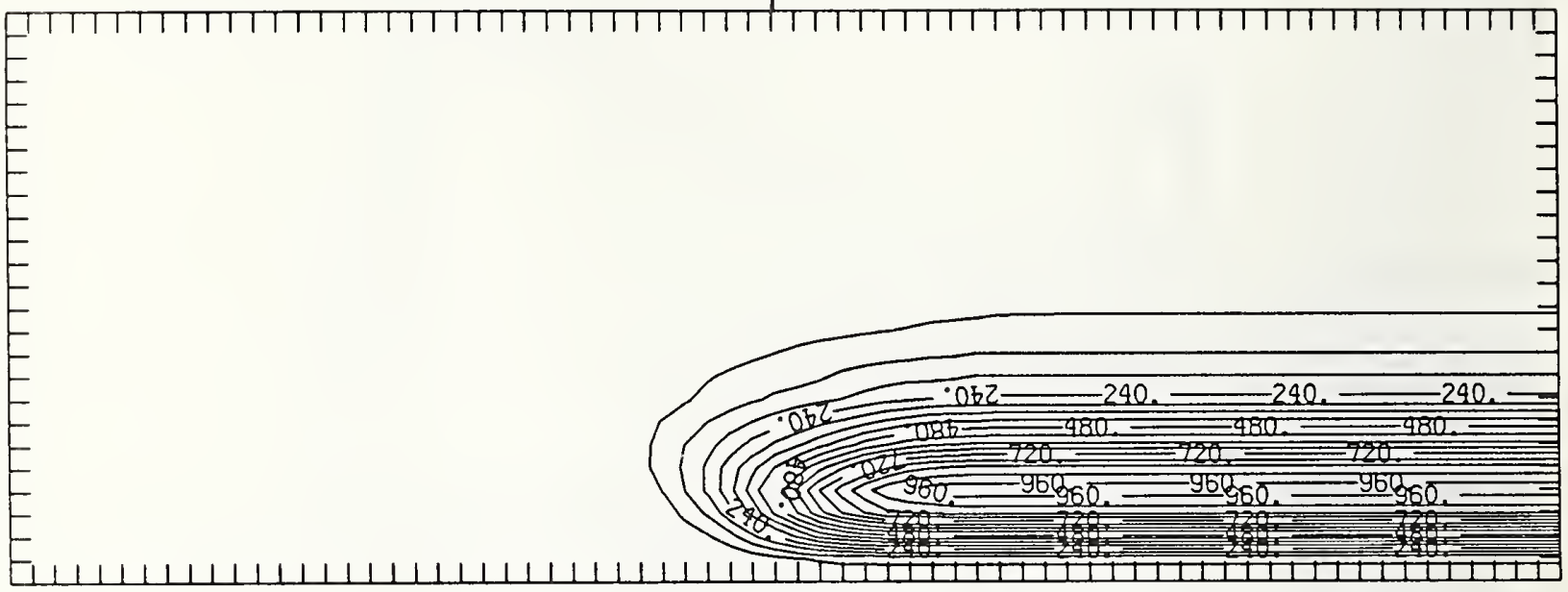

Figure B350-3 Two-dimensional distribution of $350 \mathrm{keV}$ boron implanted past a mask edge. The length increment (distance between tick marks) is $0.04 \mu \mathrm{m}$ and the scale factor is 1000 .

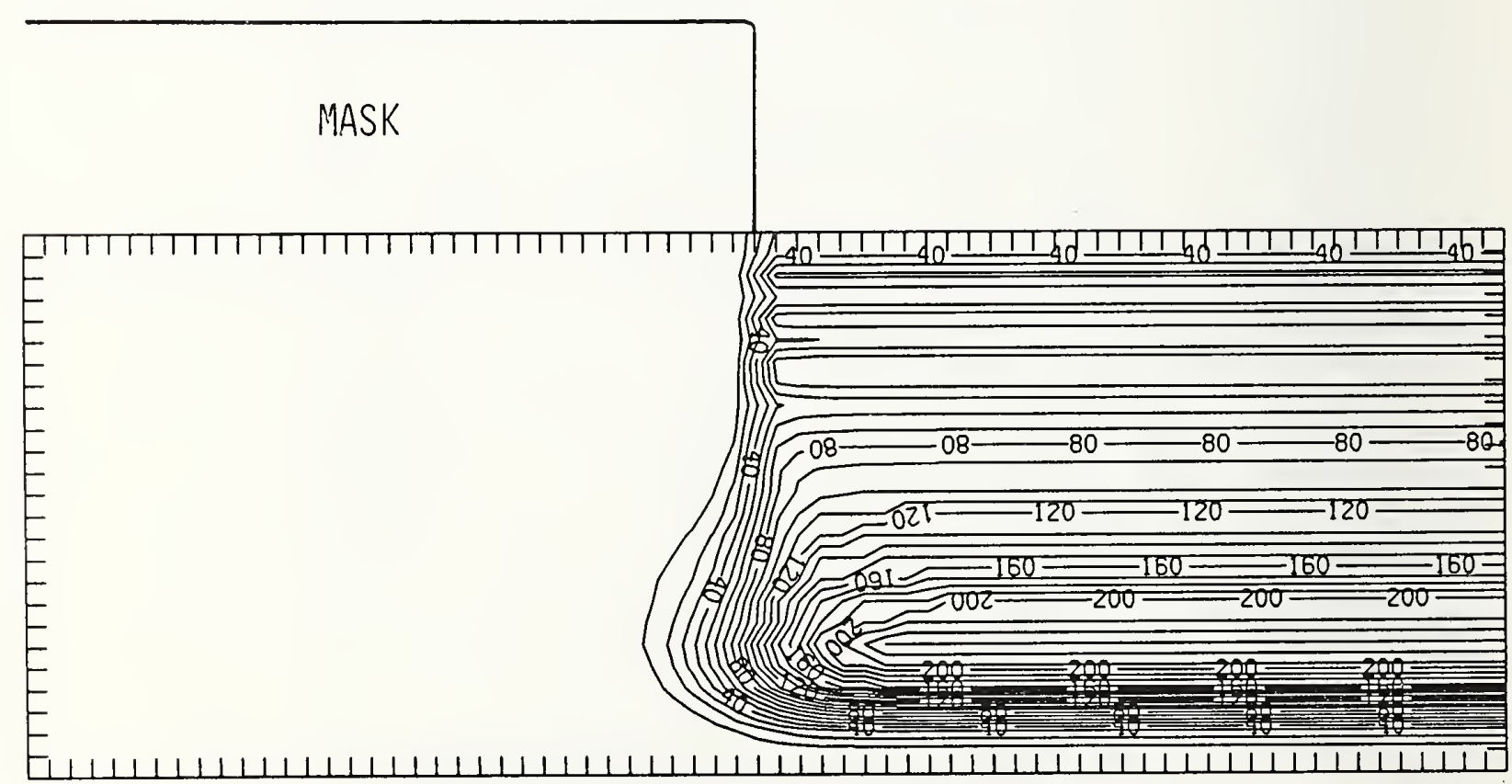

Figure B350-4 Two-dimensional distribution of Frenkel pairs created by $350 \mathrm{keV}$ boron implanted past a mask edge. The length increment (distance between tick marks) is $0.04 \mu \mathrm{m}$ and the scale factor is 1. 


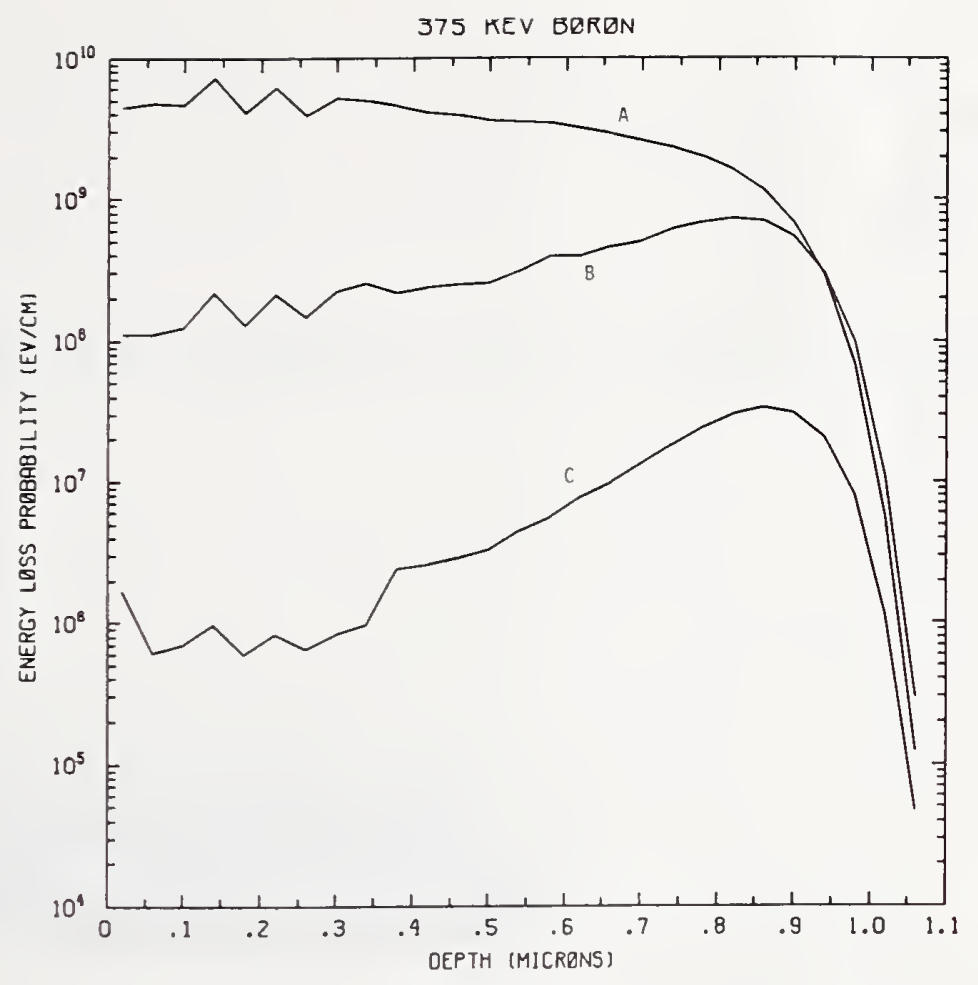

Figure B375-1 One-dimensional probability distributions for energy loss due to electronic excitation (A), displacement damage(B), and phonon excitation (C) for $375 \mathrm{keV}$ boron normally incident on a silicon target.

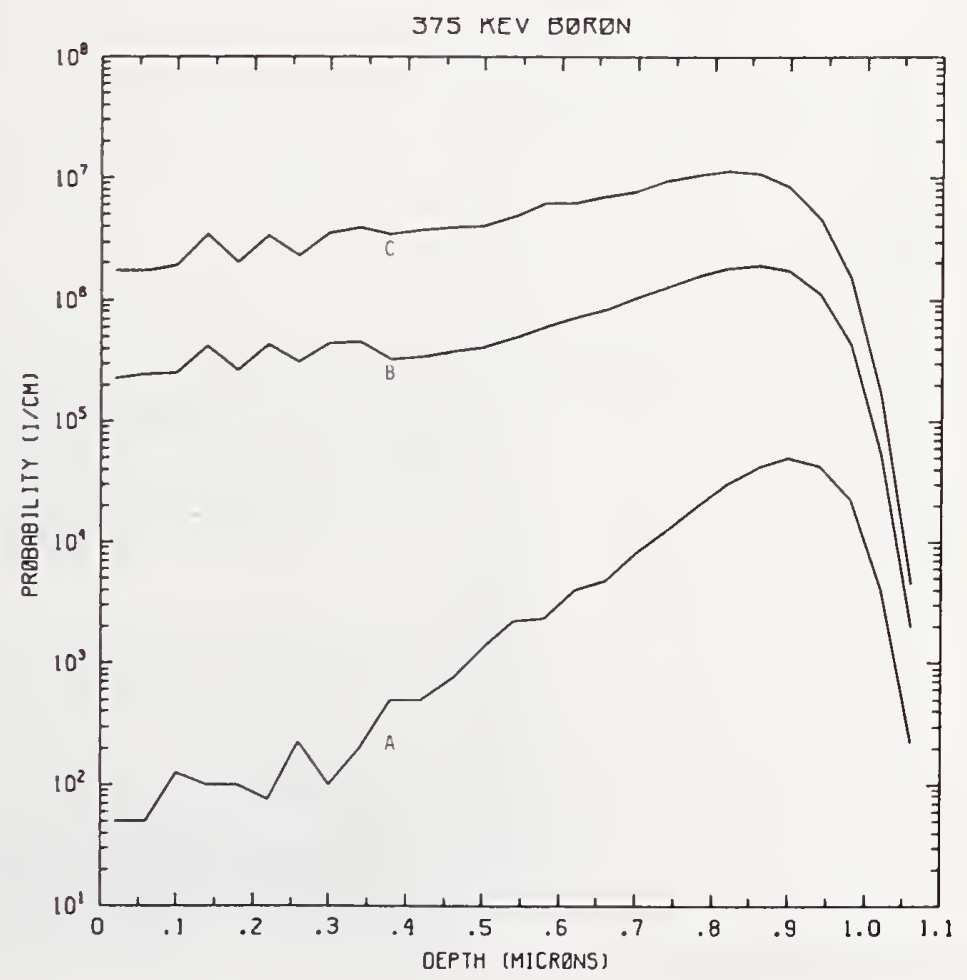

Figure B375-2 One-dimensional probability distributions for implanted boron (A), primary silicon displacements (B), and Frenkel pairs (C) for $375 \mathrm{keV}$ boron normally incident on a silicon target. 


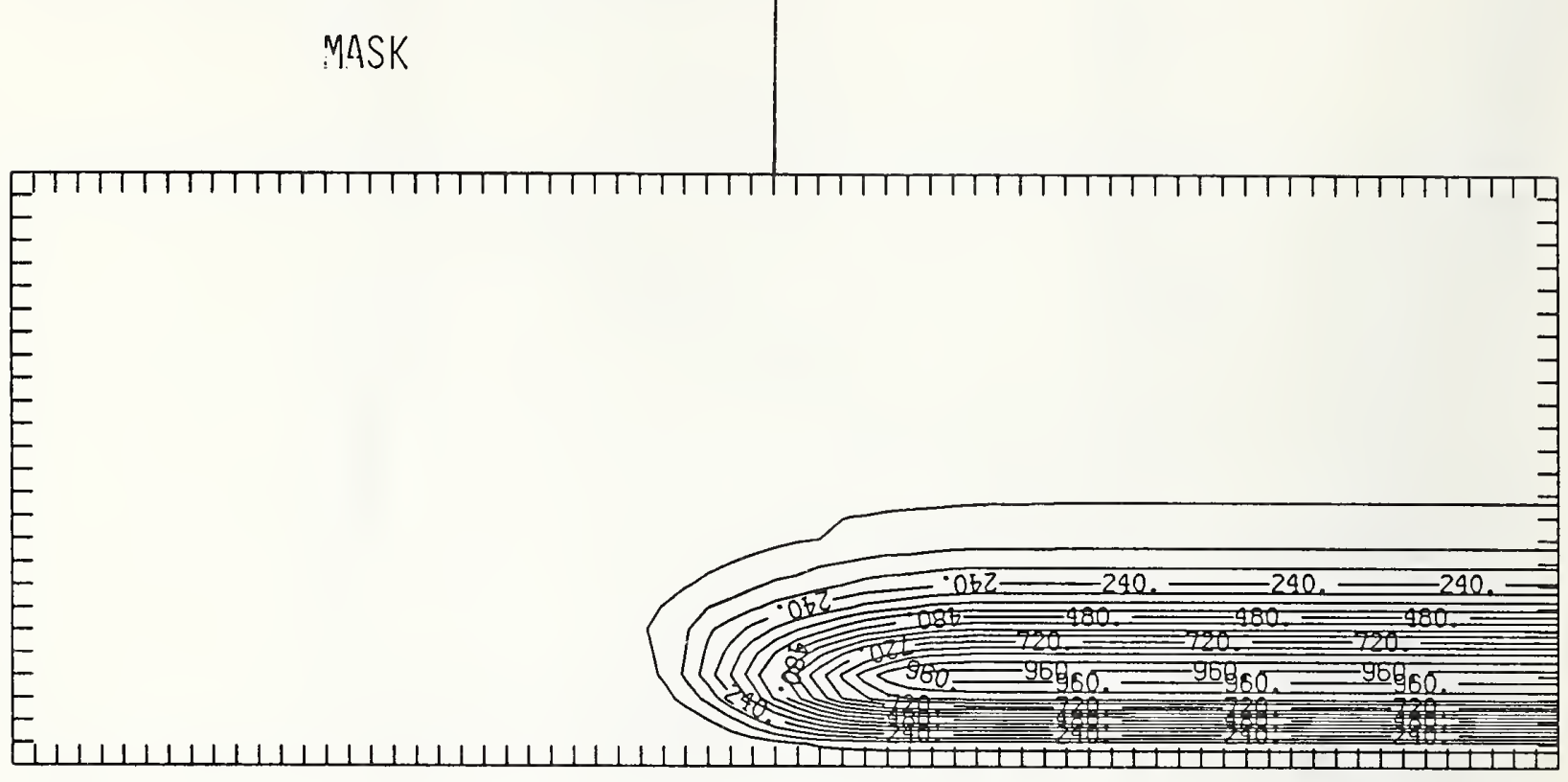

Figure B375-3 Two-dimensional distribution of $375 \mathrm{keV}$ boron implanted past a mask edge. The length increment (distance between tick marks) is $0.04 \mu \mathrm{m}$ and the scale factor is 1000 .

MASK

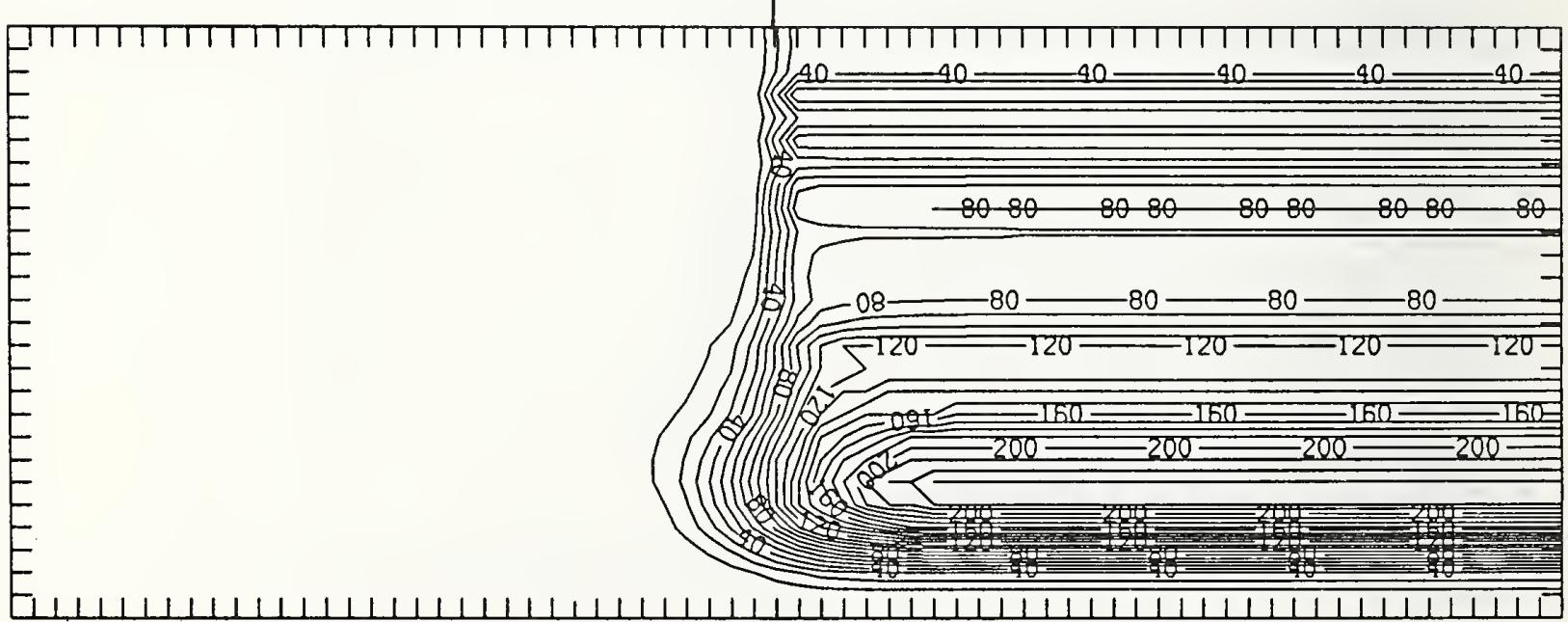

$\because$-ure B375-4 Two-dimensional distribution of Frenkel pairs created by $375 \mathrm{keV}$ boron implanted past a mask edge. The length increment (distance between tick marks) is $0.04 \mu \mathrm{m}$ and the scale factor is 1 . 


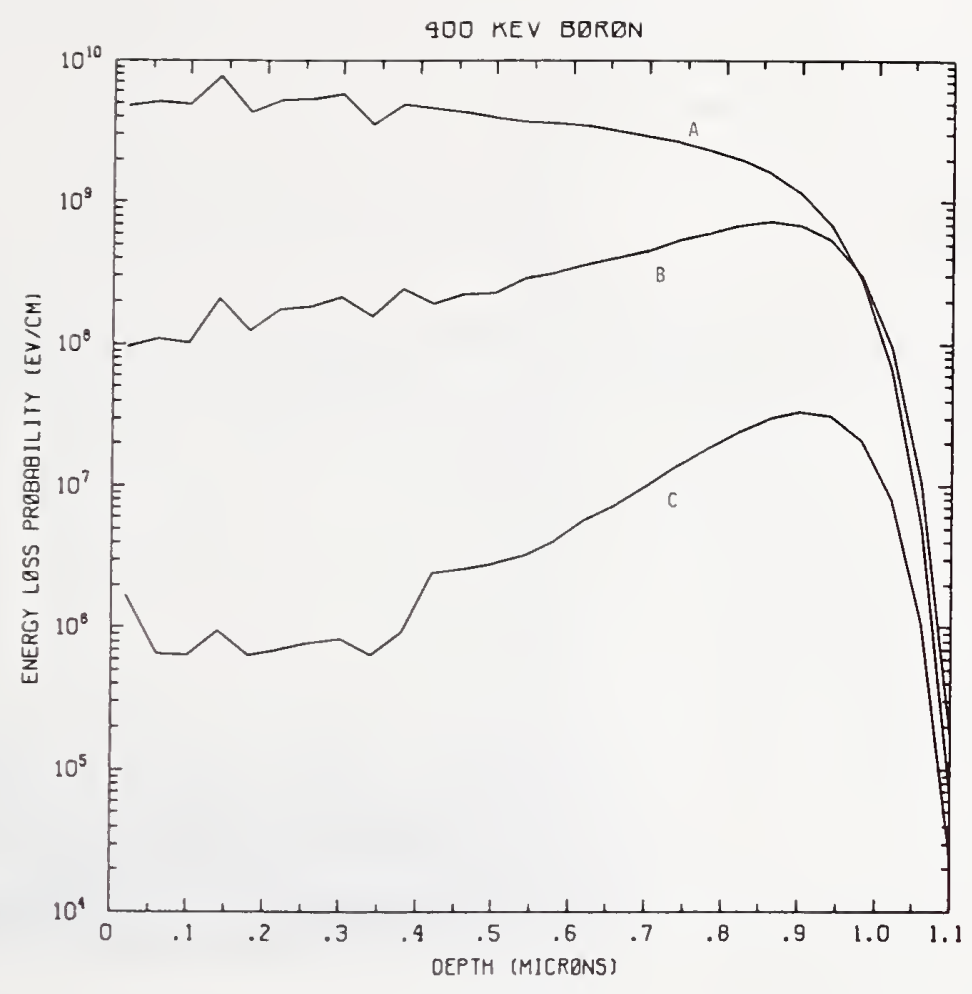

Figure B400-1 One-dimensional probability distributions for energy loss due to electronic excitation (A), displacement damage(B), and phonon excitation (C) for $400 \mathrm{keV}$ boron normally incident on a silicon target.

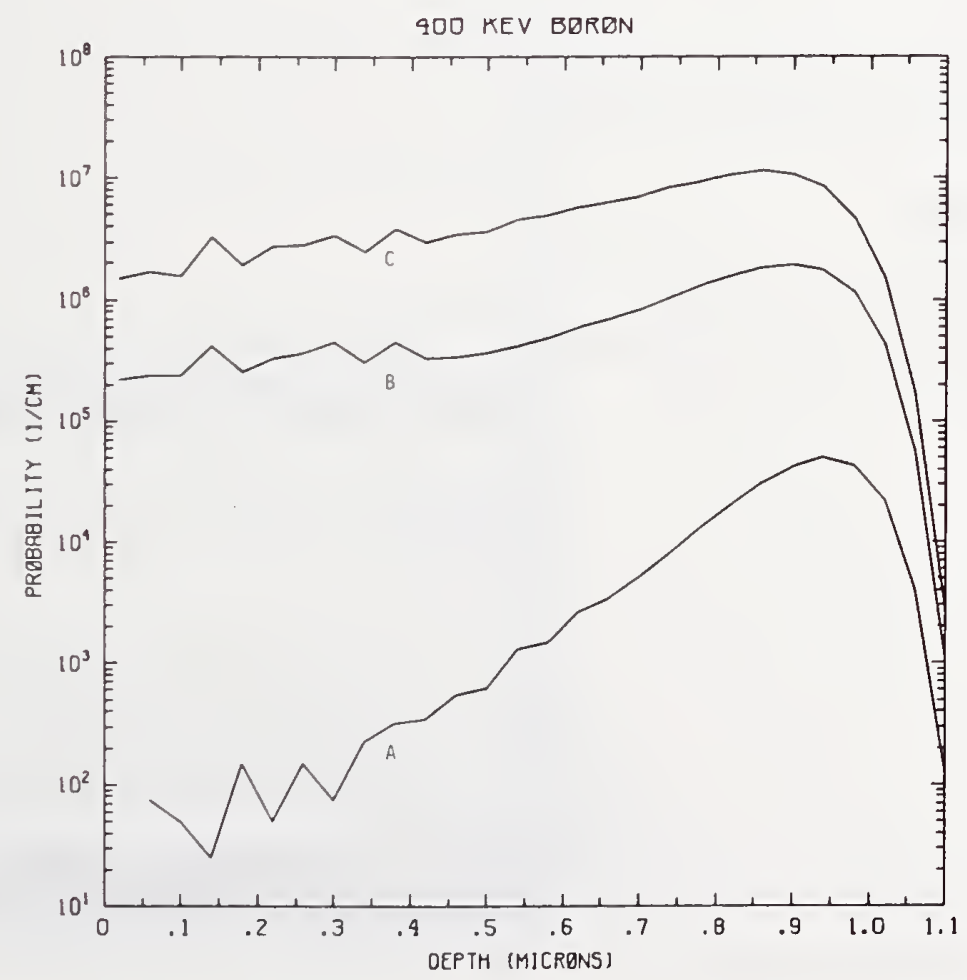

Figure B400-2 One-dimensional probability distributions for implanted boron (A), primary silicon displacements (B), and Frenkel pairs (C) for $400 \mathrm{keV}$ boron normally incident on a silicon target. 


\section{MASK}

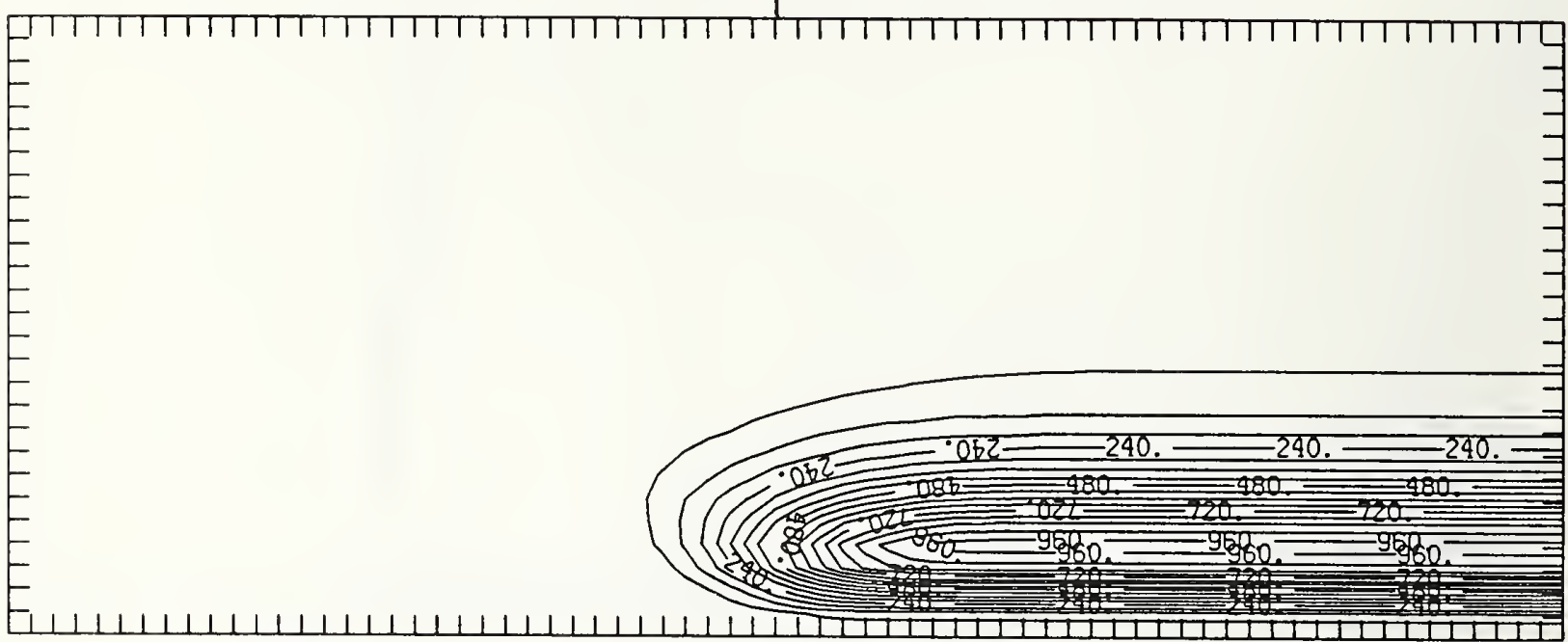

Figure B400-3 Two-dimensional distribution of $400 \mathrm{keV}$ boron implanted past a mask edge. The length increment (distance between tick marks) is $0.04 \mu \mathrm{m}$ and the scale factor is 1000.

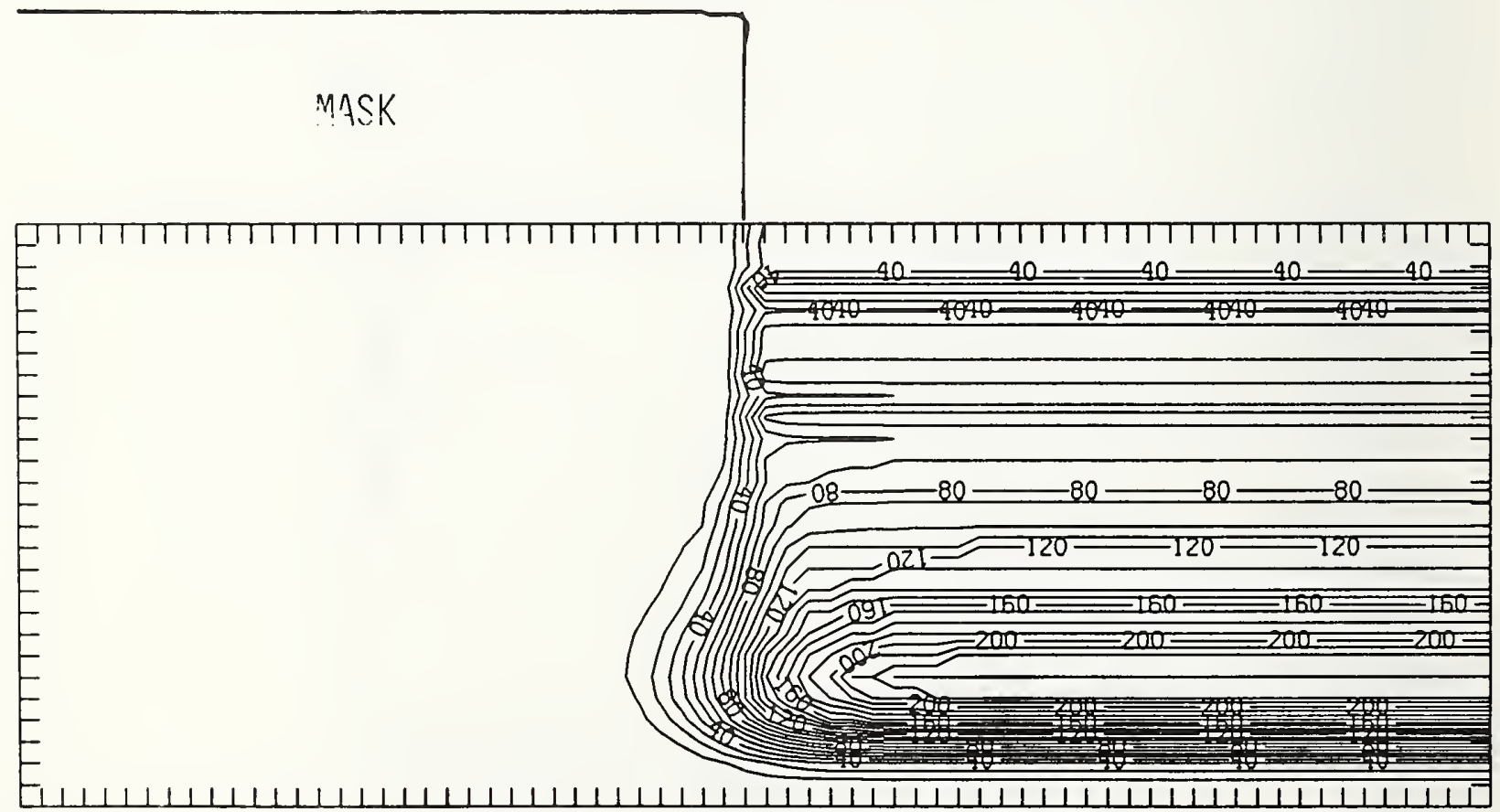

Figure B400-4 Two-dimensional distribution of Frenkel pairs created by $400 \mathrm{keV}$ boron implanted past a mask edge. The length increment (distance between tick marks) is $0.04 \mu \mathrm{m}$ and the scale factor is 1 . 


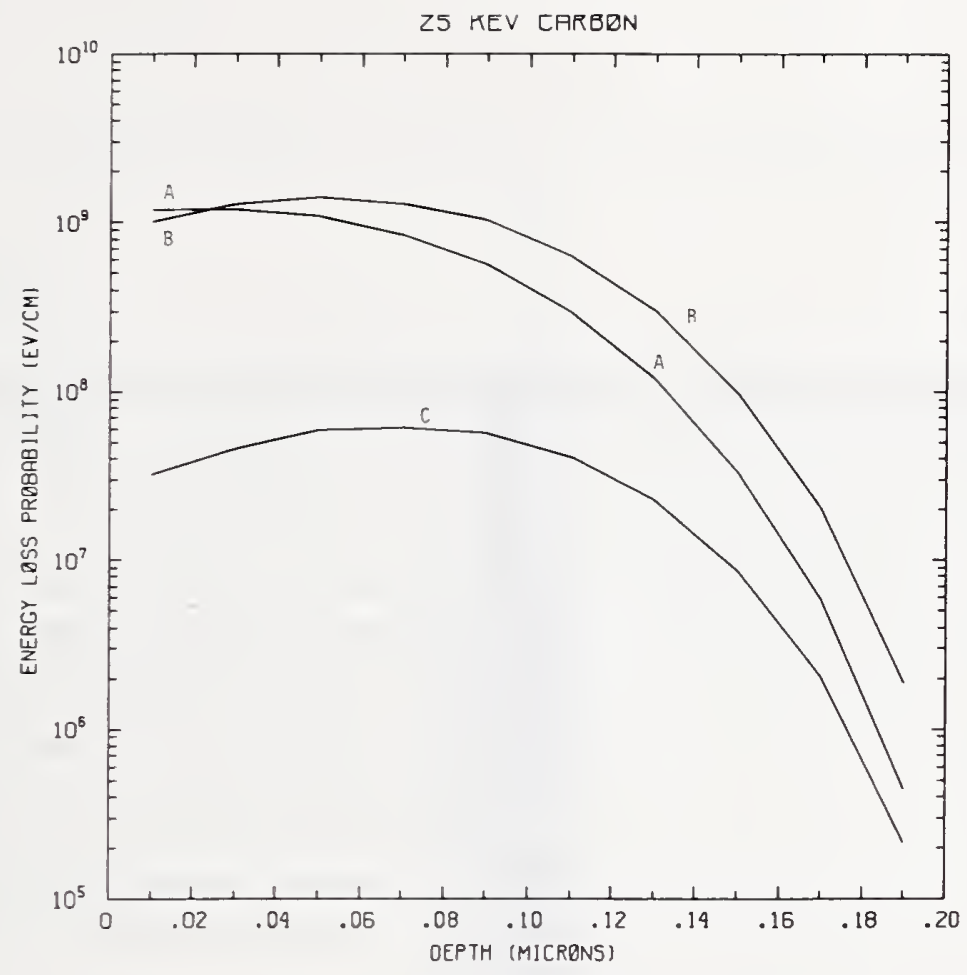

Figure C25-1 One-dimensional probability distributions for energy loss due to electronic excitation (A), displacement damage(B), and phonon excitation (C) for $25 \mathrm{keV}$ carbon normally incident on a silicon target.

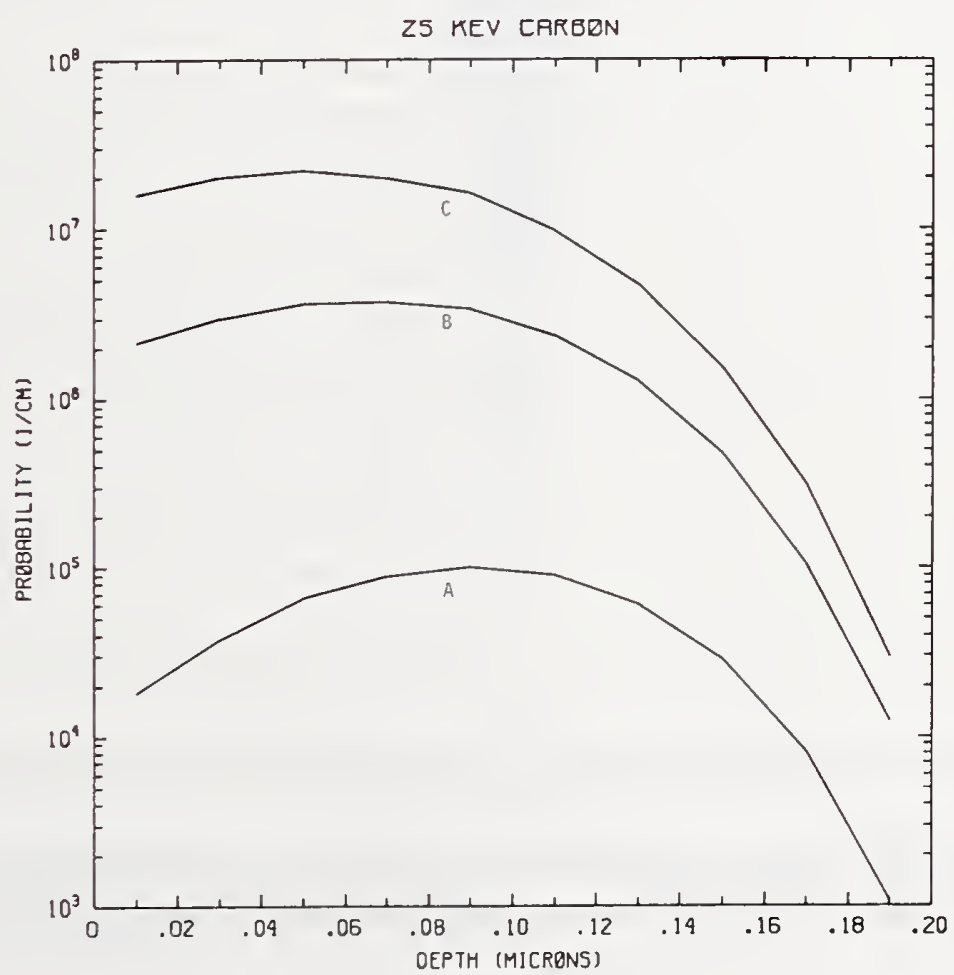

Figure C25-2 One-dimensional probability distributions for implanted carbon (A), primary silicon displacements (B), and Frenkel pairs (C) for $25 \mathrm{keV}$ carbon normally incident on a silicon target. 
MASK

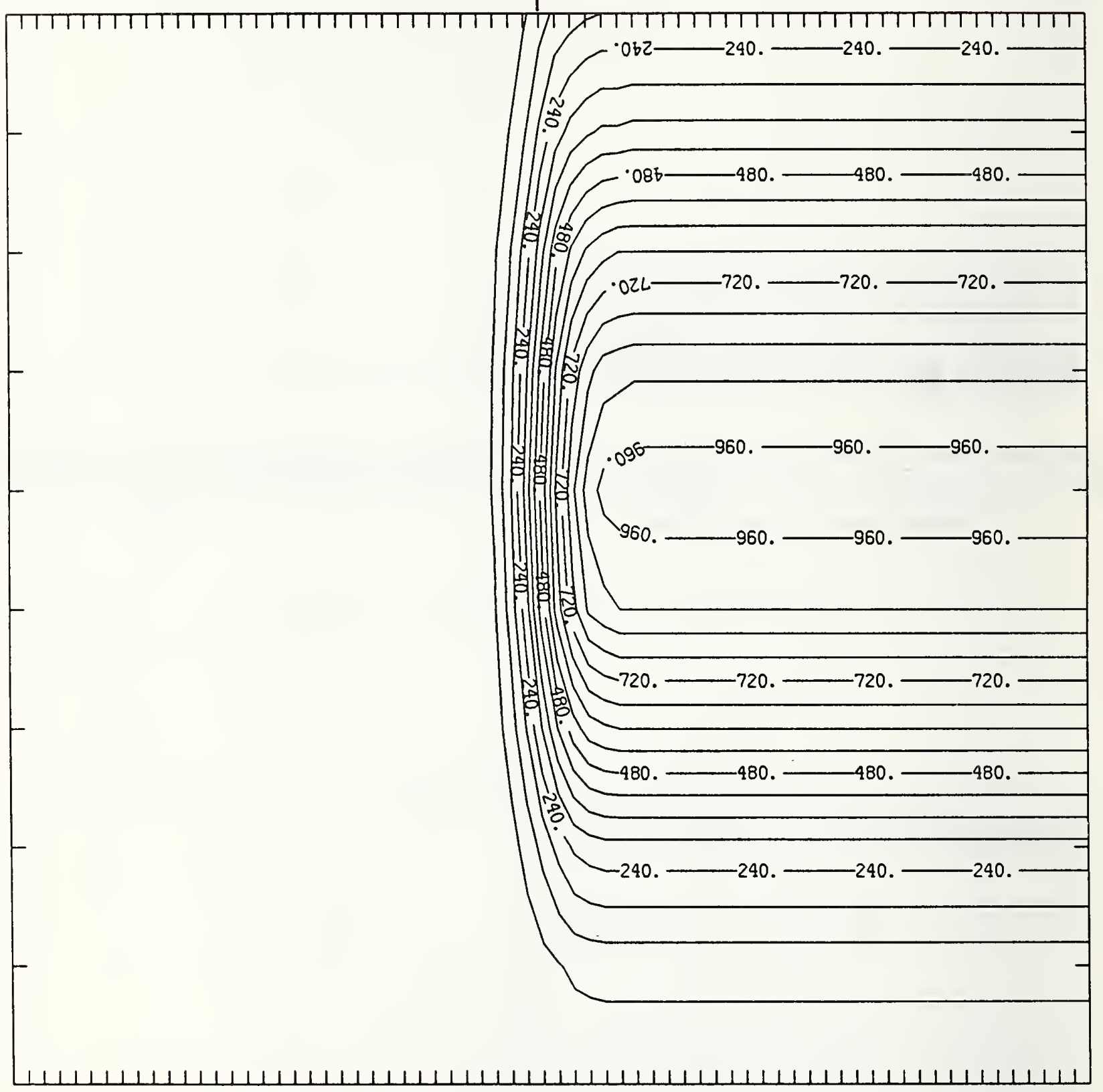

Figure C25-3 Two-dimensional distribution of $25 \mathrm{keV}$ carbon implanted past a mask edge. The length increment (distance between tick marks) is $0.02 \mu \mathrm{m}$ and the scale factor is 1000 . 
PMASK

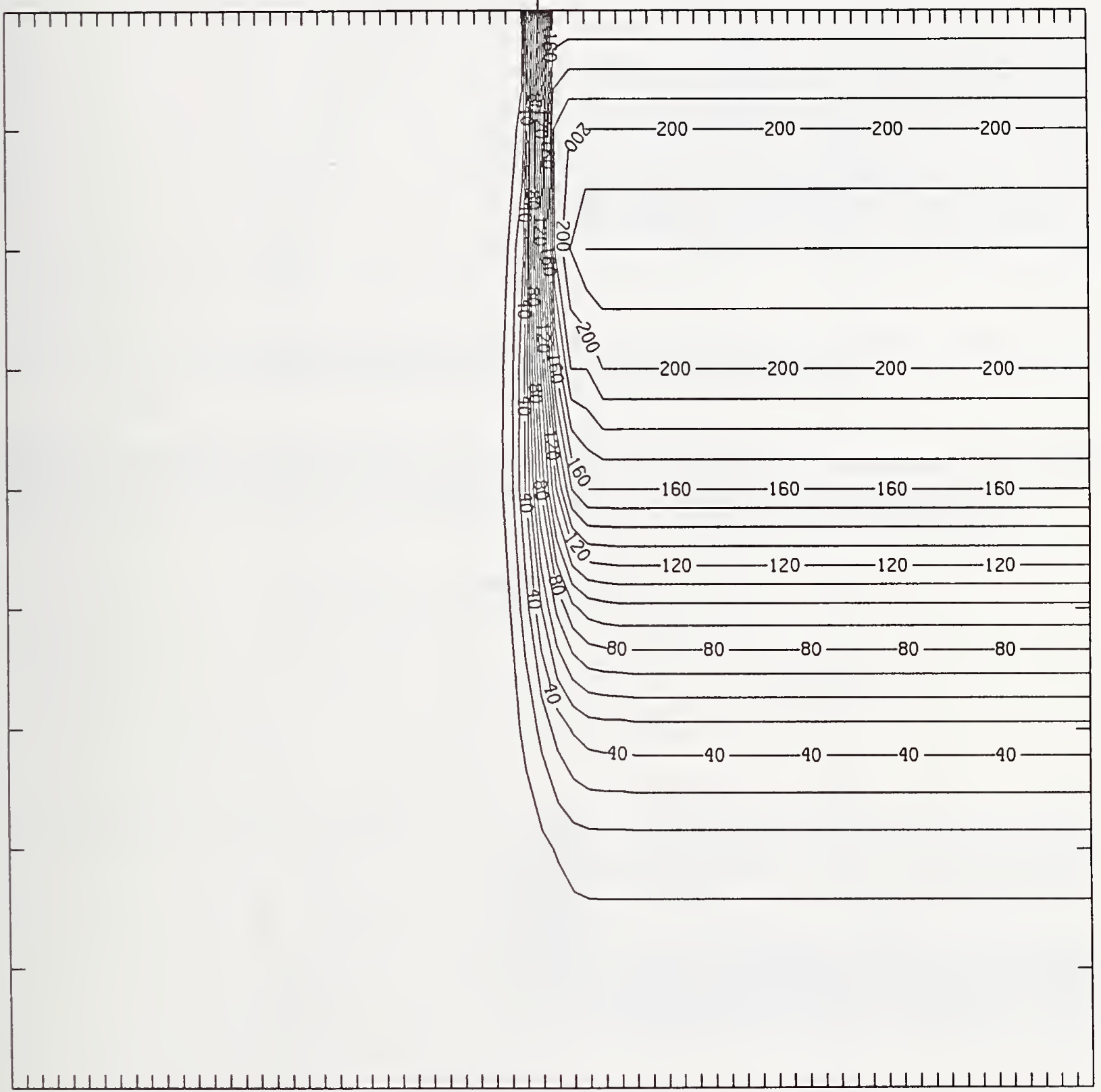

Figure C25-4 Two-dimensional distribution of Frenkel pairs created by $25 \mathrm{keV}$ carbon implanted past a mask edge. The length increment (distance between tick marks) is $0.02 \mu \mathrm{m}$ and the scale factor is 1 . 


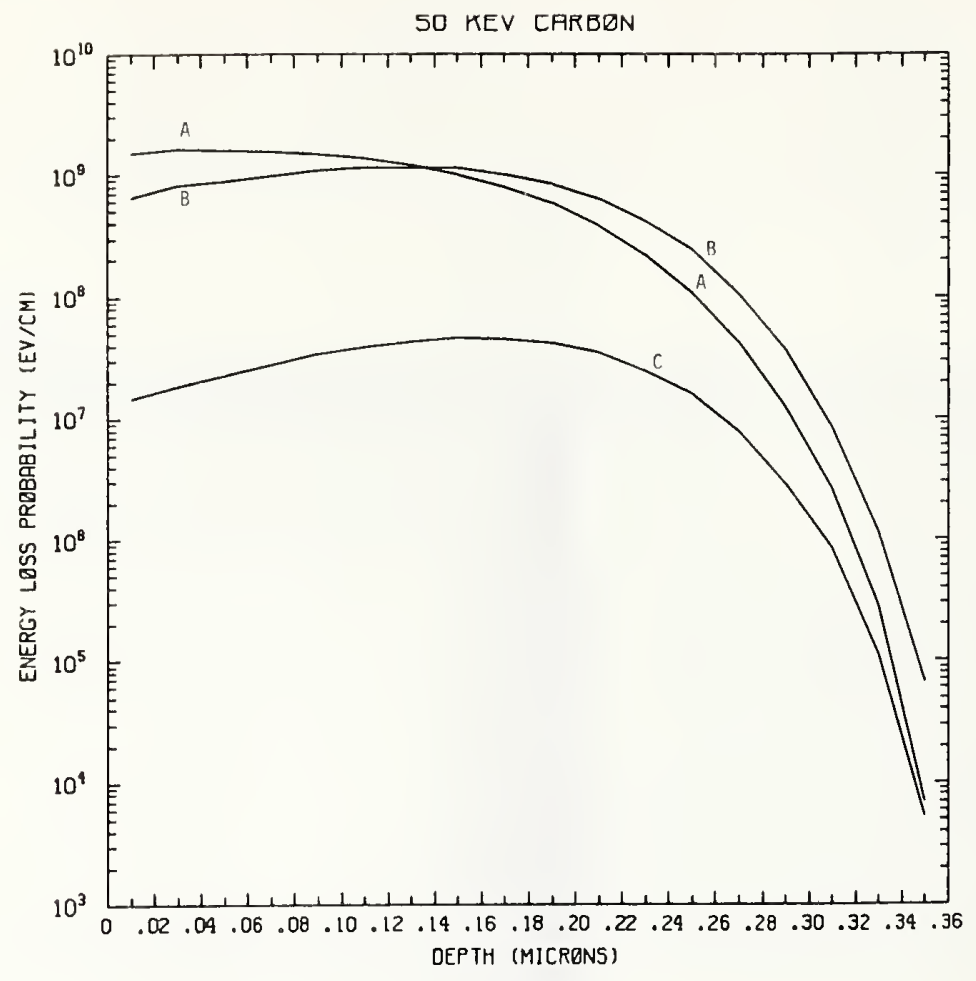

Figure C50-1 One-dimensional probability distributions for energy loss due to electronic excitation (A), displacement damage(B), and phonon excitation (C) for $50 \mathrm{keV}$ carbon normally incident on a silicon target.

50 KEV CARBDN

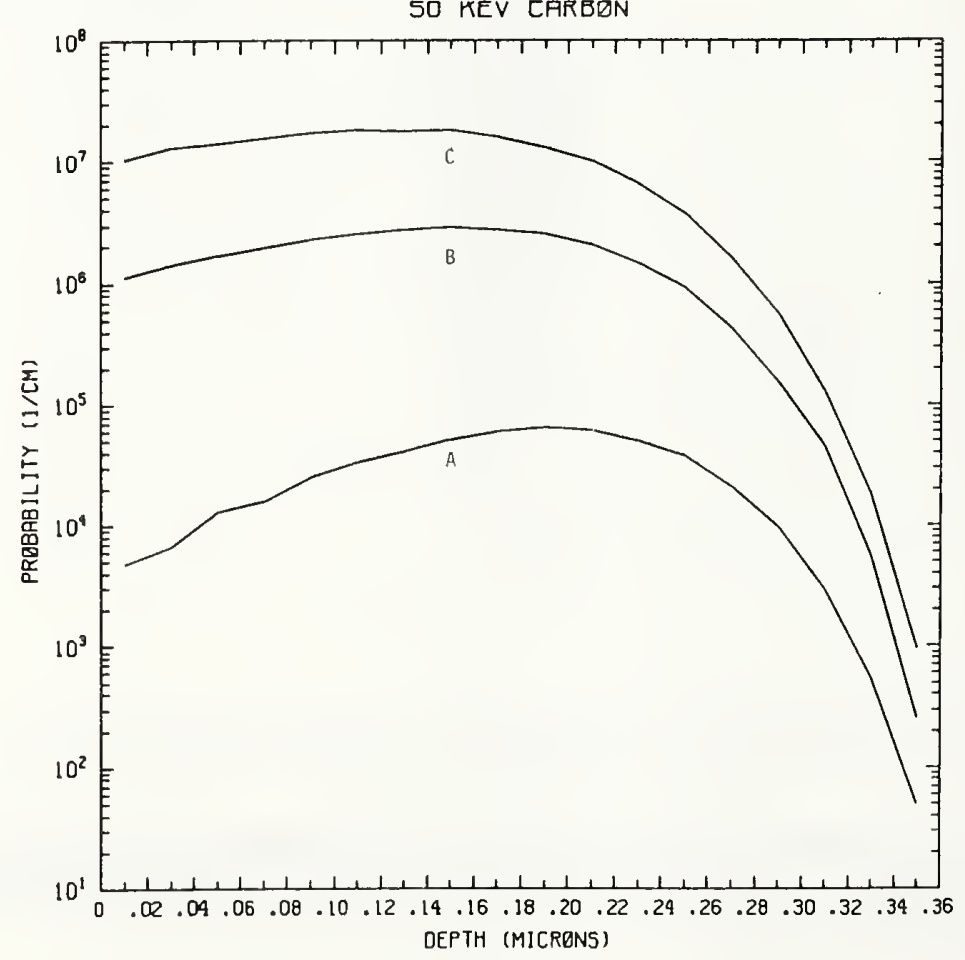

Figure C50-2 One-dimensional probability distributions for implanted carbon (A), primary silicon displacements (B), and Frenkel pairs (C) for $50 \mathrm{keV}$ carbon normally incident on a silicon target. 


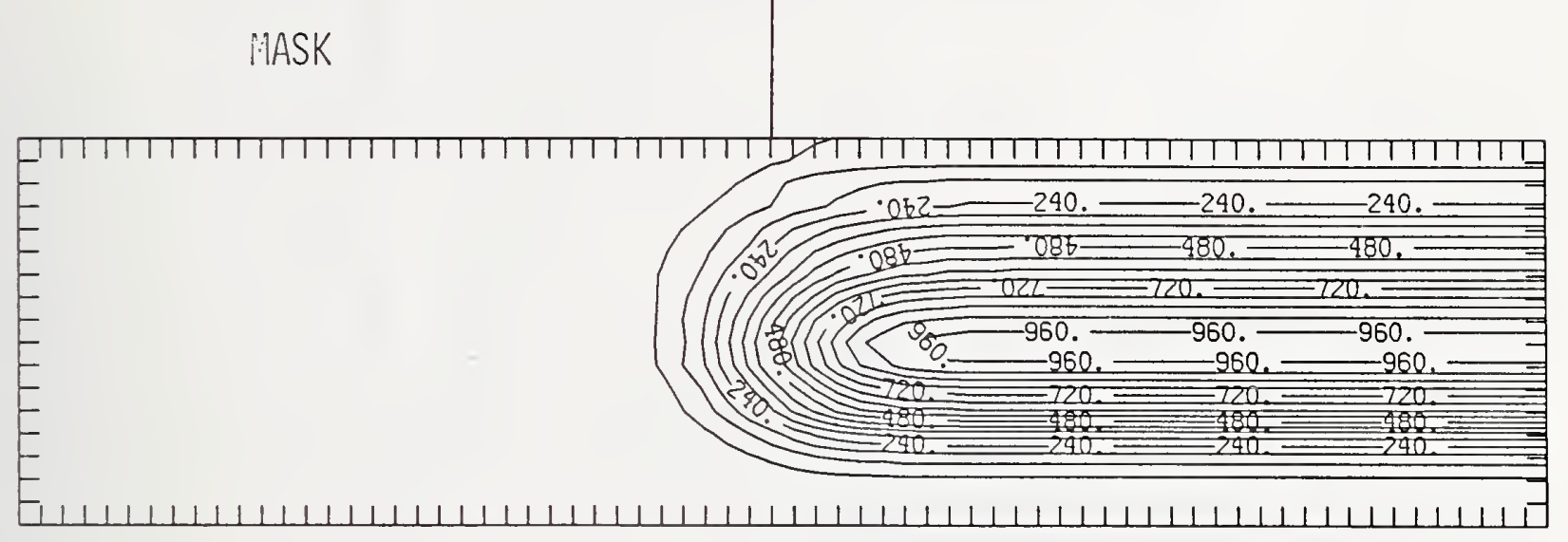

Figure C50-3 Two-dimensional distribution of $50 \mathrm{keV}$ carbon implanted past a mask edge. The length increment (distance between tick marks) is $0.02 \mu \mathrm{m}$ and the scale factor is 1000 .

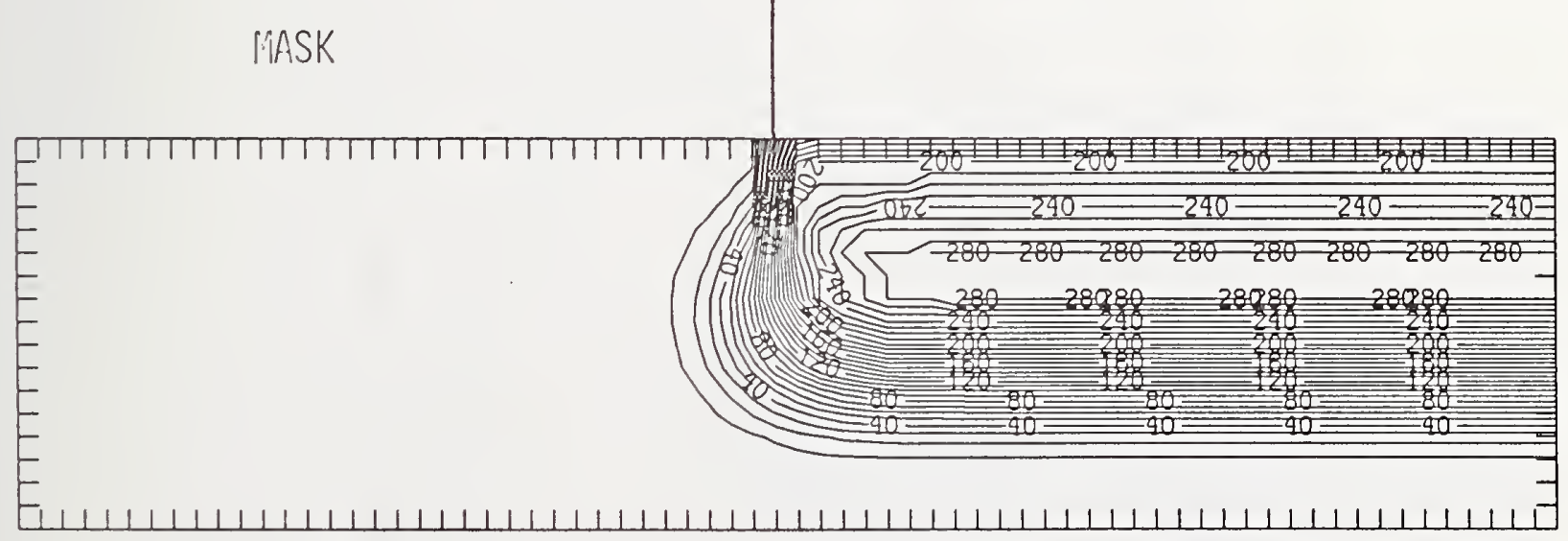

Figure C50-4 Two-dimensional distribution of Frenkel pairs created by $50 \mathrm{keV}$ carbon implanted past a mask edge. The length increment (distance between tick marks) is $0.02 \mu \mathrm{m}$ and the scale factor is 1 . 


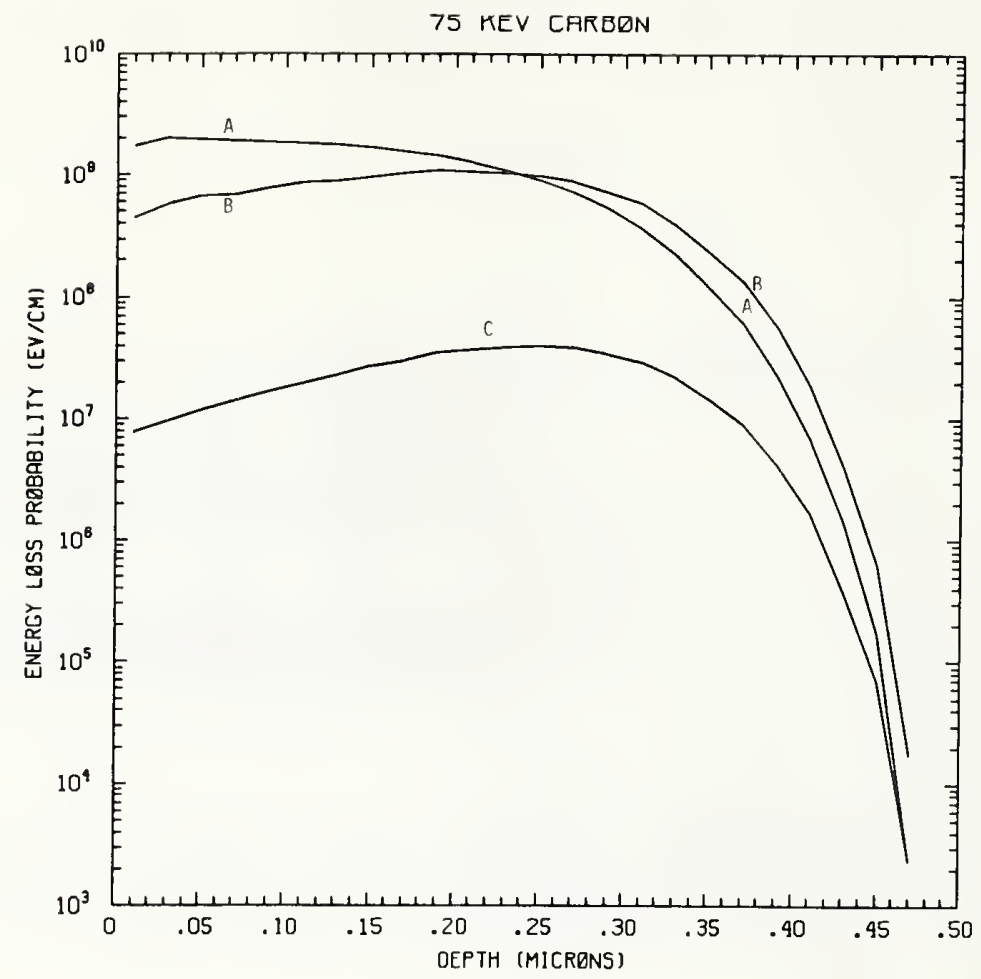

Figure C75-1 One-dimensional probability distributions for energy loss due to electronic excitation (A), displacement damage(B), and phonon excitation (C) for $75 \mathrm{keV}$ carbon normally incident on a silicon target.

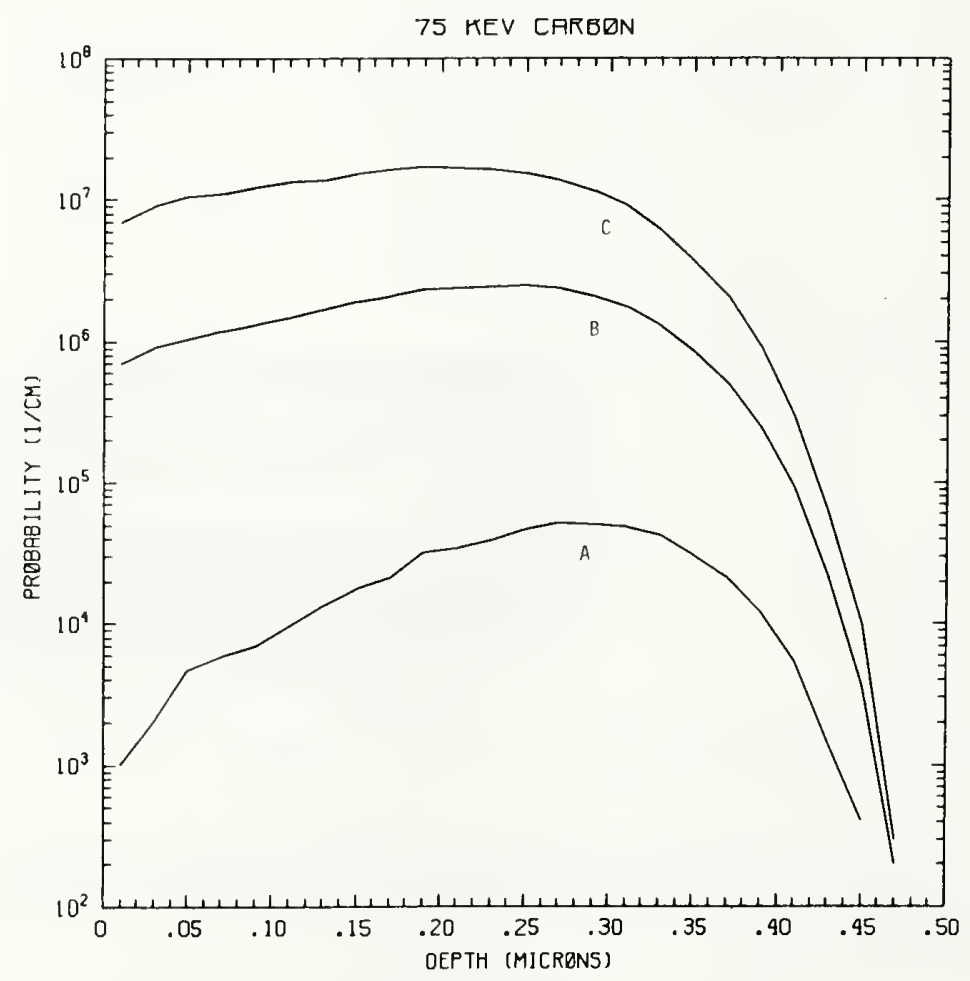

Figure C75-2 One-dimensional probability distributions for implanted carbon (A), primary silicon displacements (B), and Frenkel pairs (C) for $75 \mathrm{keV}$ carbon normally incident on a silicon target. 


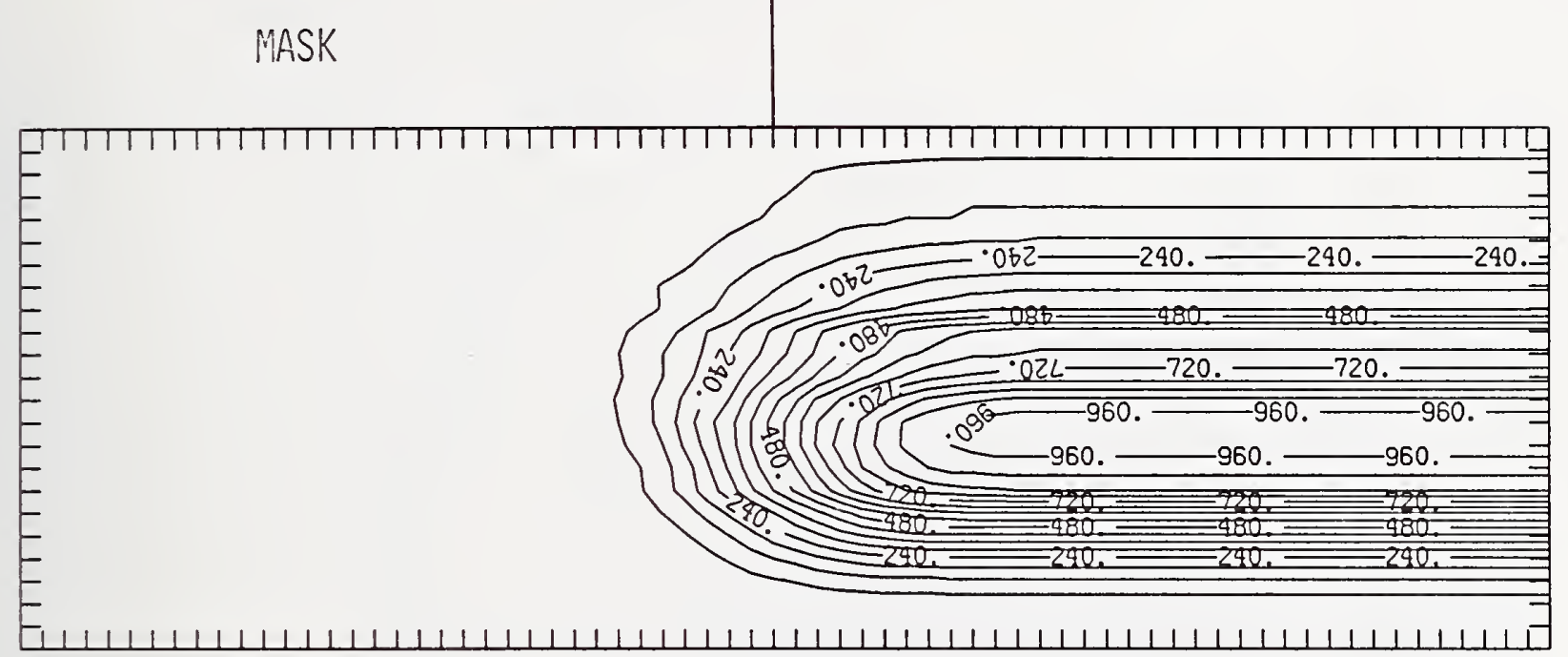

Figure C75-3 Two-dimensional distribution of $75 \mathrm{keV}$ carbon implanted past a mask edge. The length increment (distance between tick marks) is $0.02 \mu \mathrm{m}$ and the scale factor is 1000.

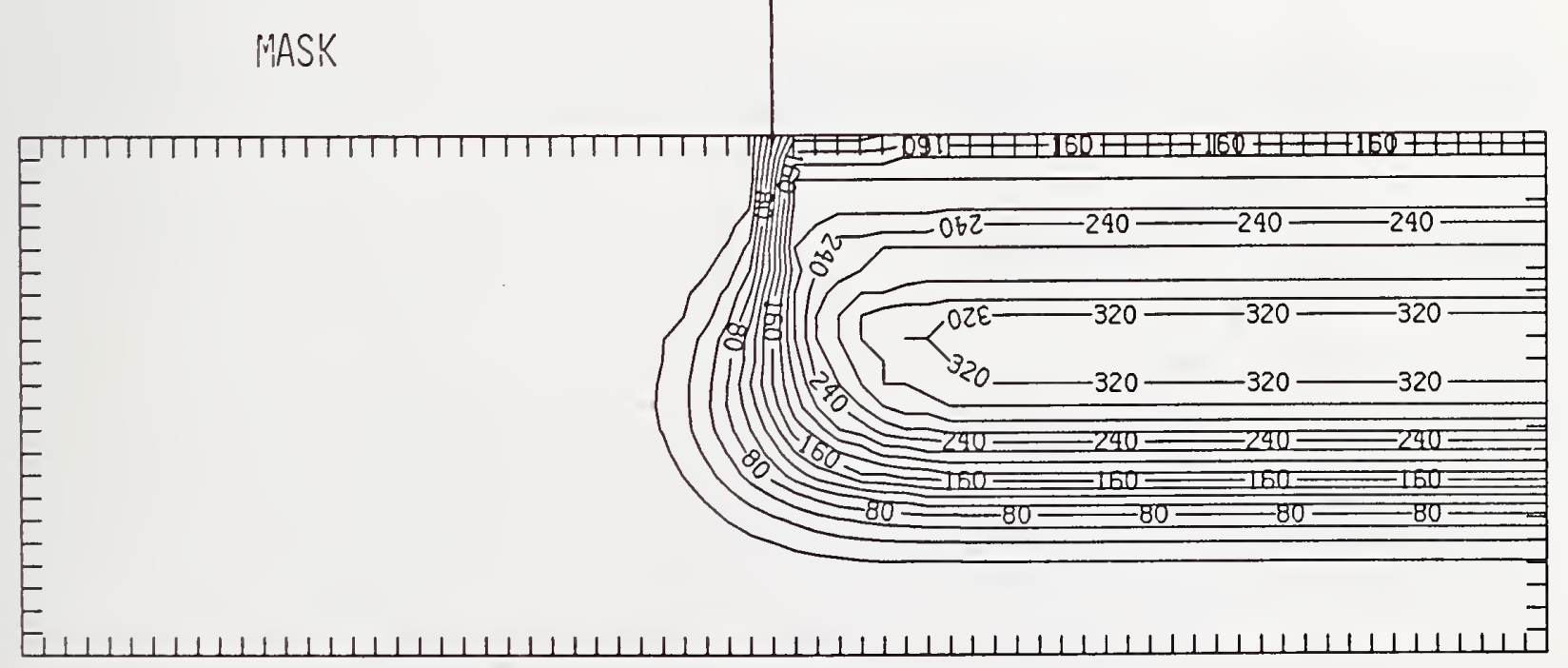

Figure C75-4 Two-dimensional distribution of Frenkel pairs created by $75 \mathrm{keV}$ carbon implanted past a mask edge. The length increment (distance between tick marks) is $0.02 \mu \mathrm{m}$ and the scale factor is 1 . 
100 KEV CARBDN

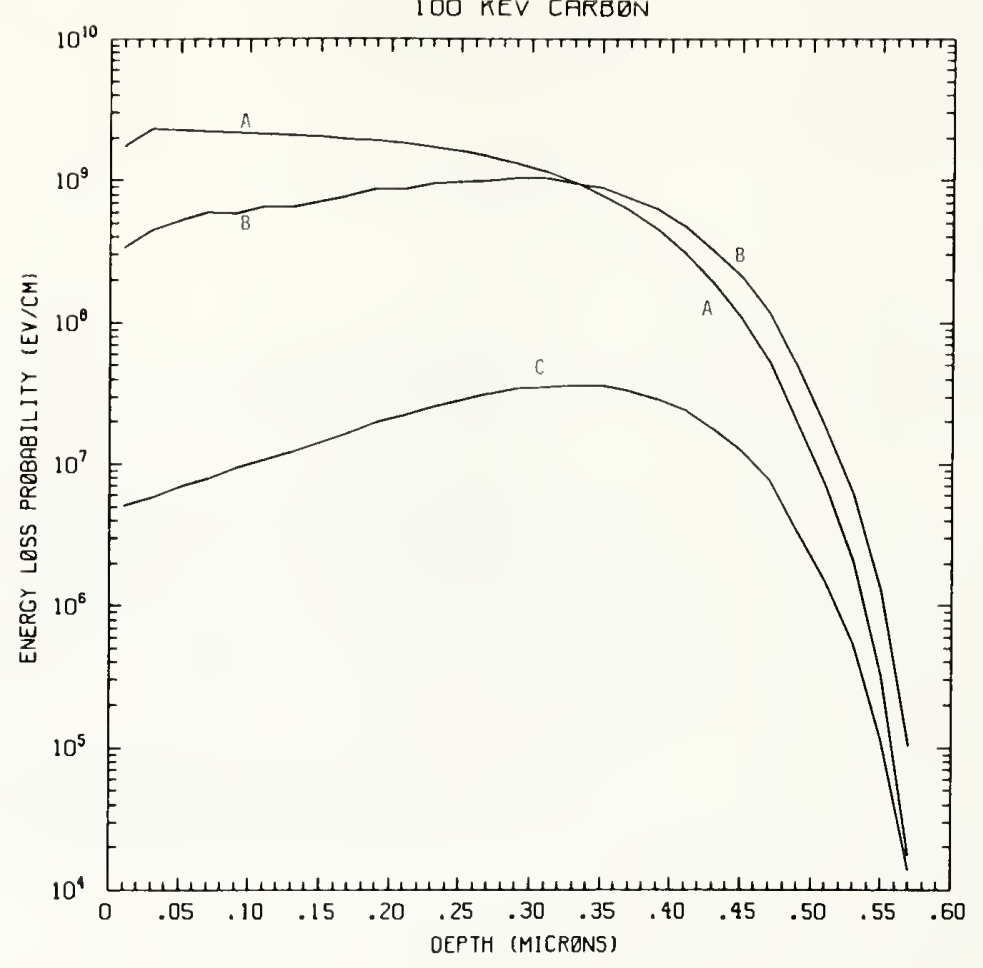

Figure C100-1 One-dimensional probability distributions for energy loss due to electronic excitation (A), displacement damage(B), and phonon excitation (C) for $100 \mathrm{keV}$ carbon normally incident on a silicon target.

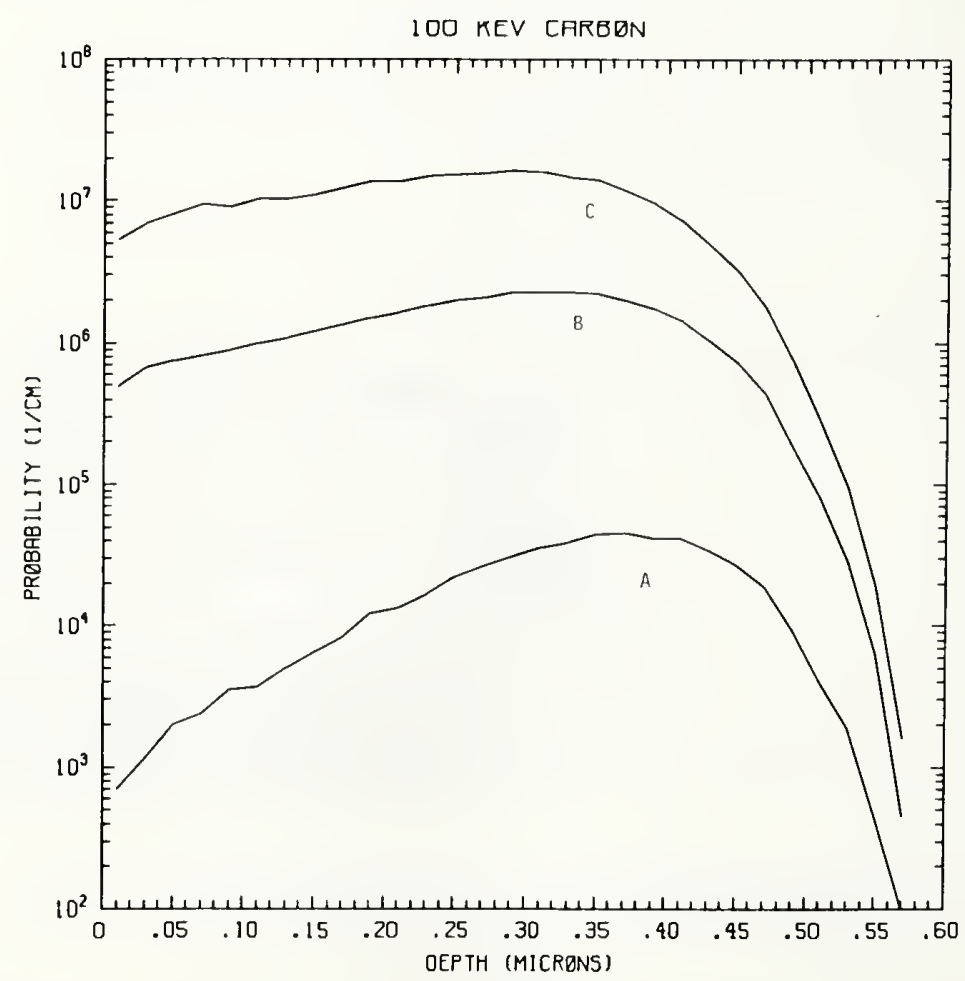

Figure C100-2 One-dimensional probability distributions for implanted carbon (A), primary silicon displacements (B), and Frenkel pairs (C) for $100 \mathrm{keV}$ carbon normally incident on a silicon target. 


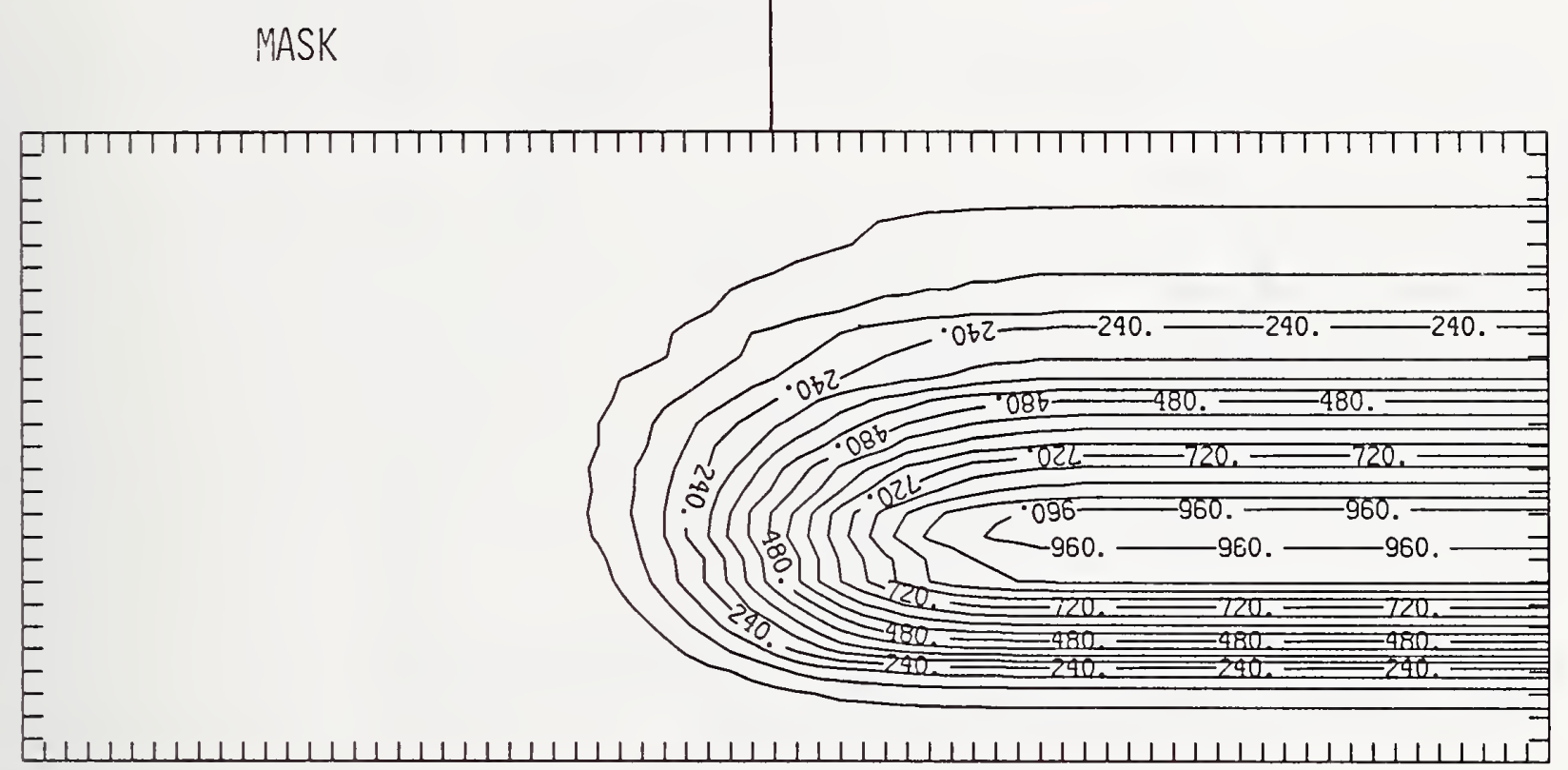

Figure C100-3 Two-dimensional distribution of $100 \mathrm{keV}$ carbon implanted past a mask edge. The length increment (distance between tick marks) is $0.02 \mu \mathrm{m}$ and the scale factor is 1000 .

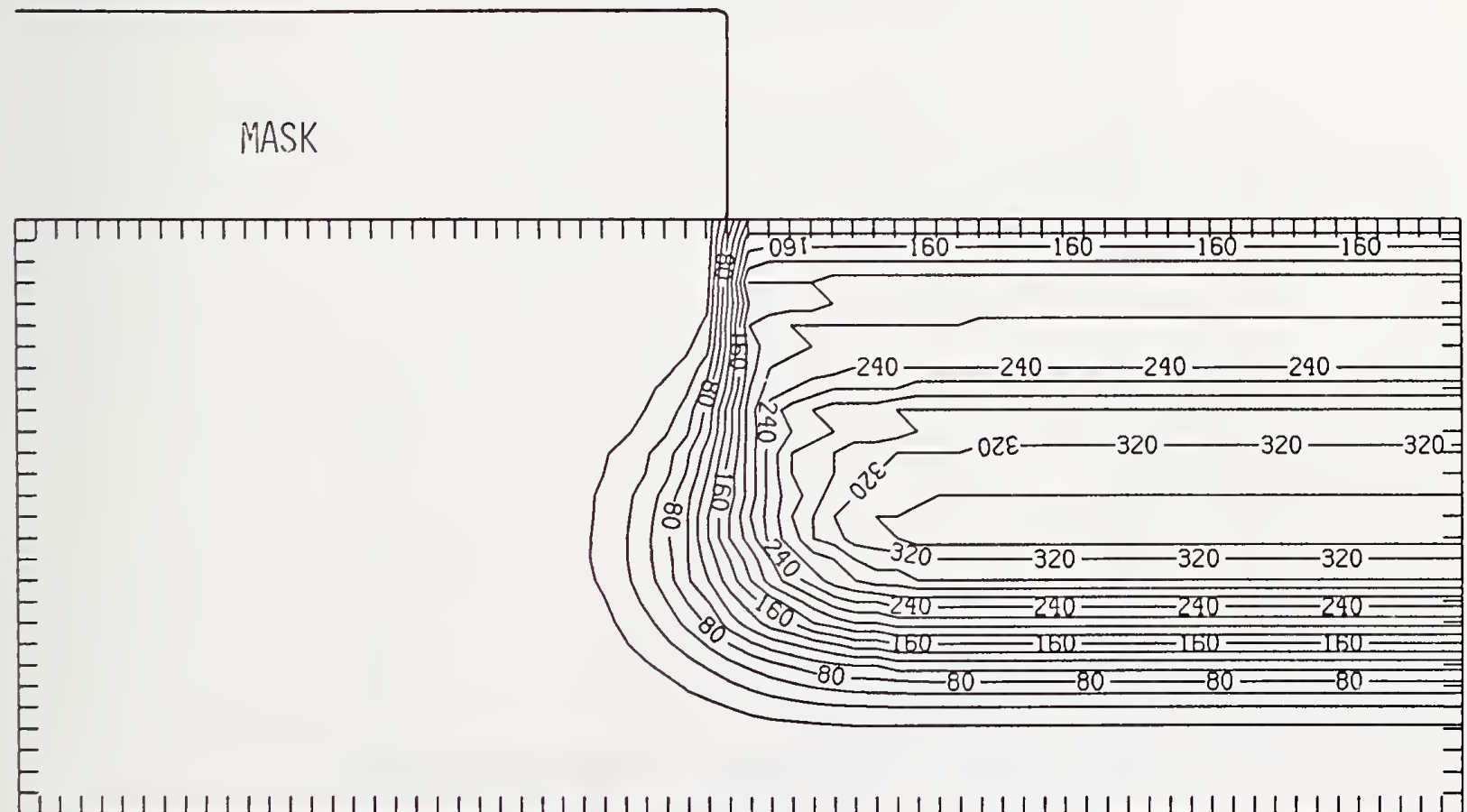

Figure C100-4 Two-dimensional distribution of Frenkel pairs created by $100 \mathrm{keV}$ carbon implanted past a mask edge. The length increment (distance between tick marks) is $0.02 \mu \mathrm{m}$ and the scale factor is 1 . 


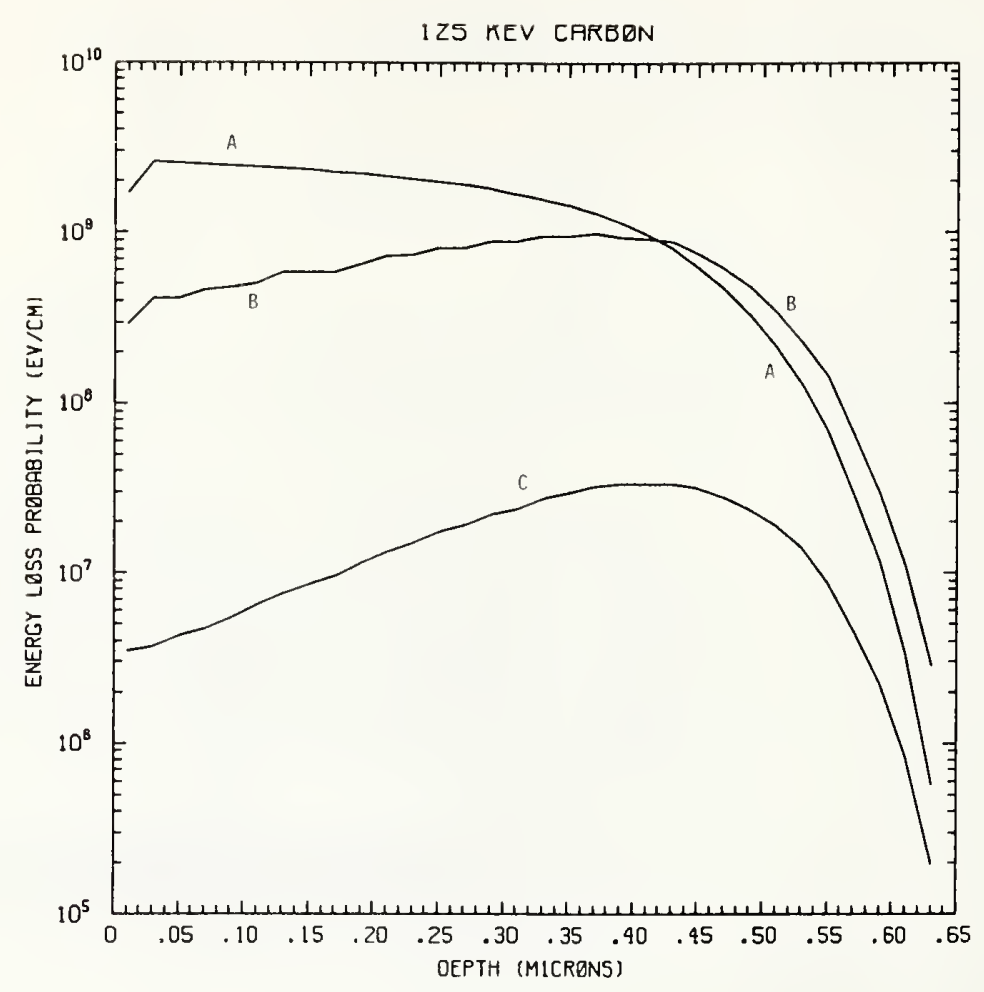

Figure C125-1 One-dimensional probability distributions for energy loss due to electronic excitation (A), displacement damage(B), and phonon excitation (C) for $125 \mathrm{keV}$ carbon normally incident on a silicon target.

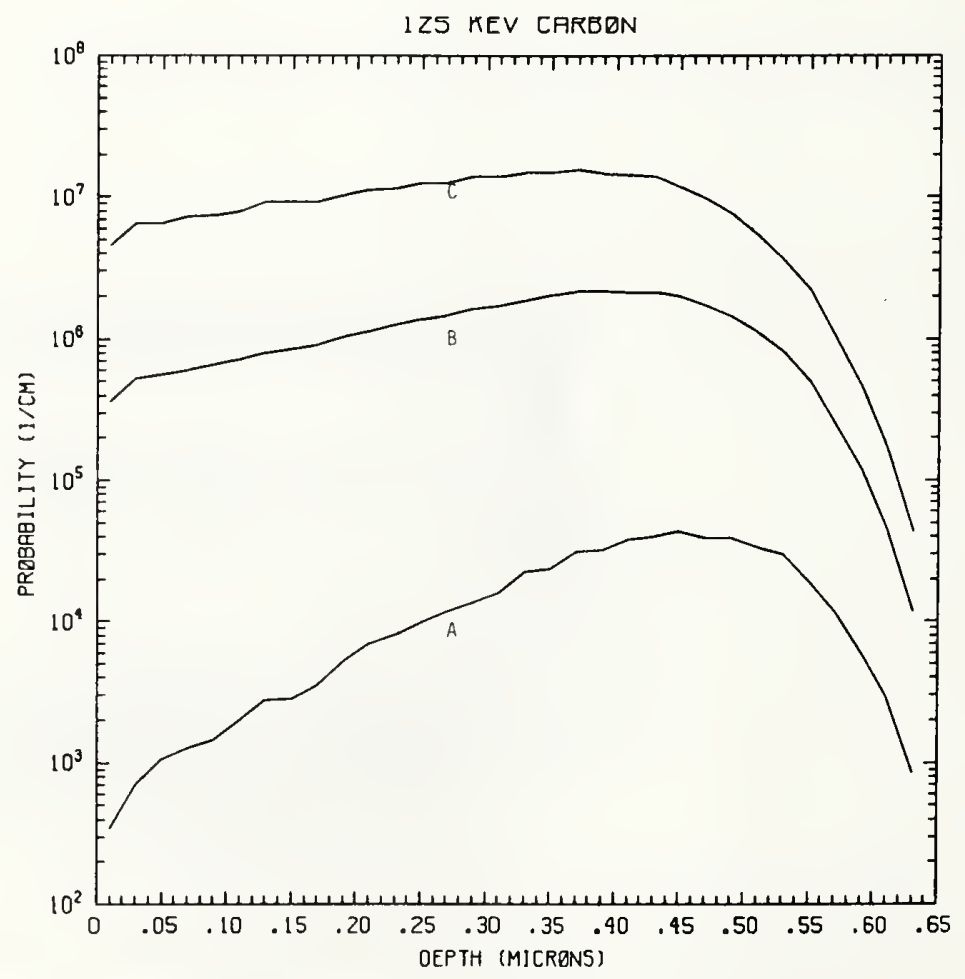

Figure C125-2 One-dimensional probability distributions for implanted carbon (A), primary silicon displacements (B), and Frenkel pairs (C) for $125 \mathrm{keV}$ carbon normally incident on a silicon target. 
MASK

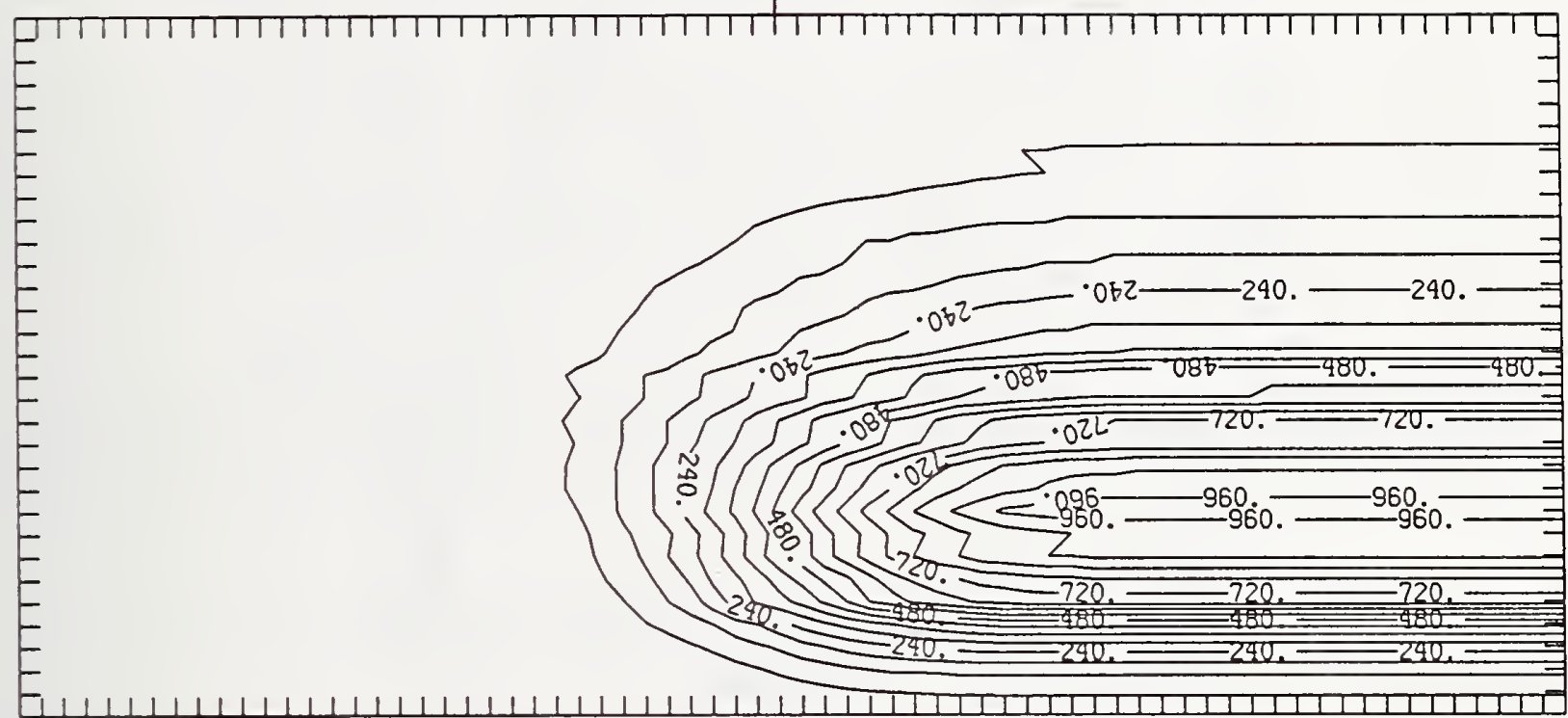

Figure C125-3 Two-dimensional distribution of $125 \mathrm{keV}$ carbon implanted past a mask edge. The length increment (distance between tick marks) is $0.02 \mu \mathrm{m}$ and the scale factor is 1000.

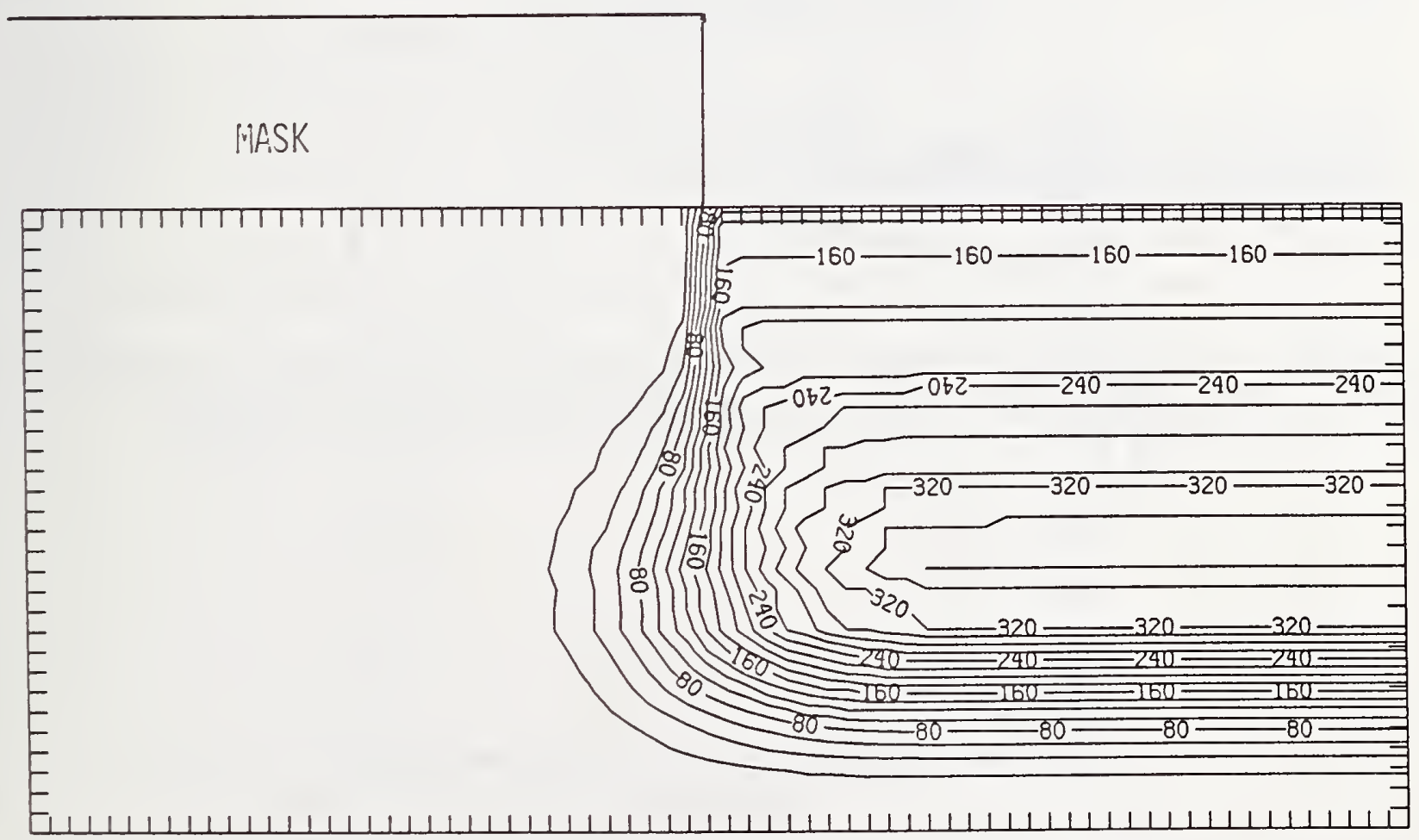

Figure C125-4 Two-dimensional distribution of Frenkel pairs created by $125 \mathrm{keV}$ carbon implanted past a mask edge. The length increment (distance between tick marks) is $0.02 \mu \mathrm{m}$ and the scale factor is 1 . 


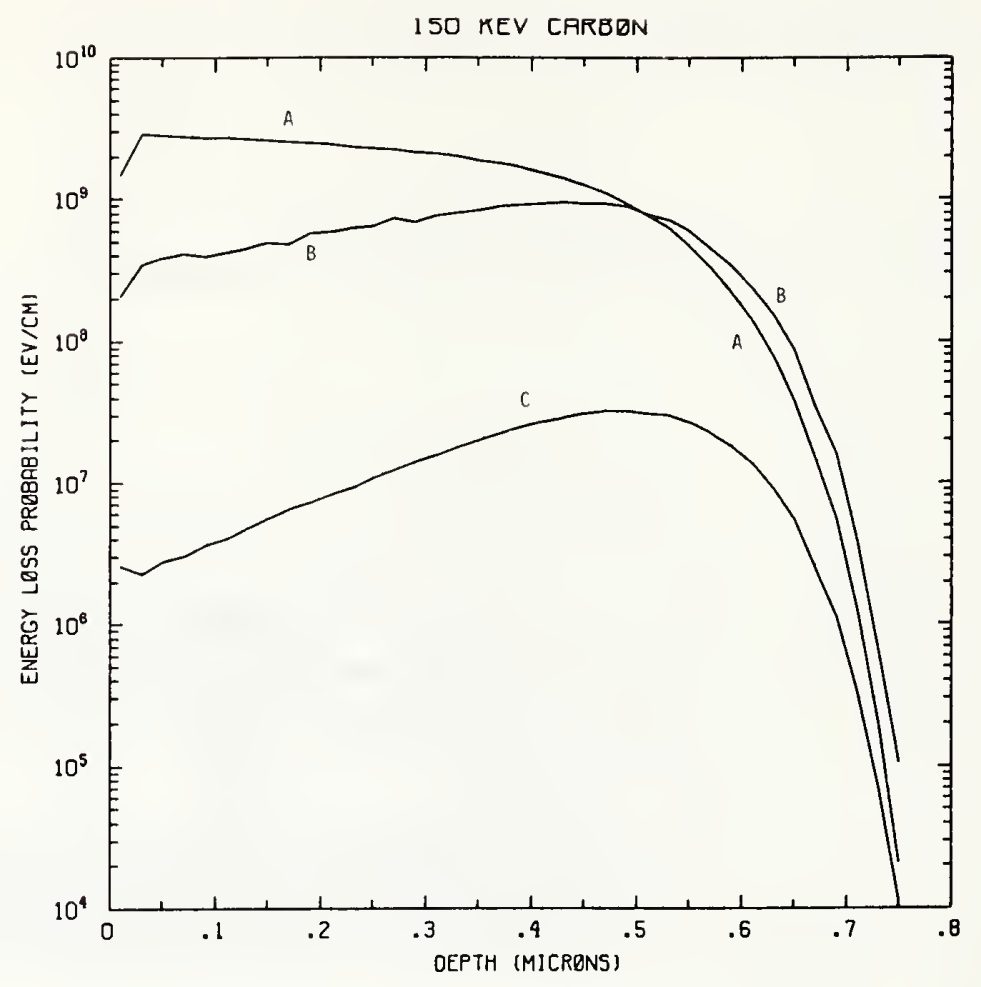

Figure C150-1 One-dimensional probability distributions for energy loss due to electronic excitation (A), displacement damage(B), and phonon excitation (C) for $150 \mathrm{keV}$ carbon normally incident on a silicon target.

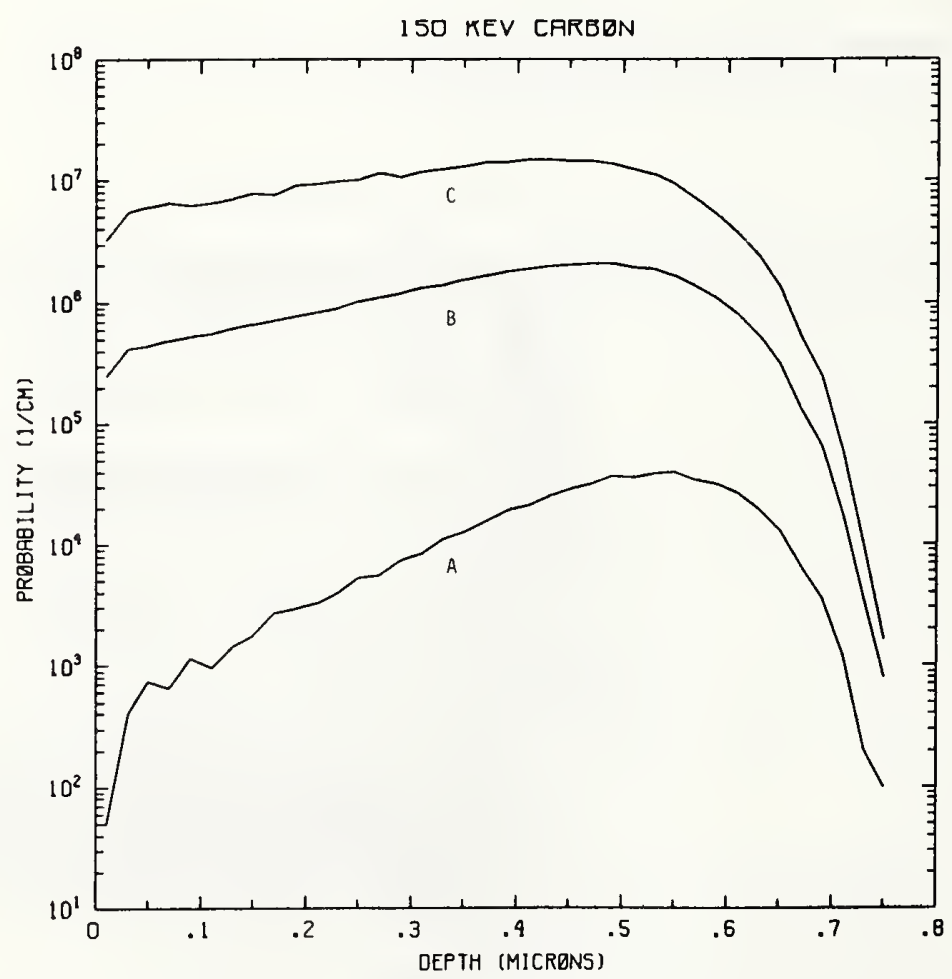

Figure C150-2 One-dimensional probability distributions for implanted carbon (A), primary silicon displacements (B), and Frenkel pairs (C) for $150 \mathrm{keV}$ carbon normally incident on a silicon target. 


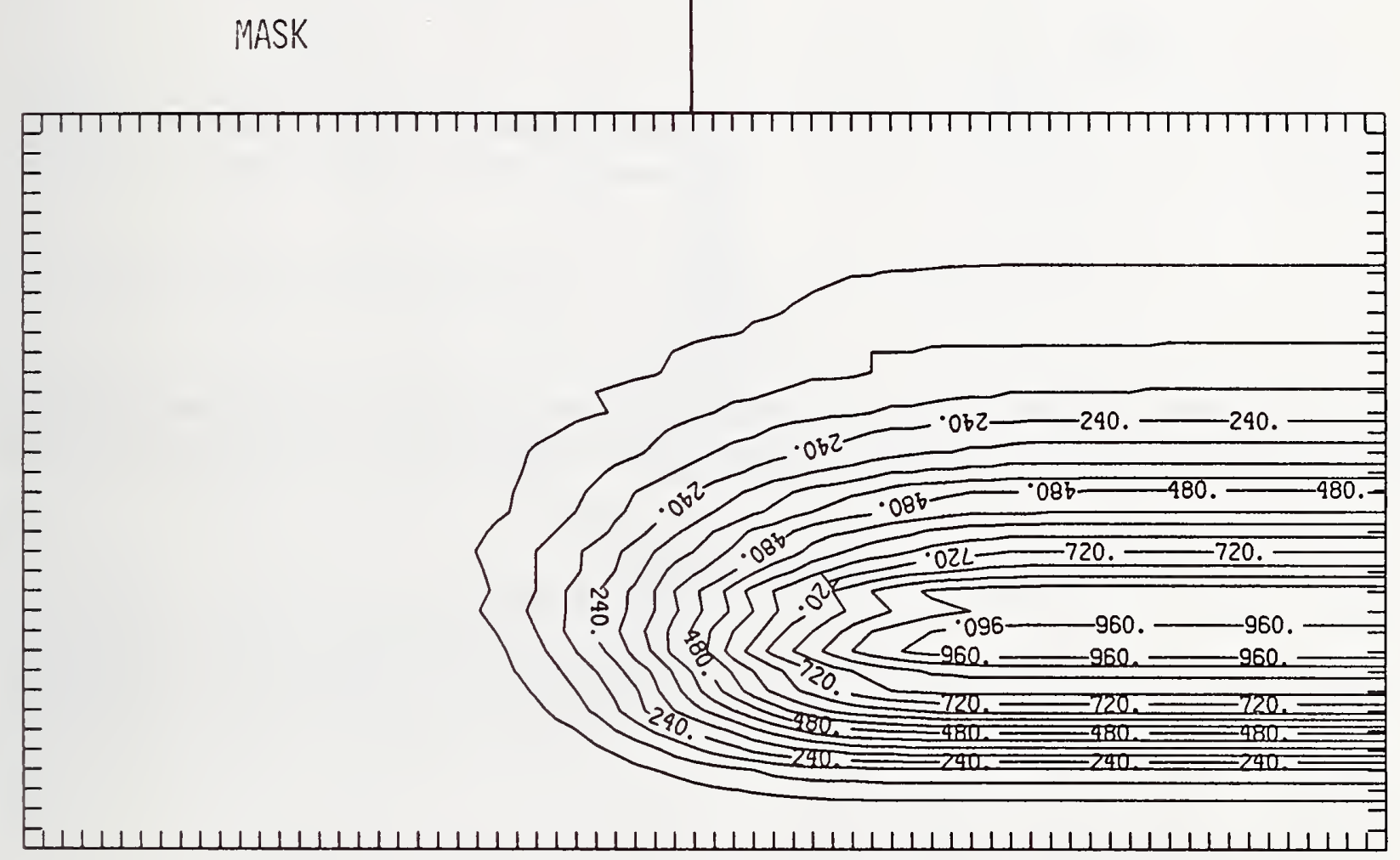

Figure C150-3 Two-dimensional distribution of $150 \mathrm{keV}$ carbon implanted past a mask edge. The length increment (distance between tick marks) is $0.02 \mu \mathrm{m}$ and the scale factor is 1000. 
MASK

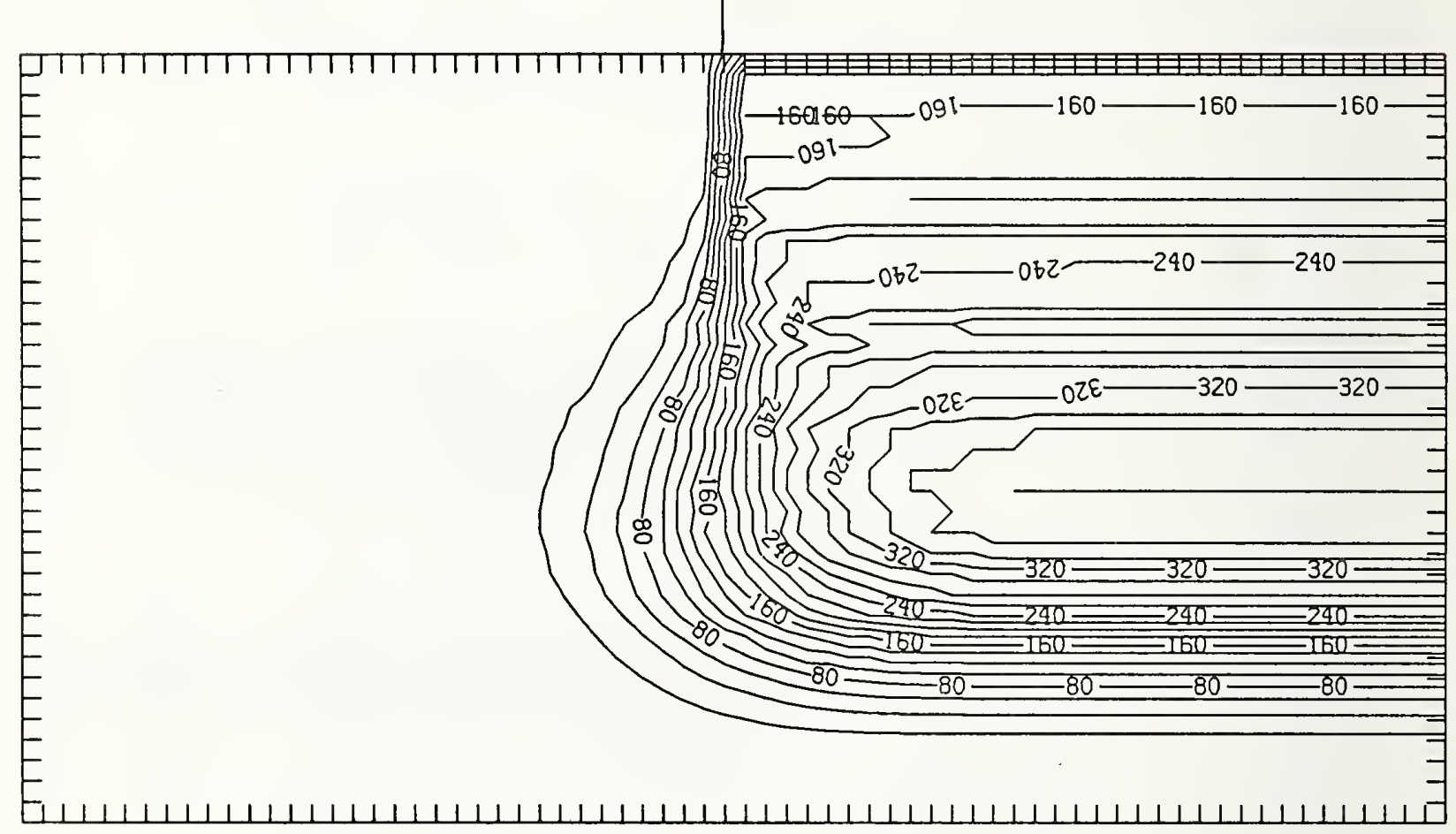

Figure C150-4 Two-dimensional distribution of Frenkel pairs created by $150 \mathrm{keV}$ carbon implanted past a mask edge. The length increment (distance between tick marks) is $0.02 \mu \mathrm{m}$ and the scale factor is 1. 


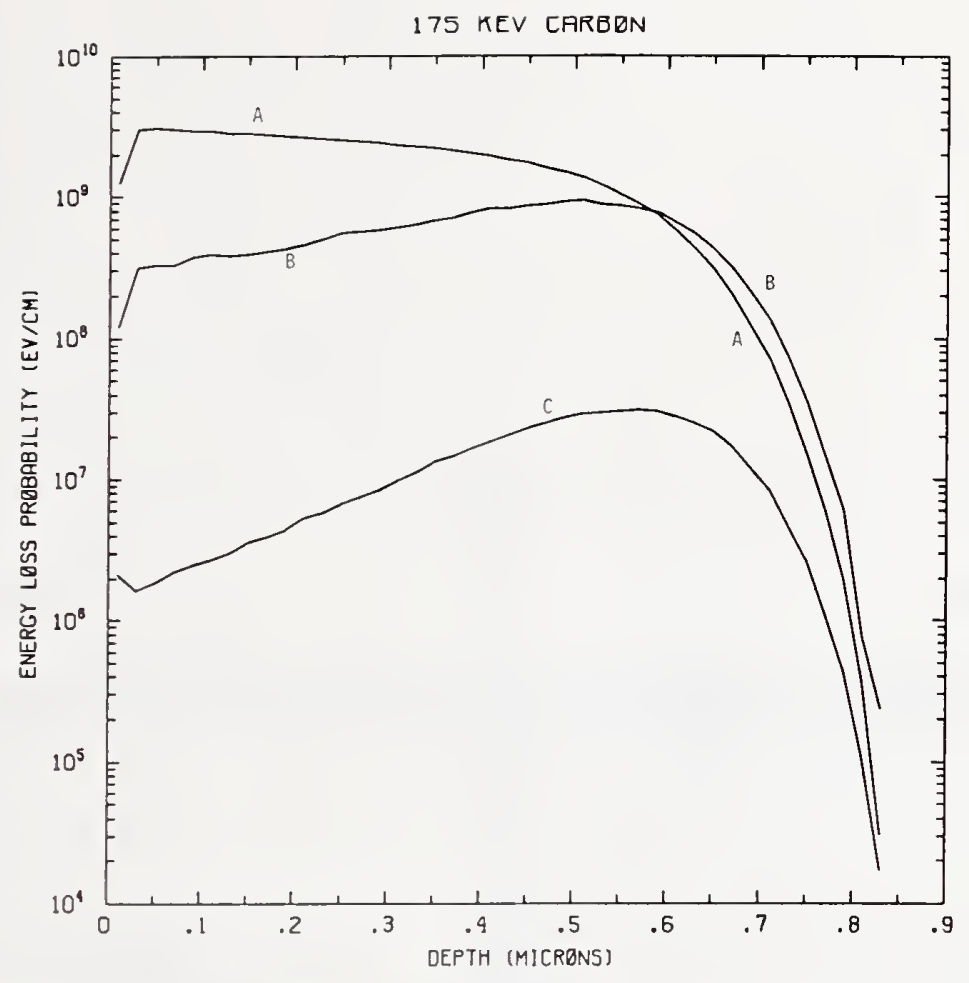

Figure C175-1 One-dimensional probability distributions for energy loss due to electronic excitation (A), displacement damage(B), and phonon excitation (C) for $175 \mathrm{keV}$ carbon normally incident on a silicon target.

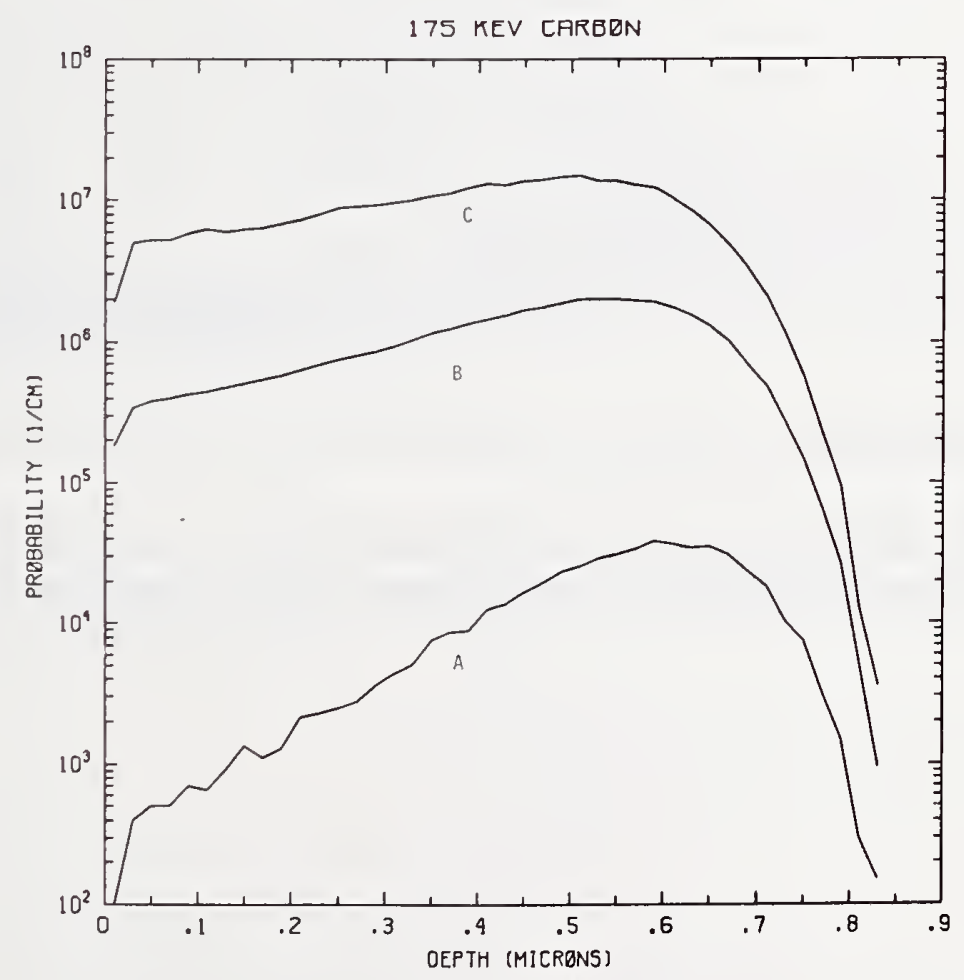

Figure C175-2 One-dimensional probability distributions for implanted carbon (A), primary silicon displacements (B), and Frenkel pairs (C) for $175 \mathrm{keV}$ carbon normally incident on a silicon target. 


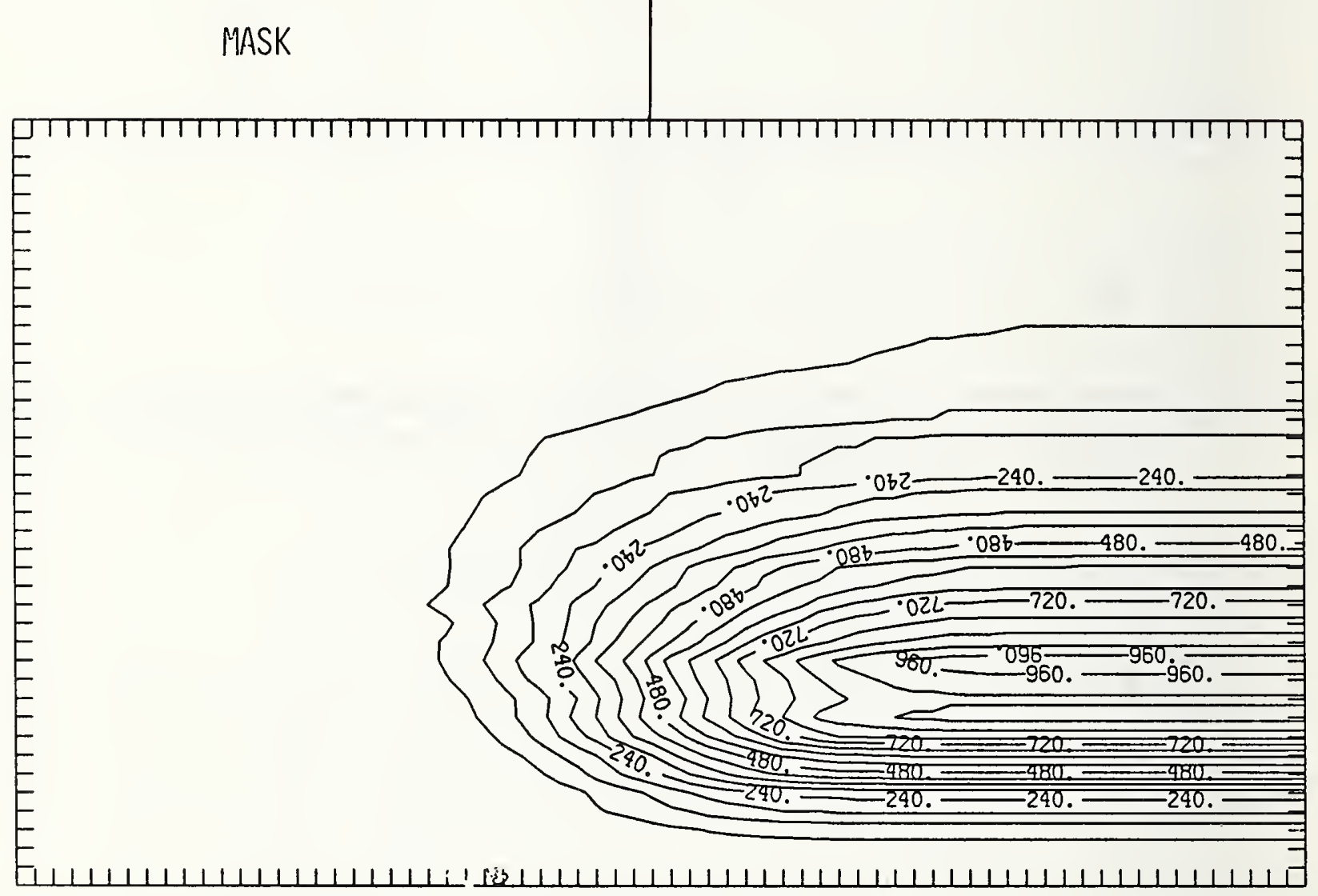

Figure C175-3 Two-dimensional distribution of $175 \mathrm{keV}$ carbon implanted past a mask edge. The length increment (distance between tick marks) is $0.02 \mu \mathrm{m}$ and the scale factor is 1000 . 


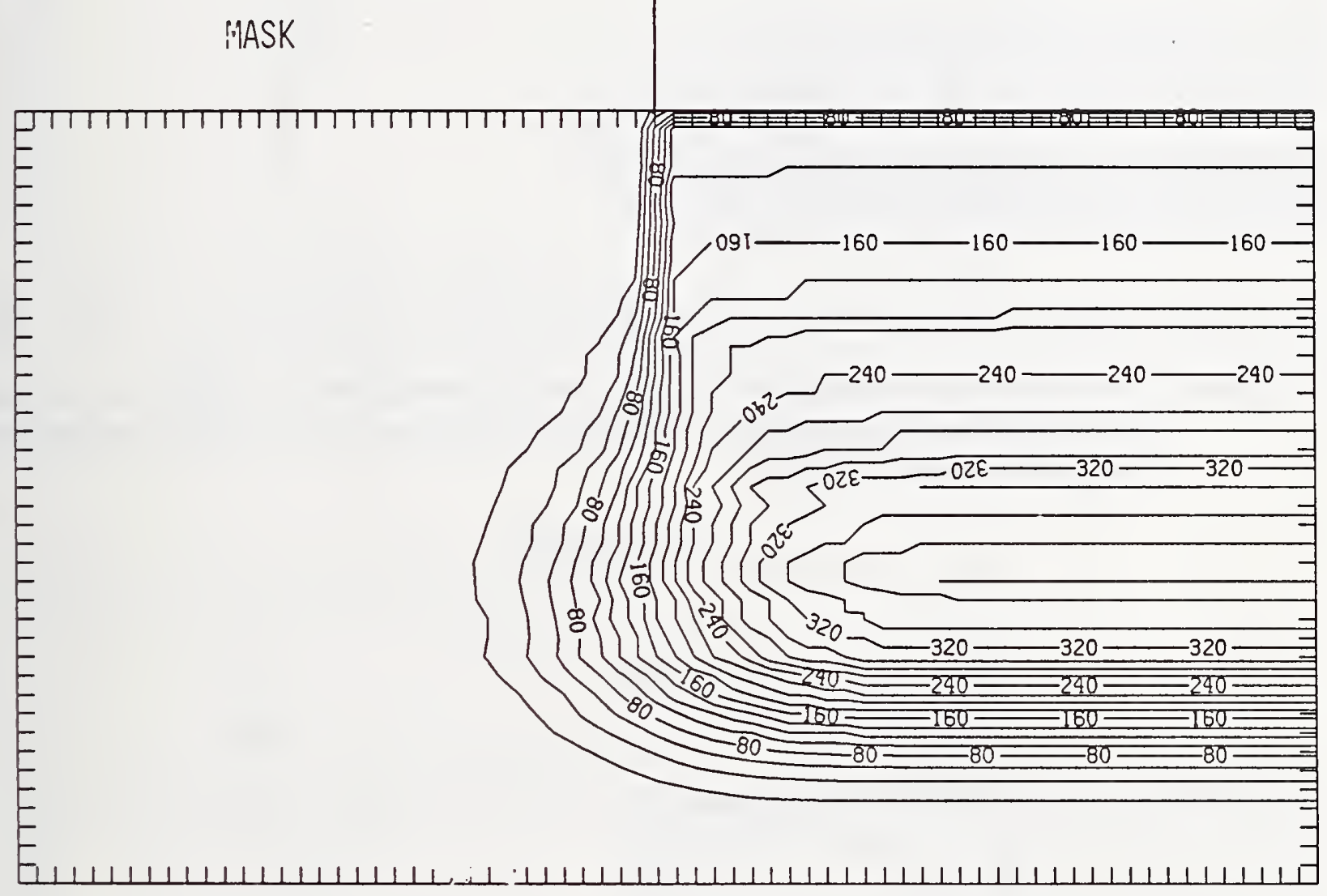

Figure C175-4 Two-dimensional distribution of Frenkel pairs created by $175 \mathrm{keV}$ carbon implanted past a mask edge. The length increment (distance between tick marks) is $0.02 \mu \mathrm{m}$ and the scale factor is 1 . 


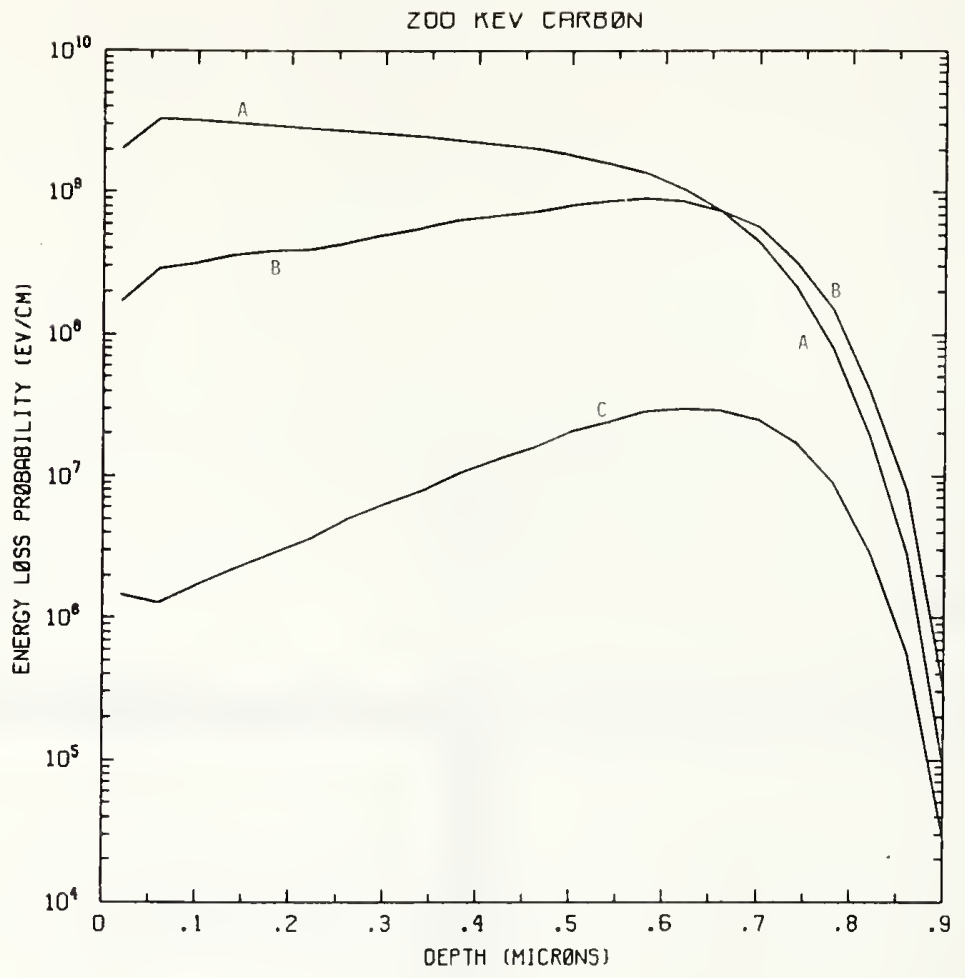

Figure C200-1 One-dimensional probability distributions for energy loss due to electronic excitation (A), displacement damage(B), and phonon excitation (C) for $200 \mathrm{keV}$ carbon normally incident on a silicon target.

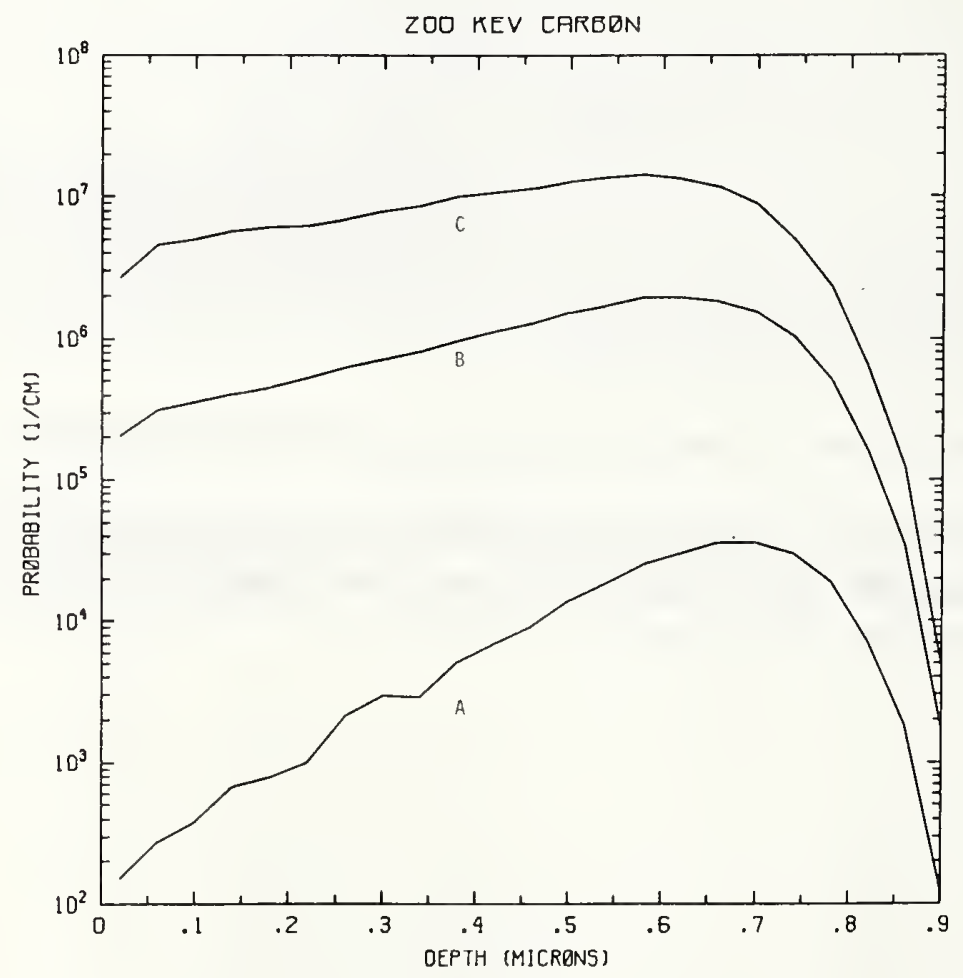

Figure C200-2 One-dimensional probability distributions for implanted carbon (A), primary silicon displacements (B), and Frenkel pairs (C) for $200 \mathrm{keV}$ carbon normally incident on a silicon target. 
MASK

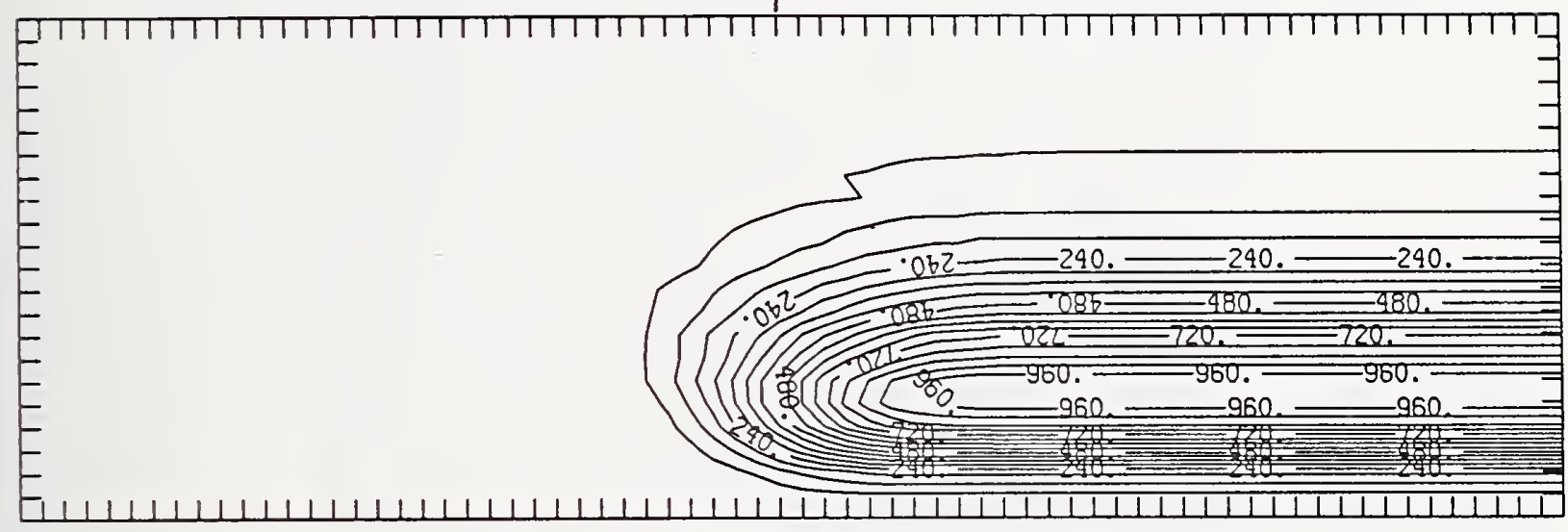

Figure C200-3 Two-dimensional distribution of $200 \mathrm{keV}$ carbon implanted past a mask edge. The length increment (distance between tick marks) is $0.04 \mu \mathrm{m}$ and the scale factor is 1000 .

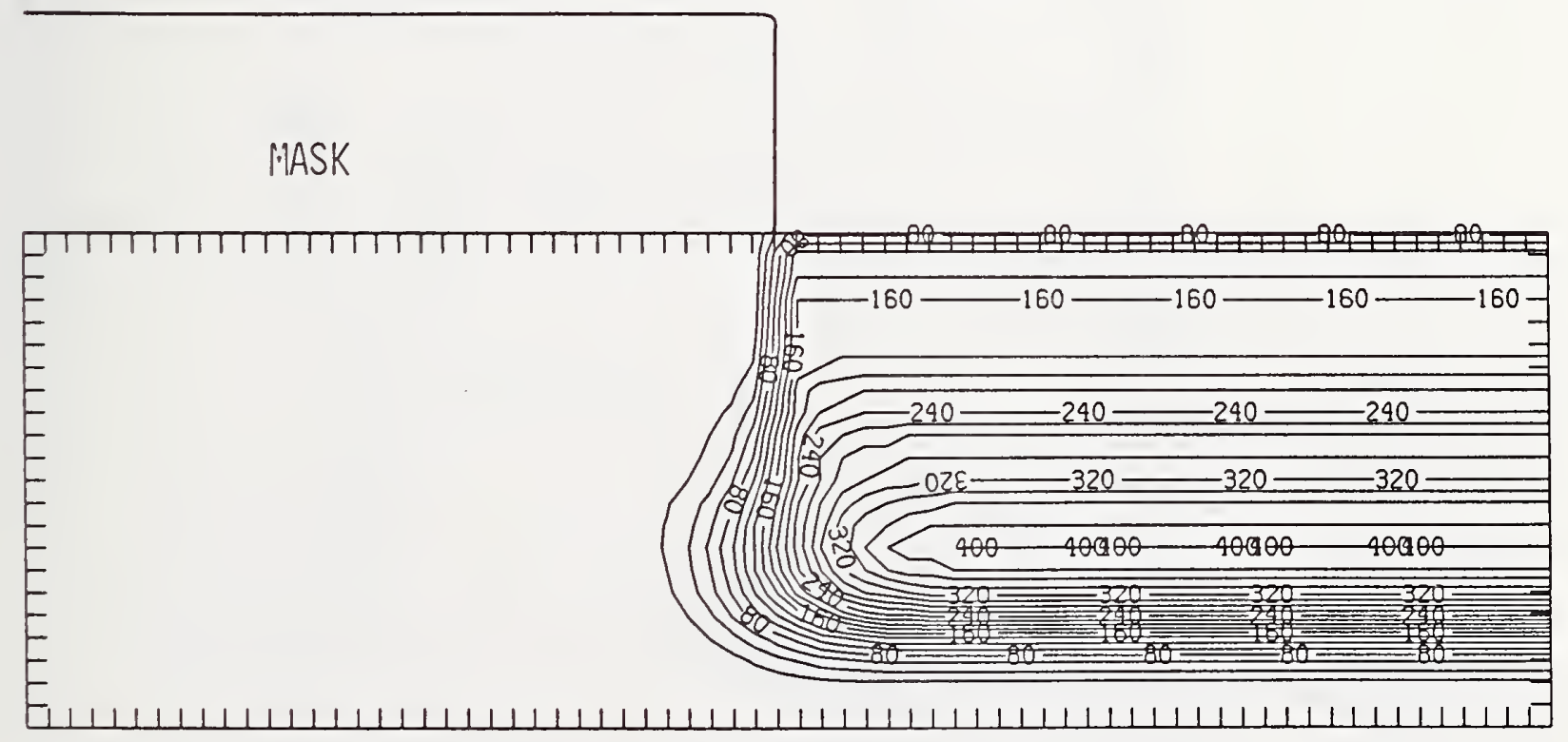

Figure C200-4 Two-dimensional distribution of Frenkel pairs created by $200 \mathrm{keV}$ carbon implanted past a mask edge. The length increment (distance between tick marks) is $0.04 \mu \mathrm{m}$ and the scale factor is 1 . 


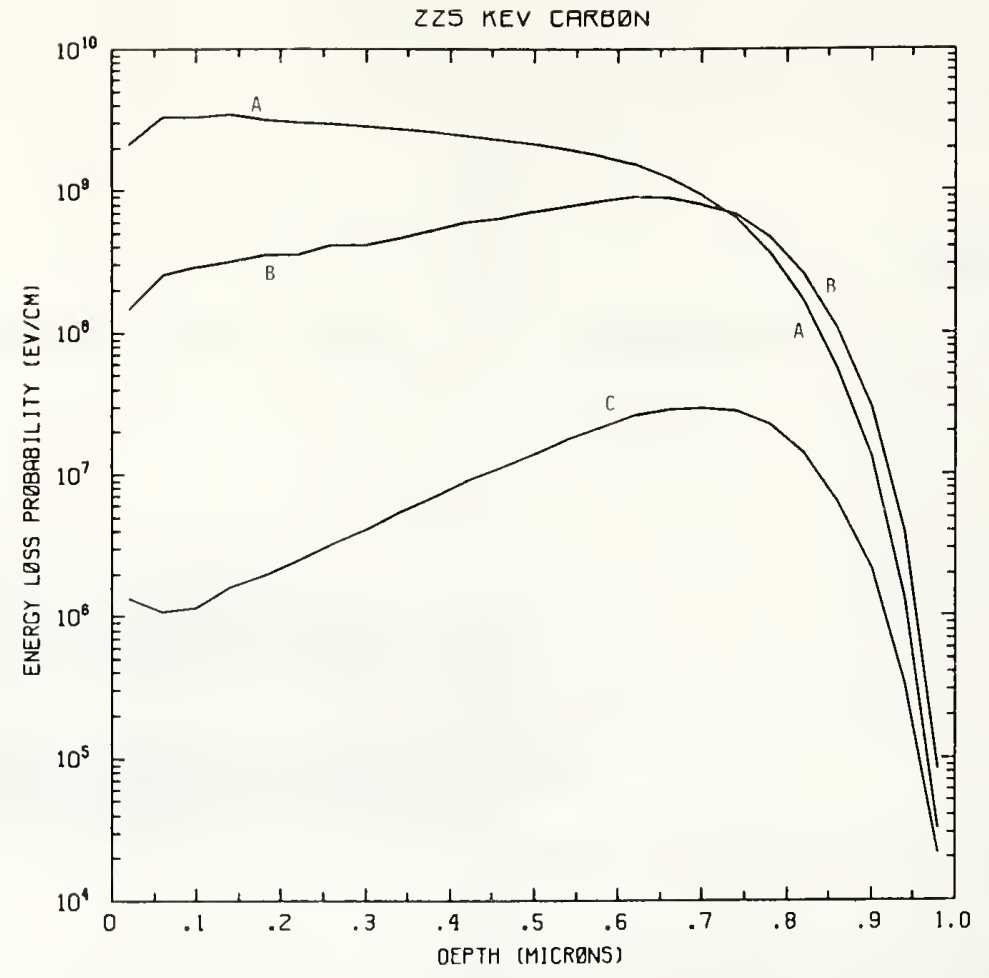

Figure C225-1 One-dimensional probability distributions for energy loss due to electronic excitation (A), displacement damage(B), and phonon excitation (C) for $225 \mathrm{keV}$ carbon normally incident on a silicon target.

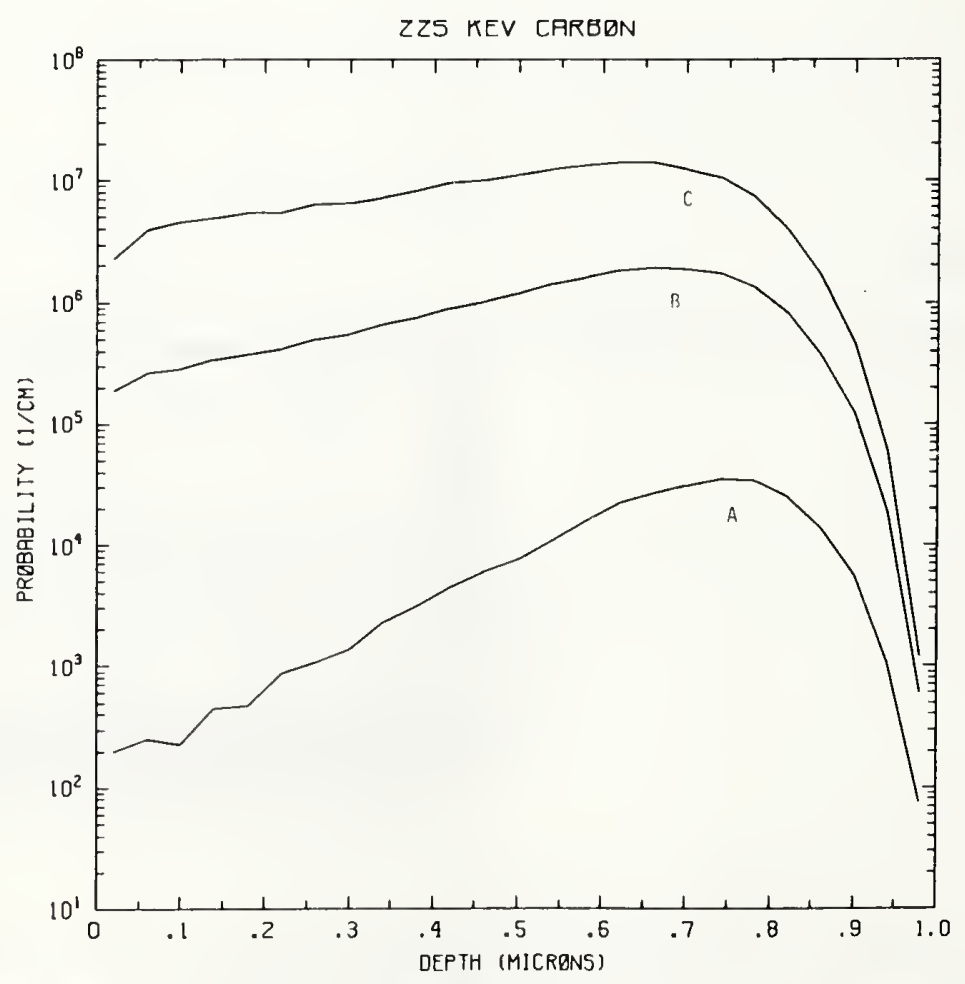

Figure C225-2 One-dimensional probability distributions for implanted carbon (A), primary silicon displacements (B), and Frenkel pairs (C) for $225 \mathrm{keV}$ carbon normally incident on a silicon target. 


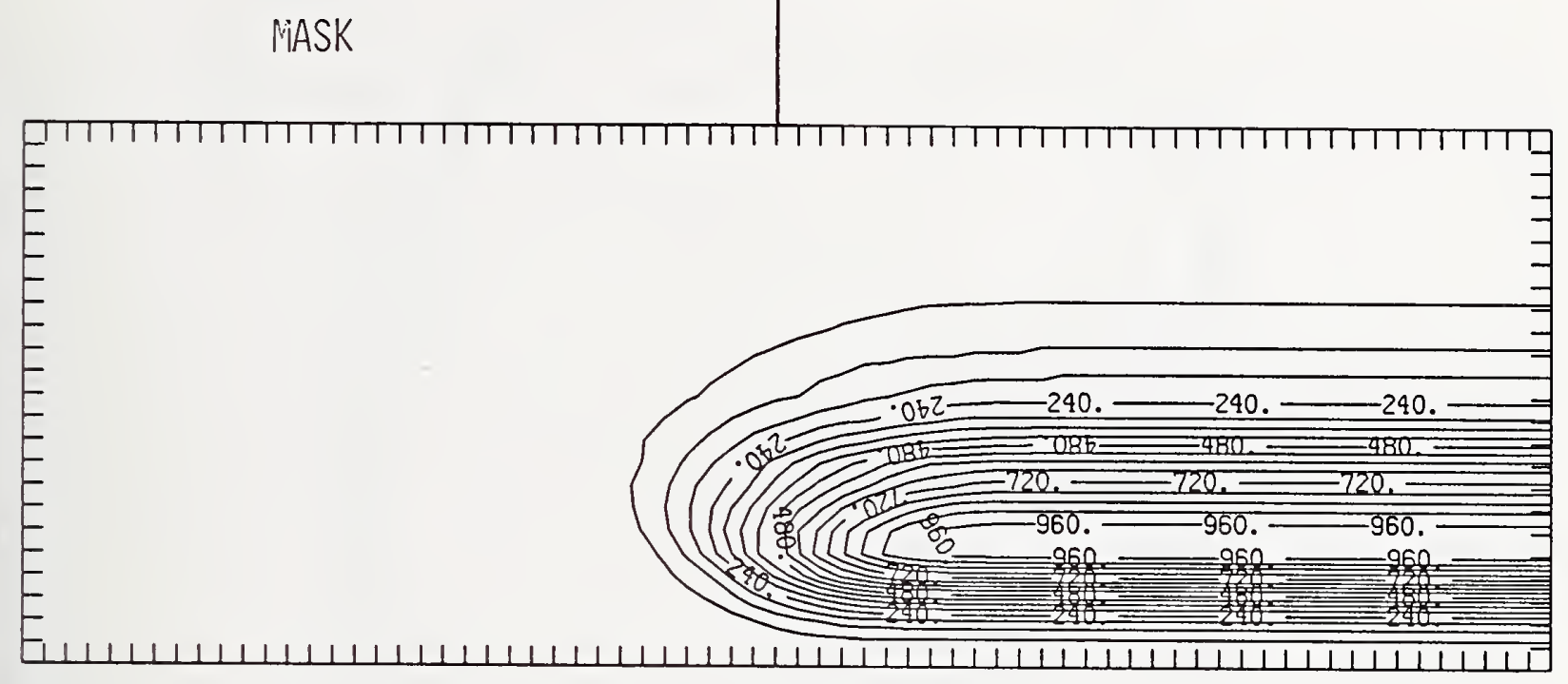

Figure C225-3 Two-dimensional distribution of $225 \mathrm{keV}$ carbon implanted past a mask edge. The length increment (distance between tick marks) is $0.04 \mu \mathrm{m}$ and the scale factor is 1000 .

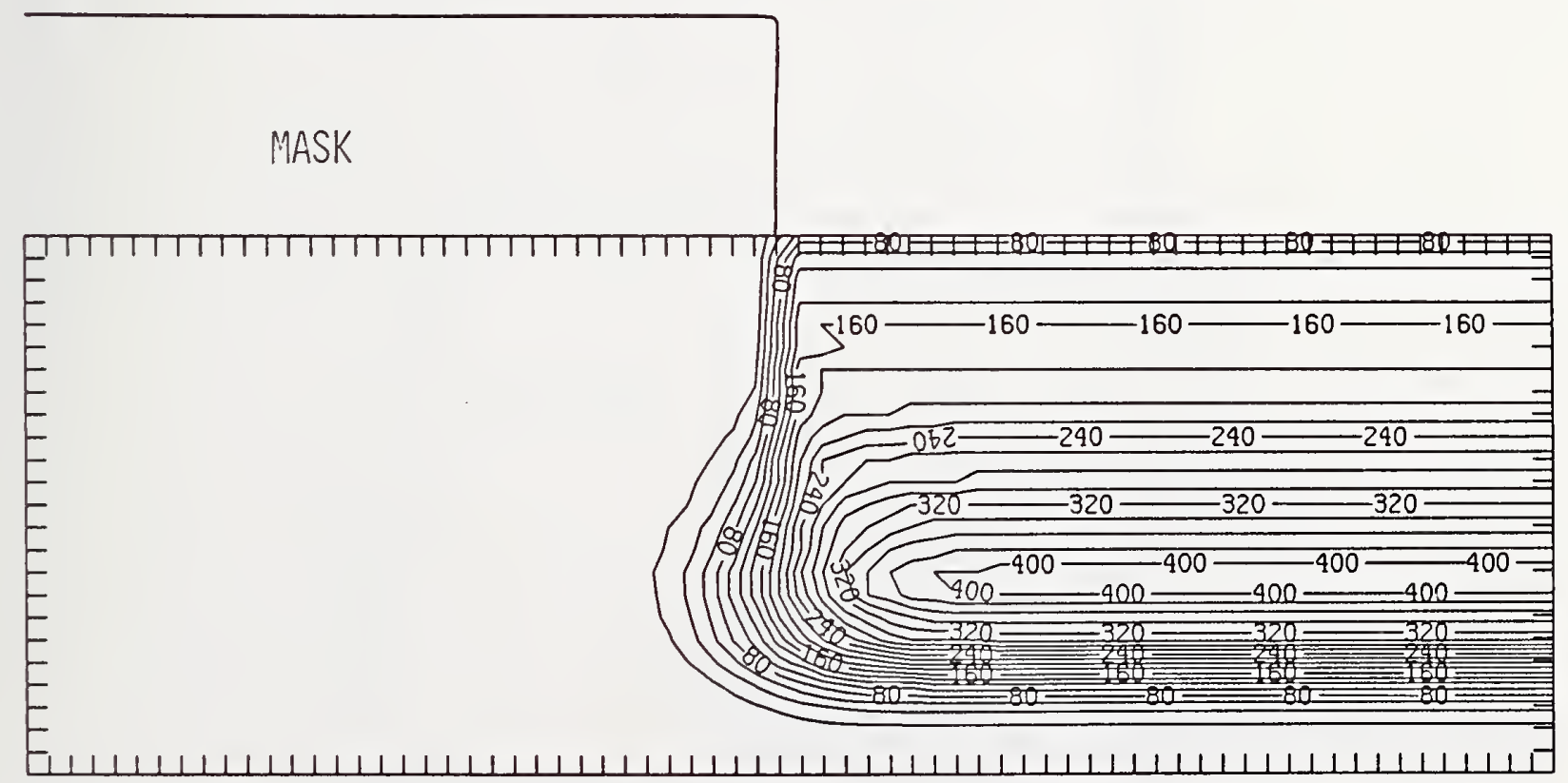

Figure C225-4 Two-dimensional distribution of Frenkel pairs created by $225 \mathrm{keV}$ carbon implanted past a mask edge. The length increment (distance between tick marks) is $0.04 \mu \mathrm{m}$ and the scale factor is 1 . 


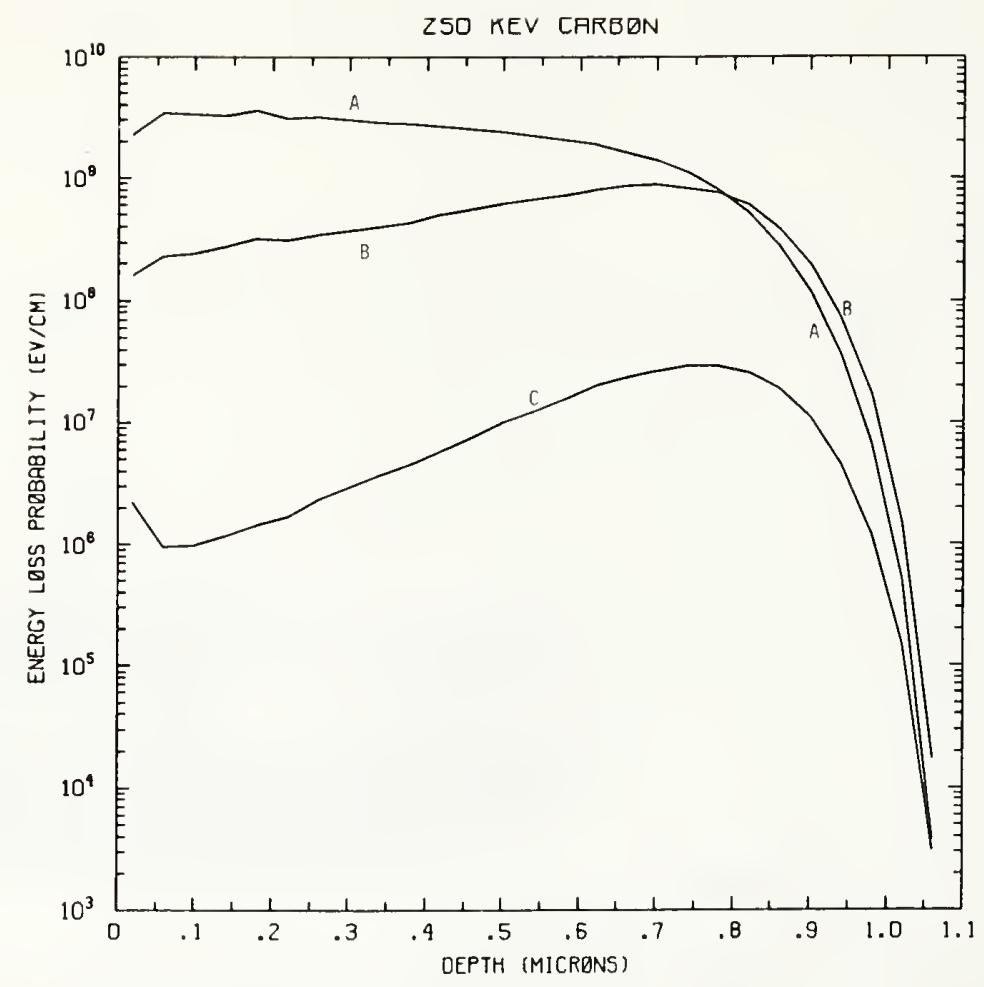

Figure C250-1 One-dimensional probability distributions for energy loss due to electronic excitation (A), displacement damage(B), and phonon excitation (C) for $250 \mathrm{keV}$ carbon normally incident on a silicon target.

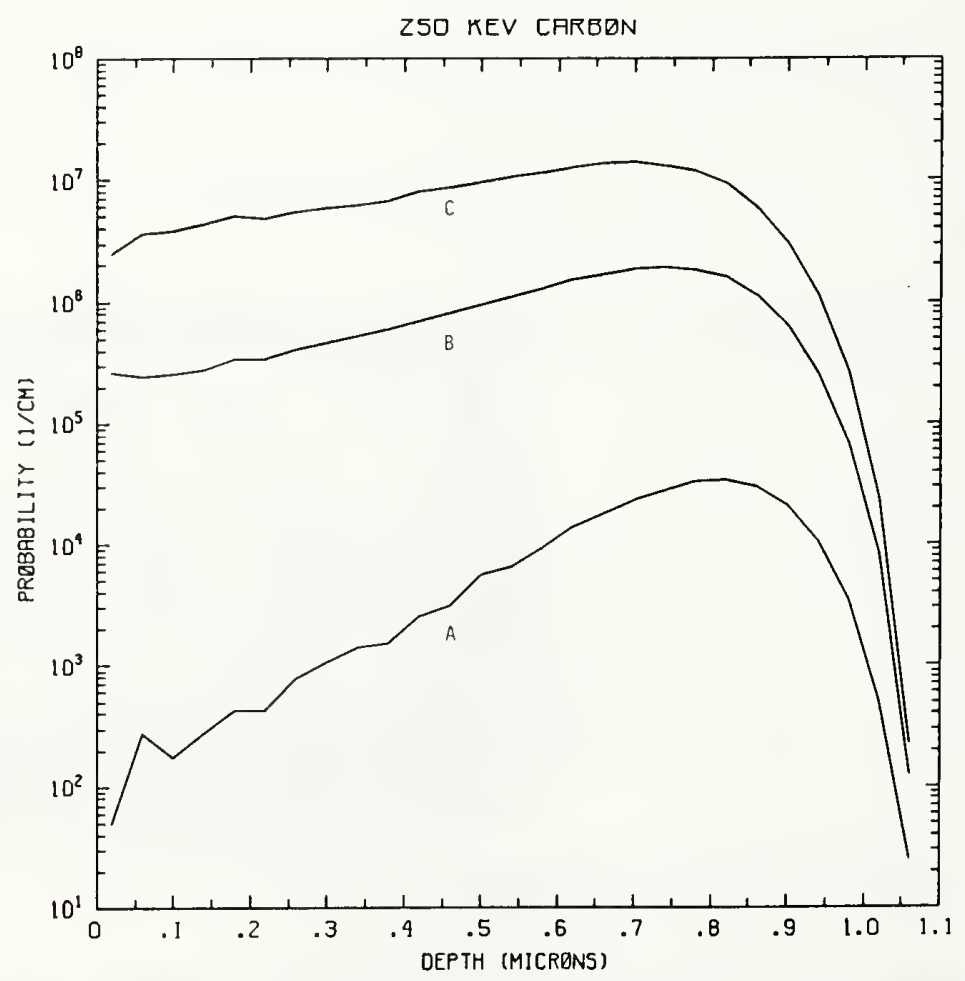

Figure C250-2 One-dimensional probability distributions for implanted carbon (A), primary silicon displacements (B), and Frenkel pairs (C) for $250 \mathrm{keV}$ carbon normally incident on a silicon target. 


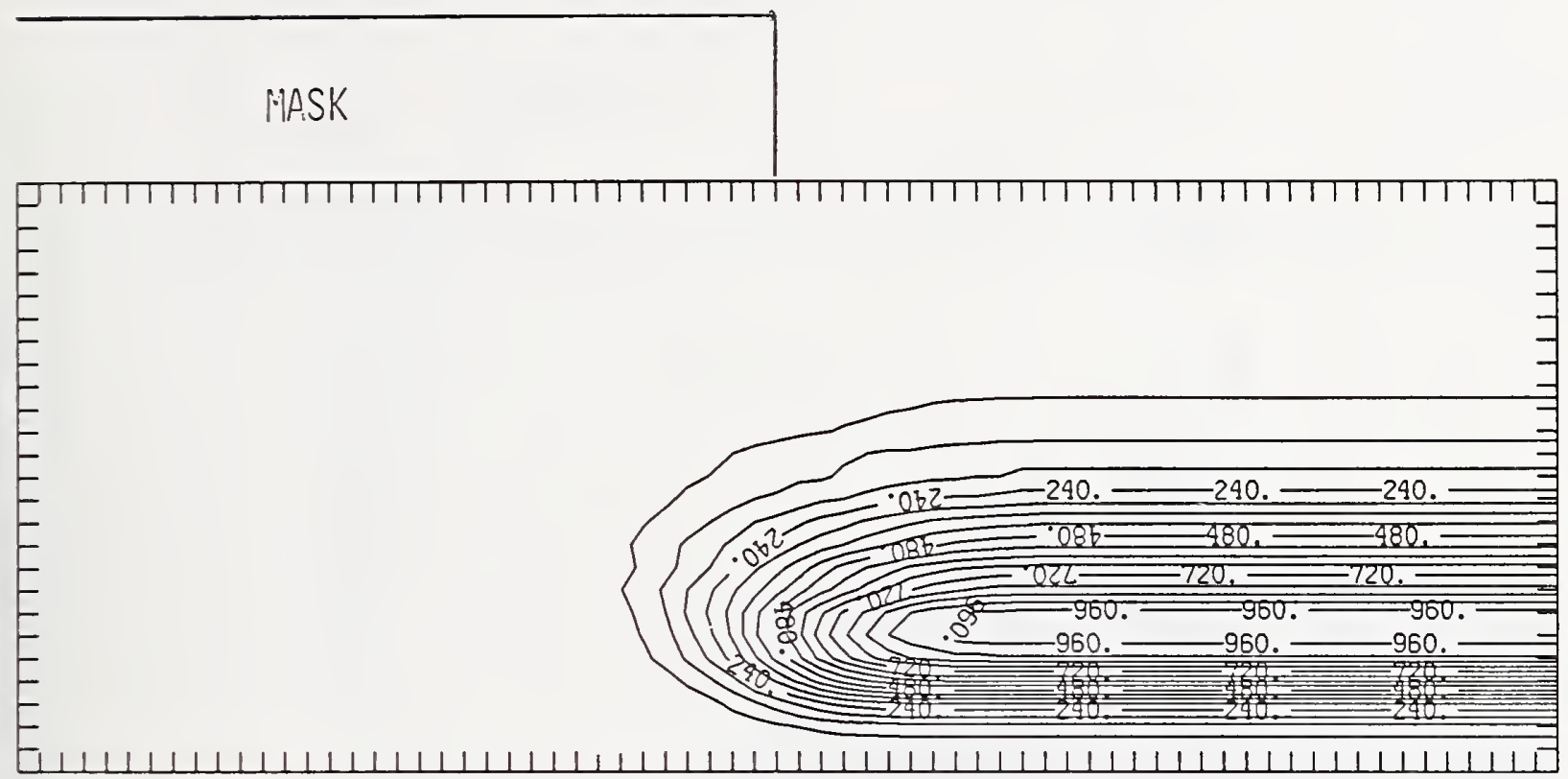

Figure C250-3 Two-dimensional distribution of $250 \mathrm{keV}$ carbon implanted past a mask edge. The length increment (distance between tick marks) is $0.04 \mu \mathrm{m}$ and the scale factor is 1000 .

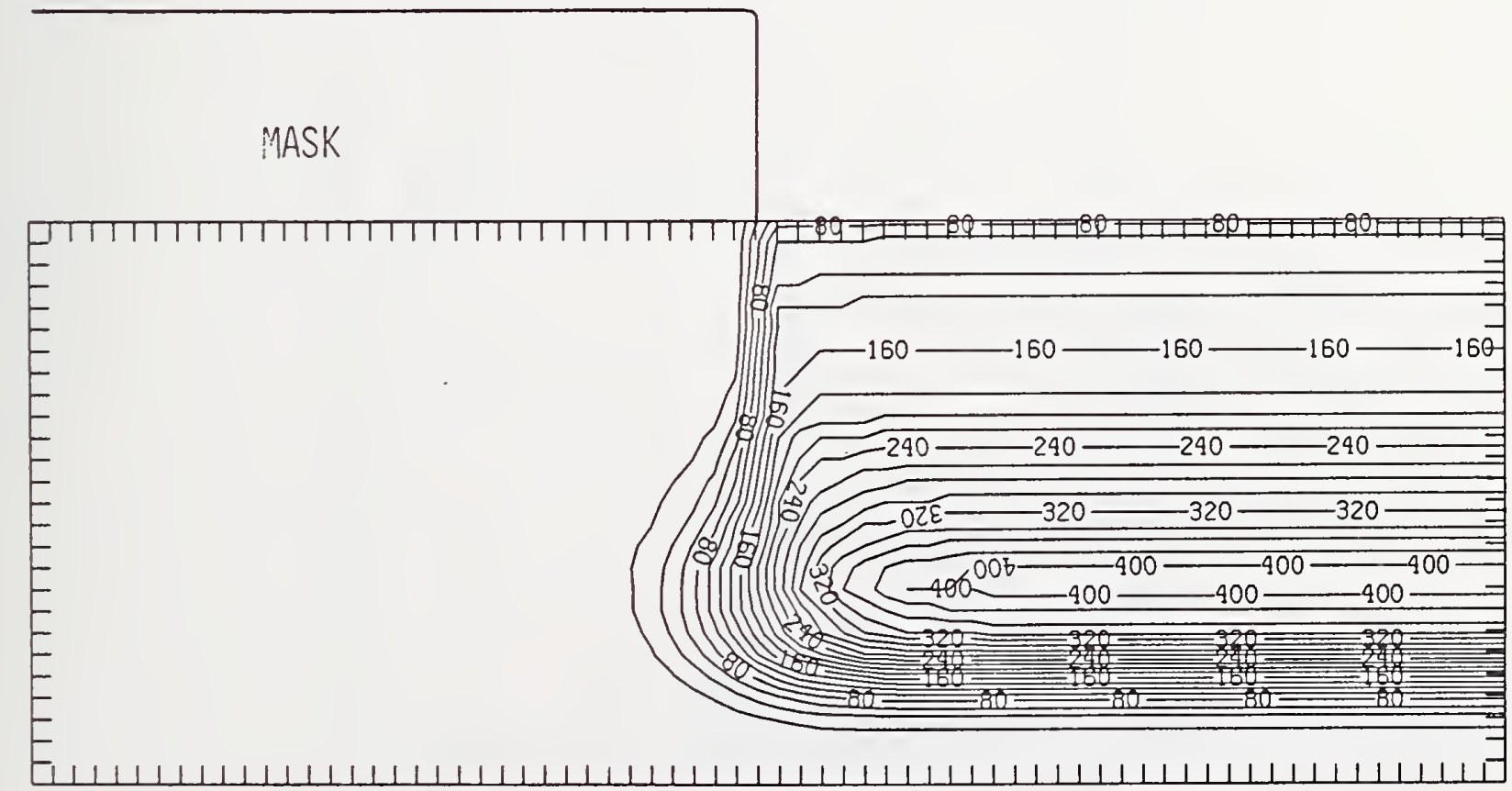

Figure C250-4 Two-dimensional distribution of Frenkel pairs created by $250 \mathrm{keV}$ carbon implanted past a mask edge. The length increment (distance between tick marks) is $0.04 \mu \mathrm{m}$ and the scale factor is 1. 


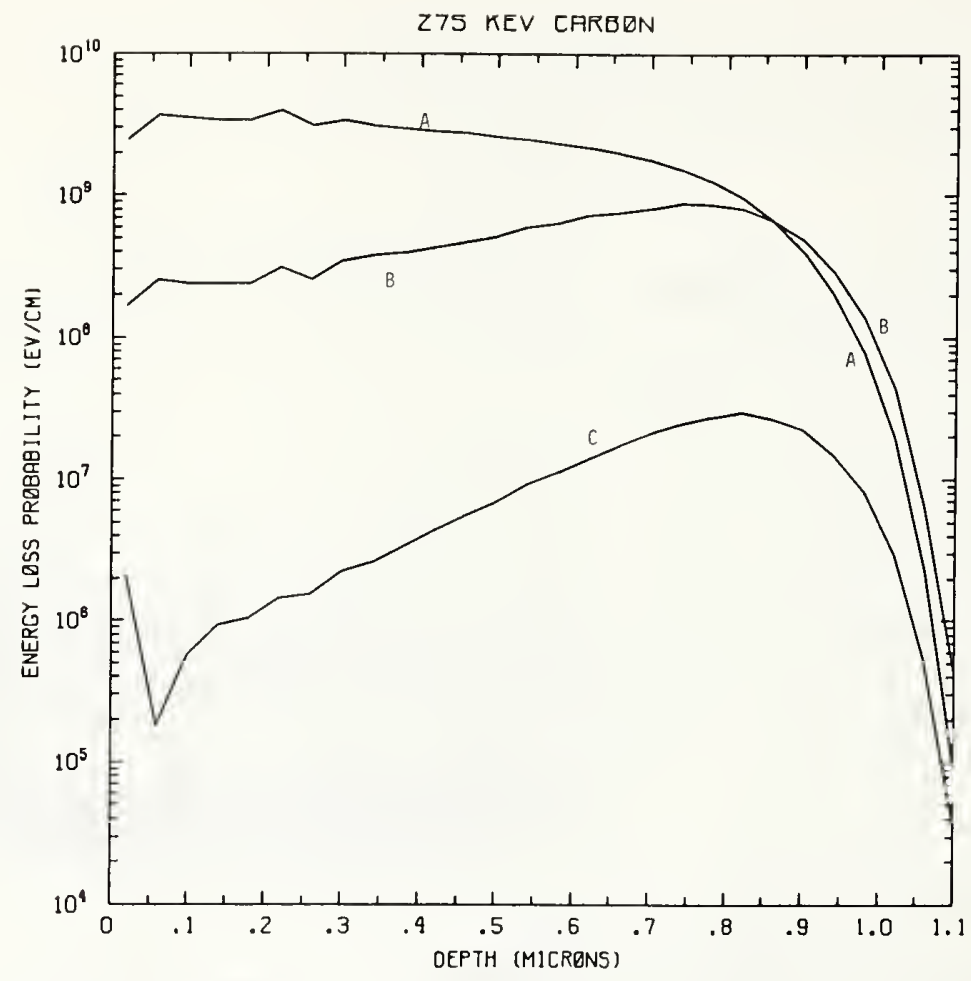

Figure C275-1 One-dimensional probability distributions for energy loss due to electronic excitation (A), displacement damage(B), and phonon excitation (C) for $275 \mathrm{keV}$ carbon normally incident on a silicon target.

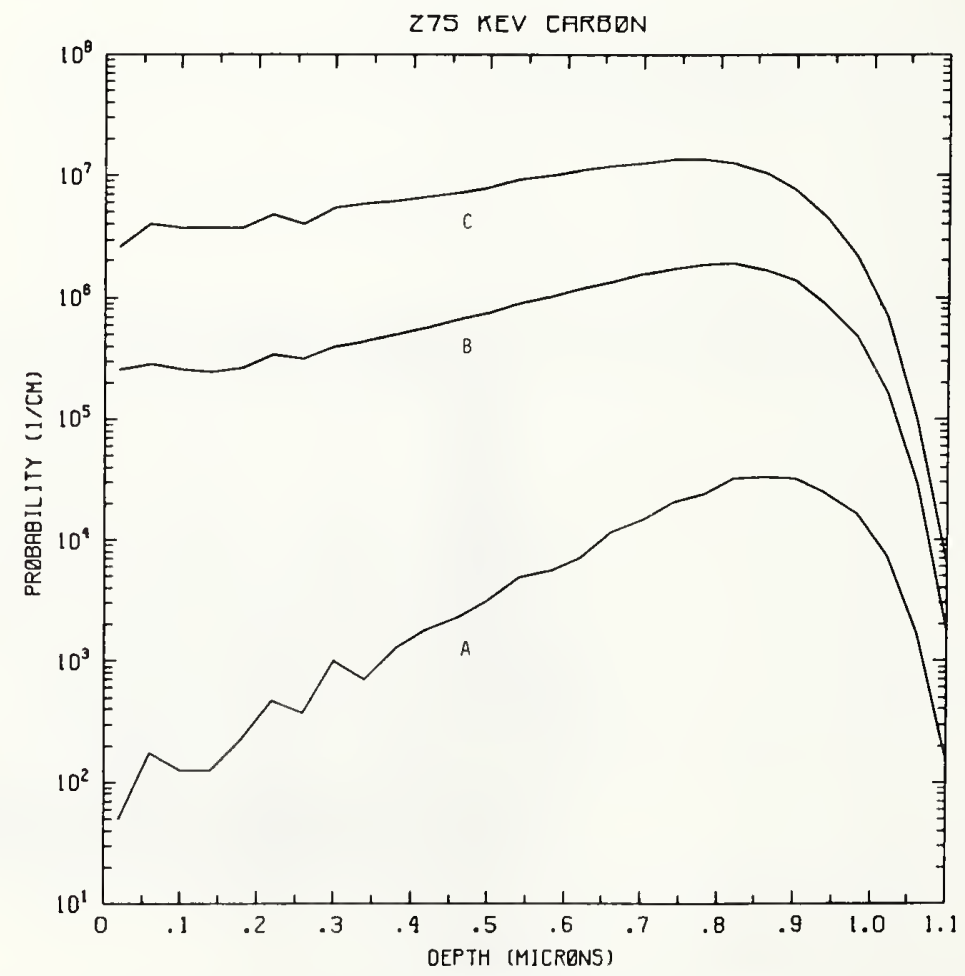

Figure C275-2 One-dimensional probability distributions for implanted carbon (A), primary silicon displacements (B), and Frenkel pairs (C) for $275 \mathrm{keV}$ carbon normally incident on a silicon target. 


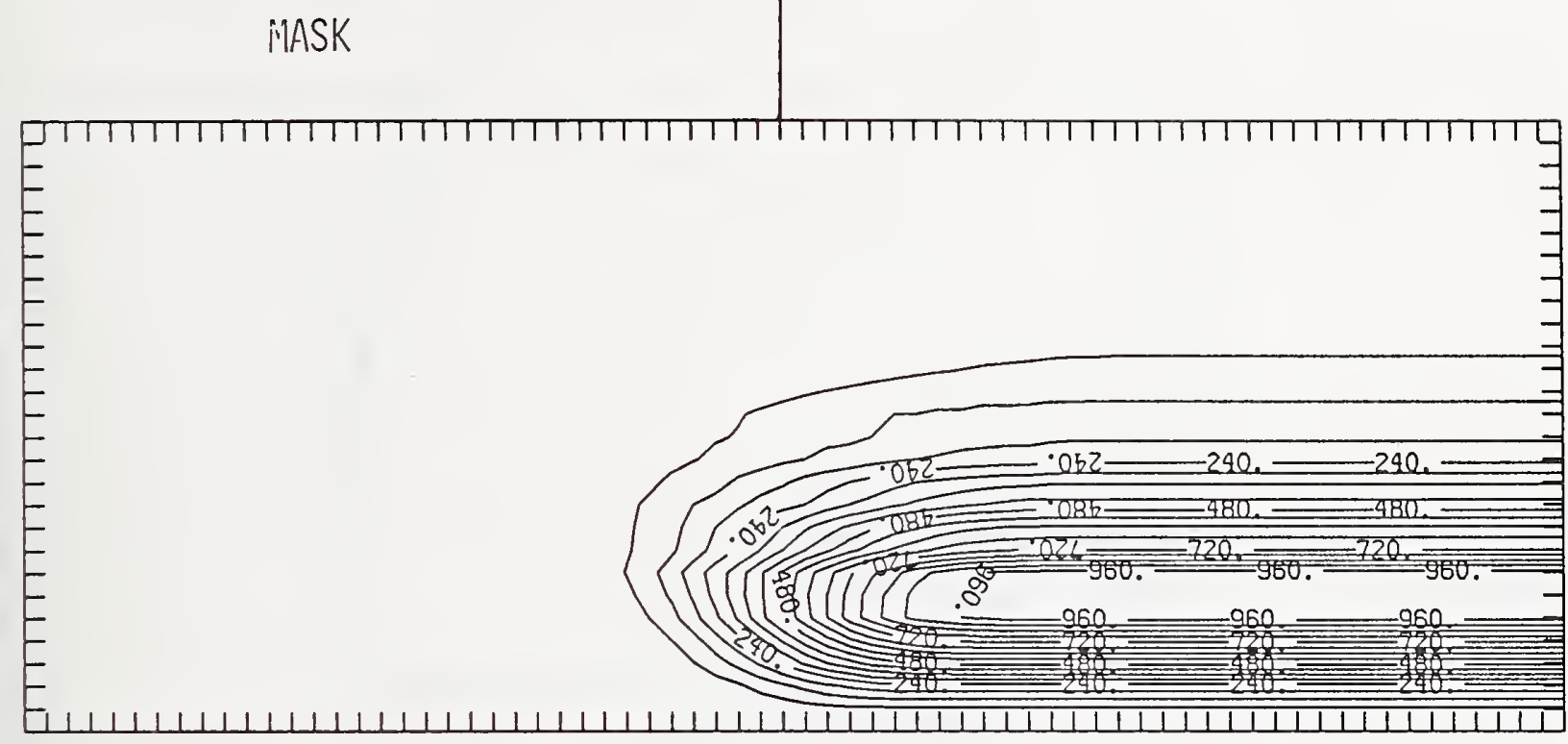

Figure C275-3 Two-dimensional distribution of $275 \mathrm{keV}$ carbon implanted past a mask edge. The length increment (distance between tick marks) is $0.04 \mu \mathrm{m}$ and the scale factor is 1000.

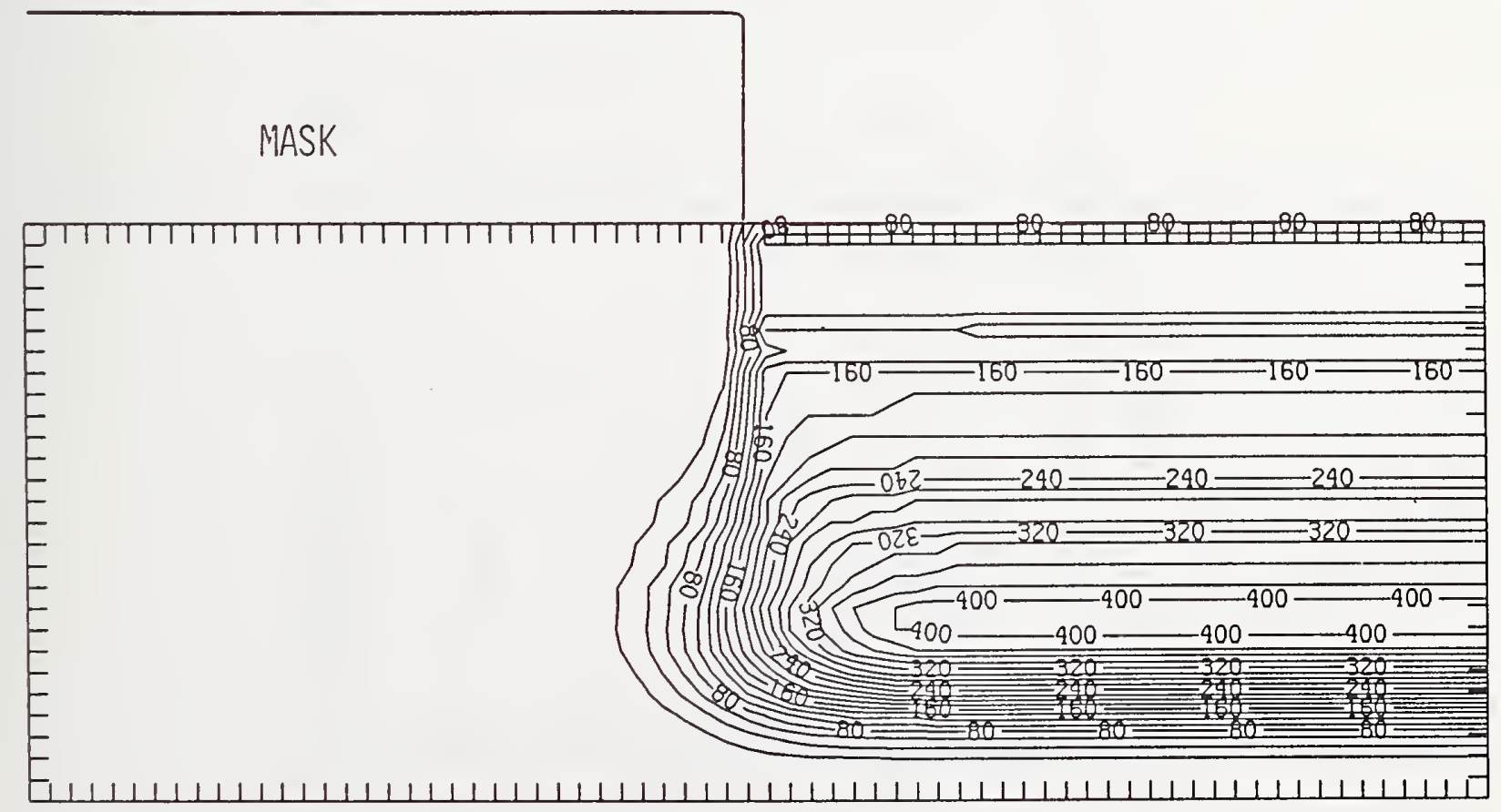

Figure C275-4 Two-dimensional distribution of Frenkel pairs created by $275 \mathrm{keV}$ carbon implanted past a mask edge. The length increment (distance between tick marks) is $0.04 \mu \mathrm{m}$ and the scale factor is 1 . 


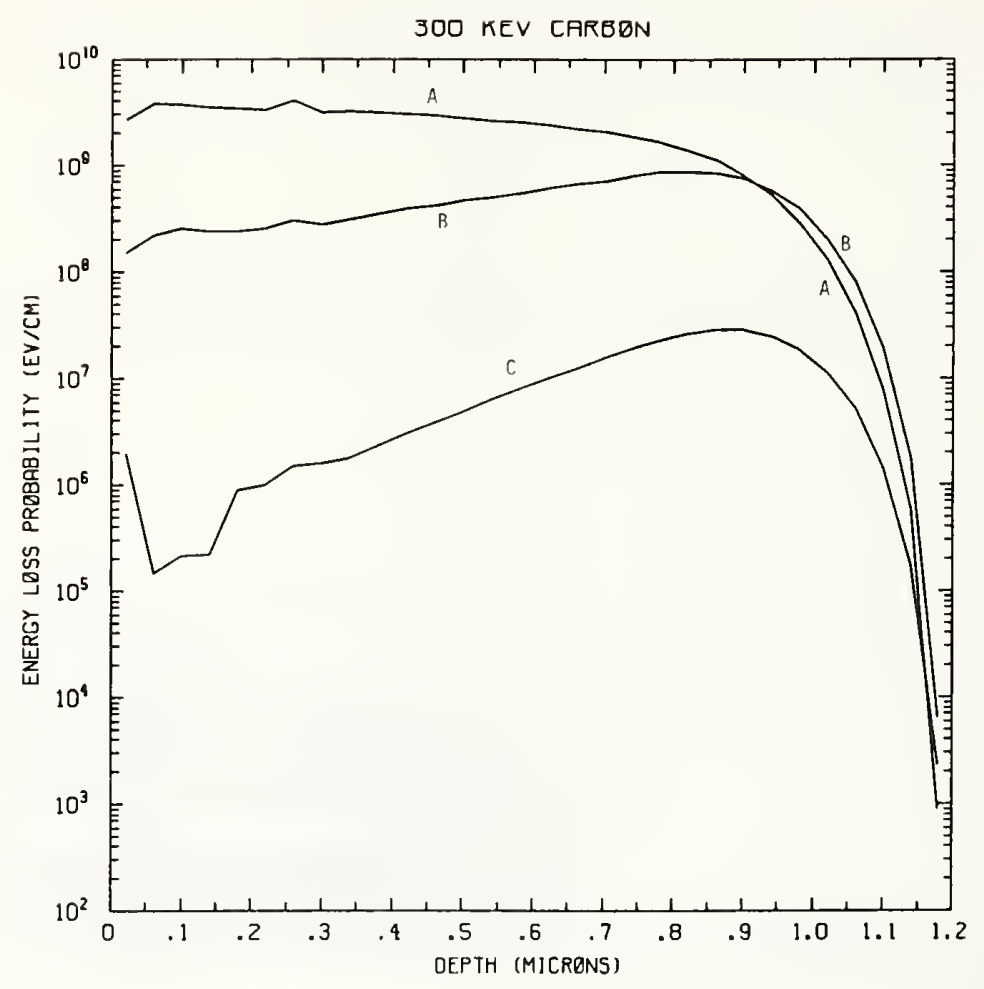

Figure C300-1 One-dimensional probability distributions for energy loss due to electronic excitation (A), displacement damage(B), and phonon excitation (C) for $300 \mathrm{keV}$ carbon normally incident on a silicon target.

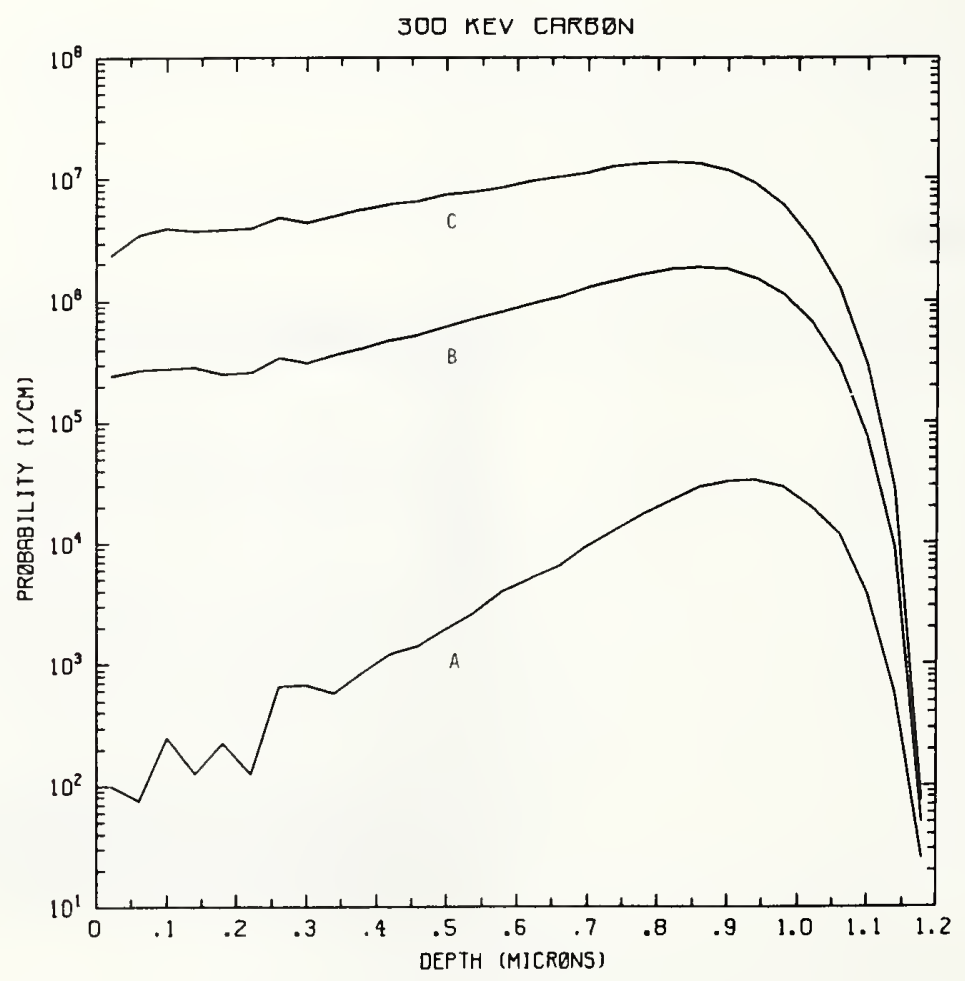

Figure C300-2 One-dimensional probability distributions for implanted carbon (A), primary silicon displacements (B), and Frenkel pairs (C) for $300 \mathrm{keV}$ carbon normally incident on a silicon target. 


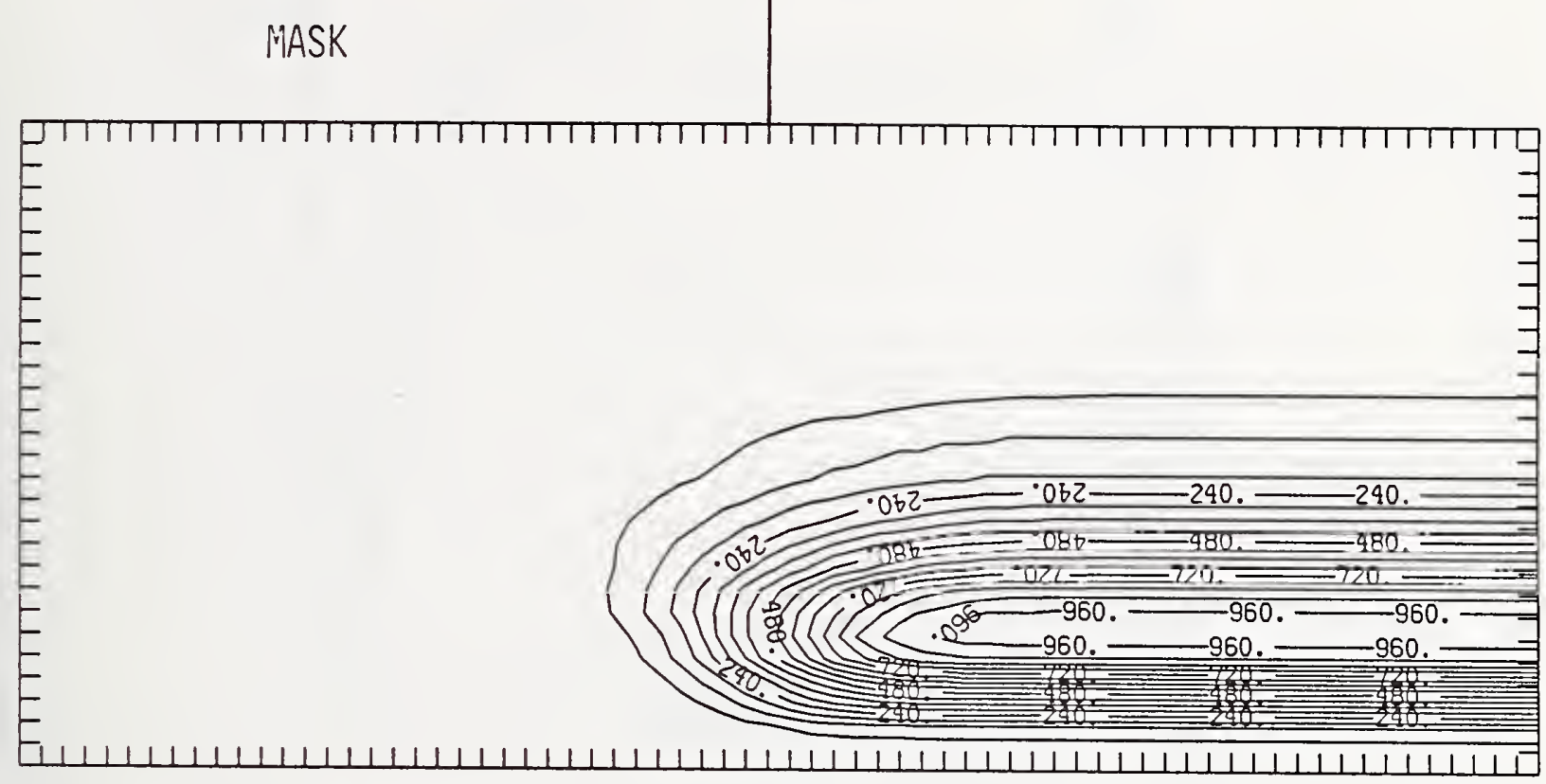

Figure C300-3 Two-dimensional distribution of $300 \mathrm{keV}$ carbon implanted past a mask edge. The length increment (distance between tick marks) is $0.04 \mu \mathrm{m}$ and the scale factor is 1000 .

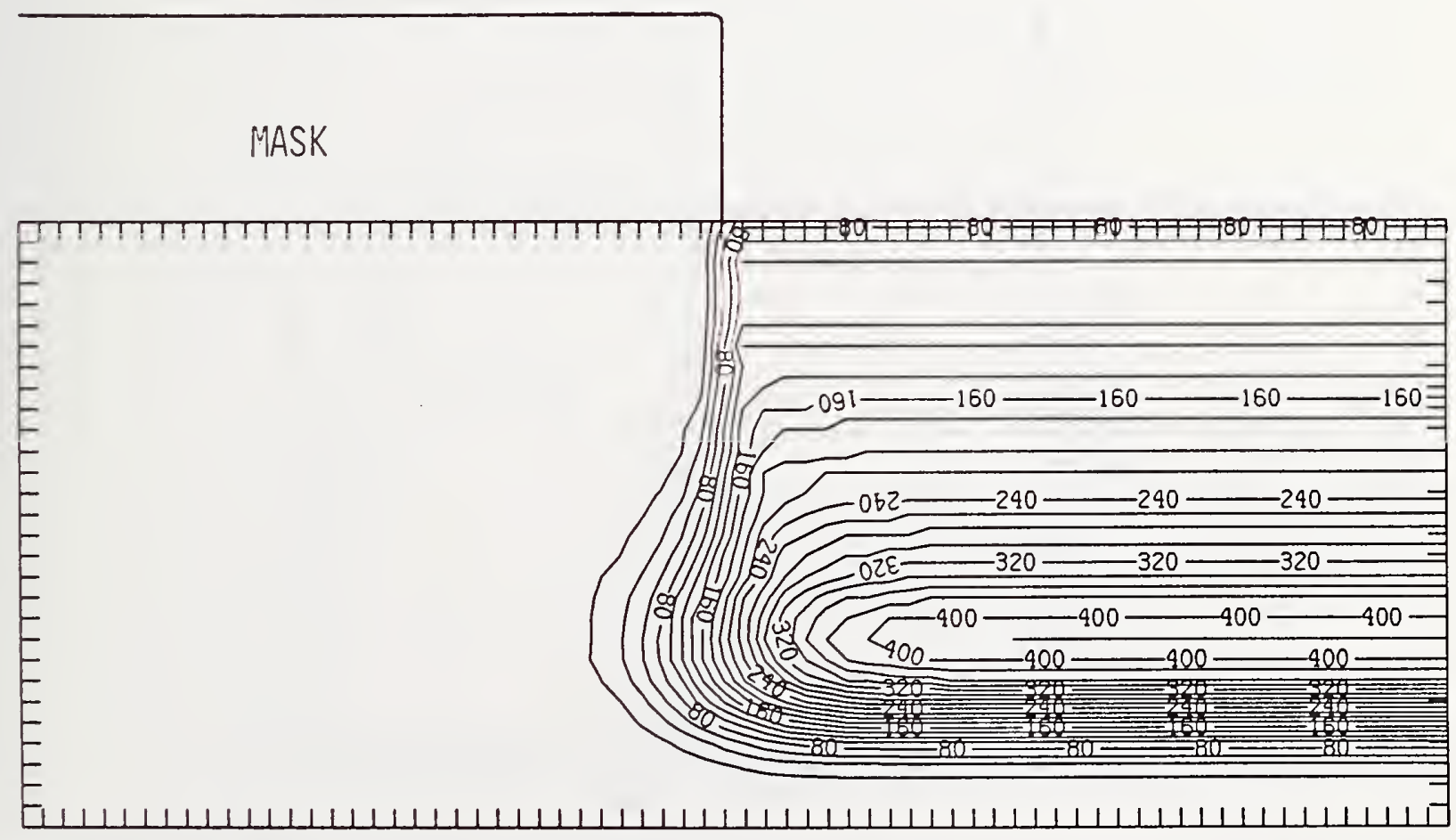

Figure C300-4 Two-dimensional distribution of Frenkel pairs created by $300 \mathrm{keV}$ carbon implanted past a mask edge. The length increment (distance between tick marks) is $0.04 \mu \mathrm{m}$ and the scale factor is 1 . 


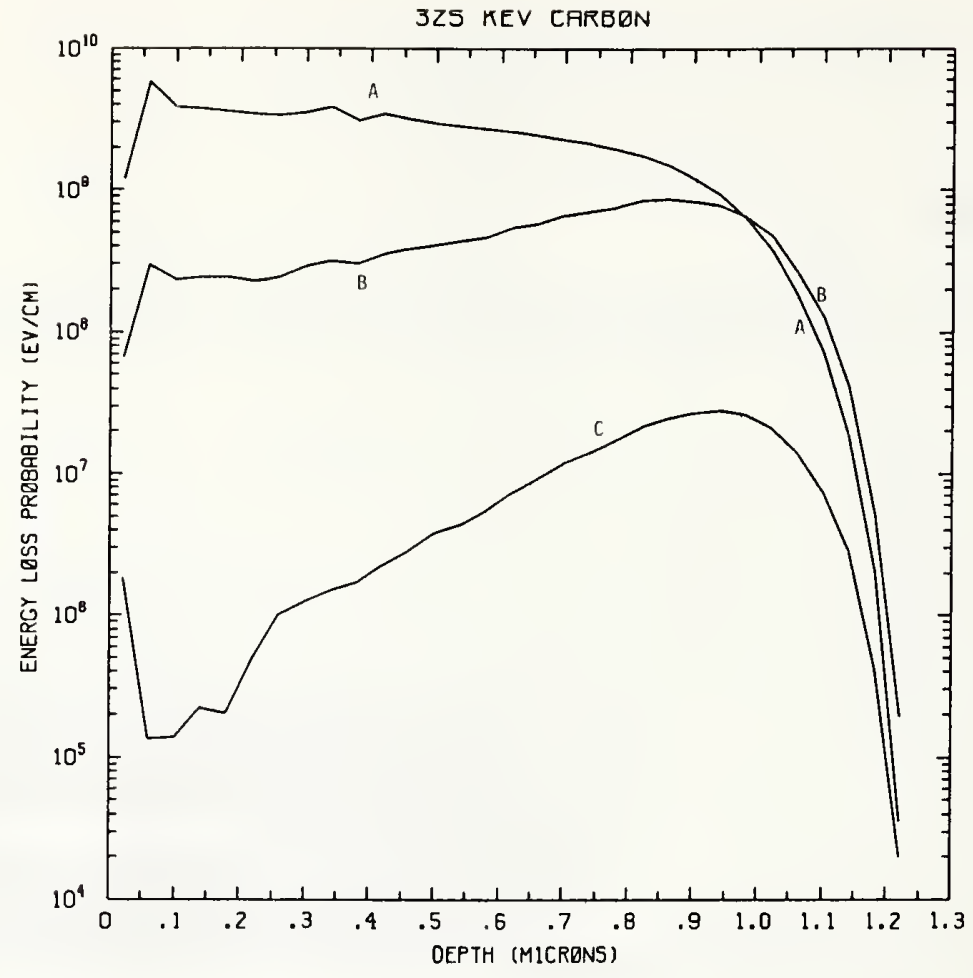

Figure C325-1 One-dimensional probability distributions for energy loss due to electronic excitation (A), displacement damage(B), and phonon excitation (C) for $325 \mathrm{keV}$ carbon normally incident on a silicon target.

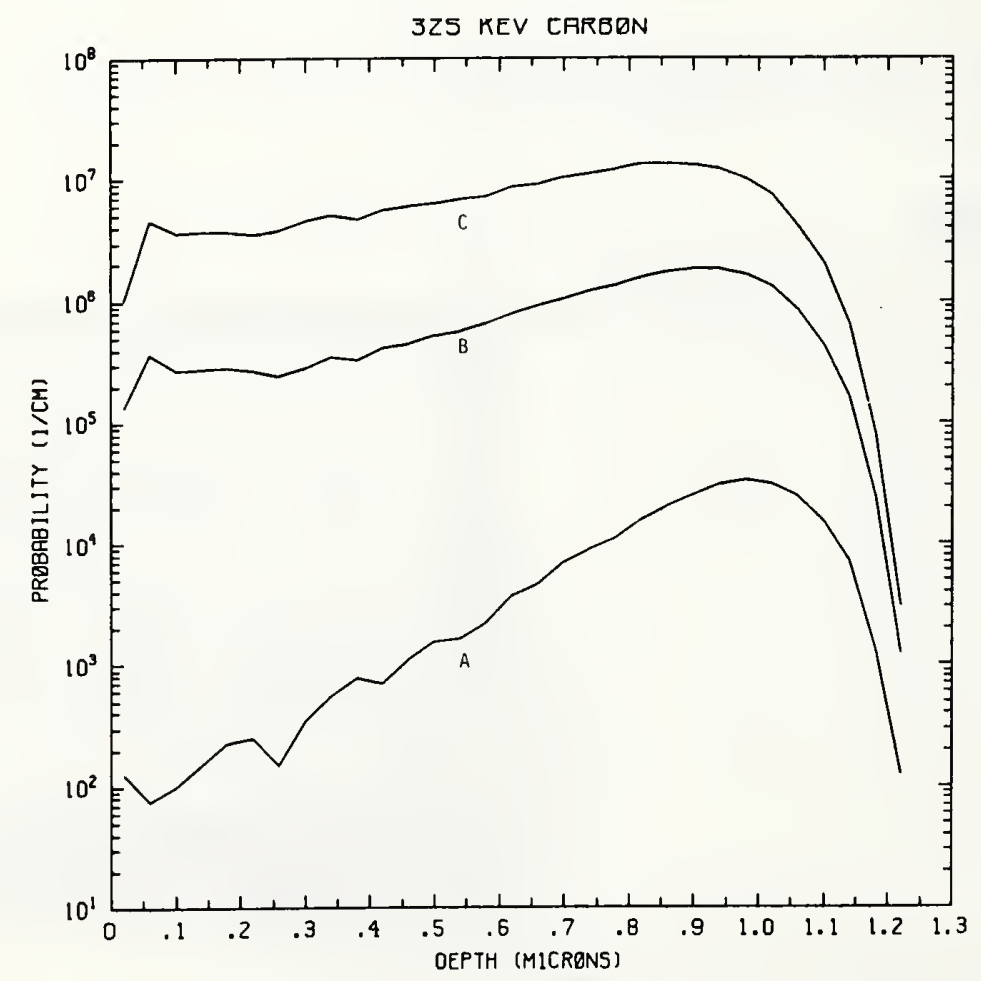

Figure C325-2 One-dimensional probability distributions for implanted carbon (A), primary silicon displacements (B), and Frenkel pairs (C) for $325 \mathrm{keV}$ carbon normally incident on a silicon target. 


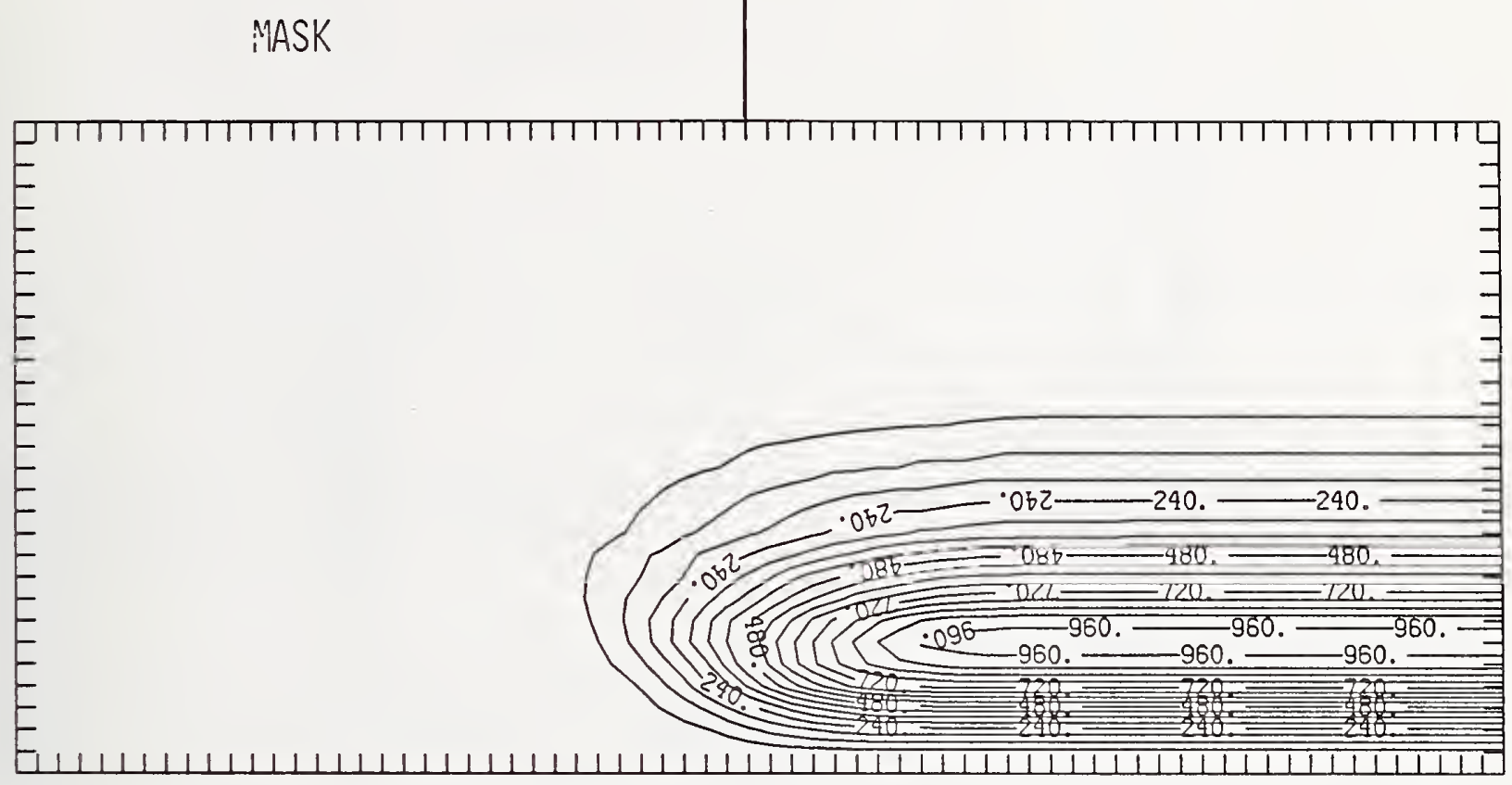

Figure C325-3 Two-dimensional distribution of $325 \mathrm{keV}$ carbon implanted past a mask edge. The length increment (distance between tick marks) is $0.04 \mu \mathrm{m}$ and the scale factor is 1000 .

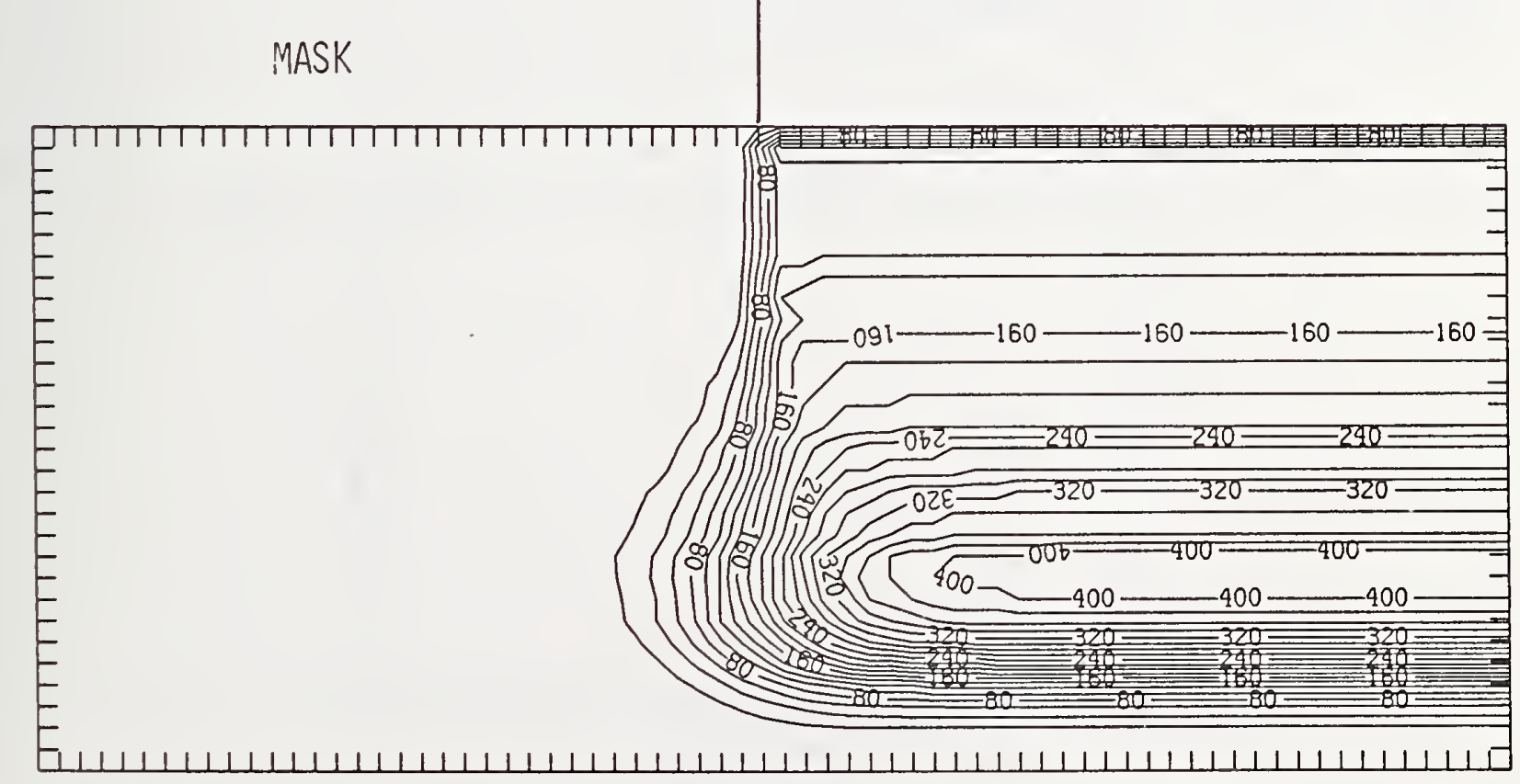

Figure C325-4 Two-dimensional distribution of Frenkel pairs created by $325 \mathrm{keV}$ carbon implanted past a mask edge. The length increment (distance between tick marks) is $0.04 \mu \mathrm{m}$ and the scale factor is 1 . 


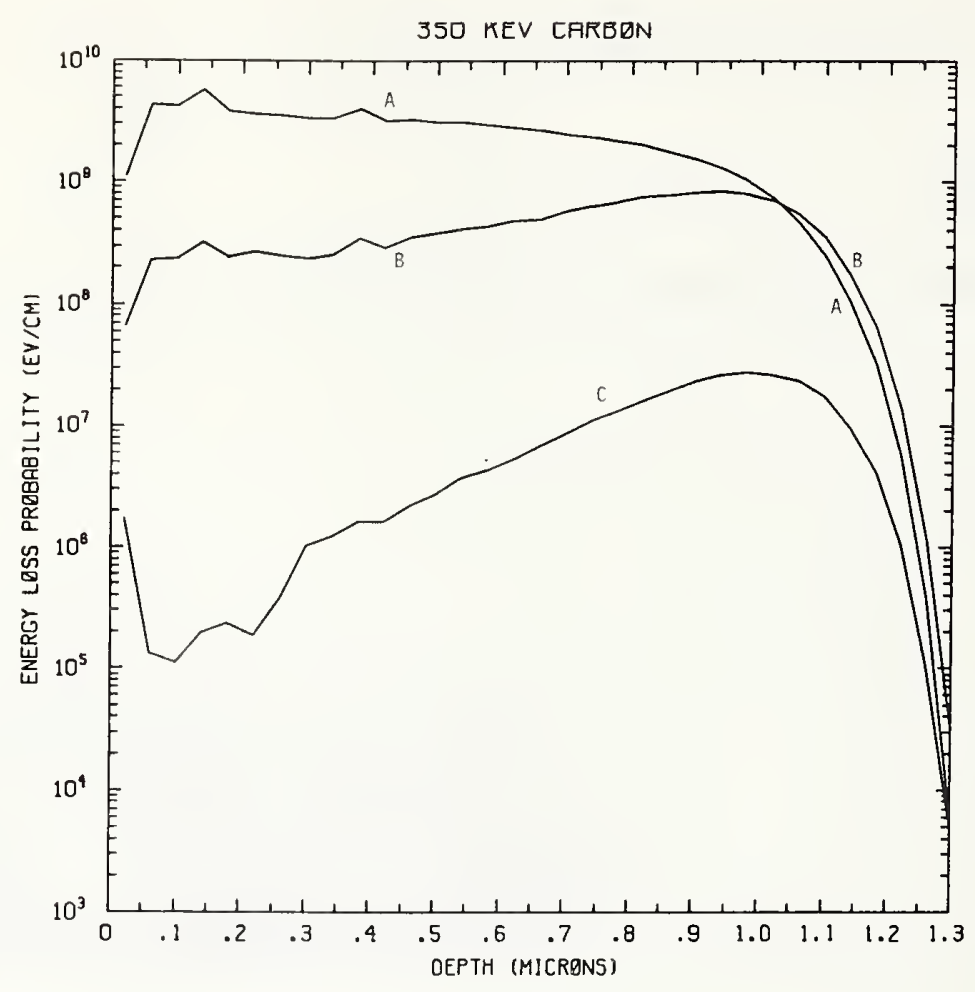

Figure C350-1 One-dimensional probability distributions for energy loss due to electronic excitation (A), displacement damage(B), and phonon excitation (C) for $350 \mathrm{keV}$ carbon normally incident on a silicon target.

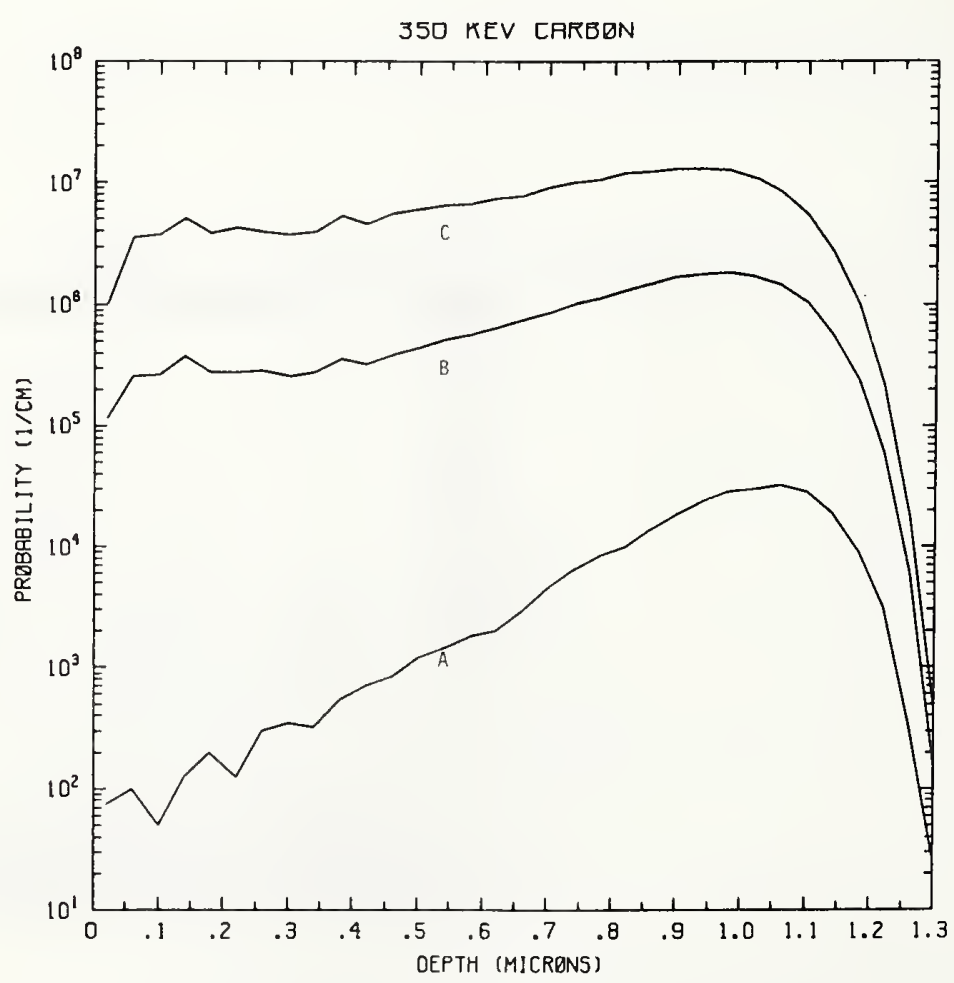

Figure C350-2 One-dimensional probability distributions for implanted carbon (A), primary silicon displacements (B), and Frenkel pairs (C) for $350 \mathrm{keV}$ carbon normally incident on a silicon target. 


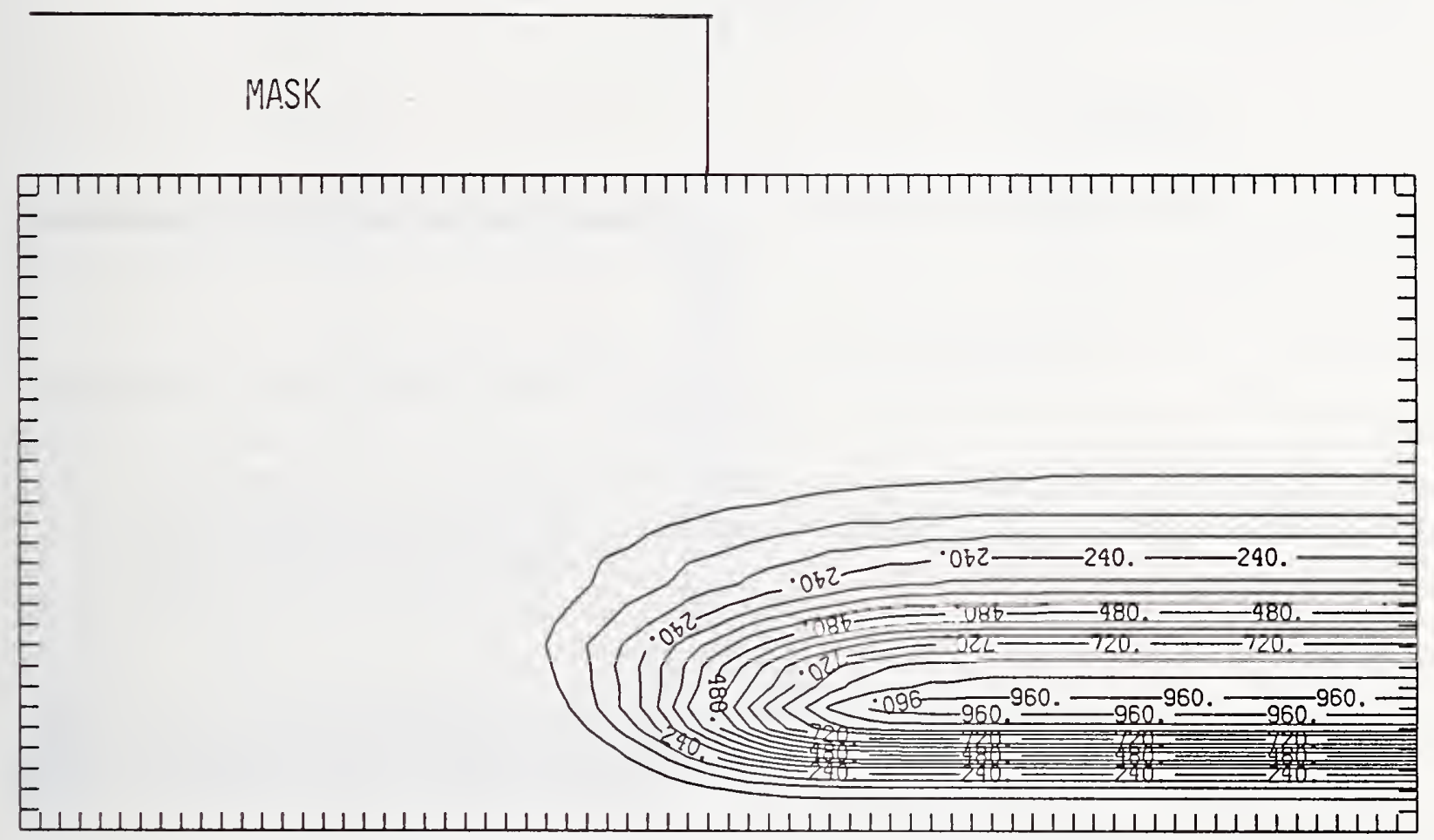

Figure C350-3 Two-dimensional distribution of $350 \mathrm{keV}$ carbon implanted past a mask edge. The length increment (distance between tick marks) is $0.04 \mu \mathrm{m}$ and the scale factor is 1000. 


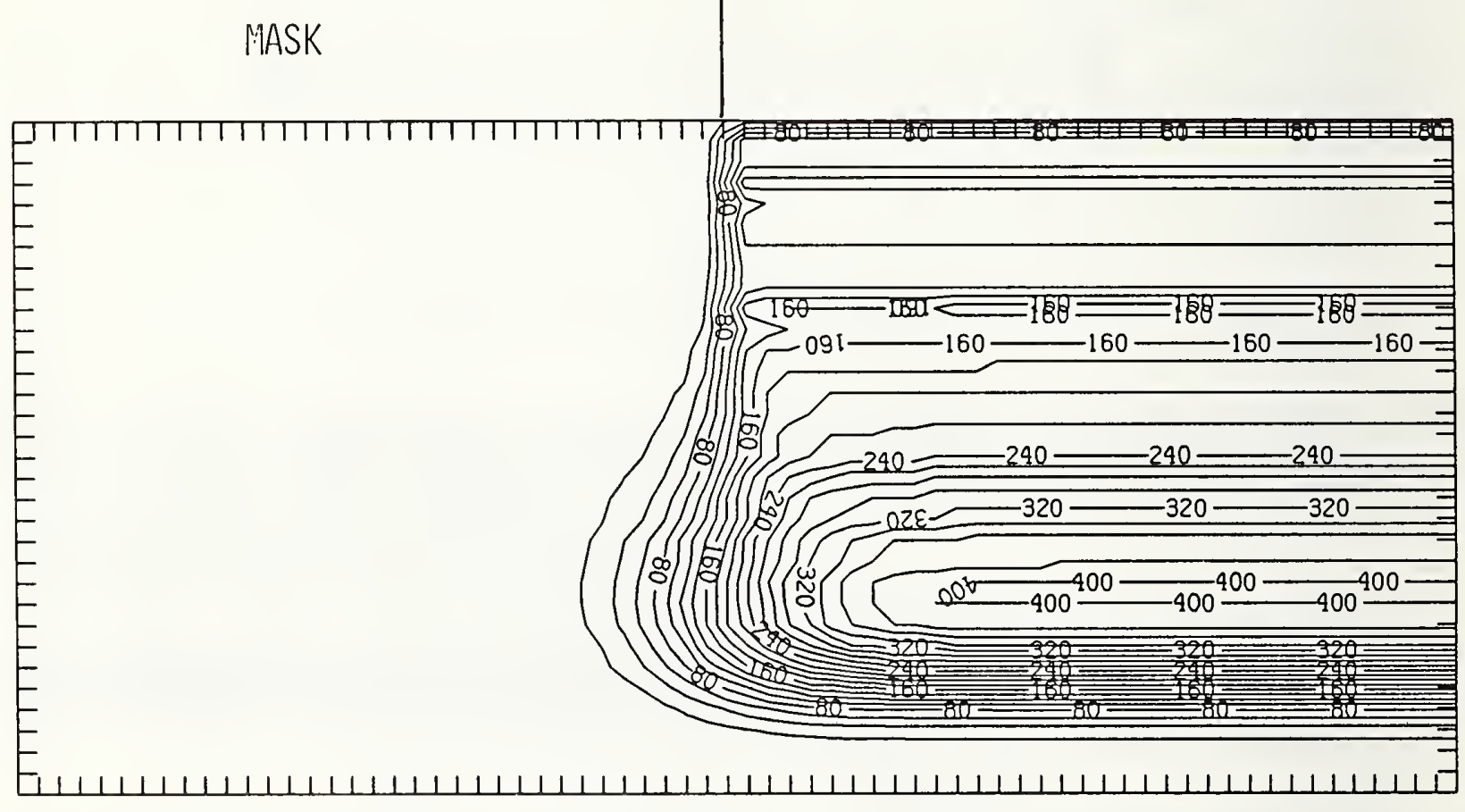

Figure C350-4 Two-dimensional distribution of Frenkel pairs created by $350 \mathrm{keV}$ carbon implanted past a mask edge. The length increment (distance between tick marks) is $0.04 \mu \mathrm{m}$ and the scale factor is 1 . 


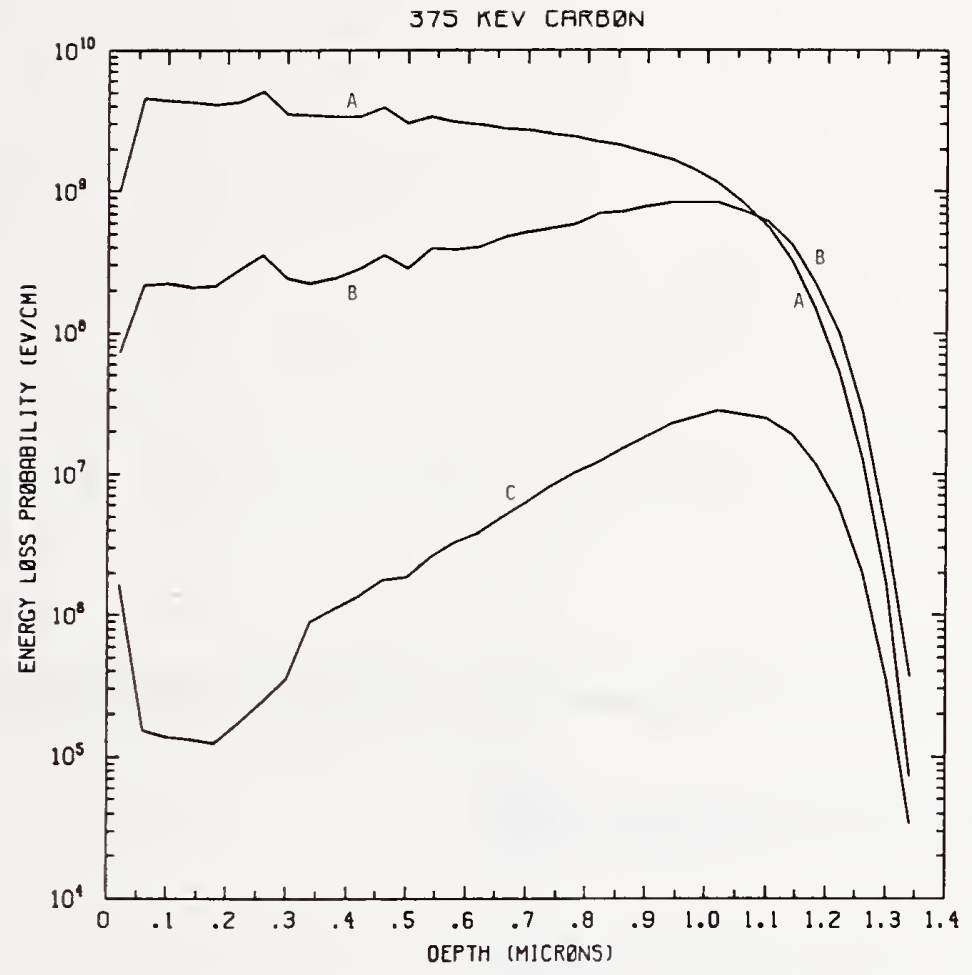

Figure C375-1 One-dimensional probability distributions for energy loss due to electronic excitation (A), displacement damage(B), and phonon excitation (C) for $375 \mathrm{keV}$ carbon normally incident on a silicon target.

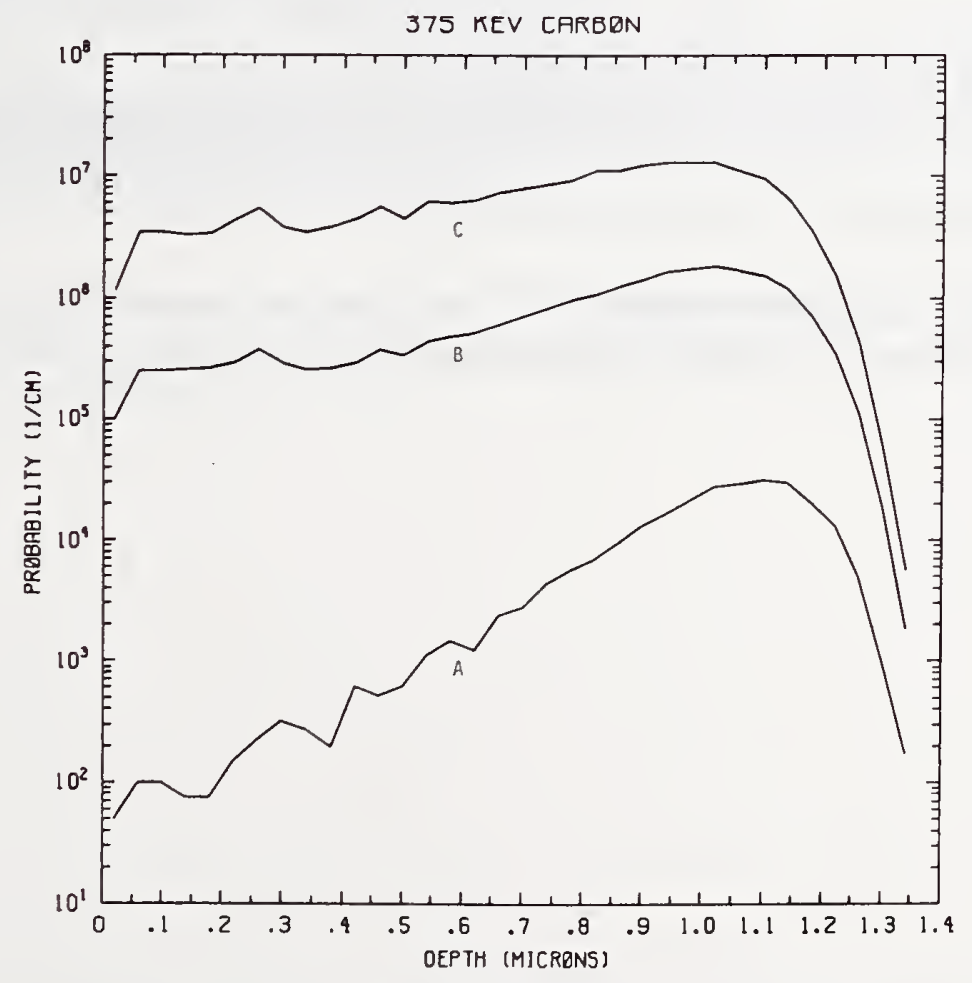

Figure C375-2 One-dimensional probability distributions for implanted carbon (A), primary silicon displacements (B), and Frenkel pairs (C) for $375 \mathrm{keV}$ carbon normally incident on a silicon target. 


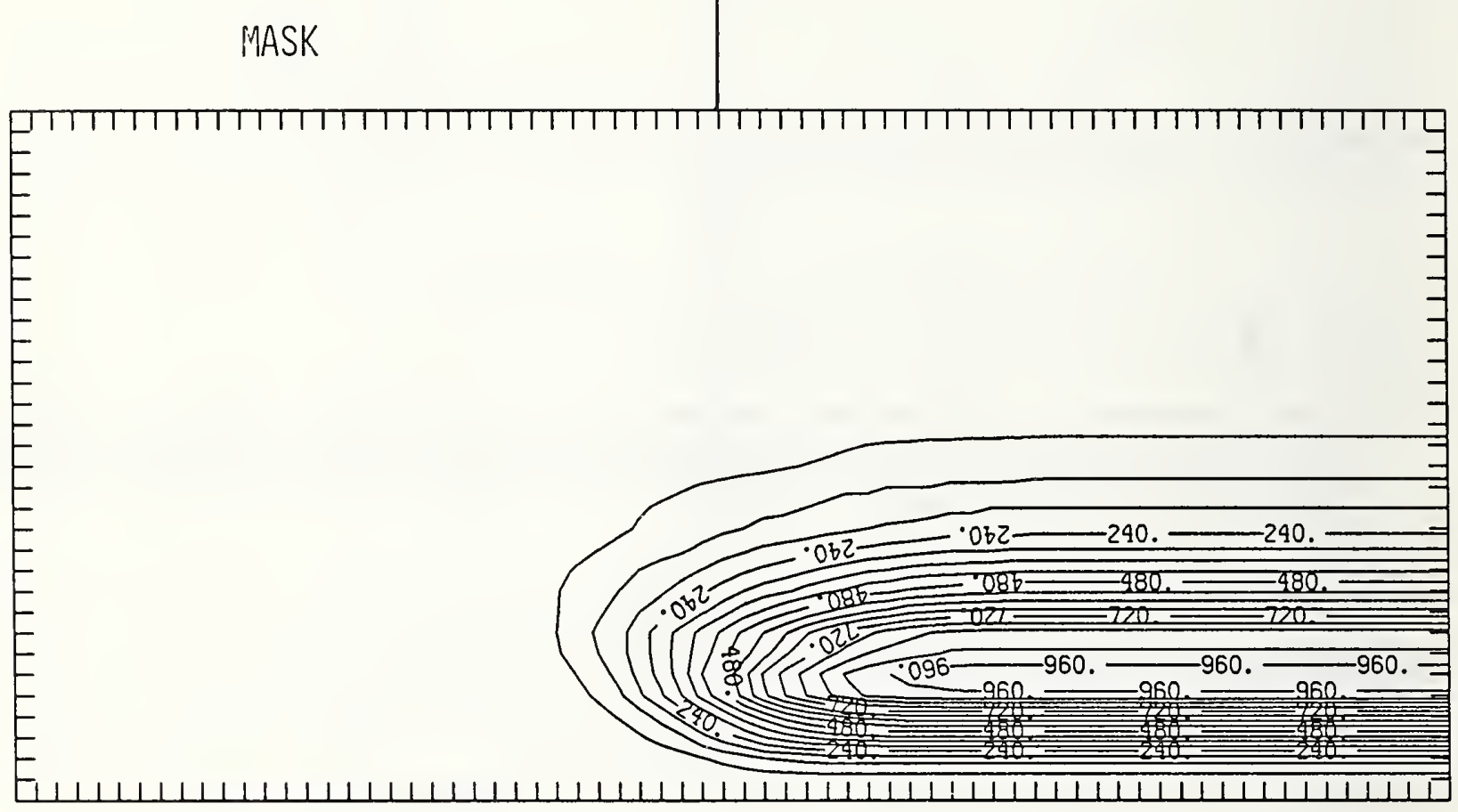

Figure C375-3 Two-dimensional distribution of $375 \mathrm{keV}$ carbon implanted past a mask edge. The length increment (distance between tick marks) is $0.04 \mu \mathrm{m}$ and the scale factor is 1000 . 


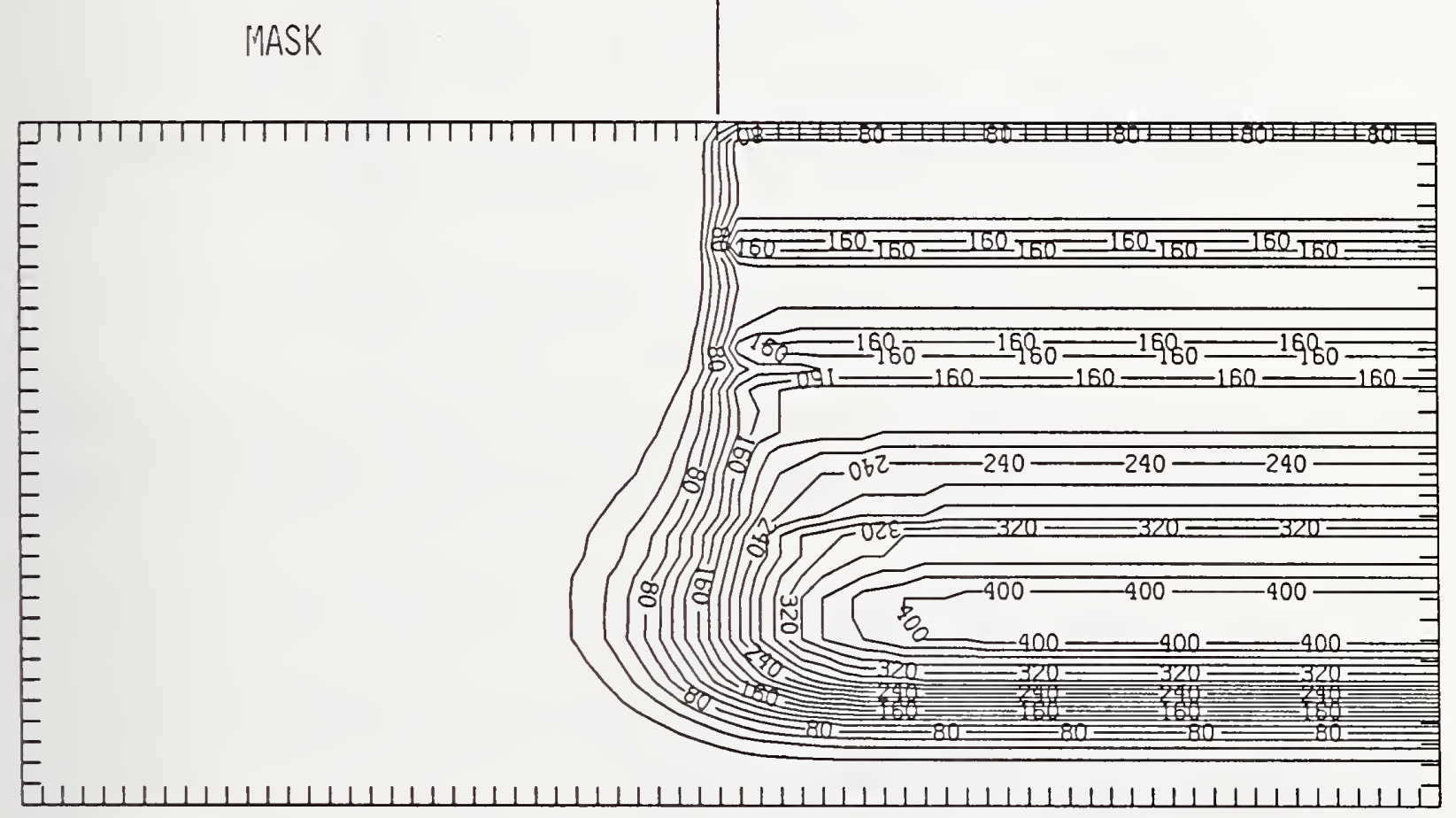

Figure C375-4 Two-dimensional distribution of Frenkel pairs created by $375 \mathrm{keV}$ carbon implanted past a mask edge. The length increment (distance between tick marks) is $0.04 \mu \mathrm{m}$ and the scale factor is 1 . 


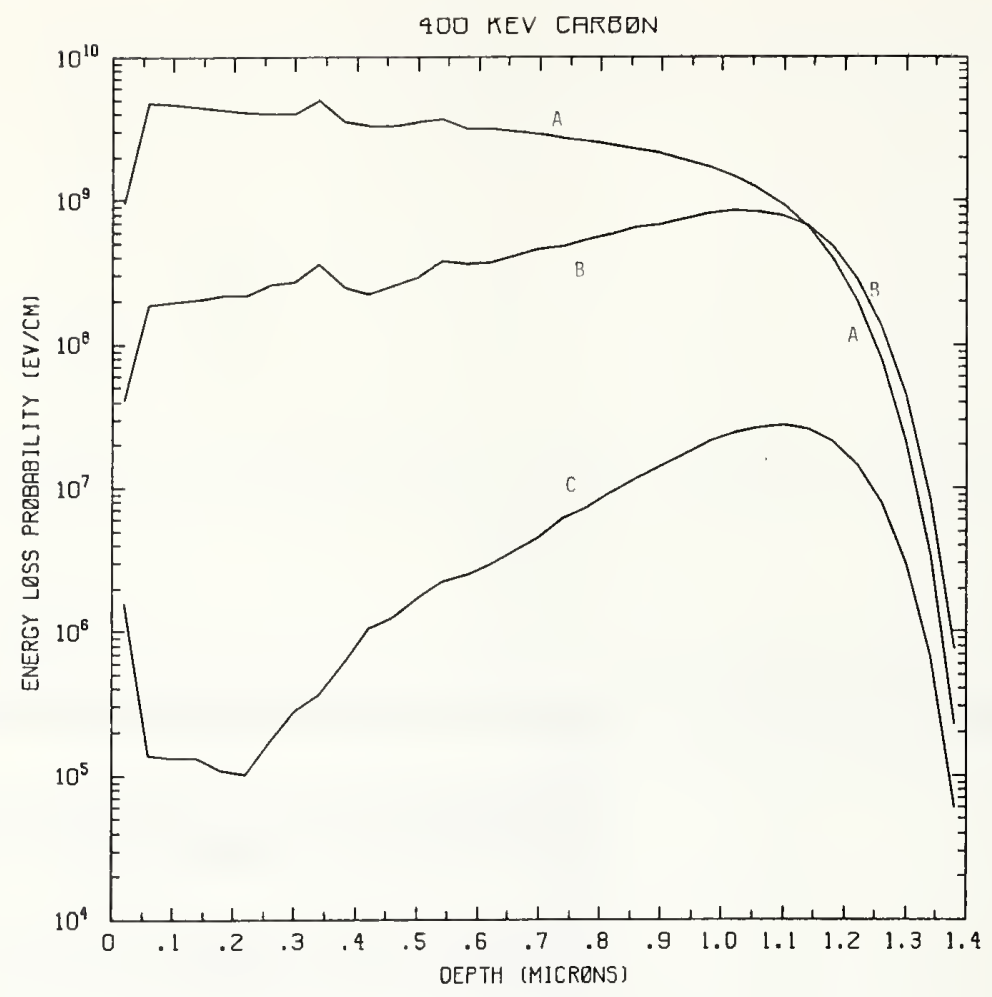

Figure C400-1 One-dimensional probability distributions for energy loss due to electronic excitation (A), displacement damage(B), and phonon excitation (C) for $400 \mathrm{keV}$ carbon normally incident on a silicon target.

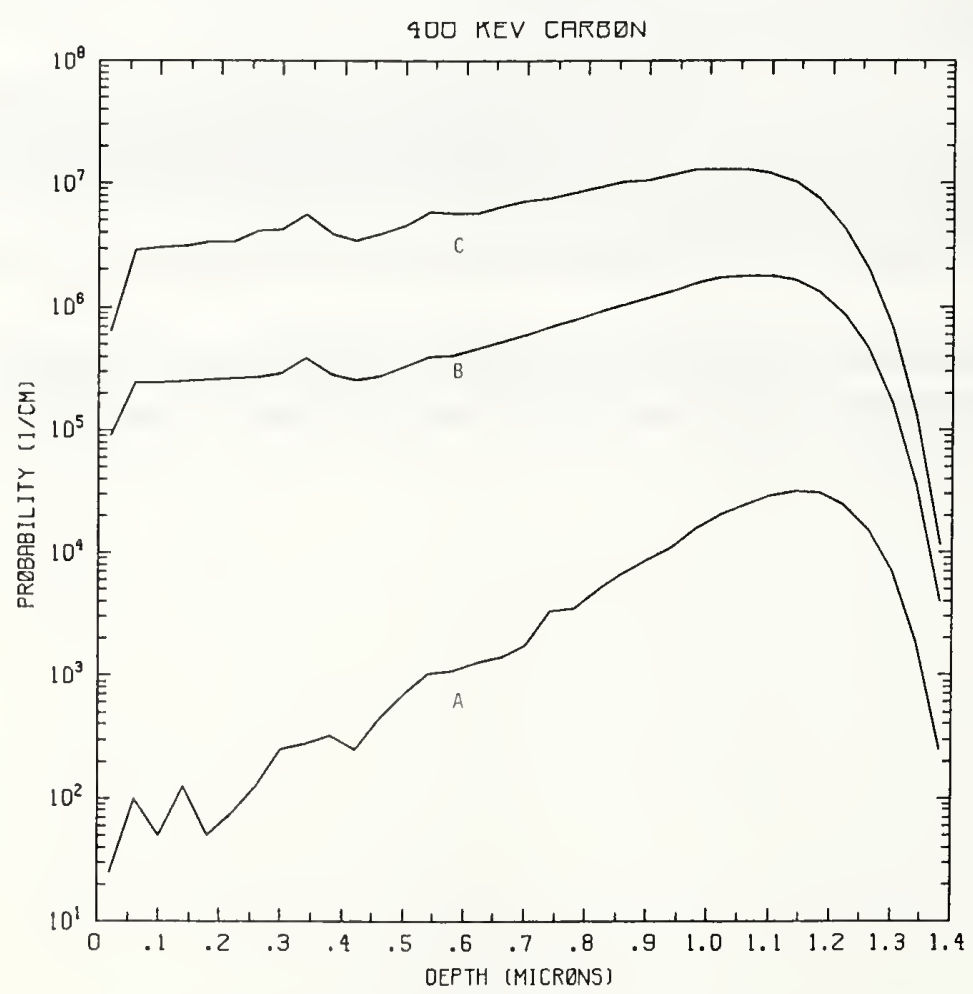

Figure C400-2 One-dimensional probability distributions for implanted carbon (A), primary silicon displacements (B), and Frenkel pairs (C) for $400 \mathrm{keV}$ carbon normally incident on a silicon target. 


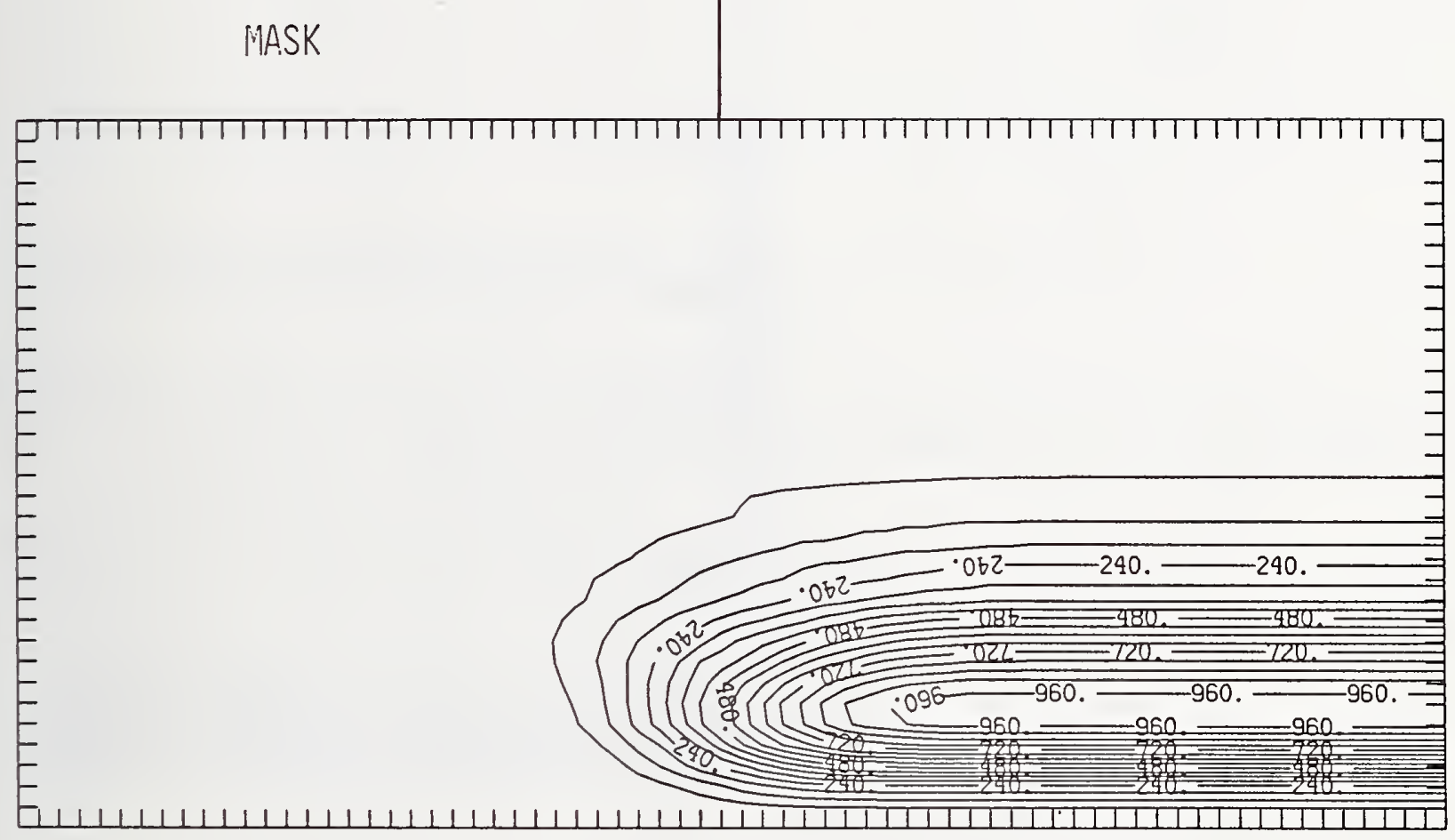

Figure C400-3 Two-dimensional distribution of $400 \mathrm{keV}$ carbon implanted past a mask edge. The length increment (distance between tick marks) is $0.04 \mu \mathrm{m}$ and the scale factor is 1000 . 


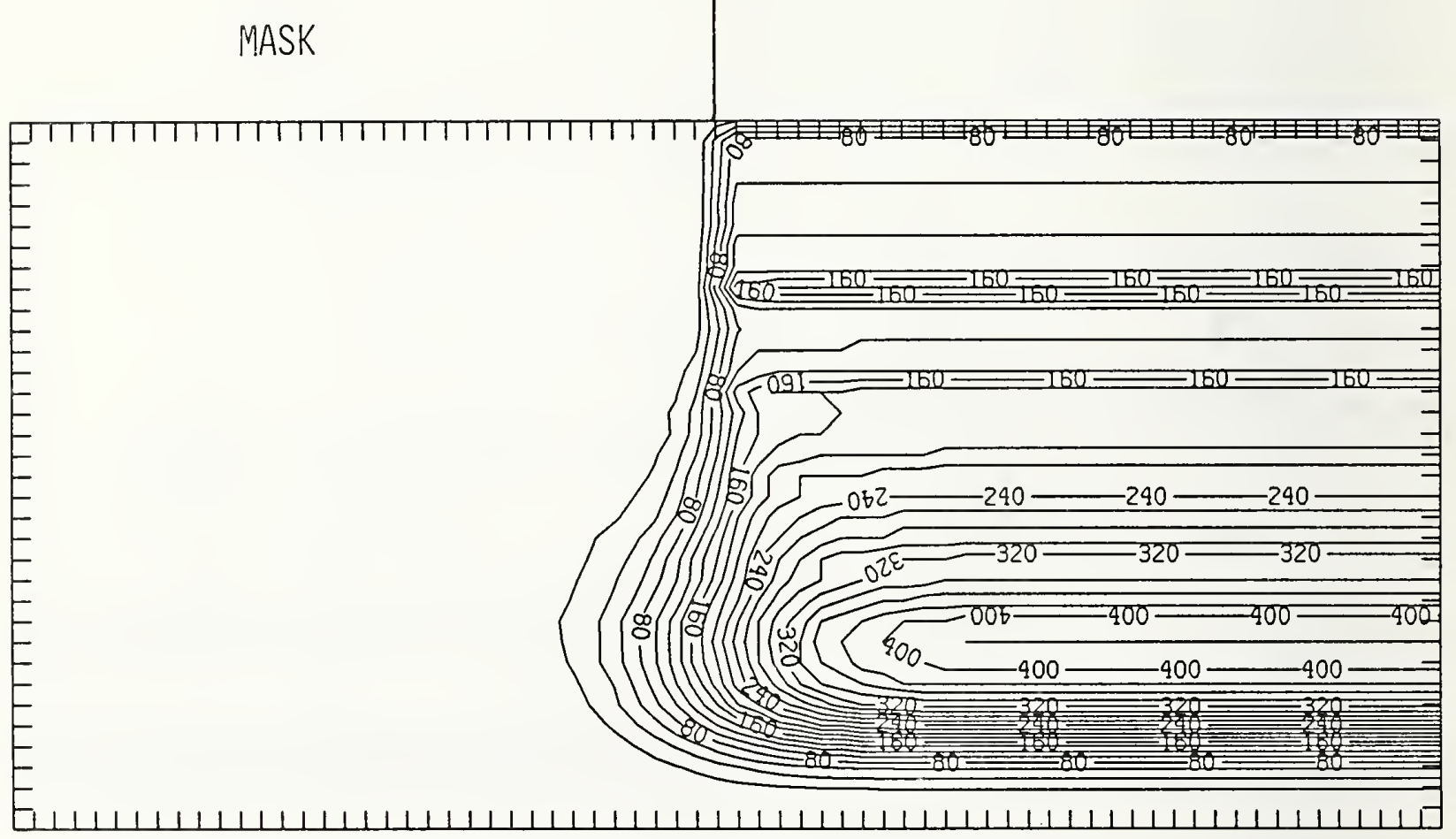

Figure C400-4 Two-dimensional distribution of Frenkel pairs created by $400 \mathrm{keV}$ carbon implanted past a mask edge. The length increment (distance between tick marks) is $0.04 \mu \mathrm{m}$ and the scale factor is 1 . 


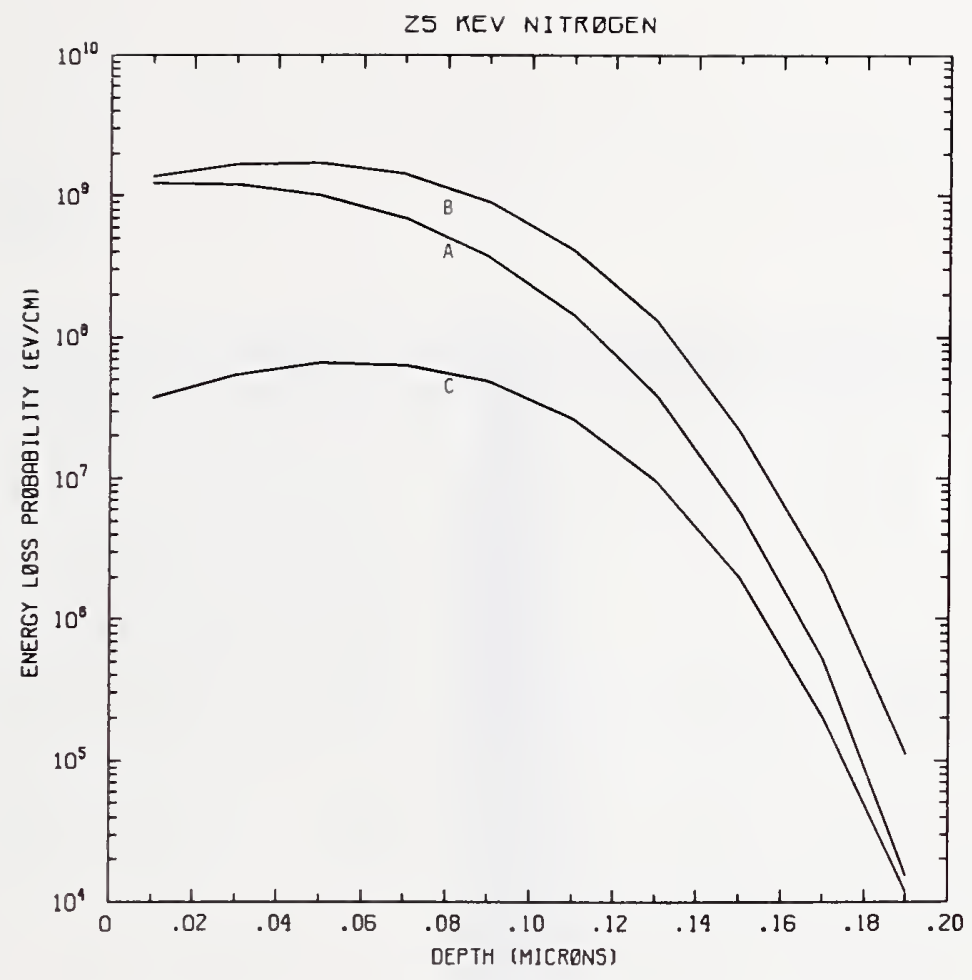

Figure N25-1 One-dimensional probability distributions for energy loss due to electronic excitation (A), displacement damage(B), and phonon excitation (C) for $25 \mathrm{keV}$ nitrogen normally incident on a silicon target.

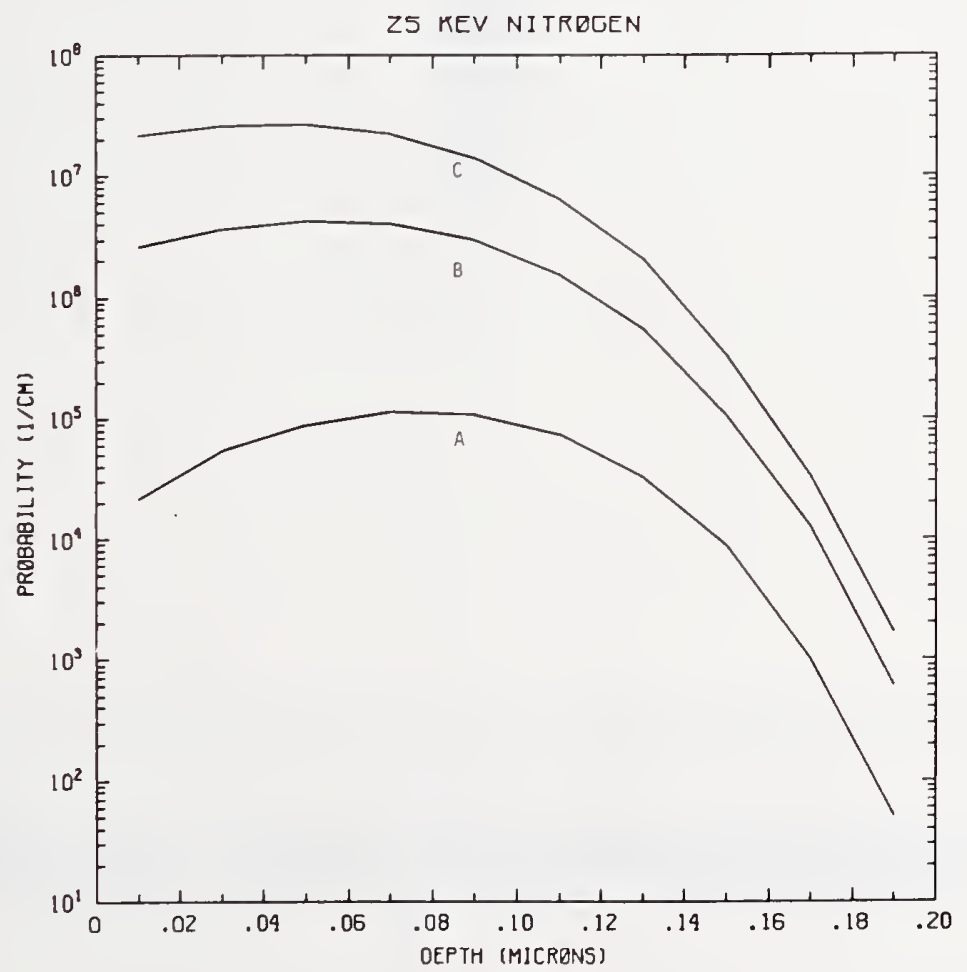

Figure N25-2 One-dimensional probability distributions for implanted nitrogen (A), primary silicon displacements (B), and Frenkel pairs (C) for $25 \mathrm{keV}$ nitrogen normally incident on a silicon target. 


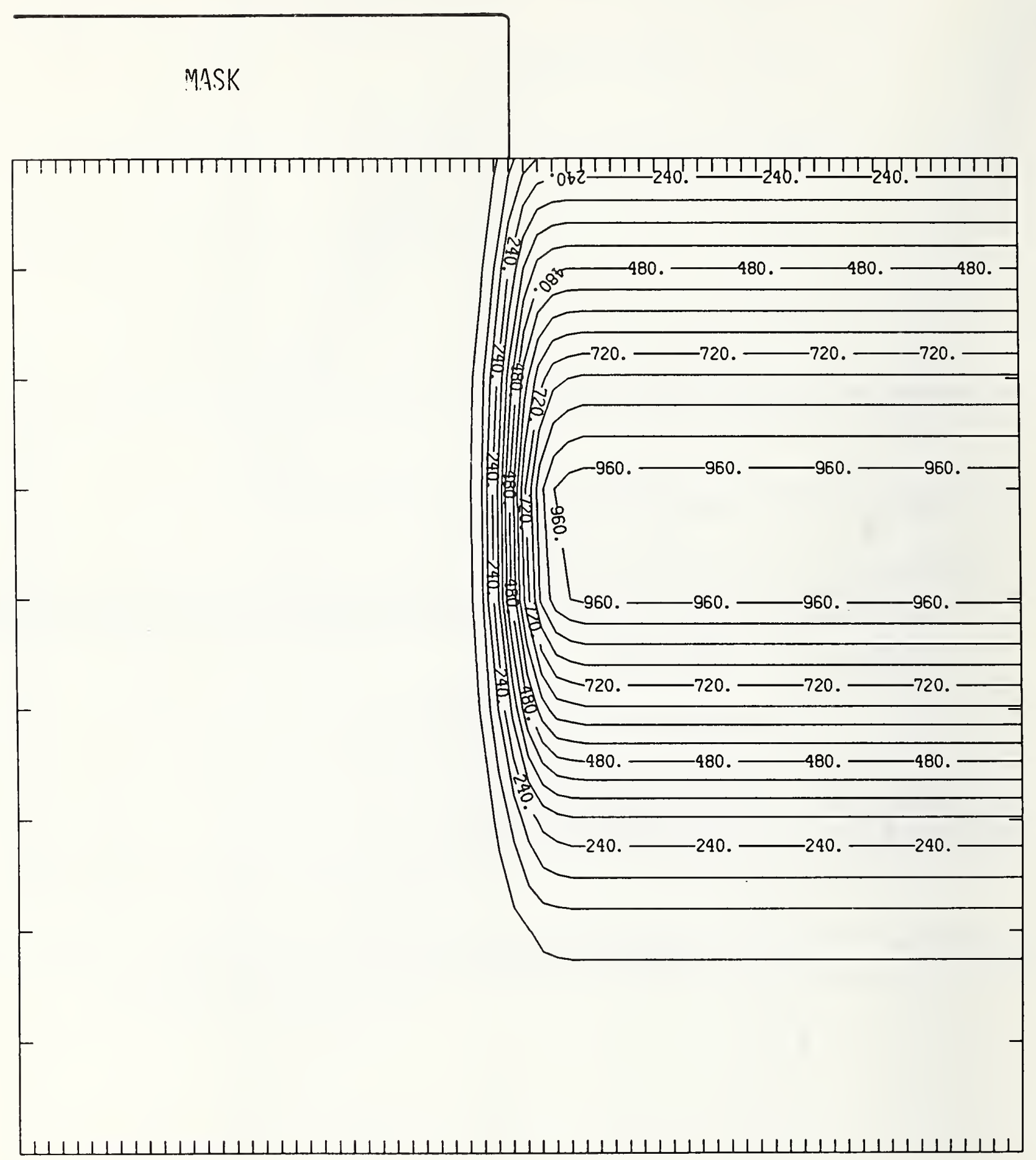

Figure N25-3 Two-dimensional distribution of $25 \mathrm{keV}$ nitrogen implanted past a mask edge. The length increment (distance between tick marks) is $0.02 \mu \mathrm{m}$ and the scale factor is 1000. 


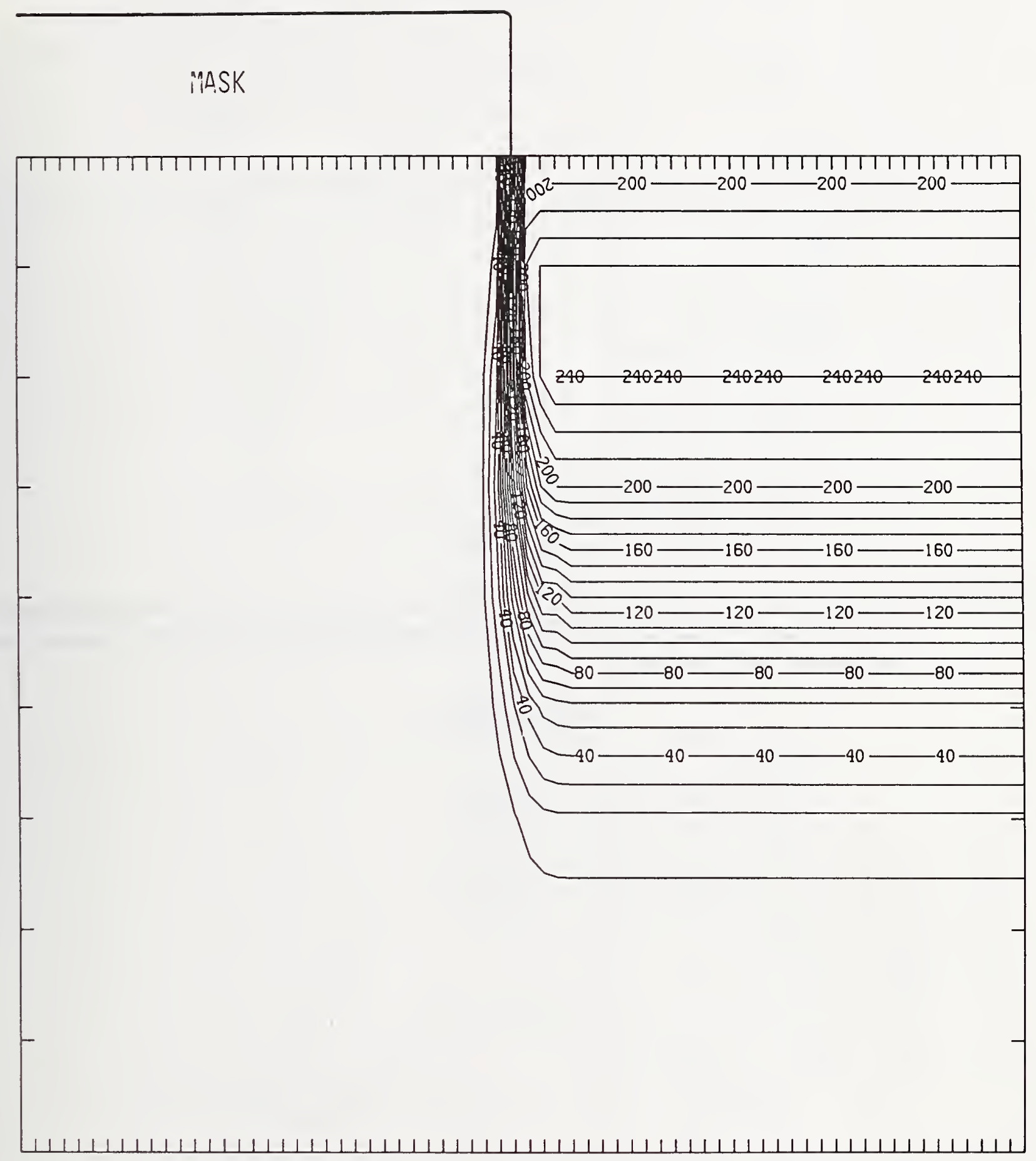

Figure N25-4 Two-dimensional distribution of Frenkel pairs created by $25 \mathrm{keV}$ nitrogen implanted past a mask edge. The length increment (distance between tick marks) is $0.02 \mu \mathrm{m}$ and the scale factor is 1 . 


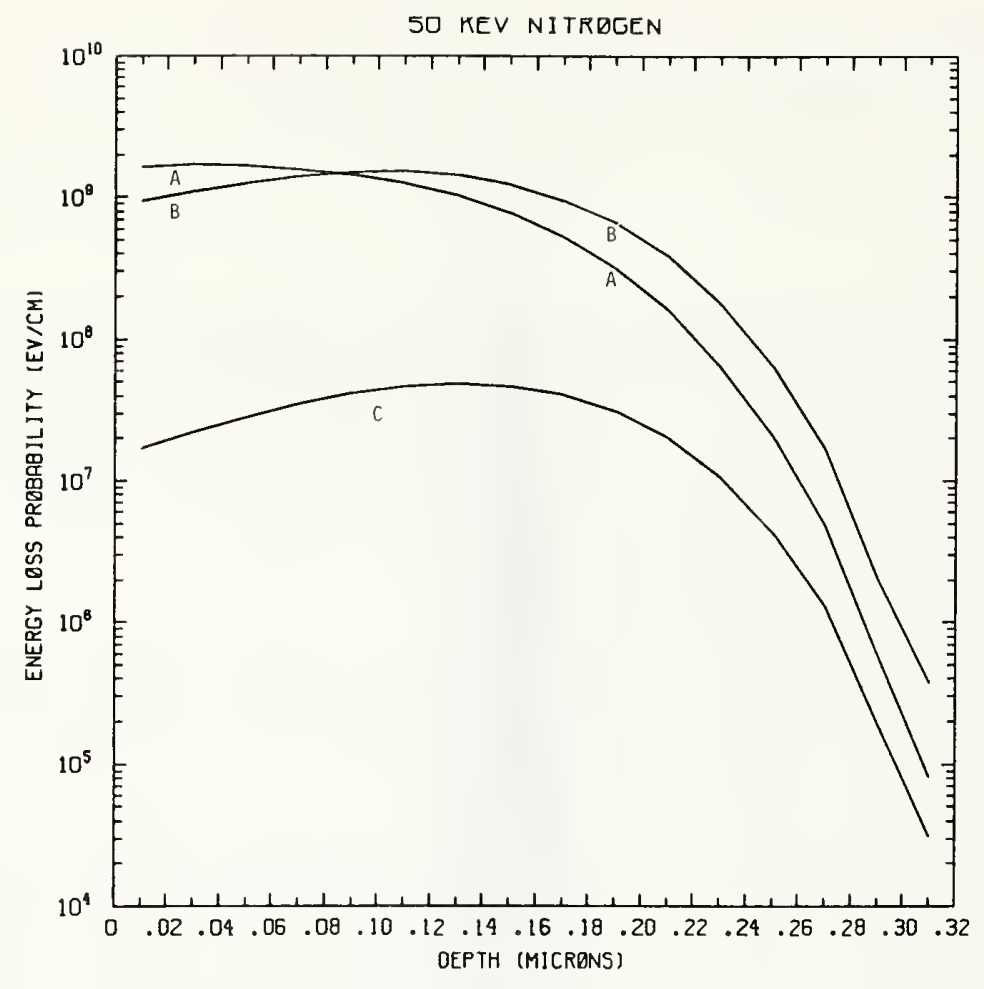

Figure N50-1 One-dimensional probability distributions for energy loss due to electronic excitation (A), displacement damage(B), and phonon excitation (C) for $50 \mathrm{keV}$ nitrogen normally incident on a silicon target.

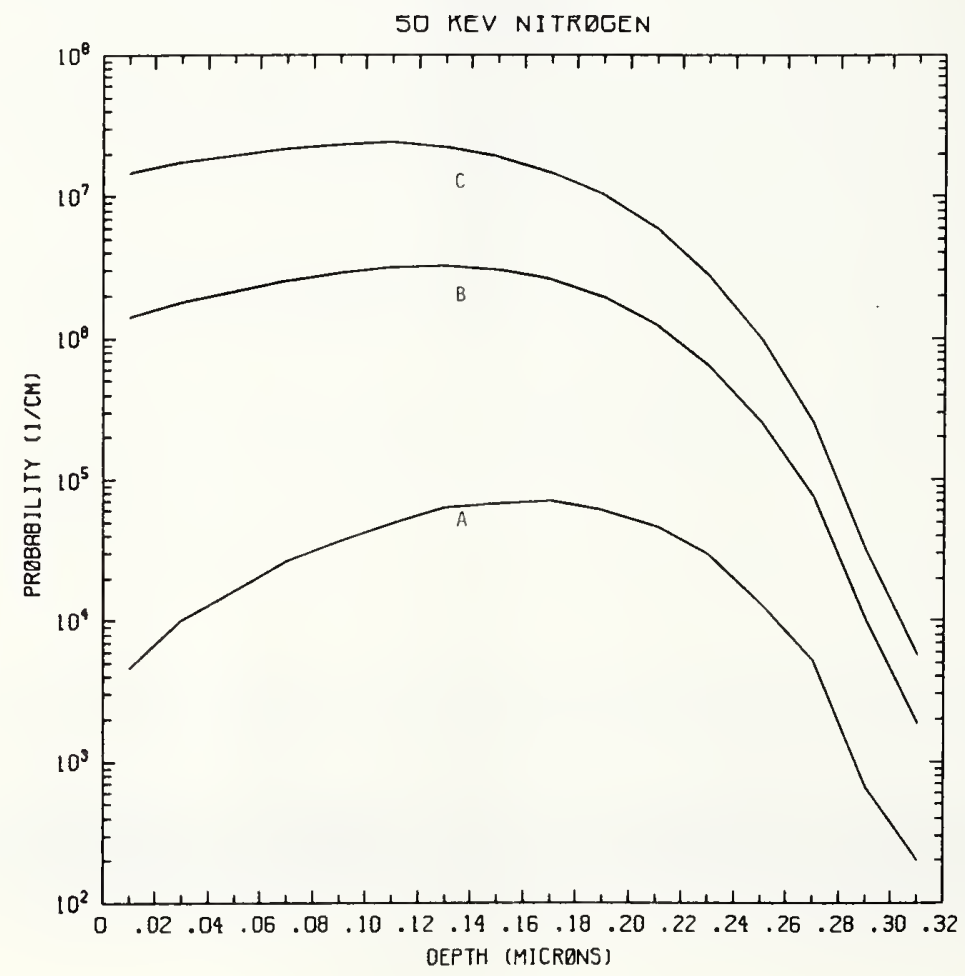

Figure N50-2 One-dimensional probability distributions for implanted nitrogen (A), primary silicon displacements (B), and Frenkel pairs (C) for $50 \mathrm{keV}$ nitrogen normally incident on a silicon target. 


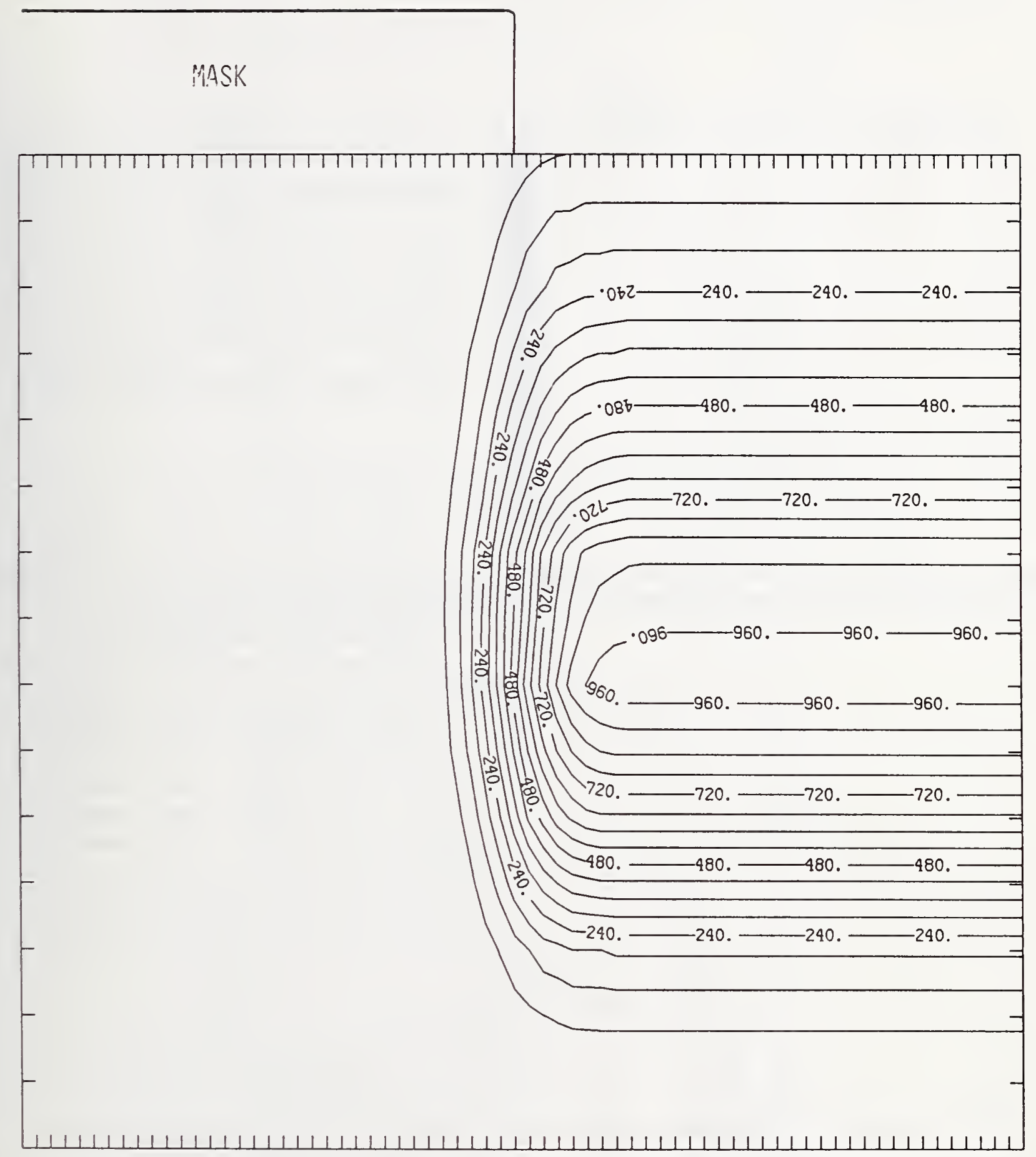

Figure N50-3 Two-dimensional distribution of $50 \mathrm{keV}$ nitrogen implanted past a mask edge. The length increment (distance between tick marks) is $0.02 \mu \mathrm{m}$ and the scale factor is 1000. 


\section{MASK}

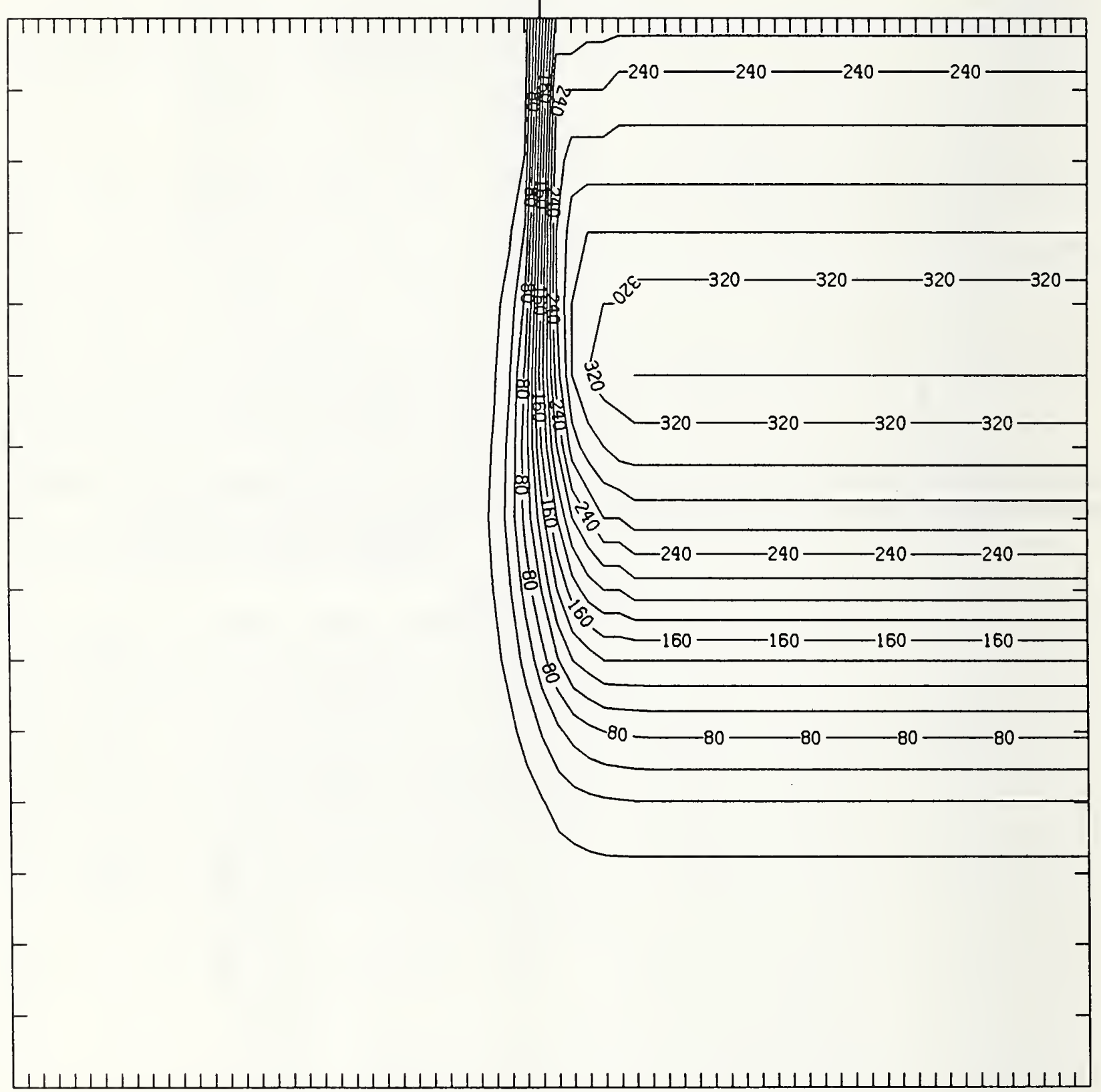

Figure N50-4 Two-dimensional distribution of Frenkel pairs created by $50 \mathrm{keV}$ nitrogen implanted past a mask edge. The length increment (distance between tick marks) is $0.02 \mu \mathrm{m}$ and the scale factor is 1 . 


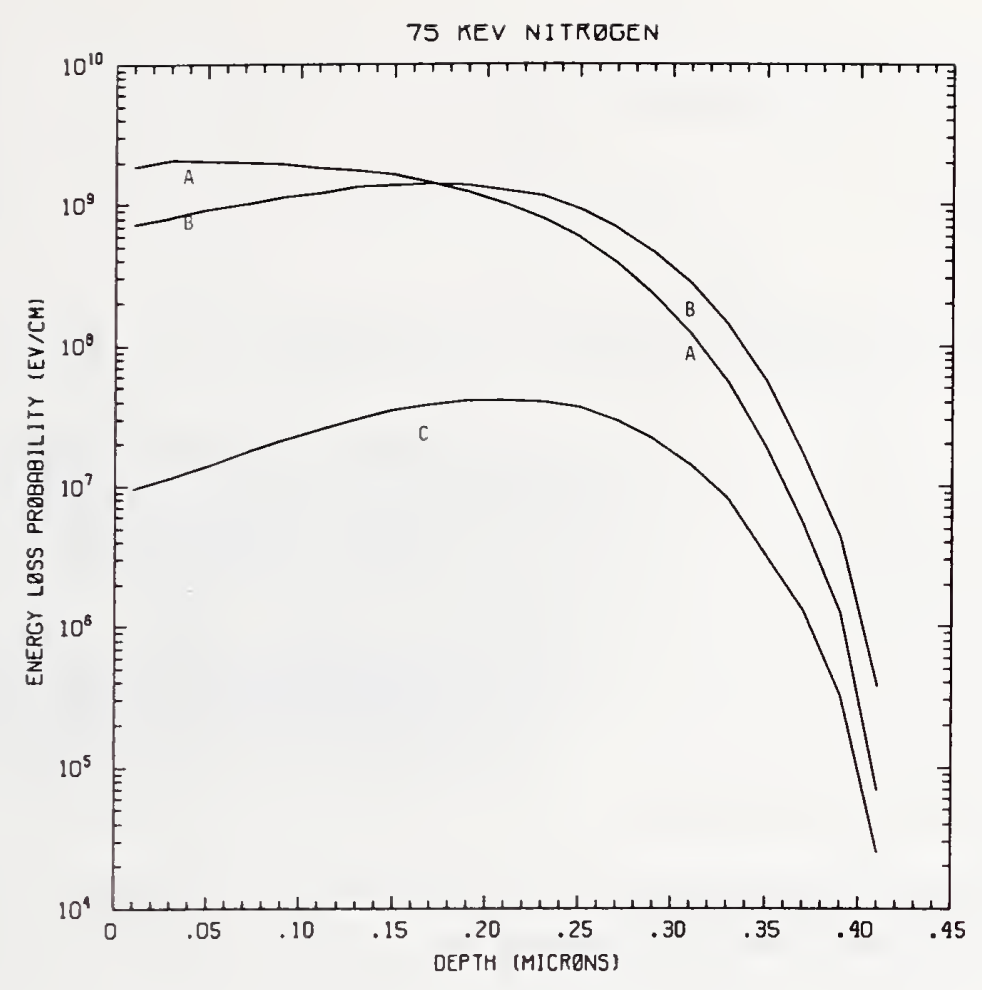

Figure N75-1 One-dimensional probability distributions for energy loss due to electronic excitation (A), displacement damage(B), and phonon excitation (C) for $75 \mathrm{keV}$ nitrogen normally incident on a silicon target.

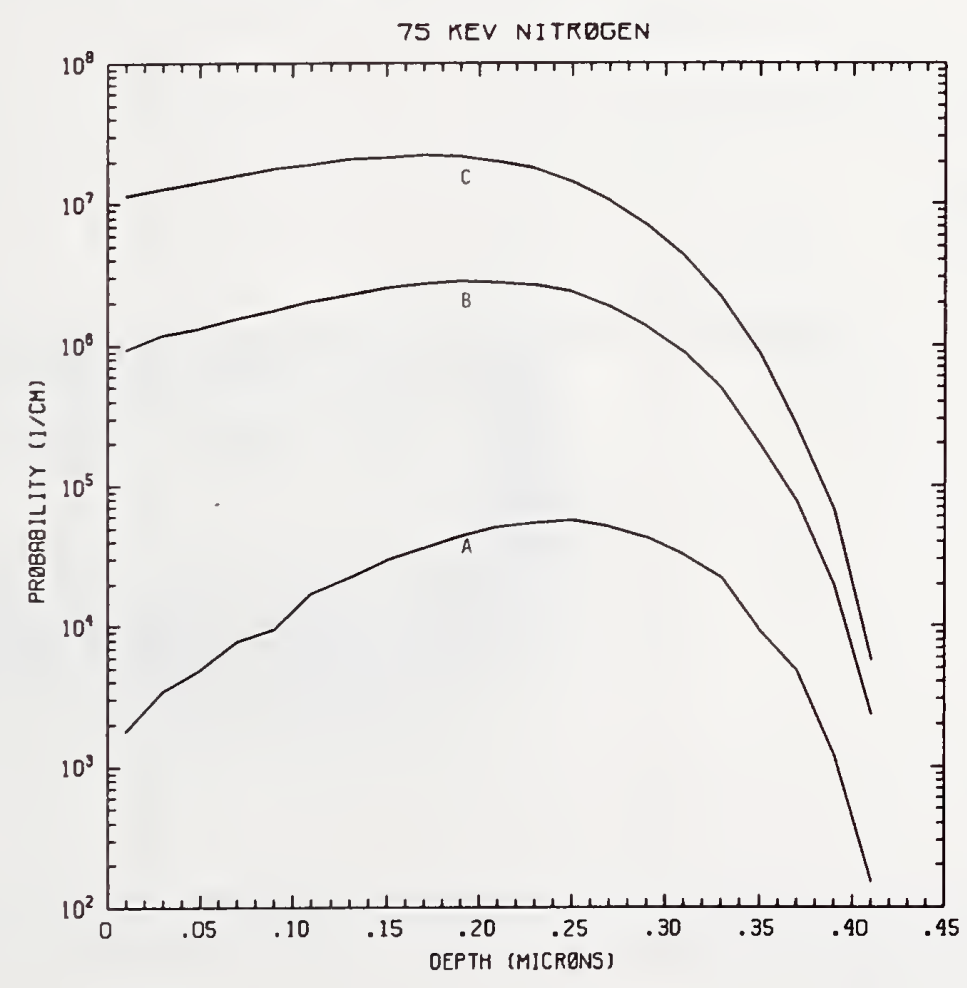

Figure N75-2 One-dimensional probability distributions for implanted nitrogen (A), primary silicon displacements (B), and Frenkel pairs (C) for $75 \mathrm{keV}$ nitrogen normally incident on a silicon target. 


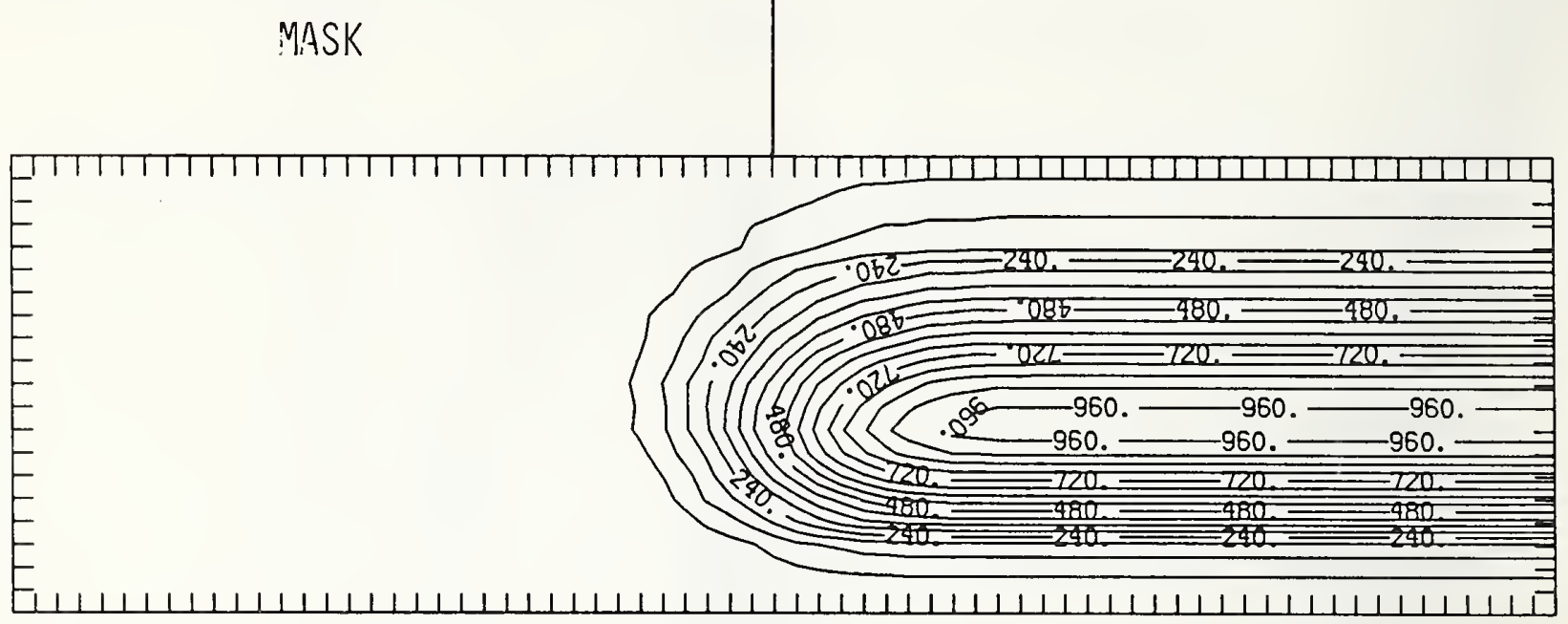

Figure N75-3 Two-dimensional distribution of $75 \mathrm{keV}$ nitrogen implanted past a mask edge. The length increment (distance between tick marks) is $0.02 \mu \mathrm{m}$ and the scale factor is 1000 .

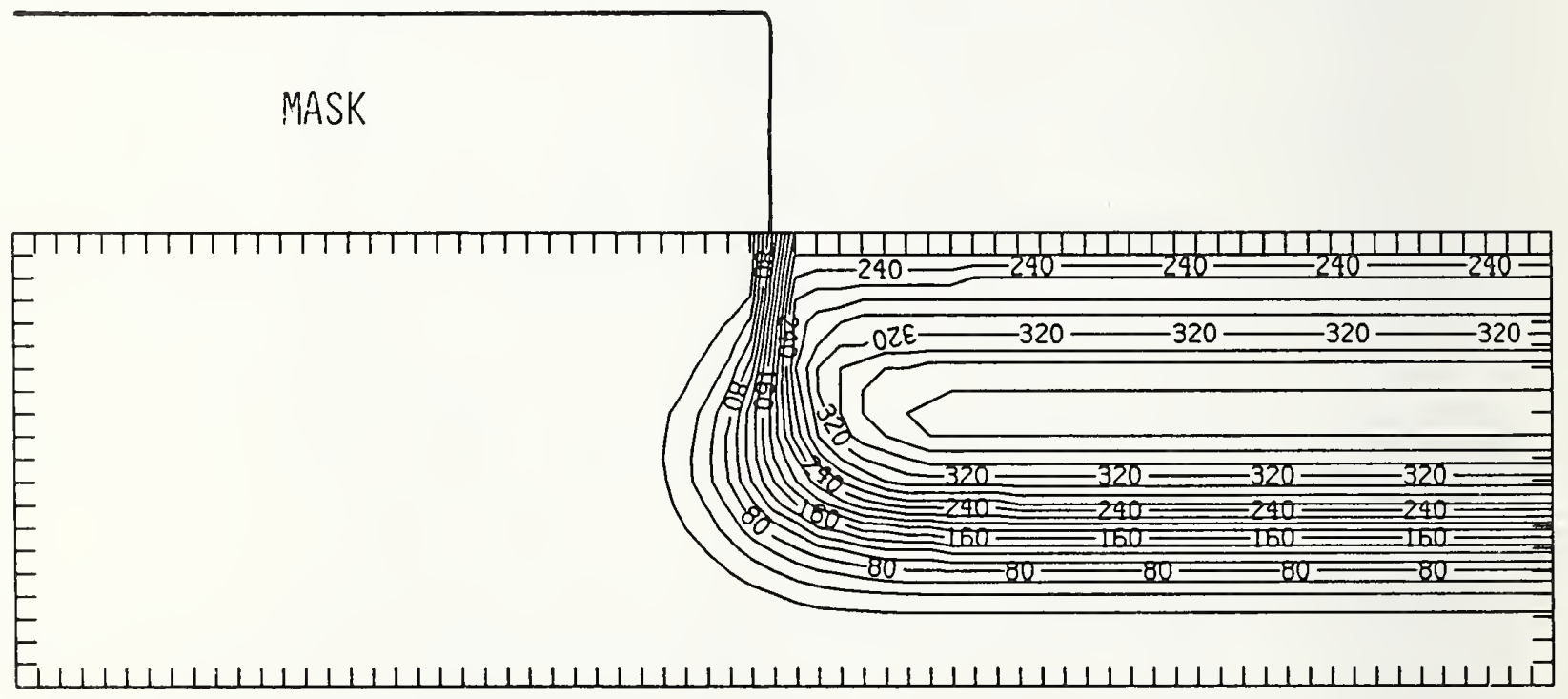

Figure N75-4 Two-dimensional distribution of Frenkel pairs created by $75 \mathrm{keV}$ nitrogen implanted past a mask edge. The length increment (distance between tick marks) is $0.02 \mu \mathrm{m}$ and the scale factor is 1 . 


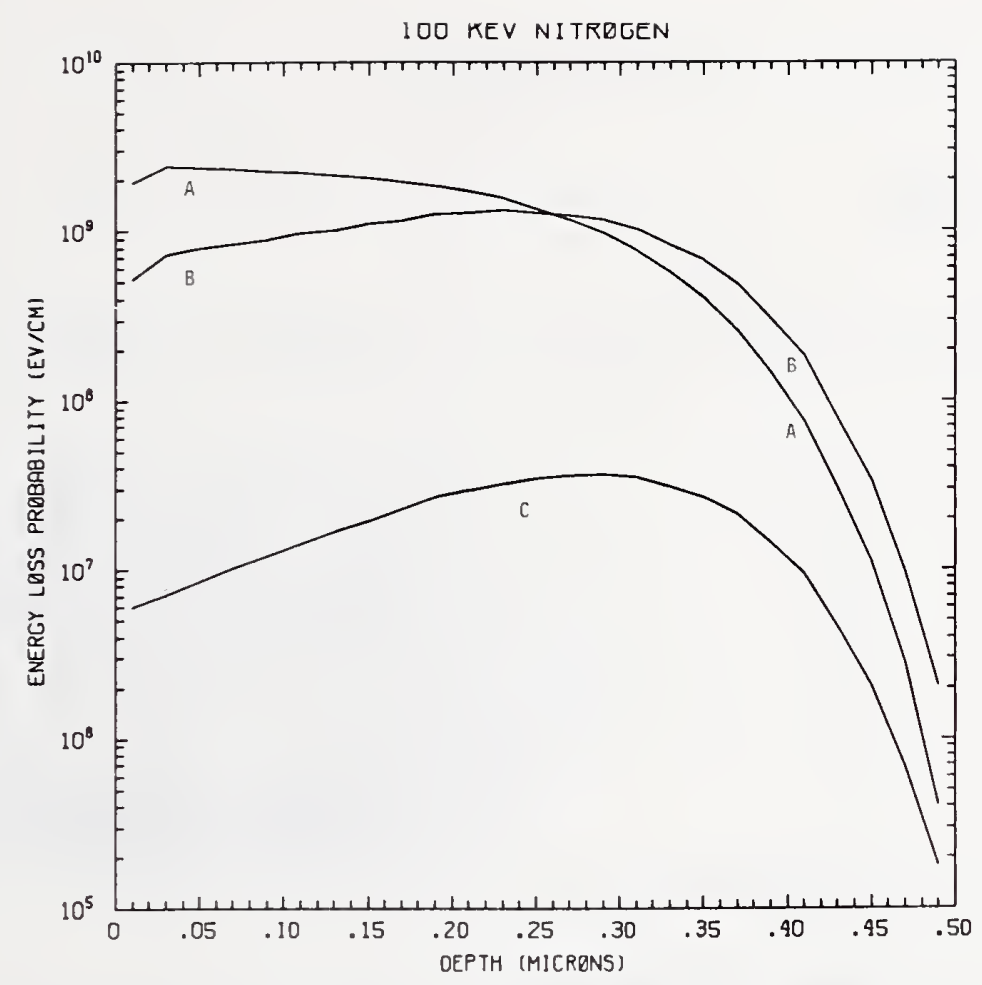

Figure N100-1 One-dimensional probability distributions for energy loss due to electronic excitation (A), displacement damage(B), and phonon excitation (C) for $100 \mathrm{keV}$ nitrogen normally incident on a silicon target.

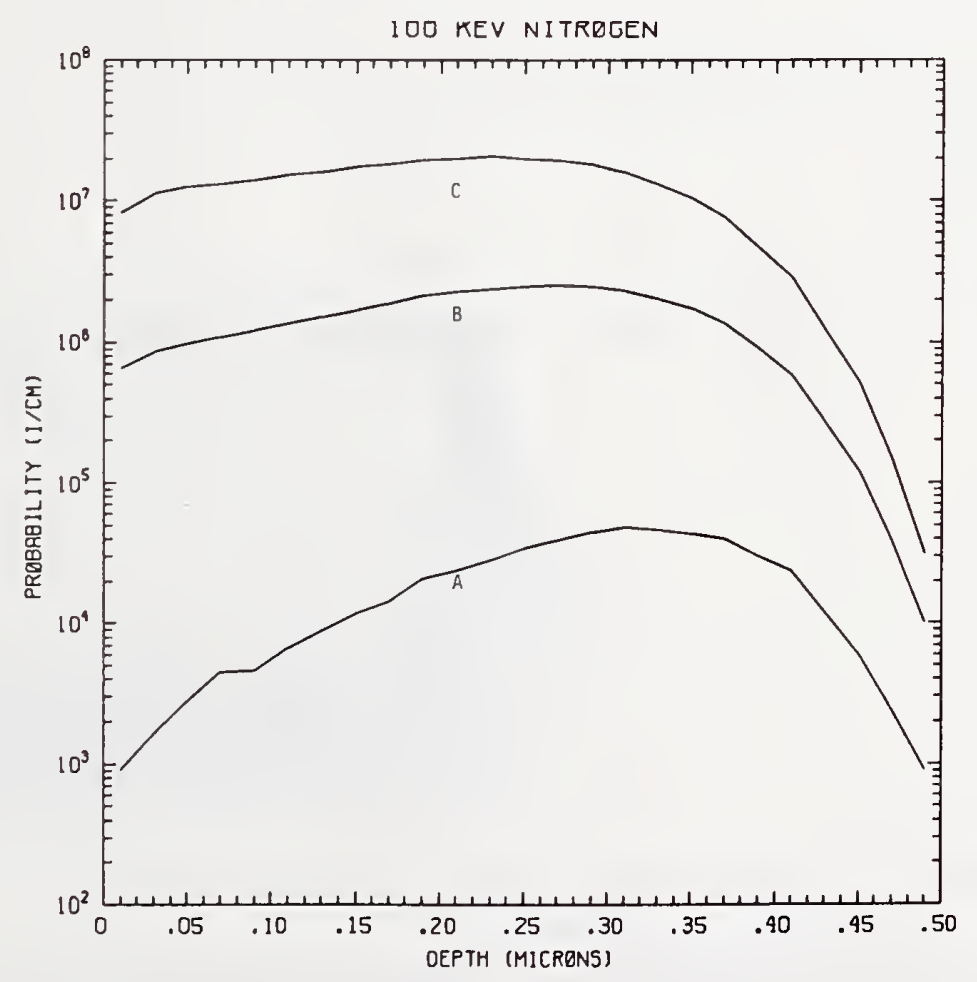

Figure N100-2 One-dimensional probability distributions for implanted nitrogen (A), primary silicon displacements (B), and Frenkel pairs (C) for $100 \mathrm{keV}$ nitrogen normally incident on a silicon target. 
IIASK

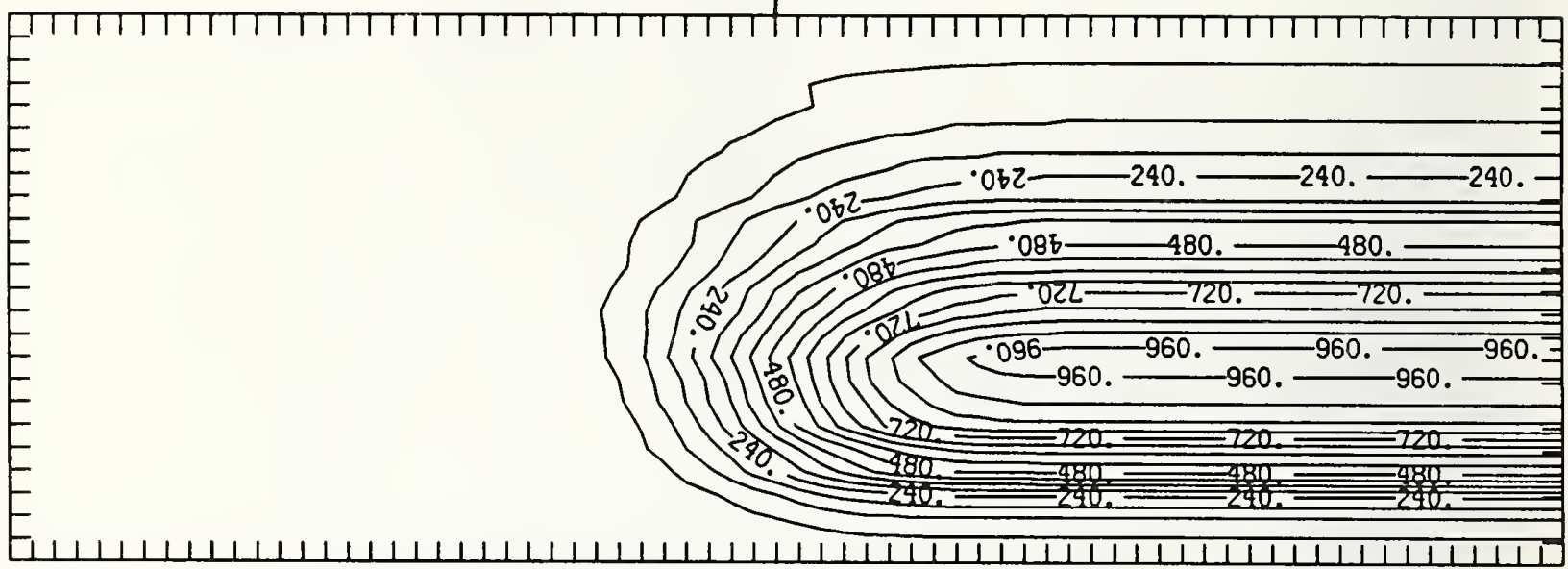

Figure N100-3 Two-dimensional distribution of $100 \mathrm{keV}$ nitrogen implanted past a mask edge. The length increment (distance between tick marks) is $0.02 \mu \mathrm{m}$ and the scale factor is 1000 .

\section{MASK}

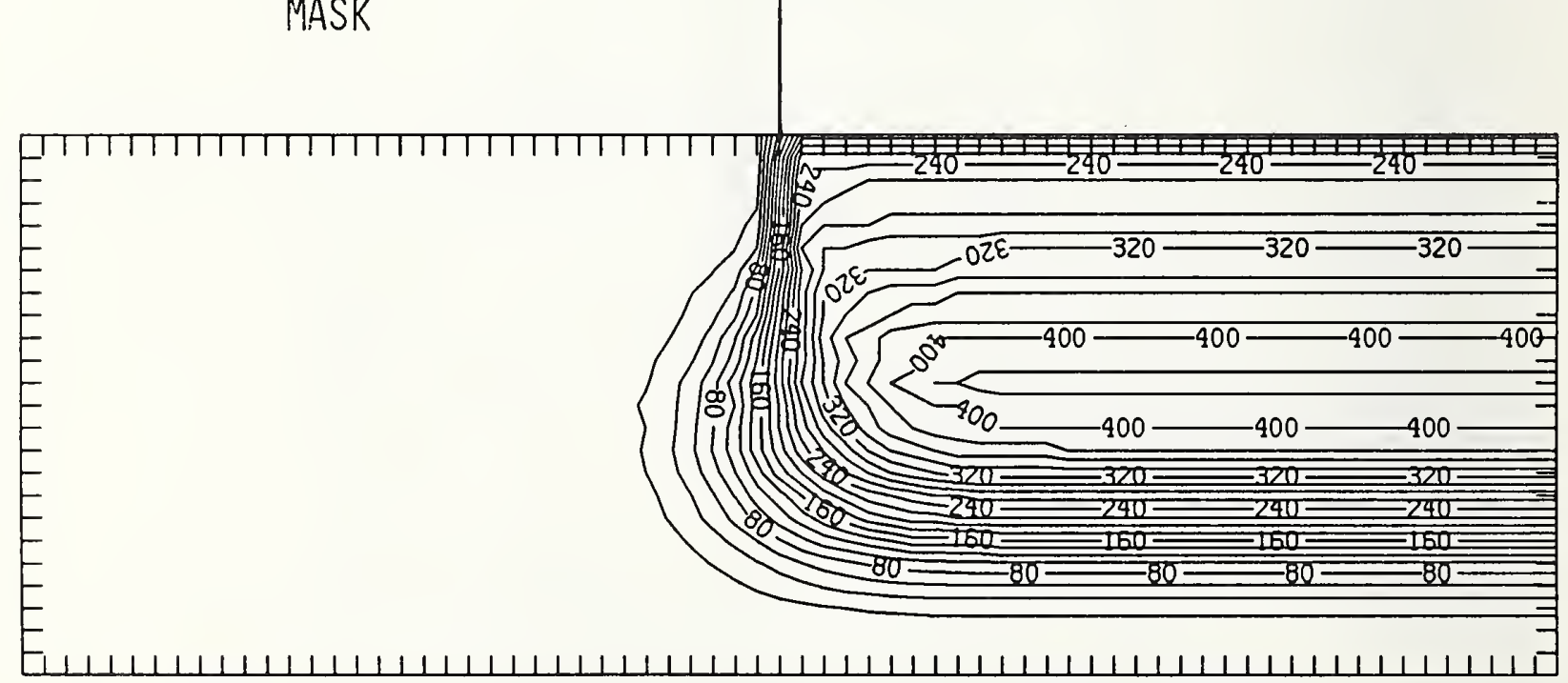

Figure N100-4 Two-dimensional distribution of Frenkel pairs created by $100 \mathrm{keV}$ nitrogen implanted past a mask edge. The length increment (distance between tick marks) is $0.02 \mu \mathrm{m}$ and the scale factor is 1 . 


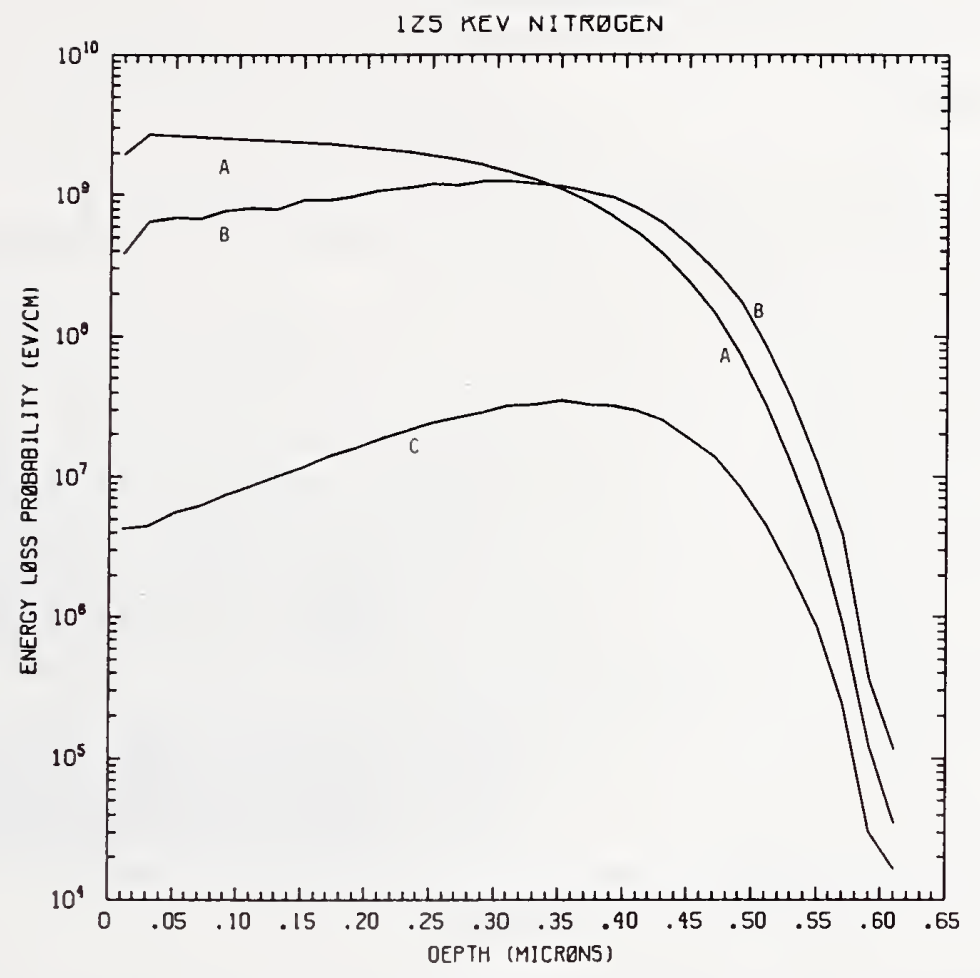

Figure N125-1 One-dimensional probability distributions for energy loss due to electronic excitation (A), displacement damage(B), and phonon excitation (C) for $125 \mathrm{keV}$ nitrogen normally incident on a silicon target.

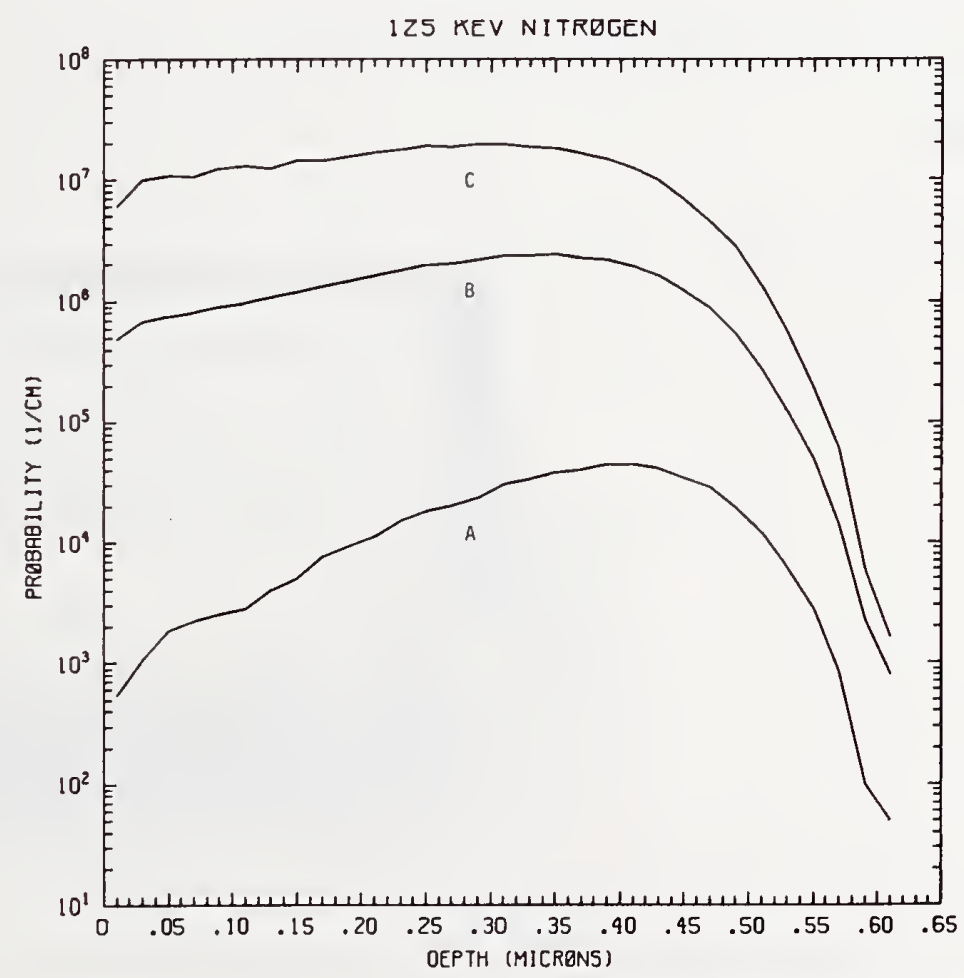

Figure N125-2 One-dimensional probability distributions for implanted nitrogen (A), primary silicon displacements (B), and Frenkel pairs (C) for $125 \mathrm{keV}$ nitrogen normally incident on a silicon target. 


\section{MASK}

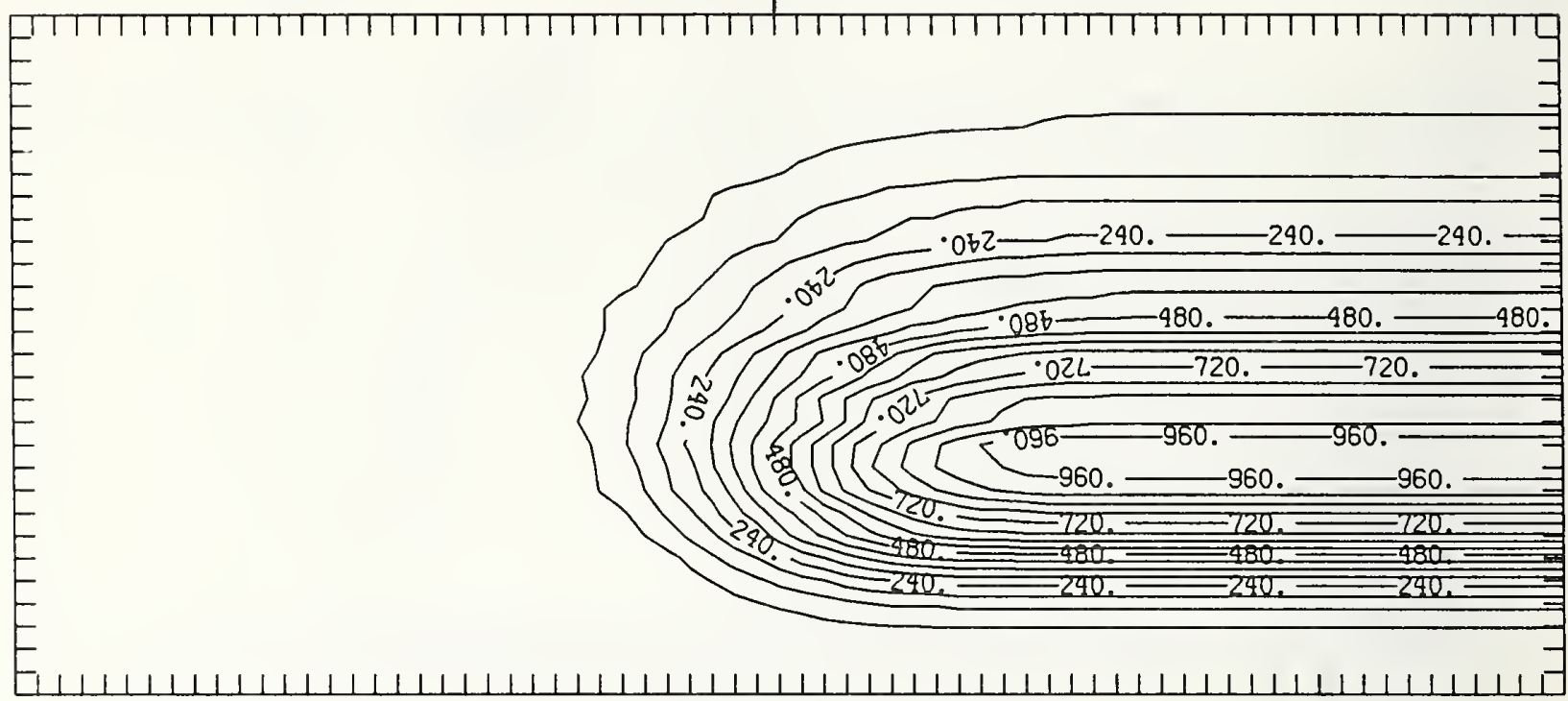

Figure N125-3 Two-dimensional distribution of $125 \mathrm{keV}$ nitrogen implanted past a mask edge. The length increment (distance between tick marks) is $0.02 \mu \mathrm{m}$ and the scale factor is 1000.

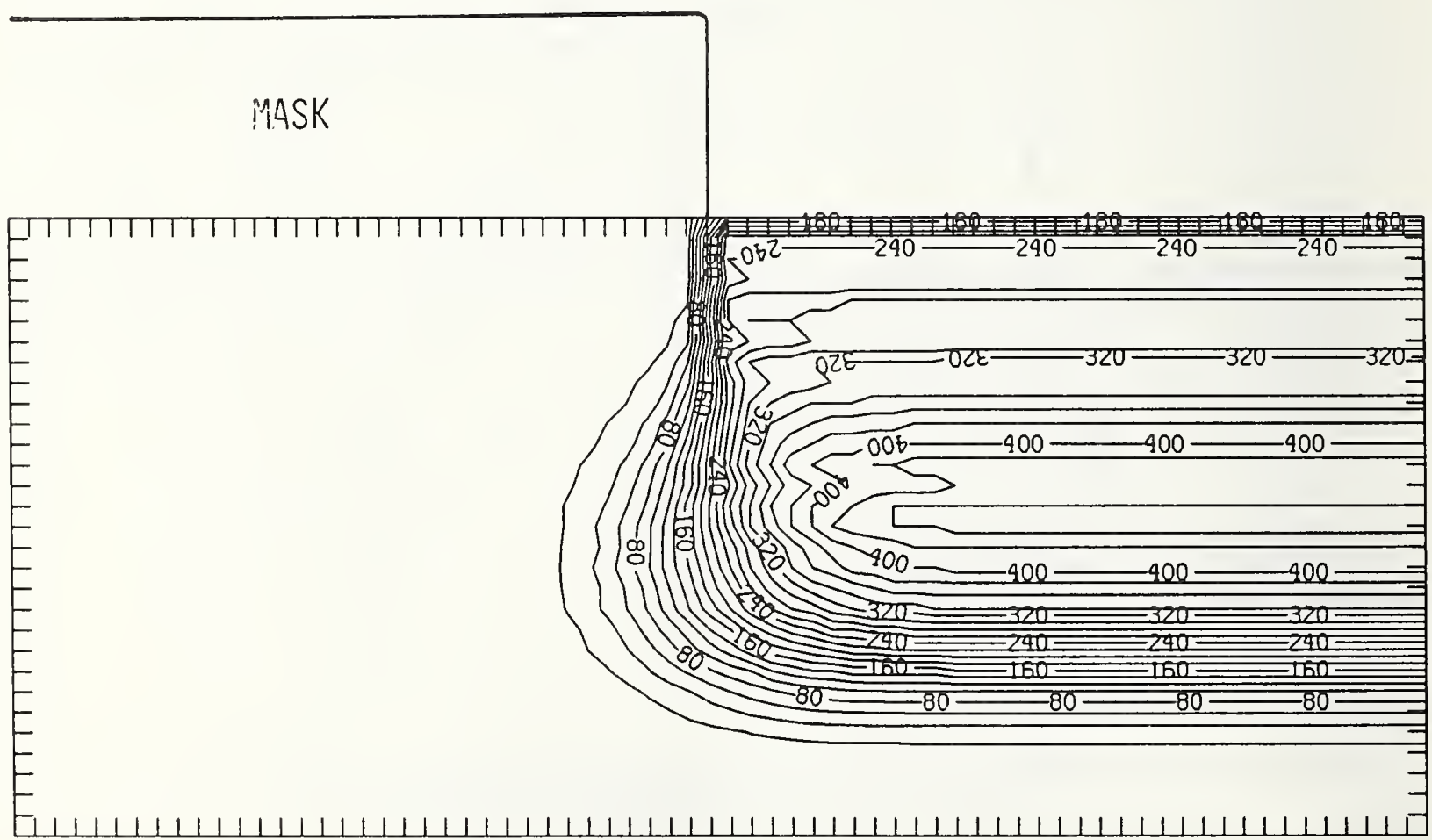

Figure N125-4 Two-dimensional distribution of Frenkel pairs created by $125 \mathrm{keV}$ nitrogen implanted past a mask edge. The length increment (distance between tick marks) is $0.02 \mu \mathrm{m}$ and the scale factor is 1 . 


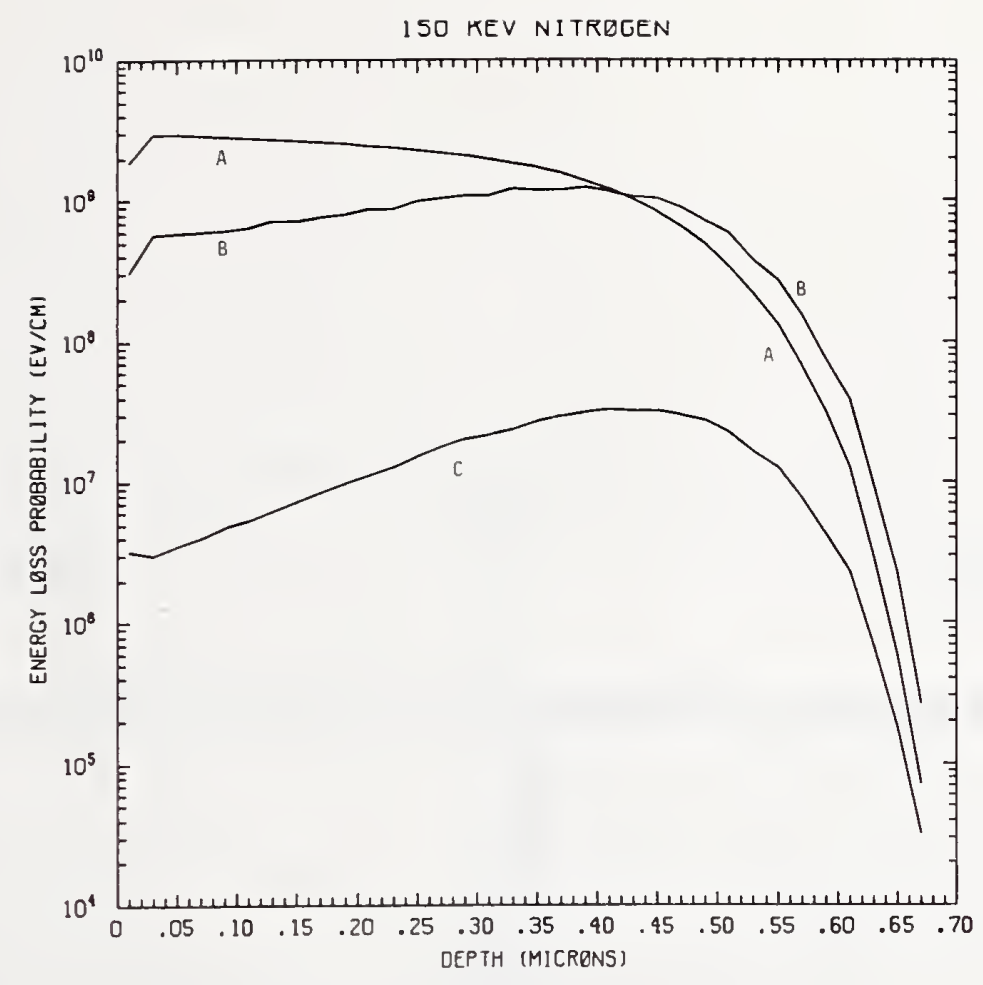

Figure N150-1 One-dimensional probability distributions for energy loss due to electronic excitation (A), displacement damage(B), and phonon excitation (C) for $150 \mathrm{keV}$ nitrogen normally incident on a silicon target.

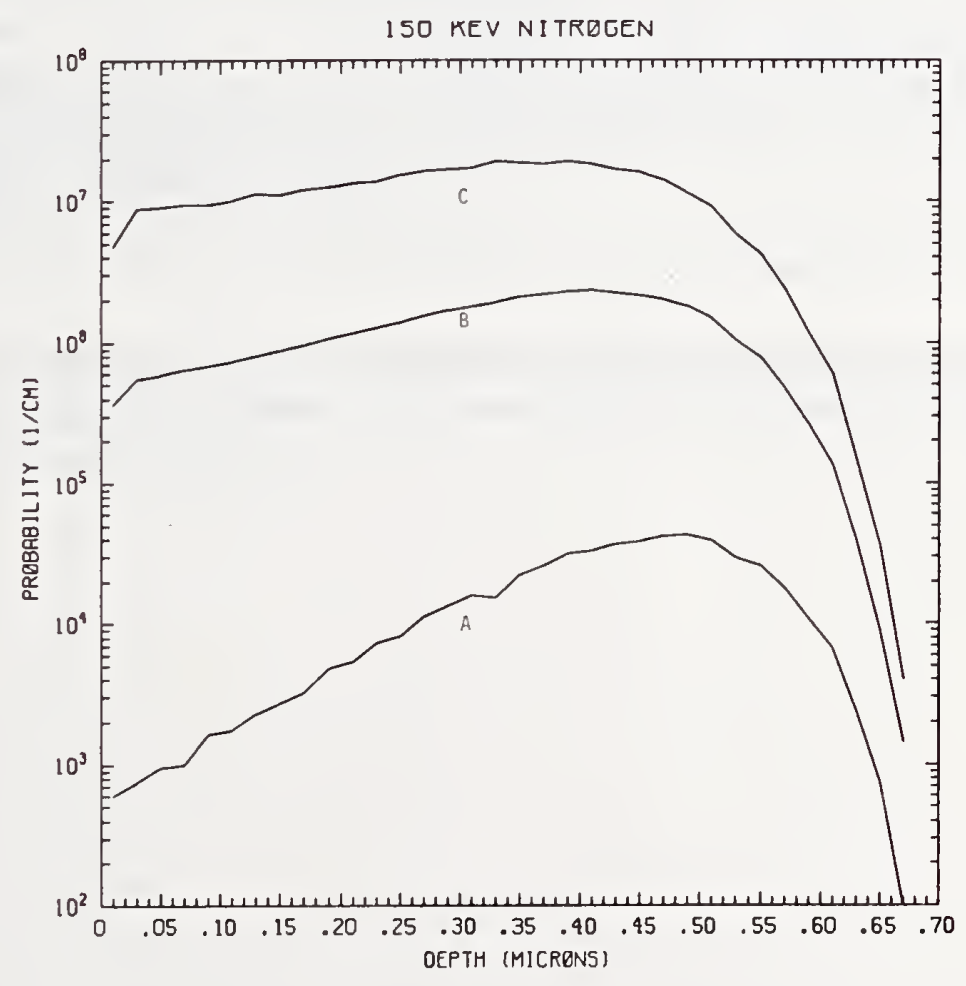

Figure N150-2 One-dimensional probability distributions for implanted nitrogen (A), primary silicon displacements (B), and Frenkel pairs (C) for $150 \mathrm{keV}$ nitrogen normally incident on a silicon target. 


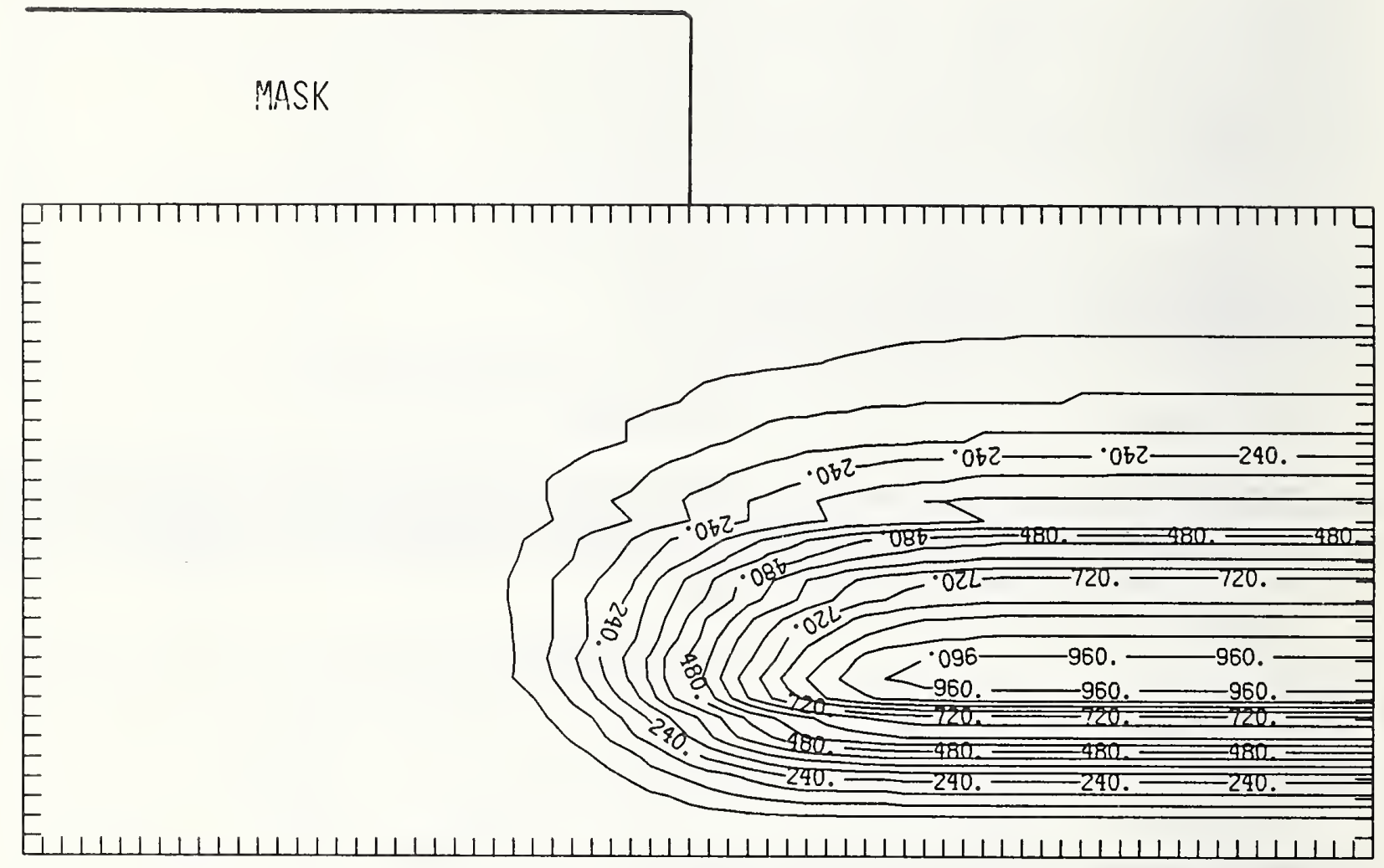

Figure N150-3 Two-dimensional distribution of $150 \mathrm{keV}$ nitrogen implanted past a mask edge. The length increment (distance between tick marks) is $0.02 \mu \mathrm{m}$ and the scale factor is 1000. 


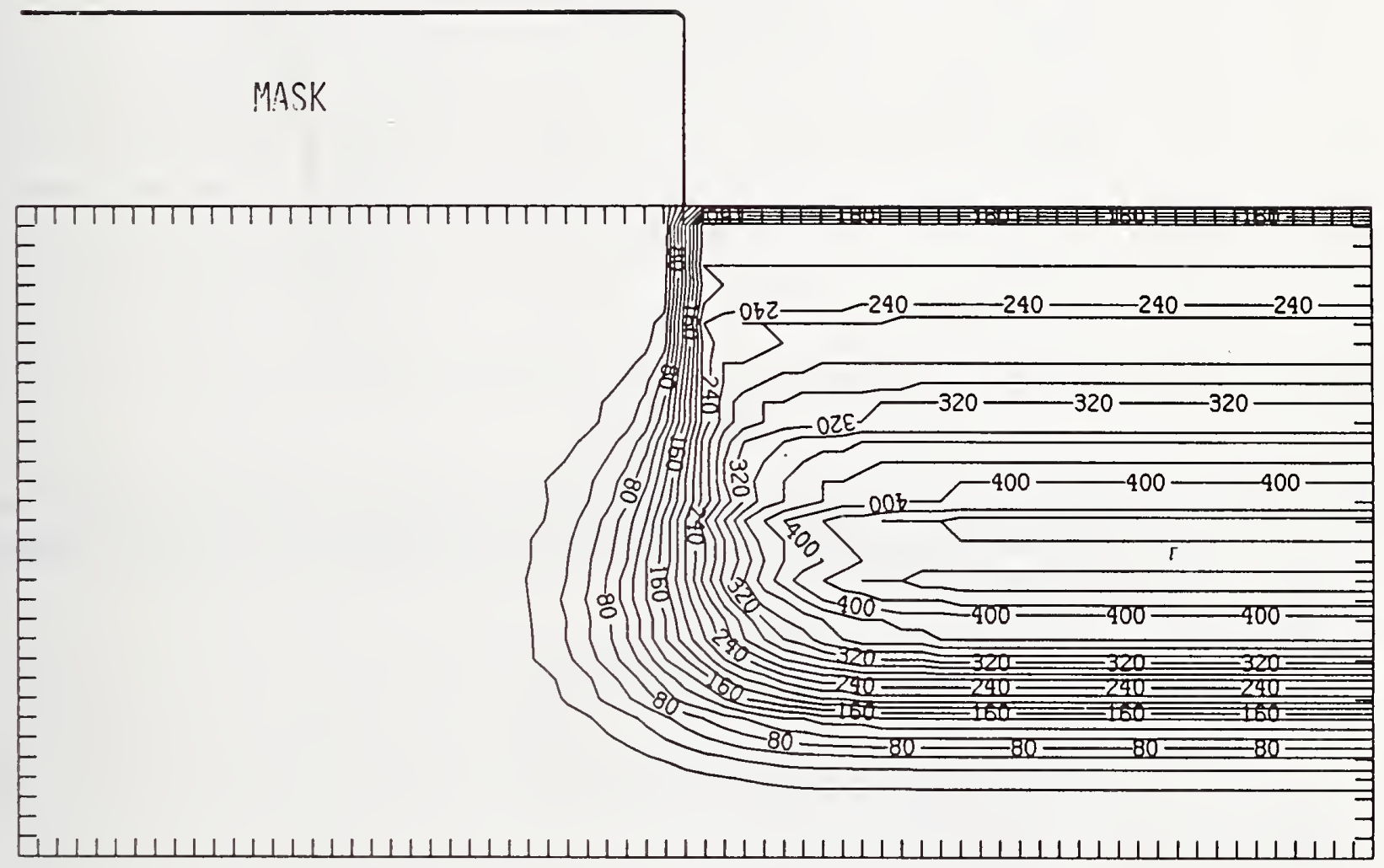

Figure N150-4 Two-dimensional distribution of Frenkel pairs created by $150 \mathrm{keV}$ nitrogen implanted past a mask edge. The length increment (distance between tick marks) is $0.02 \mu \mathrm{m}$ and the scale factor is 1 . 


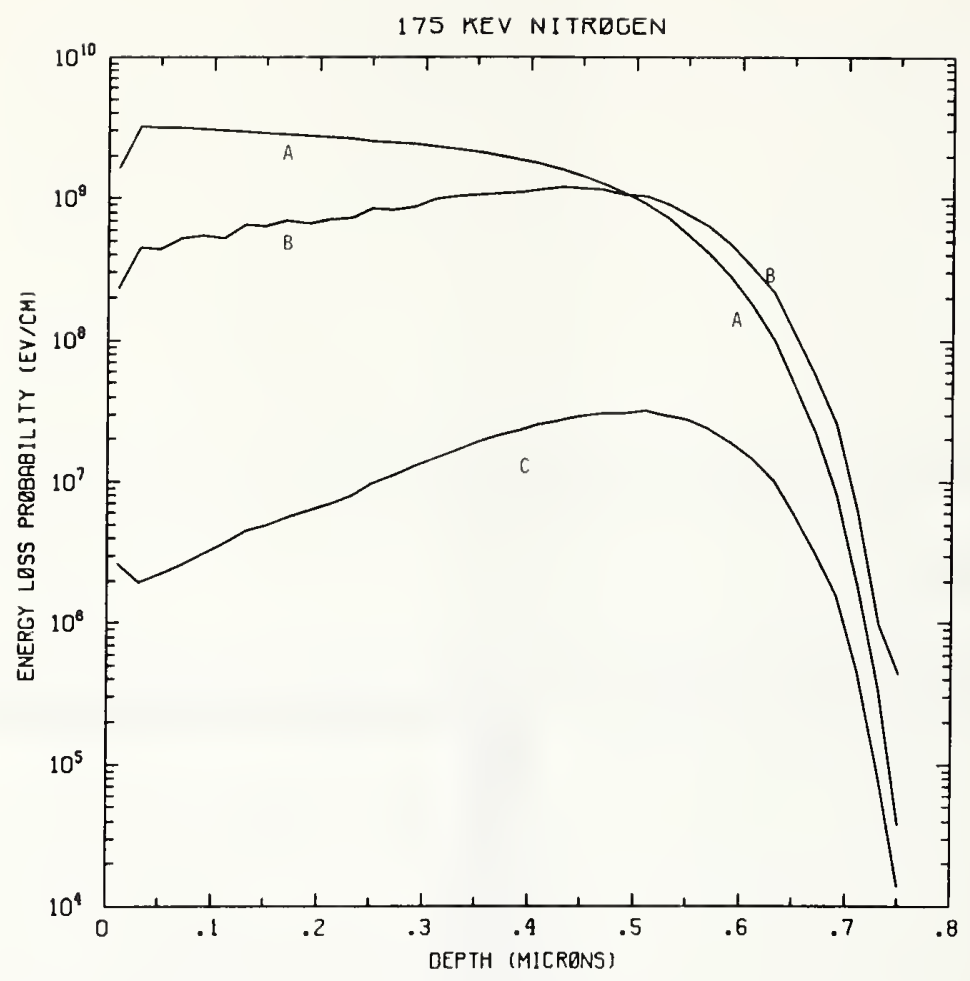

Figure N175-1 One-dimensional probability distributions for energy loss due to electronic excitation (A), displacement damage(B), and phonon excitation (C) for $175 \mathrm{keV}$ nitrogen normally incident on a silicon target.

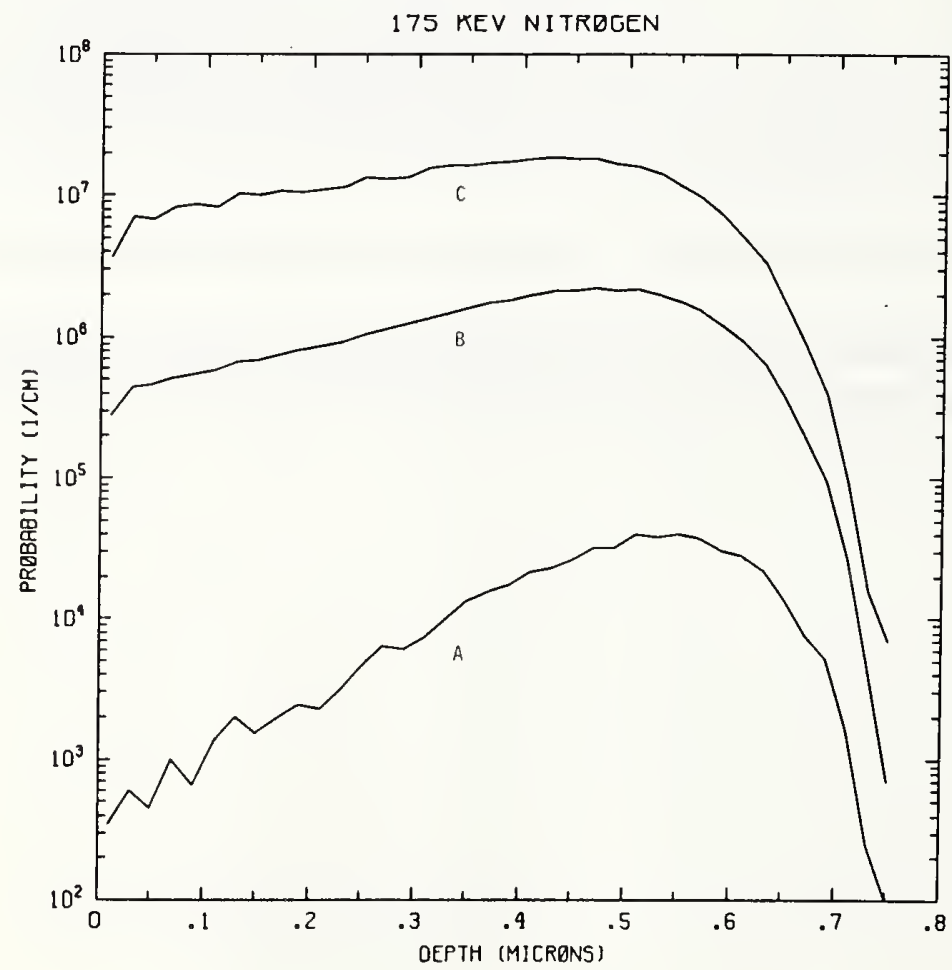

Figure N175-2 One-dimensional probability distributions for implanted nitrogen (A), primary silicon displacements (B), and Frenkel pairs (C) for $175 \mathrm{keV}$ nitrogen normally incident on a silicon target. 


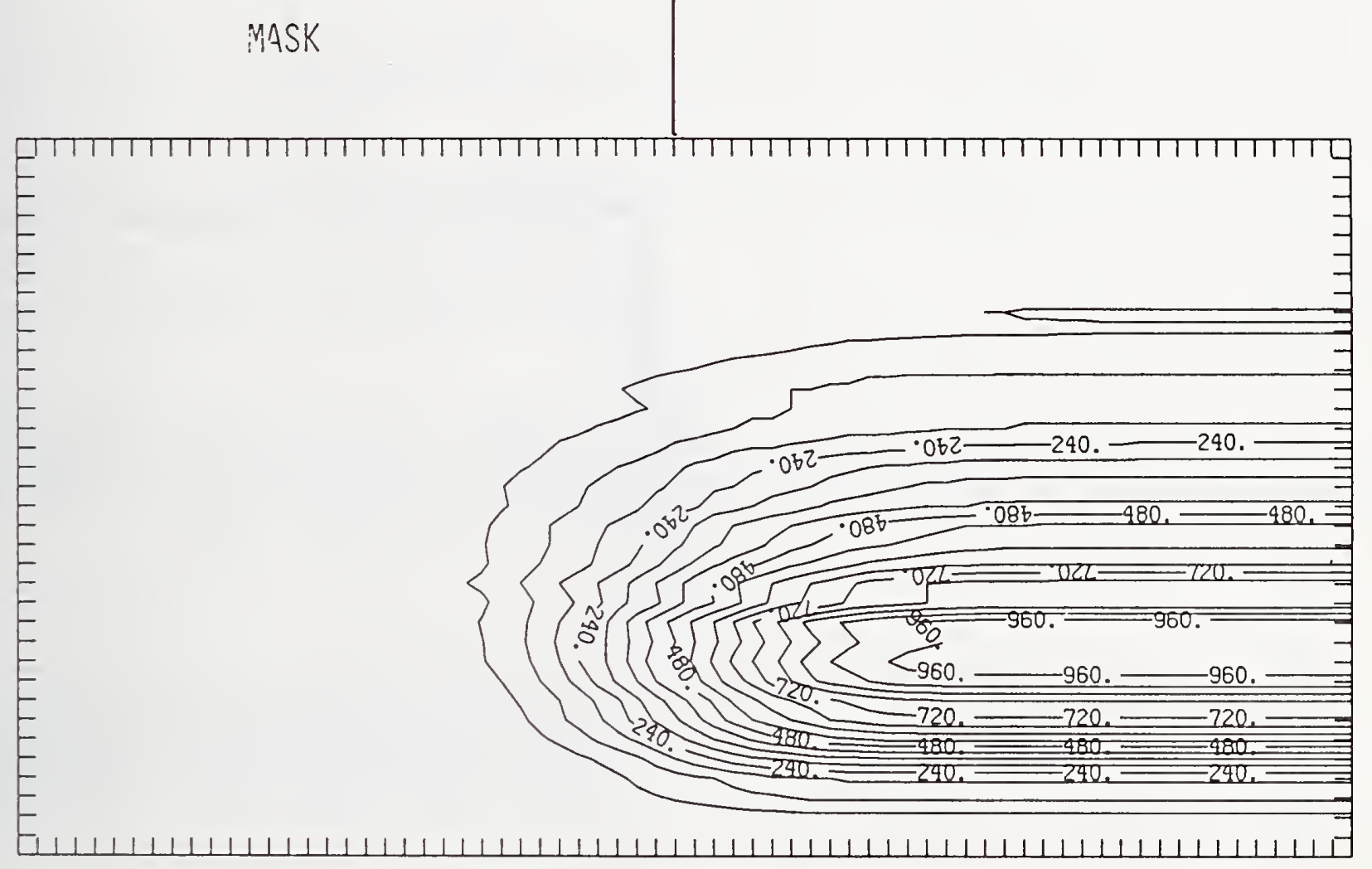

Figure N175-3 Two-dimensional distribution of $175 \mathrm{keV}$ nitrogen implanted past a mask edge. The length increment (distance between tick marks) is $0.02 \mu \mathrm{m}$ and the scale factor is 1000 . 


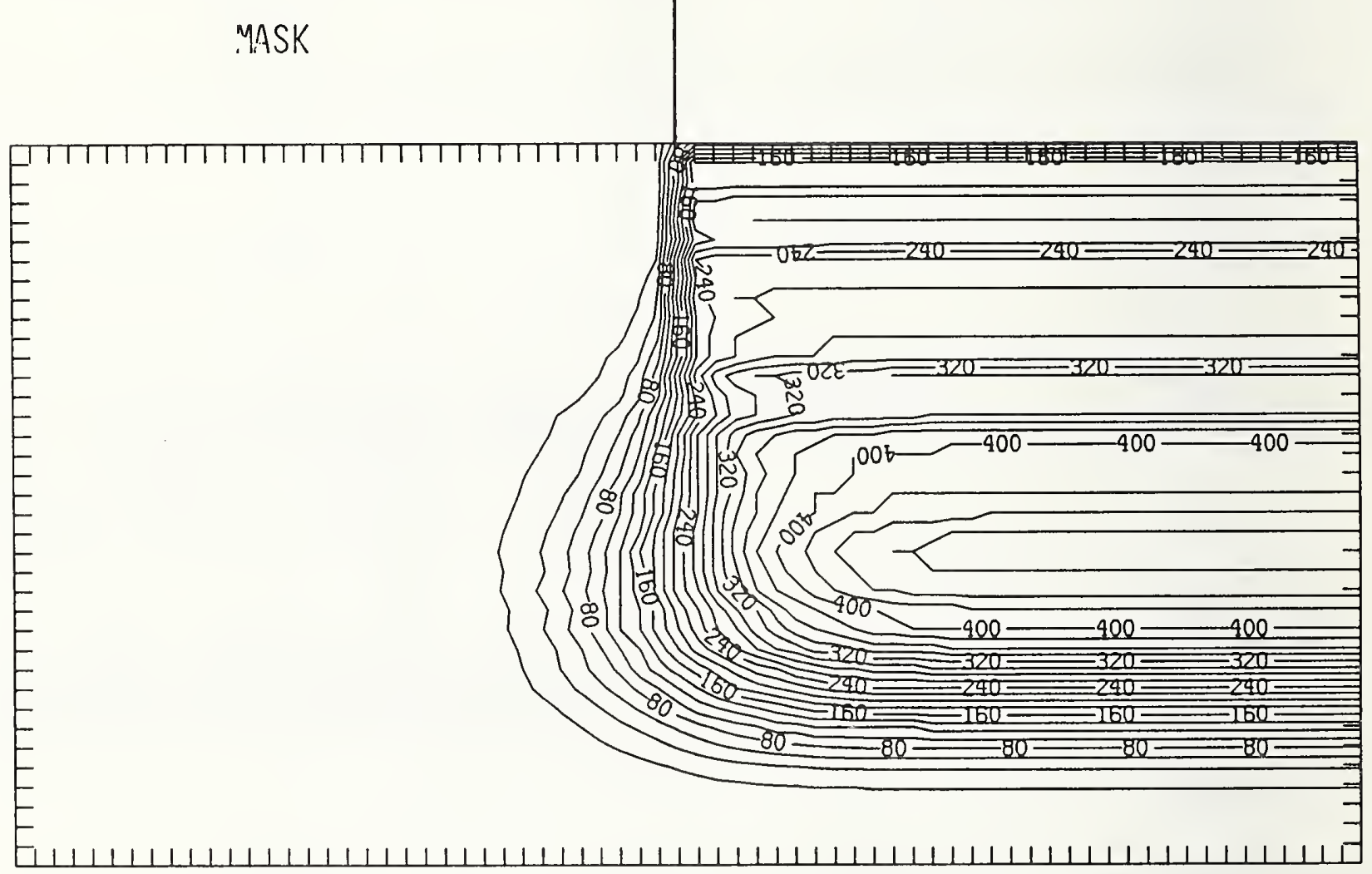

Figure N175-4 Two-dimensional distribution of Frenkel pairs created by $175 \mathrm{keV}$ nitrogen implanted past a mask edge. The length increment (distance between tick marks) is $0.02 \mu \mathrm{m}$ and the scale factor is 1 . 


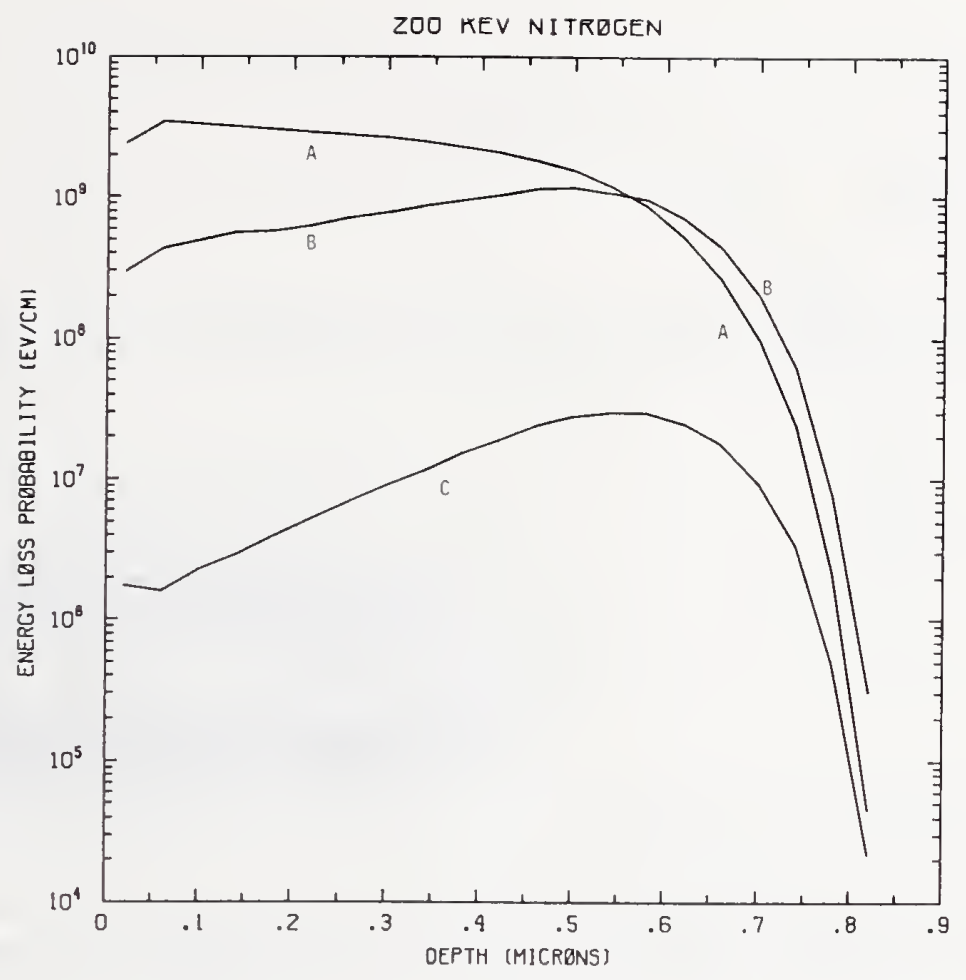

Figure N200-1 One-dimensional probability distributions for energy loss due to electronic excitation (A), displacement damage(B), and phonon excitation (C) for $200 \mathrm{keV}$ nitrogen normally incident on a silicon target.

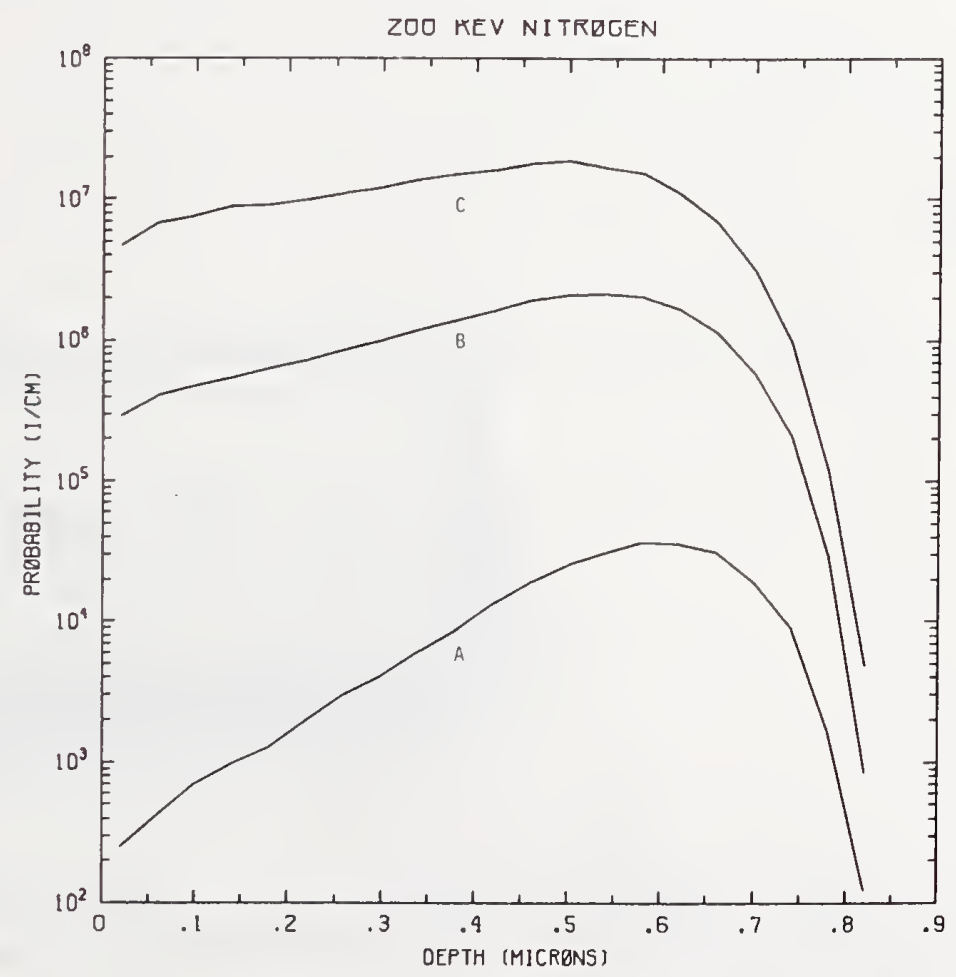

Figure N200-2 One-dimensional probability distributions for implanted nitrogen (A), primary silicon displacements (B), and Frenkel pairs (C) for $200 \mathrm{keV}$ nitrogen normally incident on a silicon target. 


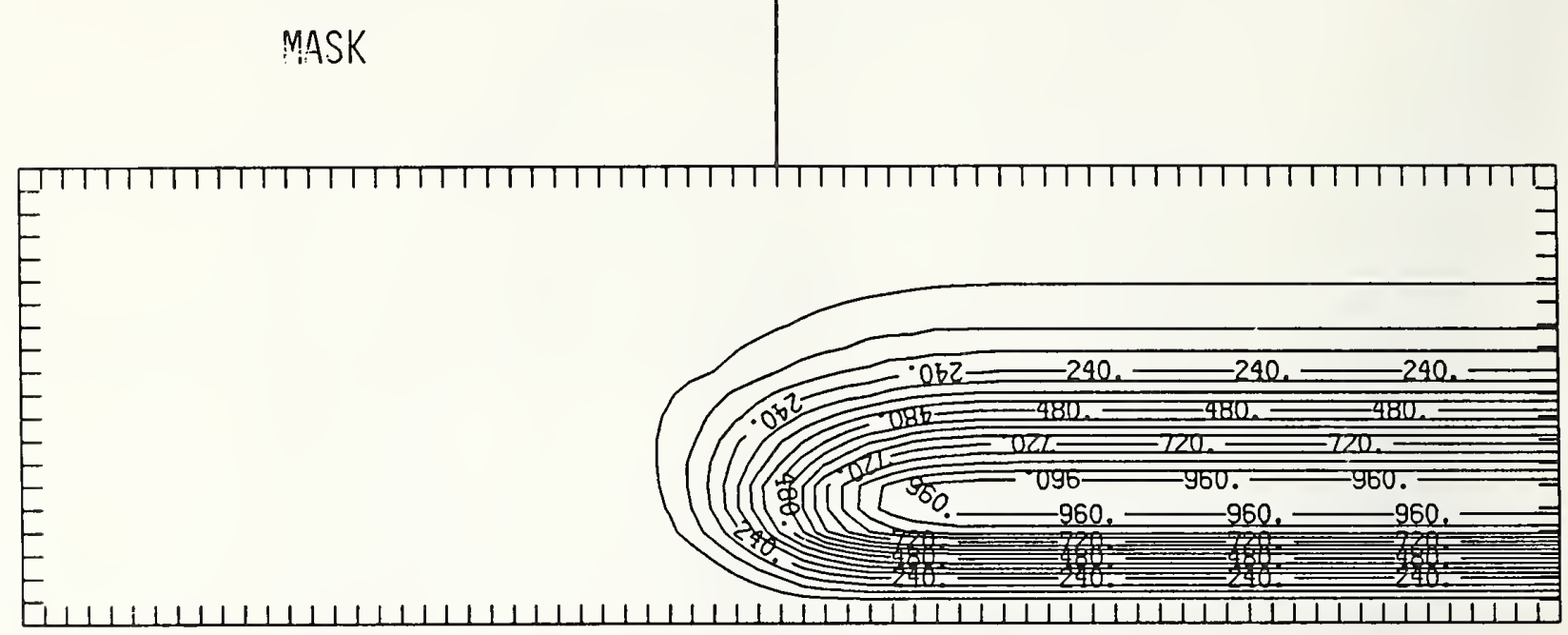

Figure N200-3 Two-dimensional distribution of $200 \mathrm{keV}$ nitrogen implanted past a mask edge. The length increment (distance between tick marks) is $0.04 \mu \mathrm{m}$ and the scale factor is 1000.

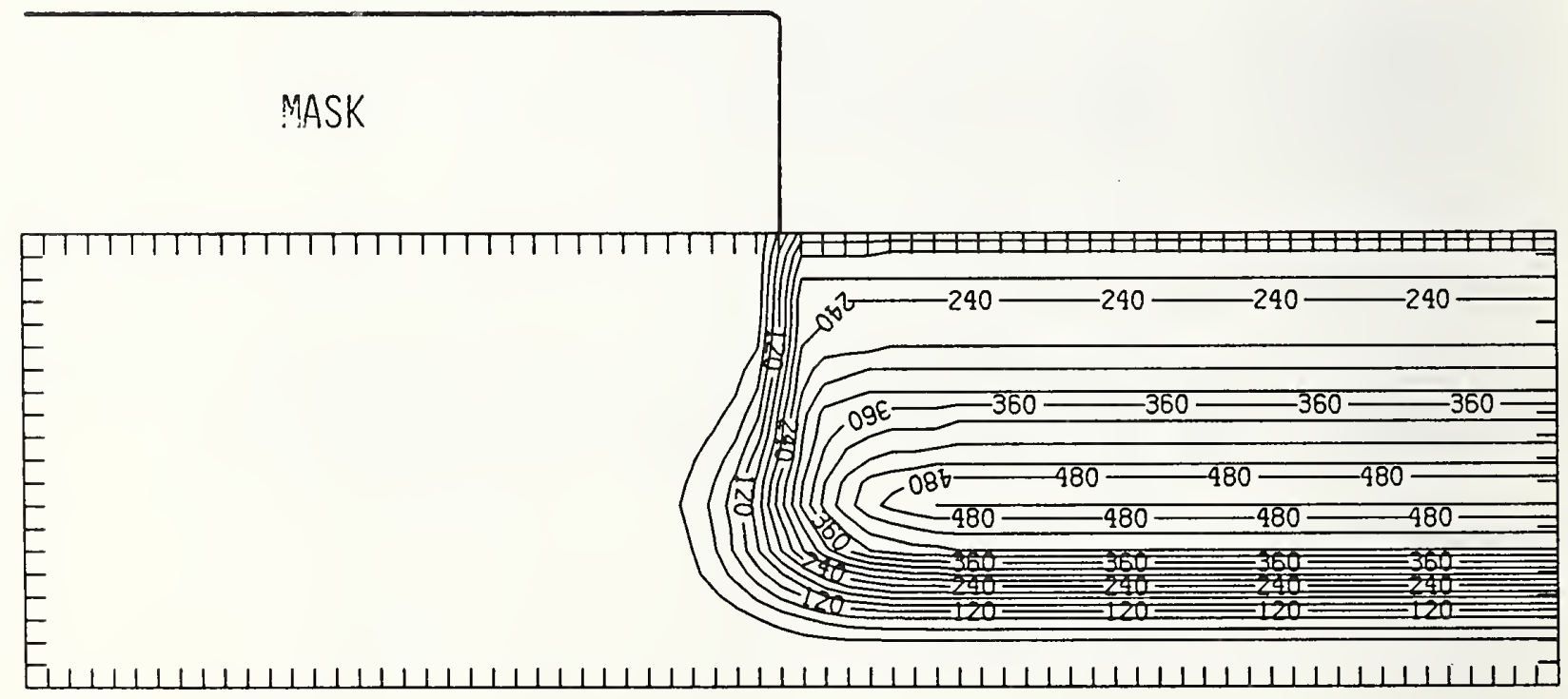

Figure N200-4 Two-dimensional distribution of Frenkel pairs created by $200 \mathrm{keV}$ nitrogen implanted past a mask edge. The length increment (distance between tick marks) is $0.04 \mu \mathrm{m}$ and the scale factor is 1 . 


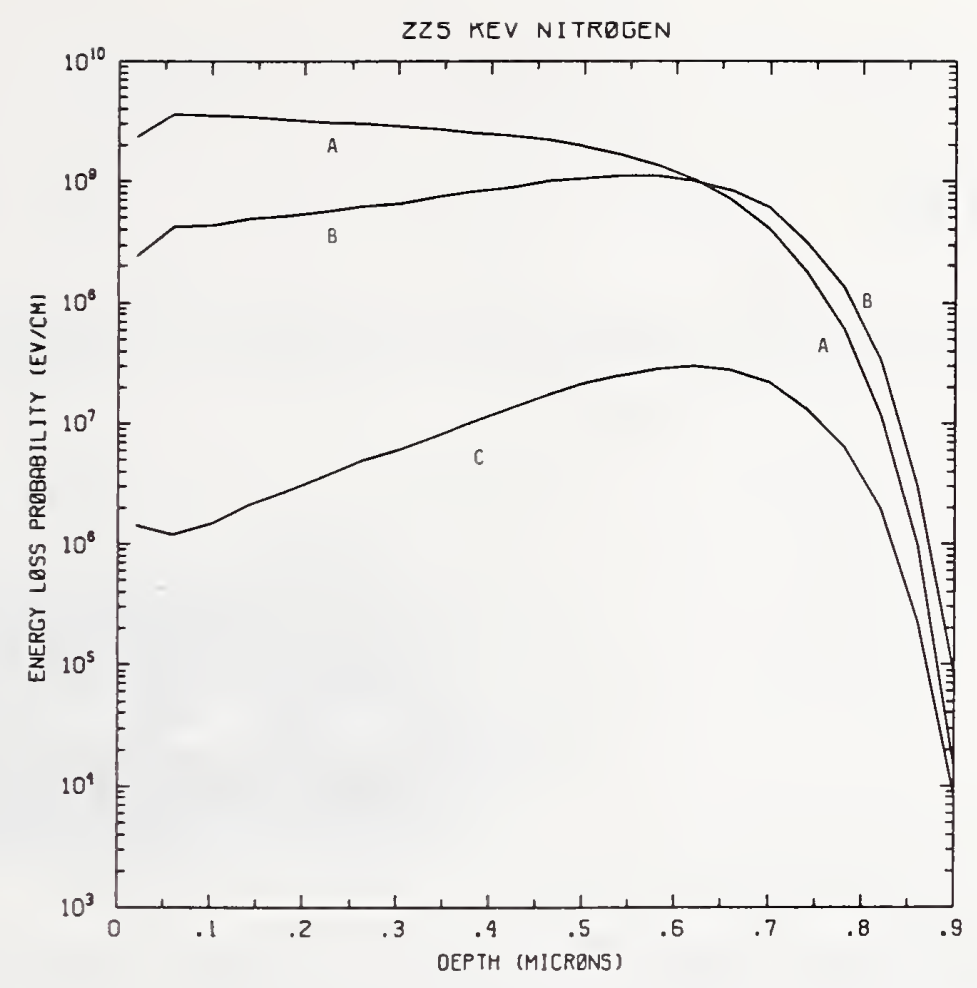

Figure N225-1 One-dimensional probability distributions for energy loss due to electronic excitation (A), displacement damage(B), and phonon excitation (C) for $225 \mathrm{keV}$ nitrogen normally incident on a silicon target.

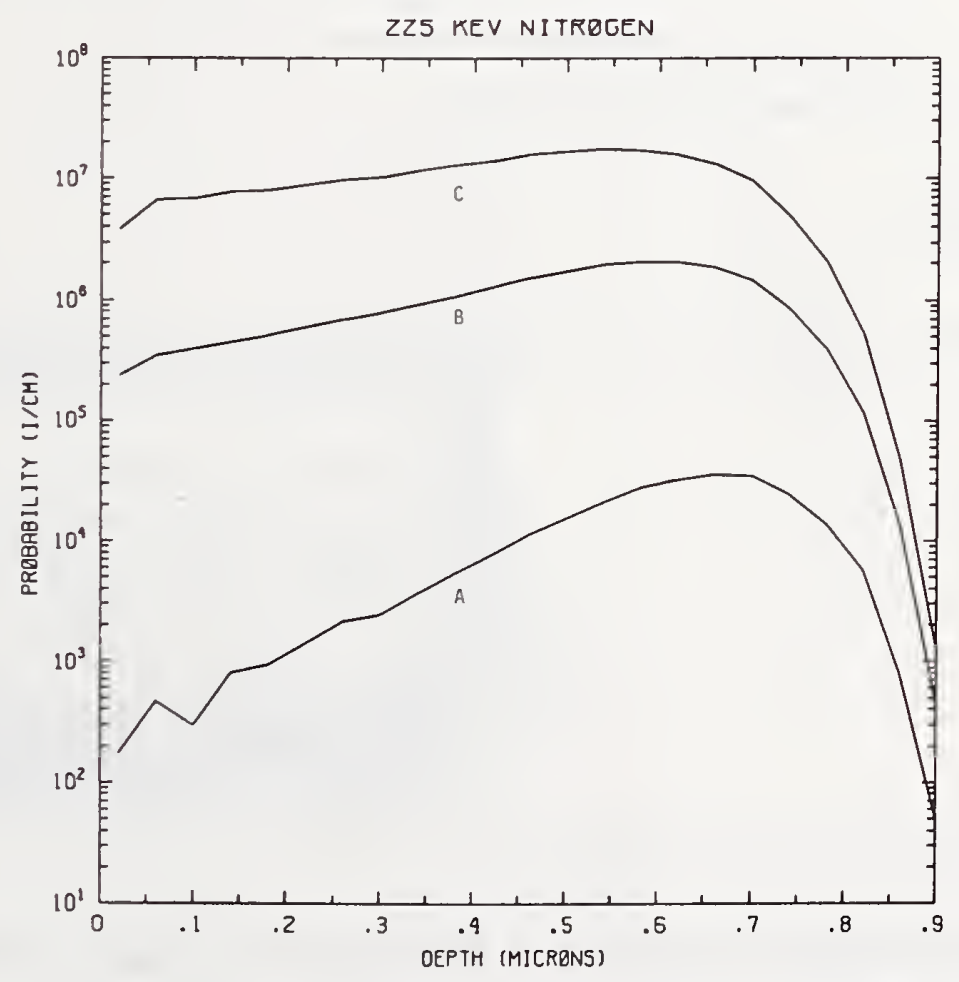

Figure N225-2 One-dimensional probability distributions for implanted nitrogen (A), primary silicon displacements (B), and Frenkel pairs (C) for $225 \mathrm{keV}$ nitrogen normally incident on a silicon target. 


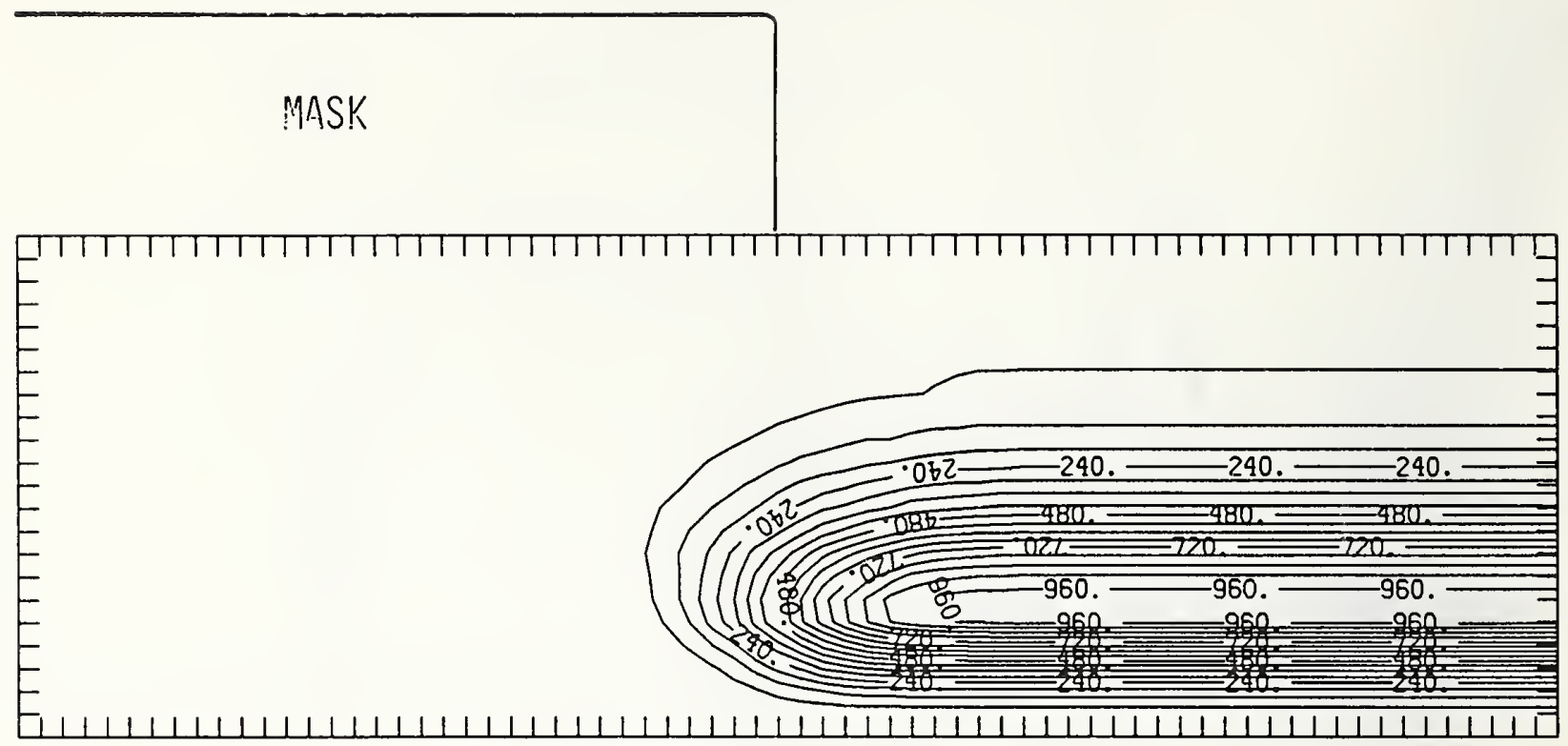

Figure N225-3 Two-dimensional distribution of $225 \mathrm{keV}$ nitrogen implanted past a mask edge. The length increment (distance between tick marks) is $0.04 \mu \mathrm{m}$ and the scale factor is 1000 .

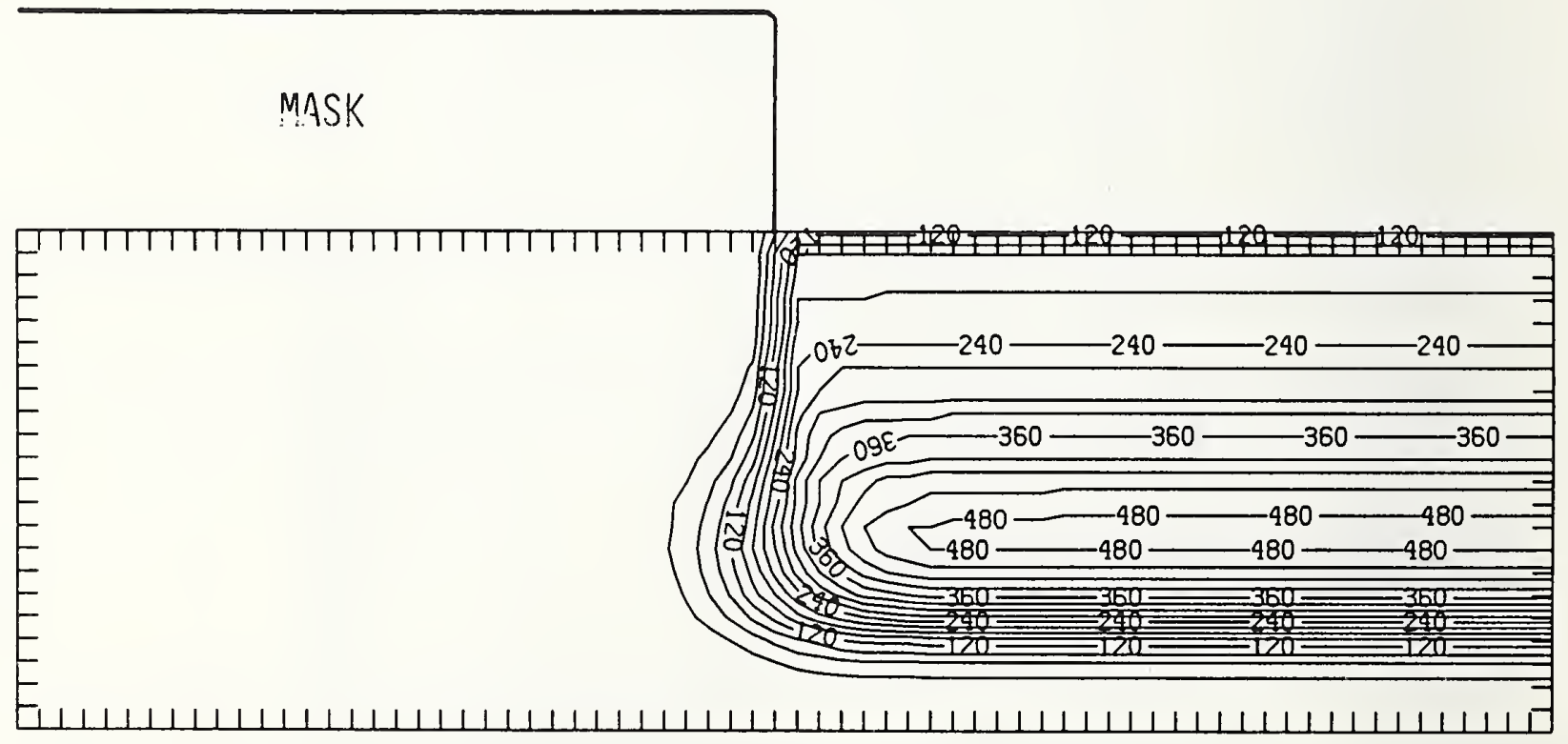

Figure N225-4 Two-dimensional distribution of Frenkel pairs created by $225 \mathrm{keV}$ nitrogen implanted past a mask edge. The length increment (distance between tick marks) is $0.04 \mu \mathrm{m}$ ( and the scale factor is 1 . 


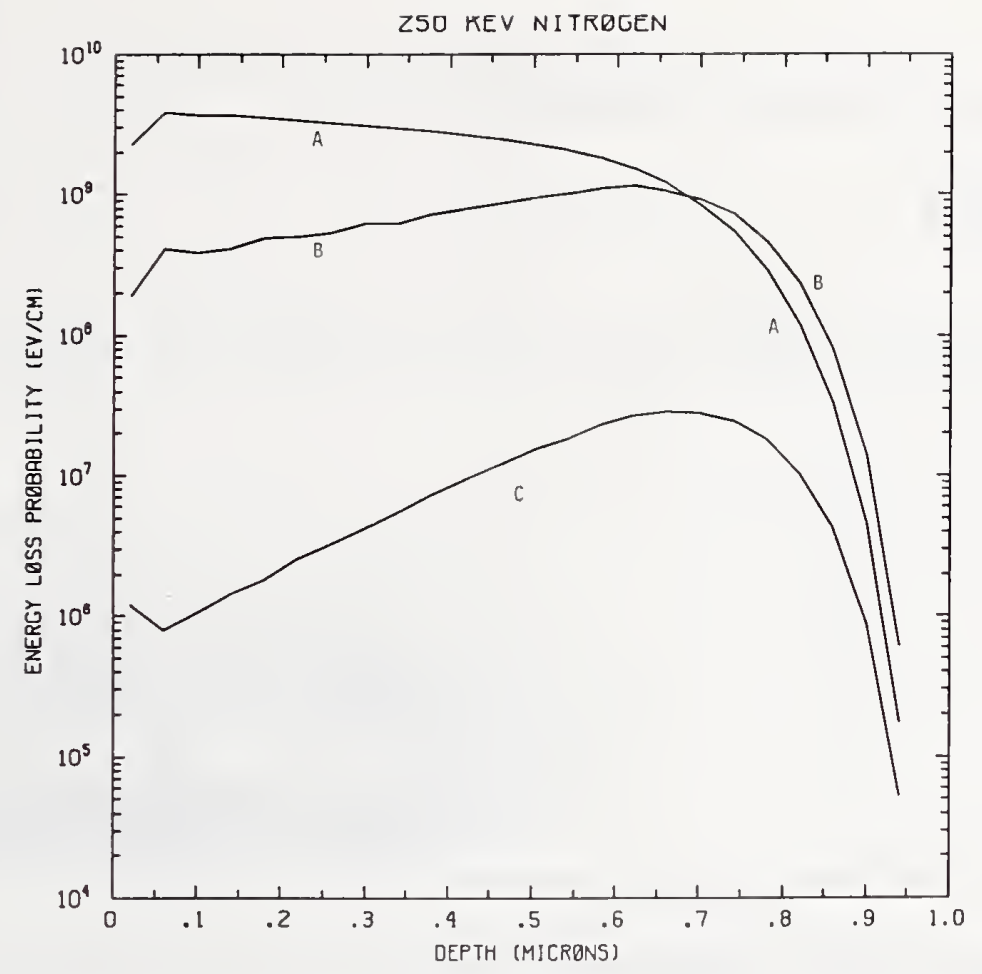

Figure N250-1 One-dimensional probability distributions for energy loss due to electronic excitation (A), displacement damage(B), and phonon excitation (C) for $250 \mathrm{keV}$ nitrogen normally incident on a silicon target.

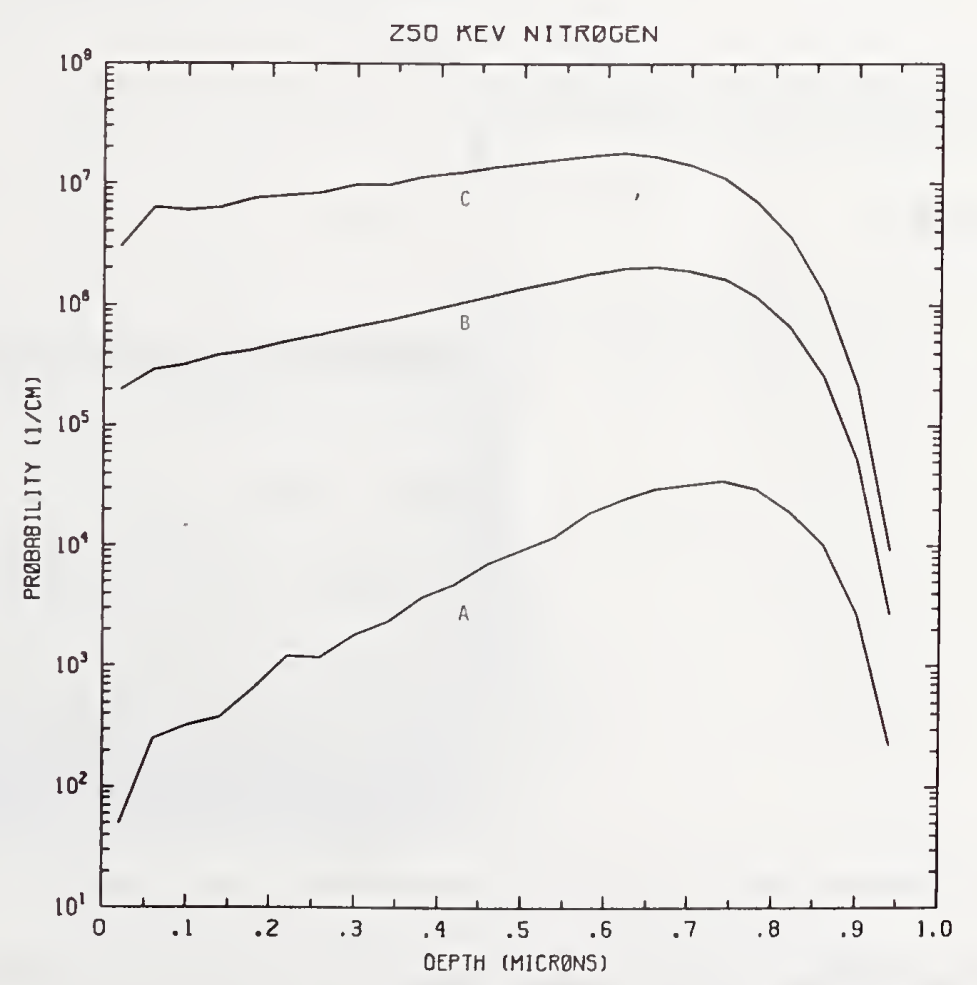

Figure N250-2 One-dimensional probability distributions for implanted nitrogen (A), primary silicon displacements (B), and Frenkel pairs (C) for $250 \mathrm{keV}$ nitrogen normally incident on a silicon target. 


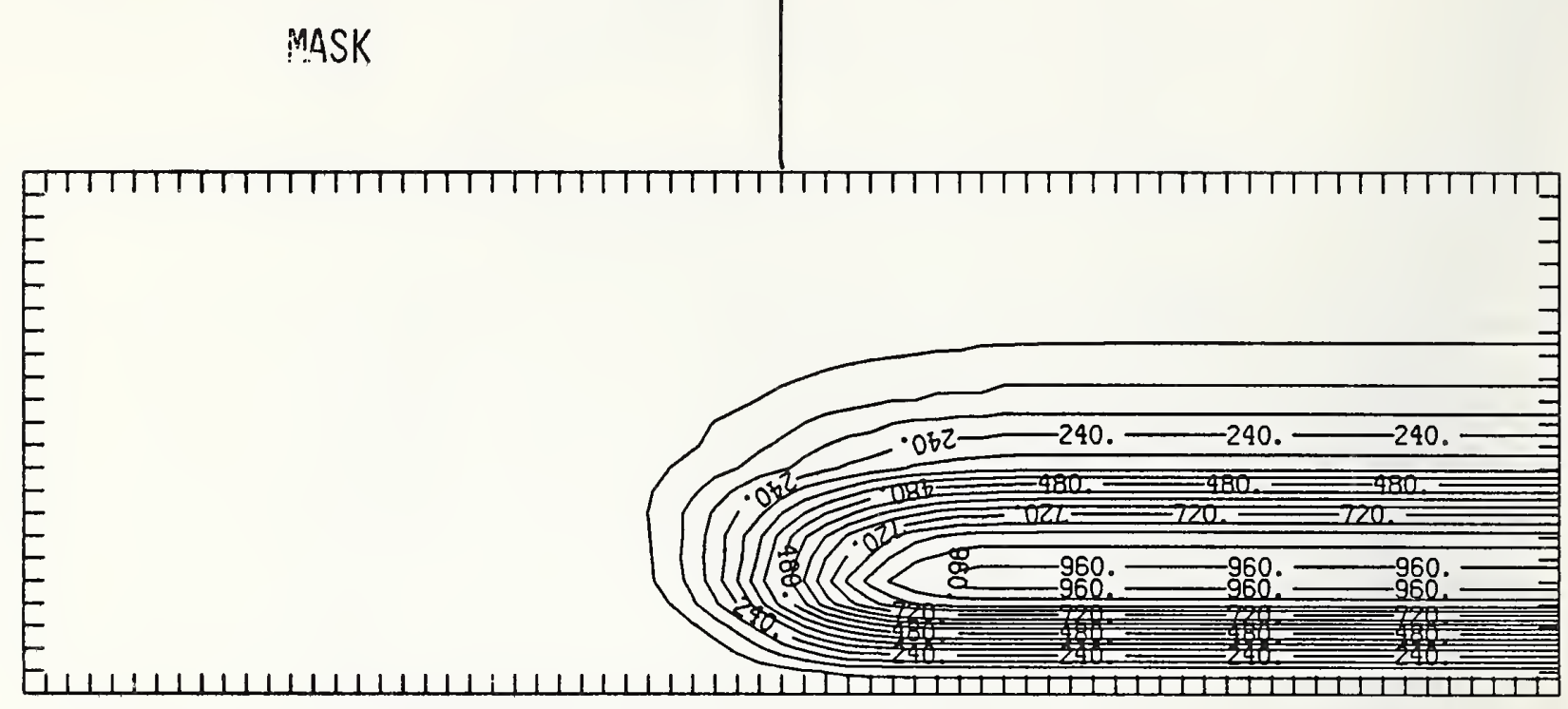

Figure N250-3 Two-dimensional distribution of $250 \mathrm{keV}$ nitrogen implanted past a mask edge. The length increment (distance between tick marks) is $0.04 \mu \mathrm{m}$ and the scale factor is 1000 .

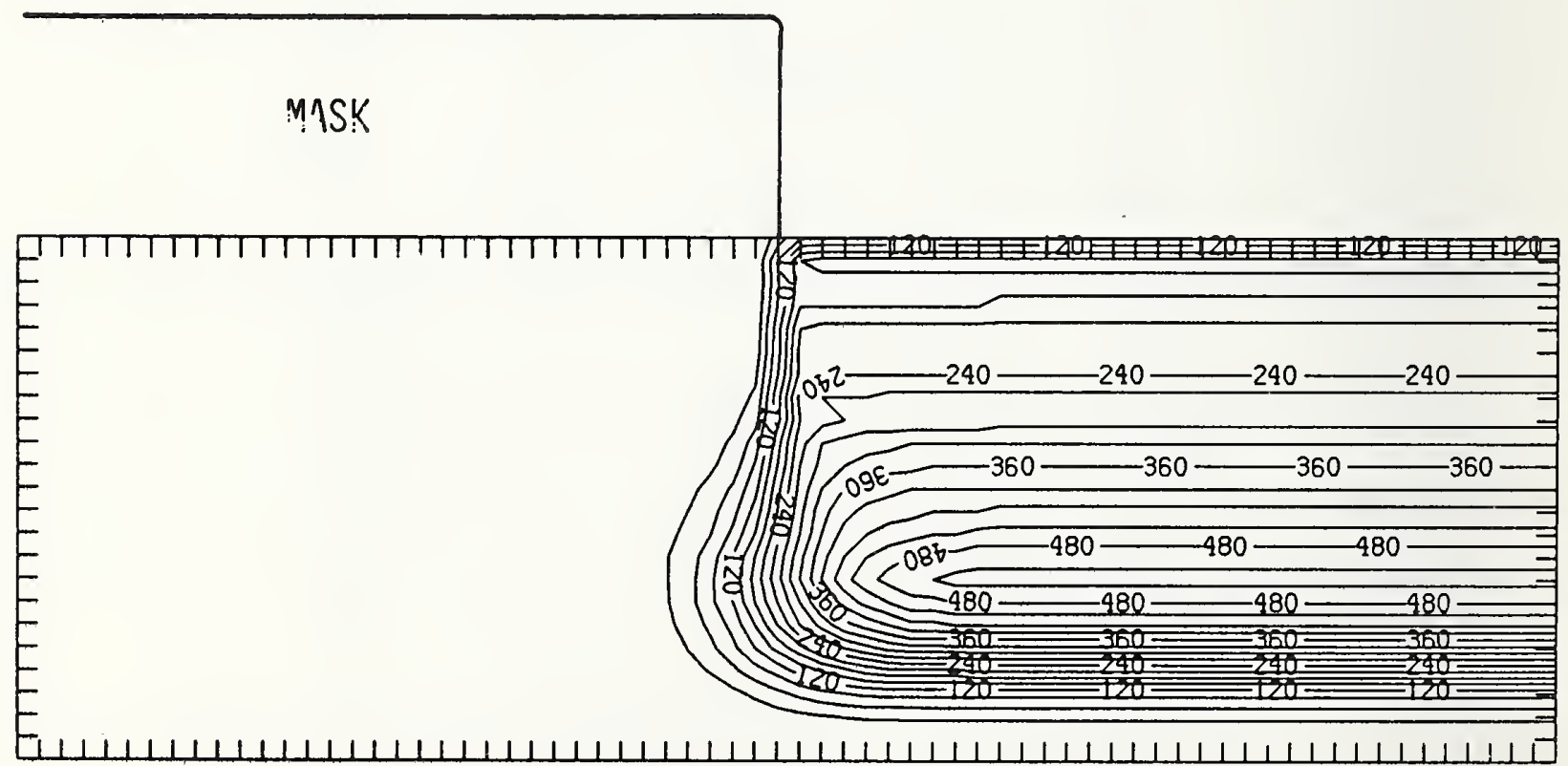

Figure N250-4 Two-dimensional distribution of Frenkel pairs created by $250 \mathrm{keV}$ nitrogen implanted past a mask edge. The length increment (distance between tick marks) is $0.04 \mu \mathrm{m}$ and the scale factor is 1 . 


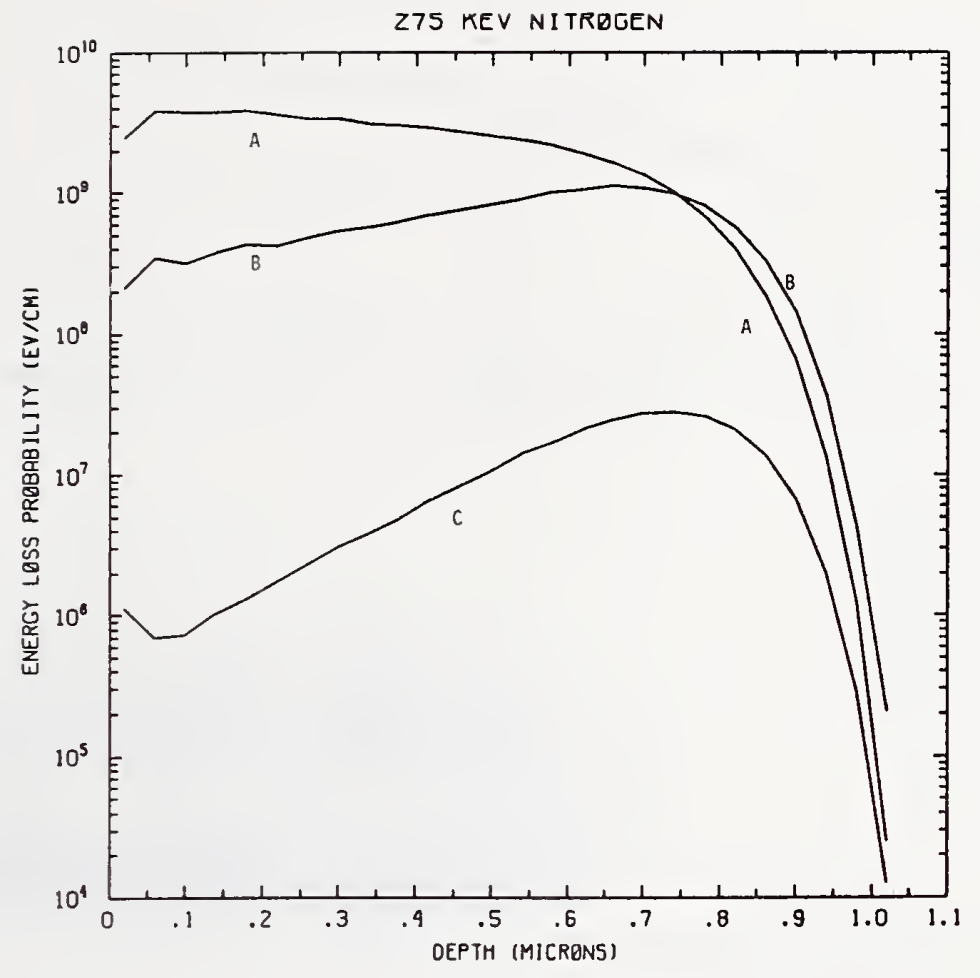

Figure N275-1 One-dimensional probability distributions for energy loss due to electronic excitation (A), displacement damage(B), and phonon excitation (C) for $275 \mathrm{keV}$ nitrogen norroally incident on a silicon target.

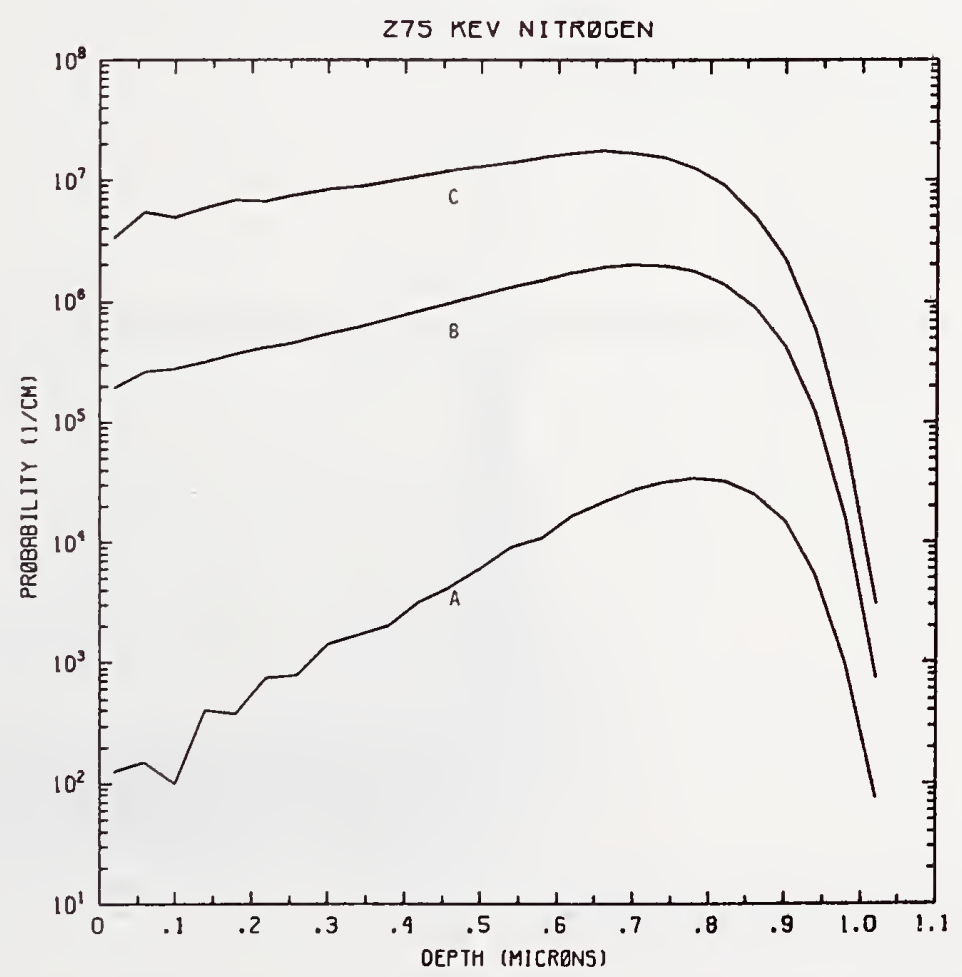

Figure N275-2 One-dimensional probability distributions for implanted nitrogen (A), primary silicon displacements (B), and Frenkel pairs (C) for $275 \mathrm{keV}$ nitrogen normally incident on a silicon target. 


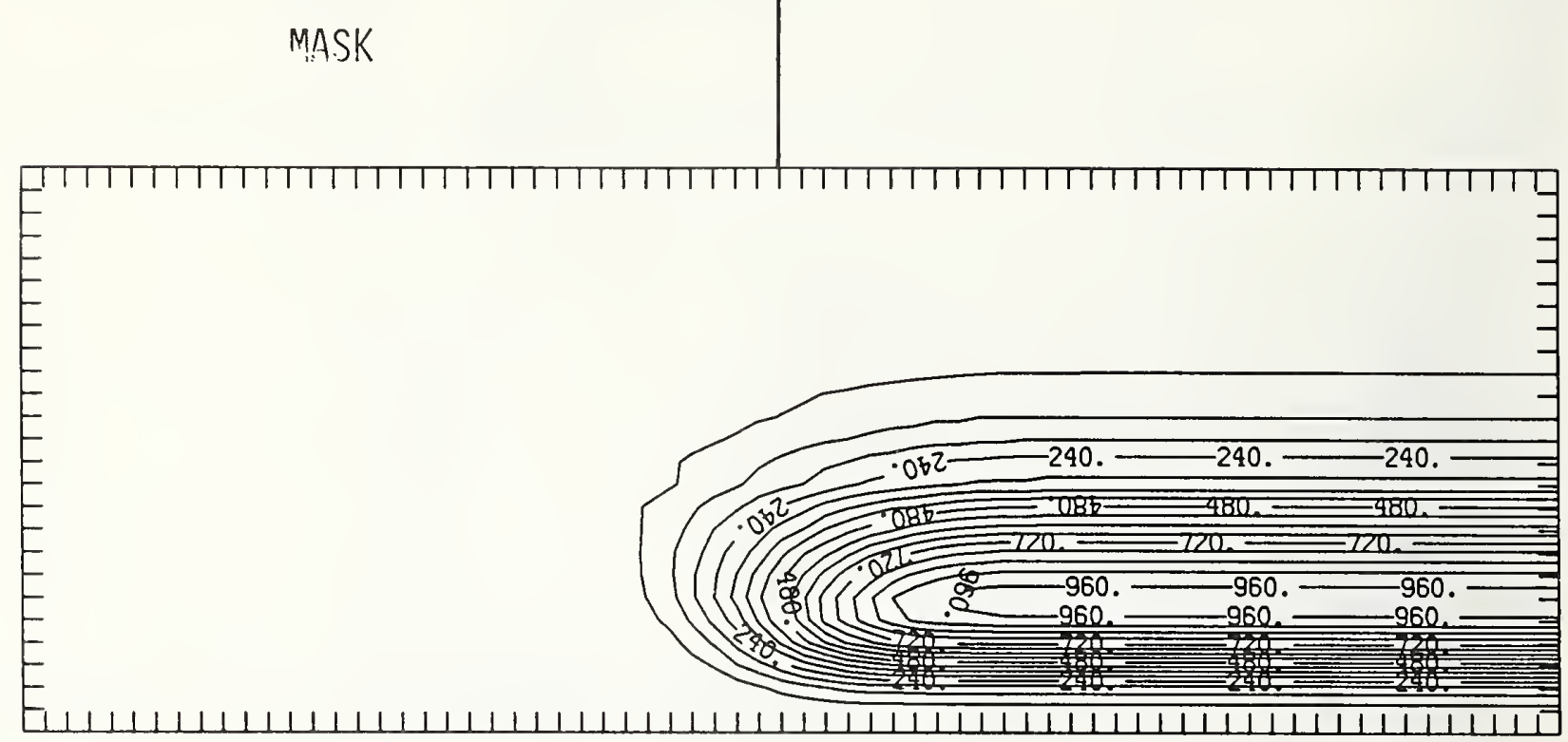

Figure N275-3 Two-dimensional distribution of $275 \mathrm{keV}$ nitrogen implanted past a mask edge. The length increment (distance between tick marks) is $0.04 \mu \mathrm{m}$ and the scale factor is 1000 .

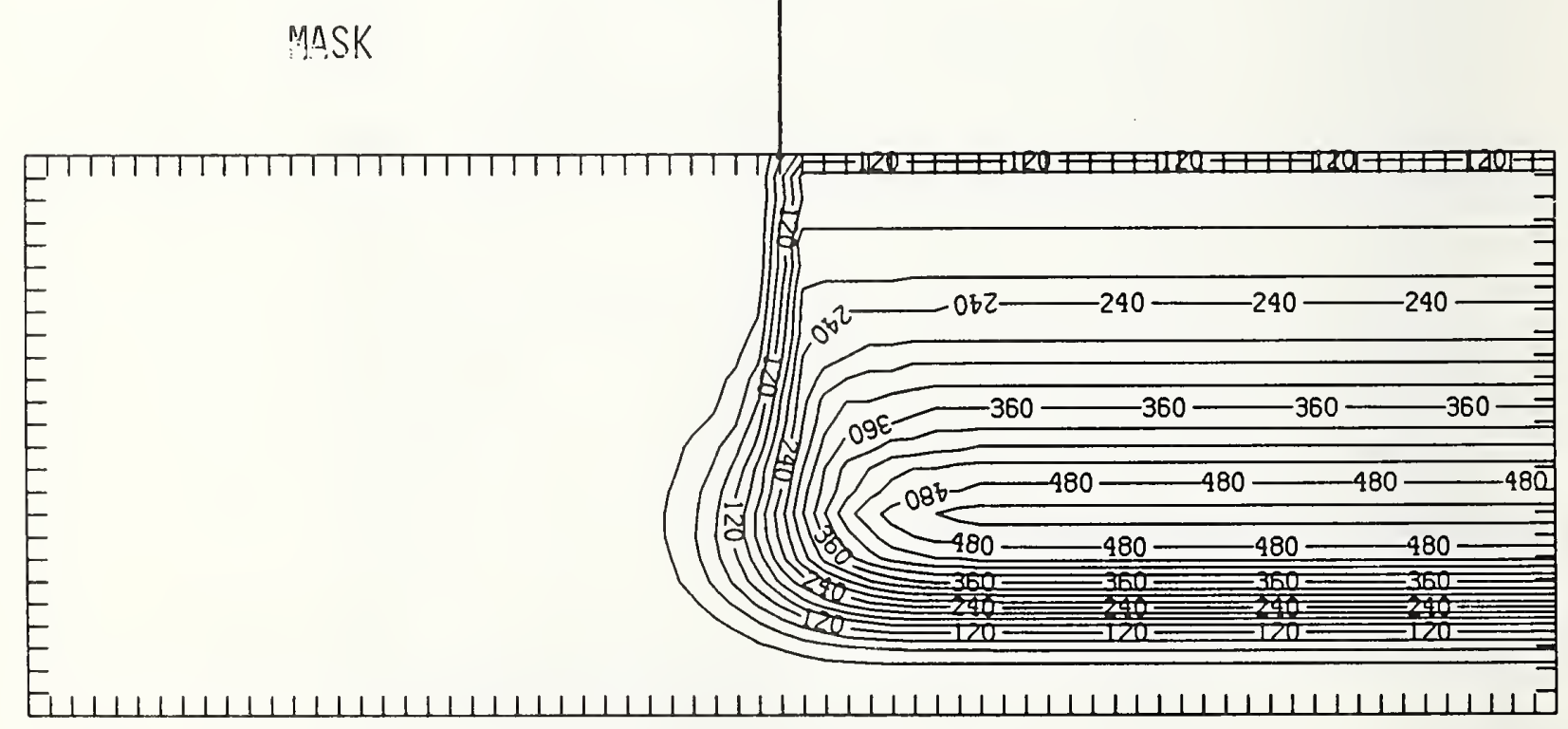

Figure N275-4 Two-dimensional distribution of Frenkel pairs created by $275 \mathrm{keV}$ nitrogen implanted past a mask edge. The length increment (distance between tick marks) is $0.04 \mu \mathrm{m}$ and the scale factor is 1 . 


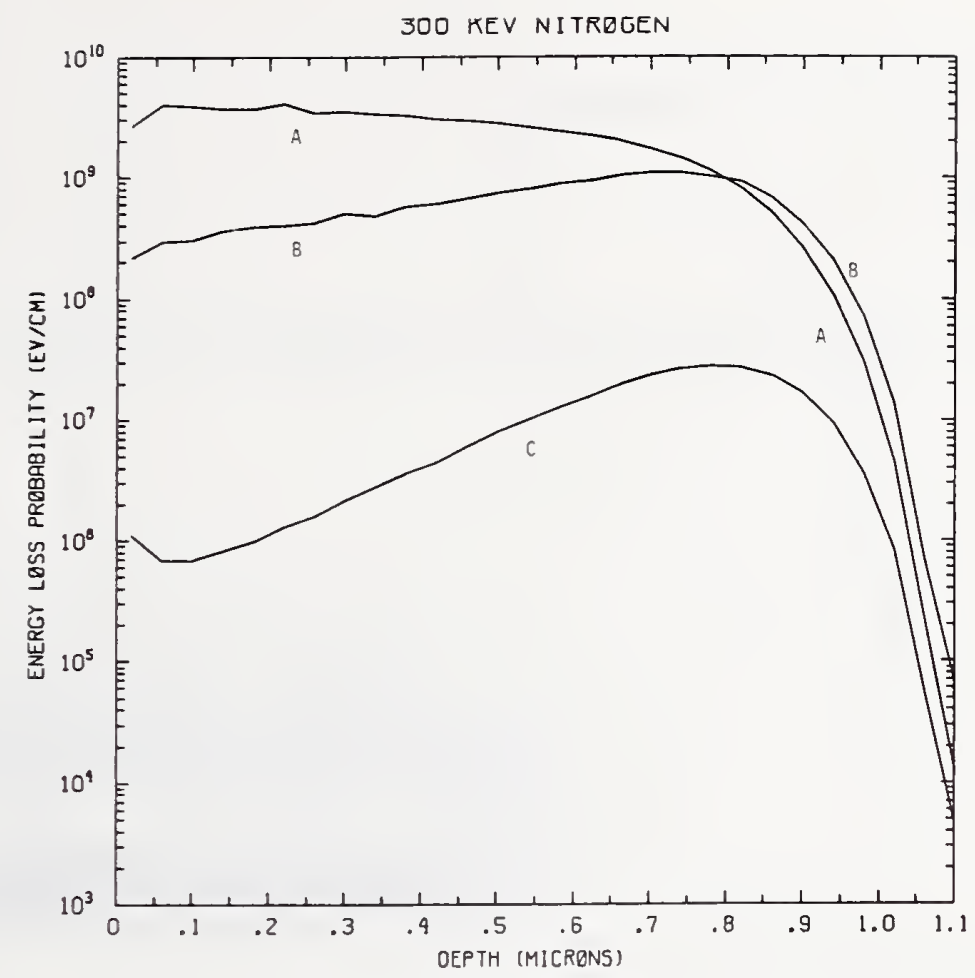

Figure N300-1 One-dimensional probability distributions for energy loss due to electronic excitation (A), displacement damage(B), and phonon excitation (C) for $300 \mathrm{keV}$ nitrogen normally incident on a silicon target.

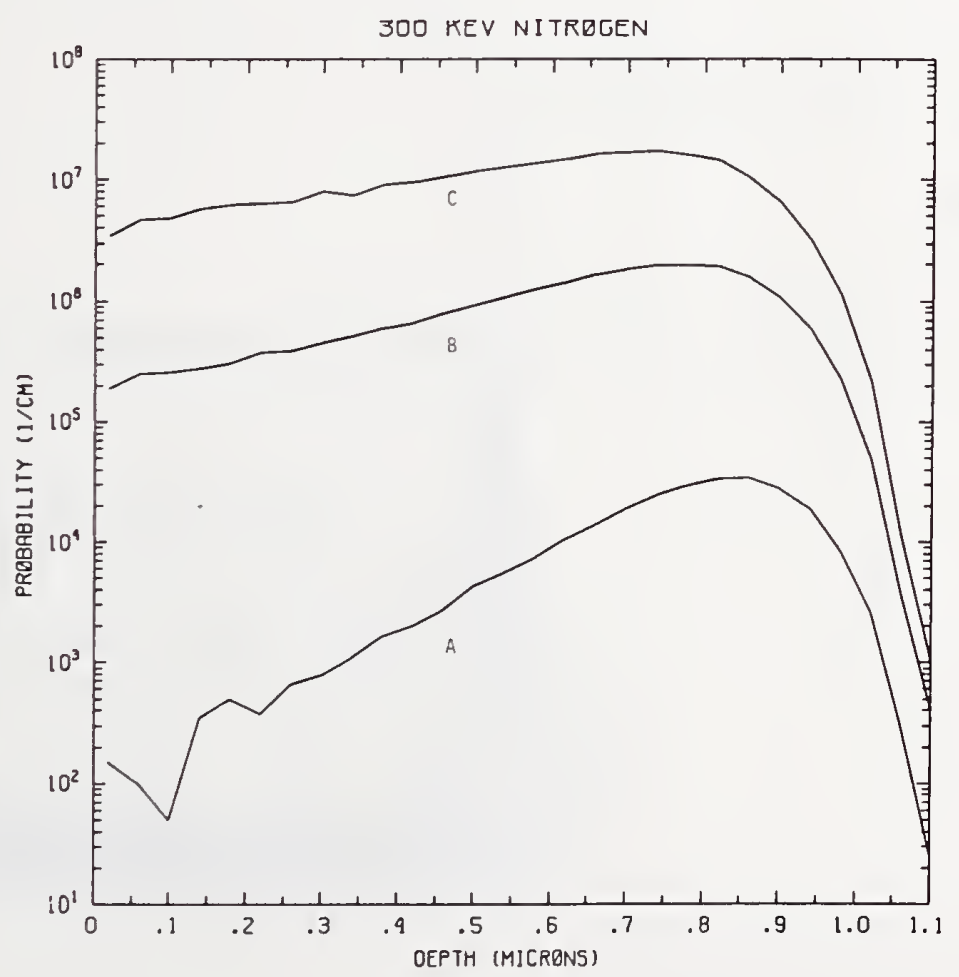

Figure N300-2 One-dimensional probability distributions for implanted nitrogen (A), primary silicon displacements (B), and Frenkel pairs (C) for $300 \mathrm{keV}$ nitrogen normally incident on a silicon target. 


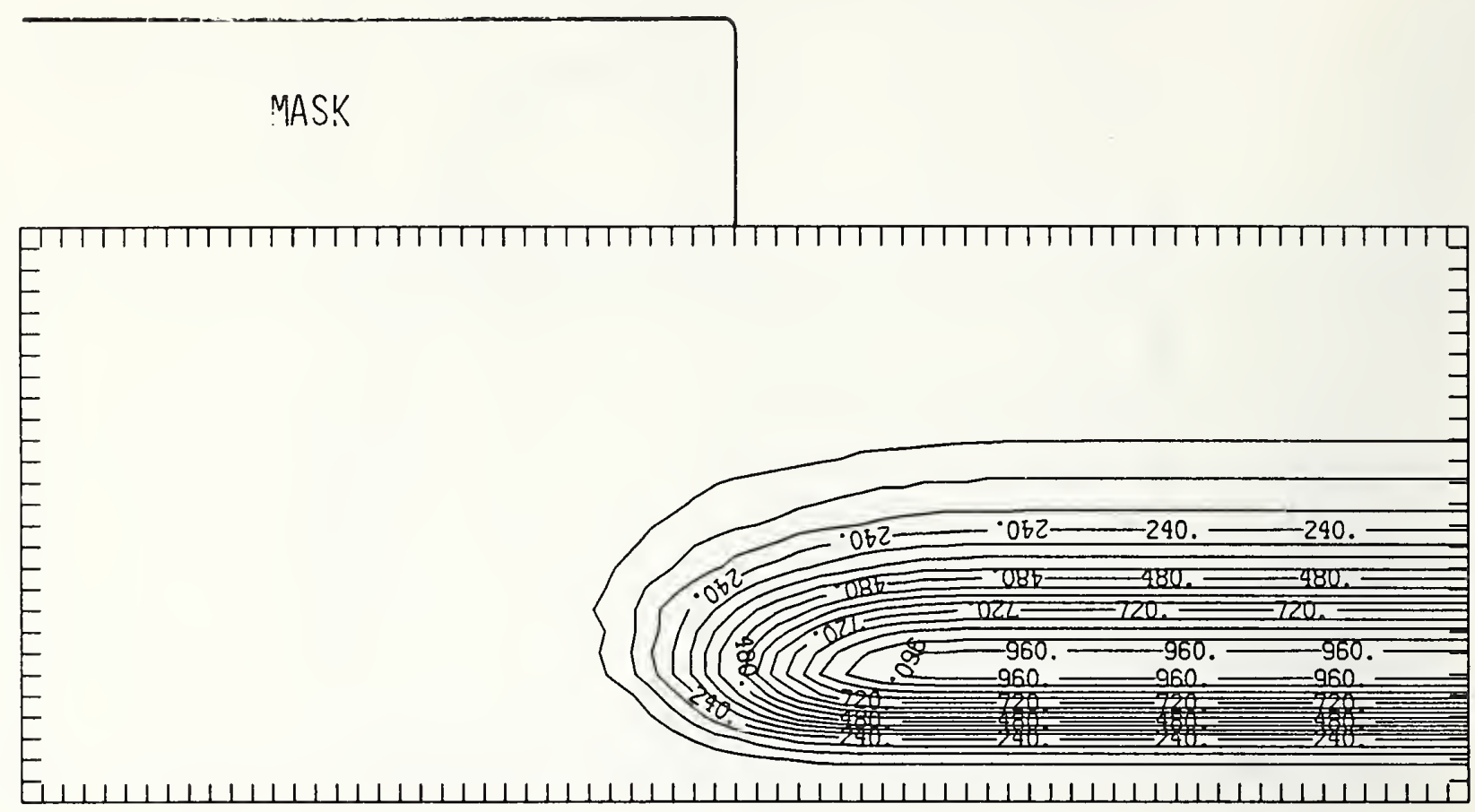

Figure N300-3 Two-dimensional distribution of $300 \mathrm{keV}$ nitrogen implanted past a mask edge. The length increment (distance between tick marks) is $0.04 \mu \mathrm{m}$ and the scale factor is 1000 .

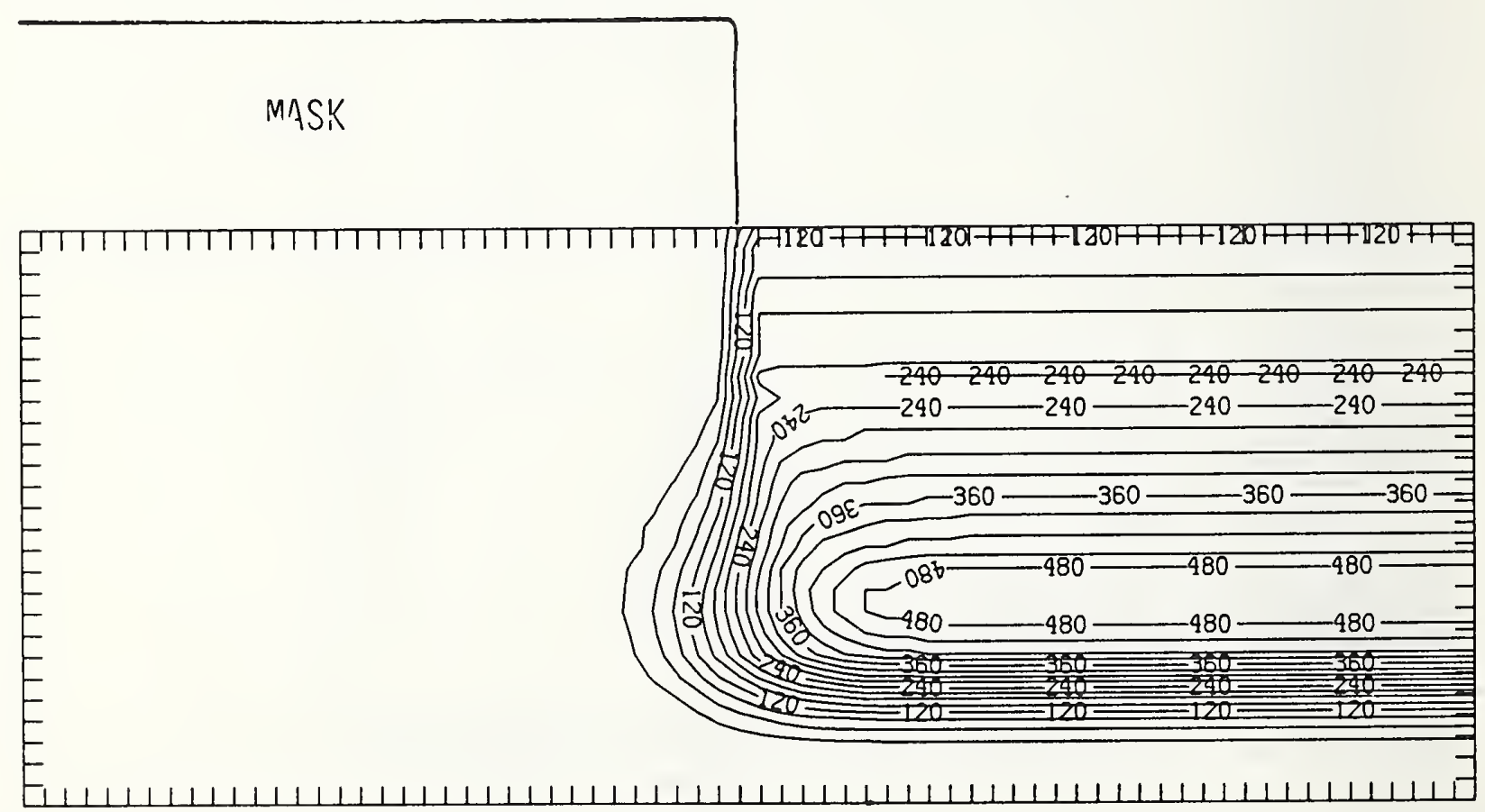

Figure N300-4 Two-dimensional distribution of Frenkel pairs created by $300 \mathrm{keV}$ nitrogen implanted past a mask edge. The length increment (distance between tick marks) is $0.04 \mu \mathrm{m}$ and the scale factor is 1 . 


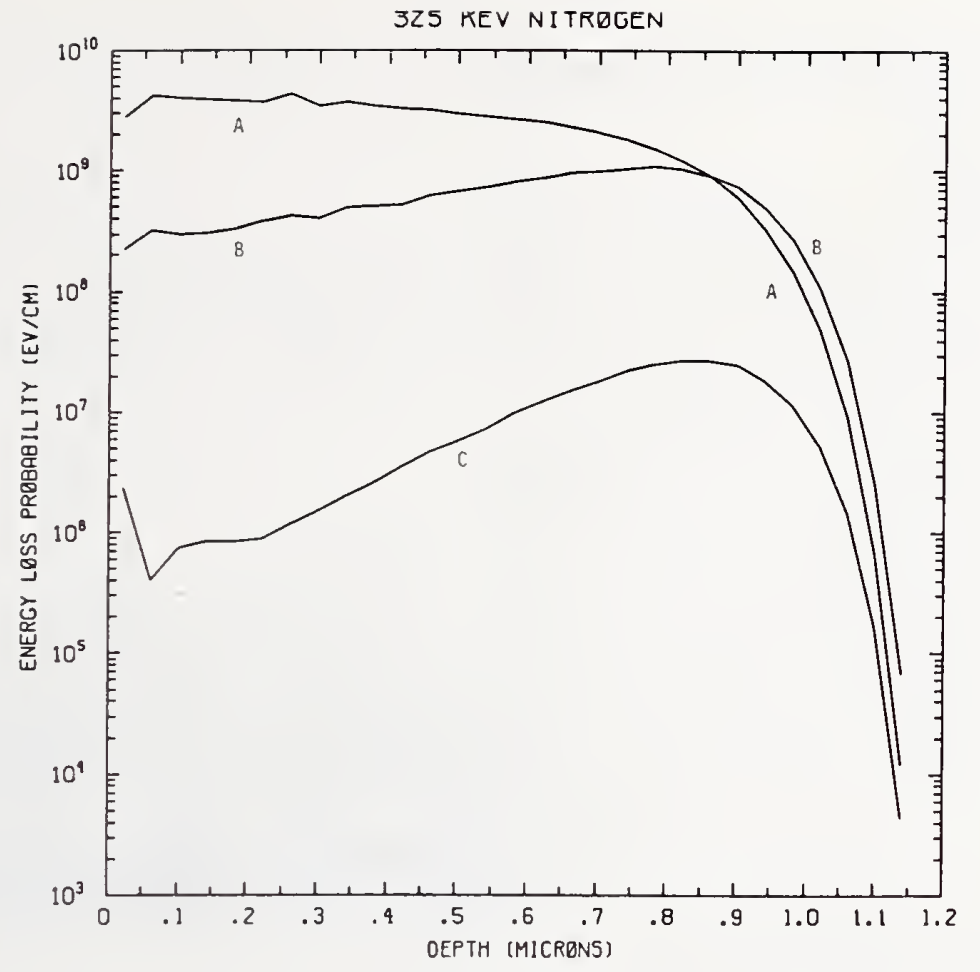

Figure N325-1 One-dimensional probability distributions for energy loss due to electronic excitation (A), displacement damage(B), and phonon excitation (C) for $325 \mathrm{keV}$ nitrogen normally incident on a silicon target.

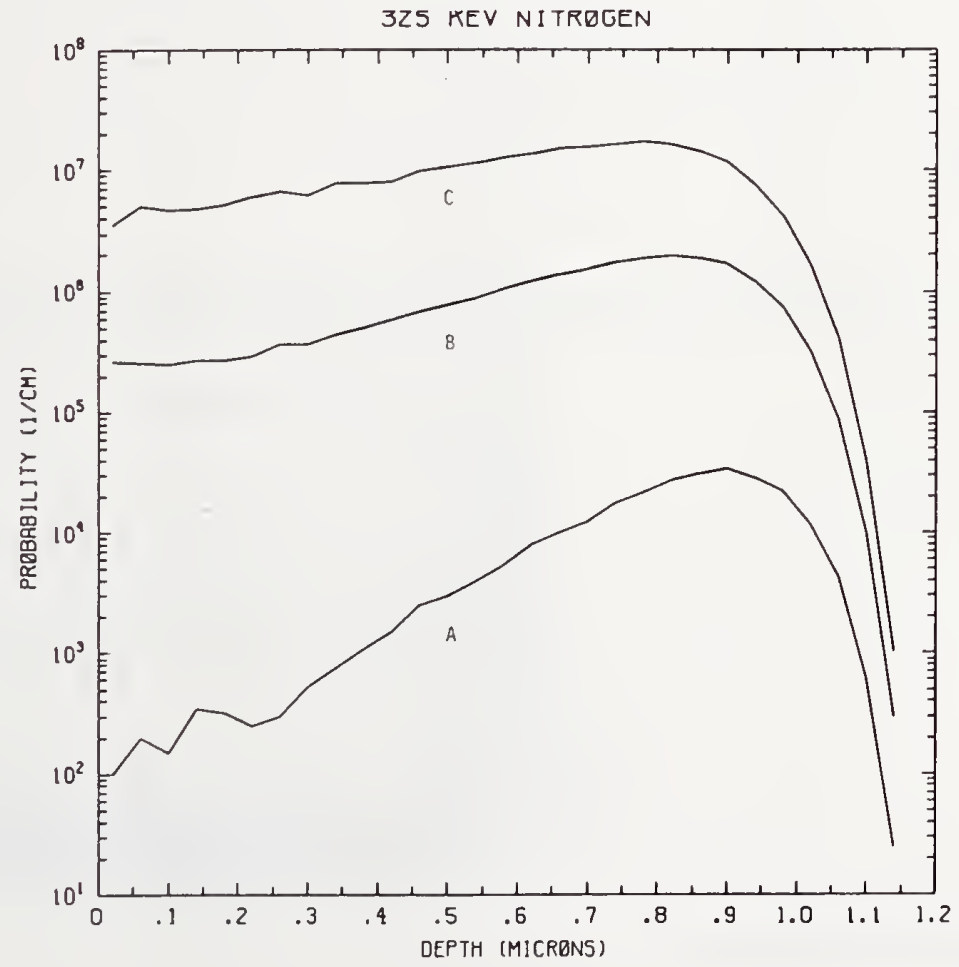

Figure N325-2 One-dimensional probability distributions for implanted nitrogen (A), primary silicon displacements (B), and Frenkel pairs (C) for $325 \mathrm{keV}$ nitrogen normally incident on a silicon target. 


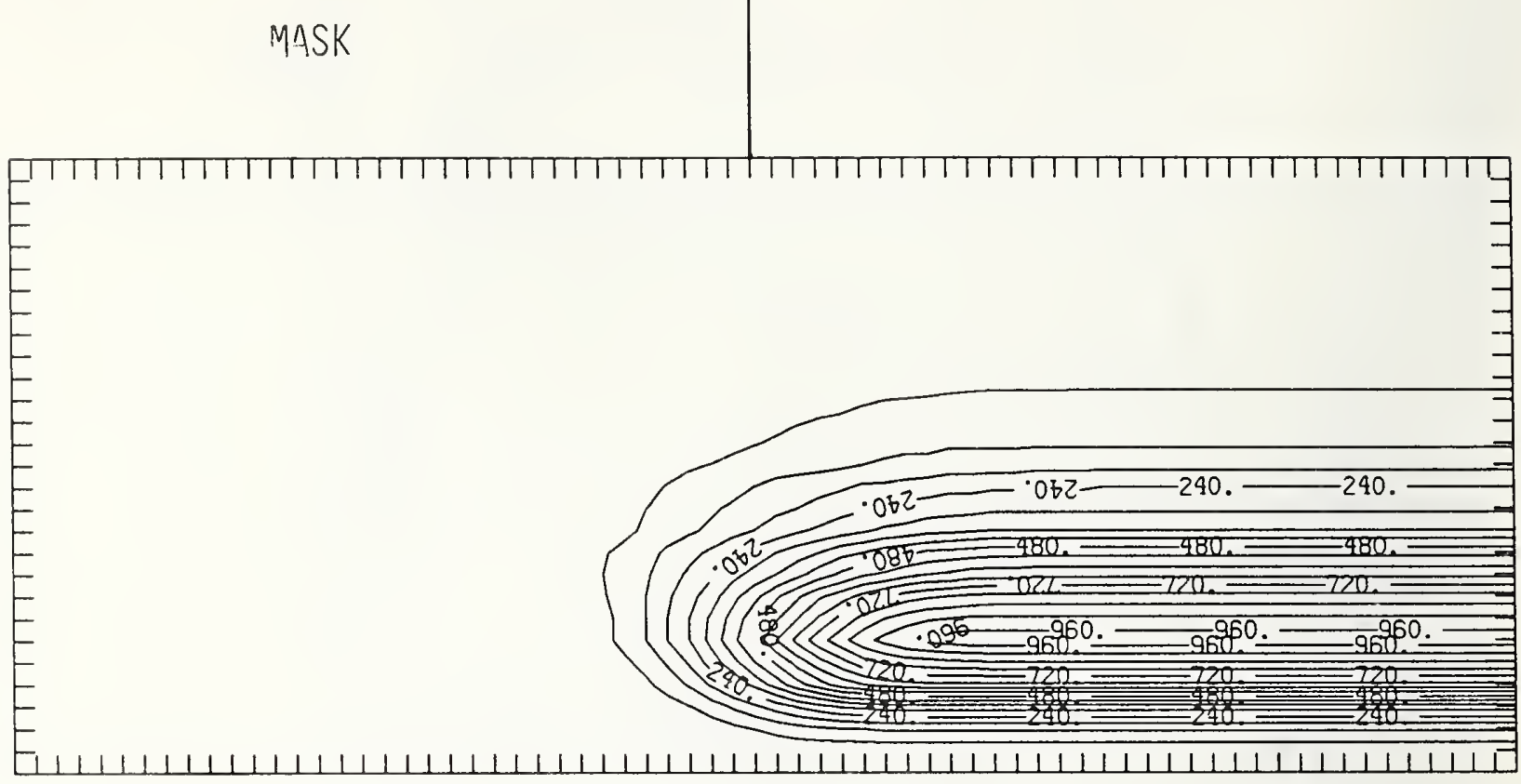

Figure N325-3 Two-dimensional distribution of $325 \mathrm{keV}$ nitrogen implanted past a mask edge. The length increment (distance between tick marks) is $0.04 \mu \mathrm{m}$ and the scale factor is 1000.

MASK

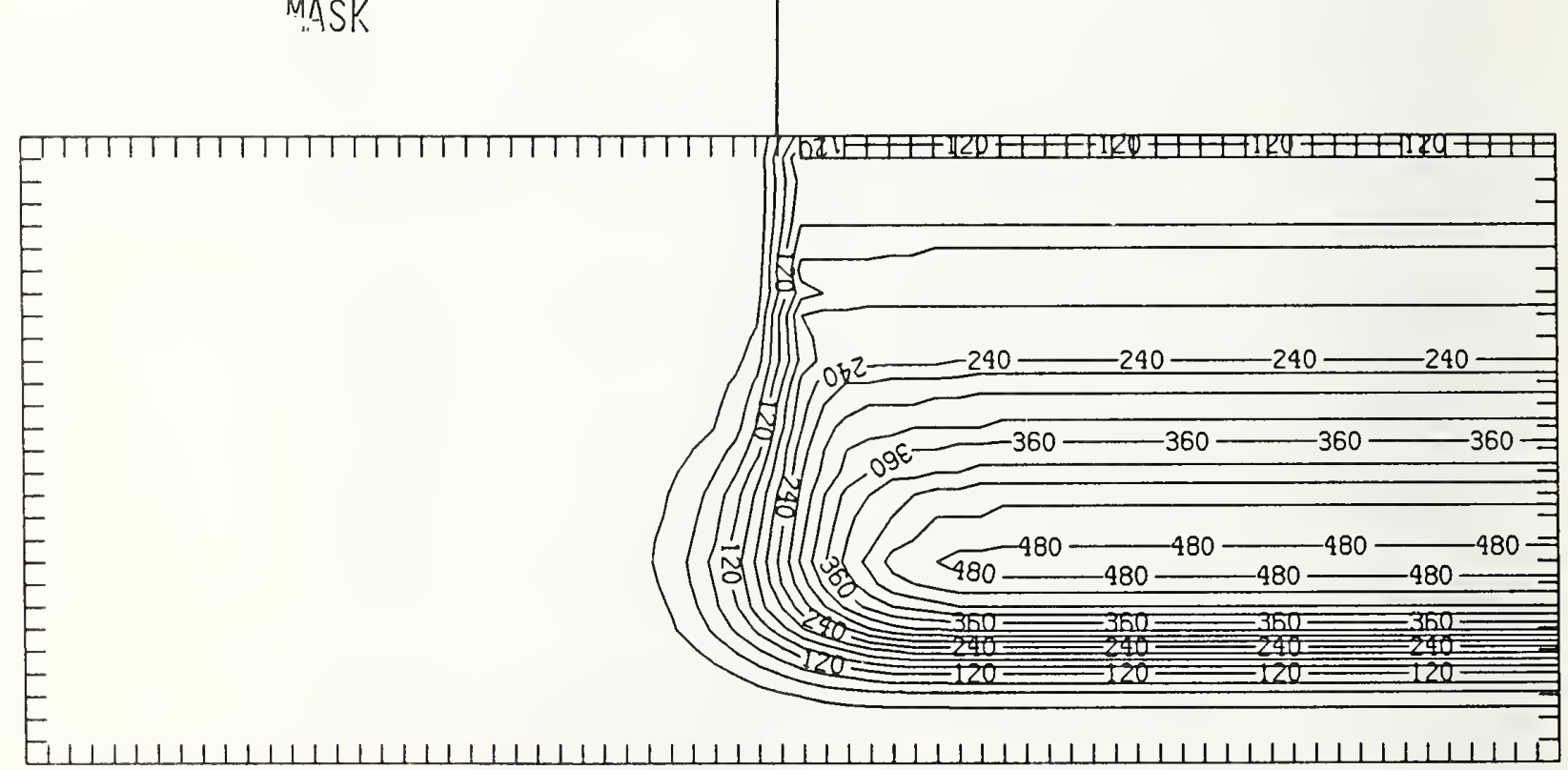

Figure N325-4 Two-dimensional distribution of Frenkel pairs created by $325 \mathrm{keV}$ nitrogen implanted past a mask edge. The length increment (distance between tick marks) is $0.04 \mu \mathrm{m}$ and the scale factor is 1 . 


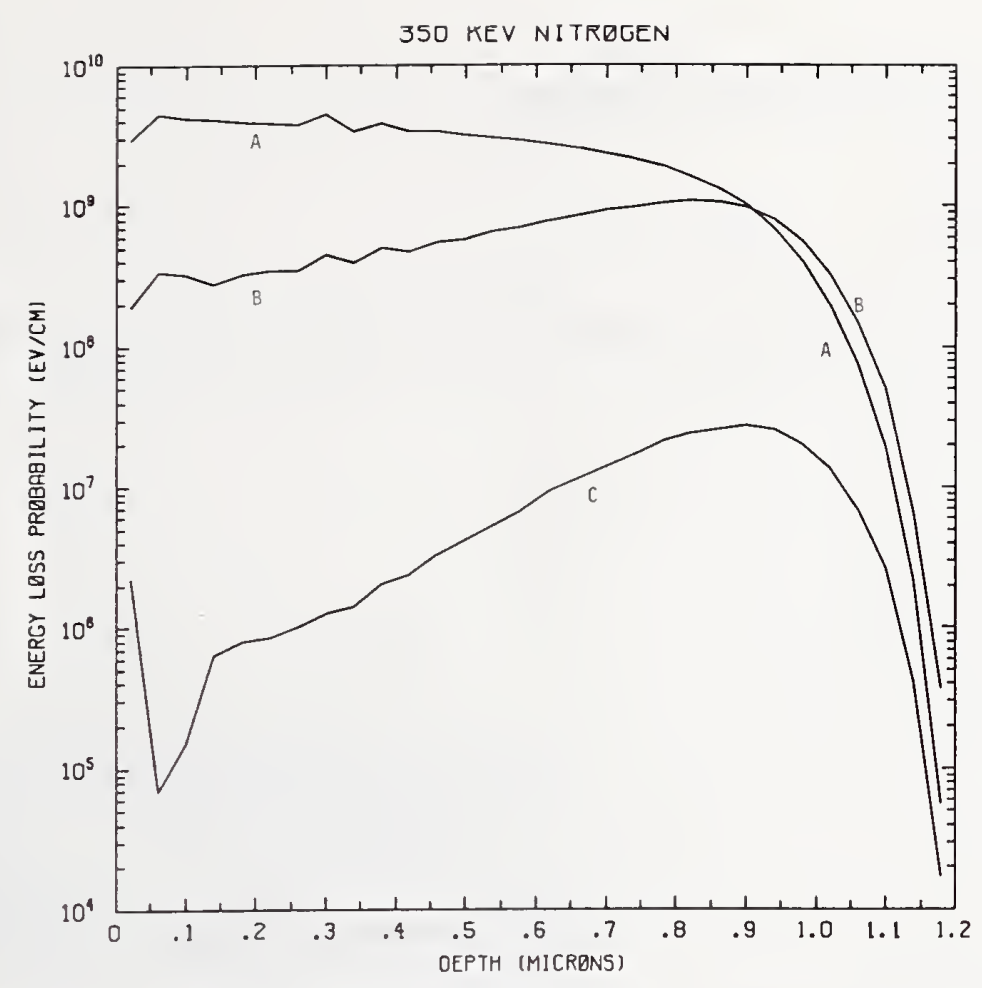

Figure N350-1 One-dimensional probability distributions for energy loss due to electronic excitation (A), displacement damage(B), and phonon excitation (C) for $350 \mathrm{keV}$ nitrogen normally incident on a silicon target.

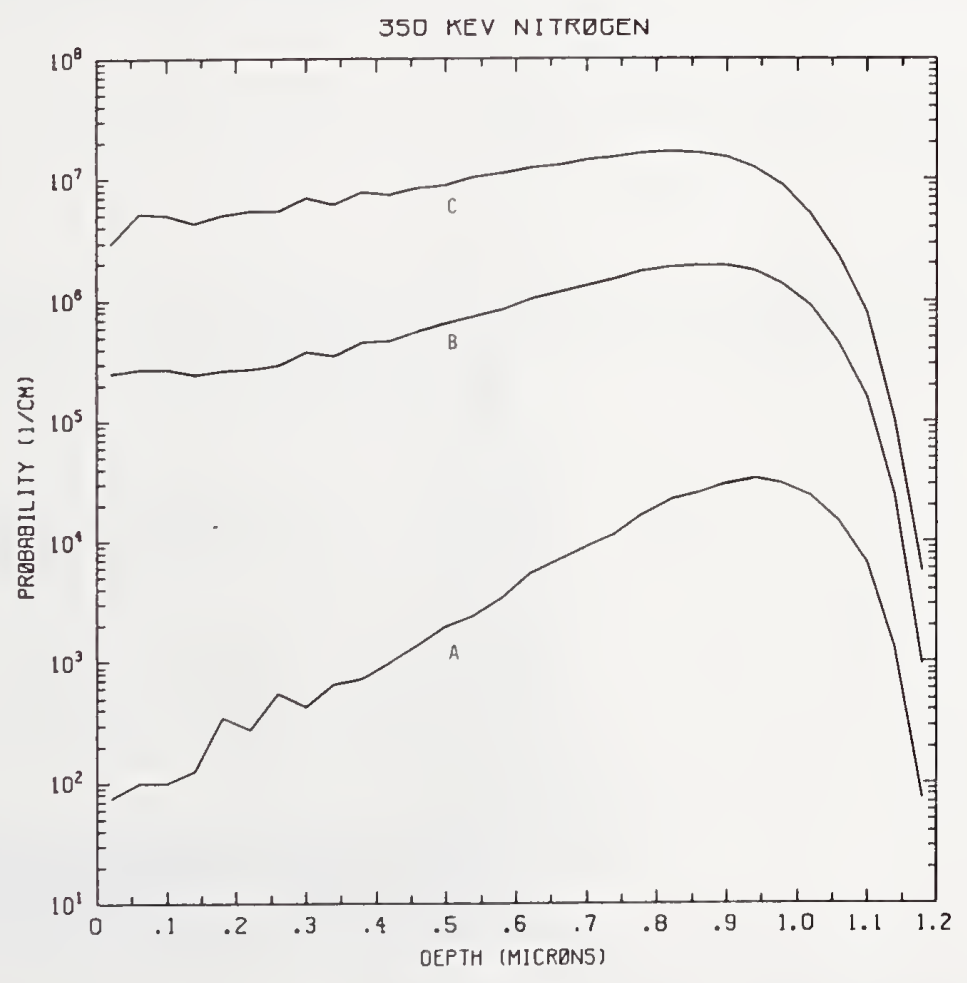

Figure N350-2 One-dimensional probability distributions for implanted nitrogen (A), primary silicon displacements (B), and Frenkel pairs (C) for $350 \mathrm{keV}$ nitrogen normally incident on a silicon target. 


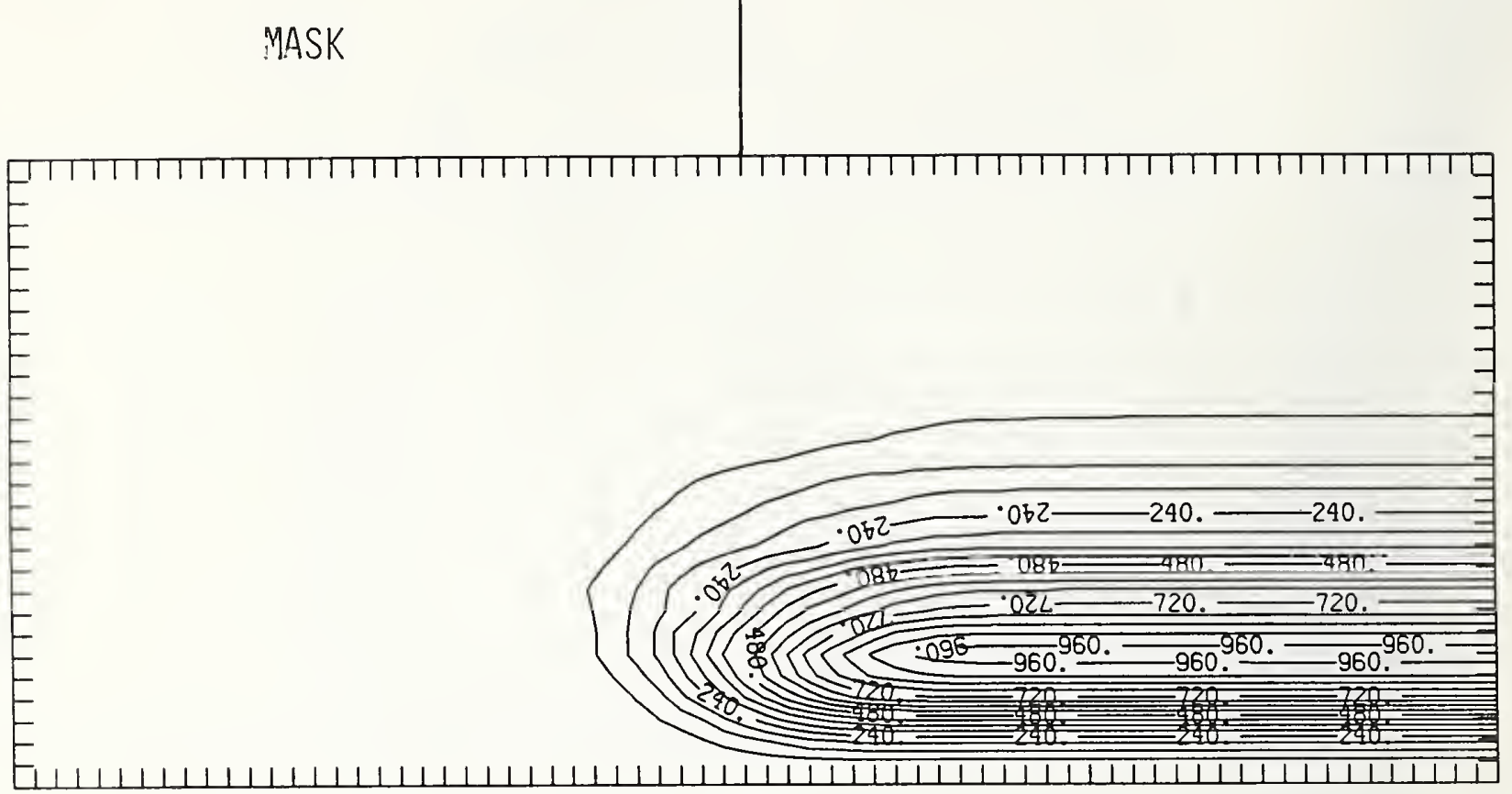

Figure N350-3 Two-dimensional distribution of $350 \mathrm{keV}$ nitrogen implanted past a mask edge. The length increment (distance between tick marks) is $0.04 \mu \mathrm{m}$ and the scale factor if 1000 .

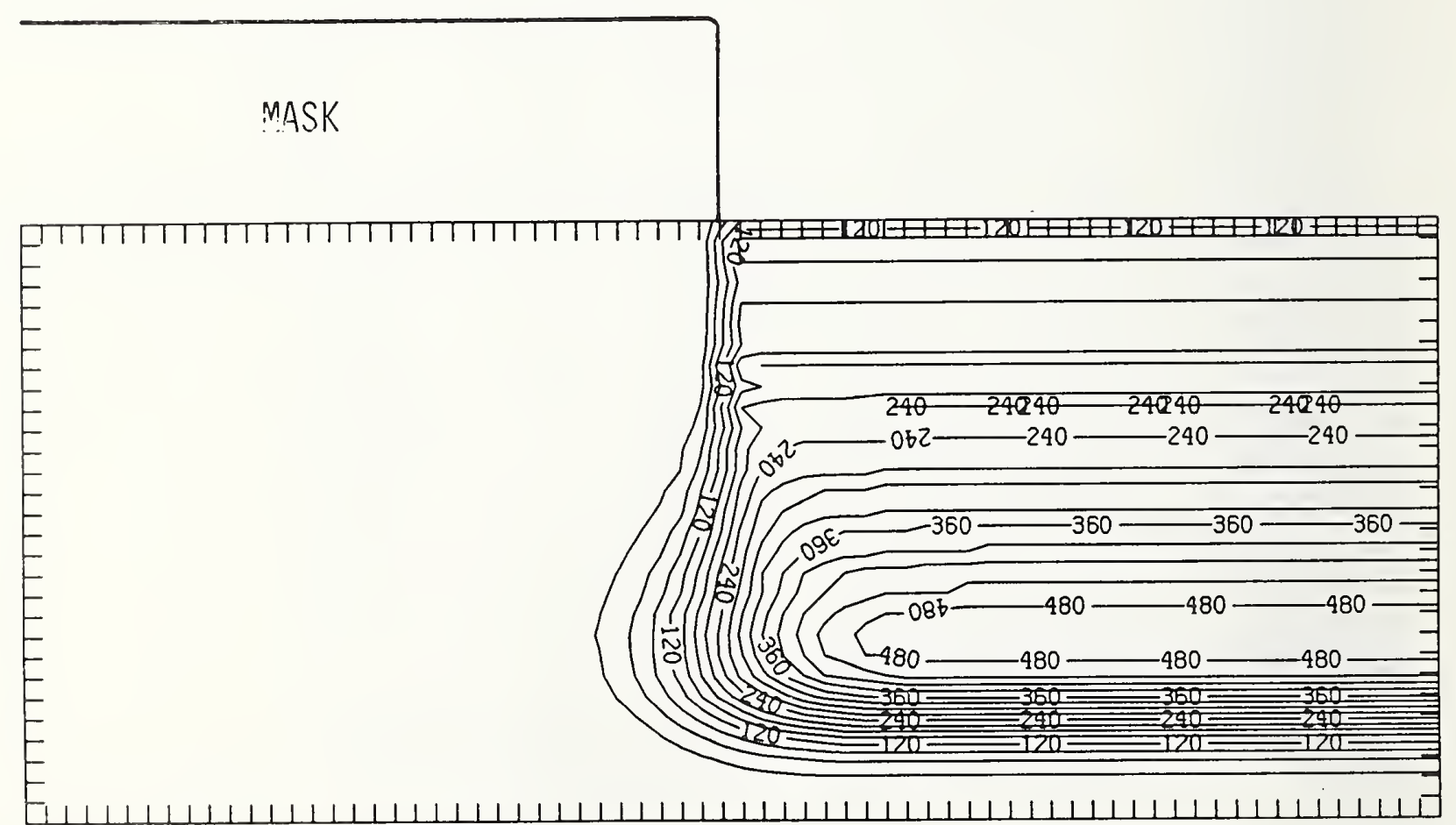

Figure N350-4 Two-dimensional distribution of Frenkel pairs created by $350 \mathrm{keV}$ nitrogen implanted past a mask edge. The length increment (distance between tick marks) is $0.04 \mu \mathrm{m}$ and the scale factor is 1 . 


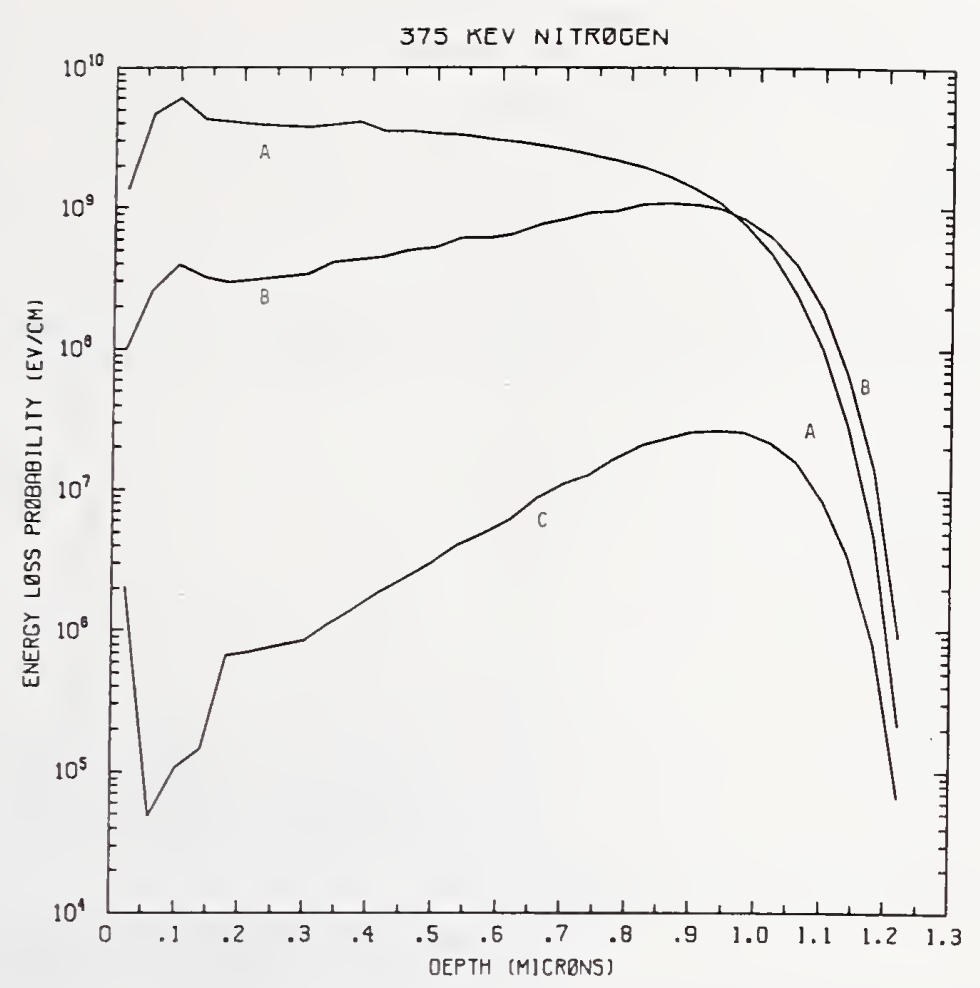

Figure N375-1 One-dimensional probability distributions for energy loss due to electronic excitation (A), displacement damage(B), and phonon excitation (C) for $375 \mathrm{keV}$ nitrogen normally incident on a silicon target.

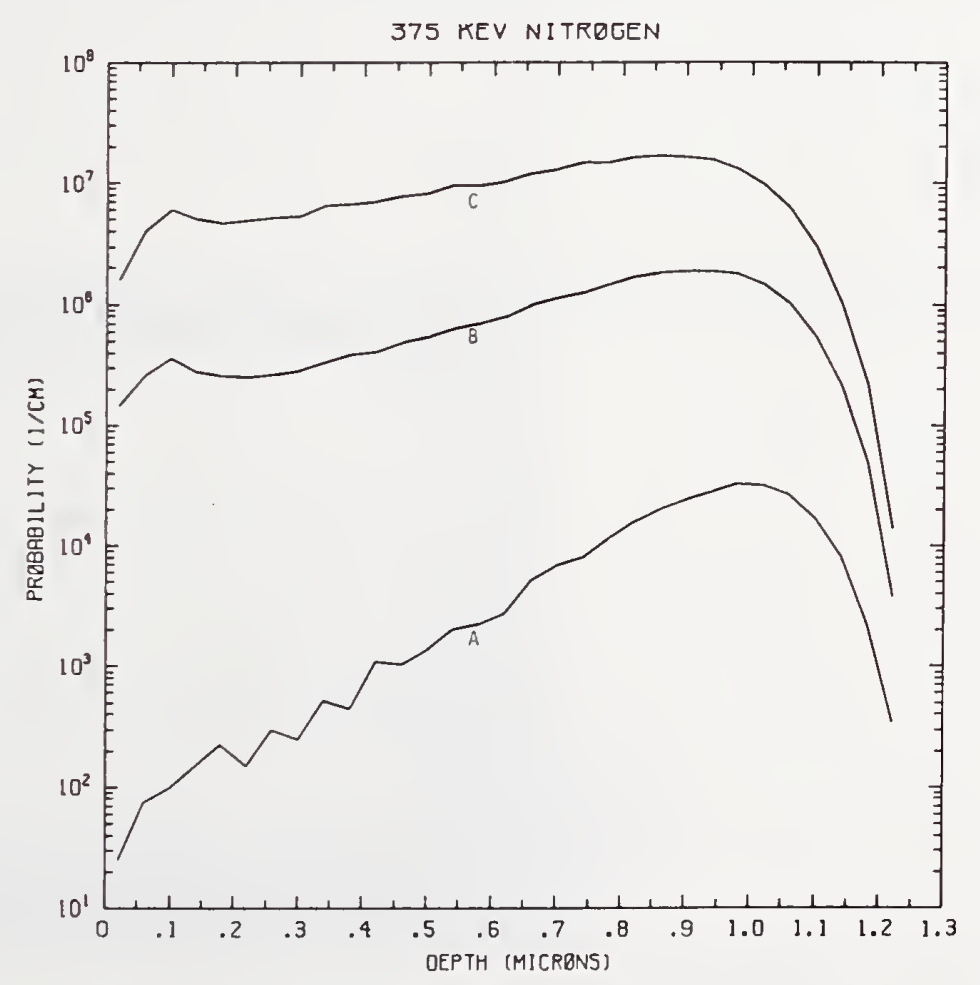

Figure N375-2 One-dimensional probability distributions for implanted nitrogen (A), primary silicon displacements (B), and Frenkel pairs (C) for $375 \mathrm{keV}$ nitrogen normally incident on a silicon target. 


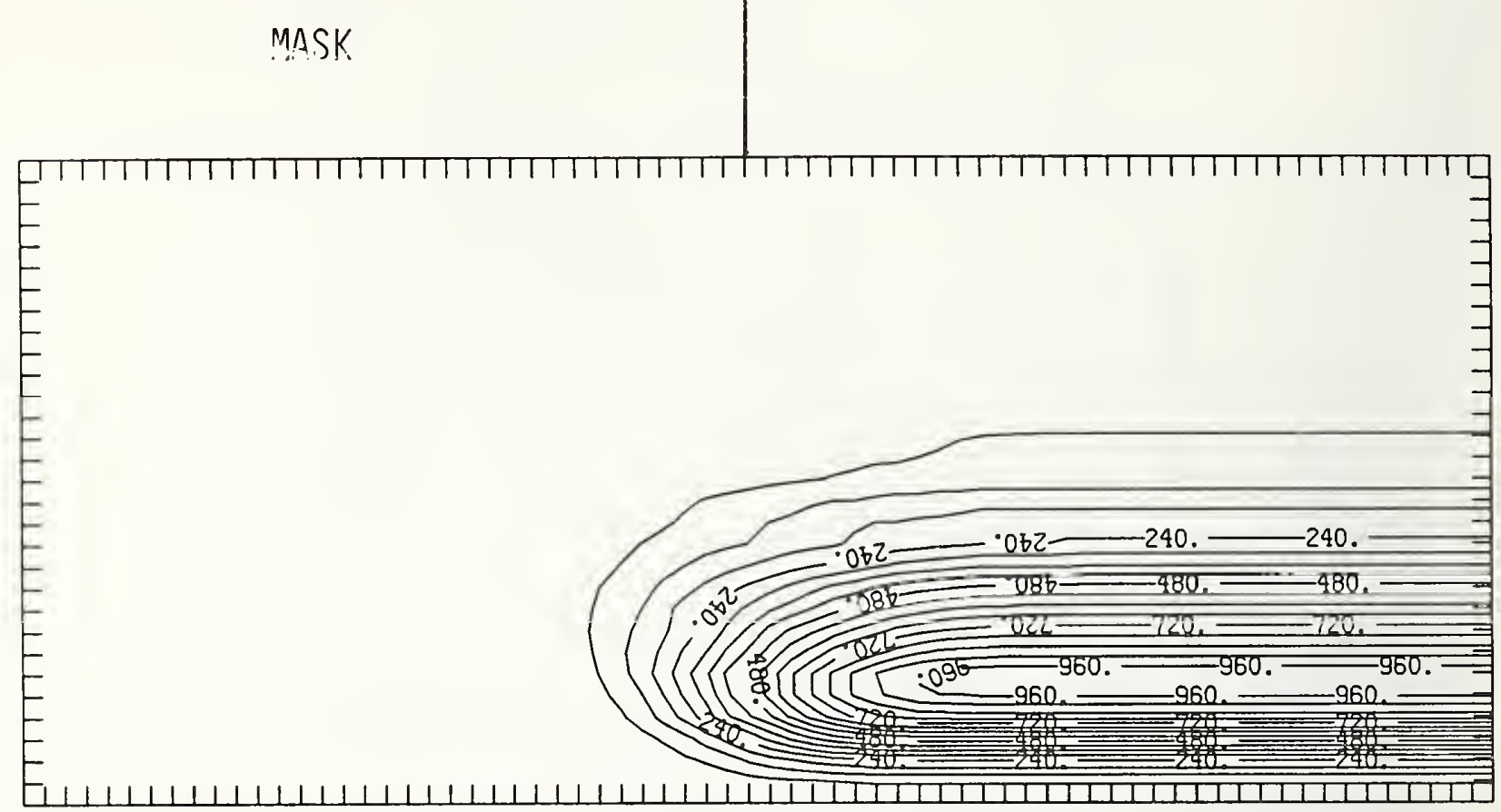

Figure N375-3 Two-dimensional distribution of $375 \mathrm{keV}$ nitrogen implanted past a mask edge. The length increment (distance between tick marks) is $0.04 \mu \mathrm{m}$ and the scale factor is 1000.

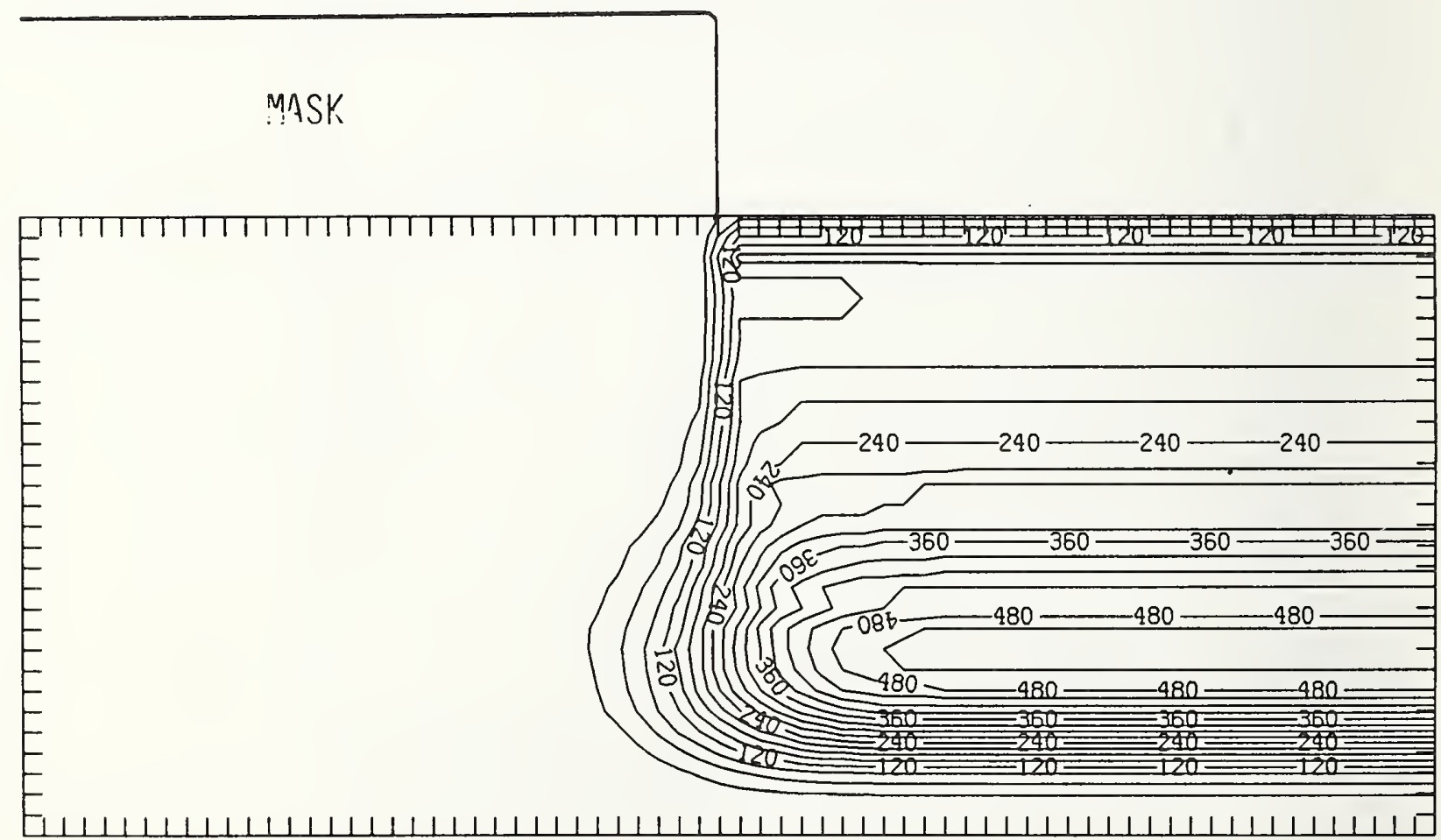

Figure N375-4 Two-dimensional distribution of Frenkel pairs created by $375 \mathrm{keV}$ nitrogen implanted past a mask edge. The length increment (distance between tick marks) is $0.04 \mu \mathrm{m}$ and the scale factor is 1 . 


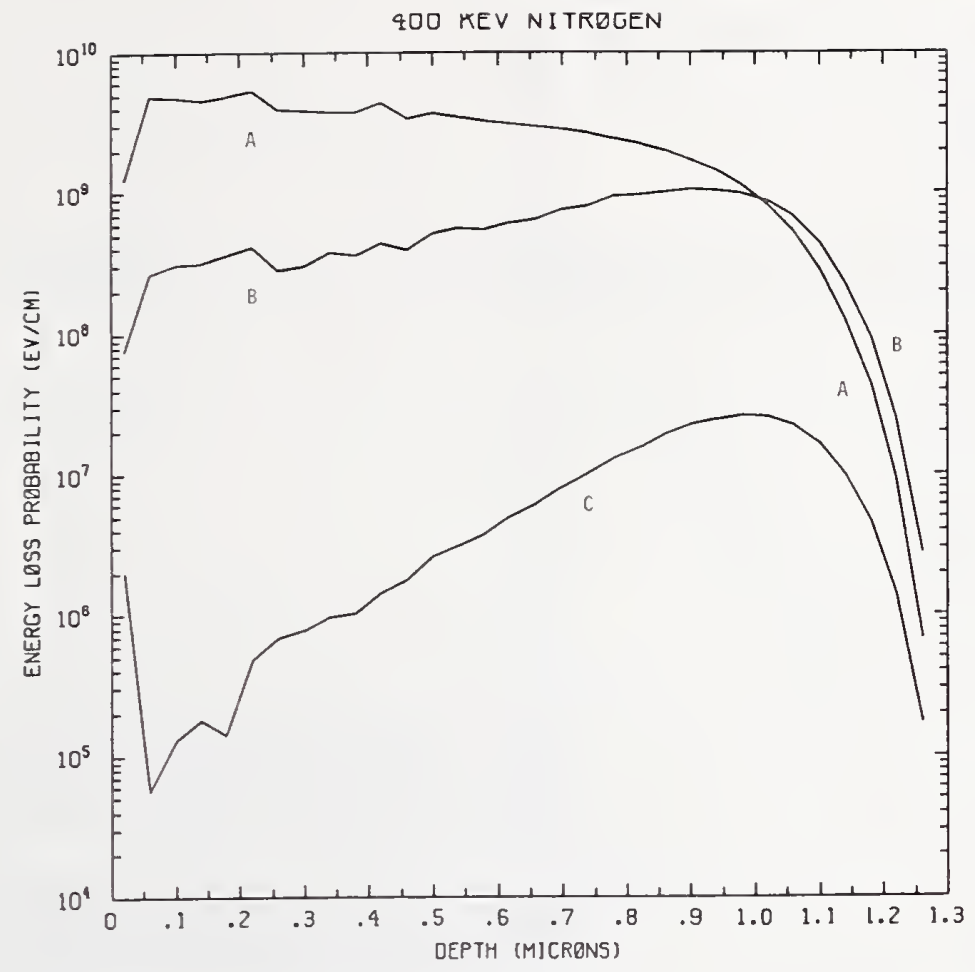

Figure N400-1 One-dimensional probability distributions for energy loss due to electronic excitation (A), displacement damage(B), and phonon excitation (C) for $400 \mathrm{keV}$ nitrogen normally incident on a silicon target.

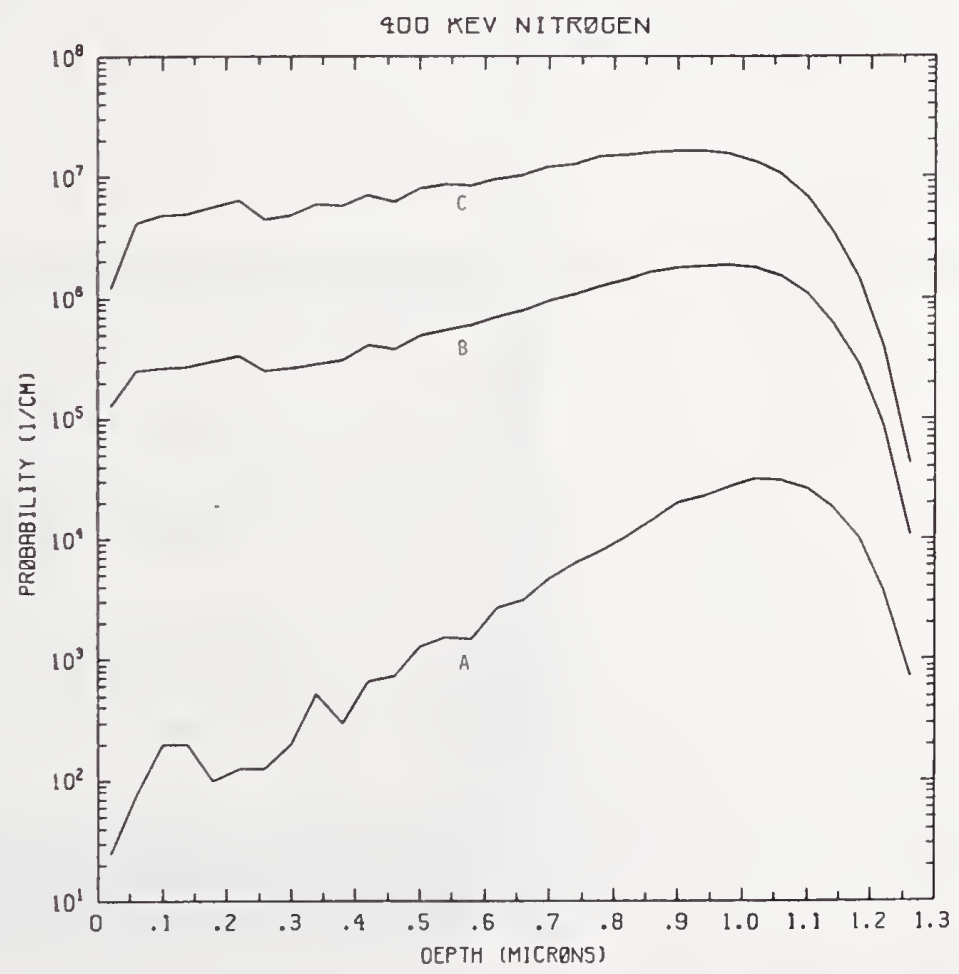

Figure N400-2 One-dimensional probability distributions for implanted nitrogen (A), primary silicon displacements (B), and Frenkel pairs (C) for $400 \mathrm{keV}$ nitrogen normally incident on a silicon target. 


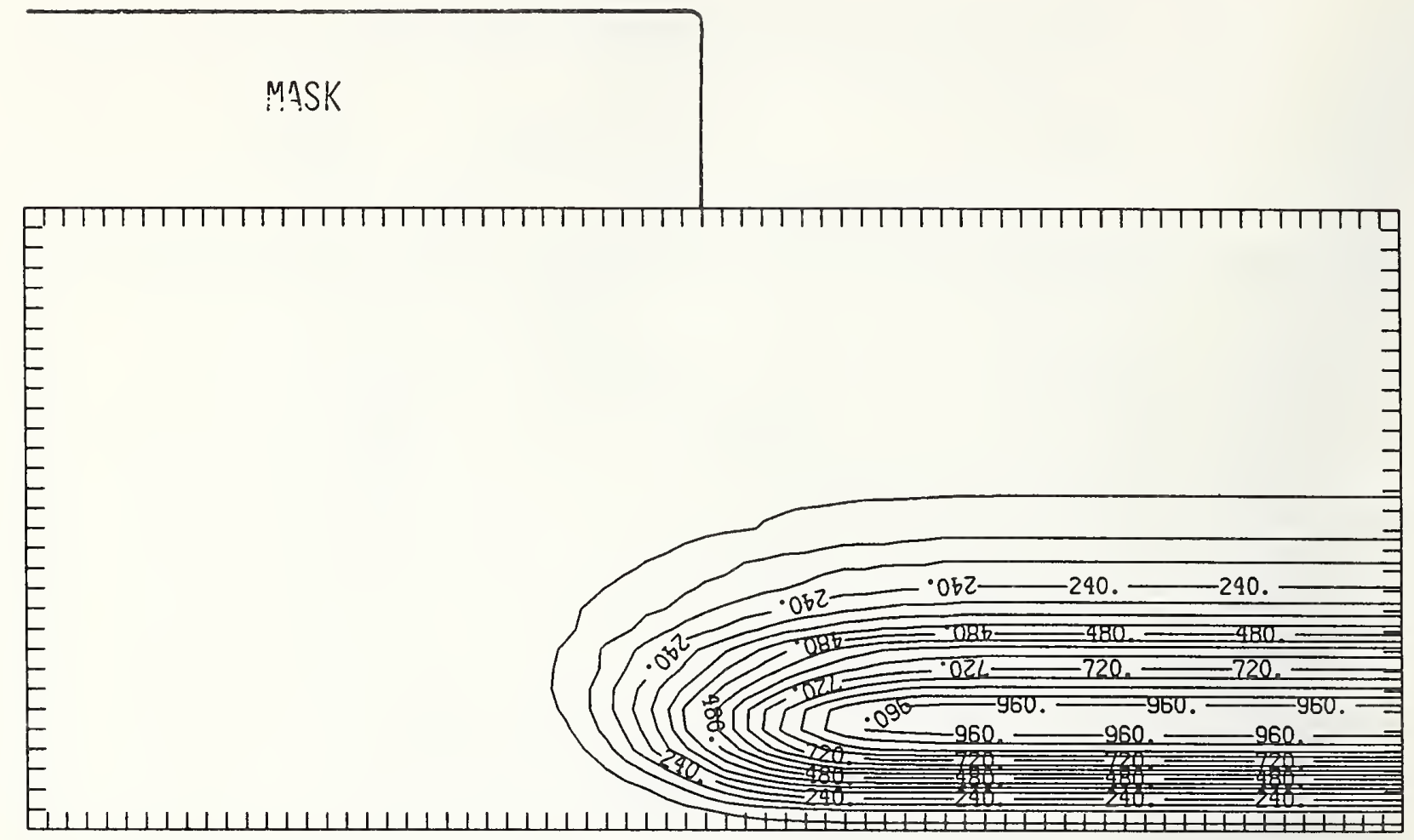

Figure N400-3 Two-dimensional distribution of $400 \mathrm{keV}$ nitrogen implanted past a mask edge. The length increment (distance between tick marks) is $0.04 \mu \mathrm{m}$ and the scale factor is 1000.

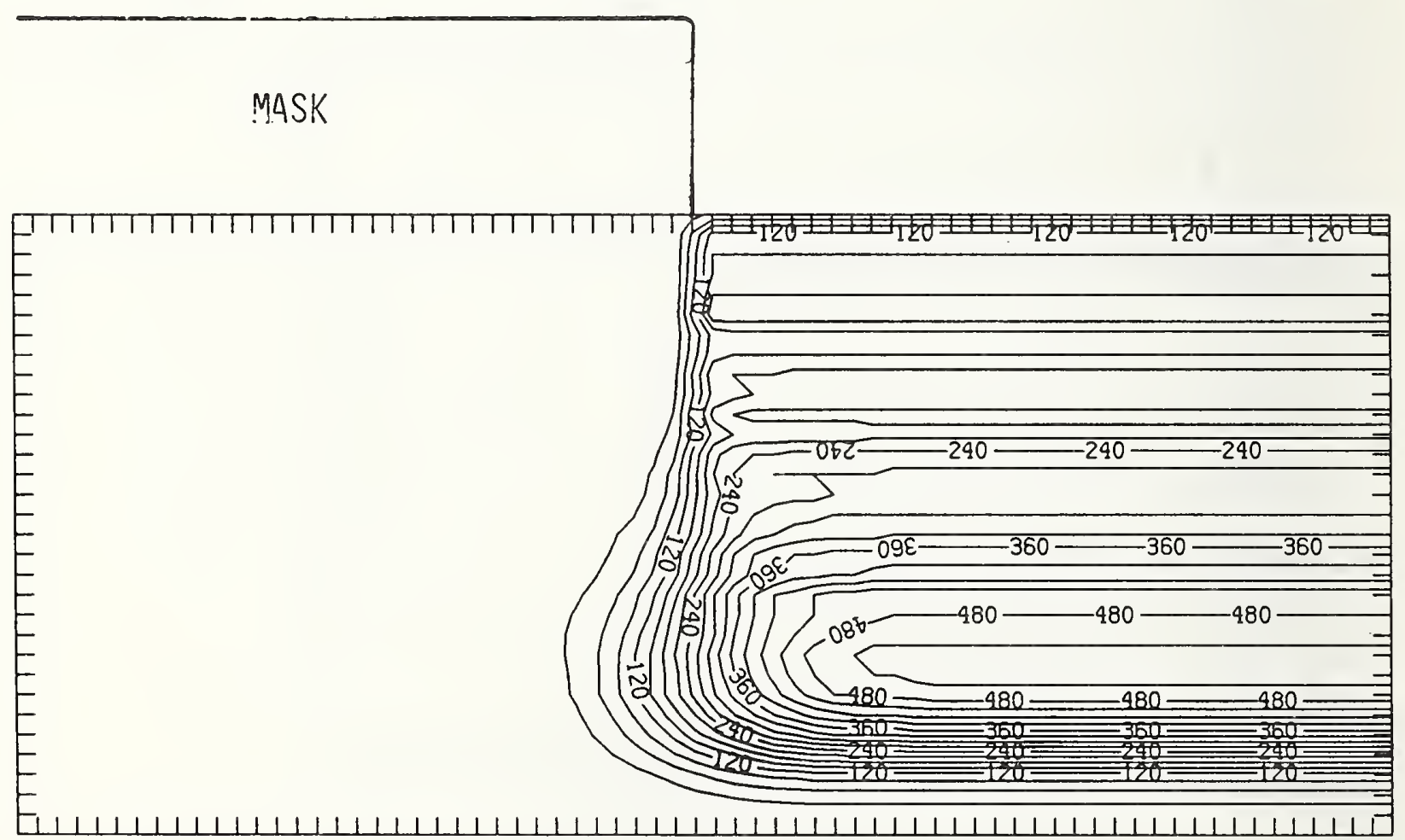

Figure N400-4 Two-dimensional distribution of Frenkel pairs created by $400 \mathrm{keV}$ nitrogen implanted past a mask edge. The length increment (distance between tick marks) is $0.04 \mu \mathrm{m}$ and the scale factor is 1. 


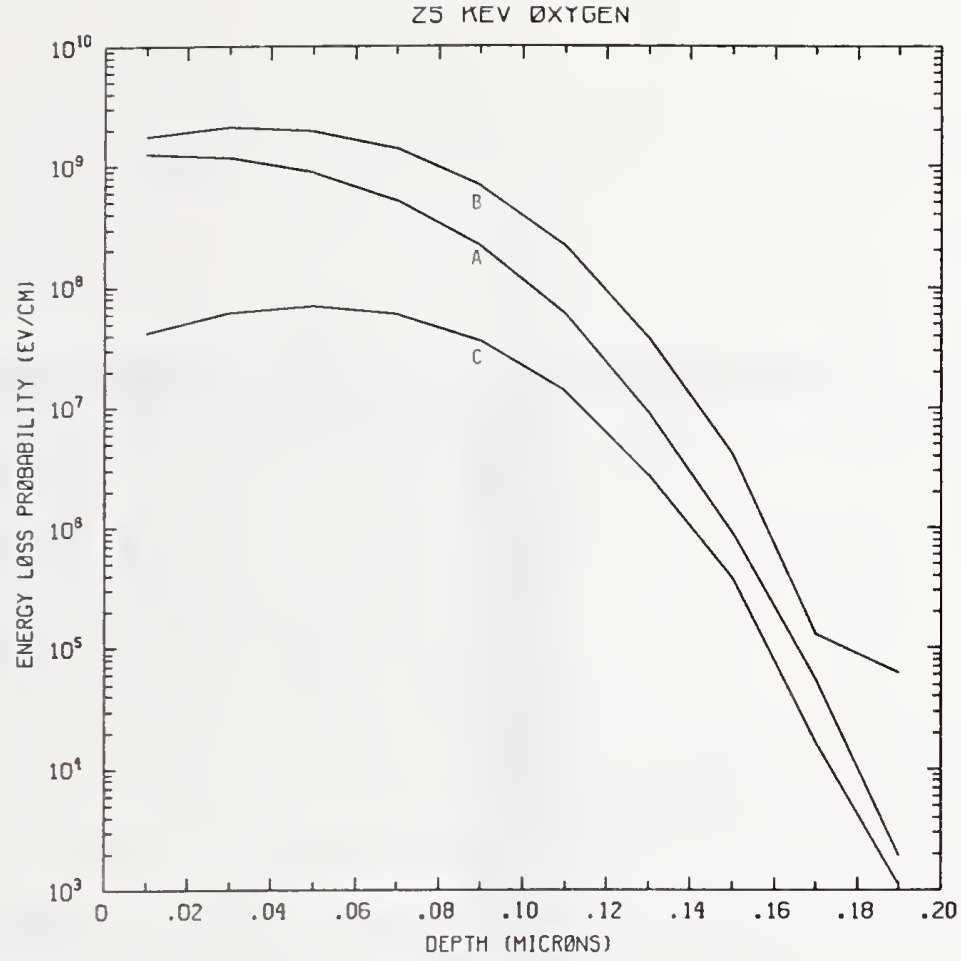

Figure 025-1 One-dimensional probability distributions for energy loss due to electronic excitation (A), displacement damage(B), and phonon excitation (C) for $25 \mathrm{keV}$ oxygen normally incident on a silicon target.

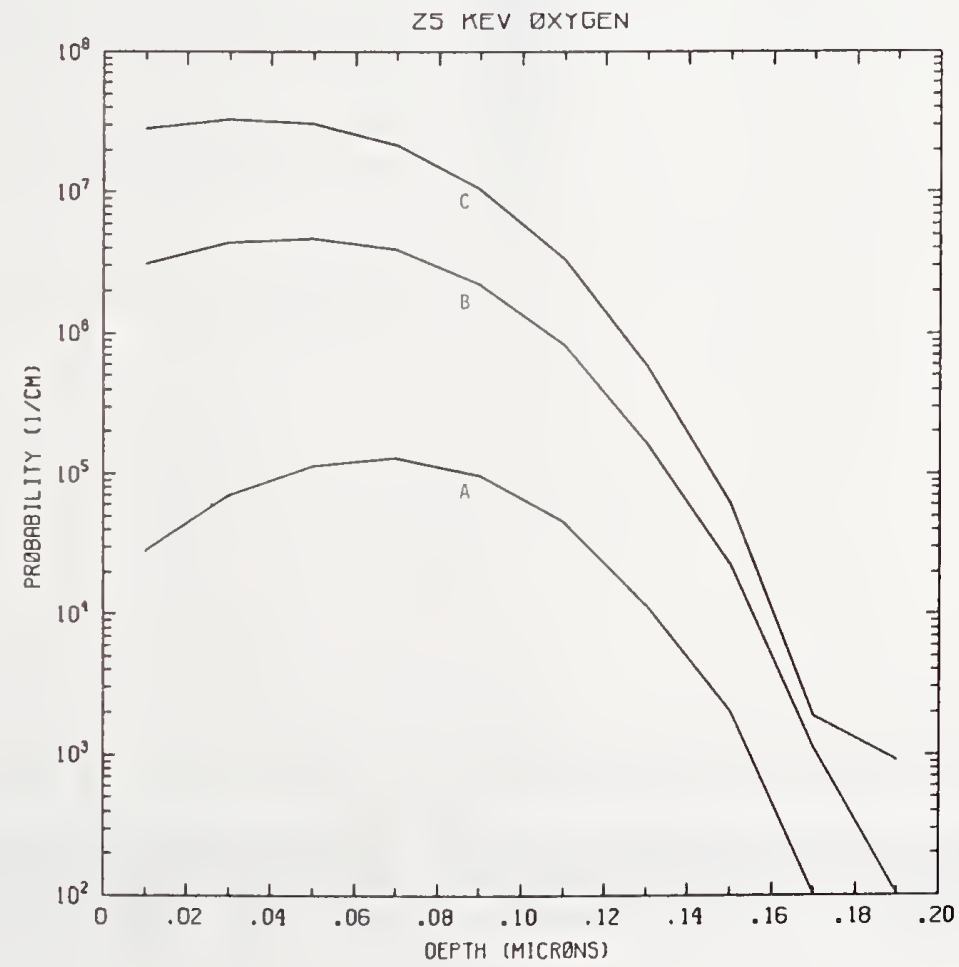

Figure 025-2 One-dimensional probability distributions for implanted oxygen (A), primary silicon displacements (B), and Frenkel pairs (C) for $25 \mathrm{keV}$ oxygen normally incident on a silicon target. 


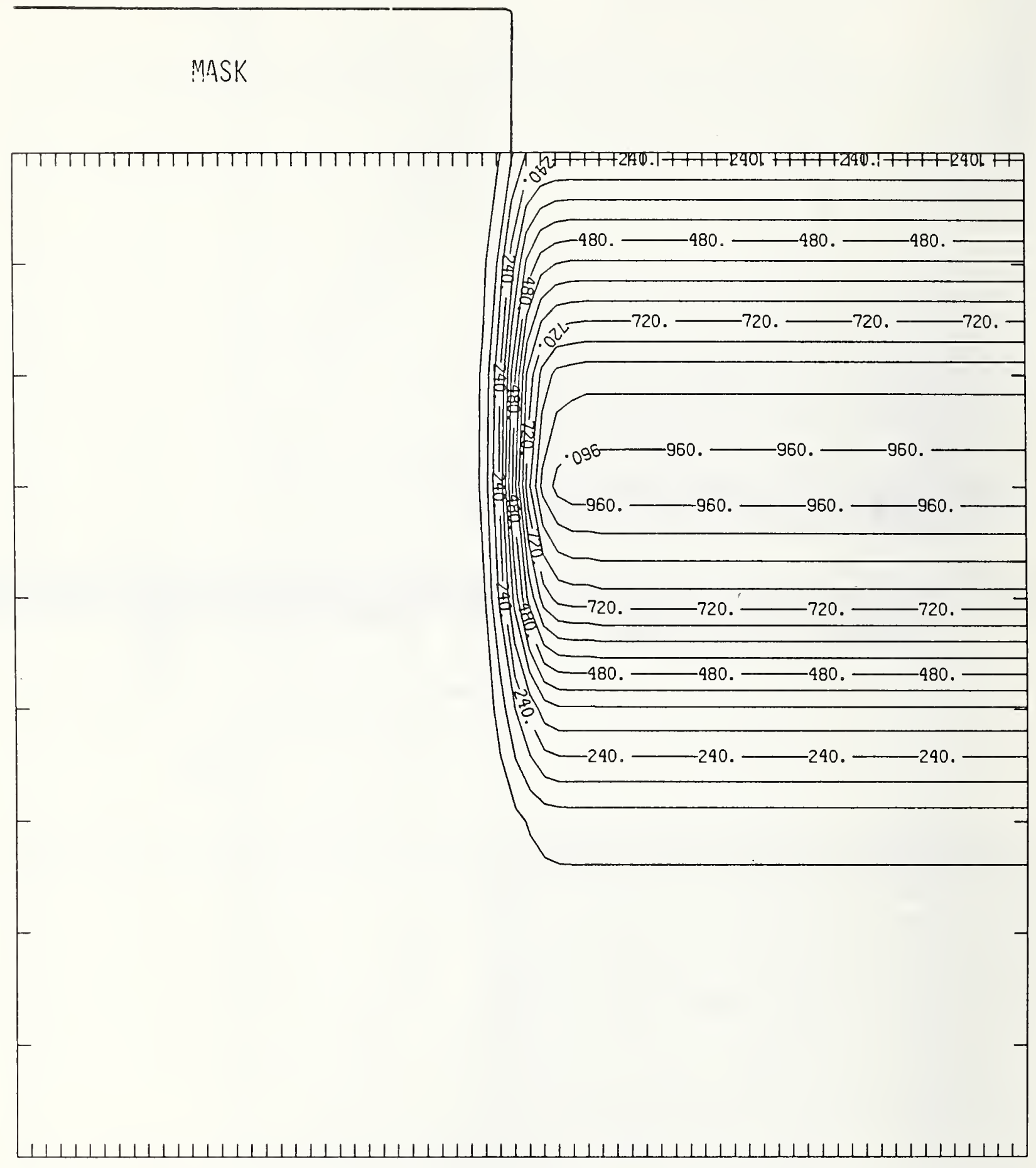

Figure O25-3 Two-dimensional distribution of $25 \mathrm{keV}$ oxygen implanted past a mask edge. The length increment (distance between tick marks) is $0.02 \mu \mathrm{m}$ and the scale factor is 1000. 


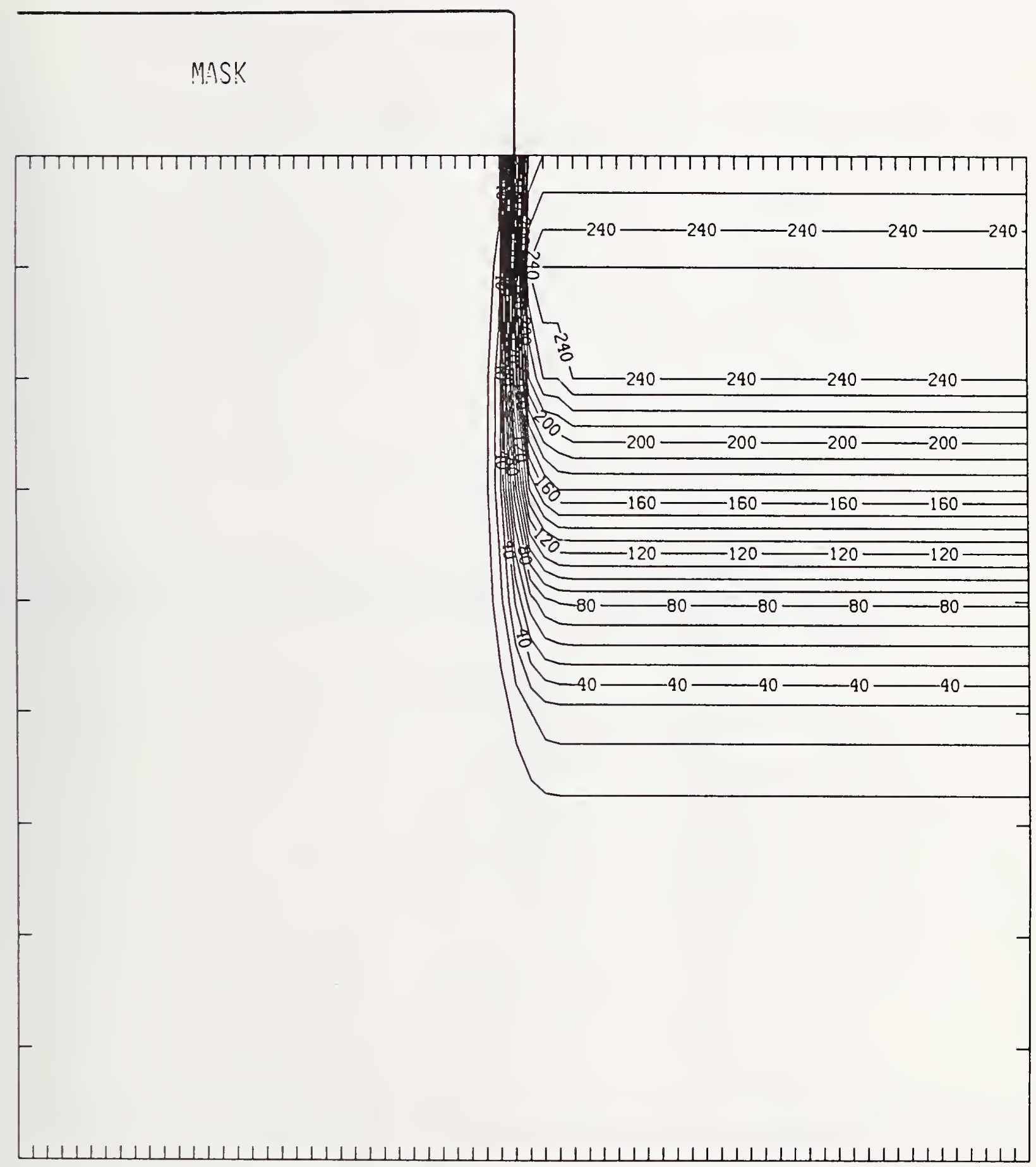

Figure 025-4 Two-dimensional distribution of Frenkel pairs created by $25 \mathrm{keV}$ oxygen implanted past a mask edge. The length increment (distance between tick marks) is $0.02 \mu \mathrm{m}$ and the scale factor is 1. 


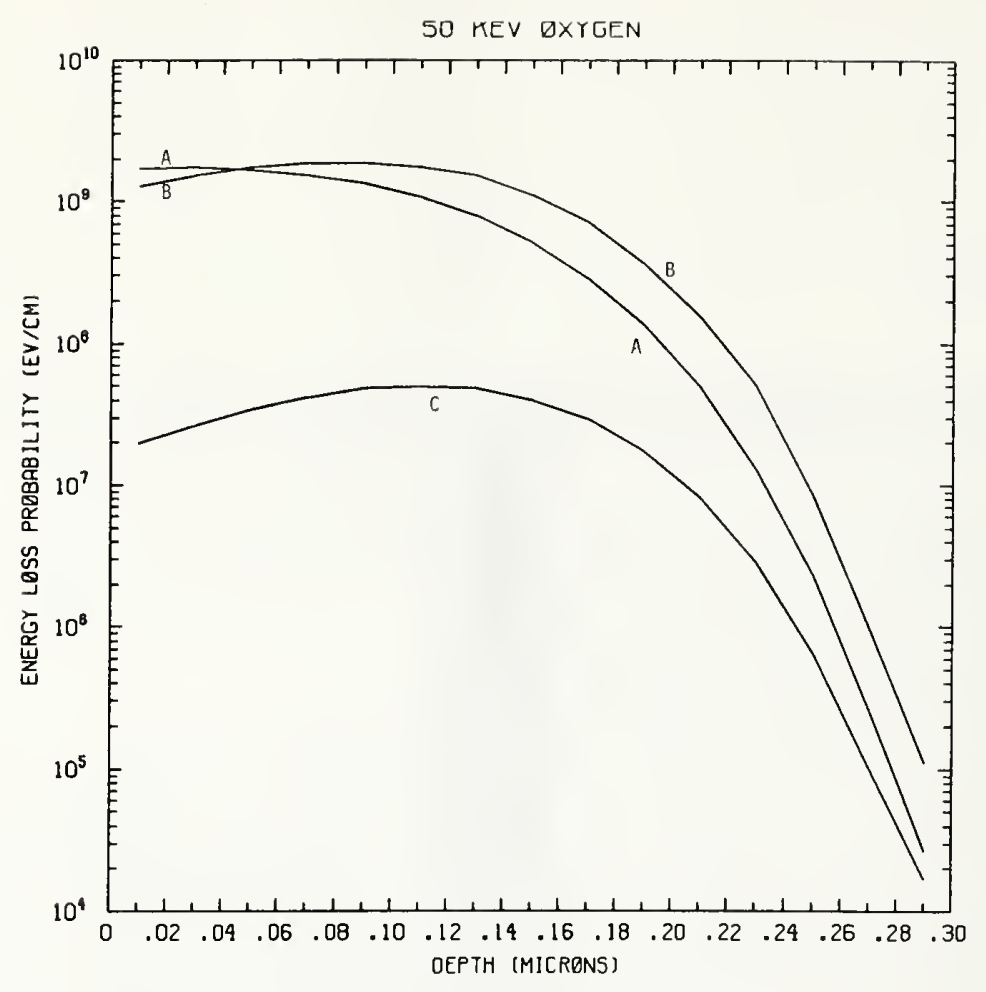

Figure 050-1 One-dimensional probability distributions for energy loss due to electronic excitation (A), displacement damage(B), and phonon excitation (C) for $50 \mathrm{keV}$ oxygen normally incident on a silicon target.

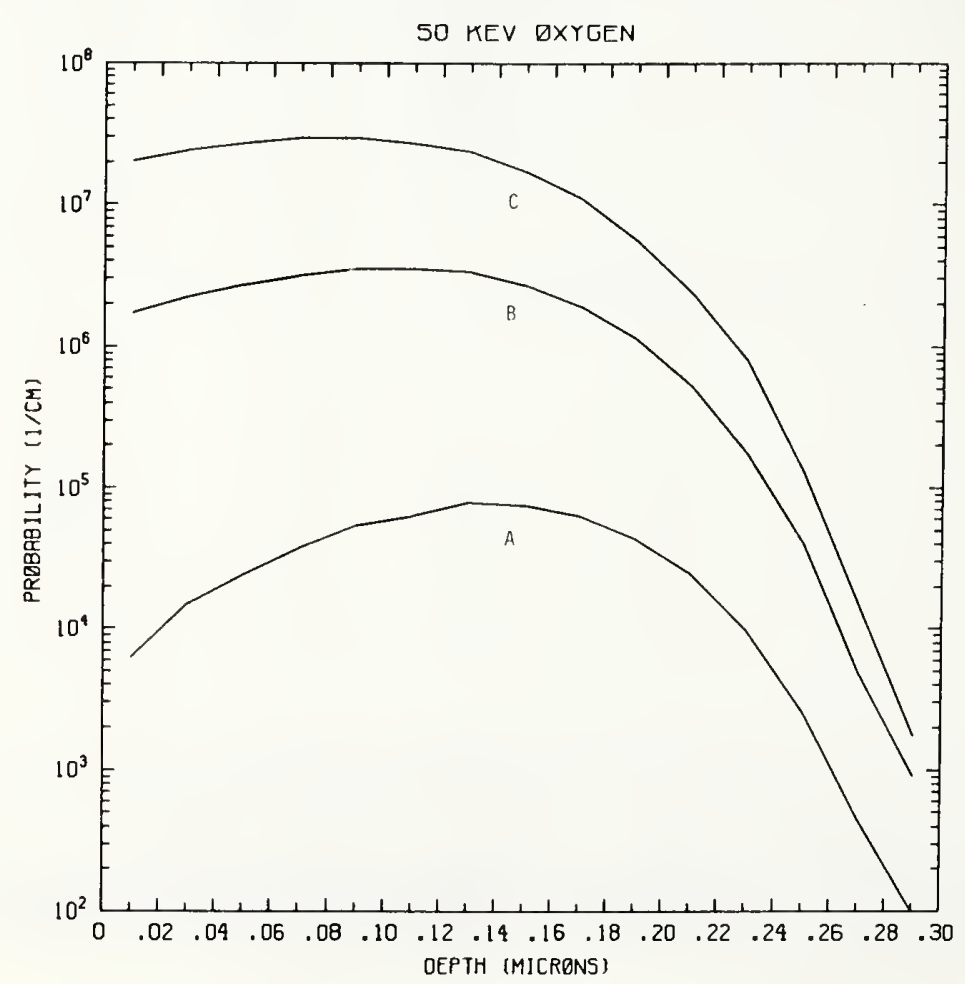

Figure 050-2 One-dimensional probability distributions for implanted oxygen (A), primary silicon displacements (B), and Frenkel pairs (C) for $50 \mathrm{keV}$ oxygen normally incident on a silicon target. 


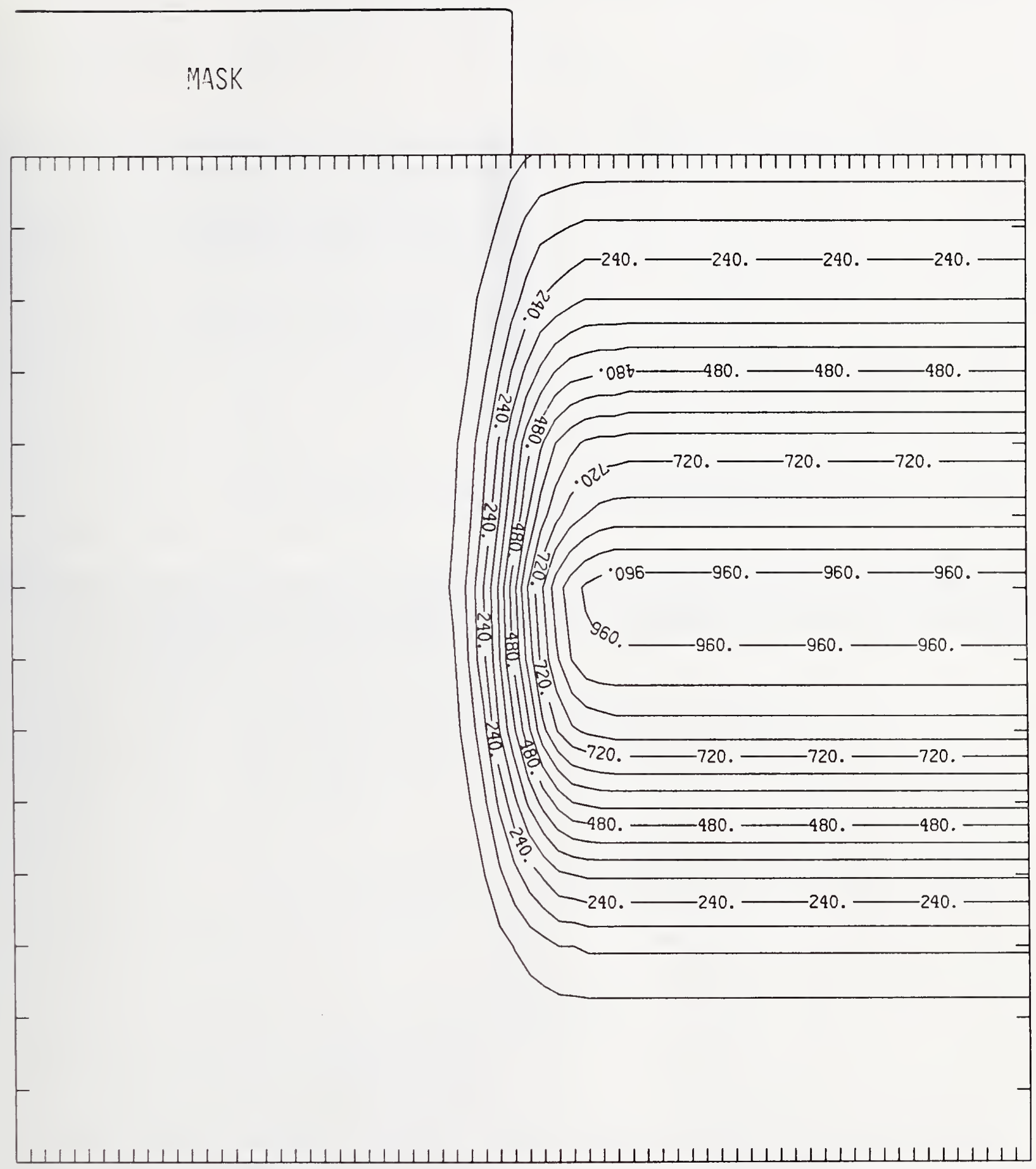

Figure O50-3 Two-dimensional distribution of $50 \mathrm{keV}$ oxygen implanted past a mask edge. The length increment (distance between tick marks) is $0.02 \mu \mathrm{m}$ and the scale factor is 1000 . 


\section{MASK}

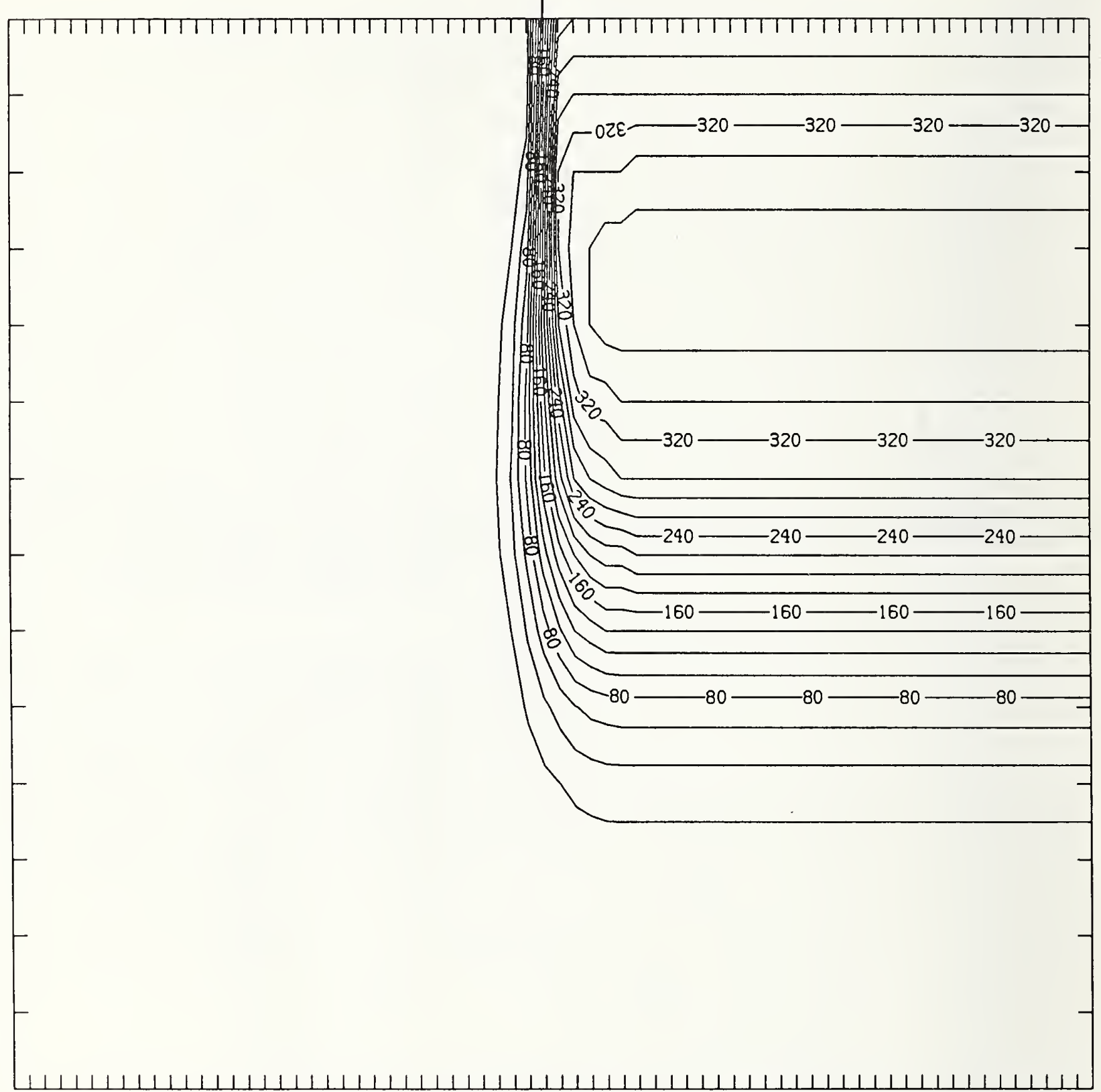

Figure 050-4 Two-dimensional distribution of Frenkel pairs created by $50 \mathrm{keV}$ oxygen implanted past a mask edge. The length increment (distance between tick marks) is $0.02 \mu \mathrm{m}$ and the scale factor is 1 . 


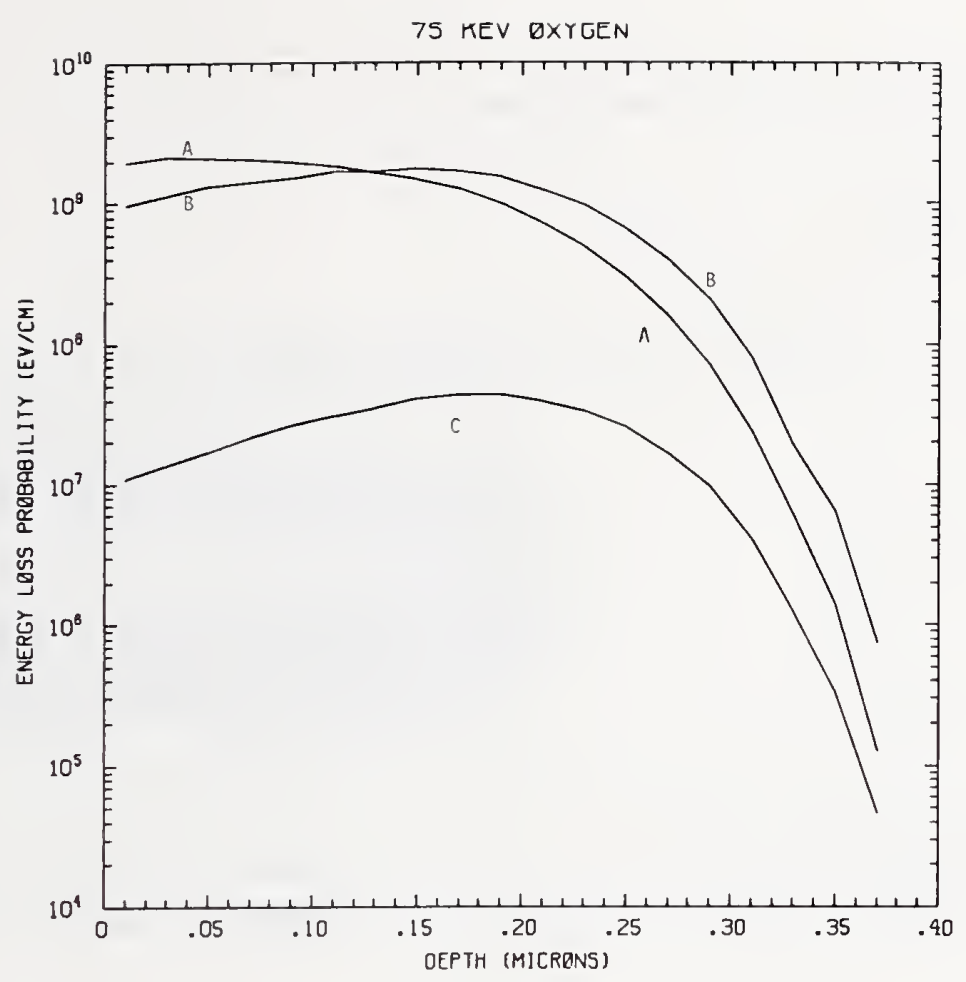

Figure 075-1 One-dimensional probability distributions for energy loss due to electronic excitation (A), displacement damage(B), and phonon excitation (C) for $75 \mathrm{keV}$ oxygen normally incident on a silicon target.

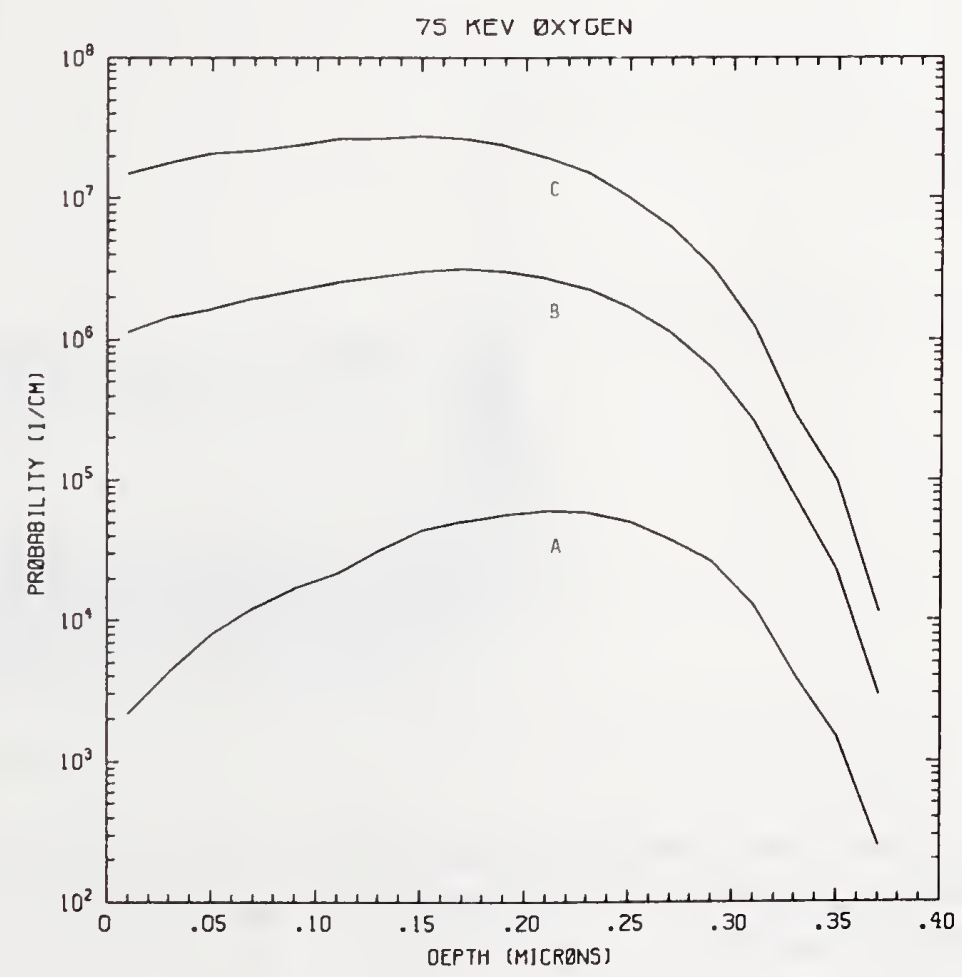

Figure 075-2 One-dimensional probability distributions for implanted oxygen (A), primary silicon displacements (B), and Frenkel pairs (C) for $75 \mathrm{keV}$ oxygen normally incident on a silicon target. 


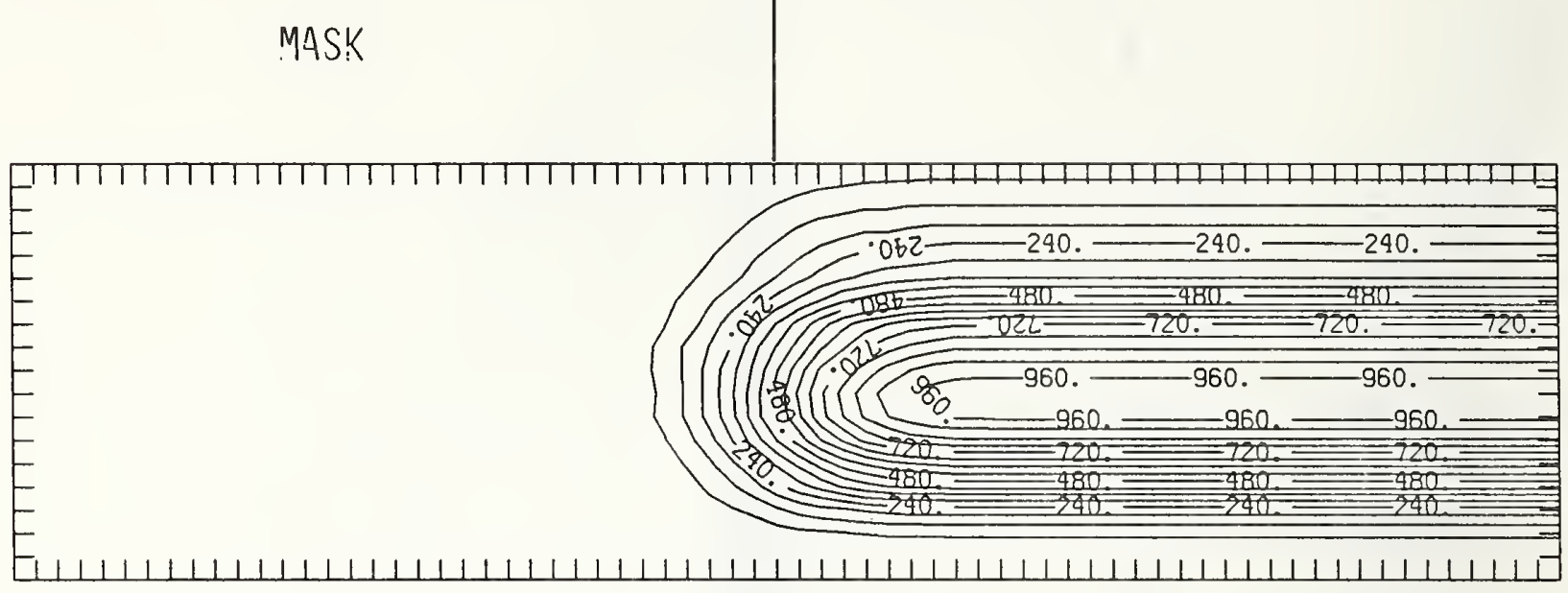

Figure 075-3 Two-dimensional distribution of $75 \mathrm{keV}$ oxygen implanted past a mask edge. The length increment (distance between tick marks) is $0.02 \mu \mathrm{m}$ and the scale factor is 1000 .

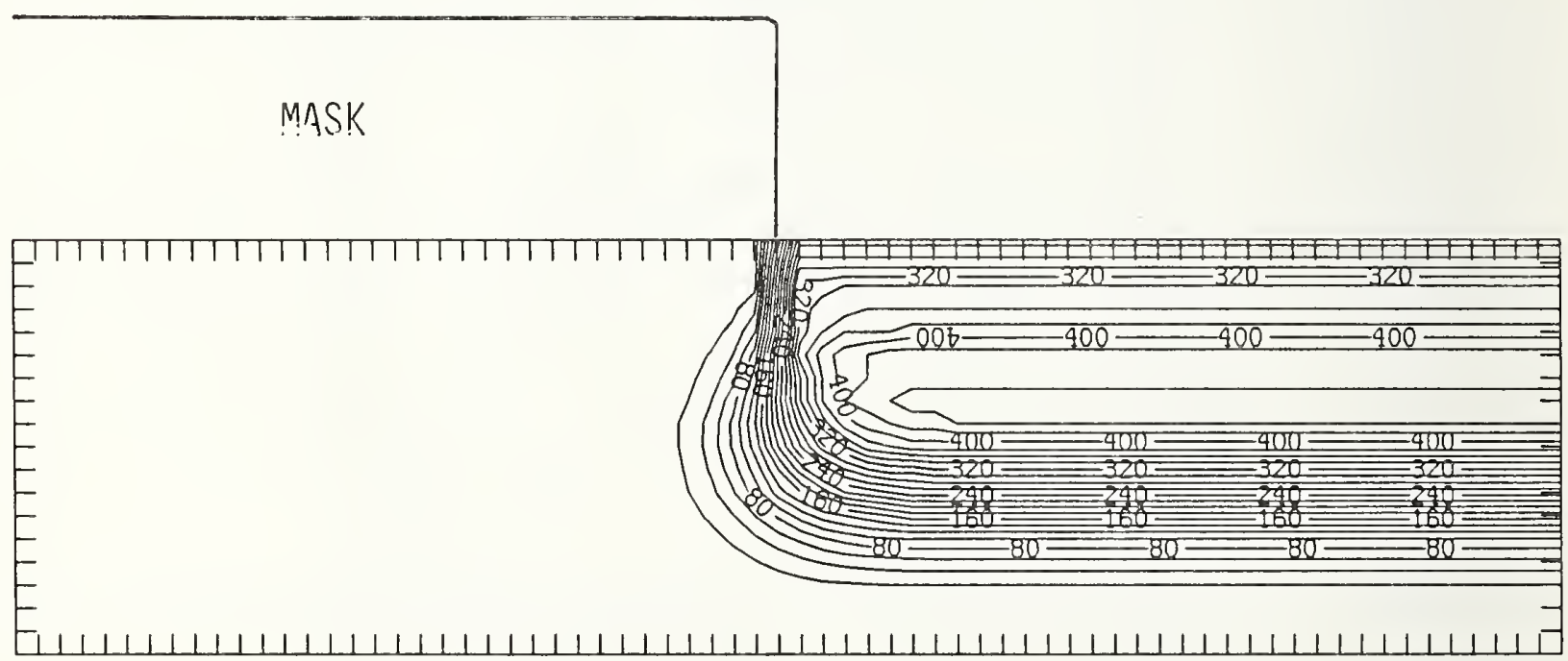

Figure 075-4 Two-dimensional distribution of Frenkel pairs created by $75 \mathrm{keV}$ oxygen implanted past a mask edge. The length increment (distance between tick marks) is $0.02 \mu \mathrm{m}$ and the scale factor is 1 . 


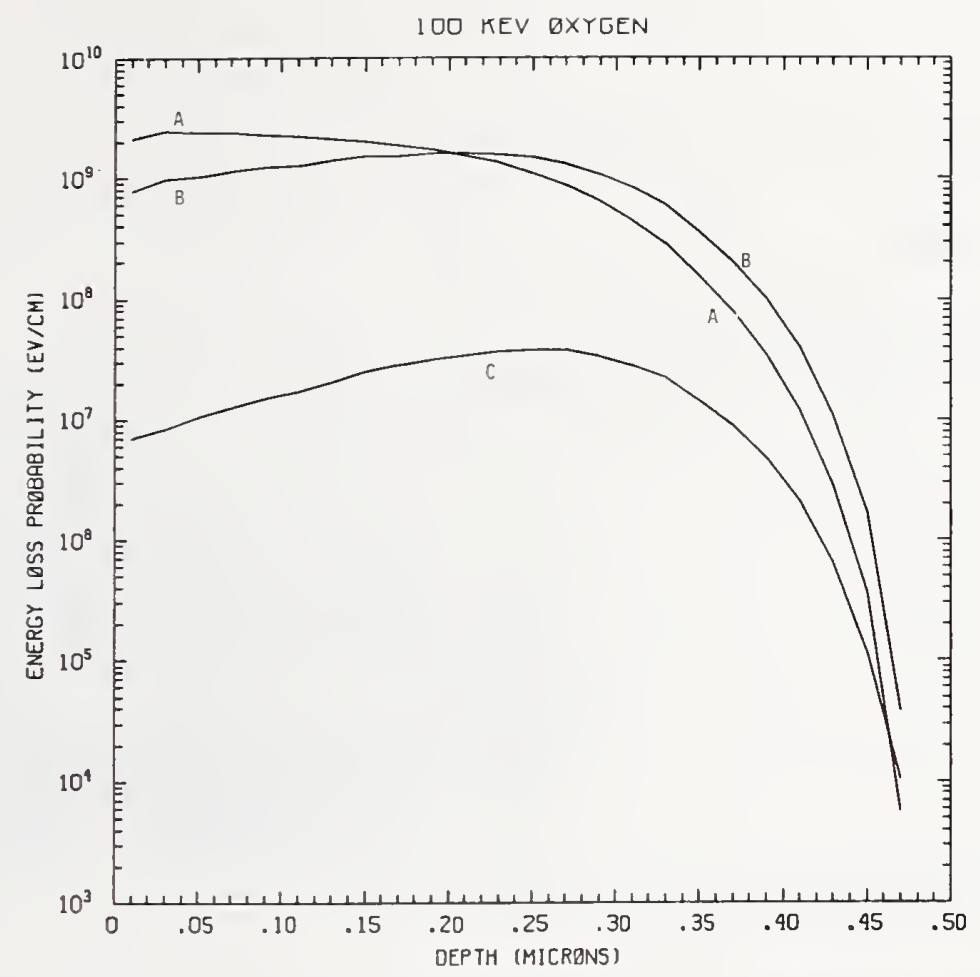

Figure 0100-1 One-dimensional probability distributions for energy loss due to electronic excitation (A), displacement damage(B), and phonon excitation (C) for $100 \mathrm{keV}$ oxygen normally incident on a silicon target.

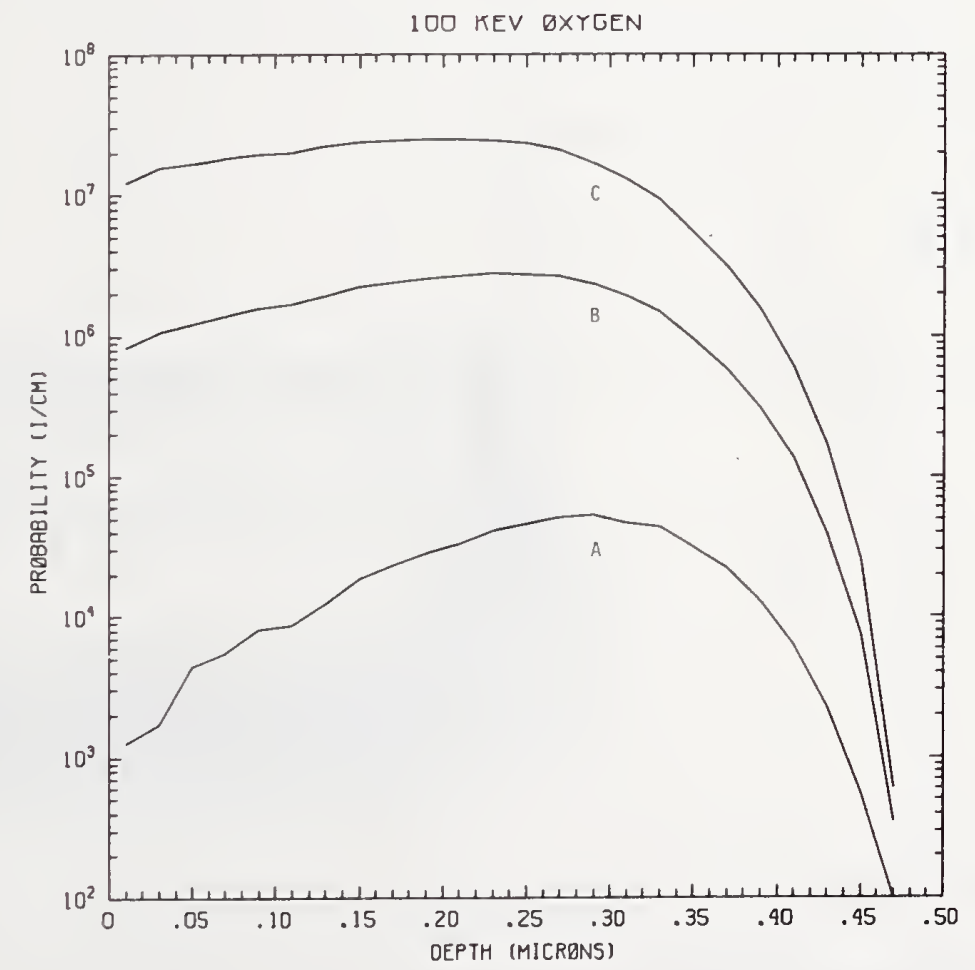

Figure O100-2 One-dimensional probability distributions for implanted oxygen (A), primary silicon displacements (B), and Frenkel pairs (C) for $100 \mathrm{keV}$ oxygen normally incident on a silicon target. 


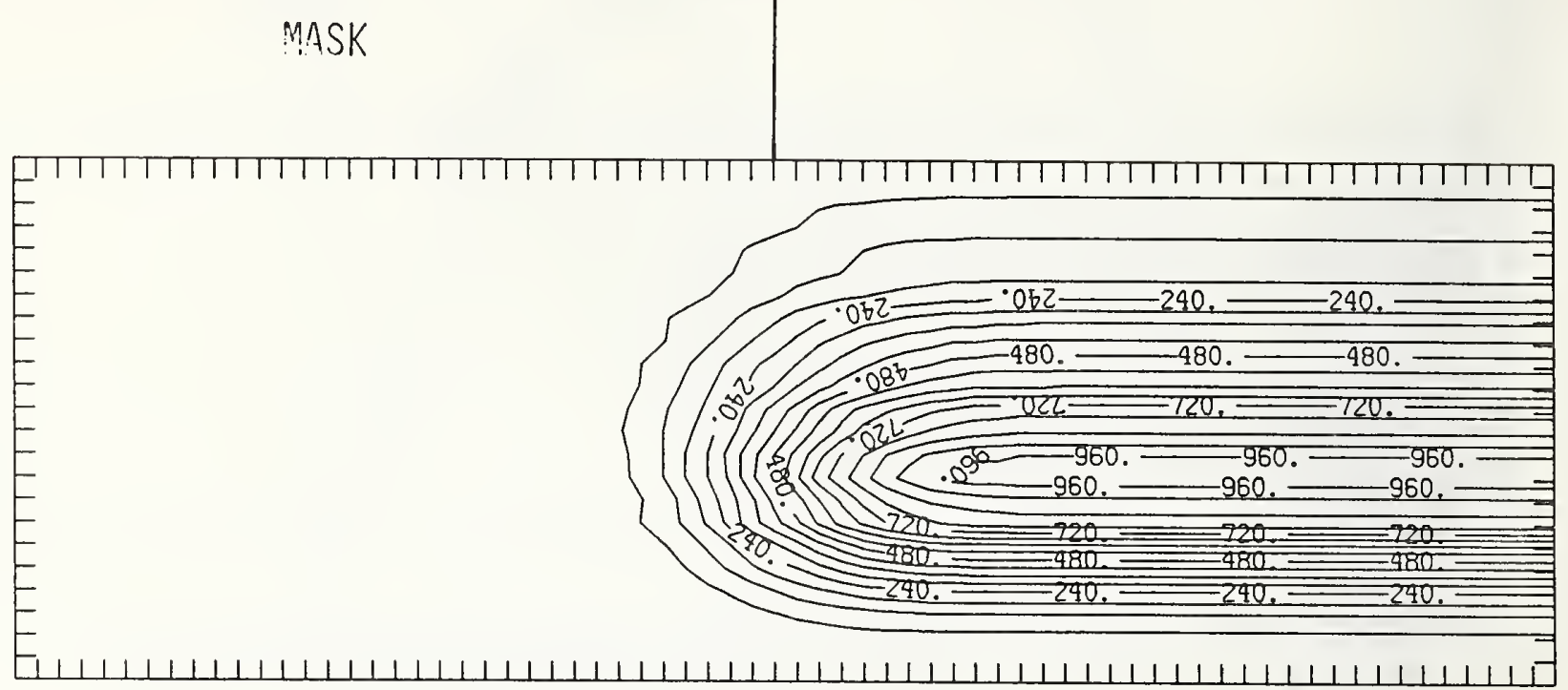

Figure 0100-3 Two-dimensional distribution of $100 \mathrm{keV}$ oxygen implanted past a mask edge. The length increment (distance between tick marks) is $0.02 \mu \mathrm{m}$ and the scale factor is 1000 .

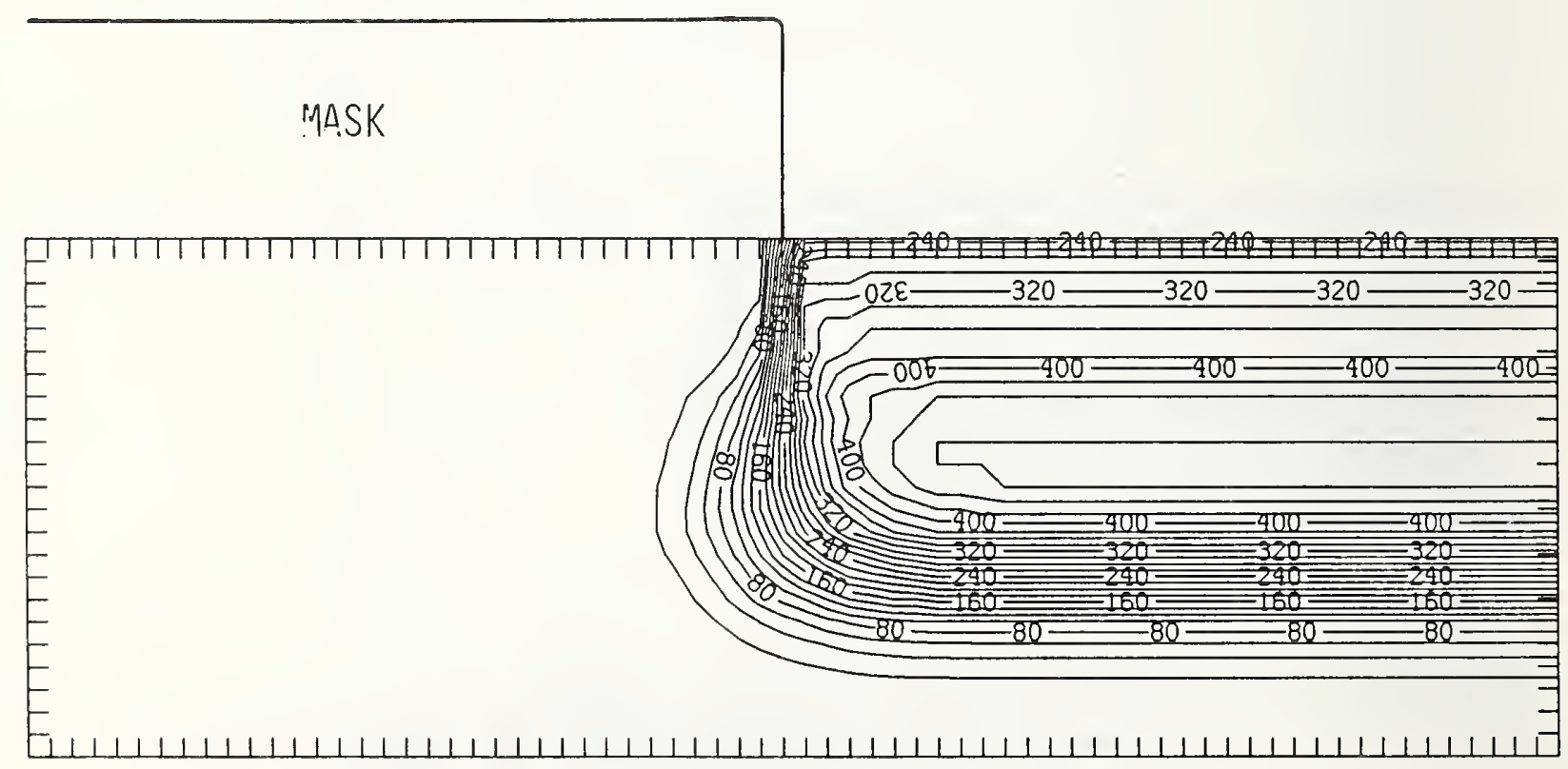

Figure 0100-4 Two-dimensional distribution of Frenkel pairs created by $100 \mathrm{keV}$ oxygen implanted( past a mask edge. The length increment (distance between tick marks) is $0.02 \mu \mathrm{m}$ and the scale factor is 1 . 


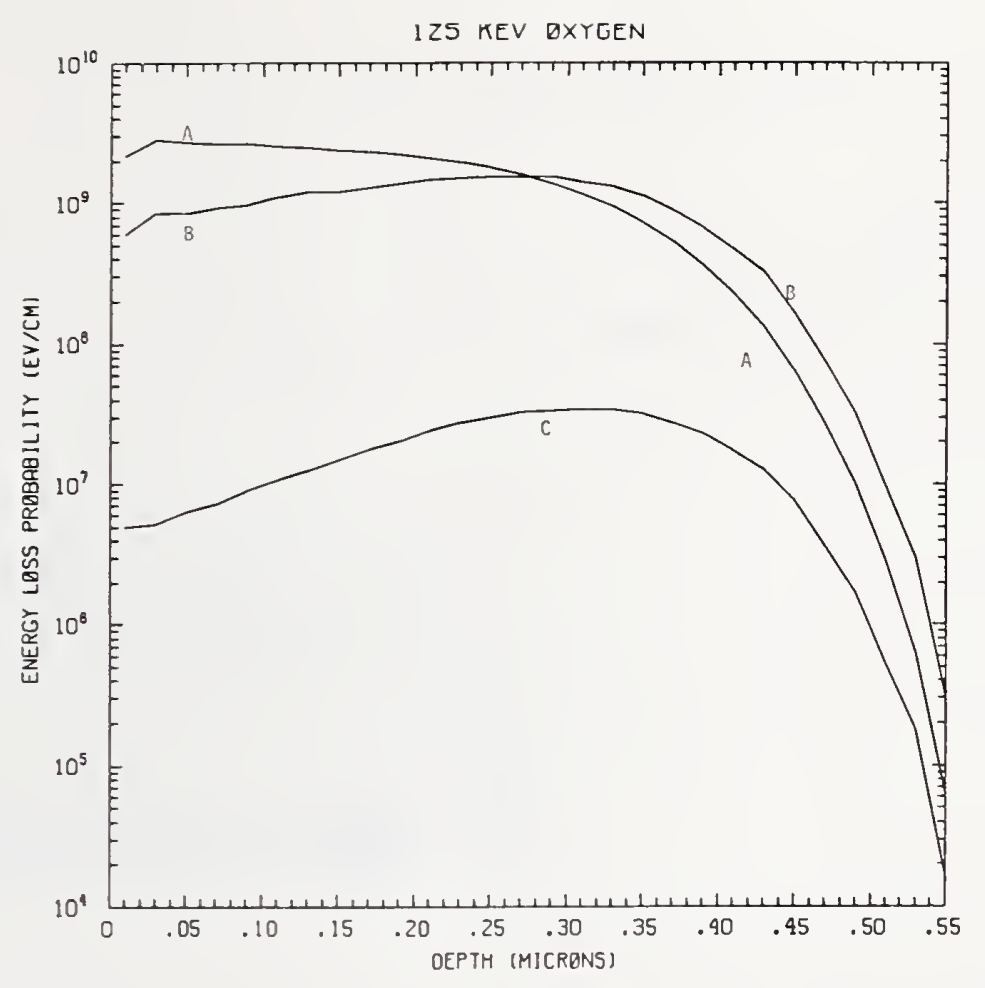

Figure 0125-1 One-dimensional probability distributions for energy loss due to electronic excitation (A), displacement damage(B), and phonon excitation (C) for $125 \mathrm{keV}$ oxygen normally incident on a silicon target.

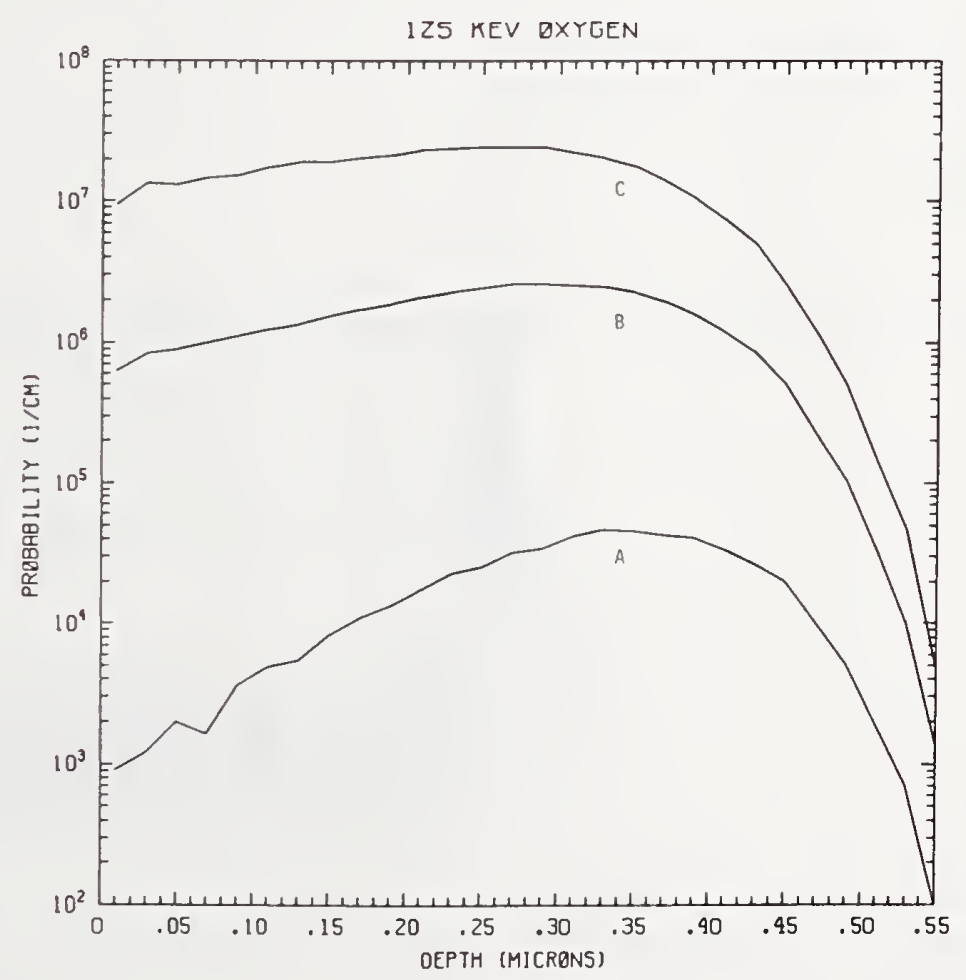

Figure 0125-2 One-dimensional probability distributions for implanted oxygen (A), primary silicon displacements (B), and Frenkel pairs (C) for $125 \mathrm{keV}$ oxygen normally incident on a silicon target. 


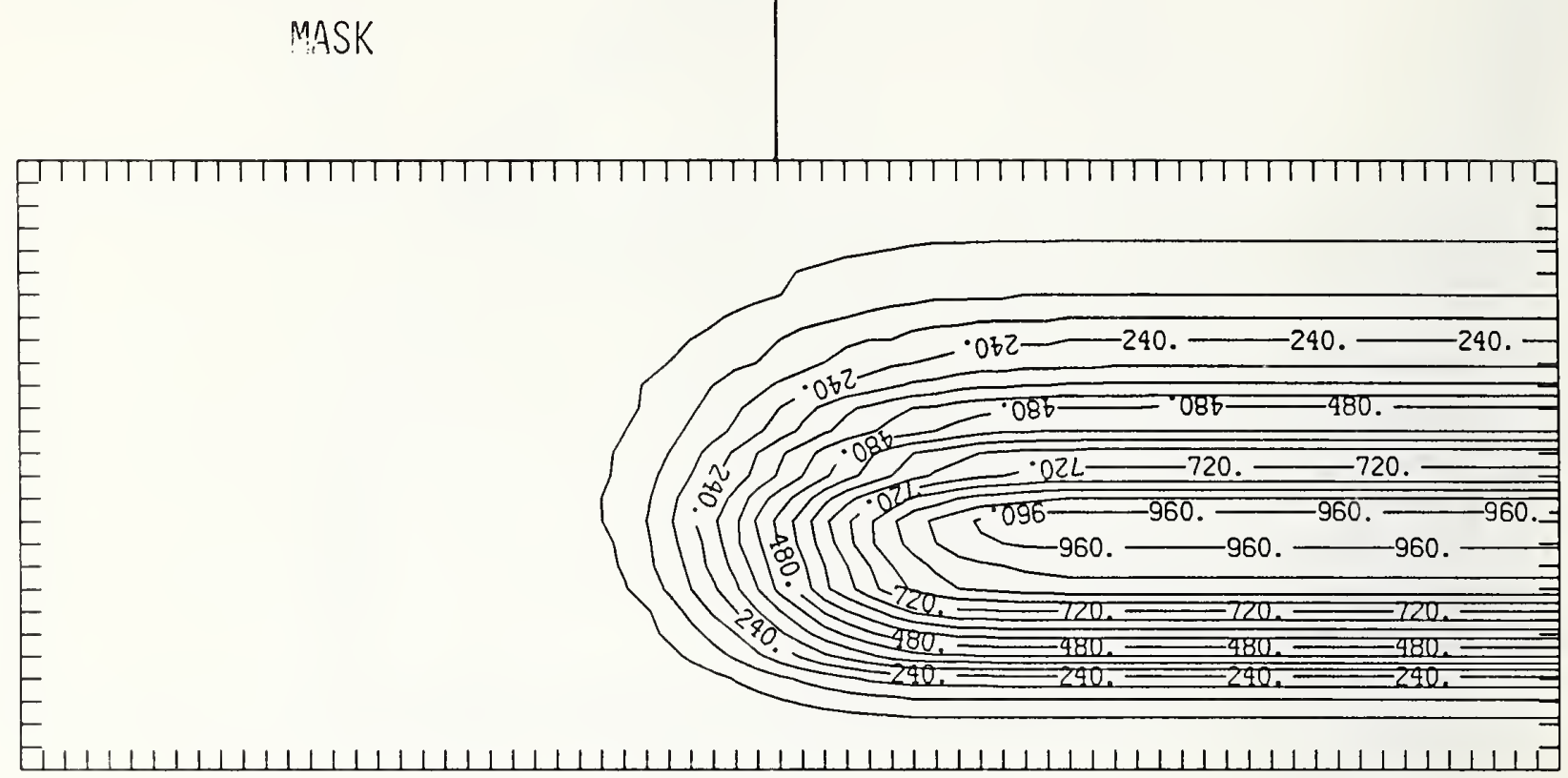

Figure 0125-3 Two-dimensional distribution of $125 \mathrm{keV}$ oxygen implanted past a mask edge. The length increment (distance between tick marks) is $0.02 \mu \mathrm{m}$ and the scale factor is 1000.

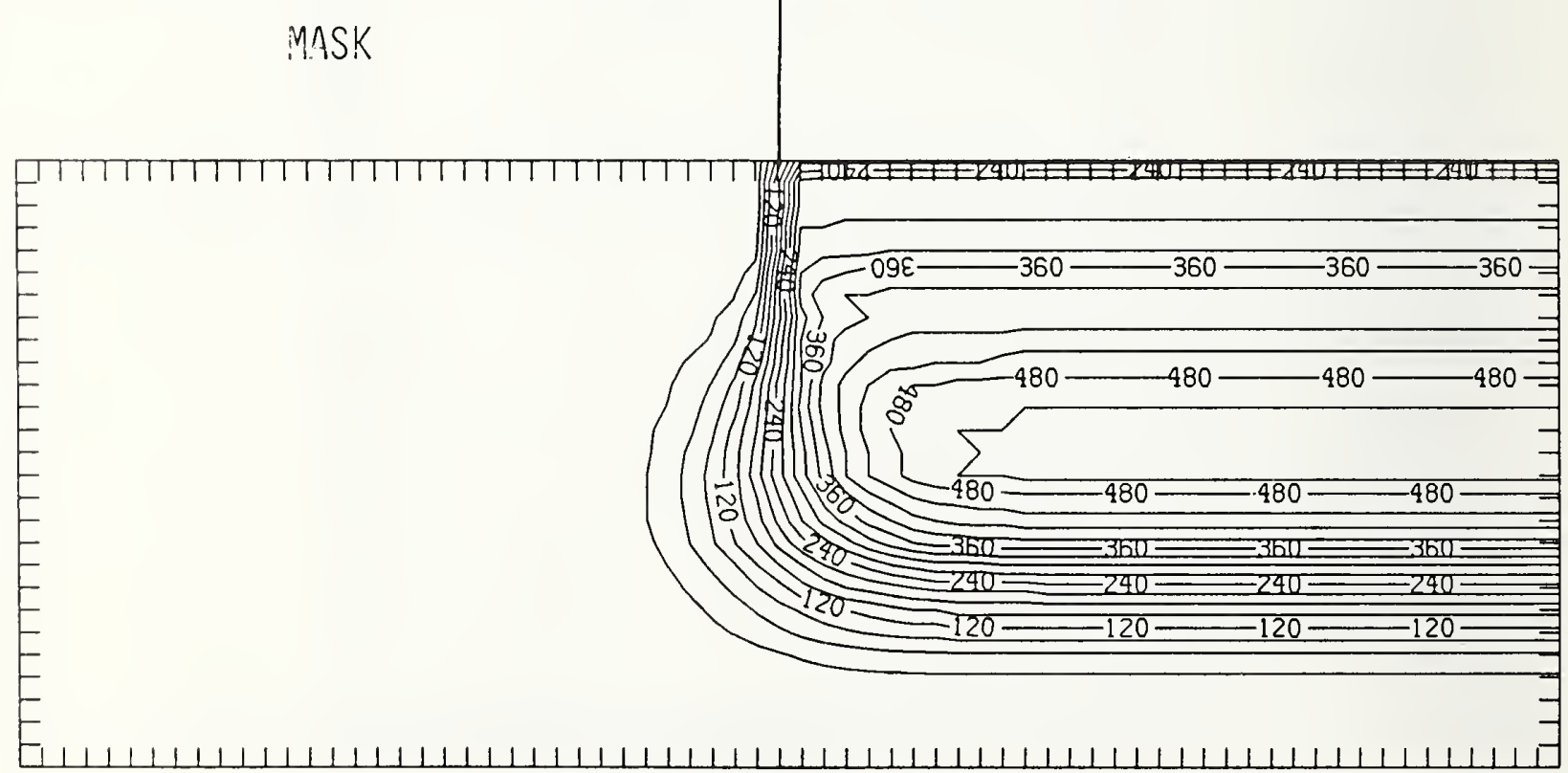

Figure 0125-4 Two-dimensional distribution of Frenkel pairs created by $125 \mathrm{keV}$ oxygen implanted past a mask edge. The length increment (distance between tick marks) is $0.02 \mu \mathrm{m}$ and the scale factor is 1 . 


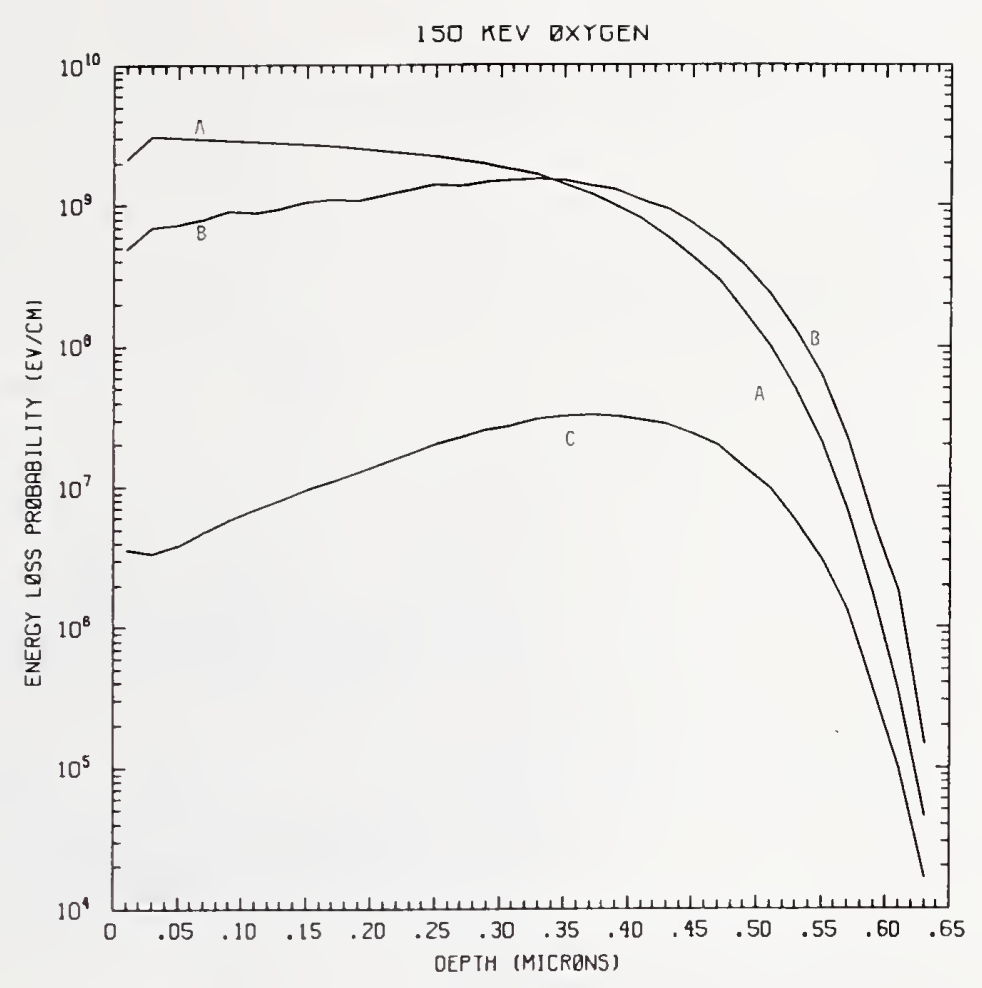

Figure O150-1 One-dimensional probability distributions for energy loss due to electronic excitation (A), displacement damage(B), and phonon excitation (C) for $150 \mathrm{keV}$ oxygen normally incident on a silicon target.

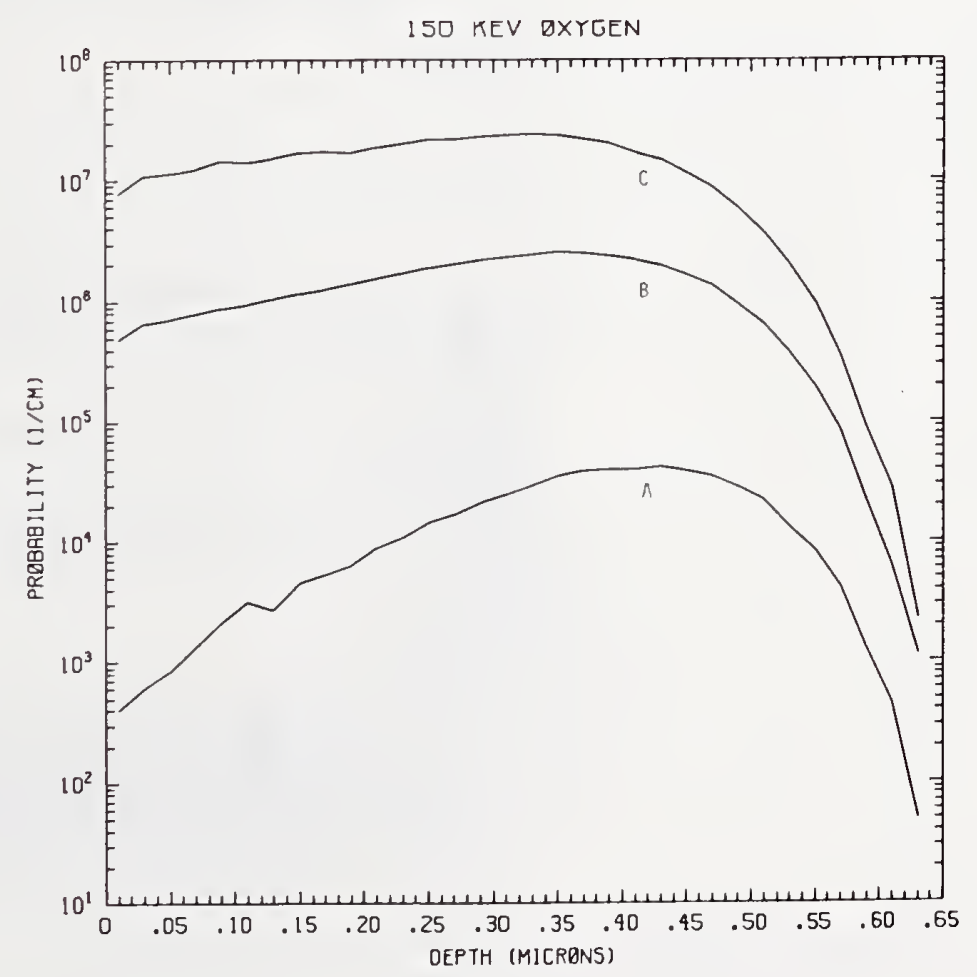

Figure 0150-2 One-dimensional probability distributions for implanted oxygen (A), primary silicon displacements (B), and Frenkel pairs (C) for $150 \mathrm{keV}$ oxygen normally incident on a silicon target. 


\section{MASK}

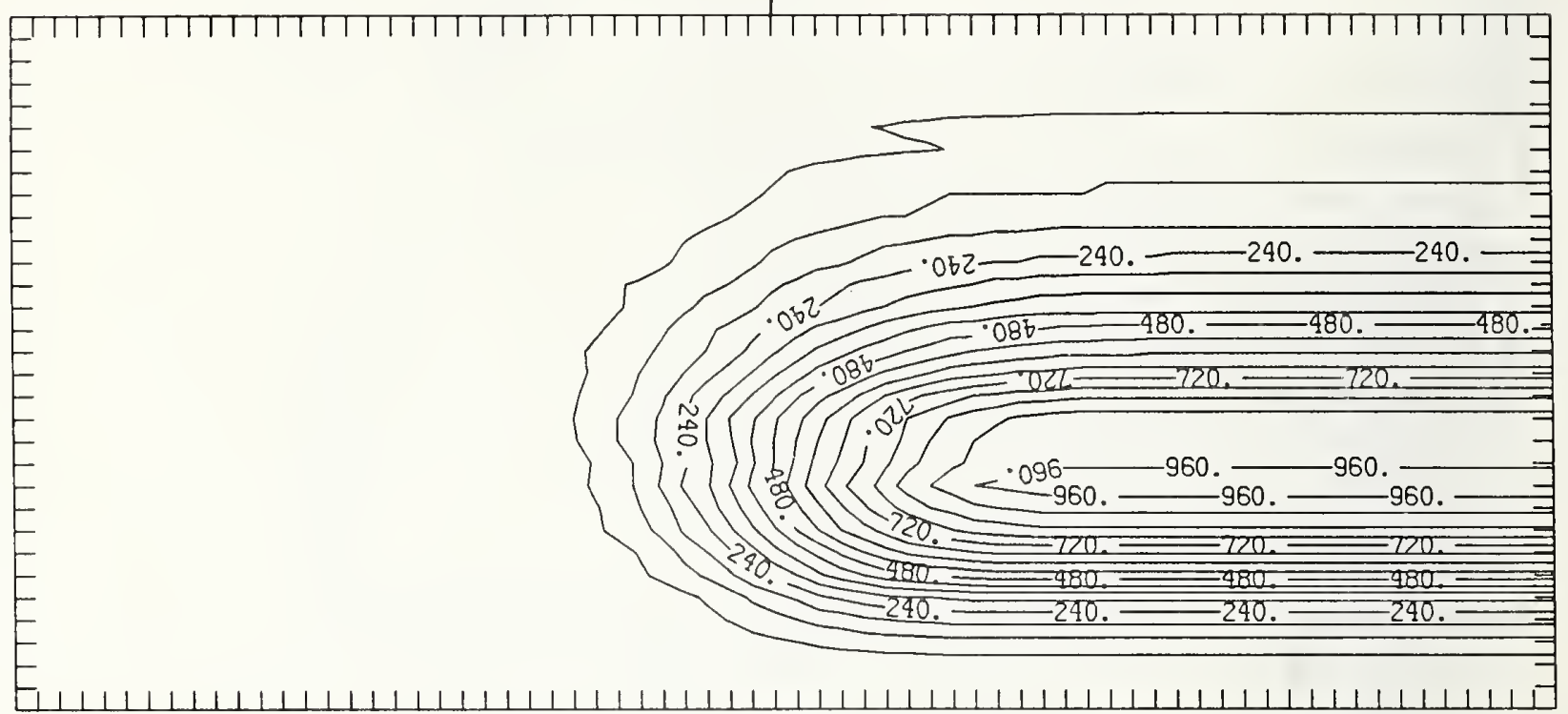

Figure 0150-3 Two-dimensional distribution of $150 \mathrm{keV}$ oxygen implanted past a mask edge. The length increment (distance between tick marks) is $0.02 \mu \mathrm{m}$ and the scale factor is 1000 .

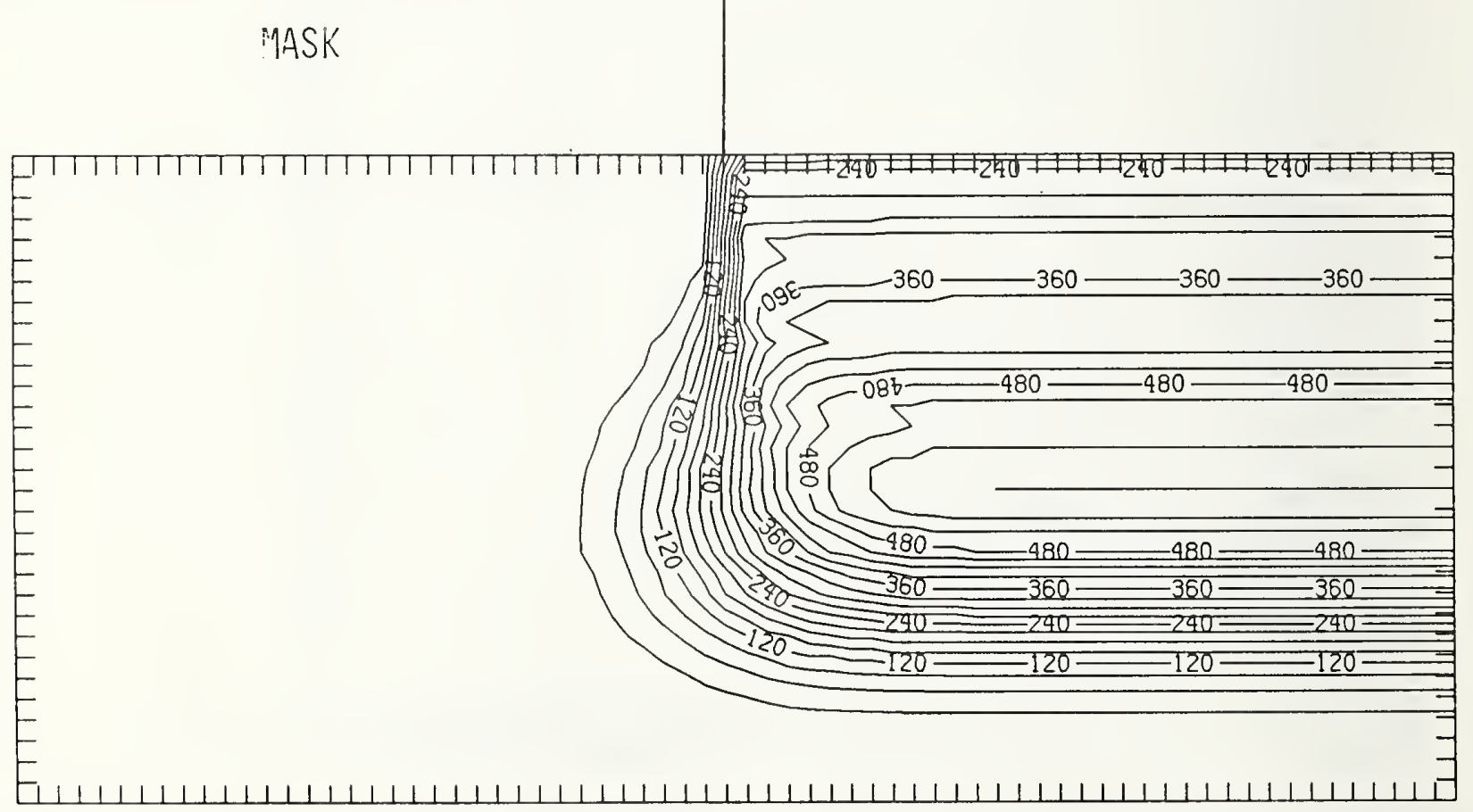

Figure O150-4 Two-dimensional distribution of Frenkel pairs created by $150 \mathrm{keV}$ oxygen implanted past a mask edge. The length increment (distance between tick marks) is $0.02 \mu \mathrm{m}$ and the scale factor is 1. 


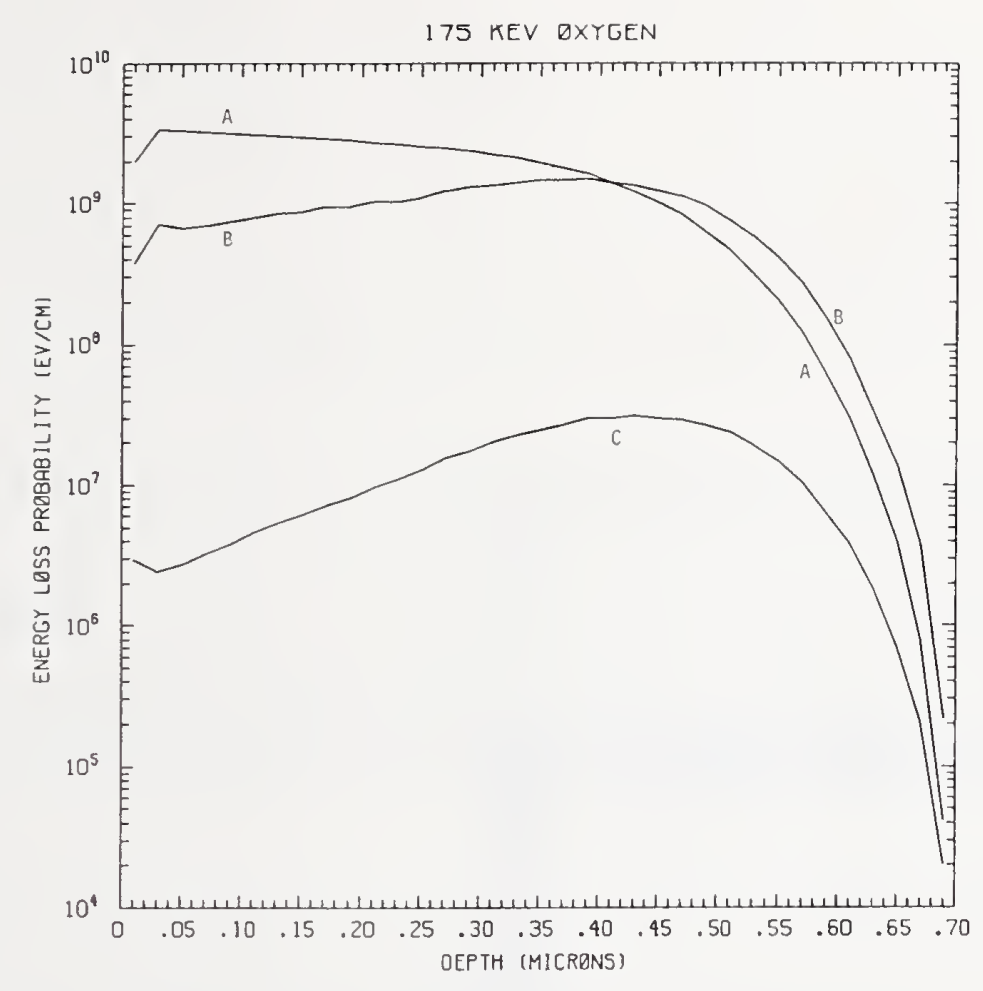

Figure 0175-1 One-dimensional probability distributions for energy loss due to electronic excitation (A), displacement damage(B), and phonon excitation (C) for $175 \mathrm{keV}$ oxygen normally incident on a silicon target.

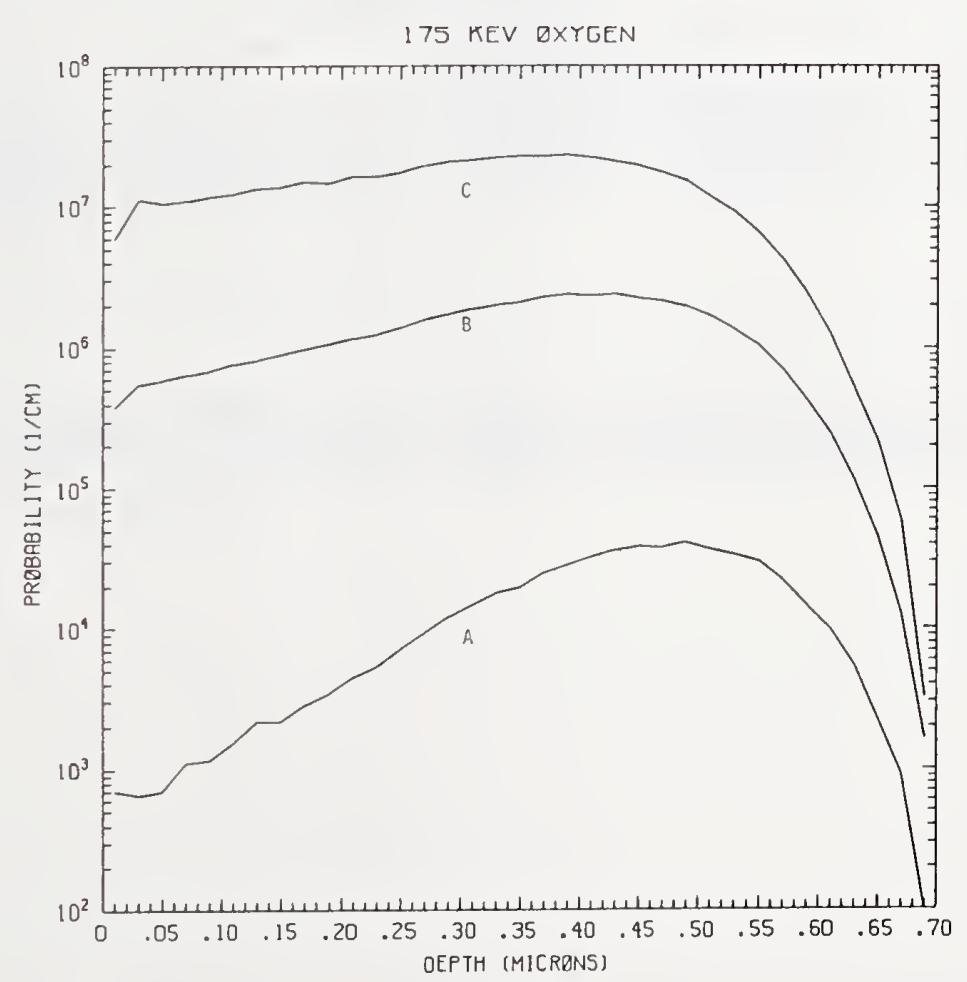

Figure 0175-2 One-dimensional probability distributions for implanted oxygen (A), primary silicon displacements (B), and Frenkel pairs (C) for $175 \mathrm{keV}$ oxygen normally incident on a silicon target. 


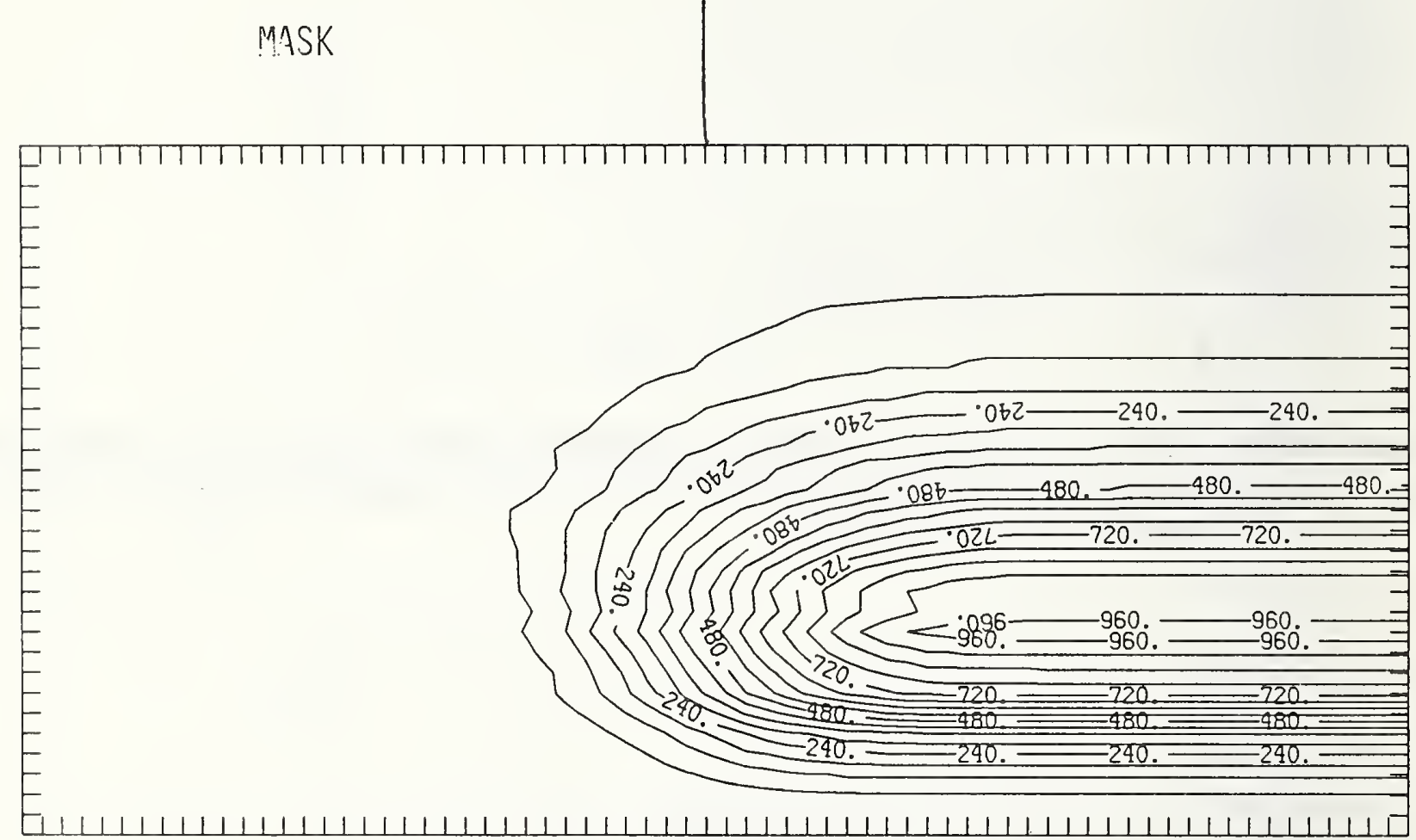

Figure 0175-3 Two-dimensional distribution of $175 \mathrm{keV}$ oxygen implanted past a mask edge. The length increment (distance between tick marks) is $0.02 \mu \mathrm{m}$ and the scale factor is 1000 . 


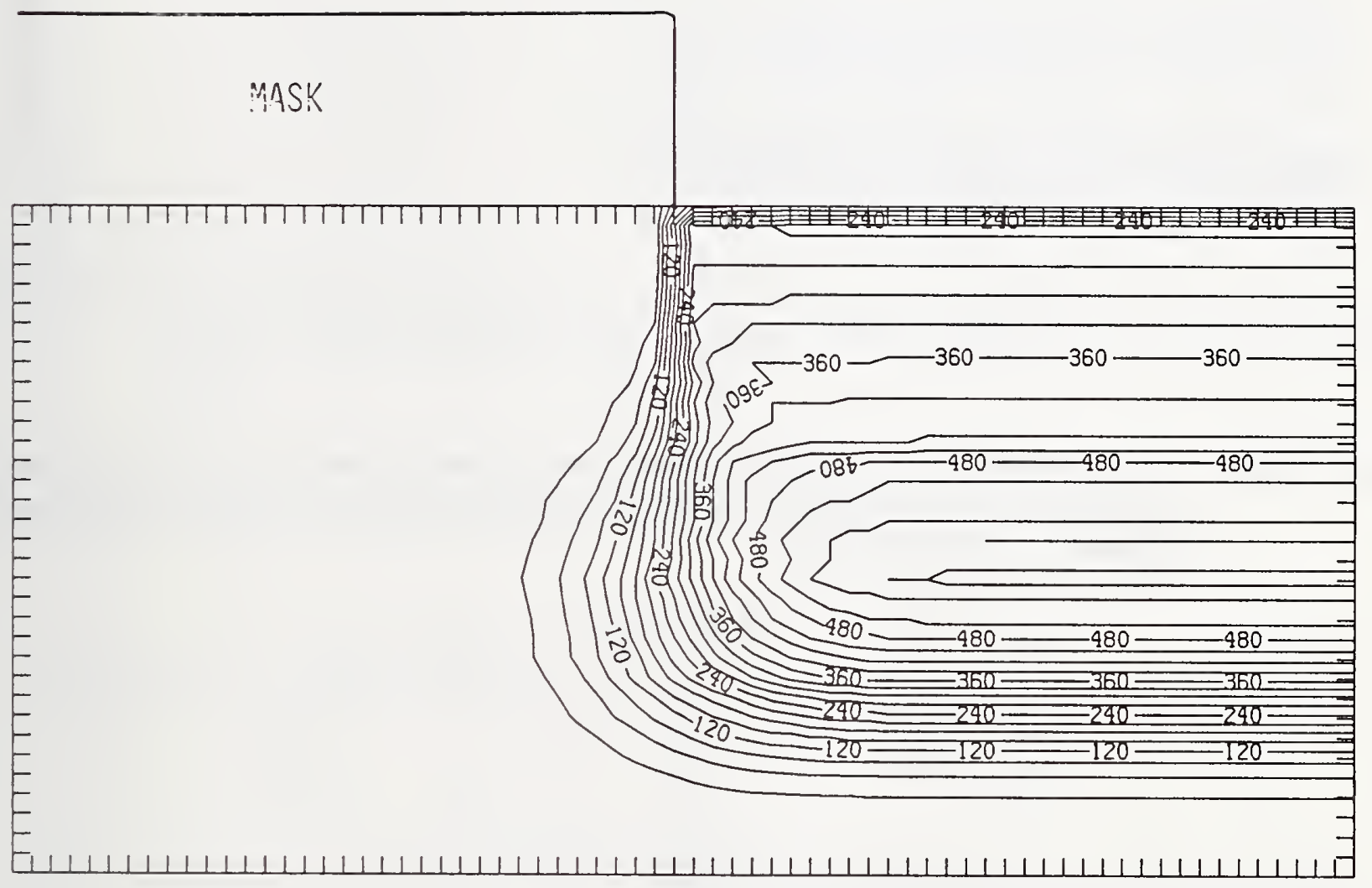

Figure 0175-4 Two-dimensional distribution of Frenkel pairs created by $175 \mathrm{keV}$ oxygen implanted past a mask edge. The length increment (distance between tick marks) is $0.02 \mu \mathrm{m}$ and the scale factor is 1 . 


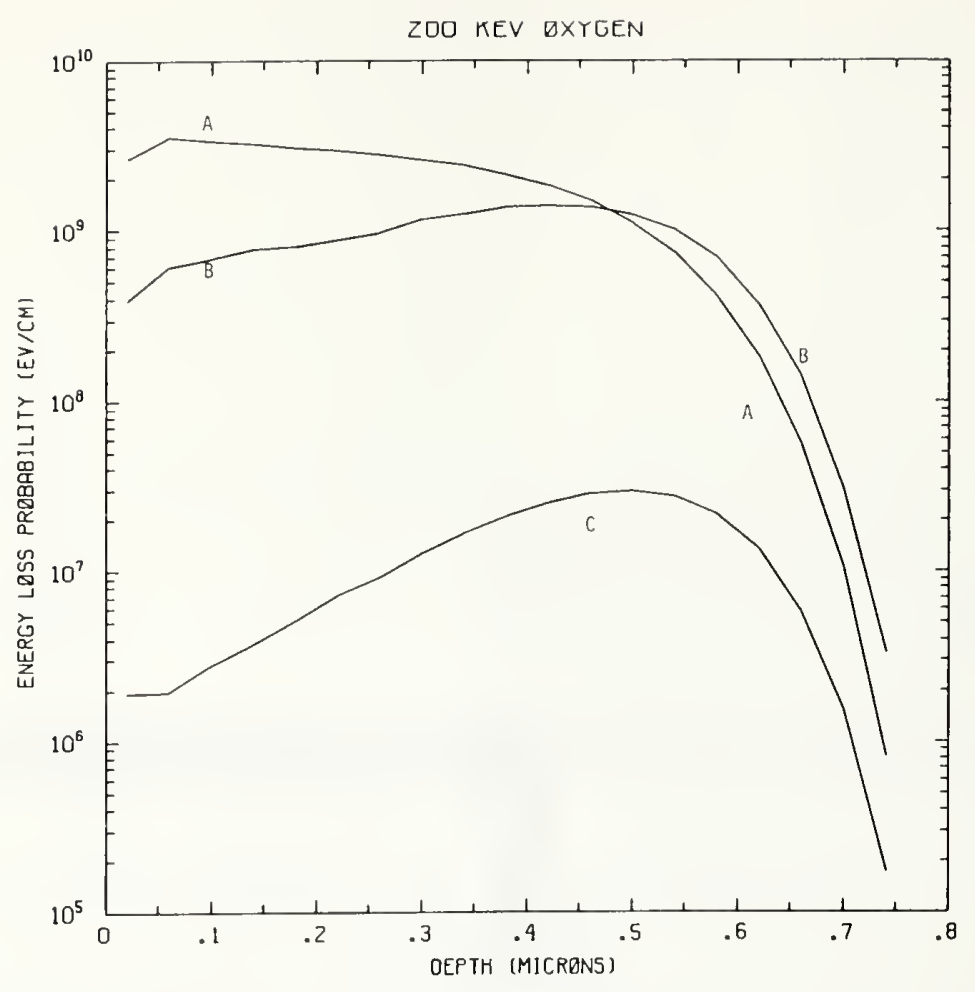

Figure 0200-1 One-dimensional probability distributions for energy loss due to electronic excitation (A), displacement damage(B), and phonon excitation (C) for $200 \mathrm{keV}$ oxygen normally incident on a silicon target.

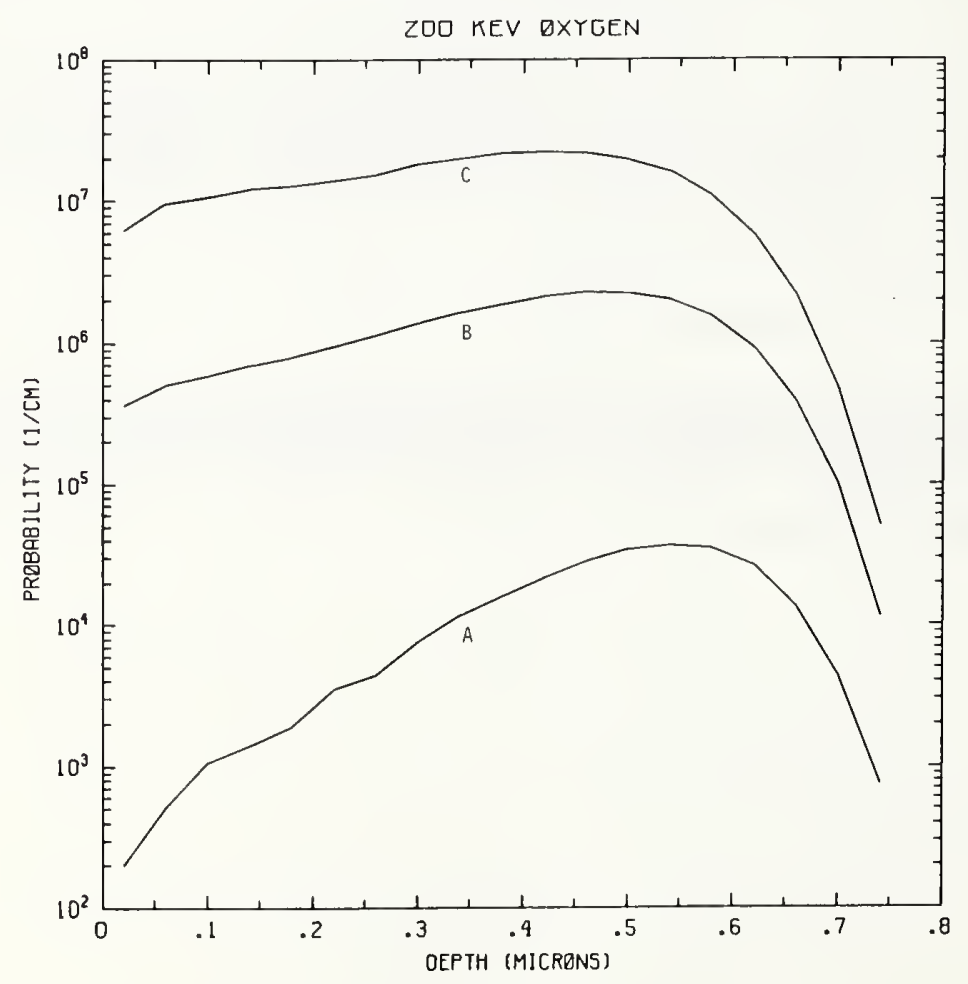

Figure O200-2 One-dimensional probability distributions for implanted oxygen (A), primary silicon displacements (B), and Frenkel pairs (C) for $200 \mathrm{keV}$ oxygen normally incident on a silicon target. 


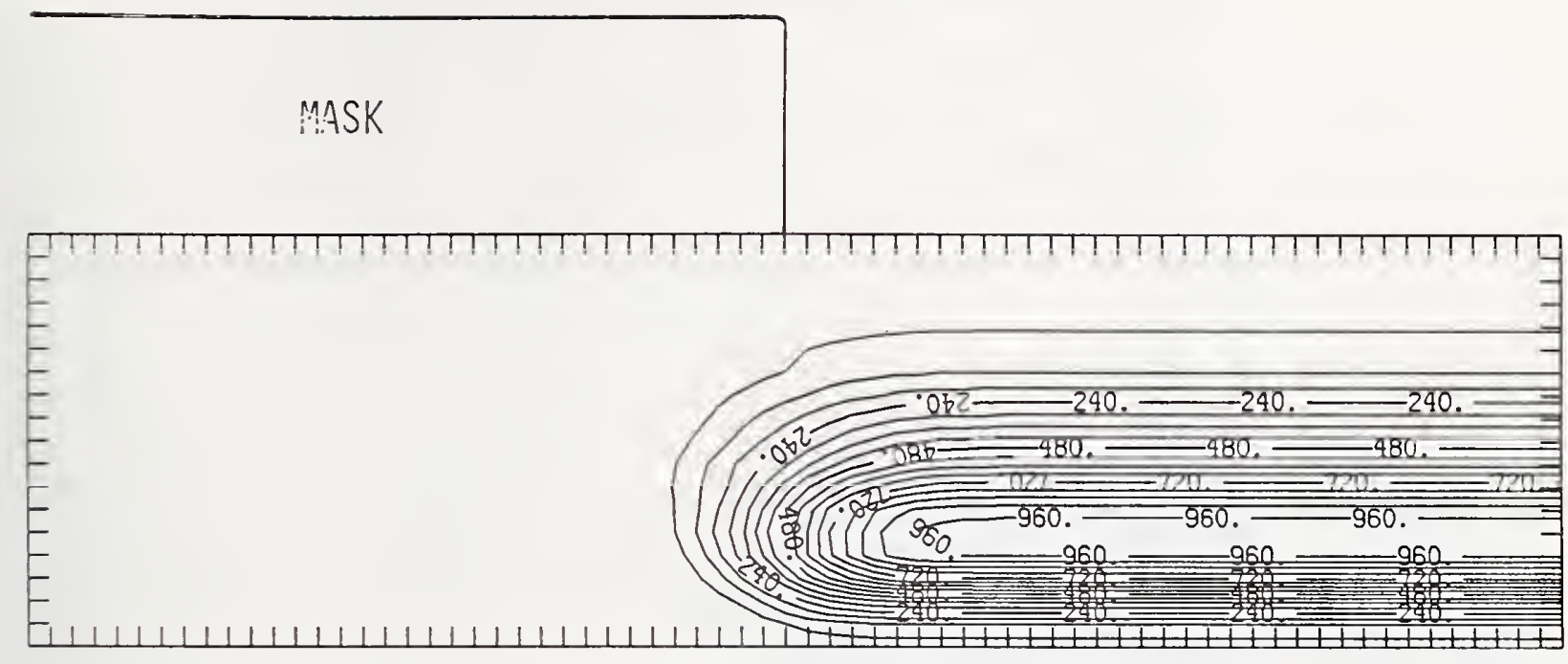

Figure O200-3 Two-dimensional distribution of $200 \mathrm{keV}$ oxygen implanted past a mask edge. The length increment (distance between tick marks) is $0.04 \mu \mathrm{m}$ and the scale factor is 1000.

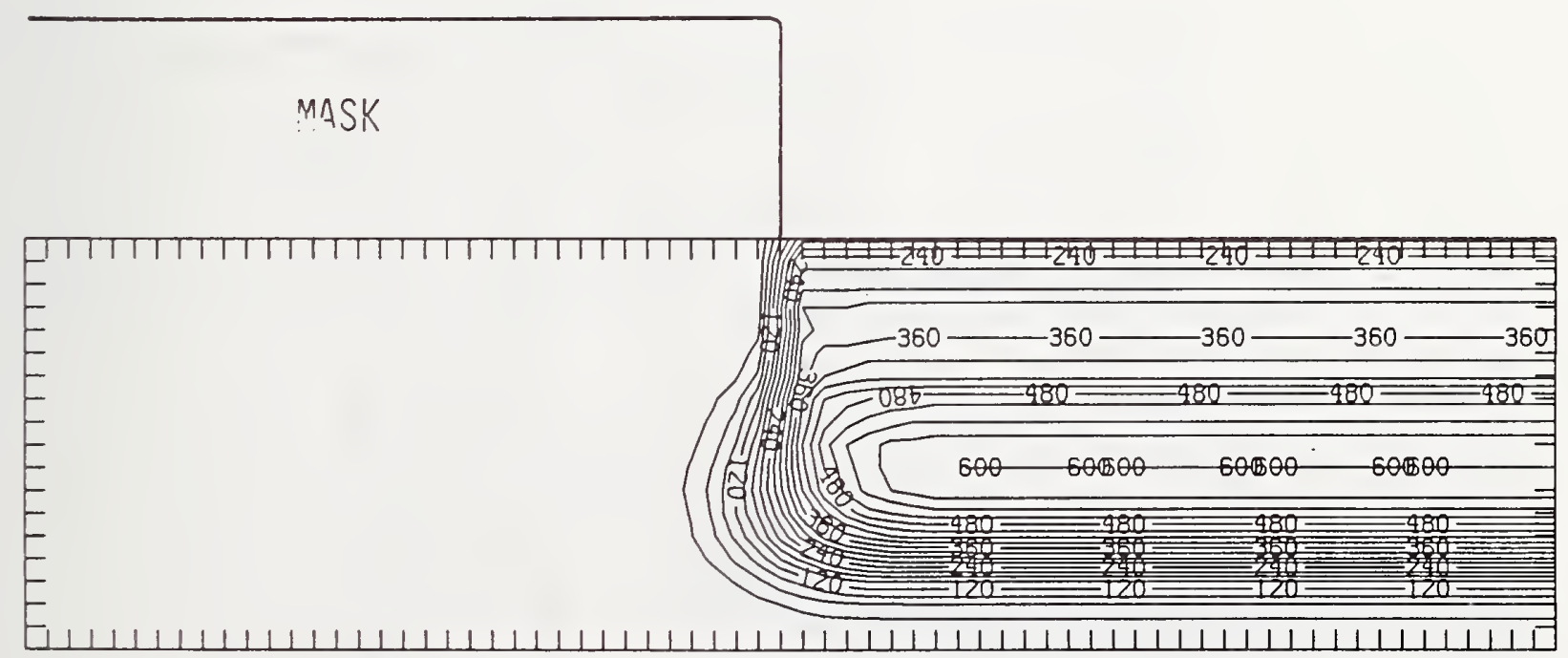

Figure 0200-4 Two-dimensional distribution of Frenkel pairs created by $200 \mathrm{keV}$ oxygen implanted past a mask edge. The length increment (distance between tick marks) is $0.04 \mu \mathrm{m}$ and the scale factor is 1. 


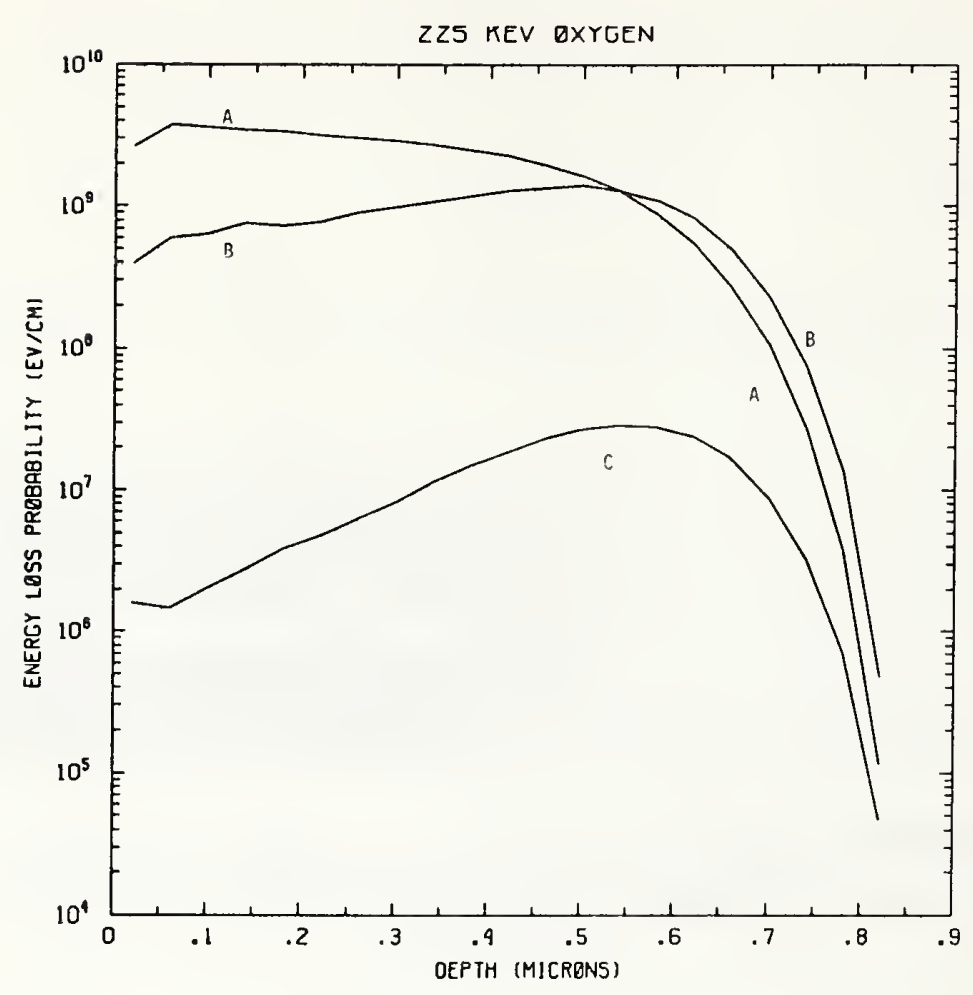

Figure 0225-1 One-dimensional probability distributions for energy loss due to electronic excitation (A), displacement damage(B), and phonon excitation (C) for $225 \mathrm{keV}$ oxygen normally incident on a silicon target.

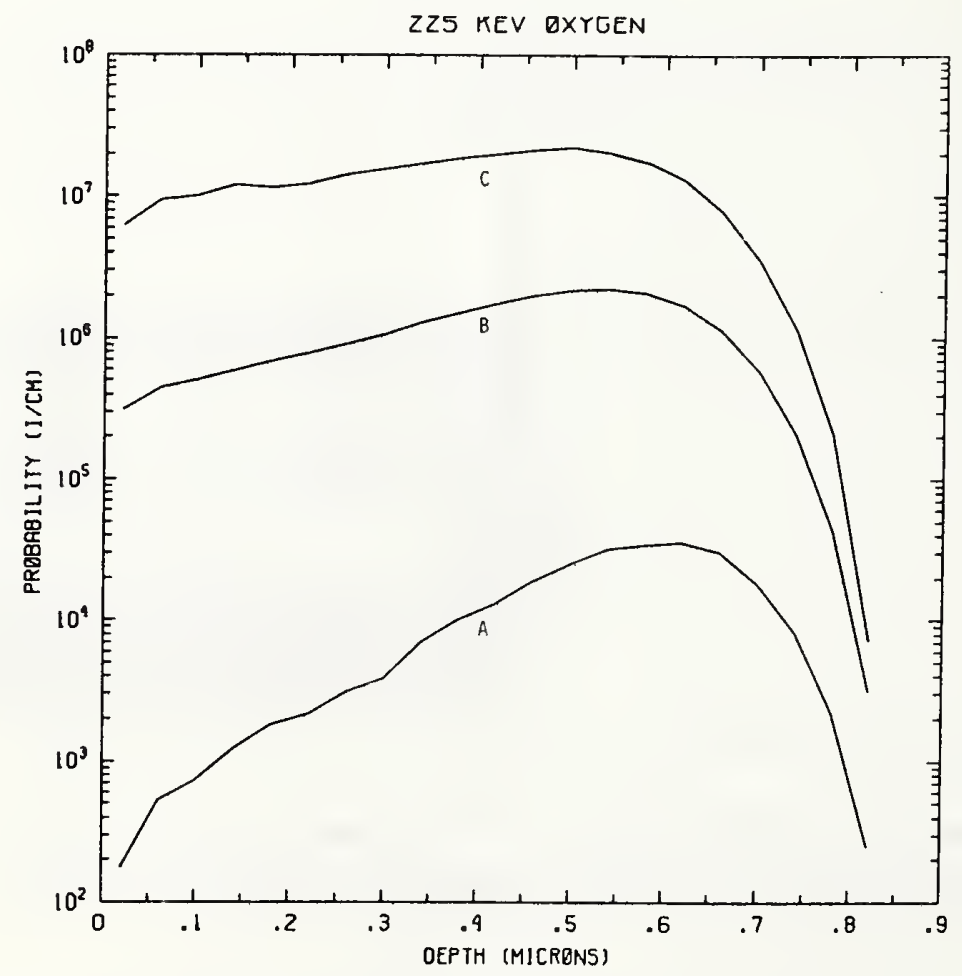

Figure 0225-2 One-dimensional probability distributions for implanted oxygen (A), primary silicon displacements (B), and Frenkel pairs (C) for $225 \mathrm{keV}$ oxygen normally incident on a silicon target. 


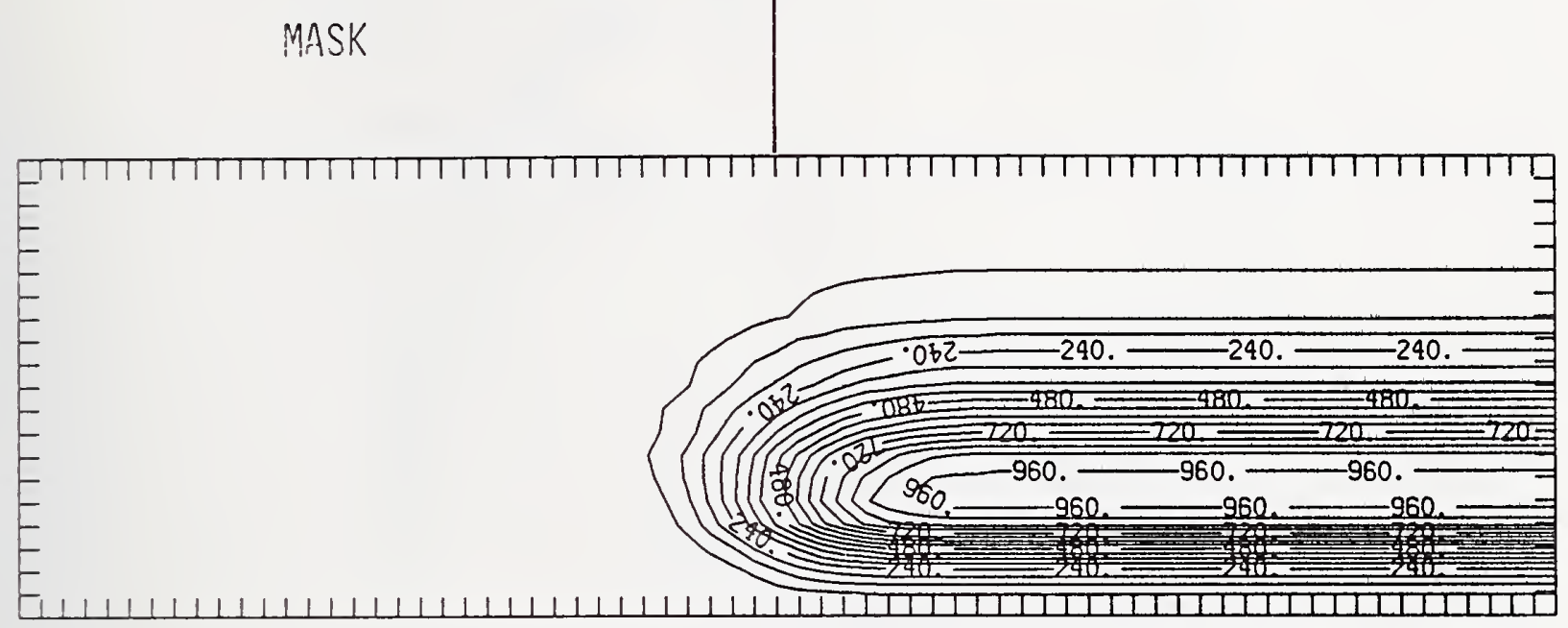

Figure 0225-3 Two-dimensional distribution of $225 \mathrm{keV}$ oxygen implanted past a mask edge. The length increment (distance between tick marks) is $0.04 \mu \mathrm{m}$ and the scale factor is 1000 .

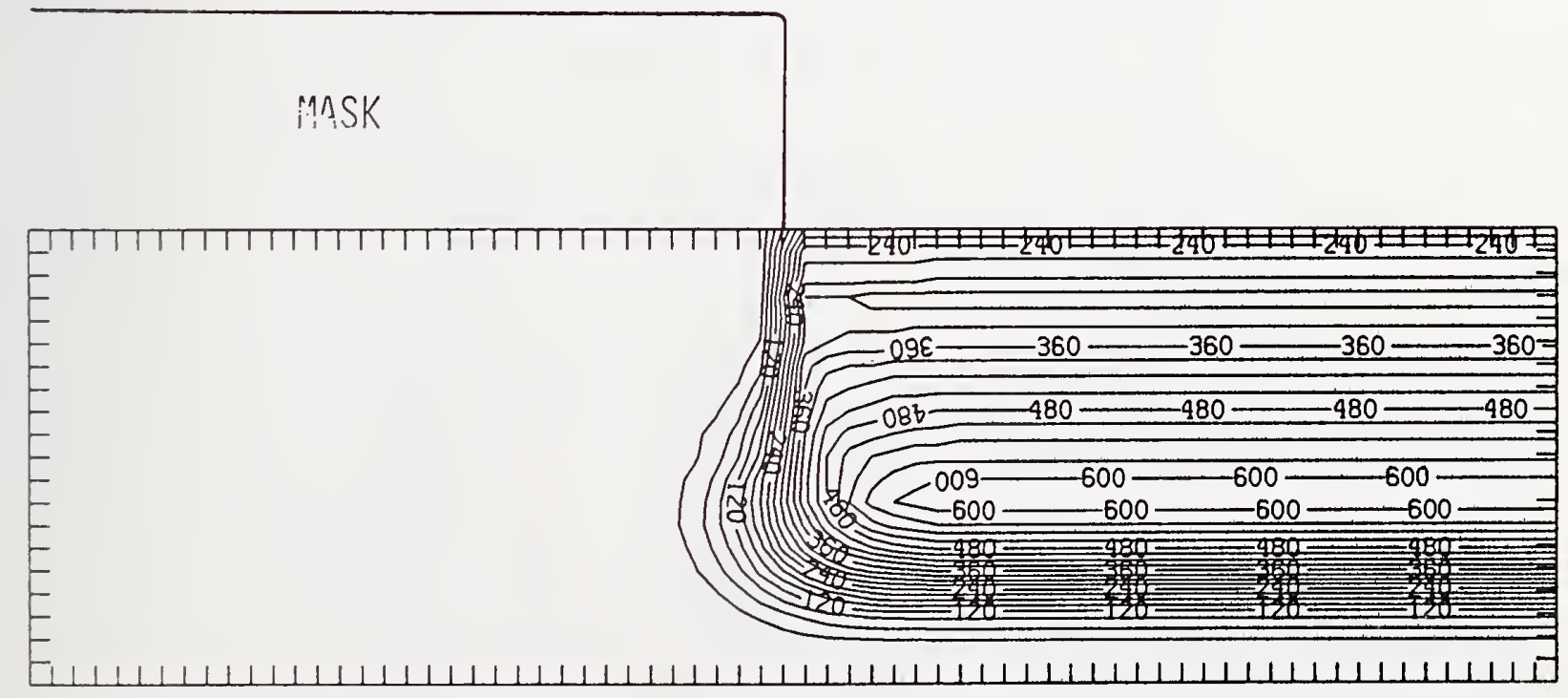

Figure 0225-4 Two-dimensional distribution of Frenkel pairs created by $225 \mathrm{keV}$ oxygen implanted past a mask edge. The length increment (distance between tick marks) is $0.04 \mu \mathrm{m}$ and the scale factor is 1. 


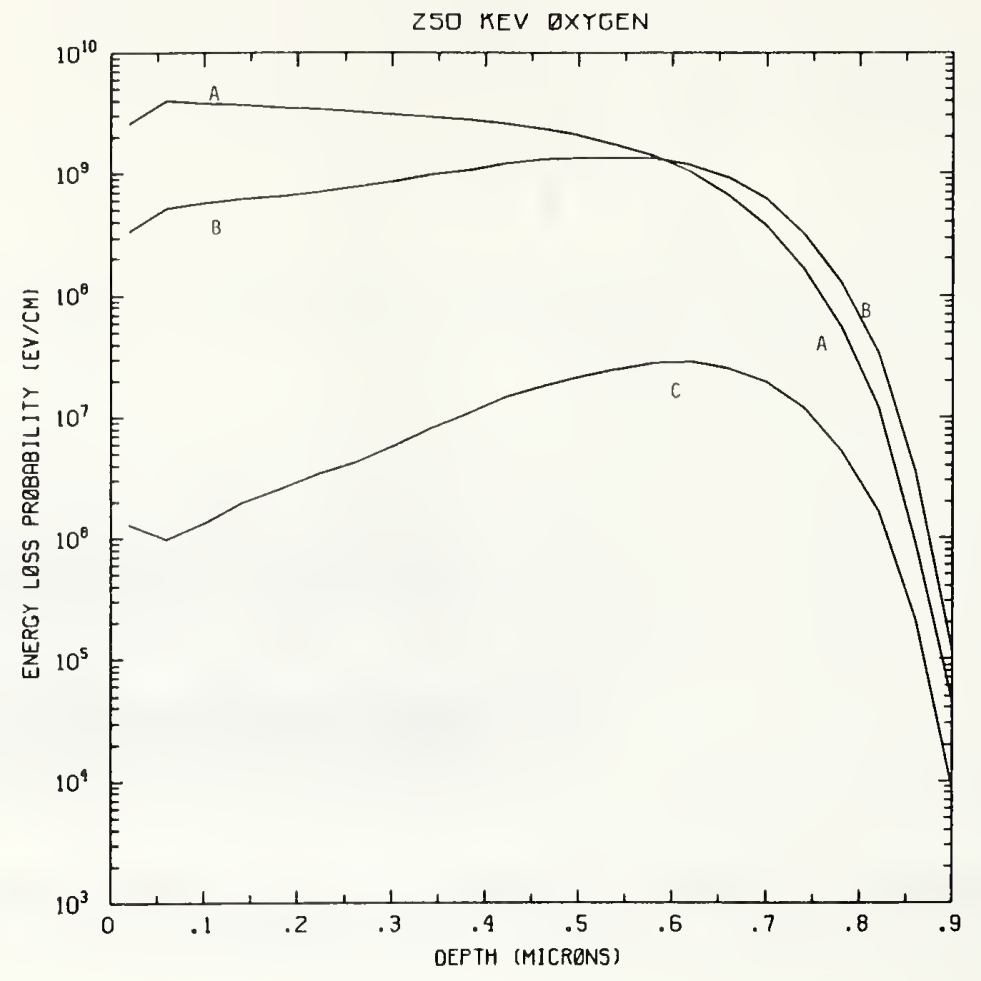

Figure O250-1 One-dimensional probability distributions for energy loss due to electronic excitation (A), displacement damage(B), and phonon excitation (C) for $250 \mathrm{keV}$ oxygen normally incident on a silicon target.

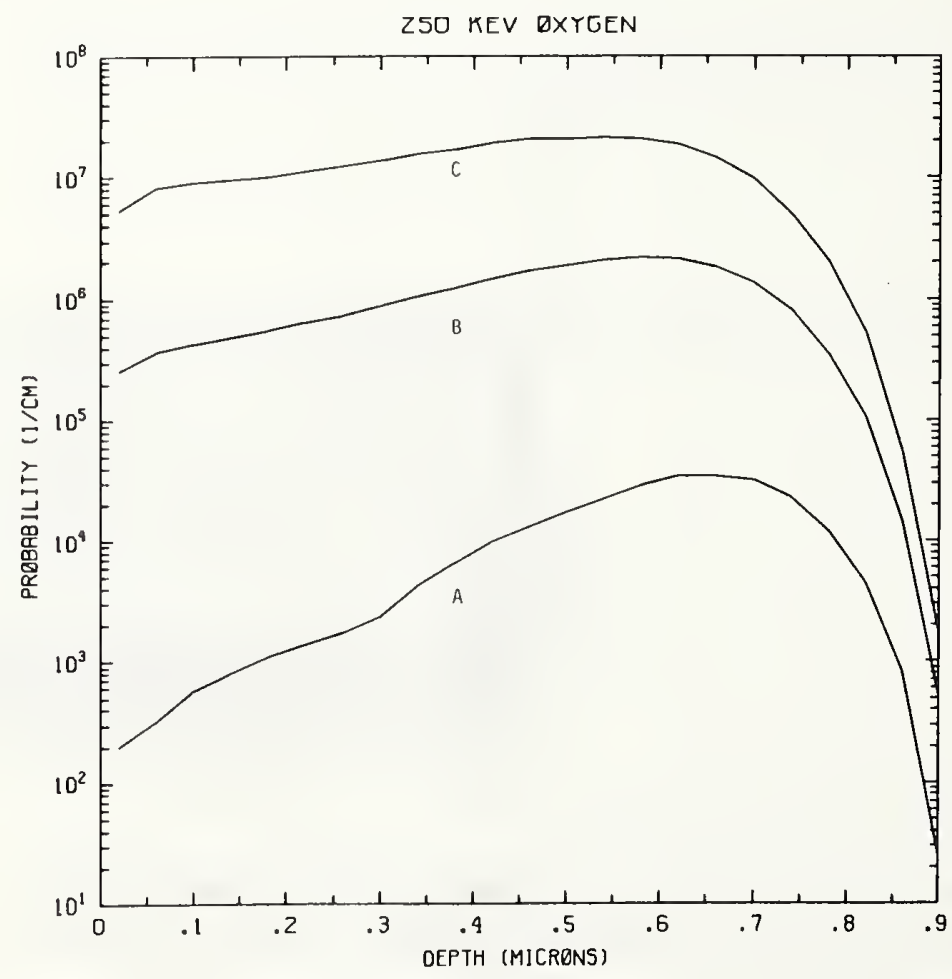

Figure 0250-2 One-dimensional probability distributions for implanted oxygen (A), primary silicon displacements (B), and Frenkel pairs (C) for $250 \mathrm{keV}$ oxygen normally incident on a silicon target. 


\section{MASK}

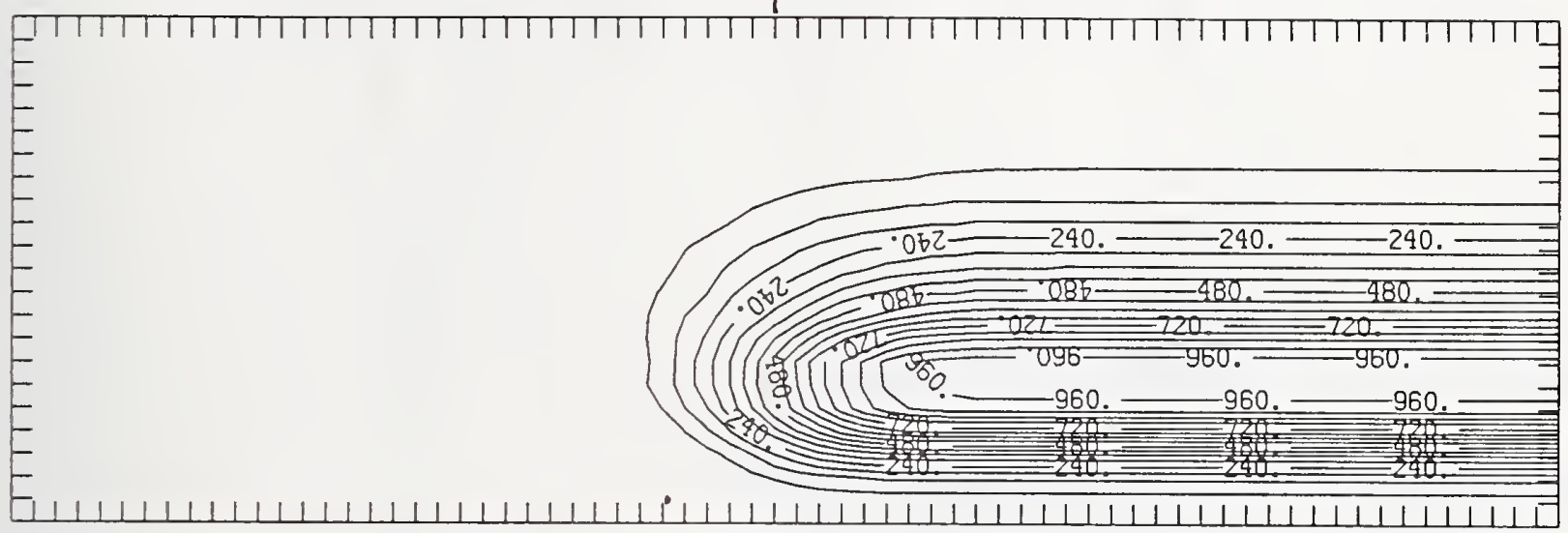

Figure 0250-3 Two-dimensional distribution of $250 \mathrm{keV}$ oxygen implanted past a mask edge. The length increment (distance between tick marks) is $0.04 \mu \mathrm{m}$ and the scale factor is 1000.

MASK

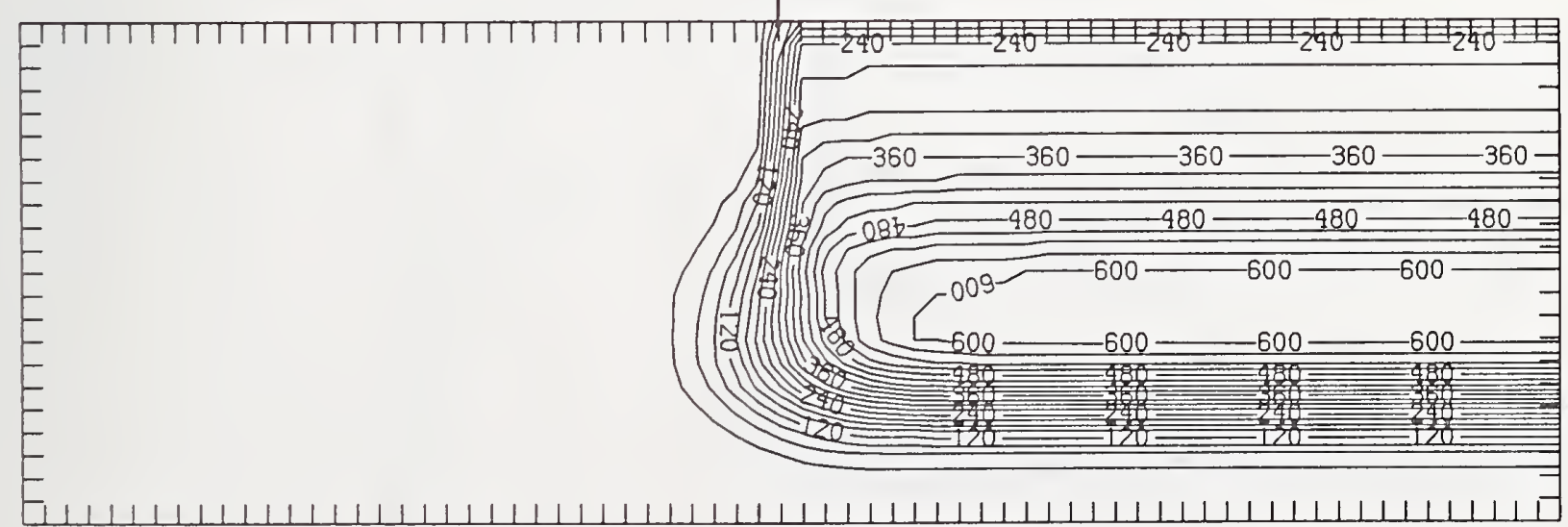

Figure 0250-4 Two-dimensional distribution of Frenkel pairs created by $250 \mathrm{keV}$ oxygen implanted past a mask edge. The length increment (distance between tick marks) is $0.04 \mu \mathrm{m}$ and the scale factor is 1. 


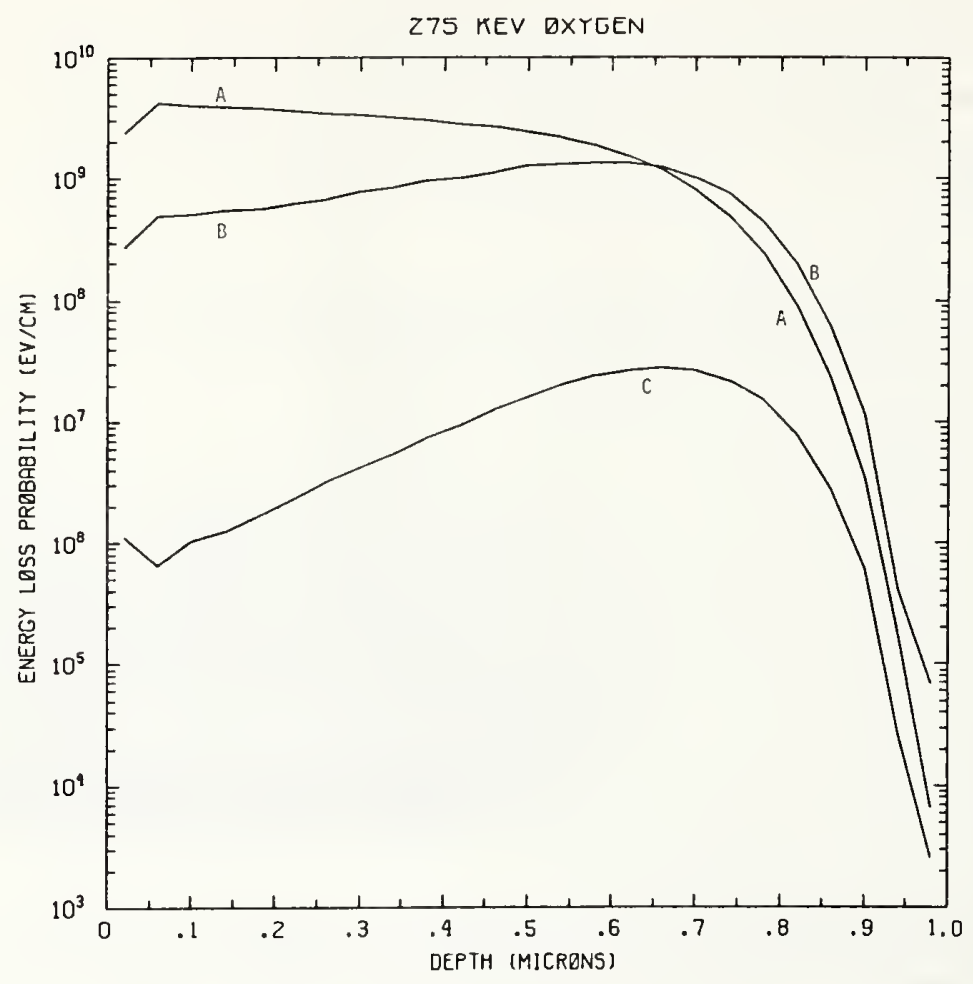

Figure 0275-1 One-dimensional probability distributions for energy loss due to electronic excitation (A), displacement damage(B), and phonon excitation (C) for $275 \mathrm{keV}$ oxygen normally incident on a silicon target.

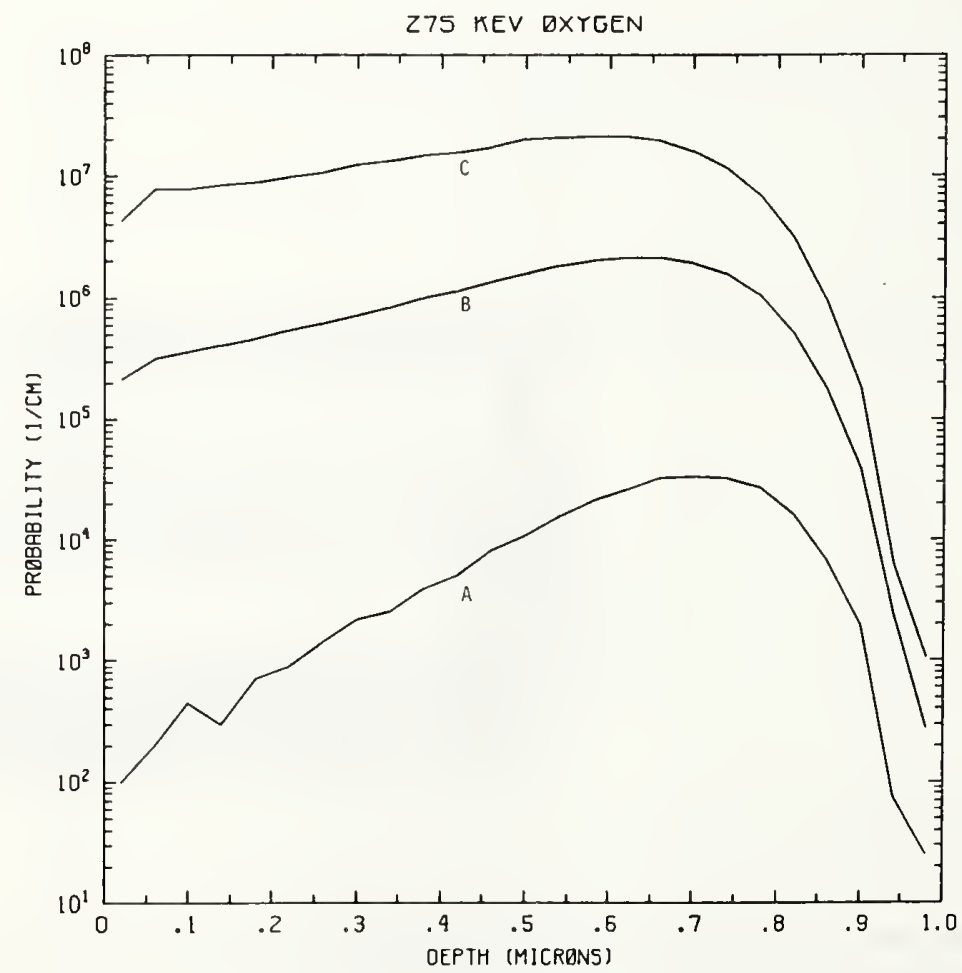

Figure 0275-2 One-dimensional probability distributions for implanted oxygen (A), primary silicon displacements (B), and Frenkel pairs (C) for $275 \mathrm{keV}$ oxygen normally incident on a silicon target. 


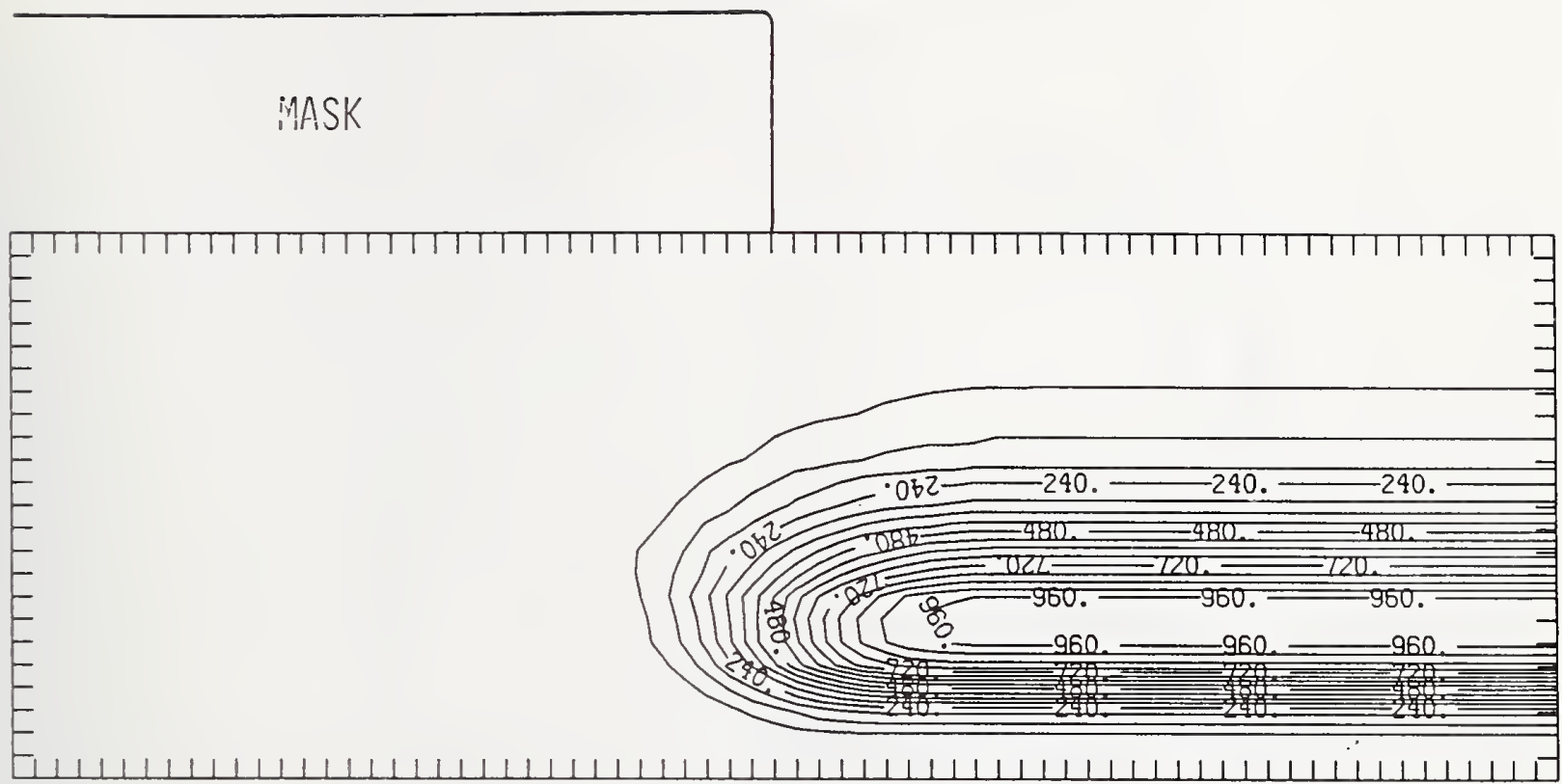

Figure 0275-3 Two-dimensional distribution of $275 \mathrm{keV}$ oxygen implanted past a mask edge. The length increment (distance between tick marks) is $0.04 \mu \mathrm{m}$ and the scale factor is 1000 .

!IASK

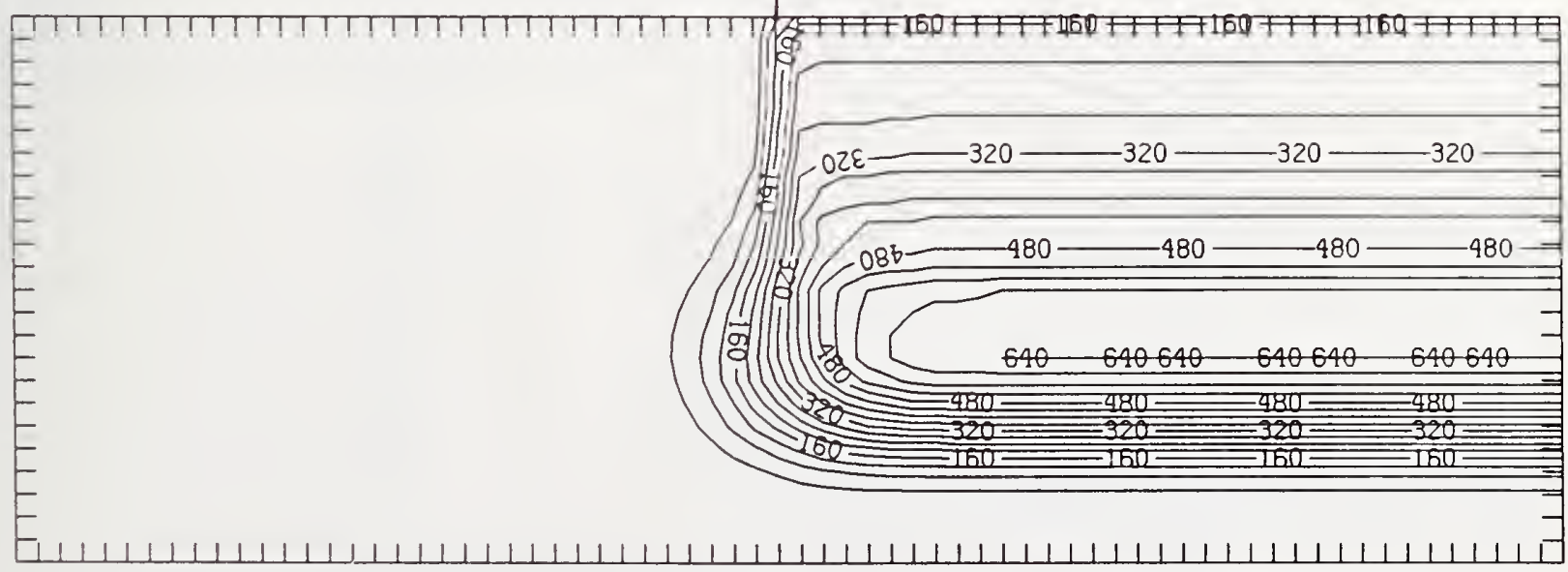

Figure 0275-4 Two-dimensional distribution of Frenkel pairs created by $275 \mathrm{keV}$ oxygen implanted past a mask edge. The length increment (distance between tick marks) is $0.04 \mu \mathrm{m}$ and the scale factor is 1 . 


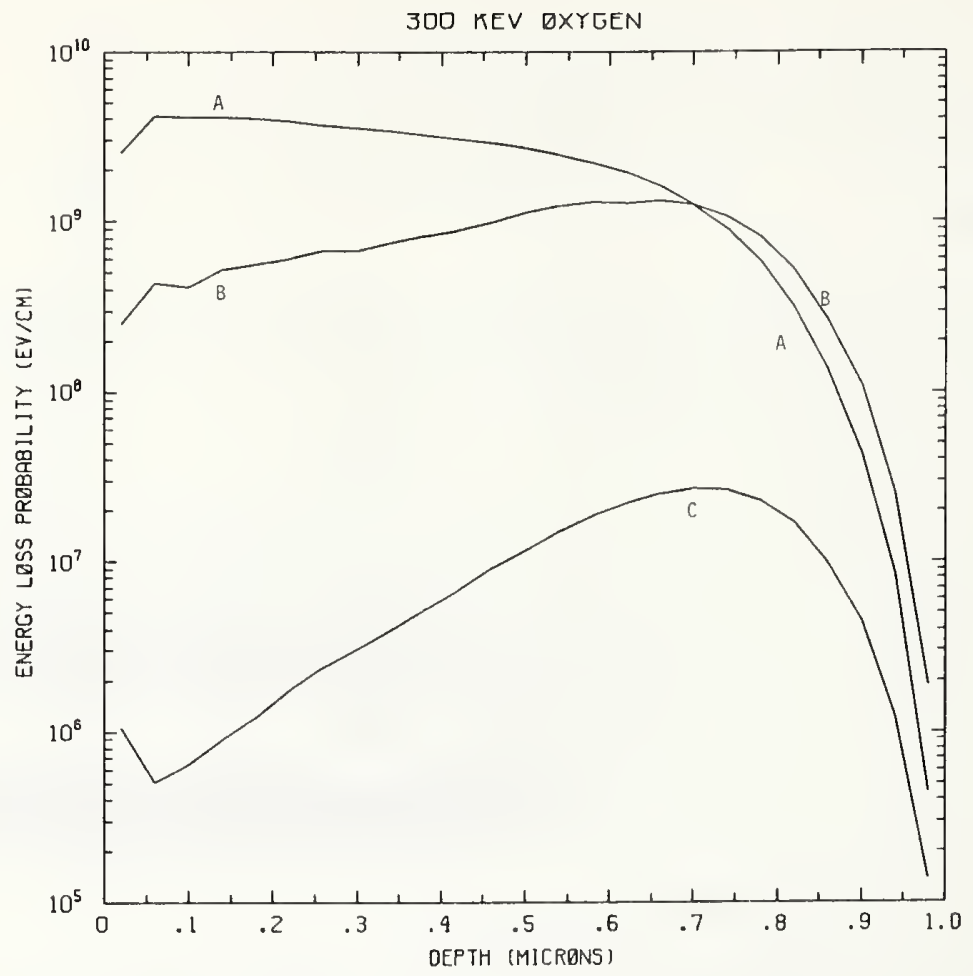

Figure O300-1 One-dimensional probability distributions for energy loss due to electronic excitation (A), displacement damage(B), and phonon excitation (C) for $300 \mathrm{keV}$ oxygen normally incident on a silicon target.

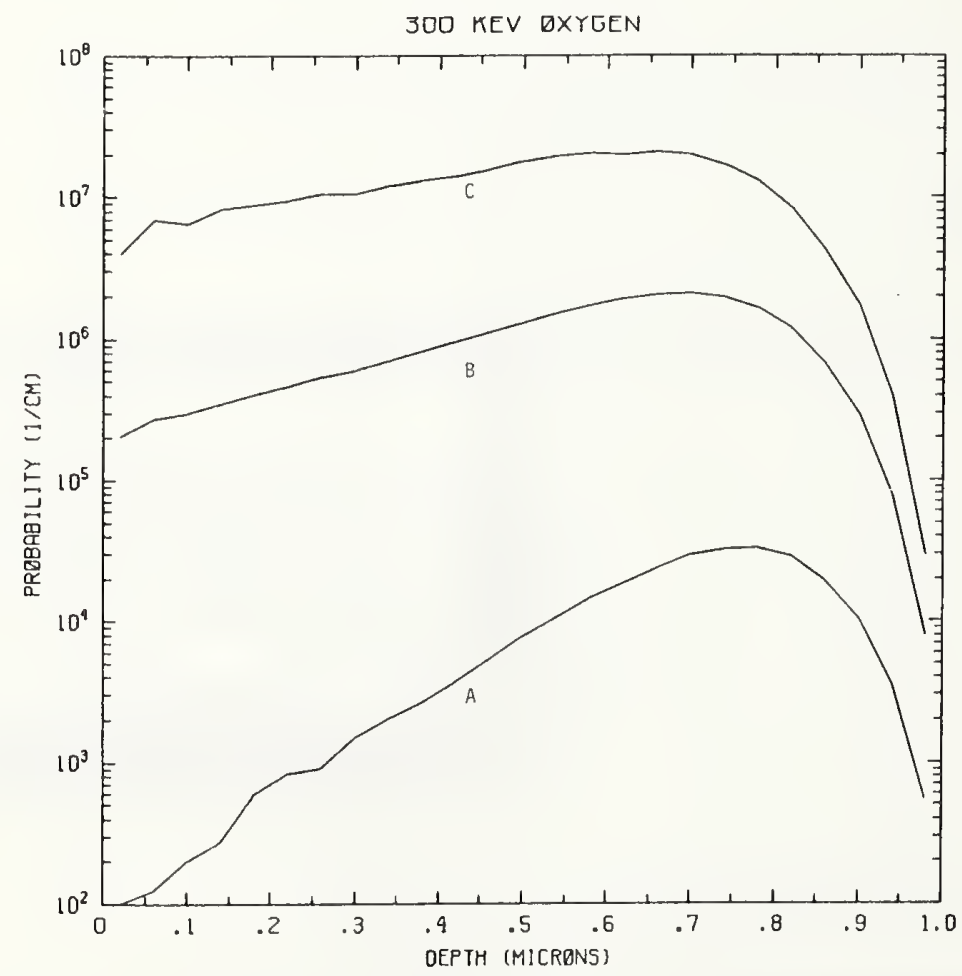

Figure O300-2 One-dimensional probability distributions for implanted oxygen (A), primary silicon displacements (B), and Frenkel pairs (C) for $300 \mathrm{keV}$ oxygen normally incident on a silicon target. 


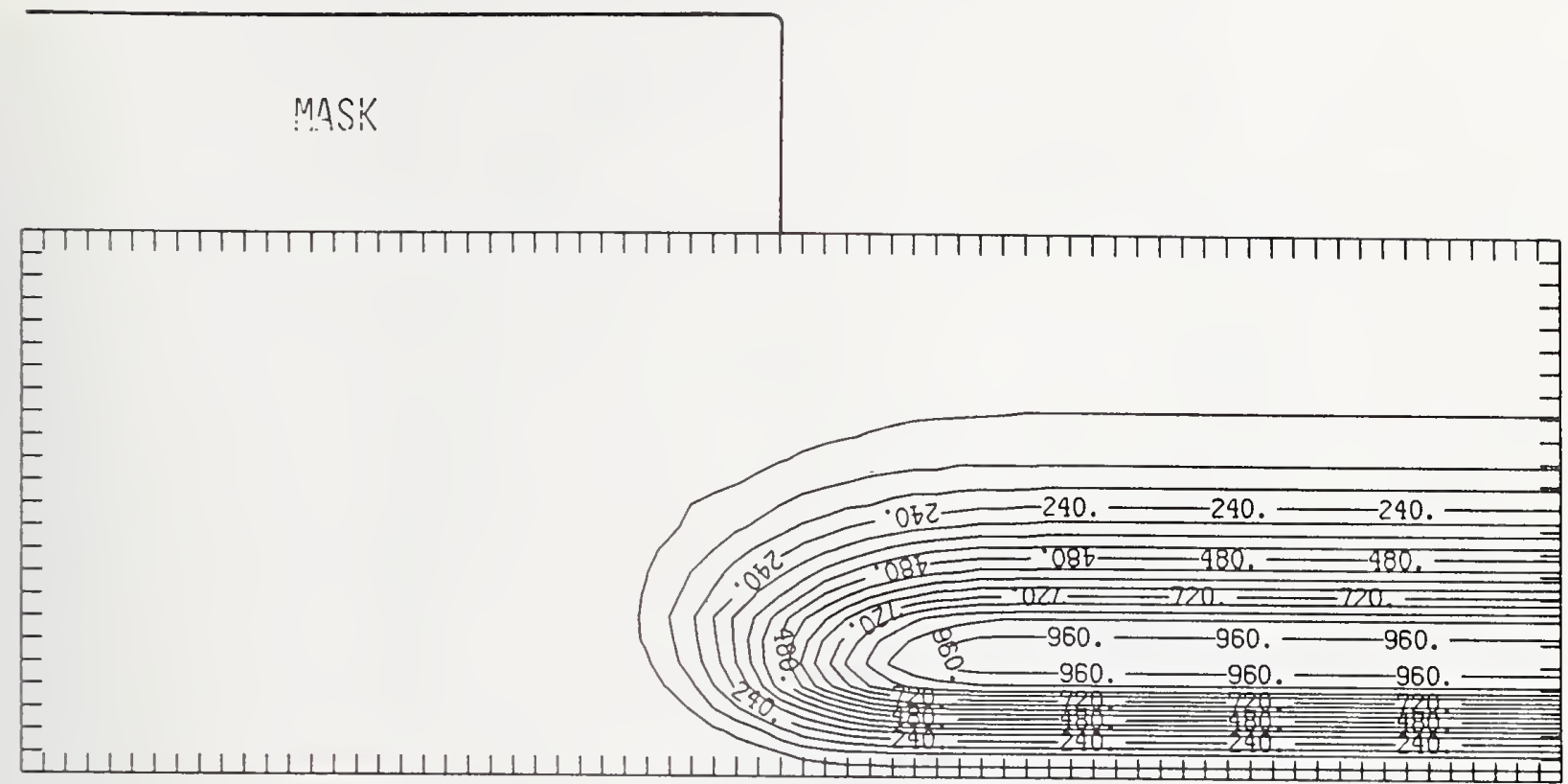

Figure 0300-3 Two-dimensional distribution of $300 \mathrm{keV}$ oxygen implanted past a mask edge. The length increment (distance between tick marks) is $0.04 \mu \mathrm{m}$ and the scale factor is 1000 .

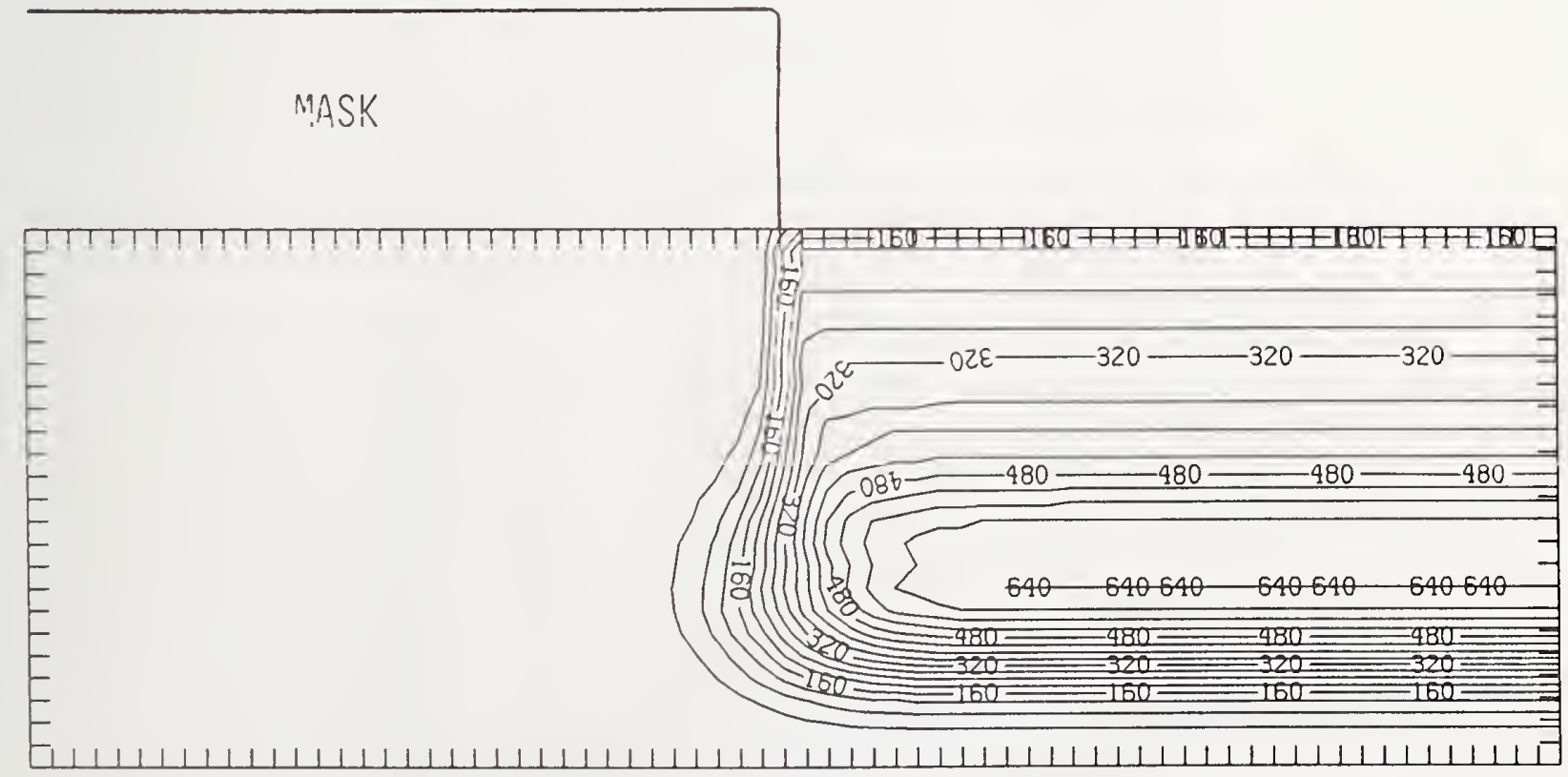

Figure 0300-4 Two-dimensional distribution of Frenkel pairs created by $300 \mathrm{keV}$ oxygen implanted past a mask edge. The length increment (distance between tick marks) is $0.04 \mu \mathrm{m}$ and the scale factor is 1 . 


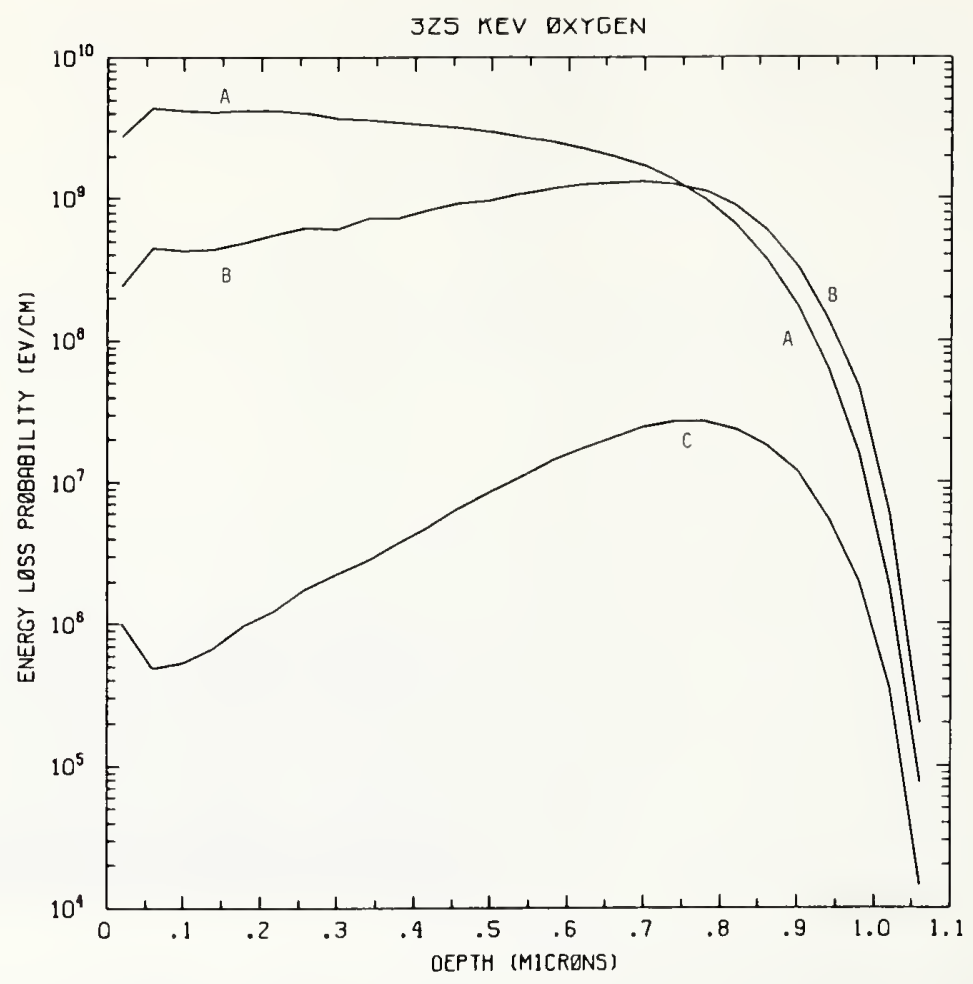

Figure 0325-1 One-dimensional probability distributions for energy loss due to electronic excitation (A), displacement damage(B), and phonon excitation (C) for $325 \mathrm{keV}$ oxygen normally incident on a silicon target.

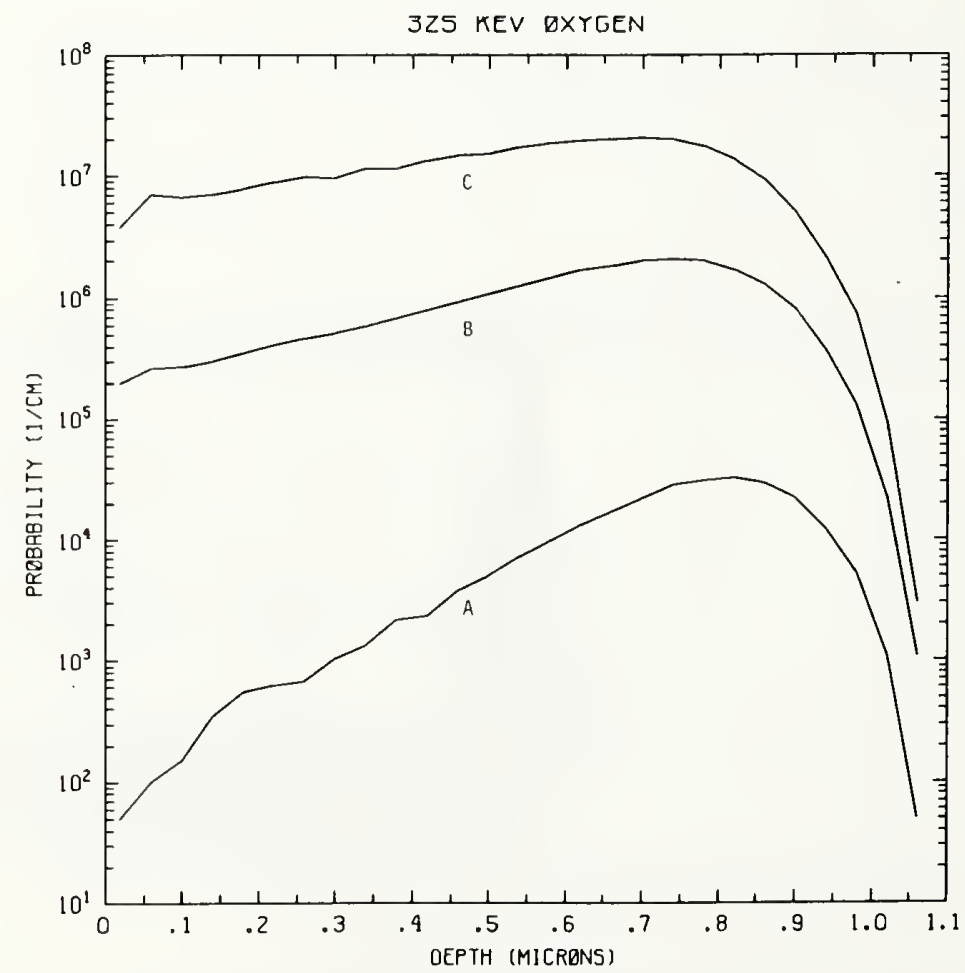

Figure 0325-2 One-dimensional probability distributions for implanted oxygen (A), primary silicon displacements (B), and Frenkel pairs (C) for $325 \mathrm{keV}$ oxygen normally incident on a silicon target. 


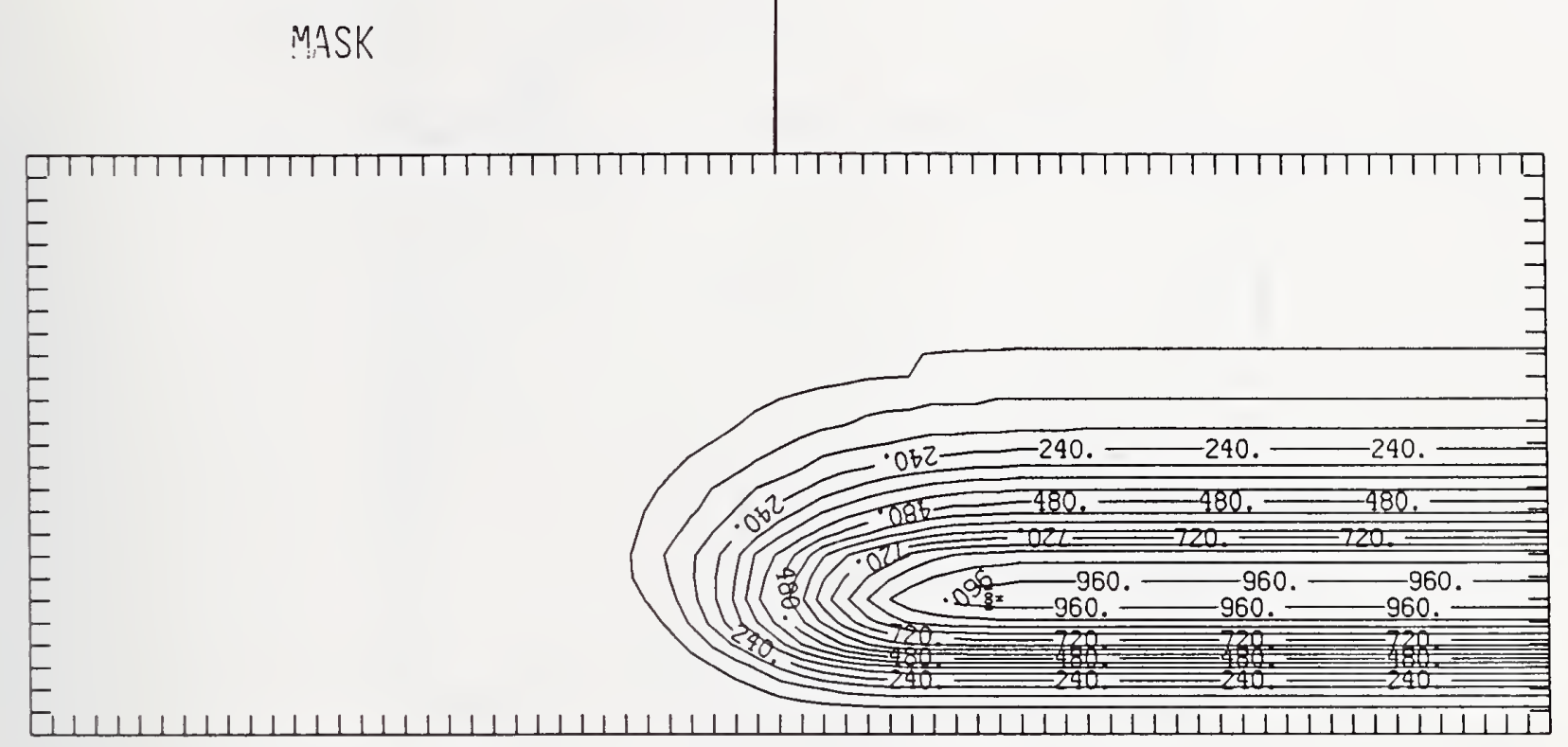

Figure 0325-3 Two-dimensional distribution of $325 \mathrm{keV}$ oxygen implanted past a mask edge. The length increment (distance between tick marks) is $0.04 \mu \mathrm{m}$ and the scale factor is 1000 .

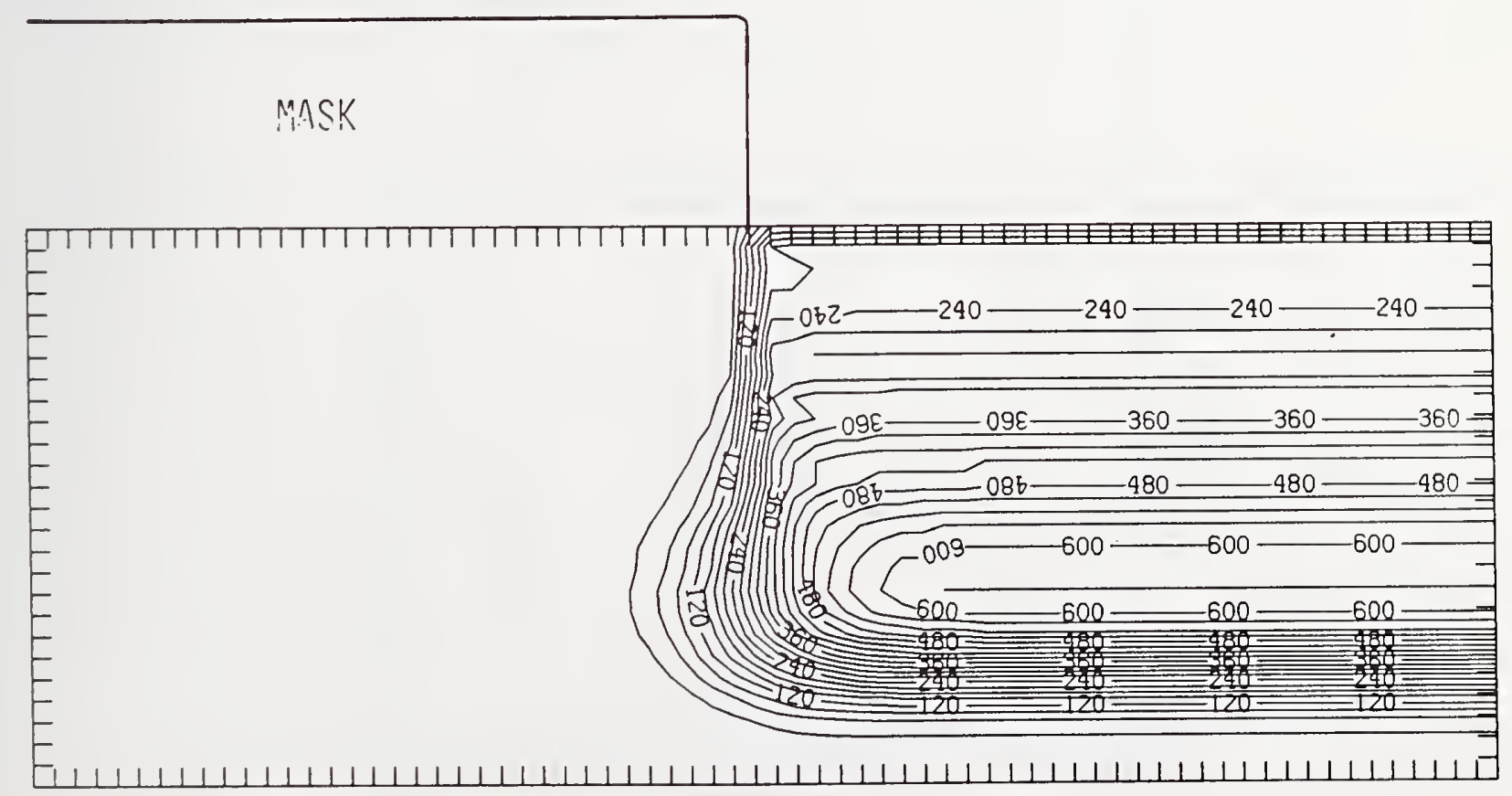

Figure 0325-4 Two-dimensional distribution of Frenkel pairs created by $325 \mathrm{keV}$ oxygen implanted past a mask edge. The length increment (distance between tick marks) is $0.04 \mu \mathrm{m}$ and the scale factor is 1 . 


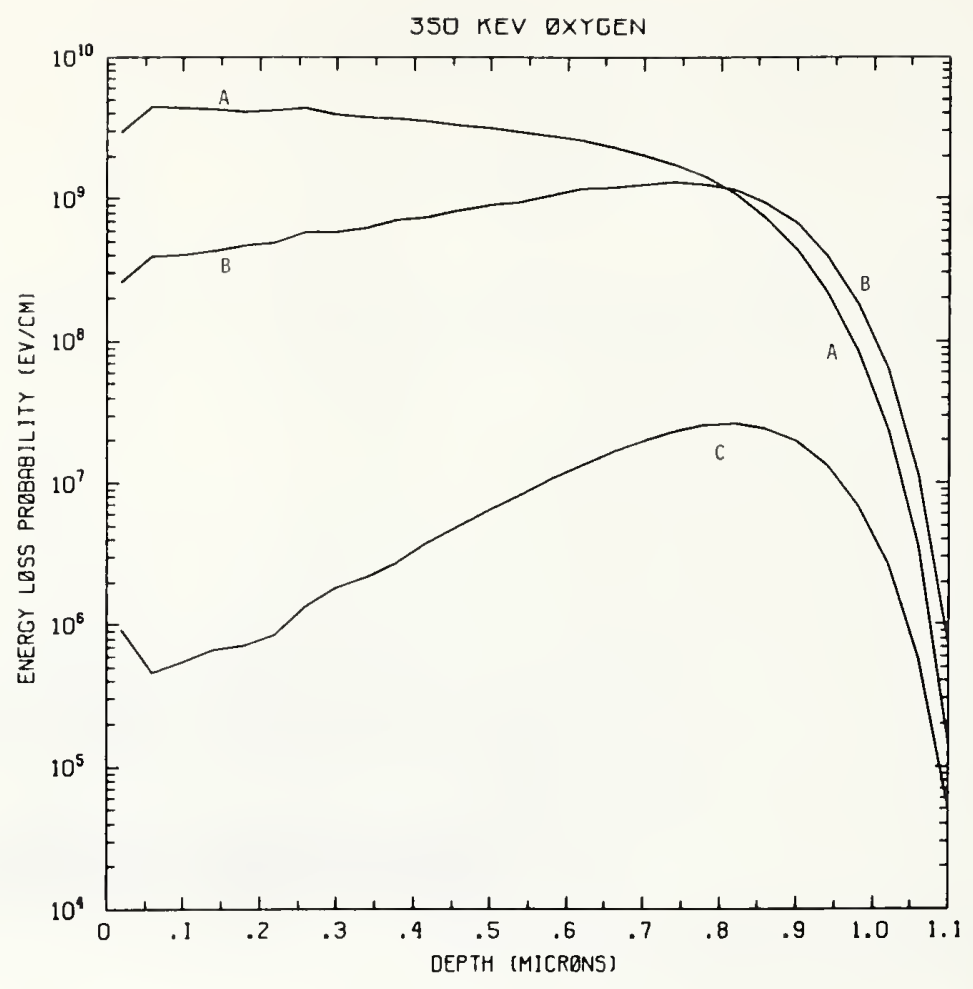

Figure 0350-1 One-dimensional probability distributions for energy loss due to electronic excitation (A), displacement damage(B), and phonon excitation (C) for $350 \mathrm{keV}$ oxygen normally incident on a silicon target.

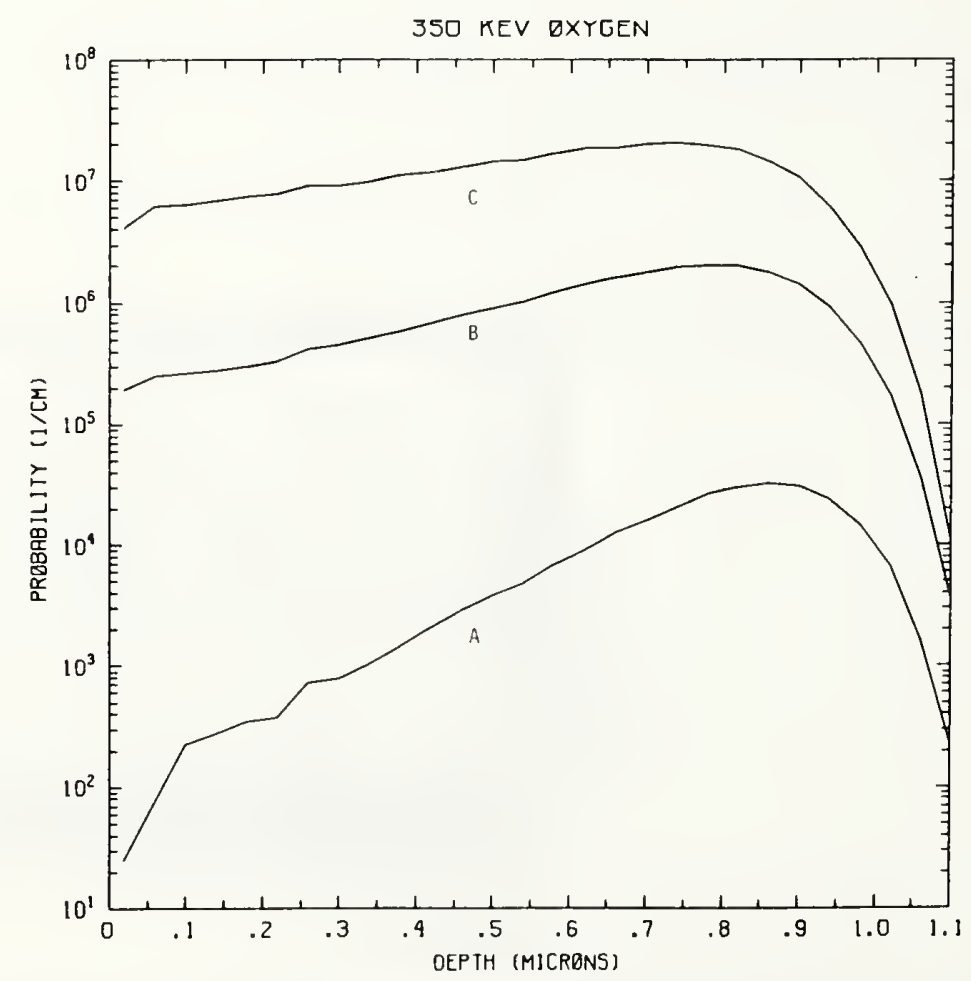

Figure O350-2 One-dimensional probability distributions for implanted oxygen (A), primary silicon displacements (B), and Frenkel pairs (C) for $350 \mathrm{keV}$ oxygen normally incident on a silicon target. 


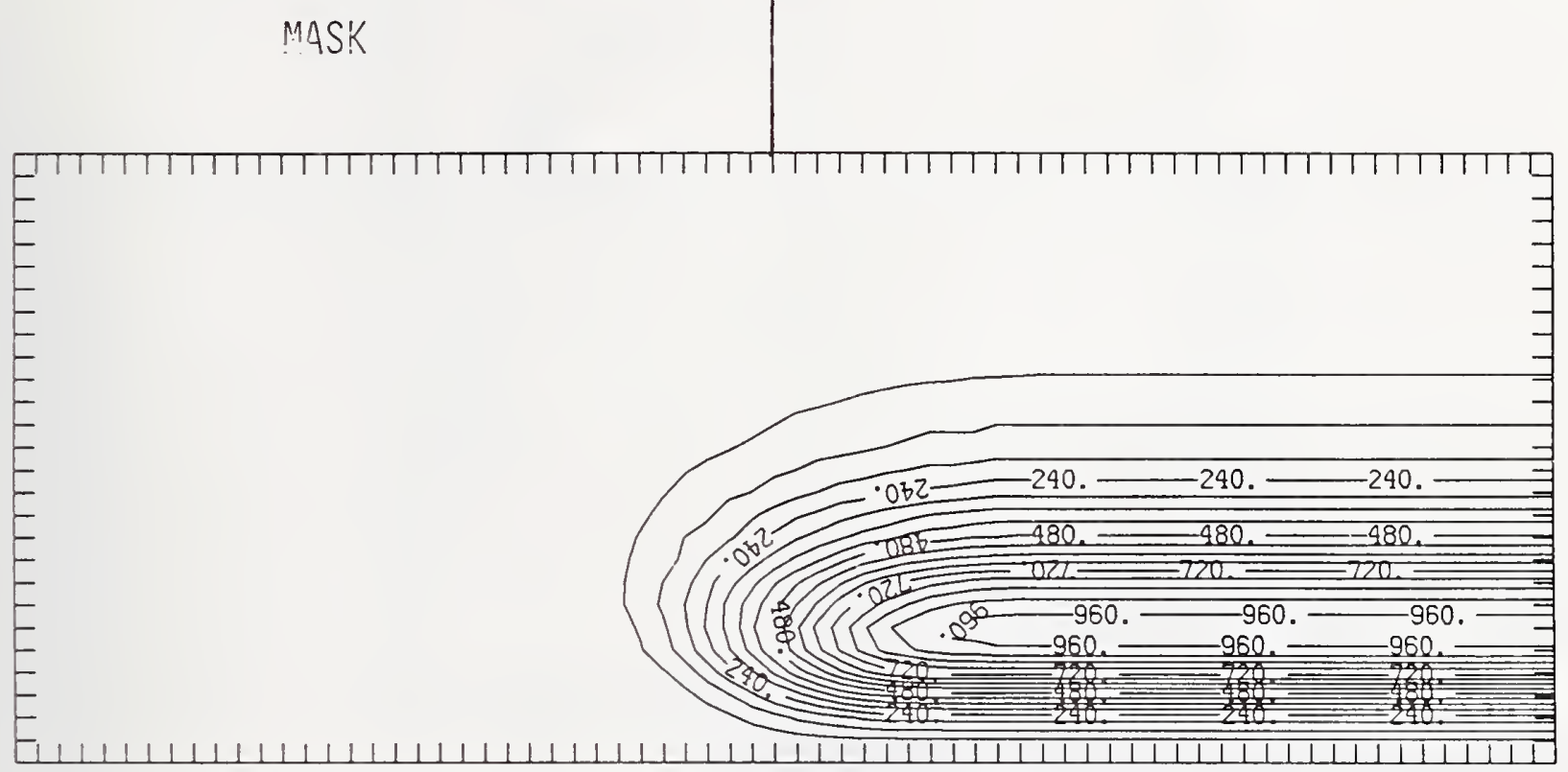

Figure 0350-3 Two-dimensional distribution of $350 \mathrm{keV}$ oxygen implanted past a mask edge. The length increment (distance between tick marks) is $0.04 \mu \mathrm{m}$ and the scale factor is 1000 .

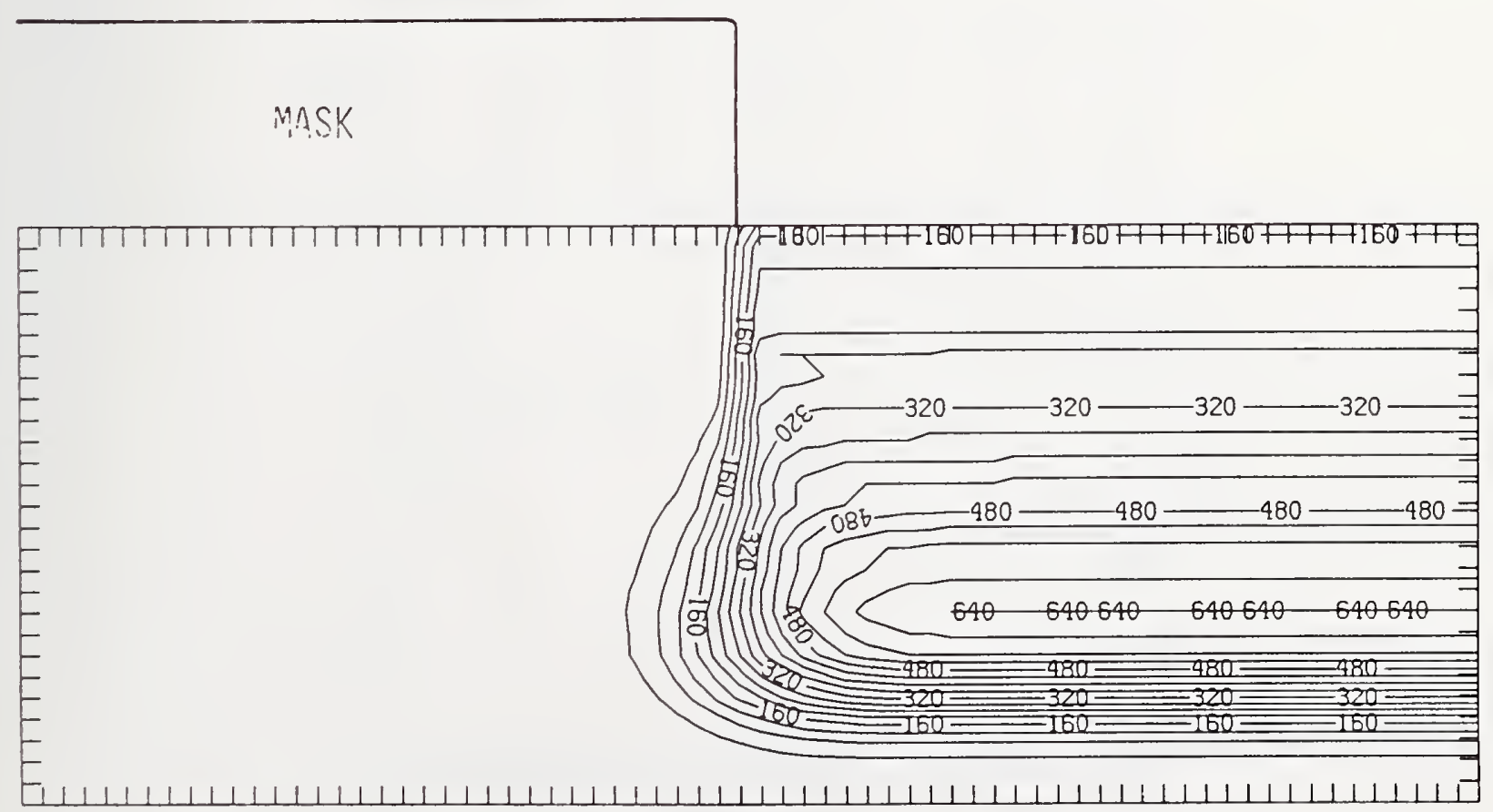

Figure 0350-4 Two-dimensional distribution of Frenkel pairs created by $350 \mathrm{keV}$ oxygen implanted past a mask edge. The length increment (distance between tick marks) is $0.04 \mu \mathrm{m}$ and the scale factor is 1 . 


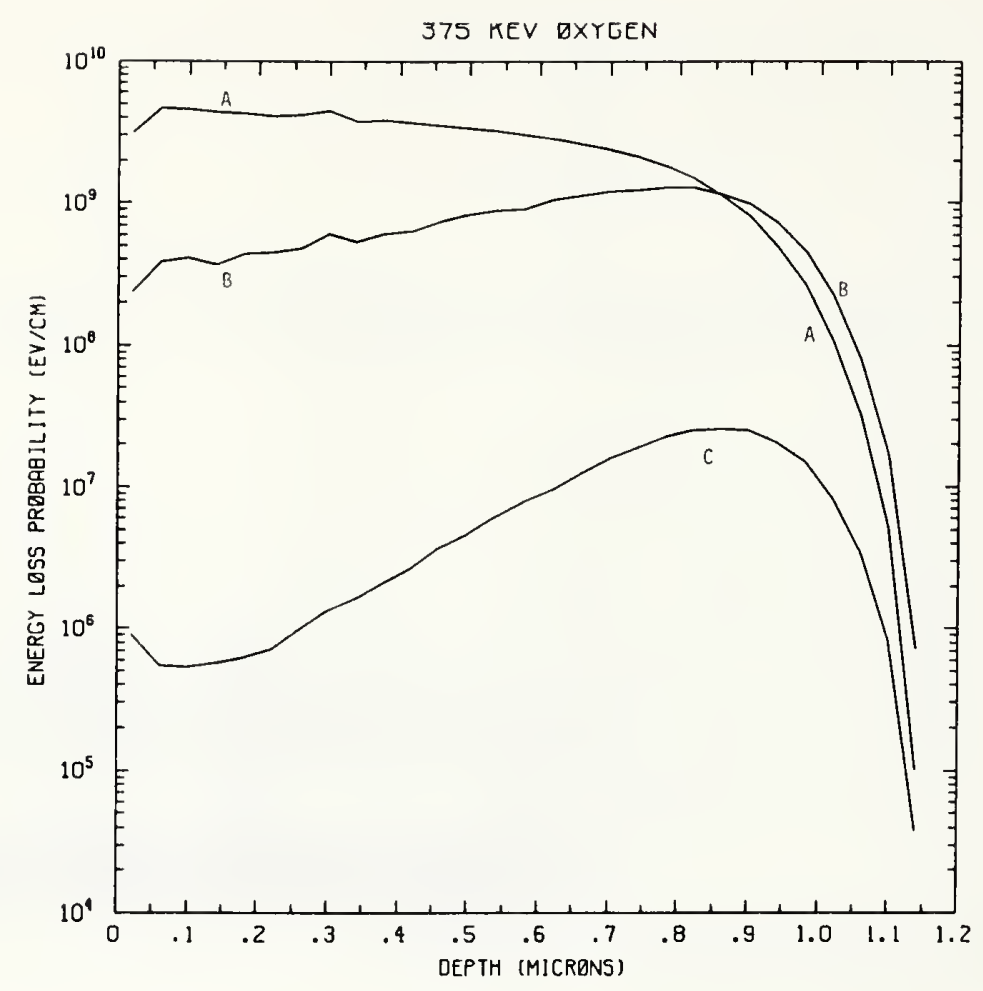

Figure 0375-1 One-dimensional probability distributions for energy loss due to electronic excitation (A), displacement damage(B), and phonon excitation (C) for $375 \mathrm{keV}$ oxygen normally incident on a silicon target.

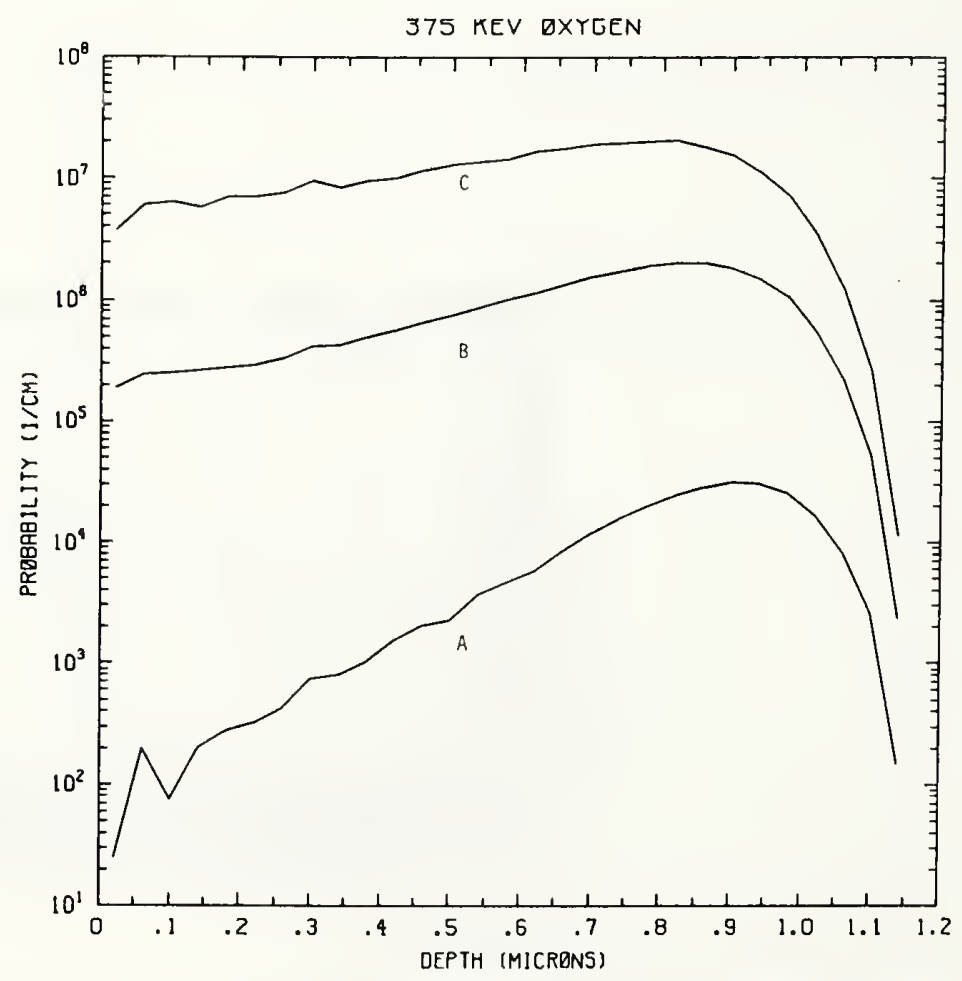

Figure 0375-2 One-dimensional probability distributions for implanted oxygen (A), primary silicon displacements (B), and Frenkel pairs (C) for $375 \mathrm{keV}$ oxygen normally incident on a silicon target. 


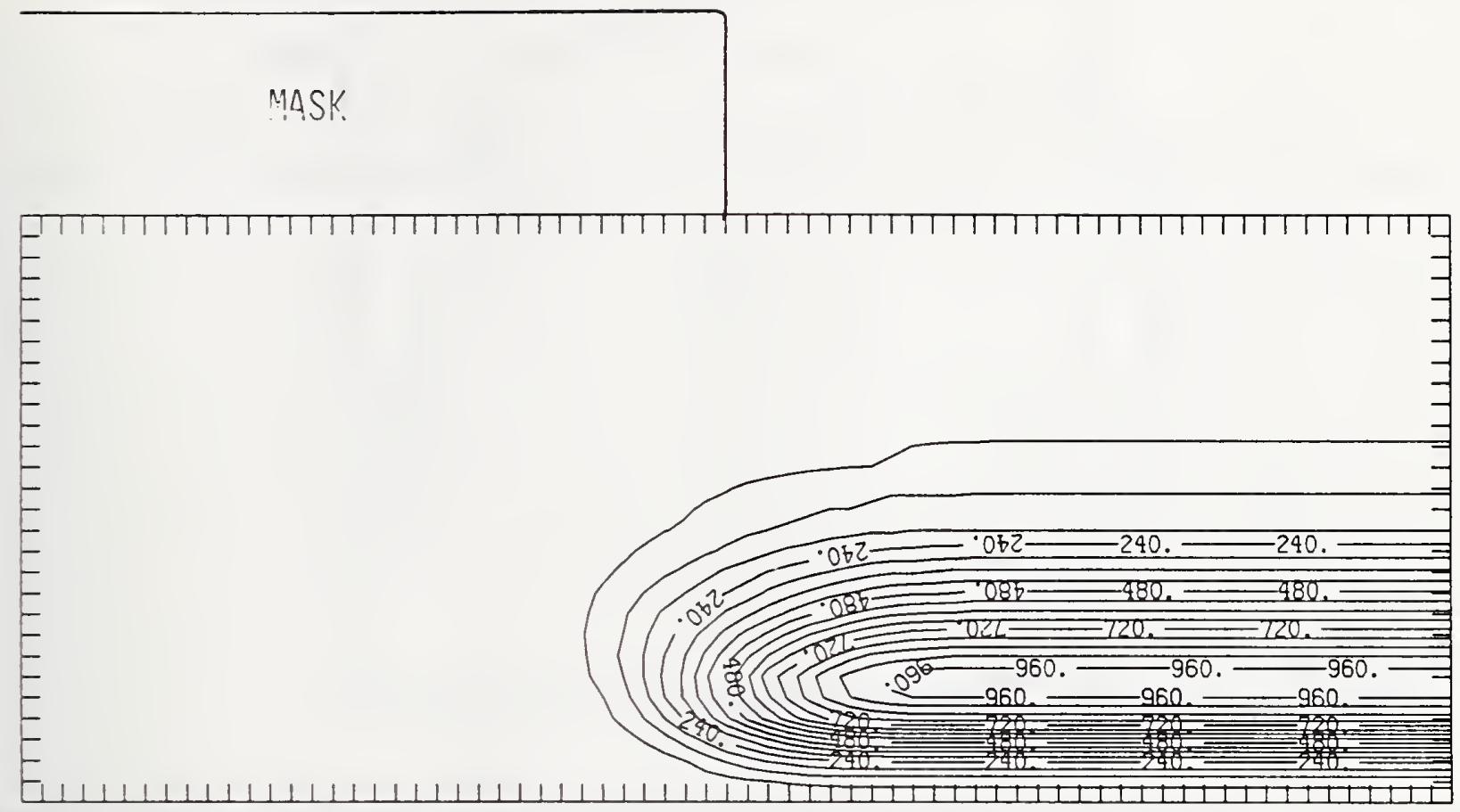

Figure 0375-3 Two-dimensional distribution of $375 \mathrm{keV}$ oxygen implanted past a mask edge. The length increment (distance between tick marks) is $0.04 \mu \mathrm{m}$ and the scale factor is 1000 .

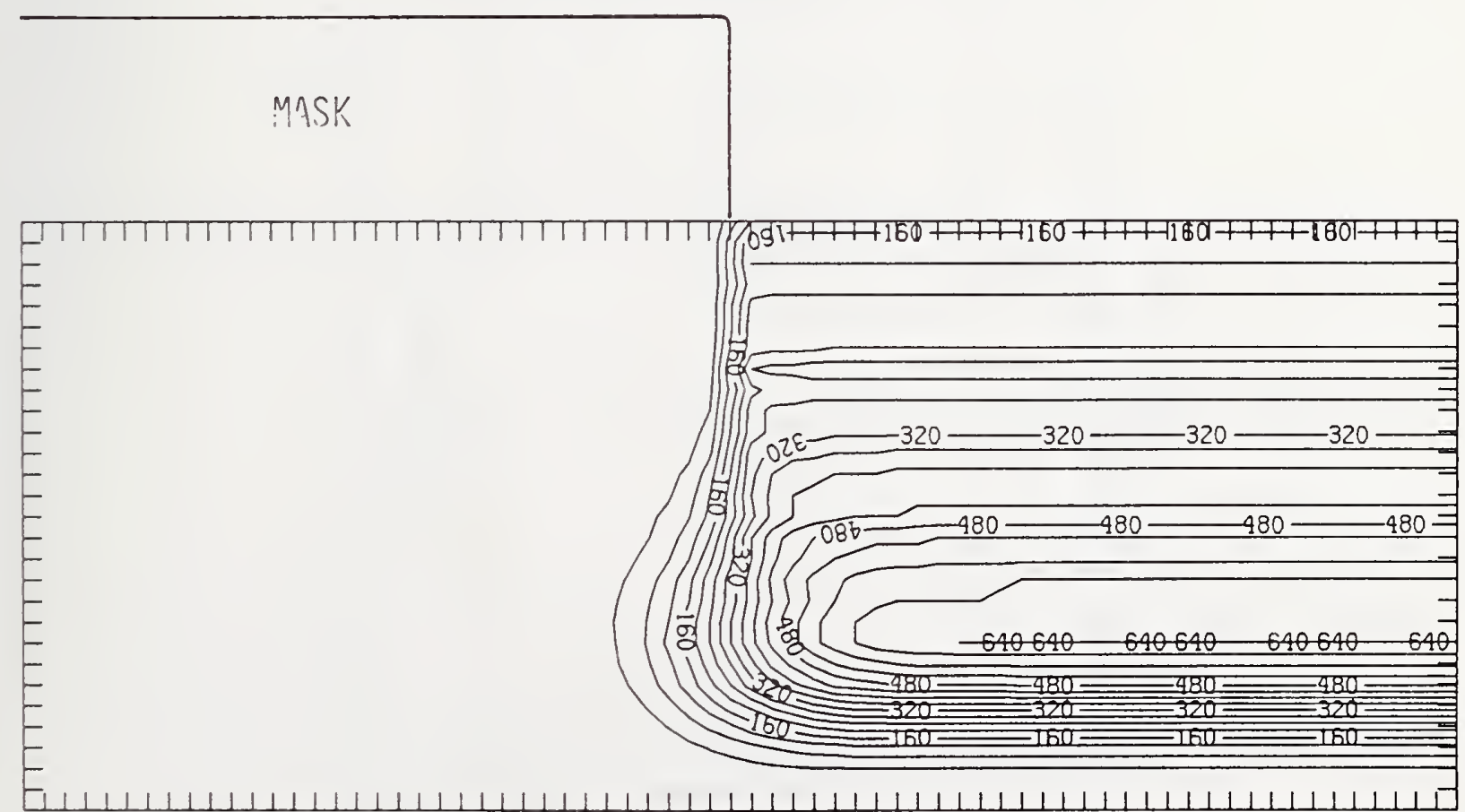

Figure 0375-4 Two-dimensional distribution of Frenkel pairs created by $375 \mathrm{keV}$ oxygen implanted past a mask edge. The length increment (distance between tick marks) is $0.04 \mu \mathrm{m}$ and the scale factor is 1 . 


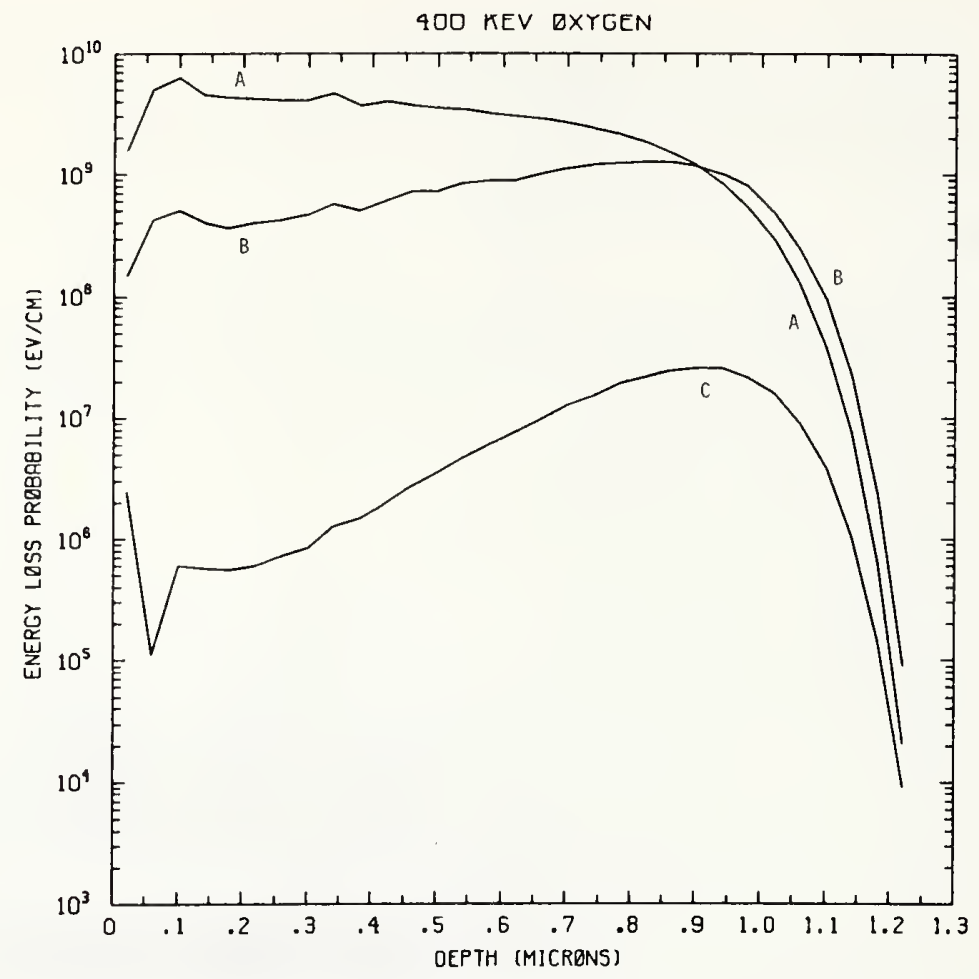

Figure 0400-1 One-dimensional probability distributions for energy loss due to electronic excitation (A), displacement damage(B), and phonon excitation (C) for $400 \mathrm{keV}$ oxygen normally incident on a silicon target.

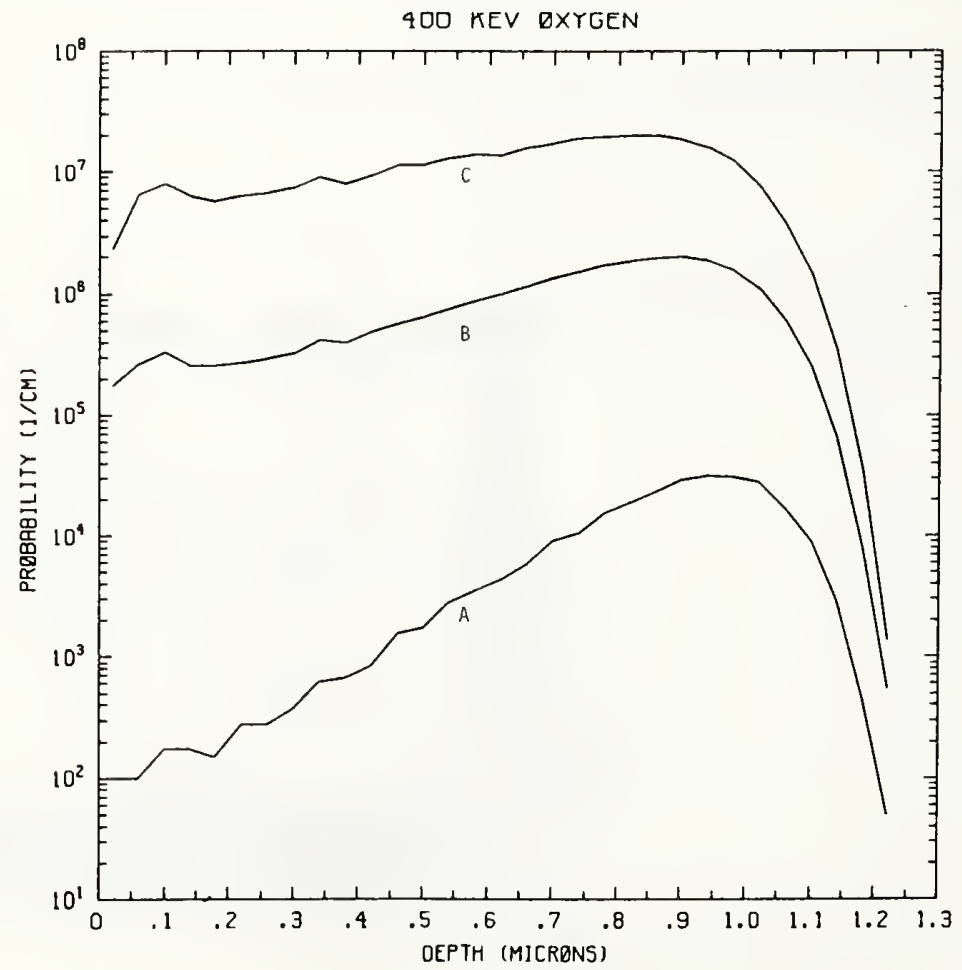

Figure 0400-2 One-dimensional probability distributions for implanted oxygen (A), primary silicon displacements (B), and Frenkel pairs (C) for $400 \mathrm{keV}$ oxygen normally incident on a silicon target. 


\section{MASK}

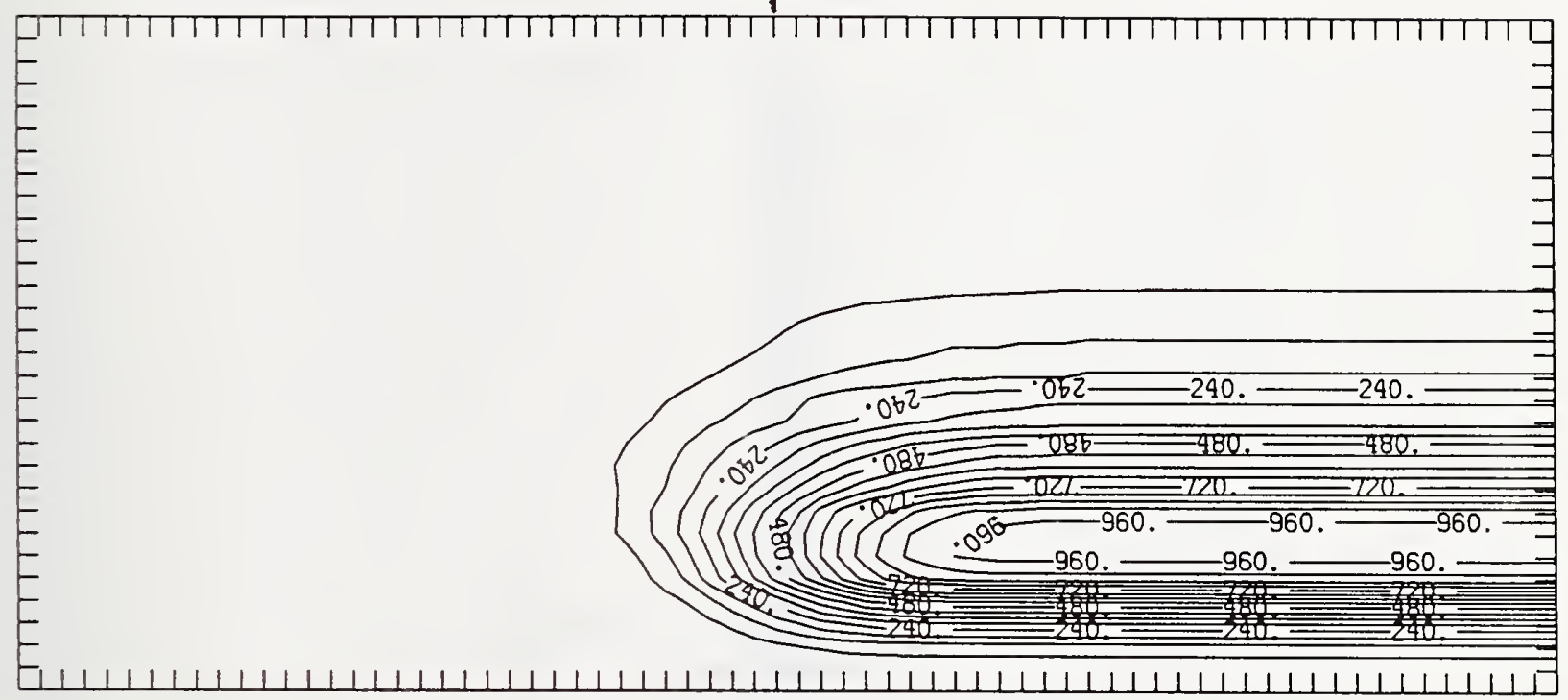

Figure 0400-3 Two-dimensional distribution of $400 \mathrm{keV}$ oxygen implanted past a mask edge. The length increment (distance between tick marks) is $0.04 \mu \mathrm{m}$ and the scale factor is 1000 .

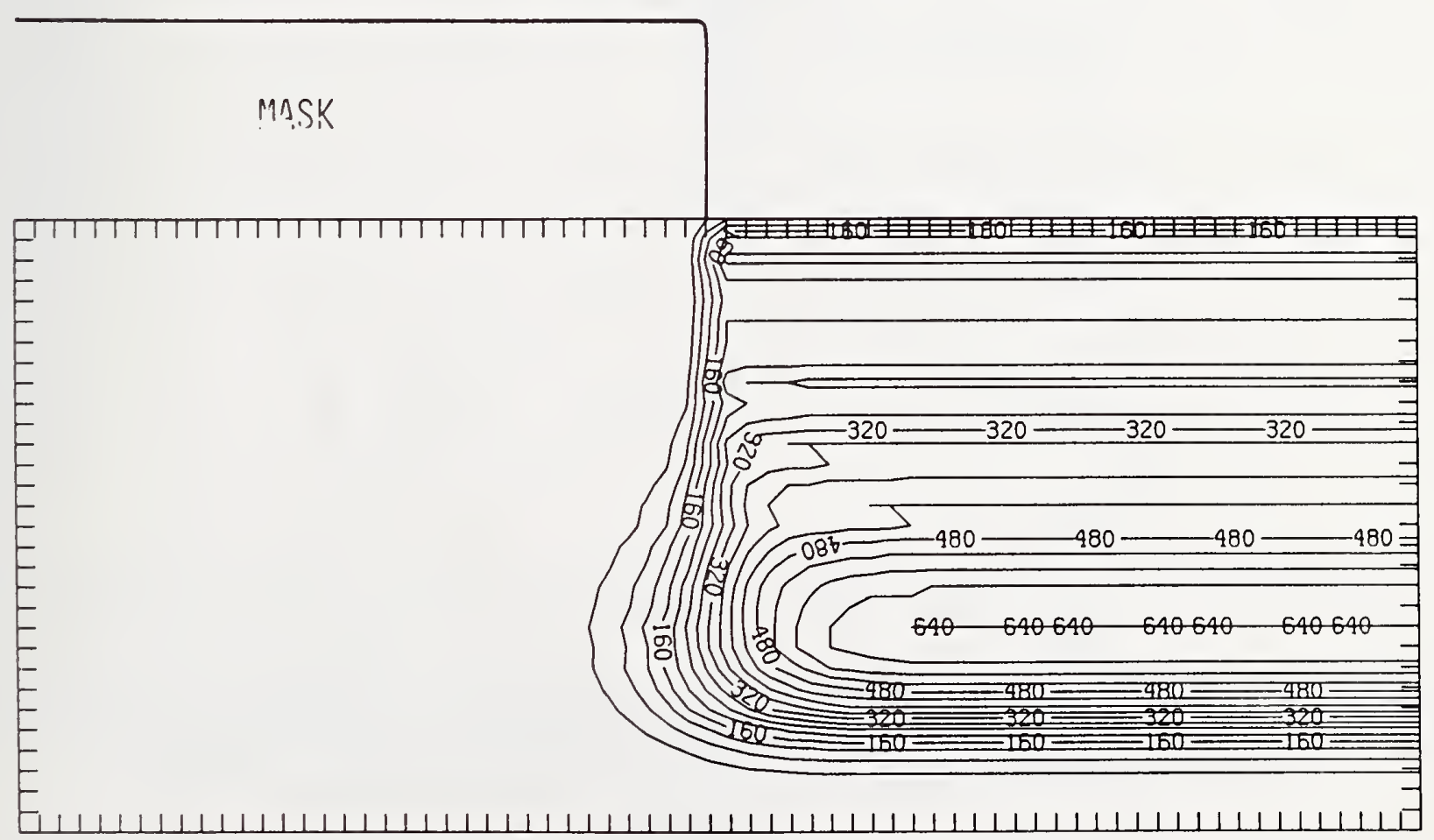

Figure 0400-4 Two-dimensional distribution of Frenkel pairs created by $400 \mathrm{keV}$ oxygen implanted past a mask edge. The length increment (distance between tick marks) is $0.04 \mu \mathrm{m}$ and the scale factor is 1 . 


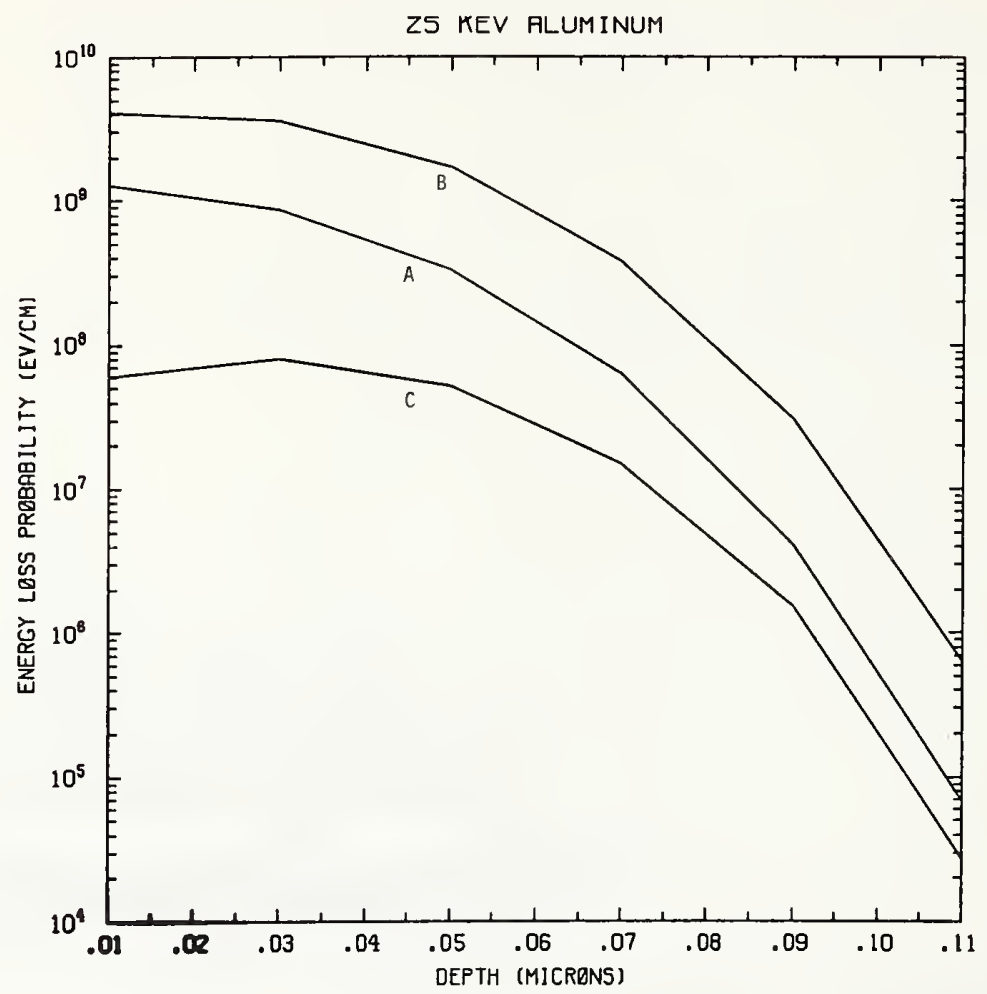

Figure Al25-1 One-dimensional probability distributions for energy loss due to electronic excitation (A), displacement damage(B), and phonon excitation (C) for $25 \mathrm{keV}$ aluminum normally incident on a silicon target.

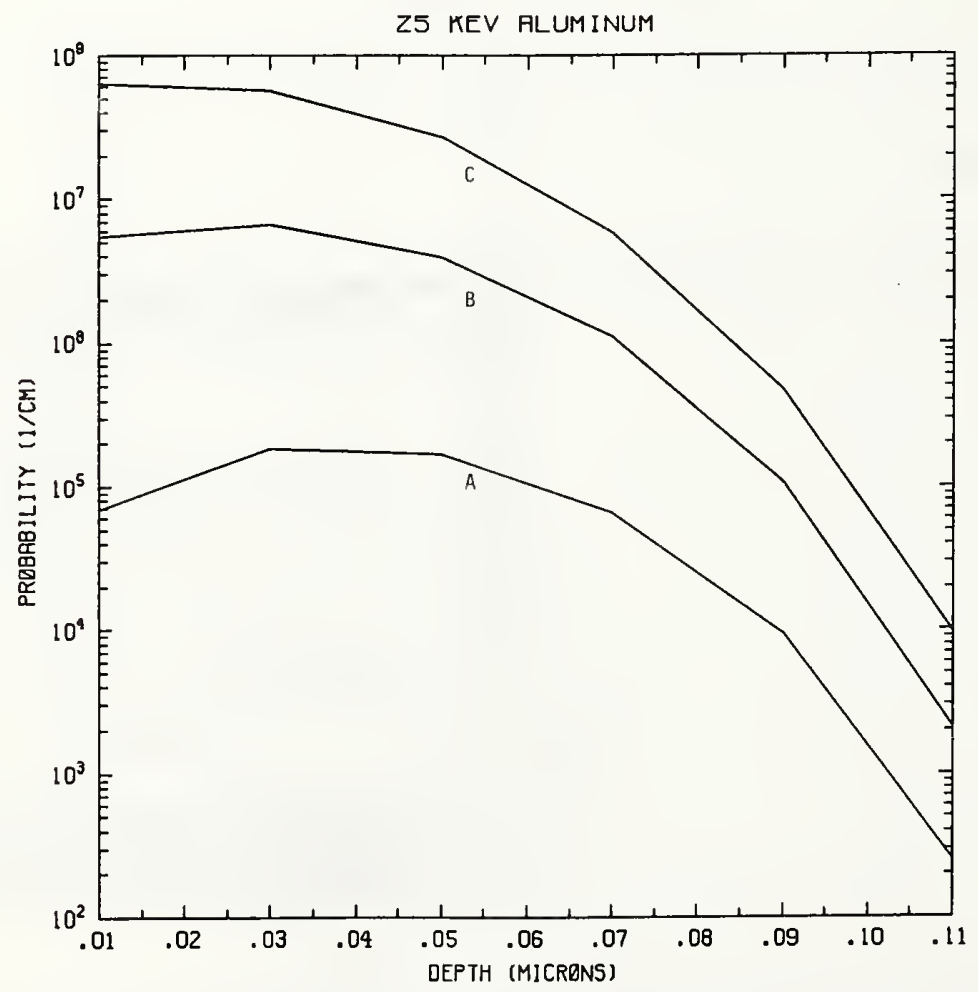

Figure Al25-2 One-dimensional probability distributions for implanted aluminum (A), primary silicon displacements (B), and Frenkel pairs (C) for $25 \mathrm{keV}$ aluminum normally incident on a silicon target. 


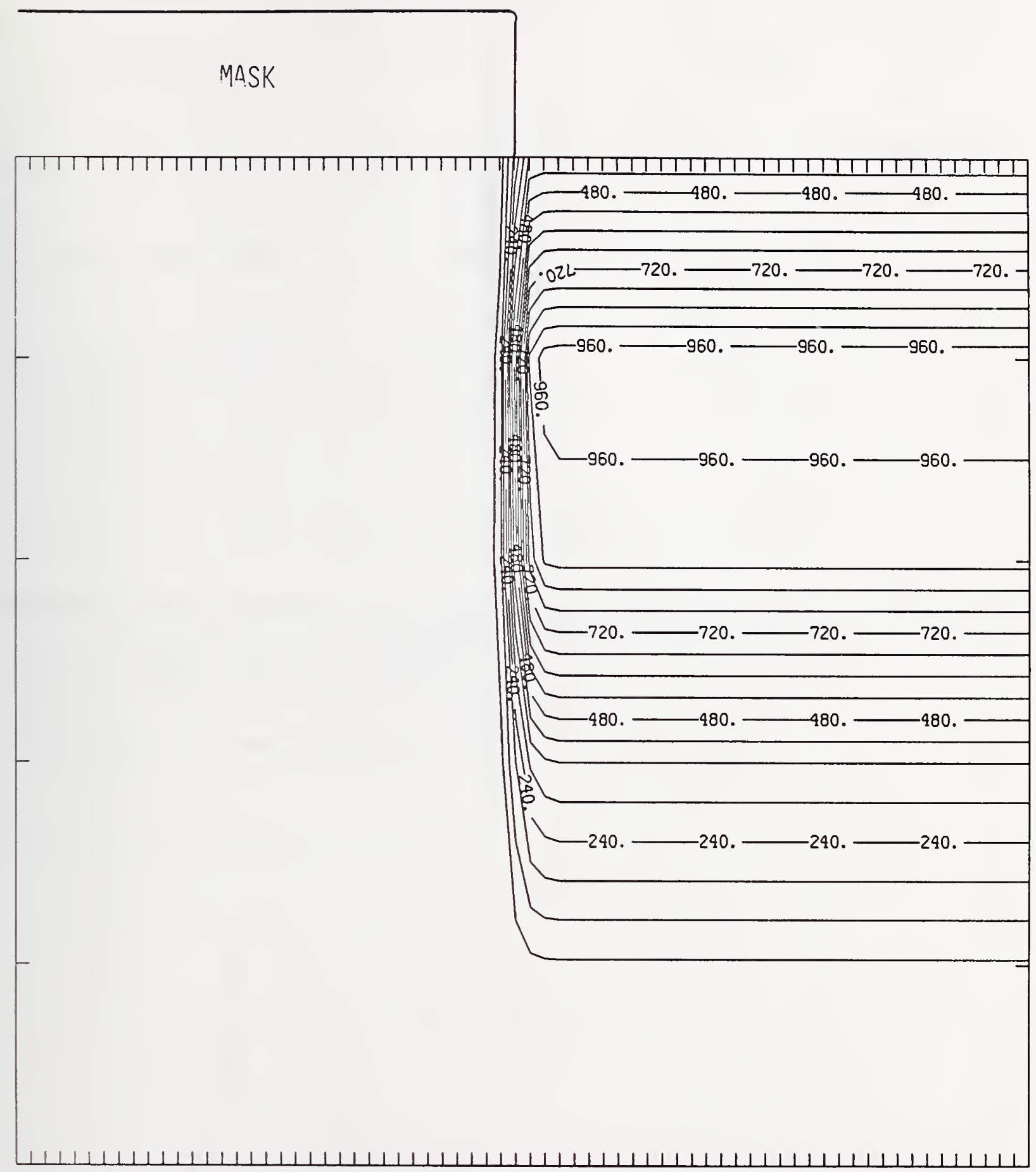

Figure Al25-3 Two-dimensional distribution of $25 \mathrm{keV}$ aluminum implanted past a mask edge. The length increment (distance between tick marks) is $0.02 \mu \mathrm{m}$ and the scale factor is 1000 . 
MASK

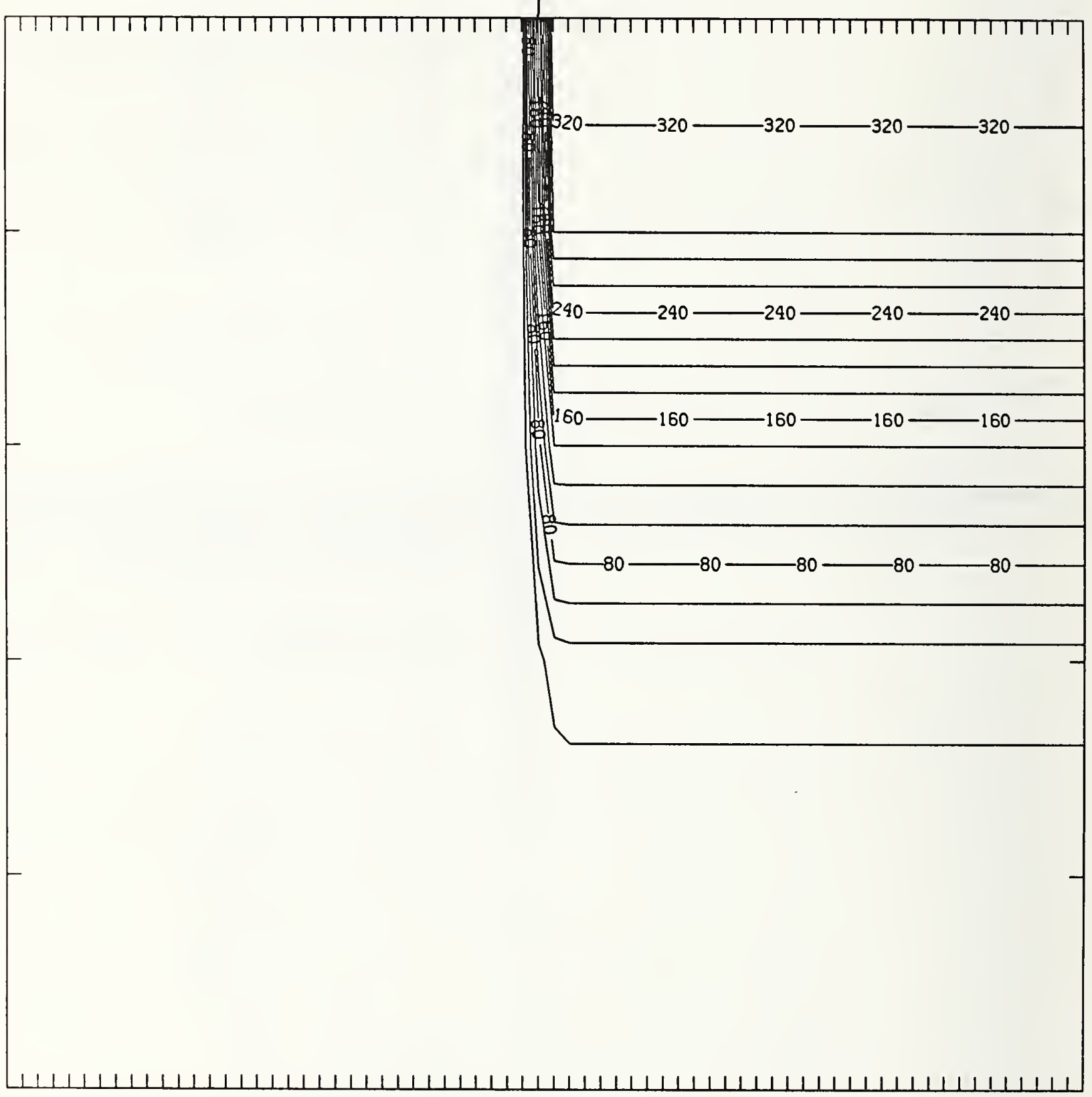

Figure Al25-4 Two-dimensional distribution of Frenkel pairs created by $25 \mathrm{keV}$ aluminum implanted past a mask edge. The length increment (distance between tick marks) is $0.02 \mu \mathrm{m}$ and the scale factor is 1 . 


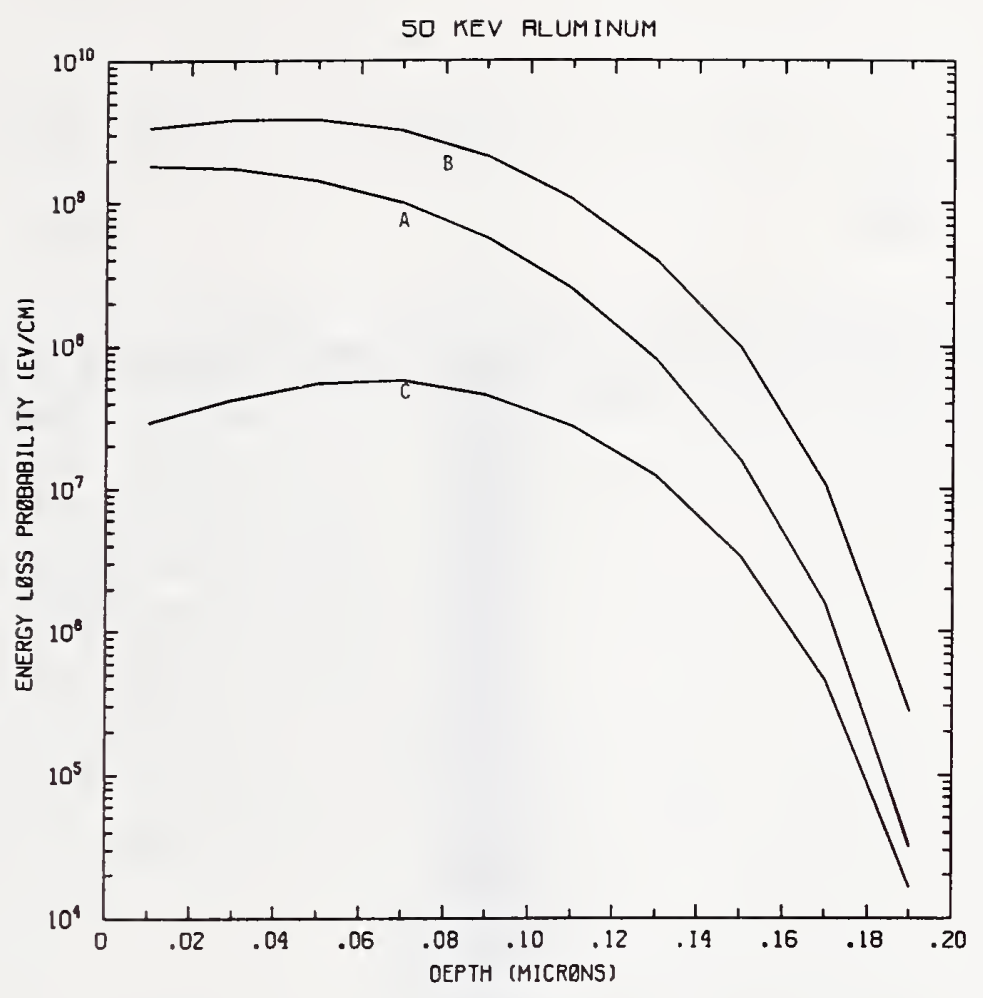

Figure Al50-1 One-dimensional probability distributions for energy loss due to electronic excitation (A), displacement damage(B), and phonon excitation (C) for $50 \mathrm{keV}$ aluminum normally incident on a silicon target.

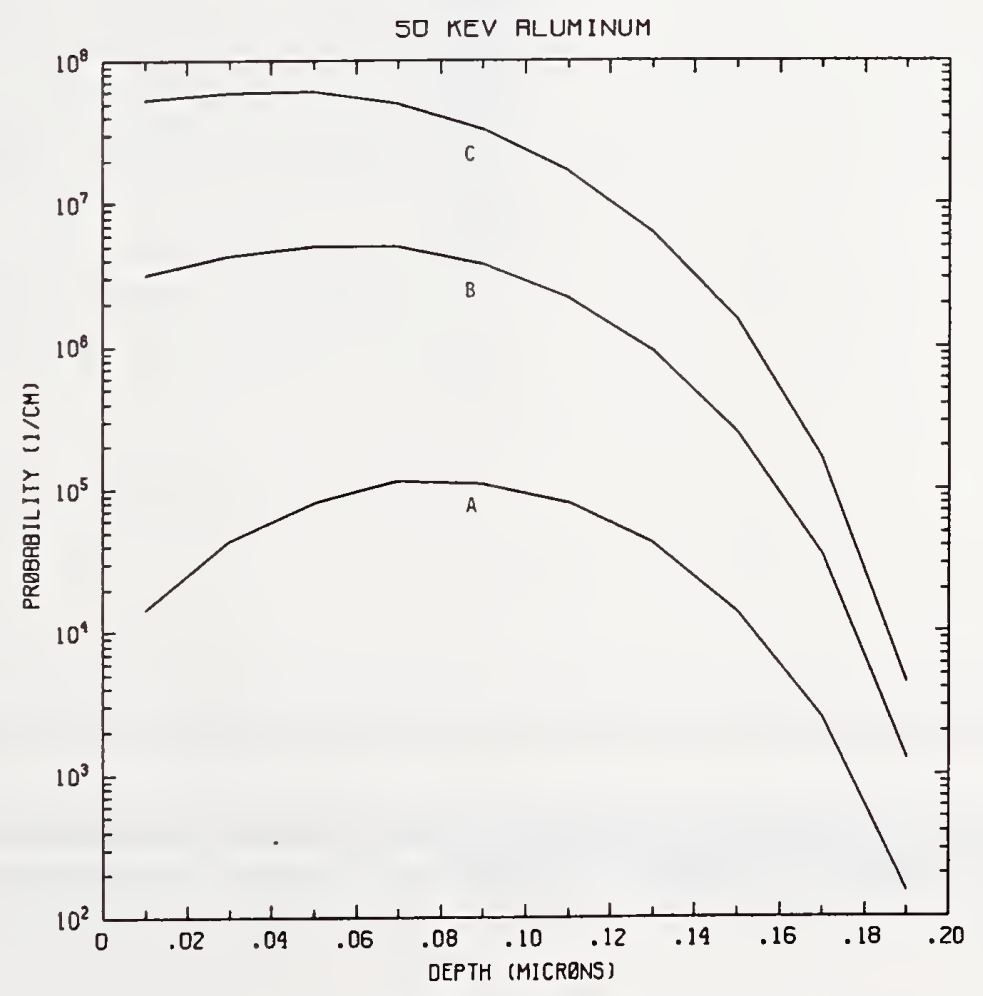

Figure Al50-2 One-dimensional probability distributions for implanted aluminum (A), primary silicon displacements (B), and Frenkel pairs (C) for $50 \mathrm{keV}$ aluminum normally incident on a silicon target. 


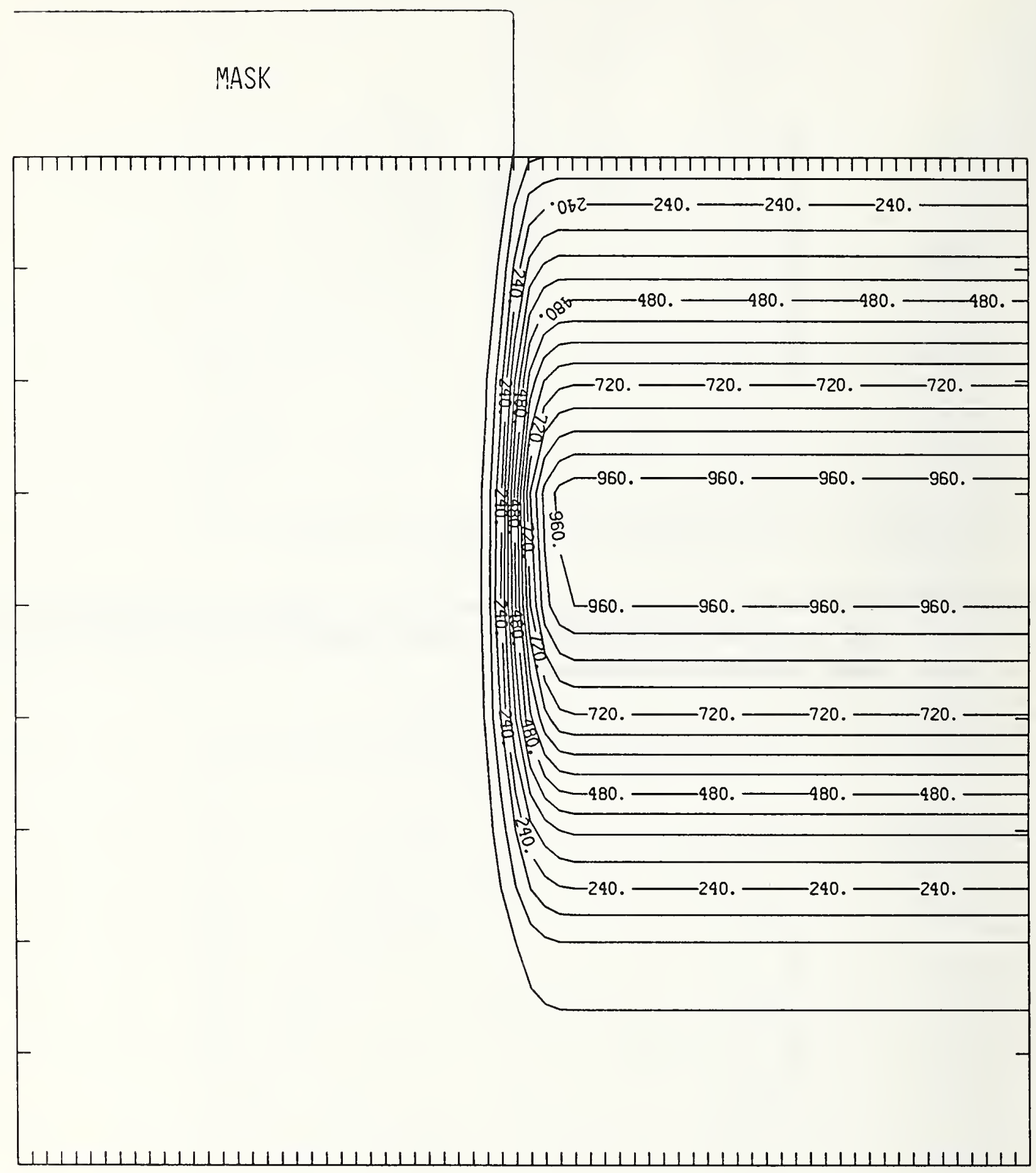

Figure A150-3 Two-dimensional distribution of $50 \mathrm{keV}$ aluminum implanted past a mask edge. The length increment (distance between tick marks) is $0.02 \mu \mathrm{m}$ and the scale factor is 1000. 


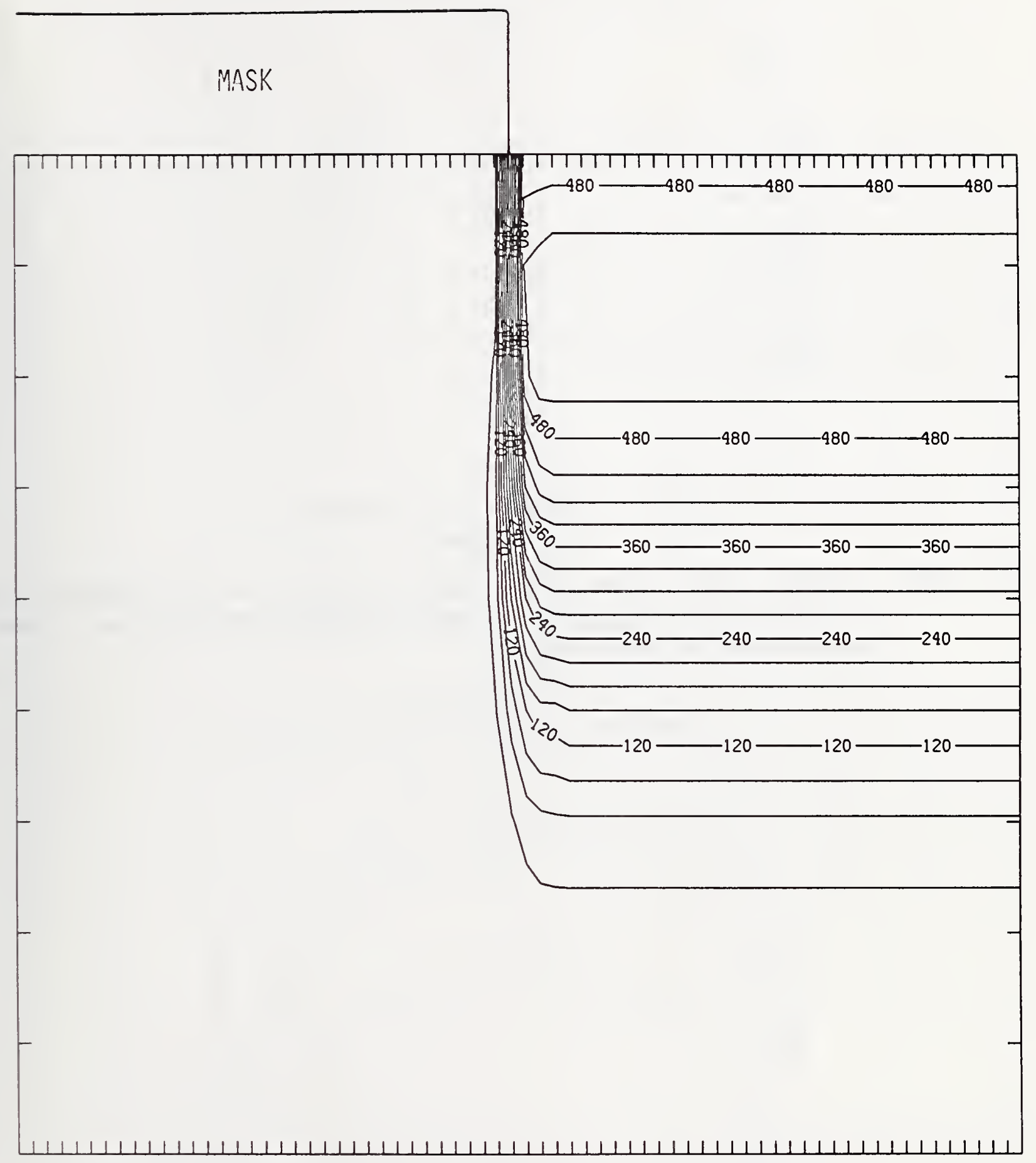

Figure Al50-4 Two-dimensional distribution of Frenkel pairs created by $50 \mathrm{keV}$ aluminum implanted past a mask edge. The length increment (distance between tick marks) is $0.02 \mu \mathrm{m}$ and the scale factor is 1 . 


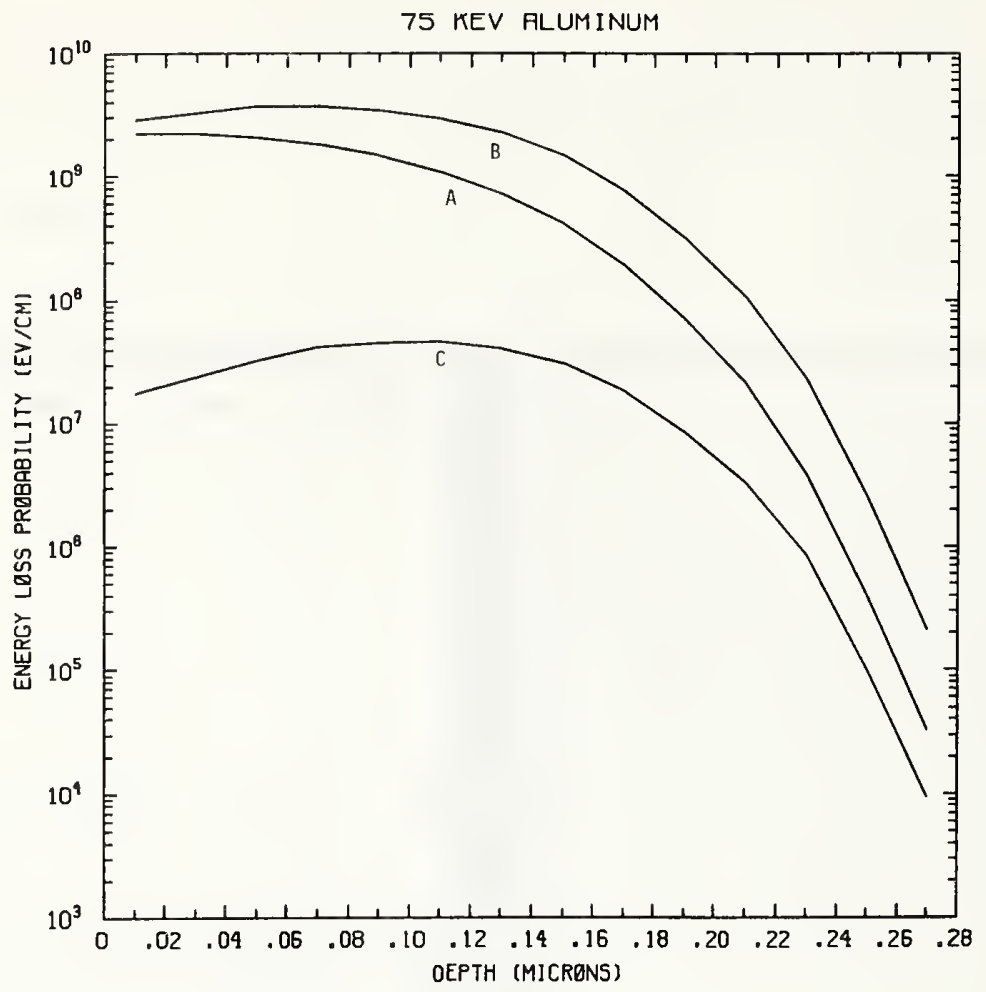

Figure A175-1 One-dimensional probability distributions for energy loss due to electronic excitation (A), displacement damage(B), and phonon excitation (C) for $75 \mathrm{keV}$ aluminum normally incident on a silicon target.

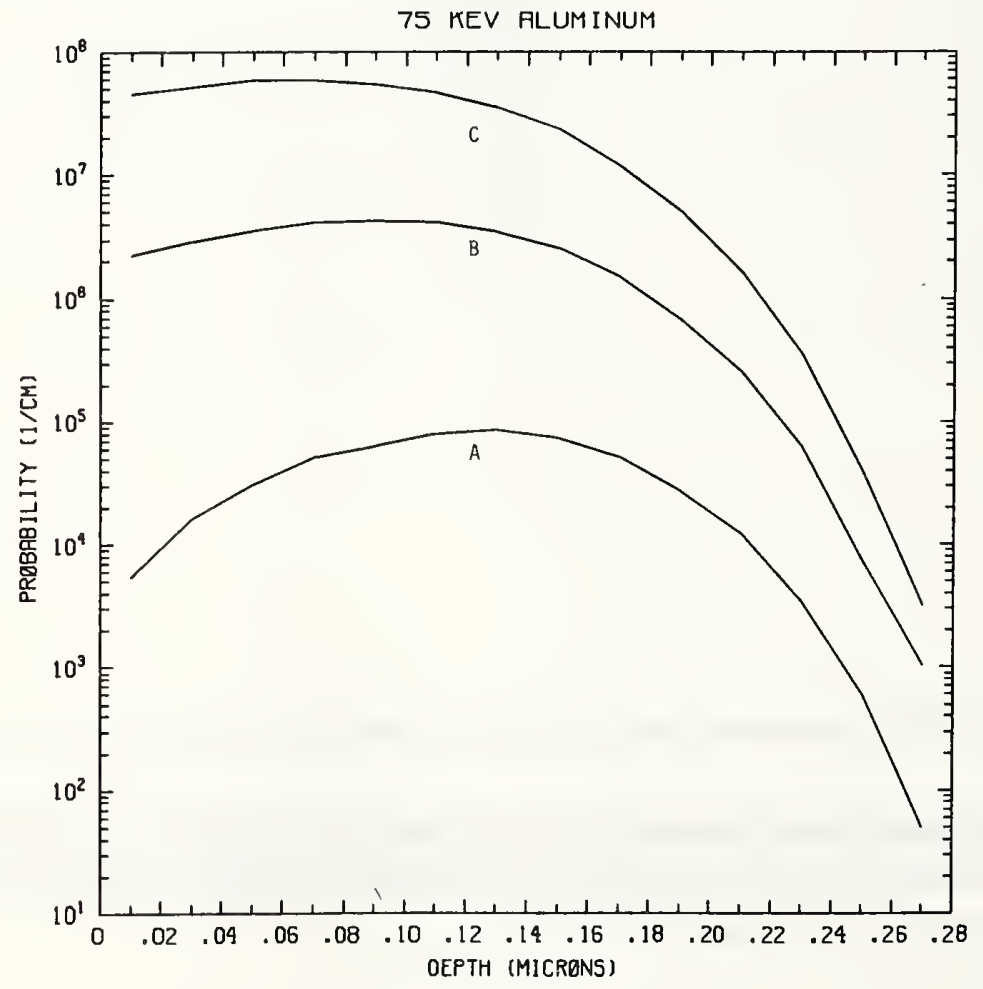

Figure A175-2 One-dimensional probability distributions for implanted aluminum (A), primary silicon displacements (B), and Frenkel pairs (C) for $75 \mathrm{keV}$ aluminum normally incident on a silicon target. 


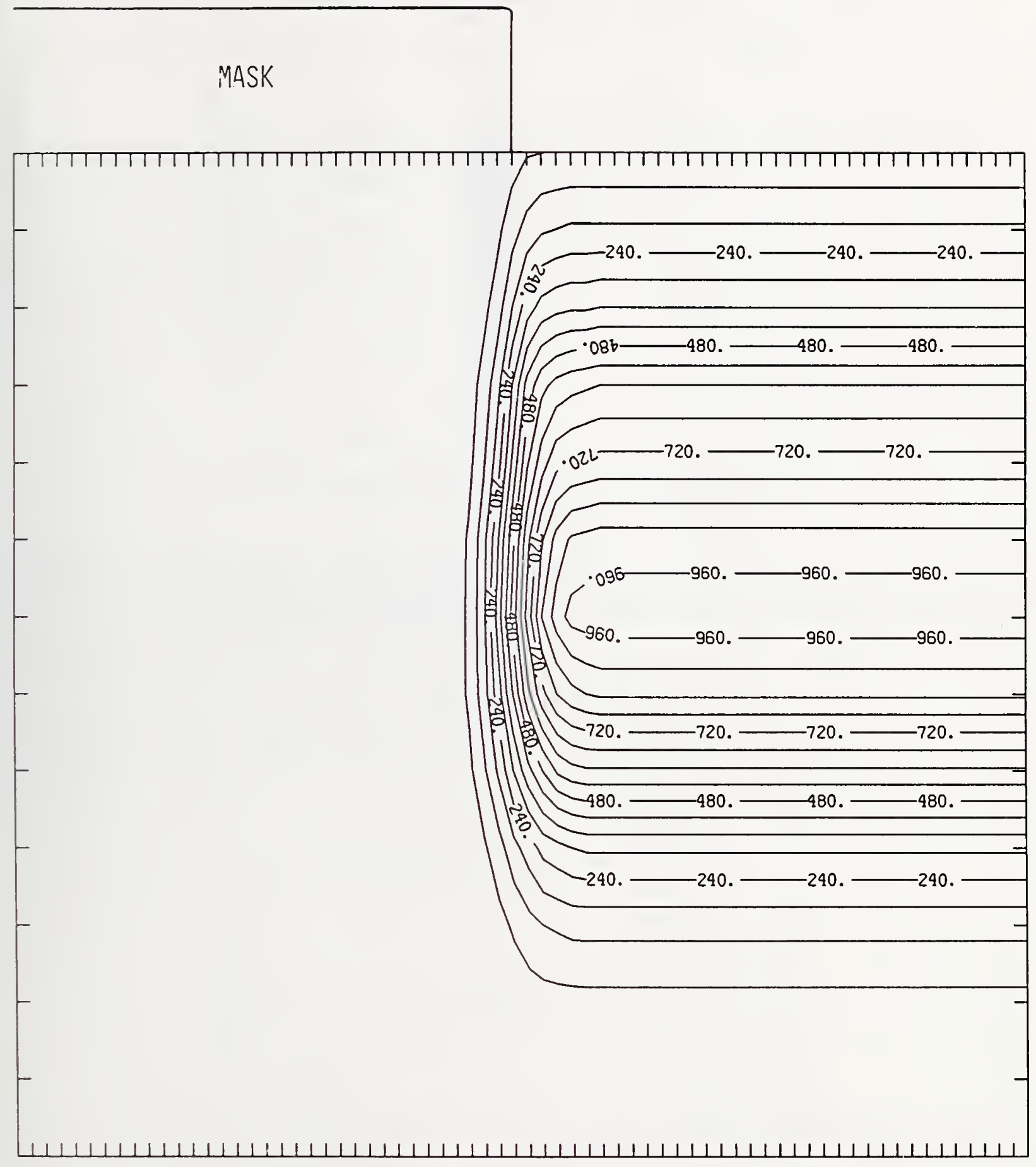

Figure A175-3 Two-dimensional distribution of $75 \mathrm{keV}$ aluminum implanted past a mask edge. The length increment (distance between tick marks) is $0.02 \mu \mathrm{m}$ and the scale factor is 1000 . 


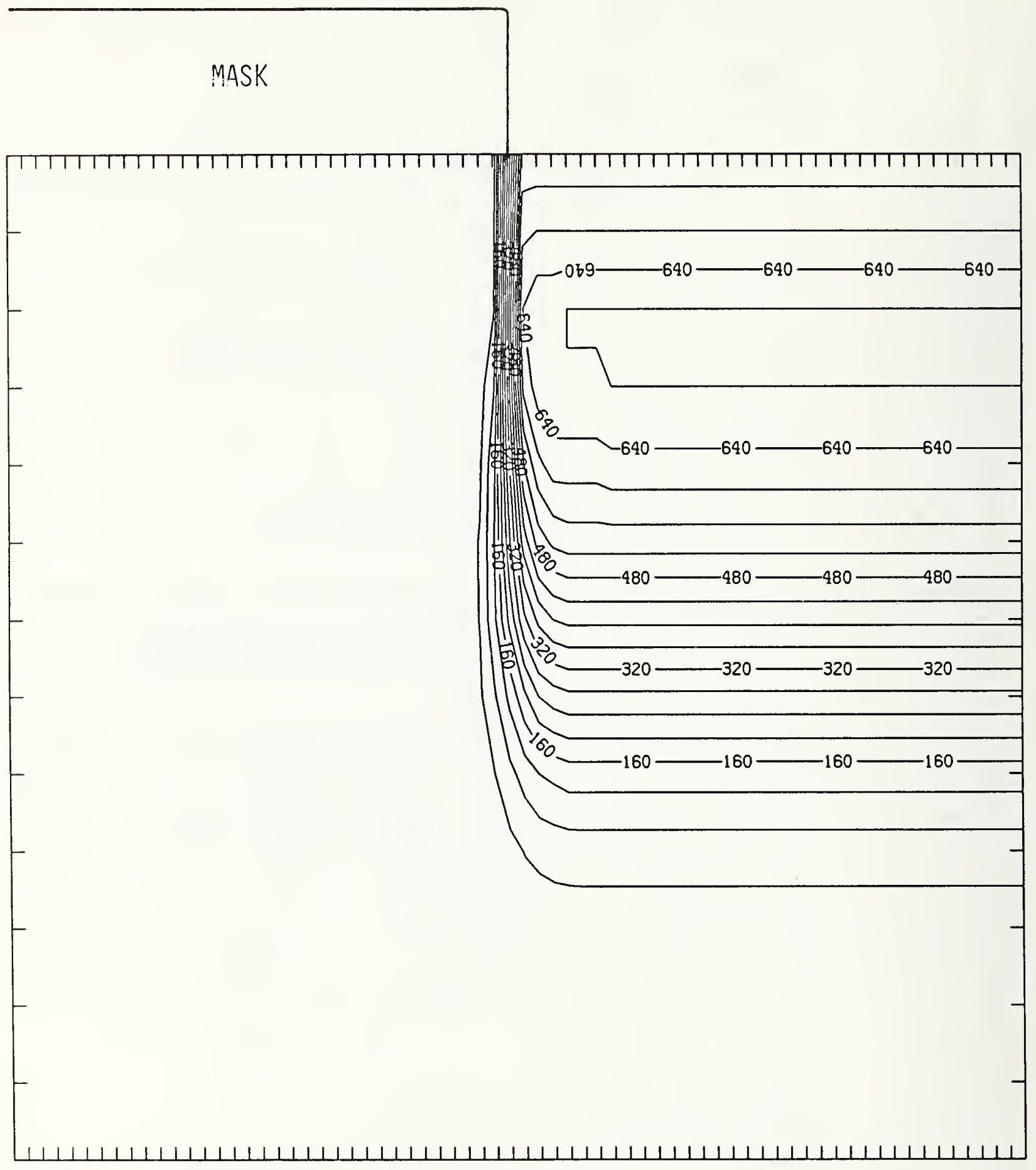

Figure Al75-4 Two-dimensional distribution of Frenkel pairs created by $75 \mathrm{keV}$ aluminum implanted past a mask edge. The length increment (distance between tick marks) is $0.02 \mu \mathrm{m}$ and the scale factor is 1 . 


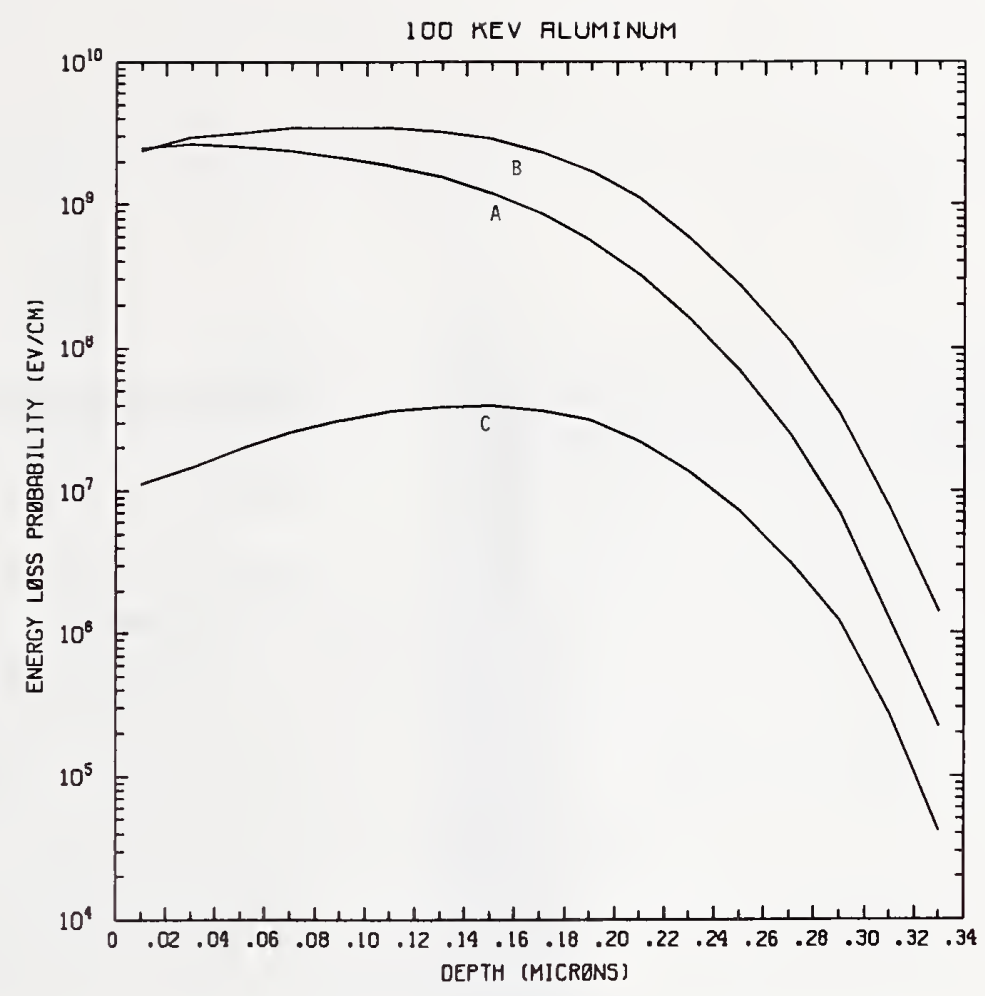

Figure Al100-1 One-dimensional probability distributions for energy loss due to electronic excitation (A), displacement damage(B), and phonon excitation (C) for $100 \mathrm{keV}$ aluminum normally incident on a silicon target.

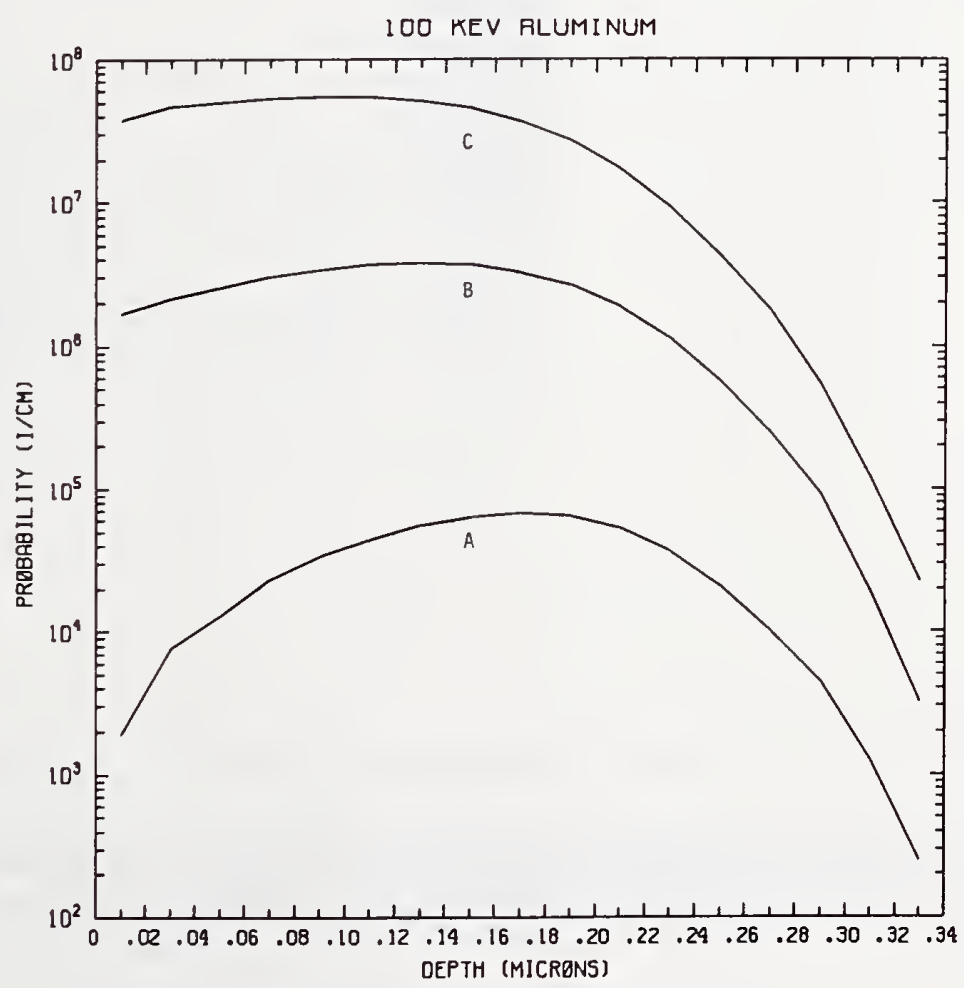

Figure Al100-2 One-dimensional probability distributions for implanted aluminum (A), primary silicon displacements (B), and Frenkel pairs (C) for $100 \mathrm{keV}$ aluminum normally incident on a silicon target. 


\section{MASK}

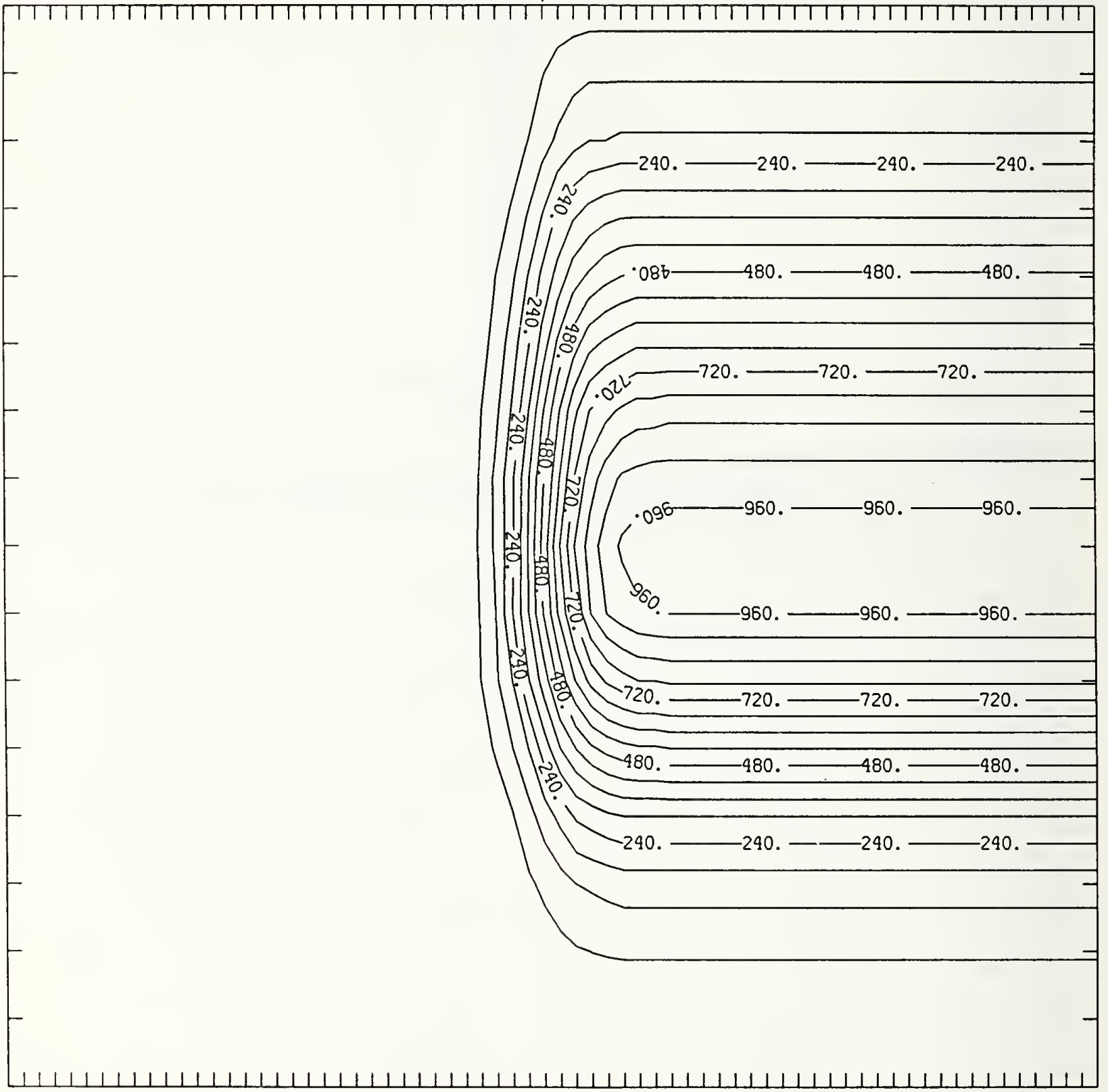

Figure Al100-3 Two-dimensional distribution of $100 \mathrm{keV}$ aluminum implanted past a mask edge. The length increment (distance between tick marks) is $0.02 \mu \mathrm{m}$ and the scale factor is 1000 . 


\section{MASK}

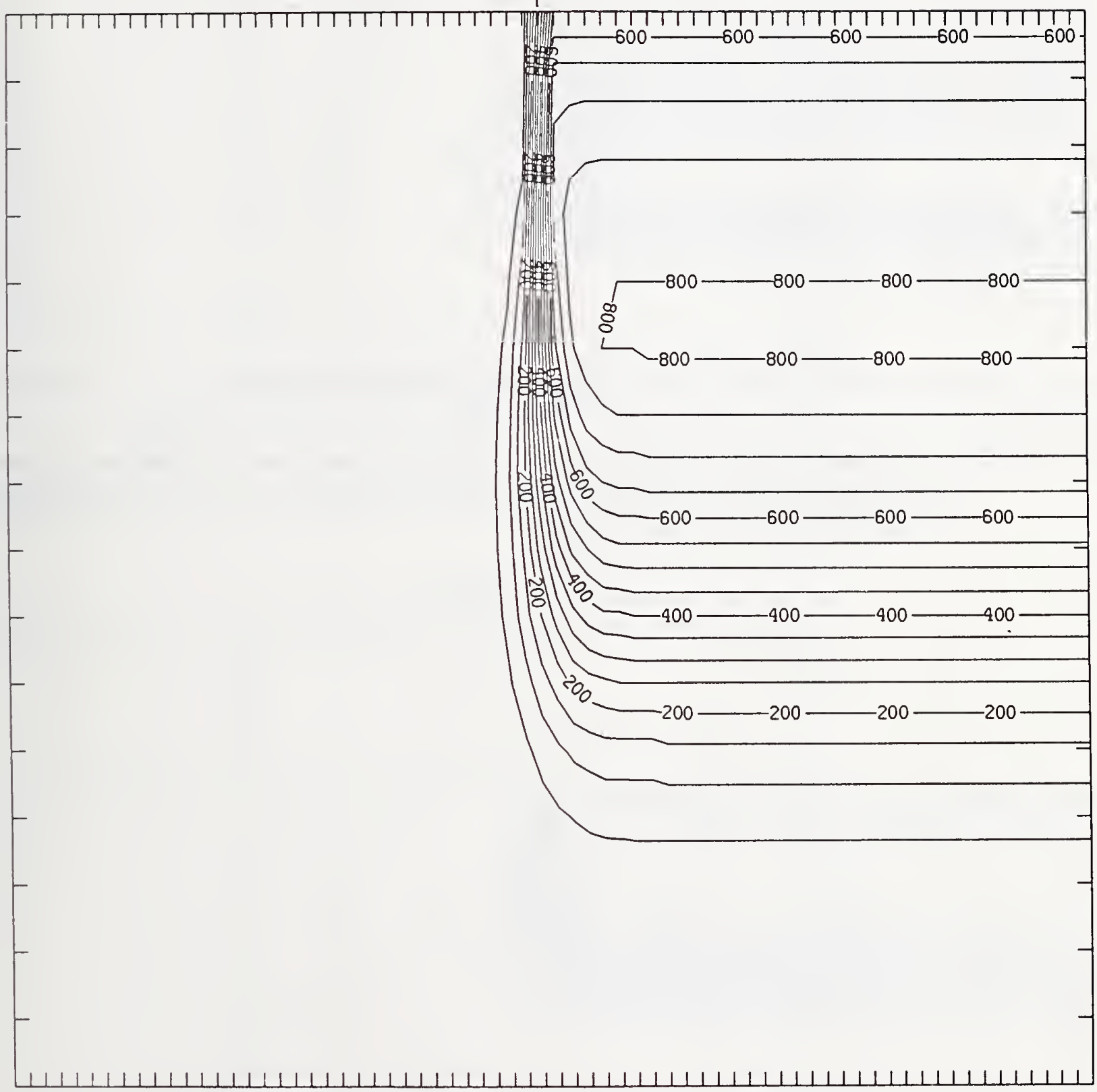

Figure Al100-4 Two-dimensional distribution of Frenkel pairs created by $100 \mathrm{keV}$ aluminum implanted past a mask edge. The length increment (distance between tick marks) is $0.02 \mu \mathrm{m}$ and the scale factor is 1. 


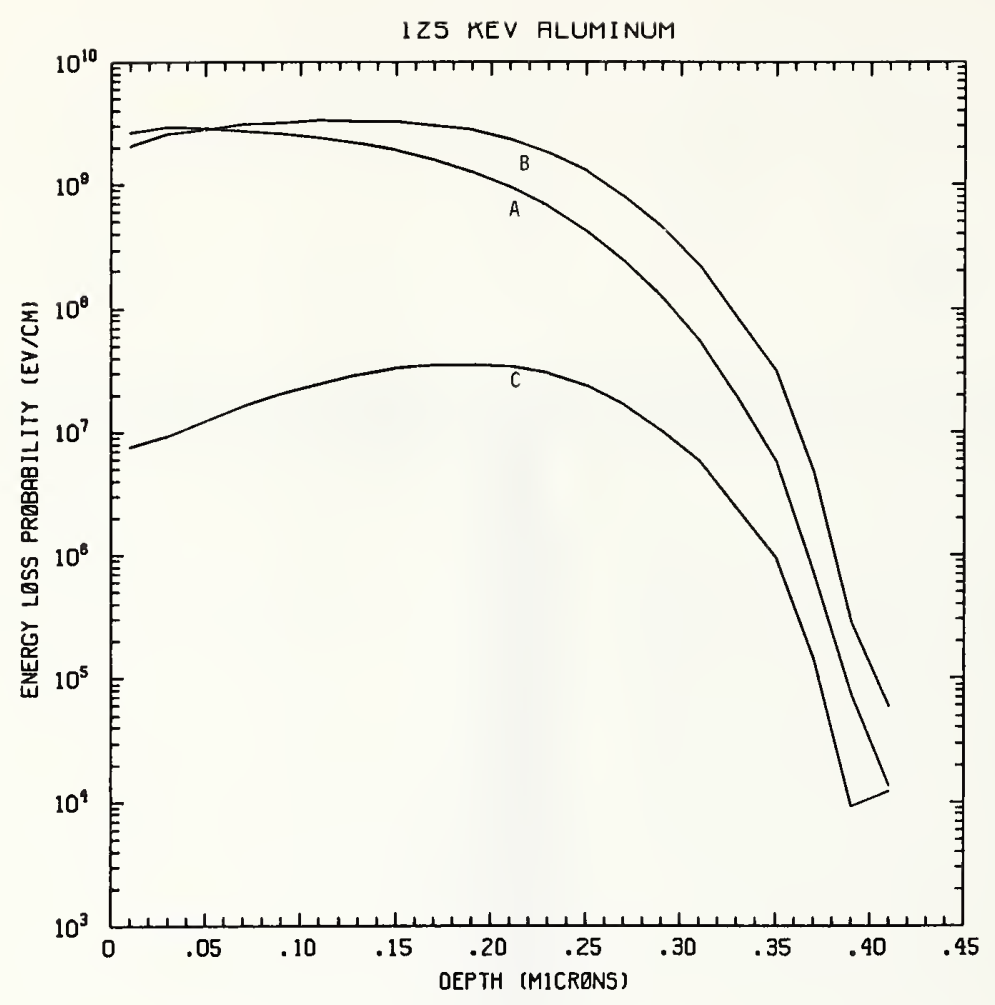

Figure Al125-1 One-dimensional probability distributions for energy loss due to electronic excitation (A), displacement damage(B), and phonon excitation (C) for $125 \mathrm{keV}$ aluminum normally incident on a silicon target.

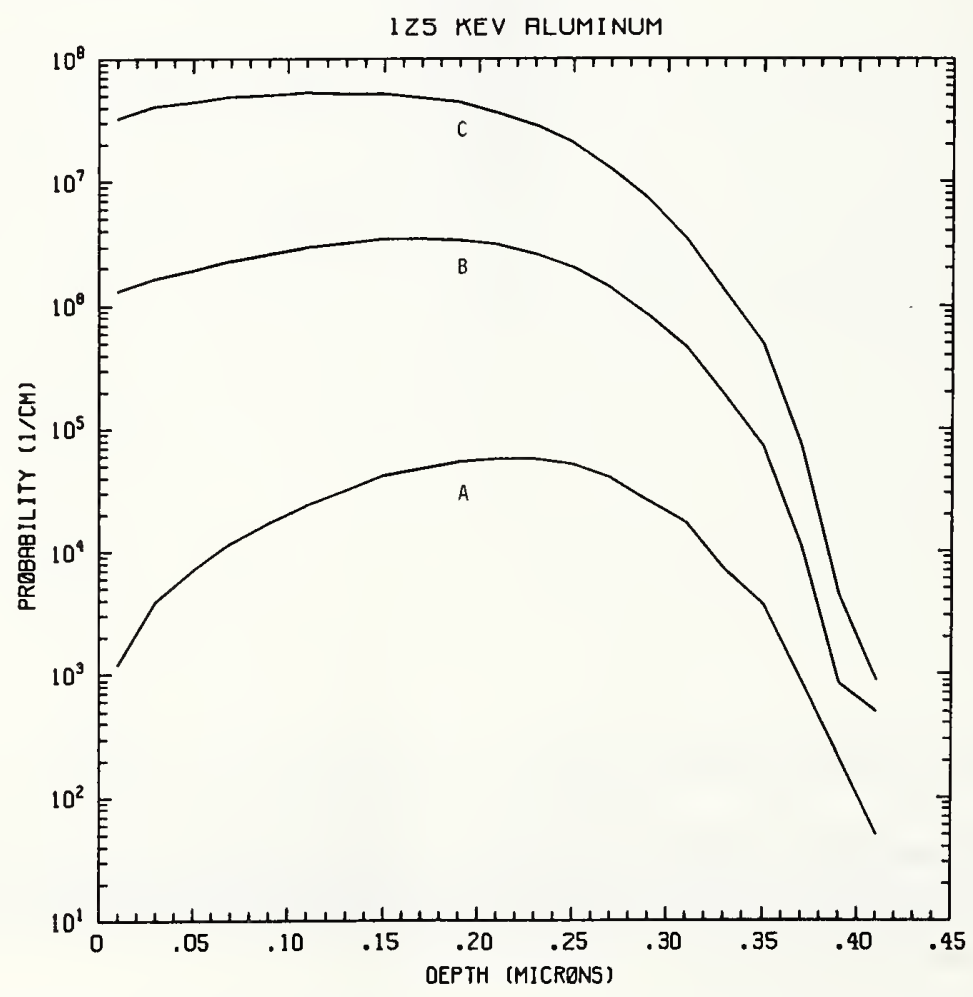

Figure Al125-2 One-dimensional probability distributions for implanted aluminum (A), primary silicon displacements (B), and Frenkel pairs (C) for $125 \mathrm{keV}$ aluminum normally incident on a silicon target. 


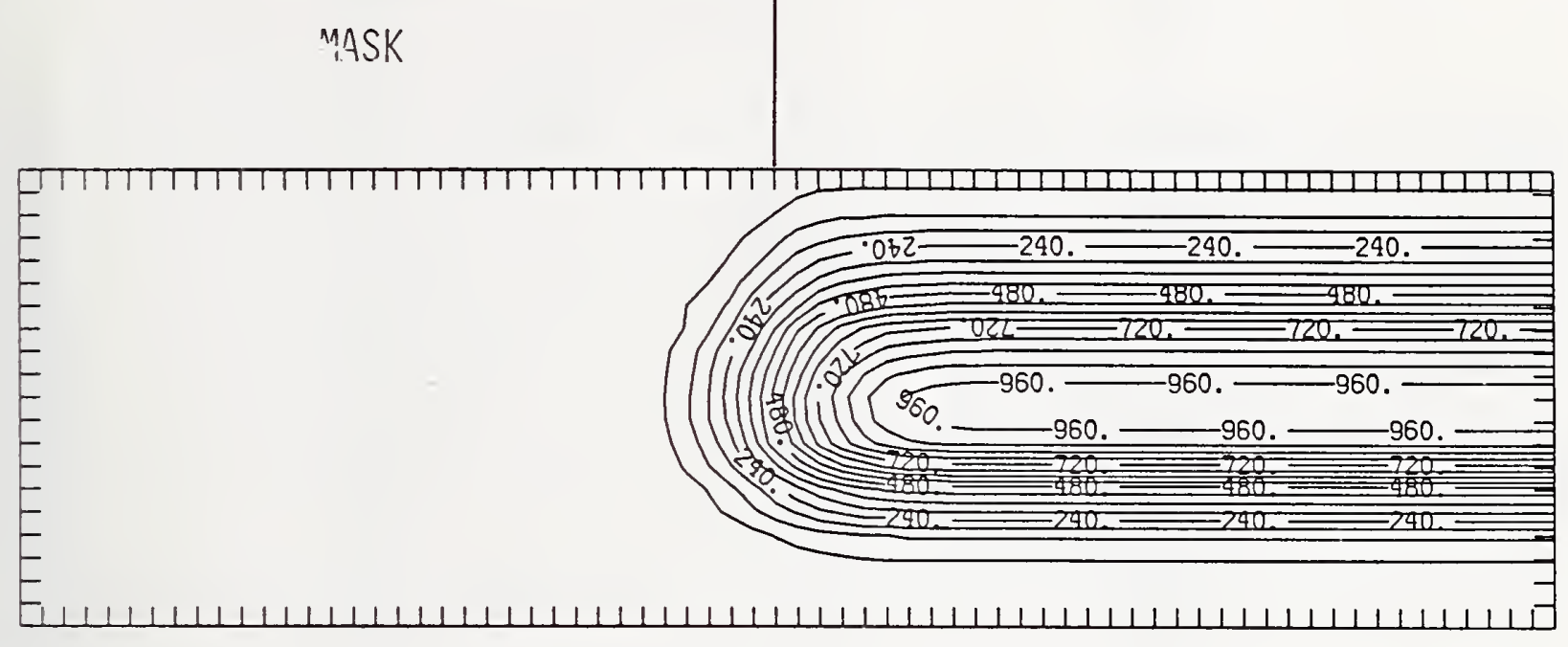

Figure Al125-3 Two-dimensional distribution of $125 \mathrm{keV}$ aluminum implanted past a mask edge. The length increment (distance between tick marks) is $0.02 \mu \mathrm{m}$ and the scale factor is 1000 .

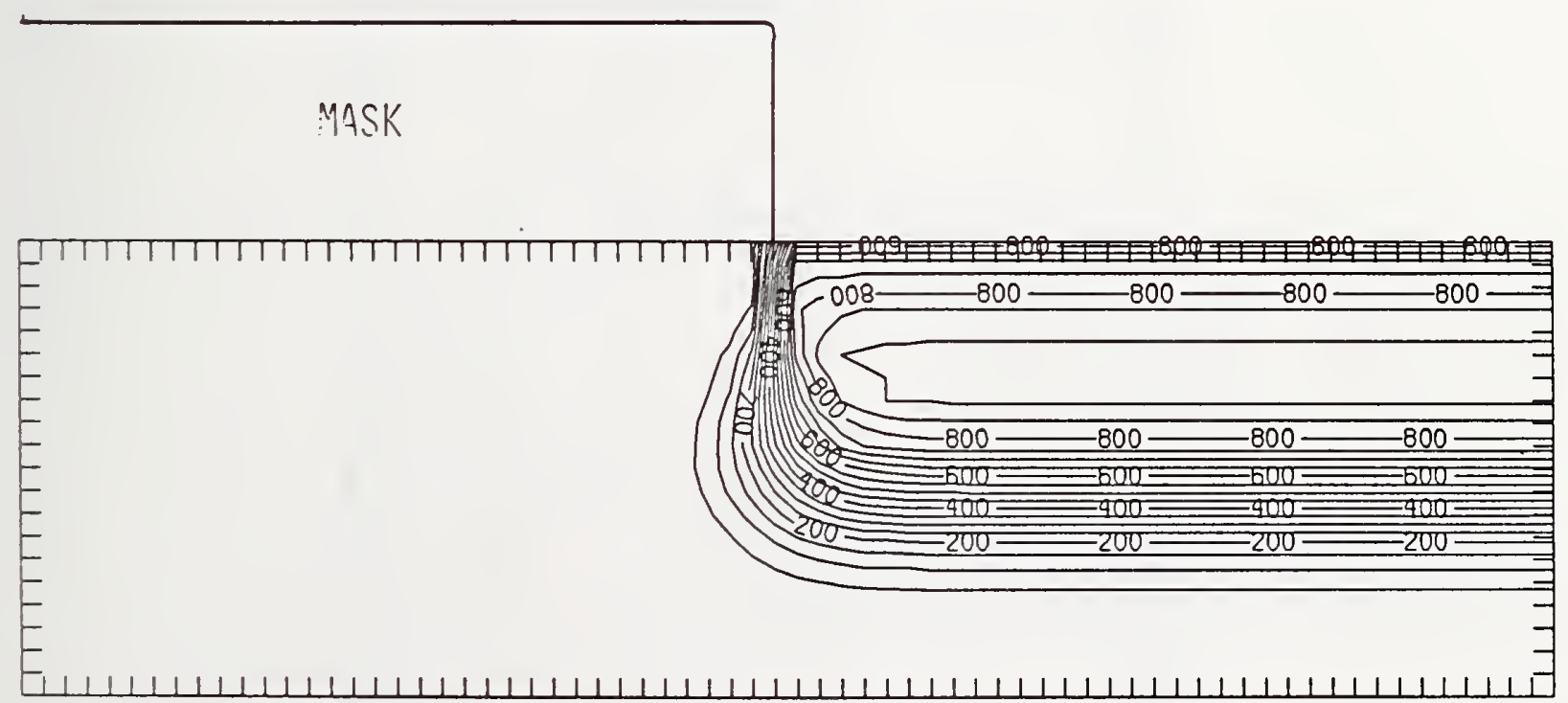

Figure Al125-4 Two-dimensional distribution of Frenkel pairs created by $125 \mathrm{keV}$ aluminum implanted past a mask edge. The length increment (distance between tick marks) is $0.02 \mu \mathrm{m}$ and the scale factor is 1. 


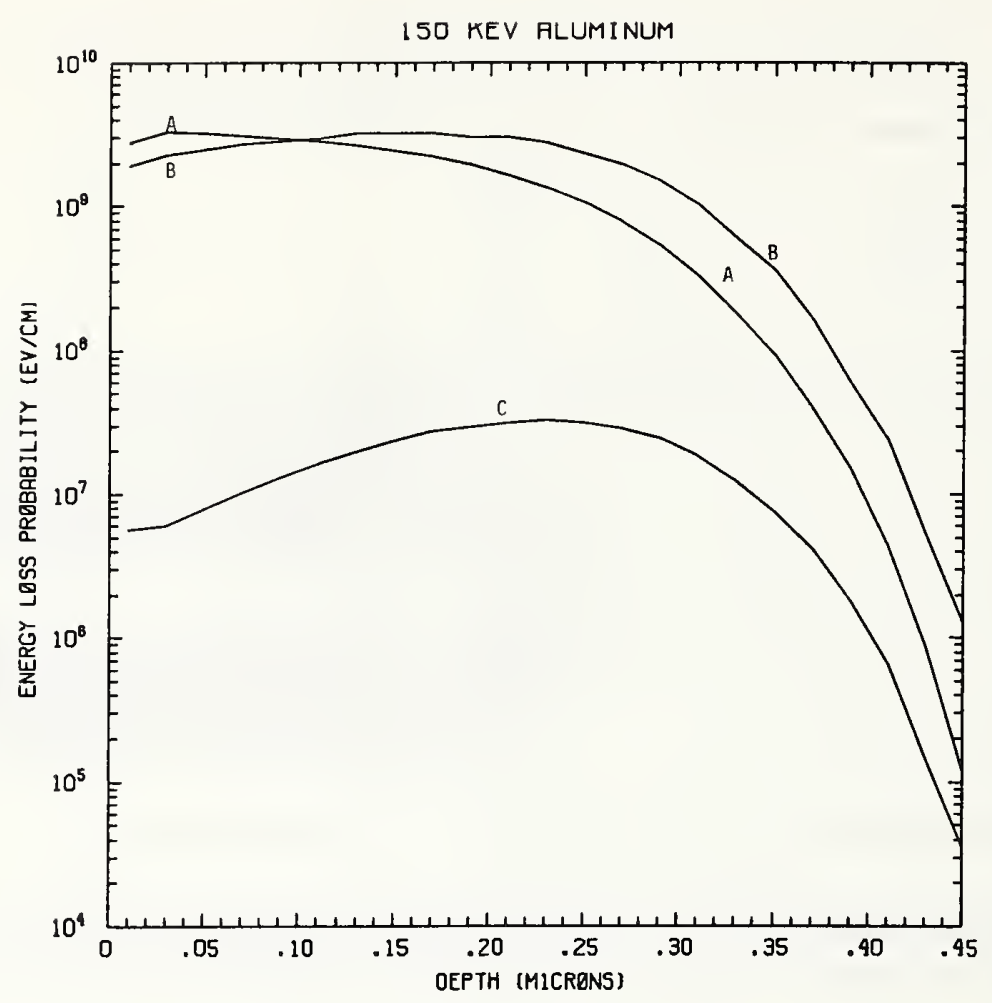

Figure Al150-1 One-dimensional probability distributions for energy loss due to electronic excitation (A), displacement damage(B), and phonon excitation (C) for $150 \mathrm{keV}$ aluminum nor mally incident on a silicon target.

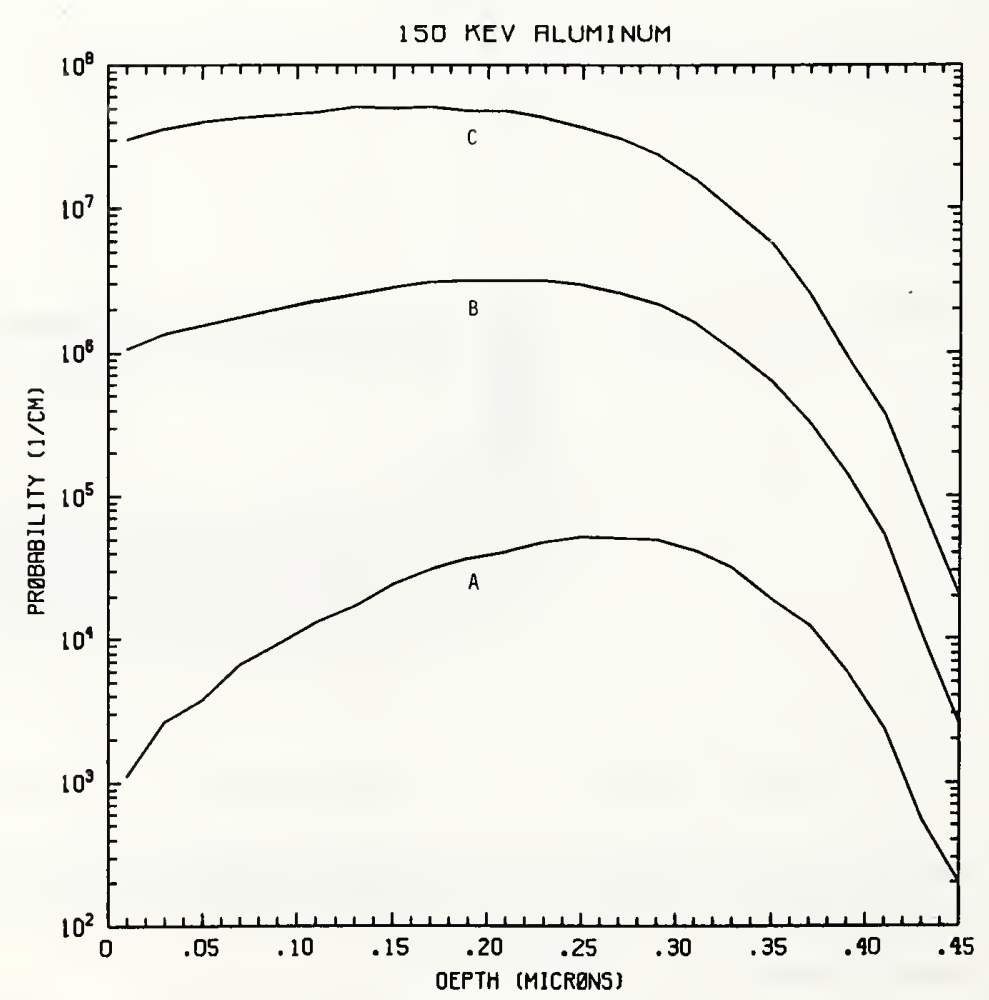

Figure Al150-2 One-dimensional probability distributions for implanted aluminum (A), primary silicon displacements (B), and Frenkel pairs (C) for $150 \mathrm{keV}$ aluminum normally incident on a silicon target. 


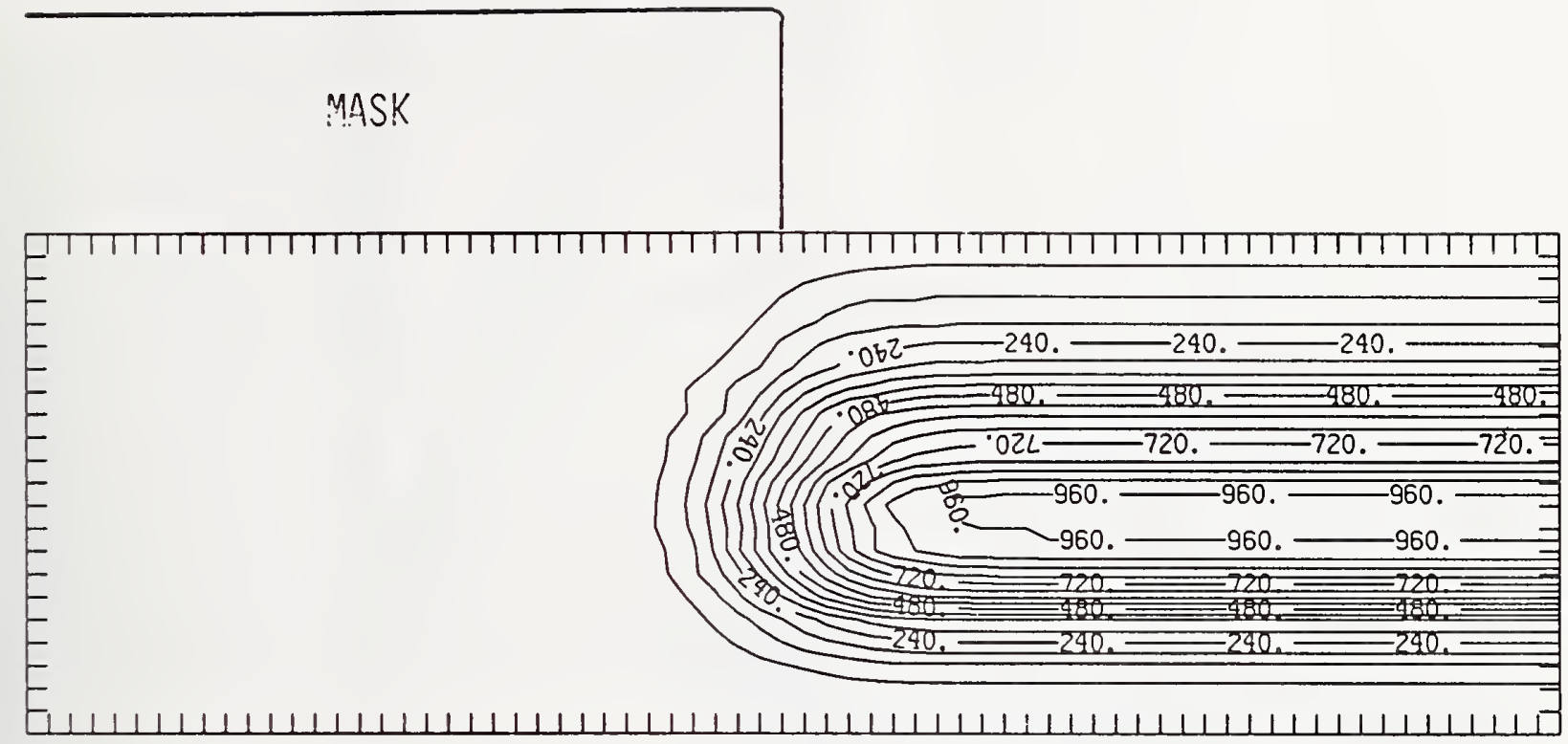

Figure Al150-3 Two-dimensional distribution of $150 \mathrm{keV}$ aluminum implanted past a mask edge. The length increment (distance between tick marks) is $0.02 \mu \mathrm{m}$ and the scale factor is 1000 .

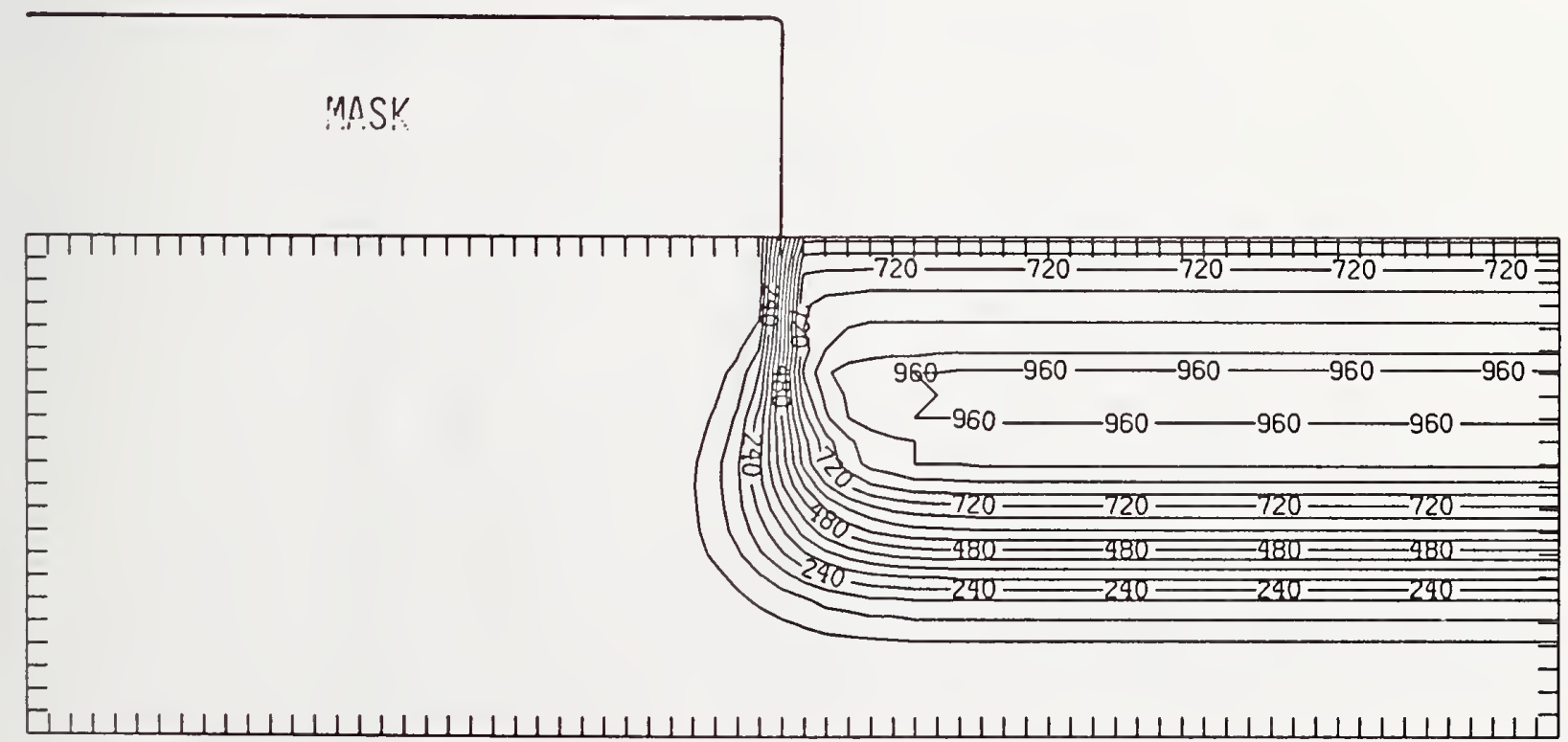

Figure Al150-4 Two-dimensional distribution of Frenkel pairs created by $150 \mathrm{keV}$ aluminum implanted past a mask edge. The length increment (distance between tick marks) is $0.02 \mu \mathrm{m}$ and the scale factor is 1 . 


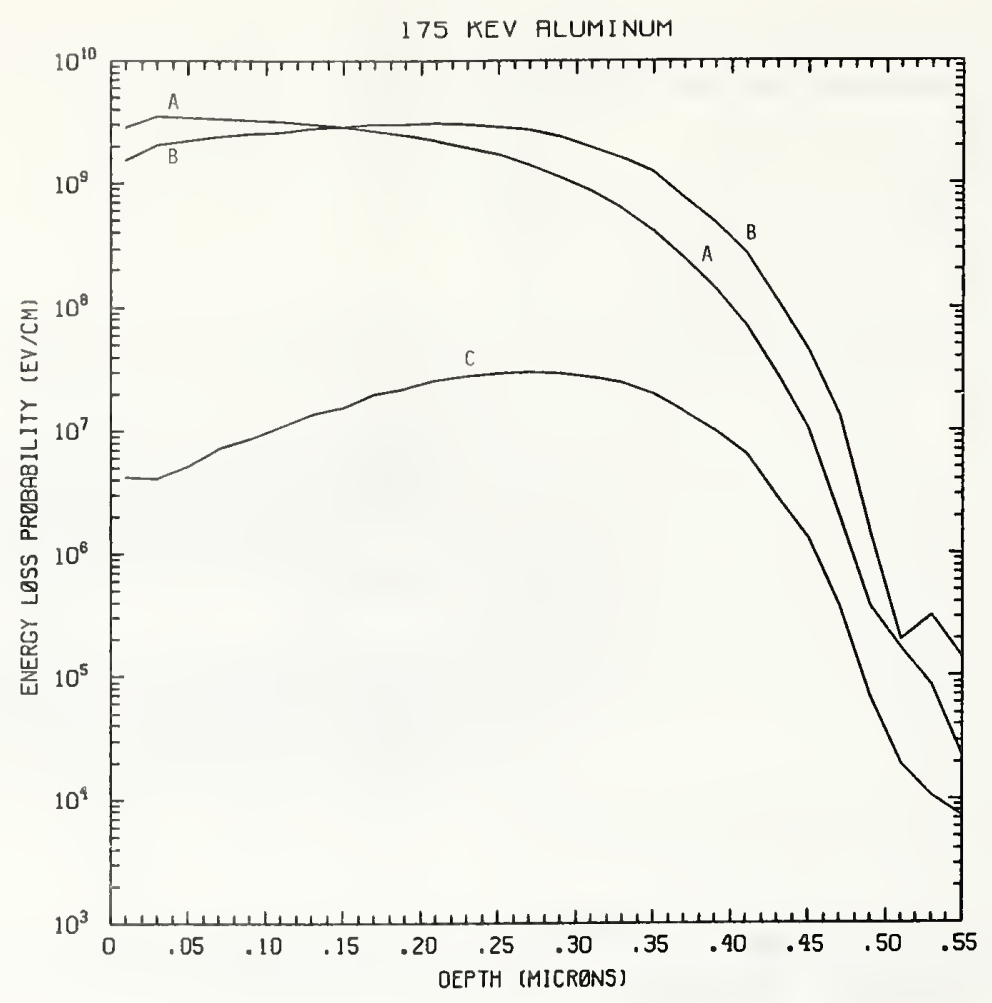

Figure Al175-1 One-dimensional probability distributions for energy loss due to electronic excitation (A), displacement damage(B), and phonon excitation (C) for $175 \mathrm{keV}$ aluminum normally incident on a silicon target.

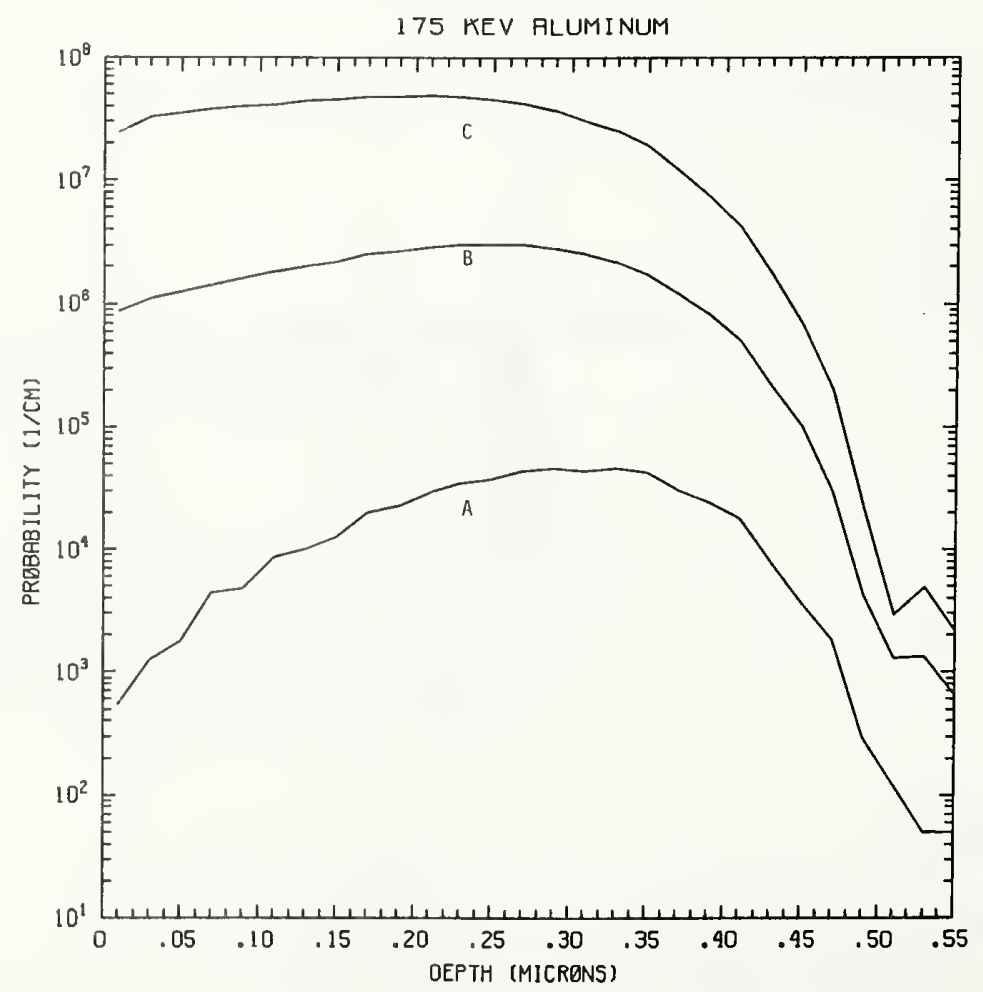

Figure Al175-2 One-dimensional probability distributions for implanted aluminum (A), primary silicon displacements (B), and Frenkel pairs (C) for $175 \mathrm{keV}$ aluminum normally incident on a silicon target. 


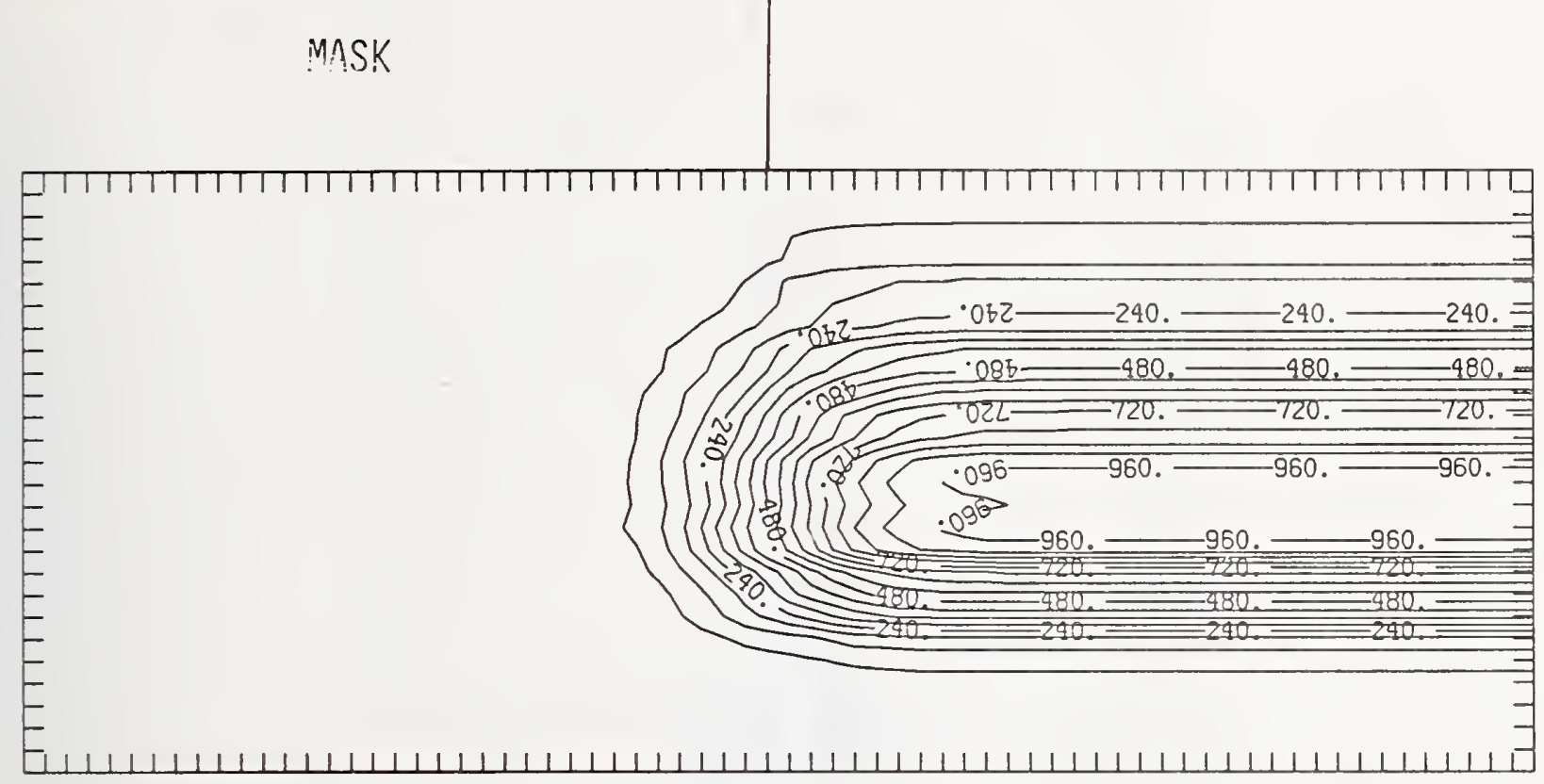

Figure Al175-3 Two-dimensional distribution of $175 \mathrm{keV}$ aluminum implanted past a mask edge. The length increment (distance between tick marks) is $0.02 \mu \mathrm{m}$ and the scale factor is 1000 .

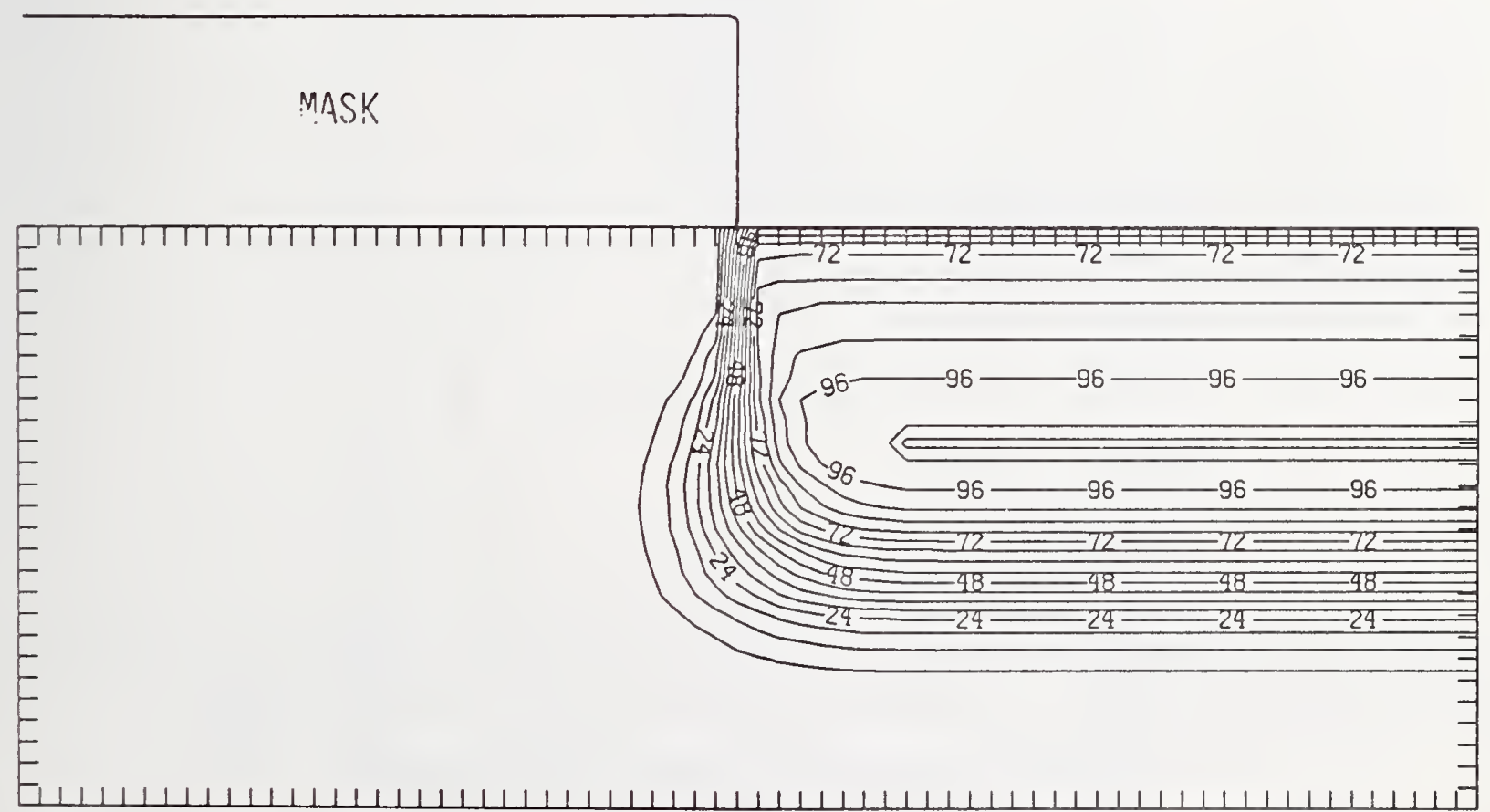

Figure Al175-4 Two-dimensional distribution of Frenkel pairs created by $175 \mathrm{keV}$ aluminum implanted past a mask edge. The length increment (distance between tick marks) is $0.02 \mu \mathrm{m}$ and the scale factor is 0.1 . 


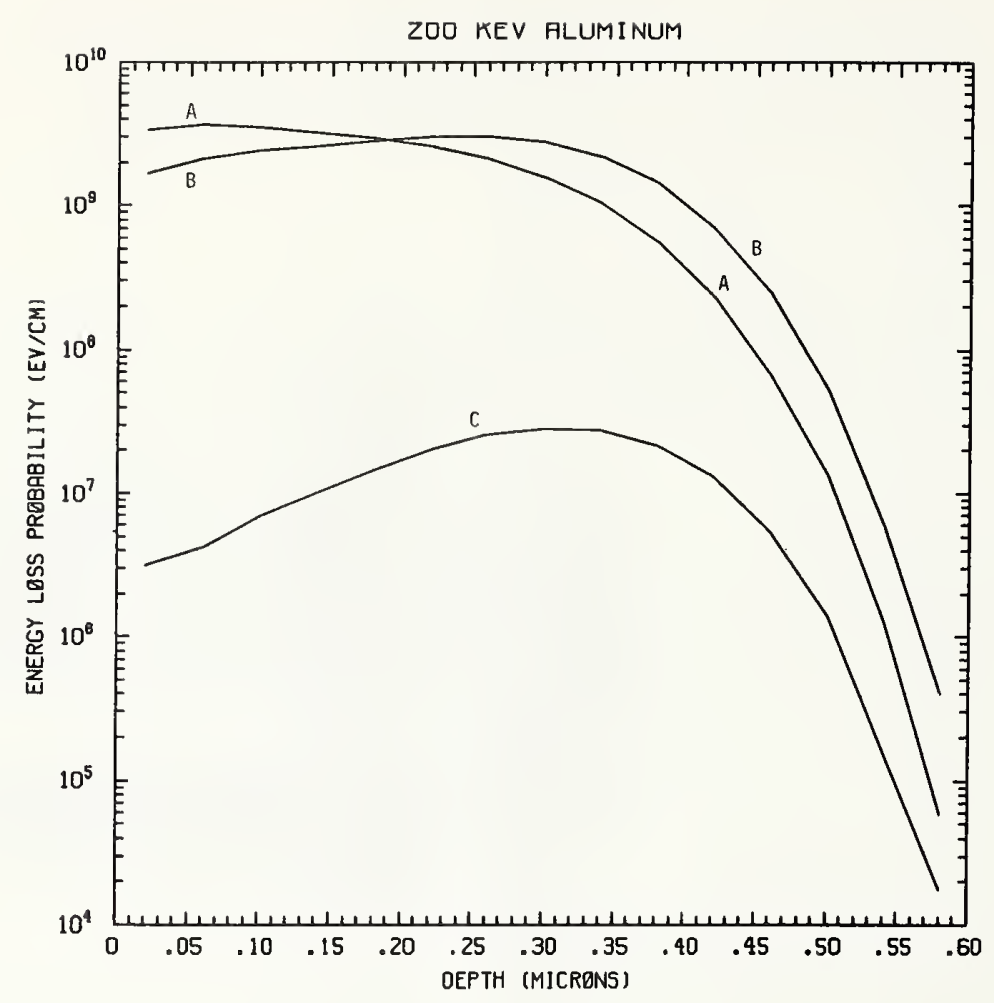

Figure Al200-1 One-dimensional probability distributions for energy loss due to electronic excitation (A), displacement damage(B), and phonon excitation (C) for $200 \mathrm{keV}$ aluminum normally incident on a silicon target.

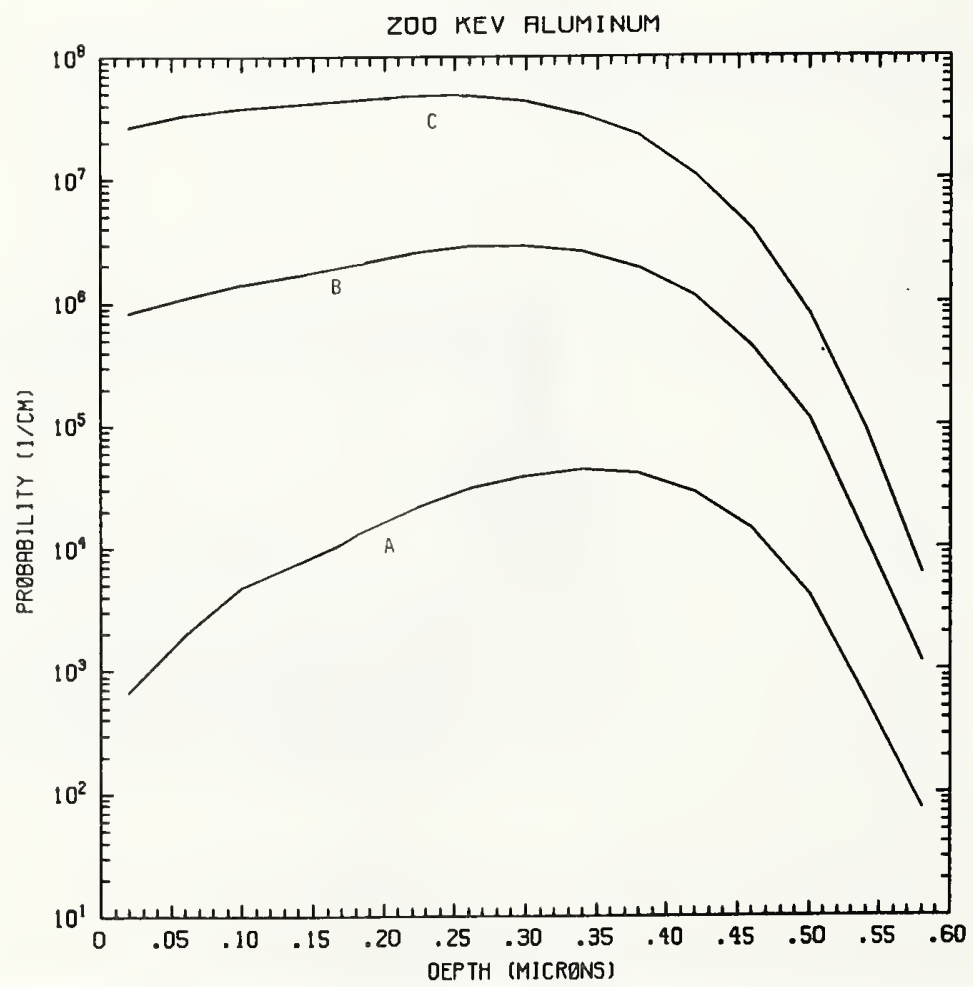

Figure Al200-2 One-dimensional probability distributions for implanted aluminum (A), primary silicon displacements (B), and Frenkel pairs (C) for $200 \mathrm{keV}$ aluminum normally incident on a silicon target. 


\section{MASK}

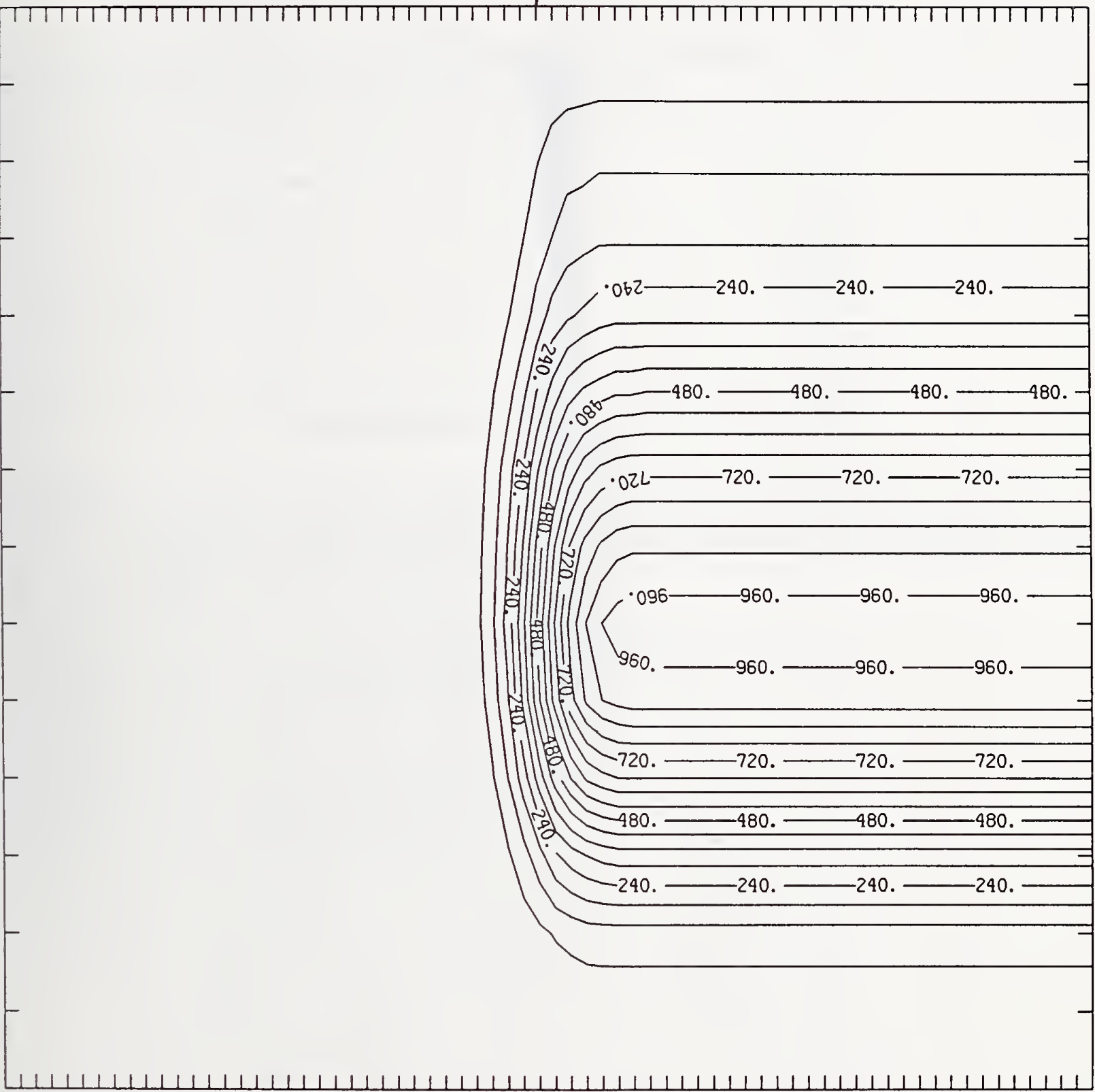

Figure Al200-3 Two-dimensional distribution of $200 \mathrm{keV}$ aluminum implanted past a mask edge. The length increment (distance between tick marks) is $0.04 \mu \mathrm{m}$ and the scale factor is 1000. 


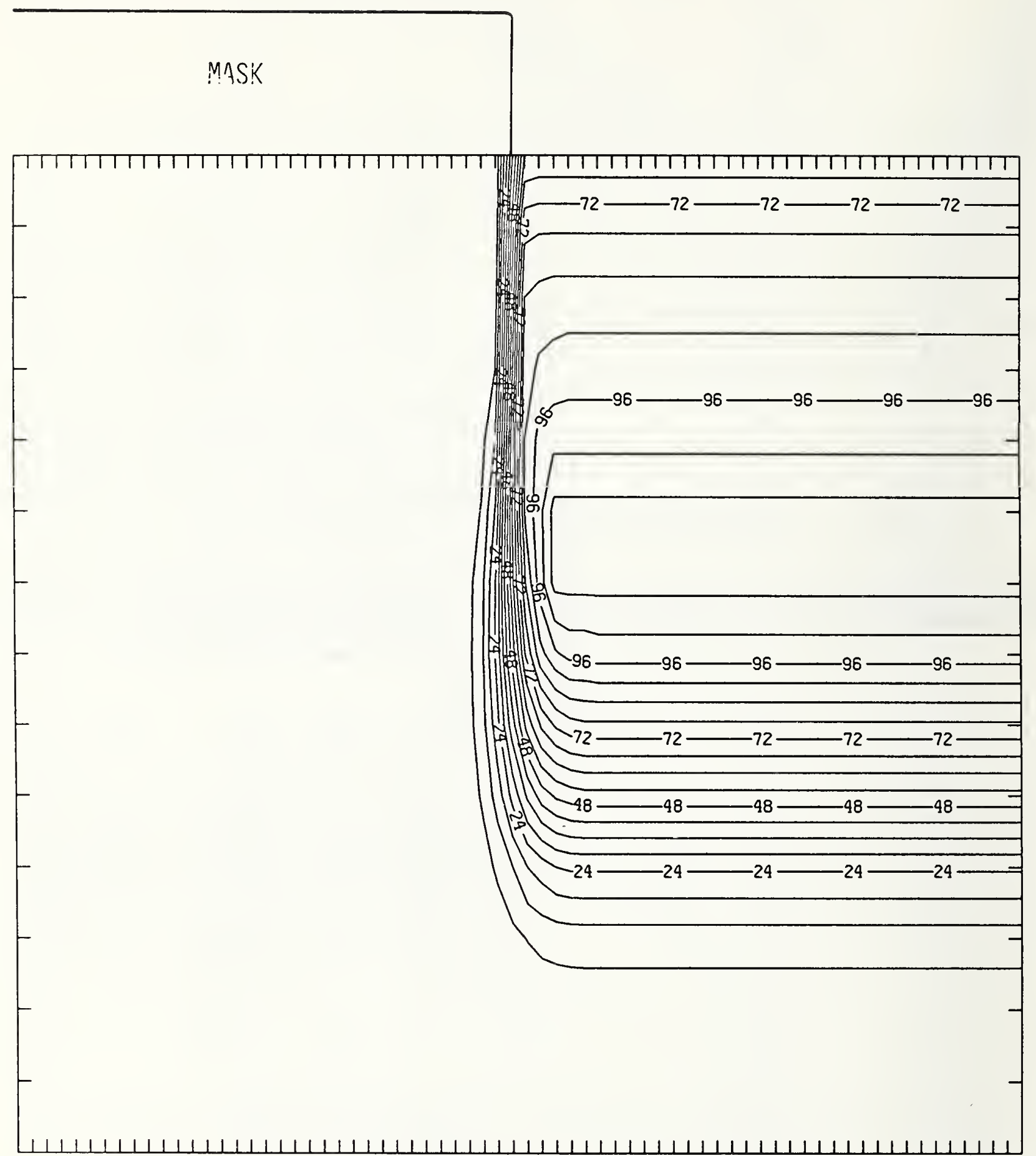

Figure Al200-4 Two-dimensional distribution of Frenkel pairs created by $200 \mathrm{keV}$ aluminum implanted past a mask edge. The length increment (distance between tick marks) is $0.04 \mu \mathrm{m}$ and the scale factor is 0.1 . 
ZZS KEV RLUMINUM

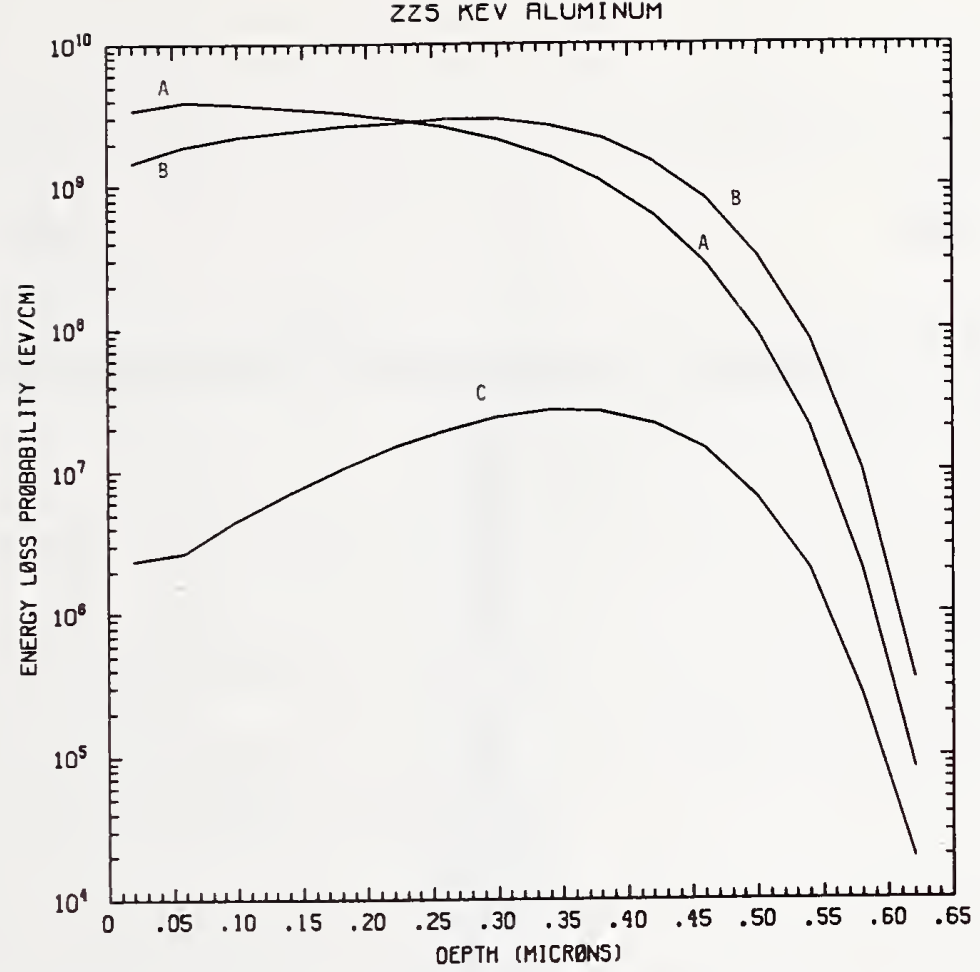

Figure Al225-1 One-dimensional probability distributions for energy loss due to electronic excitation (A), displacement damage(B), and phonon excitation (C) for $225 \mathrm{keV}$ aluminum normally incident on a silicon target.

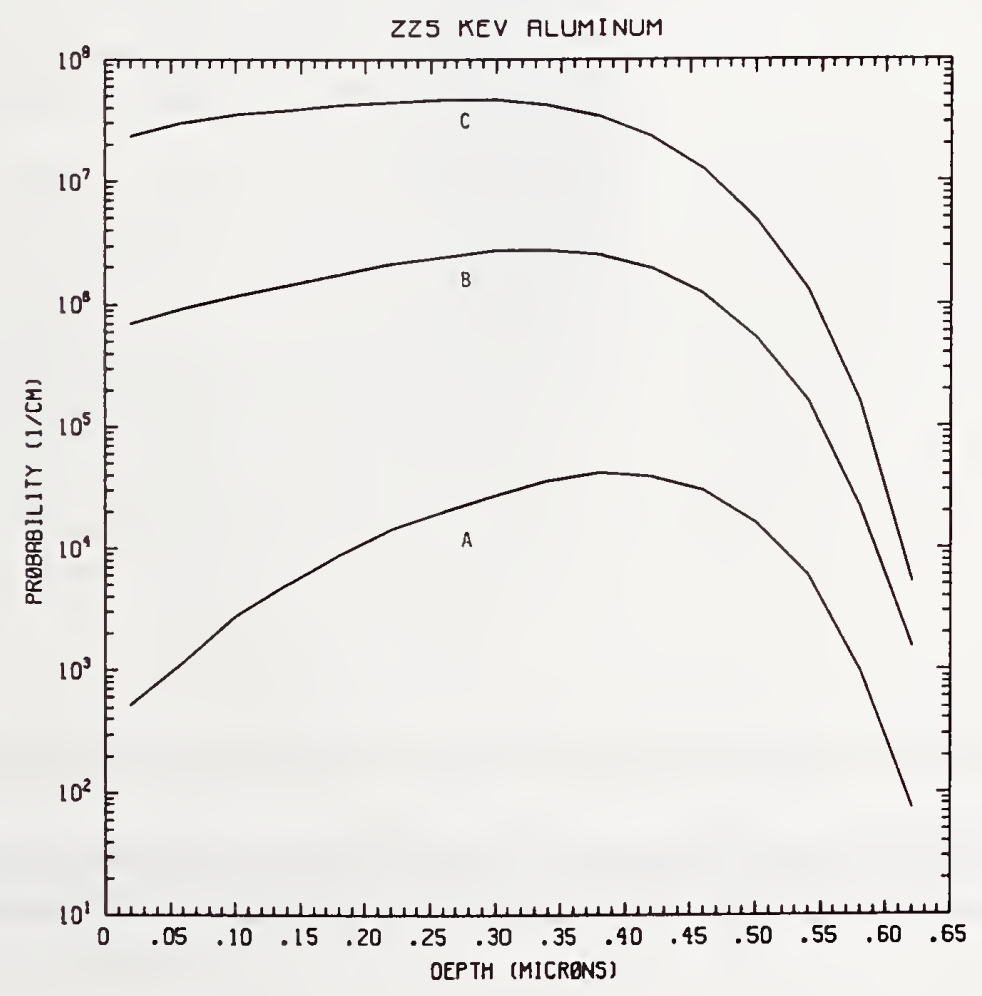

Figure Al225-2 One-dimensional probability distributions for implanted aluminum (A), primary silicon displacements (B), and Frenkel pairs (C) for $225 \mathrm{keV}$ aluminum normally incident on a silicon target. 


\section{MASK}

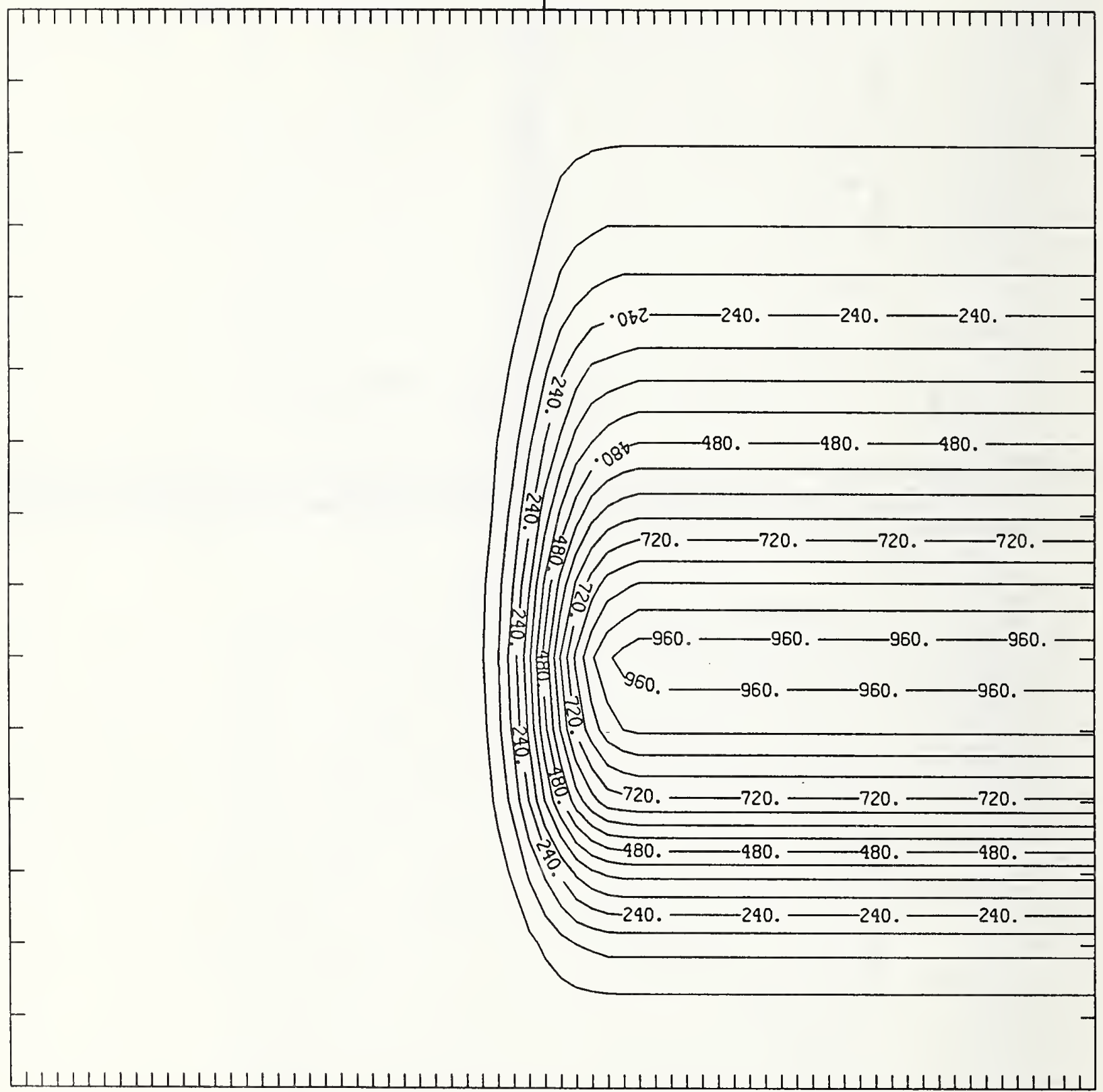

Figure Al225-3 Two-dimensional distribution of $225 \mathrm{keV}$ aluminum implanted past a mask edge. The length increment (distance between tick marks) is $0.04 \mu \mathrm{m}$ and the scale factor is 1000 . 


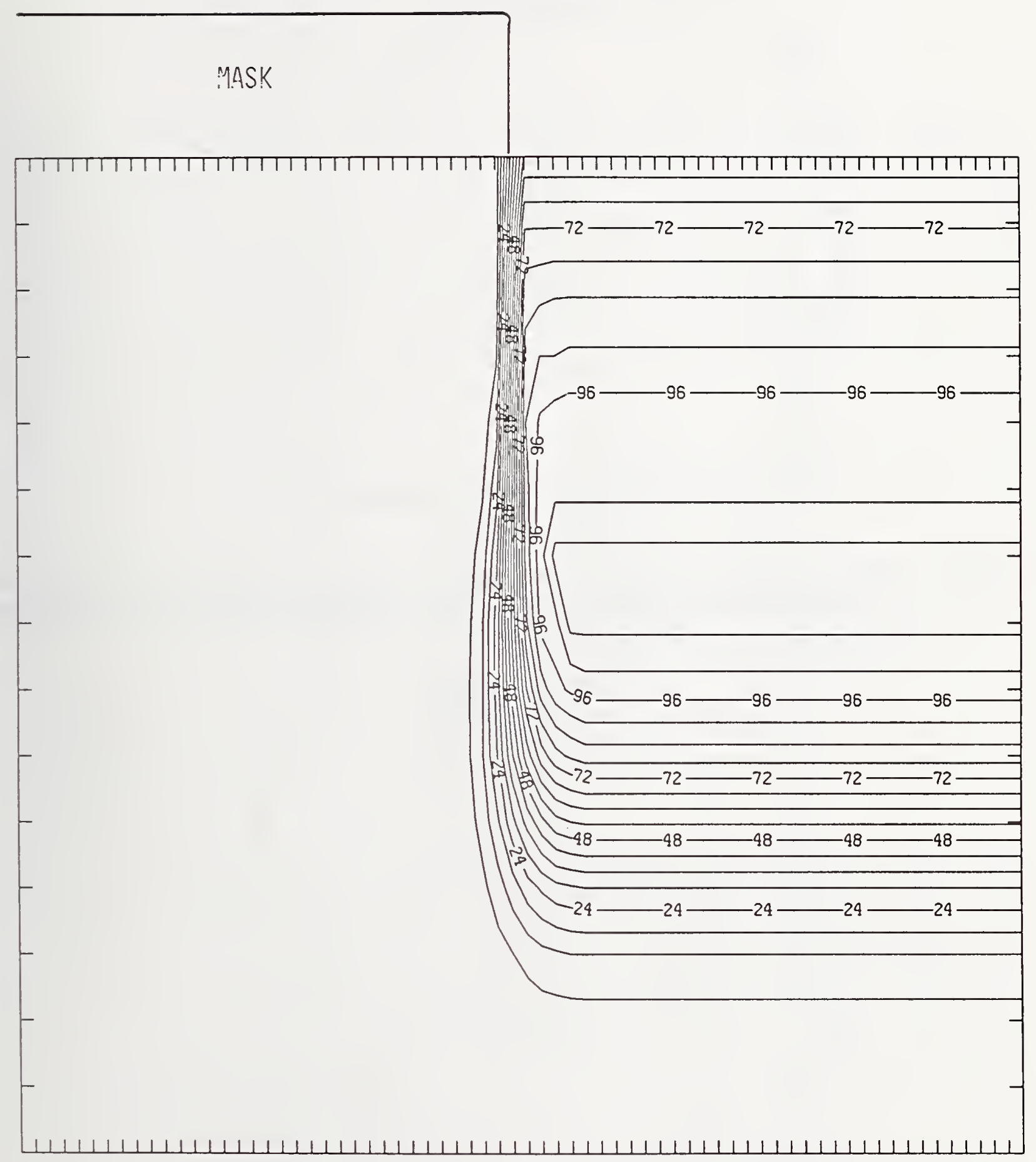

Figure A1225-4 Two-dimensional distribution of Frenkel pairs created by $225 \mathrm{keV}$ aluminum implanted past a mask edge. The length increment (distance between tick marks) is $0.04 \mu \mathrm{m}$ and the scale factor is 0.1 . 


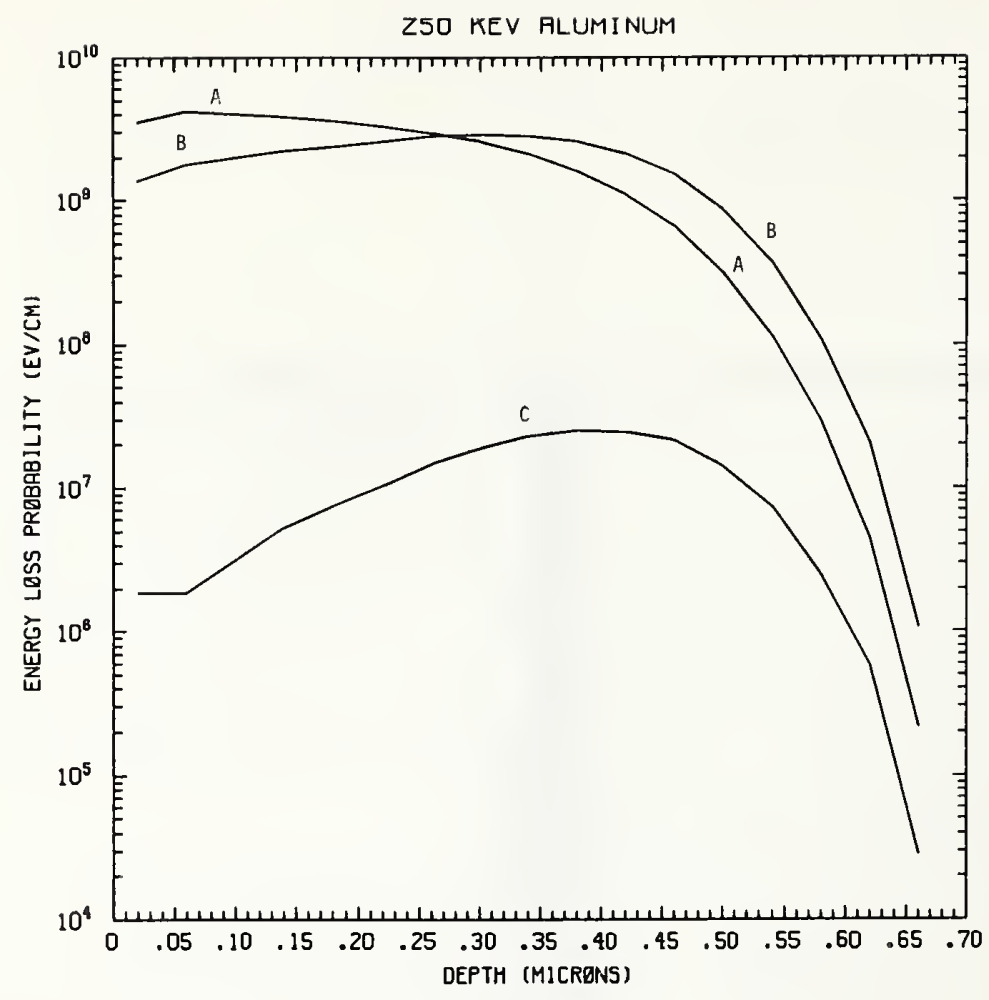

Figure Al250-1 One-dimensional probability distributions for energy loss due to electronic excitation (A), displacement damage(B), and phonon excitation (C) for $250 \mathrm{keV}$ aluminum normally incident on a silicon target.

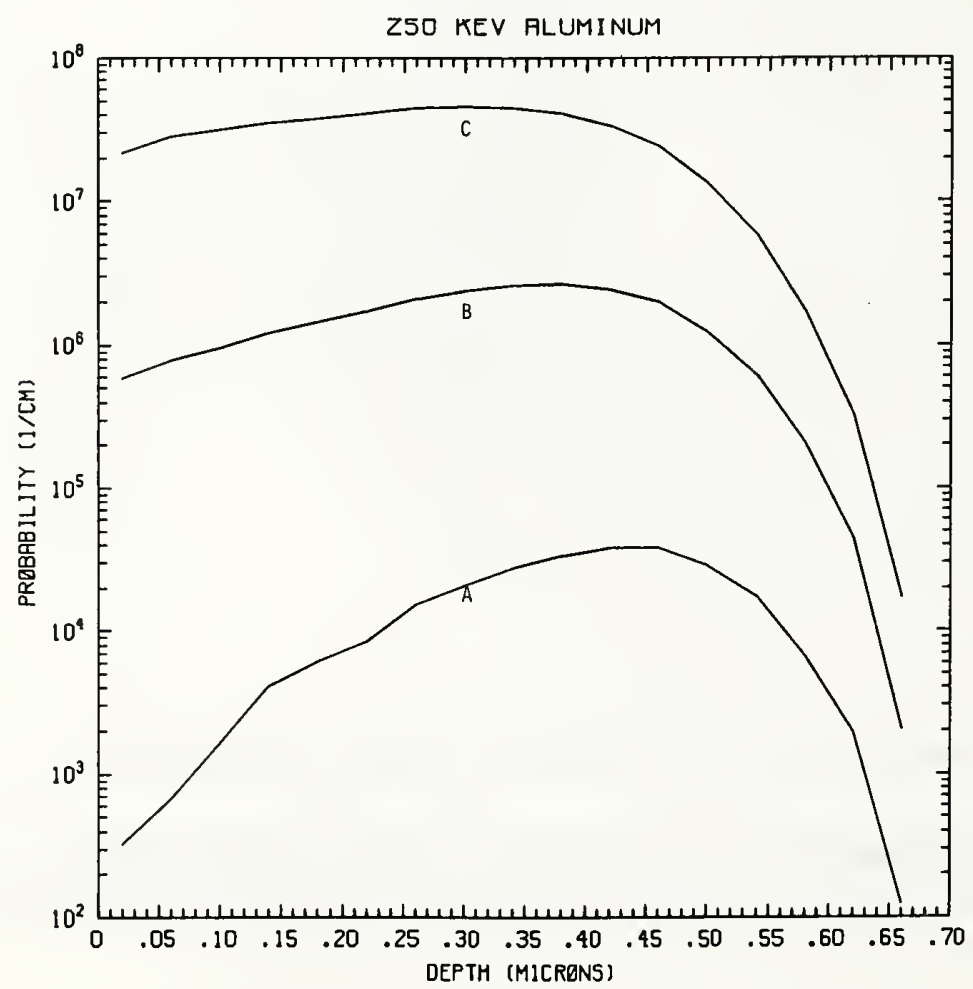

Figure Al250-2 One-dimensional probability distributions for implanted aluminum (A), primary silicon displacements (B), and Frenkel pairs (C) for $250 \mathrm{keV}$ aluminum normally incident on a silicon target. 
MASK

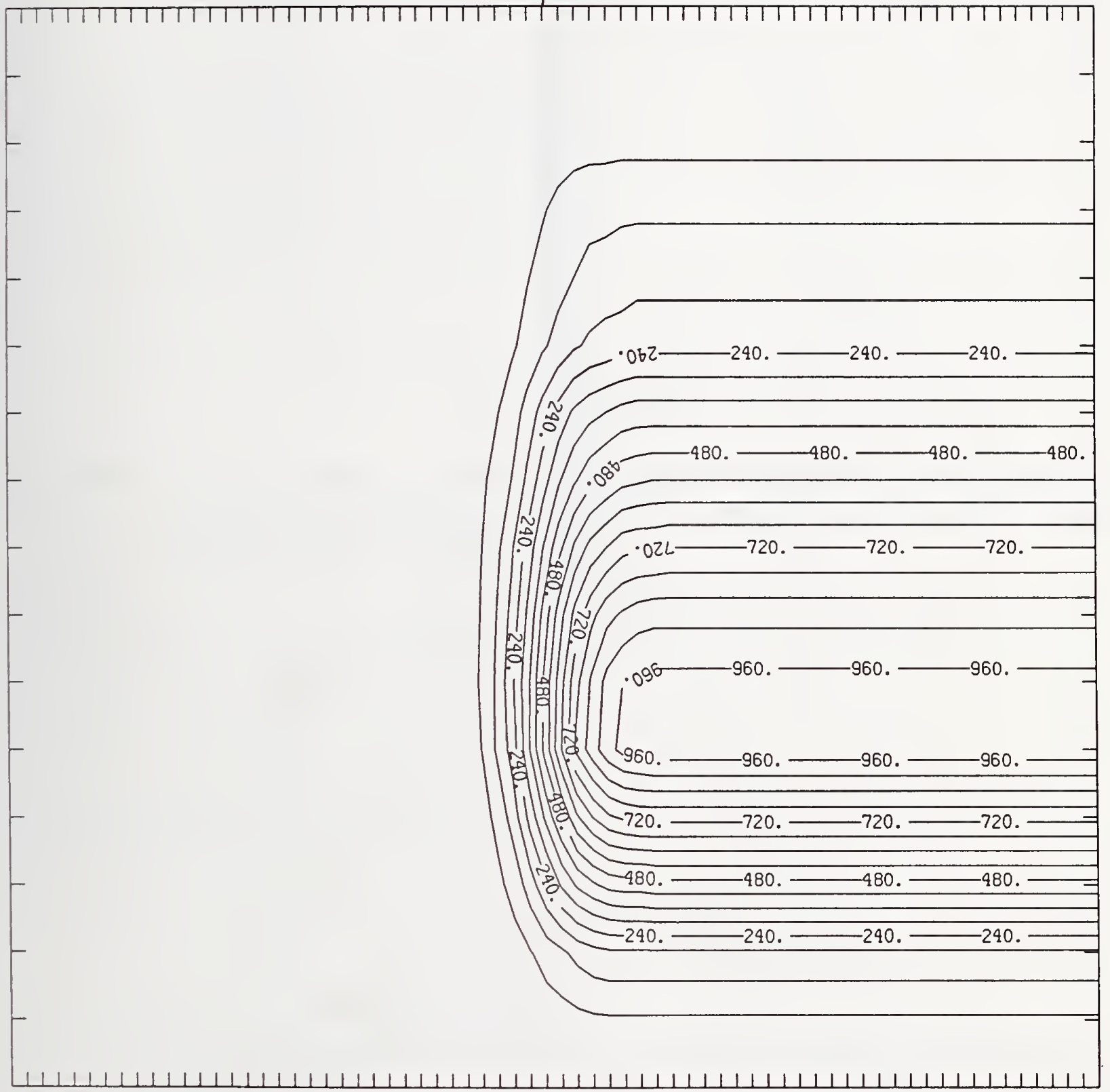

Figure A1250-3 Two-dimensional distribution of $250 \mathrm{keV}$ aluminum implanted past a mask edge. The length increment (distance between tick marks) is $0.04 \mu \mathrm{m}$ and the scale factor is 1000 . 


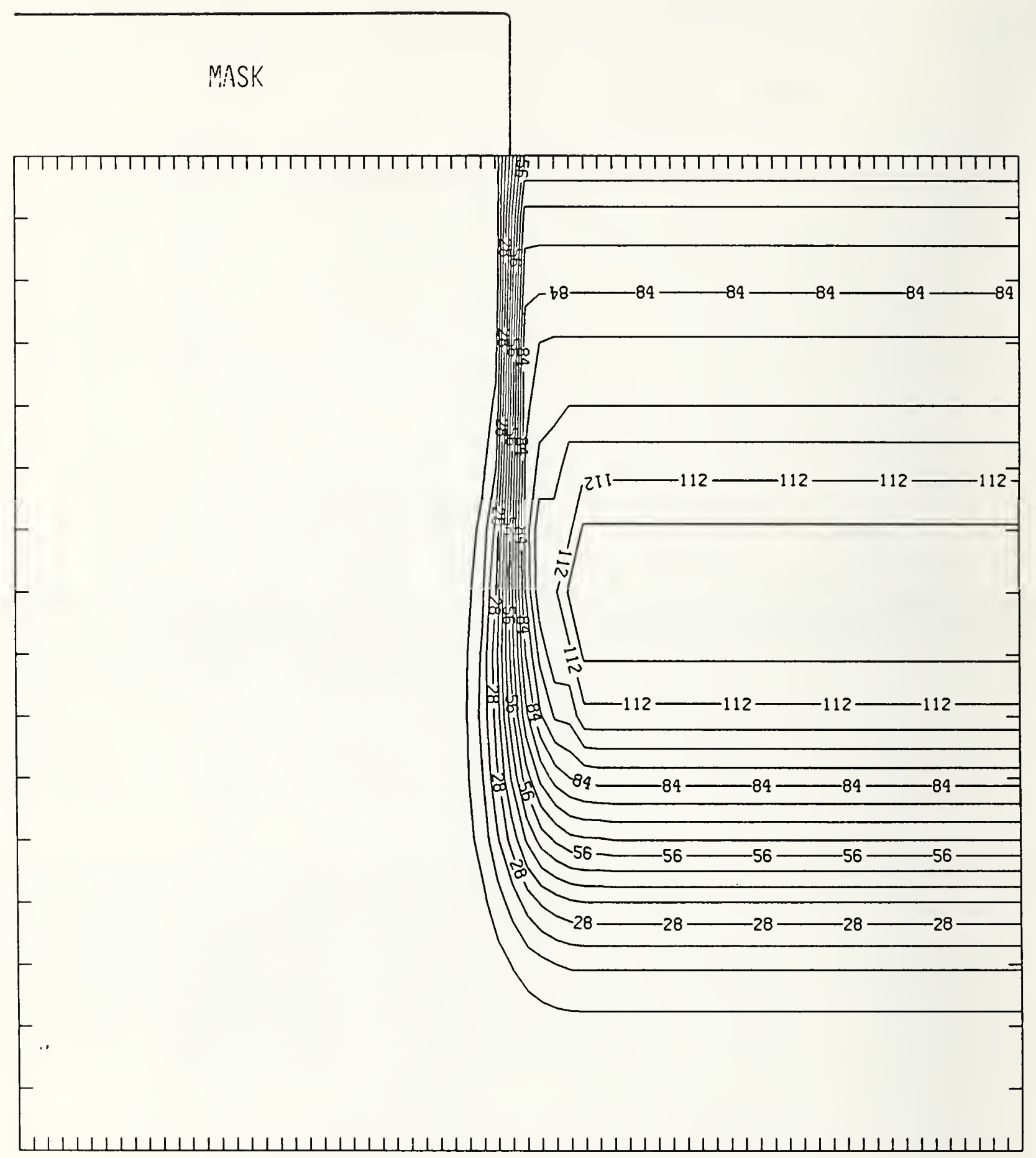

Figure Al250-4 Two-dimensional distribution of Frenkel pairs created by $250 \mathrm{keV}$ aluminum implanted past a mask edge. The length increment (distance between tick marks) is $0.04 \mu \mathrm{m}$ and the scale factor is 0.1 . 


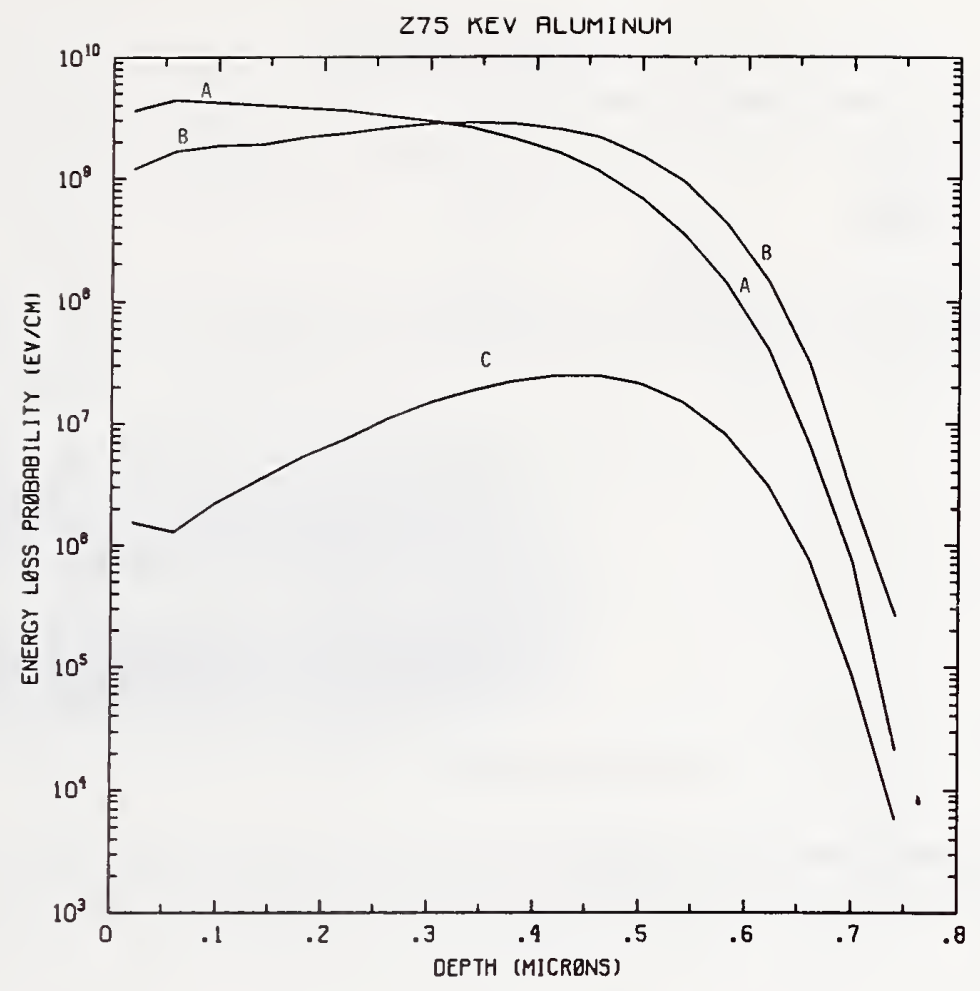

Figure Al275-1 One-dimensional probability distributions for energy loss due to electronic excitation (A), displacement damage(B), and phonon excitation (C) for $275 \mathrm{keV}$ aluminum normally incident on a silicon target.

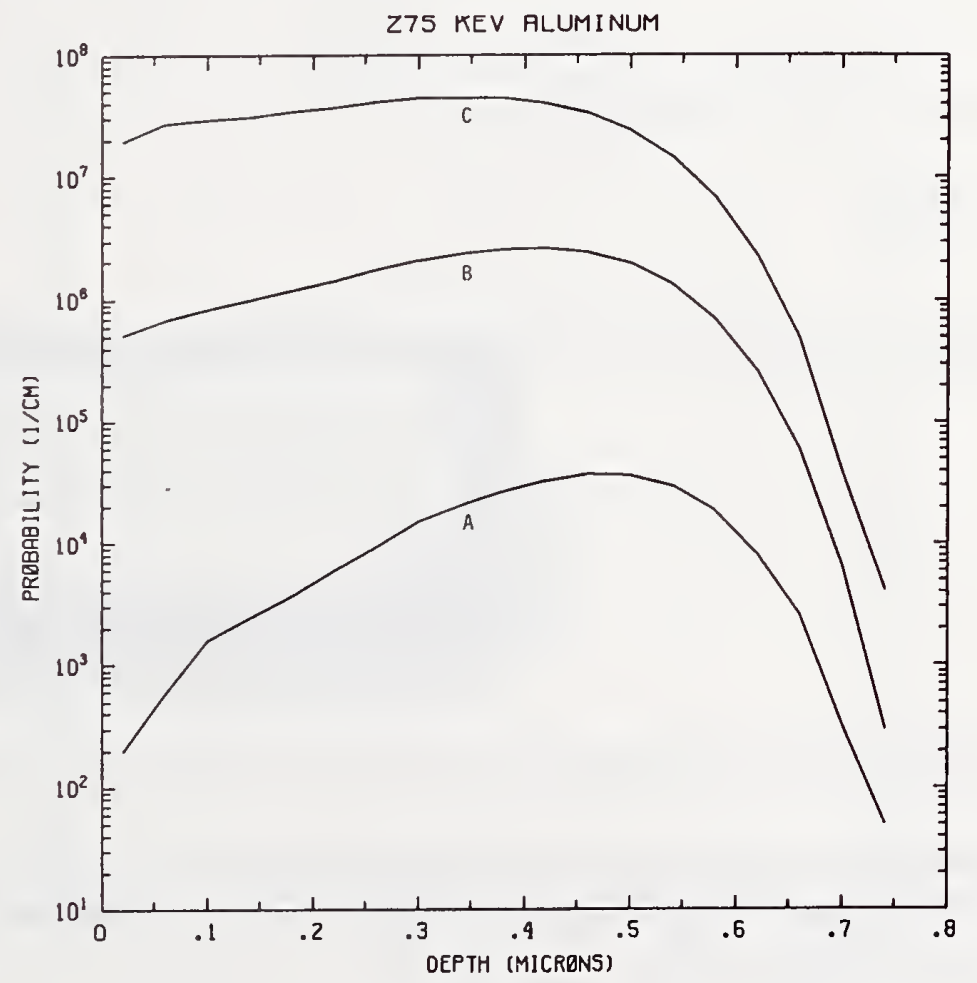

Figure Al275-2 One-dimensional probability distributions for implanted aluminum (A), primary silicon displacements (B), and Frenkel pairs (C) for $275 \mathrm{keV}$ aluminum normally incident on a silicon target. 
MASK

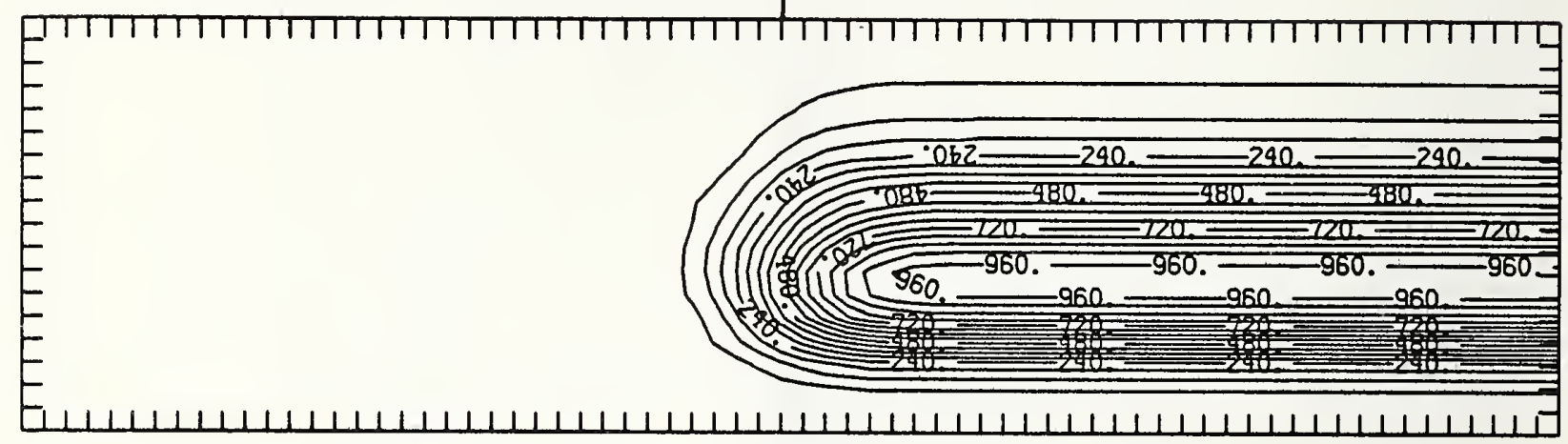

Figure Al275-3 Two-dimensional distribution of $275 \mathrm{keV}$ aluminum implanted past a mask edge. The length increment (distance between tick marks) is $0.04 \mu \mathrm{m}$ and the scale factor is 1000.

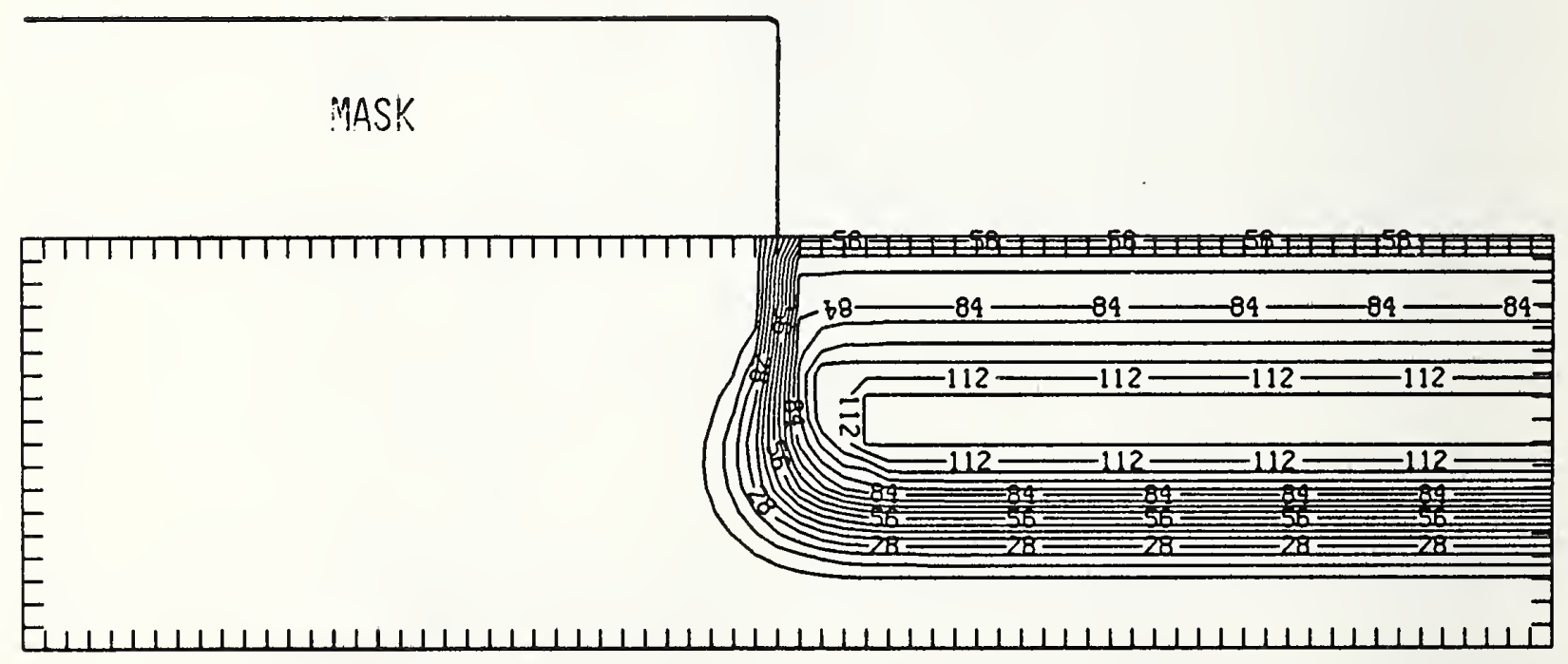

Figure Al275-4 Two-dimensional distribution of Frenkel pairs created by $275 \mathrm{keV}$ aluminum implanted past a mask edge. The length increment (distance between tick marks) is $0.04 \mu \mathrm{m}$ and the scale factor is 0.1 . 


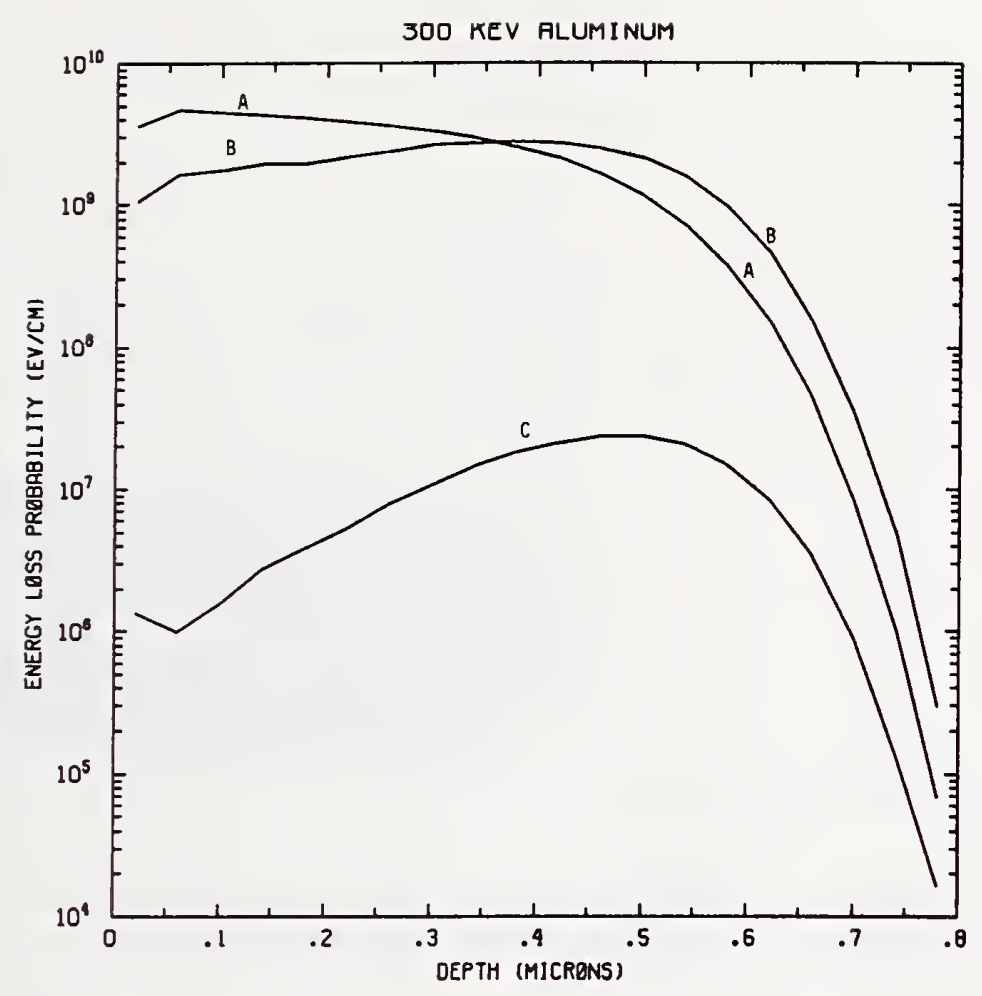

Figure Al300-1 One-dimensional probability distributions for energy loss due to electronic excitation (A), displacement damage(B), and phonon excitation (C) for $300 \mathrm{keV}$ aluminum normally incident on a silicon target.

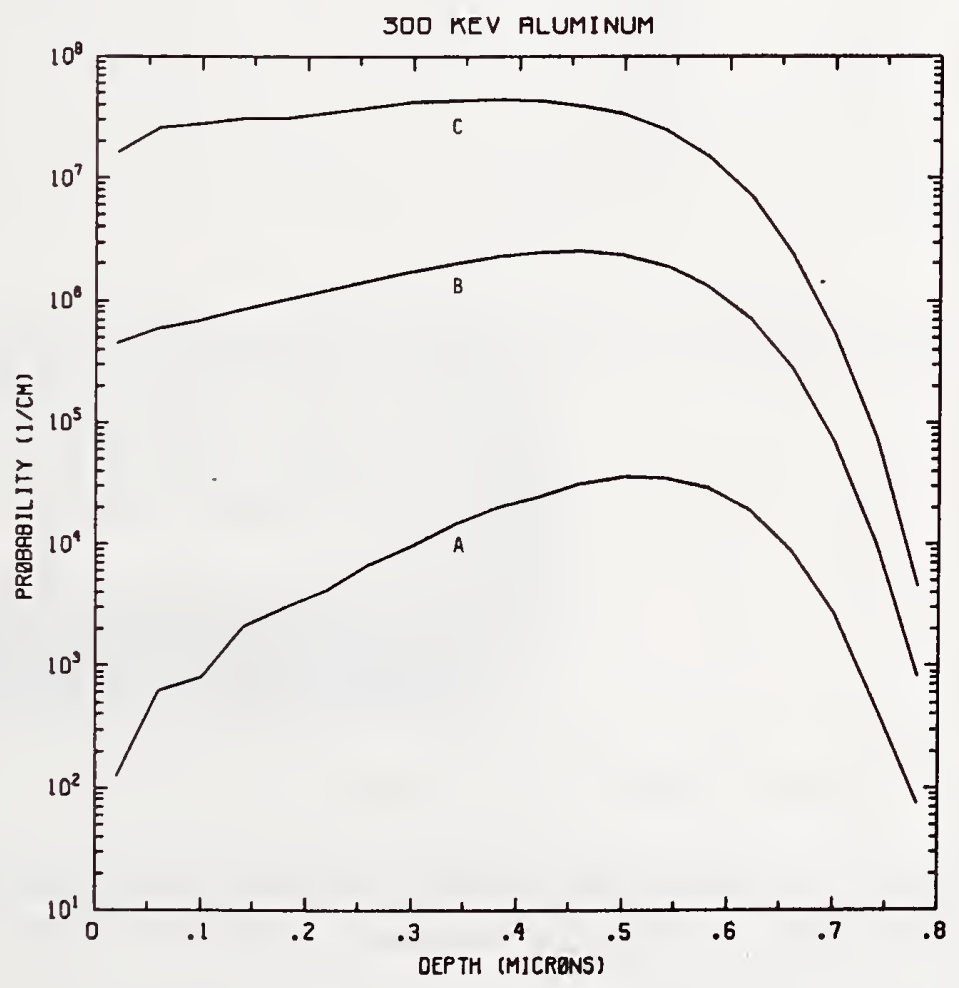

Figure A1300-2 One-dimensional probability distributions for implanted aluminum (A), primary silicon displacements (B), and Frenkel pairs (C) for $300 \mathrm{keV}$ aluminum normally incident on a silicon target. 


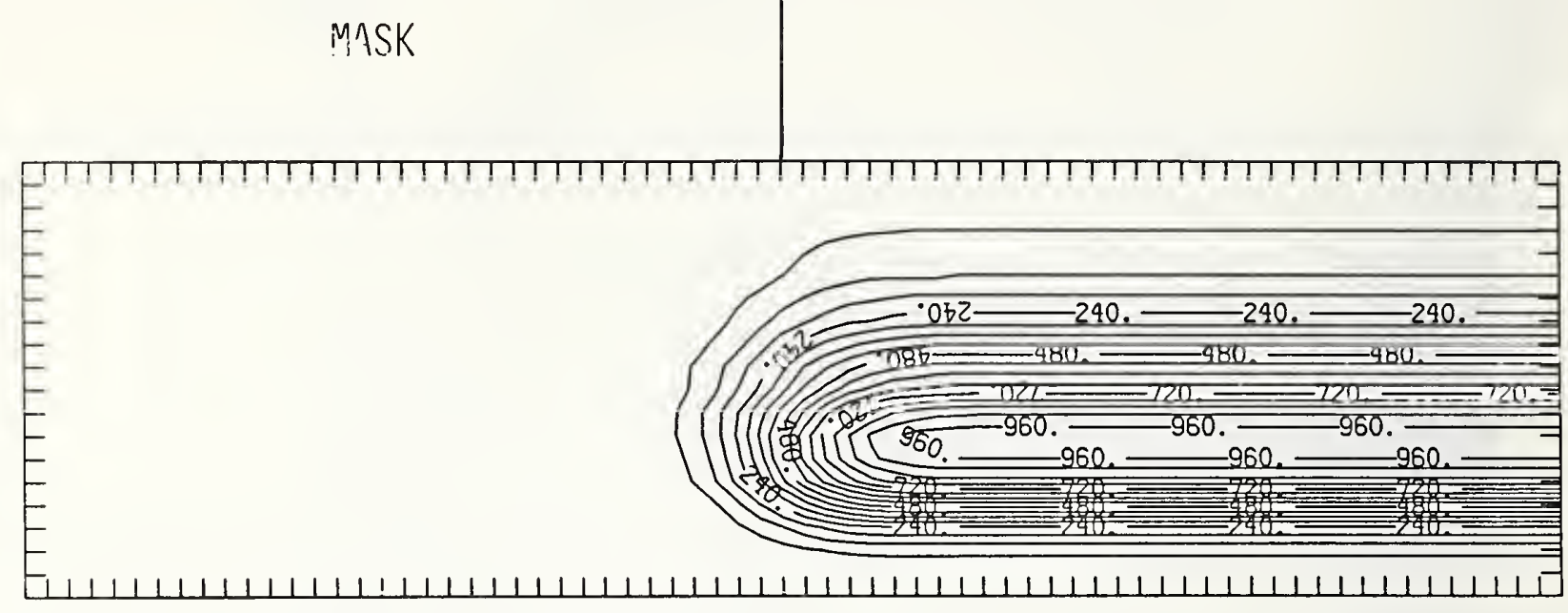

Figure Al300-3 Two-dimensional distribution of $300 \mathrm{keV}$ aluminum implanted past a mask edge. The length increment (distance between tick marks) is $0.04 \mu \mathrm{m}$ and the scale factor is 1000 .

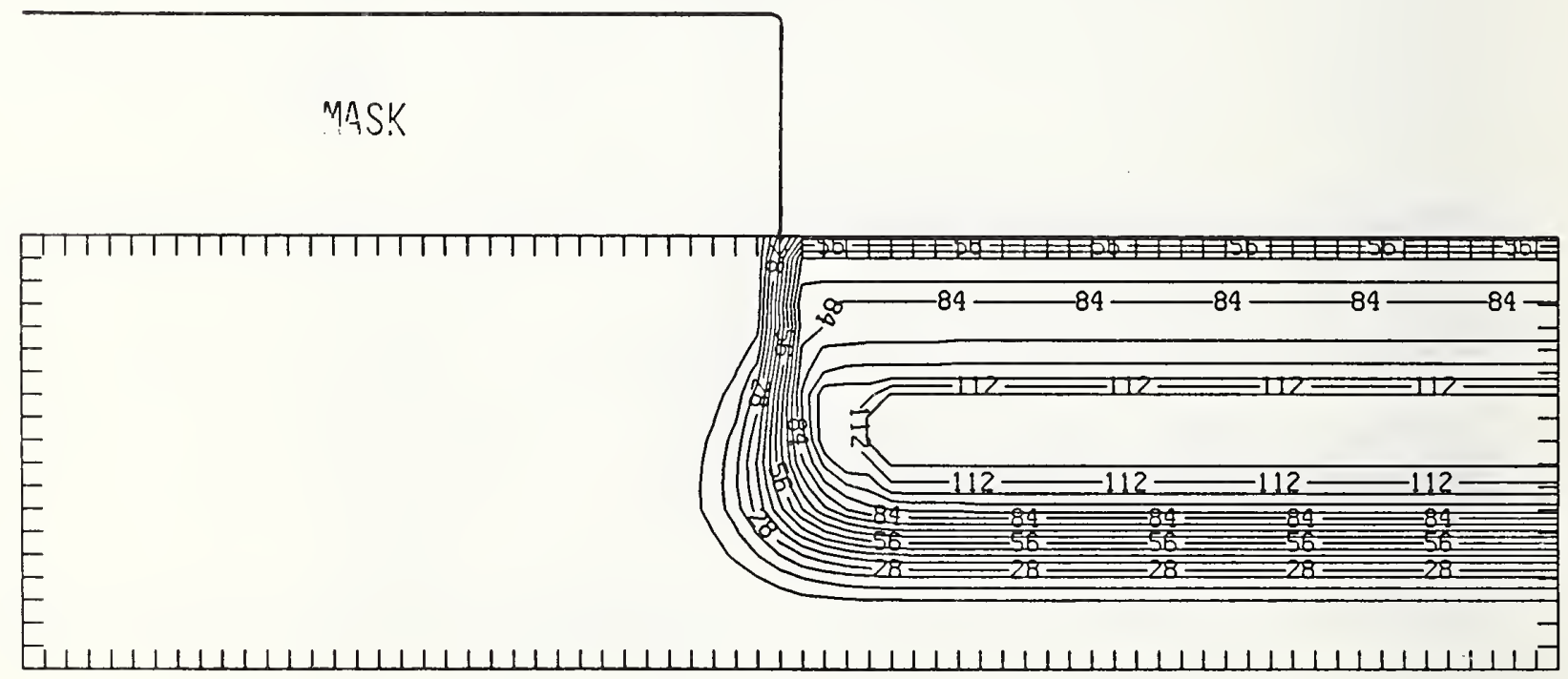

Figure Al300-4 Two-dimensional distribution of Frenkel pairs created by $300 \mathrm{keV}$ aluminum implanted past a mask edge. The length increment (distance between tick marks) is $0.04 \mu \mathrm{m}$ and the scale factor is 0.1 . 


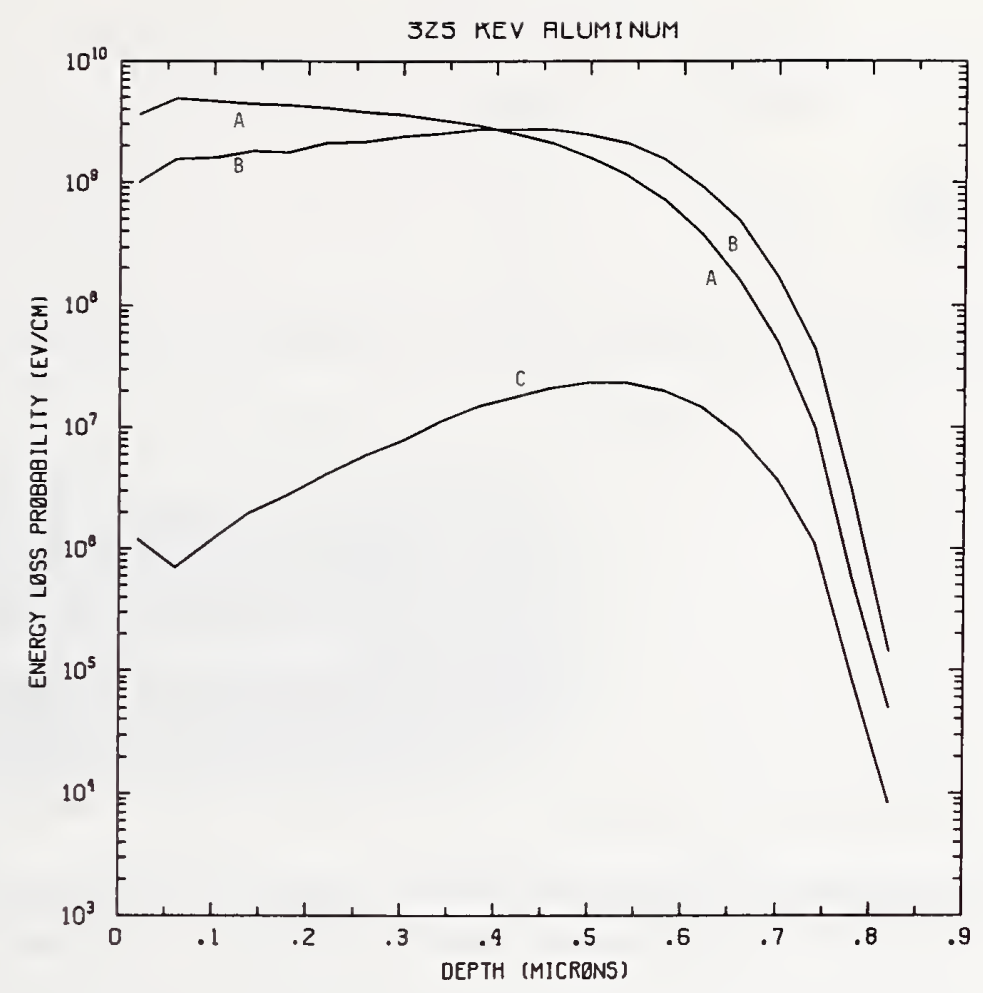

Figure Al325-1 One-dimensional probability distributions for energy loss due to electronic excitation (A), displacement damage(B), and phonon excitation (C) for $325 \mathrm{keV}$ aluminum normally incident on a silicon target.

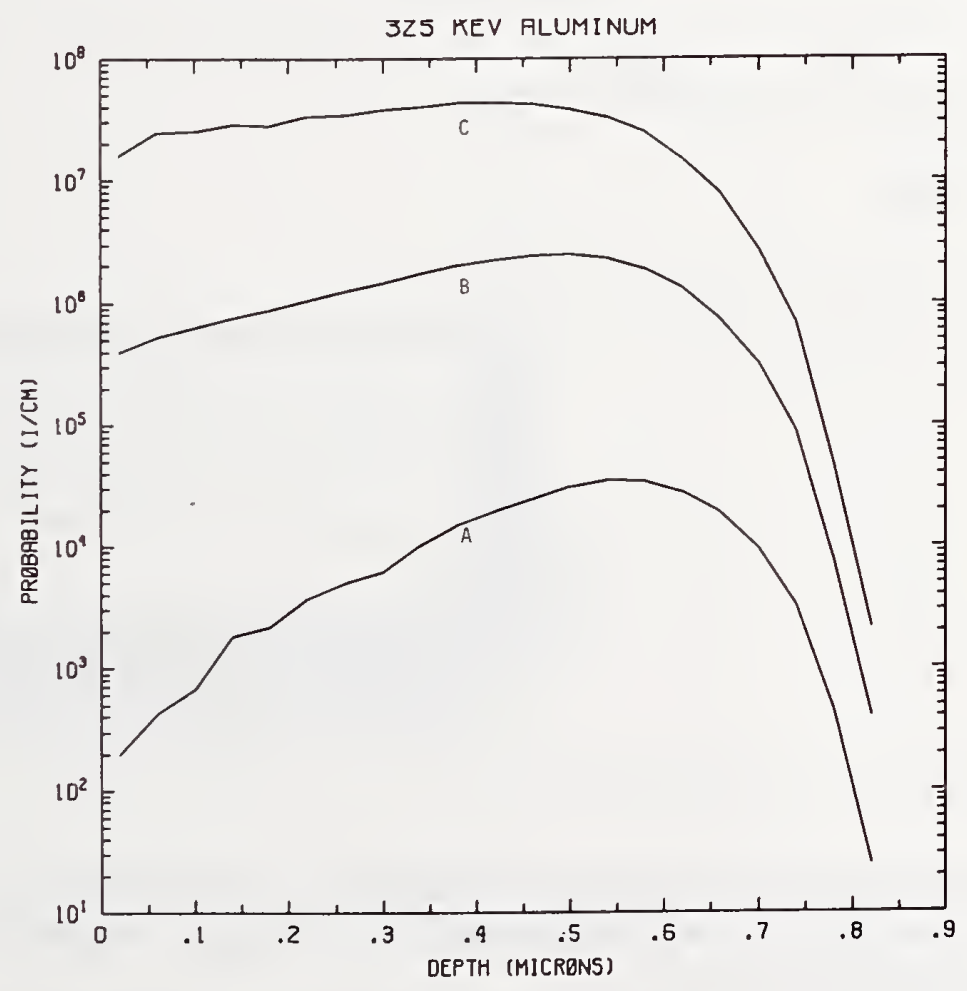

Figure Al325-2 One-dimensional probability distributions for implanted aluminum (A), primary silicon displacements (B), and Frenkel pairs (C) for $325 \mathrm{keV}$ aluminum normally incident on a silicon target. 


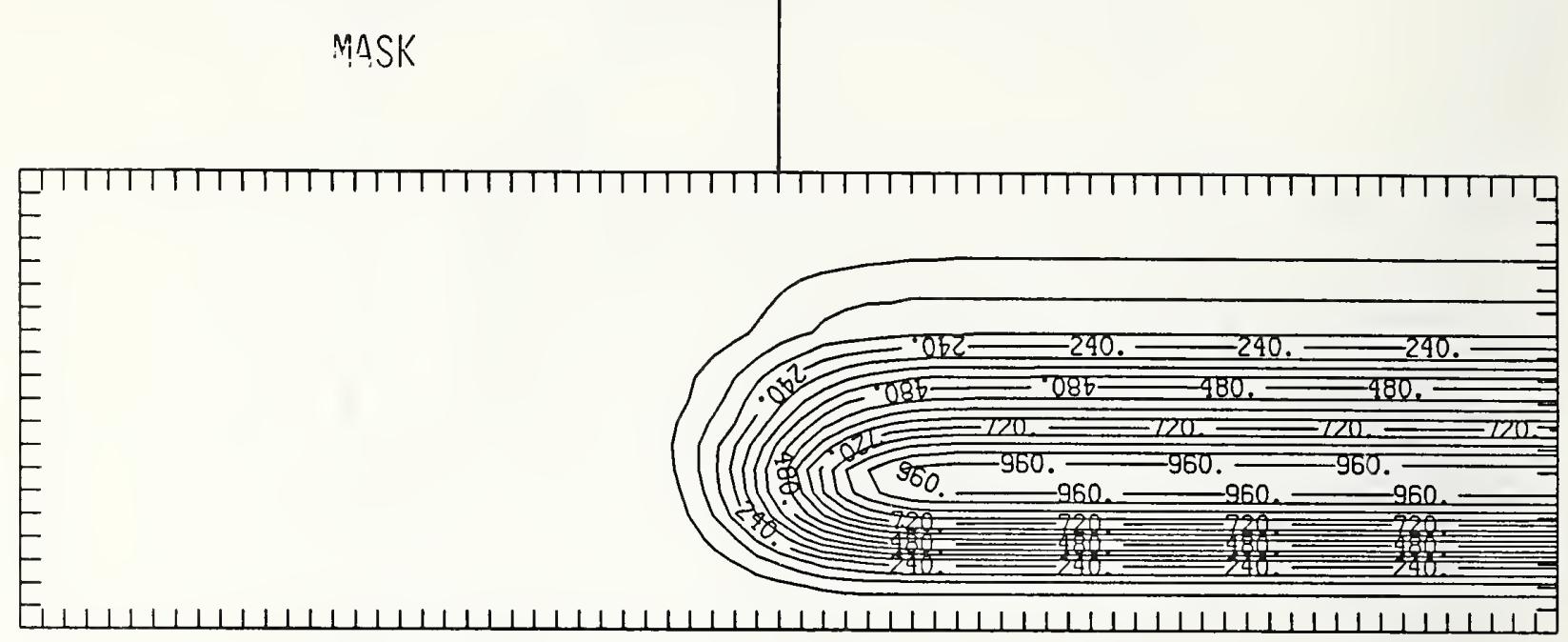

Figure Al325-3 Two-dimensional distribution of $325 \mathrm{keV}$ aluminum implanted past a mask edge. The length increment (distance between tick marks) is $0.04 \mu \mathrm{m}$ and the scale factor is 1000.

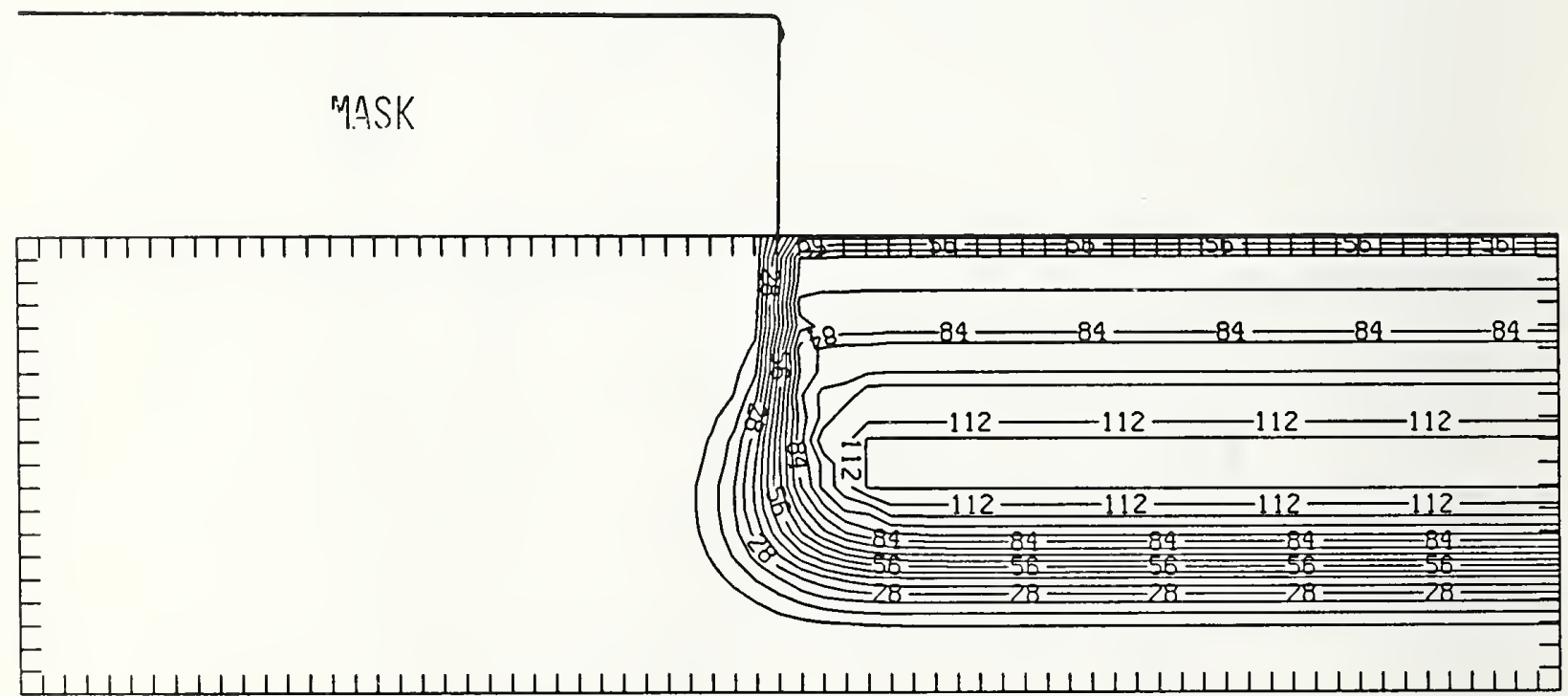

Figure Al325-4 Two-dimensional distribution of Frenkel pairs created by $325 \mathrm{keV}$ aluminum implanted past a mask edge. The length increment (distance between tick marks) is $0.04 \mu \mathrm{m}$ and the scale factor is 0.1 . 


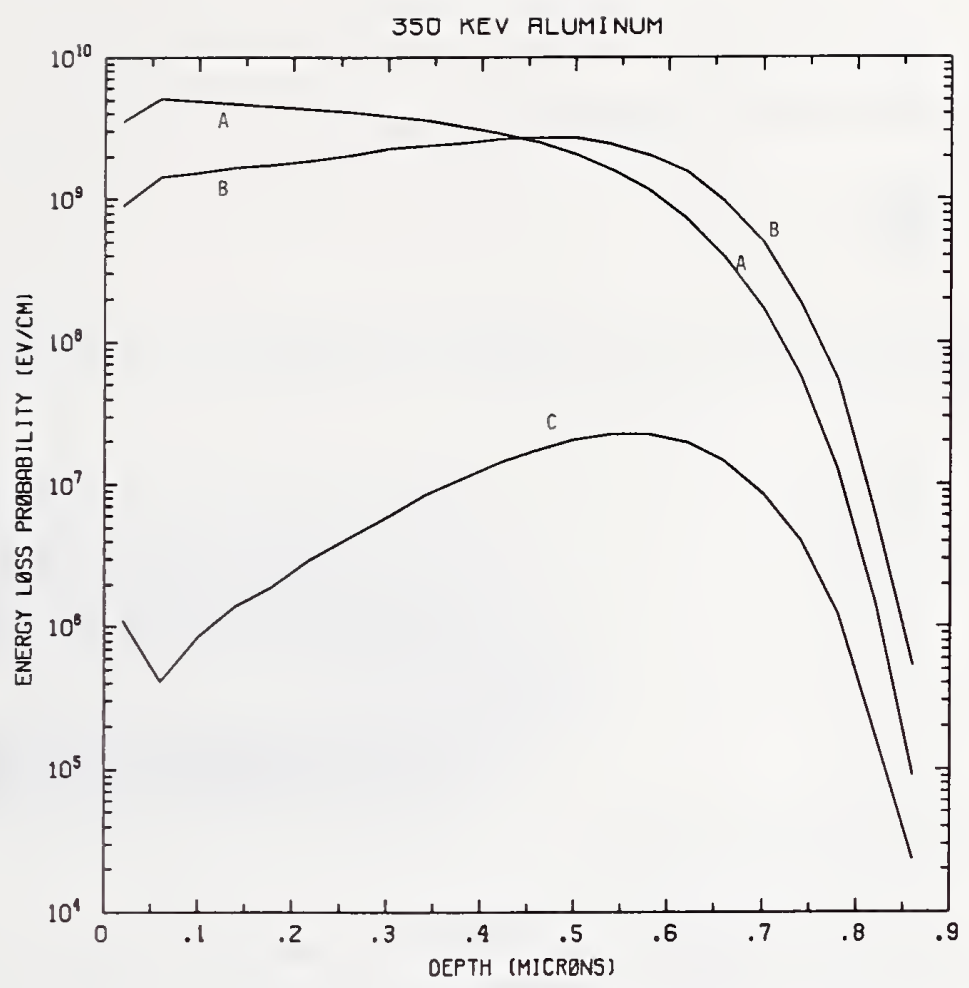

Figure Al350-1 One-dimensional probability distributions for energy loss due to electronic excitation (A), displacement damage(B), and phonon excitation (C) for $350 \mathrm{keV}$ aluminum normally incident on a silicon target.

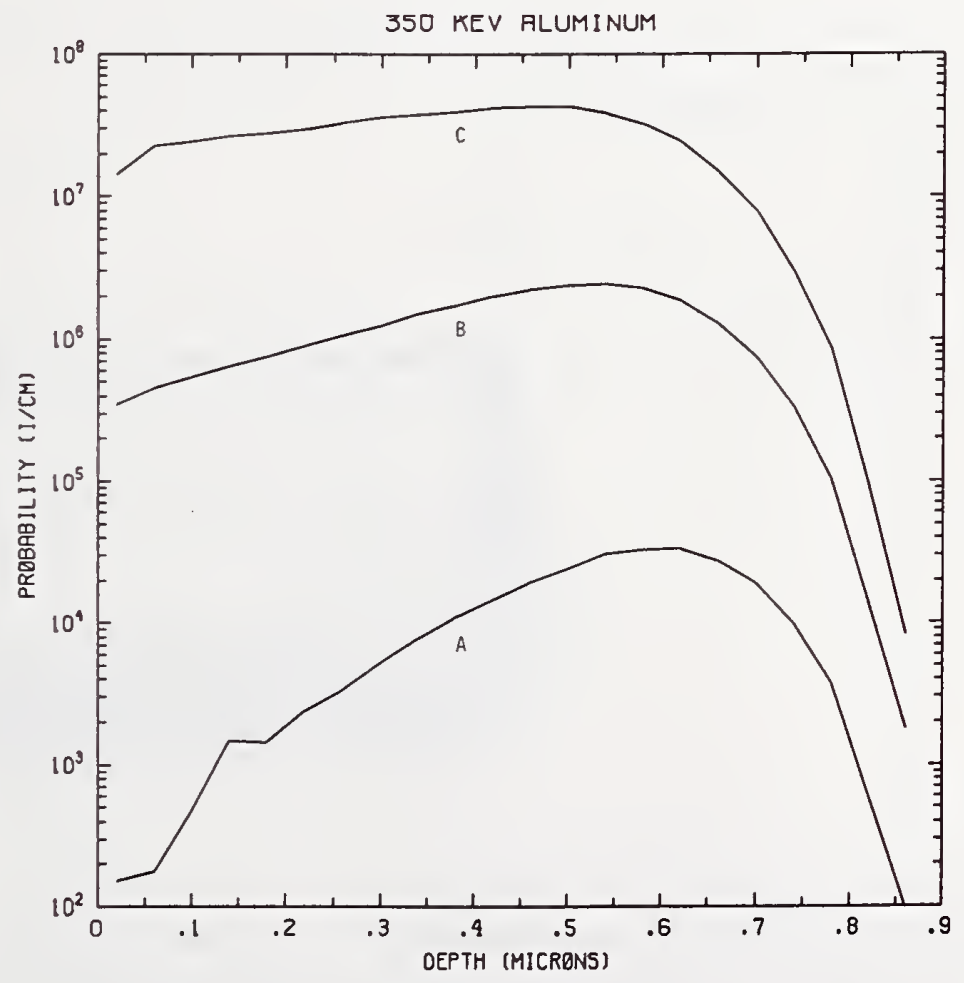

Figure Al350-2 One-dimensional probability distributions for implanted aluminum (A), primary silicon displacements (B), and Frenkel pairs (C) for $350 \mathrm{keV}$ aluminum normally incident on a silicon target. 


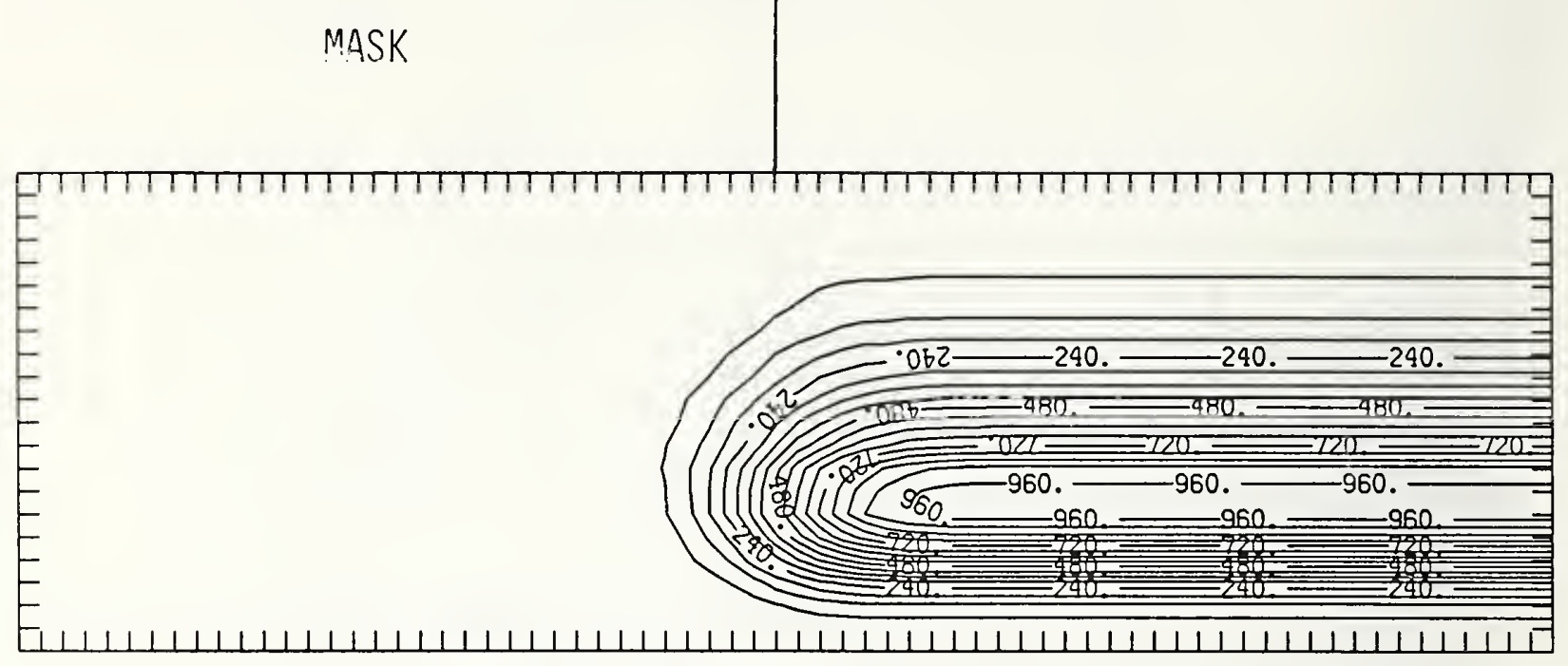

Figure Al350-3 Two-dimensional distribution of $350 \mathrm{keV}$ aluminum implanted past a mask edge. The length increment (distance between tick marks) is $0.04 \mu \mathrm{m}$ and the scale factor is 1000 .

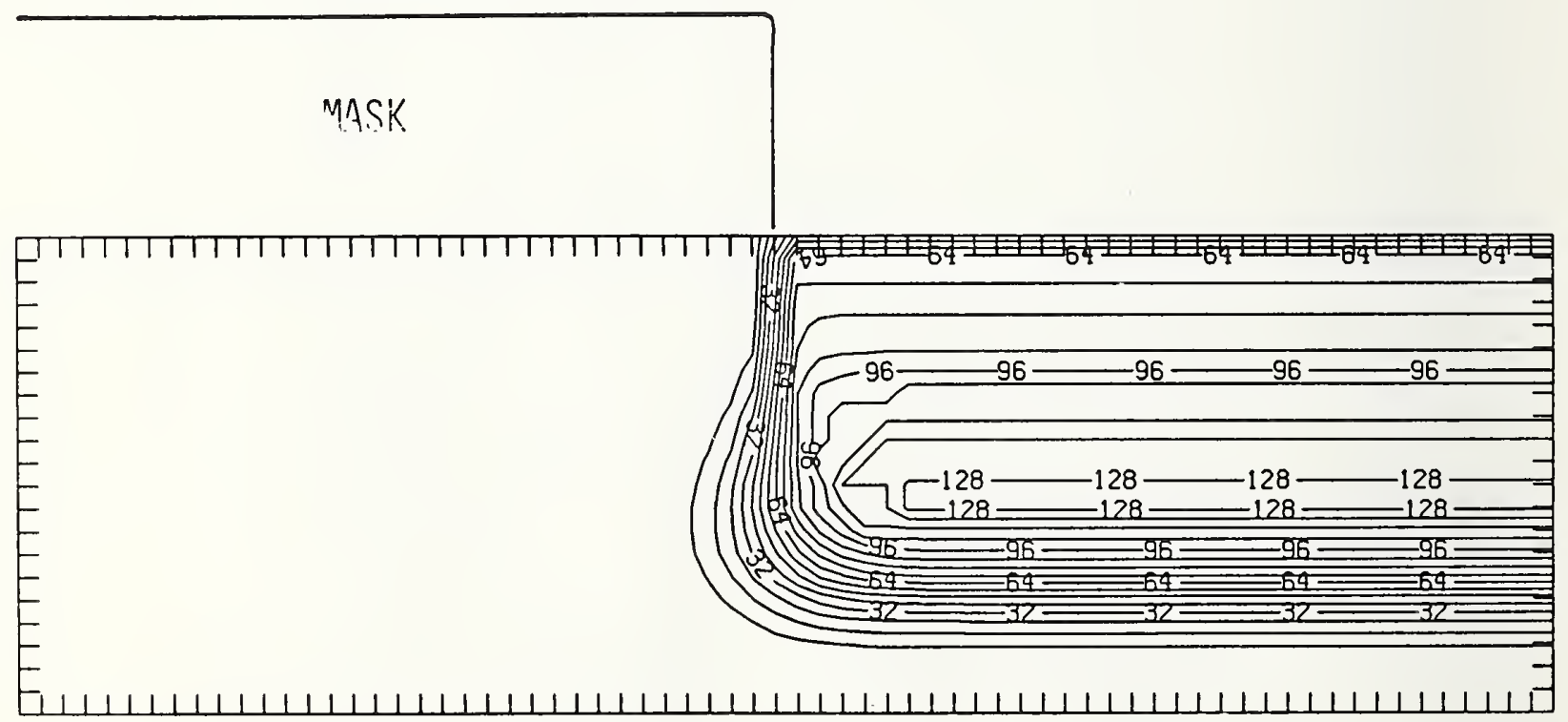

Figure A1350-4 Two-dimensional distribution of Frenkel pairs created by $350 \mathrm{keV}$ aluminum implanted past a mask edge. The length increment (distance between tick marks) is $0.04 \mu \mathrm{m}$ and the scale factor is 0.1 . 


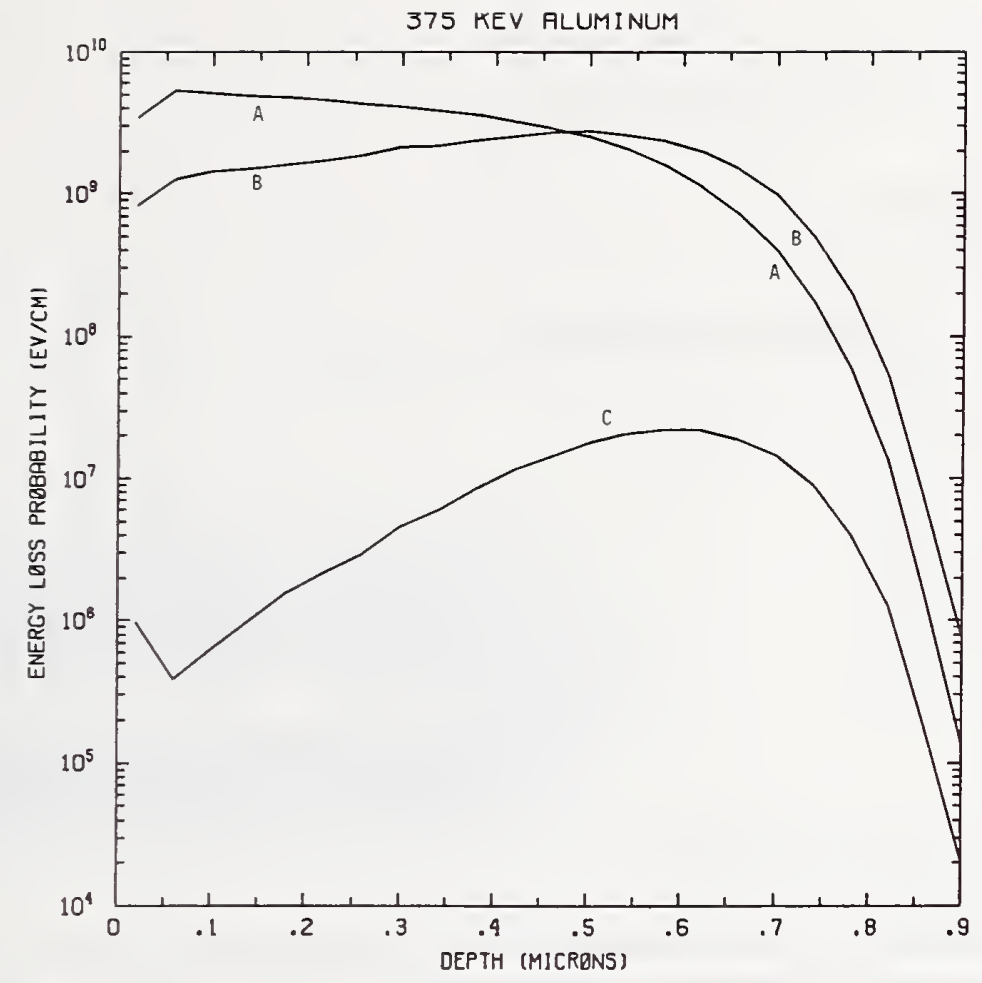

Figure Al375-1 One-dimensional probability distributions for energy loss due to electronic excitation (A), displacement damage(B), and phonon excitation (C) for $375 \mathrm{keV}$ aluminum normally incident on a silicon target.

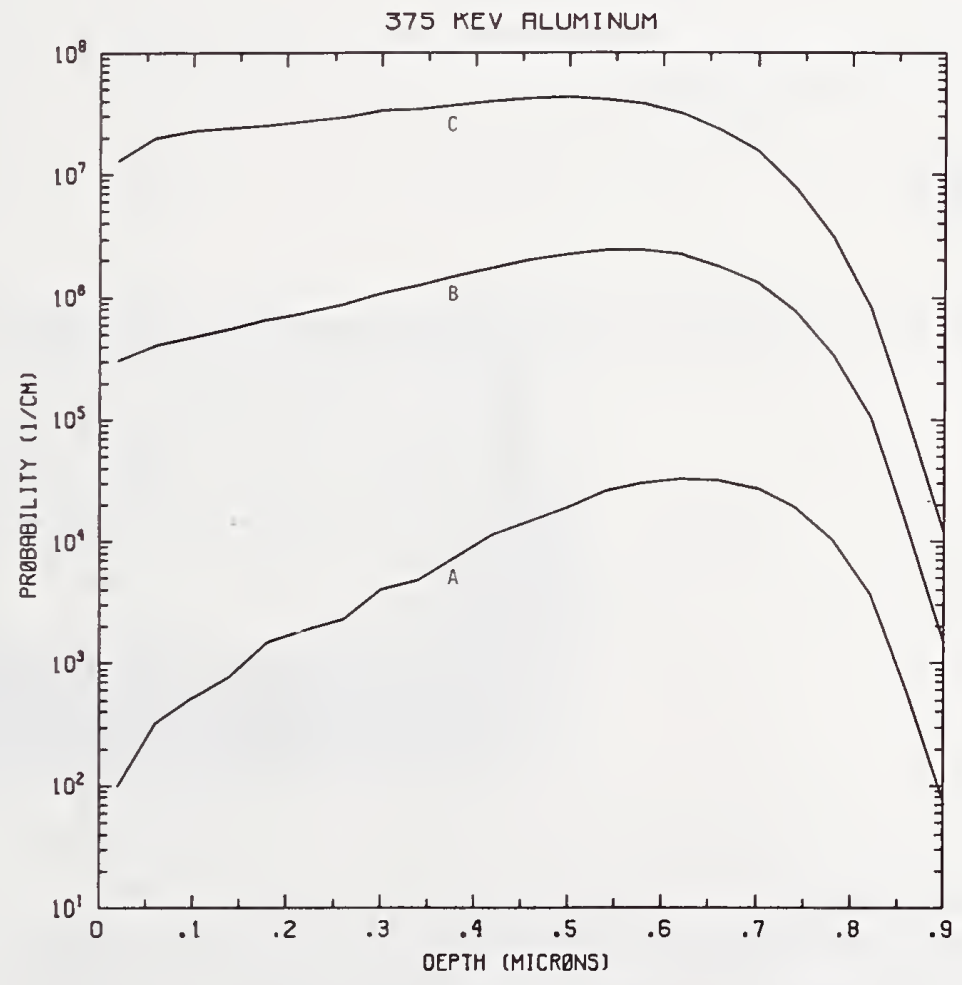

Figure Al375-2 One-dimensional probability distributions for implanted aluminum (A), primary silicon displacements (B), and Frenkel pairs (C) for $375 \mathrm{keV}$ aluminum normally incident on a silicon target. 


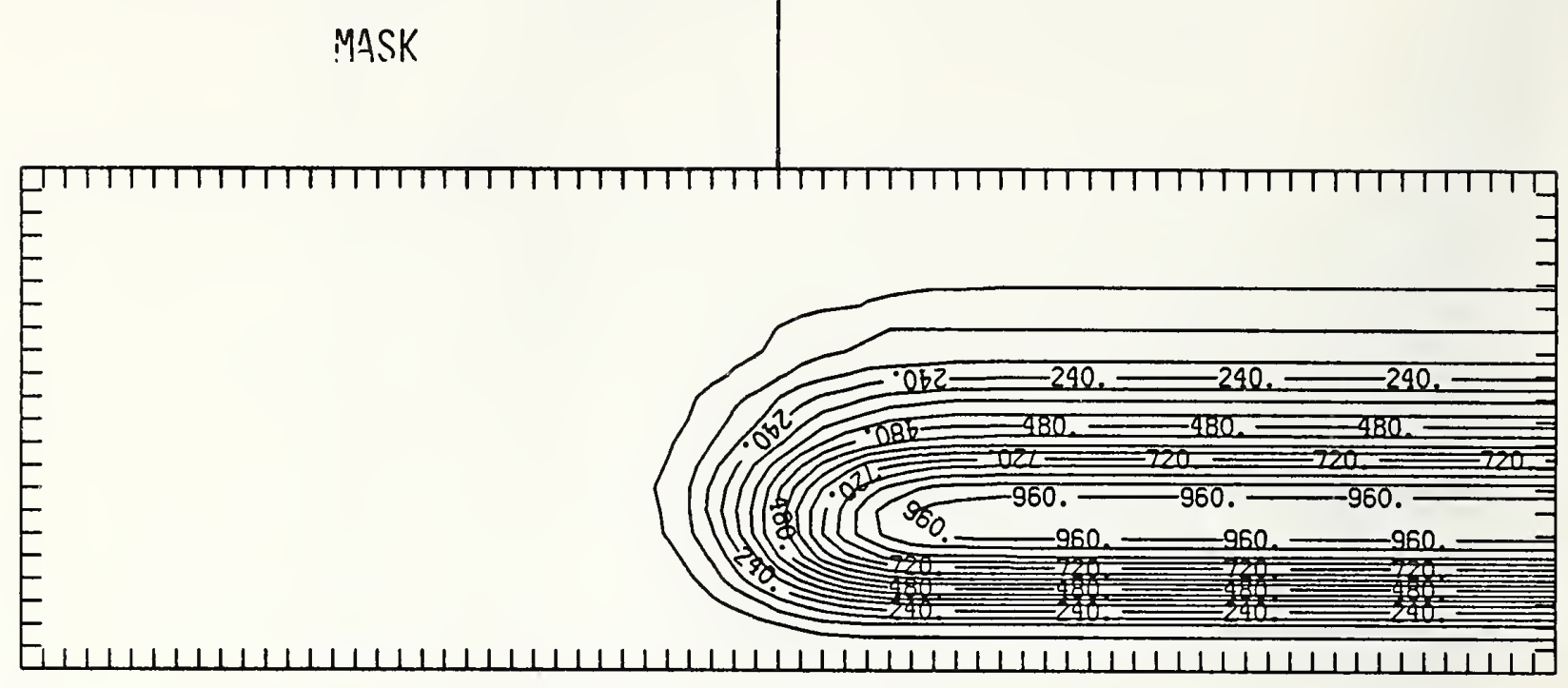

Figure Al375-3 Two-dimensional distribution of $375 \mathrm{keV}$ aluminum implanted past a mask edge. The length increment (distance between tick marks) is $0.04 \mu \mathrm{m}$ and the scale factor is 1000 .

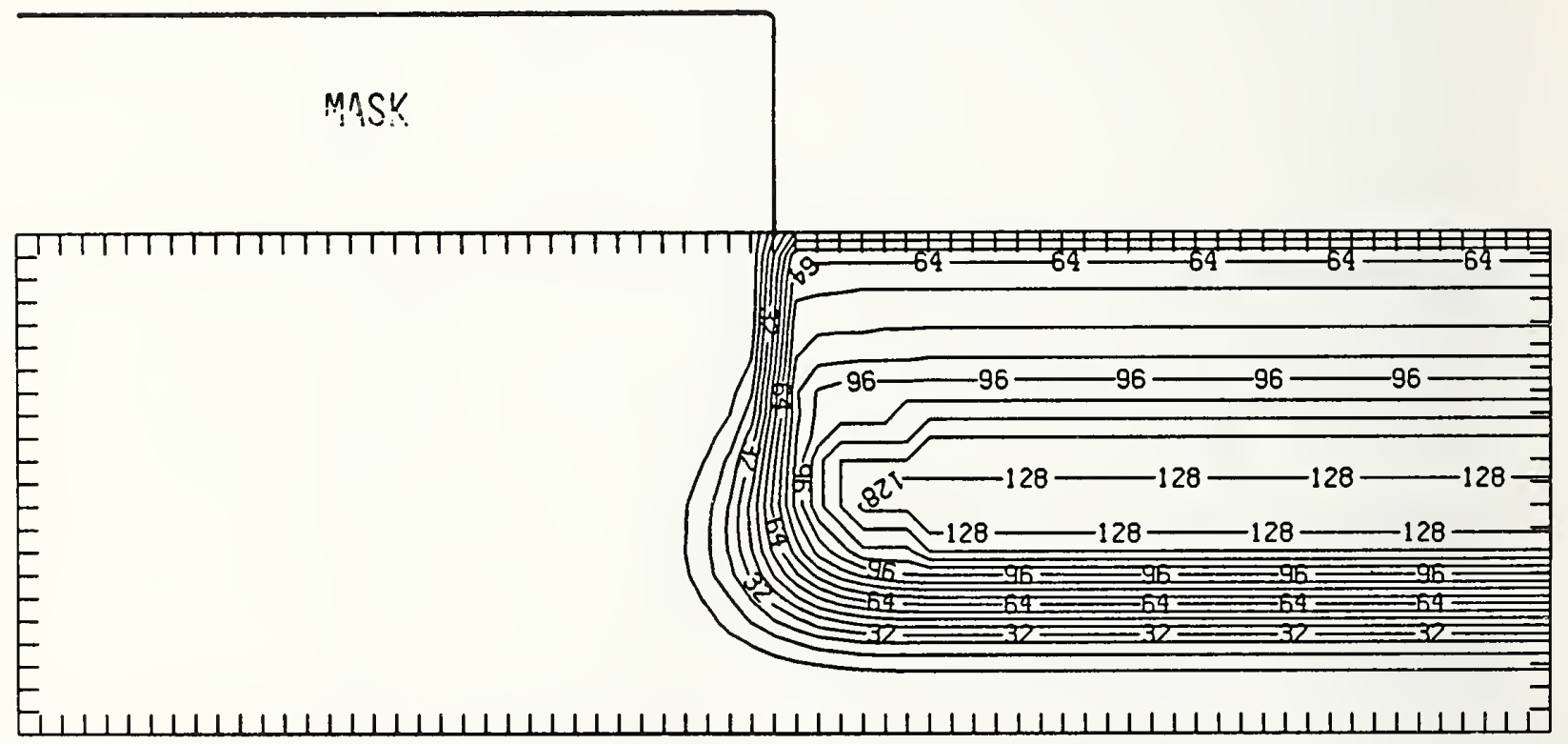

Figure Al375-4 Two-dimensional distribution of Frenkel pairs created by $375 \mathrm{keV}$ aluminum implanted past a mask edge. The length increment (distance between tick marks) is $0.04 \mu \mathrm{m}$ and the scale factor is 0.1 . 


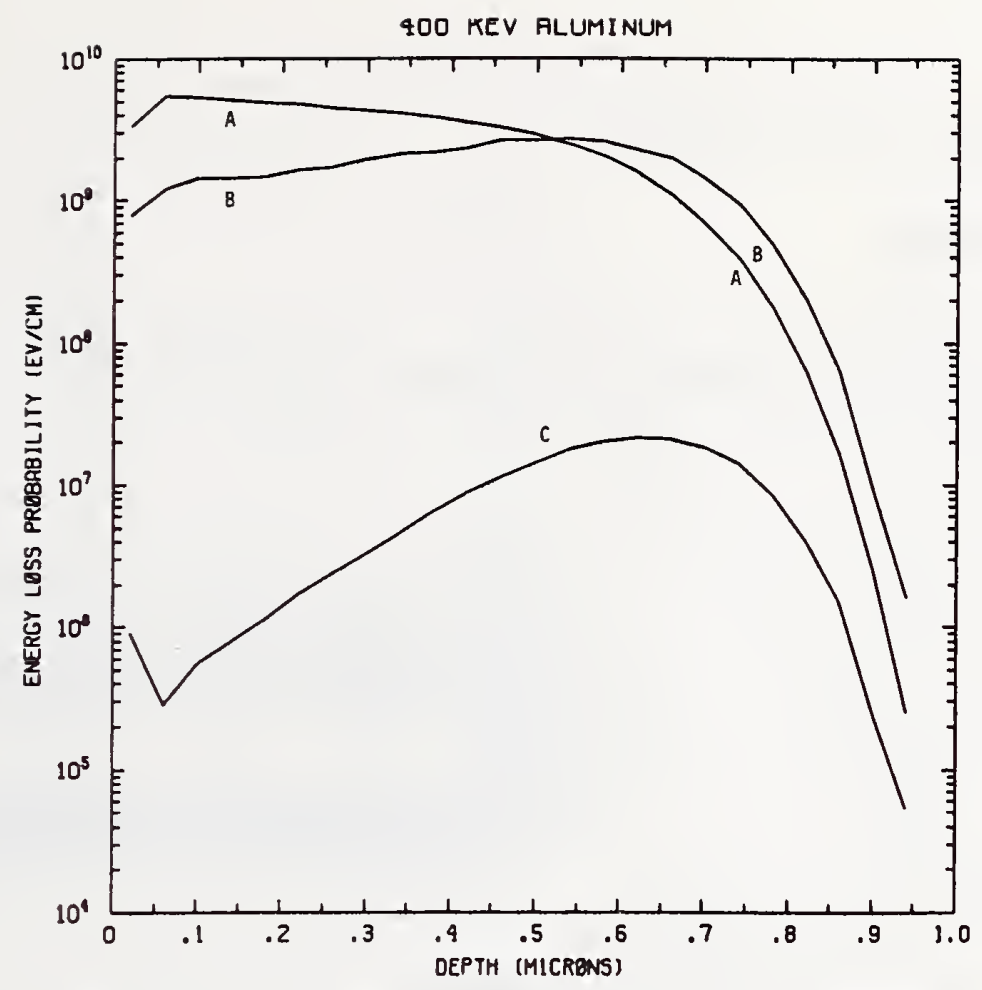

Figure Al400-1 One-dimensional probability distributions for energy loss due to electronic excitation (A), displacement damage(B), and phonon excitation (C) for $400 \mathrm{keV}$ aluminum normally incident on a silicon target.

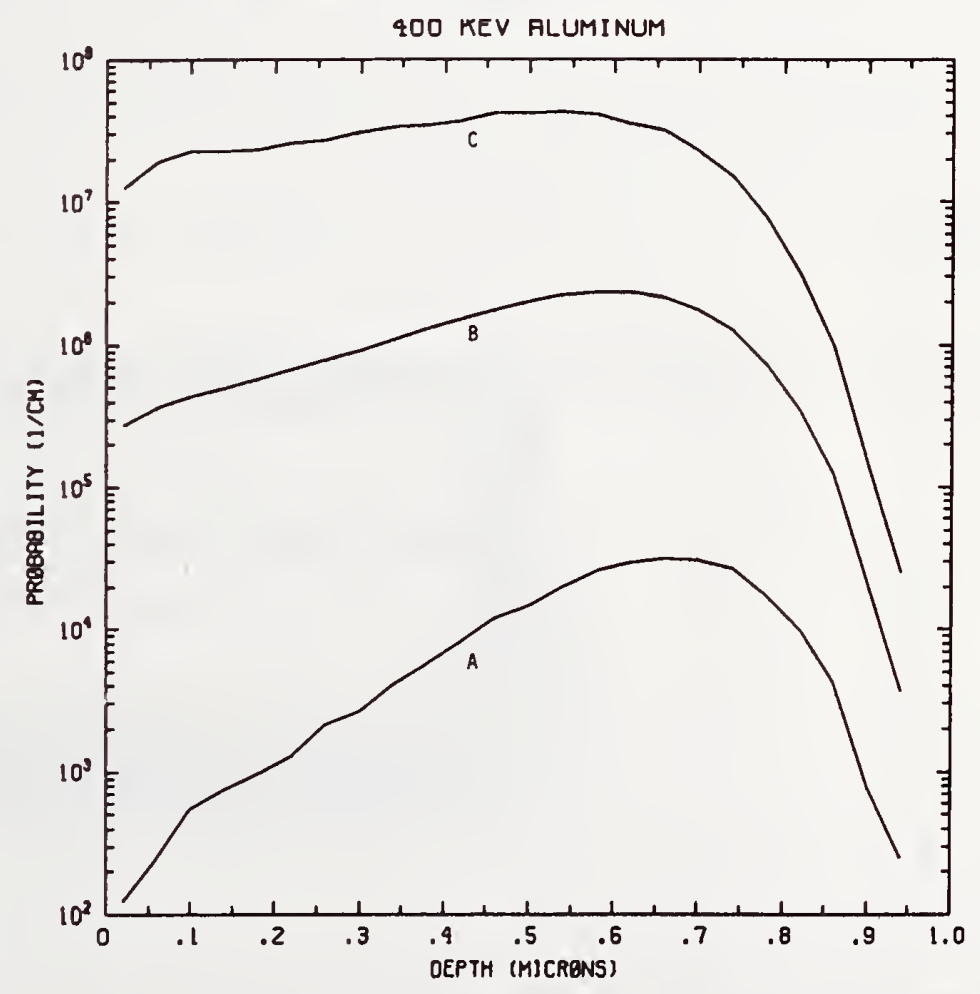

Figure Al400-2 One-dimensional probability distributions for implanted aluminum (A), primary silicon displacements (B), and Frenkel pairs (C) for $400 \mathrm{keV}$ aluminum normally incident on a silicon target. 


\section{MASK}

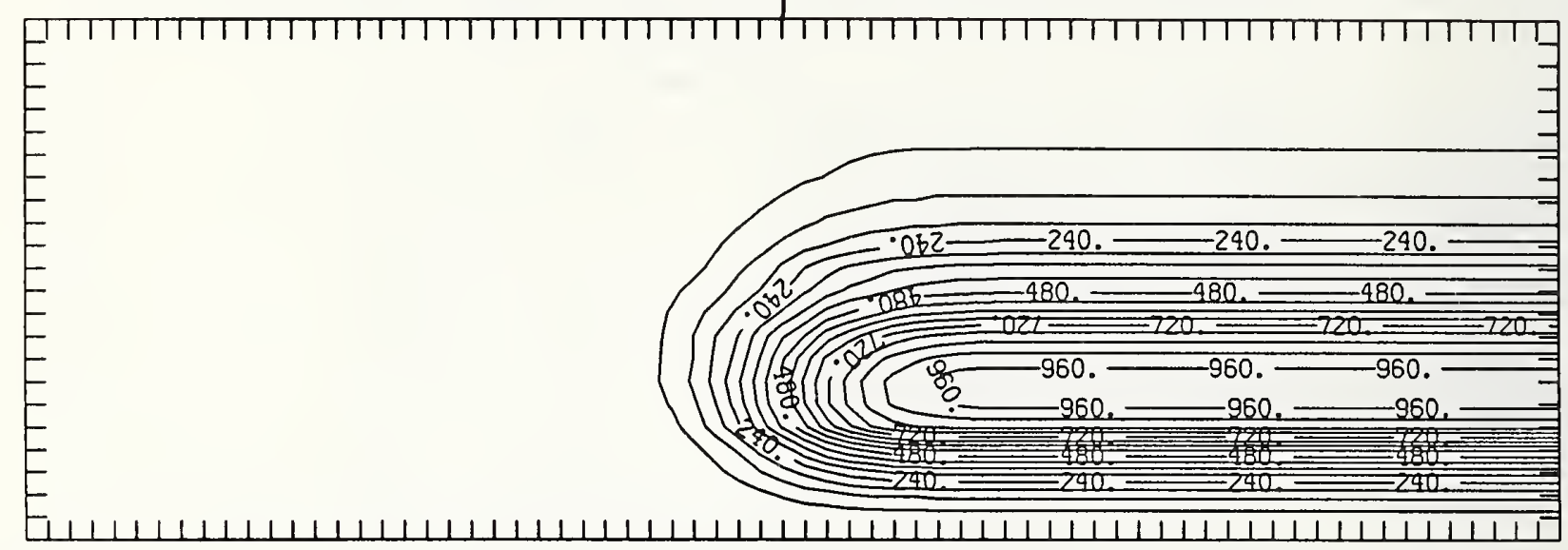

Figure Al400-3 Two-dimensional distribution of $400 \mathrm{keV}$ aluminum implanted past a mask edge. The length increment (distance between tick marks) is $0.04 \mu \mathrm{m}$ and the scale factor is 1000.

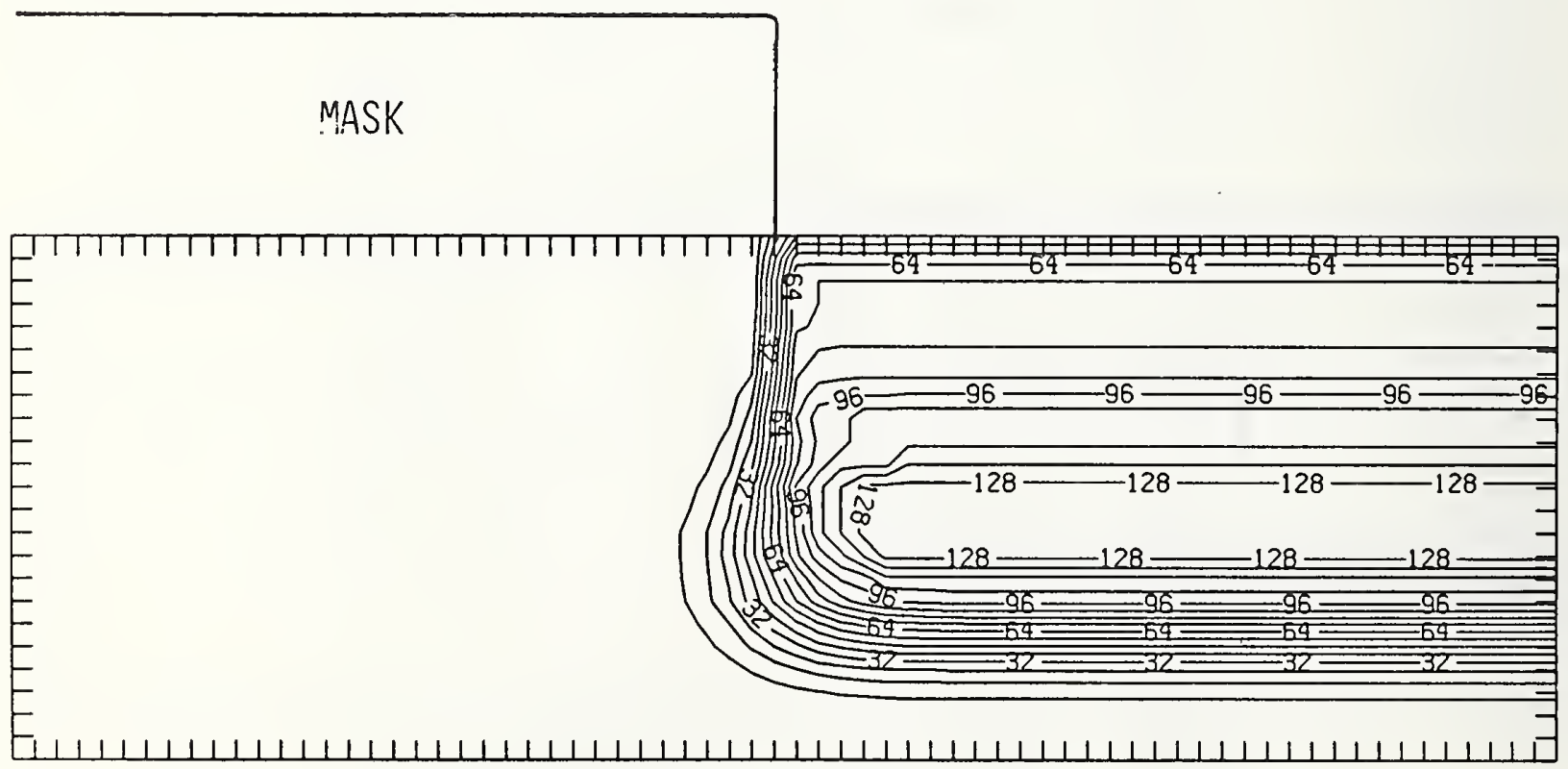

Figure A1400-4 Two-dimensional distribution of Frenkel pairs created by $400 \mathrm{keV}$ aluminum implanted past a mask edge. The length increment (distance between tick marks) is $0.04 \mu \mathrm{m}$ and the scale factor is 0.1 . 


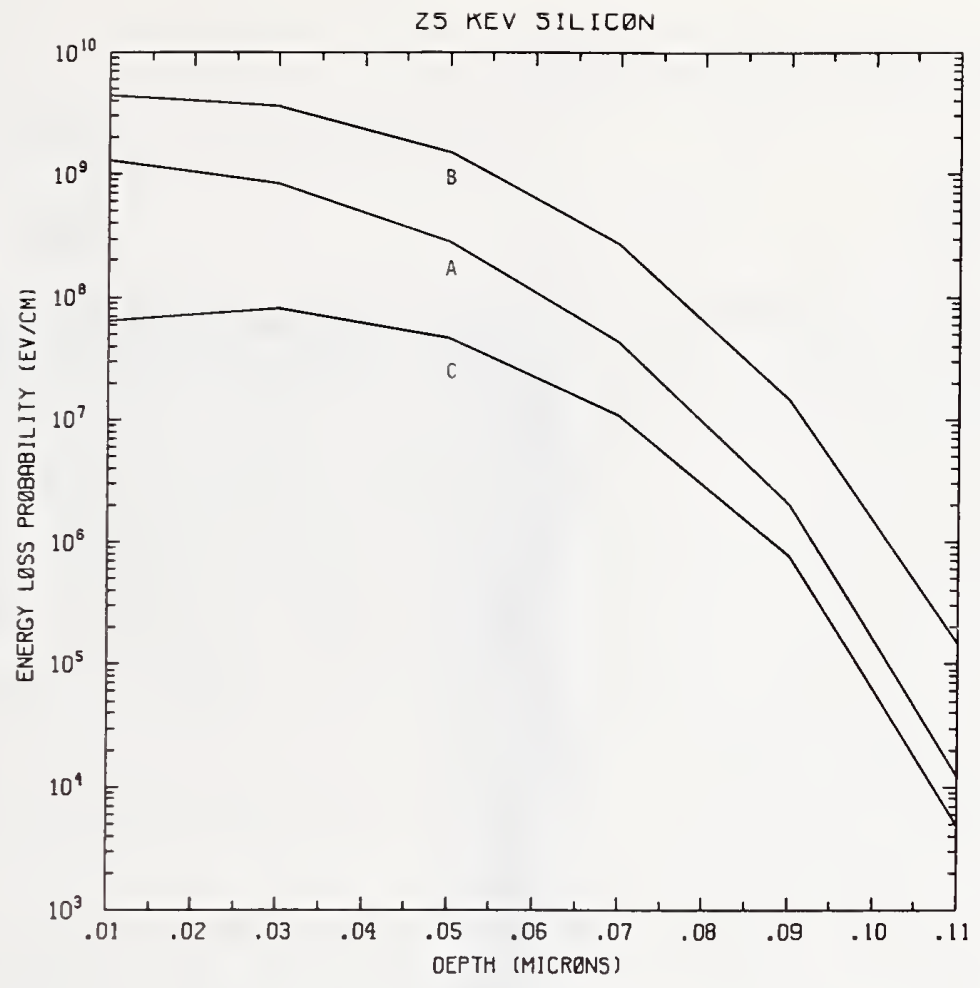

Figure Si25-1 One-dimensional probability distributions for energy loss due to electronic excitation (A), displacement damage(B), and phonon excitation (C) for $25 \mathrm{keV}$ silicon normally incident on a silicon target.

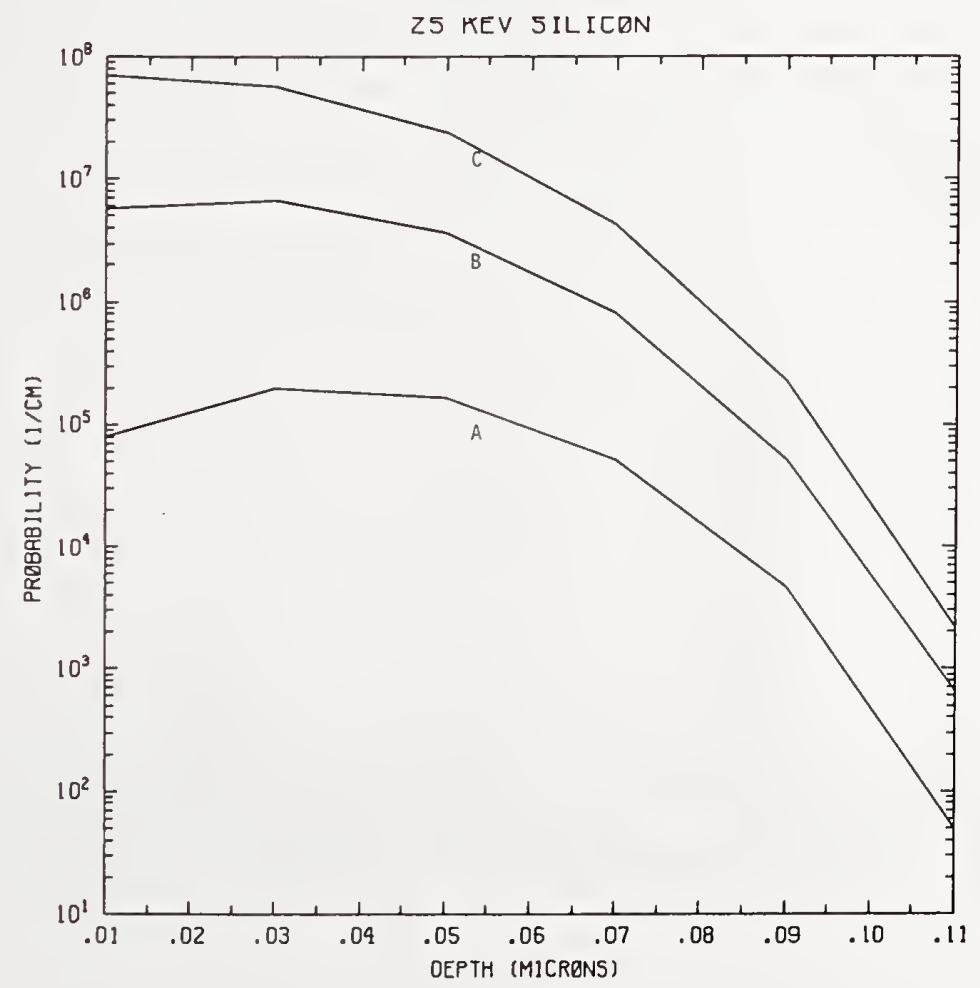

Figure Si25-2 One-dimensional probability distributions for implanted silicon (A), primary silicon displacements (B), and Frenkel pairs (C) for $25 \mathrm{keV}$ silicon normally incident on a silicon target. 


\section{MASK}

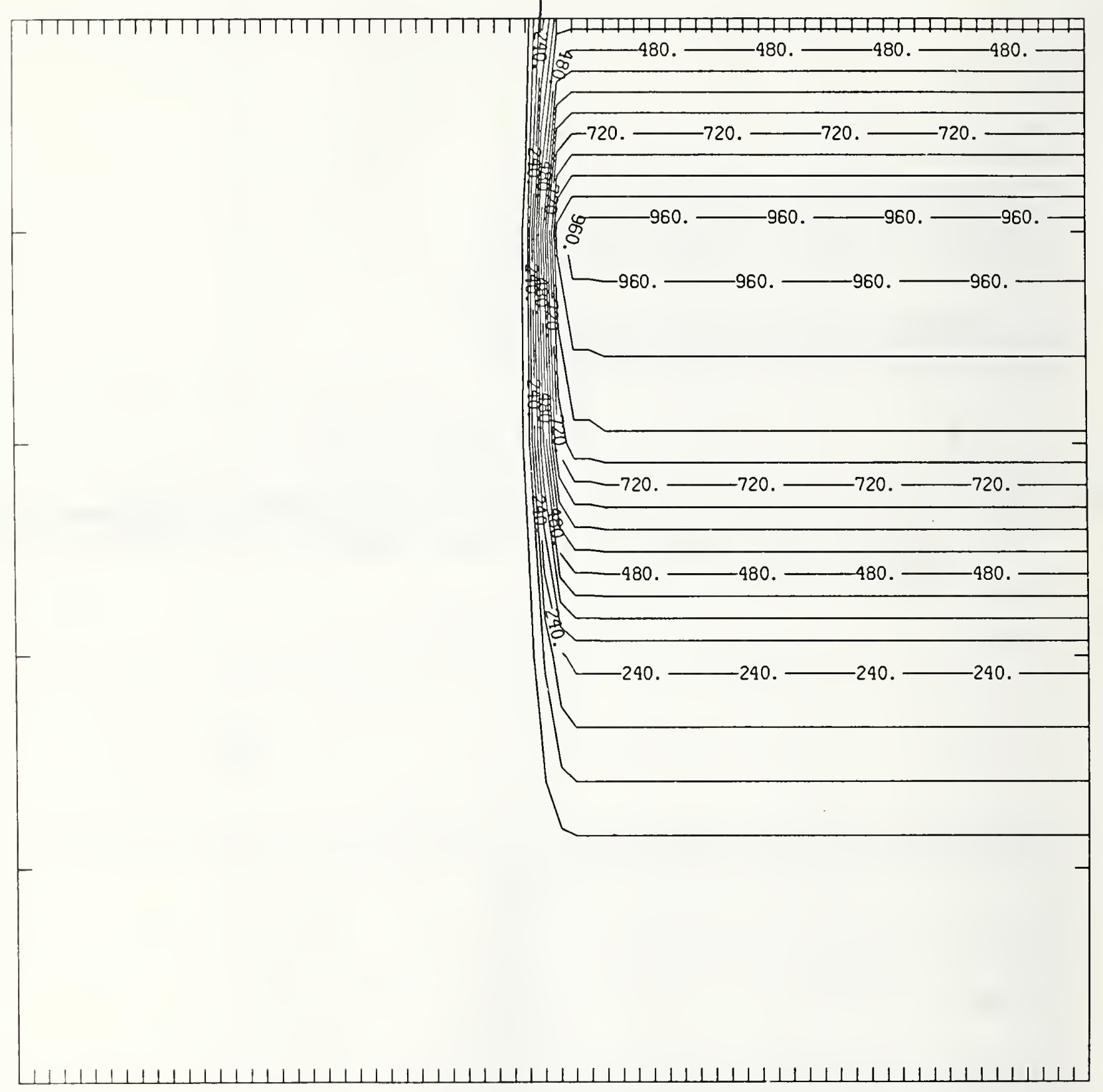

Figure Si25-3 Two-dimensional distribution of $25 \mathrm{keV}$ silicon implanted past a mask edge. The length increment (distance between tick marks) is $0.02 \mu \mathrm{m}$ and the scale factor is 1000 . 


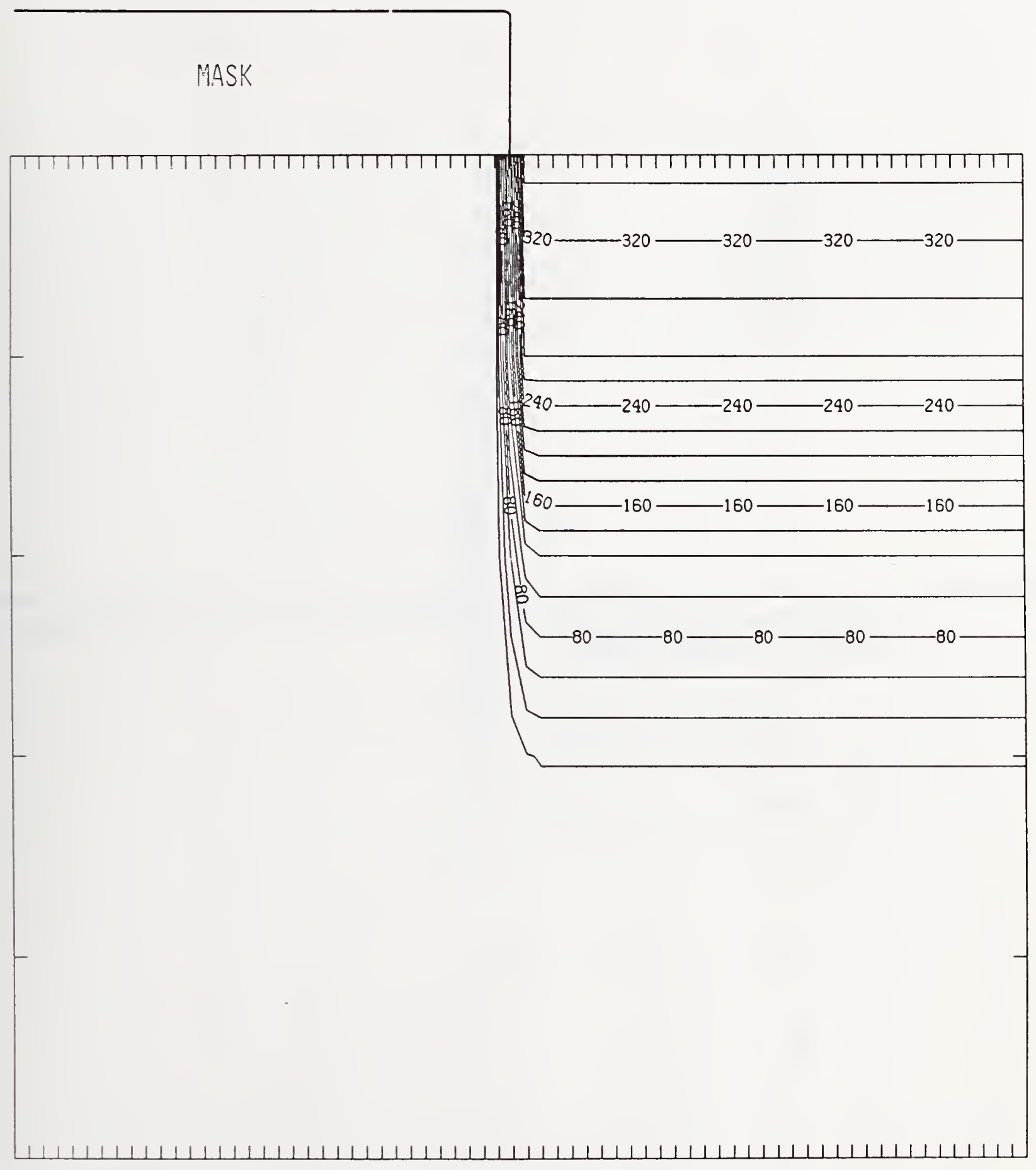

Figure Si25-4 Two-dimensional distribution of Frenkel pairs created by $25 \mathrm{keV}$ silicon implanted past a mask edge. The length increment (distance between tick marks) is $0.02 \mu \mathrm{m}$ and the scale factor is 1. 


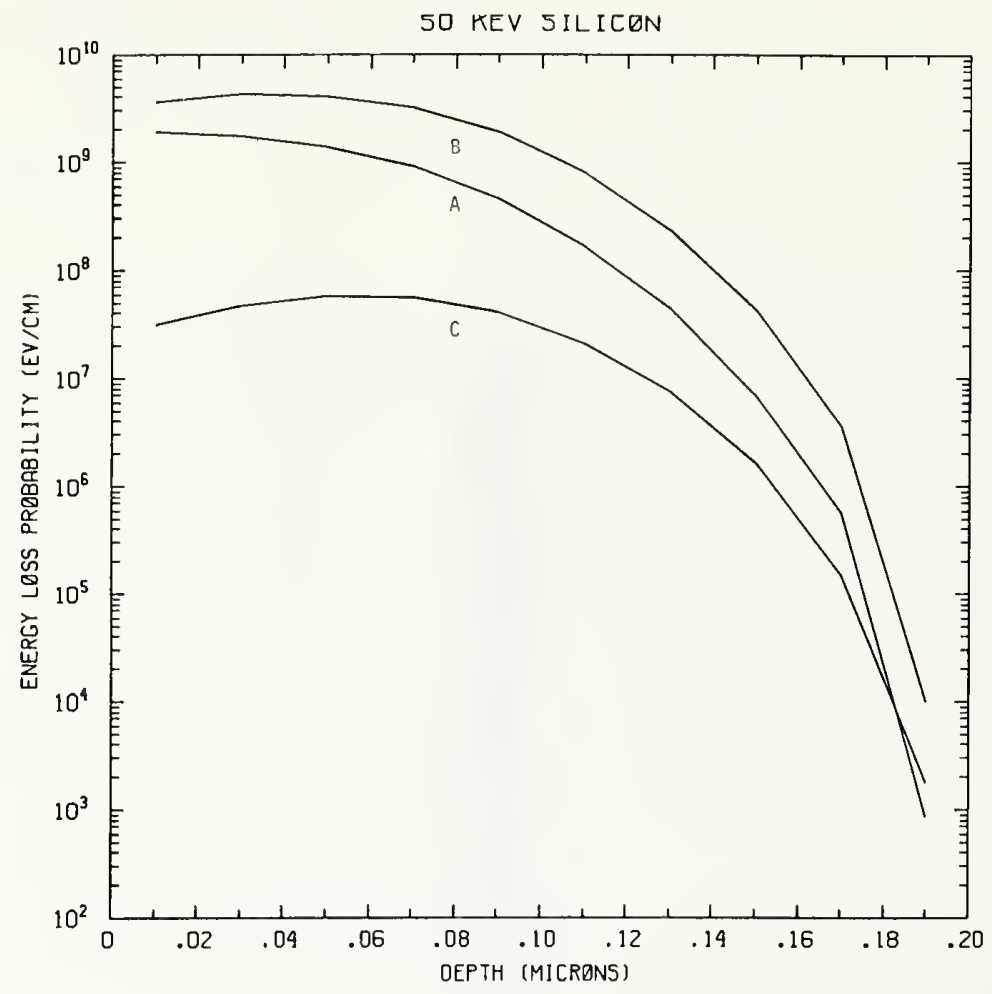

Figure Si50-1 One-dimensional probability distributions for energy loss due to electronic excitation (A), displacement damage(B), and phonon excitation (C) for $50 \mathrm{keV}$ silicon normally incident on a silicon target.

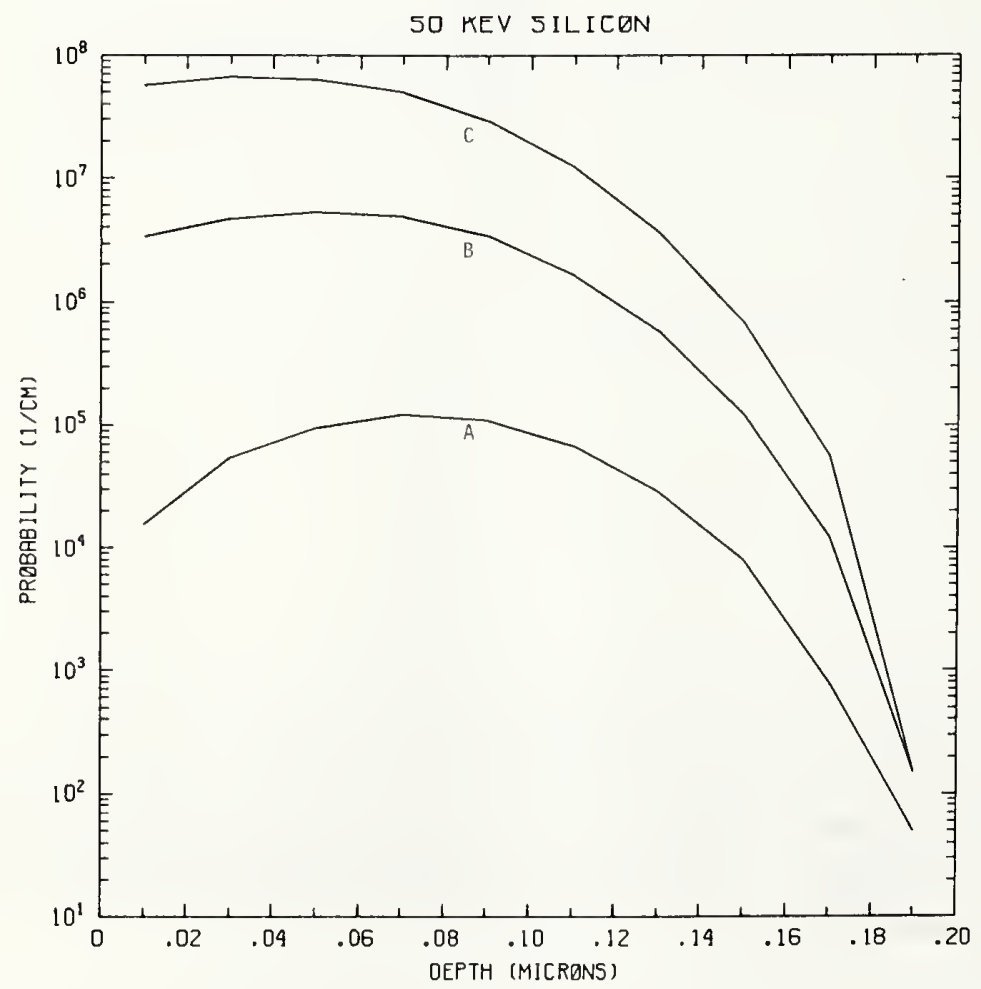

Figure Si50-2 One-dimensional probability distributions for implanted silicon (A), primary silicon displacements (B), and Frenkel pairs (C) for $50 \mathrm{keV}$ silicon normally incident on a silicon target. 
MASK

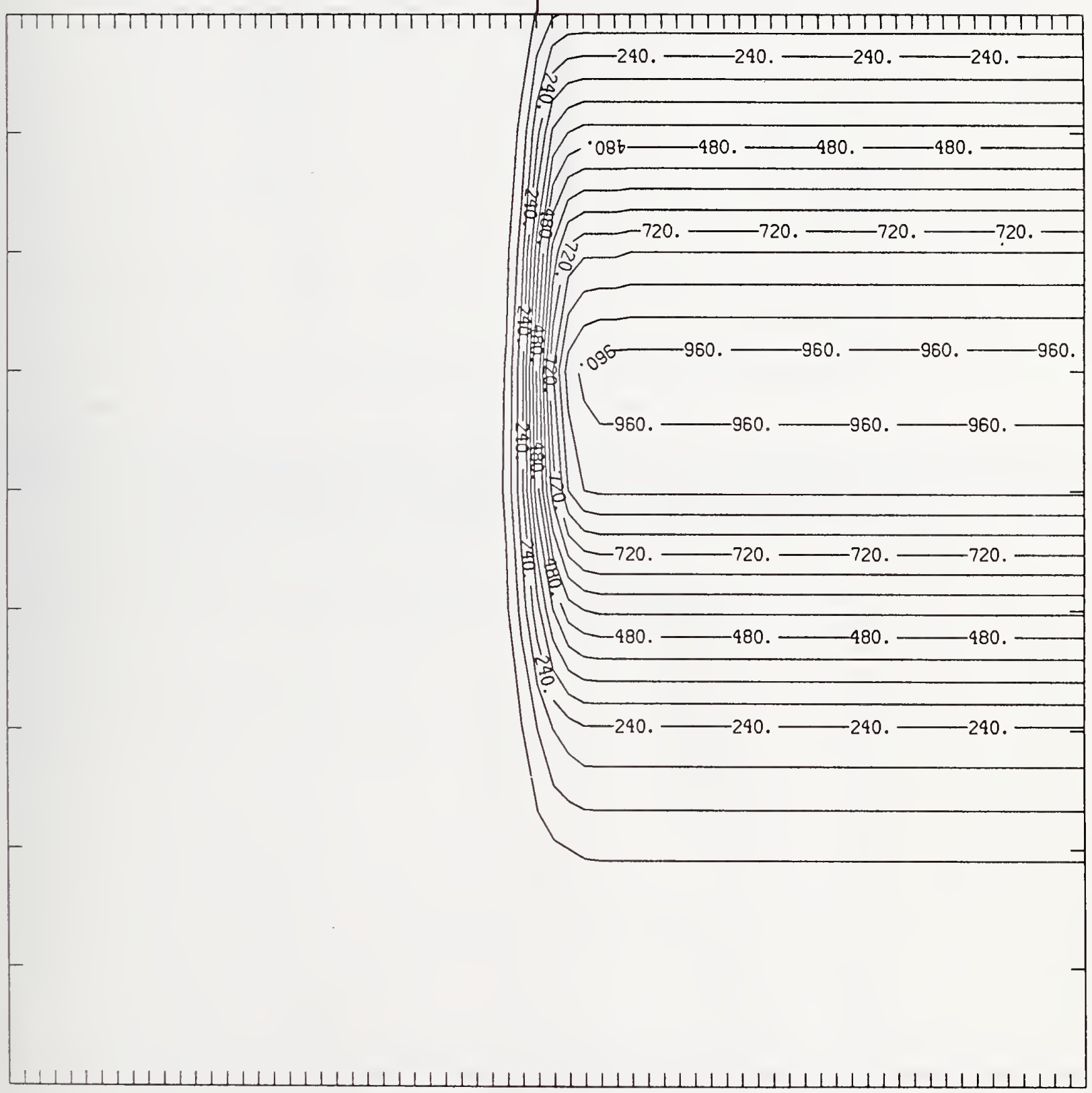

Figure Si50-3 Two-dimensional distribution of $50 \mathrm{keV}$ silicon implanted past a mask edge. The length increment (distance between tick marks) is $0.02 \mu \mathrm{m}$ and the scale factor is 1000 . 


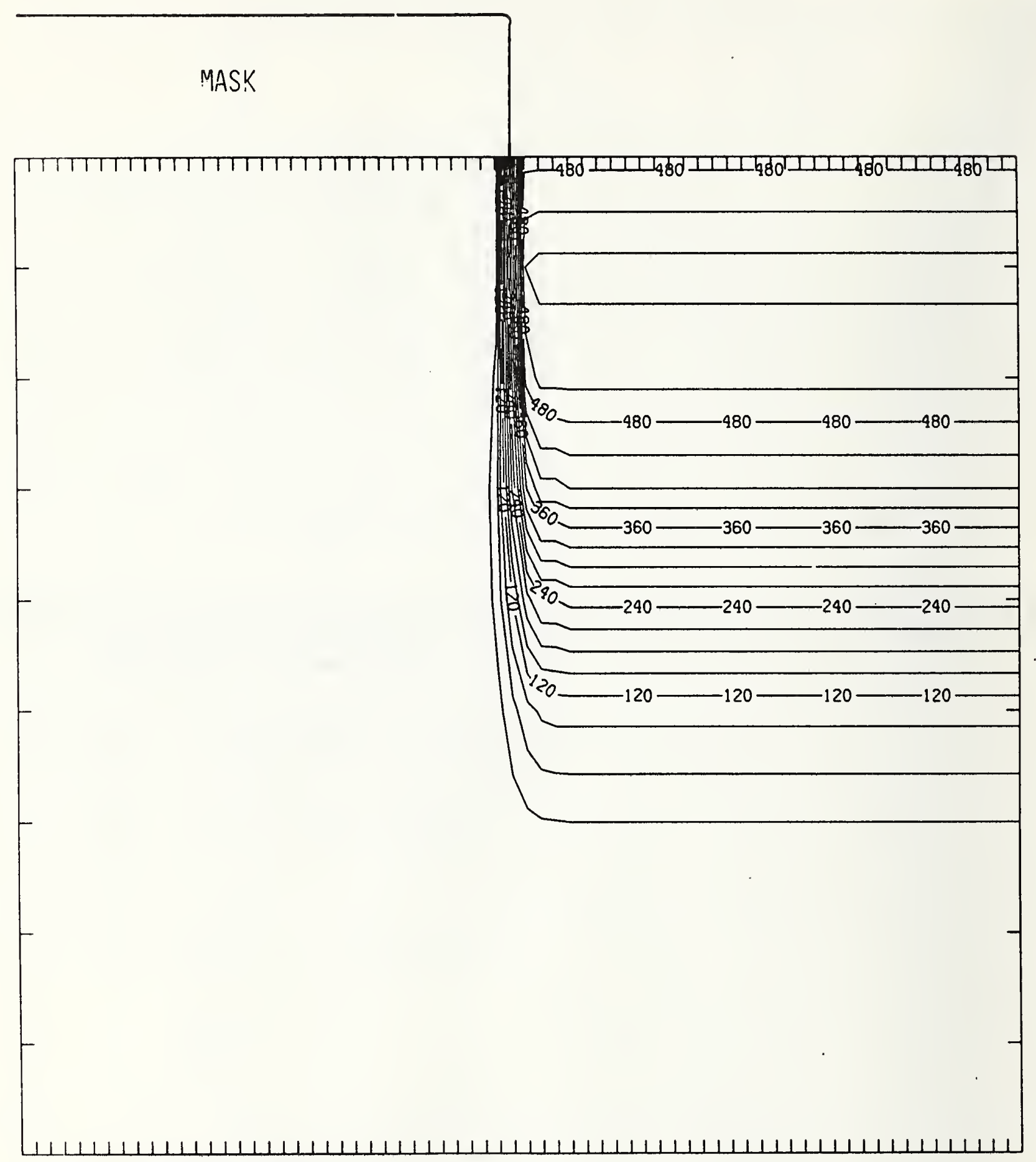

Figure Si50-4 Two-dimensional distribution of Frenkel pairs created by $50 \mathrm{keV}$ silicon implanted past a mask edge. The length increment (distance between tick marks) is $0.02 \mu \mathrm{m}$ and the scale factor is 1 . 


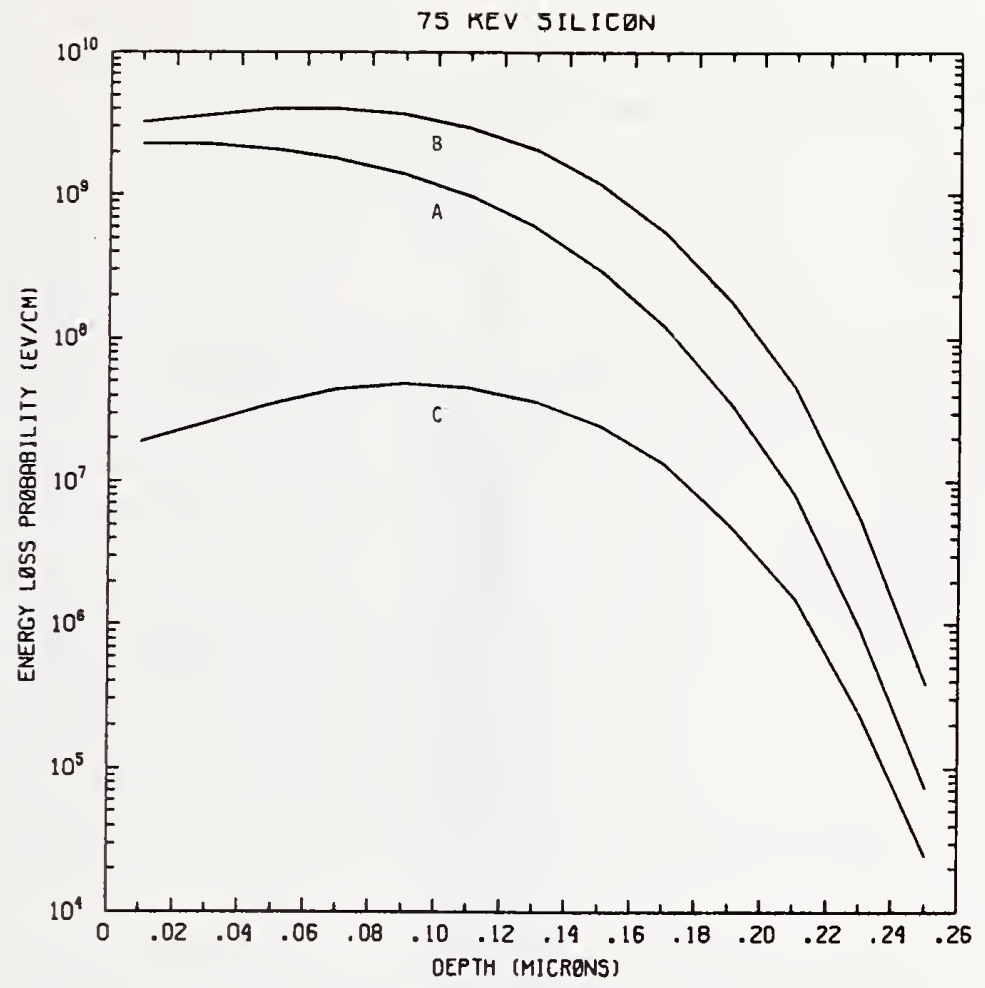

Figure Si75-1 One-dimensional probability distributions for energy loss due to electronic excitation (A), displacement damage(B), and phonon excitation (C) for $75 \mathrm{keV}$ silicon normally incident on a silicon target.

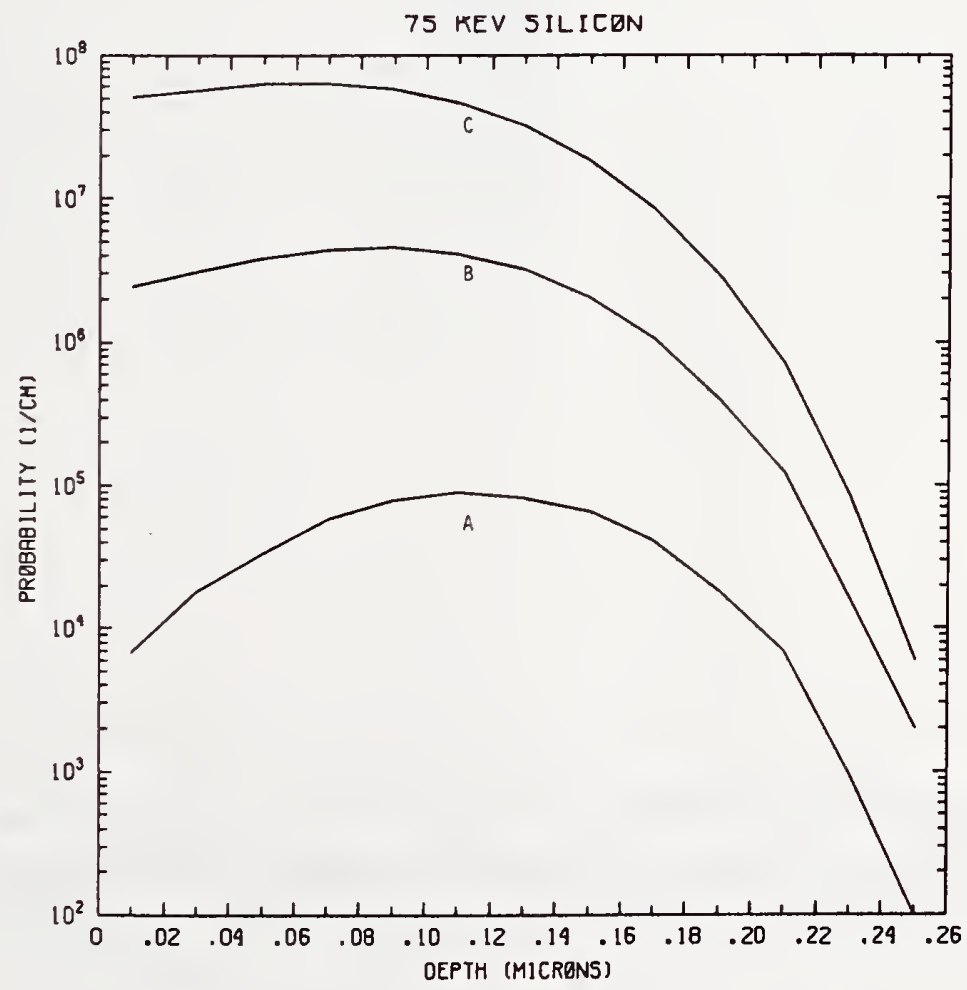

Figure Si75-2 One-dimensional probability distributions for implanted silicon (A), primary silicon displacements (B), and Frenkel pairs (C) for $75 \mathrm{keV}$ silicon normally incident on a silicon target. 
MASK

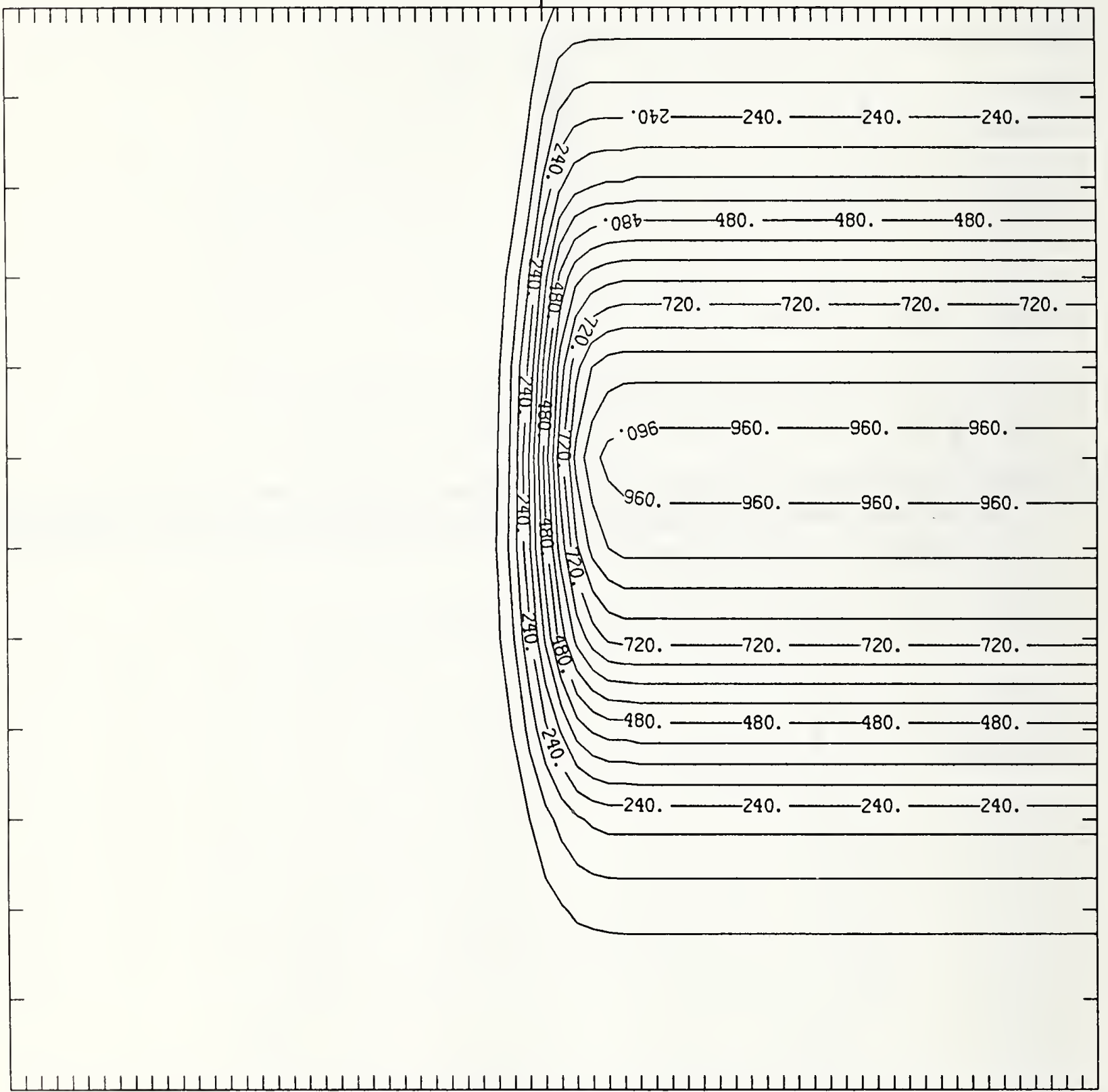

Figure Si75-3 Two-dimensional distribution of $75 \mathrm{keV}$ silicon implanted past a mask edge. The length increment (distance between tick marks) is $0.02 \mu \mathrm{m}$ and the scale factor is 1000. 


\section{MASK}

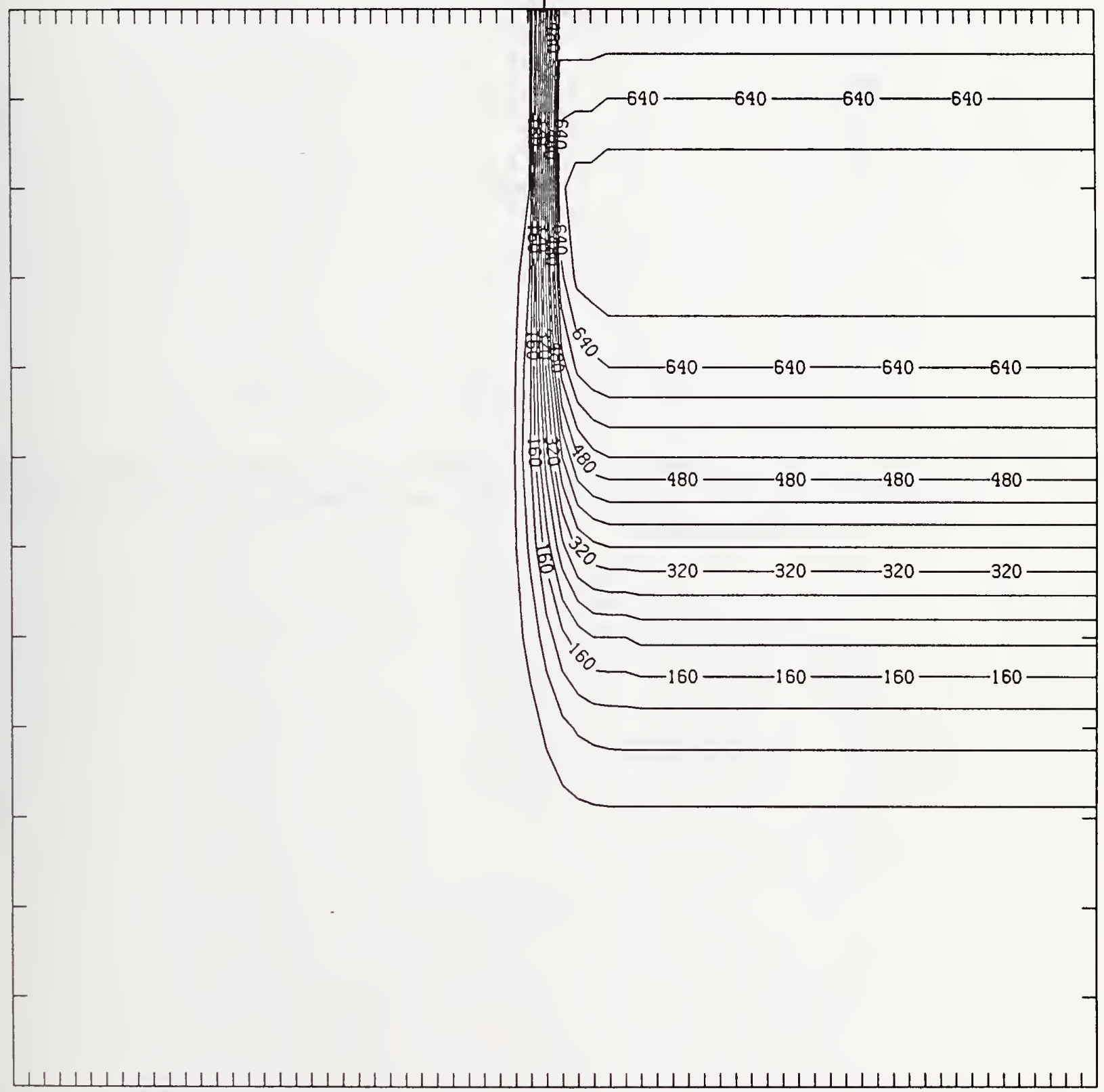

Figure Si75-4 Two-dimensional distribution of Frenkel pairs created by $75 \mathrm{keV}$ silicon implanted past a mask edge. The length increment (distance between tick marks) is $0.02 \mu \mathrm{m}$ and the scale factor is 1 . 


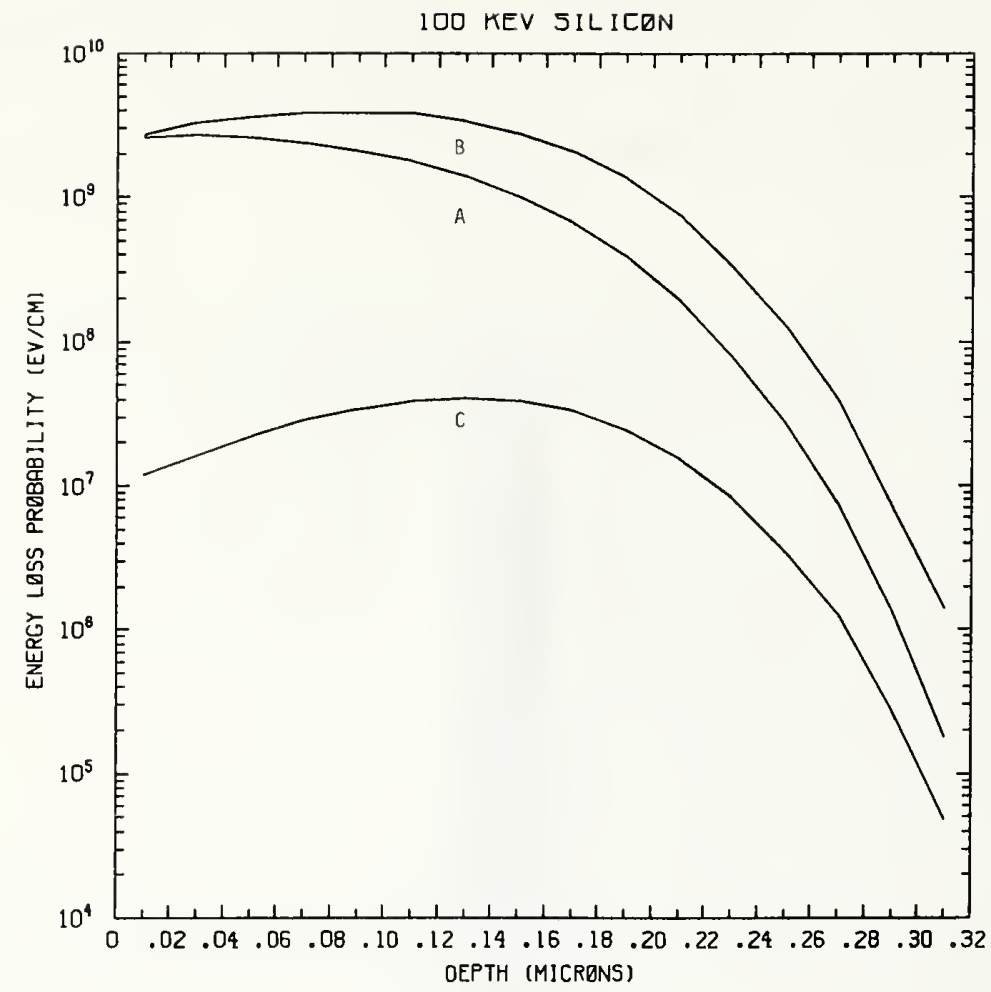

Figure Si100-1 One-dimensional probability distributions for energy loss due to electronic excitation (A), displacement damage(B), and phonon excitation (C) for $100 \mathrm{keV}$ silicon normally incident on a silicon target.

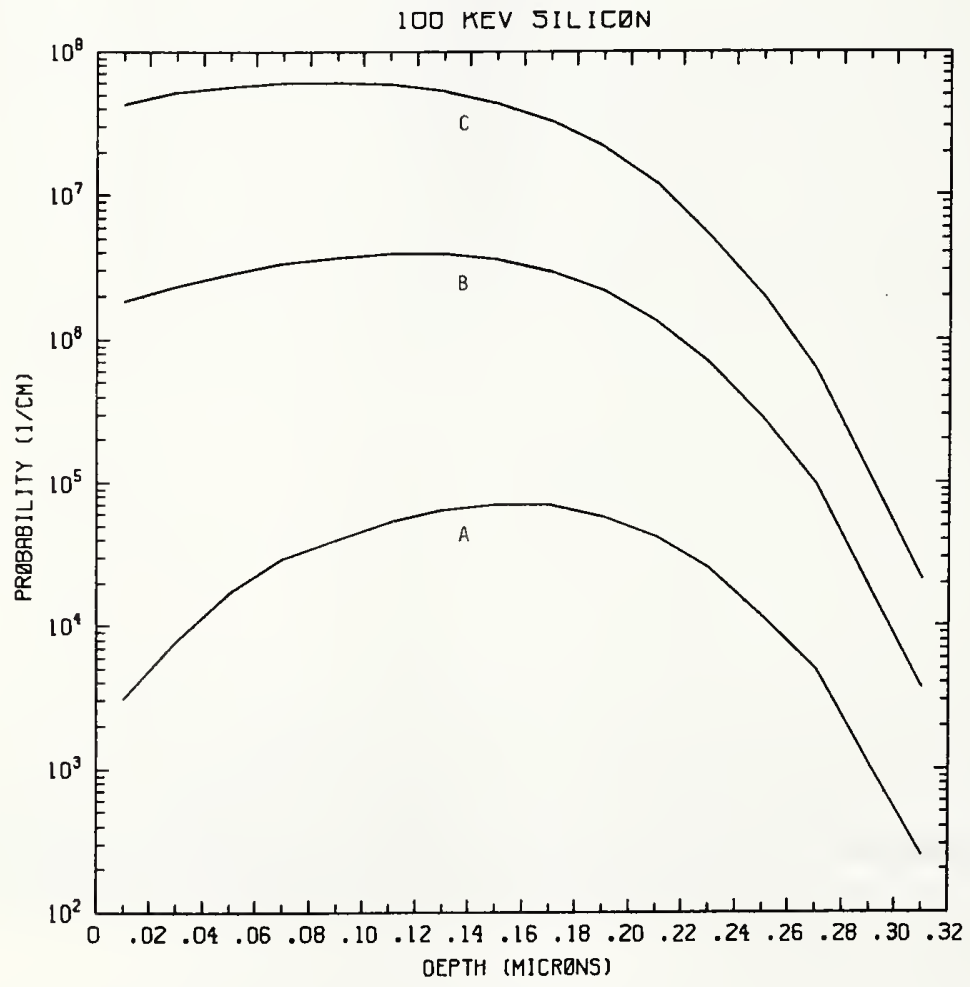

Figure Si100-2 One-dimensional probability distributions for implanted silicon (A), primary silicon displacements (B), and Frenkel pairs (C) for $100 \mathrm{keV}$ silicon normally incident on a silicon target. 
MASK

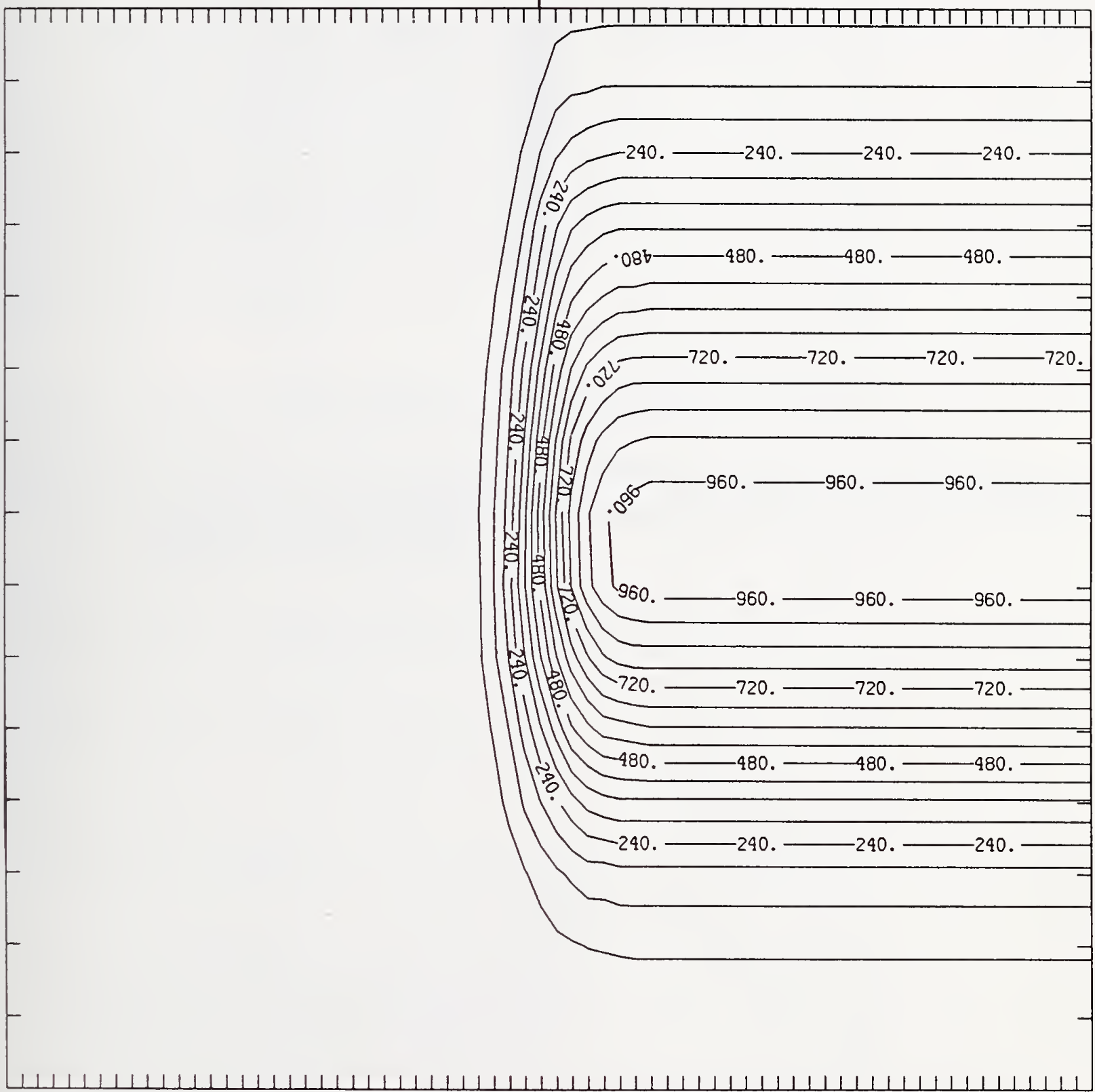

Figure Si100-3 Two-dimensional distribution of $100 \mathrm{keV}$ silicon implanted past a mask edge. The length ircrement (distance between tick marks) is $0.02 \mu \mathrm{m}$ and the scale factor is 1000 . 
MASK

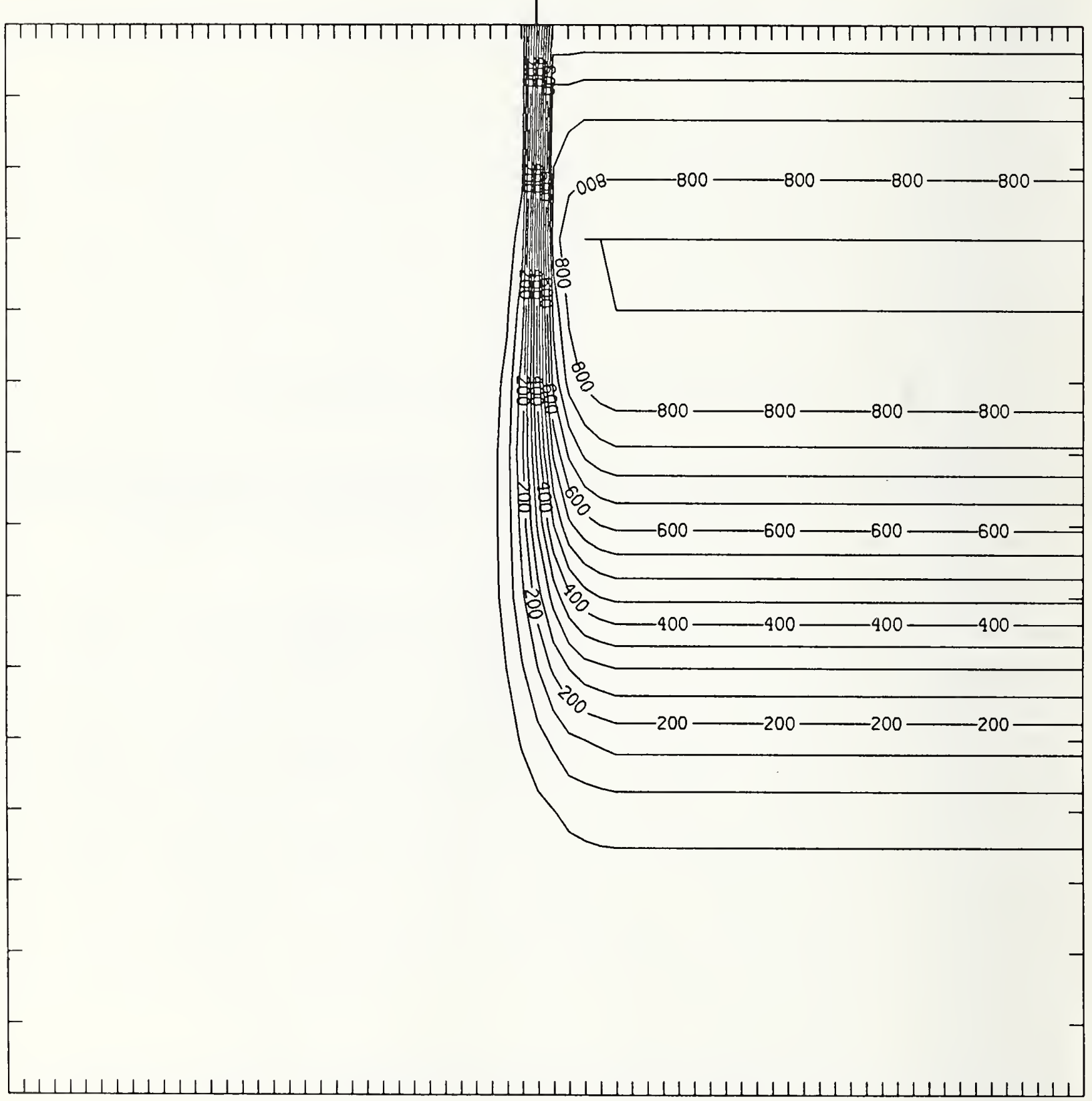

Figure Si100-4 Two-dimensional distribution of Frenkel pairs created by $100 \mathrm{keV}$ silicon implanted past a mask edge. The length increment (distance between tick marks) is $0.02 \mu \mathrm{m}$ and the scale factor is 1 . 


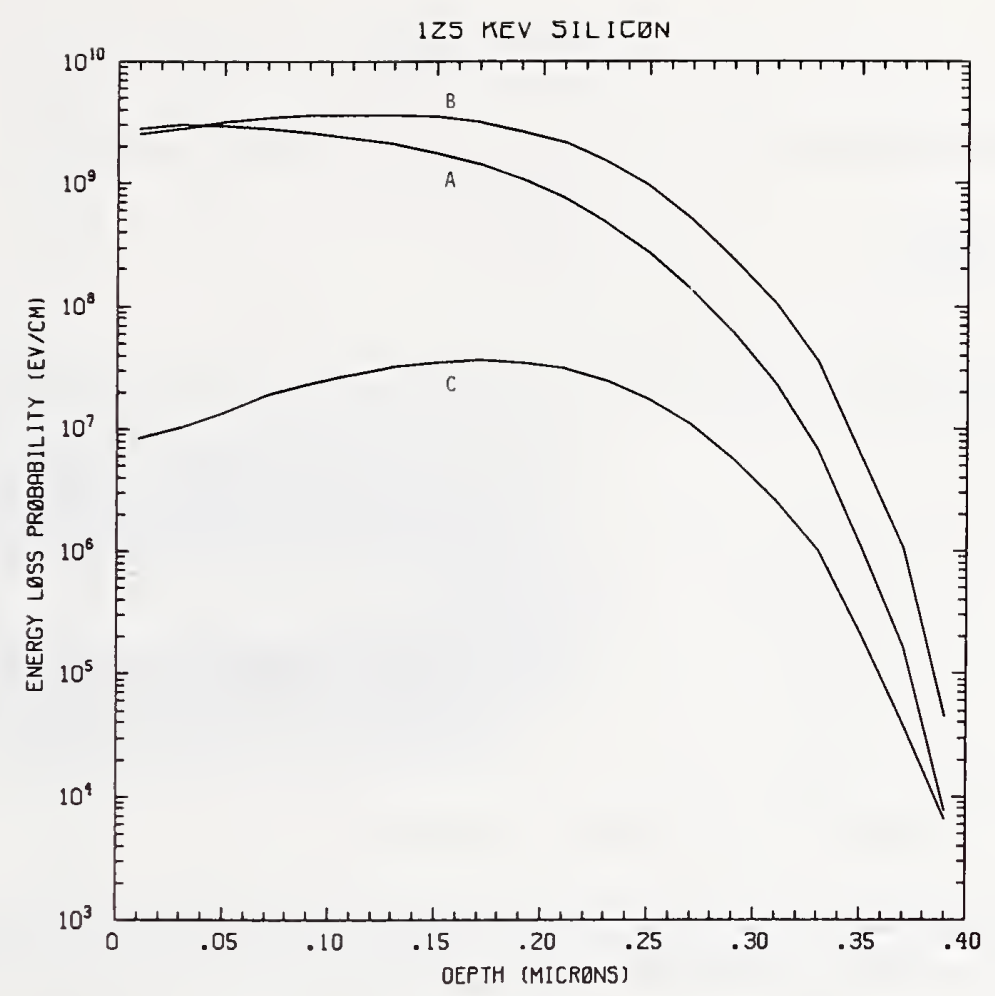

Figure Si125-1 One-dimensional probability distributions for energy loss due to electronic excitation (A), displacement damage(B), and phonon excitation (C) for $125 \mathrm{keV}$ silicon normally incident on a silicon target.

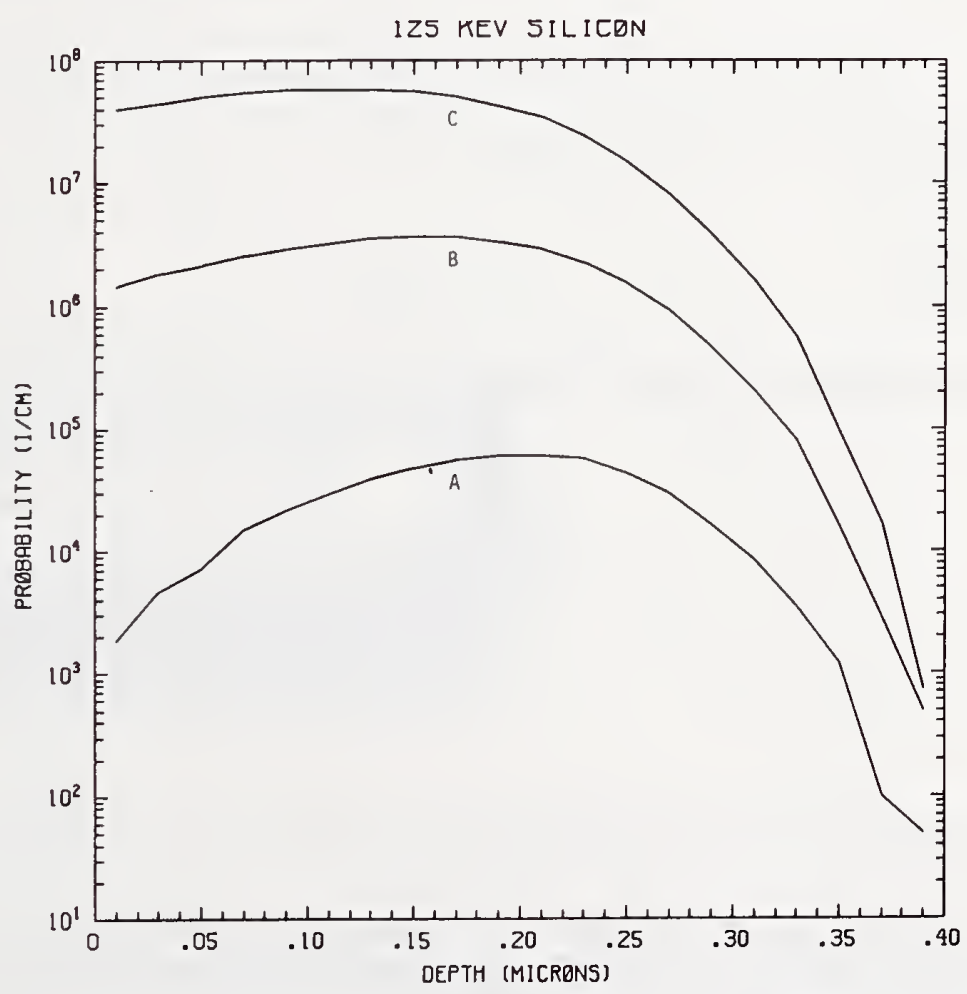

Figure Si125-2 One-dimensional probability distributions for implanted silicon (A), primary silicon displacements (B), and Frenkel pairs (C) for $125 \mathrm{keV}$ silicon normally incident on a silicon target. 


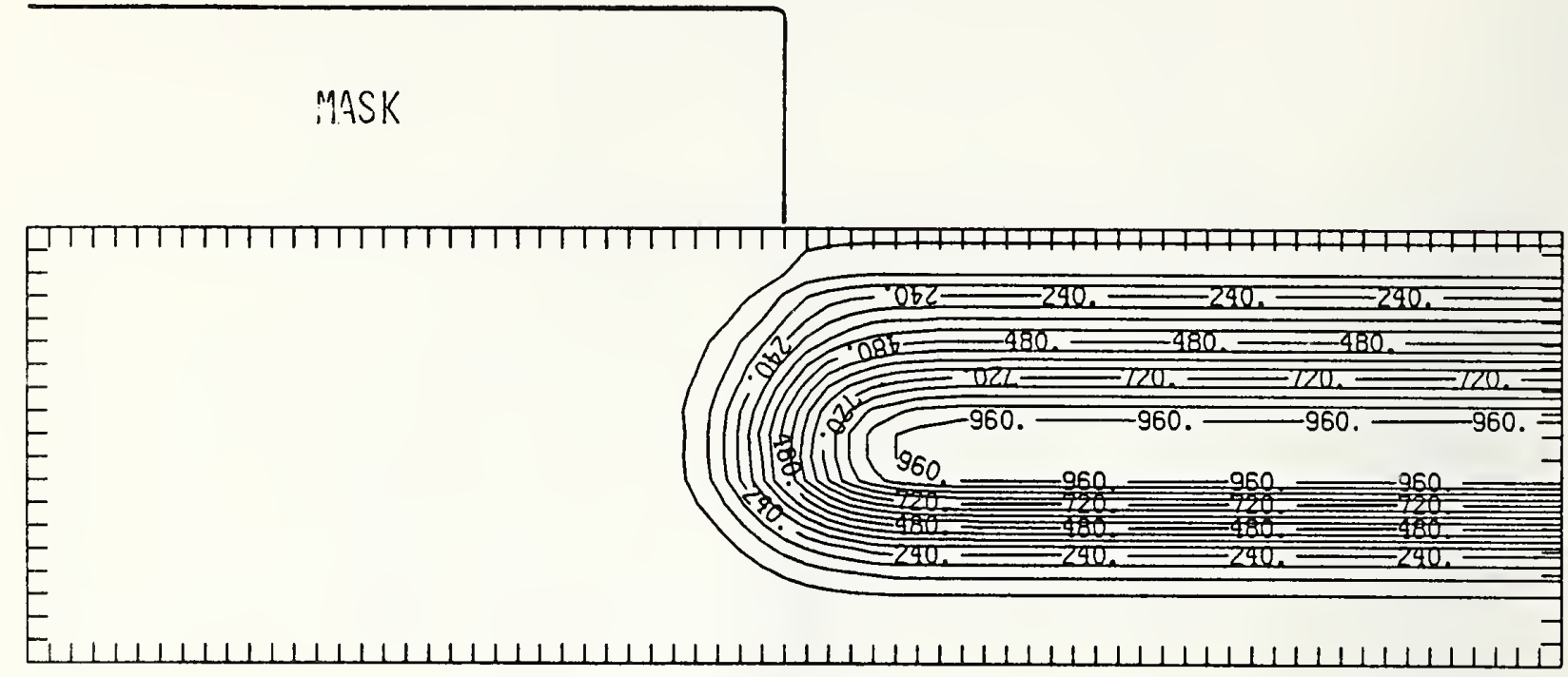

Figure Si125-3 Two-dimensional distribution of $125 \mathrm{keV}$ silicon implanted past a mask edge. The length increment (distance between tick marks) is $0.02 \mu \mathrm{m}$ and the scale factor is 1000 .

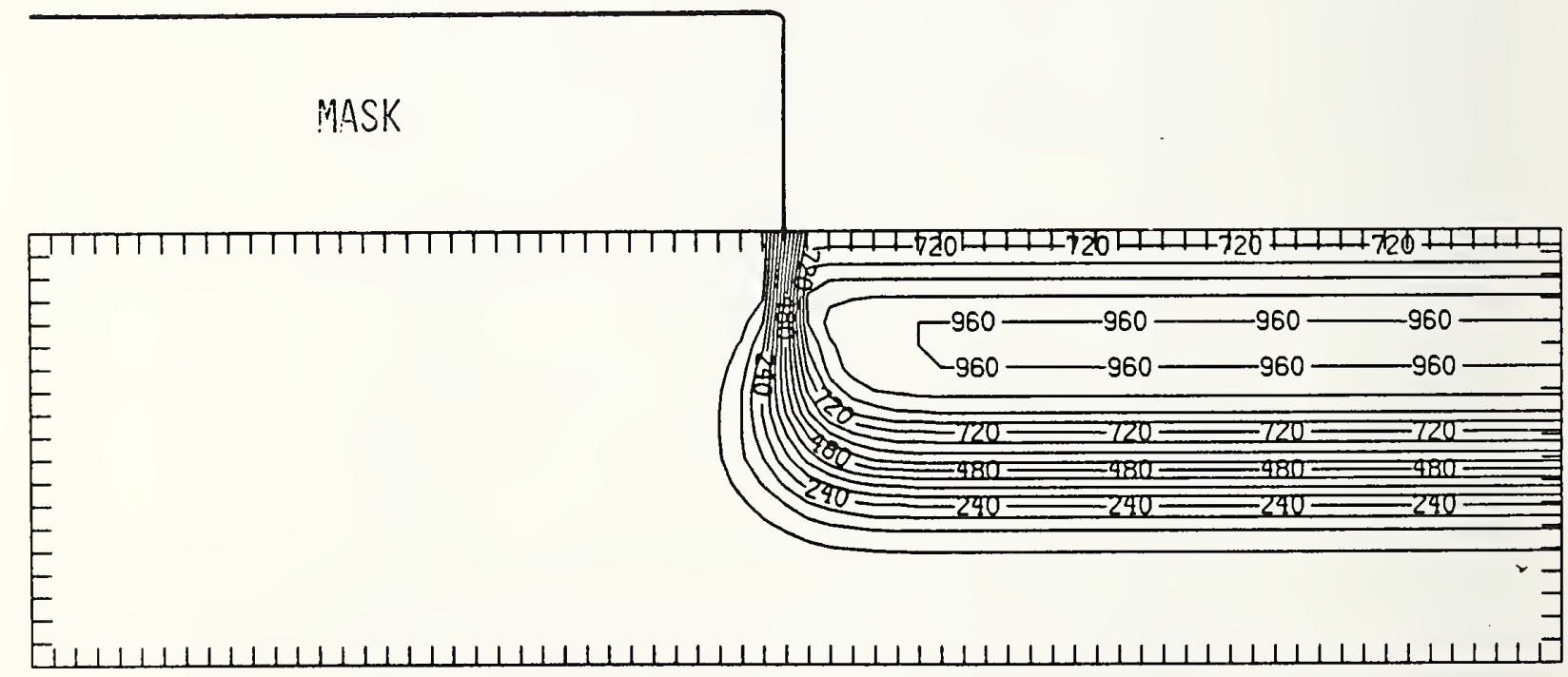

Figure Si125-4 Two-dimensional distribution of Frenkel pairs created by $125 \mathrm{keV}$ silicon implanted past a mask edge. The length increment (distance between tick marks) is $0.02 \mu \mathrm{m}$ and the scale factor is 1 . 


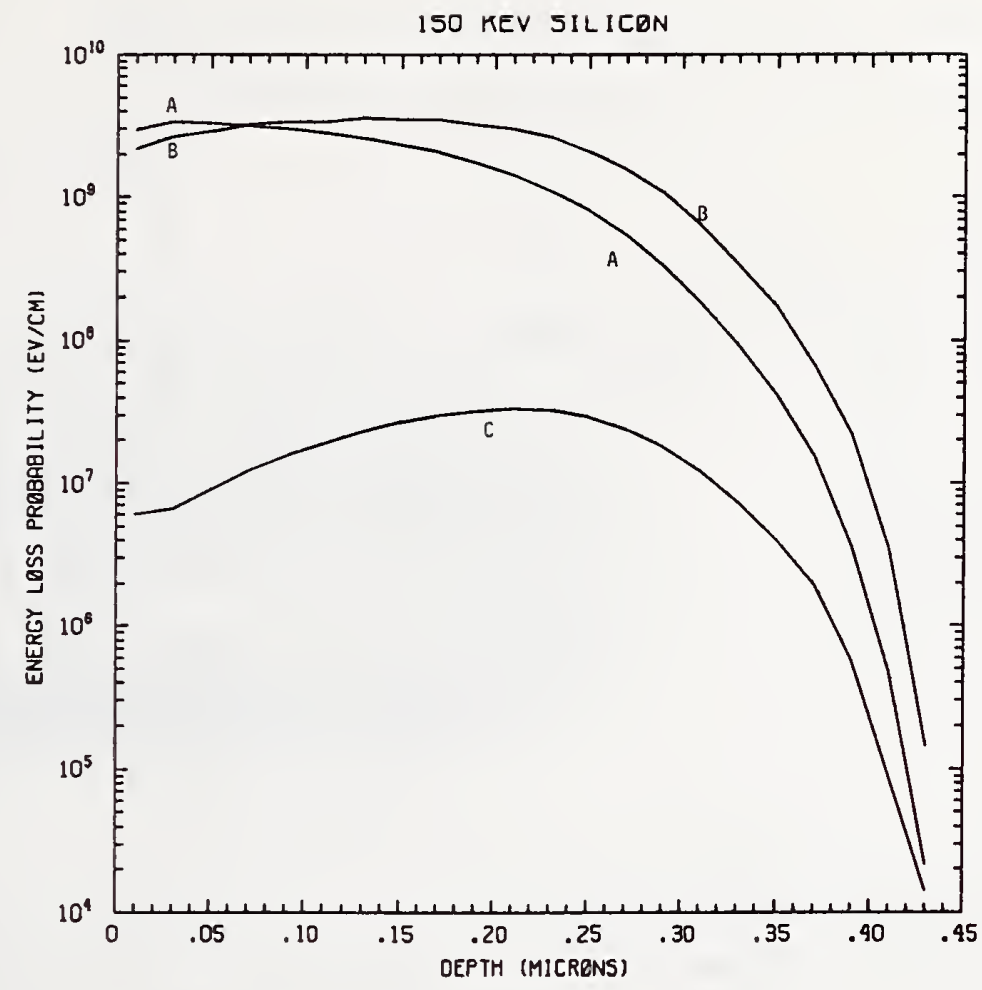

Figure Si150-1 One-dimensional probability distributions for energy loss due to electronic excitation (A), displacement damage(B), and phonon excitation (C) for $150 \mathrm{keV}$ silicon normally incident on a silicon target.

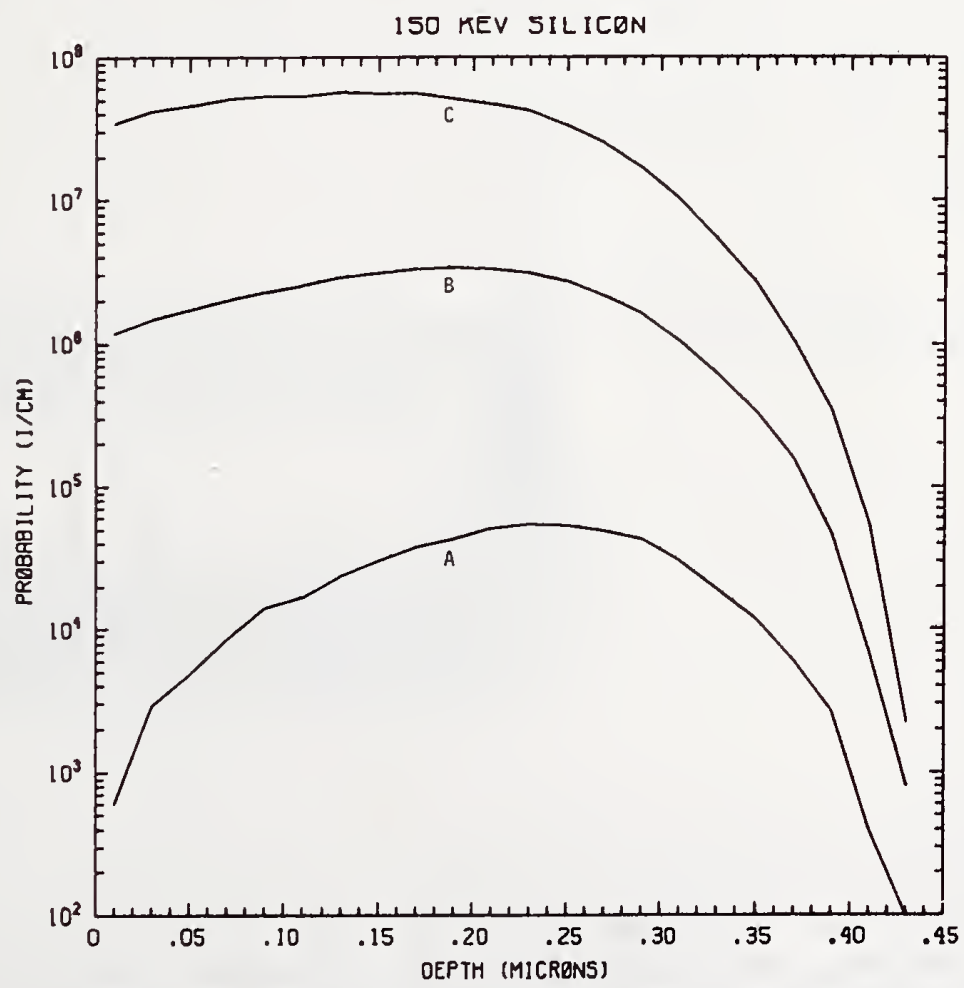

Figure Si150-2 One-dimensional probability distributions for implanted silicon (A), primary silicon displacements (B), and Frenkel pairs (C) for $150 \mathrm{keV}$ silicon normally incident on a silicon target. 
MASK

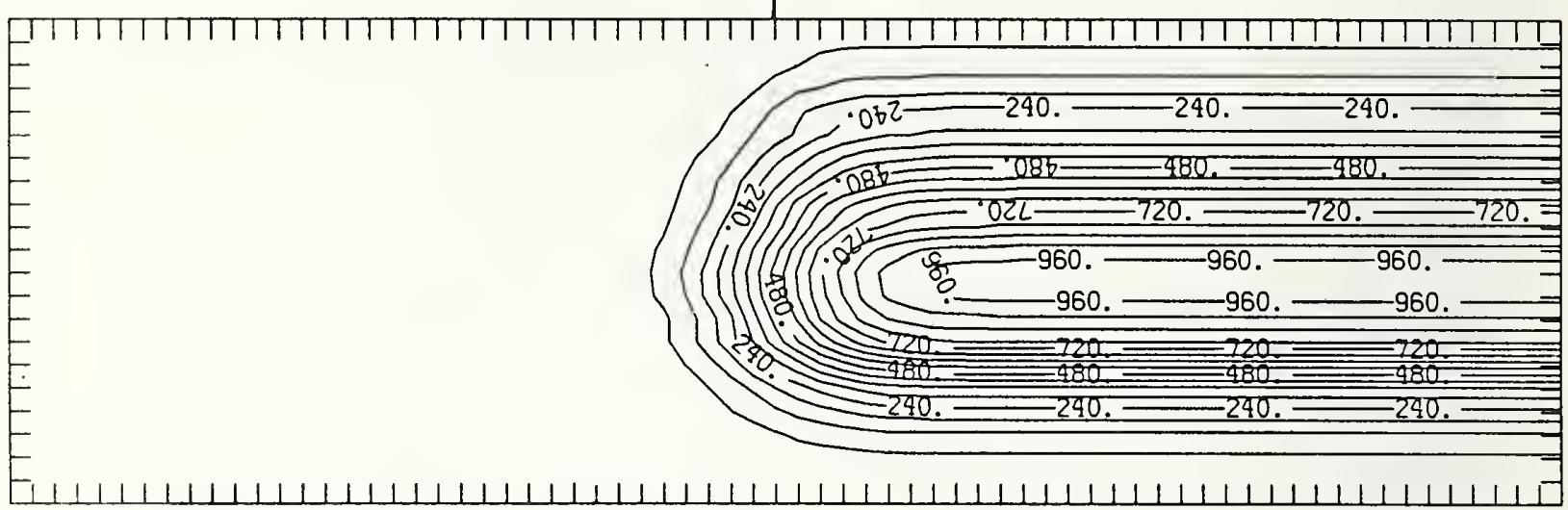

Figure Si150-3 Two-dimensional distribution of $150 \mathrm{keV}$ silicon implanted past a mask edge. The length increment (distance between tick marks) is $0.02 \mu \mathrm{m}$ and the scale factor is 1000 .

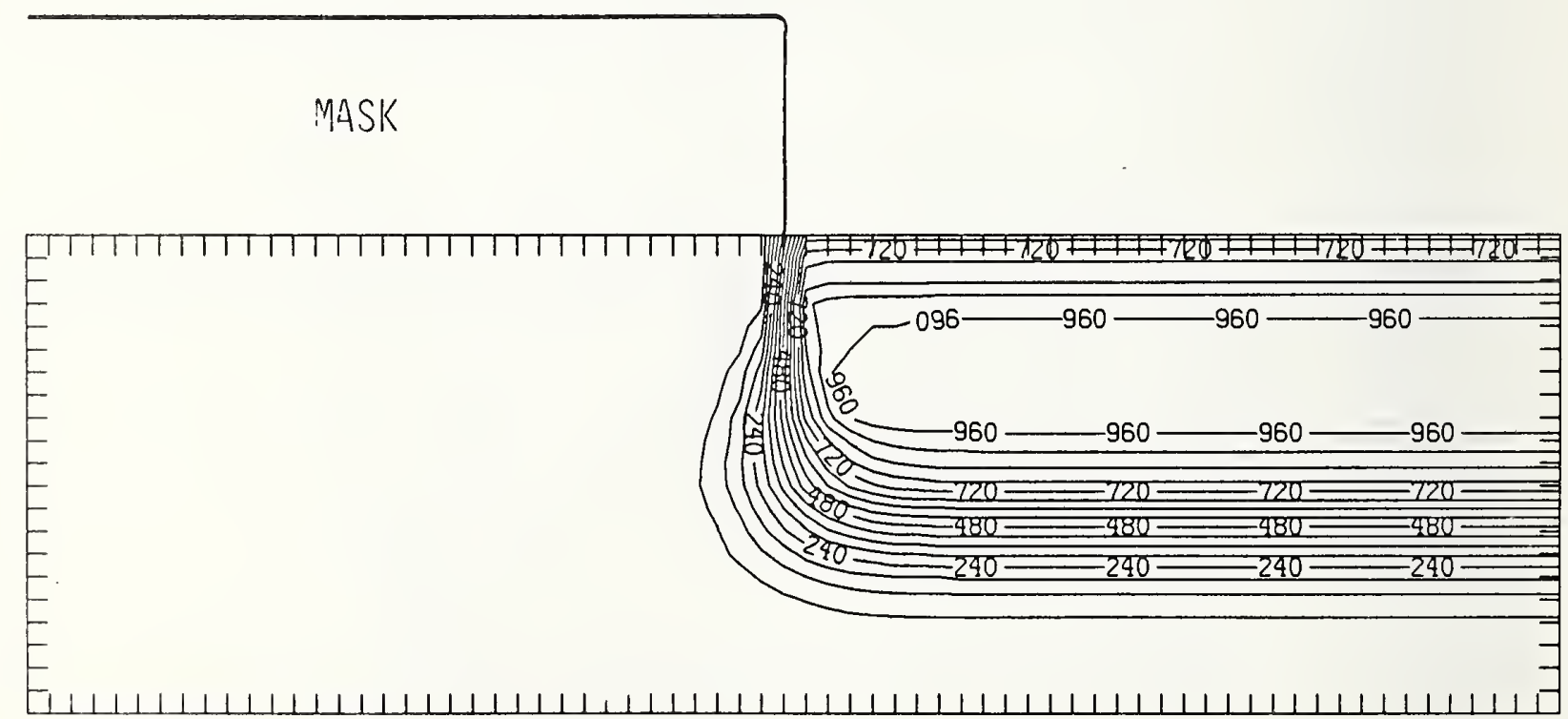

Figure Si150-4 Two-dimensional distribution of Frenkel pairs created by $150 \mathrm{keV}$ silicon implanted past a mask edge. The length increment (distance between tick marks) is $0.02 \mu \mathrm{m}$ and the scale factor is 1 . 


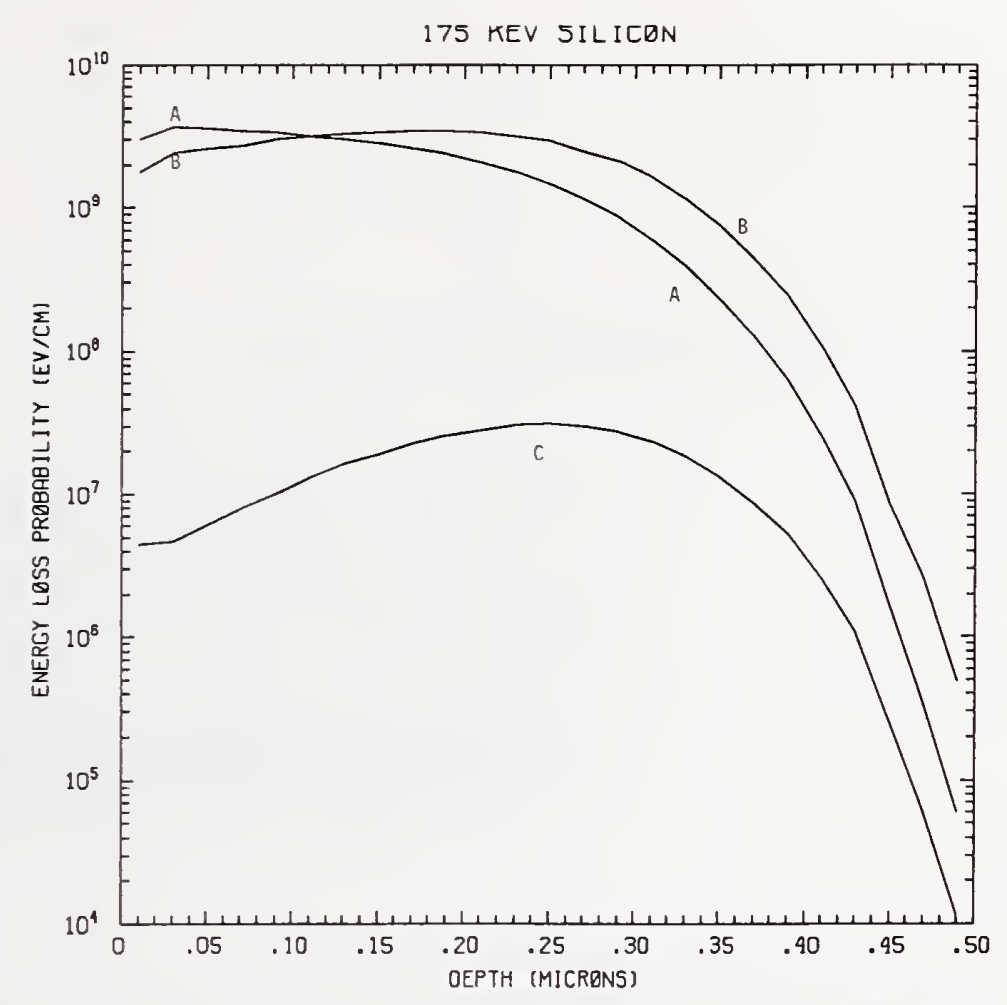

Figure Si175-1 One-dimensional probability distributions for energy loss due to electronic excitation (A), displacement damage(B), and phonon excitation (C) for $175 \mathrm{keV}$ silicon normally incident on a silicon target.

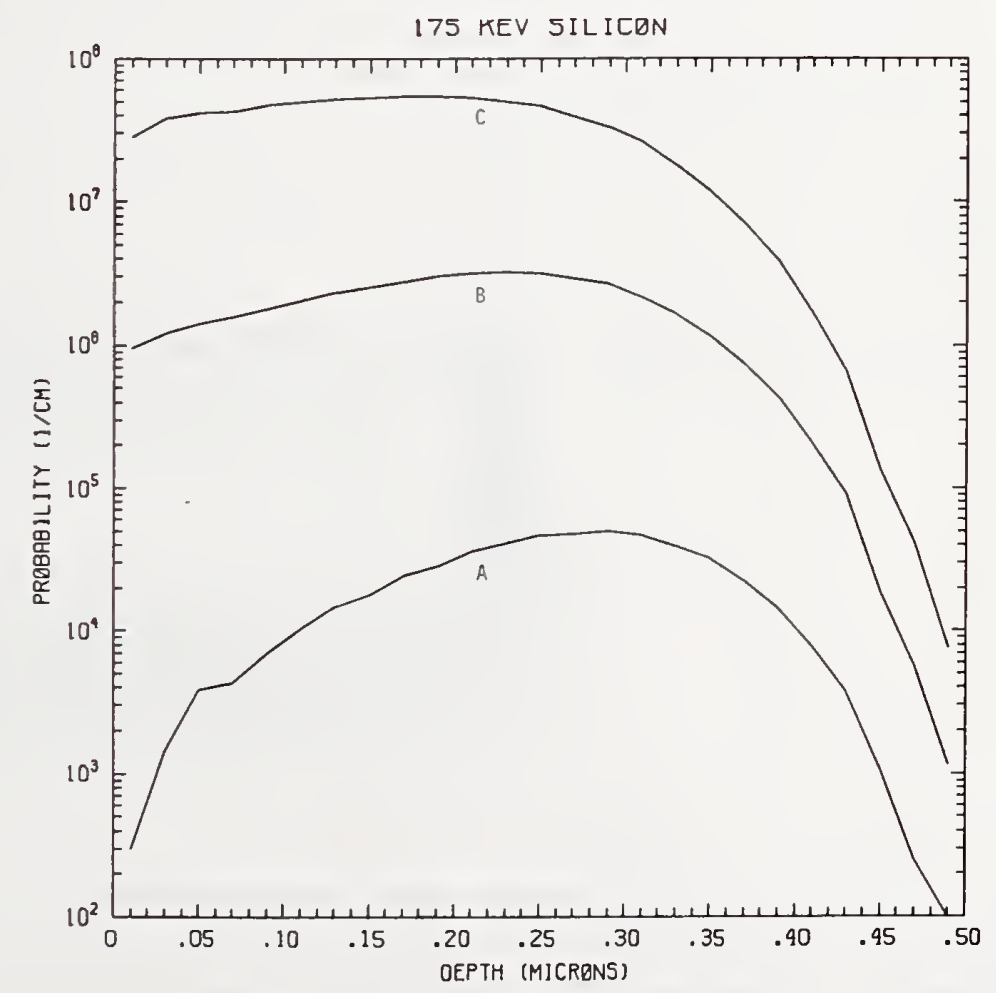

Figure Si175-2 One-dimensional probability distributions for implanted silicon (A), primary silicon displacements (B), and Frenkel pairs (C) for $175 \mathrm{keV}$ silicon normally incident on a silicon target. 


\section{MASK}

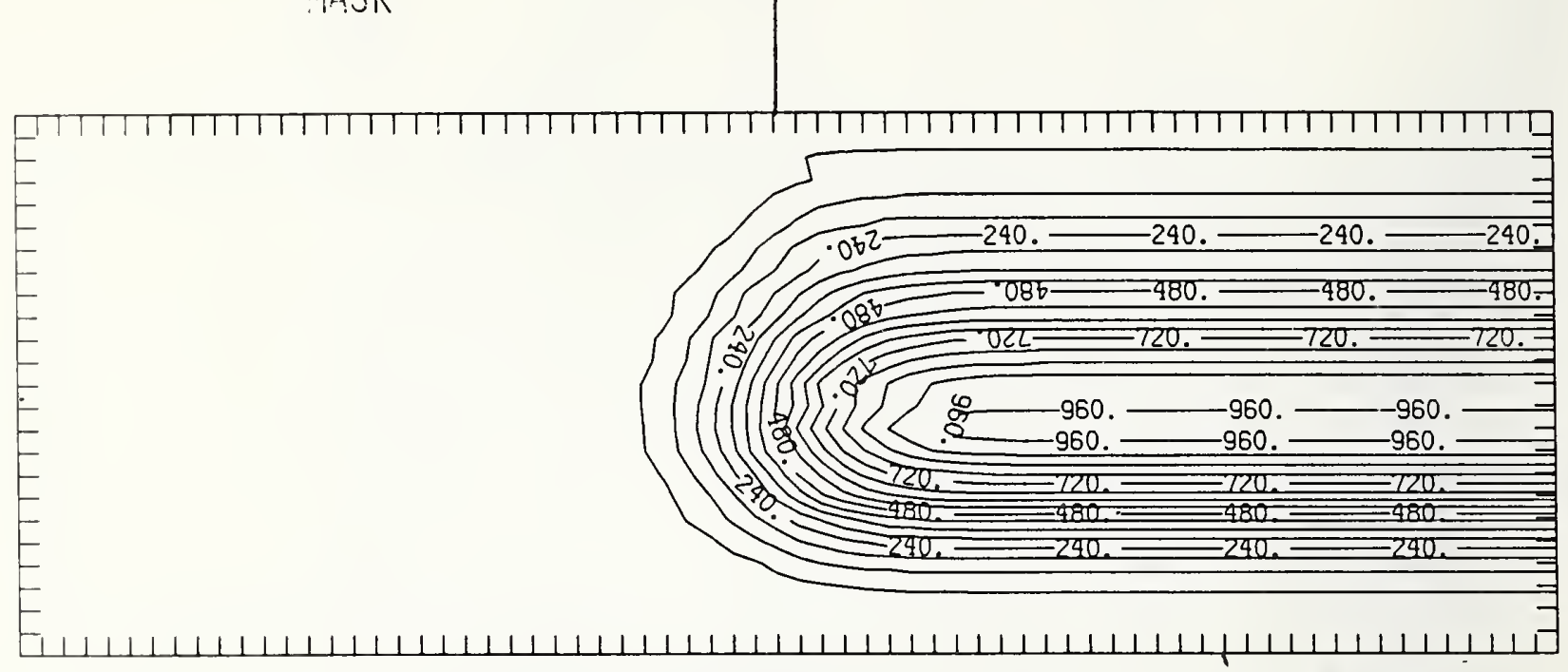

Figure Si175-3 Two-dimensional distribution of $175 \mathrm{keV}$ silicon implanted past a mask edge. The length increment (distance between tick marks) is $0.02 \mu \mathrm{m}$ and the scale factor is 1000 .

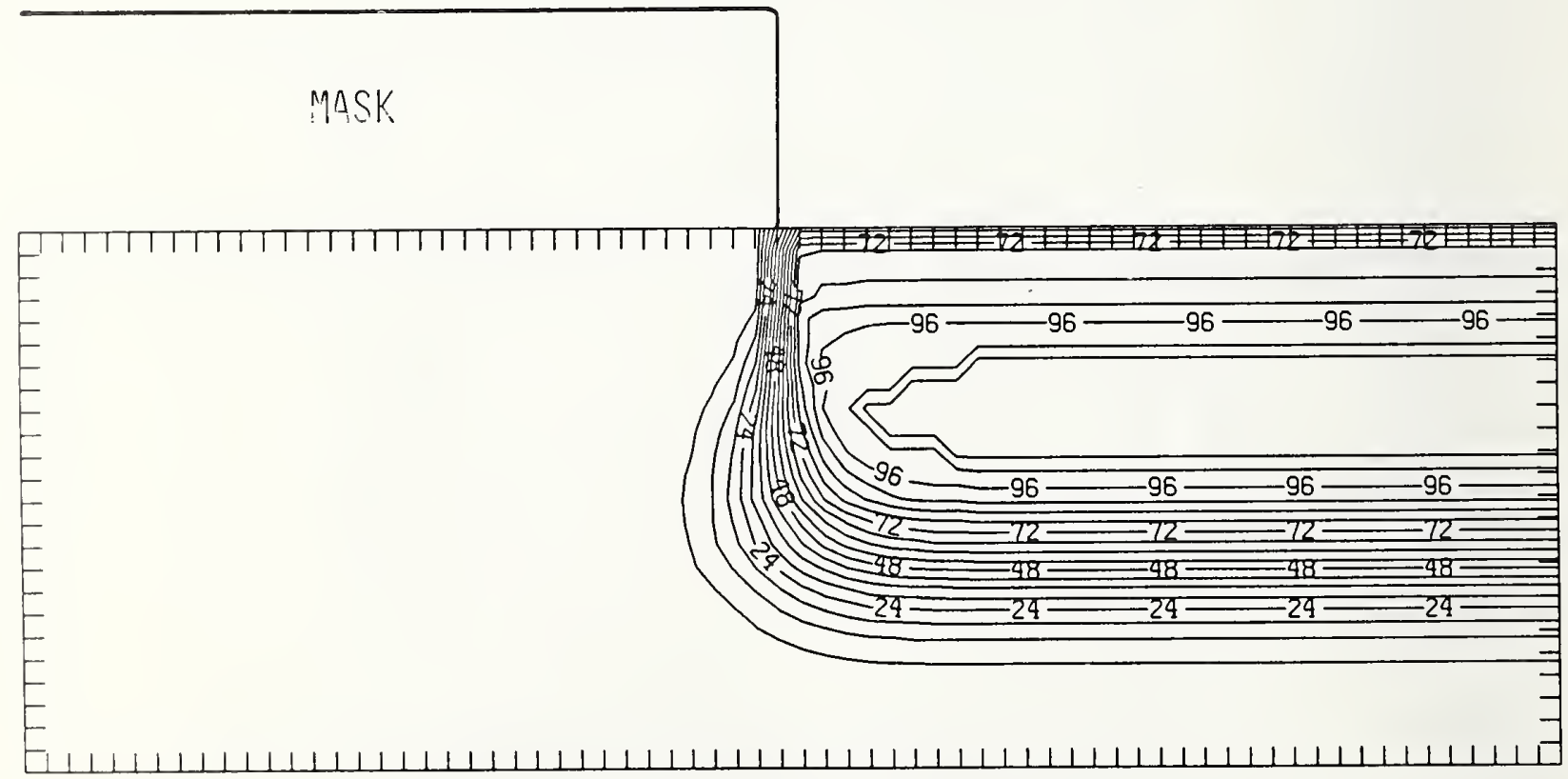

Figure Si175-4 Two-dimensional distribution of Frenkel pairs created by $175 \mathrm{keV}$ silicon implanted past a mask edge. The length increment (distance between tick marks) is $0.02 \mu \mathrm{m}$ and the scale factor is 0.1 . 


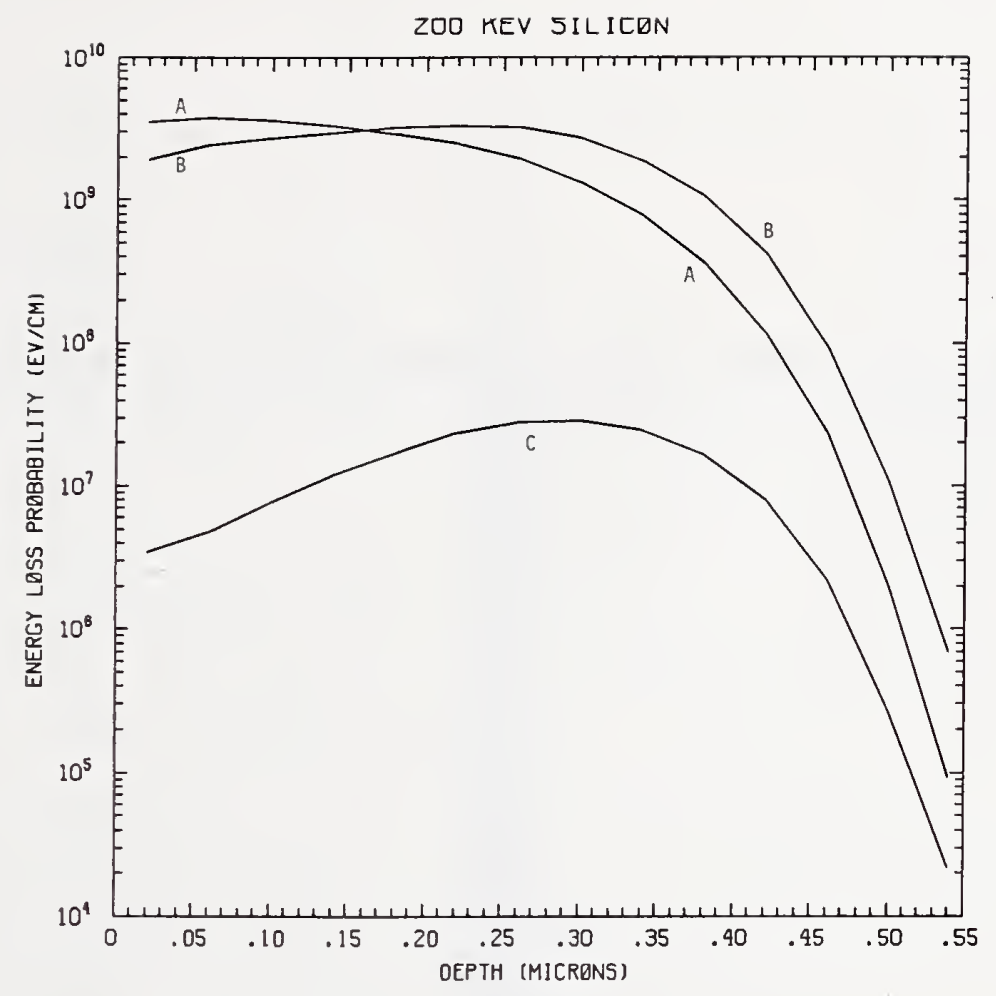

Figure Si200-1 One-dimensional probability distributions for energy loss due to electronic excitation (A), displacement damage(B), and phonon excitation (C) for $200 \mathrm{keV}$ silicon normally incident on a silicon target.

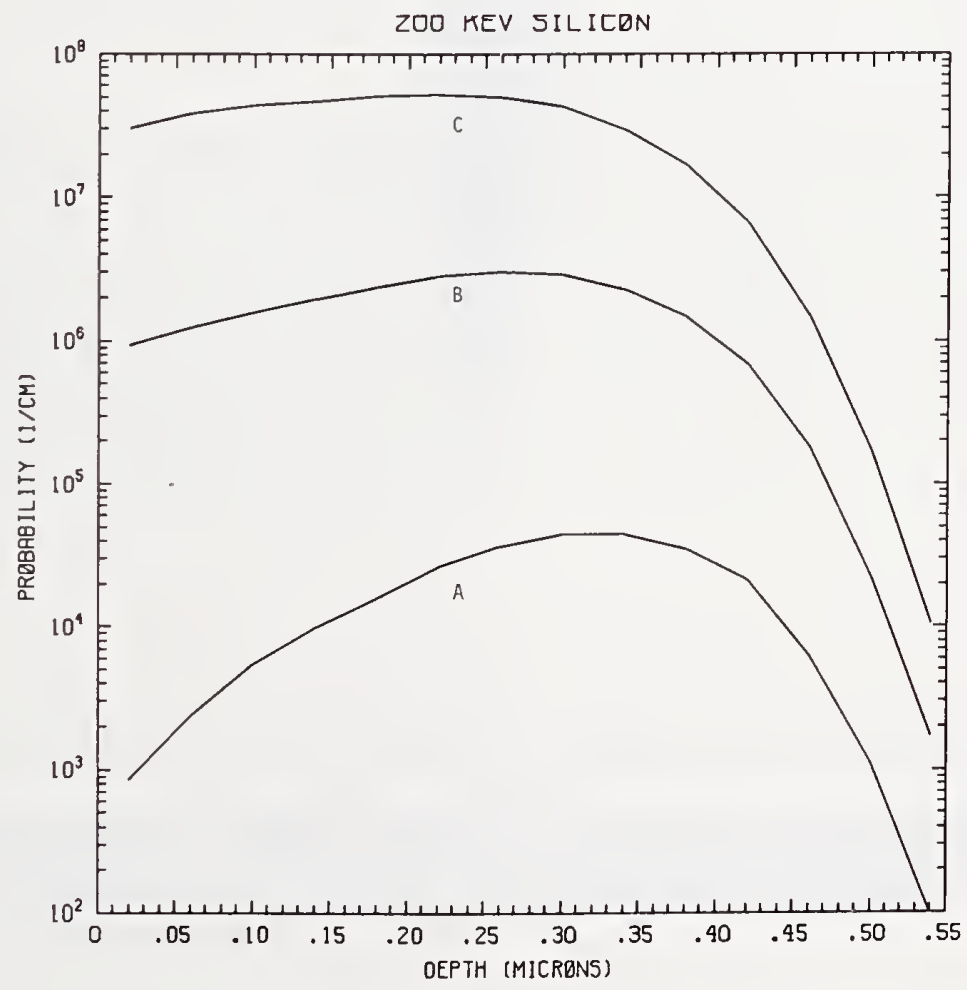

Figure Si200-2 One-dimensional probability distributions for implanted silicon (A), primary silicon displacements (B), and Frenkel pairs (C) for $200 \mathrm{keV}$ silicon normally incident on a silicon target. 


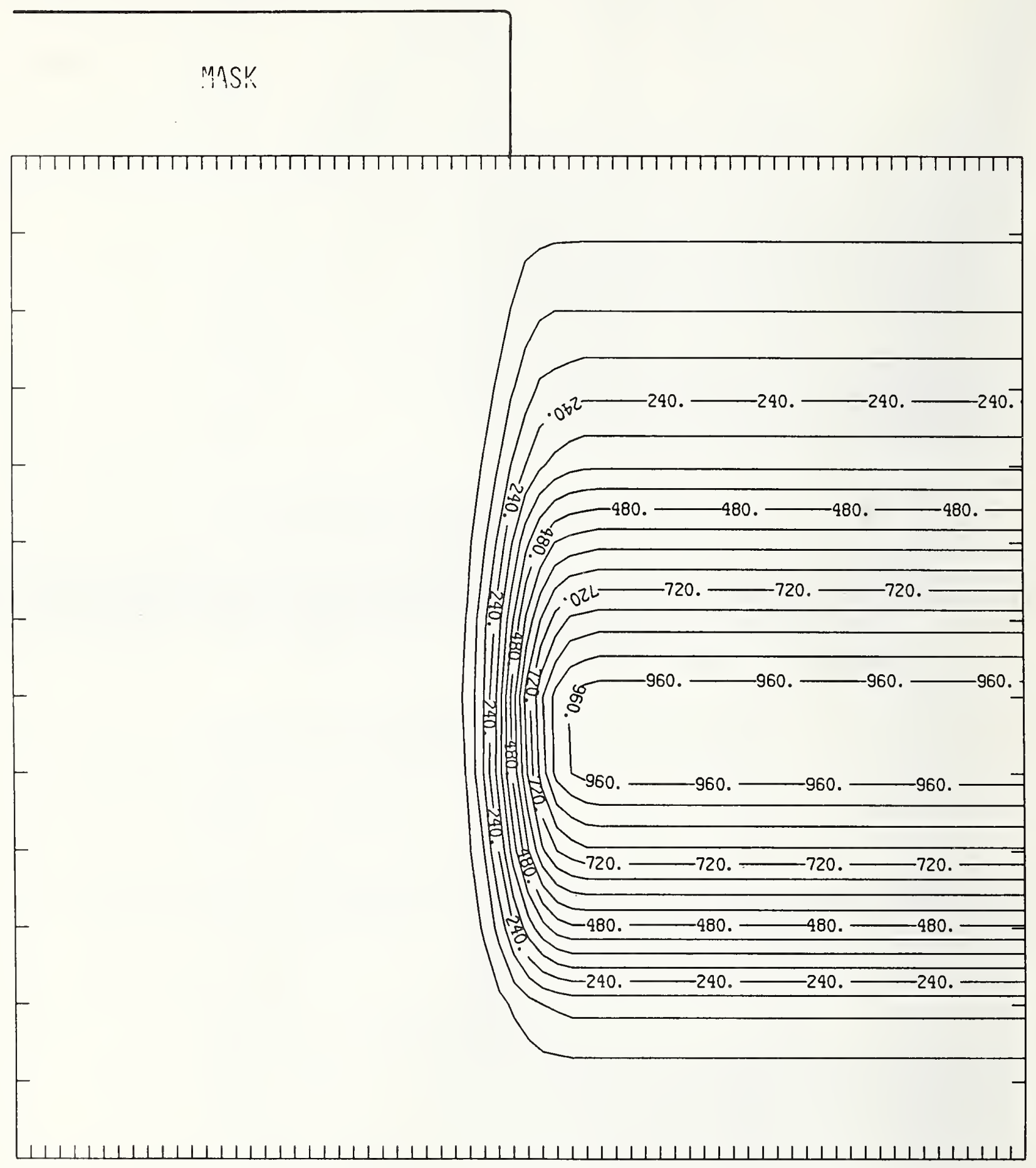

Figure Si200-3 Two-dimensional distribution of $200 \mathrm{keV}$ silicon implanted past a mask edge. The length increment (distance between tick marks) is $0.04 \mu \mathrm{m}$ and the scale factor is 1000 . 


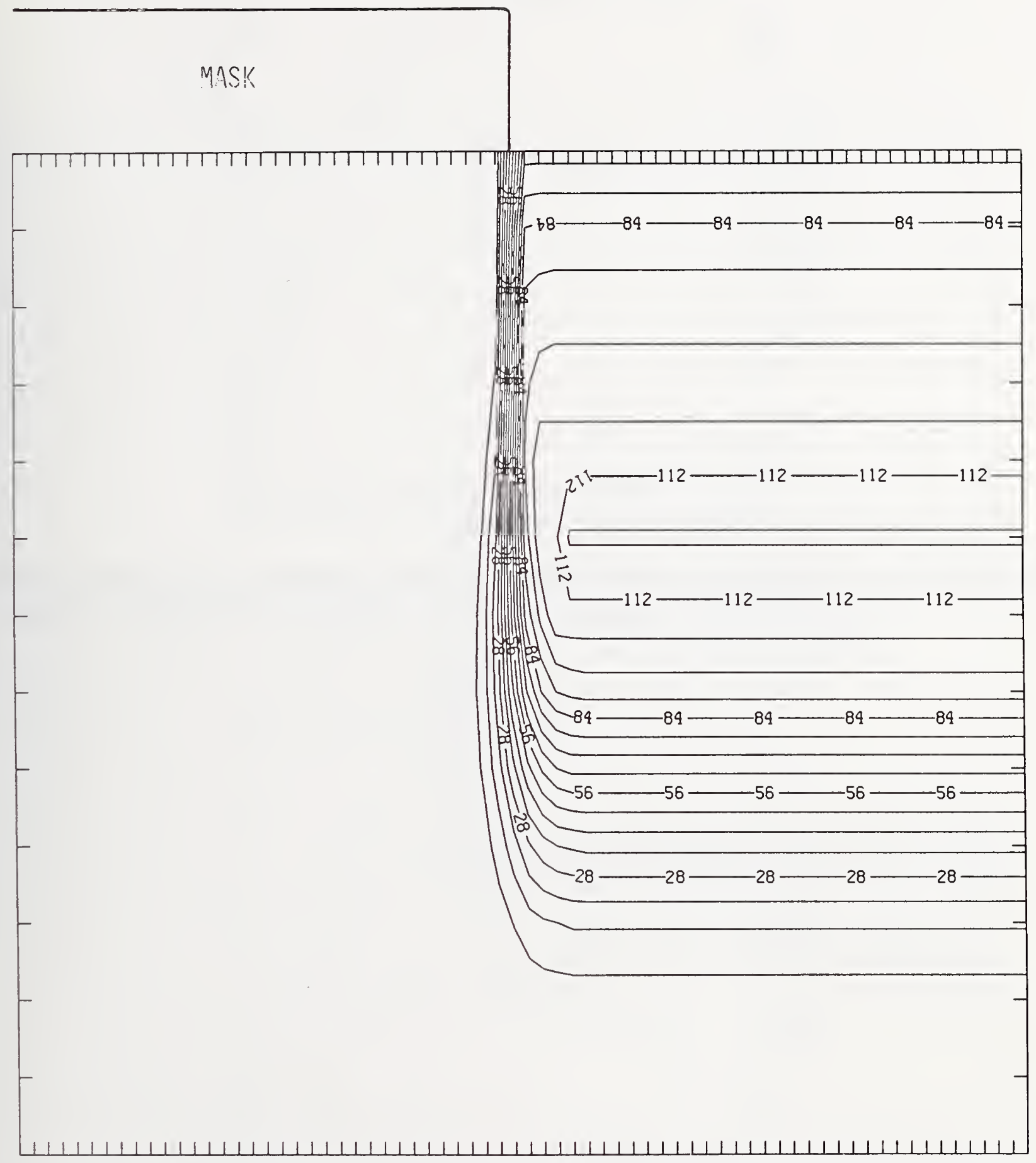

Figure Si200-4 Two-dimensional distribution of Frenkel pairs created by $200 \mathrm{keV}$ silicon implanted past a mask edge. The length increment (distance between tick marks) is $0.04 \mu \mathrm{m}$ and the scale factor is 0.1 . 


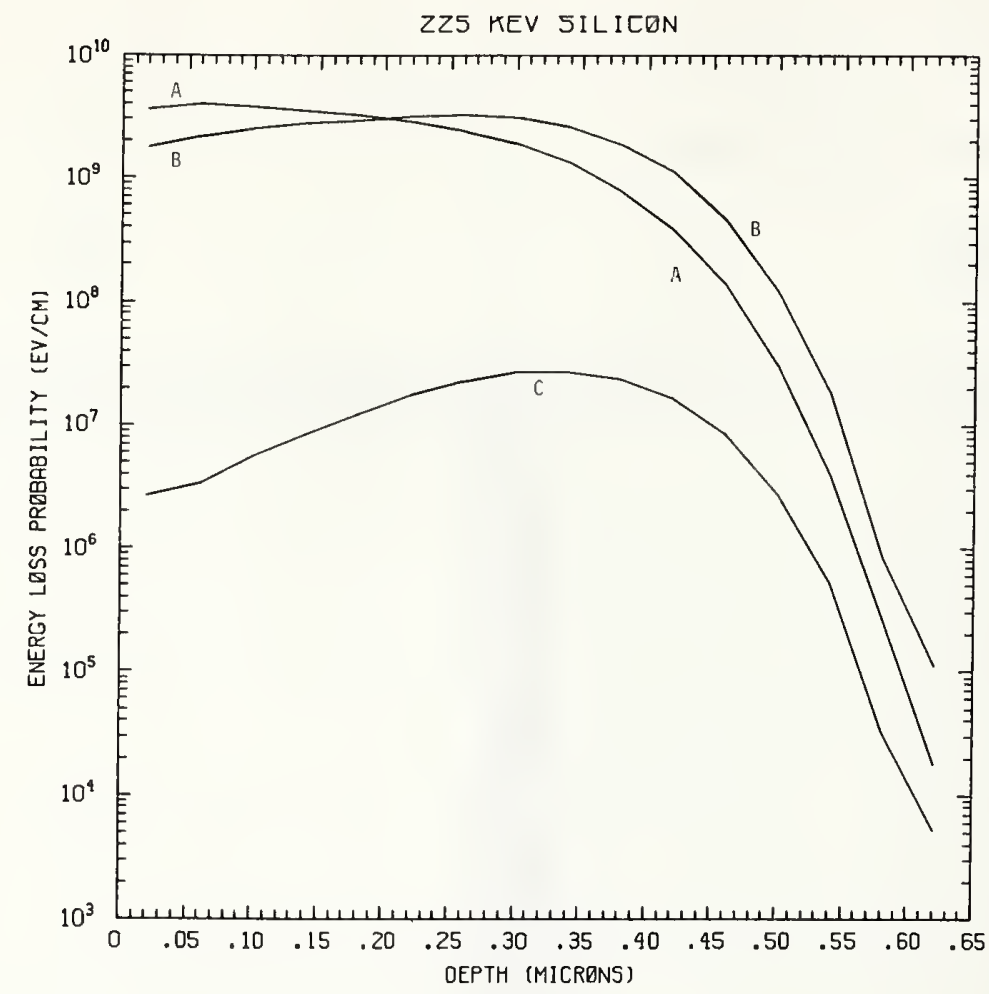

Figure Si225-1 One-dimensional probability distributions for energy loss due to electronic excitation (A), displacement damage(B), and phonon excitation (C) for $225 \mathrm{keV}$ silicon normally incident on a silicon target.

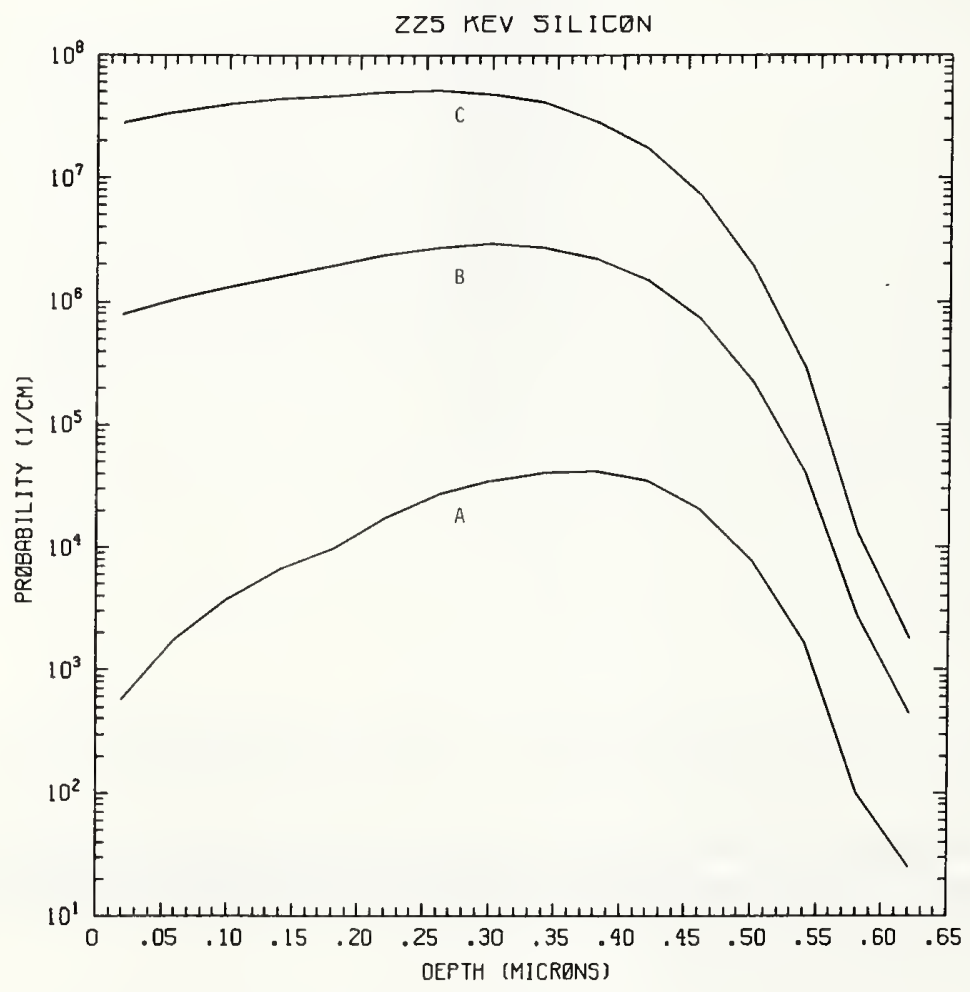

Figure Si225-2 One-dimensional probability distributions for implanted silicon (A), primary silicon displacements (B), and Frenkel pairs (C) for $225 \mathrm{keV}$ silicon normally incident on a silicon target. 


$$
M \cap S K
$$

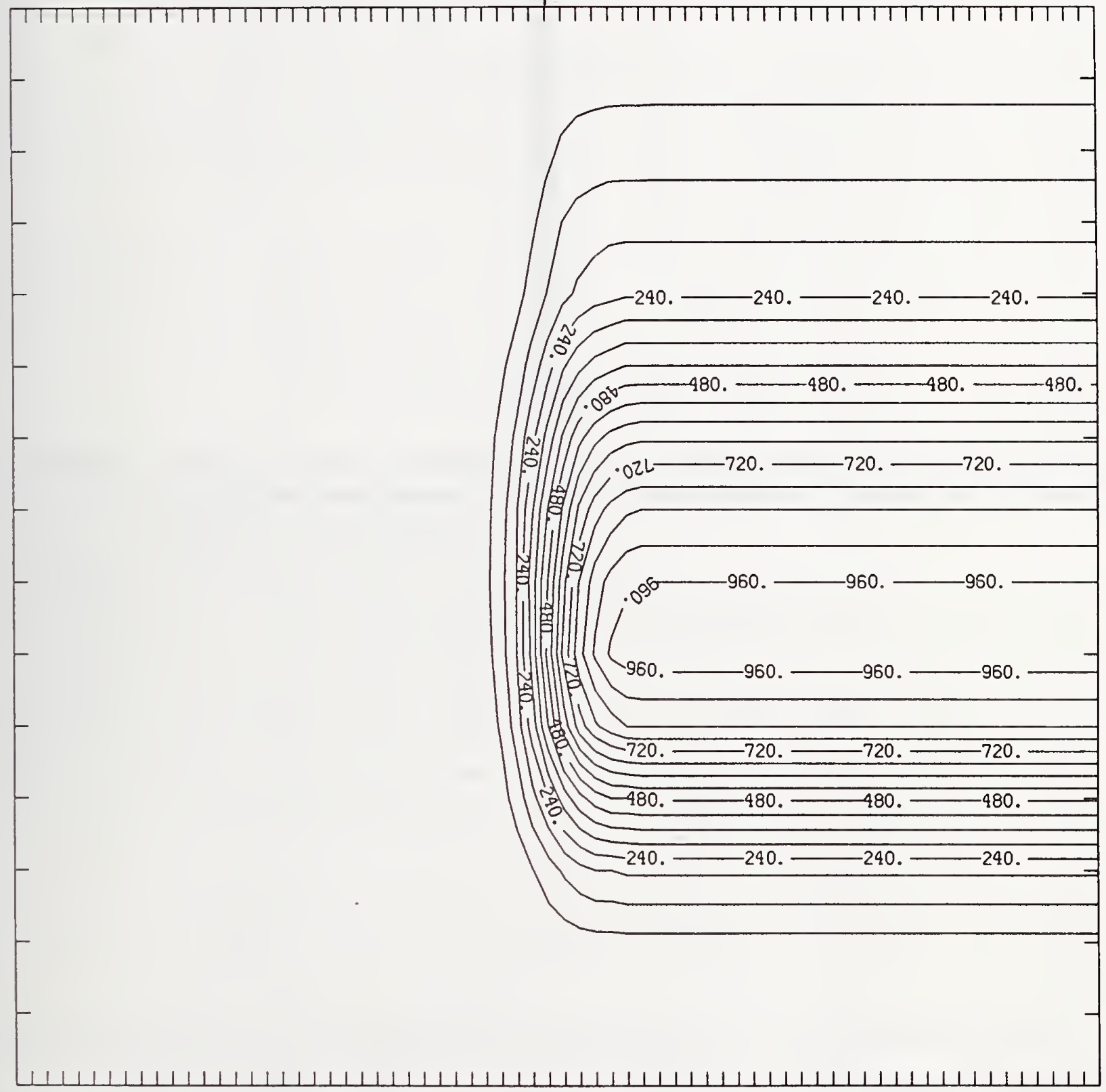

Figure Si225-3 Two-dimensional distribution of $225 \mathrm{keV}$ silicon implanted past a mask edge. The length increment (distance between tick marks) is $0.04 \mu \mathrm{m}$ and the scale factor is 1000 . 
MISK

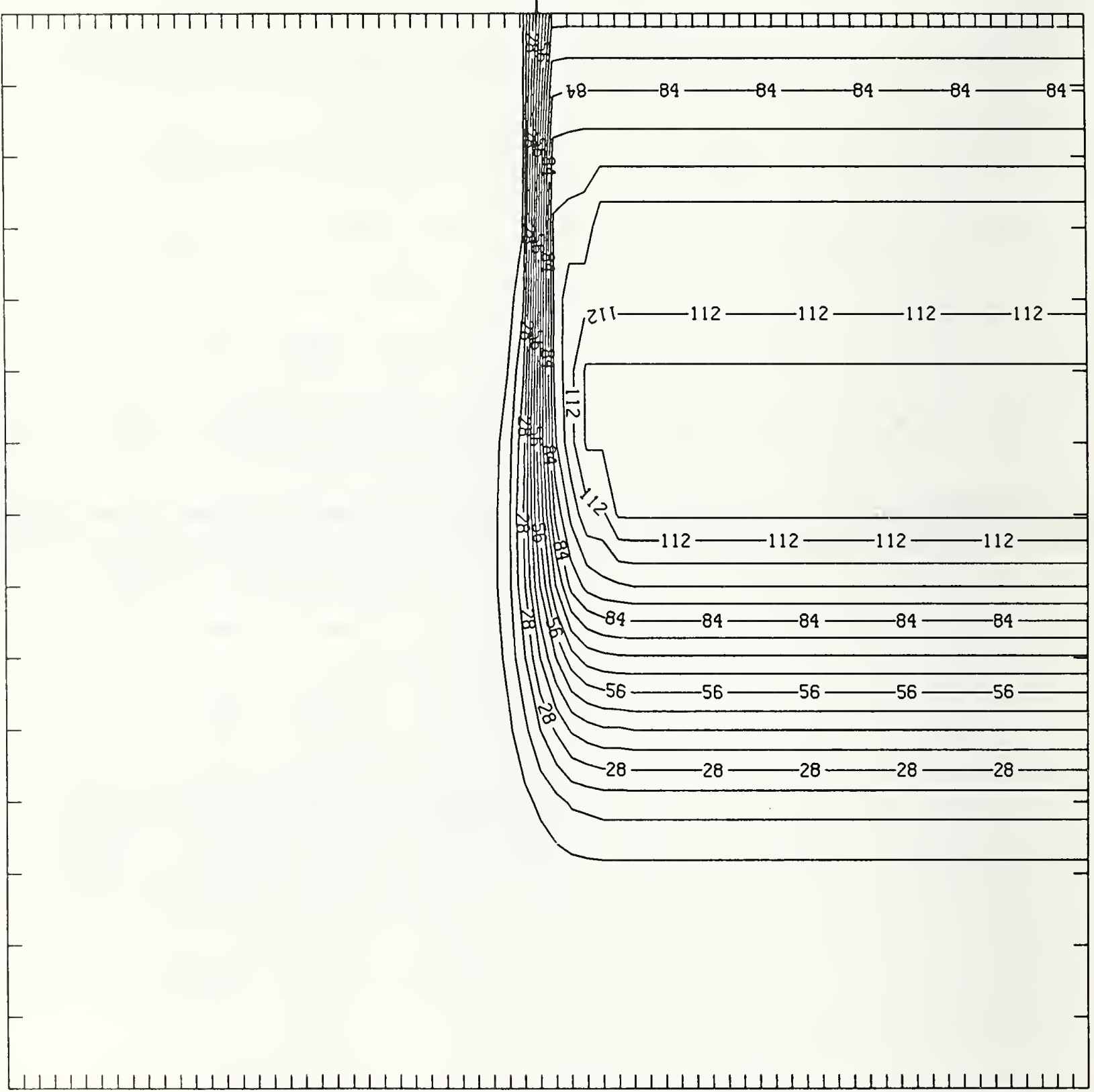

Figure Si225-4 Two-dimensional distribution of Frenkel pairs created by $225 \mathrm{keV}$ silicon implanted past a mask edge. The length increment (distance between tick marks) is $0.04 \mu \mathrm{m}$ and the scale factor is 0.1 . 


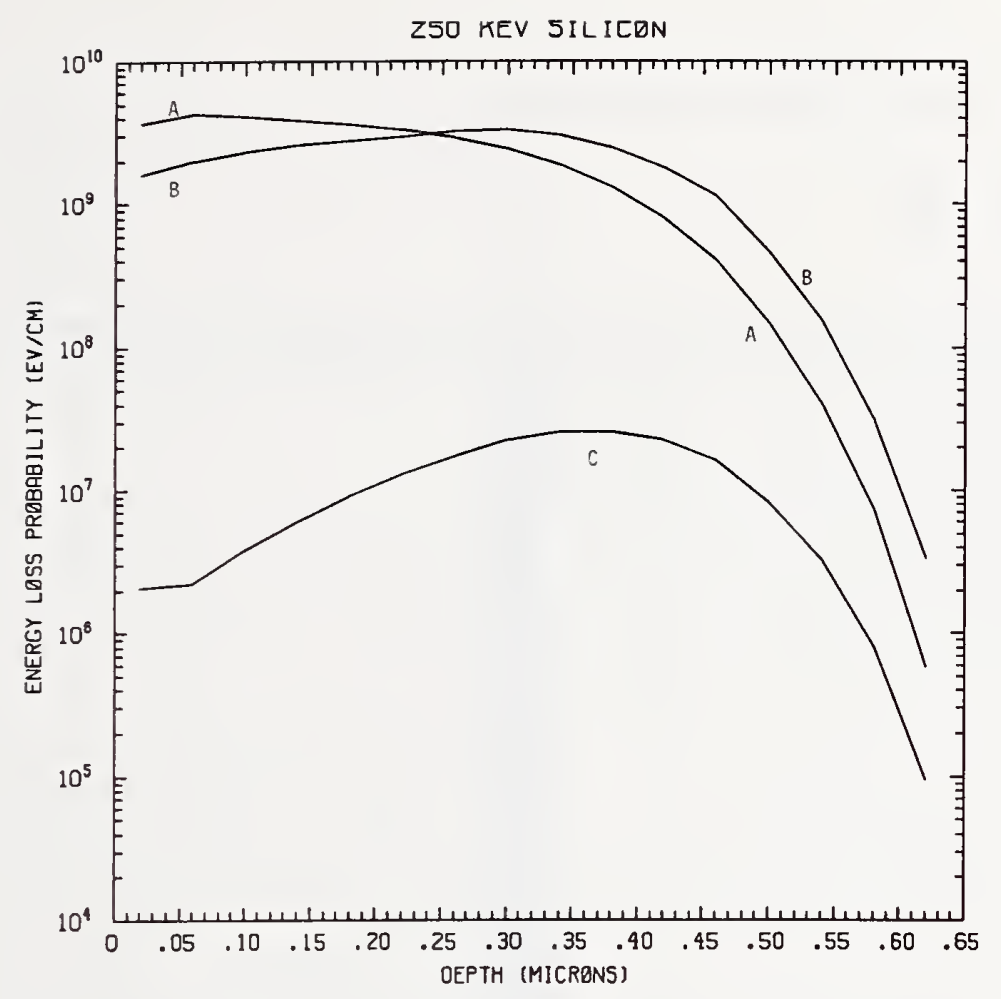

Figure Si250-1 One-dimensional probability distributions for energy loss due to electronic excitation (A), displacement damage(B), and phonon excitation (C) for $250 \mathrm{keV}$ silicon normally incident on a silicon target.

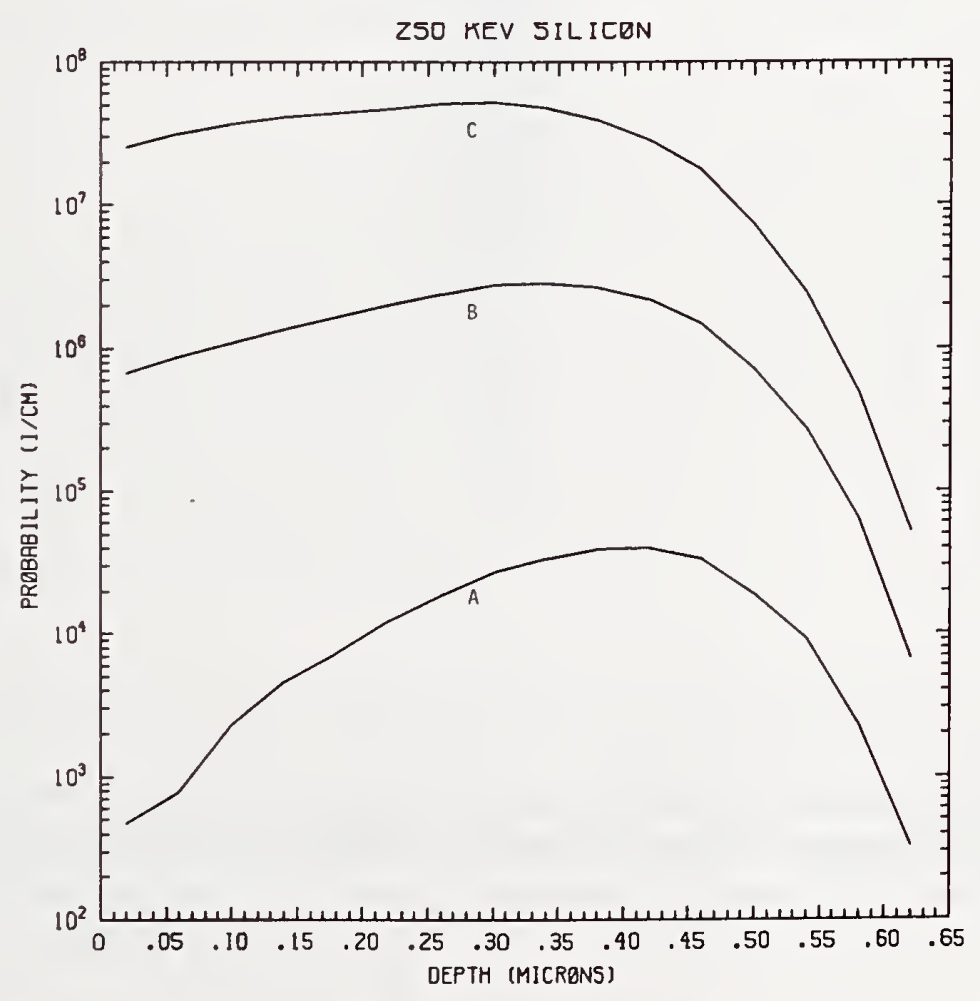

Figure Si250-2 One-dimensional probability distributions for implanted silicon (A), primary silicon displacements (B), and Frenkel pairs (C) for $250 \mathrm{keV}$ silicon normally incident on a silicon target. 


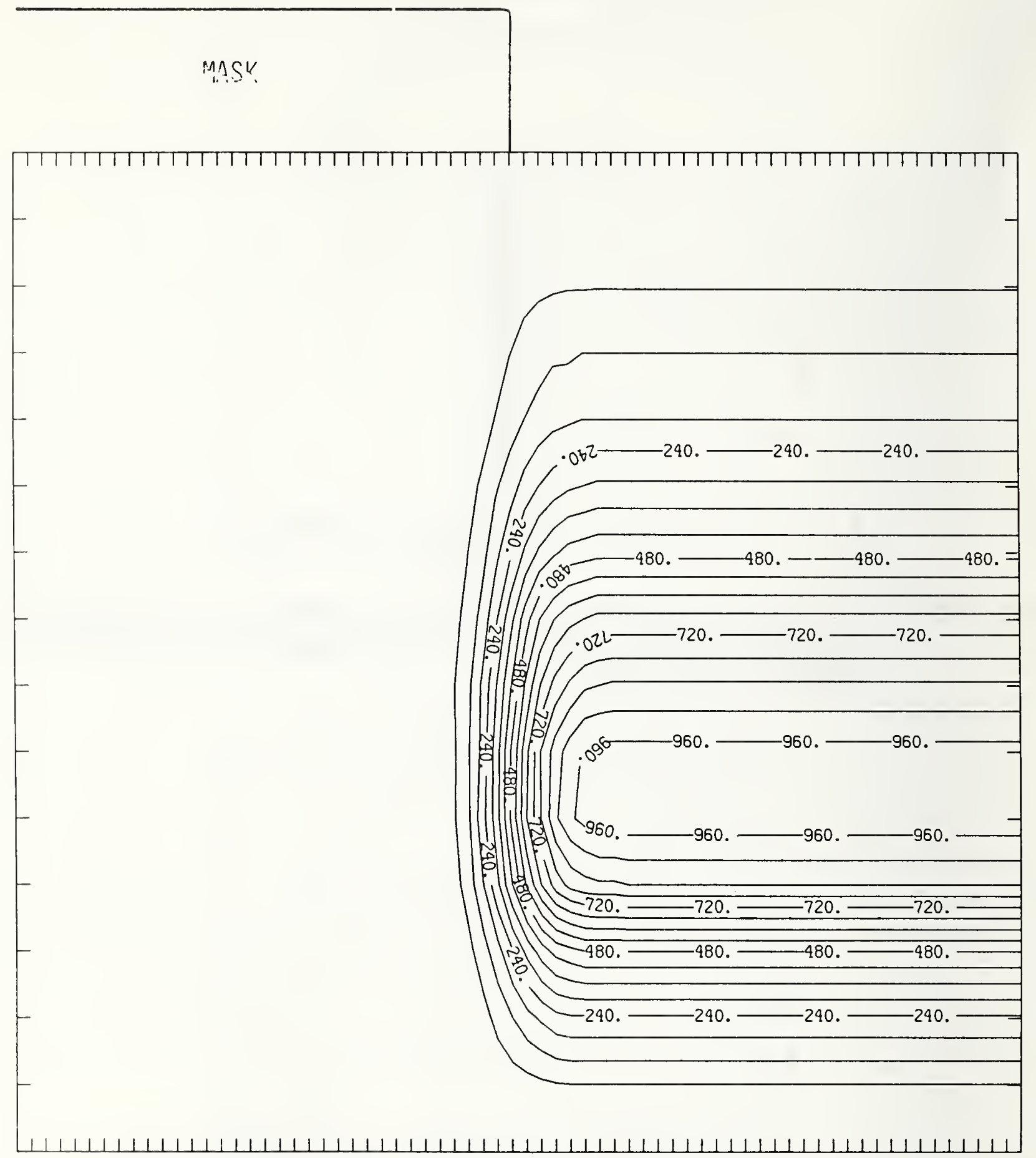

Figure Si250-3 Two-dimensional distribution of $250 \mathrm{keV}$ silicon implanted past a mask edge. The length increment (distance between tick marks) is $0.04 \mu \mathrm{m}$ and the scale factor is 1000 . 
MASK

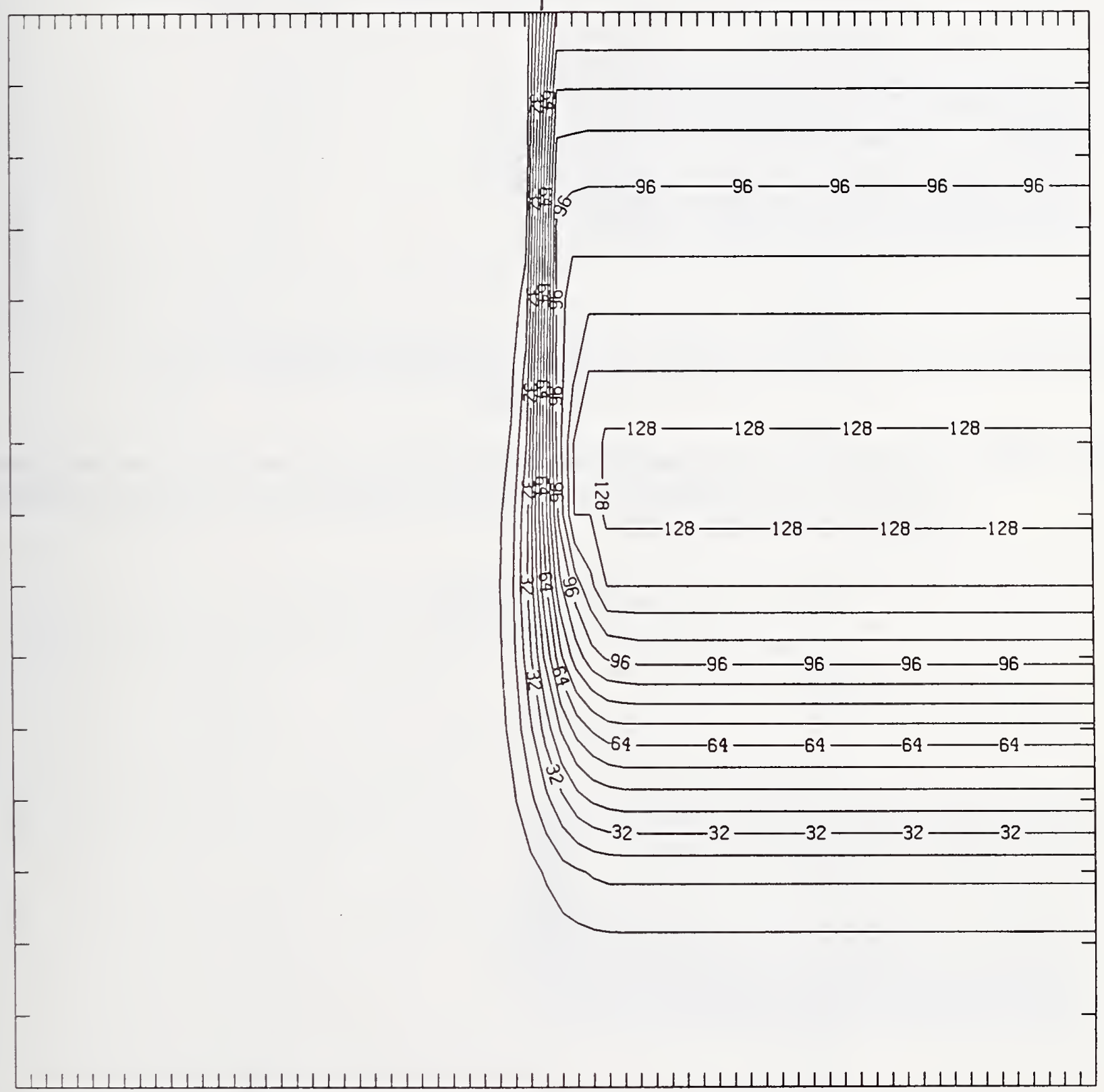

Figure Si250-4 Two-dimensional distribution of Frenkel pairs created by $250 \mathrm{keV}$ silicon implanted past a mask edge. The length increment (distance between tick marks) is $0.04 \mu \mathrm{m}$ and the scale factor is 0.1 . 
Z75 KEV SILICDN

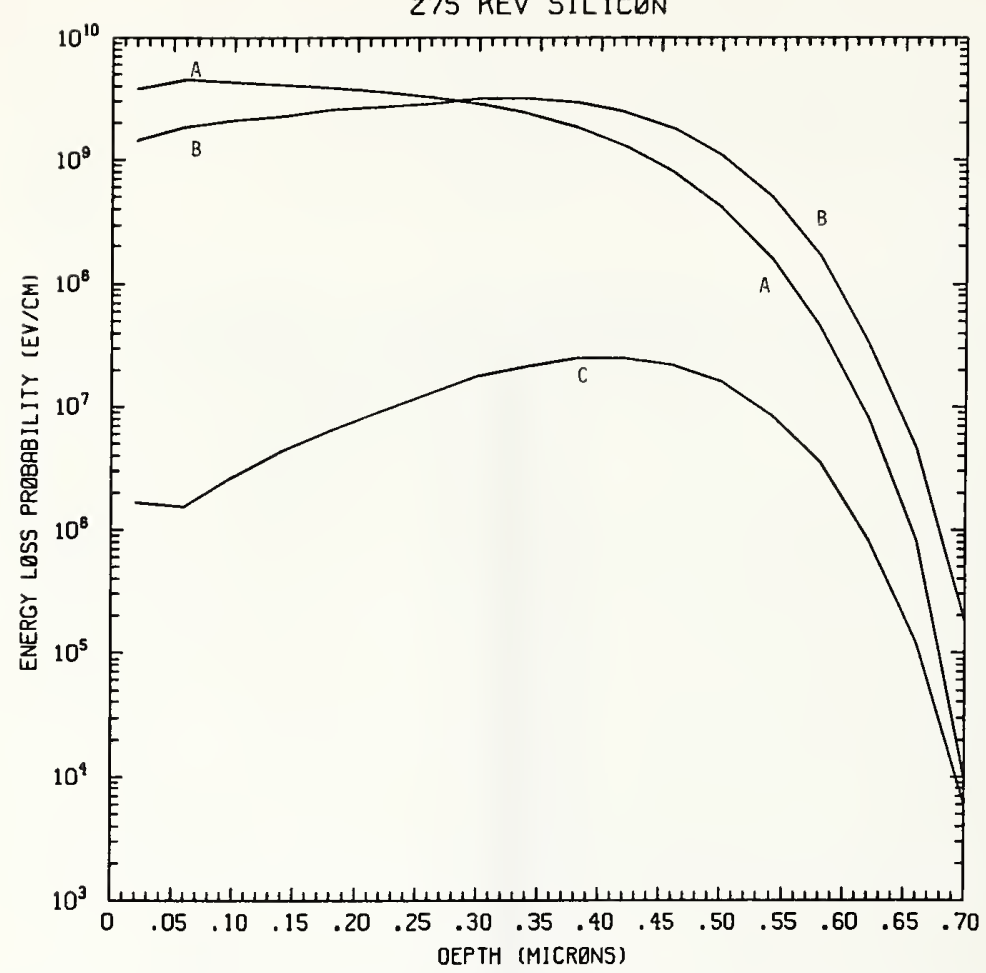

Figure Si275-1 One-dimensional probability distributions for energy loss due to electronic excitation (A), displacement damage(B), and phonon excitation (C) for $275 \mathrm{keV}$ silicon normally incident on a silicon target.

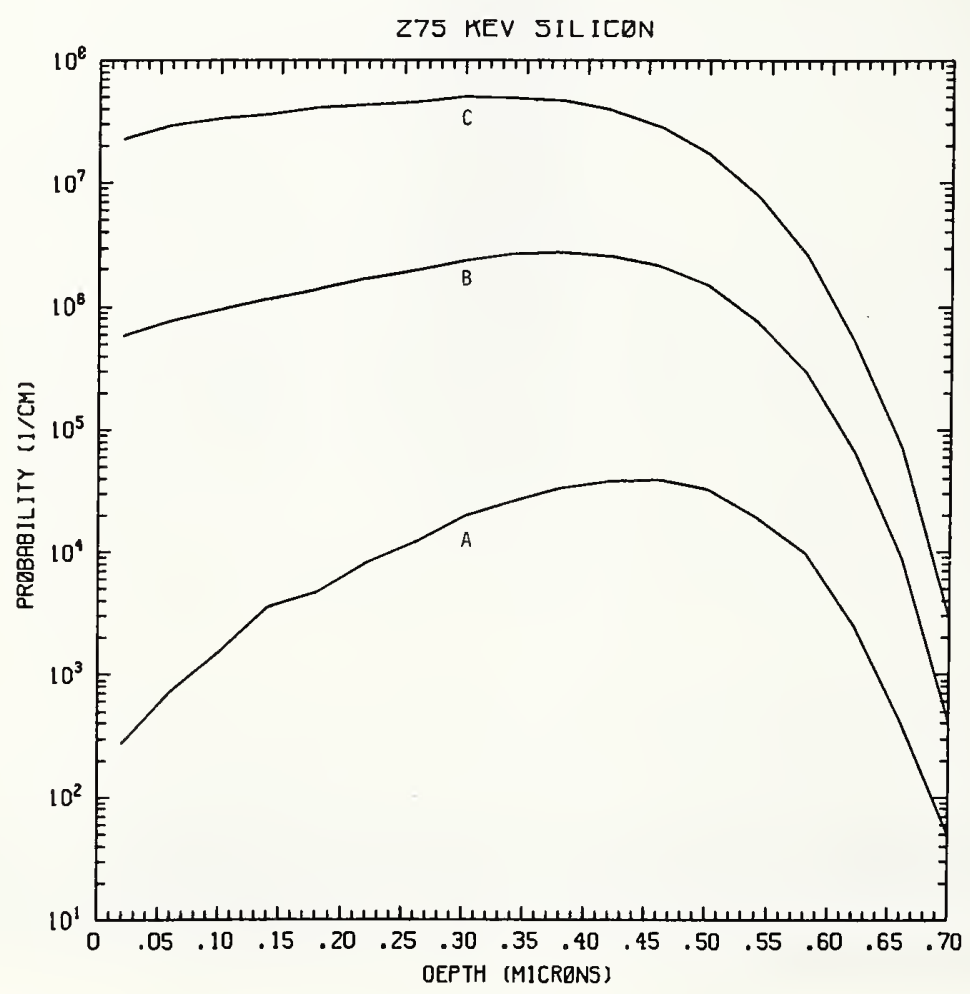

Figure Si275-2 One-dimensional probability distributions for implanted silicon (A), primary silicon displacements (B), and Frenkel pairs (C) for $275 \mathrm{keV}$ silicon normally incident on a silicon target. 
MASK

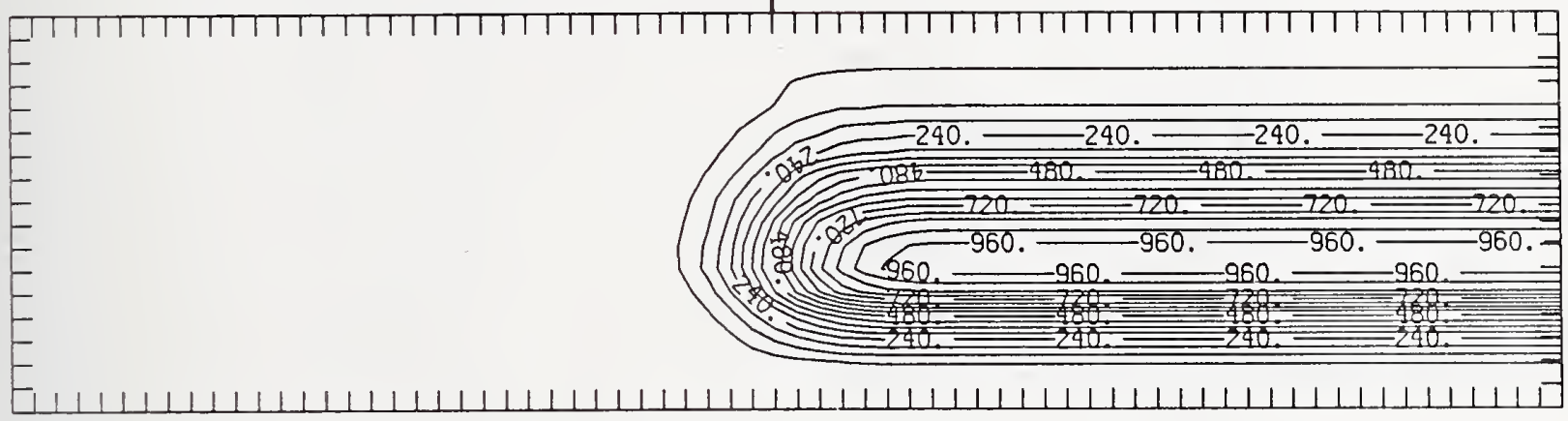

Figure Si275-3 Two-dimensional distribution of $275 \mathrm{keV}$ silicon implanted past a mask edge. The length increment (distance between tick marks) is $0.04 \mu \mathrm{m}$ and the scale factor is 1000.

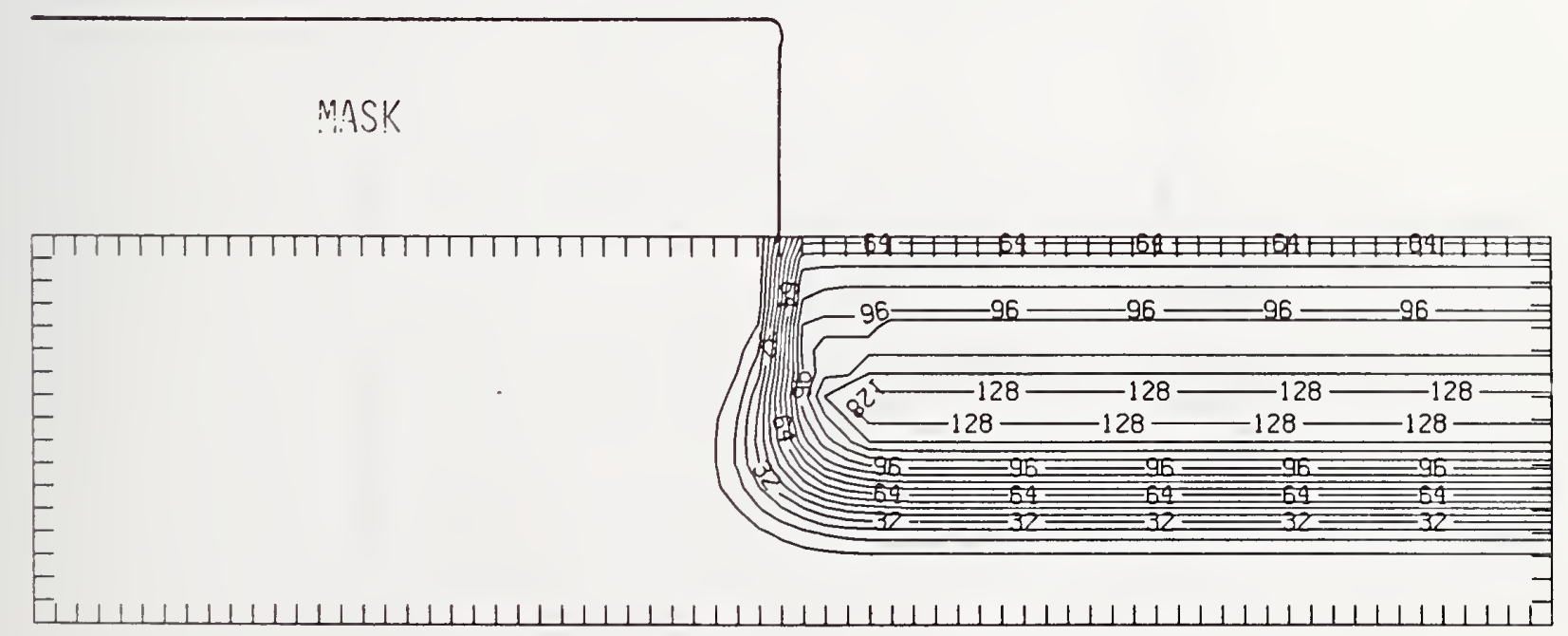

Figure Si275-4 Two-dimensional distribution of Frenkel pairs created by $275 \mathrm{keV}$ silicon implanted past a mask edge. The length increment (distance between tick marks) is $0.04 \mu \mathrm{m}$ and the scale factor is 0.1 . 


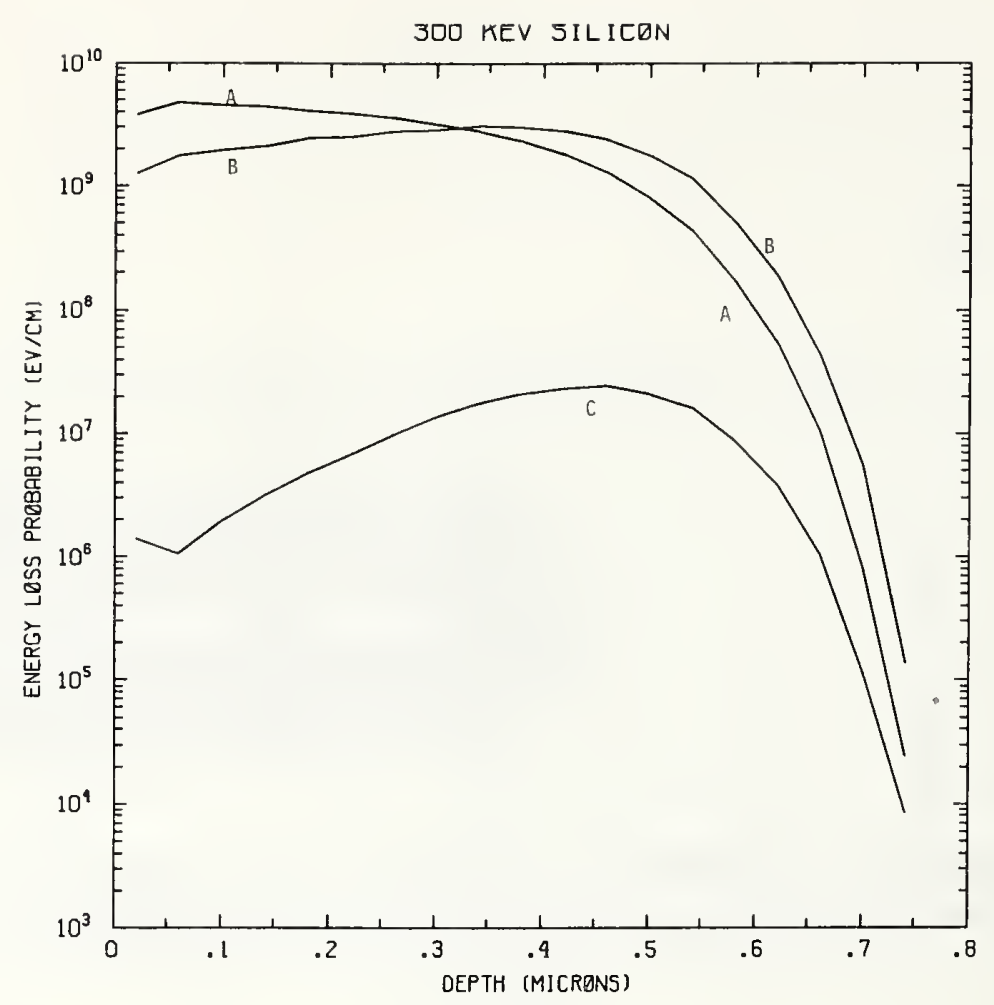

Figure Si300-1 One-dimensional probability distributions for energy loss due to electronic excitation (A), displacement damage(B), and phonon excitation (C) for $300 \mathrm{keV}$ silicon normally incident on a silicon target.

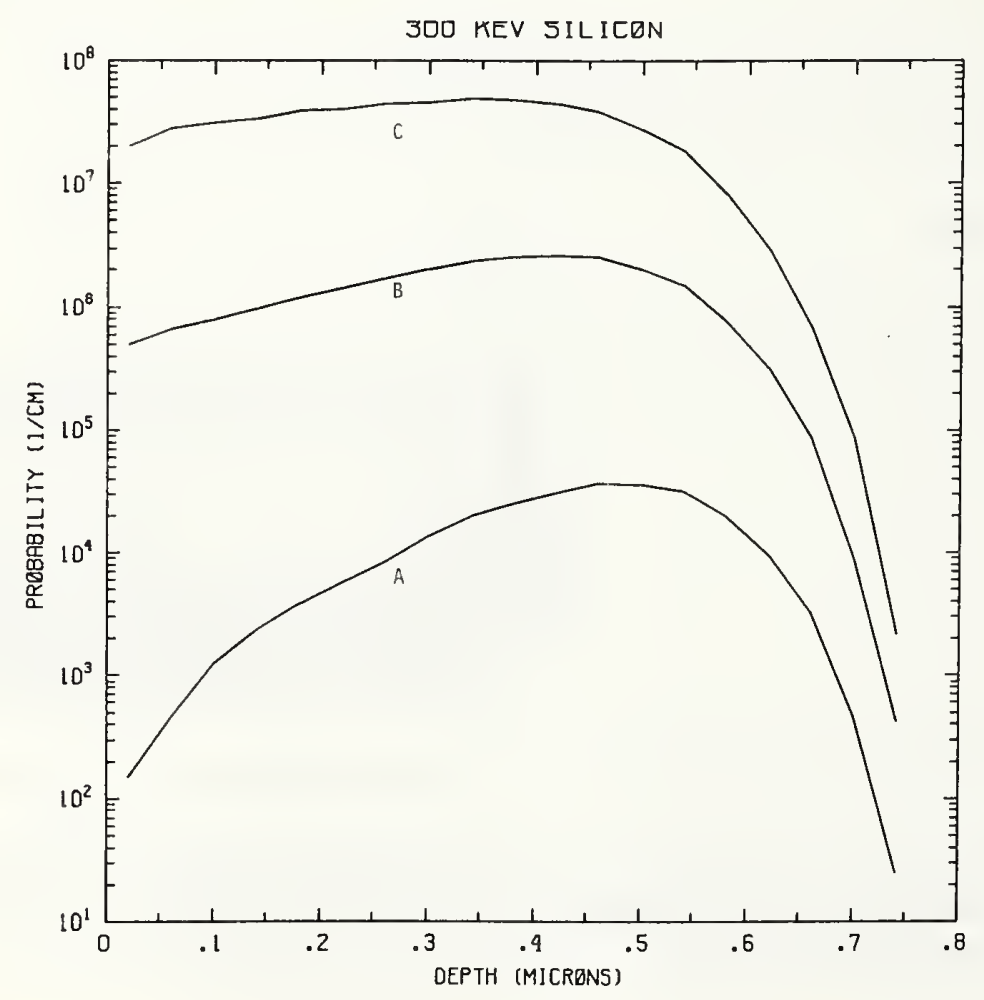

Figure Si300-2 One-dimensional probability distributions for implanted silicon (A), primary silicon displacements (B), and Frenkel pairs (C) for $300 \mathrm{keV}$ silicon normally incident on a silicon target. 


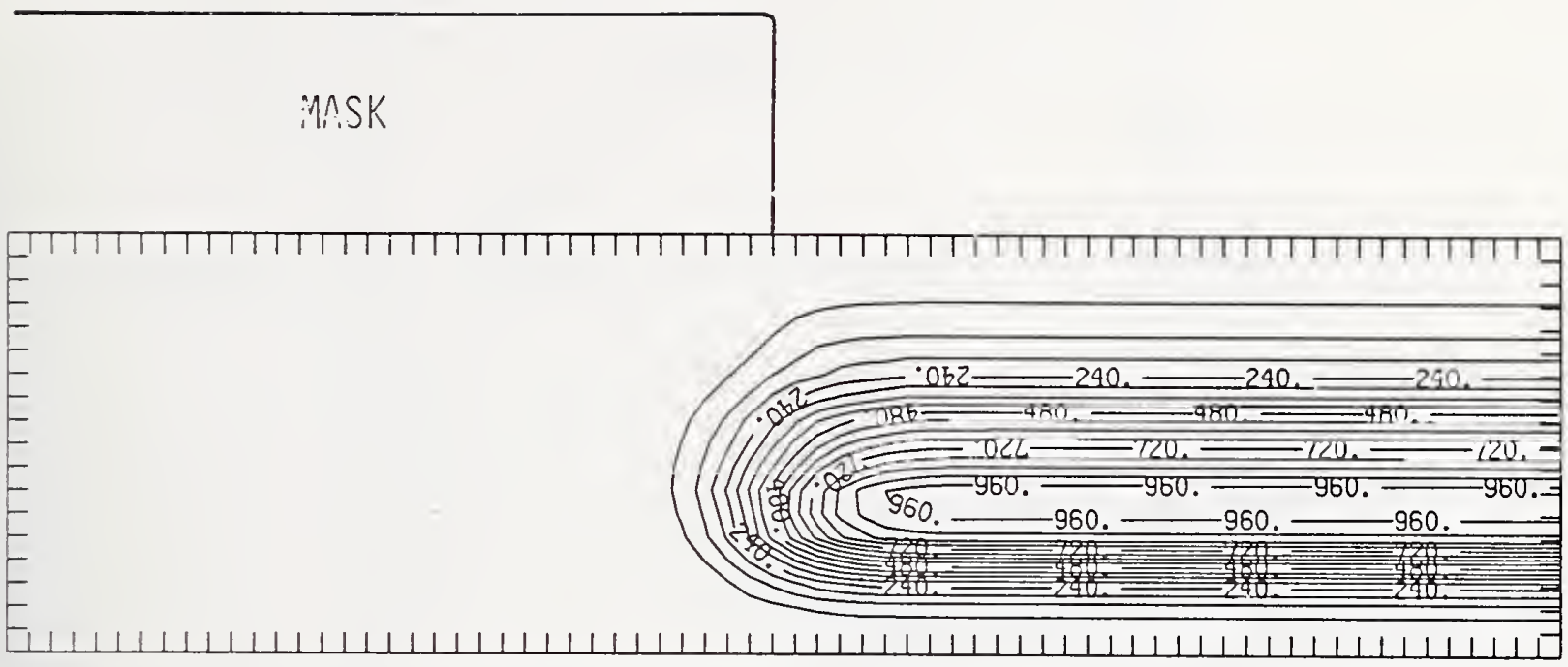

Figure Si300-3 Two-dimensional distribution of $300 \mathrm{keV}$ silicon implanted past a mask edge. The length increment (distance between tick marks) is $0.04 \mu \mathrm{m}$ and the scale factor is 1000 .

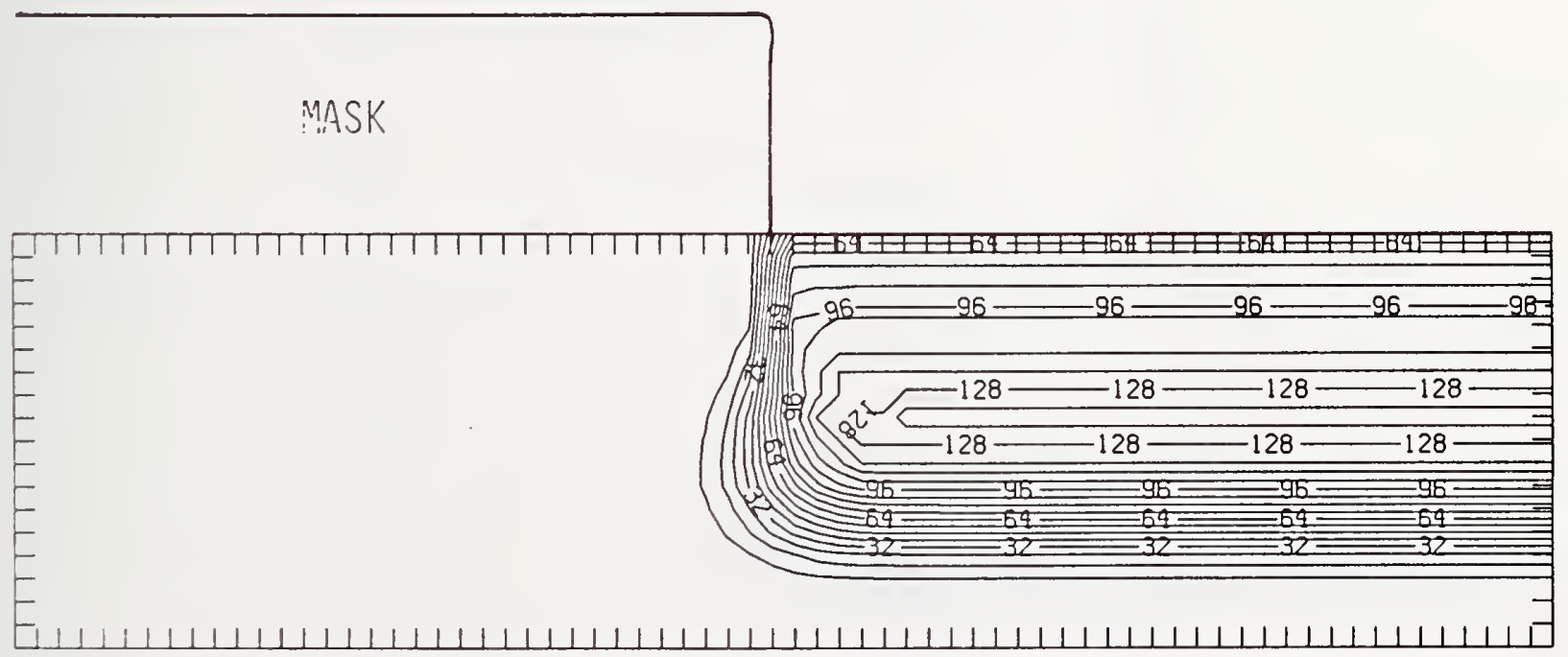

Figure Si300-4 Two-dimensional distribution of Frenkel pairs created by $300 \mathrm{keV}$ silicon implanted past a mask edge. The length increment (distance between tick marks) is $0.04 \mu \mathrm{m}$ and the scale factor is 0.1 . 


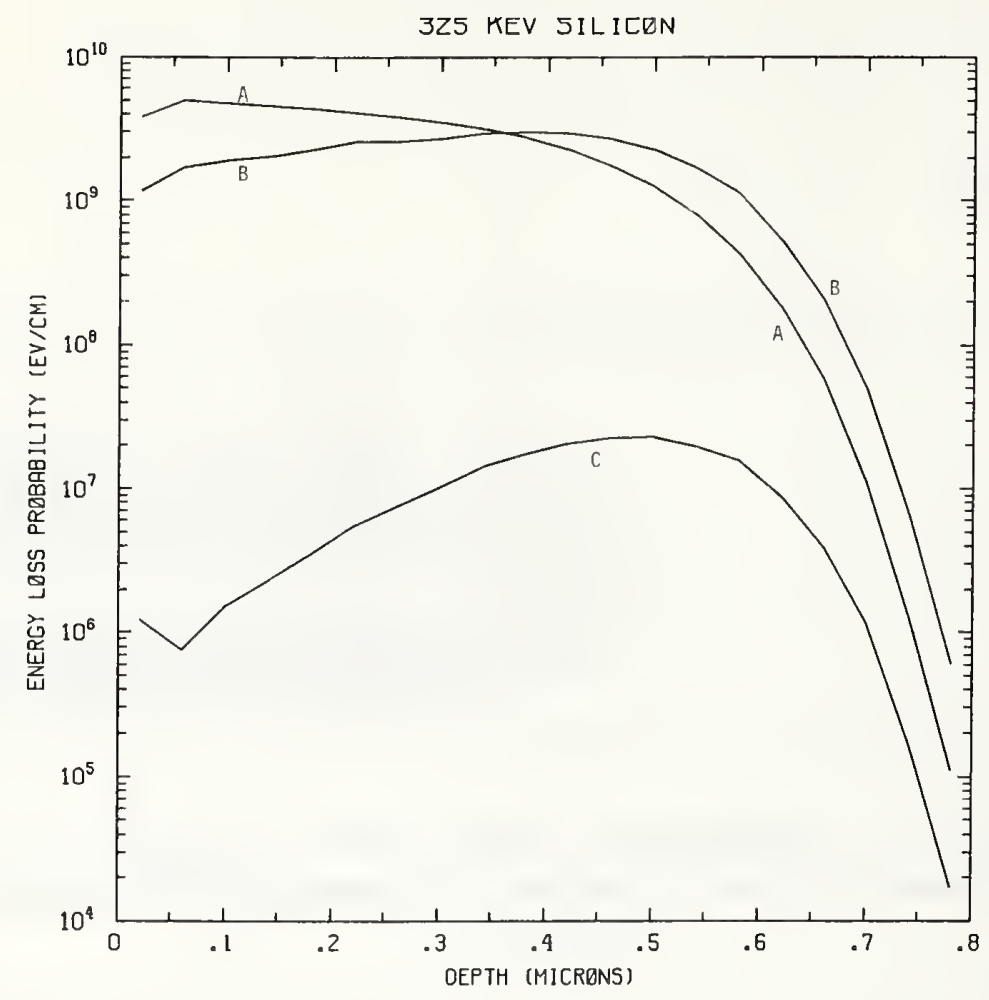

Figure Si325-1 One-dimensional probability distributions for energy loss due to electronic excitation (A), displacement damage(B), and phonon excitation (C) for $325 \mathrm{keV}$ silicon normally incident on a silicon target.

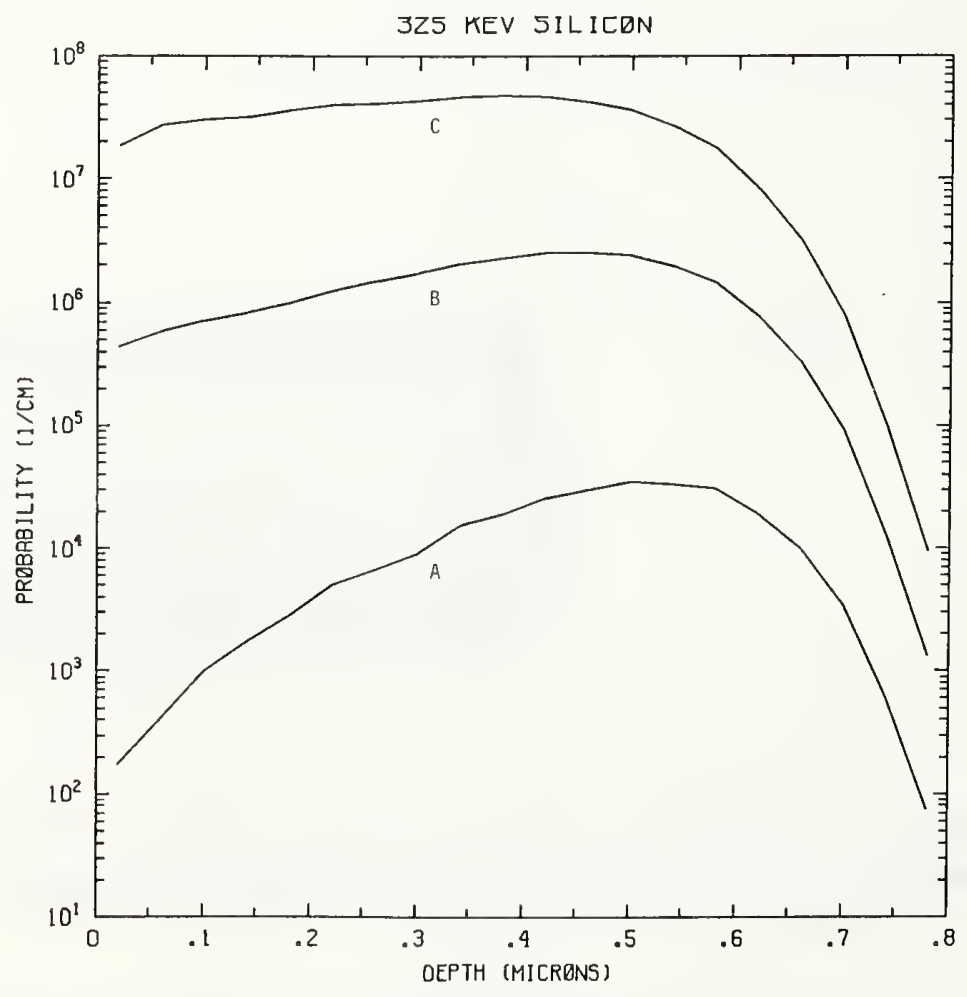

Figure Si325-2 One-dimensional probability distributions for implanted silicon (A), primary silicon displacements (B), and Frenkel pairs (C) for $325 \mathrm{keV}$ silicon normally incident on a silicon target. 


\section{MASK}

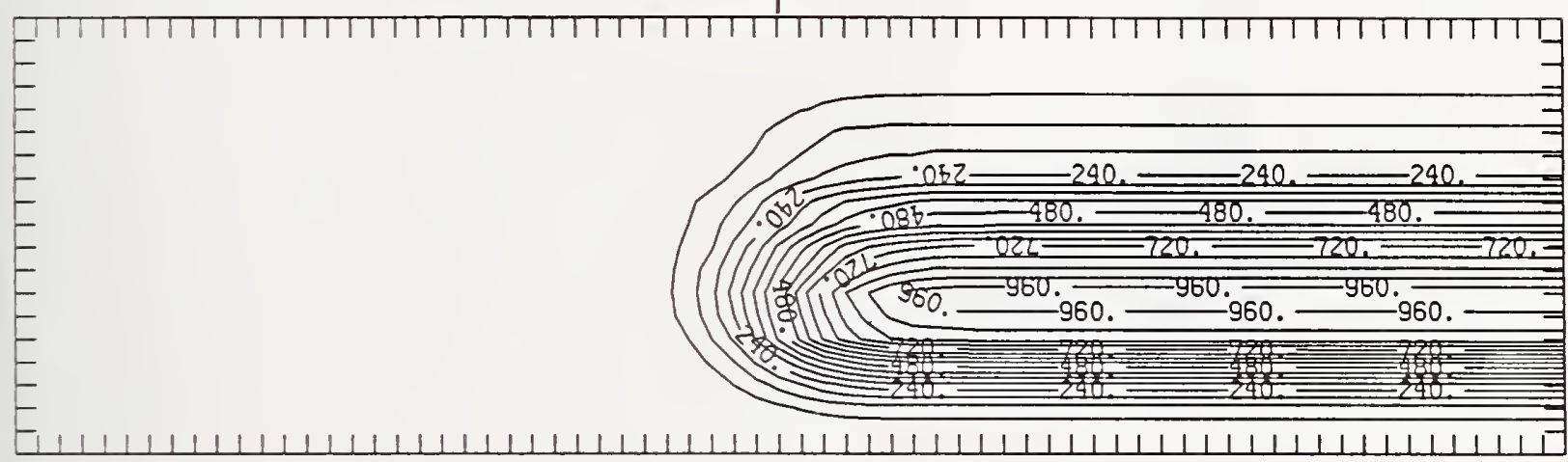

Figure Si325-3 Two-dimensional distribution of $325 \mathrm{keV}$ silicon implanted past a mask edge. The length increment (distance between tick marks) is $0.04 \mu \mathrm{m}$ and the scale factor is 1000 .

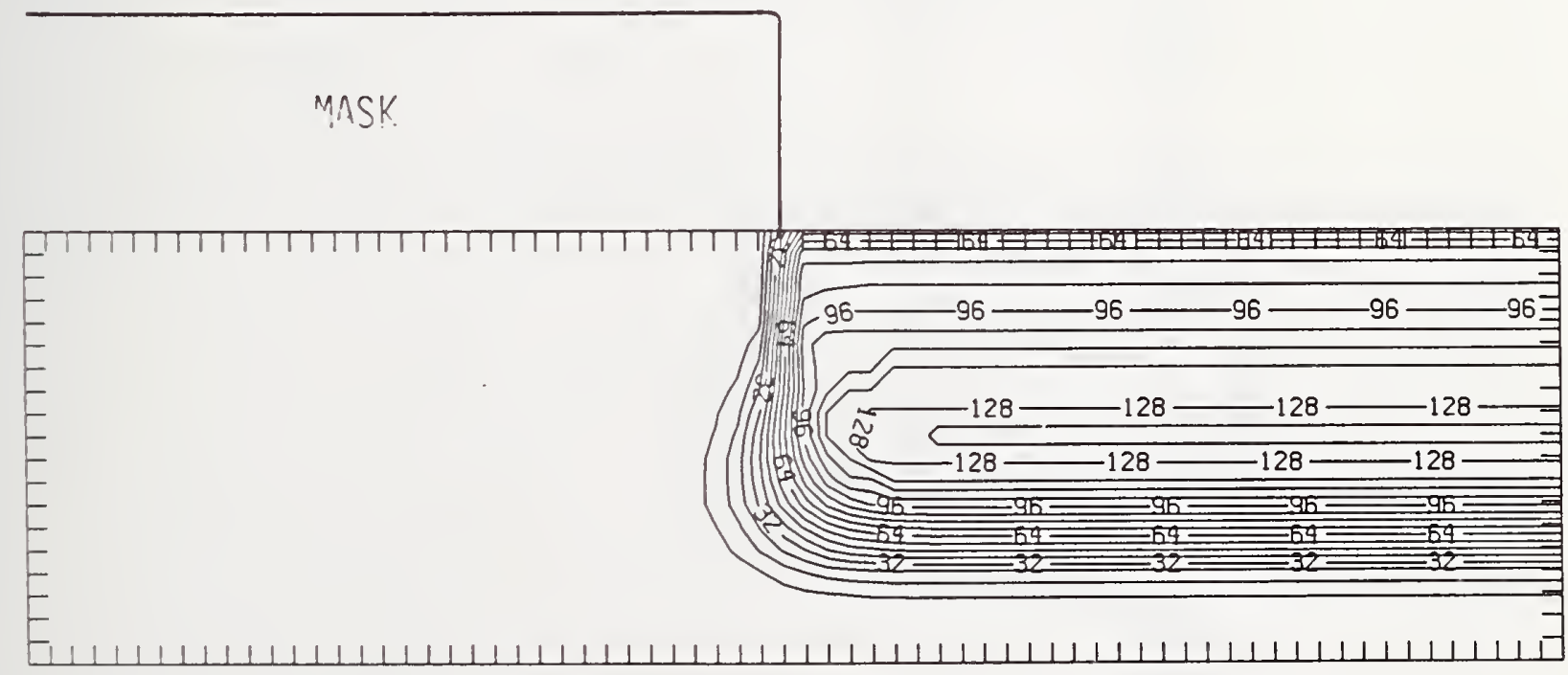

Figure Si325-4 Two-dimensional distribution of Frenkel pairs created by $325 \mathrm{keV}$ silicon implanted past a mask edge. The length increment (distance between tick marks) is $0.04 \mu \mathrm{m}$ and the scale factor is 0.1 . 


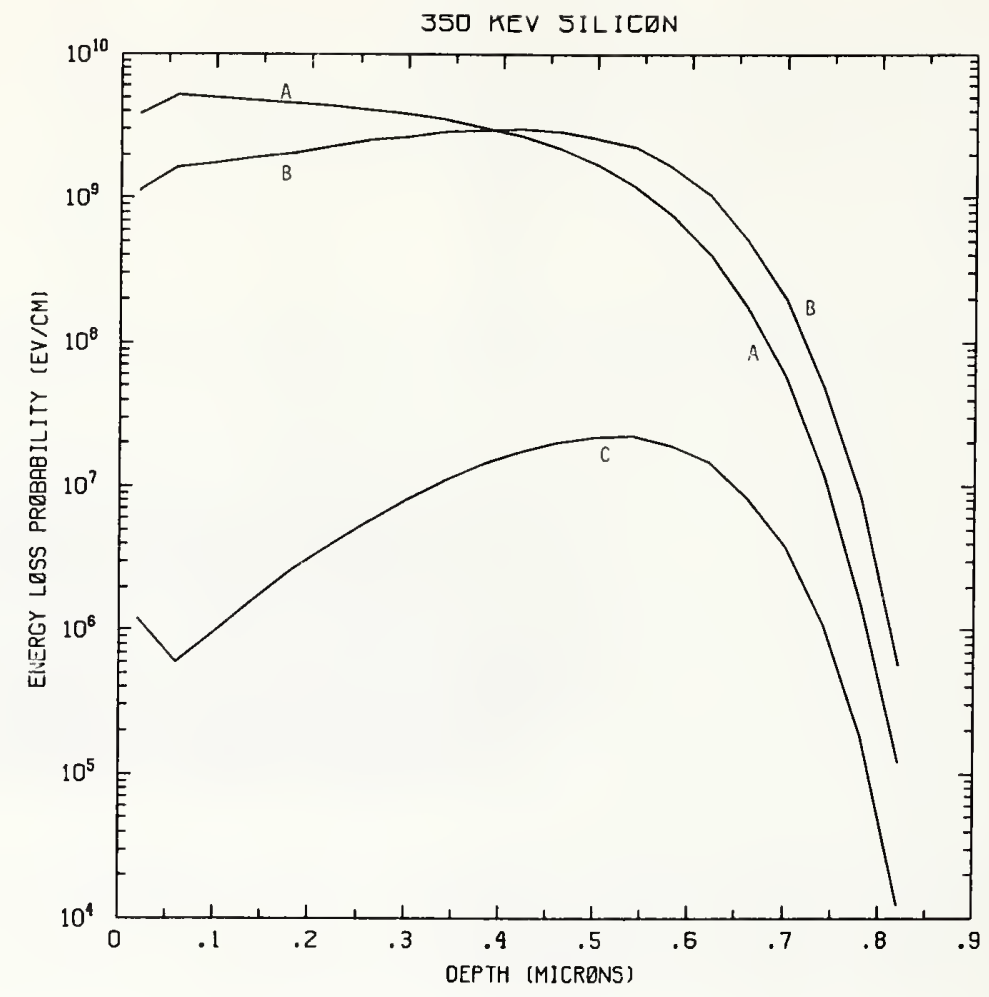

Figure Si350-1 One-dimensional probability distributions for energy loss due to electronic excitation (A), displacement damage(B), and phonon excitation (C) for $350 \mathrm{keV}$ silicon normally incident on a silicon target.

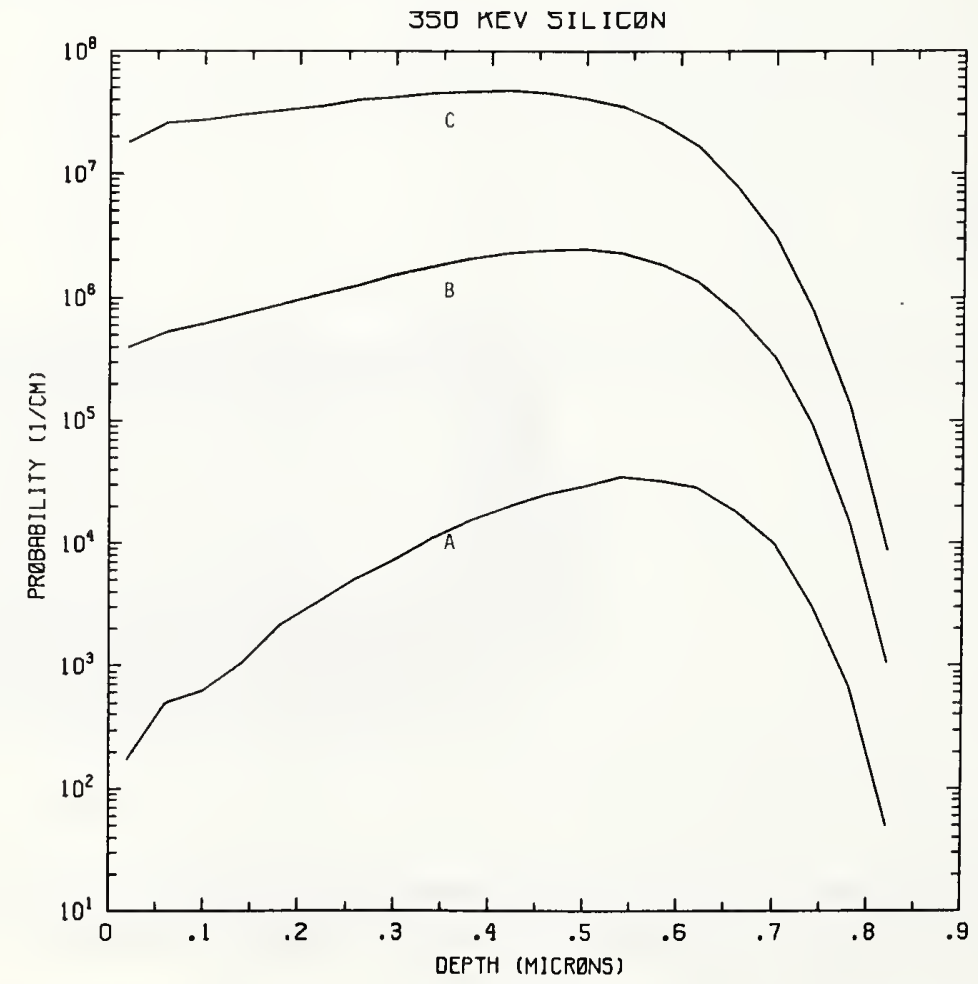

Figure Si350-2 One-dimensional probability distributions for implanted silicon (A), primary silicon displacements (B), and Frenkel pairs (C) for $350 \mathrm{keV}$ silicon normally incident on a silicon target. 
$M \triangle S K$

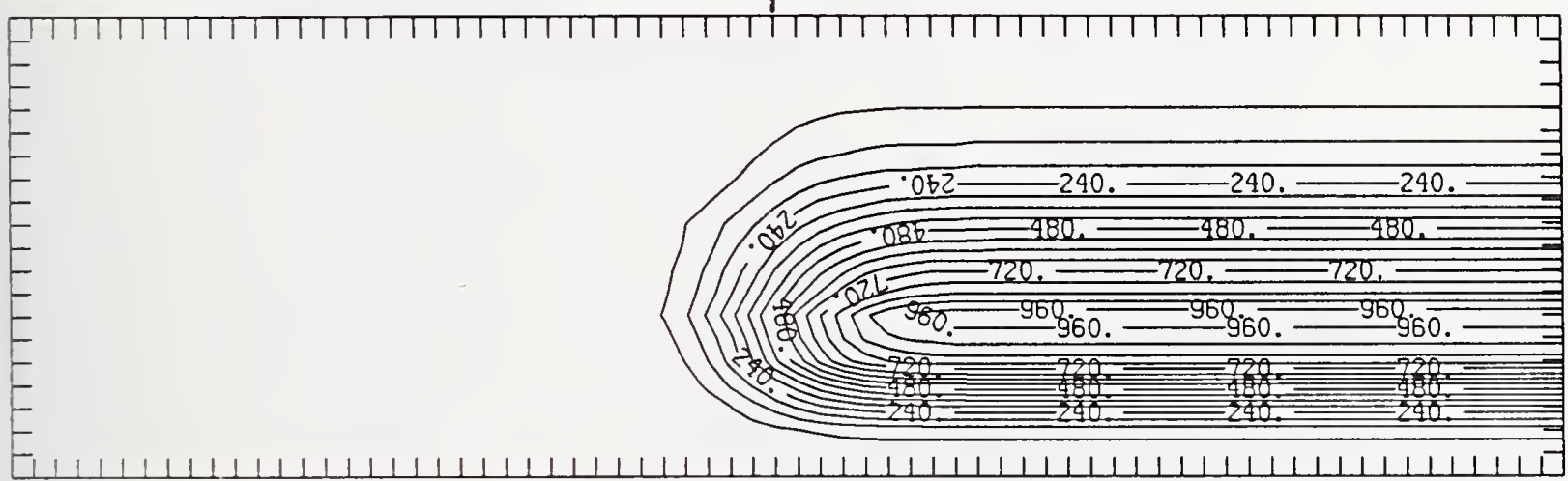

Figure Si350-3 Two-dimensional distribution of $350 \mathrm{keV}$ silicon implanted past a mask edge. The length increment (distance between tick marks) is $0.04 \mu \mathrm{m}$ and the scale factor is 1000 .

MSSK

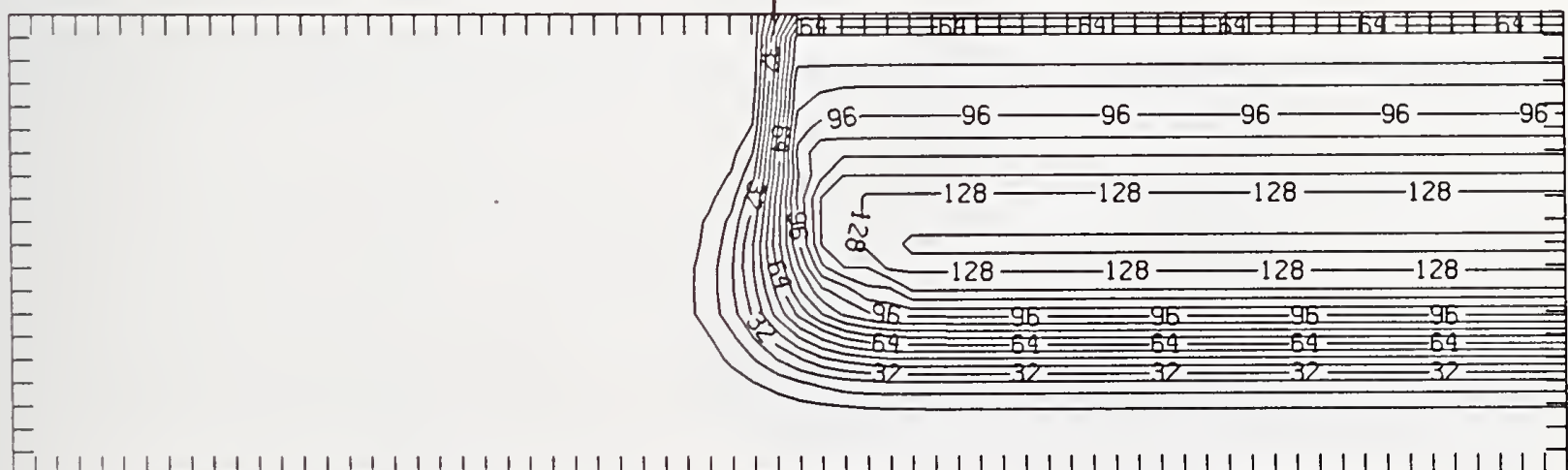

Figure Si350-4 Two-dimensional distribution of Frenkel pairs created by $350 \mathrm{keV}$ silicon implanted past a mask edge. The length increment (distance between tick marks) is $0.04 \mu \mathrm{m}$ and the scale factor is 0.1 . 


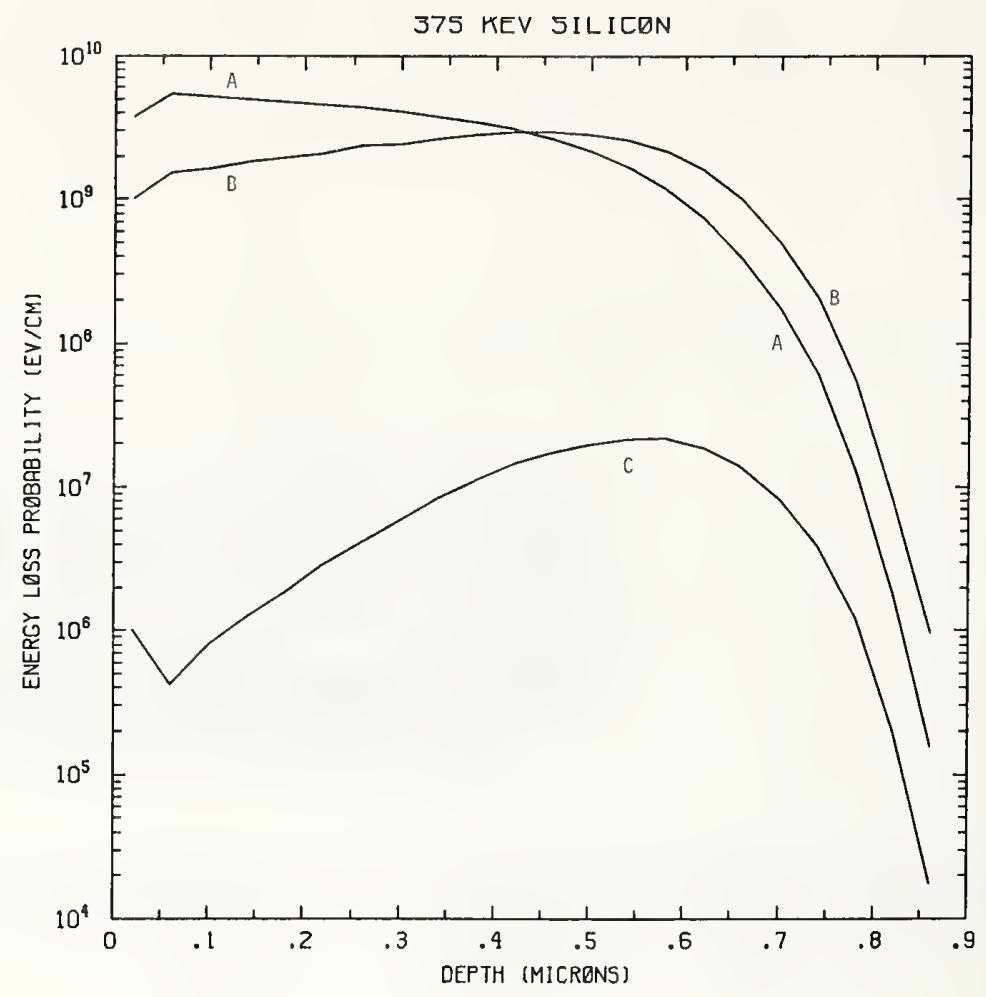

Figure Si375-1 One-dimensional probability distributions for energy loss due to electronic excitation (A), displacement damage(B), and phonon excitation (C) for $375 \mathrm{keV}$ silicon normally incident on a silicon target.

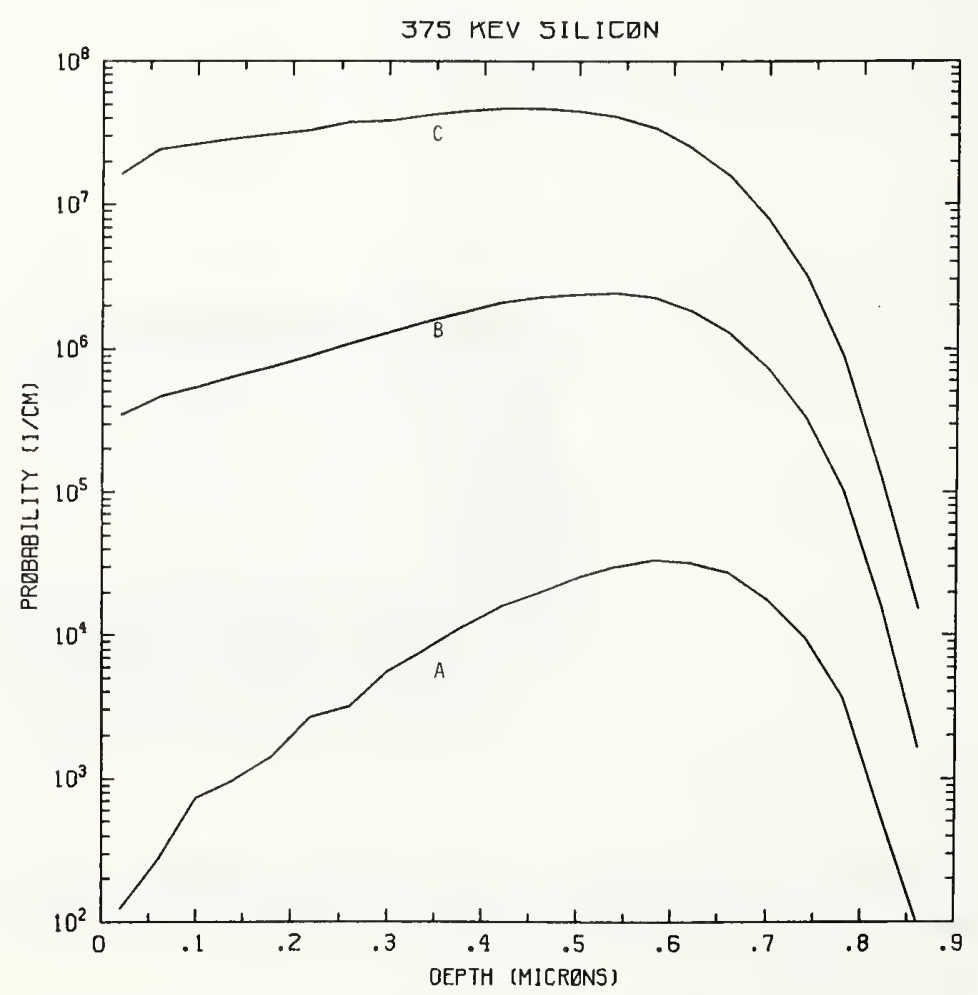

Figure Si375-2 One-dimensional probability distributions for implanted silicon (A), primary silicon displacements (B), and Frenkel pairs (C) for $375 \mathrm{keV}$ silicon normally incident on a silicon target. 
MASK

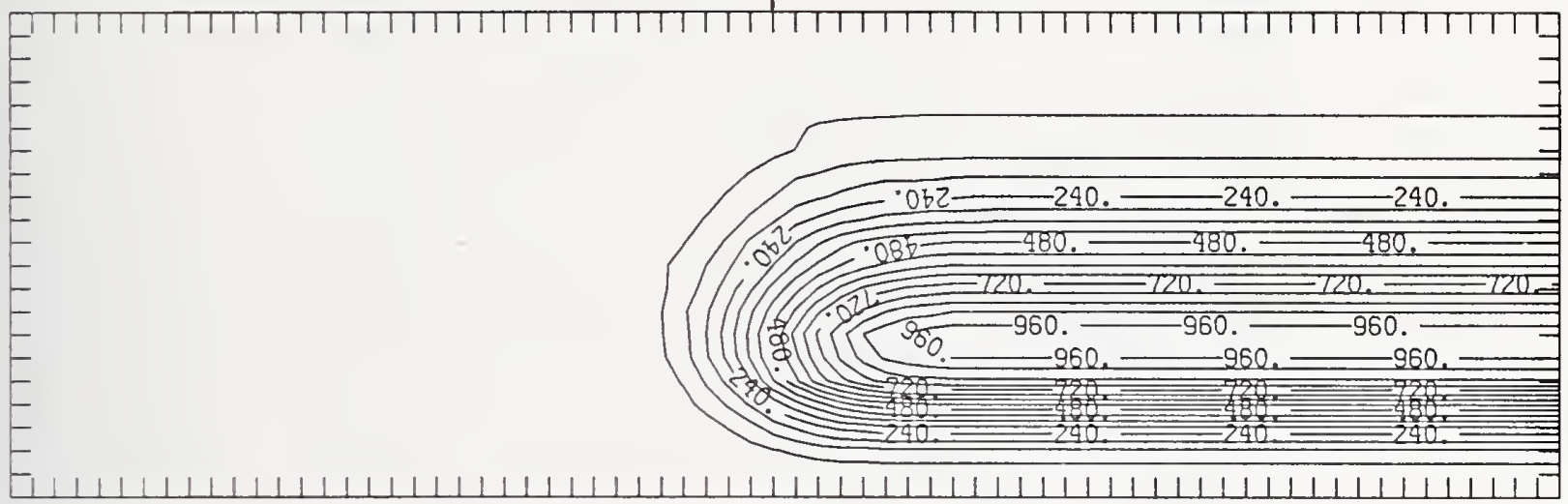

Figure Si375-3 Two-dimensional distribution of $375 \mathrm{keV}$ silicon implanted past a mask edge. The length increment (distance between tick marks) is $0.04 \mu \mathrm{m}$ and the scale factor is 1000 .

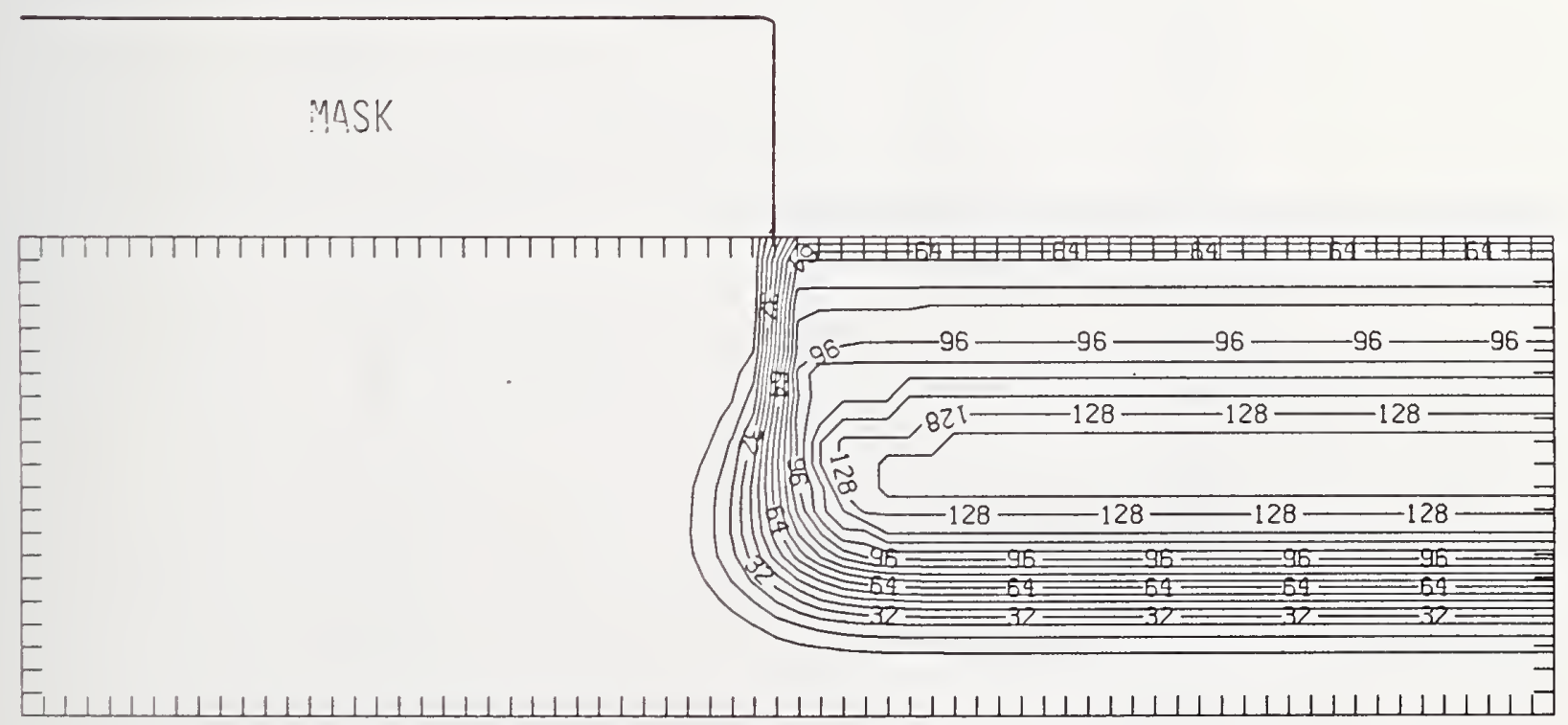

Figure Si375-4 Two-dimensional distribution of Frenkel pairs created by $375 \mathrm{keV}$ silicon implanted past a mask edge. The length increment (distance between tick marks) is $0.04 \mu \mathrm{m}$ and the scale factor is 0.1 . 


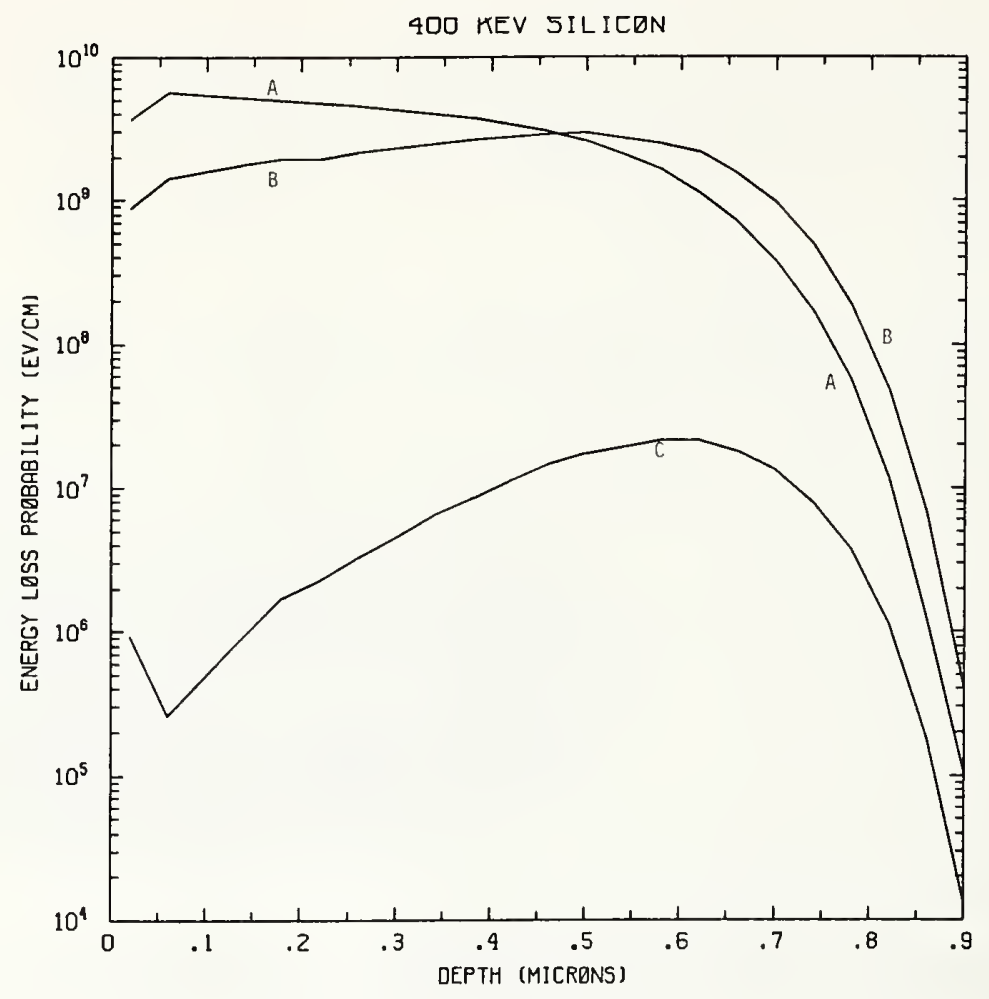

Figure Si400-1 One-dimensional probability distributions for energy loss due to electronic excitation (A), displacement damage(B), and phonon excitation (C) for $400 \mathrm{keV}$ silicon normally incident on a silicon target.

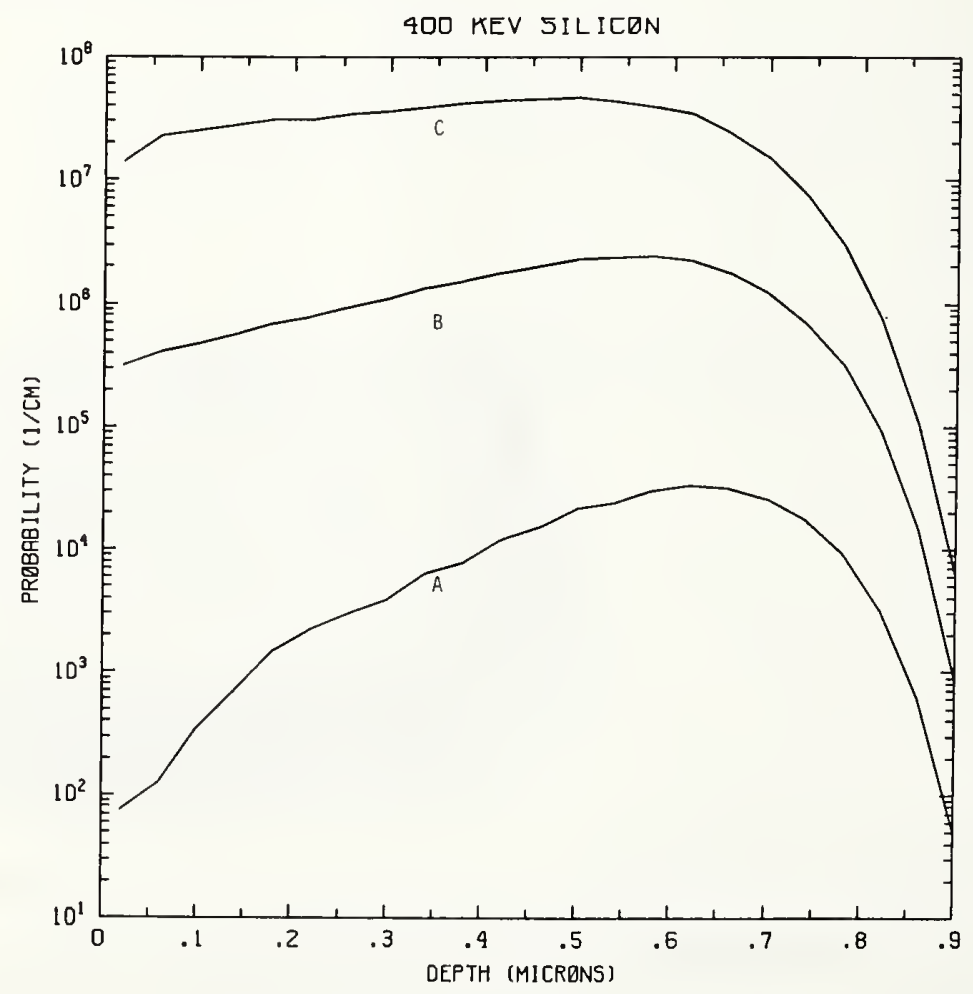

Figure Si400-2 One-dimensional probability distributions for implanted silicon (A), primary silicon displacements (B), and Frenkel pairs (C) for $400 \mathrm{keV}$ silicon normally incident on a silicon target. 


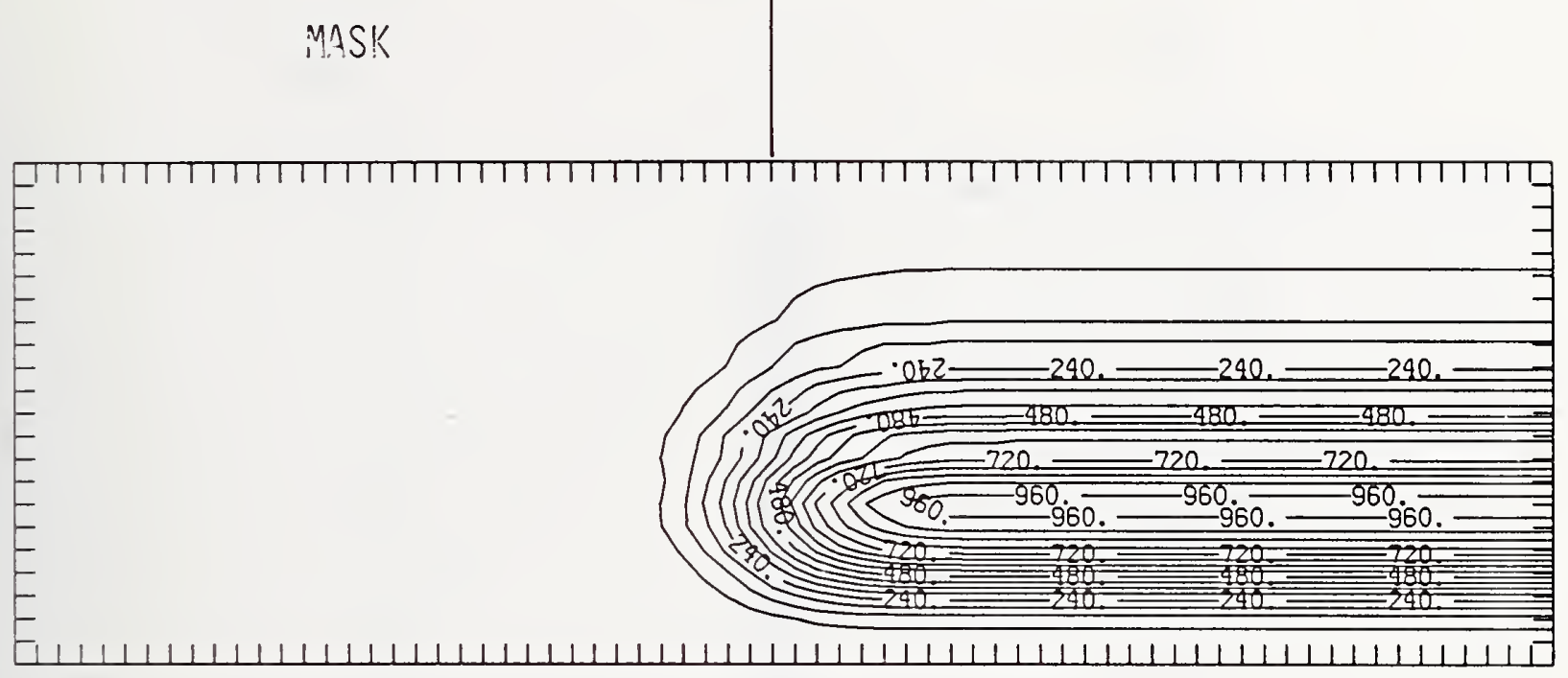

Figure Si400-3 Two-dimensional distribution of $400 \mathrm{keV}$ silicon implanted past a mask edge. The length increment (distance between tick marks) is $0.04 \mu \mathrm{m}$ and the scale factor is 1000 .

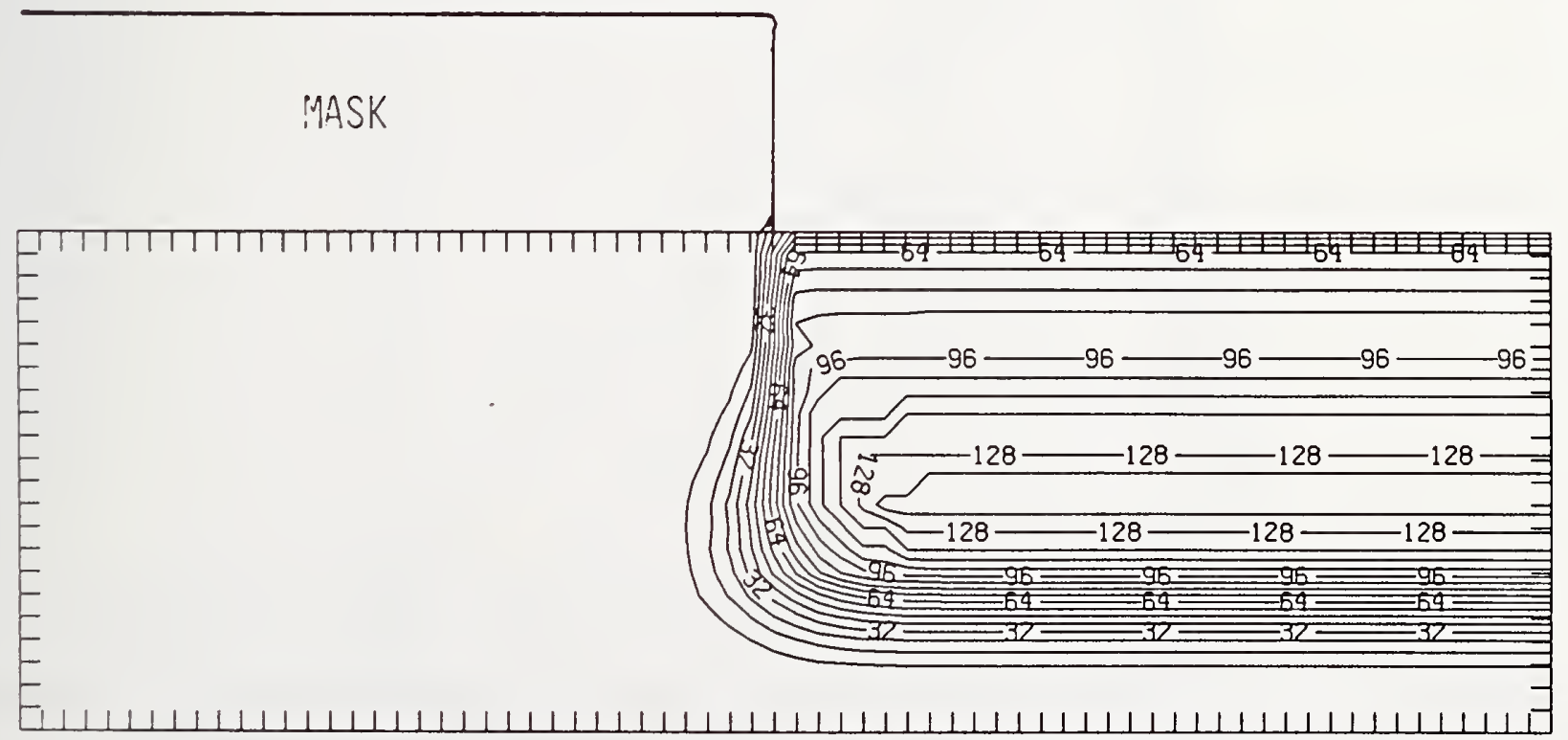

Figure Si400-4 Two-dimensional distribution of Frenkel pairs created by $400 \mathrm{keV}$ silicon implanted past a mask edge. The length increment (distance between tick marks) is $0.04 \mu \mathrm{m}$ and the scale factor is 0.1 . 


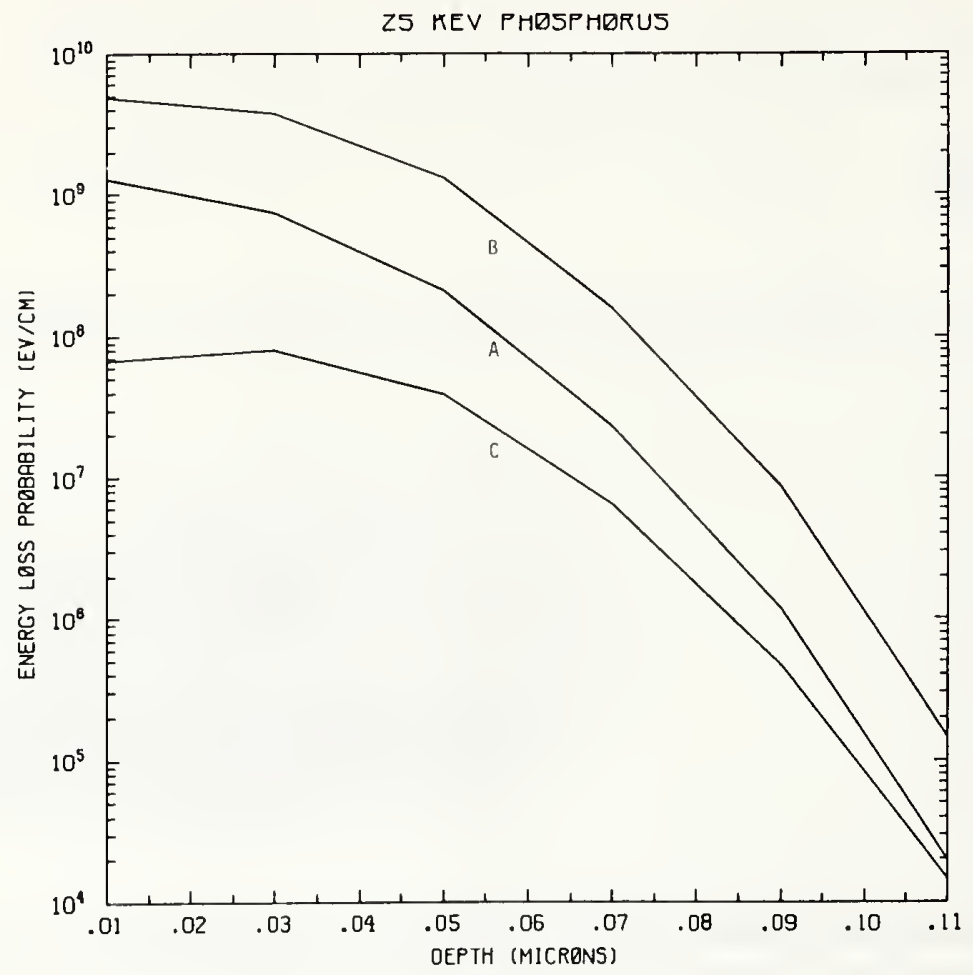

Figure P25-1 One-dimensional probability distributions for energy loss due to electronic excitation (A), displacement damage(B), and phonon excitation (C) for $25 \mathrm{keV}$ phosphorus normally incident on a silicon target.

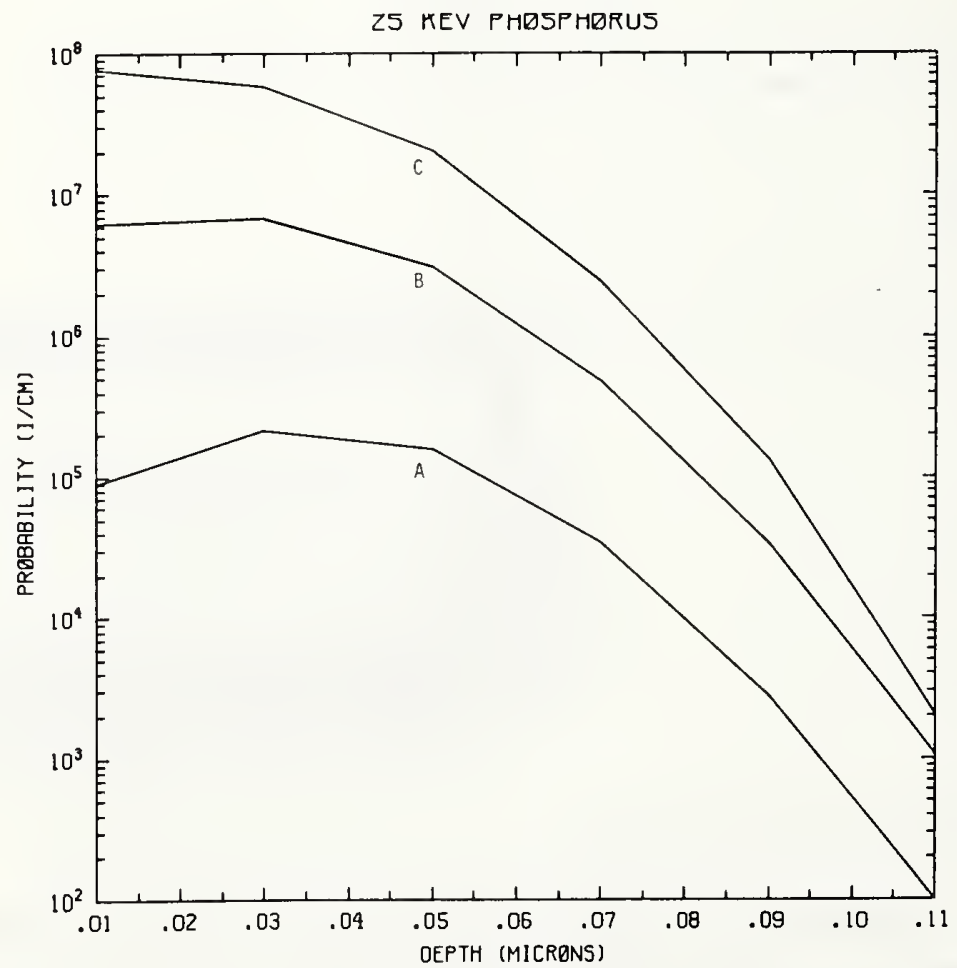

Figure P25-2 One-dimensional probability distributions for implanted phosphorus (A), primary silicon displacements (B), and Frenkel pairs (C) for $25 \mathrm{keV}$ phosphorus normally incident on a silicon target. 


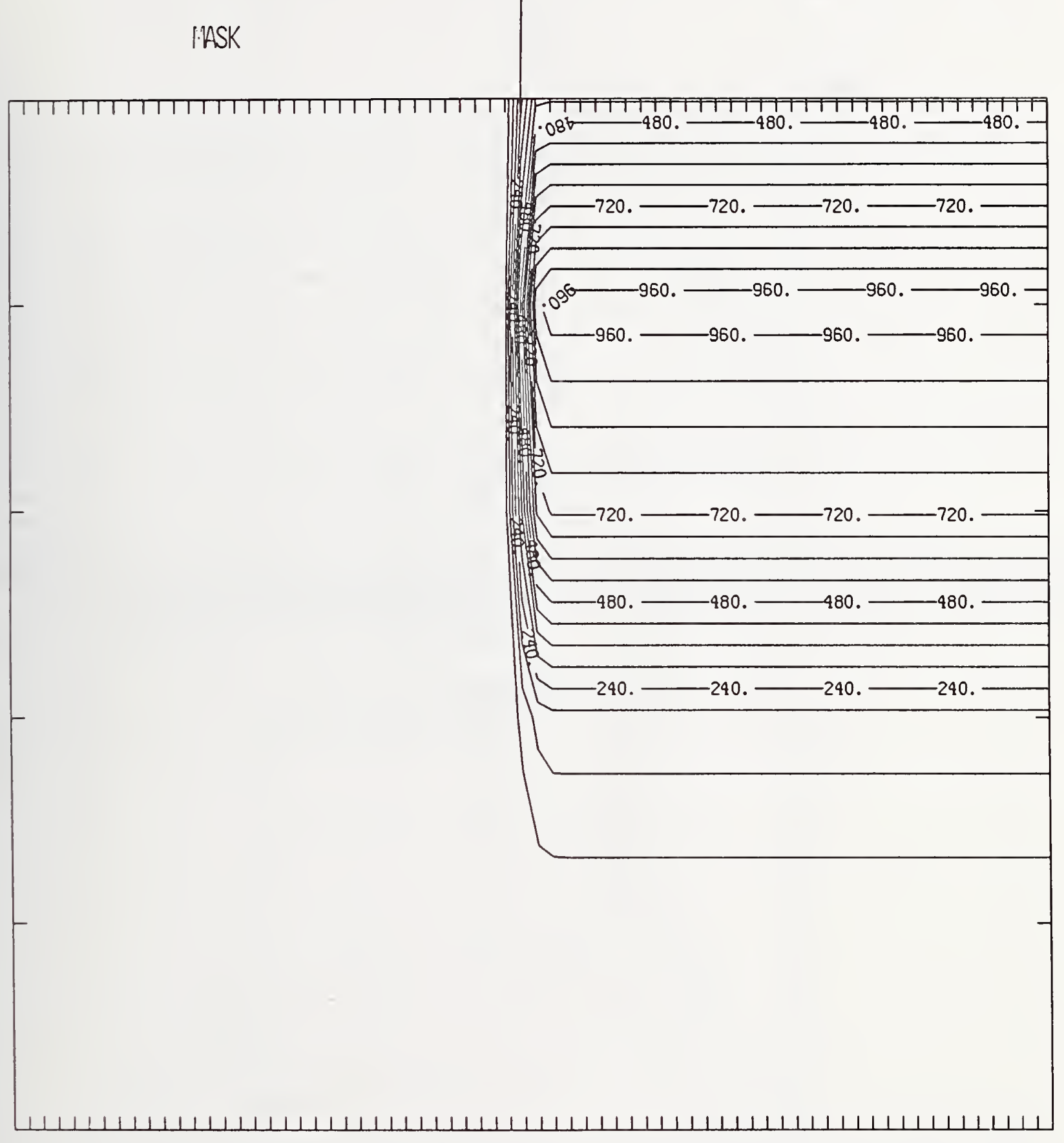

Figure P25-3 Two-dimensional distribution of $25 \mathrm{keV}$ phosphorus implanted past a mask edge. The length increment (distance between tick marks) is $0.02 \mu \mathrm{m}$ and the scale factor is 1000. 
MASK

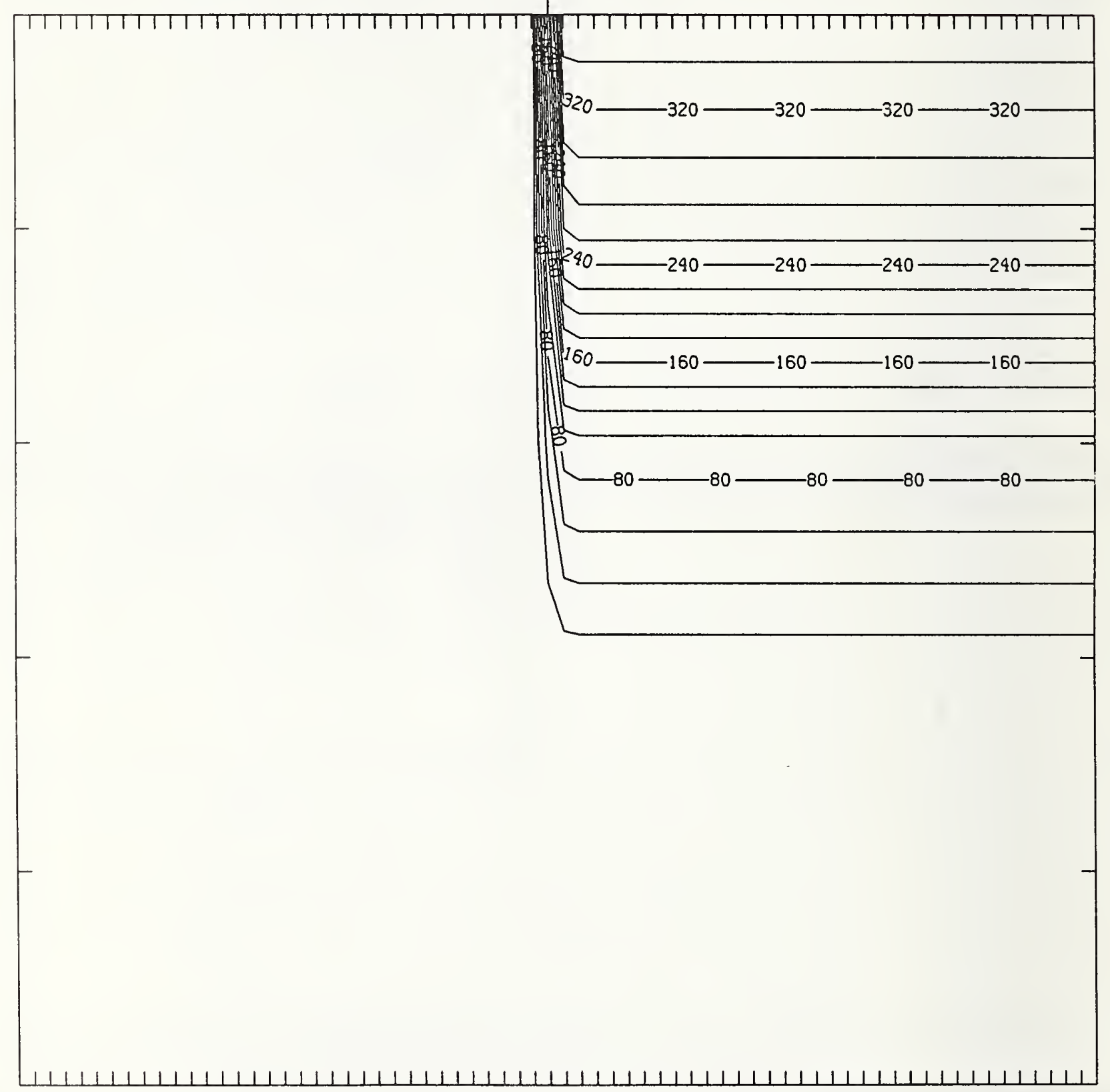

Figure P25-4 Two-dimensional distribution of Frenkel pairs created by $25 \mathrm{keV}$ phosphorus implanted past a mask edge. The length increment (distance between tick marks) is $0.02 \mu \mathrm{m}$ and the scale factor is 1 . 


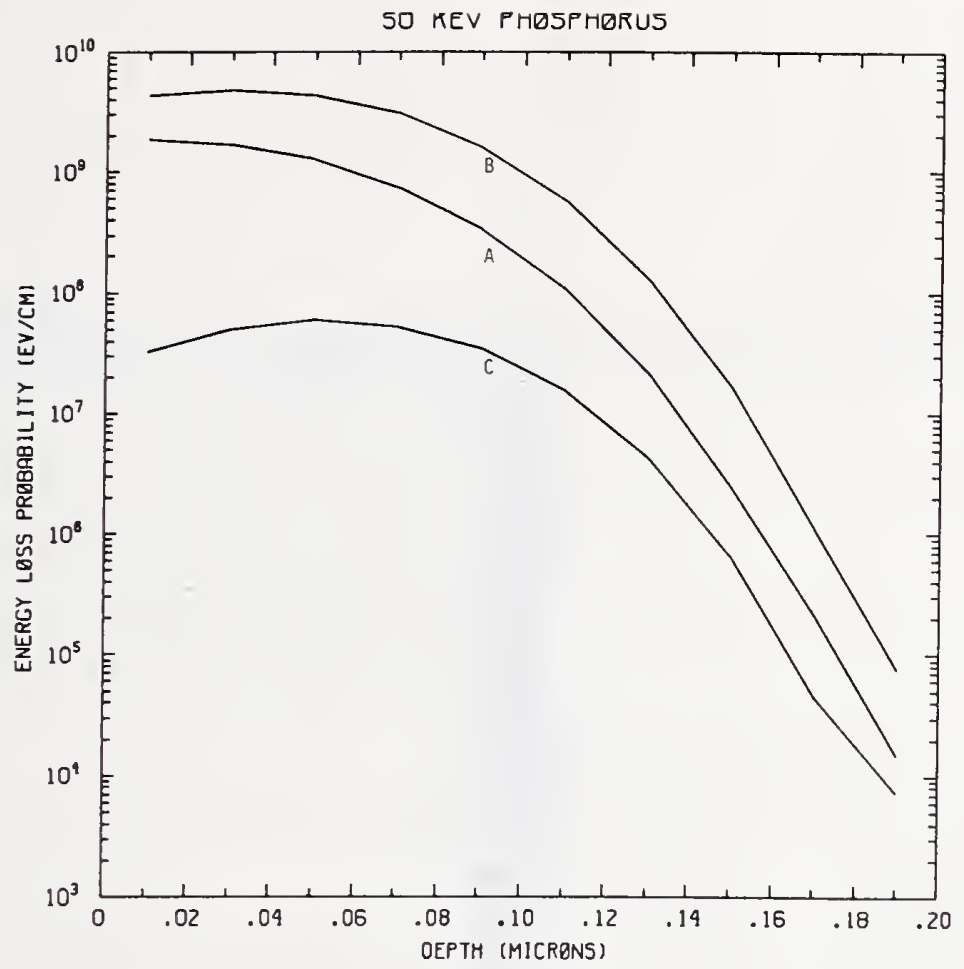

Figure P50-1 One-dimensional probability distributions for energy loss due to electronic excitation (A), displacement damage(B), and phonon excitation (C) for $50 \mathrm{keV}$ phosphorus normally incident on a silicon target.

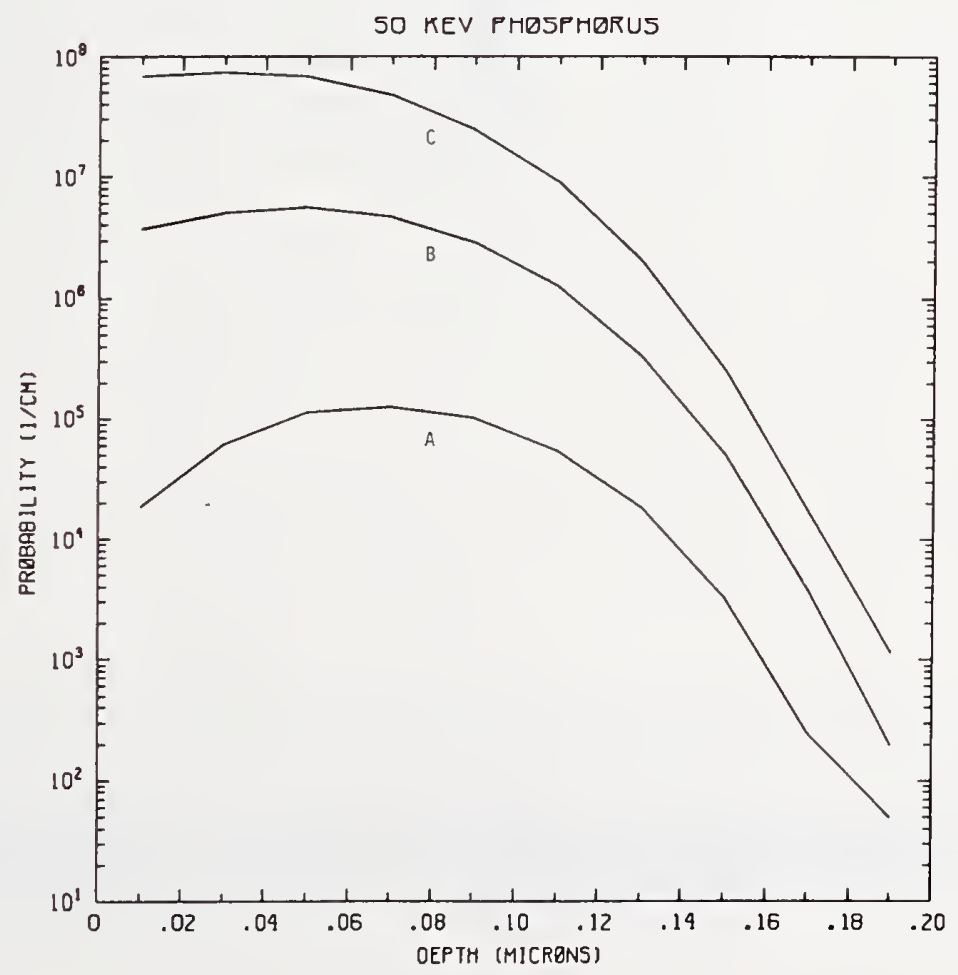

Figure P50-2 One-dimensional probability distributions for implanted phosphorus (A), primary silicon displacements (B), and Frenkel pairs (C) for $50 \mathrm{keV}$ phosphorus normally incident on a silicon target. 
P'ASK

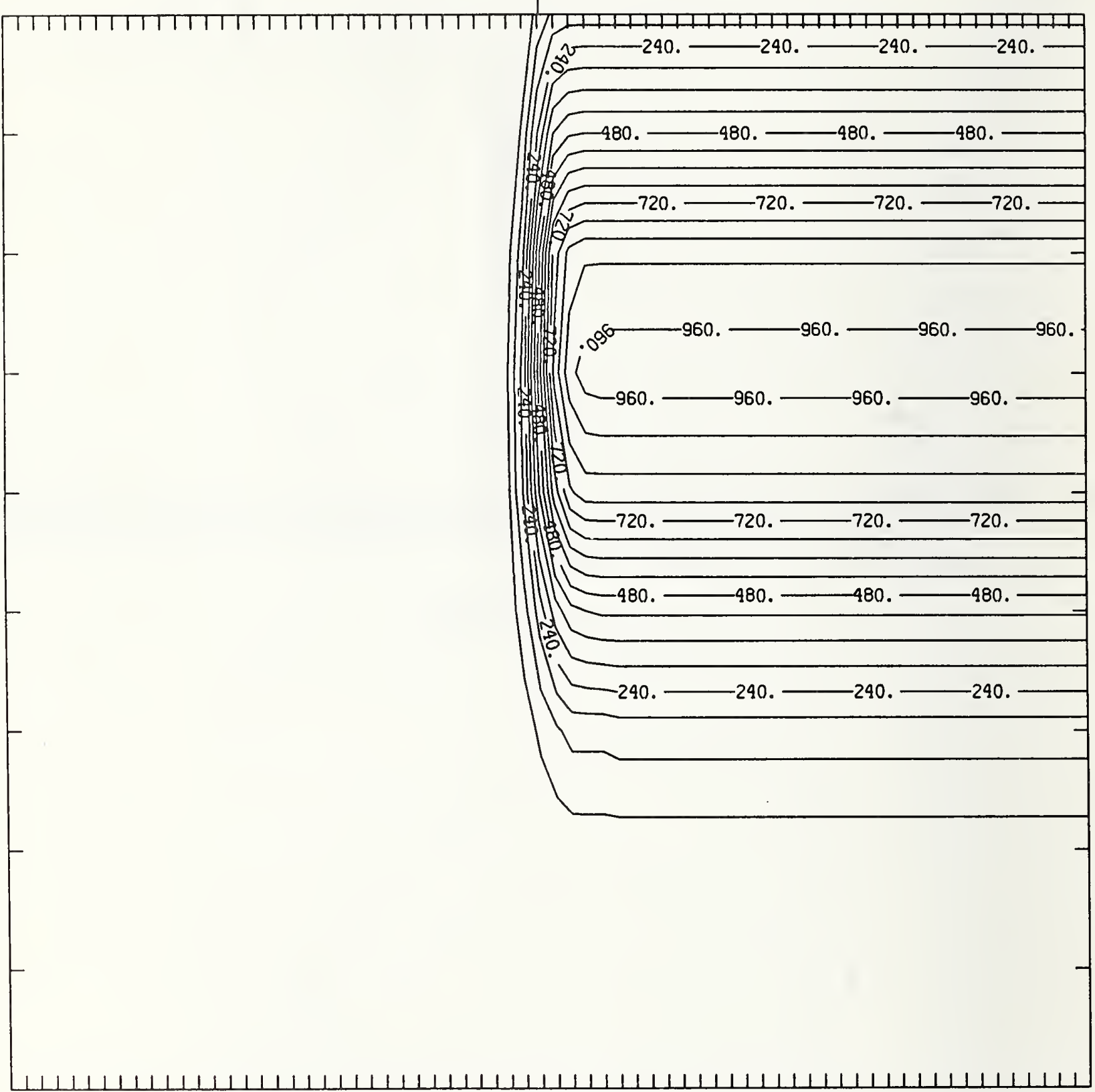

Figure P50-3 Two-dimensional distribution of $50 \mathrm{keV}$ phosphorus implanted past a mask edge. The length increment (distance between tick marks) is $0.02 \mu \mathrm{m}$ and the scale factor is 1000. 


\section{HASK}

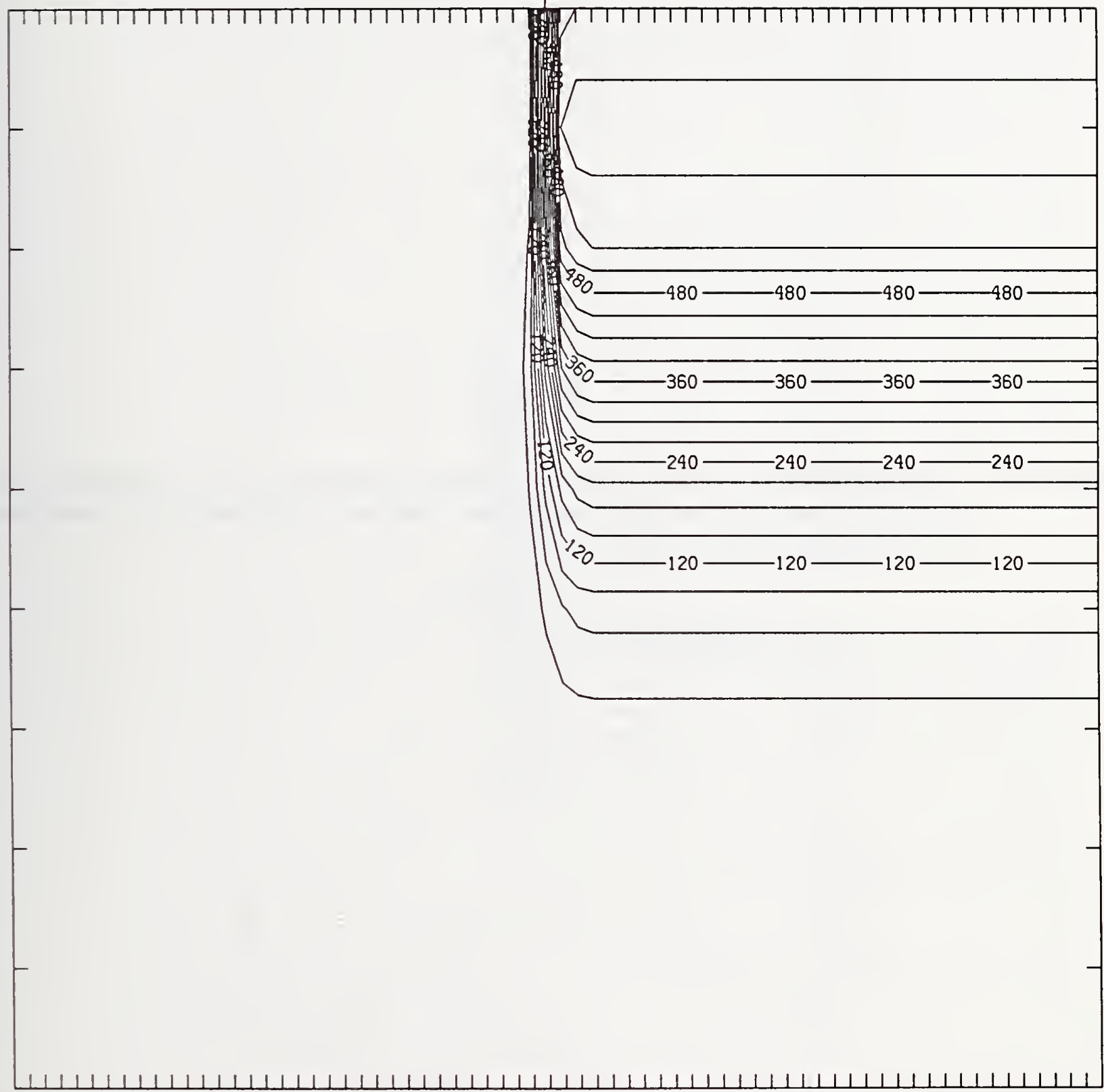

Figure P50-4 Two-dimensional distribution of Frenkel pairs created by $50 \mathrm{keV}$ phosphorus implanted past a mask edge. The length increment (distance between tick marks) is $0.02 \mu \mathrm{m}$ and the scale factor is 1 . 


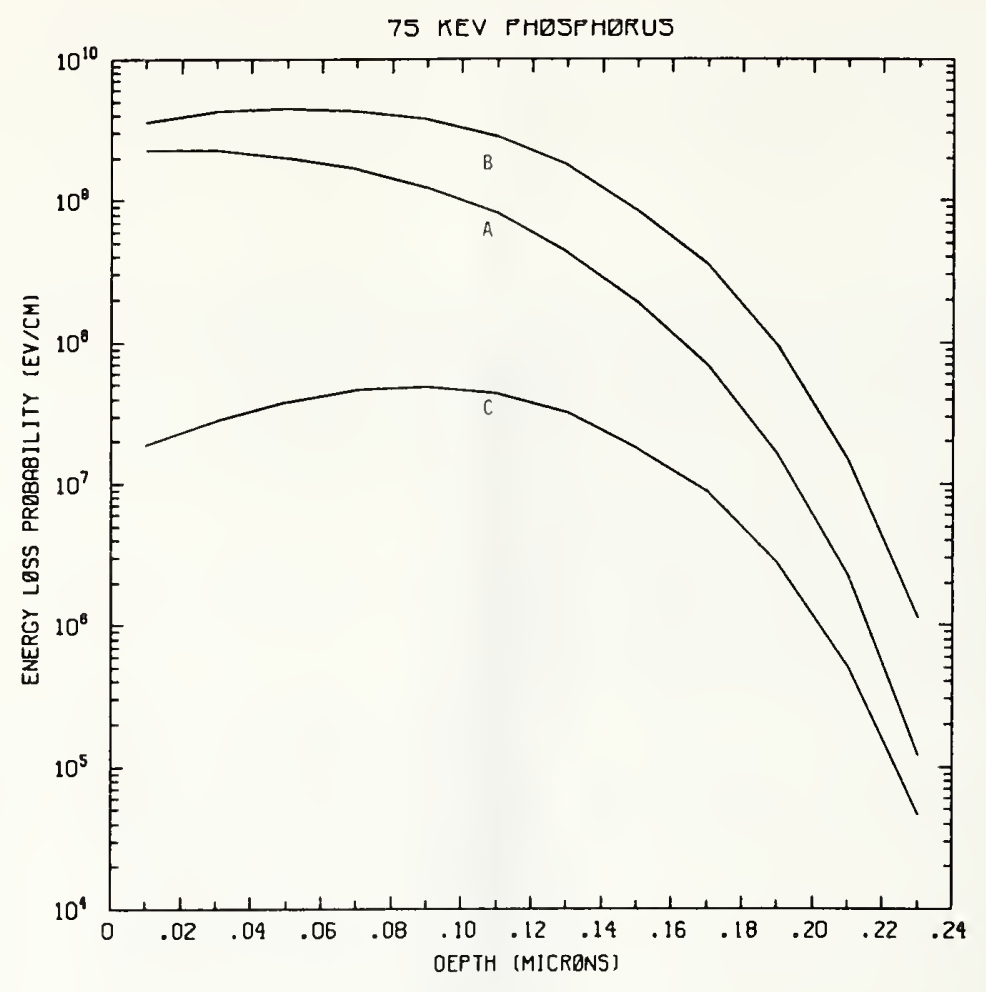

Figure P75-1 One-dimensional probability distributions for energy loss due to electronic excitation (A), displacement damage(B), and phonon excitation (C) for $75 \mathrm{keV}$ phosphorus normally incident on a silicon target.

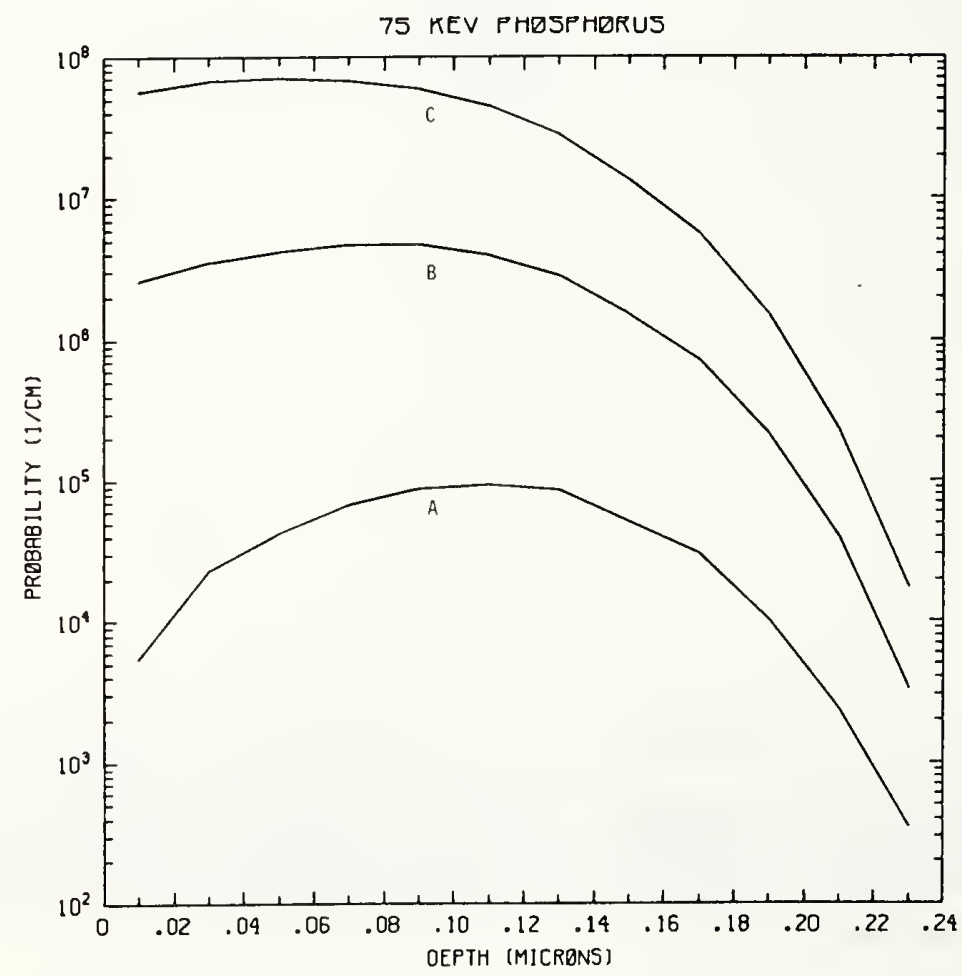

Figure P75-2 One-dimensional probability distributions for implanted phosphorus (A), primary silicon displacements (B), and Frenkel pairs (C) for $75 \mathrm{keV}$ phosphorus normally incident on a silicon target. 


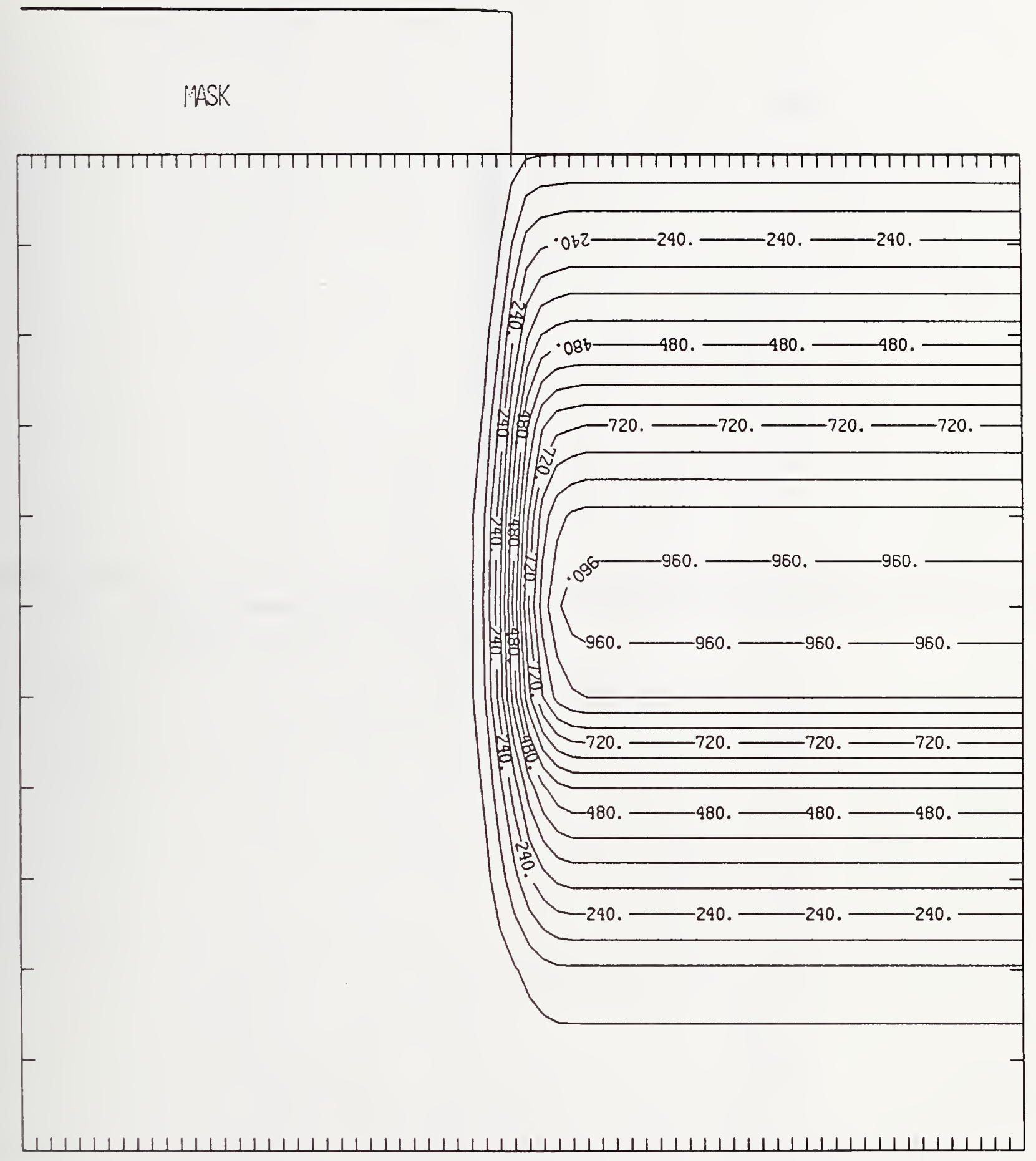

Figure P75-3 Two-dimensional distribution of $75 \mathrm{keV}$ phosphorus implanted past a mask edge. The length increment (distance between tick marks) is $0.02 \mu \mathrm{m}$ and the scale factor is 1000. 
IASK

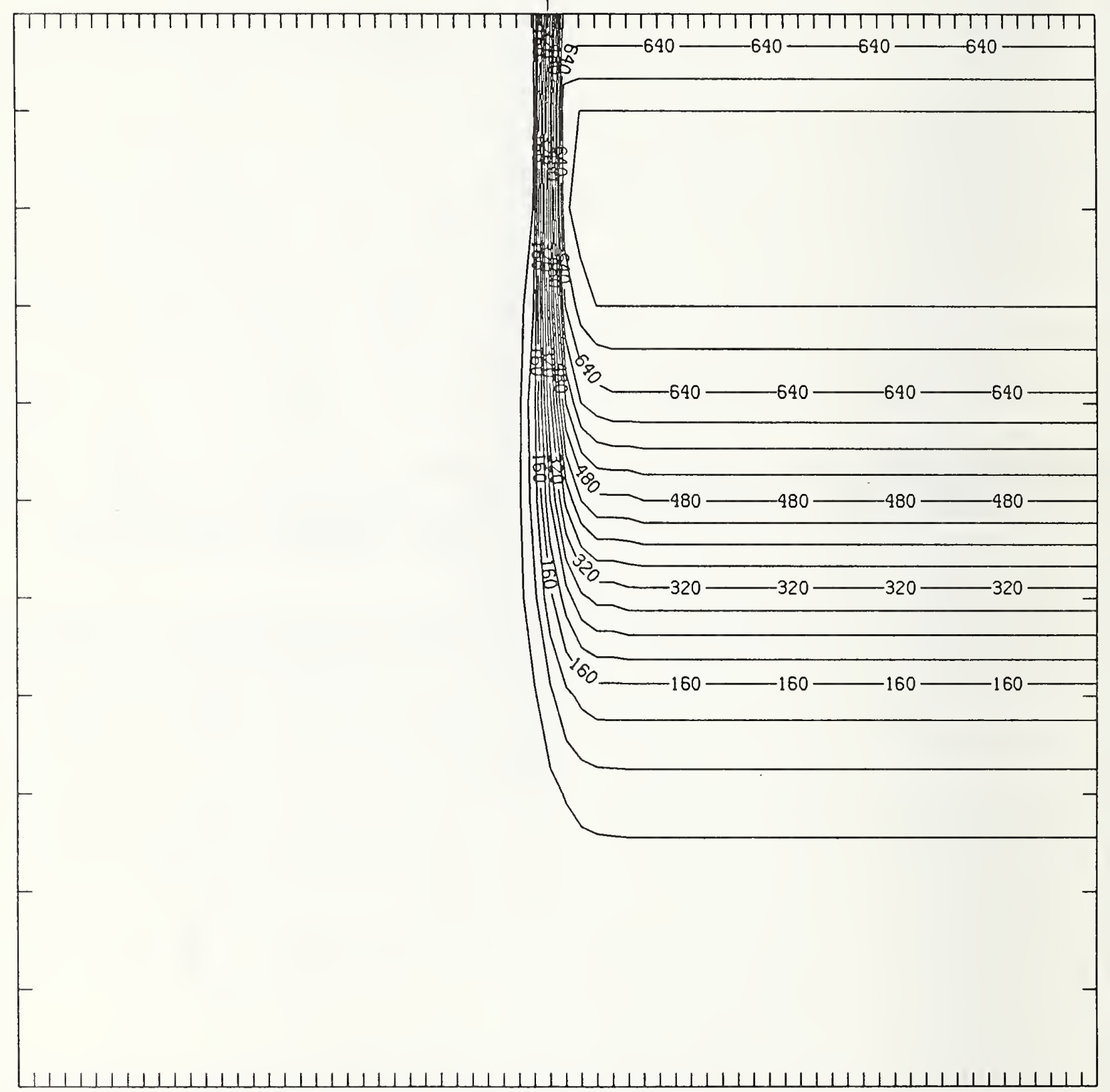

Figure P75-4 Two-dimensional distribution of Frenkel pairs created by $75 \mathrm{keV}$ phosphorus implanted past a mask edge. The length increment (distance between tick marks) is $0.02 \mu \mathrm{m}$ and the scale factor is 1. 


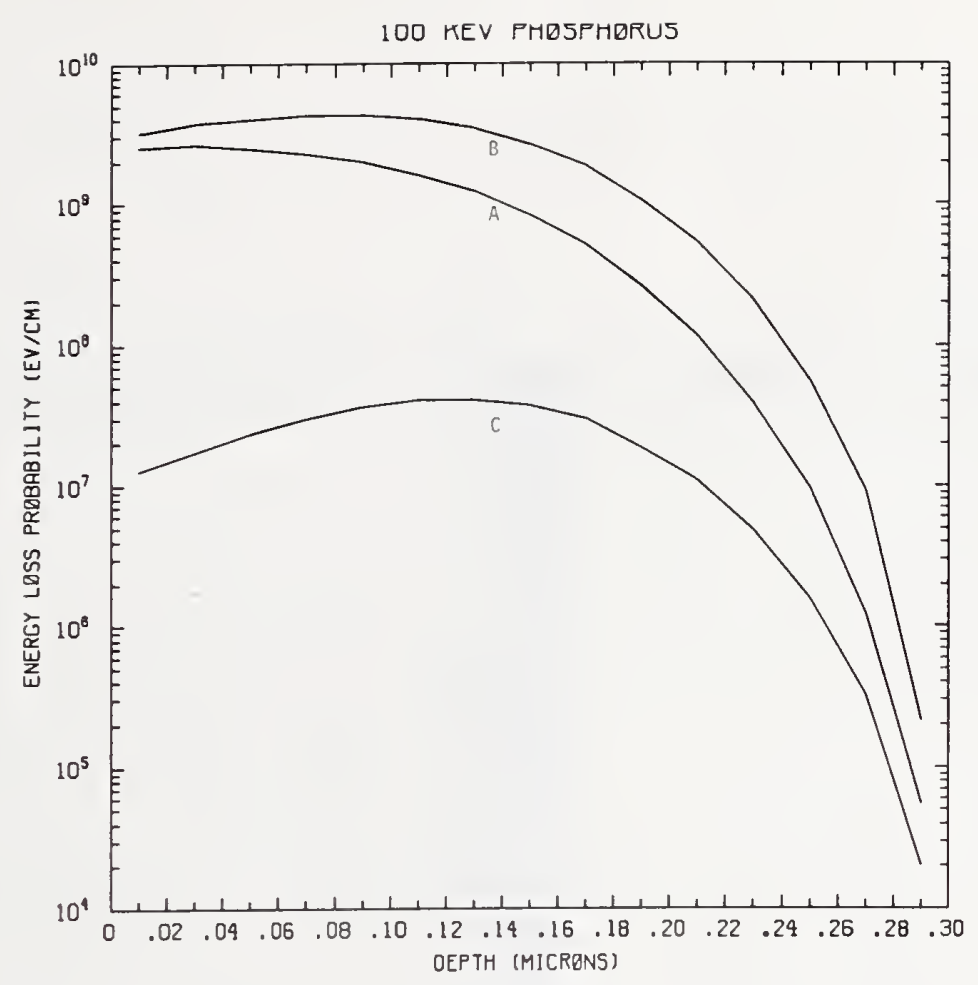

Figure P100-1 One-dimensional probability distributions for energy loss due to electronic excitation (A), displacement damage(B), and phonon excitation (C) for $100 \mathrm{keV}$ phosphorus normally incident on a silicon target.

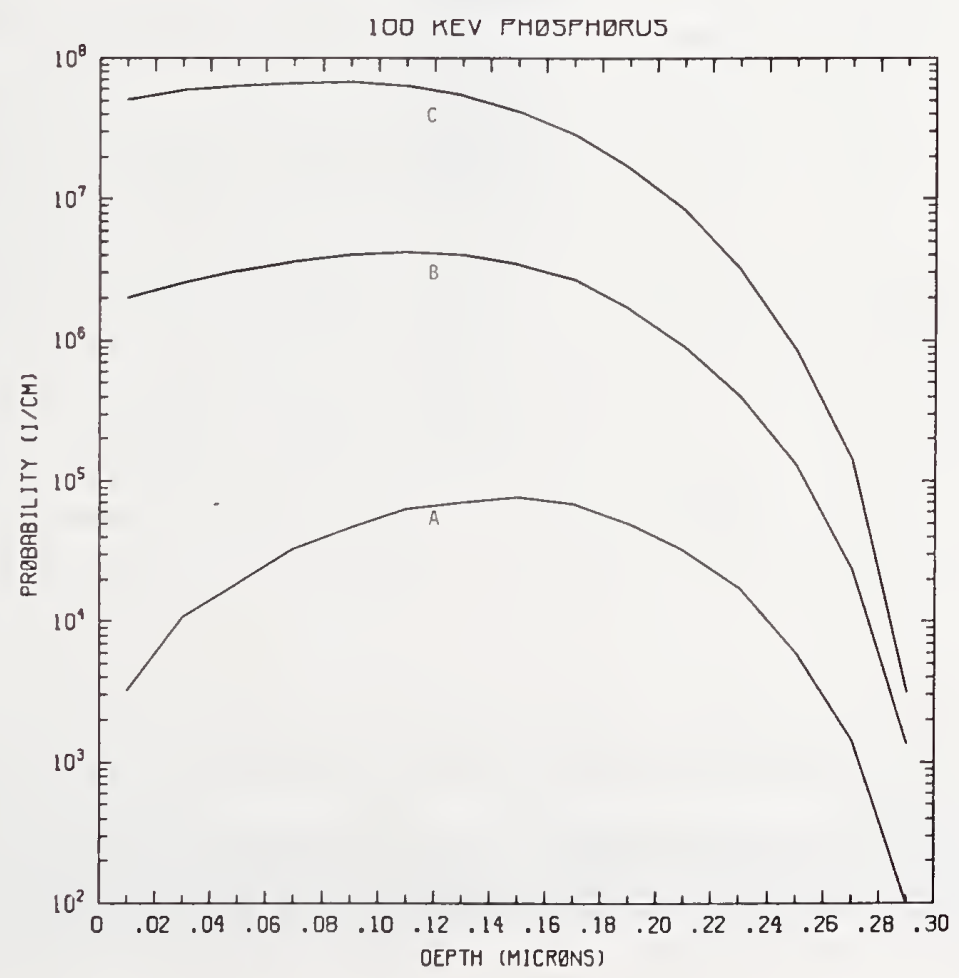

Figure P100-2 One-dimensional probability distributions for implanted phosphorus (A), primary silicon displacements (B), and Frenkel pairs (C) for $100 \mathrm{keV}$ phosphorus normally incident on a silicon target. 


\section{FASK}

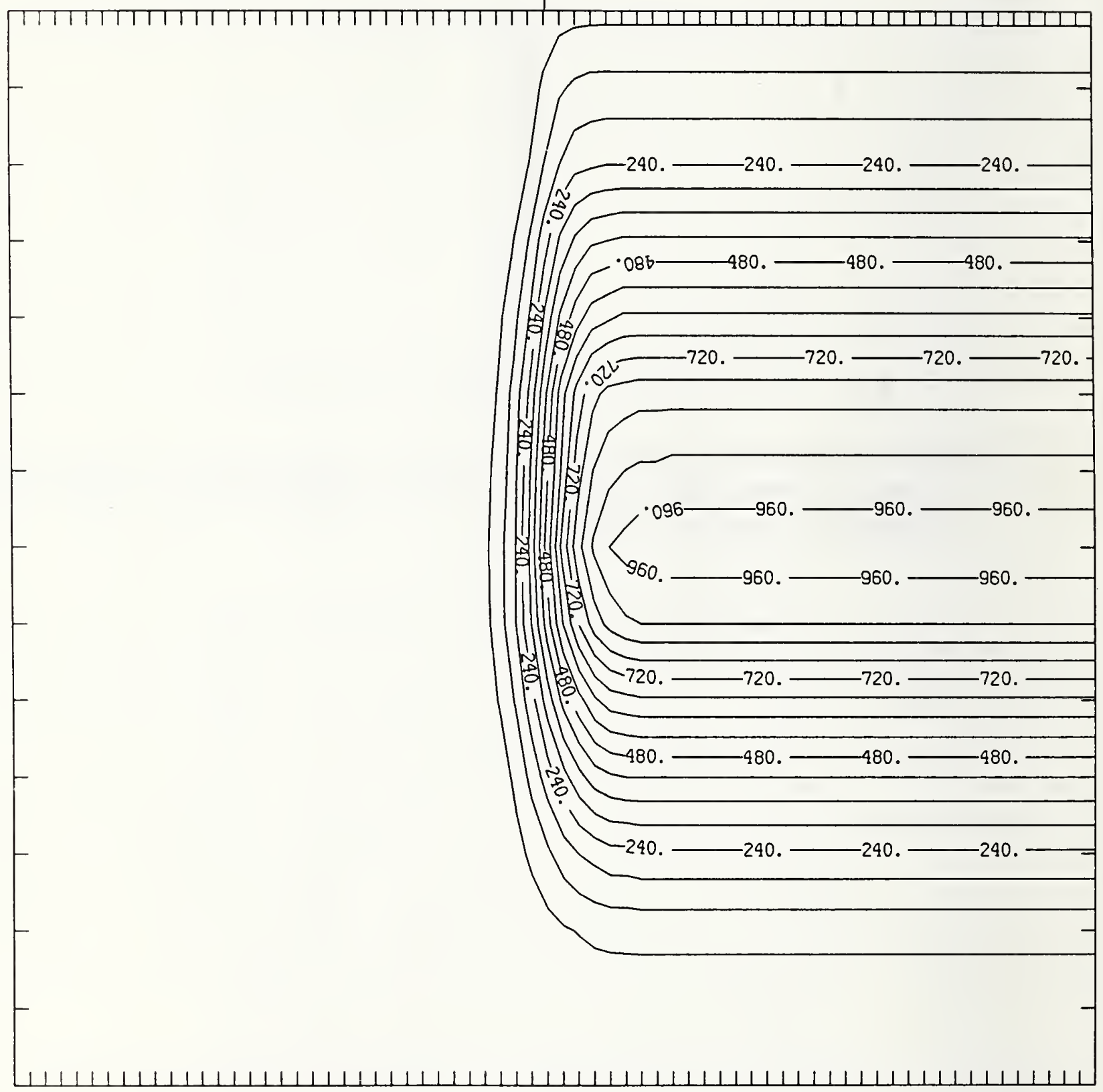

Figure P100-3 Two-dimensional distribution of $100 \mathrm{keV}$ phosphorus implanted past a mask edge. The length increment (distance between tick marks) is $0.02 \mu \mathrm{m}$ and the scale factor is 1000 . 


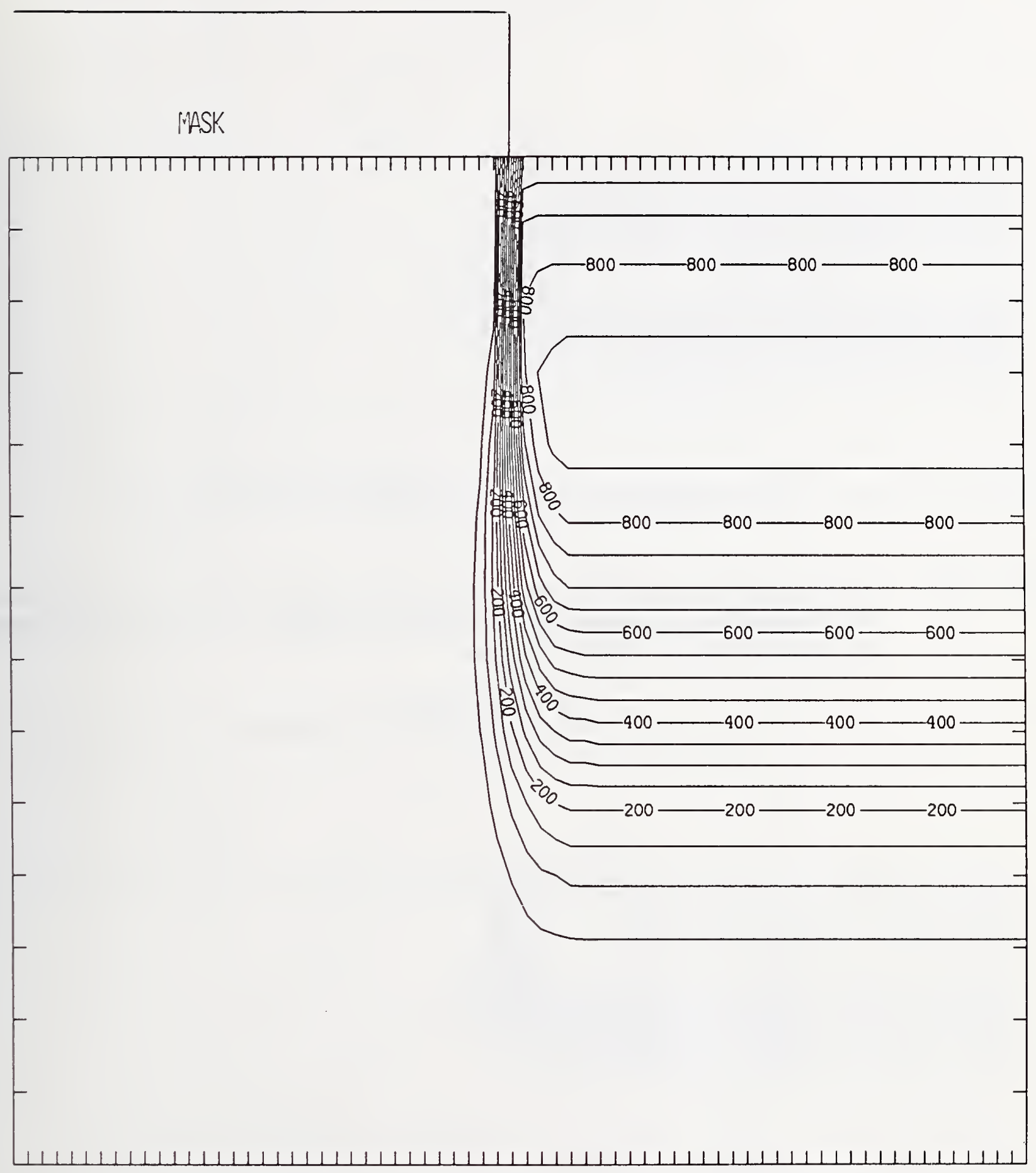

Figure P100-4 Two-dimensional distribution of Frenkel pairs created by $100 \mathrm{keV}$ phosphorus implanted past a mask edge. The length increment (distance between tick marks) is $0.02 \mu \mathrm{m}$ and the scale factor is 1 . 


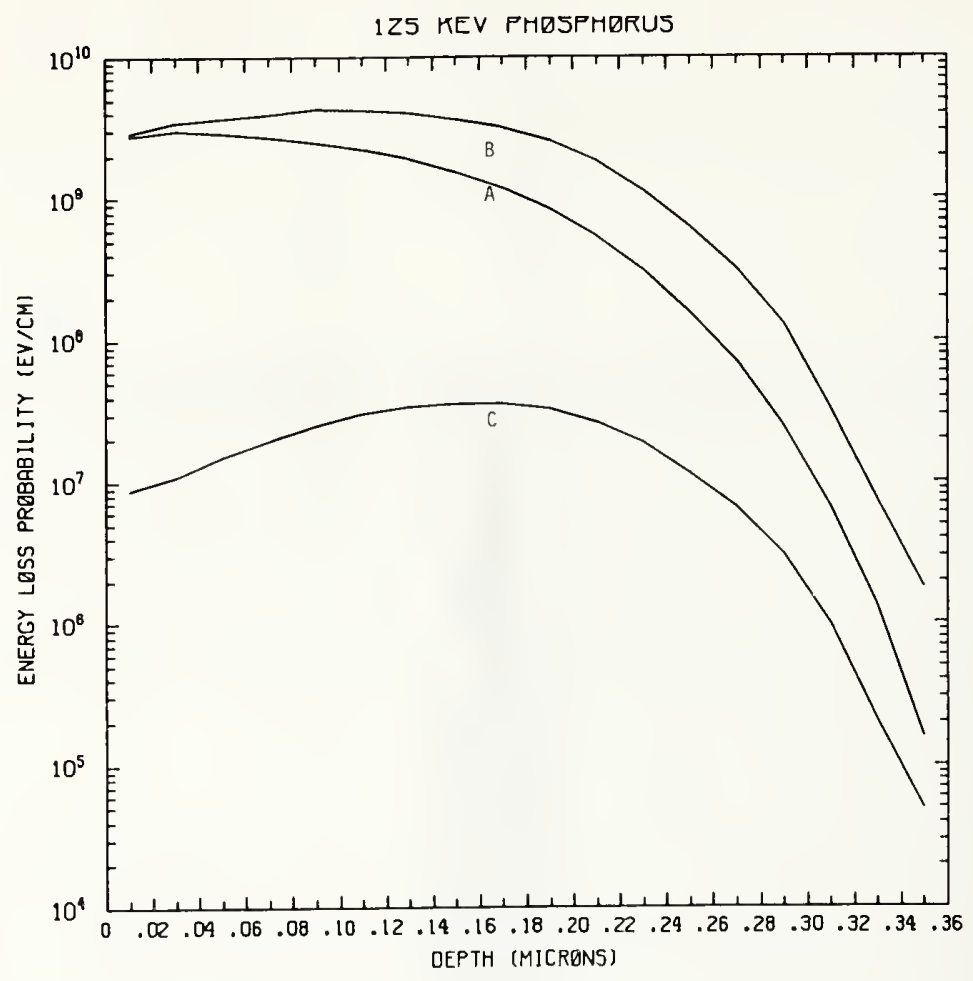

Figure P125-1 One-dimensional probability distributions for energy loss due to electronic excitation (A), displacement damage(B), and phonon excitation (C) for $125 \mathrm{keV}$ phosphorus normally incident on a silicon target.

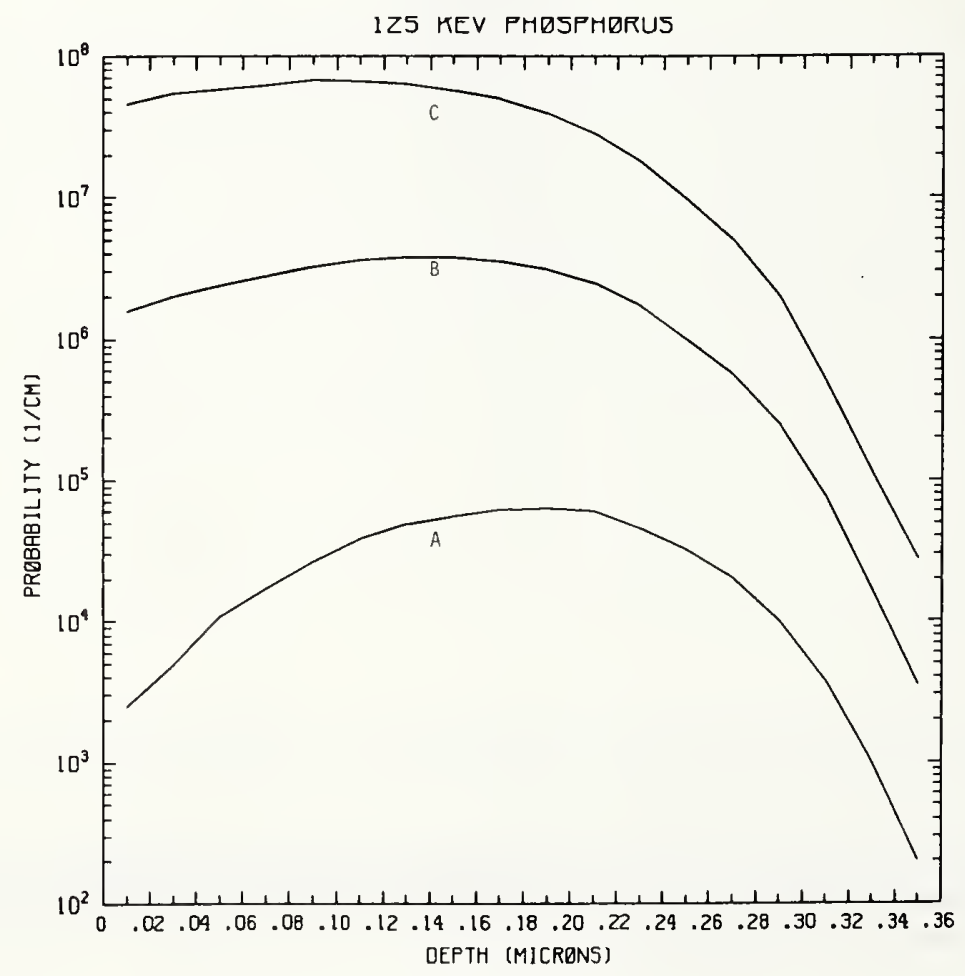

Figure P125-2 One-dimensional probability distributions for implanted phosphorus (A), primary silicon displacements (B), and Frenkel pairs (C) for $125 \mathrm{keV}$ phosphorus normally incident on a silicon target. 
MASK

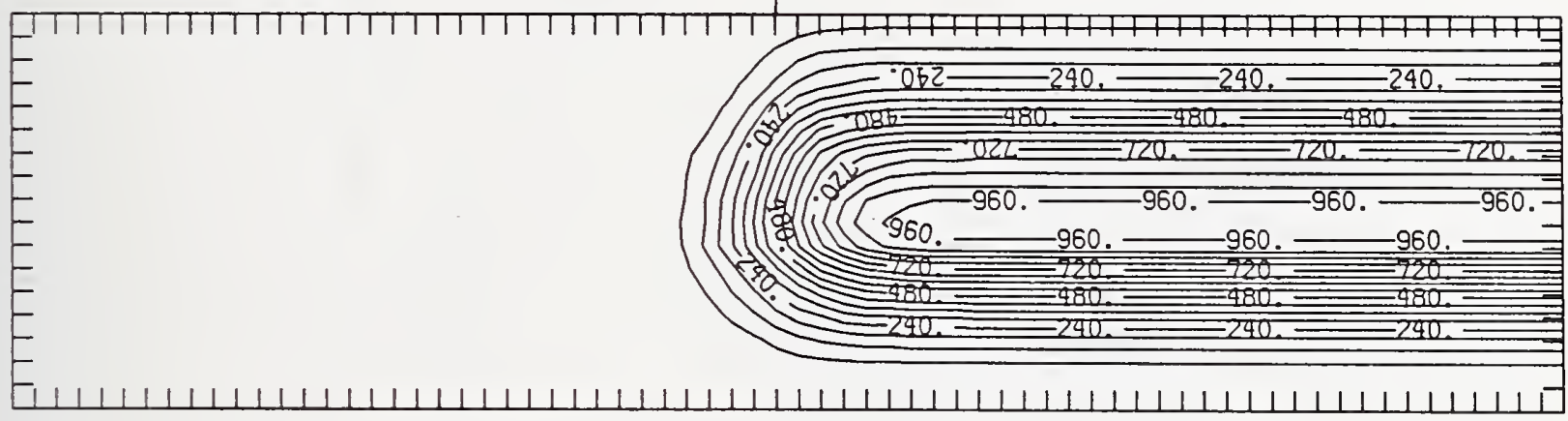

Figure P125-3 Two-dimensional distribution of $125 \mathrm{keV}$ phosphorus implanted past a mask edge. The length increment (distance between tick marks) is $0.02 \mu \mathrm{m}$ and the scale factor is 1000 .

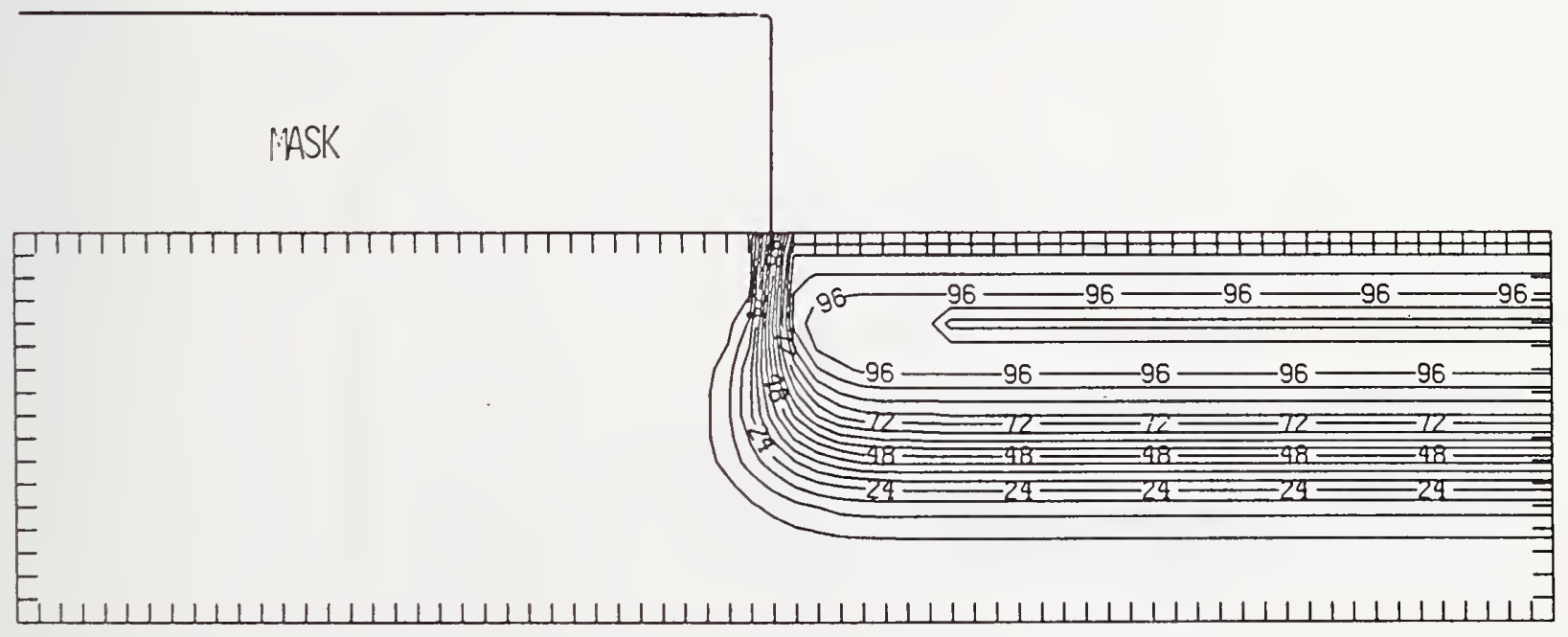

Figure P125-4 Two-dimensional distribution of Frenkel pairs created by $125 \mathrm{keV}$ phosphorus implanted past a mask edge. The length increment (distance between tick marks) is $0.02 \mu \mathrm{m}$ and the scale factor is 0.1 . 


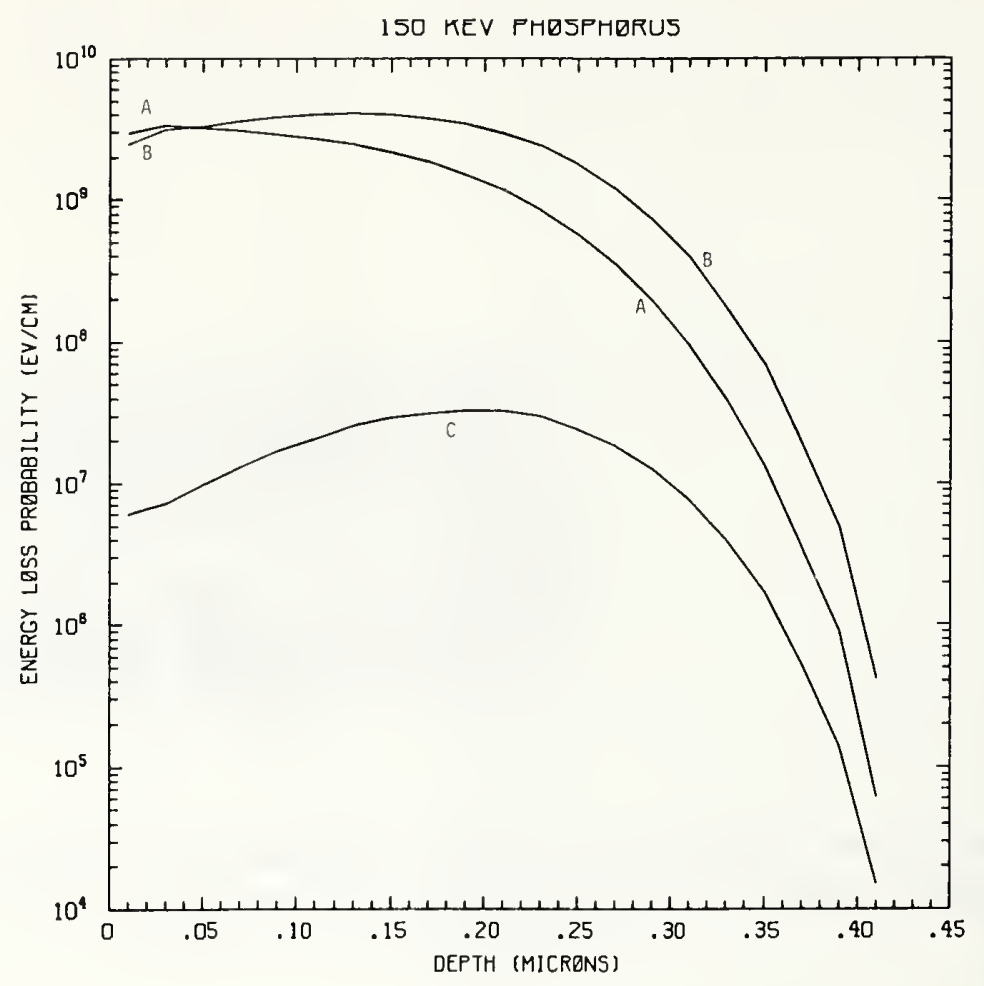

Figure P150-1 One-dimensional probability distributions for energy loss due to electronic excitation (A), displacement damage(B), and phonon excitation (C) for $150 \mathrm{keV}$ phosphorus normally incident on a silicon target.

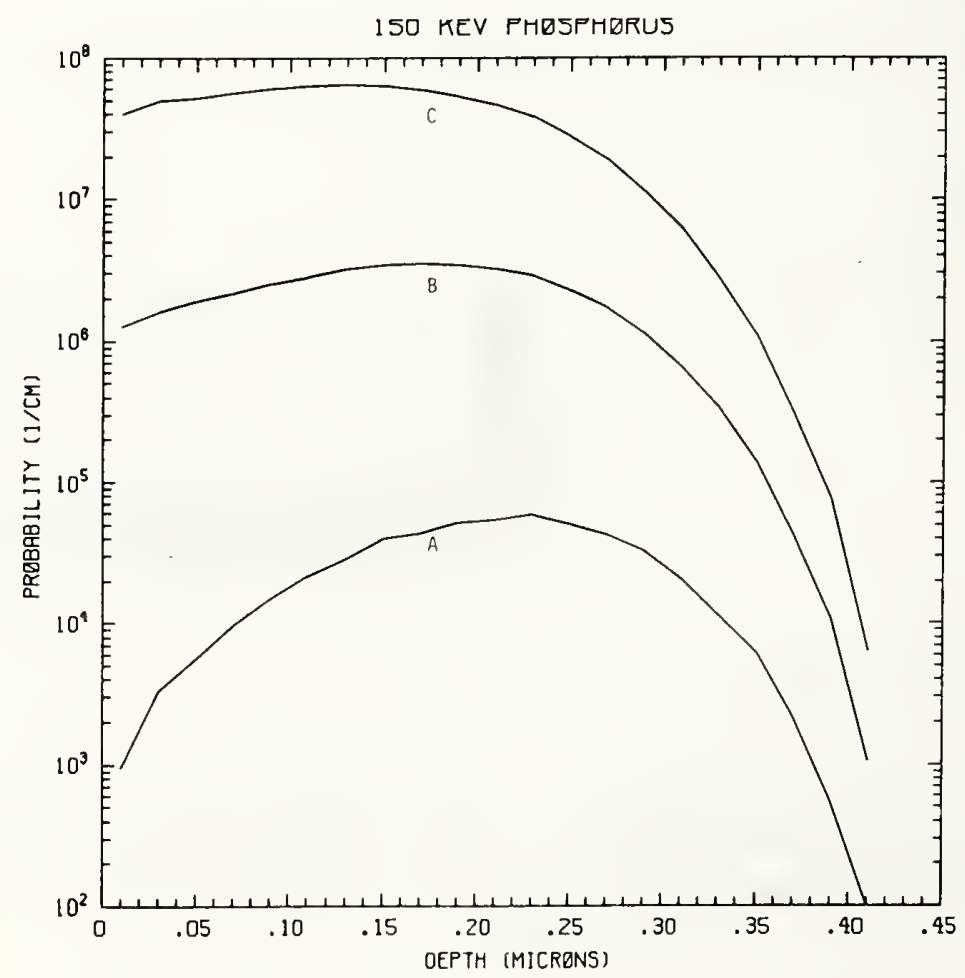

Figure P150-2 One-dimensional probability distributions for implanted phosphorus (A), primary silicon displacements (B), and Frenkel pairs (C) for $150 \mathrm{keV}$ phosphorus normally incident on a silicon target. 
Misk

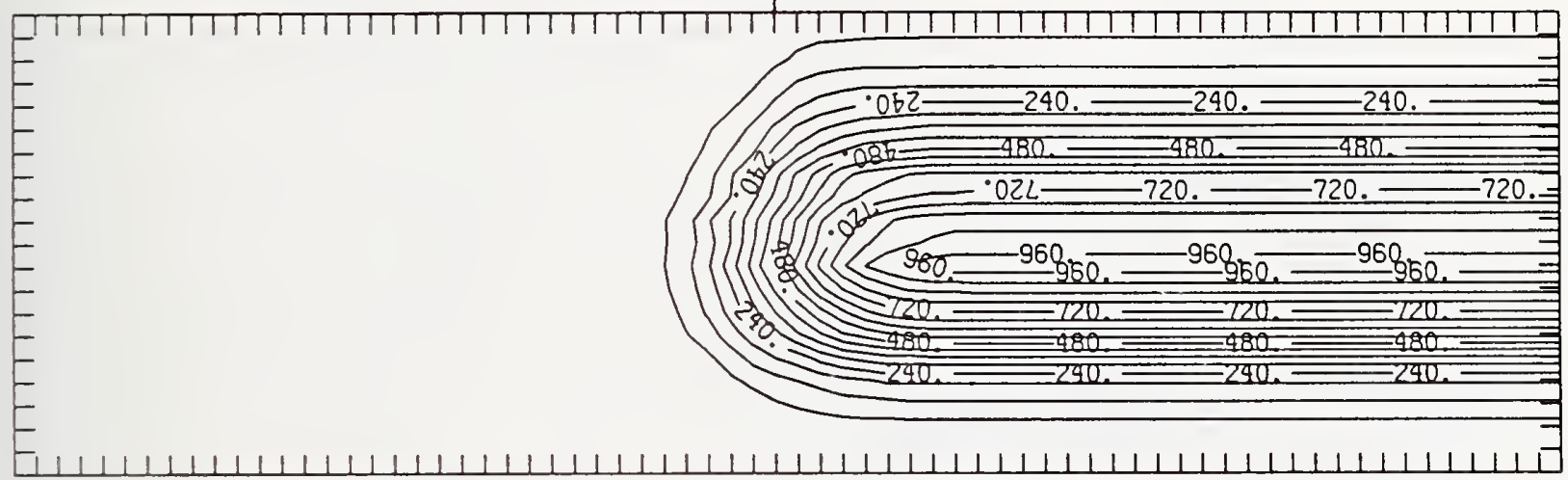

Figure P150-3 Two-dimensional distribution of $150 \mathrm{keV}$ phosphorus implanted past a mask edge. The length increment (distance between tick marks) is $0.02 \mu \mathrm{m}$ and the scale factor is 1000 .

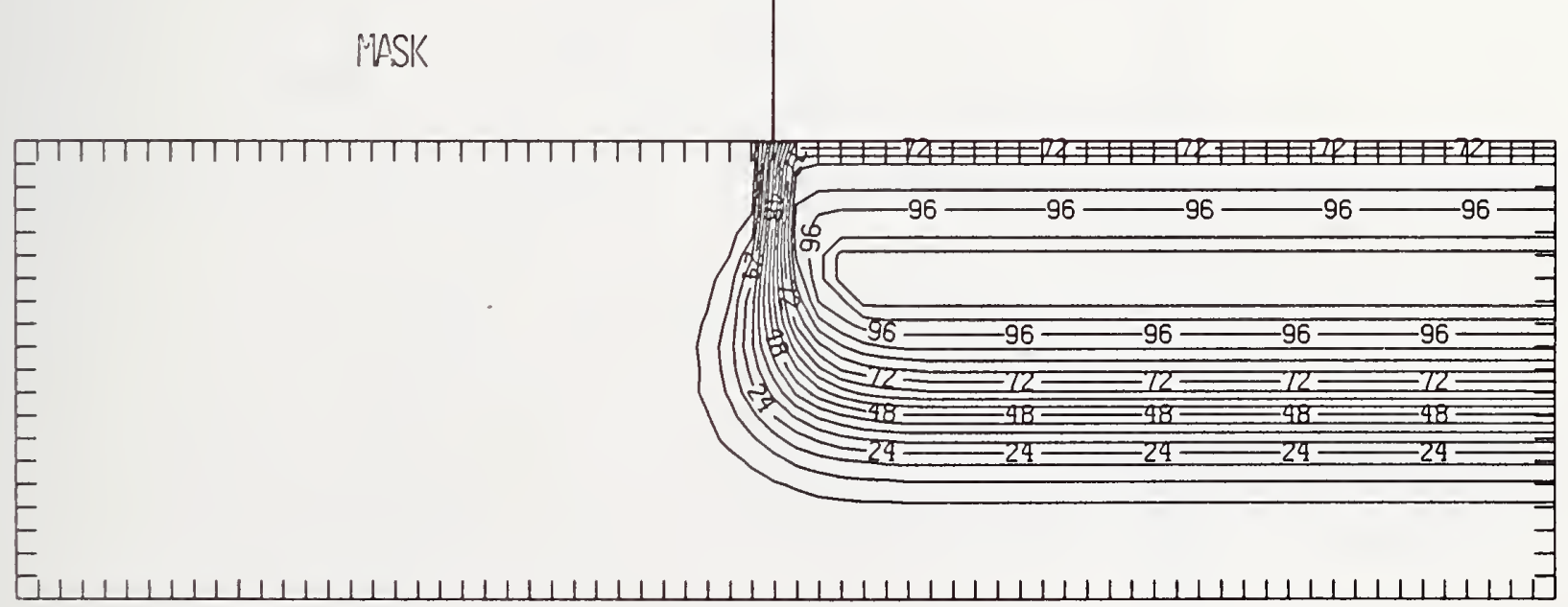

Figure P150-4 Two-dimensional distribution of Frenkel pairs created by $150 \mathrm{keV}$ phosphorus implanted past a mask edge. The length increment (distance between tick marks) is $0.02 \mu \mathrm{m}$ and the scale factor is 0.1 . 


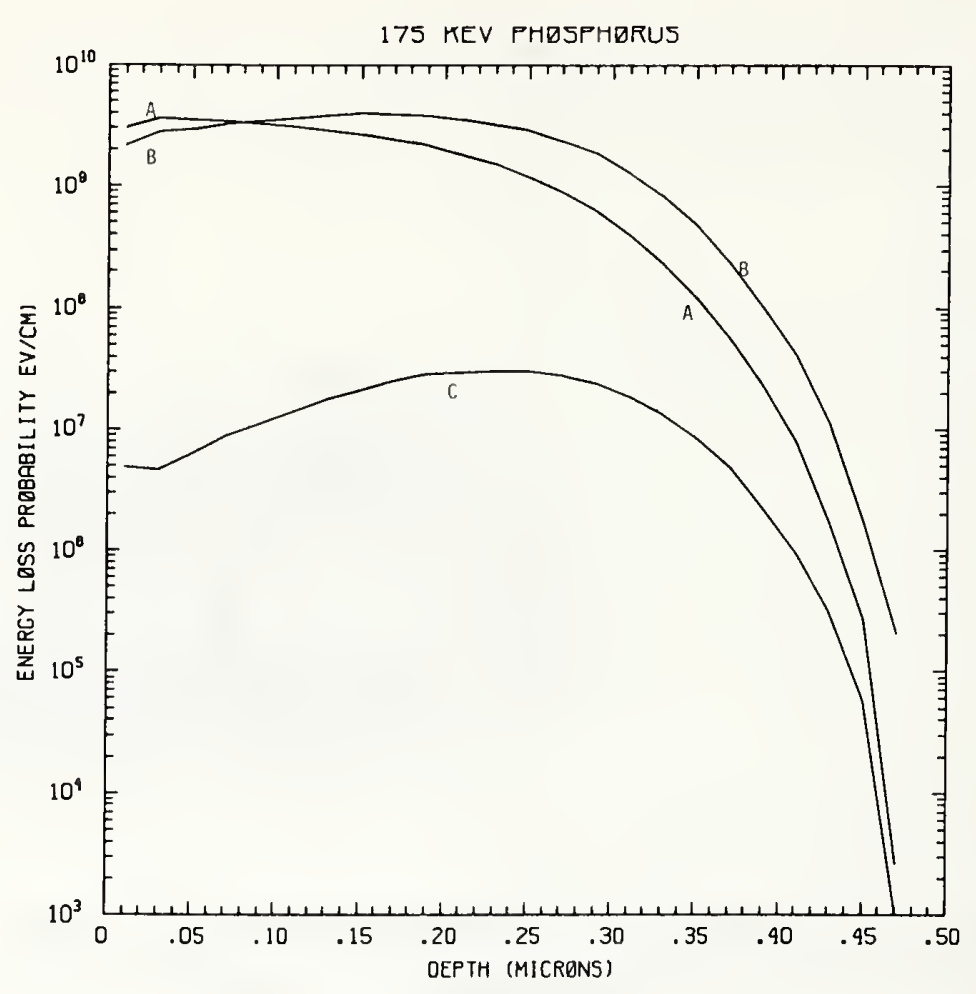

Figure P175-1 One-dimensional probability distributions for energy loss due to electronic excitation (A), displacement damage(B), and phonon excitation (C) for $175 \mathrm{keV}$ phosphorus normally incident on a silicon target.

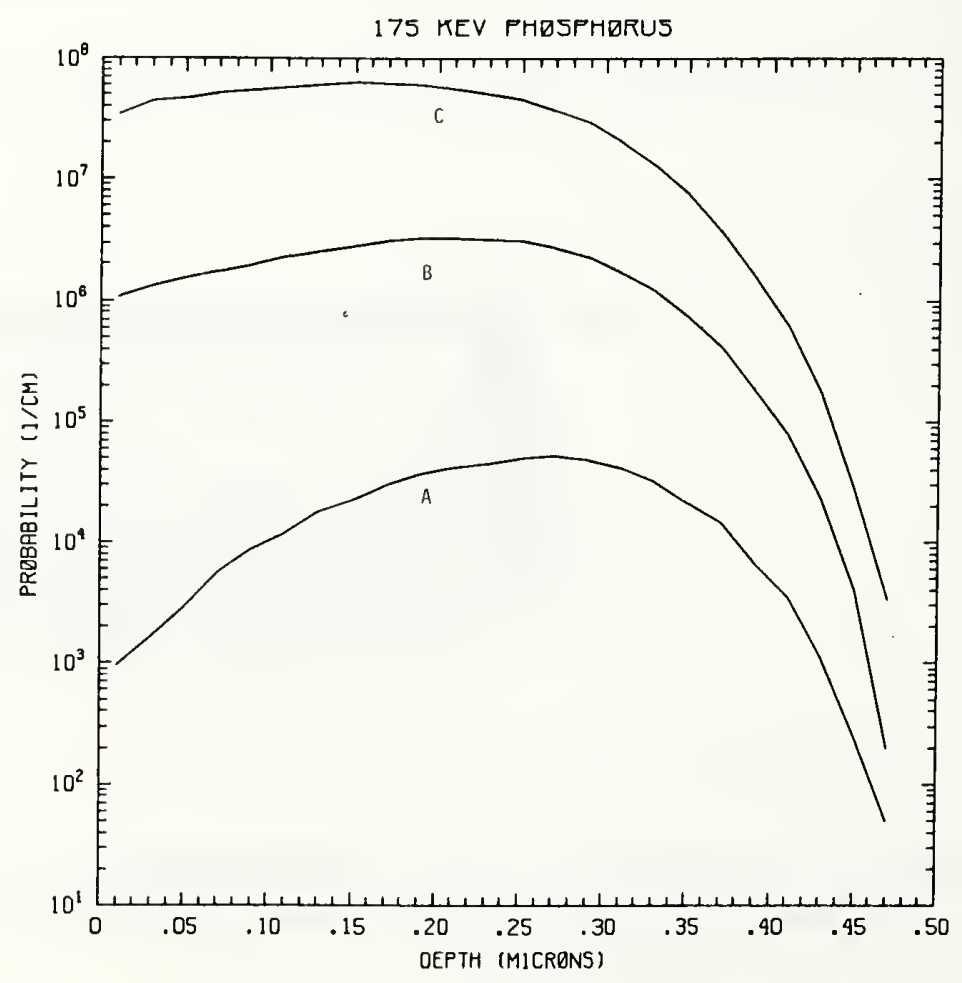

Figure P175-2 One-dimensional probability distributions for implanted phosphorus (A), primary silicon displacements (B), and Frenkel pairs (C) for $175 \mathrm{keV}$ phosphorus normally incident on a silicon target. 


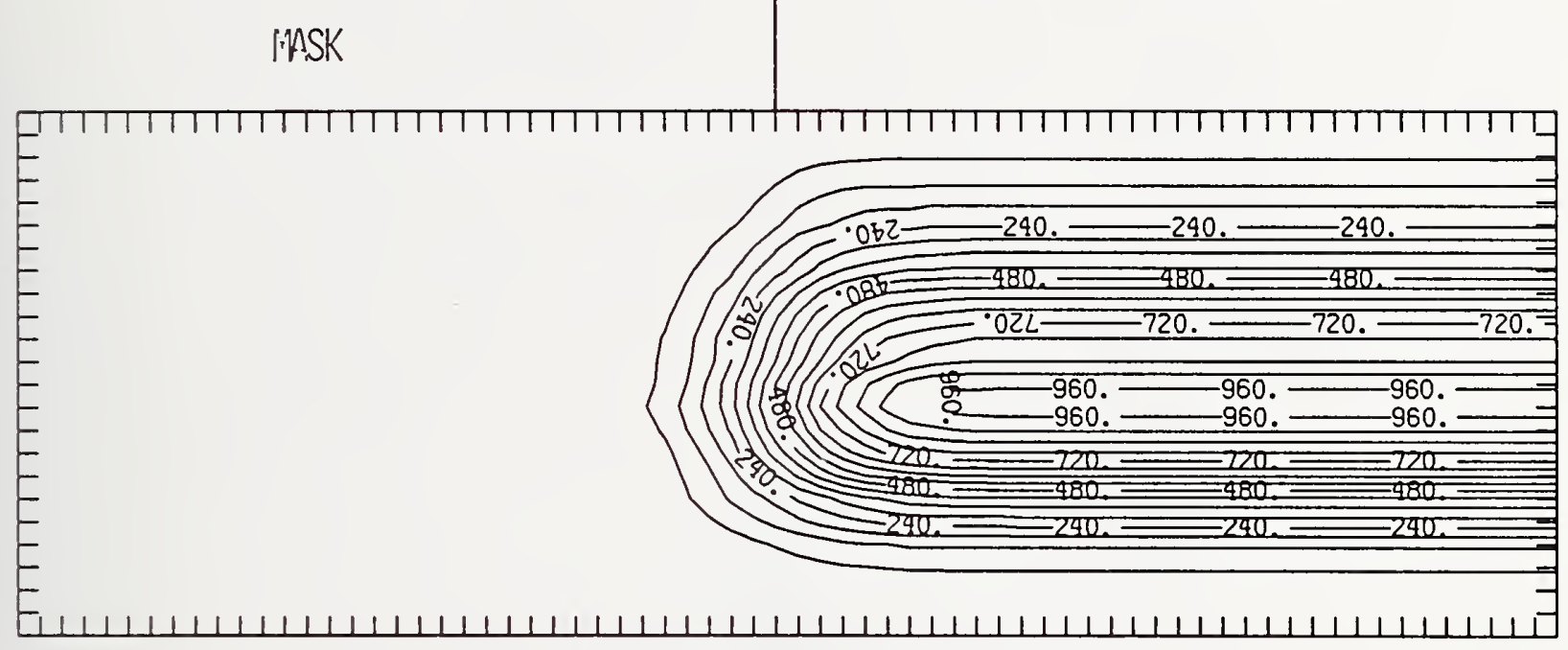

Figure P175-3 Two-dimensional distribution of $175 \mathrm{keV}$ phosphorus implanted past a mask edge. The length increment (distance between tick marks) is $0.02 \mu \mathrm{m}$ and the scale factor is 1000 .

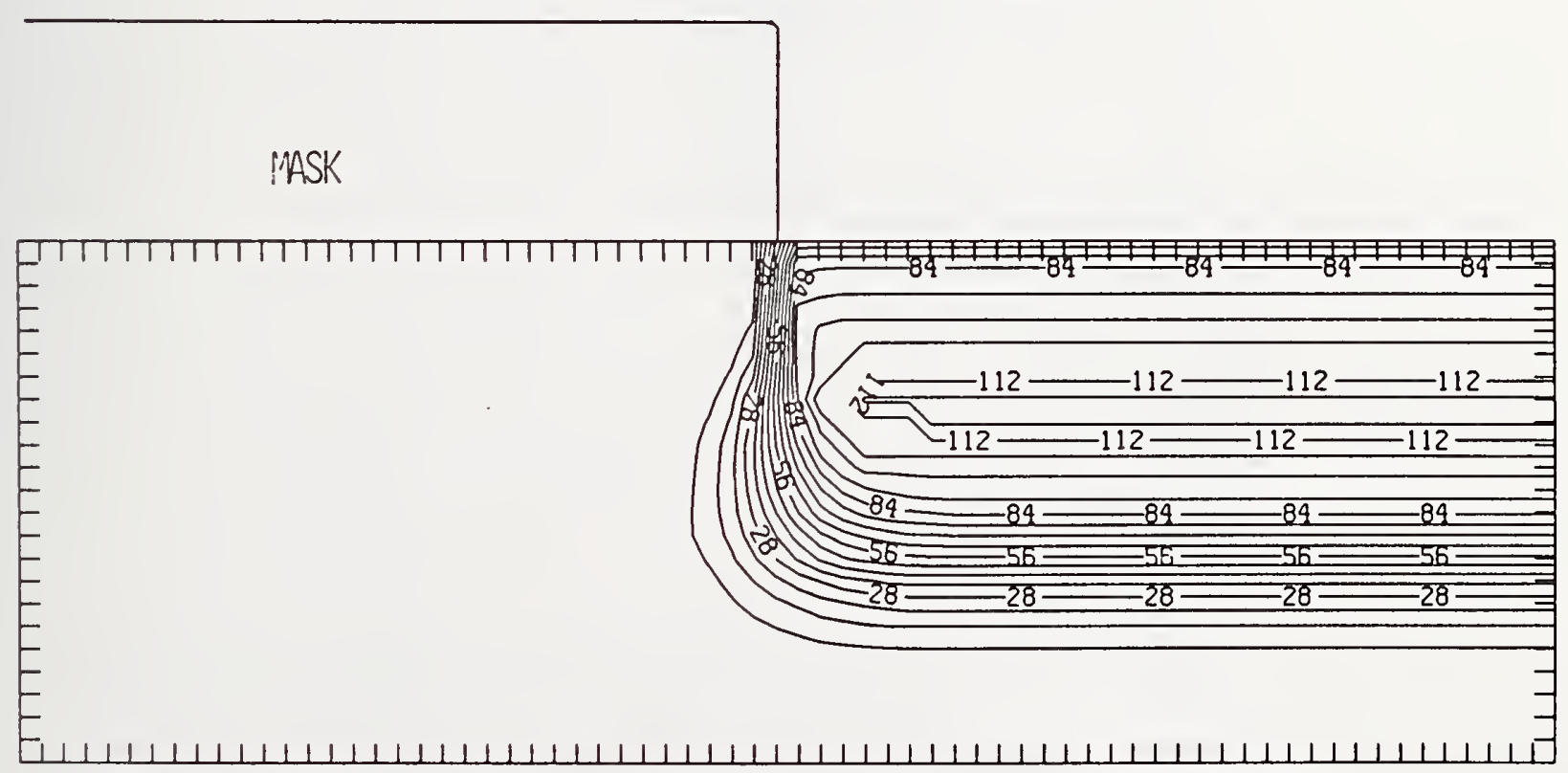

Figure P175-4 Two-dimensional distribution of Frenkel pairs created by $175 \mathrm{keV}$ phosphorus implanted past a mask edge. The length increment (distance between tick marks) is $0.02 \mu \mathrm{m}$ and the scale factor is 0.1 . 


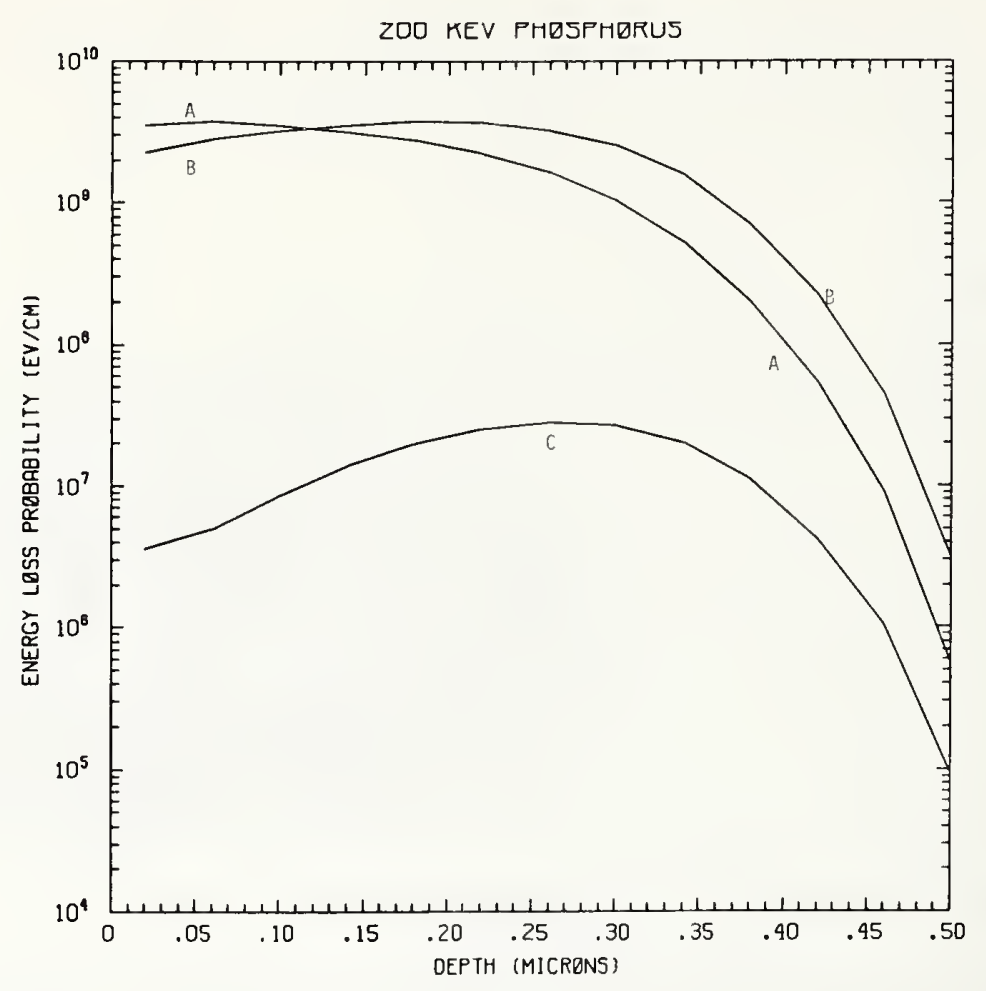

Figure P200-1 One-dimensional probability distributions for energy loss due to electronic excitation (A), displacement damage(B), and phonon excitation (C) for $200 \mathrm{keV}$ phosphorus normally incident on a silicon target.

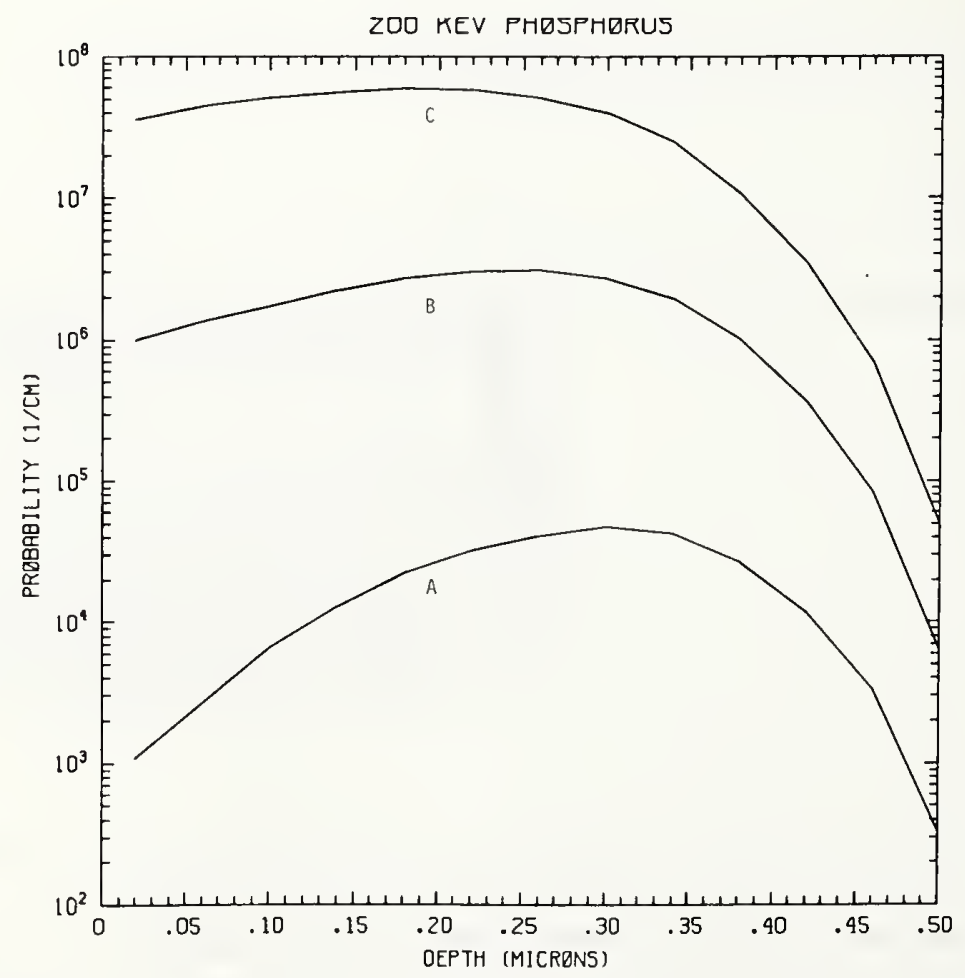

Figure P200-2 One-dimensional probability distributions for implanted phosphorus (A), primary silicon displacements (B), and Frenkel pairs (C) for $200 \mathrm{keV}$ phosphorus normally incident on a silicon target. 
I'ASK

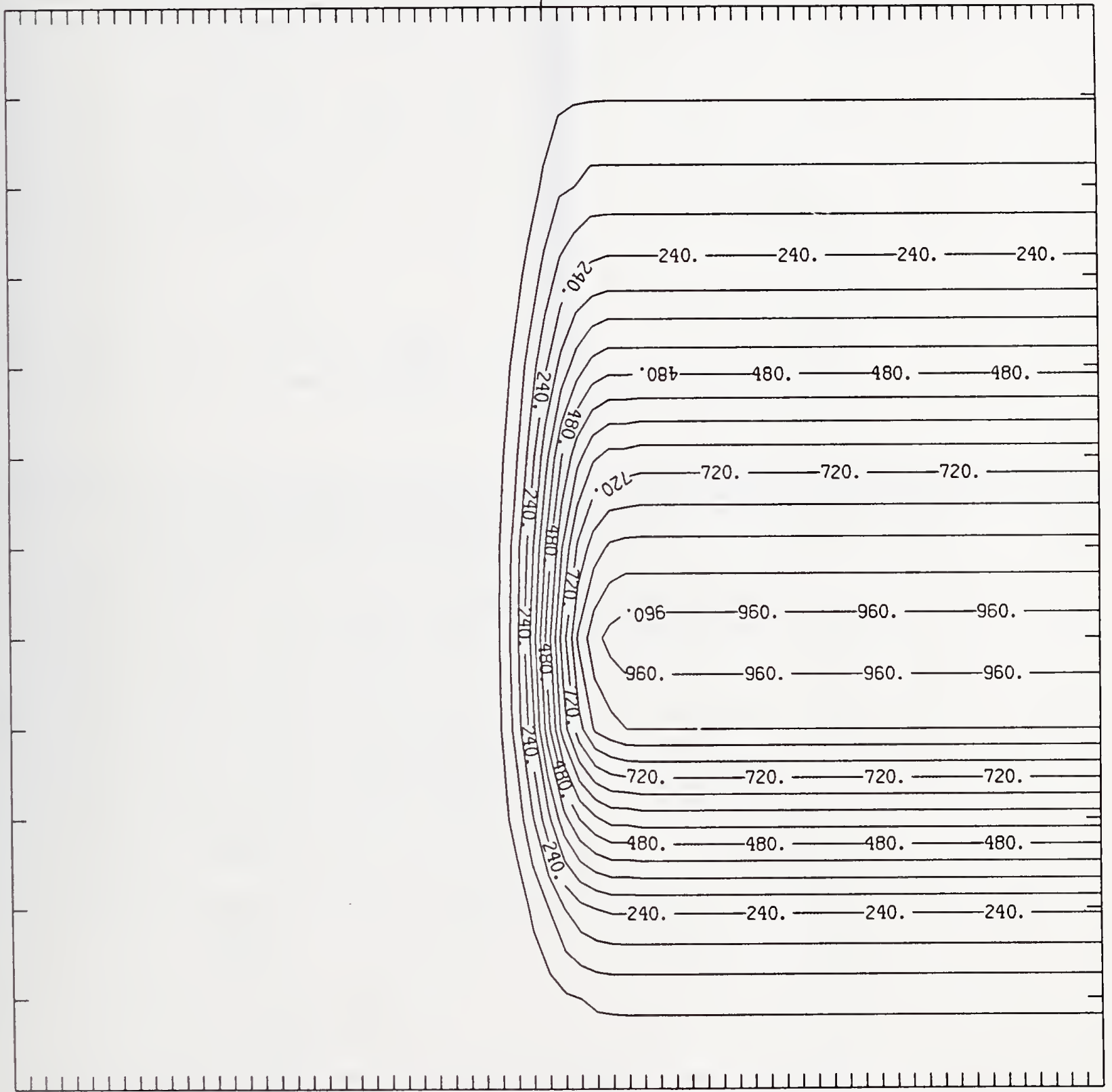

Figure P200-3 Two-dimensional distribution of $200 \mathrm{keV}$ phosphorus implanted past a mask edge. The length increment (distance between tick marks) is $0.04 \mu \mathrm{m}$ and the scale factor is 1000 . 


\section{PASK}

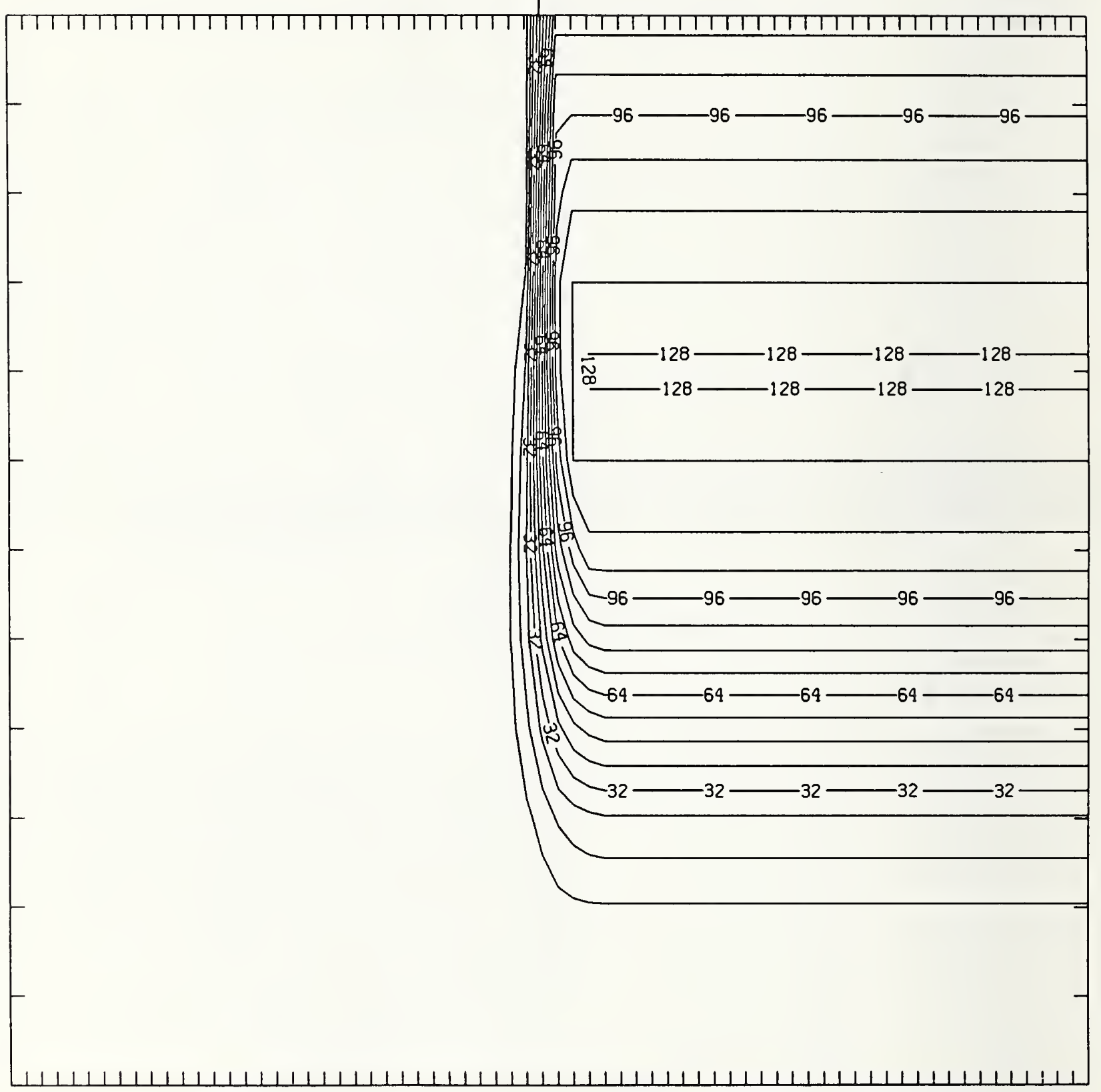

Figure P200-4 Two-dimensional distribution of Frenkel pairs created by $200 \mathrm{keV}$ phosphorus implanted past a mask edge. The length increment (distance between tick marks) is $0.04 \mu \mathrm{m}$ and the scale factor is 0.1 . 


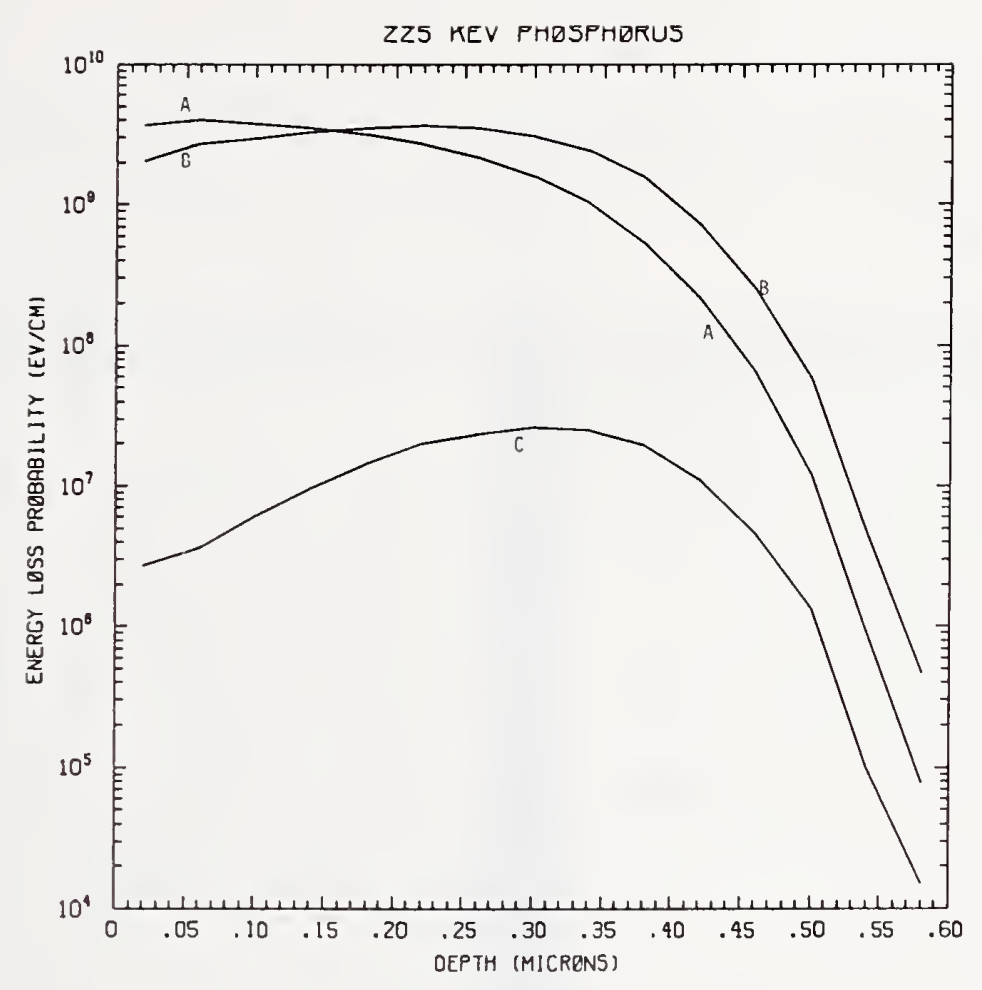

Figure P225-1 One-dimensional probability distributions for energy loss due to electronic excitation (A), displacement damage(B), and phonon excitation (C) for $225 \mathrm{keV}$ phosphorus normally incident on a silicon target.

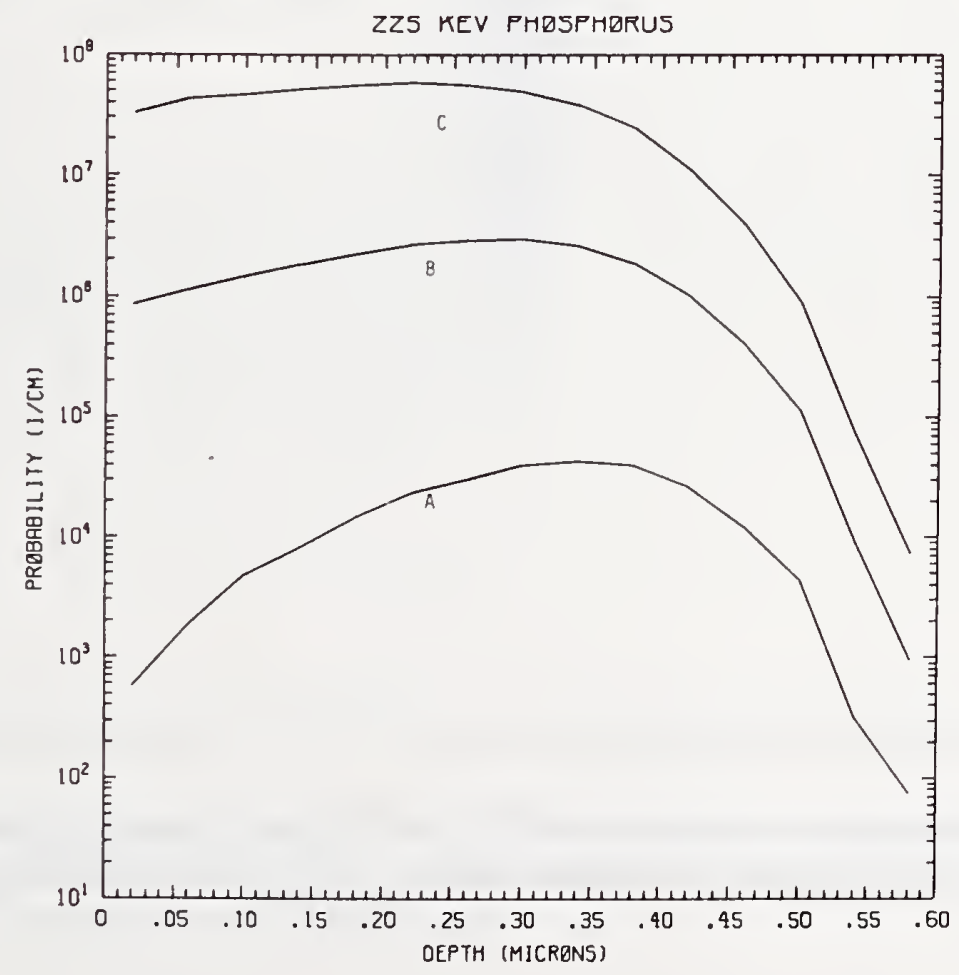

Figure P225-2 One-dimensional probability distributions for implanted phosphorus (A), primary silicon displacements (B), and Frenkel pairs (C) for $225 \mathrm{keV}$ phosphorus normally incident on a silicon target. 


\section{F'ASK}

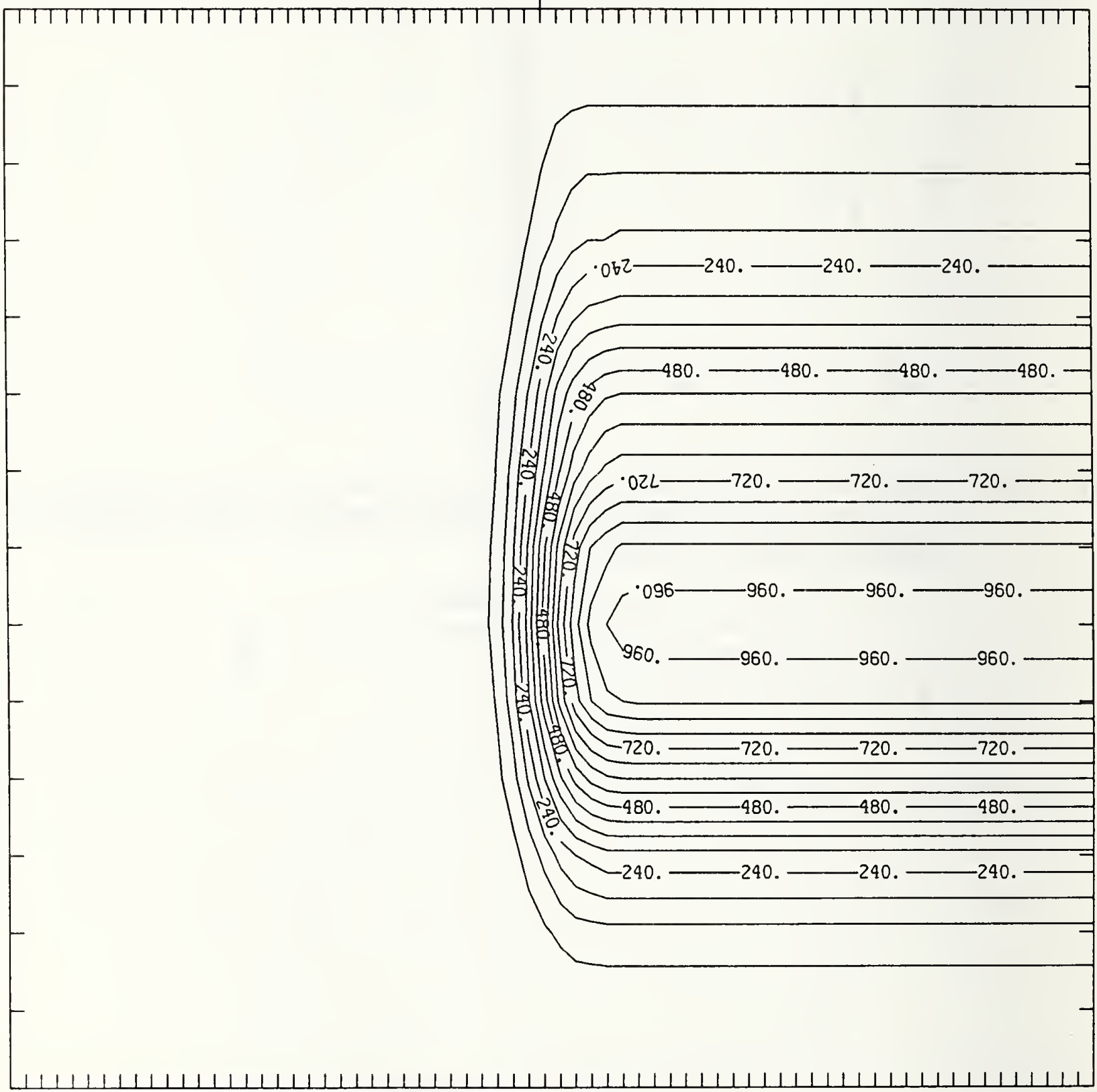

Figure P225-3 Two-dimensional distribution of $225 \mathrm{keV}$ phosphorus implanted past a mask edge. The length increment (distance between tick marks) is $0.04 \mu \mathrm{m}$ and the scale factor is 1000 . 


\section{PASK}

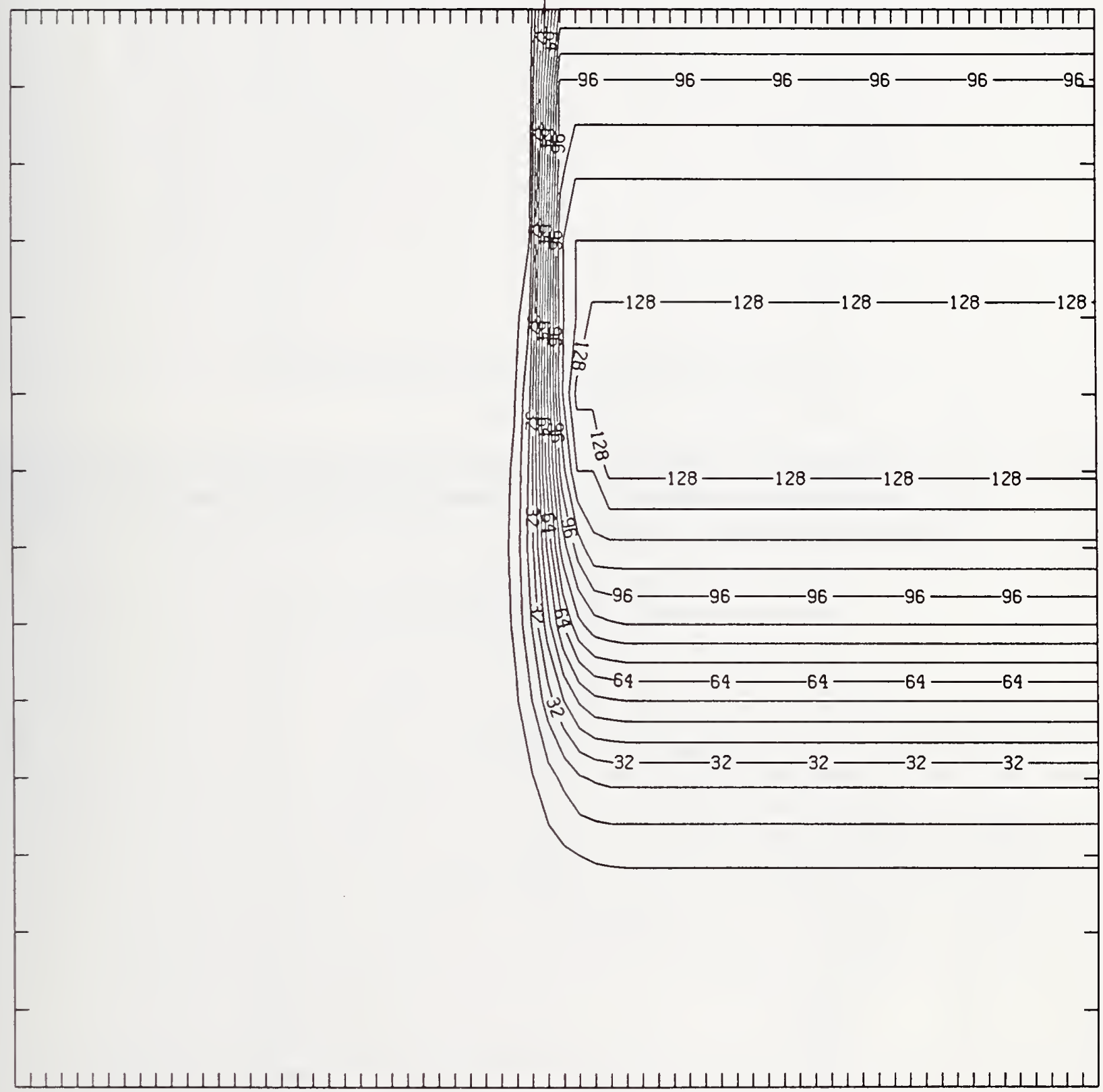

Figure P225-4 Two-dimensional distribution of Frenkel pairs created by $225 \mathrm{keV}$ phosphorus implanted past a mask edge. The length increment (distance between tick marks) is $0.04 \mu \mathrm{m}$ and the scale factor is 0.1 . 


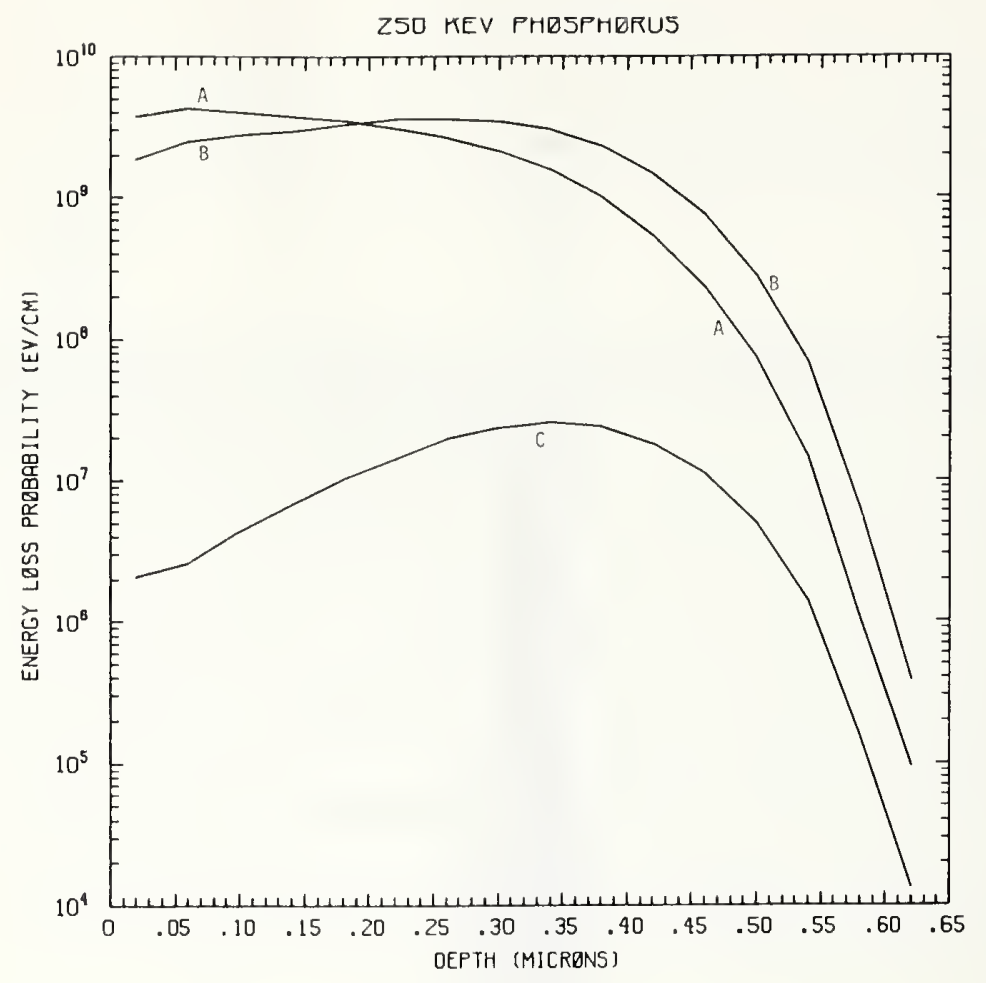

Figure P250-1 One-dimensional probability distributions for energy loss due to electronic excitation (A), displacement damage(B), and phonon excitation (C) for $250 \mathrm{keV}$ phosphorus normally incident on a silicon target.

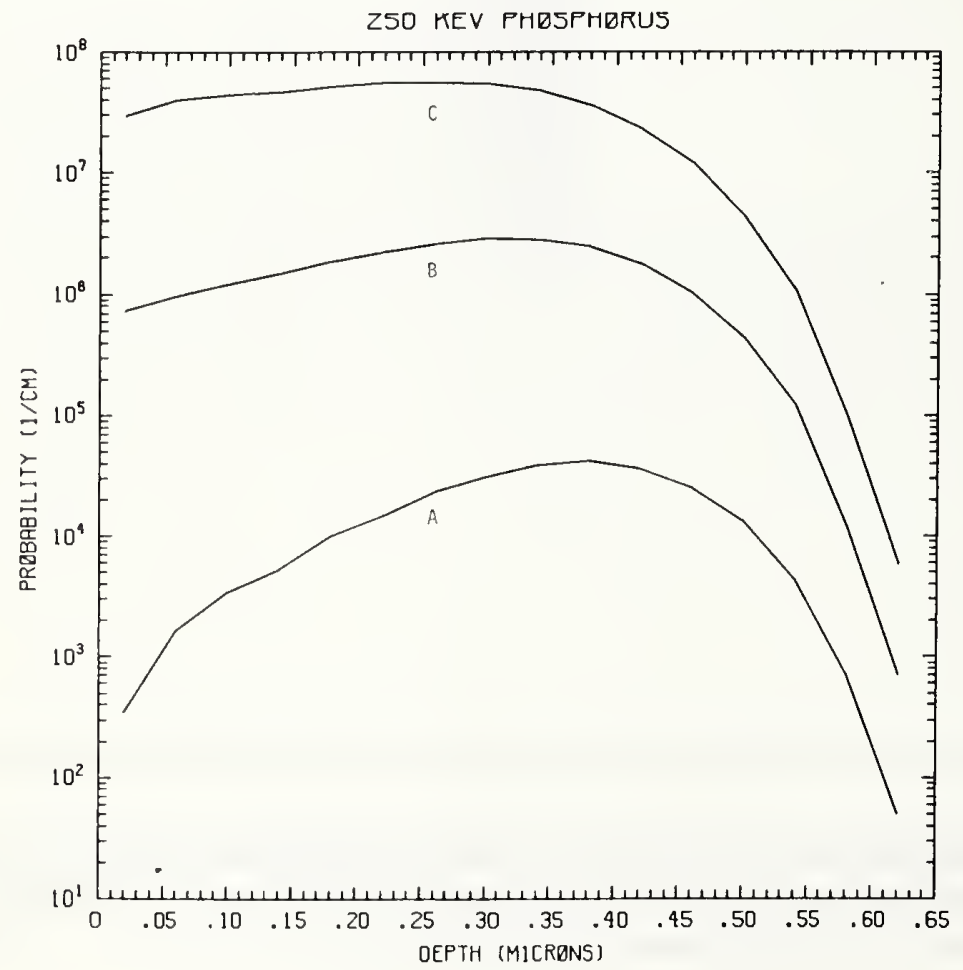

Figure P250-2 One-dimensional probability distributions for implanted phosphorus (A), primary silicon displacements (B), and Frenkel pairs (C) for $250 \mathrm{keV}$ phosphorus normally incident on a silicon target. 
MASK

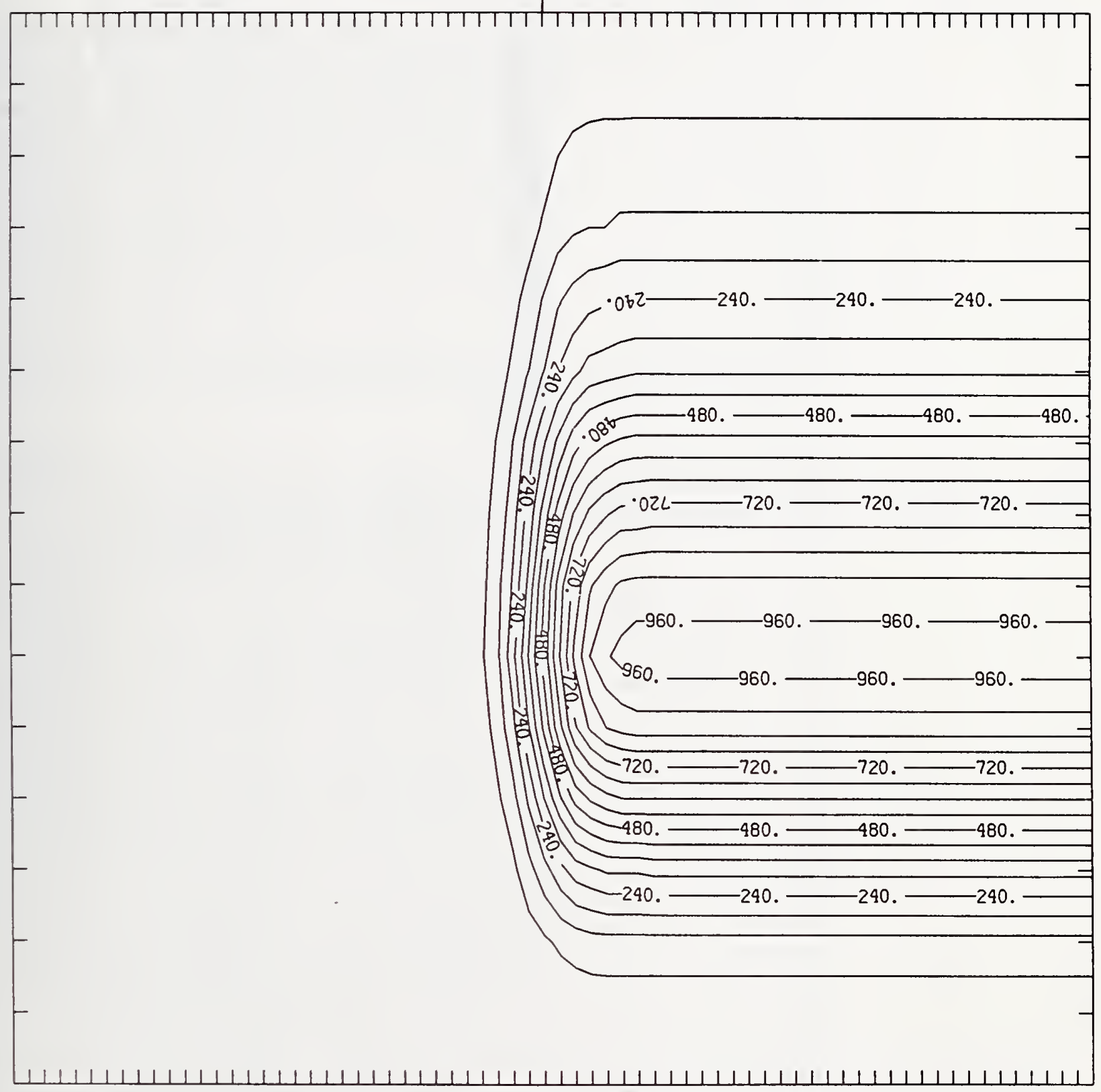

Figure P250-3 Two-dimensional distribution of $250 \mathrm{keV}$ phosphorus implanted past a mask edge. The length increment (distance between tick marks) is $0.04 \mu \mathrm{m}$ and the scale factor is 1000 . 


\section{F.ASK}

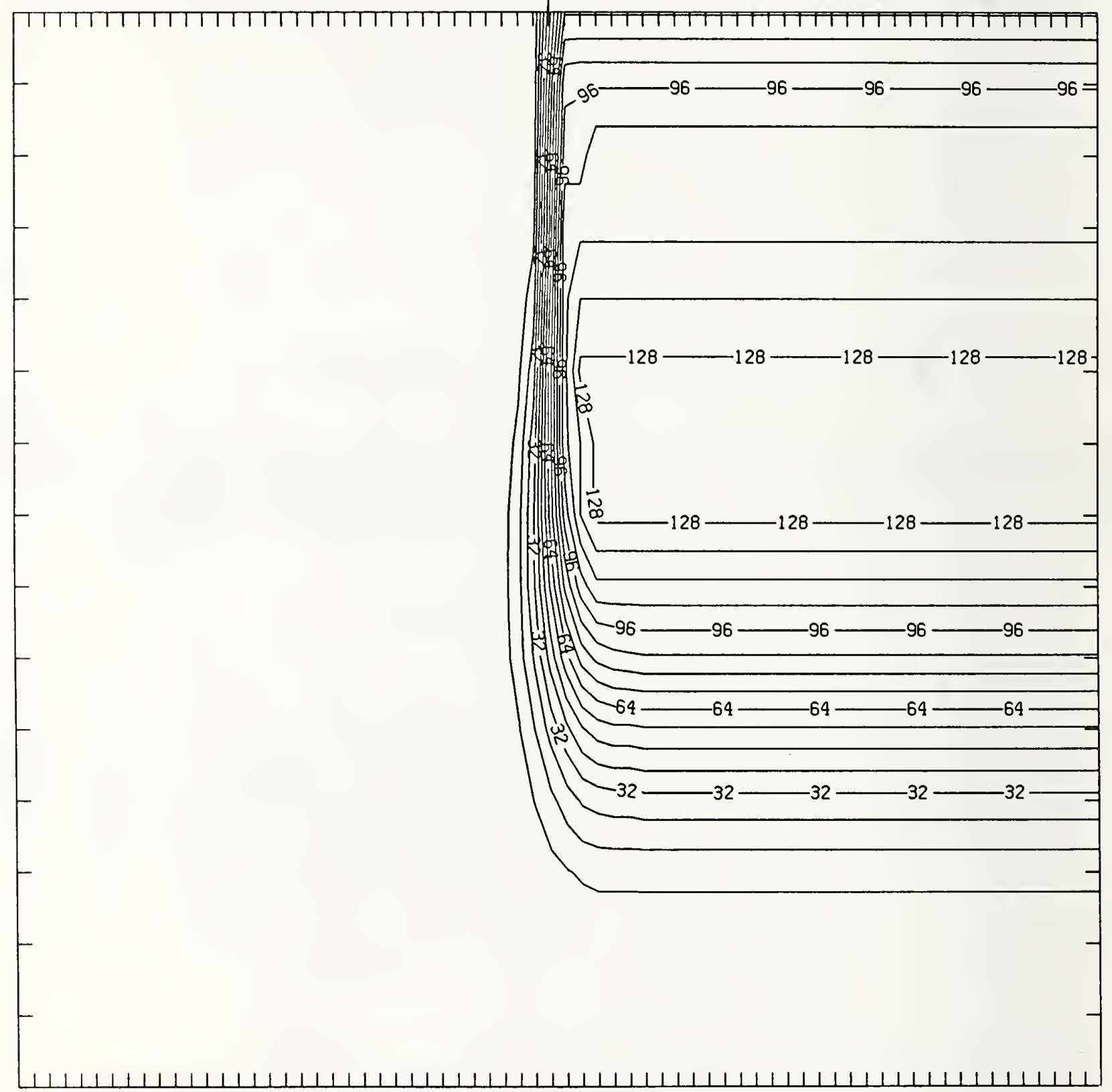

Figure P250-4 Two-dimensional distribution of Frenkel pairs created by $250 \mathrm{keV}$ phosphorus implanted past a mask edge. The length increment (distance between tick marks) is $0.04 \mu \mathrm{m}$ and the scale factor is 0.1 . 


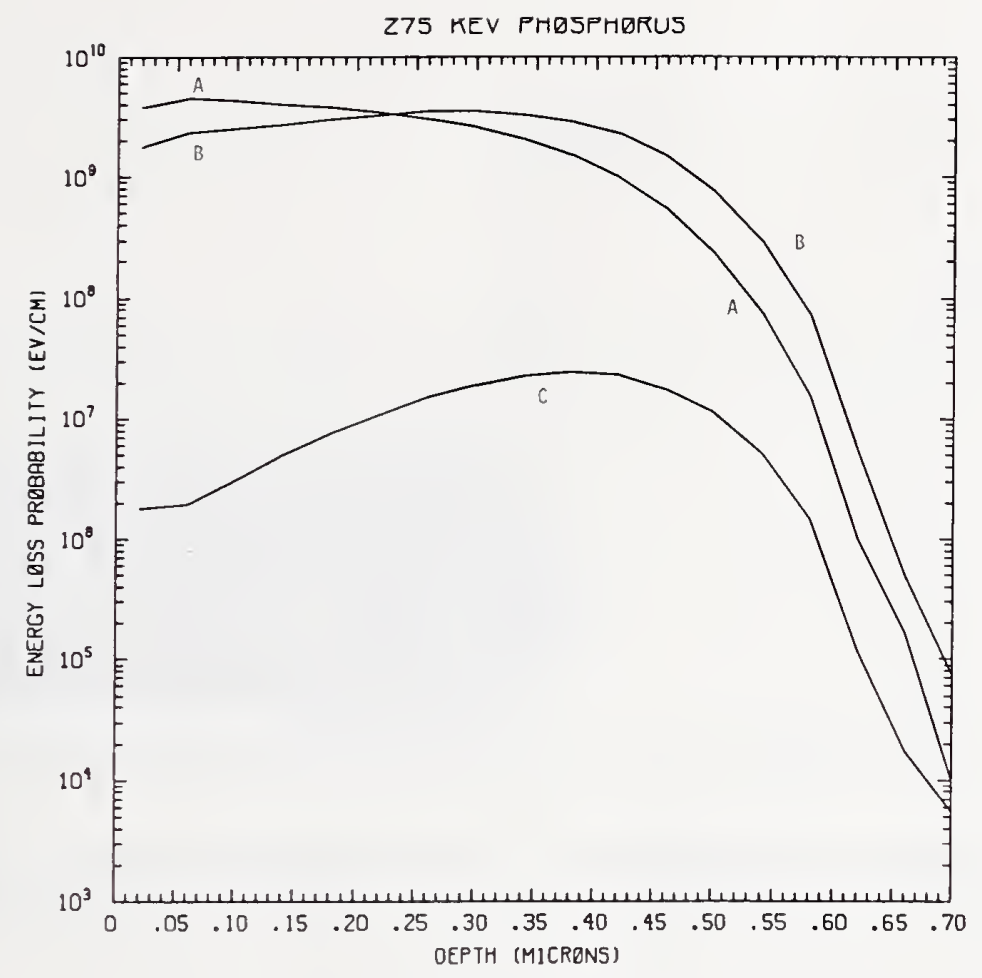

Figure P275-1 One-dimensional probability distributions for energy loss due to electronic excitation (A), displacement damage(B), and phonon excitation (C) for $275 \mathrm{keV}$ phosphorus normally incident on a silicon target.

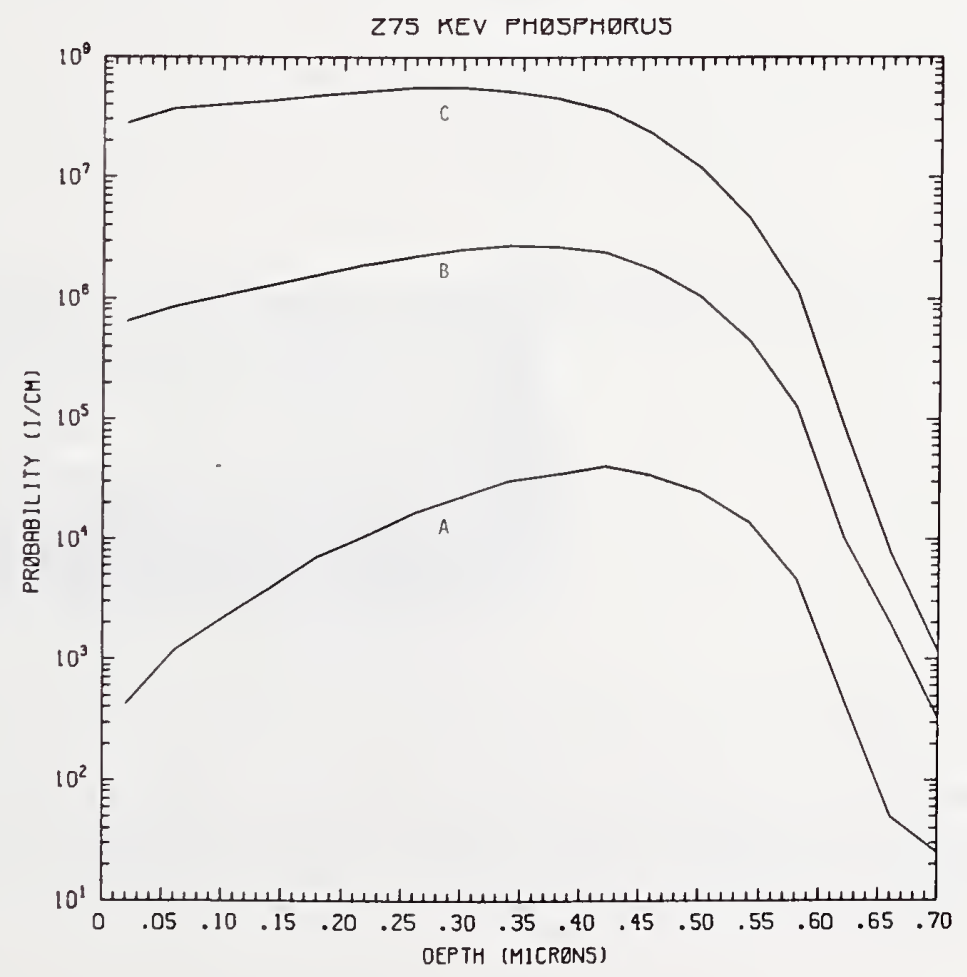

Figure P275-2 One-dimensional probability distributions for implanted phosphorus (A), primary silicon displacements (B), and Frenkel pairs (C) for $275 \mathrm{keV}$ phosphorus normally incident on a silicon target. 


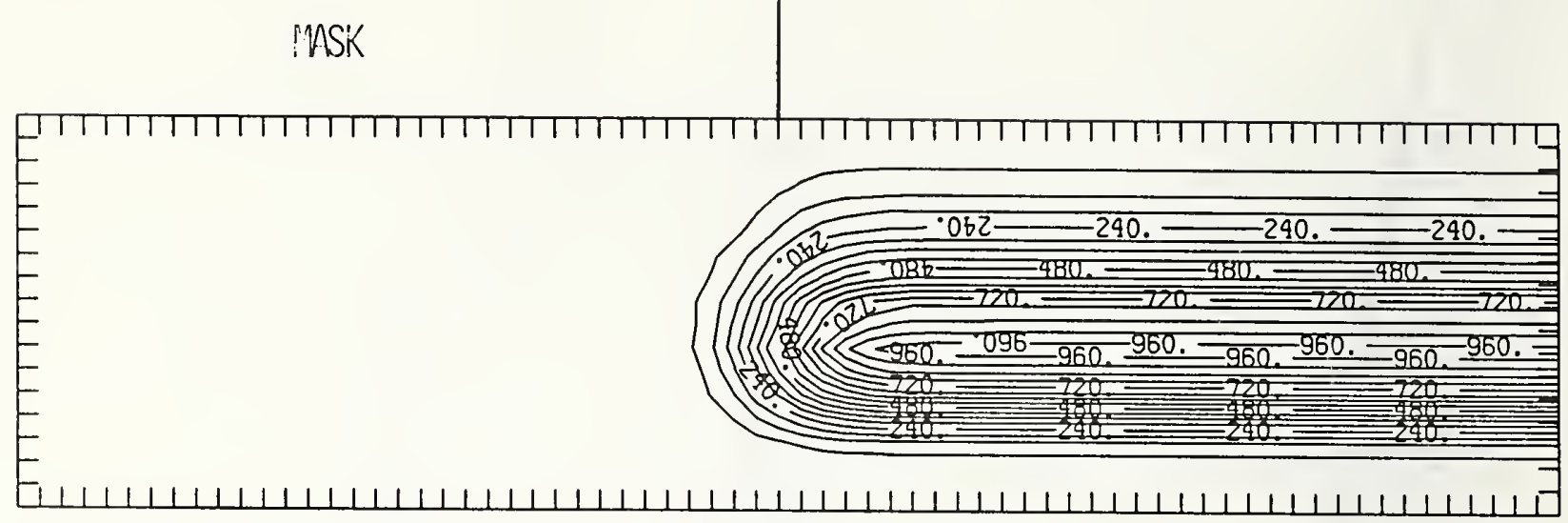

Figure P275-3 Two-dimensional distribution of $275 \mathrm{keV}$ phosphorus implanted past a mask edge. The length increment (distance between tick marks) is $0.04 \mu \mathrm{m}$ and the scale factor is 1000 .

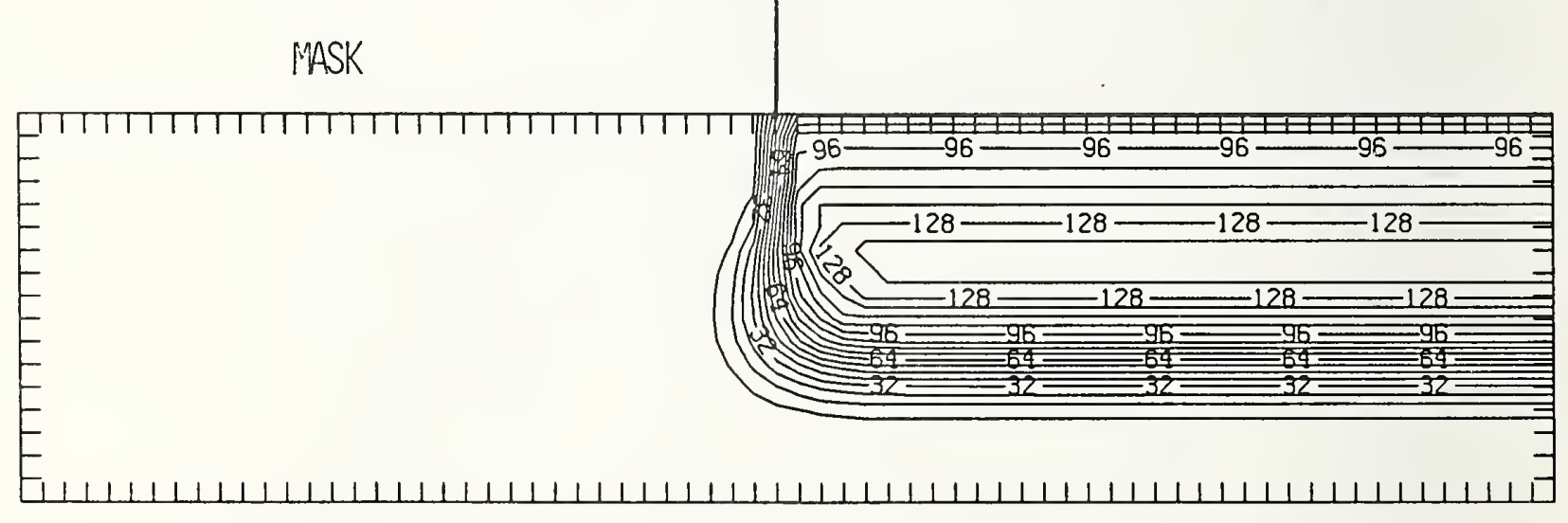

Figure P275-4 Two-dimensional distribution of Frenkel pairs created by $275 \mathrm{keV}$ phosphorus implanted past a mask edge. The length increment (distance between tick marks) is $0.04 \mu \mathrm{m}$ and the scale factor is 0.1 . 


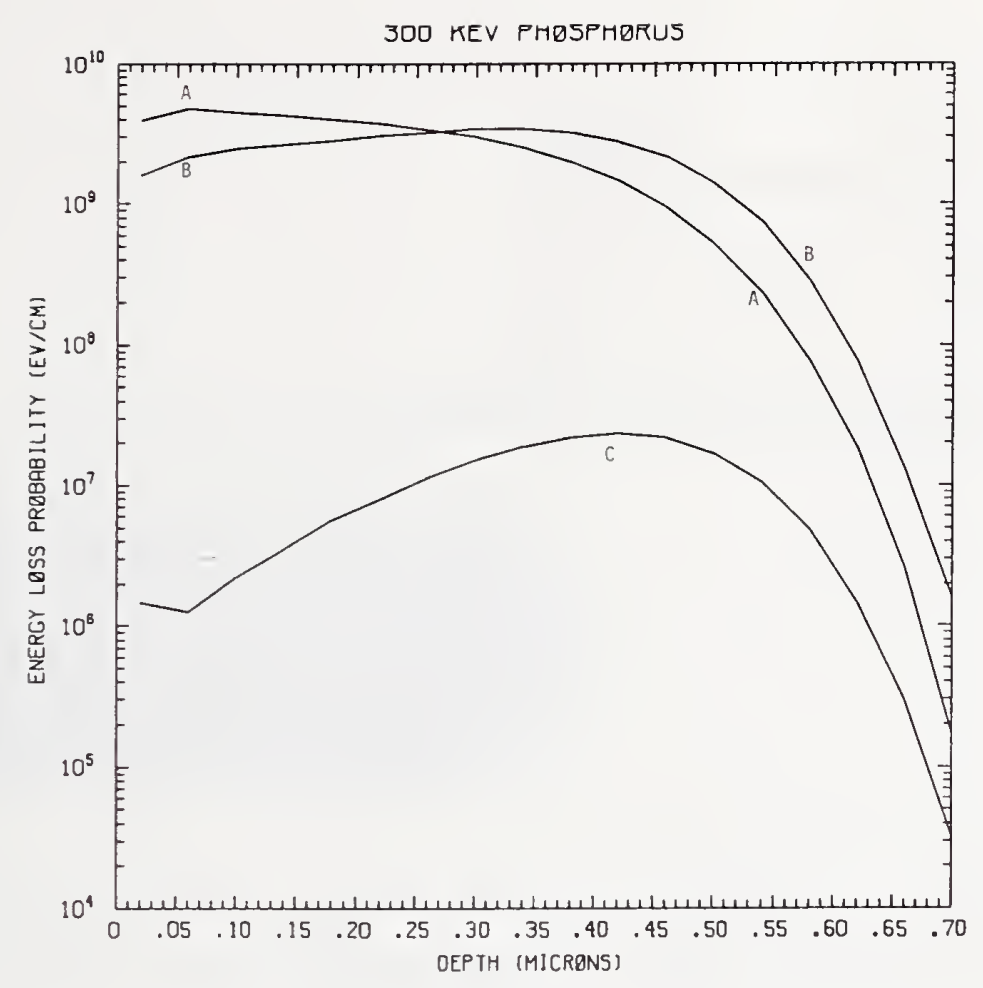

Figure P300-1 One-dimensional probability distributions for energy loss due to electronic excitation (A), displacement damage(B), and phonon excitation (C) for $300 \mathrm{keV}$ phosphorus normally incident on a silicon target.

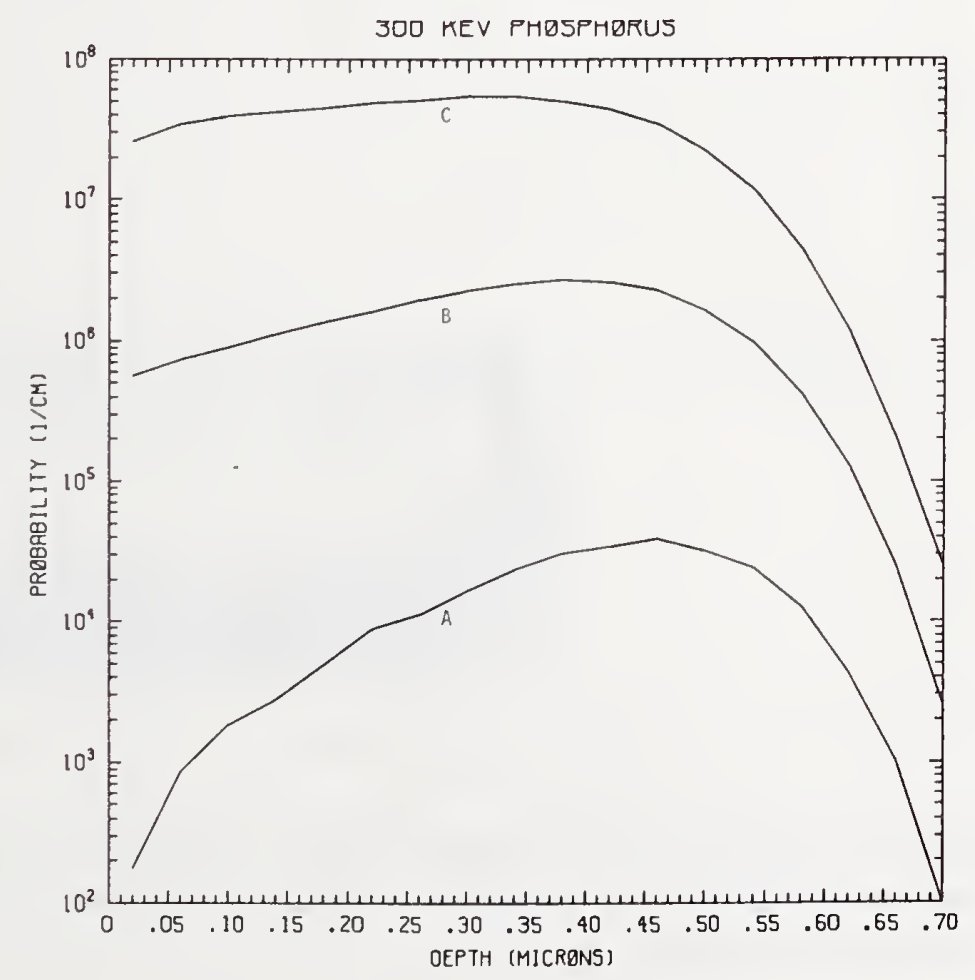

Figure P300-2 One-dimensional probability distributions for implanted phosphorus (A), primary silicon displacements (B), and Frenkel pairs (C) for $300 \mathrm{keV}$ phosphorus normally incident on a silicon target. 


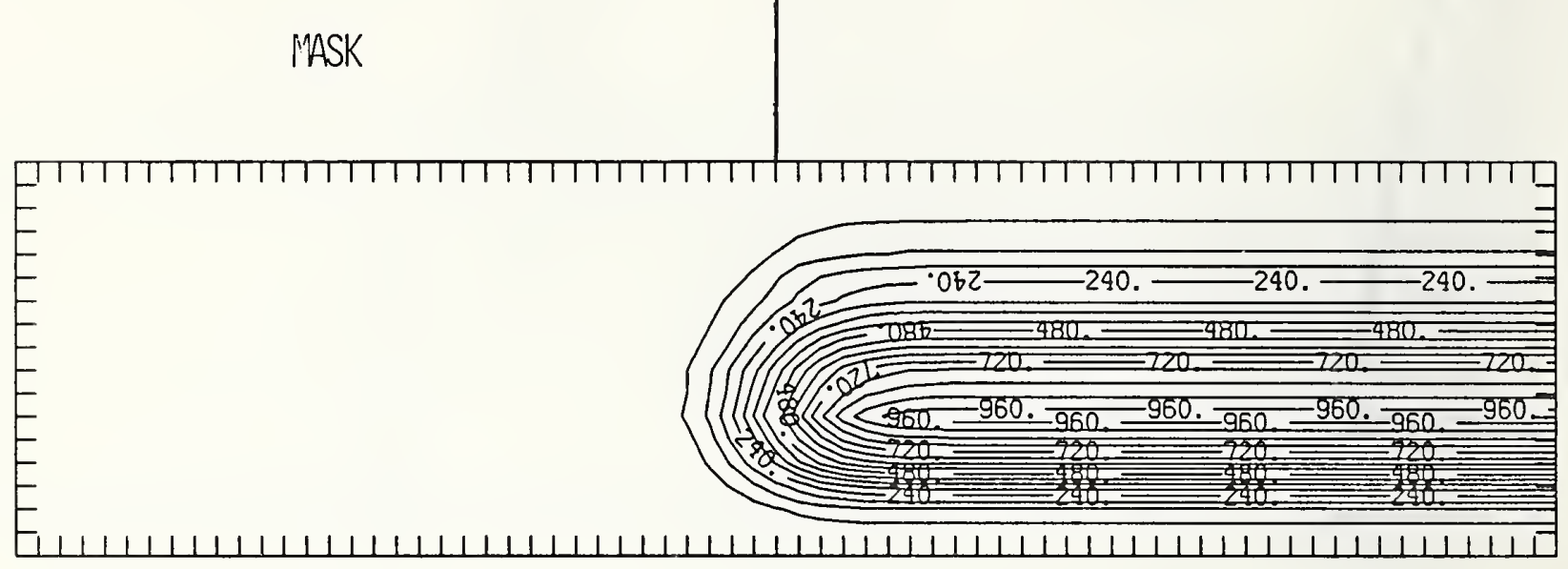

Figure P300-3 Two-dimensional distribution of $300 \mathrm{keV}$ phosphorus implanted past a mask edge. The length increment (distance between tick marks) is $0.04 \mu \mathrm{m}$ and the scale factor is 1000 .

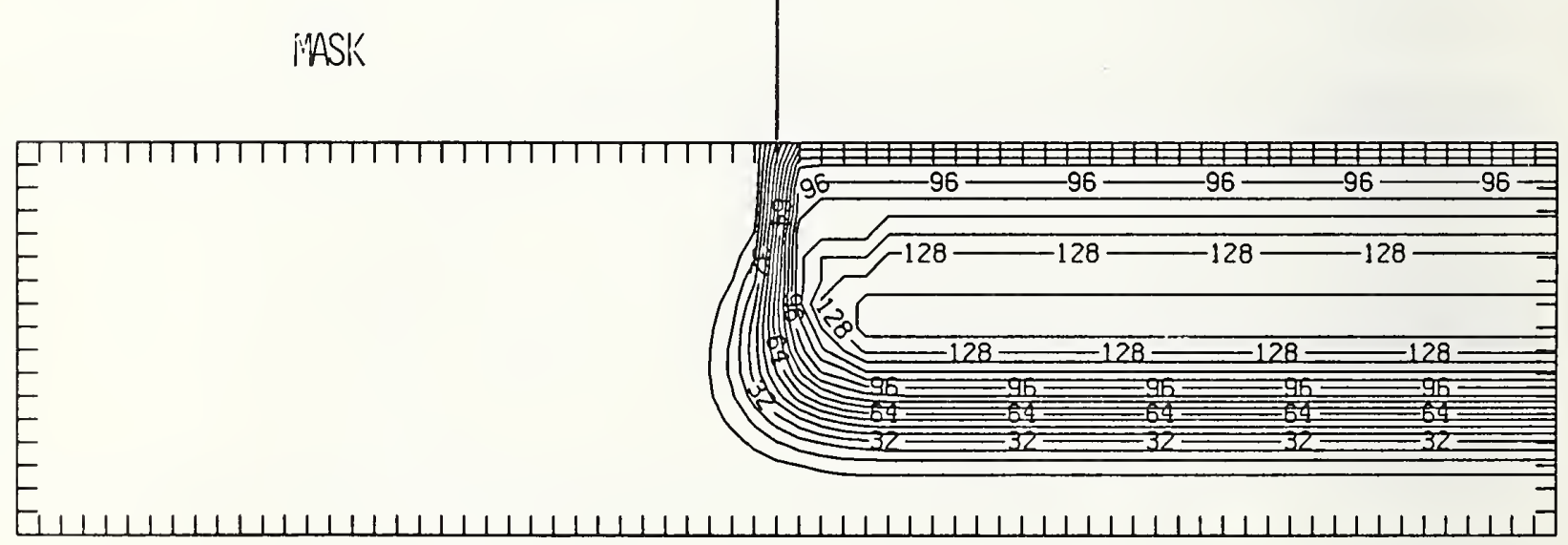

Figure P300-4 Two-dimensional distribution of Frenkel pairs created by $300 \mathrm{keV}$ phosphorus implanted past a mask edge. The length increment (distance between tick marks) is $0.04 \mu \mathrm{m}$ and the scale factor is 0.1 . 


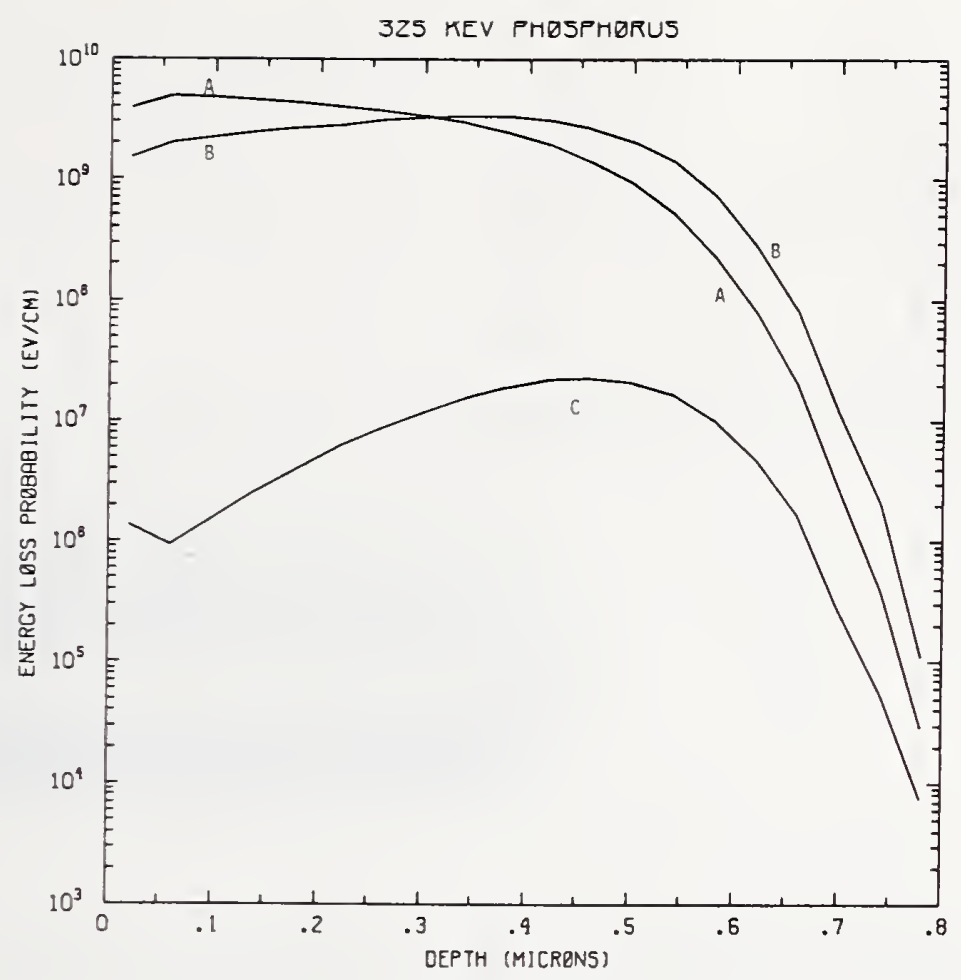

Figure P325-1 One-dimensional probability distributions for energy loss due to electronic excitation (A), displacement damage(B), and phonon excitation (C) for $325 \mathrm{keV}$ phosphorus normally incident on a silicon target.

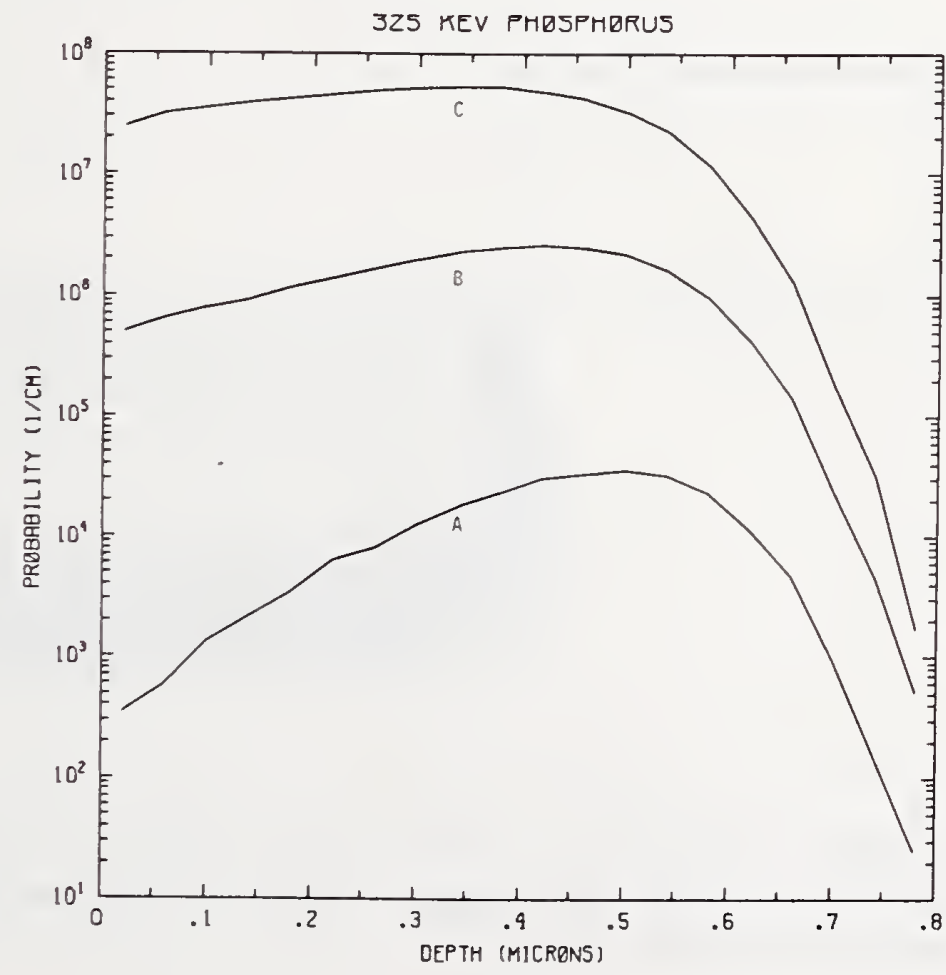

Figure P325-2 One-dimensional probability distributions for implanted phosphorus (A), primary silicon displacements (B), and Frenkel pairs (C) for $325 \mathrm{keV}$ phosphorus normally incident on a silicon target. 


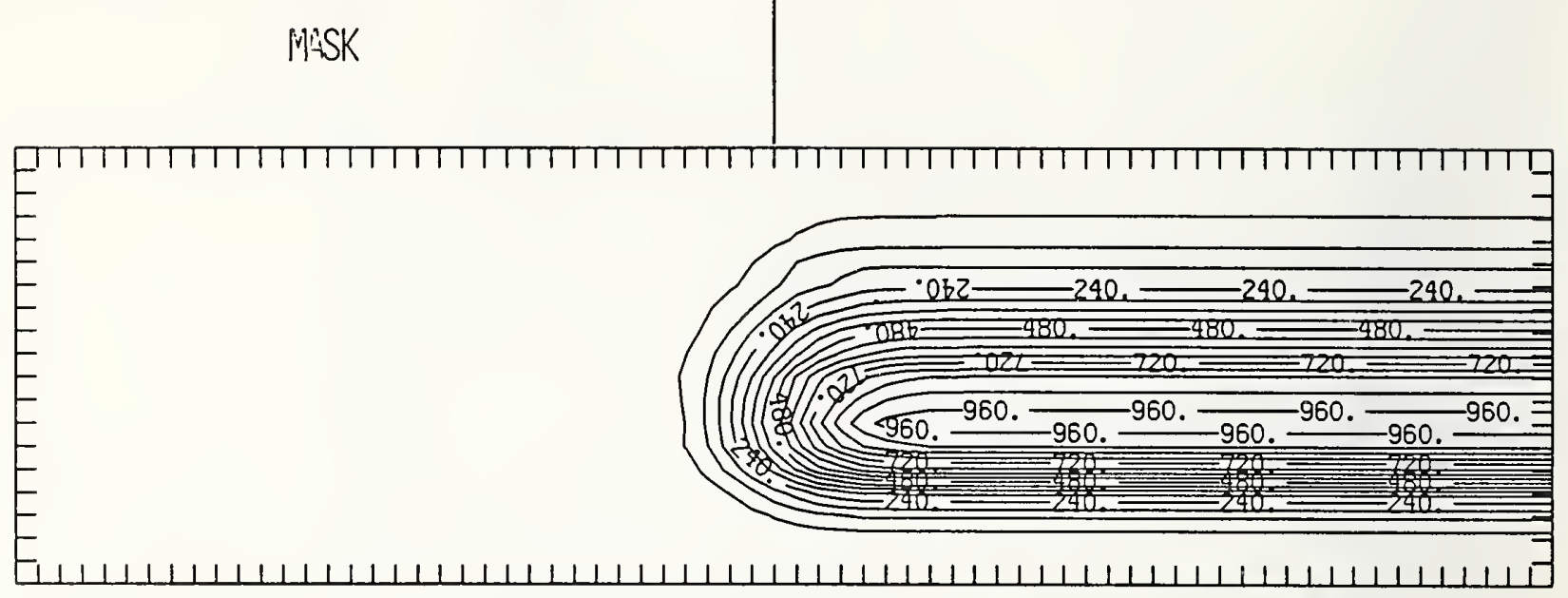

Figure P325-3 Two-dimensional distribution of $325 \mathrm{keV}$ phosphorus implanted past a mask edge. The length increment (distance between tick marks) is $0.04 \mu \mathrm{m}$ and the scale factor is 1000 .

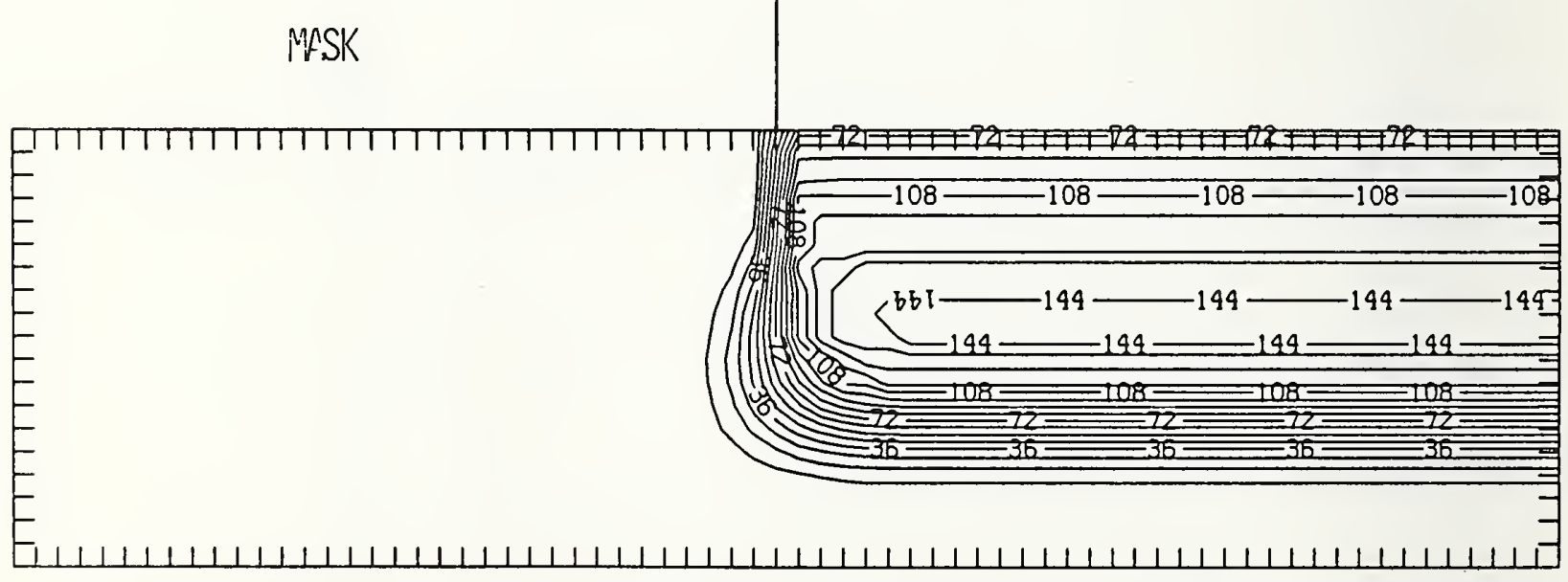

Figure P325-4 Two-dimensional distribution of Frenkel pairs created by $325 \mathrm{keV}$ phosphorus implanted past a mask edge. The length increment (distance between tick marks) is $0.04 \mu \mathrm{m}$ and the scale factor is 0.1 . 


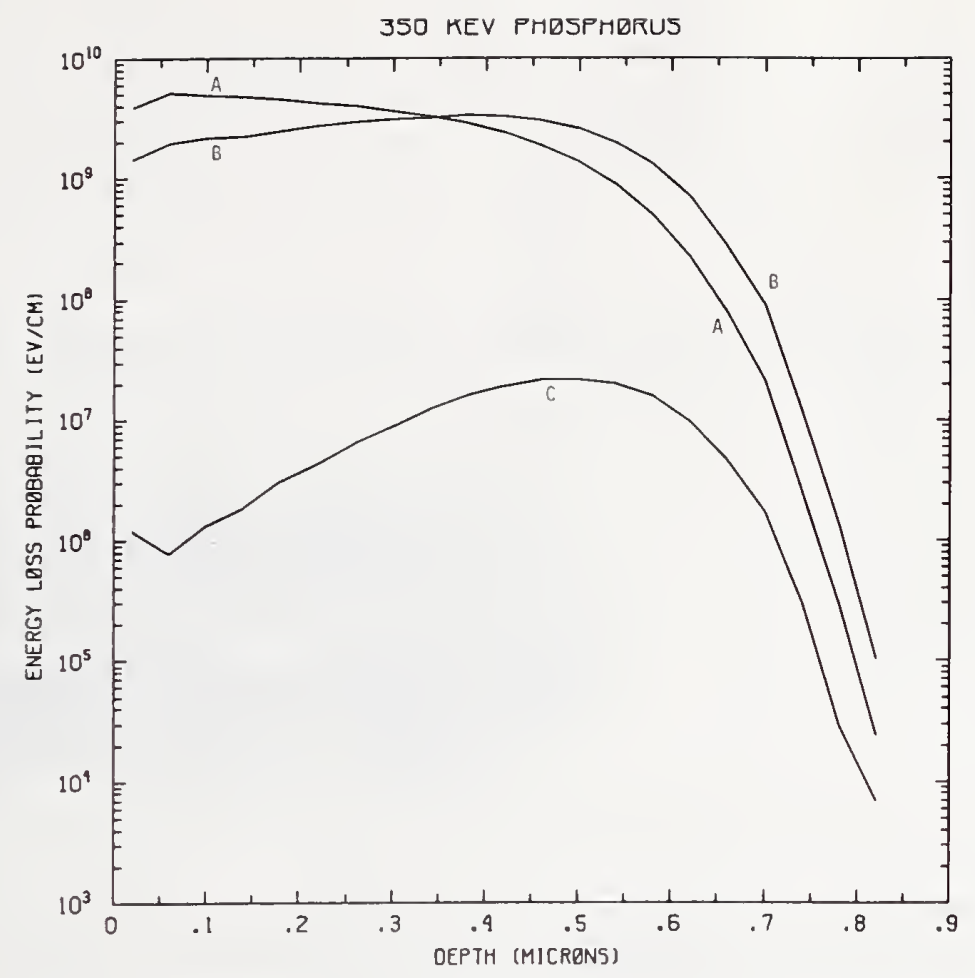

Figure P350-1 One-dimensional probability distributions for energy loss due to electronic excitation (A), displacement damage(B), and phonon excitation (C) for $350 \mathrm{keV}$ phosphorus normally incident on a silicon target.

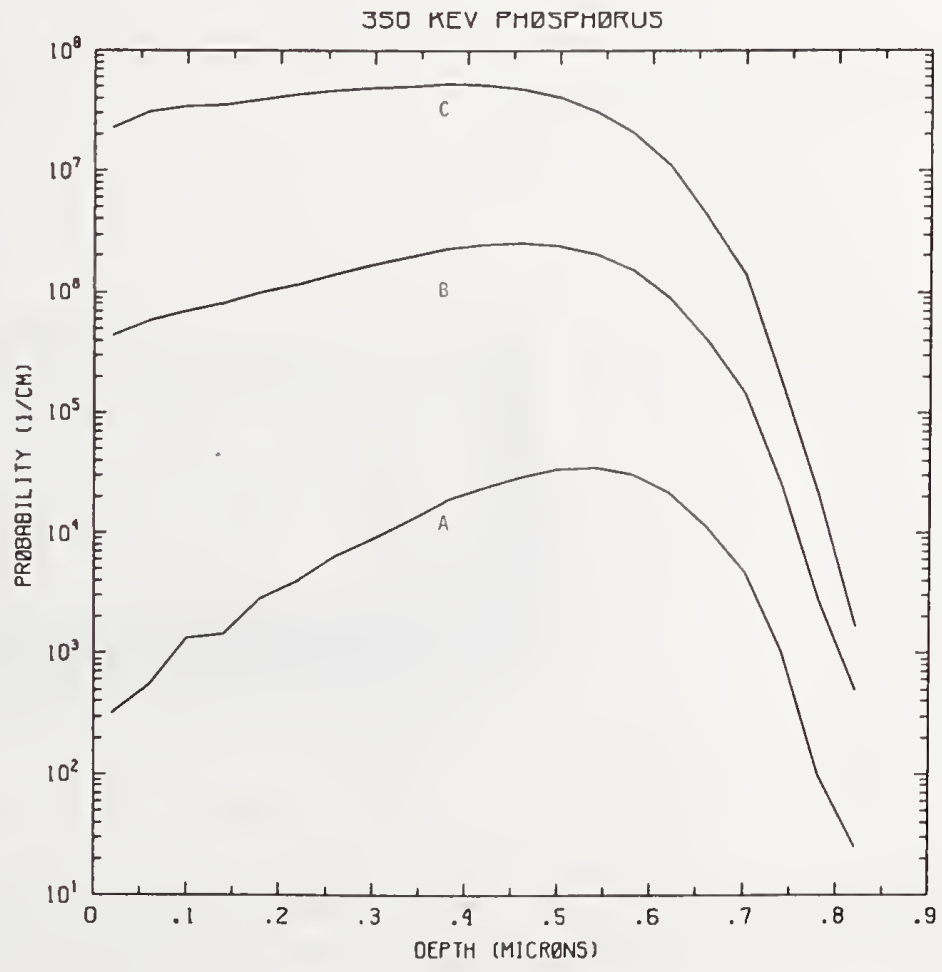

Figure P350-2 One-dimensional probability distributions for implanted phosphorus (A), primary silicon displacements (B), and Frenkel pairs (C) for $350 \mathrm{keV}$ phosphorus normally incident on a silicon target. 


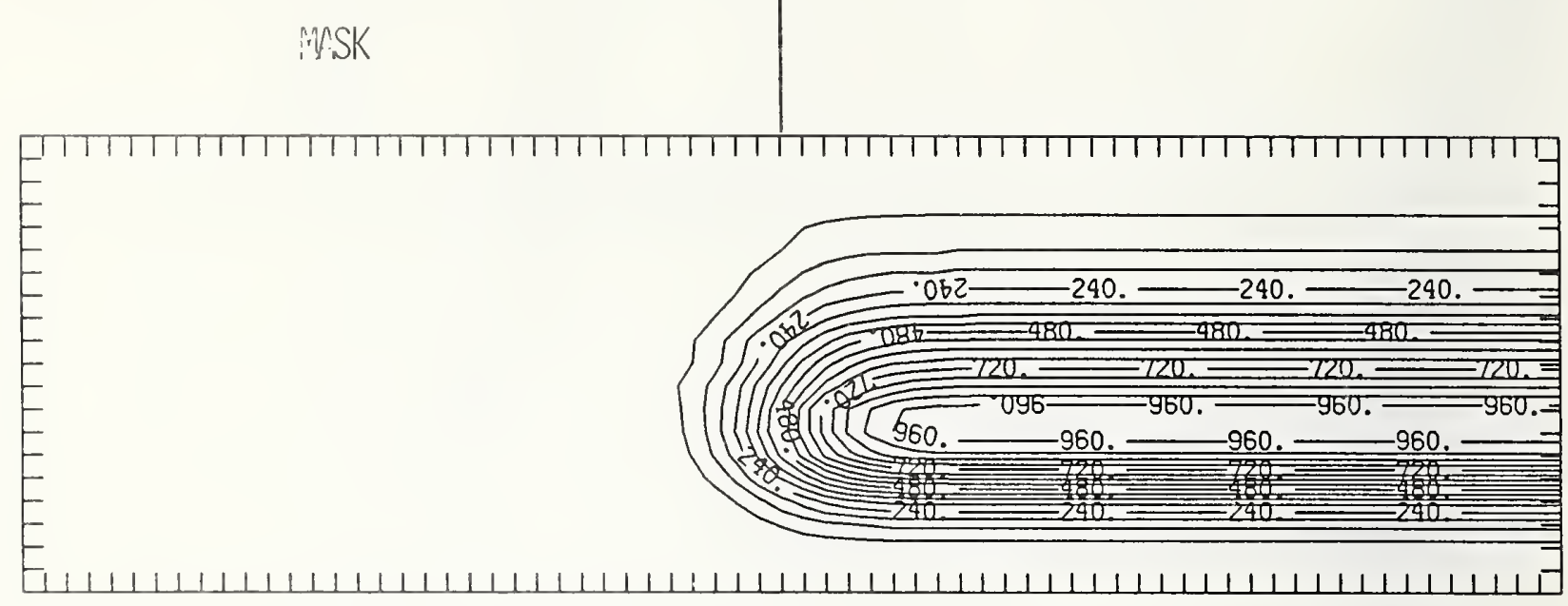

Figure P350-3 Two-dimensional distribution of $350 \mathrm{keV}$ phosphorus implanted past a mask edge. The length increment (distance between tick marks) is $0.04 \mu \mathrm{m}$ and the scale factor is 1000 .

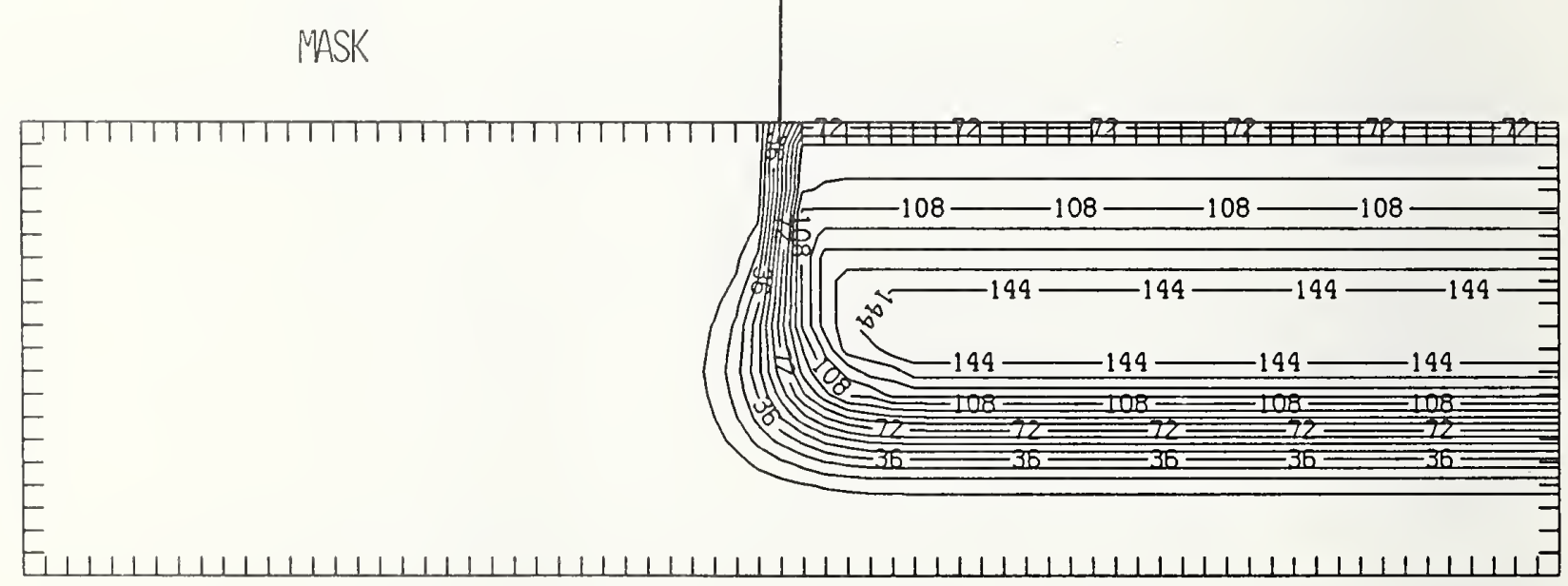

Figure P350-4 Two-dimensional distribution of Frenkel pairs created by $350 \mathrm{keV}$ phosphorus implanted past a mask edge. The length increment (distance between tick marks) is $0.04 \mu \mathrm{m}$ and the scale factor is 0.1 . 


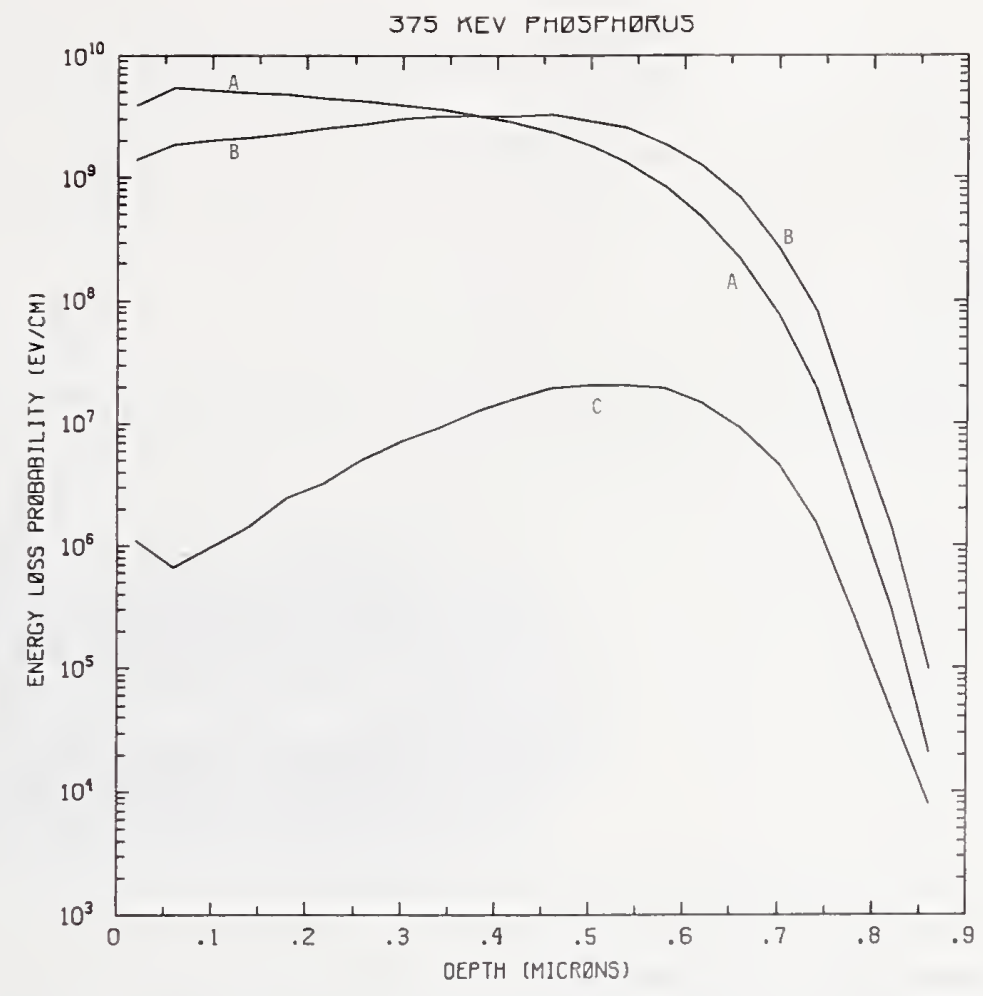

Figure P375-1 One-dimensional probability distributions for energy loss due to electronic excitation (A), displacement damage(B), and phonon excitation (C) for $375 \mathrm{keV}$ phosphorus normally incident on a silicon target.

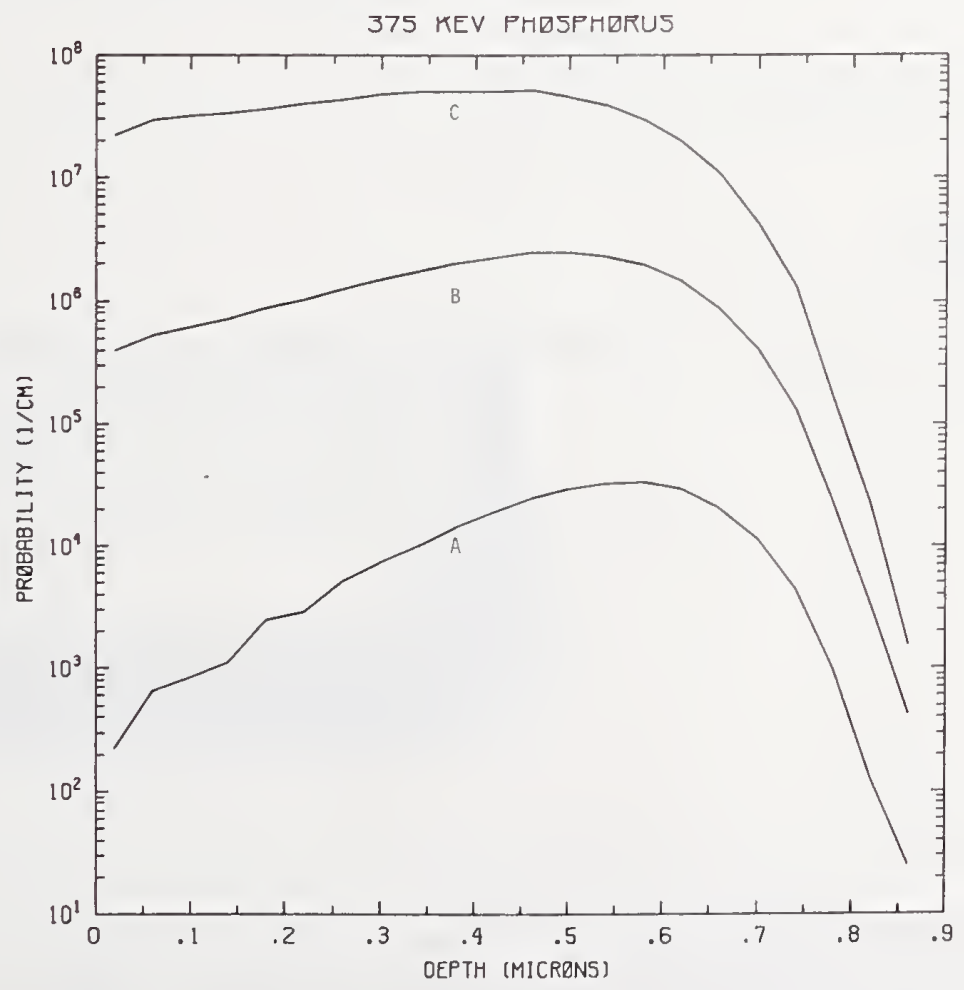

Figure P375-2 One-dimensional probability distributions for implanted phosphorus (A), primary silicon displacements (B), and Frenkel pairs (C) for $375 \mathrm{keV}$ phosphorus normally incident on a silicon target. 


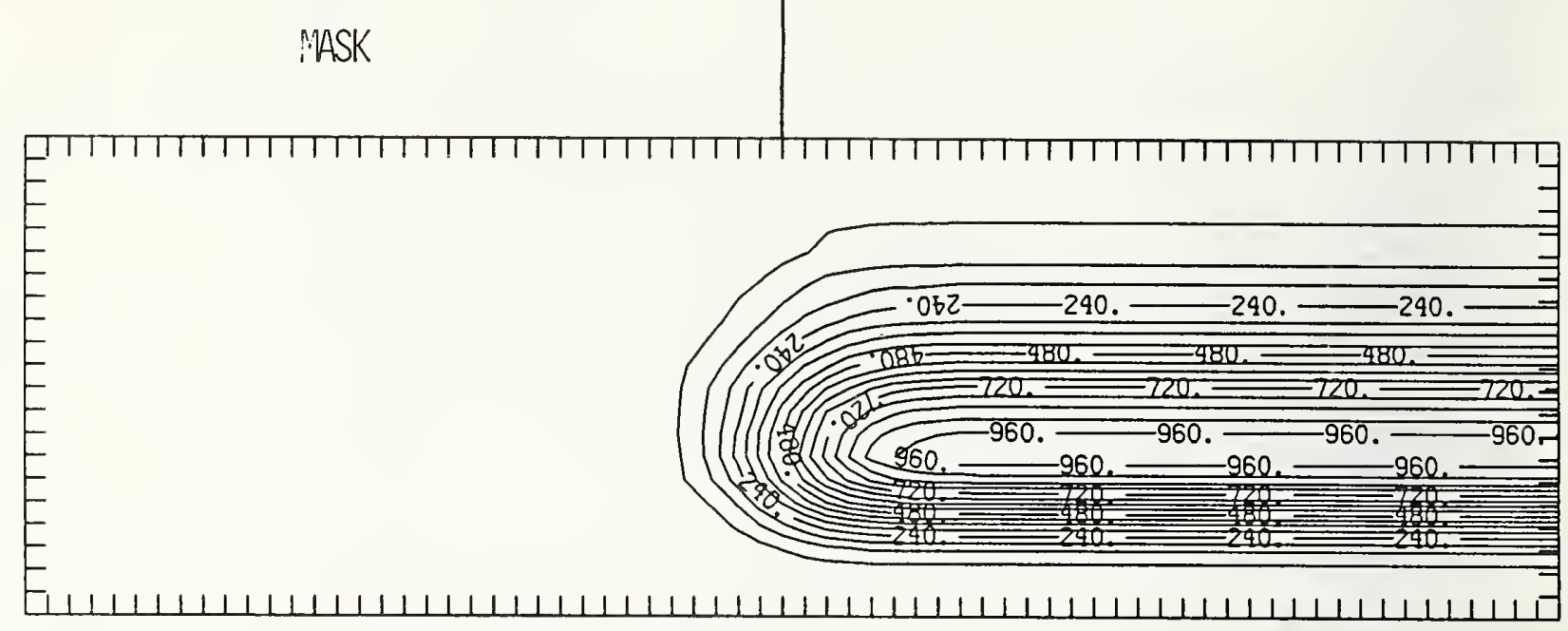

Figure P375-3 Two-dimensional distribution of $375 \mathrm{keV}$ phosphorus implanted past a mask edge. The length increment (distance between tick marks) is $0.04 \mu \mathrm{m}$ and the scale factor is 1000 .

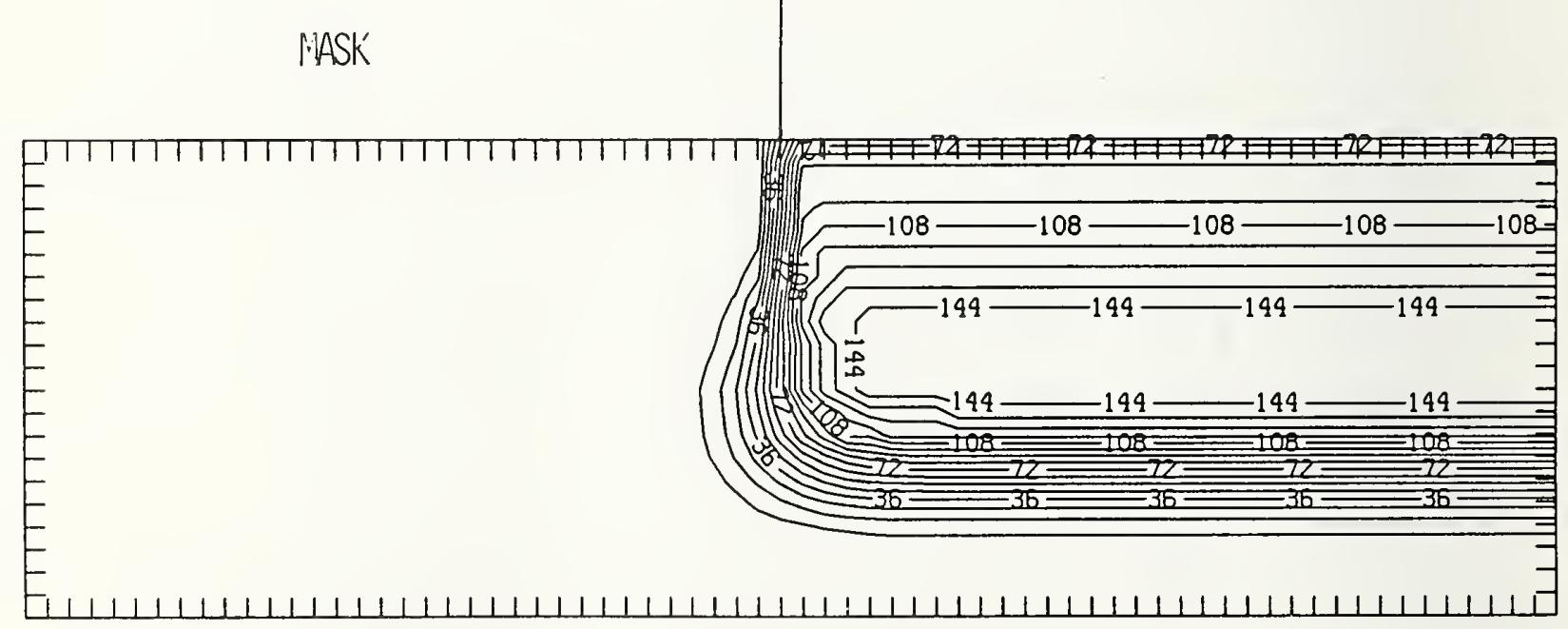

Figure P375-4 Two-dimensional distribution of Frenkel pairs created by $375 \mathrm{keV}$ phosphorus implanted past a mask edge. The length increment (distance between tick marks) is $0.04 \mu \mathrm{m}$ and the scale factor is 0.1 . 


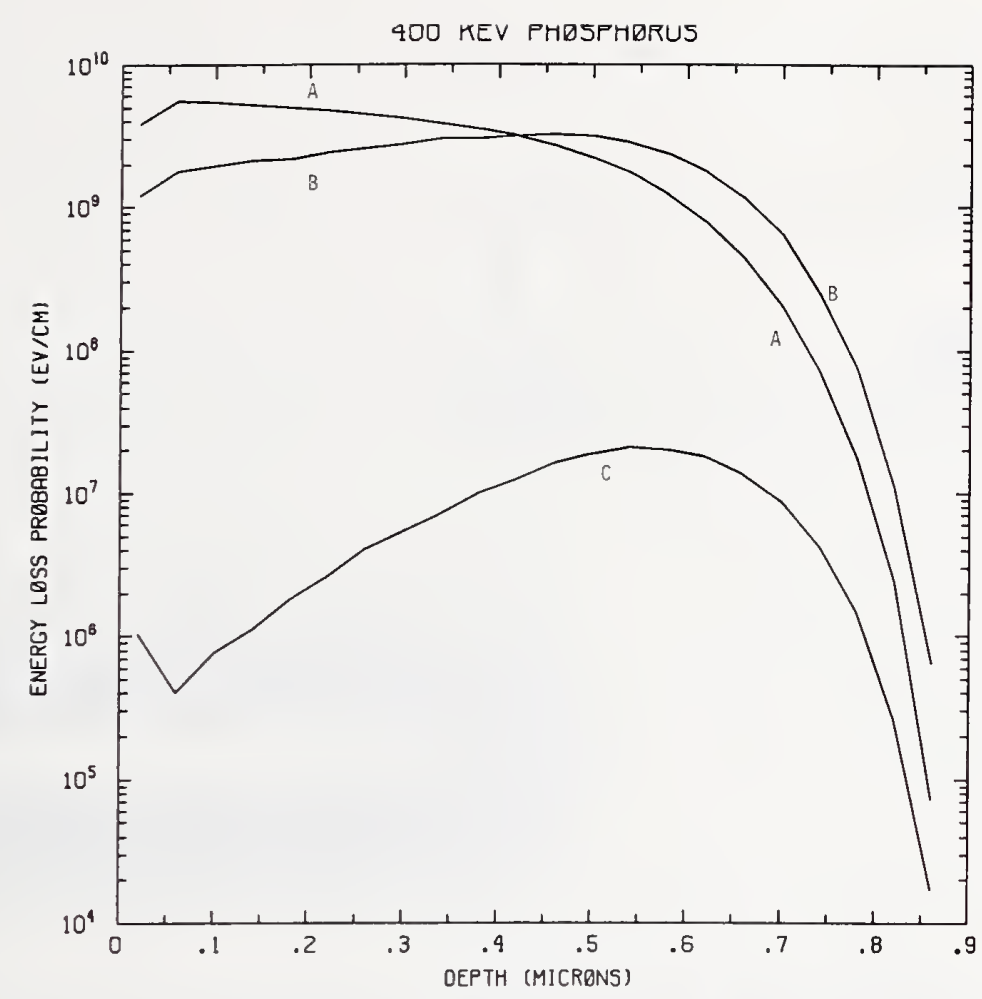

Figure P400-1 One-dimensional probability distributions for energy loss due to electronic excitation (A), displacement damage(B), and phonon excitation (C) for $400 \mathrm{keV}$ phosphorus normally incident on a silicon target.

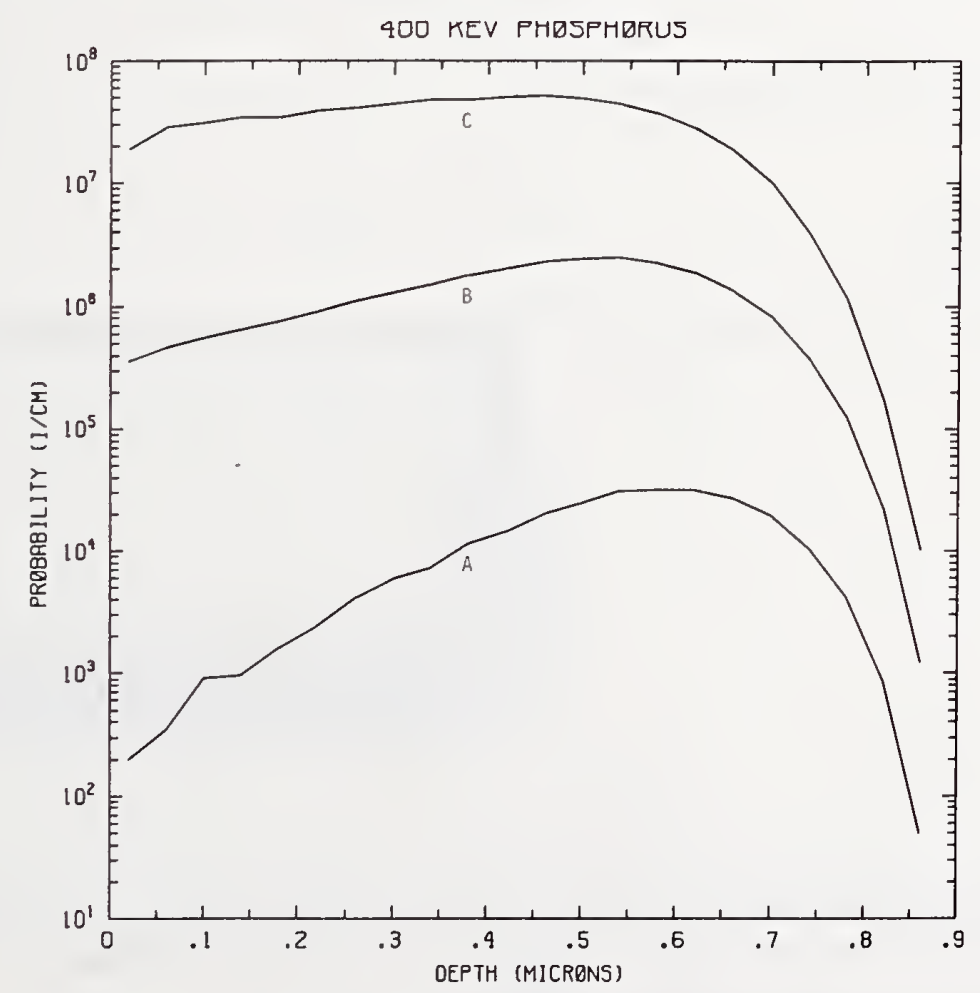

Figure P400-2 One-dimensional probability distributions for implanted phosphorus (A), primary silicon displacements (B), and Frenkel pairs (C) for $400 \mathrm{keV}$ phosphorus normally incident on a silicon target. 


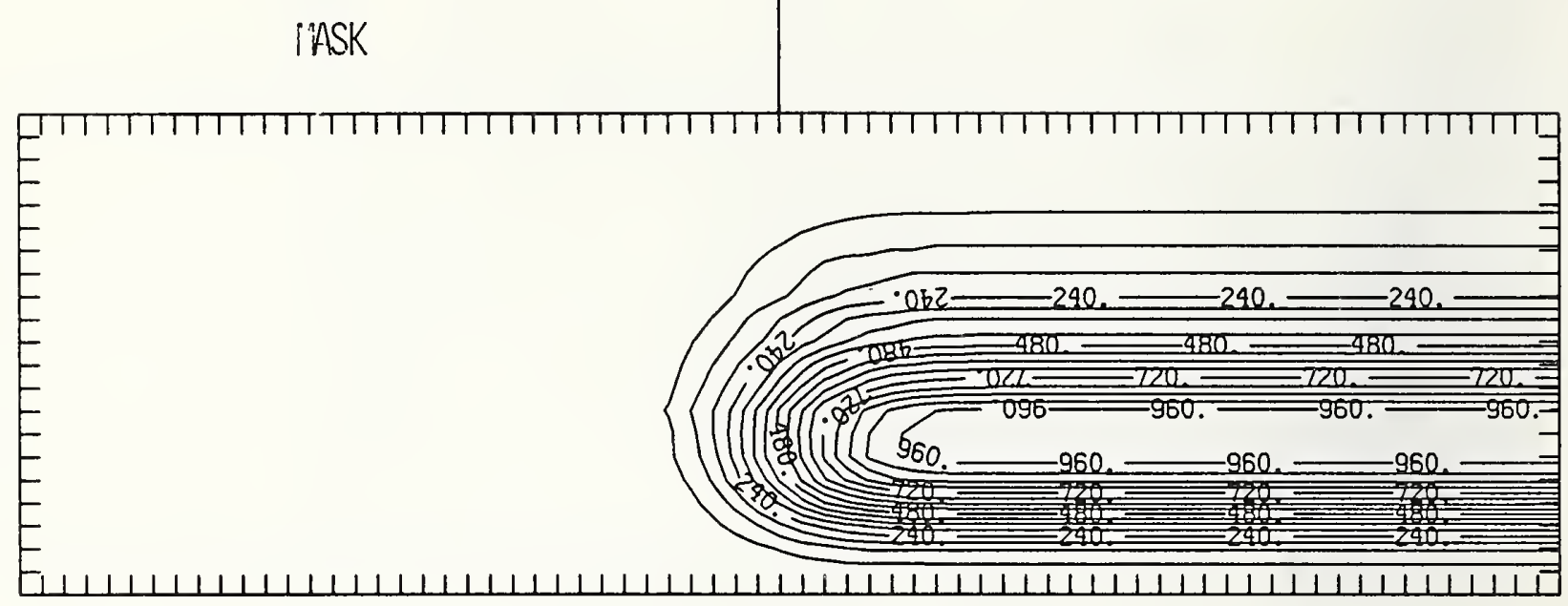

Figure P400-3 Two-dimensional distribution of $400 \mathrm{keV}$ phosphorus implanted past a mask edge. The length increment (distance between tick marks) is $0.04 \mu \mathrm{m}$ and the scale factor is 1000 .

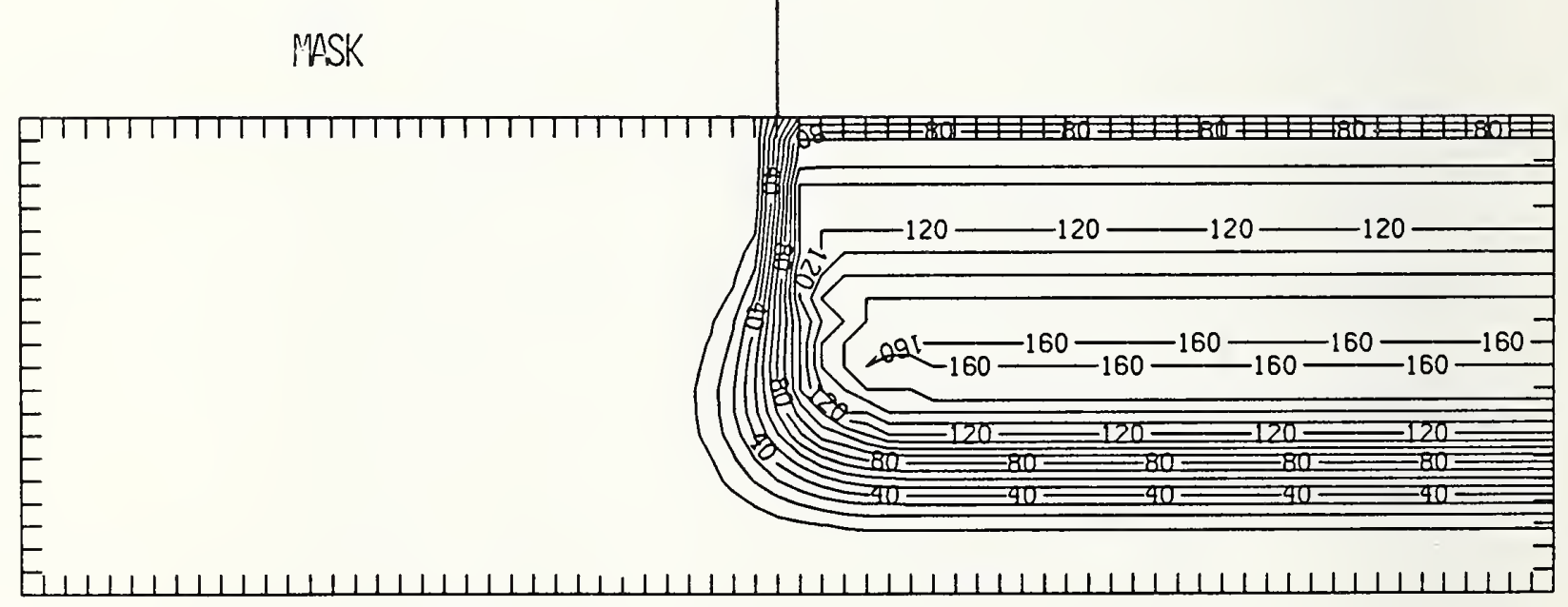

Figure P400-4 Two-dimensional distribution of Frenkel pairs created by $400 \mathrm{keV}$ phosphorus implanted past a mask edge. The length increment (distance between tick marks) is $0.04 \mu \mathrm{m}$ and the scale factor is 0.1 . 


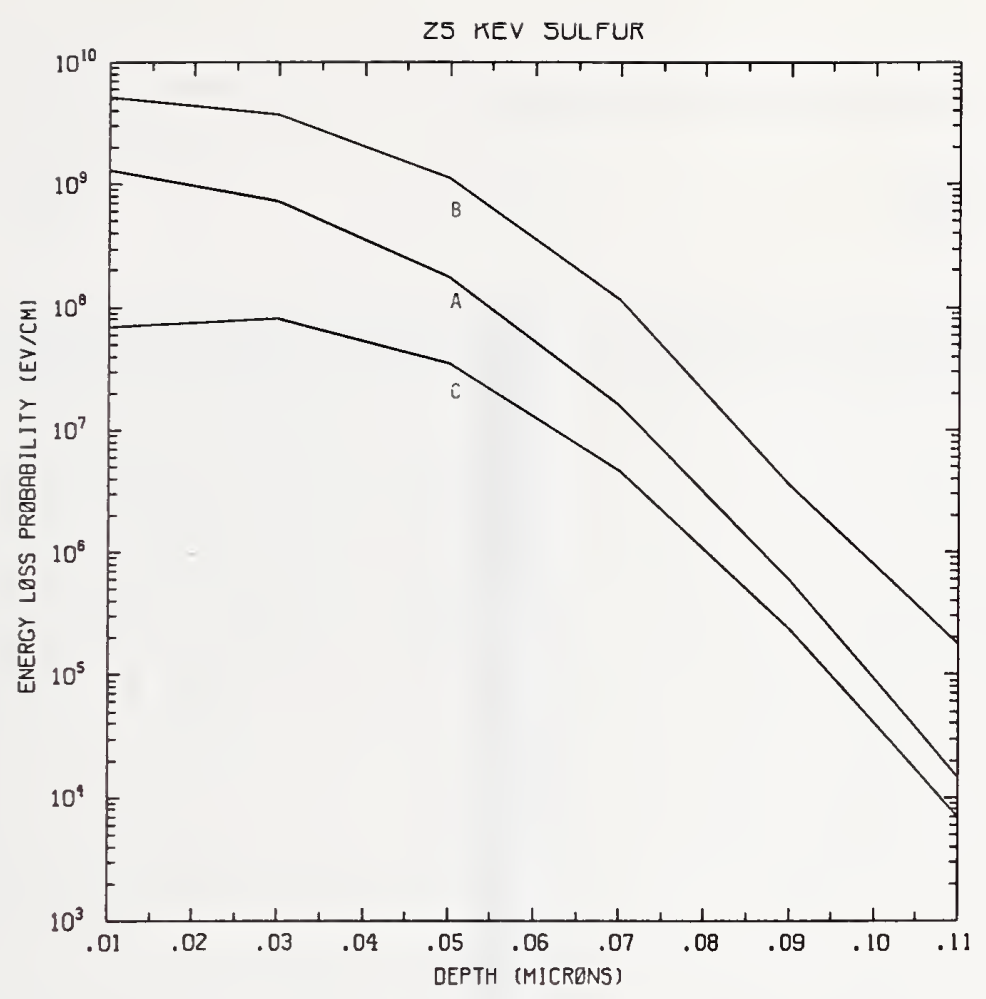

Figure S25-1 One-dimensional probability distributions for energy loss due to electronic excitation (A), displacement damage(B), and phonon excitation (C) for $25 \mathrm{keV}$ sulfur normally incident on a silicon target.

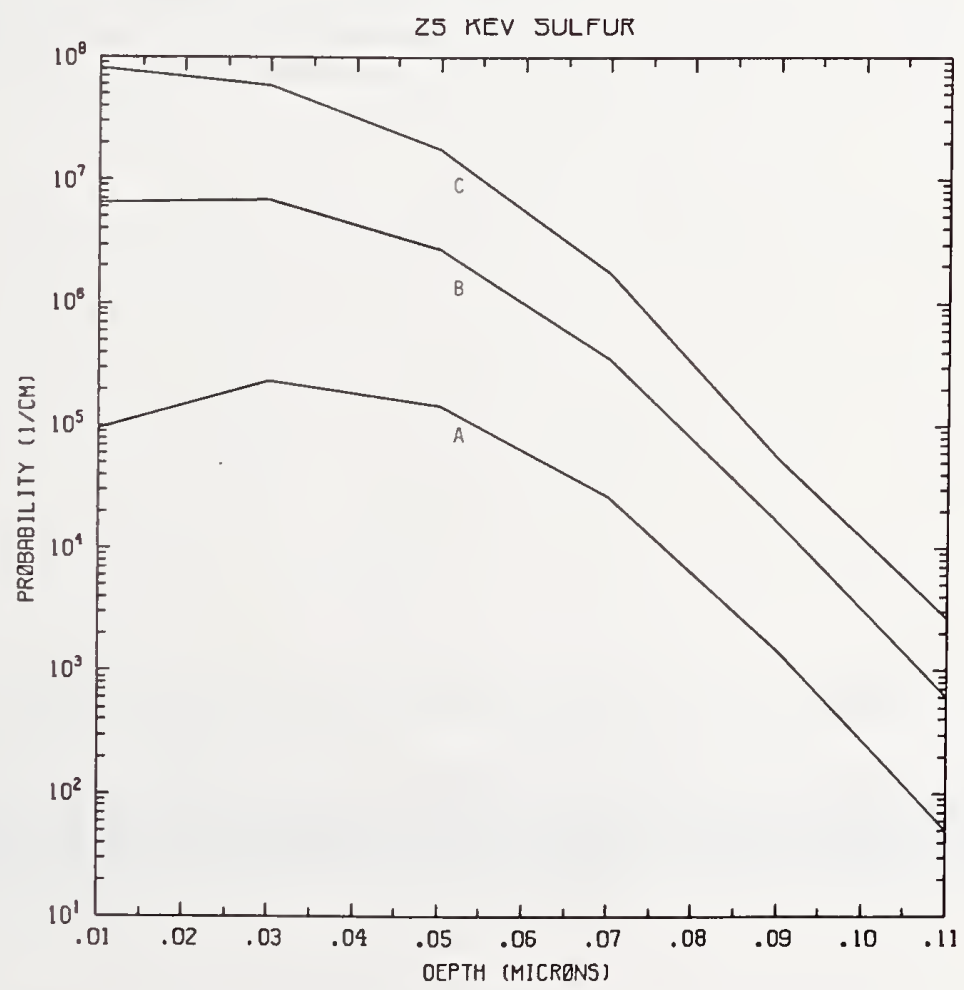

Figure S25-2 One-dimensional probability distributions for implanted sulfur (A), primary silicon displacements (B), and Frenkel pairs (C) for $25 \mathrm{keV}$ sulfur normally incident on a silicon target. 


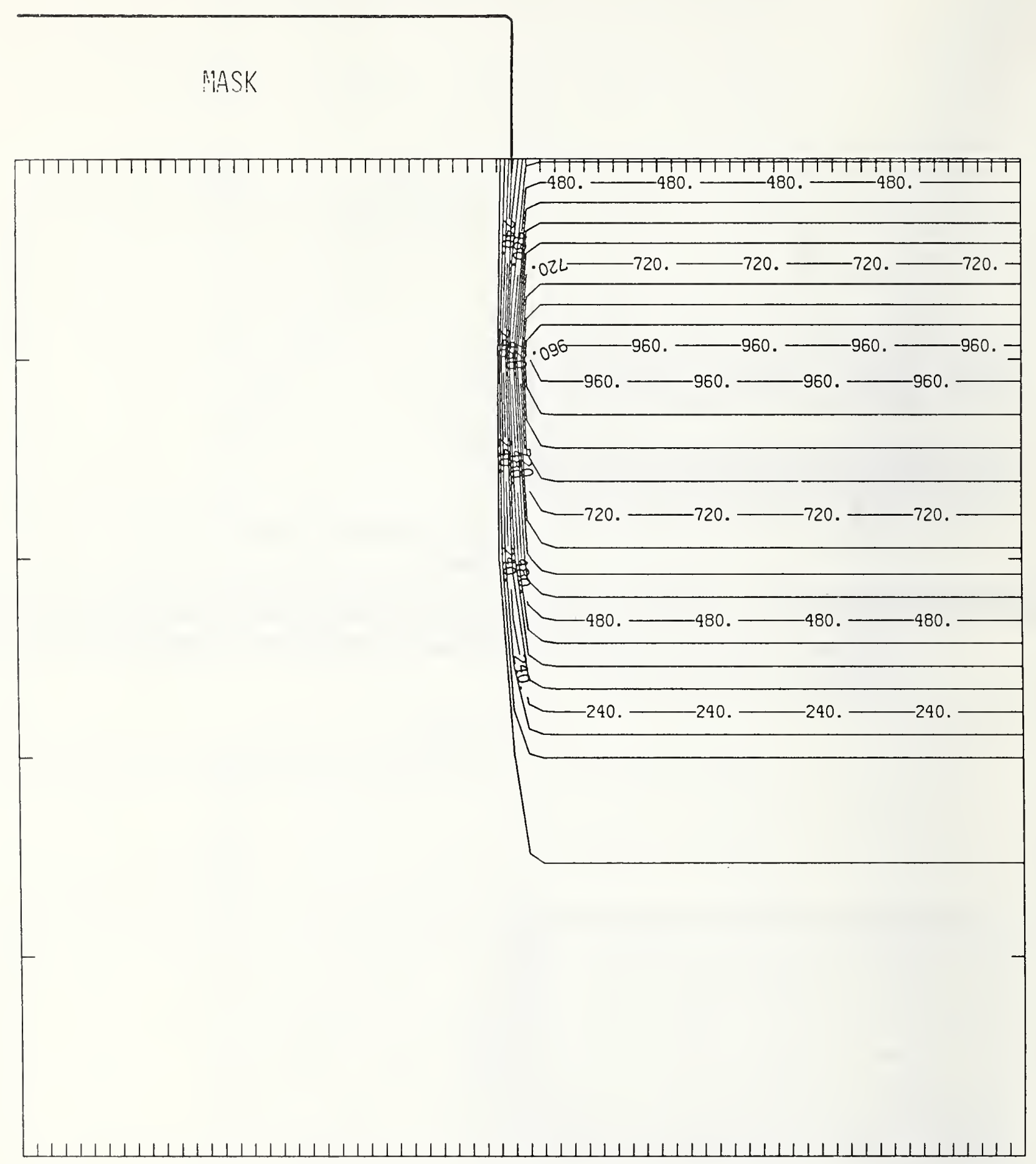

Figure S25-3 Two-dimensional distribution of $25 \mathrm{keV}$ sulfur implanted past a mask edge. The length increment (distance between tick marks) is $0.02 \mu \mathrm{m}$ and the scale factor is 1000 . 


\section{MASK}

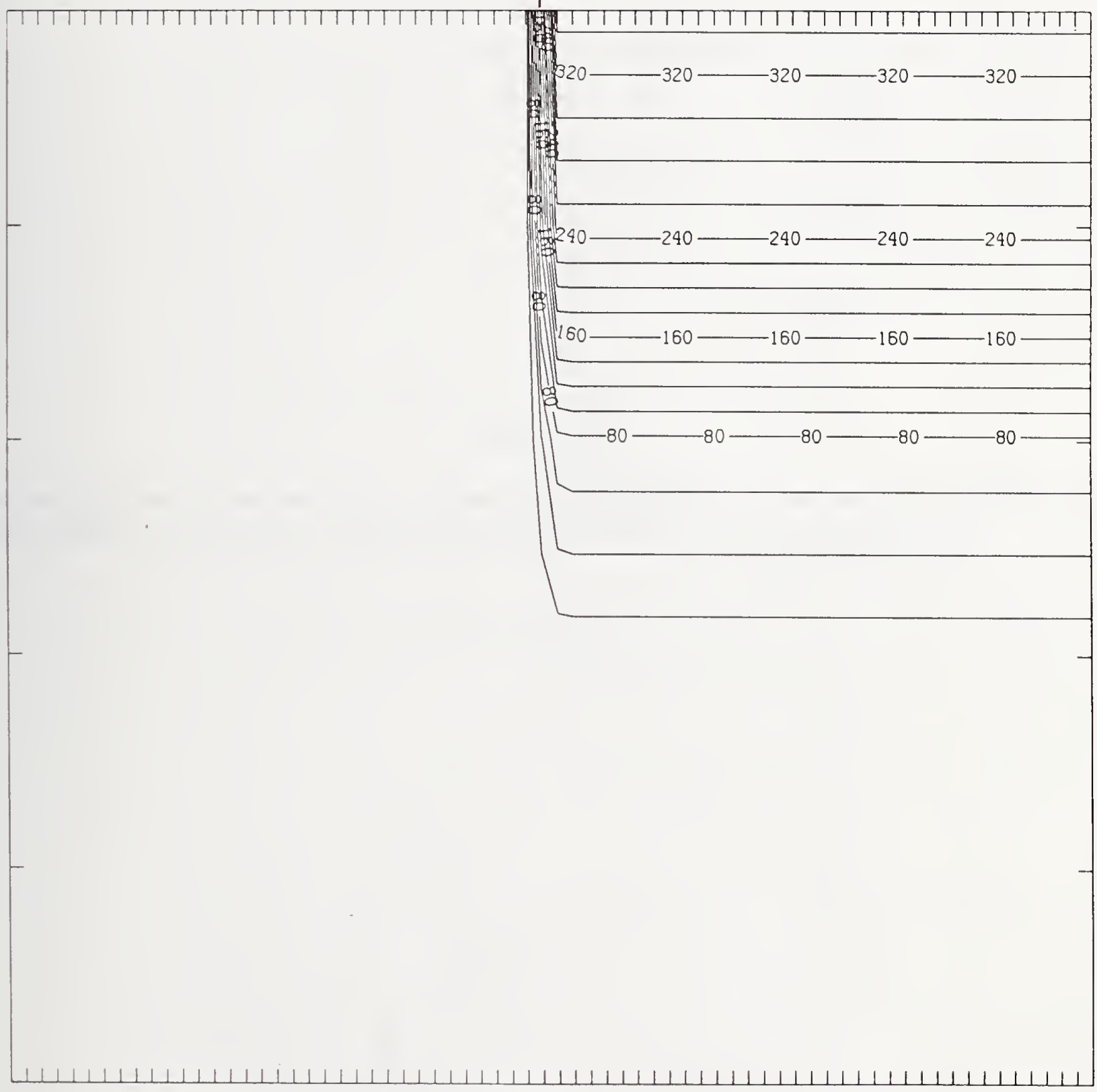

Figure S25-4 Two-dimensional distribution of Frenkel pairs created by $25 \mathrm{keV}$ sulfur implanted past a mask edge. The length increment (distance between tick marks) is $0.02 \mu \mathrm{m}$ and the scale factor is 1 . 


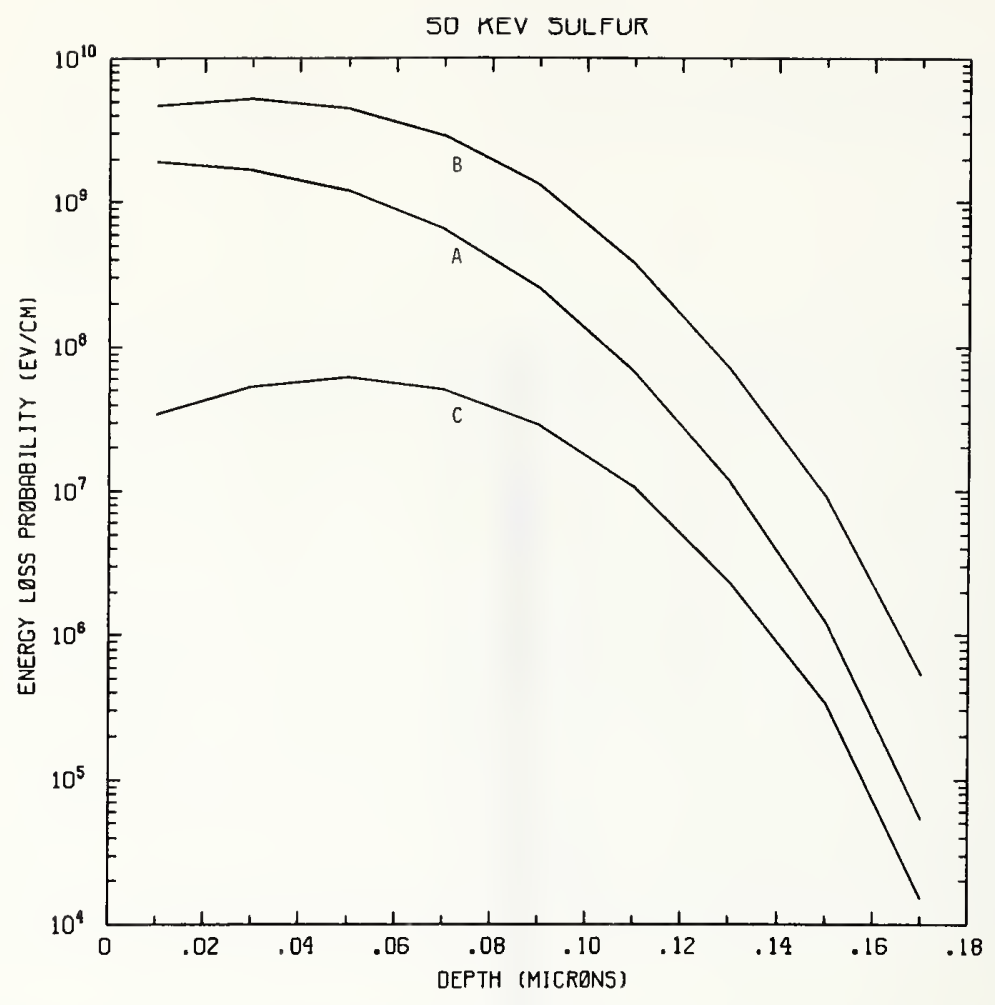

Figure S50-1 One-dimensional probability distributions for energy loss due to electronic excitation (A), displacement damage(B), and phonon excitation (C) for $50 \mathrm{keV}$ sulfur normally incident on a silicon target.

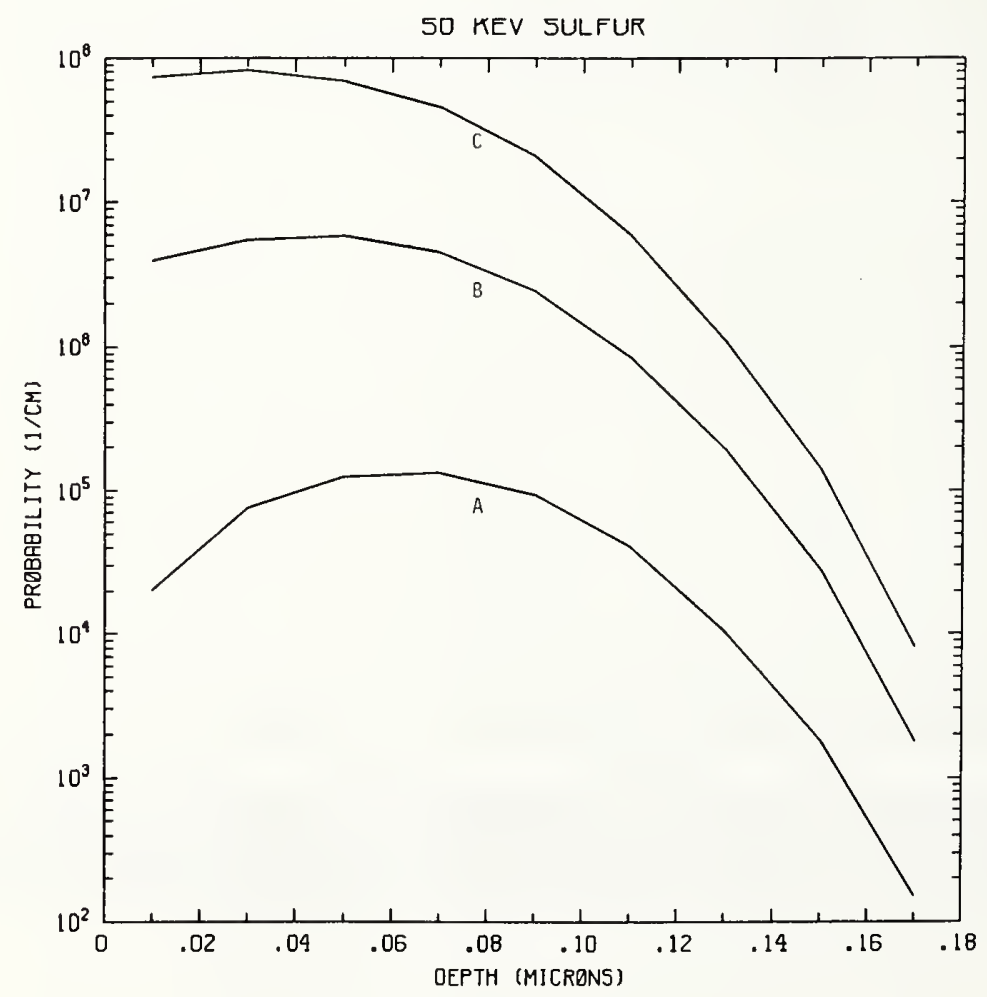

Figure S50-2 One-dimensional probability distributions for implanted sulfur (A), primary silicor displacements (B), and Frenkel pairs (C) for $50 \mathrm{keV}$ sulfur normally incident on a silicon target. 


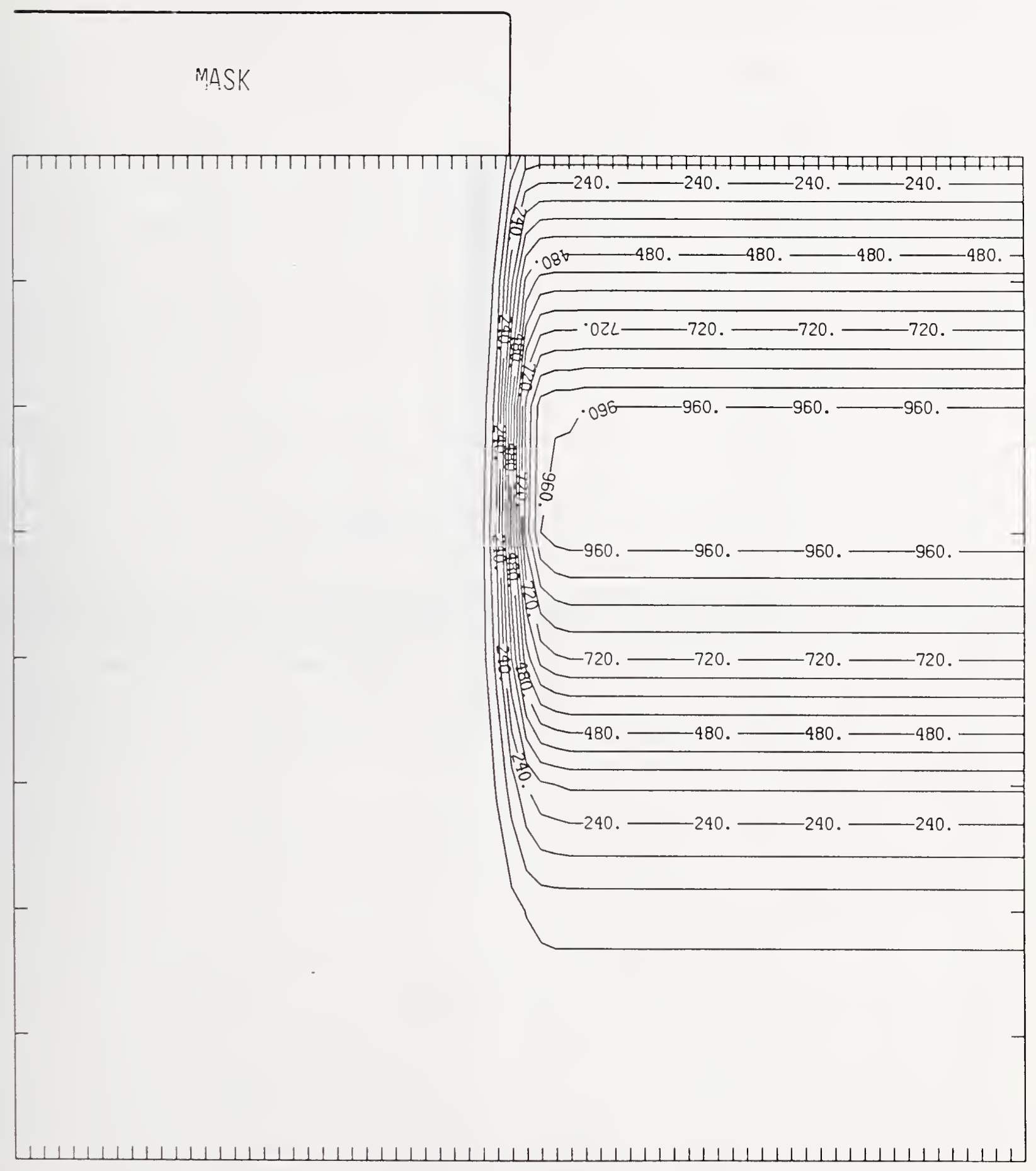

Figure S50-3 Two-dimensional distribution of $50 \mathrm{keV}$ sulfur implanted past a mask edge. The length increment (distance between tick marks) is $0.02 \mu \mathrm{m}$ and the scale factor is 1000 . 


\section{MASK}

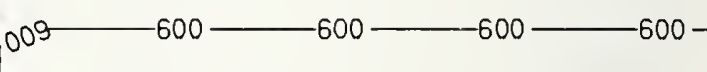

so

600 $600-600-600$

98

$-480$

$-480$

480

480

360

$-360$

360

360

240

$-240$

240

240

120

120

120

$-120$

Figure S50-4 Two-dimensional distribution of Frenkel pairs created by $50 \mathrm{keV}$ sulfur implanted past a mask edge. The length increment (distance between tick marks) is $0.02 \mu \mathrm{m}$ and the scale factor is 1 . 


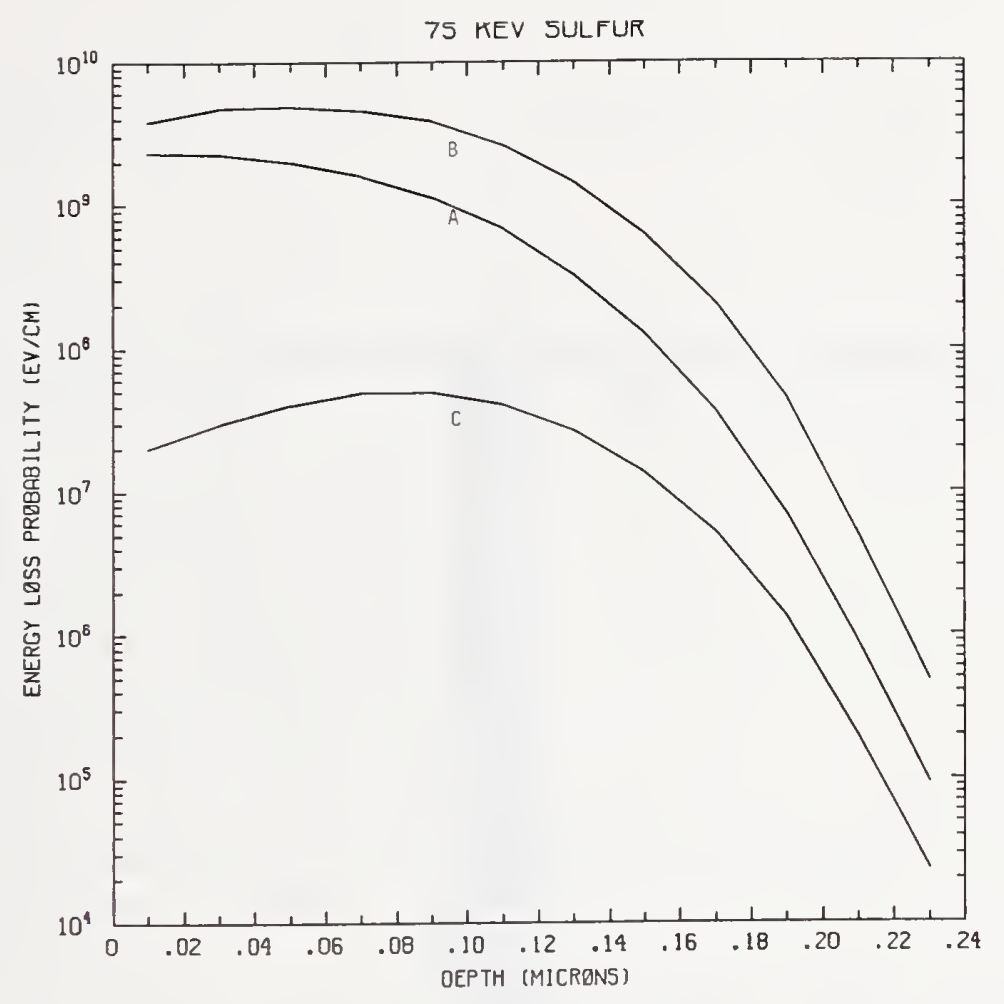

Figure S75-1 One-dimensional probability distributions for energy loss due to electronic excitation (A), displacement damage(B), and phonon excitation (C) for $75 \mathrm{keV}$ sulfur normally incident on a silicon target.

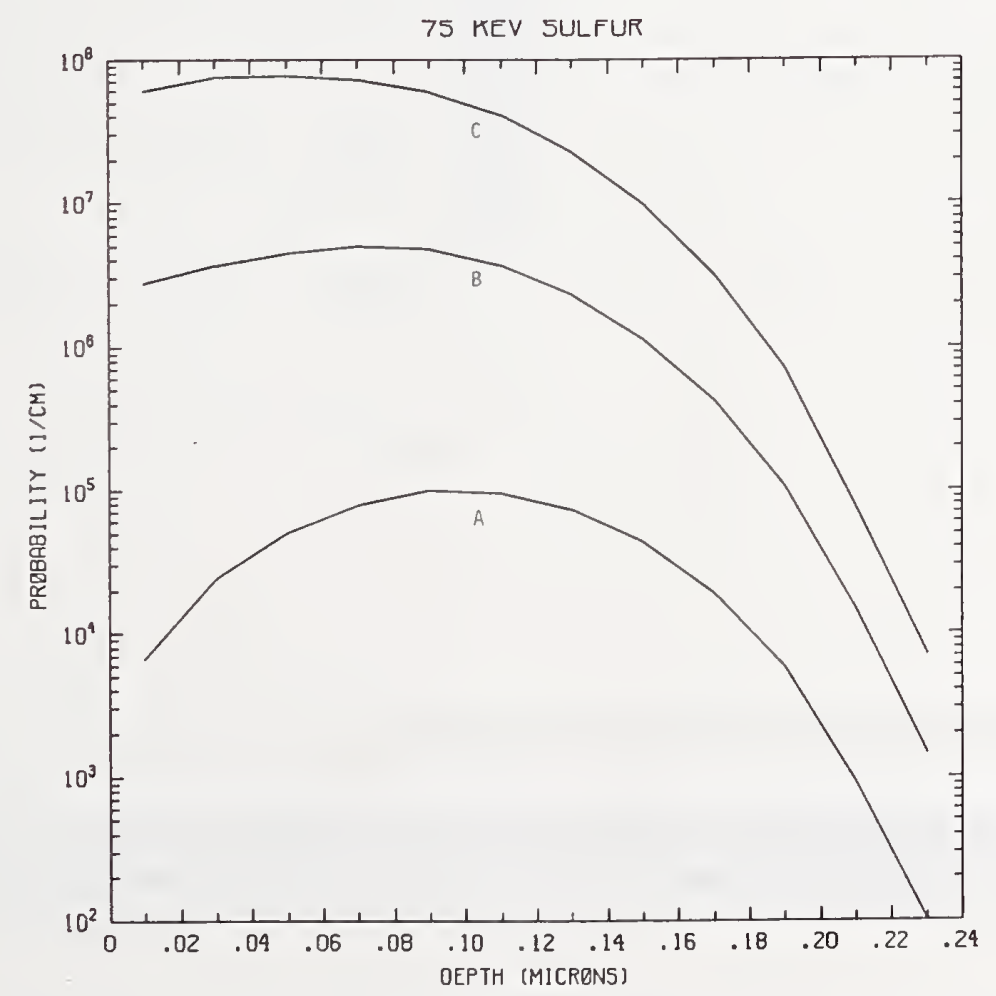

Figure S75-2 One-dimensional probability distributions for implanted sulfur (A), primary silicon displacements (B), and Frenkel pairs (C) for $75 \mathrm{keV}$ sulfur normally incident on a silicon target. 


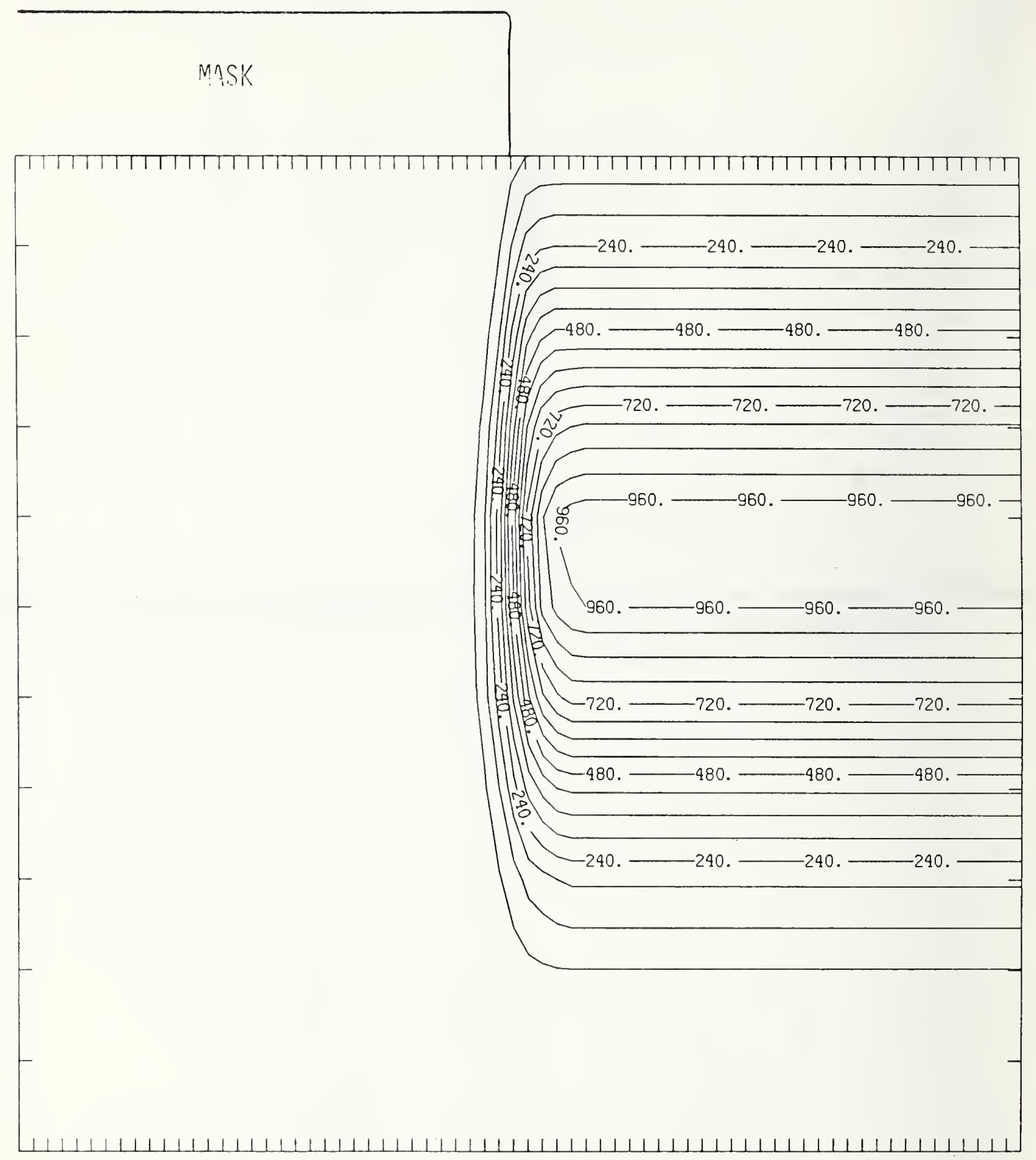

Figure S75-3 Two-dimensional distribution of $75 \mathrm{keV}$ sulfur implanted past a mask edge. The length increment (distance between tick marks) is $0.02 \mu \mathrm{m}$ and the scale factor is 1000 . 


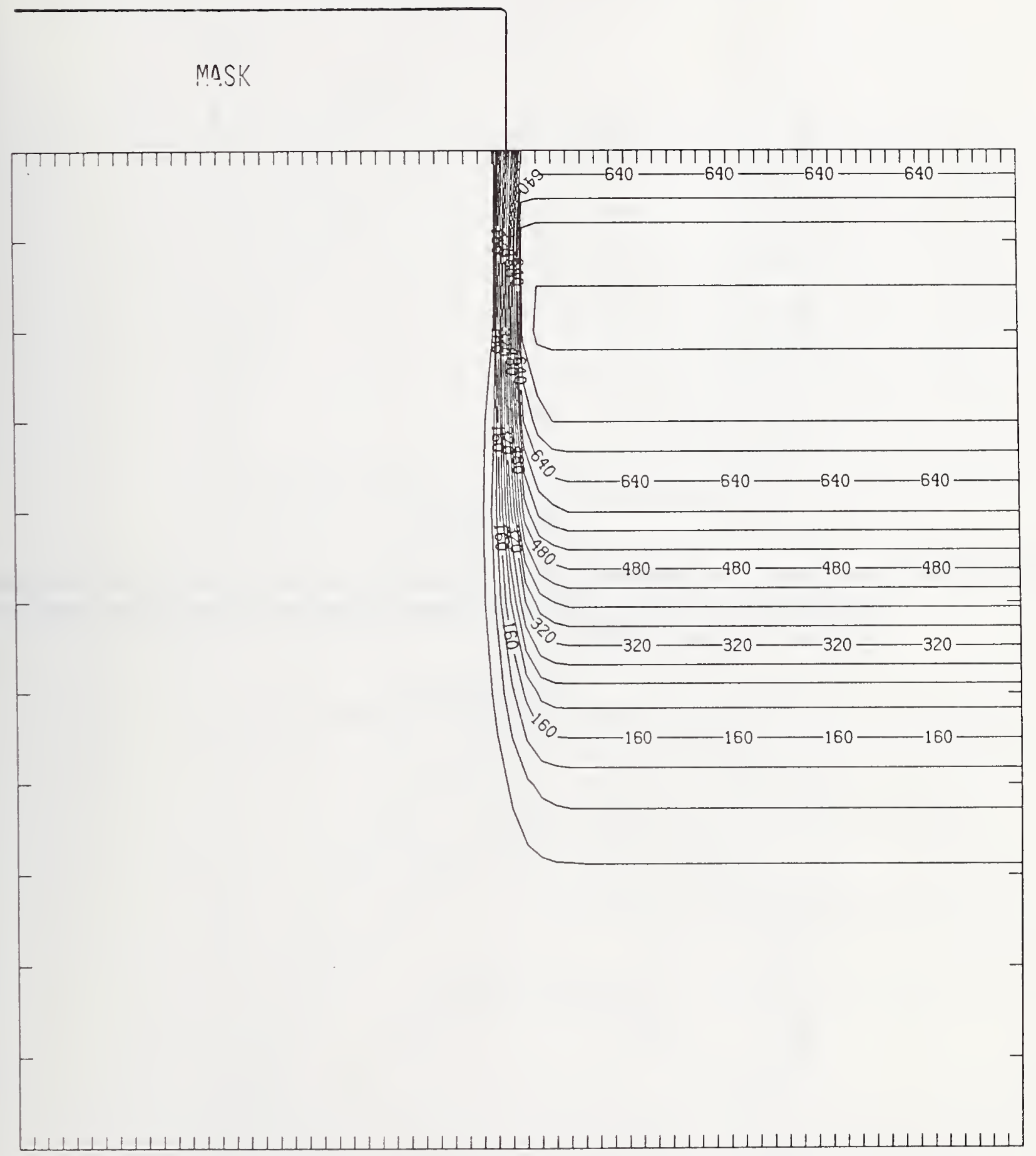

Figure S75-4 Two-dimensional distribution of Frenkel pairs created by $75 \mathrm{keV}$ sulfur implanted past a mask edge. The length increment (distance between tick marks) is $0.02 \mu \mathrm{m}$ and the scale factor is 1 . 


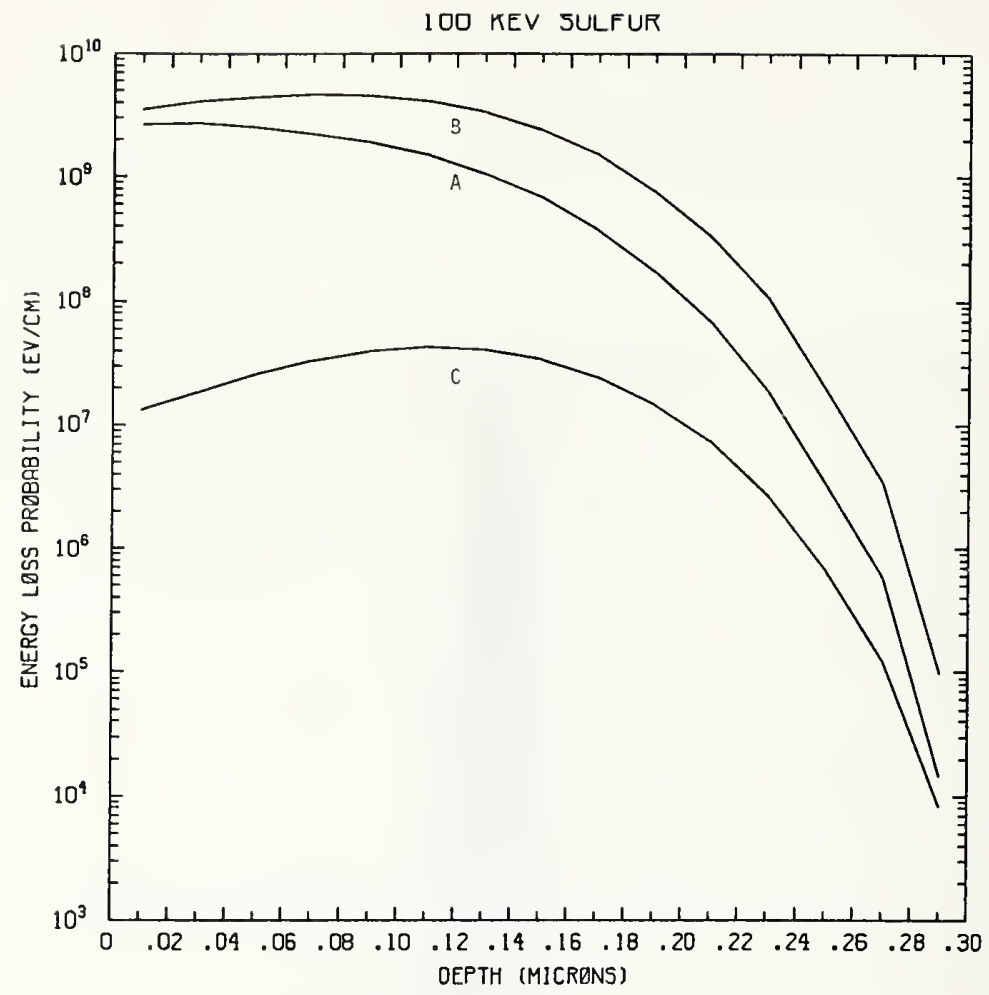

Figure S100-1 One-dimensional probability distributions for energy loss due to electronic excitatior (A), displacement damage(B), and phonon excitation (C) for $100 \mathrm{keV}$ sulfur normall incident on a silicon target.

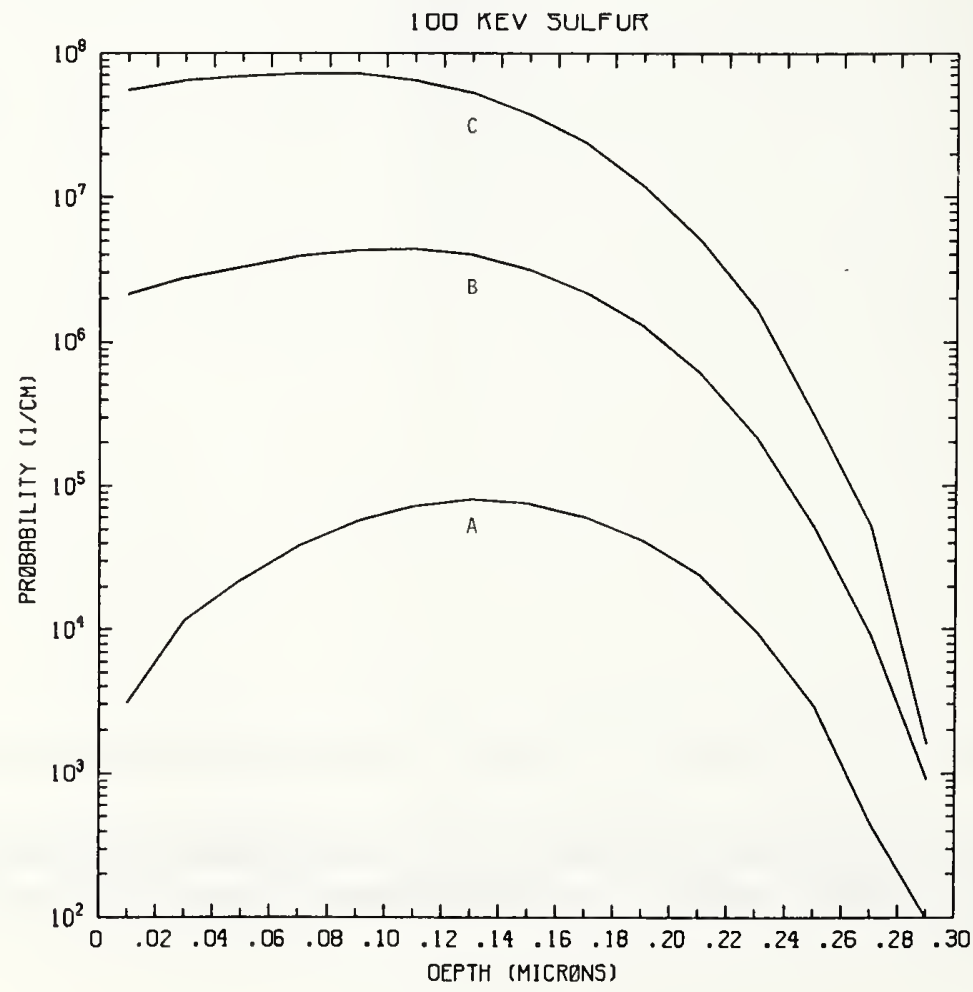

Figure S100-2 One-dimensional probability distributions for implanted sulfur (A), primary silicon displacements (B), and Frenkel pairs (C) for $100 \mathrm{keV}$ sulfur normally incident on a silicon target. 


\section{MASK}

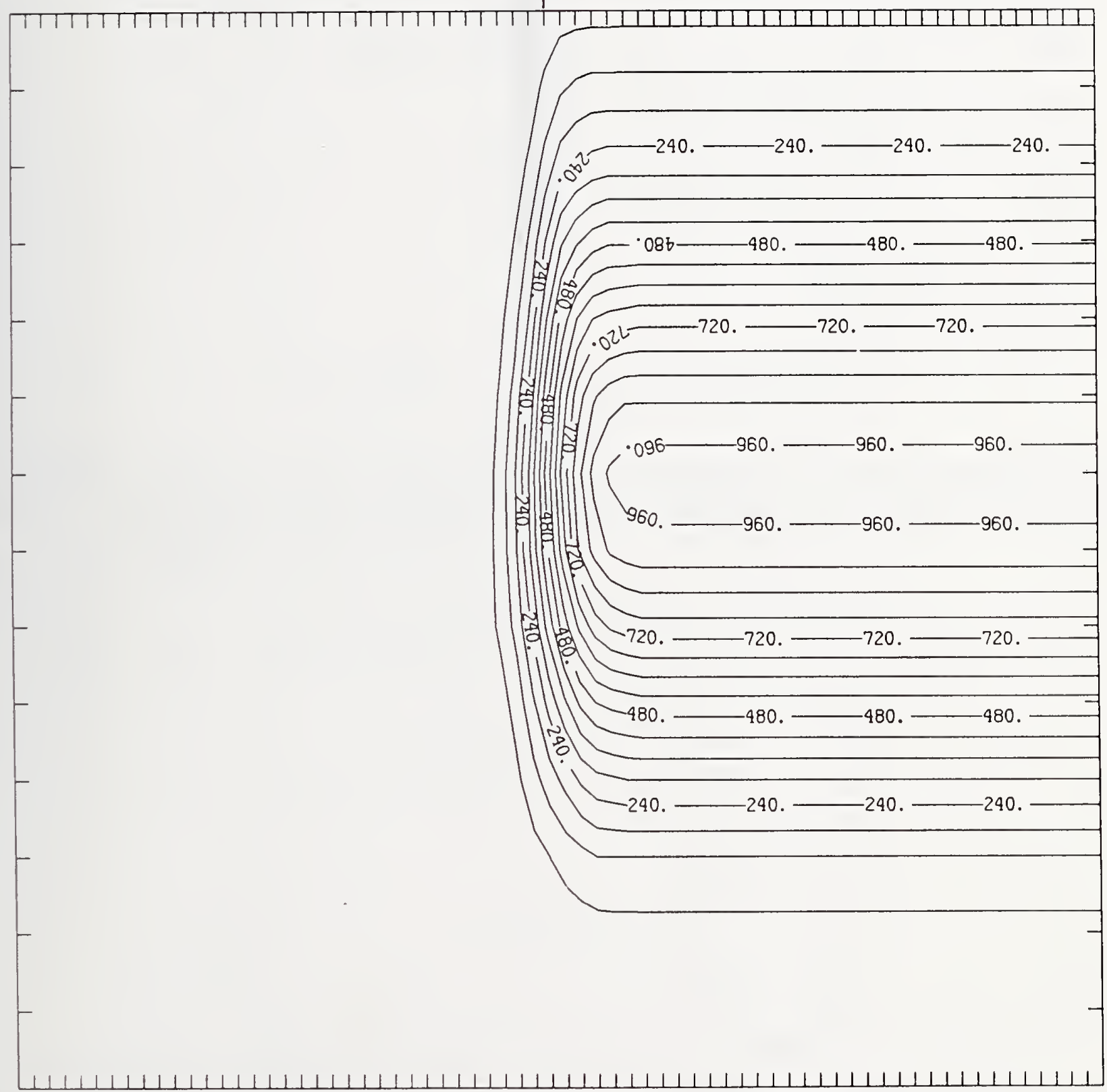

Figure S100-3 Two-dimensional distribution of $100 \mathrm{keV}$ sulfur implanted past a mask edge. The length increment (distance between tick marks) is $0.02 \mu \mathrm{m}$ and the scale factor is 1000 . 
MASK

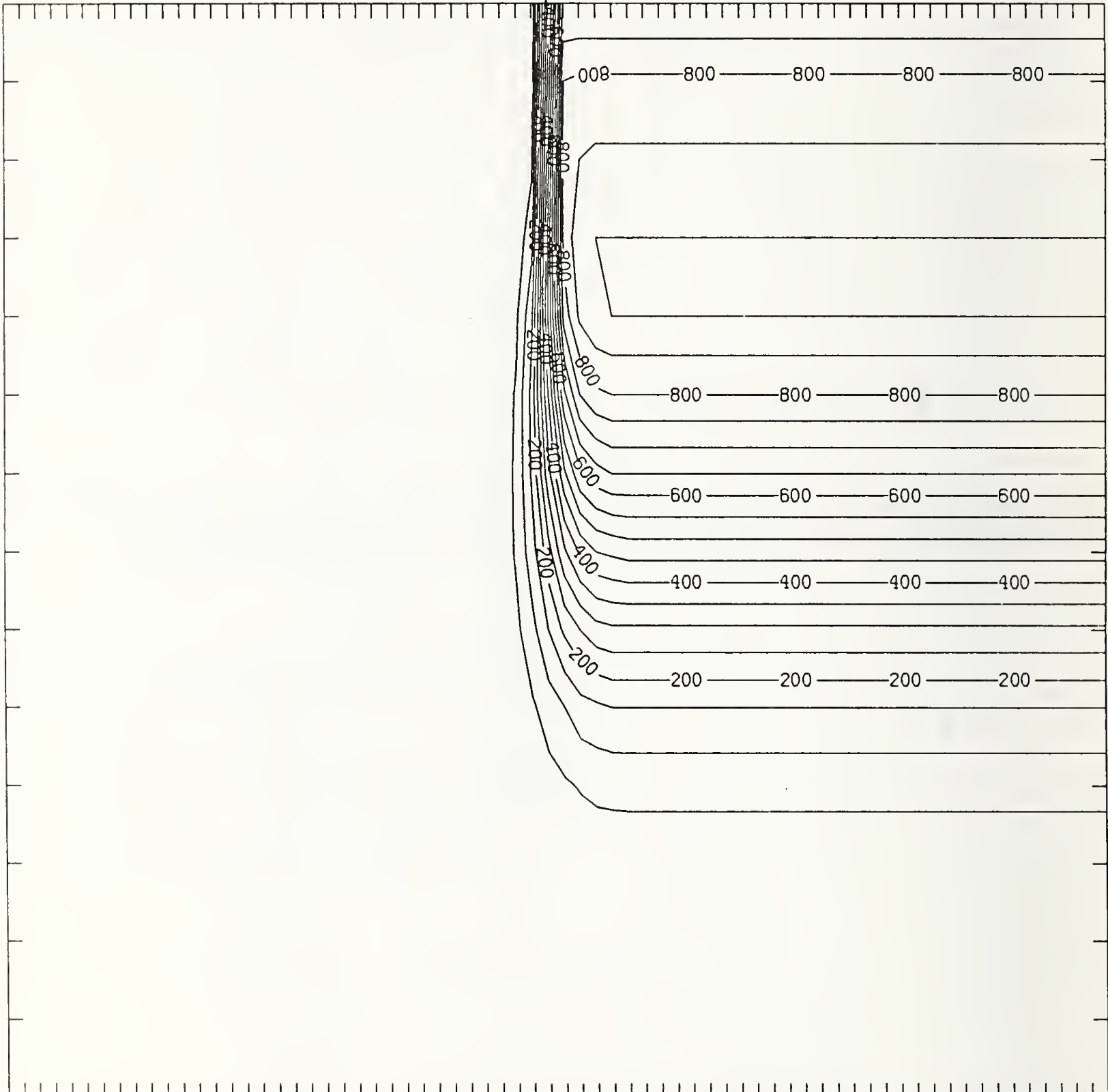

Figure S100-4 Two-dimensional distribution of Frenkel pairs created by $100 \mathrm{keV}$ sulfur implanted past a mask edge. The length increment (distance between tick marks) is $0.02 \mu \mathrm{m}$ and the scale factor is 1 . 


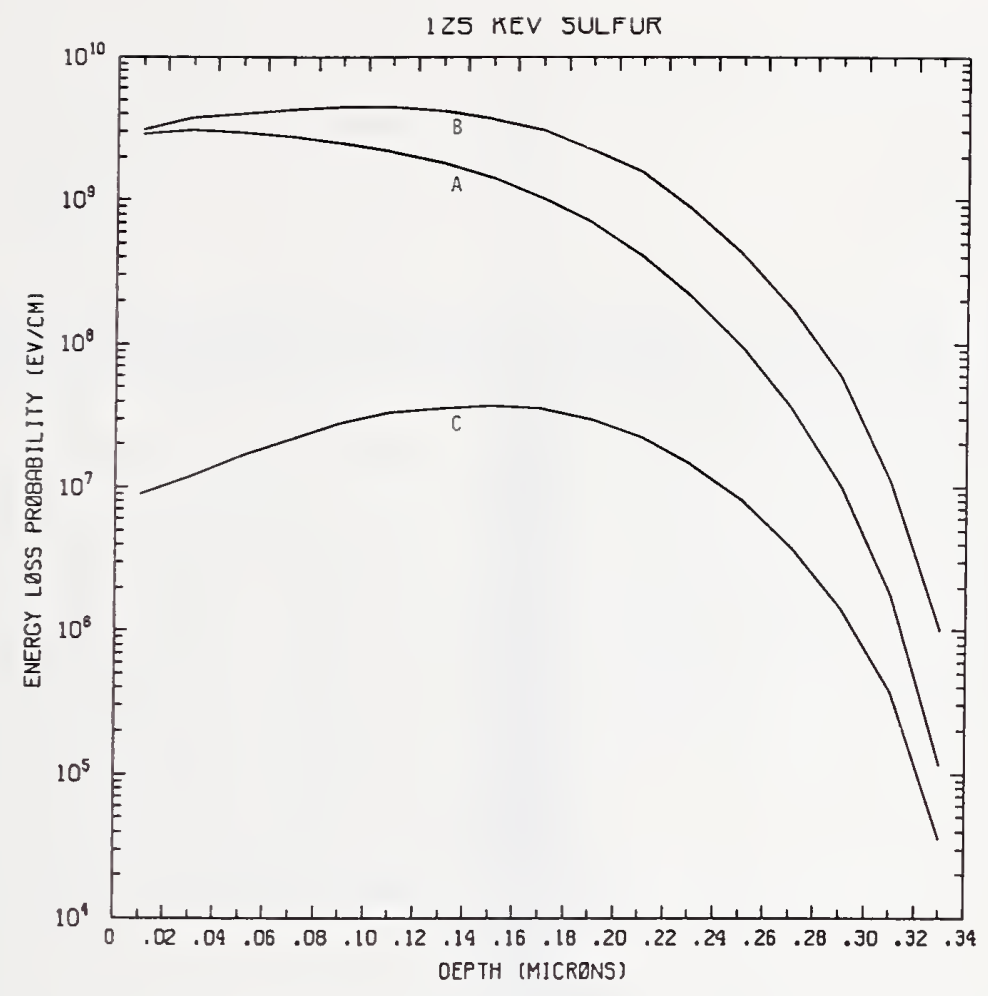

Figure S125-1 One-dimensional probability distributions for energy loss due to electronic excitation (A), displacement damage(B), and phonon excitation (C) for $125 \mathrm{keV}$ sulfur normally incident on a silicon target.

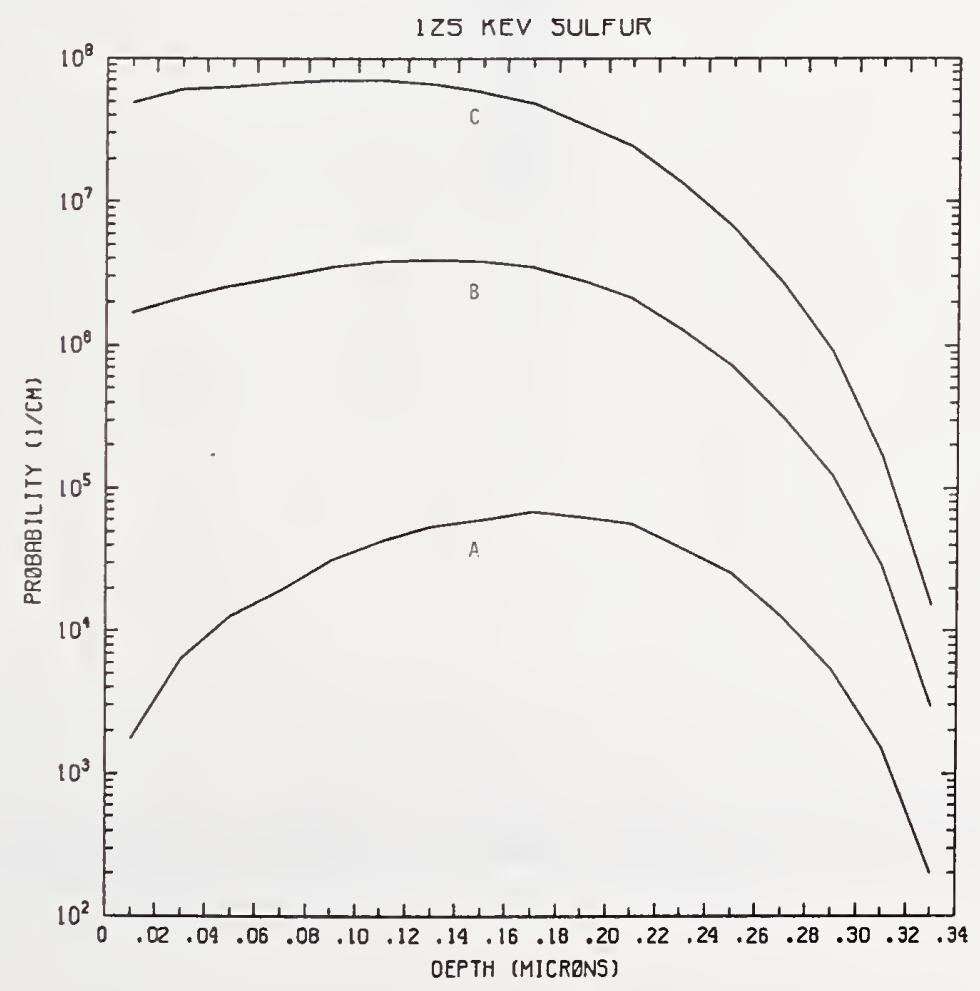

Figure S125-2 One-dimensional probability distributions for implanted sulfur (A), primary silicon displacements (B), and Frenkel pairs (C) for $125 \mathrm{keV}$ sulfur normally incident on a silicon target. 


\section{"IASK}

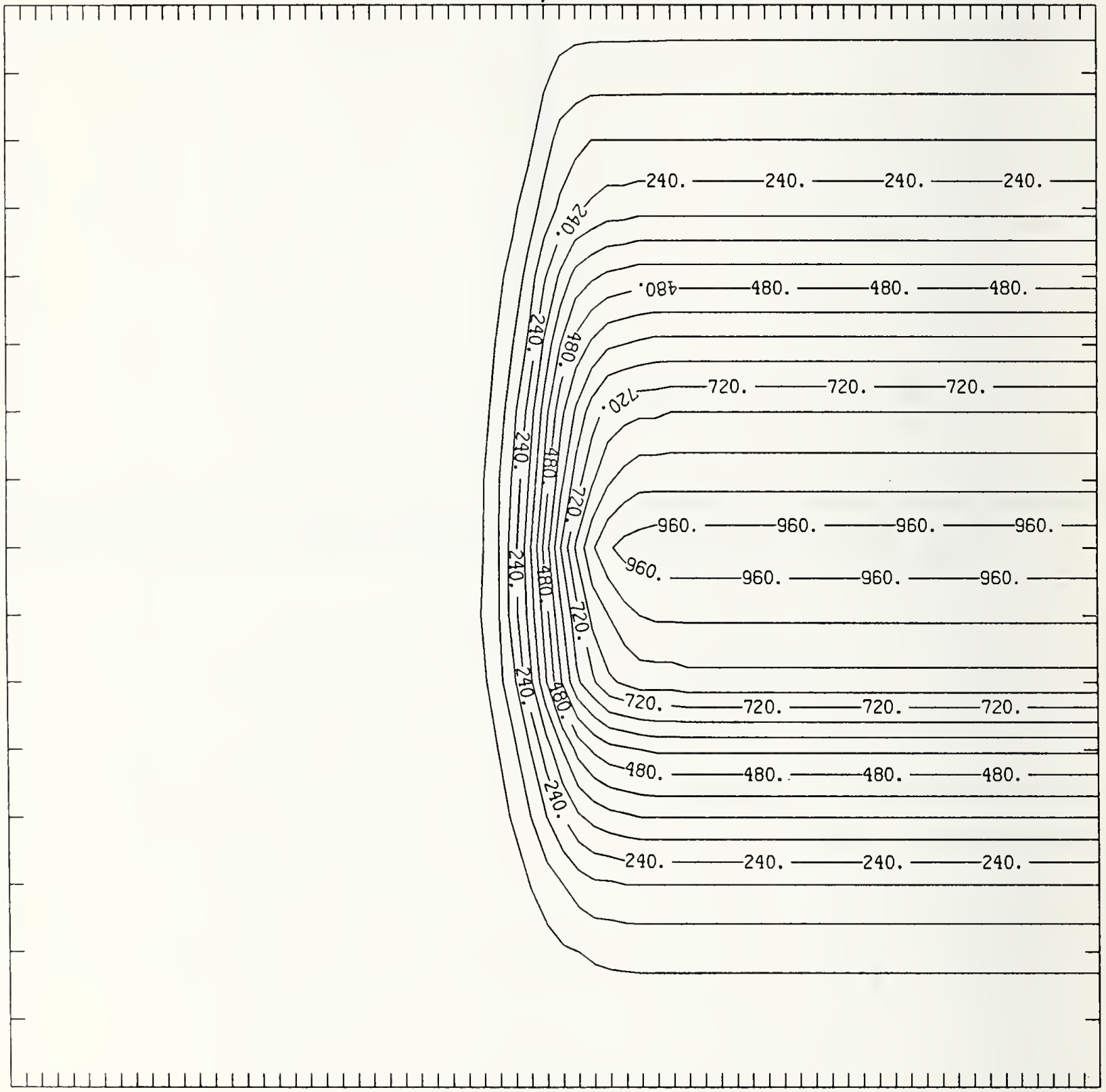

Figure S125-3 Two-dimensional distribution of $125 \mathrm{keV}$ sulfur implanted past a mask edge. The length increment (distance between tick marks) is $0.02 \mu \mathrm{m}$ and the scale factor is 1000 . 


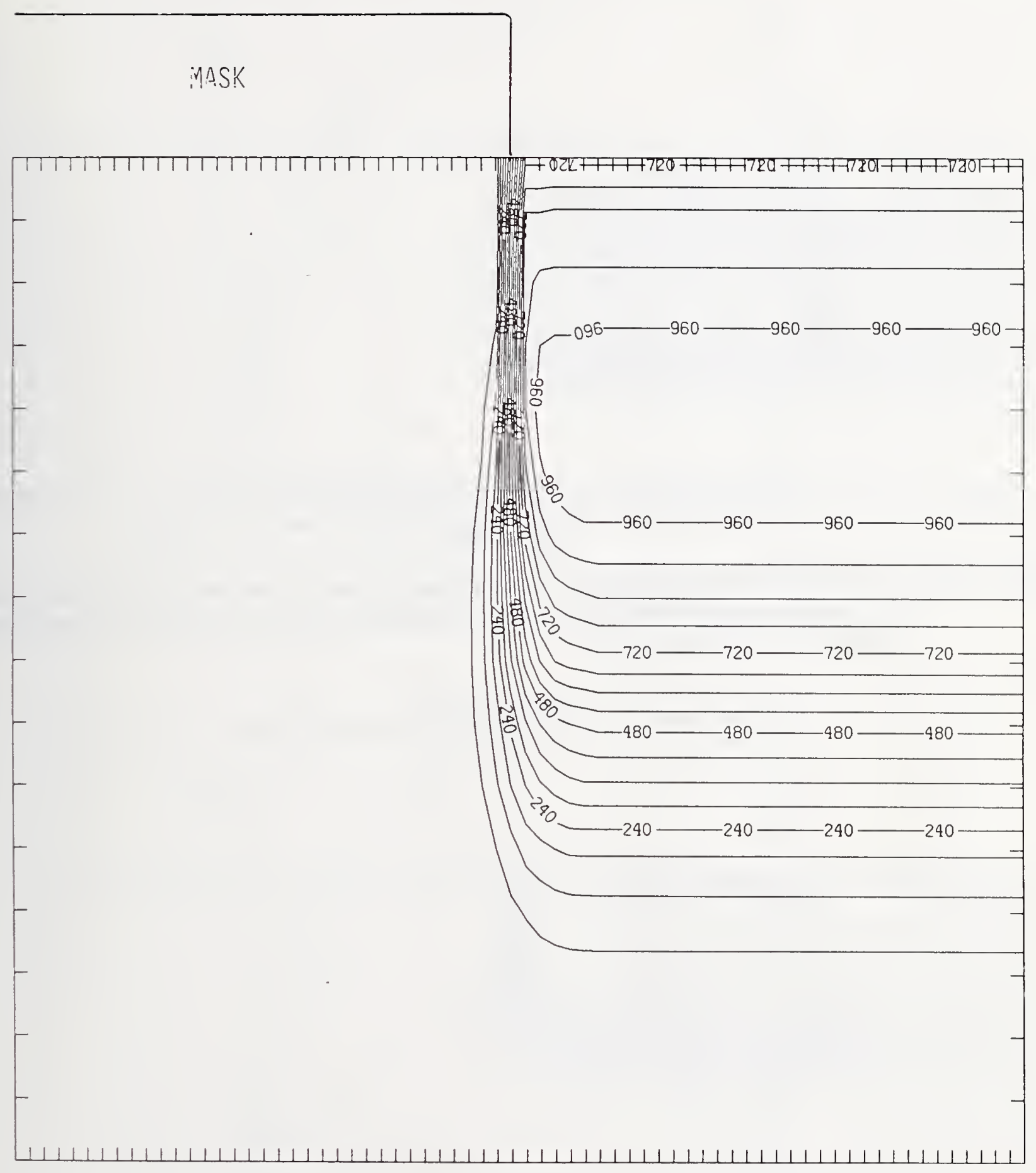

Figure S125-4 Two-dimensional distribution of Frenkel pairs created by $125 \mathrm{keV}$ sulfur implanted past a mask edge. The length increment (distance between tick marks) is $0.02 \mu \mathrm{m}$ and the scale factor is 1 . 


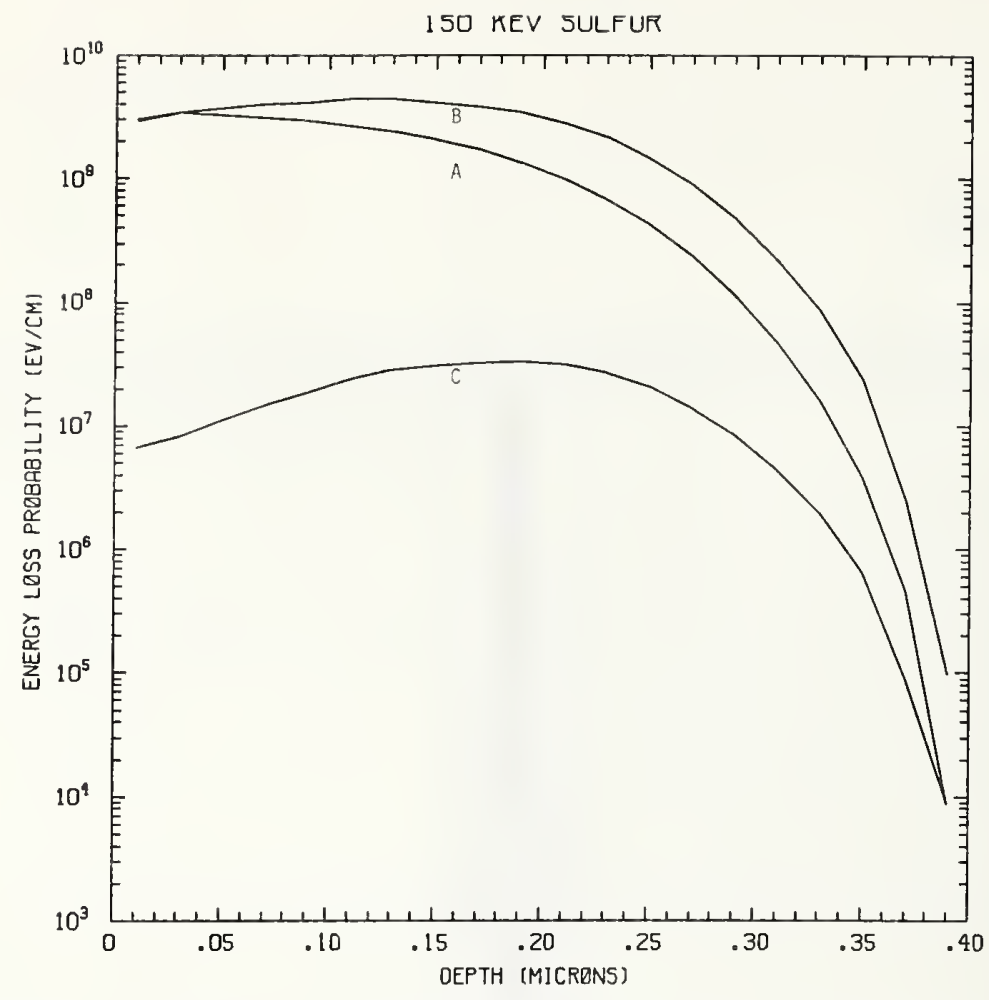

Figure S150-1 One-dimensional probability distributions for energy loss due to electronic excitation (A), displacement damage(B), and phonon excitation (C) for $150 \mathrm{keV}$ sulfur normally incident on a silicon target.

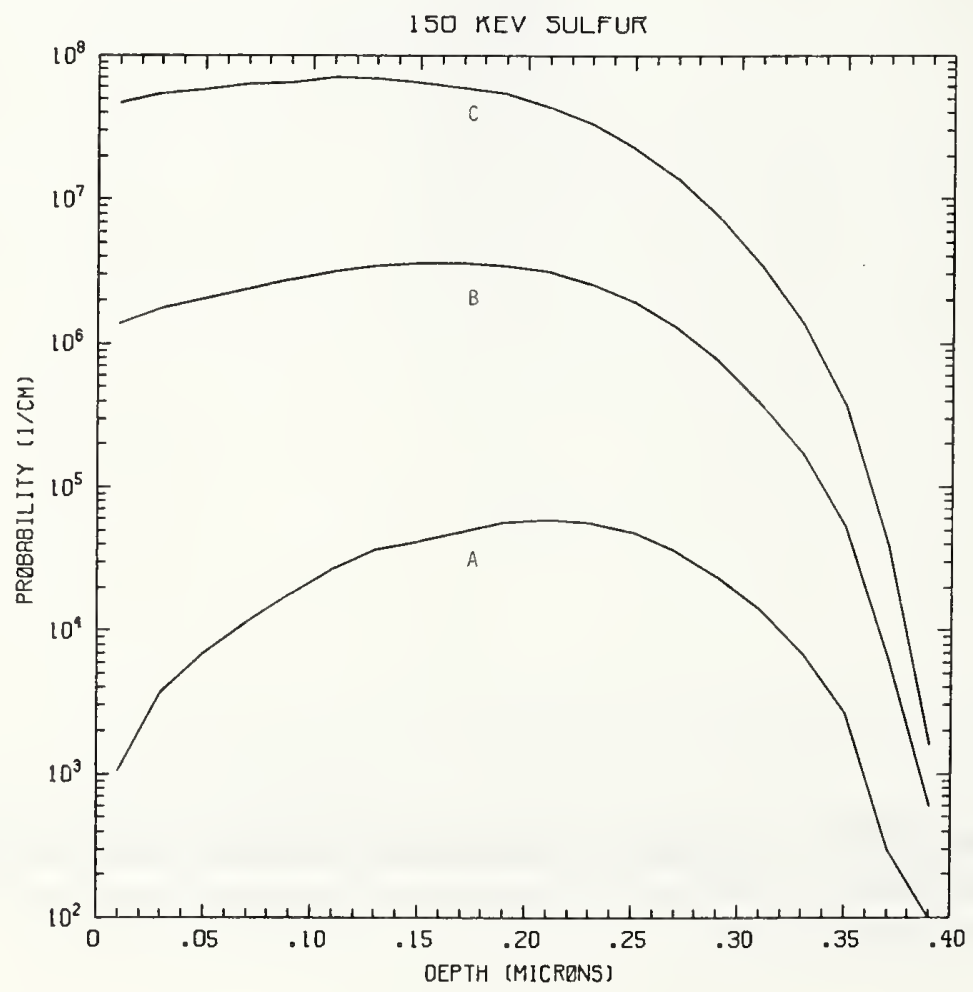

Figure S150-2 One-dimensional probability distributions for implanted sulfur (A), primary silicon displacements (B), and Frenkel pairs (C) for $150 \mathrm{keV}$ sulfur normally incident on a silicon target. 


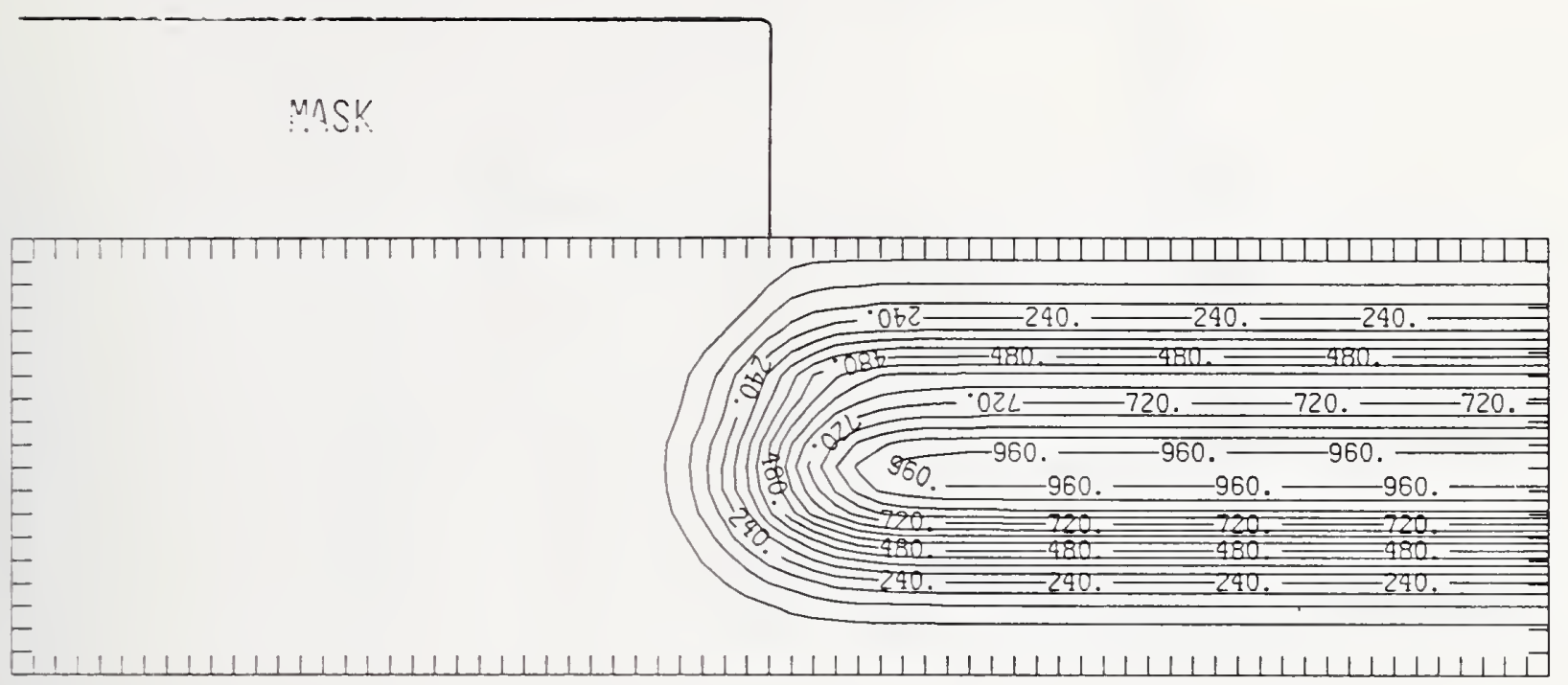

Figure S150-3 Two-dimensional distribution of $150 \mathrm{keV}$ sulfur implanted past a mask edge. The length increment (distance between tick marks) is $0.02 \mu \mathrm{m}$ and the scale factor is 1000.

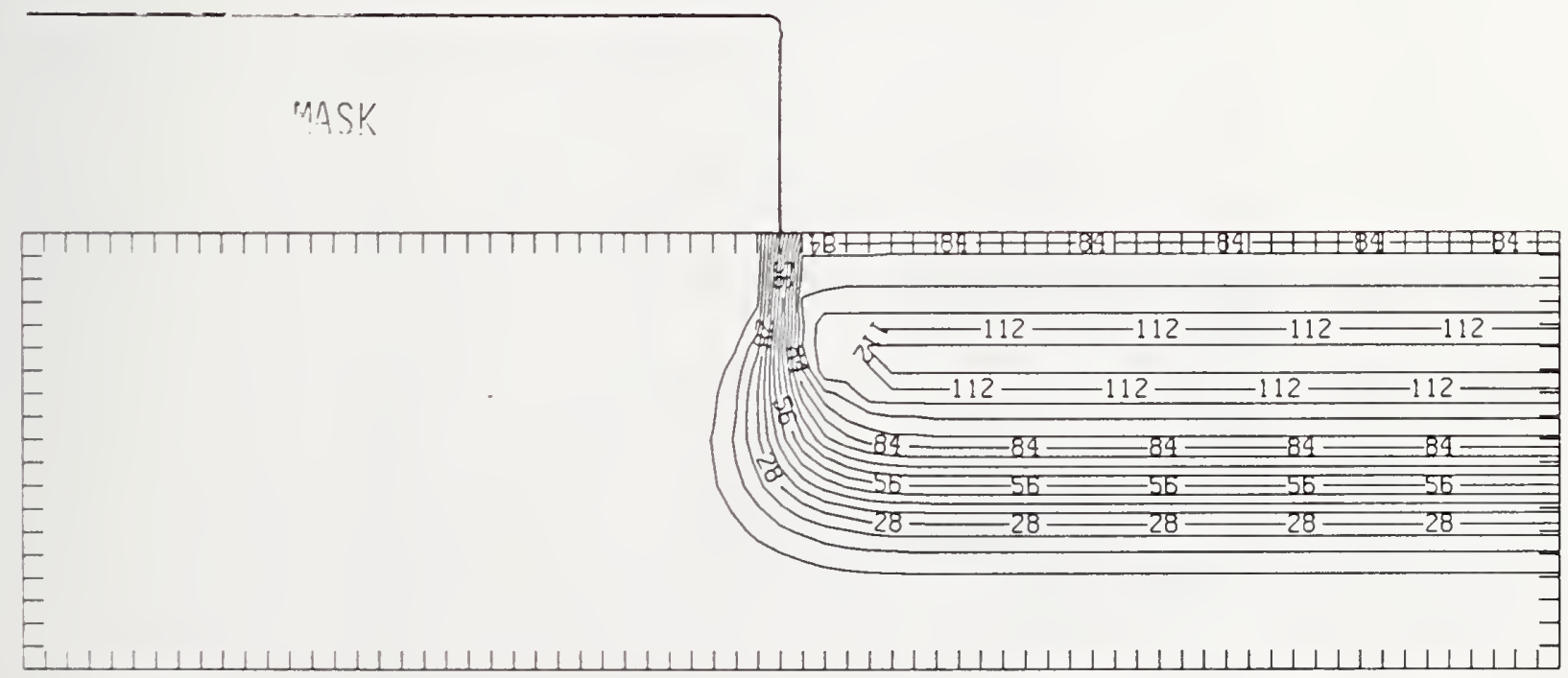

Figure S150-4 Two-dimensional distribution of Frenkel pairs created by $150 \mathrm{keV}$ sulfur implanted past a mask edge. The length increment (distance between tick marks) is $0.02 \mu \mathrm{m}$ and the scale factor is 0.1 . 


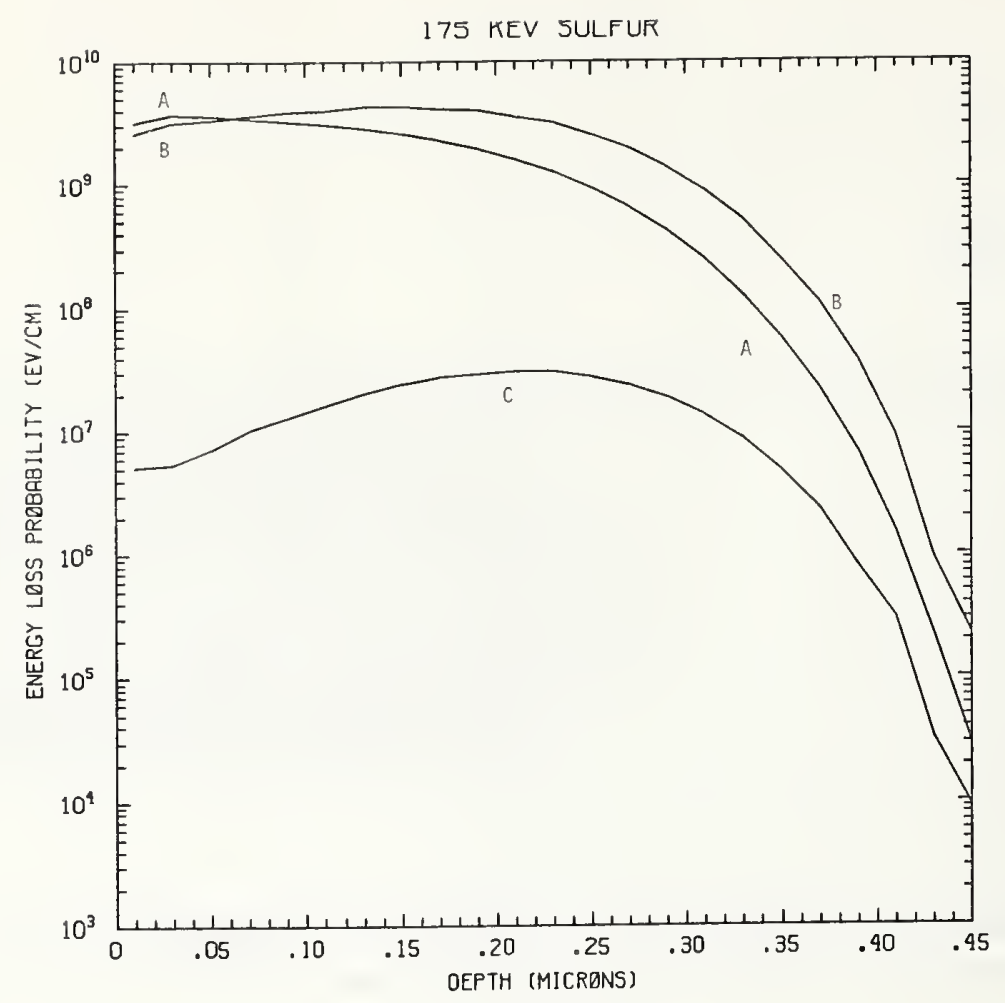

Figure S175-1 One-dimensional probability distributions for energy loss due to electronic excitation (A), displacement damage(B), and phonon excitation (C) for $175 \mathrm{keV}$ sulfur normally incident on a silicon target.

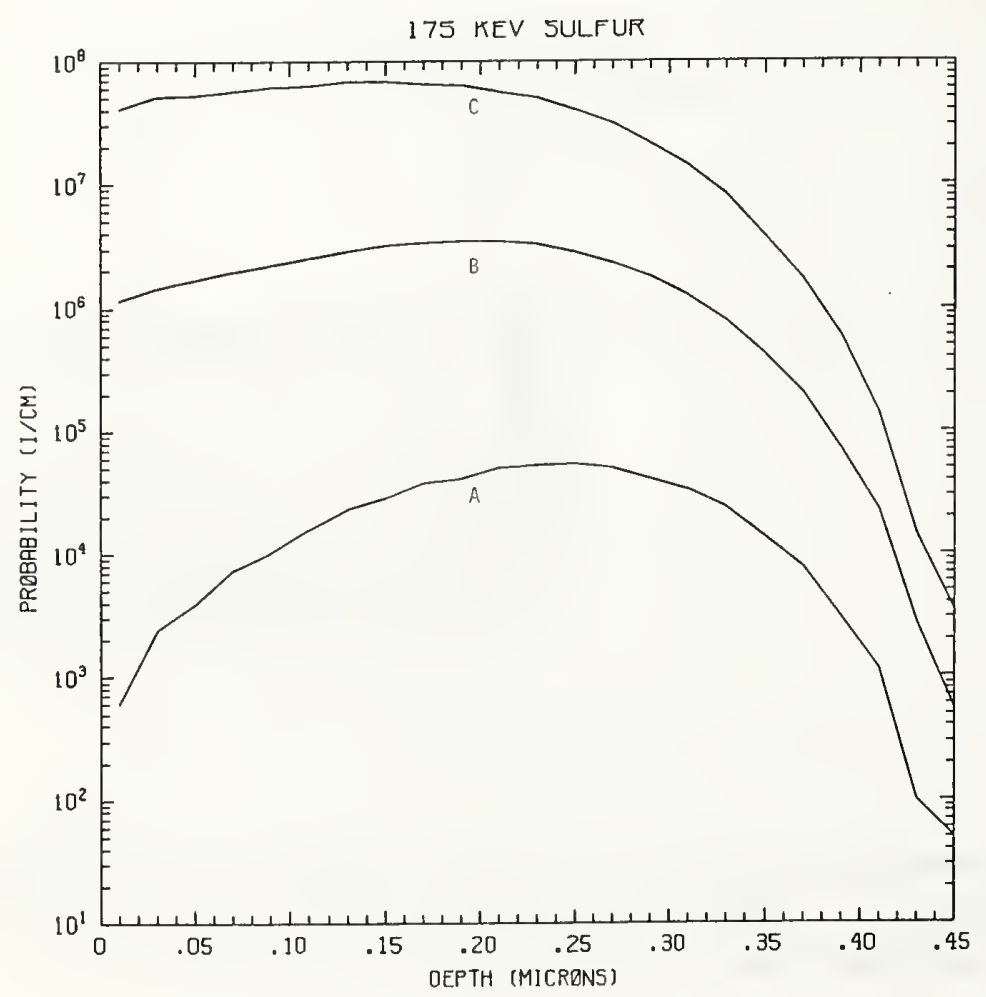

Figure S175-2 One-dimensional probability distributions for implanted sulfur (A), primary silicon displacements (B), and Frenkel pairs (C) for $175 \mathrm{keV}$ sulfur normally incident on a silicon target. 


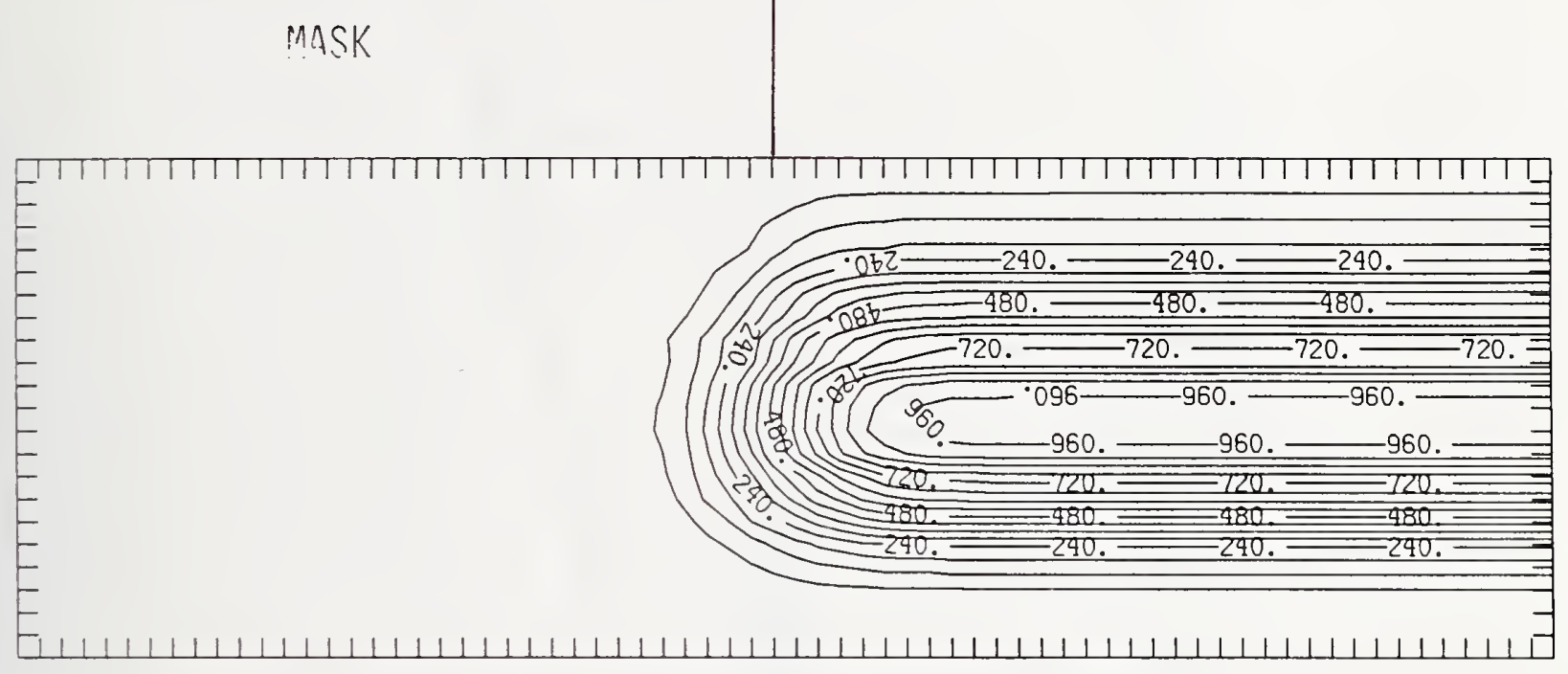

Figure S175-3 Two-dimensional distribution of $175 \mathrm{keV}$ sulfur implanted past a mask edge. The length increment (distance between tick marks) is $0.02 \mu \mathrm{m}$ and the scale factor is 1000 .

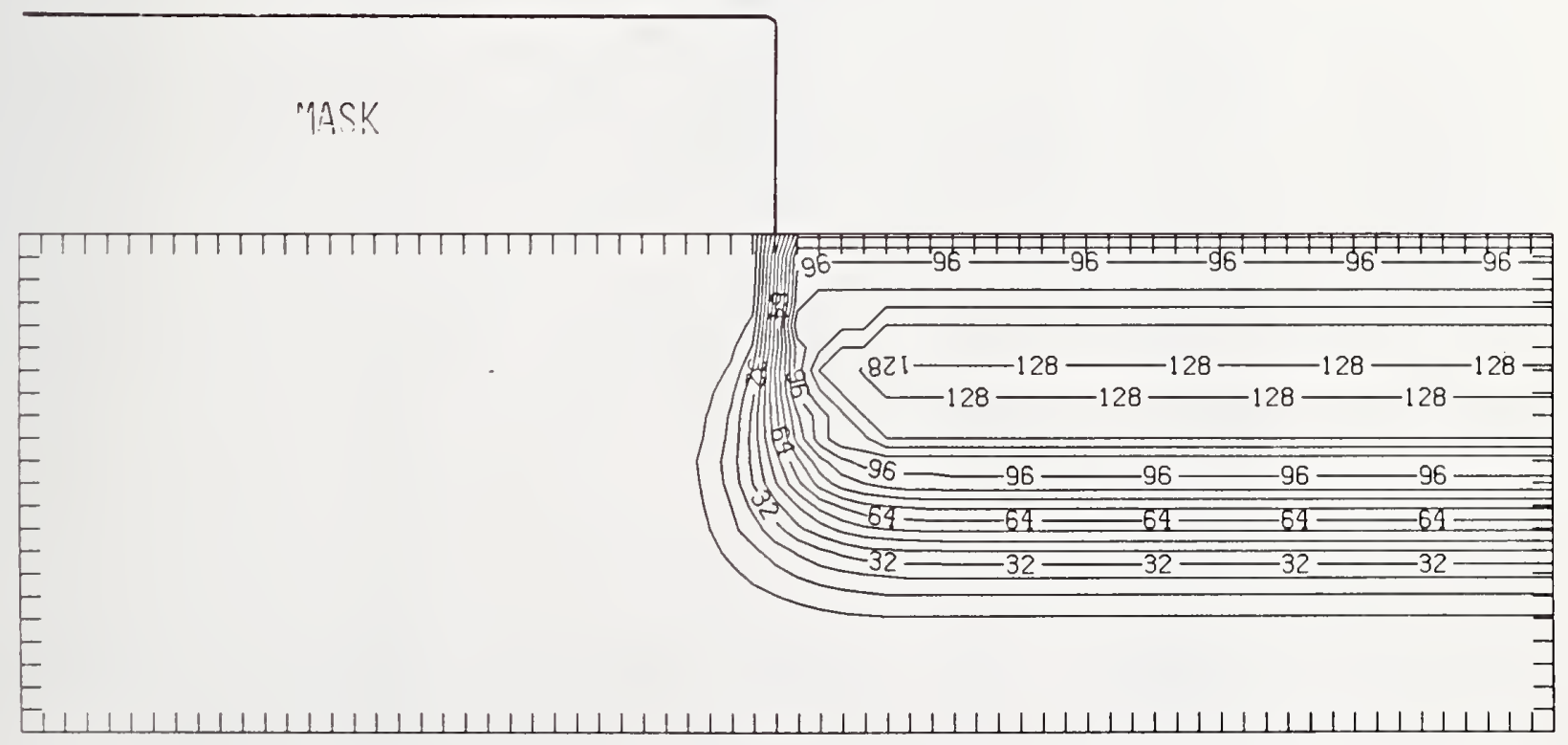

Figure S175-4 Two-dimensional distribution of Frenkel pairs created by $175 \mathrm{keV}$ sulfur implanted past a mask edge. The length increment (distance between tick marks) is $0.02 \mu \mathrm{m}$ and the scale factor is 0.1 . 


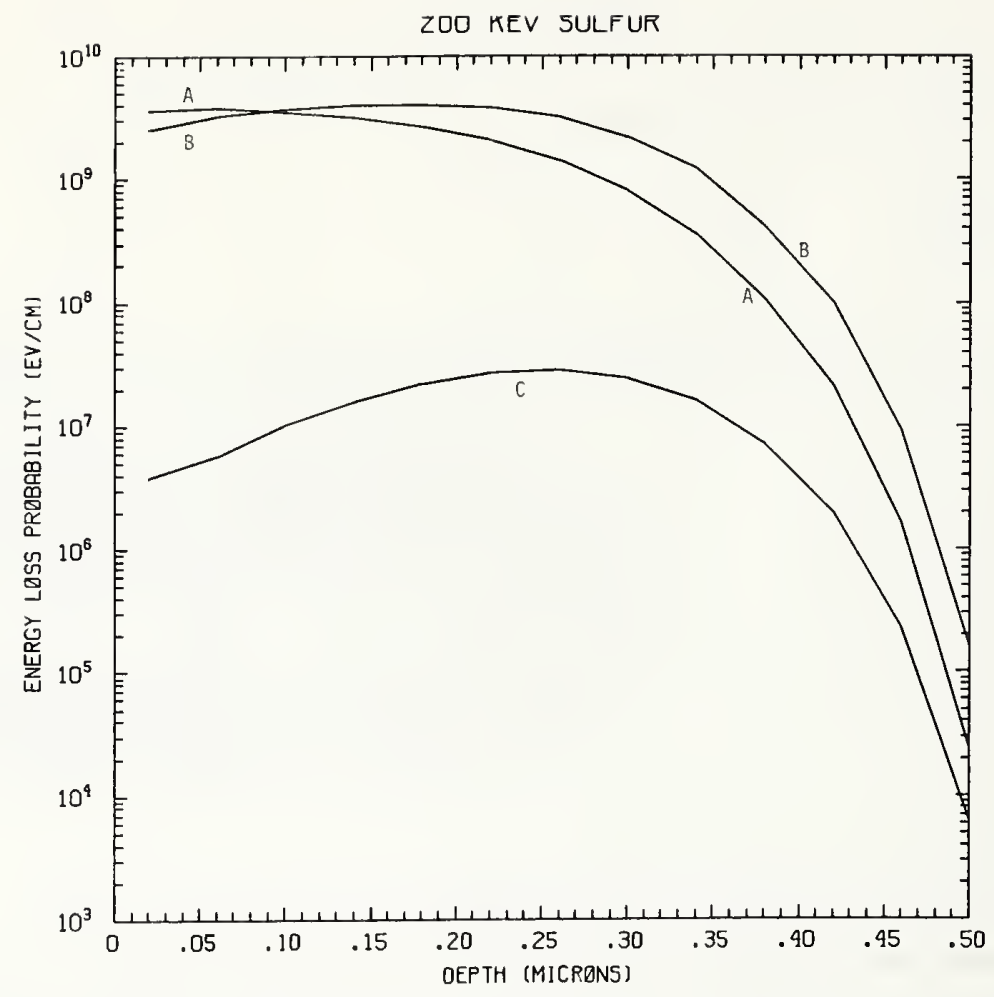

Figure S200-1 One-dimensional probability distributions for energy loss due to electronic excitation (A), displacement damage(B), and phonon excitation (C) for $200 \mathrm{keV}$ sulfur normally incident on a silicon target.

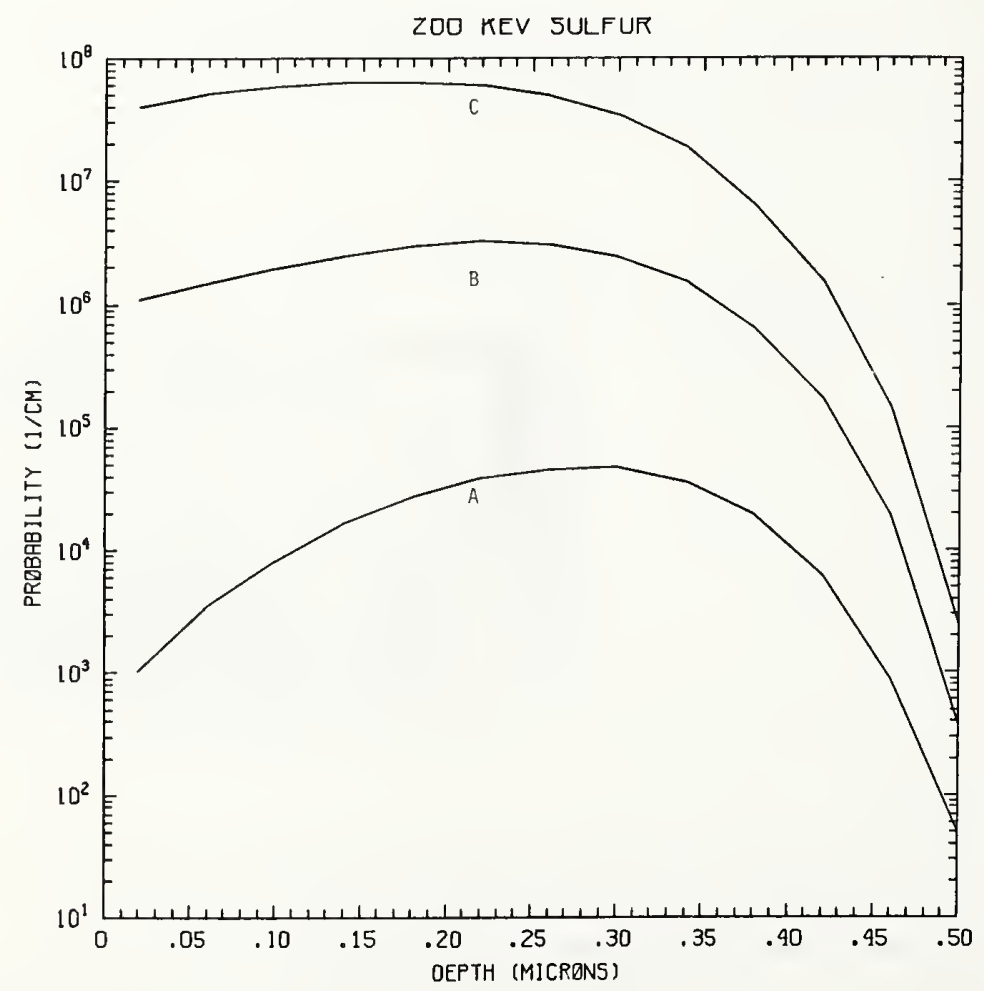

Figure S200-2 One-dimensional probability distributions for implanted sulfur (A), primary silicon displacements (B), and Frenkel pairs (C) for $200 \mathrm{keV}$ sulfur normally incident on a silicon target. 
MISK

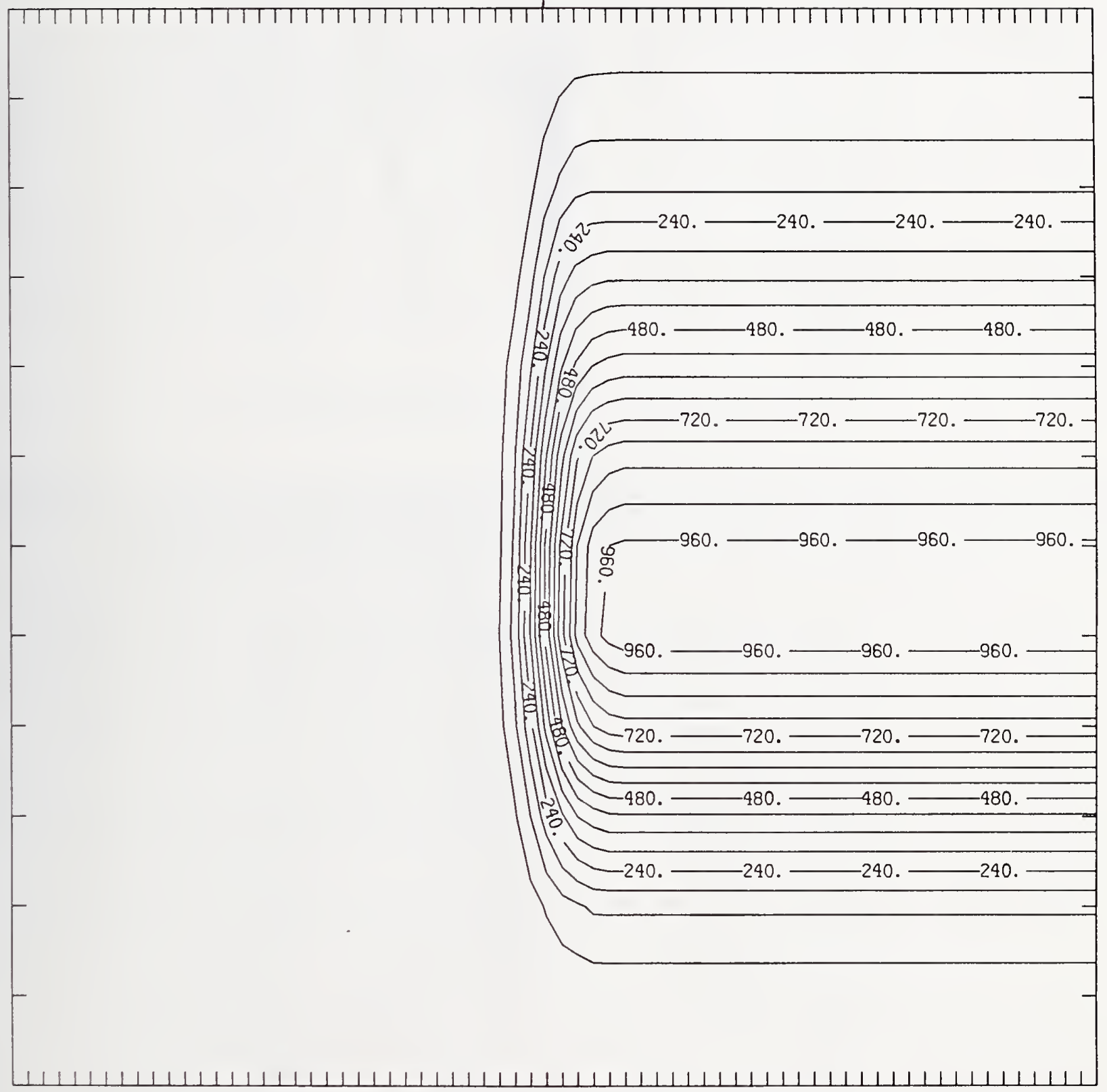

Figure S200-3 Two-dimensional distribution of $200 \mathrm{keV}$ sulfur implanted past a mask edge. The length increment (distance between tick marks) is $0.04 \mu \mathrm{m}$ and the scale factor is 1000. 


\section{MASK}

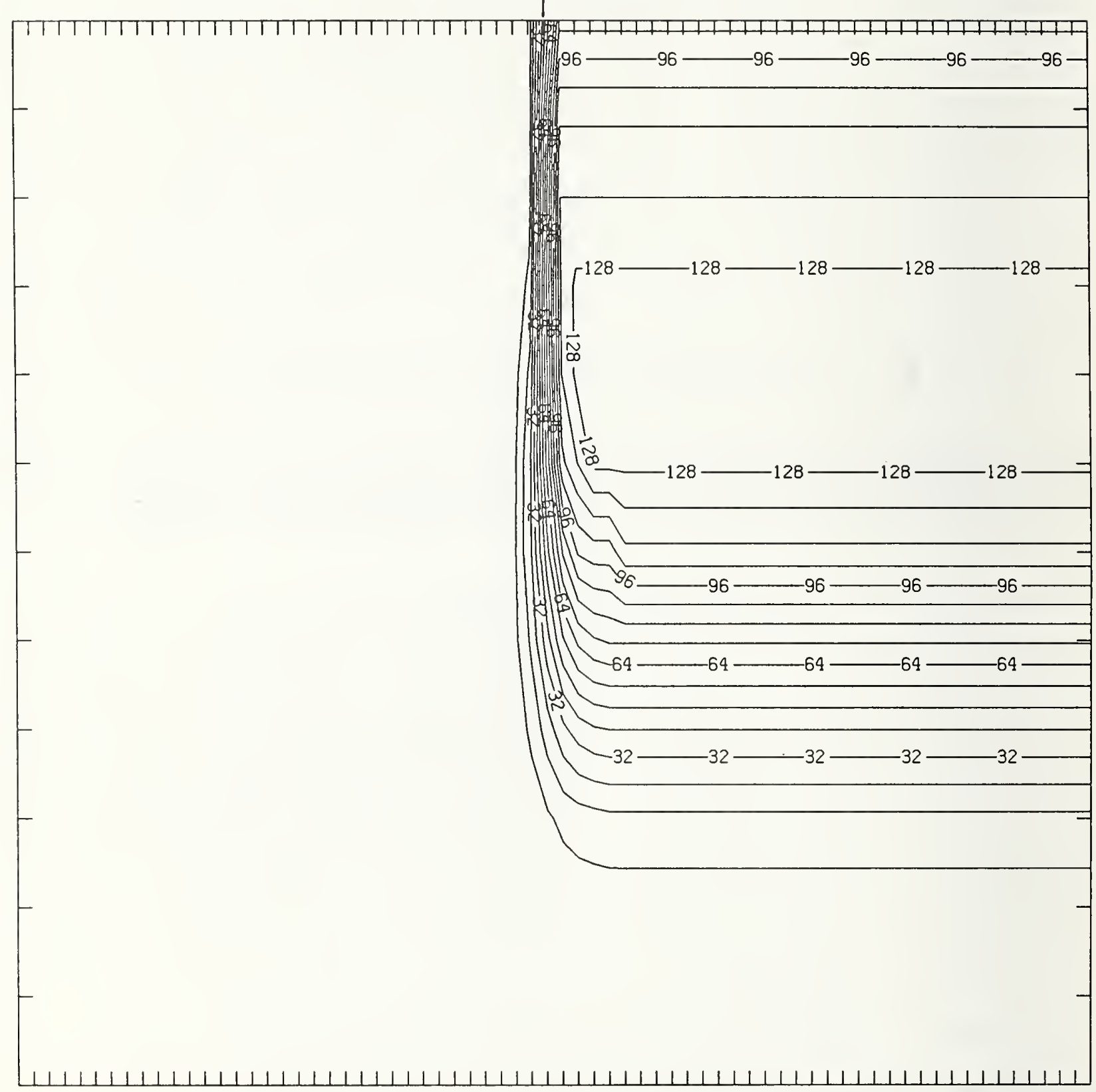

Figure S200-4 Two-dimensional distribution of Frenkel pairs created by $200 \mathrm{keV}$ sulfur implanted past a mask edge. The length increment (distance between tick marks) is $0.04 \mu \mathrm{m}$ and the scale factor is 0.1 . 


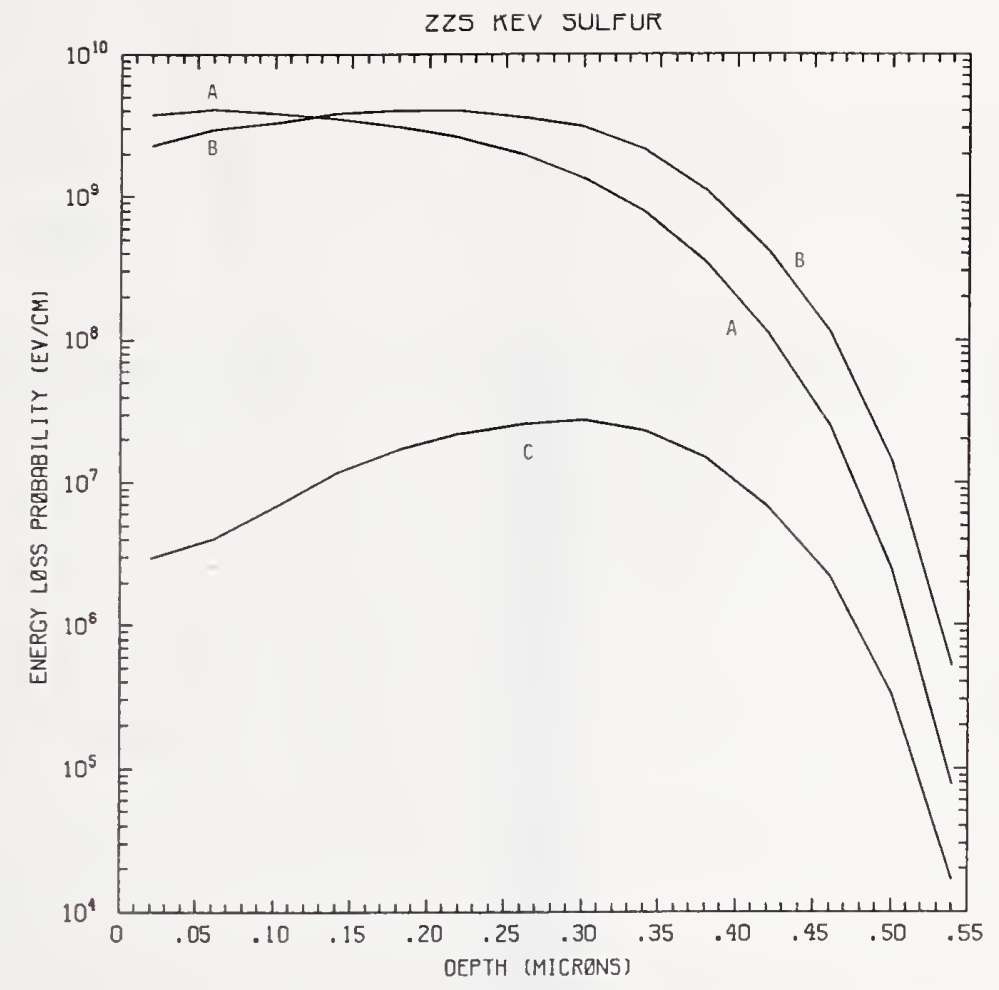

Figure S225-1 One-dimensional probability distributions for energy loss due to electronic excitation (A), displacement damage(B), and phonon excitation (C) for $225 \mathrm{keV}$ sulfur normalì incident on a silicon target.

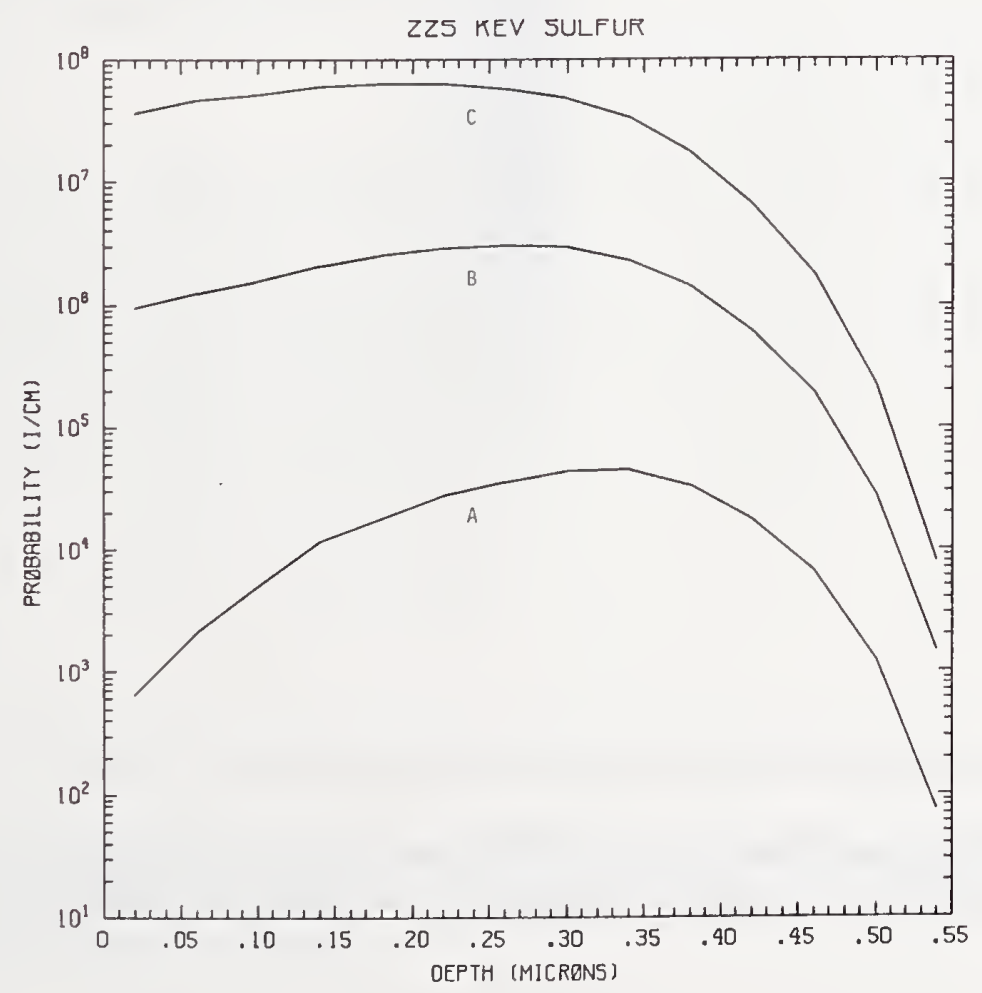

Figure S225-2 One-dimensional probability distributions for implanted sulfur (A), primary silicon displacements (B), and Frenkel pairs (C) for $225 \mathrm{keV}$ sulfur normally incident on a silicon target. 


\section{MASK}

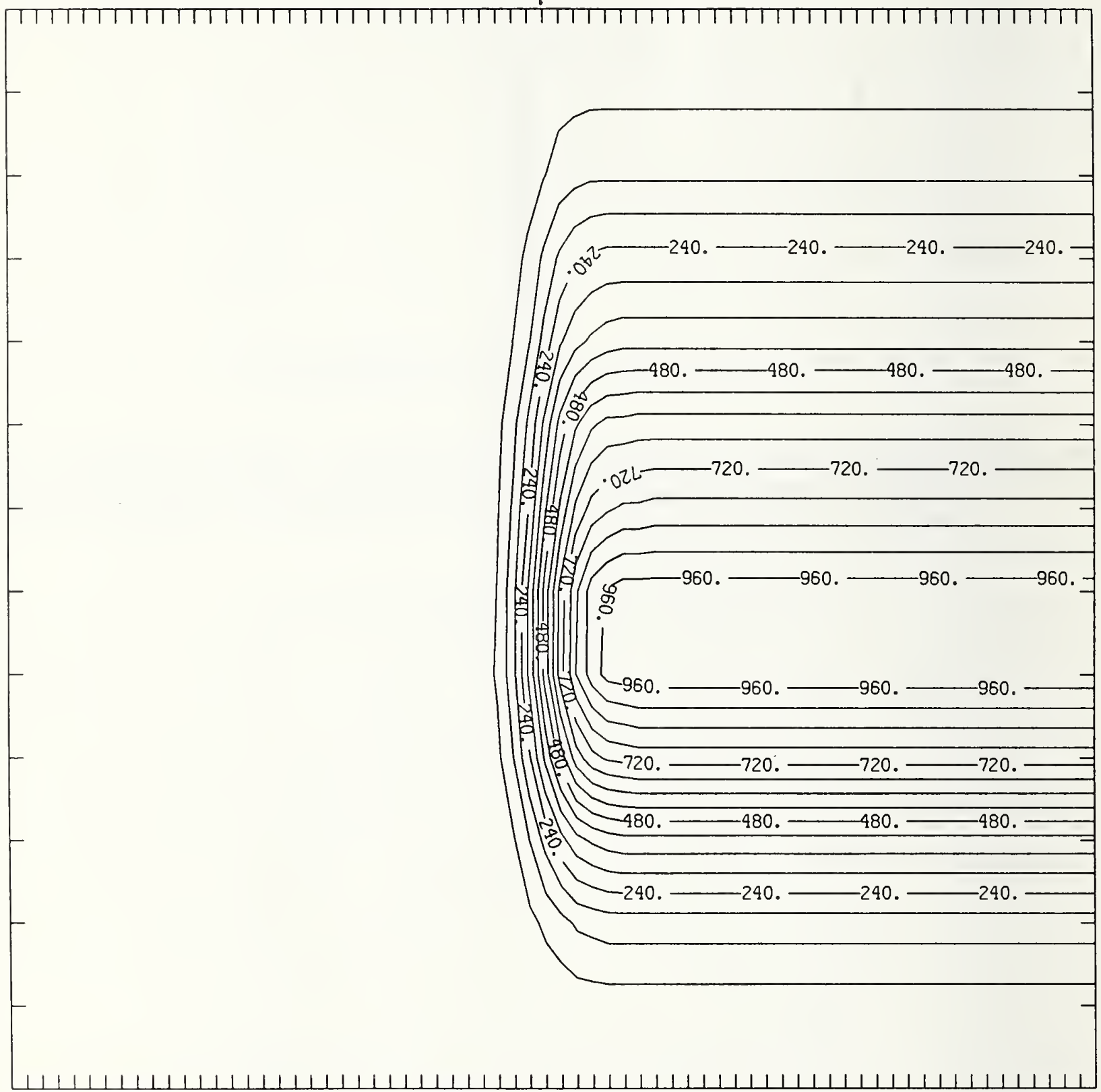

Figure S225-3 Two-dimensional distribution of $225 \mathrm{keV}$ sulfur implanted past a mask edge. The length increment (distance between tick marks) is $0.04 \mu \mathrm{m}$ and the scale factor is 1000 . 


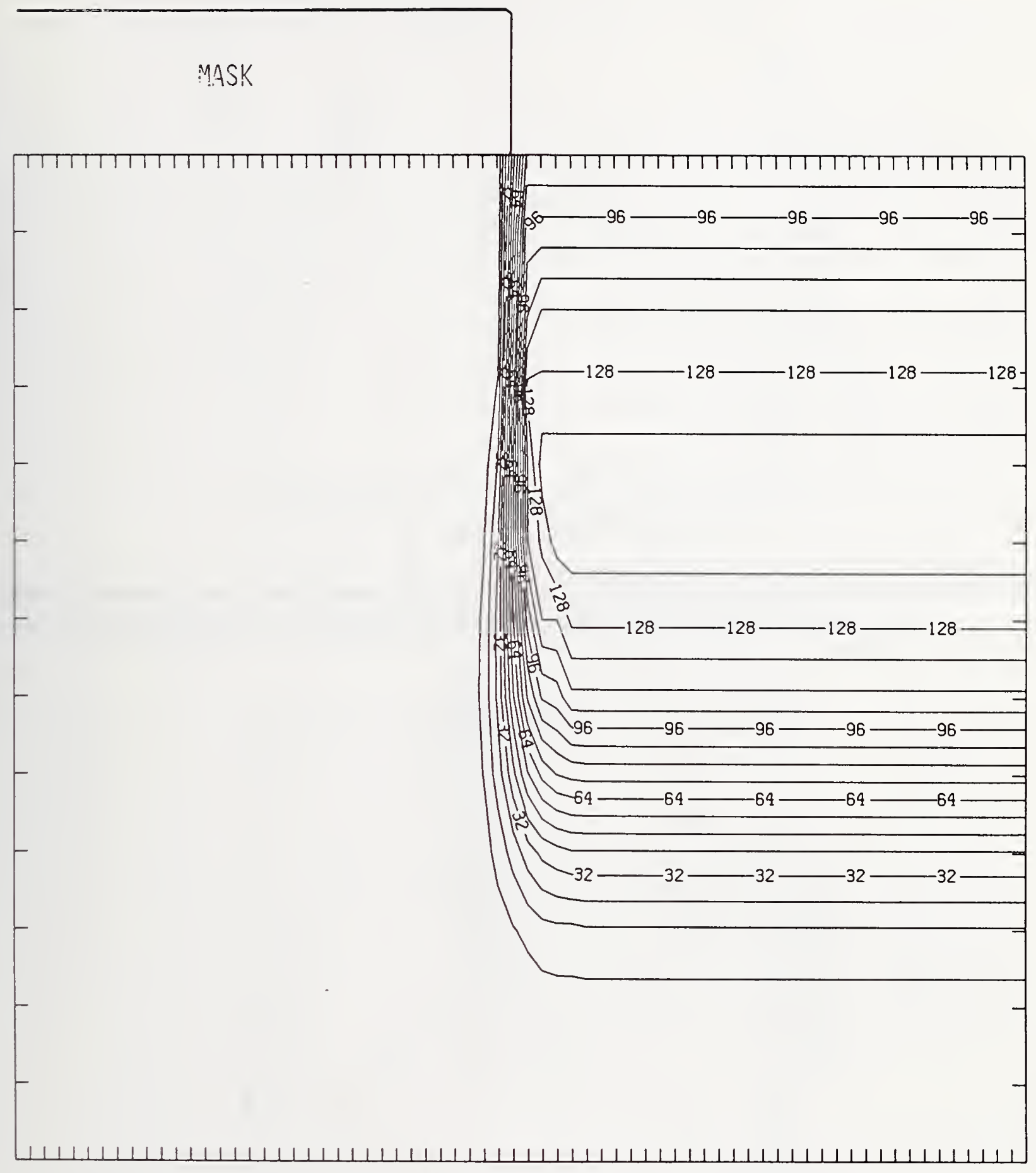

Figure S225-4 Two-dimensional distribution of Frenkel pairs created by $225 \mathrm{keV}$ sulfur implanted past a mask edge. The length increment (distance between tick marks) is $0.04 \mu \mathrm{m}$ and the scale factor is 0.1 . 


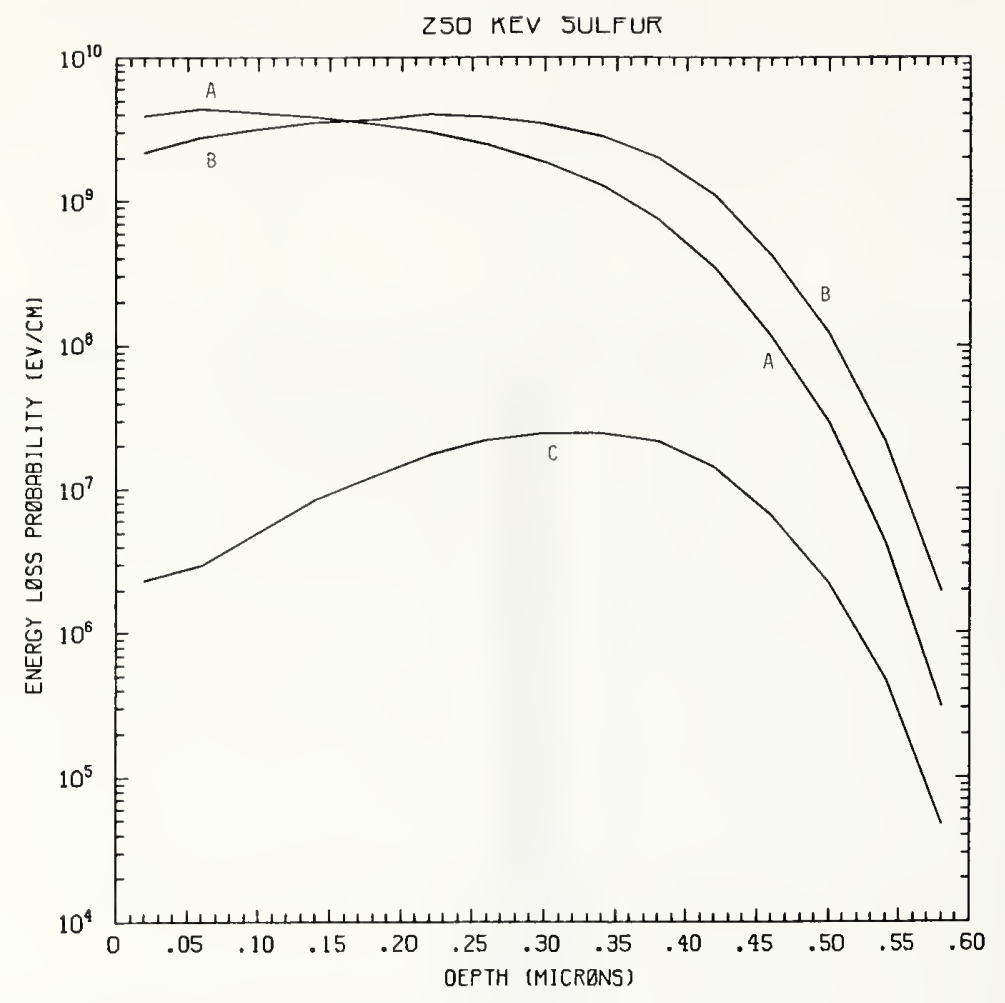

Figure S250-1 One-dimensional probability distributions for energy loss due to electronic excitation (A), displacement damage(B), and phonon excitation (C) for $250 \mathrm{keV}$ sulfur normally incident on a silicon target.

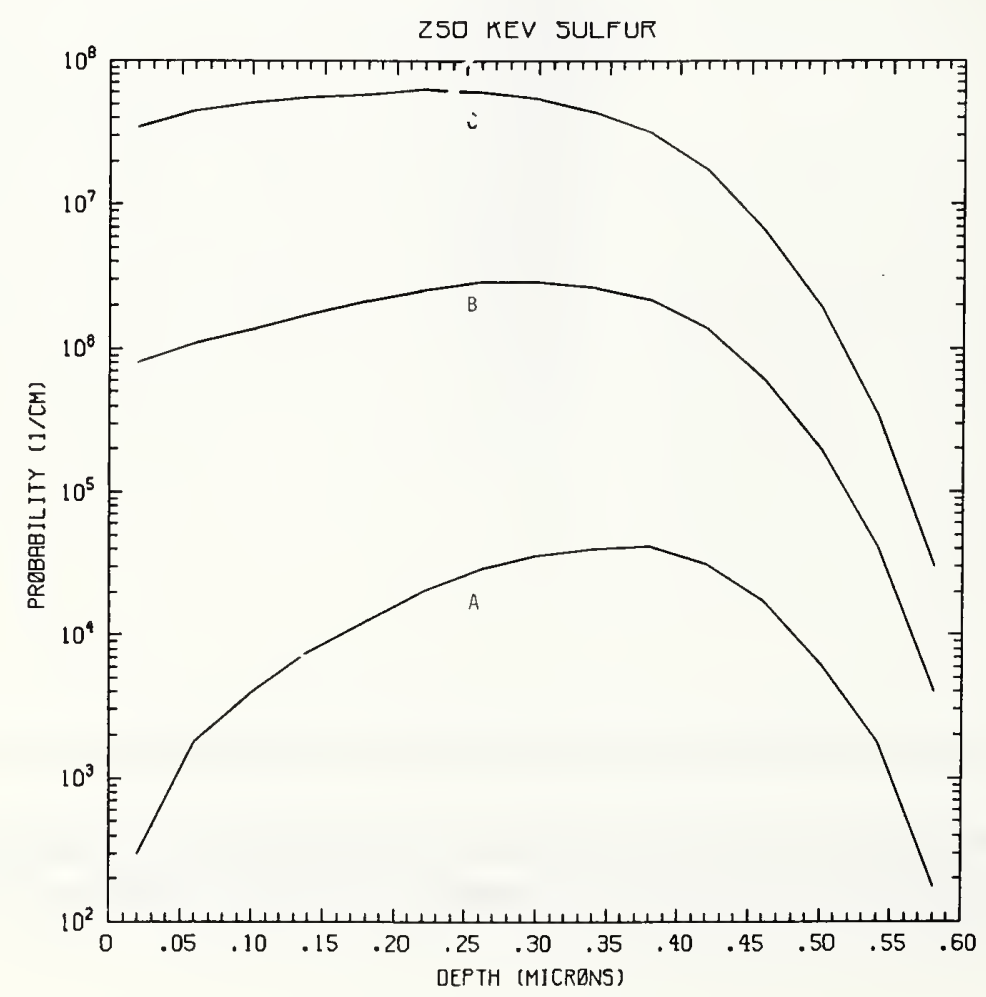

Figure S250-2 One-dimensional probability distributions for implanted sulfur (A), primary silicon displacements (B), and Frenkel pairs (C) for $250 \mathrm{keV}$ sulfur normally incident on a silicon target. 


\section{iASAK}

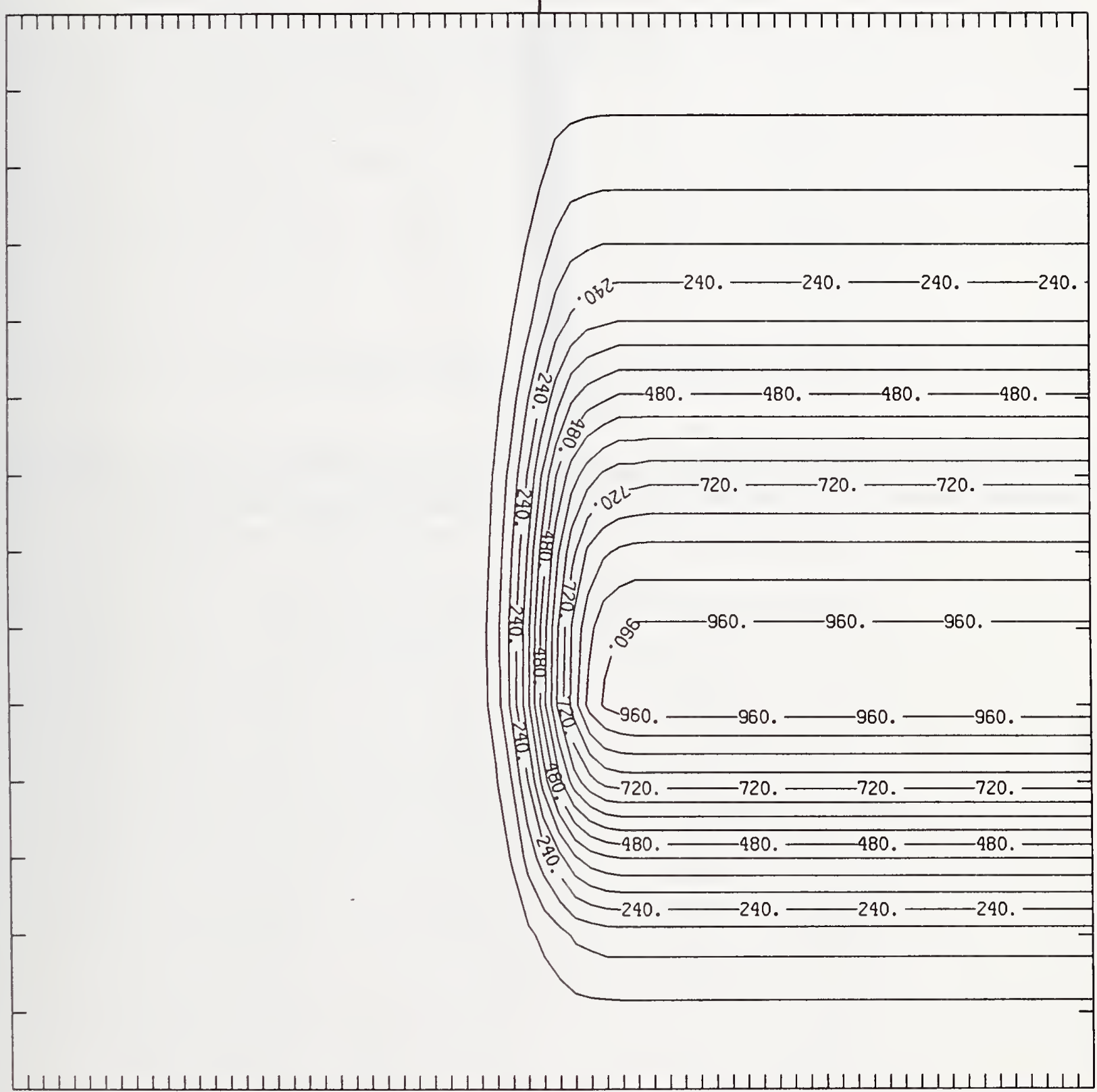

Figure S250-3 Two-dimensional distribution of $250 \mathrm{keV}$ sulfur implanted past a mask edge. The length increment (distance between tick marks) is $0.04 \mu \mathrm{m}$ and the scale factor is 1000 . 


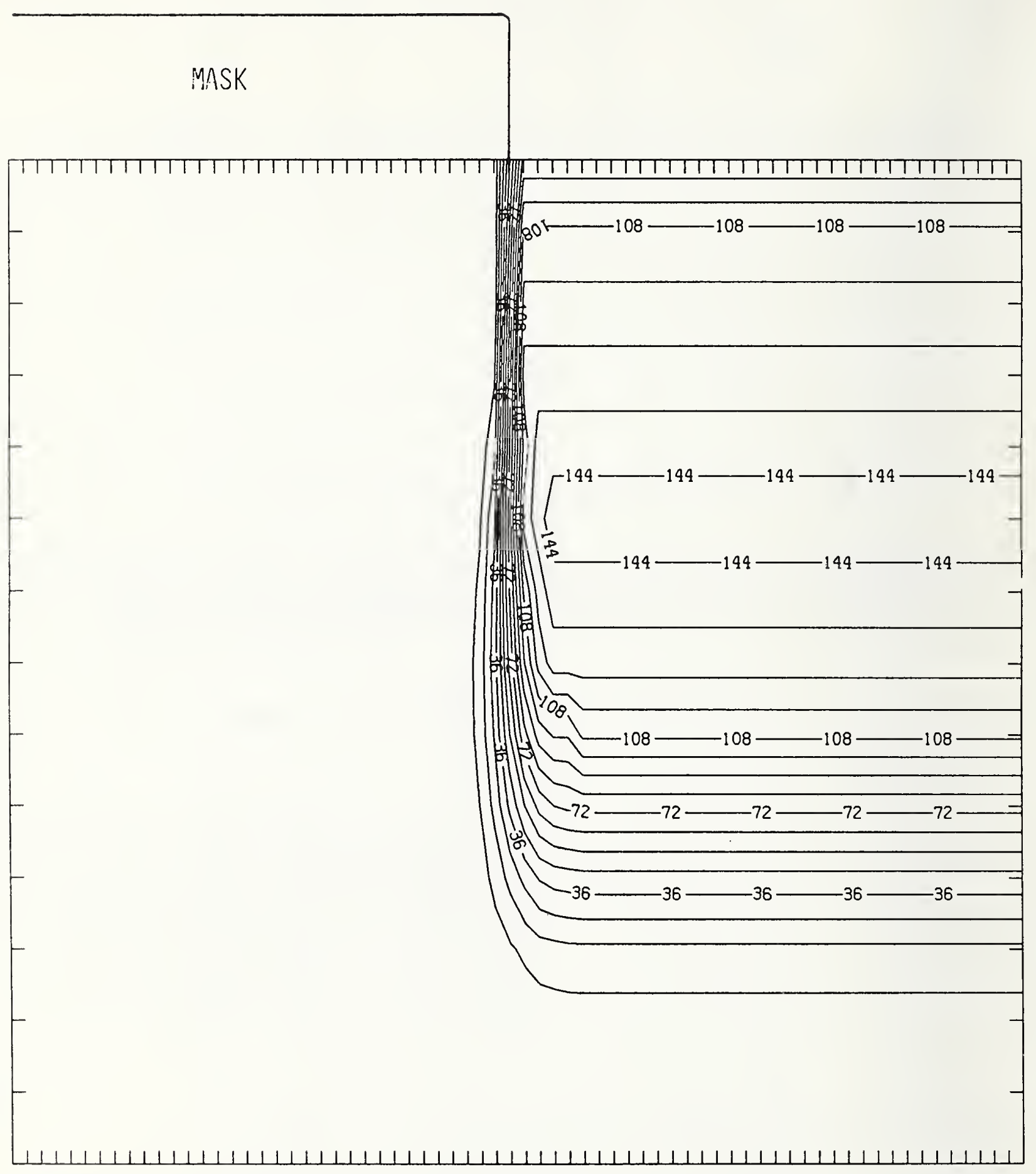

Figure S250-4 Two-dimensional distribution of Frenkel pairs created by $250 \mathrm{keV}$ sulfur implanted past a mask edge. The length increment (distance between tick marks) is $0.04 \mu \mathrm{m}$ and the scale factor is 0.1 . 


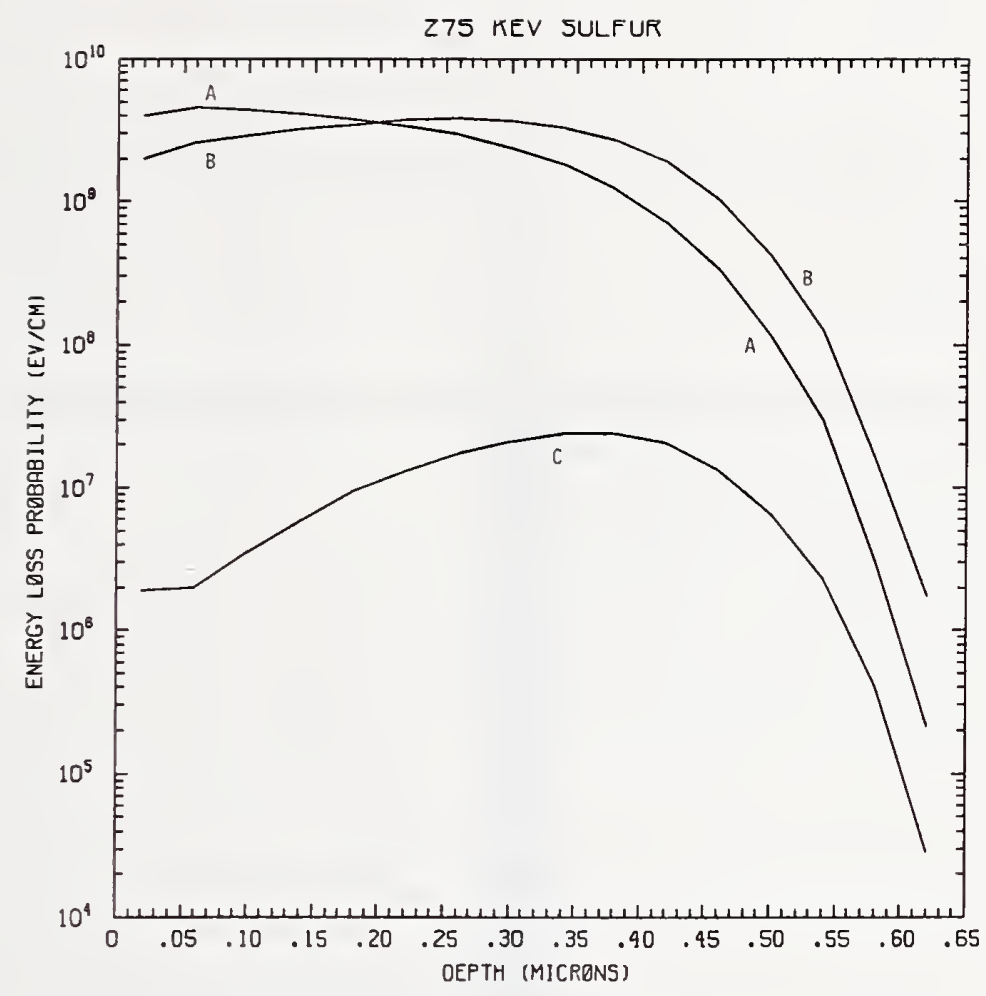

Figure S275-1 One-dimensional probability distributions for energy loss due to electronic excitation (A), displacement damage(B), and phonon excitation (C) for $275 \mathrm{keV}$ sulfur normally incident on a silicon target.

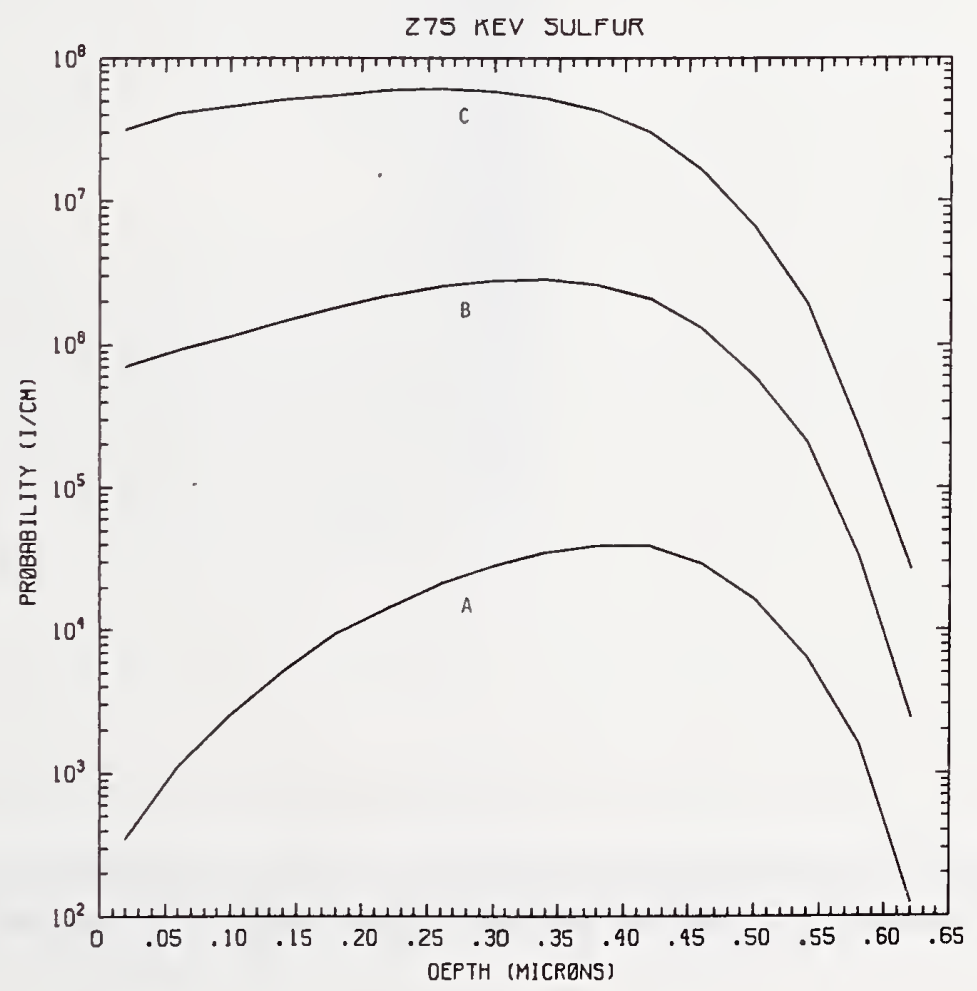

Figure S275-2 One-dimensional probability distributions for implanted sulfur (A), primary silicon displacements (B), and Frenkel pairs (C) for $275 \mathrm{keV}$ sulfur normally incident on a silicon target. 


\section{M.ASK}

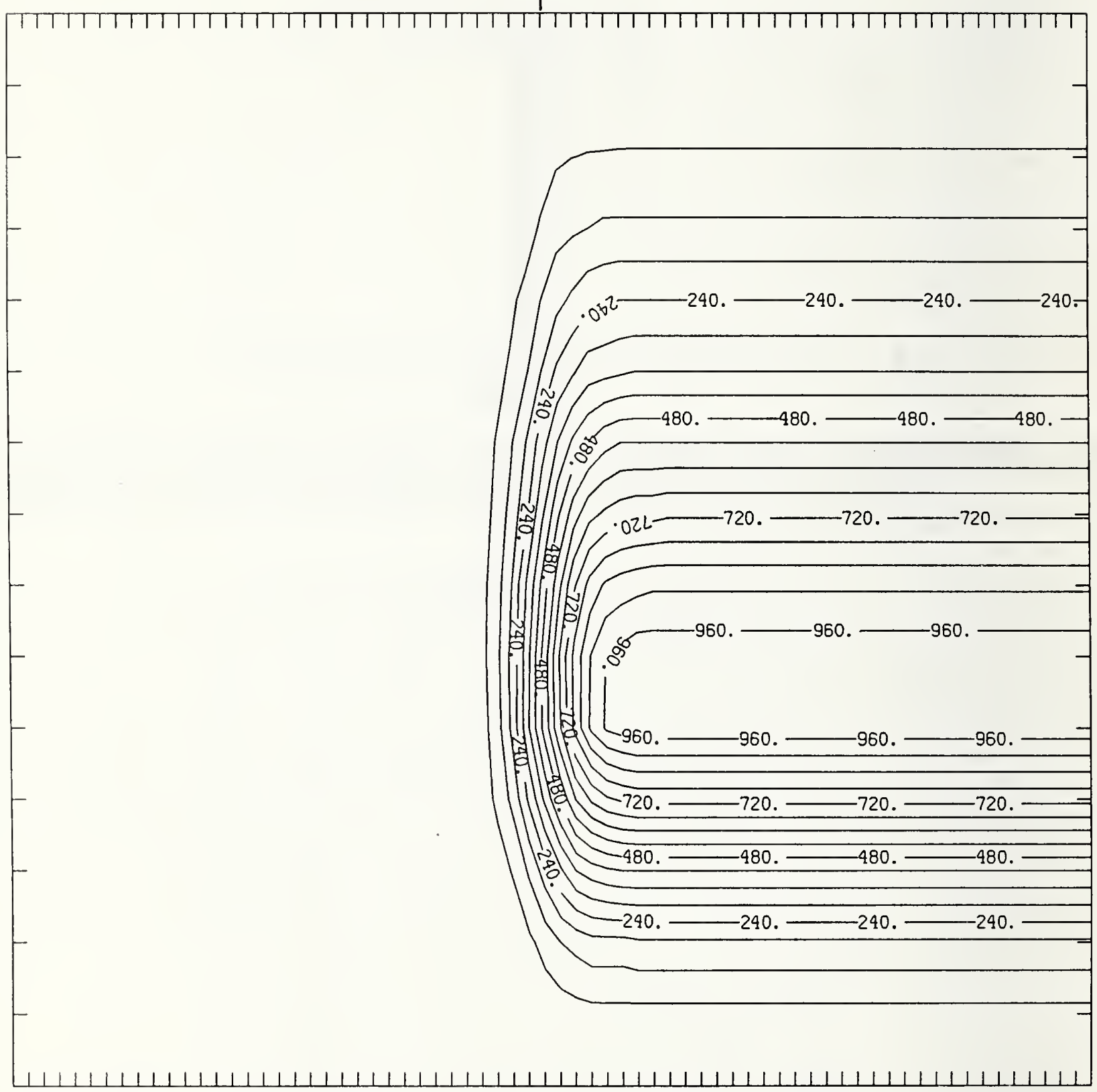

Figure S275-3 Two-dimensional distribution of $275 \mathrm{keV}$ sulfur implanted past a mask edge. The length increment (distance between tick marks) is $0.04 \mu \mathrm{m}$ and the scale factor is 1000. 


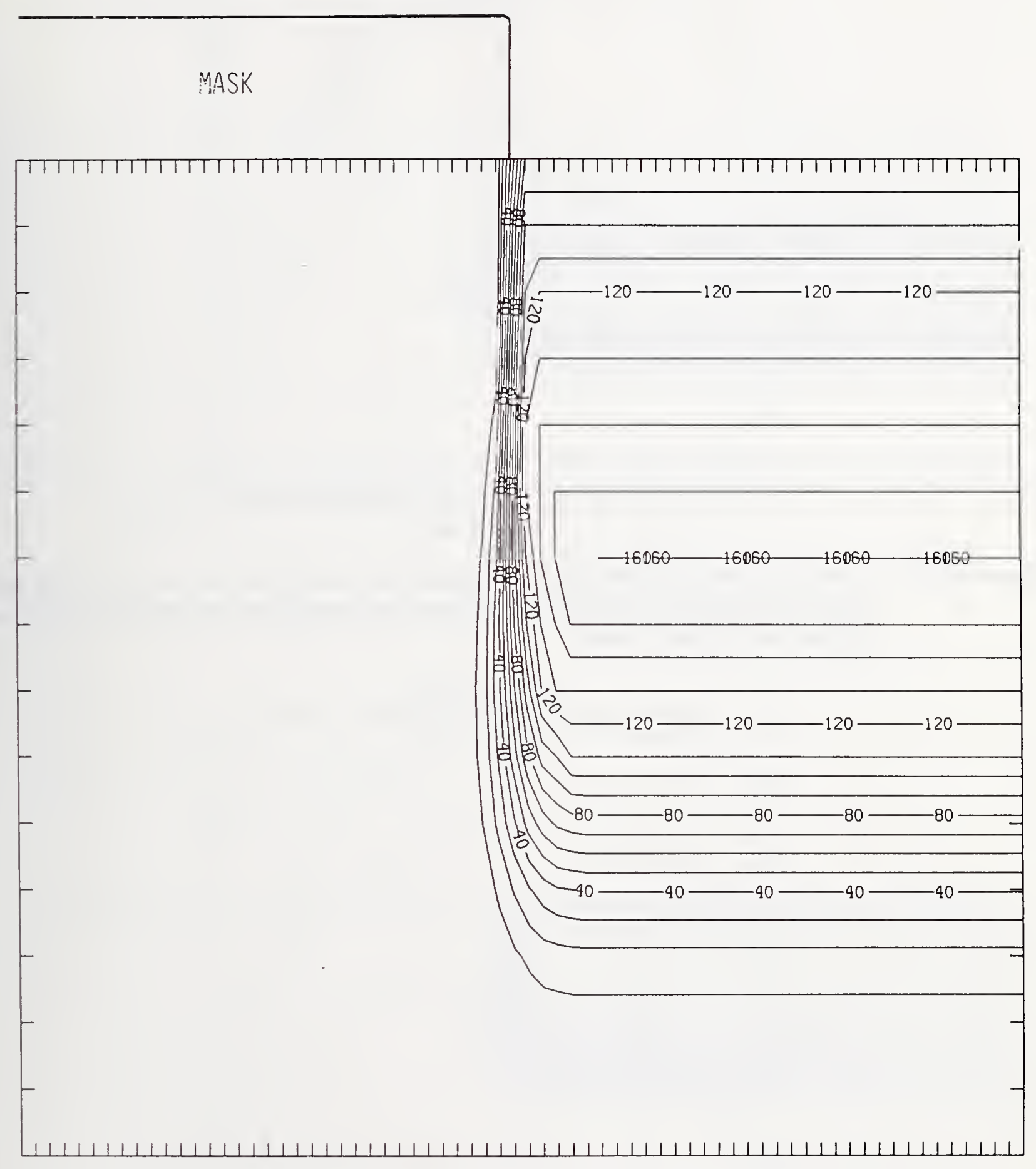

Figure S275-4 Two-dimensional distribution of Frenkel pairs created by $275 \mathrm{keV}$ sulfur implanted past a mask edge. The length increment (distance between tick marks) is $0.04 \mu \mathrm{m}$ and the scale factor is 0.1 . 


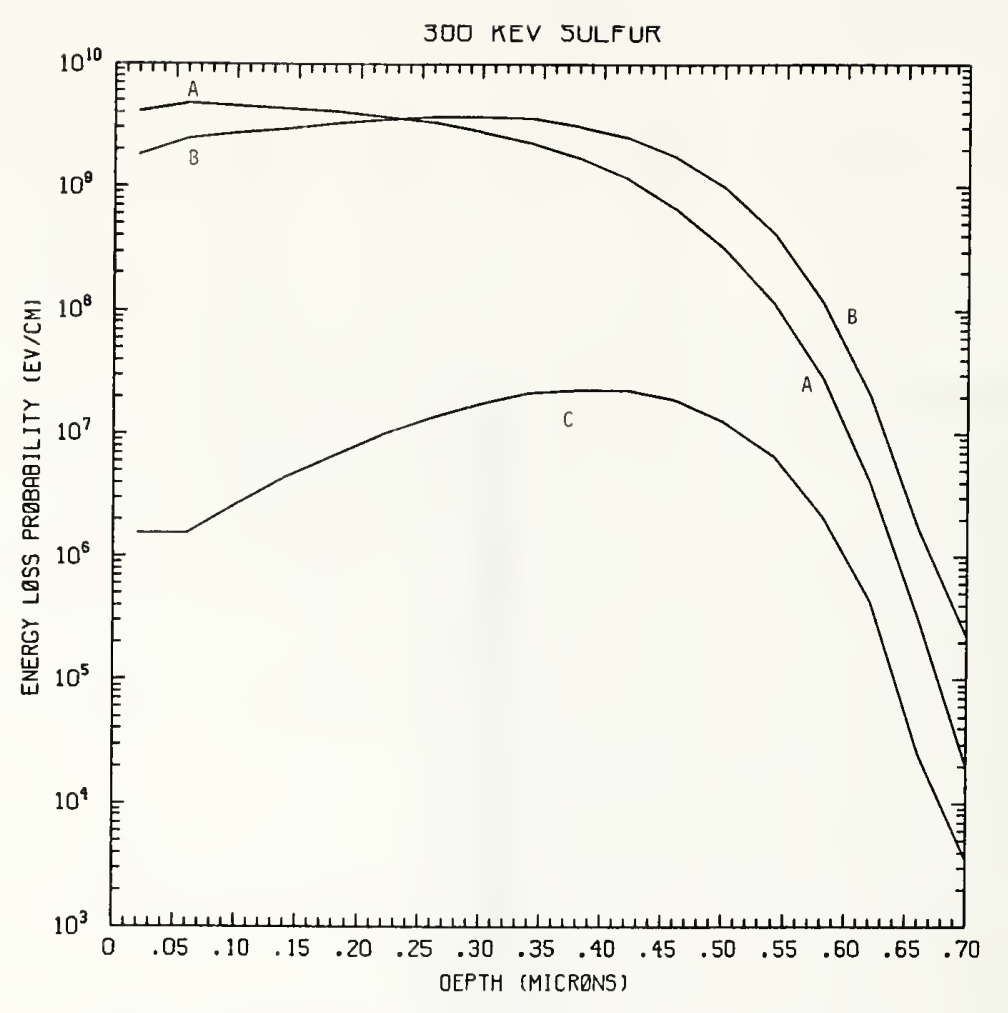

Figure S300-1 One-dimensional probability distributions for energy loss due to electronic excitation (A), displacement damage(B), and phonon excitation (C) for $300 \mathrm{keV}$ sulfur normally incident on a silicon target.

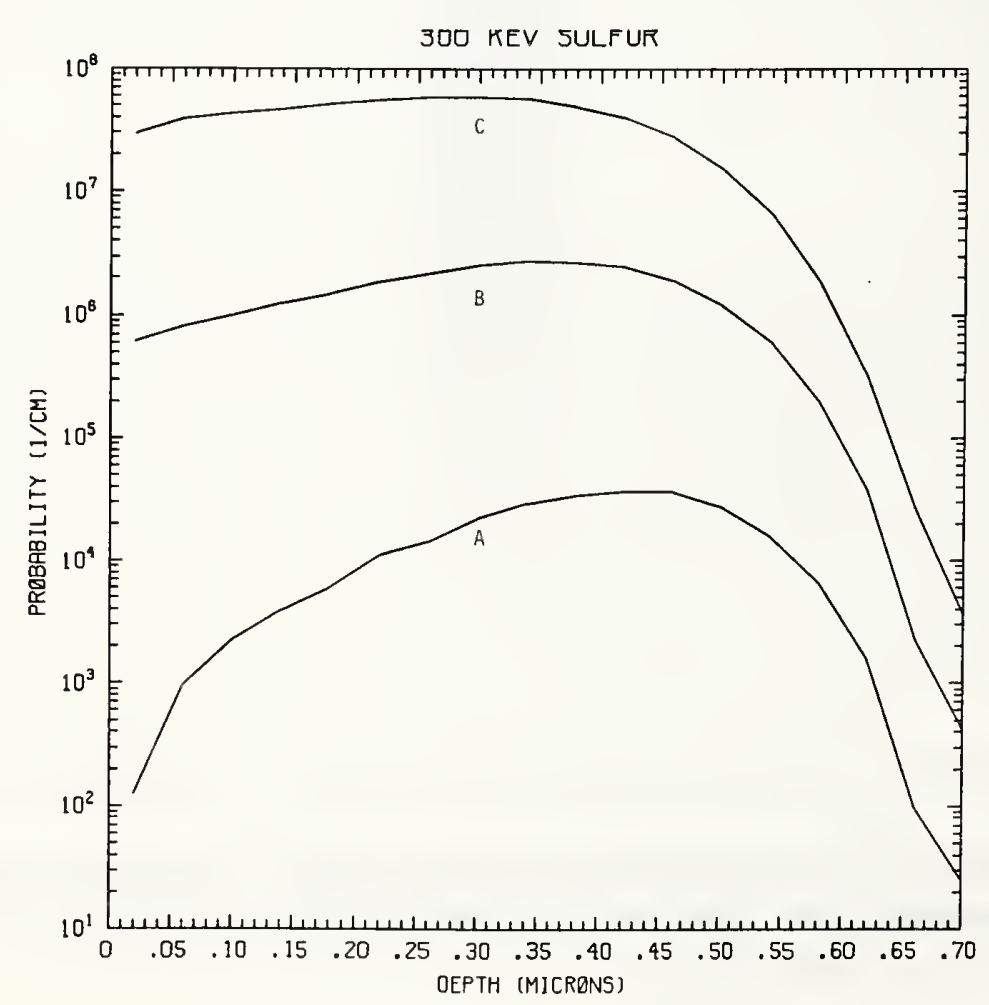

Figure S300-2 One-dimensional probability distributions for implanted sulfur (A), primary silicon displacements (B), and Frenkel pairs (C) for $300 \mathrm{keV}$ sulfur normally incident on a silicon target. 


\section{MASK}

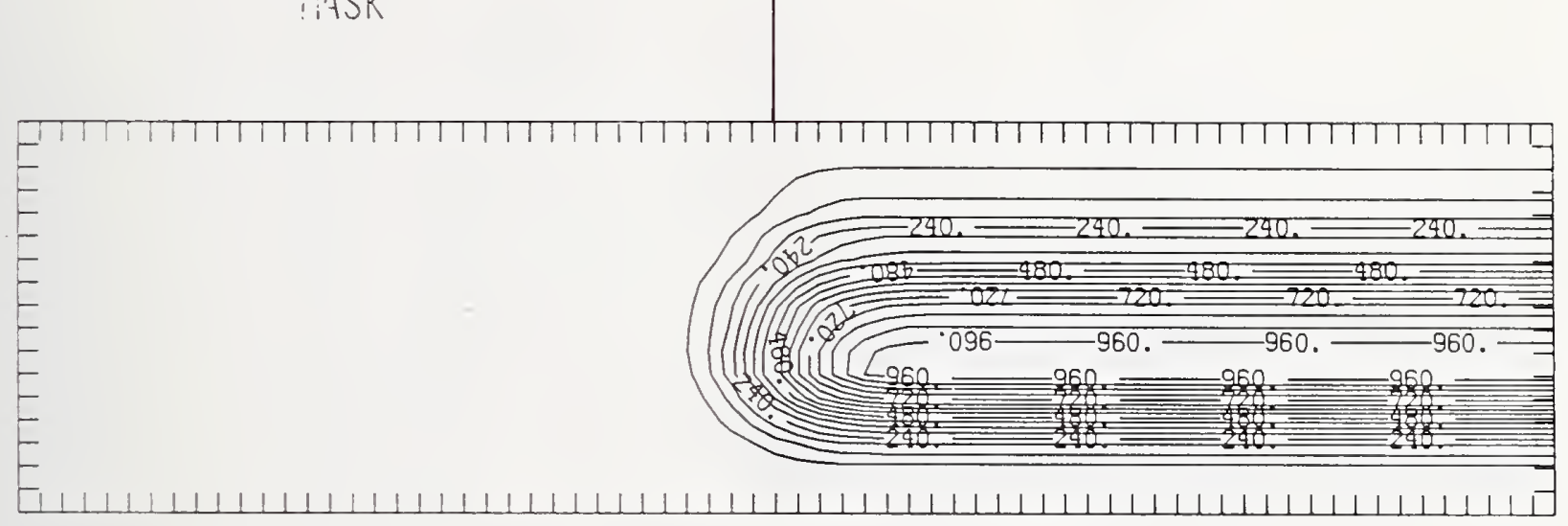

Figure S300-3 Two-dimensional distribution of $300 \mathrm{keV}$ sulfur implanted past a mask edge. The length increment (distance between tick marks) is $0.04 \mu \mathrm{m}$ and the scale factor is 1000 .

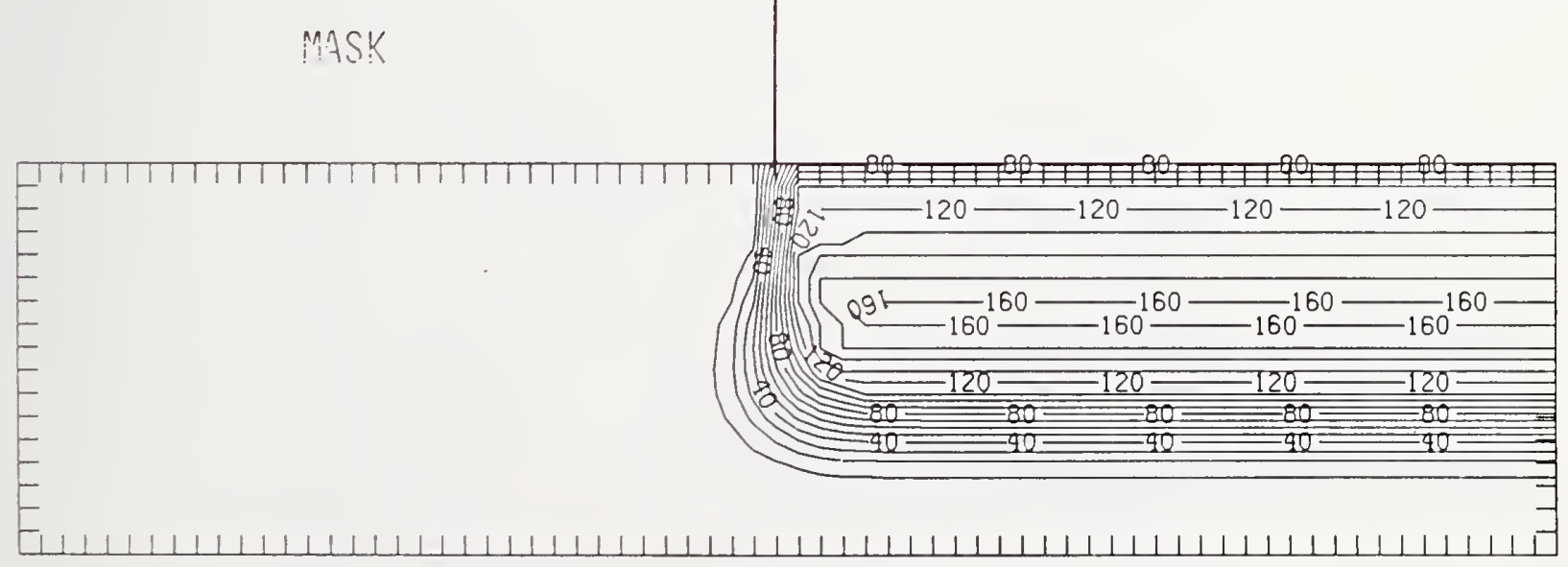

Figure S300-4 Two-dimensional distribution of Frenkel pairs created by $300 \mathrm{keV}$ sulfur implanted past a mask edge. The length increment (distance between tick marks) is $0.04 \mu \mathrm{m}$ and the scale factor is 0.1 . 


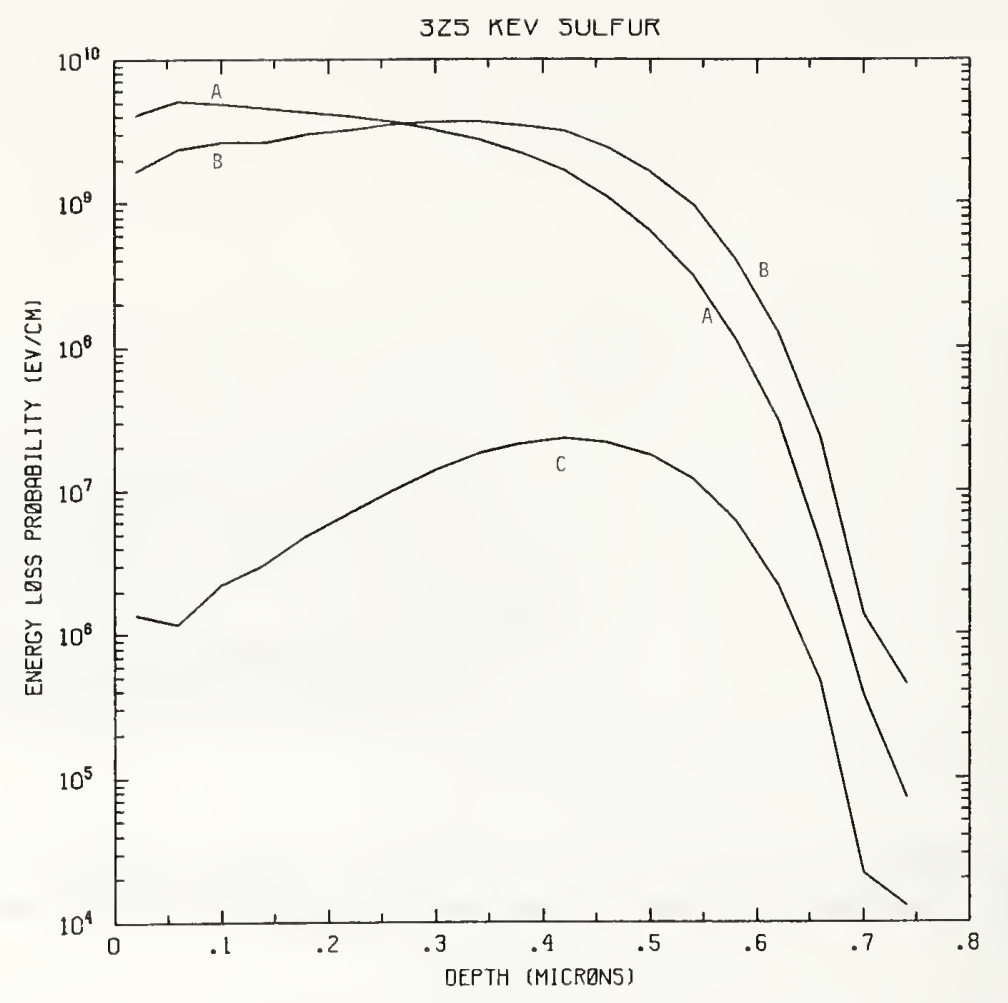

Figure S325-1 One-dimensional probability distributions for energy loss due to electronic excitatios (A), displacement damage(B), and phonon excitation (C) for $325 \mathrm{keV}$ sulfur normall incident on a silicon target.

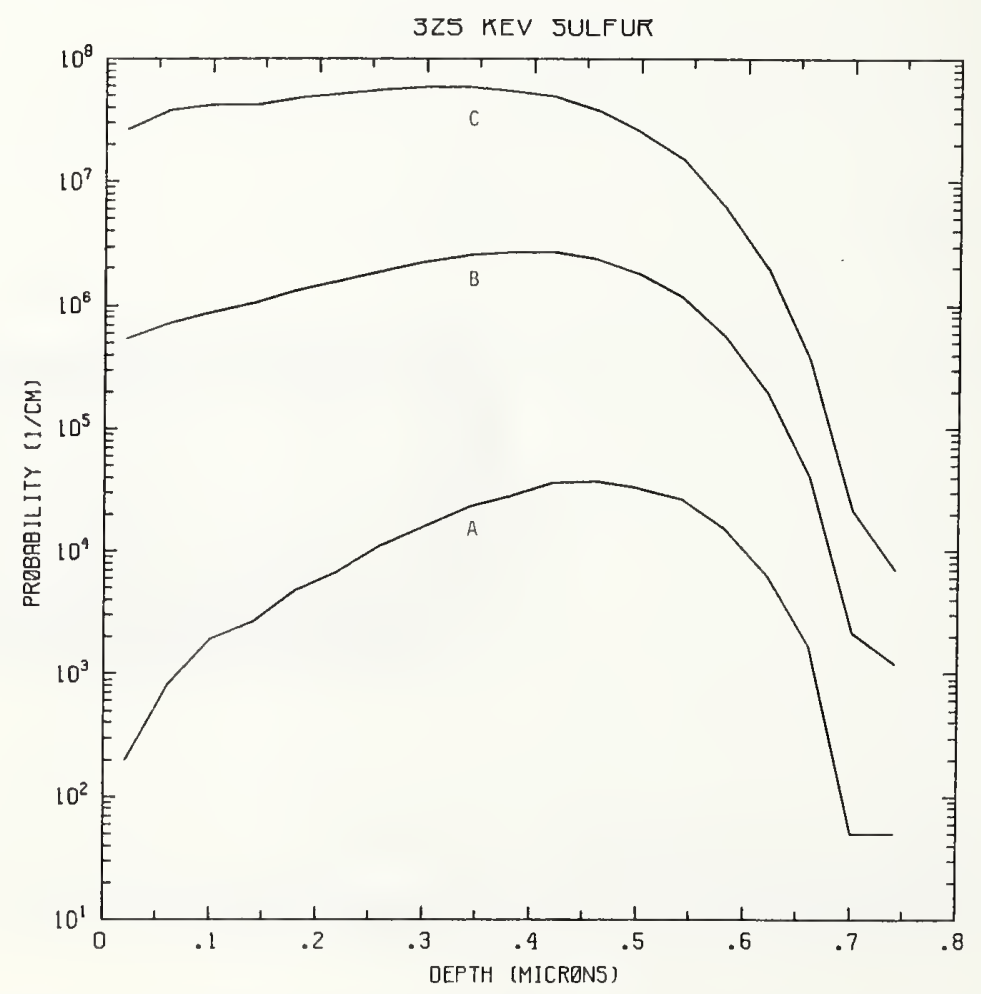

Figure S325-2 One-dimensional probability distributions for implanted sulfur (A), primary silicon displacements (B), and Frenkel pairs (C) for $325 \mathrm{keV}$ sulfur normally incident on a silicon target. 


\section{MASK}

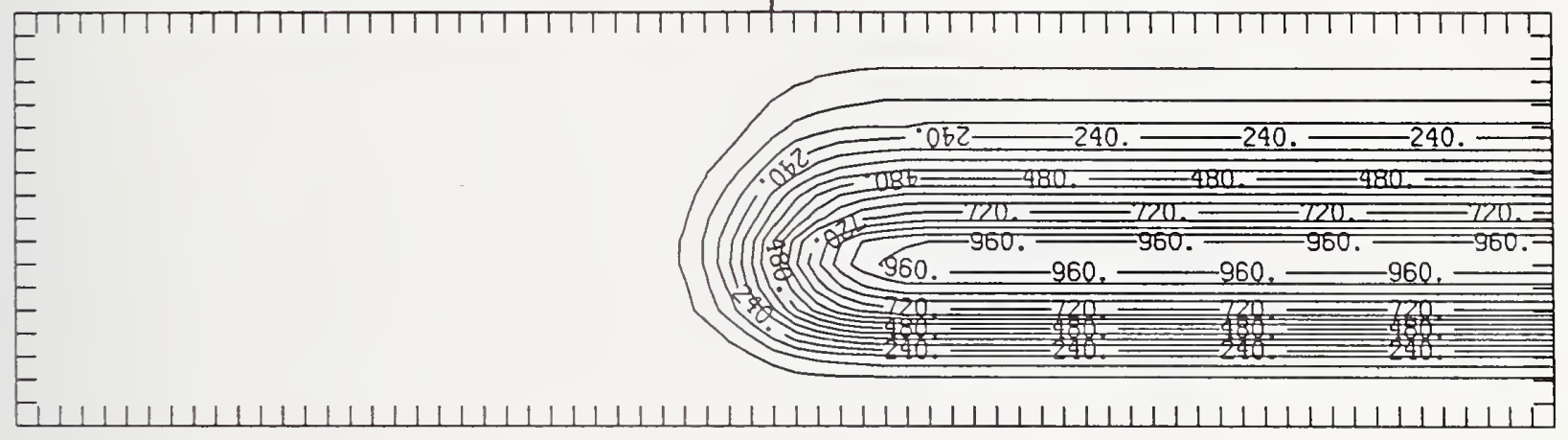

Figure S325-3 Two-dimensional distribution of $325 \mathrm{keV}$ sulfur implanted past a mask edge. The length increment (distance between tick marks) is $0.04 \mu \mathrm{m}$ and the scale factor is 1000.

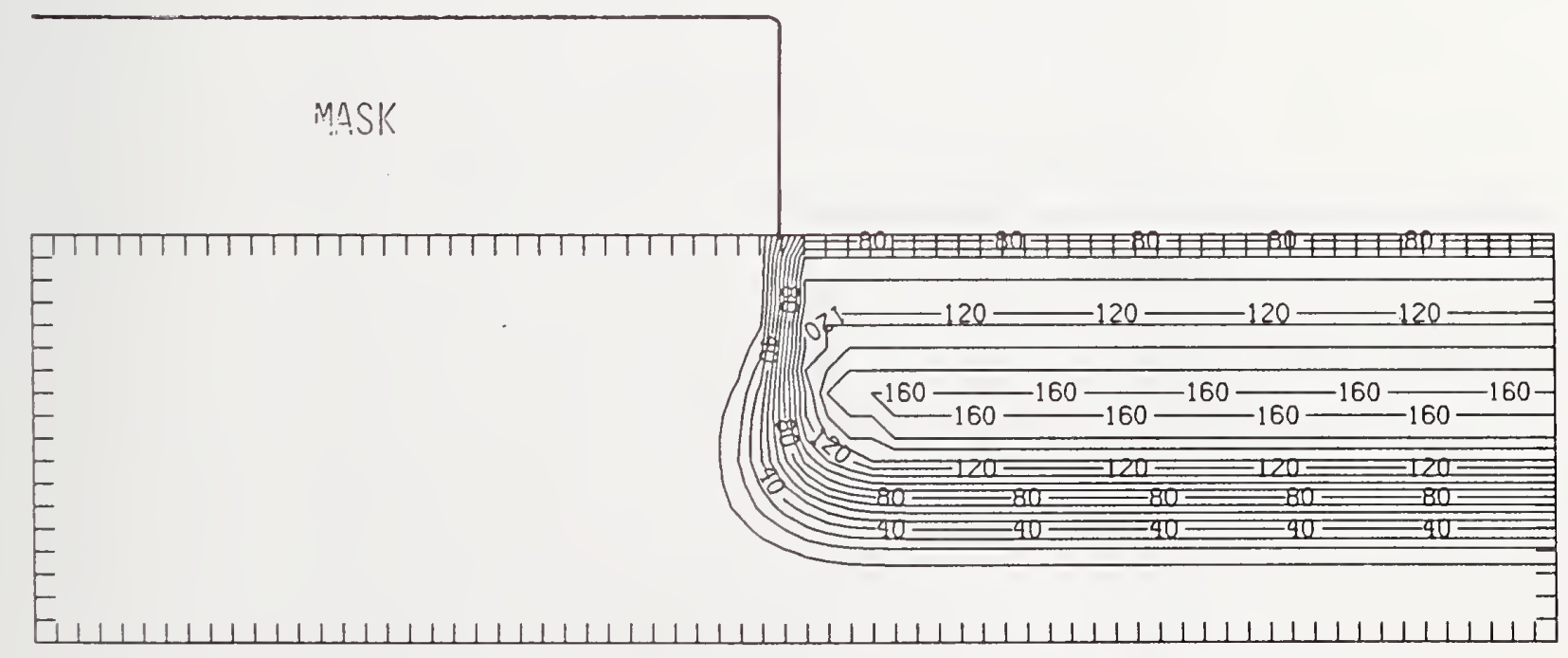

Figure S325-4 Two-dimensional distribution of Frenkel pairs created by $325 \mathrm{keV}$ sulfur implanted past a mask edge. The length increment (distance between tick marks) is $0.04 \mu \mathrm{m}$ and the scale factor is 0.1 . 


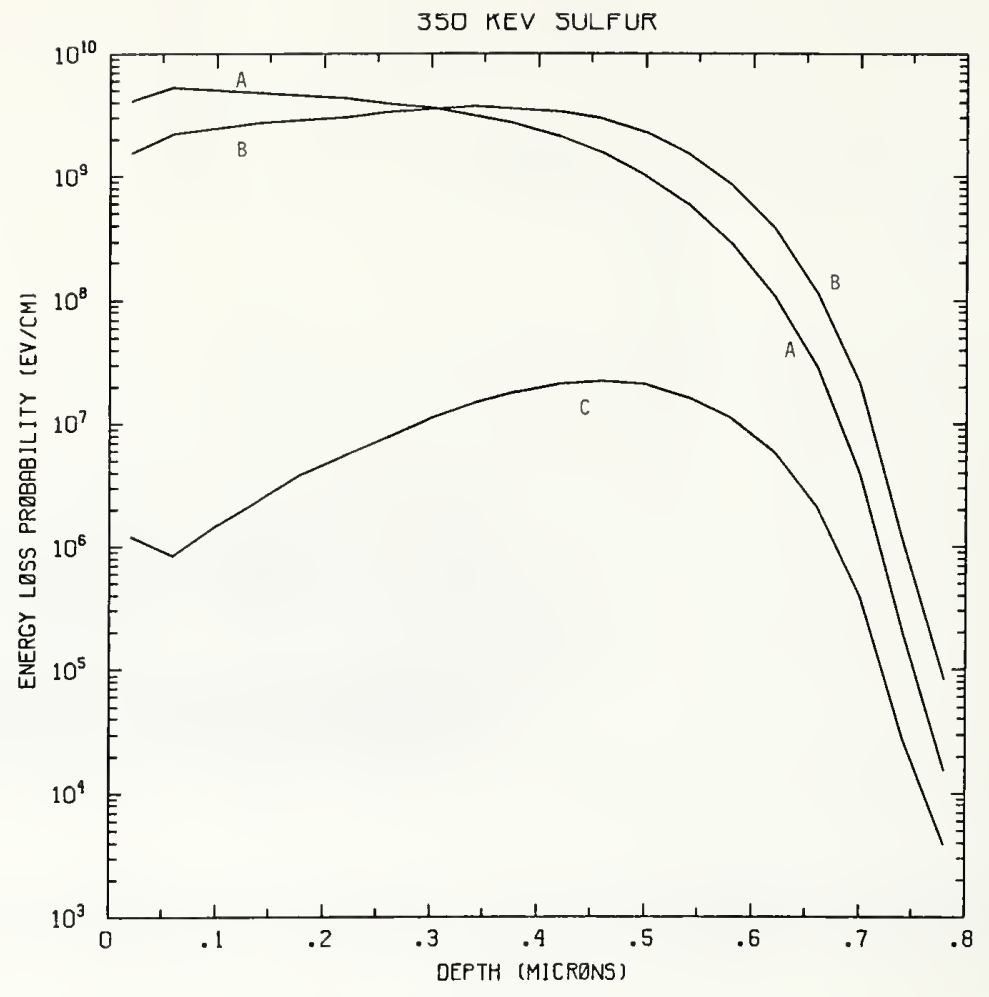

Figure S350-1 One-dimensional probability distributions for energy loss due to electronic excitation (A), displacement damage(B), and phonon excitation (C) for $350 \mathrm{keV}$ sulfur normally incident on a silicon target.

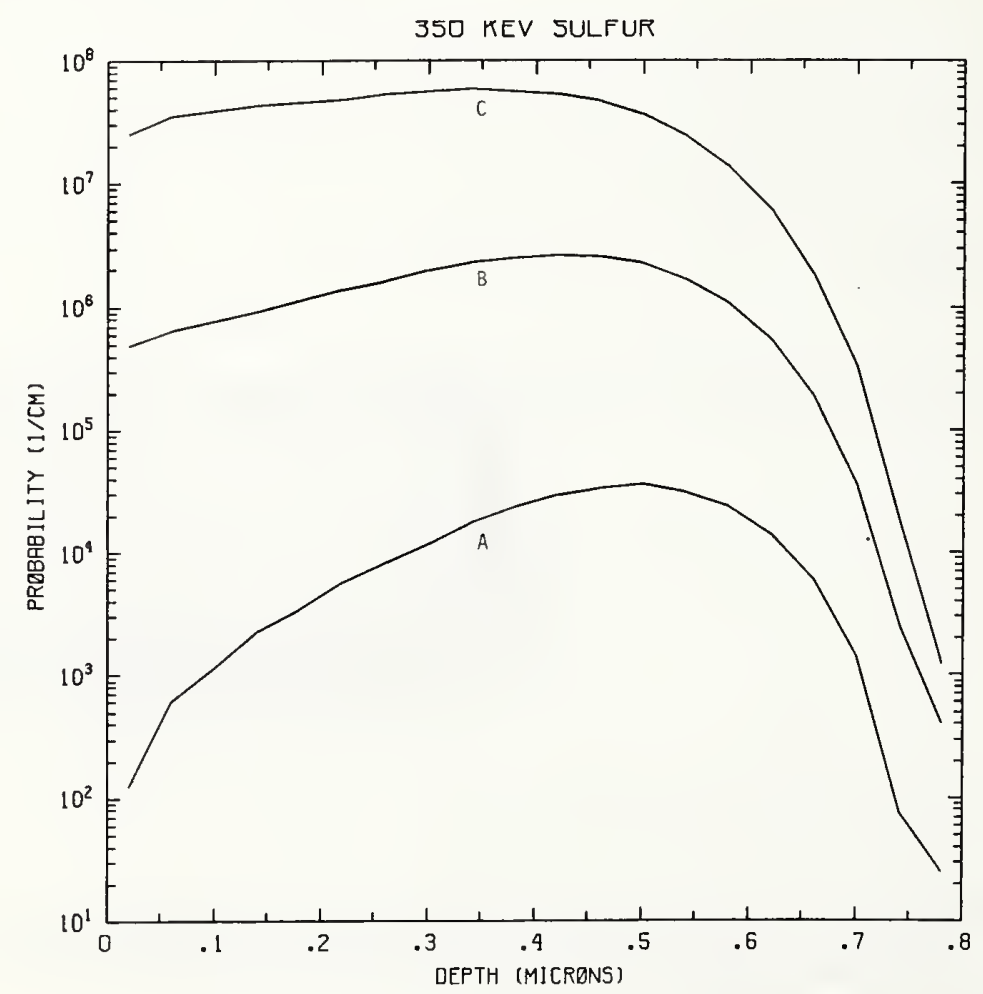

Figure S350-2 One-dimensional probability distributions for implanted sulfur (A), primary silicon displacements (B), and Frenkel pairs (C) for $350 \mathrm{keV}$ sulfur normally incident on a silicon target. 


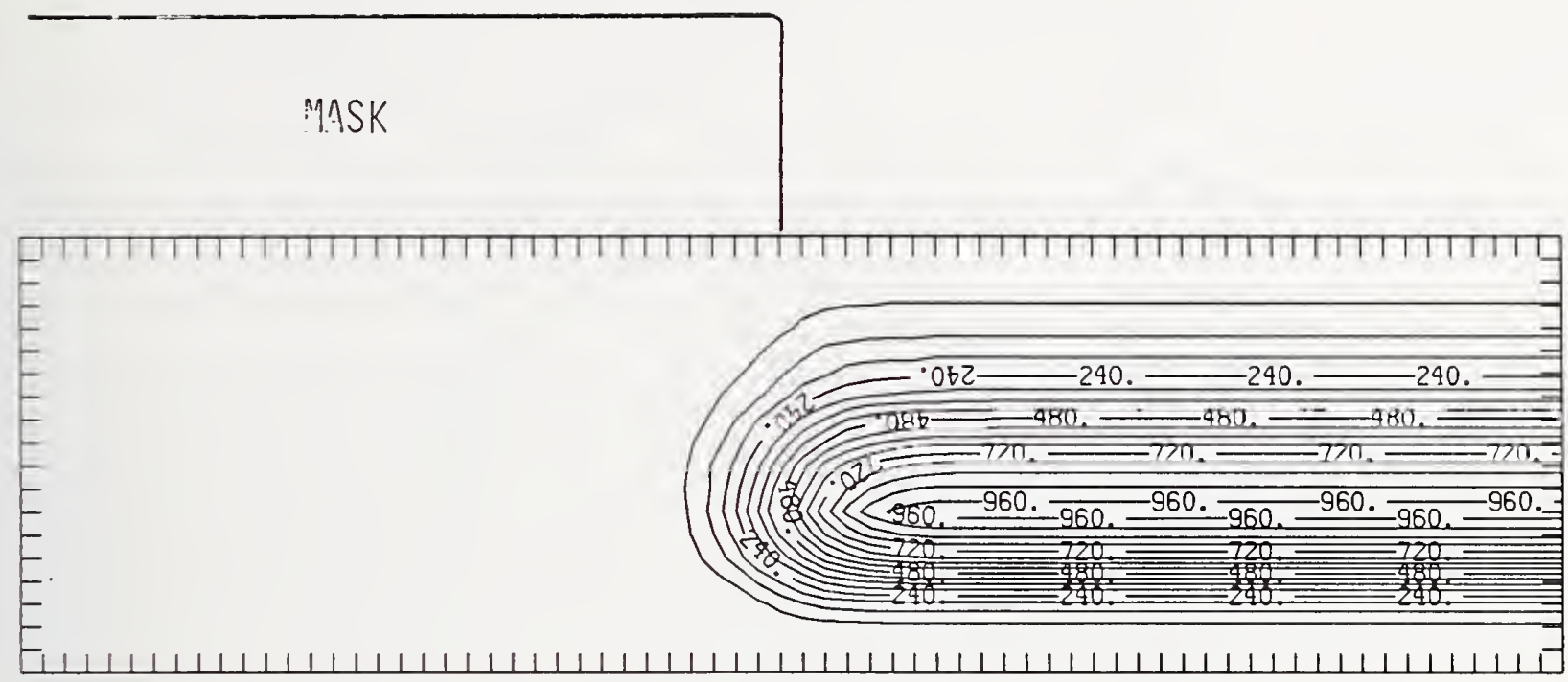

Figure S350-3 Two-dimensional distribution of $350 \mathrm{keV}$ sulfur implanted past a mask edge. The length increment (distance between tick marks) is $0.04 \mu \mathrm{m}$ and the scale factor is 1000 .

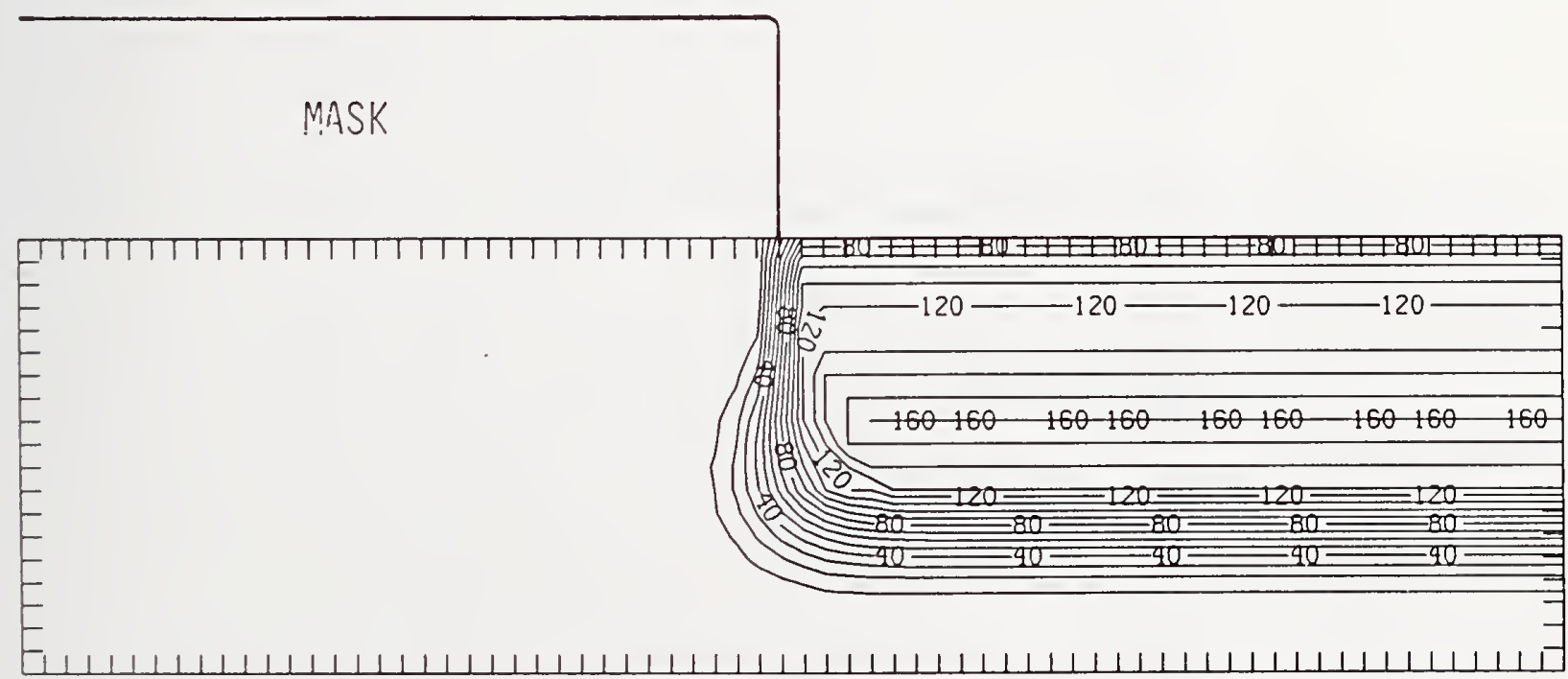

Figure S350-4 Two-dimensional distribution of Frenkel pairs created by $350 \mathrm{keV}$ sulfur implanted past a mask edge. The length increment (distance between tick marks) is $0.04 \mu \mathrm{m}$ and the scale factor is 0.1 . 


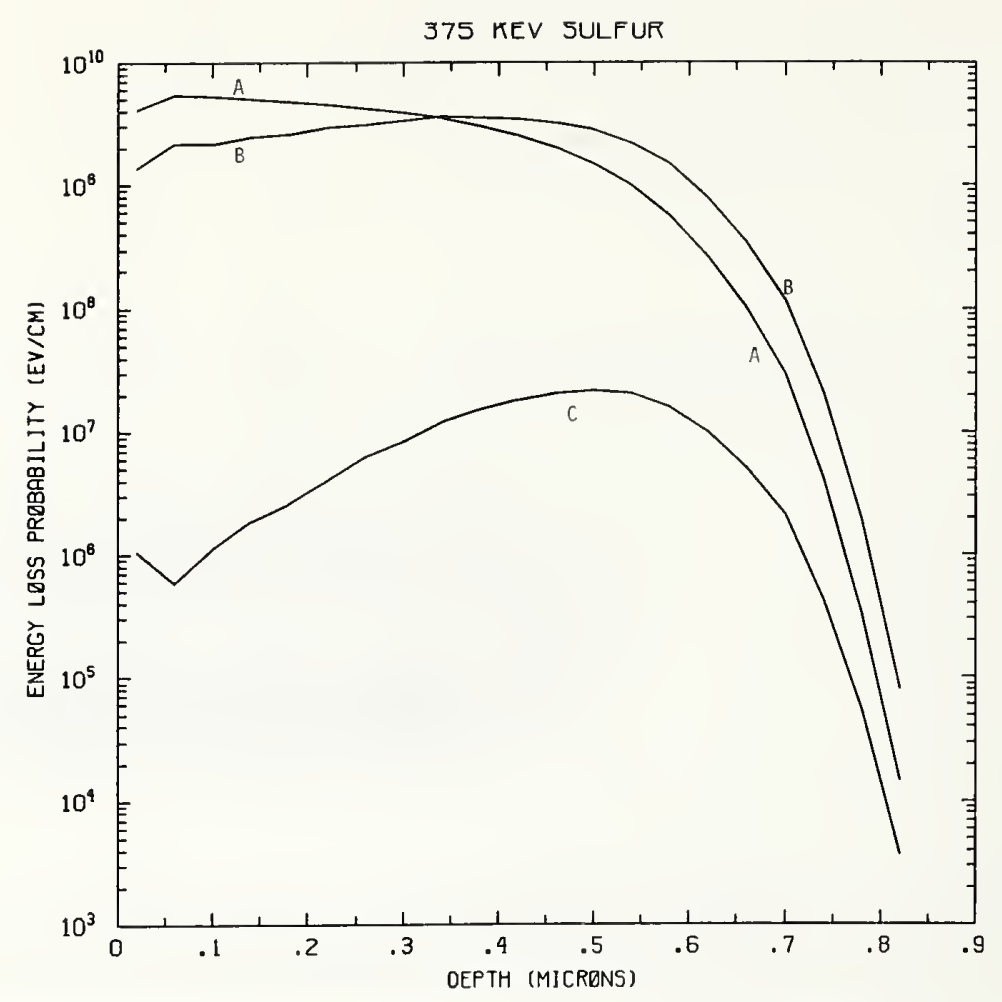

Figure S375-1 One-dimensional probability distributions for energy loss due to electronic excitation (A), displacement damage(B), and phonon excitation (C) for $375 \mathrm{keV}$ sulfur normally incident on a silicon target.

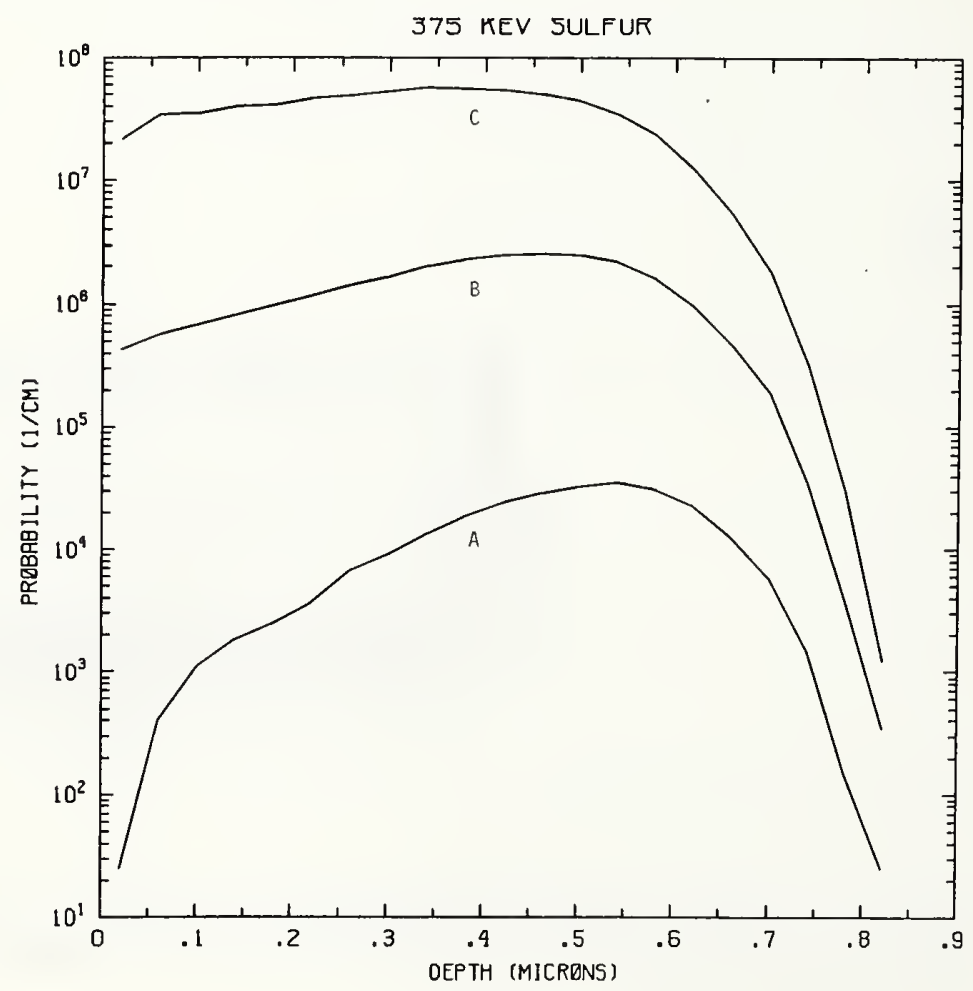

Figure S375-2 One-dimensional probability distributions for implanted sulfur (A), primary silicon displacements (B), and Frenkel pairs (C) for $375 \mathrm{keV}$ sulfur normally incident on a silicon target. 


\section{MASK}

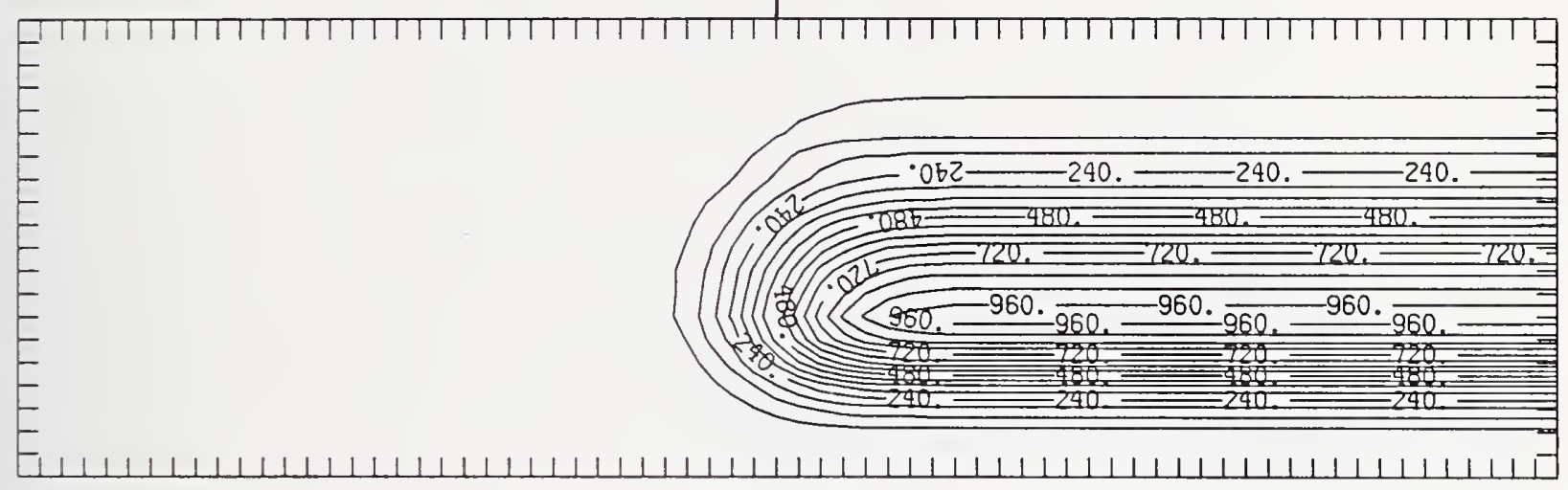

Figure S375-3 Two-dimensional distribution of $375 \mathrm{keV}$ sulfur implanted past a mask edge. The length increment (distance between tick marks) is $0.04 \mu \mathrm{m}$ and the scale factor is 1000.

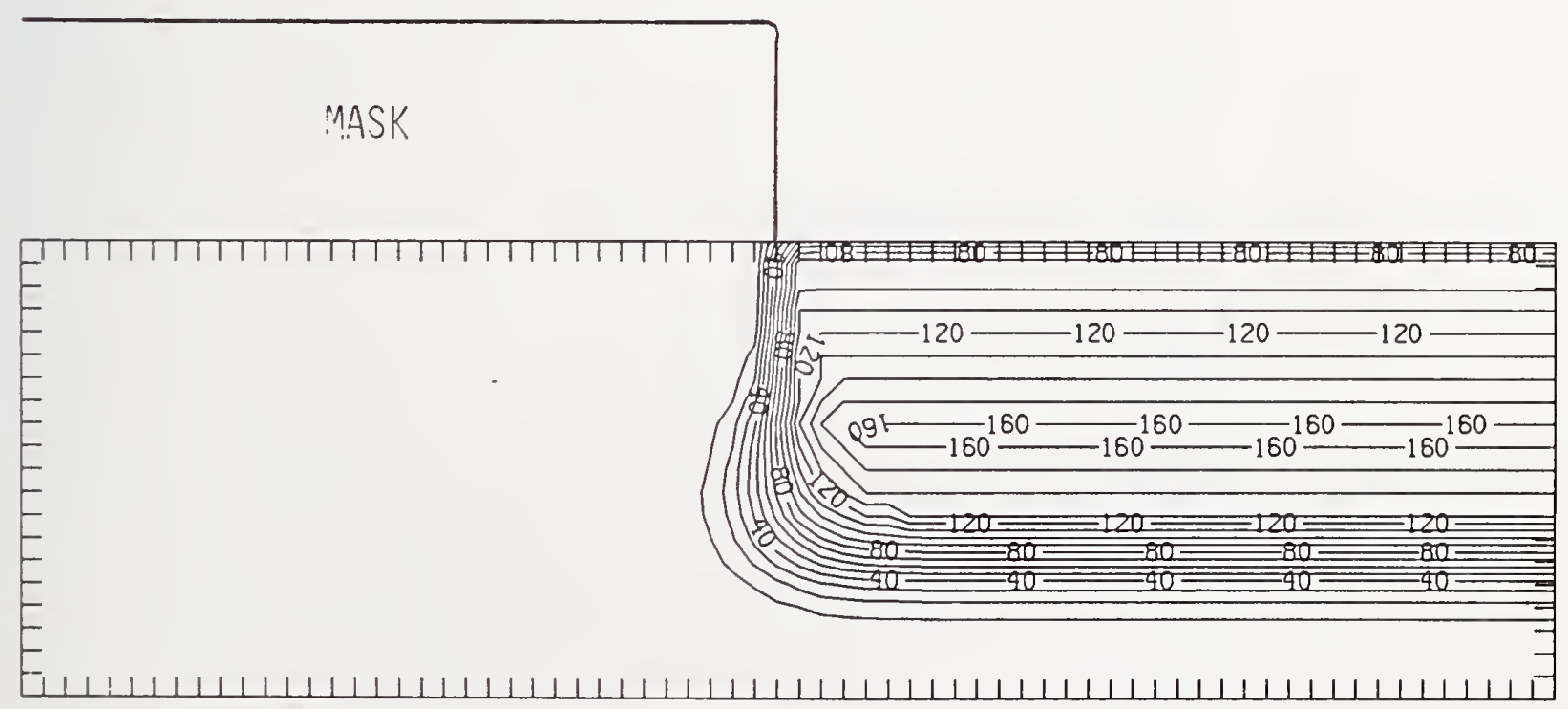

Figure S375-4 Two-dimensional distribution of Frenkel pairs created by $375 \mathrm{keV}$ sulfur implanted past a mask edge. The length increment (distance between tick marks) is $0.04 \mu \mathrm{m}$ and the scale factor is 0.1 . 


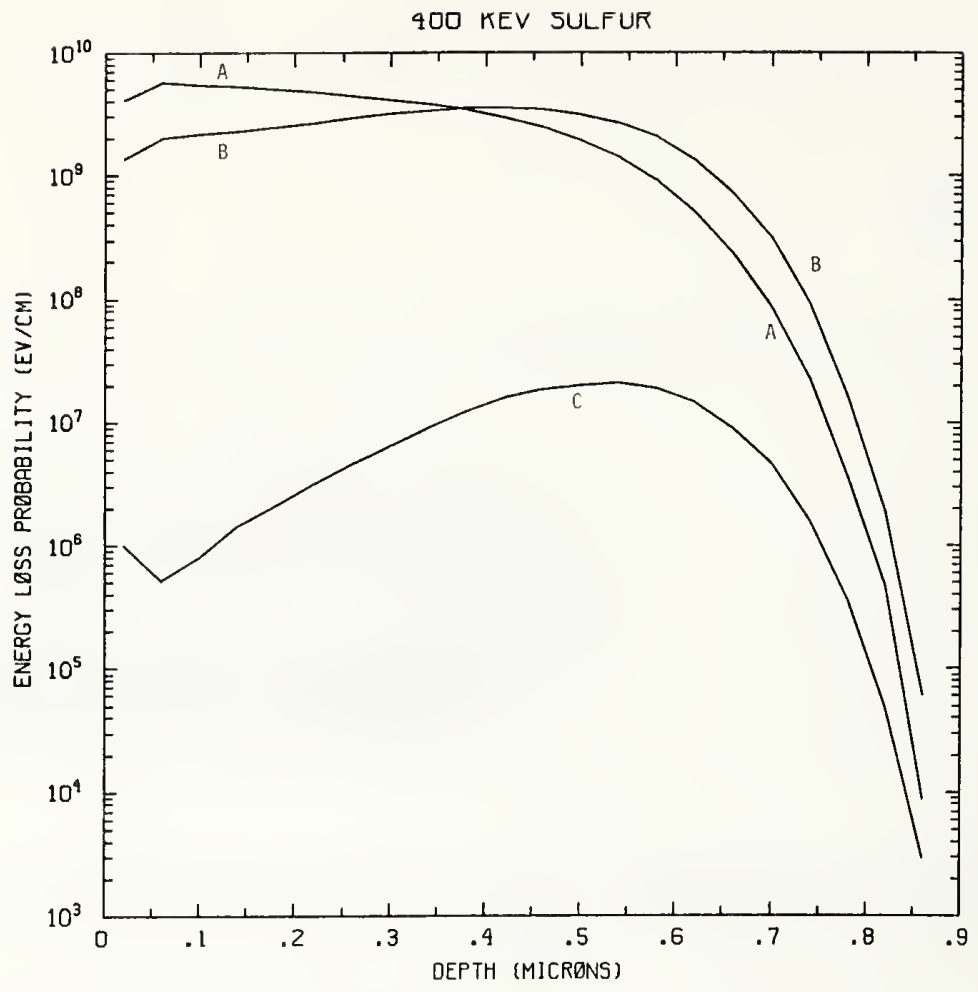

Figure S400-1 One-dimensional probability distributions for energy loss due to electronic excitation (A), displacement damage(B), and phonon excitation (C) for $400 \mathrm{keV}$ sulfur normally incident on a silicon target.

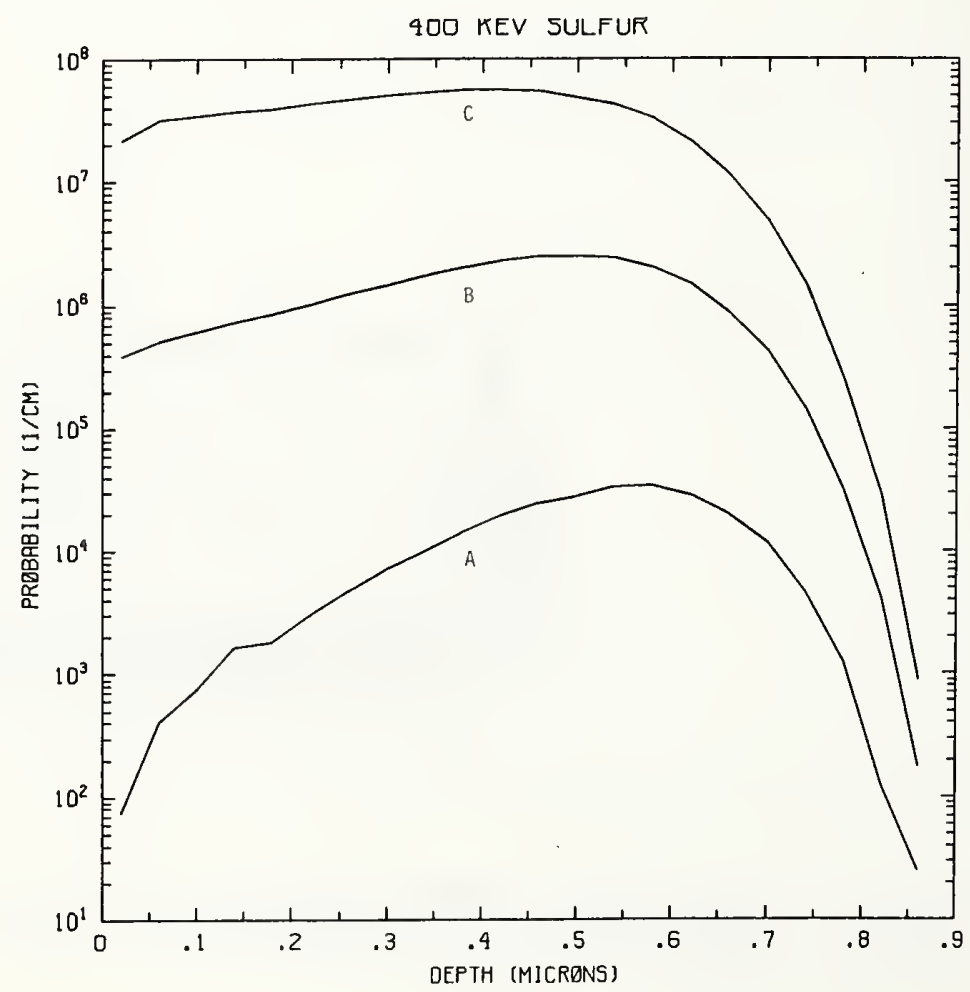

Figure S400-2 One-dimensional probability distributions for implanted sulfur (A), primary silicon displacements (B), and Frenkel pairs (C) for $400 \mathrm{keV}$ sulfur normally incident on a silicon target. 


\section{MASK}

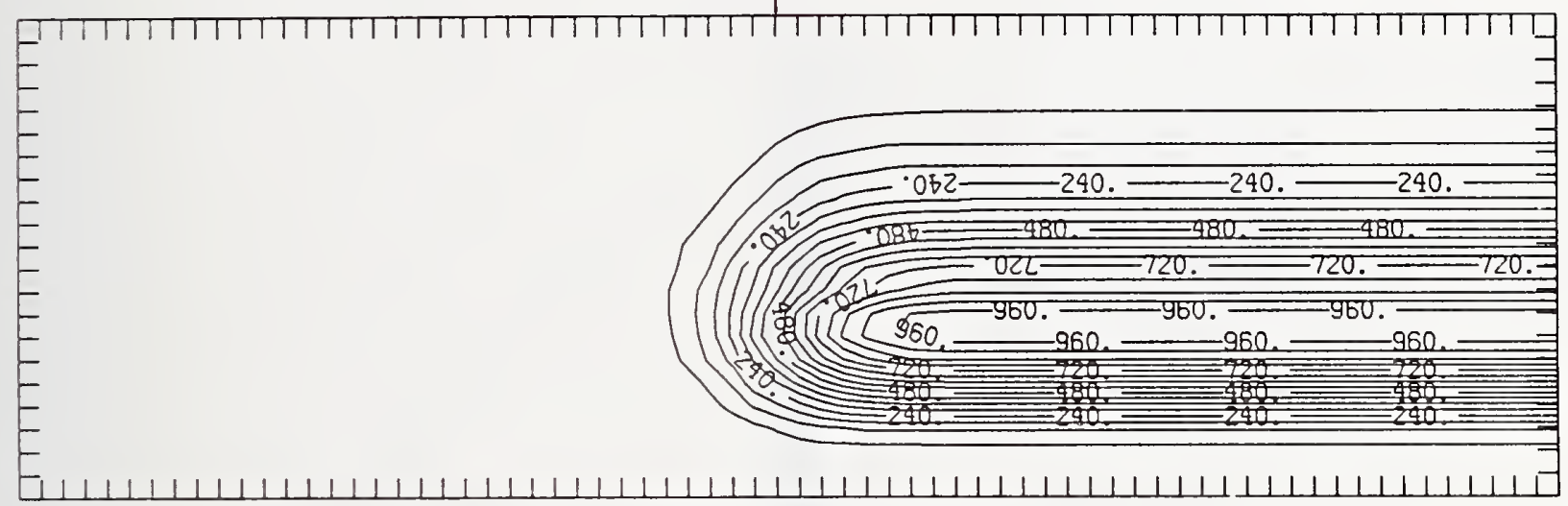

Figure S400-3 Two-dimensional distribution of $400 \mathrm{keV}$ sulfur implanted past a mask edge. The length increment (distance between tick marks) is $0.04 \mu \mathrm{m}$ and the scale factor is 1000 .

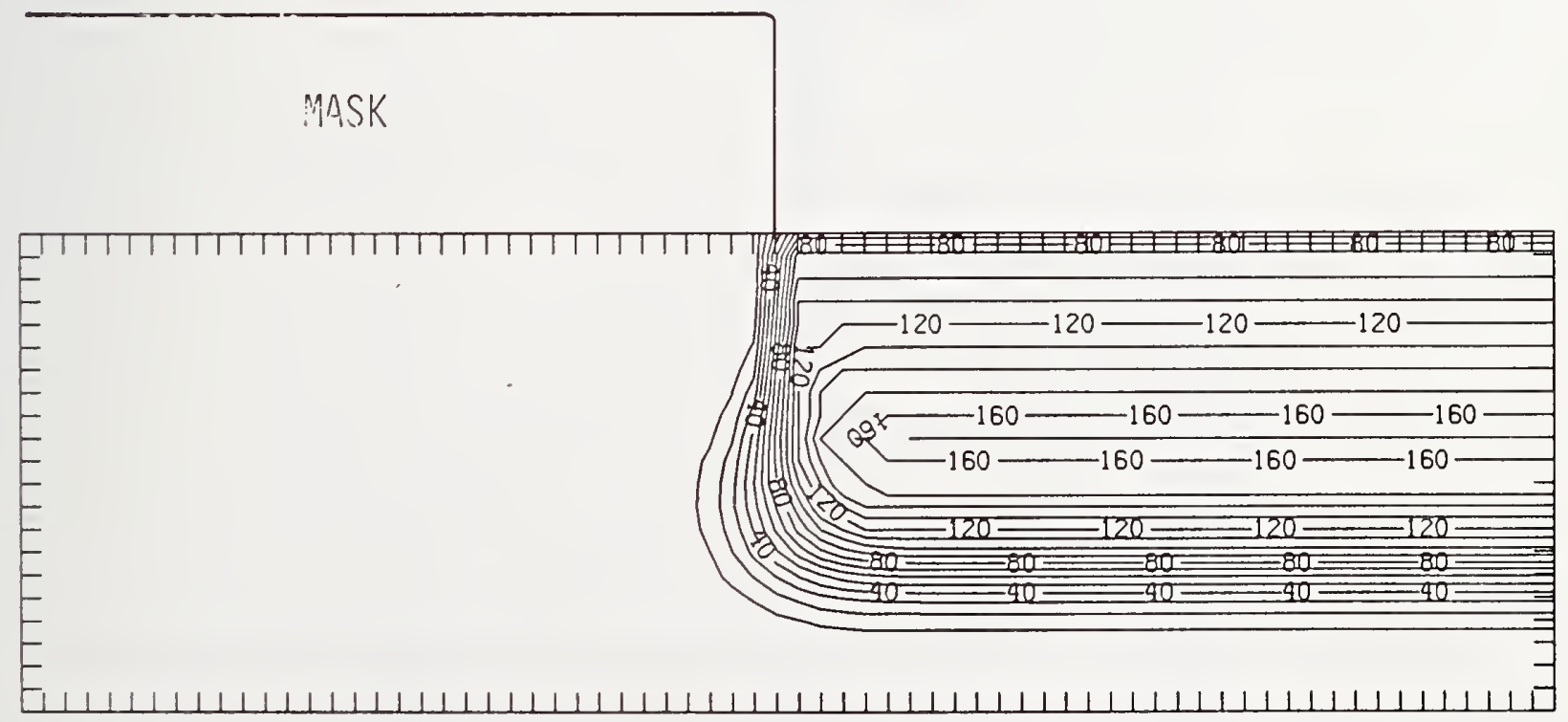

Figure S400-4 Two-dimensional distribution of Frenkel pairs created by $400 \mathrm{keV}$ sulfur implanted past a mask edge. The length increment (distance between tick marks) is $0.04 \mu \mathrm{m}$ and the scale factor is 0.1 . 


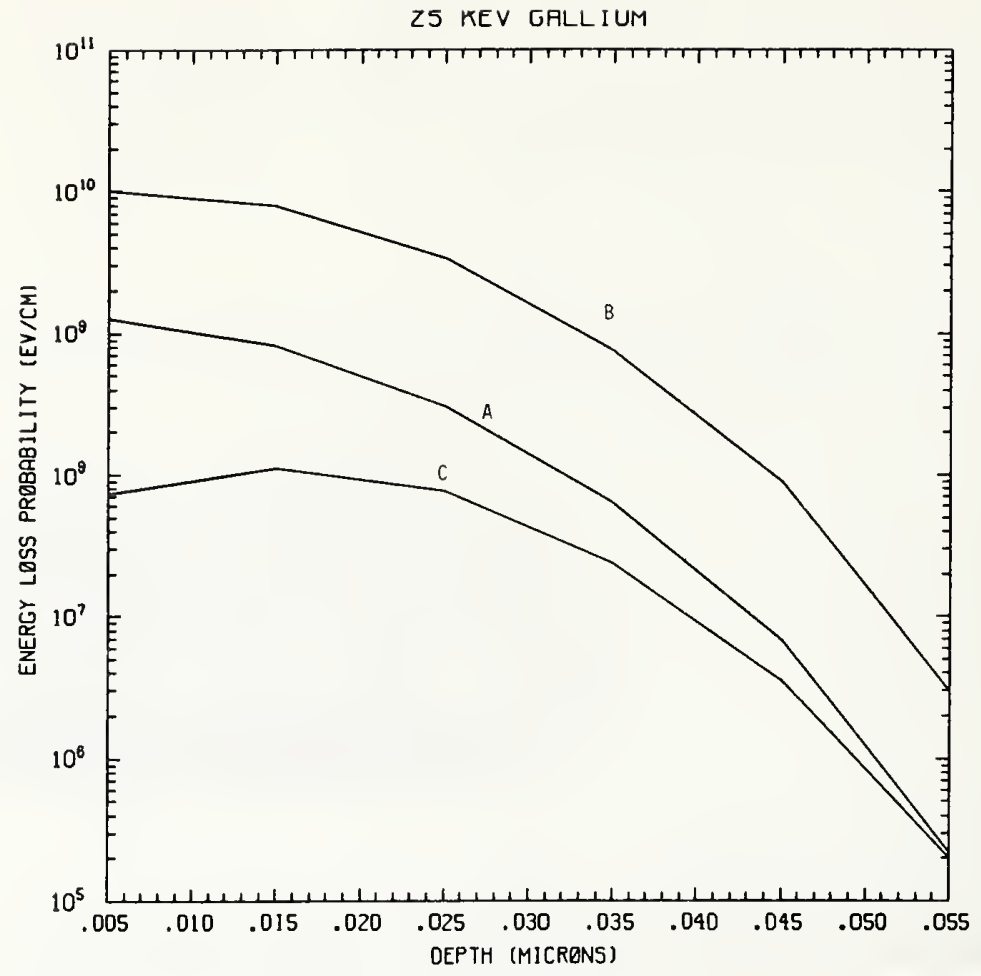

Figure Ga25-1 One-dimensional probability distributions for energy loss due to electronic excitation (A), displacement damage(B), and phonon excitation (C) for $25 \mathrm{keV}$ gallium normally incident on a silicon target.

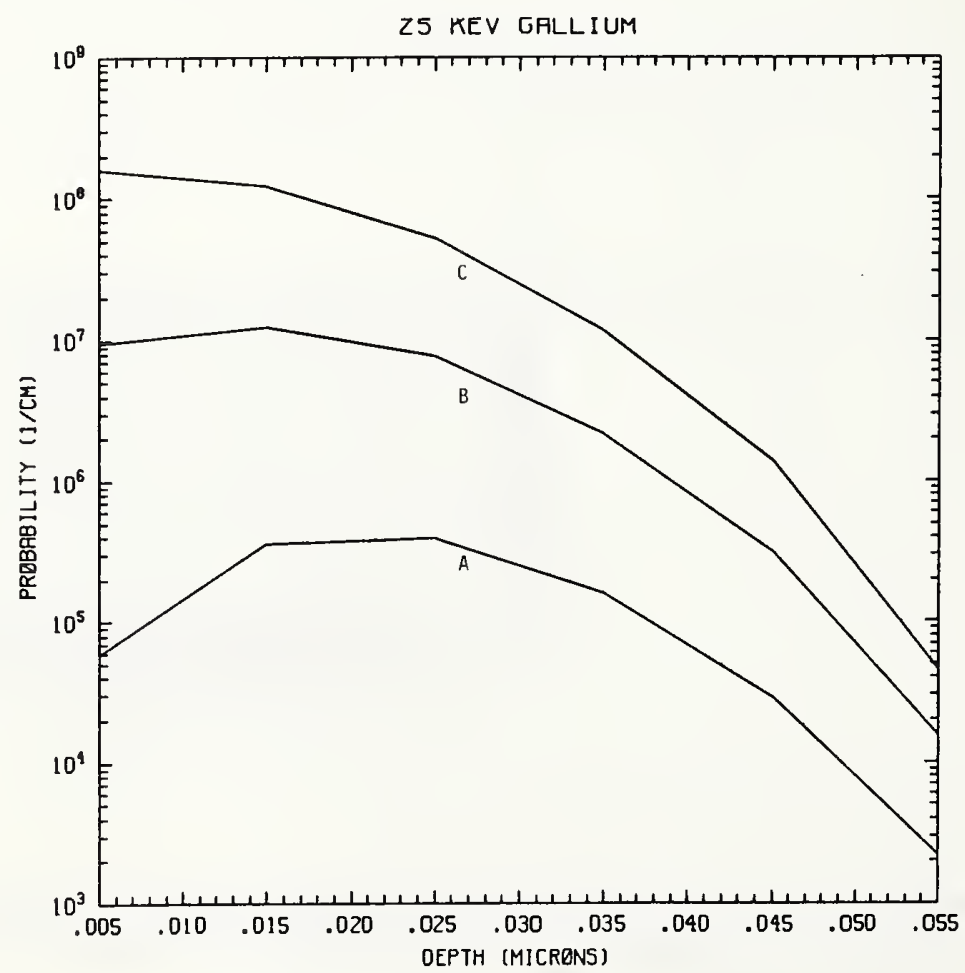

Figure Ga25-2 One-dimensional probability distributions for implanted gallium (A), primary silicon displacements (B), and Frenkel pairs (C) for $25 \mathrm{keV}$ gallium normally incident on a silicon target. 


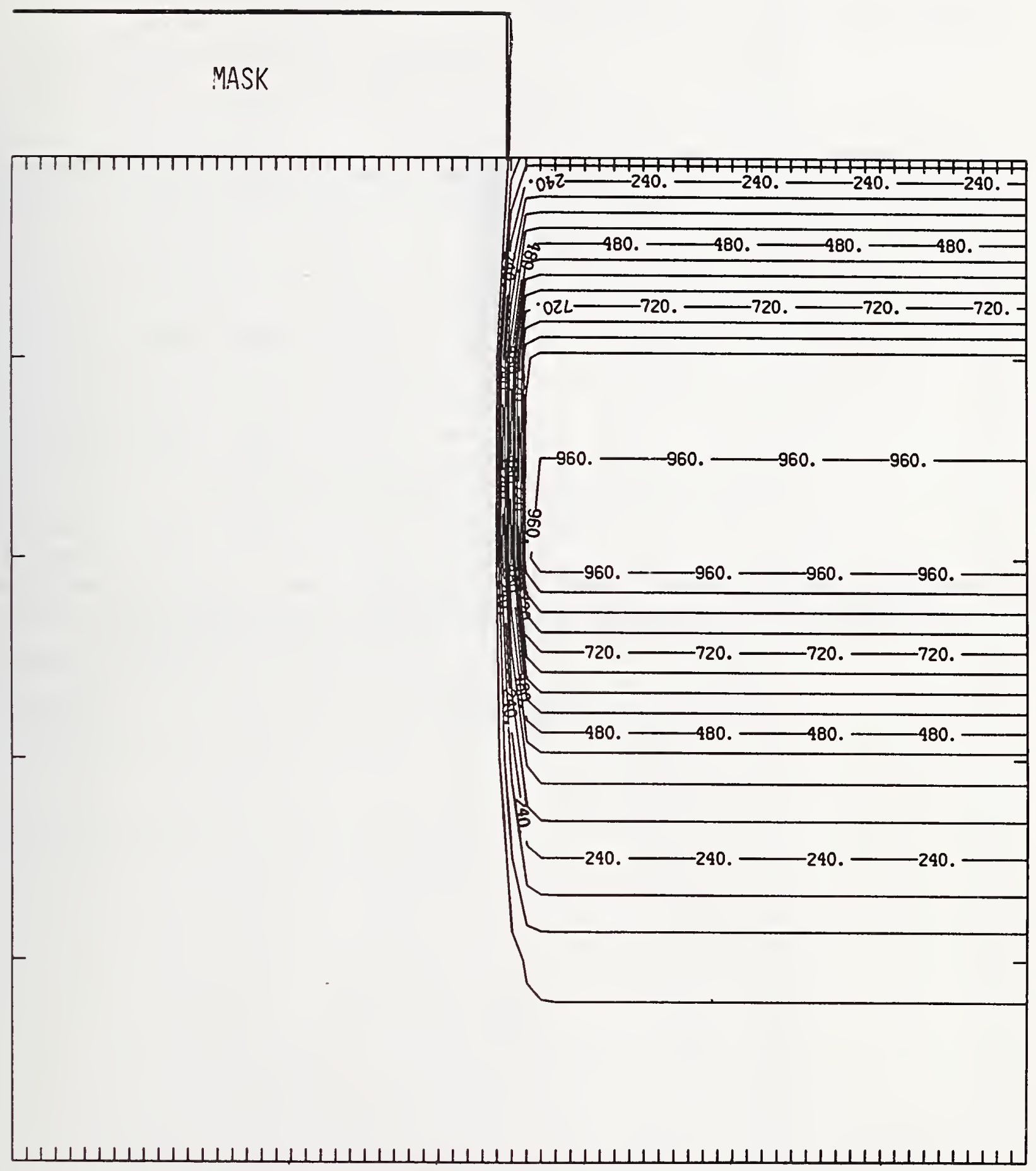

Figure Ga25-3 Two-dimensional distribution of $25 \mathrm{keV}$ gallium implanted past a mask edge. The length increment (distance between tick marks) is $0.01 \mu \mathrm{m}$ and the scale factor is 1000. 


\section{MASK}

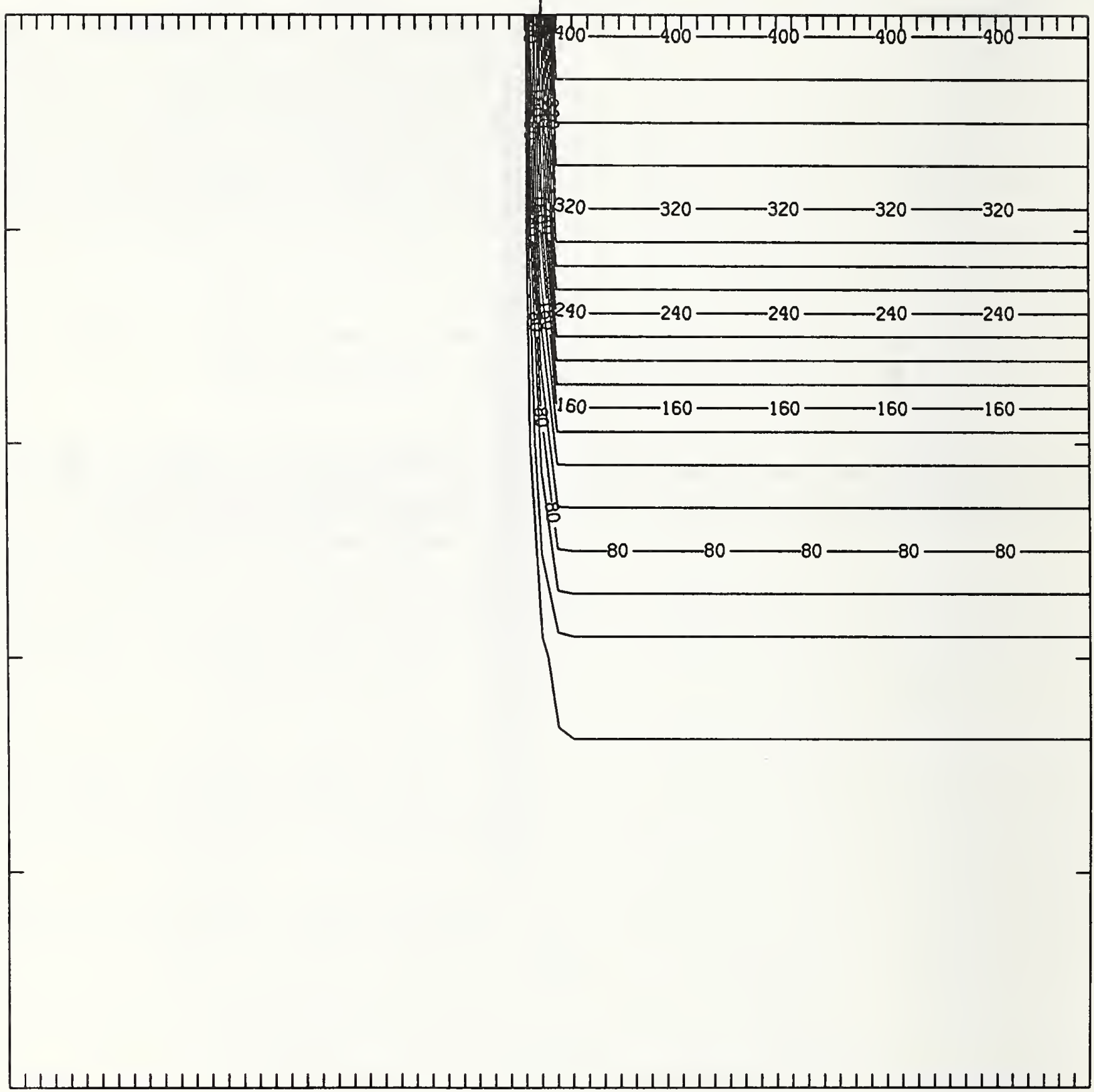

Figure Ga25-4 Two-dimensional distribution of Frenkel pairs created by $25 \mathrm{keV}$ gallium implanted past a mask edge. The length increment (distance between tick marks) is $0.01 \mu \mathrm{m}$ and the scale factor is 1. 


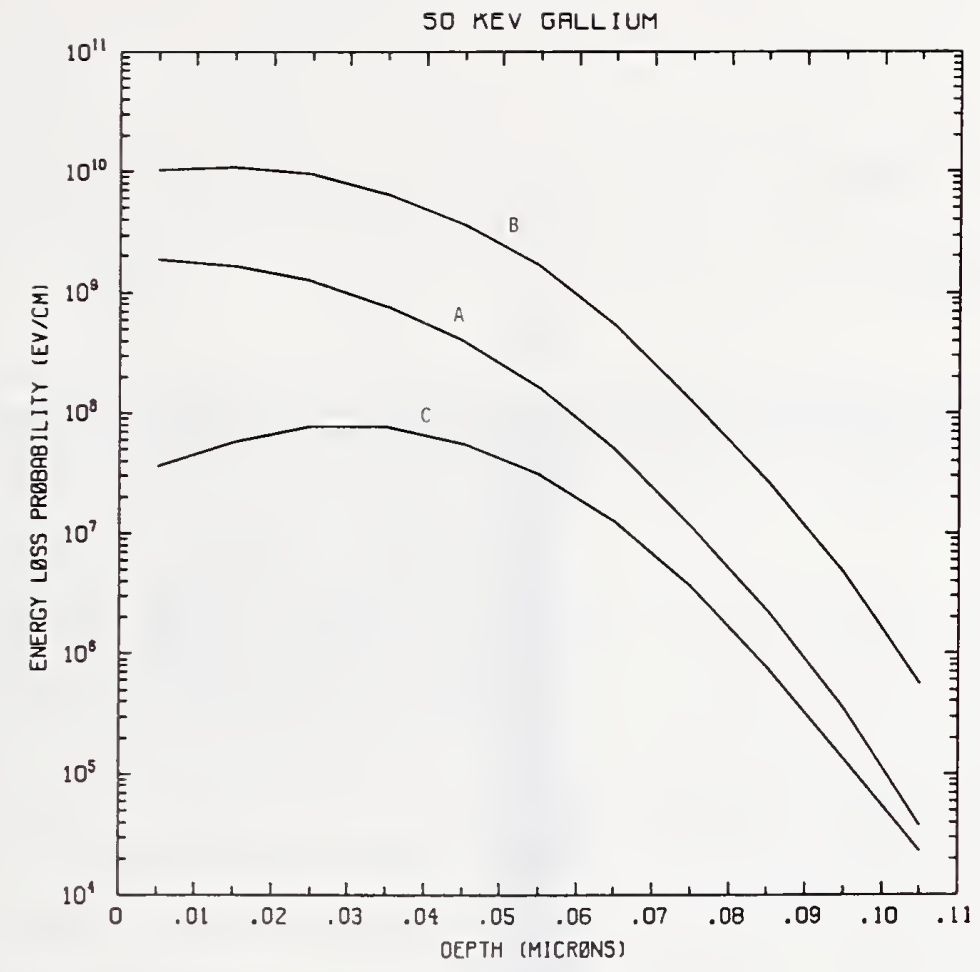

Figure Ga50-1 One-dimensional probability distributions for energy loss due to electronic excitation (A), displacement damage(B), and phonon excitation (C) for $50 \mathrm{keV}$ gallium normally incident on a silicon target.

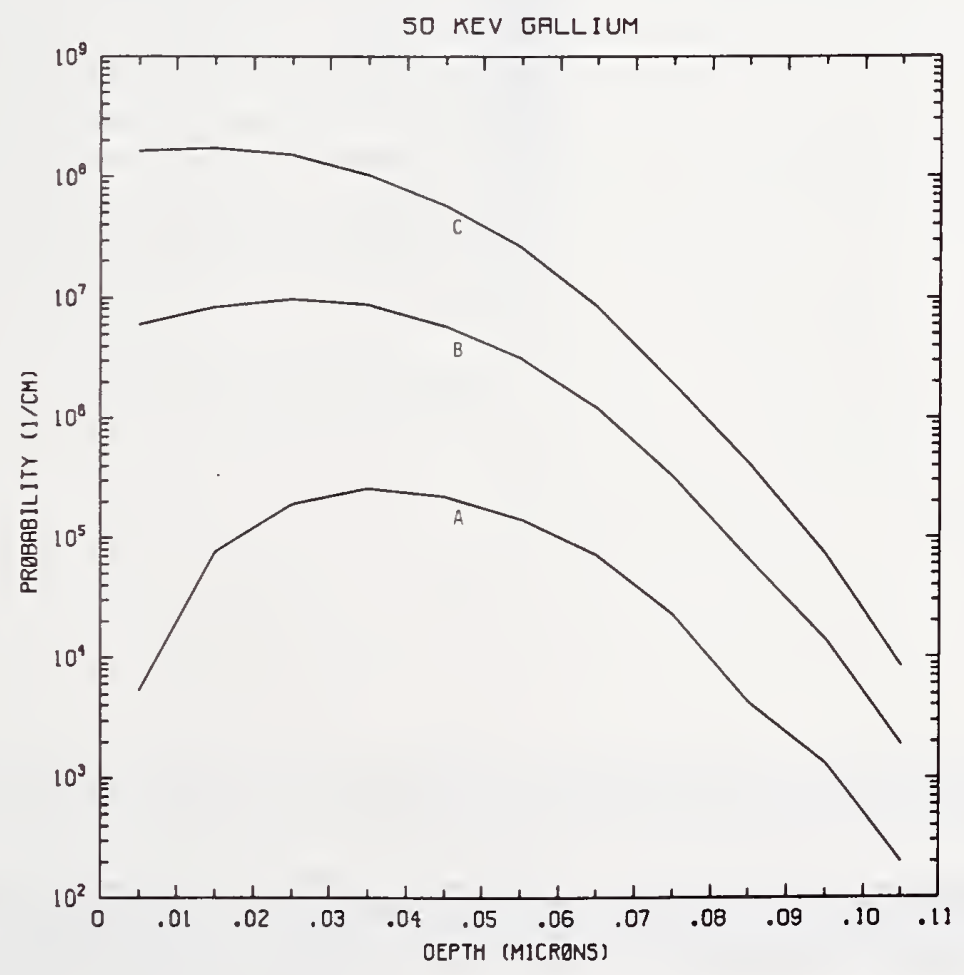

Figure Ga50-2 One-dimensional probability distributions for implanted gallium (A), primary silicon displacements (B), and Frenkel pairs (C) for $50 \mathrm{keV}$ gallium normally incident on a silicon target. 


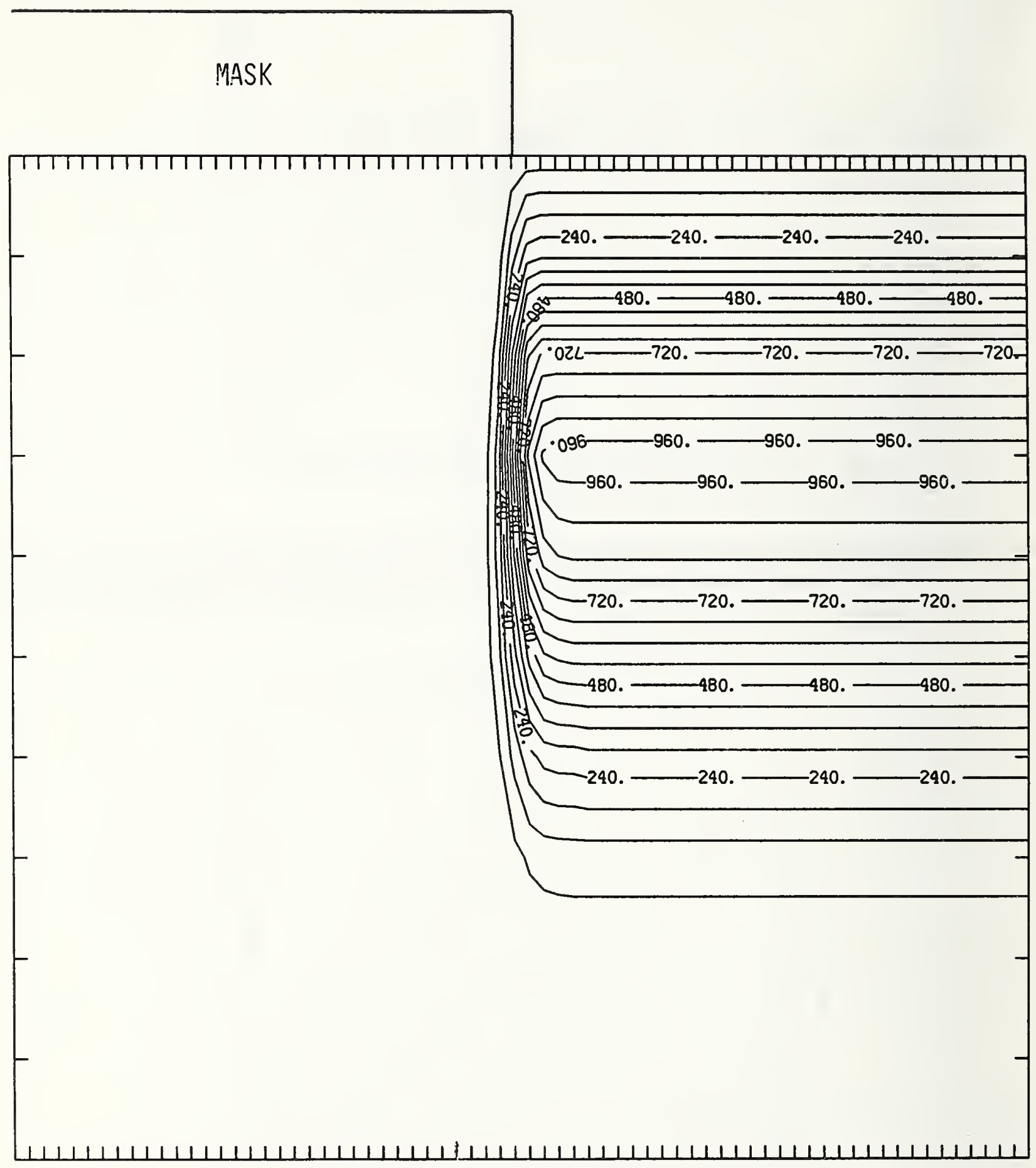

Figure Ga50-3 Two-dimensional distribution of $50 \mathrm{keV}$ gallium implanted past a mask edge. The length increment (distance between tick marks) is $0.01 \mu \mathrm{m}$ and the scale factor is 1000. 


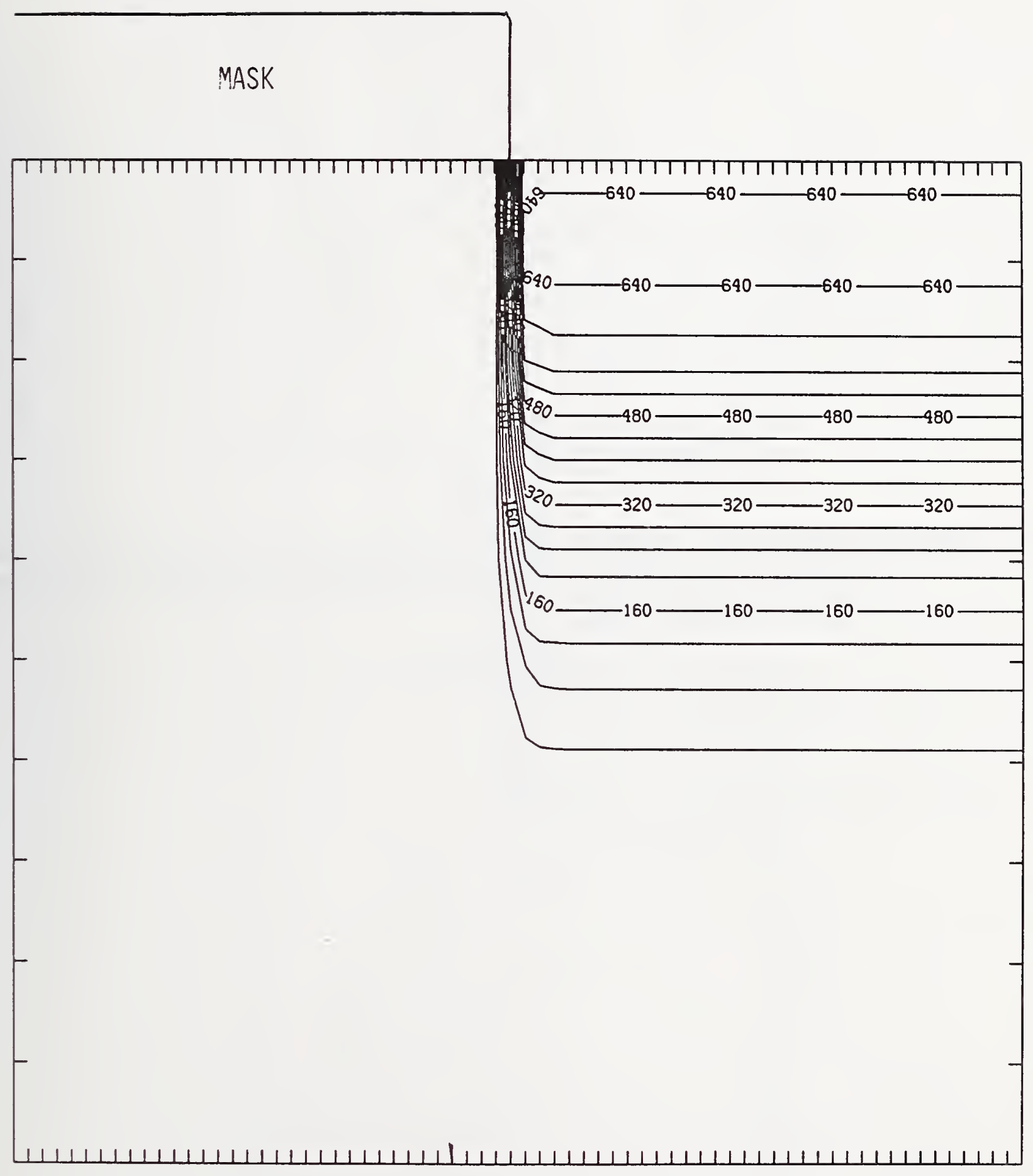

Figure Ga50-4 Two-dimensional distribution of Frenkel pairs created by $50 \mathrm{keV}$ gallium implanted past a mask edge. The length increment (distance between tick marks) is $0.01 \mu \mathrm{m}$ and the scale factor is 1 . 


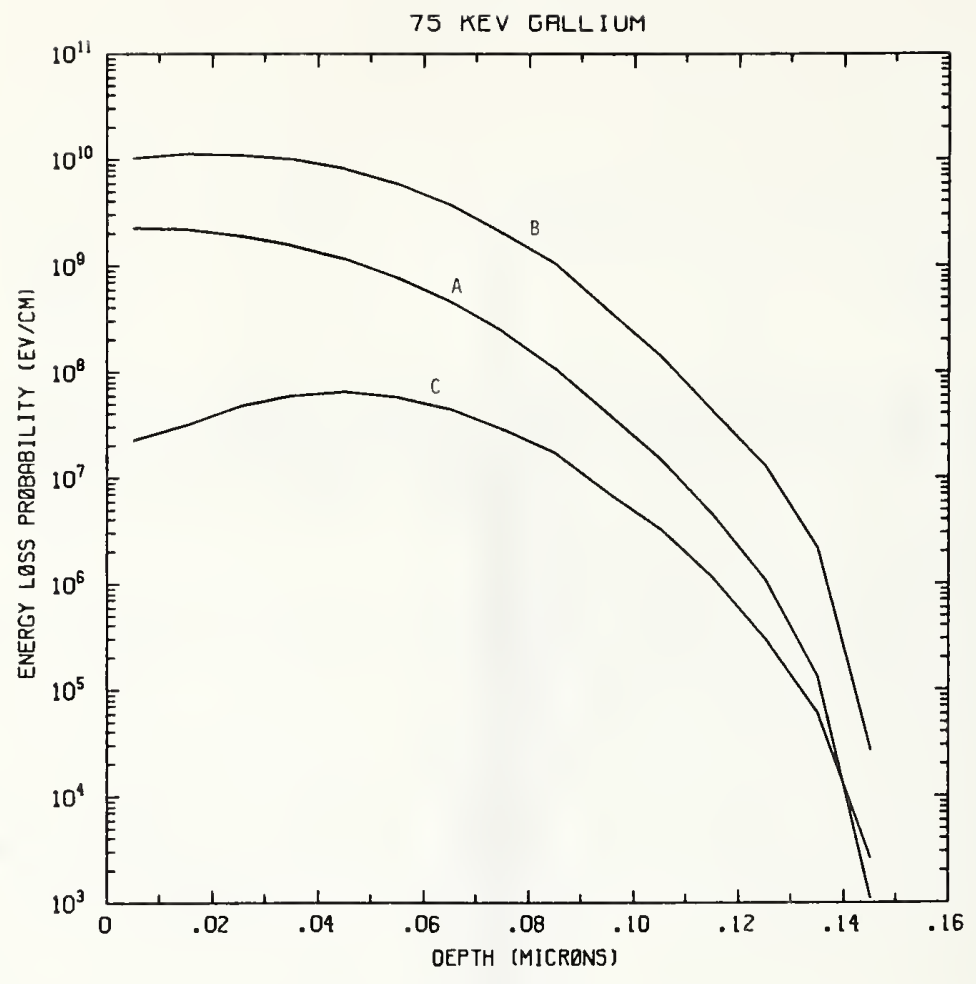

Figure Ga75-1 One-dimensional probability distributions for energy loss due to electronic excitation (A), displacement damage(B), and phonon excitation (C) for $75 \mathrm{keV}$ gallium normally incident on a silicon target.

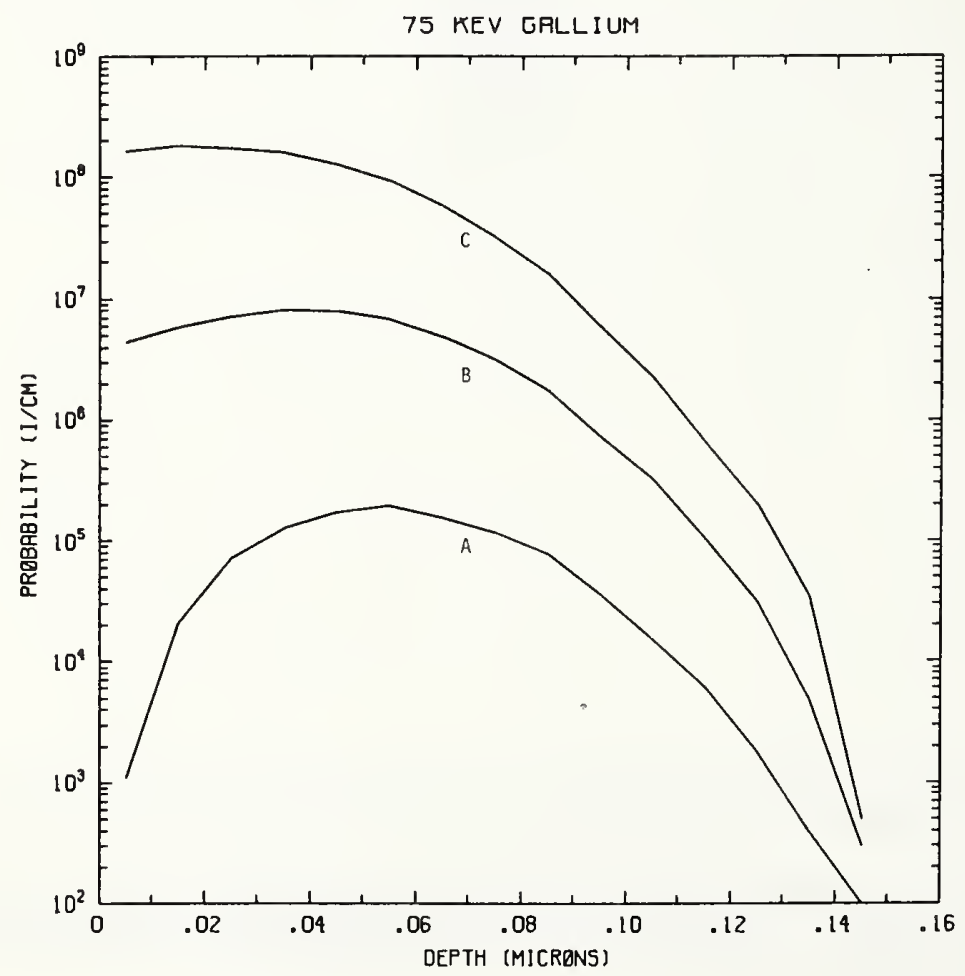

Figure Ga75-2 One-dimensional probability distributions for implanted gallium (A), primary silicon displacements (B), and Frenkel pairs (C) for $75 \mathrm{keV}$ gallium normally incident on a silicon target. 


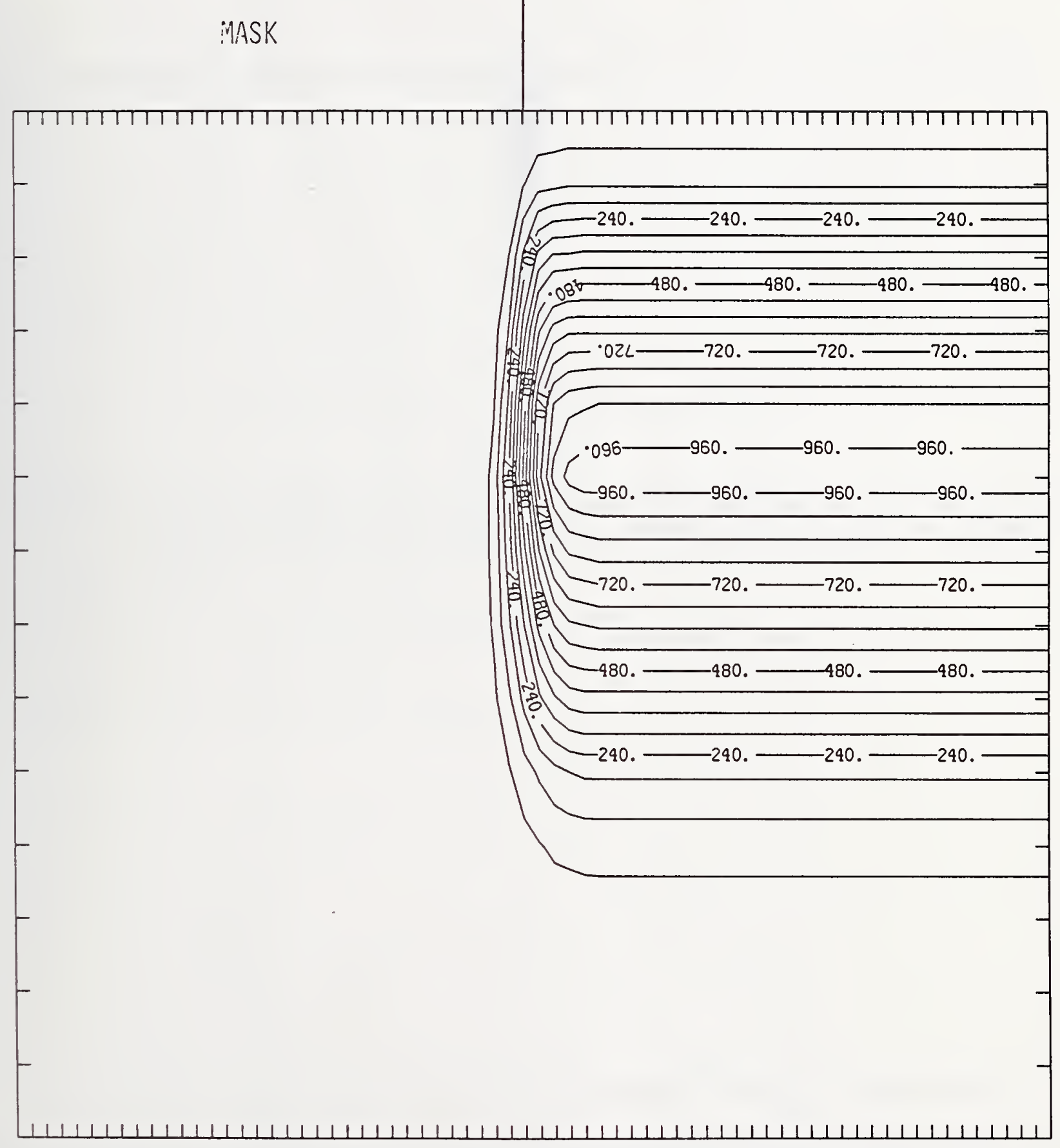

Figure Ga75-3 Two-dimensional distribution of $75 \mathrm{keV}$ gallium implanted past a mask edge. The length increment (distance between tick marks) is $0.01 \mu \mathrm{m}$ and the scale factor is 1000 . 


\section{MASK}

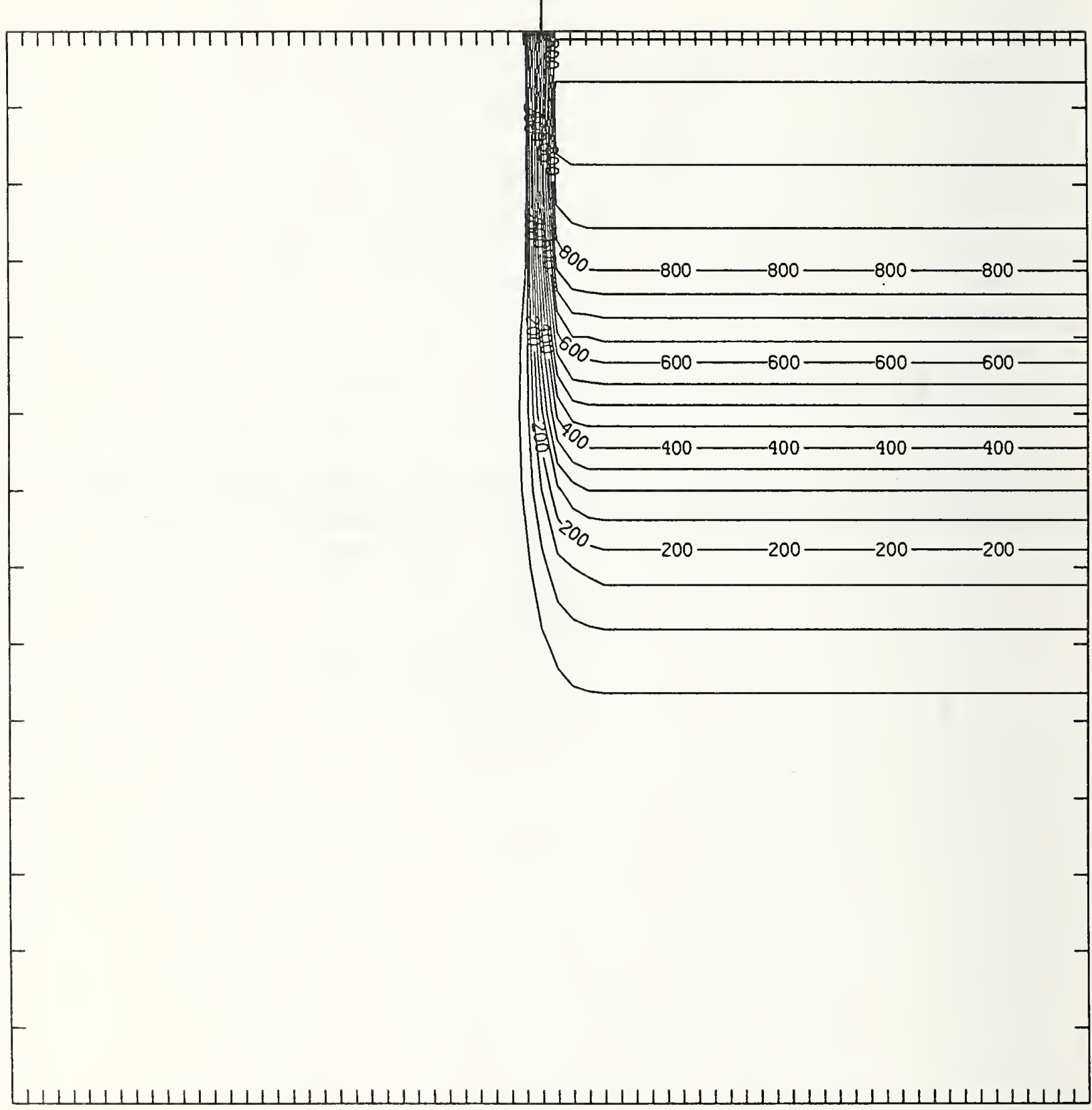

Figure Ga75-4 Two-dimensional distribution of Frenkel pairs created by $75 \mathrm{keV}$ gallium implanted past a mask edge. The length increment (distance between tick marks) is $0.01 \mu \mathrm{m}$ and the scale factor is 1 . 


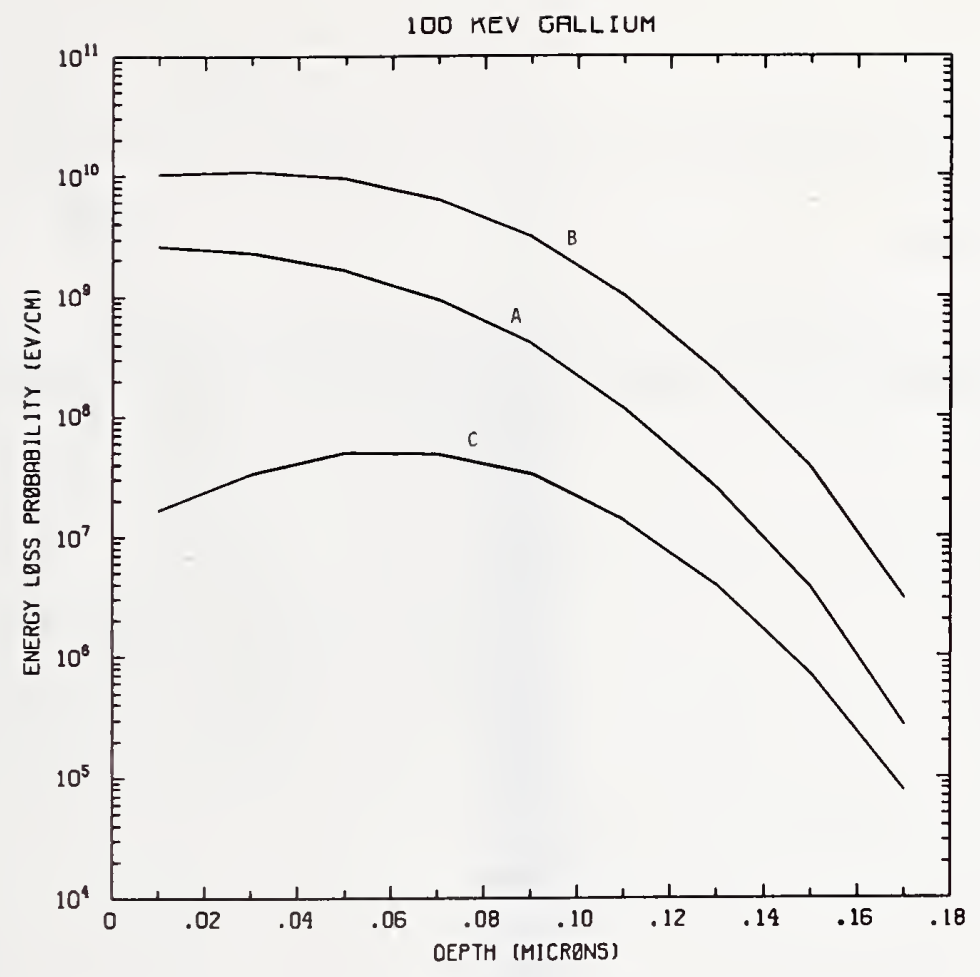

Figure Ga100-1 One-dimensional probability distributions for energy loss due to electronic excitation (A), displacement damage(B), and phonon excitation (C) for $100 \mathrm{keV}$ gallium normally incident on a silicon target.

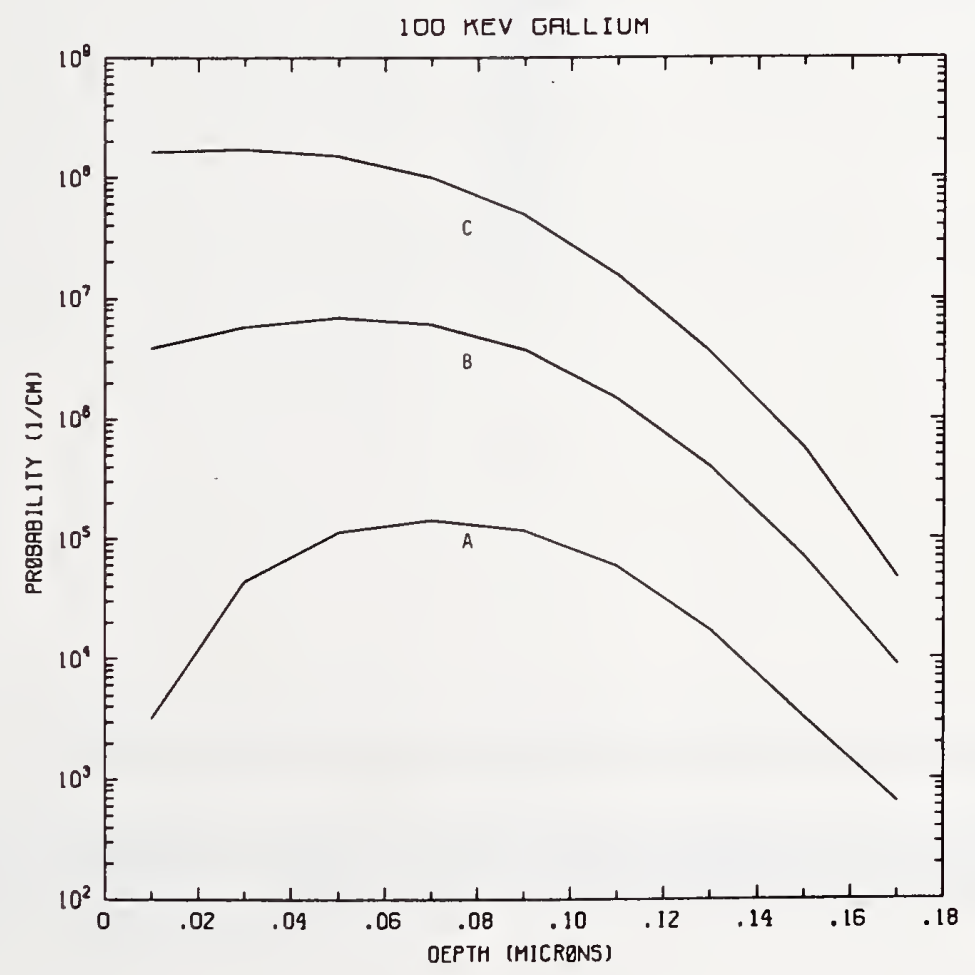

Figure Ga100-2 One-dimensional probability distributions for implanted gallium (A), primary silicon displacements (B), and Frenkel pairs (C) for $100 \mathrm{keV}$ gallium normally incident on a silicon target. 


\section{M.ASK}

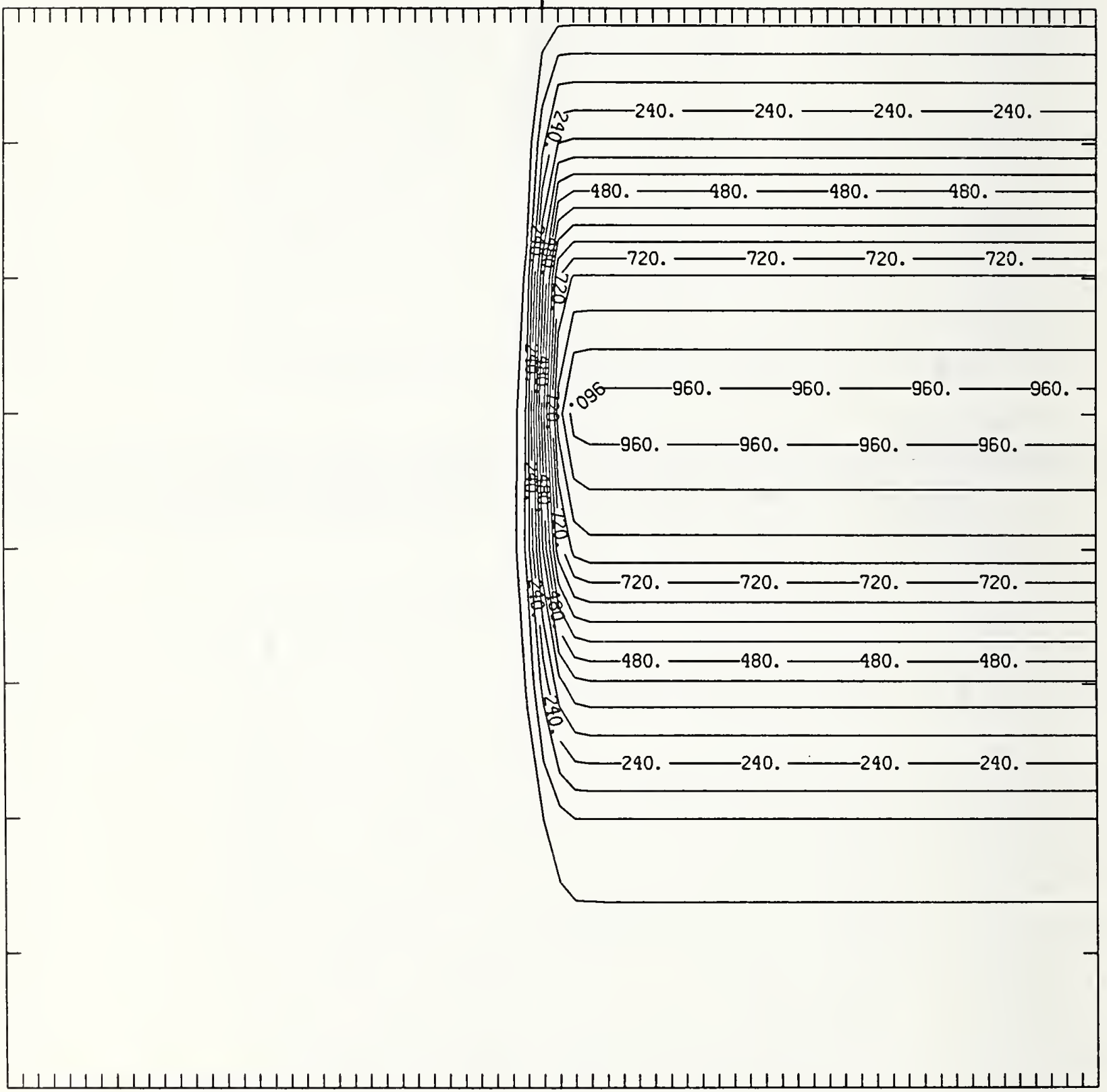

Figure Ga100-3 Two-dimensional distribution of $100 \mathrm{keV}$ gallium implanted past a mask edge. The length increment (distance between tick marks) is $0.02 \mu \mathrm{m}$ and the scale factor is 1000 . 


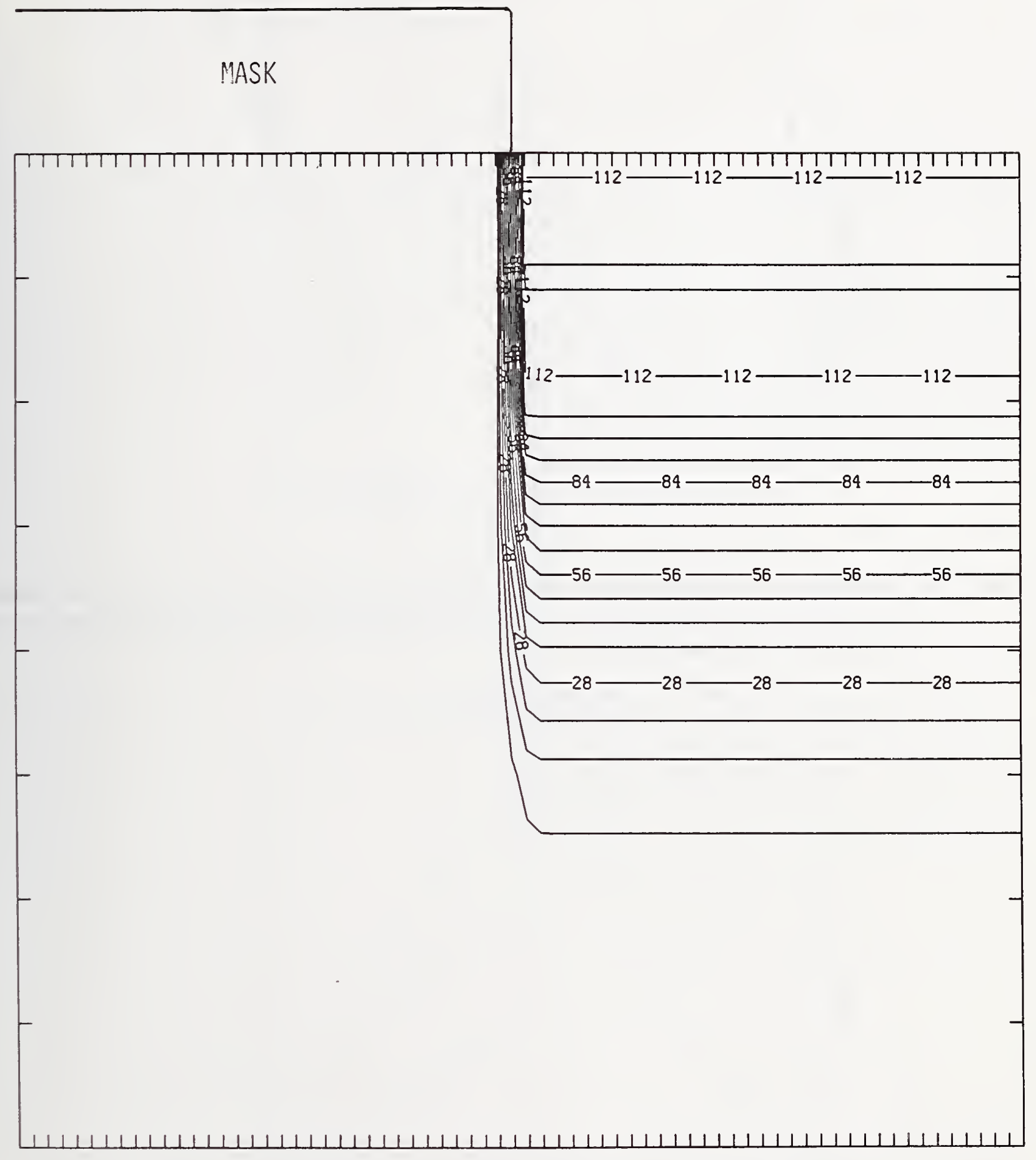

Figure Ga100-4 Two-dimensional distribution of Frenkel pairs created by $100 \mathrm{keV}$ gallium implanted past a mask edge. The length increment (distance between tick marks) is $0.02 \mu \mathrm{m}$ and the scale factor is 0.1 . 


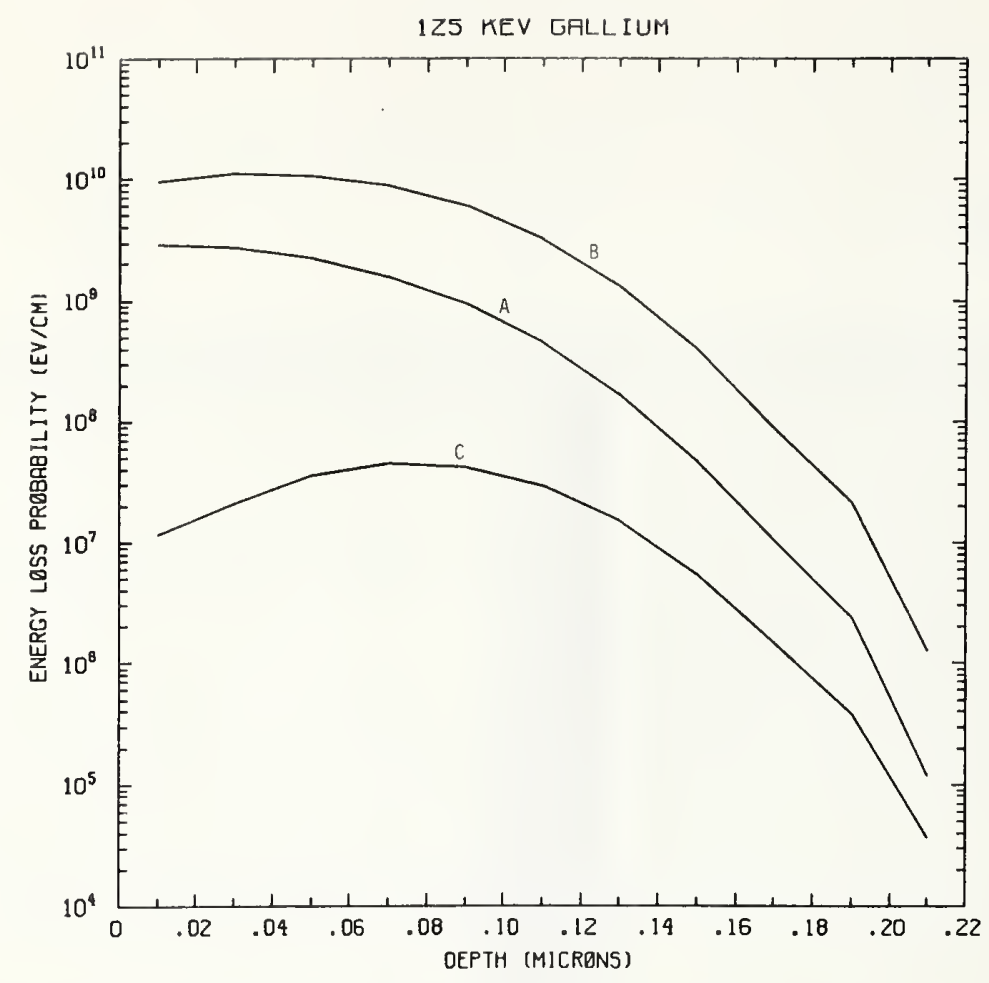

Figure Ga125-1 One-dimensional probability distributions for energy loss due to electronic excitation (A), displacement damage(B), and phonon excitation (C) for $125 \mathrm{keV}$ gallium normally incident on a silicon target.

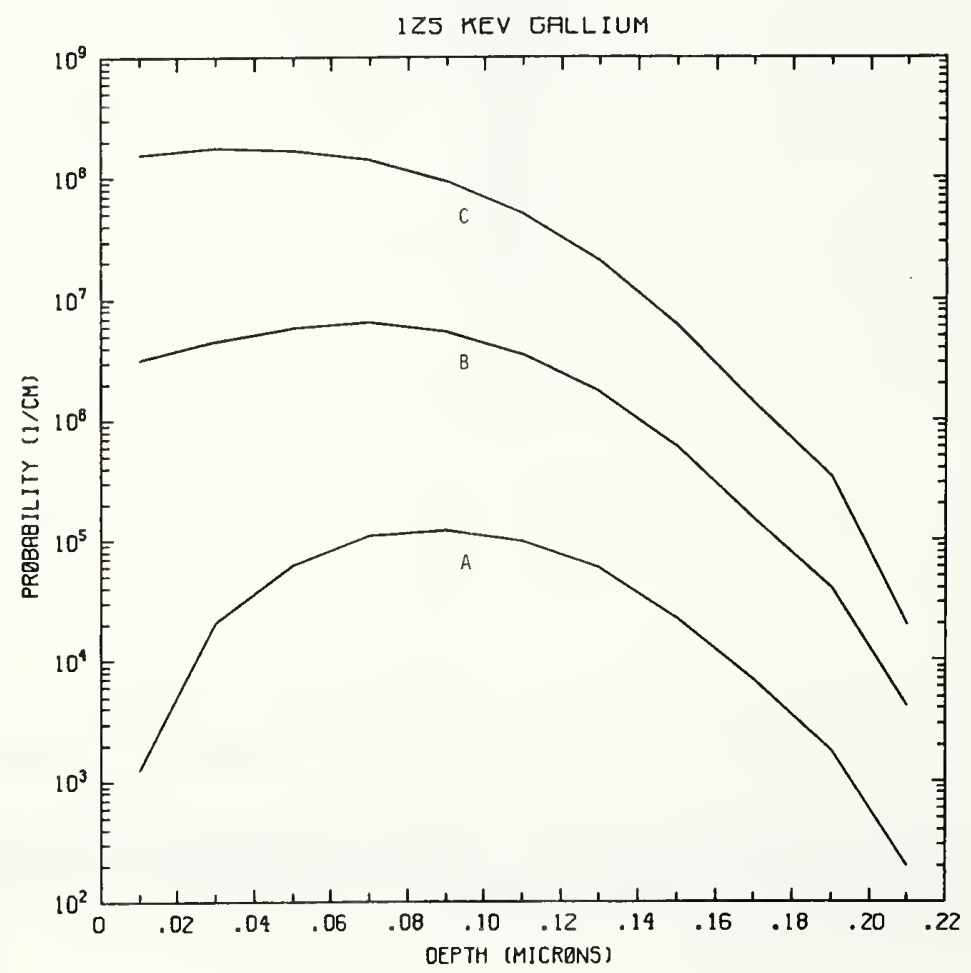

Figure Ga125-2 One-dimensional probability distributions for implanted gallium (A), primary silicon displacements (B), and Frenkel pairs (C) for $125 \mathrm{keV}$ gallium normally incident on a silicon target. 


\section{M.ASK}

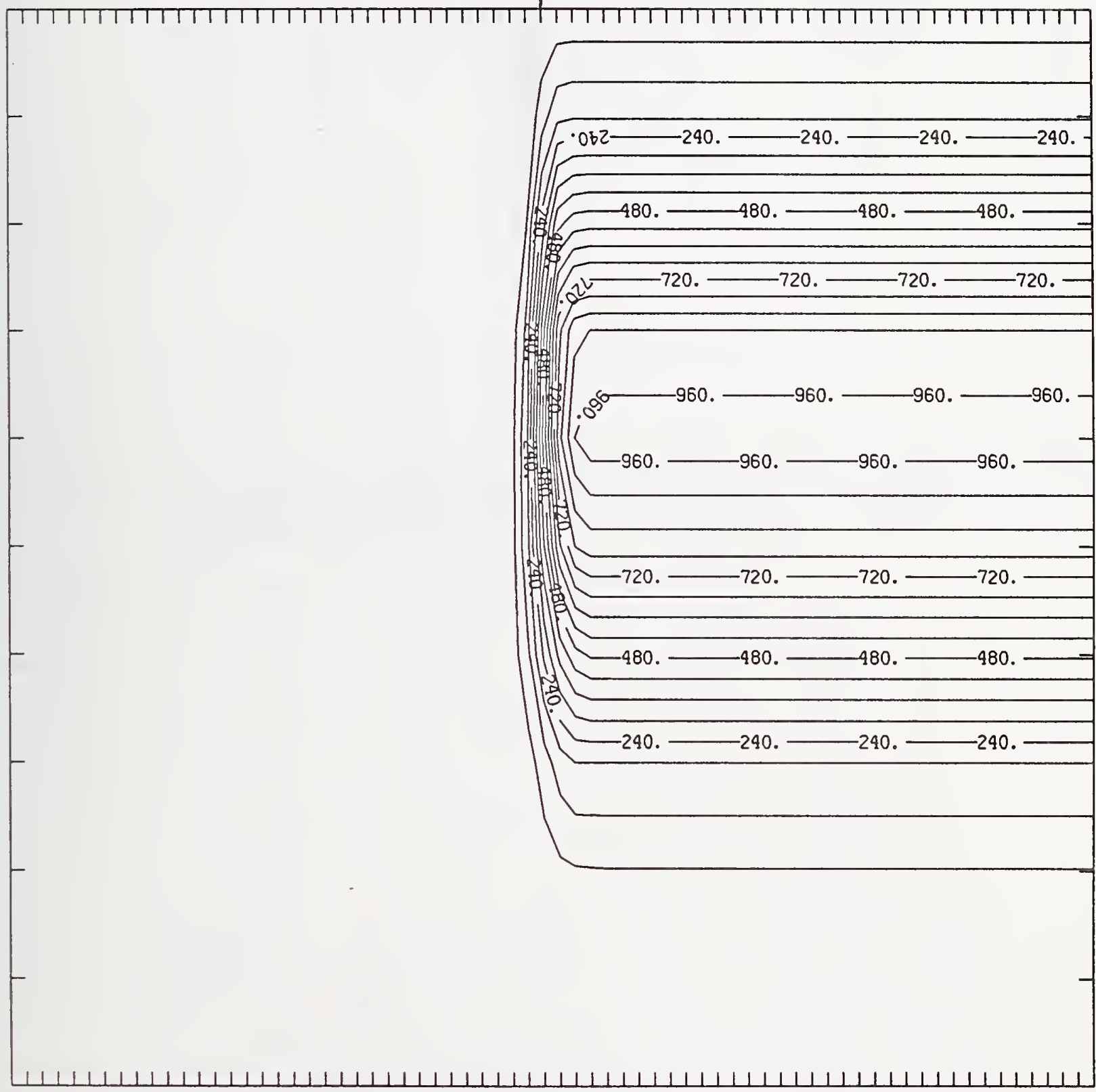

Figure Ga125-3 Two-dimensional distribution of $125 \mathrm{keV}$ gallium implanted past a mask edge. The length increment (distance between tick marks) is $0.02 \mu \mathrm{m}$ and the scale factor is 1000 . 


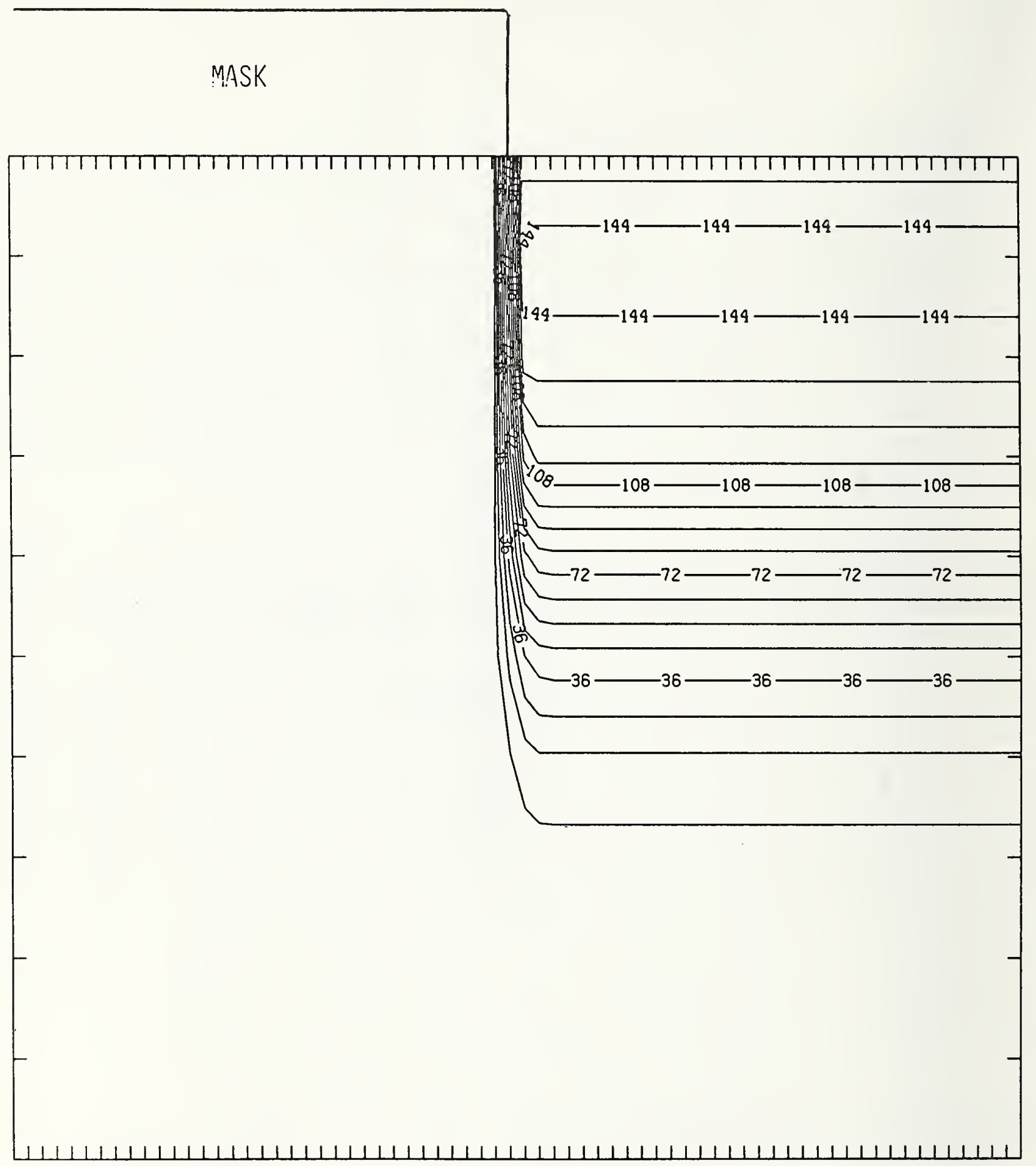

Figure Ga125-4 Two-dimensional distribution of Frenkel pairs created by $125 \mathrm{keV}$ gallium implanted past a mask edge. The length increment (distance between tick marks) is $0.02 \mu \mathrm{m}$ and the scale factor is 0.1 . 


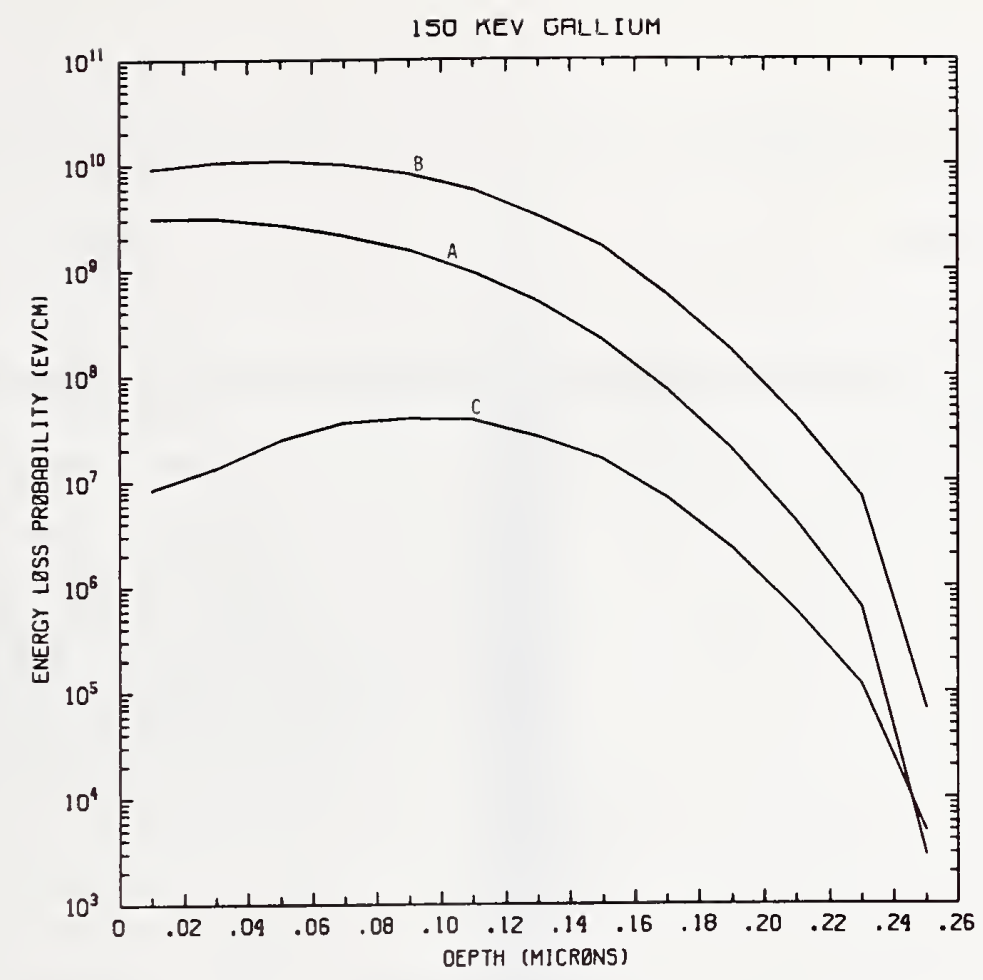

Figure Ga150-1 One-dimensional probability distributions for energy loss due to electronic excitation (A), displacement damage(B), and phonon excitation (C) for $150 \mathrm{keV}$ gallium normally incident on a silicon target.

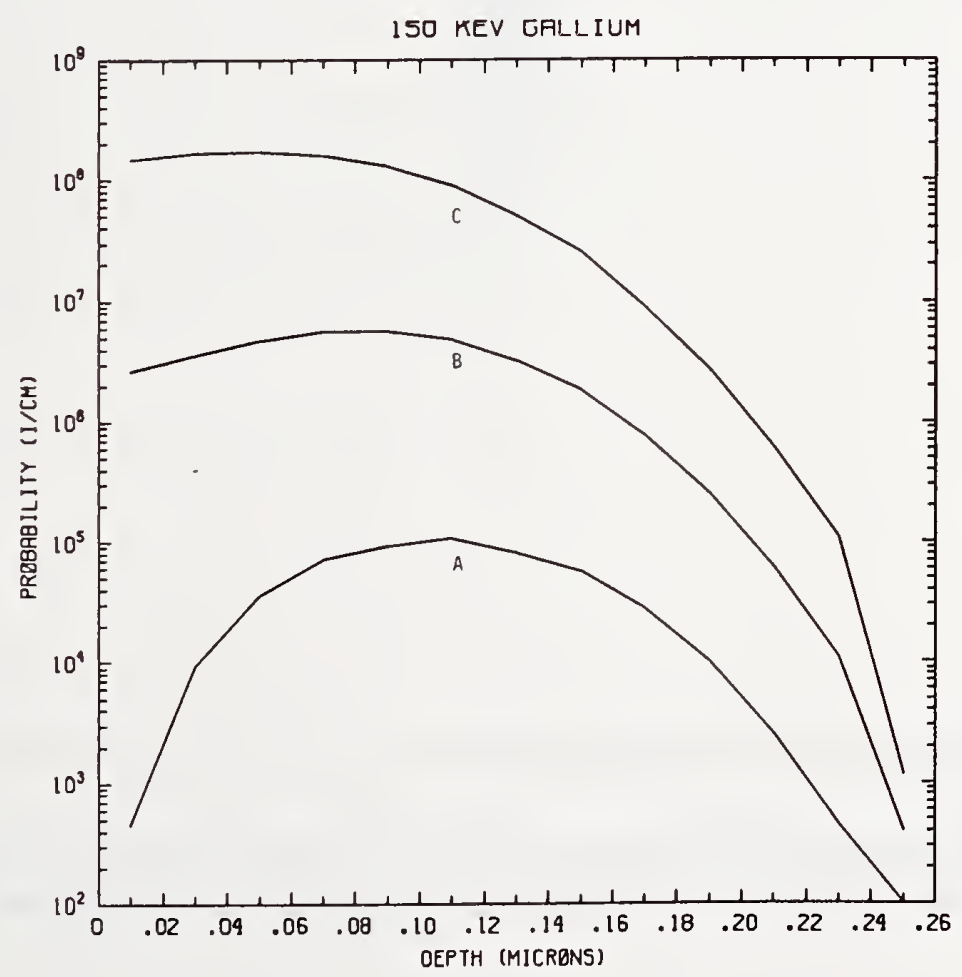

Figure Ga150-2 One-dimensional probability distributions for implanted gallium (A), primary silicon displacements (B), and Frenkel pairs (C) for $150 \mathrm{keV}$ gallium normally incident on a silicon target. 


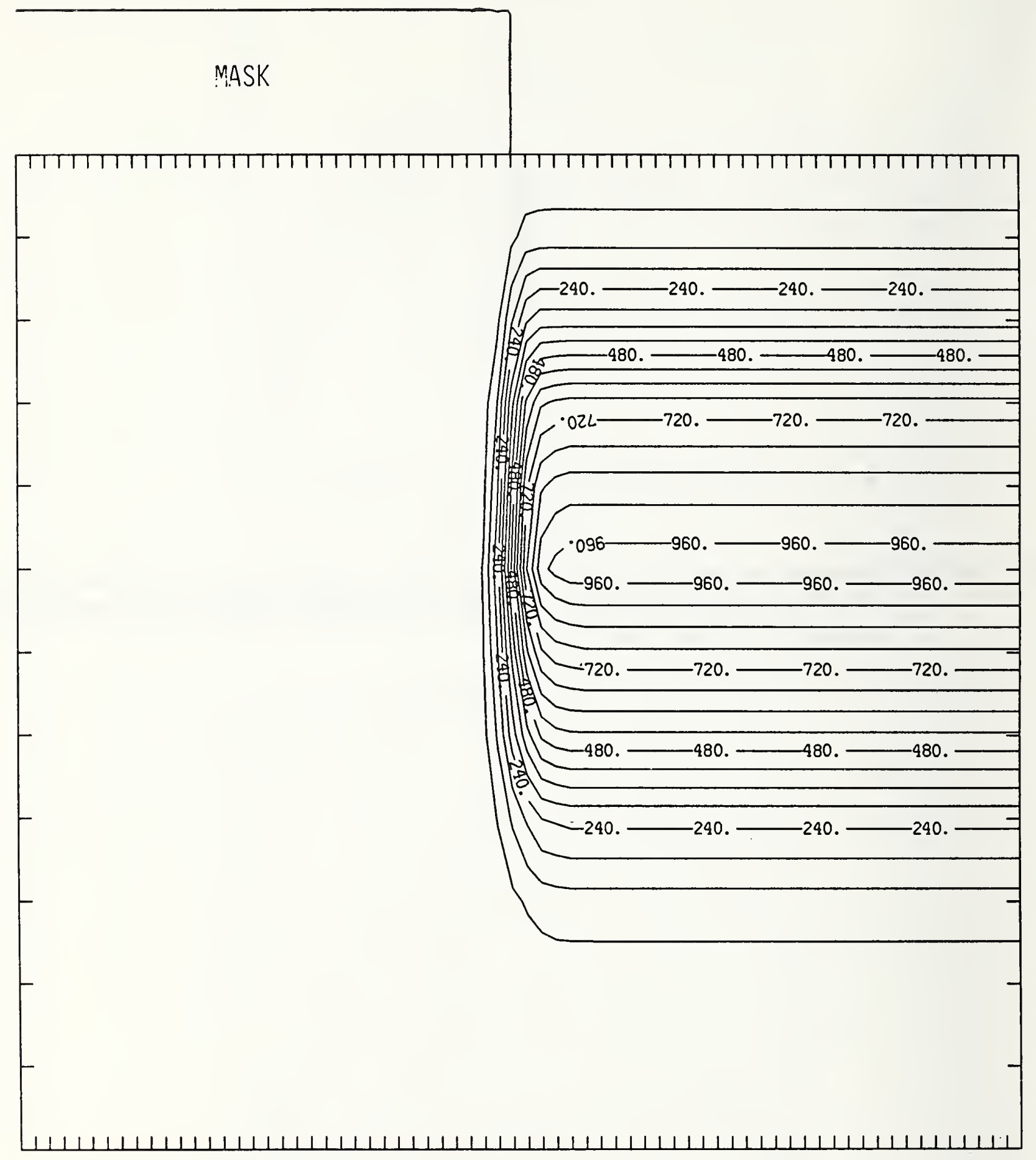

Figure Ga150-3 Two-dimensional distribution of $150 \mathrm{keV}$ gallium implanted past a mask edge. The length increment (distance between tick marks) is $0.02 \mu \mathrm{m}$ and the scale factor is 1000. 


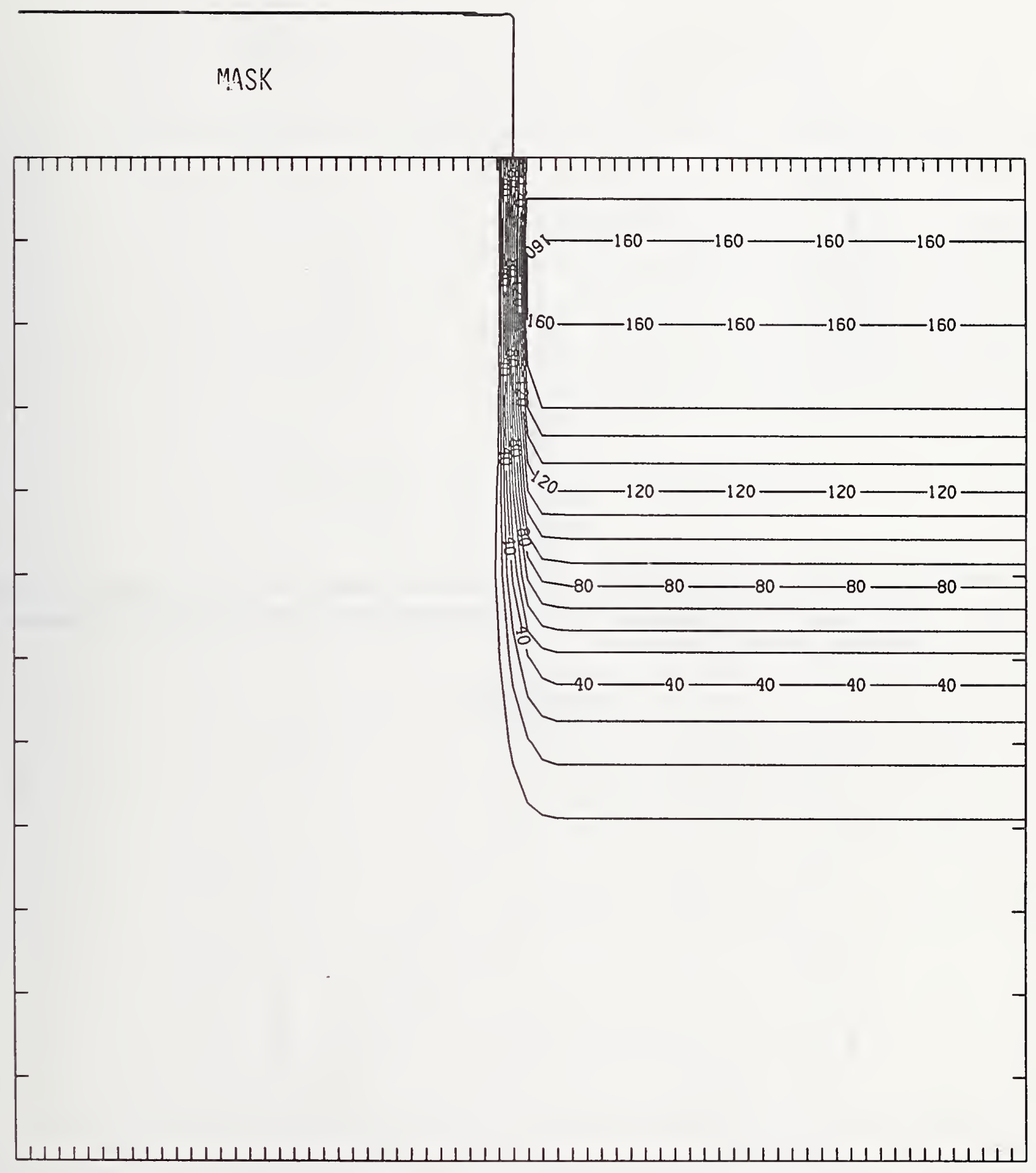

Figure Ga150-4 Two-dimensional distribution of Frenkel pairs created by $150 \mathrm{keV}$ gallium implanted past a mask edge. The length increment (distance between tick marks) is $0.02 \mu \mathrm{m}$ and the scale factor is 0.1 . 


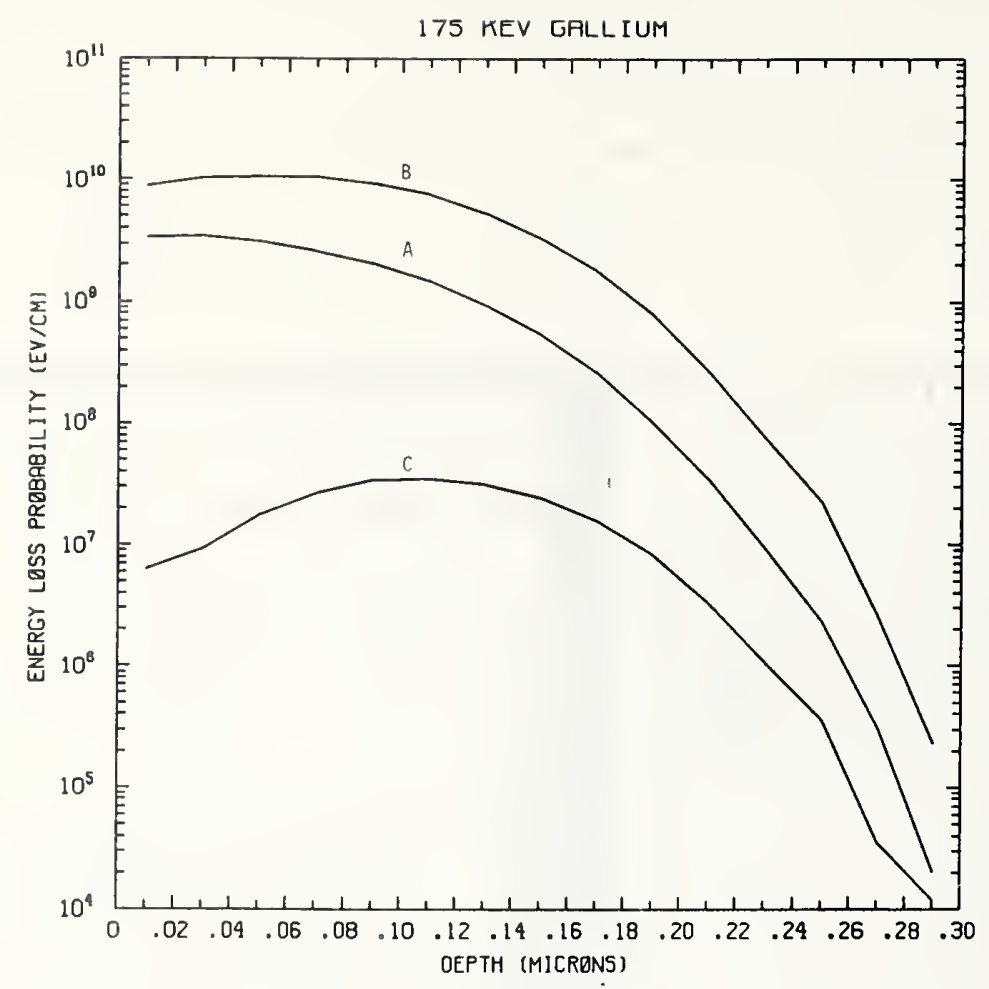

Figure Ga175-1 One-dimensional probability distributions for energy loss due to electronic excitation (A), displacement damage(B), and phonon excitation (C) for $175 \mathrm{keV}$ gallium normally incident on a silicon target.

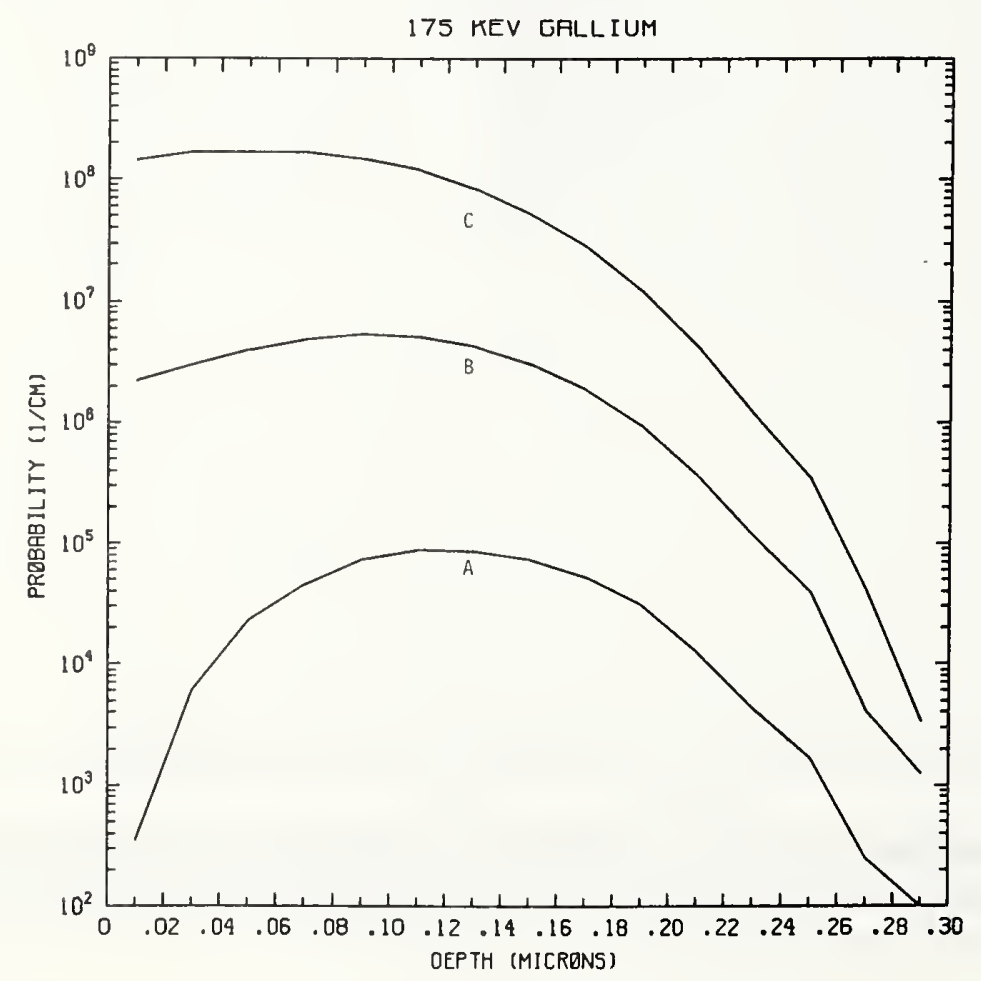

Figure Ga175-2 One-dimensional probability distributions for implanted gallium (A), primary silicon displacements (B), and Frenkel pairs (C) for $175 \mathrm{keV}$ gallium normally incident on a silicon target. 


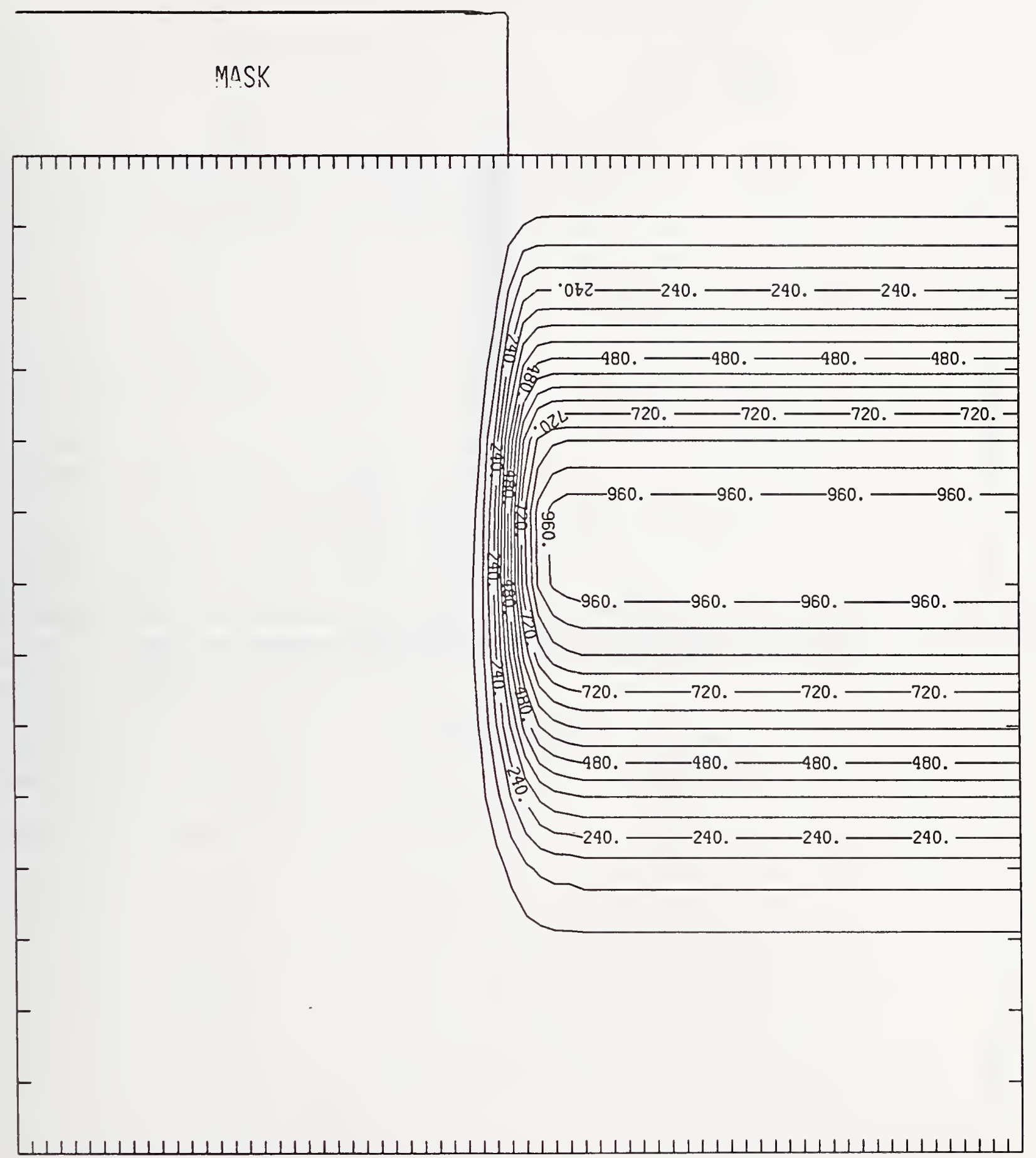

Figure Ga175-3 Two-dimensional distribution of $175 \mathrm{keV}$ gallium implanted past a mask edge. The length increment (distance between tick marks) is $0.02 \mu \mathrm{m}$ and the scale factor is 1000 . 


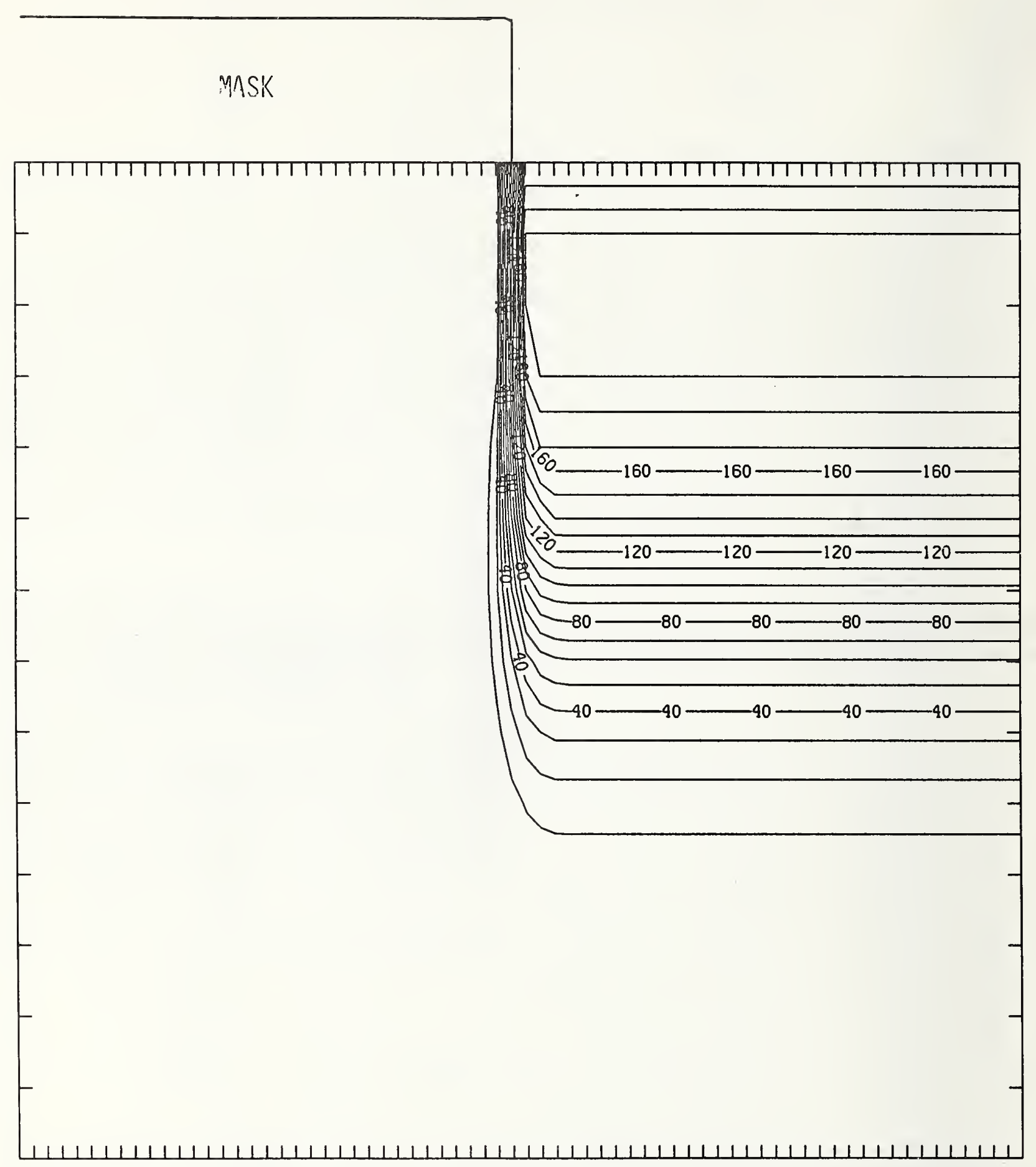

Figure Ga175-4 Two-dimensional distribution of Frenkel pairs created by $175 \mathrm{keV}$ gallium implanted past a mask edge. The length increment (distance between tick marks) is $0.02 \mu \mathrm{m}$ and the scale factor is 0.1 . 


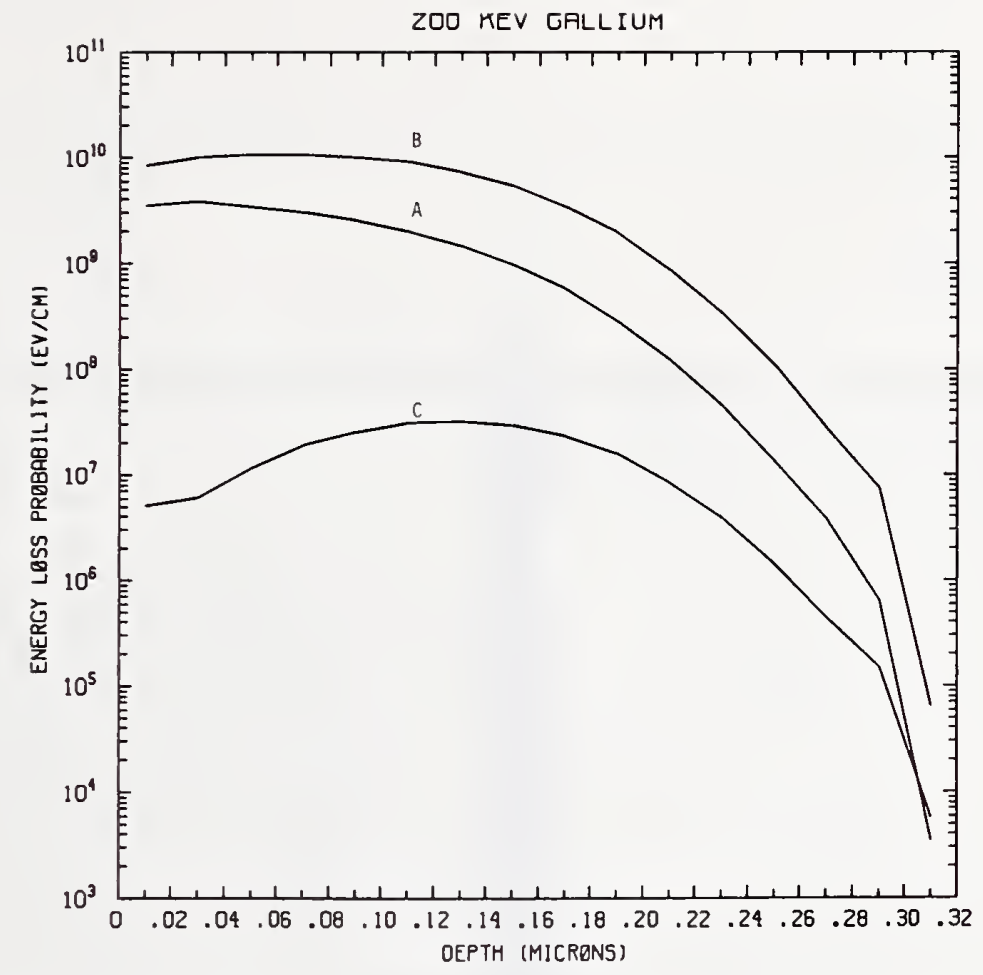

Figure Ga200-1 One-dimensional probability distributions for energy loss due to electronic excitation (A), displacement damage(B), and phonon excitation (C) for $200 \mathrm{keV}$ gallium normally incident on a silicon target.

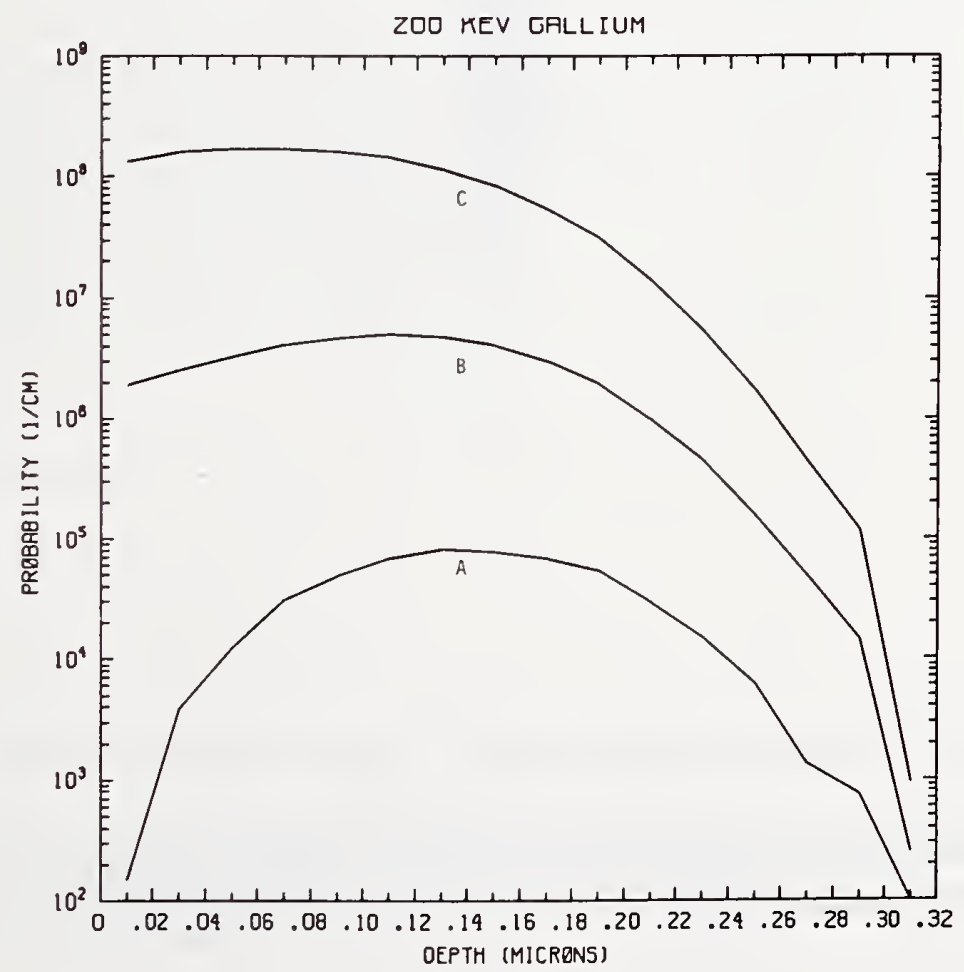

Figure Ga200-2 One-dimensional probability distributions for implanted gallium (A), primary silicon displacements (B), and Frenkel pairs (C) for $200 \mathrm{keV}$ gallium normally incident on a silicon target. 


\section{MASK}

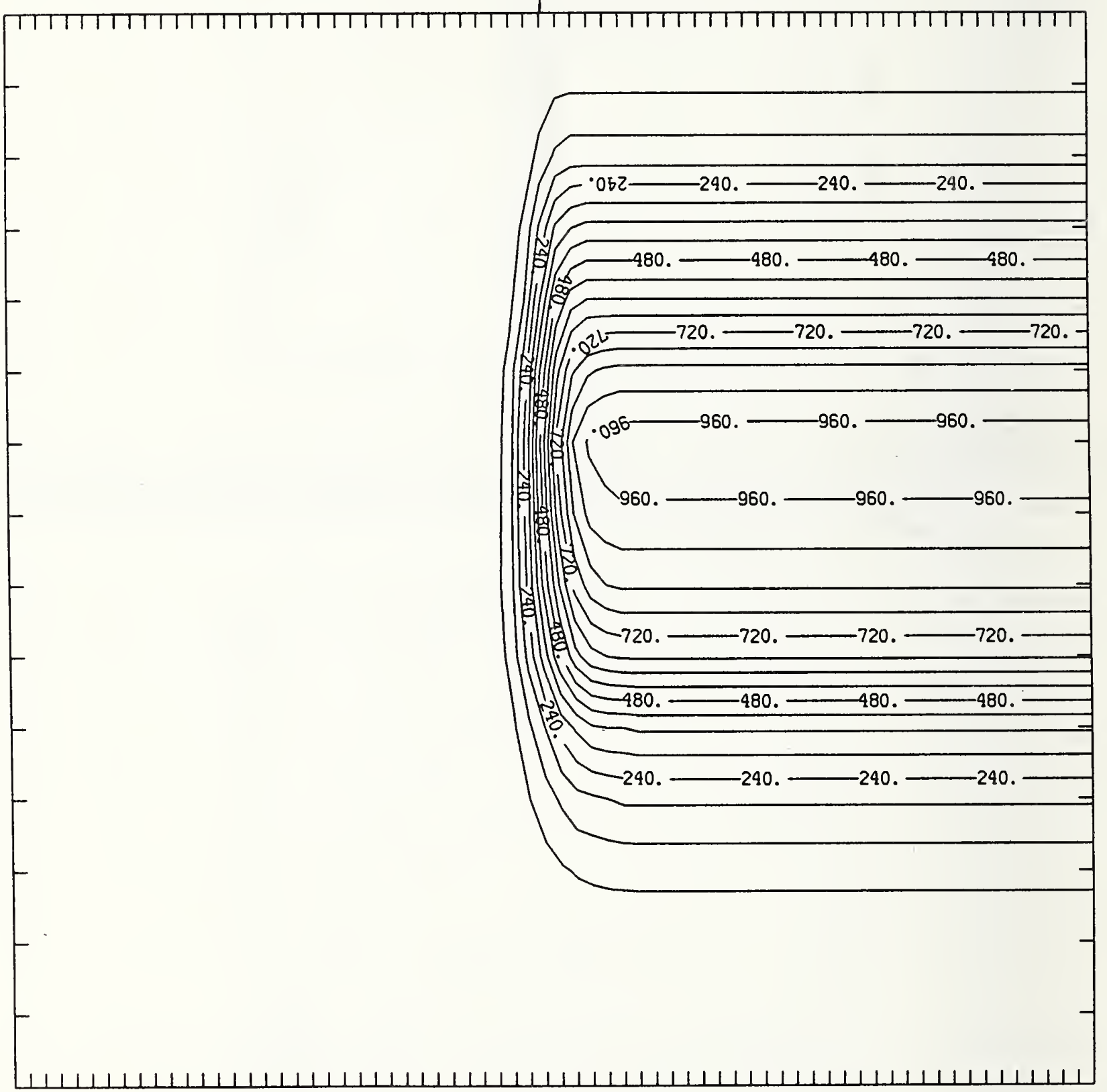

Figure Ga200-3 Two-dimensional distribution of $200 \mathrm{keV}$ gallium implanted past a mask edge. The length increment (distance between tick marks) is $0.02 \mu \mathrm{m}$ and the scale factor is 1000 . 


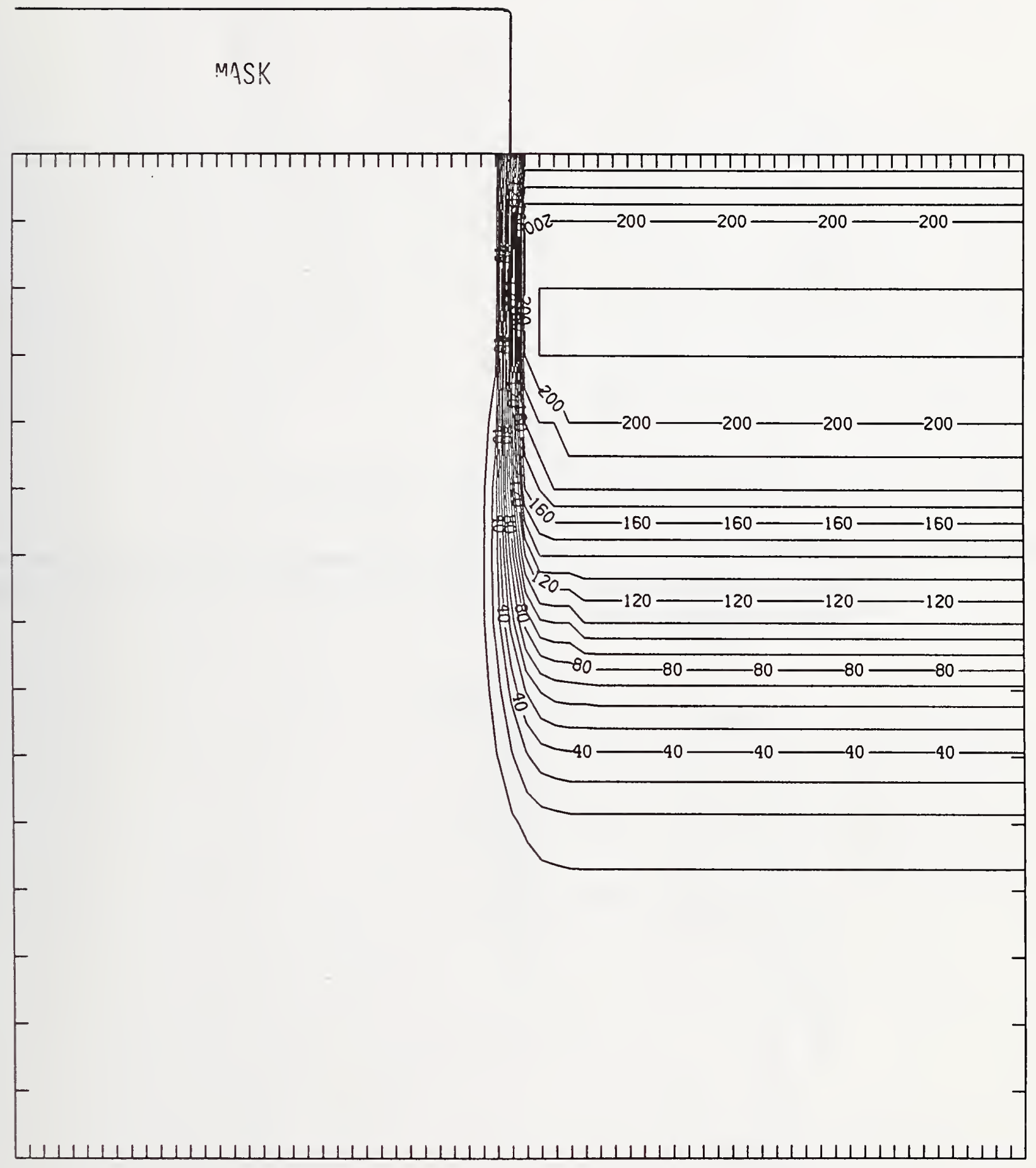

Figure Ga200-4 Two-dimensional distribution of Frenkel pairs created by $200 \mathrm{keV}$ gallium implanted past a mask edge. The length increment (distance between tick marks) is $0.02 \mu \mathrm{m}$ and the scale factor is 0.1 . 


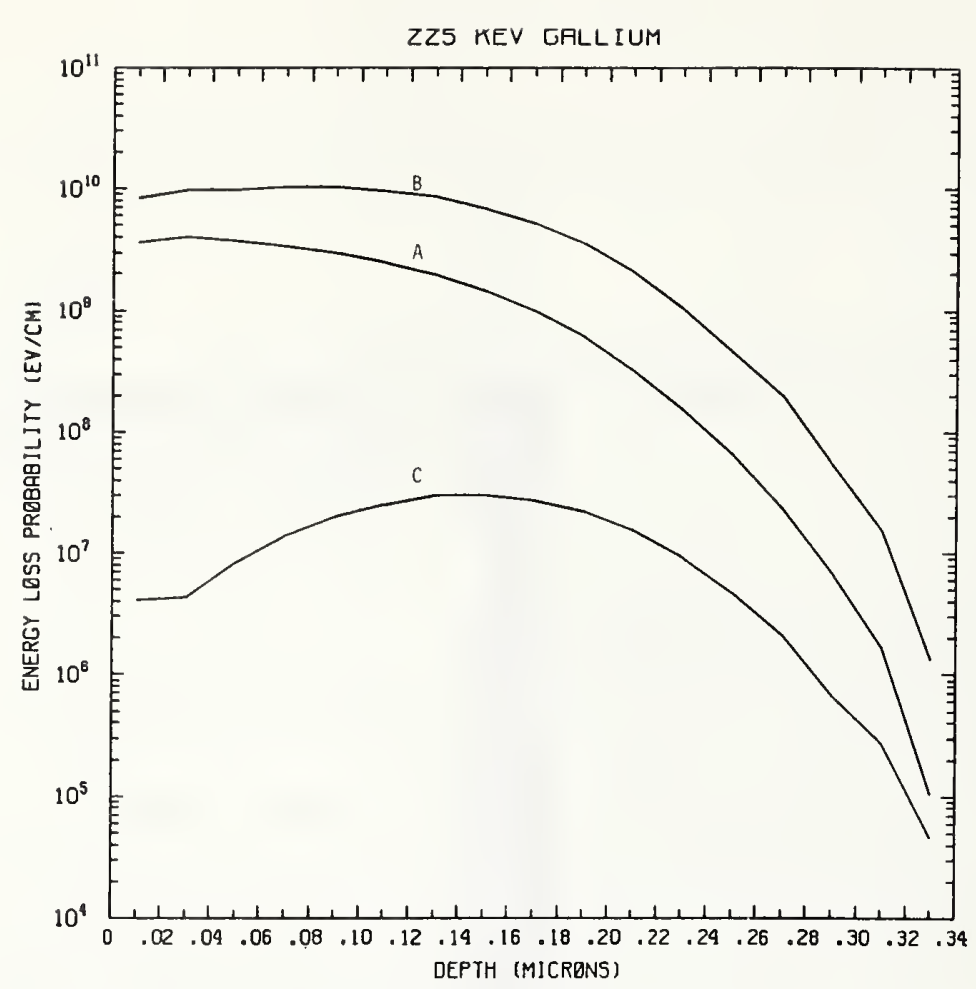

Figure Ga225-1 One-dimensional probability distributions for energy loss due to electronic excitation (A), displacement damage(B), and phonon excitation (C) for $225 \mathrm{keV}$ gallium normally incident on a silicon target.

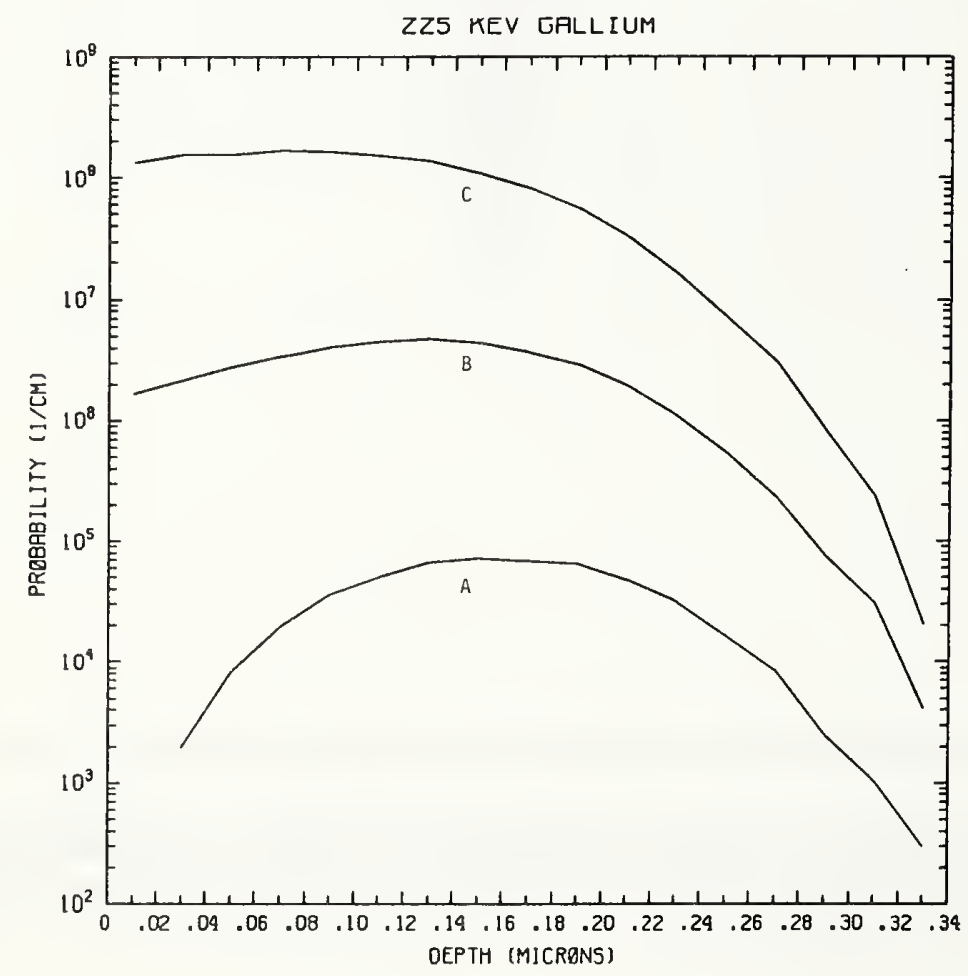

Figure Ga225-2 One-dimensional probability distributions for implanted gallium (A), primary silicon displacements (B), and Frenkel pairs (C) for $225 \mathrm{keV}$ gallium normally incident on a silicon target. 


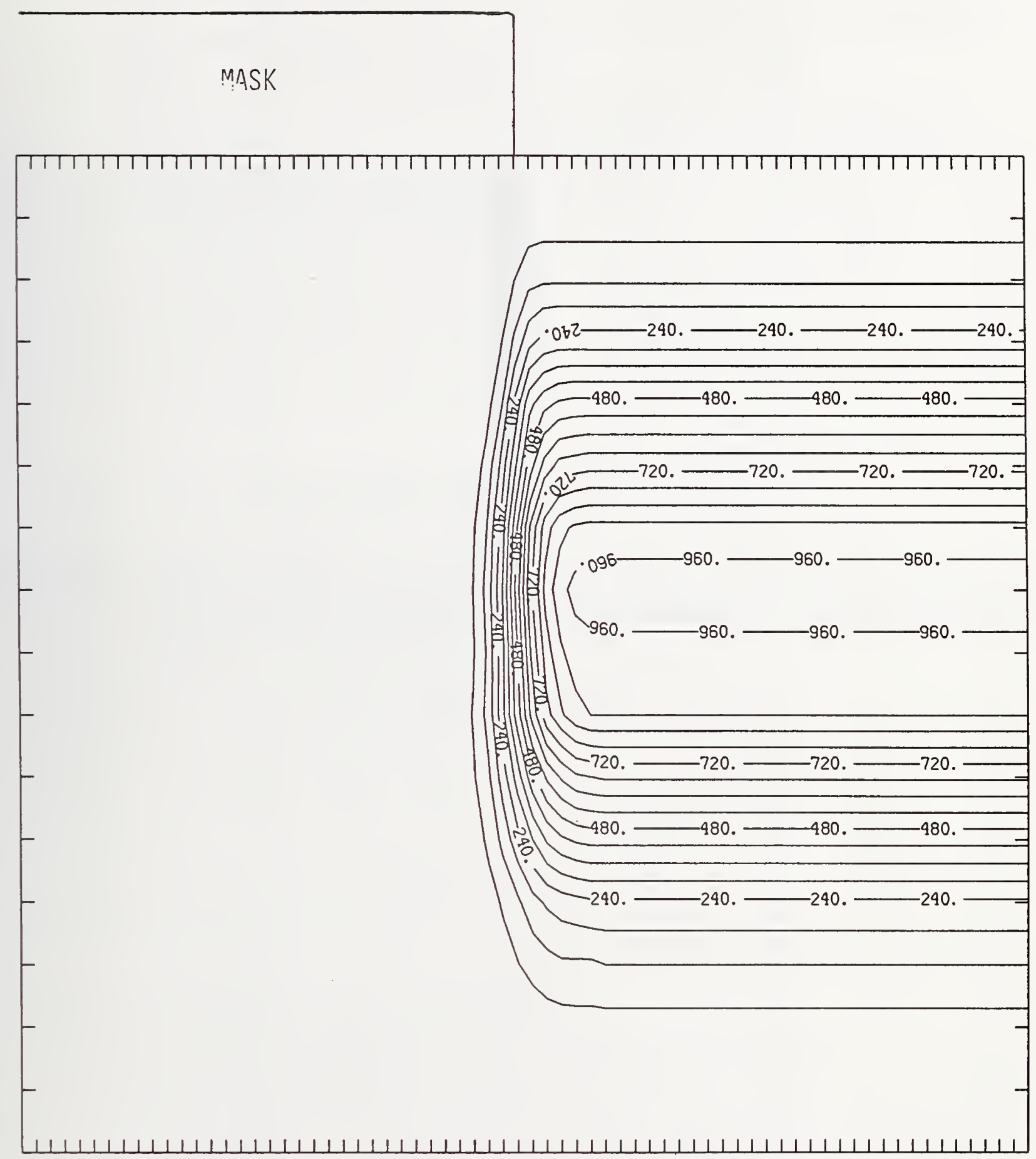

Figure Ga225-3 Two-dimensional distribution of $225 \mathrm{keV}$ gallium implanted past a mask edge. The length increment (distance between tick marks) is $0.02 \mu \mathrm{m}$ and the scale factor is 1000 . 


\section{MASK}

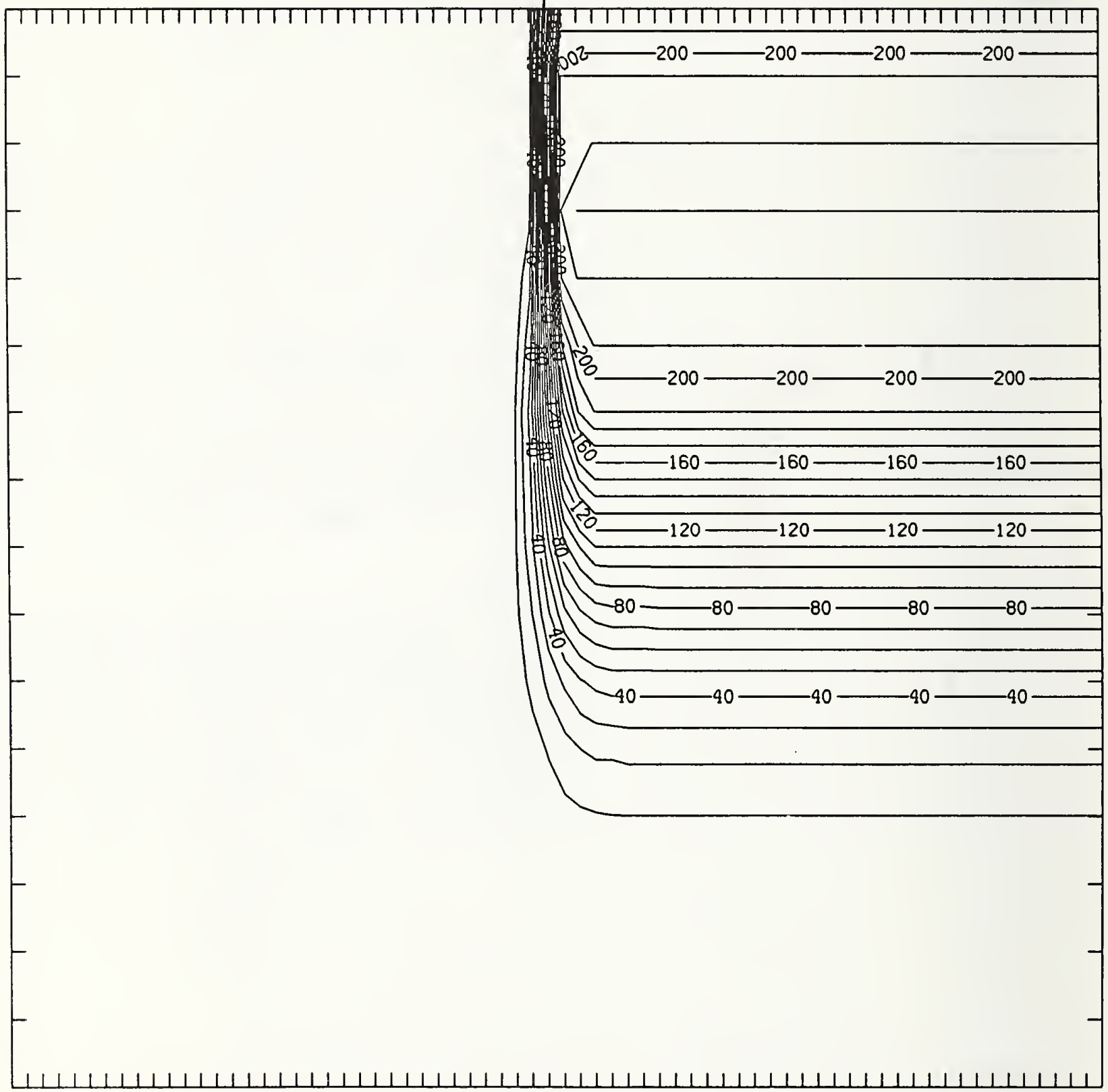

Figure Ga225-4 Two-dimensional distribution of Frenkel pairs created by $225 \mathrm{keV}$ gallium implanted past a mask edge. The length increment (distance between tick marks) is $0.02 \mu \mathrm{m}$ and the scale factor is 0.1 . 


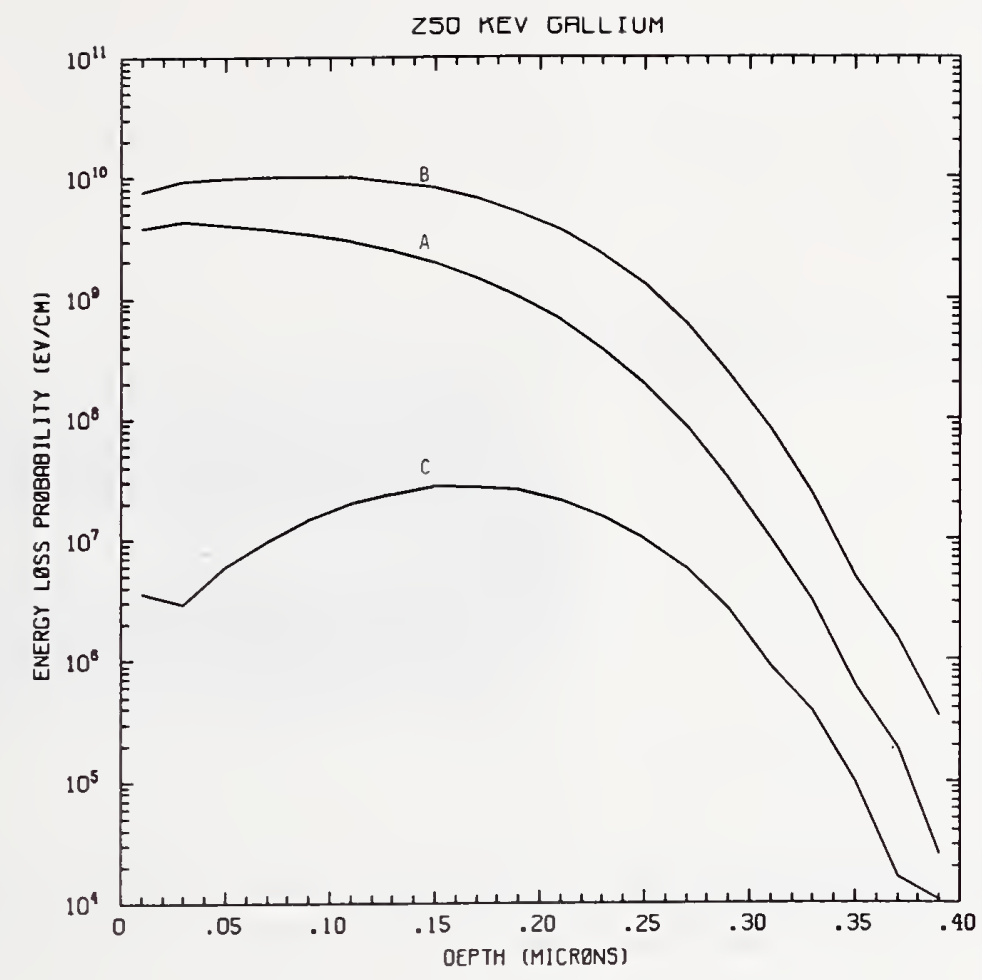

Figure Ga250-1 One-dimensional probability distributions for energy loss due to electronic excitation (A), displacement damage(B), and phonon excitation (C) for $250 \mathrm{keV}$ gallium normally incident on a silicon target.

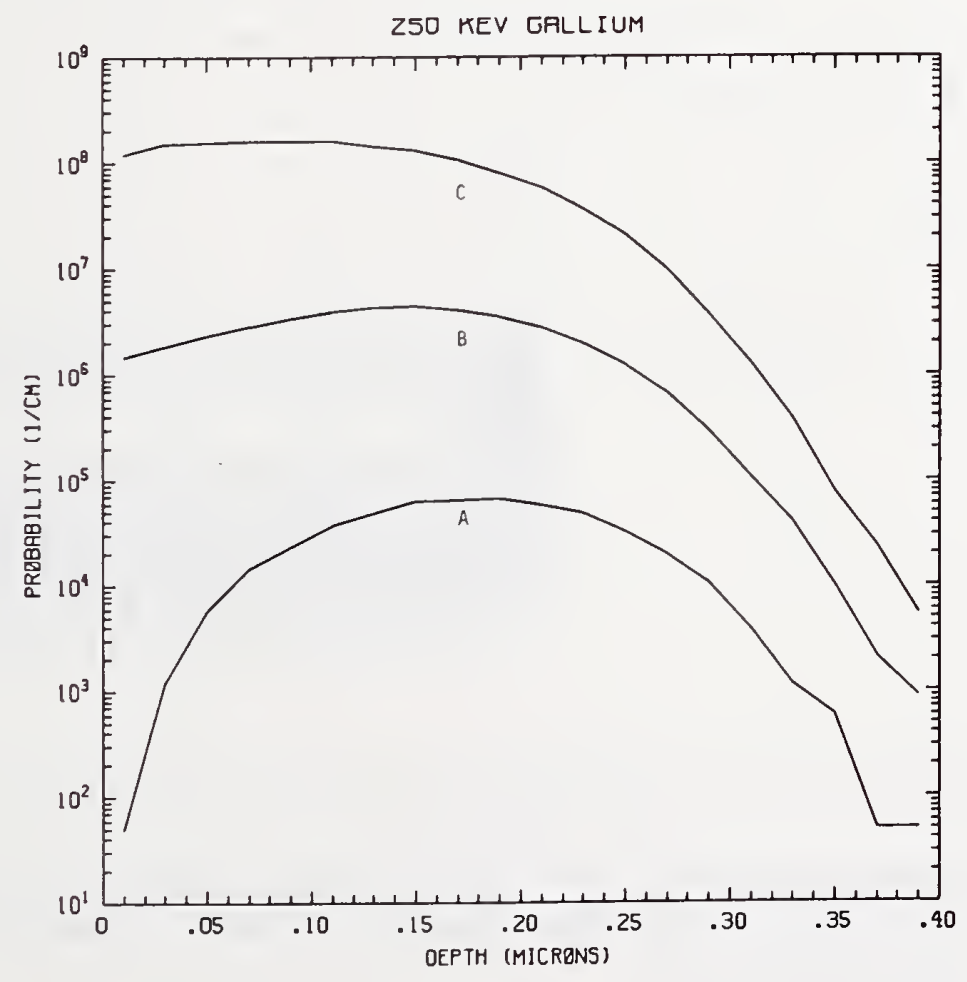

Figure Ga250-2 One-dimensional probability distributions for implanted gallium (A), primary silicon displacements (B), and Frenkel pairs (C) for $250 \mathrm{keV}$ gallium normally incident on a . silicon target. 


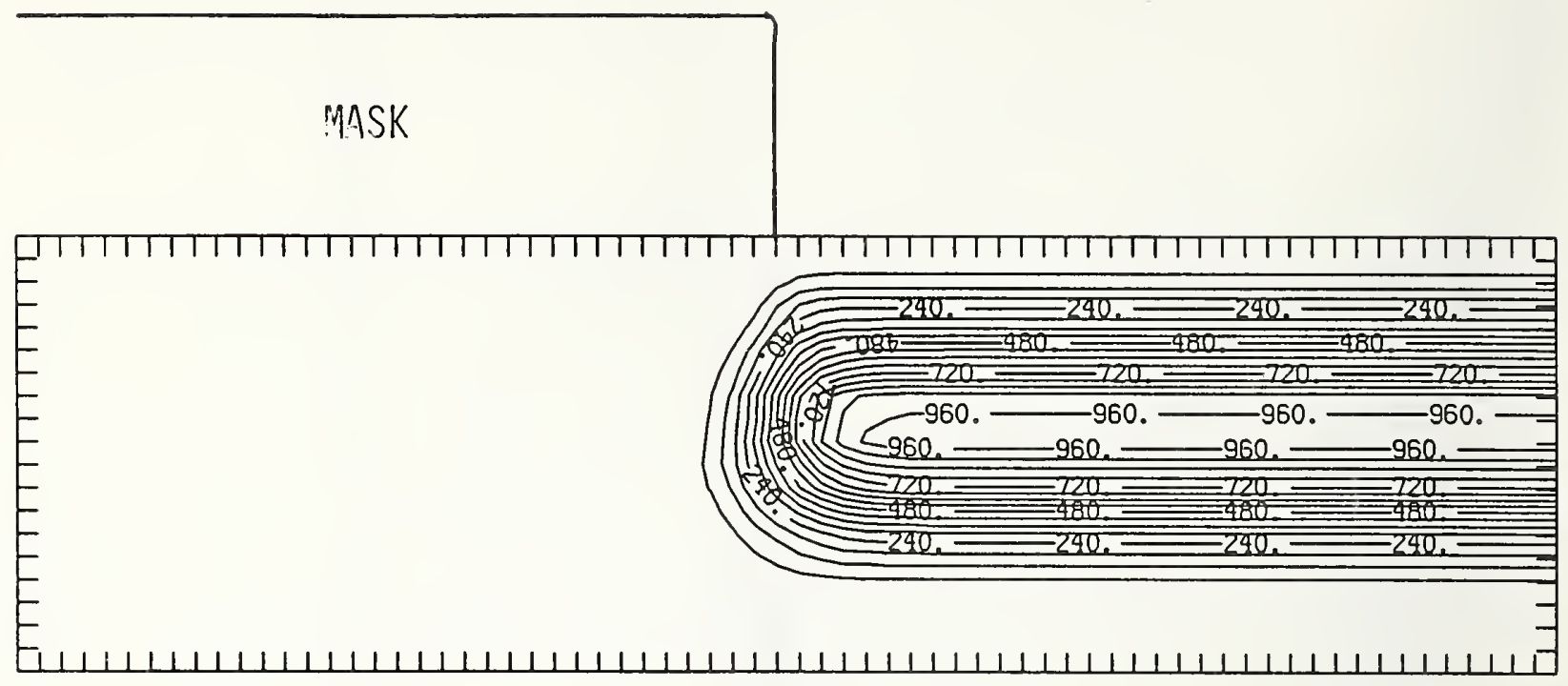

Figure Ga250-3 Two-dimensional distribution of $250 \mathrm{keV}$ gallium implanted past a mask edge. The length increment (distance between tick marks) is $0.02 \mu \mathrm{m}$ and the scale factor is 1000 .

M.ASK

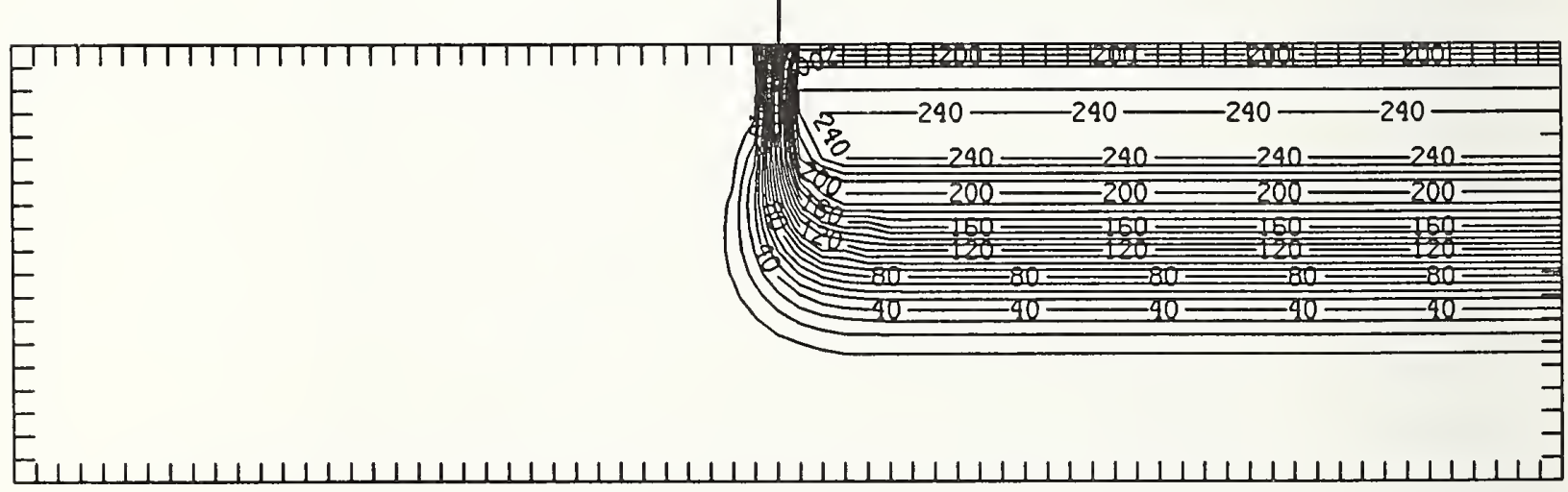

Figure Ga250-4 Two-dimensional distribution of Frenkel pairs created by $250 \mathrm{keV}$ gallium implanted past a mask edge. The length increment (distance between tick marks) is $0.02 \mu \mathrm{m}$ and the scale factor is 0.1 . 


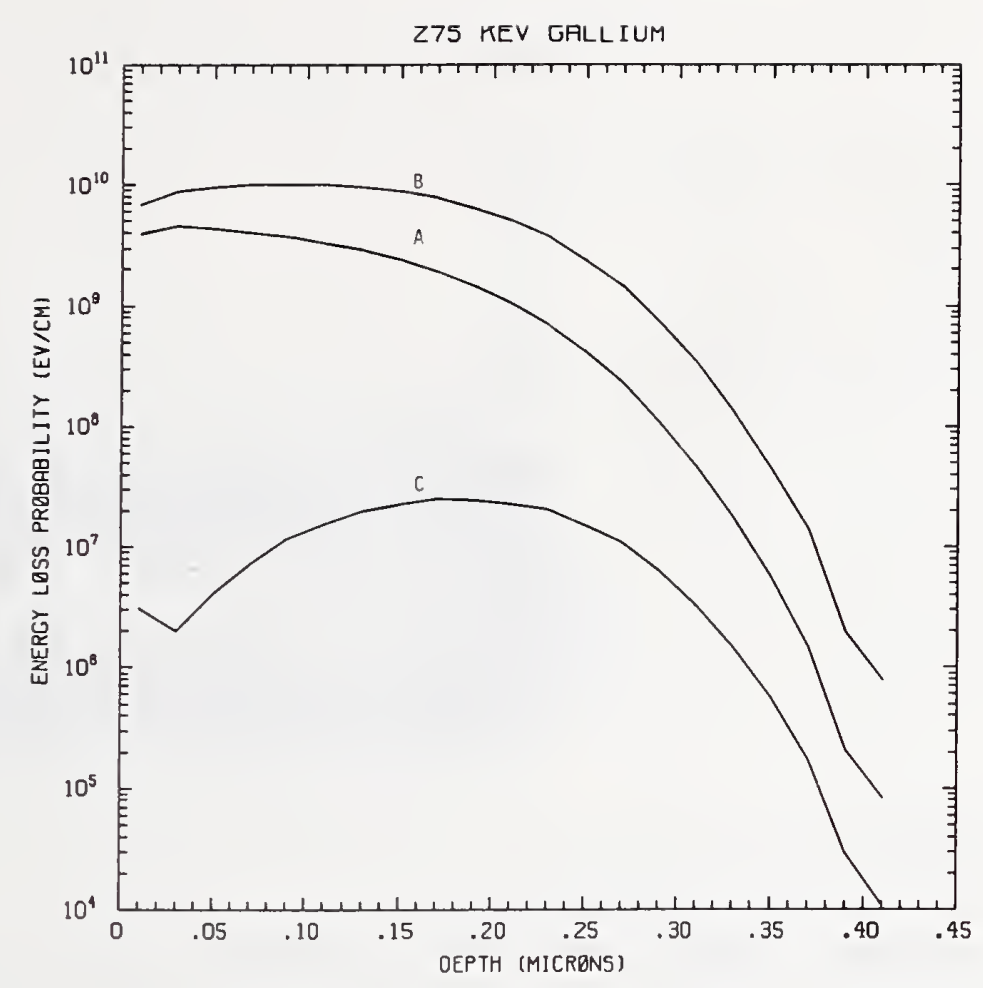

Figure Ga275-1 One-dimensional probability distributions for energy loss due to electronic excitation (A), displacement damage(B), and phonon excitation (C) for $275 \mathrm{keV}$ gallium normally incident on a silicon target.

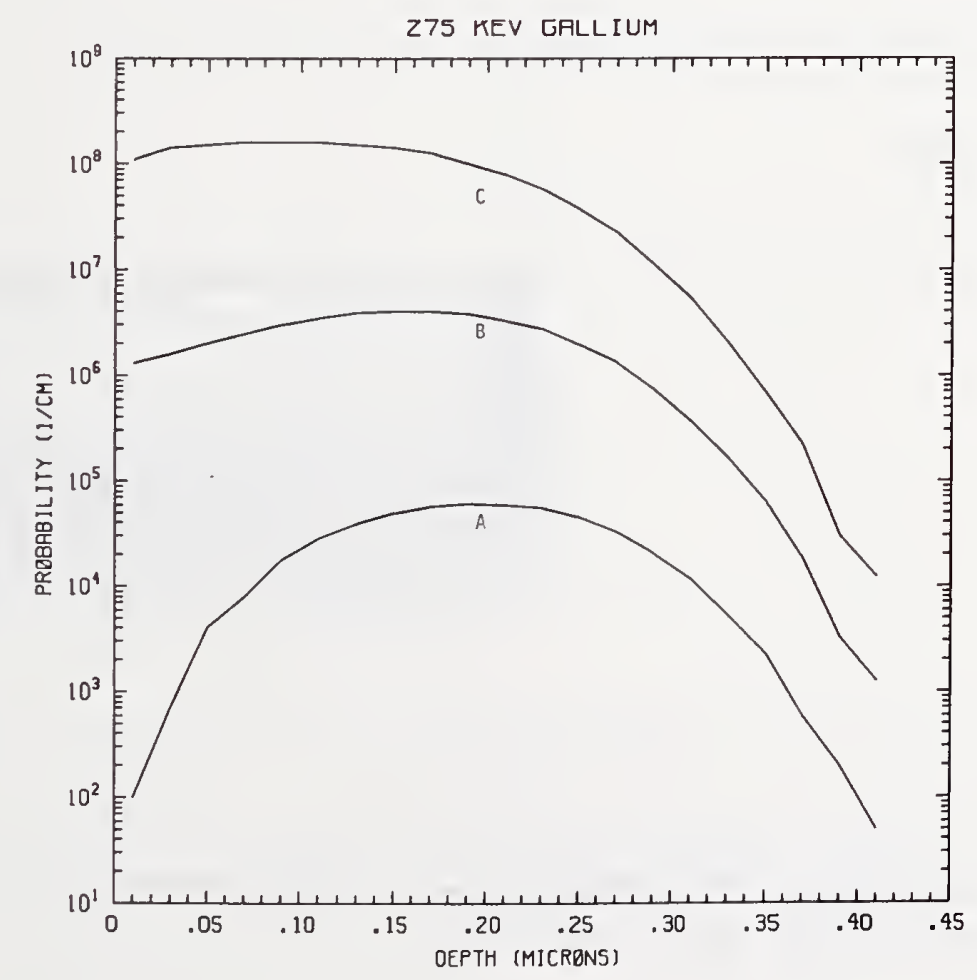

Figure Ga275-2 One-dimensional probability distributions for implanted gallium (A), primary silicon displacements (B), and Frenkel pairs (C) for $275 \mathrm{keV}$ gallium normally incident on a silicon target. 


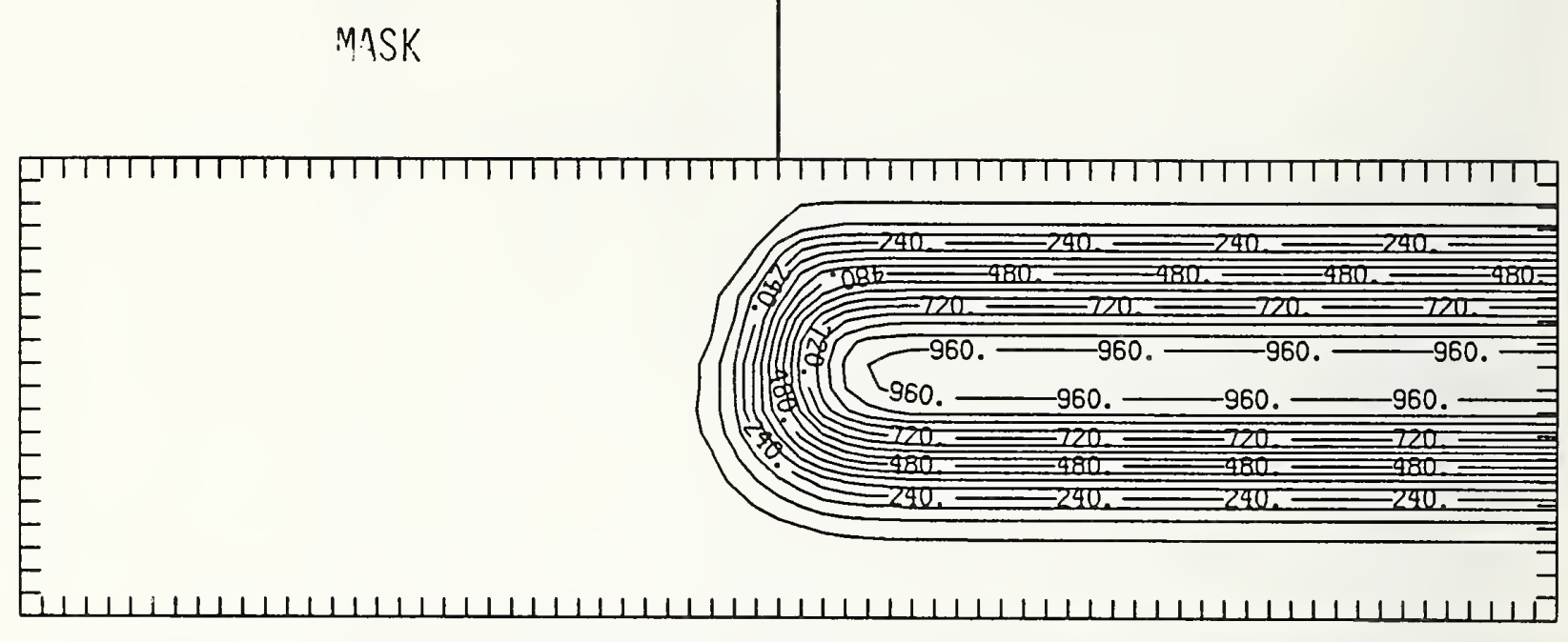

Figure Ga275-3 Two-dimensional distribution of $275 \mathrm{keV}$ gallium implanted past a mask edge. The length increment (distance between tick marks) is $0.02 \mu \mathrm{m}$ and the scale factor is 1000 .

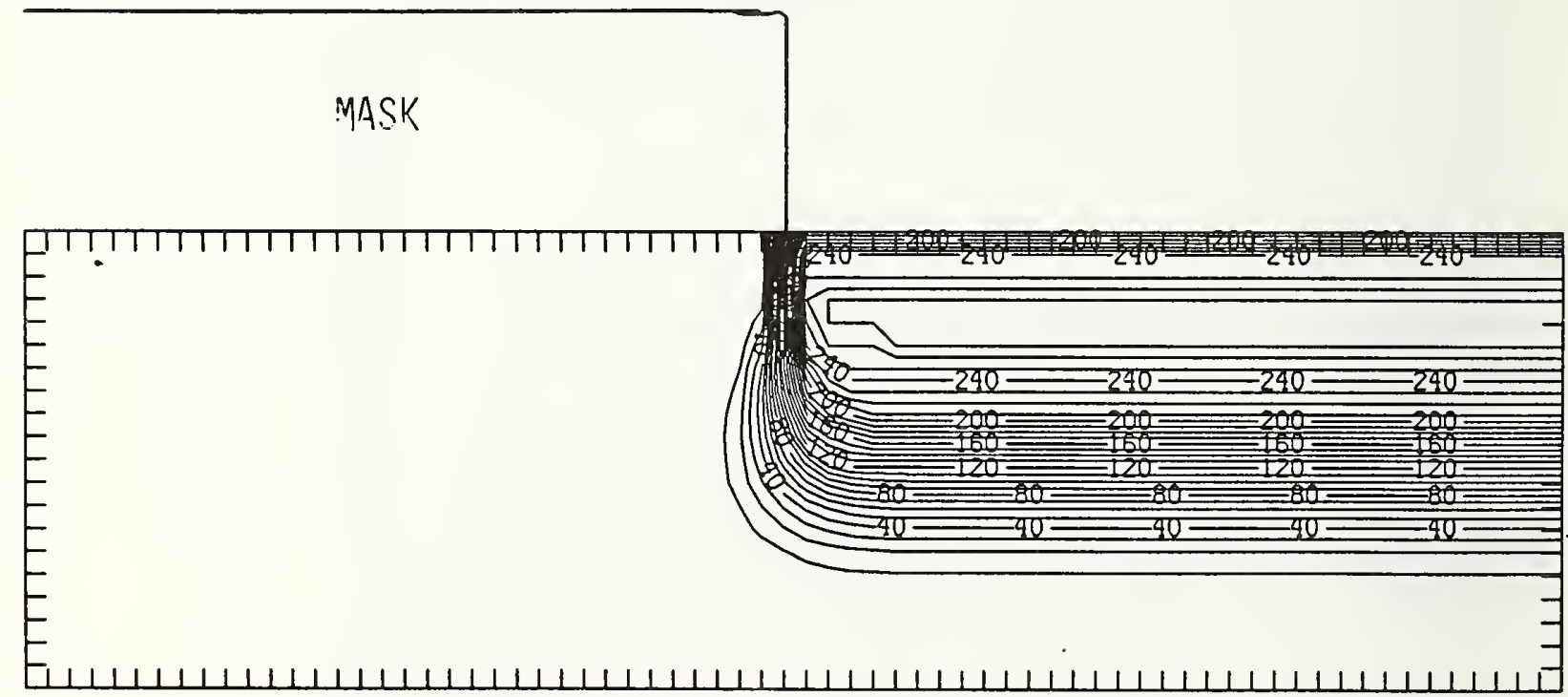

Figure Ga275-4 Two-dimensional distribution of Frenkel pairs created by $275 \mathrm{keV}$ gallium implanted past a mask edge. The length increment (distance between tick marks) is $0.02 \mu \mathrm{m}$ and the scale factor is 0.1 . 


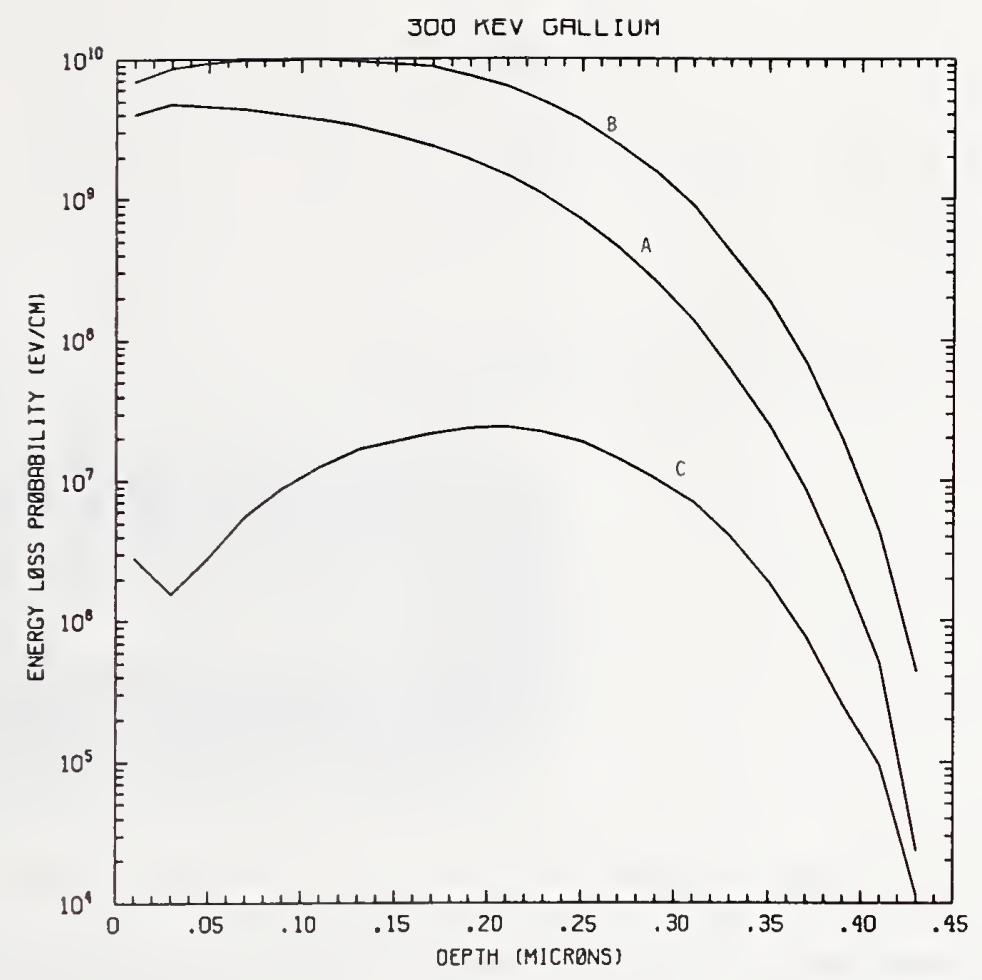

Figure Ga300-1 One-dimensional probability distributions for energy loss due to electronic excitation (A), displacement damage(B), and phonon excitation (C) for $300 \mathrm{keV}$ gallium normally incident on a silicon target.

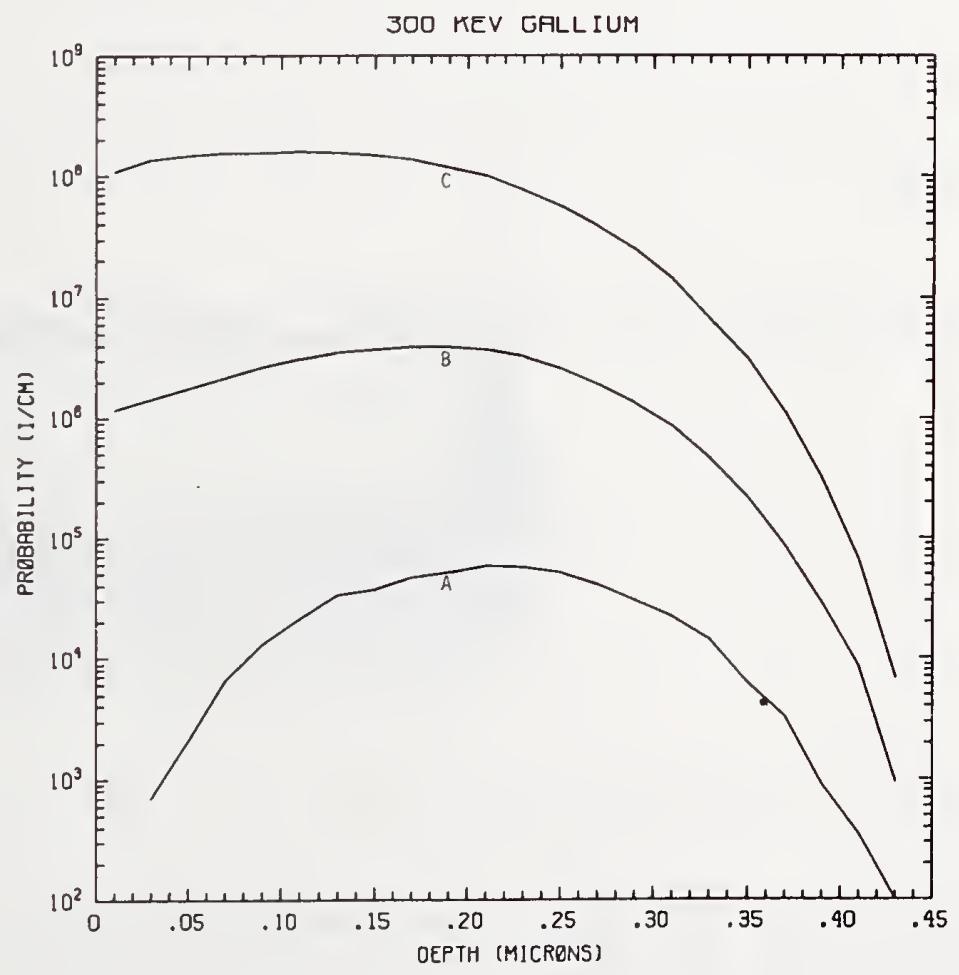

Figure Ga300-2 One-dimensional probability distributions for implanted gallium (A), primary silicon displacements (B), and Frenkel pairs (C) for $300 \mathrm{keV}$ gallium normally incident on a silicon target. 


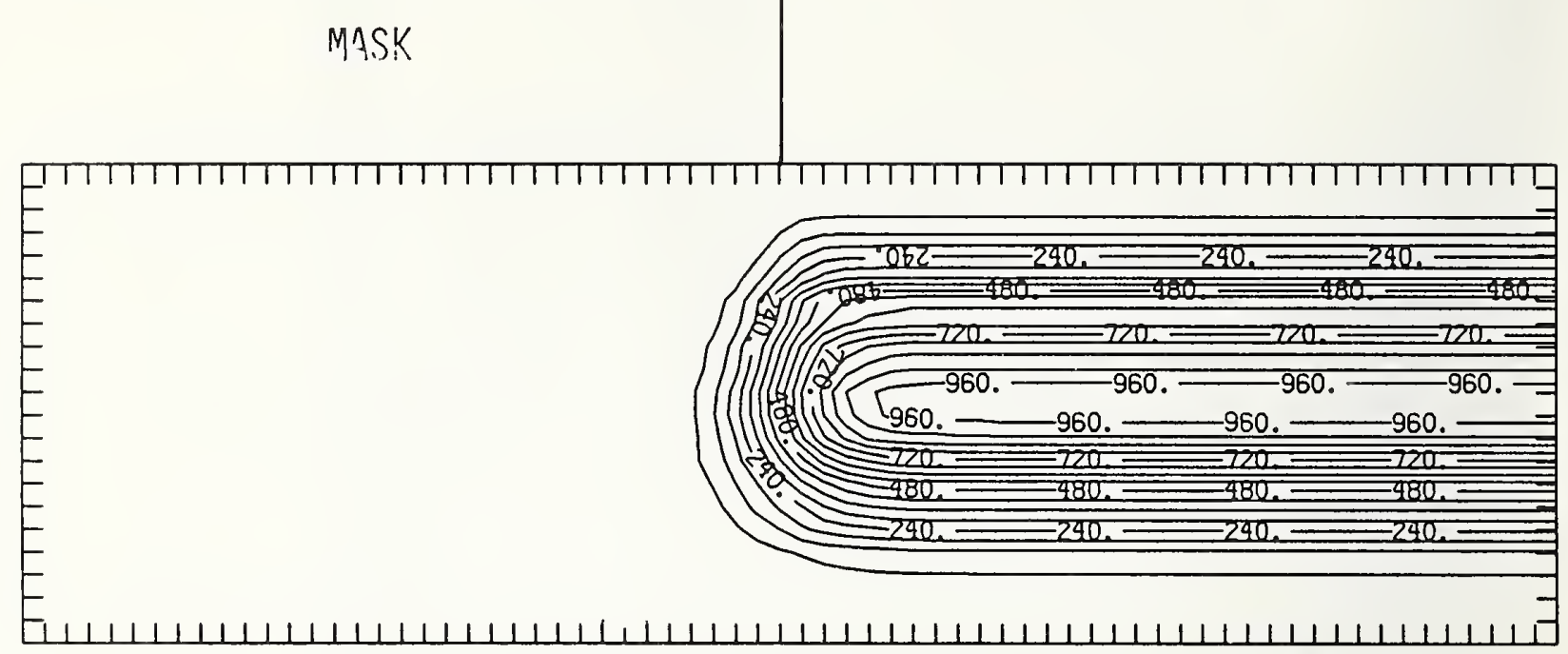

Figure Ga300-3 Two-dimensional distribution of $300 \mathrm{keV}$ gallium implanted past a mask edge. The length increment (distance between tick marks) is $0.02 \mu \mathrm{m}$ and the scale factor is 1000.

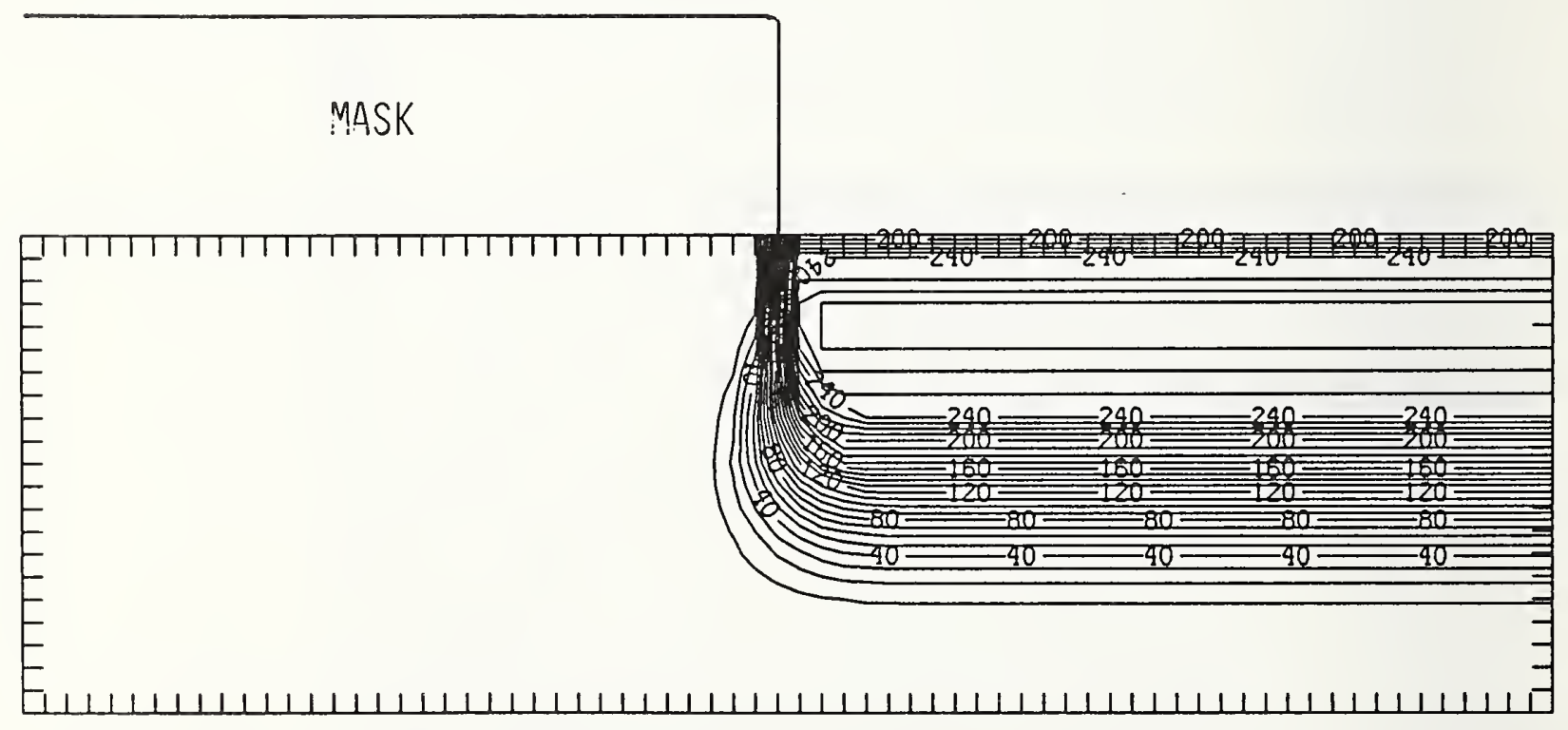

Figure Ga300-4 Two-dimensional distribution of Frenkel pairs created by $300 \mathrm{keV}$ gallium implanted past a mask edge. The length increment (distance between tick marks) is $0.02 \mu \mathrm{m}$ and the scale factor is 0.1 . 


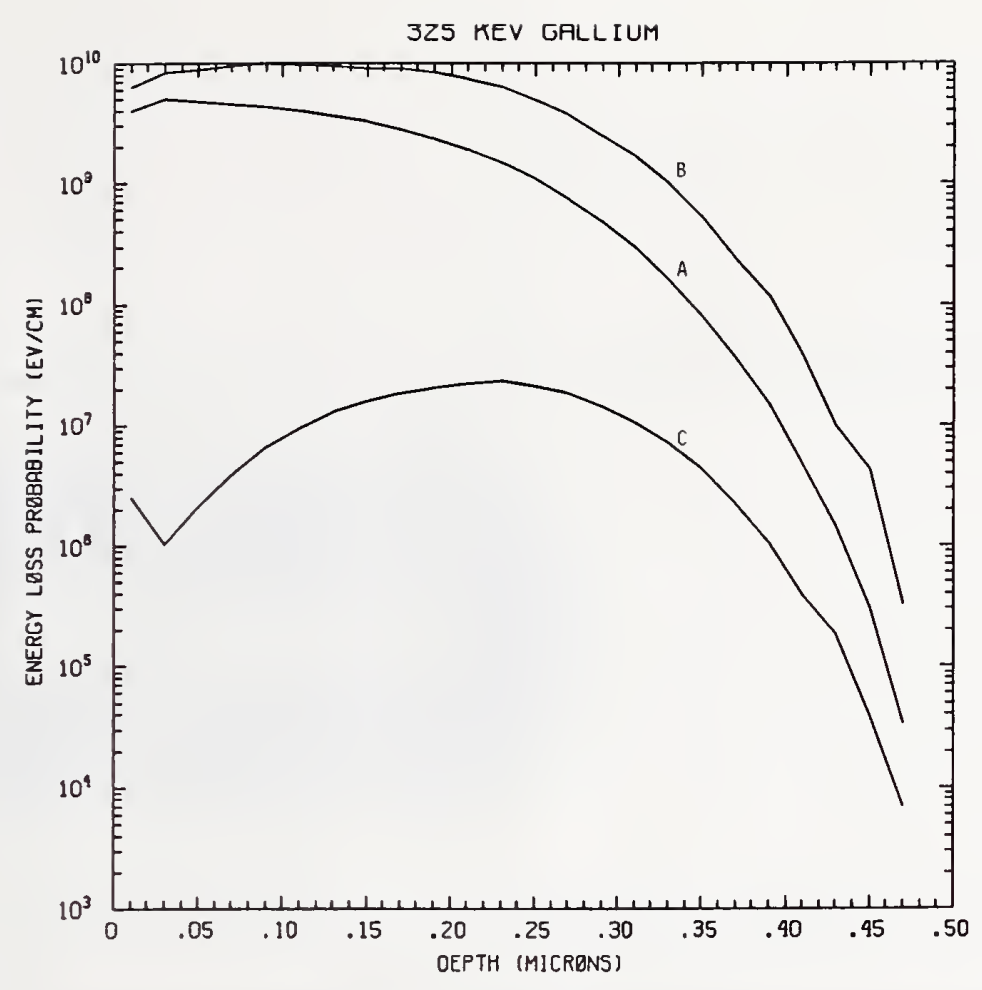

Figure Ga325-1 One-dimensional probability distributions for energy loss due to electronic excitation (A), displacement damage(B), and phonon excitation (C) for $325 \mathrm{keV}$ gallium normally incident on a silicon target.

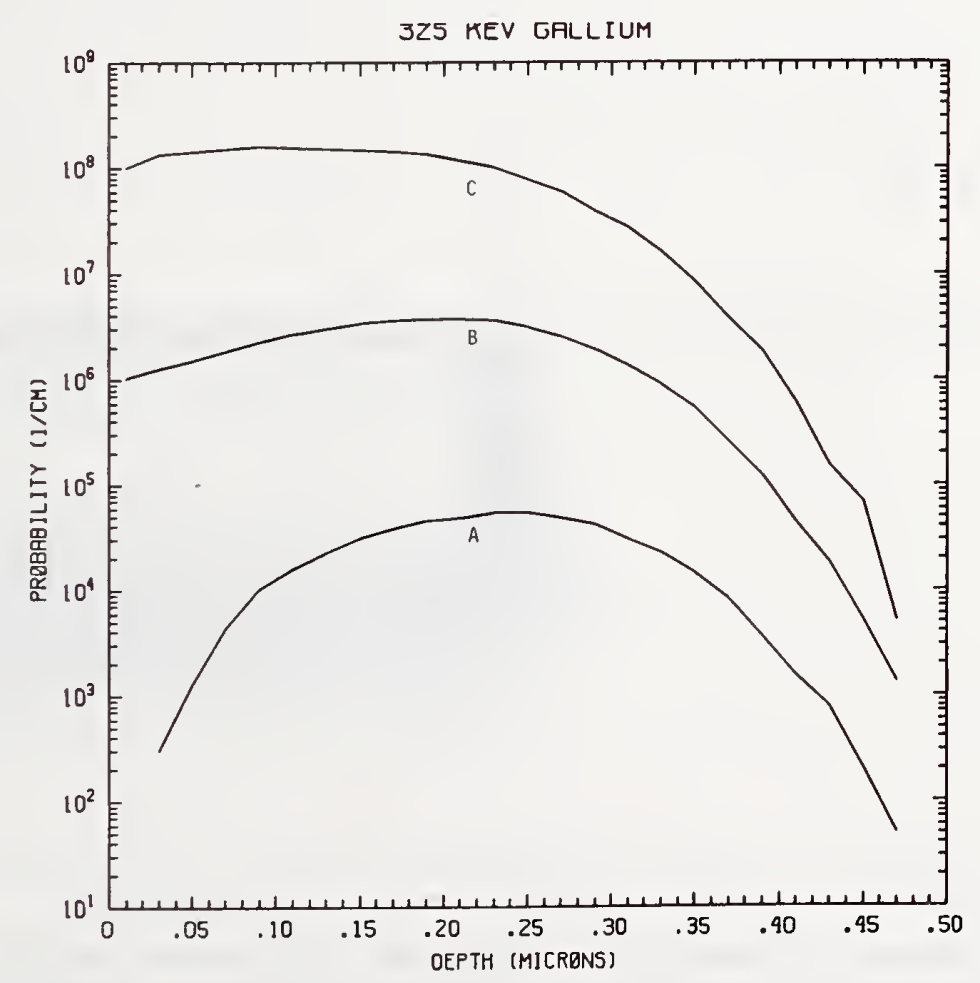

Figure Ga325-2 One-dimensional probability distributions for implanted gallium (A), primary silicon displacements (B), and Frenkel pairs (C) for $325 \mathrm{keV}$ gallium normally incident on a silicon target. 


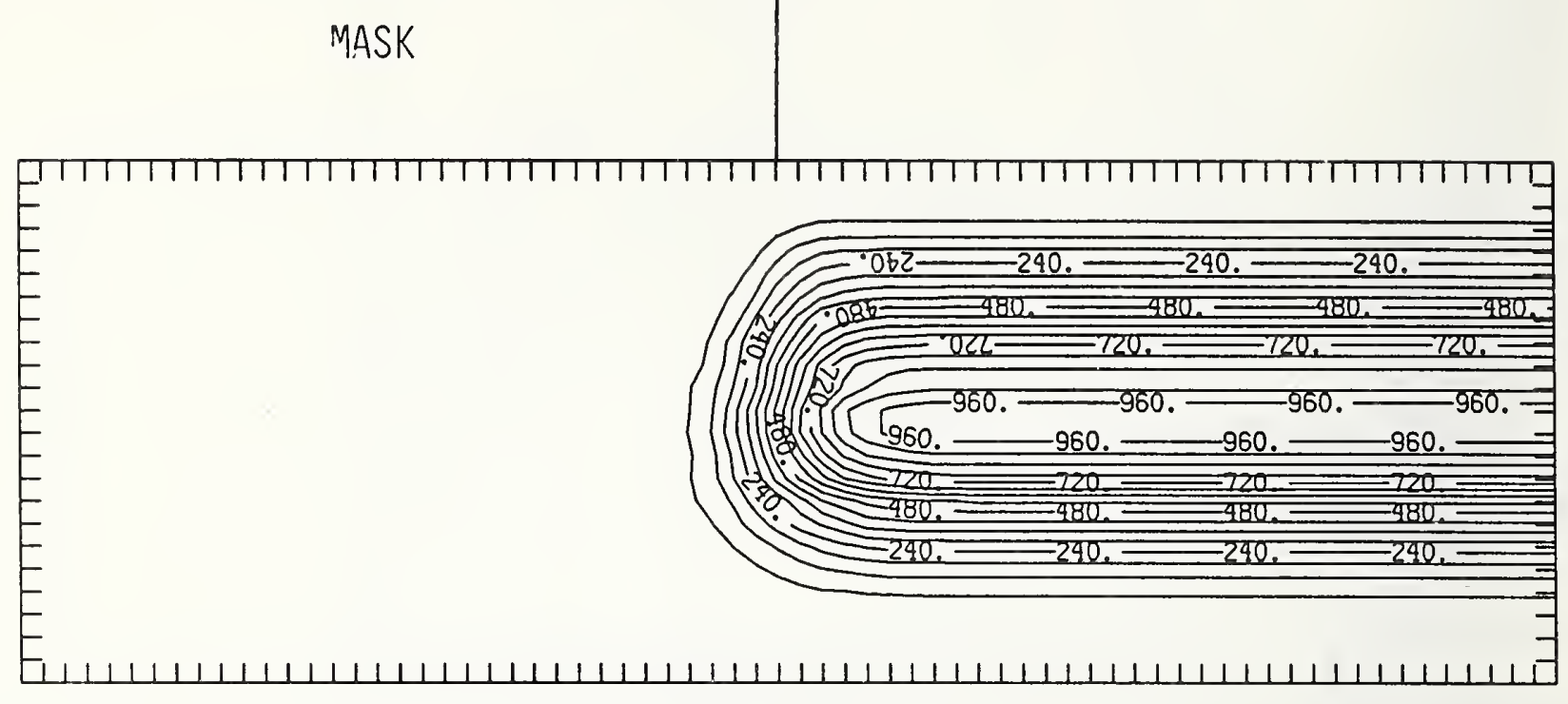

Figure Ga325-3 Two-dimensional distribution of $325 \mathrm{keV}$ gallium implanted past a mask edge. The length increment (distance between tick marks) is $0.02 \mu \mathrm{m}$ and the scale factor is 1000 .

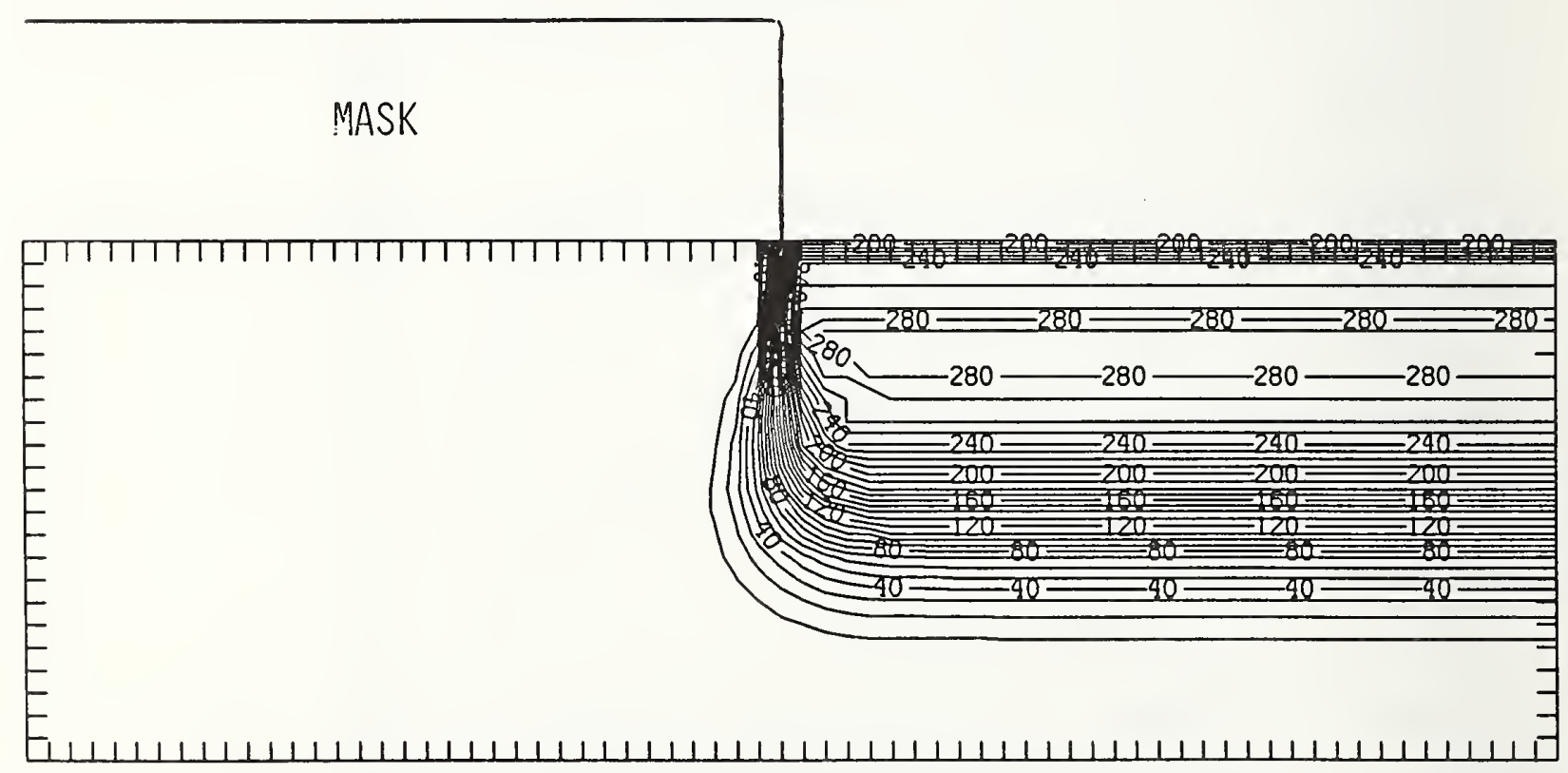

Figure Ga325-4 Two-dimensional distribution of Frenkel pairs created by $325 \mathrm{keV}$ gallium implanted past a mask edge. The length increment (distance between tick marks) is $0.02 \mu \mathrm{m}$ and the scale factor is 0.1 . 


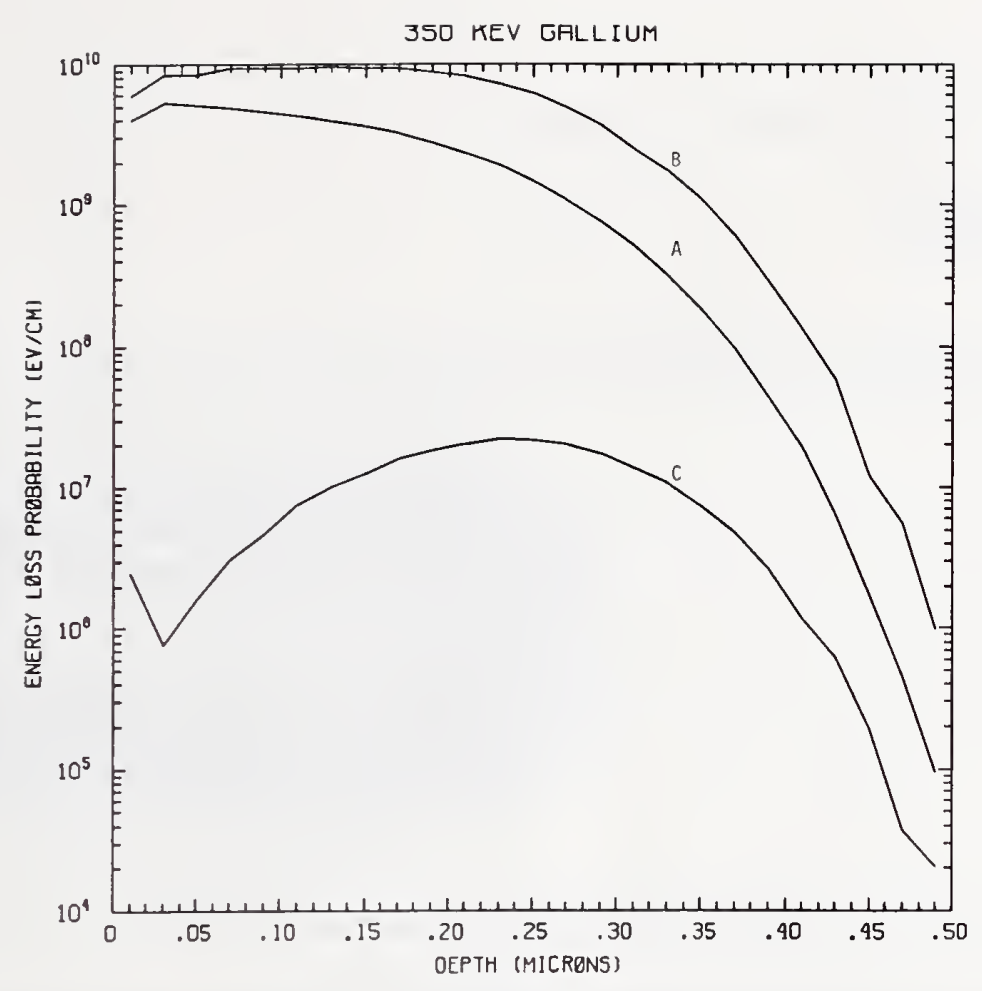

Figure Ga350-1 One-dimensional probability distributions for energy loss due to electronic excitation (A), displacement damage(B), and phonon excitation (C) for $350 \mathrm{keV}$ gallium normally incident on a silicon target.

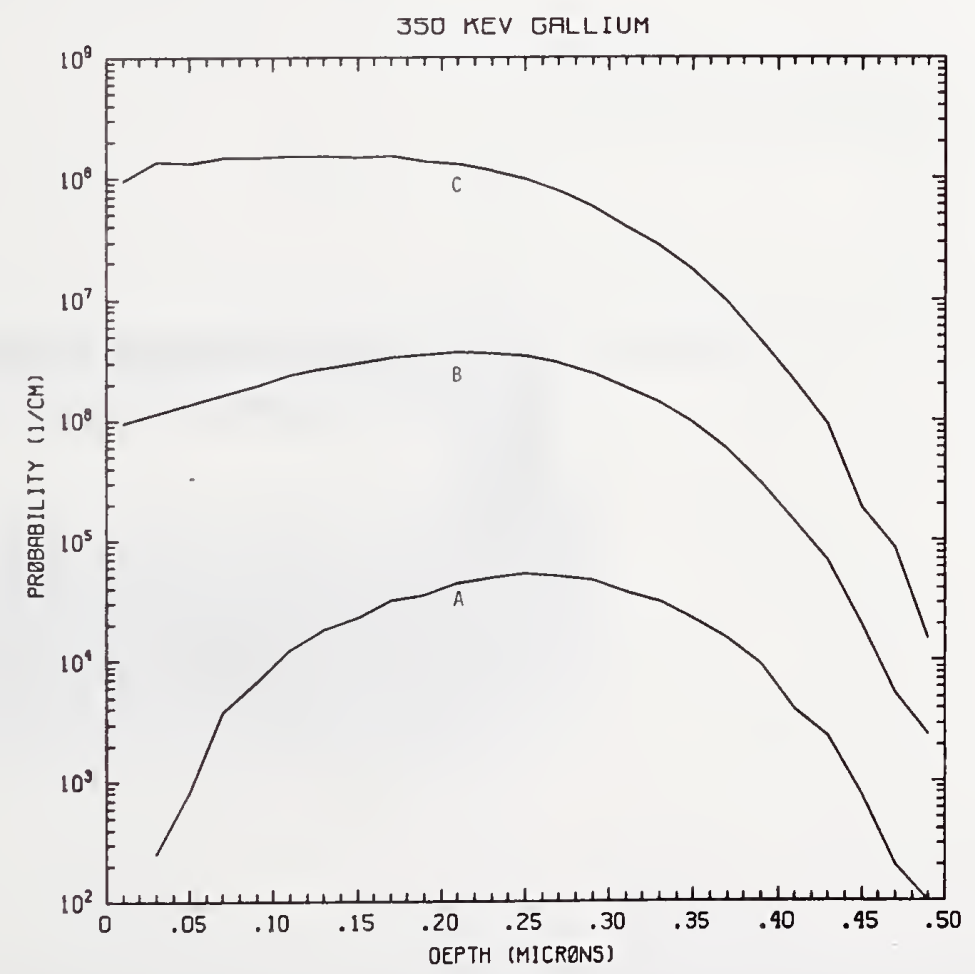

Figure Ga350-2 One-dimensional probability distributions for implanted gallium (A), primary silicon displacements (B), and Frenkel pairs (C) for $350 \mathrm{keV}$ gallium normally incident on a silicon target. 


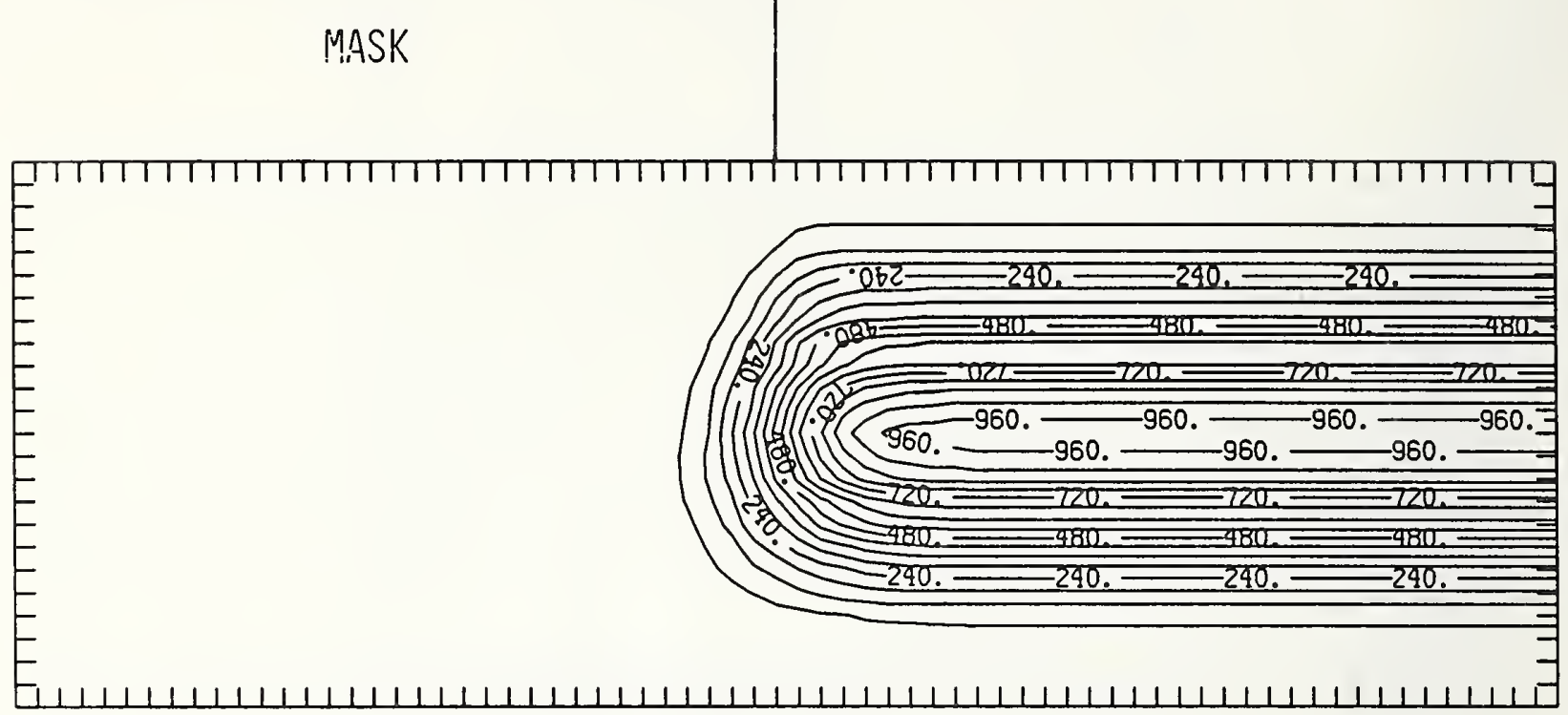

Figure Ga350-3 Two-dimensional distribution of $350 \mathrm{keV}$ gallium implanted past a mask edge. The length increment (distance between tick marks) is $0.02 \mu \mathrm{m}$ and the scale factor is 1000.

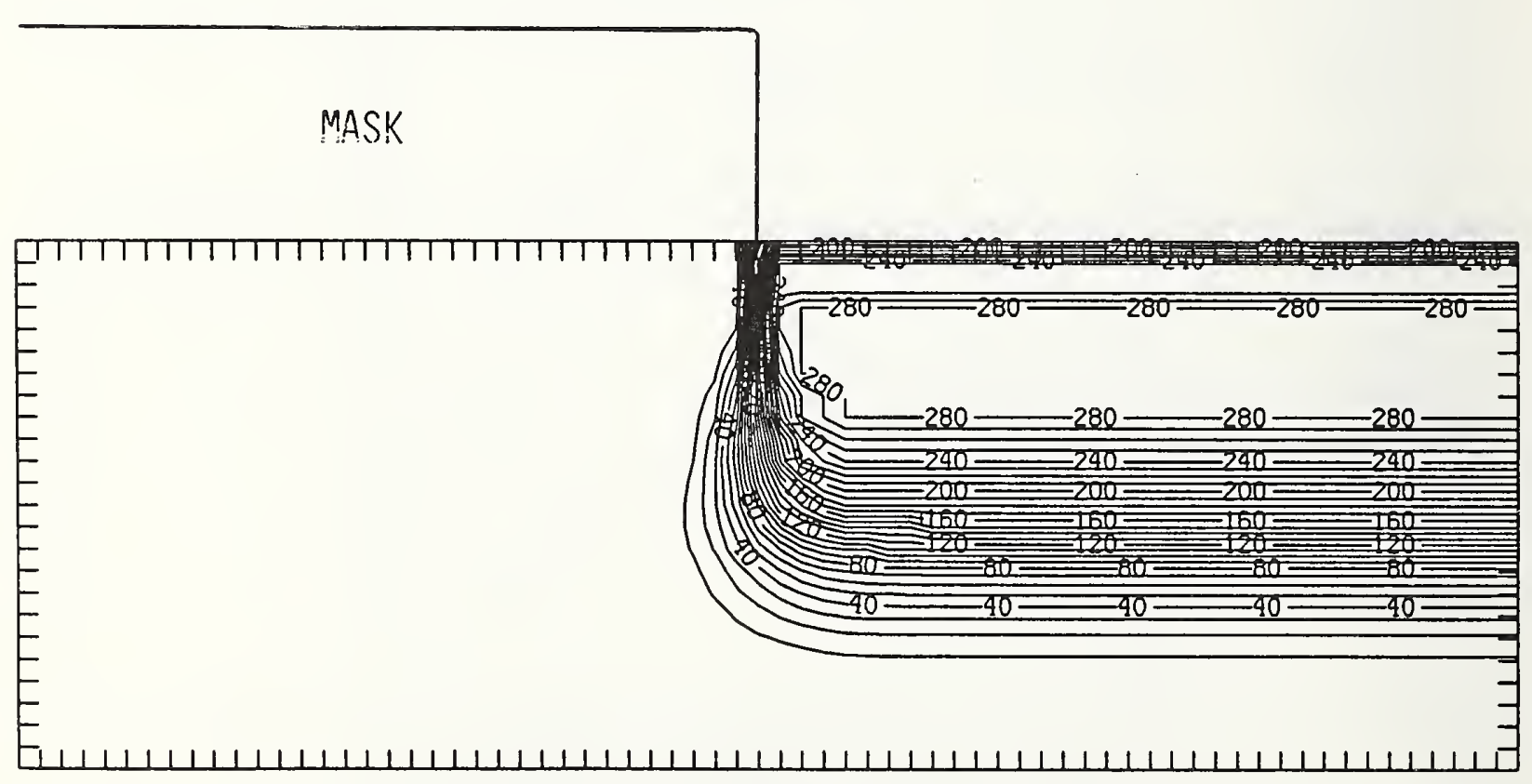

Figure Ga350-4 Two-dimensional distribution of Frenkel pairs created by $350 \mathrm{keV}$ gallium implanted past a mask edge. The length increment (distance between tick marks) is $0.02 \mu \mathrm{m}$ and the scale factor is 0.1 . 


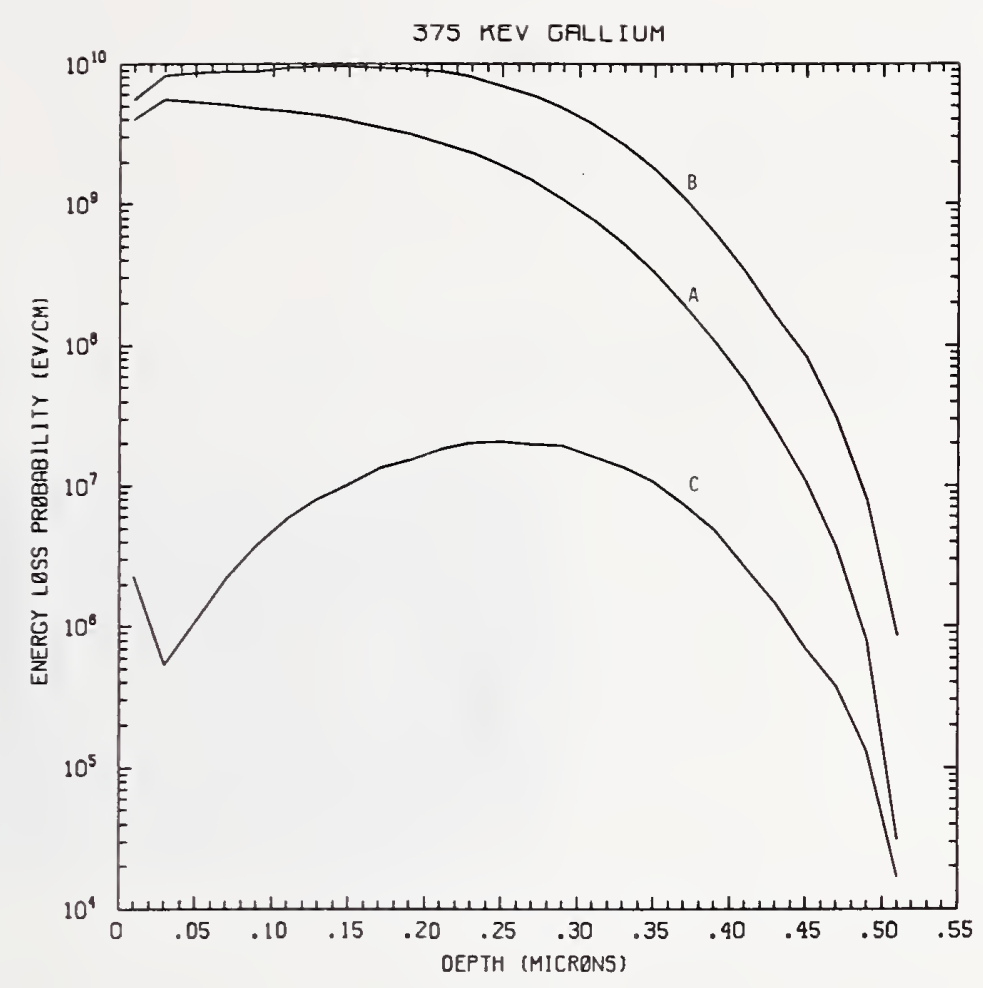

Figure Ga375-1 One-dimensional probability distributions for energy loss due to electronic excitation (A), displacement damage(B), and phonon excitation (C) for $375 \mathrm{keV}$ gallium normally incident on a silicon target.

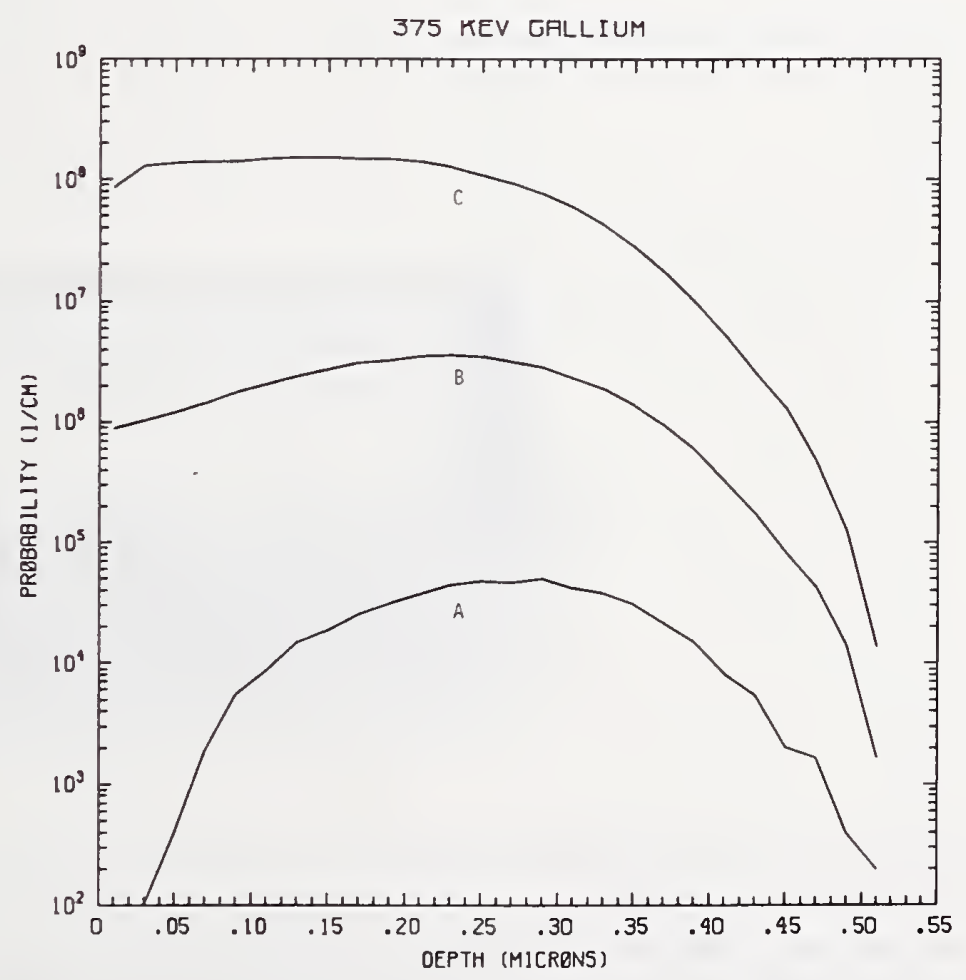

Figure Ga375-2 One-dimensional probability distributions for implanted gallium (A), primary silicon displacements (B), and Frenkel pairs (C) for $375 \mathrm{keV}$ gallium normally incident on a silicon target. 


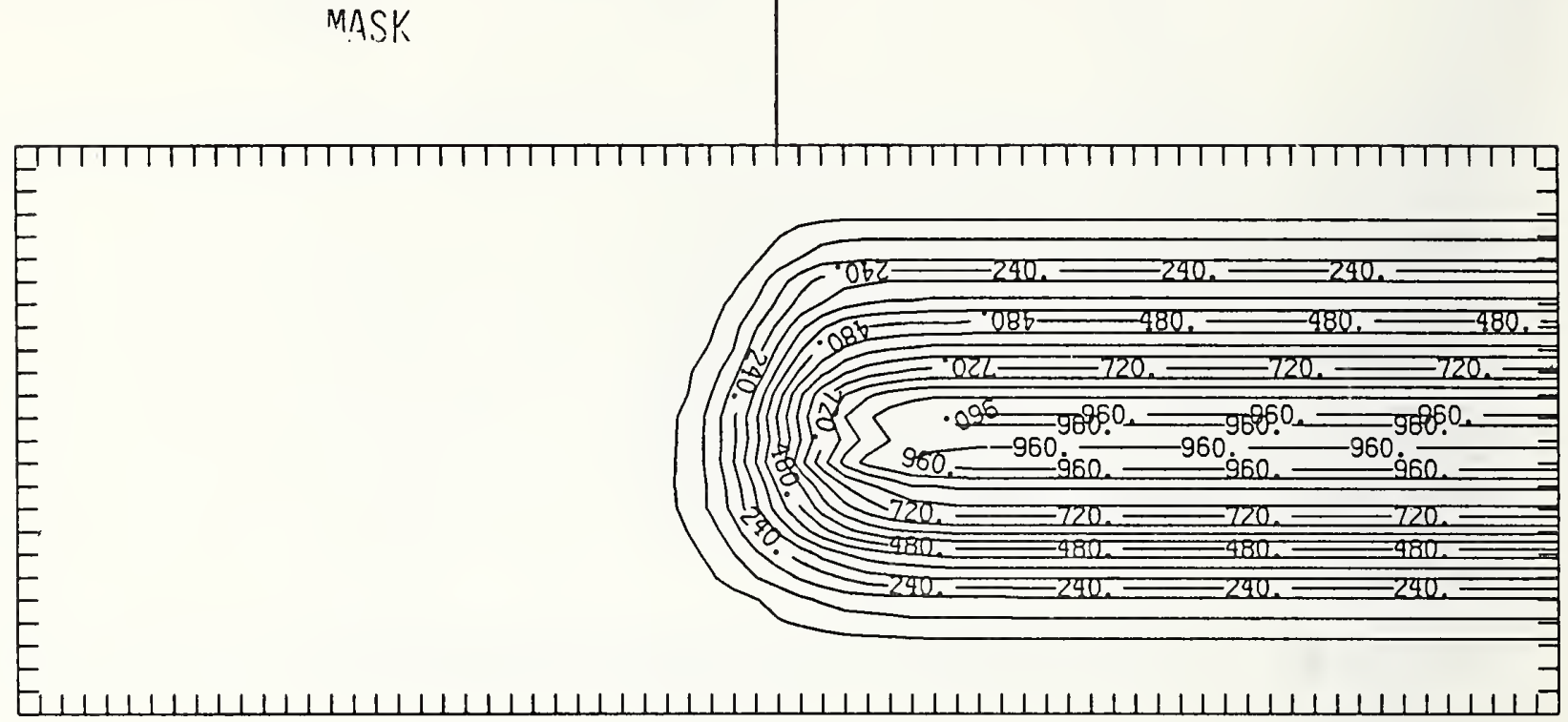

Figure Ga375-3 Two-dimensional distribution of $375 \mathrm{keV}$ gallium implanted past a mask edge. The length increment (distance between tick marks) is $0.02 \mu \mathrm{m}$ and the scale factor is 1000.

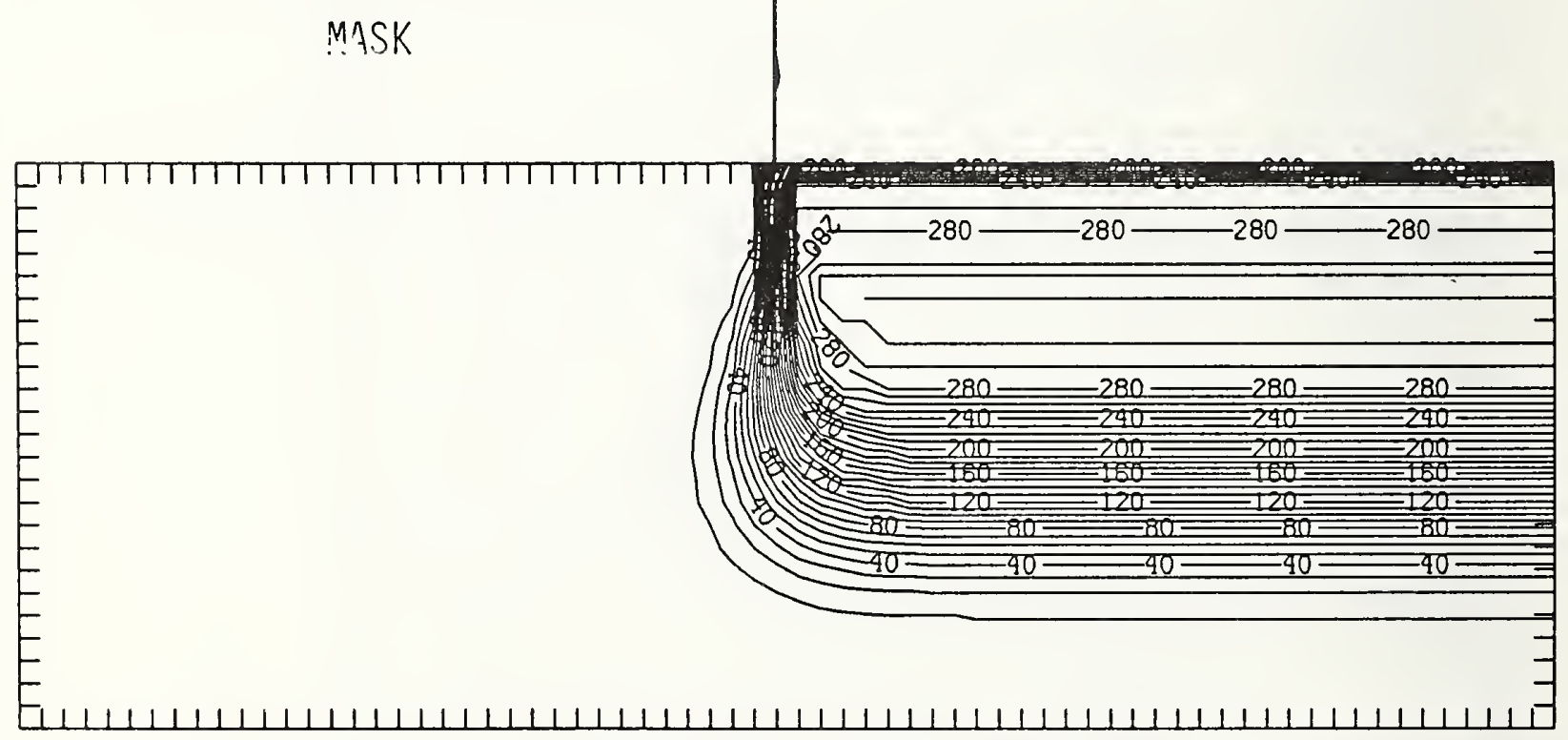

Figure Ga375-4 Two-dimensional distribution of Frenkel pairs created by $375 \mathrm{keV}$ gallium implanted past a mask edge. The length increment (distance between tick marks) is $0.02 \mu \mathrm{n}$ and the scale factor is 0.1 . 


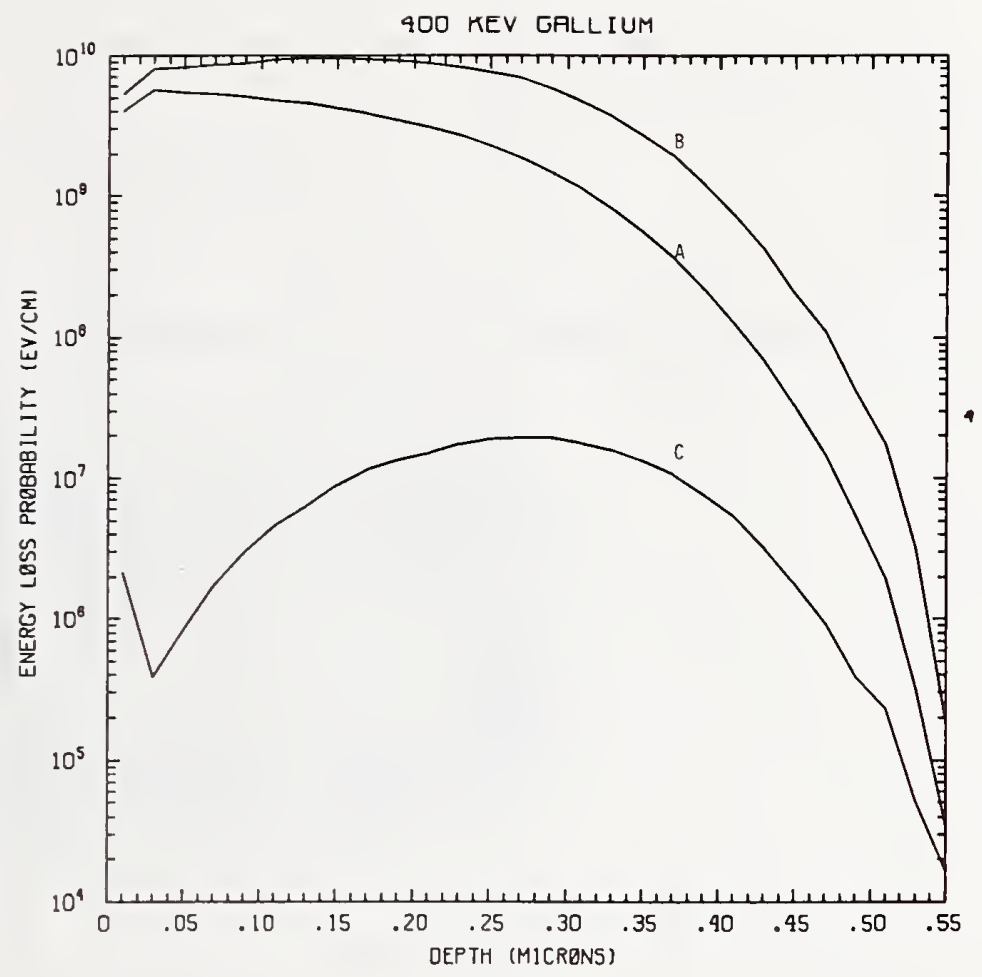

Figure Ga400-1 One-dimensional probability distributions for energy loss due to electronic excitation (A), displacement damage(B), and phonon excitation (C) for $400 \mathrm{keV}$ gallium normally incident on a silicon target.

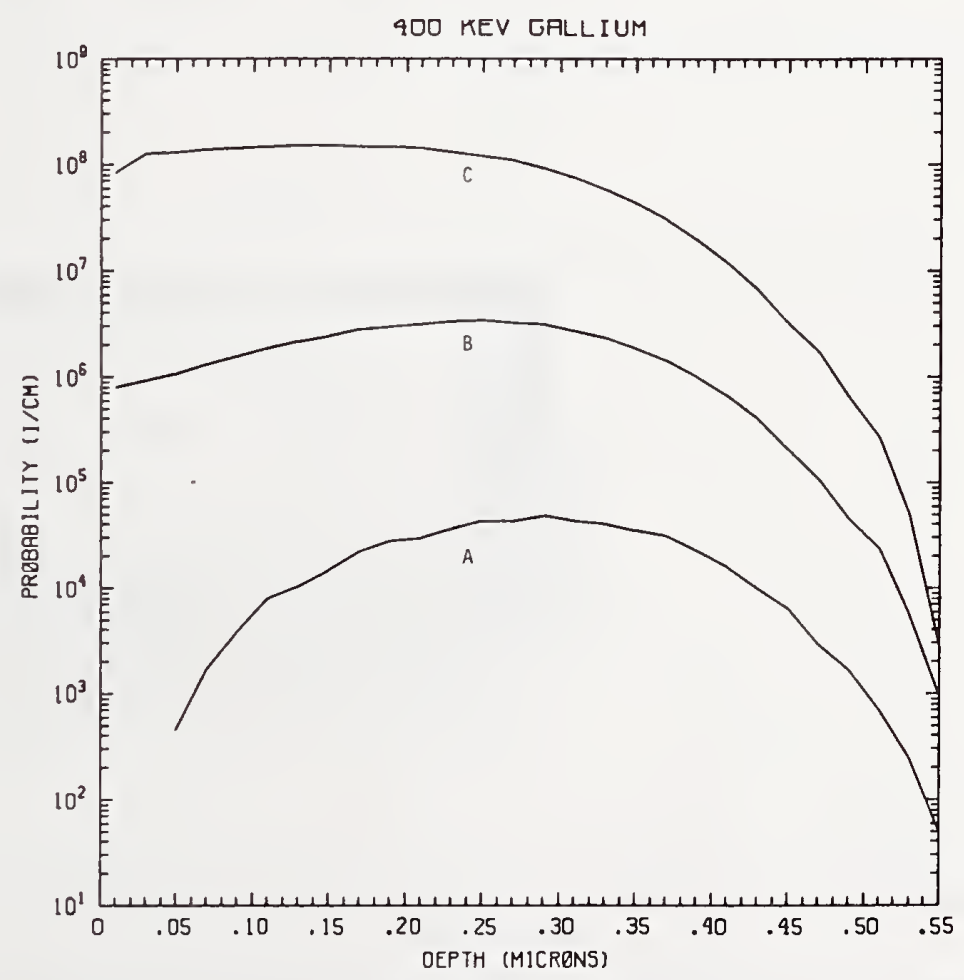

Figure Ga400-2 One-dimensional probability distributions for implanted gallium (A), primary silicon displacements (B), and Frenkel pairs (C) for $400 \mathrm{keV}$ gallium normally incident on a silicon target. 


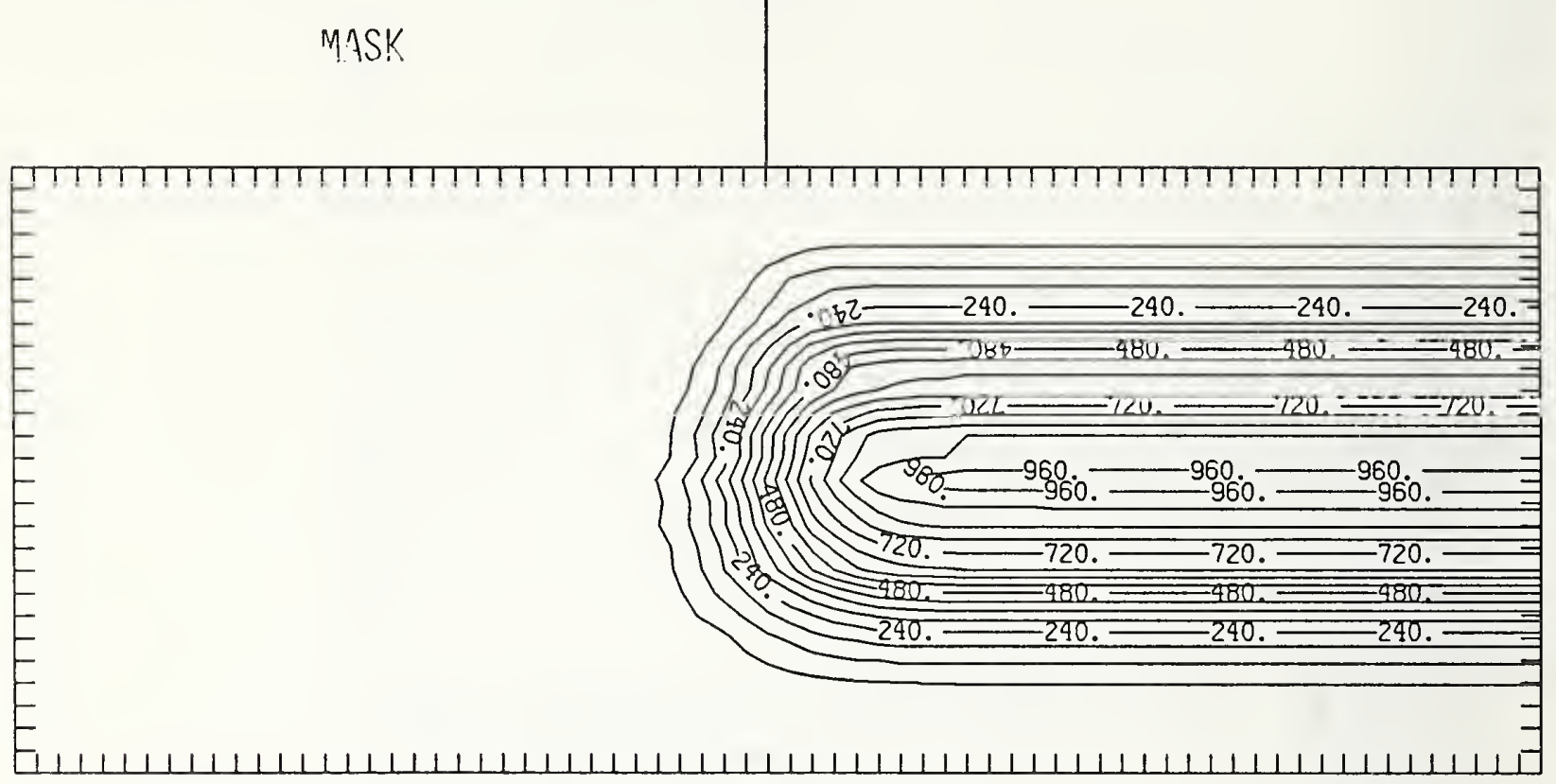

Figure Ga400-3 Two-dimensional distribution of $400 \mathrm{keV}$ gallium implanted past a mask edge. The length increment (distance between tick marks) is $0.02 \mu \mathrm{m}$ and the scale factor is 1000 .

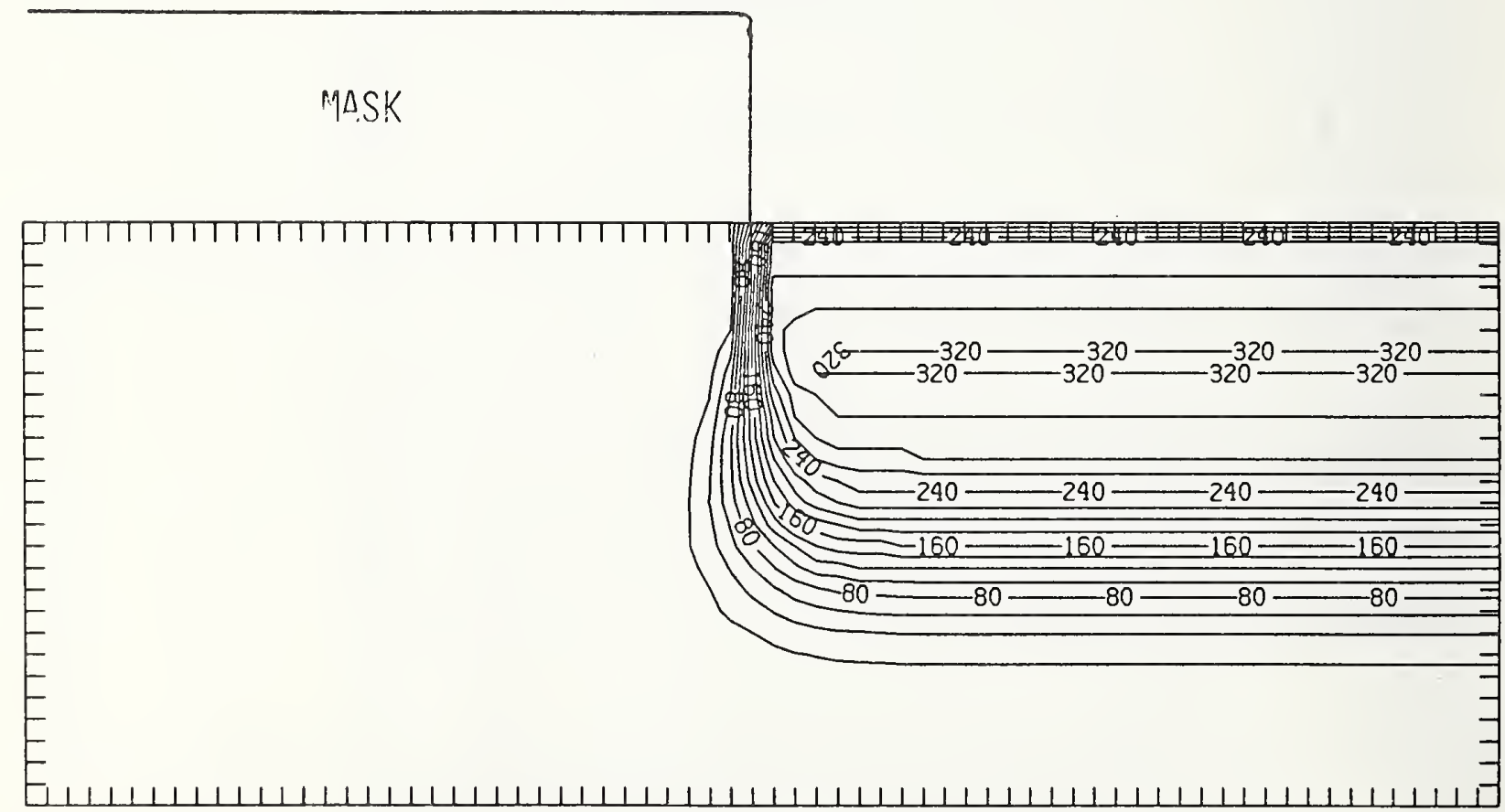

Figure Ga400-4 Two-dimensional distribution of Frenkel pairs created by $400 \mathrm{keV}$ gallium implanted past a mask edge. The length increment (distance between tick marks) is $0.02 \mu \mathrm{m}$ and the scale factor is 0.1 . 


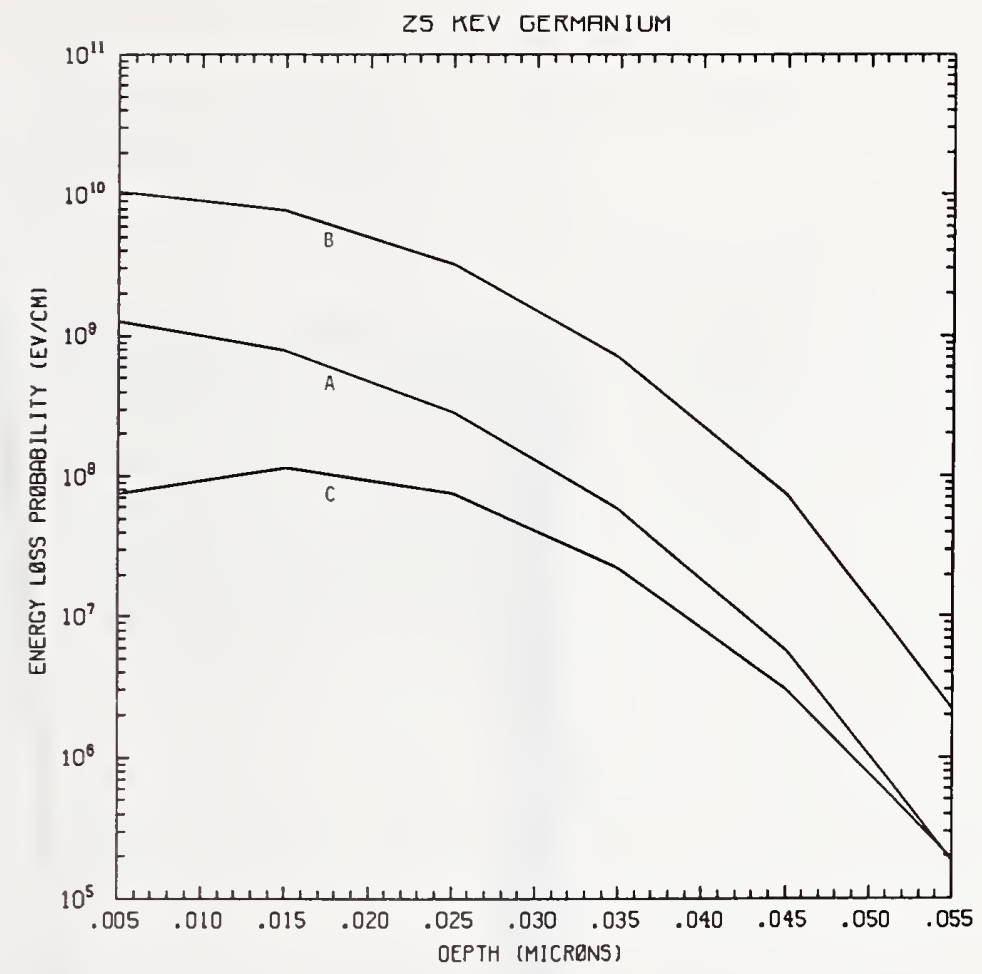

Figure Ge25-1 One-dimensional probability distributions for energy loss due to electronic excitation (A), displacement damage(B), and phonon excitation (C) for $25 \mathrm{keV}$ germanium normally incident on a silicon target.

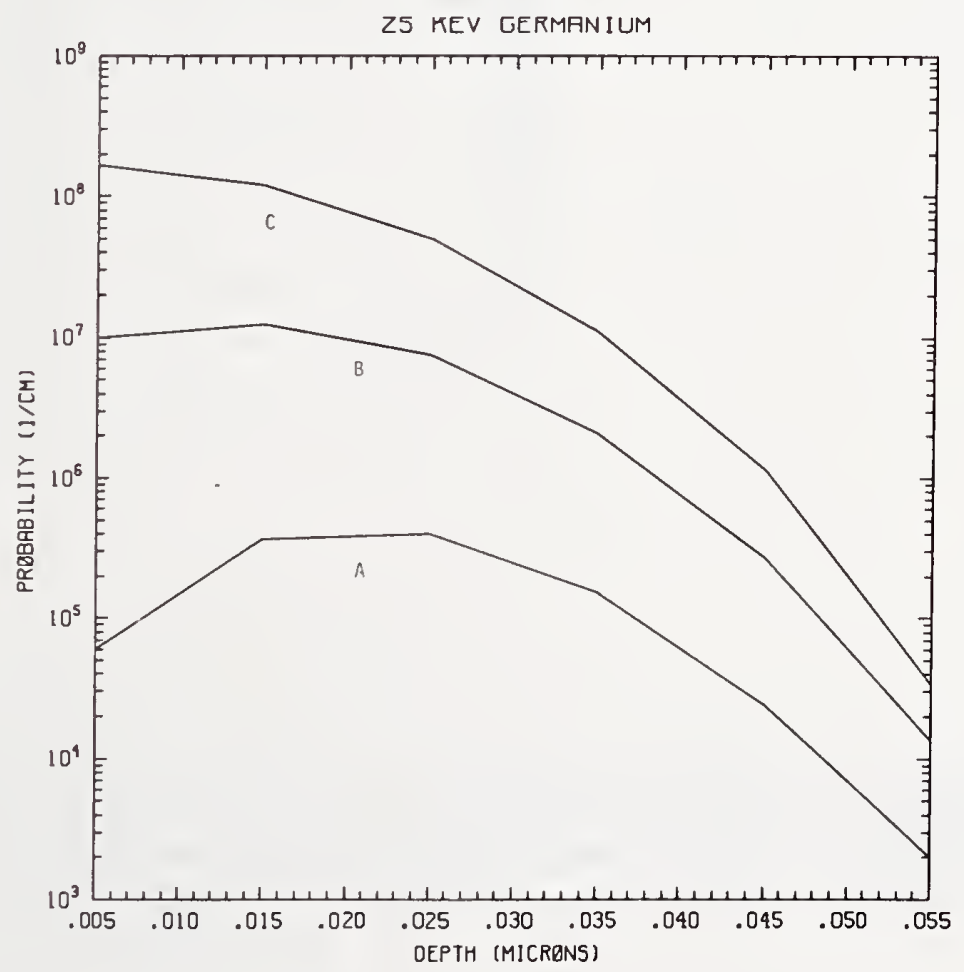

Figure Ge25-2 One-dimensional probability distributions for implanted germanium (A), primary silicon displacements (B), and Frenkel pairs (C) for $25 \mathrm{keV}$ germanium normally incident on a silicon target. 


\section{MASK}

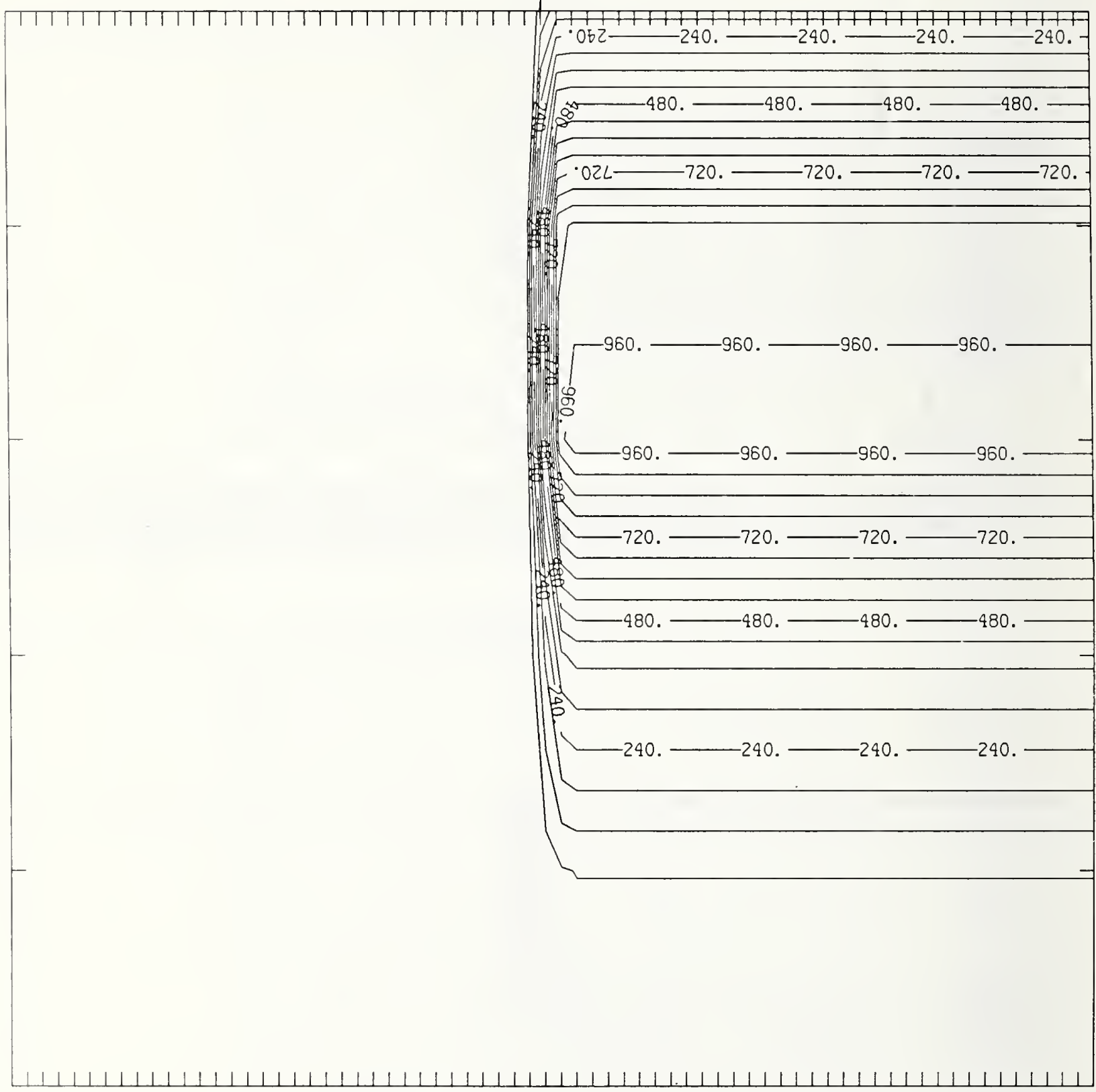

Figure Ge25-3 Two-dimensional distribution of $25 \mathrm{keV}$ germanium implanted past a mask edge. The length increment (distance between tick marks) is $0.01 \mu \mathrm{m}$ and the scale factor is 1000. 
MASK

00

400

$-400$

400

$-400$

20

320

$-320$

320

$-320$

240

$240-240-240-240$

160

$-160$

160

$-160$

160

$-80$

$-80$

80

80

$-80$

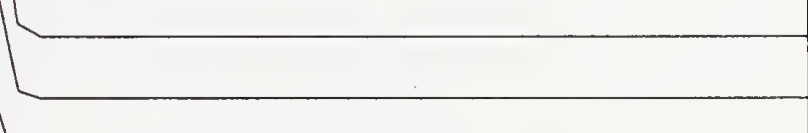

Figure Ge25-4 Two-dimensional distribution of Frenkel pairs created by $25 \mathrm{keV}$ germanium implanted past a mask edge. The length increment (distance between tick marks) is $0.01 \mu \mathrm{m}$ and the scale factor is 1 . 


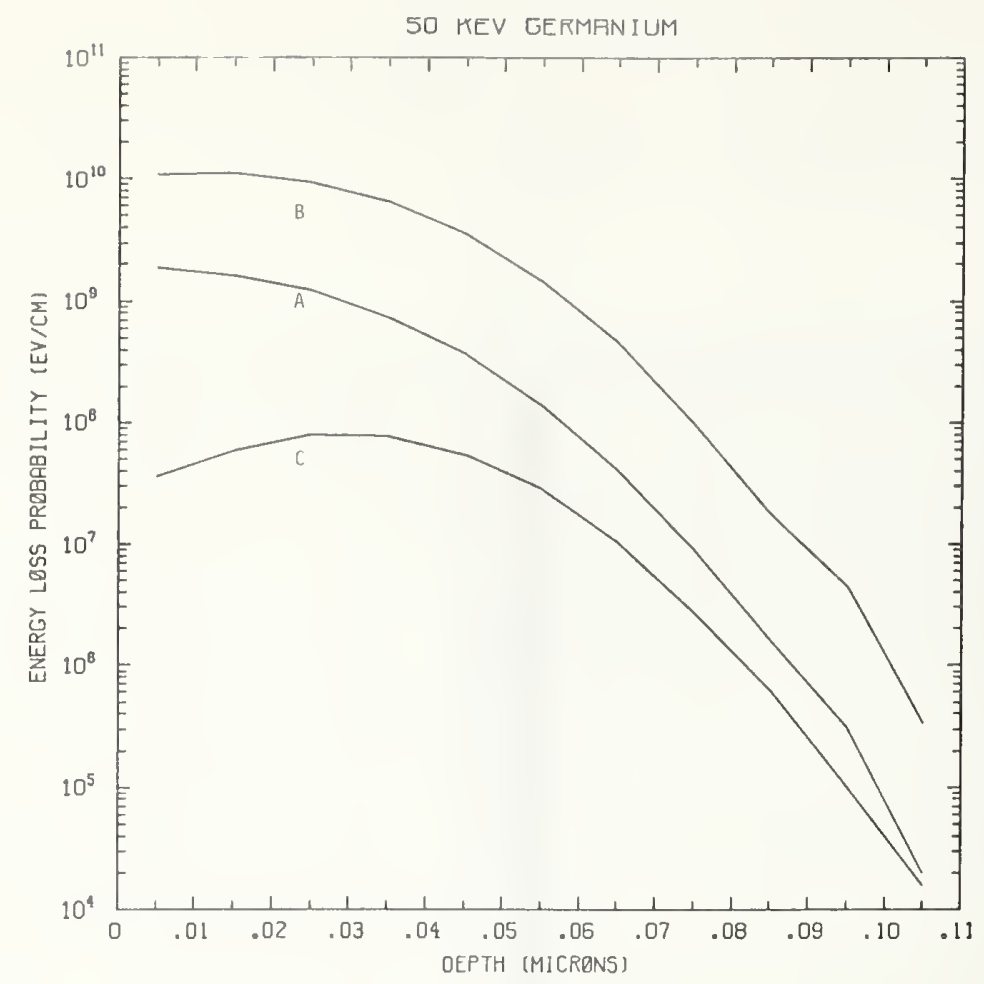

Figure Ge50-1 One-dimensional probability distributions for energy loss due to electronic excitation (A), displacement damage(B), and phonon excitation (C) for $50 \mathrm{keV}$ germanium normally incident on a silicon target.

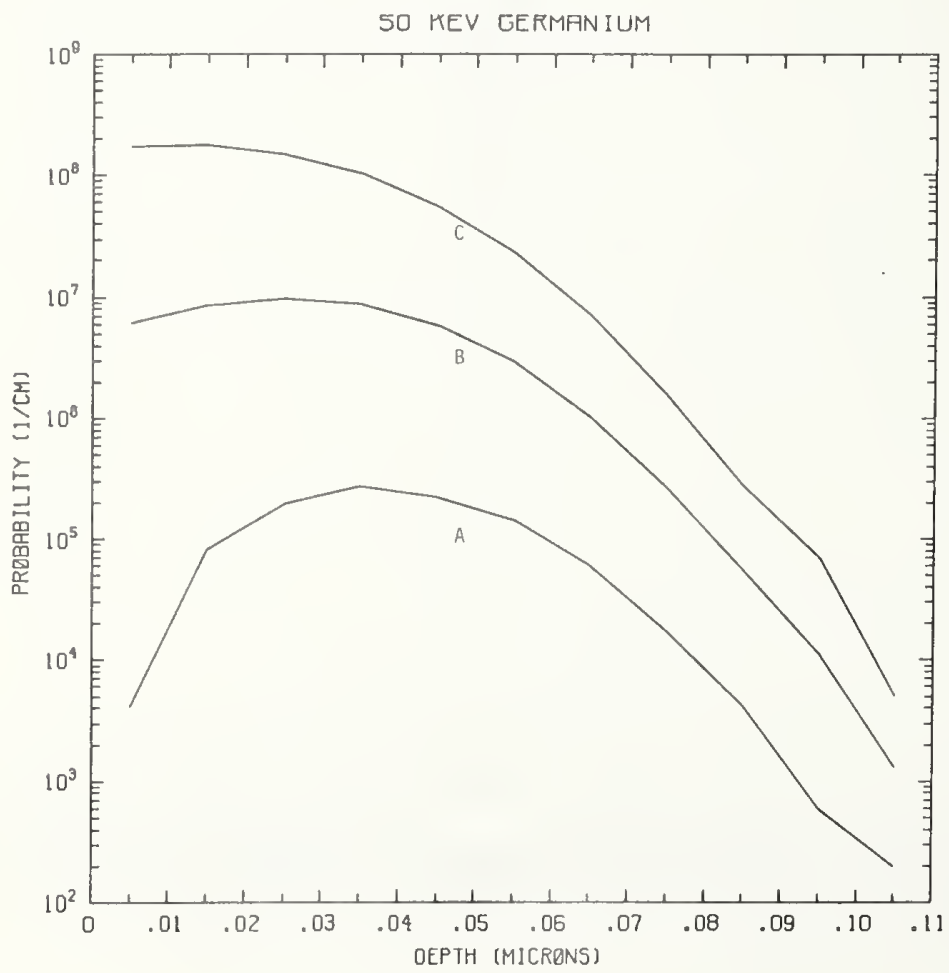

Figure Ge50-2 One-dimensional probability distributions for implanted germanium (A), primary silicon displacements (B), and Frenkel pairs (C) for $50 \mathrm{keV}$ germanium normally incident on a silicon target. 


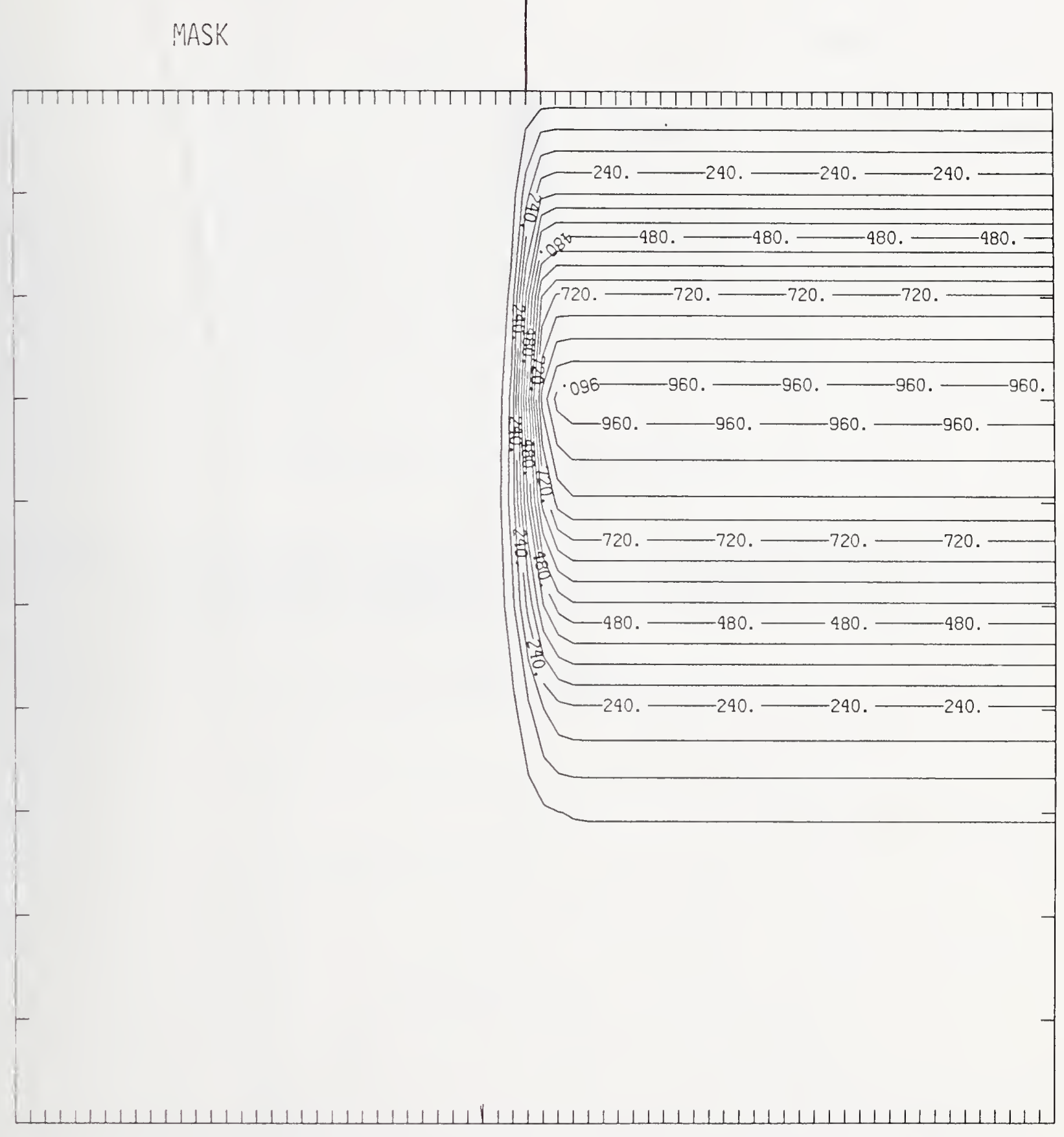

Figure Ge50-3 Two-dimensional distribution of $50 \mathrm{keV}$ germanium implanted past a mask edge. The length increment (distance between tick marks) is $0.01 \mu \mathrm{m}$ and the scale factor is 1000 . 


\section{MASK}

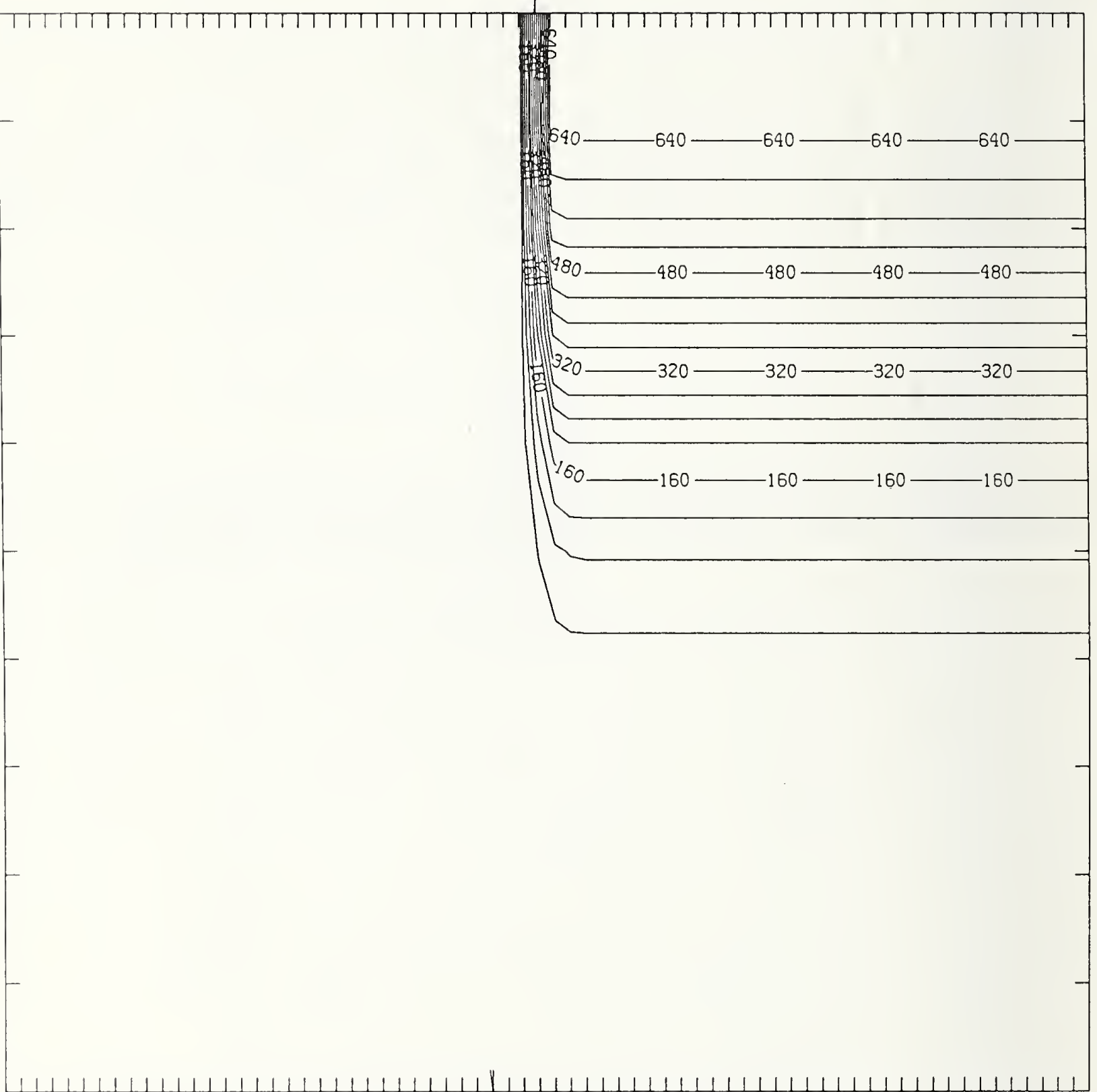

Figure Ge50-4 Two-dimensional distribution of Frenkel pairs created by $50 \mathrm{keV}$ germanium implanted past a mask edge. The length increment (distance between tick marks) is $0.01 \mu \mathrm{m}$ and the scale factor is 1 . 


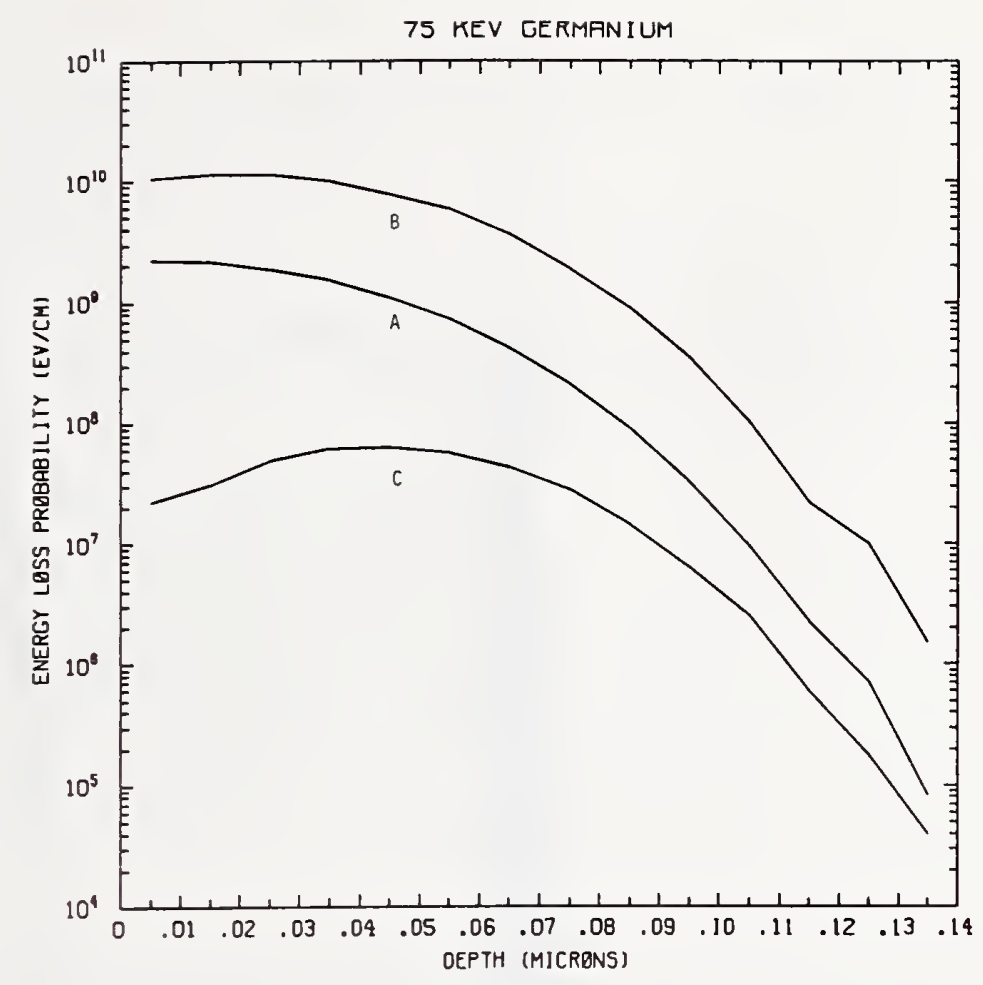

Figure Ge75-1 One-dimensional probability distributions for energy loss due to electronic excitation (A), displacement damage(B), and phonon excitation (C) for $75 \mathrm{keV}$ germanium normally incident on a silicon target.

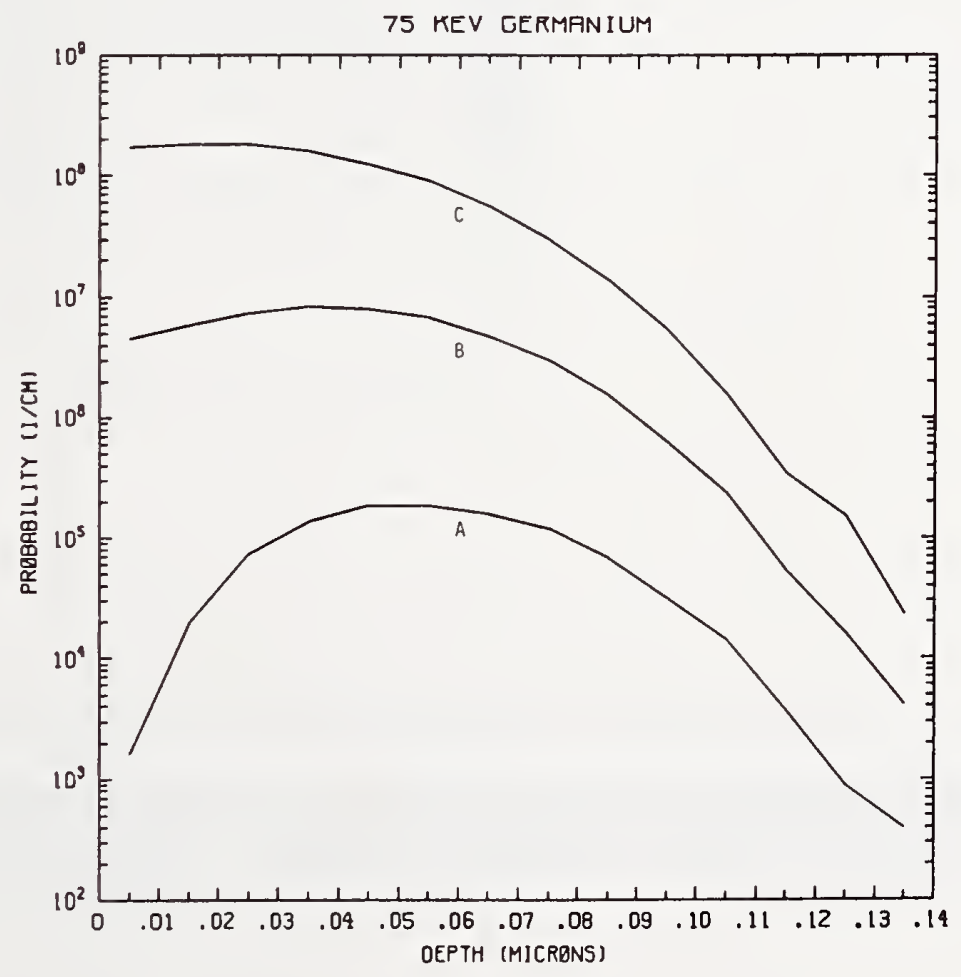

Figure Ge75-2 One-dimensional probability distributions for implanted germanium (A), primary silicon displacements (B), and Frenkel pairs (C) for $75 \mathrm{keV}$ germanium normally incident on a silicon target. 
MASK

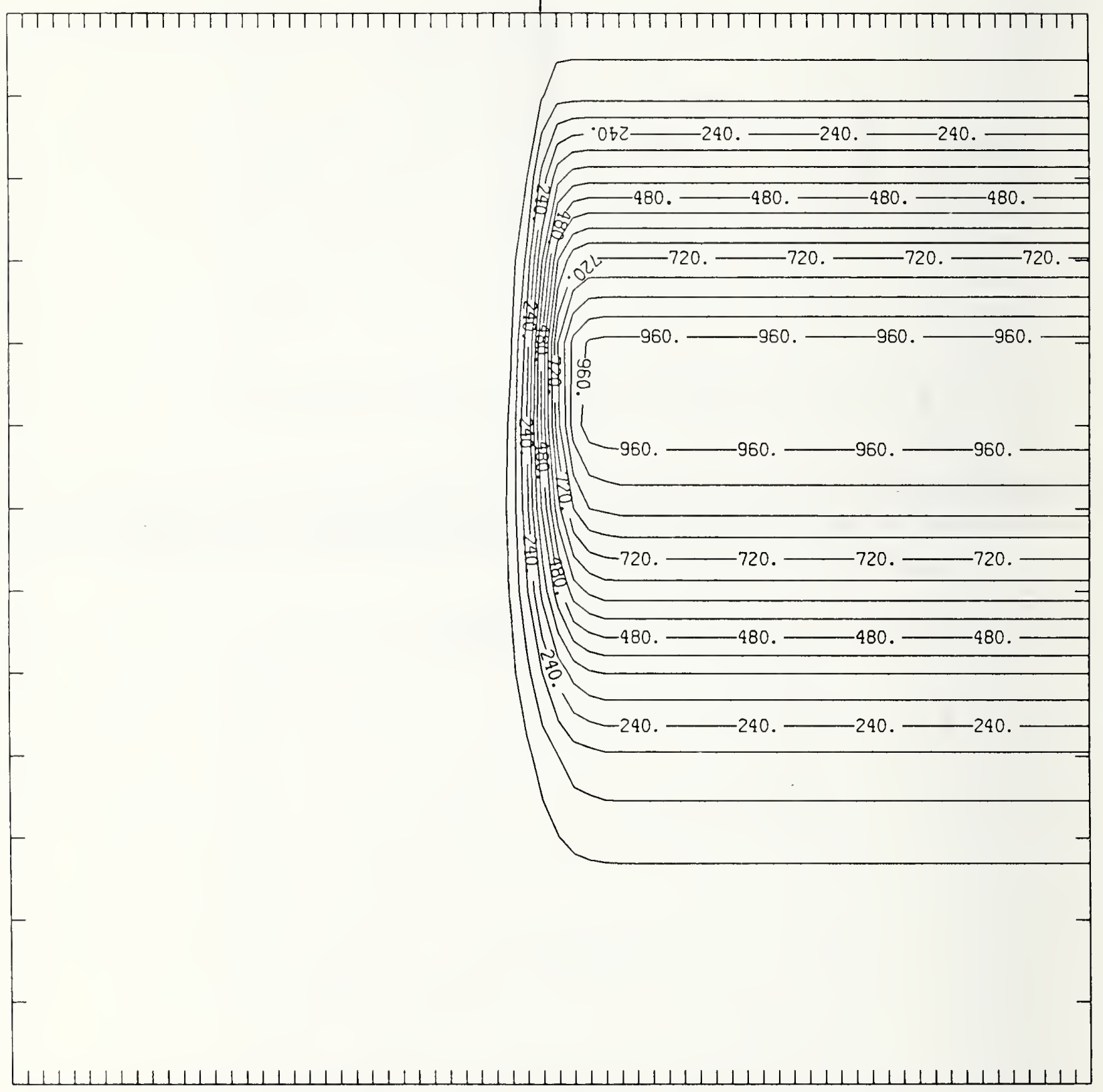

Figure Ge75-3 Two-dimensional distribution of $75 \mathrm{keV}$ germanium implanted past a mask edge. The length increment (distance between tick marks) is $0.01 \mu \mathrm{m}$ and the scale factor is 1000. 


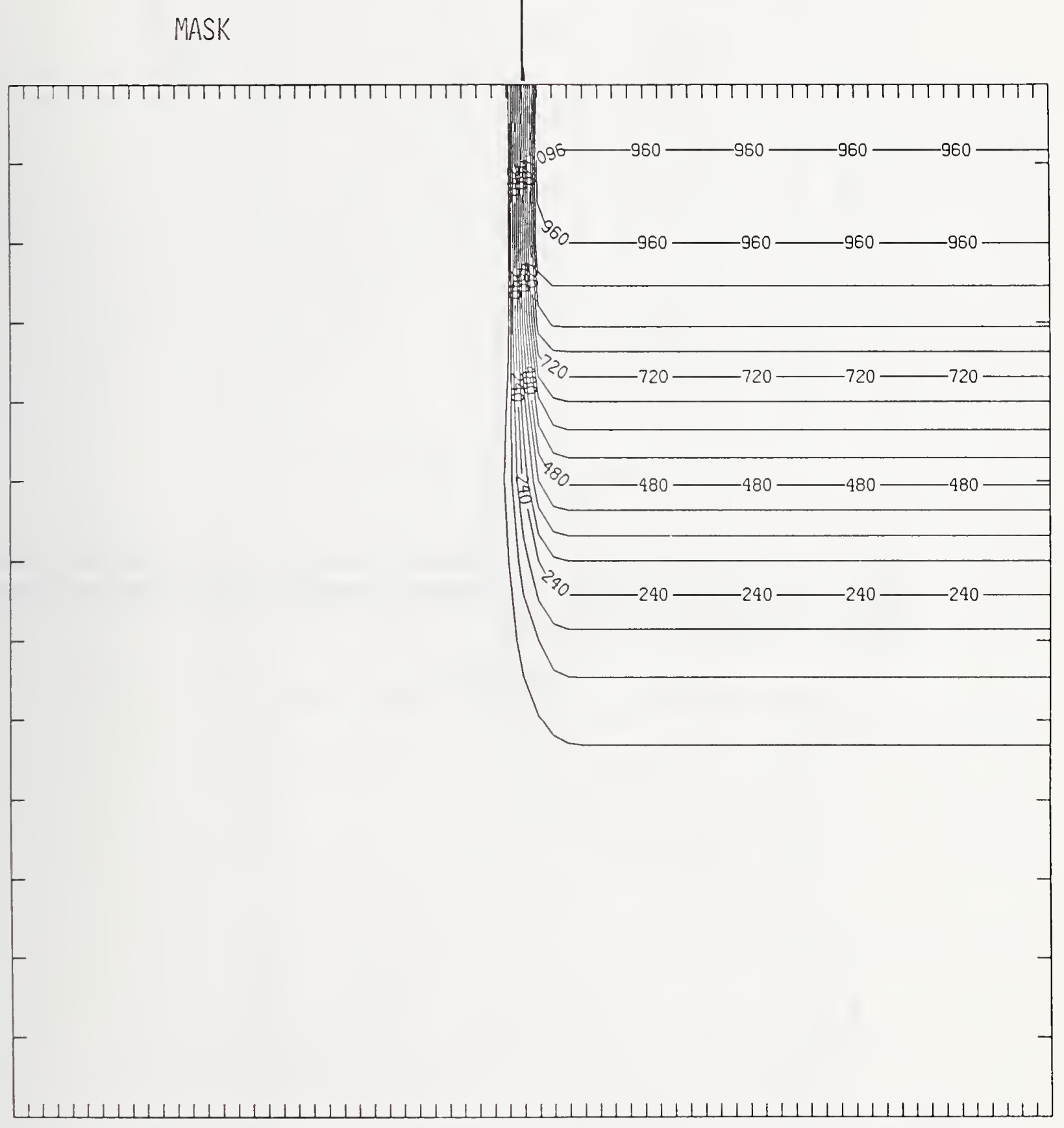

Figure Ge75-4 Two-dimensional distribution of Frenkel pairs created by $75 \mathrm{keV}$ germanium implanted past a mask edge. The length increment (distance between tick marks) is $0.01 \mu \mathrm{m}$ and the scale factor is 1 . 


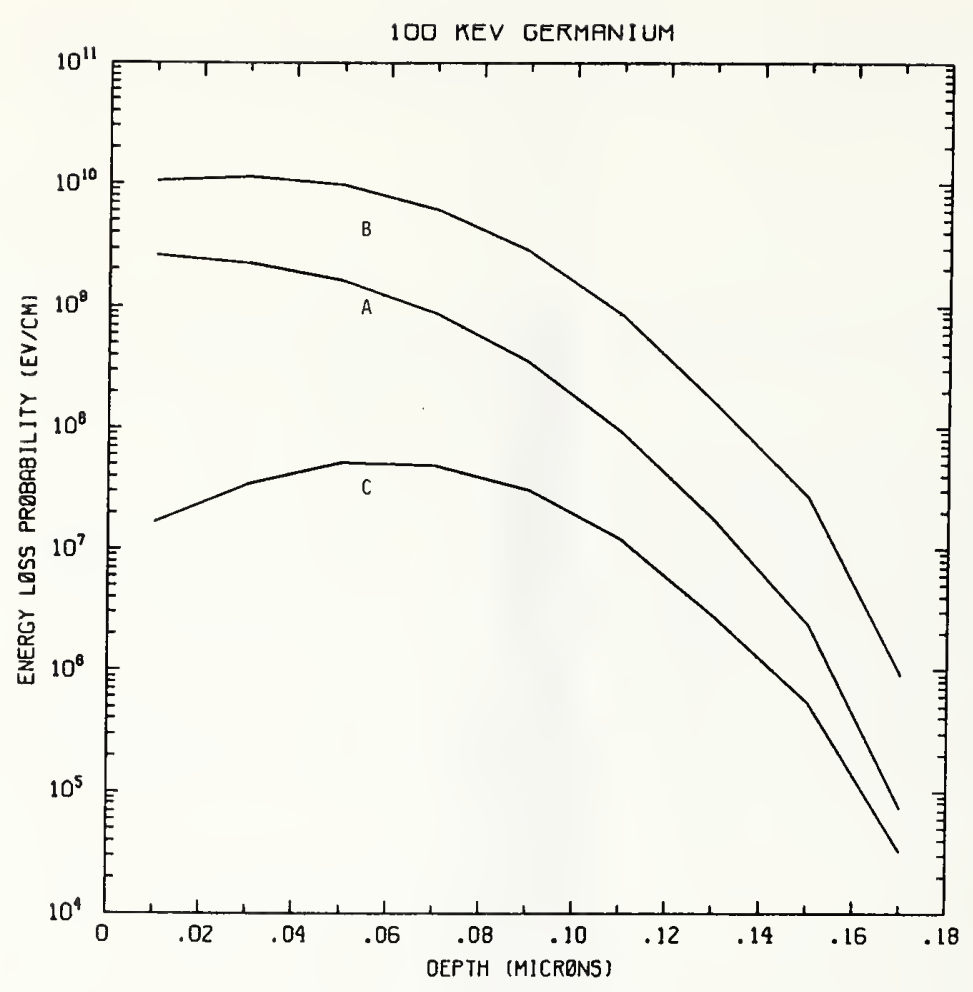

Figure Ge100-1 One-dimensional probability distributions for energy loss due to electronic excitation (A), displacement damage(B), and phonon excitation (C) for $100 \mathrm{keV}$ germanium normally incident on a silicon target.

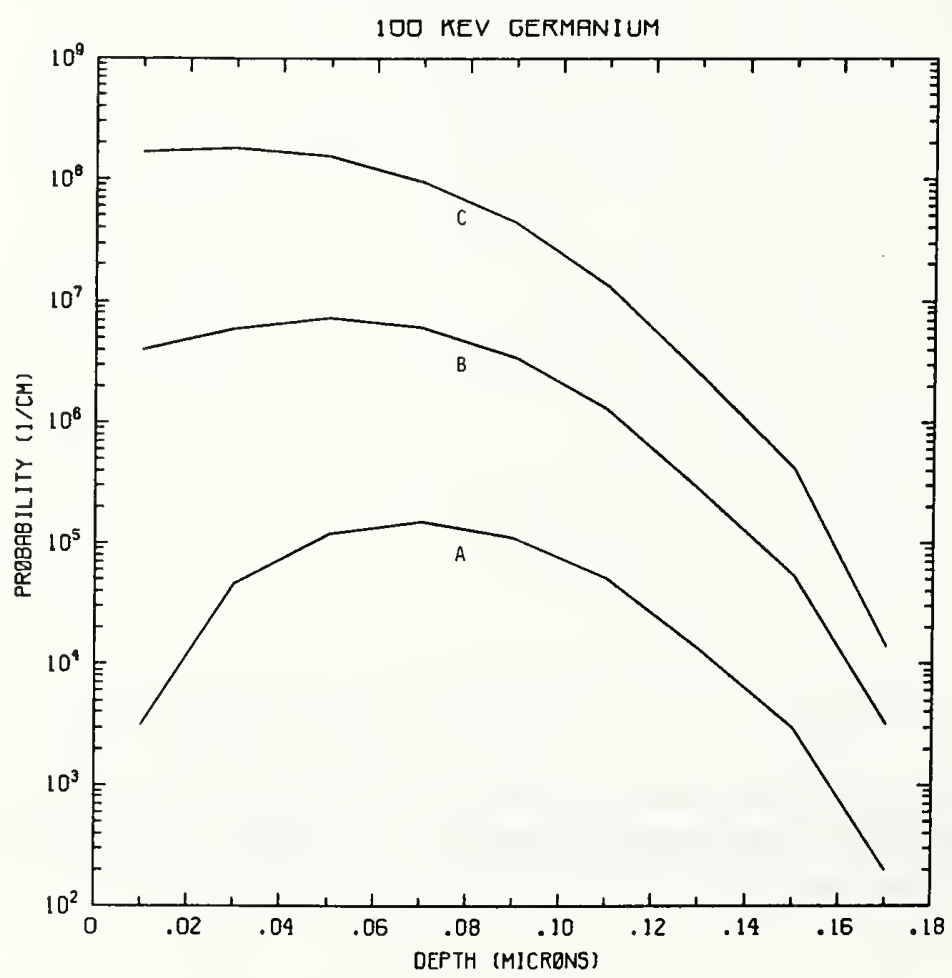

Figure Ge100-2 One-dimensional probability distributions for implanted germanium (A), primary silicon displacements (B), and Frenkel pairs (C) for $100 \mathrm{keV}$ germanium normally incident on a silicon target. 
IMASK

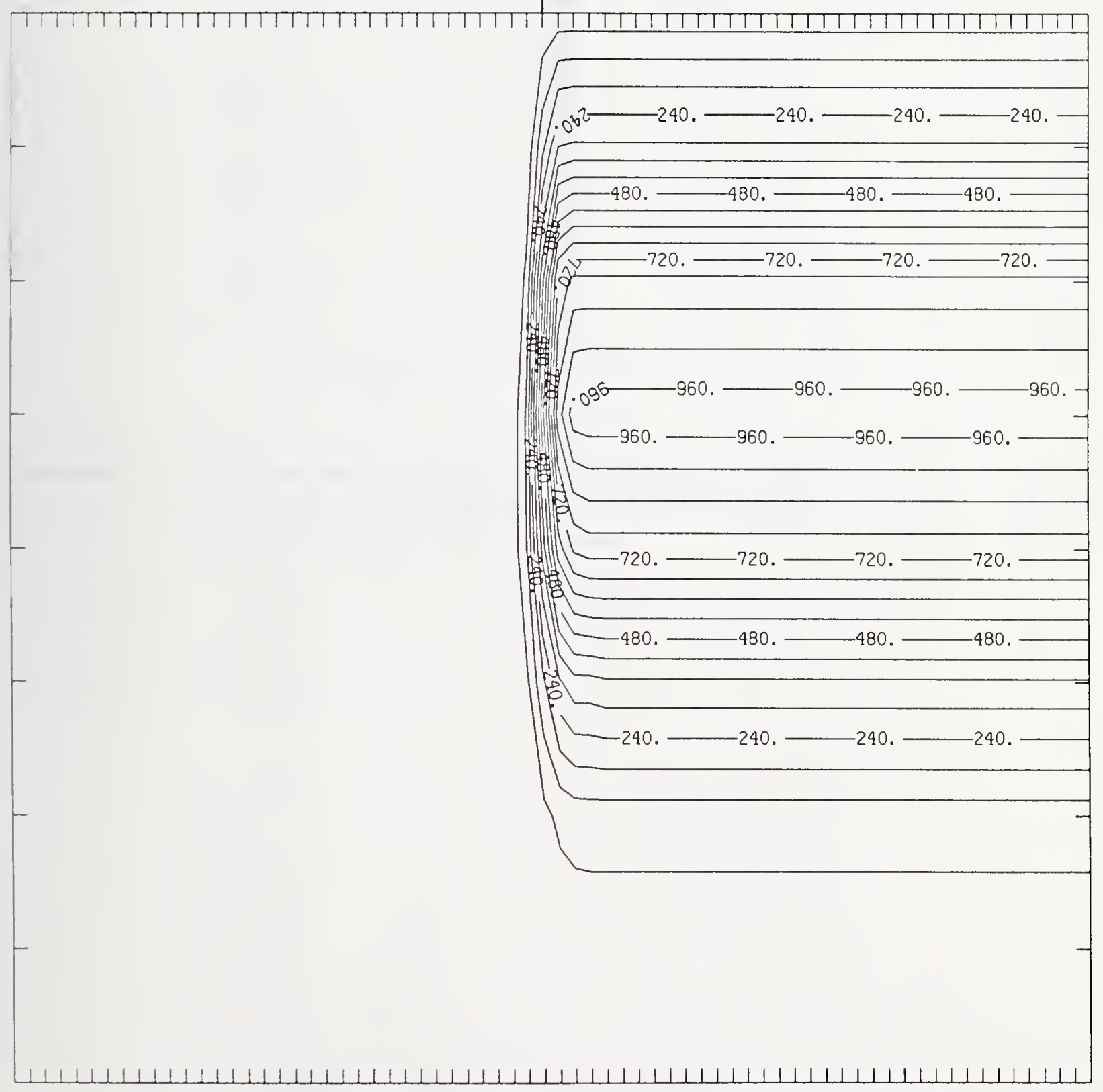

Figure Ge100-3 Two-dimensional distribution of $100 \mathrm{keV}$ germanium implanted past a mask edge. The length increment (distance between tick marks) is $0.02 \mu \mathrm{m}$ and the scale factor is 1000 . 


\section{MASK}

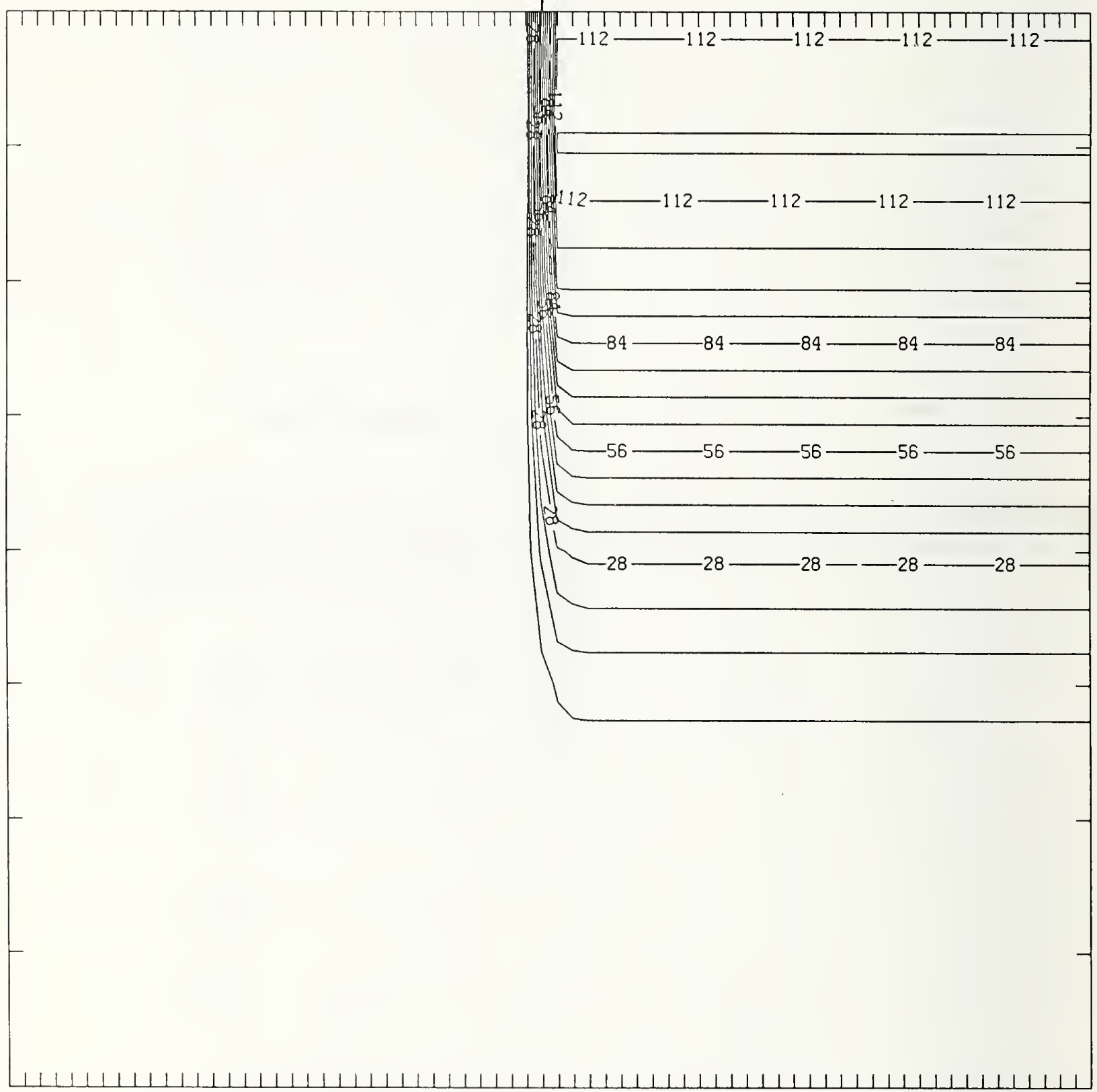

Figure Ge100-4 Two-dimensional distribution of Frenkel pairs created by $100 \mathrm{keV}$ germanium implanted past a mask edge. The length increment (distance between tick marks) is $0.02 \mu \mathrm{m}$ and the scale factor is 0.1 . 


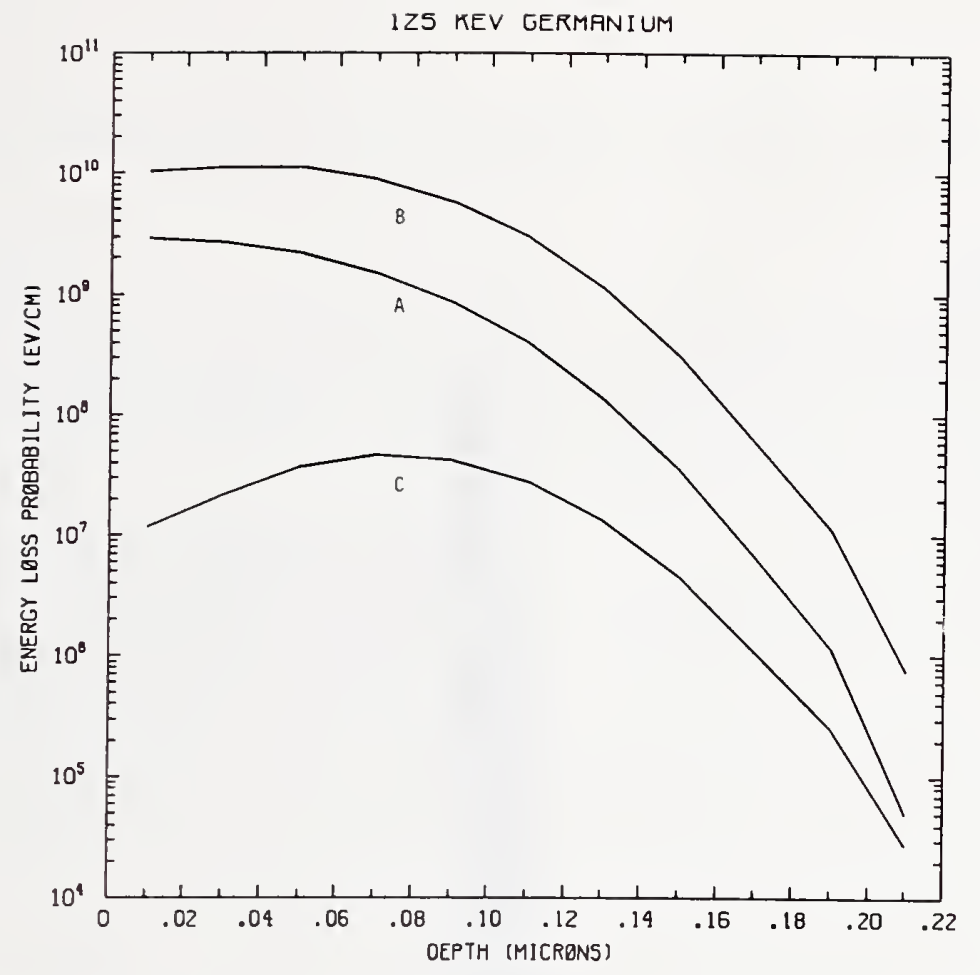

Figure Ge125-1 One-dimensional probability distributions for energy loss due to electronic excitation (A), displacement damage(B), and phonon excitation (C) for $125 \mathrm{keV}$ germanium normally incident on a silicon target.

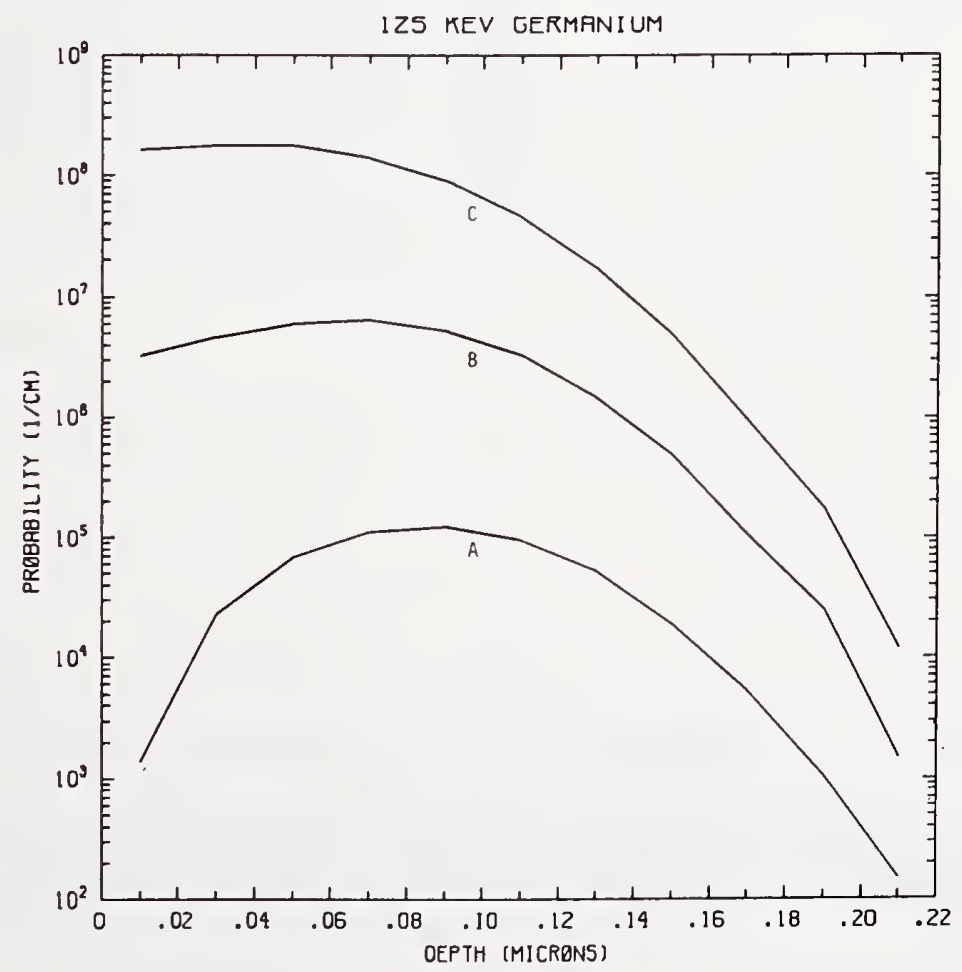

Figure Ge125-2 One-dimensional probability distributions for implanted germanium (A), primary silicon displacements (B), and Frenkel pairs (C) for $125 \mathrm{keV}$ germanium normally incident on a silicon target. 
MASK

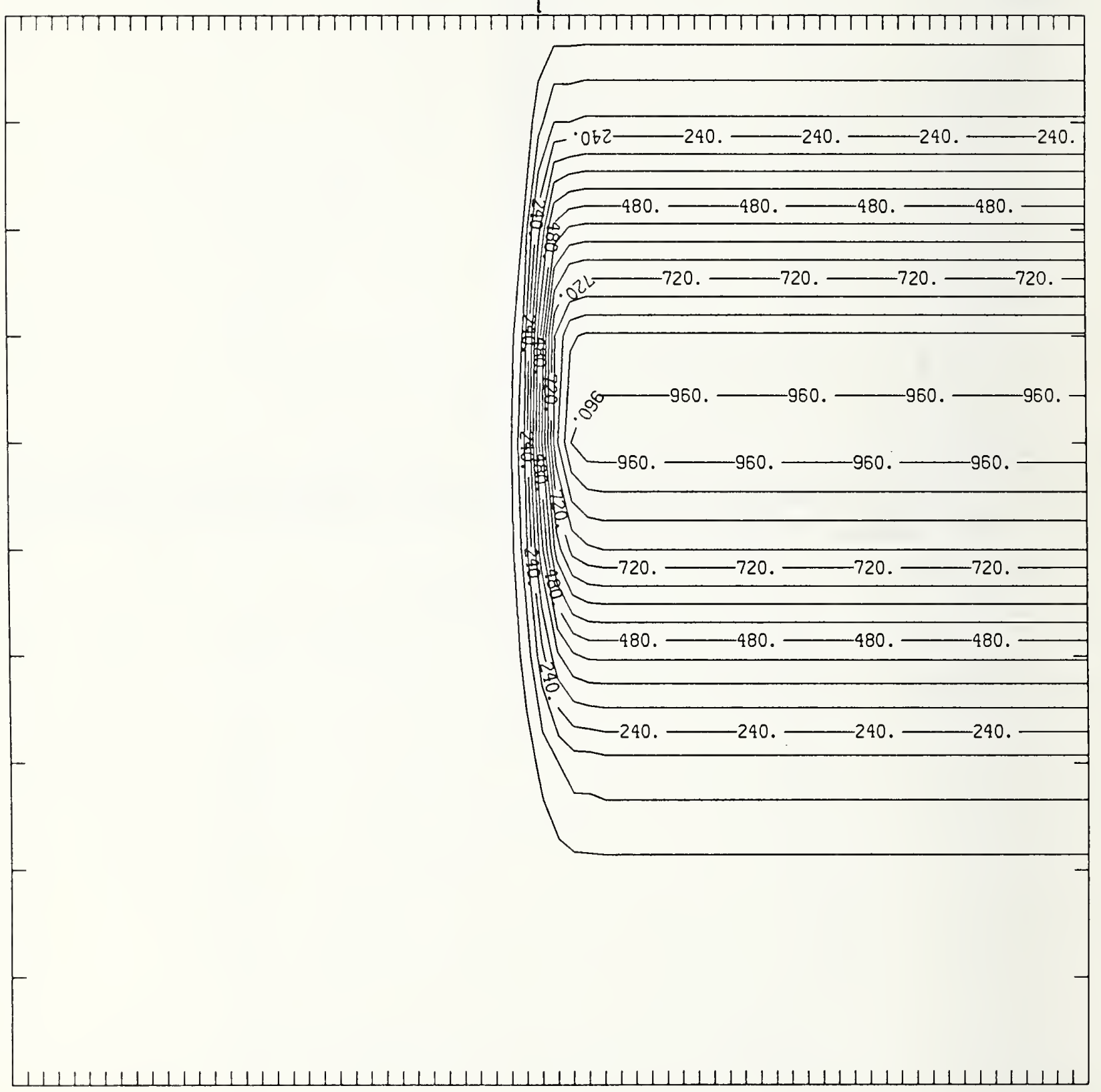

Figure Ge125-3 Two-dimensional distribution of $125 \mathrm{keV}$ germanium implanted past a mask edge. The length increment (distance between tick marks) is $0.02 \mu \mathrm{m}$ and the scale factor is 1000 . 


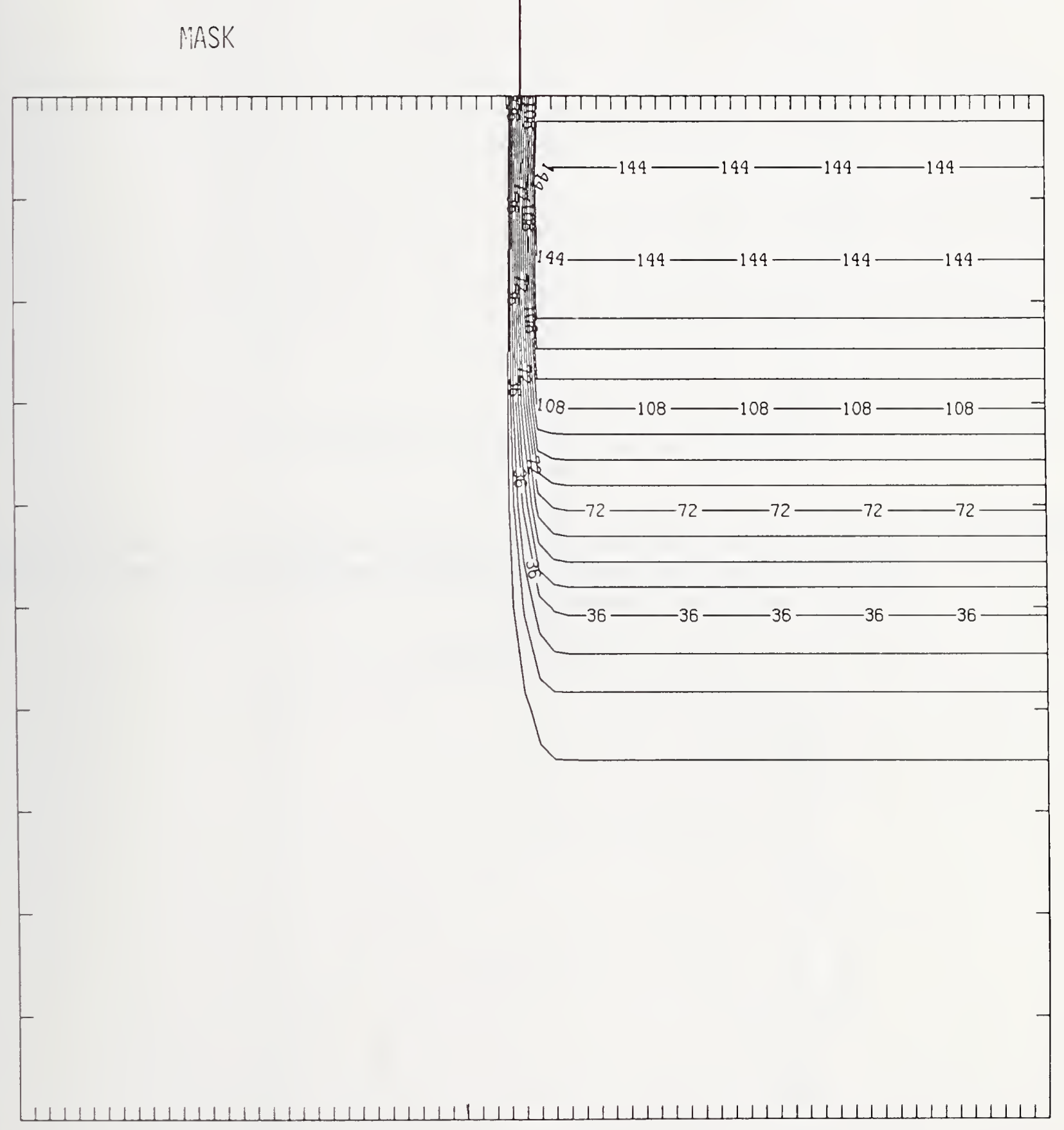

Figure Ge125-4 Two-dimensional distribution of Frenkel pairs created by $125 \mathrm{keV}$ germanium implanted past a mask edge. The length increment (distance between tick marks) is $0.02 \mu \mathrm{m}$ and the scale factor is 0.1 . 


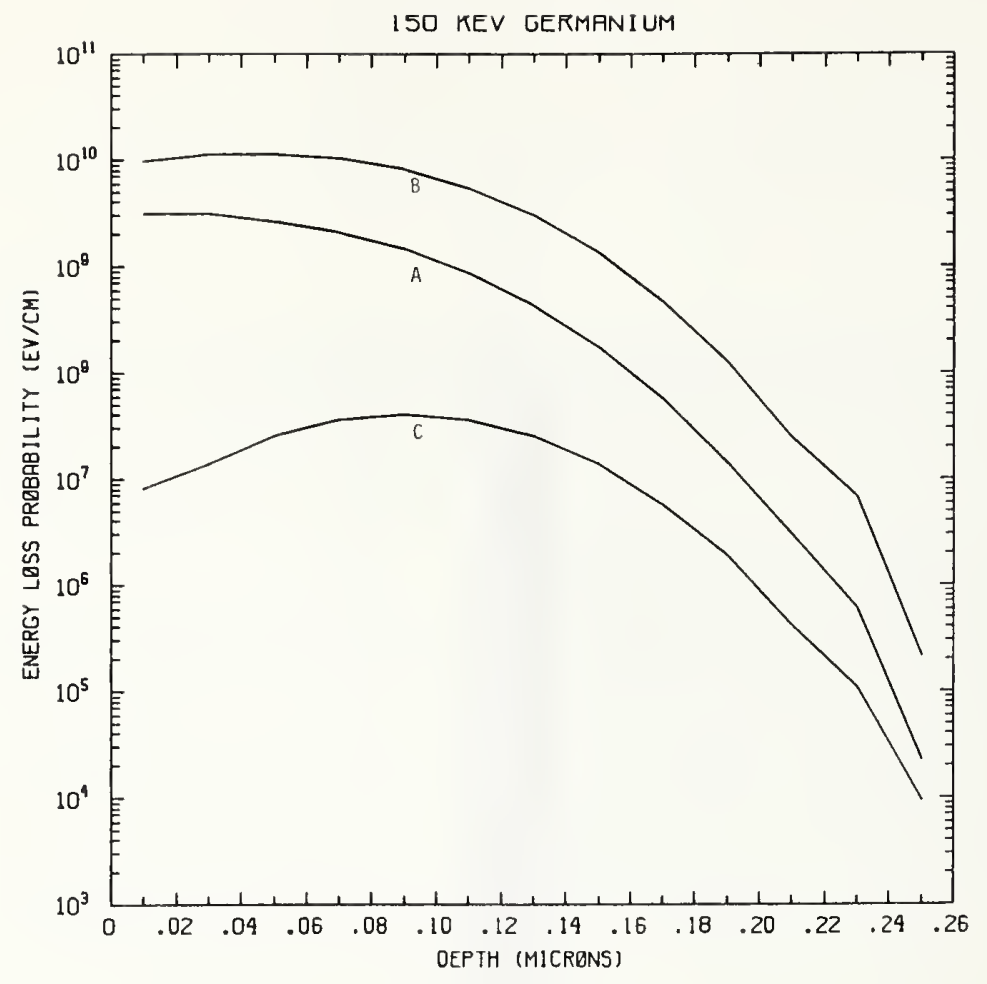

Figure Ge150-1 One-dimensional probability distributions for energy loss due to electronic excitation (A), displacement damage(B), and phonon excitation (C) for $150 \mathrm{keV}$ germanium normally incident on a silicon target.

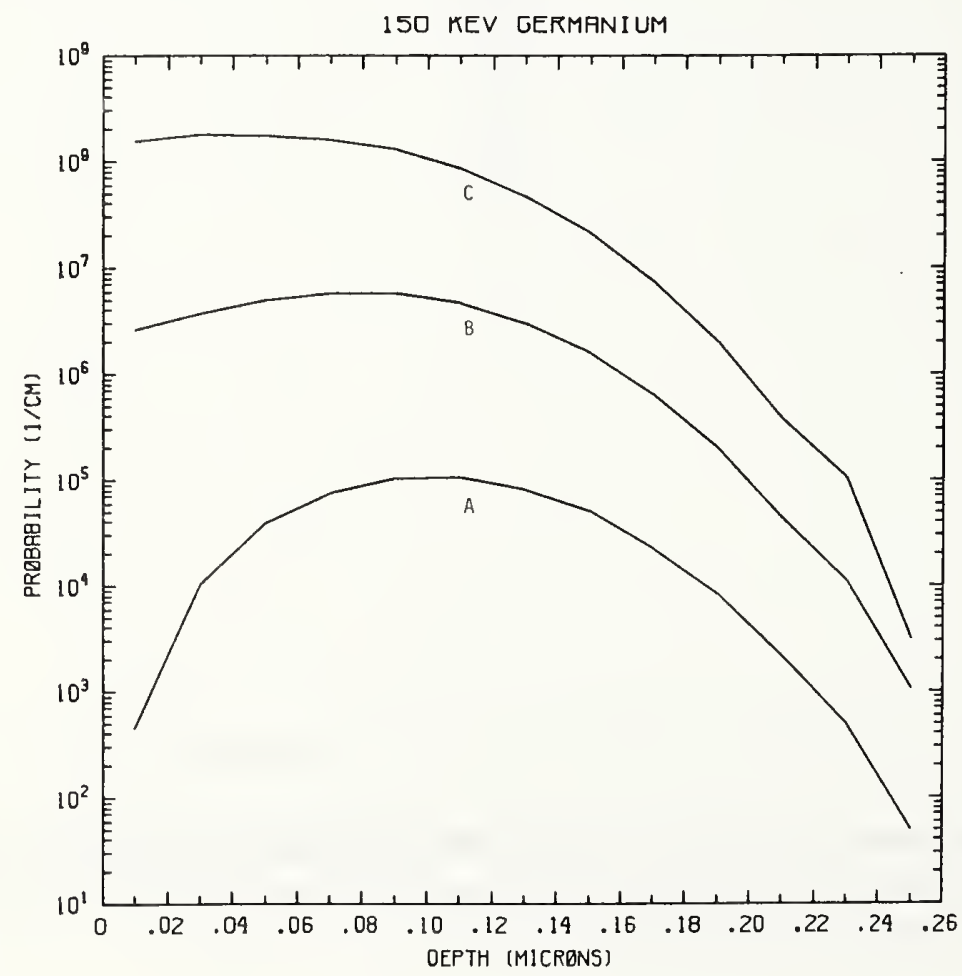

Figure Ge150-2 One-dimensional probability distributions for implanted germanium (A), primary silicon displacements (B), and Frenkel pairs (C) for $150 \mathrm{keV}$ germanium normally incident on a silicon target. 


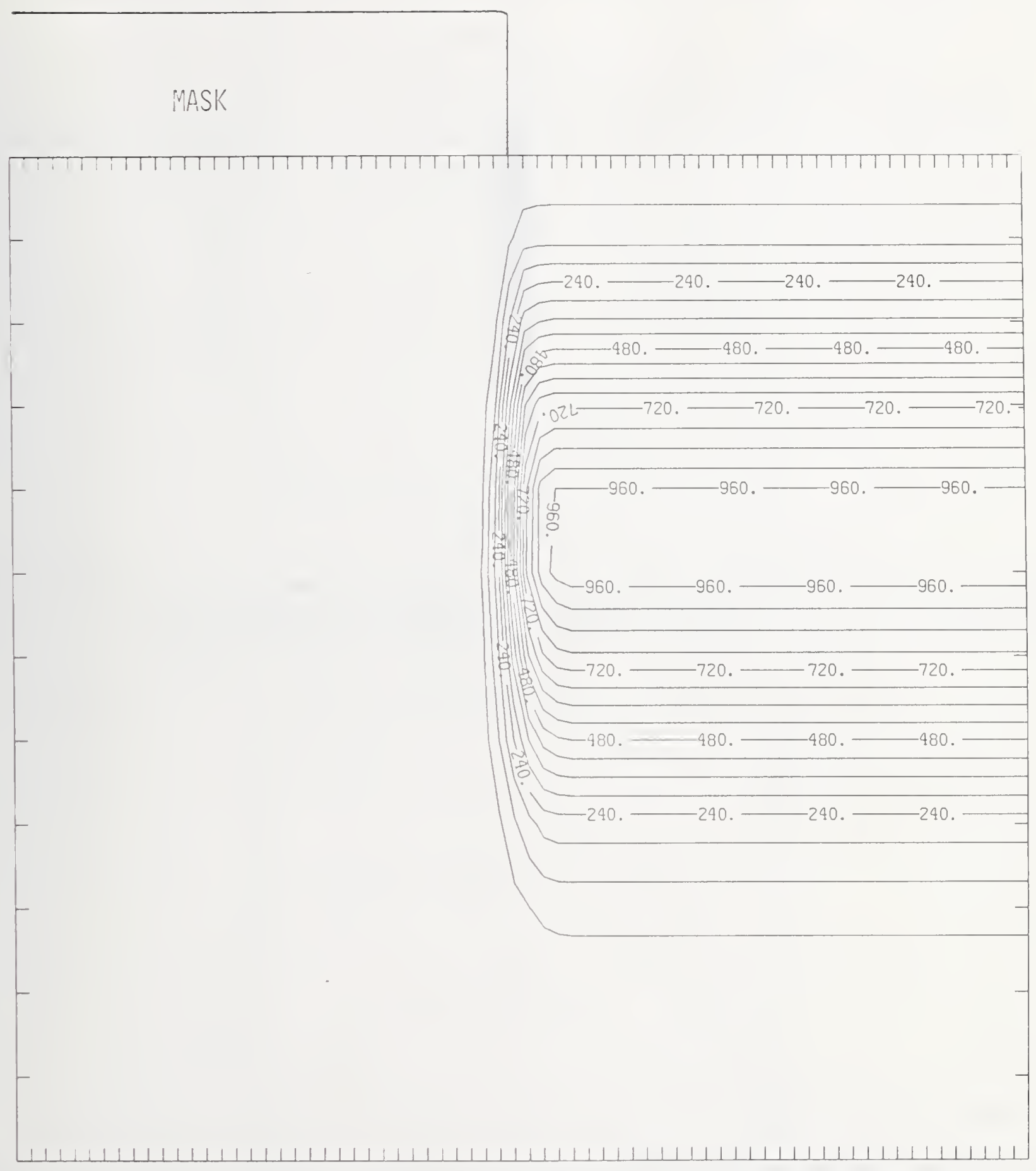

Figure Ge150-3 Two-dimensional distribution of $150 \mathrm{keV}$ germanium implanted past a mask edge. The length increment (distance between tick marks) is $0.02 \mu \mathrm{m}$ and the scale factor is 1000 . 
MASK

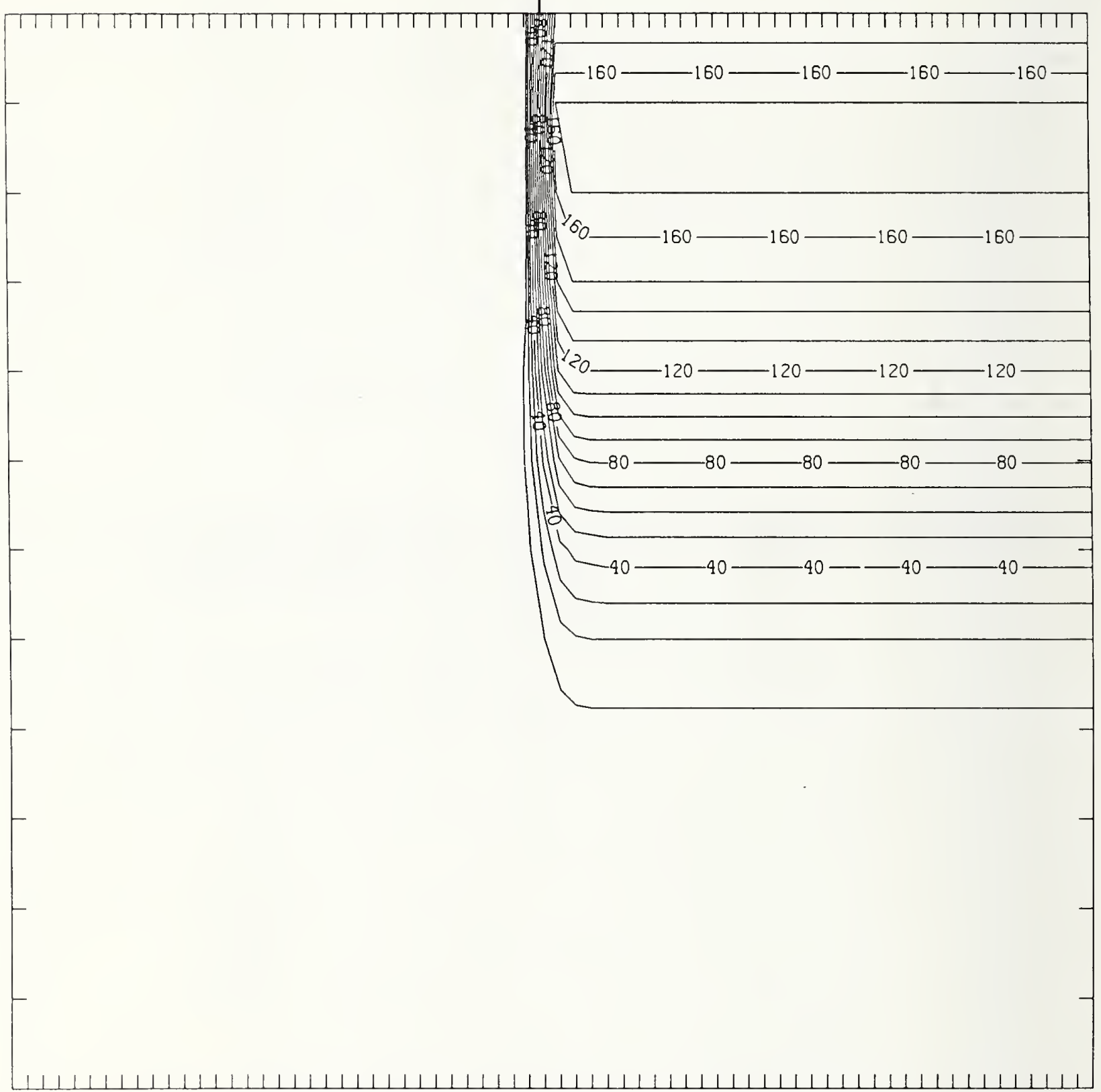

Figure Ge150-4 Two-dimensional distribution of Frenkel pairs created by $150 \mathrm{keV}$ germanium implanted past a mask edge. The length increment (distance between tick marks) is $0.02 \mu \mathrm{m}$ and the scale factor is 0.1 . 


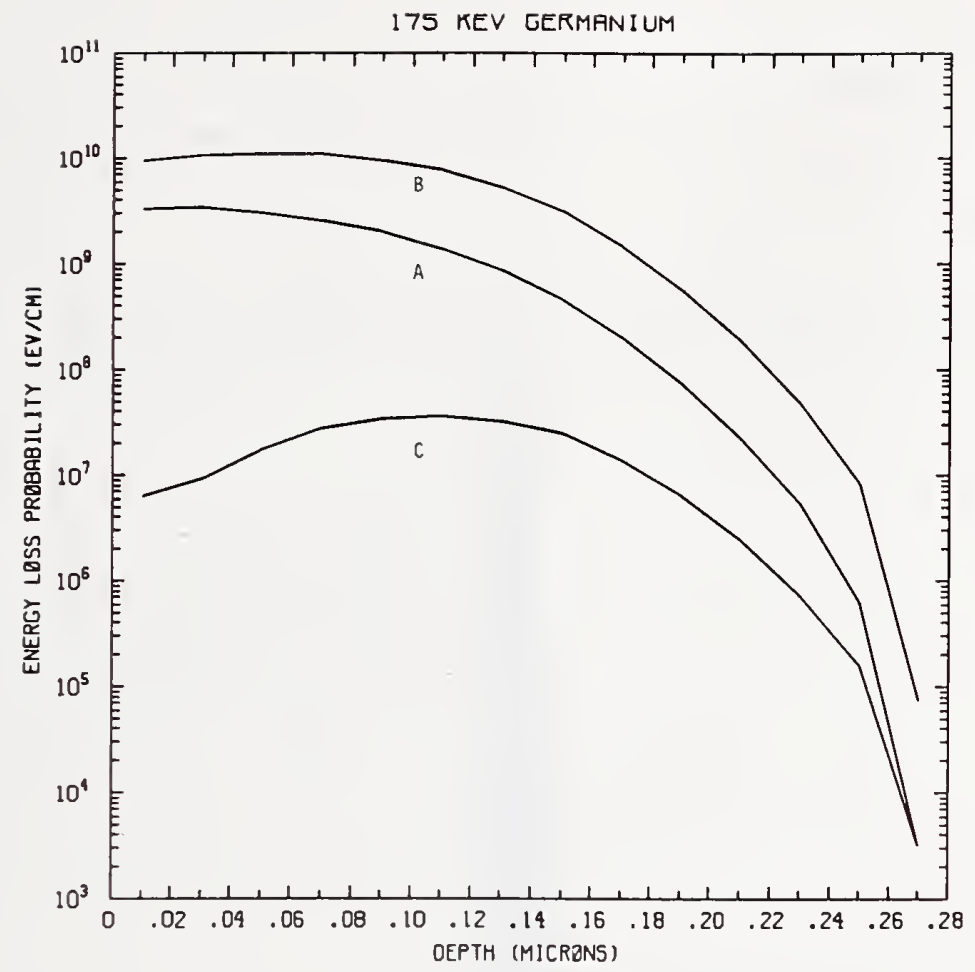

Figure Ge175-1 One-dimensional probability distributions for energy loss due to electronic excitation (A), displacement damage(B), and phonon excitation (C) for $175 \mathrm{keV}$ germanium normally incident on a silicon target.

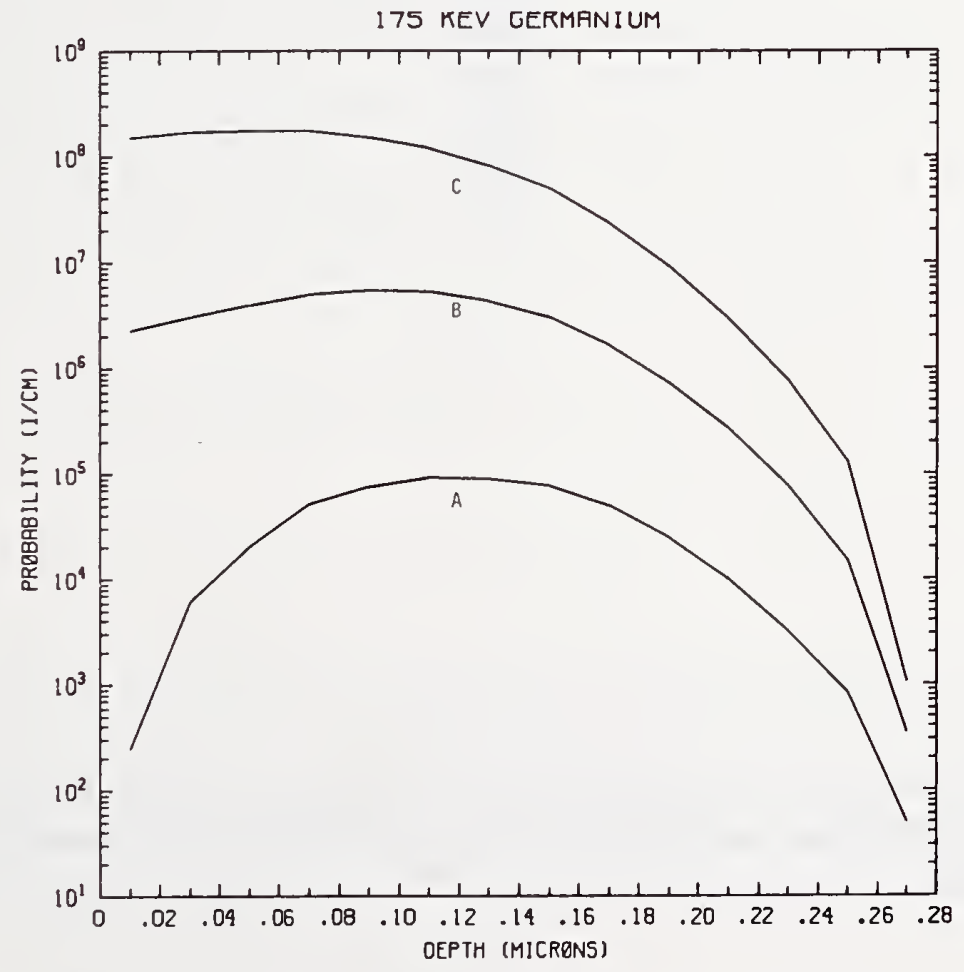

Figure Ge175-2 One-dimensional probability distributions for implanted germanium (A), primary silicon displacements (B), and Frenkel pairs (C) for $175 \mathrm{keV}$ germanium normally incident on a silicon target. 


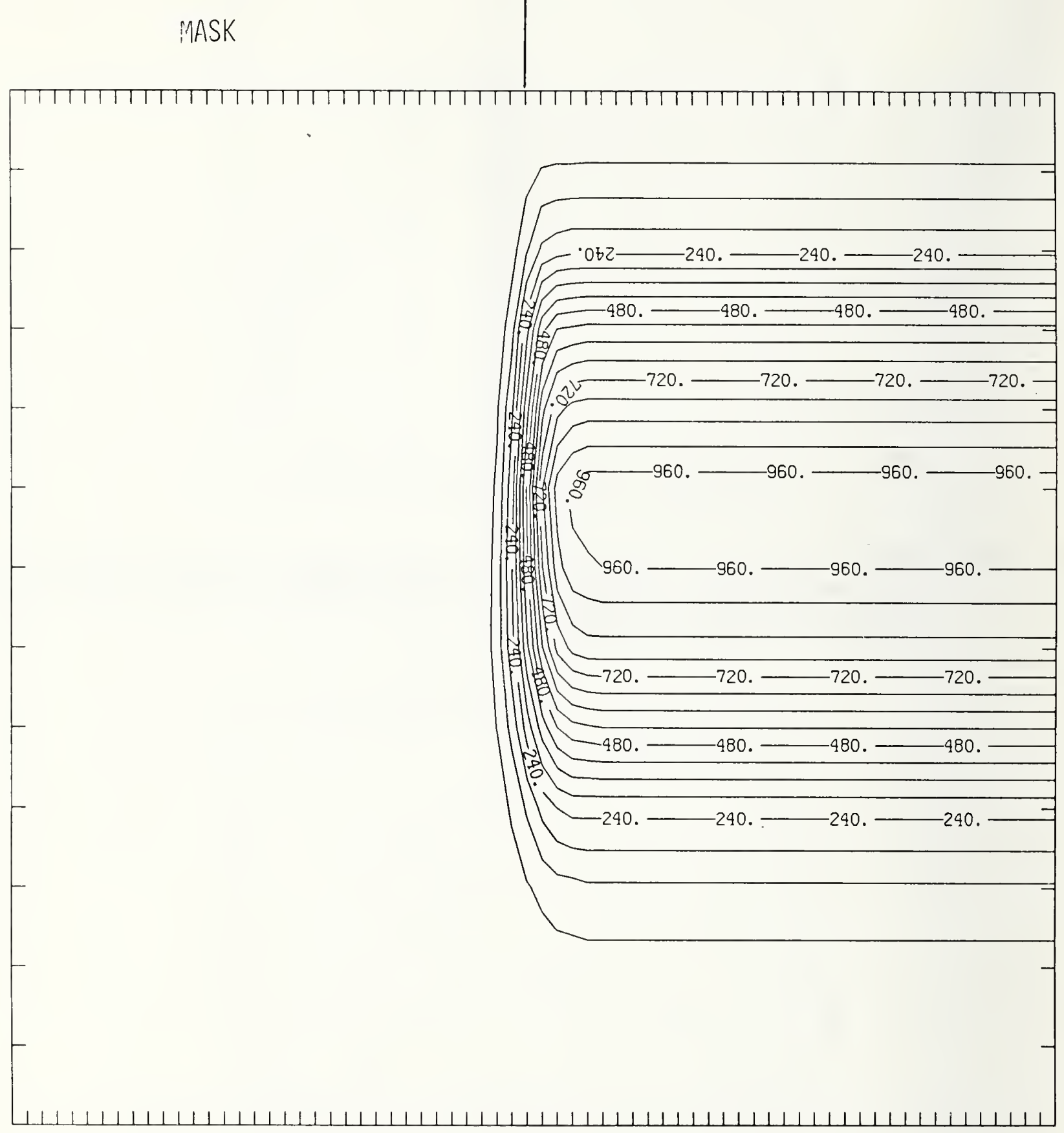

Figure Ge175-3 Two-dimensional distribution of $175 \mathrm{keV}$ germanium implanted past a mask edge. The length increment (distance between tick marks) is $0.02 \mu \mathrm{m}$ and the scale factor is 1000 . 


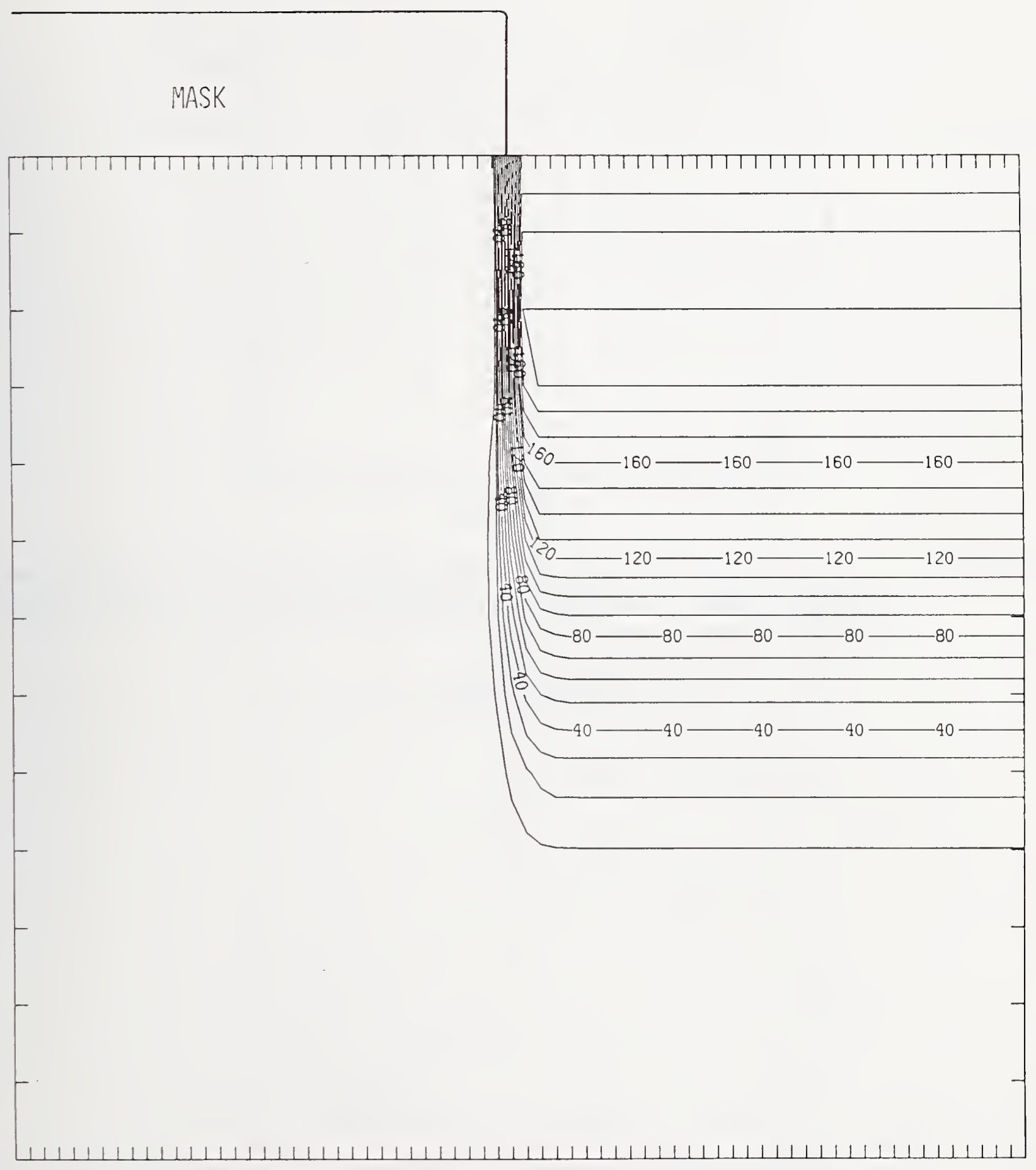

Figure Ge175-4 Two-dimensional distribution of Frenkel pairs created by $175 \mathrm{keV}$ germanium implanted past a mask edge. The length increment (distance between tick marks) is $0.02 \mu \mathrm{m}$ and the scale factor is 0.1 . 


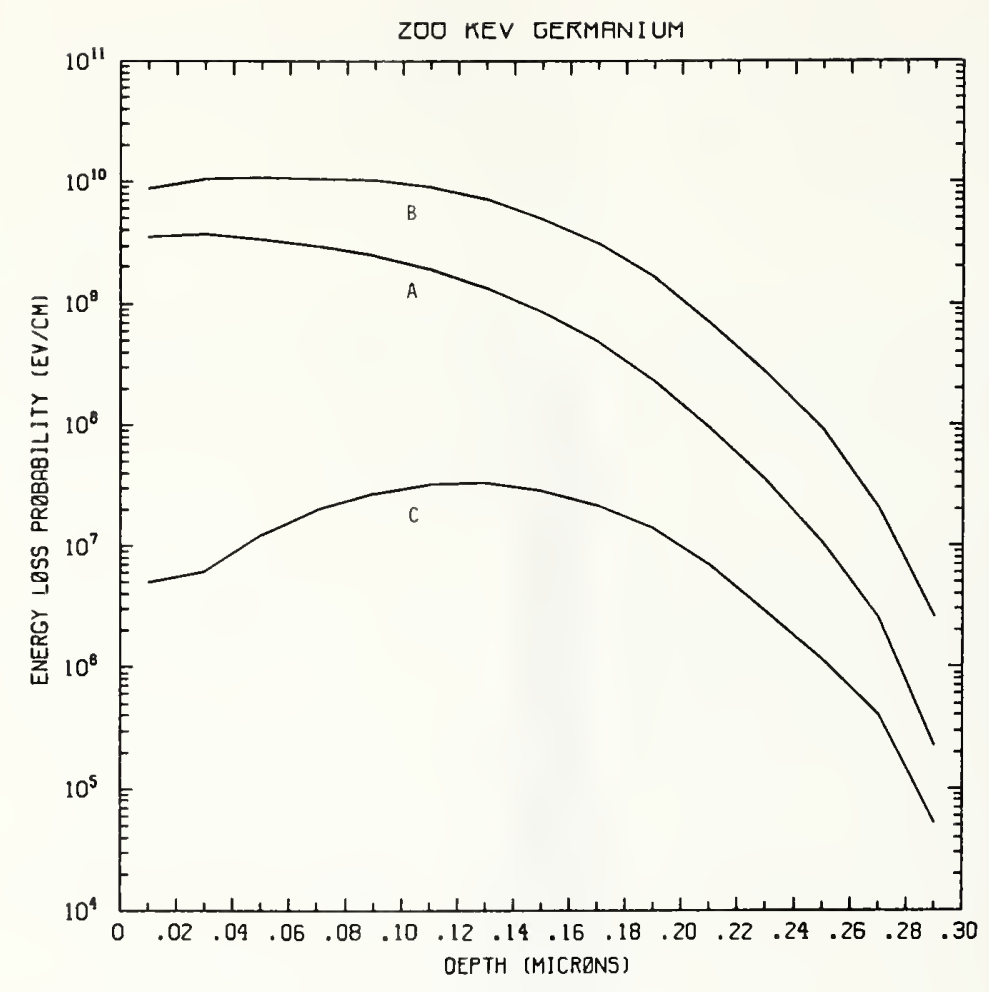

Figure Ge200-1 One-dimensional probability distributions for energy loss due to electronic excitation (A), displacement damage(B), and phonon excitation (C) for $200 \mathrm{keV}$ germanium normally incident on a silicon target.

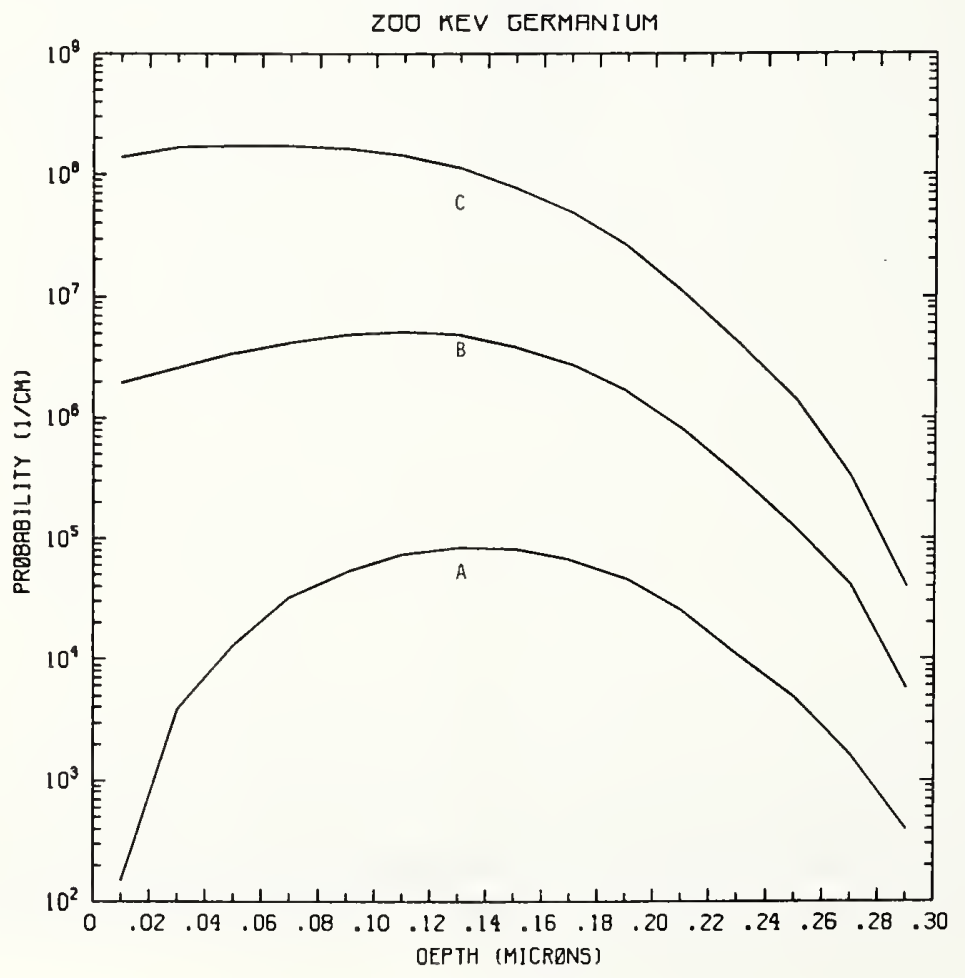

Figure Ge200-2 One-dimensional probability distributions for implanted germanium (A), primary silicon displacements (B), and Frenkel pairs (C) for $200 \mathrm{keV}$ germanium normally incident on a silicon target. 


\section{MASK}

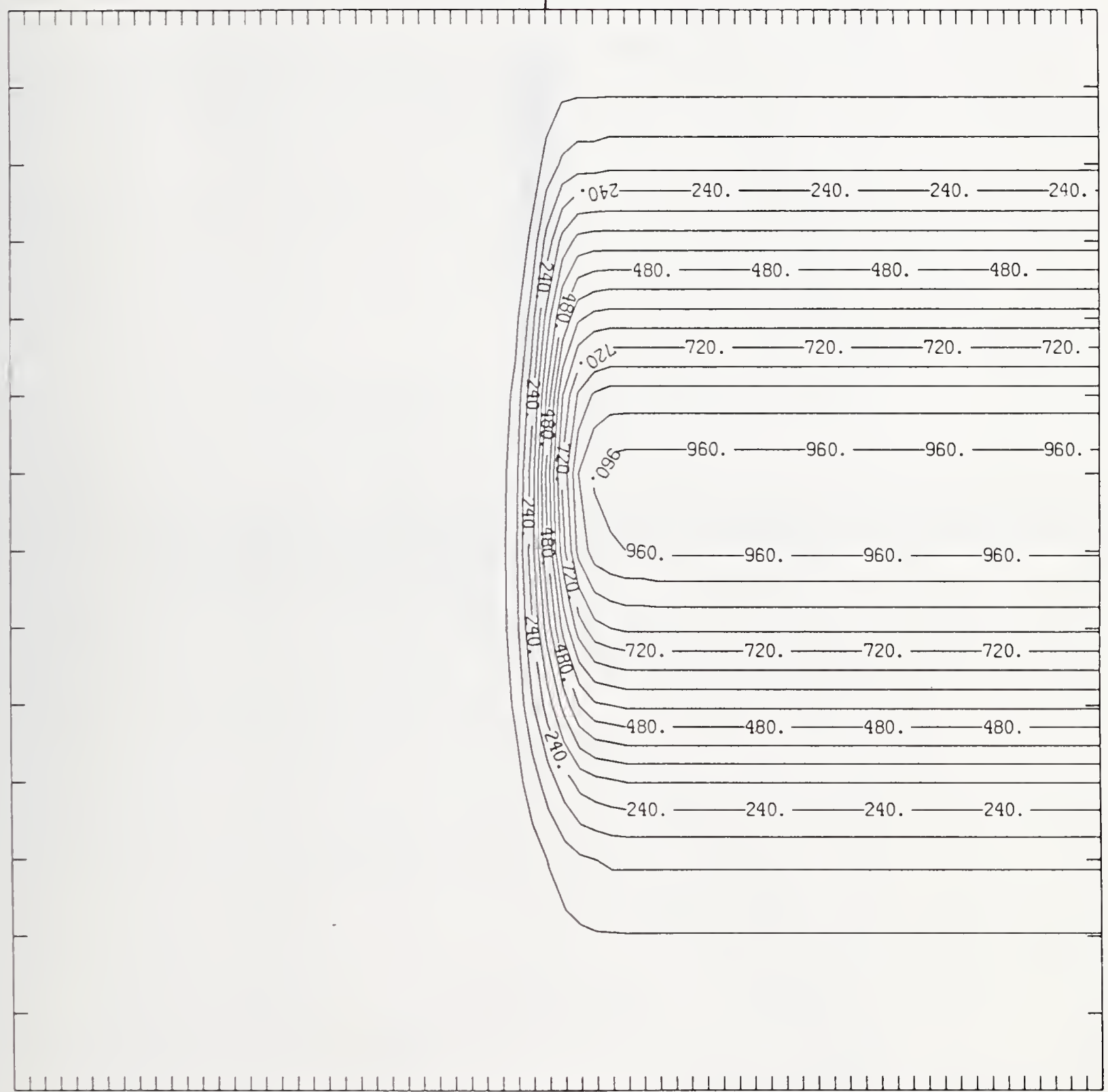

Figure Ge200-3 Two-dimensional distribution of $200 \mathrm{keV}$ germanium implanted past a mask edge. The length increment (distance between tick marks) is $0.02 \mu \mathrm{m}$ and the scale factor is 1000 . 
MASK

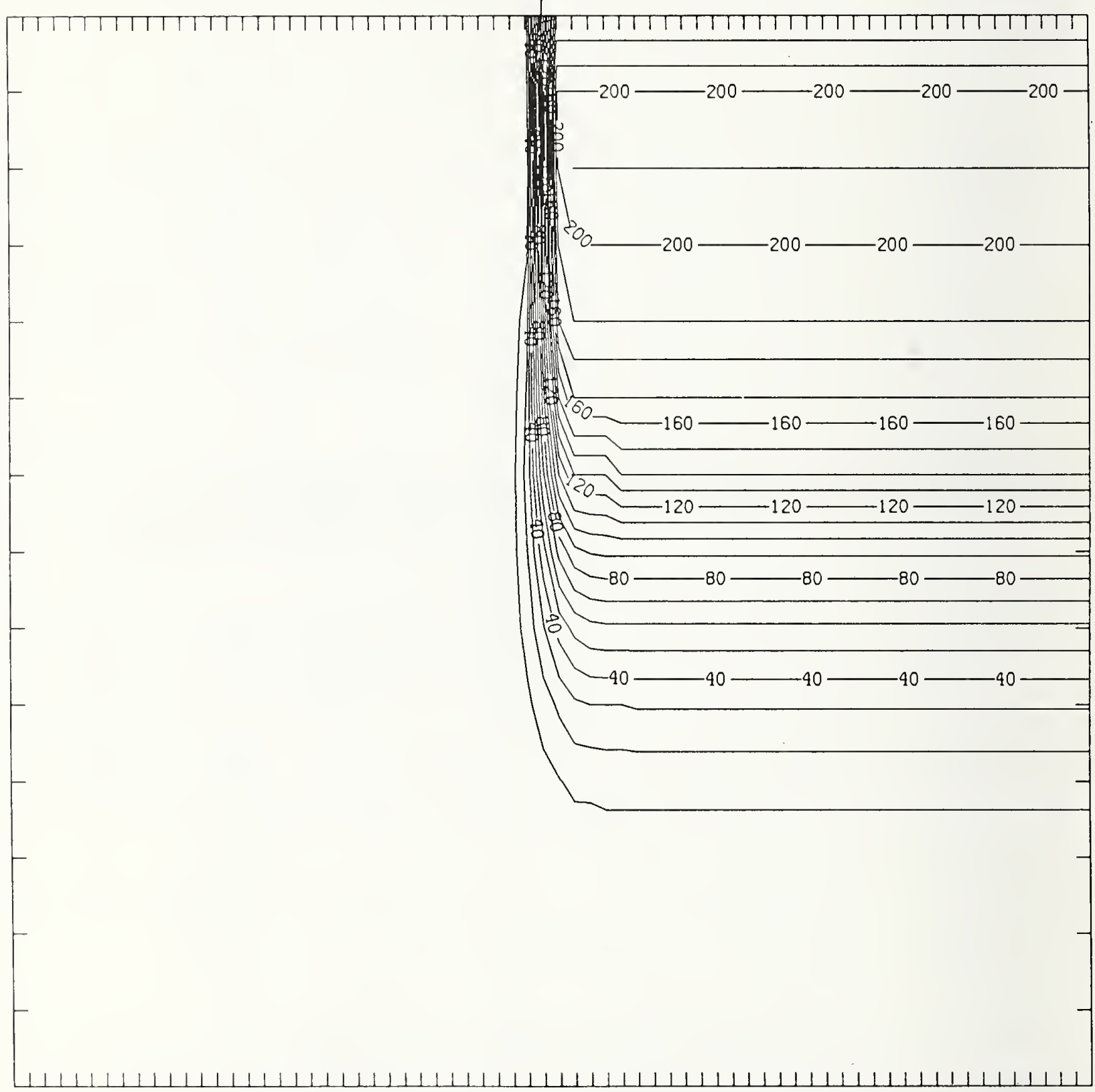

Figure Ge200-4 Two-dimensional distribution of Frenkel pairs created by $200 \mathrm{keV}$ germanium implanted past a mask edge. The length increment (distance between tick marks) is $0.02 \mu \mathrm{m}$ and the scale factor is 0.1 . 


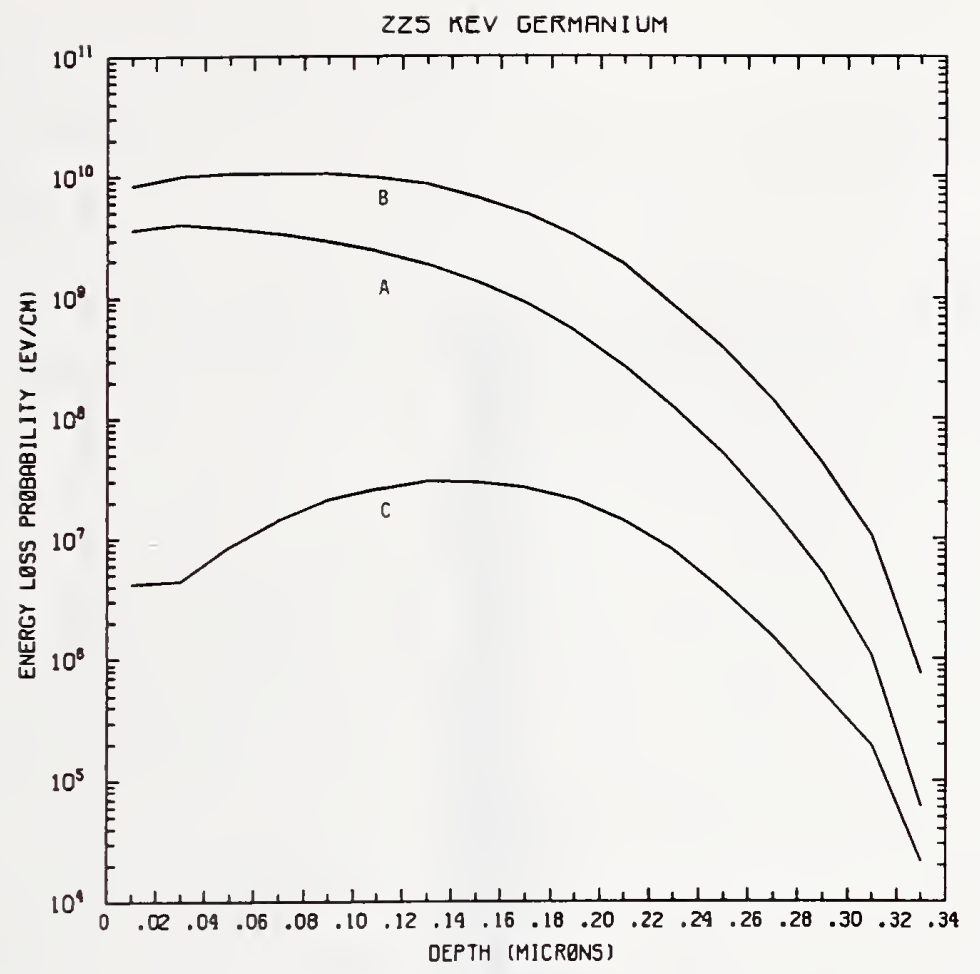

Figure Ge225-1 One-dimensional probability distributions for energy loss due to electronic excitation (A), displacement damage(B), and phonon excitation (C) for $225 \mathrm{keV}$ germanium normally incident on a silicon target.

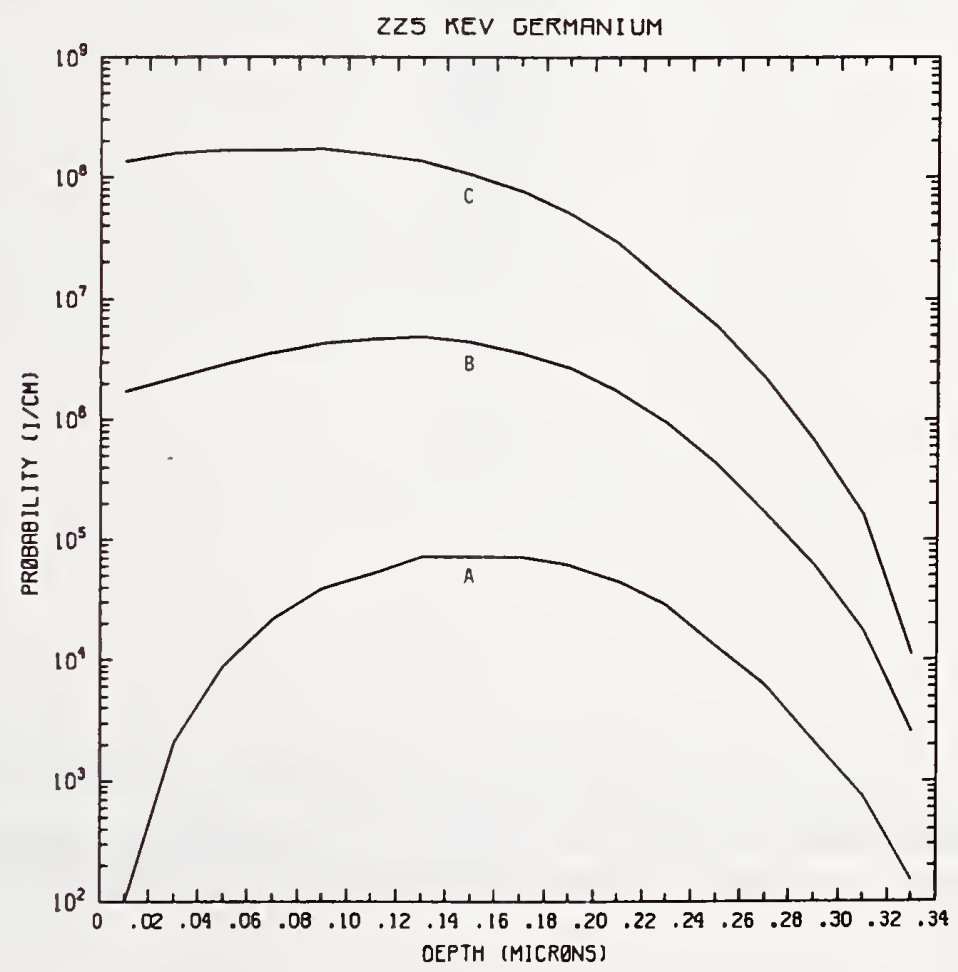

Figure Ge225-2 One-dimensional probability distributions for implanted germanium (A), primary silicon displacements (B), and Frenkel pairs (C) for $225 \mathrm{keV}$ germanium normally incident on a silicon target. 
MASK

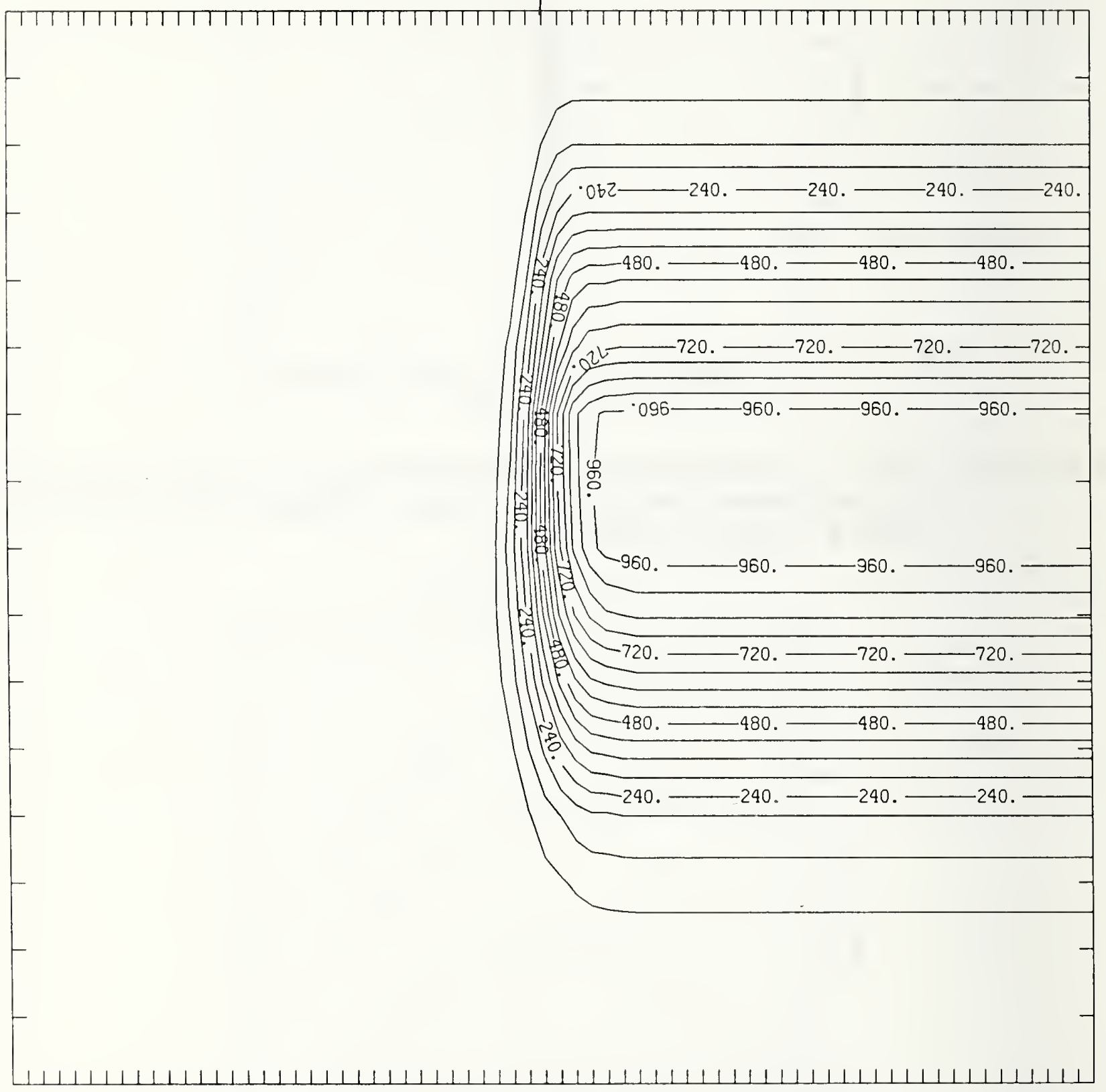

Figure Ge225-3 Two-dimensional distribution of $225 \mathrm{keV}$ germanium implanted past a mask edge. The length increment (distance between tick marks) is $0.02 \mu \mathrm{m}$ and the scale factor is 1000 . 


\section{MASK}

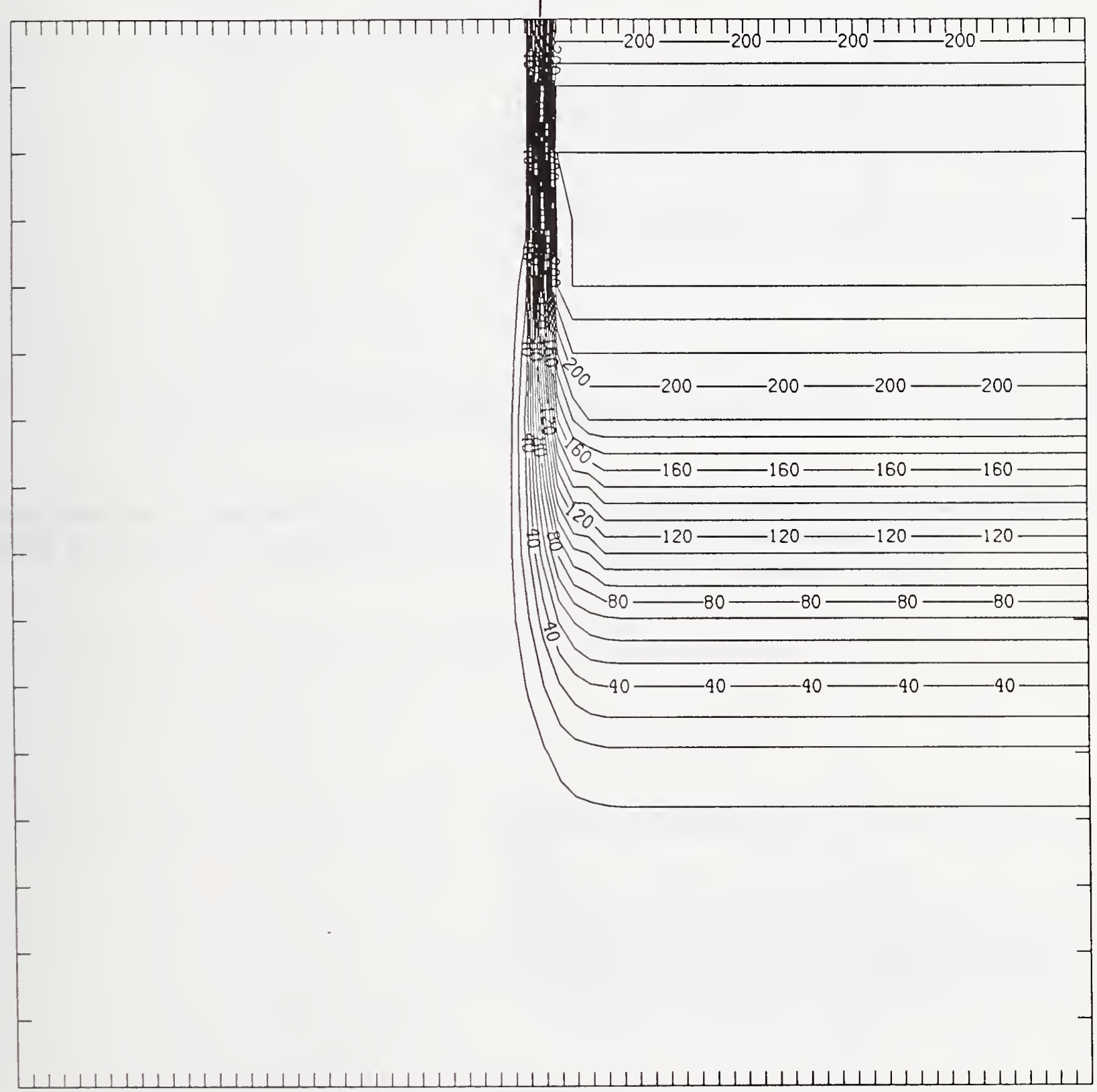

Figure Ge225-4 Two-dimensional distribution of Frenkel pairs created by $225 \mathrm{keV}$ germanium implanted past a mask edge. The length increment (distance between tick marks) is $0.02 \mu \mathrm{m}$ and the scale factor is 0.1 . 


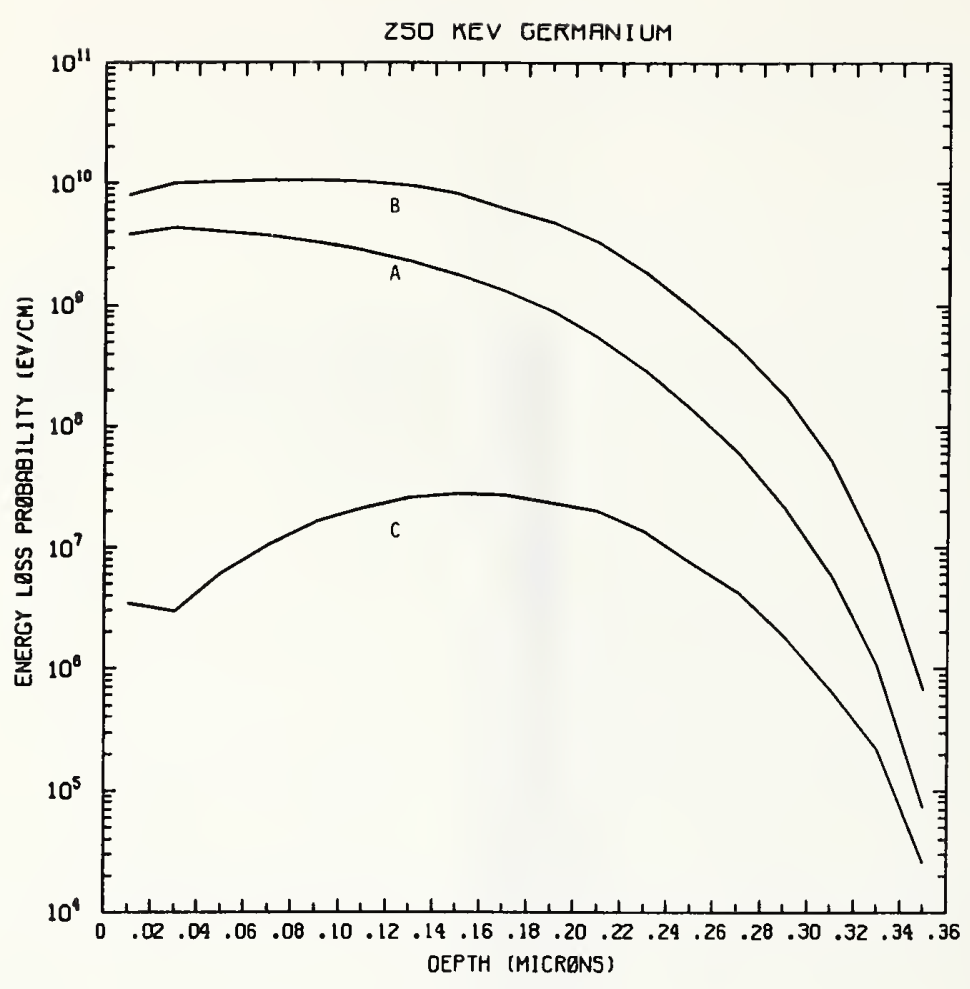

Figure Ge250-1 One-dimensional probability distributions for energy loss due to electronic excitation (A), displacement damage(B), and phonon excitation (C) for $250 \mathrm{keV}$ germanium normally incident on a silicon target.

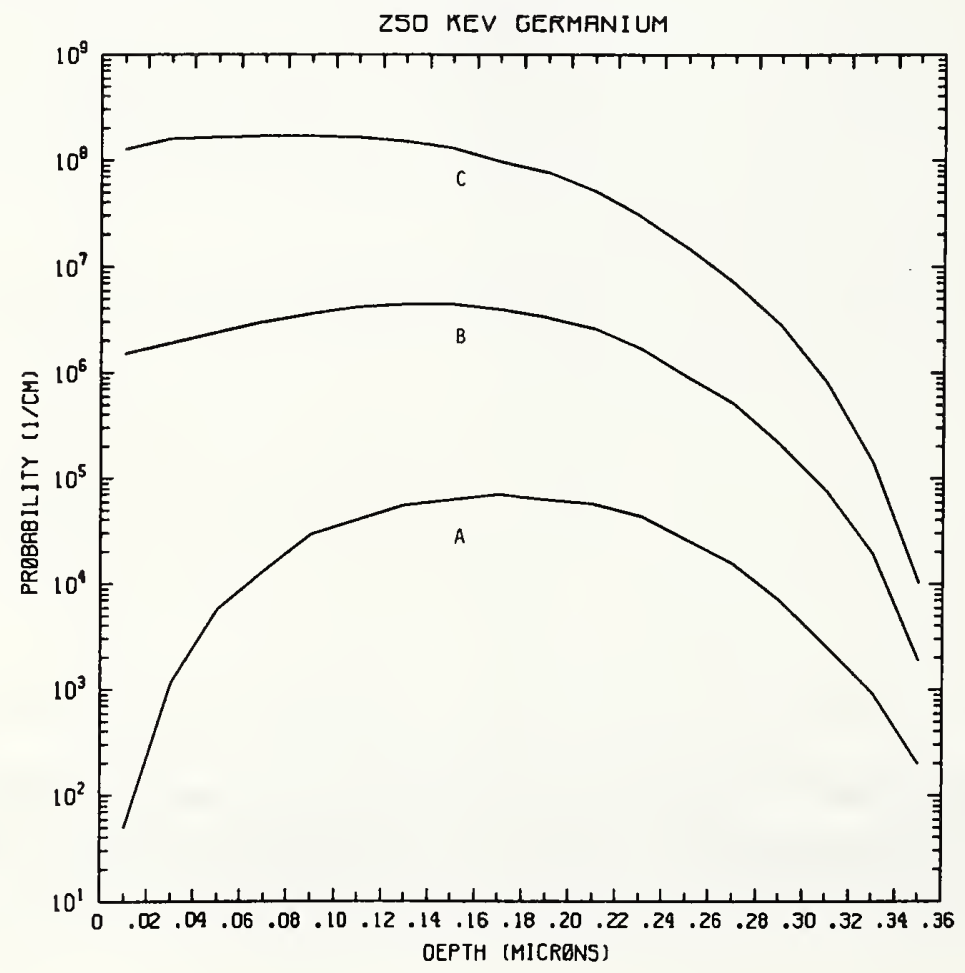

Figure Ge250-2 One-dimensional probability distributions for implanted germanium (A), primary silicon displacements (B), and Frenkel pairs (C) for $250 \mathrm{keV}$ germanium normally incident on a silicon target. 
MASK

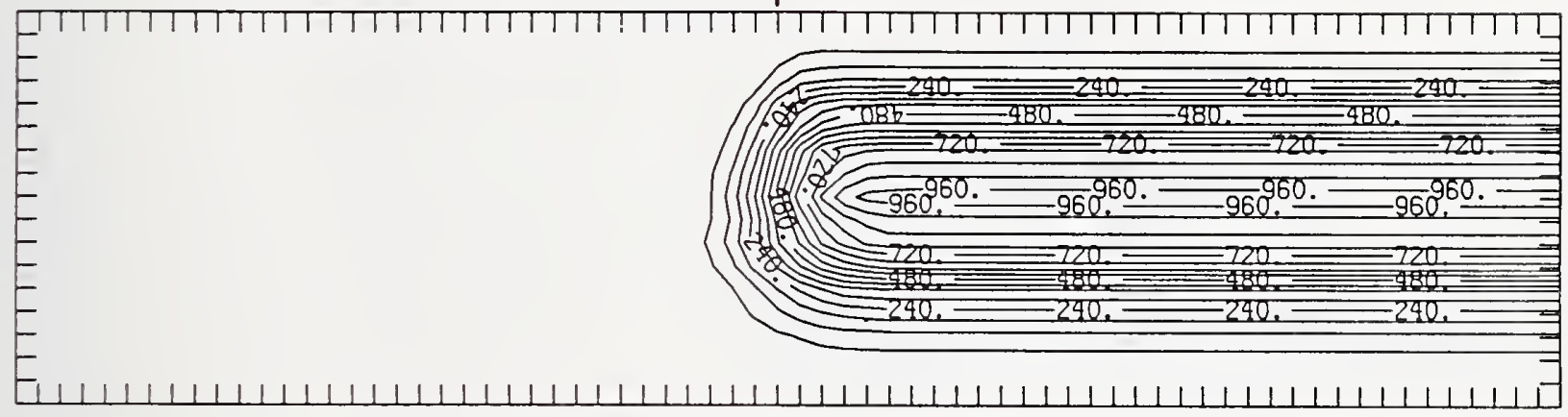

Figure Ge250-3 Two-dimensional distribution of $250 \mathrm{keV}$ germanium implanted past a mask edge. The length increment (distance between tick marks) is $0.02 \mu \mathrm{m}$ and the scale factor is 1000 .

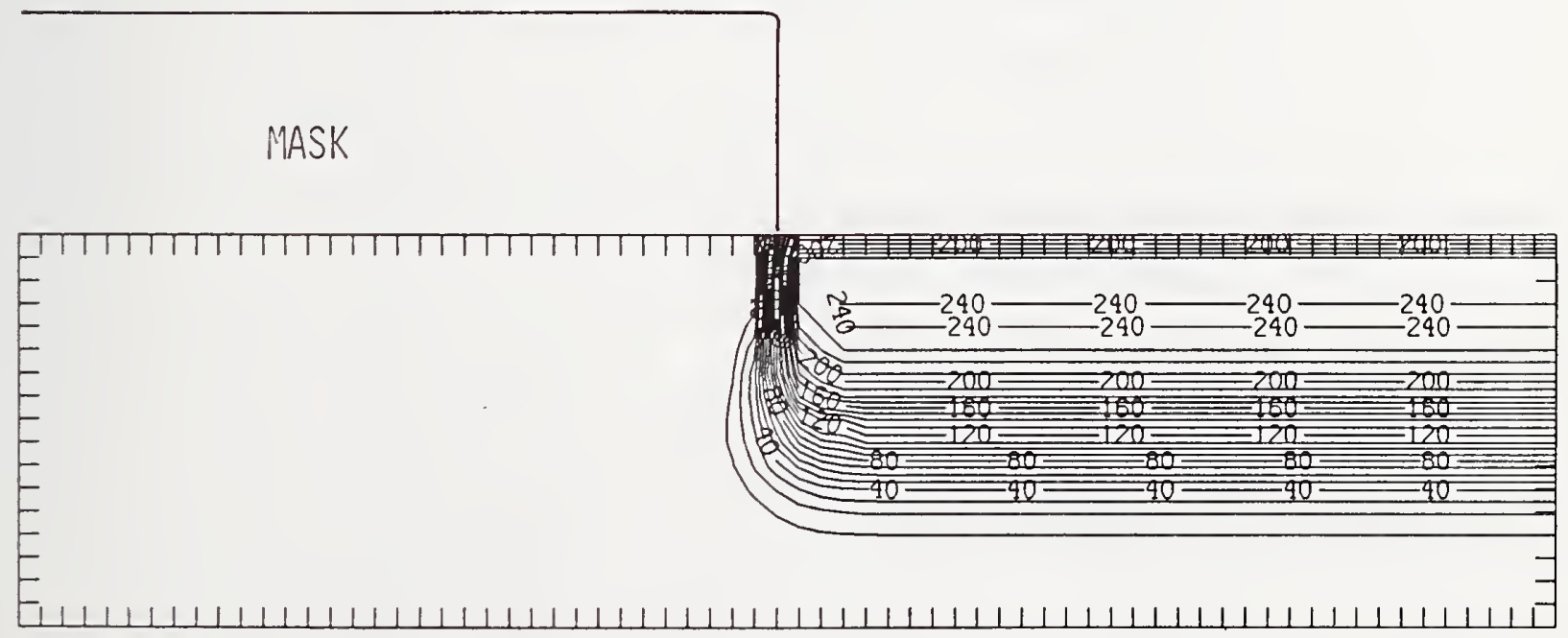

Figure Ge250-4 Two-dimensional distribution of Frenkel pairs created by $250 \mathrm{keV}$ germanium implanted past a mask edge. The length increment (distance between tick marks) is $0.02 \mu \mathrm{m}$ and the scale factor is 0.1 . 


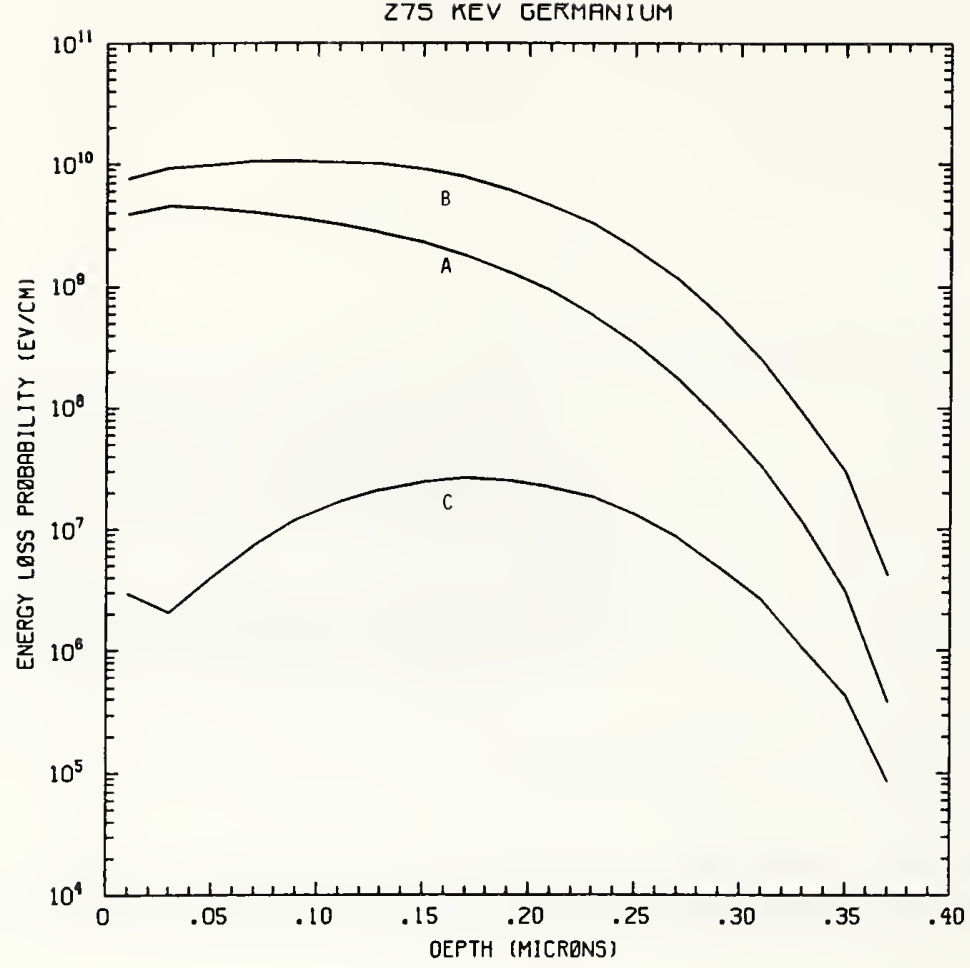

Figure Ge275-1 One-dimensional probability distributions for energy loss due to electronic excitation (A), displacement damage(B), and phonon excitation (C) for $275 \mathrm{keV}$ germanium normally incident on a silicon target.

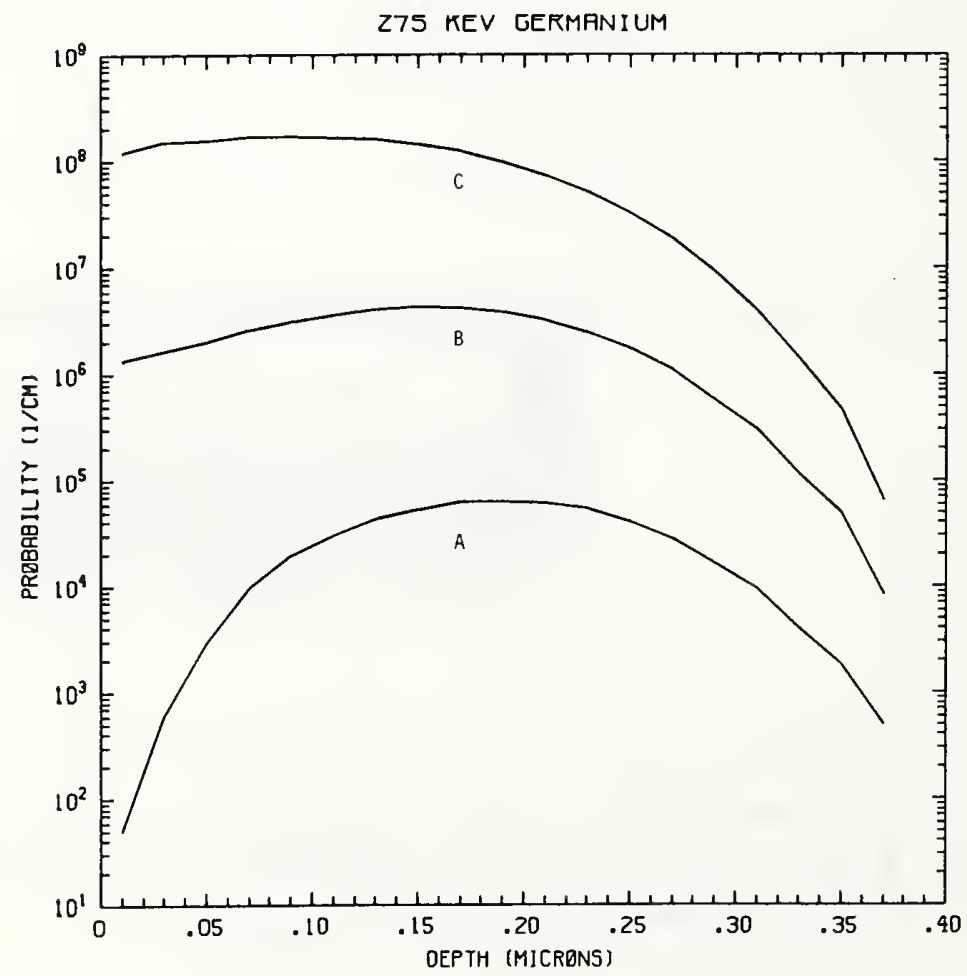

Figure Ge275-2 One-dimensional probability distributions for implanted germanium (A), primary silicon displacements (B), and Frenkel pairs (C) for $275 \mathrm{keV}$ germanium normally incident on a silicon target. 


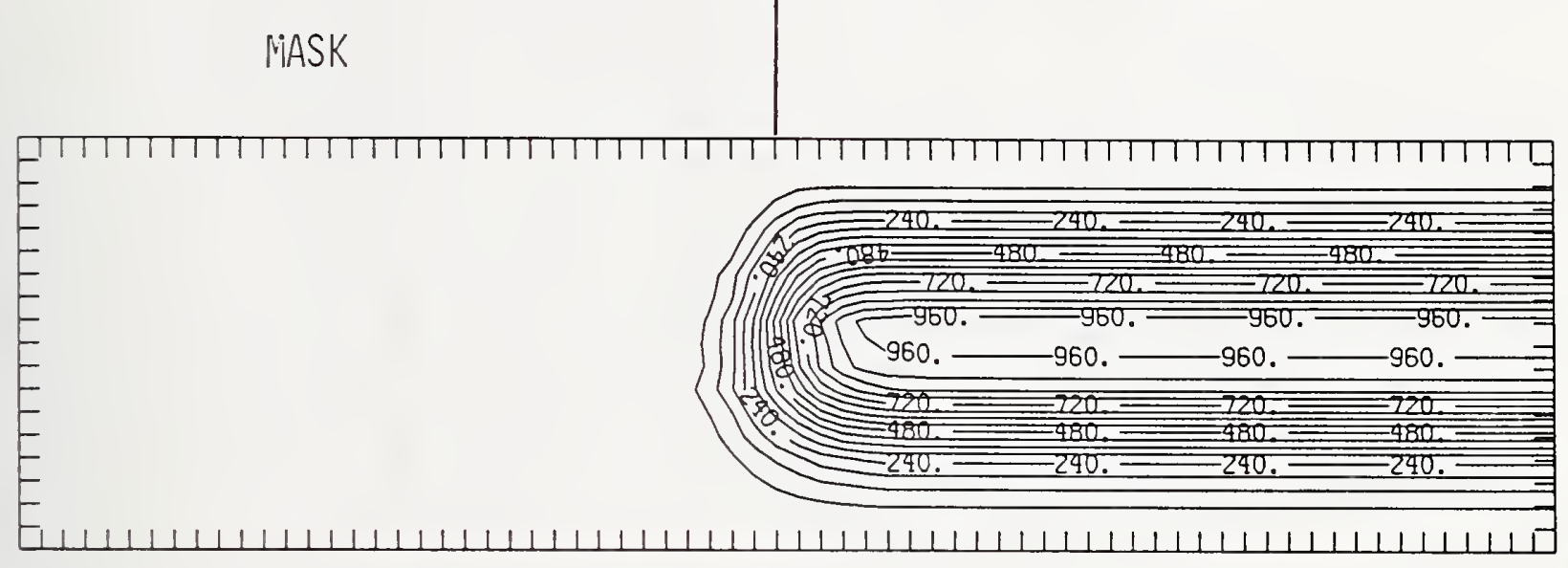

Figure Ge275-3 Two-dimensional distribution of $275 \mathrm{keV}$ germanium implanted past a mask edge. The length increment (distance between tick marks) is $0.02 \mu \mathrm{m}$ and the scale factor is 1000 .

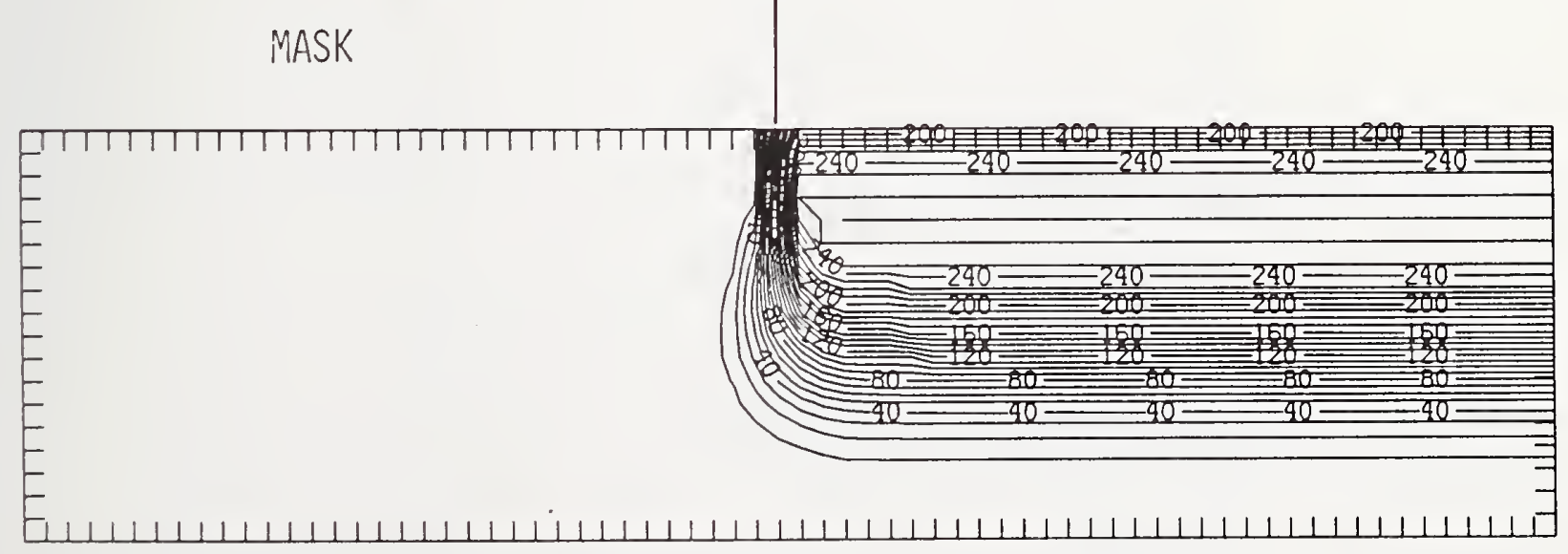

Figure Ge275-4 Two-dimensional distribution of Frenkel pairs created by $275 \mathrm{keV}$ germanium implanted past a mask edge. The length increment (distance between tick marks) is $0.02 \mu \mathrm{m}$ and the scale factor is 0.1 . 


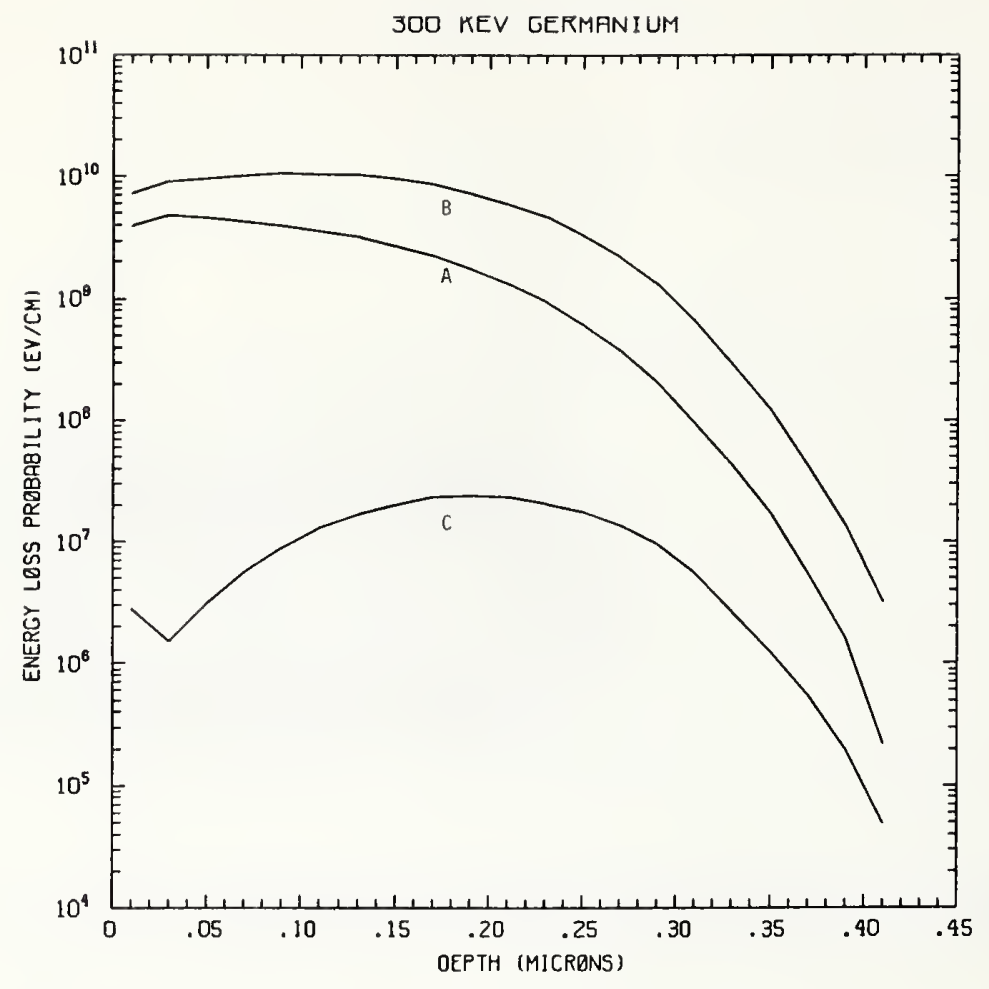

Figure Ge300-1 One-dimensional probability distributions for energy loss due to electronic excitation (A), displacement damage(B), and phonon excitation (C) for $300 \mathrm{keV}$ germanium normally incident on a silicon target.

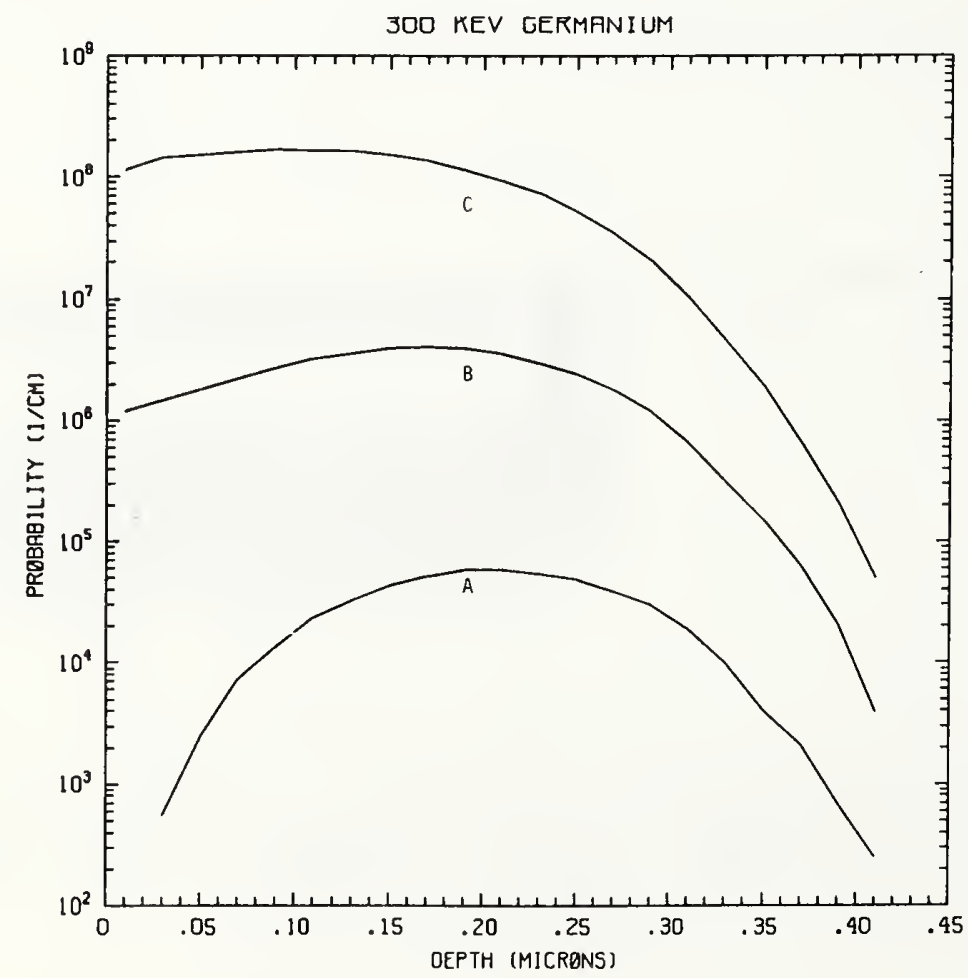

Figure Ge300-2 One-dimensional probability distributions for implanted germanium (A), primary silicon displacements (B), and Frenkel pairs (C) for $300 \mathrm{keV}$ germanium normally incident on a silicon target. 


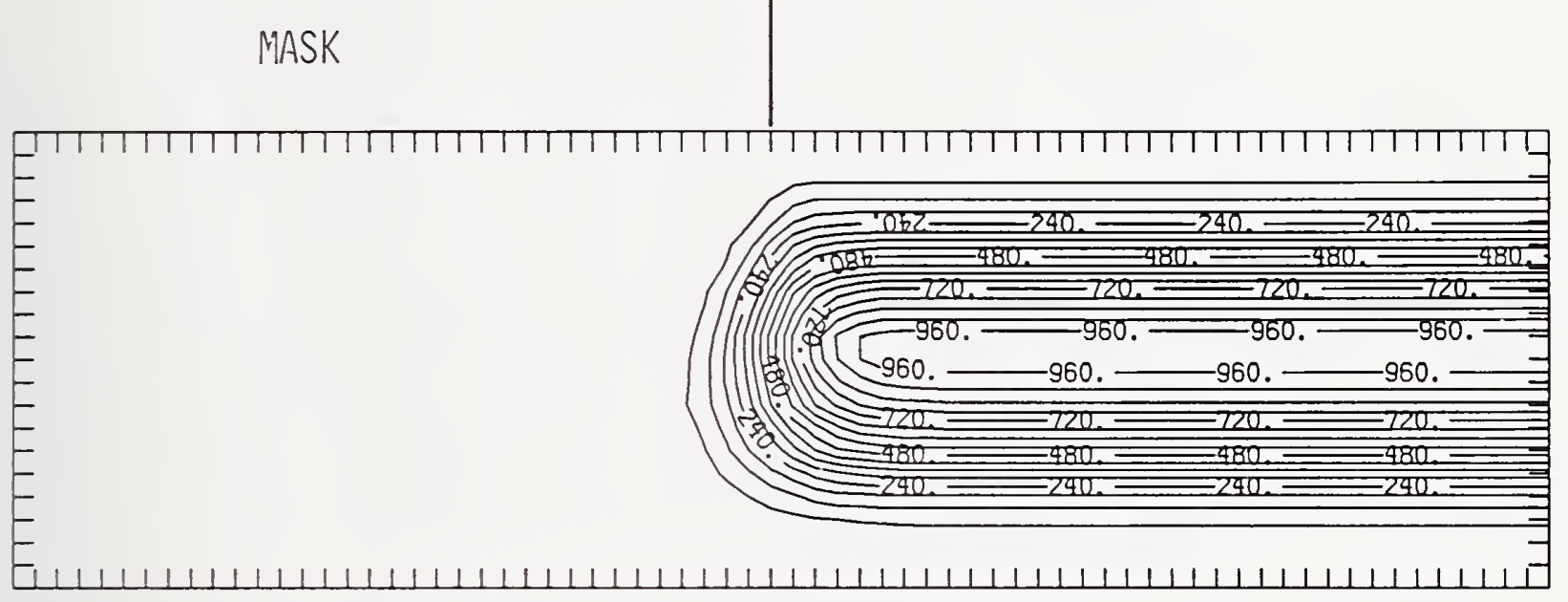

Figure Ge300-3 Two-dimensional distribution of $300 \mathrm{keV}$ germanium implanted past a mask edge. The length increment (distance between tick marks) is $0.02 \mu \mathrm{m}$ and the scale factor is 1000 .

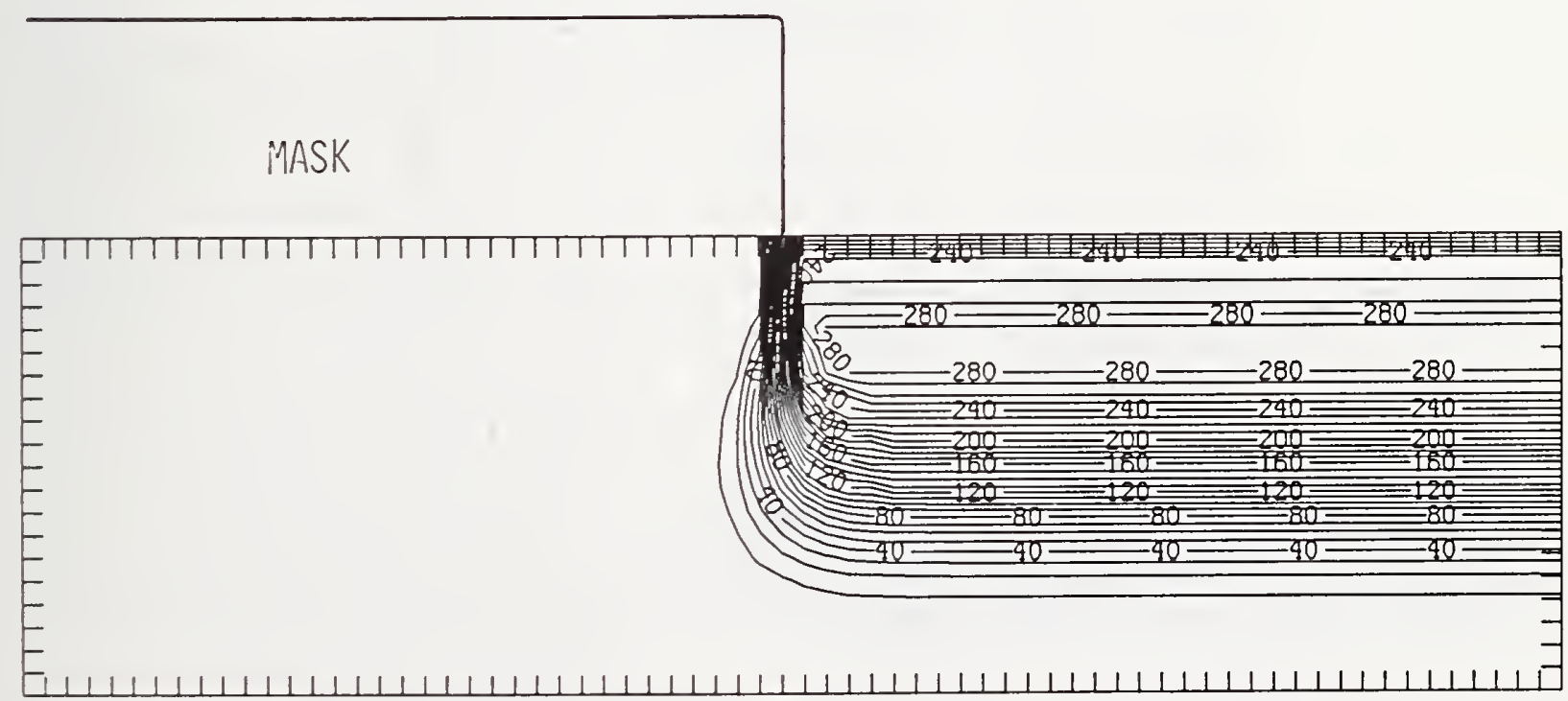

Figure Ge300-4 Two-dimensional distribution of Frenkel pairs created by $300 \mathrm{keV}$ germanium implanted past a mask edge. The length increment (distance between tick marks) is $0.02 \mu \mathrm{m}$ and the scale factor is 0.1 . 


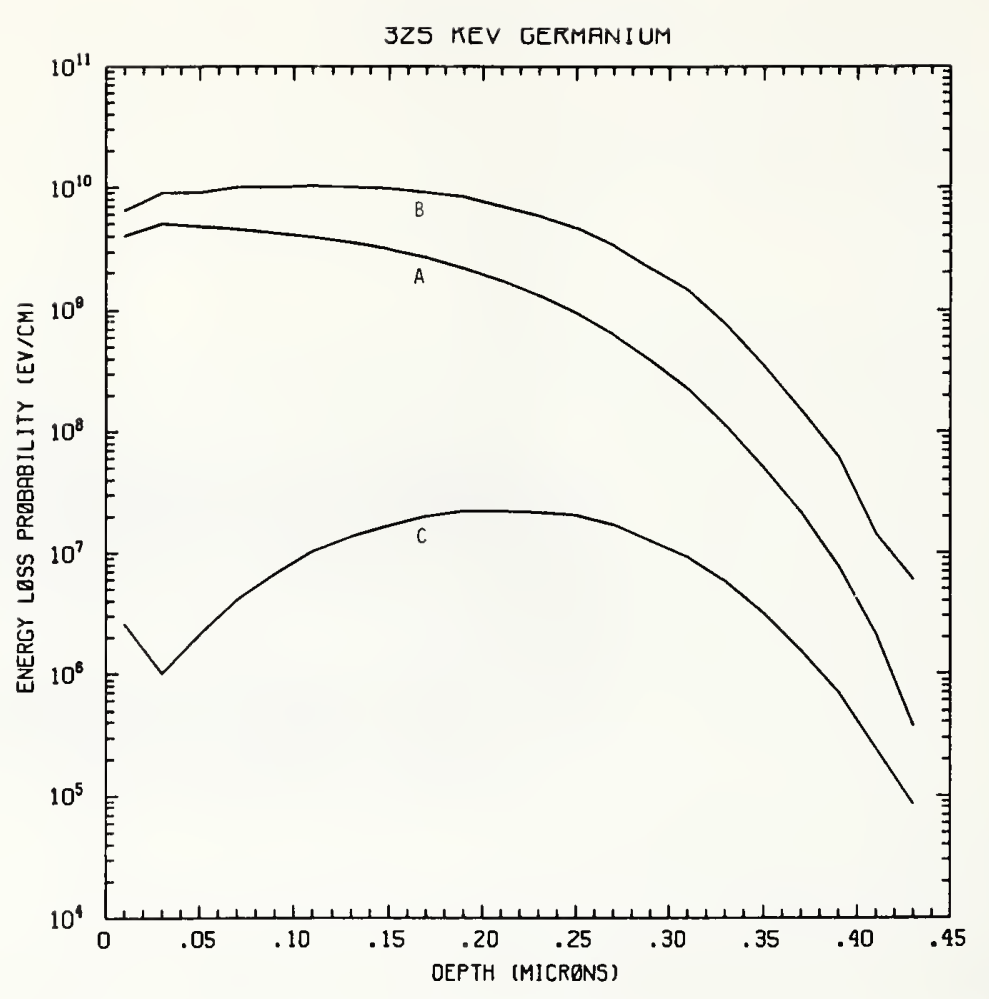

Figure Ge325-1 One-dimensional probability distributions for energy loss due to electronic excitation (A), displacement damage(B), and phonon excitation (C) for $325 \mathrm{keV}$ germanium normally incident on a silicon target.

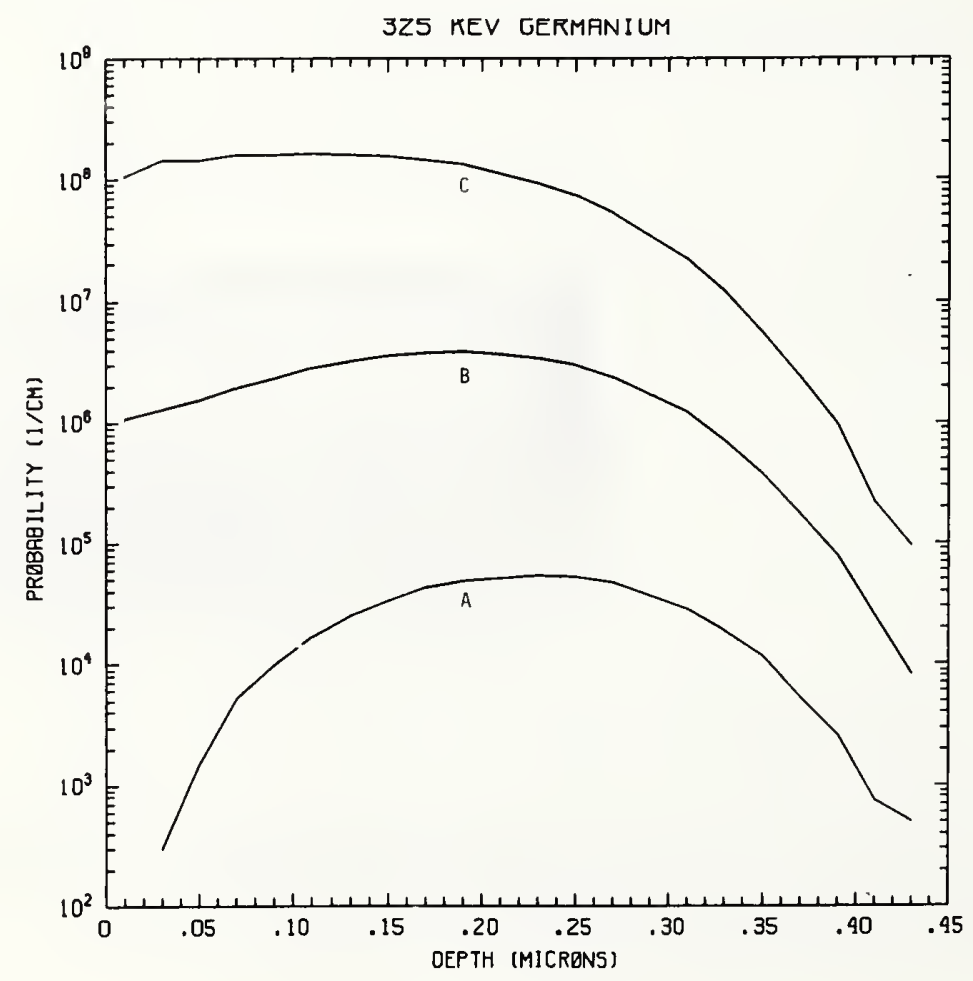

Figure Ge325-2 One-dimensional probability distributions for implanted germanium (A), primary silicon displacements (B), and Frenkel pairs (C) for $325 \mathrm{keV}$ germanium normally incident on a silicon target. 


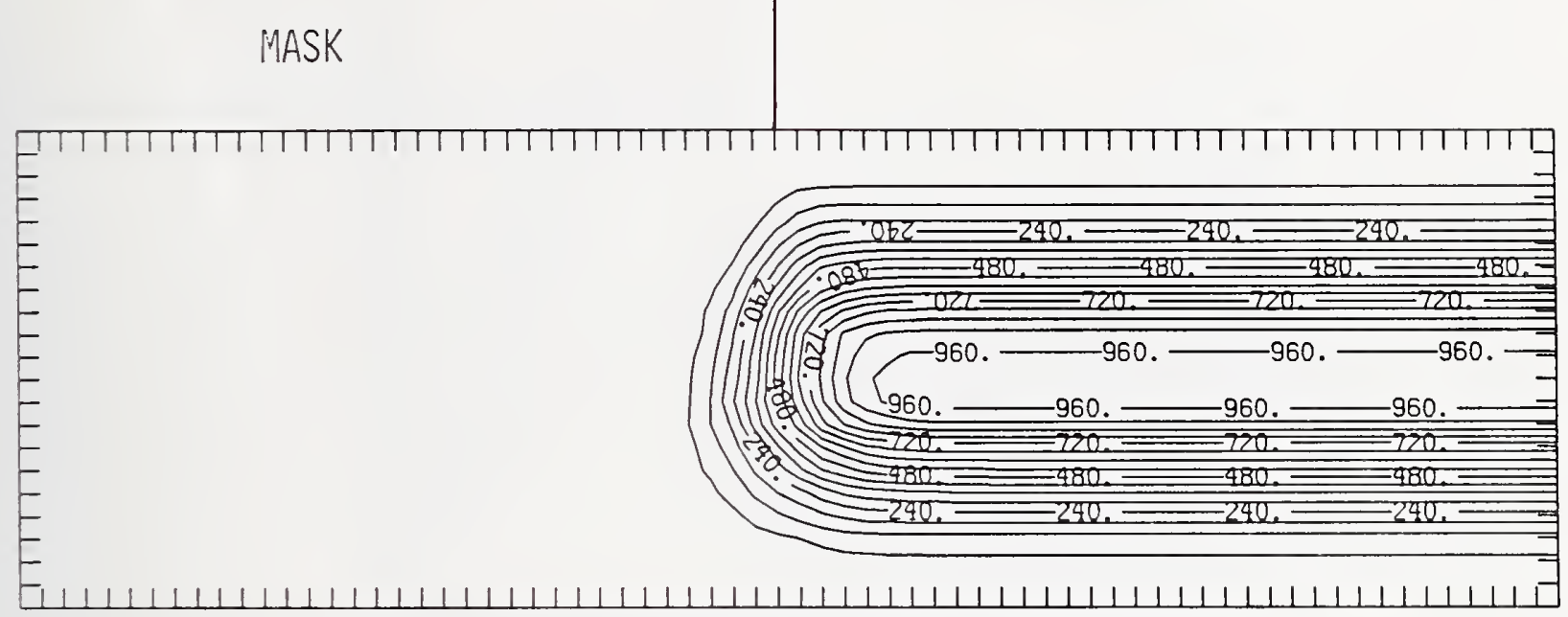

Figure Ge325-3 Two-dimensional distribution of $325 \mathrm{keV}$ germanium implanted past a mask edge. The length increment (distance between tick marks) is $0.02 \mu \mathrm{m}$ and the scale factor is 1000 .

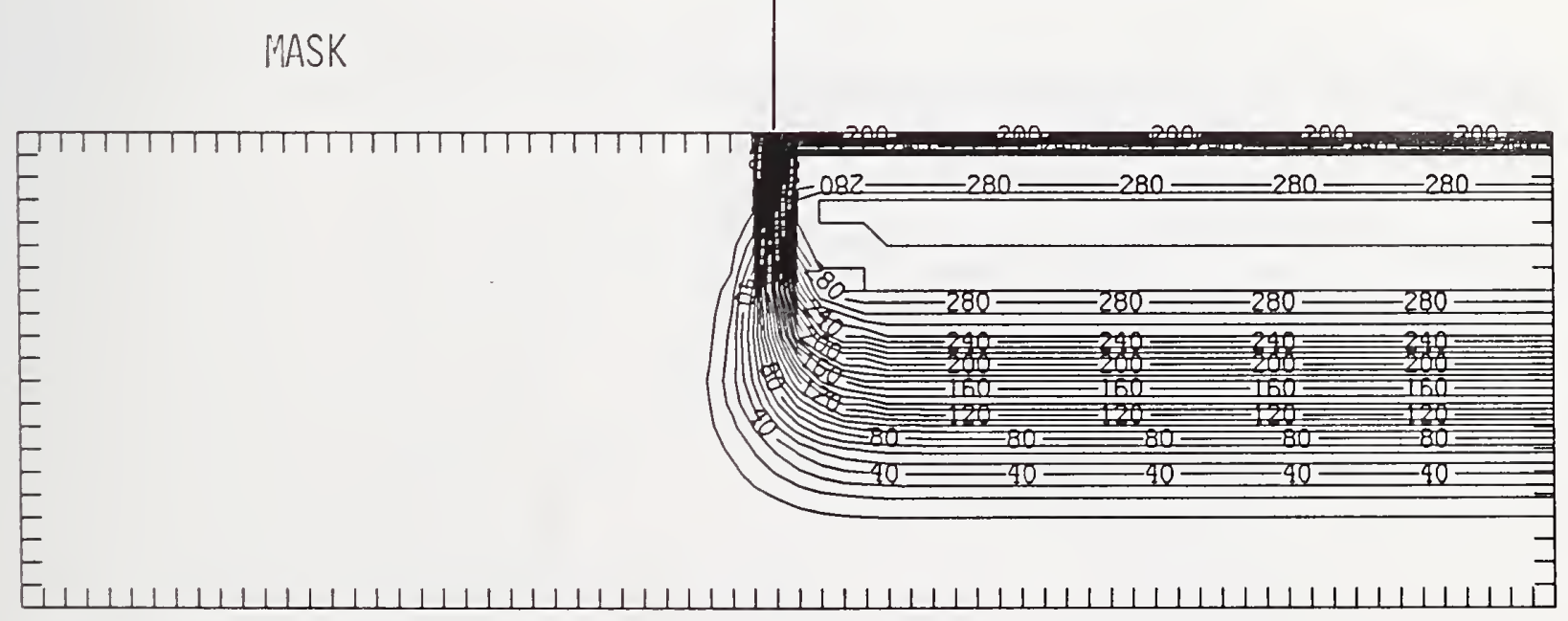

Figure Ge325-4 Two-dimensional distribution of Frenkel pairs created by $325 \mathrm{keV}$ germanium implanted past a mask edge. The length increment (distance between tick marks) is $0.02 \mu \mathrm{m}$ and the scale factor is 0.1 . 


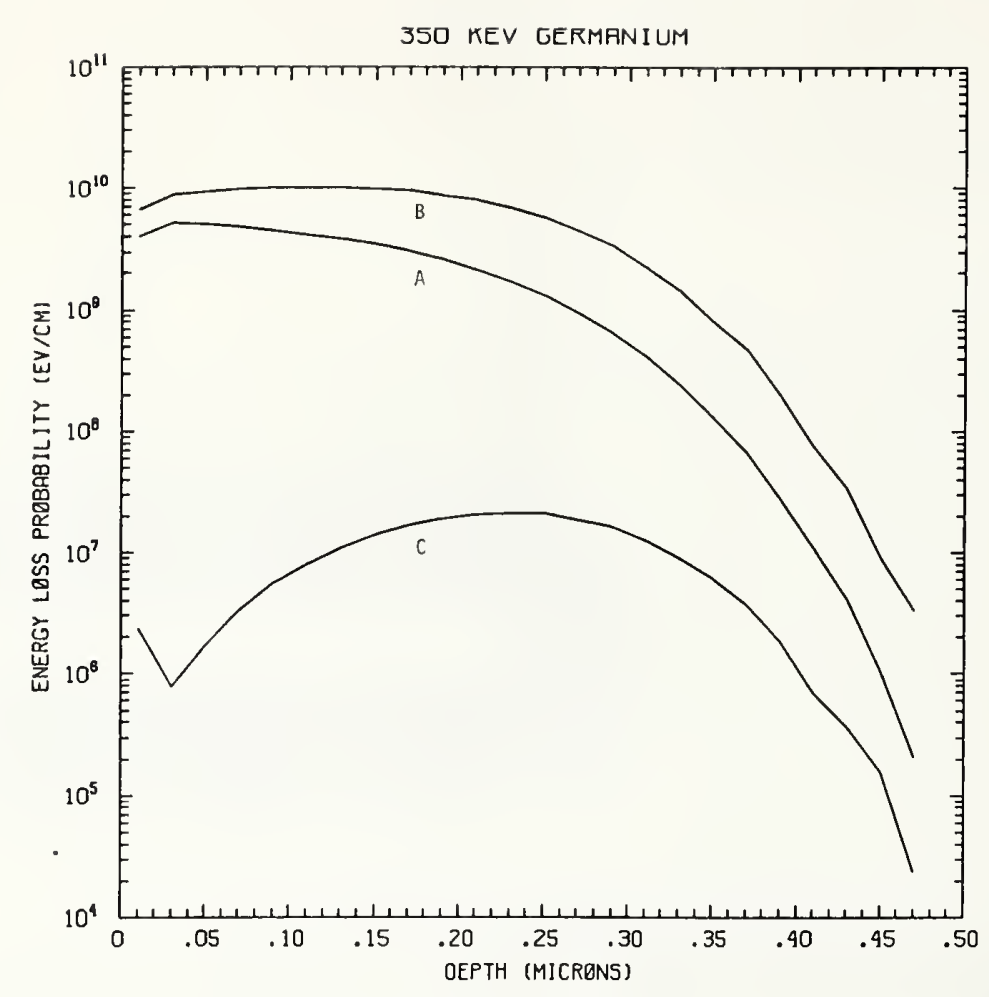

Figure Ge350-1 One-dimensional probability distributions for energy loss due to electronic excitation (A), displacement damage(B), and phonon excitation (C) for $350 \mathrm{keV}$ germanium normally incident on a silicon target.

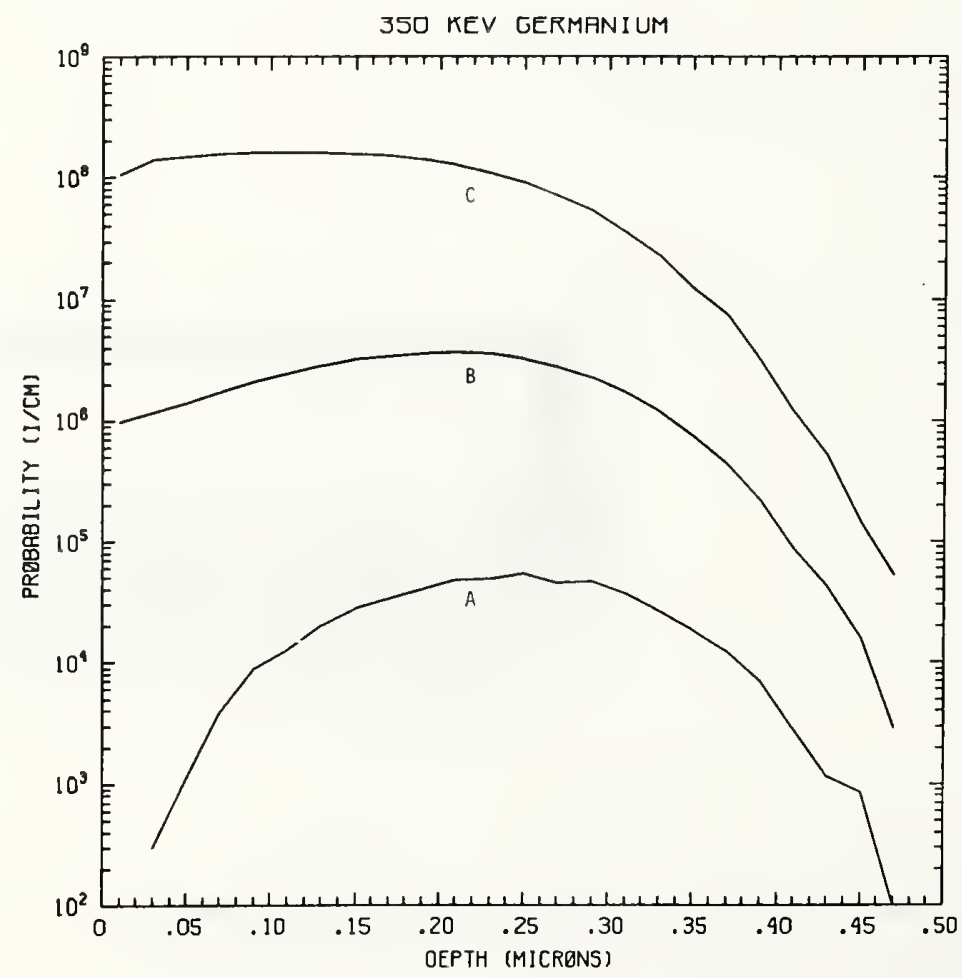

Figure Ge350-2 One-dimensional probability distributions for implanted germanium (A), primary silicon displacements (B), and Frenkel pairs (C) for $350 \mathrm{keV}$ germanium normally incident on a silicon target. 


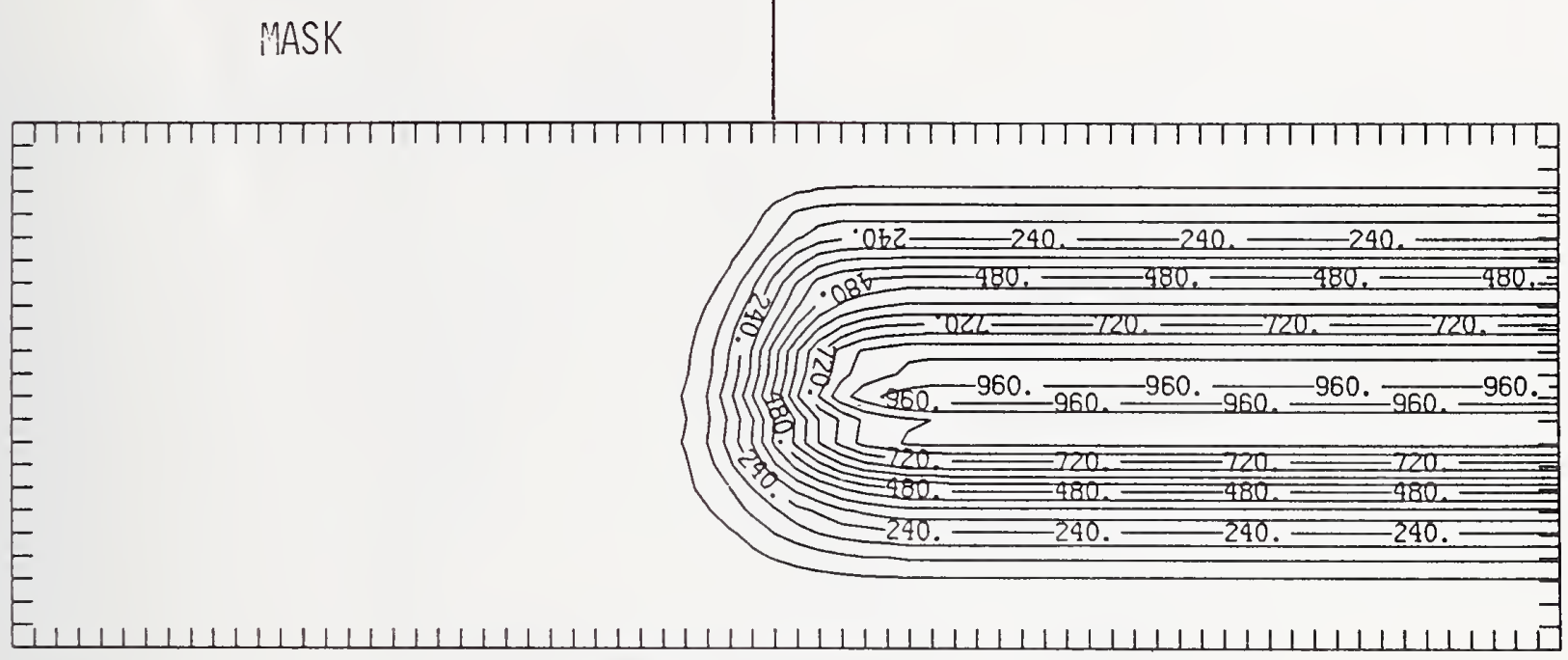

Figure Ge350-3 Two-dimensional distribution of $350 \mathrm{keV}$ germanium implanted past a mask edge. The length increment (distance between tick marks) is $0.02 \mu \mathrm{m}$ and the scale factor is 1000 .

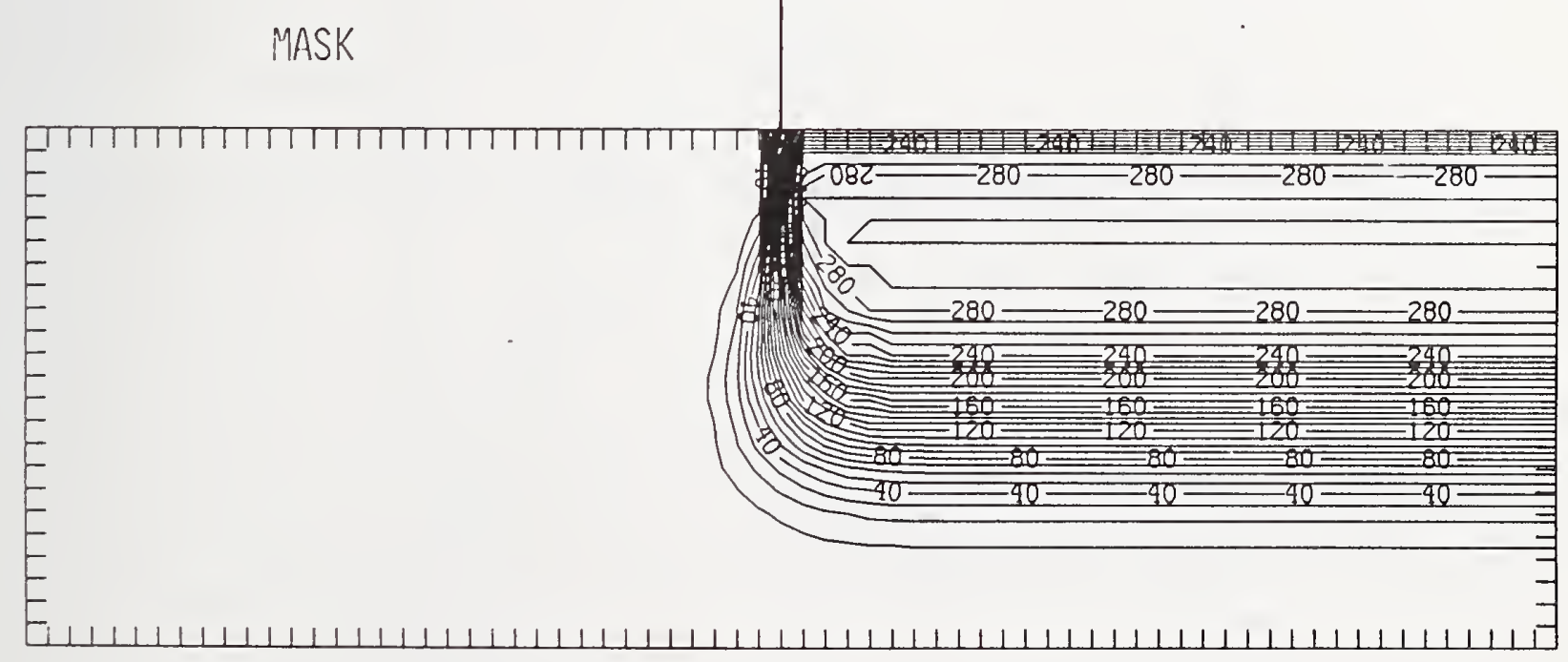

Figure Ge350-4 Two-dimensional distribution of Frenkel pairs created by $350 \mathrm{keV}$ germanium implanted past a mask edge. The length increment (distance between tick marks) is $0.02 \mu \mathrm{m}$ and the scale factor is 0.1 . 


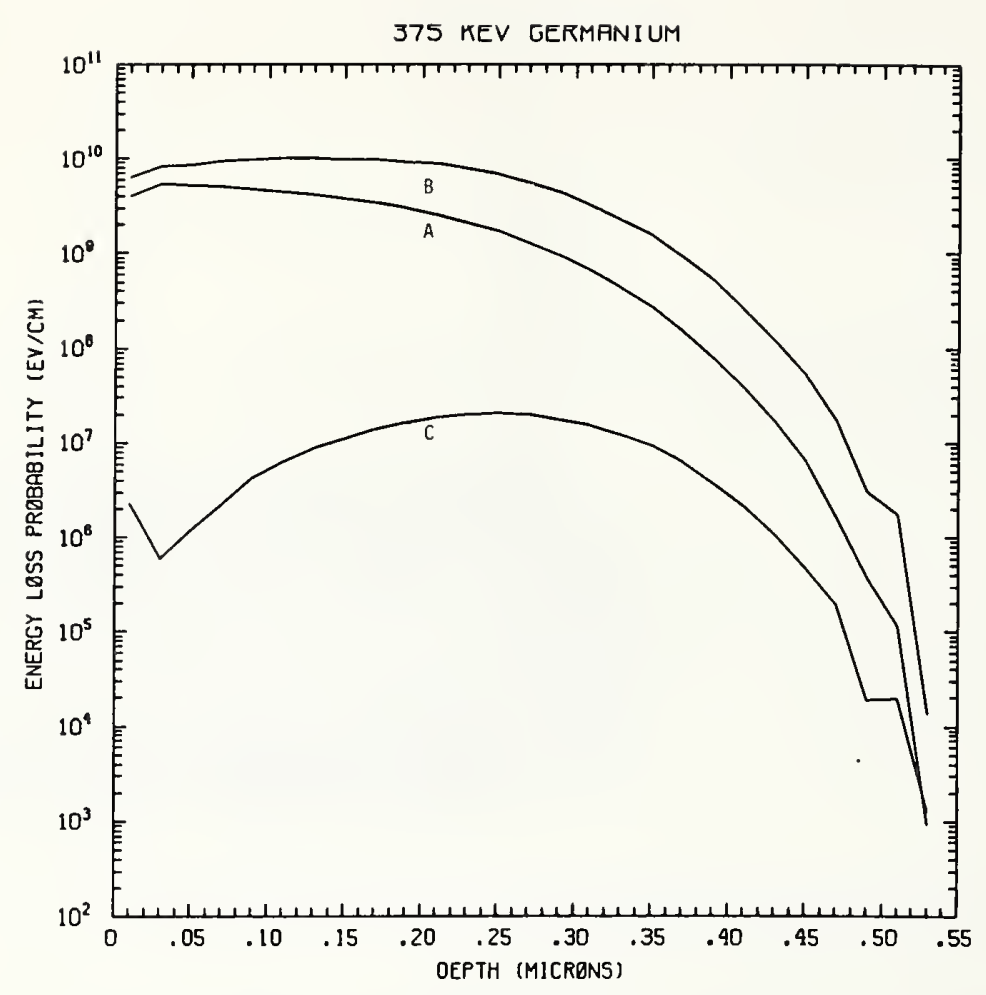

Figure Ge375-1 One-dimensional probability distributions for energy loss due to electronic excitation (A), displacement damage(B), and phonon excitation (C) for $375 \mathrm{keV}$ germanium normally incident on a silicon target.

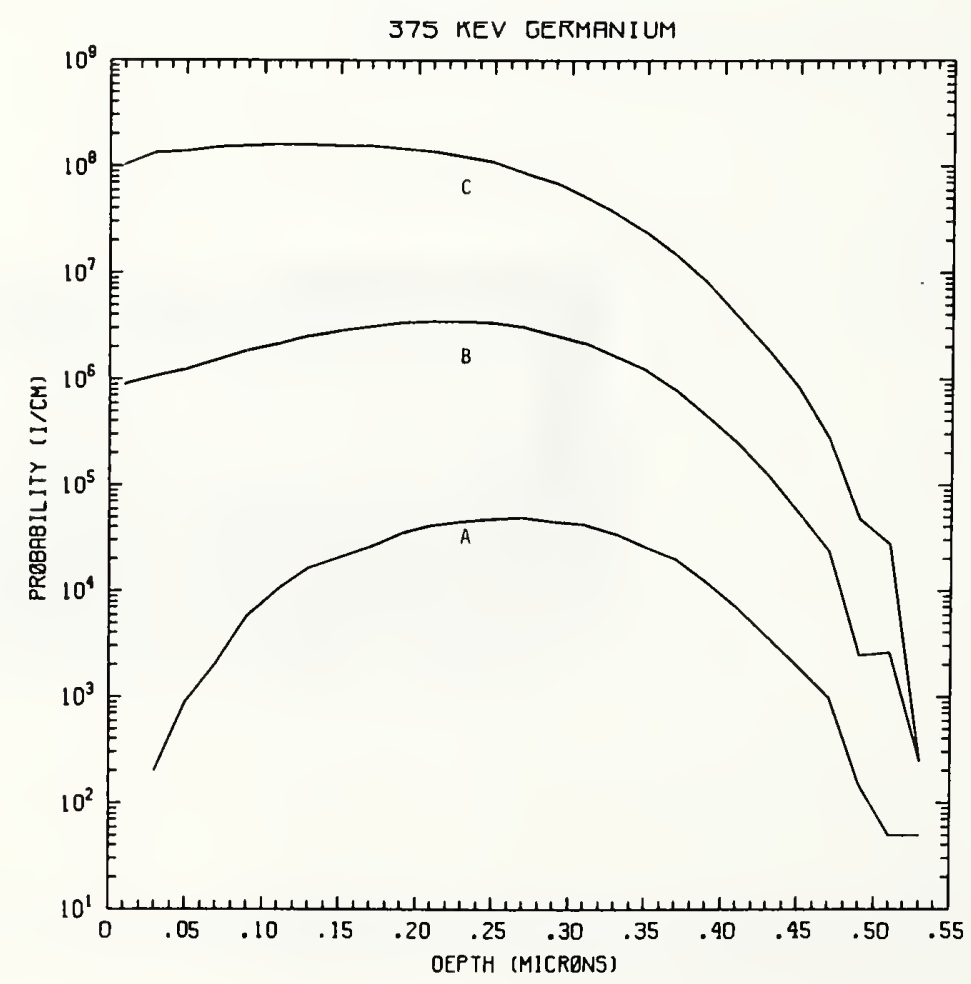

Figure Ge375-2 One-dimensional probability distributions for implanted germanium (A), primary silicon displacements (B), and Frenkel pairs (C) for $375 \mathrm{keV}$ germanium normally incident on a silicon target. 
MASK

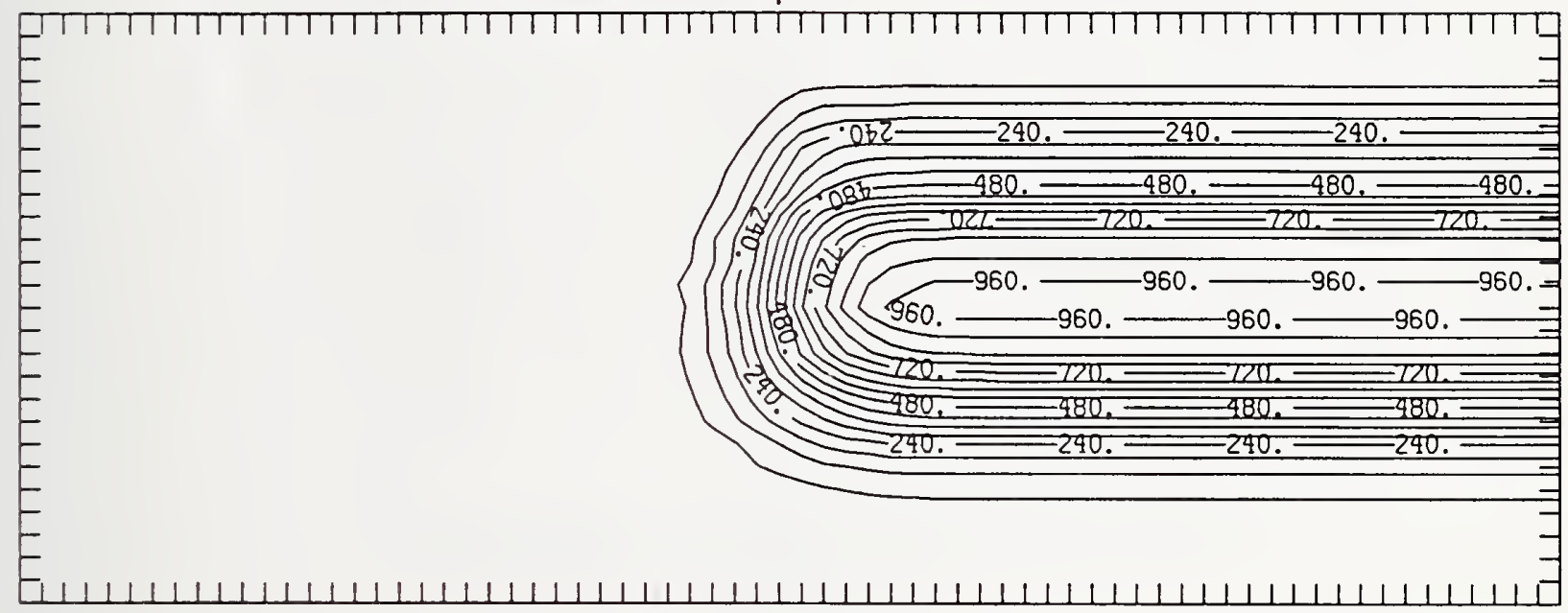

Figure Ge375-3 Two-dimensional distribution of $375 \mathrm{keV}$ germanium implanted past a mask edge. The length increment (distance between tick marks) is $0.02 \mu \mathrm{m}$ and the scale factor is 1000 .

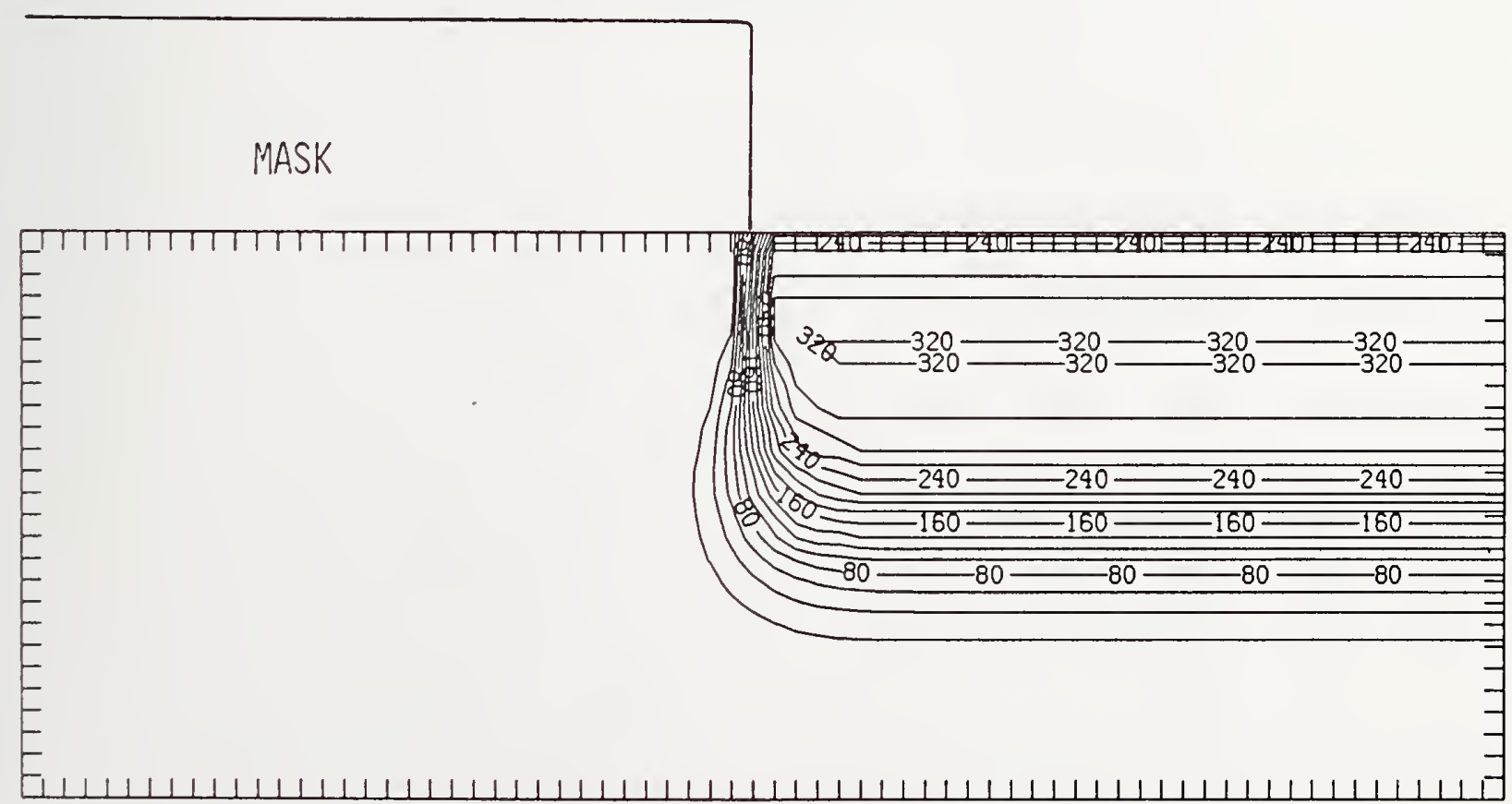

Figure Ge375-4 Two-dimensional distribution of Frenkel pairs created by $375 \mathrm{keV}$ germanium implanted past a mask edge. The length increment (distance between tick marks) is $0.02 \mu \mathrm{m}$ and the scale factor is 0.1 . 


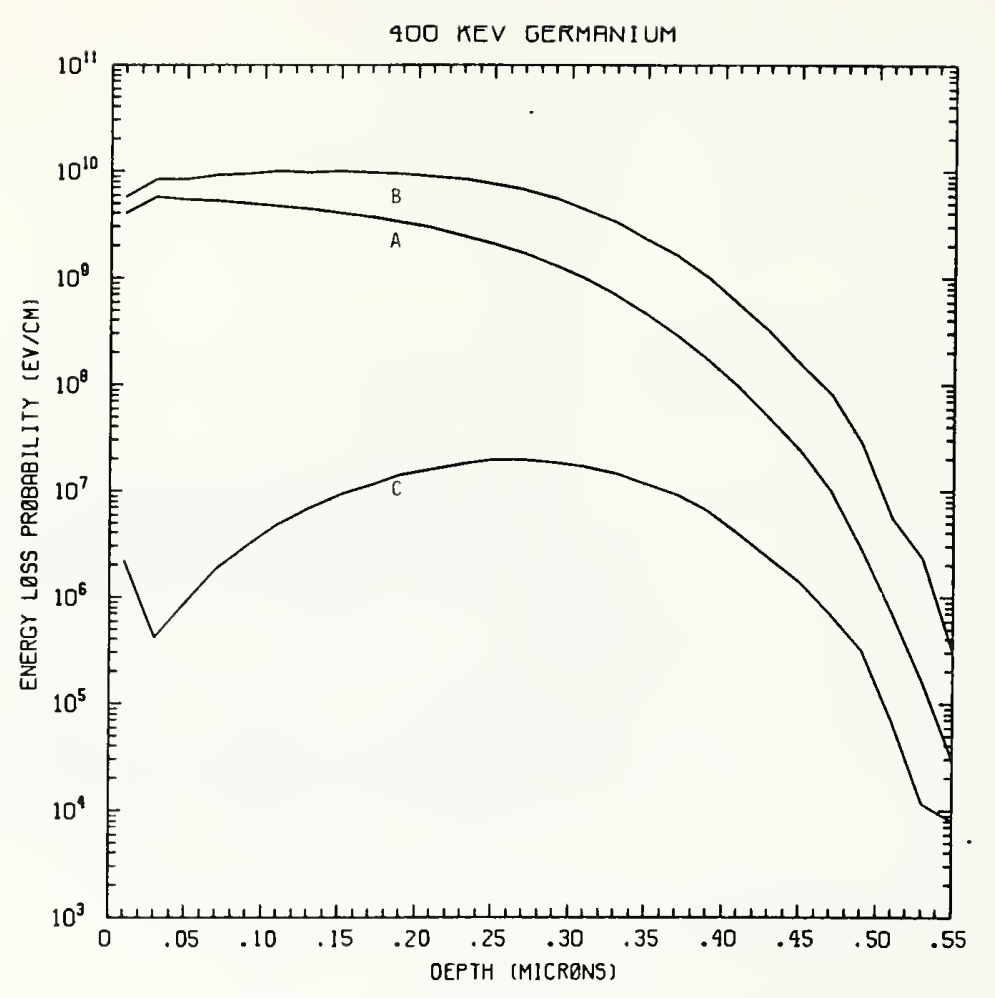

Figure Ge400-1 One-dimensional probability distributions for energy loss due to electronic excitation (A), displacement damage(B), and phonon excitation (C) for $400 \mathrm{keV}$ germanium normally incident on a silicon target.

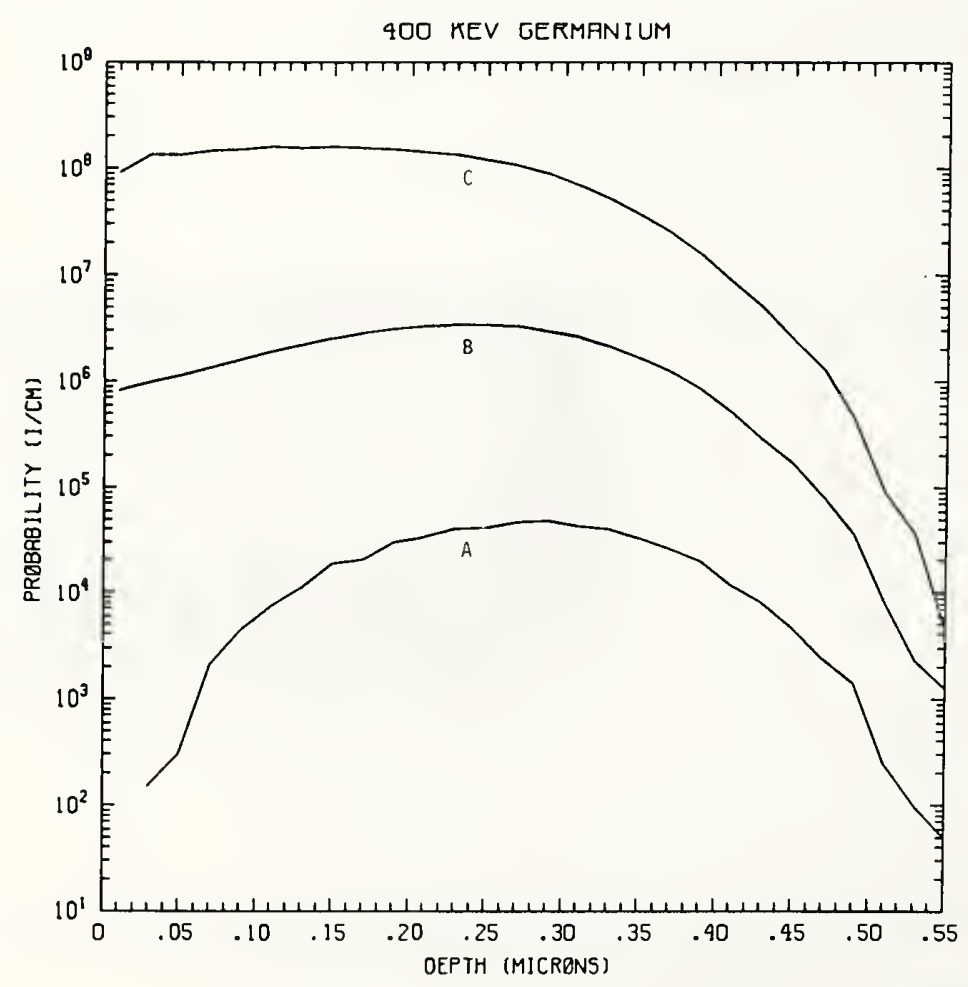

Figure Ge400-2 One-dimensional probability distributions for implanted germanium (A), primary silicon displacements (B), and Frenkel pairs (C) for $400 \mathrm{keV}$ germanium normally incident on a silicon target. 


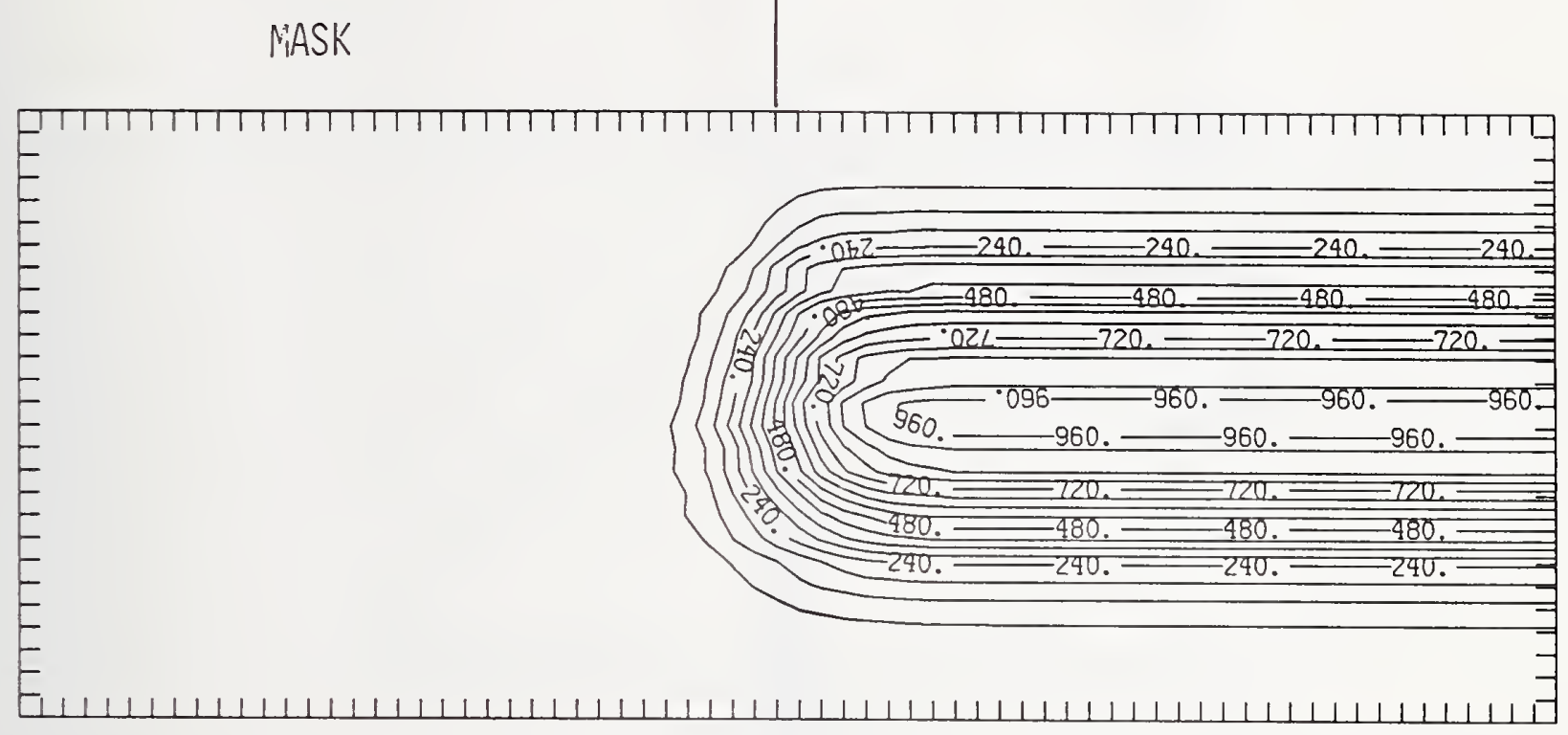

Figure Ge400-3 Two-dimensional distribution of $400 \mathrm{keV}$ germanium implanted past a mask edge. The length increment (distance between tick marks) is $0.02 \mu \mathrm{m}$ and the scale factor is 1000 .

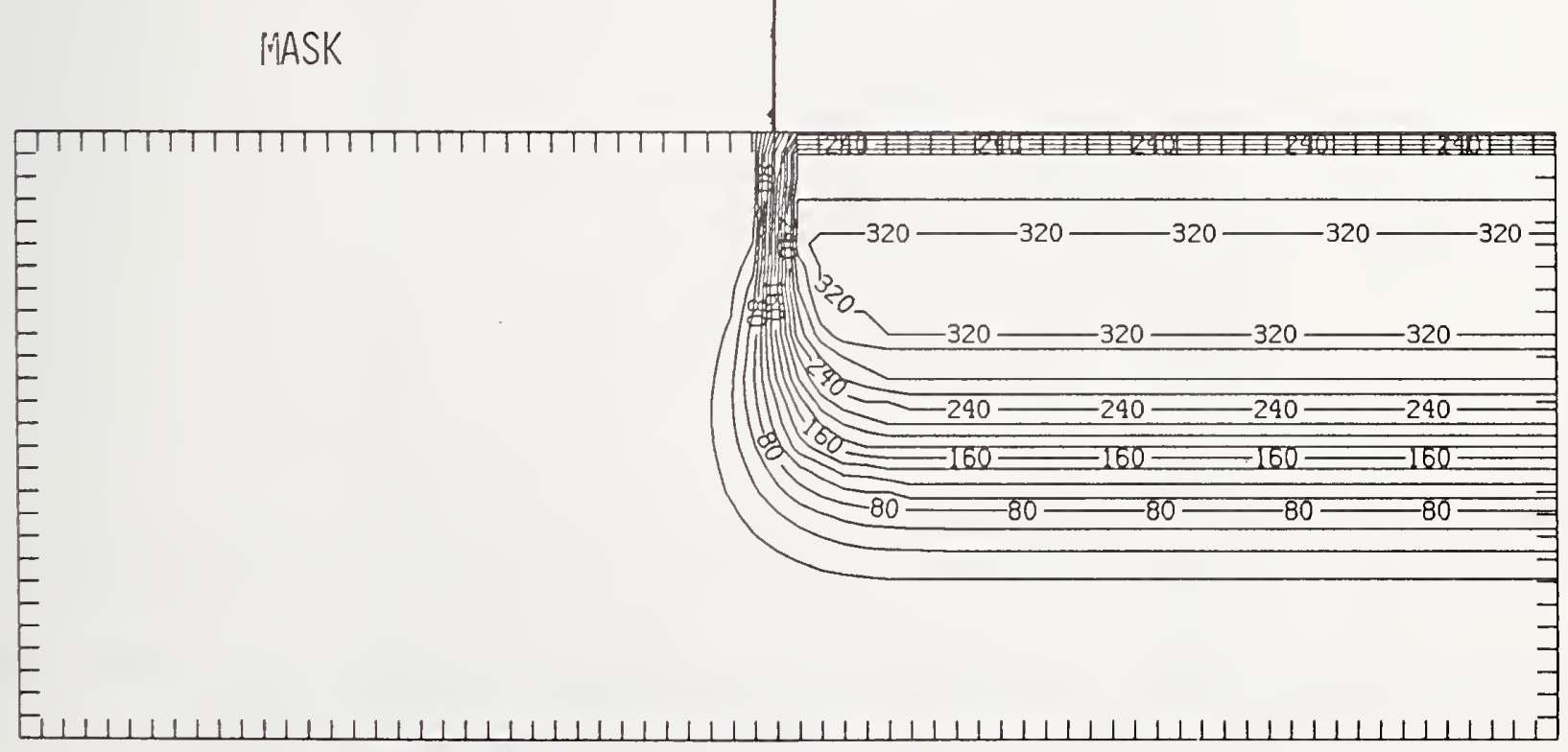

Figure Ge400-4 Two-dimensional distribution of Frenkel pairs created by $400 \mathrm{keV}$ germanium implanted past a mask edge. The length increment (distance between tick marks) is $0.02 \mu \mathrm{m}$ and the scale factor is 0.1 . 


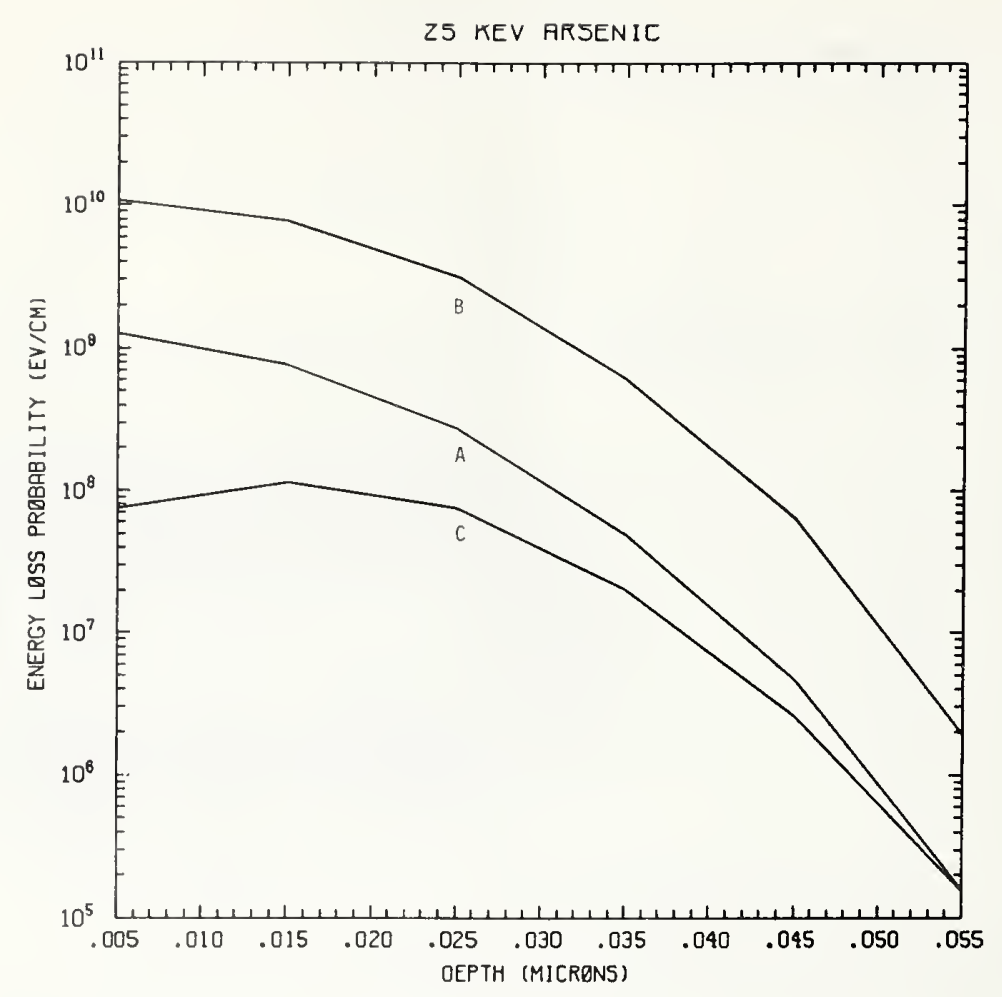

Figure As25-1 One-dimensional probability distributions for energy loss due to electronic excitation (A), displacement damage(B), and phonon excitation (C) for $25 \mathrm{keV}$ arsenic normally incident on a silicon target.

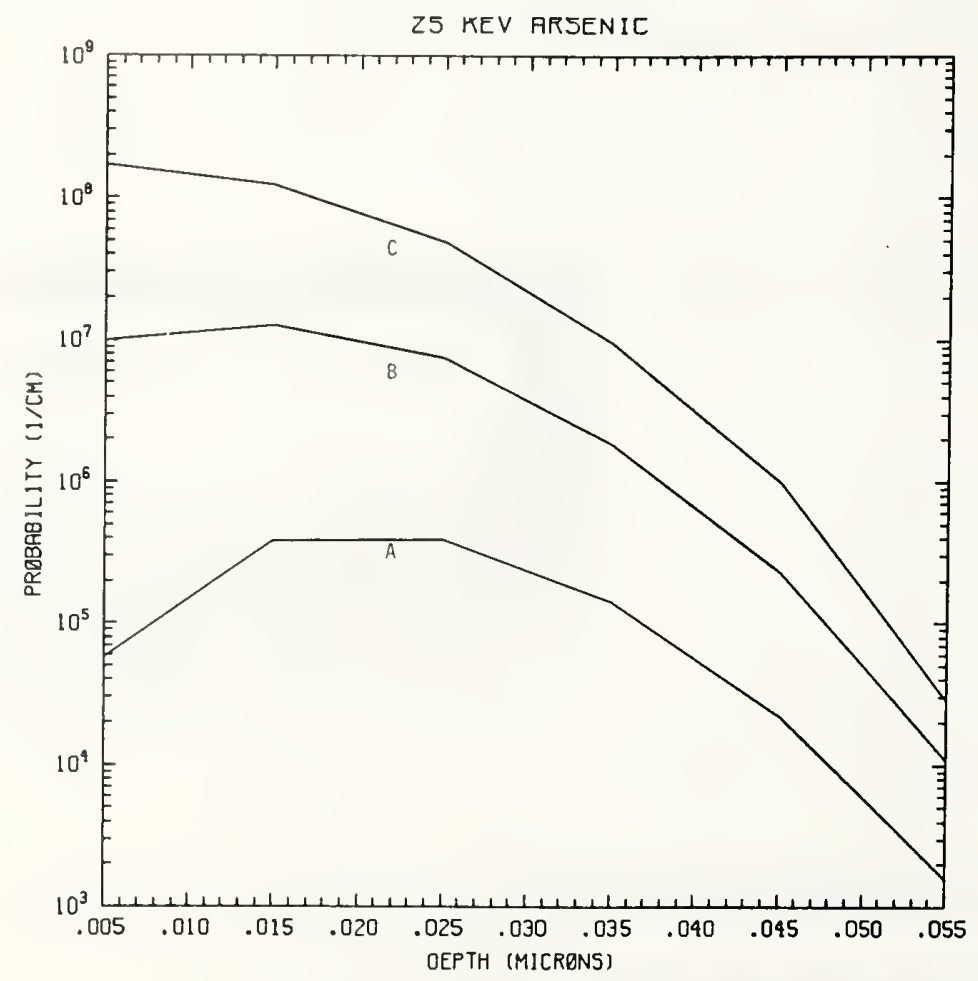

Figure As25-2 One-dimensional probability distributions for implanted arsenic (A), primary silicon displacements (B), and Frenkel pairs (C) for $25 \mathrm{keV}$ arsenic normally incident on a silicon target. 
MASK

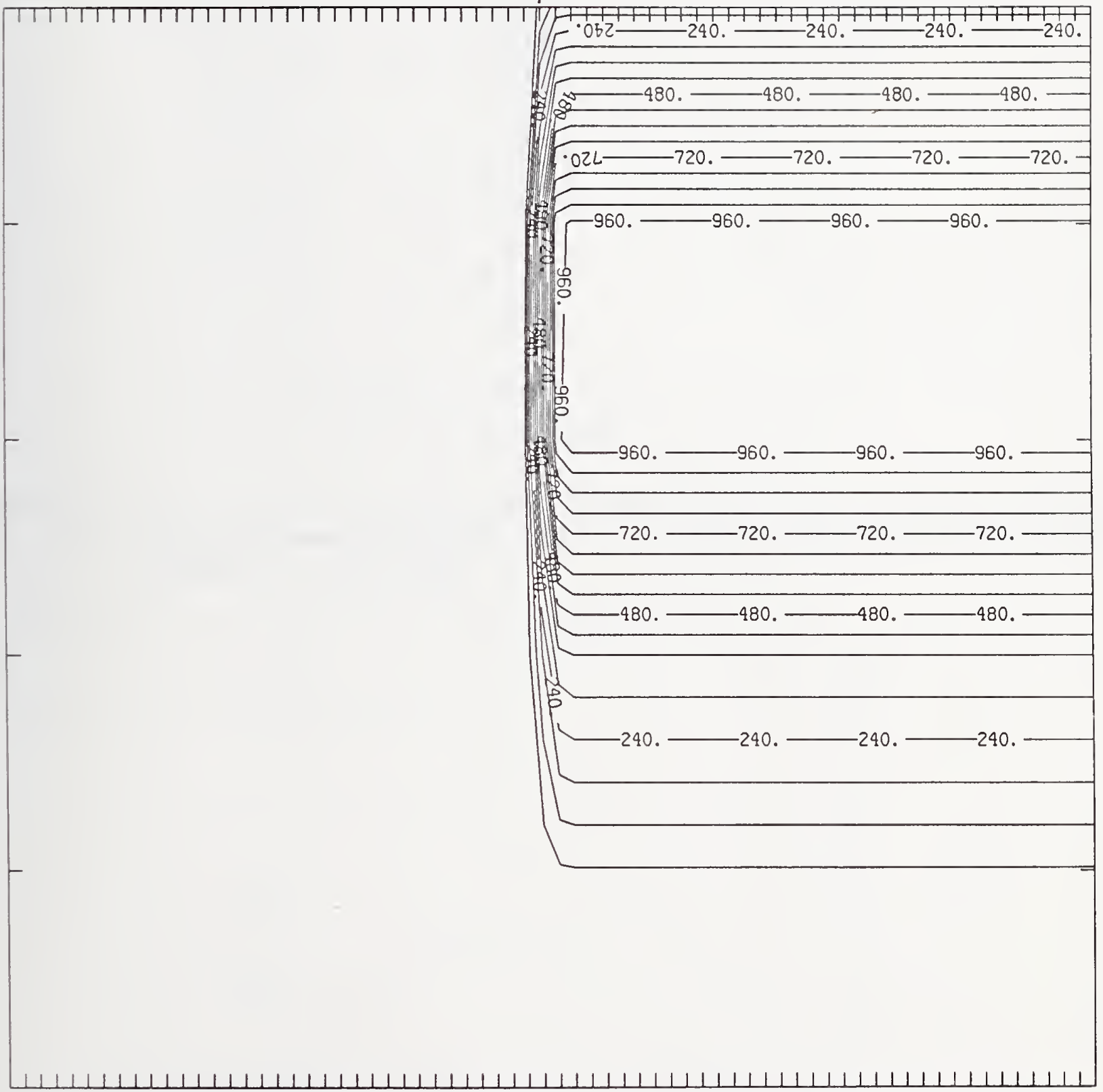

Figure As25-3 Two-dimensional distribution of $25 \mathrm{keV}$ arsenic implanted past a mask edge. The length increment (distance between tick marks) is $0.01 \mu \mathrm{m}$ and the scale factor is 1000 . 


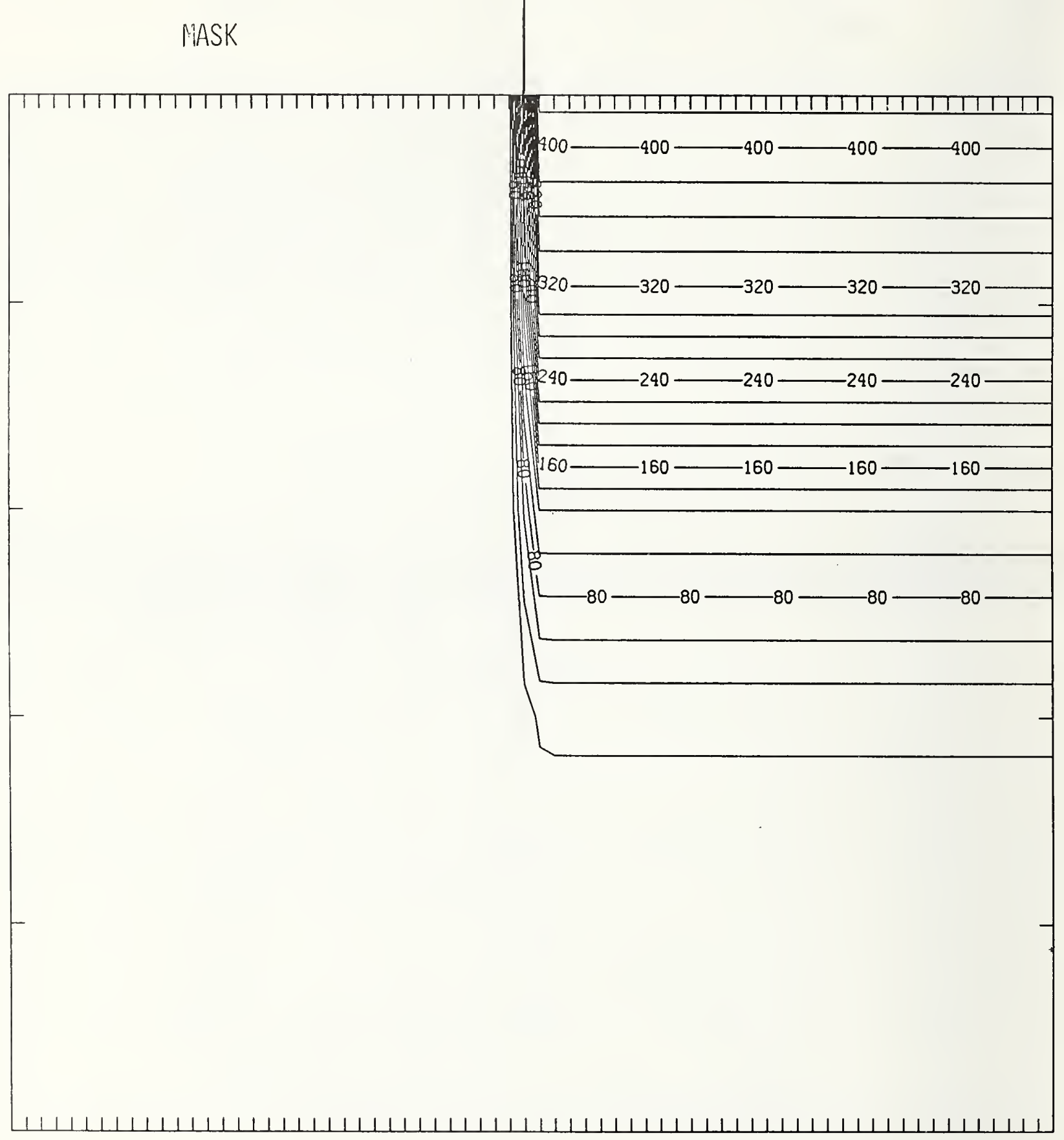

Figure As25-4 Two-dimensional distribution of Frenkel pairs created by $25 \mathrm{keV}$ arsenic implanted past a mask edge. The length increment (distance between tick marks) is $0.01 \mu \mathrm{m}$ and the scale factor is 1 . 


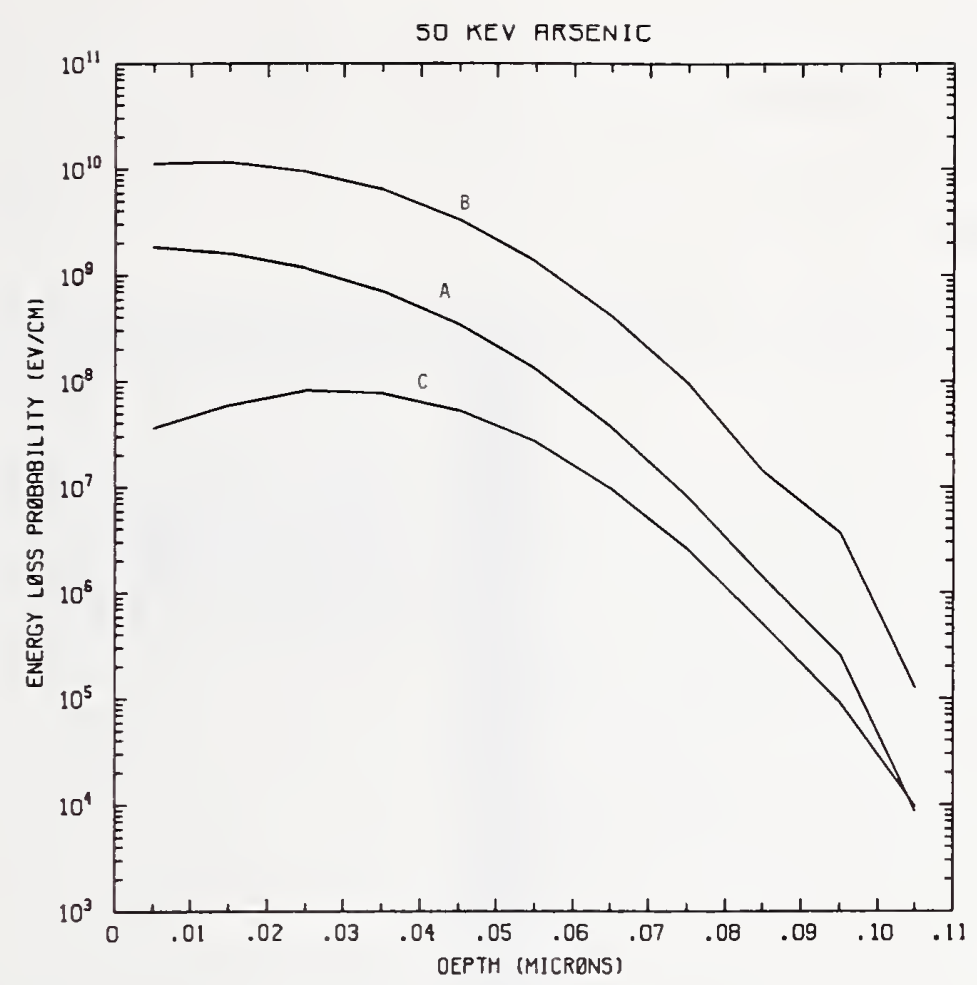

Figure As50-1 One-dimensional probability distributions for energy loss due to electronic excitation (A), displacement damage(B), and phonon excitation (C) for $50 \mathrm{keV}$ arsenic normally incident on a silicon target.

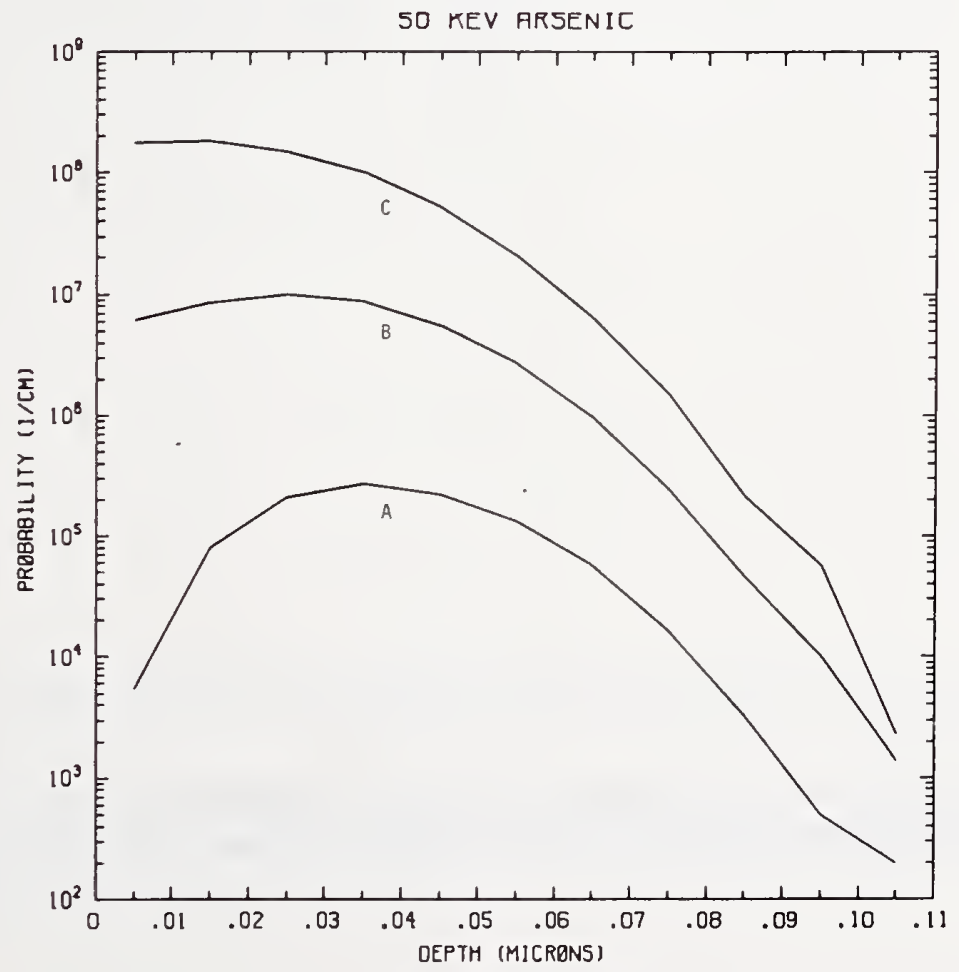

Figure As50-2 One-dimensional probability distributions for implanted arsenic (A), primary silicon displacements (B), and Frenkel pairs (C) for $50 \mathrm{keV}$ arsenic normally incident on a silicon target. 


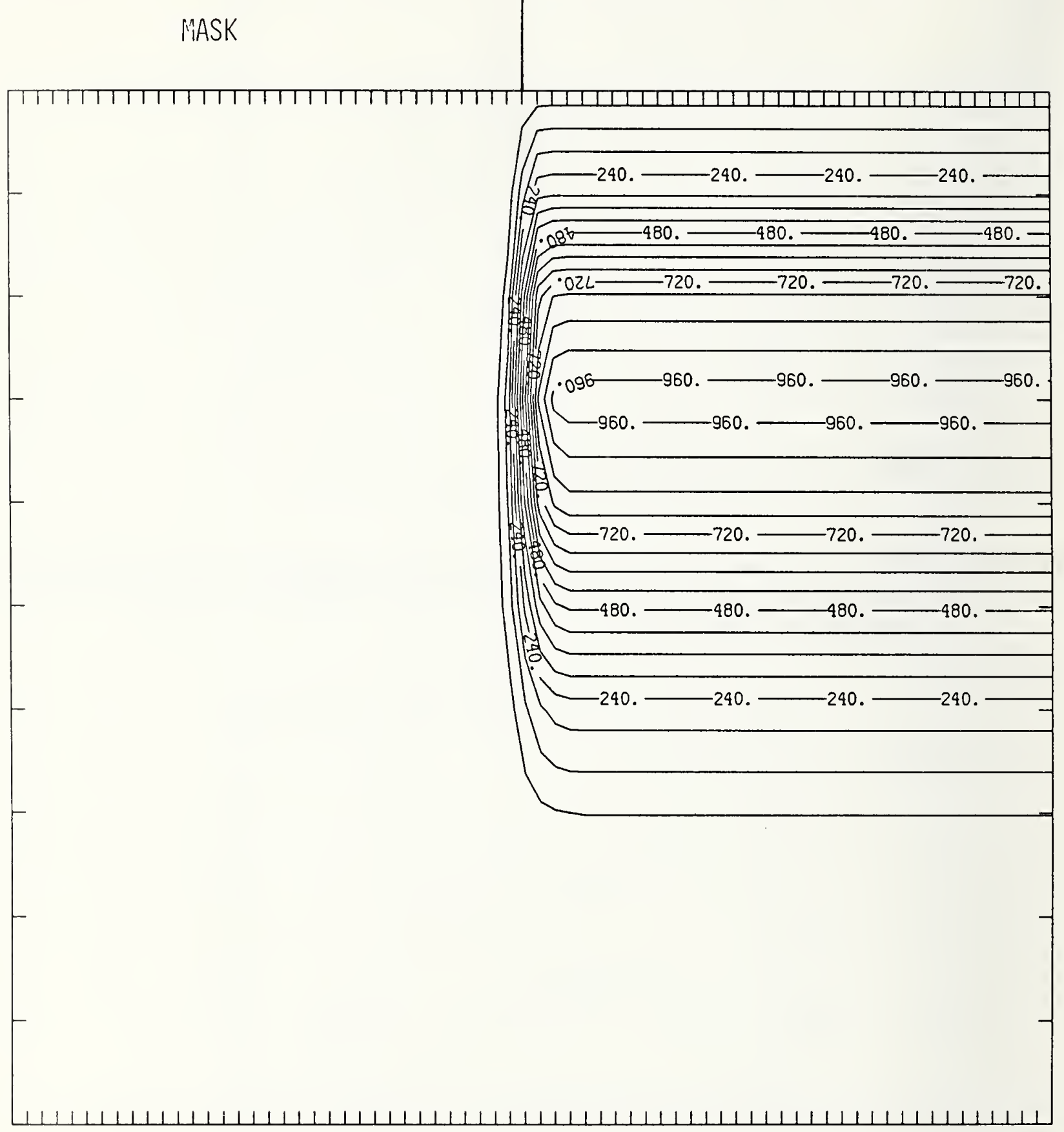

Figure As50-3 Two-dimensional distribution of $50 \mathrm{keV}$ arsenic implanted past a mask edge. The length increment (distance between tick marks) is $0.01 \mu \mathrm{m}$ and the scale factor is 1000 . 


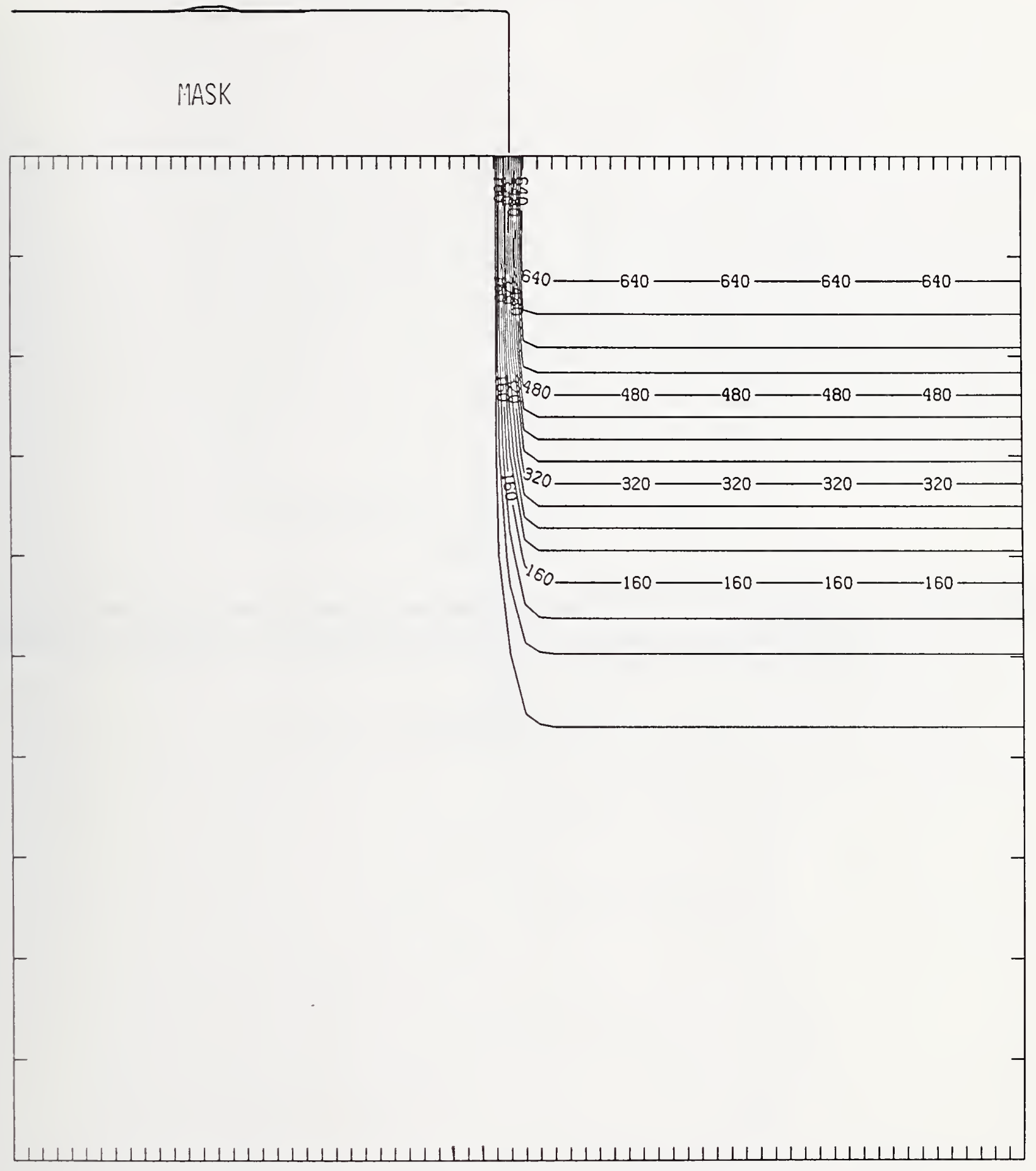

Figure As50-4 Two-dimensional distribution of Frenkel pairs created by $50 \mathrm{keV}$ arsenic implanted past a mask edge. The length increment (distance between tick marks) is $0.01 \mu \mathrm{m}$ and the scale factor is 1 . 


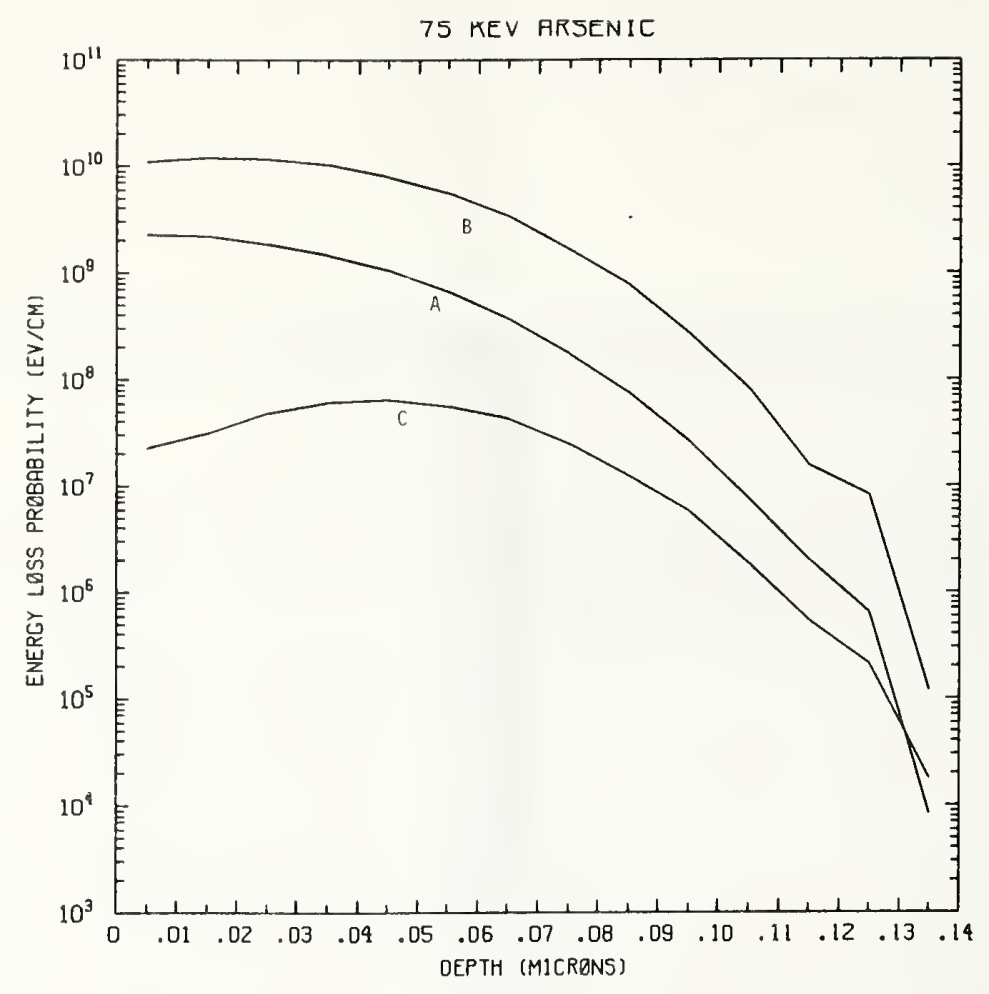

Figure As75-1 One-dimensional probability distributions for energy loss due to electronic excitation (A), displacement damage(B), and phonon excitation (C) for $75 \mathrm{keV}$ arsenic normally incident on a silicon target.

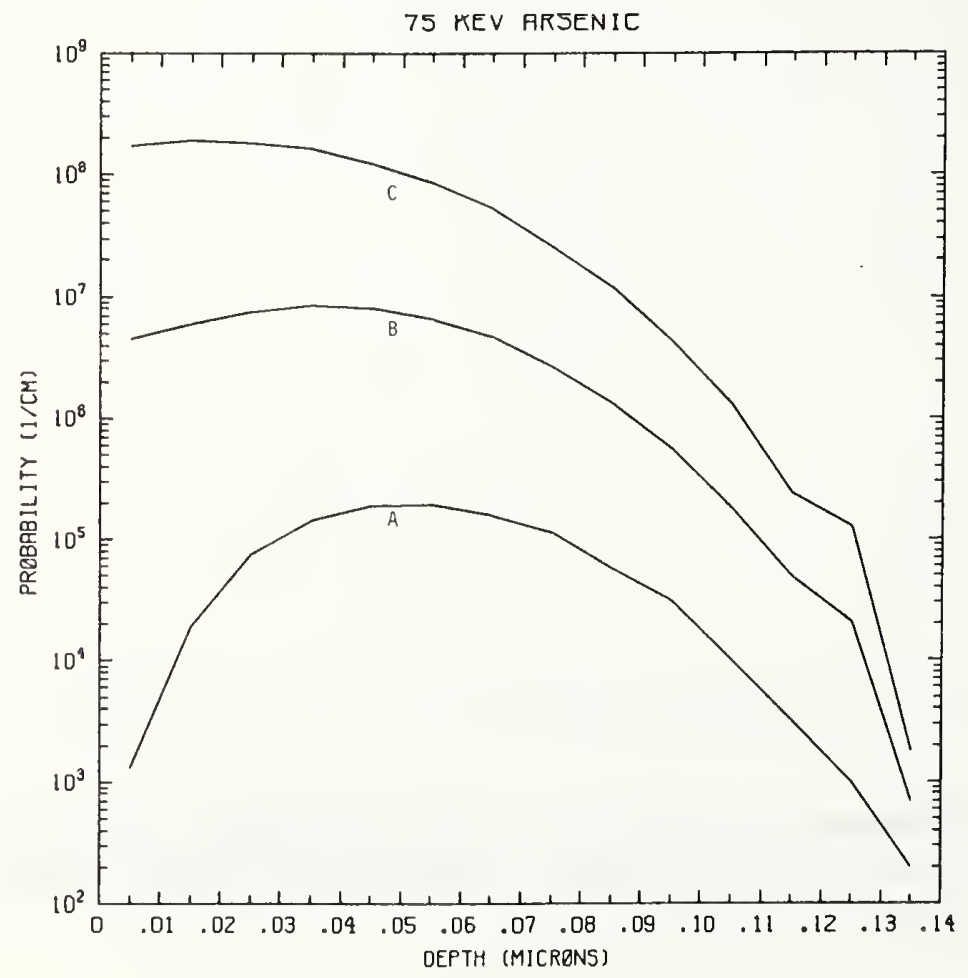

Figure As75-2 One-dimensional probability distributions for implanted arsenic (A), primary silicon displacements (B), and Frenkel pairs (C) for $75 \mathrm{keV}$ arsenic normally incident on a silicon target. 


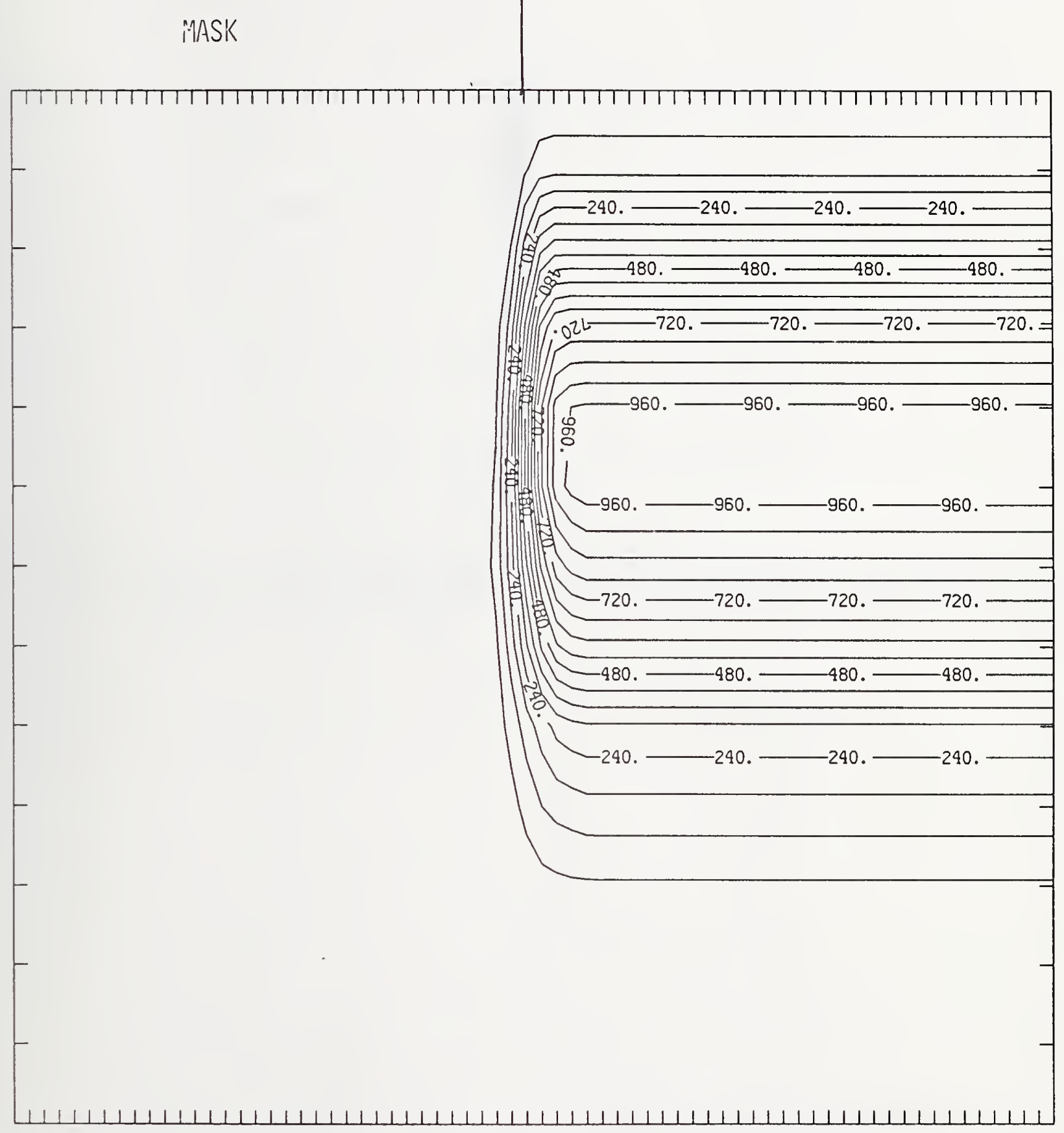

Figure As75-3 Two-dimensional distribution of $75 \mathrm{keV}$ arsenic implanted past a mask edge. The length increment (distance between tick marks) is $0.01 \mu \mathrm{m}$ and the scale factor is 1000 . 
MASK

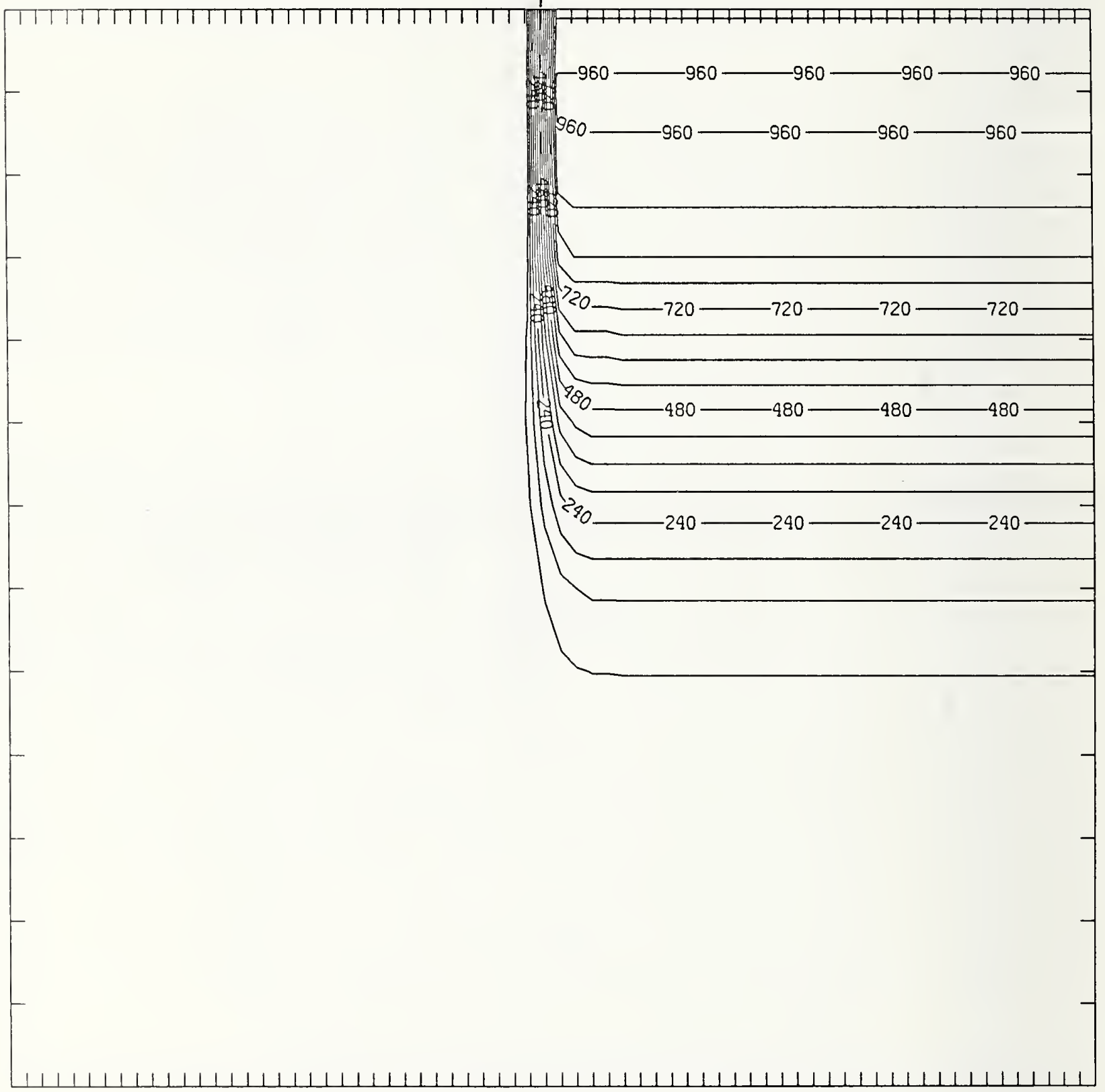

Figure As75-4 Two-dimensional distribution of Frenkel pairs created by $75 \mathrm{keV}$ arsenic implanted past a mask edge. The length increment (distance between tick marks) is $0.01 \mu \mathrm{m}$ and the scale factor is 1 . 


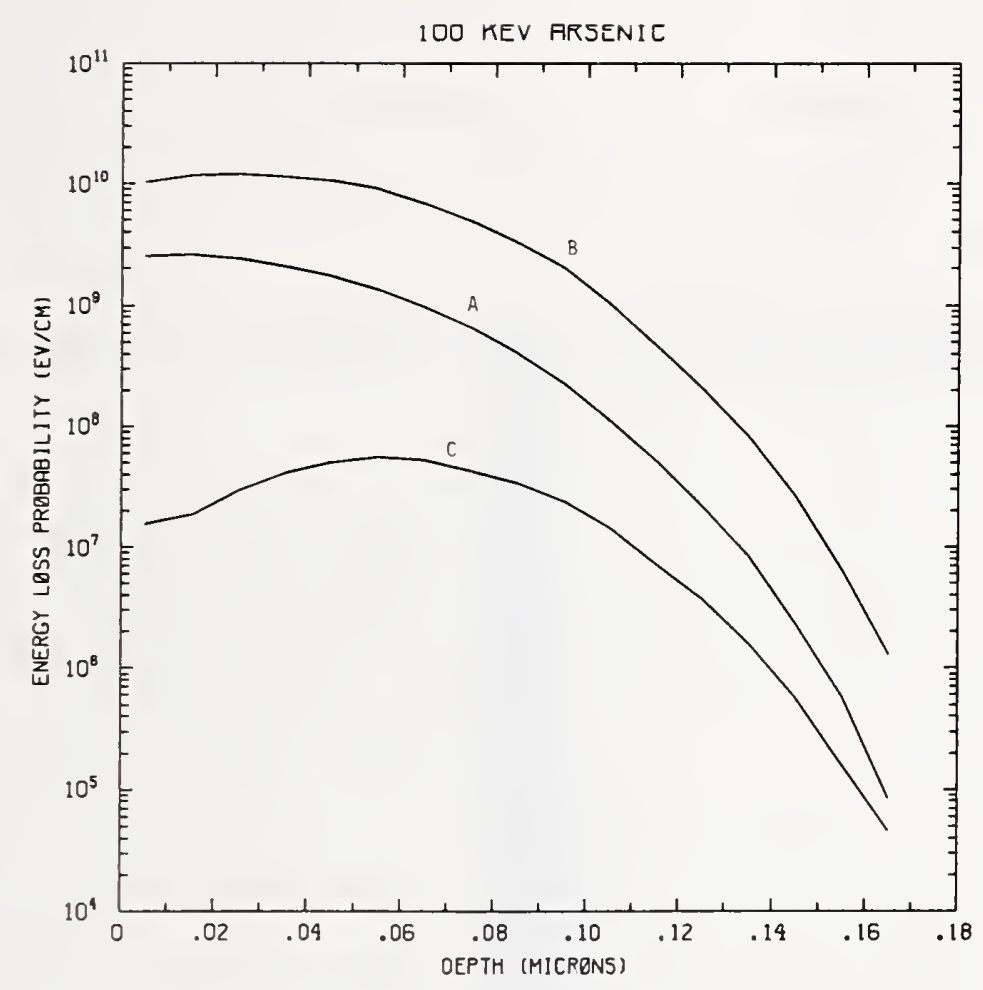

Figure As100-1 One-dimensional probability distributions for energy loss due to electronic excitation (A), displacement damage(B), and phonon excitation (C) for $100 \mathrm{keV}$ arsenic normally incident on a silicon target.

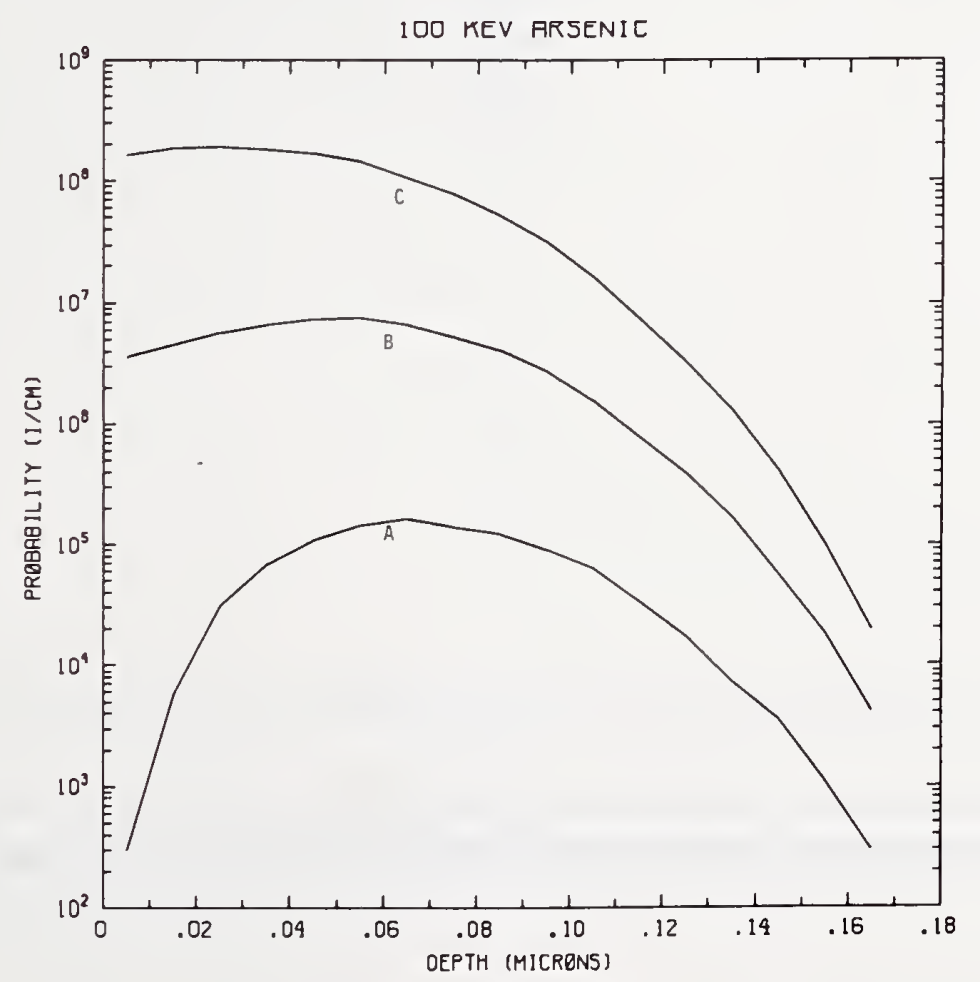

Figure As100-2 One-dimensional probability distributions for implanted arsenic (A), primary silicon displacements (B), and Frenkel pairs (C) for $100 \mathrm{keV}$ arsenic normally incident on a silicon target. 


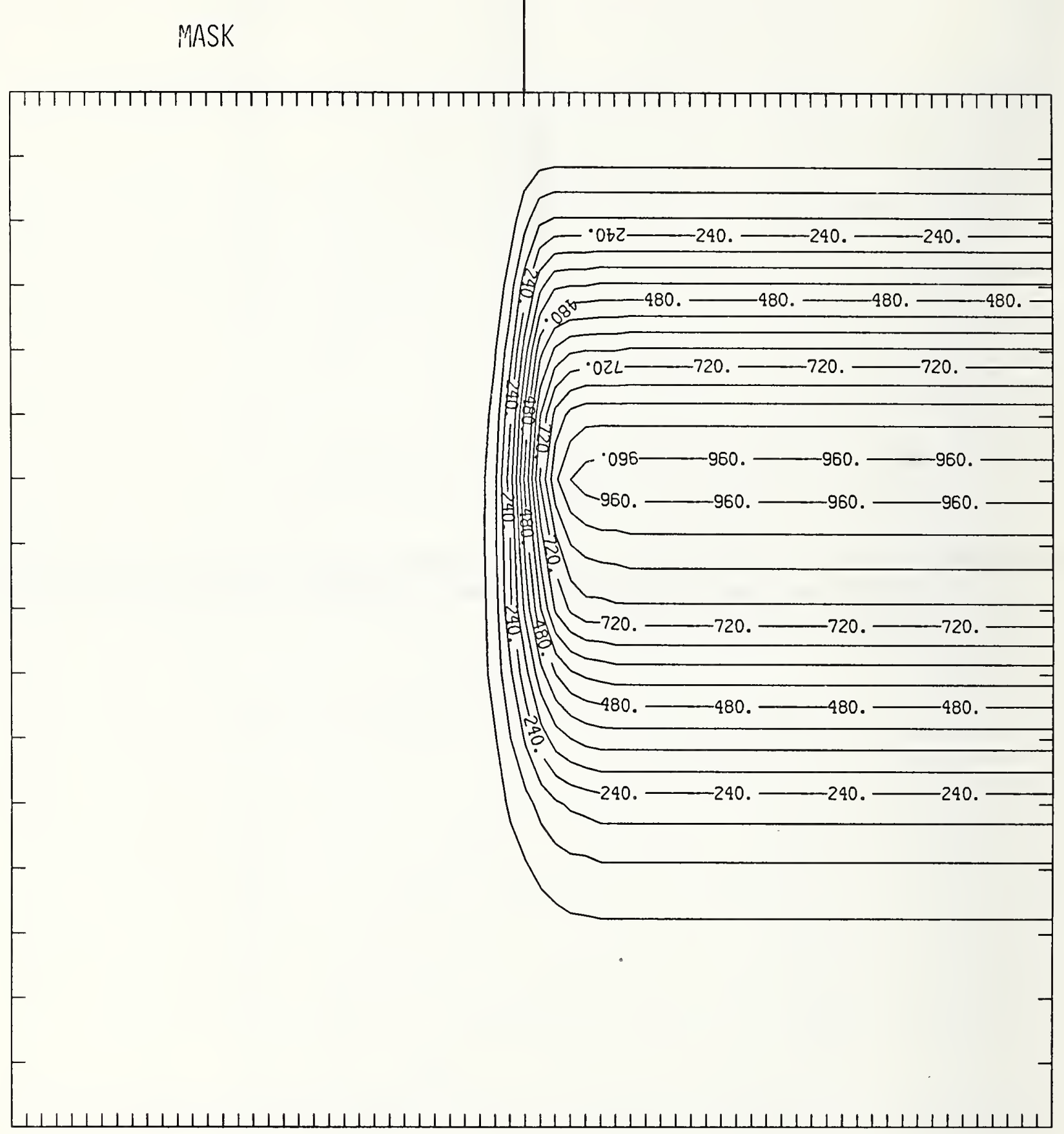

Figure As100-3 Two-dimensional distribution of $100 \mathrm{keV}$ arsenic implanted past a mask edge. The length increment (distance between tick marks) is $0.01 \mu \mathrm{m}$ and the scale factor is 1000. 


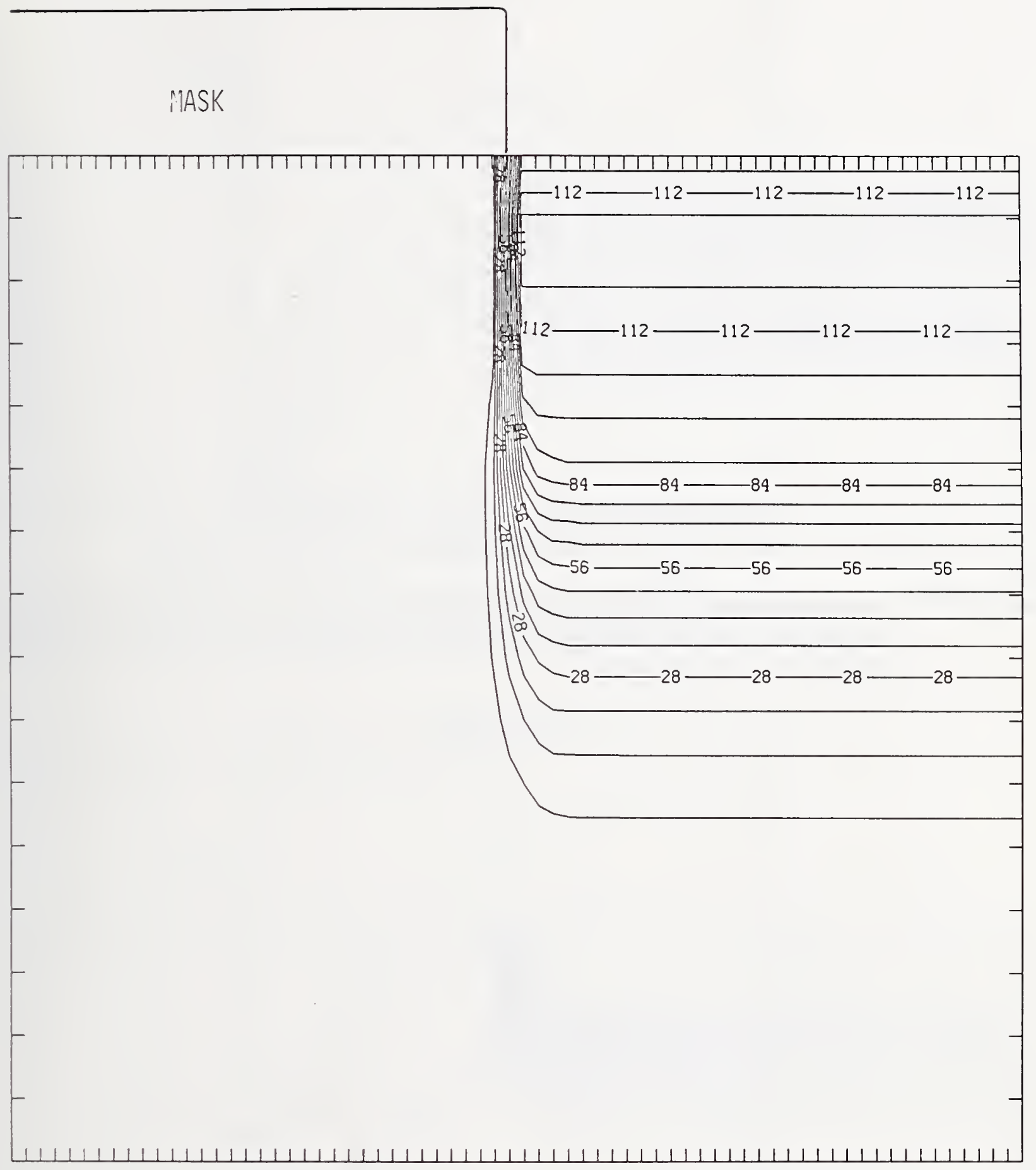

Figure As100-4 Two-dimensional distribution of Frenkel pairs created by $100 \mathrm{keV}$ arsenic implanted past a mask edge. The length increment (distance between tick marks) is $0.01 \mu \mathrm{m}$ and the scale factor is 0.1 . 


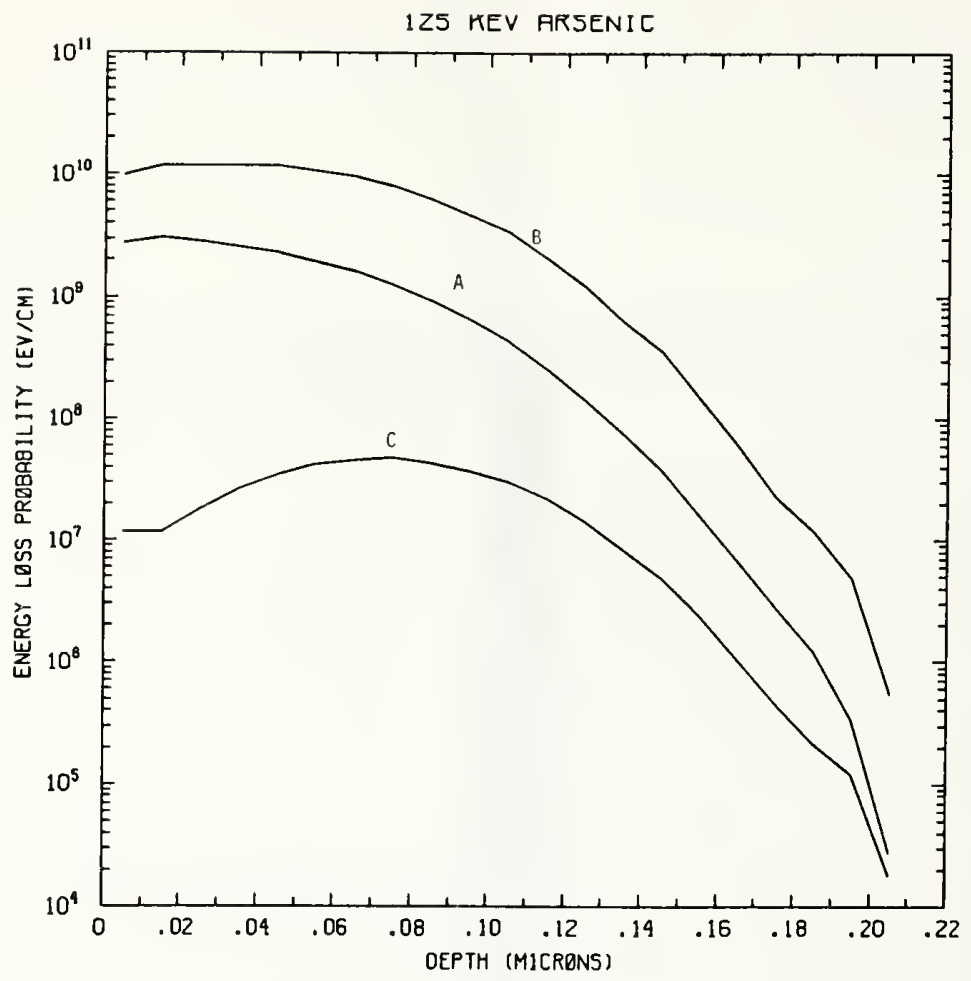

Figure As125-1 One-dimensional probability distributions for energy loss due to electronic excitation (A), displacement damage(B), and phonon excitation (C) for $125 \mathrm{keV}$ arsenic normally incident on a silicon target.

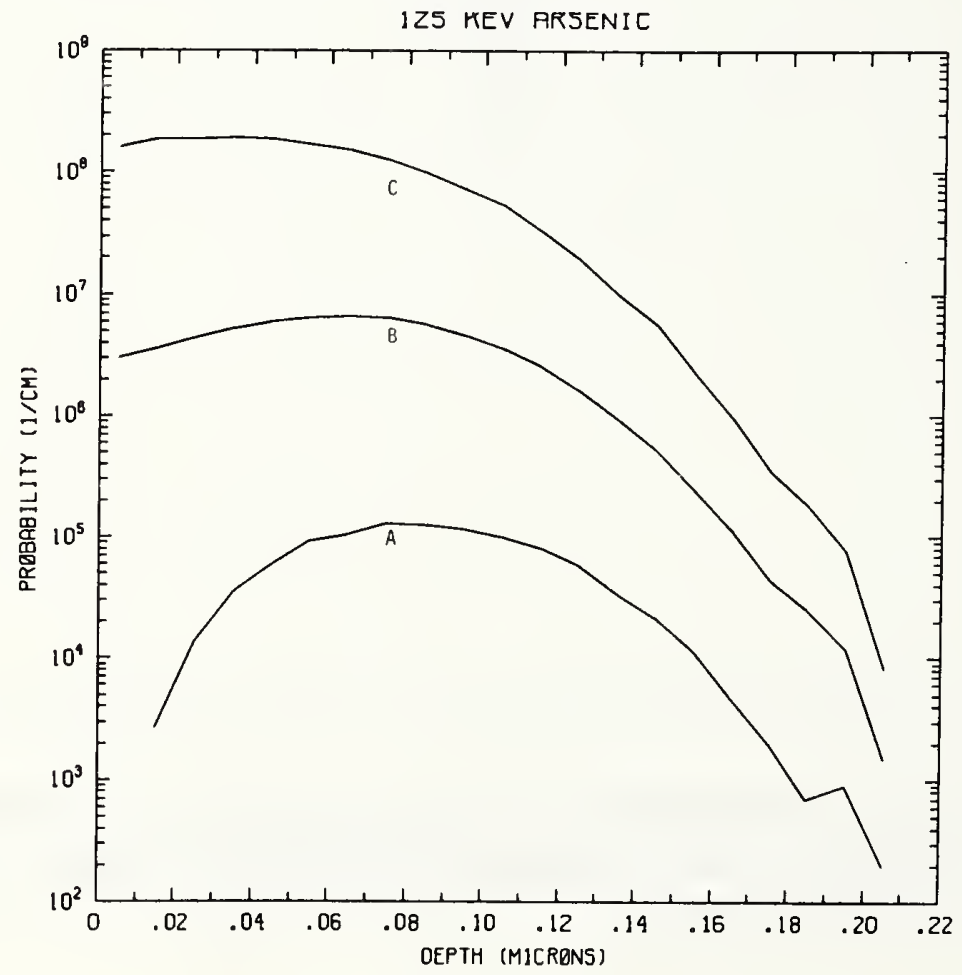

Figure As125-2 One-dimensional probability distributions for implanted arsenic (A), primary silicon displacements (B), and Frenkel pairs (C) for $125 \mathrm{keV}$ arsenic normally incident on a silicon target. 


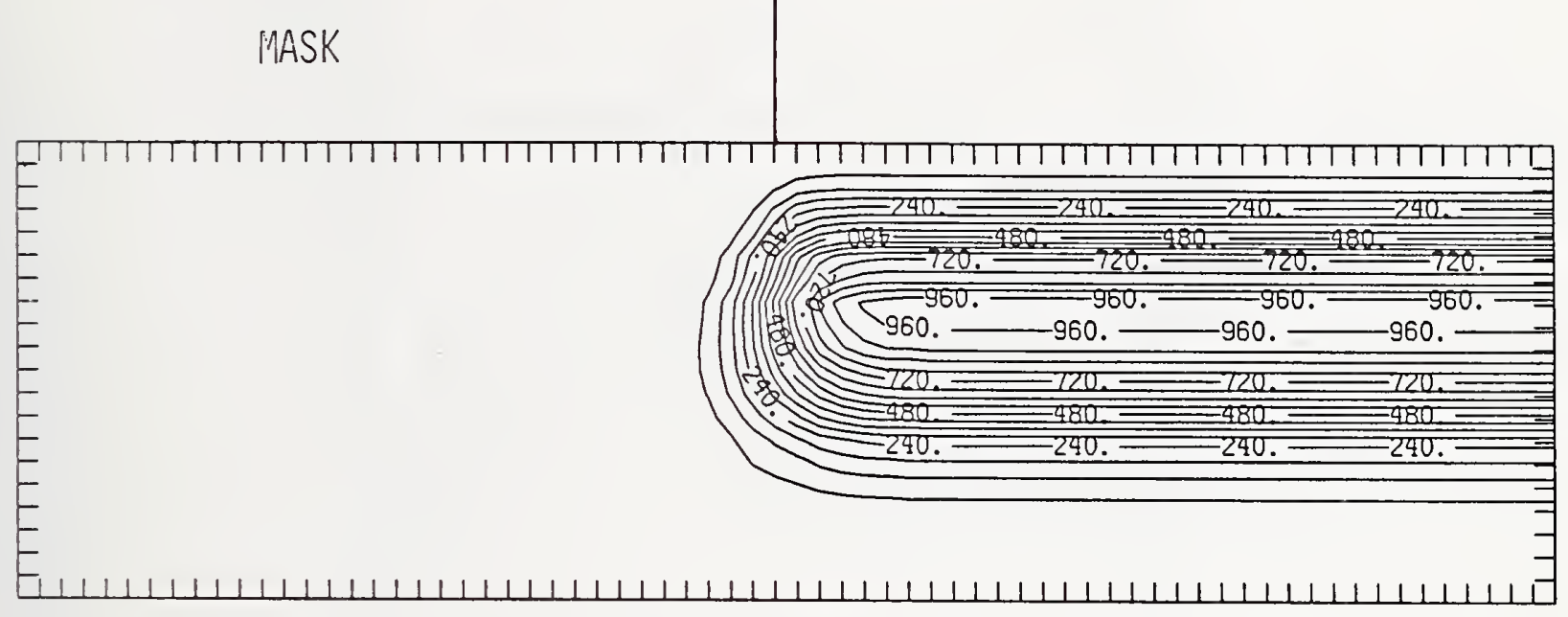

Figure As125-3 Two-dimensional distribution of $125 \mathrm{keV}$ arsenic implanted past a mask edge. The length increment (distance between tick marks) is $0.01 \mu \mathrm{m}$ and the scale factor is 1000 .

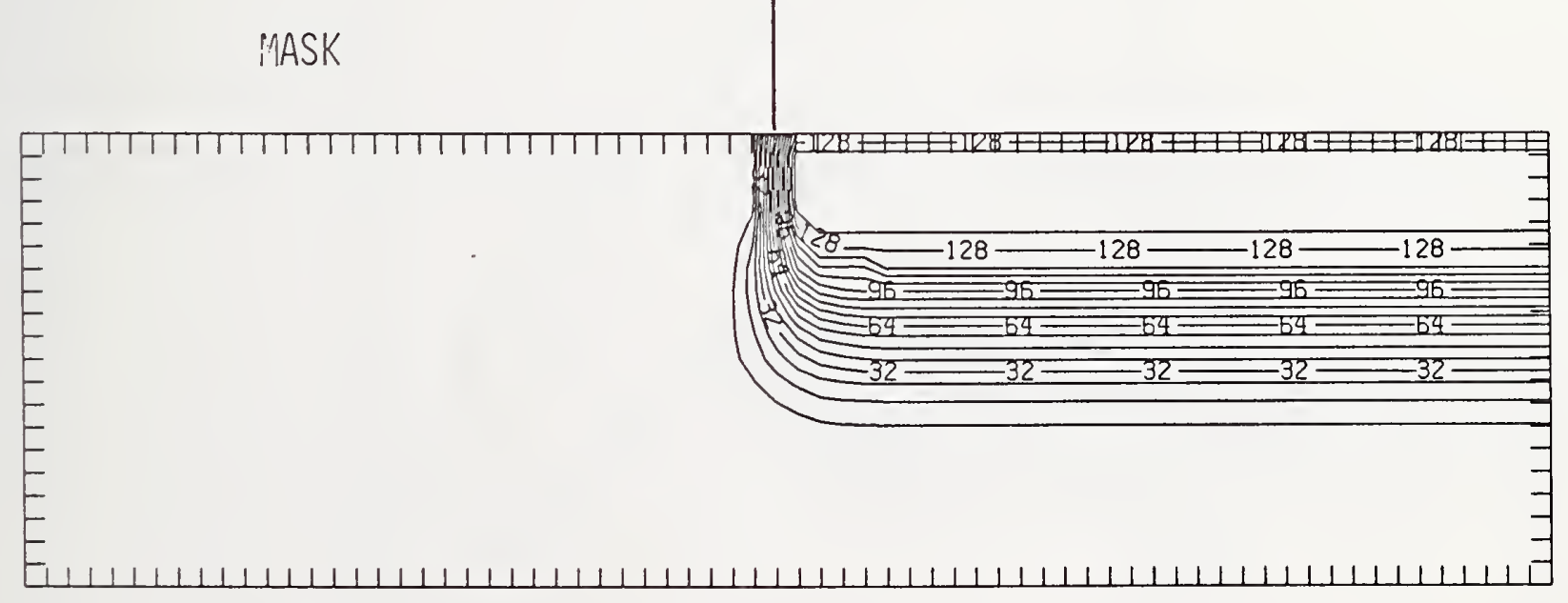

Figure As125-4 Two-dimensional distribution of Frenkel pairs created by $125 \mathrm{keV}$ arsenic implanted past a mask edge. The length increment (distance between tick marks) is $0.01 \mu \mathrm{m}$ and the scale factor is 0.1 . 


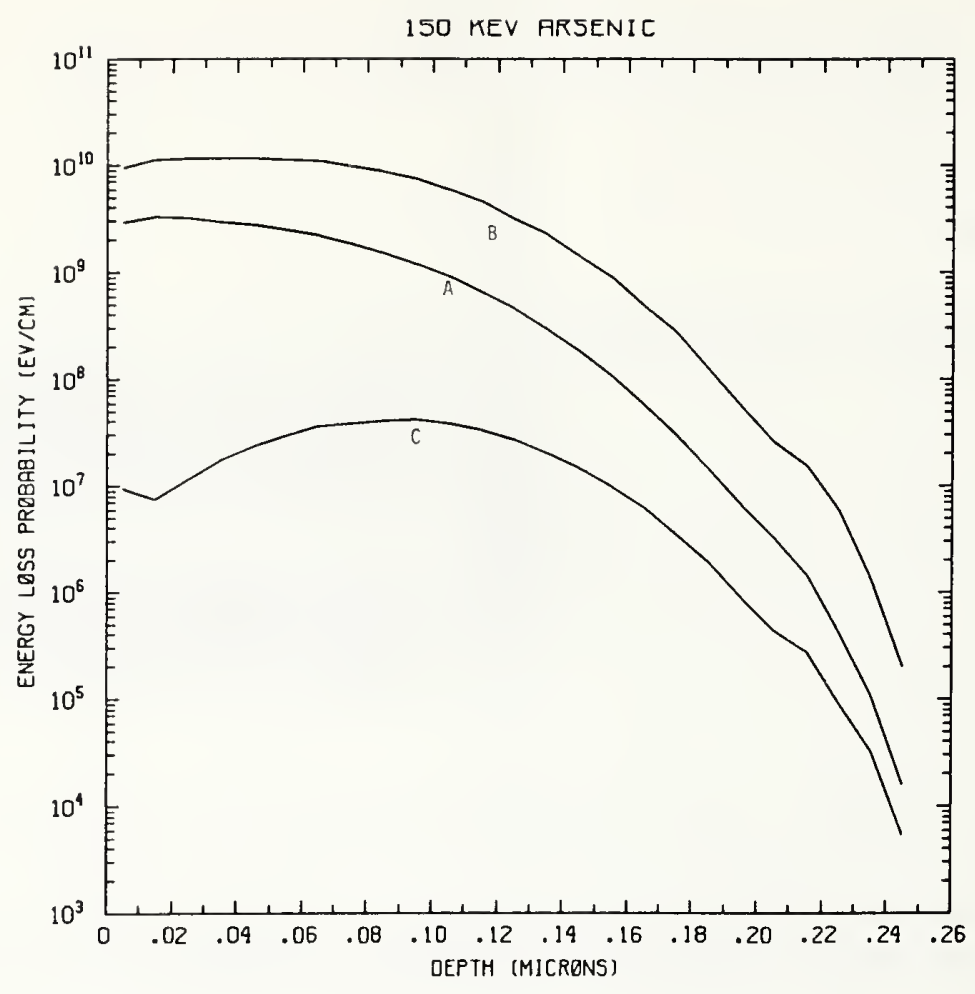

Figure As150-1 One-dimensional probability distributions for energy loss due to electronic excitation (A), displacement damage(B), and phonon excitation (C) for $150 \mathrm{keV}$ arsenic normally incident on a silicon target.

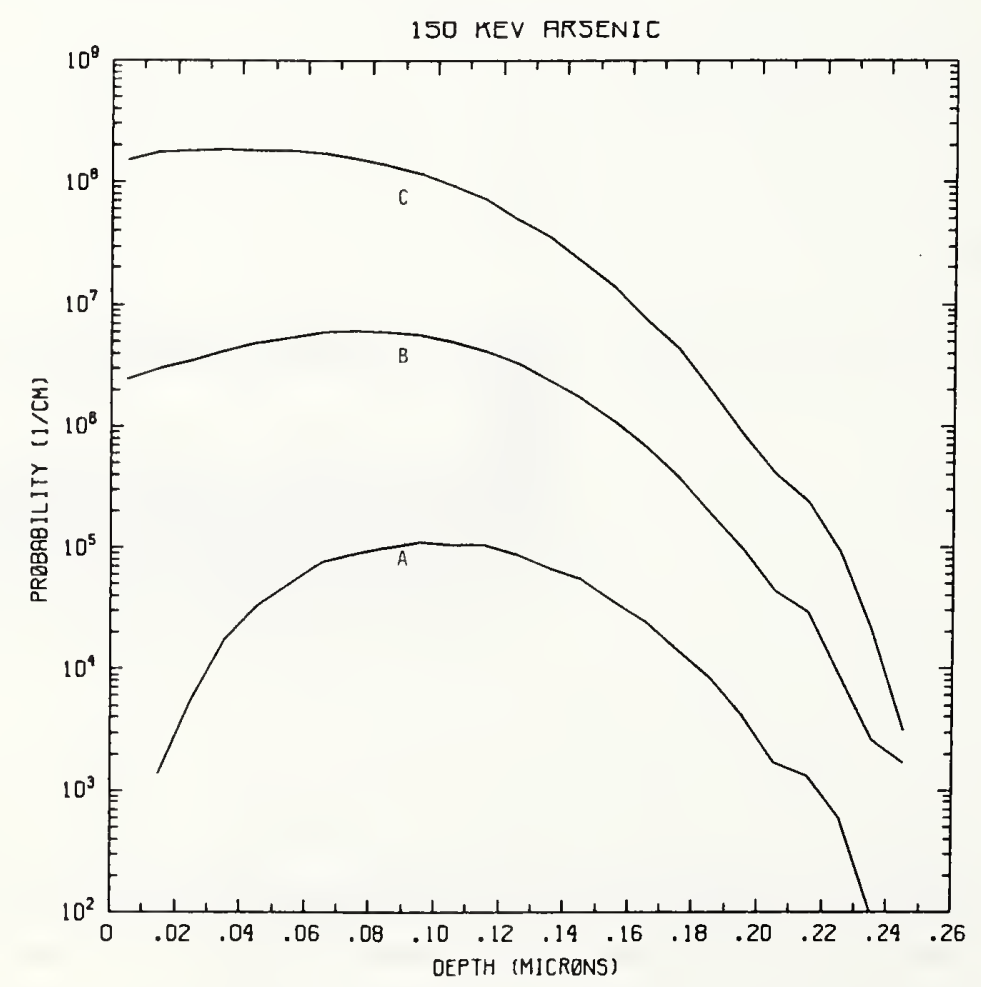

Figure As 150-2 One-dimensional probability distributions for implanted arsenic (A), primary silicon displacements (B), and Frenkel pairs (C) for $150 \mathrm{keV}$ arsenic normally incident on a silicon target. 


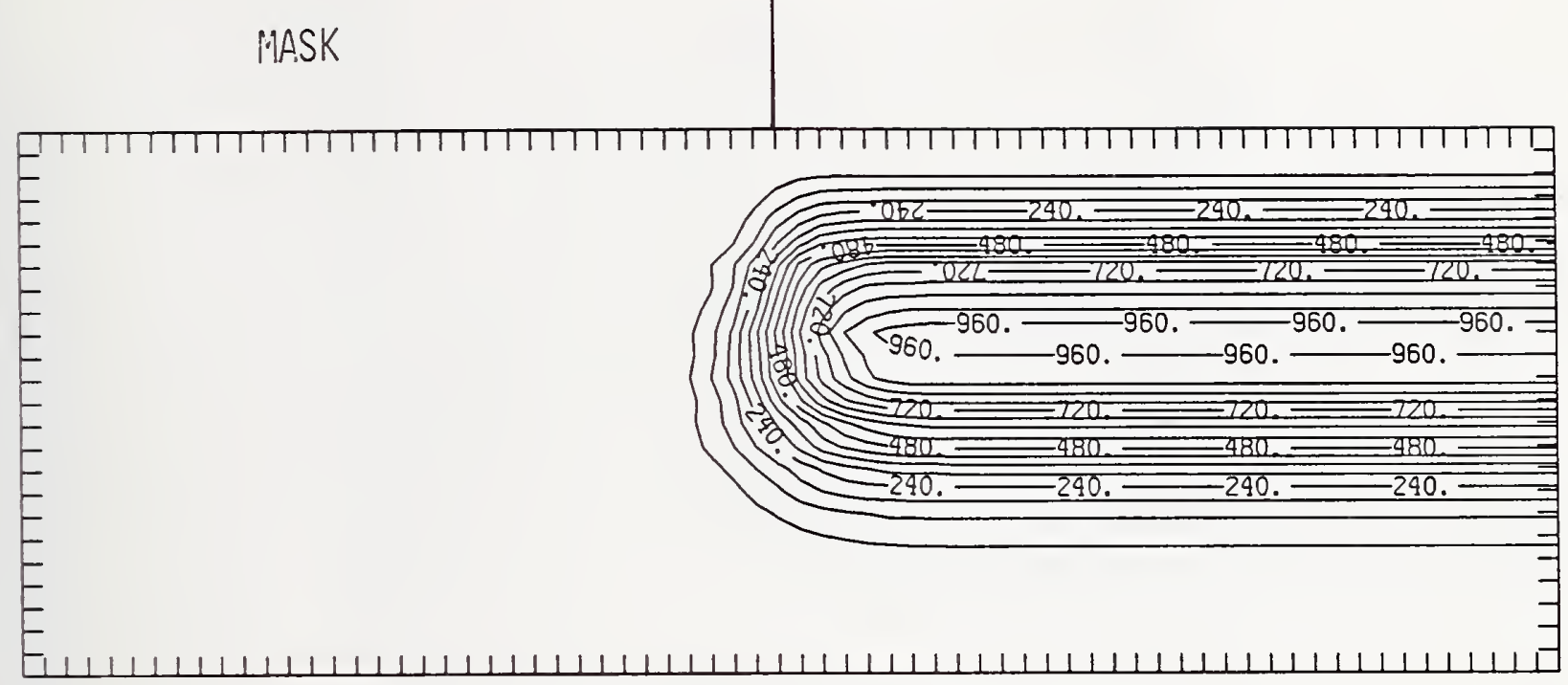

Figure As150-3 Two-dimensional distribution of $150 \mathrm{keV}$ arsenic implanted past a mask edge. The length increment (distance between tick marks) is $0.01 \mu \mathrm{m}$ and the scale factor is 1000 .

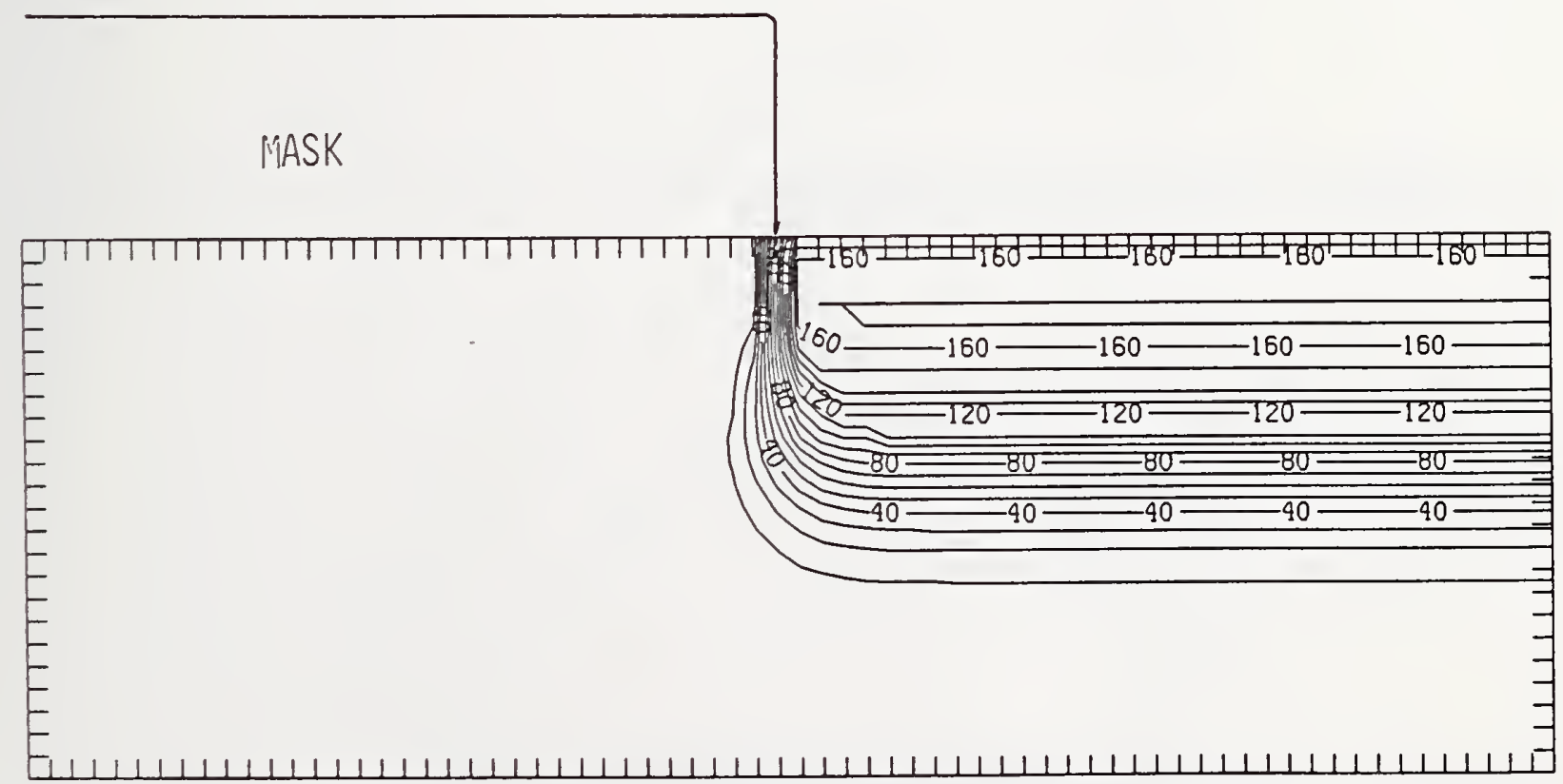

Figure As150-4 Two-dimensional distribution of Frenkel pairs created by $150 \mathrm{keV}$ arsenic implanted past a mask edge. The length increment (distance between tick marks) is $0.01 \mu \mathrm{m}$ and the scale factor is 0.1 . 
175 KEV ARSENIC

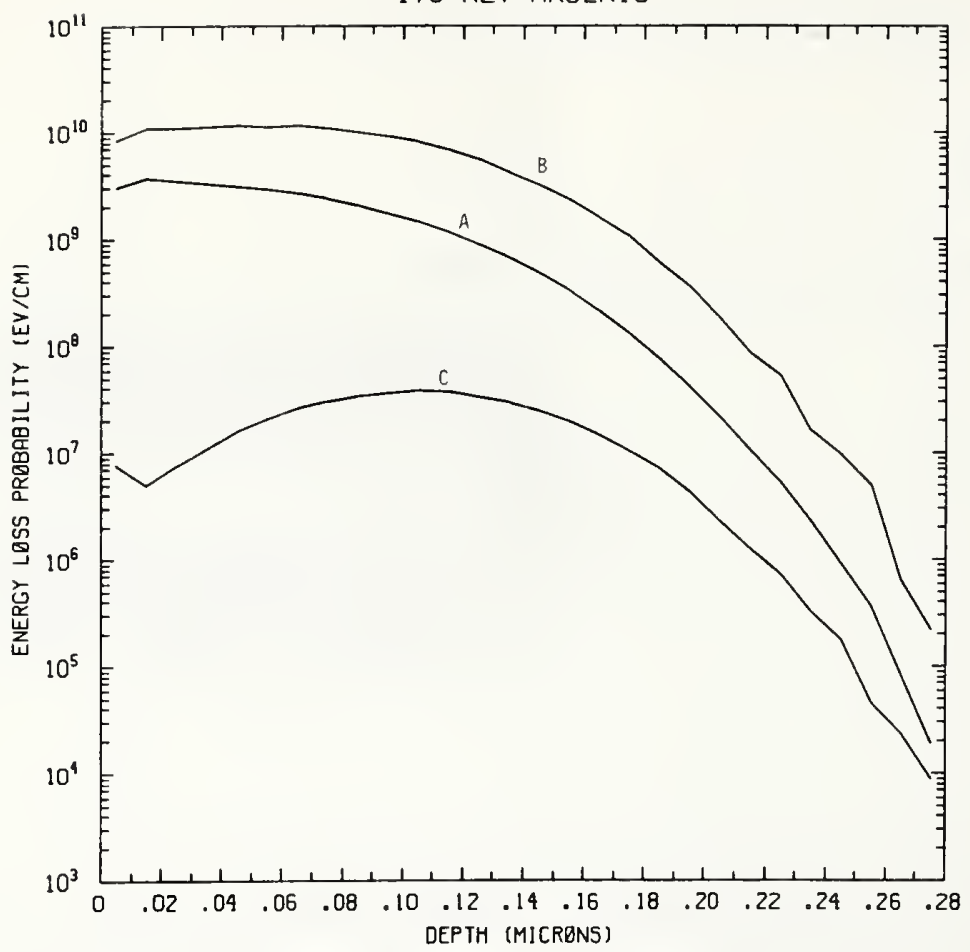

Figure As175-1 One-dimensional probability distributions for energy loss due to electronic excitation (A), displacement damage(B), and phonon excitation (C) for $175 \mathrm{keV}$ arsenic normally incident on a silicon target.

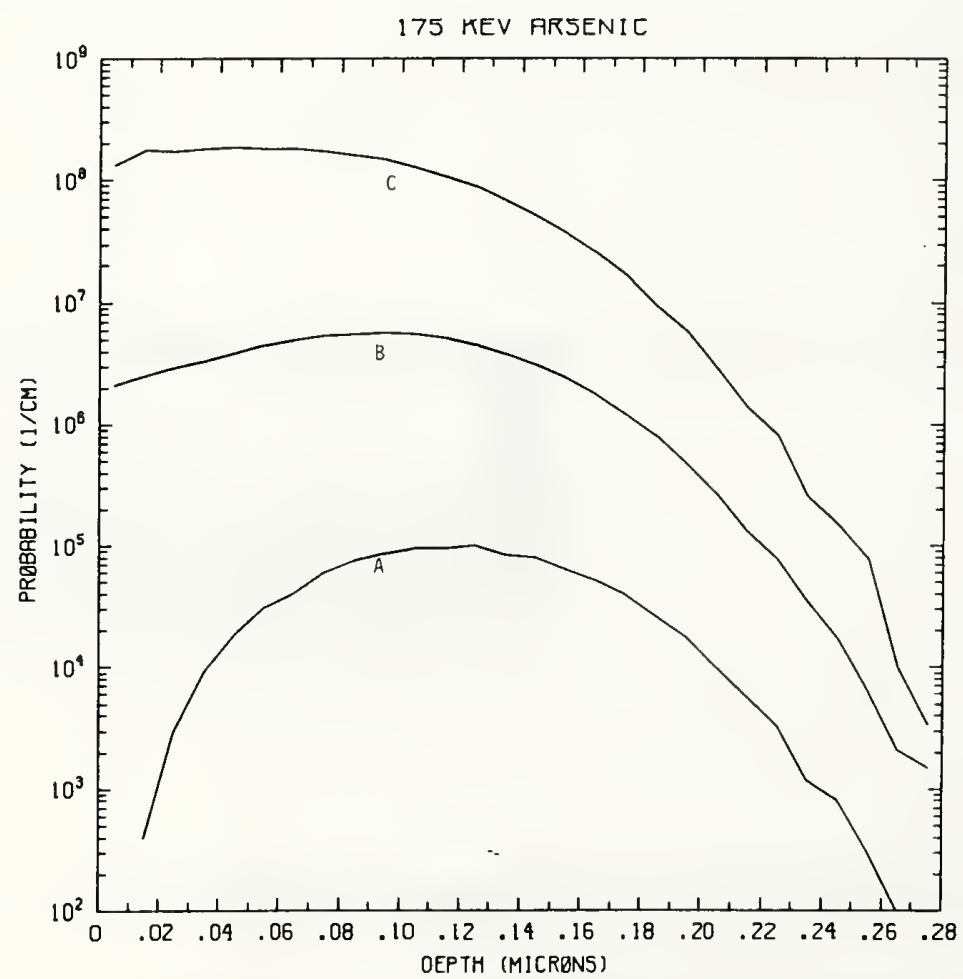

Figure As175-2 One-dimensional probability distributions for implanted arsenic (A), primary silicon displacements (B), and Frenkel pairs (C) for $175 \mathrm{keV}$ arsenic normally incident on a silicon target. 


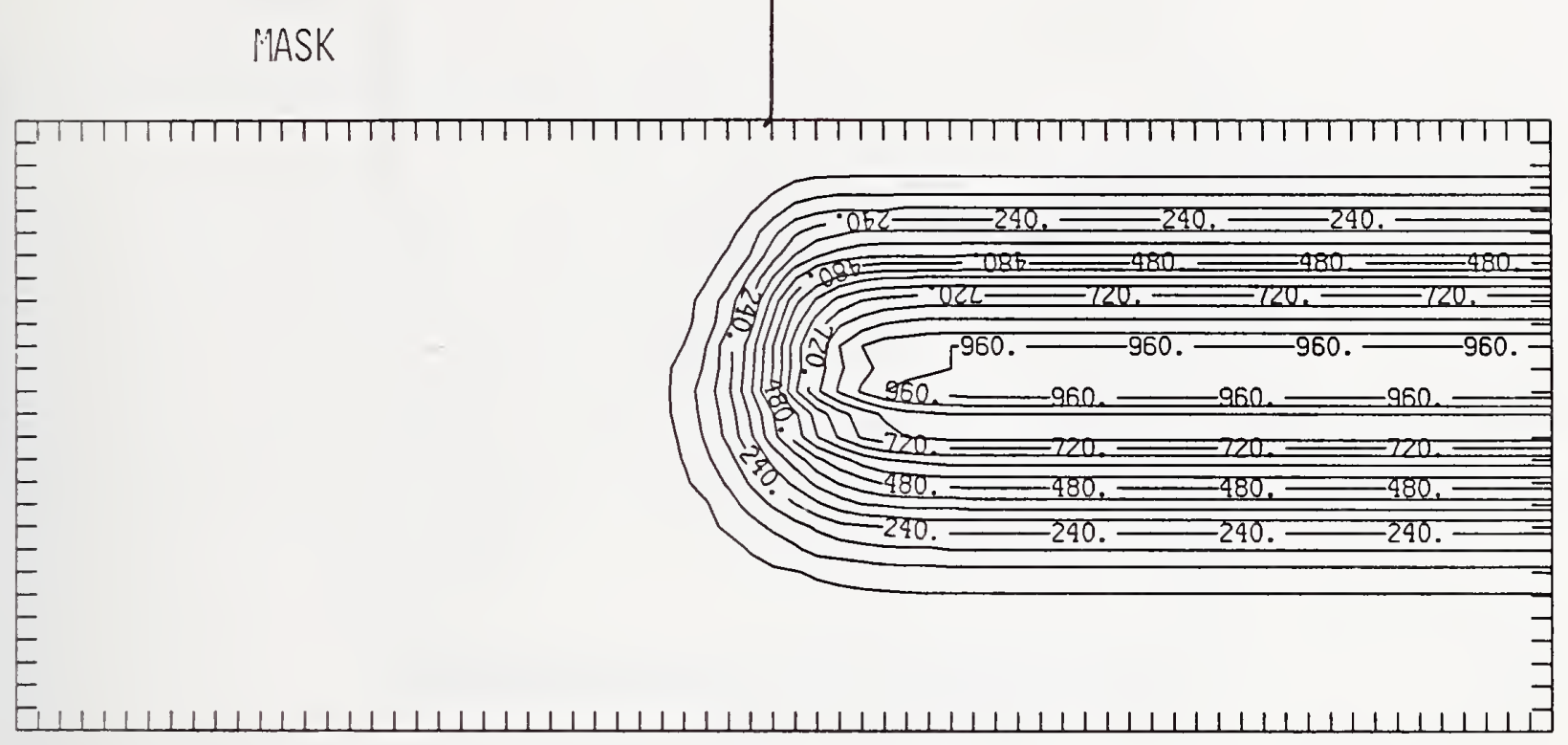

Figure As175-3 Two-dimensional distribution of $175 \mathrm{keV}$ arsenic implanted past a mask edge. The length increment (distance between tick marks) is $0.01 \mu \mathrm{m}$ and the scale factor is 1000 .

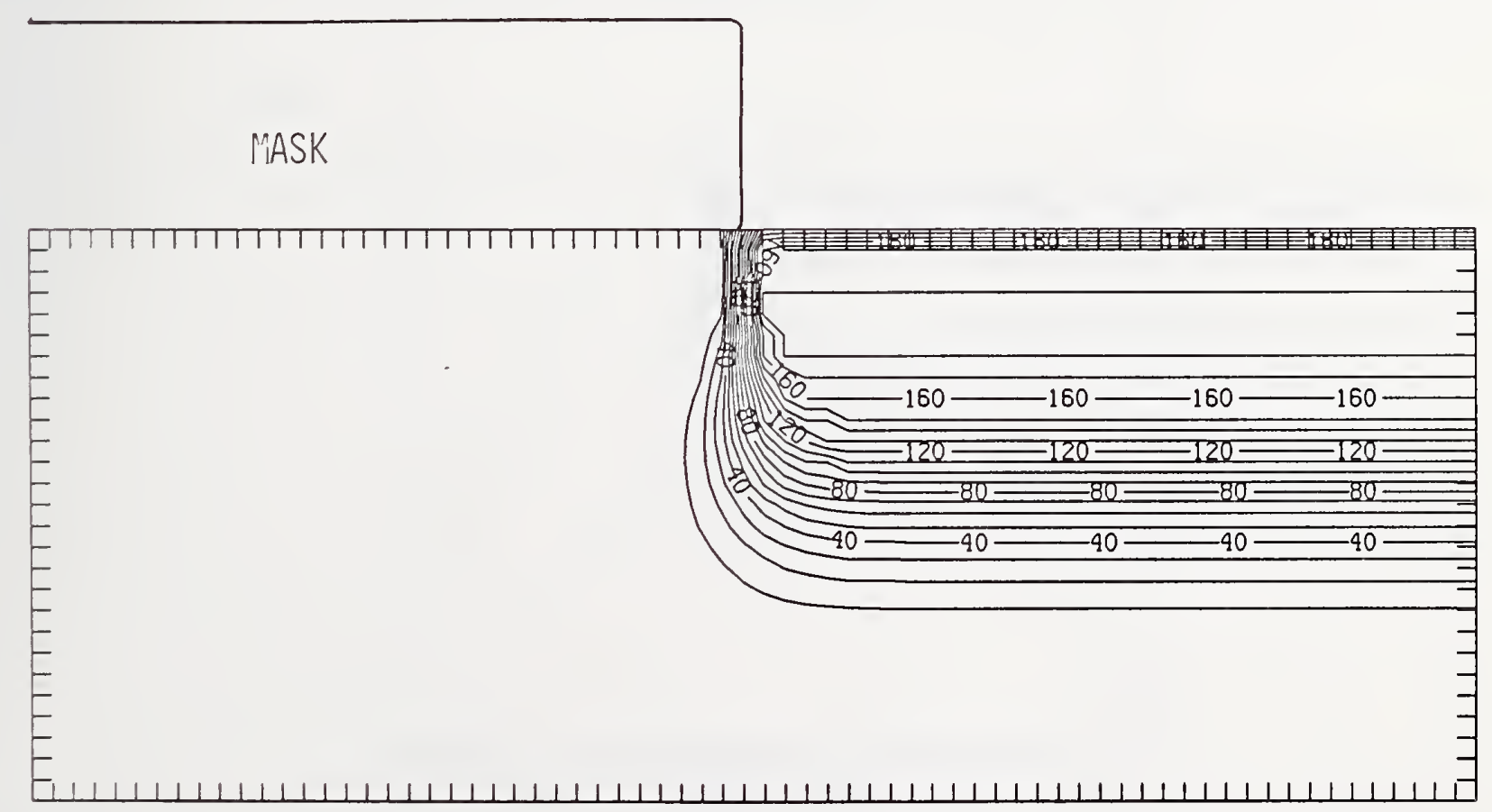

Figure As175-4 Two-dimensional distribution of Frenkel pairs created by $175 \mathrm{keV}$ arsenic implanted past a mask edge. The length increment (distance between tick marks) is $0.01 \mu \mathrm{m}$ and the scale factor is 0.1 . 


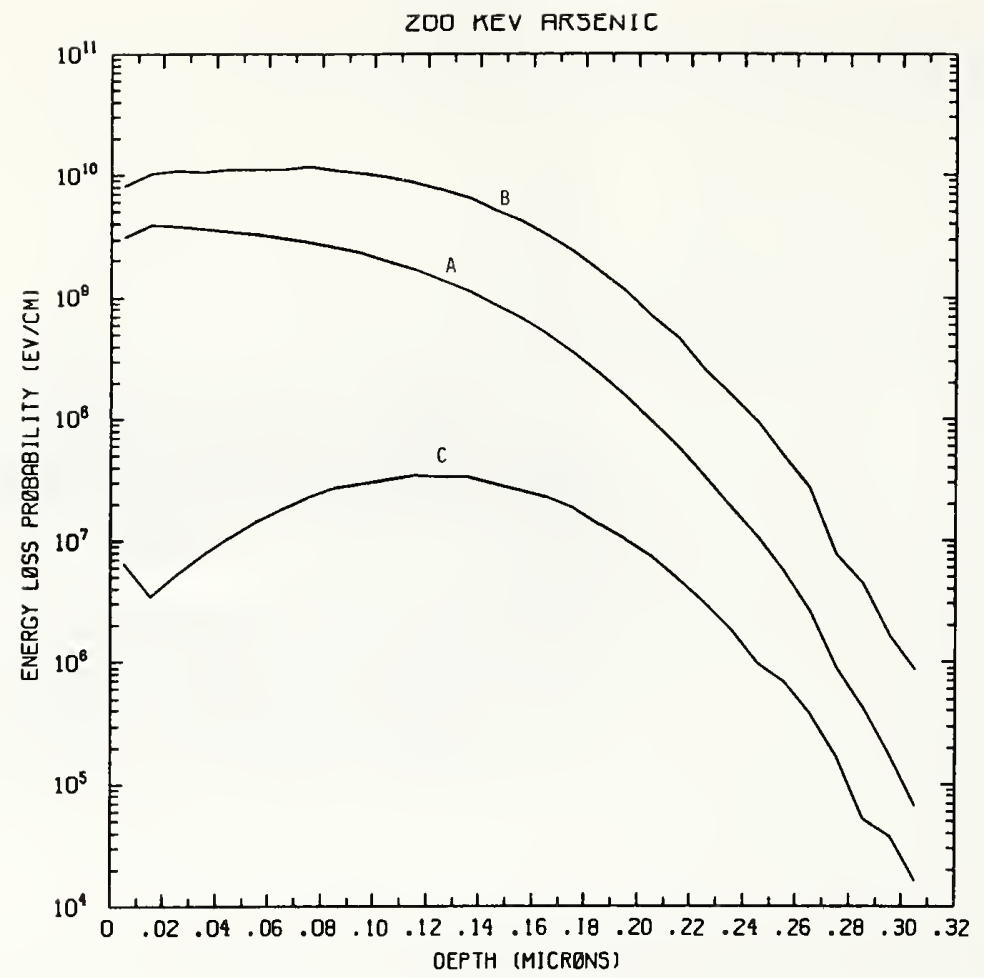

Figure As200-1 One-dimensional probability distributions for energy loss due to electronic excitation (A), displacement damage(B), and phonon excitation (C) for $200 \mathrm{keV}$ arsenic normally incident on a silicon target.

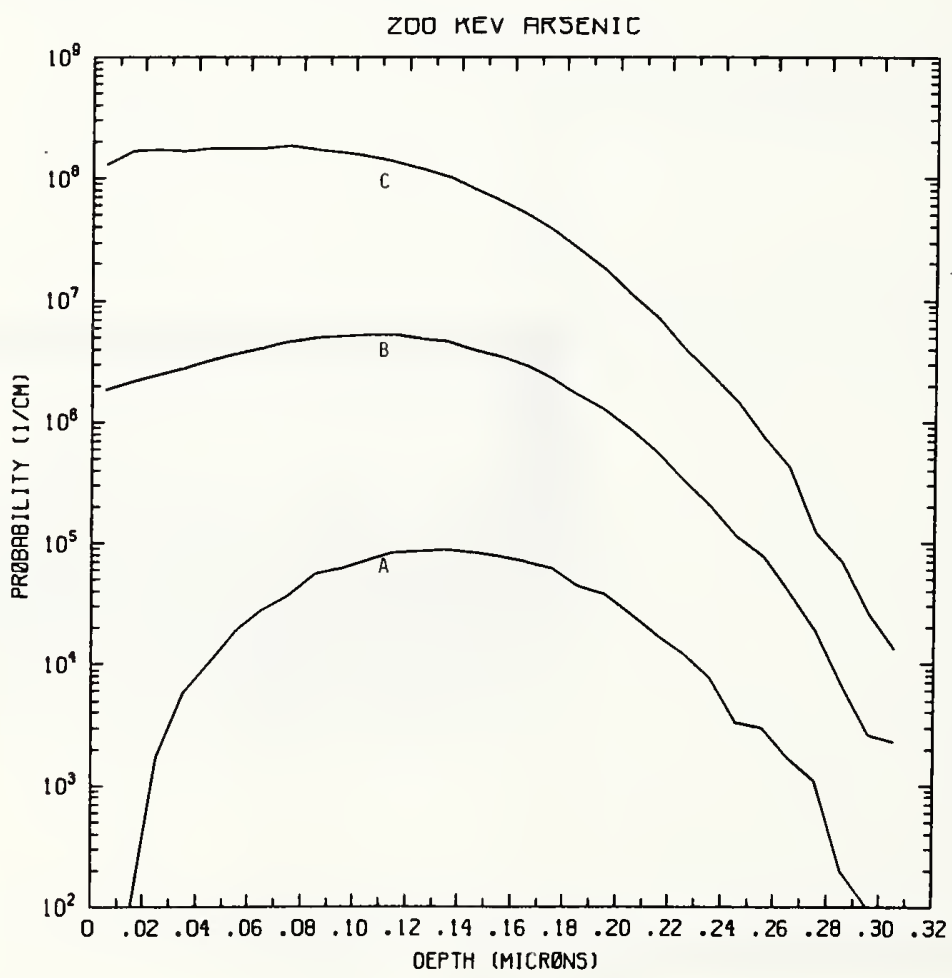

Figure As200-2 One-dimensional probability distributions for implanted arsenic (A), primary silicon displacements (B), and Frenkel pairs (C) for $200 \mathrm{keV}$ arsenic normally incident on a silicon target. 


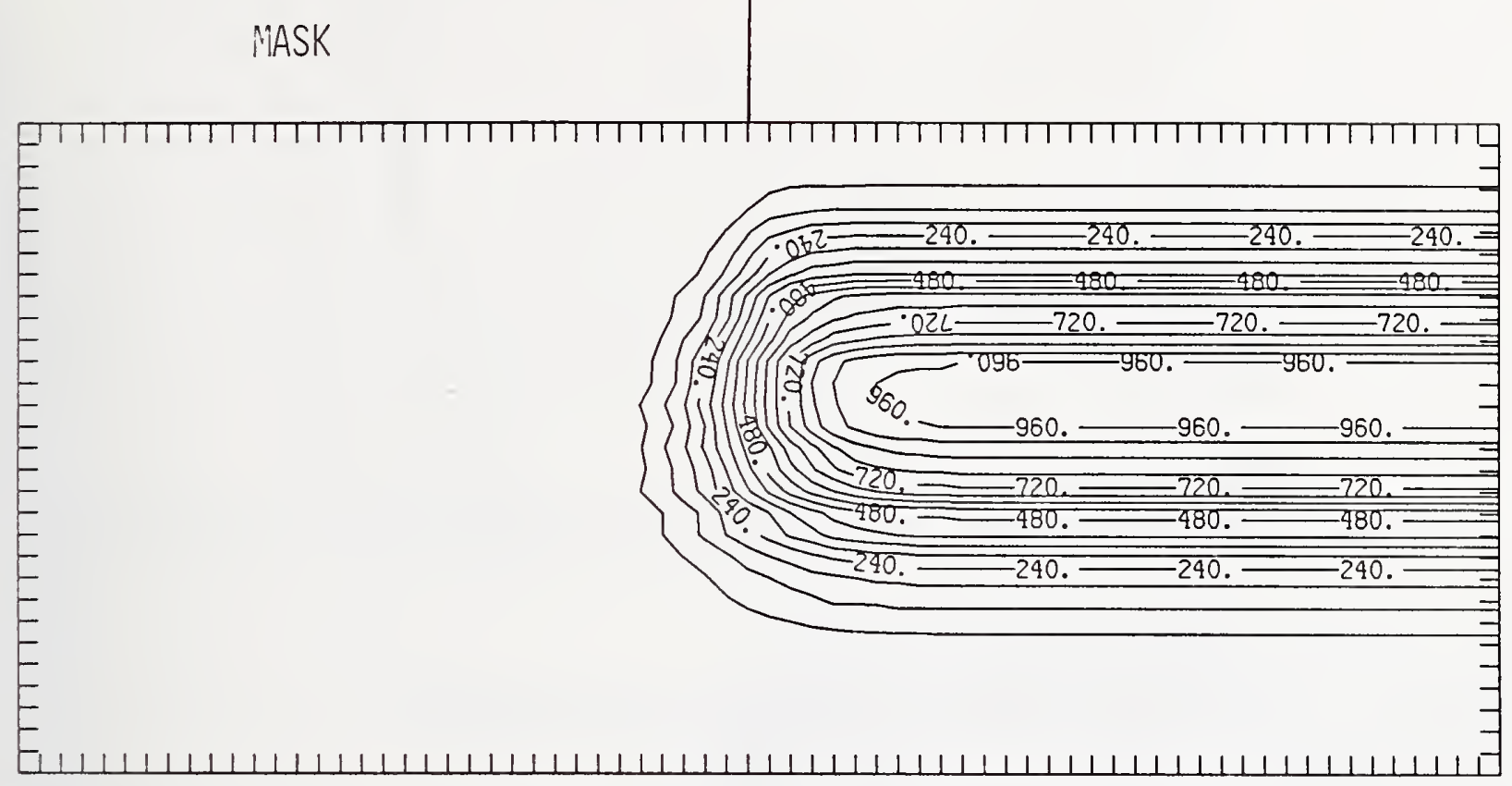

Figure As200-3 Two-dimensional distribution of $200 \mathrm{keV}$ arsenic implanted past a mask edge. The length increment (distance between tick marks) is $0.01 \mu \mathrm{m}$ and the scale factor is 1000 .

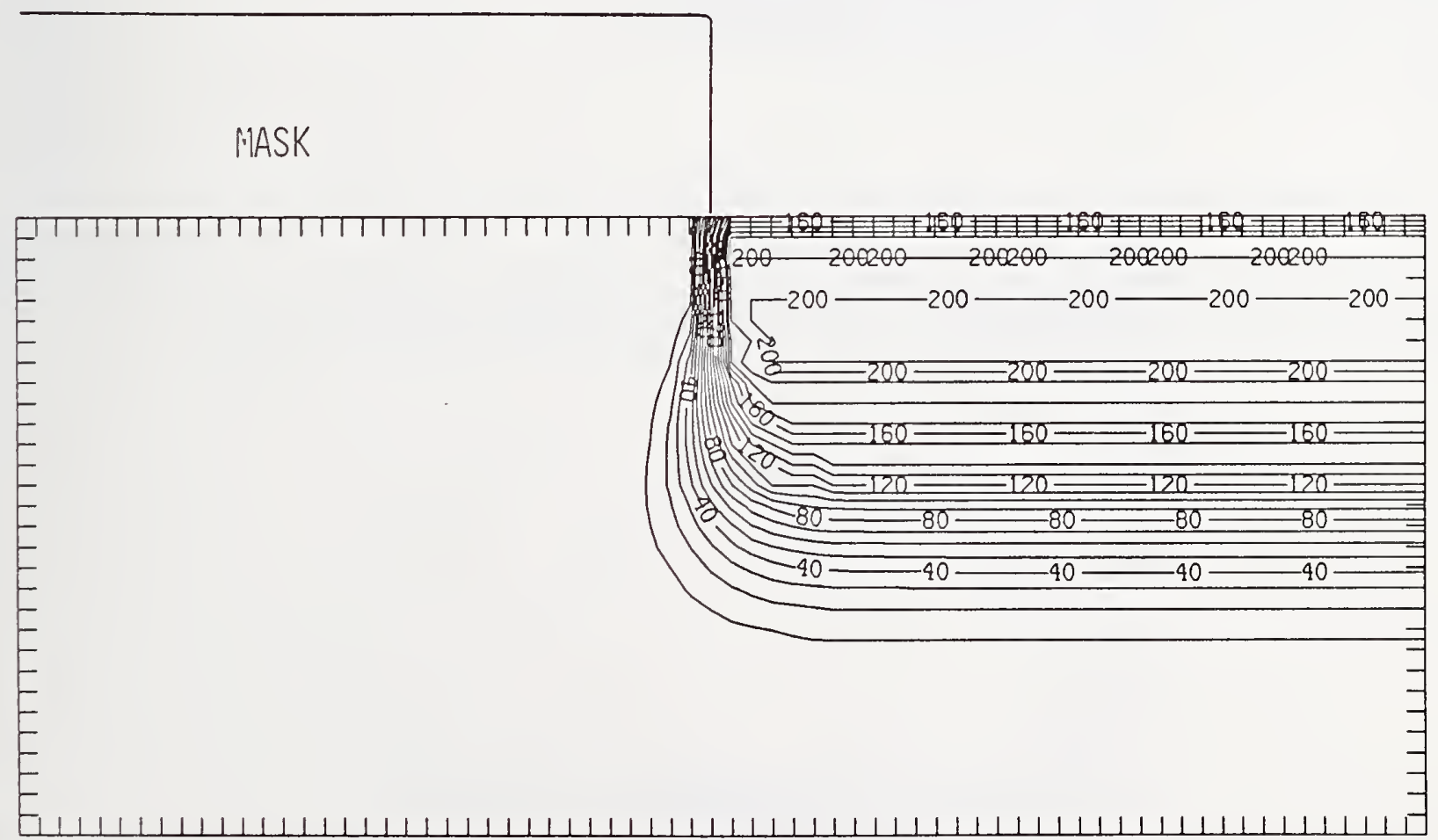

Figure As200-4 Two-dimensional distribution of Frenkel pairs created by $200 \mathrm{keV}$ arsenic implanted past a mask edge. The length increment (distance between tick marks) is $0.01 \mu \mathrm{m}$ and the scale factor is 0.1 . 


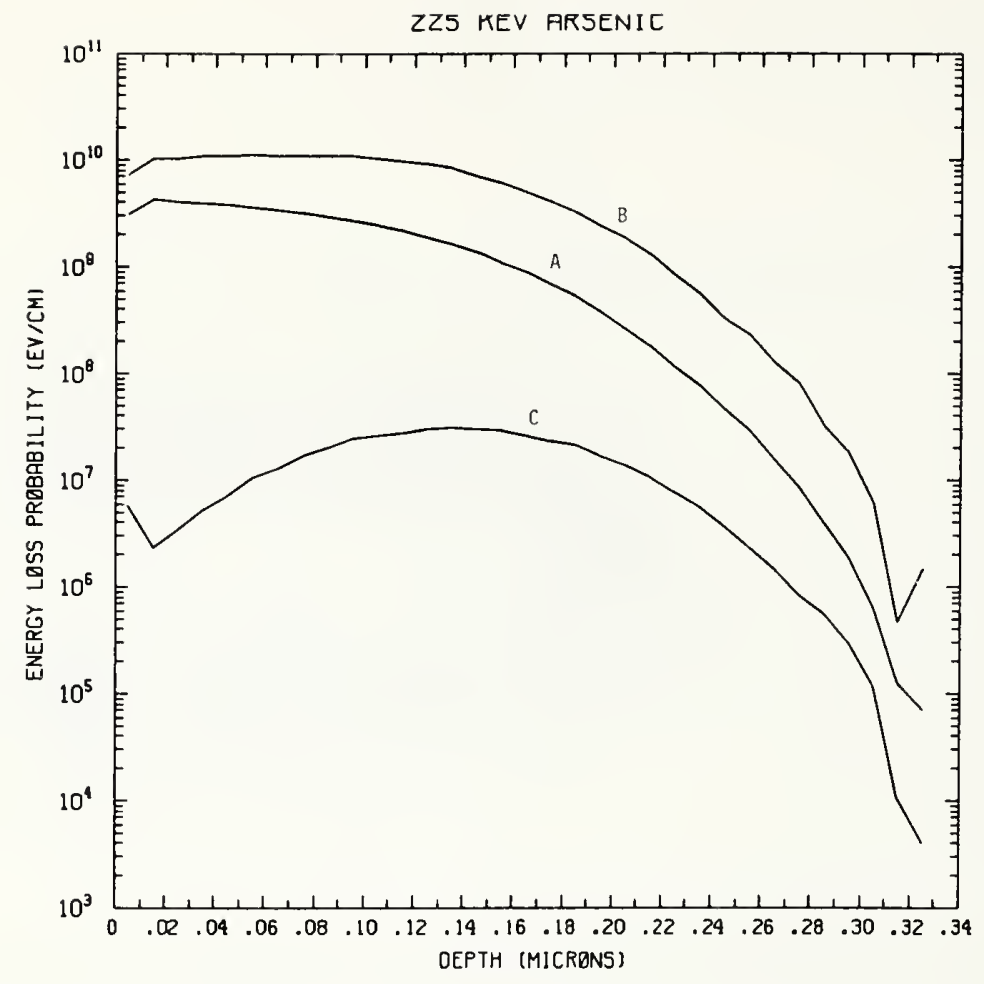

Figure As225-1 One-dimensional probability distributions for energy loss due to electronic excitation (A), displacement damage(B), and phonon excitation (C) for $225 \mathrm{keV}$ arsenic normally incident on a silicon target.

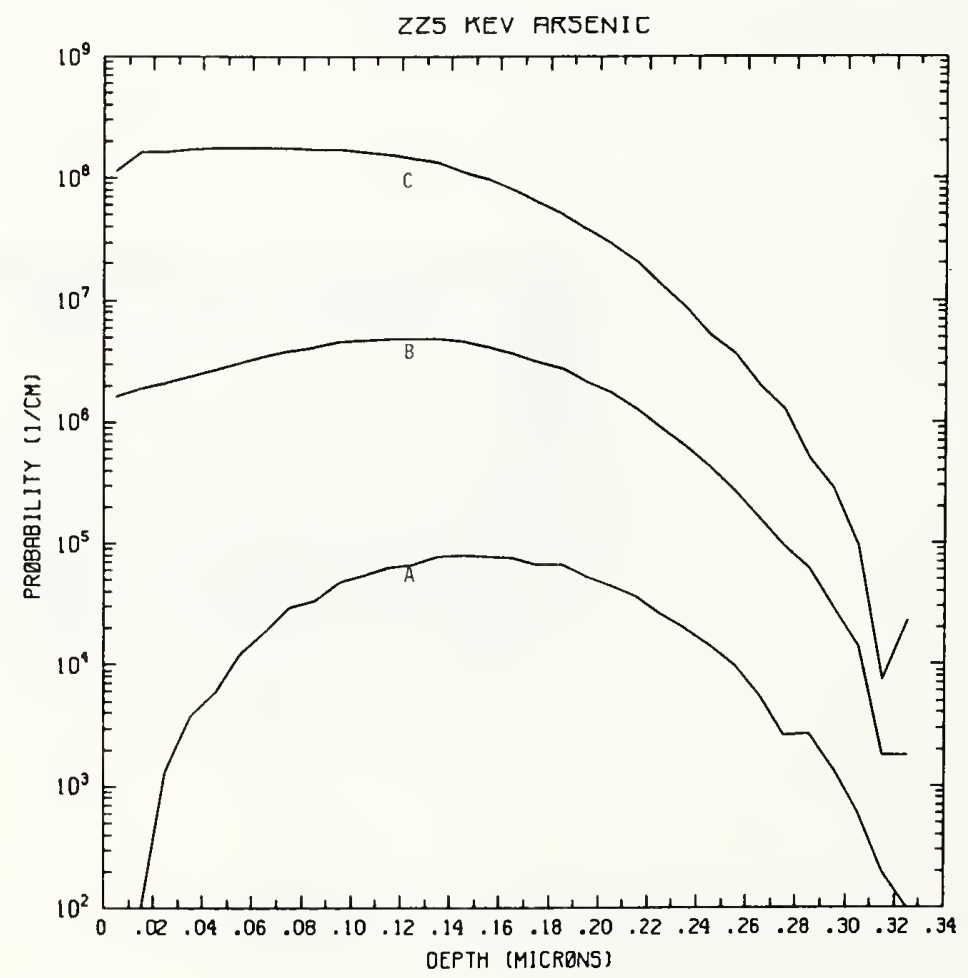

Figure As225-2 One-dimensional probability distributions for implanted arsenic (A), primary silicon displacements (B), and Frenkel pairs (C) for $225 \mathrm{keV}$ arsenic normally incident on a silicon target. 


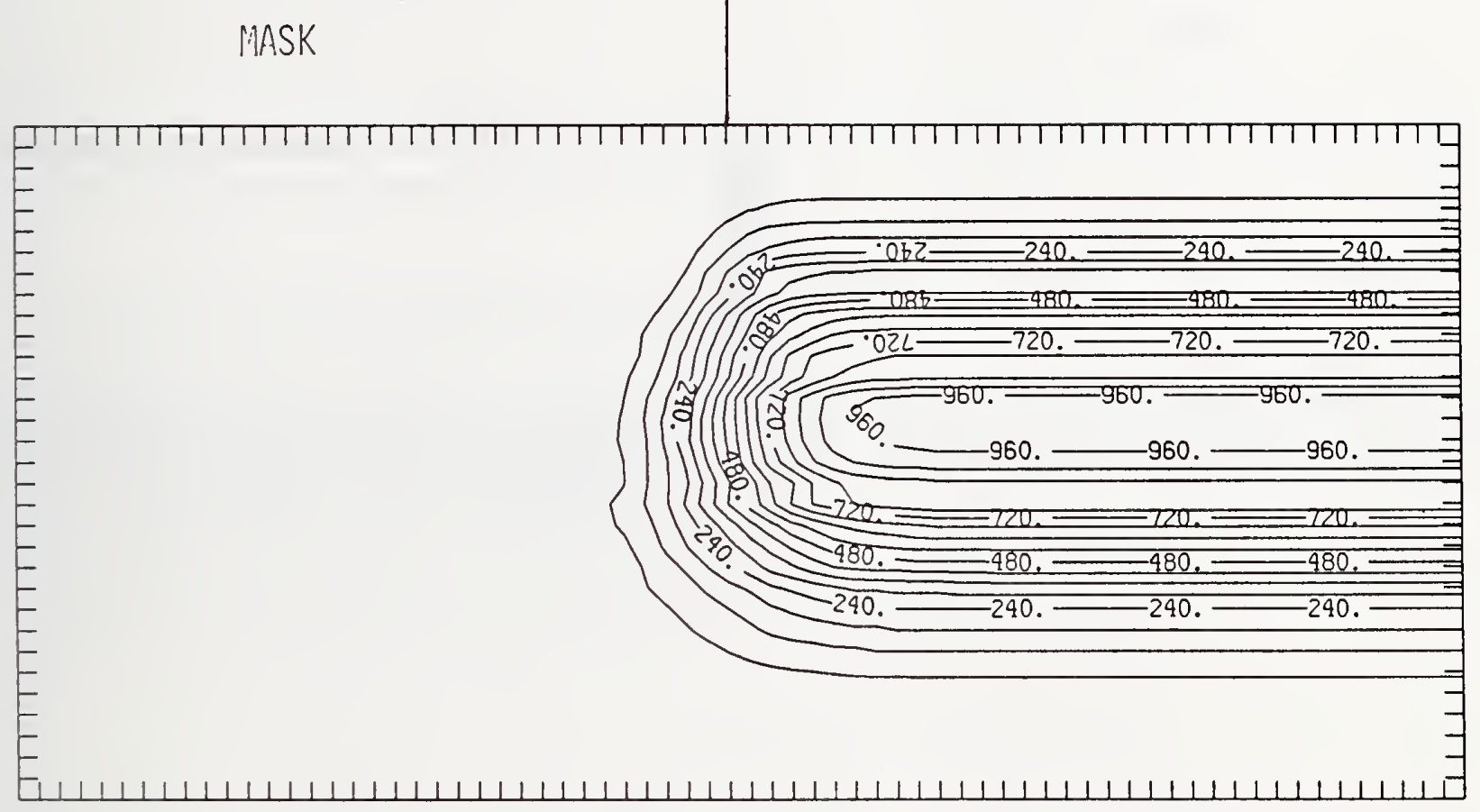

Figure As225-3 Two-dimensional distribution of $225 \mathrm{keV}$ arsenic implanted past a mask edge. The length increment (distance between tick marks) is $0.01 \mu \mathrm{m}$ and the scale factor is 1000. 


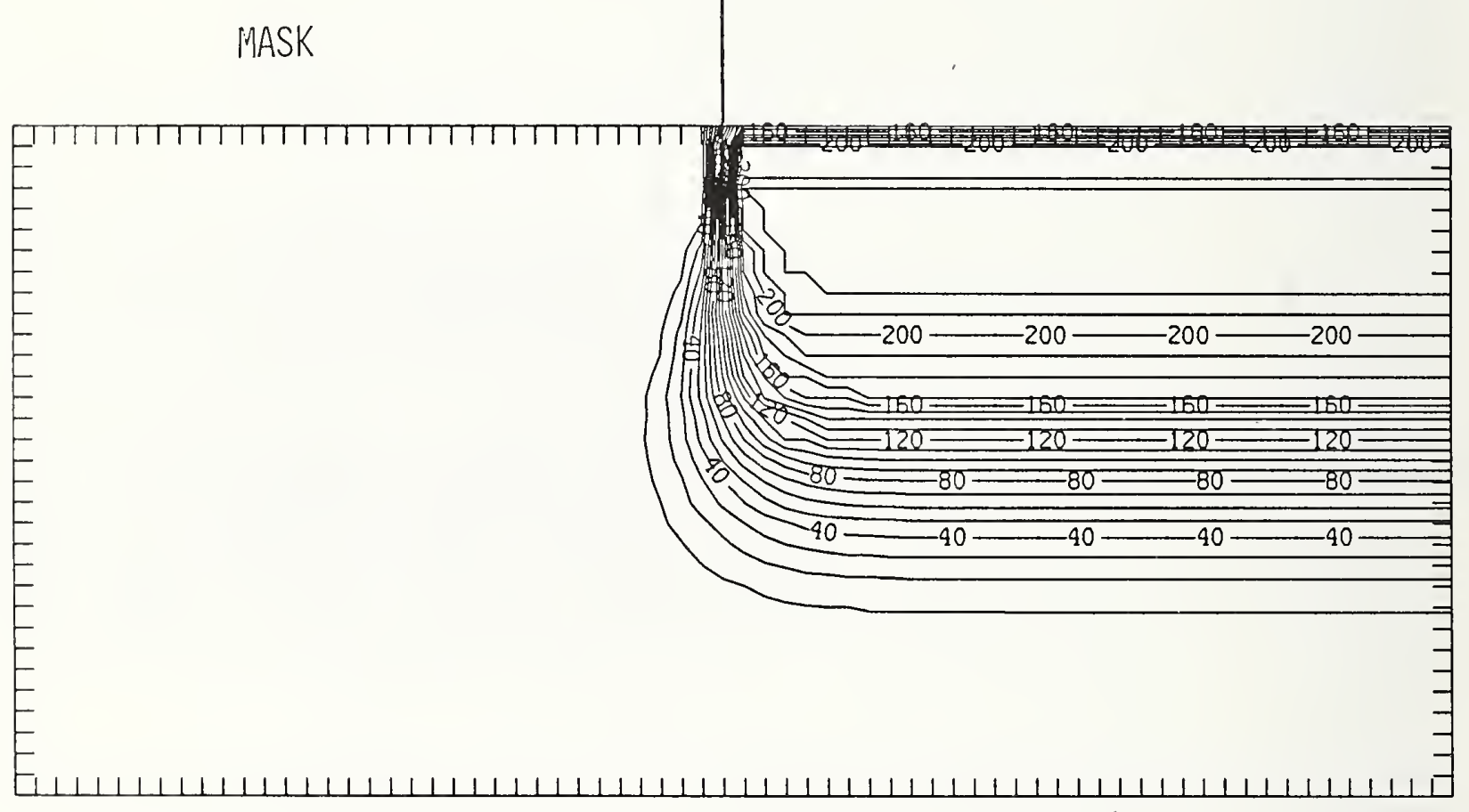

Figure As225-4 Two-dimensional distribution of Frenkel pairs created by $225 \mathrm{keV}$ arsenic implanted past a mask edge. The length increment (distance between tick marks) is $0.01 \mu \mathrm{m}$ and the scale factor is 0.1 . 


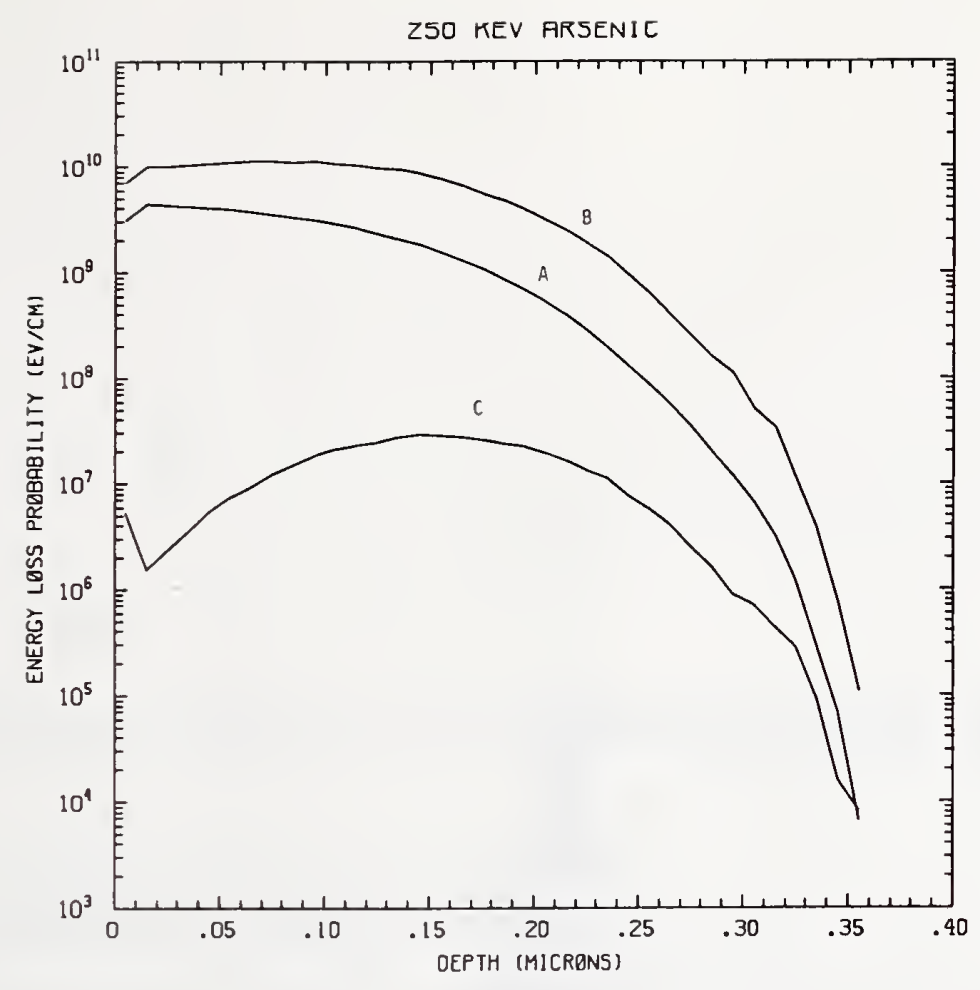

Figure As250-1 One-dimensional probability distributions for energy loss due to electronic excitation (A), displacement damage(B), and phonon excitation (C) for $250 \mathrm{keV}$ arsenic normally incident on a silicon target.

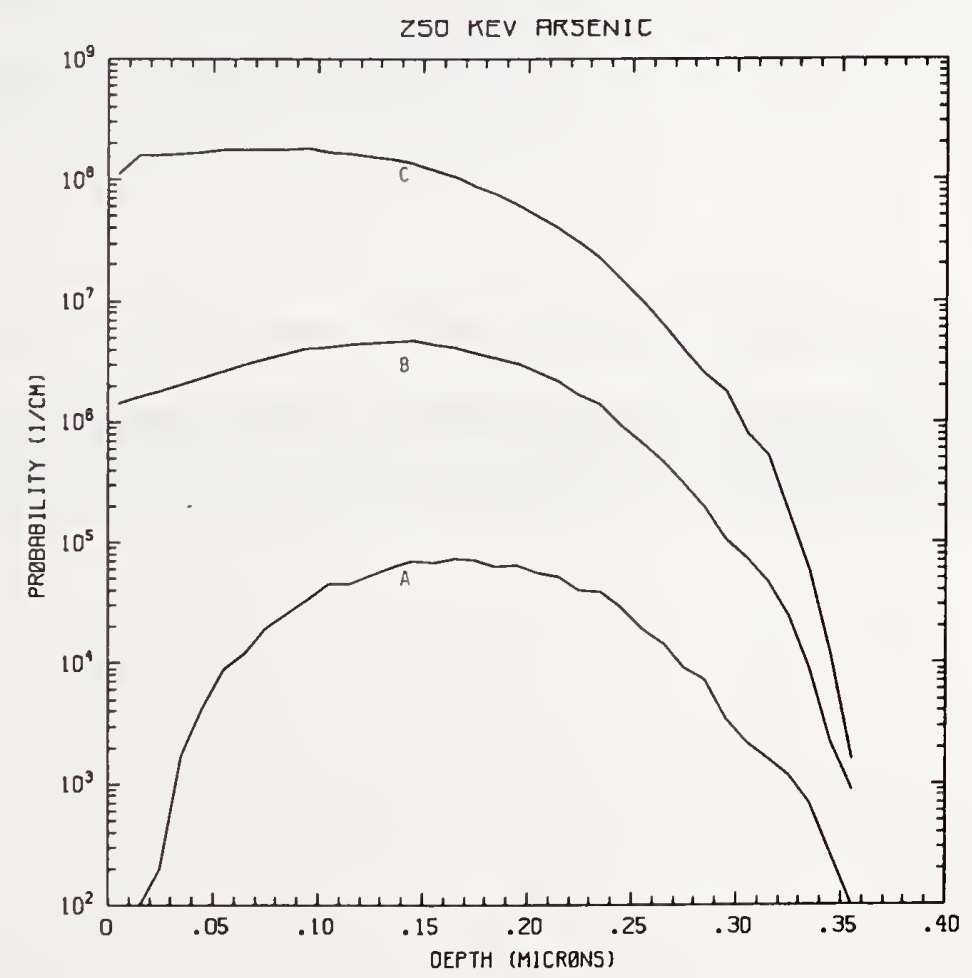

Figure As250-2 One-dimensional probability distributions for implanted arsenic (A), primary silicon displacements (B), and Frenkel pairs (C) for $250 \mathrm{keV}$ arsenic normally incident on a silicon target. 


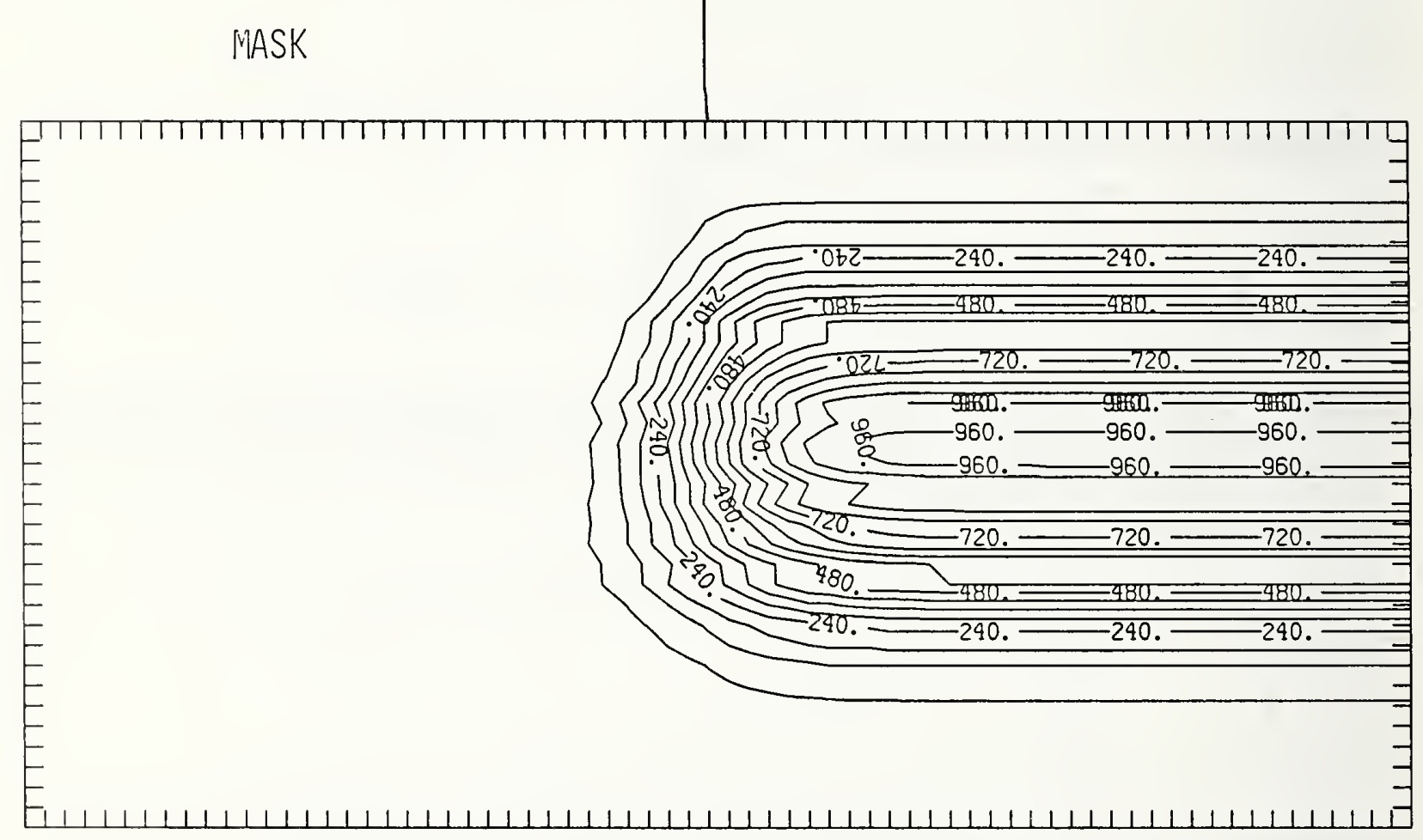

Figure As250-3 Two-dimensional distribution of $250 \mathrm{keV}$ arsenic implanted past a mask edge. The length increment (distance between tick marks) is $0.01 \mu \mathrm{m}$ and the scale factor is 1000 . 


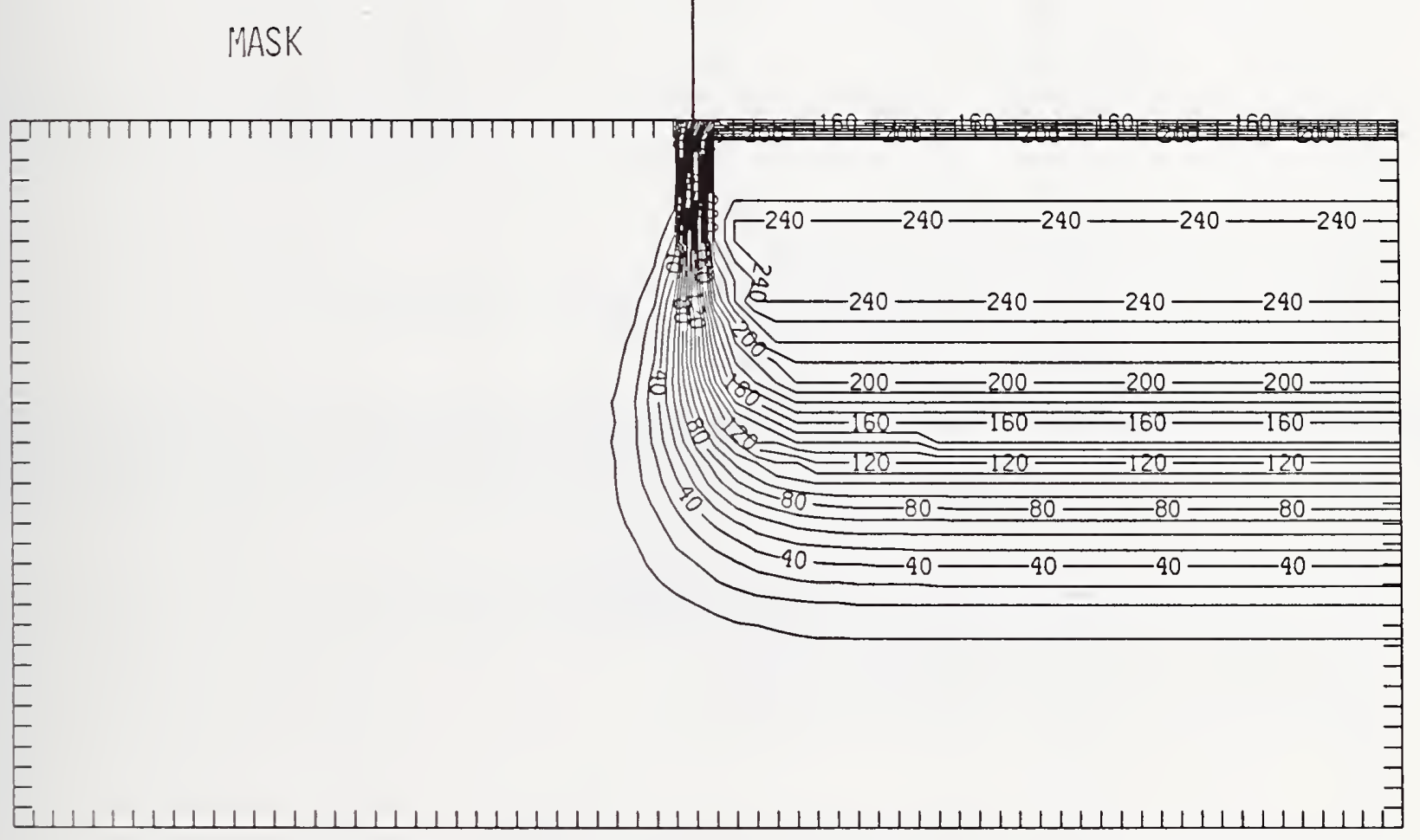

Figure As250-4 Two-dimensional distribution of Frenkel pairs created by $250 \mathrm{keV}$ arsenic implanted past a mask edge. The length increment (distance between tick marks) is $0.01 \mu \mathrm{m}$ and the scale factor is 0.1 . 


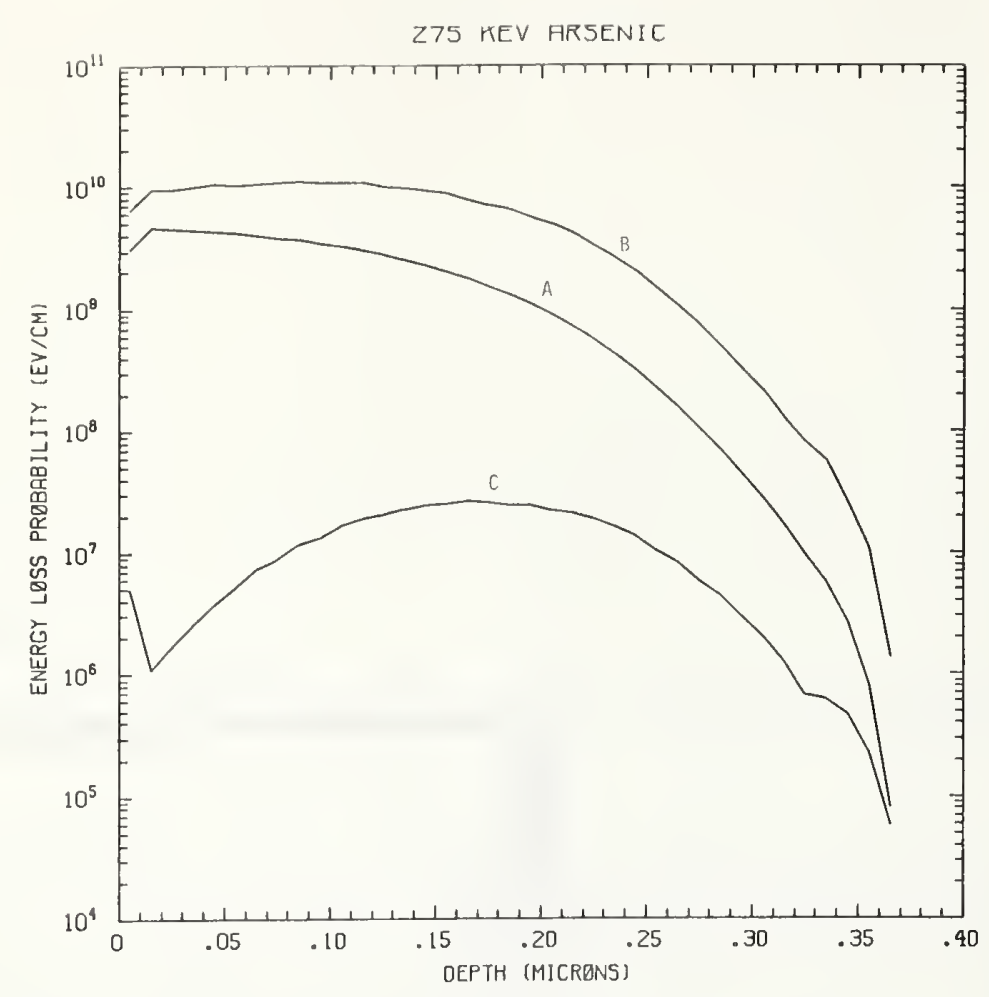

Figure As275-1 One-dimensional probability distributions for energy loss due to electronic excitation (A), displacement damage(B), and phonon excitation (C) for $275 \mathrm{keV}$ arsenic normally incident on a silicon target.

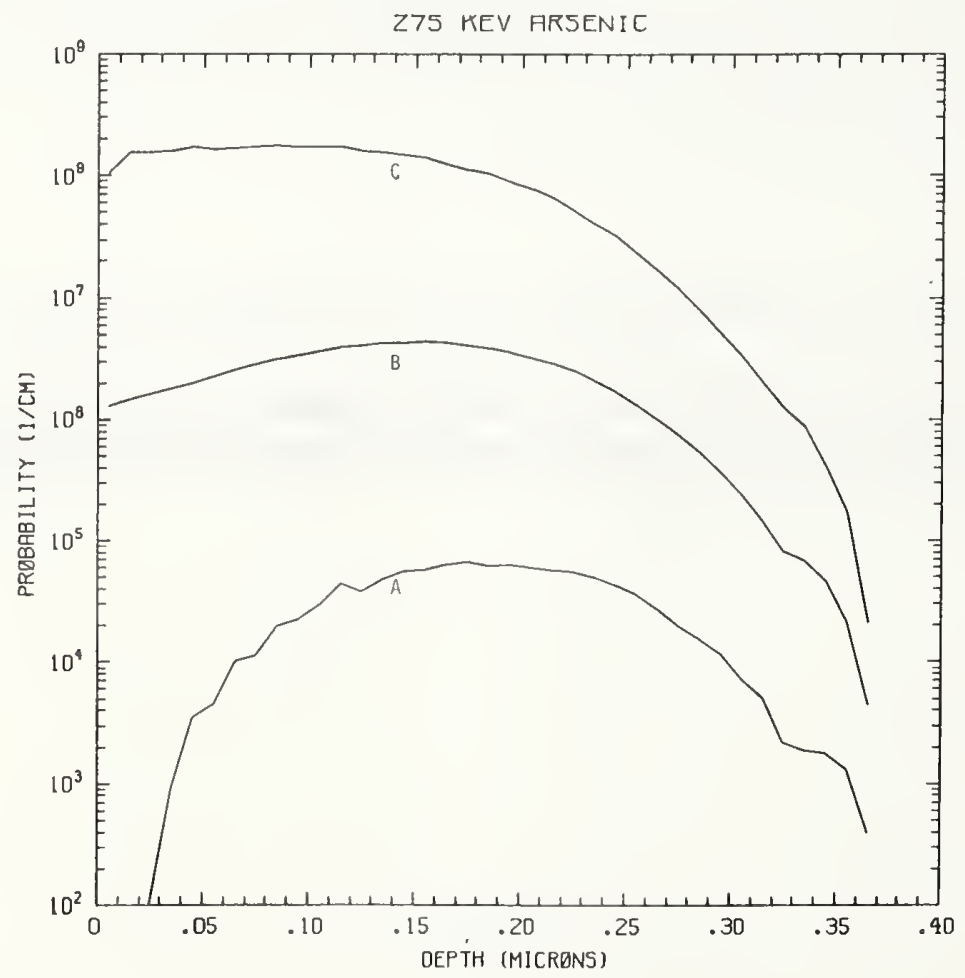

Figure As275-2 One-dimensional probability distributions for implanted arsenic (A), primary silicon displacements (B), and Frenkel pairs (C) for $275 \mathrm{keV}$ arsenic normally incident on a silicon target. 


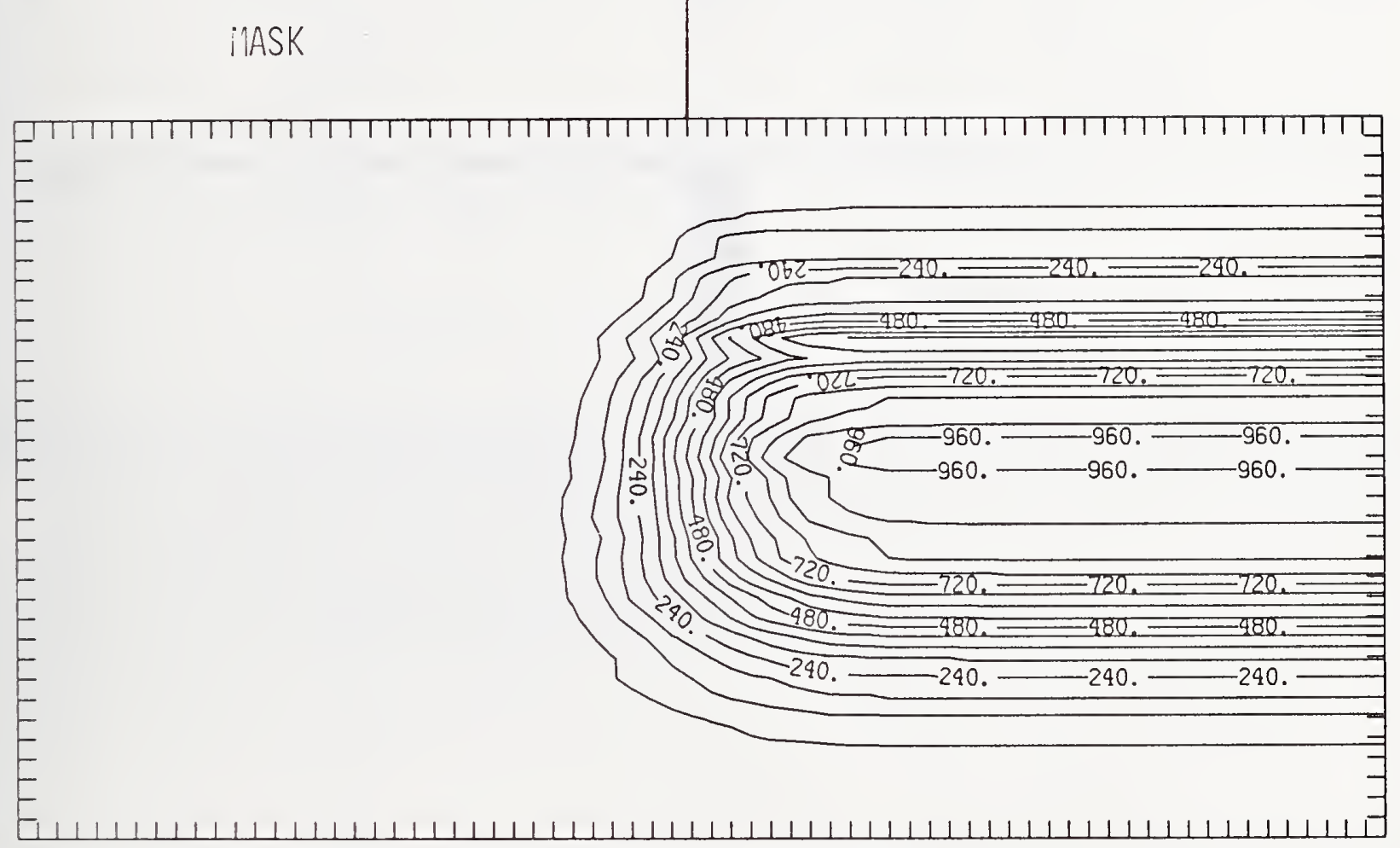

Figure As275-3 Two-dimensional distribution of $275 \mathrm{keV}$ arsenic implanted past a mask edge. The length increment (distance between tick marks) is $0.01 \mu \mathrm{m}$ and the scale factor is 0.1 . 


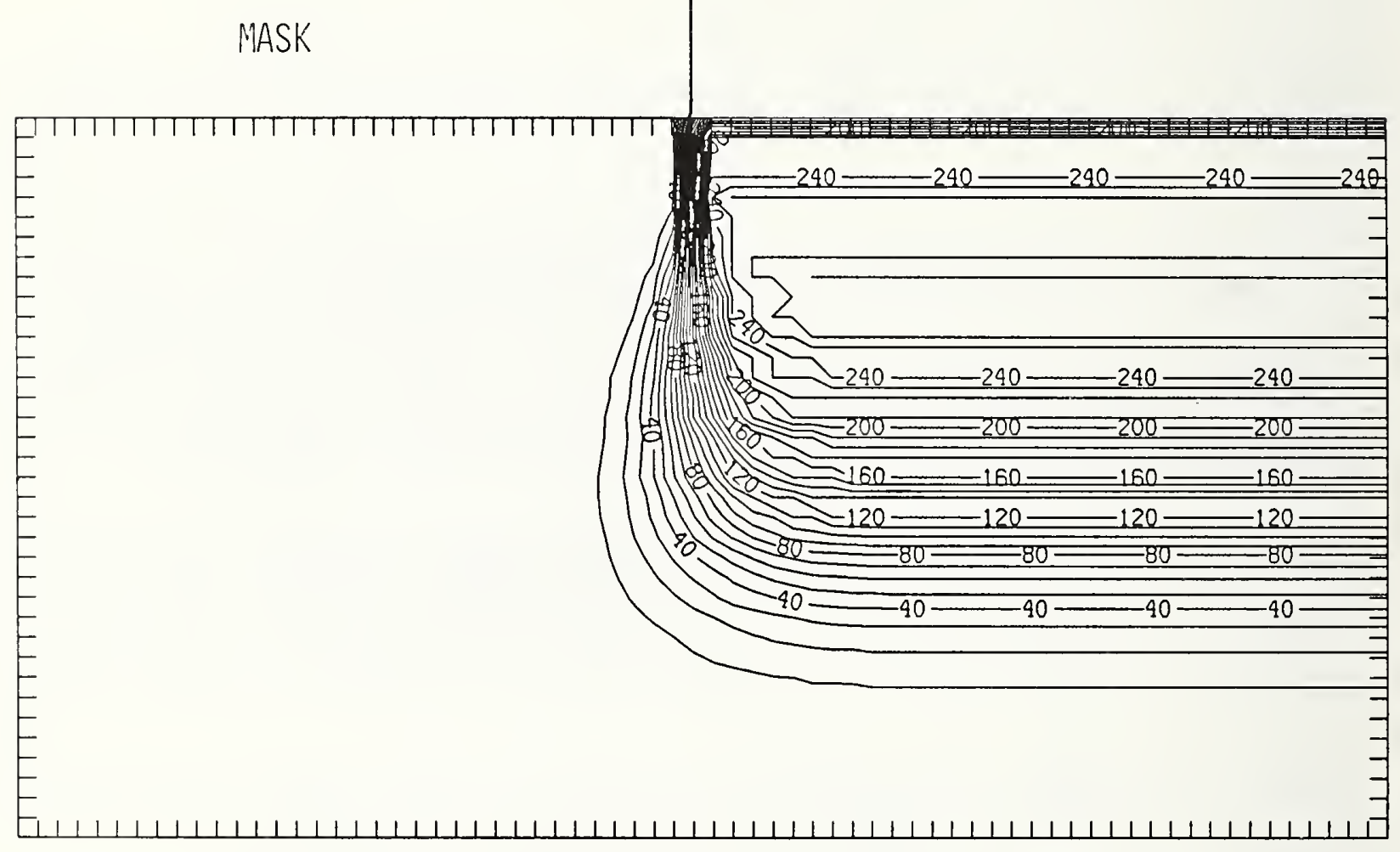

Figure As275-4 Two-dimensional distribution of Frenkel pairs created by $275 \mathrm{keV}$ arsenic implanted past a mask edge. The length increment (distance between tick marks) is $0.01 \mu \mathrm{m}$ and the scale factor is 1000 . 


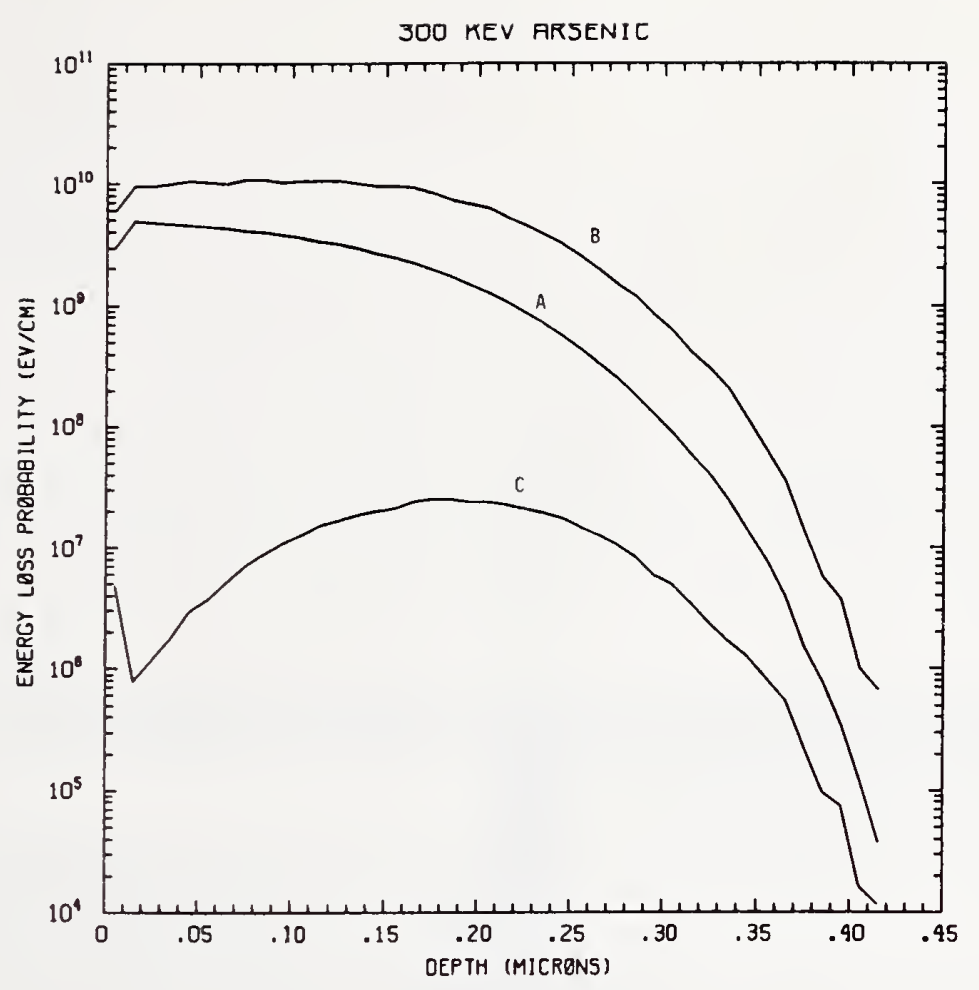

Figure As300-1 One-dimensional probability distributions for energy loss due to electronic excitation (A), displacement damage(B), and phonon excitation (C) for $300 \mathrm{keV}$ arsenic normally incident on a silicon target.

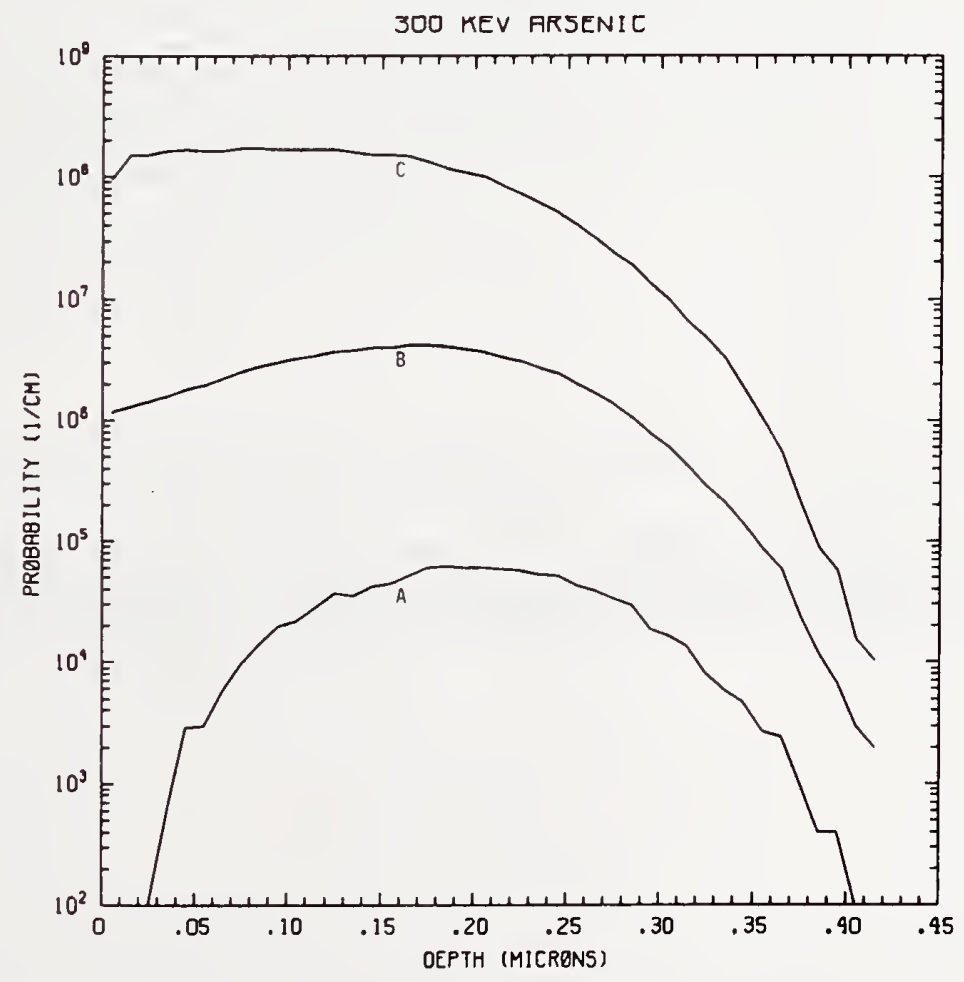

Figure As300-2 One-dimensional probability distributions for implanted arsenic (A), primary silicon displacements (B), and Frenkel pairs (C) for $300 \mathrm{keV}$ arsenic normally incident on a silicon target. 


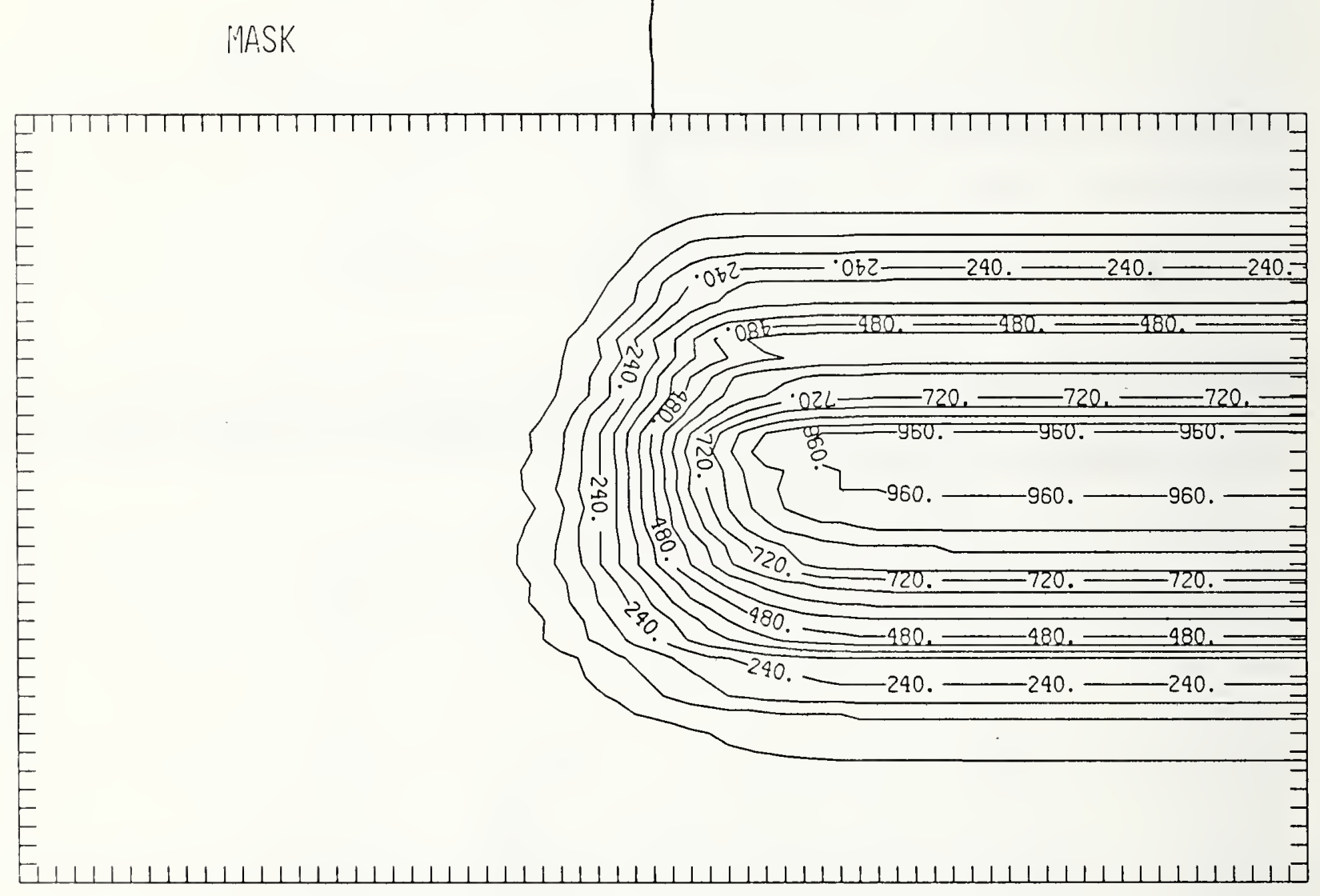

Figure As300-3 Two-dimensional distribution of $300 \mathrm{keV}$ arsenic implanted past a mask edge. The length increment (distance between tick marks) is $0.01 \mu \mathrm{m}$ and the scale factor is 1000. 


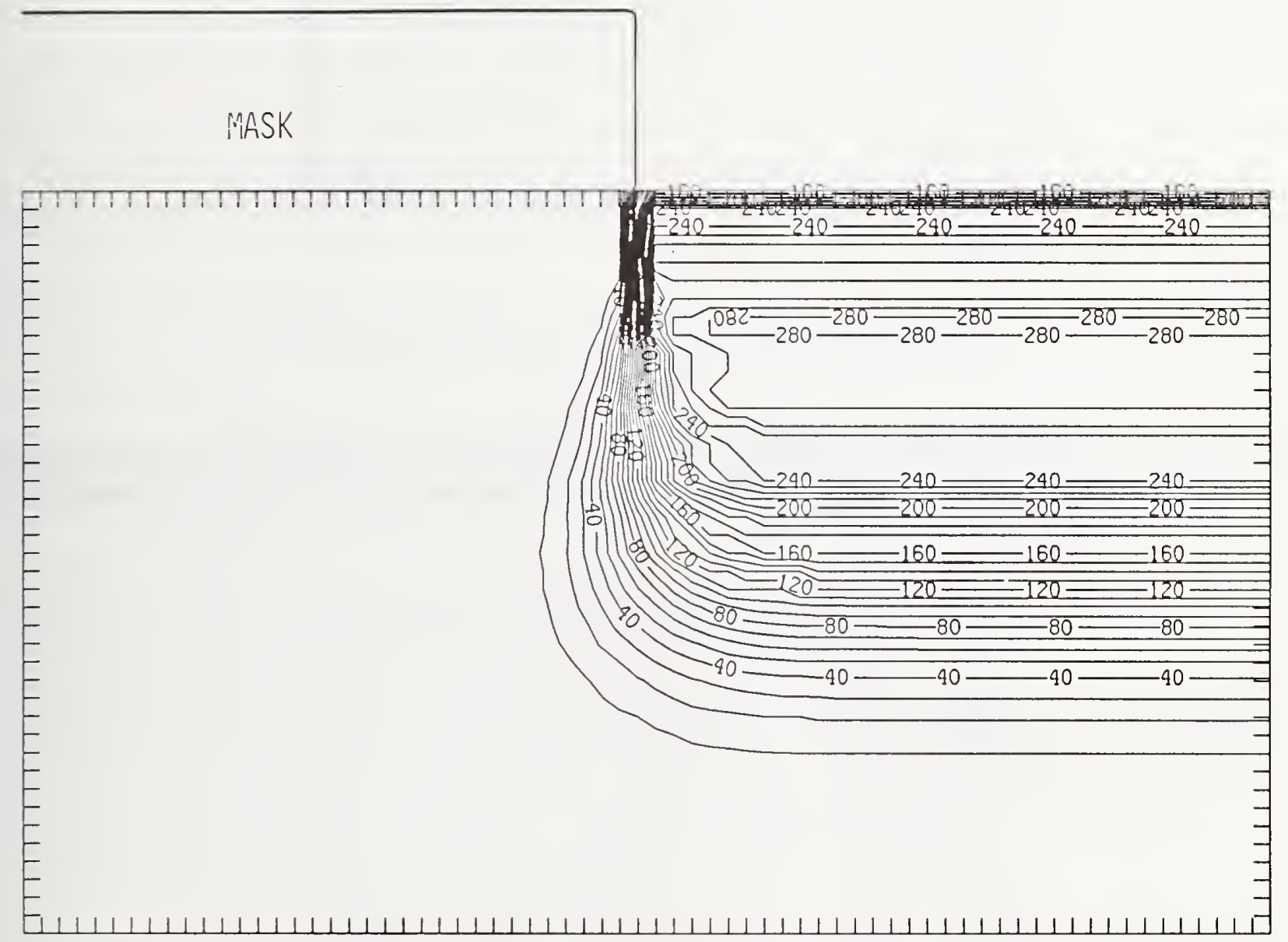

Figure As300-4 Two-dimensional distribution of Frenkel pairs created by $300 \mathrm{keV}$ arsenic implanted past a mask edge. The length increment (distance between tick marks) is $0.01 \mu \mathrm{m}$ and the scale factor is 0.1 . 


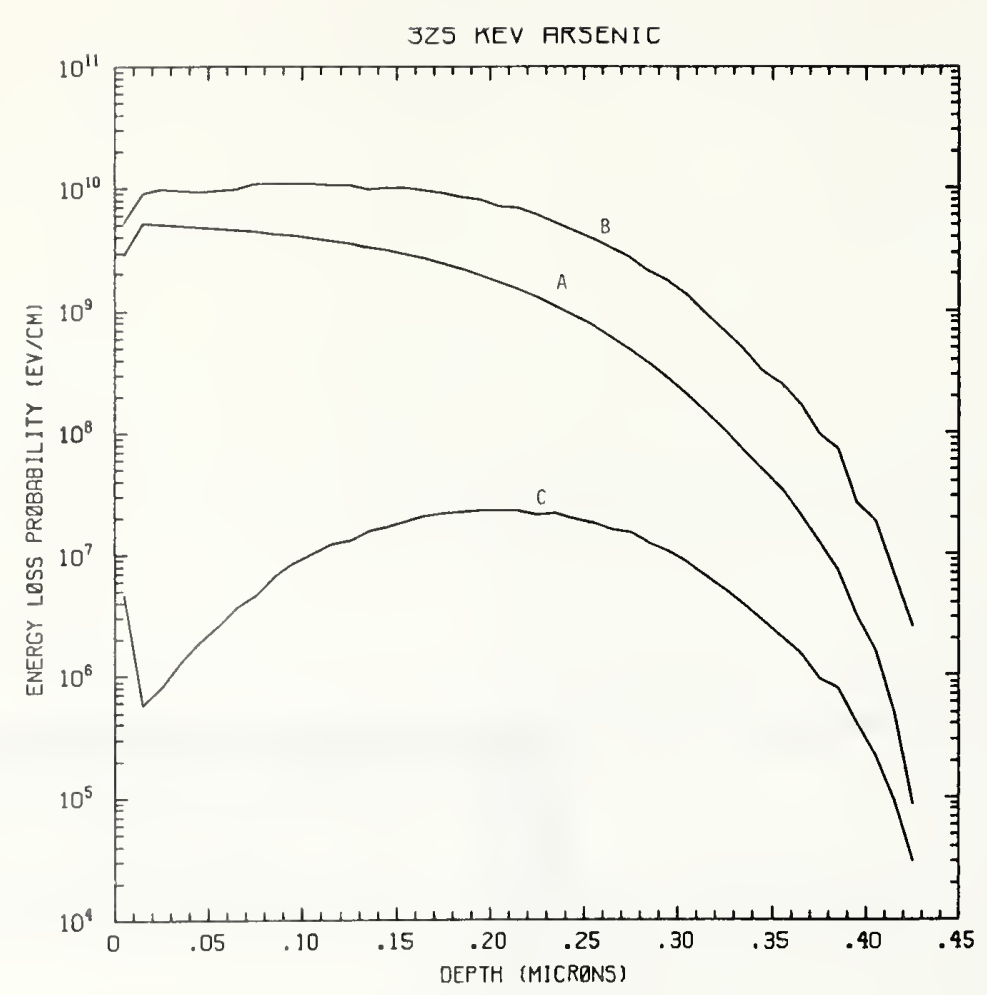

Figure As325-1 One-dimensional probability distributions for energy loss due to electronic excitation (A), displacement damage(B), and phonon excitation (C) for $325 \mathrm{keV}$ arsenic normally incident on a silicon target.

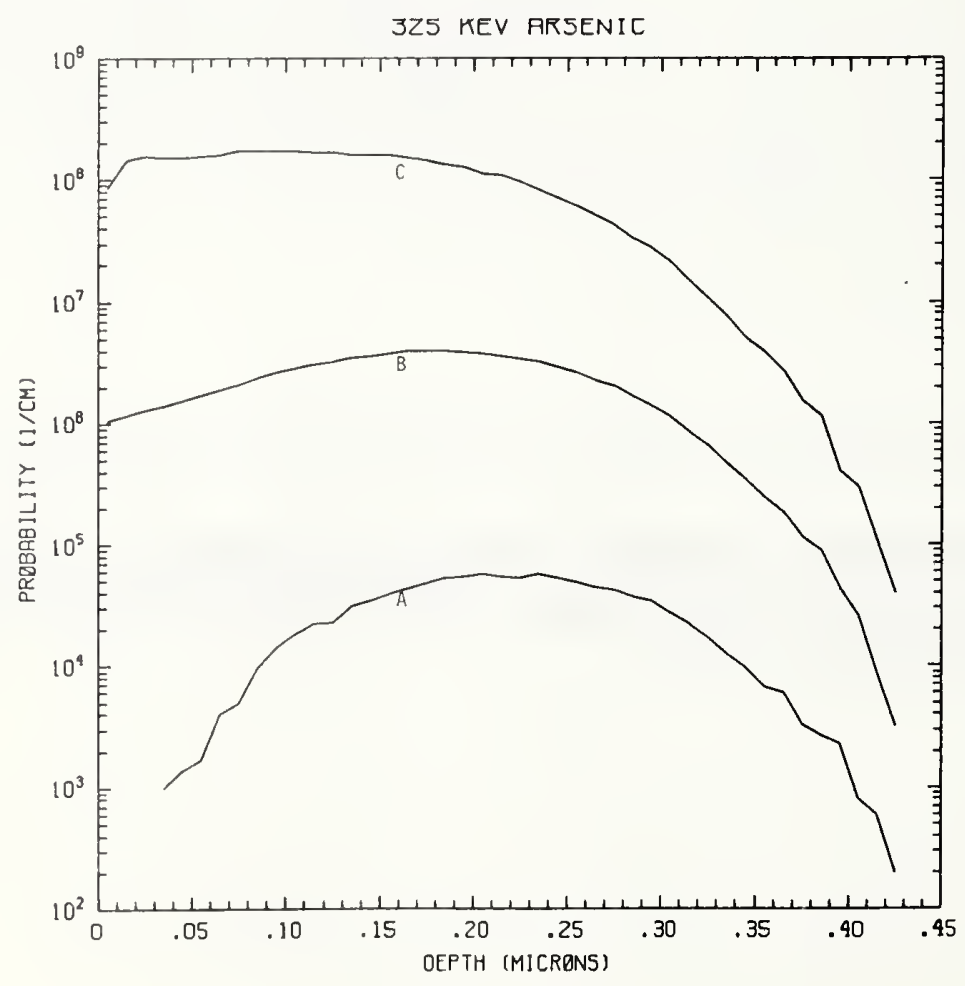

Figure As325-2 One-dimensional probability distributions for implanted arsenic (A), primary silicon displacements (B), and Frenkel pairs (C) for $325 \mathrm{keV}$ arsenic normally incident on a silicon target. 


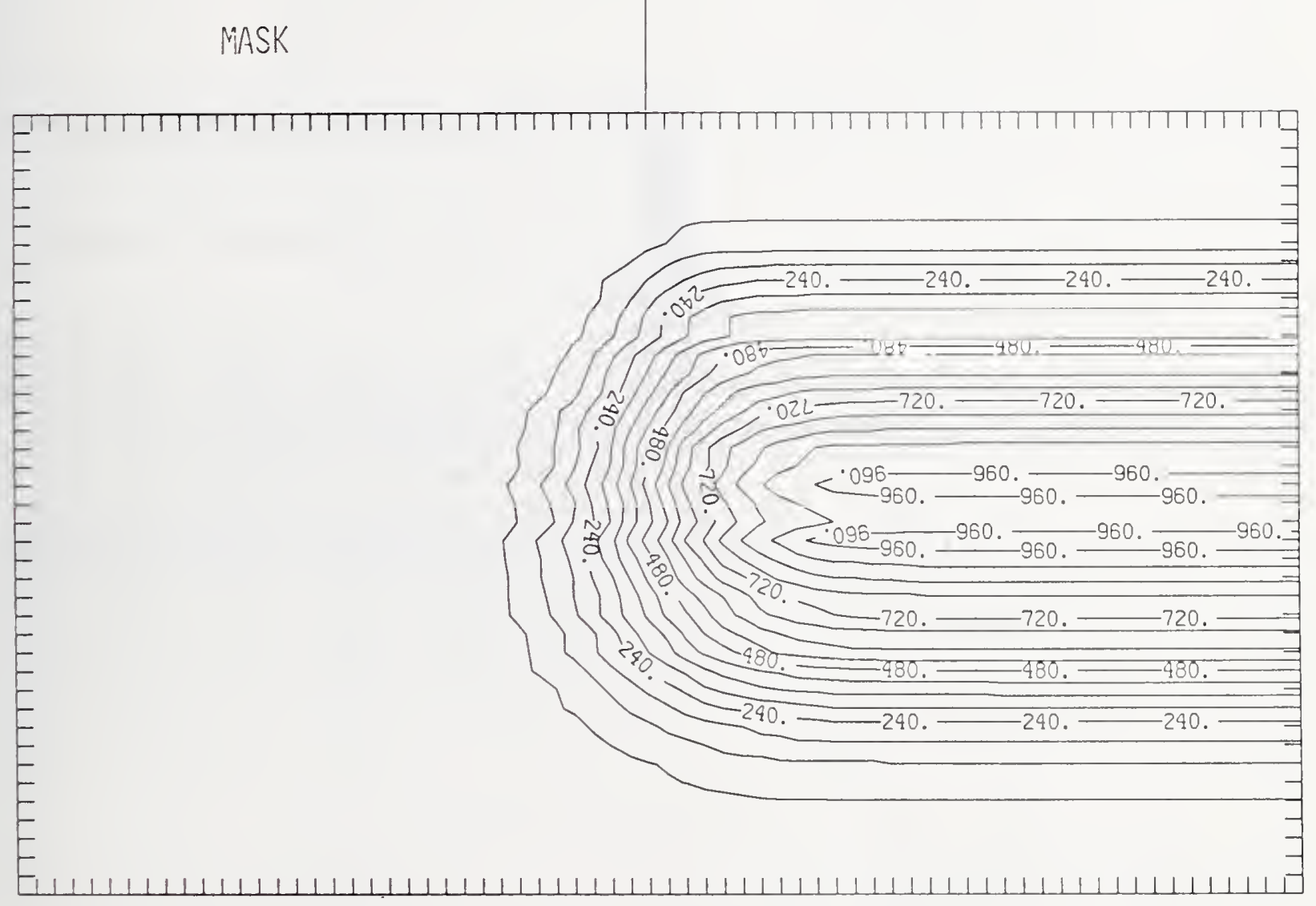

Figure As325-3 Two-dimensional distribution of $325 \mathrm{keV}$ arsenic implanted past a mask edge. The length increment (distance between tick marks) is $0.01 \mu \mathrm{m}$ and the scale factor is 1000 . 


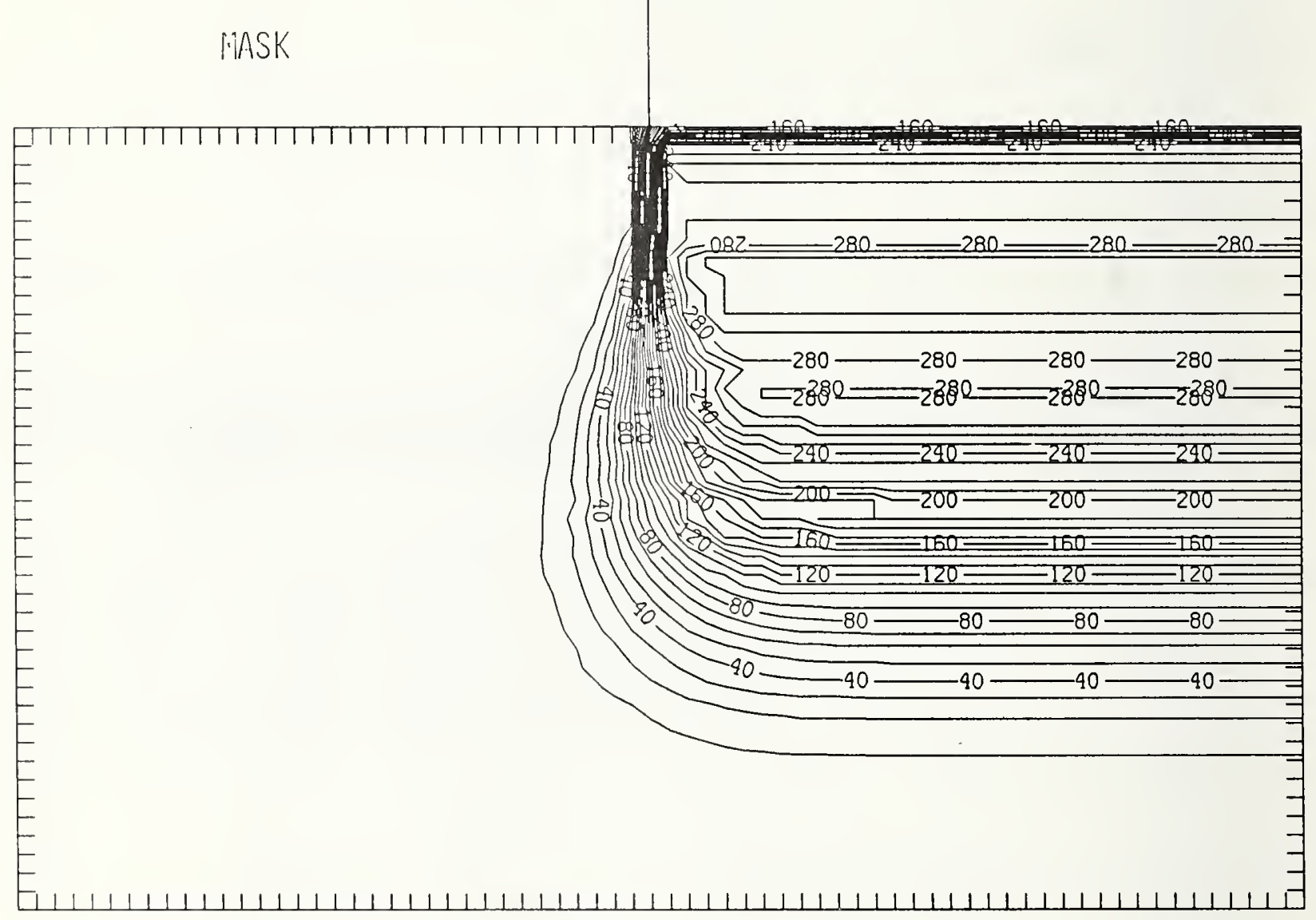

Figure As325-4 Two-dimensional distribution of Frenkel pairs created by $325 \mathrm{keV}$ arsenic implanted past a mask edge. The length increment (distance between tick marks) is $0.01 \mu \mathrm{m}$ and the scale factor is 1000 . 


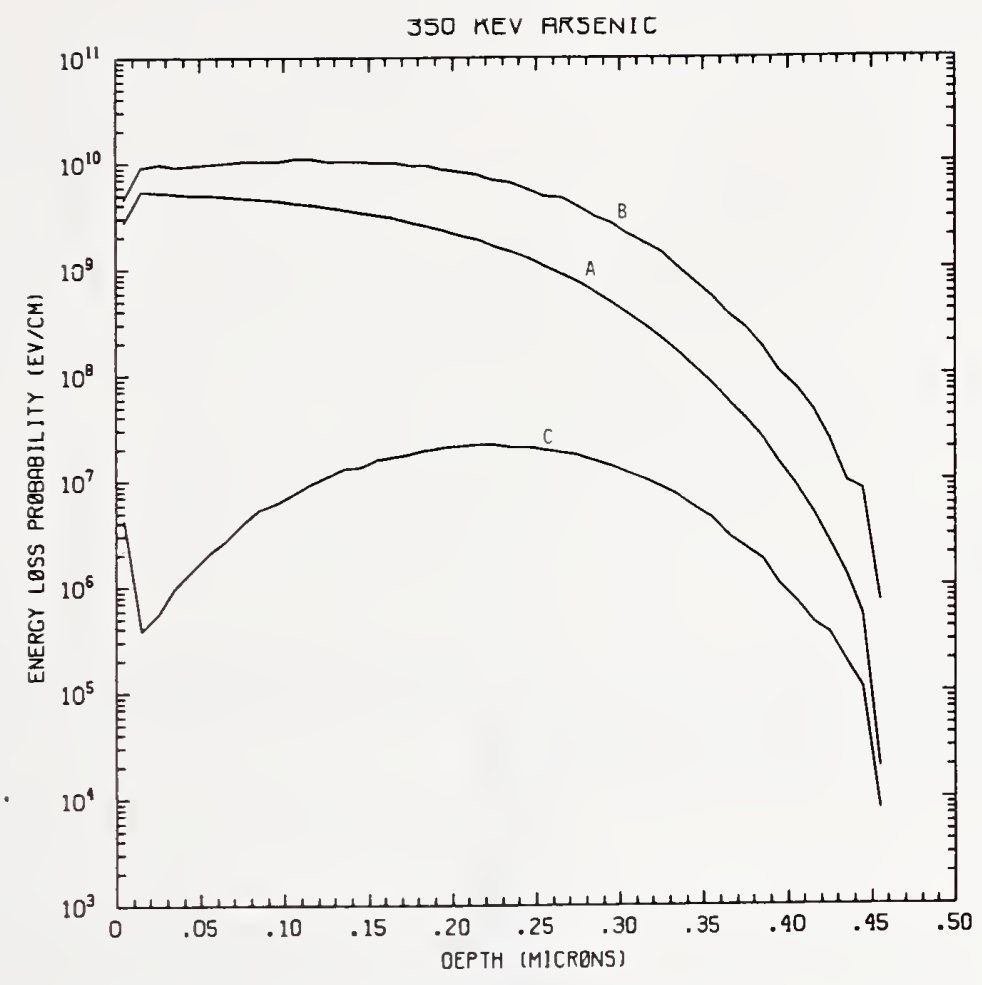

Figure As350-1 One-dimensional probability distributions for energy loss due to electronic excitation (A), displacement damage(B), and phonon excitation (C) for $350 \mathrm{keV}$ arsenic normally incident on a silicon target.

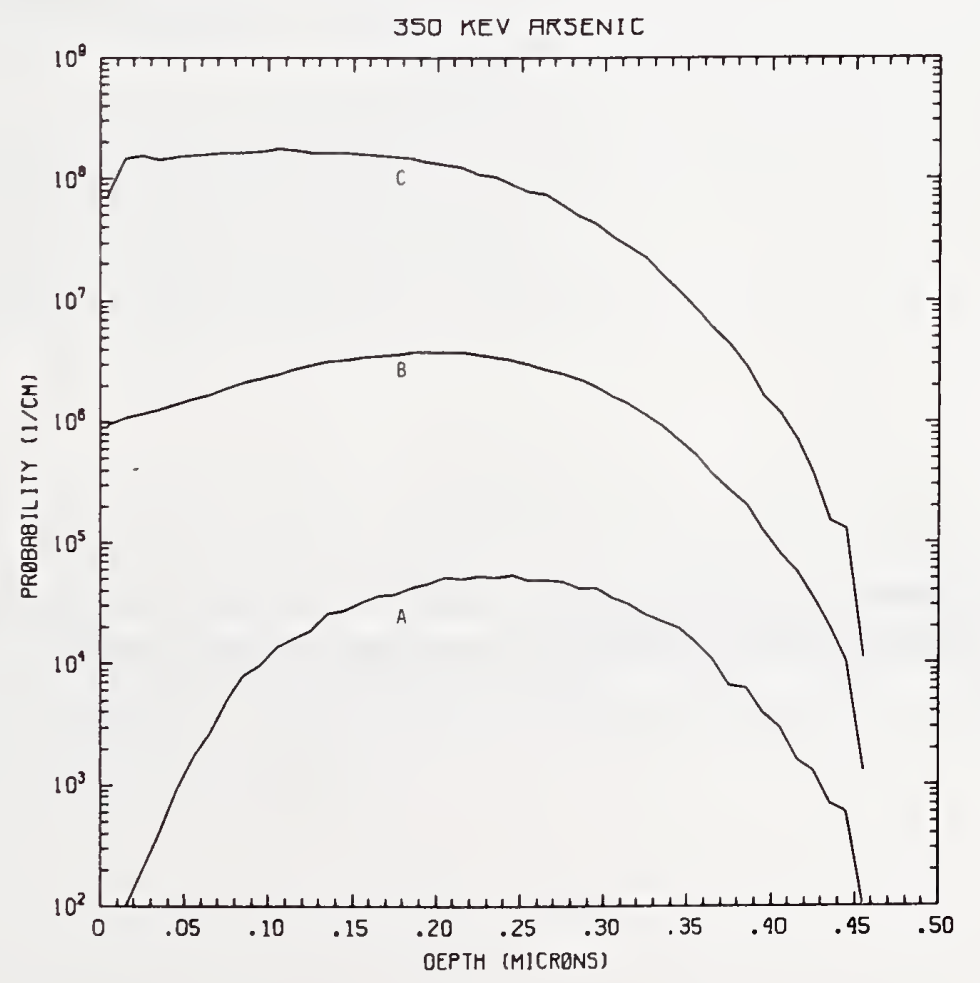

Figure As350-2 One-dimensional probability distributions for implanted arsenic (A), primary silicon displacements (B), and Frenkel pairs (C) for $350 \mathrm{keV}$ arsenic normally incident on a silicon target. 
MASK

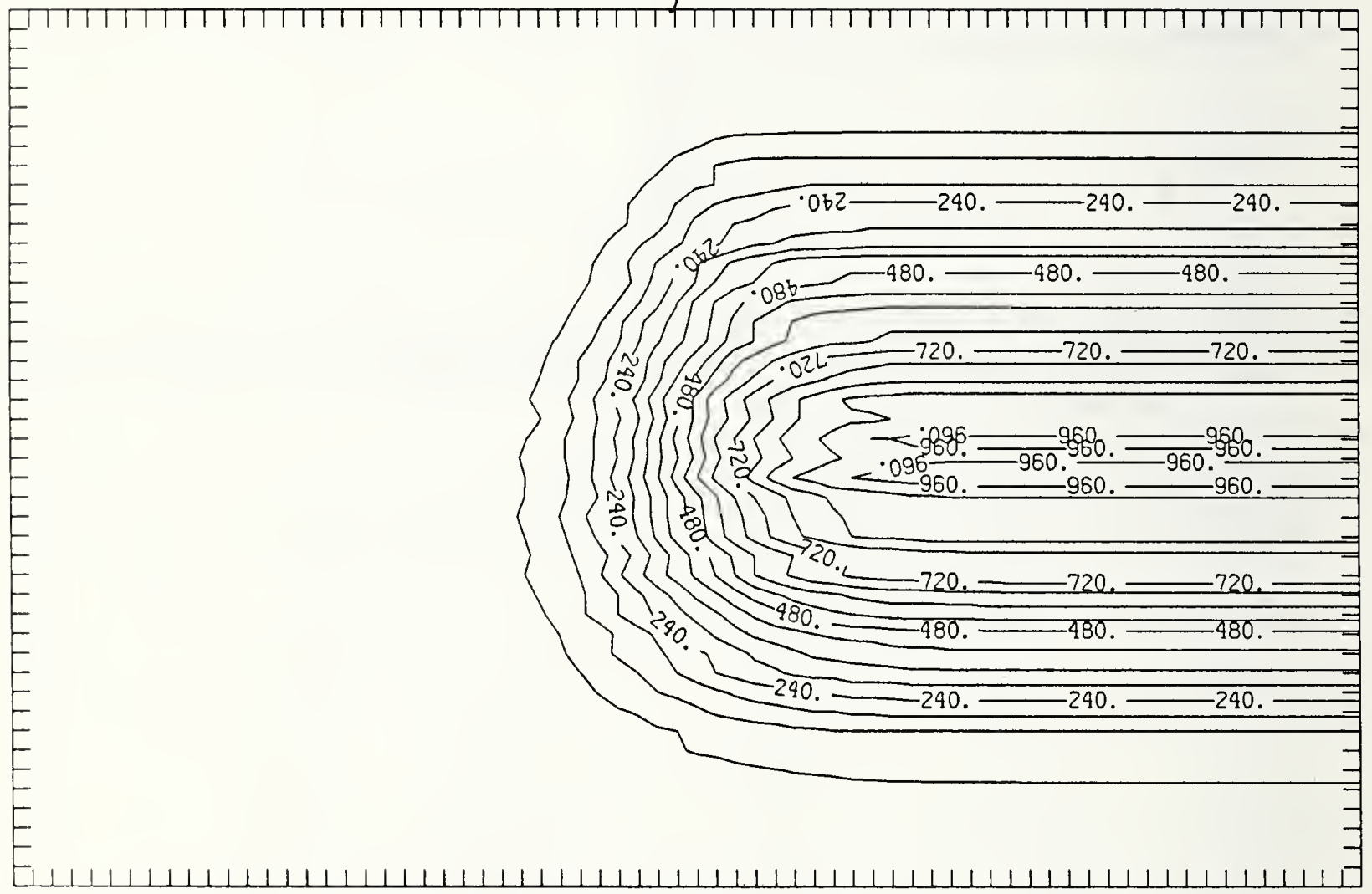

Figure As350-3 Two-dimensional distribution of $350 \mathrm{keV}$ arsenic implanted past a mask edge. The length increment (distance between tick marks) is $0.01 \mu \mathrm{m}$ and the scale factor is 1000 . 


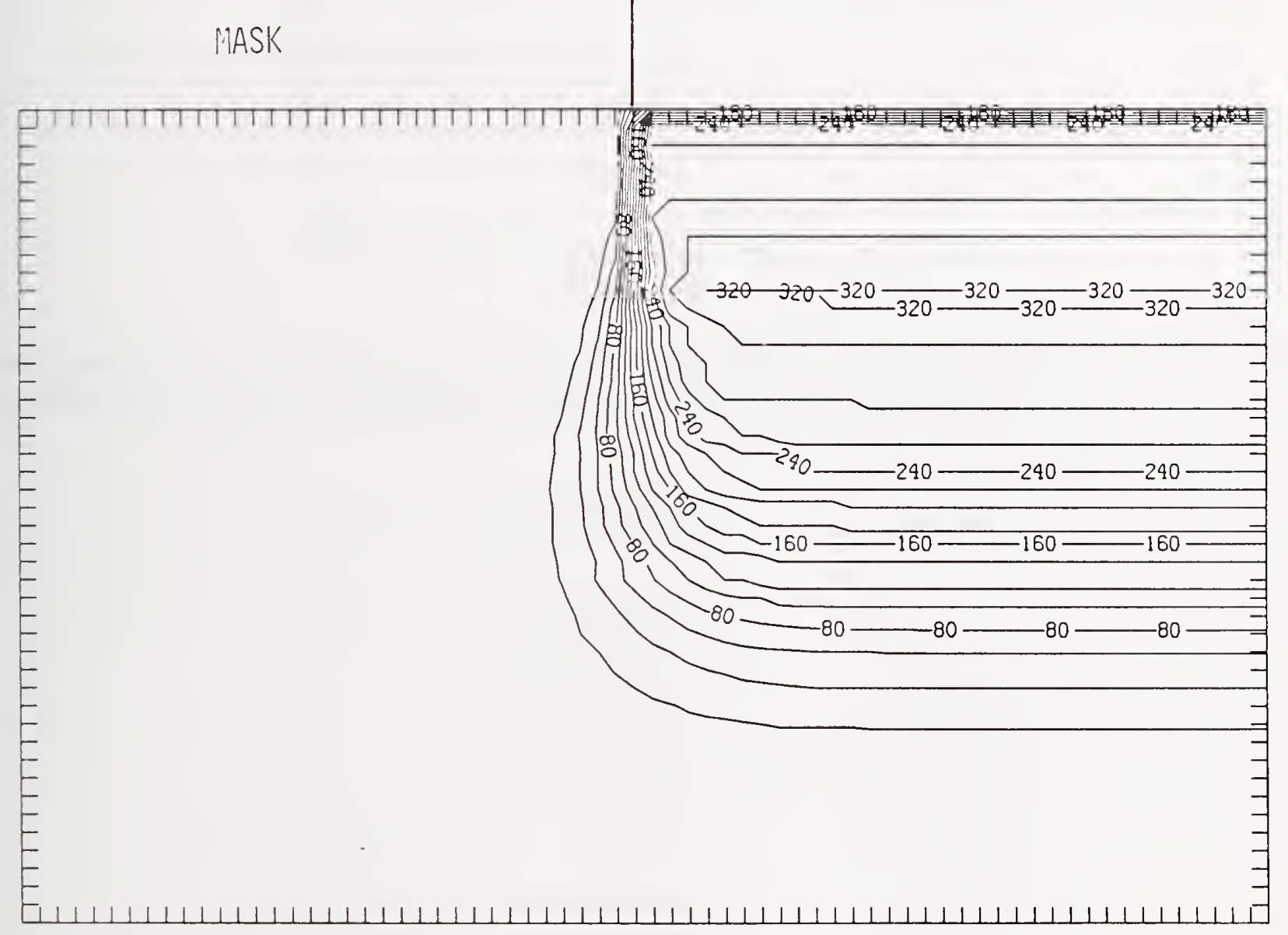

Figure As350-4 Two-dimensional distribution of Frenkel pairs created by $350 \mathrm{keV}$ arsenic implanted past a mask edge. The length increment (distance between tick marks) is $0.01 \mu \mathrm{m}$ and the scale factor is 0.1 . 


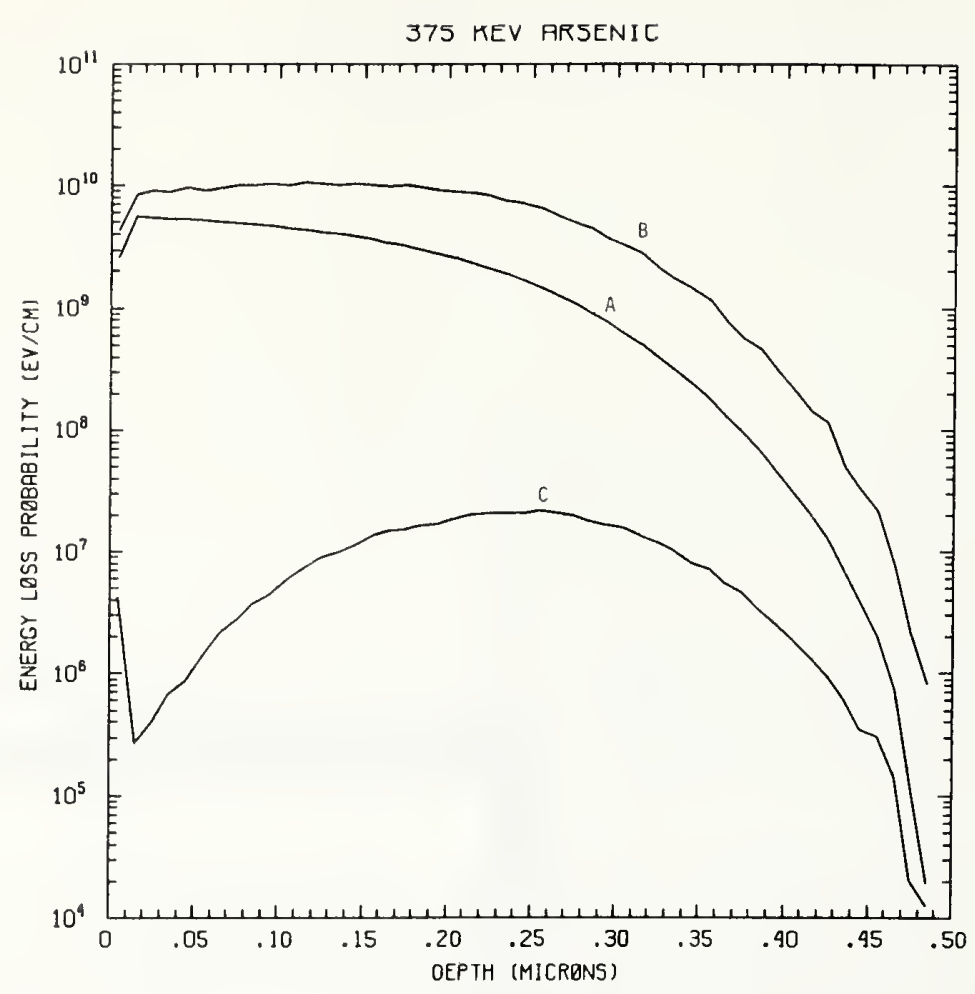

Figure As375-1 One-dimensional probability distributions for energy loss due to electronic excitation (A), displacement damage(B), and phonon excitation (C) for $375 \mathrm{keV}$ arsenic normally incident on a silicon target.

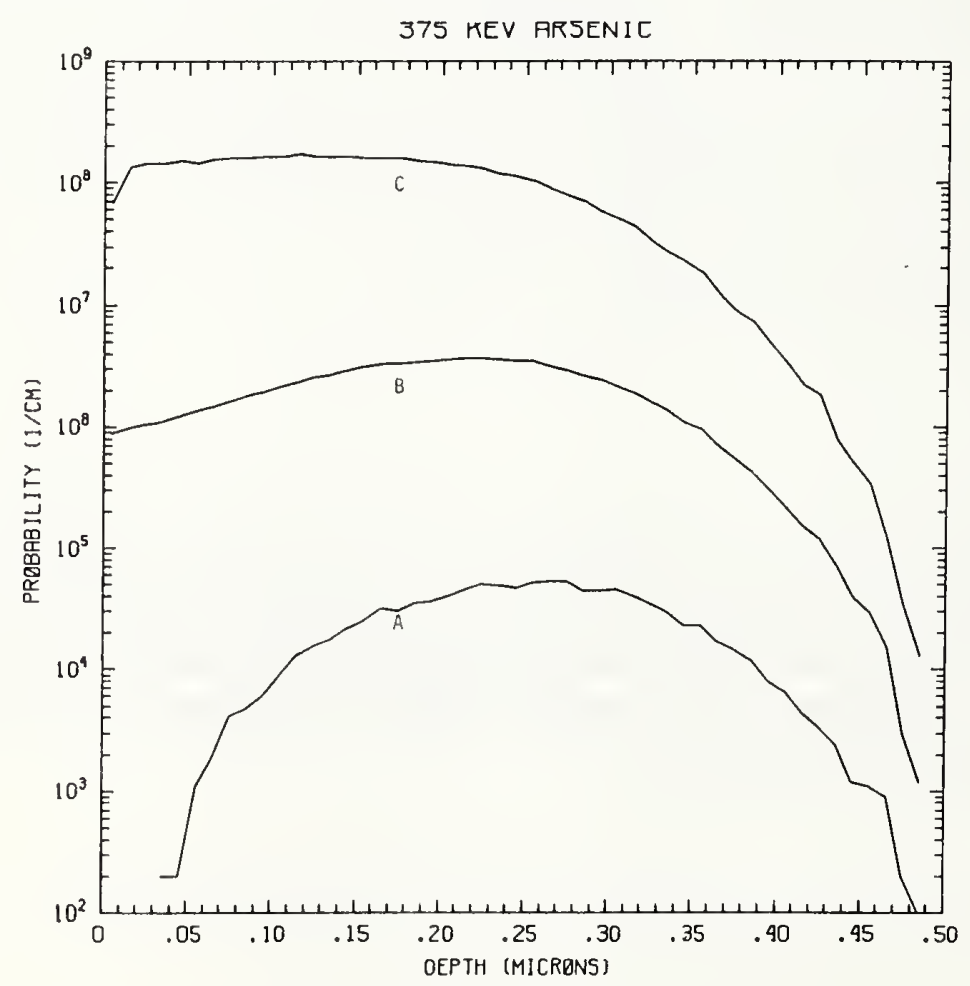

Figure As375-2 One-dimensional probability distributions for implanted arsenic (A), primary silicor displacements (B), and Frenkel pairs (C) for $375 \mathrm{keV}$ arsenic normally incident on a silicon target. 


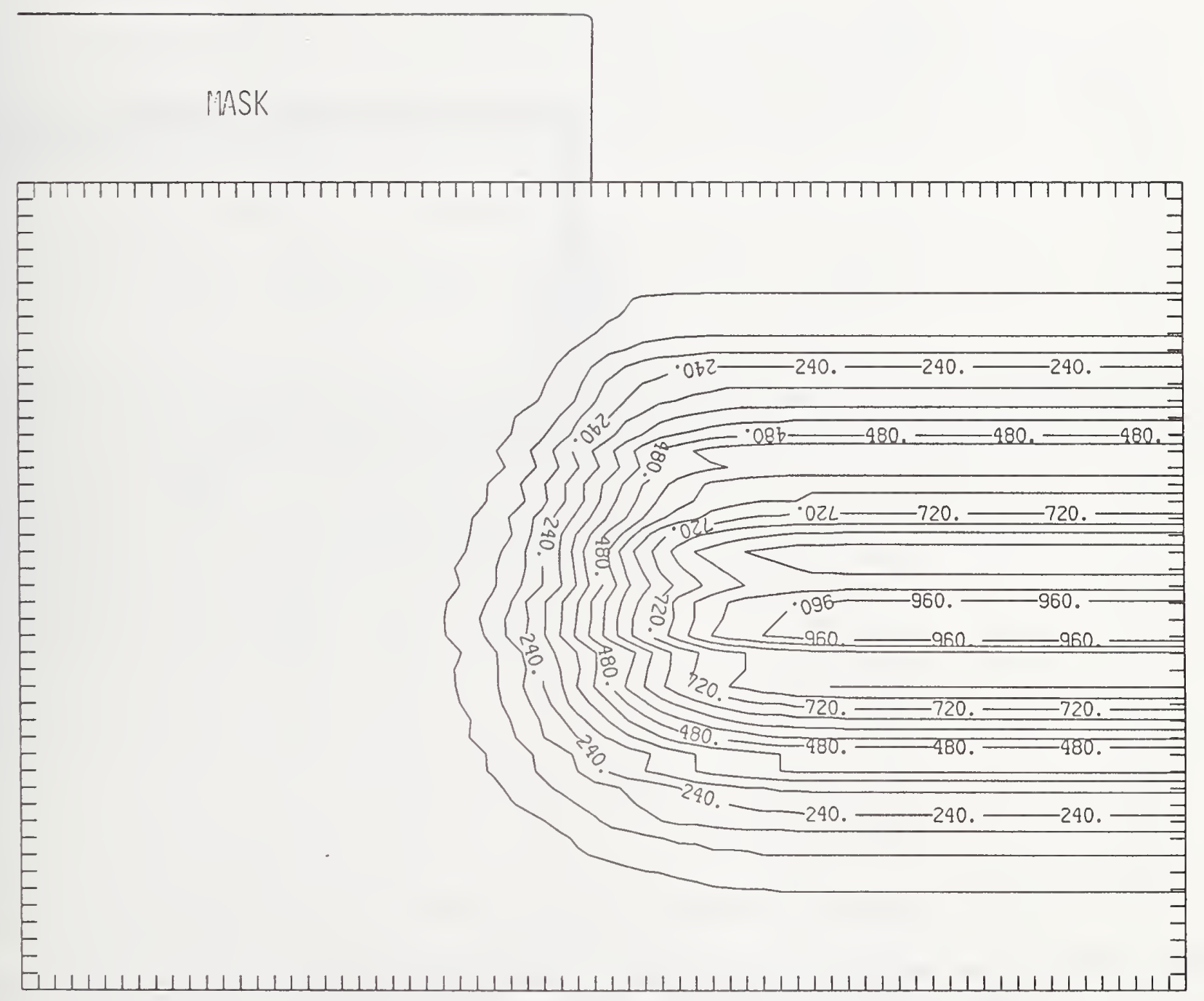

Figure As375-3 Two-dimensional distribution of $375 \mathrm{keV}$ arsenic implanted past a mask edge. The length increment (distance between tick marks) is $0.01 \mu \mathrm{m}$ and the scale factor is 1000 . 
MASK

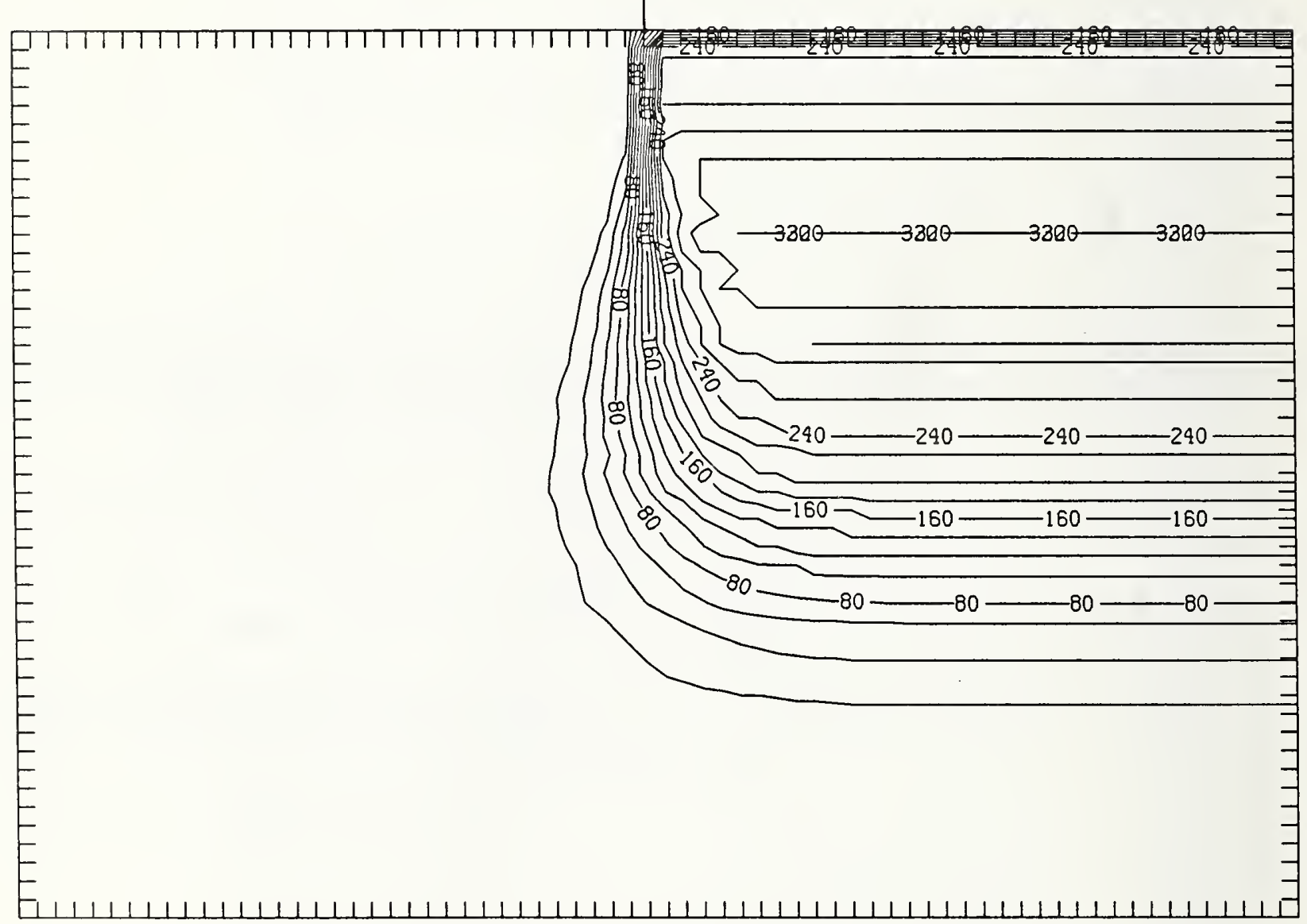

Figure As375-4 Two-dimensional distribution of Frenkel pairs created by $375 \mathrm{keV}$ arsenic implanted past a mask edge. The length increment (distance between tick marks) is $0.01 \mu \mathrm{m}$ and the scale factor is 0.1 . 


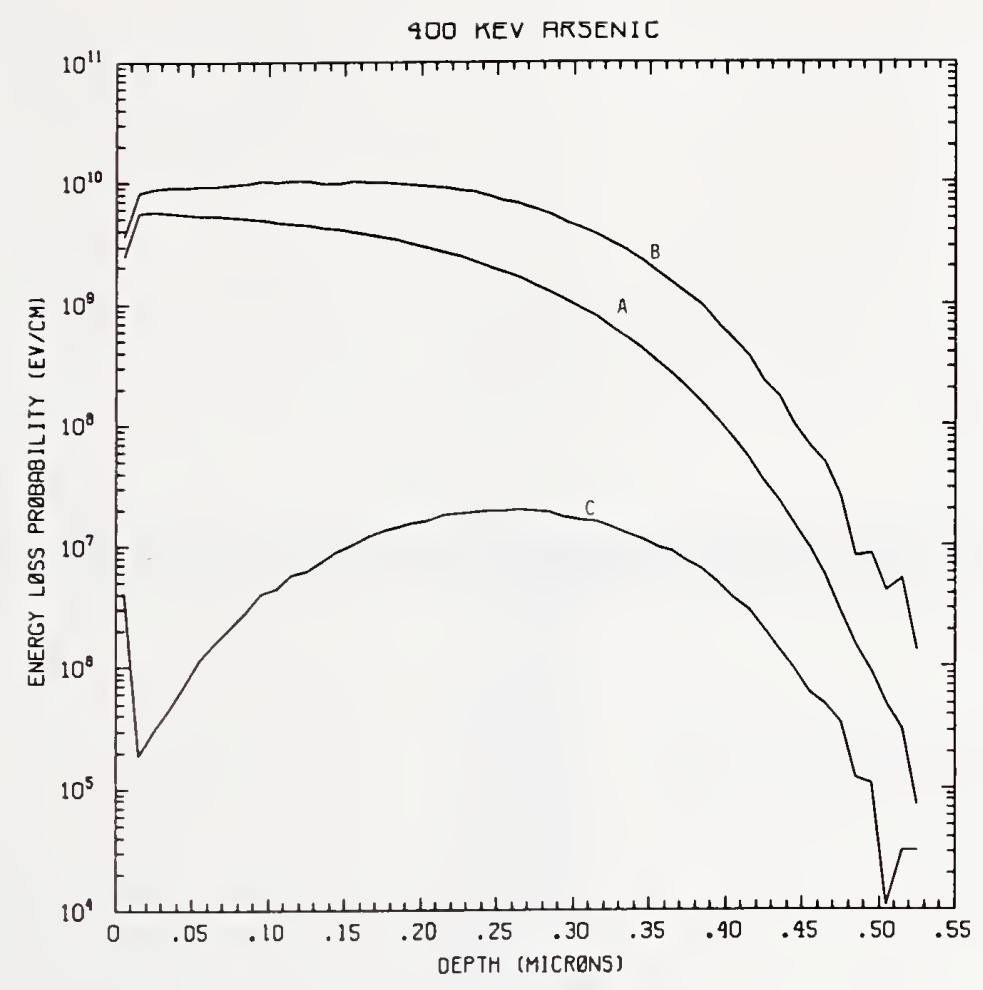

Figure As400-1 One-dimensional probability distributions for energy loss due to electronic excitation (A), displacement damage(B), and phonon excitation (C) for $400 \mathrm{keV}$ arsenic normally incident on a silicon target.

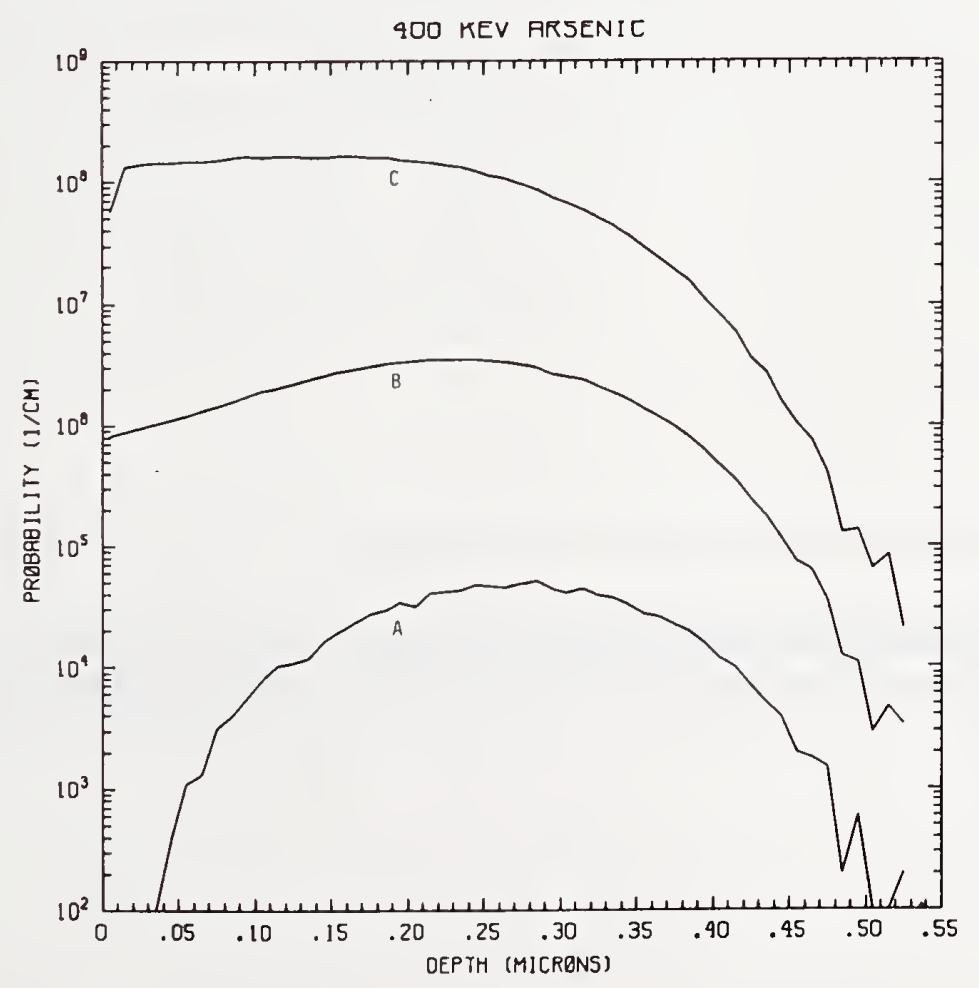

Figure As400-2 One-dimensional probability distributions for implanted arsenic (A), primary silicon displacements (B), and Frenkel pairs (C) for $400 \mathrm{keV}$ arsenic normally incident on a silicon target. 


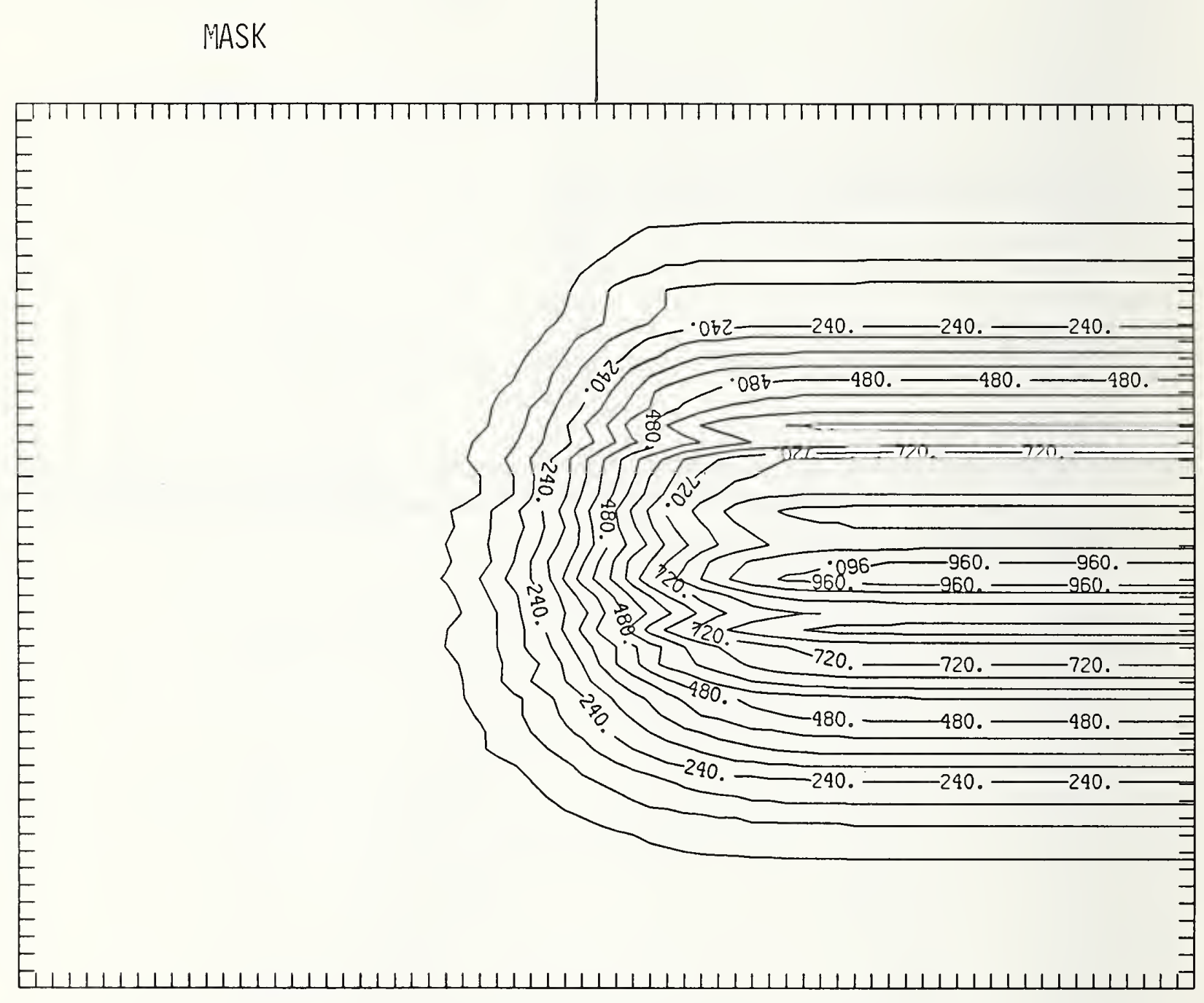

Figure As400-3 Two-dimensional distribution of $400 \mathrm{keV}$ arsenic implanted past a mask edge. The length increment (distance between tick marks) is $0.01 \mu \mathrm{m}$ and the scale factor is 1000 . 


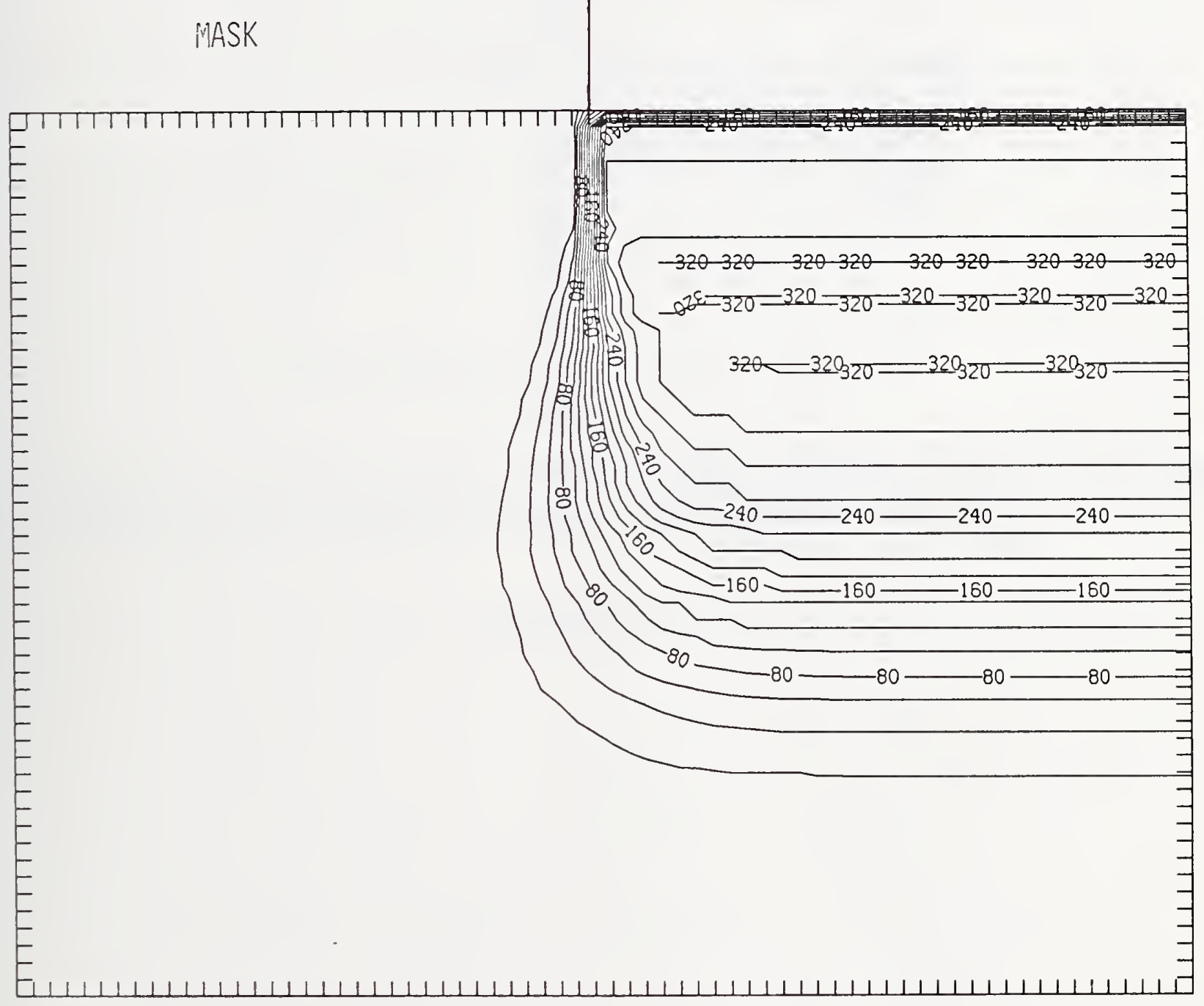

Figure As400-4 Two-dimensional distribution of Frenkel pairs created by $400 \mathrm{keV}$ arsenic implanted past a mask edge. The length increment (distance between tick marks) is $0.01 \mu \mathrm{m}$ and the scale factor is 0.1 . 


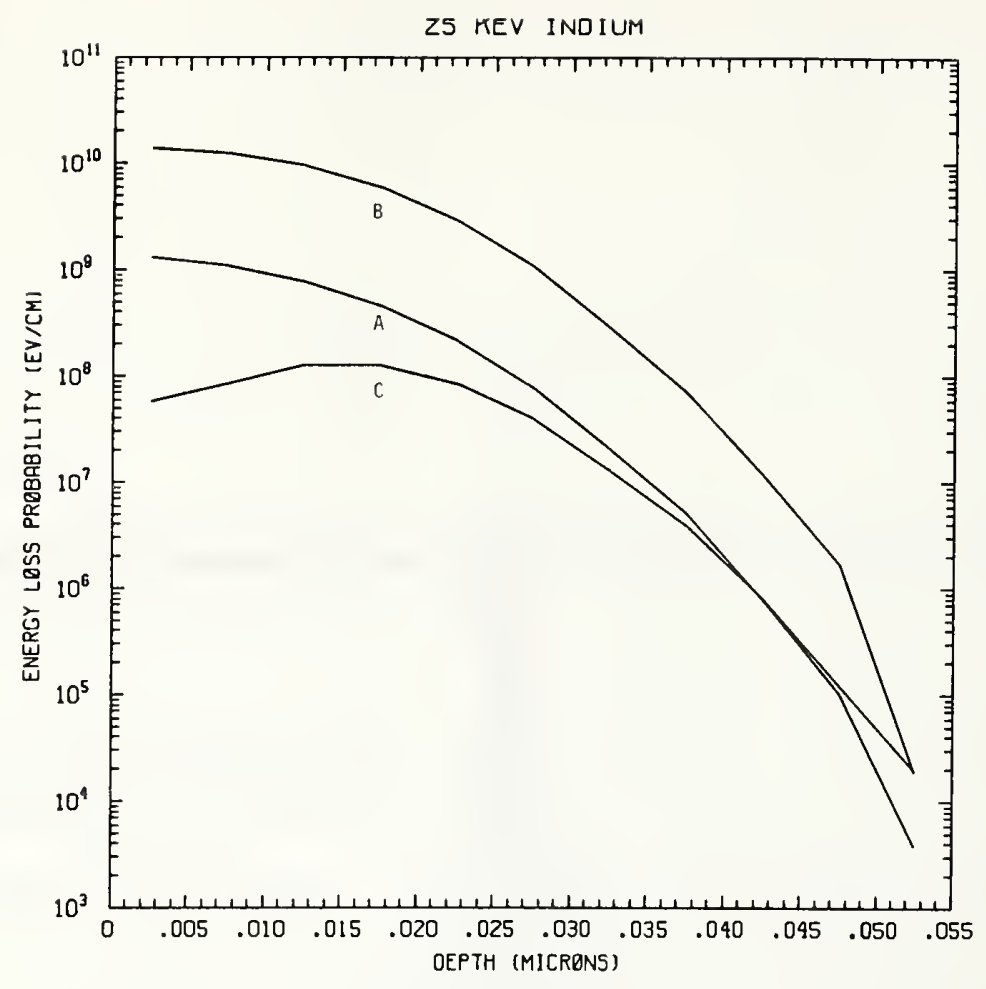

Figure In25-1 One-dimensional probability distributions for energy loss due to electronic excitation (A), displacement damage(B), and phonon excitation (C) for $25 \mathrm{keV}$ indium normally incident on a silicon target.

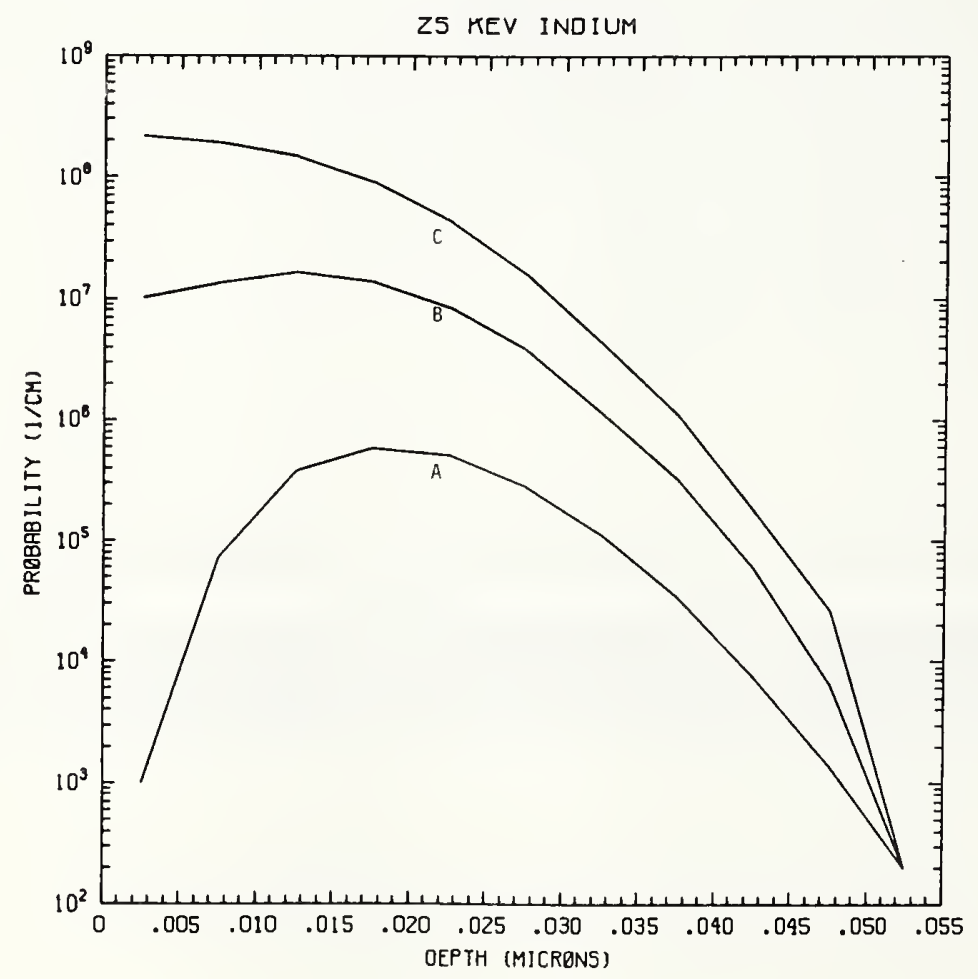

Figure In25-2 One-dimensional probability distributions for implanted indium (A), primary silicon displacements (B), and Frenkel pairs (C) for $25 \mathrm{keV}$ indium normally incident on a silicon target. 
MASK

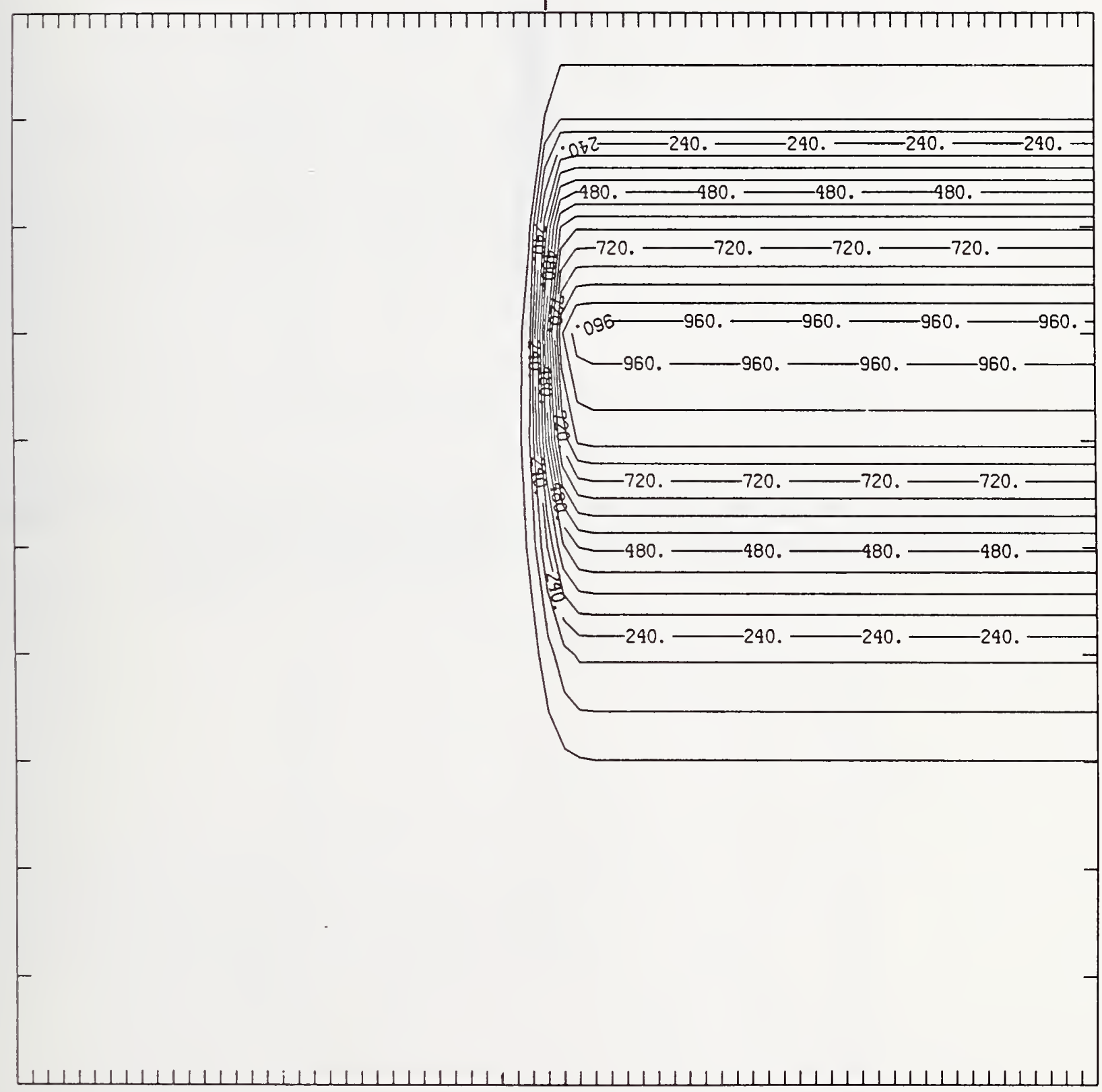

Figure In25-3 Two-dimensional distribution of $25 \mathrm{keV}$ indium implanted past a mask edge. The length increment (distance between tick marks) is $0.005 \mu \mathrm{m}$ and the scale factor is 1000 . 
MASK

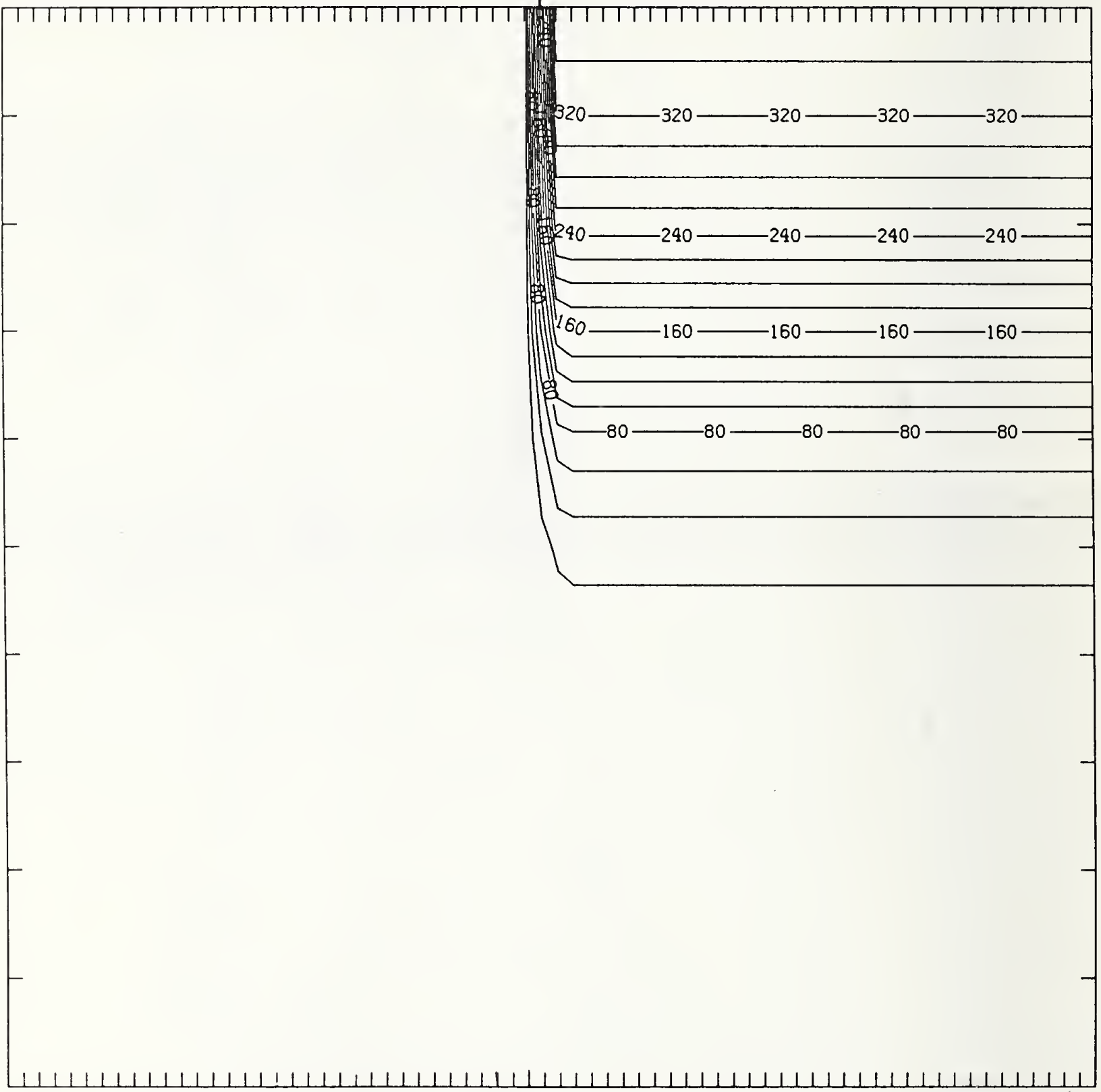

Figure In25-4 Two-dimensional distribution of Frenkel pairs created by $25 \mathrm{keV}$ indium implanted past a mask edge. The length increment (distance between tick marks) is $0.005 \mu \mathrm{m}$ and the scale factor is 1 . 


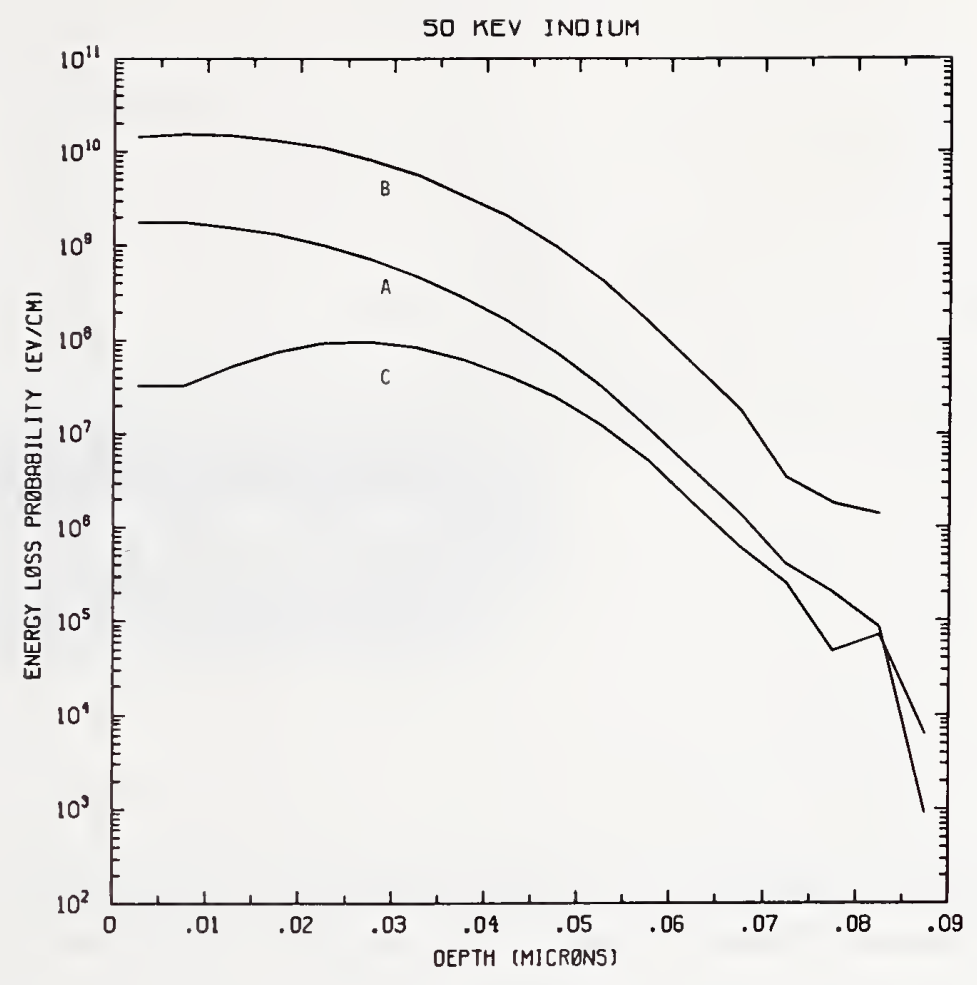

Figure In50-1 One-dimensional probability distributions for energy loss due to electronic excitation (A), displacement damage(B), and phonon excitation (C) for $50 \mathrm{keV}$ indium normally incident on a silicon target.

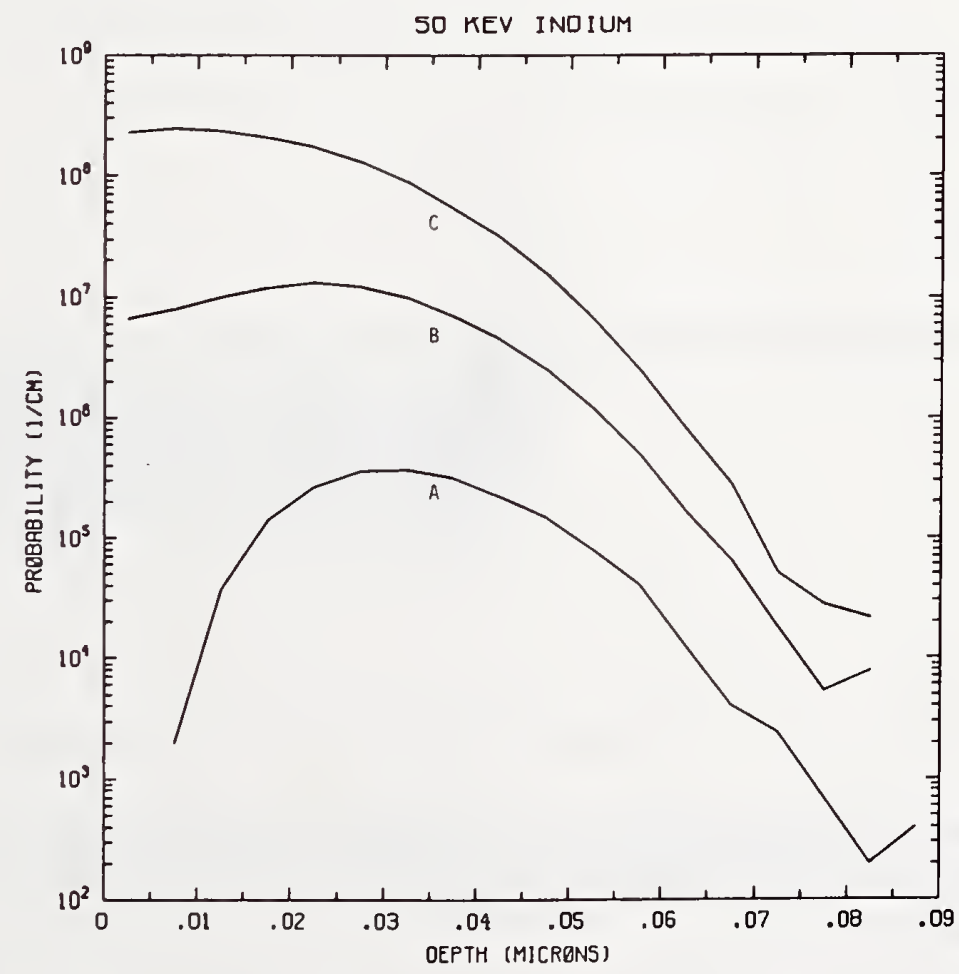

Figure In50-2 One-dimensional probability distributions for implanted indium (A), primary silicon displacements (B), and Frenkel pairs (C) for $50 \mathrm{keV}$ indium normally incident on a silicon target. 


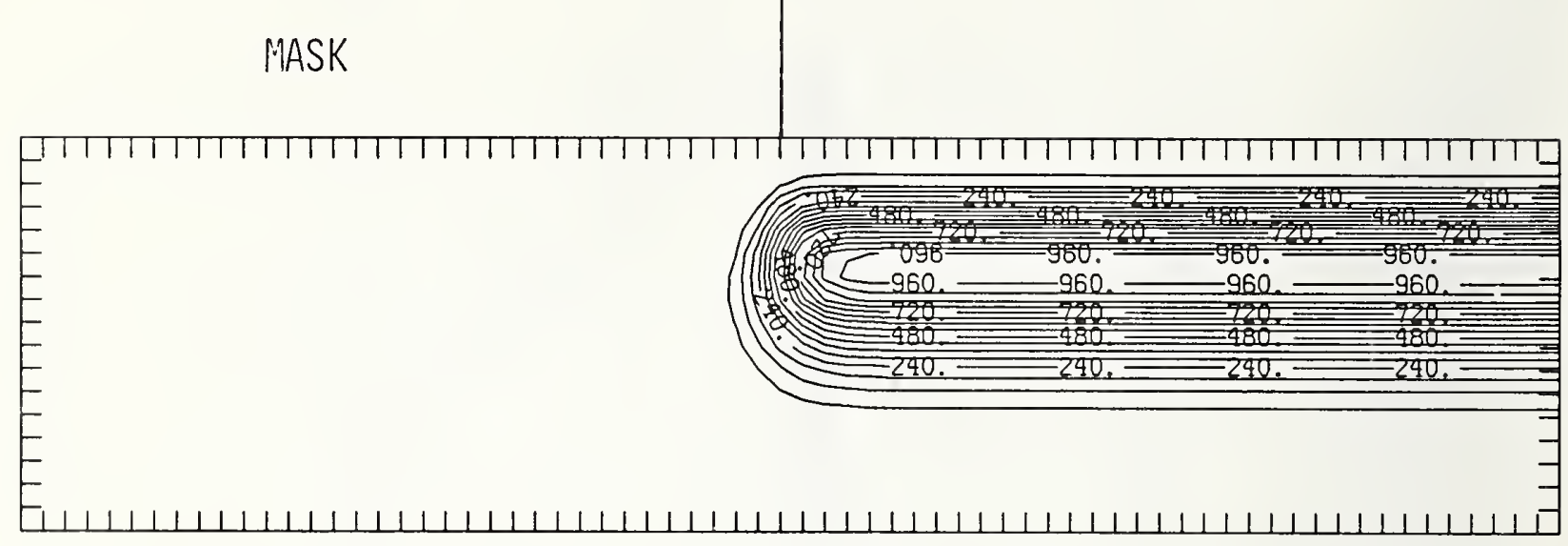

Figure In50-3 Two-dimensional distribution of $50 \mathrm{keV}$ indium implanted past a mask edge. The length increment (distance between tick marks) is $0.005 \mu \mathrm{m}$ and the scale factor is 1000 .

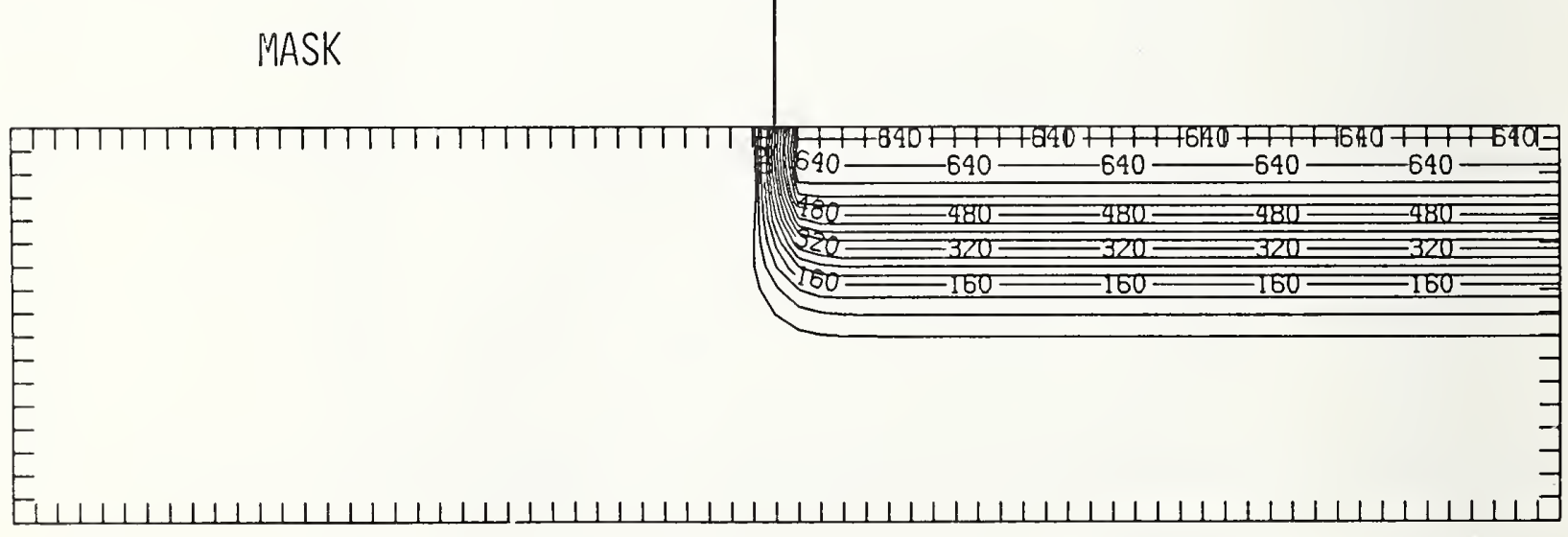

Figure In50-4 Two-dimensional distribution of Frenkel pairs created by $50 \mathrm{keV}$ indium implanted past a mask edge. The length increment (distance between tick marks) is $0.005 \mu \mathrm{m}$ and the scale factor is 1 . 


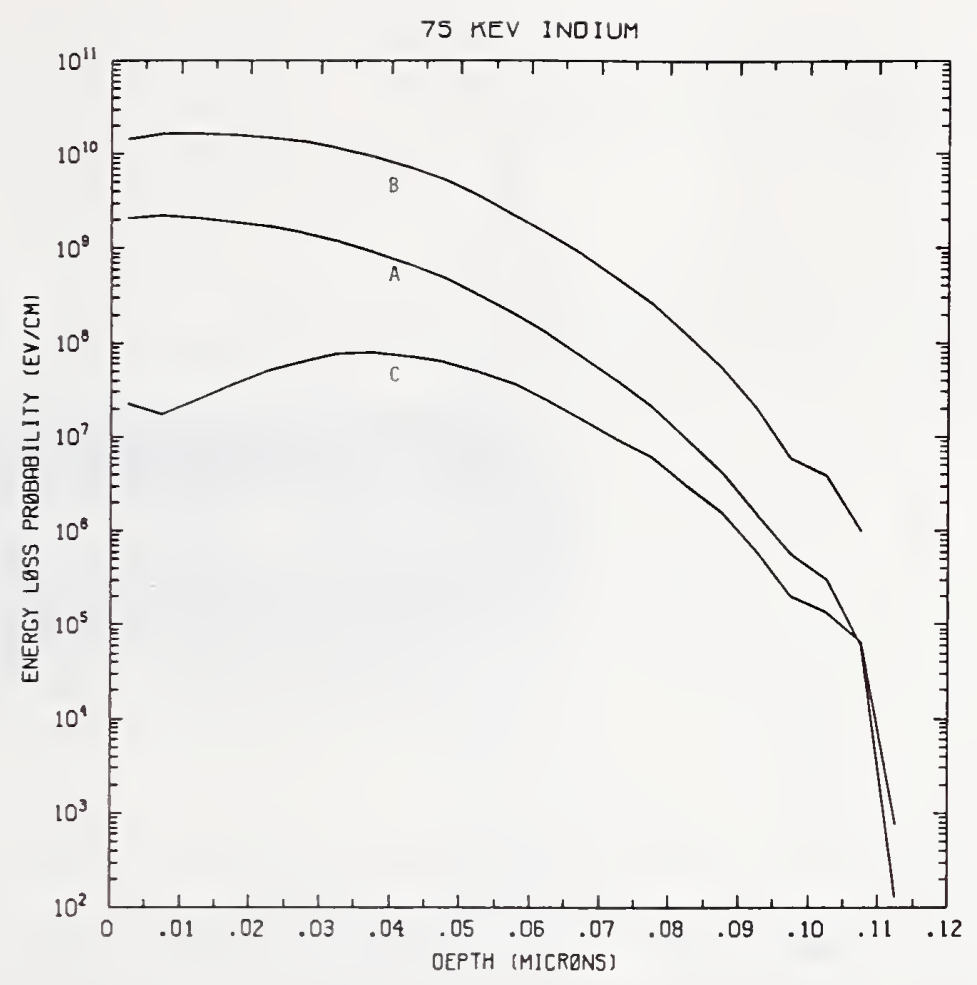

Figure In75-1 One-dimensional probability distributions for energy loss due to electronic excitation (A), displacement damage(B), and phonon excitation (C) for $75 \mathrm{keV}$ indium normally incident on a silicon target.

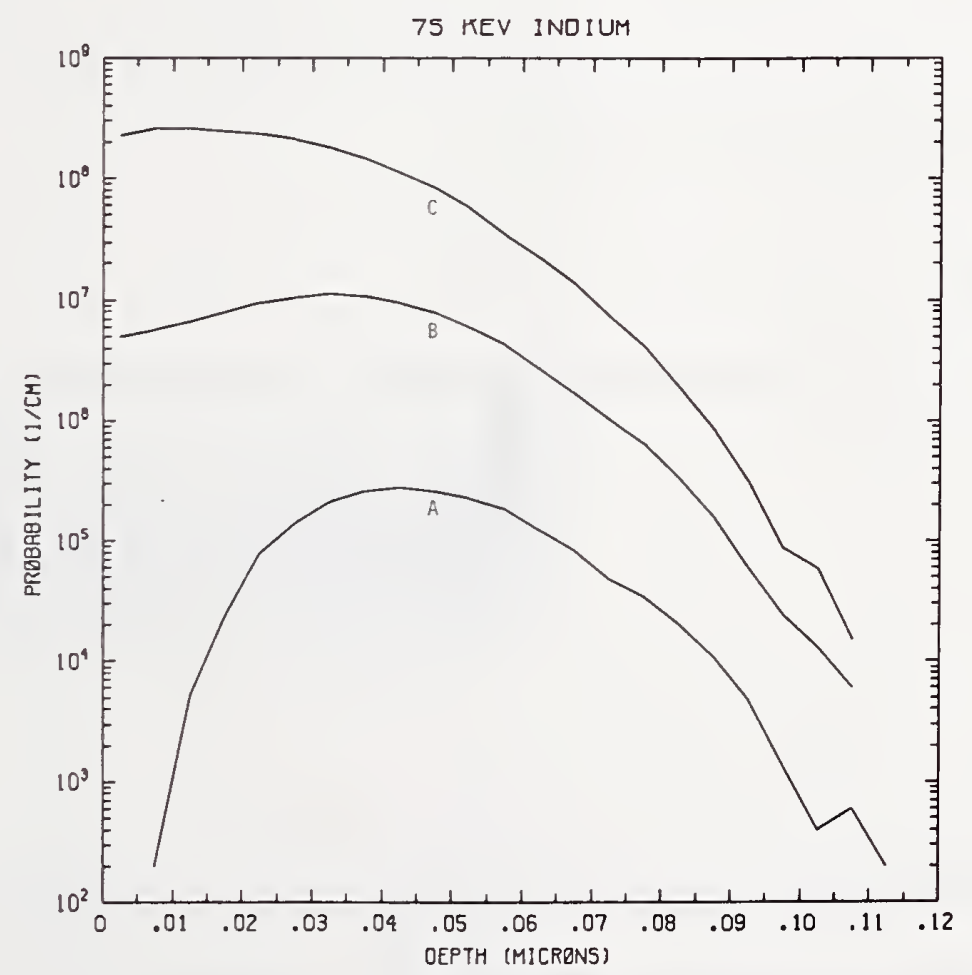

Figure In75-2 One-dimensional probability distributions for implanted indium (A), primary silicon displacements (B), and Frenkel pairs (C) for $75 \mathrm{keV}$ indium normally incident on a silicon target. 


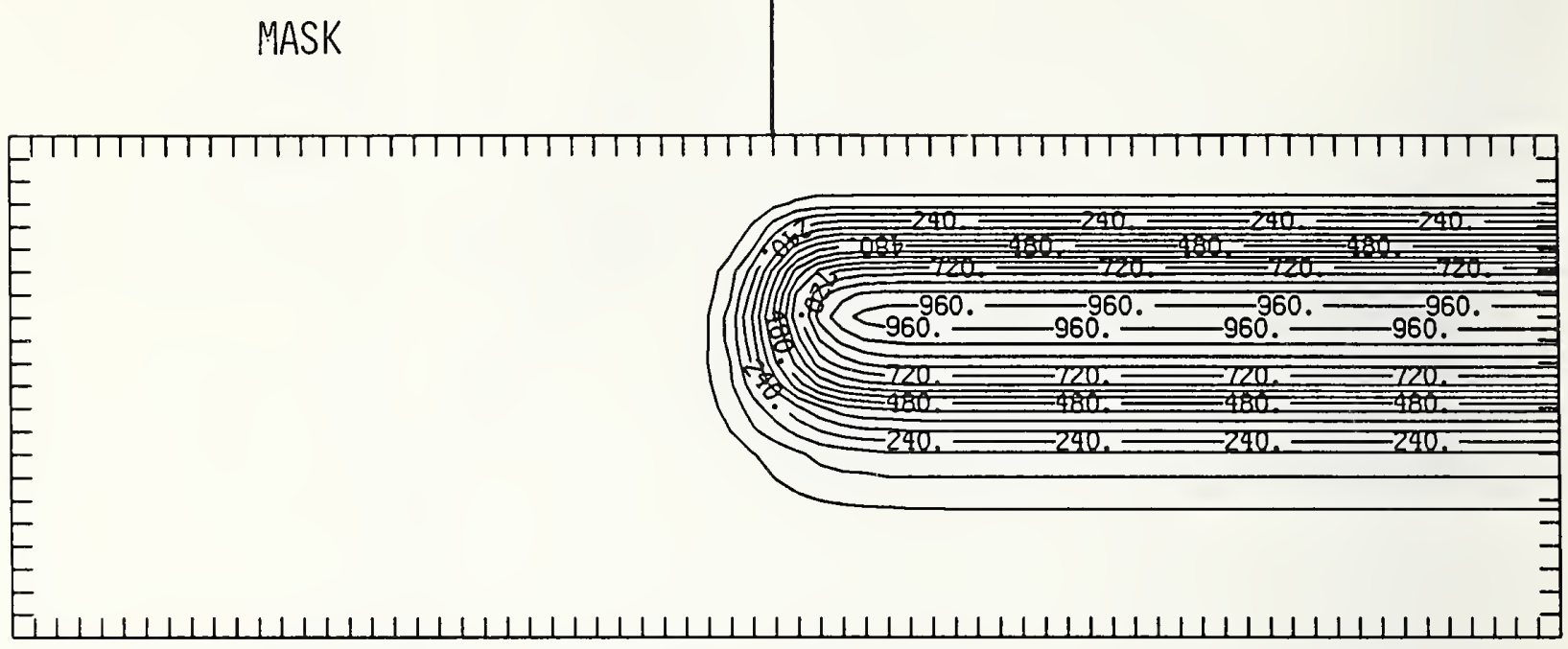

Figure In75-3 Two-dimensional distribution of $75 \mathrm{keV}$ indium implanted past a mask edge. The length increment (distance between tick marks) is $0.005 \mu \mathrm{m}$ and the scale factor is 1000.

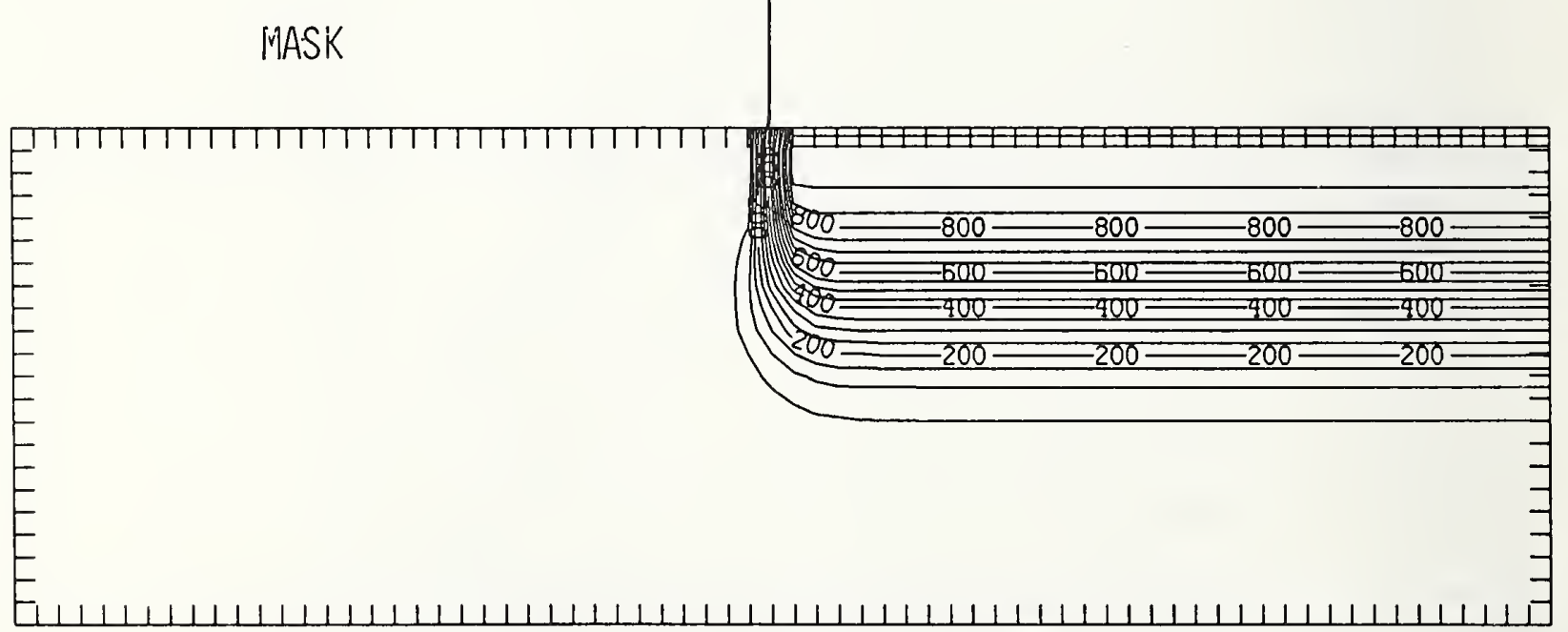

Figure In75-4 Two-dimensional distribution of Frenkel pairs created by $75 \mathrm{keV}$ indium implanted past a mask edge. The length increment (distance between tick marks) is $0.005 \mu \mathrm{m}$ and the scale factor is 1. 


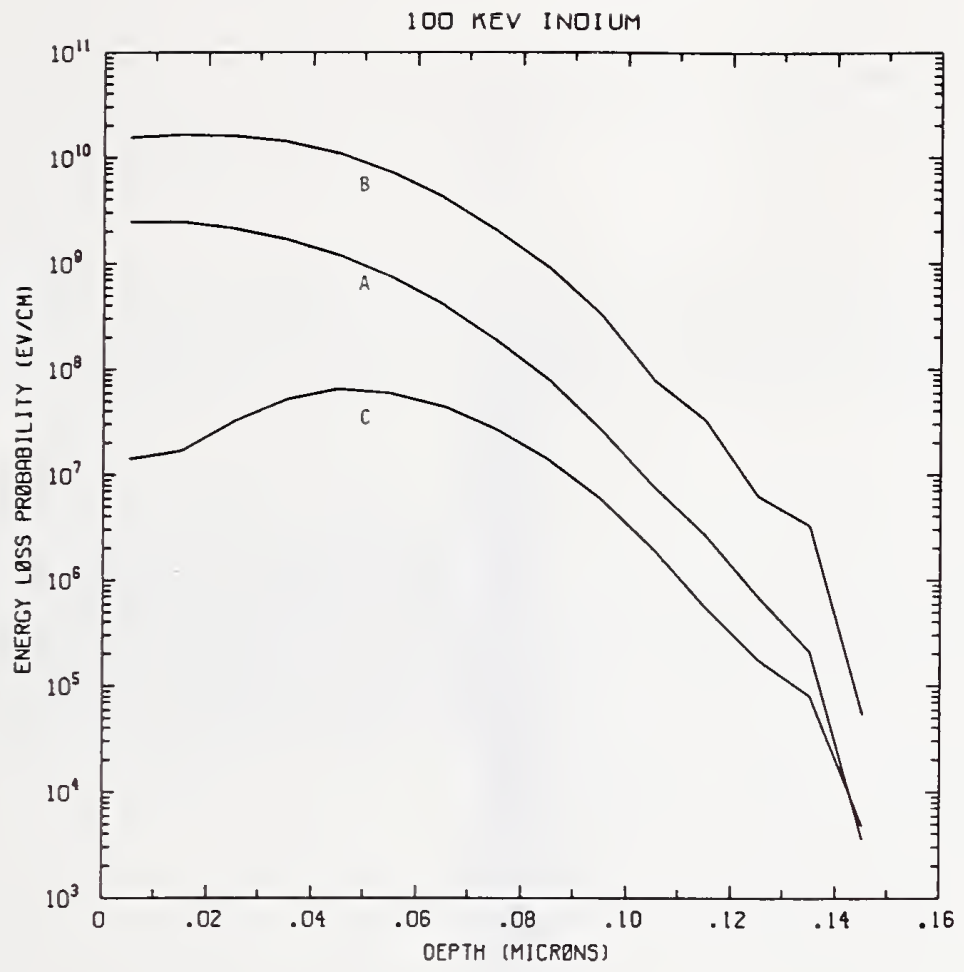

Figure In100-1 One-dimensional probability distributions for energy loss due to electronic excitation (A), displacement damage(B), and phonon excitation (C) for $100 \mathrm{keV}$ indium normally incident on a silicon target.

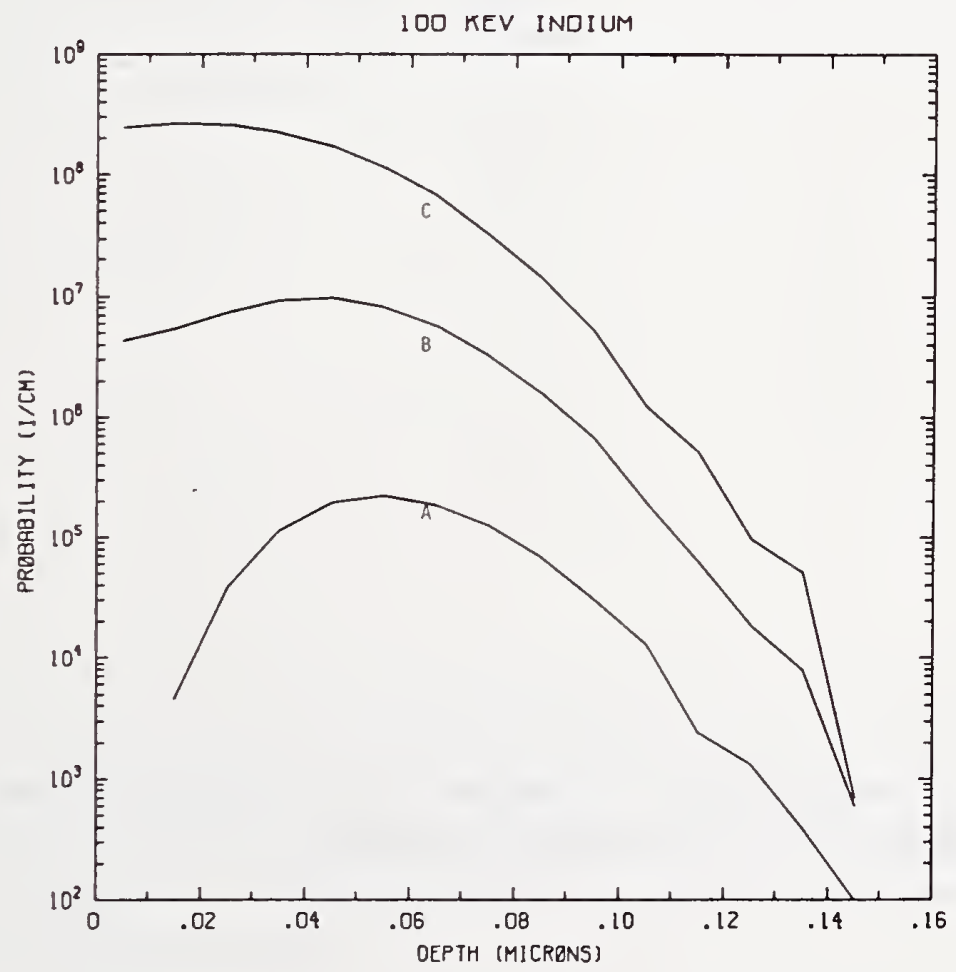

Figure In100-2 One-dimensional probability distributions for implanted indium (A), primary silicon displacements (B), and Frenkel pairs (C) for $100 \mathrm{keV}$ indium normally incident on a silicon target. 
MASK

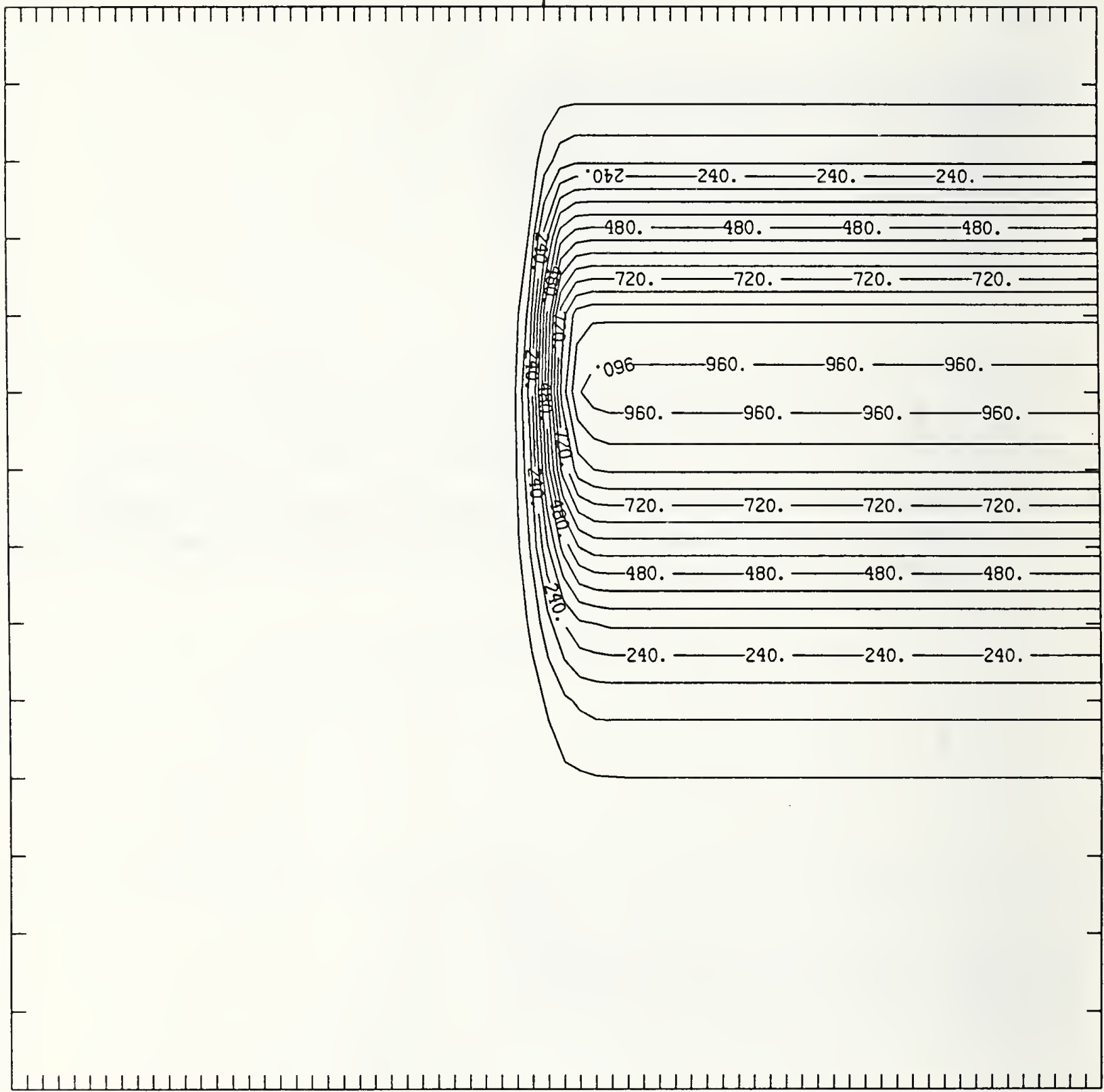

Figure In100-3 Two-dimensional distribution of $100 \mathrm{keV}$ indium implanted past a mask edge. The length increment (distance between tick marks) is $0.01 \mu \mathrm{m}$ and the scale factor is 1000. 


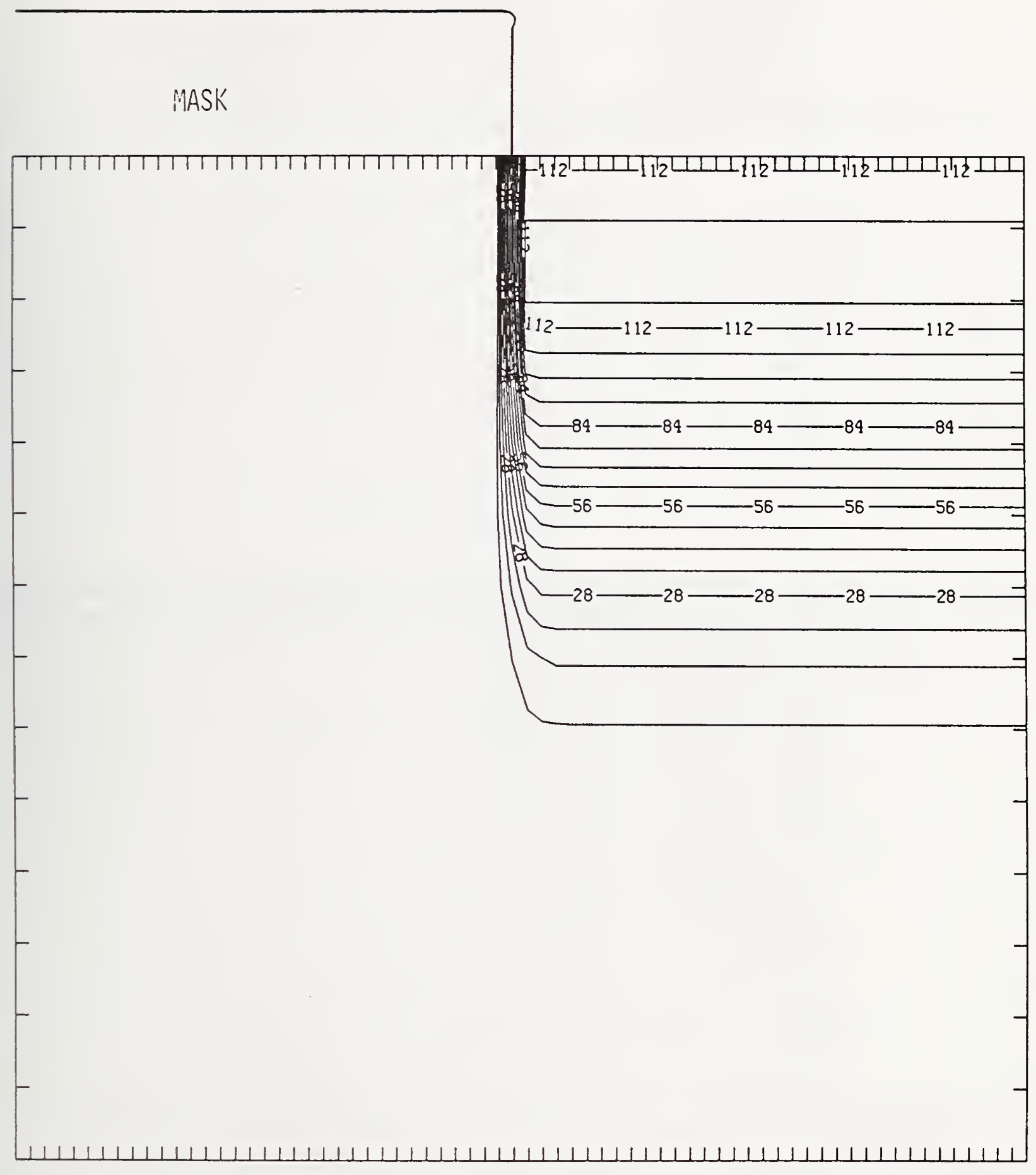

Figure In100-4 Two-dimensional distribution of Frenkel pairs created by $100 \mathrm{keV}$ indium implanted past a mask edge. The length increment (distance between tick marks) is $0.01 \mu \mathrm{m}$ and the scale factor is 0.1 . 


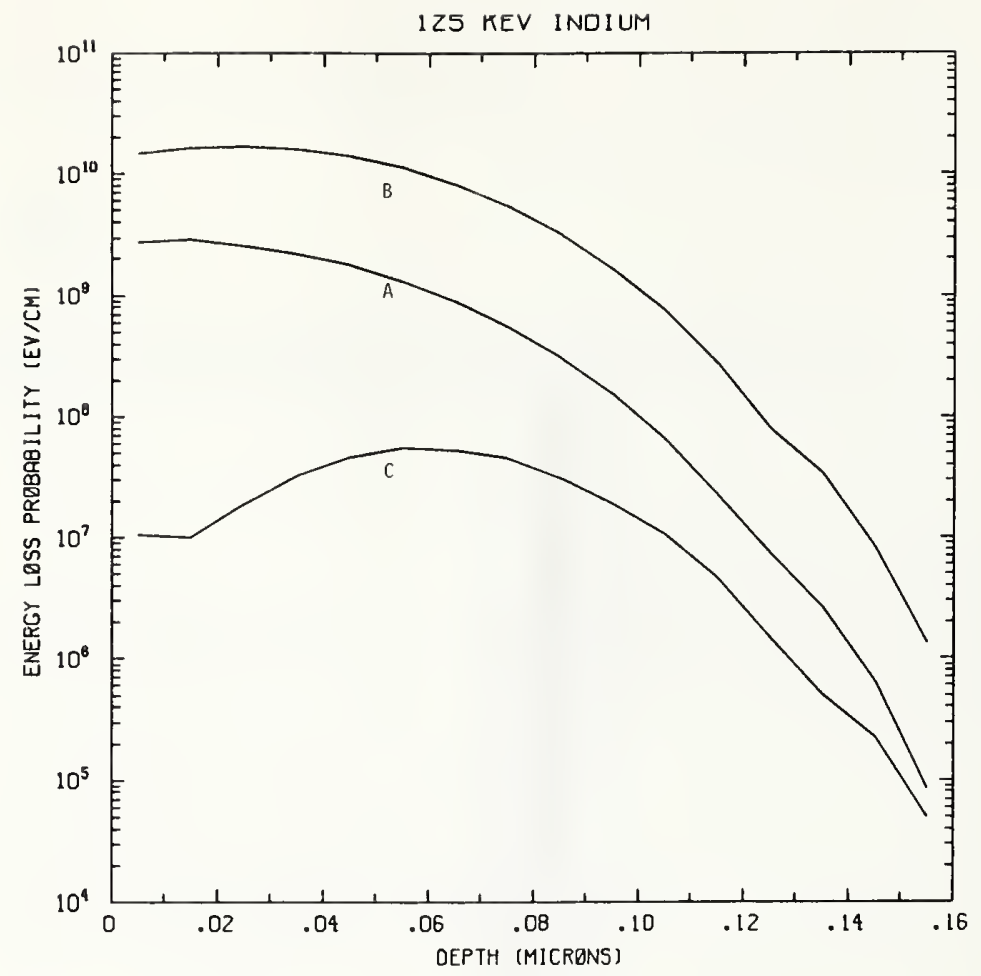

Figure In125-1 One-dimensional probability distributions for energy loss due to electronic excitation (A), displacement damage(B), and phonon excitation (C) for $125 \mathrm{keV}$ indium normally incident on a silicon target.

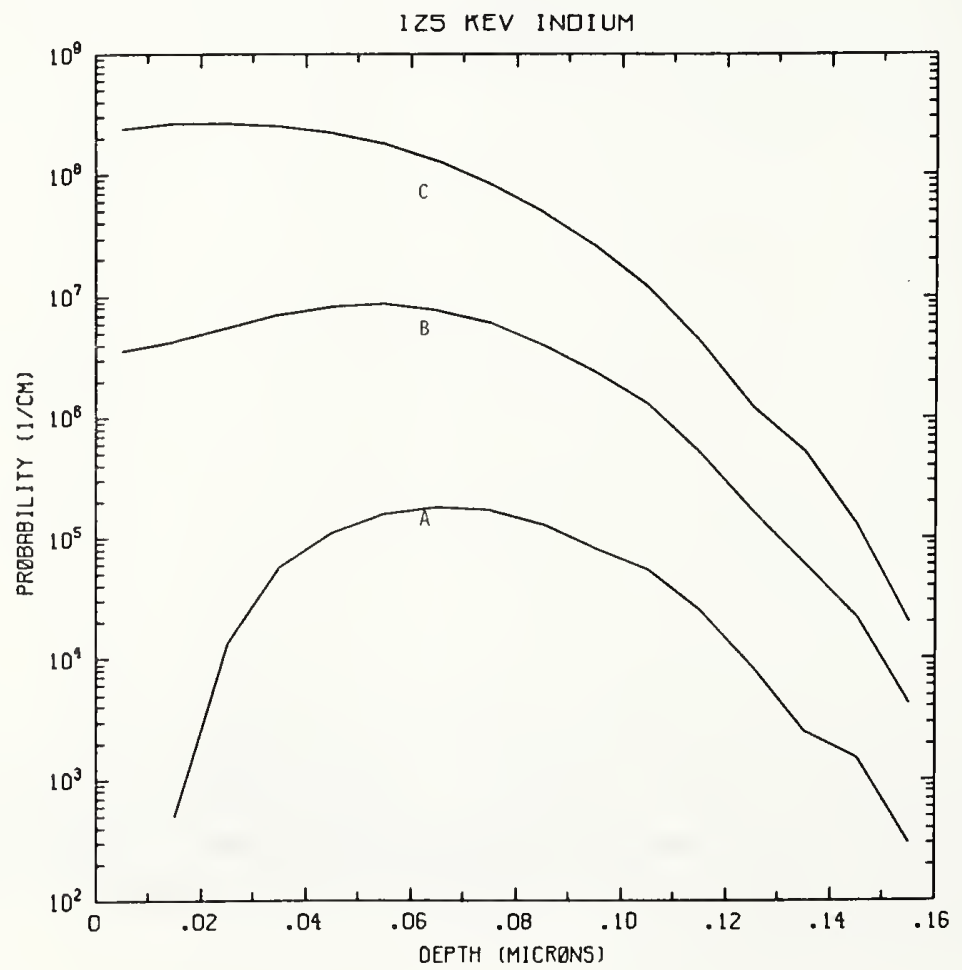

Figure In125-2 One-dimensional probability distributions for implanted indium (A), primary silicon displacements (B), and Frenkel pairs (C) for $125 \mathrm{keV}$ indium normally incident on a silicon target. 


\section{MASK}

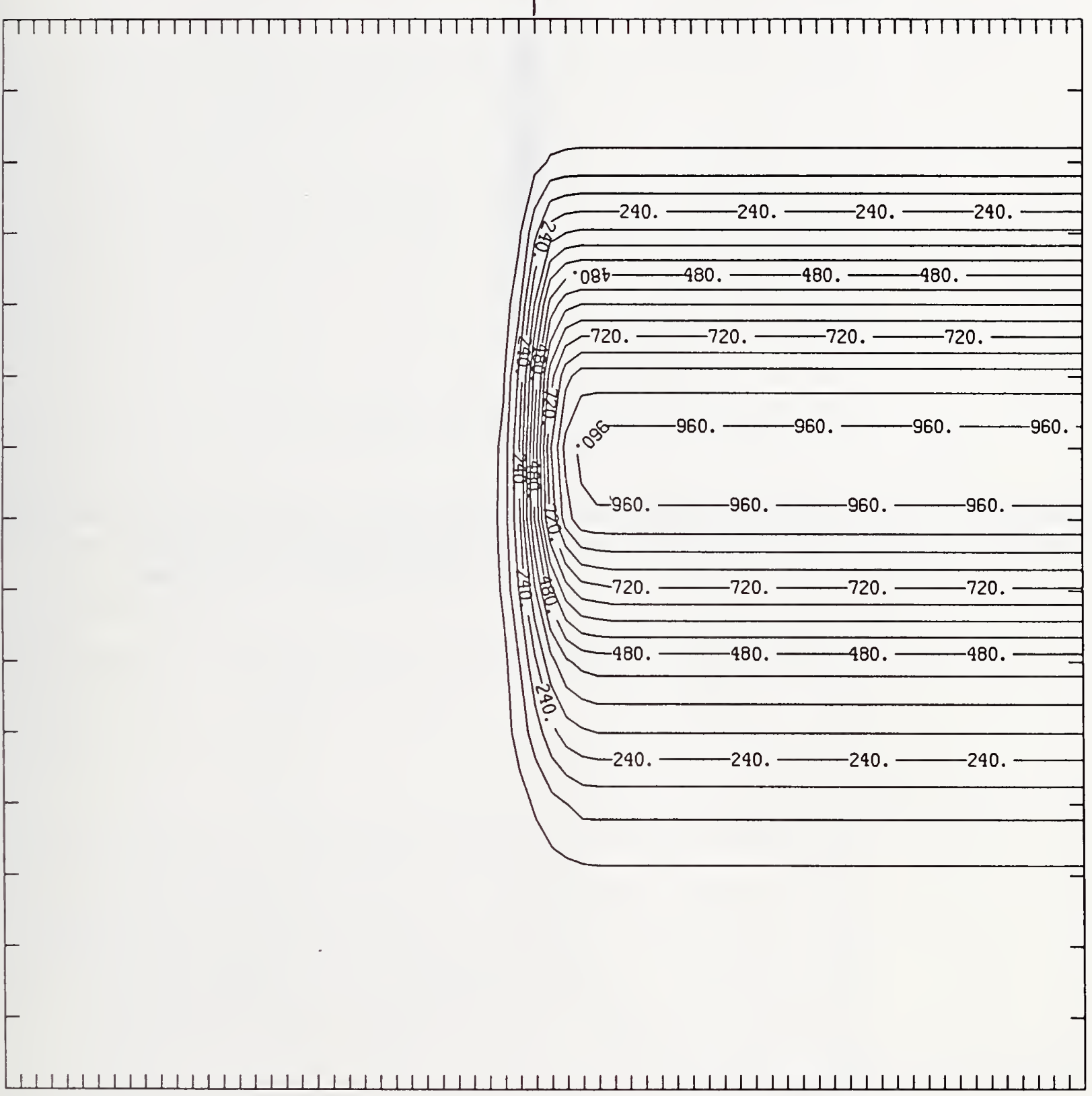

Figure In125-3 Two-dimensional distribution of $125 \mathrm{keV}$ indium implanted past a mask edge. The length increment (distance between tick marks) is $0.01 \mu \mathrm{m}$ and the scale factor is 1000 . 


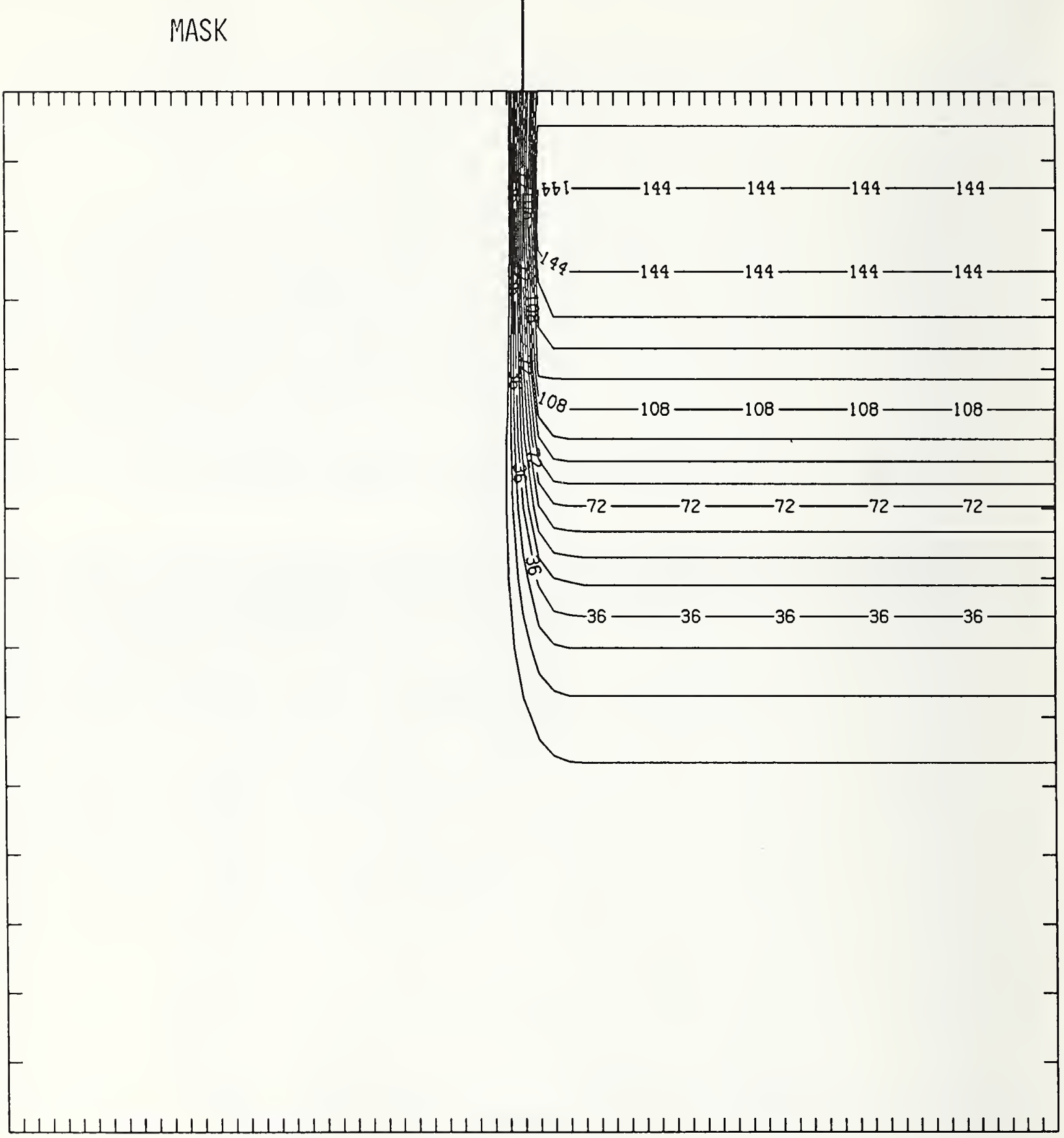

Figure In125-4 Two-dimensional distribution of Frenkel pairs created by $125 \mathrm{keV}$ indium implanted past a mask edge. The length increment (distance between tick marks) is $0.01 \mu \mathrm{m}$ and the scale factor is 0.1 . 


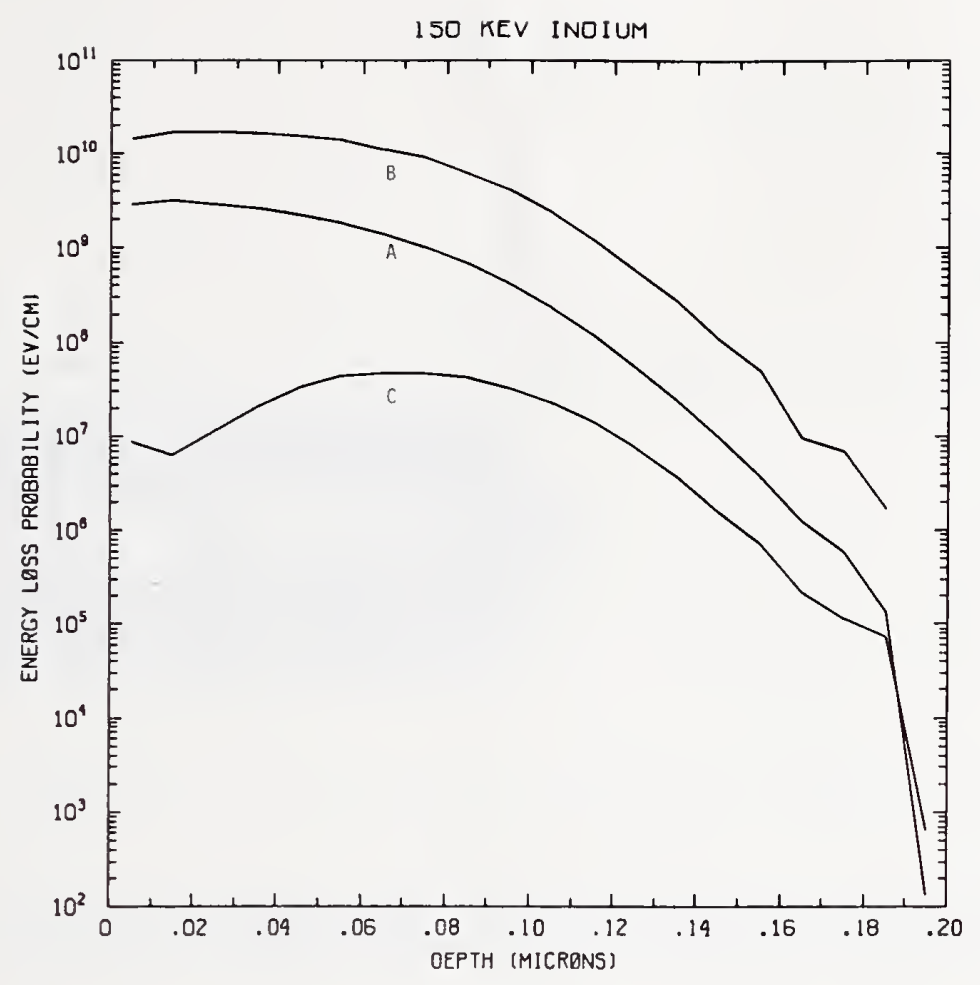

Figure In150-1 One-dimensional probability distributions for energy loss due to electronic excitation (A), displacement damage(B), and phonon excitation (C) for $150 \mathrm{keV}$ indium normally incident on a silicon target.

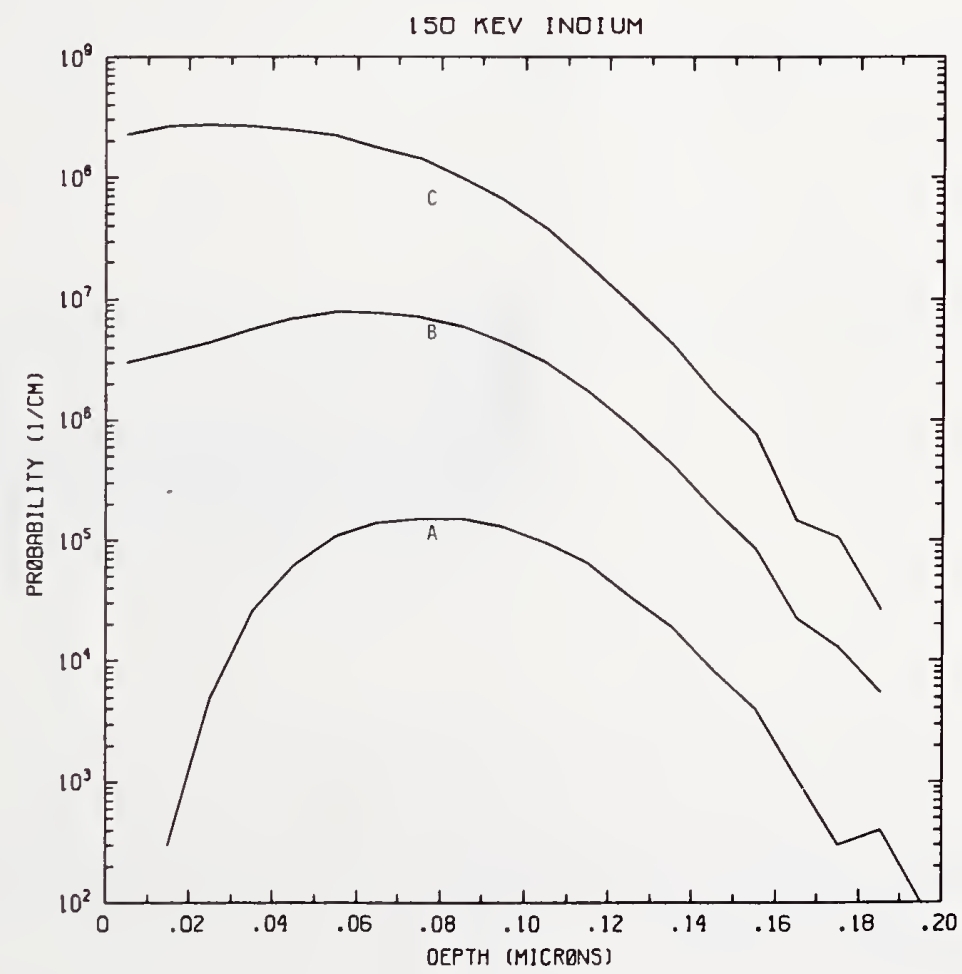

Figure In150-2 One-dimensional probability distributions for implanted indium (A), primary silicon displacements (B), and Frenkel pairs (C) for $150 \mathrm{keV}$ indium normally incident on a silicon target. 


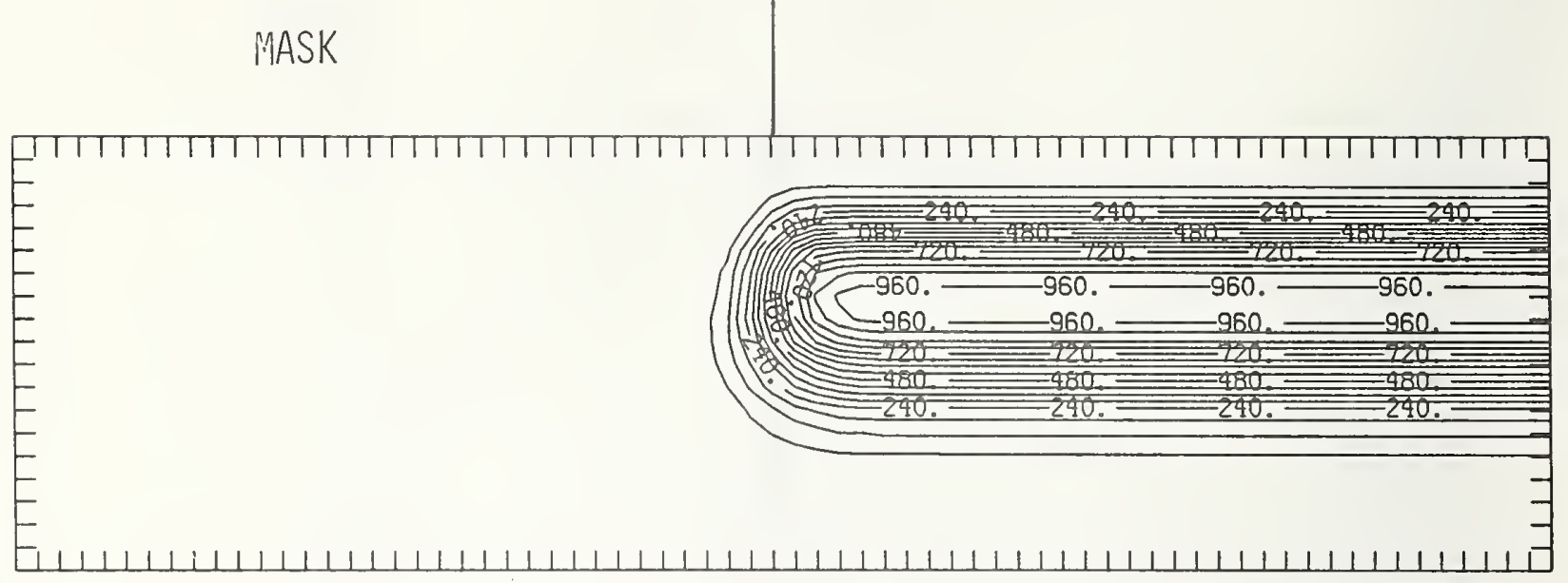

Figure In150-3 Two-dimensional distribution of $150 \mathrm{keV}$ indium implanted past a mask edge. The length increment (distance between tick marks) is $0.01 \mu \mathrm{m}$ and the scale factor is 1000 .

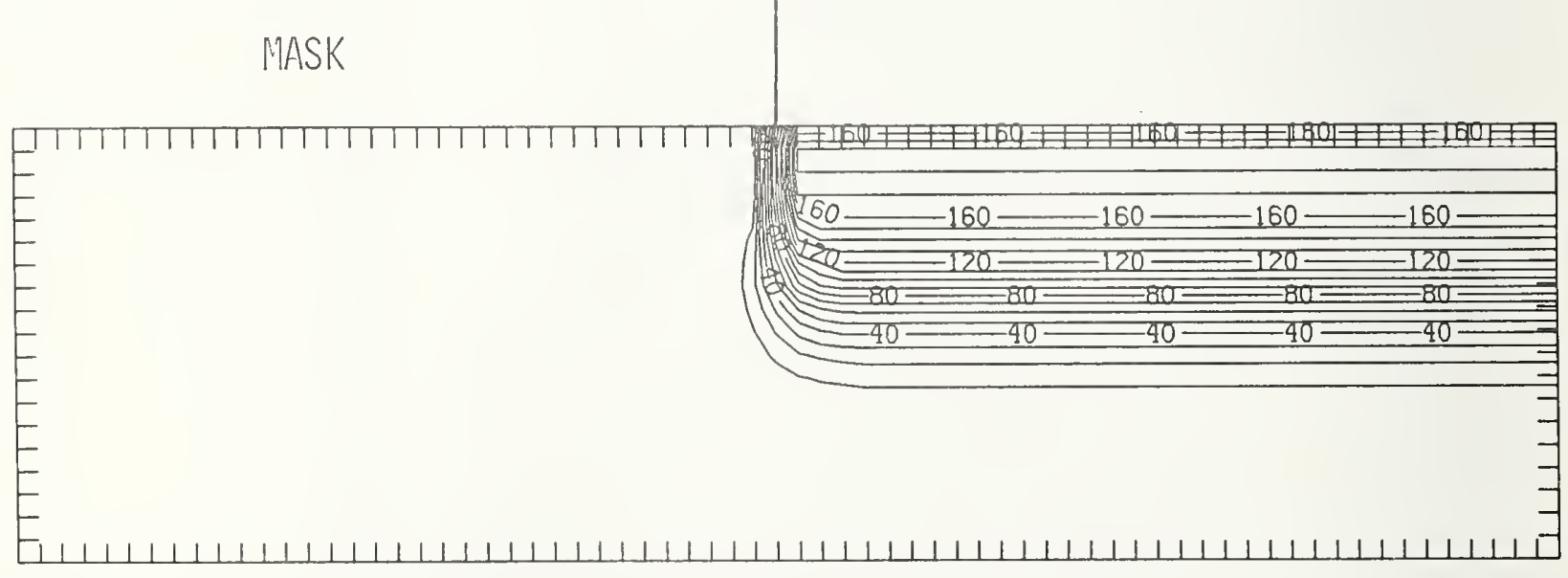

Figure In150-4 Two-dimensional distribution of Frenkel pairs created by $150 \mathrm{keV}$ indium implanted past a mask edge. The length increment (distance between tick marks) is $0.01 \mu \mathrm{m}$ and the scale factor is 0.1 . 


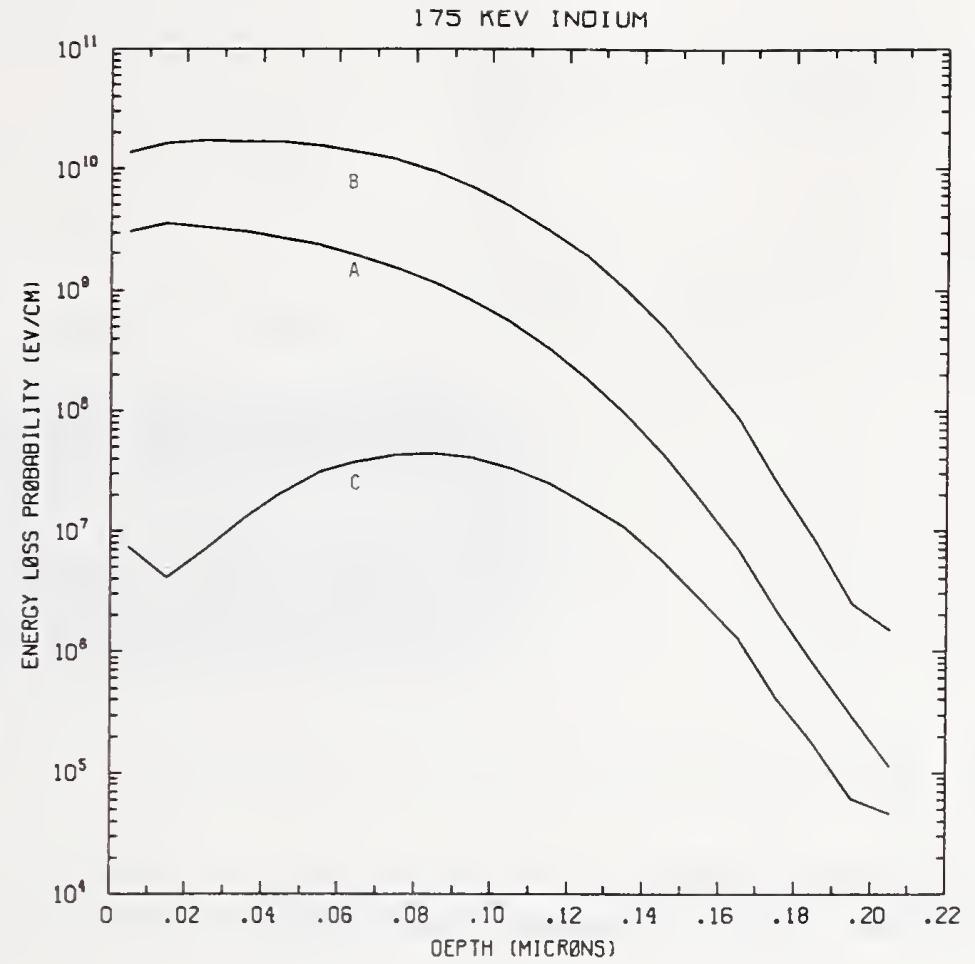

Figure In175-1 One-dimensional probability distributions for energy loss due to electronic excitation (A), displacement damage(B), and phonon excitation (C) for $175 \mathrm{keV}$ indium normally incident on a silicon target.

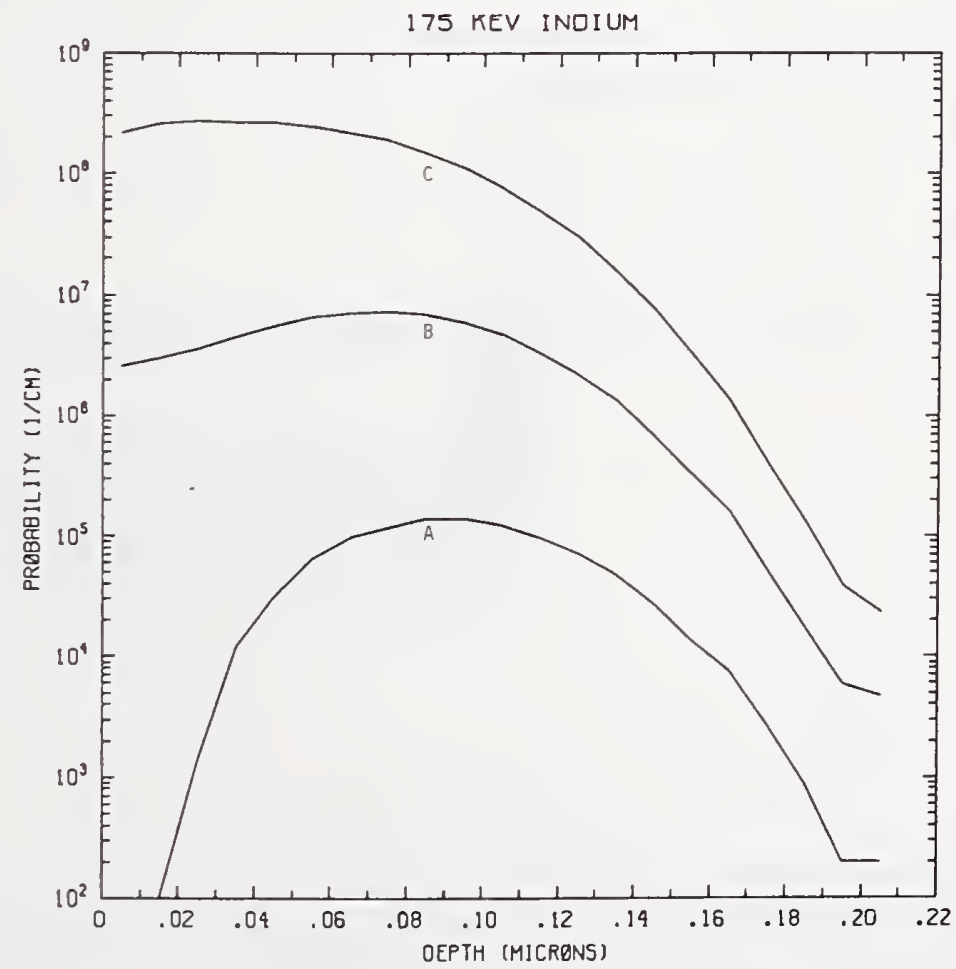

Figure In175-2 One-dimensional probability distributions for implanted indium (A), primary silicon displacements (B), and Frenkel pairs (C) for $175 \mathrm{keV}$ indium normally incident on a silicon target. 


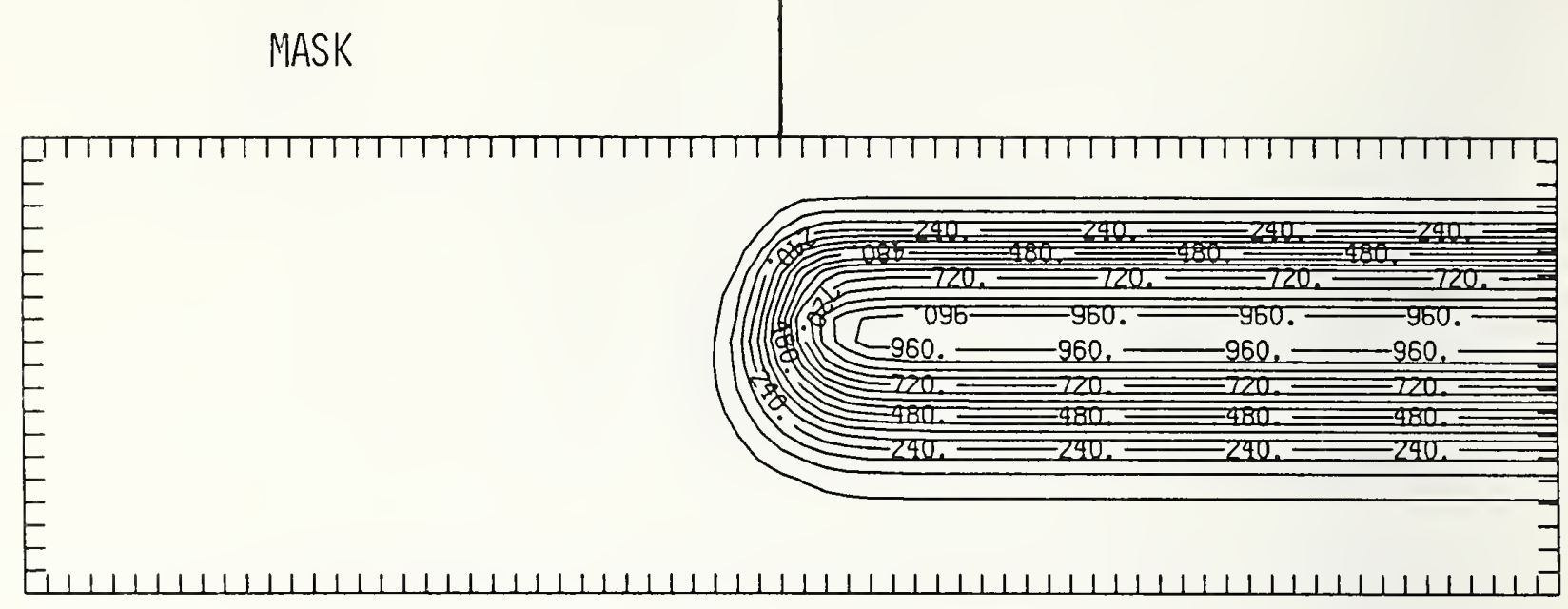

Figure In175-3 Two-dimensional distribution of $175 \mathrm{keV}$ indium implanted past a mask edge. The length increment (distance between tick marks) is $0.01 \mu \mathrm{m}$ and the scale factor is 1000 .

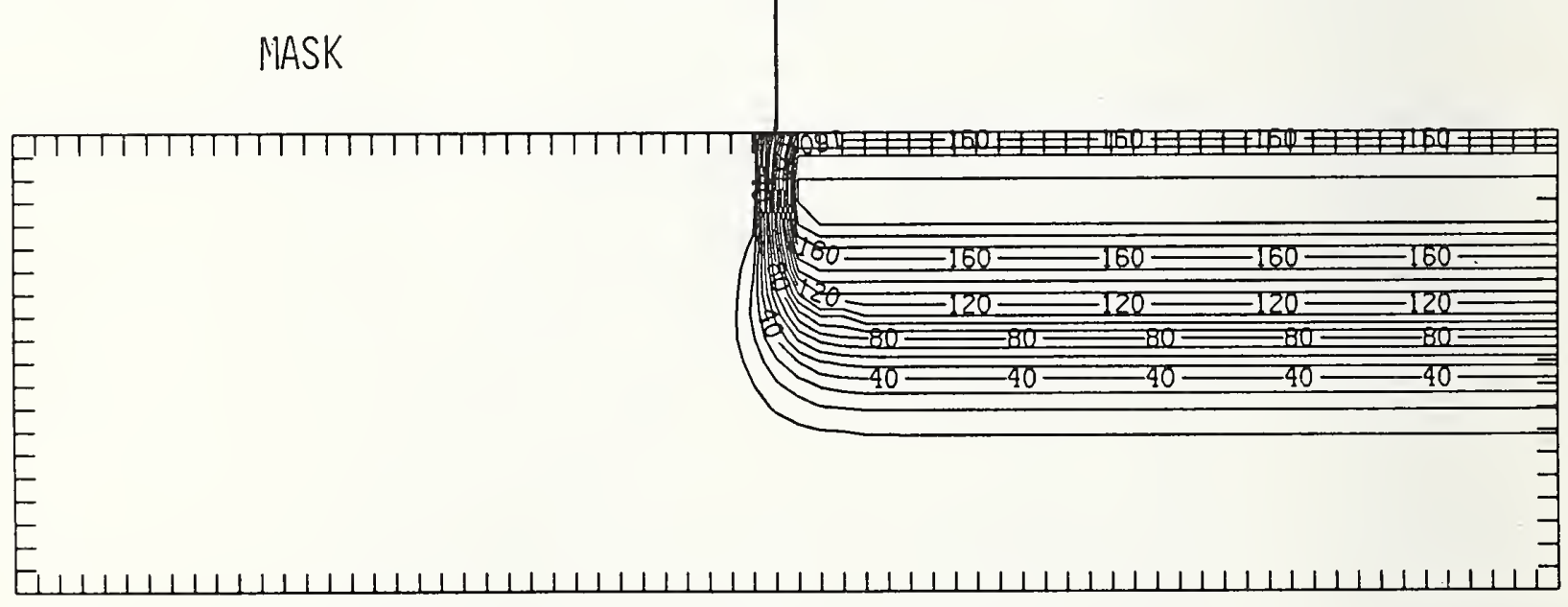

Figure In175-4 Two-dimensional distribution of Frenkel pairs created by $175 \mathrm{keV}$ indium implanted past a mask edge. The length increment (distance between tick marks) is $0.01 \mu \mathrm{m}$ and the scale factor is 0.1 . 


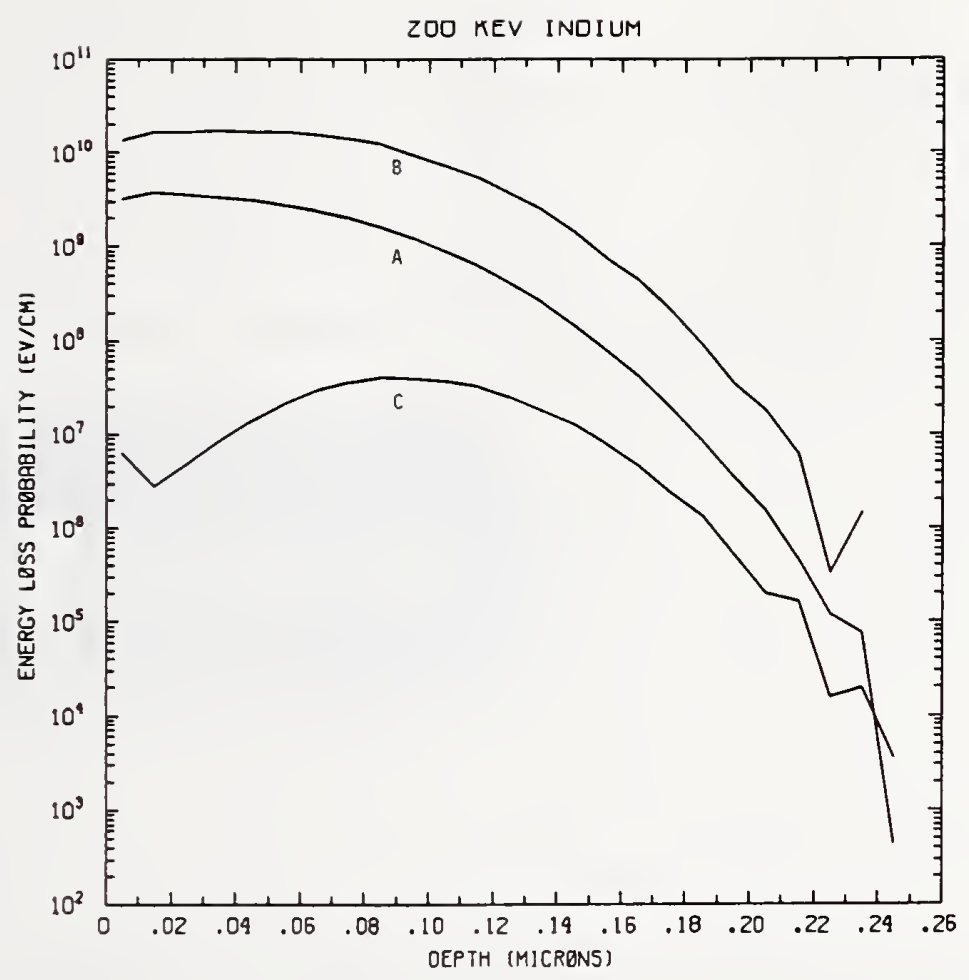

Figure In200-1 One-dimensional probability distributions for energy loss due to electronic excitation (A), displacement damage(B), and phonon excitation (C) for $200 \mathrm{keV}$ indium normally incident on a silicon target.

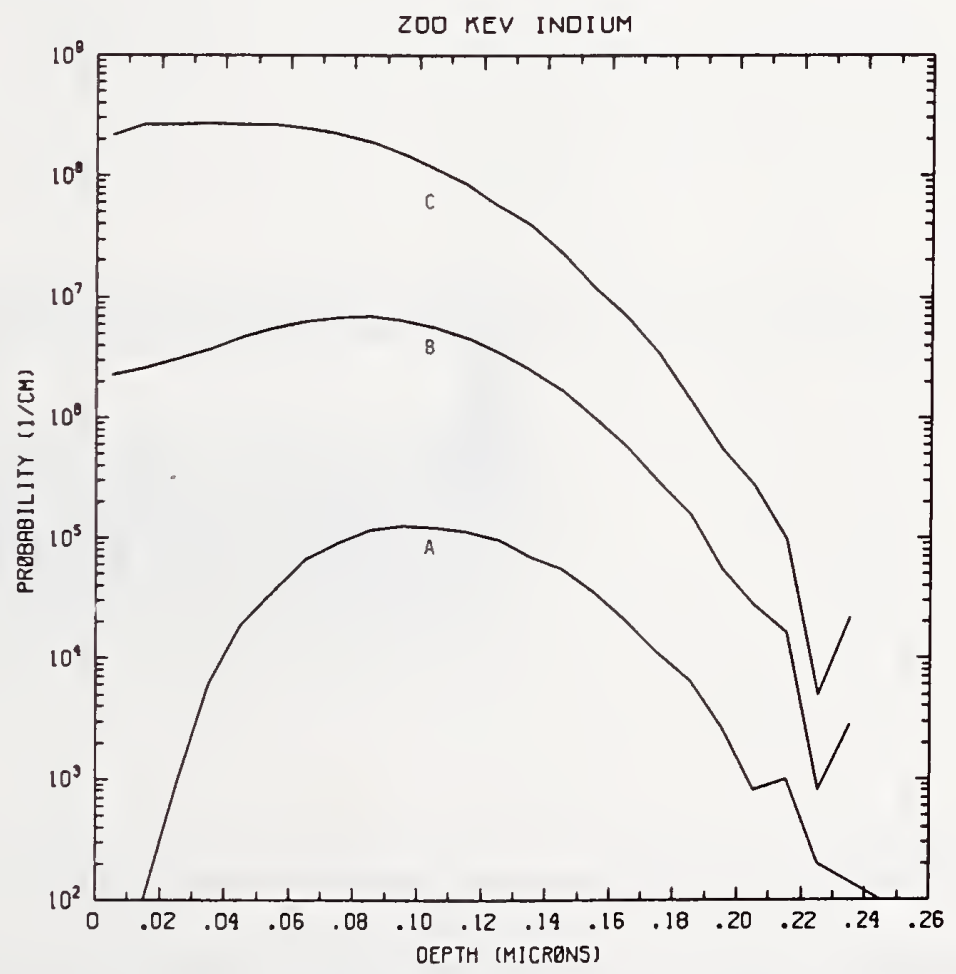

Figure In200-2 One-dimensional probability distributions for implanted indium (A), primary silicon displacements (B), and Frenkel pairs (C) for $200 \mathrm{keV}$ indium normally incident on a silicon target. 


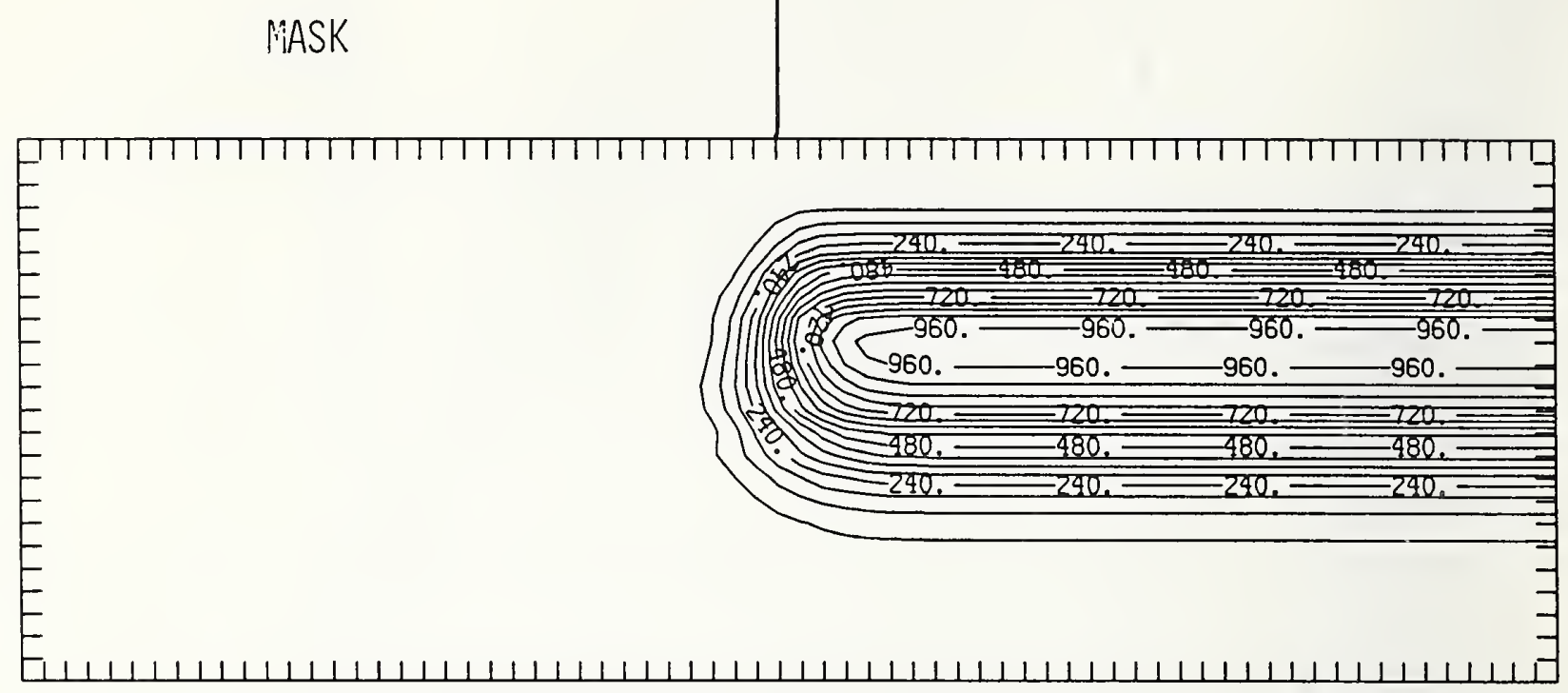

Figure In200-3 Two-dimensional distribution of $200 \mathrm{keV}$ indium implanted past a mask edge. The length increment (distance between tick marks) is $0.01 \mu \mathrm{m}$ and the scale factor is 1000.

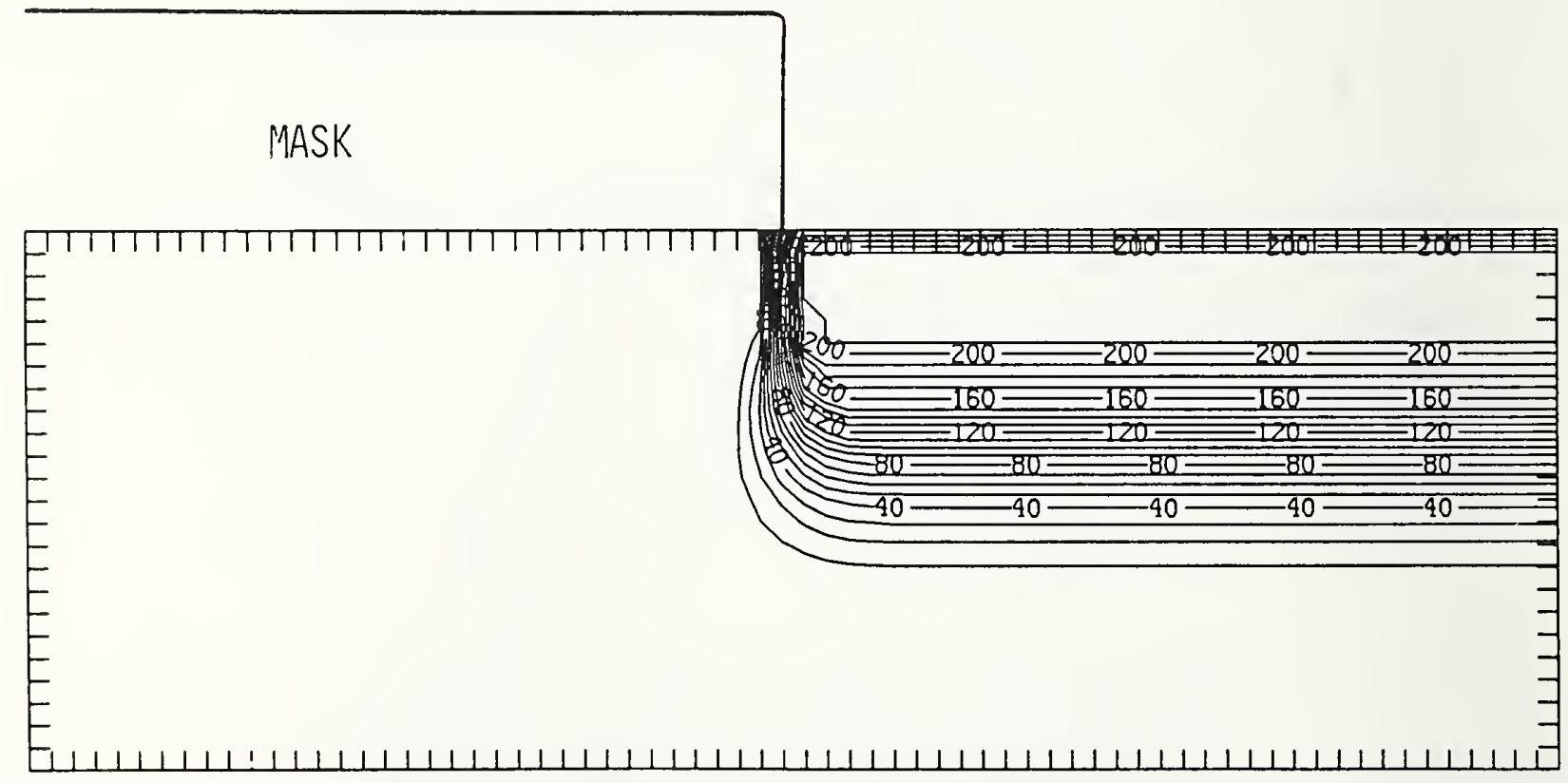

Figure In200-4 Two-dimensional distribution of Frenkel pairs created by $200 \mathrm{keV}$ indium implanted past a mask edge. The length increment (distance between tick marks) is $0.01 \mu \mathrm{m}$ and the scale factor is 0.1 . 


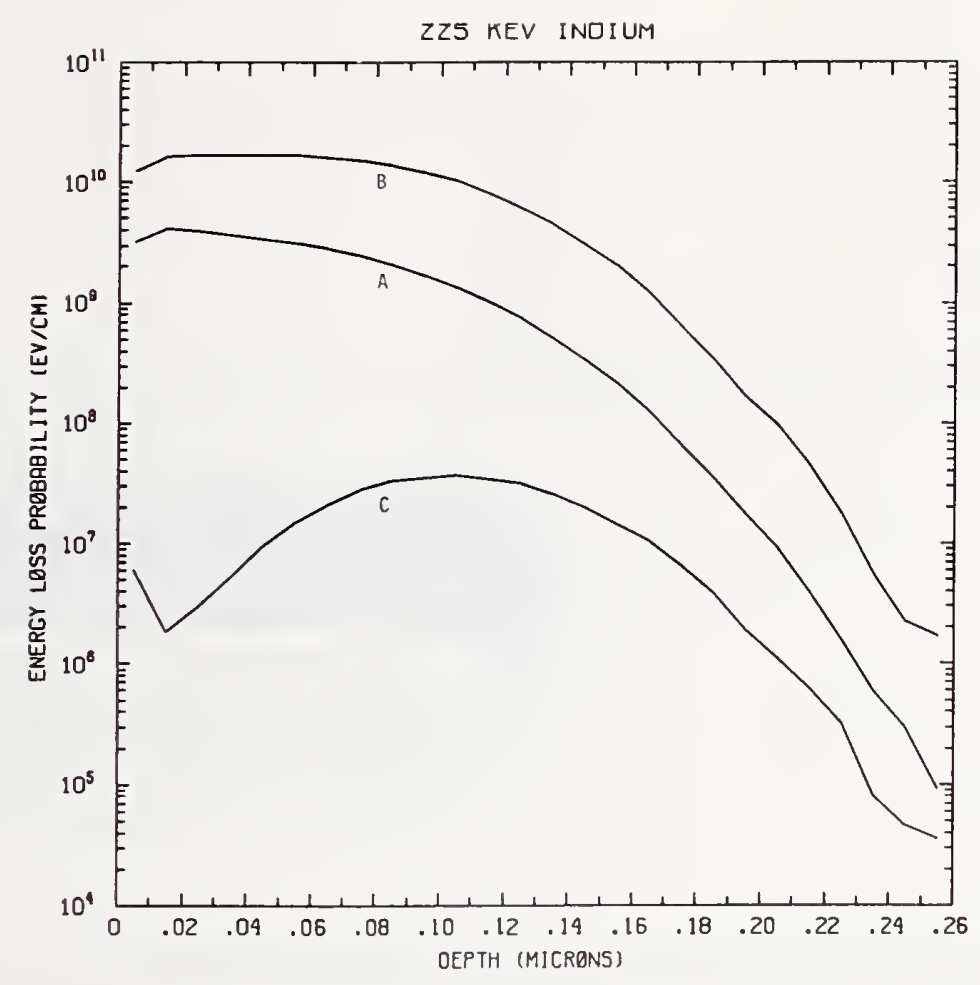

Figure In225-1 One-dimensional probability distributions for energy loss due to electronic excitation (A), displacement damage(B), and phonon excitation (C) for $225 \mathrm{keV}$ indium normally incident on a silicon target.

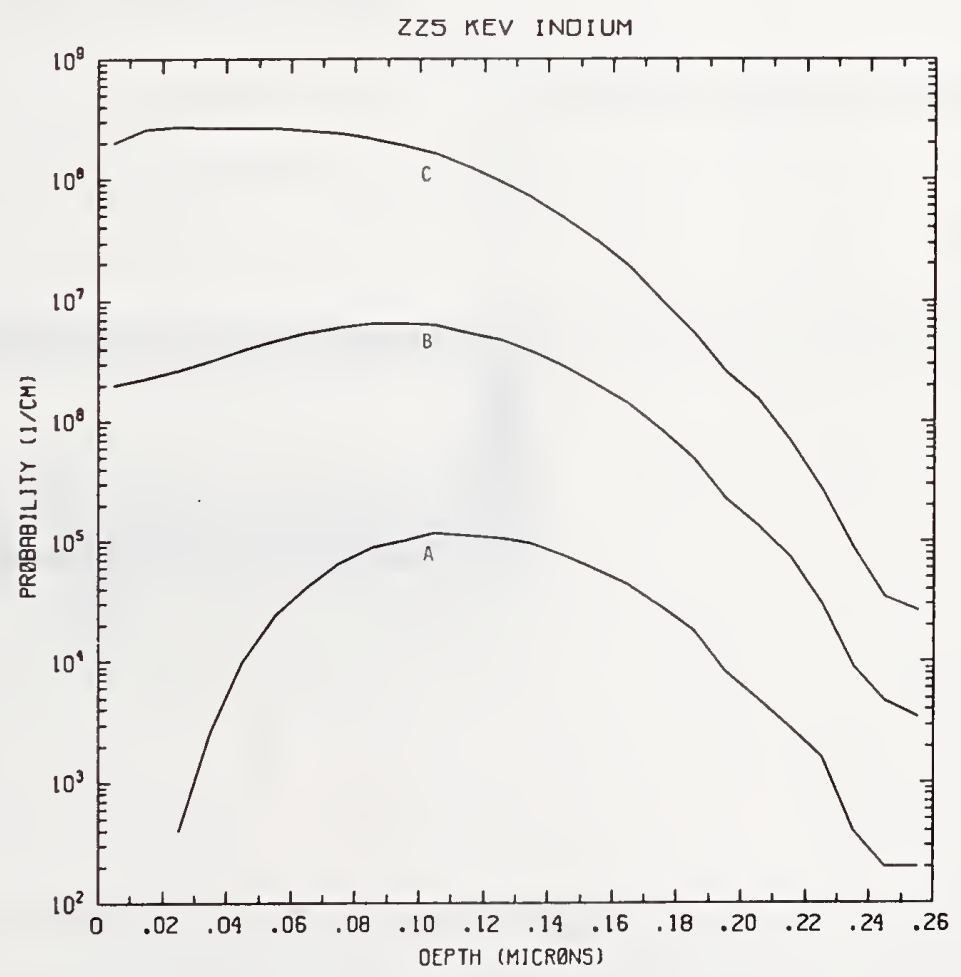

Figure In225-2 One-dimensional probability distributions for implanted indium (A), primary silicon displacements (B), and Frenkel pairs (C) for $225 \mathrm{keV}$ indium normally incident on a silicon target. 


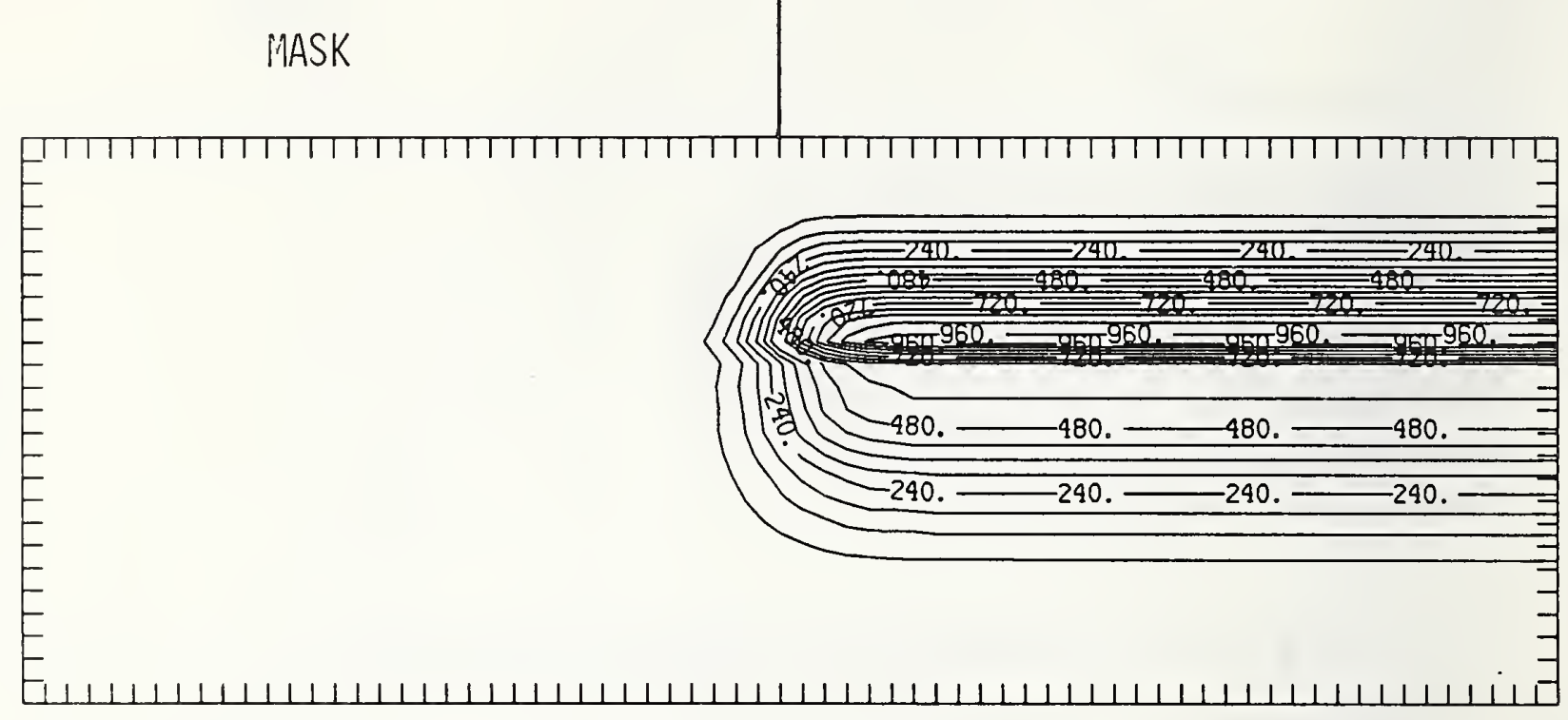

Figure In225-3 Two-dimensional distribution of $225 \mathrm{keV}$ indium implanted past a mask edge. The length increment (distance between tick marks) is $0.01 \mu \mathrm{m}$ and the scale factor is 1000.

\section{MASK}

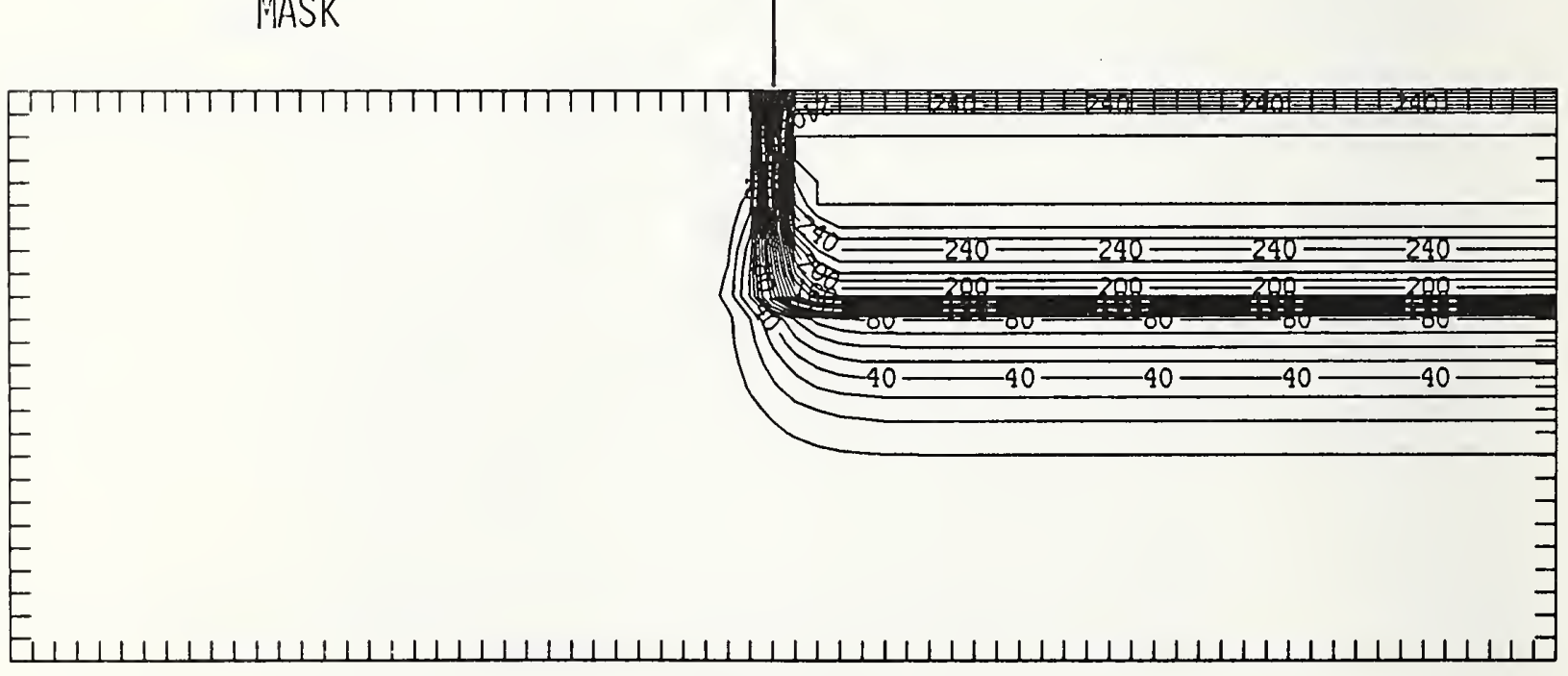

Figure In225-4 Two-dimensional distribution of Frenkel pairs created by $225 \mathrm{keV}$ indium implanted past a mask edge. The length increment (distance between tick marks) is $0.01 \mu \mathrm{m}$ and the scale factor is 0.1 . 


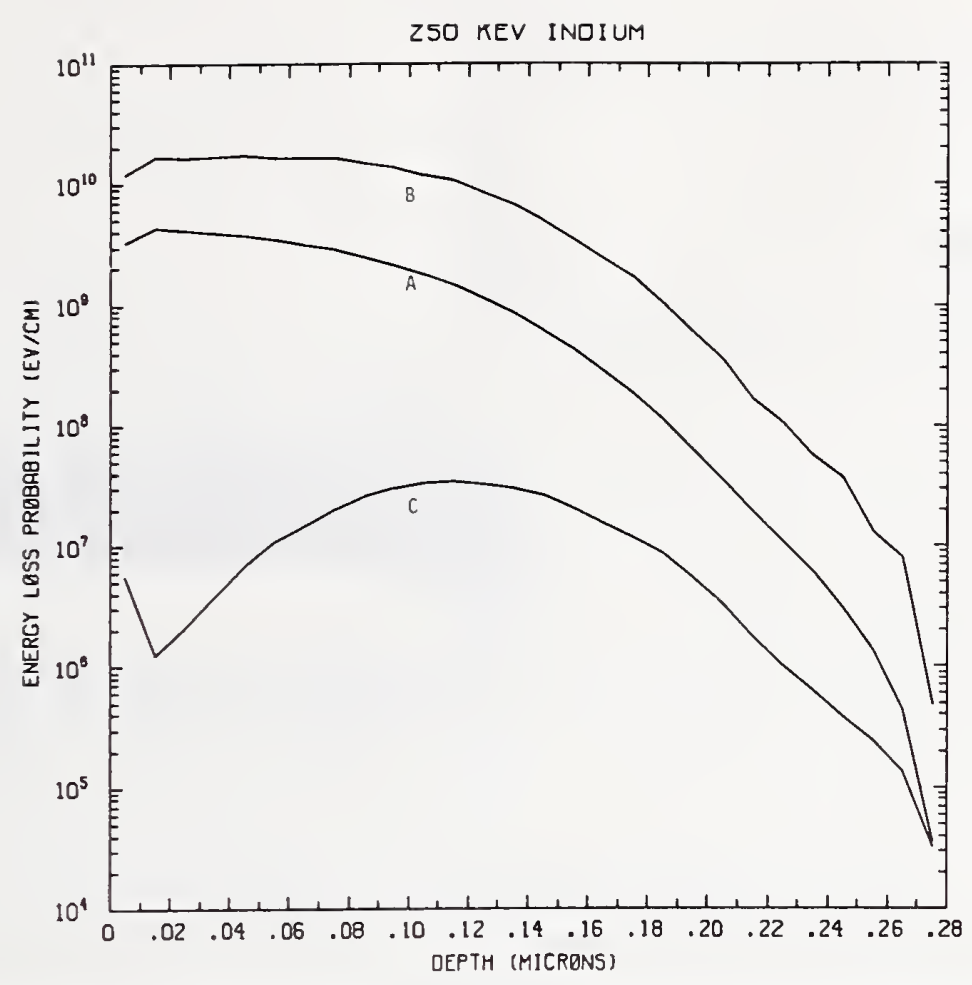

Figure In250-1 One-dimensional probability distributions for energy loss due to electronic excitation (A), displacement damage(B), and phonon excitation (C) for $250 \mathrm{keV}$ indium normally incident on a silicon target.

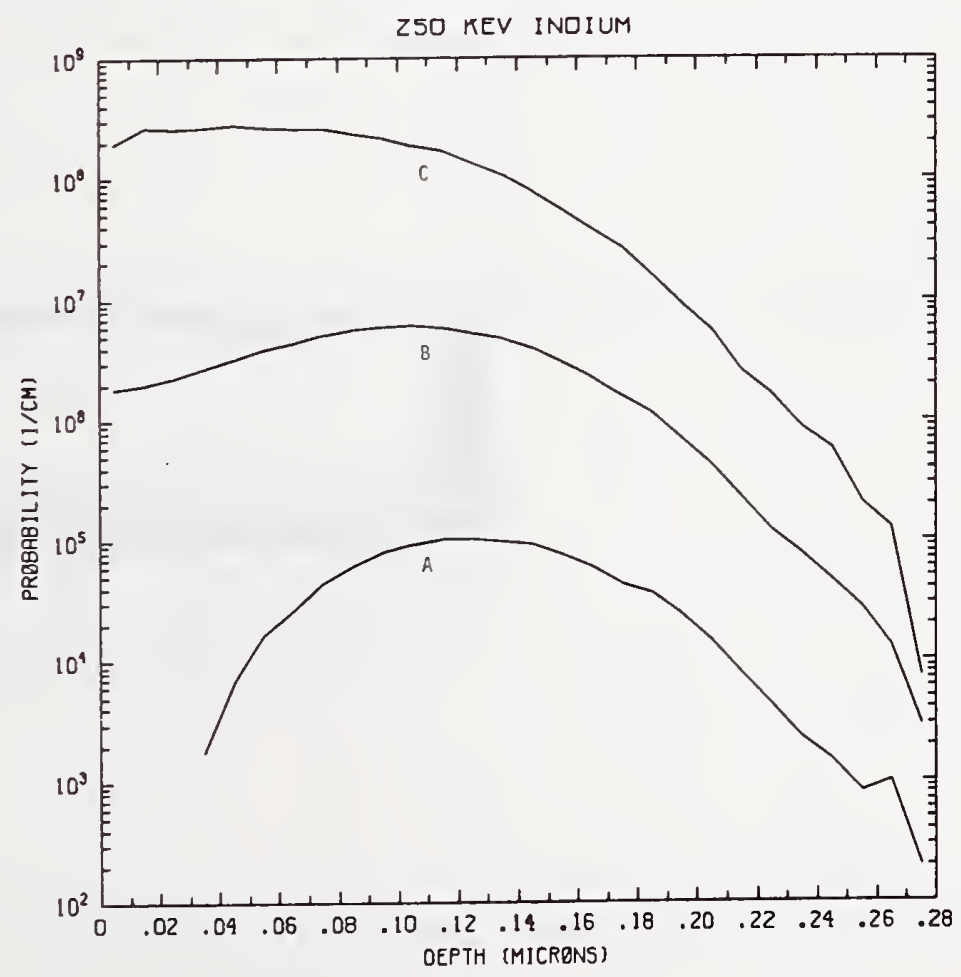

Figure In250-2 One-dimensional probability distributions for implanted indium (A), primary silicon displacements (B), and Frenkel pairs (C) for $250 \mathrm{keV}$ indium normally incident on a silicon target. 


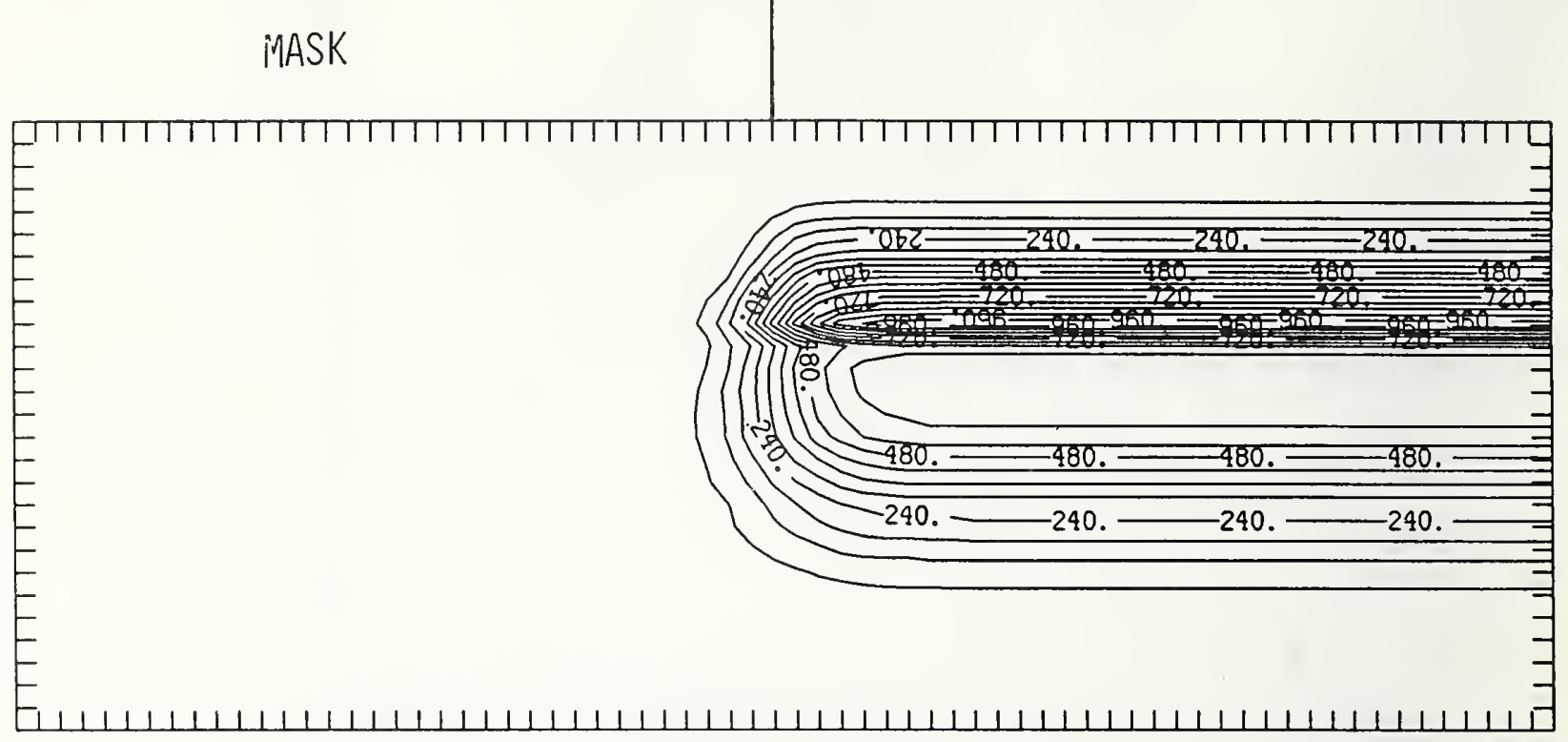

Figure In250-3 Two-dimensional distribution of $250 \mathrm{keV}$ indium implanted past a mask edge. The length increment (distance between tick marks) is $0.01 \mu \mathrm{m}$ and the scale factor is 1000 .

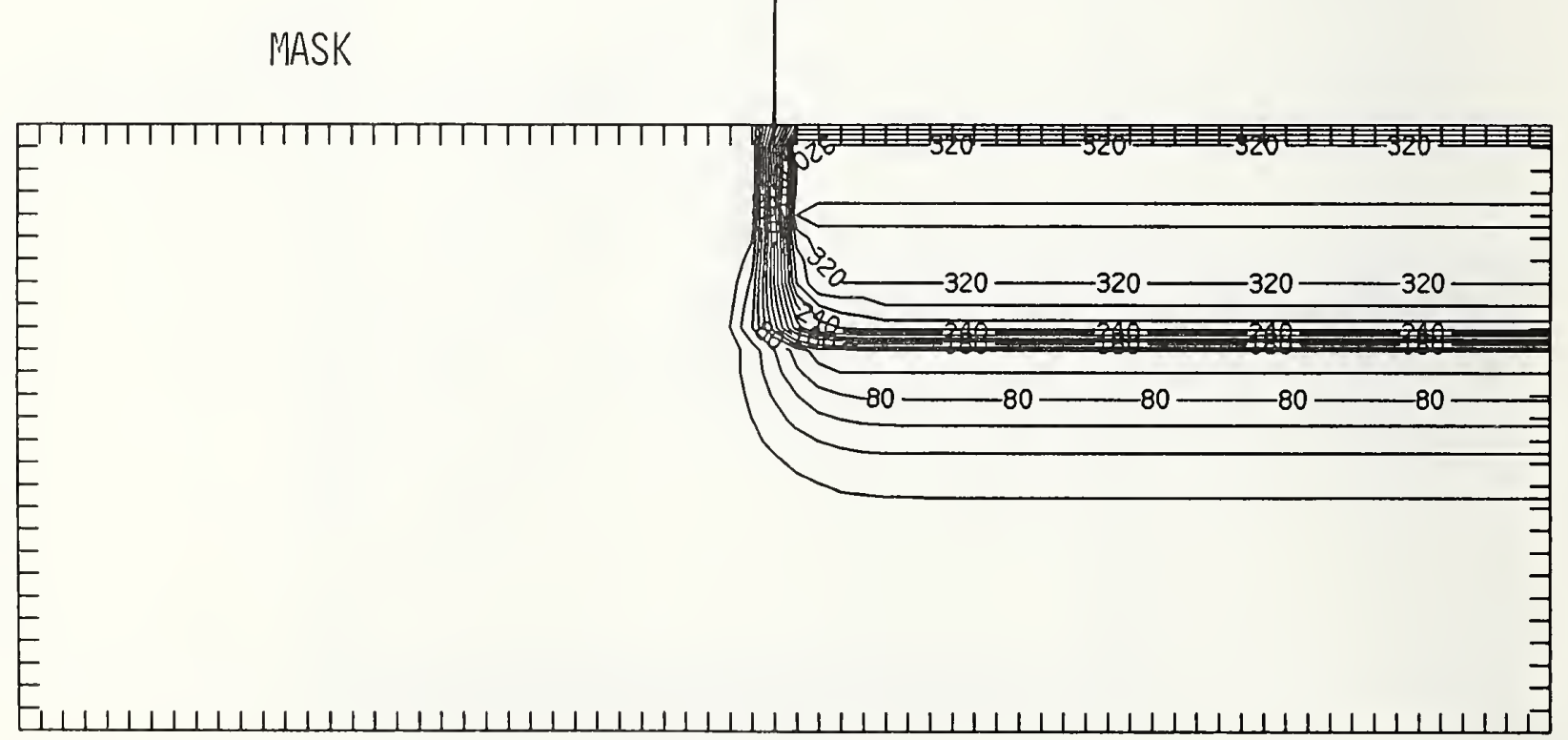

Figure In250-4 Two-dimensional distribution of Frenkel pairs created by $250 \mathrm{keV}$ indium implanted past a mask edge. The length increment (distance between tick marks) is $0.01 \mu \mathrm{m}$ and the scale factor is 0.1 . 


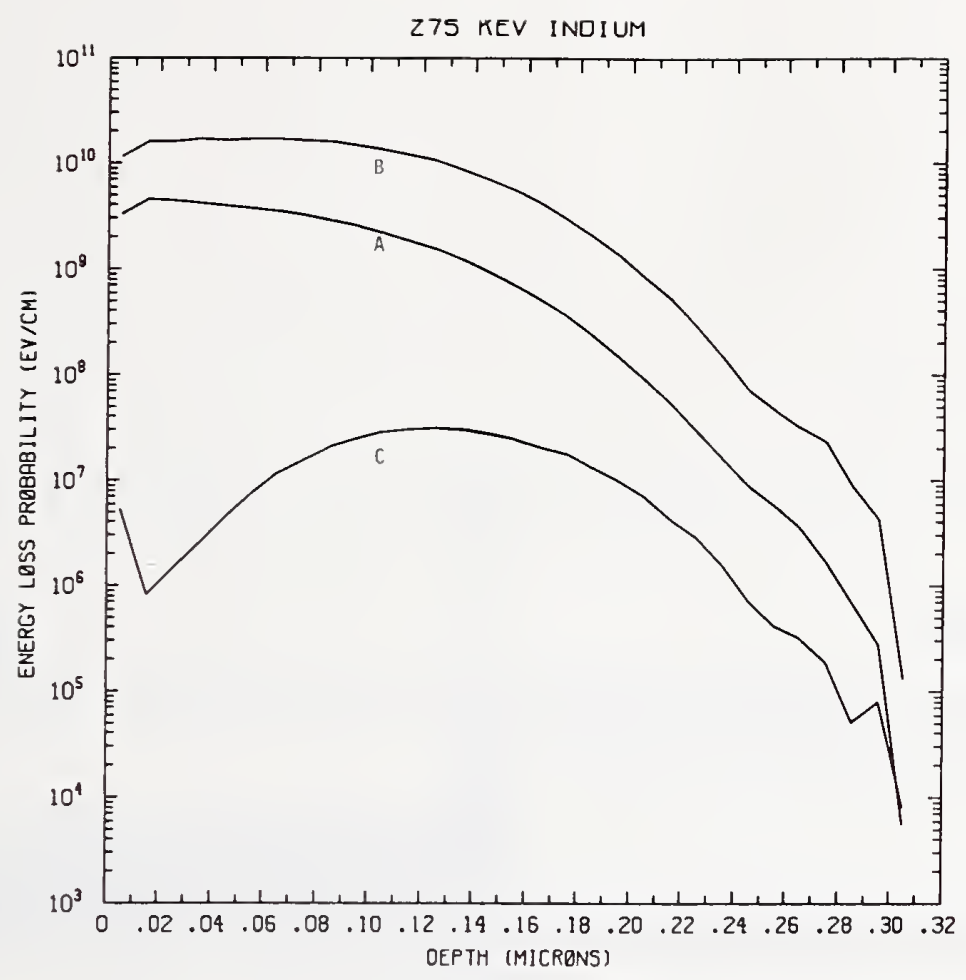

Figure In275-1 One-dimensional probability distributions for energy loss due to electronic excitation (A), displacement damage(B), and phonon excitation (C) for $275 \mathrm{keV}$ indium normally incident on a silicon target.

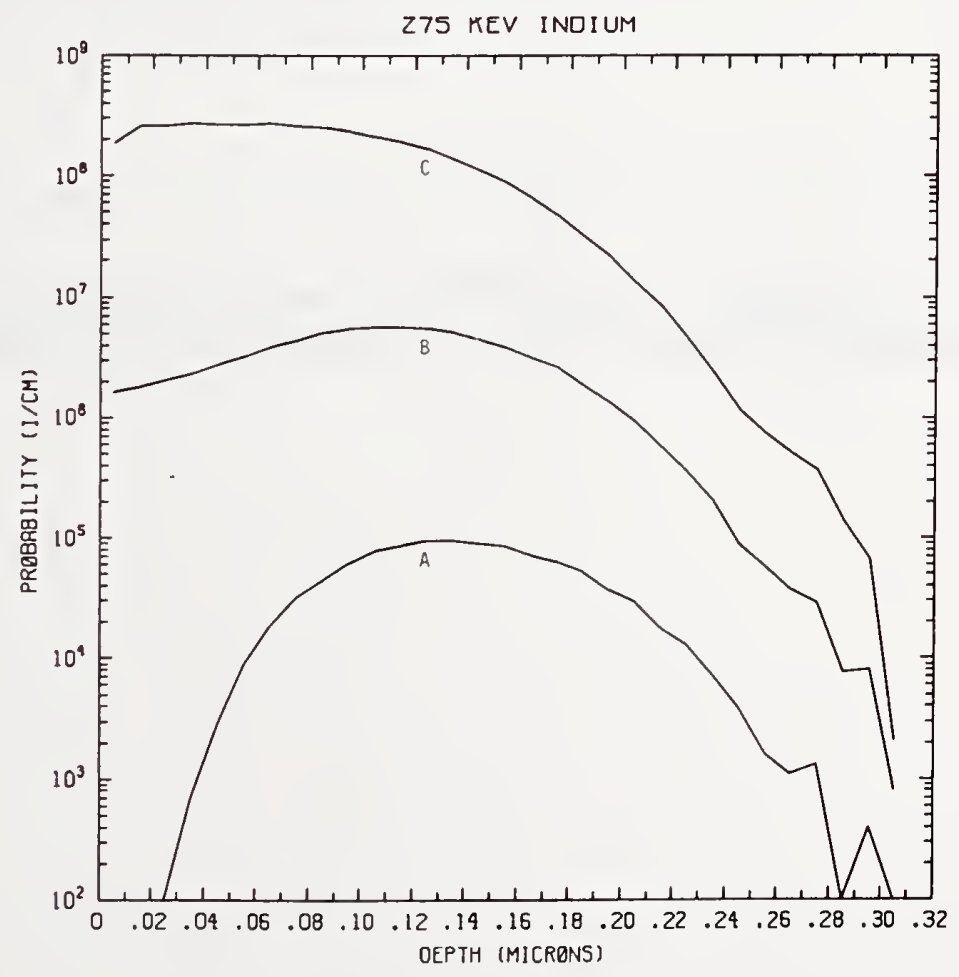

Figure In275-2 One-dimensional probability distributions for implanted indium (A), primary silicon displacements (B), and Frenkel pairs (C) for $275 \mathrm{keV}$ indium normally incident on a silicon target. 


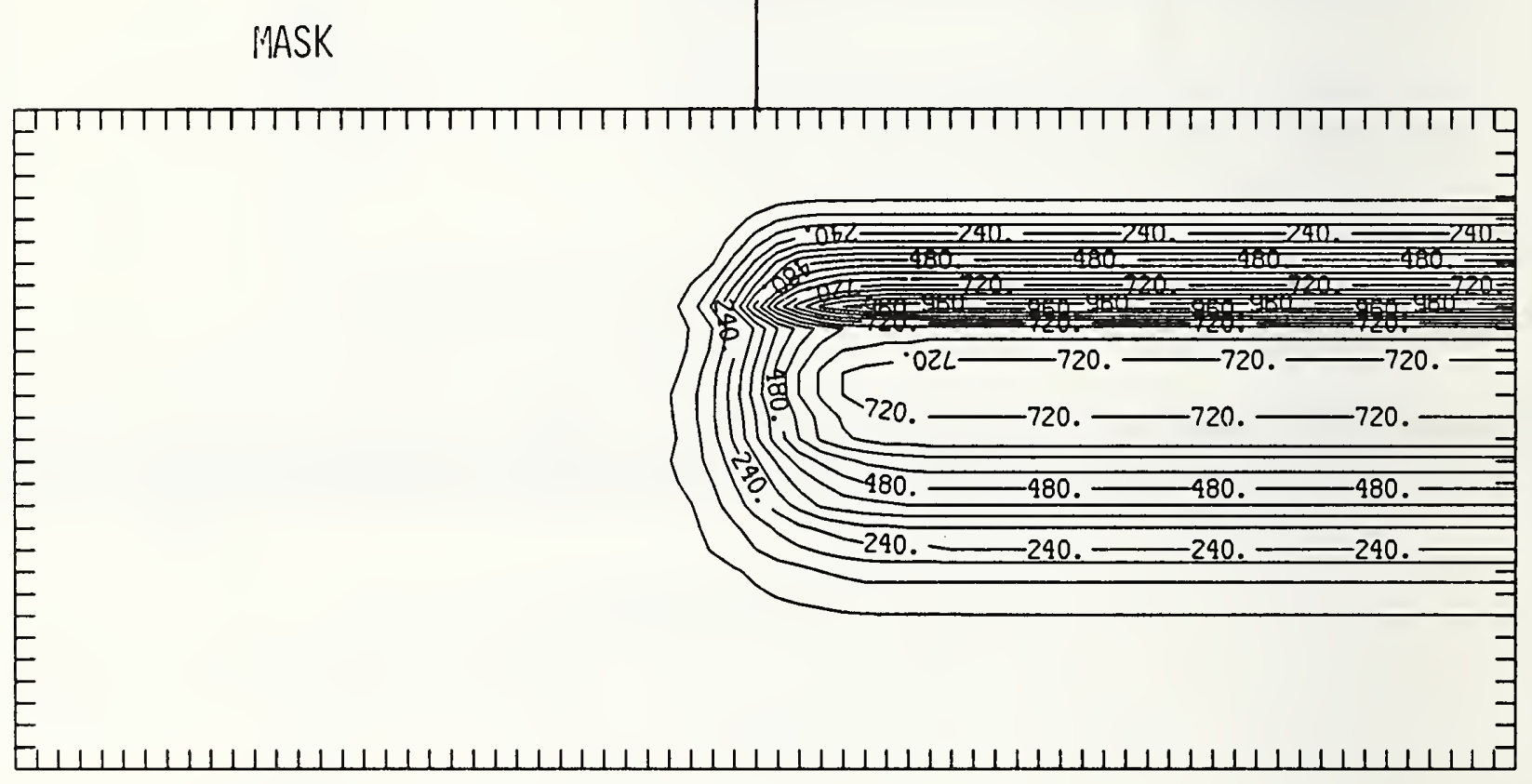

Figure In275-3 Two-dimensional distribution of $275 \mathrm{keV}$ indium implanted past a mask edge. The length increment (distance between tick marks) is $0.01 \mu \mathrm{m}$ and the scale factor is 1000 . 


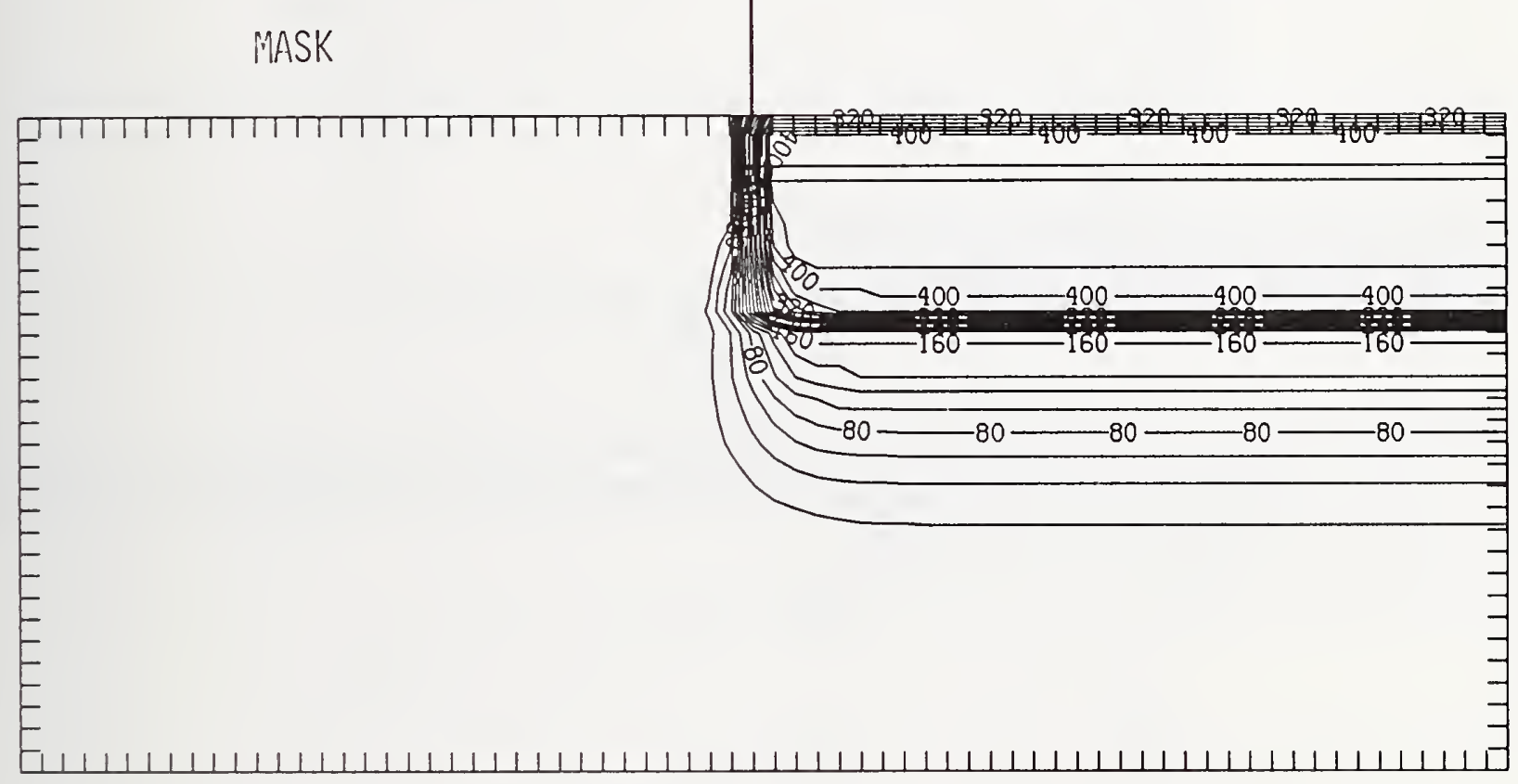

Figure In275-4 Two-dimensional distribution of Frenkel pairs created by $275 \mathrm{keV}$ indium implanted past a mask edge. The length increment (distance between tick marks) is $0.01 \mu \mathrm{m}$ and the scale factor is 0.1 . 


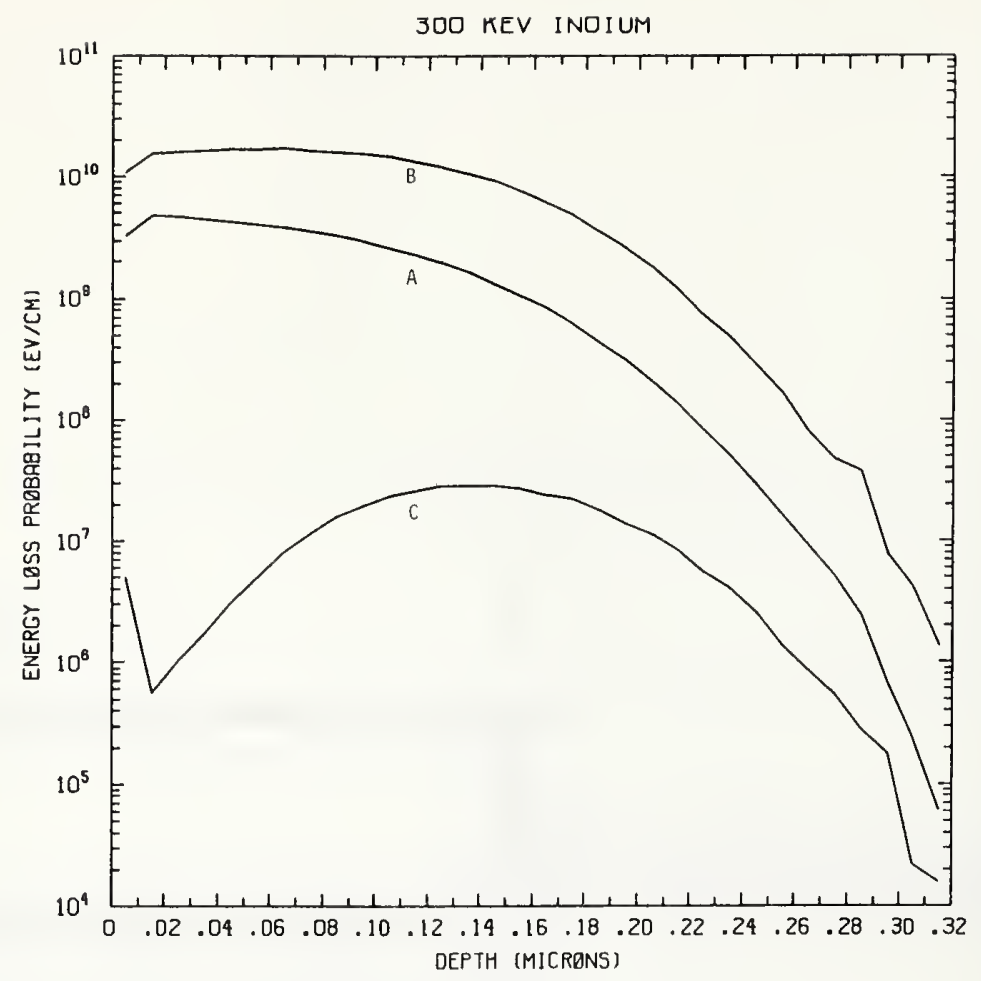

Figure In300-1 One-dimensional probability distributions for energy loss due to electronic excitation (A), displacement damage(B), and phonon excitation (C) for $300 \mathrm{keV}$ indium normall incident on a silicon target.

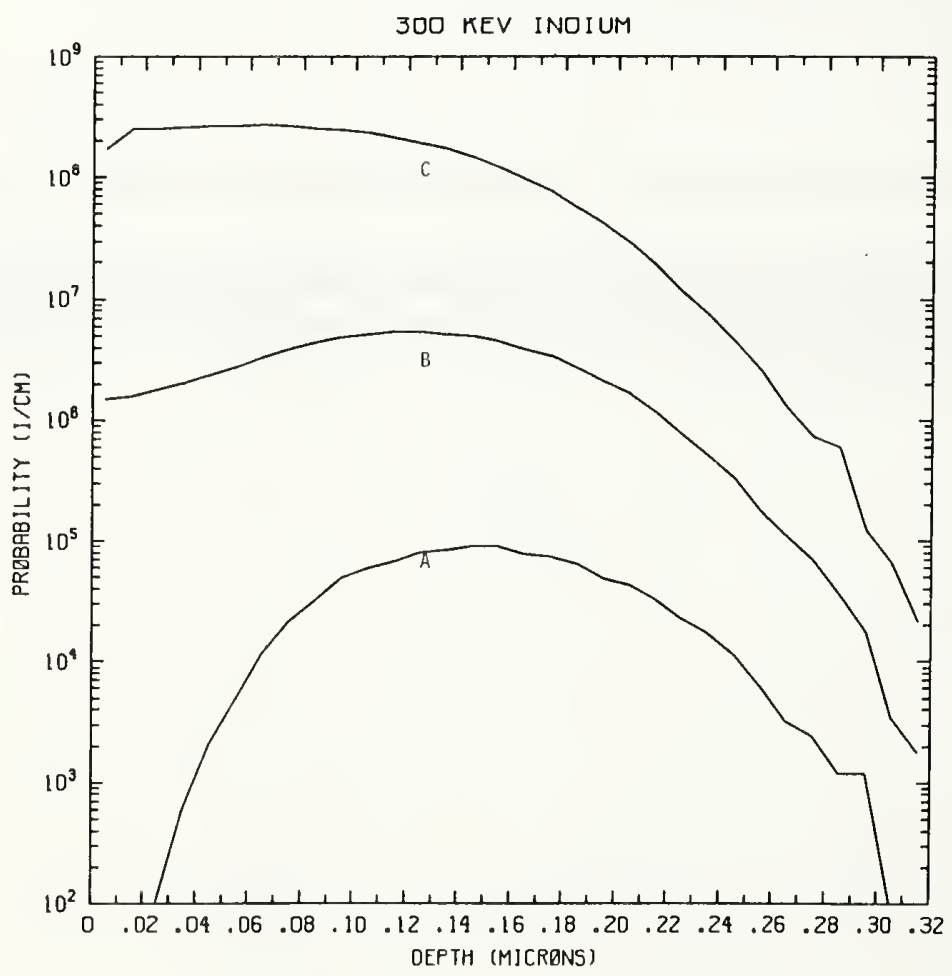

Figure In300-2 One-dimensional probability distributions for implanted indium (A), primary silicon displacements (B), and Frenkel pairs (C) for $300 \mathrm{keV}$ indium normally incident on a silicon target. 


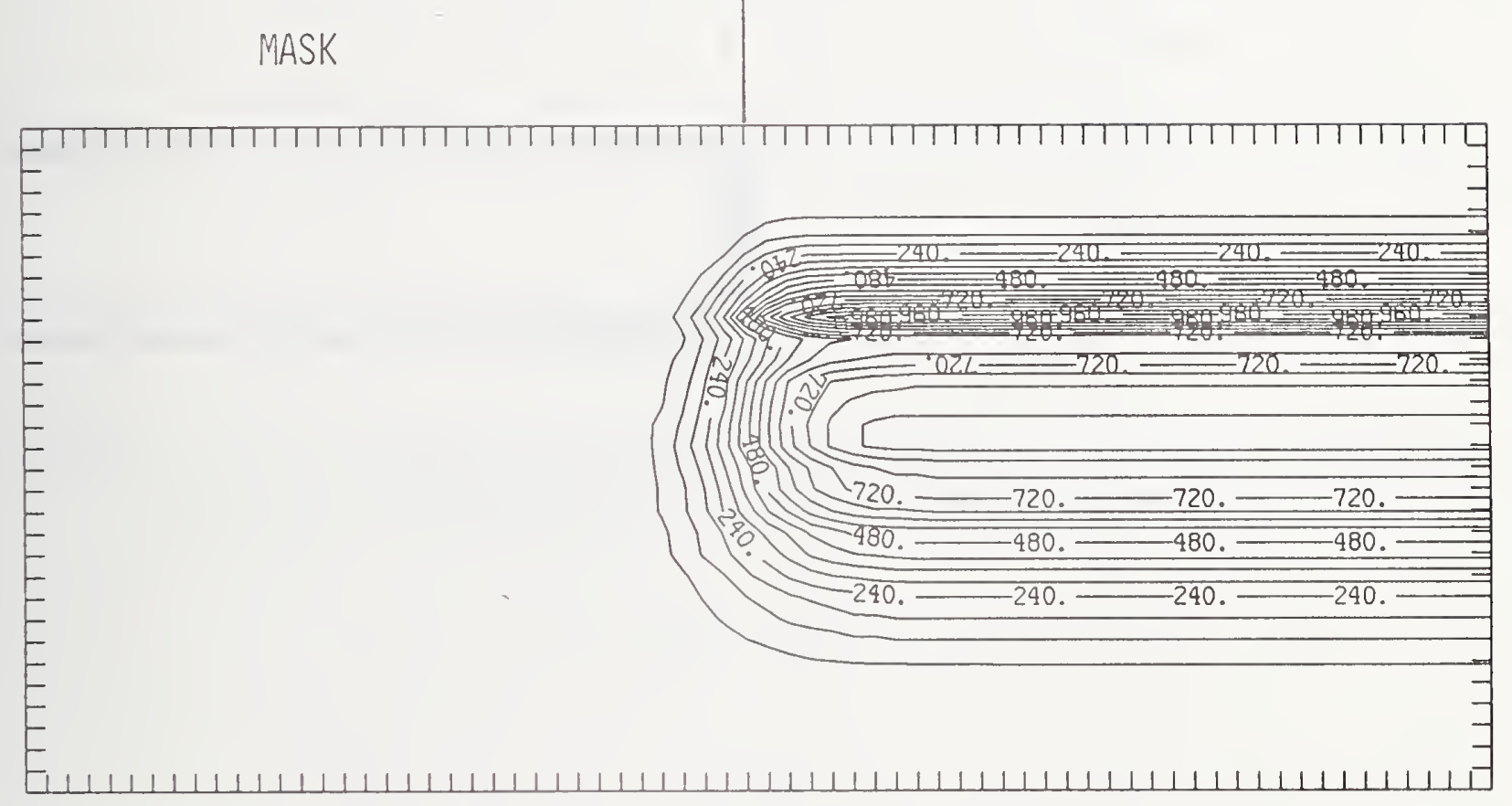

Figure In300-3 Two-dimensional distribution of $300 \mathrm{keV}$ indium implanted past a mask edge. The length increment (distance between tick marks) is $0.01 \mu \mathrm{m}$ and the scale factor is 1000 . 


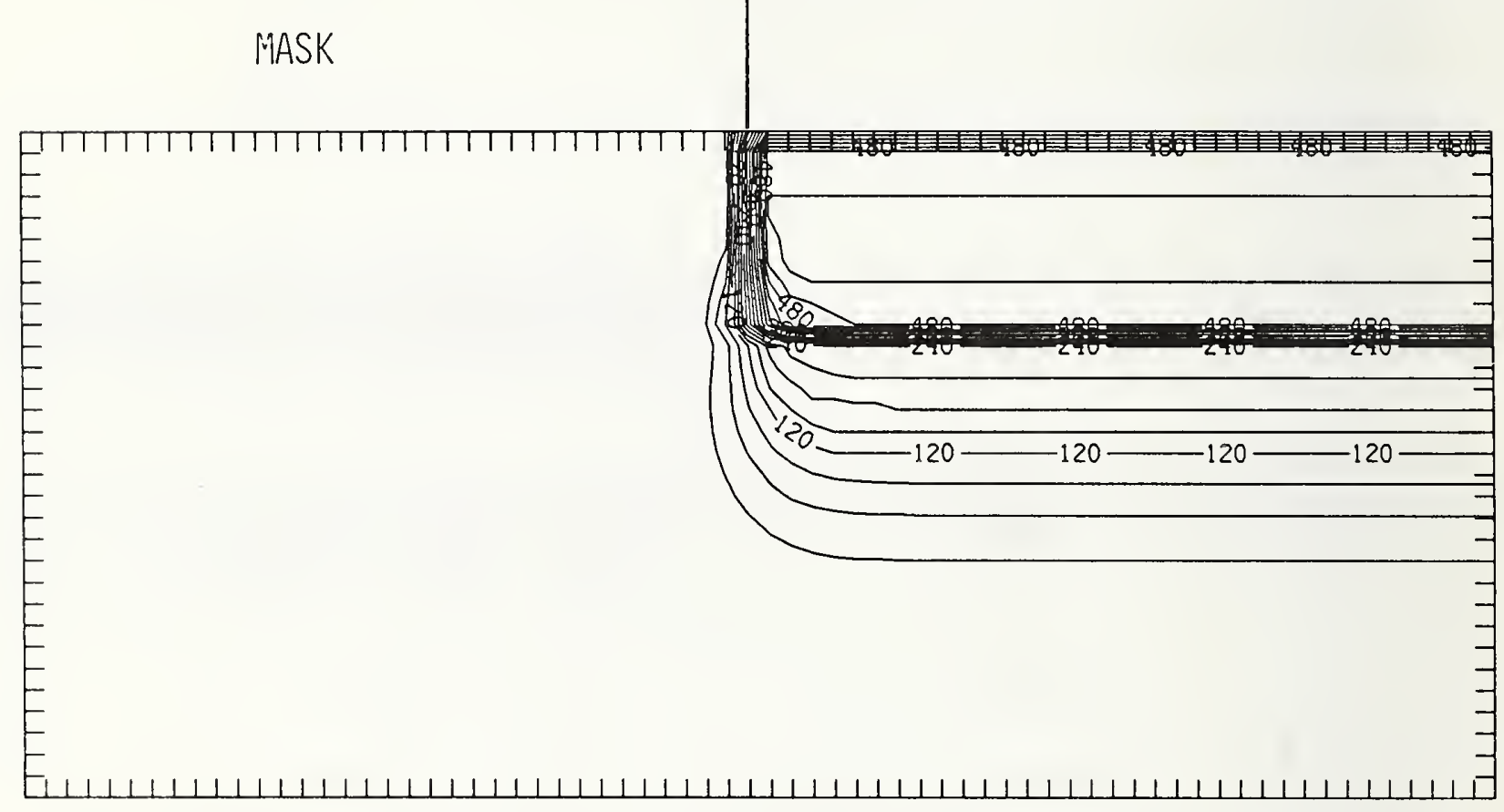

Figure In300-4 Two-dimensional distribution of Frenkel pairs created by $300 \mathrm{keV}$ indium implanted past a mask edge. The length increment (distance between tick marks) is $0.01 \mu \mathrm{m}$ and the scale factor is 0.1 . 


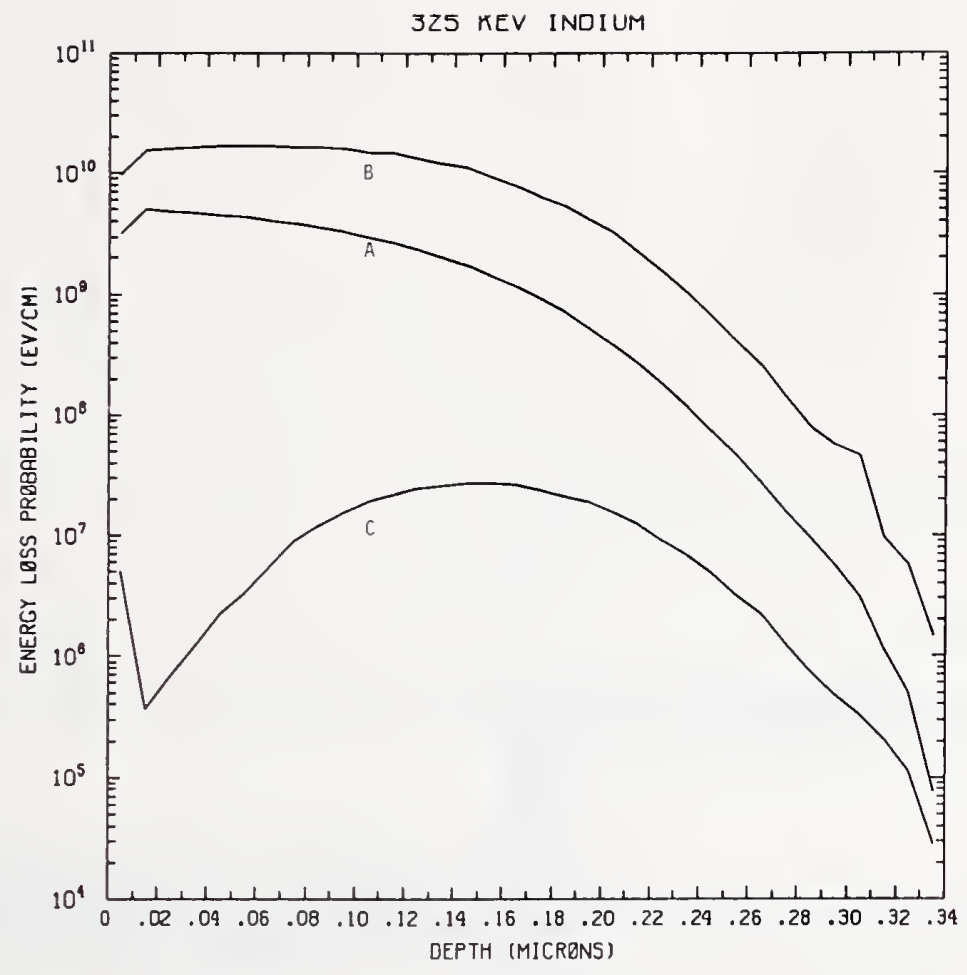

Figure In325-1 One-dimensional probability distributions for energy loss due to electronic excitation (A), displacement damage(B), and phonon excitation (C) for $325 \mathrm{keV}$ indium normally incident on a silicon target.

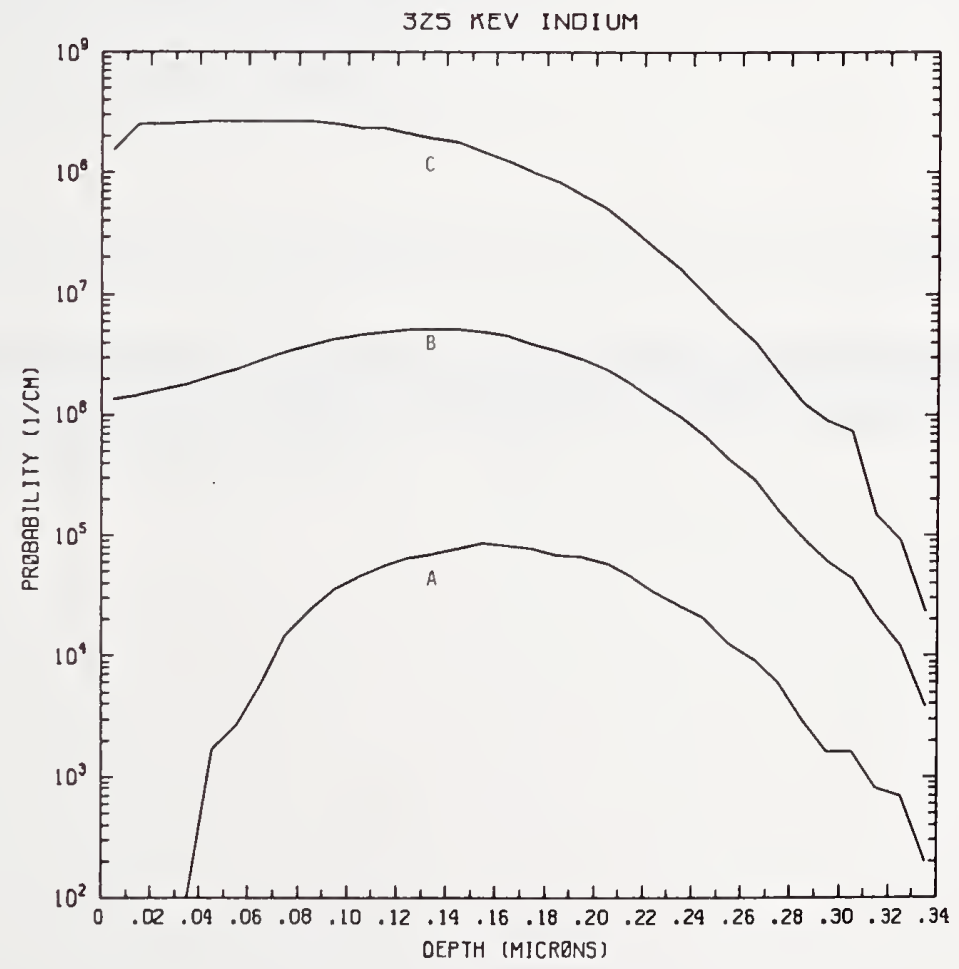

Figure In325-2 One-dimensional probability distributions for implanted indium (A), primary silicon displacements (B), and Frenkel pairs (C) for $325 \mathrm{keV}$ indium normally incident on a silicon target. 


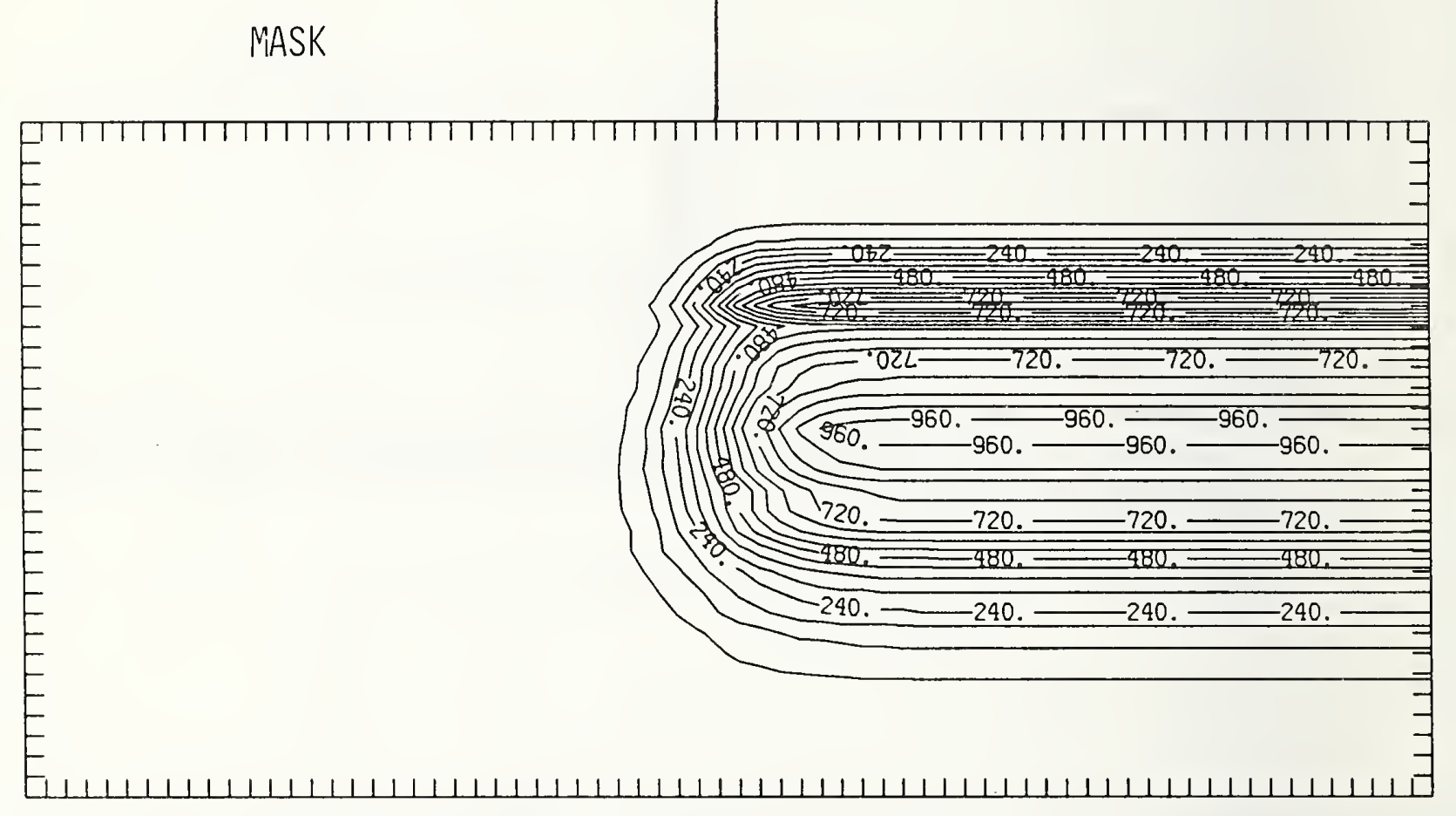

Figure In325-3 Two-dimensional distribution of $325 \mathrm{keV}$ indium implanted past a mask edge. The length increment (distance between tick marks) is $0.01 \mu \mathrm{m}$ and the scale factor is 1000 . 


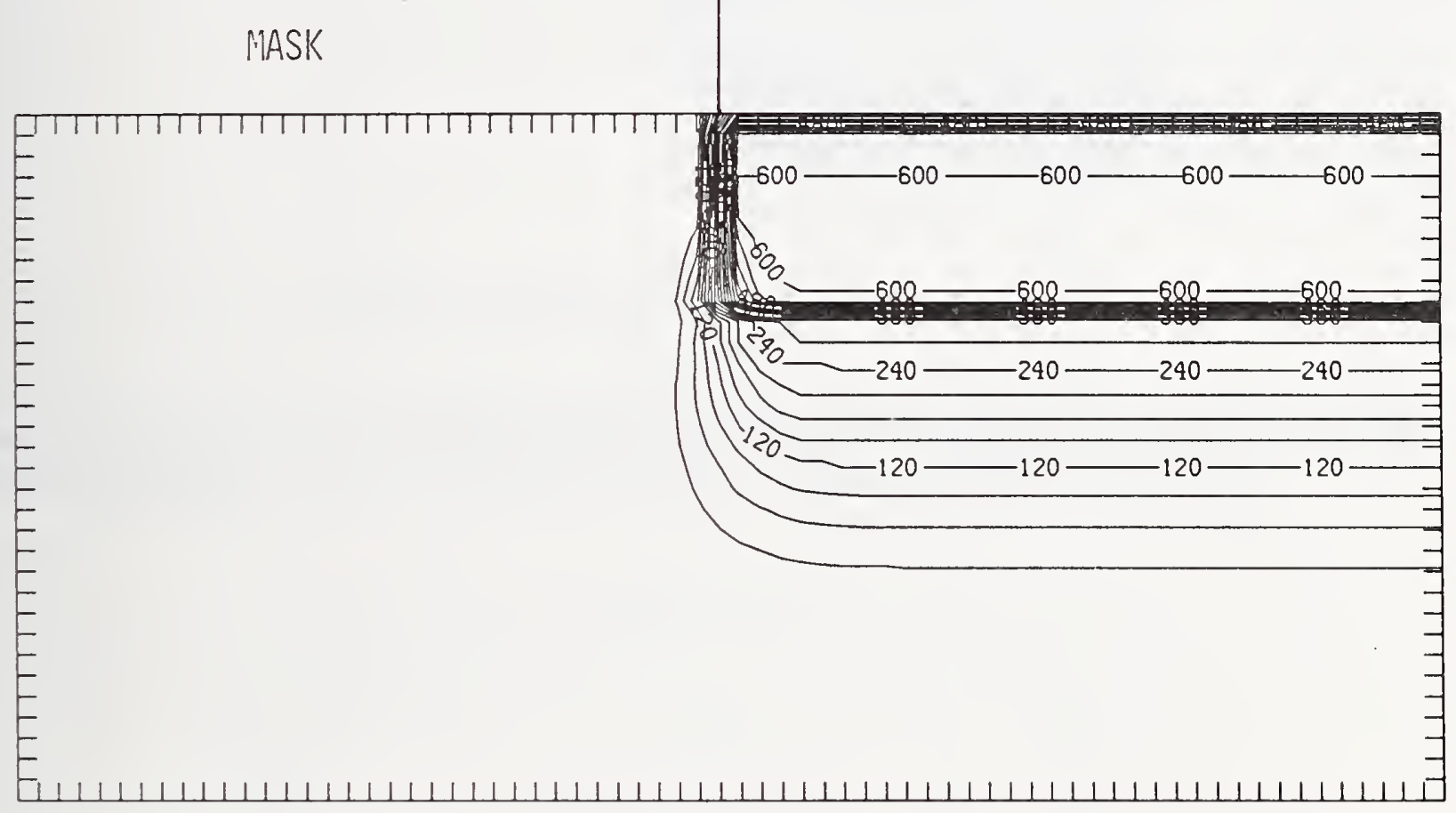

Figure In325-4 Two-dimensional distribution of Frenkel pairs created by $325 \mathrm{keV}$ indium implanted past a mask edge. The length increment (distance between tick marks) is $0.01 \mu \mathrm{m}$ and the scale factor is 0.1 . 


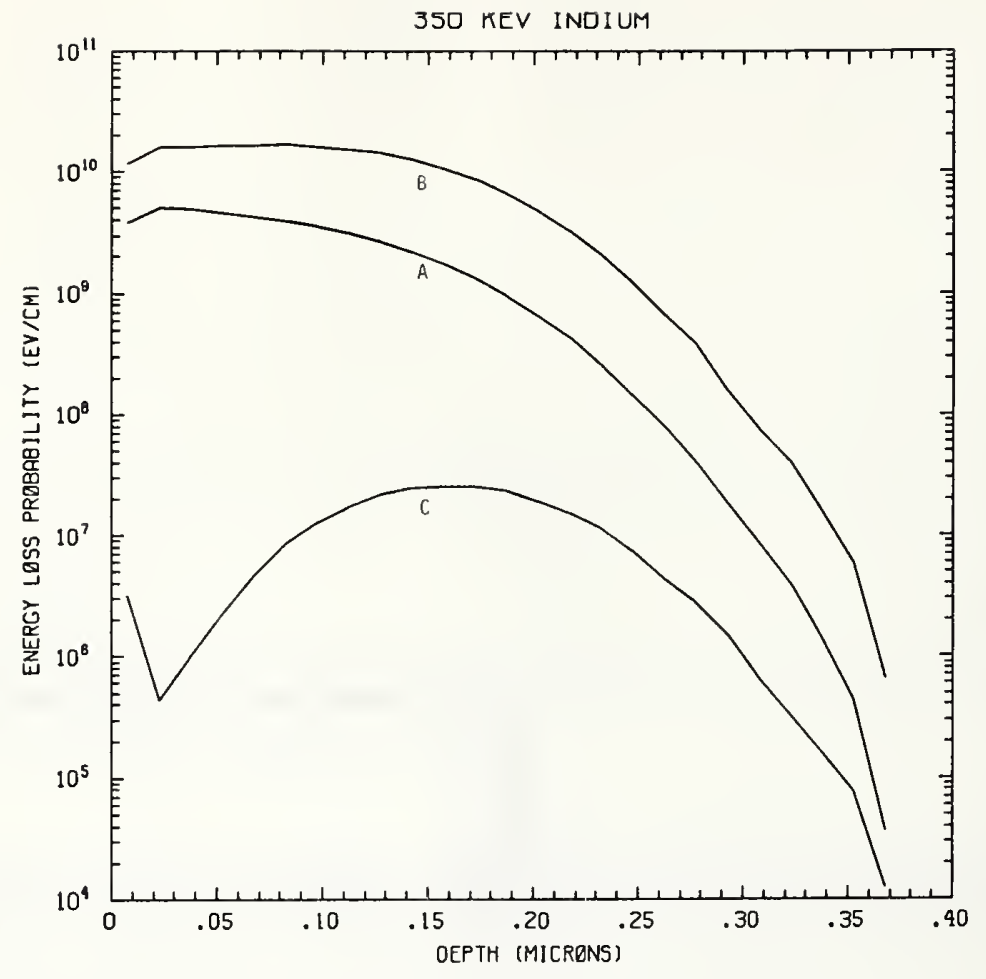

Figure In350-1 One-dimensional probability distributions for energy loss due to electronic excitation (A), displacement damage(B), and phonon excitation (C) for $350 \mathrm{keV}$ indium normally incident on a silicon target.

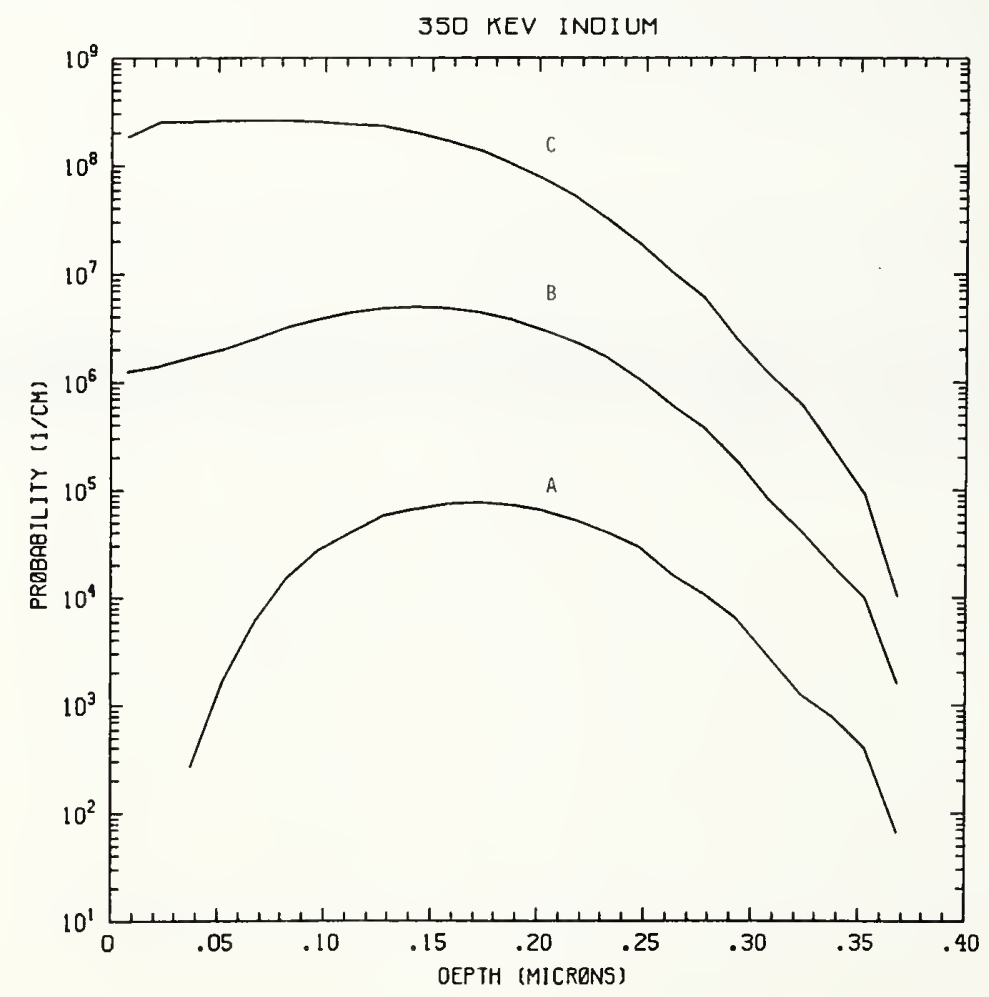

Figure In350-2 One-dimensional probability distributions for implanted indium (A), primary silicon displacements (B), and Frenkel pairs (C) for $350 \mathrm{keV}$ indium normally incident on a silicon target. 


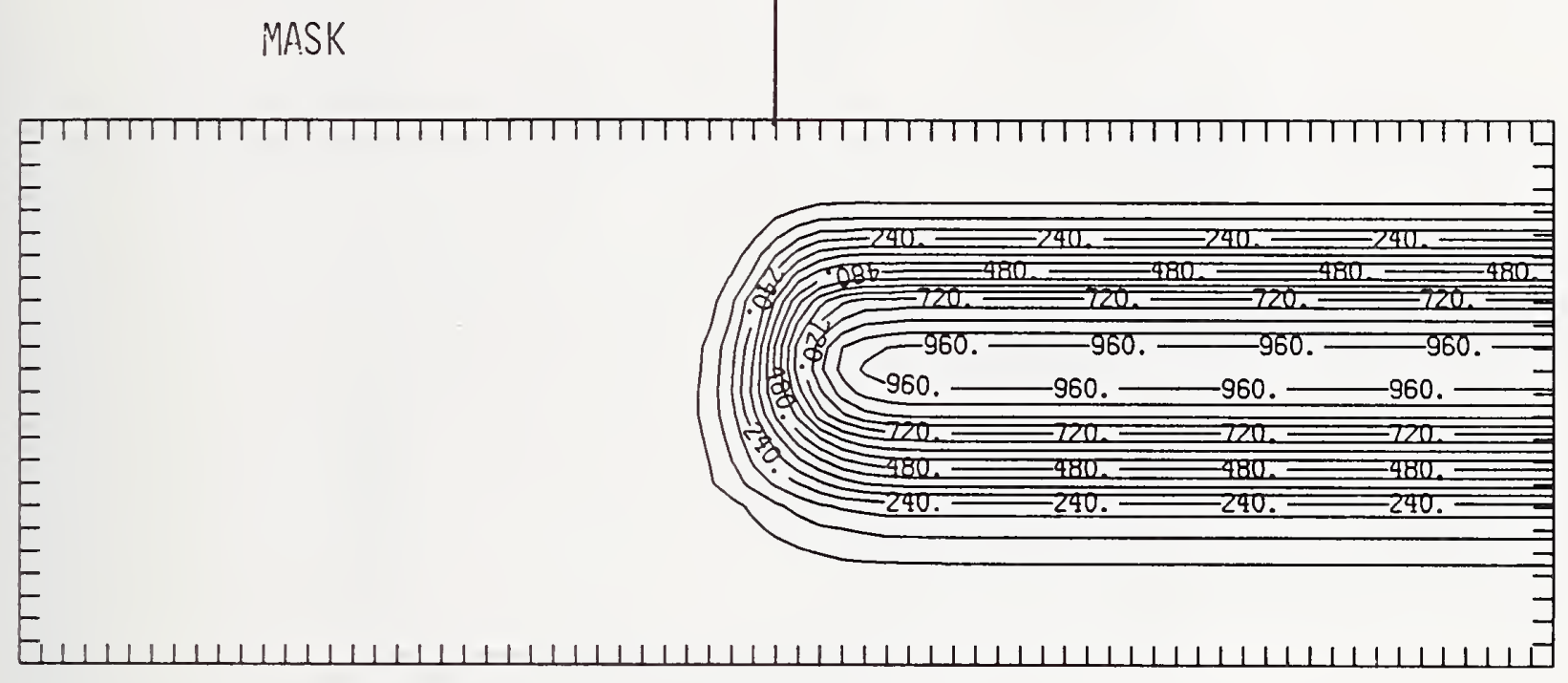

Figure In350-3 Two-dimensional distribution of $350 \mathrm{keV}$ indium implanted past a mask edge. The length increment (distance between tick marks) is $0.015 \mu \mathrm{m}$ and the scale factor is 1000 .

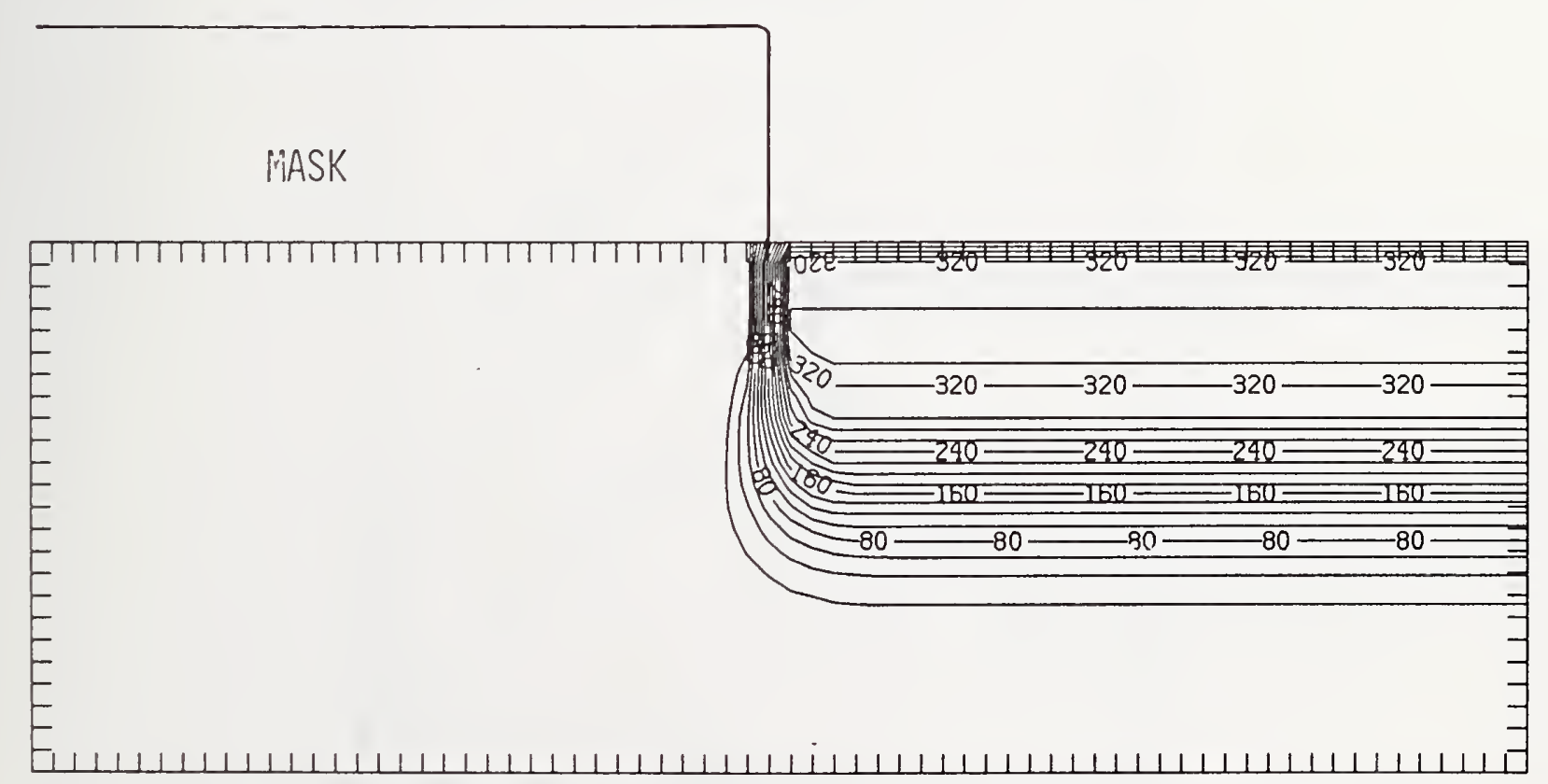

Figure In350-4 Two-dimensional distribution of Frenkel pairs created by $350 \mathrm{keV}$ indium implanted past a mask edge. The length increment (distance between tick marks) is $0.015 \mu \mathrm{m}$ and the scale factor is 0.1 . 


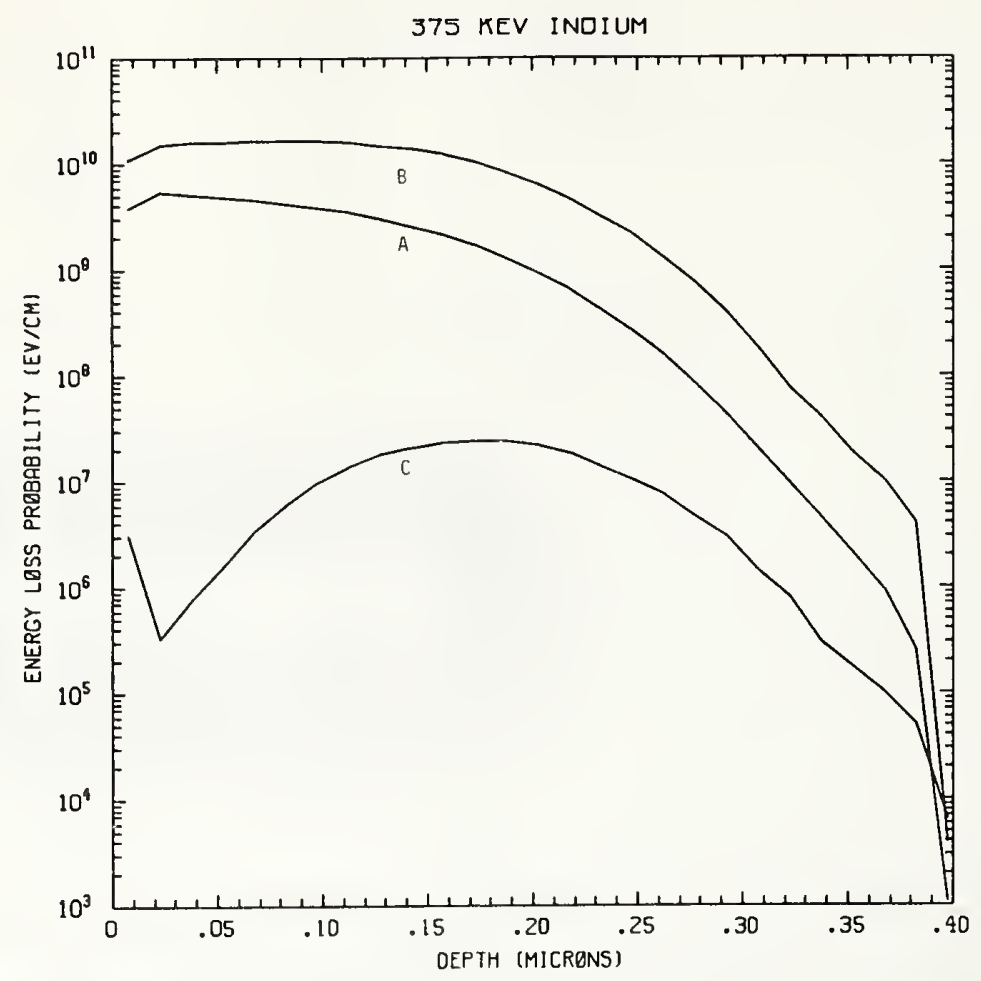

Figure In375-1 One-dimensional probability distributions for energy loss due to electronic excitatior (A), displacement damage(B), and phonon excitation (C) for $375 \mathrm{keV}$ indium normall incident on a silicon target.

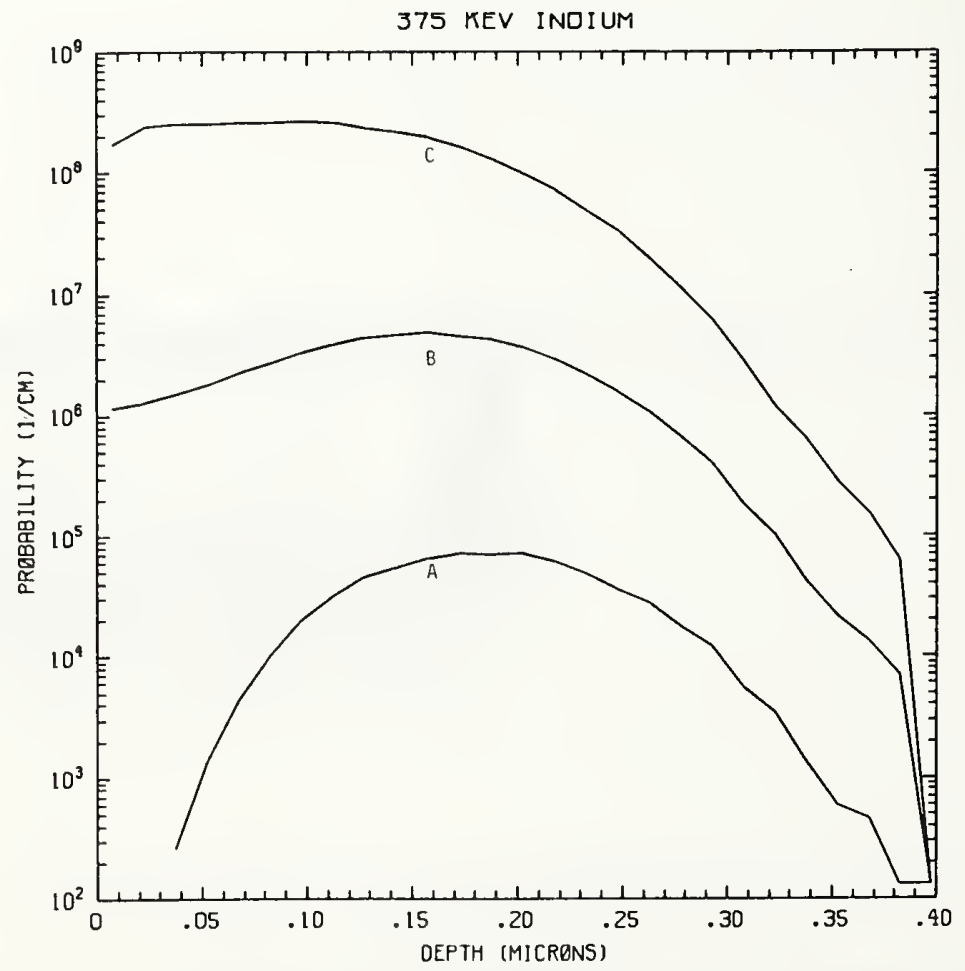

Figure In375-2 One-dimensional probability distributions for implanted indium (A), primary silicon displacements (B), and Frenkel pairs (C) for $375 \mathrm{keV}$ indium normally incident on a silicon target. 


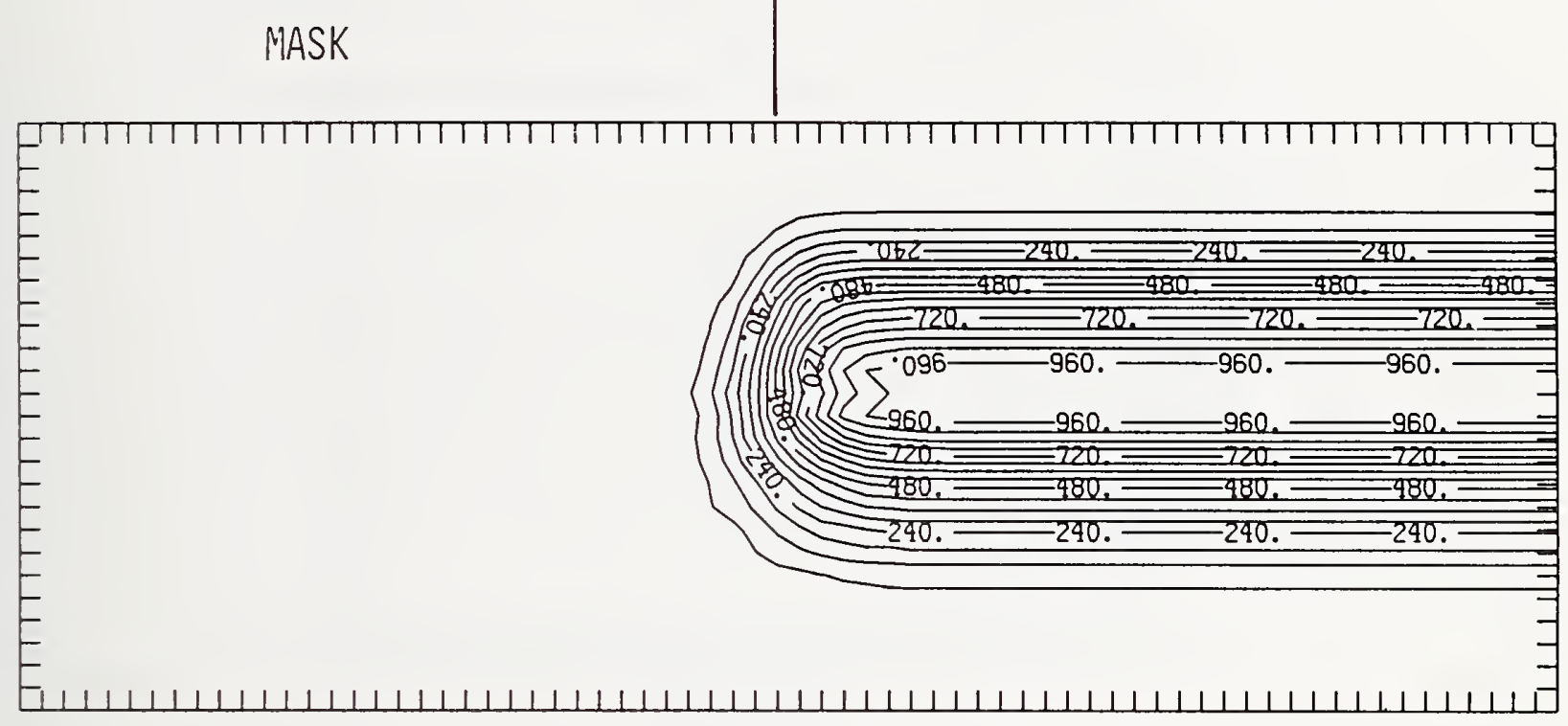

Figure In 375-3 Two-dimensional distribution of $375 \mathrm{keV}$ indium implanted past a mask edge. The length increment (distance between tick marks) is $0.015 \mu \mathrm{m}$ and the scale factor is 1000 .

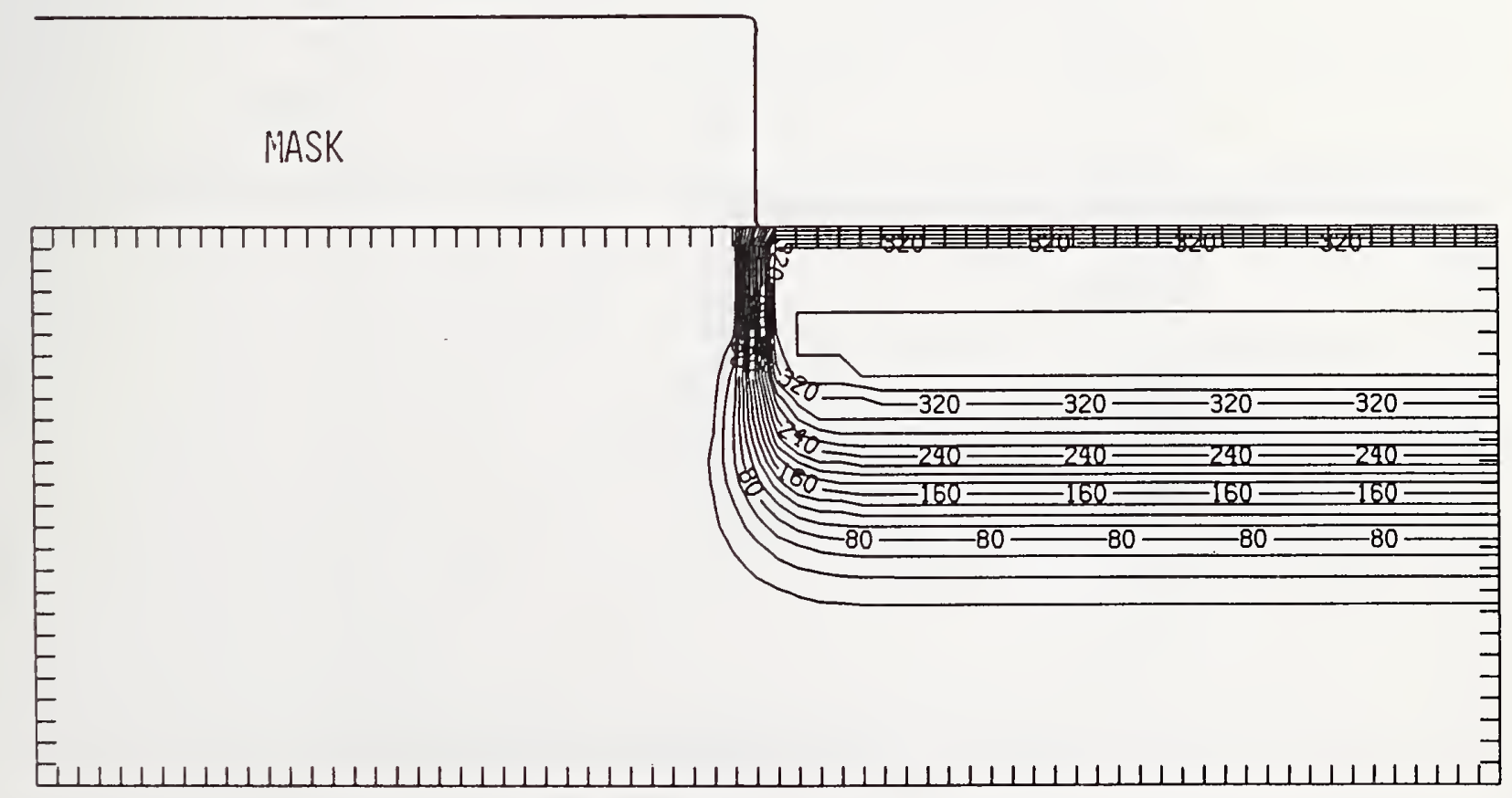

Figure In 375-4 Two-dimensional distribution of Frenkel pairs created by $375 \mathrm{keV}$ indium implanted past a mask edge. The length increment (distance between tick marks) is $0.015 \mu \mathrm{m}$ and the scale factor is 0.1 . 


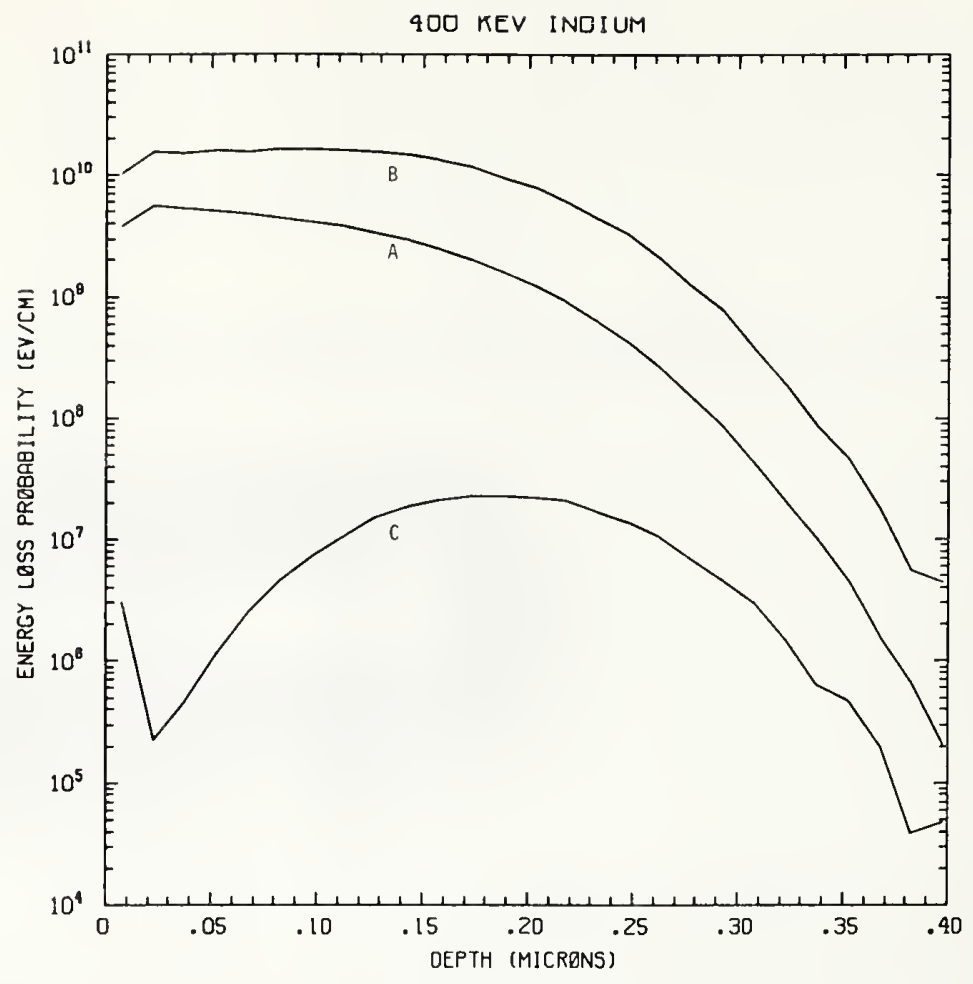

Figure In400-1 One-dimensional probability distributions for energy loss due to electronic excitation (A), displacement damage(B), and phonon excitation (C) for $400 \mathrm{keV}$ indium normally incident on a silicon target.

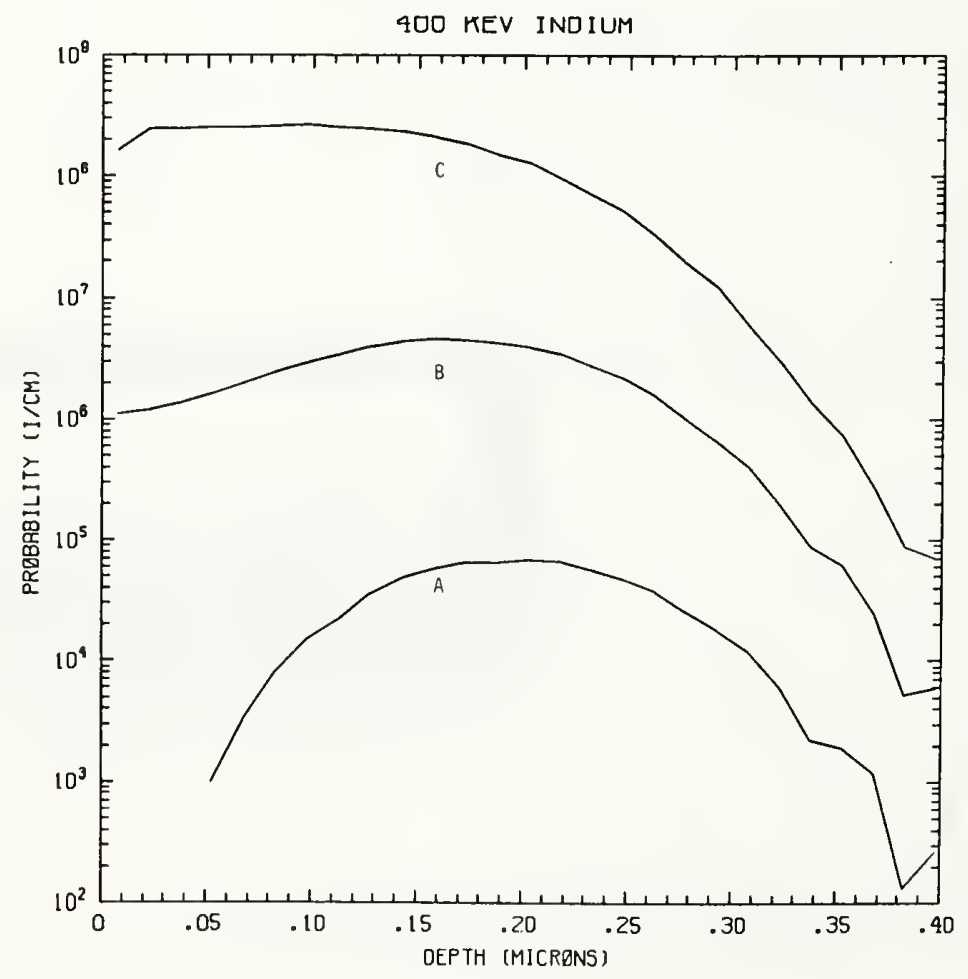

Figure In400-2 One-dimensional probability distributions for implanted indium (A), primary silicon displacements (B), and Frenkel pairs (C) for $400 \mathrm{keV}$ indium normally incident on a silicon target. 


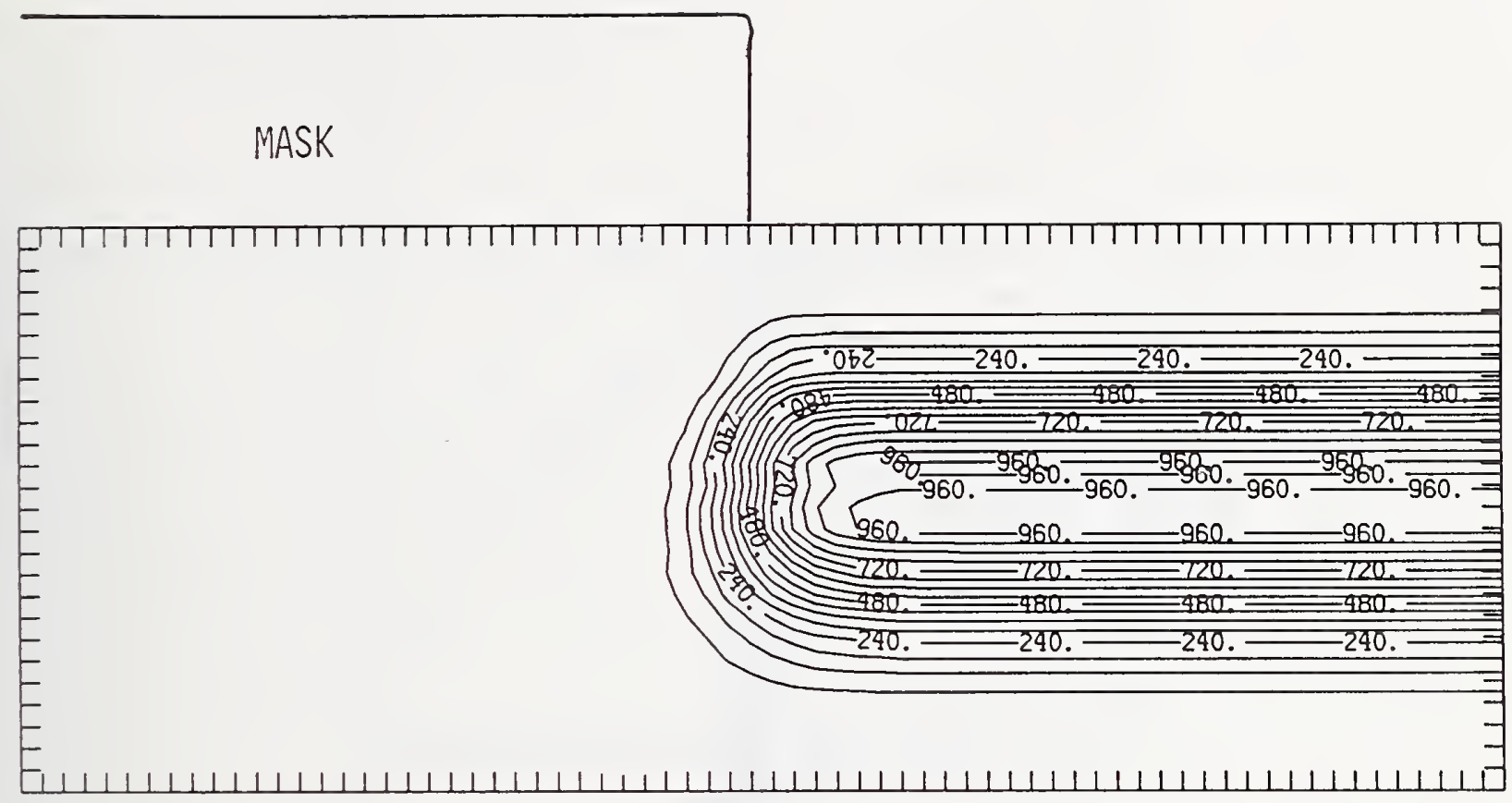

Figure In400-3 Two-dimensional distribution of $400 \mathrm{keV}$ indium implanted past a mask edge. The length increment (distance between tick marks) is $0.015 \mu \mathrm{m}$ and the scale factor is 1000.

MASK

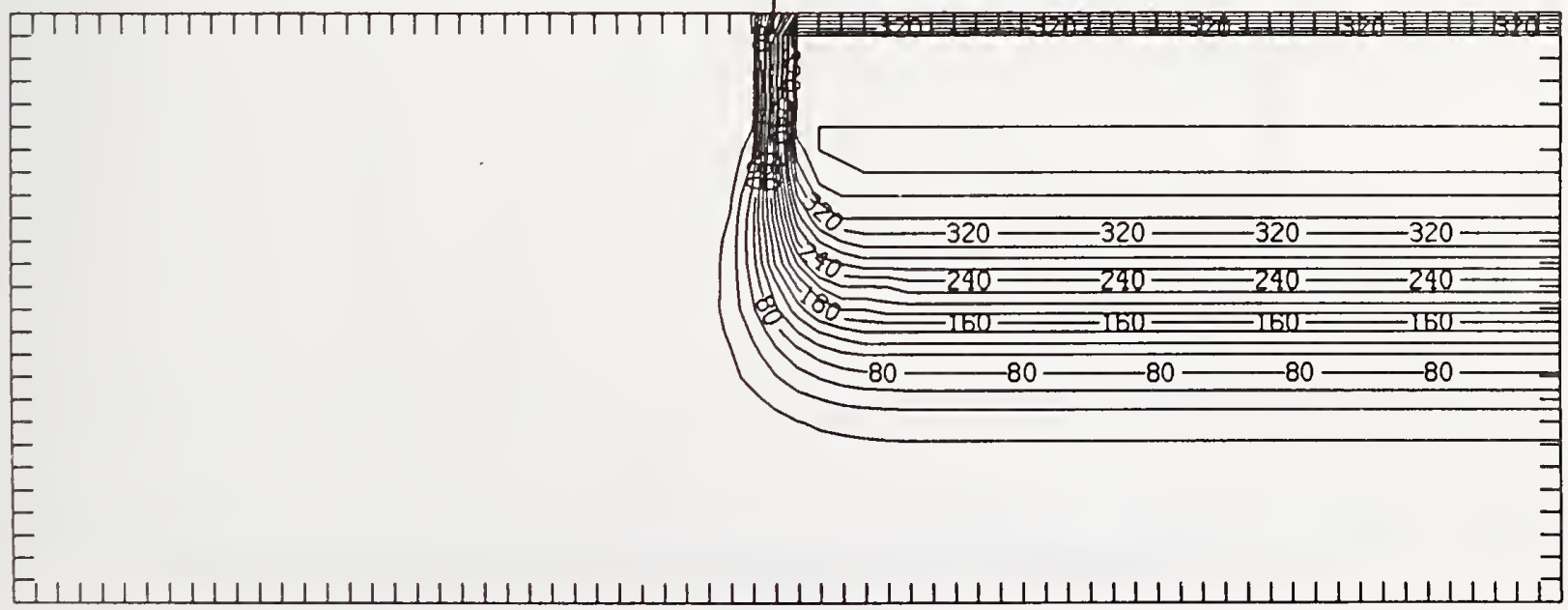

Figure In 400-4 Two-dimensional distribution of Frenkel pairs created by $400 \mathrm{keV}$ indium implanted past a mask edge. The length increment (distance between tick marks) is $0.015 \mu \mathrm{m}$ and the scale factor is 0.1 . 


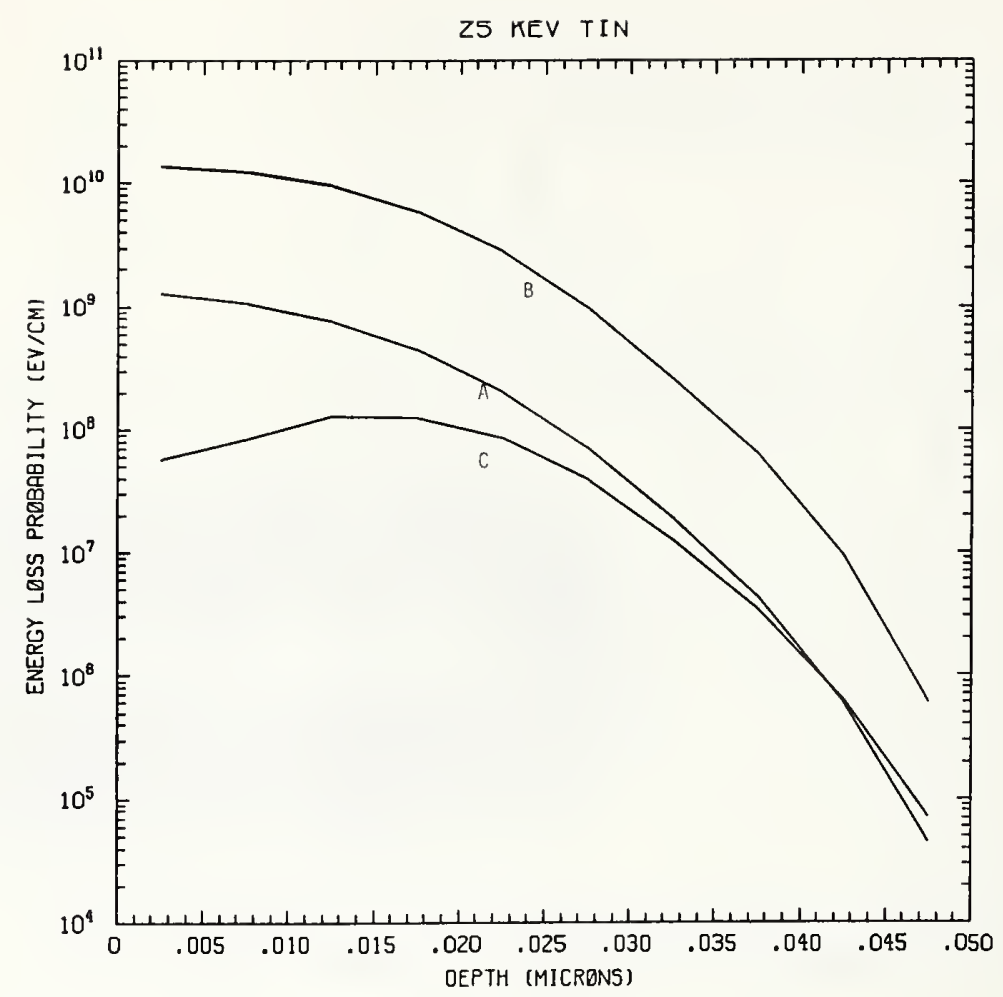

Figure Sn25-1 One-dimensional probability distributions for energy loss due to electronic excitation (A), displacement damage(B), and phonon excitation (C) for $25 \mathrm{keV}$ tin normally incident on a silicon target.

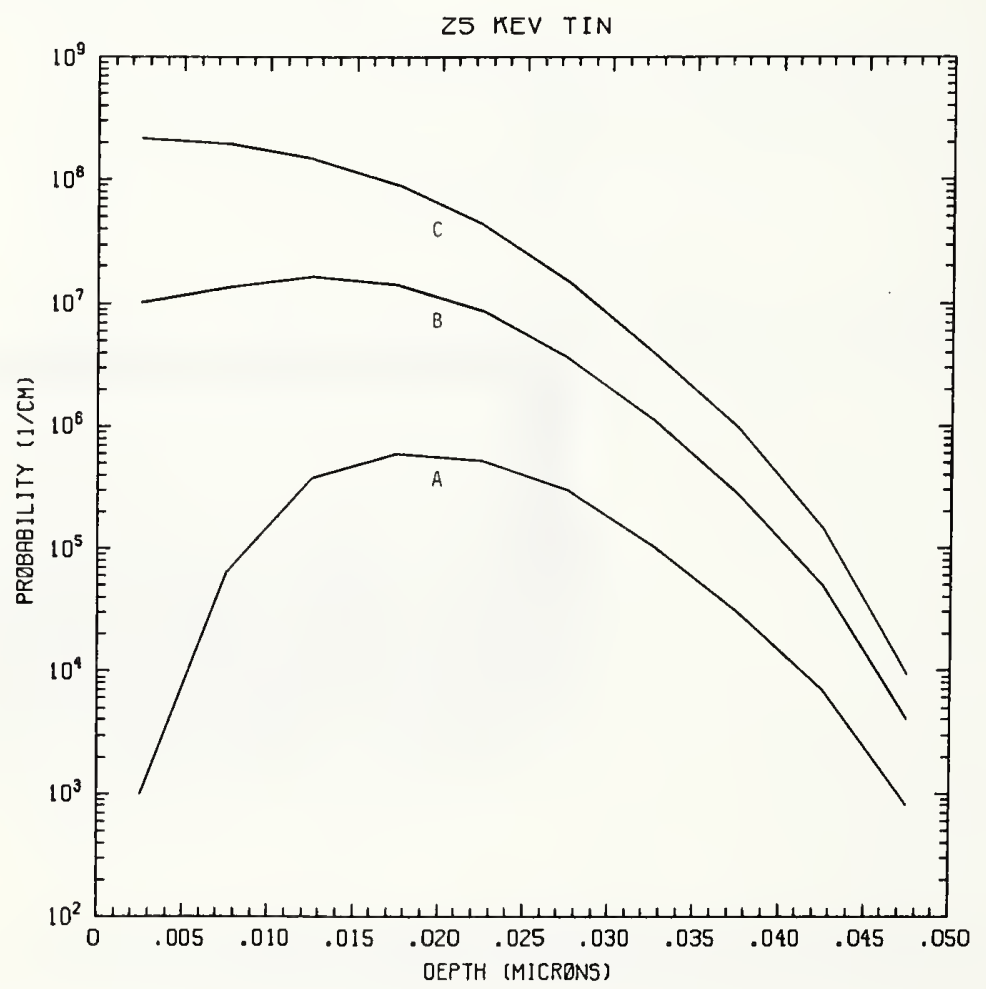

Figure Sn25-2 One-dimensional probability distributions for implanted tin (A), primary silicon displacements (B), and Frenkel pairs (C) for $25 \mathrm{keV}$ tin normally incident on a silicon target. 
MASK

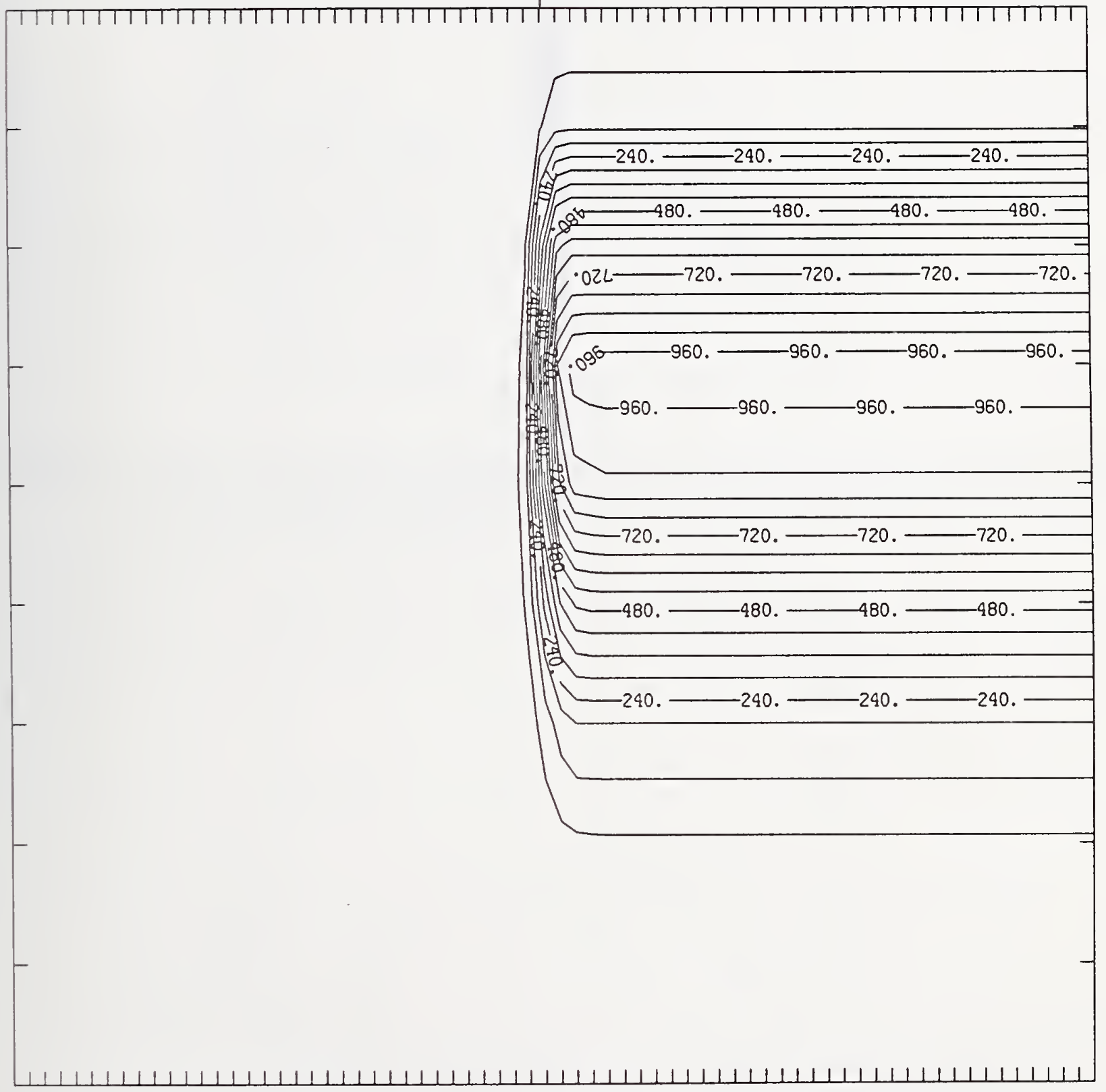

Figure Sn25-3 Two-dimensional distribution of $25 \mathrm{keV}$ tin implanted past a mask edge. The length increment (distance between tick marks) is $0.005 \mu \mathrm{m}$ and the scale factor is 1000 . 


\section{MASK}

IIIIIITTIIIIIIITITTTITITTI

$320-320-320-320-320-$

in

$240-240-240-240 \_-1$

$160-160-160-160-160+10$

$160-160-160-7$

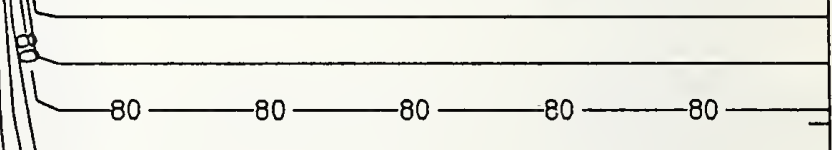

.

80

80

$-$ 


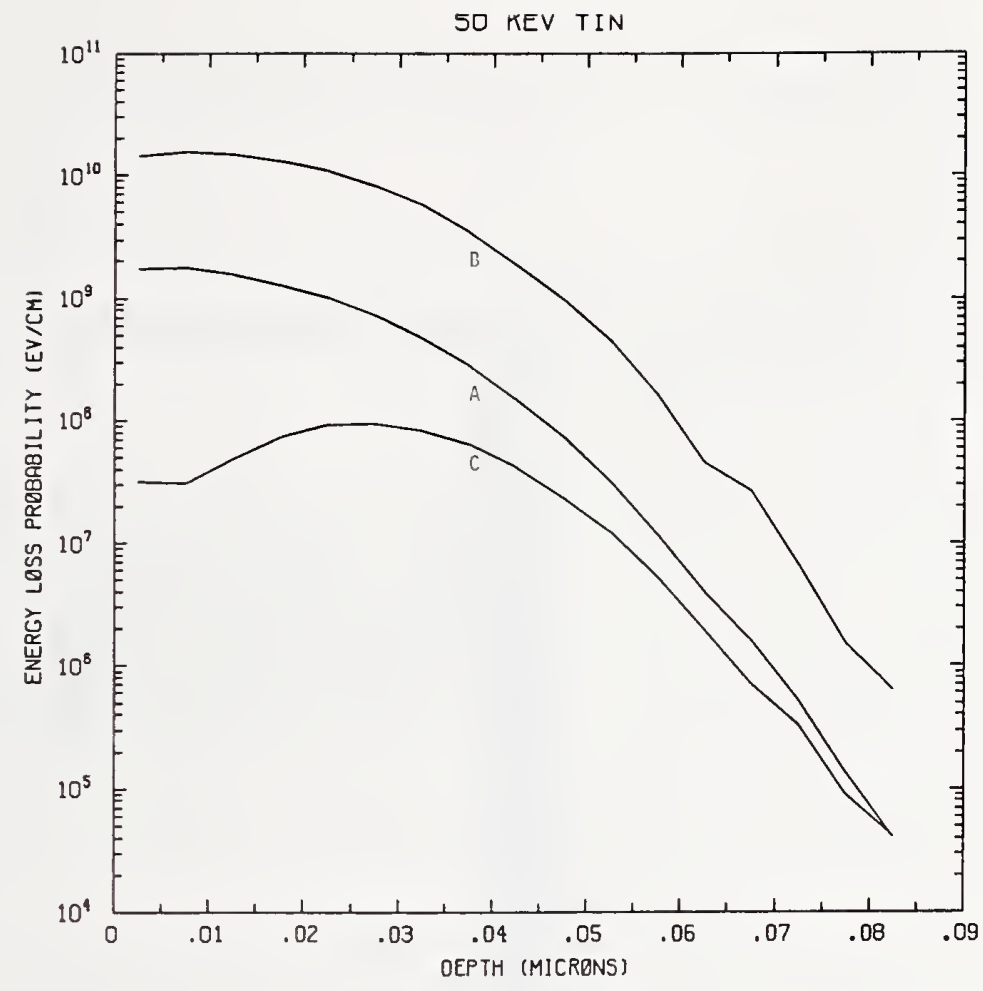

Figure Sn50-1 One-dimensional probability distributions for energy loss due to electronic excitation (A), displacement damage(B), and phonon excitation (C) for $50 \mathrm{keV}$ tin normally incident on a silicon target.

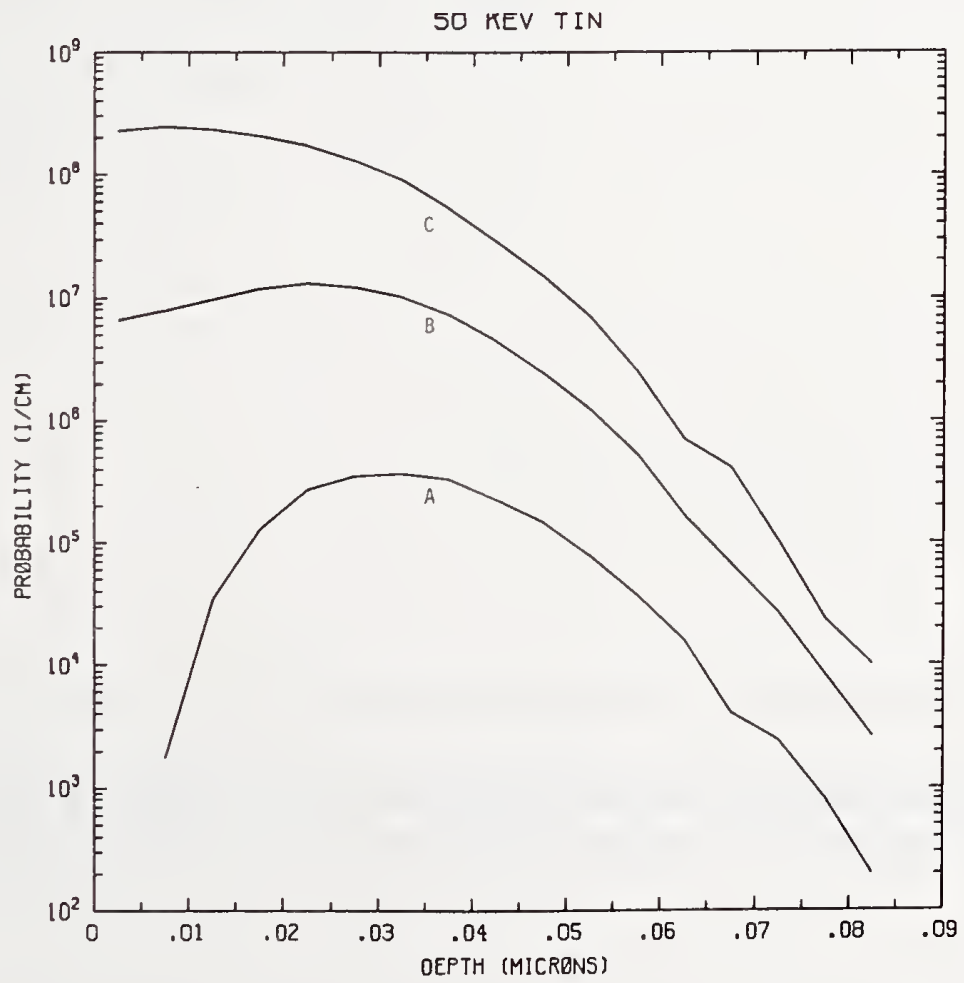

Figure Sn50-2 One-dimensional probability distributions for implanted tin (A), primary silicon displacements (B), and Frenkel pairs (C) for $50 \mathrm{keV}$ tin normally incident on a silicon target. 
MASK

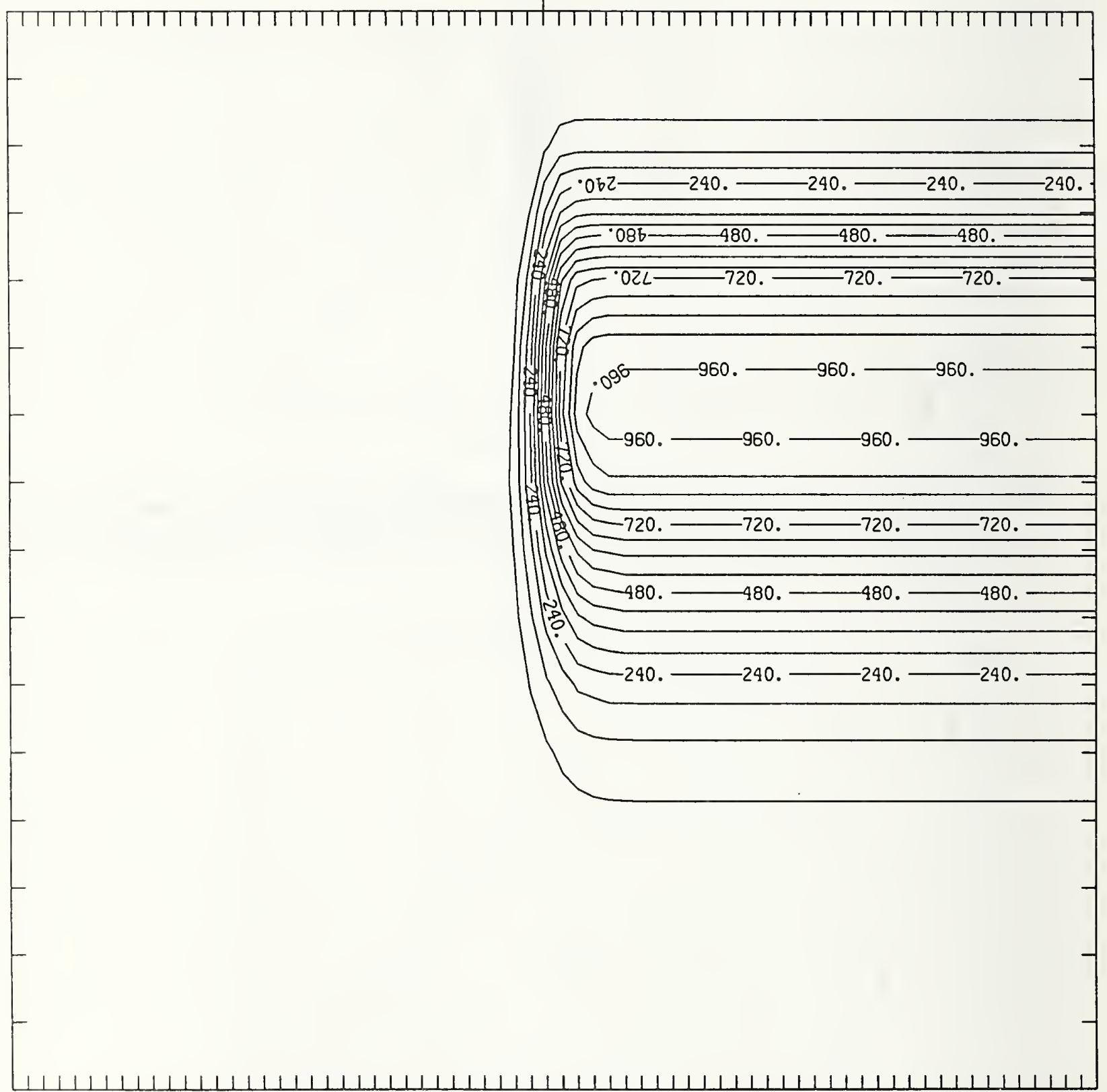

Figure Sn50-3 Two-dimensional distribution of $50 \mathrm{keV}$ tin implanted past a mask edge. The length increment (distance between tick marks) is $0.005 \mu \mathrm{m}$ and the scale factor is 1000 . 


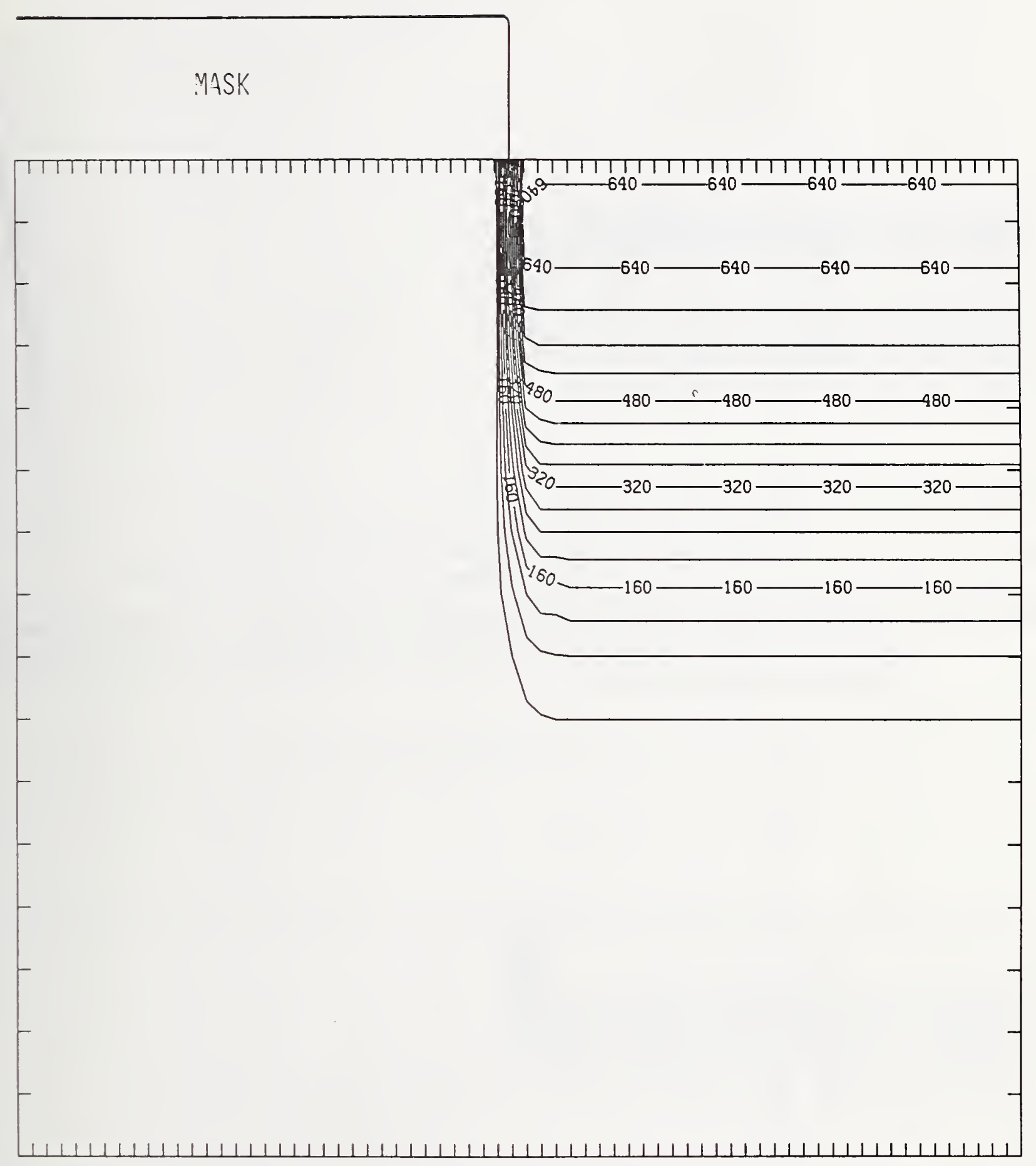

Figure Sn50-4 Two-dimensional distribution of Frenkel pairs created by $50 \mathrm{keV}$ tin implanted past a mask edge. The length increment (distance between tick marks) is $0.005 \mu \mathrm{m}$ and the scale factor is 1 . 


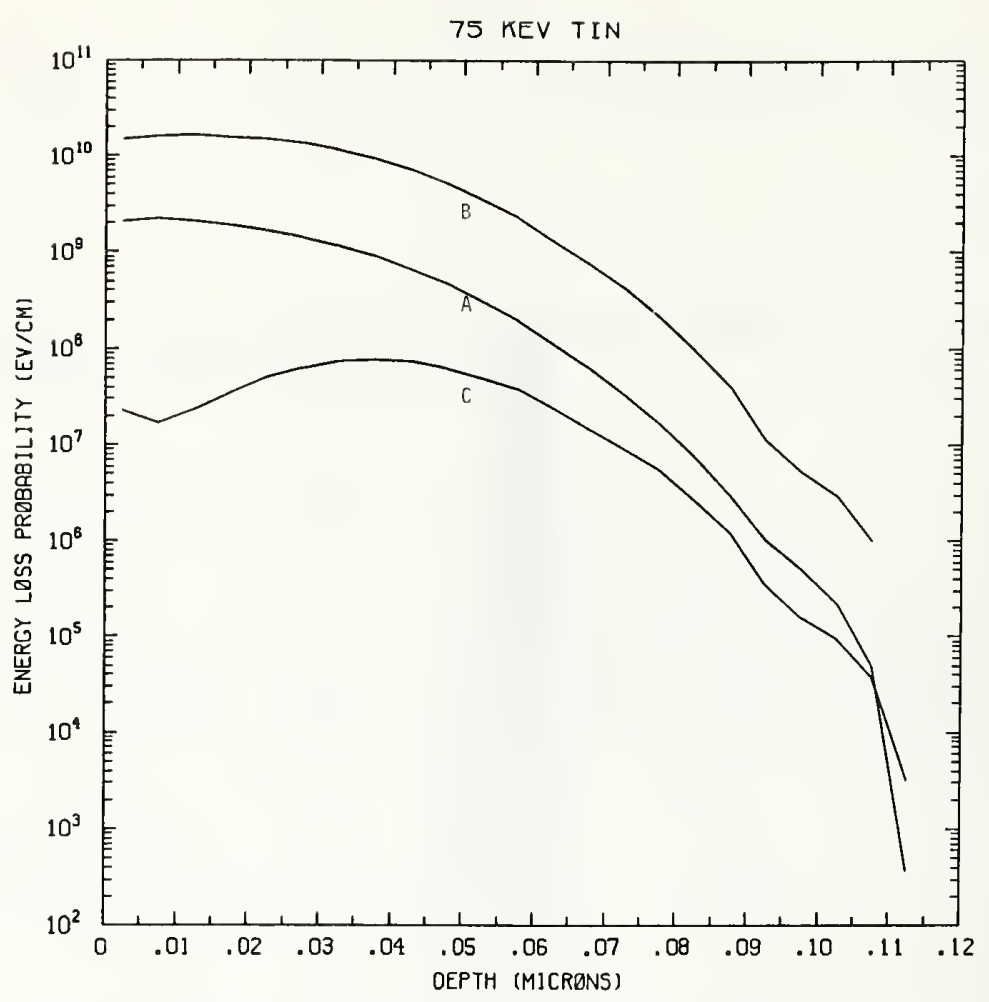

Figure Sn75-1 One-dimensional probability distributions for energy loss due to electronic excitation (A), displacement damage(B), and phonon excitation (C) for $75 \mathrm{keV}$ tin normally incident on a silicon target.

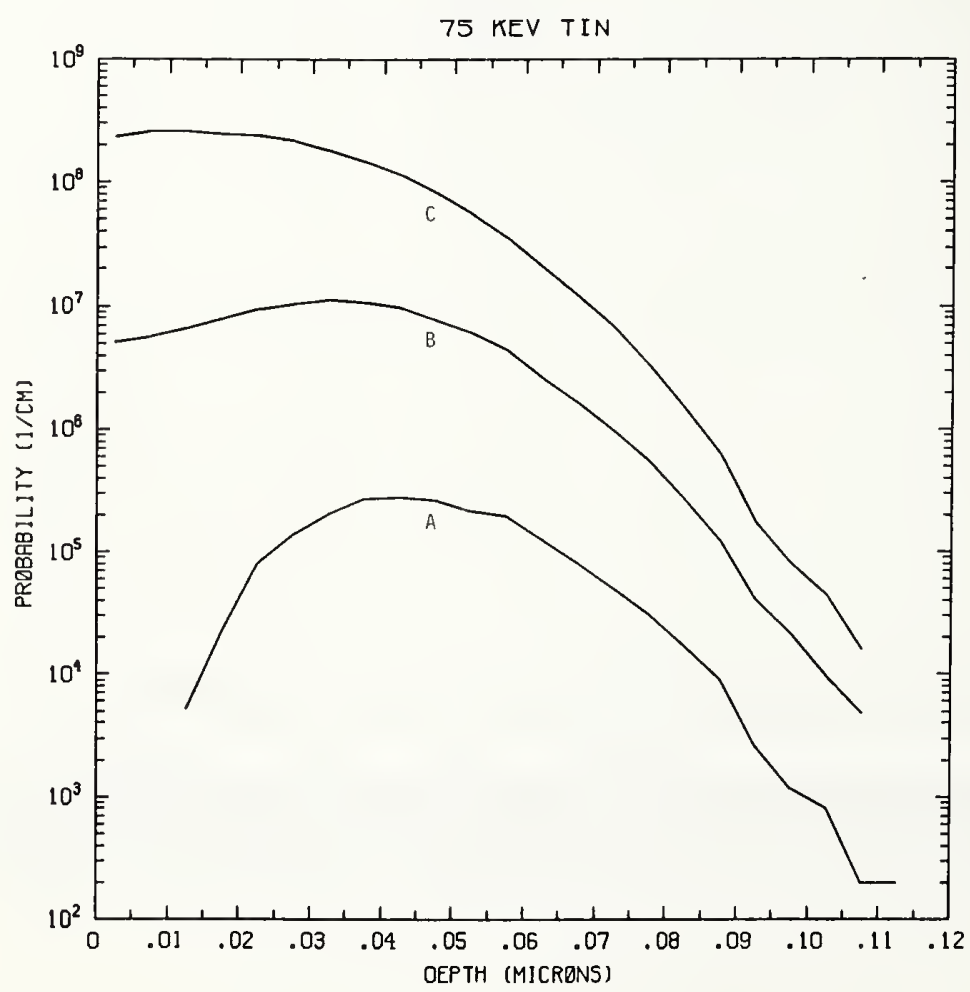

Figure Sn75-2 One-dimensional probability distributions for implanted tin (A), primary silicon displacements (B), and Frenkel pairs (C) for $75 \mathrm{keV}$ tin normally incident on a silicon target. 


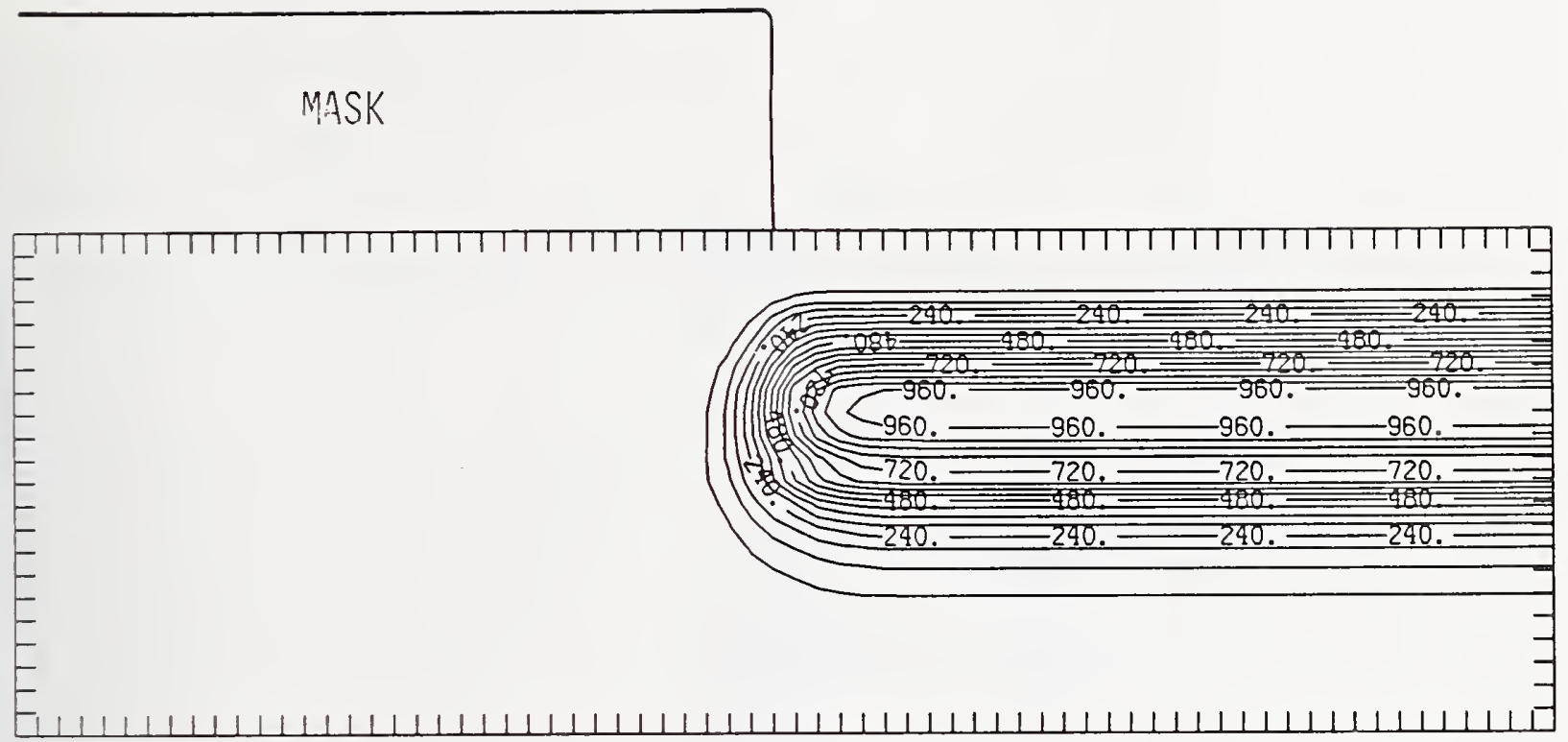

Figure Sn75-3 Two-dimensional distribution of $75 \mathrm{keV}$ tin implanted past a mask edge. The length increment (distance between tick marks) is $0.005 \mu \mathrm{m}$ and the scale factor is 1000 .

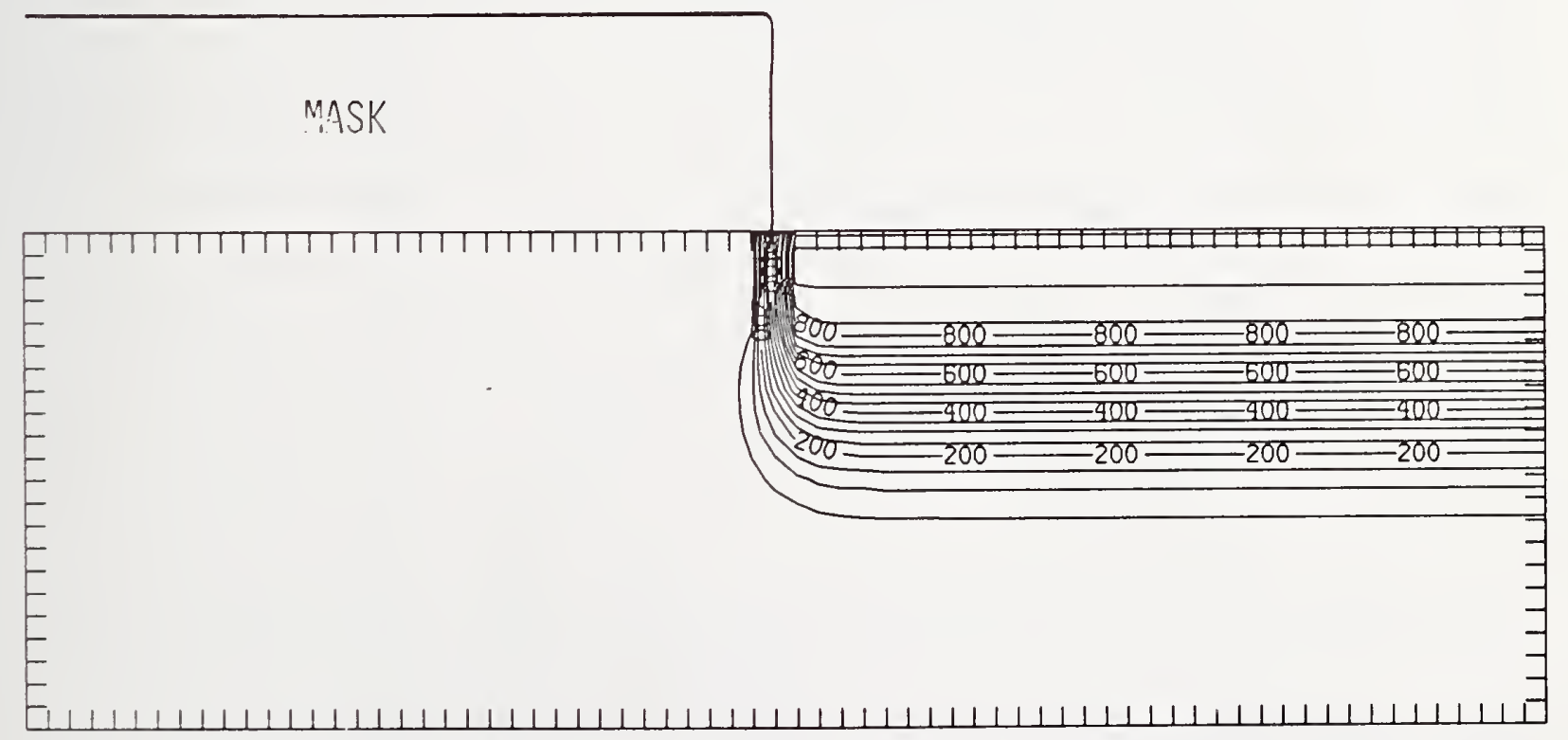

Figure Sn75-4 Two-dimensional distribution of Frenkel pairs created by $75 \mathrm{keV}$ tin implanted past a mask edge. The length increment (distance between tick marks) is $0.005 \mu \mathrm{m}$ and the scale factor is 1 . 


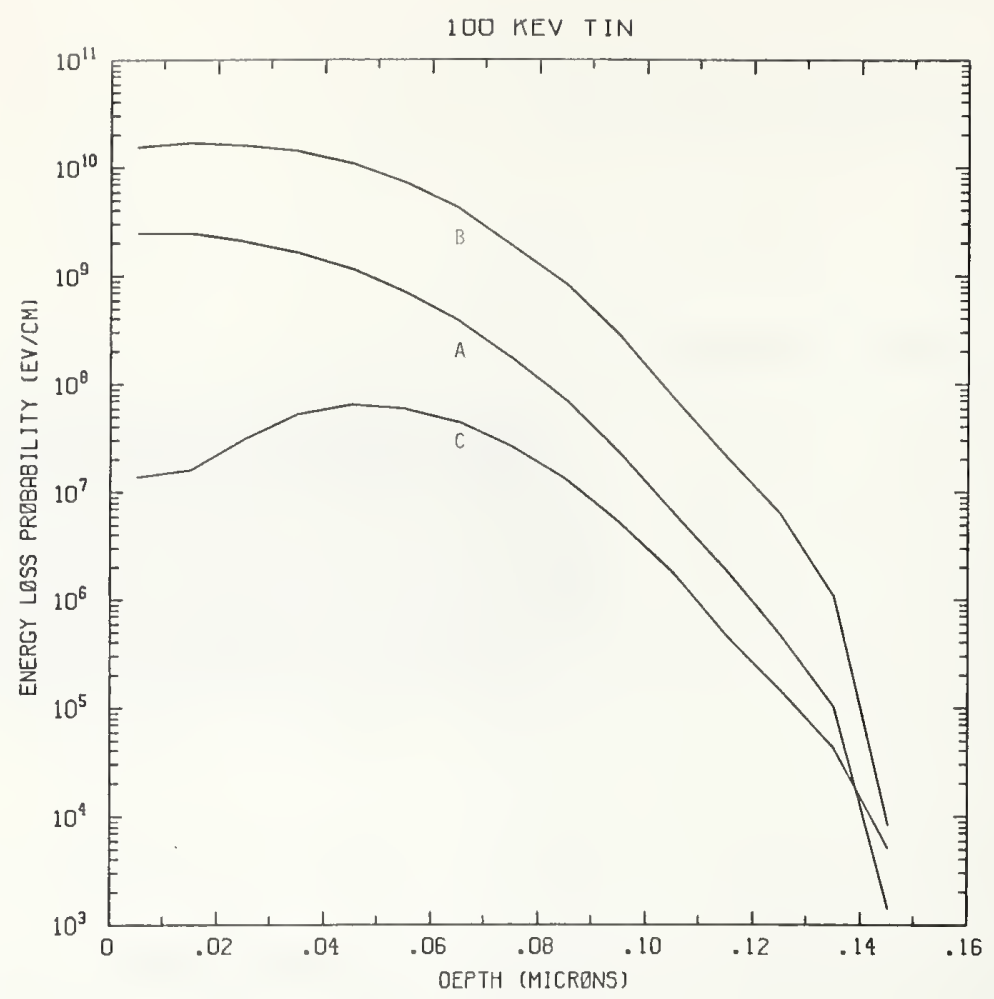

Figure Sn100-1 One-dimensional probability distributions for energy loss due to electronic excitation (A), displacement damage(B), and phonon excitation (C) for $100 \mathrm{keV}$ tin normally incident on a silicon target.

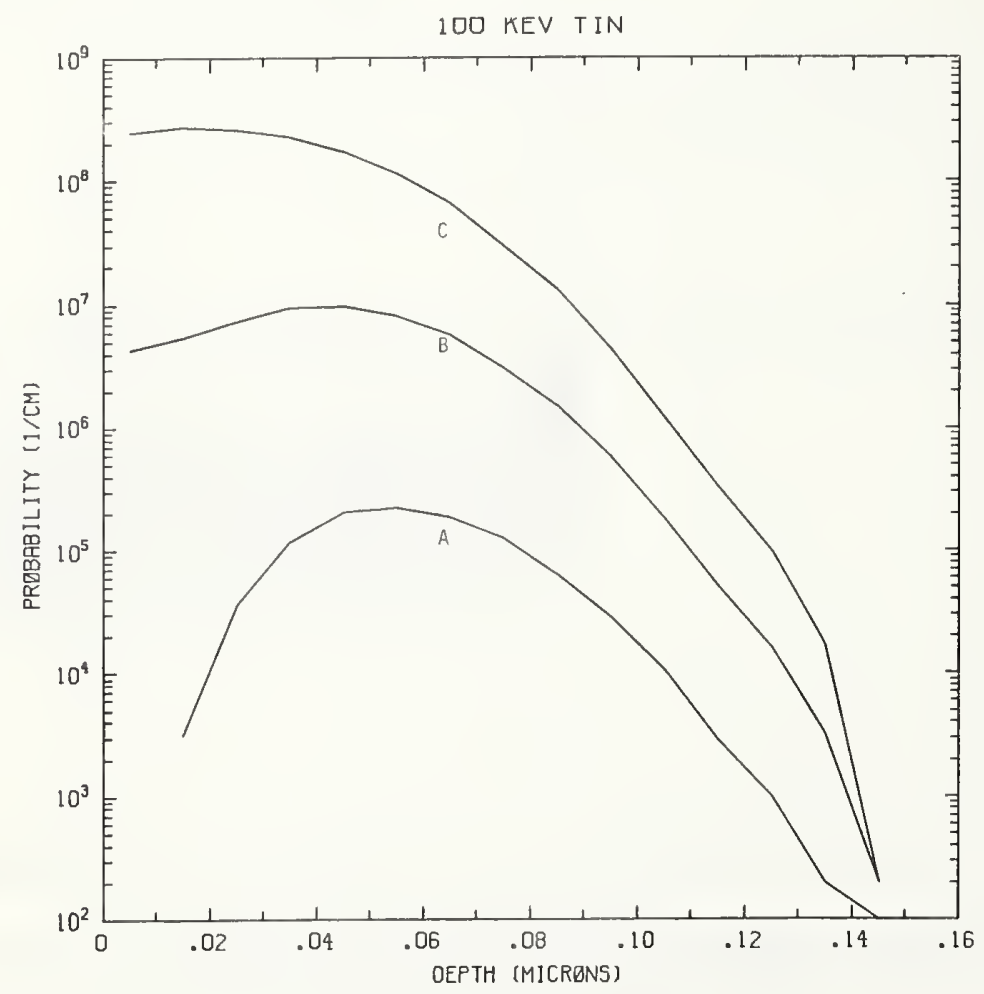

Figure Sn100-2 One-dimensional probability distributions for implanted tin (A), primary silicon displacements (B), and Frenkel pairs (C) for $100 \mathrm{keV}$ tin normally incident on a silicon target. 


\section{MISK}

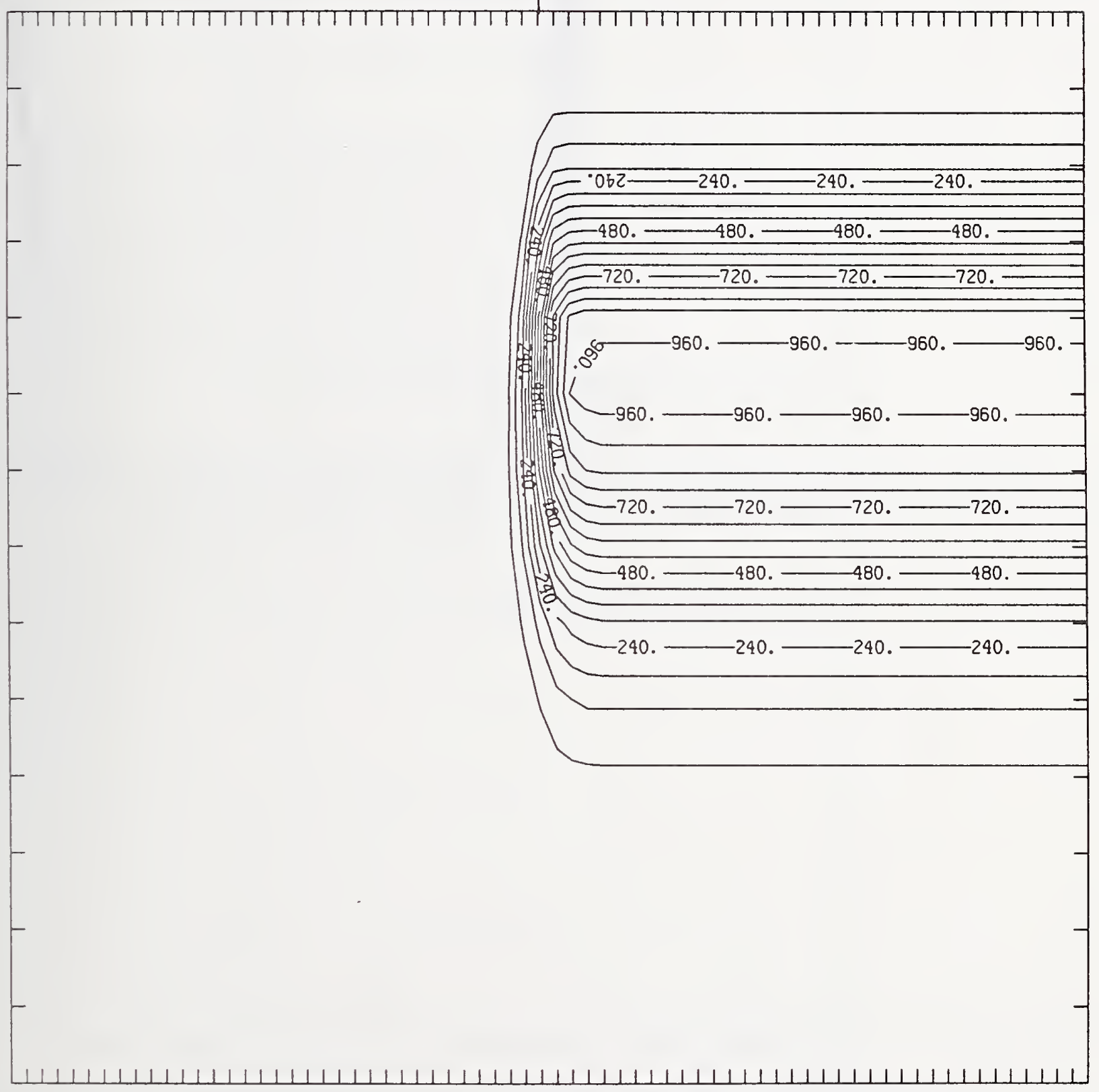

Figure Sn100-3 Two-dimensional distribution of $100 \mathrm{keV}$ tin implanted past a mask edge. The length increment (distance between tick marks) is $0.01 \mu \mathrm{m}$ and the scale factor is 1000 . 


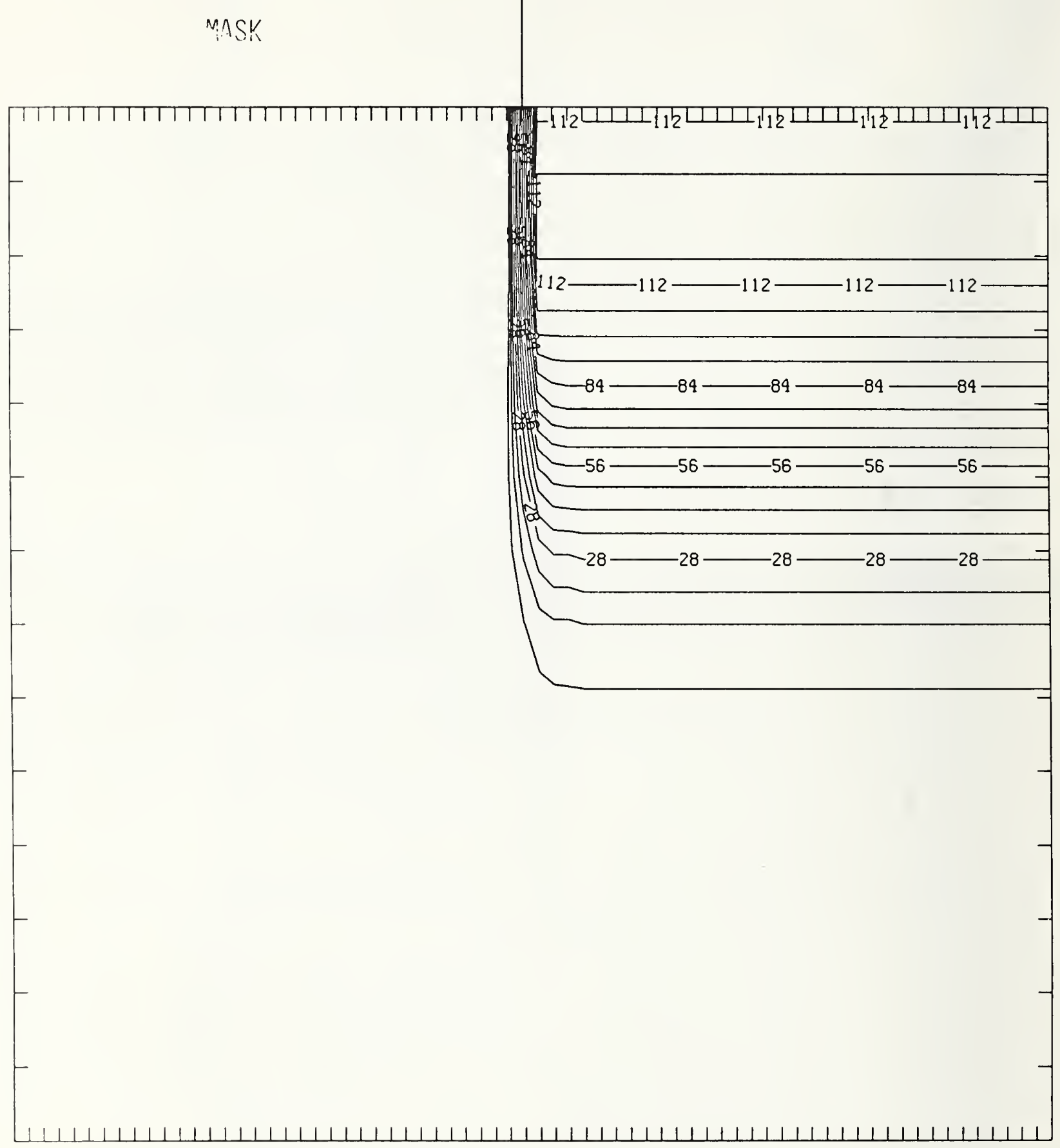

Figure Sn100-4 Two-dimensional distribution of Frenkel pairs created by $100 \mathrm{keV}$ tin implanted past a mask edge. The length increment (distance between tick marks) is $0.01 \mu \mathrm{m}$ and the scale factor is 0.1 . 


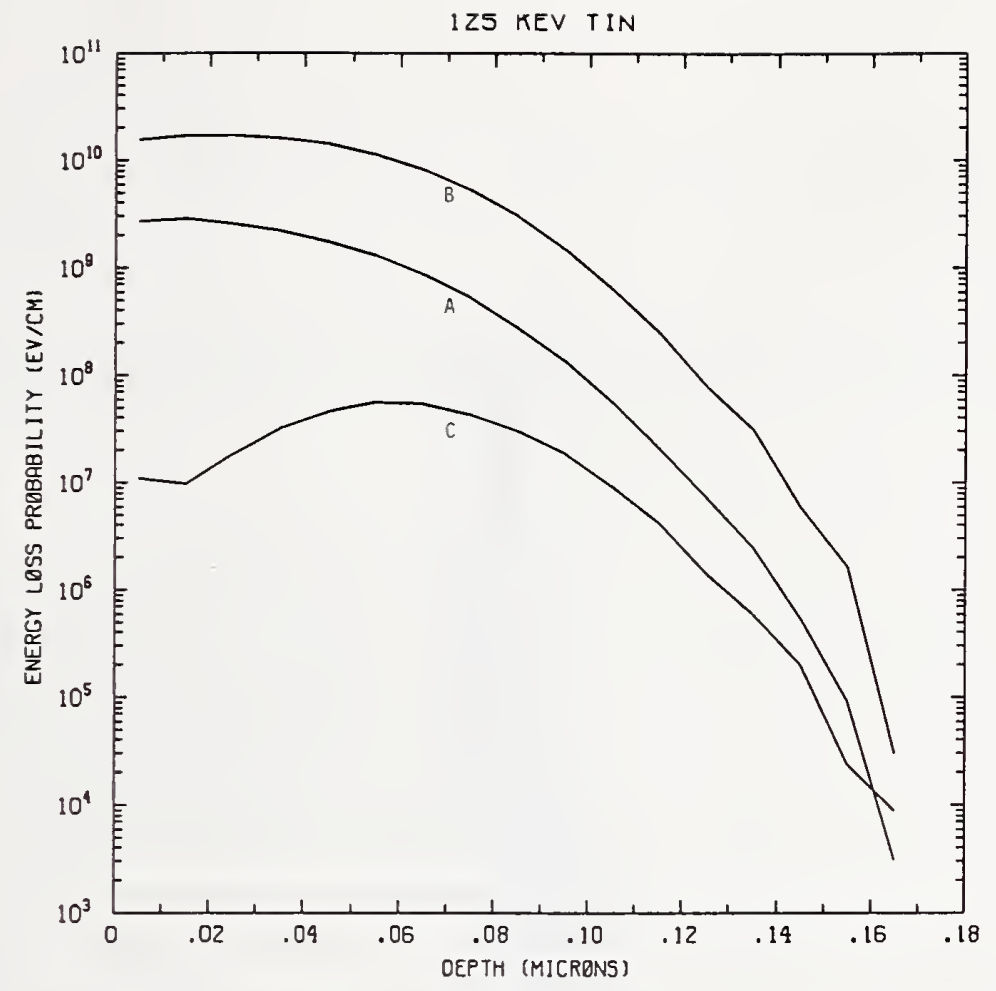

Figure Sn125-1 One-dimensional probability distributions for energy loss due to electronic excitation (A), displacement damage(B), and phonon excitation (C) for $125 \mathrm{keV}$ tin normally incident on a silicon target.

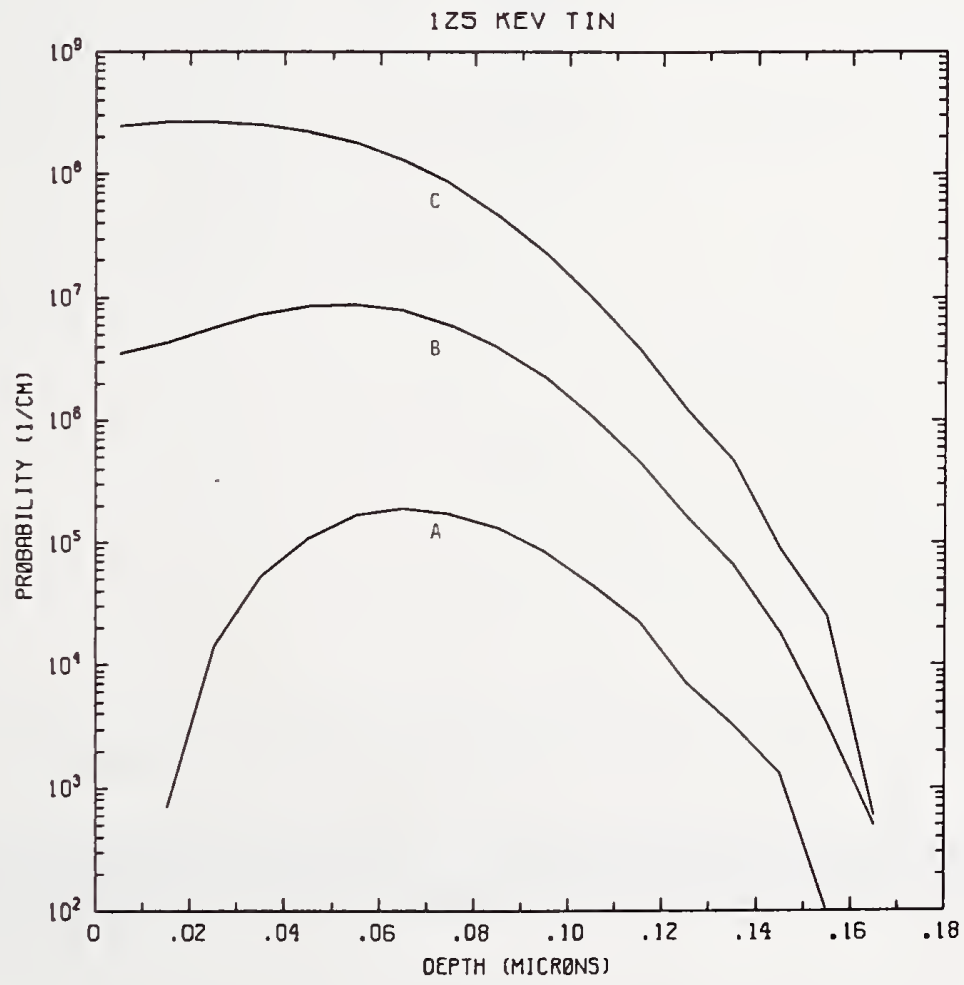

Figure Sn125-2 One-dimensional probability distributions for implanted tin (A), primary silicon displacements (B), and Frenkel pairs (C) for $125 \mathrm{keV}$ tin normally incident on a silicon target. 


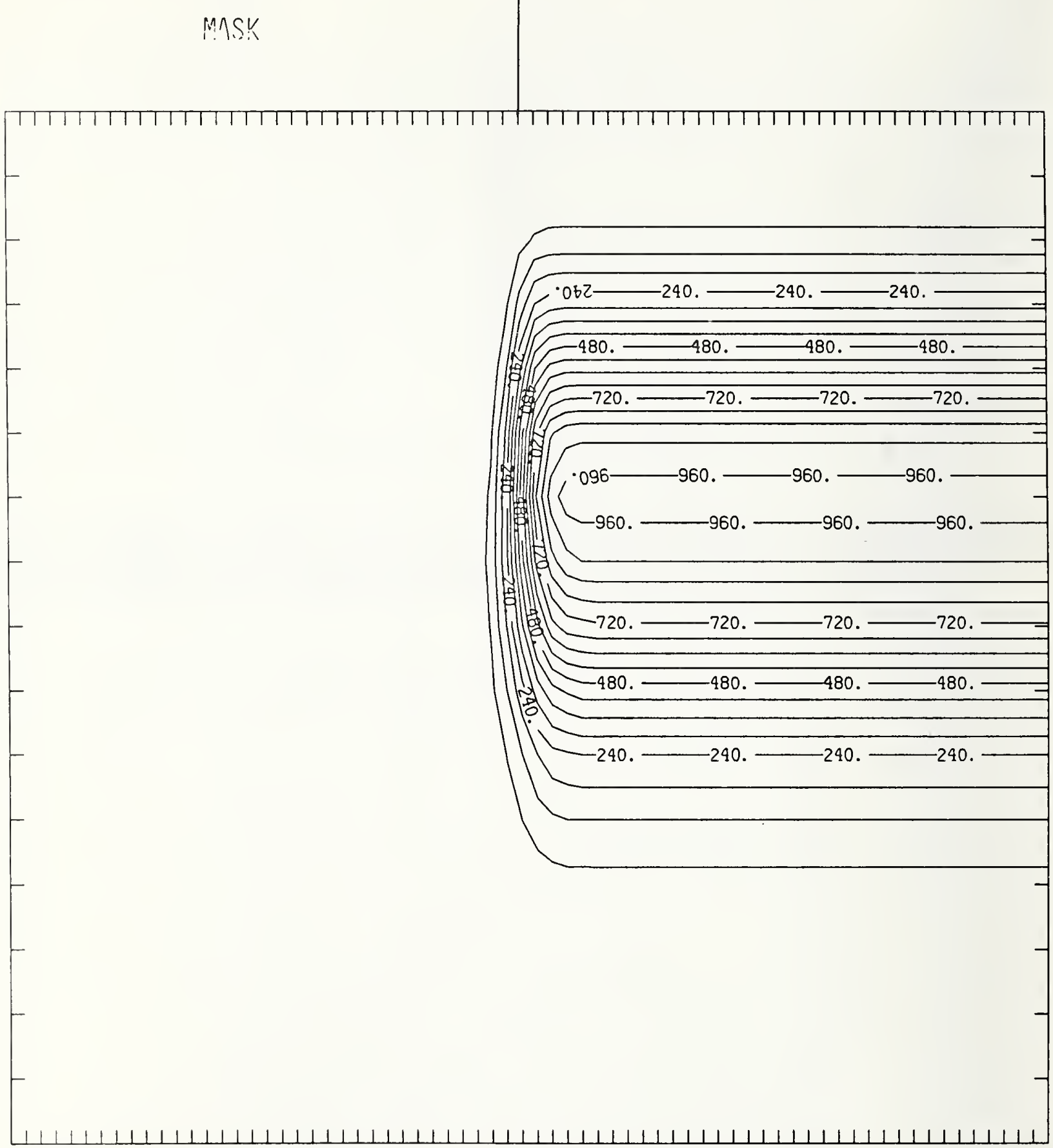

Figure Sn125-3 Two-dimensional distribution of $125 \mathrm{keV}$ tin implanted past a mask edge. The length increment (distance between tick marks) is $0.01 \mu \mathrm{m}$ and the scale factor is 1000 . 


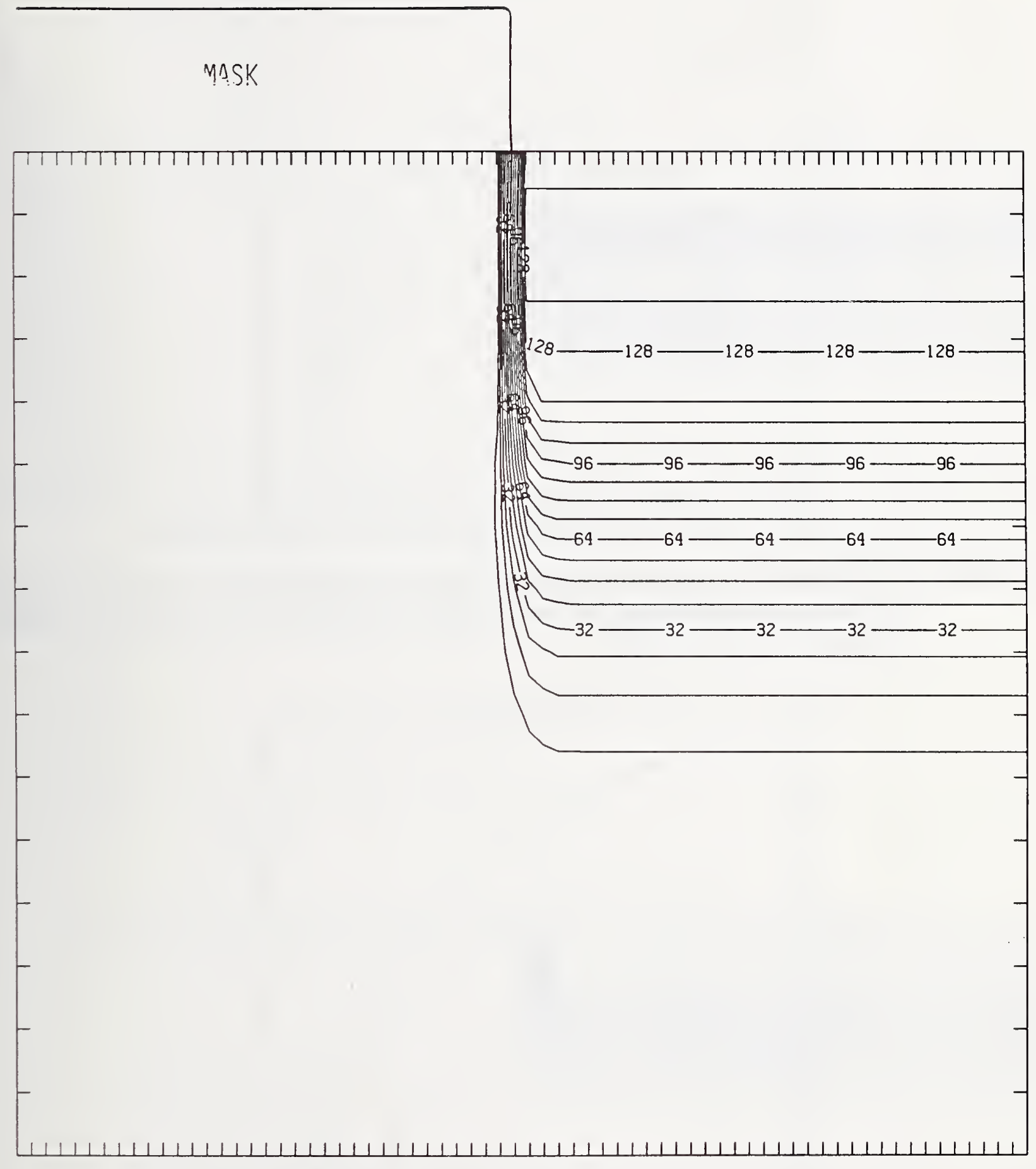

Figure Sn125-4 Two-dimensional distribution of Frenkel pairs created by $125 \mathrm{keV}$ tin implanted past a mask edge. The length increment (distance between tick marks) is $0.01 \mu \mathrm{m}$ and the scale factor is 0.1 . 


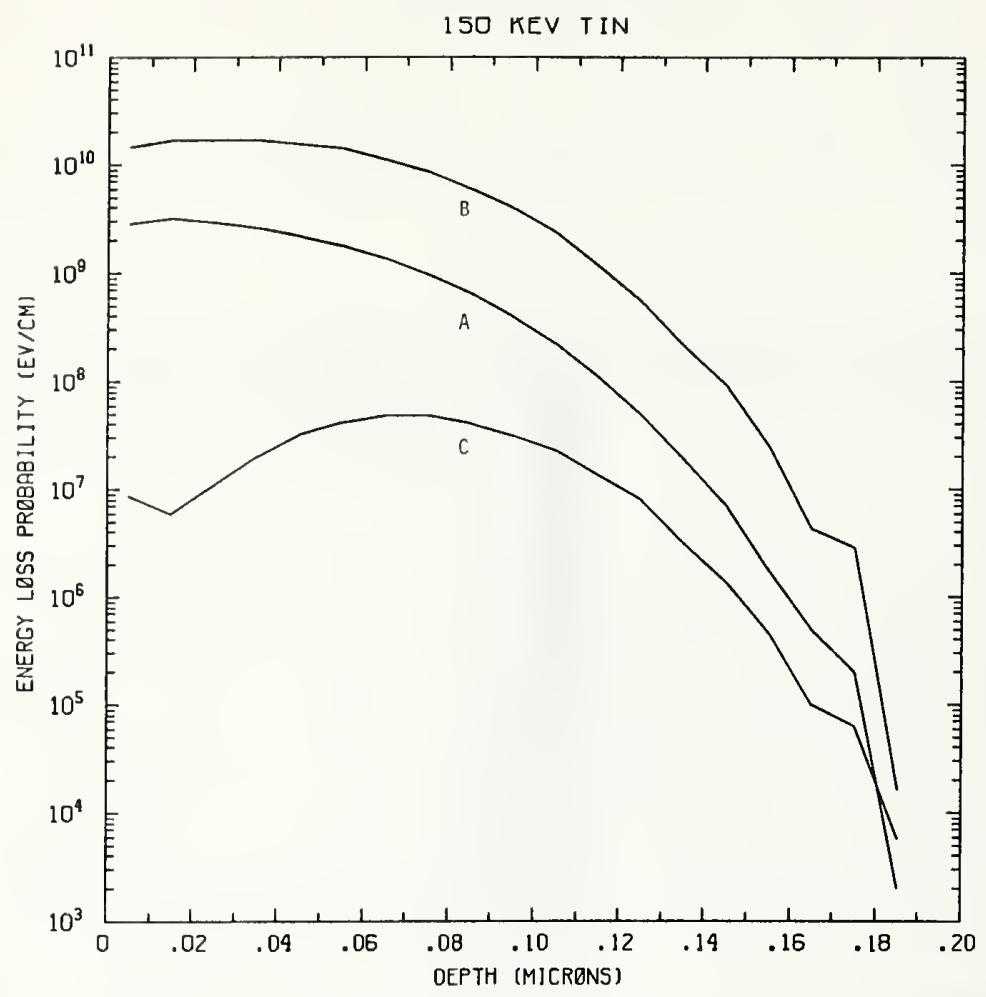

Figure Sn150-1 One-dimensional probability distributions for energy loss due to electronic excitation (A), displacement damage(B), and phonon excitation (C) for $150 \mathrm{keV}$ tin normally incident on a silicon target.

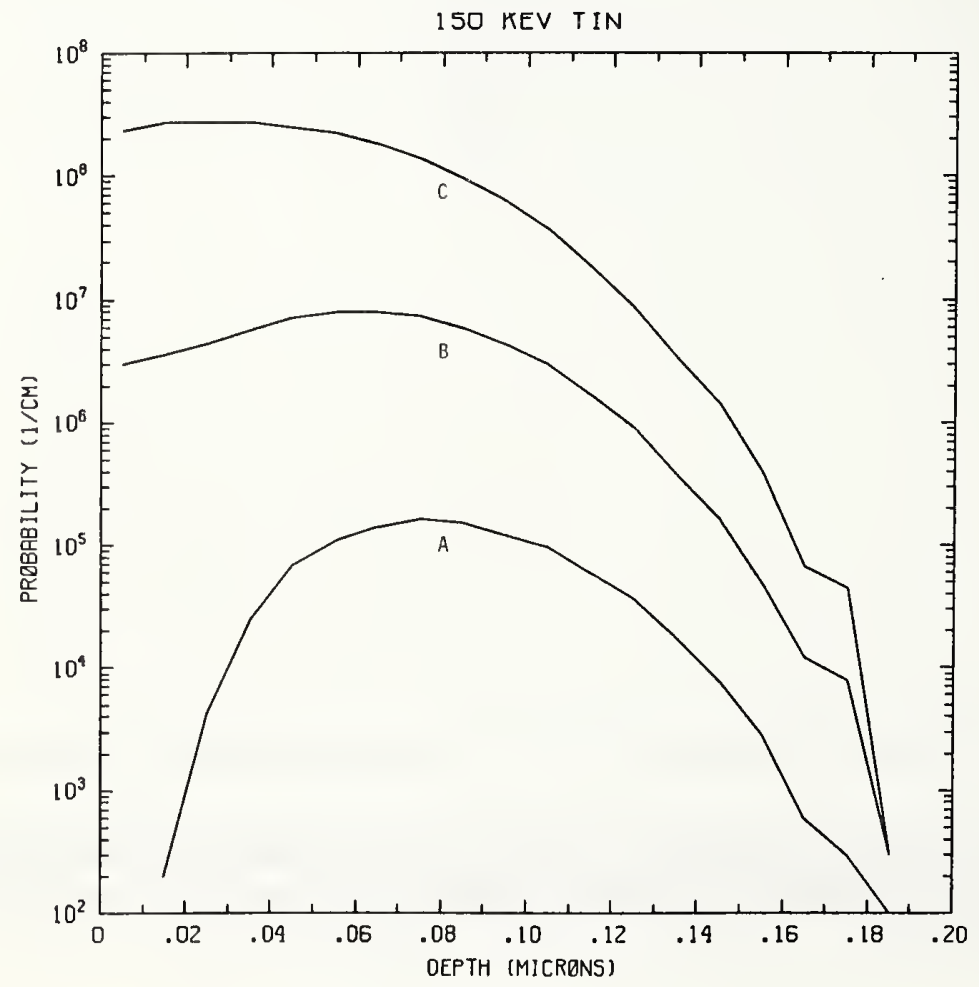

Figure Sn150-2 One-dimensional probability distributions for implanted tin (A), primary silicon displacements (B), and Frenkel pairs (C) for $150 \mathrm{keV}$ tin normally incident on a silicon target. 


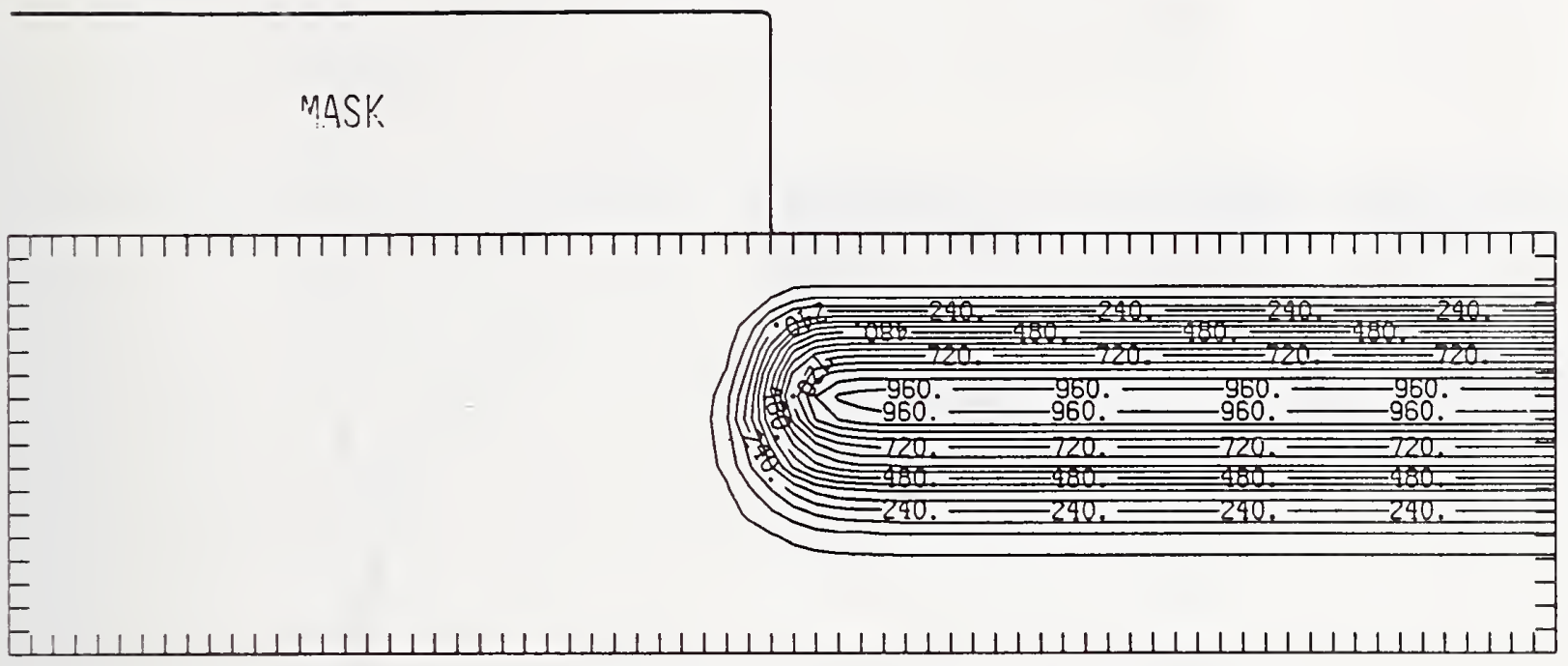

Figure Sn150-3 Two-dimensional distribution of $150 \mathrm{keV}$ tin implanted past a mask edge. The length increment (distance between tick marks) is $0.01 \mu \mathrm{m}$ and the scale factor is 1000 .

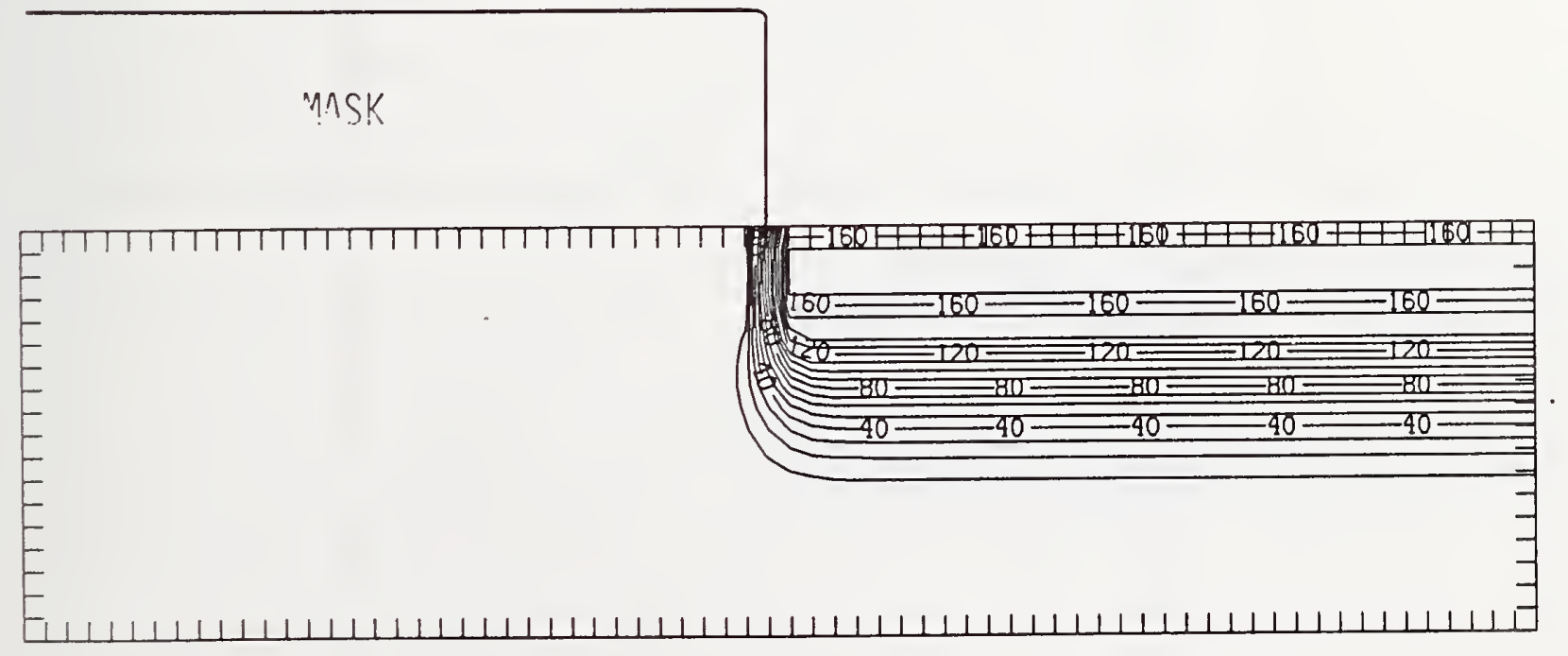

Figure Sn150-4 Two-dimensional distribution of Frenkel pairs created by $150 \mathrm{keV}$ tin implanted past a mask edge. The length increment (distance between tick marks) is $0.01 \mu \mathrm{m}$ and the scale factor is 0.1 . 


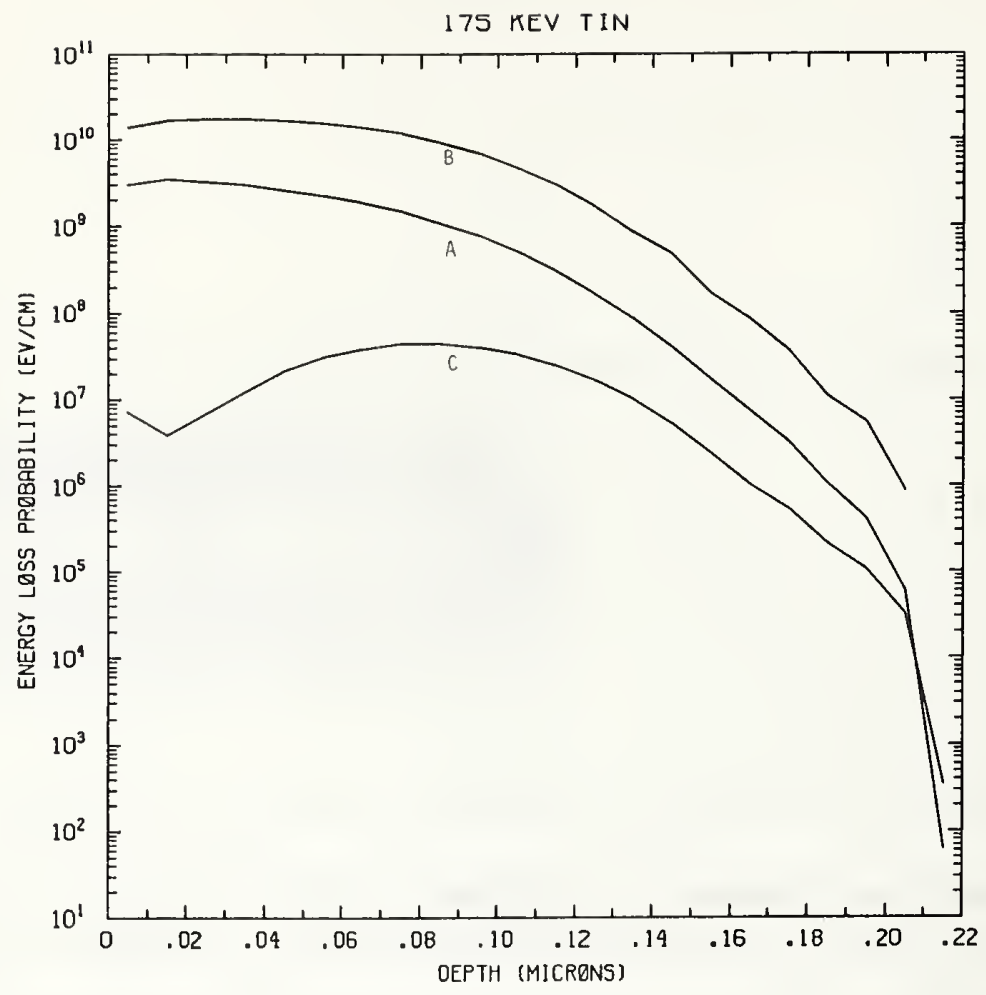

Figure Sn175-1 One-dimensional probability distributions for energy loss due to electronic excitation (A), displacement damage(B), and phonon excitation (C) for $175 \mathrm{keV}$ tin normall incident on a silicon target.

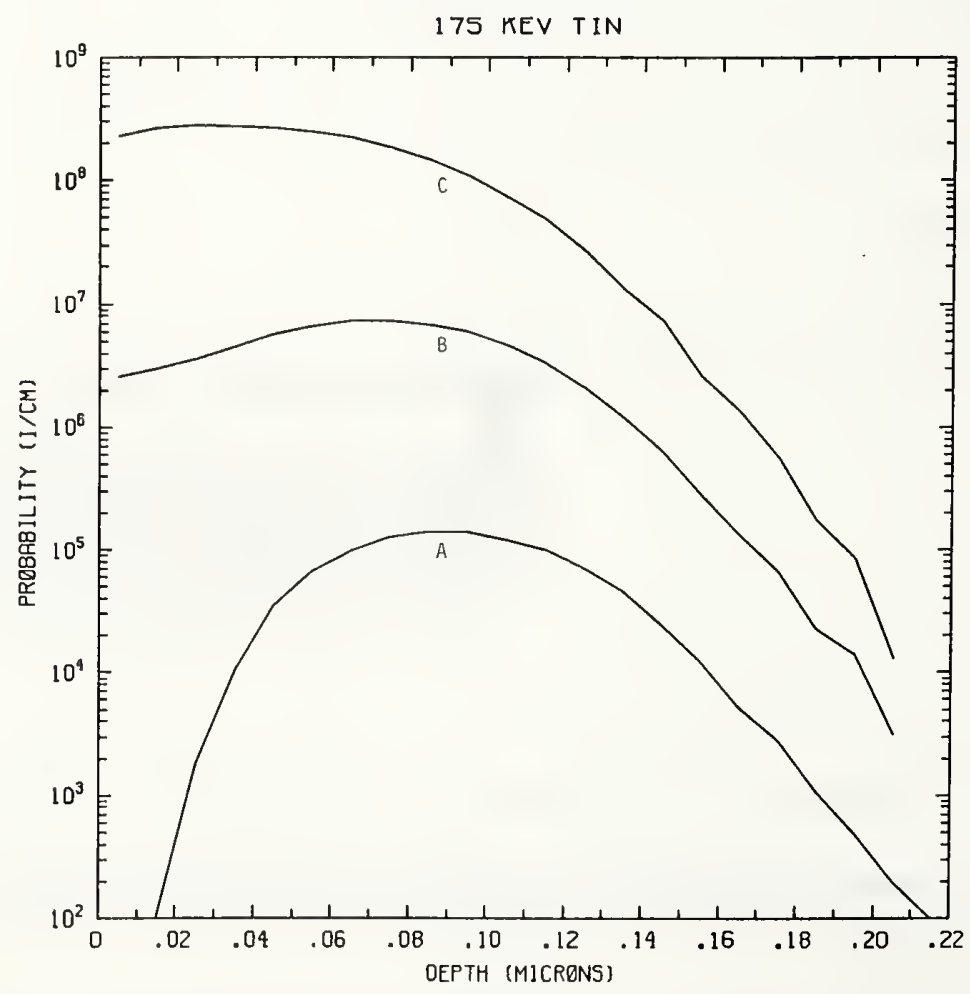

Figure Sn175-2 One-dimensional probability distributions for implanted tin (A), primary silicon displacements (B), and Frenkel pairs (C) for $175 \mathrm{keV}$ tin normally incident on a silicon target. 


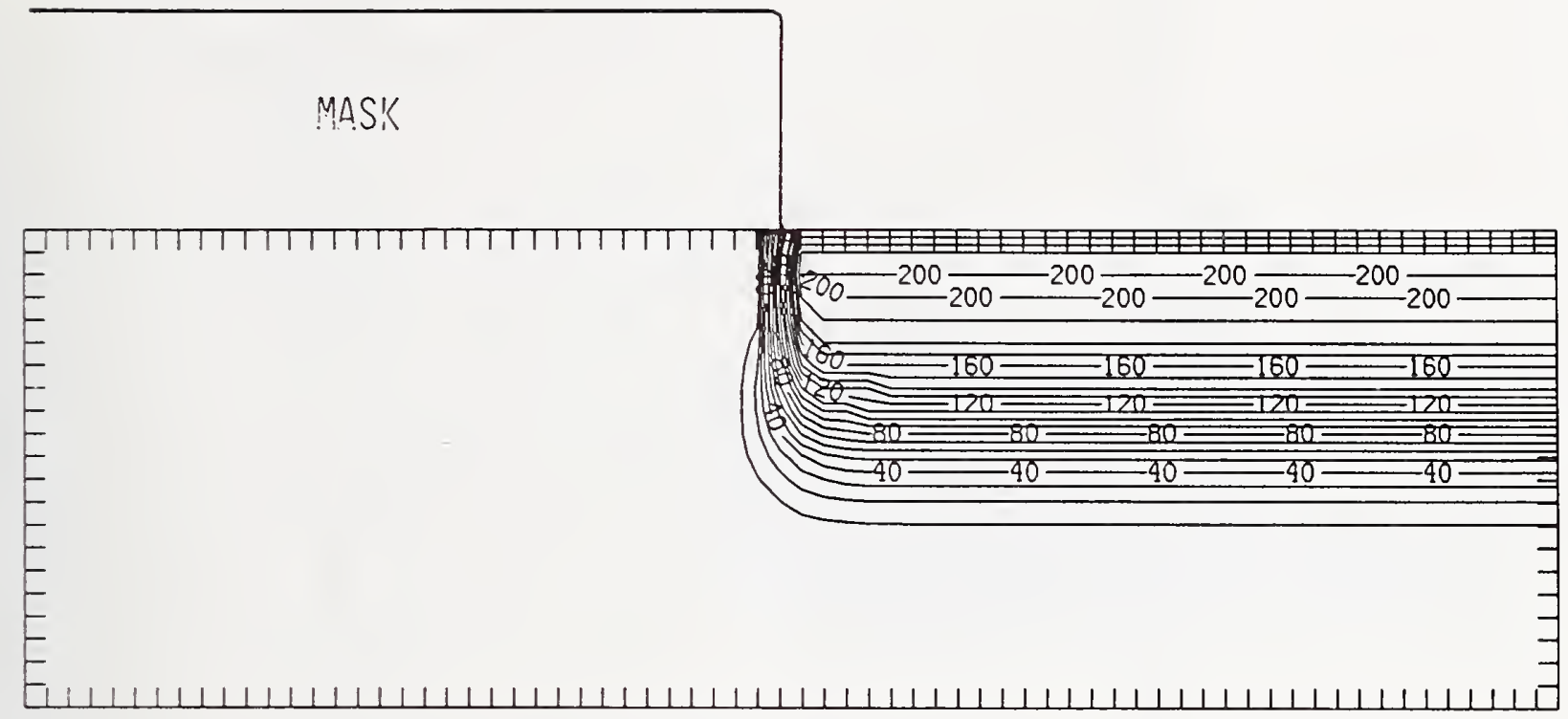

Figure Sn175-3 Two-dimensional distribution of $175 \mathrm{keV}$ tin implanted past a mask edge. The length increment (distance between tick marks) is $0.01 \mu \mathrm{m}$ and the scale factor is 1000 .

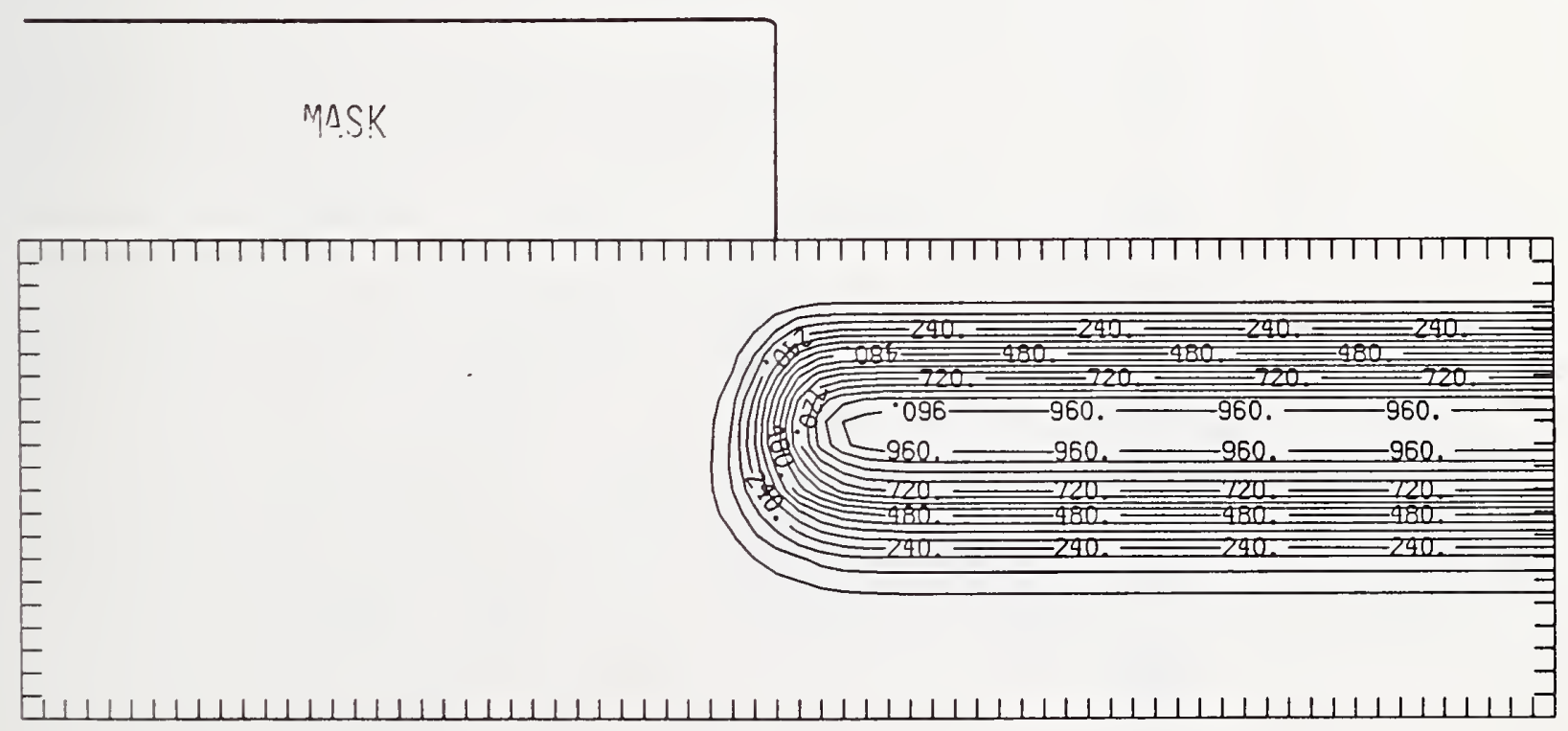

Figure Sn175-4 Two-dimensional distribution of Frenkel pairs created by $175 \mathrm{keV}$ tin implanted past a mask edge. The length increment (distance between tick marks) is $0.01 \mu \mathrm{m}$ and the scale factor is 0.1 . 


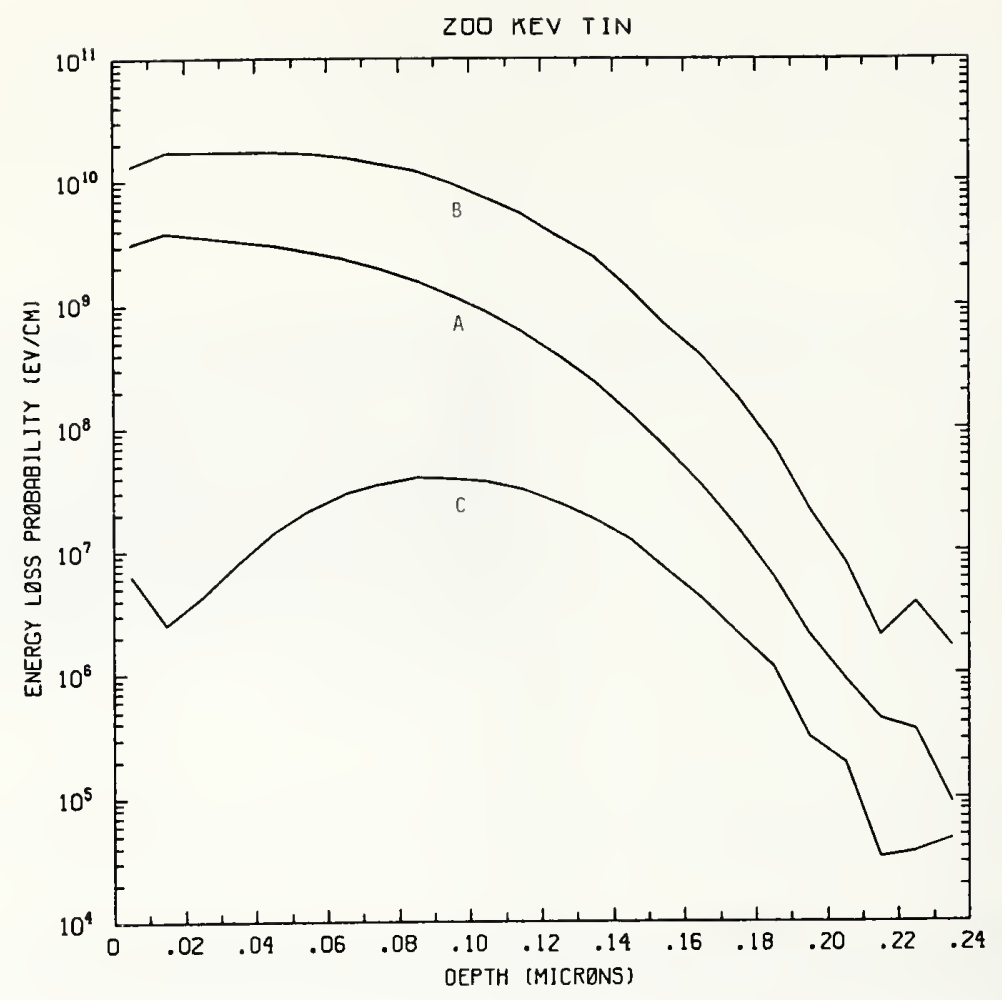

Figure Sn200-1 One-dimensional probability distributions for energy loss due to electronic excitation (A), displacement damage(B), and phonon excitation (C) for $200 \mathrm{keV}$ tin normally incident on a silicon target.

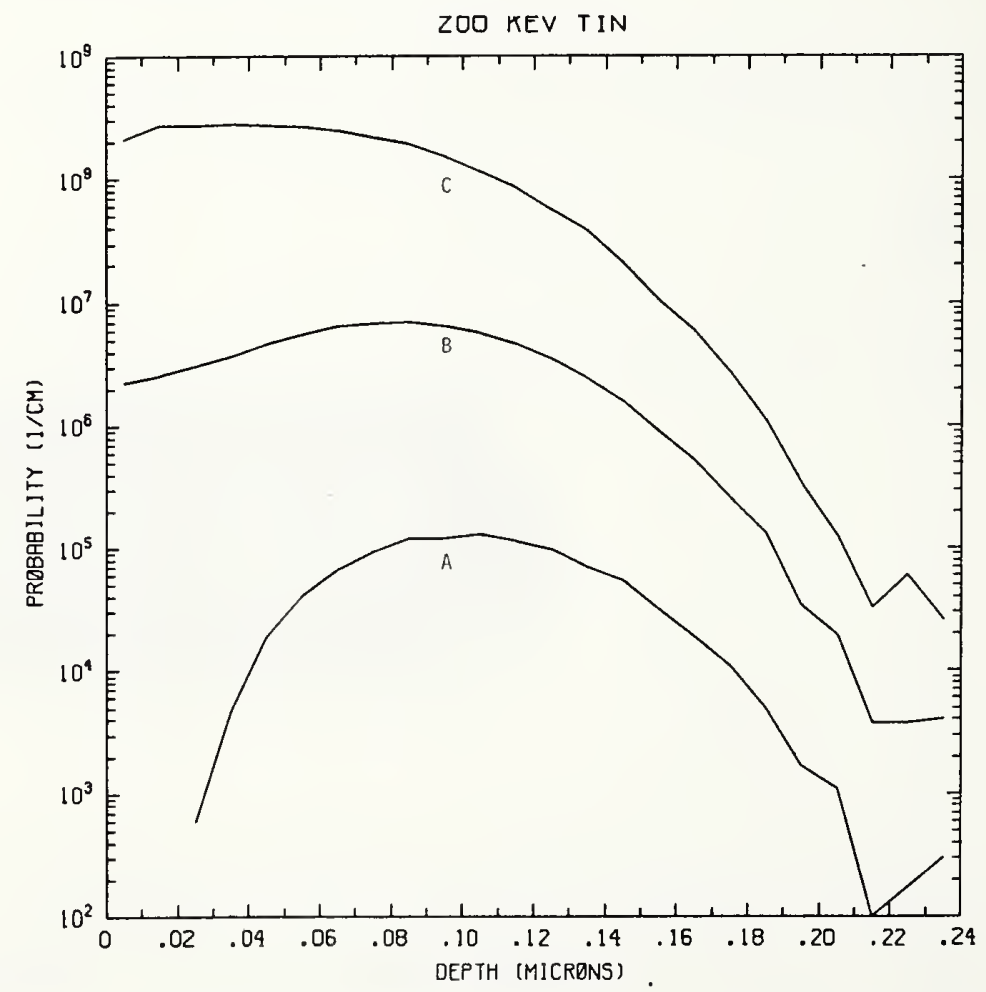

Figure Sn200-2 One-dimensional probability distributions for implanted tin (A), primary silicon displacements (B), and Frenkel pairs (C) for $200 \mathrm{keV}$ tin normally incident on a silicon target. 


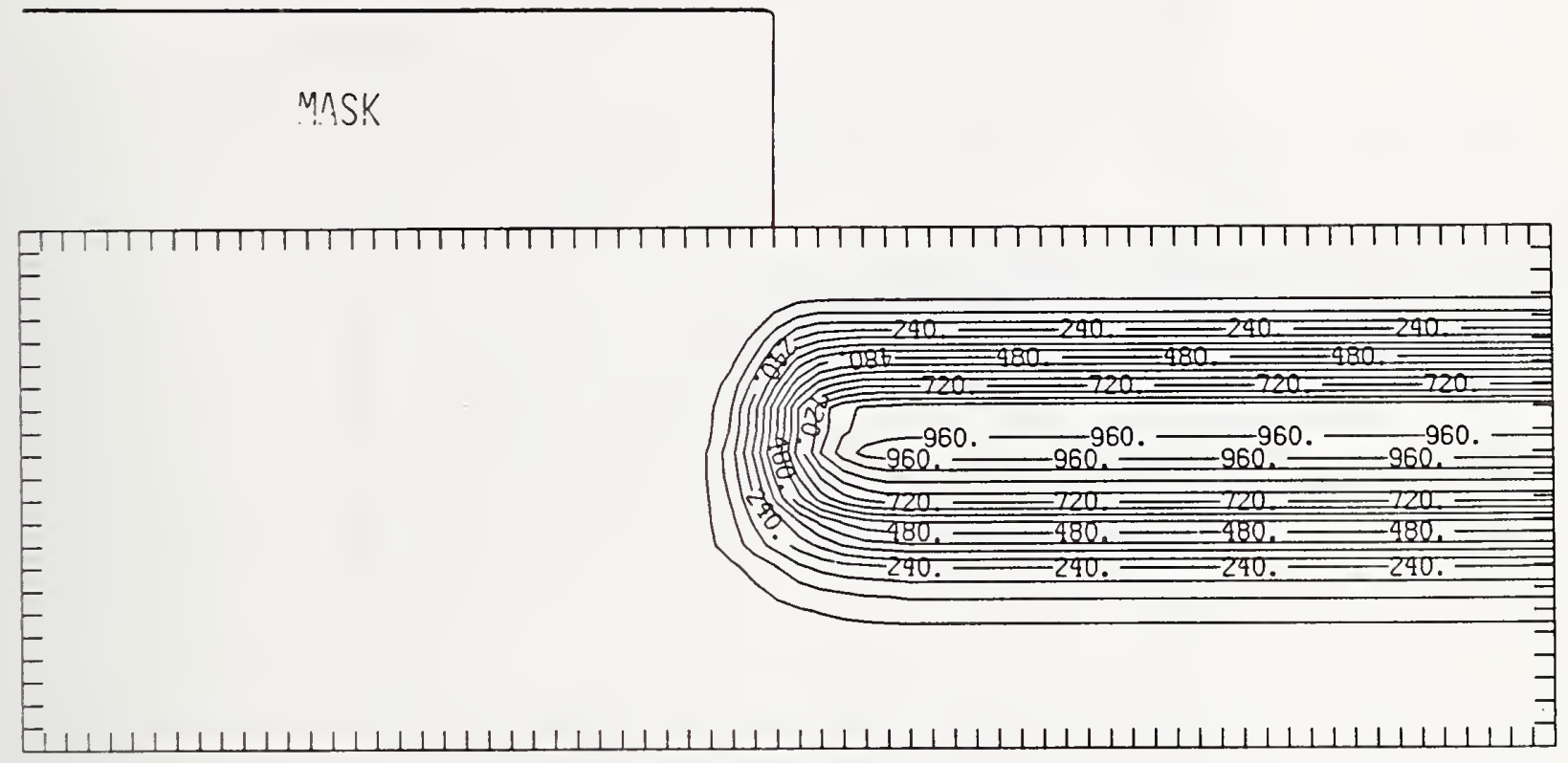

Figure Sn200-3 Two-dimensional distribution of $200 \mathrm{keV}$ tin implanted past a mask edge. The length increment (distance between tick marks) is $0.01 \mu \mathrm{m}$ and the scale factor is 1000 .

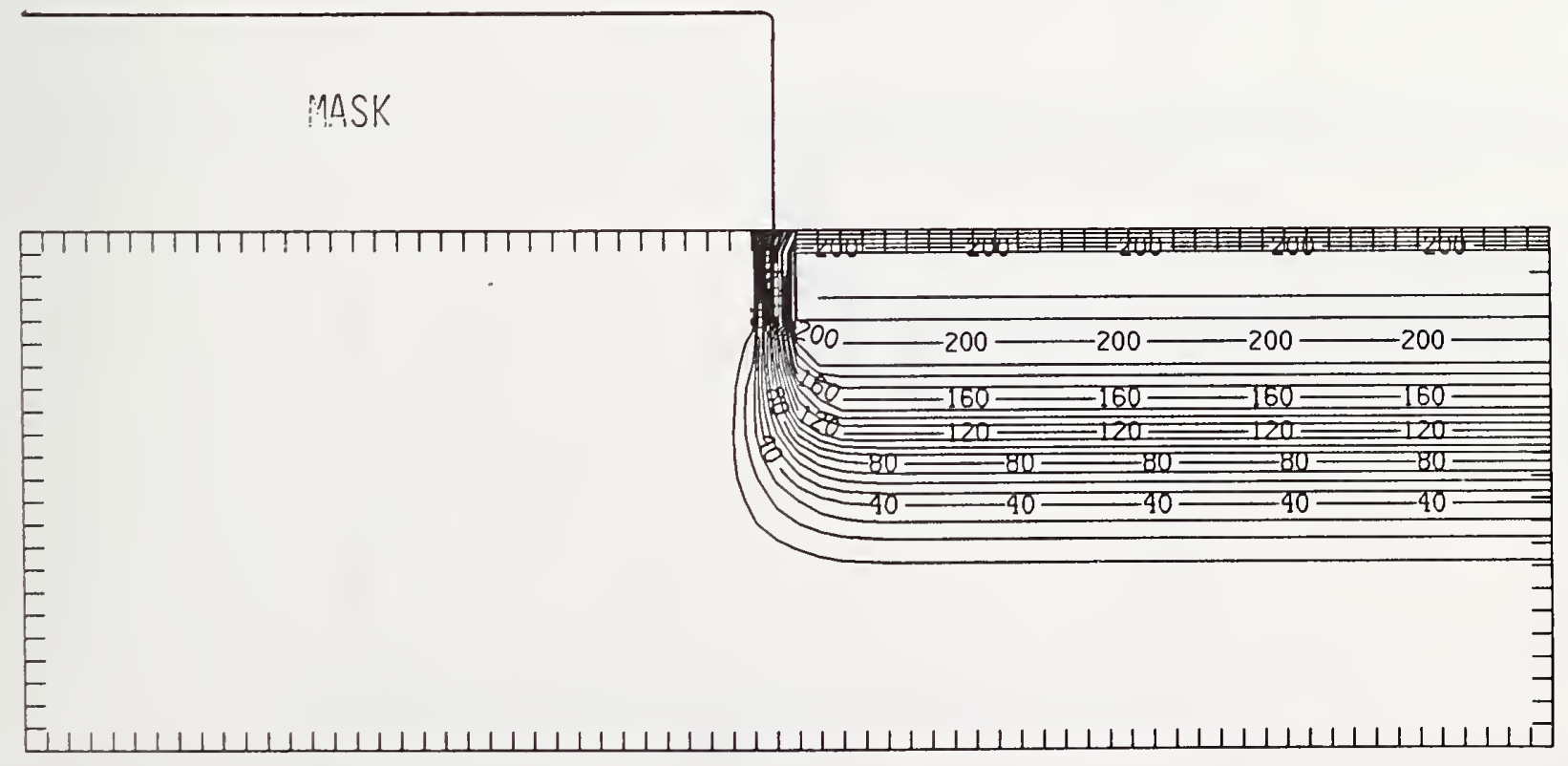

Figure Sn200-4 Two-dimensional distribution of Frenkel pairs created by $200 \mathrm{keV}$ tin implanted past a mask edge. The length increment (distance between tick marks) is $0.01 \mu \mathrm{m}$ and the scale factor is 0.1 . 


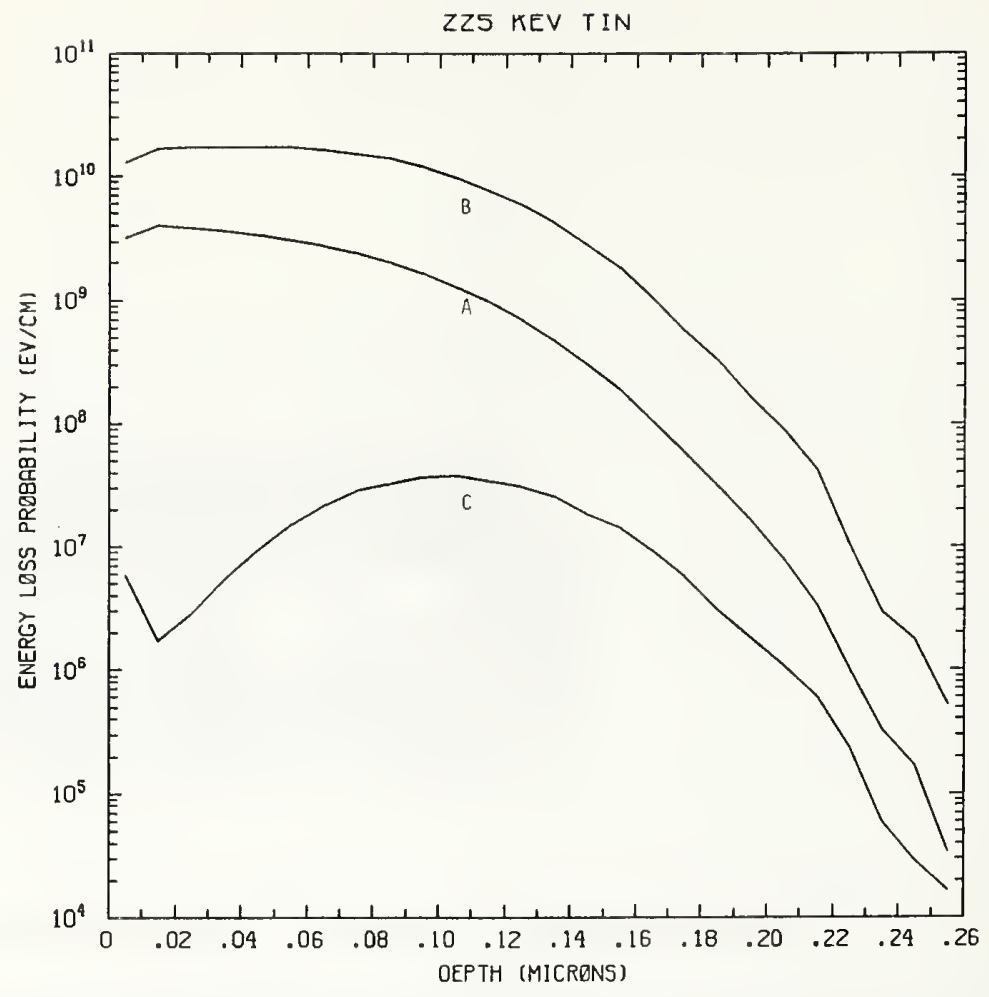

Figure Sn225-1 One-dimensional probability distributions for energy loss due to electronic excitation (A), displacement damage(B), and phonon excitation (C) for $225 \mathrm{keV}$ tin normally incident on a silicon target.

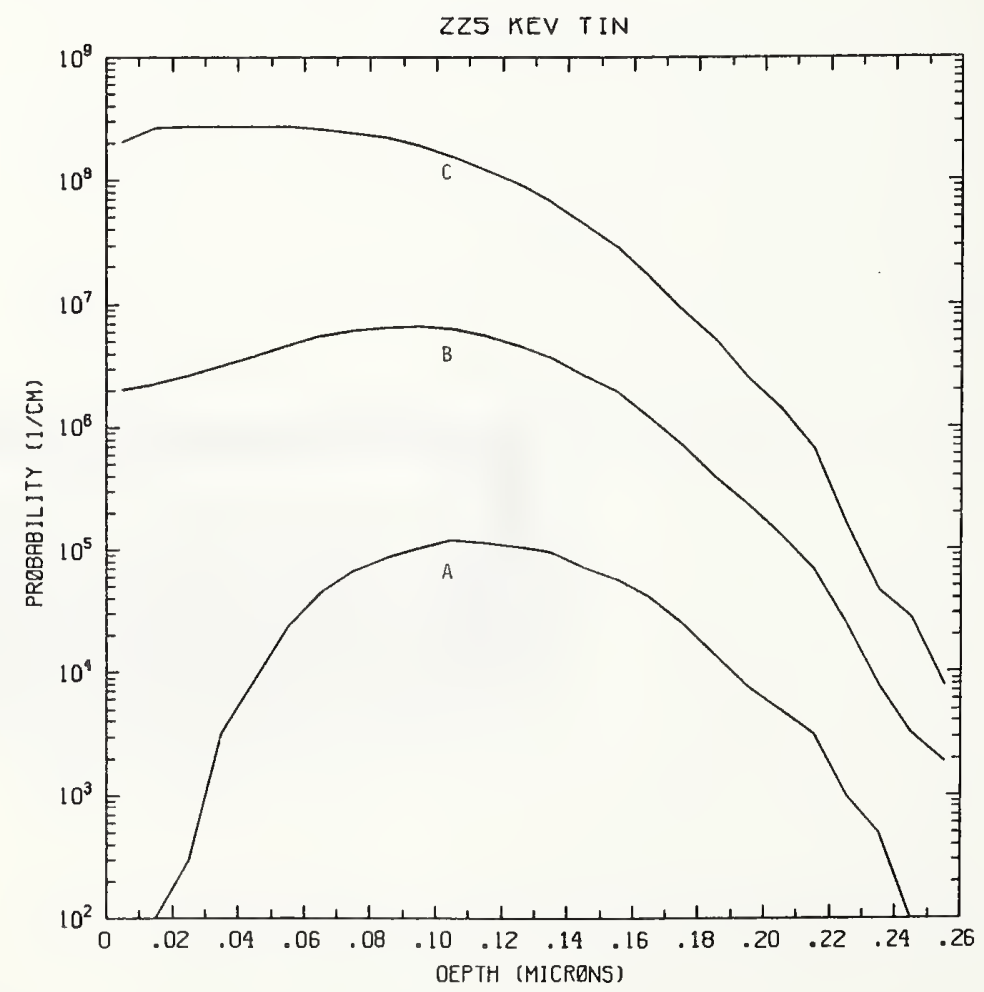

Figure Sn225-2 One-dimensional probability distributions for implanted tin (A), primary silicon displacements (B), and Frenkel pairs (C) for $225 \mathrm{keV}$ tin normally incident on a silicon target. 


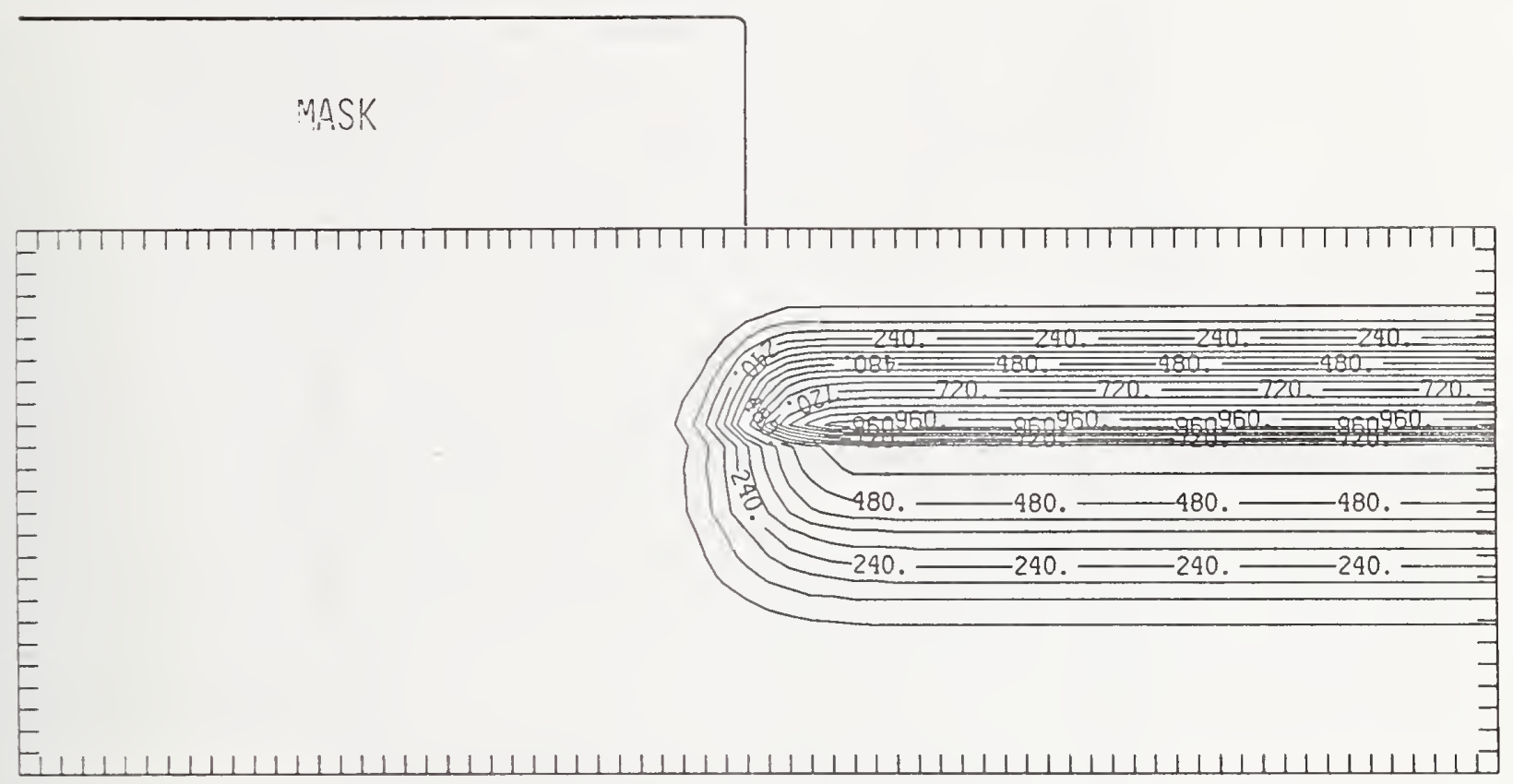

Figure Sn225-3 Two-dimensional distribution of $225 \mathrm{keV}$ tin implanted past a mask edge. The length increment (distance between tick marks) is $0.01 \mu \mathrm{m}$ and the scale factor is 1000 .

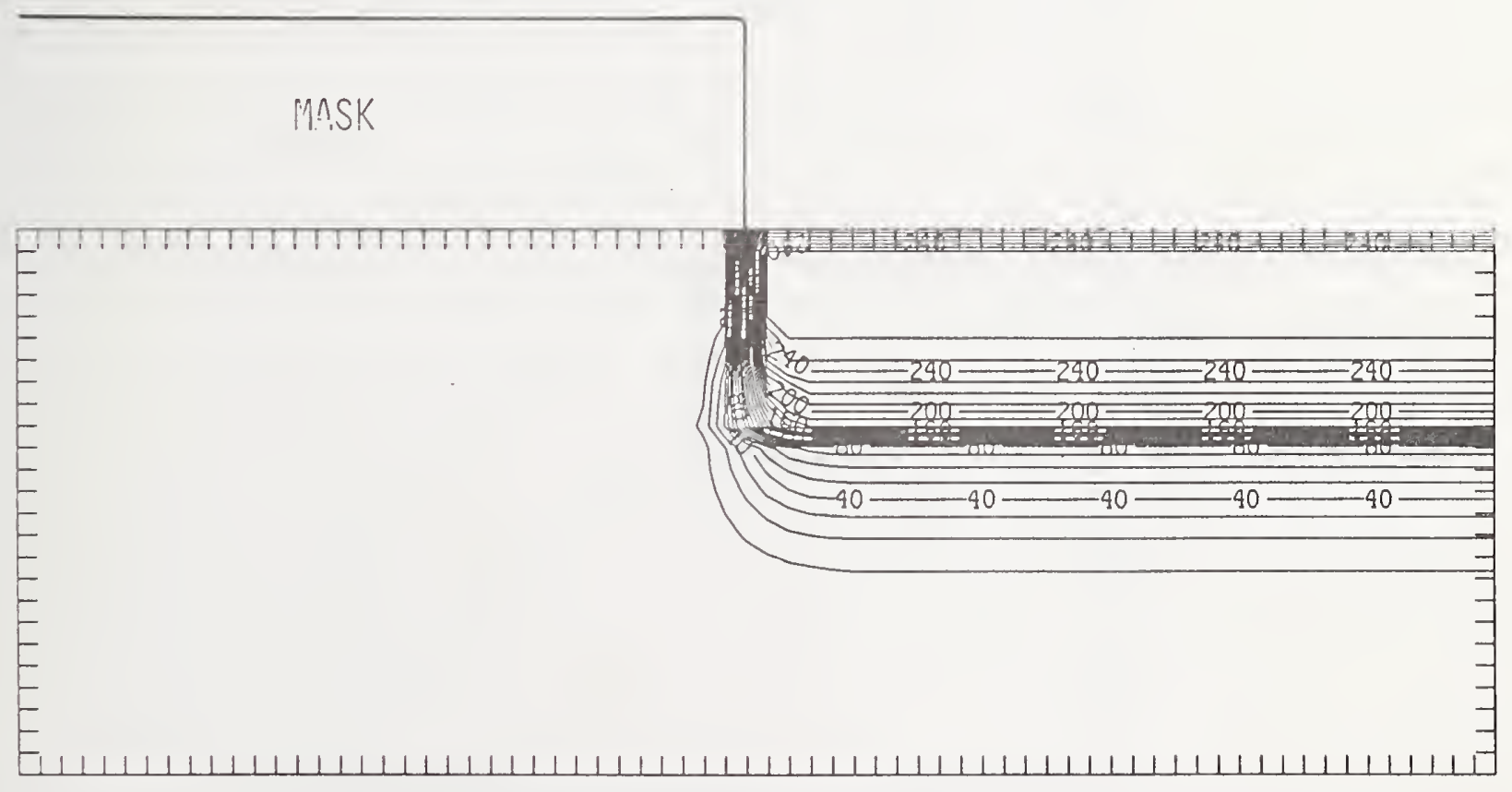

Figure Sn225-4 Two-dimensional distribution of Frenkel pairs created by $225 \mathrm{keV}$ tin implanted past a mask edge. The length increment (distance between tick marks) is $0.01 \mu \mathrm{m}$ and the scale factor is 0.1 . 


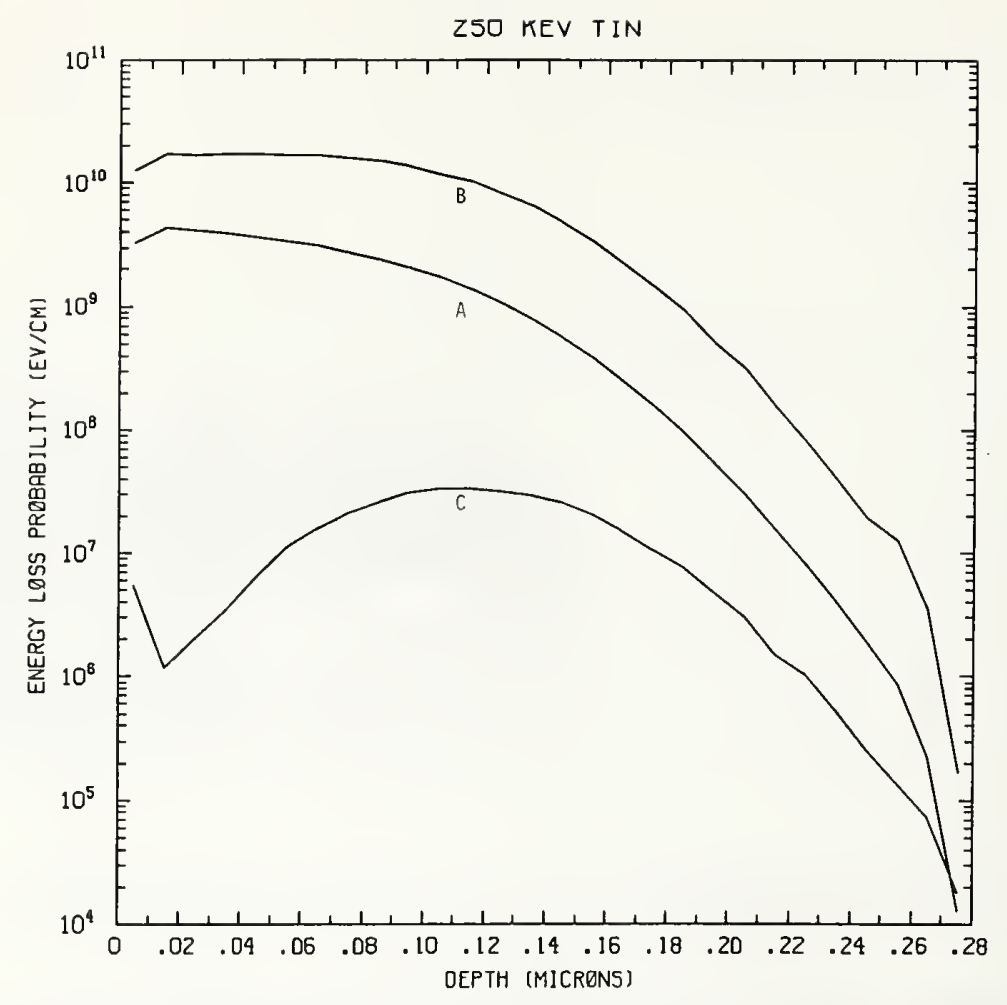

Figure Sn250-1 One-dimensional probability distributions for energy loss due to electronic excitation (A), displacement damage(B), and phonon excitation (C) for $250 \mathrm{keV}$ tin normally incident on a silicon target.

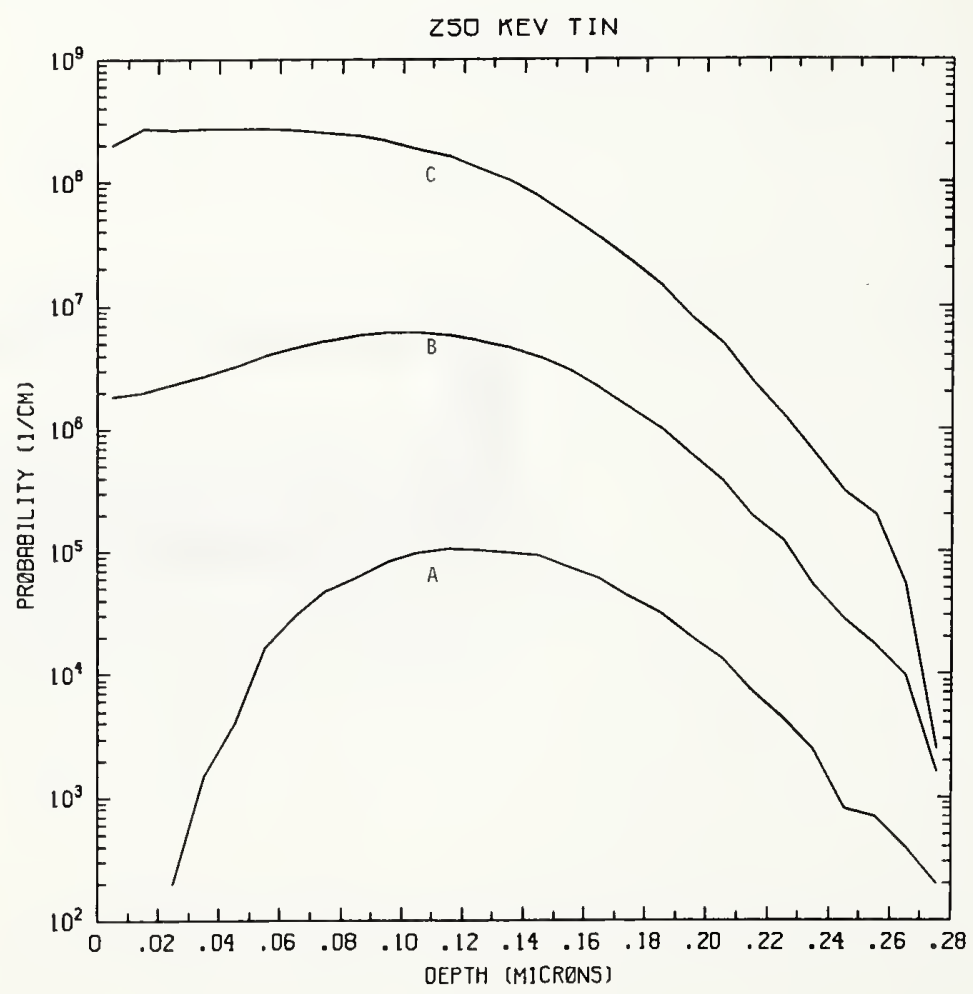

Figure Sn250-2 One-dimensional probability distributions for implanted tin (A), primary silicon displacements (B), and Frenkel pairs (C) for $250 \mathrm{keV}$ tin normally incident on a silicon target. 


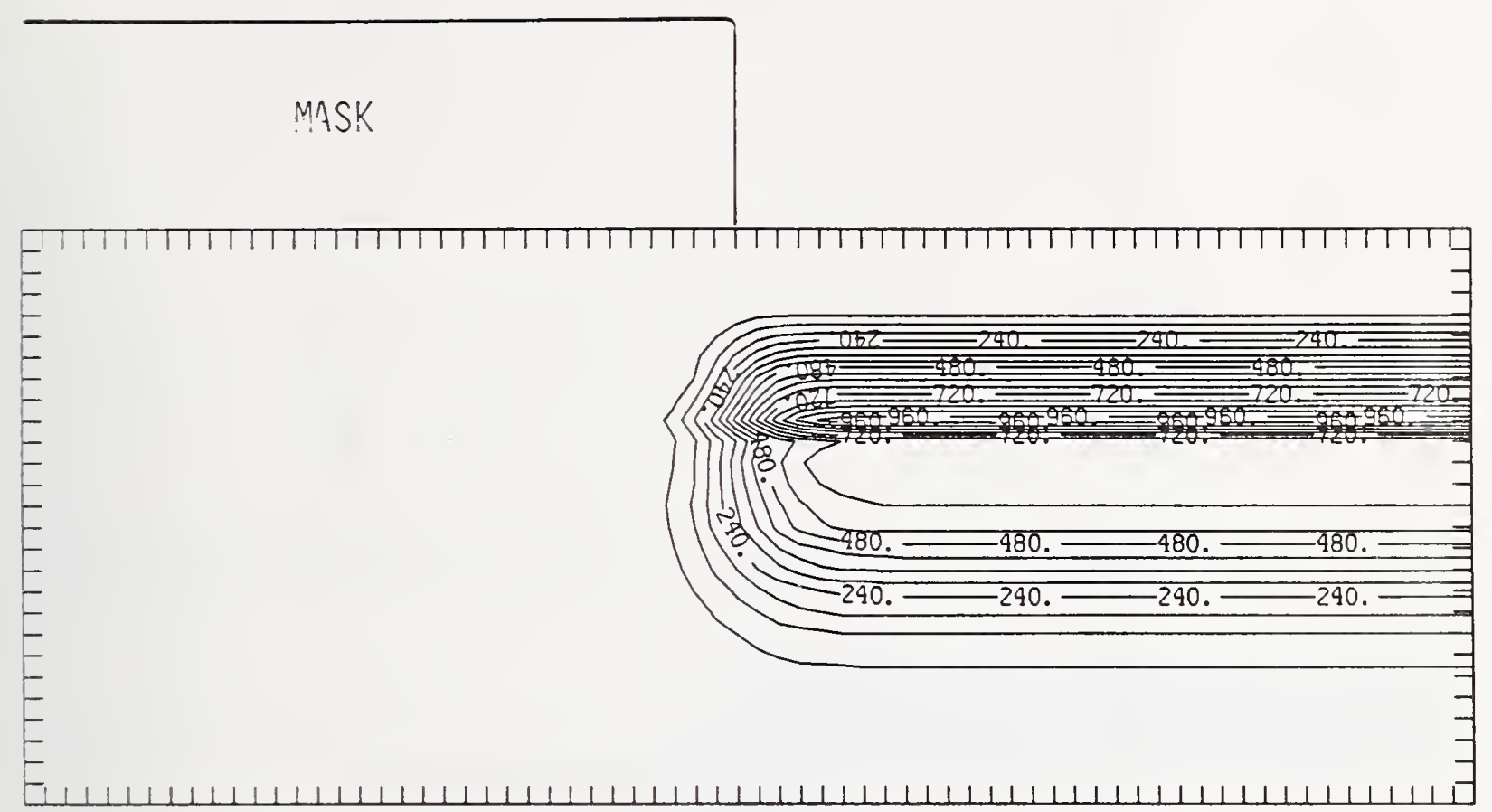

Figure Sn250-3 Two-dimensional distribution of $250 \mathrm{keV}$ tin implanted past a mask edge. The length increment (distance between tick marks) is $0.01 \mu \mathrm{m}$ and the scale factor is 1000 .

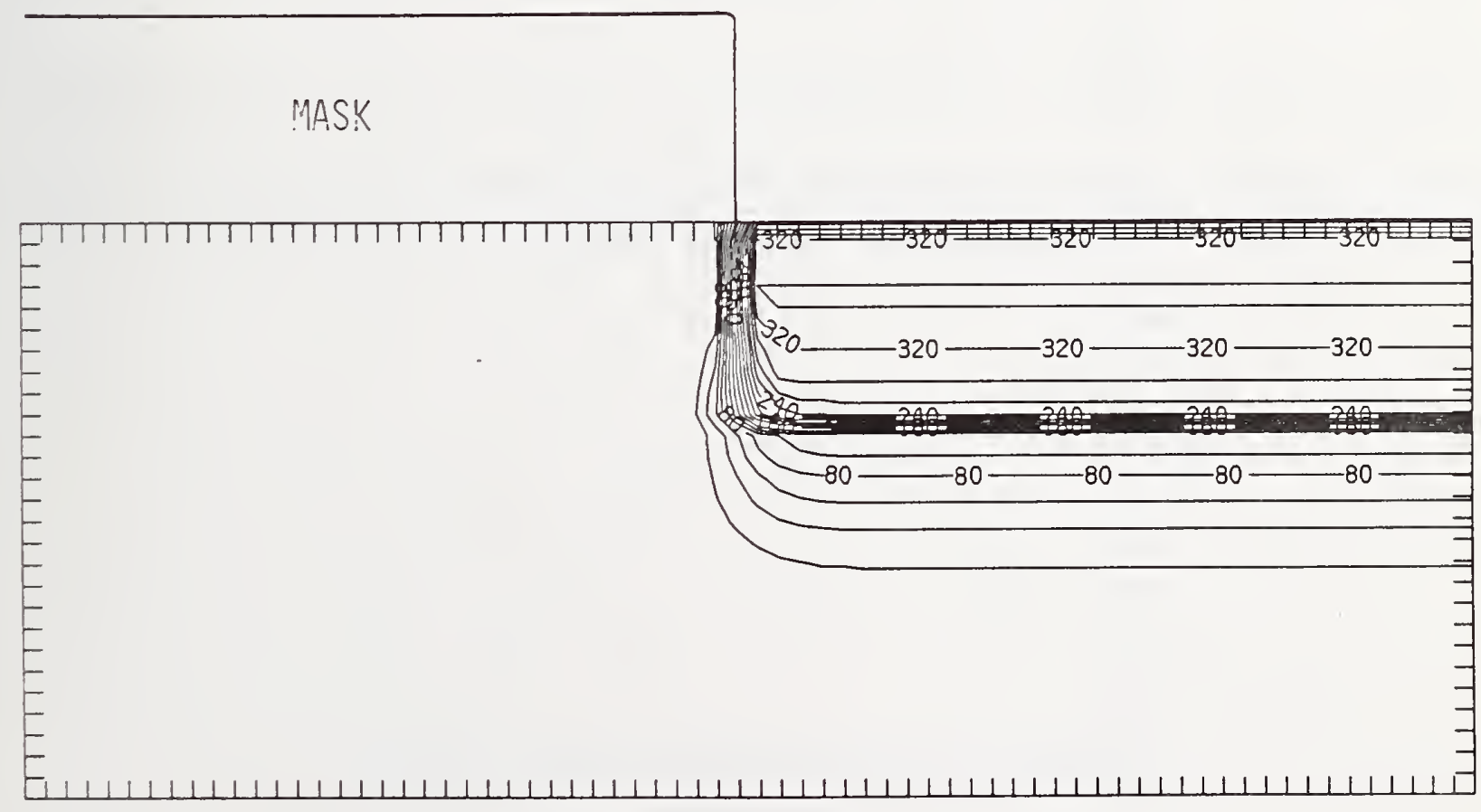

Figure Sn250-4 Two-dimensional distribution of Frenkel pairs created by $250 \mathrm{keV}$ tin implanted past a mask edge. The length increment (distance between tick marks) is $0.01 \mu \mathrm{m}$ and the scale factor is 0.1 . 


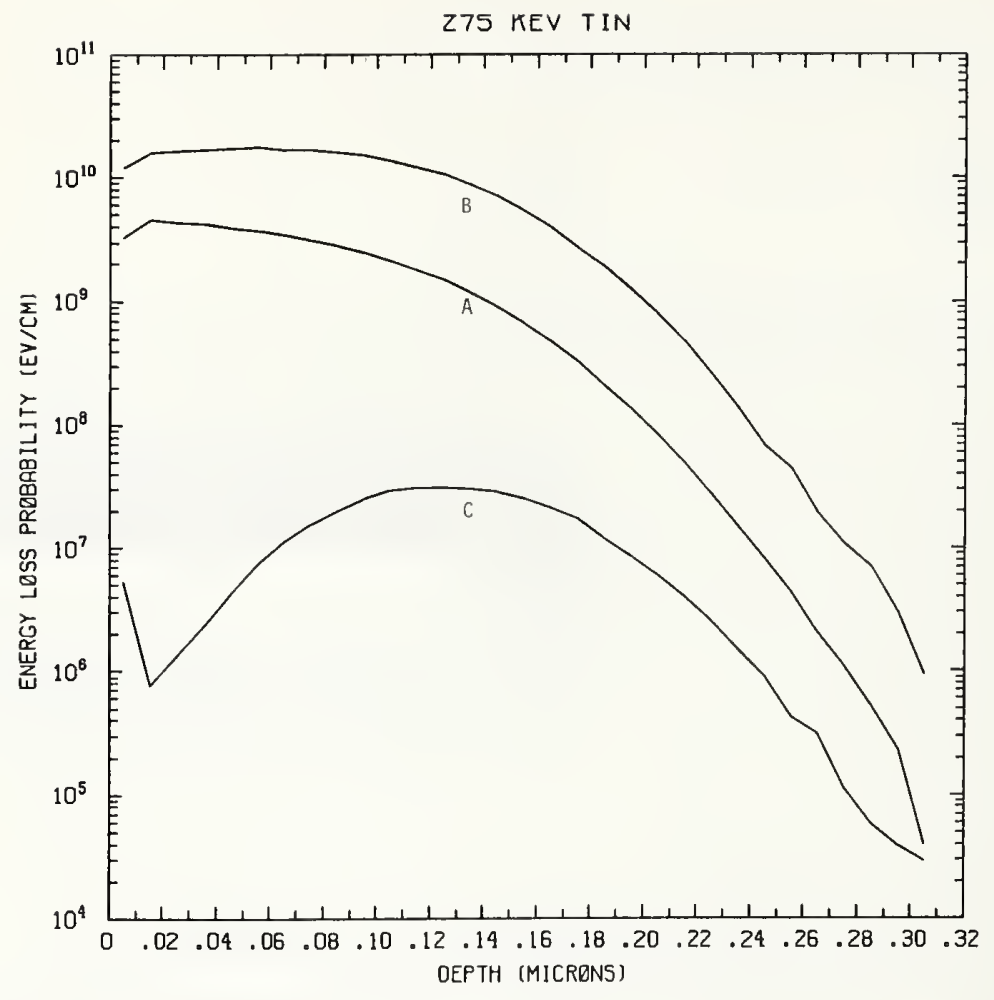

Figure Sn275-1 One-dimensional probability distributions for energy loss due to electronic excitation (A), displacement damage(B), and phonon excitation (C) for $275 \mathrm{keV}$ tin normally incident on a silicon target.

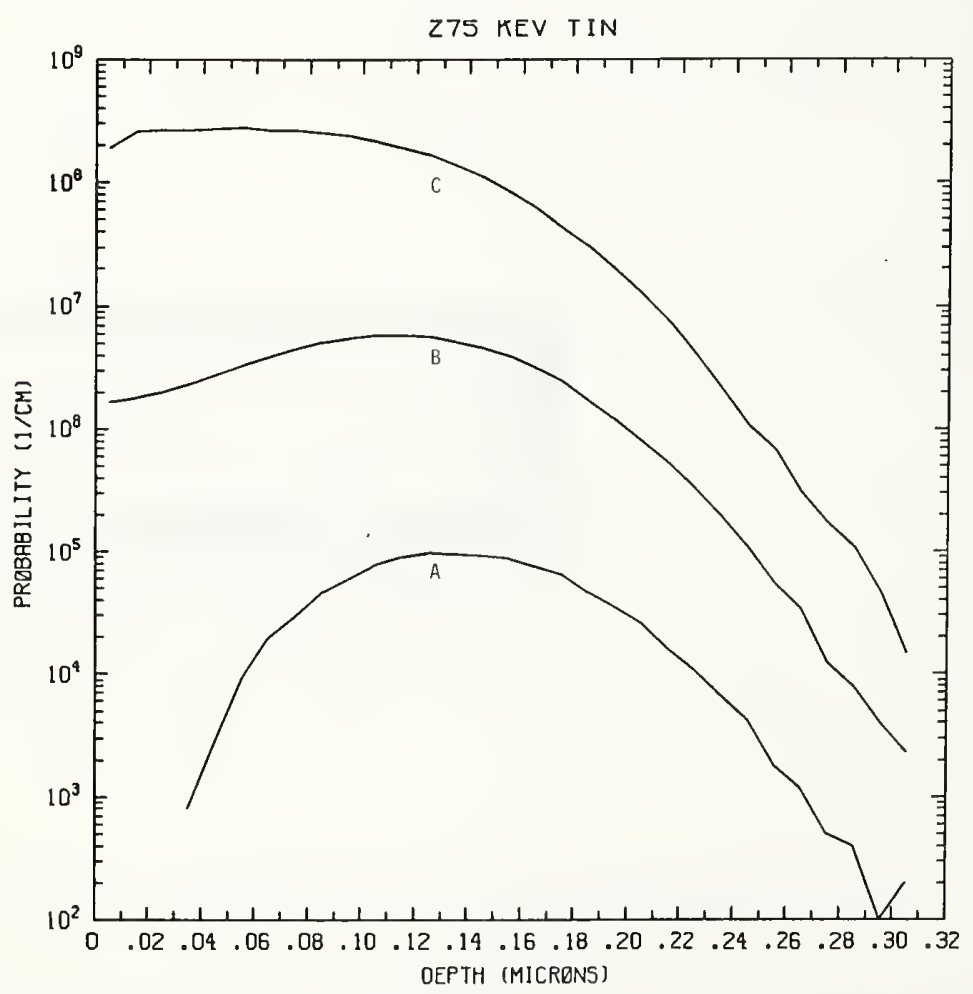

Figure Sn275-2 One-dimensional probability distributions for implanted tin (A), primary silicon displacements (B), and Frenkel pairs (C) for $275 \mathrm{keV}$ tin normally incident on a silicon target. 


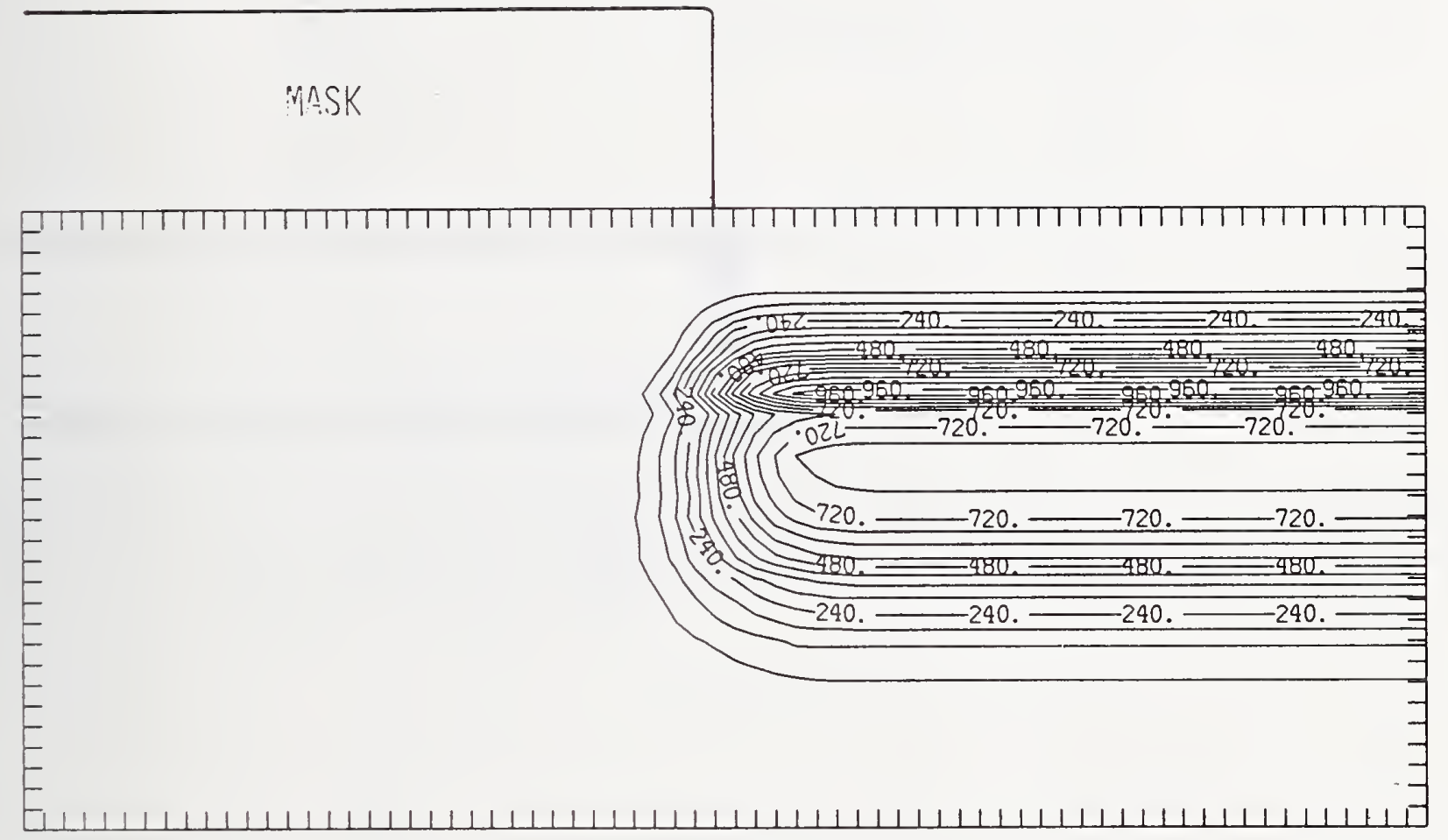

Figure Sn275-3 Two-dimensional distribution of $275 \mathrm{keV}$ tin implanted past a mask edge. The length increment (distance between tick marks) is $0.01 \mu \mathrm{m}$ and the scale factor is 1000 . 


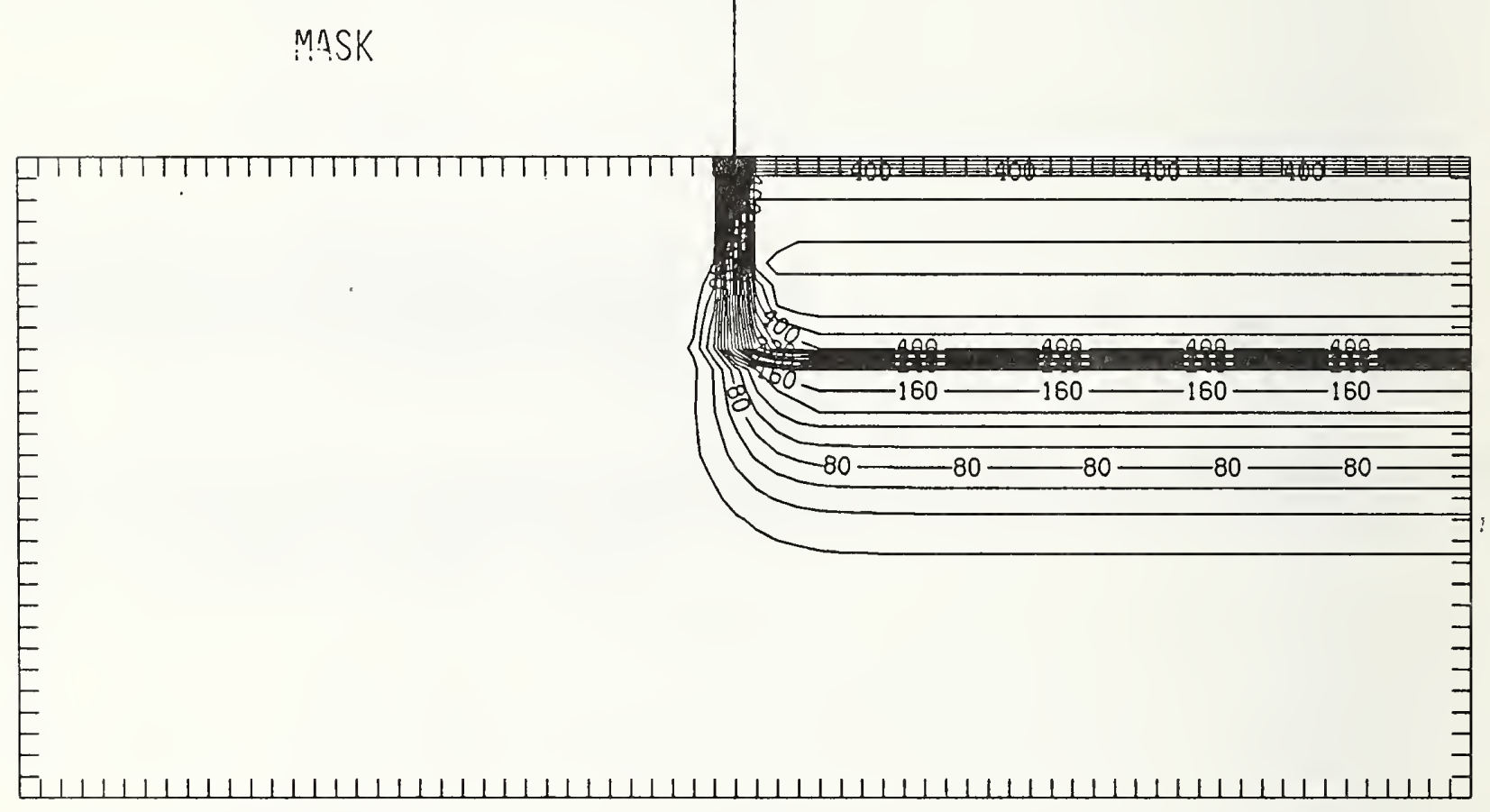

Figure Sn275-4 Two-dimensional distribution of Frenkel pairs created by $275 \mathrm{keV}$ tin implanted past a mask edge. The length increment (distance between tick marks) is $0.01 \mu \mathrm{m}$ and the scale factor is 0.1 . 


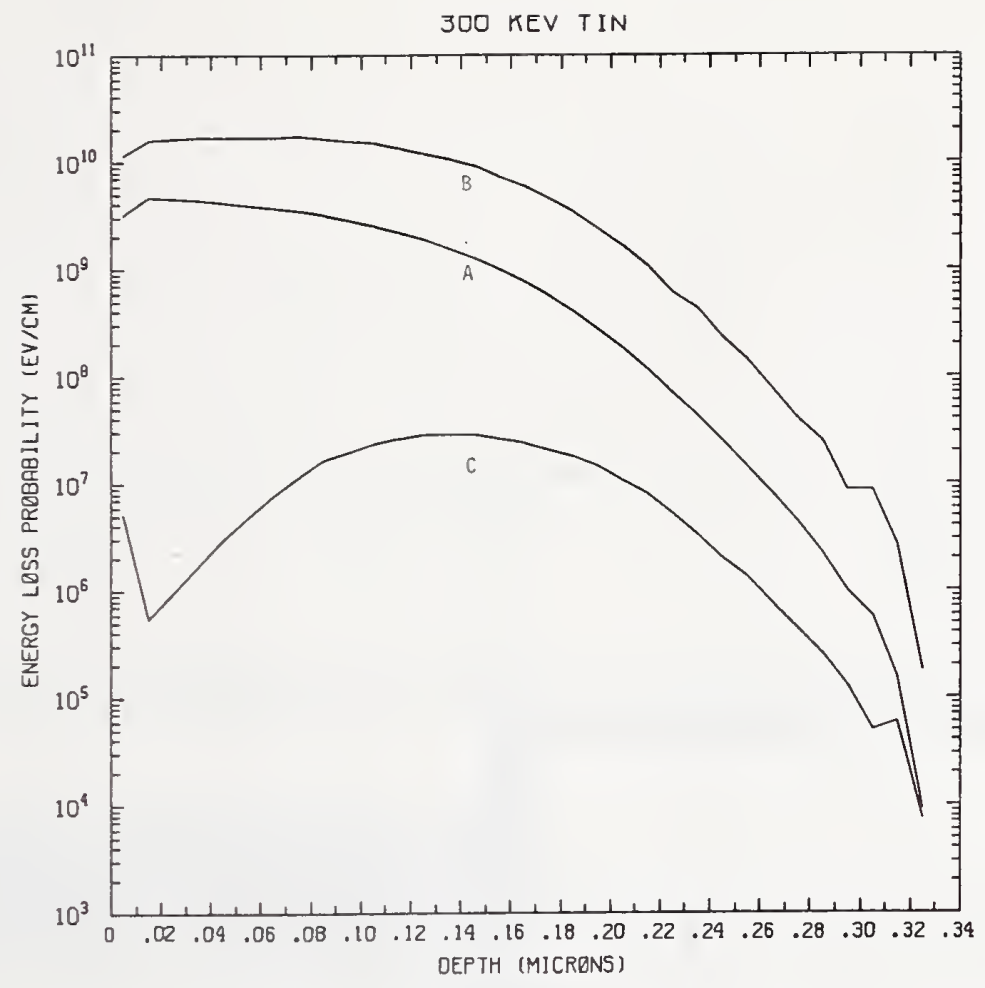

Figure Sn300-1 One-dimensional probability distributions for energy loss due to electronic excitation (A), displacement damage(B), and phonon excitation (C) for $300 \mathrm{keV}$ tin normally incident on a silicon target.

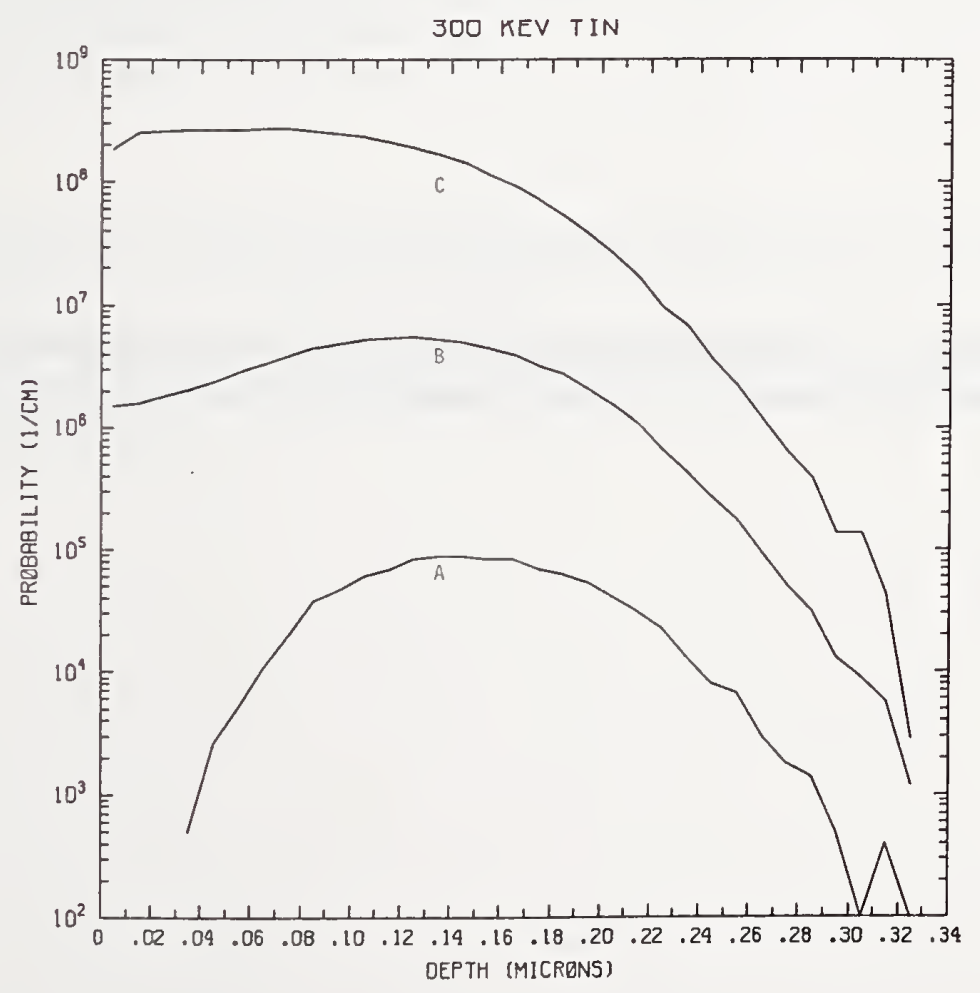

Figure Sn300-2 One-dimensional probability distributions for implanted tin (A), primary silicon displacements (B), and Frenkel pairs (C) for $300 \mathrm{keV}$ tin normally incident on a silicon target. 


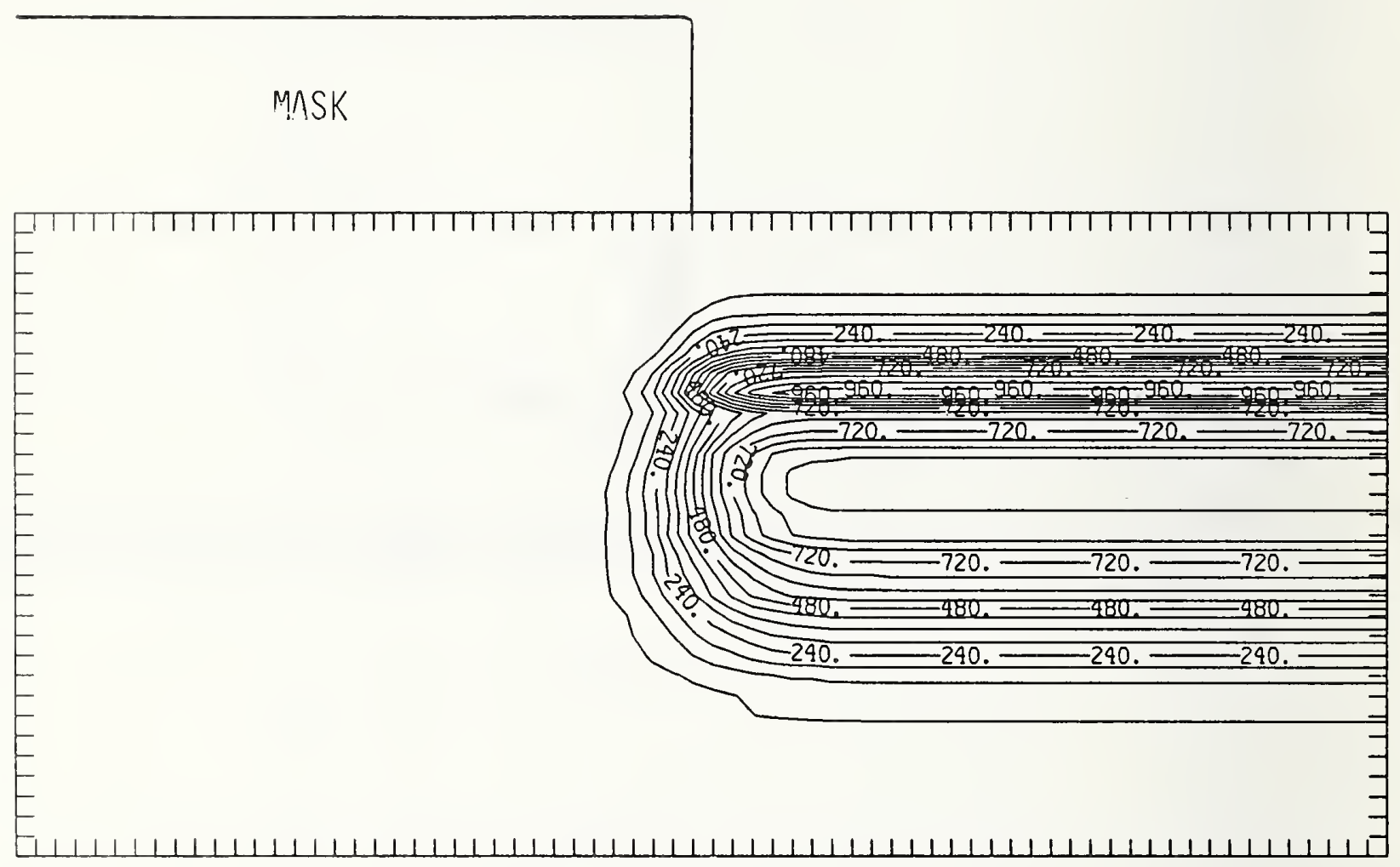

Figure Sn300-3 Two-dimensional distribution of $300 \mathrm{keV}$ tin implanted past a mask edge. The length increment (distance between tick marks) is $0.01 \mu \mathrm{m}$ and the scale factor is 1000 . 


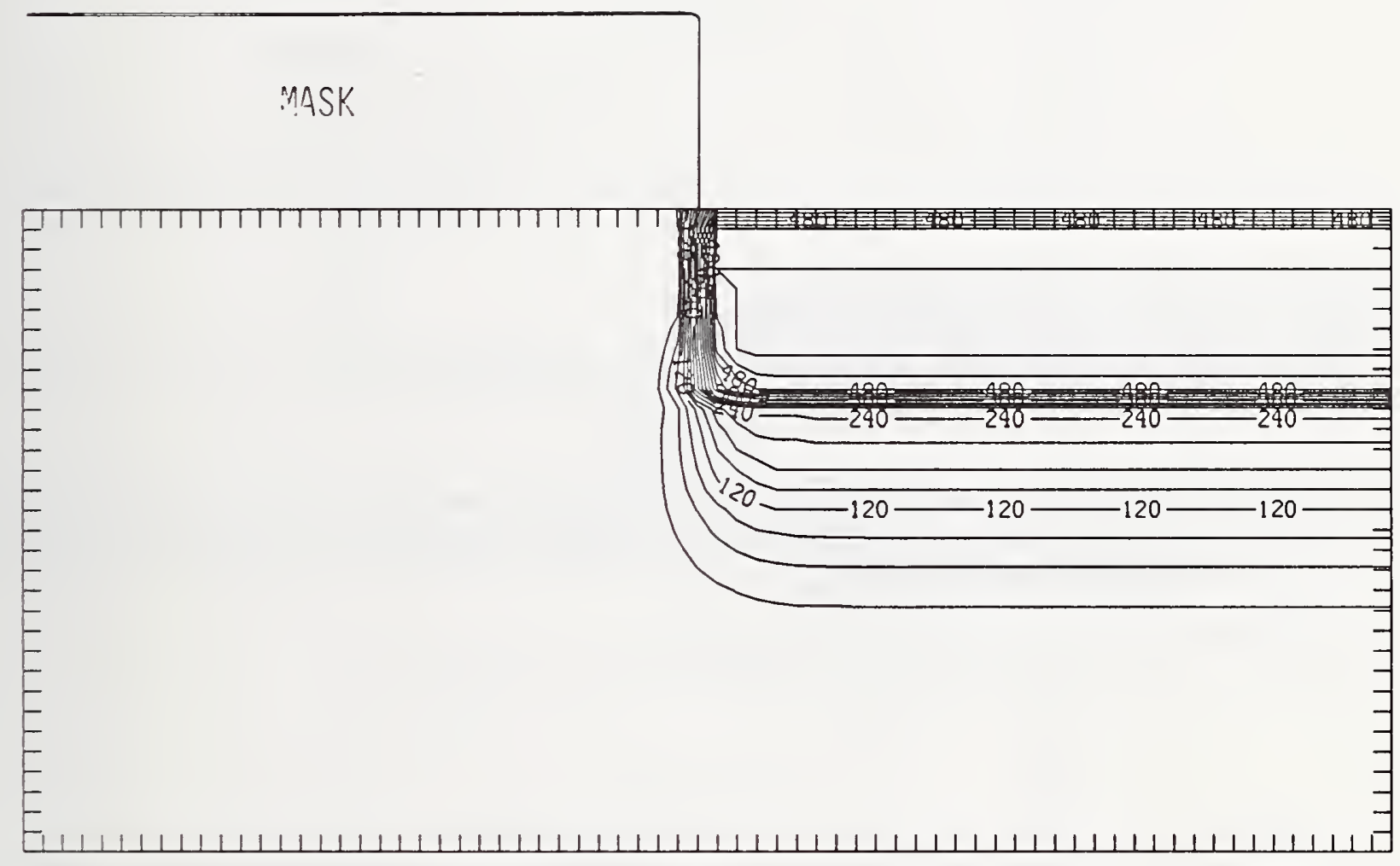

Figure Sn300-4 Two-dimensional distribution of Frenkel pairs created by $300 \mathrm{keV}$ tin implanted past a mask edge. The length increment (distance between tick marks) is $0.01 \mu \mathrm{m}$ and the scale factor is 0.1 . 


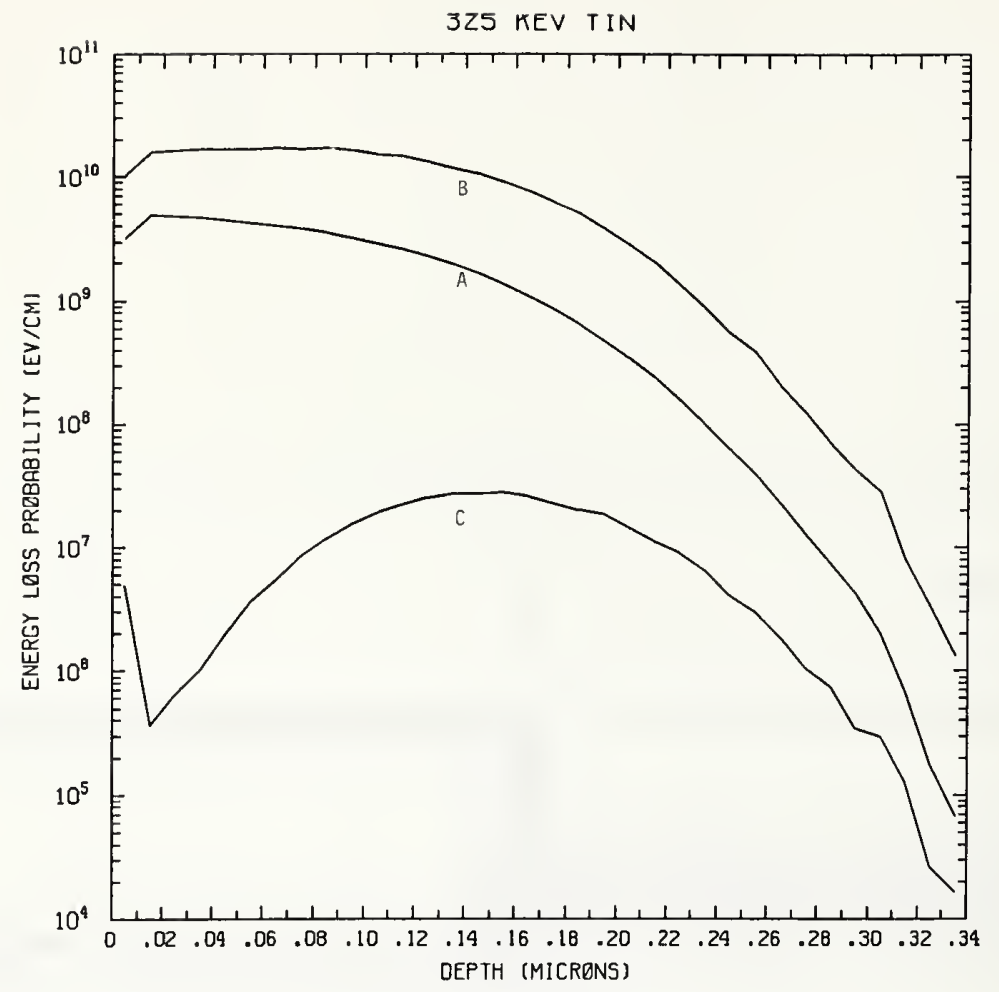

Figure Sn325-1 One-dimensional probability distributions for energy loss due to electronic excitation (A), displacement damage(B), and phonon excitation (C) for $325 \mathrm{keV}$ tin normally incident on a silicon target.

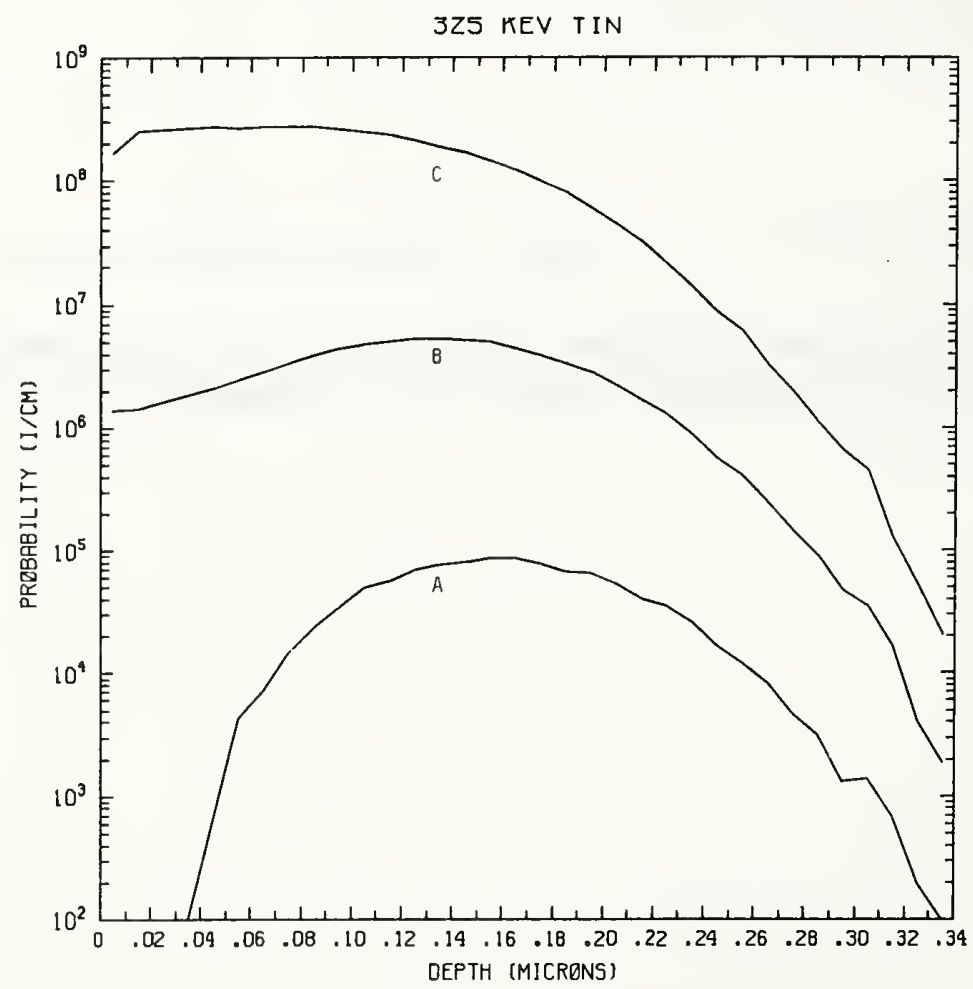

Figure Sn325-2 One-dimensional probability distributions for implanted tin (A), primary silicon displacements (B), and Frenkel pairs (C) for $325 \mathrm{keV}$ tin normally incident on a silicon target. 


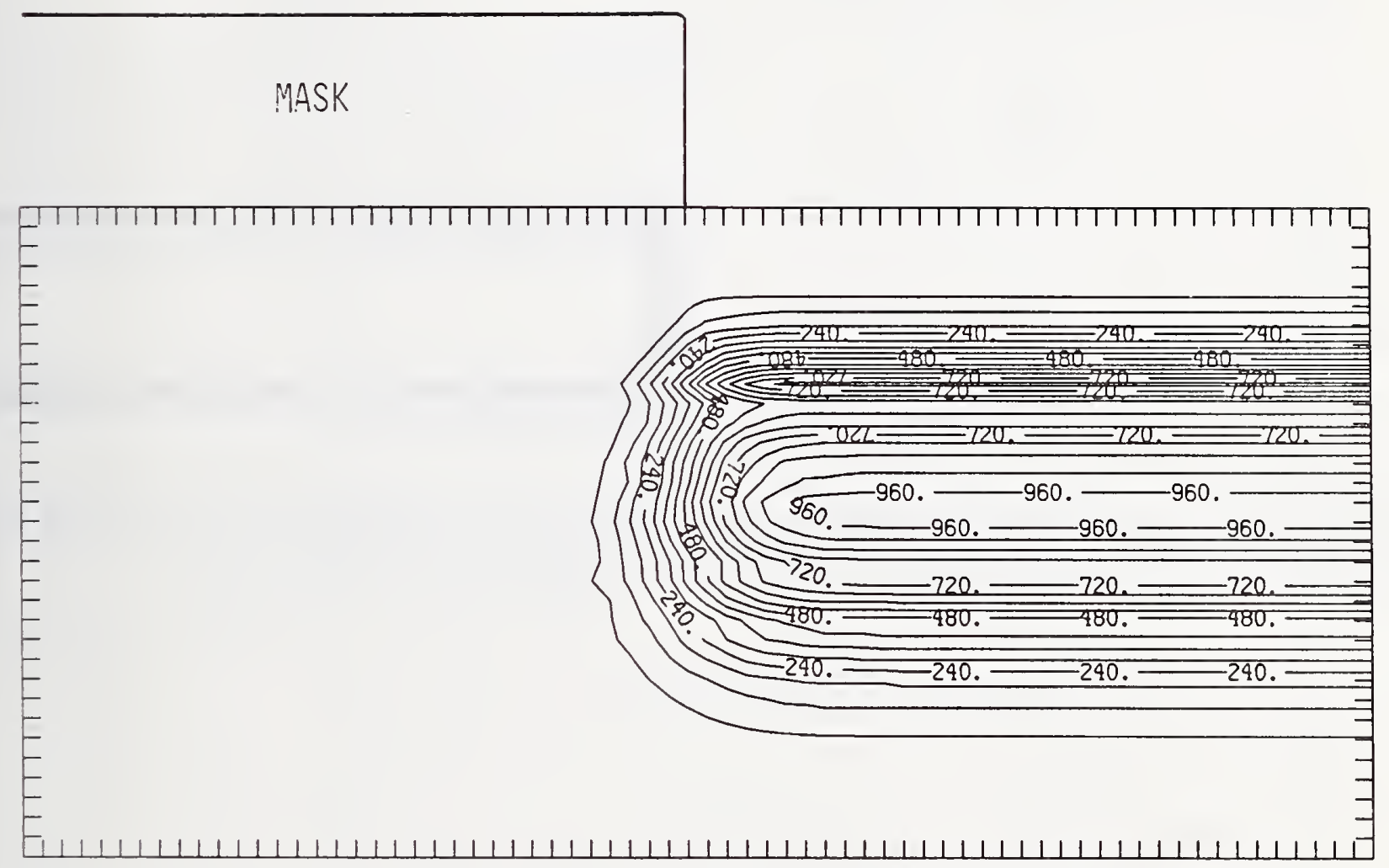

Figure Sn325-3 Two-dimensional distribution of $325 \mathrm{keV}$ tin implanted past a mask edge. The length increment (distance between tick marks) is $0.01 \mu \mathrm{m}$ and the scale factor is 1000 . 


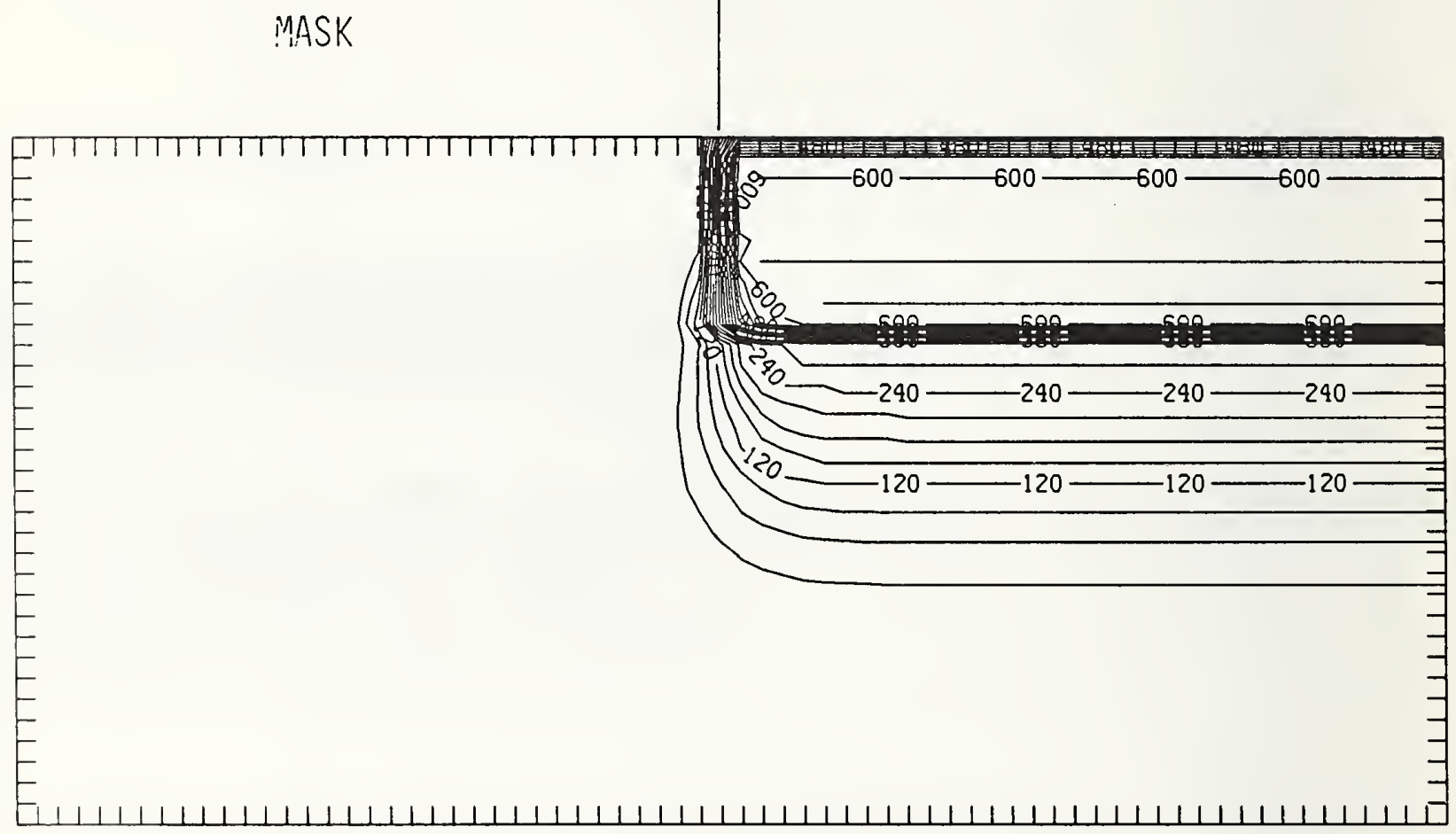

Figure Sn325-4 Two-dimensional distribution of Frenkel pairs created by $325 \mathrm{keV}$ tin implanted past a mask edge. The length increment (distance between tick marks) is $0.01 \mu \mathrm{m}$ and the scale factor is 0.1 . 


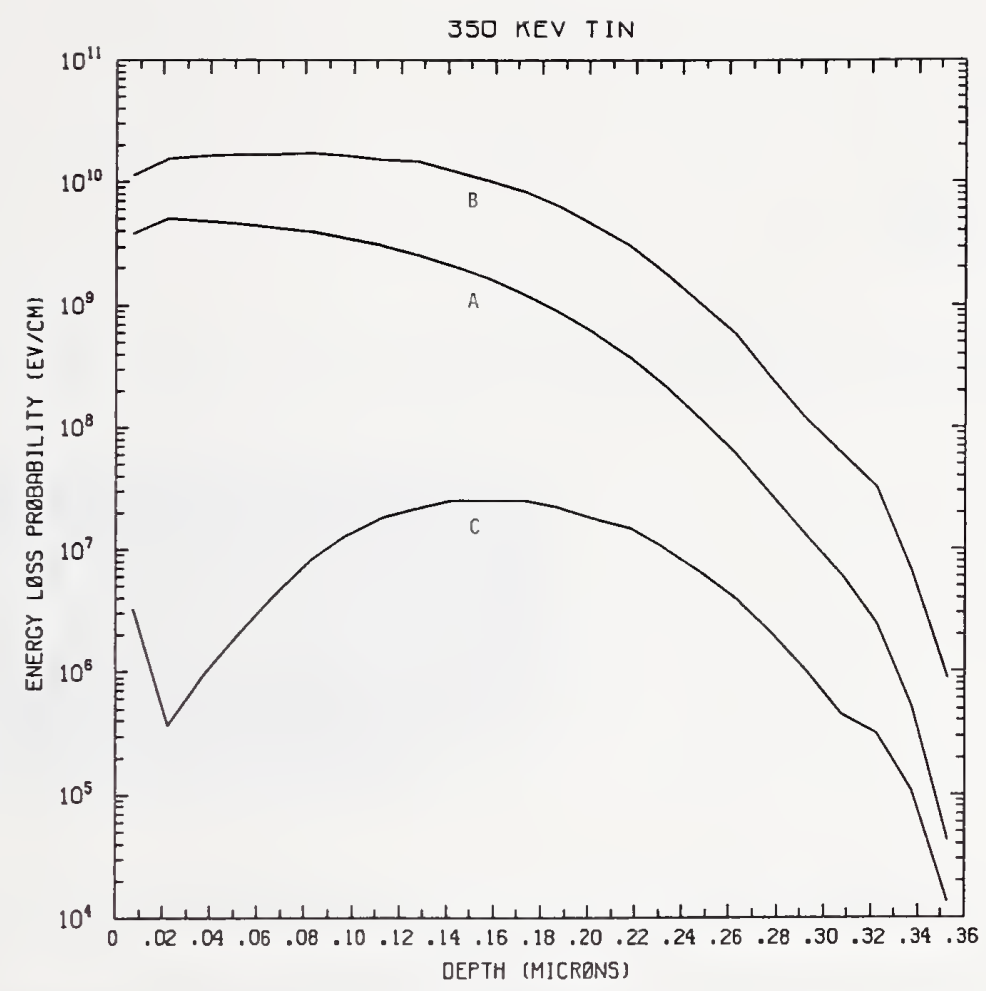

Figure Sn350-1 One-dimensional probability distributions for energy loss due to electronic excitation (A), displacement damage(B), and phonon excitation (C) for $350 \mathrm{keV}$ tin normally incident on a silicon target.

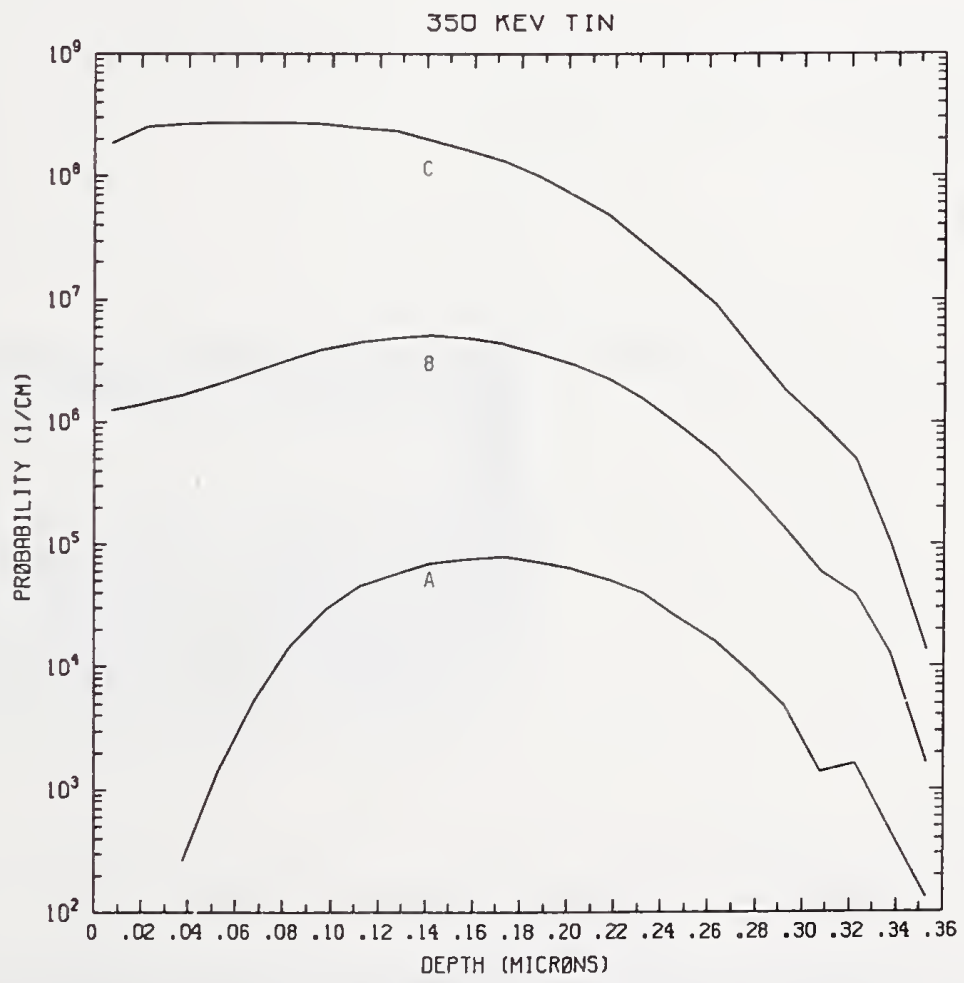

Figure Sn350-2 One-dimensional probability distributions for implanted tin (A), primary silicon displacements (B), and Frenkel pairs (C) for $350 \mathrm{keV}$ tin normally incident on a silicon target. 


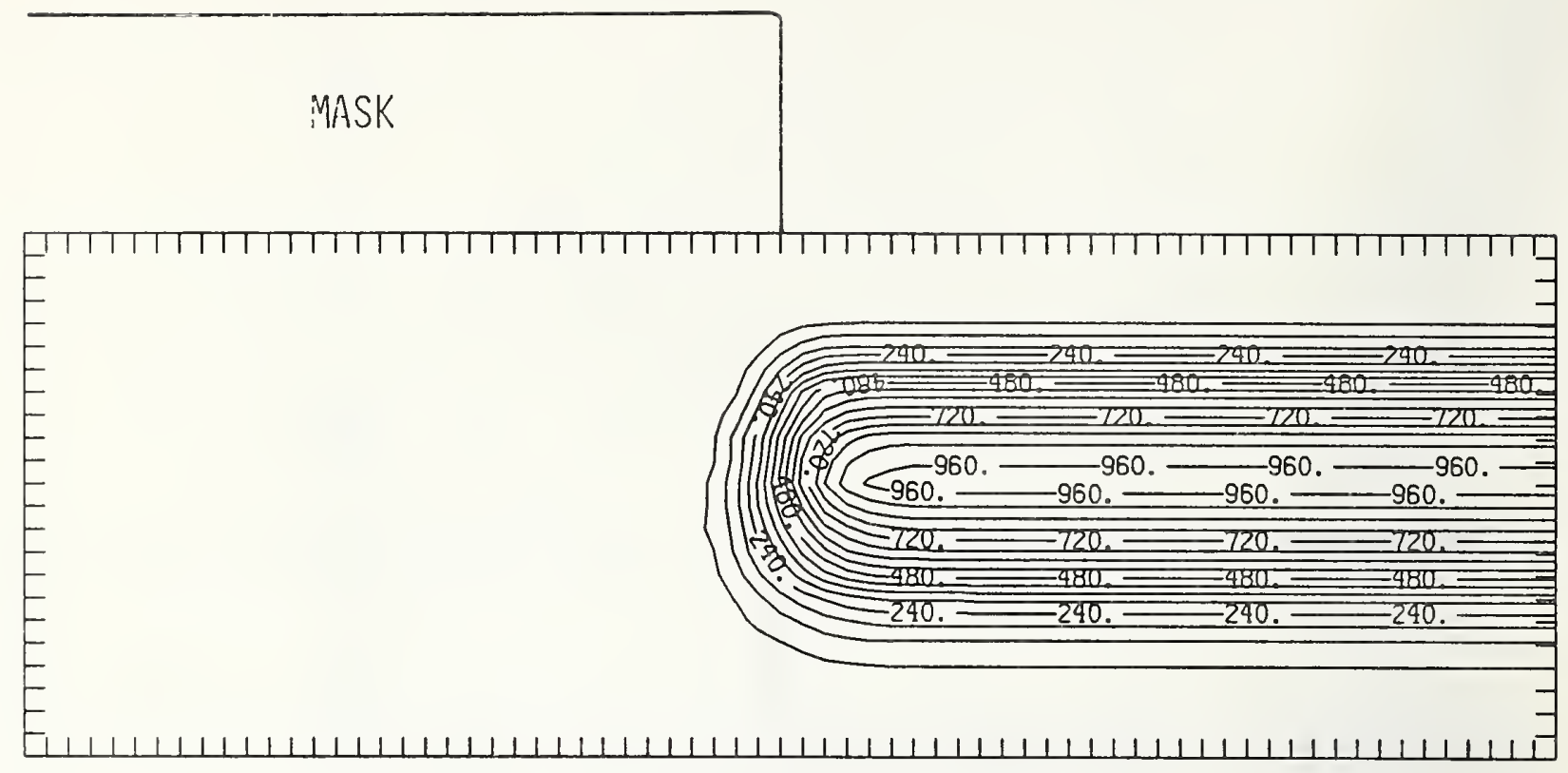

Figure Sn350-3 Two-dimensional distribution of $350 \mathrm{keV}$ tin implanted past a mask edge. The length increment (distance between tick marks) is $0.015 \mu \mathrm{m}$ and the scale factor is 1000 .

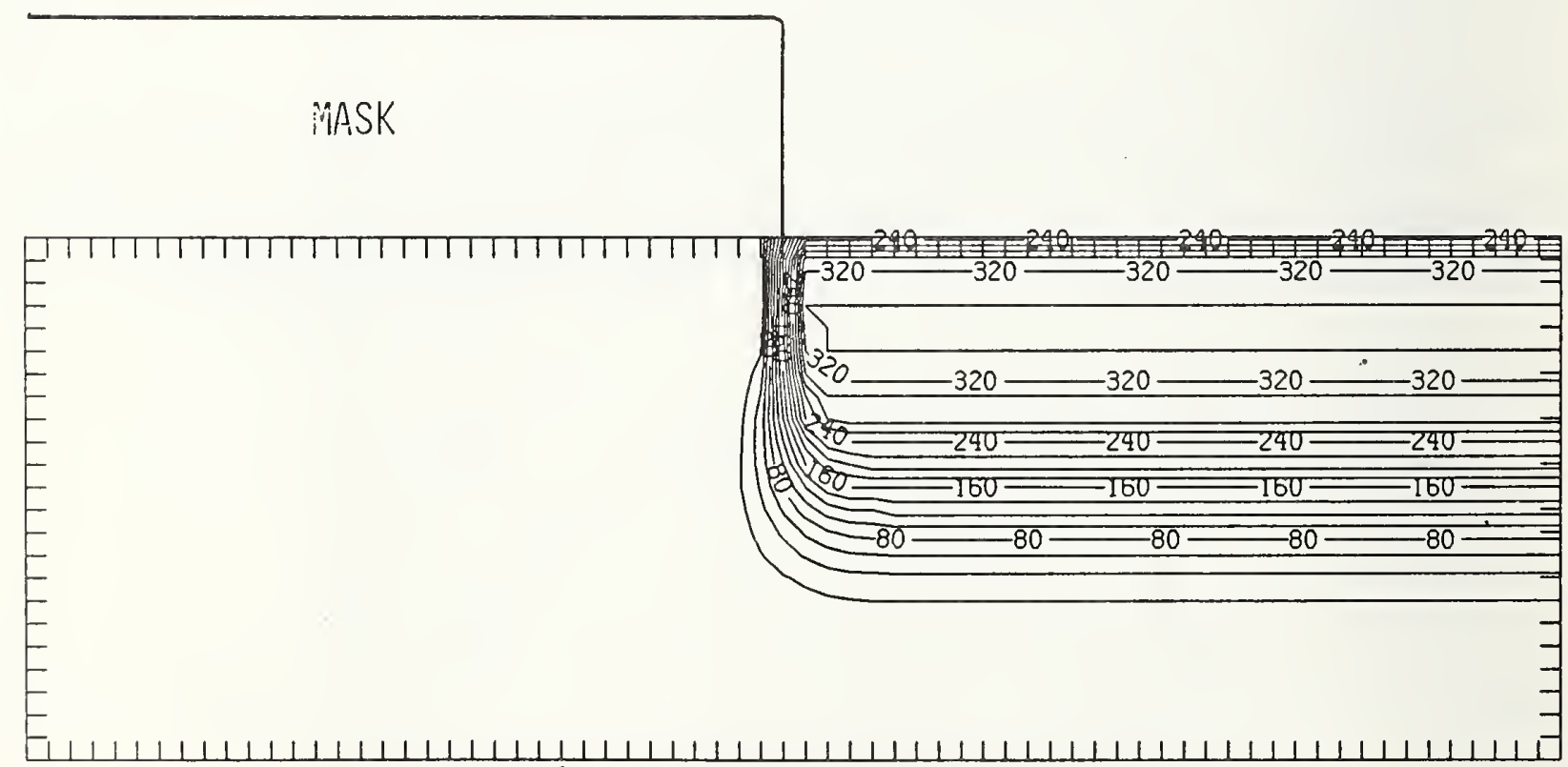

Figure Sn350-4 Two-dimensional distribution of Frenkel pairs created by $350 \mathrm{keV}$ tin implanted past a mask edge. The length increment (distance between tick marks) is $0.015 \mu \mathrm{m}$ and the scale factor is 0.1 . 
375 KEV TIN

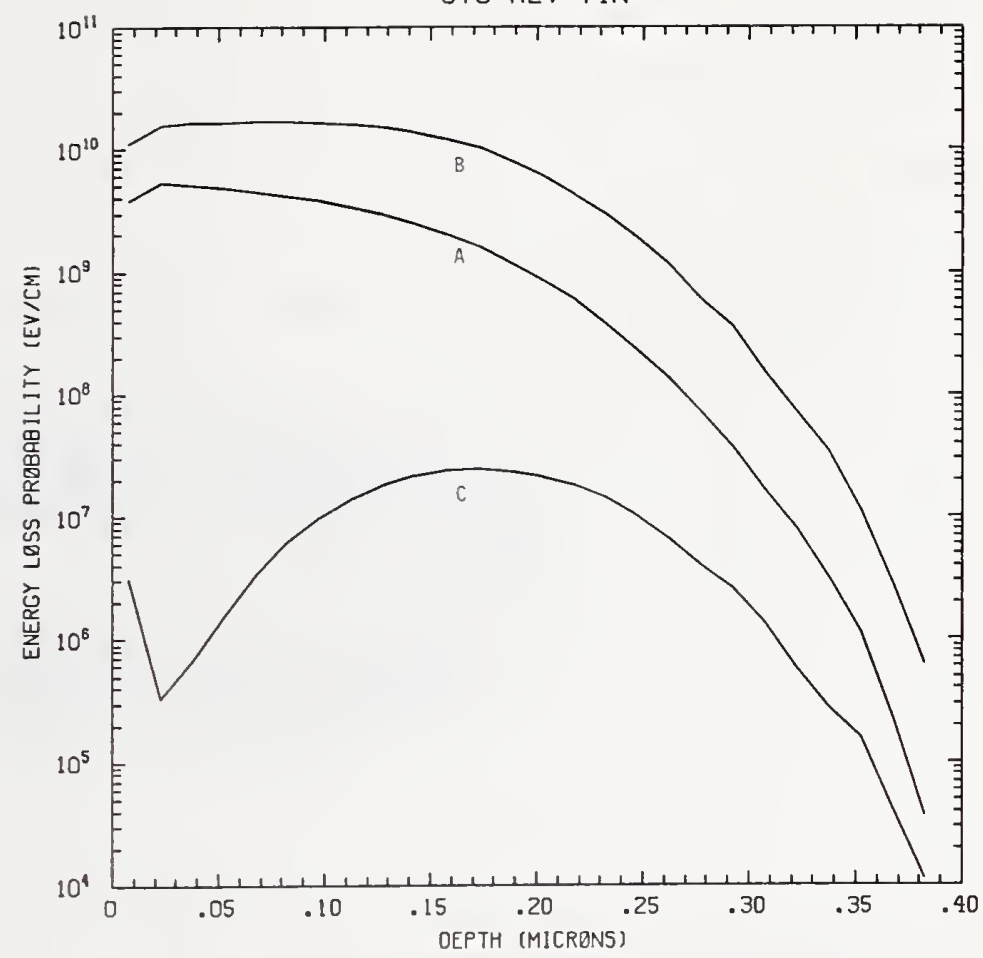

Figure Sn375-1 One-dimensional probability distributions for energy loss due to electronic excitation (A), displacement damage(B), and phonon excitation (C) for $375 \mathrm{keV}$ tin normally incident on a silicon target.

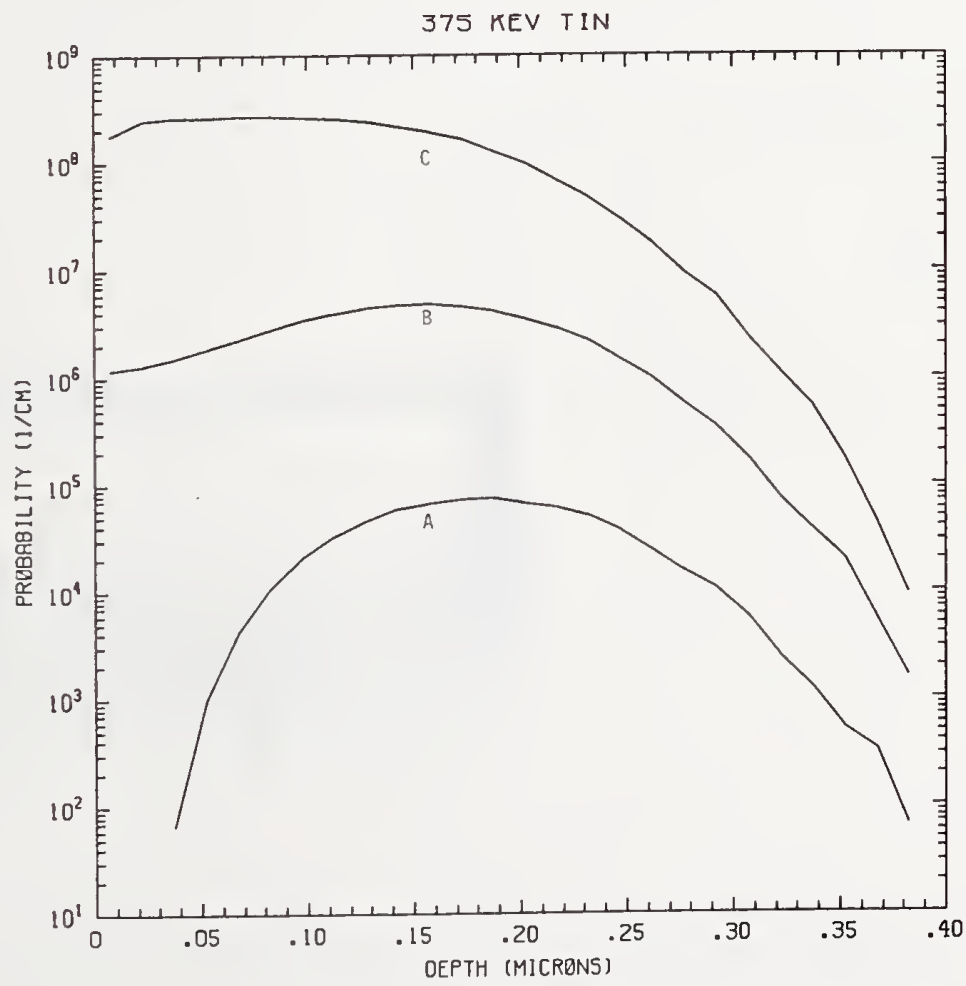

Figure Sn375-2 One-dimensional probability distributions for implanted tin (A), primary silicon displacements (B), and Frenkel pairs (C) for $375 \mathrm{keV}$ tin normally incident on a silicon target. 


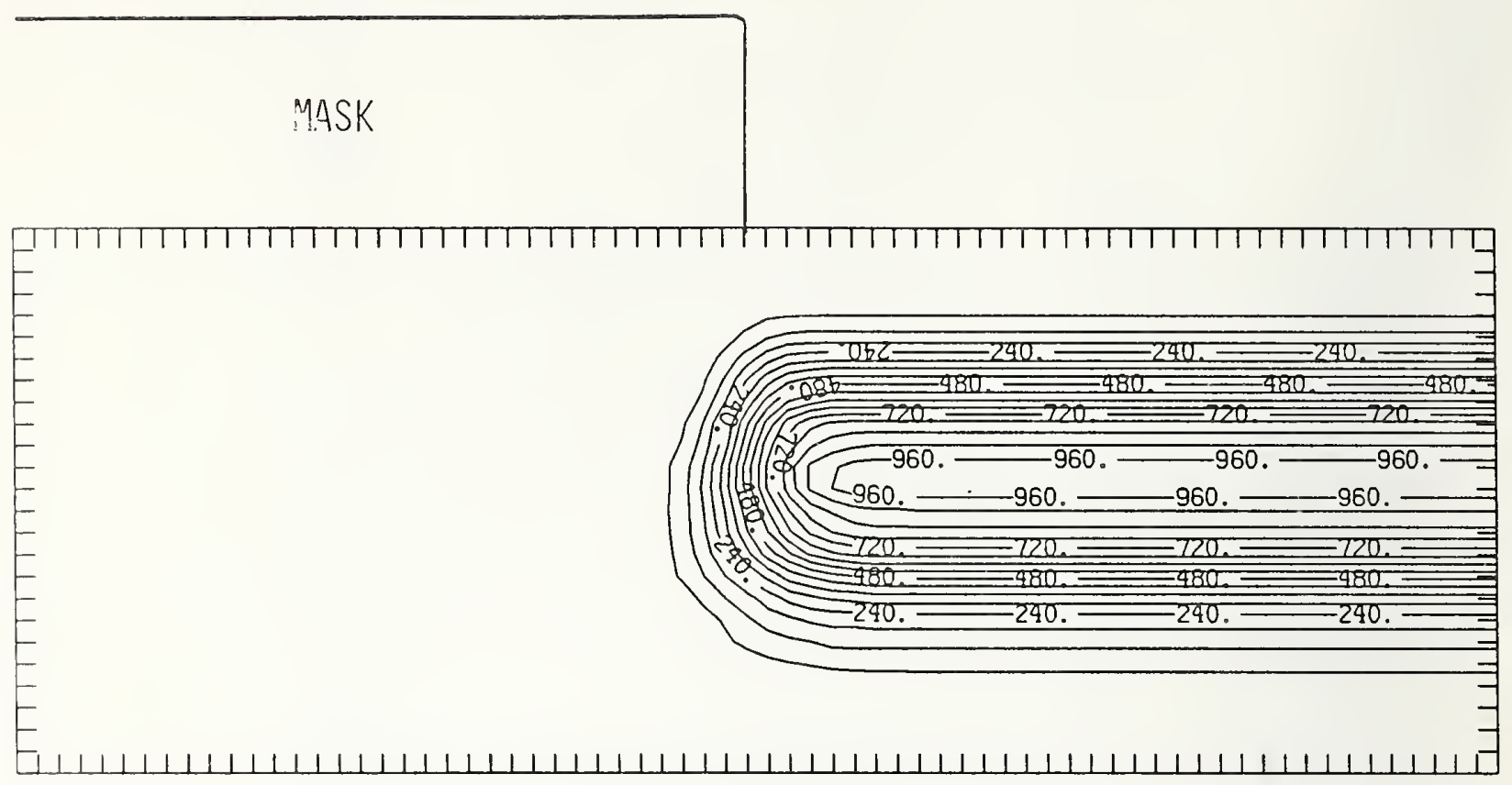

Figure Sn375-3 Two-dimensional distribution of $375 \mathrm{keV}$ tin implanted past a mask edge. The length increment (distance between tick marks) is $0.015 \mu \mathrm{m}$ and the scale factor is 1000 .

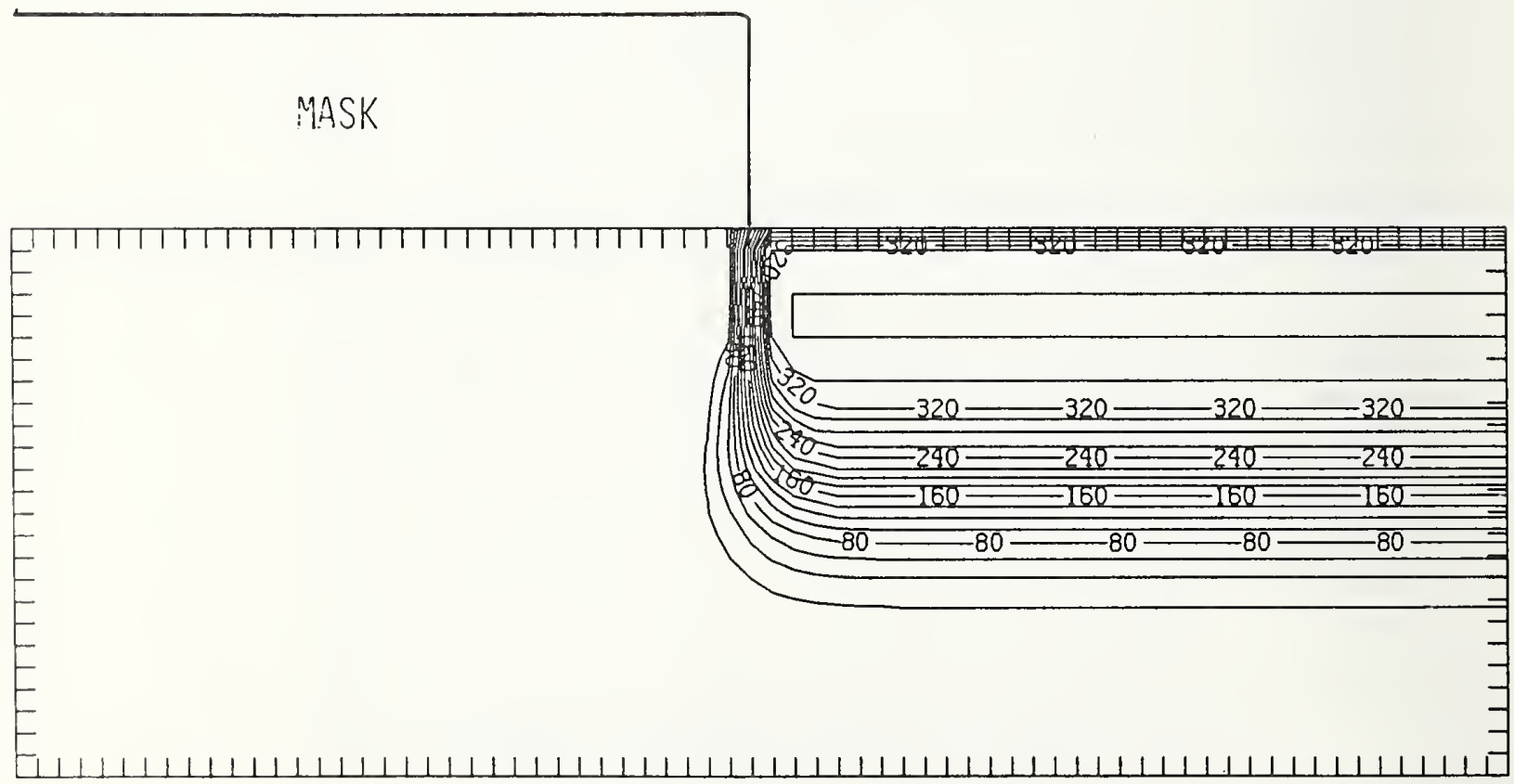

Figure Sn375-4 Two-dimensional distribution of Frenkel pairs created by $375 \mathrm{keV}$ tin implanted past a mask edge. The length increment (distance between tick marks) is $0.015 \mu \mathrm{m}$ and the scale factor is 0.1 . 


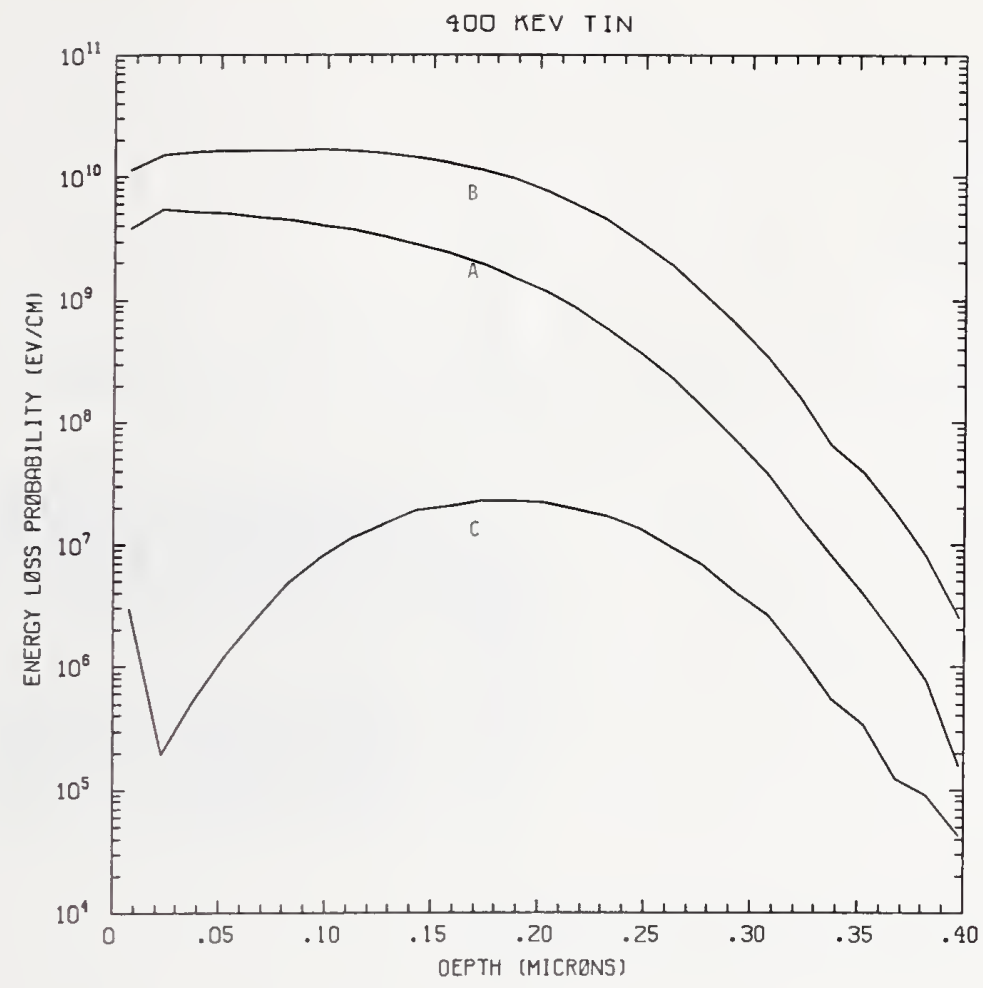

Figure Sn400-1 One-dimensional probability distributions for energy loss due to electronic excitation (A), displacement damage(B), and phonon excitation (C) for $400 \mathrm{keV}$ tin normally incident on a silicon target.

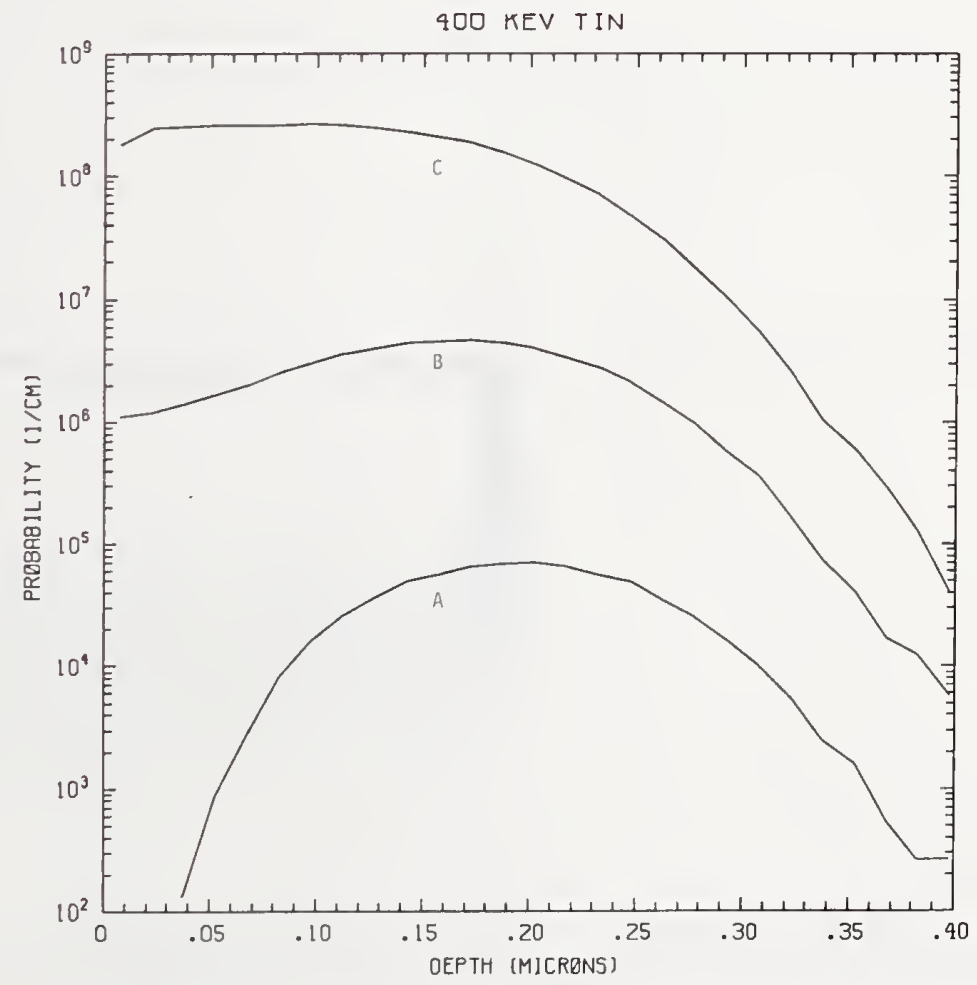

Figure Sn400-2 One-dimensional probability distributions for implanted tin (A), primary silicon displacements (B), and Frenkel pairs (C) for $400 \mathrm{keV}$ tin normally incident on a silicon target. 


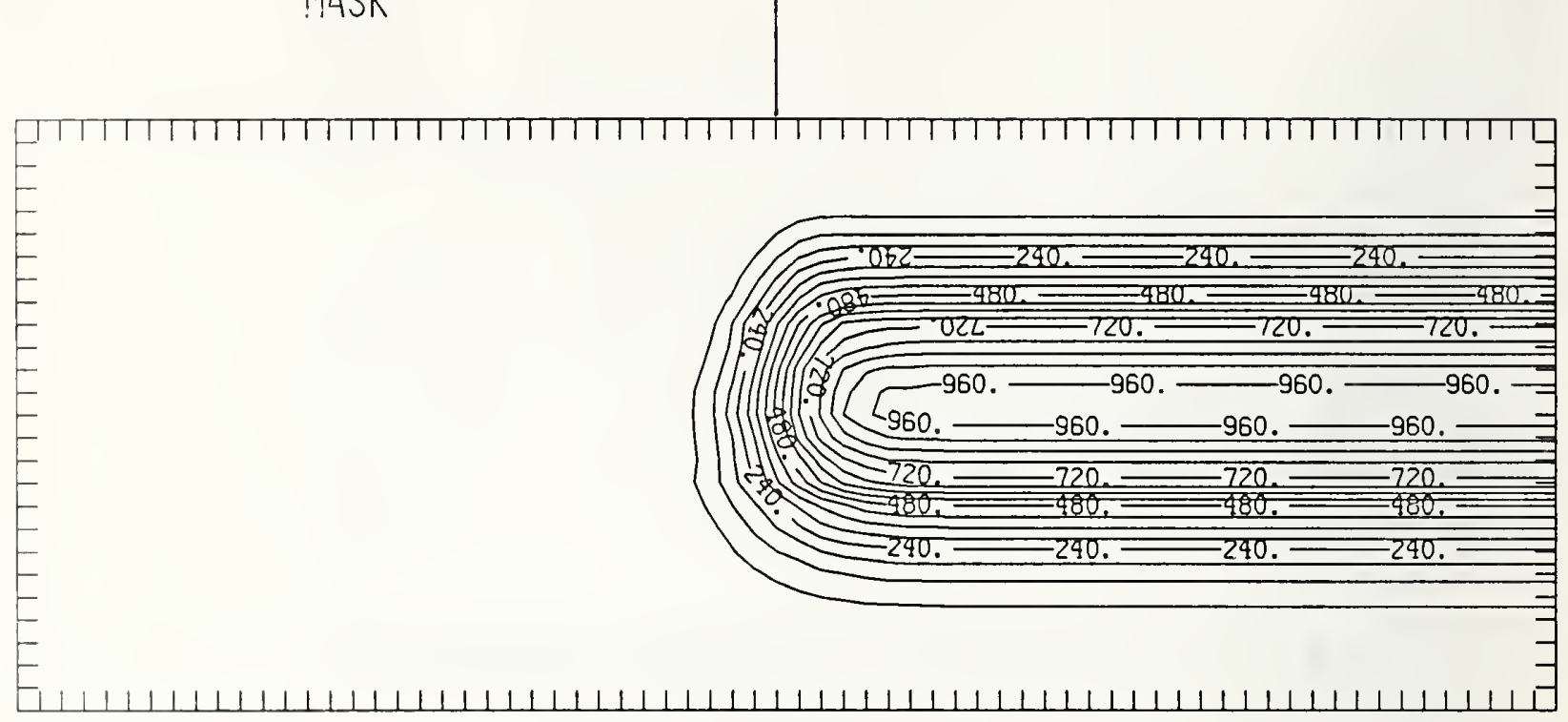

Figure Sn400-3 Two-dimensional distribution of $400 \mathrm{keV}$ tin implanted past a mask edge. The length increment (distance between tick marks) is $0.015 \mu \mathrm{m}$ and the scale factor is 1000 .

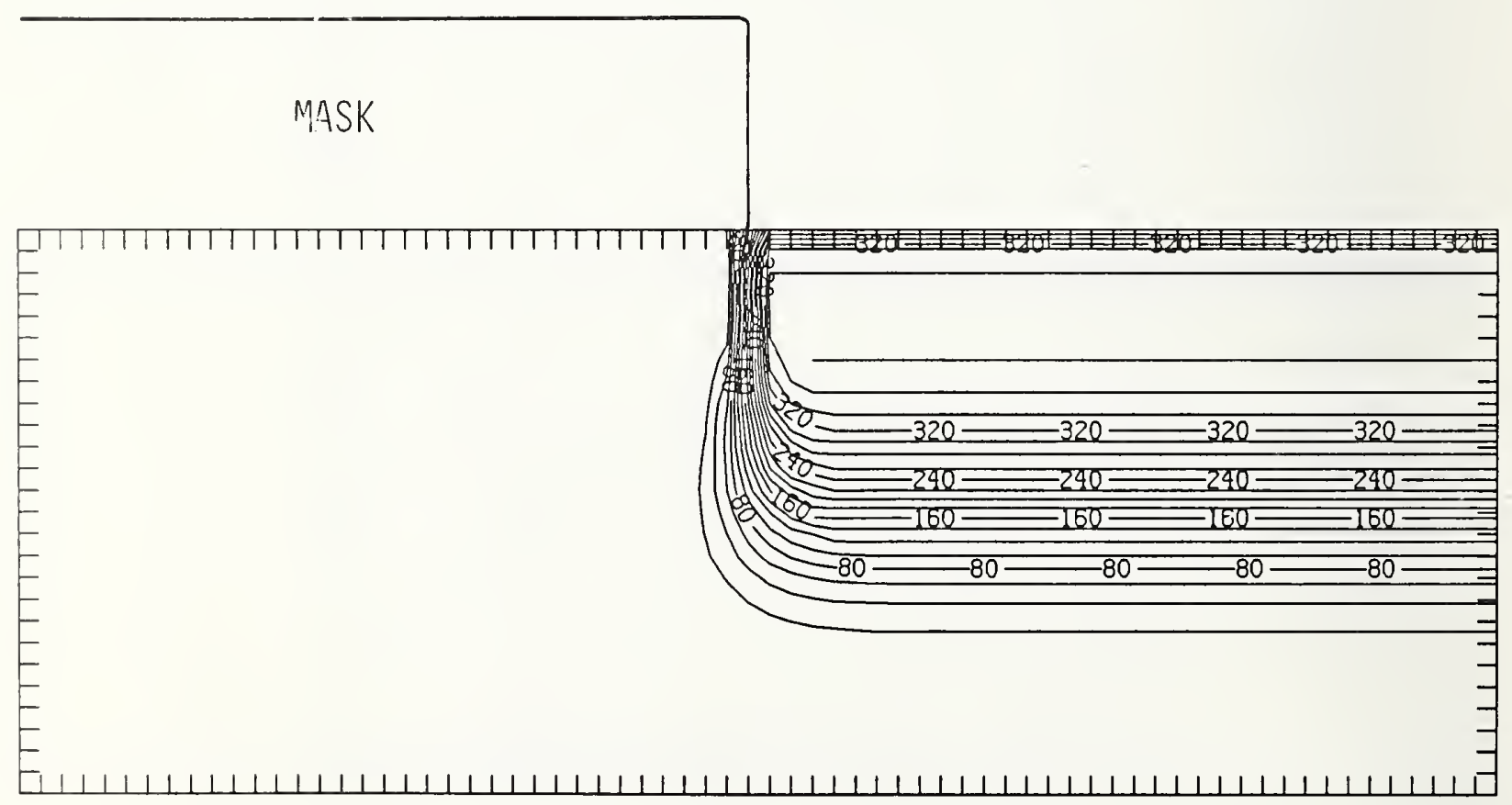

Figure Sn400-4 Two-dimensional distribution of Frenkel pairs created by $400 \mathrm{keV}$ tin implanted past a mask edge. The length increment (distance between tick marks) is $0.015 \mu \mathrm{m}$ and the scale factor is 0.1 . 


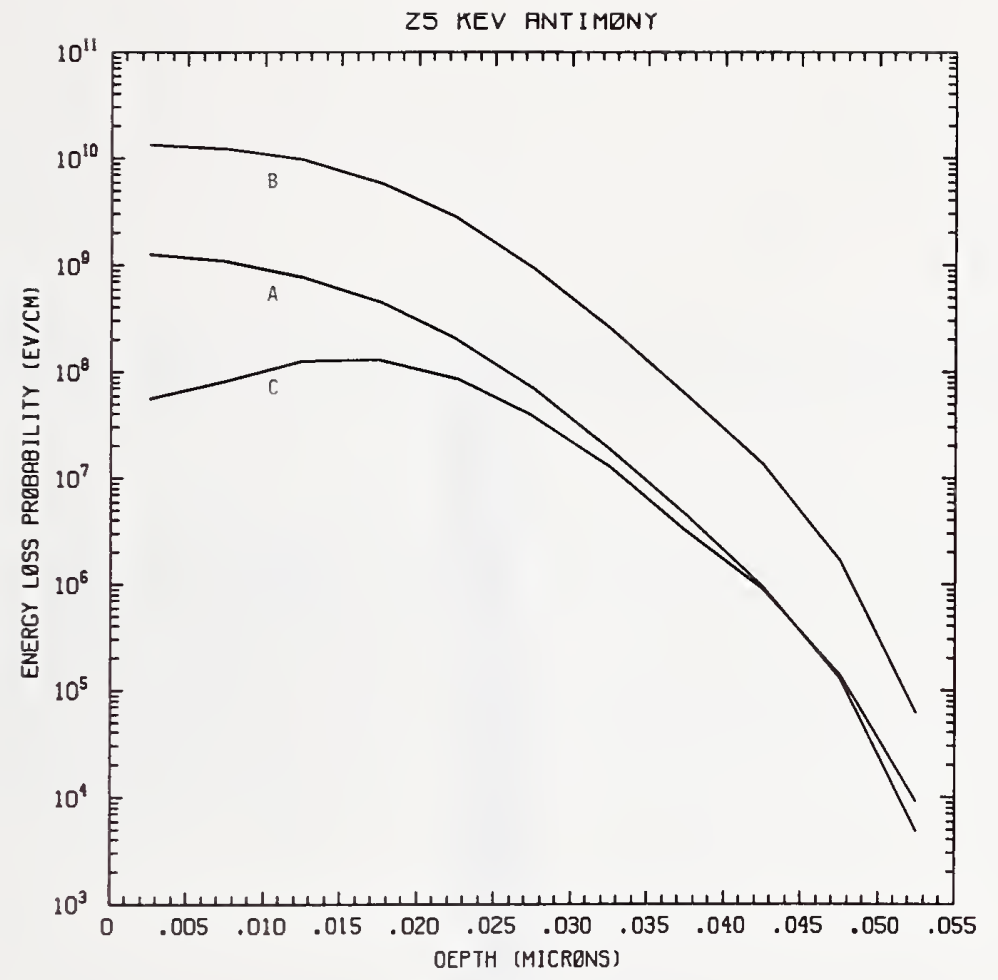

Figure Sb25-1 One-dimensional probability distributions for energy loss due to electronic excitation (A), displacement damage(B), and phonon excitation (C) for $25 \mathrm{keV}$ antimony normally incident on a silicon target.

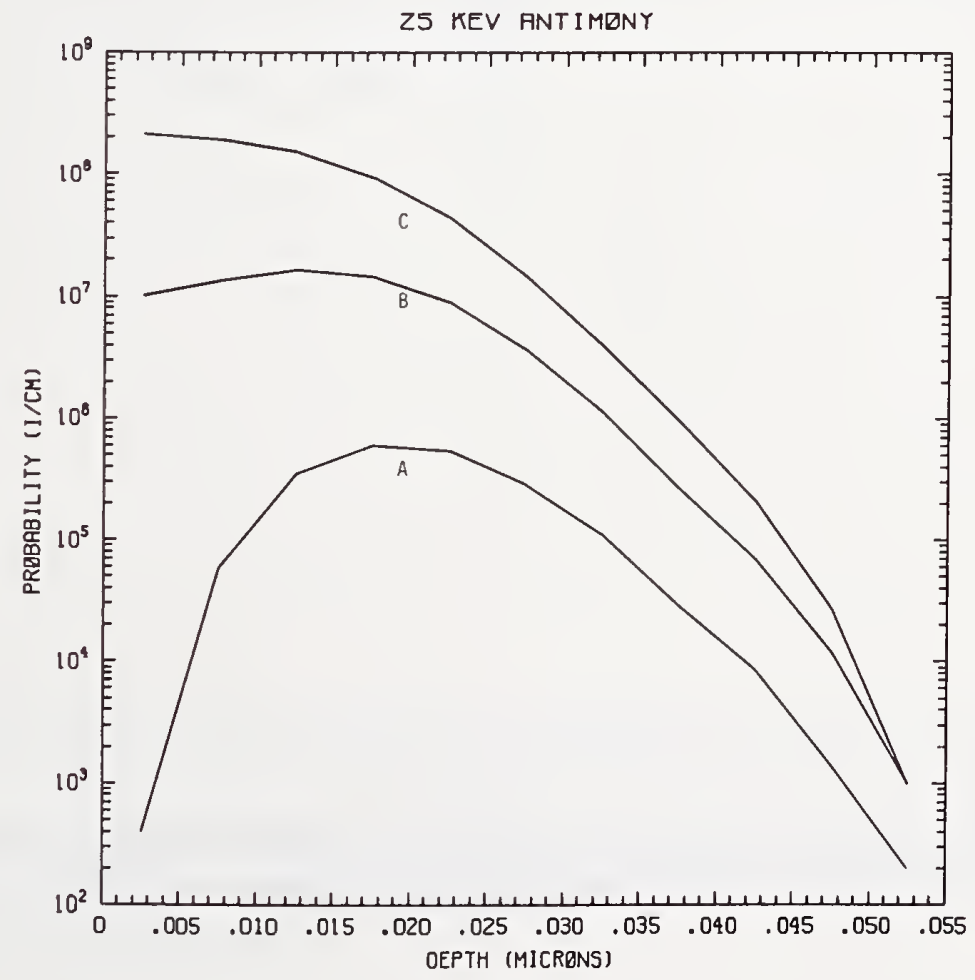

Figure Sb25-2 One-dimensional probability distributions for implanted antimony (A), primary silicon displacements (B), and Frenkel pairs (C) for $25 \mathrm{keV}$ antimony normally incident on a silicon target. 


\section{MASK}

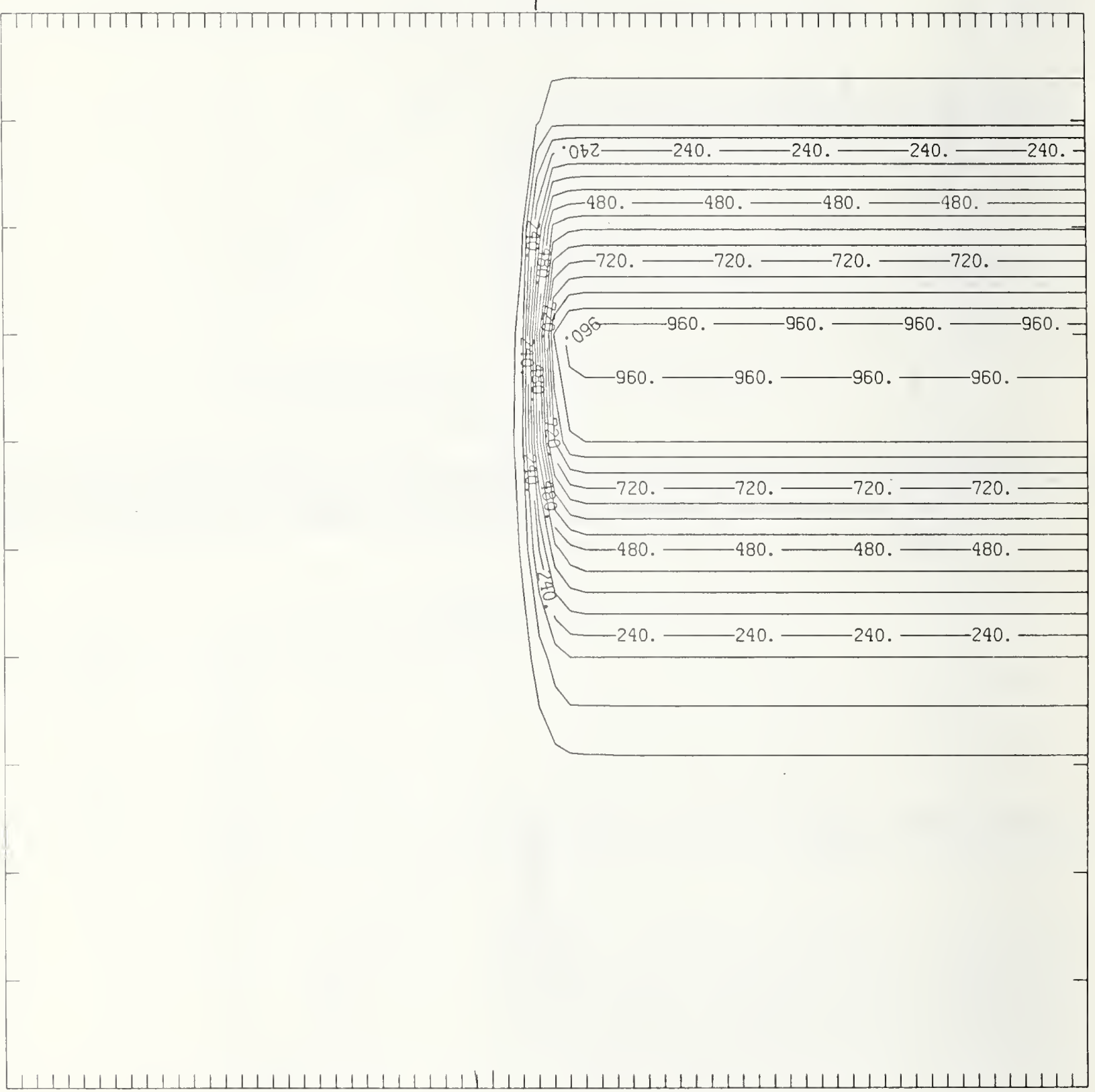

Figure Sb25-3 Two-dimensional distribution of $25 \mathrm{keV}$ antimony implanted past a mask edge. The length increment (distance between tick marks) is $0.005 \mu \mathrm{m}$ and the scale factor is 1000 . 
MASK

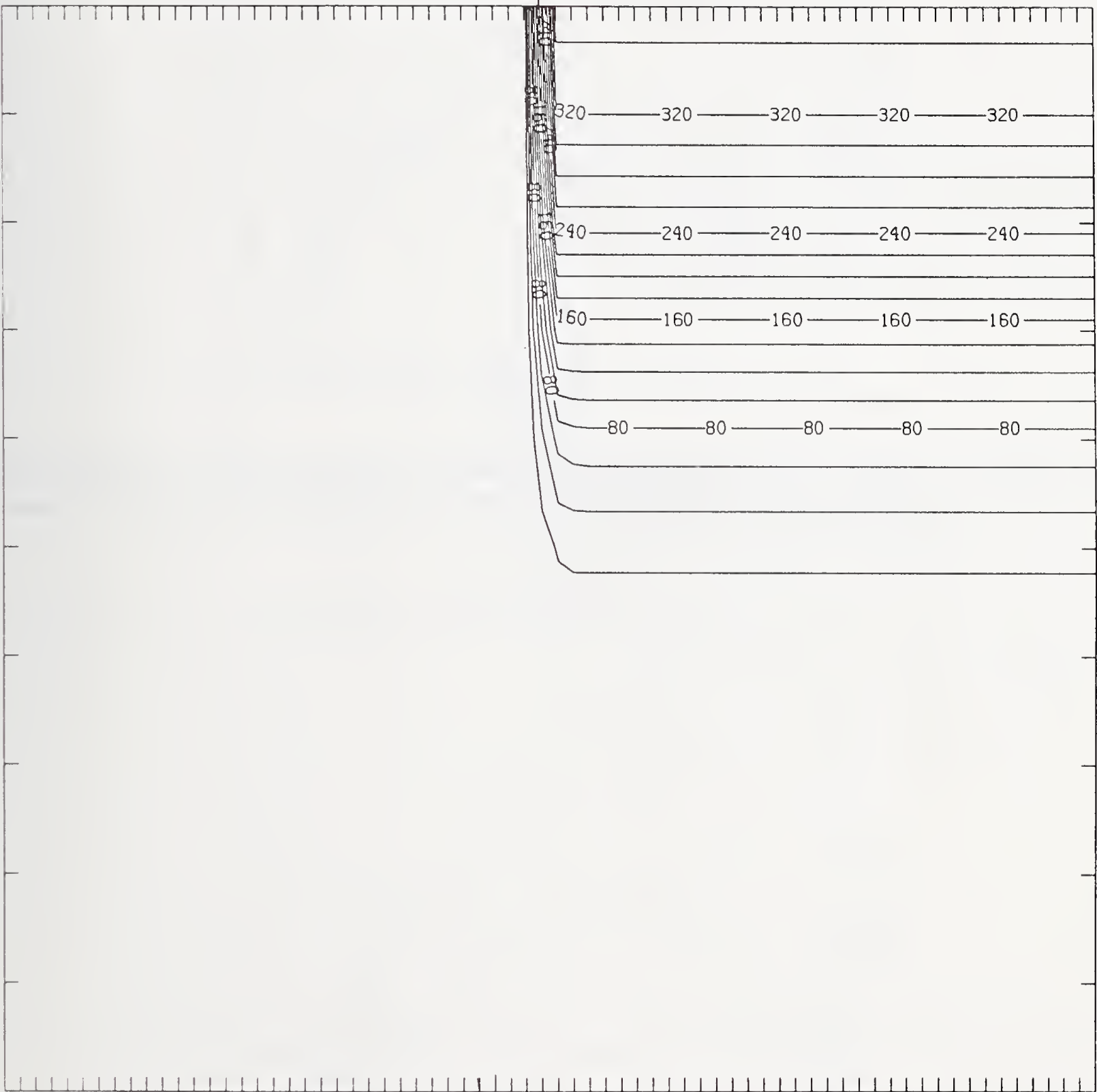

Figure Sb25-4 Two-dimensional distribution of Frenkel pairs created by $25 \mathrm{keV}$ antimony implanted past a mask edge. The length increment (distance between tick marks) is $0.005 \mu \mathrm{m}$ and the scale factor is 1 . 


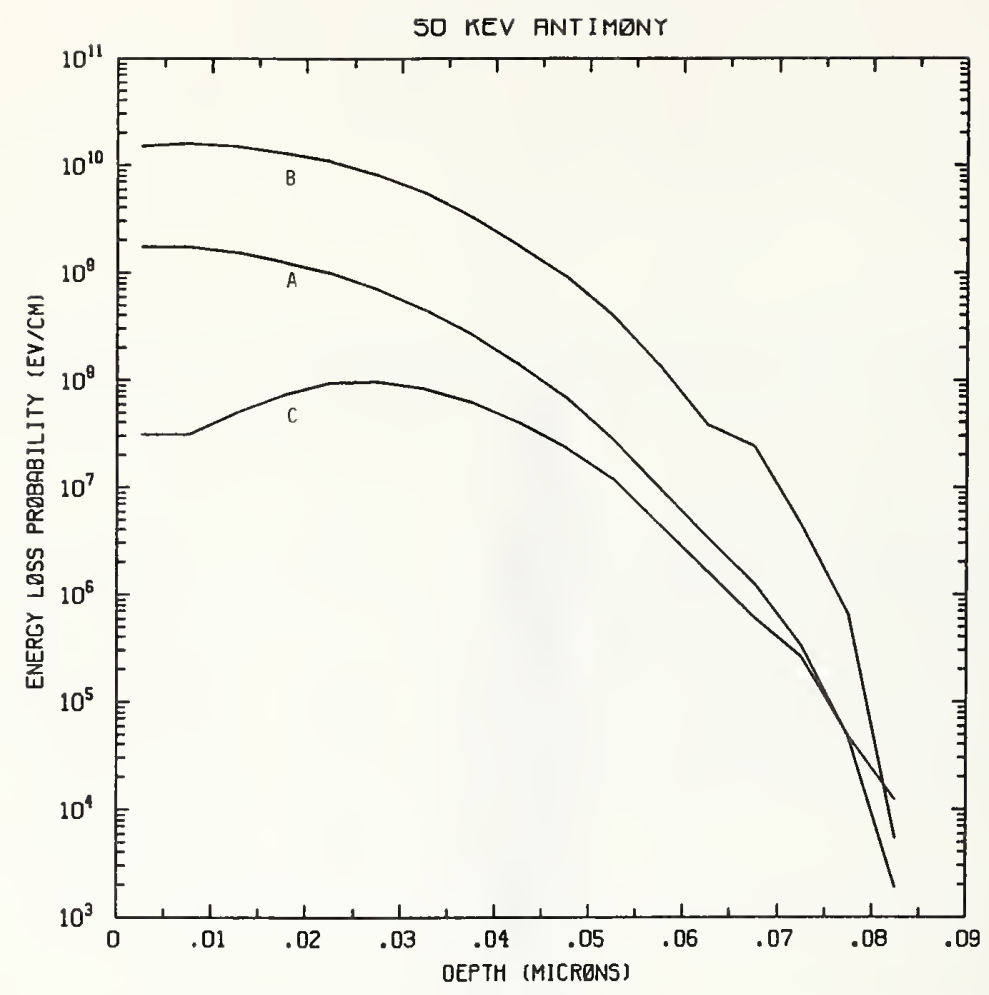

Figure Sb50-1 One-dimensional probability distributions for energy loss due to electronic excitation (A), displacement damage(B), and phonon excitation (C) for $50 \mathrm{keV}$ antimony normally incident on a silicon target.

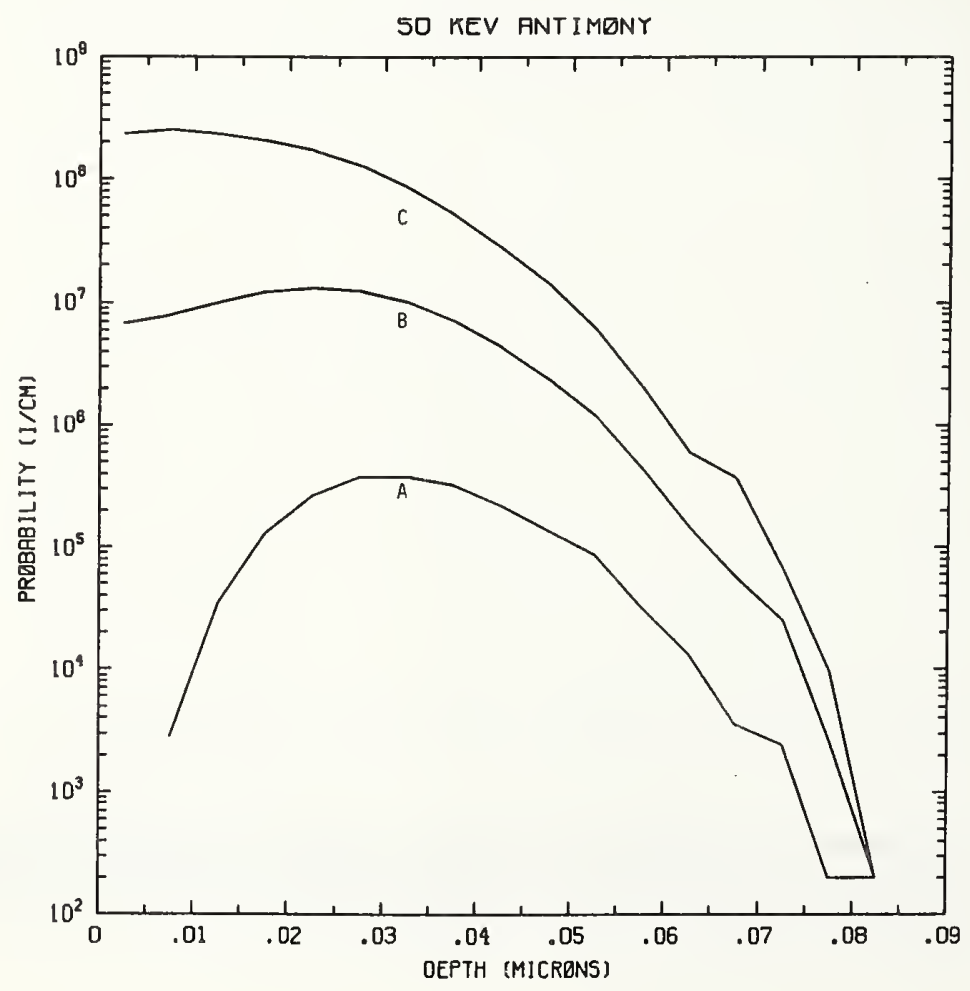

Figure Sb50-2 One-dimensional probability distributions for implanted antimony (A), primary silicon displacements (B), and Frenkel pairs (C) for $50 \mathrm{keV}$ antimony normally incident on a silicon target. 


\section{PIASK}

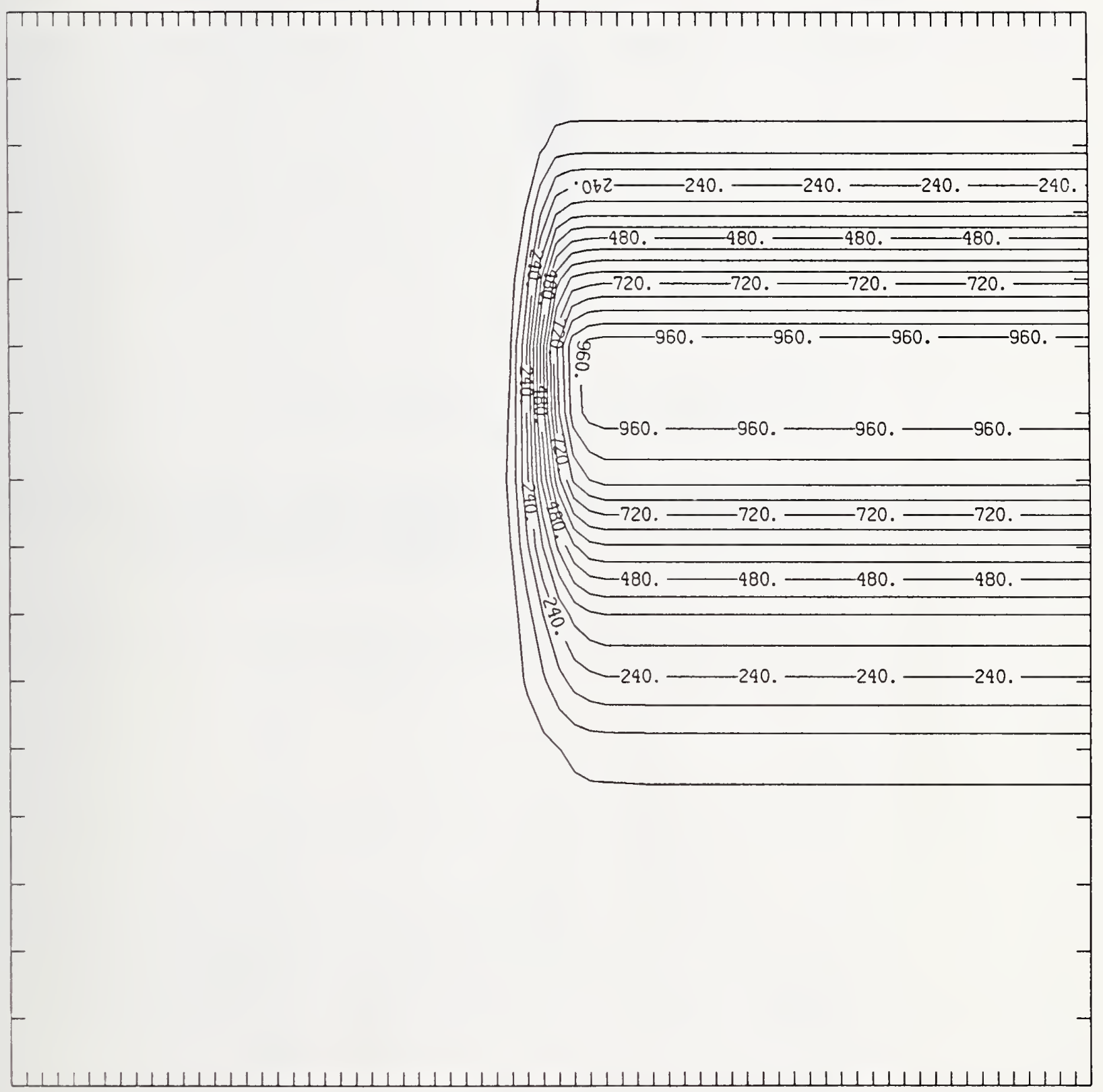

Figure Sb50-3 Two-dimensional distribution of $50 \mathrm{keV}$ antimony implanted past a mask edge. The length increment (distance between tick marks) is $0.005 \mu \mathrm{m}$ and the scale factor is 1000 . 
MASK

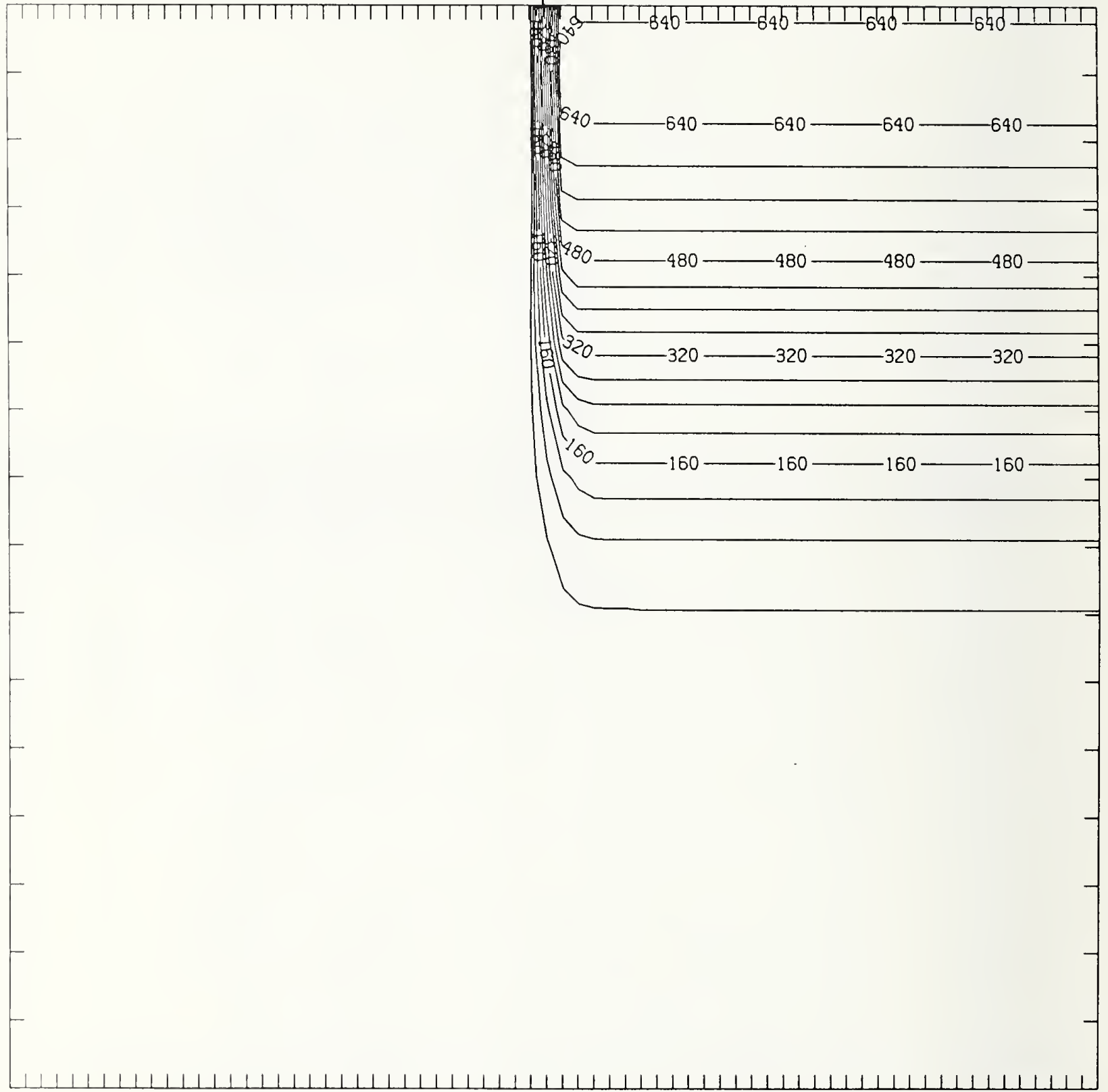

Figure Sb50-4 Two-dimensional distribution of Frenkel pairs created by $50 \mathrm{keV}$ antimony implanted past a mask edge. The length increment (distance between tick marks) is $0.005 \mu \mathrm{m}$ and the scale factor is 1. 


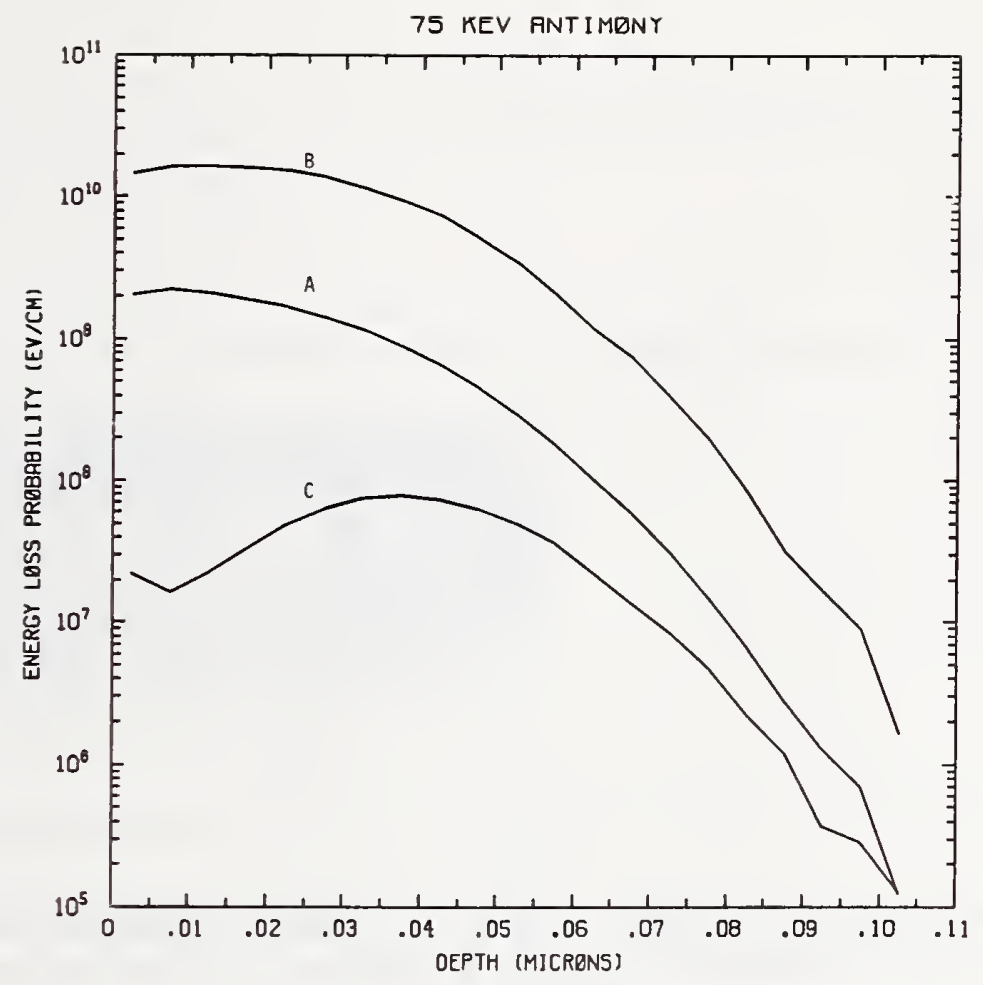

Figure Sb75-1 One-dimensional probability distributions for energy loss due to electronic excitation (A), displacement damage(B), and phonon excitation (C) for $75 \mathrm{keV}$ antimony normally incident on a silicon target.

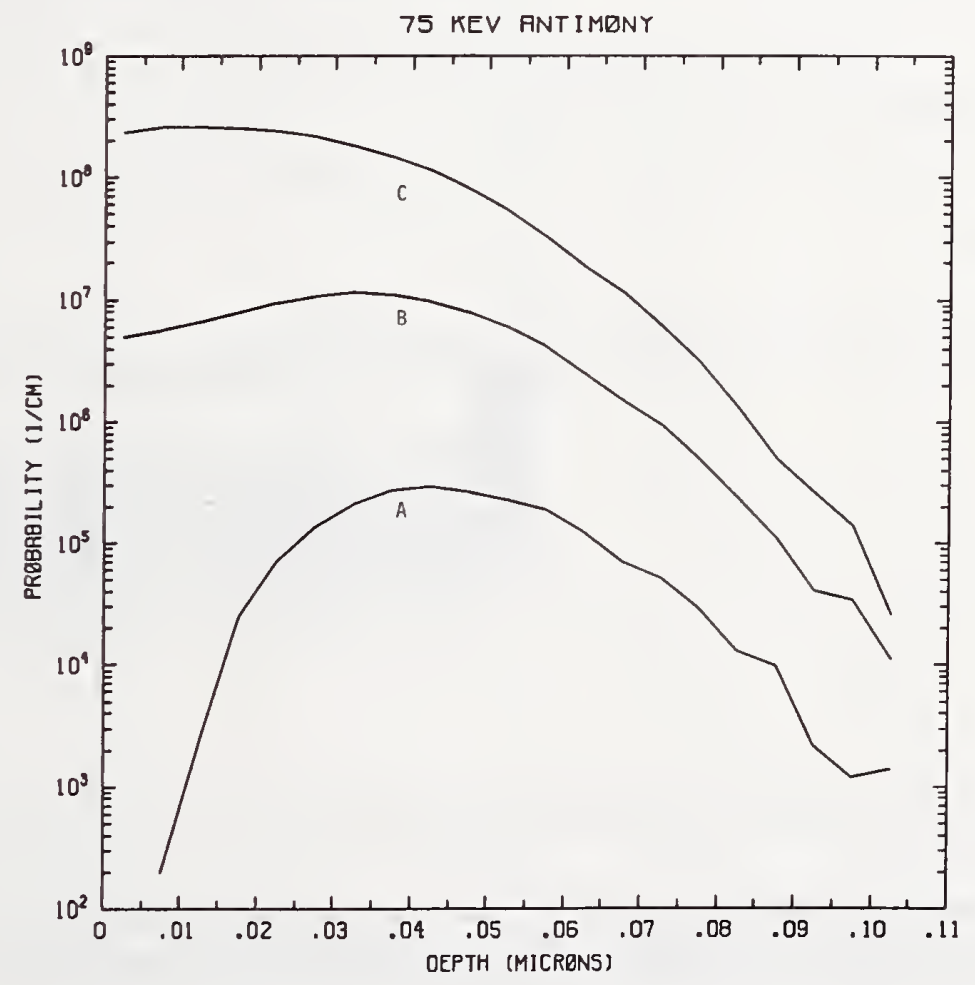

Figure Sb75-2 One-dimensional probability distributions for implanted antimony (A), primary silicon displacements (B), and Frenkel pairs (C) for $75 \mathrm{keV}$ antimony normally incident on a silicon target. 


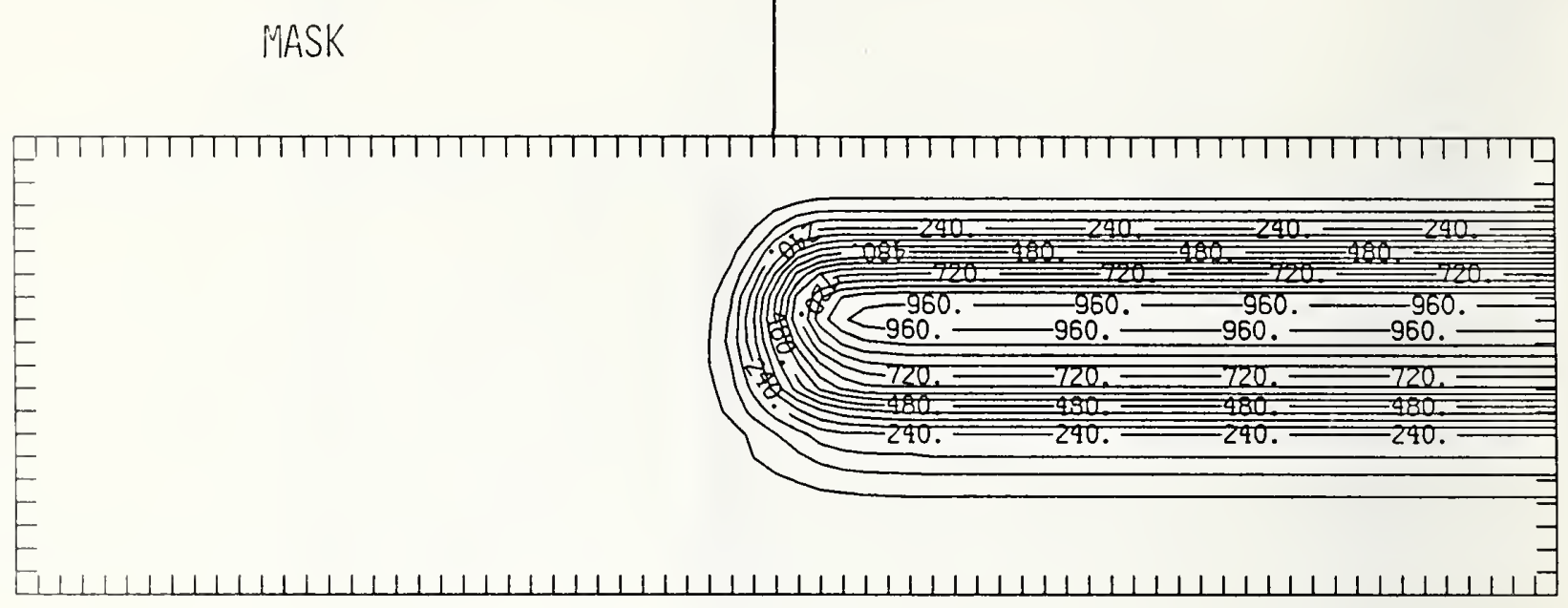

Figure Sb75-3 Two-dimensional distribution of $75 \mathrm{keV}$ antimony implanted past a mask edge. The length increment (distance between tick marks) is $0.005 \mu \mathrm{m}$ and the scale factor is 1000 .

MASK

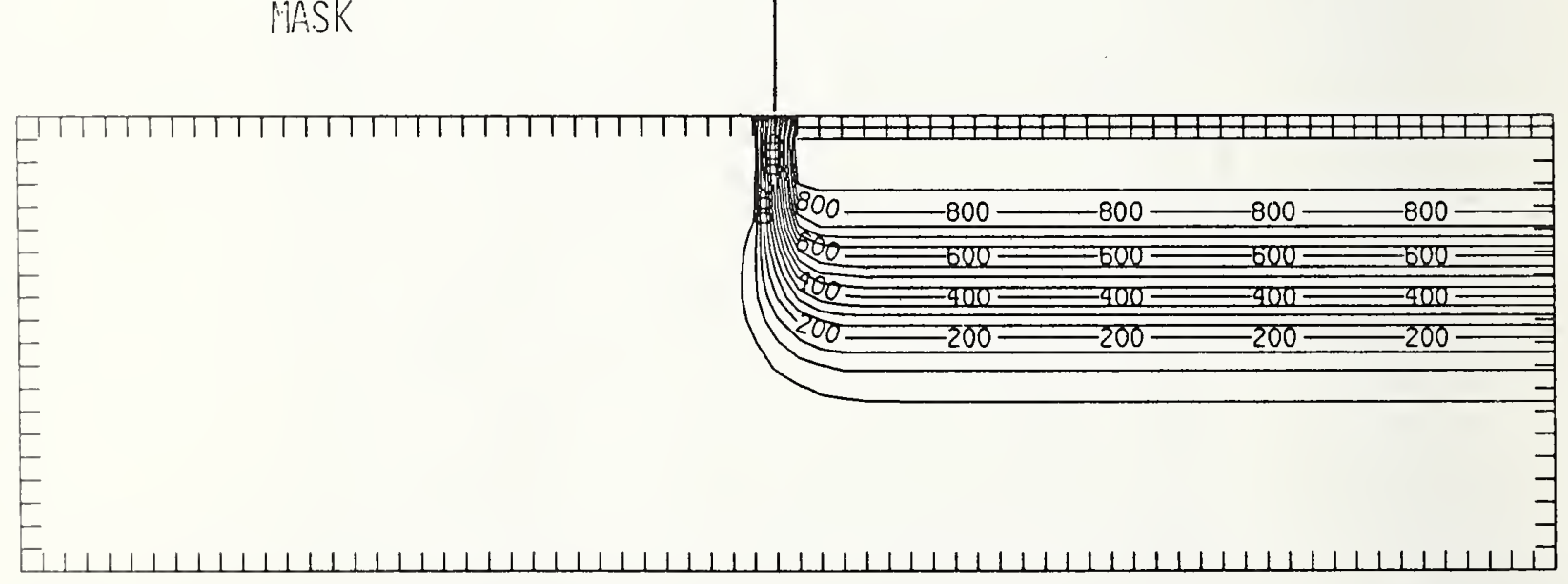

Figure Sb75-4 Two-dimensional distribution of Frenkel pairs created by $75 \mathrm{keV}$ antimony implanted past a mask edge. The length increment (distance between tick marks) is $0.005 \mu \mathrm{m}$ and the scale factor is 1 . 


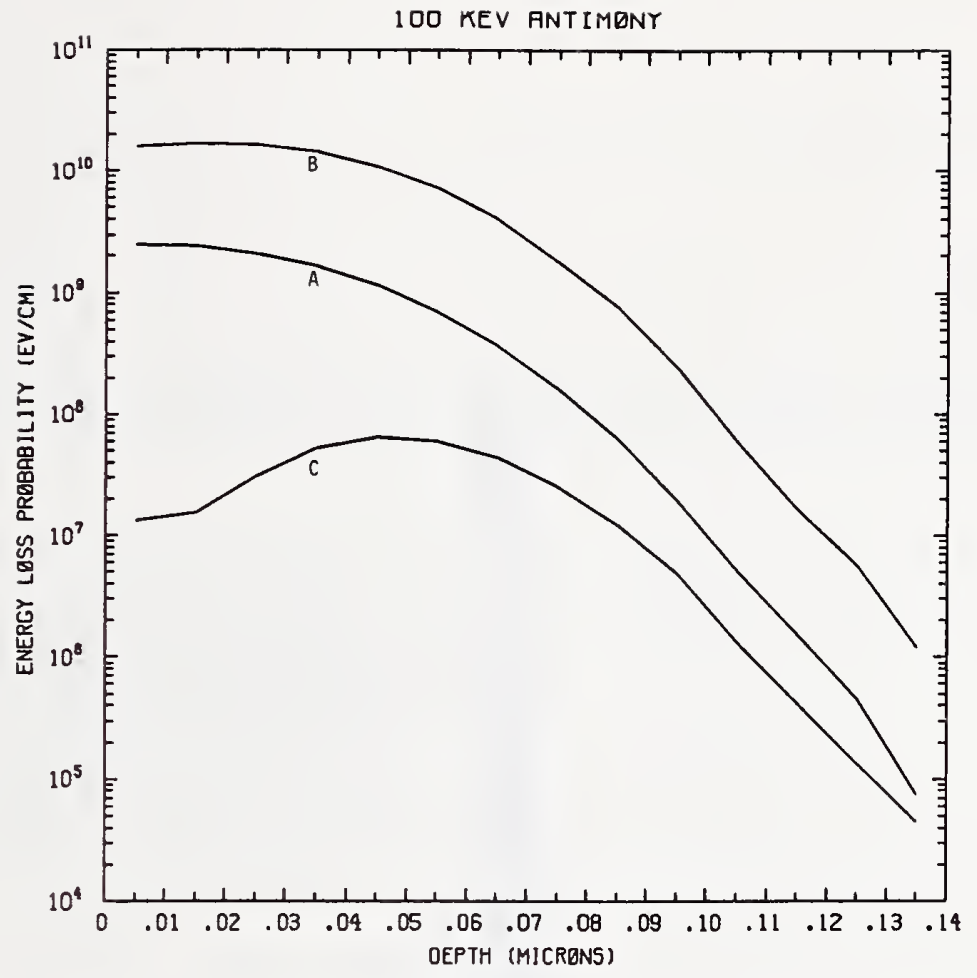

Figure Sb100-1 One-dimensional probability distributions for energy loss due to electronic excitation (A), displacement damage(B), and phonon excitation (C) for $100 \mathrm{keV}$ antimony normally incident on a silicon target.

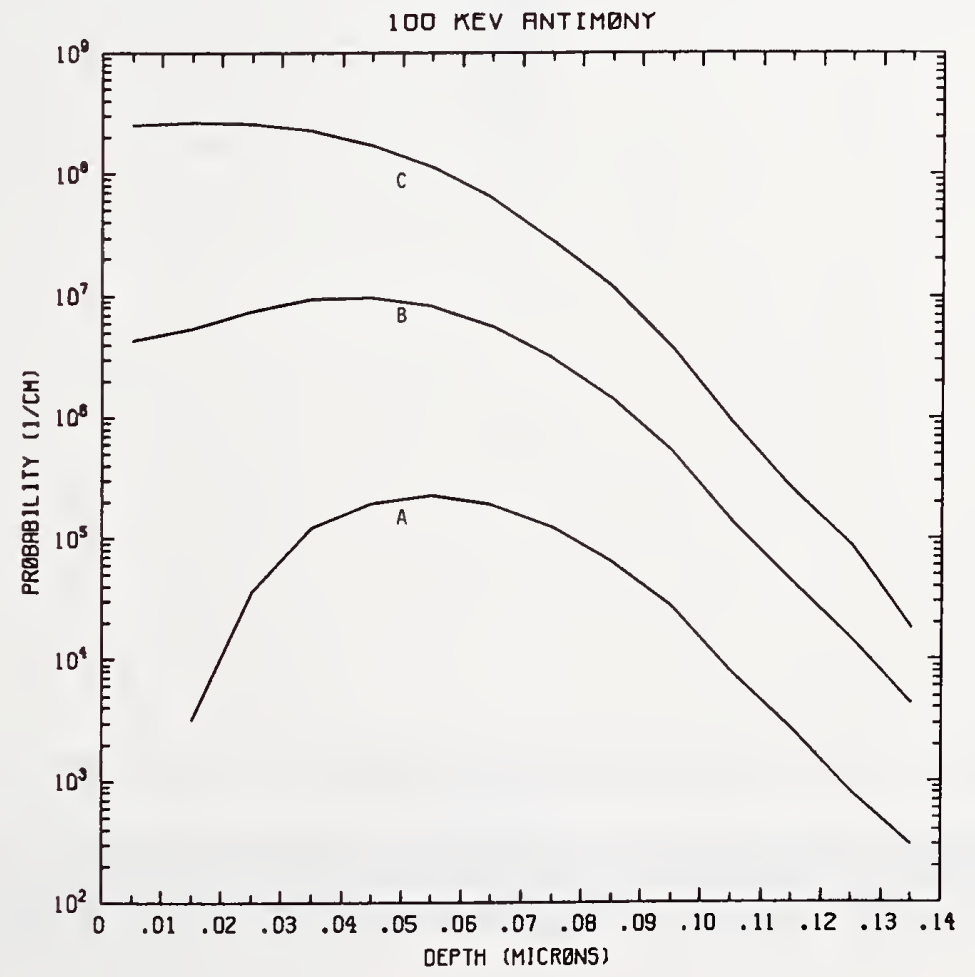

Figure Sb100-2 One-dimensional probability distributions for implanted antimony (A), primary silicon displacements (B), and Frenkel pairs (C) for $100 \mathrm{keV}$ antimony normally incident on a silicon target. 


\section{IIASK}

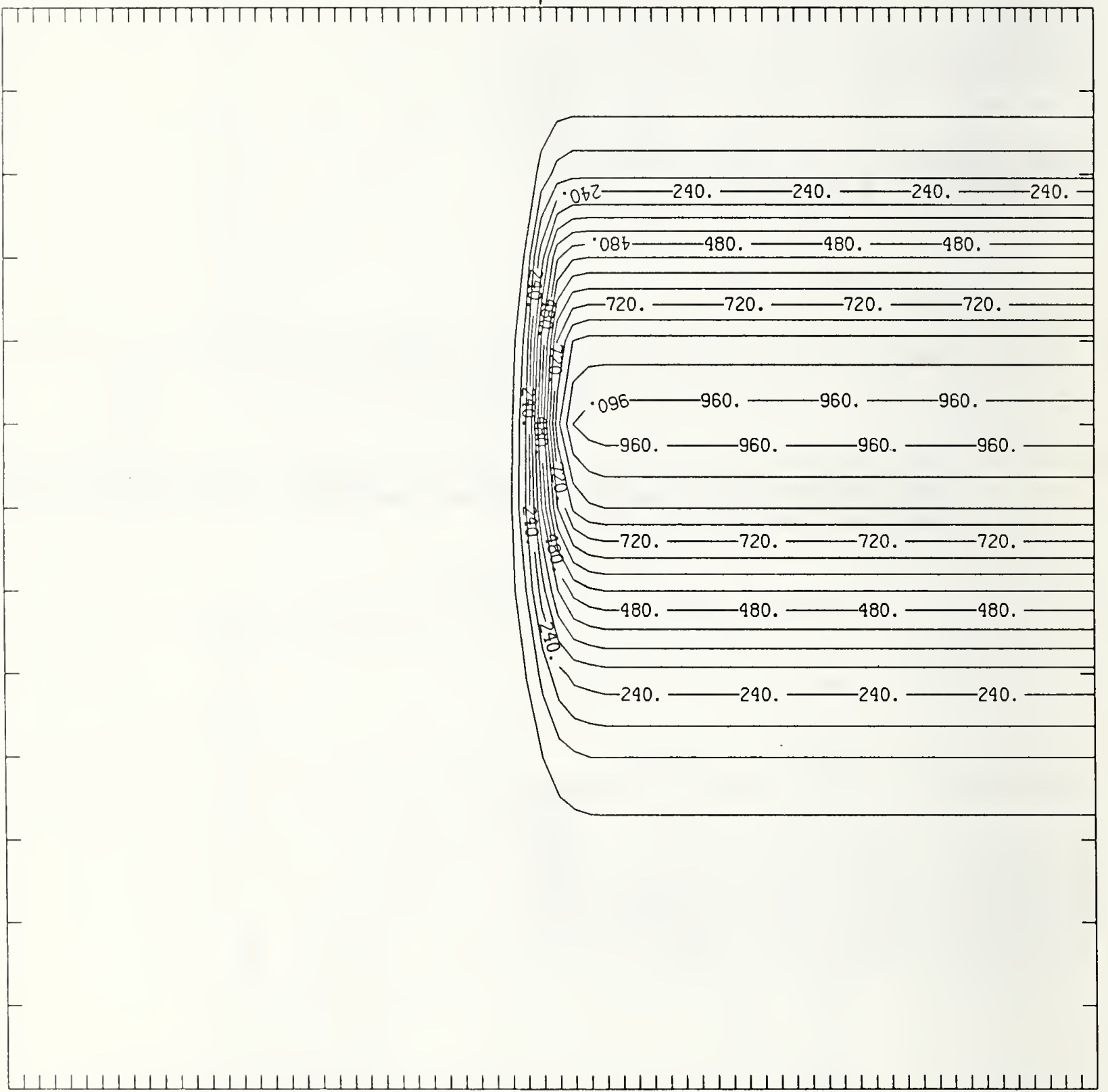

Figure Sb100-3 Two-dimensional distribution of $100 \mathrm{keV}$ antimony implanted past a mask edge. The length increment (distance between tick marks) is $0.01 \mu \mathrm{m}$ and the scale factor is 1000. 


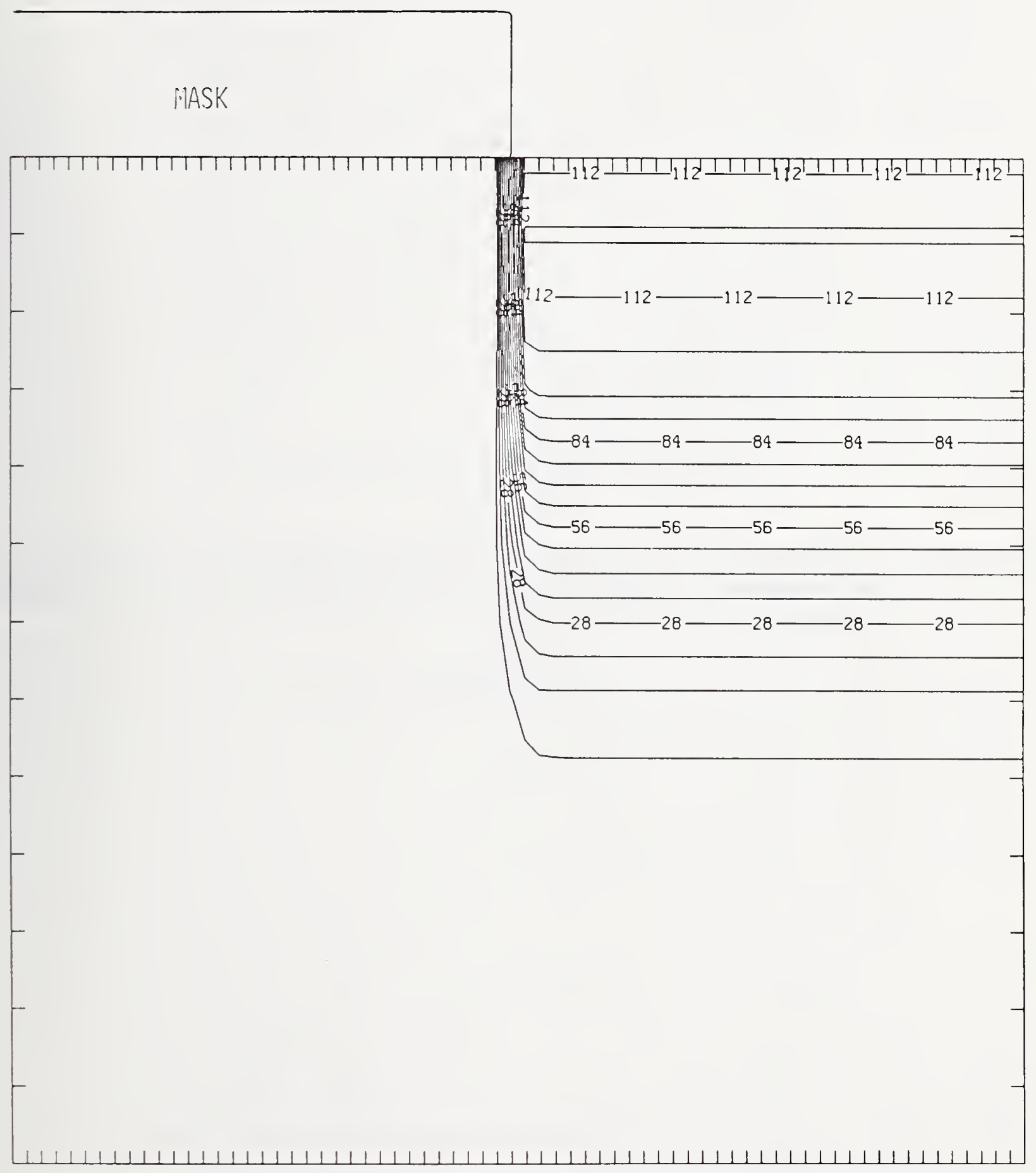

Figure Sb100-4 Two-dimensional distribution of Frenkel pairs created by $100 \mathrm{keV}$ antimony implanted past a mask edge. The length increment (distance between tick marks) is $0.01 \mu \mathrm{m}$ and the scale factor is 0.1 . 


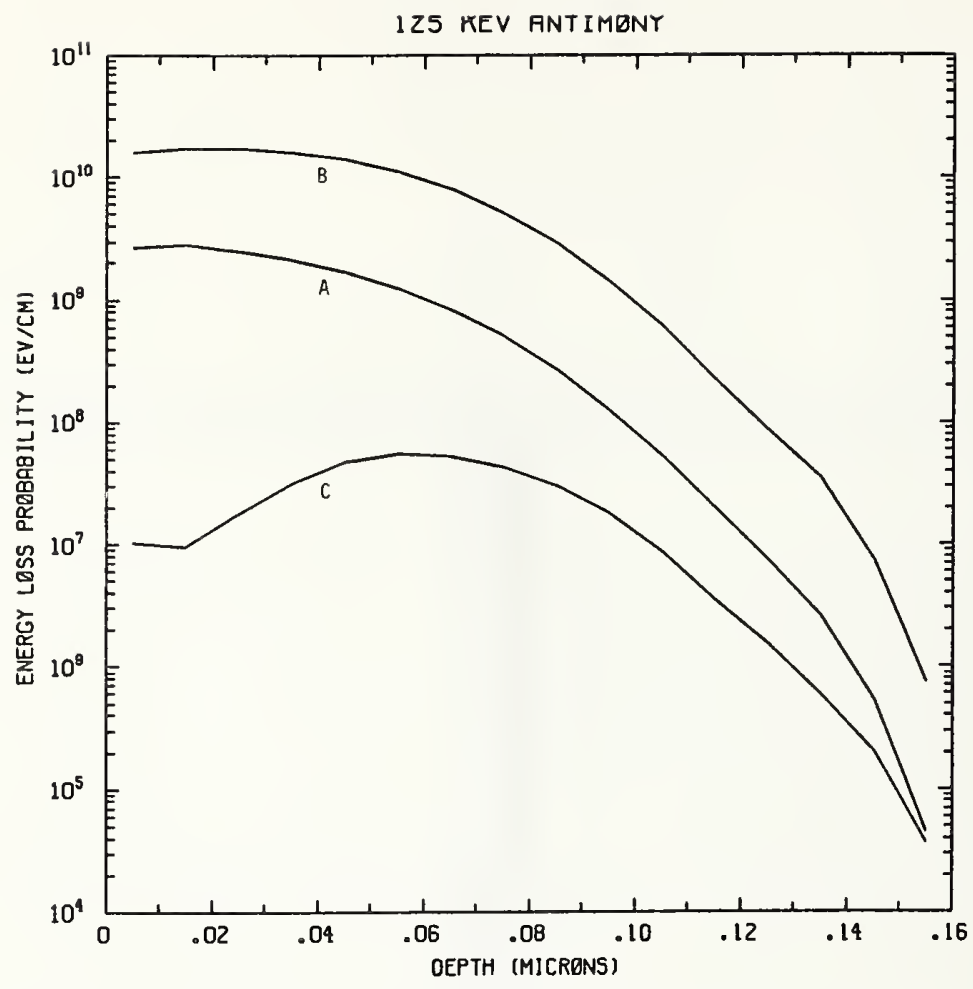

Figure Sb125-1 One-dimensional probability distributions for energy loss due to electronic excitation (A), displacement damage(B), and phonon excitation (C) for $125 \mathrm{keV}$ antimony normally incident on a silicon target.

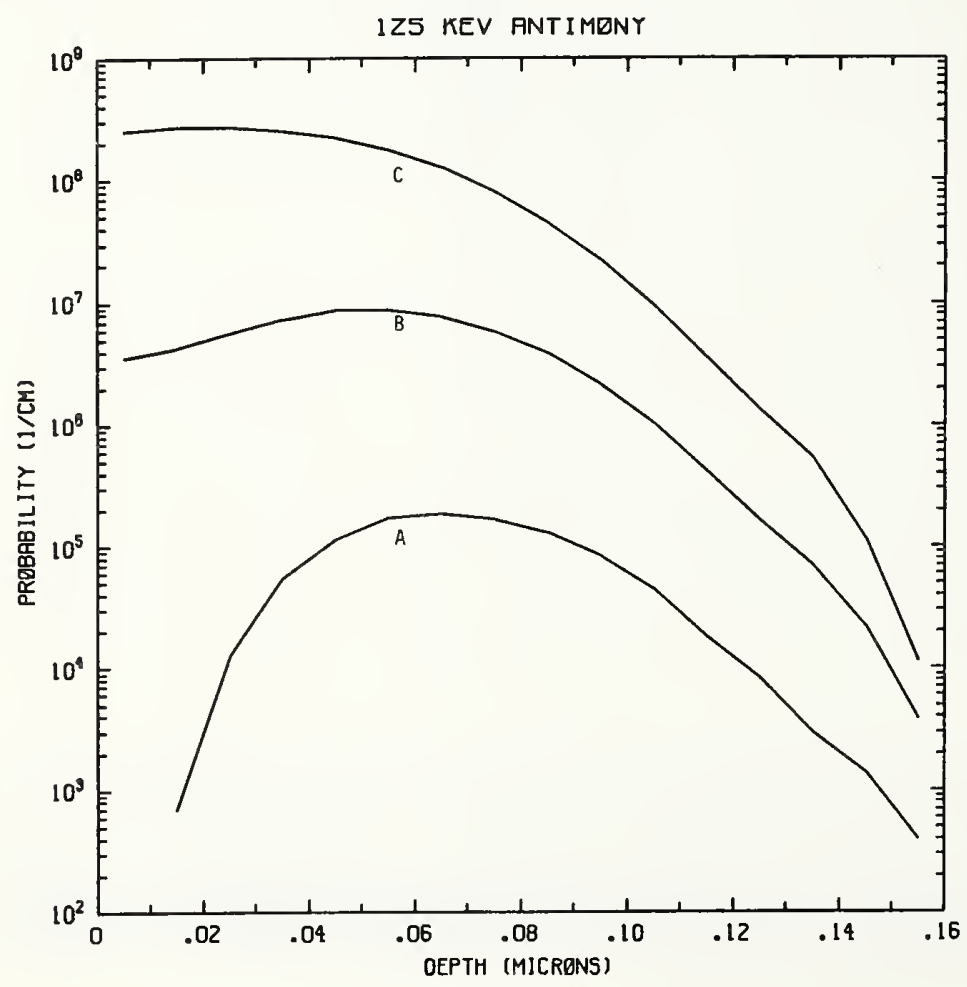

Figure Sb125-2 One-dimensional probability distributions for implanted antimony (A), primary silicon displacements (B), and Frenkel pairs (C) for $125 \mathrm{keV}$ antimony normally incident on a silicon target. 


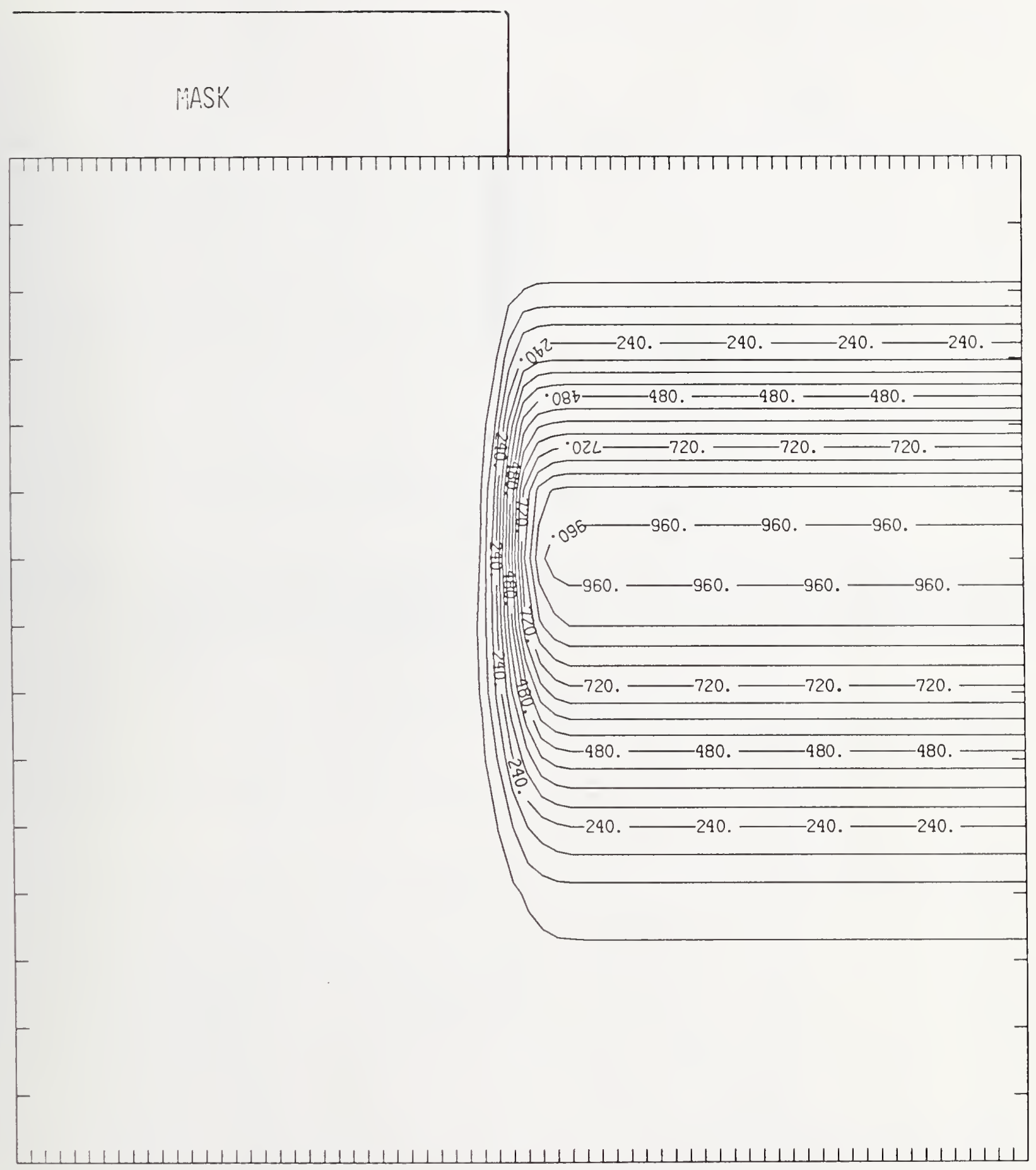

Figure Sb125-3 Two-dimensional distribution of $125 \mathrm{keV}$ antimony implanted past a mask edge. The length increment (distance between tick marks) is $0.01 \mu \mathrm{m}$ and the scale factor is 1000. 


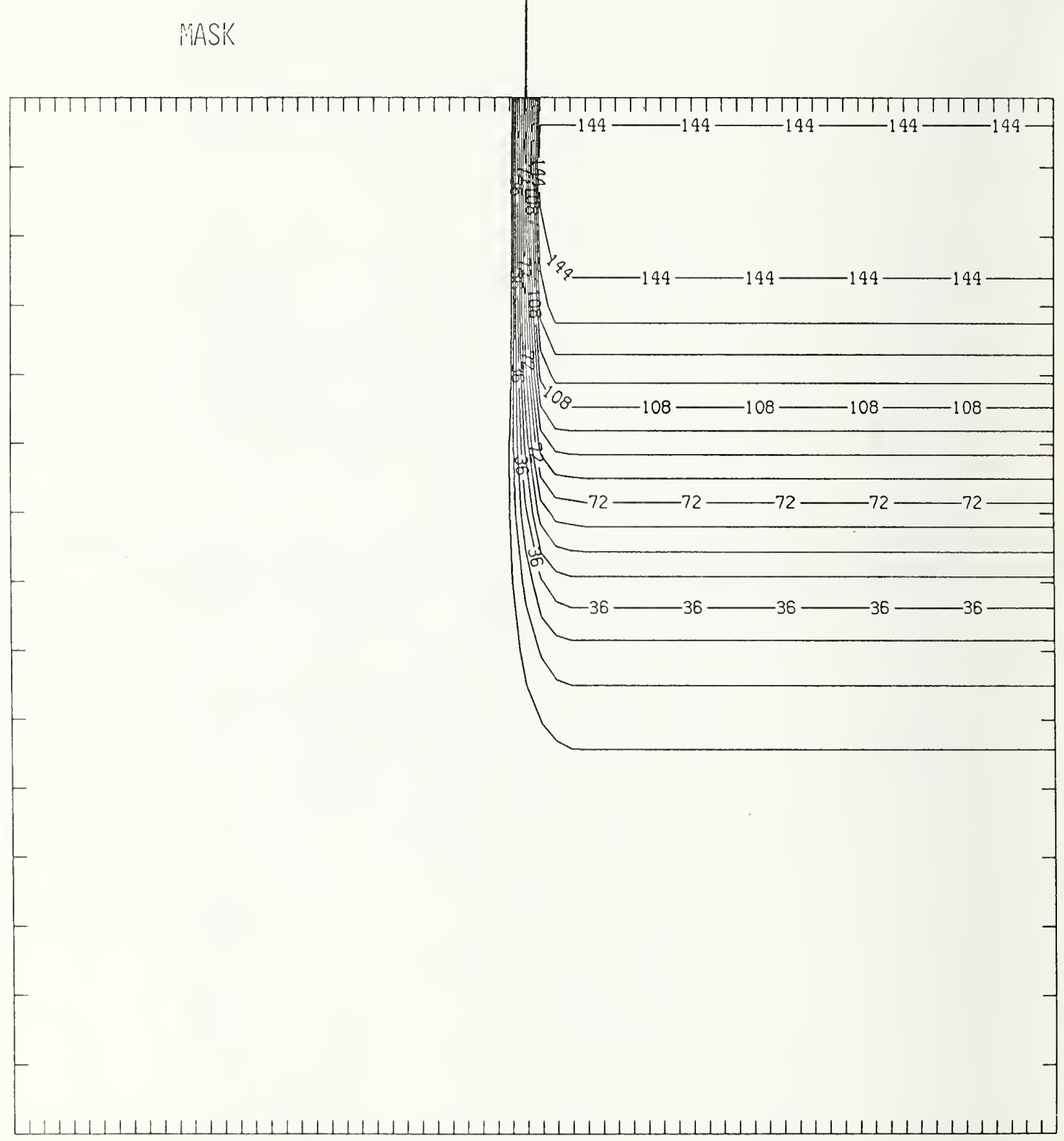

Figure Sb125-4 Two-dimensional distribution of Frenkel pairs created by $125 \mathrm{keV}$ antimony implanted past a mask edge. The length increment (distance between tick marks) is $0.01 \mu \mathrm{m}$ and the scale factor is 0.1 . 


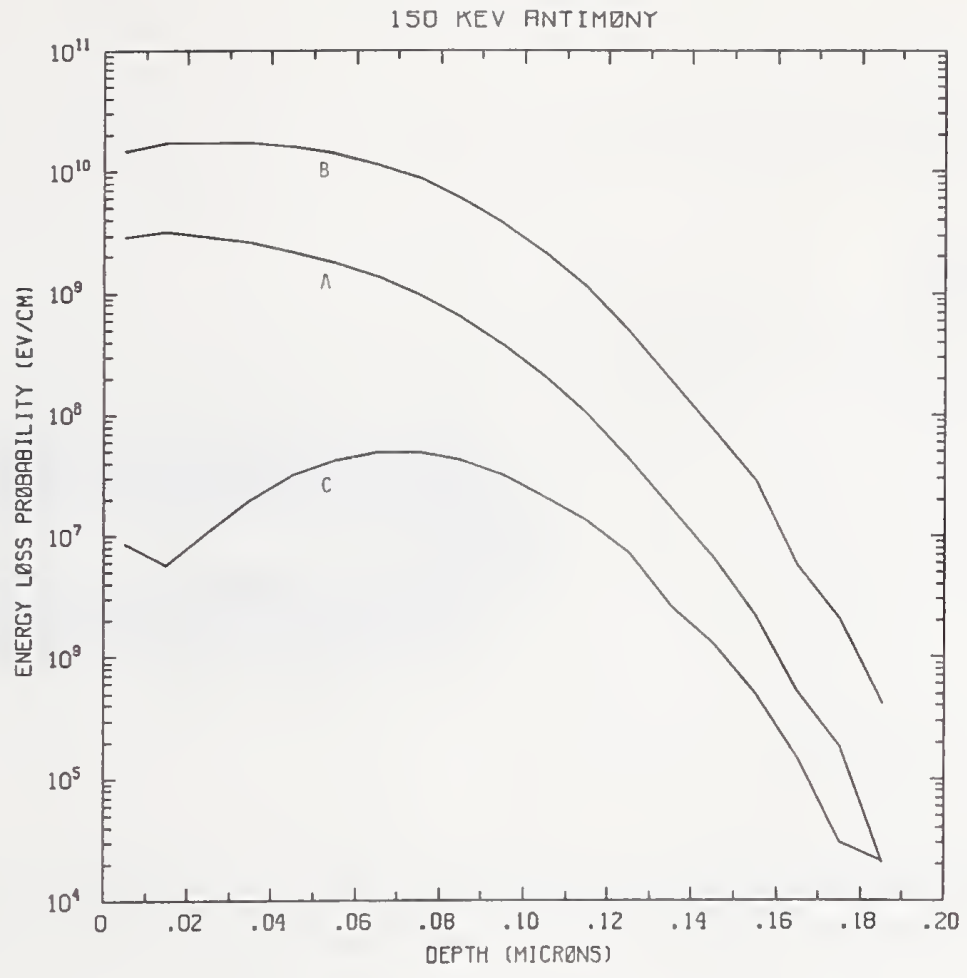

Figure Sb150-1 One-dimensional probability distributions for energy loss due to electronic excitation (A), displacement damage(B), and phonon excitation (C) for $150 \mathrm{keV}$ antimony normally incident on a silicon target.

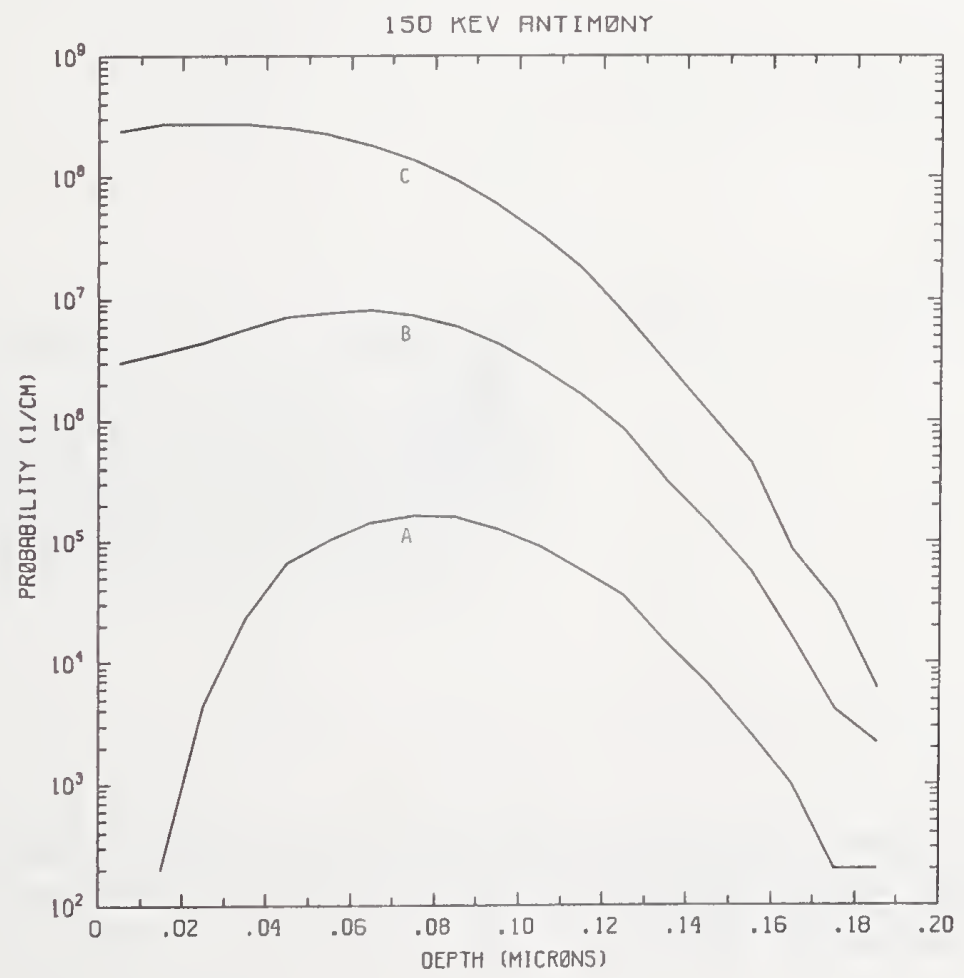

Figure Sb150-2 One-dimensional probability distributions for implanted antimony (A), primary silicon displacements (B), and Frenkel pairs (C) for $150 \mathrm{keV}$ antimony normally incident on a silicon target. 


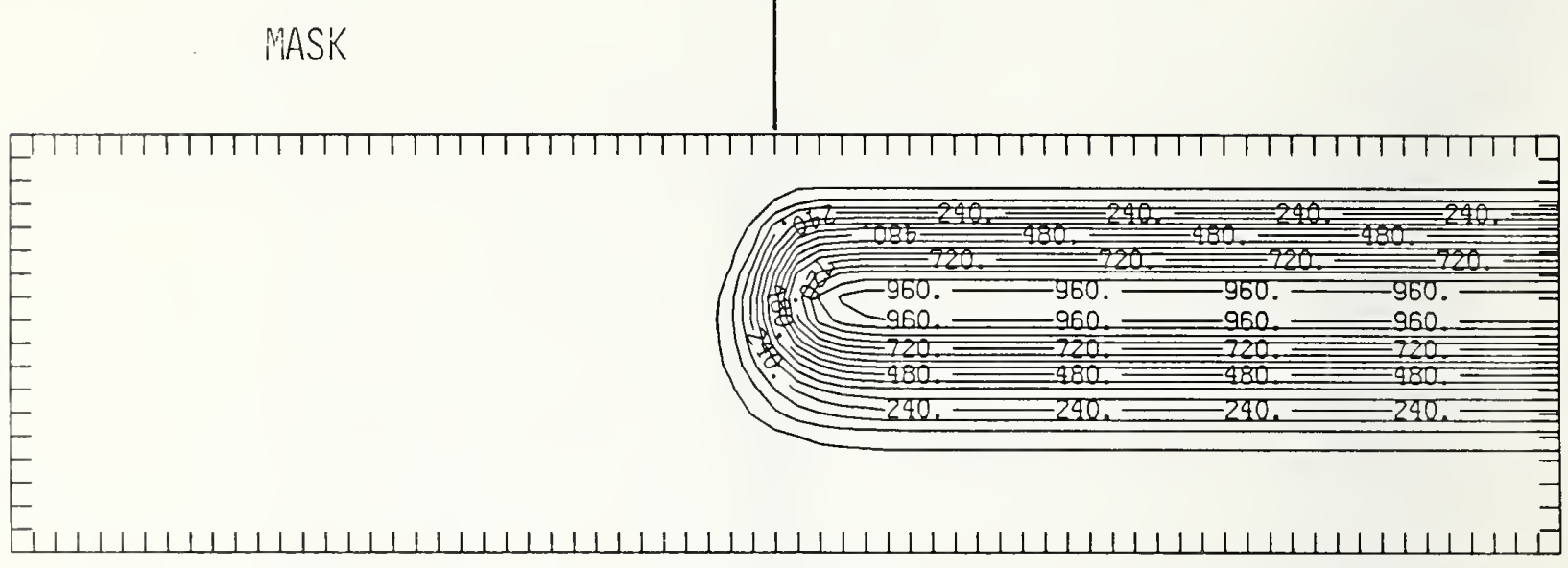

Figure Sb150-3 Two-dimensional distribution of $150 \mathrm{keV}$ antimony implanted past a mask edge. The length increment (distance between tick marks) is $0.01 \mu \mathrm{m}$ and the scale factor is 1000 .

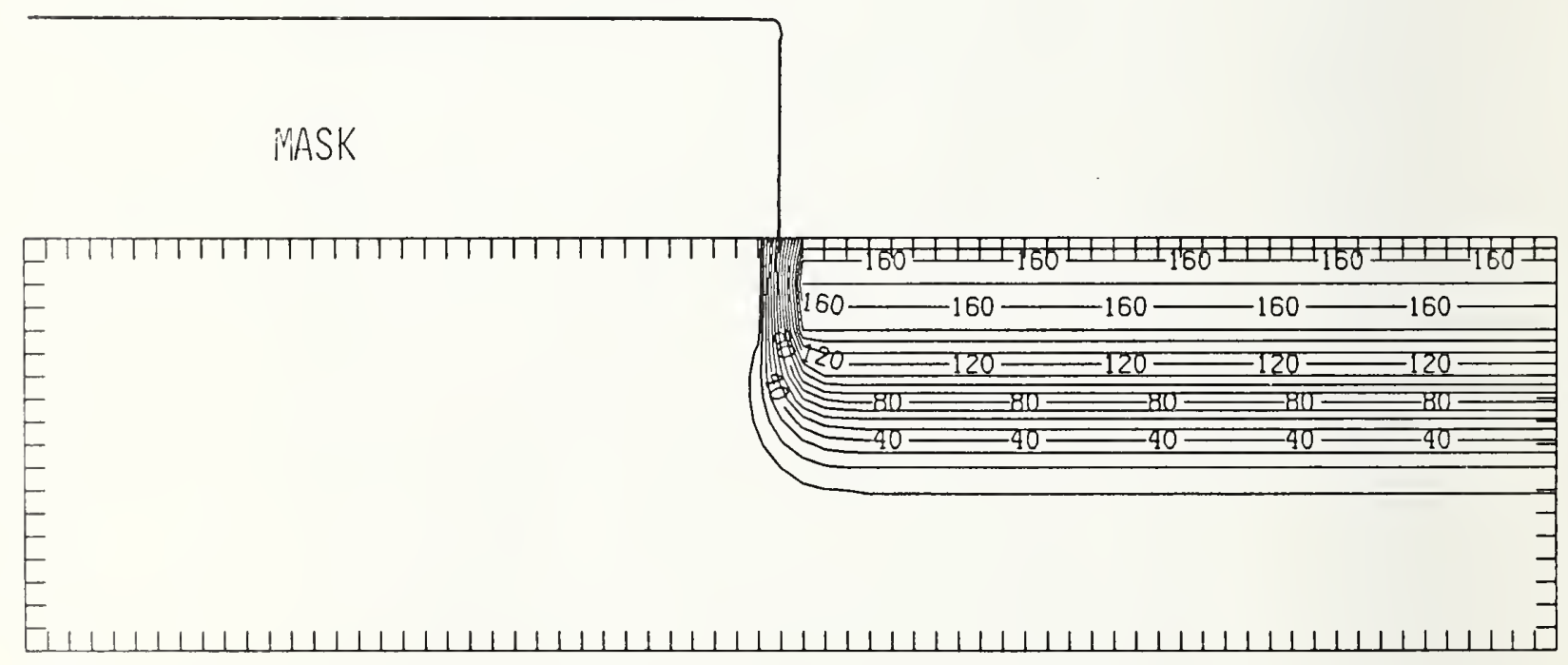

Figure Sb150-4 Two-dimensional distribution of Frenkel pairs created by $150 \mathrm{keV}$ antimony implanted past a mask edge. The length increment (distance between tick marks) is $0.01 \mu \mathrm{m}$ and the scale factor is 0.1 . 


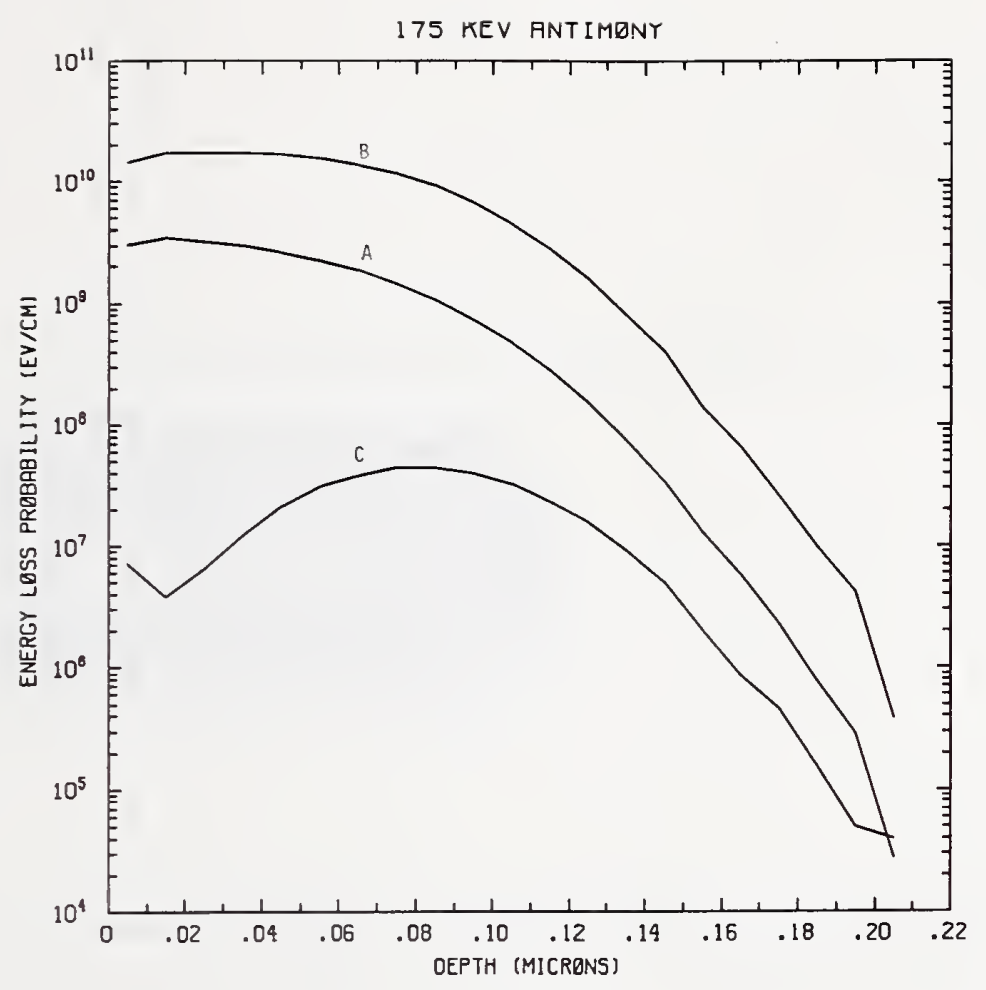

Figure Sb175-1 One-dimensional probability distributions for energy loss due to electronic excitation (A), displacement damage(B), and phonon excitation (C) for $175 \mathrm{keV}$ antimony normally incident on a silicon target.

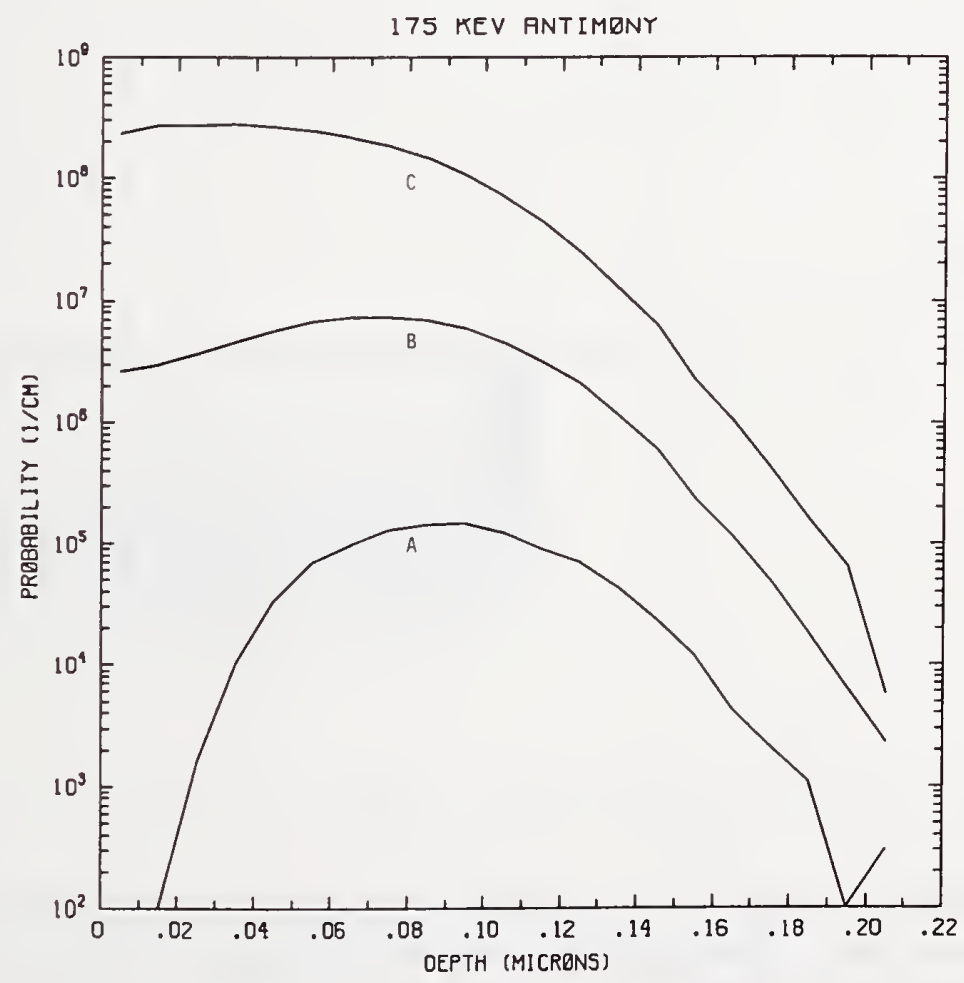

Figure Sb175-2 One-dimensional probability distributions for implanted antimony (A), primary silicon displacements (B), and Frenkel pairs (C) for $175 \mathrm{keV}$ antimony normally incident on a silicon target. 


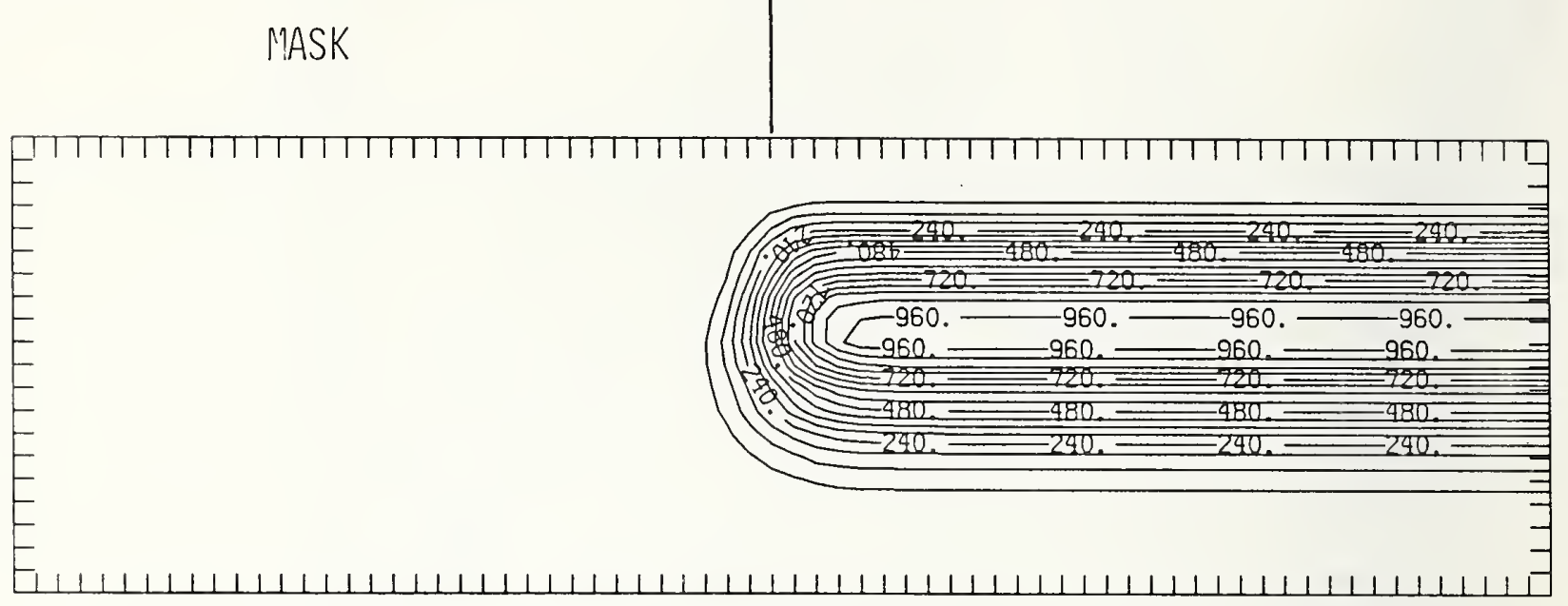

Figure Sb175-3 Two-dimensional distribution of $175 \mathrm{keV}$ antimony implanted past a mask edge. The length increment (distance between tick marks) is $0.01 \mu \mathrm{m}$ and the scale factor is 1000 .

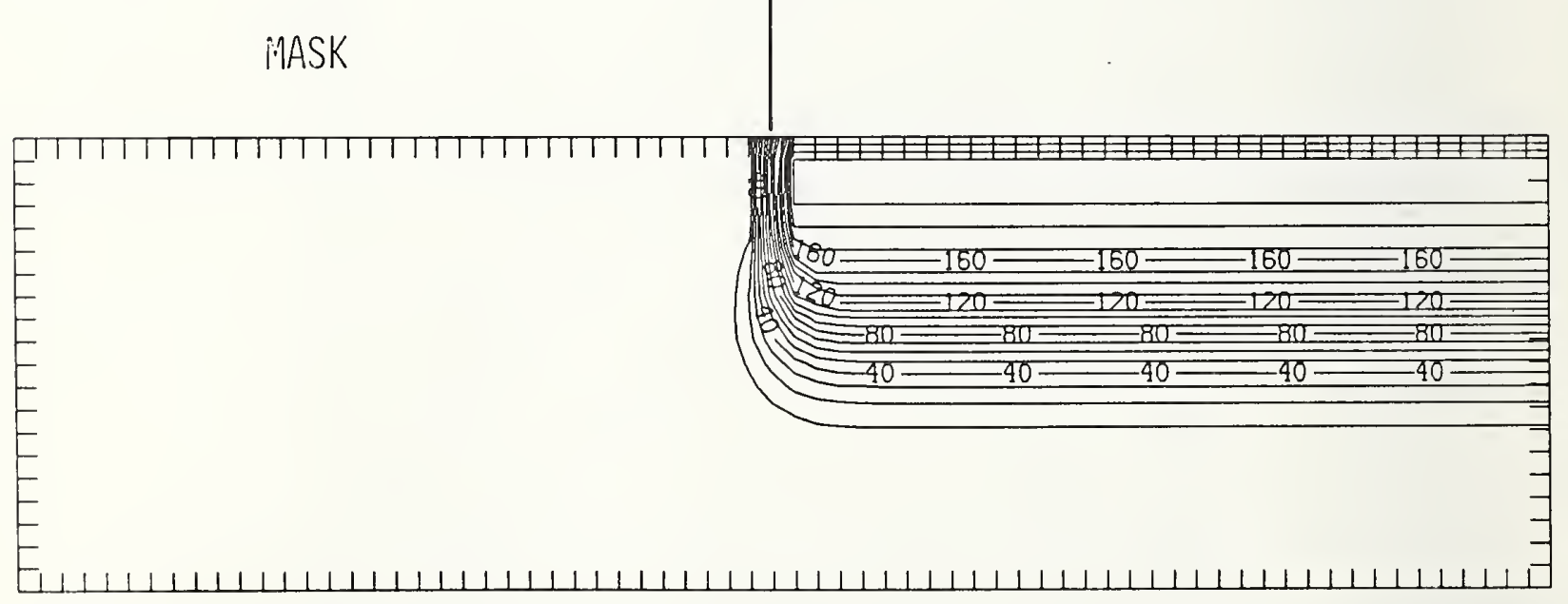

Figure Sb175-4 Two-dimensional distribution of Frenkel pairs created by $175 \mathrm{keV}$ antimony implanted past a mask edge. The length increment (distance between tick marks) is $0.01 \mu \mathrm{m}$ and the scale factor is 0.1 . 


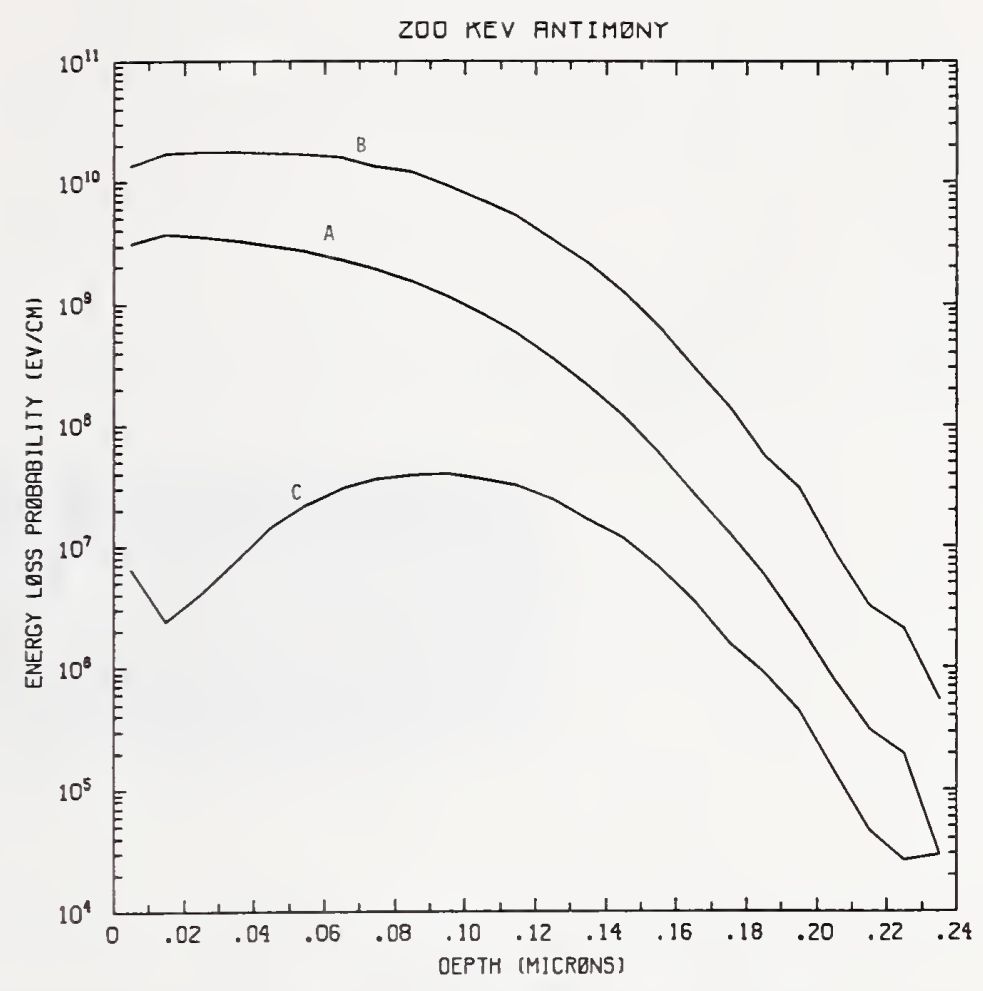

Figure Sb200-1 One-dimensional probability distributions for energy loss due to electronic excitation (A), displacement damage(B), and phonon excitation (C) for $200 \mathrm{keV}$ antimony normally incident on a silicon target.

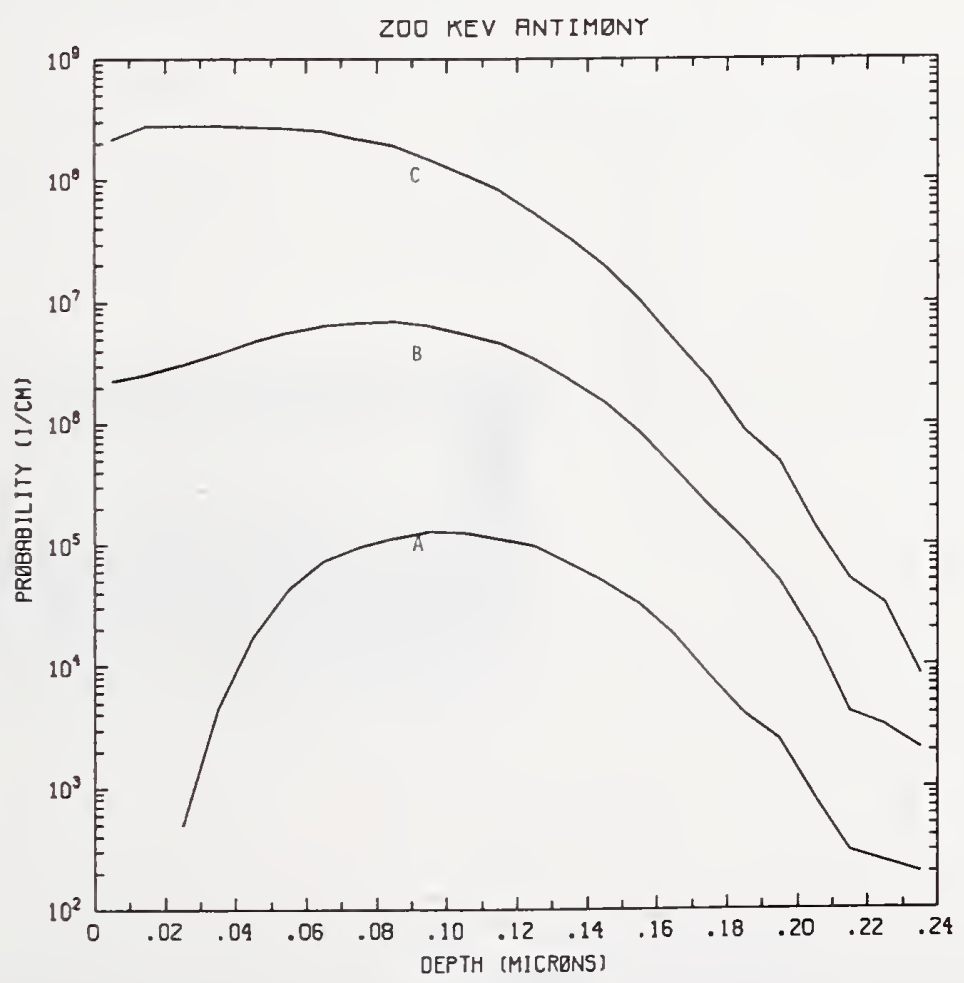

Figure Sb200-2 One-dimensional probability distributions for implanted antimony (A), primary silicon displacements (B), and Frenkel pairs (C) for $200 \mathrm{keV}$ antimony normally incident on a silicon target. 


\section{MASK}

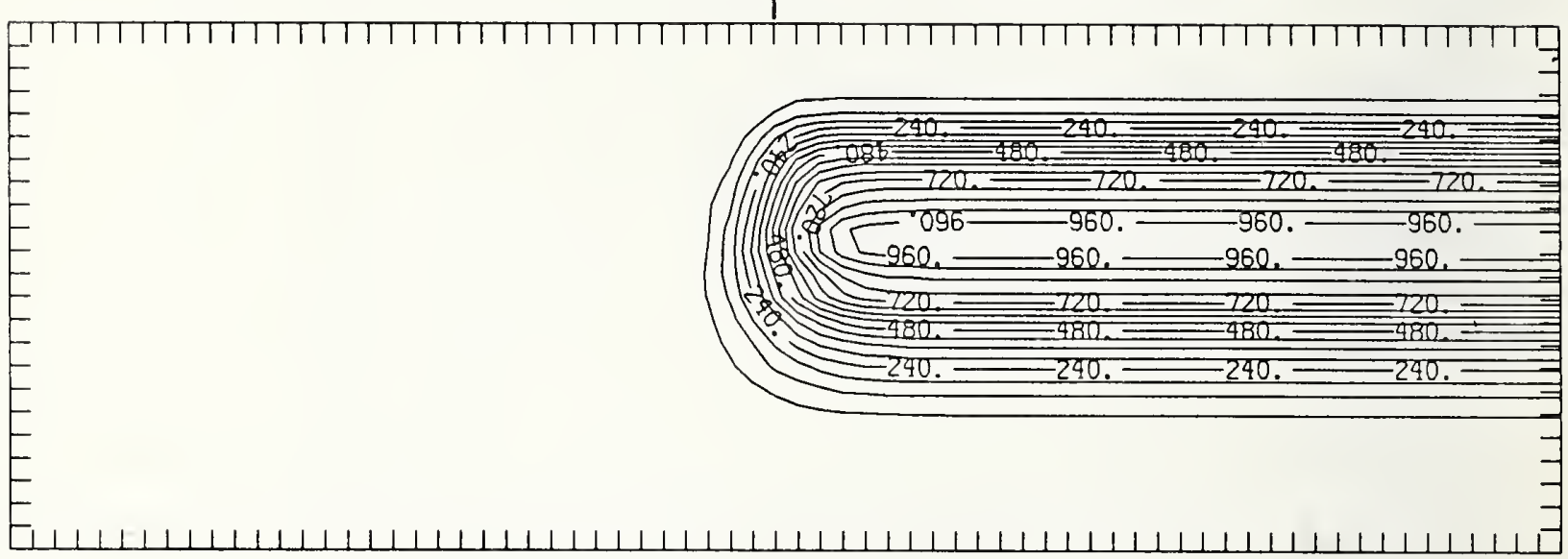

Figure Sb200-3 Two-dimensional distribution of $200 \mathrm{keV}$ antimony implanted past a mask edge. The length increment (distance between tick marks) is $0.01 \mu \mathrm{m}$ and the scale factor is 1000.

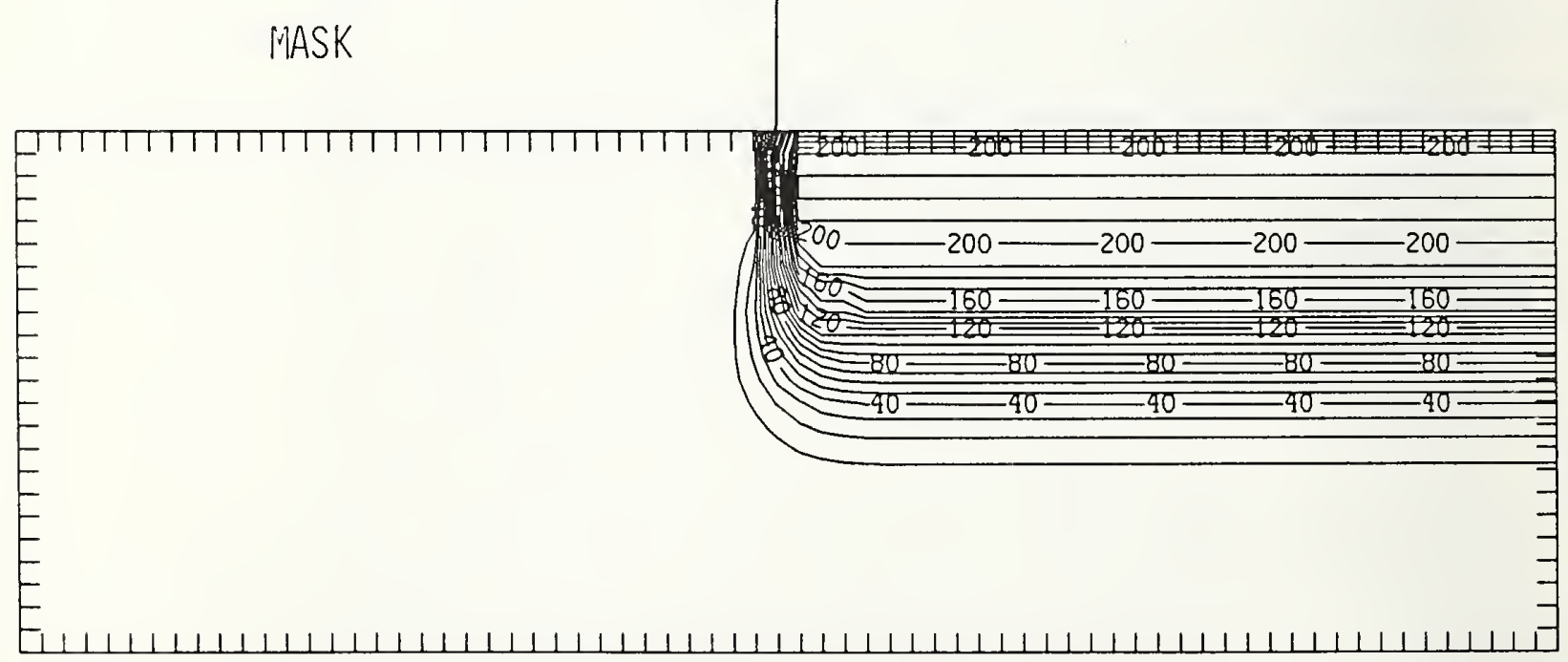

Figure Sb200-4 Two-dimensional distribution of Frenkel pairs created by $200 \mathrm{keV}$ antimony implanted past a mask edge. The length increment (distance between tick marks) is $0.01 \mu \mathrm{m}$ and the scale factor is 0.1 . 


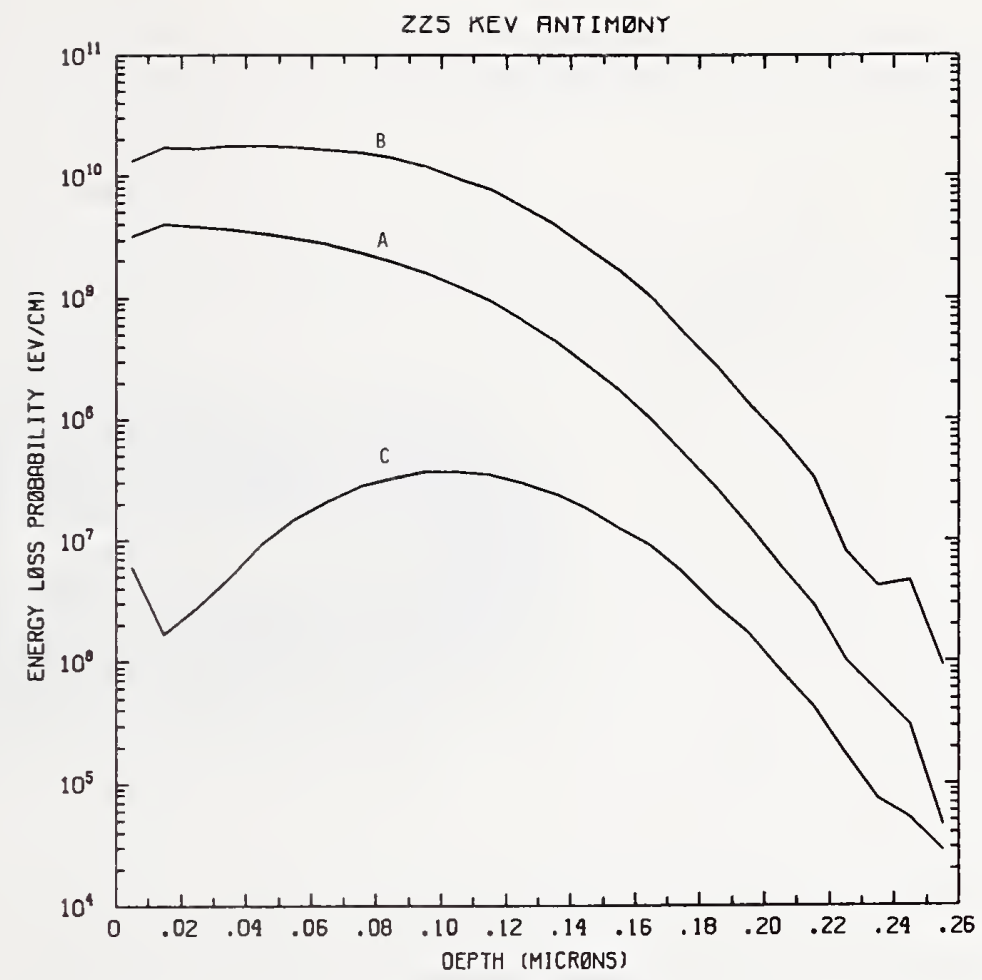

Figure Sb225-1 One-dimensional probability distributions for energy loss due to electronic excitation (A), displacement damage(B), and phonon excitation (C) for $225 \mathrm{keV}$ antimony normally incident on a silicon target.

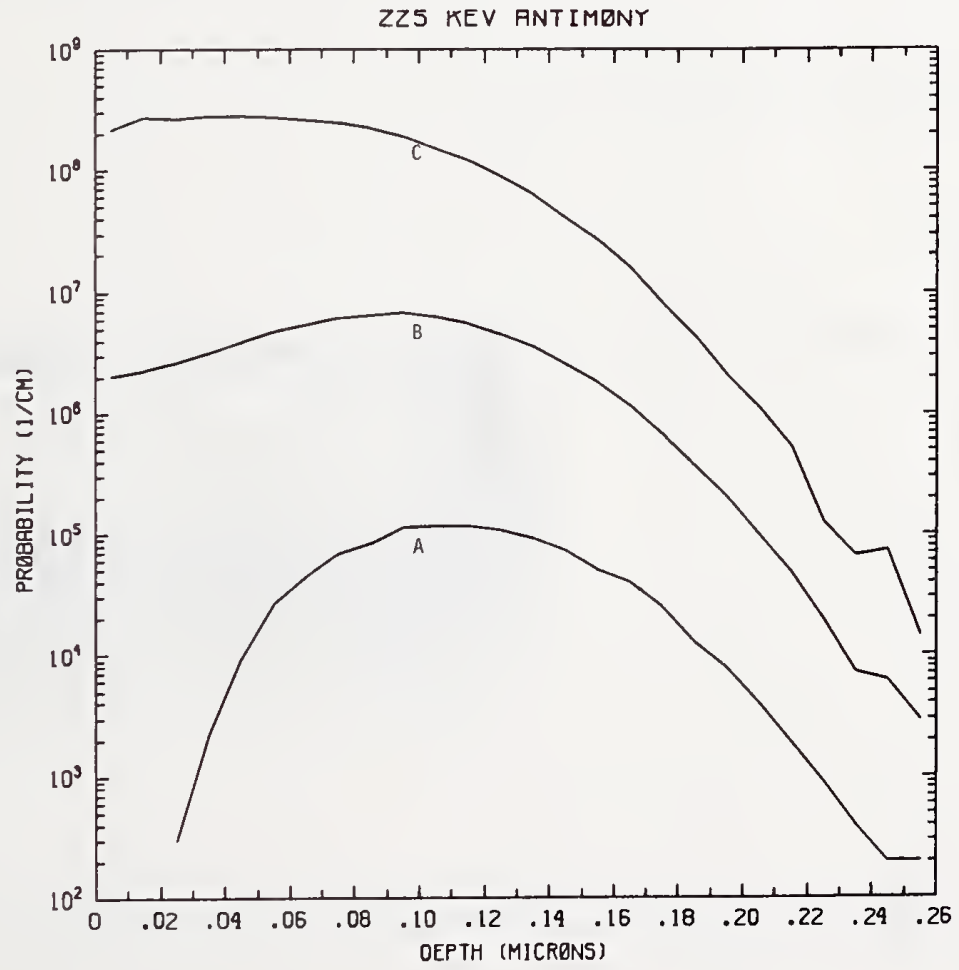

Figure Sb225-2 One-dimensional probability distributions for implanted antimony (A), primary silicon displacements (B), and Frenkel pairs (C) for $225 \mathrm{keV}$ antimony normally incident on a silicon target. 


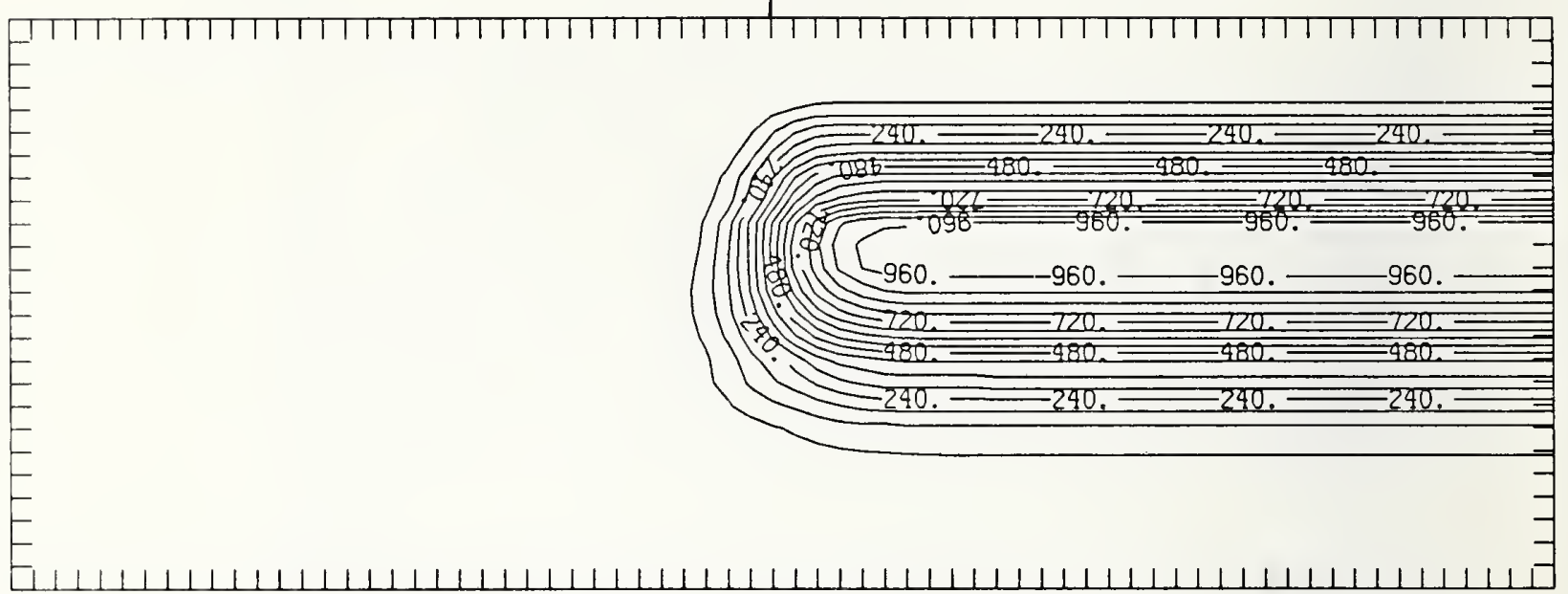

Figure Sb225-3 Two-dimensional distribution of $225 \mathrm{keV}$ antimony implanted past a mask edge. The length increment (distance between tick marks) is $0.01 \mu \mathrm{m}$ and the scale factor is 1000 .

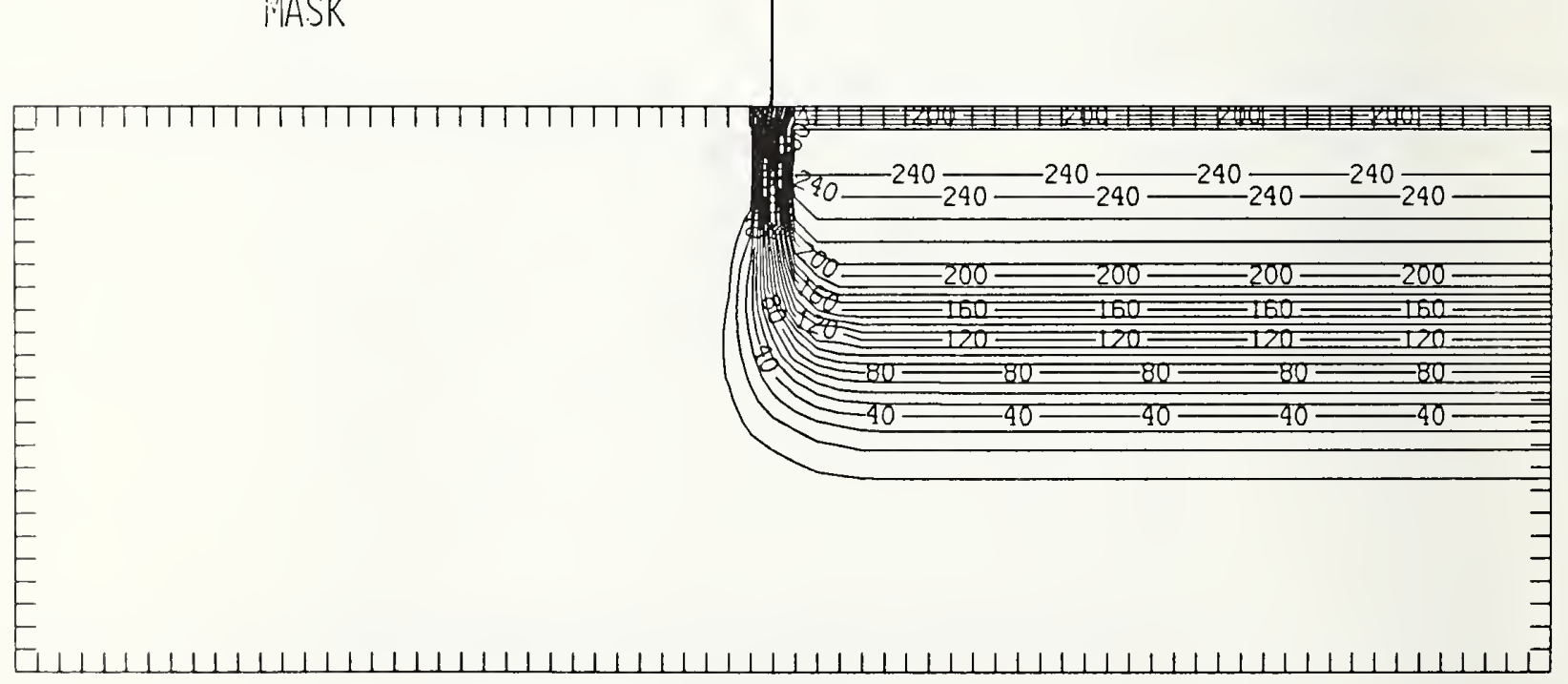

Figure Sb225-4 Two-dimensional distribution of Frenkel pairs created by $225 \mathrm{keV}$ antimony implanted past a mask edge. The length increment (distance between tick marks) is $0.01 \mu \mathrm{m}$ and the scale factor is 0.1 . 


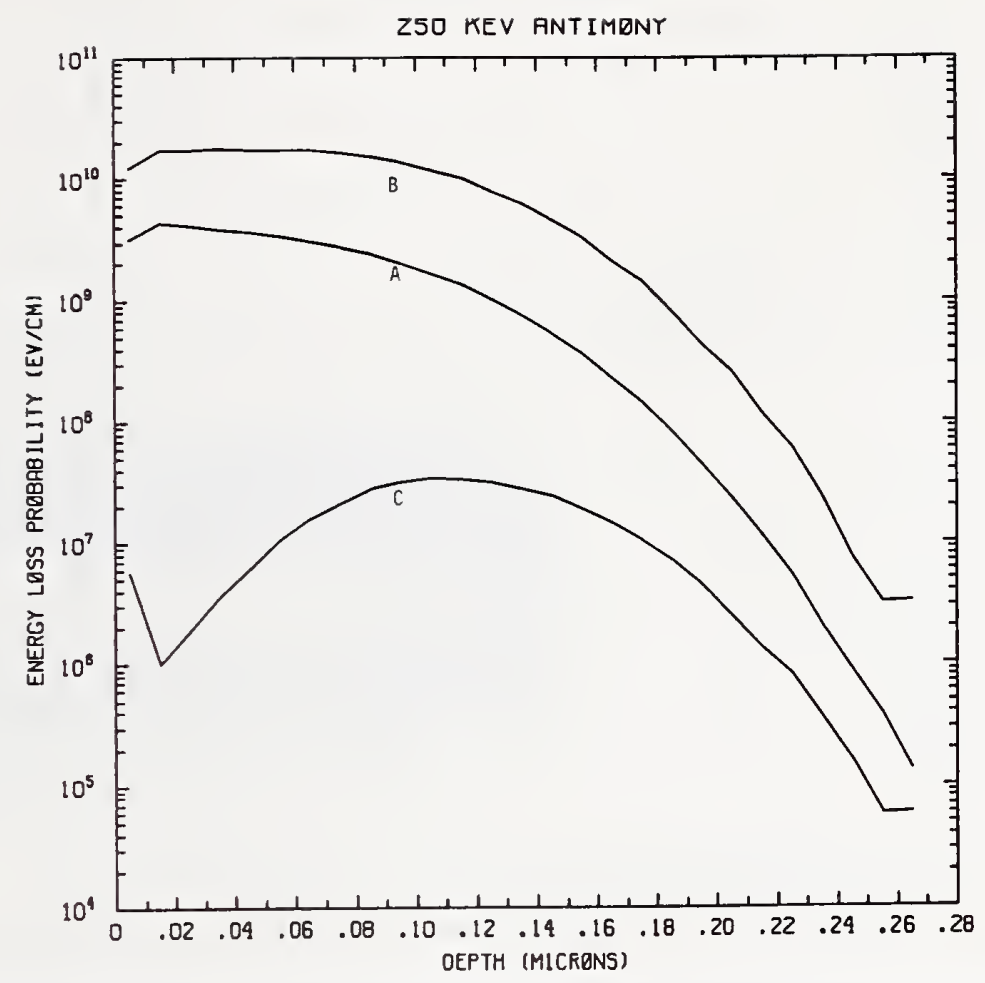

Figure Sb250-1 One-dimensional probability distributions for energy loss due to electronic excitation (A), displacement damage(B), and phonon excitation (C) for $250 \mathrm{keV}$ antimony normally incident on a silicon target.

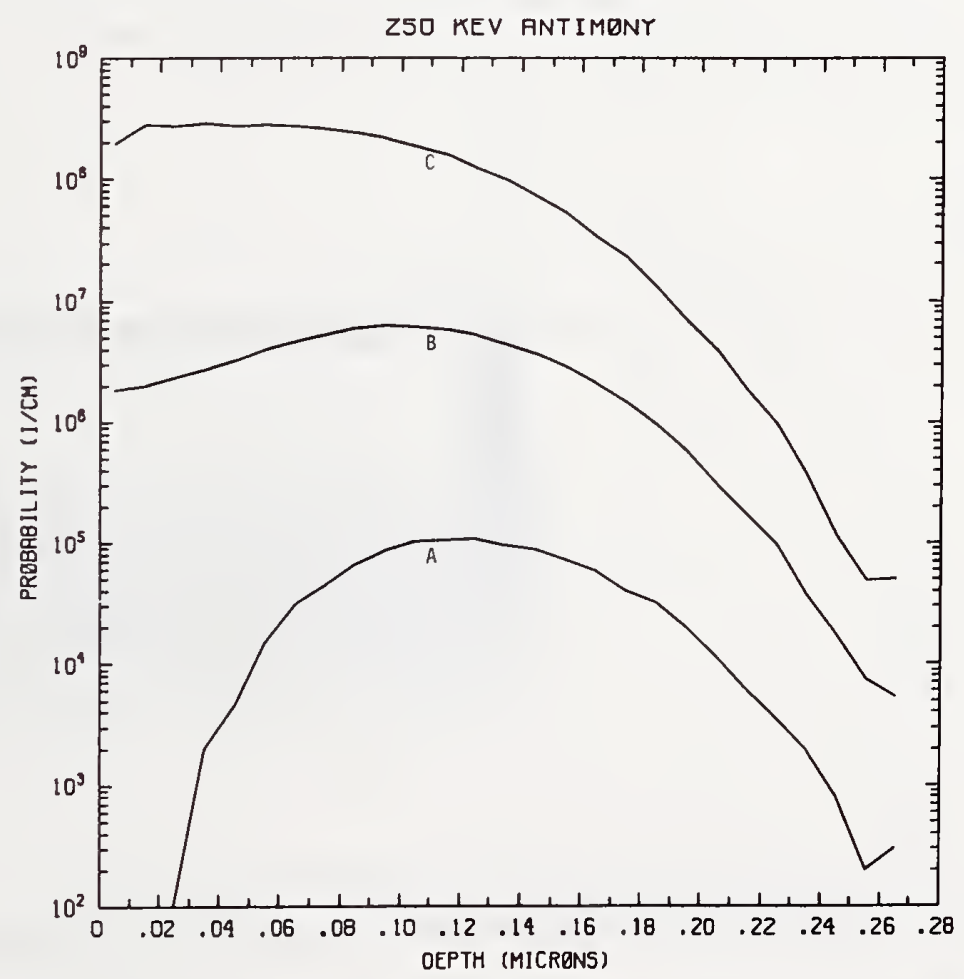

Figure Sb250-2 One-dimensional probability distributions for implanted antimony (A), primary silicon displacements (B), and Frenkel pairs (C) for $250 \mathrm{keV}$ antimony normally incident on a silicon target. 
MA.SK

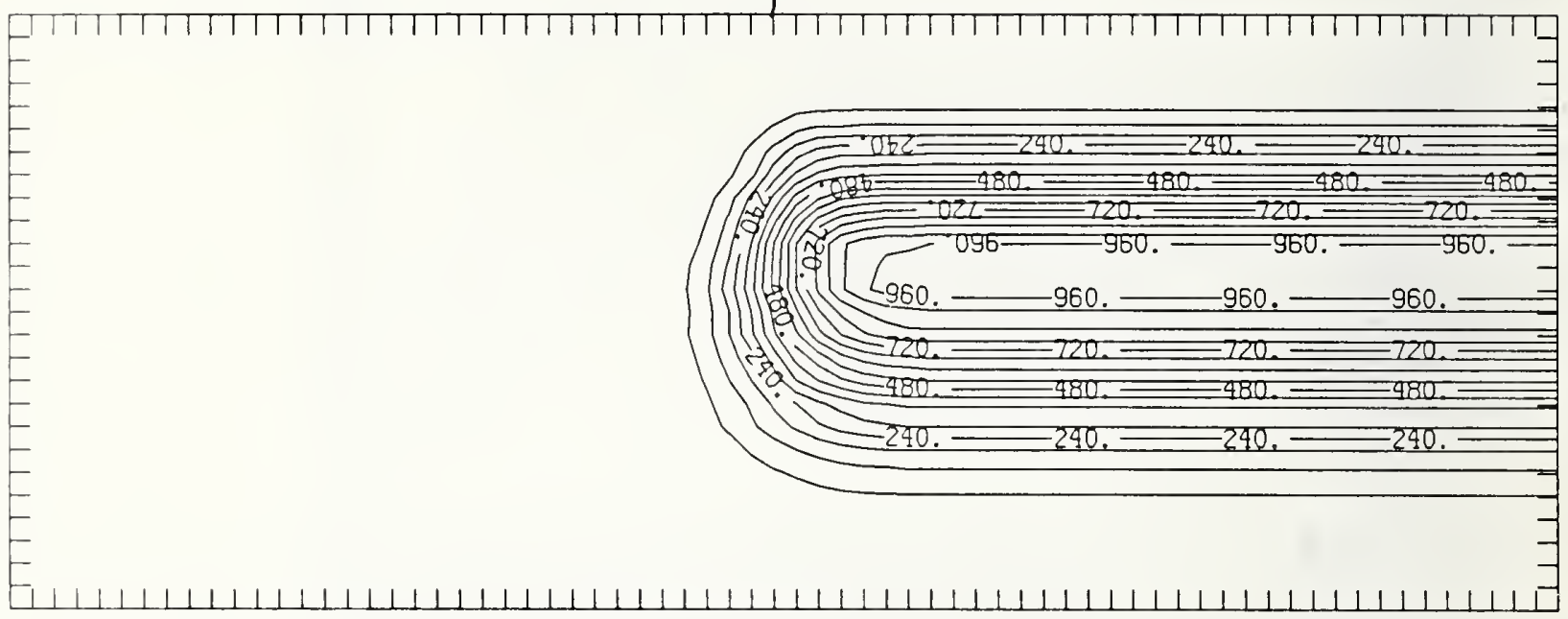

Figure Sb250-3 Two-dimensional distribution of $250 \mathrm{keV}$ antimony implanted past a mask edge. The length increment (distance between tick marks) is $0.01 \mu \mathrm{m}$ and the scale factor is 1000 .

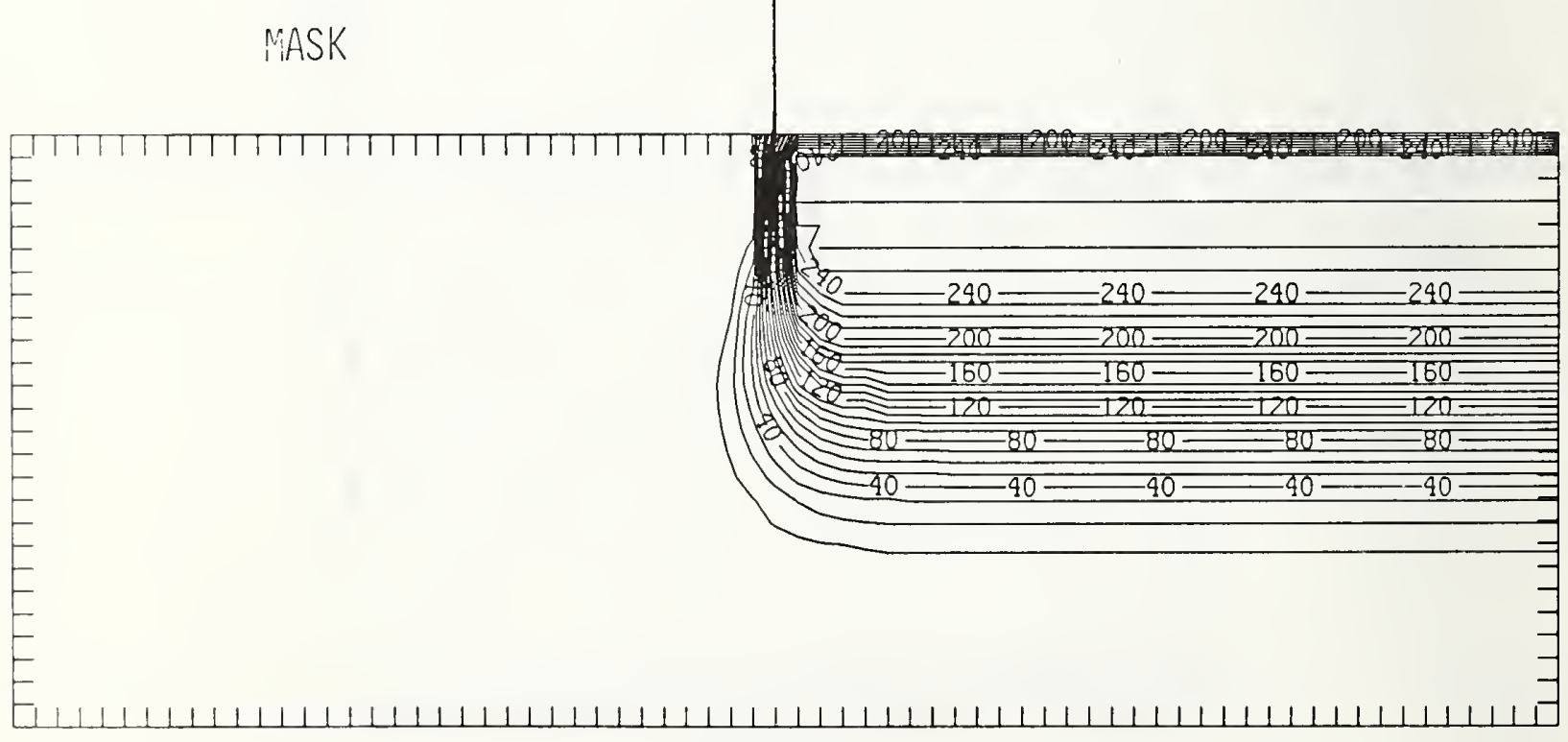

Figure Sb250-4 Two-dimensional distribution of Frenkel pairs created by $250 \mathrm{keV}$ antimony implanted past a mask edge. The length increment (distance between tick marks) is $0.01 \mu \mathrm{m}$ and the scale factor is 0.1 . 


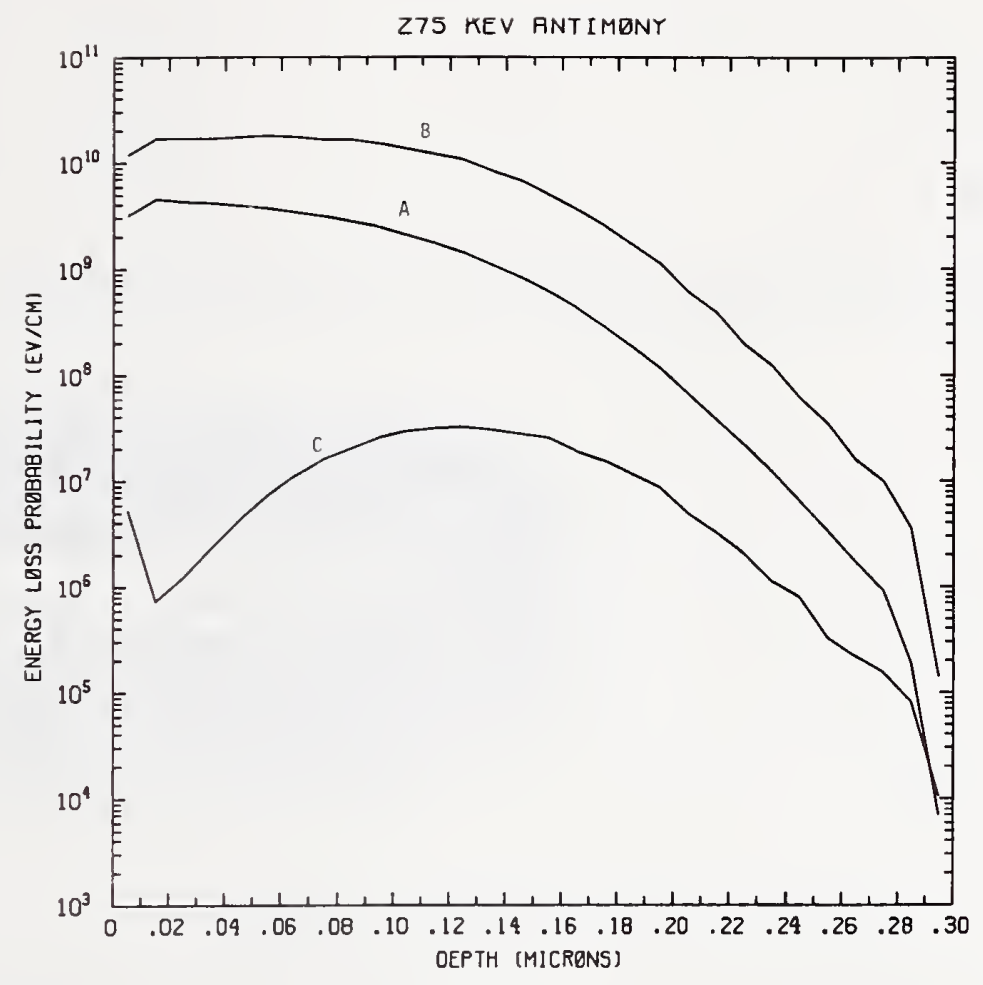

Figure Sb275-1 One-dimensional probability distributions for energy loss due to electronic excitation (A), displacement damage(B), and phonon excitation (C) for $275 \mathrm{keV}$ antimony normally incident on a silicon target.

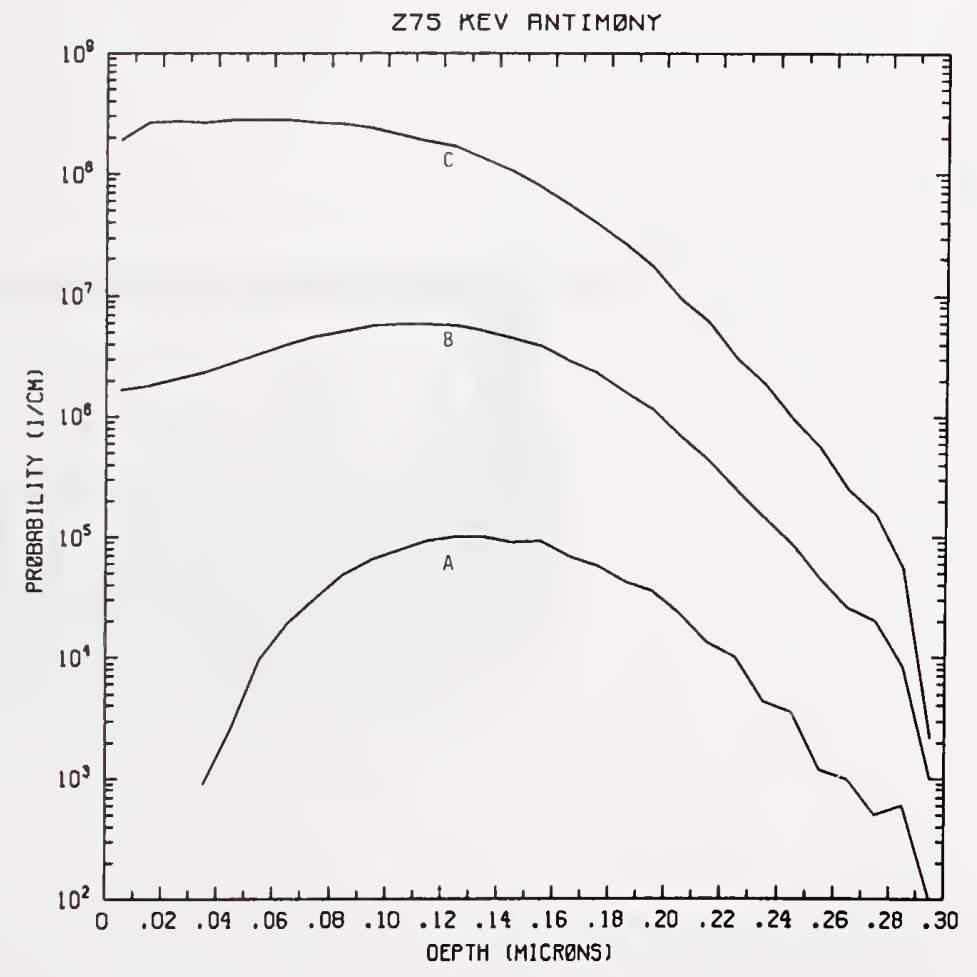

Figure Sb275-2 One-dimensional probability distributions for implanted antimony (A), primary silicon displacements (B), and Frenkel pairs (C) for $275 \mathrm{keV}$ antimony normally incident on a silicon target. 


\section{MASK}

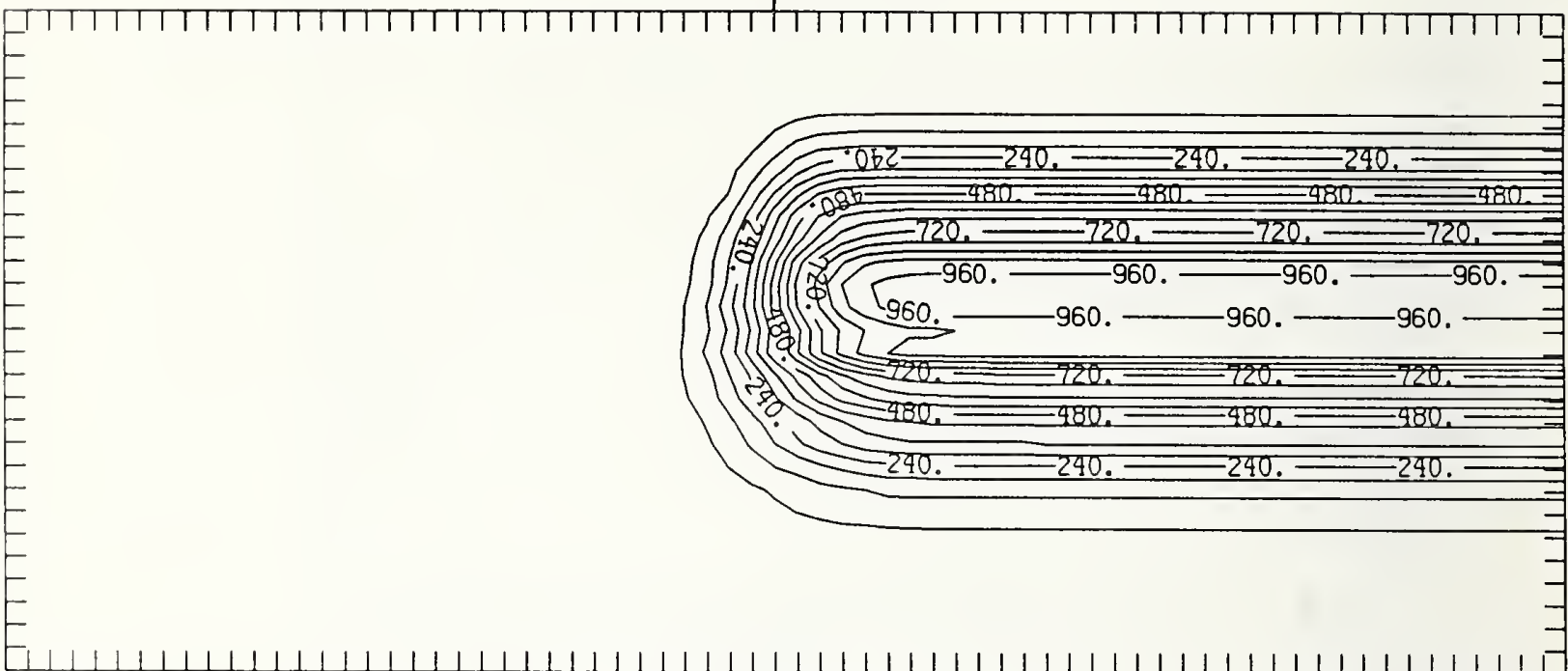

Figure Sb275-3 Two-dimensional distribution of $275 \mathrm{keV}$ antimony implanted past a mask edge. The length increment (distance between tick marks) is $0.01 \mu \mathrm{m}$ and the scale factor is 1000 .

MASK

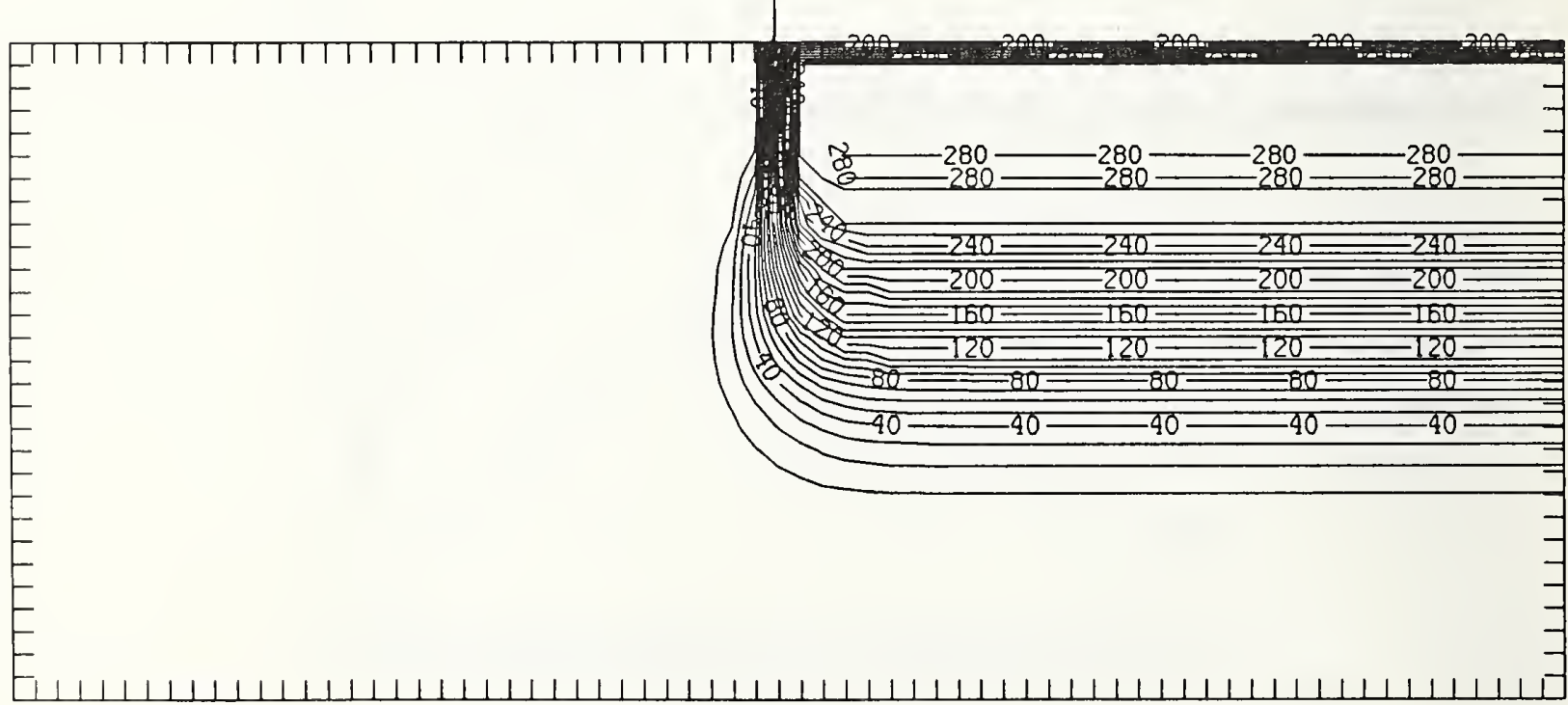

Figure Sb275-4 Two-dimensional distribution of Frenkel pairs created by $275 \mathrm{keV}$ antimony implanted past a mask edge. The length increment (distance between tick marks) is $0.01 \mu \mathrm{m}$ and the scale factor is 0.1 . 


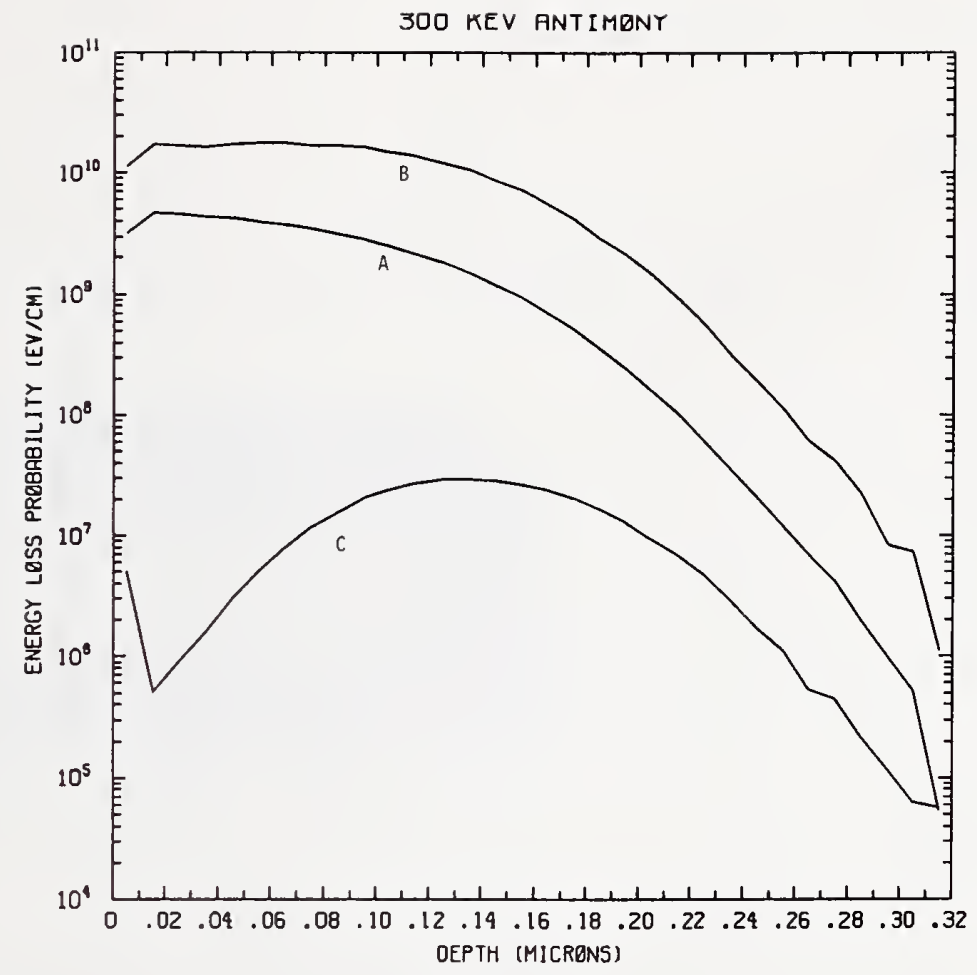

Figure Sb300-1 One-dimensional probability distributions for energy loss due to electronic excitation (A), displacement damage(B), and phonon excitation (C) for $300 \mathrm{keV}$ antimony normally incident on a silicon target.

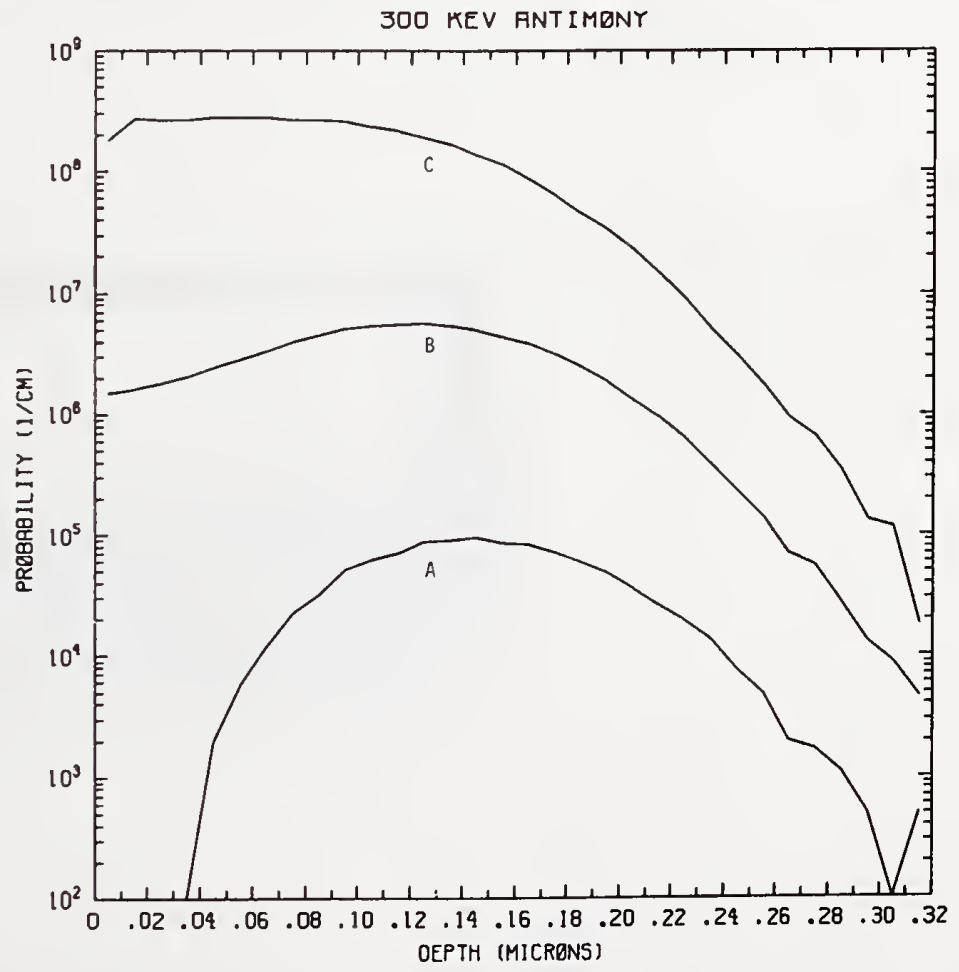

Figure Sb300-2 One-dimensional probability distributions for implanted antimony (A), primary silicon displacements (B), and Frenkel pairs (C) for $300 \mathrm{keV}$ antimony normally incident on a silicon target. 


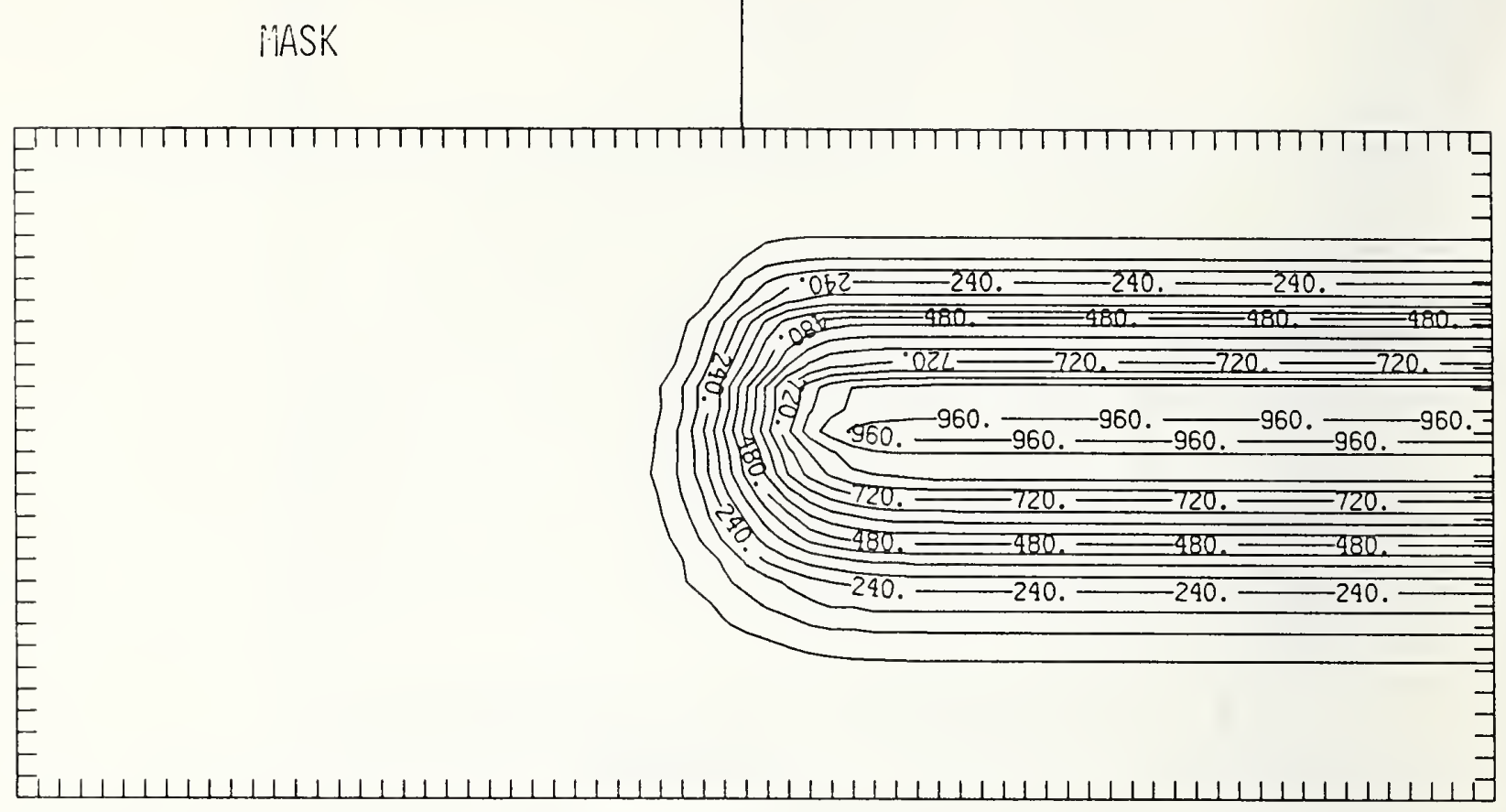

Figure Sb300-3 Two-dimensional distribution of $300 \mathrm{keV}$ antimony implanted past a mask edge. The length increment (distance between tick marks) is $0.01 \mu \mathrm{m}$ and the scale factor is 1000.

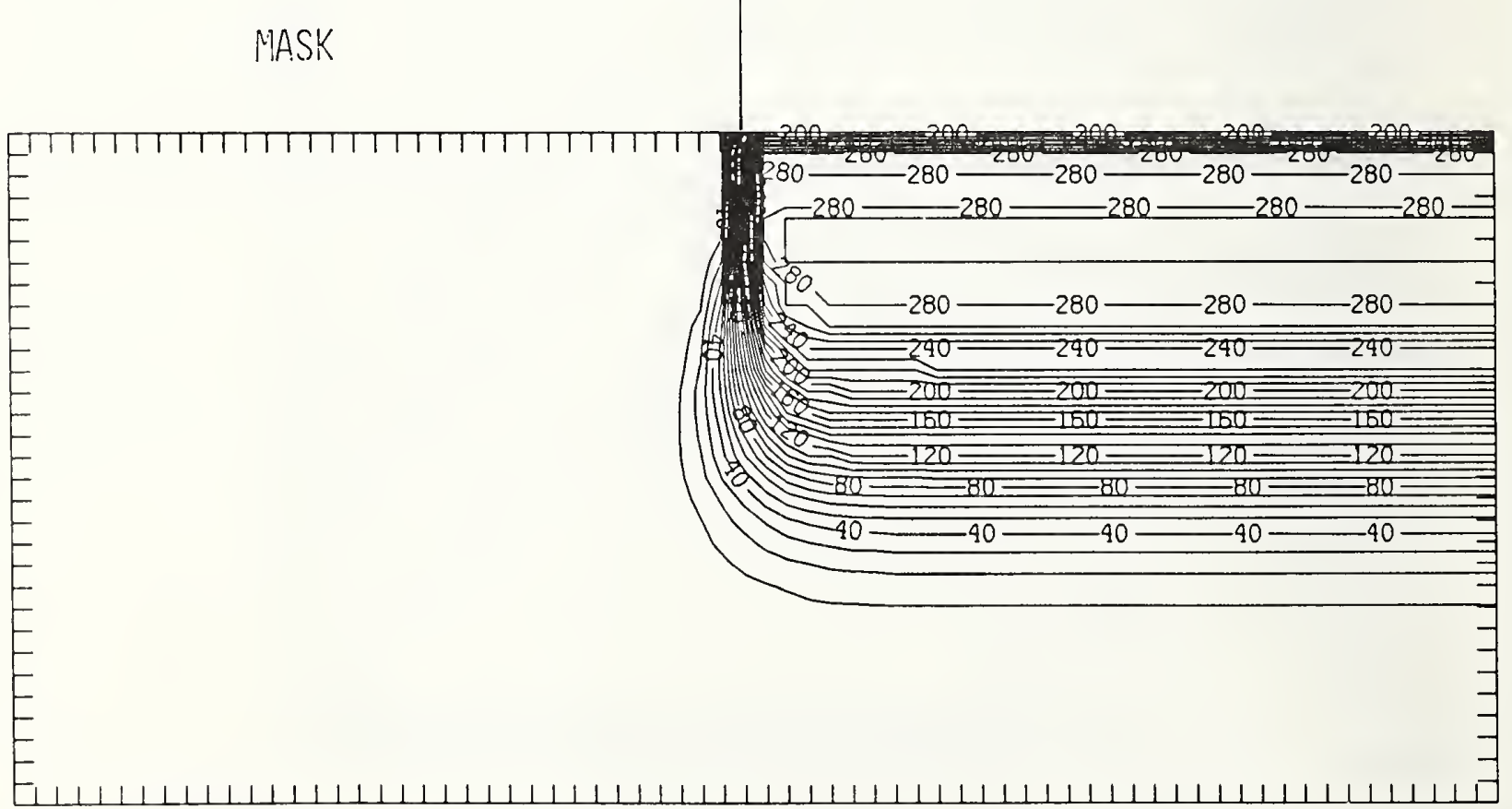

Figure Sb300-4 Two-dimensional distribution of Frenkel pairs created by $300 \mathrm{keV}$ antimony implanted past a mask edge. The length increment (distance between tick marks) is $0.01 \mu \mathrm{m}$ and the scale factor is 0.1 . 


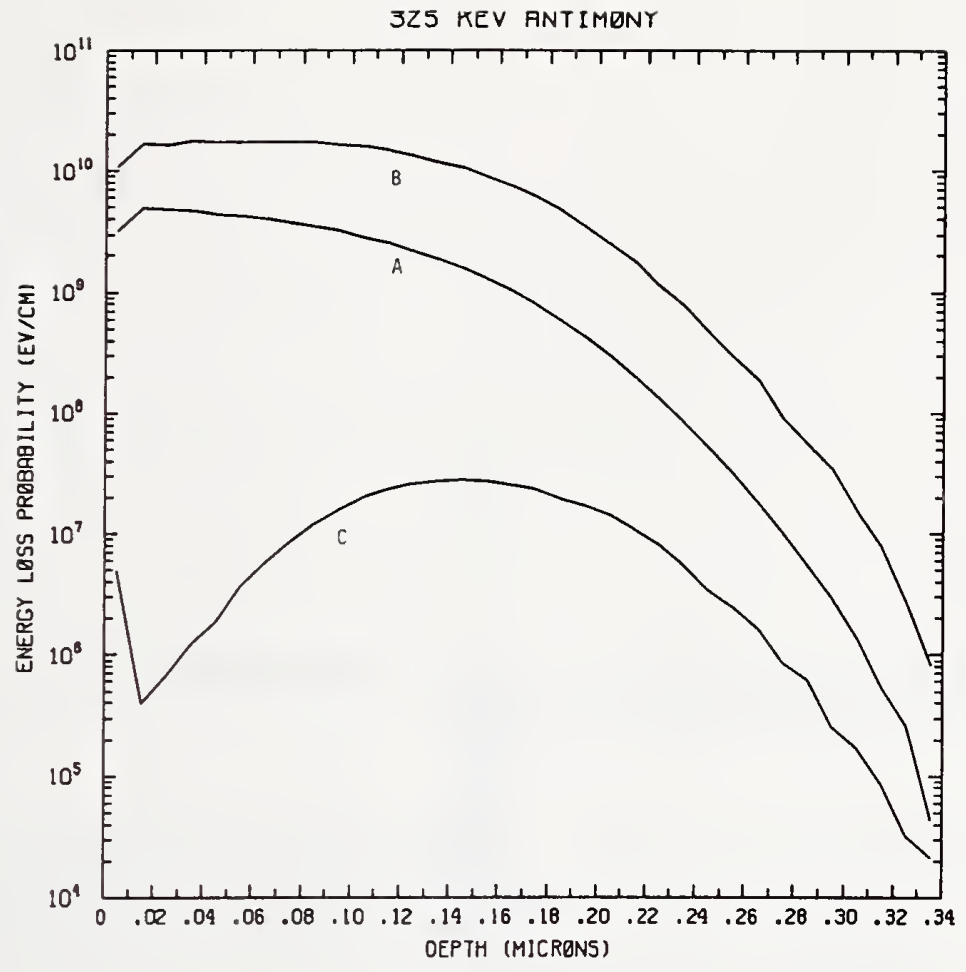

Figure Sb325-1 One-dimensional probability distributions for energy loss due to electronic excitation (A), displacement damage(B), and phonon excitation (C) for $325 \mathrm{keV}$ antimony normally incident on a silicon target.

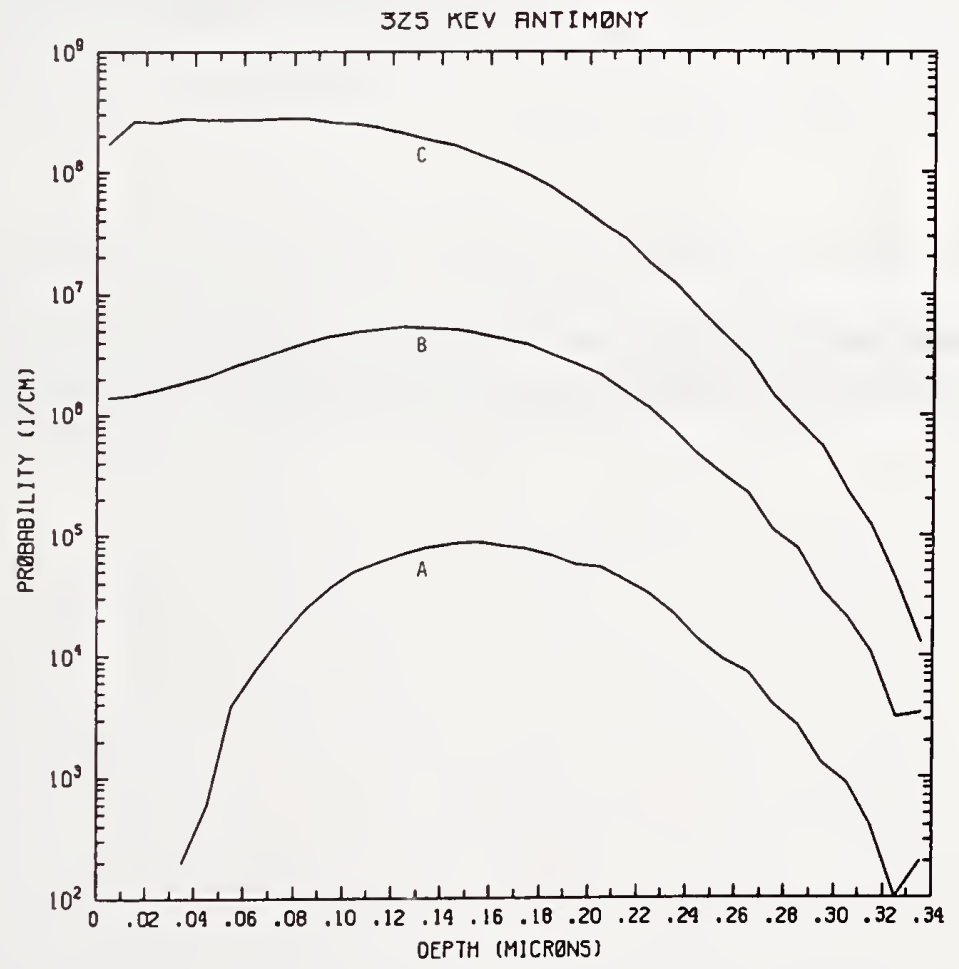

Figure Sb325-2 One-dimensional probability distributions for implanted antimony (A), primary silicon displacements (B), and Frenkel pairs (C) for $325 \mathrm{keV}$ antimony normally incident on a silicon target. 


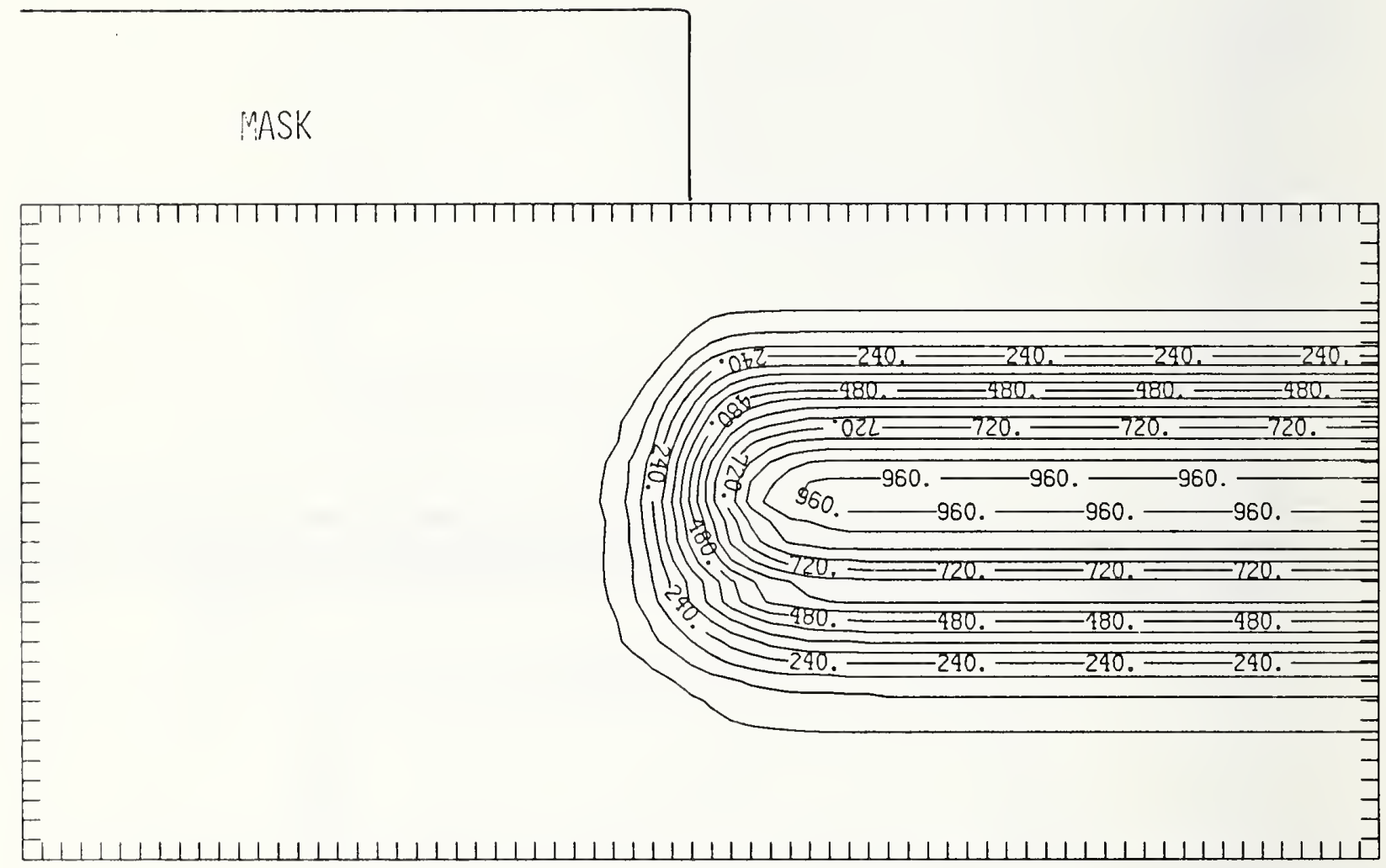

Figure Sb325-3 Two-dimensional distribution of $325 \mathrm{keV}$ antimony implanted past a mask edge. The length increment (distance between tick marks) is $0.01 \mu \mathrm{m}$ and the scale factor is 1000 . 


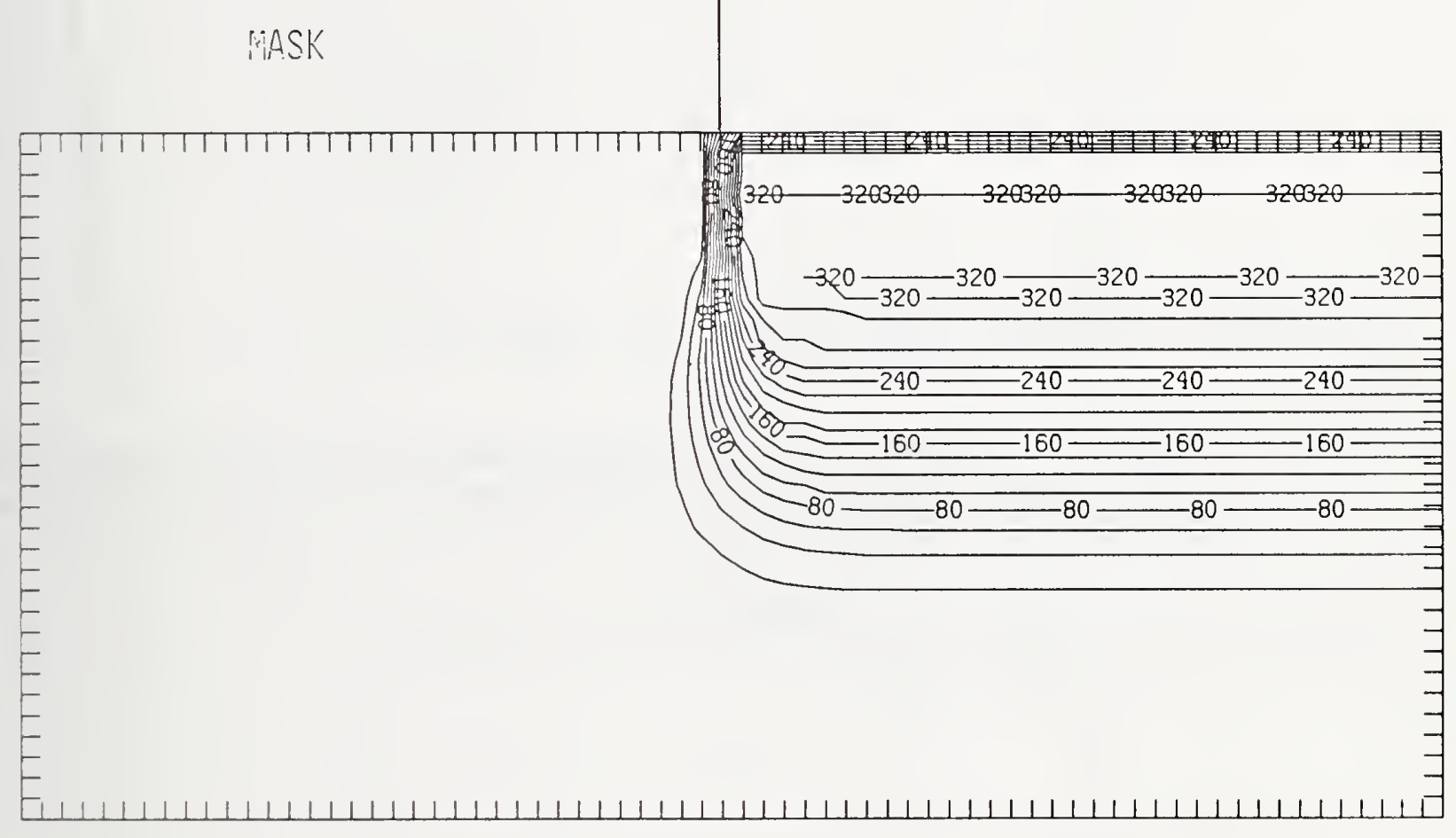

Figure Sb325-4 Two-dimensional distribution of Frenkel pairs created by $325 \mathrm{keV}$ antimony implanted past a mask edge. The length increment (distance between tick marks) is $0.01 \mu \mathrm{m}$ and the scale factor is 0.1 . 


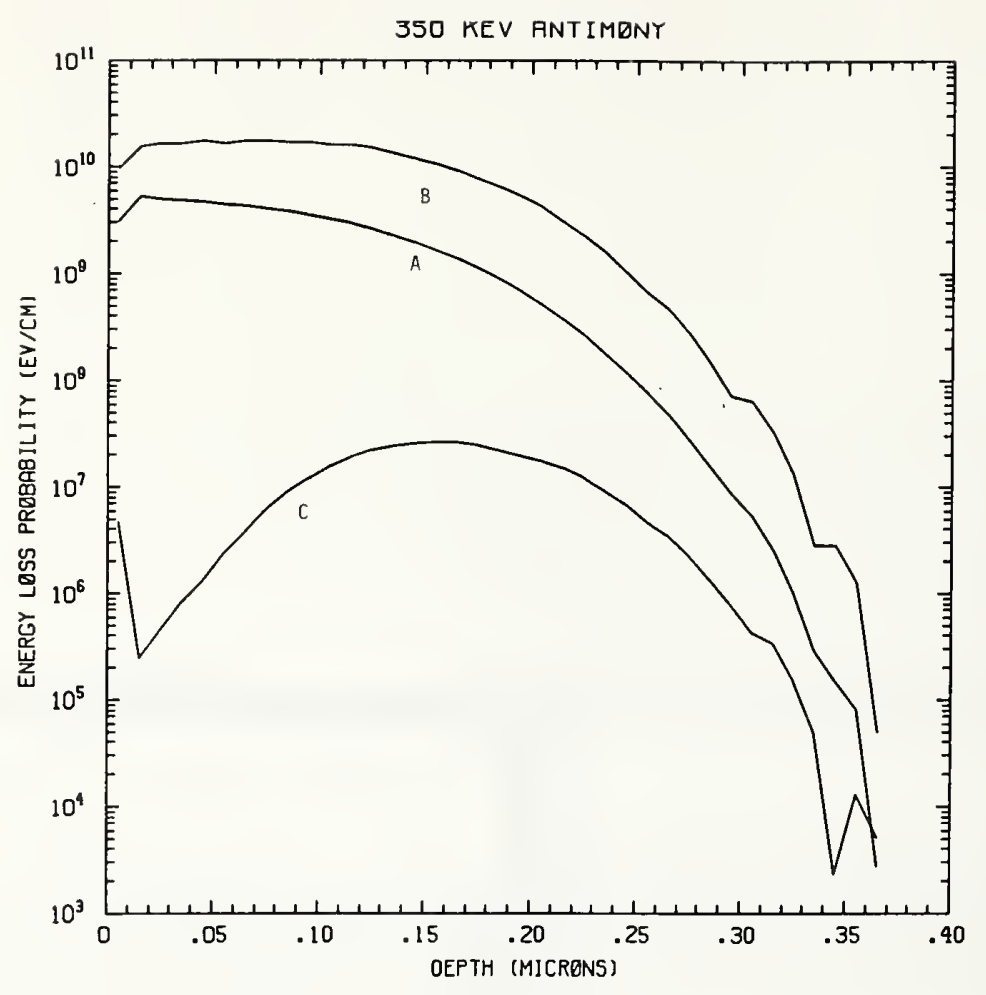

Figure Sb350-1 One-dimensional probability distributions for energy loss due to electronic excitation (A), displacement damage(B), and phonon excitation (C) for $350 \mathrm{keV}$ antimony normally incident on a silicon target.

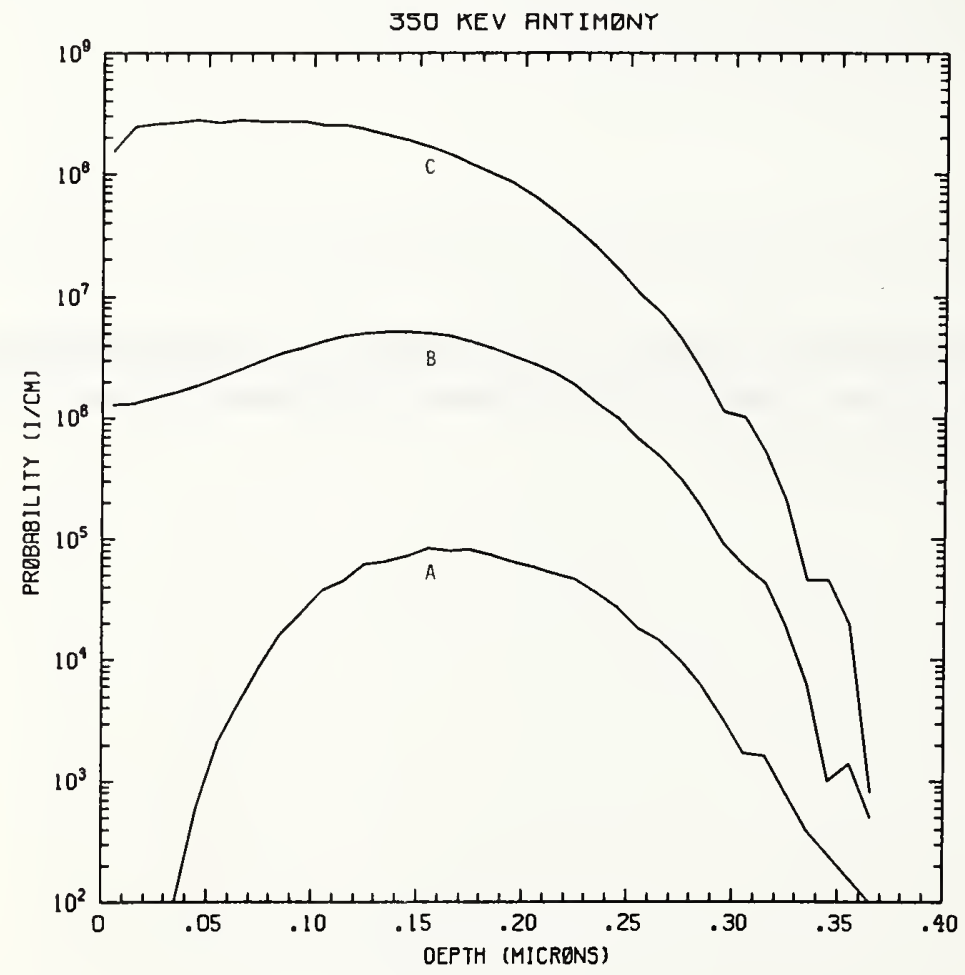

Figure Sb350-2 One-dimensional probability distributions for implanted antimony (A), primary silicon displacements (B), and Frenkel pairs (C) for $350 \mathrm{keV}$ antimony normally incident on a silicon target. 


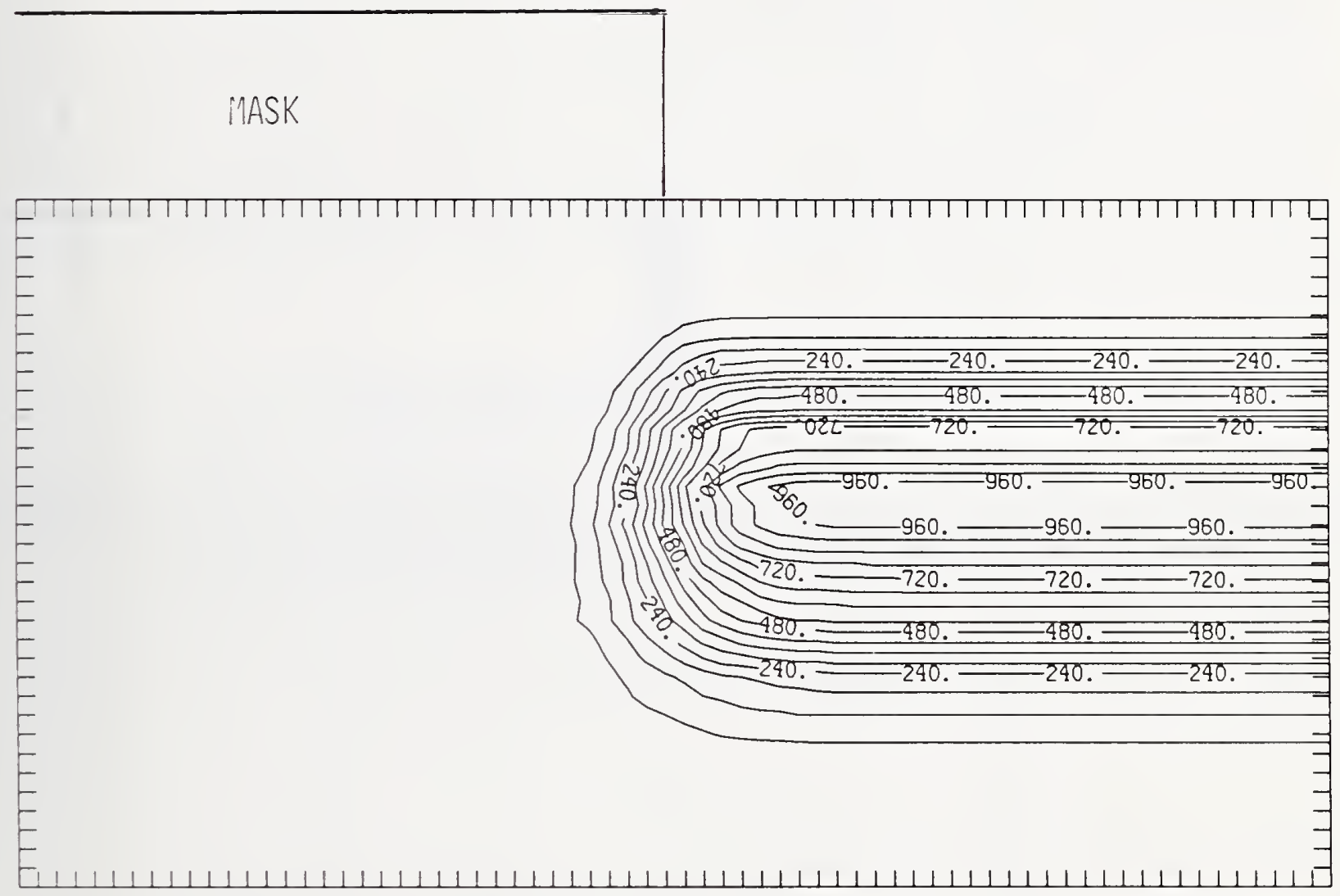

Figure Sb350-3 Two-dimensional distribution of $350 \mathrm{keV}$ antimony implanted past a mask edge. The length increment (distance between tick marks) is $0.01 \mu \mathrm{m}$ and the scale factor is 1000 . 


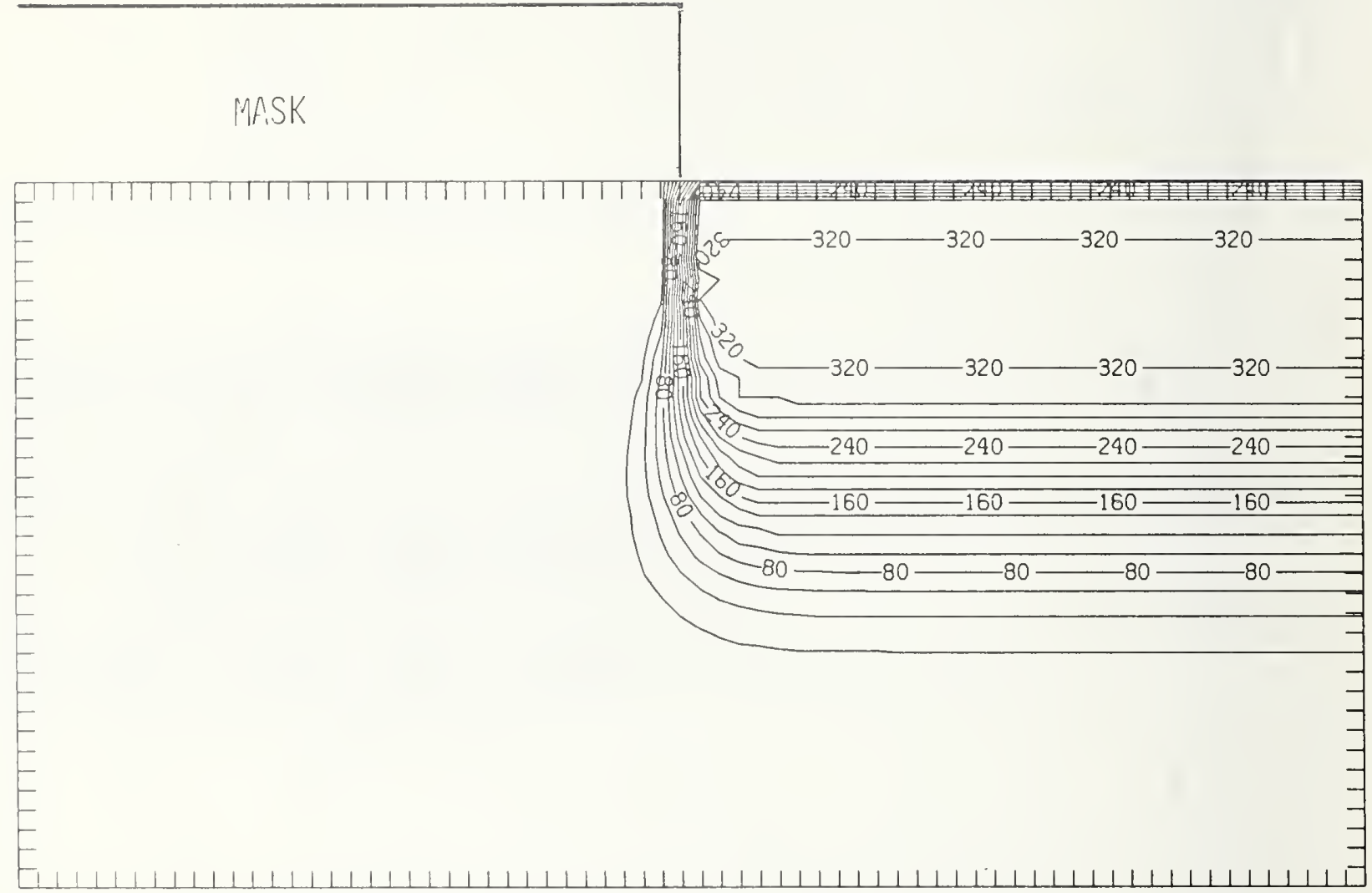

Figure Sb350-4 Two-dimensional distribution of Frenkel pairs created by $350 \mathrm{keV}$ antimony implanted past a mask edge. The length increment (distance between tick marks) is $0.01 \mu \mathrm{m}$ and the scale factor is 0.1 . 


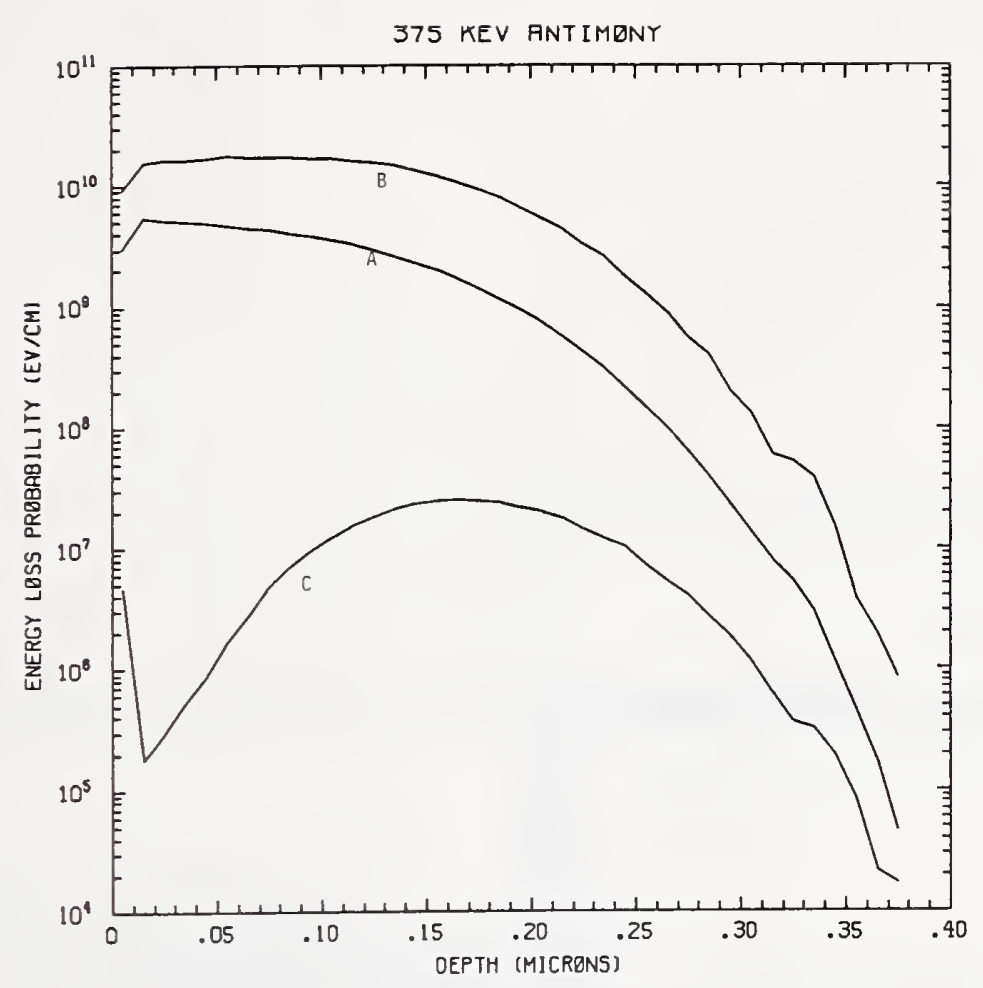

Figure Sb375-1 One-dimensional probability distributions for energy loss due to electronic excitation (A), displacement damage(B), and phonon excitation (C) for $375 \mathrm{keV}$ antimony normally incident on a silicon target.

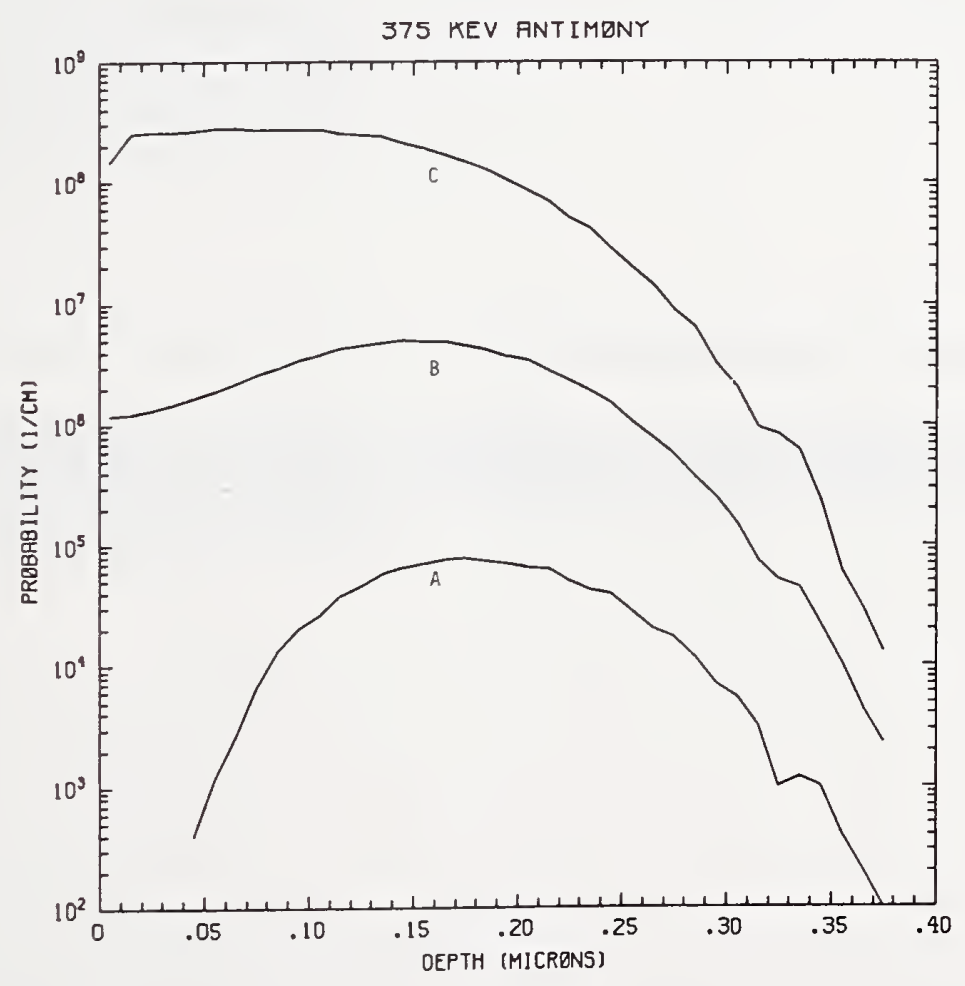

Figure Sb375-2 One-dimensional probability distributions for implanted antimony (A), primary silicon displacements (B), and Frenkel pairs (C) for $375 \mathrm{keV}$ antimony normally incident on a silicon target. 


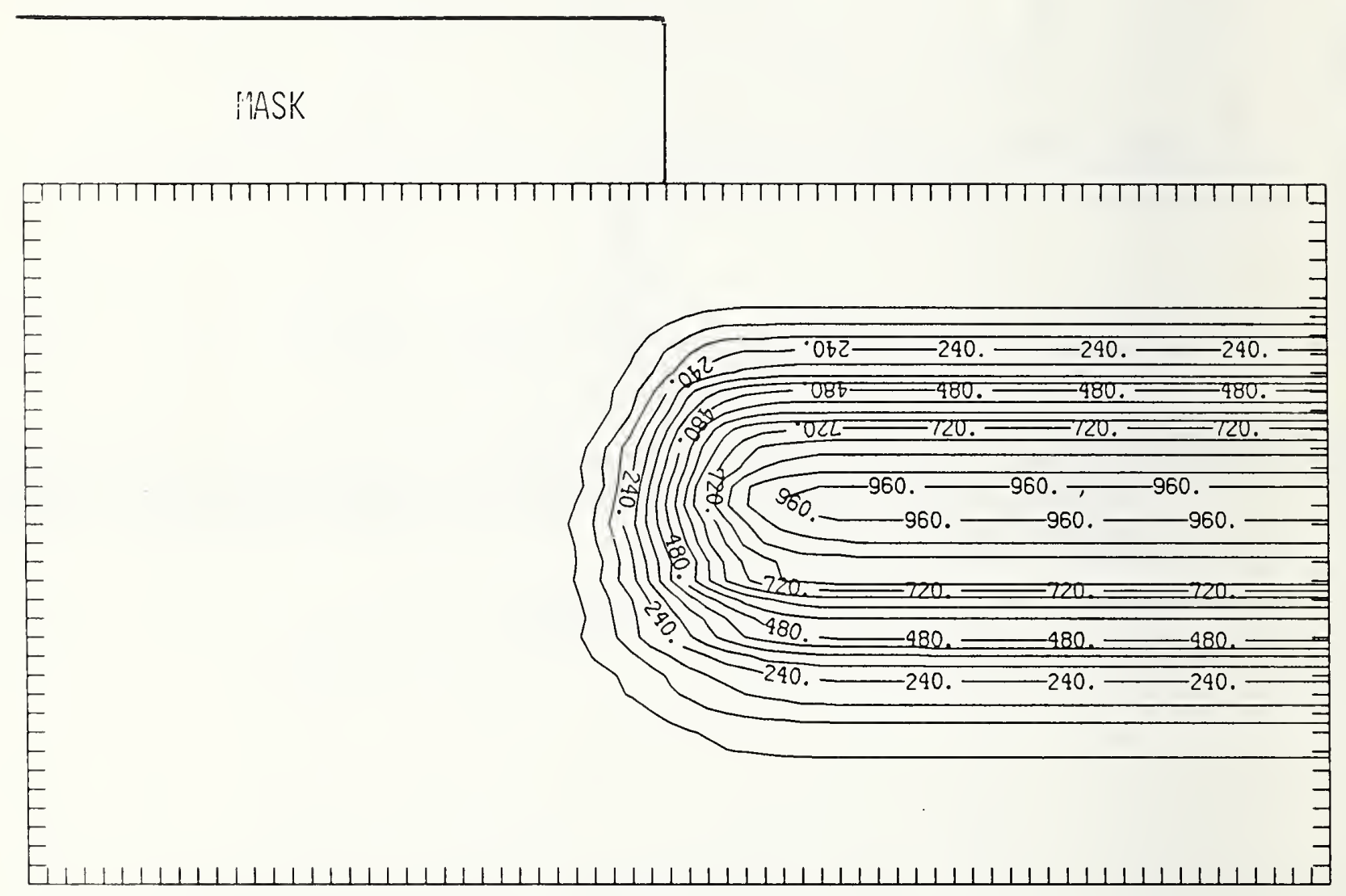

Figure Sb375-3 Two-dimensional distribution of $375 \mathrm{keV}$ antimony implanted past a mask edge. The length increment (distance between tick marks) is $0.01 \mu \mathrm{m}$ and the scale factor is 1000 . 


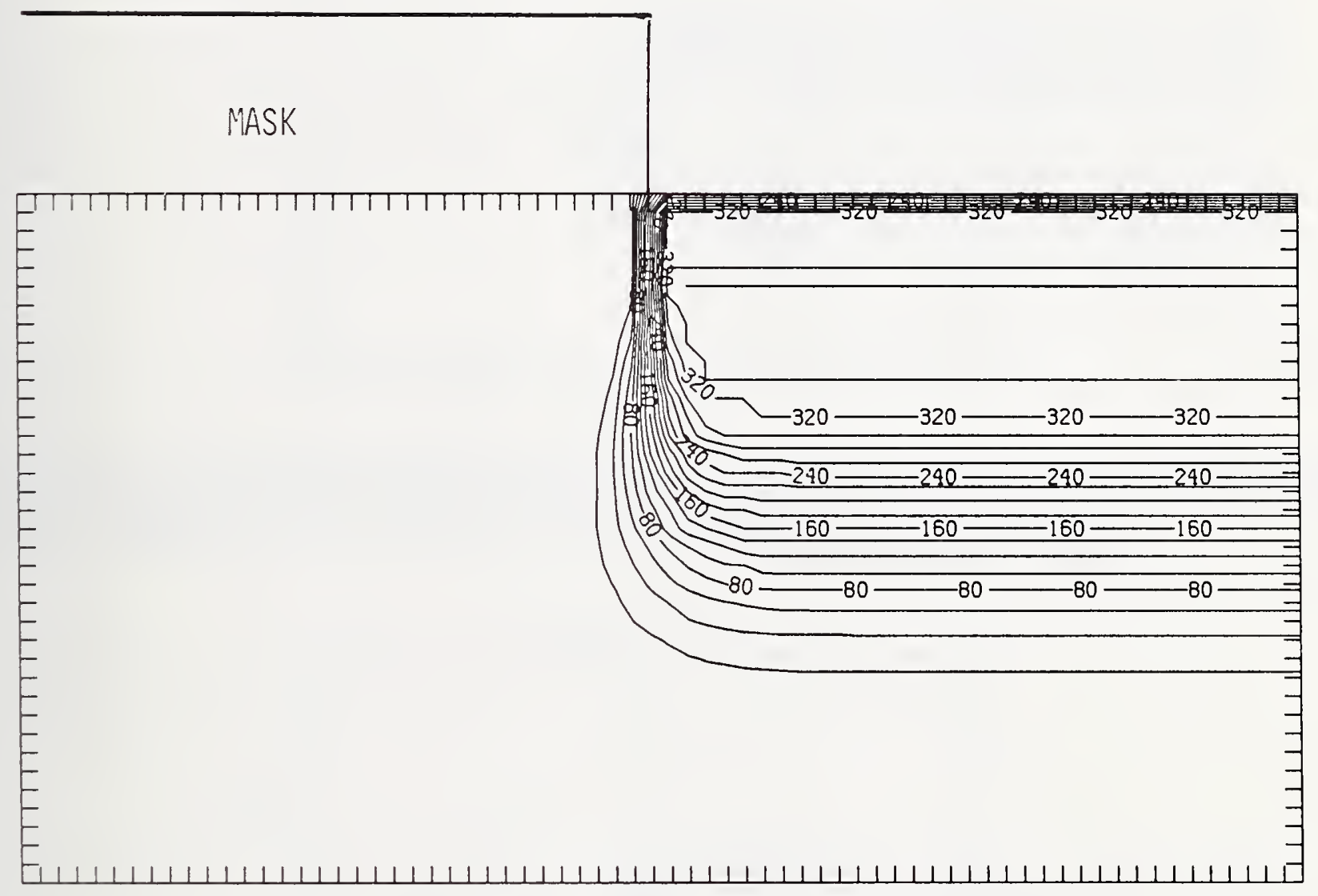

Figure Sb375-4 Two-dimensional distribution of Frenkel pairs created by $375 \mathrm{keV}$ antimony implanted past a mask edge. The length increment (distance between tick marks) is $0.01 \mu \mathrm{m}$ and the scale factor is 0.1 . 


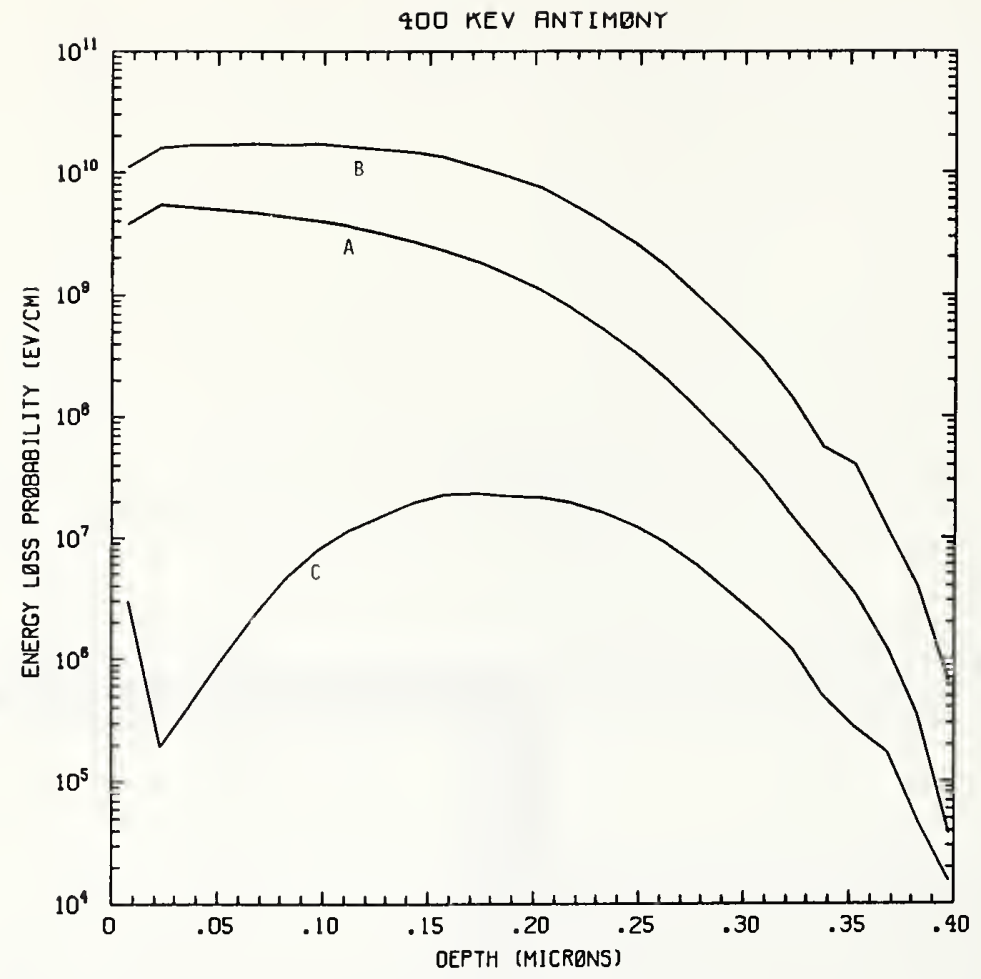

Figure Sb400-1 One-dimensional probability distributions for energy loss due to electronic excitation (A), displacement damage(B), and phonon excitation (C) for $400 \mathrm{keV}$ antimony normally incident on a silicon target.

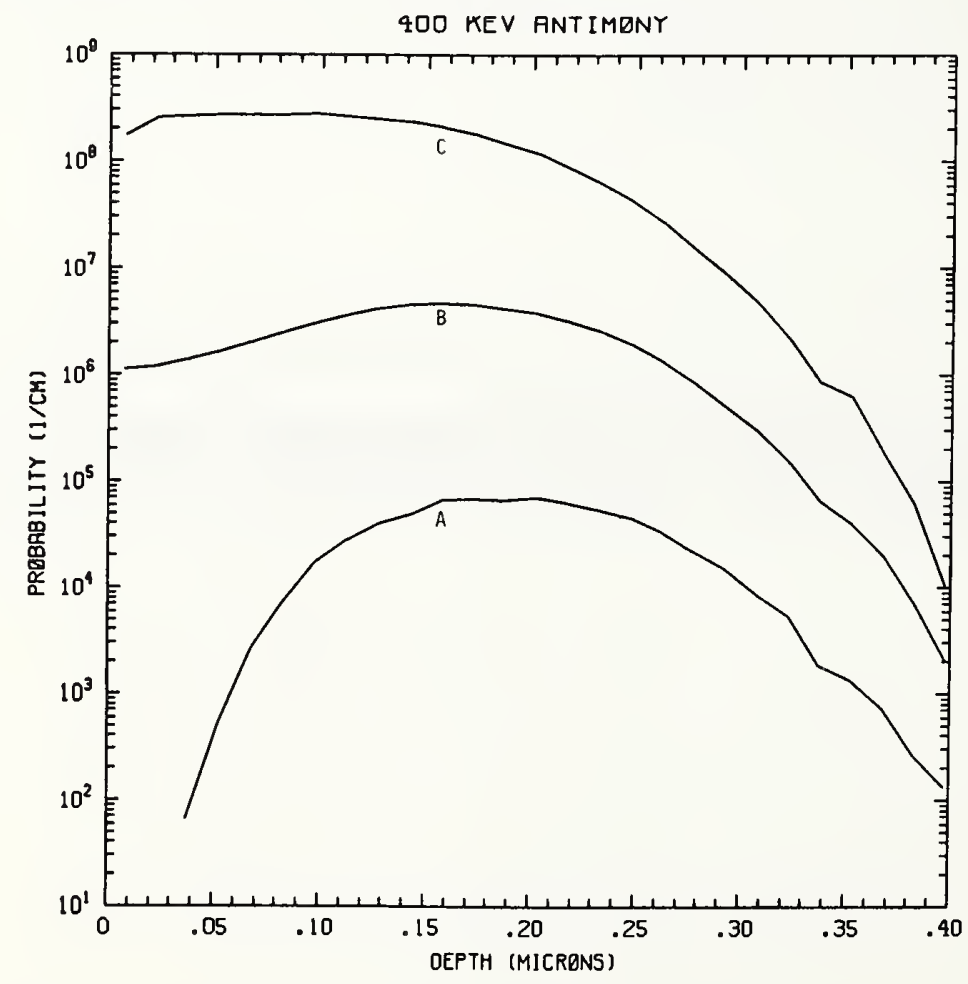

Figure Sb400-2 One-dimensional probability distributions for implanted antimony (A), primary silicon displacements (B), and Frenkel pairs (C) for $400 \mathrm{keV}$ antimony normally incident on a silicon target. 


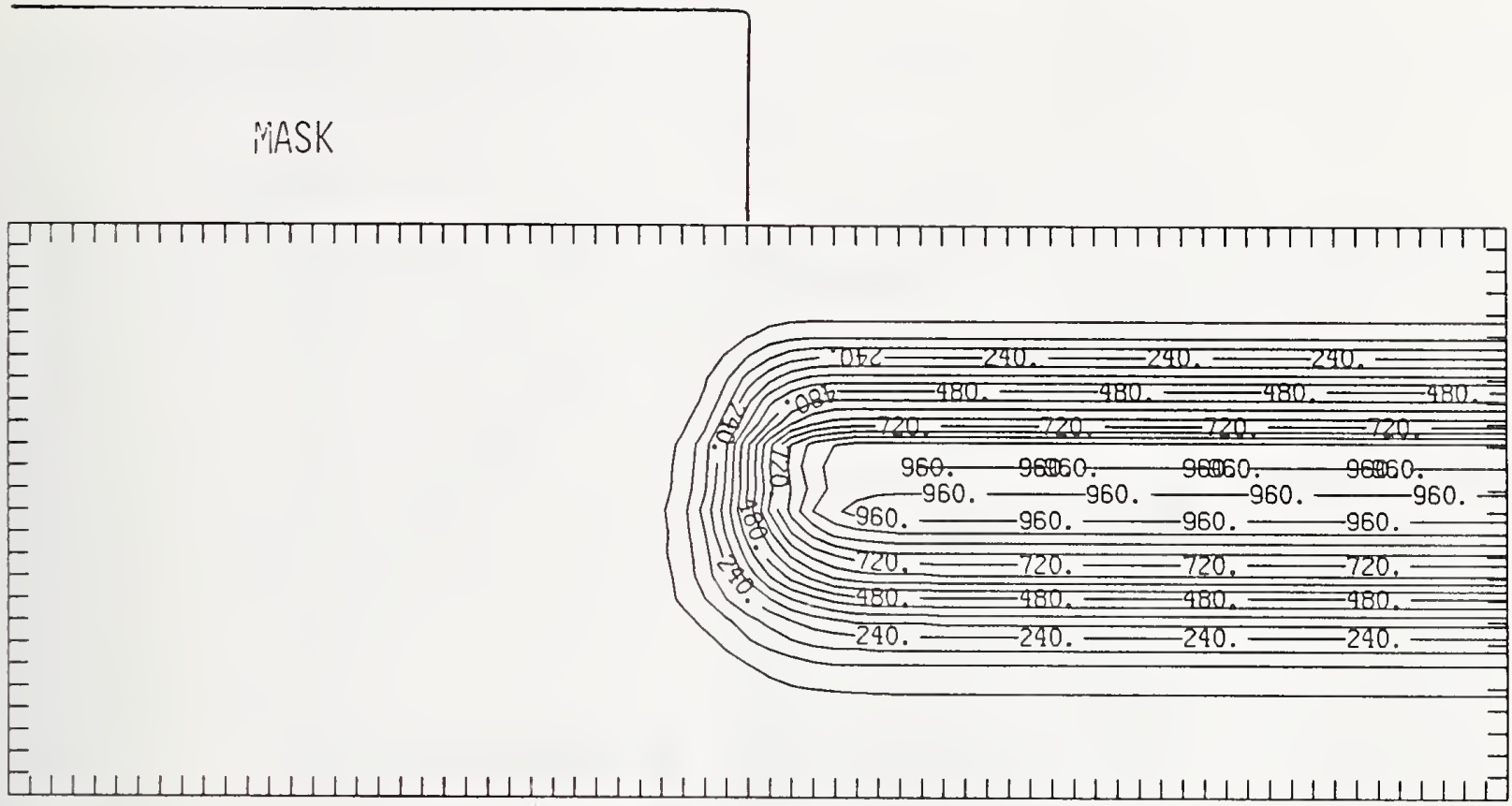

Figure Sb400-3 Two-dimensional distribution of $400 \mathrm{keV}$ antimony implanted past a mask edge. The length increment (distance between tick marks) is $0.015 \mu \mathrm{m}$ and the scale factor is 1000.

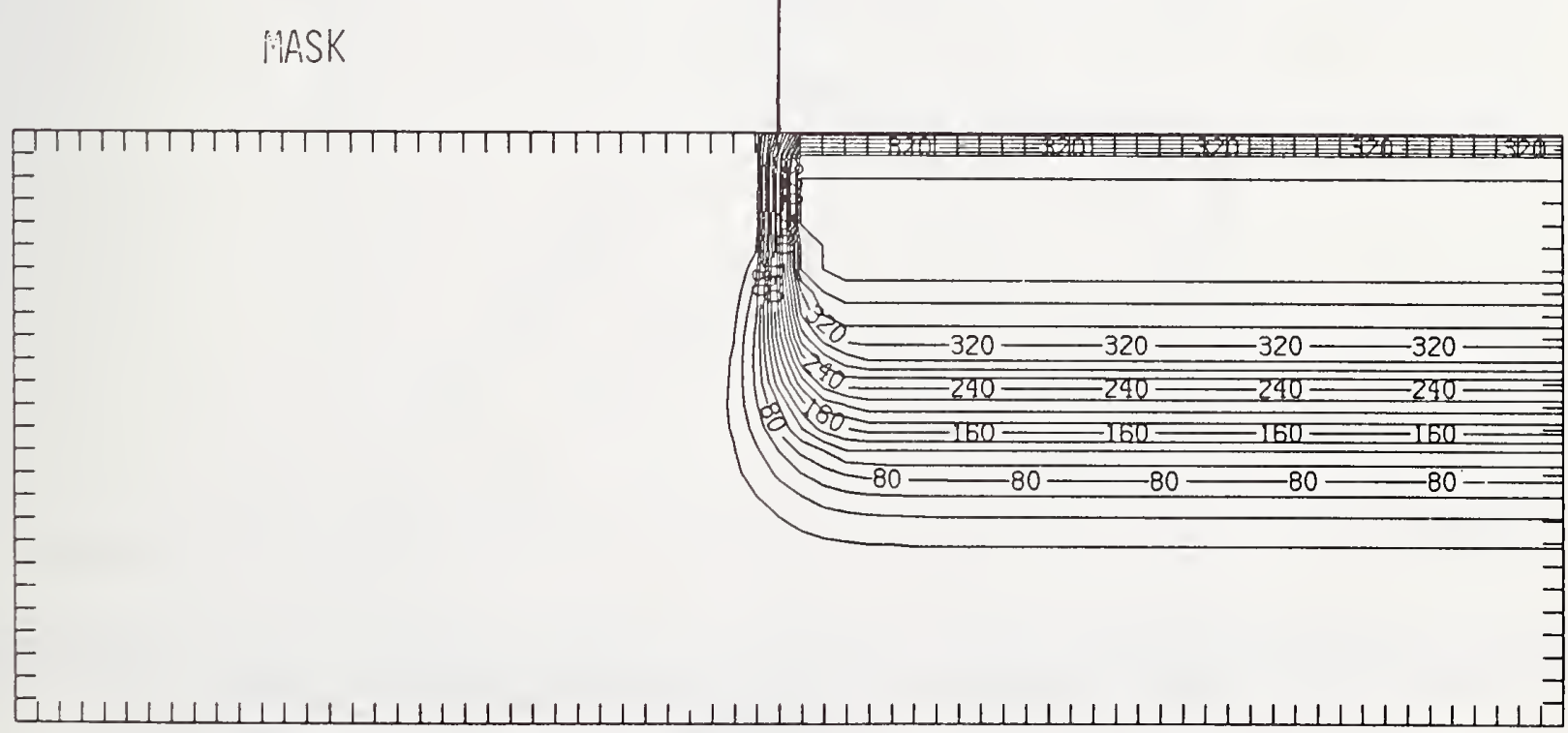

Figure Sb400-4 Two-dimensional distribution of Frenkel pairs created by $400 \mathrm{keV}$ antimony implanted past a mask edge. The length increment (distance between tick marks) is $0.015 \mu \mathrm{m}$ and the scale factor is 0.1 . 


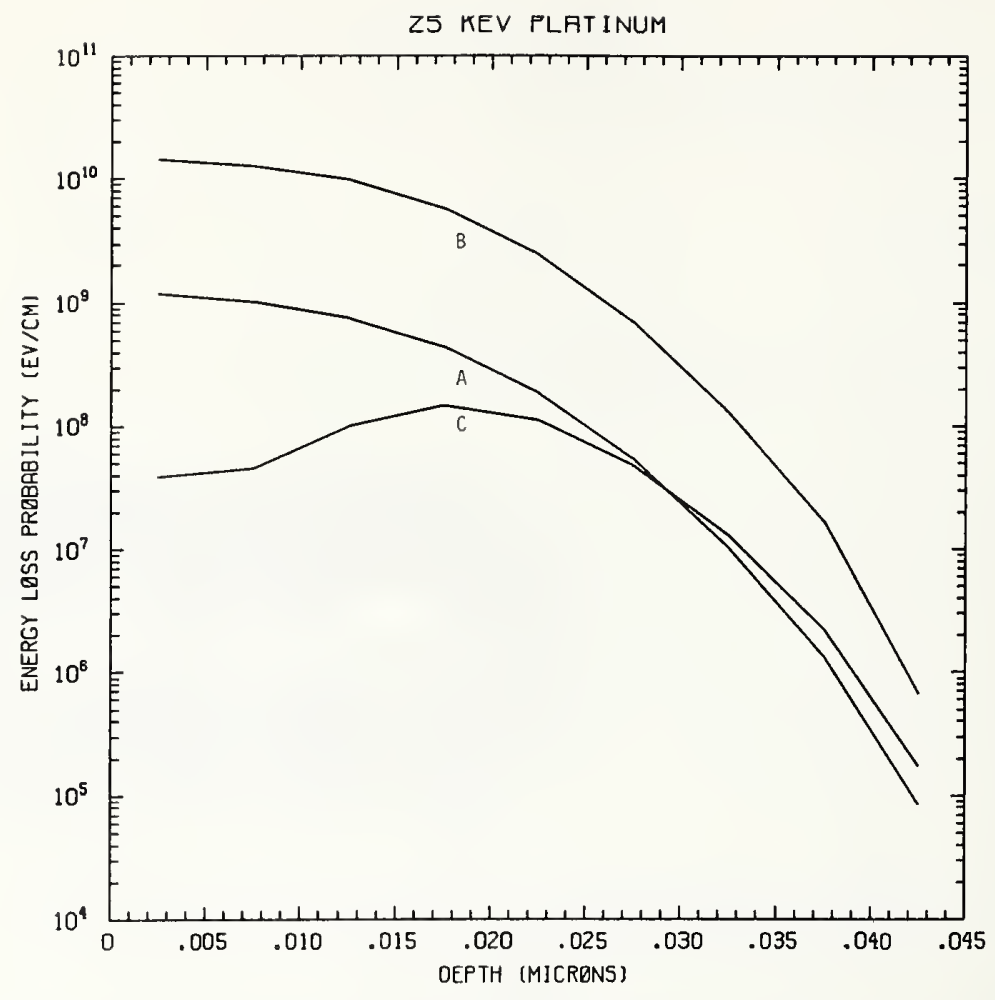

Figure Pt25-1 One-dimensional probability distributions for energy loss due to electronic excitation (A), displacement damage(B), and phonon excitation (C) for $25 \mathrm{keV}$ platinum normally incident on a silicon target.

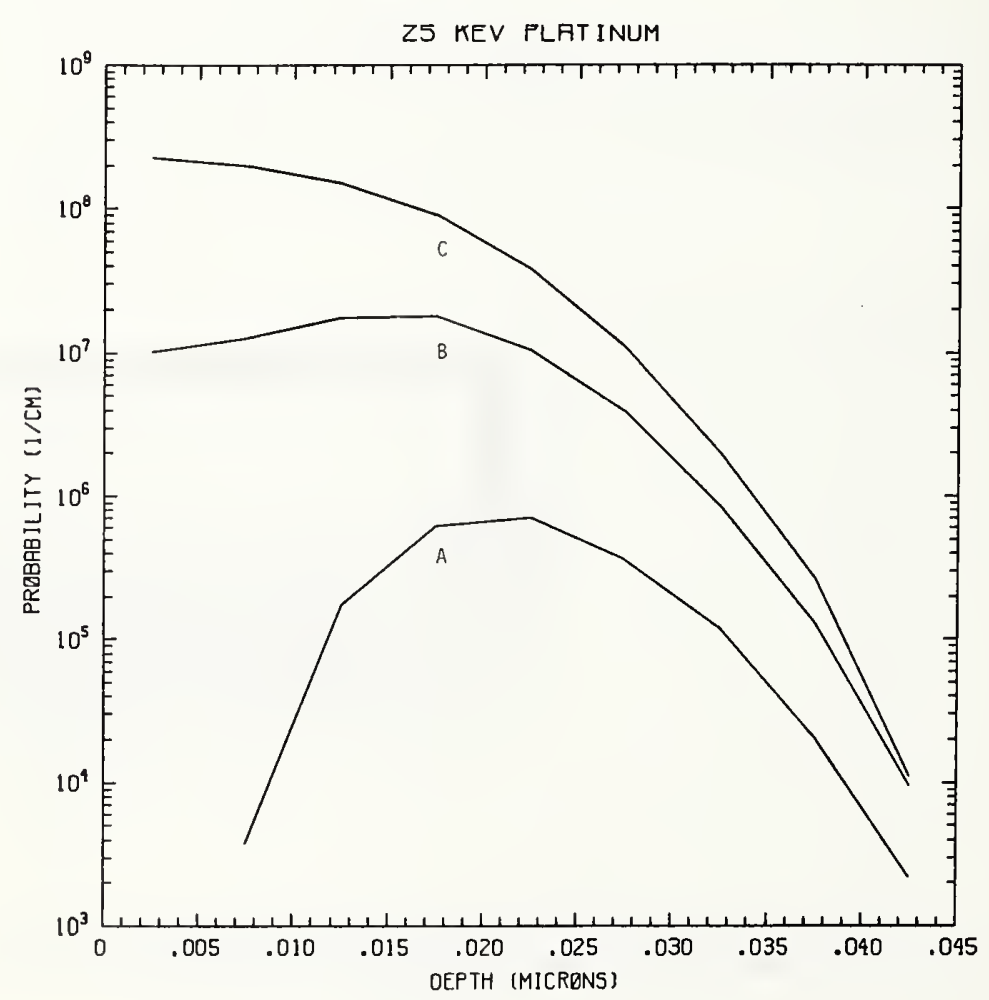

Figure Pt25-2 One-dimensional probability distributions for implanted platinum (A), primary silicon displacements (B), and Frenkel pairs (C) for $25 \mathrm{keV}$ platinum normally incident on a silicon target. 
MASK

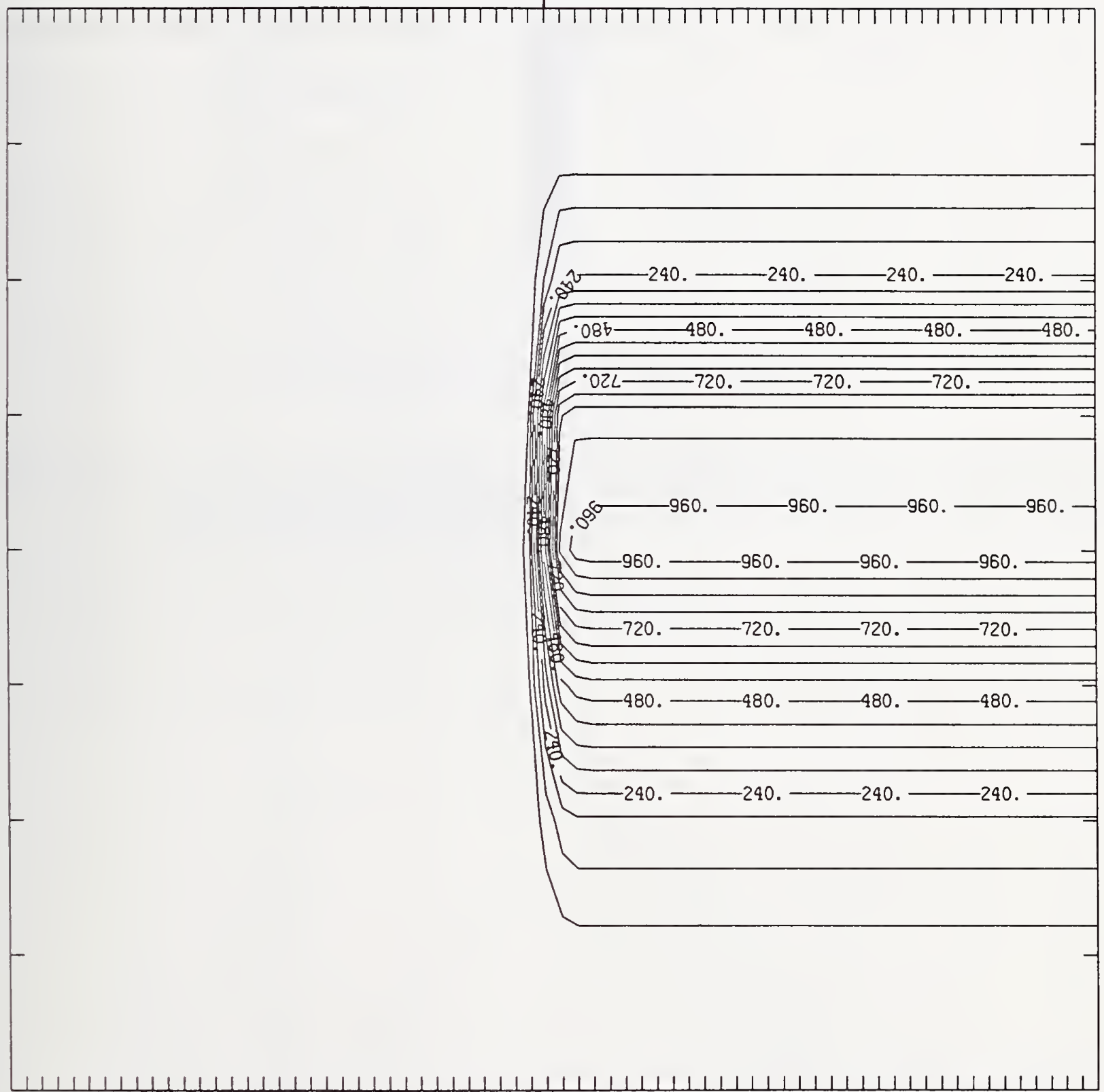

Figure Pt25-3 Two-dimensional distribution of $25 \mathrm{keV}$ platinum implanted past a mask edge. The length increment (distance between tick marks) is $0.005 \mu \mathrm{m}$ and the scale factor is 1000 . 


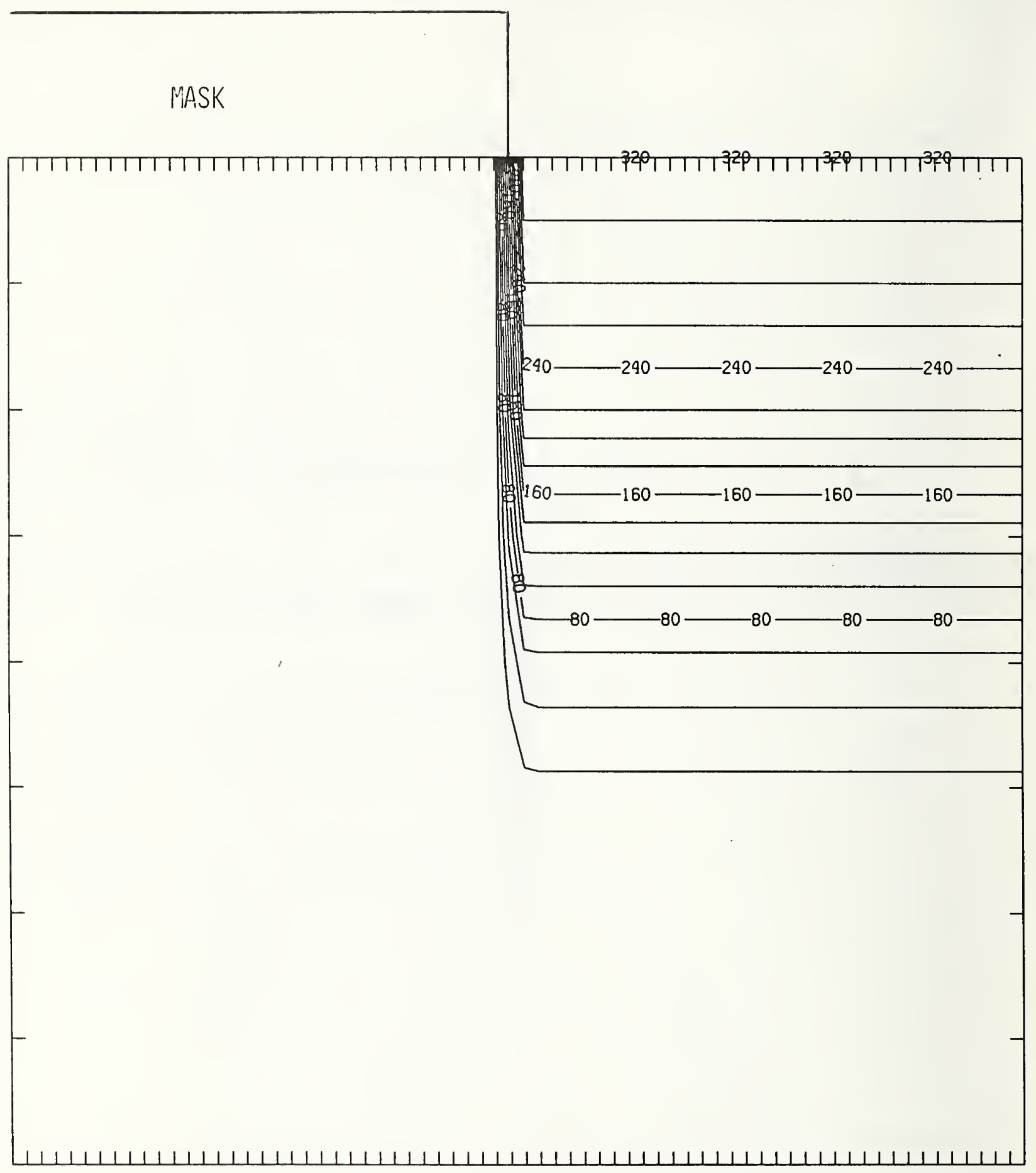

Figure Pt25-4 Two-dimensional distribution of Frenkel pairs created by $25 \mathrm{keV}$ platinum implanted past a mask edge. The length increment (distance between tick marks) is $0.005 \mu \mathrm{m}$ and the scale factor is 1 . 


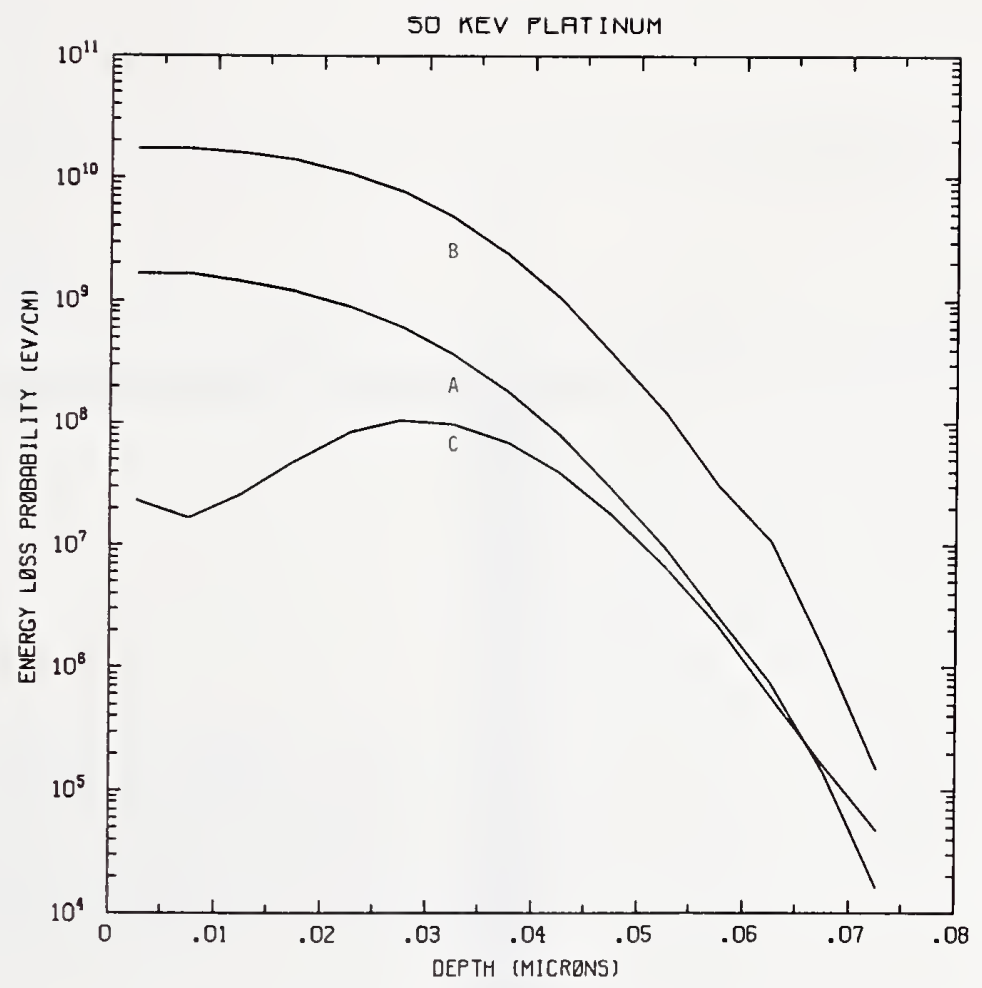

Figure Pt50-1 One-dimensional probability distributions for energy loss due to electronic excitation (A), displacement damage(B), and phonon excitation (C) for $50 \mathrm{keV}$ platinum normally incident on a silicon target.

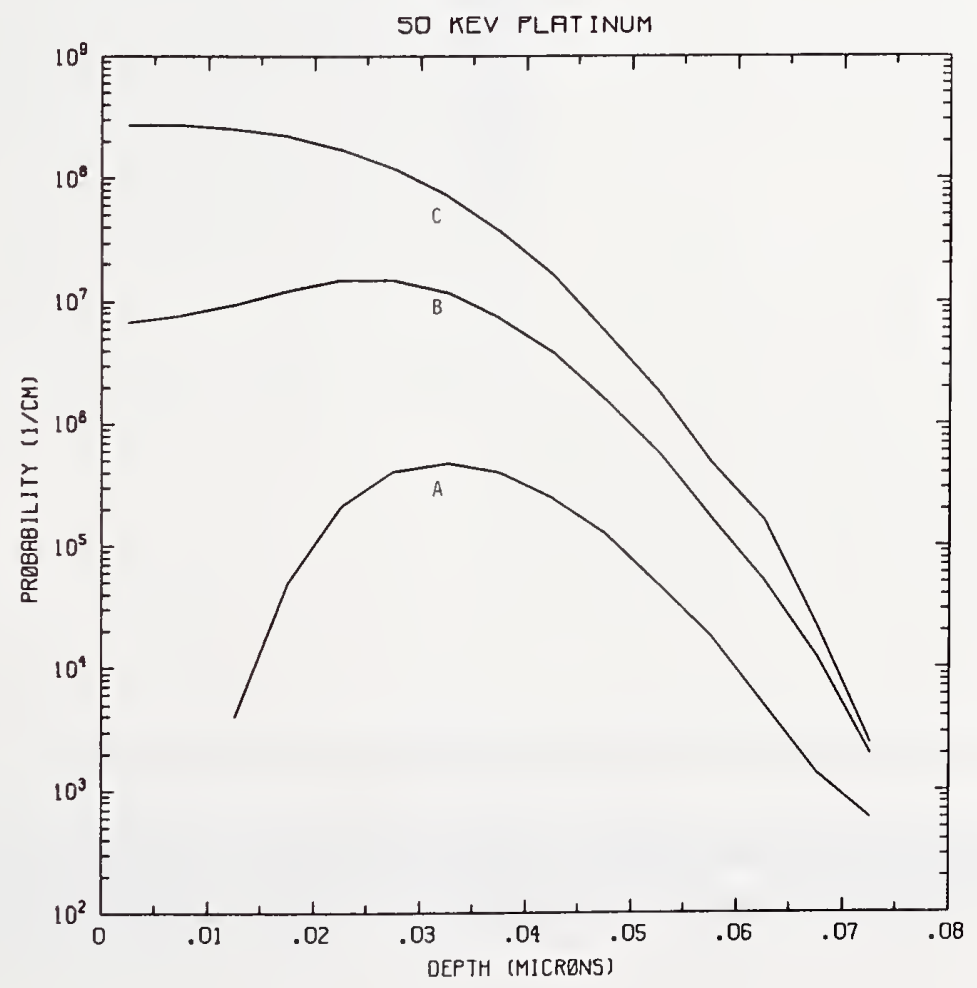

Figure Pt50-2 One-dimensional probability distributions for implanted platinum (A), primary silicon displacements (B), and Frenkel pairs (C) for $50 \mathrm{keV}$ platinum normally incident on a silicon target. 


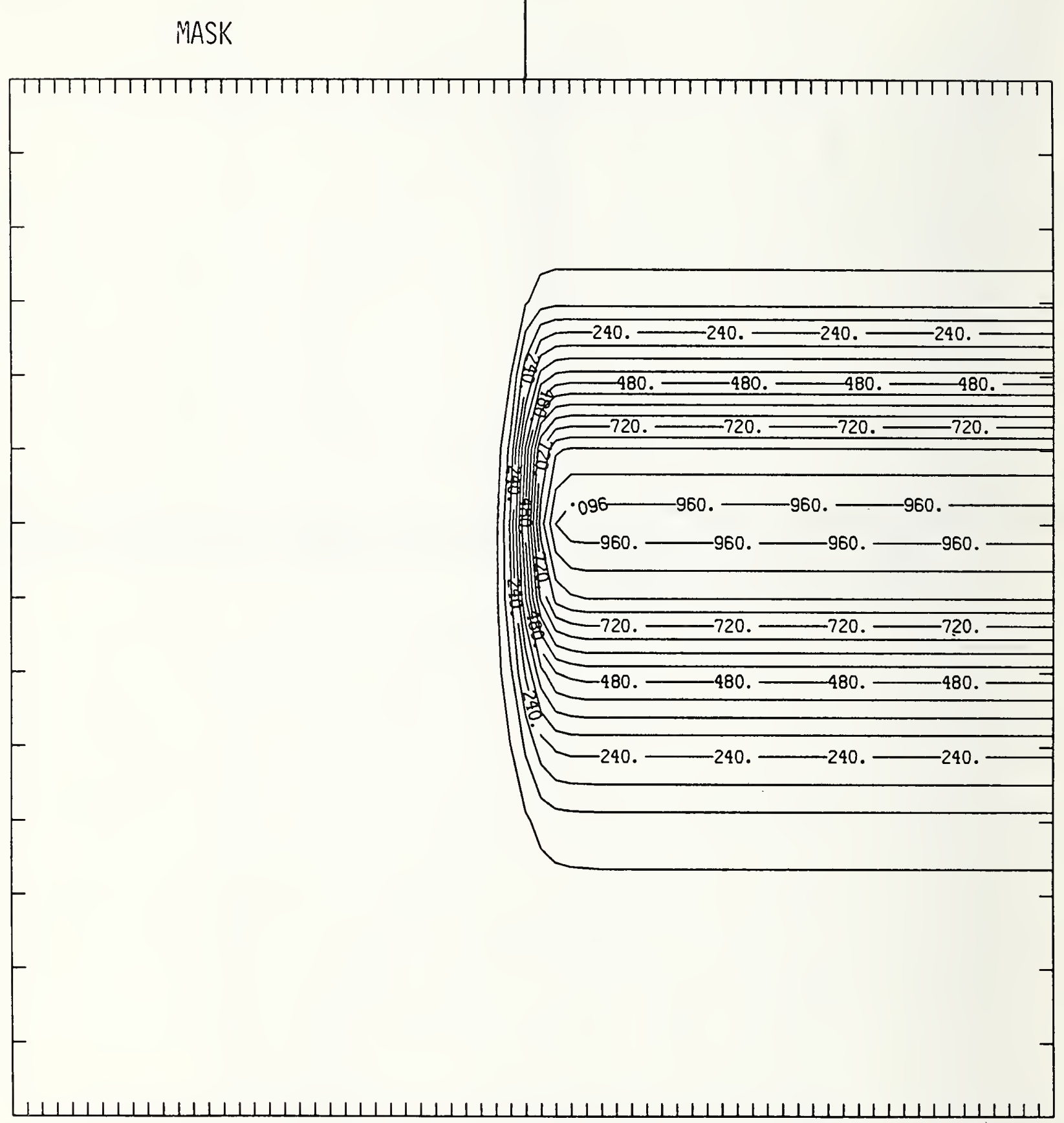

Figure Pt50-3 Two-dimensional distribution of $50 \mathrm{keV}$ platinum implanted past a mask edge. The length increment (distance between tick marks) is $0.005 \mu \mathrm{m}$ and the scale factor is 1000 . 


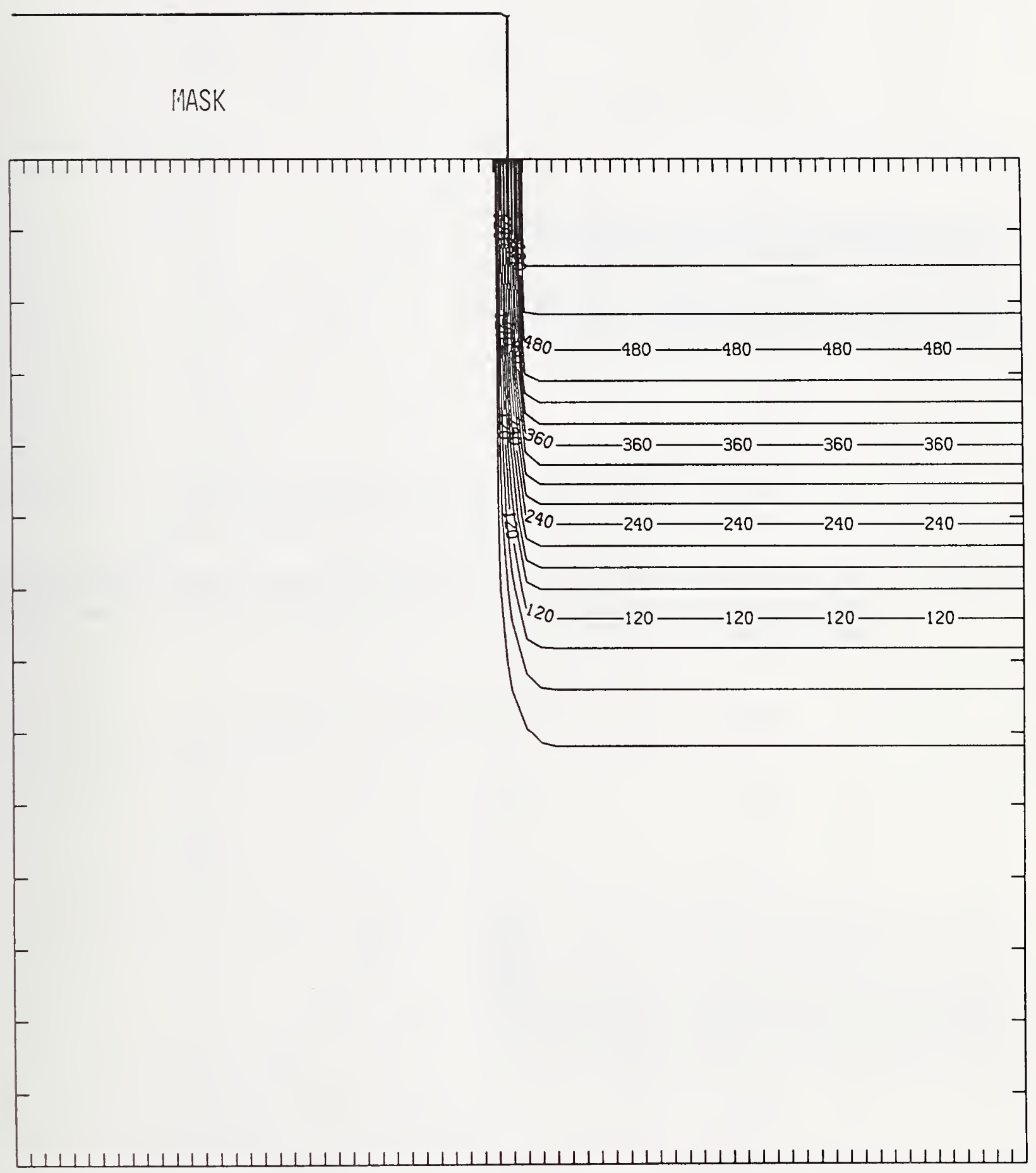

Figure Pt50-4 Two-dimensional distribution of Frenkel pairs created by $50 \mathrm{keV}$ platinum implanted past a mask edge. The length increment (distance between tick marks) is $0.005 \mu \mathrm{m}$ and the scale factor is 1 . 


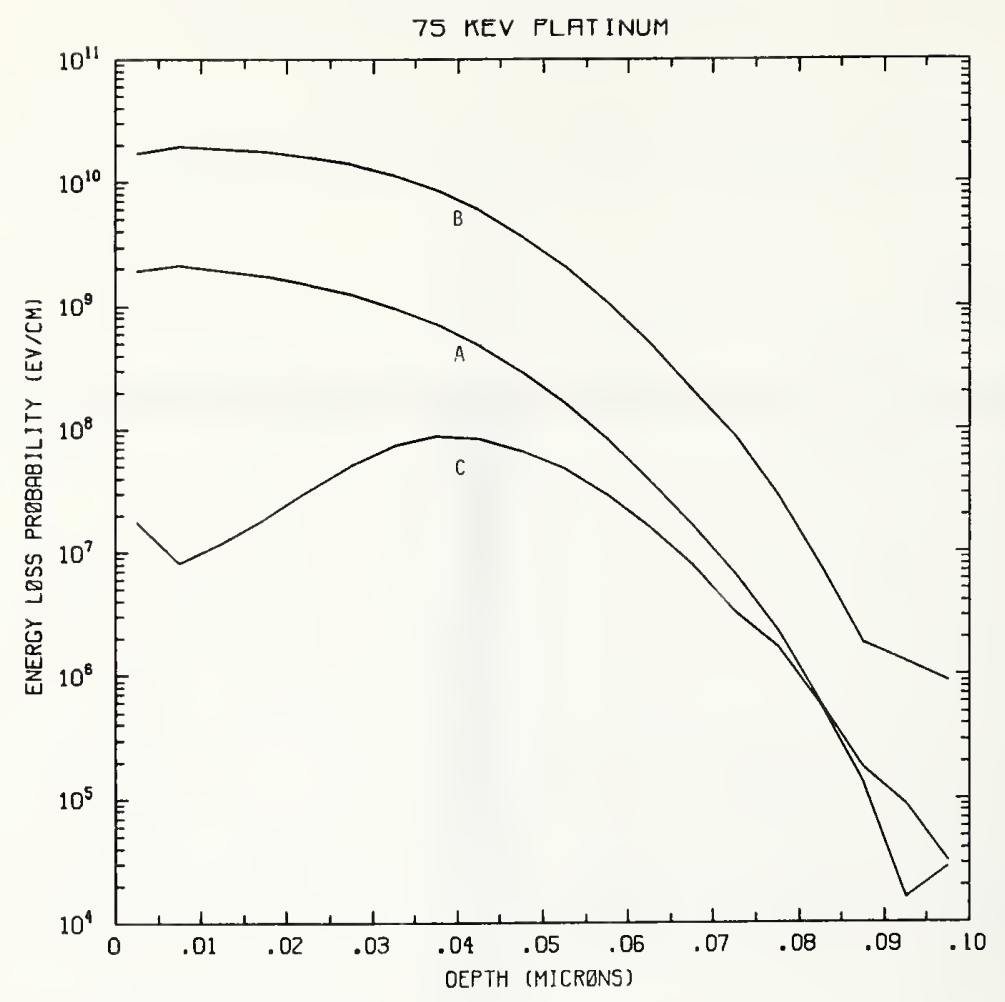

Figure Pt75-1 One-dimensional probability distributions for energy loss due to electronic excitation (A), displacement damage(B), and phonon excitation (C) for $75 \mathrm{keV}$ platinum normally incident on a silicon target.

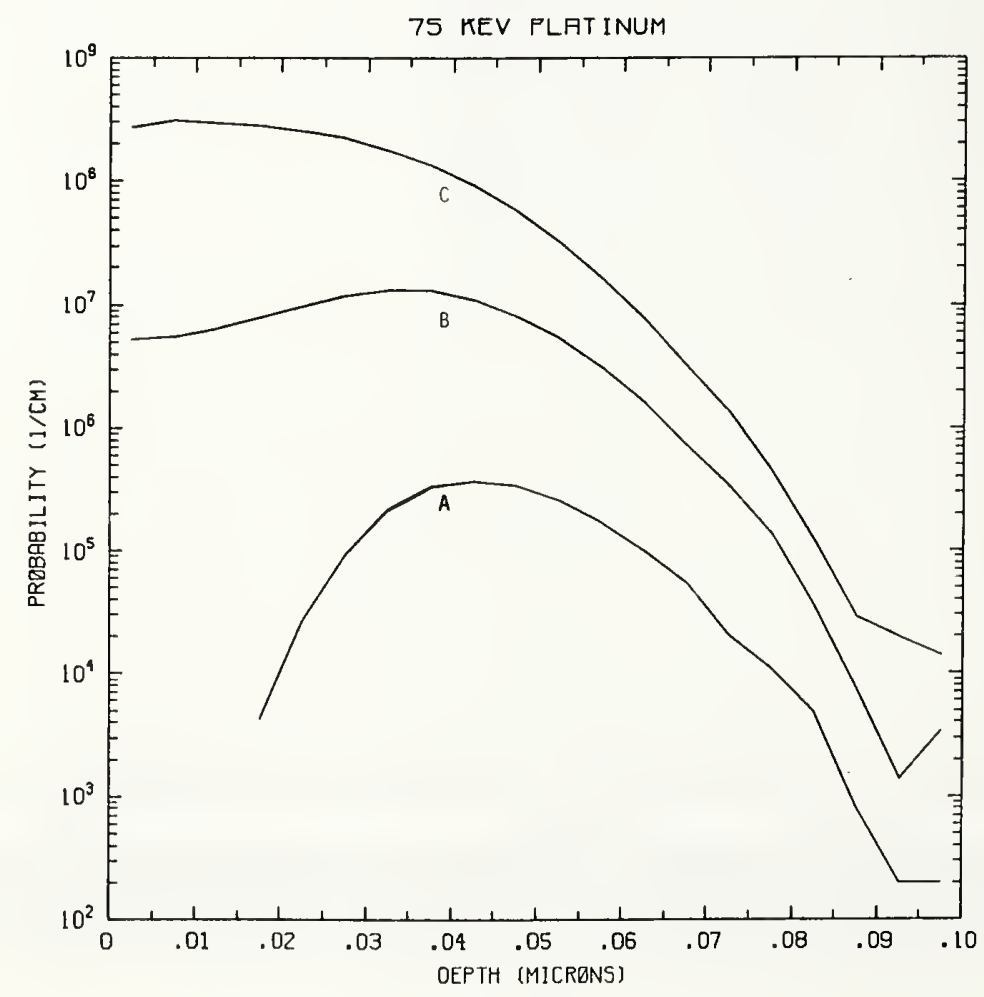

Figure Pt75-2 One-dimensional probability distributions for implanted platinum (A), primary silicon displacements (B), and Frenkel pairs (C) for $75 \mathrm{keV}$ platinum normally incident on a silicon target. 


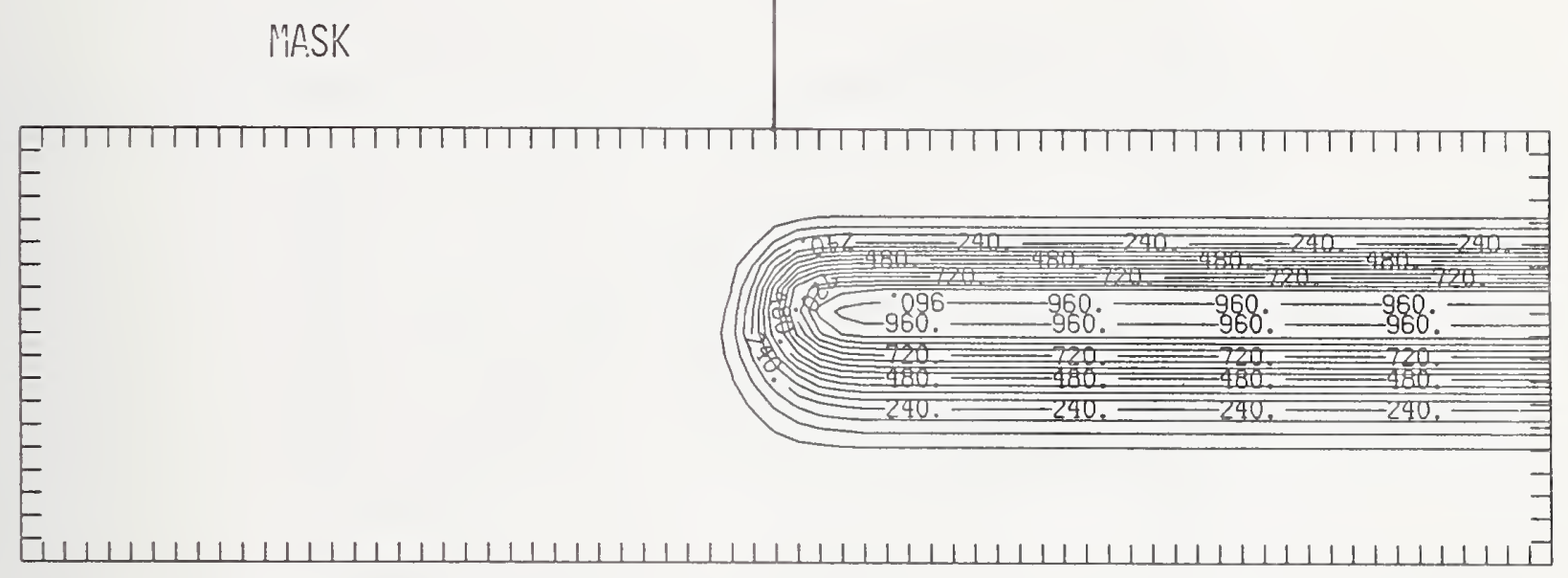

Figure Pt75-3 Two-dimensional distribution of $75 \mathrm{keV}$ platinum implanted past a mask edge. The length increment (distance between tick marks) is $0.005 \mu \mathrm{m}$ and the scale factor is 1000.

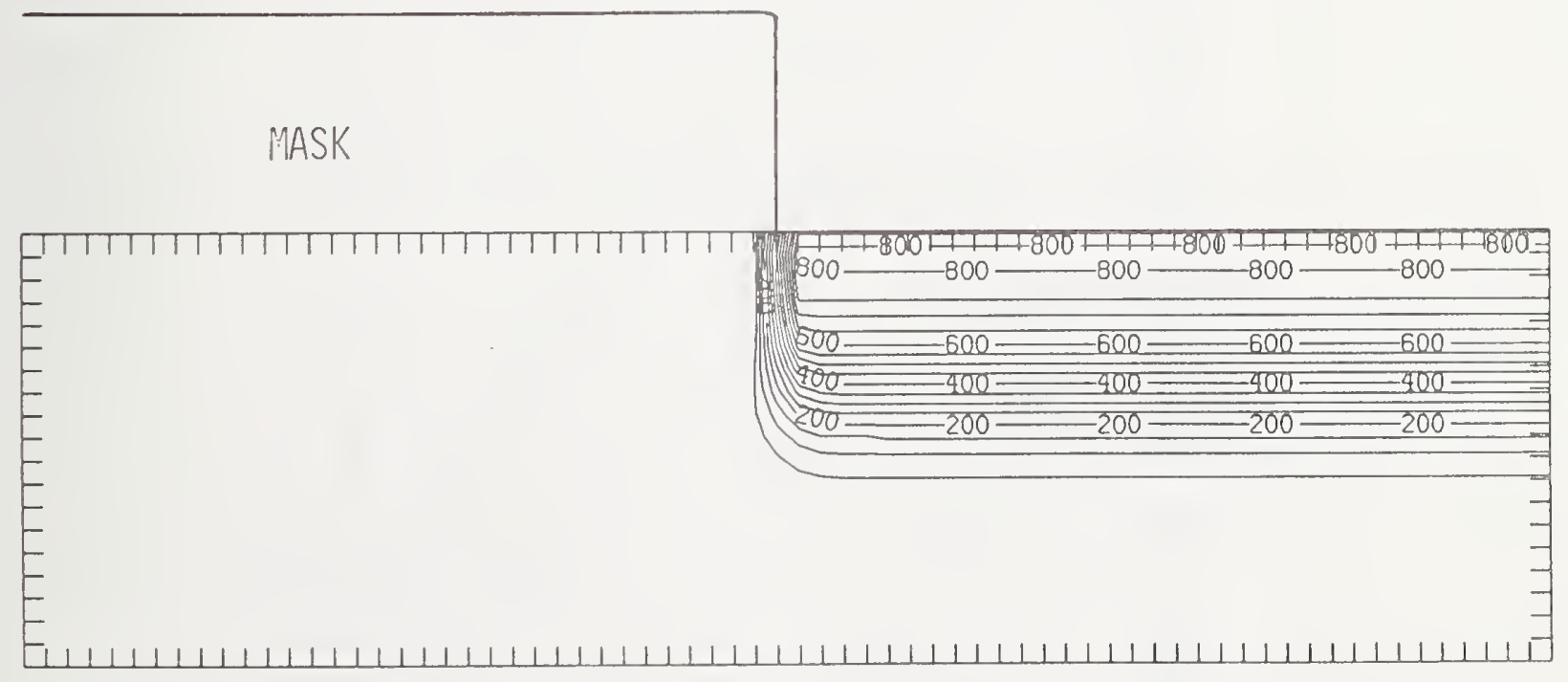

Figure Pt75-4 Two-dimensional distribution of Frenkel pairs created by $75 \mathrm{keV}$ platinum implanted past a mask edge. The length increment (distance between tick marks) is $0.005 \mu \mathrm{m}$ and the scale factor is 1. 


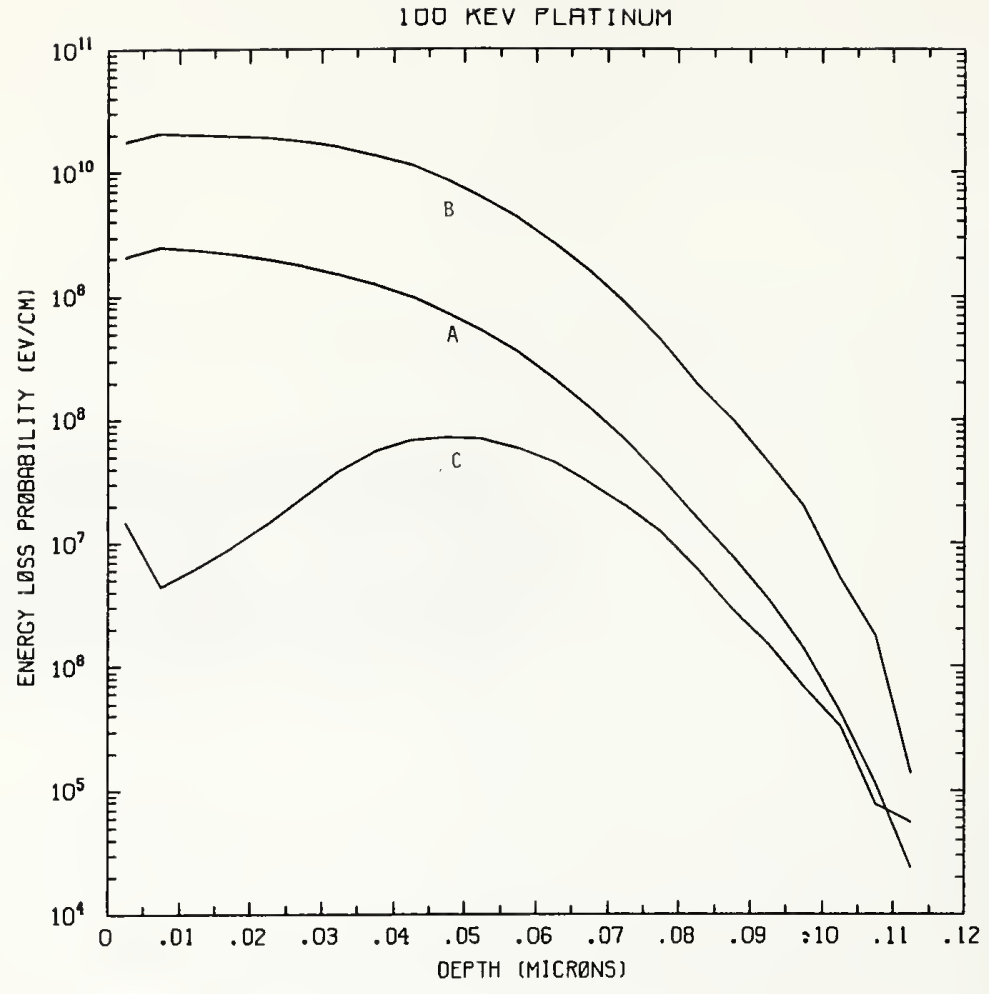

Figure Pt100-1 One-dimensional probability distributions for energy loss due to electronic excitation (A), displacement damage(B), and phonon excitation (C) for $100 \mathrm{keV}$ platinum normally incident on a silicon target.

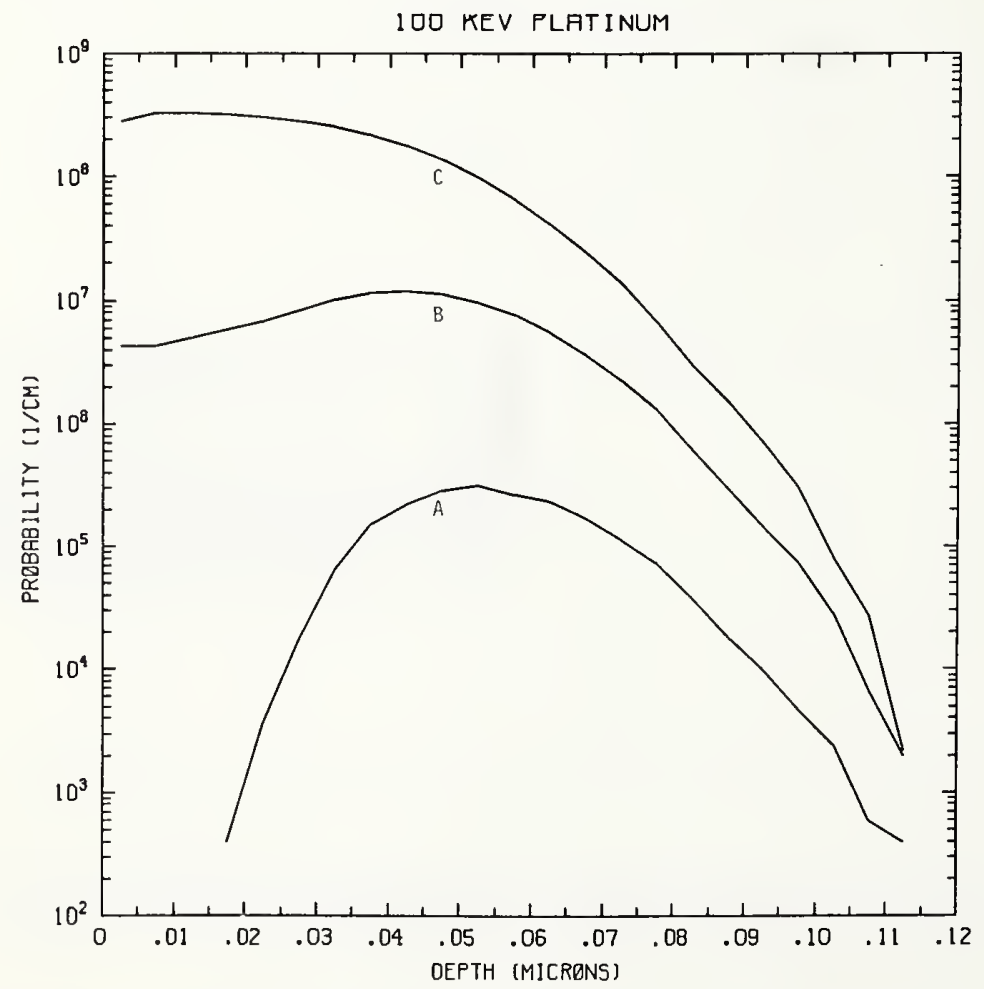

Figure Pt100-2 One-dimensional probability distributions for implanted platinum (A), primary silicon displacements (B), and Frenkel pairs (C) for $100 \mathrm{keV}$ platinum normally incident on a silicon target. 


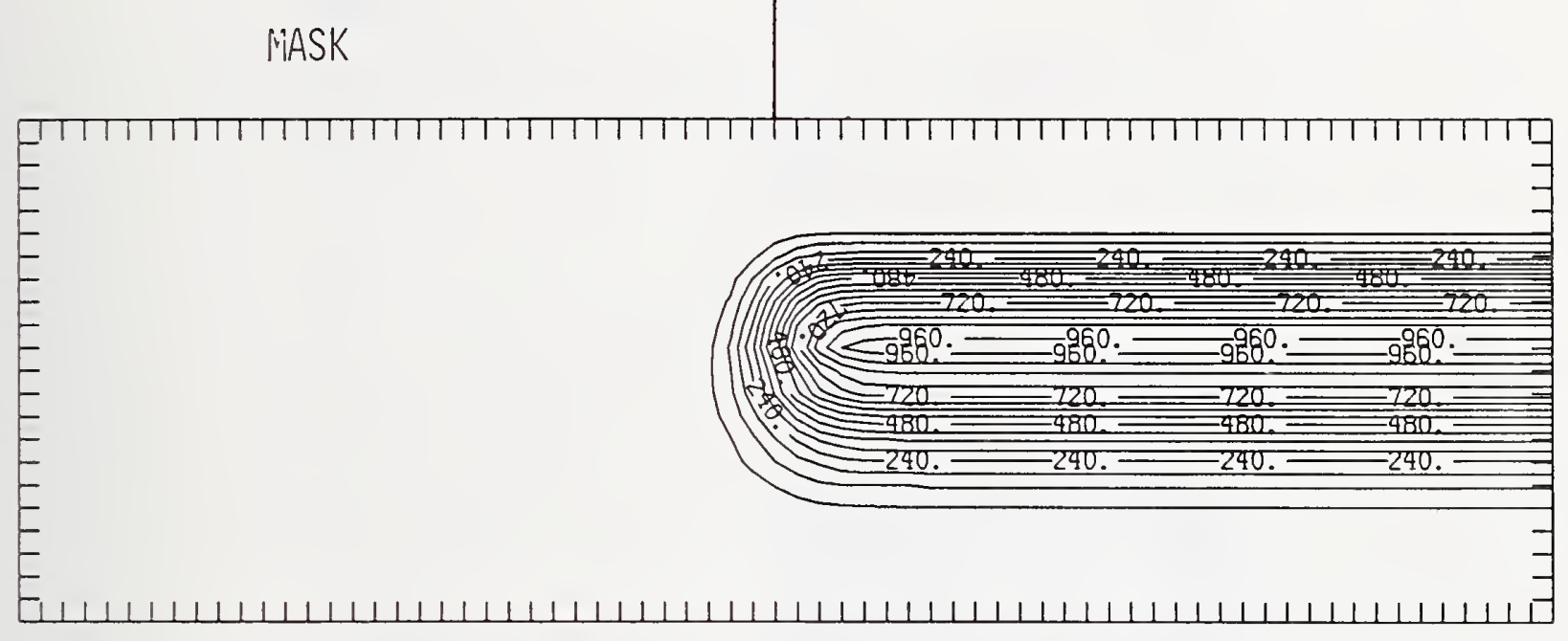

Figure Pt100-3 Two-dimensional distribution of $100 \mathrm{keV}$ platinum implanted past a mask edge. The length increment (distance between tick marks) is $0.005 \mu \mathrm{m}$ and the scale factor is 1000 .

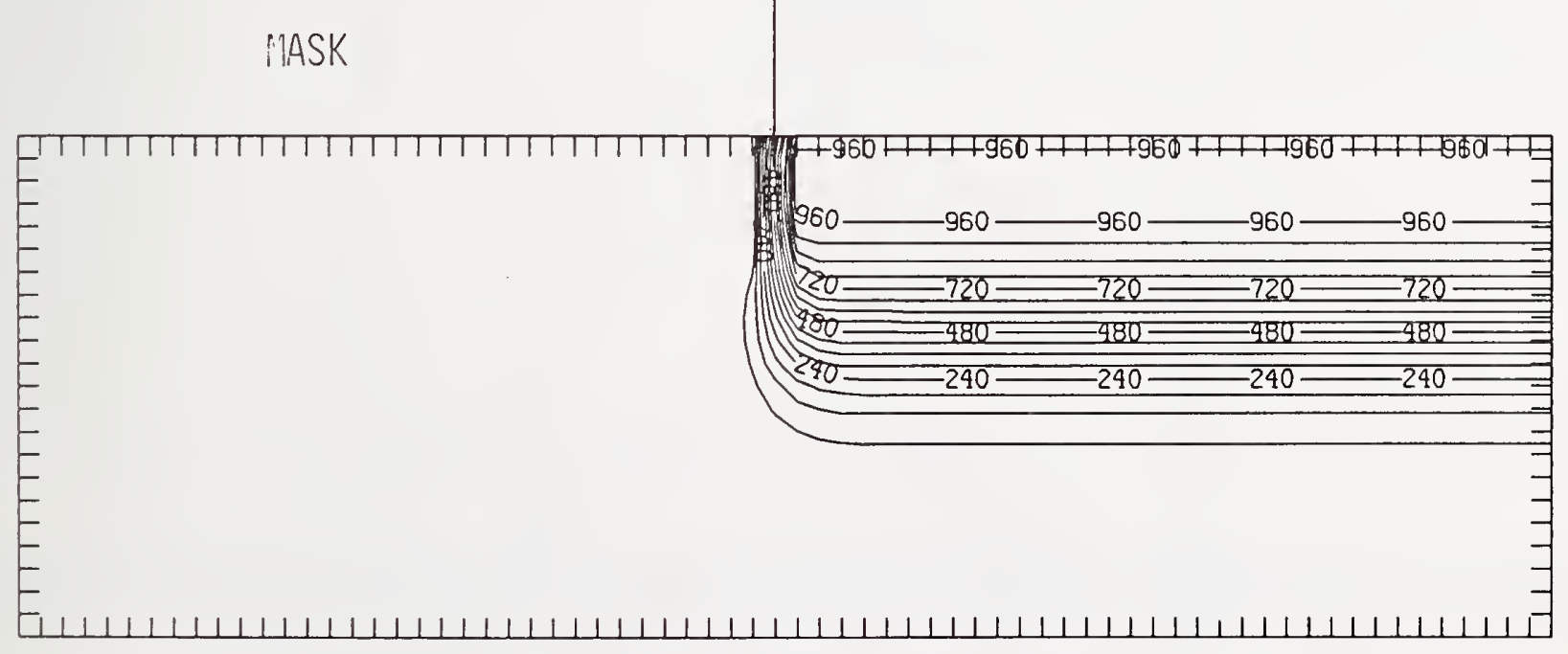

Figure Pt100-4 Two-dimensional distribution of Frenkel pairs created by $100 \mathrm{keV}$ platinum implanted past a mask edge. The length increment (distance between tick marks) is $0.005 \mu \mathrm{m}$ and the scale factor is 1 . 


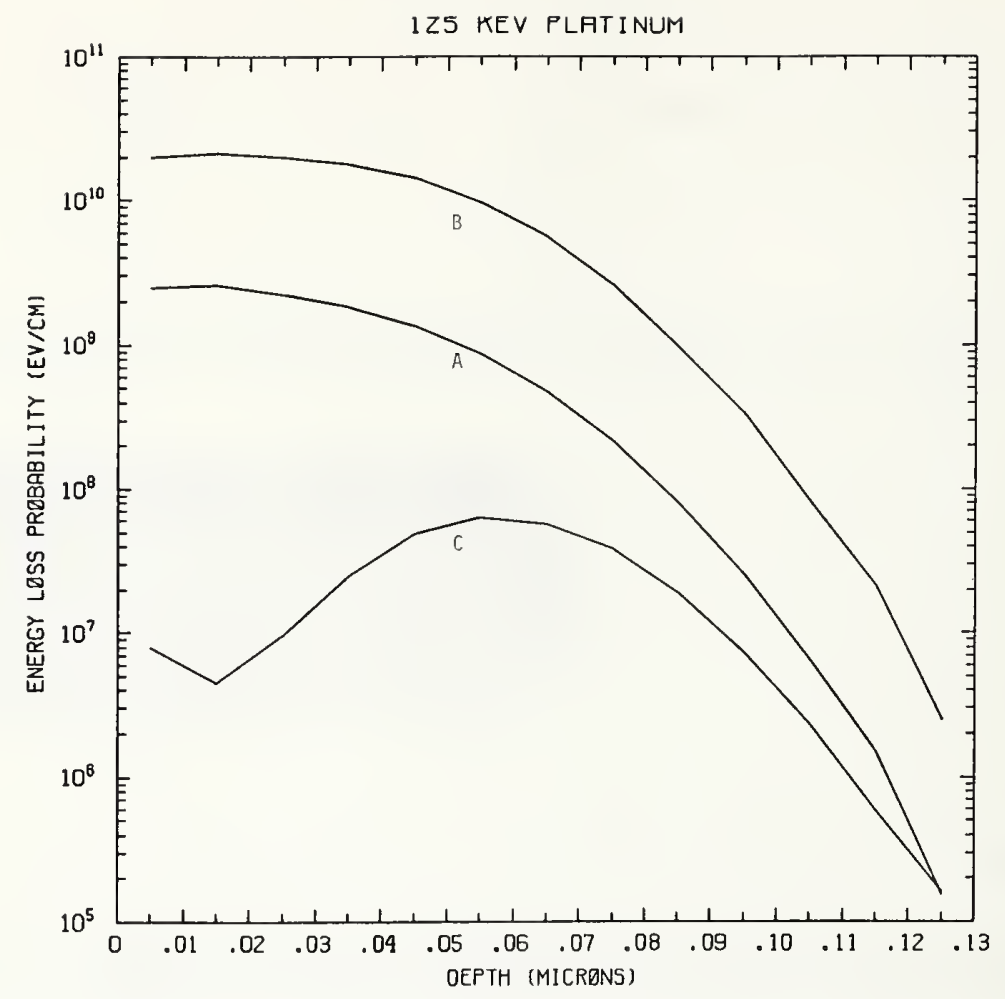

Figure Pt125-1 One-dimensional probability distributions for energy loss due to electronic excitation (A), displacement damage(B), and phonon excitation (C) for $125 \mathrm{keV}$ platinum normally incident on a silicon target.

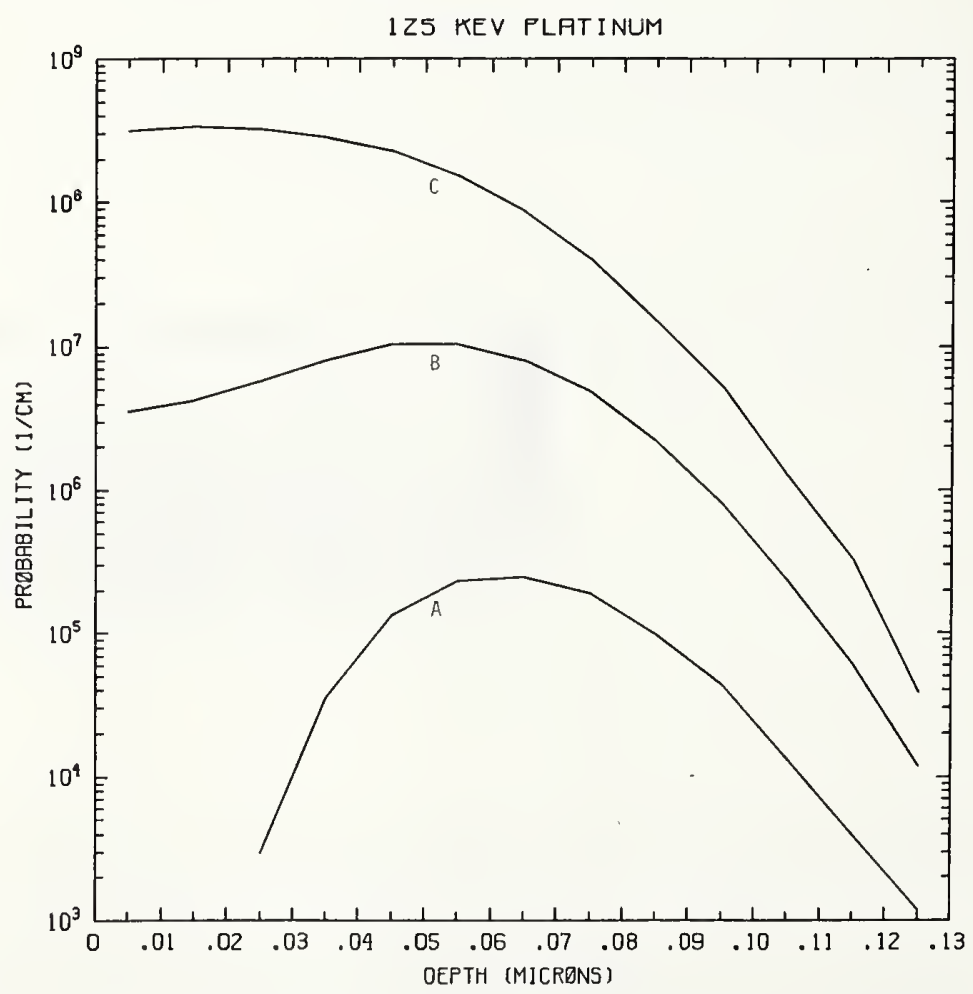

Figure Pt125-2 One-dimensional probability distributions for implanted platinum (A), primary silicon displacements (B), and Frenkel pairs (C) for $125 \mathrm{keV}$ platinum normally incident on a silicon target. 


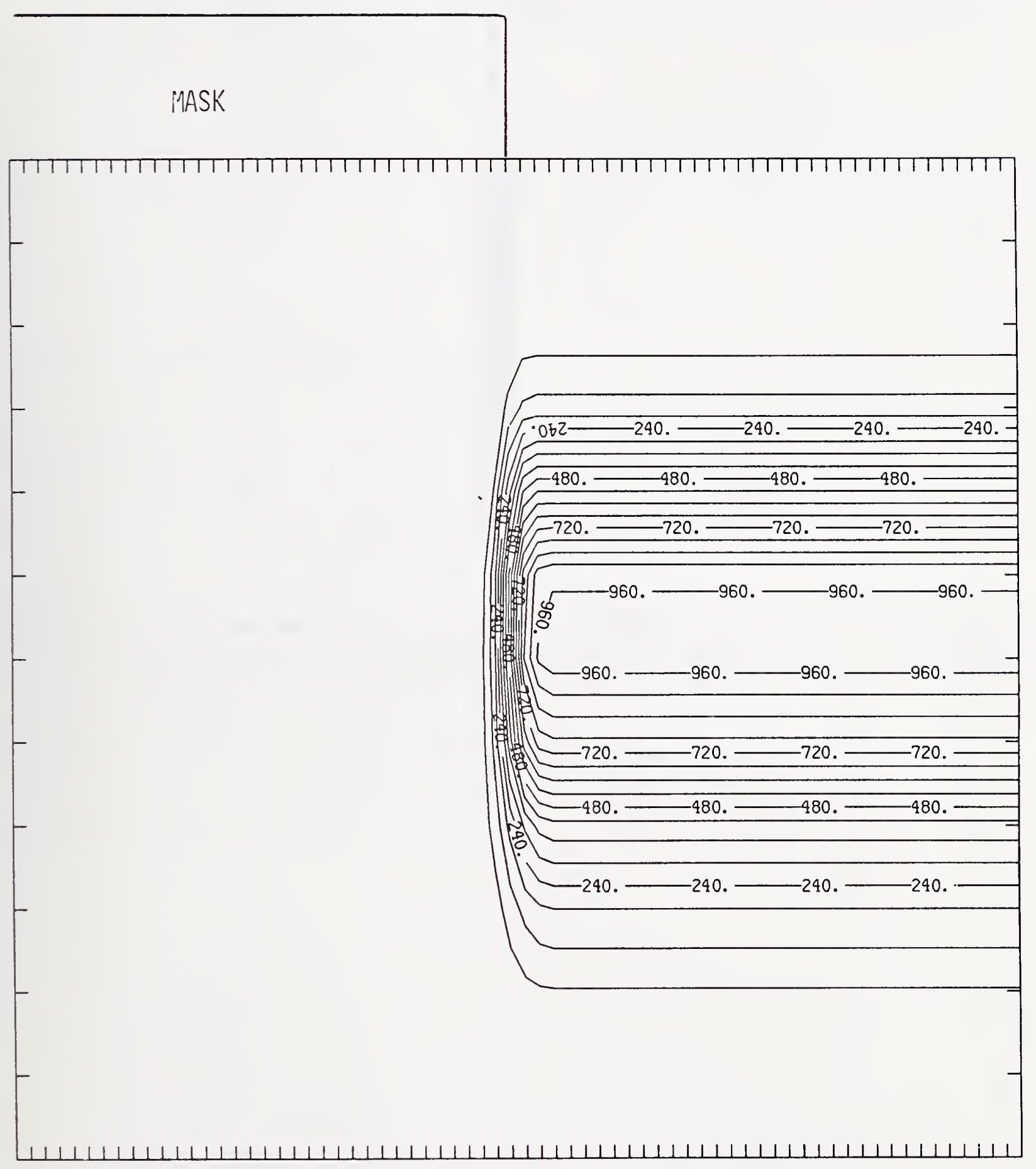

Figure Pt125-3 Two-dimensional distribution of $125 \mathrm{keV}$ platinum implanted past a mask edge. The length increment (distance between tick marks) is $0.01 \mu \mathrm{m}$ and the scale factor is 1000. 
MASK

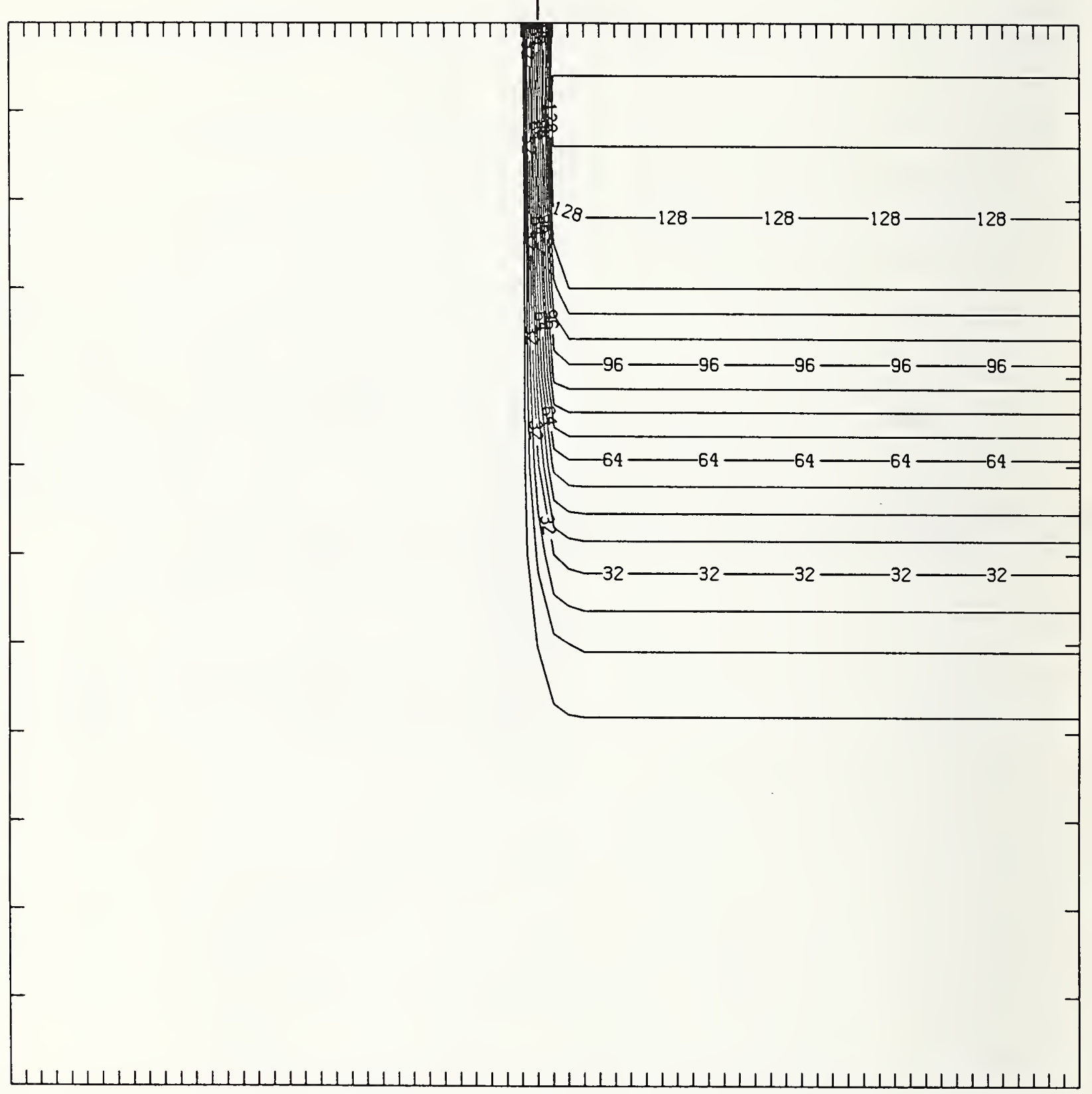

Figure Pt125-4 Two-dimensional distribution of Frenkel pairs created by $125 \mathrm{keV}$ platinum implanted past a mask edge. The length increment (distance between tick marks) is $0.01 \mu \mathrm{m}$ and the scale factor is 0.1 . 


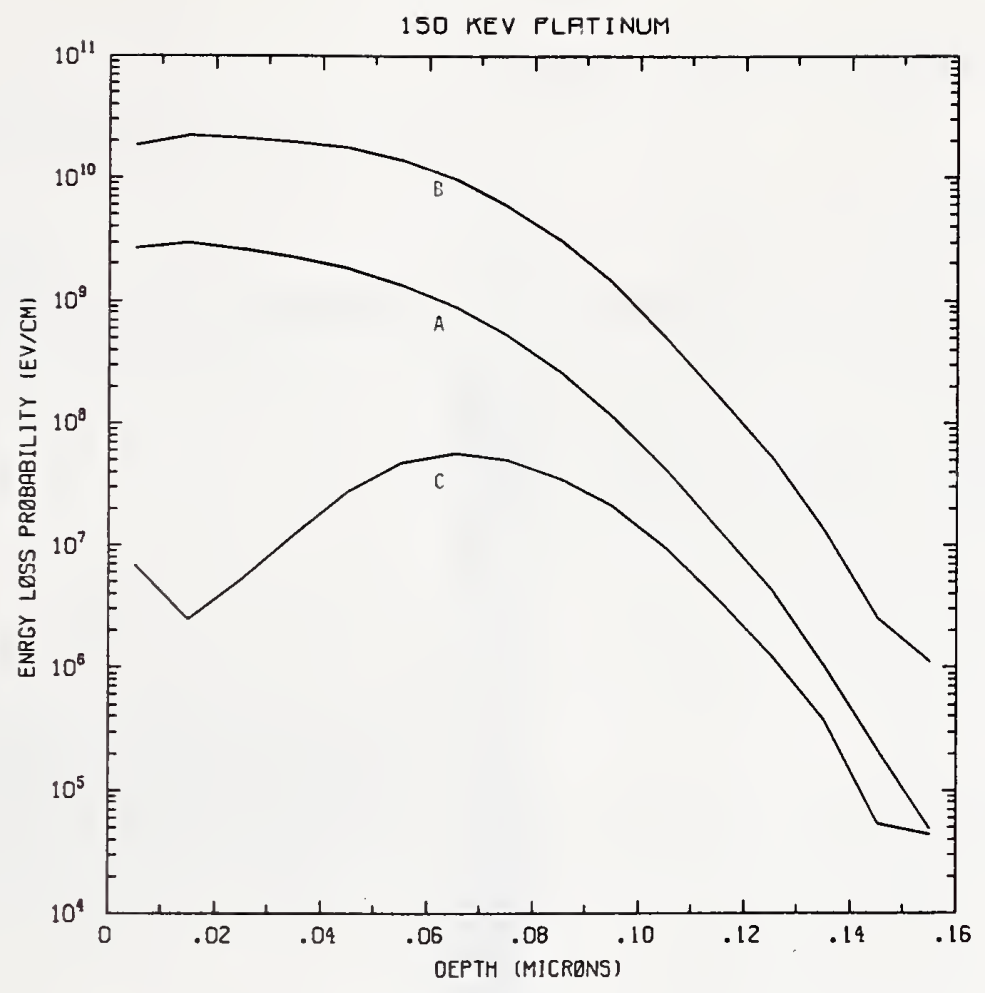

Figure Pt150-1 One-dimensional probability distributions for energy loss due to electronic excitation (A), displacement damage(B), and phonon excitation (C) for $150 \mathrm{keV}$ platinum normally incident on a silicon target.

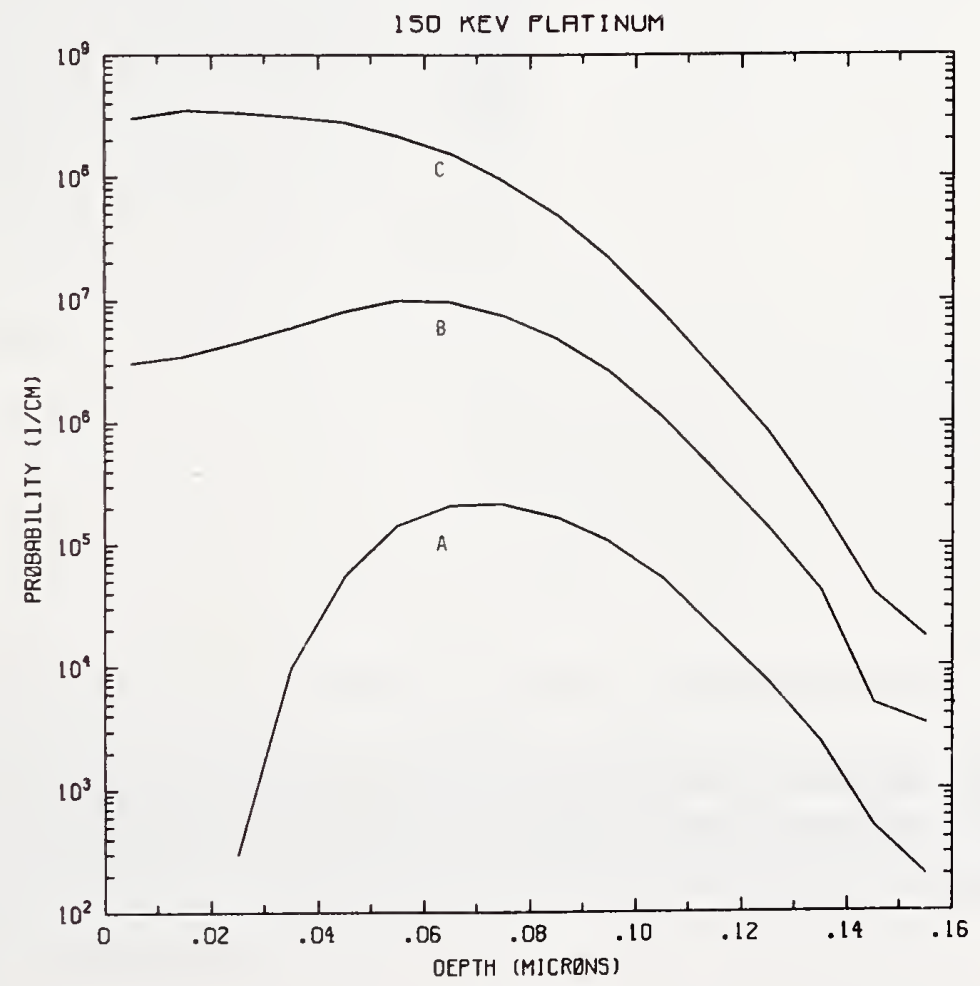

Figure Pt150-2 One-dimensional probability distributions for implanted platinum (A), primary silicon displacements (B), and Frenkel pairs (C) for $150 \mathrm{keV}$ platinum normally incident on a silicon target. 
l'ASK

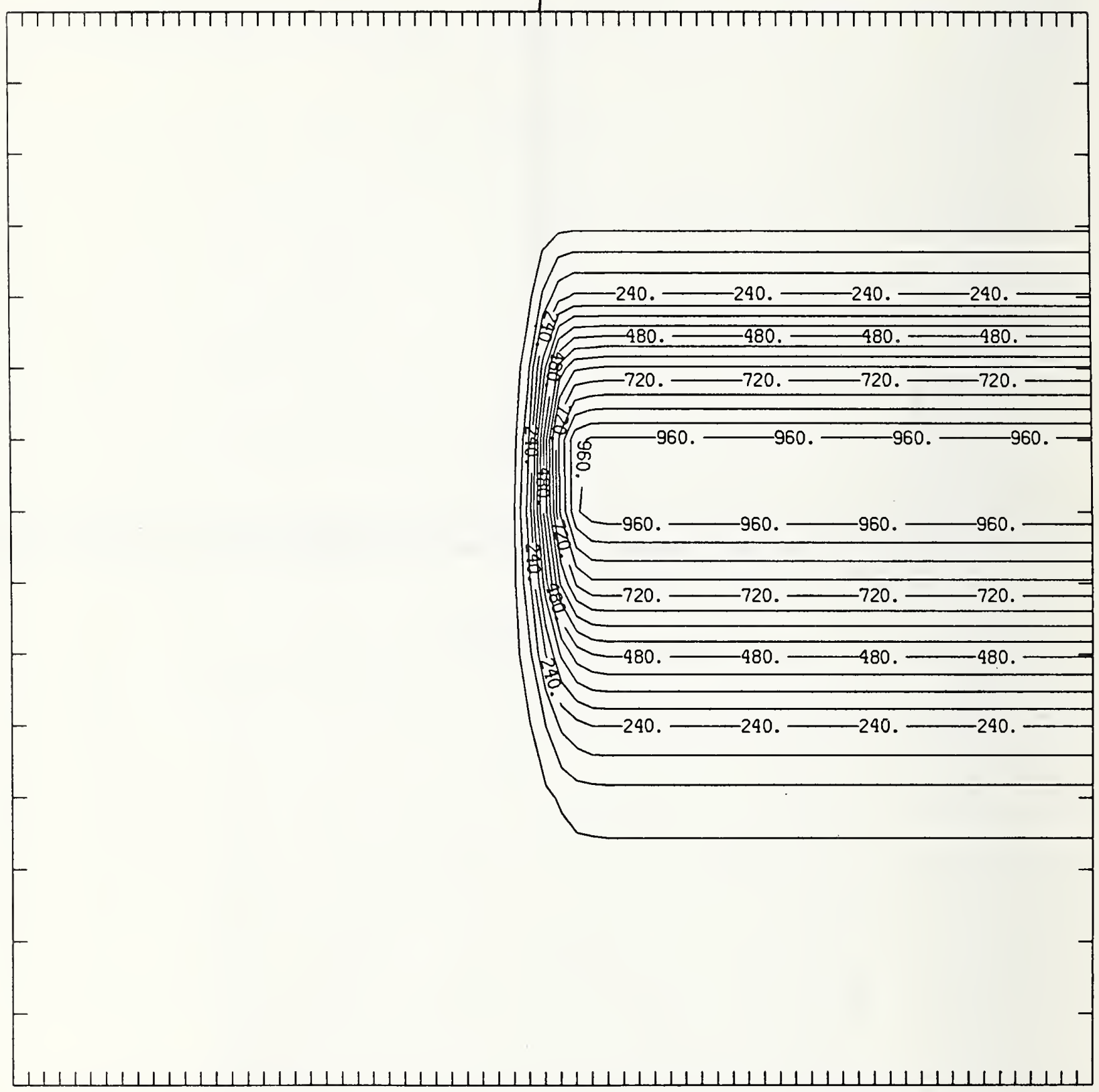

Figure Pt150-3 Two-dimensional distribution of $150 \mathrm{keV}$ platinum implanted past a mask edge. The length increment (distance between tick marks) is $0.01 \mu \mathrm{m}$ and the scale factor is 1000 . 
MASK

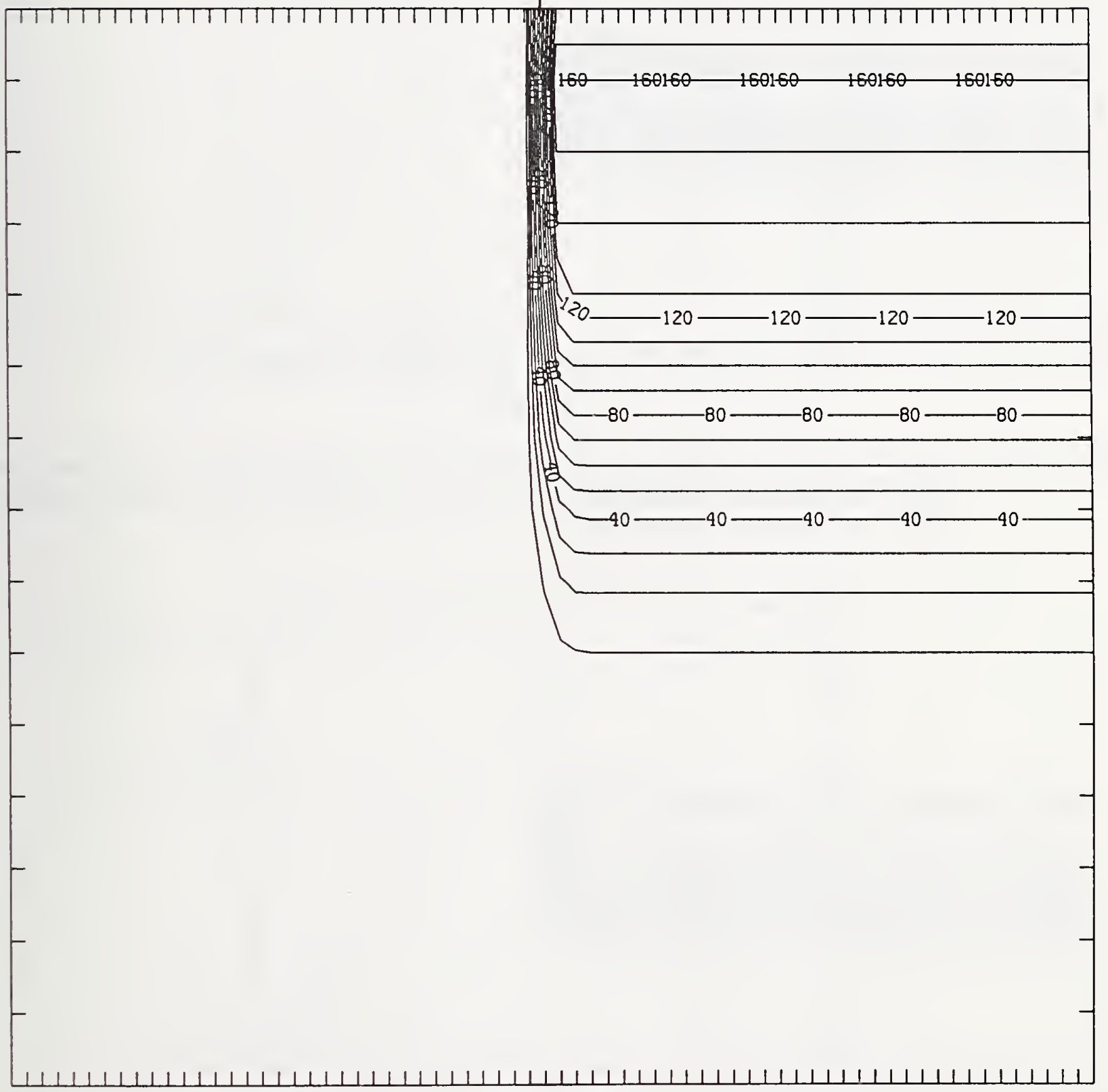

Figure Pt150-4 Two-dimensional distribution of Frenkel pairs created by $150 \mathrm{keV}$ platinum implanted past a mask edge. The length increment (distance between tick marks) is $0.01 \mu \mathrm{m}$ and the scale factor is 0.1 . 


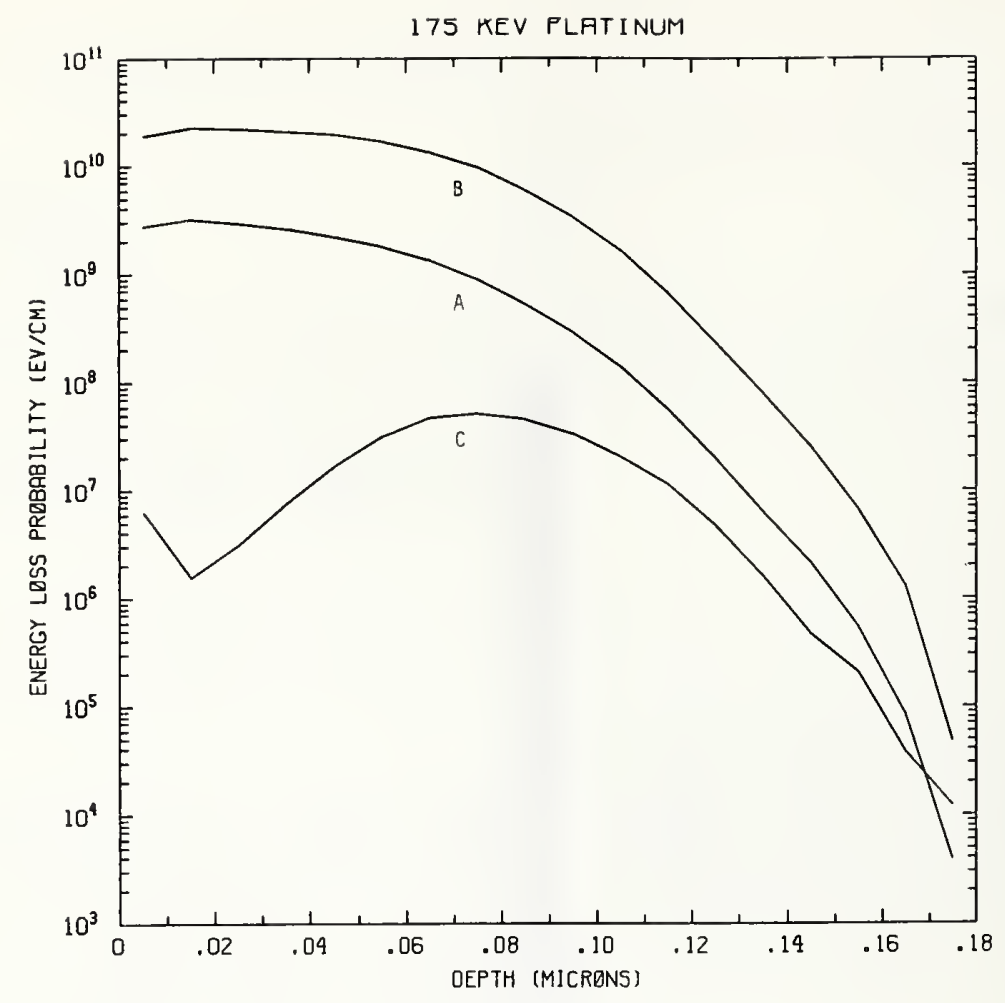

Figure Pt175-1 One-dimensional probability distributions for energy loss due to electronic excitation (A), displacement damage(B), and phonon excitation (C) for $175 \mathrm{keV}$ platinum normally incident on a silicon target.

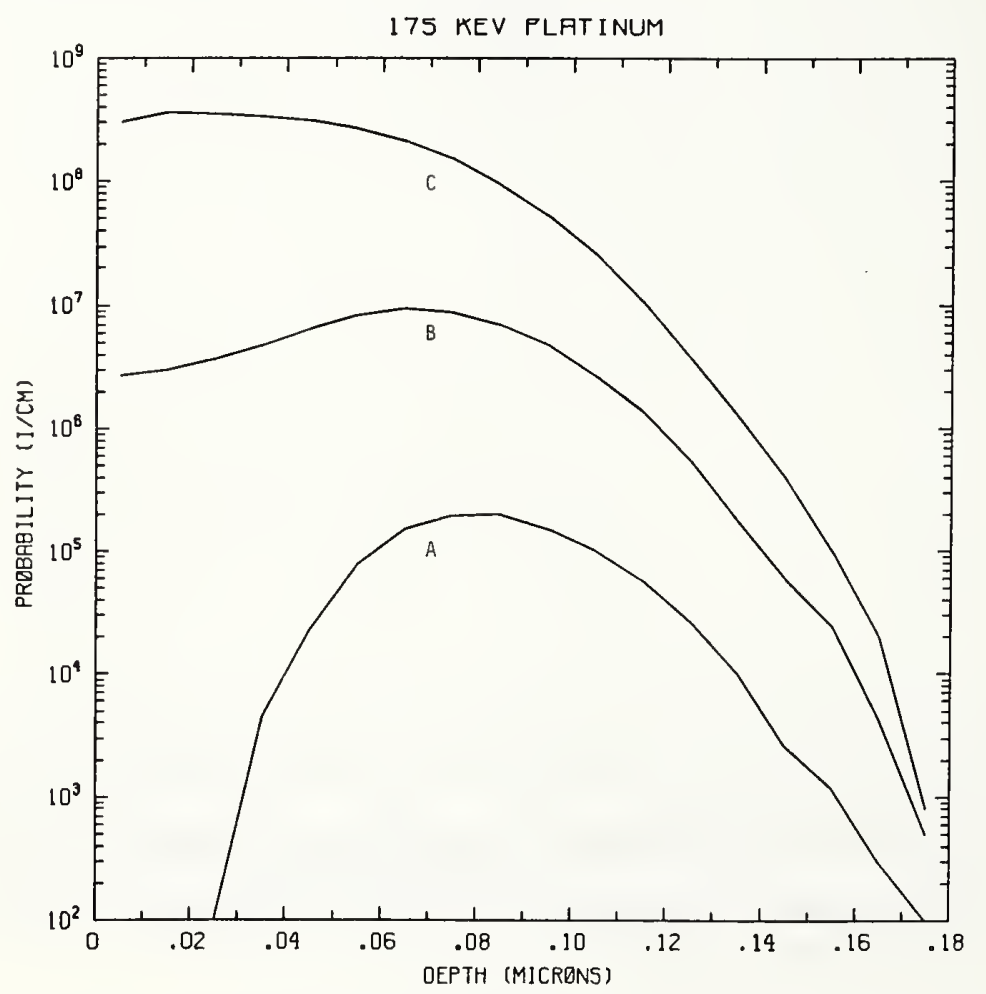

Figure Pt175-2 One-dimensional probability distributions for implanted platinum (A), primary silicon displacements (B), and Frenkel pairs (C) for $175 \mathrm{keV}$ platinum normally incident on a silicon target. 
MASK

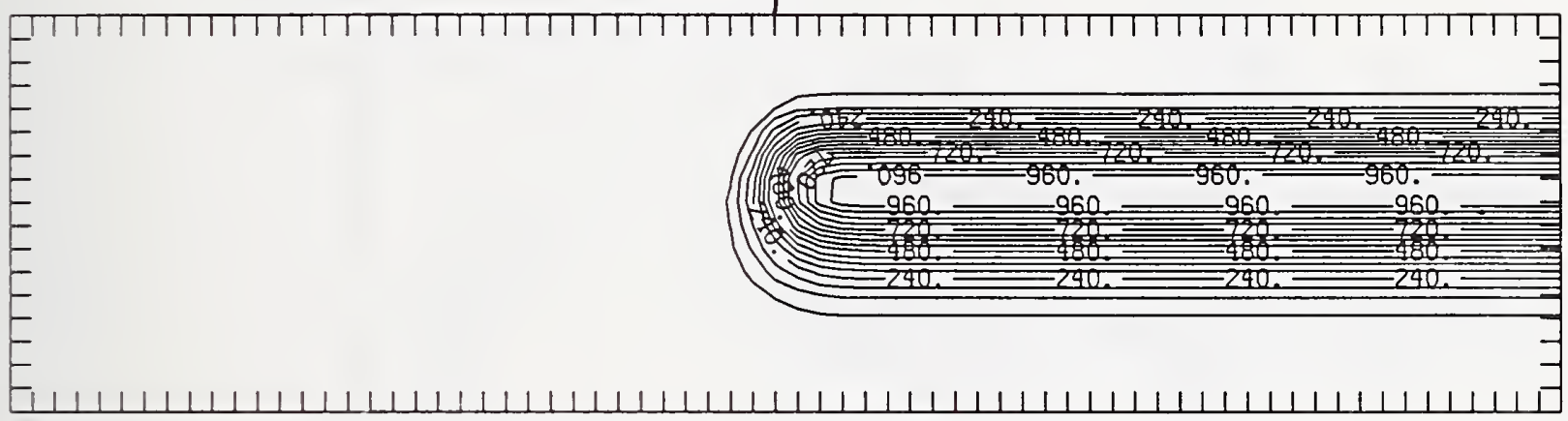

Figure Pt175-3 Two-dimensional distribution of $175 \mathrm{keV}$ platinum implanted past a mask edge. The length increment (distance between tick marks) is $0.01 \mu \mathrm{m}$ and the scale factor is 1000 .

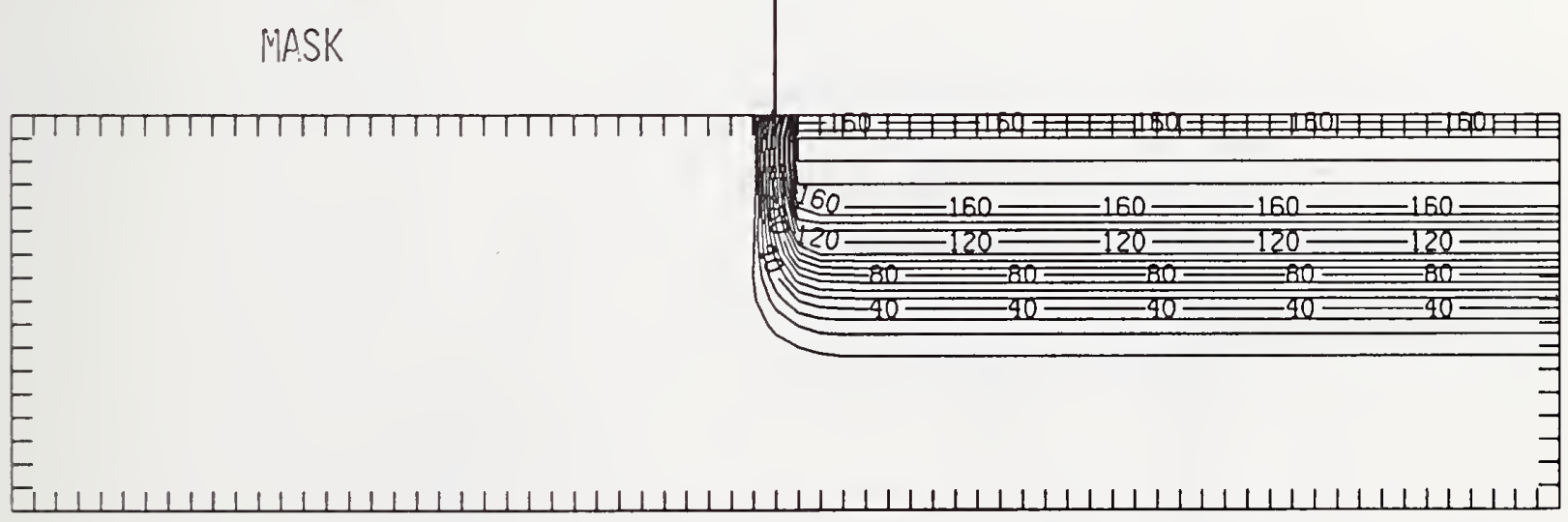

Figure Pt175-4 Two-dimensional distribution of Frenkel pairs created by $175 \mathrm{keV}$ platinum implanted past a mask edge. The length increment (distance between tick marks) is $0.01 \mu \mathrm{m}$ and the scale factor is 0.1 . 


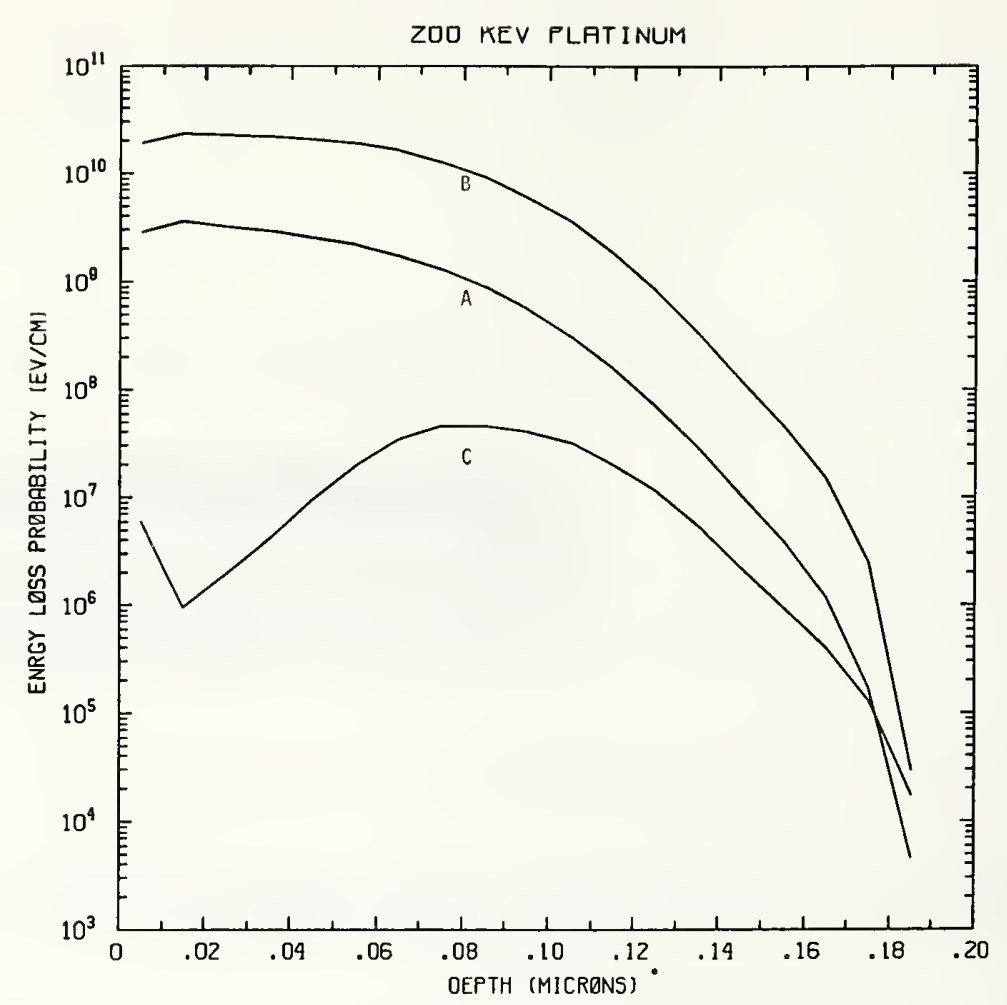

Figure Pt200-1 One-dimensional probability distributions for energy loss due to electronic excitation (A), displacement damage(B), and phonon excitation (C) for $200 \mathrm{keV}$ platinum normally incident on a silicon target.

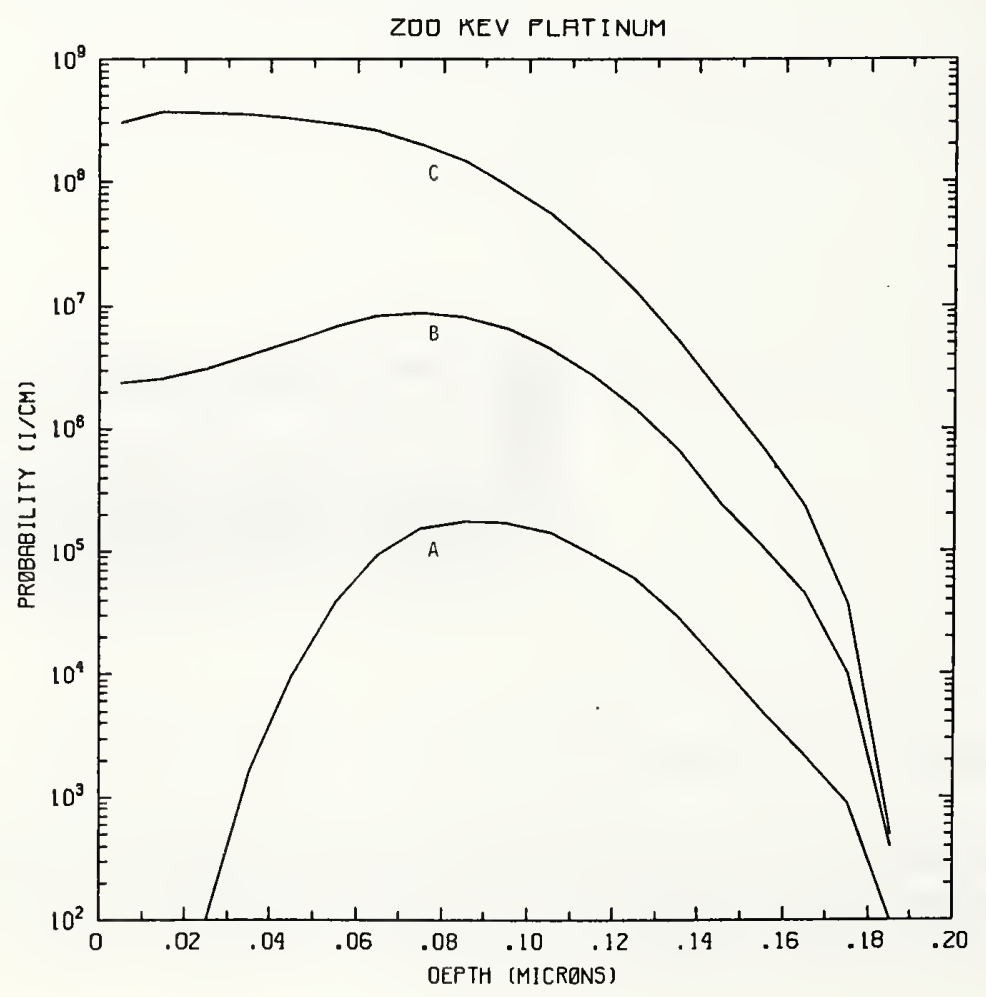

Figure Pt200-2 One-dimensional probability distributions for implanted platinum (A), primary silicon displacements (B), and Frenkel pairs (C) for $200 \mathrm{keV}$ platinum normally incident on a silicon target. 


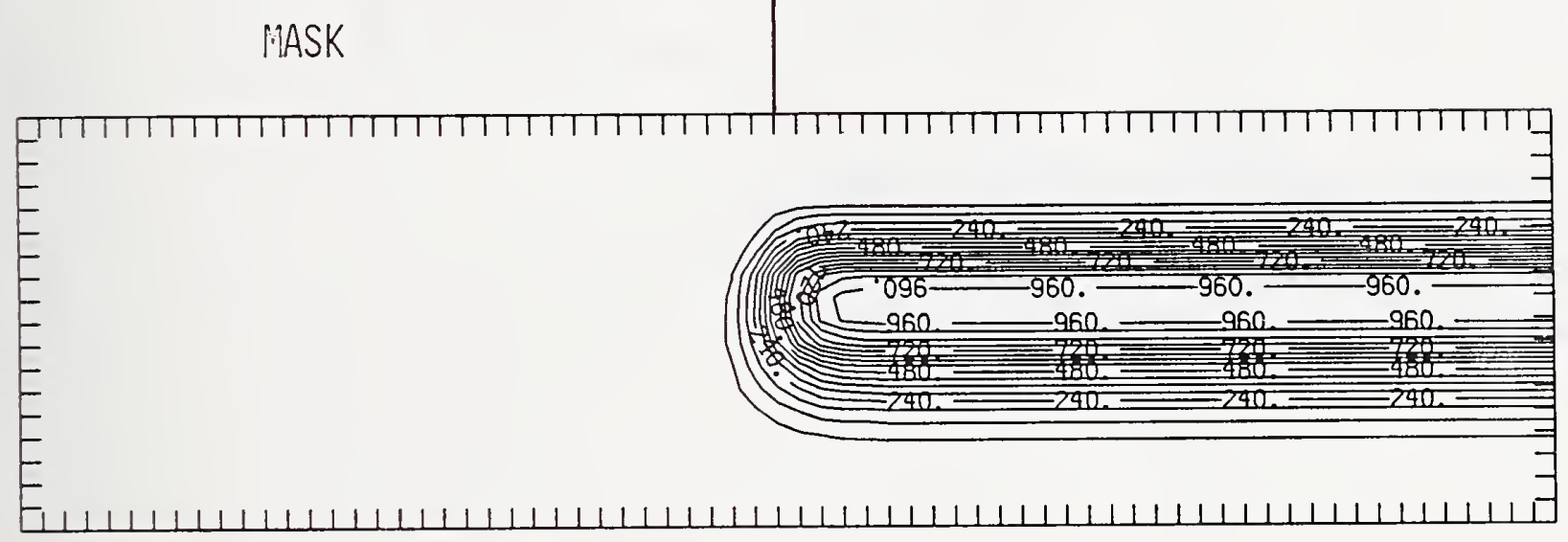

Figure Pt200-3 Two-dimensional distribution of $200 \mathrm{keV}$ platinum implanted past a mask edge. The length increment (distance between tick marks) is $0.01 \mu \mathrm{m}$ and the scale factor is 1000.

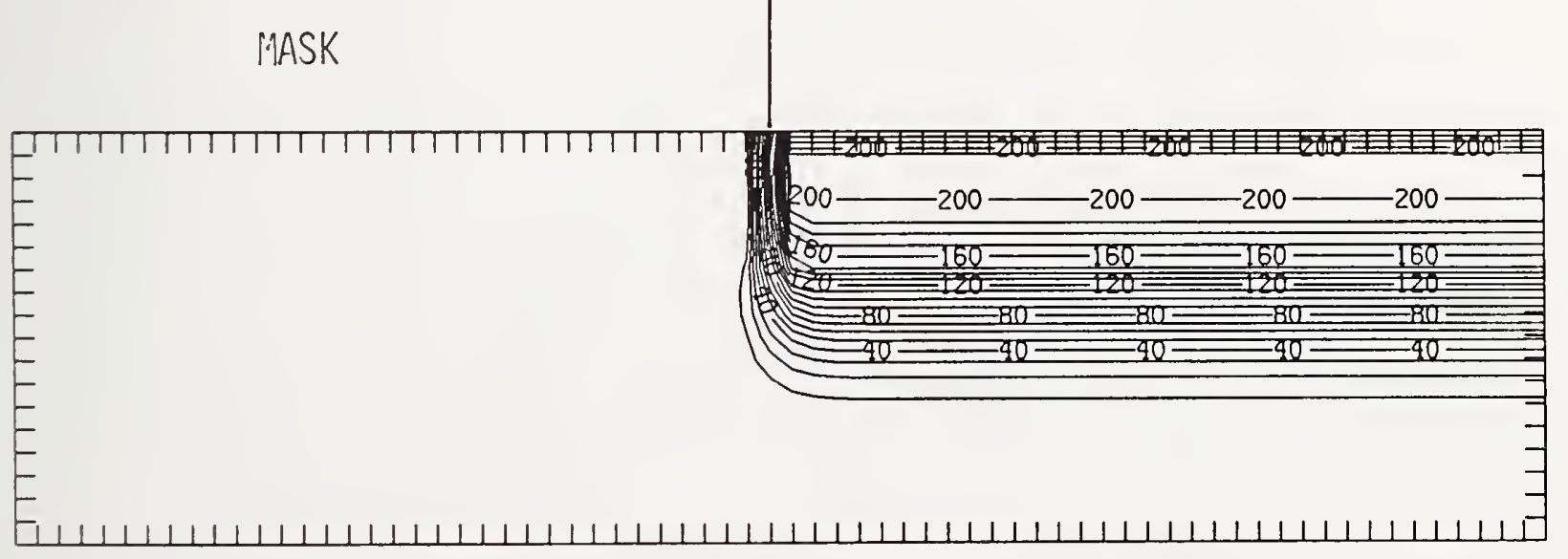

Figure Pt200-4 Two-dimensional distribution of Frenkel pairs created by $200 \mathrm{keV}$ platinum implanted past a mask edge. The length increment (distance between tick marks) is $0.01 \mu \mathrm{m}$ and the scale factor is 0.1 . 


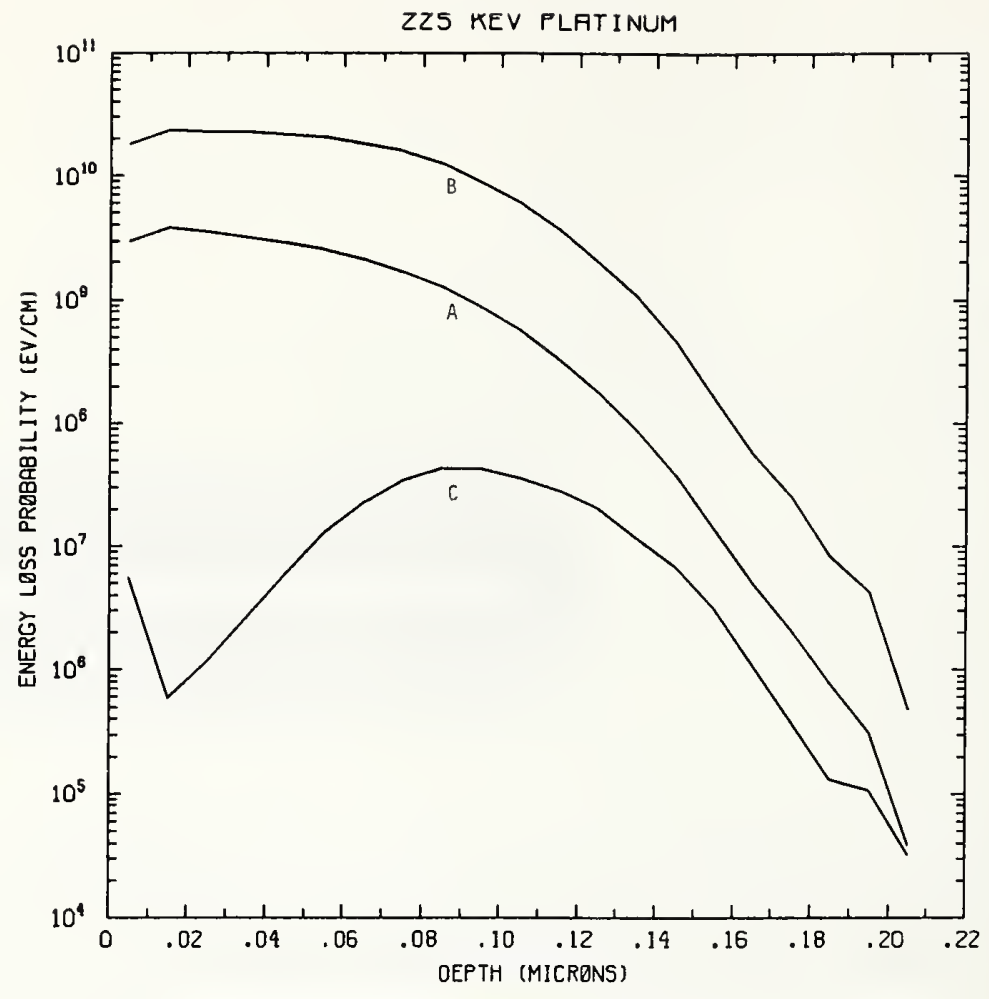

Figure Pt225-1 One-dimensional probability distributions for energy loss due to electronic excitation (A), displacement damage(B), and phonon excitation (C) for $225 \mathrm{keV}$ platinum normally incident on a silicon target.

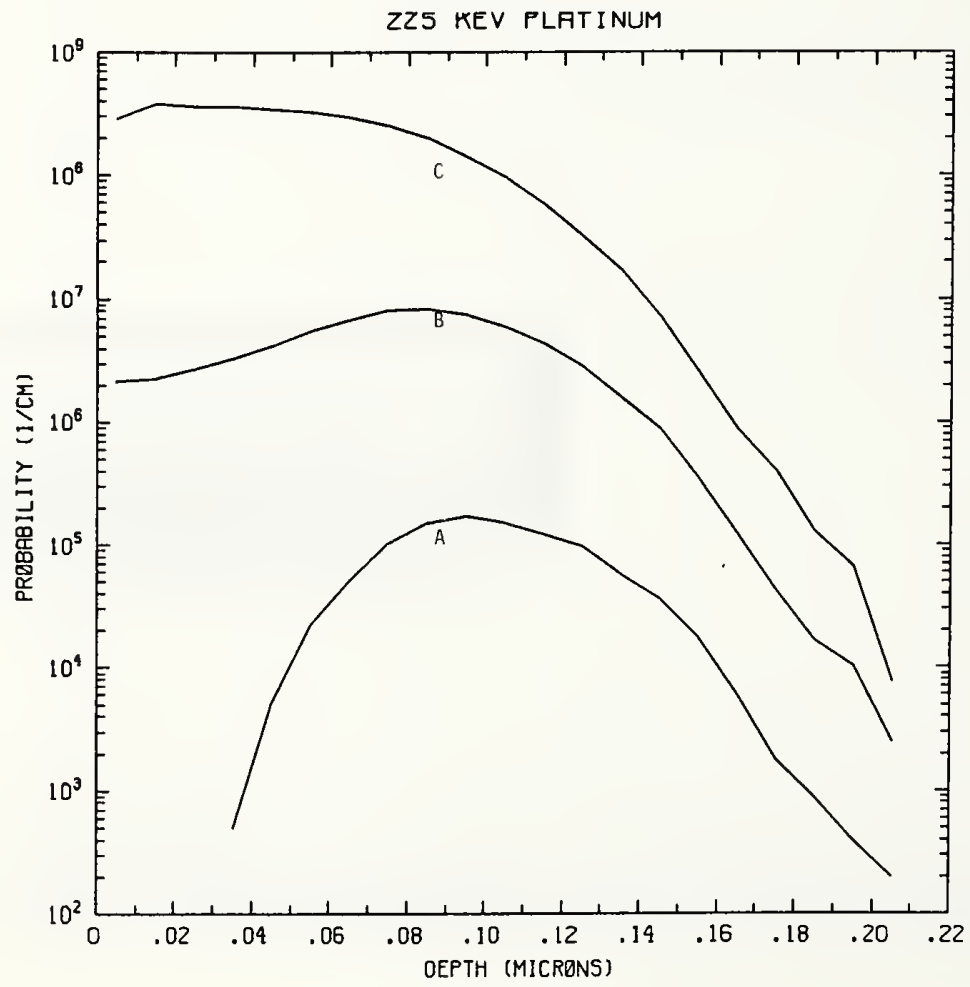

Figure Pt225-2 One-dimensional probability distributions for implanted platinum (A), primary silicon displacements (B), and Frenkel pairs (C) for $225 \mathrm{keV}$ platinum normally incident on a silicon target. 


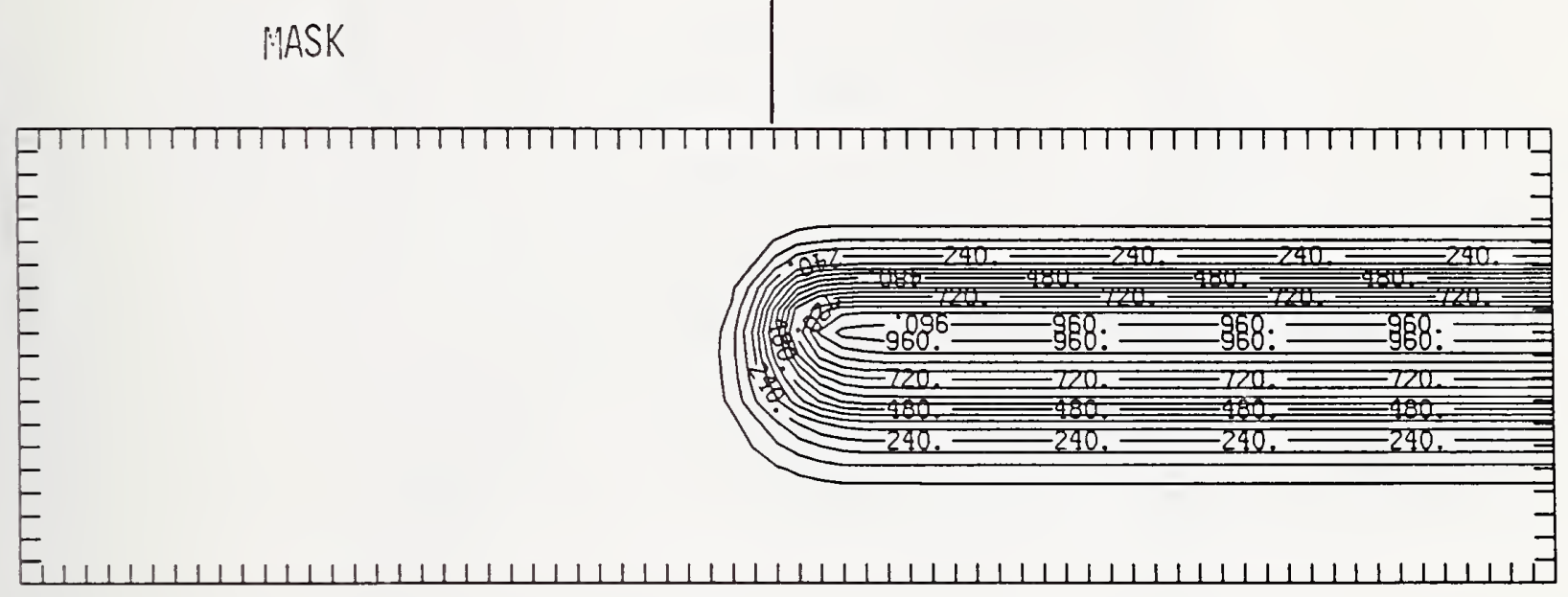

Figure Pt225-3 Two-dimensional distribution of $225 \mathrm{keV}$ platinum implanted past a mask edge. The length increment (distance between tick marks) is $0.01 \mu \mathrm{m}$ and the scale factor is 1000 .

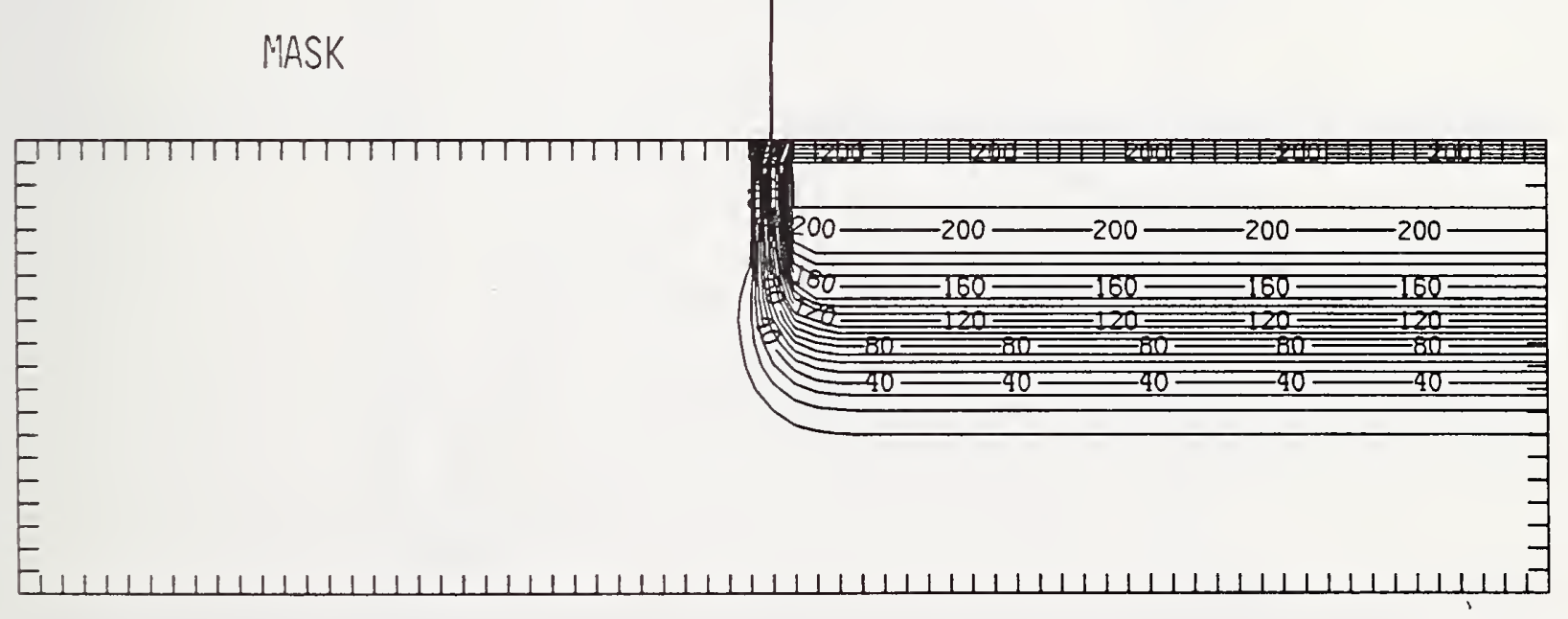

Figure Pt225-4 Two-dimensional distribution of Frenkel pairs created by $225 \mathrm{keV}$ platinum implanted past a mask edge. The length increment (distance between tick marks) is $0.01 \mu \mathrm{m}$ and the scale factor is 0.1 . 


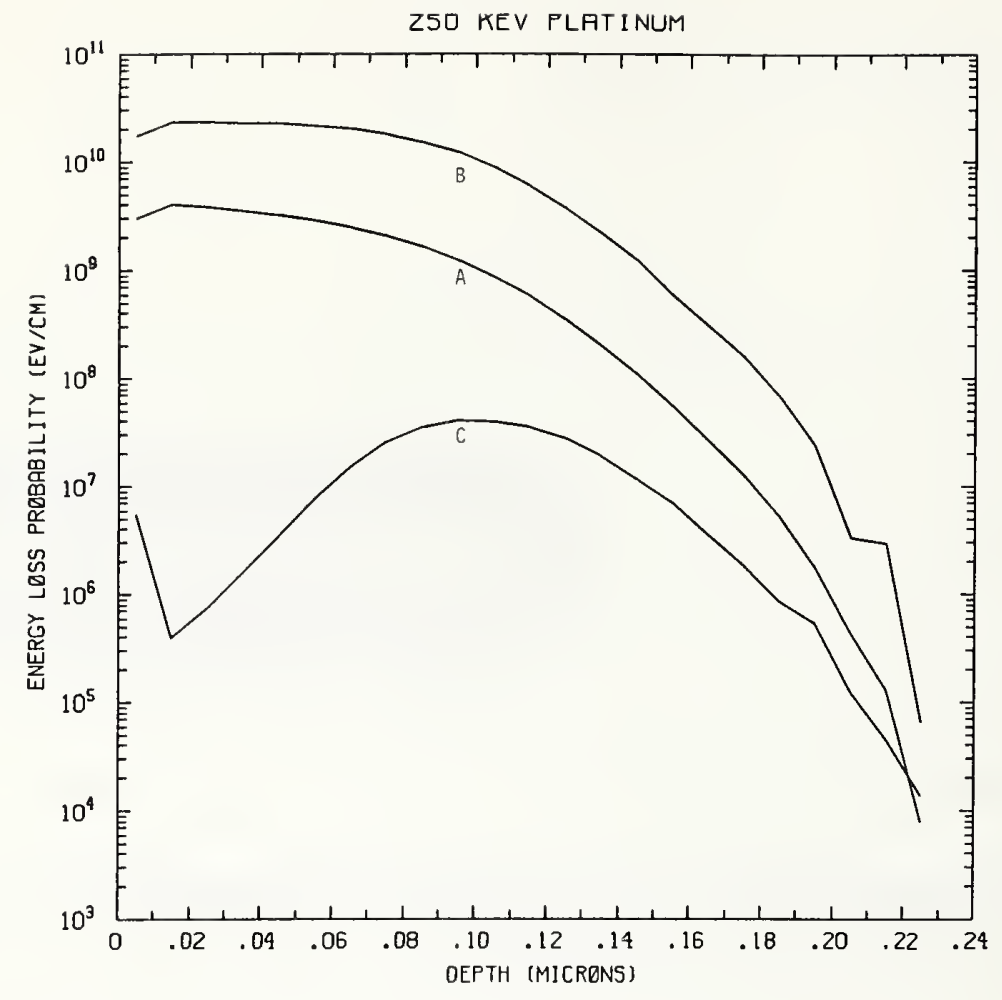

Figure Pt250-1 One-dimensional probability distributions for energy loss due to electronic excitation (A), displacement damage(B), and phonon excitation (C) for $250 \mathrm{keV}$ platinum normally incident on a silicon target.

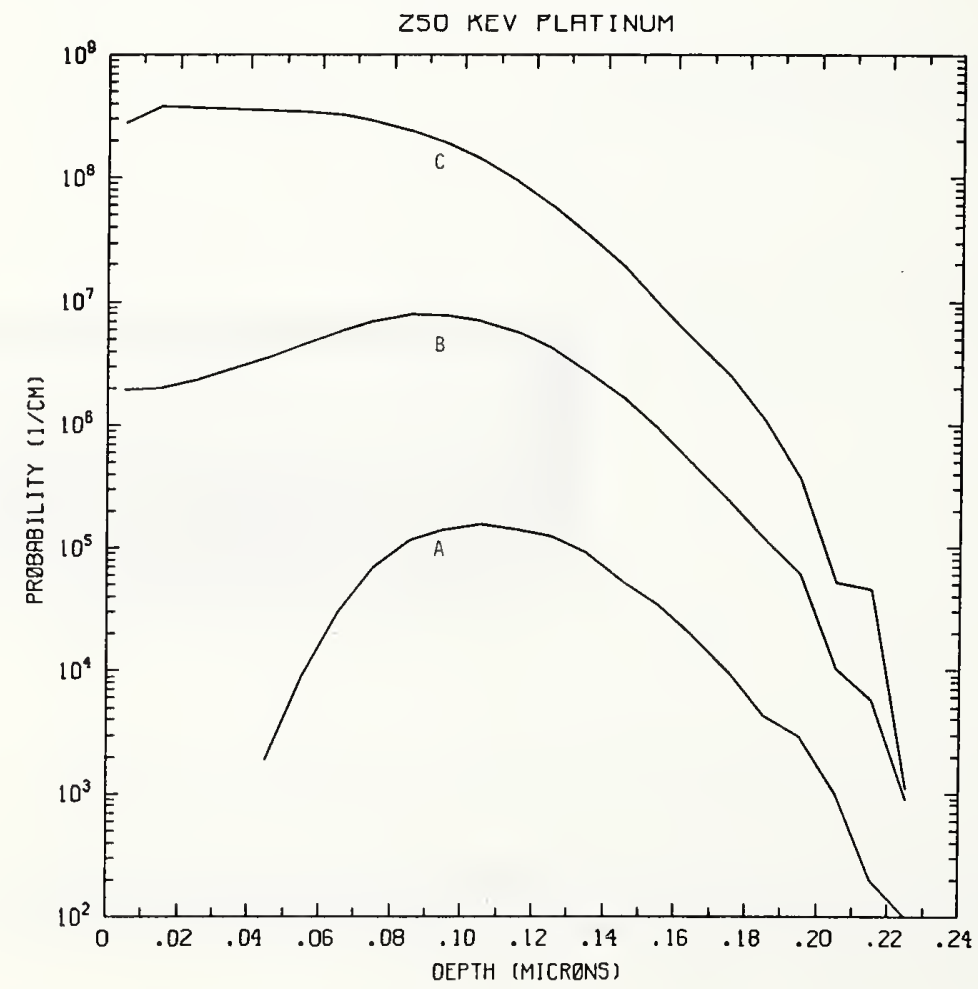

Figure Pt250-2 One-dimensional probability distributions for implanted platinum (A), primary silicon displacements (B), and Frenkel pairs (C) for $250 \mathrm{keV}$ platinum normally incident on a silicon target. 


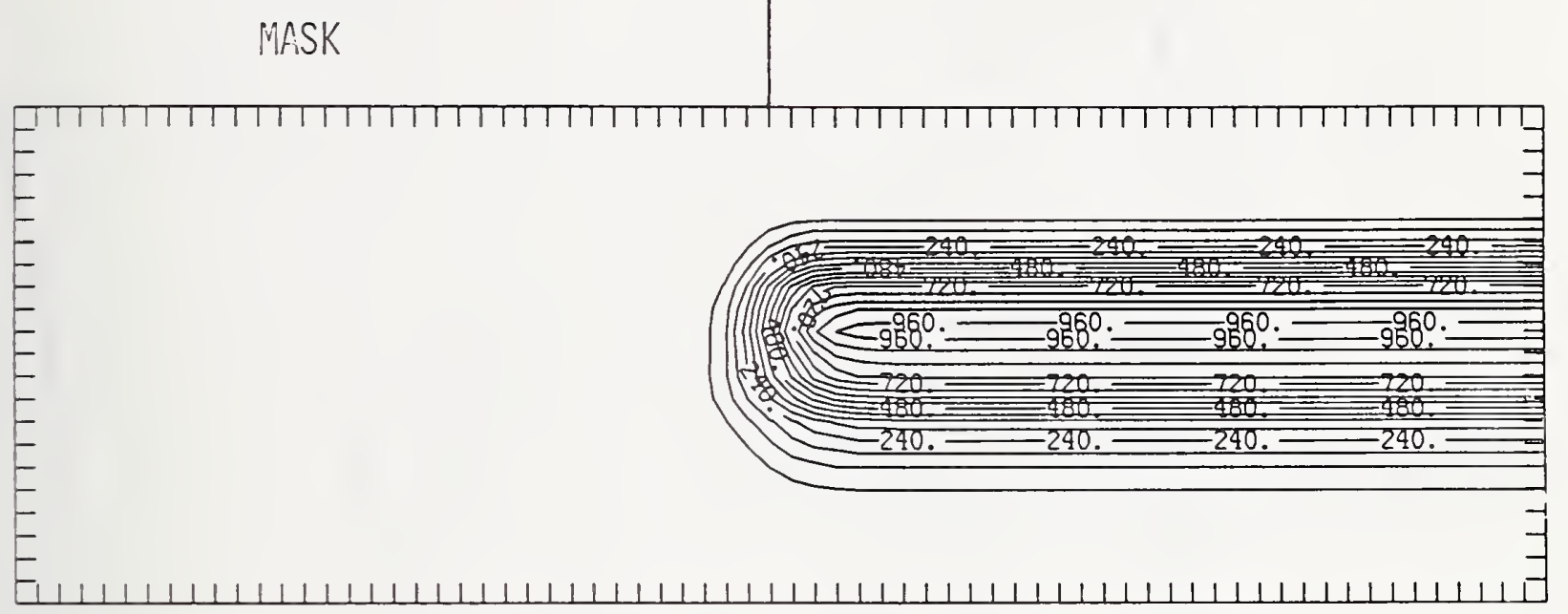

Figure Pt250-3 Two-dimensional distribution of $250 \mathrm{keV}$ platinum implanted past a mask edge. The length increment (distance between tick marks) is $0.01 \mu \mathrm{m}$ and the scale factor is 1000 .

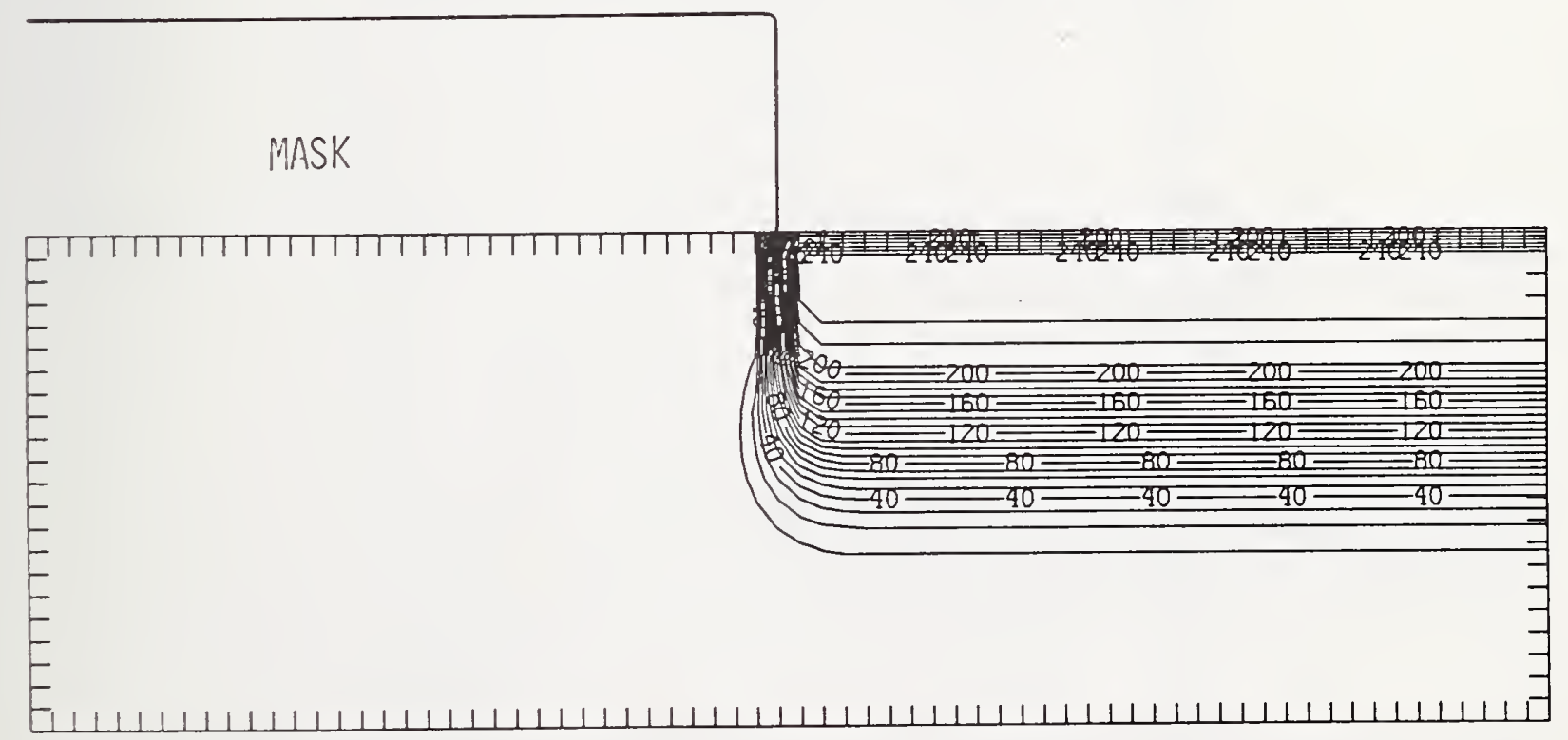

Figure Pt250-4 Two-dimensional distribution of Frenkel pairs created by $250 \mathrm{keV}$ platinum implanted past a mask edge. The length increment (distance between tick marks) is $0.01 \mu \mathrm{m}$ and the scale factor is 0.1 . 


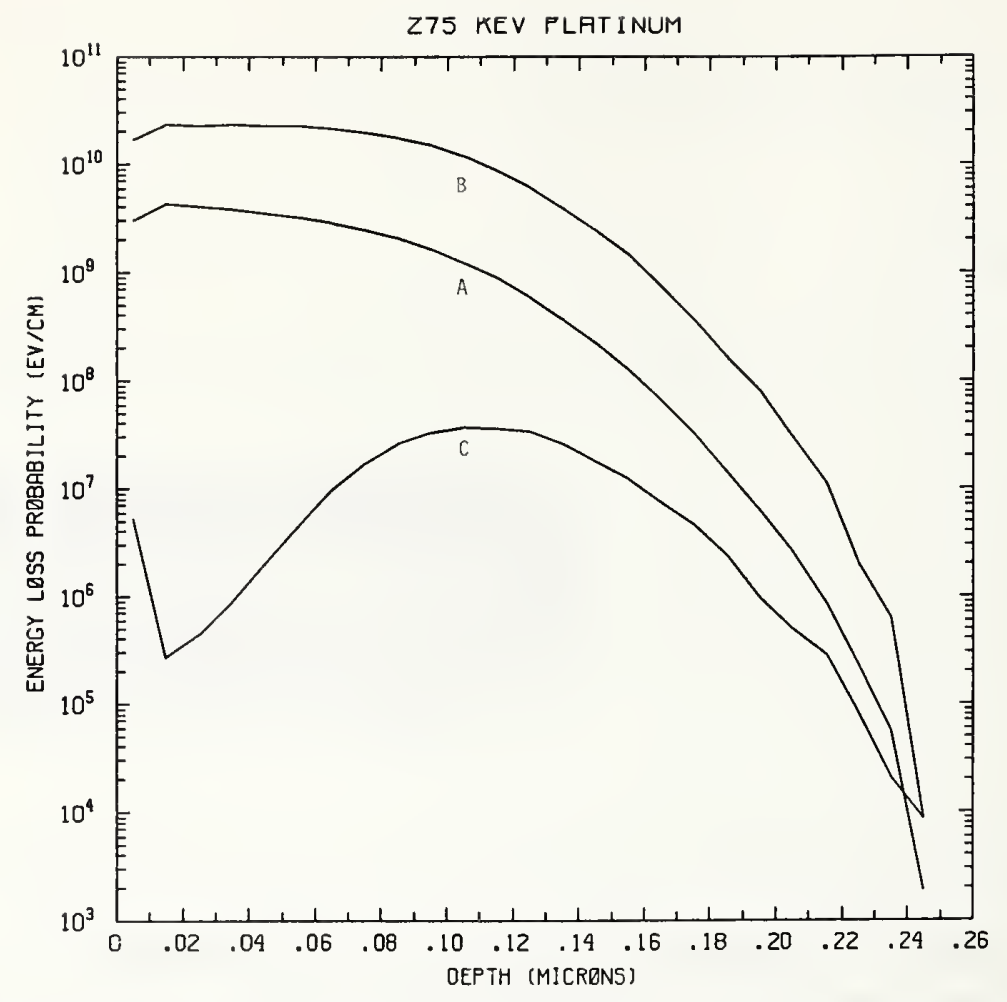

Figure Pt275-1 One-dimensional probability distributions for energy loss due to electronic excitation (A), displacement damage(B), and phonon excitation (C) for $275 \mathrm{keV}$ platinum normally incident on a silicon target.

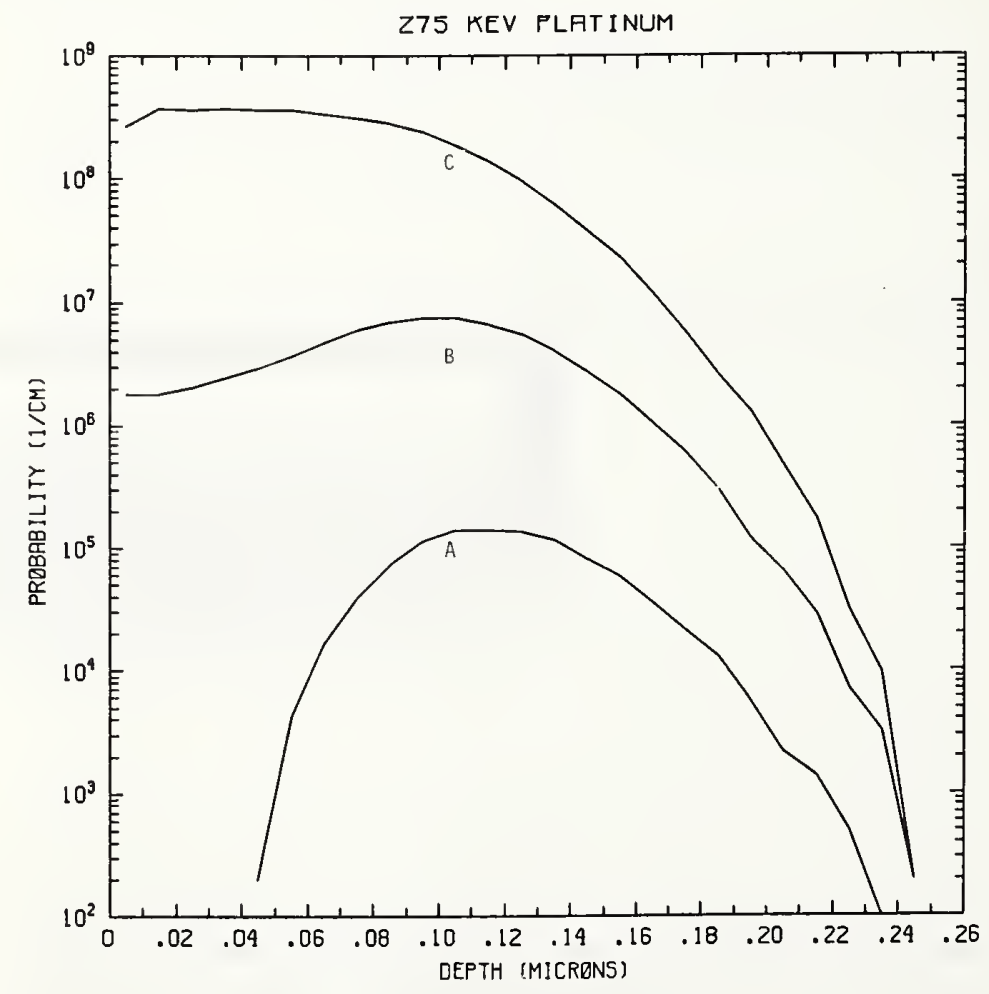

Figure Pt275-2 One-dimensional probability distributions for implanted platinum (A), primary silicon displacements (B), and Frenkel pairs (C) for $275 \mathrm{keV}$ platinum normally incident on a silicon target. 


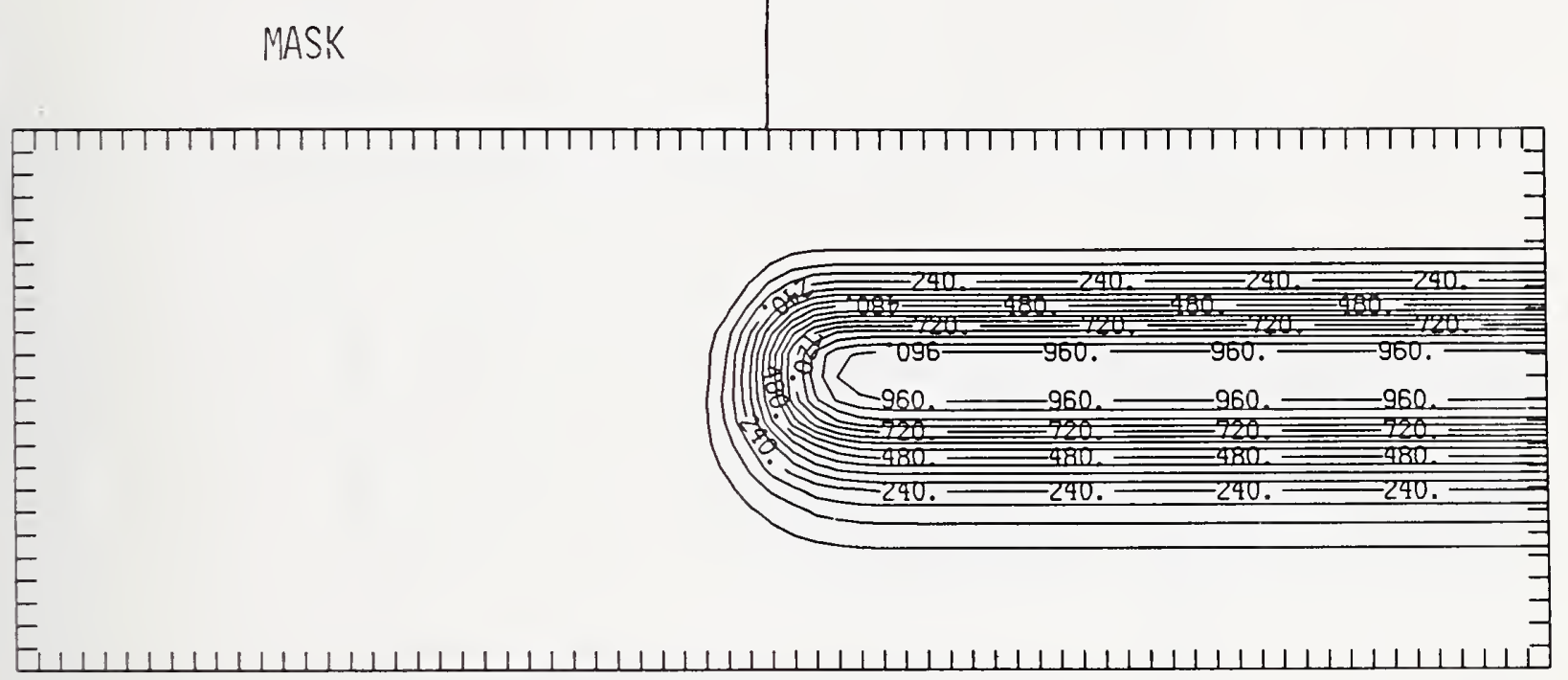

Figure Pt275-3 Two-dimensional distribution of $275 \mathrm{keV}$ platinum implanted past a mask edge. The length increment (distance between tick marks) is $0.01 \mu \mathrm{m}$ and the scale factor is 1000 .

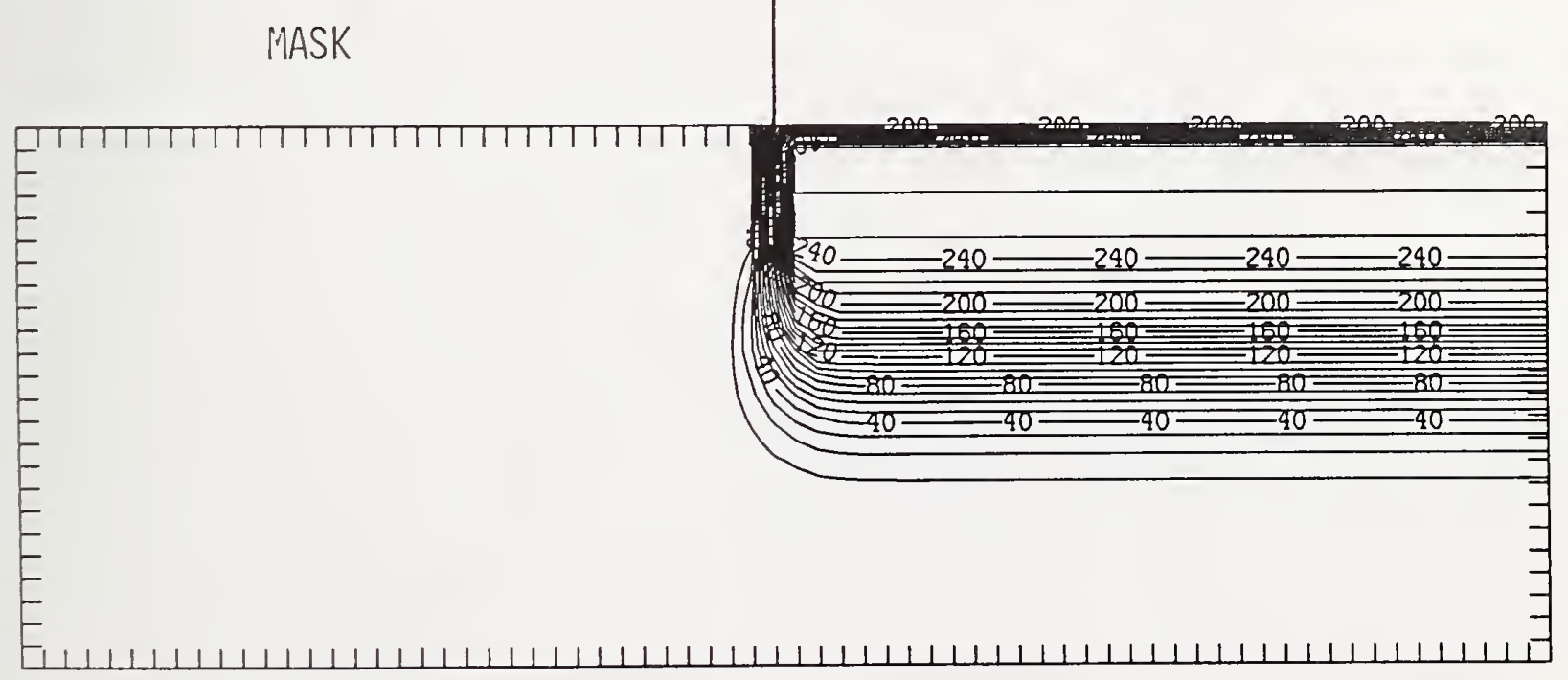

Figure Pt275-4 Two-dimensional distribution of Frenkel pairs created by $275 \mathrm{keV}$ platinum implanted past a mask edge. The length increment (distance between tick marks) is $0.01 \mu \mathrm{m}$ and the scale factor is 0.1 . 


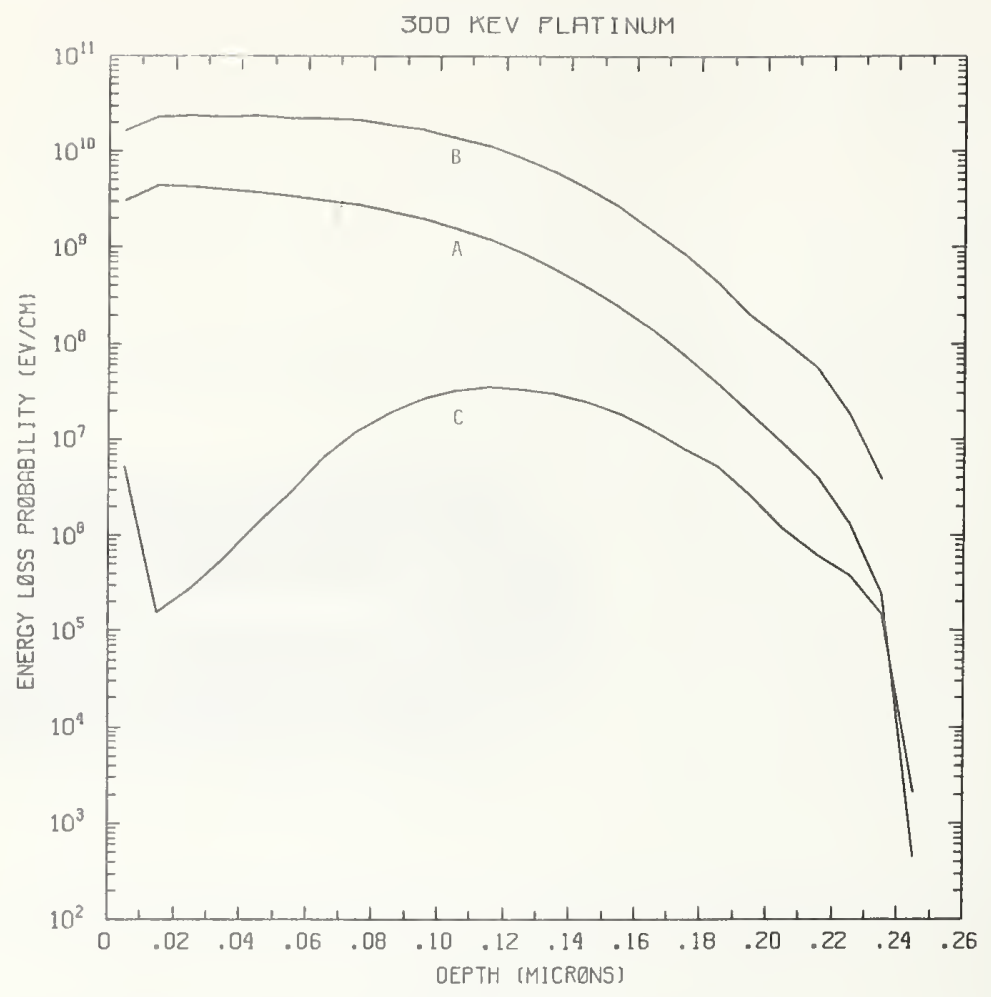

Figure Pt300-1 One-dimensional probability distributions for energy loss due to electronic excitation $(A)$, displacement damage(B), and phonon excitation (C) for $300 \mathrm{keV}$ platinum normally incident on a silicon target.

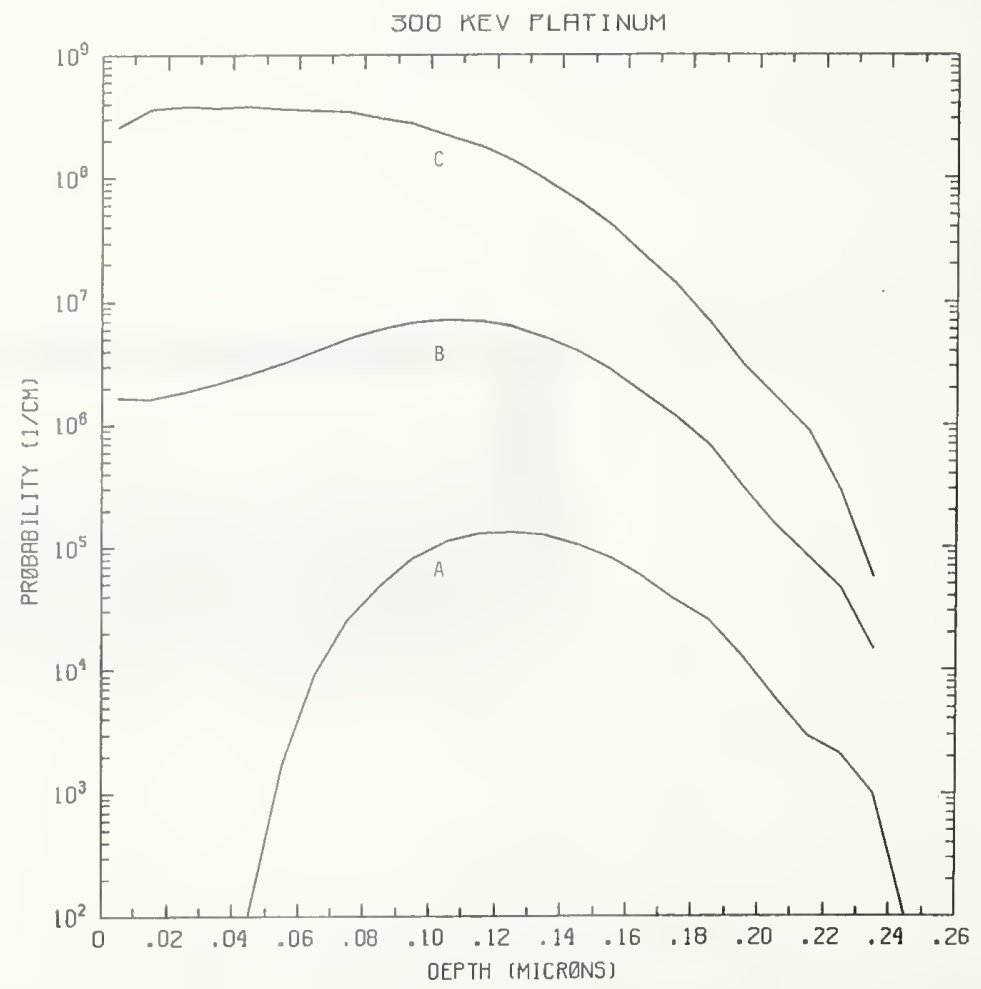

Figure Pt300-2 One-dimensional probability distributions for implanted platinum (A), primary silicon displacements (B), and Frenkel pairs (C) for $300 \mathrm{keV}$ platinum normally incident on a silicon target. 


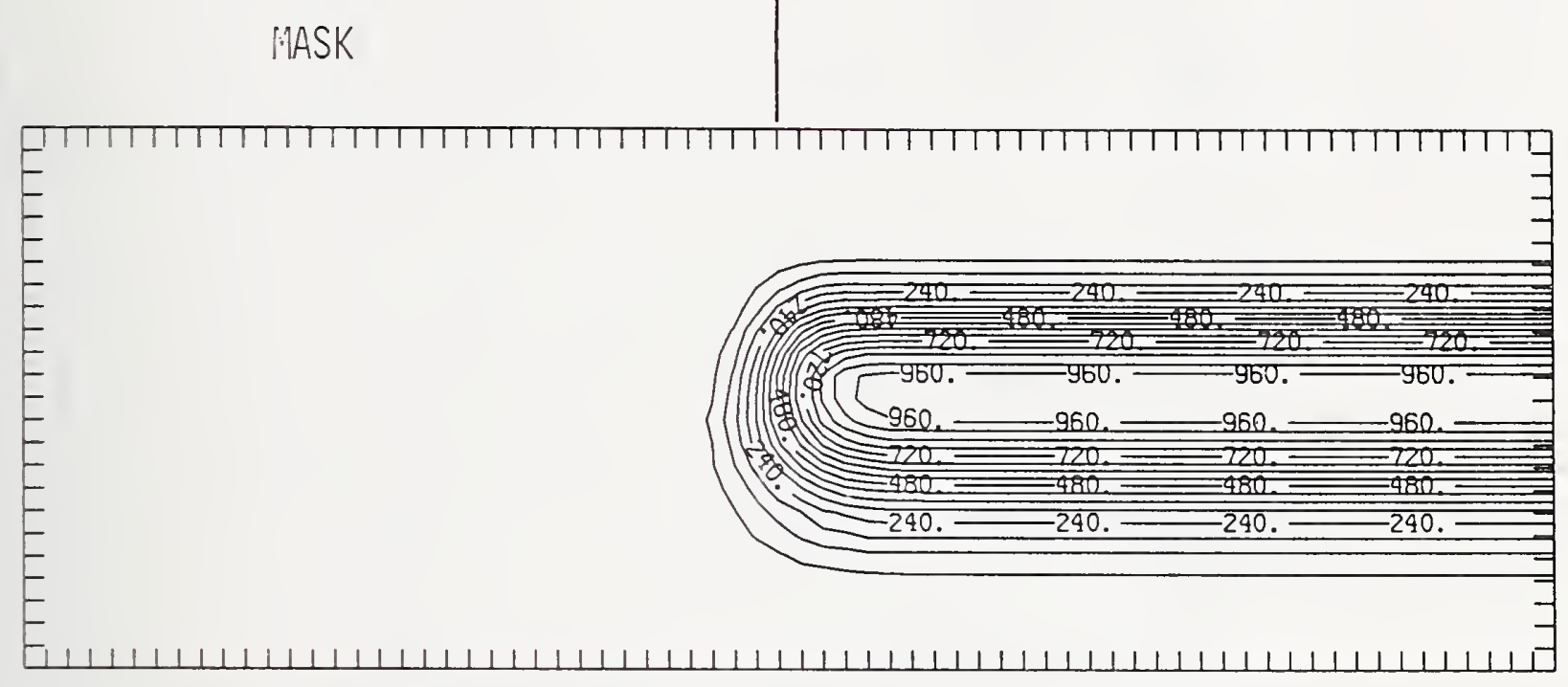

Figure Pt300-3 Two-dimensional distribution of $300 \mathrm{keV}$ platinum implanted past a mask edge. The length increment (distance between tick marks) is $0.01 \mu \mathrm{m}$ and the scale factor is 1000 .

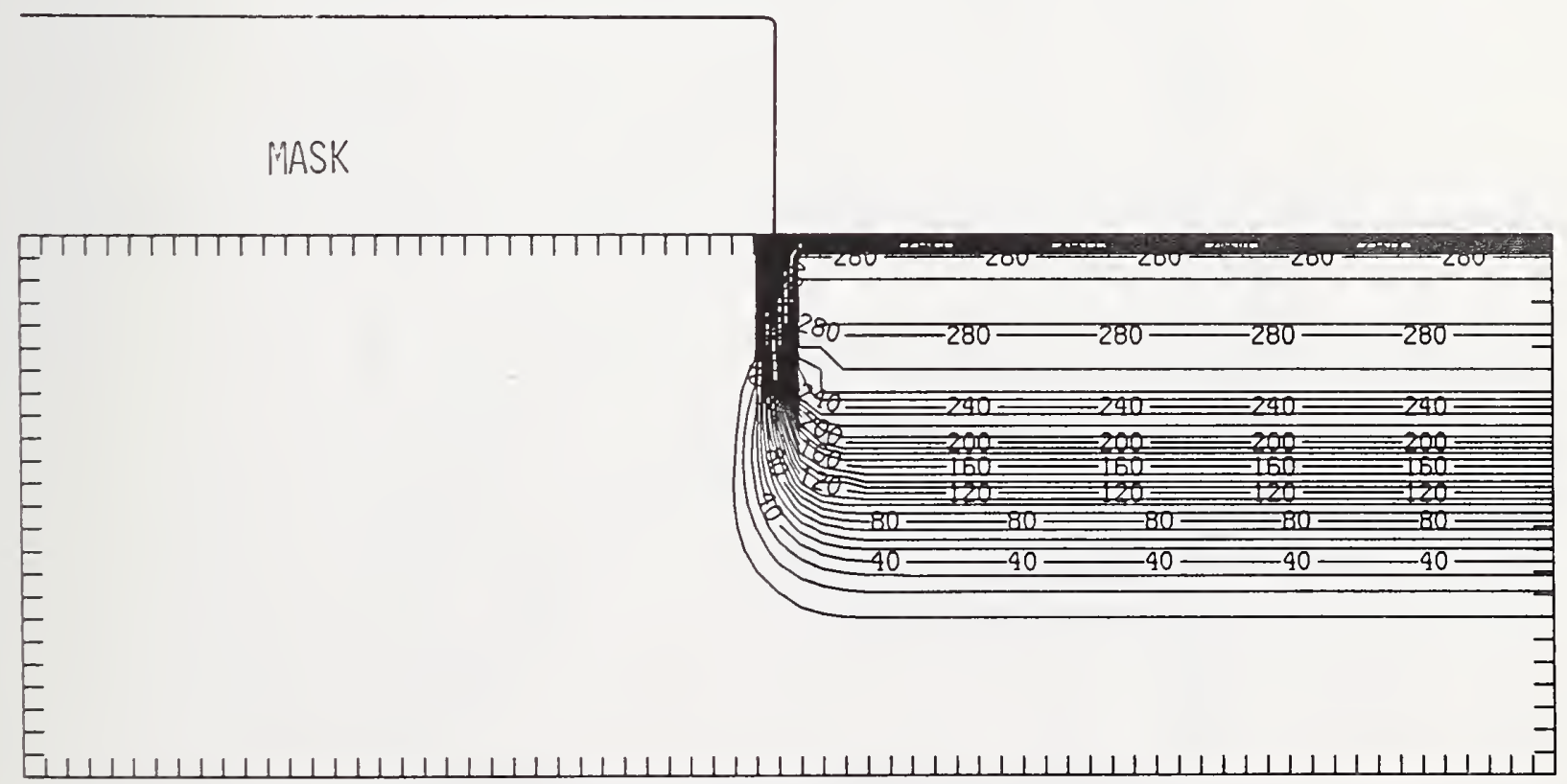

Figure Pt300-4 Two-dimensional distribution of Frenkel pairs created by $300 \mathrm{keV}$ platinum implanted past a mask edge. The length increment (distance between tick marks) is $0.01 \mu \mathrm{m}$ and the scale factor is 0.1 . 


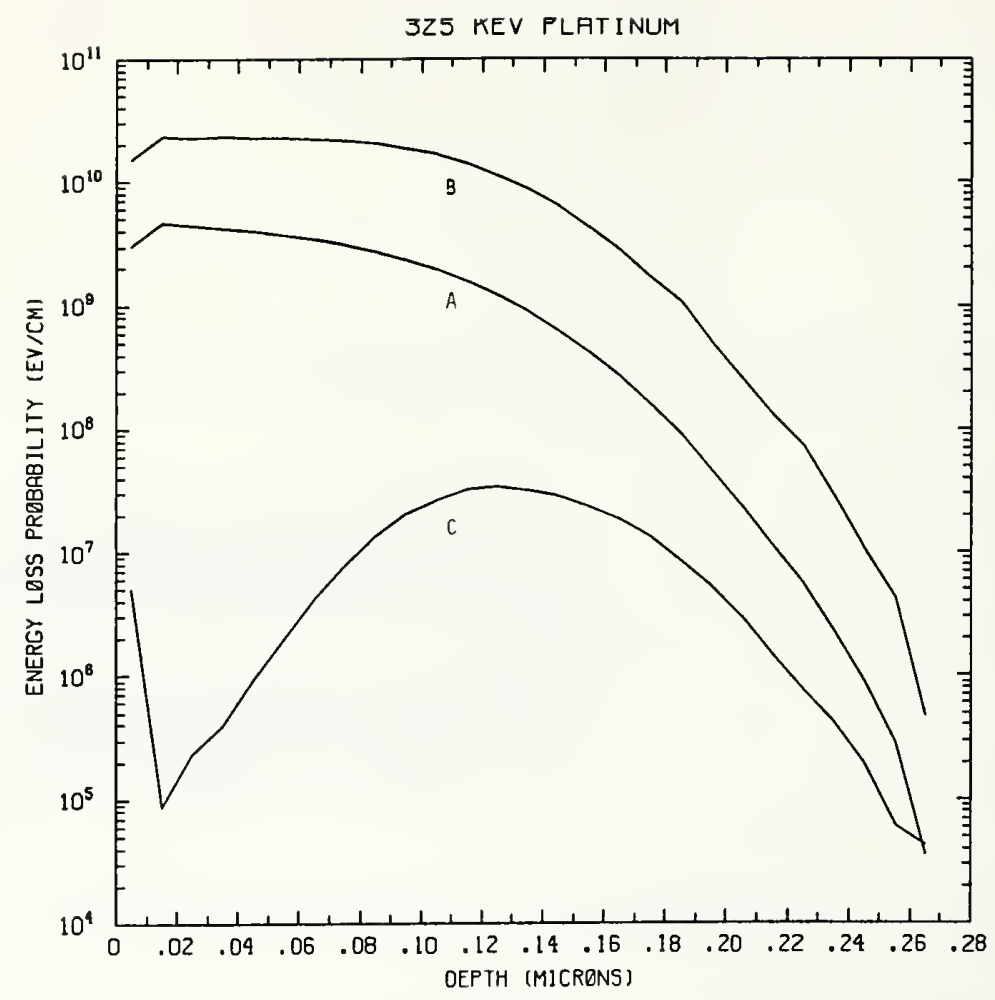

Figure Pt325-1 One-dimensional probability distributions for energy loss due to electronic excitation (A), displacement damage(B), and phonon excitation (C) for $325 \mathrm{keV}$ platinum normally incident on a silicon target.

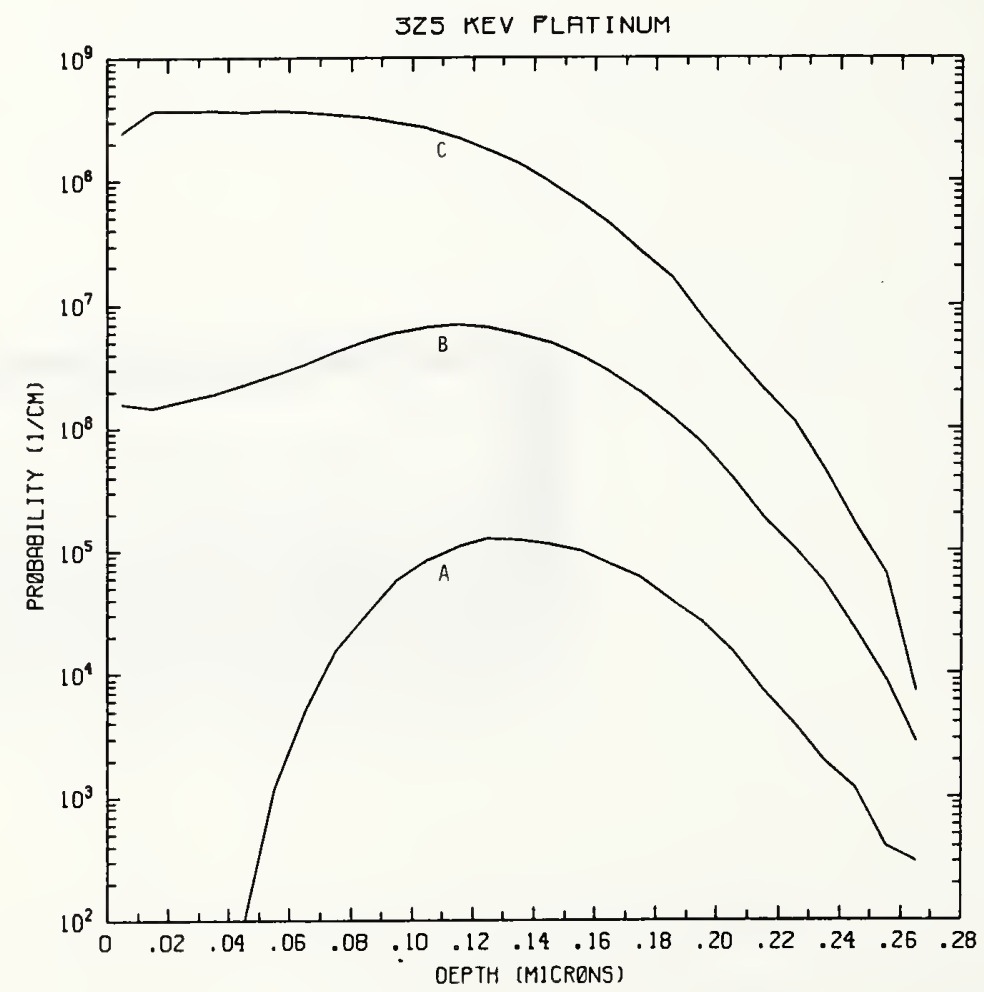

Figure Pt325-2 One-dimensional probability distributions for implanted platinum (A), primary silicon displacements (B), and Frenkel pairs (C) for $325 \mathrm{keV}$ platinum normally incident on a silicon target. 


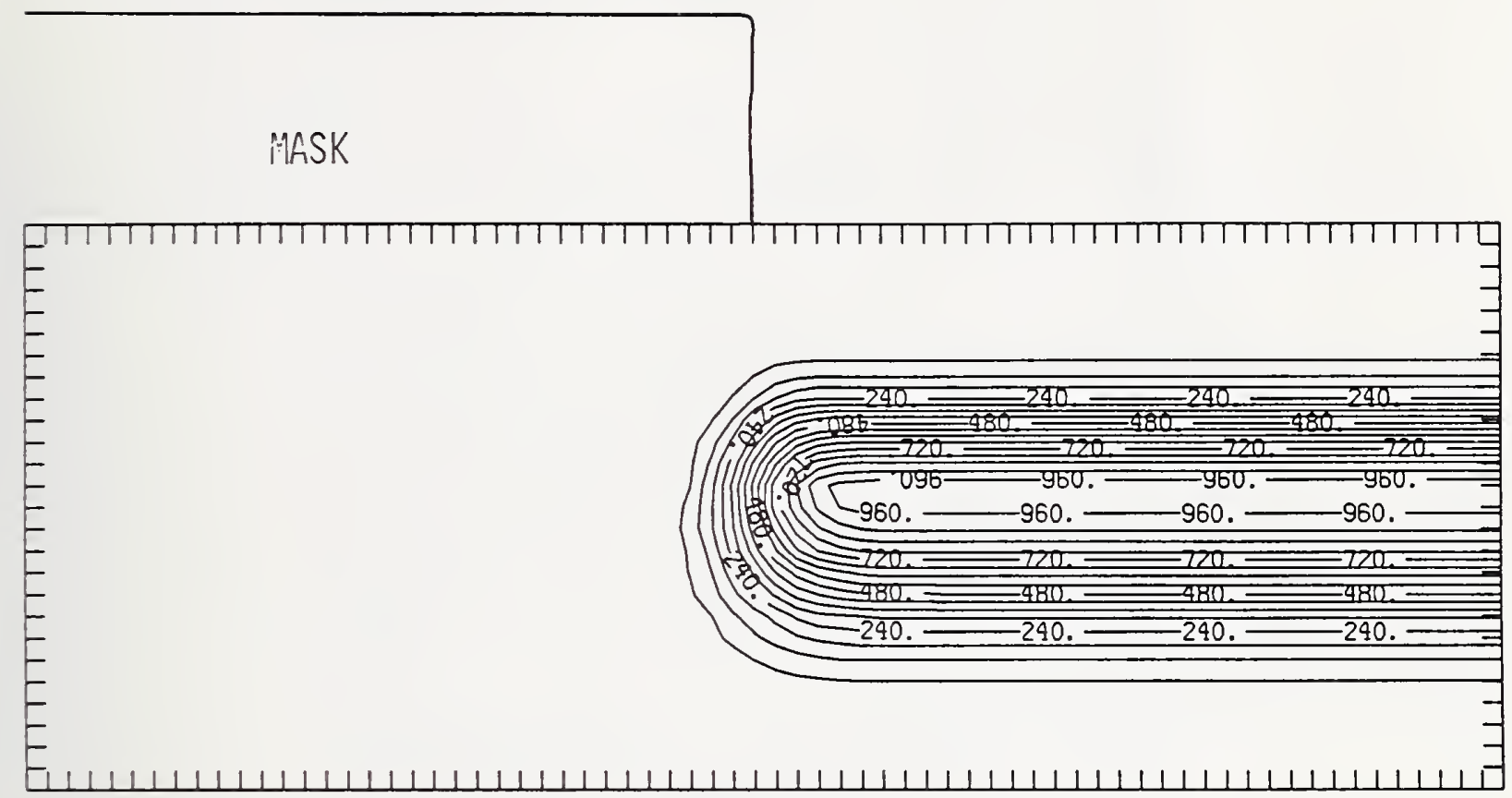

Figure Pt325-3 Two-dimensional distribution of $325 \mathrm{keV}$ platinum implanted past a mask edge. The length increment (distance between tick marks) is $0.01 \mu \mathrm{m}$ and the scale factor is 1000 .

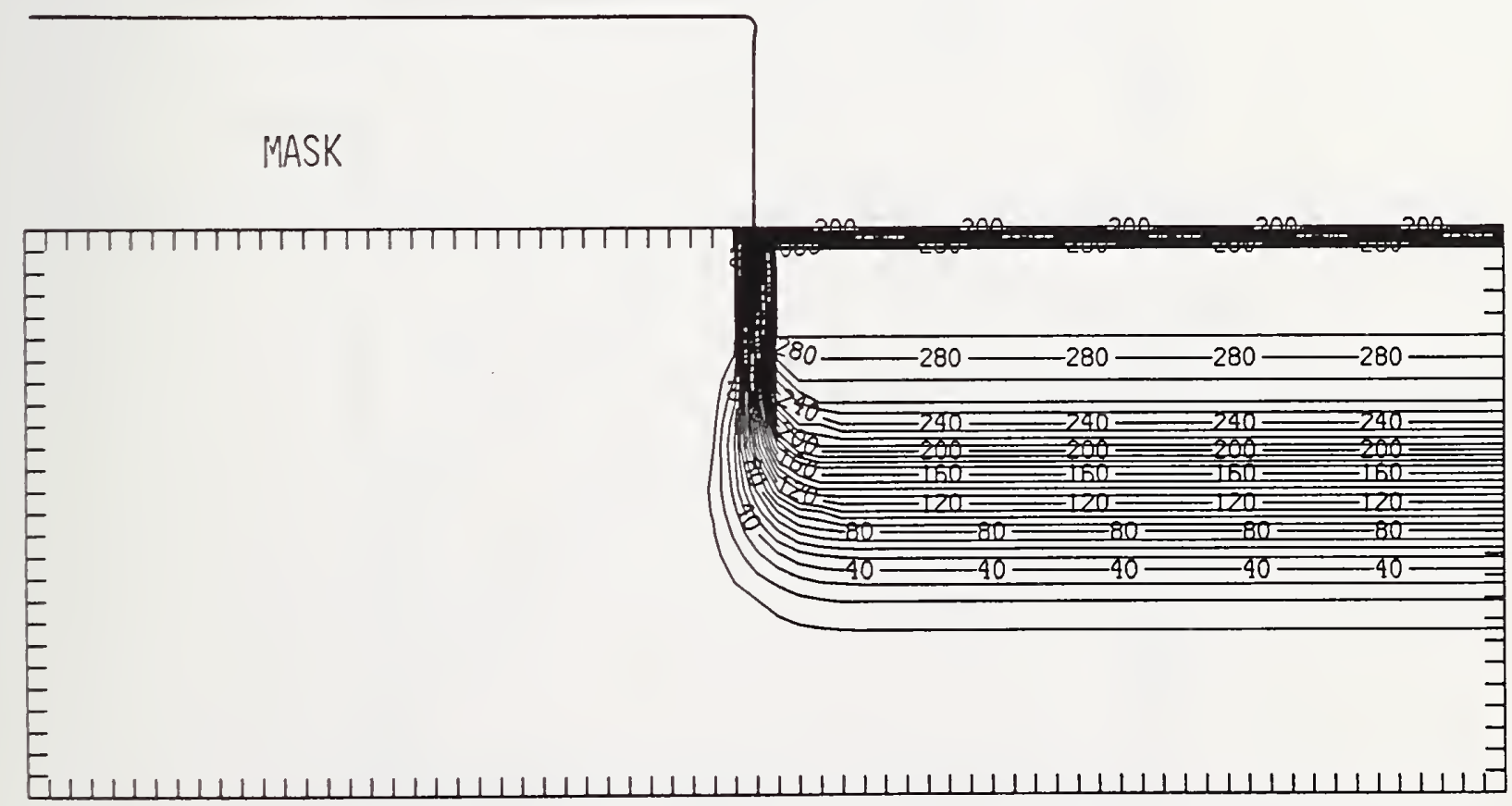

Figure Pt325-4 Two-dimensional distribution of Frenkel pairs created by $325 \mathrm{keV}$ platinum implanted past a mask edge. The length increment (distance between tick marks) is $0.01 \mu \mathrm{m}$ and the scale factor is 0.1 . 


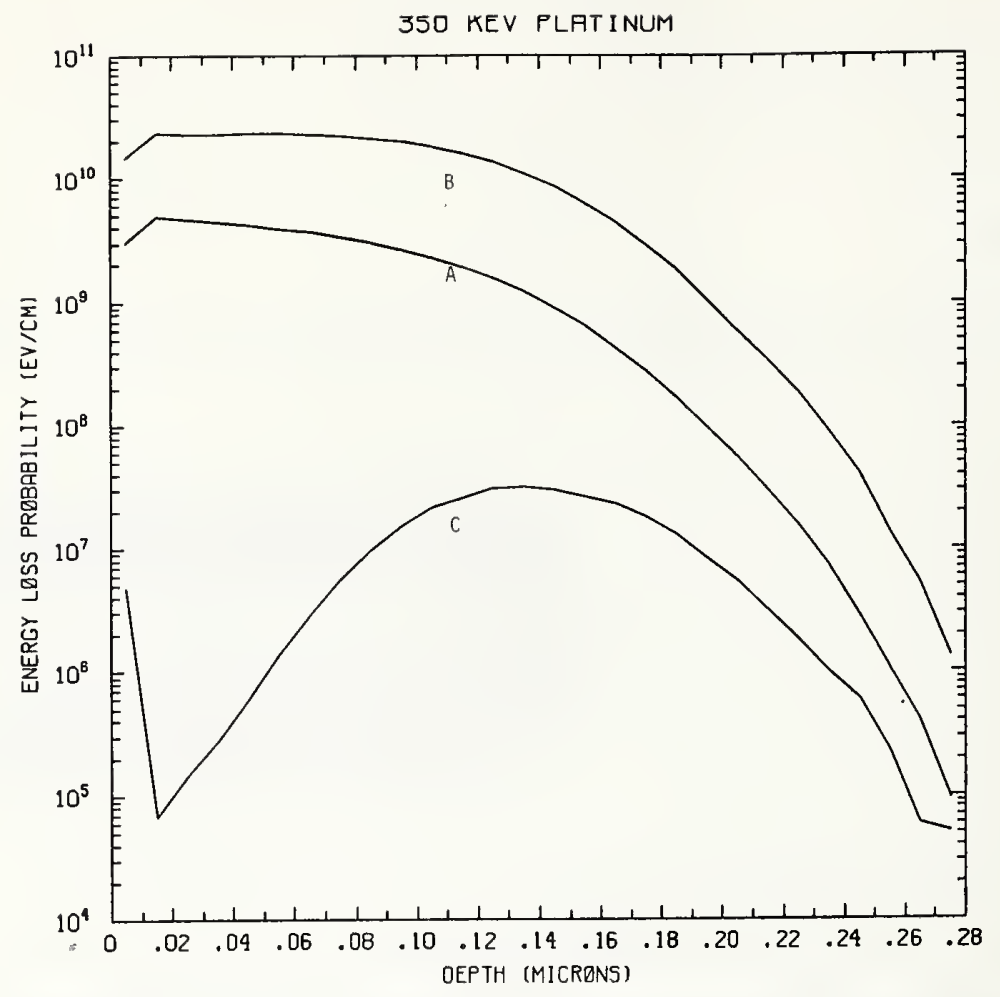

Figure Pt350-1 One-dimensional probability distributions for energy loss due to electronic excitation (A), displacement damage(B), and phonon excitation (C) for $350 \mathrm{keV}$ platinum normally incident on a silicon target.

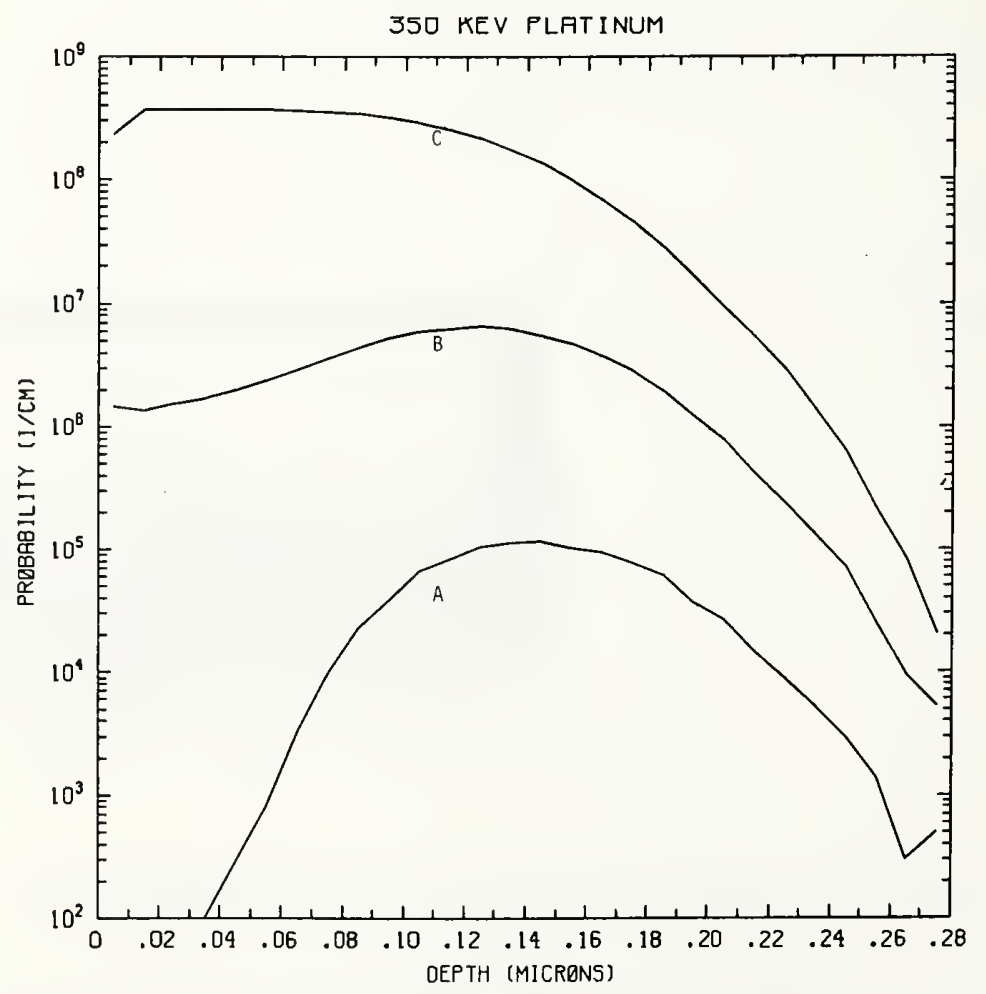

Figure Pt350-2 One-dimensional probability distributions for implanted platinum (A), primary silicon displacements (B), and Frenkel pairs (C) for $350 \mathrm{keV}$ platinum normally incident on a silicon target. 
MASK

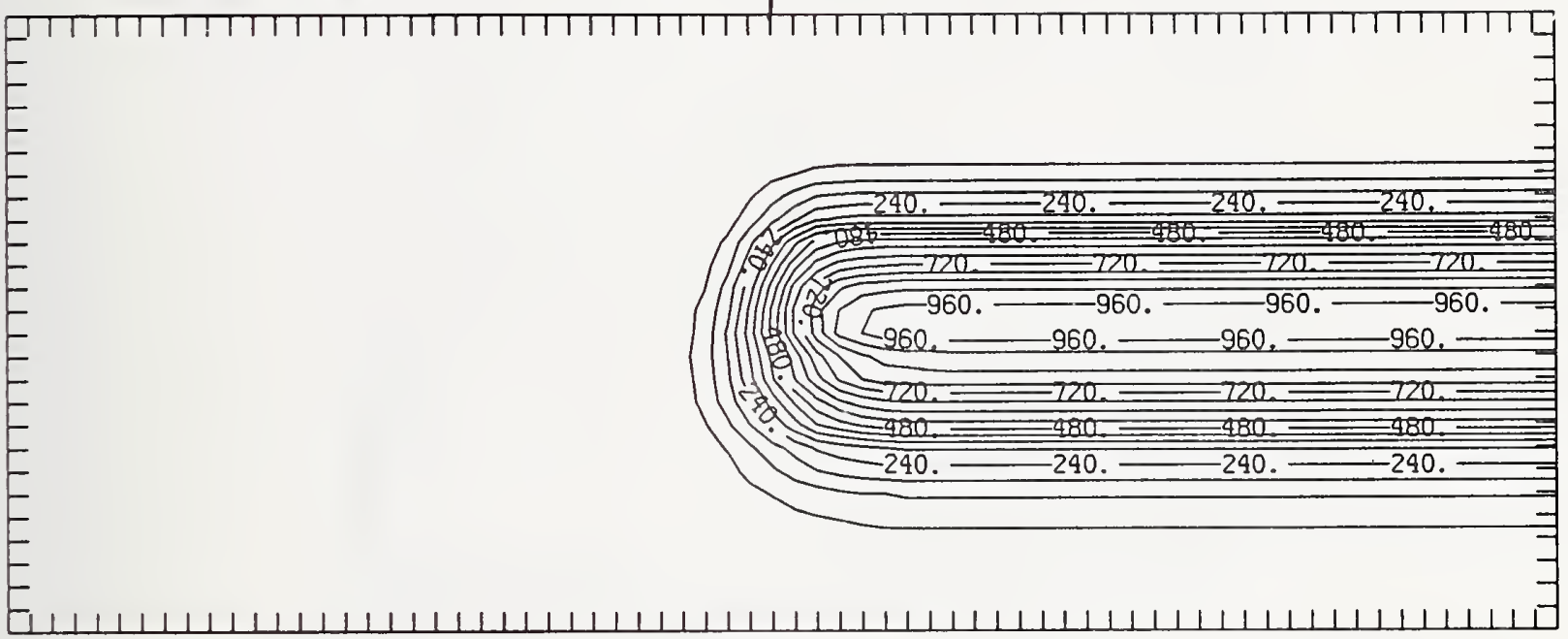

Figure Pt350-3 Two-dimensional distribution of $350 \mathrm{keV}$ platinum implanted past a mask edge. The length increment (distance between tick marks) is $0.01 \mu \mathrm{m}$ and the scale factor is 1000 .

MASK

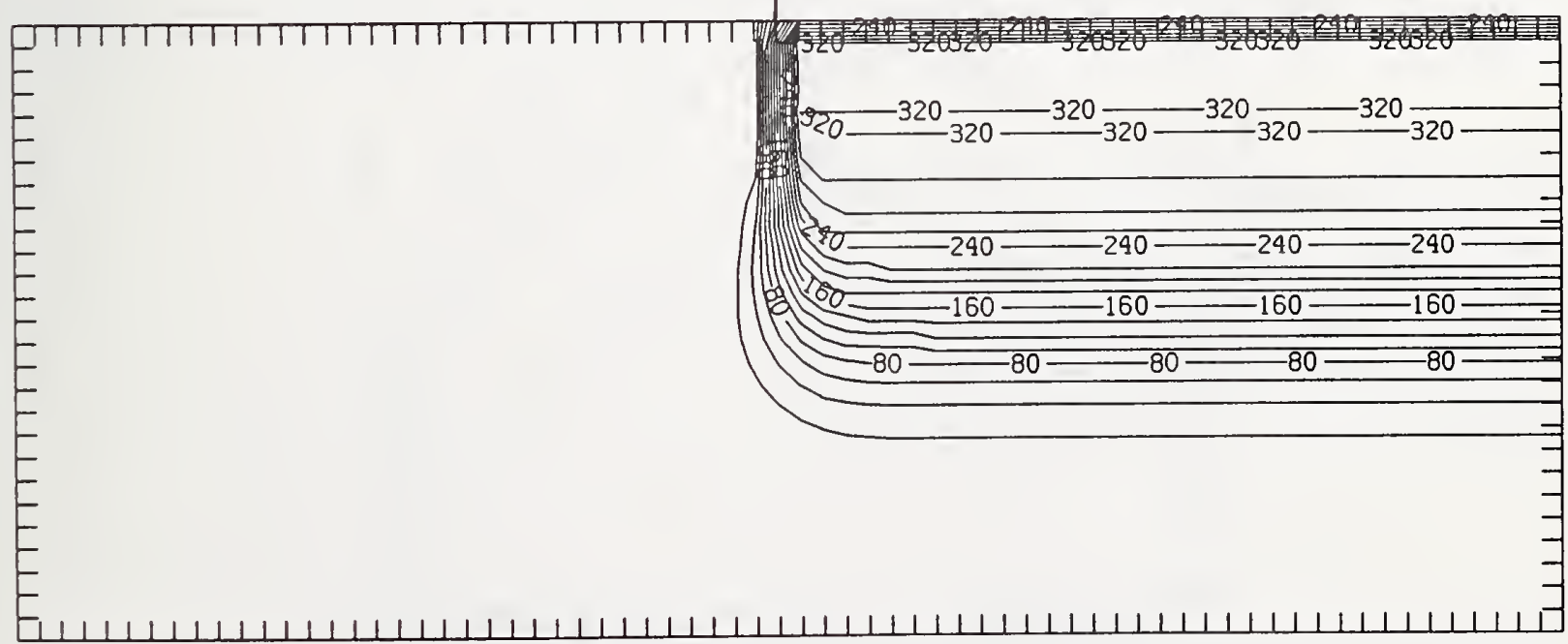

Figure P t350-4 Two-dimensional distribution of Frenkel pairs created by $350 \mathrm{keV}$ platinum implanted past a mask edge. The length increment (distance between tick marks) is $0.01 \mu \mathrm{m}$ and the scale factor is 0.1 . 


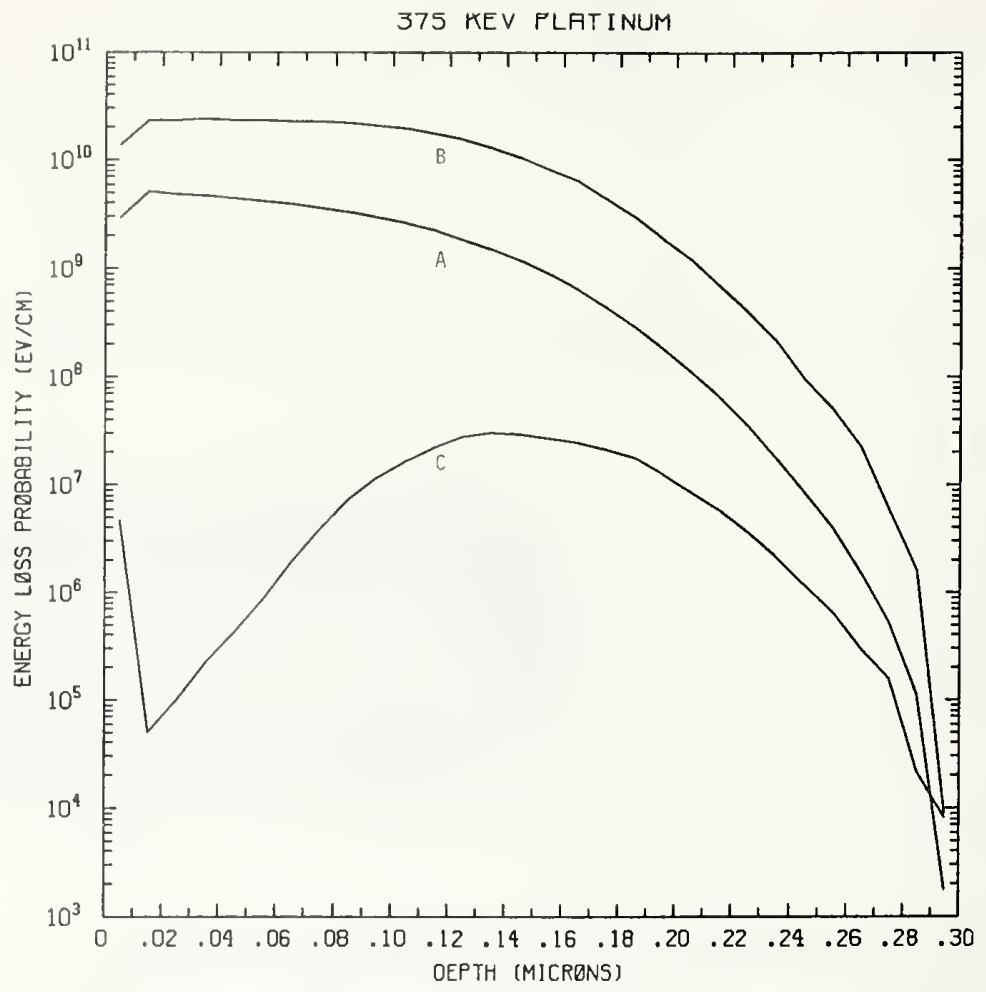

Figure Pt375-1. One-dimensional probability distributions for energy loss due to electronic excitation (A), displacement damage(B), and phonon excitation (C) for $375 \mathrm{keV}$ platinum normally incident on a silicon target.

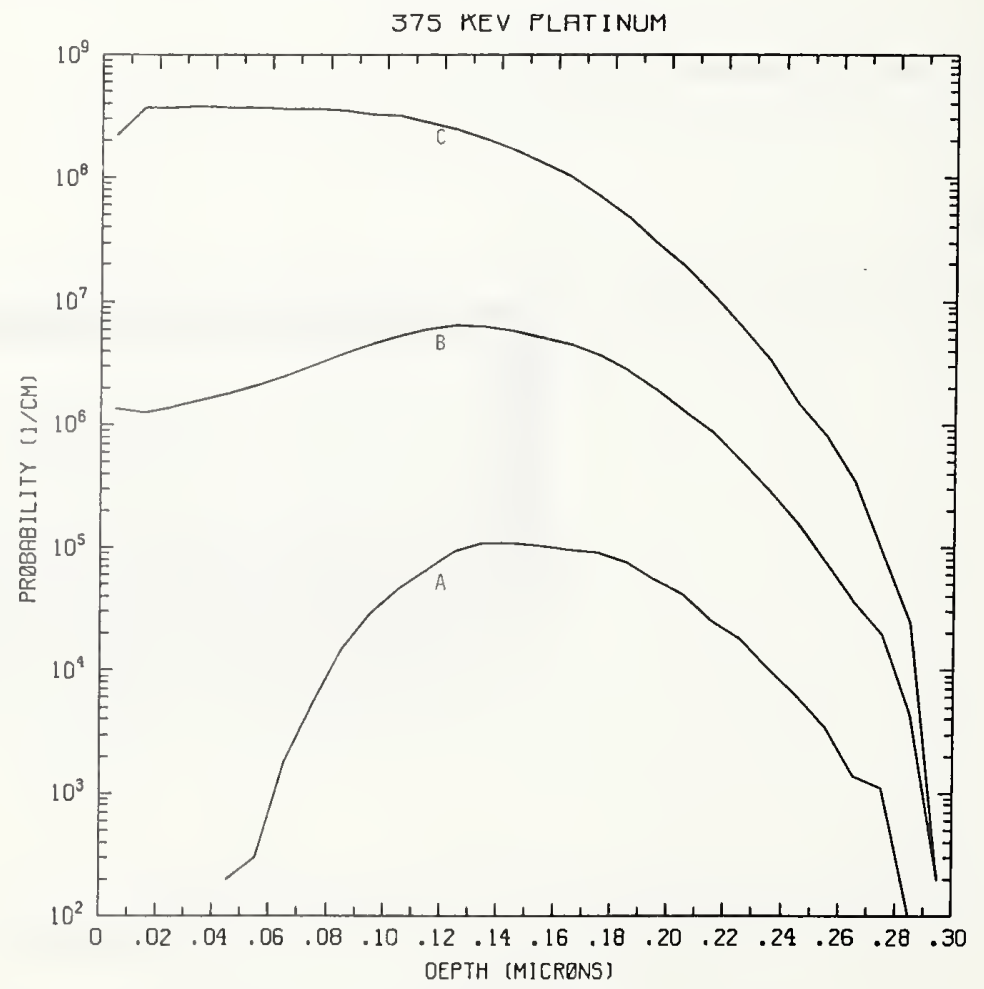

Figure Pt375-2 One-dimensional probability distributions for implanted platinum (A), primary silicon displacements (B), and Frenkel pairs (C) for $375 \mathrm{keV}$ platinum normally incident on a silicon target. 


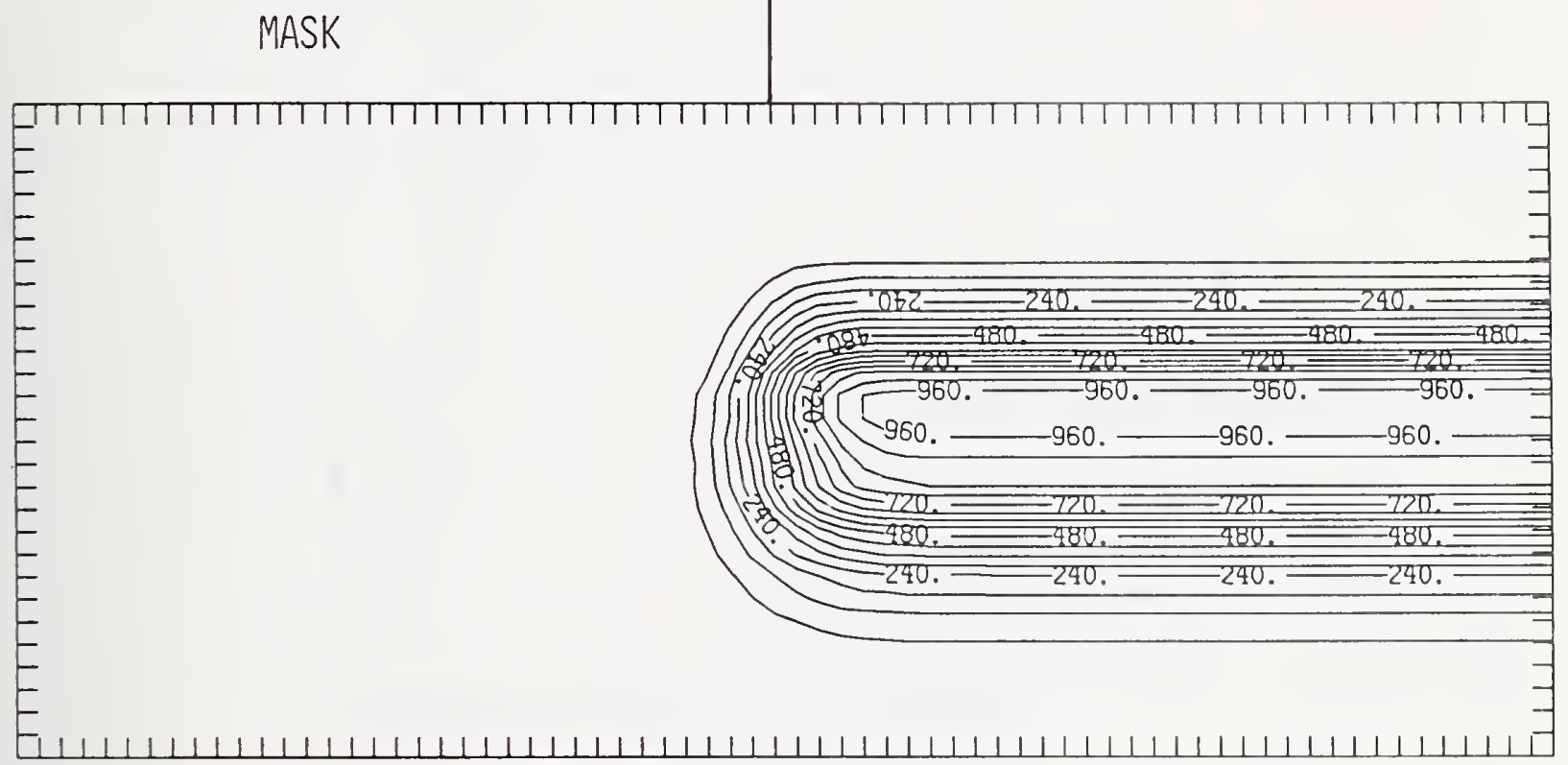

Figure Pt375-3 Two-dimensional distribution of $375 \mathrm{keV}$ platinum implanted past a mask edge. The length increment (distance between tick marks) is $0.01 \mu \mathrm{m}$ and the scale factor is 1000 .

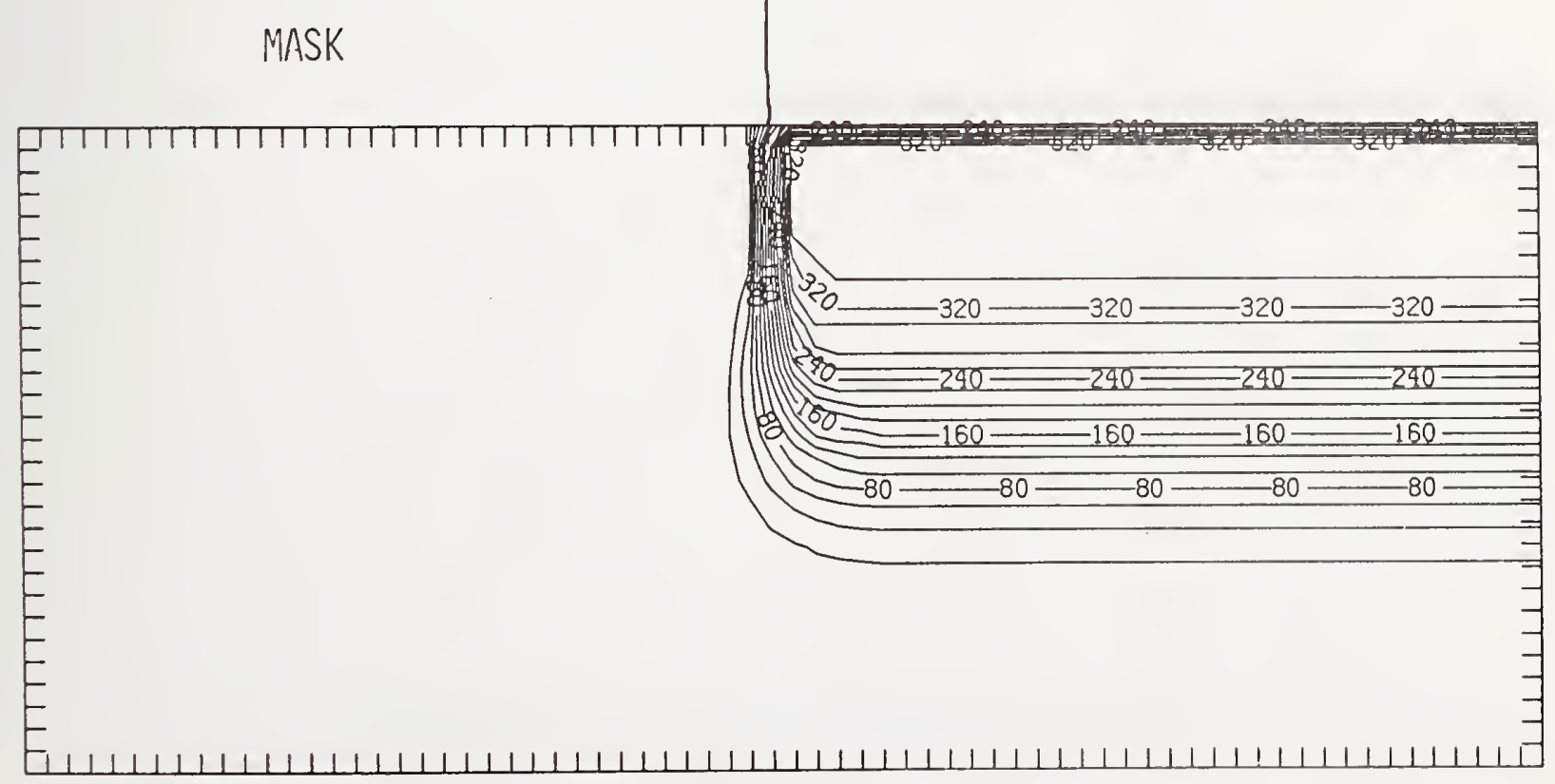

Figure Pt375-4 Two-dimensional distribution of Frenkel pairs created by $375 \mathrm{keV}$ platinum implanted past a mask edge. The length increment (distance between tick marks) is $0.01 \mu \mathrm{m}$ and the scale factor is 0.1 . 


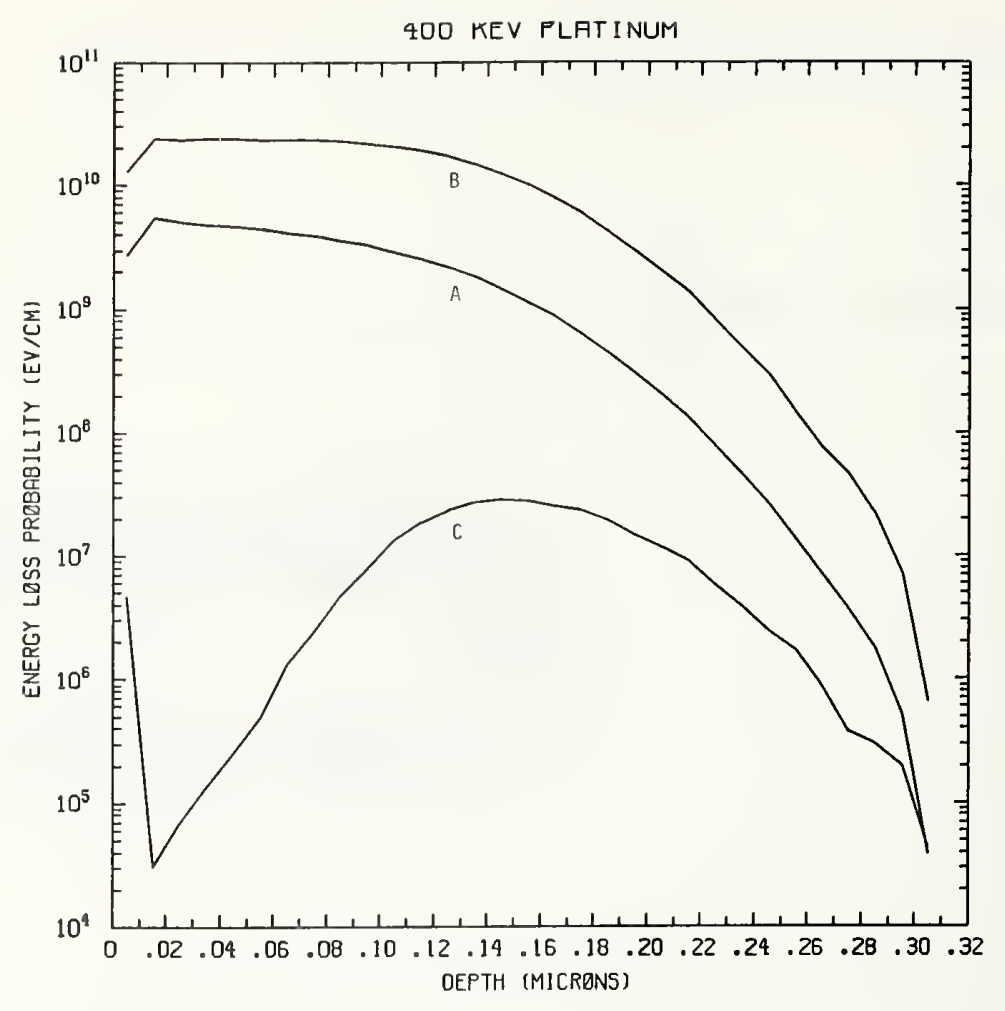

Figure Pt400-1 One-dimensional probability distributions for energy loss due to electronic excitation (A), displacement damage(B), and phonon excitation (C) for $400 \mathrm{keV}$ platinum normally incident on a silicon target.

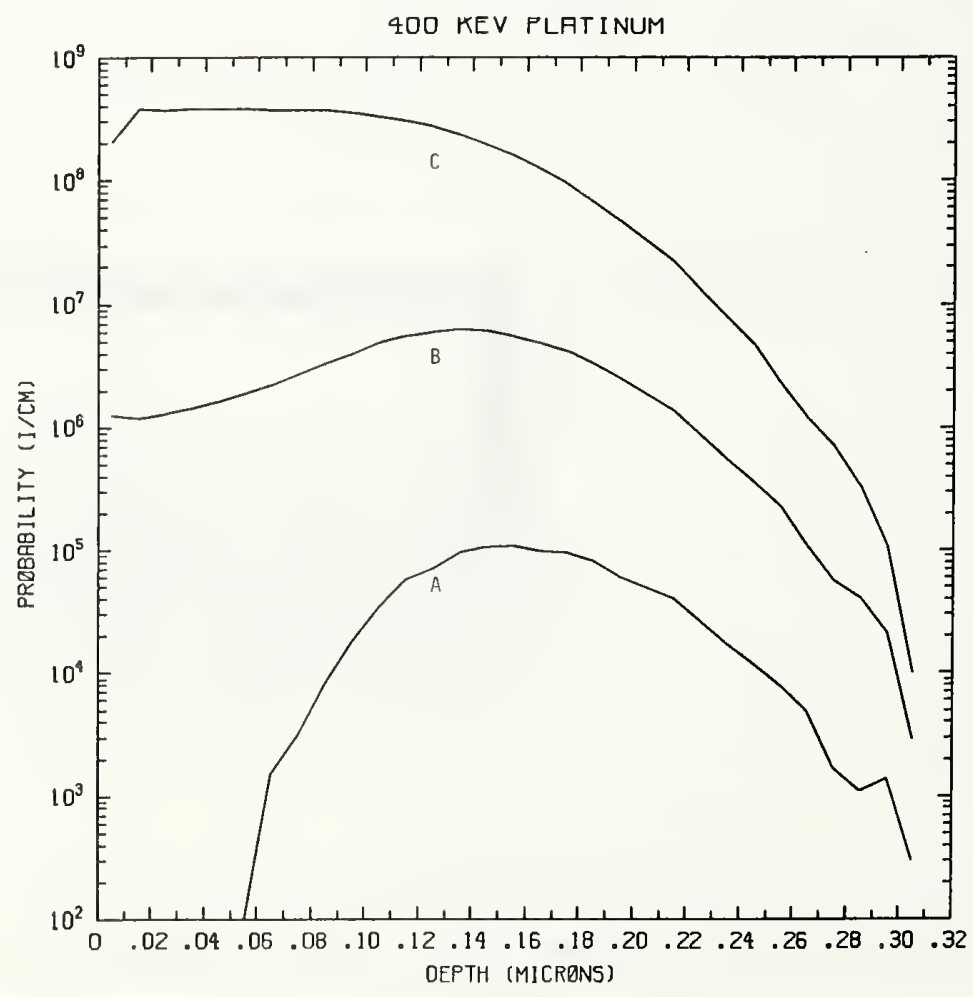

Figure Pt400-2 One-dimensional probability distributions for implanted platinum (A), primary silicon displacements (B), and Frenkel pairs (C) for $400 \mathrm{keV}$ platinum normally incident on a silicon target. 


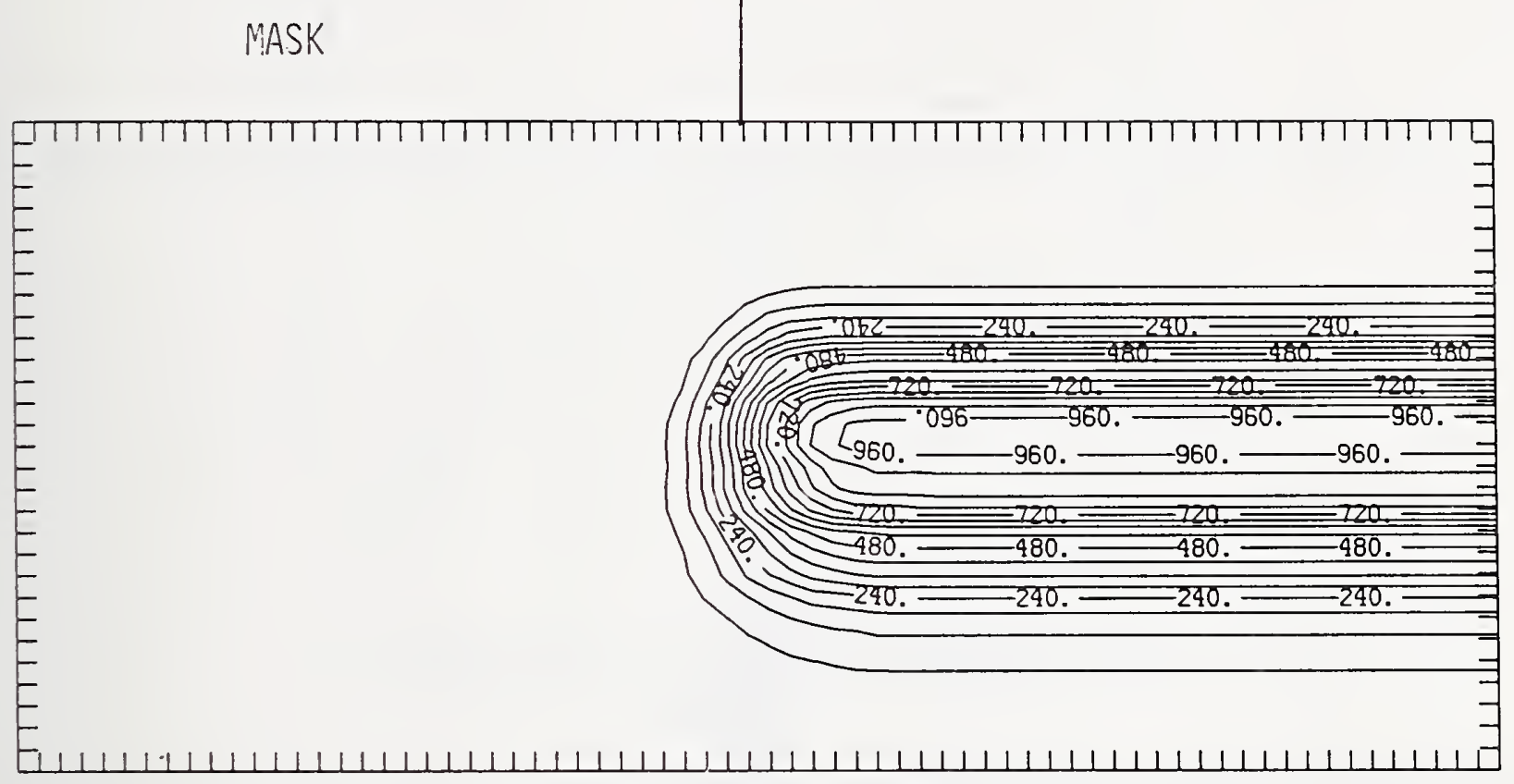

Figure Pt400-3 Two-dimensional distribution of $400 \mathrm{keV}$ platinum implanted past a mask edge. The length increment (distance between tick marks) is $0.01 \mu \mathrm{m}$ and the scale factor is 1000 .

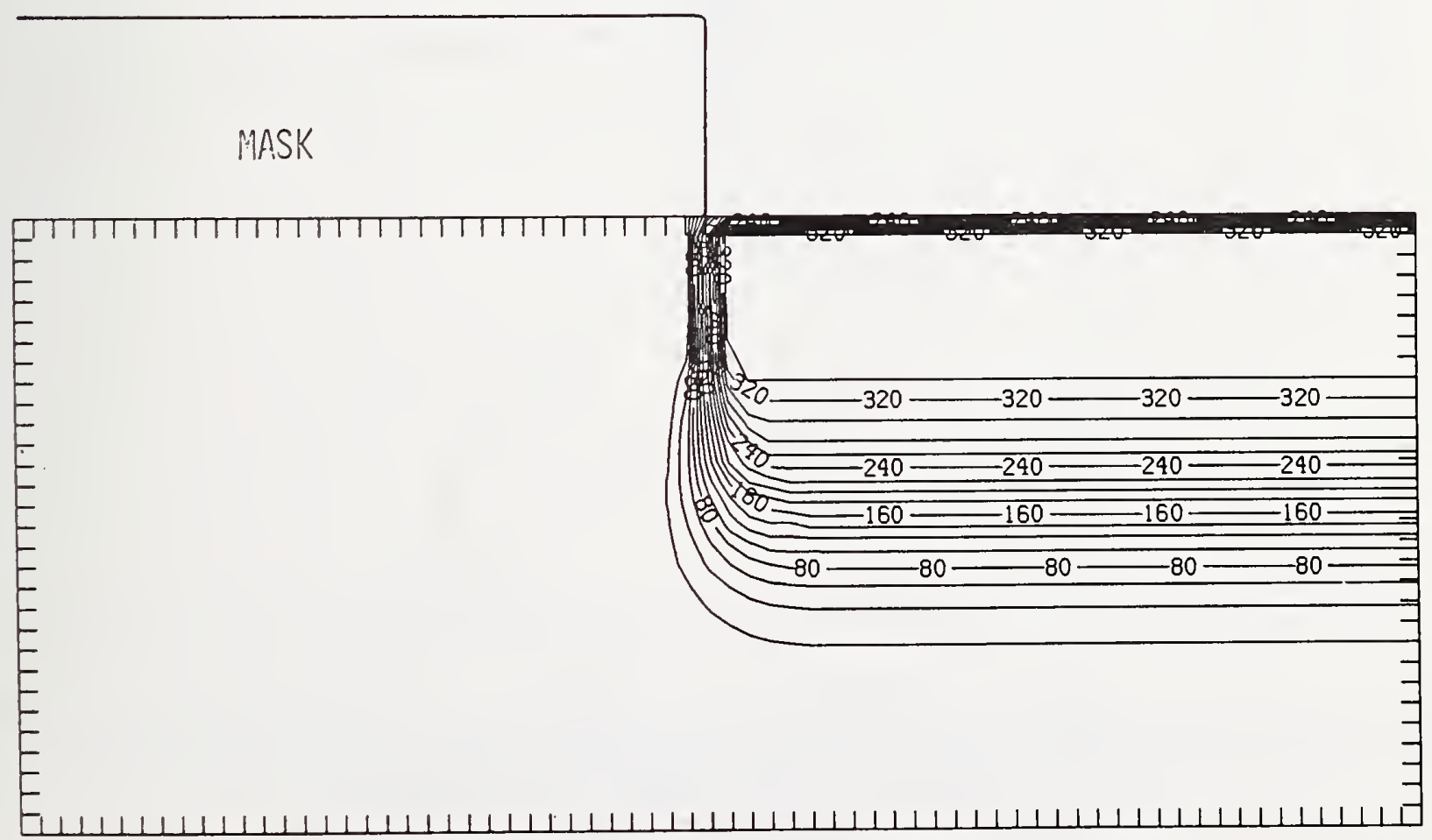

Figure Pt400-4 Two-dimensional distribution of Frenkel pairs created by $400 \mathrm{keV}$ platinum implanted past a mask edge. The length increment (distance between tick marks) is $0.01 \mu \mathrm{m}$ and the scale factor is 0.1 . 


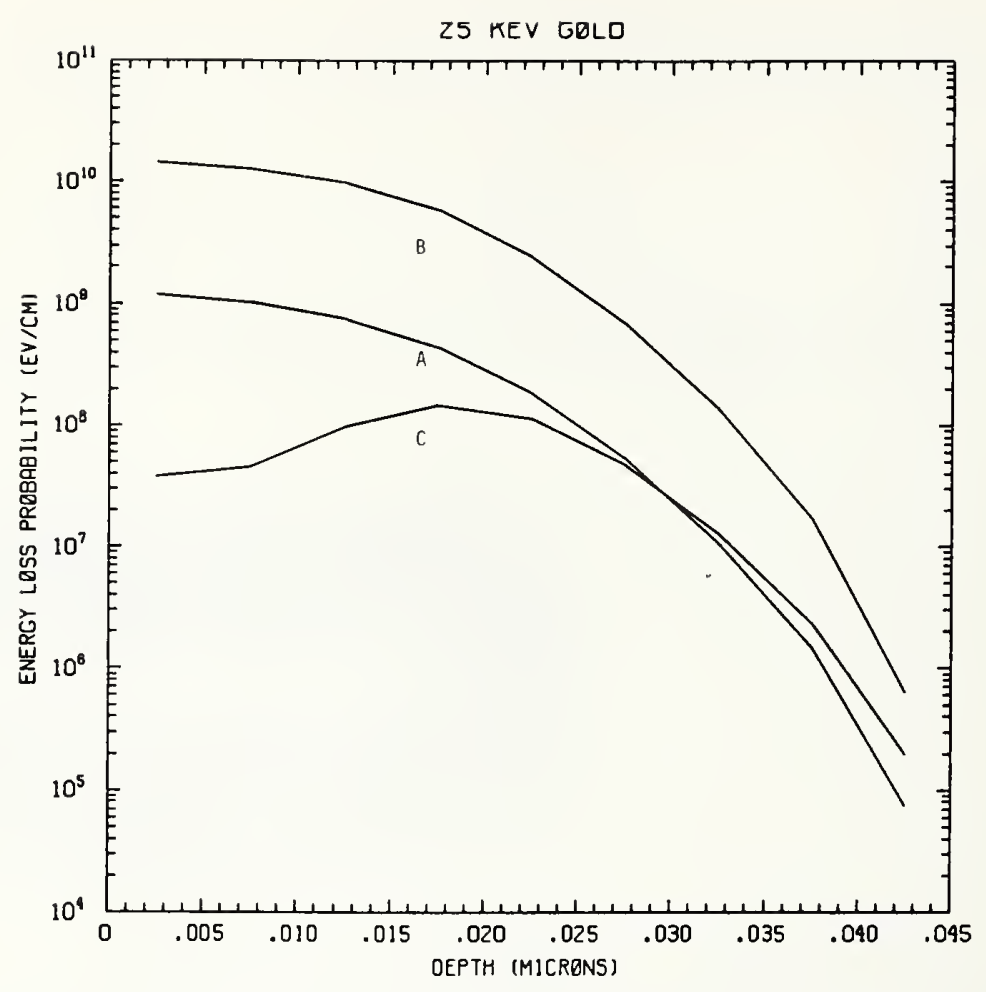

Figure Au25-1 One-dimensional probability distributions for energy loss due to electronic excitation (A), displacement damage(B), and phonon excitation (C) for $25 \mathrm{keV}$ gold normally incident on a silicon target.

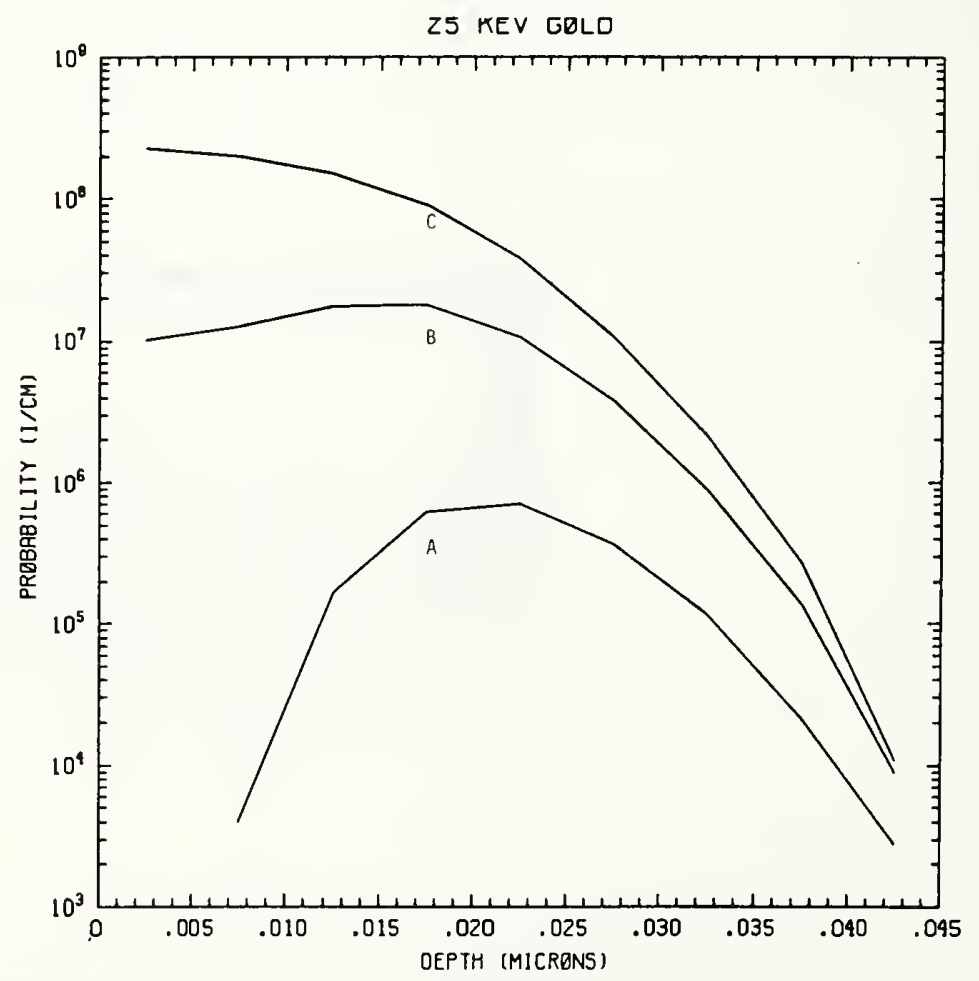

Figure Au25-2 One-dimensional probability distributions for implanted gold (A), primary silicon displacements (B), and Frenkel pairs (C) for $25 \mathrm{keV}$ gold normally incident on a silicon target. 


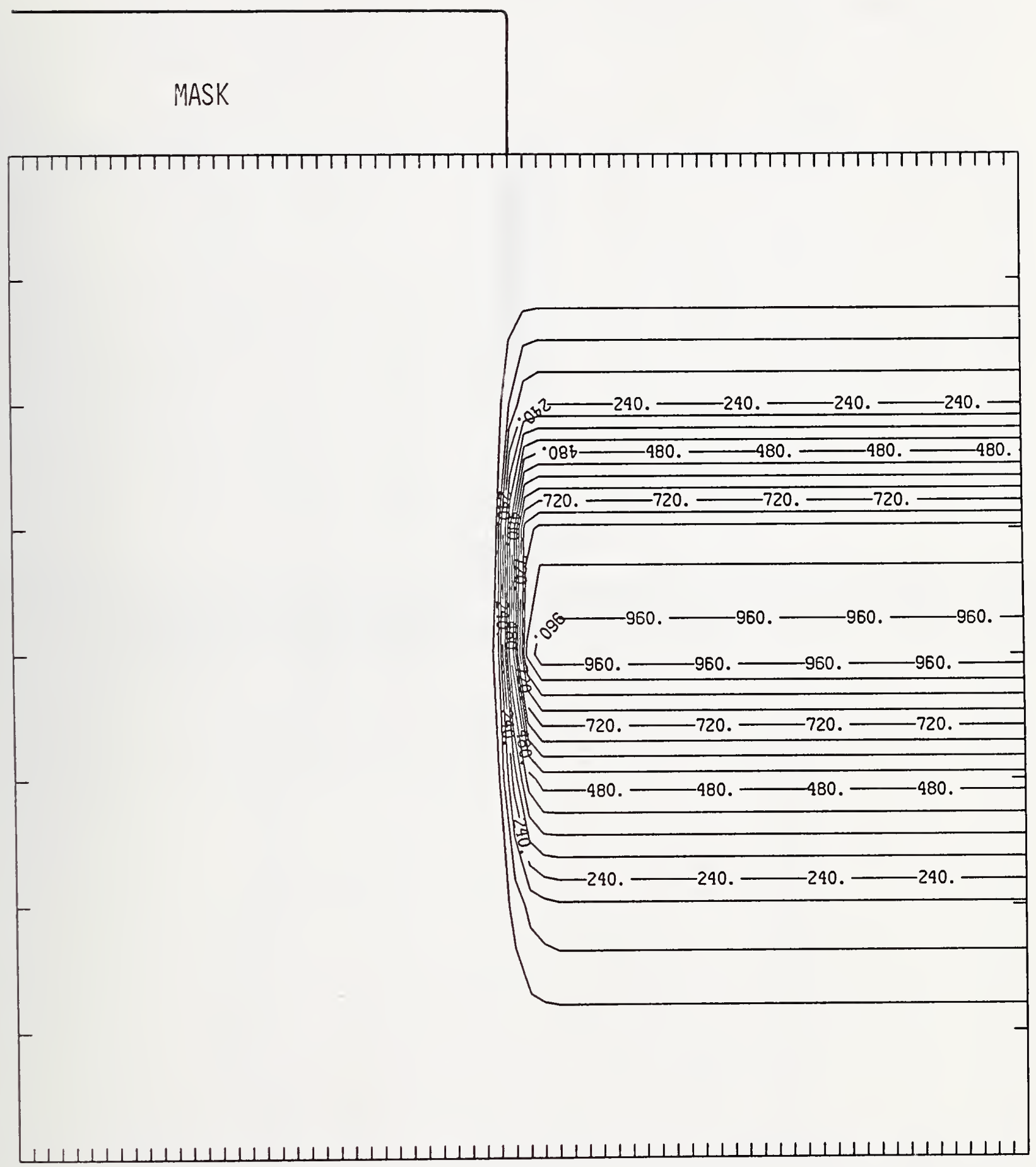

Figure Au25-3 Two-dimensional distribution of $25 \mathrm{keV}$ gold implanted past a mask edge. The length increment (distance between tick marks) is $0.005 \mu \mathrm{m}$ and the scale factor is 1000 . 
MASK

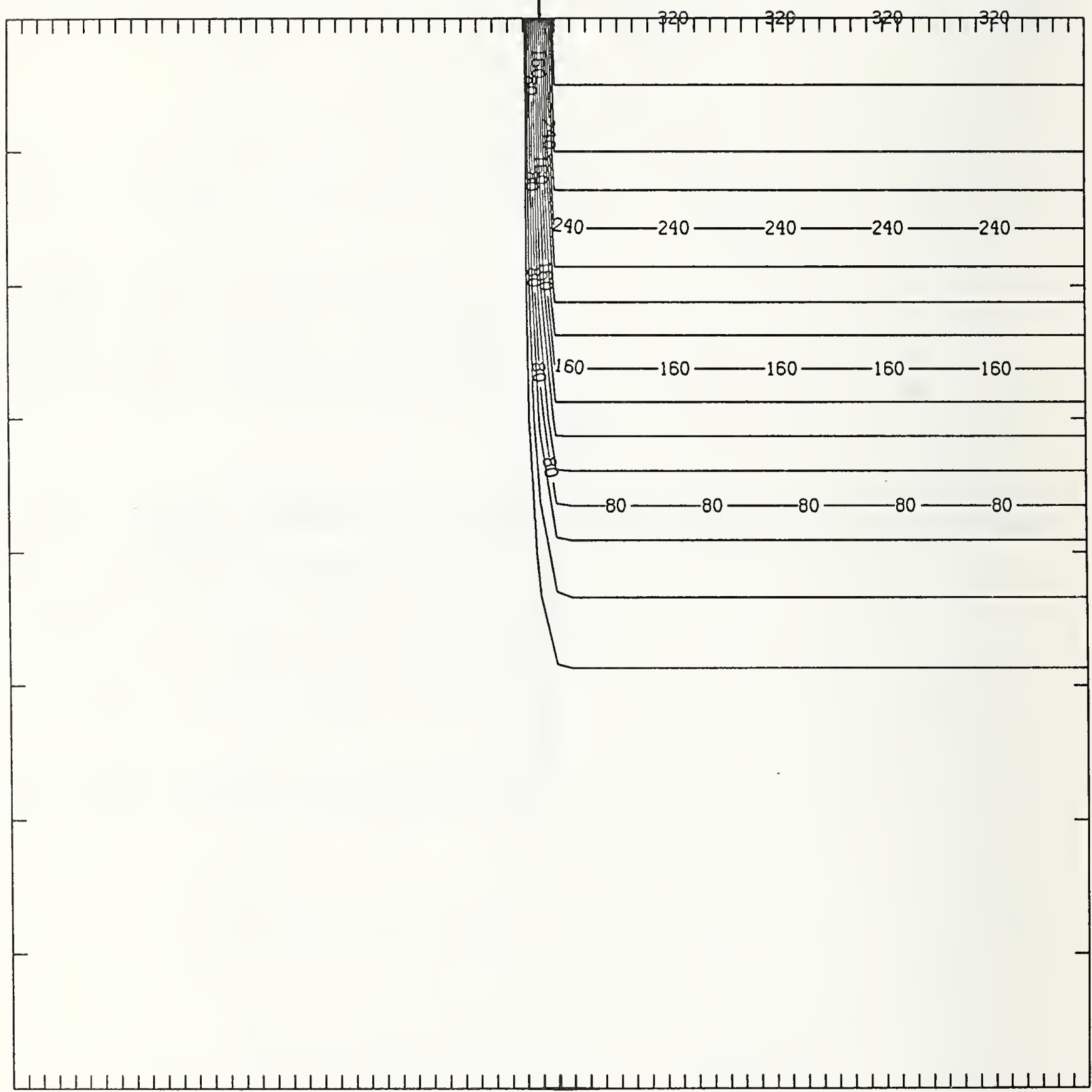

Figure Au25-4 Two-dimensional distribution of Frenkel pairs created by $25 \mathrm{keV}$ gold implanted past a mask edge. The length increment (distance between tick marks) is $0.005 \mu \mathrm{m}$ and the scale factor is 1 . 


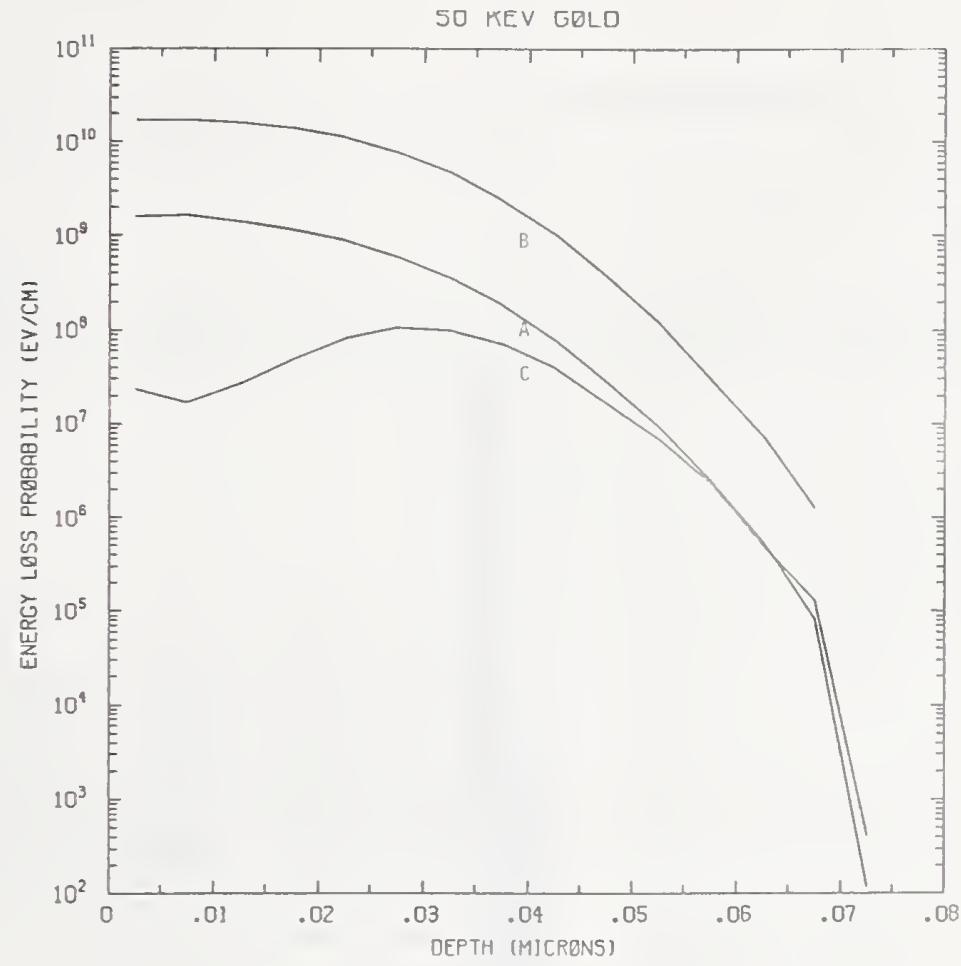

Figure Au50-1 One-dimensional probability distributions for energy loss due to electronic excitation (A), displacement damage(B), and phonon excitation (C) for $50 \mathrm{keV}$ gold normally incident on a silicon target.

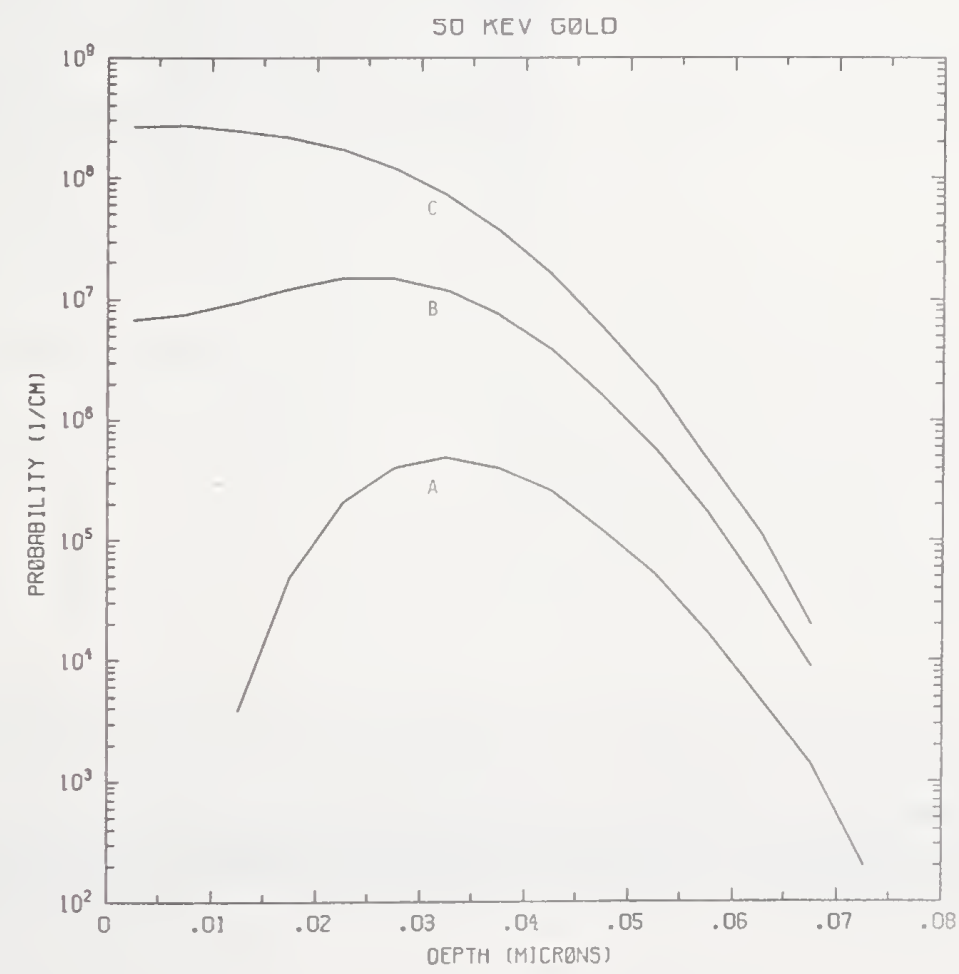

Figure Au50-2 One-dimensional probability distributions for implanted gold (A), primary silicon displacements (B), and Frenkel pairs (C) for $50 \mathrm{keV}$ gold normally incident on a silicon target. 
MASK

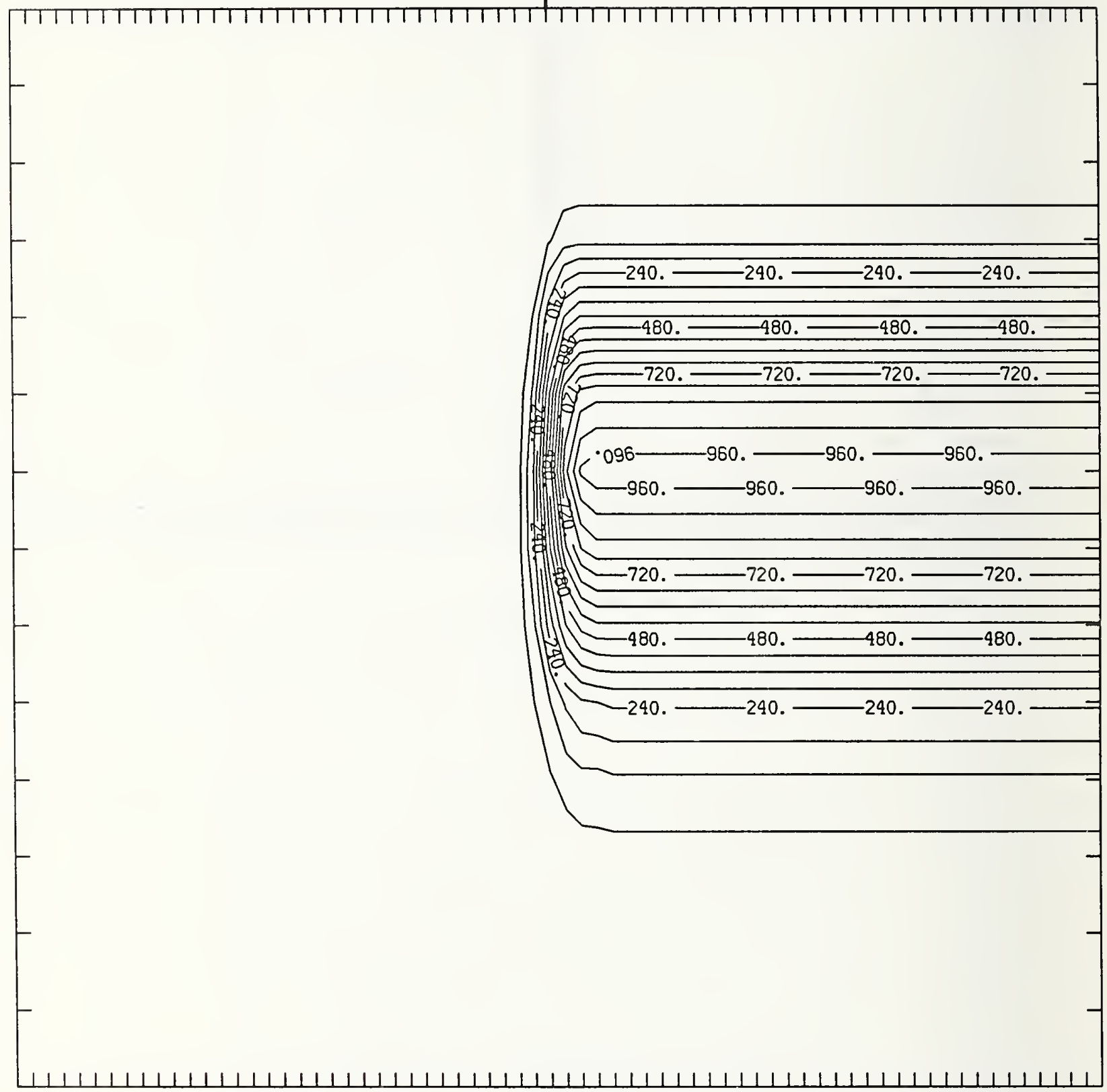

Figure Au50-3 Two-dimensional distribution of $50 \mathrm{keV}$ gold implanted past a mask edge. The length increment (distance between tick marks) is $0.005 \mu \mathrm{m}$ and the scale factor is 1000. 
MASK

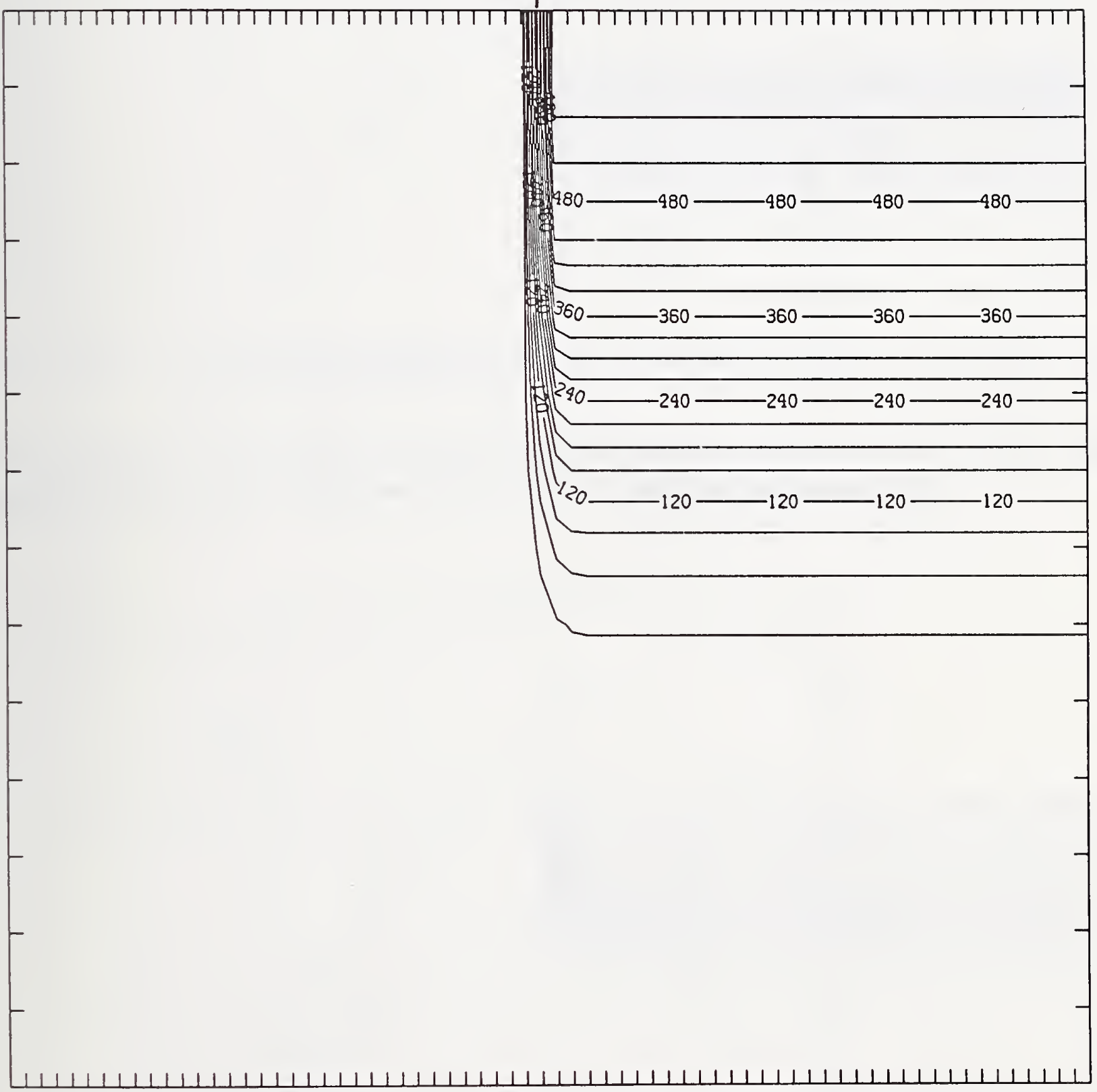

Figure Au50-4 Two-dimensional distribution of Frenkel pairs created by $50 \mathrm{keV}$ gold implanted past a mask edge. The length increment (distance between tick marks) is $0.005 \mu \mathrm{m}$ and the scale factor is 1 . 


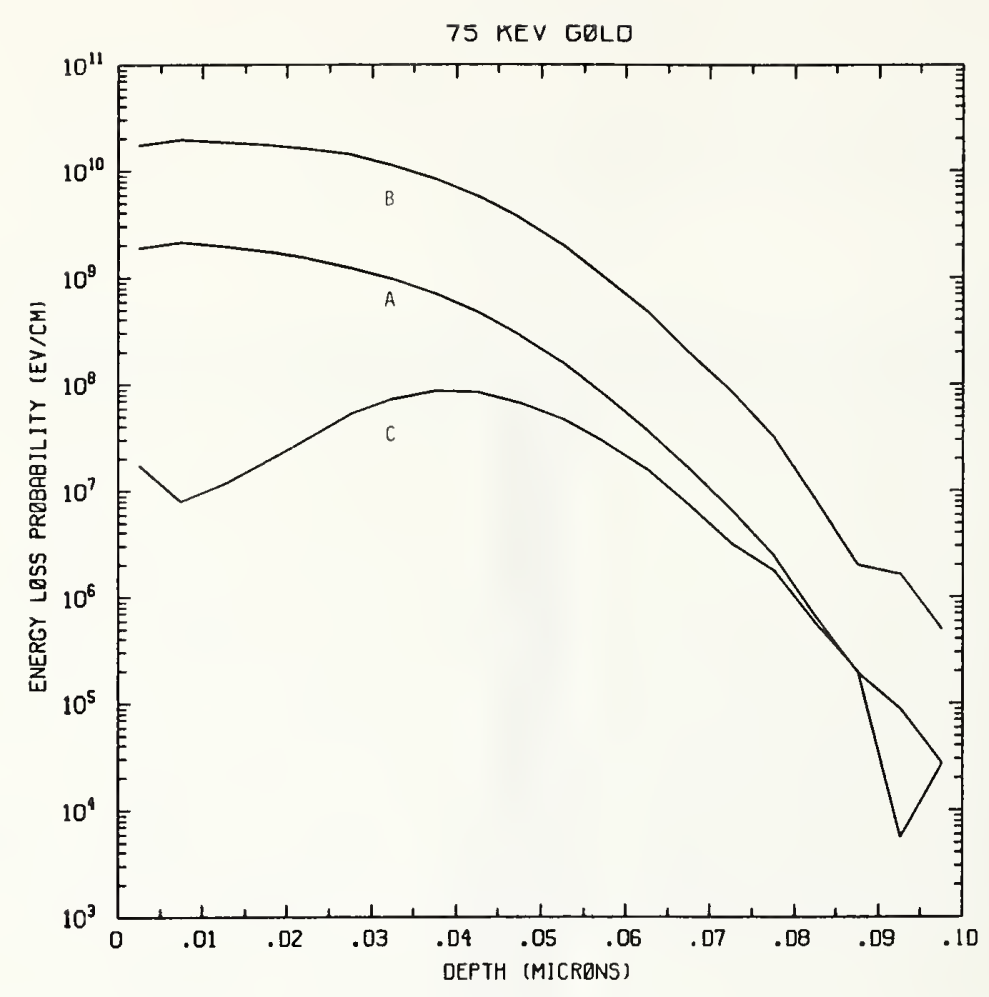

Figure Au75-1 One-dimensional probability distributions for energy loss due to electronic excitation (A), displacement damage(B), and phonon excitation (C) for $75 \mathrm{keV}$ gold normally incident on a silicon target.

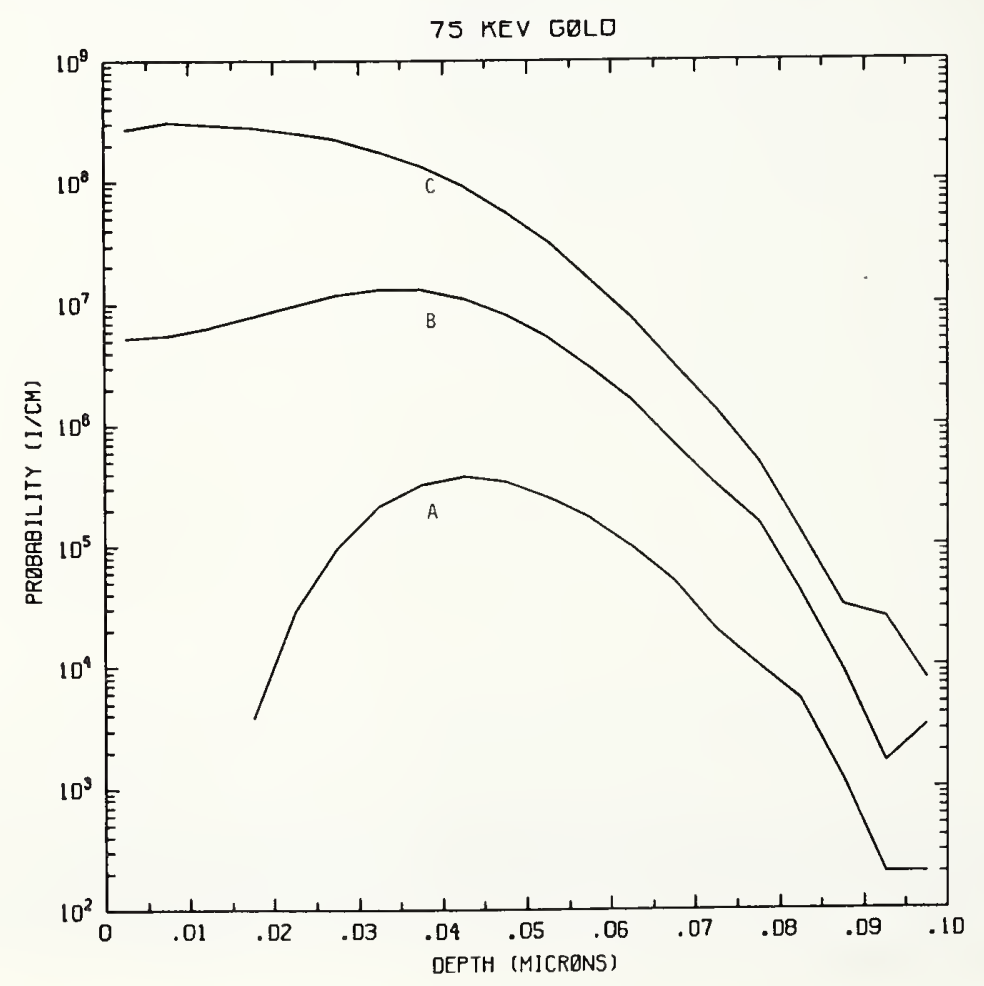

Figure Au75-2 One-dimensional probability distributions for implanted gold (A), primary silicon displacements (B), and Frenkel pairs (C) for $75 \mathrm{keV}$ gold normally incident on a silicon target. 


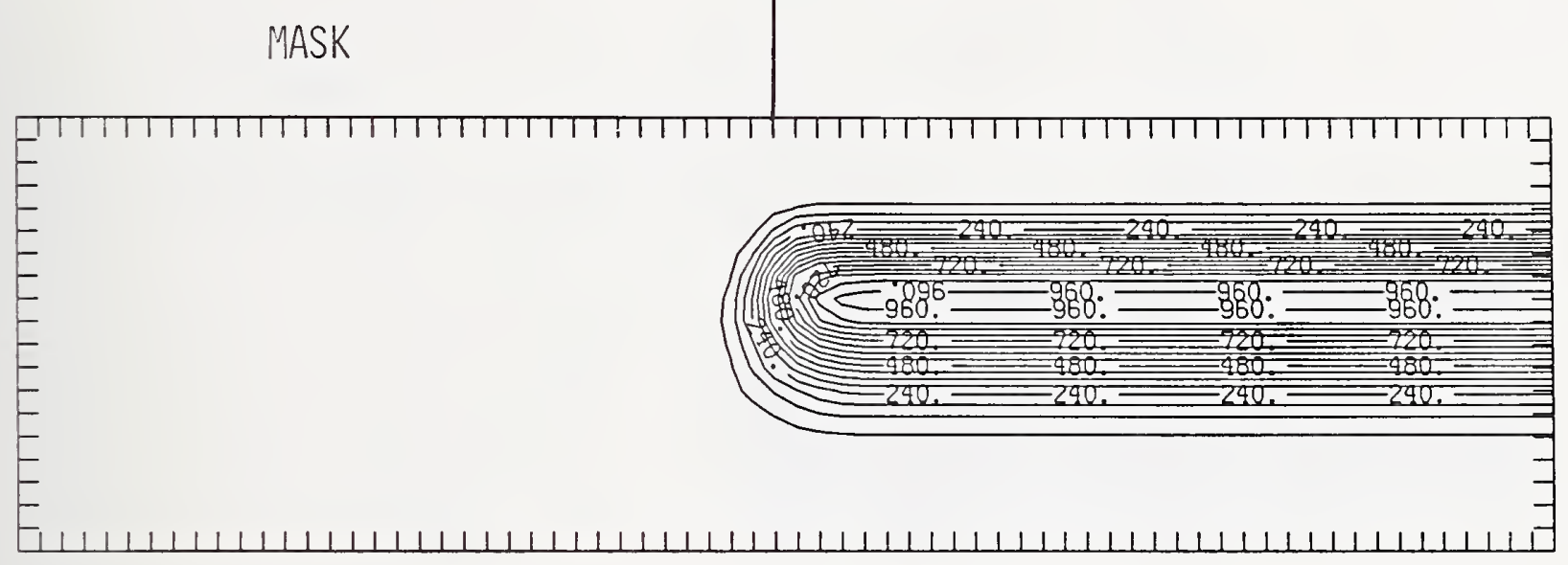

Figure Au75-3 Two-dimensional distribution of $75 \mathrm{keV}$ gold implanted past a mask edge. The length increment (distance between tick marks) is $0.005 \mu \mathrm{m}$ and the scale factor is 1000 .

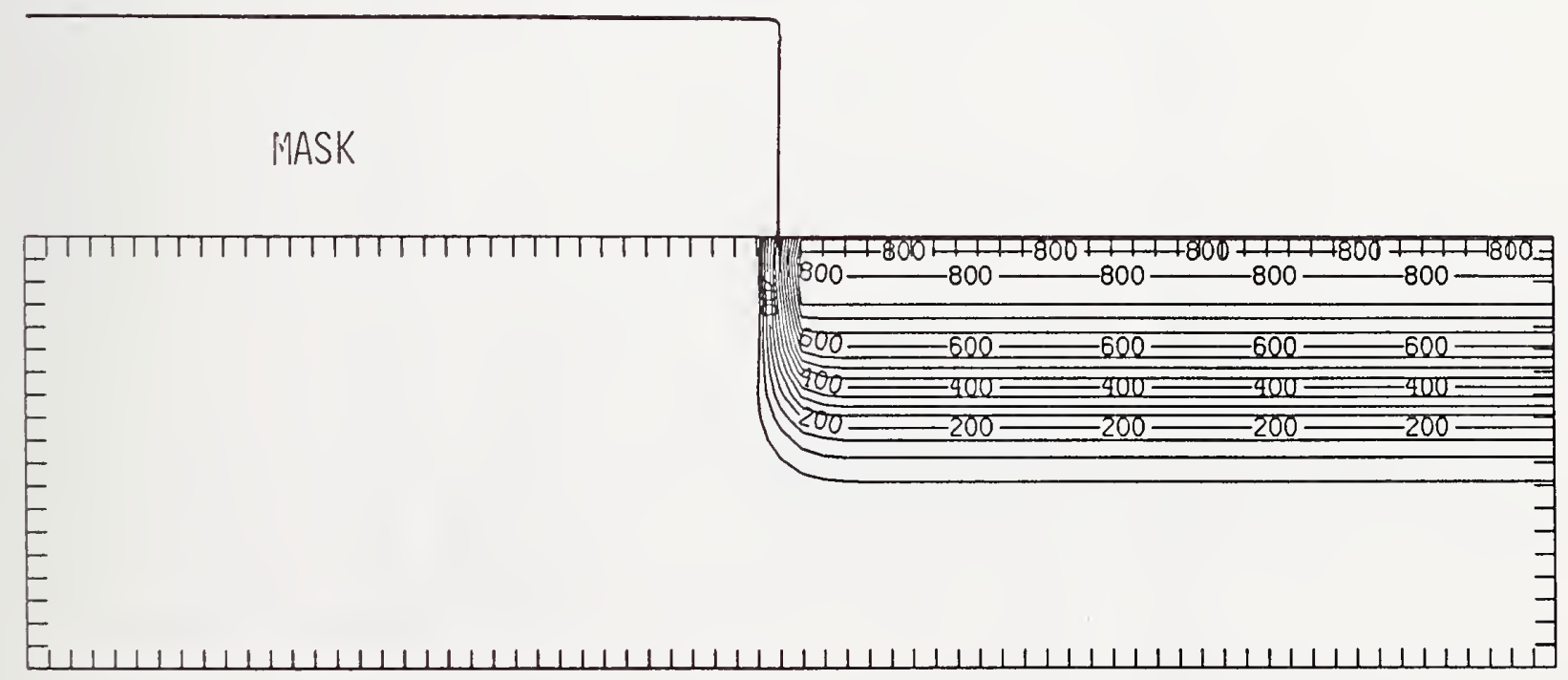

Figure Au75-4 Two-dimensional distribution of Frenkel pairs created by $75 \mathrm{keV}$ gold implanted past a mask edge. The length increment (distance between tick marks) is $0.005 \mu \mathrm{m}$ and the scale factor is 1 . 


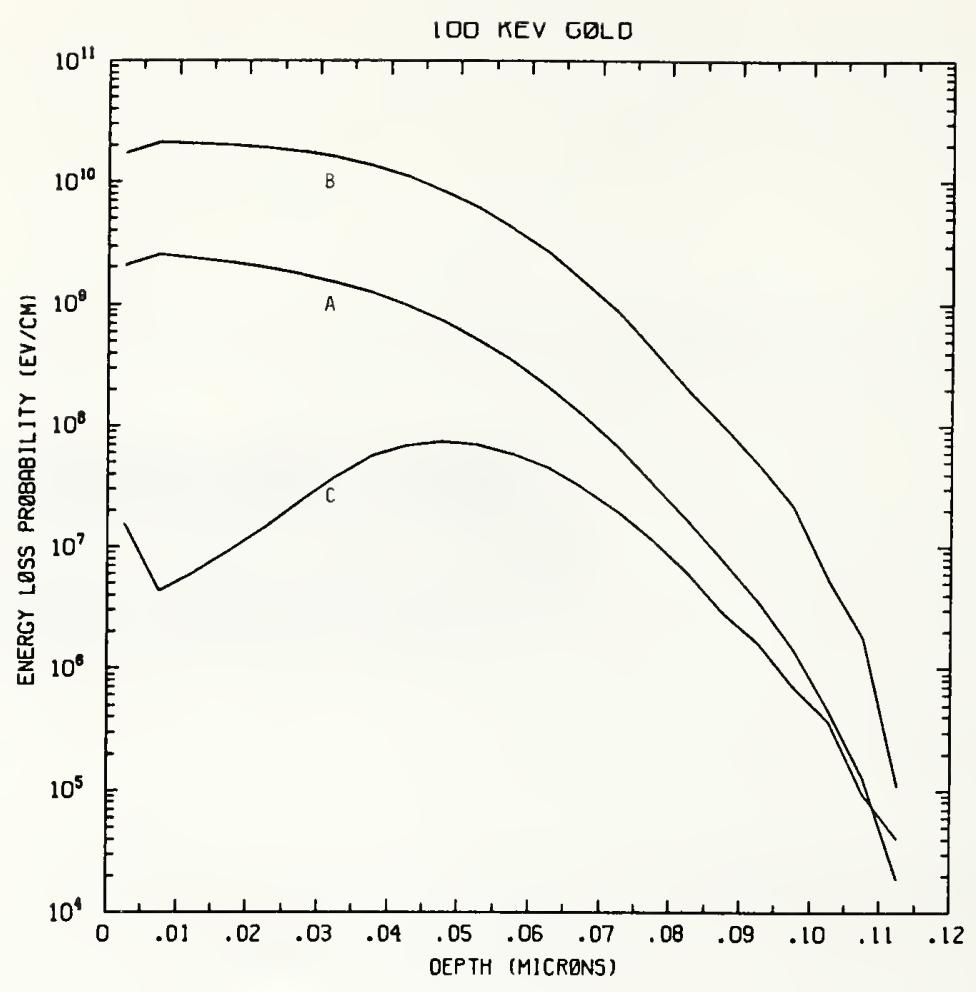

Figure Au100-1 One-dimensional probability distributions for energy loss due to electronic excitation (A), displacement damage(B), and phonon excitation (C) for $100 \mathrm{keV}$ gold normally incident on a silicon target.

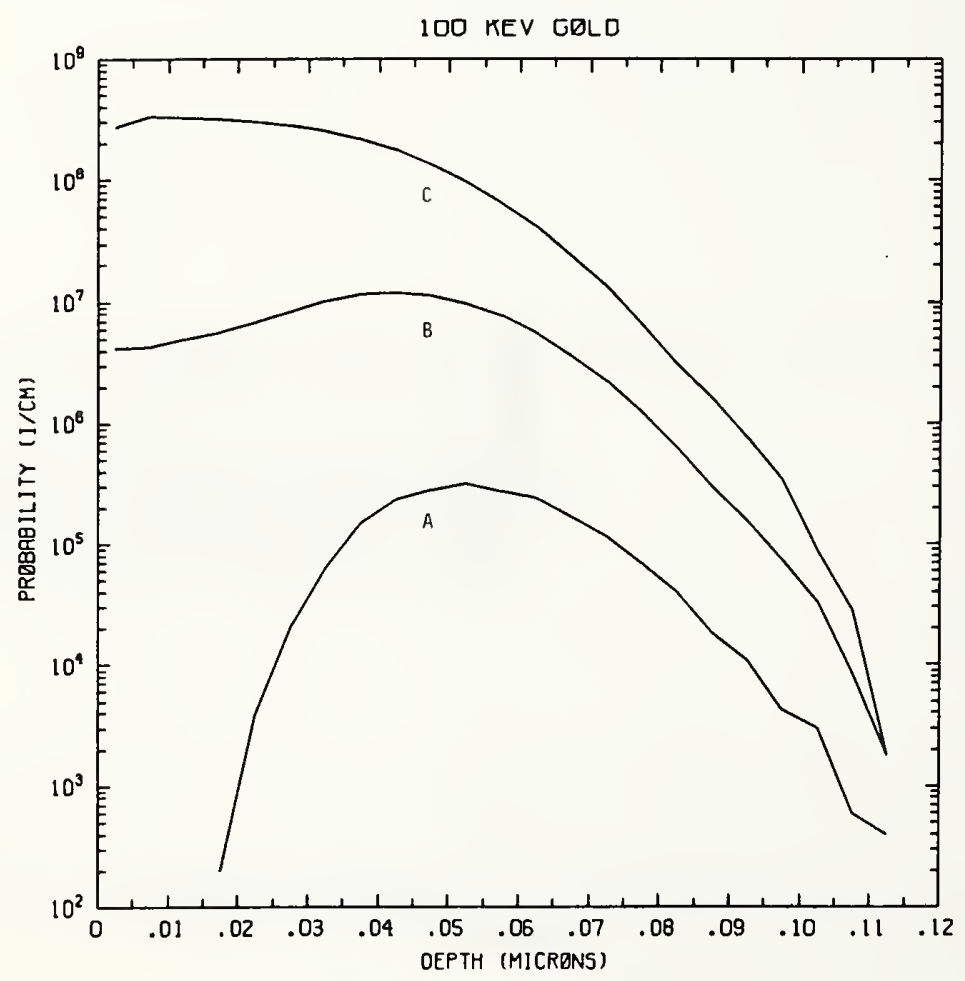

Figure Au100-2 One-dimensional probability distributions for implanted gold (A), primary silicon displacements (B), and Frenkel pairs (C) for $100 \mathrm{keV}$ gold normally incident on a silicon target. 


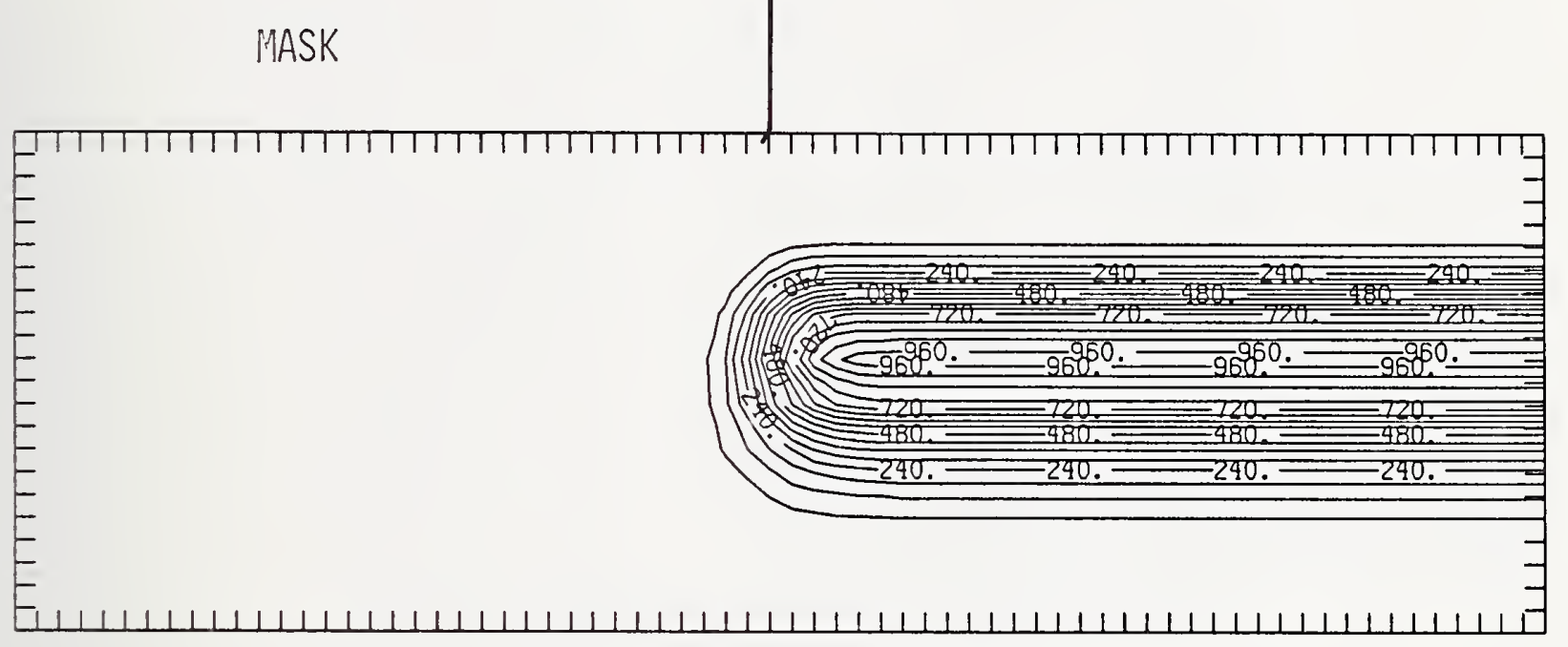

Figure Au100-3 Two-dimensional distribution of $100 \mathrm{keV}$ gold implanted past a mask edge. The length increment (distance between tick marks) is $0.005 \mu \mathrm{m}$ and the scale factor is 1000 .

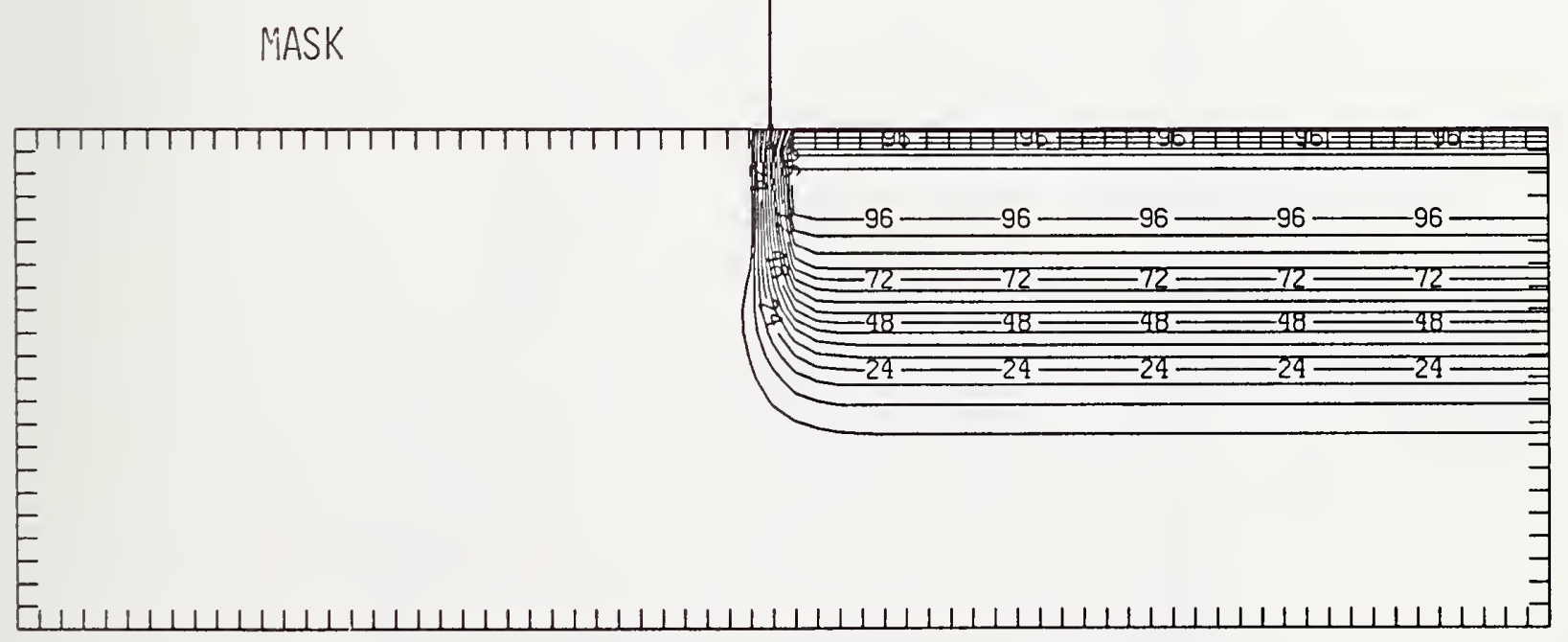

Figure Au100-4 Two-dimensional distribution of Frenkel pairs created by $100 \mathrm{keV}$ gold implanted past a mask edge. The length increment (distance between tick marks) is $0.005 \mu \mathrm{m}$ and the scale factor is 0.1 . 


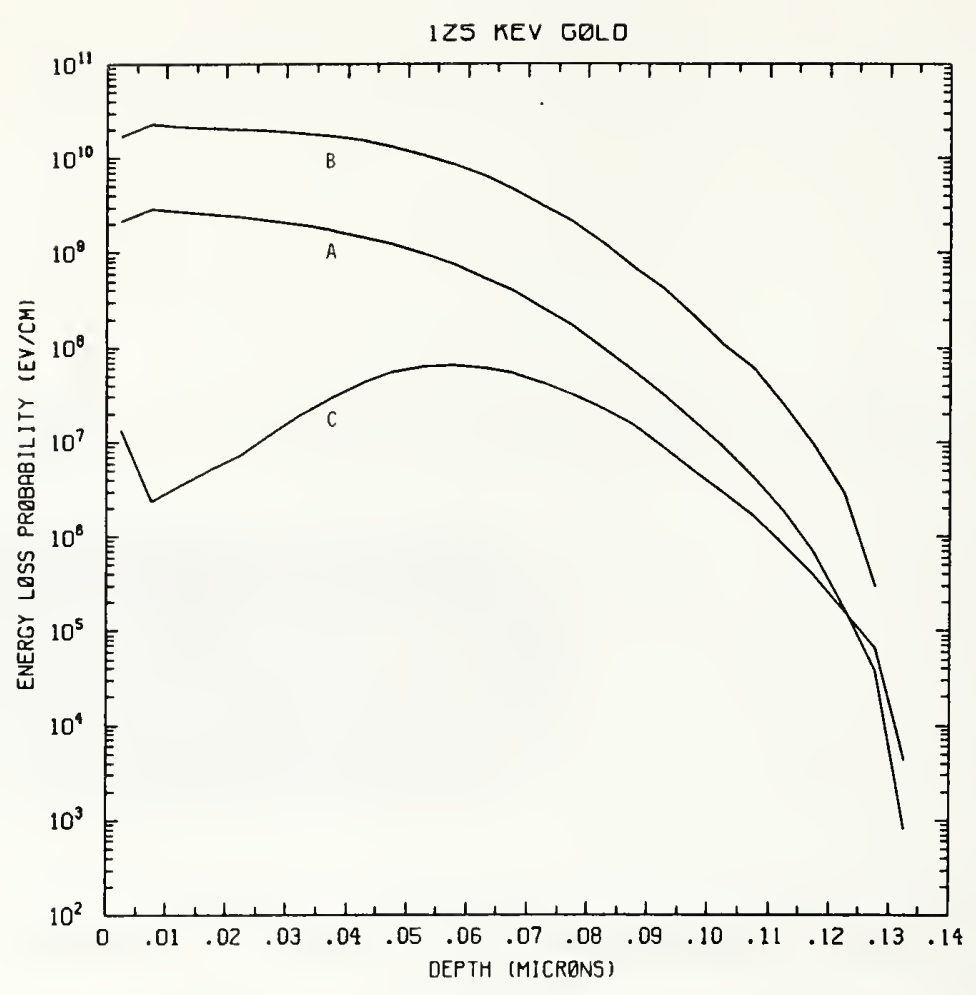

Figure Au125-1 One-dimensional probability distributions for energy loss due to electronic excitation (A), displacement damage(B), and phonon excitation (C) for $125 \mathrm{keV}$ gold normally incident on a silicon target.

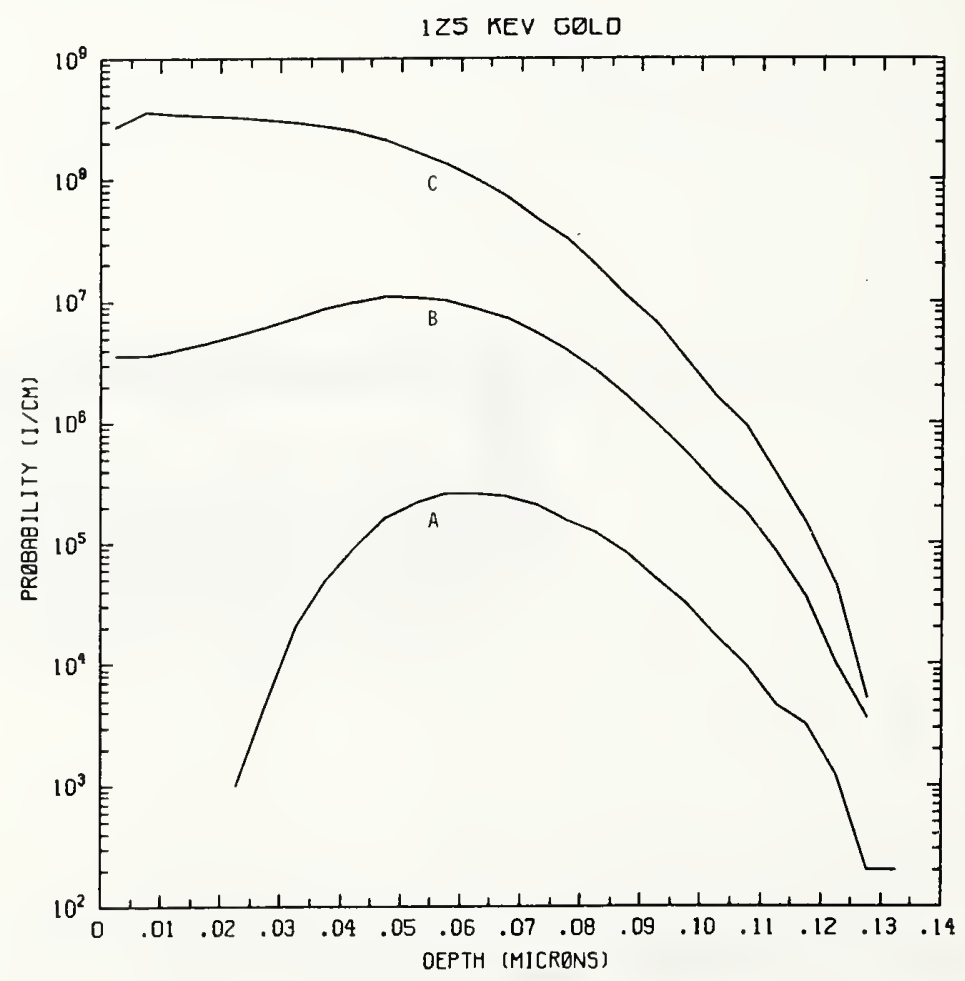

Figure Au125-2 One-dimensional probability distributions for implanted gold (A), primary silicon displacements (B), and Frenkel pairs (C) for $125 \mathrm{keV}$ gold normally incident on a silicon target. 
MASK

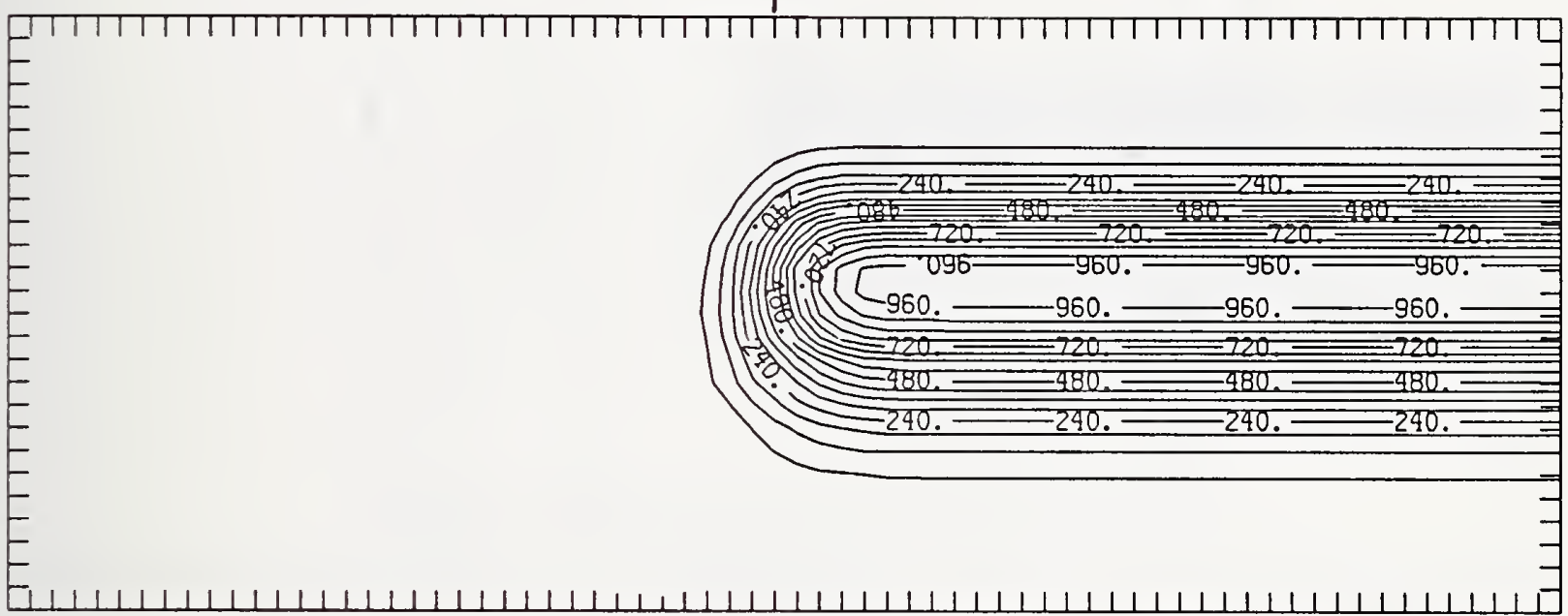

Figure Au125-3 Two-dimensional distribution of $125 \mathrm{keV}$ gold implanted past a mask edge. The length increment (distance between tick marks) is $0.005 \mu \mathrm{m}$ and the scale factor is 1000 .

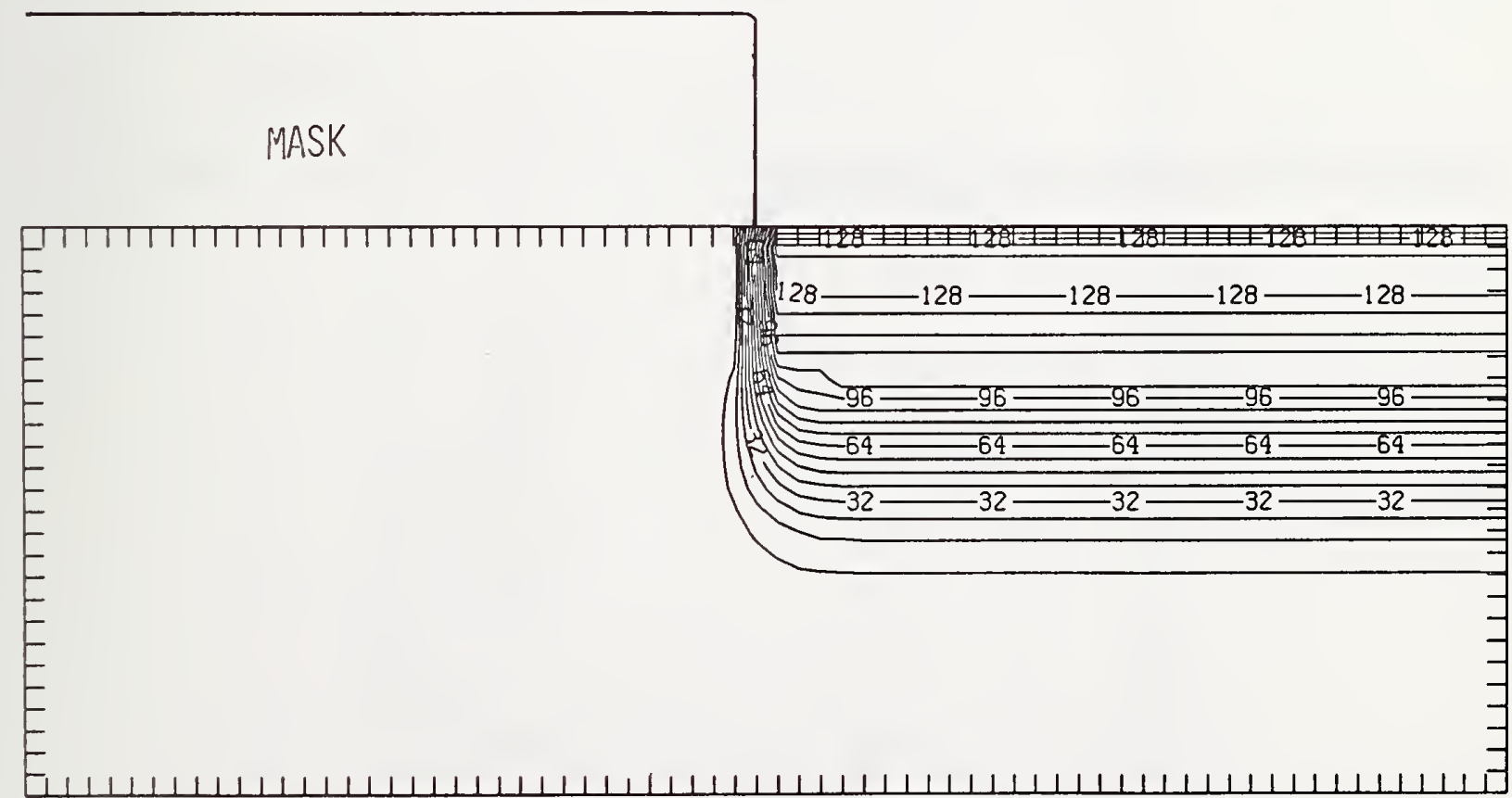

Figure Au125-4 Two-dimensional distribution of Frenkel pairs created by $125 \mathrm{keV}$ gold implanted past a mask edge. The length increment (distance between tick marks) is $0.005 \mu \mathrm{m}$ and the scale factor is 0.1 . 


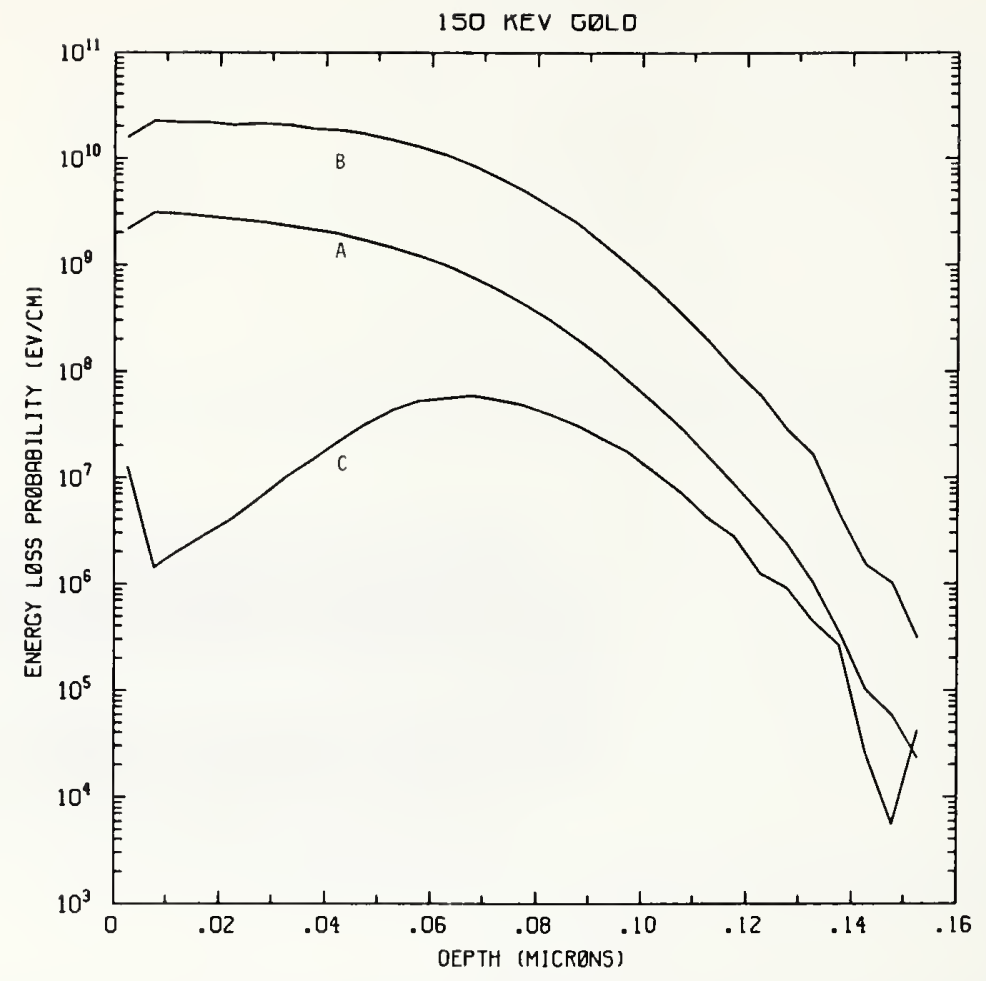

Figure Aul50-1 One-dimensional probability distributions for energy loss due to electronic excitation (A), displacement damage(B), and phonon excitation (C) for $150 \mathrm{keV}$ gold normally incident on a silicon target.

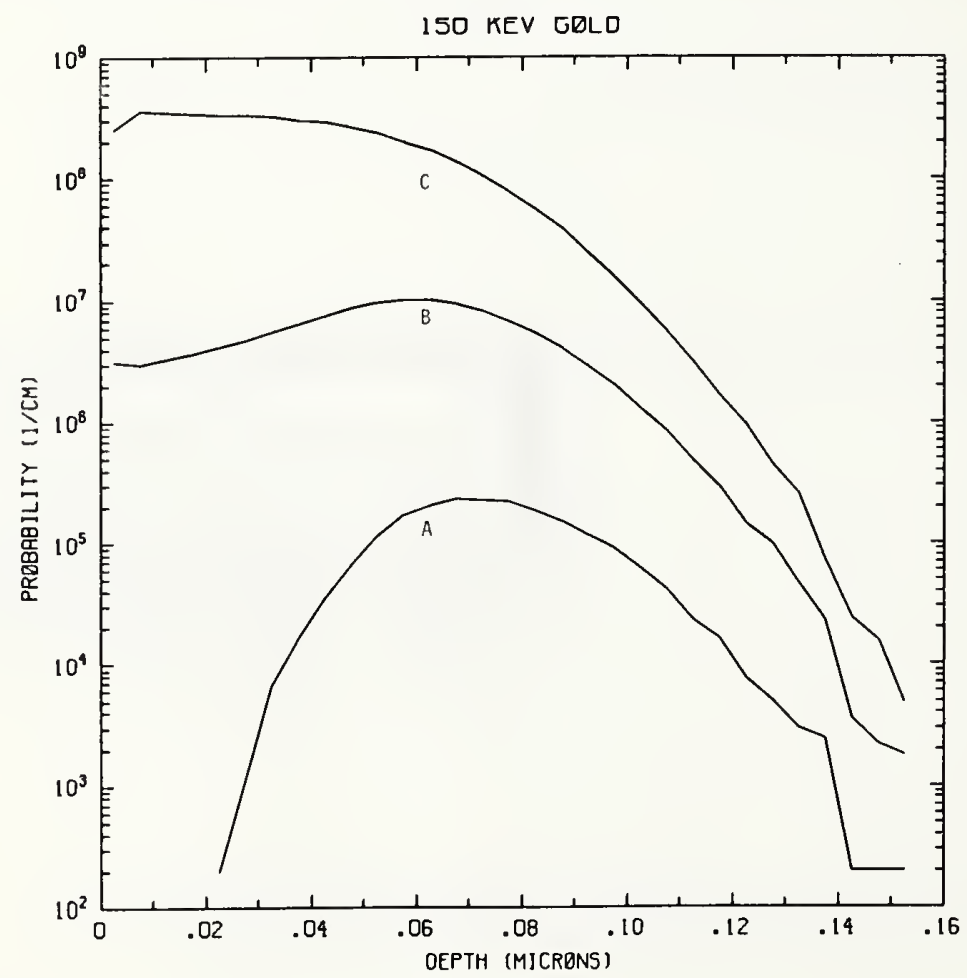

Figure Au150-2 One-dimensional probability distributions for implanted gold (A), primary silicon displacements (B), and Frenkel pairs (C) for $150 \mathrm{keV}$ gold normally incident on a silicon target. 
MASK

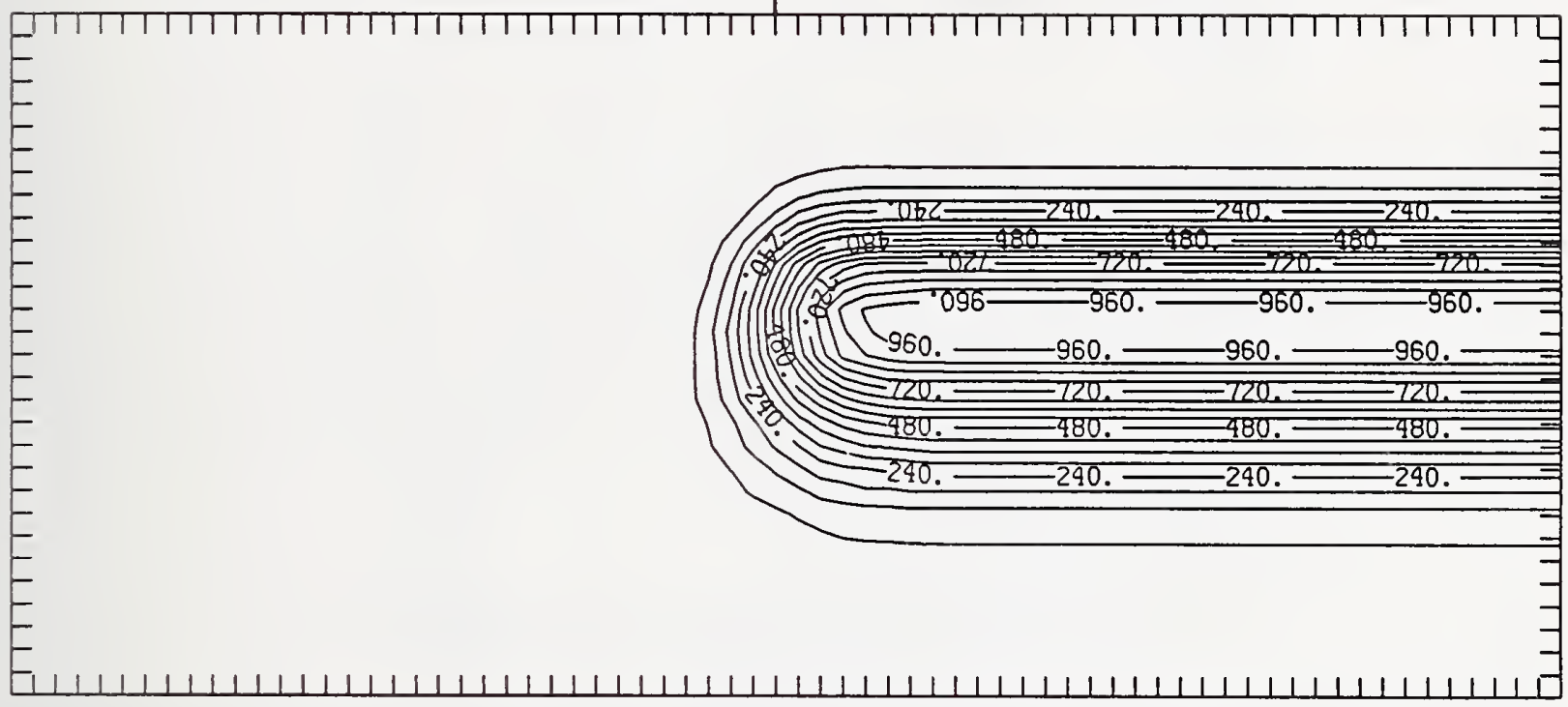

Figure Au150-3 Two-dimensional distribution of $150 \mathrm{keV}$ gold implanted past a mask edge. The length increment (distance between tick marks) is $0.005 \mu \mathrm{m}$ and the scale factor is 1000 .

MASK

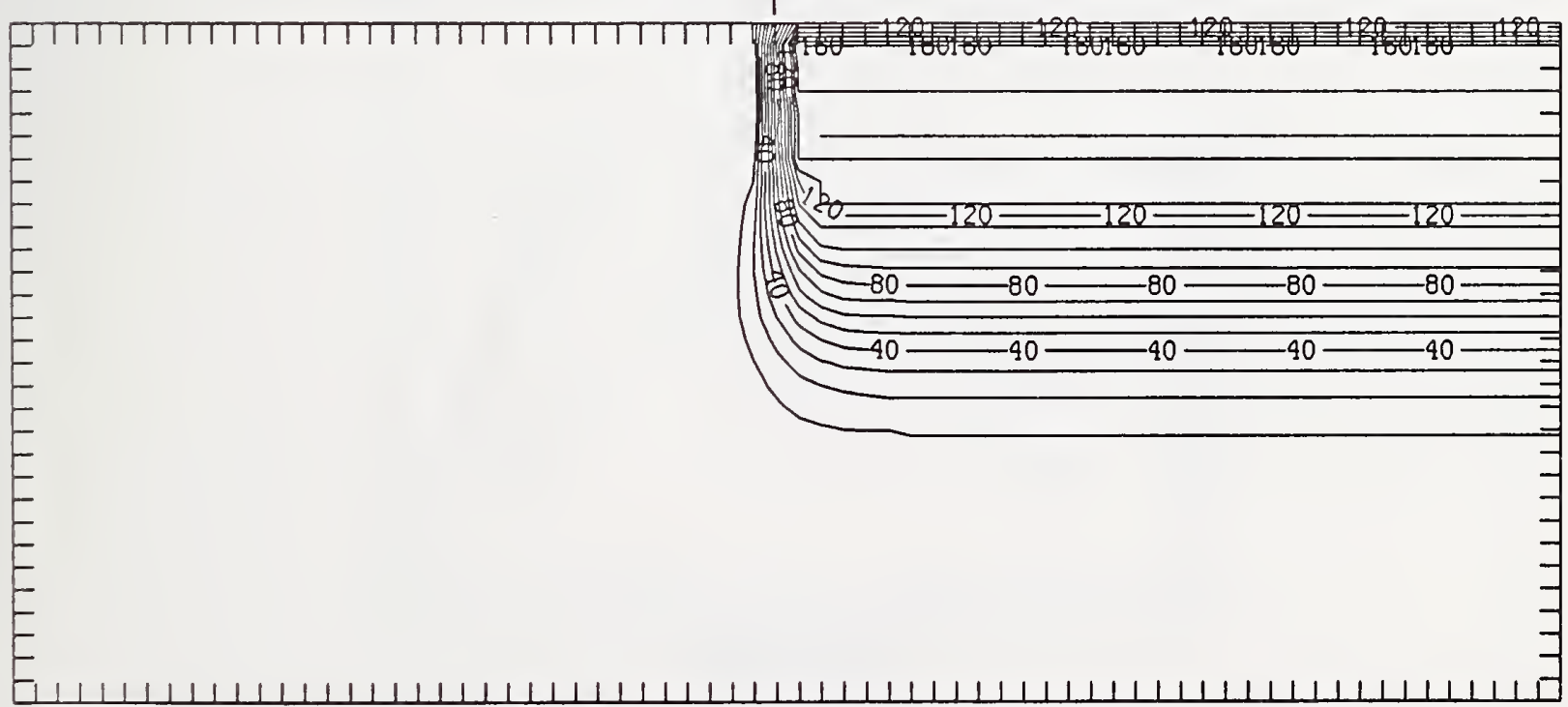

Figure Au150-4 Two-dimensional distribution of Frenkel pairs created by $150 \mathrm{keV}$ gold implanted past a mask edge. The length increment (distance between tick marks) is $0.005 \mu \mathrm{m}$ and the scale factor is 0.1 . 


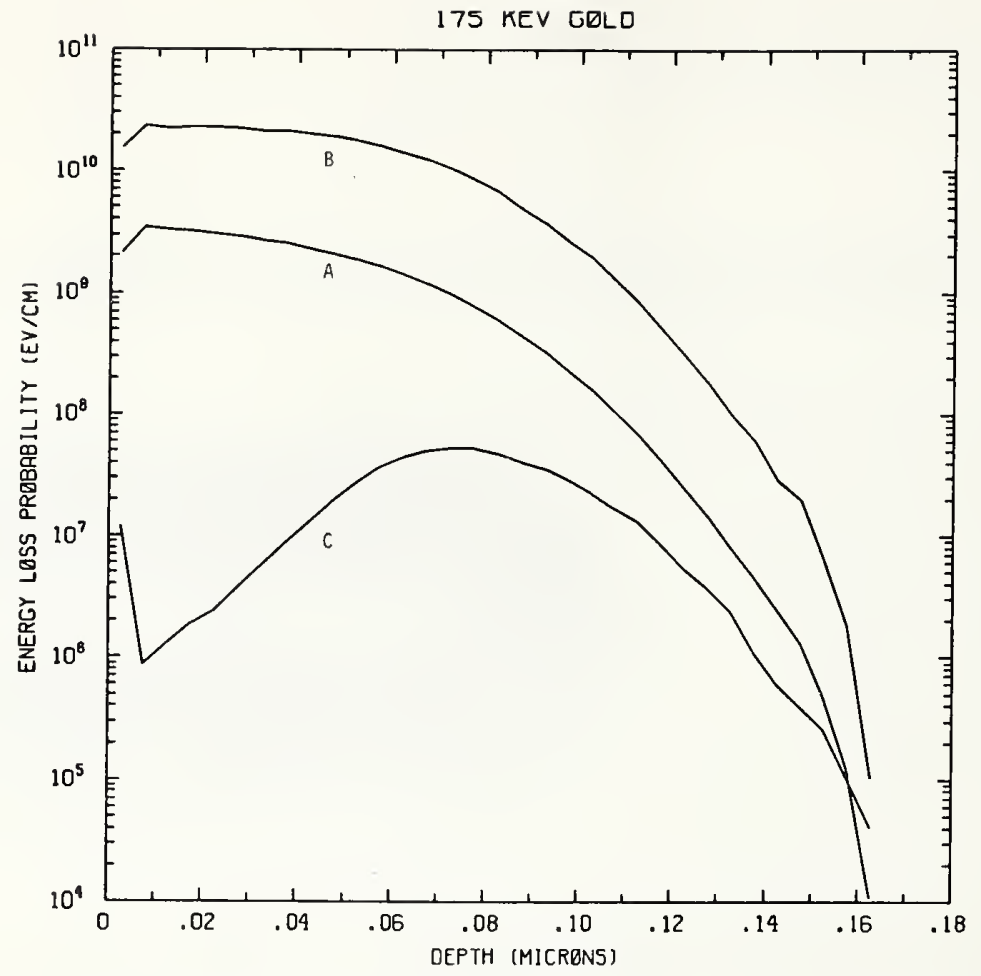

Figure Au175-1 One-dimensional probability distributions for energy loss due to electronic excitation (A), displacement damage(B), and phonon excitation (C) for $175 \mathrm{keV}$ gold normally incident on a silicon target.

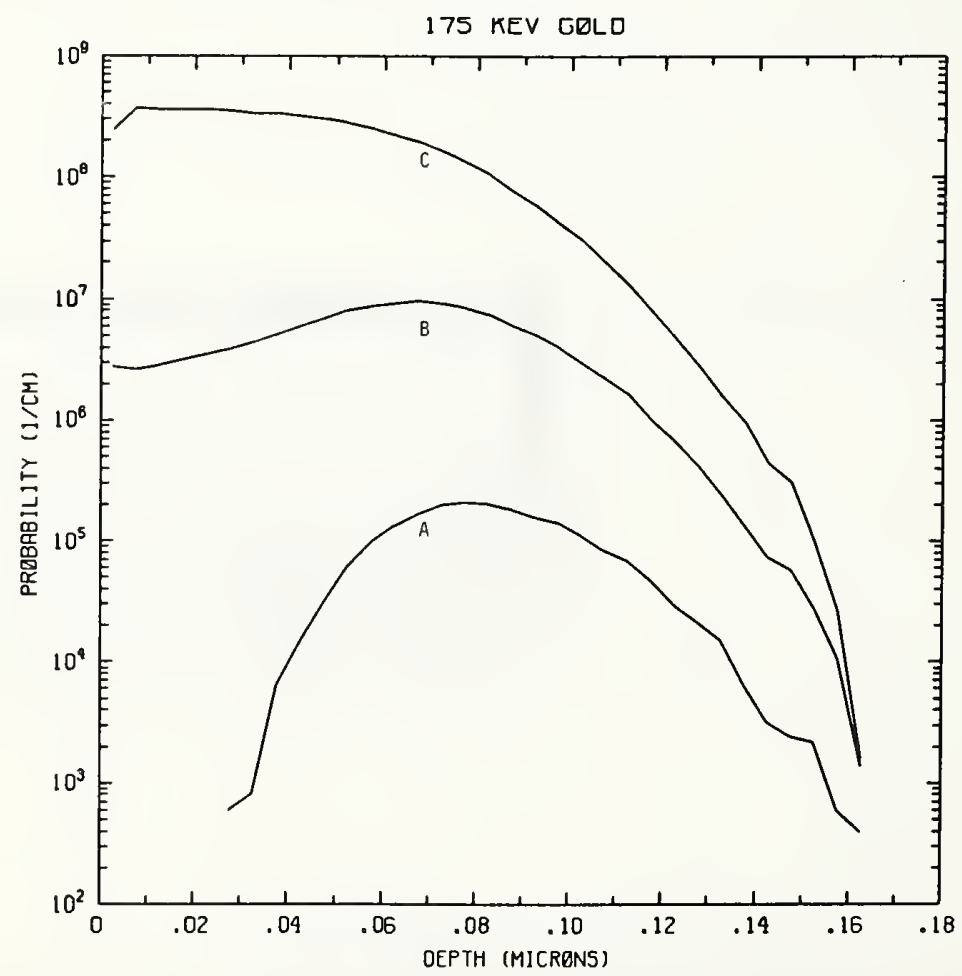

Figure Au175-2 One-dimensional probability distributions for implanted gold (A), primary silicon displacements (B), and Frenkel pairs (C) for $175 \mathrm{keV}$ gold normally incident on a silicon target. 
MASK

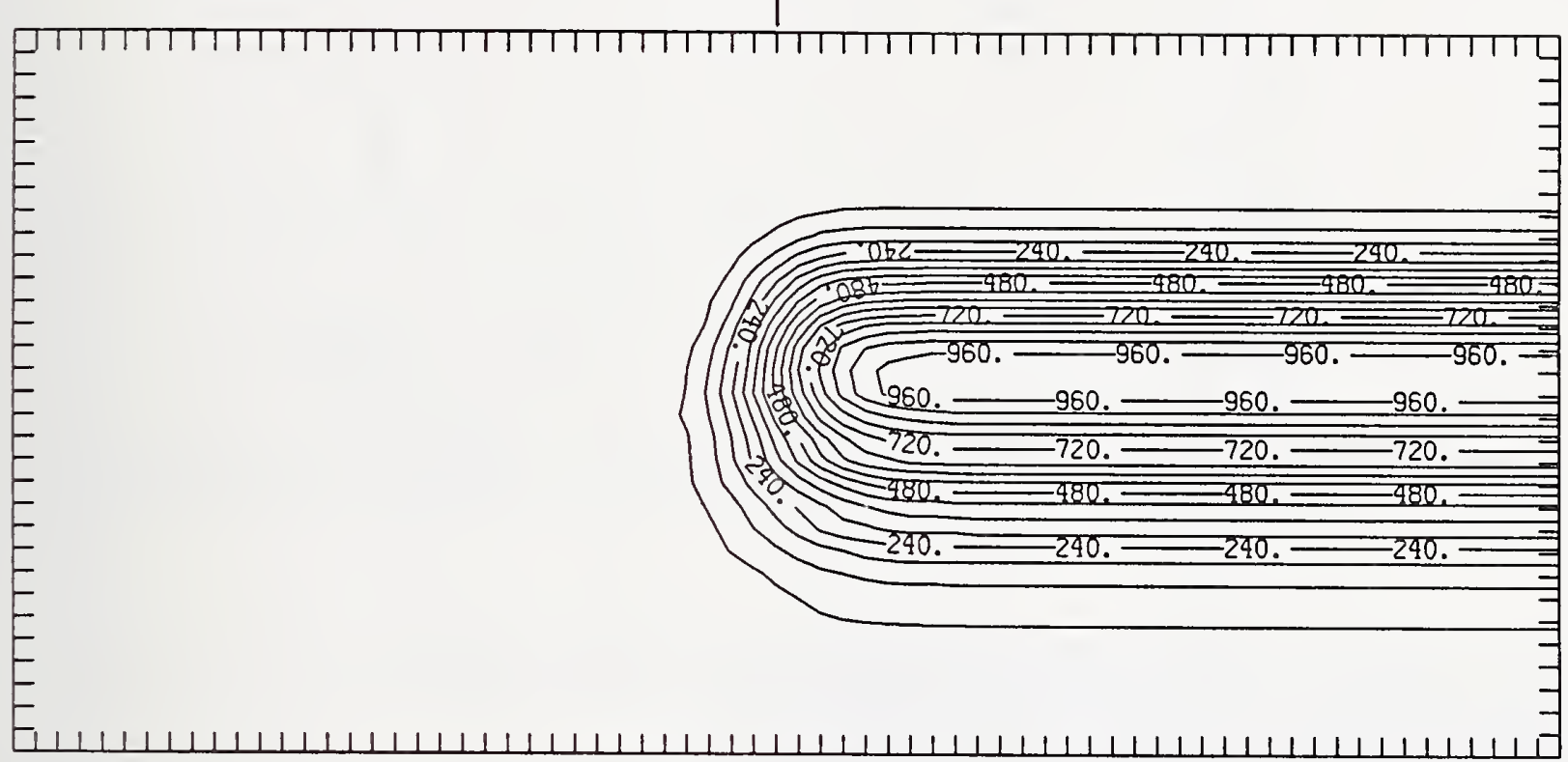

Figure Au175-3 Two-dimensional distribution of $175 \mathrm{keV}$ gold implanted past a mask edge. The length increment (distance between tick marks) is $0.005 \mu \mathrm{m}$ and the scale factor is 1000 .

MASK

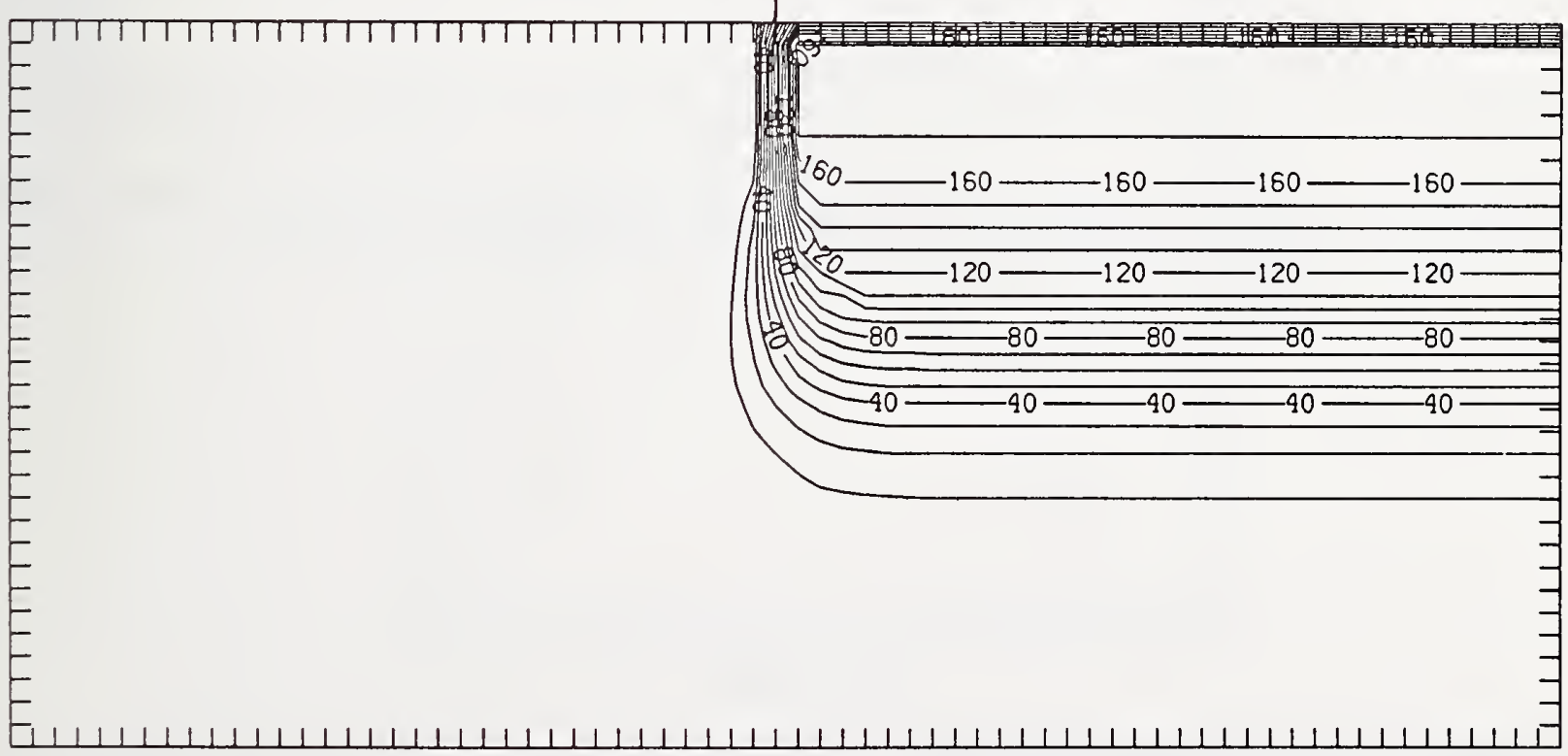

Figure Au175-4 Two-dimensional distribution of Frenkel pairs created by $175 \mathrm{keV}$ gold implanted past a mask edge. The length increment (distance between tick marks) is $0.005 \mu \mathrm{m}$ and the scale factor is 0.1 . 


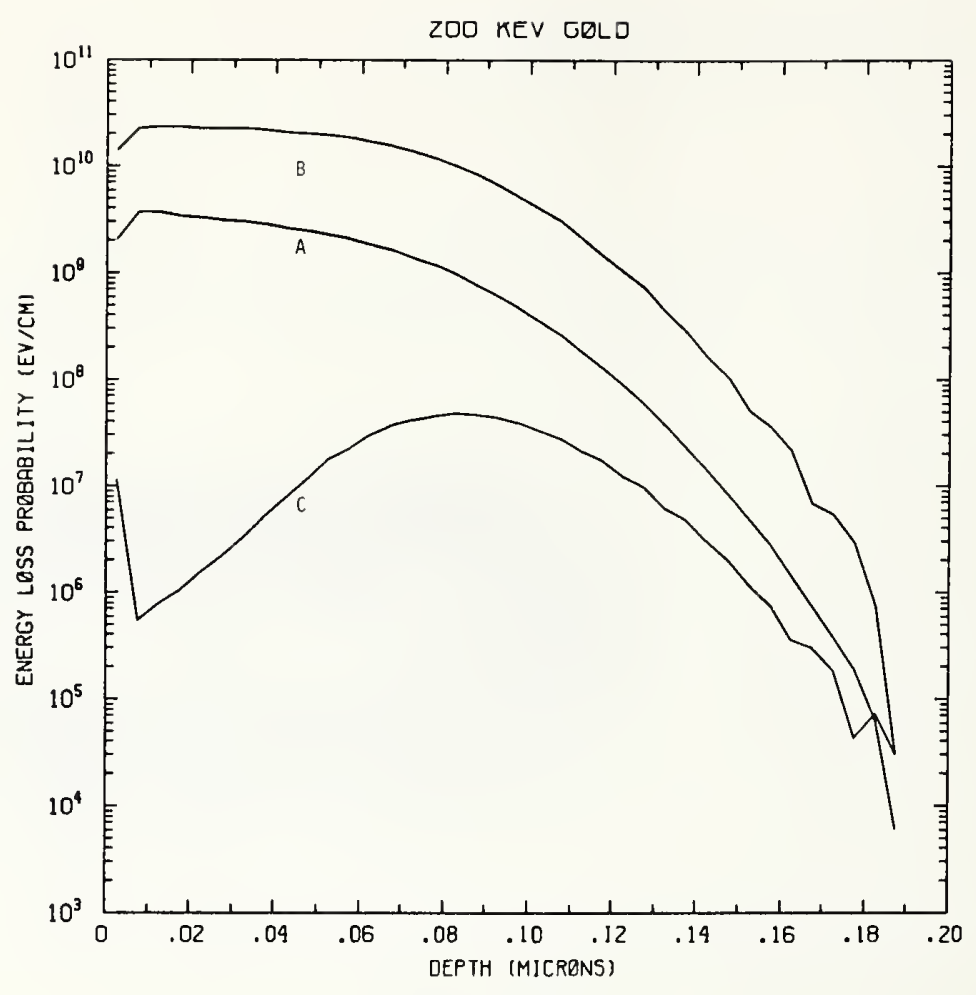

Figure Au200-1 One-dimensional probability distributions for energy loss due to electronic excitation (A), displacement damage(B), and phonon excitation (C) for $200 \mathrm{keV}$ gold normally incident on a silicon target.

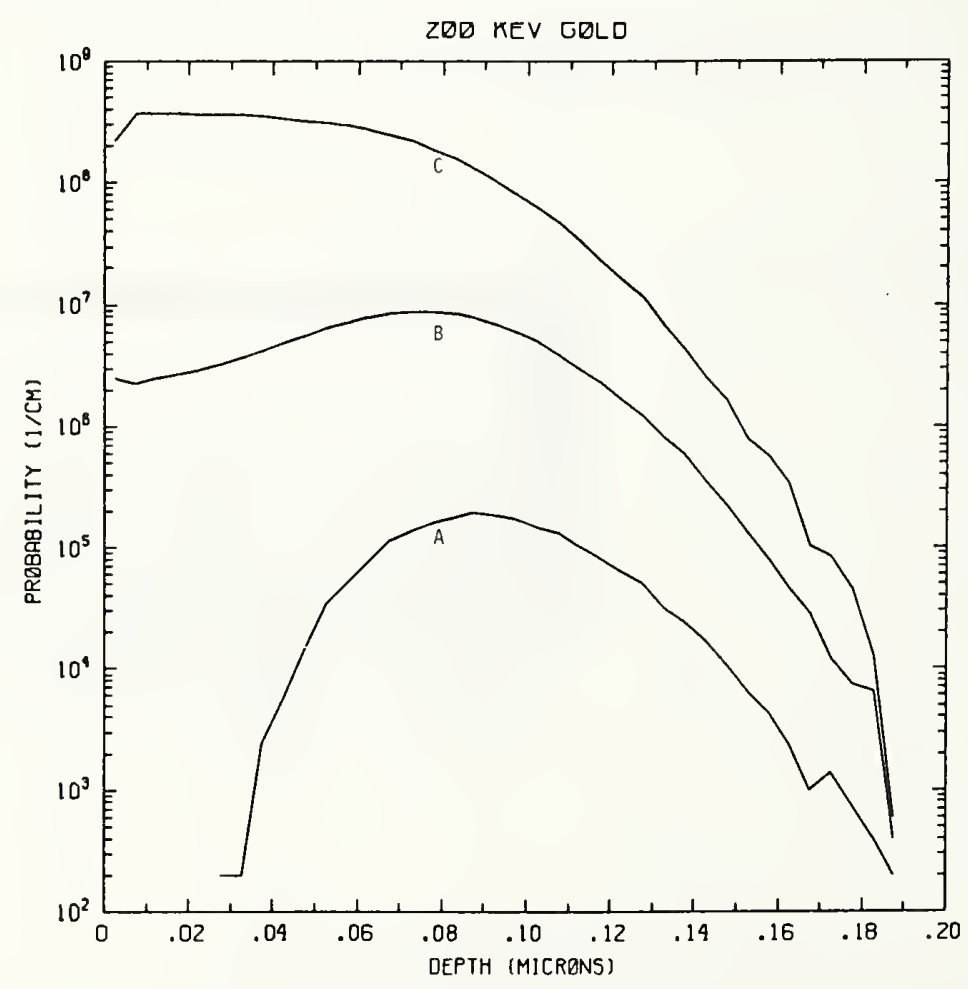

Figure Au200-2 One-dimensional probability distributions for implanted gold (A), primary silicon displacements (B), and Frenkel pairs (C) for $200 \mathrm{keV}$ gold normally incident on a silicon target. 


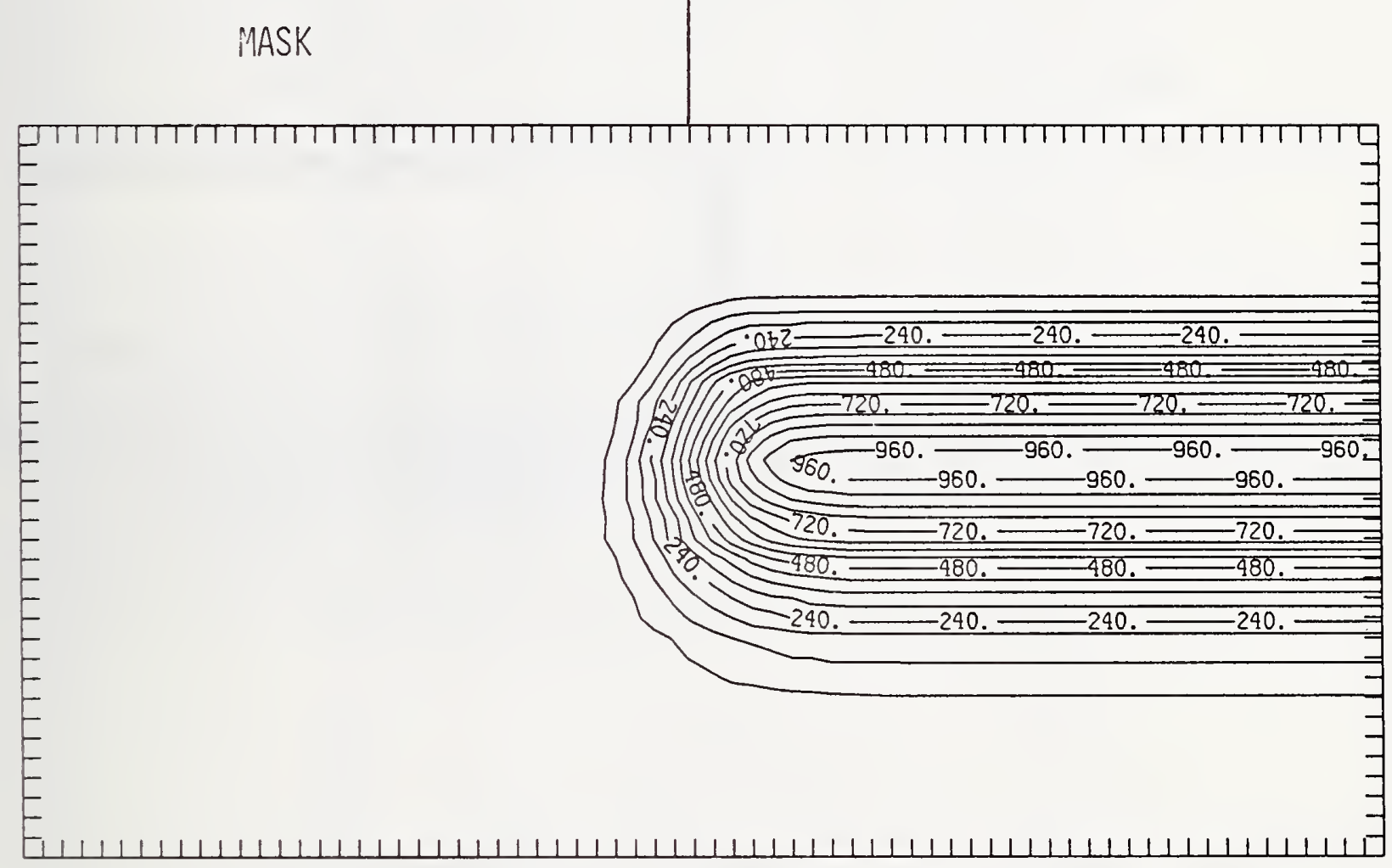

Figure Au200-3 Two-dimensional distribution of $200 \mathrm{keV}$ gold implanted past a mask edge. The length increment (distance between tick marks) is $0.005 \mu \mathrm{m}$ and the scale factor is 1000 . 


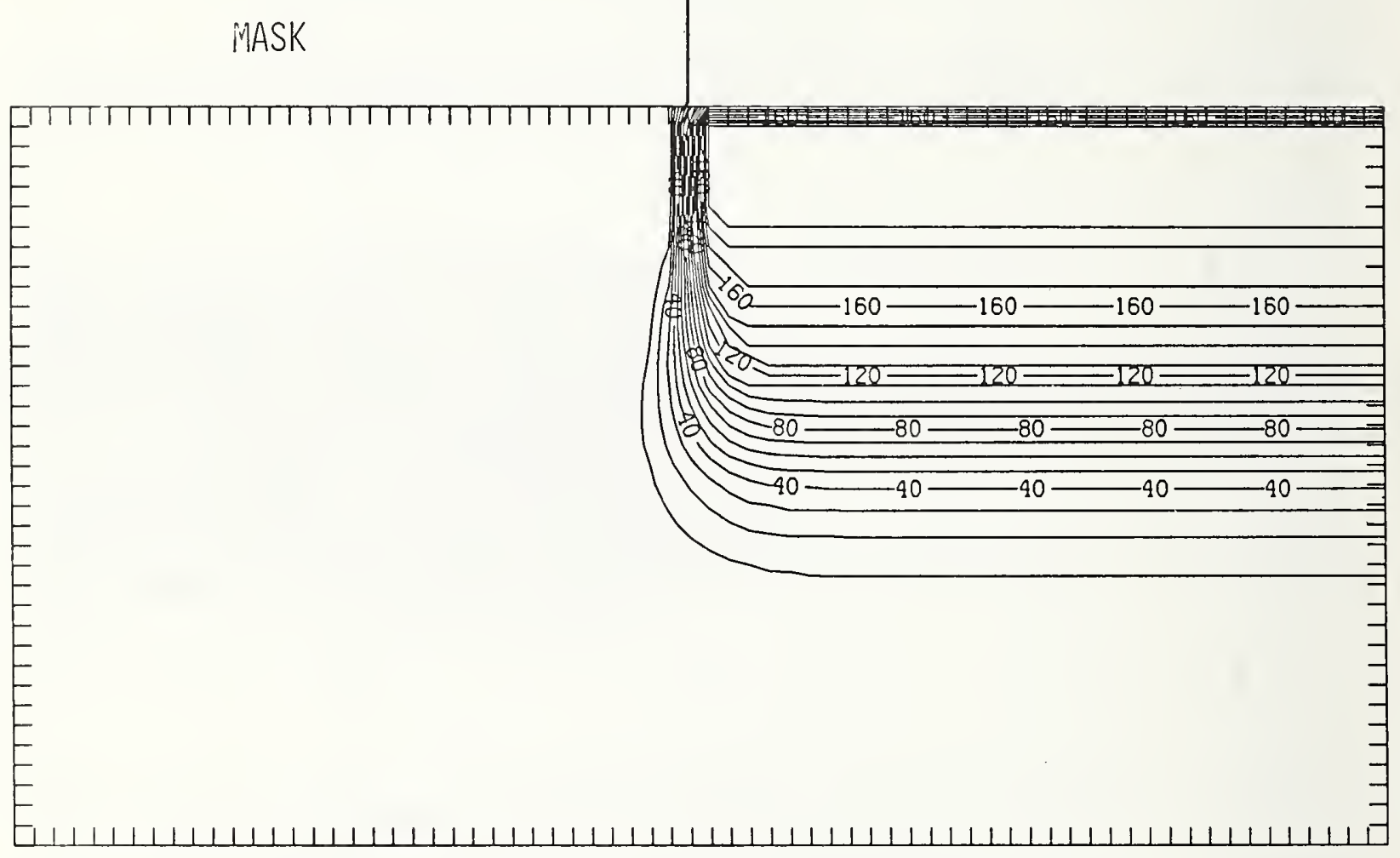

Figure Au200-4 Two-dimensional distribution of Frenkel pairs created by $200 \mathrm{keV}$ gold implanted past a mask edge. The length increment (distance between tick marks) is $0.005 \mu \mathrm{m}$ and the scale factor is 0.1 . 


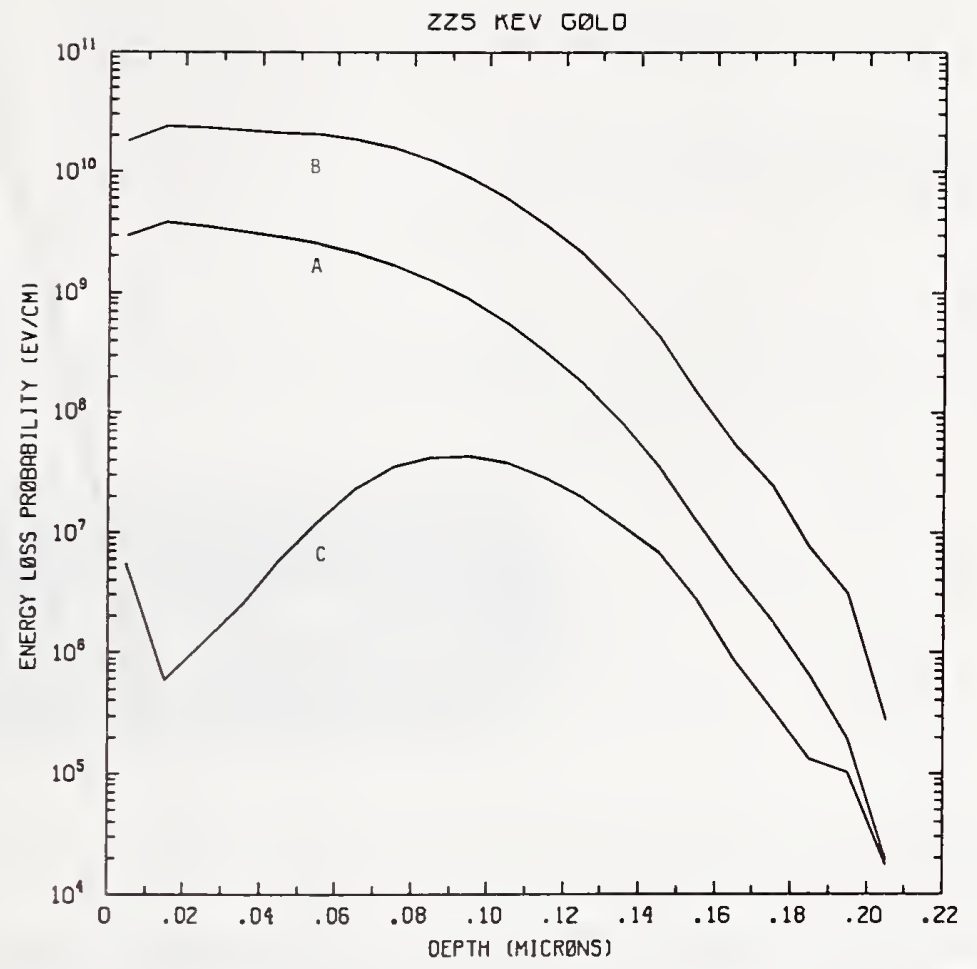

Figure Au225-1 One-dimensional probability distributions for energy loss due to electronic excitation (A), displacement damage(B), and phonon excitation (C) for $225 \mathrm{keV}$ gold normally incident on a silicon target.

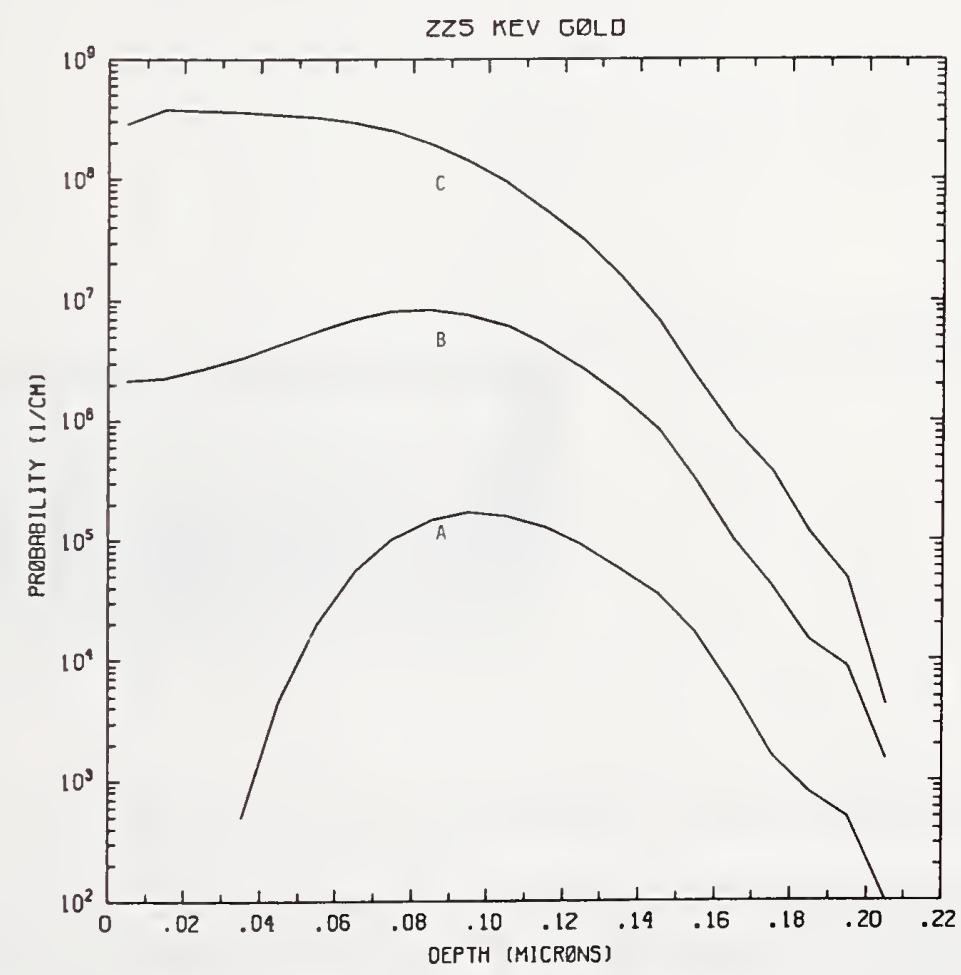

Figure Au225-2 One-dimensional probability distributions for implanted gold (A), primary silicon displacements (B), and Frenkel pairs (C) for $225 \mathrm{keV}$ gold normally incident on a silicon target. 


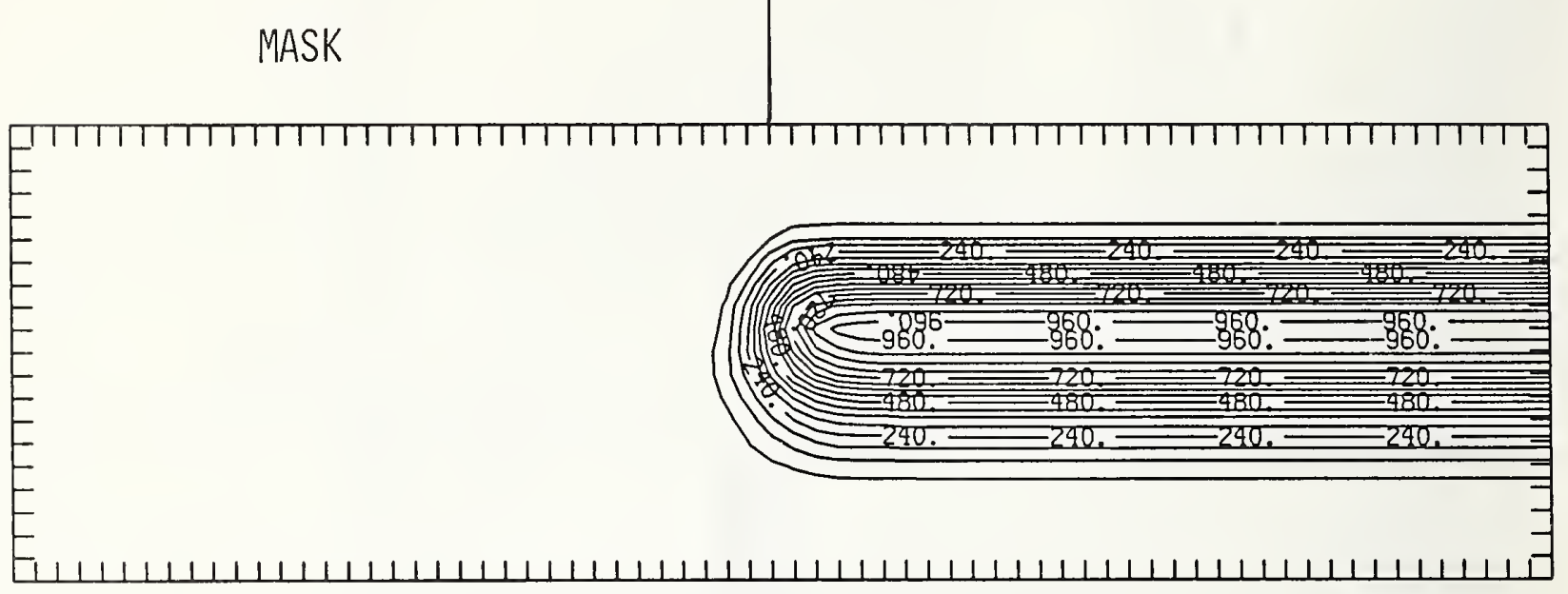

Figure Au225-3 Two-dimensional distribution of $225 \mathrm{keV}$ gold implanted past a mask edge. The length increment (distance between tick marks) is $0.01 \mu \mathrm{m}$ and the scale factor is 1000 .

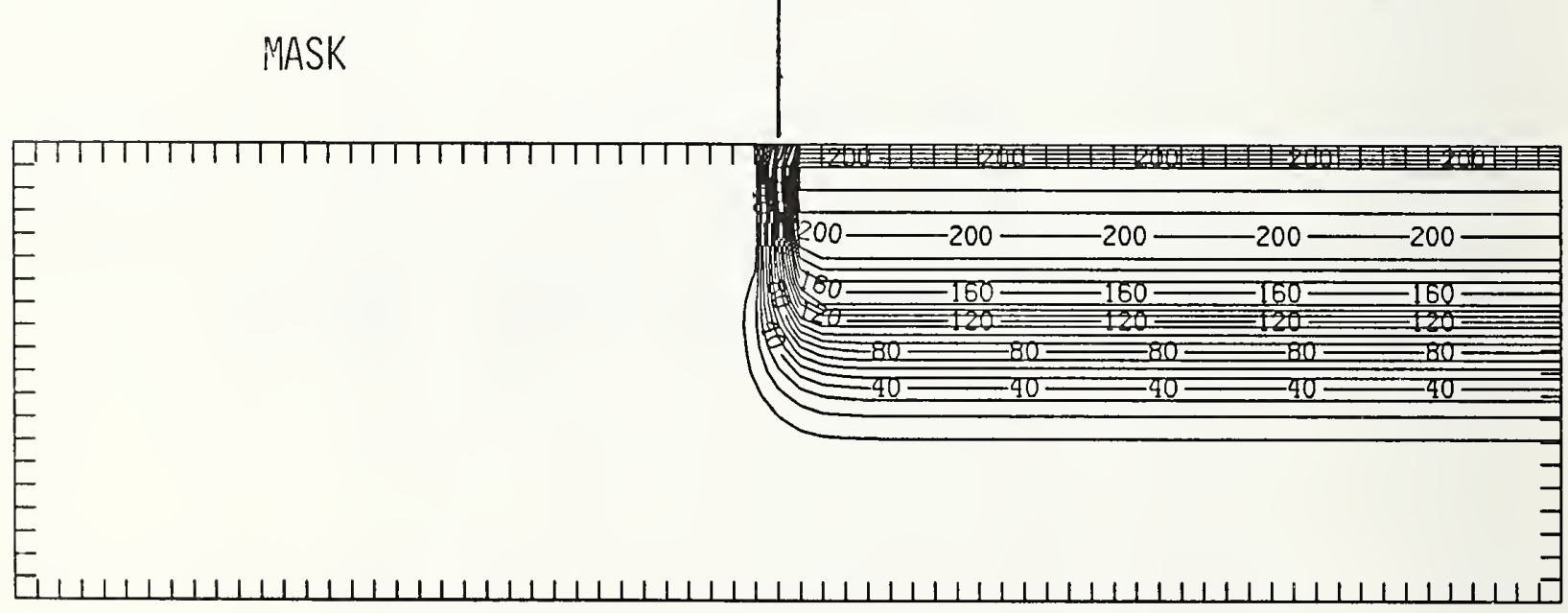

Figure Au225-4 Two-dimensional distribution of Frenkel pairs created by $225 \mathrm{keV}$ gold implanted past a mask edge. The length increment (distance between tick marks) is $0.01 \mu \mathrm{m}$ and the scale factor is 0.1 . 


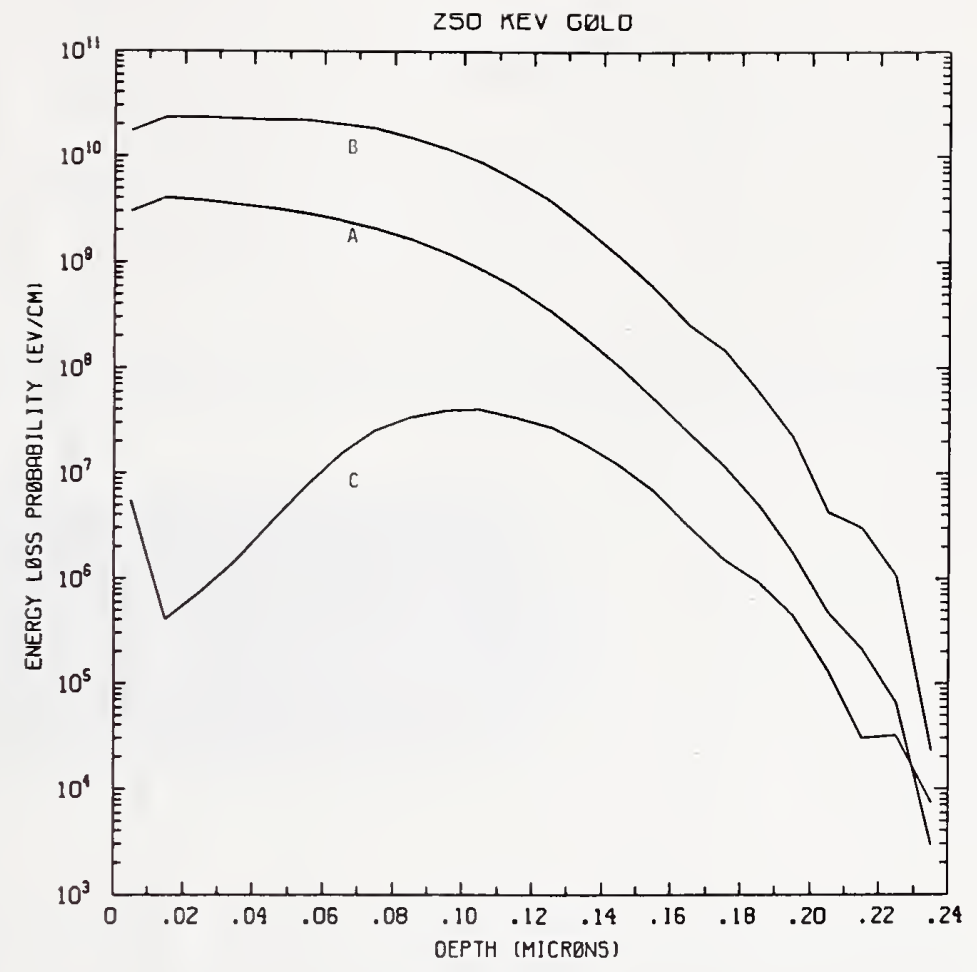

Figure Au250-1 One-dimensional probability distributions for energy loss due to electronic excitation (A), displacement damage(B), and phonon excitation (C) for $250 \mathrm{keV}$ gold normally incident on a silicon target.

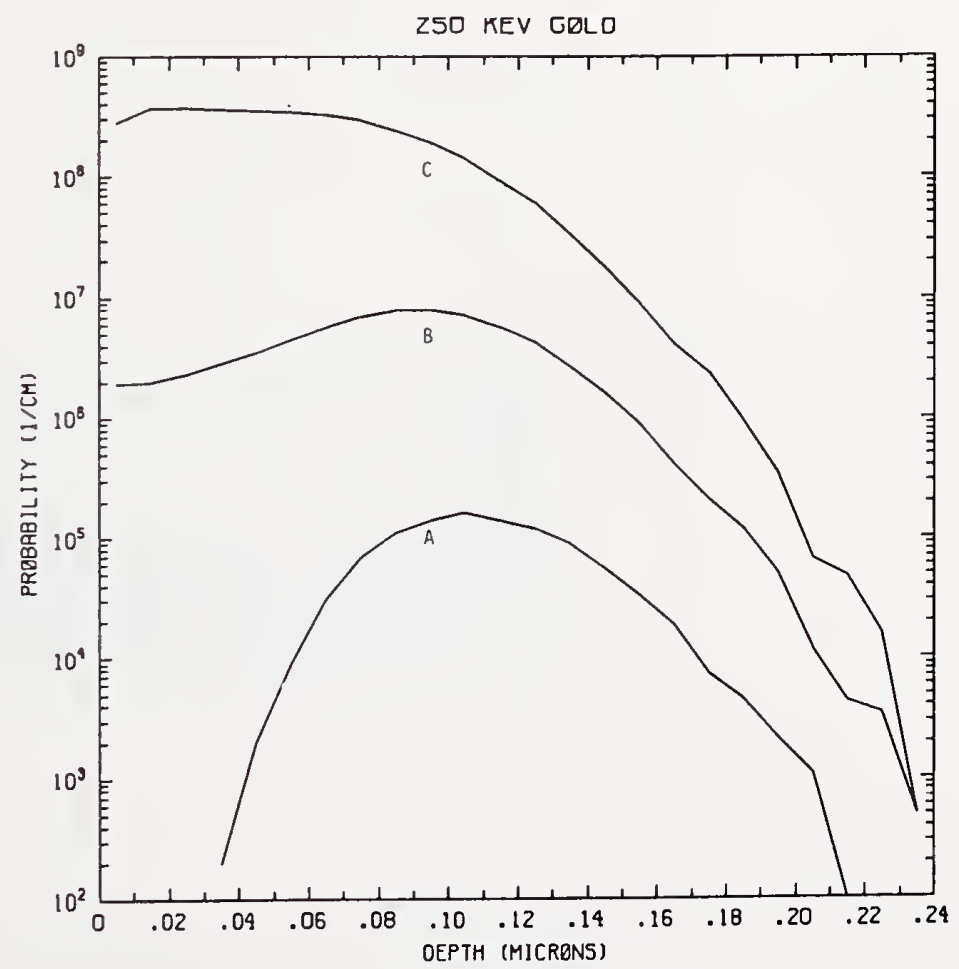

Figure Au250-2 One-dimensional probability distributions for implanted gold (A), primary silicon displacements (B), and Frenkel pairs (C) for $250 \mathrm{keV}$ gold normally incident on a silicon target. 


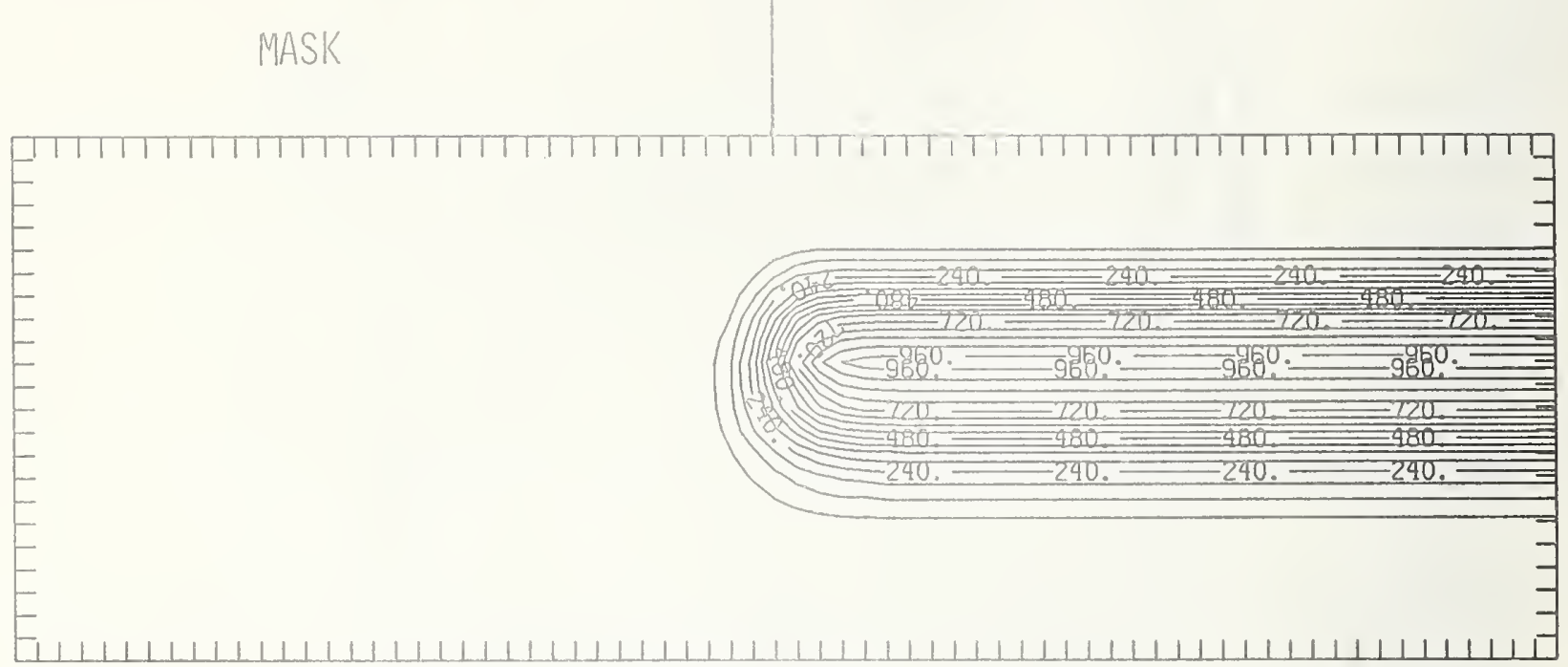

Figure Au250-3 Two-dimensional distribution of $250 \mathrm{keV}$ gold implanted past a mask edge. The length increment (distance betwcen tick marl ss) is $0.01 \mu \mathrm{m}$ and the scale factor is 1000 .

\section{MASK}

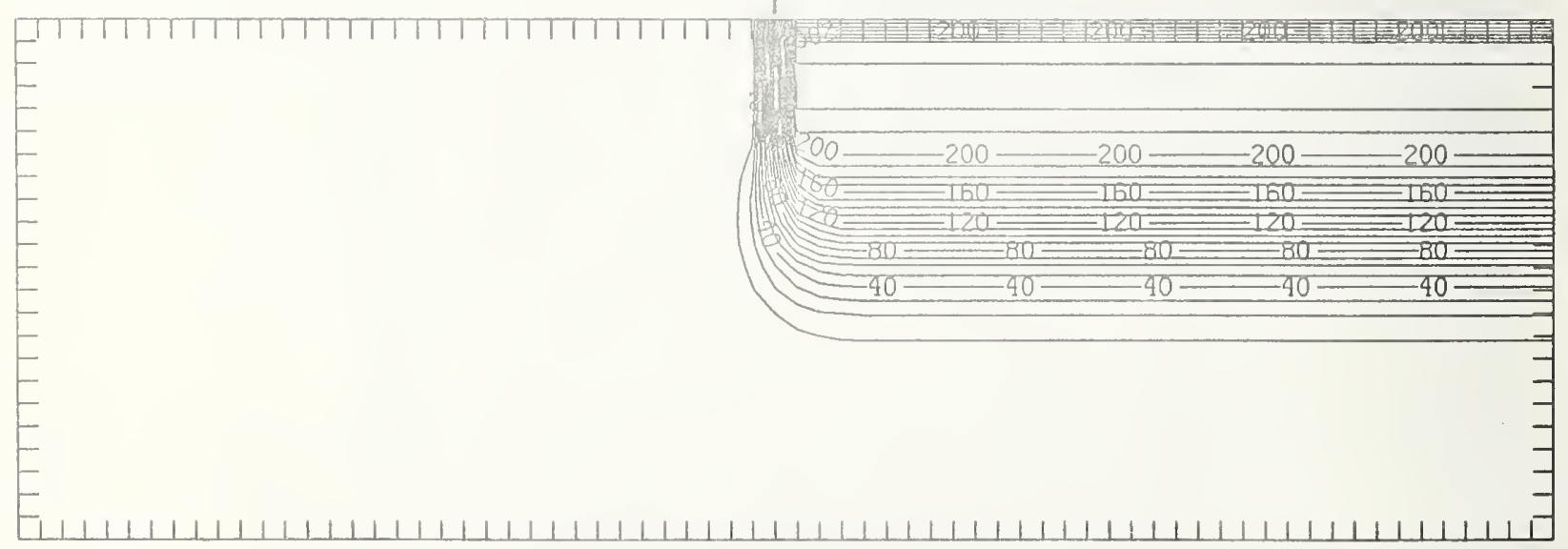

Figure Au250-4 Two-dimensional distribution of Frenkel pairs created by $250 \mathrm{keV}$ gold implanted past a mask edge. The length increment (distance between tick marks) is $0.01 \mu \mathrm{m}$ and the scale factor is 0.1 . 


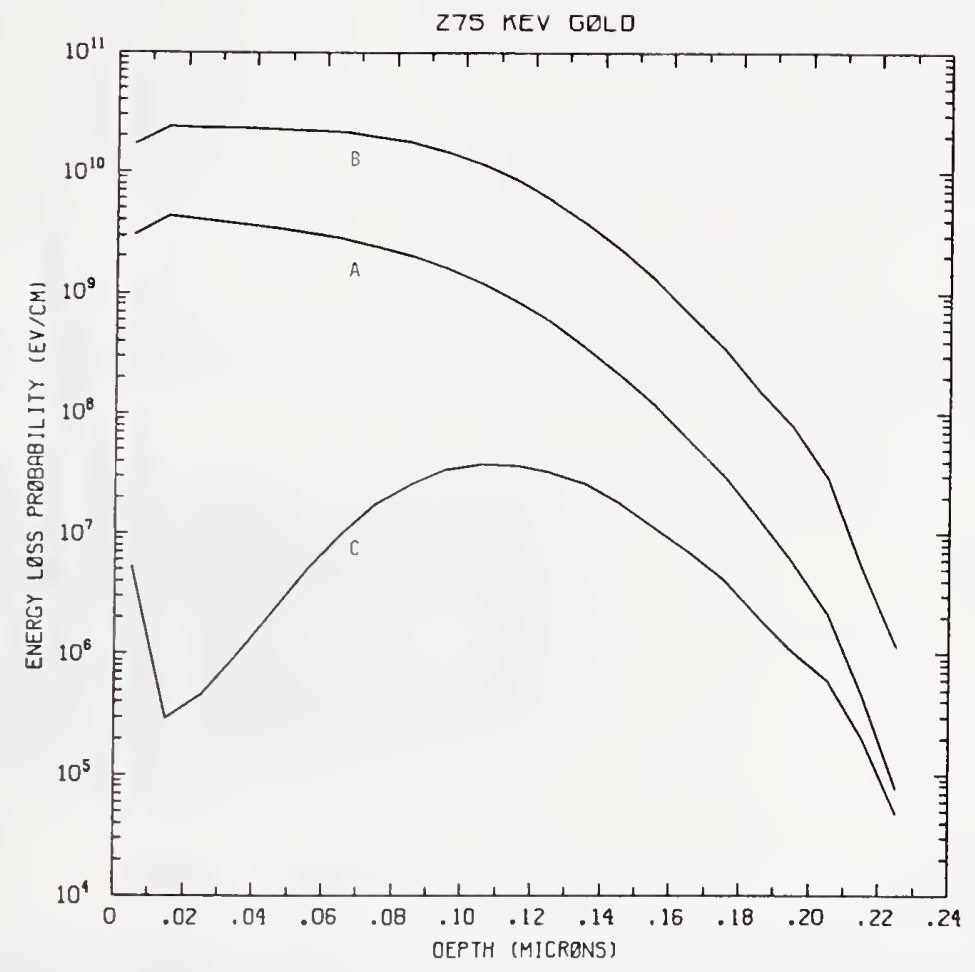

Figure Au275-1 One-dimensional probability distributions for energy loss due to electronic excitation (A), displacement damage(B), and phonon excitation (C) for $275 \mathrm{keV}$ gold normally incident on a silicon target.

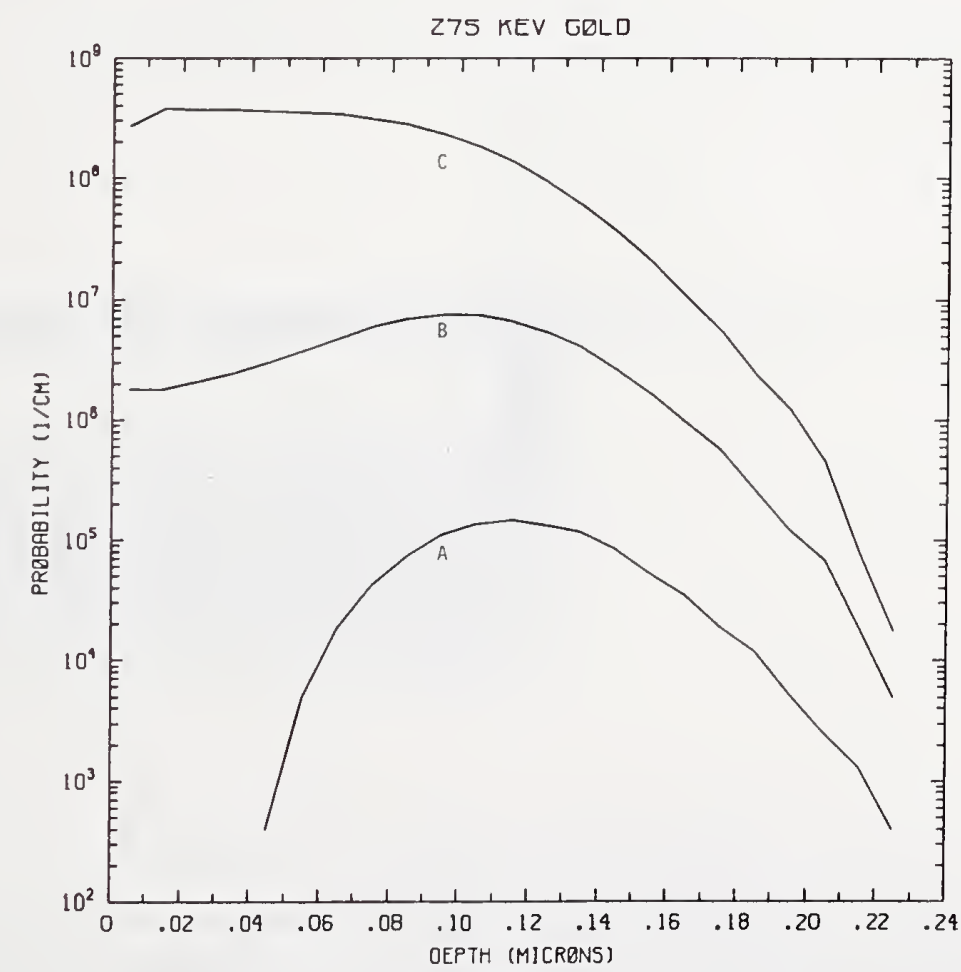

Figure Au275-2 One-dimensional probability distributions for implanted gold (A), primary silicon displacements (B), and Frenkel pairs (C) for $275 \mathrm{keV}$ gold normally incident on a silicon target. 


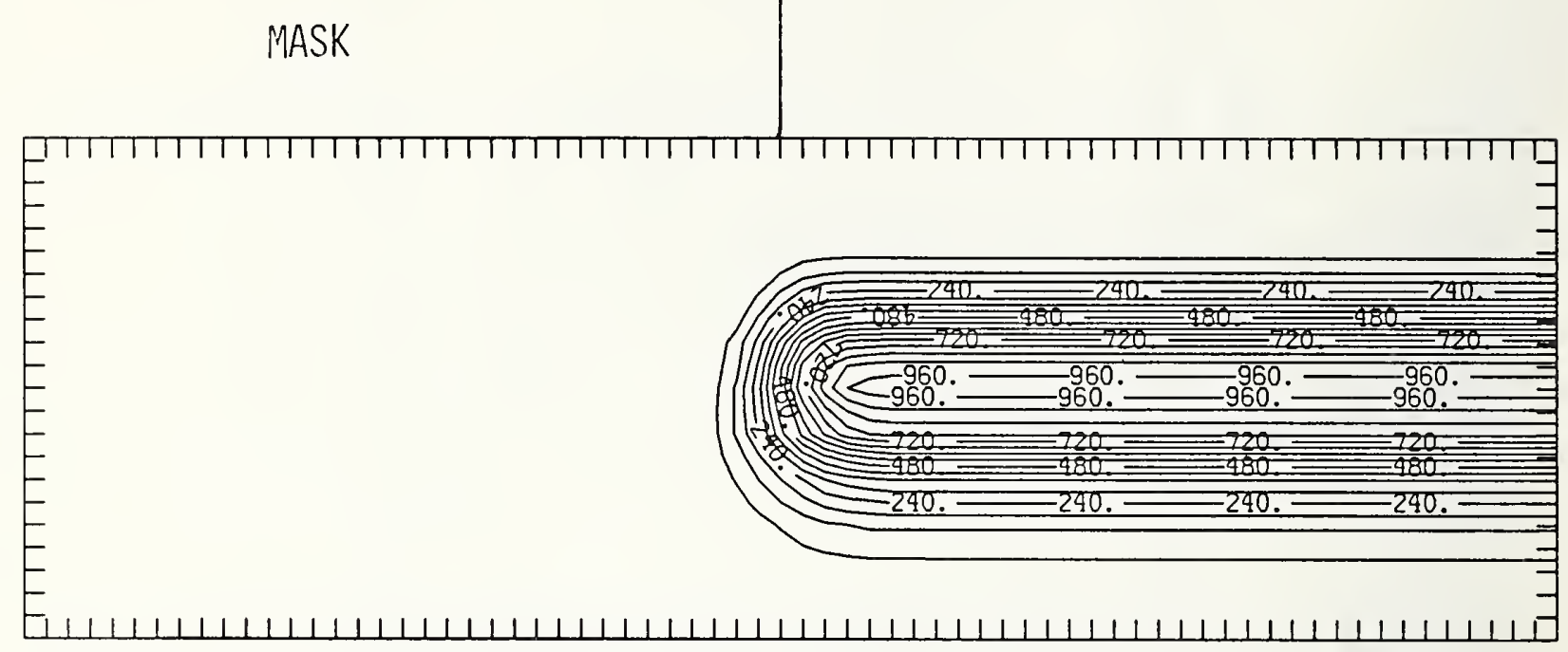

Figure Au275-3 Two-dimensional distribution of $275 \mathrm{keV}$ gold implanted past a mask edge. The length increment (distance between tick marks) is $0.01 \mu \mathrm{m}$ and the scale factor is 1000 .

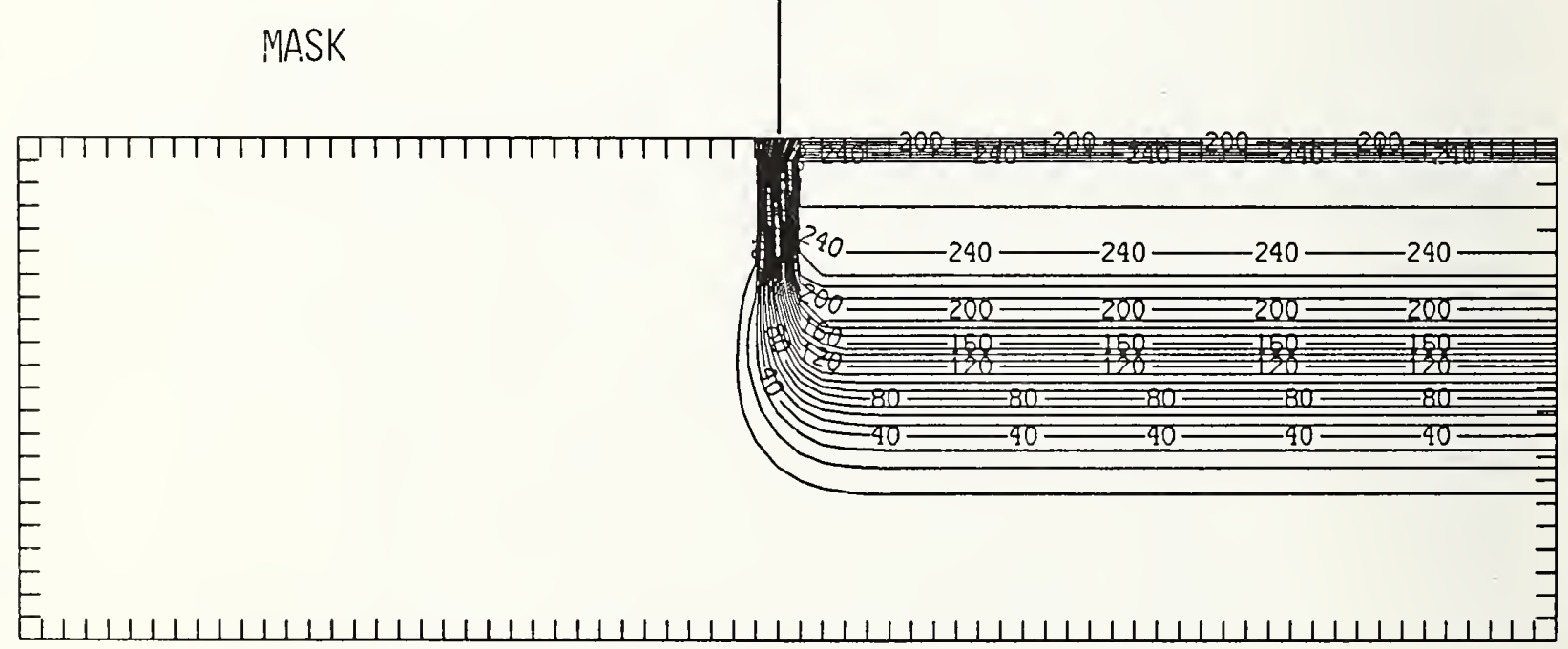

Figure Au275-4 Two-dimensional distribution of Frenkel pairs created by $275 \mathrm{keV}$ gold implanted past a mask edge. The length increment (distance between tick marks) is $0.01 \mu \mathrm{m}$ and the scale factor is 0.1 . 


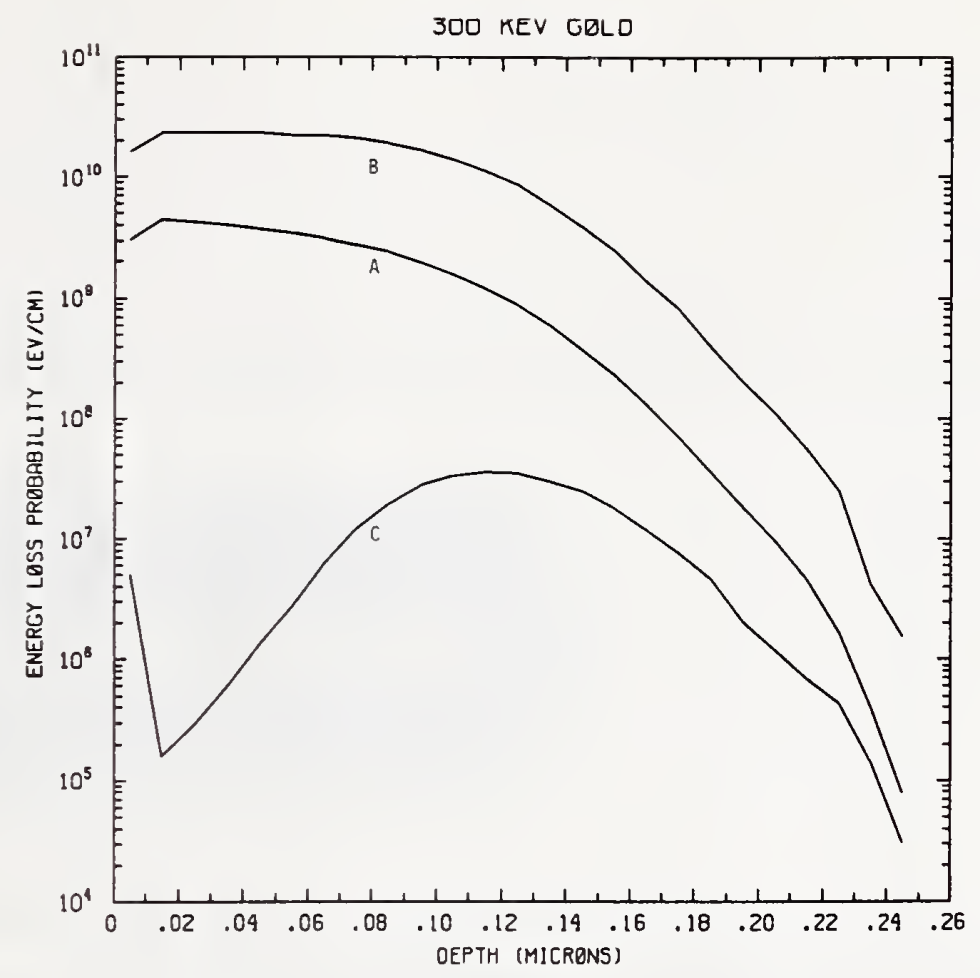

Figure Au300-1 One-dimensional probability distributions for energy loss due to electronic excitation (A), displacement damage(B), and phonon excitation (C) for $300 \mathrm{keV}$ gold normally incident on a silicon target.

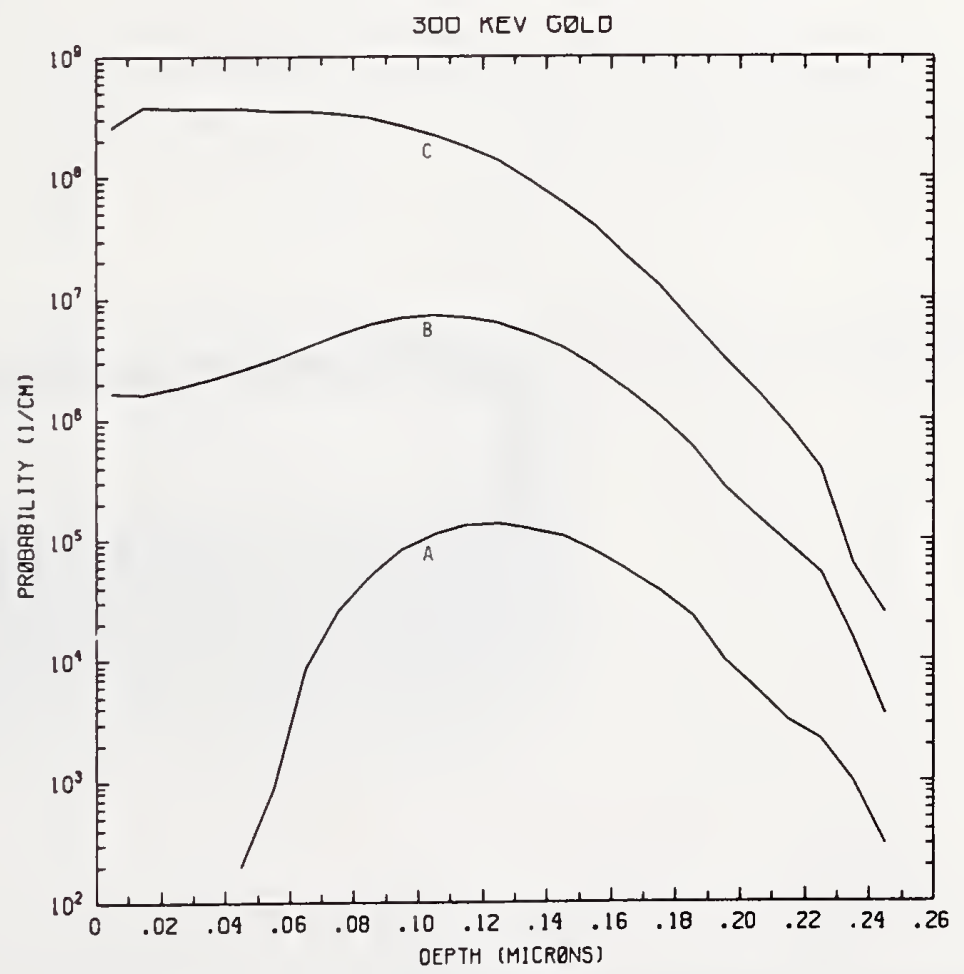

Figure Au300-2 One-dimensional probability distributions for implanted gold (A), primary silicon displacements (B), and Trenkel pairs (C) for $300 \mathrm{keV}$ gold normally incident on a silicon target. 


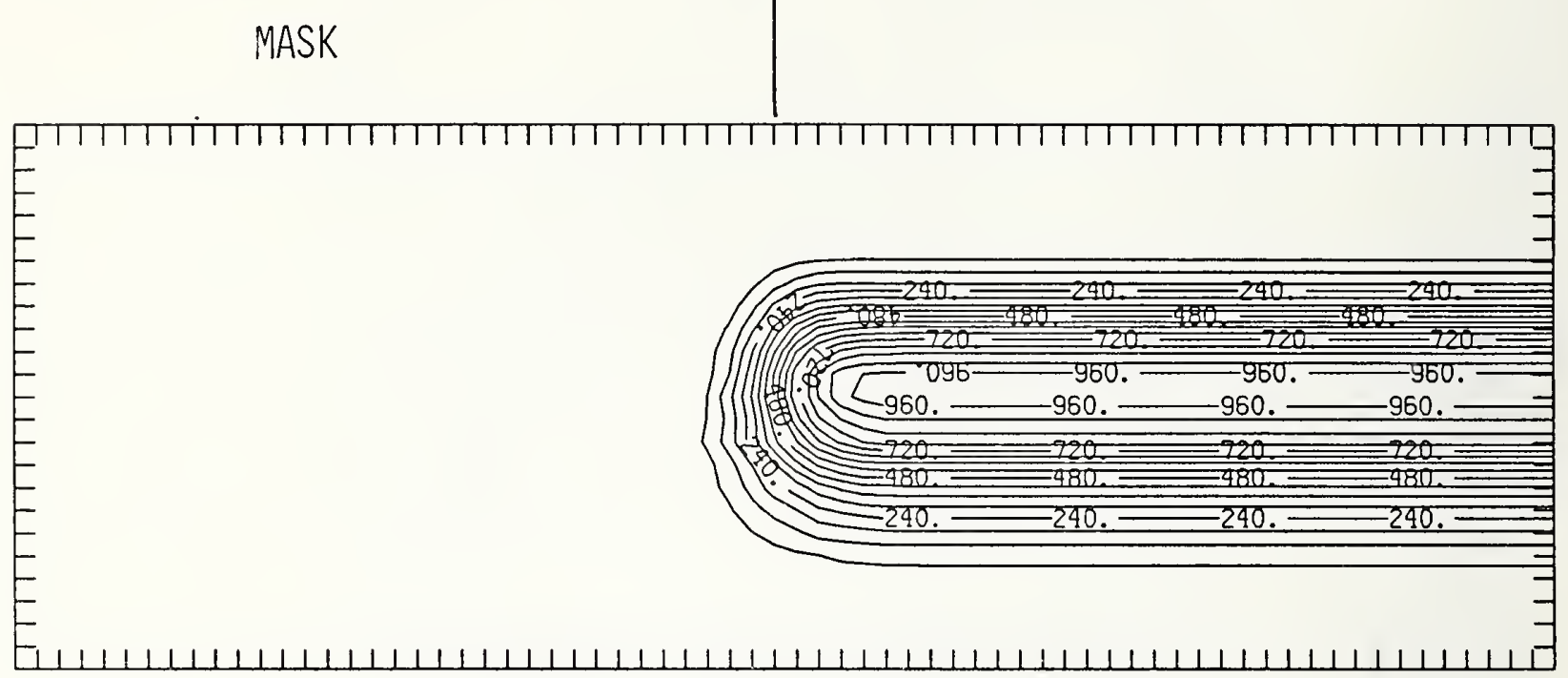

Figure Au300-3 Two-dimensional distribution of $300 \mathrm{keV}$ gold implanted past a mask edge. The length increment (distance between tick marks) is $0.01 \mu \mathrm{m}$ and the scale factor is 1000 .

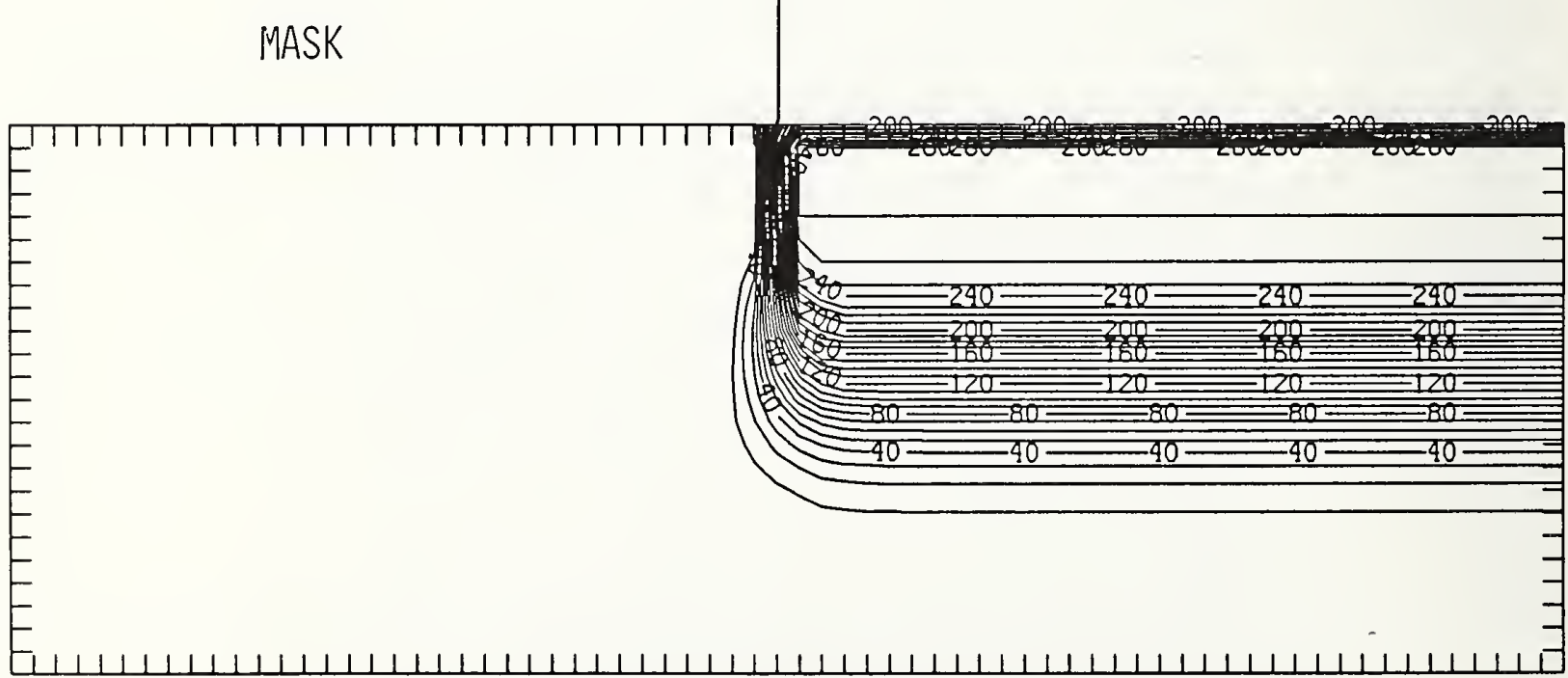

Figure Au300-4 Two-dimensional distribution of Frenkel pairs created by $300 \mathrm{keV}$ gold implanted past a mask edge. The length increment (distance between tick marks) is $0.01 \mu \mathrm{m}$ and the scale factor is 0.1 . 


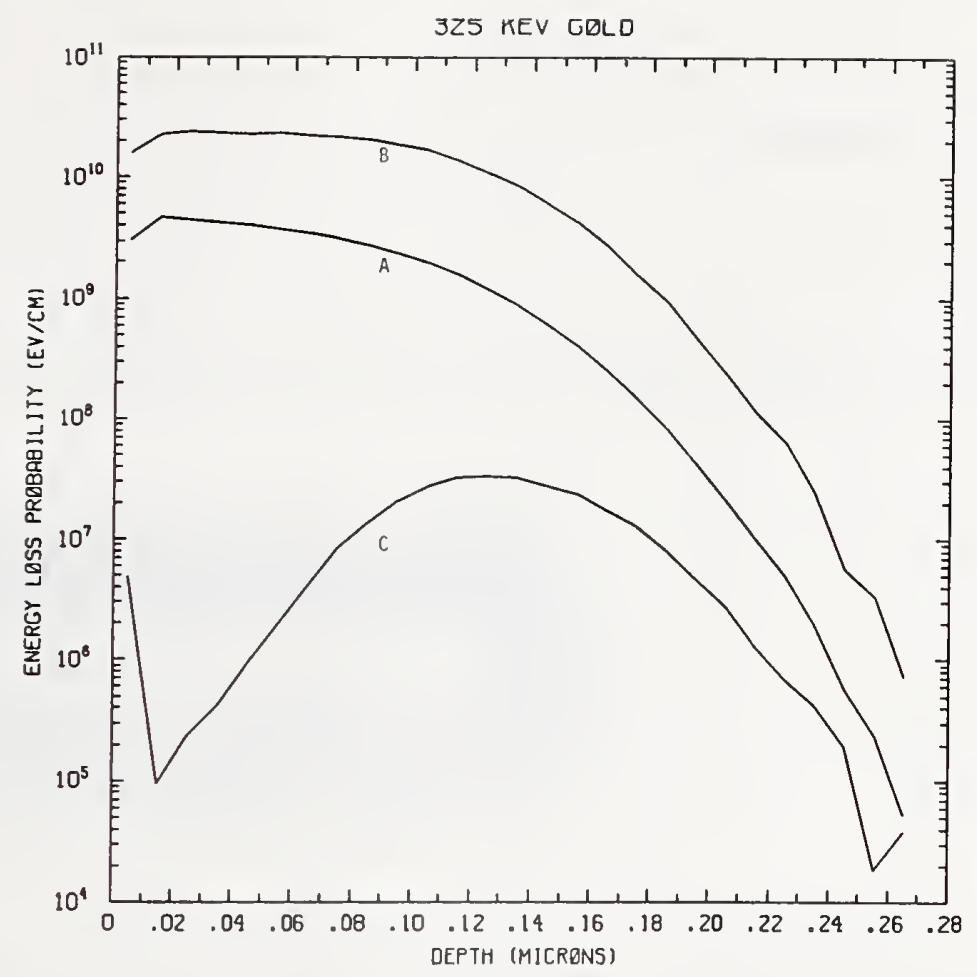

Figure Au325-1 One-dimensional probability distributions for energy loss due to electronic excitation (A), displacement damage(B), and phonon excitation (C) for $325 \mathrm{keV}$ gold normally incident on a silicon target.

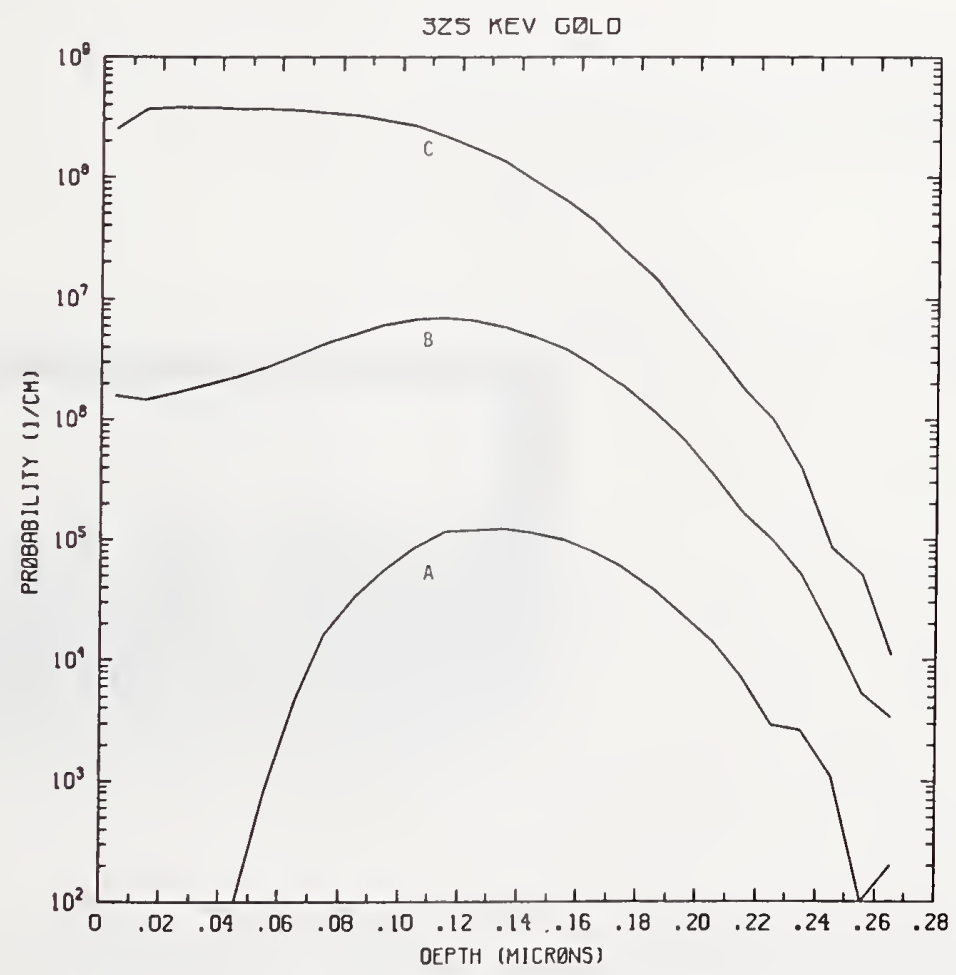

Figure Au325-2 One-dimensional probability distributions for implanted gold (A), primary silicon displacements (B), and Frenkel pairs (C) for $325 \mathrm{keV}$ gold normally incident on a silicon target. 


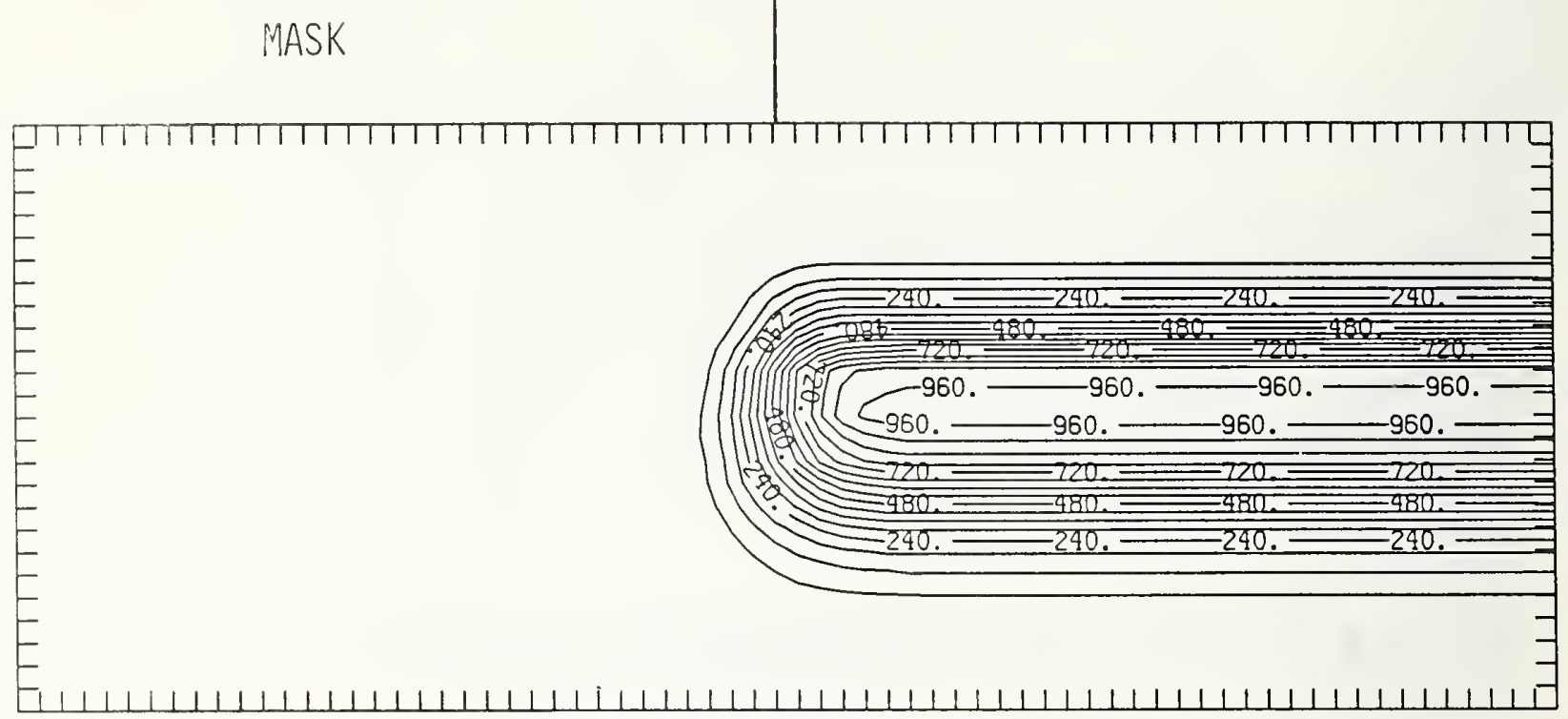

Figure Au325-3 Two-dimensional distribution of $325 \mathrm{keV}$ gold implanted past a mask edge. The length increment (distance between tick marks) is $0.01 \mu \mathrm{m}$ and the scale factor is 1000 .

MASK

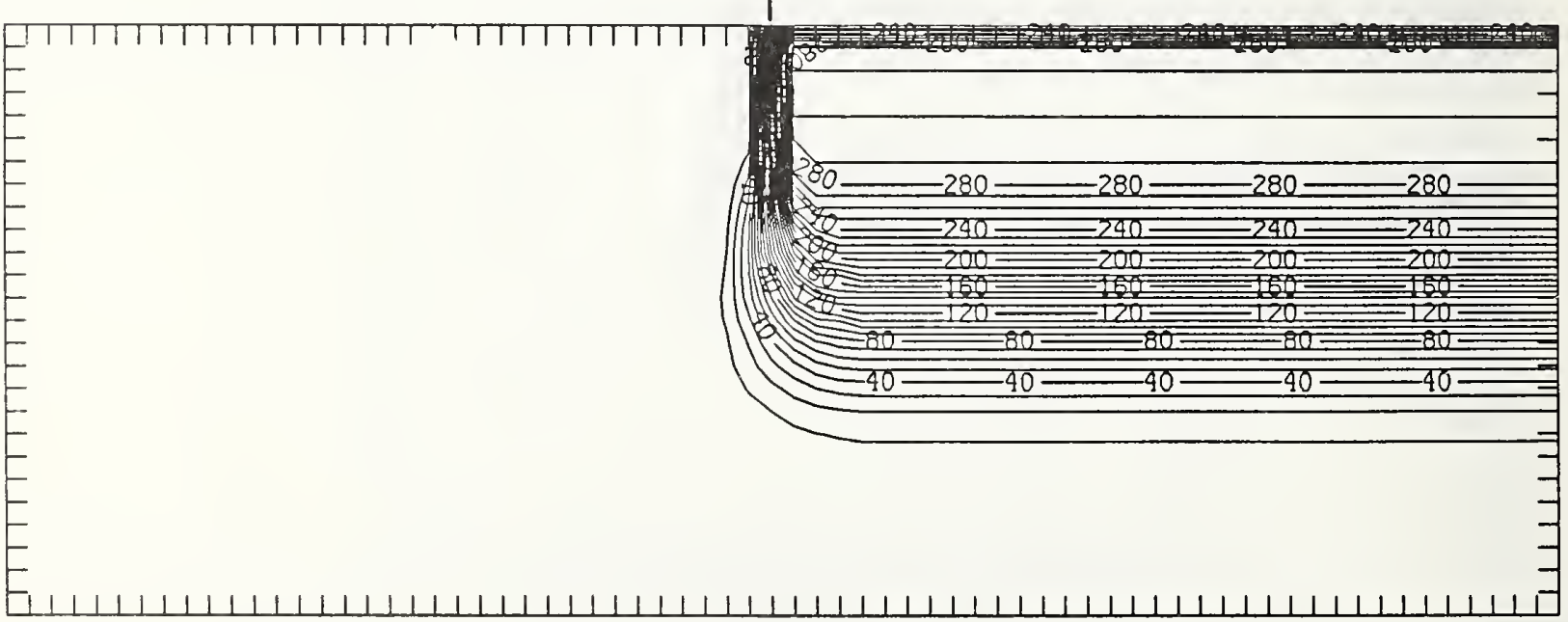

Figure Au325-4 Two-dimensional distribution of Frenkel pairs created by $325 \mathrm{keV}$ gold implanted past a mask edge. The length increment (distance between tick marks) is $0.01 \mu \mathrm{m}$ and the scale factor is 0.1 . 


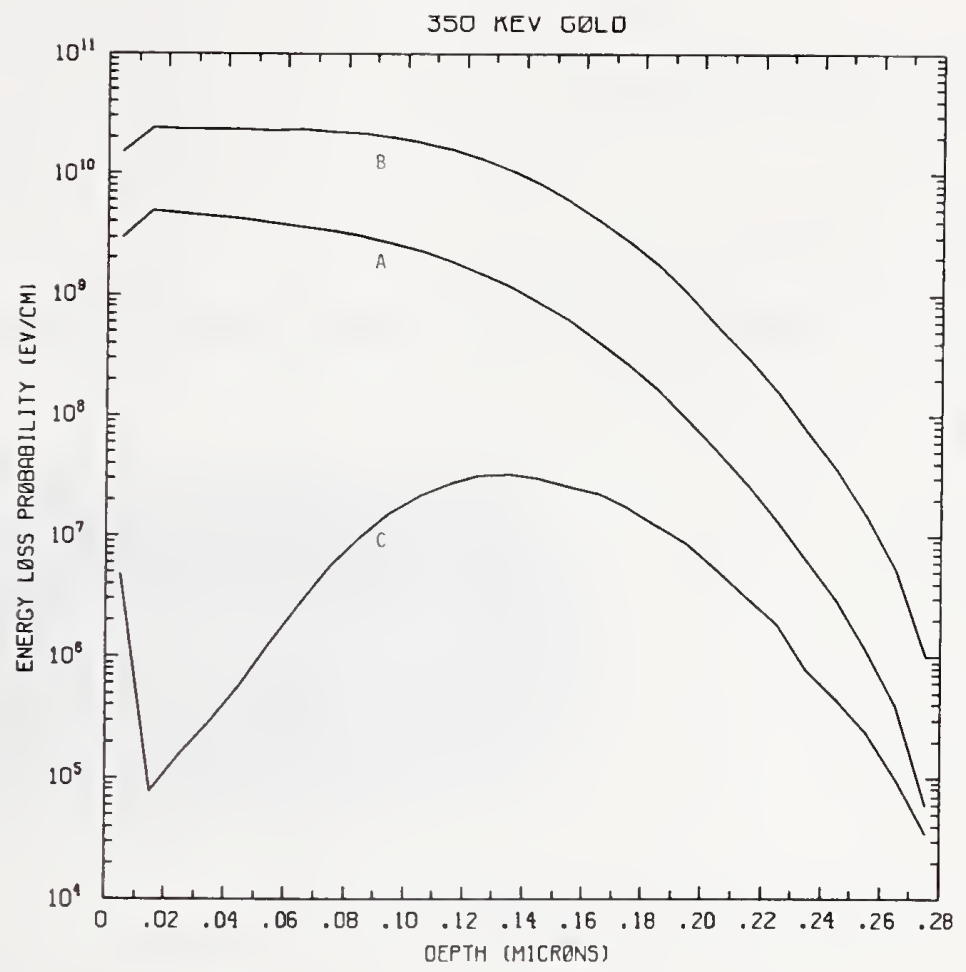

Figure Au350-1 One-dimensional probability distributions for energy loss due to electronic excitation (A), displacement damage(B), and phonon excitation (C) for $350 \mathrm{keV}$ gold normally incident on a silicon target.

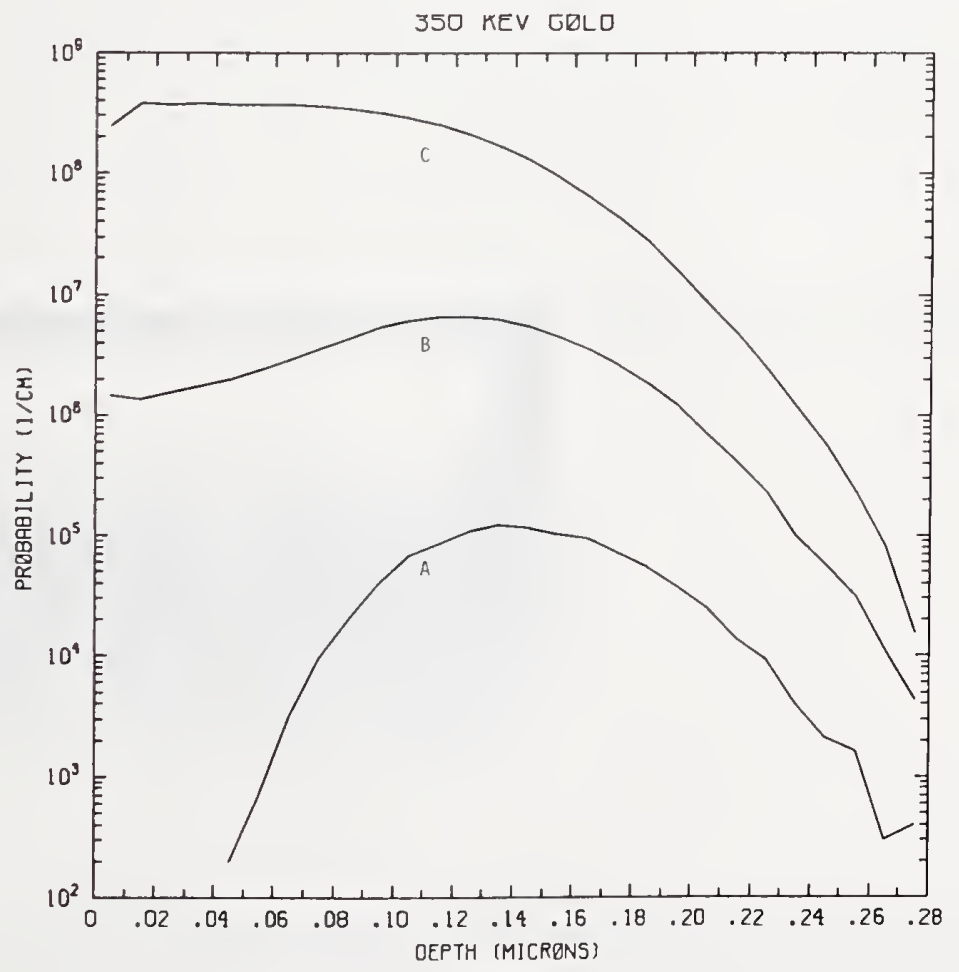

Figure Au350-2 One-dimensional probability distributions for implanted gold (A), primary silicon displacements (B), and Frenkel pairs (C) for $350 \mathrm{keV}$ gold normally incident on a silicon target. 


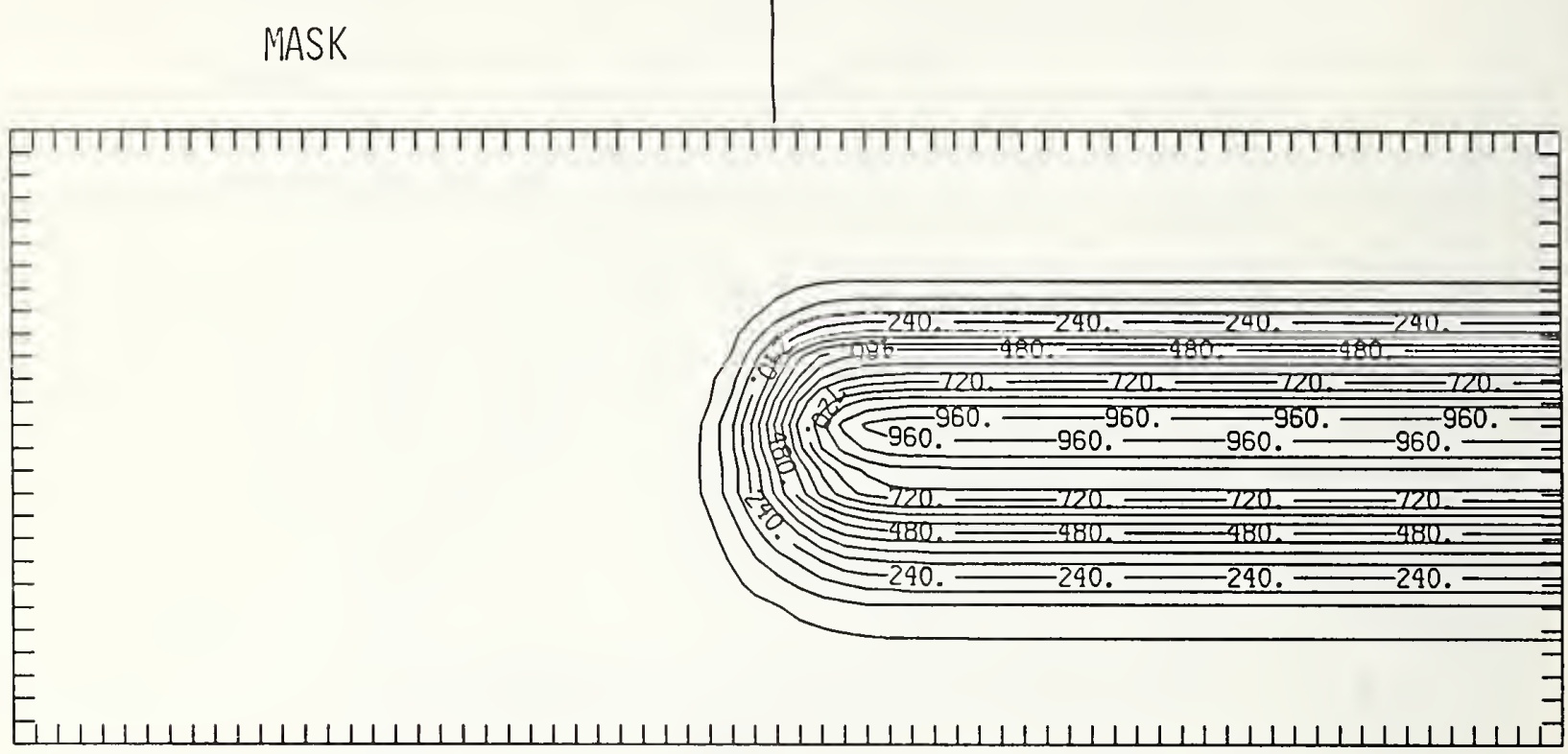

Figure Au350-3 Two-dimensional distribution of $350 \mathrm{keV}$ gold implanted past a mask edge. The length increment (distance between tick marks) is $0.01 \mu \mathrm{m}$ and the scale factor is 1000 .

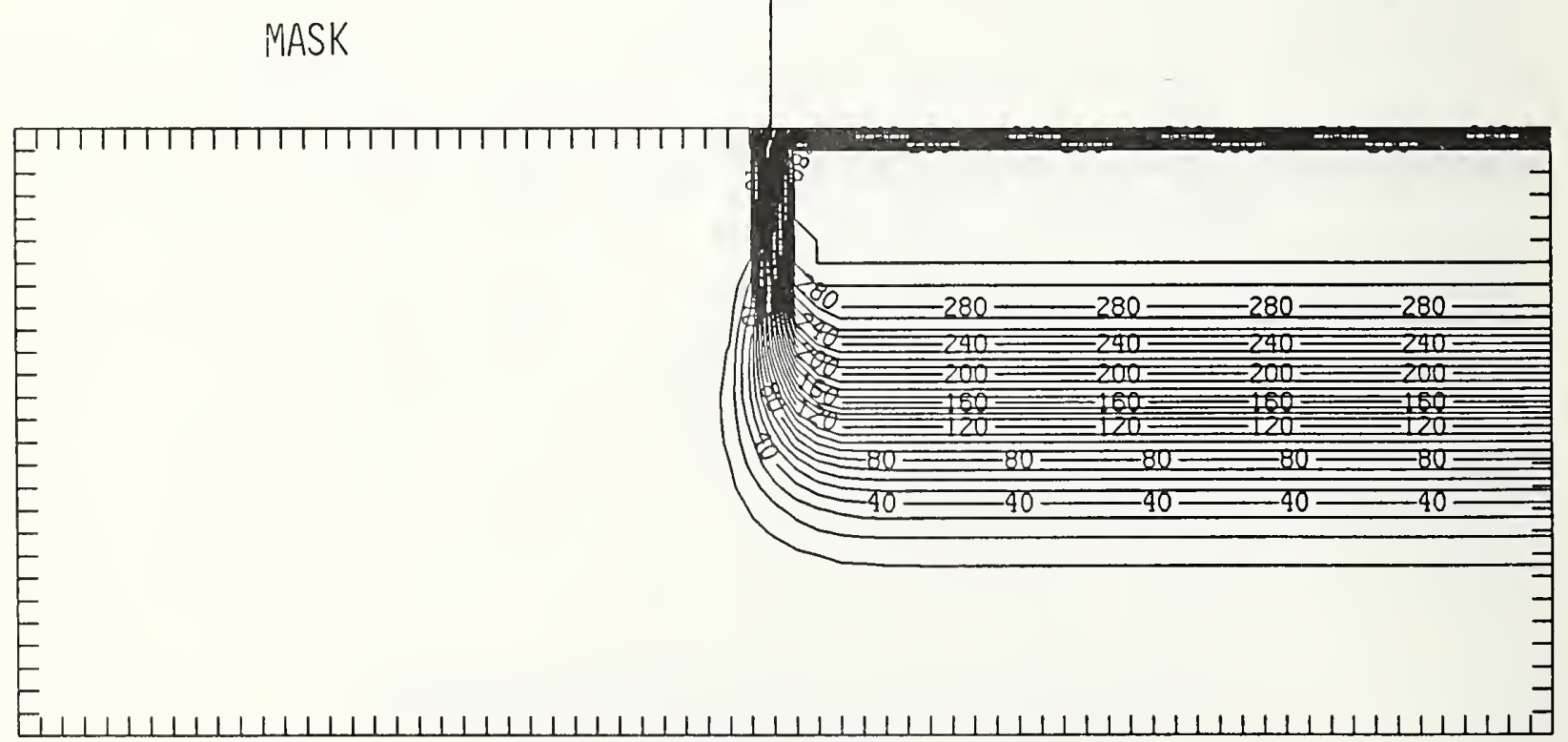

Figure Au350-4 Two-dimensional distribution of Frenkel pairs created by $350 \mathrm{keV}$ gold implanted past a mask edge. The length increment (distance between tick marks) is $0.01 \mu \mathrm{m}$ and the scale factor is 0.1 . 


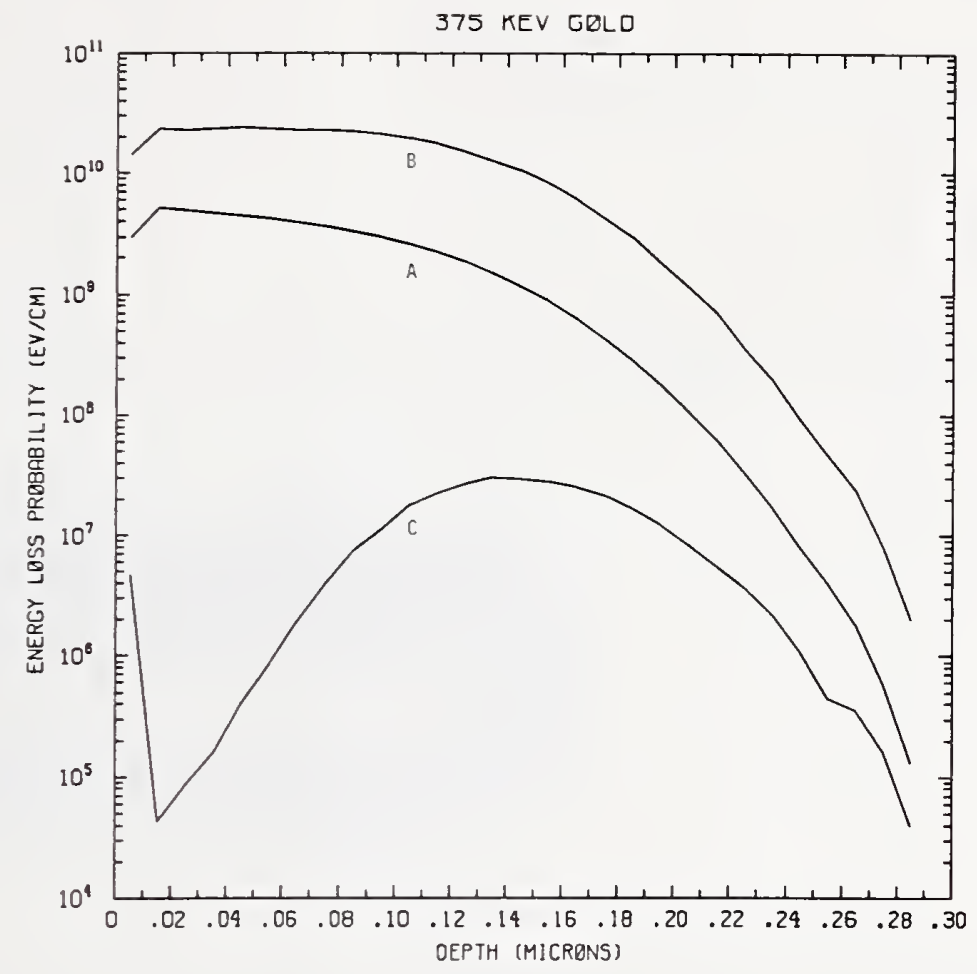

Figure Au375-1 One-dimensional probability distributions for energy loss due to electronic excitation (A), displacement damage(B), and phonon excitation (C) for $375 \mathrm{keV}$ gold normally incident on a silicon target.

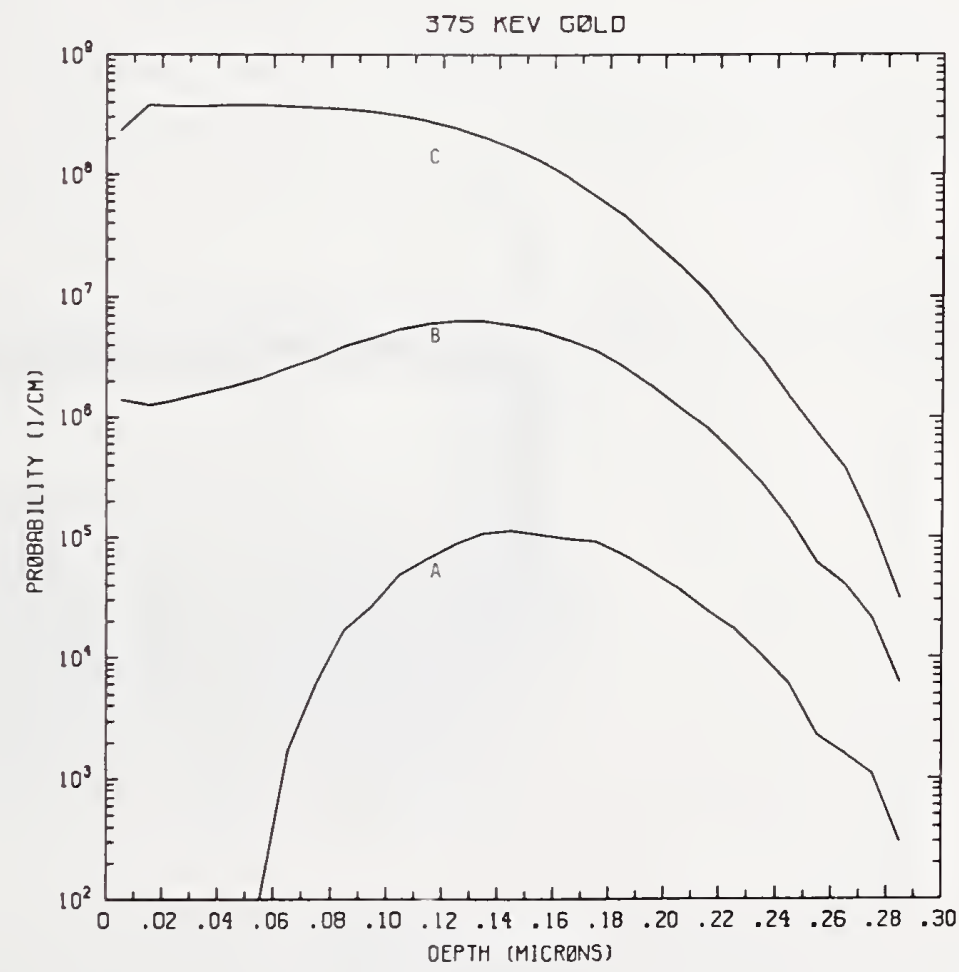

Figure Au375-2 One-dimensional probability distributions for implanted gold (A), primary silicon displacements (B), and Frenkel pairs (C) for $375 \mathrm{keV}$ gold normally incident on a silicon target. 
MASK

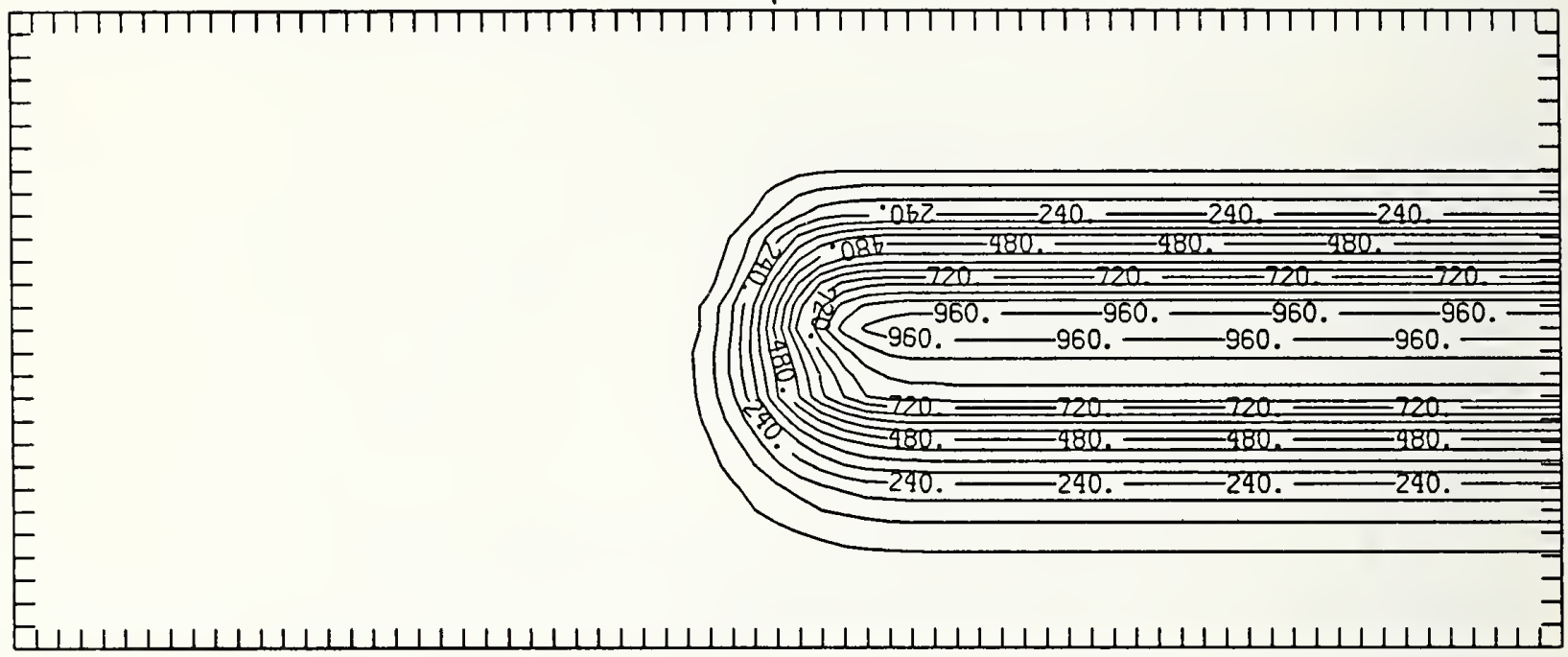

Figure Au375-3 Two-dimensional distribution of $375 \mathrm{keV}$ gold implanted past a mask edge. The length increment (distance between tick marks) is $0.01 \mu \mathrm{m}$ and the scale factor is 1000 .

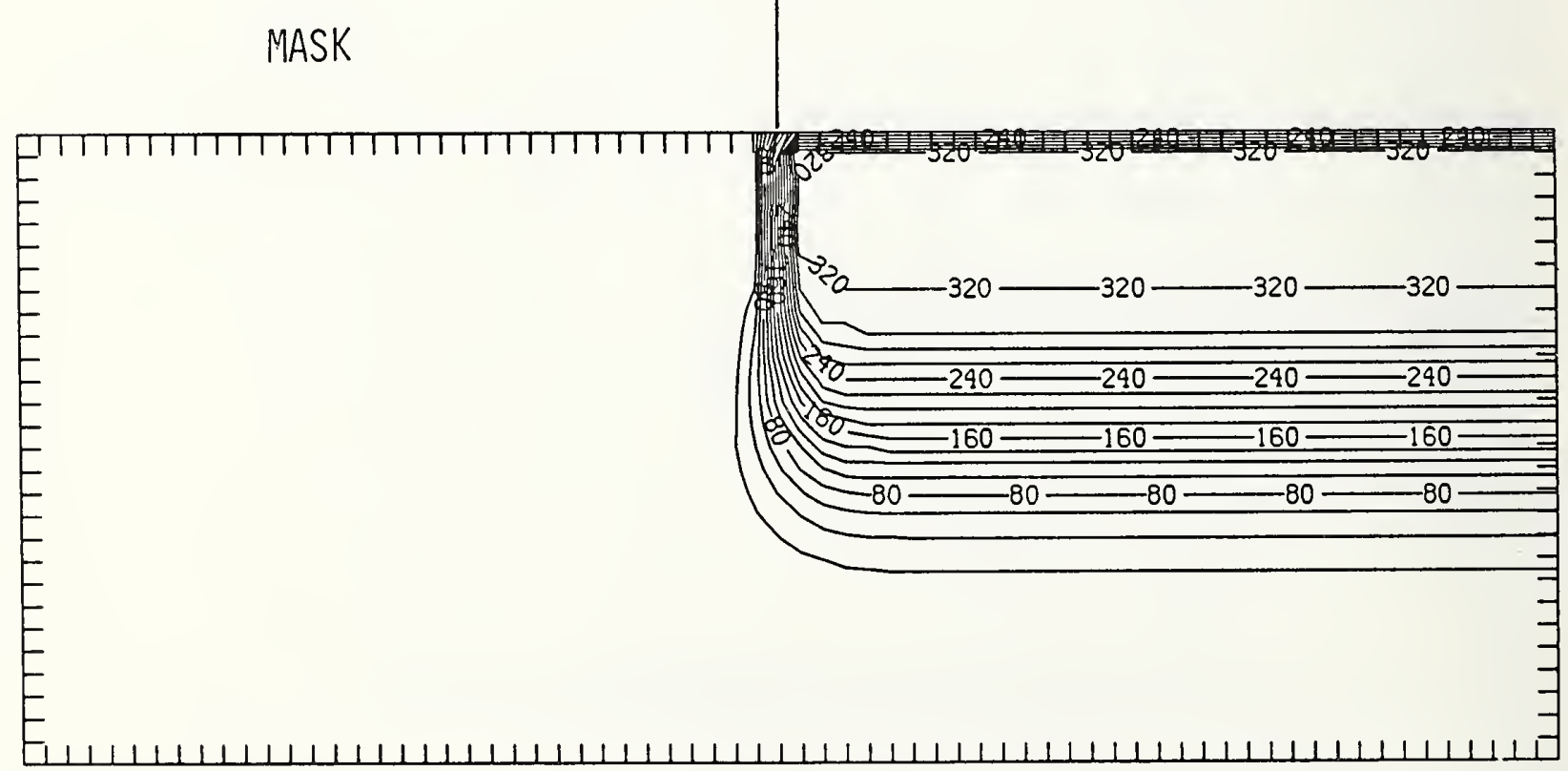

Figure Au375-4 Two-dimensional distribution of Frenkel pairs created by $375 \mathrm{keV}$ gold implanted past a mask edge. The length increment (distance between tick marks) is $0.01 \mu \mathrm{m}$ and the scale factor is 0.1 . 


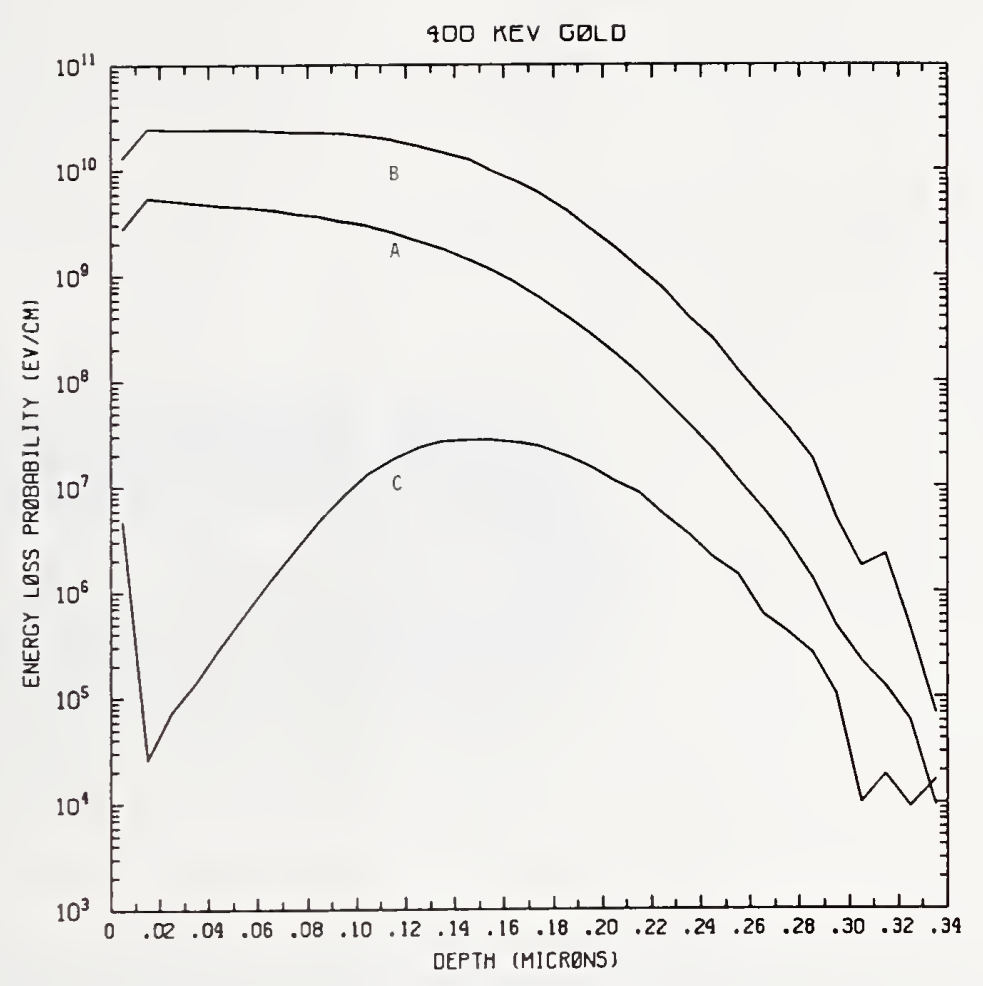

Figure Au400-1 One-dimensional probability distributions for energy loss due to electronic excitation (A), displacement damage(B), and phonon excitation (C) for $400 \mathrm{keV}$ gold normally incident on a silicon target.

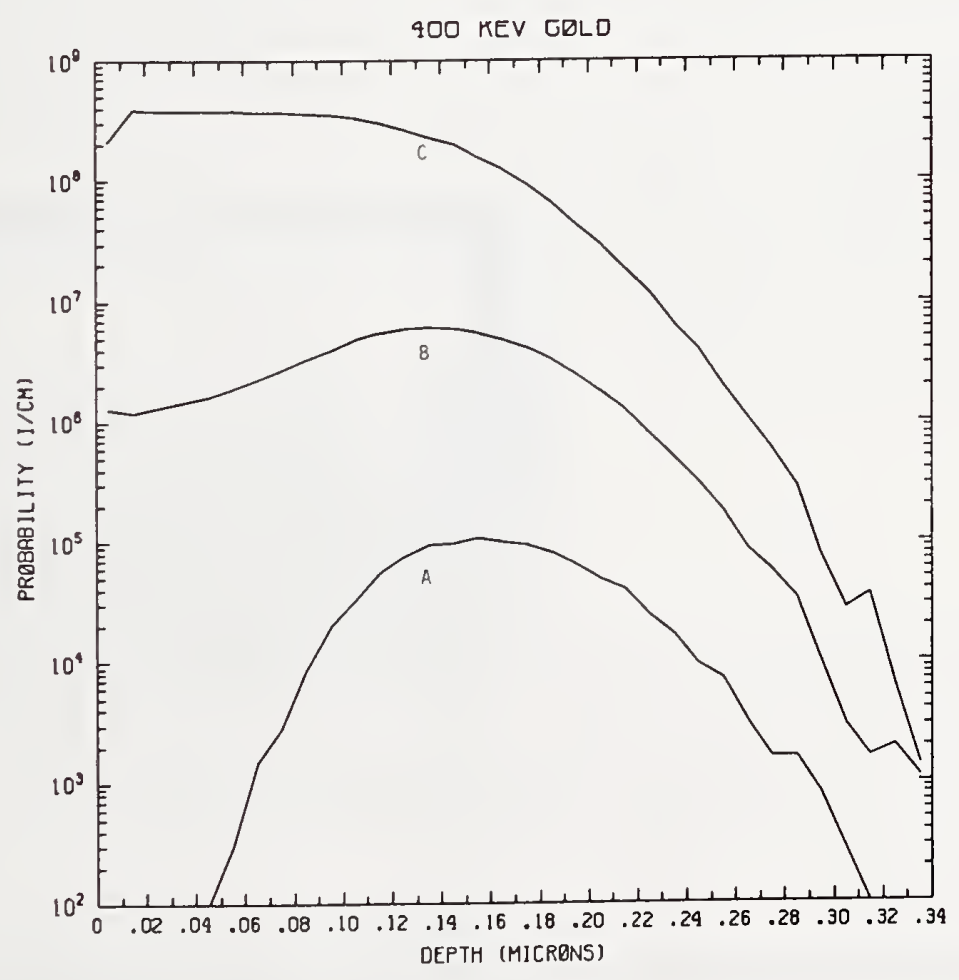

Figure Au400-2 One-dimensional probability distributions for implanted gold (A), primary silicon displacements (B), and Frenkel pairs (C) for $400 \mathrm{keV}$ gold normally incident on a silicon target. 


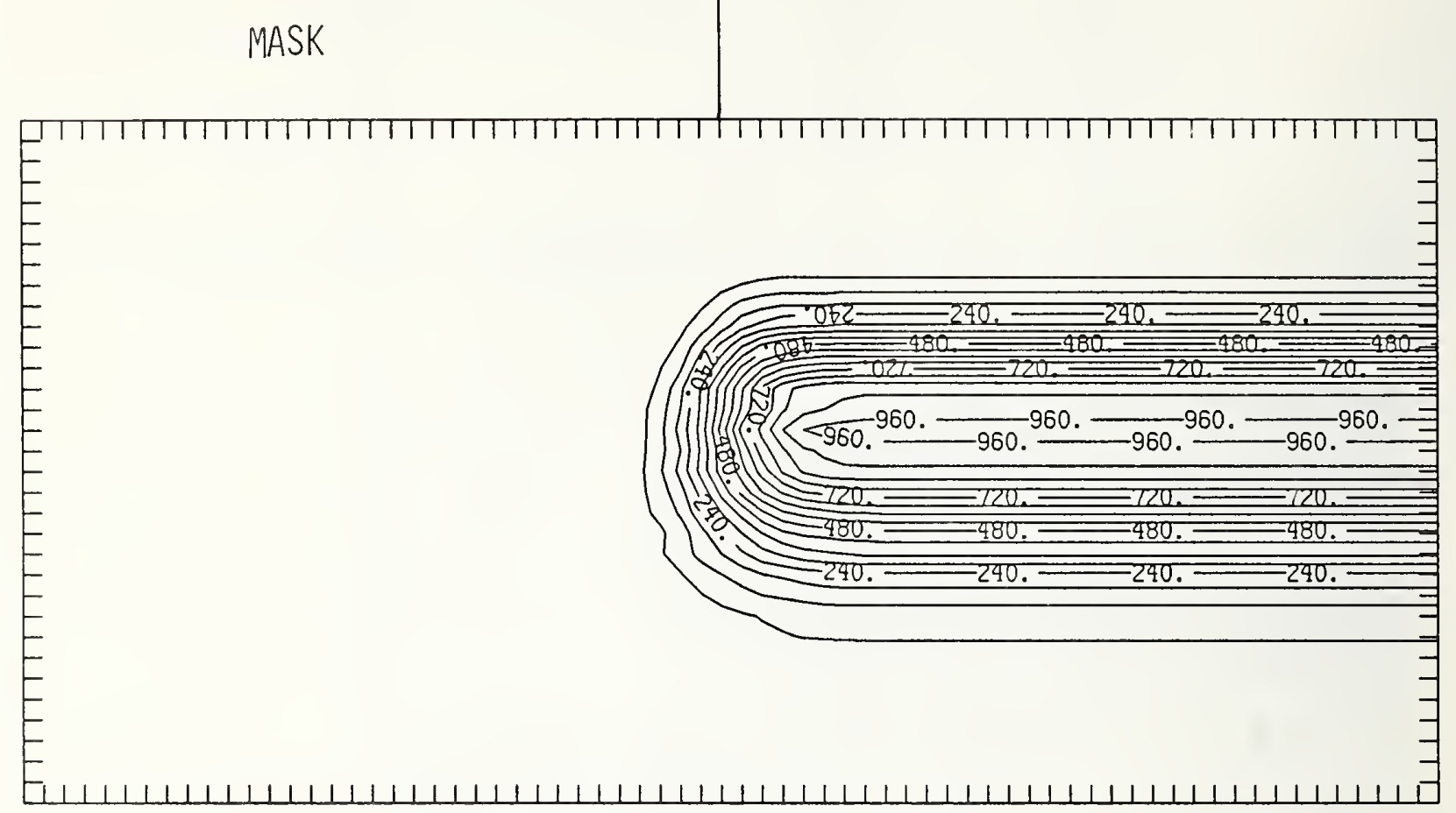

Figure Au400-3 Two-dimensional distribution of $400 \mathrm{keV}$ gold implanted past a mask edge. The length increment (distance between tick marks) is $0.01 \mu \mathrm{m}$ and the scale factor is 1000 .

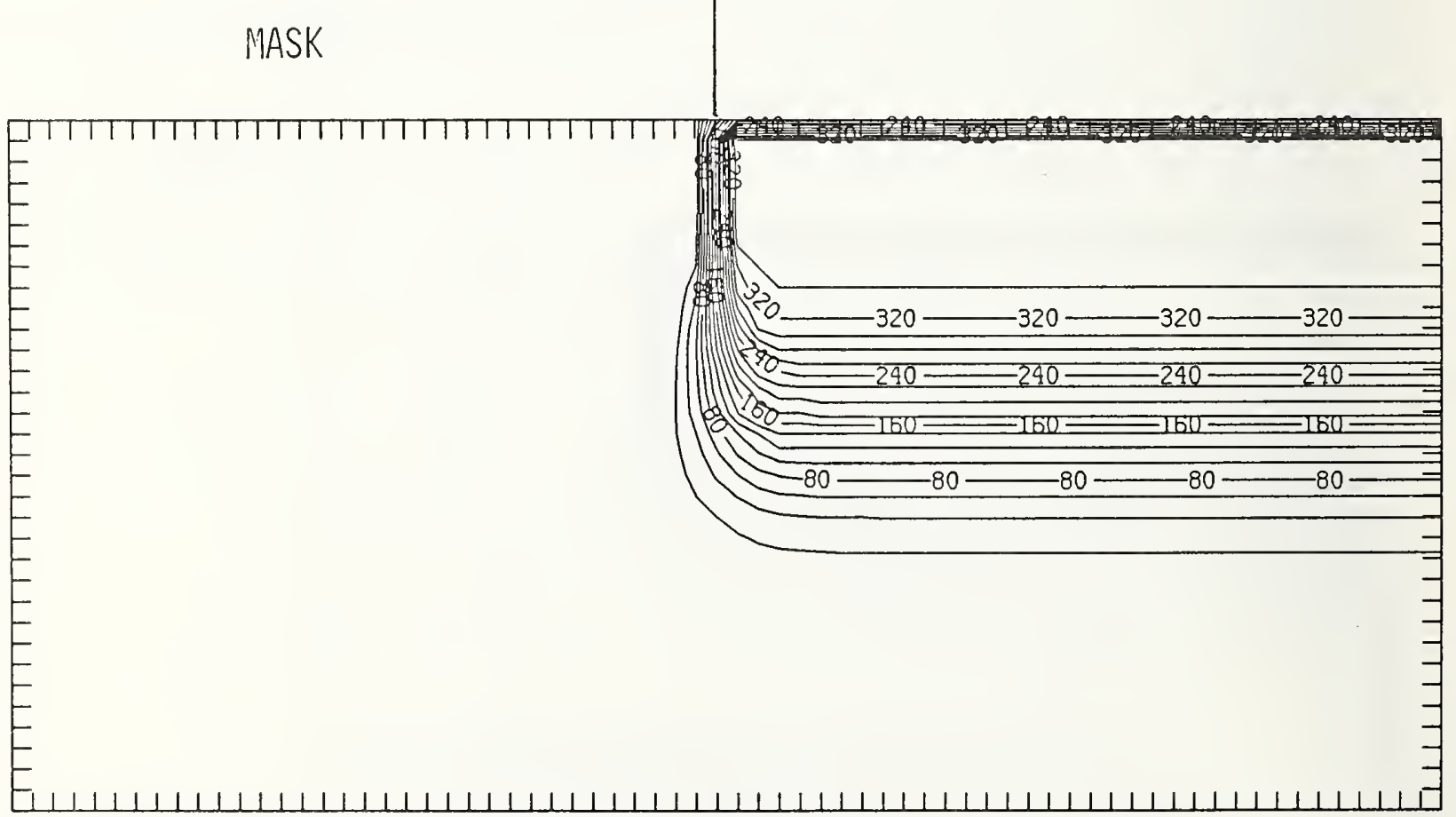

Figure Au400-4 Two-dimensional distribution of Frenkel pairs created by $400 \mathrm{keV}$ gold implanted past a mask edge. The length increment (distance between tick marks) is $0.01 \mu \mathrm{m}$ and the scale factor is 0.1 . 
NBS.114A (REV. 2.8C)

U.S. DEPT. OF COMM.

BIBLIOGRAPHIC DATA

1. PUBLICATION OR

REPORT NO.

SHEET (See instructions)

NBS / SP $-400 / 79$

4. TITLE AND SUBTITLE

Semiconductor Measurement Technology: Results of the Monte Carlo Calculation of One- and Two-Dimensional Distributions of Particles and Damage: Ion Implanted Dopants in Silicon

\section{AUTHOR(S)}

John Albers

6. PERFORMING ORGANIZATION (If joint or oth than NBS, see instructions)

NATIONAL BUREAU OF STAID

U.S. DEPARTMENT OF COMIAET

GAITHERSBURG, MD 2089 ?

9. SPONSORING ORGANIZATION NAME AIND COMPL

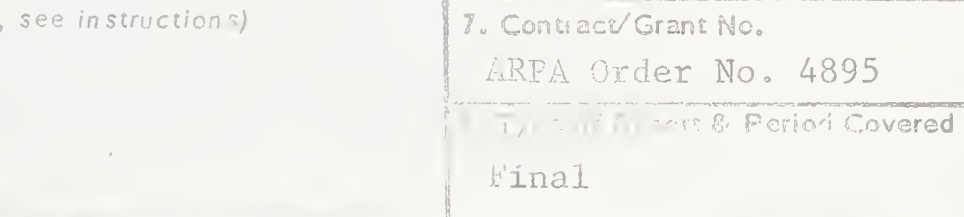

Defense Advanced Research Proje

1400 Wilson Boulevard

Arlington, VA 22209

10. SUPPLEMENTARY NOTES

Library of Congress Catalog Catd Numberi 87-619864

$\square$ Document describes a computer program; SF-185, FIPS Software Summary, is attached.

11. ABSTRACT (A 200-word or less factual summary of most significant information. If document includes a significant bibliography or literature survey, mention it here)

The TRansport of Ions in Matter (IRIM) Monte Carlo code was used to calculate the two-dimensional distributions of particlos, primary damage, and electronic and nuclear energy loss for implantation of a line beam source into silicon targets. Approximate two-dimensional distributions of the Frenkel pairs (vacancy-interstitial) created by the primary displacem-nt dange of the target atoms were calculated by means of the Kinchin-Pease equation. These particle, damage, and energy loss distributions allowed for the calculation of the one-dimensional distributions of these quantities for implantation into umanul targets. A superposition technique was used to construct the two-dinension: I pricicle and approximate Frenkel pairs distributions for implantation pat 1 m. The energetic ions used in the cal-

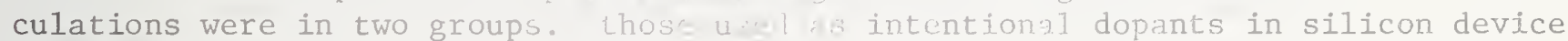
fabrication and those which either limited lifetime or acted as gettering sites. The particle distributions were paramcterized by means of standard polynomial fitting techniques.

12. KEY WORDS (Six to twelve entries, alphabe al orde: displacement damage; electronic energy only proper names: and separate key words by semicolons) implantation; Monte Carlo calculation; nulear energy loss; silicon device fabrication; two-dimensional djstributions: vacancies

13. AVAILABILITY

[X] Unlimited

For Official Distribution. Do Not Ruleas: to NTIS

X Order From Superintendent of Documents, U.S. Government Prifiting Offics Wastington, D.C. 20402.

$\square$ Order From National Technical Info mation servic: (MTIS), Springfield, V/ .. 2?.' I

1.4. NO. OF
PFINTED PAGES
671
1.5. Price




\section{Periodical}

Journal of Research-The Journal of Research of the National Bureau of Standards reports NBS research and development in those disciplines of the physical and engineering sciences in which the Bureau is active. These include physics, chemistry, engineering, mathematics, and computer sciences. Papers cover a broad range of subjects, with major emphasis on measurement methodology and the basic technology underlying standardization. Also included from time to time are survey articles on topics closely related to the Bureau's technical and scientific programs. Issued six limes a year.

\section{Nonperiodicals}

Monographs-Major contributions to the technical literature on various subjects related to the Bureau's scientific and technical activities.

Handbooks-Recommended codes of engineering and industrial practice (including safety codes) developed in cooperation with interested industries, professional organizations, and regulatory bodies.

Special Publications-Include proceedings of conferences sponsored by NBS, NBS annual reports, and other special publications appropriate to this grouping such as wall charts, pocket cards, and bibliographies.

Applied Mathematics Series-Mathematical tables, manuals, and studies of special interest to physicists, engineers, chemists, biologists, mathematicians, computer programmers, and others engaged in scientific and technical work.

Vational Standard Reference Data Series-Provides quantitative data on the physical and chemical properties of materials, compiled from the world's literature and critically evaluated. Developed under a worldwide program coordinated by NBS under the authority of the National Standard Data Act (Public Law 90-396).

NOTE: The Journal of Physical and Chemical Reference Data (JPCRD) is published quarterly for NBS by the American Chemical Society (ACS) and the American Institute of Physics (AIP). Subscriptions, reprints, and supplements are available from ACS, 1155 Sixteenth St., NW, Washington, DC 20056.

Building Science Series-Disseminates technical information developed at the Bureau on building materials, components, systems, and whole structures. The series presents research results, test methods, and performance criteria related to the structural and environmental functions and the durability and safety characteristics of building elements and sysiems.

Technical Votes-Siudies or repofts which are complete in themselves but restrictive in their treatment of a subject. Analogous to monographs but not so comprehensive in scope or definitive in treatment of the subject area. Often serve as a vehicle for final reports of work performed at NBS under the sponsorship of other government agencies.

Voluntary Product Standards-Developed under procedures published by the Department of Commerce in Part 10, Title 15, of the Code of Federal Regulations. The standards establish nationally recognized requirements for products, and provide all concerned interests with a basis for common understanding of the characteristics of the products. NBS administers this program as a supplement to the activities of the private sector standardizing organizations.

Consumer Information Series-Practical information, based on NBS research and experience, covering areas of interest to the consumer. Easily understandable language and illustrations provide useful background knowledge for shopping in today's technological marketplace.

Order the above NBS publications from: Superintendent of Docurnents, Government Printing Office, Washington, DC 20402.

Order the following NBS publications-FIPS and NBSIR's-from the National Technical Inforination Service, Springfield, VA 22161.

Federal Information Processing Standards Publications (FIPS PLB)-Publications in this series collectively constitute the Federal Information Processing Standards Register. The Register serves as the official source of information in the Federal Government regarding standards issued by NBS pursuant to the Federal Property and Administraive Services Act of 1949 as amended, Public Law 89-306 (79 Stat. 1127), and as implemented by: Executive Order 11717 (38 FR 12315, dated May 11, 1973) and Part 6 of Title 15 CFR (Code of Federal Regulations).

VBS Interagency Reports (NBSIR)-A special series of interim or final reports on work performed by NBS for outside sponsors (both government and non-government). In general, initial distribution is handled by the sponsor; public distribution is by the National Technical Information Service, Springfield, VA 22161, in paper copy or microfiche form. 
U.S. Department of Commerce

National Bureau of Standards

Gaithersburg, MD 20899

Official Business

Penalty for Private Use $\$ 300$ 